\title{
RECEIVEO
}

BEC $10 \mathrm{gm}$

OSTI

NUREG-0540

Vol. 19, No. 9

Title List of Documents

Made Publicly Available

September 1-30, 1997

U.S. Nuclear Regulatory Commission

Office of the Chief Information Officer

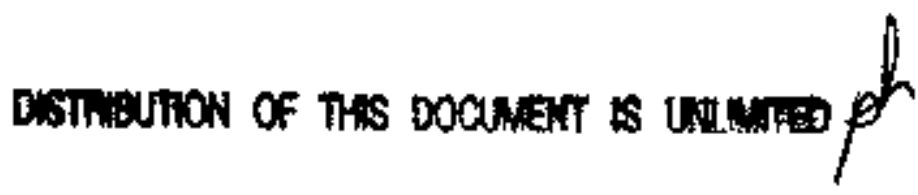

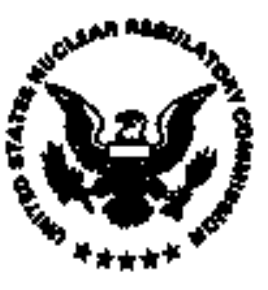




\section{AYAILABILTY NOTKCE}

\section{Avaliability of Reference Materials Cited in NAC Publications}

Wost documents clted In NRC publications will be avelitable from one of the following sources:

1. The Nfoc Public Document Room, 2120 L Streat, NW., Lower Level, Weshington, DC 20555-0001

2. The Superintendemt of Documents, U.S. Government Printing Office, P. O. Box 37682, Waskington, DC 20402-932g

3. The Notional Technical Information Service, Springffeld, VA 22161-0002

Although the fisting that follows represents the majoxity of documents cited in NAC publicstions, it is not Intended to be extusugtlva.

Referenced documents available for inspection end copying for a fee from the NRC Rublic Document Room irxclude NRC corresponderxe and internal NAC memoranda; NFC bulletins, clrculars, intormetion notices, inspection and isvestigstion notices; licensee event reports; vendor reponts and correspondence; Commission papers; and applicant and licensee docr ments and correspondence.

The following documents in the NUPES enries are avaliable for purchase from the Govermment Printing Office: format NAC staff and contractor reports, NRBC-sponsored conference pro-

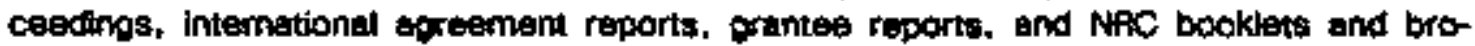
chures. Also availabie are regulatory guides. NRC regulations in the Corte of Fecteral Regudetions, und Nuelear Requetory Commission issuances.

Documents avaltable from the Natkonel Technical Information Service tnclude MPEG-8exies reports and techriticel reporta prepered by other federal aoencies and reports prepared by the Atomic Emergy Commiasion, forenunner agency to the Nuclear Regultory Commtssion.

Documents avaikabie from public and speciel technical libraries include ell open literature items, such as books, foumal artictes. and transactions. Federal Register notices, Federal and State legistation, and congressional reponts can usually be obtained from thest libreries.

Documents such es theses, dlssertations, foreign reports and transiations, and non-NAC conference procendings are avaluble for purchase from the organization sponsoring the publication cted.

Singlis coples of NFC dratt reports are avaliable free, to the extent of supply. upon written request to the Ofilice of Adrrinisaration. Dtsarlbution and Mal Services Section. U.S. Nuclear Regulatory Commlsalon, Washington, DC 20565-0007.

Coptes of industry codes and standerds used in a substantive manner th the NAC regulatory process are maintelned at the NAC Ltbrary, Two White Flint Nonth, 11545 Rockville Pike. Aockvilte, MO 20852-2738, for use by the public. Codes and standards are usuelly copyriotited and mey be purchased from the originating orgenization $\alpha$, if they are Amertcan National Standards, from the American National Standerds Institute, 1430 Broadway, New York, NY $10018-3308$.

A year's subscription of this report consists of 12 monthly issues. 


\section{DIECLAIMER}

This report was preparod as an rocount of wokk sponsored by as spency of the Urited Slates Gorernmeat. Ntither the Urited Stales Government nor any agtoci

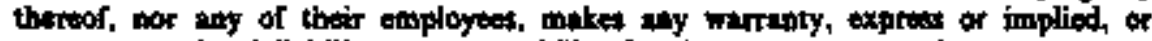

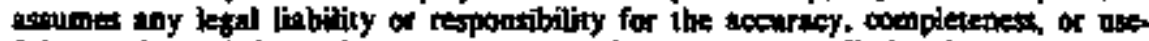
fuliness of eny information, apperatus, peoduct, or process bisclosed, or represeats

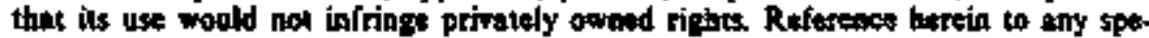
citis compereiat produch process, or service by trade name, trademark, manyfoc-

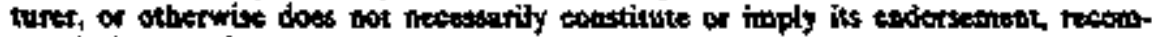

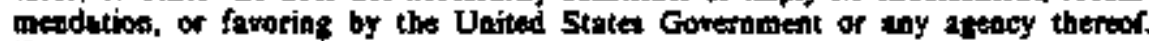
The views and opinions of authors expresed hereid do pot necestatity stute or reflect thoce of the Utited States Covertituent of say ageiry thereol. 


\section{DISCLAIMER}

Portions of this document may be illegible electronic image products. Images are produced from the best available original document. 
NUREG-0540

Vol. 19, No. 9

\section{Title List of Documents Made Publicly Available}

September 1-30, 1997

Date Publlined: Norember 1997

E B. Morrin, NRC Project Manager

Otilice of the Chitf Information Oflicer

U.S. Nuclear Regulatory Commission

Washfngton, DC 20555-0001

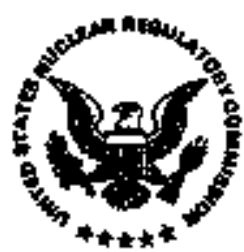




\section{CONTENTS}

Page

Preface $\ldots \ldots \ldots \ldots \ldots \ldots \ldots, \ldots, \ldots \ldots, \ldots, \ldots, \ldots, \ldots, \ldots, \ldots, v$

Arrangement of Items by Docket Number $\ldots \ldots \ldots \ldots \ldots \ldots \ldots \ldots \ldots \ldots \ldots$ xi

Alphabetic Arrangement of Docket Items by Facility Name $\ldots \ldots \ldots \ldots \ldots \ldots \ldots$ xvii

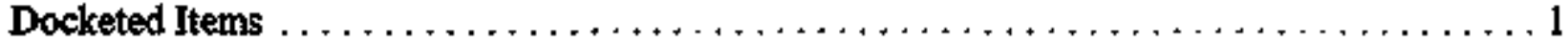

Low-Lewel Radwaste Disposal Sites - Docket $27 \ldots \ldots \ldots \ldots \ldots \ldots \ldots$

Domestic Licensing of Source Material - Docket $40 \ldots \ldots \ldots \ldots \ldots \ldots \ldots \ldots \ldots$

Domestic Licensing of Production and Utilization Facilities -Docket $50 \ldots \ldots \ldots \ldots, 14$

Standardized Nuclear Power Plant Design - Docket $52 \ldots \ldots \ldots \ldots \ldots \ldots \ldots \ldots, 138$

Domestic Licensing of Special Nuclear Material --Docket $70 \ldots \ldots \ldots \ldots \ldots \ldots 141$

Packaging of Radioactive Material for Transport and Transportation of Radioactive

Material Under Certain Conditions - Docket $71 \ldots \ldots \ldots \ldots \ldots \ldots \ldots \ldots \ldots, 148$

Licensing of Independent Spent Fuel Storage - Docket $72 \ldots \ldots \ldots \ldots \ldots \ldots \ldots$ ss

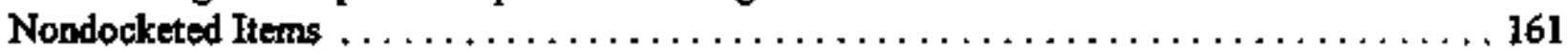

$10 \mathrm{CFR}$ - Commission Meeting Transcripts and Reports . . . . . . . . . . . 161

ACNW - Advisory Committee on Nuclear Waste $\ldots \ldots \ldots \ldots \ldots \ldots \ldots \ldots 161$

ACRS - Advisory Committe on Reactor Safeguards Documents , . . . . . . . . 162

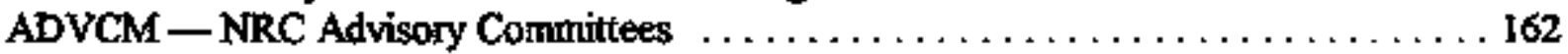

COMMISSION - NRC Commissioner Correspondence $\ldots \ldots \ldots \ldots \ldots \ldots \ldots \ldots . \ldots \ldots 2$

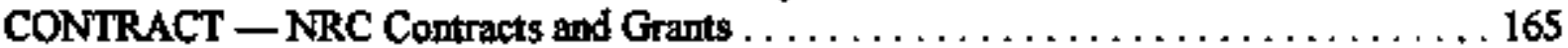

FOIA - Freedom of Information Act Requests and Appeals $\ldots \ldots \ldots \ldots \ldots \ldots 169$

GEN TECH IS - Generic Technical Issues $\ldots \ldots \ldots \ldots \ldots \ldots \ldots \ldots \ldots \ldots \ldots$ I80

L\&E - Inspection and Enforcement Issuances $\ldots \ldots \ldots \ldots \ldots \ldots \ldots \ldots \ldots \ldots \ldots, 180$

MISC - Public Document Room Miscellaneous $\ldots \ldots \ldots \ldots \ldots \ldots \ldots \ldots \ldots \ldots, 182$

NUREG - NUREG Reports and Related Comespondence $\ldots \ldots \ldots \ldots \ldots \ldots \ldots \ldots 2$

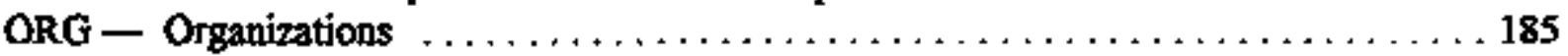

PR — Proposed and Final Rules $\ldots \ldots \ldots \ldots \ldots \ldots \ldots \ldots \ldots \ldots \ldots \ldots \ldots \ldots \ldots, \ldots \ldots \ldots$

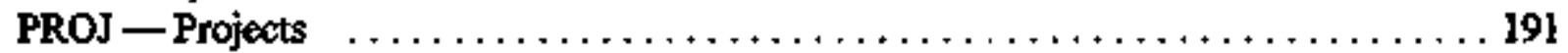

PT21 - 10 CFR Part 21 Deficiency Reports $\ldots \ldots \ldots \ldots \ldots \ldots \ldots \ldots \ldots \ldots \ldots . \ldots \ldots$

QA999 - Vendor Quality Assurance Inspections . . . . . . . . . . . . . . . . 193

REG CERT - Registration of Certificates for Sealed Sources and Devices . . . . . . . 193

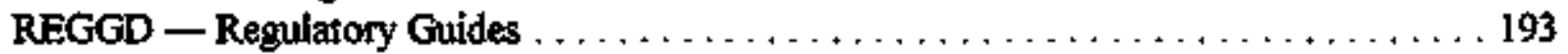

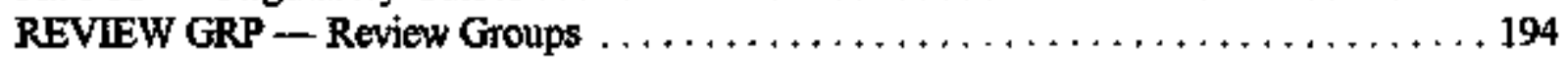

SECY - Staff Position Papers Prepared for the Commission . . . . . . . . . . . . 194

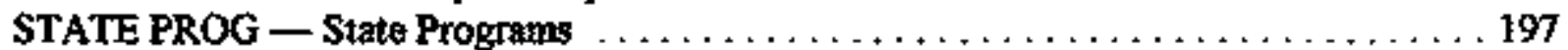

TOPREP — Topical Reports and Related Correspondence $\ldots \ldots \ldots \ldots \ldots \ldots \ldots \ldots 20 \ldots$

WASTE MGMT — Waste Management Documents . . . . . . . . . . . . . . 204 


\section{CONTENTS}

Page

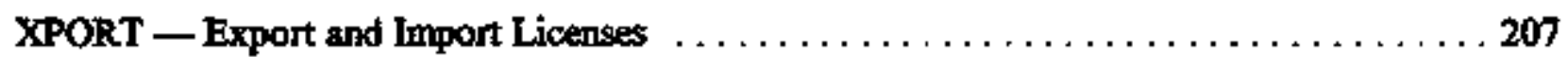

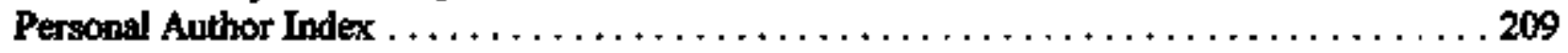

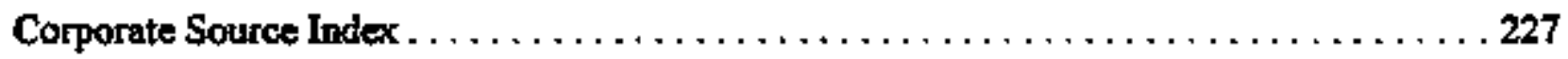

Report Number Index $\ldots \ldots \ldots \ldots \ldots \ldots \ldots \ldots \ldots \ldots \ldots \ldots \ldots \ldots \ldots \ldots \ldots \ldots$

Cross Reference of Enclosures to Principal Documents $\ldots \ldots \ldots \ldots \ldots \ldots \ldots \ldots \ldots$

Appendix A - Definitions of Docket 50 Categories $\ldots \ldots \ldots \ldots \ldots \ldots \ldots \ldots \ldots \ldots$

Appendix B - Definitions of Nondocketed Categories $\ldots \ldots \ldots \ldots \ldots \ldots \ldots \ldots \ldots \ldots$ B - 1 


\section{PREFACE}

The Title List of Documents Mode Publicly Avoulable is a monthly publication. It describes the information received and published by the U.S. Nuclear Regulatory Commission (NRC). This information inchudes (1) docketed material associated with civilian nuclear power plants and other uses of radioactive materials and (2) nondocketed material received and published by NRC pertinent to its role as a regulatory agency. As used here, docketed does not refer to Court dockets; it refers to the system by which NRC maintains its regulatory reconds. This series of documents is indexed by a Personal Author Index, a Corporate Soutec Index, and a Report Number Index.

The docketed information in the Title List includies the information formerly issued through the Department of Energy publication Power Reactor Docket Informution, last published in January 1979. NRC documents that are publicly available may be examined without charge at the NRC Public Doctument Room (PDR). Duplicate copies in paper, microfiche, or (selectively) diskette, may be obtained for a fee.
Standing orders for certain categories of docuinents are also available. Clients may search for and order desired titles through the PDR computerized Bibliographic Retrieval System (BRS), which is accessible both at the PDR and temotely. BRS aiso has some types of doctuments in full text. Other recent, selected oxcuments, as well as information about the PDR, may be viewed/downloaded from an electronic bulletin board. The PDR is staffed by professional technical fibrarians, who provide teference assistance to users. See NOTES at the end of the preface for information about reaching the PDR. Microfiche of the docketed information listed in the Title $L$ ist is available for saie on a subscription basis from the National Technical Information Service (NTTS). See NOTES at the end of the Preface for the complete NITS address.

We encourage your comments, criticisms, and suggestions. In particular, if there are title descriptions that are not meaningful, please let us know so that we can correct the database. Insofar as passible, we would like this document to be a valuable part of your reference material.

\section{DOCKETED ITEMS}

Each utility ot institution engaged in a nuclear activity licensed and regutated by the U.S. Nuclear Regulatory Conmission (NRC) is assigned a docket number. As used by NRC, docket numbers refer to the particular systen by which NRC maintains its regulatory recosds, not to law court dockets. The first two figits of a docket number identify the type of muclear activity that NRC regulates. These docket categories are as follows:

\author{
27 - Low-Level Radioactive Waste Disposal Sites \\ 30 - Byproduct Material Licenses \\ 40 - Nuclear Source Material Facilities \\ 50 - Nuclear Reactors and Fuel Reprocessing \\ Plants \\ 52 - Standard Design Certification, Early Site \\ Permit, and Combined Licenses for Nuclear \\ Power Plants \\ 70 - Special Nuclear Materials
}




\section{DOCKETED ITEMS (cont.)}

71 - Design of Packages for Transport of Radioactive Material and Quality Assurance Programs

Except for Docket 27, these numbers contespond to the parts of 'Title 10 in the Code of Federul Rezulations (10 CFR) that cover regulations pertaining to those types of licenses. The

\section{2 - Independent Spent Fuel Storage Installations}

Iemaining digits of a docket number uniquely identify a specific facility or institution. For example, Docket 50-317 identifies the Calvert Chiffs Nuclear Power Plant, Unit 1.

\section{(1) P. Operating license stage doevments \& correspondence}

\section{(2)}

8907570172 Commends util for exmplary hospitality extended to visiting Soviet technical porking grourp on 890608 .<smiles>[CH]</smiles>

(5)

6) (7)
1 NRC/PDR Document category (see Appen$\operatorname{dix} A)$.

2. NRC/PDR Accession Number (year, month, day, and seguence number of item).

3. Description of information contained in the document.

4. Personal author(s), An asterisk in this position indicates that there is no personal author.

5. Author affiliation (NRC, corporate, agency, or nonindividual source).

6. Date of document described.

7. Recipient of the document.

8. Recipient affiliation (NRC or cotporate).

9. Pages in this document.

10. This five-digit number is the NRC $48 \mathrm{X}$ microfiche designation.
11. The three-digit portion of this entry is the number of the frame on which this document starts.

12. This five-digit number is the microfiche on which this document ends.

13. The thre-digit portion of this entry is the number of the frame on which this document ends.

The Docketed Items list is arranged sequentially by Docket Number, category, and then by date of principal items. Entries indented and preceded by a dash are items that were submitted as enclosures to principal items. In general, indented items are not in accession number sequence. If the docketed document has a contract (job code) number of a formal report nturber, it will appear in the last line of the item following entry 5. 


\section{NONDOCKETED ITEMS}

The nondocketed items are divided into the catepories used for filing and searching in the NRC Public Document Room. These categories and their symbols are as follows:

10 CFR 9.7-Conmission Meeting Transeripts and Reposts

ACRS-Advisory Committee on Reactor Safeguards Doctuments

ADVCM-NRC Advisory Committees

COMMISSION-NRC Commissioner

Correspondence

CONIRACT-NRC Contracts and Grants

FOLA-Freedom of Information Act

Requiests and Appeals

GEN TECH IS-Generic Technical Isstses

I\&E-Inspection and Enforcement Issuances

I\&E Manual-NRC Inspection Manual

I\&E MISC-Draft Generic

Communications

MISC-Public Document Room

Miscellaneous

NUREG--NRC Staff and Contractor Formal Reports, Conference Proceedings, Brochures, International Agreement Reports, and Related Correspondence

ORG-Organizations

PR-Proposed and Final Rules

PRESS-Press Releases

PRM-Petitions for Rulemaking

PROJ-Projects
PT 21-10 CFR Part 21 Deficiency Reports

QA 999-Vendor Quality Assurance Inspections

RCSSD-Registration of Certificates for Sealed Sources and Devices

REGGD-Regulatory Güdes

RES-Regearch and Technical Assistance

Reports (1974 through 1988)

REVIEW GRP-Review Groups

RM-Rulemakings

SAI - Strategic Assessment Issue Papers

SECY-Staff Position Papers Prepared for the Commission

SPCH-Speeches

STATE PROG-State Programs

TOPREP-Topical Reports and Related Comrespondence

WASH-AEC-Issued Formal Reports and

Related Correspondence

WM-Waste Management Documents

WMRES - Waste Management Contract

Research Doctments

XPORTLIC-Export and Import Licenses

Appendix B lists these categories and describes the material available in each. Omission of a category in the listing for a docket indicates that no documents in that category were filed in the time period of the report.

The principal elements of the nondocketed items are- 


\section{NONDOCKETED ITEMS (cont.)}

(1) NUREG $\rightarrow$ NRC Staff and Contractor Formal Reports, Conference Proceedings, Brochures, Interrational Agreement Reports, and Related Correspondence

(2)

(4) 8910100307 NUREG/CR-5340 DATA SUMMARY REPORT FOR FISSION PRODUCT
RELEASE TEST V-2

(5)

(6)

OSBORNE, M.F, COLLINS, J.L, LORENZ, R.A. Oak Ridge National Laboratory.<smiles>[C+]=Cc1ccccc1</smiles>

FIN B-0127. ORNL/M-11105. 89/09/30. Division of Systems Rexearch (Post 880717) 69 pp.

(1) (1) (1)

$51409: \sqrt{6} 65-51409+134$.

1. Nondocket antegory.

2. NRC/PDR Accession Number (year, month, day, and sequence number of item).

3. NRC Report Namber.

4. Title and description of information contained in the document.

5. Personal author(s). An asterisk in this position indicates that there is no personal author.

6. Corporate, agency, or other nonindividual source.

7. Contract or financial identification number source.

8. Secondary report number, if any,

9. Date of document described

10. NRC organizational recipient (or sponsor).

11. Pages in this document.

12. This five-digit number is the NRC $48 \mathrm{X}$ microfiche designation.

13. The three-digit portion of this entry is the number of the frame on which this document starts.

15. The three-digit portion of this entry is the number of the frame on which this document ends.

The Nondocketed Items list is arranged sequentially by Accession Number within category. Indented items preceded by a dash are enclostares to unindented items.

Ordering and Viewing Documents:

1. The address for GPO is:

Superintendent of Documents

U.S. Government Printing Office

PO. Box 37082

Washington, DC 20402-9328

To place orders, send a check or money order payable to the Superintendent of Documents. GPO Deposit Account holders may charge their orders by calling 202-512-2249. 


\section{NONDOCKETED ITEMS (cont.)}

2. The address for NTIS is:

National Technical Information Service 5285 Port Royal Road

Springfield, Virginia 22161

To place orders for or to obtain price information about NRC publications at NTIS, elther write to NTIS or call the Sales Desk at 703-487-4650. Specify the NRC report number and title when you place your order. You may charge publications to American Express, Visa, or Mastercard credit cards; supply the charge card number when placing your order.

3. The location of the PDR is:

$2120 \mathrm{~L}$ Street, NW,

Waşinington, DC

The mailing adidress is:

U.S. Nuclear Regulatory Commission

Public Document Room

Mail Stop LI-6

Washington, DC 20555-0001
Telefacsimile

202-634-3343

Telephone

202-634-3273 or 1-800-397-4209

Internet e-mail

\section{PDR@NRC.GOV}

For more information about PDR services, for reference assistance, to place an order for documents, or to arrange for access to the PDR computer retrieval system, write, e-mail, fax or phone. When ordering documents listed in this publication, please identify the Doctiment Accession Number for each citation. Reproduction charges and method of payment, based on the current reproduction contract, may be obtained from the PDR.

To access the PDR electronic bulketin board on FedWorld, contact the FedWorld Help Desk at $703-487-4086$. 


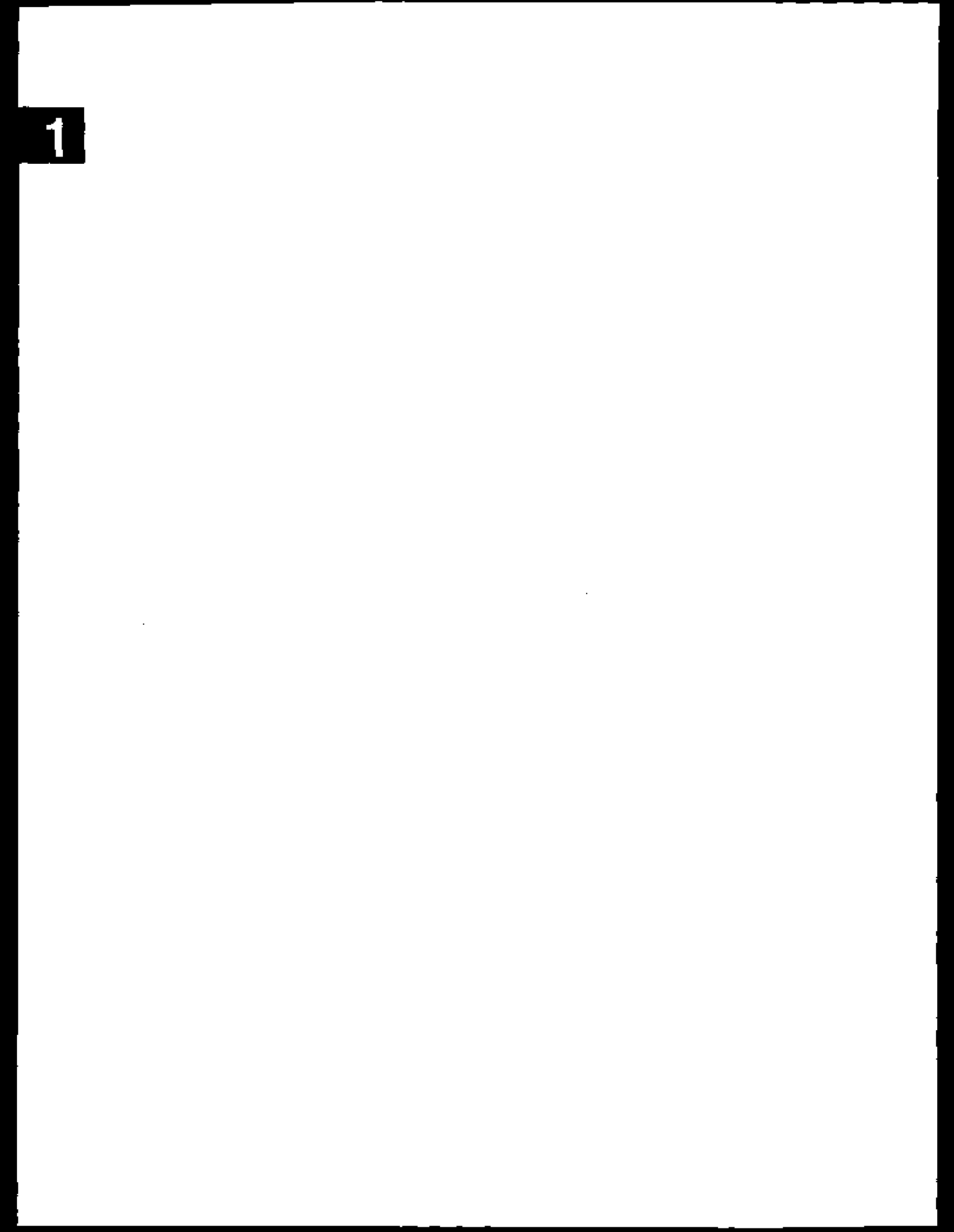




\section{ARRANCEMENT OF ITEMS BY DOCKET NUMBER}

\begin{tabular}{|c|c|}
\hline \multicolumn{2}{|c|}{ 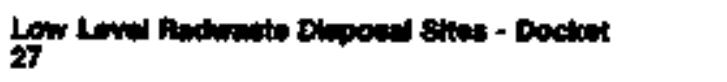 } \\
\hline $27-049$ & LOUISYLE KY, US. ECOLOGY, NC. \\
\hline DOCKET & LANanowin \\
\hline \multicolumn{2}{|c|}{ 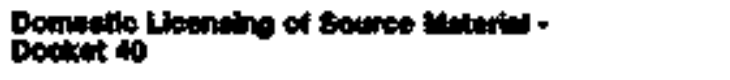 } \\
\hline $40-0235$ & 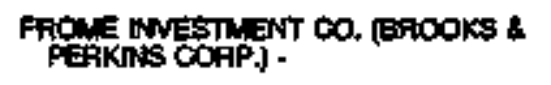 \\
\hline $40-0099$ & UATCO WISERLS CORP, RWERTON, \\
\hline $40-1162$ & WESTERM MUKCEAR, WW. LAKEWOOD. \\
\hline $40-2259$ & PATHFINDEA NINES CORP, RMERTON, \\
\hline $40-2994$ & Fin $\infty_{n}$ ASHTABULA, OH \\
\hline 403982 & AUEOSIGNLL, WC., WETPOPOLIS, \\
\hline 40.3450 & ATLAS CORP, DENMER, CO \\
\hline $40-4492$ & AHZFICAN MUCAEAR CORP, CASPAR, \\
\hline $40-6802$ & $\begin{array}{l}\text { PATHFINOER WINES COAP., SHALEY } \\
\text { EASIN, WY }\end{array}$ \\
\hline $40-0050$ & 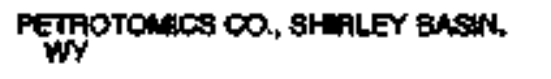 \\
\hline $40-040$ & CABGT CORP. BOYERTONN, PA \\
\hline 40.7102 & 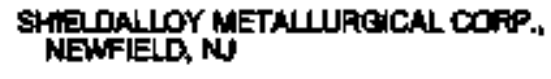 \\
\hline $40-7597$ & FOOTE WNERAL CO. EXTON, PA \\
\hline 40.7455 & WHITT AKEA COFP. $10 S$ ANEELES, CA \\
\hline $40-75 \% 6$ & FANSTEE, WHC+* NOTTH CHICAGO, IL \\
\hline $40-7604$ & $\begin{array}{l}\text { BP CHENILALS ANERLCh, INC, LIMA, } \\
\text { OH }\end{array}$ \\
\hline $40-8027$ & SECUOYAH FUELS COMP., GOFE, OK \\
\hline $40=004$ & RIO ALCON MiN"WG COAP, MOA, UT \\
\hline 408102 & EXXN COFP, HOLISTON, TX \\
\hline $40-6002$ & TOTAL MINERALS COFP, CASPER, WY \\
\hline 40-8584 & KENECOTT UAANUM CO., AAWHINS, \\
\hline 40-8681 & MTEFNATXONAL UFANIUM USA COAF. \\
\hline 40-8724 & CHבMETON CORP, PROMDENCE Ai \\
\hline $40-9767$ & 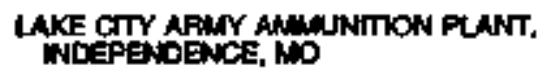 \\
\hline $40-8779$ & MOLYCOAP, WK, LOS ANLEEES, CA \\
\hline $40-6779$ & AFMY, DEFT. OF, TOOELE, UT \\
\hline $40-6789$ & AFMY, DEPT. OF, SAVANINA, IL \\
\hline $40-6005$ & CEPAG, NG, MLWAUKEE, W \\
\hline $40-6814$ & MPMY. DEFT. OF, YUMA, AZ \\
\hline 406888 & AFKY, DEPT, OF, MADISON, IN \\
\hline
\end{tabular}

\begin{tabular}{|c|c|}
\hline Bookst & Nimine \\
\hline $40-005$ & FOWER RESOUACES, WC, GLENROCK, \\
\hline $40-8002$ & ATLANTIC RACHFIELD CO. GRAMTS, $\mathrm{NM}$ \\
\hline 40.8000 & HOMESTAKE HINWG CO, GRNTS, NH \\
\hline $40-8904$ & $\begin{array}{l}\text { SOHQ WESTERN MMENG CO., SALT } \\
\text { WAKE CITY, UT }\end{array}$ \\
\hline $40-9005$ & OUNIAA MIIING CO., GAANTS, MU \\
\hline $40-8 \div 07$ & 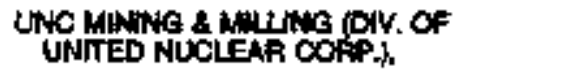 \\
\hline $40-0040$ & 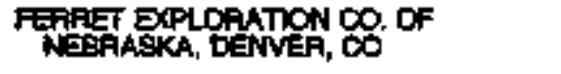 \\
\hline 40-648 & 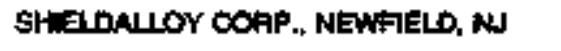 \\
\hline 40.0064 & 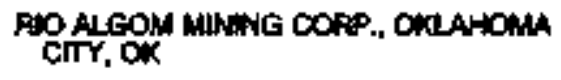 \\
\hline $40-489$ & HYOAO RESOUNCES, ING, DALLAS, TX \\
\hline 40-essi & PATHFINDEA KUNES COAP. MALS, WY \\
\hline 40-4ass & $\begin{array}{l}\text { ENVIROCAFE OF UTAH, INK., SALT } \\
\text { LAKE CITY, UT }\end{array}$ \\
\hline $40-0015$ & $\begin{array}{l}\text { HAFTLEY A HAFTEY LANDFLL } \\
\text { MONA), BAY COINTY, MI }\end{array}$ \\
\hline 40-90ez & $\begin{array}{l}\text { HAFTLFY \& HAFTUEY LANDFILI (SCA). } \\
\text { BAY COUNTY, WH }\end{array}$ \\
\hline
\end{tabular}

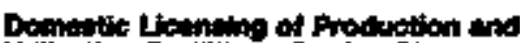
Utimation Faction - Docket of

\begin{tabular}{|c|c|}
\hline $50-2$ & FOAO NUCLEAA REACTOA \\
\hline 500 & HWIAN POINT STATONG, UNT : \\
\hline $50-5$ & $\begin{array}{l}\text { PENWSYLVANAA STATE LNYY. } \\
\text { AESEARCH REAGTOA }\end{array}$ \\
\hline $50 \times 10$ & $\begin{array}{l}\text { OFESDEN NUCLEAR POWEA STATMON, } \\
\text { UNIT I }\end{array}$ \\
\hline $60-16$ & $\begin{array}{l}\text { ENPICO FEFINI ATONHC POWER PLANT, } \\
\text { UNTT } 1\end{array}$ \\
\hline $50-27$ & $\begin{array}{l}\text { WASHINGTCN STAFE UNY. RESEAPCH } \\
\text { REACTOR (TRIGA) }\end{array}$ \\
\hline $50-29$ & $\begin{array}{l}\text { YMNKEEROWE NUCLEAR FOWZT } \\
\text { STATKON }\end{array}$ \\
\hline $50-30$ & $\begin{array}{l}\text { FLUA BROOK REEEAFCH FEACTOA } \\
\text { FACHTY }\end{array}$ \\
\hline
\end{tabular}

59.54

50-89

Bo-e?

50-11k

$50-116$

50.128

$50-130$

50-146

\section{CINTICHEM}

UNY. OF FLOAIDA TRAINANE REACTOR EENERAL ATONICS

UNDV. OF AFUZONA REZSEAACH GEACTOF

IOWA STATE WWV, RESEARCH REACTOR

TEXAS A\&H UNN, RESEAACH AEMGTOF

HUMBOLO BAY PONEA PLANT, UNT I SAXTON NUCAEAR EOFERHENTAL FACILITY
Pog

5

5

6

6

6

6

6

6

8

6

7

14

14

14

14

15

15

15

15

15

16

16

16

16

16

17

17

17

17 


\begin{tabular}{|c|c|}
\hline Dochit & Non \\
\hline $50-150$ & $\begin{array}{l}\text { OHWO STATE UNN. RESSARCH } \\
\text { WACTOF }\end{array}$ \\
\hline $50-155$ & BIG POCK POMT NUCLEAR PLANT \\
\hline 50.156 & $\begin{array}{l}\text { UNIY. OF WISCONSN RESEAFCH } \\
\text { PtEACTOF }\end{array}$ \\
\hline $50-160$ & $\begin{array}{l}\text { GEORGIA INSTIJUTE OF TECHNOLOGY } \\
\text { FESEARCH IEACTOA }\end{array}$ \\
\hline $50-160$ & OENEFAL ATOMKS \\
\hline $50-1=6$ & $\begin{array}{l}\text { UNY, OF MARYLAM RESTEARCH } \\
\text { RE AGTOR }\end{array}$ \\
\hline $\operatorname{so-171}$ & $\begin{array}{l}\text { PEACH EOTTOM ATCWIC POMEA } \\
\text { STATION, UNIT I }\end{array}$ \\
\hline $60-184$ & $\begin{array}{l}\text { NATICHAL INSTITUTE OF STANDARDS } \\
\text { \& TECHNOLOBY FEACTOA }\end{array}$ \\
\hline $50-188$ & PLUM EAOOK REACTOA - MOCKUP \\
\hline $50-168$ & 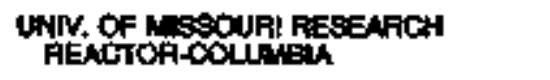 \\
\hline $50-199$ & $\begin{array}{l}\text { FHOCE ISLANO \& FFOVDENCE } \\
\text { PLANTATIONS AEC }\end{array}$ \\
\hline $50-200$ & SAN ONOFAE NULCLEAR STATION, LNIT \\
\hline 50.219 & HADDAM NECK PLNT \\
\hline $50-219$ & $\begin{array}{l}\text { OYSTER CREEK NUCLEAA PONEA } \\
\text { PUANT }\end{array}$ \\
\hline 50.20 & NINE MUE FOWT MUCLEAR STATRON, \\
\hline $50-203$ & $\begin{array}{l}\text { UWIERSTY OF WASSACHNSETS, } \\
\text { LOWELL RESE APH REACTOR }\end{array}$ \\
\hline $50-207$ & $\begin{array}{l}\text { DPESOEN NUCAEAR POWER STATION, } \\
\text { UHTT } 3\end{array}$ \\
\hline 50.243 & $\begin{array}{l}\text { OAECGN STATE UNN, RESEARCN } \\
\text { REACTOR }\end{array}$ \\
\hline $50-244$ & 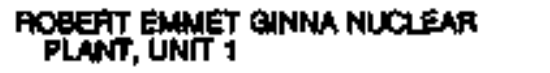 \\
\hline $50-245$ & $\begin{array}{l}\text { MILLSTONE NUCLEAR POWEA } \\
\text { STATION, UNIT } 1\end{array}$ \\
\hline $50-247$ & NOLAN FOANT STATION, UNIT 2 \\
\hline $50-240$ & $\begin{array}{l}\text { DFESOEN NUCHEAR POWEA STATION, } \\
\text { UNIT } 3\end{array}$ \\
\hline $50-200$ & TURKEY POINT PLANT, UNT a \\
\hline $50-251$ & TUAKEY POWT PUANT, LAWT 4 \\
\hline $50-20$ & $\begin{array}{l}\text { UNy, OF NEW MEACO RESEAFCA } \\
\text { REATOR }\end{array}$ \\
\hline $50-2 \$ 4$ & QUAD-CITES STATOOL LNW : \\
\hline 50-255 & PALSADES NLCLEAR PLANT \\
\hline $60-250$ & $\begin{array}{l}\text { BAONAS FEFRY NUCLEAR POWEA } \\
\text { STATON, UNT } 1\end{array}$ \\
\hline $50-200$ & $\begin{array}{l}\text { EAOWNS FERFY NUCLEAF POWEA } \\
\text { STATKON, UNIY } 2\end{array}$ \\
\hline $50-261$ & H.B. ROEINSON ELANT, UNTT 2 \\
\hline $60-205$ & 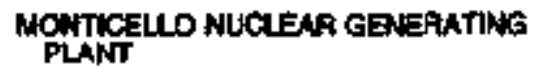 \\
\hline $50-204$ & \\
\hline
\end{tabular}

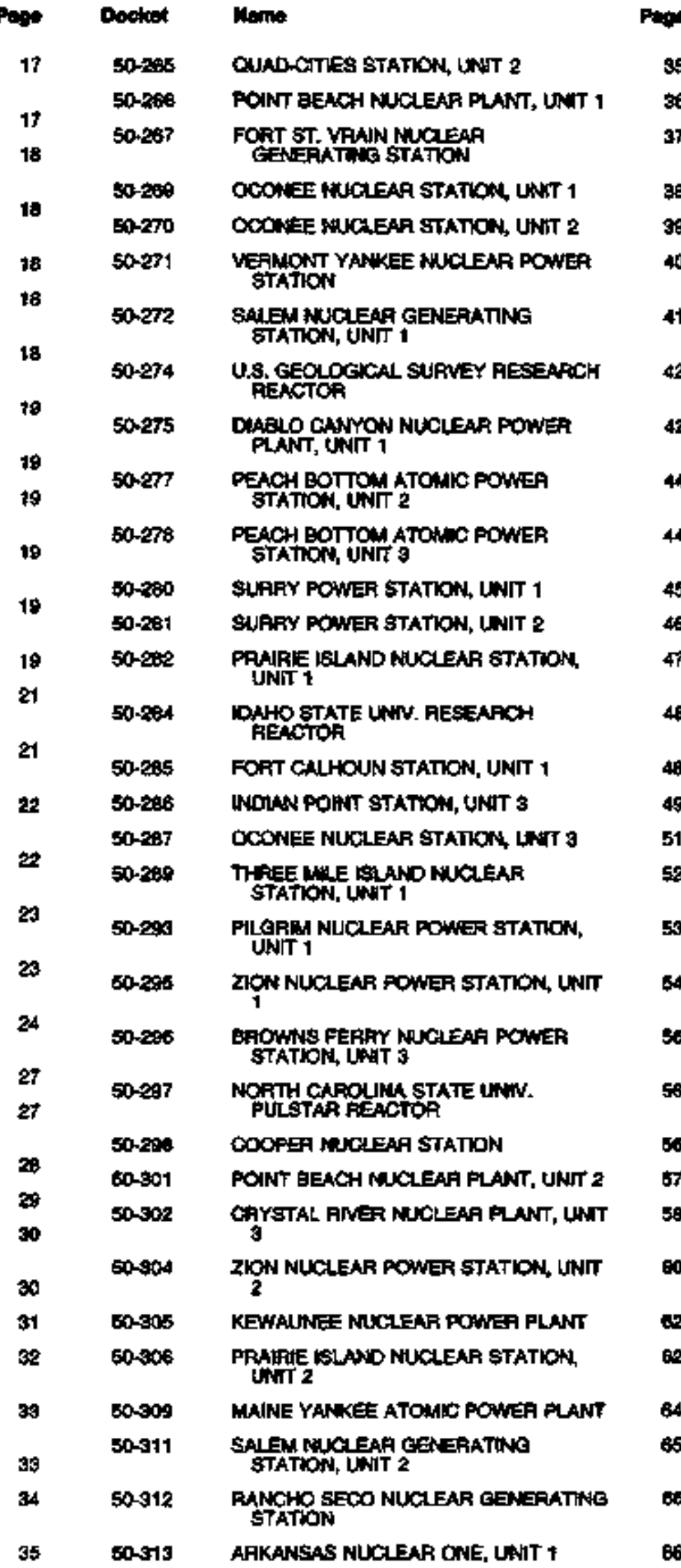




\begin{tabular}{|c|c|c|c|c|c|}
\hline Dediret & Neme & phos & Dectet & finge & Page \\
\hline S0-sts & $\begin{array}{l}\text { DONLAID C. COOK NULCEAA FONAR } \\
\text { PLANT, ENTT } 1\end{array}$ & 67 & $50-380$ & ARKANSAS MUCLEAA OHE, UNT 2 & 96 \\
\hline $50-018$ & $\begin{array}{l}\text { DOMALD C. COOK NUCLEAR FOWEF } \\
\text { FLANT, UNT } 2\end{array}$ & B8 & $50-309$ & $\begin{array}{l}\text { WHAMA, WCOUHRE NJCLEAF } \\
\text { STATION, UNT } 1\end{array}$ & 97 \\
\hline $50-917$ & $\begin{array}{l}\text { CALVEAT CLFTS NUCLENA POWER } \\
\text { PLANT, UNT I }\end{array}$ & e9 & $50-570$ & $\begin{array}{l}\text { WLLUAM B MOSUIRE NUCLEAR } \\
\text { STATION, UNT } 2\end{array}$ & $\theta$ \\
\hline $60-318$ & $\begin{array}{l}\text { CALVEAT CLFFS NLCLEAF POWER } \\
\text { PLAN, UNT } 2\end{array}$ & 70 & $\begin{array}{l}50-973 \\
50-374\end{array}$ & $\begin{array}{l}\text { LASAUE COUNTY \$TATION, UNIT I } \\
\text { LASALEF COUNTY STATION, UNIT } 2\end{array}$ & 100 \\
\hline $50-320$ & $\begin{array}{l}\text { THFEE WUE ISLAND NUCAEAR } \\
\text { STATON, WITIT }\end{array}$ & 71 & $50-382$ & $\begin{array}{l}\text { WATEFFORD GENGFATING STATION, } \\
\text { UNIT }\end{array}$ & 101 \\
\hline $50-31$ & EDWWN I. MATCH ANCLEAR FLANT, UNTT & 71 & $50<87$ & $\begin{array}{l}\text { SUBCUDHANNA STEN ELECTR:C } \\
\text { STATON, LAT } 1\end{array}$ & 102 \\
\hline so-sen & BHORGHAN MUCAEAA PONIEA STATION & 72 & 50-369 & $\begin{array}{l}\text { SUSOKEHANNA STEAM ELECTRKC } \\
\text { STATION, U:NT? }\end{array}$ & 103 \\
\hline $50-923$ & $\begin{array}{l}\text { DANBLO CANMON NUCLEAFI POWER } \\
\text { FUAN, UNH' } 2\end{array}$ & 72 & $50-368$ & ST. LUCTE PLANT, UNT 2 & 104 \\
\hline $50-324$ & $\begin{array}{l}\text { BRUNSWHCK STEAN EESTRIC PLANT. } \\
\text { UNIT } 2\end{array}$ & 74 & $50-390$ & WATTS EAT NUCAEAA FLANT, UNIT I & 105 \\
\hline $50-807$ & 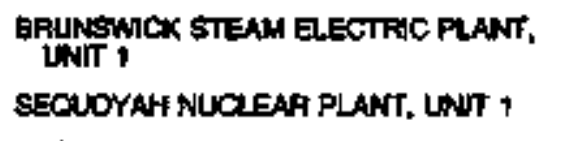 & 76 & $\begin{array}{l}50-391 \\
50005\end{array}$ & $\begin{array}{l}\text { WATTS BAF NUCEEAR FLANT, UNWT } 2 \\
\text { VRER C. SUMMER MUCAENF STATION, } \\
\text { UNT I }\end{array}$ & tos \\
\hline $50-328$ & SECUOYAH NUCLEAA PLANT, UNWT 2 & 77 & $50-397$ & WPPSS NUCLEAF PROUECT, UNIT 2 & 107 \\
\hline $50-89$ & MOLANO PLANT, UNT † & 78 & $50-460$ & $\begin{array}{l}\text { SHEAAON HARRIS NUCLEAR POWEA } \\
\text { FLANT, UNHT } 1\end{array}$ & 107 \\
\hline s0-331 & $\begin{array}{l}\text { WLLAND FLANT, UAT } 2 \\
\text { DUANE ARNOLO ENEAGY CENTER }\end{array}$ & 76 & 50.407 & UNN, OF UTAH RESEAPCH REACTOR & 109 \\
\hline $50-393$ & $\begin{array}{l}\text { JNAES A FITPATAKCK NUCLEAR } \\
\text { POWA PLANT }\end{array}$ & 79 & $\begin{array}{l}50-409 \\
50-410\end{array}$ & $\begin{array}{l}\text { WA CROSSE BOILNS WATER REACTOA } \\
\text { MNE WHLE PONT NUCOEAR STATION, } \\
\text { UNWT } 2\end{array}$ & $\begin{array}{l}108 \\
109\end{array}$ \\
\hline 50-70s & $\begin{array}{l}\text { BSAVEA VAUEY POWEA STATION, } \\
\text { UNIT I } \\
\text { ST. LUCIE PLANT, UNT } 1\end{array}$ & Bo & $50-12$ & $\begin{array}{l}\text { BSAYEA VALIEY PCWER STAKION, } \\
\text { LHWT } 2\end{array}$ & 109 \\
\hline $50-305$ & $\begin{array}{l}\text { MUSTONE NUCLEA FOWIR } \\
\text { STATON UNIT } 2\end{array}$ & 82 & $\begin{array}{l}50-413 \\
50-414\end{array}$ & $\begin{array}{l}\text { CATAWBA MUCLEAP STATION, UNIT } 1 \\
\text { CATAWBA NUCLEAP STATKN, UWT } 2\end{array}$ & $\begin{array}{l}110 \\
111\end{array}$ \\
\hline $60-338$ & NORTH ANNA PONER STATION, LNIT I & 86 & $50-416$ & EAHAND GULF RUCLEAR STATLN, UNIT & 112 \\
\hline $50-339$ & NORTH ANNA FOHAA STATION, UNT 2 & 88 & & & \\
\hline $50-341$ & 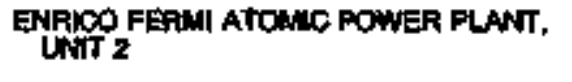 & 66 & $\infty 0-420$ & $\begin{array}{l}\text { MILLSTONE NUCLEAR POALA } \\
\text { STATON UNAT } 3\end{array}$ & 113 \\
\hline $50-344$ & TEOUAN NUCLEAR PLANT & $\mathbf{8 7}$ & $50-424$ & $\begin{array}{l}\text { ALVN W. VOGTLE AUCLEAA PAANT, } \\
\text { WNIT } 1\end{array}$ & 117 \\
\hline $50-346$ & 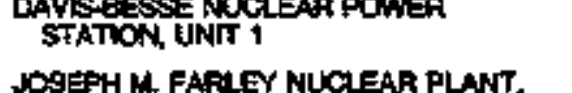 & 88 & $50-426$ & $\begin{array}{l}\text { ALVN W. YOGTLE NUGSEAA FLANT. } \\
\text { UHIT } 2\end{array}$ & 118 \\
\hline Darso & 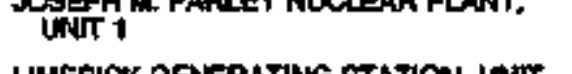 & $\infty$ & $\begin{array}{r}\text { STN } \\
4 \$ 37\end{array}$ & PLOATING NUCLEAR PLANT & 118 \\
\hline $50-352$ & UMEAICK OENERATING STATION, LAII & 69 & $50-430$ & EEUEFONTE NUCLEAP PLANT, UNIT 1 & 119 \\
\hline $50-353$ & UWEAICK GENEAATING STATION, JNTT & 99 & $50-498$ & BELLEFONTE NUCLEAR PLANT, UNIT 2 & 119 \\
\hline 50054 & HOPE CFEEK NUCLEAR STATON, UNT & 91 & $50-440$ & PEARY NGCLEAR FOWER PLANT, GNI & 118 \\
\hline 80.381 & $\underset{2}{\text { SAN ONOFAE NUCLEAP STATION UNT }}$ & pr & $\$ 0-441$ & $\begin{array}{l}\text { PERARY MUCLEAR POWEA PLANT, UNIT } \\
\mathbf{2}\end{array}$ & 121 \\
\hline $80-352$ & SAN CNKFFE NUCLEAR STATION, UNT & $\mathbf{m}$ & $\begin{array}{l}60-443 \\
50-444\end{array}$ & $\begin{array}{l}\text { SEABAOOK NUCLEAR STATION, UNIT } 1 \\
\text { SEAGFOOK NUCLEAR STATION, UNHT } 2\end{array}$ & 121 \\
\hline 50-364 & $\begin{array}{l}\text { WOEPH H. FAFLFY NUCLEAH PLANT, } \\
\text { UNIT } 2\end{array}$ & 94 & $50-445$ & $\begin{array}{l}\text { CONANCHE PEAK STEAMA ERCTRIC } \\
\text { STATKON, URT } 1\end{array}$ & 121 \\
\hline $50-399$ & EDMIN I, HATCH NUCLEAA PLANT, UNTT & 85 & $50-446$ & $\begin{array}{l}\text { DOMANCHE PEAK STEAM EECTRK } \\
\text { STATKON, UNAT }\end{array}$ & 122 \\
\hline
\end{tabular}




\begin{tabular}{|c|c|}
\hline Dockit & Mnen \\
\hline $\operatorname{stn}+40$ & BYRON STATON, LNT 1 \\
\hline STNS 留 & BYFON STATION, UNT 2 \\
\hline $5 T+50$. & BRANWOOD STATION, UNIT I \\
\hline STN 50 SOF & BFAIDWOOO STATIN, INT 2 \\
\hline $50-459$ & FIVEA BEND STATKON, UNT 1 \\
\hline $50-481$ & CLHSTON POWEA STATION, UNIT 1 \\
\hline STR+50- & WOLF CPEEK GENEFATING STATHON \\
\hline STR 460. & CALAWAY PLANT, UNIT 1 \\
\hline STN- & SOUTH TEXAS PRONECT, UNTT I \\
\hline STH +50. & SOUTH TEXAS PROAECT, UNT 2 \\
\hline STN-so- & WPPSS MLCLEAA PAOJECT. UNIT H \\
\hline STN-so & $\begin{array}{l}\text { PALO VEFUE NUCLEAR STATION, UNTT } \\
1\end{array}$ \\
\hline STN-so- & PALO VEFIDE NUCLEAR STATRON, UNET \\
\hline STN-50 & $\underset{3}{P A L O}$ VERDE MULCEAA STATION, UNIT \\
\hline $52-002$ & $\begin{array}{l}\text { SYSTEM EOP STANDAADIED } \\
\text { NLCLEAR POWEF PLANT DES. }\end{array}$ \\
\hline $52-008$ & 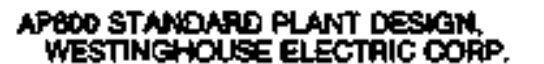 \\
\hline
\end{tabular}

\begin{tabular}{|c|c|c|c|}
\hline me & Docket & Mante & \\
\hline 123 & $70-0754$ & GENERAL ELECTAIG CO., PLEASANTON, & \\
\hline 124 & $70-0828$ & WEST UPERTY STATE COLLEE, WEST & \\
\hline 125 & $70-09999$ & $\begin{array}{l}\text { FOFIDA INSTITUTE OF TECHWNOLGY. } \\
\text { MELOSOUANE, FL }\end{array}$ & \\
\hline 128 & $70-0926$ & $\begin{array}{l}\text { CIMARRON COAP., OKLAHOMA GTY, } \\
\text { OK }\end{array}$ & \\
\hline 128 & $70-00085$ & $\begin{array}{l}\text { WASACHUSETTS INSTIJUTE OF } \\
\text { TECHNOLOGY, CAMBREE WA }\end{array}$ & \\
\hline 1 & 70.1069 & FOFIDA, URY, OF, GANESMLE, 凡L & \\
\hline 131 & $70-1100$ & $\begin{array}{l}\text { COMEUSTION ENGINEERWWG, INC. } \\
\text { MINDSOA, CT }\end{array}$ & \\
\hline 132 & $70-1113$ & GENEAAL ELEGTRIC CO., MLMANGTON & \\
\hline 133 & $70-1151$ & $\begin{array}{l}\text { WESTINGHOUSE ELECTRIC CONP, } \\
\text { PIIISELAEH, PA }\end{array}$ & \\
\hline 134 & $72-1204$ & 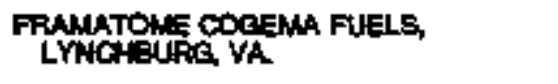 & \\
\hline 134 & $70-1252$ & ELOW NTERATIONAL CO. & \\
\hline 136 & $70-1257$ & $\begin{array}{l}\text { SIERENS NUCLEAA POWEA CORP. } \\
\text { AKCHLAND, WA }\end{array}$ & \\
\hline & $70-1974$ & DAHO STATE UNN, POCATELLO, ID & \\
\hline 137 & 70.1426 & TRANSNIXCLEAR, WC., FALLS CHISACH, & \\
\hline 138 & $70-1703$ & EASTMAN KODAK CO., ROCHESTER, NY & \\
\hline 13: & 70.1799 & $\begin{array}{l}\text { AUEGHENY HEATH, EO. \& RESEARCH } \\
\text { CORP, PITSBUTAG, PA }\end{array}$ & \\
\hline & $70-2009$ & GENZRAL ELECTRIC CO., SNN JOSE CA & \\
\hline & $70 \leq 025$ & $\begin{array}{l}\text { FRONTEP TECHMOLOGY DOPF. } \\
\text { XENIA, OH }\end{array}$ & \\
\hline 141 & $70-3070$ & $\begin{array}{l}\text { LOULSANA ENGBGY SERVICES. } \\
\text { WASHNGTON, DC }\end{array}$ & \\
\hline 141 & 70,3095 & $\begin{array}{l}\text { BAW SHENLOW LAYB OSPOSAL AREA } \\
\text { (SDA). PAFS TOWNSHIP, }\end{array}$ & \\
\hline 142 & $70-5049$ & $\begin{array}{l}\text { U.S. ENFAGHMENT COAP. (AVLIS } \\
\text { GNRICHMENT PLWND }\end{array}$ & \\
\hline $\begin{array}{l}142 \\
142\end{array}$ & $70-7001$ & 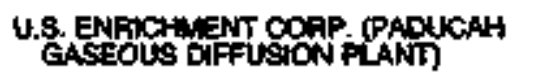 & \\
\hline 142 & 70.7002 & $\begin{array}{l}\text { U.S. ENRAYHENT COAP. } \\
\text { PORTSHOUTH GASEOUS DFFUSION }\end{array}$ & \\
\hline 142 & DOCKET & UNKNOWN & \\
\hline 149 & \multicolumn{2}{|c|}{ 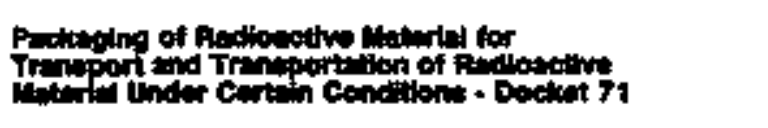 } & \\
\hline $14 \$$ & m-0023 & TENNECO BAS & \\
\hline 149 & $71-0040$ & AMERSHAM CORP. & \\
\hline & $71 \cdot 0044$ & LMTED ARRLINES, INC. & \\
\hline & $71-0000$ & THANS WORLO AIRLENES, WC. & \\
\hline 140 & $71-0102$ & $\begin{array}{l}\text { SOURCE PAODUCTION \& EOUIPUENT } \\
\text { D., ING. }\end{array}$ & \\
\hline 143 & $71-0131$ & TING INSTITUTE OF ALASKA & \\
\hline
\end{tabular}

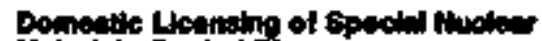
Dintind - Dookst to

70-0027 BAPCOCK \& WLCOX CO., ETNCHOURG,

14

700036 COMBUSTION ENGNEERNG, WC. HEMATTTE, HO

700113 PENSYCVAMA STATE UNIV,

70-0121 NTEAIOR, CEPT. OF, DENVER, $\infty$

70-0T33 CLEVITE COFP. CLFELAN, OH

70-0135 BABCOCK \& WILCOX CO. APCuLO, PA

70.0143

MUCIFAR FUE SEAVCES, ANG. ERWMN, DN

70-0159 PUADUE UNIV., WEST LAFAYETTE, IN

700157 TEXAS, UNIY. OF, AUSTHL TX

70-0SB4 BAECOCK \& WLCOX CO, PARKS TOWNSHF, PA

70-OBSH COMHERCE, DEPT. OF, NATIOWAL WSTIUTE OE STANDAFDS :

$70-0600$ SEATTLE UNIV, SEATTE, WA

$70-0002$

70-0eg7

AHIY, DEPT. OF, DOVEF, NJ

$70-674$

CNTICHEM, INKE, TUXEDO, NY

GENERAL ATOMICS, SAN DIEEO, CA 


\begin{tabular}{|c|c|c|c|c|c|}
\hline Docket & Mane & Pege & Docket & Nome & Pege \\
\hline $7 t-0139$ & TULSA GANMA RAY, INC. & 140 & $71-0 \$ 52$ & $\begin{array}{l}\text { SAM-SON NSPECTION \& TECHWNCAL } \\
\text { SERVEES, INC. }\end{array}$ & 180 \\
\hline $\begin{array}{l}71-0168 \\
71.0762\end{array}$ & $\begin{array}{l}X \text {-A-I TESTMG OF MICMUAN } \\
\text { ERASCO SEAVEES, NC. }\end{array}$ & $\begin{array}{l}148 \\
149\end{array}$ & $71-0050$ & $\begin{array}{l}\text { PRCFESSONAL WEONNG ASSOCIATES, } \\
\text { INC. }\end{array}$ & 180 \\
\hline $\begin{array}{l}71-0215 \\
71-0221 \\
71-0222\end{array}$ & 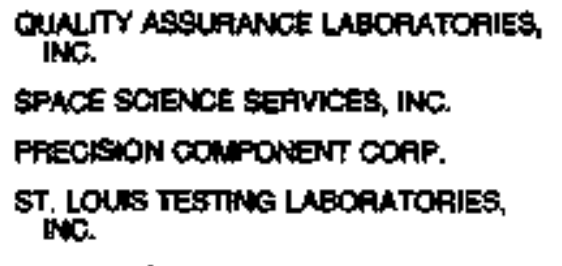 & $\begin{array}{l}149 \\
149 \\
149\end{array}$ & $\begin{array}{l}71-0607 \\
71-0073 \\
71-0077 \\
71-0679 \\
71-0082\end{array}$ & $\begin{array}{l}\text { PITT-DES HOINES, HVC. } \\
\text { THIELSCH ENGINEERING, INC. } \\
\text { ANYLL COFF. } \\
\text { TESTMASTER INSPECTKON } \infty \text {. } \\
\text { PACIFIC TECHINICAL INDUUSTRIES, WC. }\end{array}$ & $\begin{array}{l}150 \\
151 \\
151 \\
151 \\
151\end{array}$ \\
\hline $71-0234$ & XRAY, INC. & 149 & 71-06es & TESTING TECHNOLOQIES, INC. & 157 \\
\hline $71-0242$ & LAW ENGINEEAINS TESTINO $\infty$. & 148 & $71-0685$ & SIERFA TESTIMG, HC. & 151 \\
\hline $71-40250$ & TRANSWNUCEEAA, INC. & 149 & 71.0601 & TE ANALYTICAL SEAVICES, INC. & 151 \\
\hline $71-0253$ & TWIN FOATS TESTIWG, WNG. & 140 & $71-0689$ & TEK INSPECTICN & 151 \\
\hline 71.0254 & GENGAAL EECTRIC $C O$. & 149 & 71.0689 & OUALTY INSPECTION SERVCES, WC. & 151 \\
\hline $71-0262$ & WECONESIN INDUSTRZAL TESTING INC. & 149 & 710706 & SK. MGERYDE, INC. & 151 \\
\hline $71-0253$ & YLEA HEAT TRANAFER COAF. & 148 & $71-0721$ & OUAUTY SYSTEMS NDE, LTO. & 161 \\
\hline $71-0359$ & WSCONSIN CENTRIFUEAL, INC. & 149 & $71-0724$ & VALLY WSPECTION SERVKE, INC. & 151 \\
\hline $71-0346$ & PUB $\amalg C$ SERVICE $C O$. OF COLOFADO & 148 & $71-0727$ & CONTNENTAL ARLINES, WK. & 151 \\
\hline $71-0380$ & EPNEAAL ELECTFAC CO. & 149 & $71-0728$ & $\begin{array}{l}\text { ACCW-TECH EVALUATION SGTVICES, } \\
\text { WC. }\end{array}$ & 151 \\
\hline 71-0393 & VOTH HYOAO, ING. & 148 & $71-0729$ & PAUL SINN TESTMG SEFMCES & 181 \\
\hline $71-0429$ & OCLABOAATORIES, INC. & 108 & $71-0734$ & DUALTY INSPECTKON \& TESTHNG & 151 \\
\hline $71-0440$ & $\begin{array}{l}\text { PROFESSTOMAL SERVICE INDUSTRES. } \\
\text { NC. }\end{array}$ & 160 & $71-0733$ & 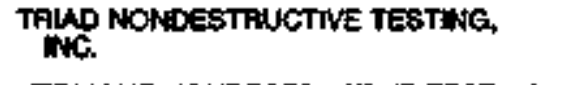 & 151 \\
\hline $71-0482$ & UNIV. OF MASSACHUSETTS & 150 & $71-0738$ & $\begin{array}{l}\text { VERUCONT NONDESTRACTME TESTING, } \\
\text { WC. }\end{array}$ & t51 \\
\hline $71-0485$ & SOUTHWEST X+1AY COFP. & 160 & $71-0740$ & MOLAND INSPECTION \& ENGINEERING, & $15 t$ \\
\hline $7+0514$ & 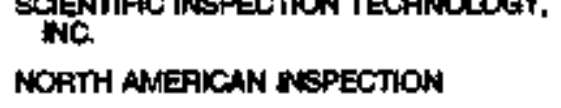 & 160 & $71+0746$ & TEGHNCAL WELDNG LABORATOFY, & 151 \\
\hline $74-0.692$ & WESTERN X-RAY & 160 & 71-0749 & GUATS INSPECTION SERVICES, NC. & 181 \\
\hline 71-0658 & WALASWEK ENTERPAISES, WNC. & 180 & $71-0761$ & 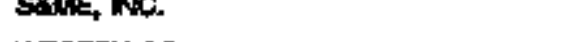 & 151 \\
\hline $71-0568$ & CRAMEA \& LINDEAL ENGEEEAS, INC. & 150 & $7 f-076$ & WESTEXO. & 151 \\
\hline $71-0579$ & SENOA ENGINEERANG CO. & 150 & nedres & 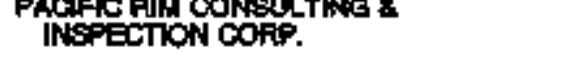 & 132 \\
\hline $71-0583$ & 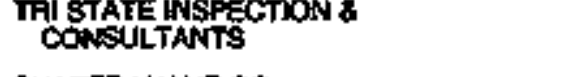 & 150 & $71-0777$ & VAUEY NOUSTRAA XVAY & 152 \\
\hline $71-0800$ & SHAFER VALVE $\infty$. & 150 & $71-0779$ & 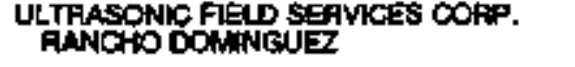 & 152 \\
\hline $71-0609$ & $\begin{array}{l}\text { SCIENTFIC WSASUFEMINT SYSTENS, } \\
\text { WC. }\end{array}$ & 150 & $71-0780$ & WESTERAN INDUSTRLAL X+AAY & 152 \\
\hline $\begin{array}{l}71-0672 \\
71-0029\end{array}$ & $\begin{array}{l}\text { SPEC CONSULTANTS, NOC. } \\
\text { NONA DATA TESTING LAgS, INC. }\end{array}$ & $\begin{array}{l}150 \\
150\end{array}$ & 71-0781 & 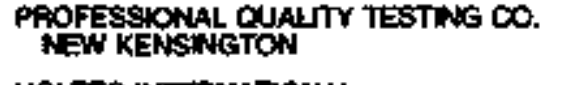 & 152 \\
\hline $71-0600$ & BCIENTIFC TECHNKCAL, INC. & 150 & $71-0784$ & HOLTEC INTEFAATIONAL & 182 \\
\hline 714600 & PADCHESS SERVICES, INC. & 150 & $71-0.005$ & OCEAM STATE TESTING & 152 \\
\hline 710032 & $\begin{array}{l}\text { OUALITY ENEREY SERVICES \& TESTS } \\
\text { CONF. }\end{array}$ & 160 & $\begin{array}{l}71-0325 \\
71-00025\end{array}$ & $\begin{array}{l}\text { PEASACOLA TESTING LABS, INC. } \\
\text { OCEANSTATE TECH. SVCS }\end{array}$ & $\begin{array}{l}152 \\
152\end{array}$ \\
\hline $71-08 \times 8$ & WOS TESTING CO, NC. & 150 & $71-0692$ & DCEANEEFiNG - SOLUS SCHAL1 & 152 \\
\hline 71.0640 & PSI ENEFSY, NC. & 150 & 71-0ecs & FEINHAFT \& ASSOCLATES, NC. & 152 \\
\hline $71-0641$ & UNITED STATES TESTINS $\infty_{4}$ ING. & 150 & $71-08357$ & ICF KAISER HANFOAD CONPANY & 152 \\
\hline
\end{tabular}




\begin{tabular}{|c|c|c|c|c|}
\hline Docket & Nmen & Paqu & Deelat & hen \\
\hline $71+0638$ & PACAFYC TESTING LABORATORIES & 152 & $71-9194$ & NODEL PAS-1, NUCAEAR PACKACINO, \\
\hline $71-0.841$ & TDELLNOS TESTMNG, INC. & 158 & & Пस6 \\
\hline $71-0842$ & PANHANDLE NDT AND INSFECTION & 152 & 71 gate & MODELA: TNAFF, ENERGY, DEPT, OF \\
\hline $71-0043$ & TESTING SERVICES \& INSPECTION INKC. & 152 & $71-9200$ & MODEL: DHF, EAW FuEl $\infty$. \\
\hline $71-0848$ & 自 PASO NGPECTION & 152 & $71-9208$ & MODEL: TNAEG, ENERGY, DEPT. OF \\
\hline $71-0854$ & X-CEL NDE & 159 & $71-9809$ & WODEL: 10-142, MUPAC SEAMCES, INC. \\
\hline $71-00860$ & TECHNOLOCY PULS INKORPORATED & 152 & $71-0215$ & 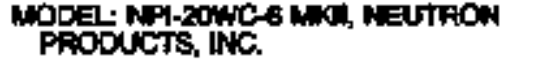 \\
\hline $71-0871$ & THE ROAEFTS CONMANY & 162 & 71.9217 & MOOEL ANF 25O, ADNANOED NUCLEA9 \\
\hline $71-0881$ & FRANKLN \& MAFSHALL COLLECE & 152 & & FUELS CORP. \\
\hline $71-0003$ & COLUMBA INMEFSTY & 152 & $71-9218$ & $\begin{array}{l}\text { MODE: TFUPACTH, ENEROY, DEFT. } \\
\text { OF }\end{array}$ \\
\hline 713010 & $\begin{array}{l}\text { MODEL NTAX, TRANSPOFTATION, } \\
\text { DEPT. OF }\end{array}$ & 162 & 71402022 & $\begin{array}{l}\text { MODEL: 14.216, SCIENTIFW ECOLOOY } \\
\text { GROUP, INC. }\end{array}$ \\
\hline $71-3057$ & $\begin{array}{l}\text { WOOEL: 뱀, IRANSPOATATION, } \\
\text { DEPT. OF }\end{array}$ & $15 \times$ & $71-9289$ & MOOEL: CAA, CaNGAL ATOMKS \\
\hline $71+3009$ & WODEL: RSE (FFENCH) CASK & 158 & $71-0203$ & NODE: TNAAM, TRANSNUCLEAR, NV. \\
\hline $71-4996$ & $\begin{array}{l}\text { MOCG: RA-2, RA-3, GENERAL } \\
\text { ELECTRAC } 6 \text {. }\end{array}$ & 150 & $71-9239$ & $\begin{array}{l}\text { MOELL: NCO-3, WESTIKGHOUSE } \\
\text { ELECTRW CONP. }\end{array}$ \\
\hline 71.5450 & 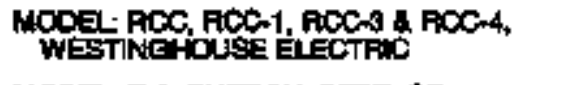 & 150 & $71-026$ & 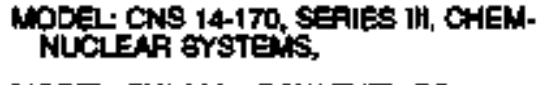 \\
\hline $74-6007$ & MOCEL T-2 ENERGY, DEPT, OF & 163 & 71.9251 & MOOEL: BW-2901, BaW FUEL CO. \\
\hline $71-6797$ & $\begin{array}{l}\text { MODEL: INKAB \& OUTEA HEIR UM, } \\
\text { ENERGY, DEPT. OF }\end{array}$ & 159 & $71-0281$ & $\begin{array}{l}\text { WOOEL: HISTAR 100, HCLTEC } \\
\text { INTERINATIOWL }\end{array}$ \\
\hline 71.6089 & MODE: 1500 , GENERAL ELECTRIC CO. & 159 & $71-8289$ & $\begin{array}{l}\text { MODE: TRANSTOA SIJARA NUCLEAR } \\
\text { COF. }\end{array}$ \\
\hline r1-3ipis & MODEL: DRE POWEA US, ENERGY, & 153 & $71-9899$ & MOOEL: Q5OL \\
\hline & DEPT. OF & & $71-0270$ & $\begin{array}{l}\text { MODEL: UMS - UNMERSAL } \\
\text { INTERATKNAL }\end{array}$ \\
\hline $11+\infty 00$ & DEPT. OF & 163 & $71-9271$ & MODEL THOLAN REACTOA VESEEL \\
\hline $71-9028$ & MOOEL $676 \& 676 E$, ANIRSHAM CORP, & 160 & $71+9276$ & MODEL: WESFEX \\
\hline $71-0083$ & $\begin{array}{l}\text { MODEL 660, 690A, 660AE, 6608, 660BE. } \\
\text { GOE, AWEFHAN }\end{array}$ & 150 & \multicolumn{2}{|c|}{ 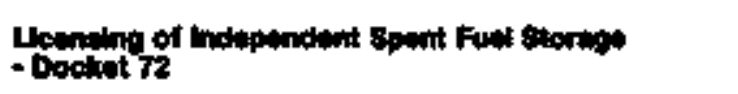 } \\
\hline $71-9000$ & 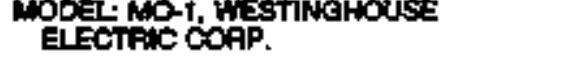 & 153 & $72-0001$ & MPARE OPEAATONS, KFEI \\
\hline $71-0079$ & $\begin{array}{l}\text { WDGL NUPAC 14D2.0, NUPAC } \\
\text { SEFVICES, DNC. }\end{array}$ & 153 & $\begin{array}{l}72-0002 \\
7204004\end{array}$ & $\begin{array}{l}\text { SURAY ISFSI 50-280 a 50-201 } \\
\text { H.B. RCBINSON LSF9, 50-281 }\end{array}$ \\
\hline $7 /-9004$ & $\begin{array}{l}\text { WOPZL: CNS 14-196H, CHEMANUCLEAR } \\
\text { SYSTIEN, INK. }\end{array}$ & 154 & $72-0004$ & OCONEE IEFS, $80 \times 266,50-270$ \& $60-267$ \\
\hline $71-0006$ & $\begin{array}{l}\text { MODEL: CNS 21-300, CHENANIFCLEAR } \\
\text { SY'STEMS, INC. }\end{array}$ & 154 & $72-0005$ & PONT BEACH ISFGA CEN LKC, 50-899 \\
\hline $71-9145$ & MODEL+ MPAC 50-1.0L NUPAC S0-25L & 154 & $72-000 t$ & $\begin{array}{l}\text { PALISADES LSFSI GEN LIC, 50-255 } \\
\text { CALVERT CUFS ISFSL, } 50-317 \text { \& 50-516 }\end{array}$ \\
\hline $71-0151$ & $\begin{array}{l}\text { WOEFL: HY100 SEAIES 3, ET AL, } \\
\text { SCENTTIC ECOLOGY }\end{array}$ & 154 & $72-0000$ & FOAT ST. VRAN LSFA, 50-257 \\
\hline 71.9159 & 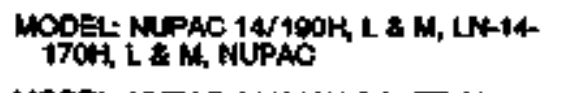 & 154 & $\begin{array}{l}72-0010 \\
72-0013\end{array}$ & 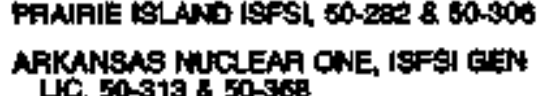 \\
\hline $71-9178$ & 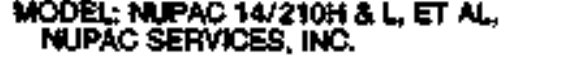 & 154 & $72-0016$ & NORTH ANNA ISFSA - 50-358 4 E0-859 \\
\hline $71-9172$ & $\begin{array}{l}\text { WODELA NUPAC 10/140 \& LN 10-195A, } \\
\text { WIPAC EARVICSS, ING. }\end{array}$ & 154 & $72-0,017$ & TROAAN ISFB, BO-S44 \\
\hline 71-917R & $\begin{array}{l}\text { MOOEL: NUPAC 7/TOO E LN 7-100. } \\
\text { HPAC SEPVICES, INC. }\end{array}$ & 154 & $72-00020$ & $\begin{array}{l}\text { COODHUE COUNTY ISFS: } \\
\text { NE ThU1-2 LFFA }\end{array}$ \\
\hline $31-9170$ & 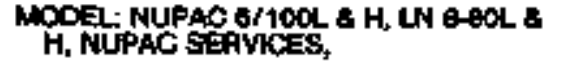 & 154 & 720022 & $\begin{array}{l}\text { WODEL: PAIVATE FLE STOAAGE } \\
\text { FACLITY }\end{array}$ \\
\hline
\end{tabular}




\begin{tabular}{|c|c|c|c|c|}
\hline Docket & Nand & $\operatorname{Pas}$ & Dotent & Nums \\
\hline $72-1000$ & $\begin{array}{l}\text { CASK CASTOR V/21, GENERAL } \\
\text { NUCLEAR SYSTENS }\end{array}$ & $16 \mathrm{~B}$ & $72-1018$ & $\begin{array}{l}\text { CASK: CASTOA X/29.33, GENERAL } \\
\text { NUCUEAR GYSTENS, WNC. }\end{array}$ \\
\hline $72-1004$ & 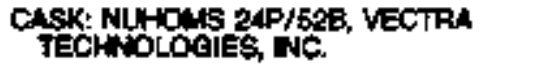 & 168 & $72-1021$ & $\begin{array}{l}\text { CASK: TNSE DRY STOFARE CASK, } \\
\text { TRANSNUCLEAR, INC. }\end{array}$ \\
\hline $72+1007$ & CASK: VSC-24, SIERRA NUCLEAR, INKC. & 15 & $72-1023$ & $\begin{array}{l}\text { OASK: TAANSTOR CASK, SIERAA } \\
\text { NCLEAP OOPF. }\end{array}$ \\
\hline $72-1009$ & $\begin{array}{l}\text { CASK: HLSTAF 100, HOLTEC } \\
\text { WTERATKNAL }\end{array}$ & 158 & $72-1024$ & GASK, EPAI DAY TRNWSFEA SYSTEM \\
\hline $72-1014$ & $\begin{array}{l}\text { CASK H. STOPWH 100, HOLTEC } \\
\text { WTEFATIONAL }\end{array}$ & 158 & $72-1005$ & CABK NACHAC \\
\hline $72-1015$ & CASK UNS UNMEFBAL WPC SYSTEN, & 159 & $72-1020$ & $\begin{array}{l}\text { MOPEL TRANGPOFTABLE STORACE } \\
\text { SYYSTEH DESICNA }\end{array}$ \\
\hline
\end{tabular}


2 


\section{ALPHABETIC ARRANEEMENT OF DOCKET ITEMS BY FACILITY NAME}

nime

Low Lewel Redwete Olepoud Stiss . Dacilest 27

LOUSBVLEE, KY, U.S. ECOLOGY, NC.

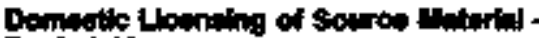
Dochent 40

ALLEDSFGNAL INC., METAOPOLS, IL AMEAICNN NUCLEAR COFP., CASPER, WN ARMY, DEPT. OF, MADESON, IN AFAY, DEPT. OF, SAVNNNA, IL AFAM, DEPT, OF, TOOELE, UT AAMY, DEPT. OF, YUNA, 17 ATLANTKC RHCHFELD CO, BRANTS, NH ATLAS CORP., DENER, CO

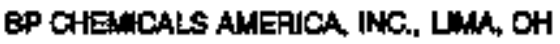
CABOT COAP DOYERTOWN, PA CERAC, WC, NLWAUKEE, W CHENETRON COAP, PAOVIDENCE, FA ENVIROCARE OF UTAK, WC., SALT LANE GTY, UT

EXXON CORP HOUSTON, TX

FANSTEEL WHC, NORTH CHKCACO, IL.

FEFRET EXPLORATION OO. OF NIZARASIKA, DENV로, $\mathbf{C O}$

FOOTE UIRERAL CO, EXTONL PA

PAOHE INNESTMENT CO. (BFOOKS : PERKINS (OAP.) -

HAATLEY A HAFTEY LNDFHL (MDNA). BAY DOUNTY, WI

HATLYY \& HAFTLEY LANDFUL (BCA), BAY CONTY, Mi

hOHESTAKE MaNiNg CO. GRANTS, NM HYORO RESOUACES, INC., DAHAS, TX INTEFWATIONAL UFANILM USA COAP. KENNECOTT UFANHAN CO, RAWLNS, wr LAKE GTY APHY ANMUNITON PLANT, TNDEPENDENCE, MO

MOLYOOAP, MC, LOS ANCELES, GA

PATHFWDER MINES CORP., MULS, WY

PATHFLOER WINES CORP, FIVETTON, WY

PATHFWDS MINES CORP., SHALYY BAST, WY

PETfOTOMICS $\infty$., shIFLF BASIN, WY

POWEA RESOUACES, INC., GLEAROCK, WV

OUMRA MINING CO, GRAATS, NM

RIO ALGOM MINING CORP, MOAB, UT
Dowent Pays

$27-046$

$40-3302$

40-44ing

40-8ess

40-8789

40-8779

40-8314

$40-9002$

40.3463

40-7604

40-6940

$40-9005$

$40-9724$

$40-8899$

40-8108

40.7560

$40-6049$

40-7397

40-0285

$40-9015$

$40-9002$

409003

40-8968

40-981

40-6584

40-8767

$40-8778$

4D-8961

40-2250

40-6022

$40-665$

40-4657

40-8000

+0-60e4
Ninge

RIO ALCOM MNINB COAP OKLAHOWA GTY, OK

FMI CO, ASHTABULA, OH

SEQUOYAH FUELS OOAP., GOAE OK

SHIJDALLOY CORP, NEWFELD, 낙

SHELDALOY MTALLIRGIGAL COFP. NEWFied N

SOHD WESTEAN MINNNE OO. SALT LAKE CFT, UT

TOTAL MHERALS COAP ${ }_{+}$CASPEF. WY

IMETCO MNEAMLS COAP., AMERTON, WY

UNC MUNANG \& MLUNG (ONV. OF UNTEO NUCUEAR COAP.H.

WESTEPN NUCLEAR, INC., LAKEWOOD, 0

WITTMKEA CORP, LOS ANGELES, CA

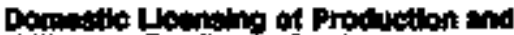

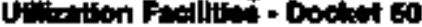

ALVN W. VOETLE NUCLEAF PLANT, UNTT 1 BO-424 I1T ALVN W. VOGTLE NUCLEAP FLANT, UNT 2

ARKANSAS NUICLEAP ONE UNIT 1

ARKANSAS MUCLEAR ONE, UNT 2

$50-25$

$50-819$

50359 16

BEAVER VALEY ROWEA STATION, UNIT 1

BEAVEA VAUEY POMUA STATIN, UNIT 2

BELUEONTE NUCLENR PLANT, UNIT 1

GEUEFONTE NUCEEAR PLANT, UNT 2

BIG ROCK PONNT AUCAEAR PLNT

BAAIDWCOD STATION, UNIT 1

GAADWOOD STATION, UNTT 2

BAOWNS FEFRY NUCLEAR FOWEA STATON, UAT 1

BAONNS FERAY NUCLEAR POWER STATKN, LAT 2

BAONNS FEPAY NUCLEAR POWER STATION, LifT 3

BRUTEWRK STEAN ELCTRAC PLANT, LNIT 1

BAUNSWICK STEAM ELECTRK PLANT, UNIT 2

BYRON STATIOH, UNIT 1

$60-334 \quad 60$

$50412 \quad 109$

$50-439$

$50-496 \quad \$ 10$

SO-155 17

STM $50-458 \quad 126$

STI $120-457$

$50.259 \quad 22$

$50-800 \quad 33$

$50-29056$

50.925 $\quad 75$

$50-324 \quad 74$

STN-50-454 123

BYRON STATION, UNIT \& STN-SO-155 124

CALLAWAY PLANT, UNTT $1 \quad$ STN-SO-483 131

CALVEAT CUFFS NUCLEAR POWEA PLANT, $50-317$ O UNIT 1

CALVETT CANFS NUCLEAF PCWEA PLANT,

$50-31870$

CATAWBA MUCLEAR STATKON, UNT 1

50-413 110

CATAWBA WUCLEAP STATION, UNTT 2

50.414 111 
Dowakt Pow

CiNTECHEM

CLNTON POWEA STATION, UNIT 1

COMANCHE PEAK STEAM ELECTFIC STATON, UNT 1

COMWNCHE PEAK \&TEAM ELECTFIC STATION, UNIT 2

COOFER NUCAEAP STATION

GRYSTAL ANEA MLCLEAR PLANT, UNIT 3

DAVS-BESSE NUCAEAA POMEA STATION, UNTा 1

DHALOO CANYON NUCLEAR POWEA FLANT, LNTT 1

DIABLO CANYON NUCAEAR POHEA PLANT, UNT 2

DONALC DOOK NUCLEAF POWEA FUNT, URT 1

DONAMO C. COOK NUCLEAP POWER PLANT, LHT 2

DOW OHABNAL CO.

DFESDEN MLCLEAR POWIG STATION U.HT 1

CFESOEN NUCLEAR POWIA STATION, DNIT 2

DAESDEN NUCAENA POWER STATION, UNIT 9

DUANE ANNOLD ENERGY CENTEA

EDWN E. HATCH MUCLEAR PLANT, UNIT 1

EDWN L, HATCH NUCLEAR PLNNT, UNIT 2

ENAICO FEAMI ATCHIC PONER PLANT, UNIT I

ENFCO FEANI ATCAIC POWER PLANT, UNIT 2

FLATTMS NUCLEAR PLANT

FORD NUCL SAP FEACTOA

FORT CALHOLN STATION, LINIT 1

FOFT ST. VRAN NUCLEAR GENEAATING STATION

GENERAL ATOMOS

GENERAL ATOMCS

GECRGIA INSTITUTE OF TECHNOLOGY FESSEAFCH REACTOR

GRAND GLAF NUCLEAR STATION, UNTT 1

HB. FOEINESON PLANT, UNIT 2

HADOAN HECK FLANT

HOPE CFEEK NUCLEAR STATIONL UNTT 1

HWHBOLD BAY FOWIA PLANT, LNNT 3

DAHO STATE UNIY, PISEAFCH FEMCTOA

INDAAN ROINT STATION, INT I

WDIN POINT STATION, UNT 2

INOLAN POWT STAJONW, UNIT a
50.54

E0-461 120

$50-445 \quad 131$

$50-446 \quad 129$

$50-2035$

$50-302 \quad 58$

$50-346 \quad 90$

50-275

$50-202072$

$50-315 \quad 67$

$50316 \quad 6$

$50-254 \quad 35$

$50-10 \quad 15$

$50+297 \quad 22$

$50-849 \quad 27$

$50-93178$

60-321 7

soss 25

50-16 15

$50-341$

STN-50-497 116

50-2 14

60-285 48

50-257 $\quad 37$

$50-99 \quad 16$

50-1Es 18

60-160 18

50-416 112

60-201 39

c0-213 18

$50-3549$

50.139 17

50.294 $4 \mathrm{a}$

50-3 14

50-247 27

$50-298$
Name

WONA STATE UNV. FESEARCH REACTOF

JHSO A FIZPATRKK NUCLEAR POWER PLANT

JOSGPH M. FAFAEY MUCLEAP PLANT, UNHT t

WOSEPH M FAFLEY NUCLEAR PLANT, UNIT

KEWALNEE NUCLEAF POMEER PLANT

LA CROSSE BOILNWS WATER REAOTOF

LASALLE COUNTY STATION, UNT 1

LASALE COUNTY STATION, UNT 2

LW:בRKK GENERATMG STATKON, UNIT 1

LRFAKK GENERATHAB STATION, UNIT 2

MANE YANKEE ATOAIC FONER PLANT

MIOLAND PLANT, UNT 1

MDLAND PLANT, UNT 2

MILLSTONE NUCLEAA PONEA STATION, UNT!

MILLSTONE NUCLEAR PONEA STATION, thit 2 MILSTONE NUCUEAR PCWEA STATKON,

MONTKCELLO NUCEEAR CENERATING PLANT

NATIONAL INSTTIJTE OF STANDARDS \& TECHNOLSY REACTO

MNE MLE POINT NUCESAR STATION, UNIT

TWNE MILE POINT NIXCLEAF STATON, UNIT 2

NORTH NMNA POWER STATION, LNIT * NORTH ANANA POWER STATION, UNIT 2 NORTH GAROUNA STATE UNUY. PULSTAR FEACTOR

COONEE MUCLEAR STATION, UNIT I

OCONEE NUCLEAR STATION, UNIT 2

OCONEE MUCLEAA STATION, UNT 3

OHO STATE UNN. RESEAFCH FEACTOR

OAERON STATE UNIY. RESEARCH FEACTOA

OYSTEA GREEK NUCAEAA POWEA FLANT

PALSADES NUCLEAR PLANT

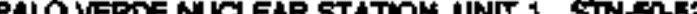

PALO VERDE NUCLEAR STATONA, UHAT 2 STH-60-929

PALO VEADE NUCLEAR STATION, UNIT a STN\$0.530 PEACH B

PEACH BOTTOM ATCUC ROWER STATION, SORT Unis 2

PENCY BOTTOM ATCANC POWEA STATIONS BO-278 UNiT 3
$0-171$

Dootret Pape

50-116 17

50-999 79

0-348 89

so-so4 24

$50-305 \quad 62$

50-409 100

B0073 99

$50-574 \quad 100$

$50.352 \quad 99$

$50250 \quad 90$

$50-400 \quad 64$

60020 T

50-39A 78

$50.245 \quad 24$

$00-336 \quad 92$

$50-483 \quad 113$

$30203 \quad 24$

$50-184 \quad 19$

$30-21$

$50-410 \quad 100$

60-339 $\quad 65$

$50-35986$

50.49756

60-200

$60.270 \quad 39$

$50-287 \quad 51$

$0.150 \quad 17$

$50-243 \quad 23$

\$0-219 21

so-ass 3

194

135

137

18

4

4 
$N=0$

PENNSYLVANIA STATE UNIY, AESEARCH AEACTOF

PEAPY NUCLEAH POWEA PLANT, UNIT 1 PEARY NUCAEAR PCWER PLANT, UtTT 2 PILGFIM MUCEAP PONEA STATON, UNIT 1

PLITH BFOOK REACTOR - HOCKAY

PUUM BAOOK RESEARCH REACTOA FACIUTY

POANT EEACH NINCLEAP PLANT, UNIT 1

PONT EENCH NUCJEAR PLANT, UNIT 2

PAARIE ISLAND NUCEEAR STATION, UNIT 1

PAAIRIE ISLAND NUCLEAR STATION, UNIT

2

OUAD-CInES STATION, UNT 1

OUADCTIES STATION, UNT 2

RANCTO SECO NUCUEAR GENERATING STATION

RHODE ISLAND \& PRONDENCE PLANTATKONS AEC

AIVEA GEND STATKON, UNIT 1

ROgEAT EMHET GIANA NUCUEAF PLANT. UNIT 1

SALEM NUCLEAP CENERATWG STATION, UNIT 1

SMLEM NUCLEAR EENERATWE STATKN, UNIT 2

SAN CNOFAE NUCLEAR STATIOK, UNT 1

SAN ONOFAE NUCLEAR STATIONS LNIT 2

SAN ONCFFE NUCLEAR STATICN, LNTT O

SNXTON NHYCLAA EXPERIMENTAL FACILITY

SEABROCK NUCLEAP STATION, UHTT 1

SEAGROOK NUCLEAF STATION, UNT 2

SECLOYAH NUCLEAR PLANT, UNT 1

SECUOYAH NUCAEAR FLANT, UNGT 2

SHEARON HAFAIS NUCLEAR FCWWER PLANT, UNTT I

SHOPEHAM NUGEAP FOWER STATION

SOUNH TEXAS PAQVET, UNT 1

SOUTH TEXAS PRONECT, UNT 2

ST. LUCAE PLANT, UNIT 1

ST. LUCGE PLWT, UNIT 2

SURFY PCWER STATION, UNAT 1

SUFRY POWER STATHON, UNT 2

SUSOUEHANNA STEAN ELECTFAC STATON, UNIT 1

SUSOUEHANAA STEAM ELECTR:C STATIOM LNIT 2

\begin{tabular}{|c|c|}
\hline bockit & pare \\
\hline $50-5$ & 15 \\
\hline $50-40$ & 118 \\
\hline $50-441$ & 121 \\
\hline $50-293$ & 59 \\
\hline $50-168$ & 10 \\
\hline $50-30$ & 16 \\
\hline $50-290$ & a3 \\
\hline $50-301$ & 67 \\
\hline so-282 & 47 \\
\hline $50-300$ & $B 2$ \\
\hline $50-254$ & 30 \\
\hline 60-965 & 95 \\
\hline $50-312$ & 68 \\
\hline 50-193 & 19 \\
\hline SO-ABB & 128 \\
\hline $50-244$ & 20 \\
\hline $50-272$ & 41 \\
\hline $50-311$ & 85 \\
\hline $50-206$ & 18 \\
\hline 50-381 & $m$ \\
\hline $50-362$ & 9 \\
\hline $50-+46$ & 17 \\
\hline $60-443$ & 121 \\
\hline $50-144$ & 2121 \\
\hline $50-327$ & 78 \\
\hline $50-328$ & 77 \\
\hline $50-400$ & 107 \\
\hline $50-322$ & 72 \\
\hline$+50-498$ & 139 \\
\hline 1.59 .490 & 138 \\
\hline $50-395$ & at \\
\hline $50-390$ & 104 \\
\hline $50-290$ & 45 \\
\hline $50-281$ & 46 \\
\hline 60-3e7 & 102 \\
\hline $50-300$ & 108 \\
\hline
\end{tabular}

Noine

Dockit Pace

TEXAS AMM UNN, RESENACH RENGTOR \$0.126 7

THAEE MILE ISLAND NUCLEAP STATIONh $\quad 50-289$ UNIT $\dagger$

THFEE MIE ISTAND NLCLEAR STATON, BOS20 71 UNT 2

TROUANAUCEEAR PLANT

50-344 87

TUFKEY POWT PLANT, UNIT 3

$50-25020$

TUFKEY POWT PLANT, UNT 4

50-251 20

U.S. GEOLOEGAL SUPVEY RESEARCH $\quad 50-274 \quad 42$ REACTCA

UNN. OF APIZONM RESEAACH REACTOR 50-113 16

UNN, OF FLOAIDA TRANING AEACTOA

UNN. OF MARYLAND RESEARCH REACTCR 50-165 18

UNY, OF MBSOUAI RESEARCH FEACTOACOLUMBA

UNN, OF NEW MEXCO RESEAPCH REACTOR

UNN, OF UTAH RESEARCH REACTOA

$50-252$

30

UNW, OF WISCONSN AESEARCH REACTOA

UNVERST OF MASSACHUSETTS, LOWEIL RESEAACH AEACTOA

VERMONT YANKEE NUCAEAR PONER STATNON

VACCL C. SUMUEA NUCUEAA STATION, UNT 1

WASHWGTON STATE UNWV. RESEAPCH AEACTOA (TRKA)

WATERFOAD GENERATINC STATION, UNIT 3

WATTS EAF MUCLEAR PLANT, UNIT I

WATTS EAF NUCLEAP FLANT, VNIT 2

50-185 10

50.407

109

WIHAAM B. MCOURE NUCLEAR STATION, UNT 1 WHLAAM B. MCONIFE MUCLEAR STATION,

$50.156 \quad 18$

$50-223 \quad 22$

$50-271 \quad 40$

50-sis 106

$50-27 \quad 16$

$50-382101$

$50-500 \quad 105$

$50-391 \quad 105$

$50569 \quad 97$

D0-370 9

WOLF CREEK GENERATINE STATION STN-50.482 130

WPPSS MUCLEAR PAOJECT, UNT 2

$60-997 \quad 107$

WPPSS NUCLEAR PFOJECT. UNIT a STNFO-50S 134

YANKEE-ROWE MUCLEAA FOWEA STATION EO-29 15

ZOM NLOEEAP FOWER STATION, UNIT 1 BO-295 54

ZON MUCLEAR FOWEA STATION UNT 2 SO-3OA

APBDO STANDAFD PLANT DESKEN. WESTINGHOUSE ELECTRC CORP.

SYSTEM BO+ STANDAPDIZTE MUCLEAR POWEA FLANT DES.

$52-000138$

$52-002 \quad 139$

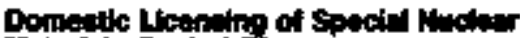
Prinew- Docket of

ALEGHENY WEALTH, ED. \& RESEARACH COAP, PTTSEURCH, Ph

AFMY, DEFT. OF, DOVER, NW

70-173

146

70-0028

143 
Lim

B:W GZAALLW LAND ORPCSAL AFEA

(SLDA), PAFKS TOWNSHP.

GABCOCK \& WLCOX CO, APOLLO, PA

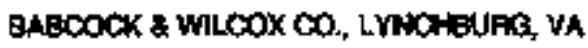

BABCOCK \& WILOX CO., PAFKS TOWNSSIP, PA

CUAARON CORP., OKLAHOMA CTY, OK

CINTICAEM, NA, TUXEOO, NY

CLEVTE COPP, CUEVELAO, OH

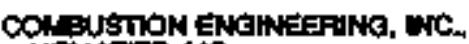
HEMATTIE, MO

COMgustow ENGINEAPING, UNC. WINDSOR, GT

COMMEACE, CEPT. OF, NATIONAL INSTITUE OE STANGARDS \&

EASTIUAN KODAK $\infty 0$. ROCHESTER, NY

EDLOW INTEFYATIONAL $\infty$. WASHAGTok, oc

FLOFUDA ASTITUTE OF TECHNOLOEY, MVLBDUPE, $\mathrm{FL}$

FLORLA, LNIV, GE, GANESVLLE, F.

PRAMKTOME DOEEM FUELS. LYNCFEUAG, VA

FRONTIER TECHNOLOCY COFF., XERHA, EENERAL ATCMICS, SAN DIEGO, CA GENERAt GLCTRIC CO., PUEASANTON, CA GENEFAL ELECTFHC OO. SAN JOSE CA GENERAL EIECTACC CO. WHMMMTON, NC IDAFO STATE UNIV, FOCATELLO, ID INTEAIOF, DEPT. OF, DENYER, 00

Loulspha ENTGY sEAVCES. WASHATGTON, DC

MASSACHUSETTS WSTTUTE OF TECHNOLOGY, CAMBAIDGE, MA

MUCLEAF FULL SERVCES, NC., ERWIN, TN

PENMEYLVANIA STATE UNN, UNIVERSTY PAFT, PA

PUFOUE UNW, WEST LAFAYETIE, W

SEATTL UNW. SEATTE WA

SIENENS NUCLEAT POWEA COAF, AICHUAD, WA

TEXAS, UNV. OF, AUSTIN, TX

TRANENUCOEAR, INC. FALS CHUPCH, VA

US. BNRCHMENT CORP. (AVLS ENRACHAIENT PLANT)

U.S. ENGICHMENT CORP, PADUCAH BASEOUS D:FLSFON PLAN

U.S. ENAWCHWFNT CORP. FOATEMONTH CASEOUS DAFUSION

MEST LIPERTY STATE COUERE, WEST UEERT, W

\begin{tabular}{|c|c|}
\hline Dociknt & Para \\
\hline $70-3065$ & 148 \\
\hline $70-0135$ & 142 \\
\hline $70-0027$ & 141 \\
\hline $70-0064$ & 148 \\
\hline $70-0925$ & 148 \\
\hline $70-0697$ & 143 \\
\hline $70-0193$ & 142 \\
\hline 70.0038 & 141 \\
\hline $70-1100$ & 144 \\
\hline 700009 & 140 \\
\hline $70-1703$ & 146 \\
\hline $70-1282$ & 145 \\
\hline $70-0 \sin$ & 149 \\
\hline $70-1068$ & 144 \\
\hline $70-1201$ & 145 \\
\hline $70-3005$ & 146 \\
\hline $70-0734$ & 140 \\
\hline $70-0754$ & 143 \\
\hline $70-2 \operatorname{ses} 3$ & 140 \\
\hline 70.3313 & 144 \\
\hline $70-1 \$ 74$ & 148 \\
\hline $70-0121$ & 142 \\
\hline 70.0070 & 148 \\
\hline $70-0098$ & 144 \\
\hline $70-0149$ & 14 \\
\hline $70-0113$ & 142 \\
\hline $70-0159$ & 143 \\
\hline $70-0000$ & 140 \\
\hline $70-1257$ & 145 \\
\hline 70.0157 & 149 \\
\hline $70-1426$ & $14 t$ \\
\hline $70-3989$ & 146 \\
\hline $70-7001$ & 148 \\
\hline 70.7002 & 147 \\
\hline 704025 & 143 \\
\hline
\end{tabular}

Him

Popentiol Pagu

WESTONEYOUSE EIECTRW OORP., PITISBURAT, PA

$70-1161 \quad 144$

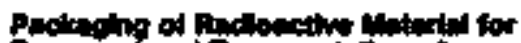

Trangent and Trateportion of

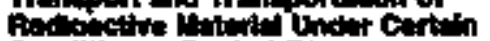

conditont - Dooket 7

ACCUTECA EVALUATION BEAVCES, INT.

71.5720 151

AMERSHMH CORP.

$74-0040 \quad 148$

ANVIL DOPP.

COLVAEA LINVERSTY

$71-0.67 \% \quad 151$

CONTISENTAL NRLINES, NEC.

$71-6083$

71-0727 151

$71-0058 \quad 150$

710746 161

71-0168 149

71-0048 152

$71-0$ ed 152

$71-0064 \quad 149$

$71-00000$

71-0784 152

$71.0657 \quad 152$

$71-0249 \quad 149$

$71-0740 \quad 151$

MDLLAND INSPECTION \& ENENNEERING,

$71-0000 \quad 150$

MOOEL+ 10-142, NUPAC SEFMCES, INC.

$71-40$ and 165

MOOEL: 14-215, SCAENTIFC ECOLOGY IAOLP, NG.

MODEl: 1500, EENGAAL ELECTFOC $\infty$.

MODE른 $65 \mathrm{AC}$

71-5oge 159

MODE- 60, 60A, 6604E, 6606, 660EE, 6 BDE, AMERBHAH

MODEl: BTS \& 6706, ANERSHAM COAP. 71-8028 159

MODE: ANF-OSO, ADVANCEO MUCLEAR 71-9217 155 FUés corp.

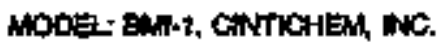

$714057 \quad 150$ MOCEZL: BUD, TRANSPOFTATION, DEFT. 71-3037 188

MODOEL: BW-2901, B\$W FLE CO.

$71-9201155$

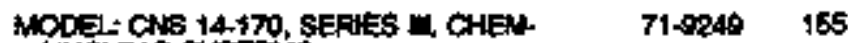
NUCLENR SYSTEMS.

MODEL: GNS 14-1OS-H, OHEMNUCLEAR SVSTENS, AC.

$7 t-9094 \quad 154$

MOEL, CNS 21+300, CHEMHUCAEAR

$71-9008+134$ STSTEMS. WC.

MODE: DAG POWER UNT, ENERGY, OFPT.

$7 t+441 \quad 168$

AODEL DHTF, BWW FEL CO.

$71-2003154$

MODELL BH-4, GENERAL ATOMCS

71-9ast

15

MOOEL: H.STAR 100, HOETEC MTEFNATRONAL

$71 \operatorname{tan1} 15$ 


\begin{tabular}{|c|c|}
\hline rete & Dockent \\
\hline $\begin{array}{l}\text { MODE: HN-100 SFA1ES, 3, ET AL, } \\
\text { SCIENTIF ECOLOEY }\end{array}$ & $71-8151$ \\
\hline 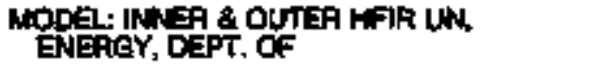 & $7 i-5797$ \\
\hline 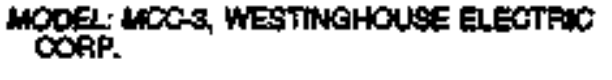 & $7+-0099$ \\
\hline $\begin{array}{l}\text { MODS: MO-1, WESTTNGHOUSE EUECTRK } \\
\text { COFP. }\end{array}$ & $71-0009$ \\
\hline 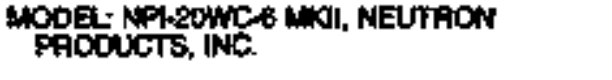 & 71.8215 \\
\hline $\begin{array}{l}\text { MODEL MTAX, TAANSPOATATION, DEPT. } \\
\text { OF }\end{array}$ & 71.3010 \\
\hline $\begin{array}{l}\text { WOOEL: KUPAC 10/140 \& UN 10-135A, } \\
\text { MUPAC SERVIGES, WKC. }\end{array}$ & $71-9177$ \\
\hline 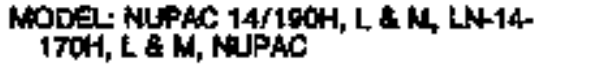 & $7 t-0150$ \\
\hline NODGL: WWPAC 14/210N \& L ET AL, MUPAC & $71-8176$ \\
\hline $\begin{array}{l}\text { NODEL NUPAC 14DE.0, NUPAC SERVICES, } \\
\text { INC. }\end{array}$ & $71-9079$ \\
\hline $\begin{array}{l}\text { MDPEL: NUPAC EO-1,5L, NHJAC 50-2-5L, ET } \\
\text { AL, NMPAC }\end{array}$ & $71-0145$ \\
\hline $\begin{array}{l}\text { MOE- NUPAC G/10OL \& H, LN GBOL \& H, } \\
\text { MLPAC SEAMCES, }\end{array}$ & $71-9+79$ \\
\hline $\begin{array}{l}\text { MODEL: NUPAC } 7 / 100 \text { \& LN 7-100, NUPAC } \\
\text { SEFMCES, INC. }\end{array}$ & $71-9170$ \\
\hline $\begin{array}{l}\text { MOOEL: PADUCAH TKGEA, WNEAGY, DEPT. } \\
\text { OF }\end{array}$ & $71-\operatorname{tos} 3$ \\
\hline MOCEL: PAS-1, NUCLEAP PACKAGING, INC. & 71.9184 \\
\hline MODEL: R-52 (FPENCH) CASK & 7120000 \\
\hline $\begin{array}{l}\text { MOCGLi RA-3, RA-3, GENEAAL ELECTRKC } \\
\text { CO. }\end{array}$ & $71-4906$ \\
\hline $\begin{array}{l}\text { MODE: } R C C, A C C-1, R C O A \& \text { ACC-4, } \\
\text { WESTINOHOUSE EIECTRIC }\end{array}$ & $71-5450$ \\
\hline MODEL T-2, ENETGY, DEPT. OF & $71-5607$ \\
\hline MODEL: THERP, ENEAGY, DEFT. OF & $71-9002$ \\
\hline MODEL: TNFAM, TRANSNLCLEAR, INK. & $71-0238$ \\
\hline HOOEL TNAREG, ENEFGY, OEPT. OF & 71.0208 \\
\hline $\begin{array}{l}\text { MOOEL: TRANSTOA SUERRA NUCLEAA } \\
\text { CORP. }\end{array}$ & $71-9286$ \\
\hline MODG: TROANAN REACTOR VESBEL & $71-9271$ \\
\hline MODEE: TRUPACT-\#, ENEARCY, DEPT. OF & 710218 \\
\hline MODEL UMS - UNNEASAL INTEANATIONAL & T1-8270 \\
\hline MODA: MESFLEX & $71-9270$ \\
\hline MORTH AMEPUCAN HASPECTION & $71-0514$ \\
\hline NONA DNTA TESTING UABS, WL. & $71-0,28$ \\
\hline CCEAN STATE TESTRE & 71-0005 \\
\hline OCEANEARING - SCUUS SCHAL & $71-0027$ \\
\hline OCEANSTATE TECH. SYCS. & $71-0025$ \\
\hline $\begin{array}{l}\text { PAGFC RHA CONEULTING \& ANGPECTION } \\
\text { COFP. }\end{array}$ & $7+0773$ \\
\hline PACAFIC TECHNICAL INDUSTFIES, HW. & $7 t-0682$ \\
\hline
\end{tabular}

Nate

Dooknt Pape

PACAFKC TESTING LABORATORIES

PANHANDLE NDT AND NSPECTION

$71-0689 \quad 157$

$710948 \quad 152$

PALL SINW TESTWHG SEAVICES

$71.0728 \quad 151$

71-0021 162

$7 t-0687 \quad 180$

$71-029140$

$71-0701 \quad 162$

PAOFESSIONAL OUALTY TESTING to.

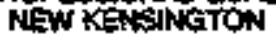

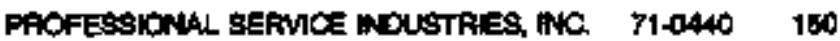

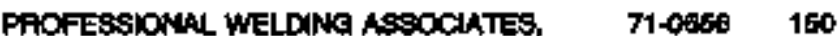
Inc.

PAOCRESS SERMCES, INC.

$71-0080 \quad 180$

PSA ENEROY, INK.

PUBLIC SERVICE CO. OF COLORADO

OC LABCRATORIES, UNC.

$71-0640 \quad 180$

$71-00465149$

$71-0429 \quad 149$

$71-0168 \quad 149$

ac.

CUAUTY ENEAGY SERVICES \& TESTS 71-0002 150 COAP.

QUALTY INSPECTION \& TESTWG

CUALTY IMAECTION SERVICES, HOC. T1-0099 161

QUALTY SYSTEMS NDEF LTD.

PENHART \& ASSOCATES, INC.

$71-0721 \quad 151$

$71-0939 \quad 152$

$71-0751 \quad 15 \%$

SESWE WH.

71.0706 15:

$71-0059 \quad 150$

SAM-SON WSPECTION a TECHNICAL SERVICES, HKG.

SCIENJIFN INSPECTION TECHNOLOQY, 71-0601 160 INC.

SCIENTIFC MEASUAENENT SYSTEMS, INC. 71.0609 150

SCIENTIFX TECHNALL, INC.

71-0629 150

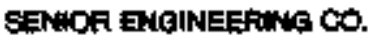

$71-0579 \$ 50$

SHAFER VALVE CO.

$71-0600 \quad 150$

SERFA TESTING, JNC.

$71-0 \operatorname{ses} 151$

SOUACE PAODLCTLN \& EOULWEAT CO.

71-otog 148

SOUTHWEST KAAY CORP.

$71-0496 \quad 160$

SPACE SGENCE BEAVICES, INC.

$71-0215$

149

SPEC CONSULTANTE, MC.

$71-0,12150$

ST, LOUIS TESTING LAEDIATTORIES, WiC.

$71-0202$

149

TAK NSPECTRON

$7 \uparrow-0,992 \quad 151$

TECHNICAL WELNNG LABOPATOFY, WC. 71-0746 151

TECHNOLOOY PLUS MEOPPOAATED

$71-0060$

152

TEI ANALYTICAL SEAVCES, INC.

710001151

$71-0028 \quad 148$

$71-0131 \quad 14 \%$

TESTWNG PSITUTE OF ALASKA

14

$71-0.49 \quad 159$ 
Hene

TESTNG TECHNOLOGIES, NC.

TEETMASTER MSPECTION $C O$.

THE ROEERTS CONPANY

THFELSCH ENGINEERING, INC.

TIDELANDS TESTING, INIC.

TRANS WORLD AVFLUNES, INC.

TRANSNUSCLEAR, NC.

TAI STATE DNSPECTION \& CONGARTANTS

TAIAD NONDESTRUYCTIVE TESTING, WC.

TRUTOW NU.S., LTD.

TULSA GMUMA RAY, INC.

TWMN PORTS TESTMGG, INC.

ULTFASONC FELD GERVICES COAP.
PANCHO DOWINGIEZ

UNITEO ANRLUES, WC.

UNITED STATES TESTING CO., WNC.

UNN, OF MASSACHIJEETTS

VAUEY HNDUSTRIAL X-RAY \& BNSPECTION SWC

VALLEY WHPPECTION SERVIE, WNO. VEFMONT NONDESTRECTNE TESTING.

VOTH HYORO, WNC.

WALASHEK ENTERPFISES, INC.

WESTERN INDUSTFAAL $X$-RAY

WESTEFN $X$-AAY

WESTEX CO.

MISCONSIN CENTHIFUEAL, INC.

WSCONGIN INDLSTRLAL TESTHAS, INC.

WOS TESTING $\mathrm{CO}_{-}$EXC.

$X$ CEEL NDE

$X$ AH TESTING OF HUCHIGNN

X.PAY, INK.

YUBA HEAT TRANSFEA COFP.

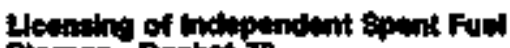

storimin - Dochet 72

AFKANSAS MUCLEAR CNE, ISFSI BEN LC. so-313 \& E0-360

\begin{tabular}{|c|c|}
\hline Docketst & Past \\
\hline $71-0983$ & 151 \\
\hline $71-0070$ & 151 \\
\hline 71.0871 & 152 \\
\hline $71+0673$ & 151 \\
\hline $71-0641$ & 182 \\
\hline $71-0000$ & 148 \\
\hline $7:-0200$ & 140 \\
\hline $71-0003$ & 150 \\
\hline $71-0739$ & 151 \\
\hline 7120431 & 150 \\
\hline 71.0139 & 149 \\
\hline 71.0253 & 149 \\
\hline $71-0779$ & 152 \\
\hline 71.0044 & 148 \\
\hline 71-Be41 & 150 \\
\hline 71.04882 & 150 \\
\hline $71-0777$ & 152 \\
\hline $71-0724$ & 151 \\
\hline $71-0758$ & 151 \\
\hline $71-06983$ & 149 \\
\hline $71-\infty 638$ & 150 \\
\hline $71-6780$ & 182 \\
\hline $71-05682$ & 150 \\
\hline $71-0705$ & 151 \\
\hline $71-0030$ & 140 \\
\hline $71-0262$ & 149 \\
\hline 71-0639 & 150 \\
\hline 71-0e64 & 182 \\
\hline $71-6158$ & 149 \\
\hline $71+6234$ & $14^{\mathrm{D}}$ \\
\hline $71-0263$ & 149 \\
\hline
\end{tabular}

$72-00 \div 9$ 157
Ham

Dooket Pege

CALVEAT CUFFS ISFS, 50-317 $\$$ 50-318

$720008 \quad 168$

CASY: CASTOA VI21, GENHAAL WMCLEAR BYSTEMS

CASK CNSTOF $X / 2 \mathrm{~B}-39$, GENEAAL MUCLAR SYSTEMS, WN.

CASK EPFI DAY TAANSFEA SYSTEM

GASE HSTAR 100, HOLTEC HTE HINATKONAL

CASK: HLSTOFH 100, HOLTEC MTERNATIONAL

CASK NACAPC

CASK: NUYONS 24P/6ZO, VECTAA TECHINGHóles, INC.

CASK: THSE DFY STOAACE CASK, TRNNISNICLEAR, WG.

CASK: TRANSTOA CASK, SEFRA NUCLEAT COAP.

CASK UNS UNIVERSAL MPC SYSTEM, NAC INTERNATIONAL, INC.

CASK: VSC-24, SUARA NUCAEAR, HC. 72.1007 158

FORT 8T. VRAW ISFS, 50-207

GOODHUE COUNTY LSFE

$72-0000 \quad 185$

$72-0018 \quad 157$

H.P. RObinson IsFs, 50-261

720009

15

INEL TMl2 ISFE'

72-0020

157

MODEL PFIVATE RUEL STORAGE FACHLITY

$72-0022$

157

MOOEL JFANSPORTAELE STORAGE

$72 \cdot 1026$

159

MORPUS OPERATIONS, ISFSI

$72-0001$

15

NORTH ANNA ISFS| - 50-358 \& 50-309

72-0016

157

OCONEE ISFS, 50-289, 50-270 \& 50-267

72-0004

158

PALISADES ISFS ENEN LIC, 50-255

72,0007

158

POINT GEACH LSFA GEN LC, $50-200$ \& LO30t

$72-0005$

156

PRARIE ISLAND TSFSI, 60-28R \& 80-300

$72-0010 \quad 166$

SUPRY LSF\$ 60-200 \& 50-281

720000 156

THOLAN ISFSI, 60344

72-0017 157

UNTWONN

DOCKET 149

URMTOWN

DOCKET 1 


\section{DOCKETED ITEMS}

\section{LOW LEVEL AMOWASTE DAsPosel SITES}

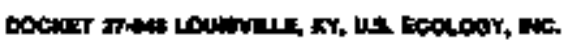

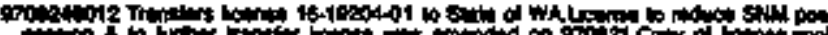

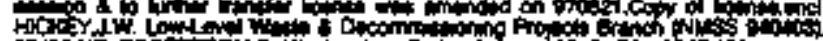

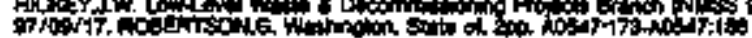

\section{Docket oocextr unawonts}

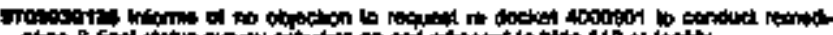

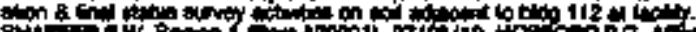
(1) conoges 101.

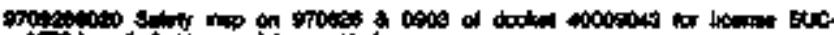

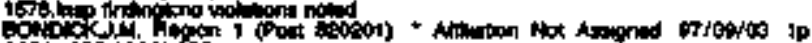

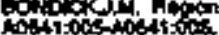

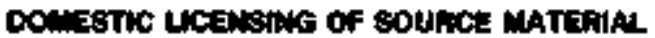

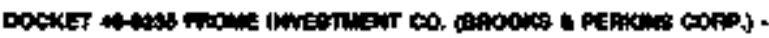

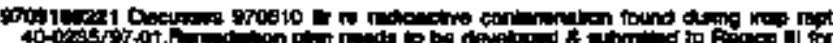

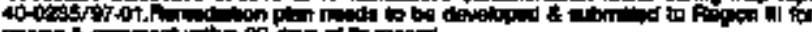

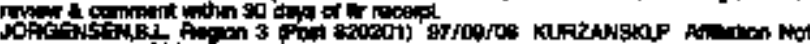

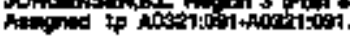

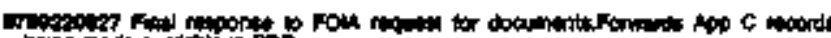

PQW

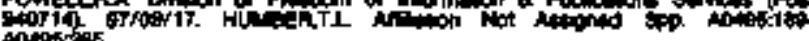
A040050.5

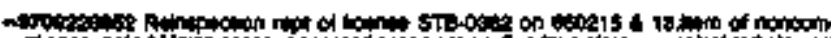

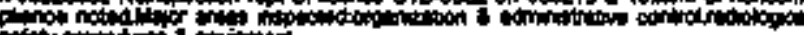

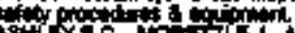

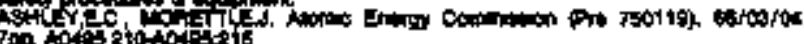

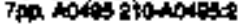

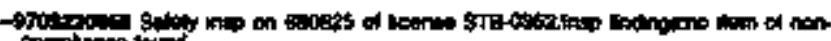

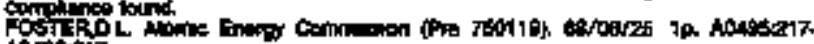

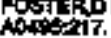

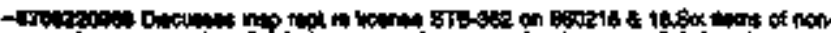

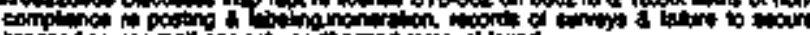

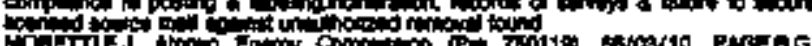

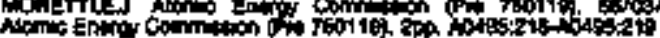

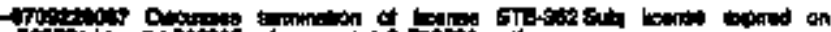
700 is

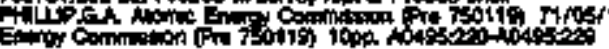

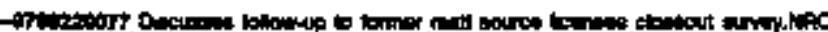

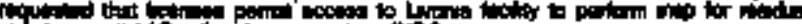

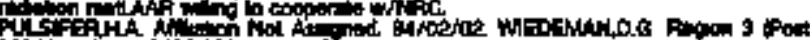

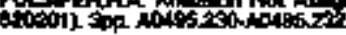

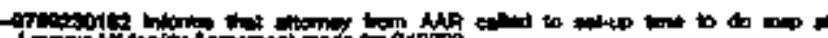

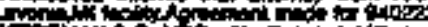
H

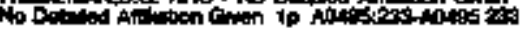

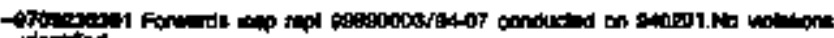

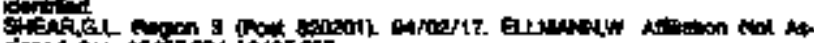

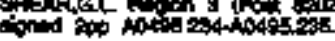

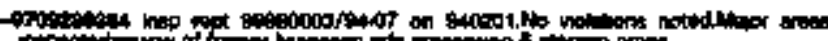

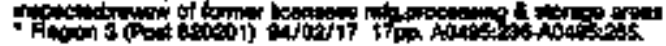

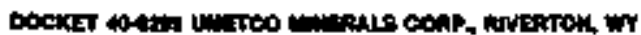

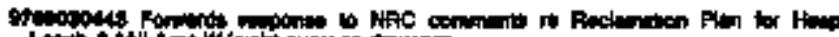

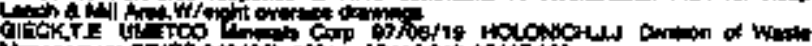

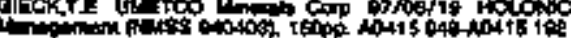

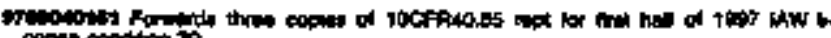
in

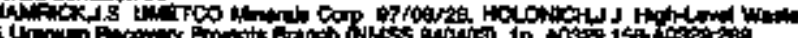

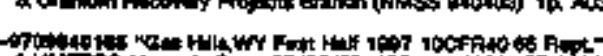

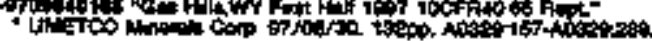

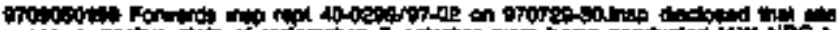

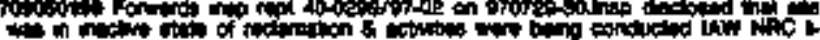

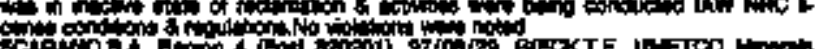
SCH

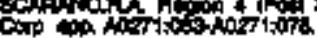

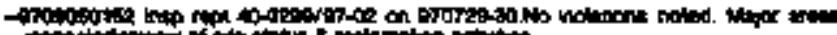

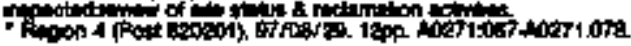

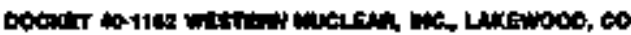

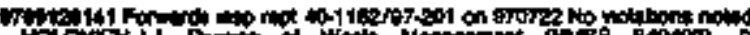

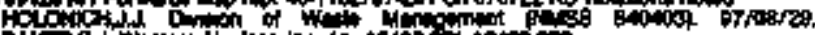

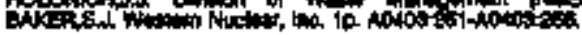

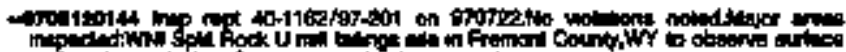

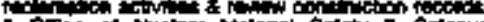

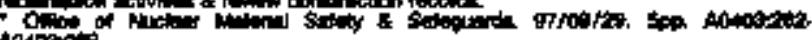
noworate

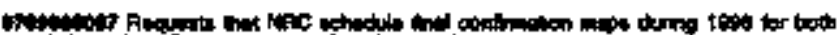

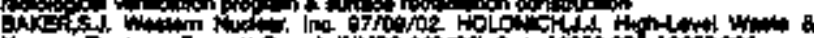

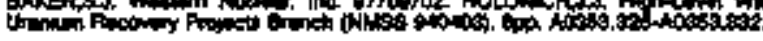

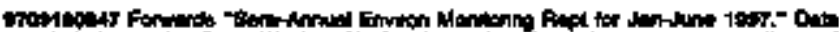

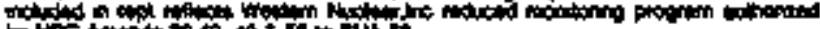

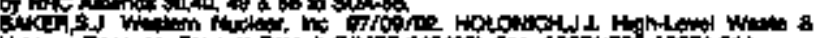

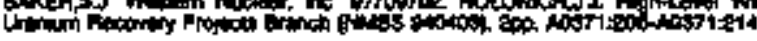

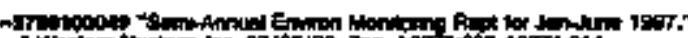

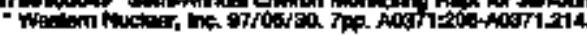

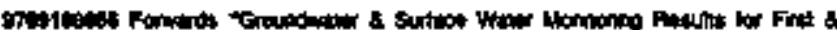

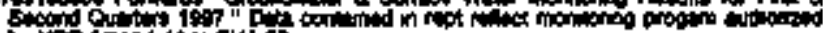

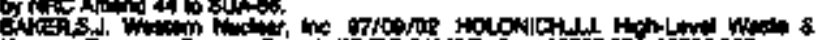

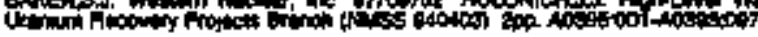

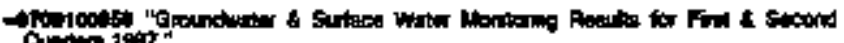

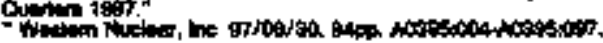

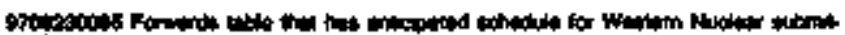

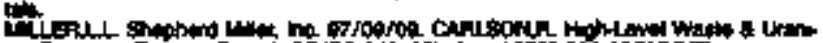

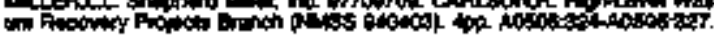

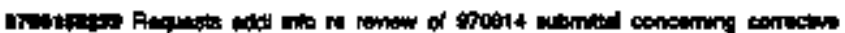

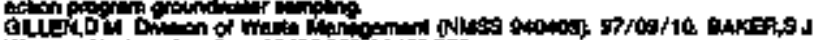

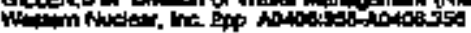

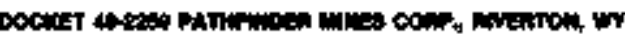

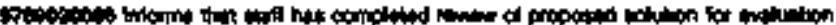

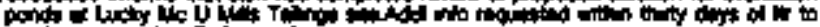

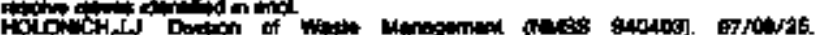

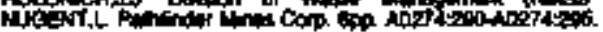

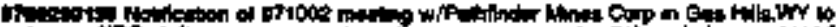

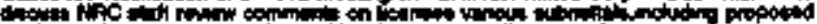

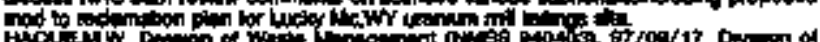

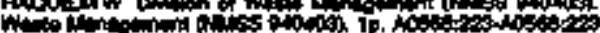

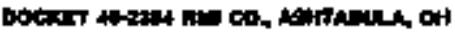

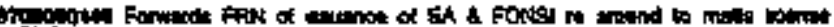
stite-

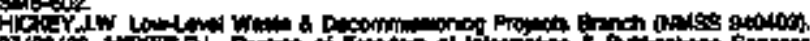

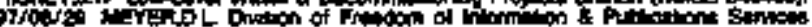

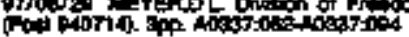

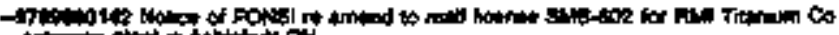

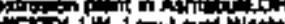

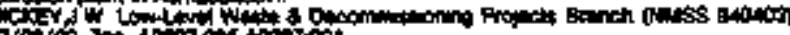

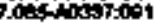

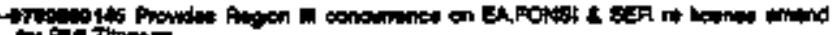

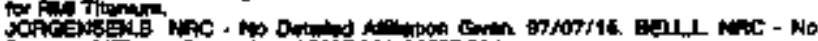

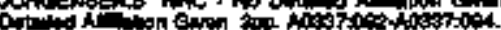

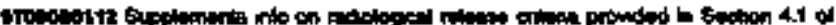

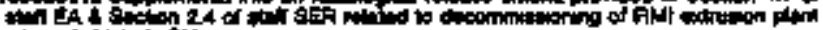

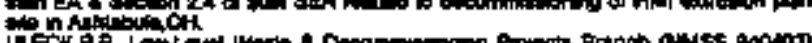

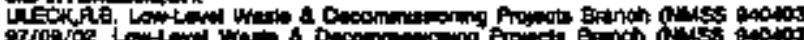

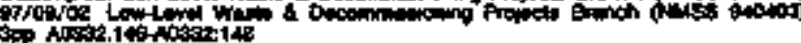




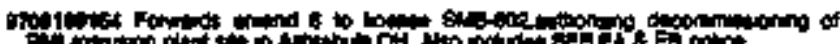

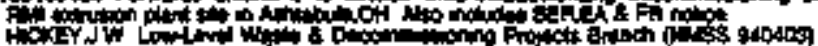
of

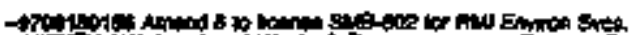

HCYJW L

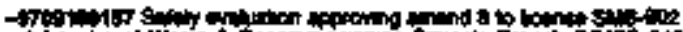

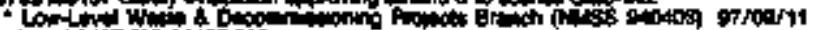

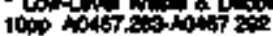

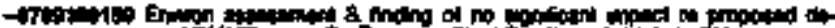

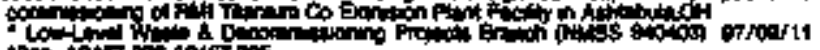
1300 100.7.

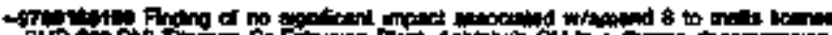

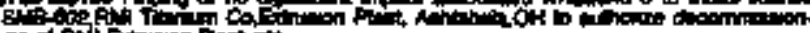

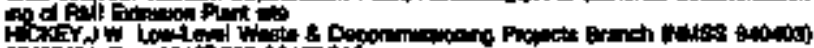

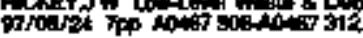

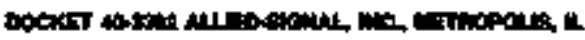

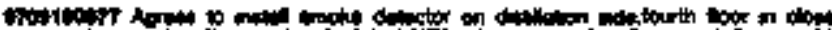

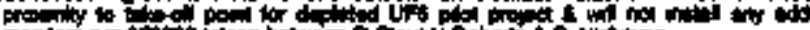

mogen

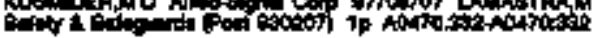

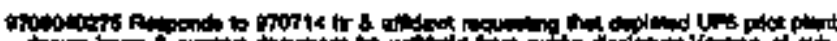

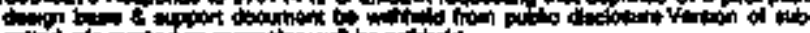

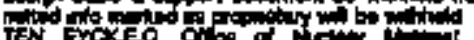

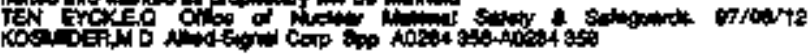

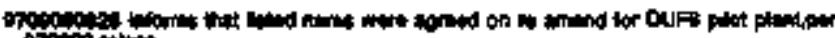

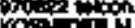

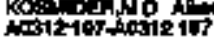

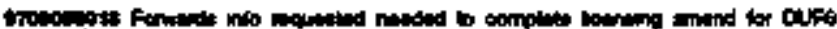

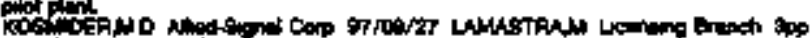

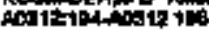

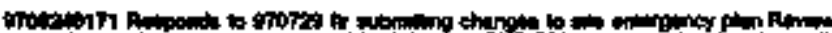
he land do

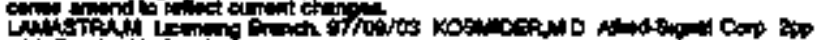

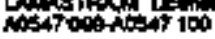

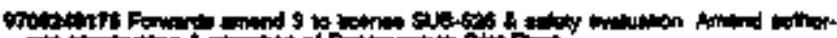
W

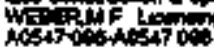

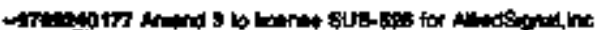

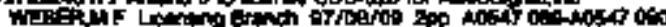

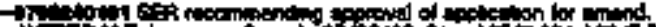

Wi:

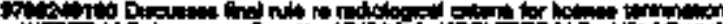

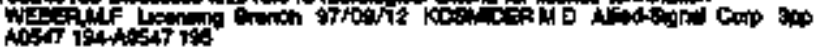

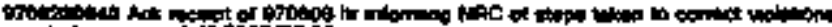

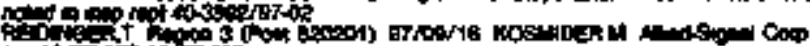
in animition

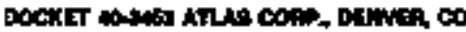

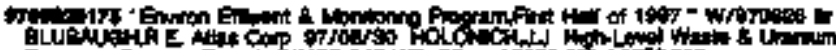

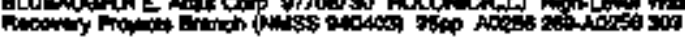

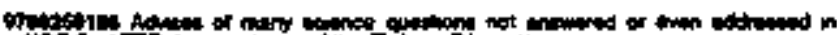
Hor How

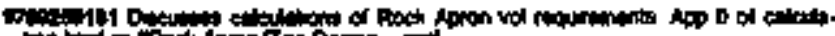

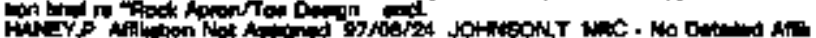

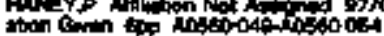

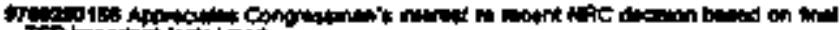
Tif

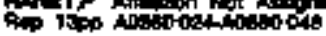

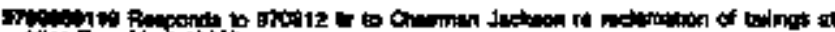

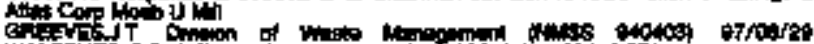

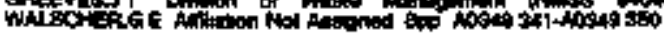

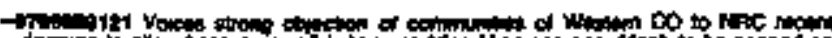

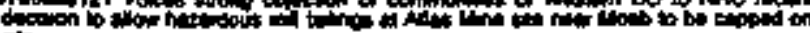

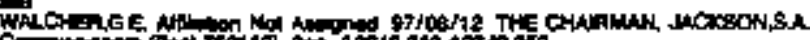

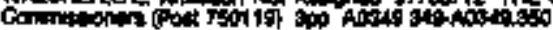

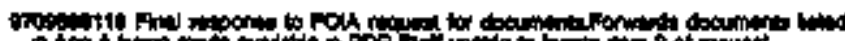

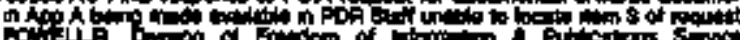

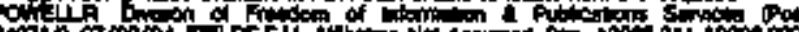

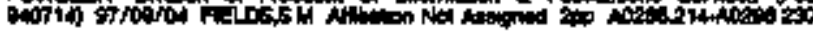

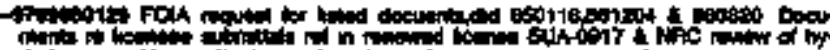

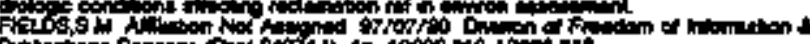

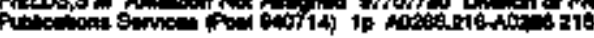

-7.

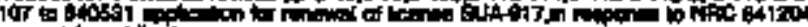

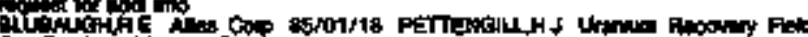

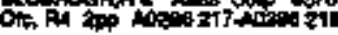

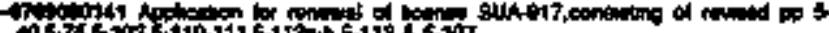

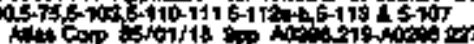

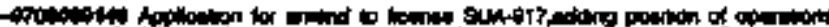

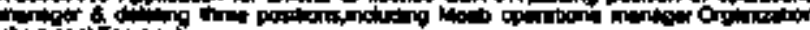
th

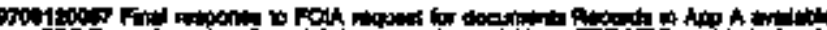

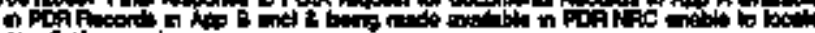
ming

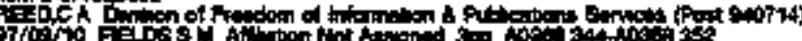

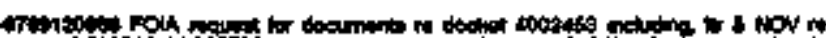

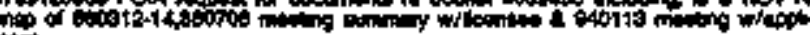

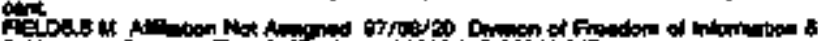

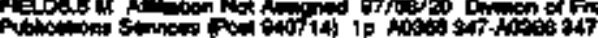

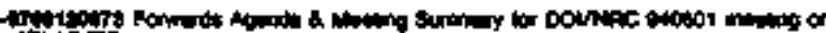
trites

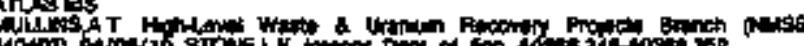

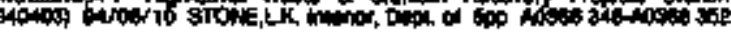

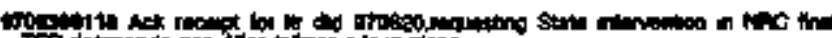

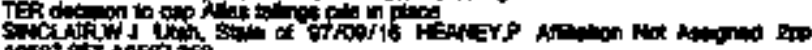

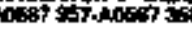

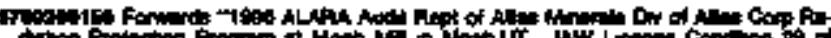

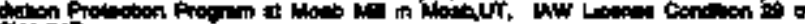

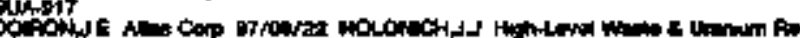

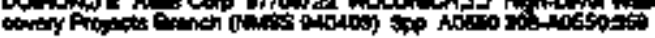

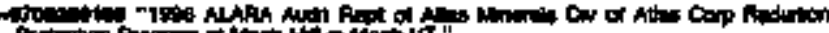

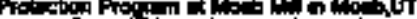

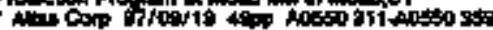

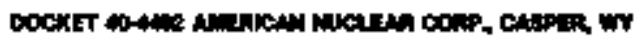

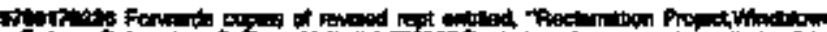

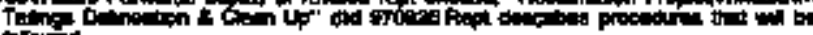

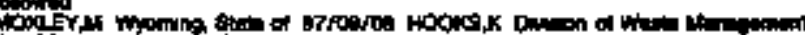

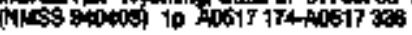

"

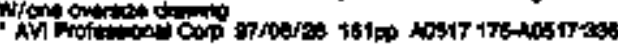

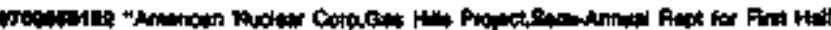

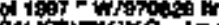

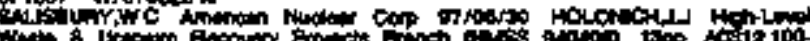

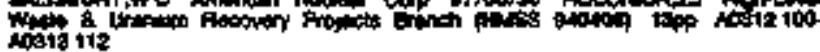

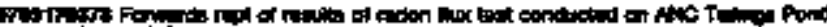

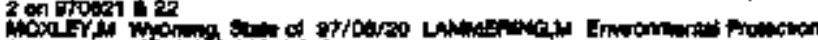

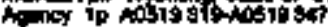

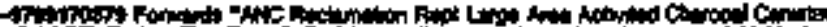

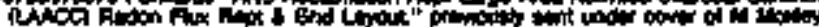
10 into

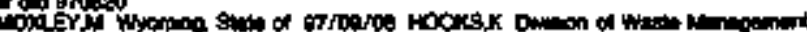

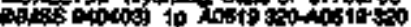

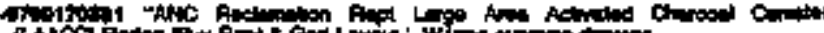

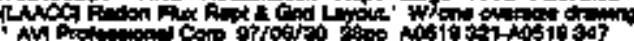

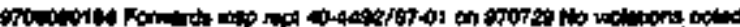

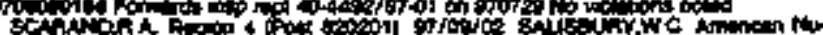

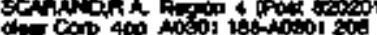

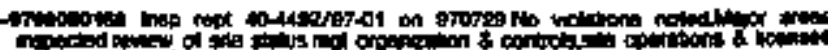

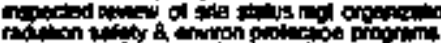

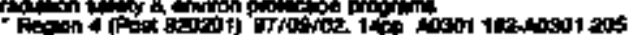




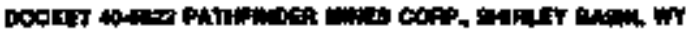

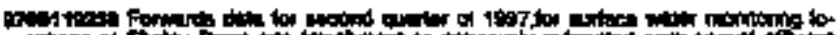
c...ons a

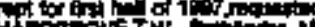

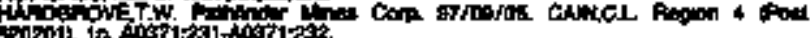

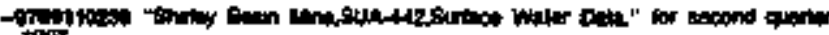
tgig

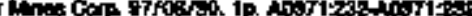

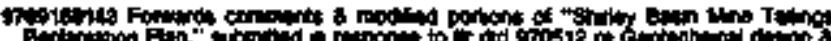

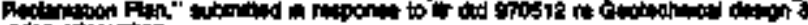

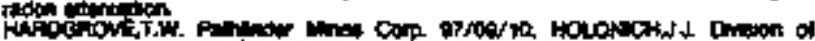

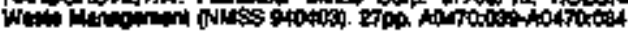

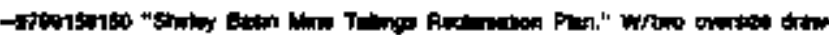

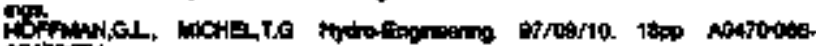

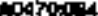

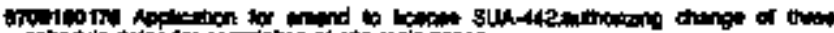

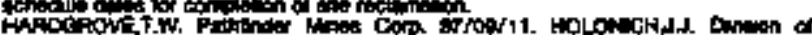

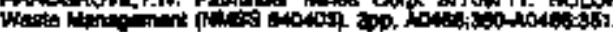

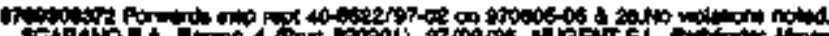

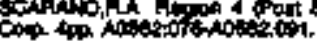

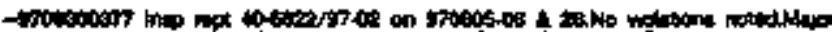

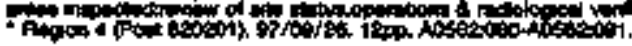

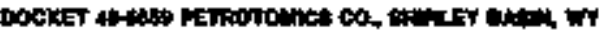

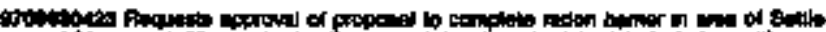

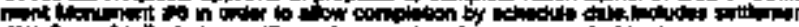

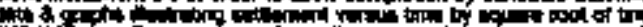

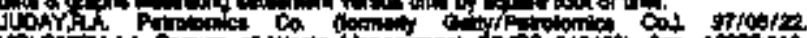

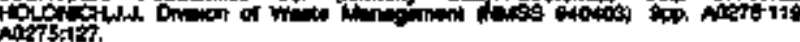

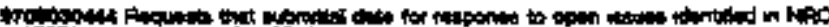

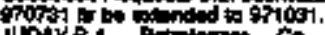

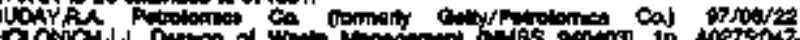
Ators on?

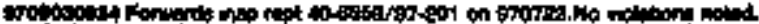

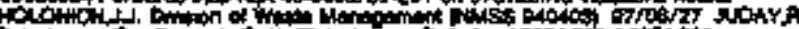

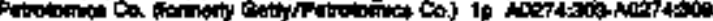

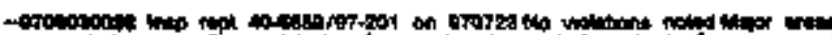

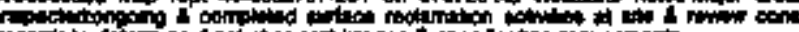

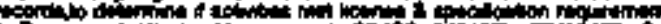

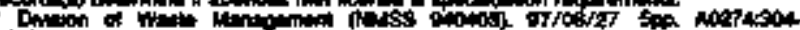
notiona.

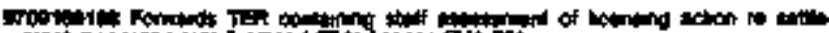

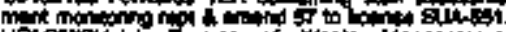

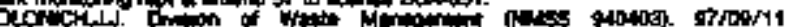

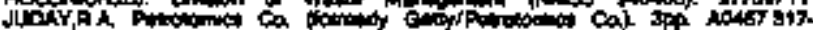

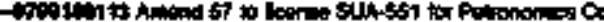

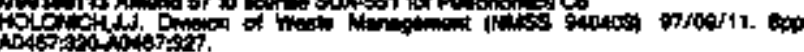

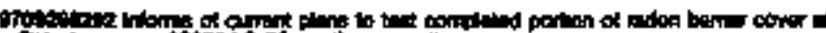

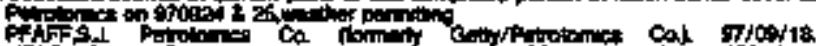

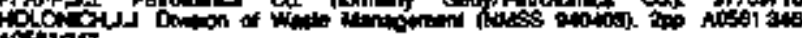
Astist

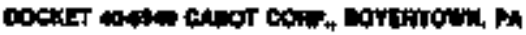

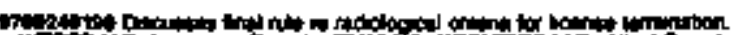

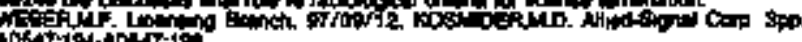

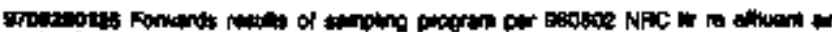

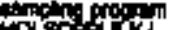
$x_{\text {ton }}$

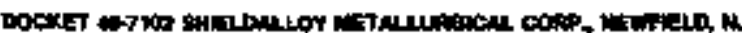

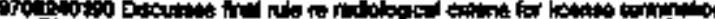

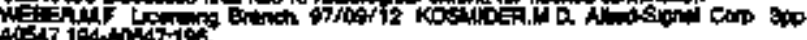

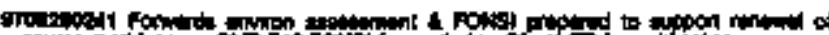

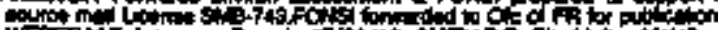

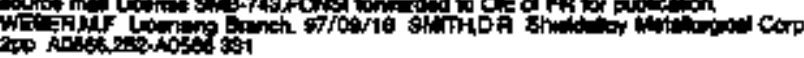

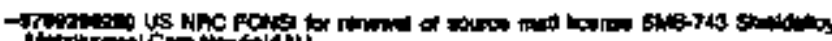

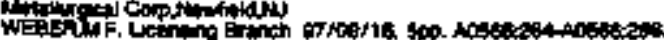

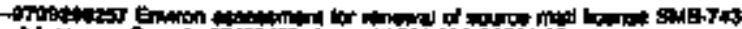

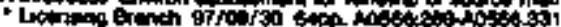

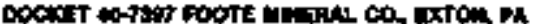

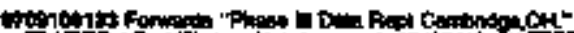

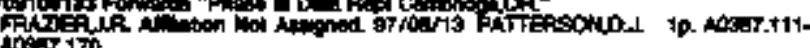

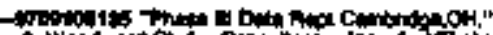

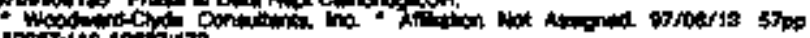

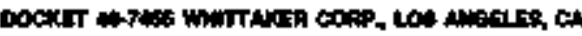

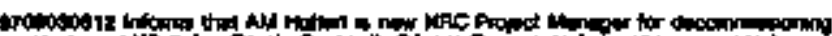

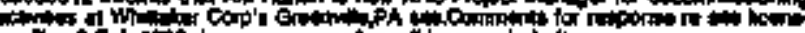

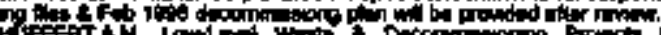

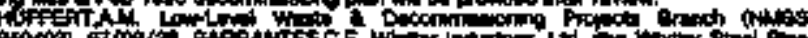

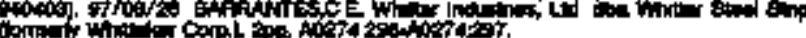

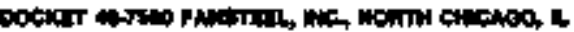

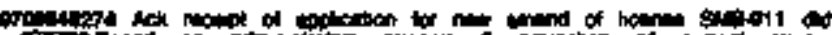

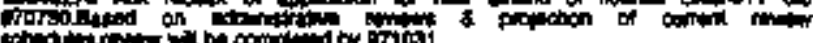

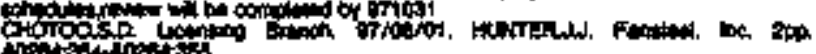

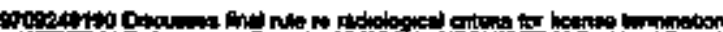

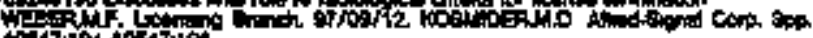

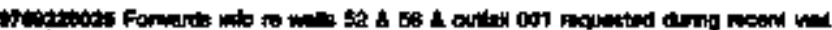

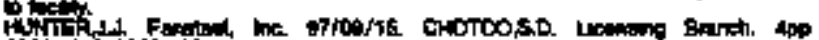

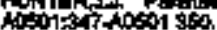

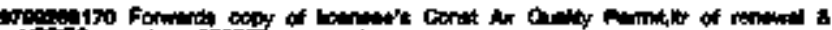

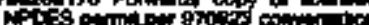

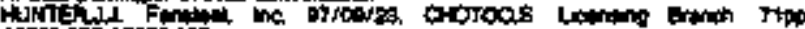

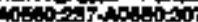

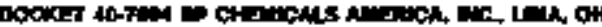

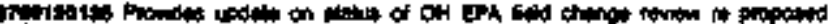
Fid cl

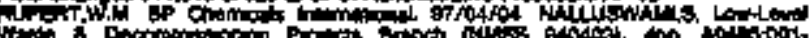
1040904

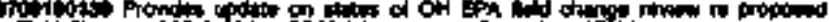

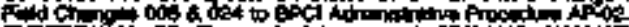

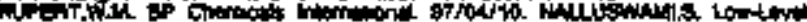

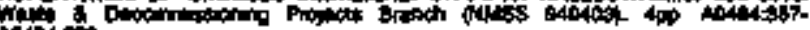
tomis

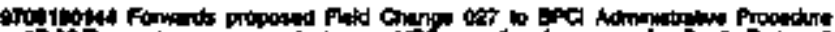

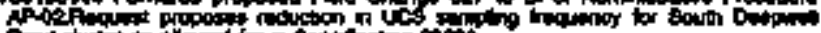

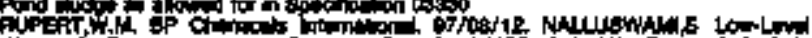

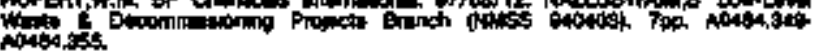

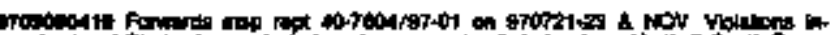

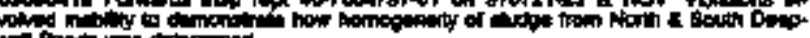

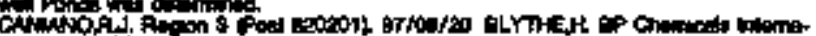

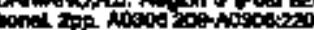

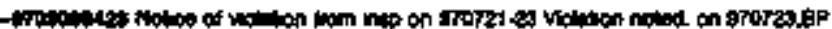

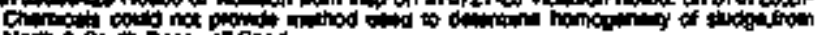

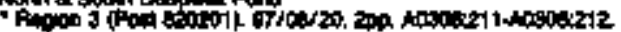

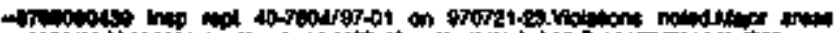

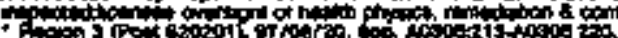

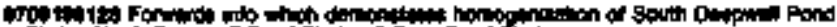

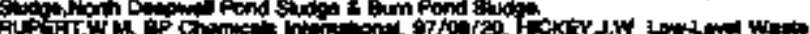

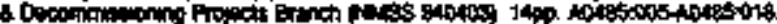

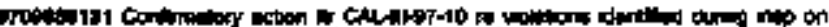

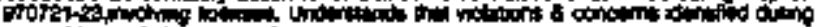

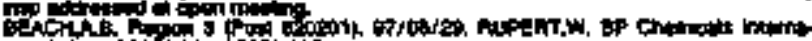
ind and

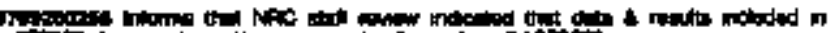

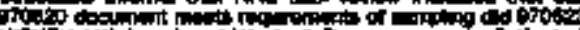

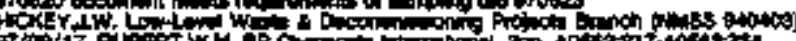

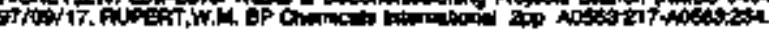

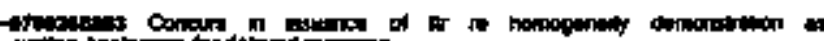

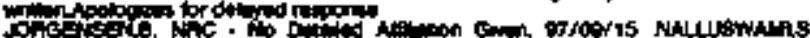

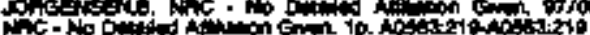

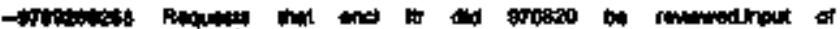

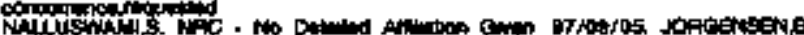

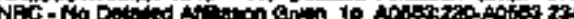




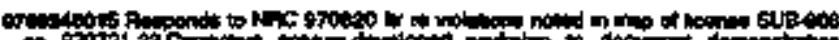

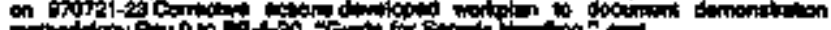

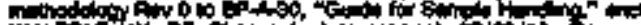

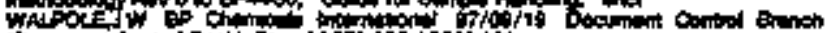

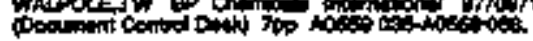

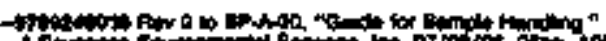

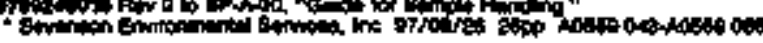

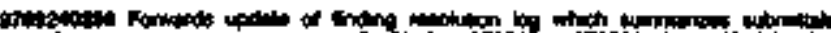

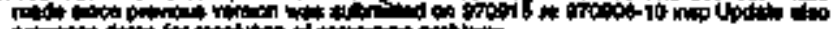

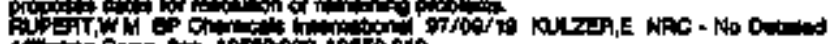
Ningon

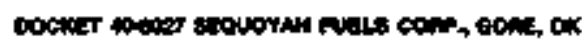

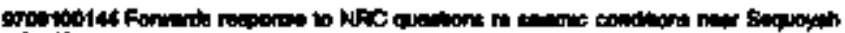

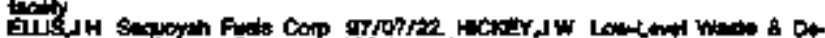

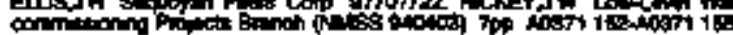

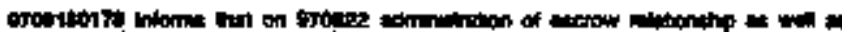

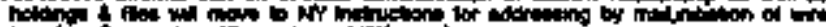

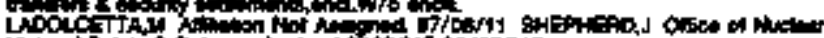

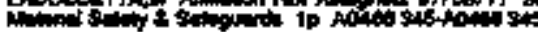

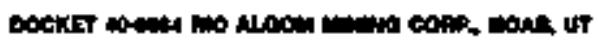

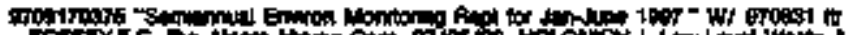

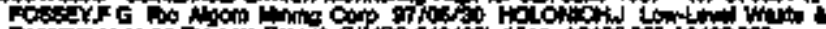

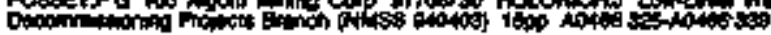

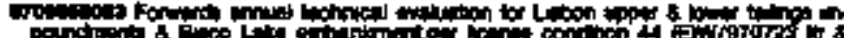

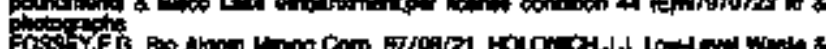

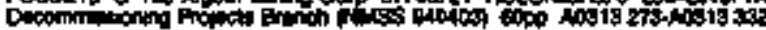

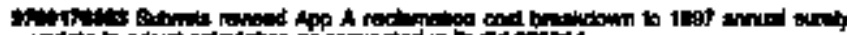

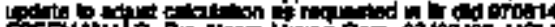

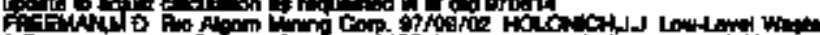

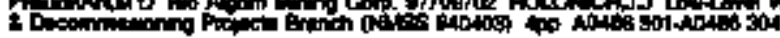

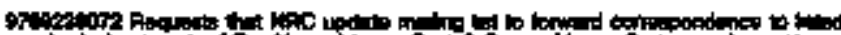

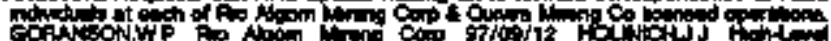

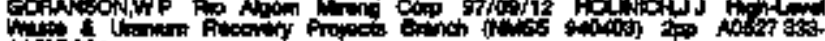
tosis

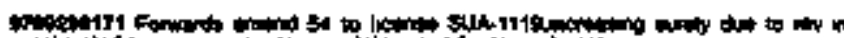

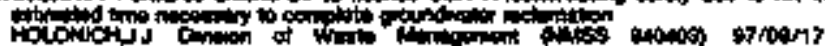

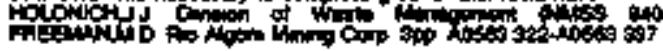

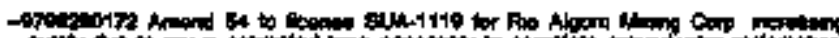

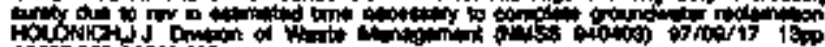

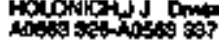

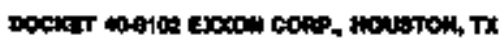

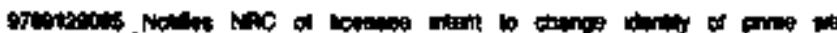

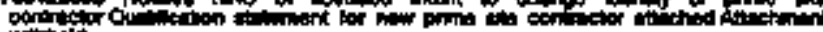

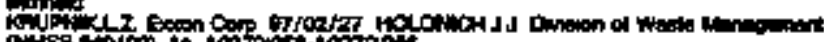

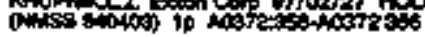

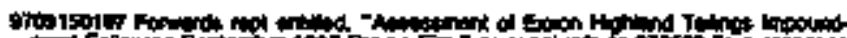

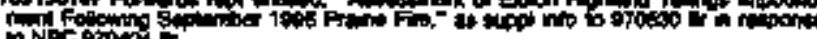
WNAC OTOM E

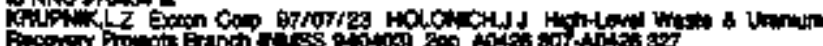

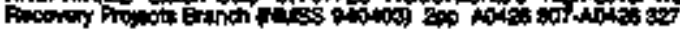

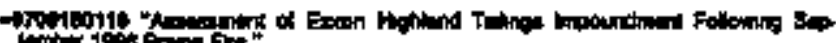

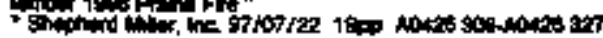

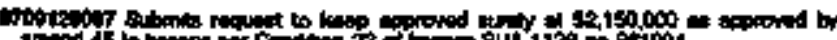

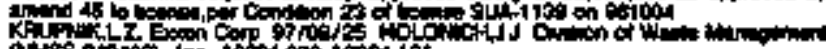

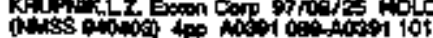

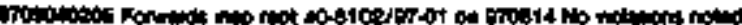

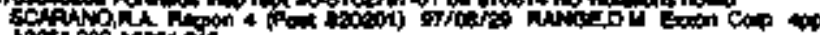

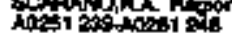

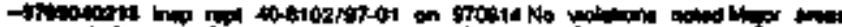

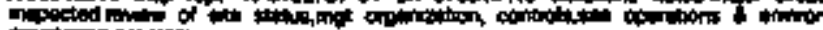

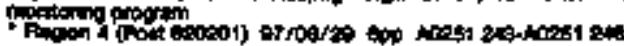

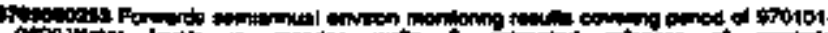

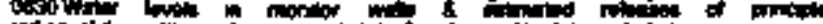

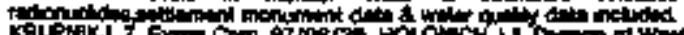

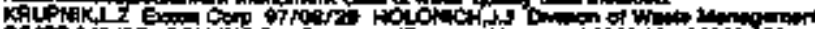

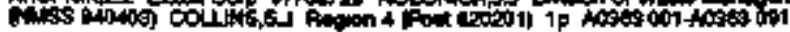

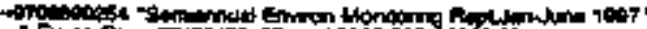

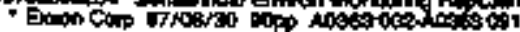

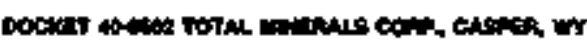

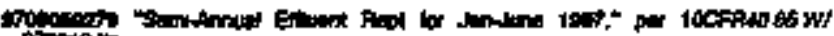

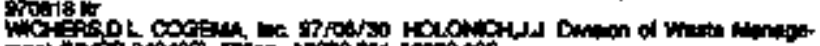

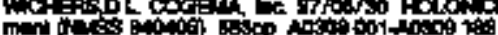

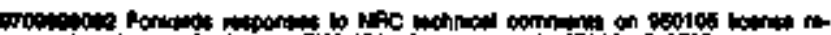

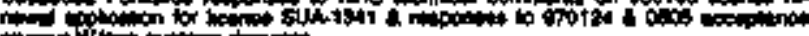

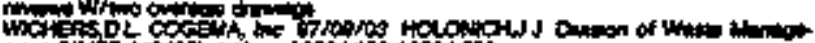

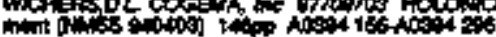

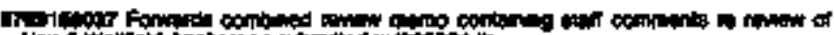
In

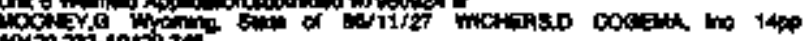

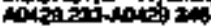

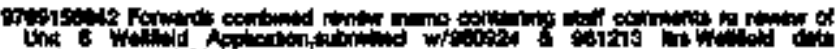

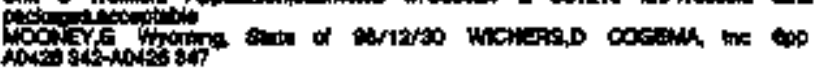

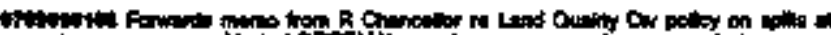

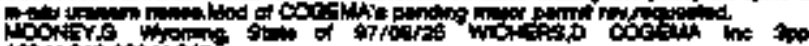

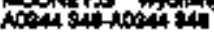

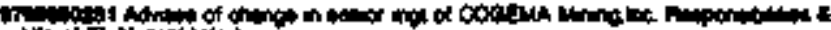

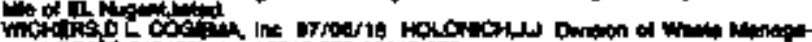

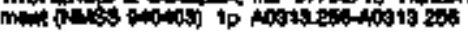

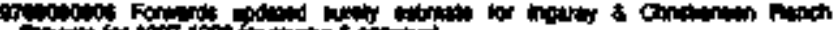

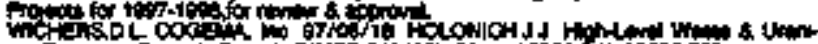

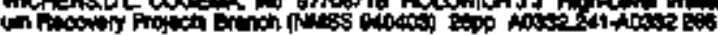

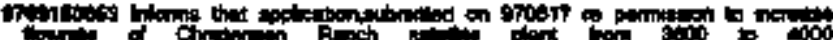

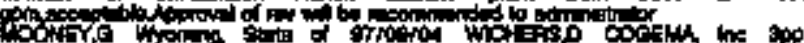

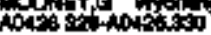

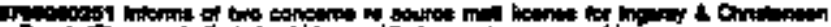

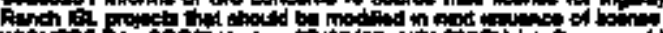

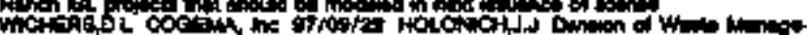
ment oN

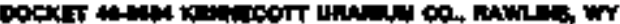

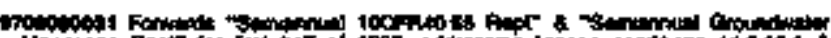

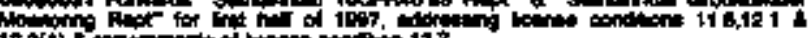

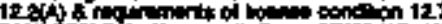

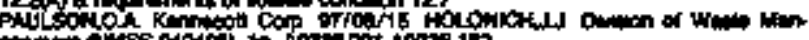

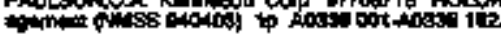

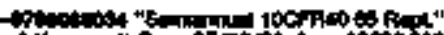

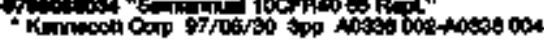

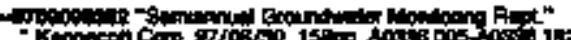

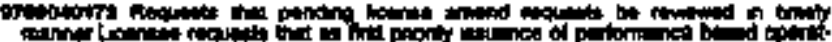

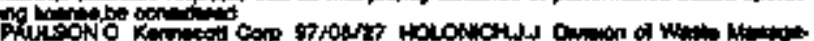

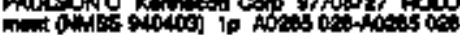

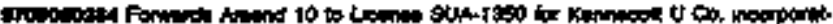

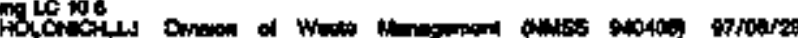

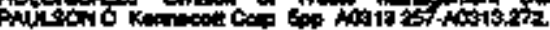

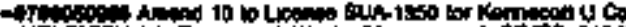

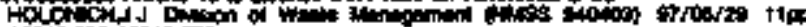

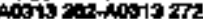

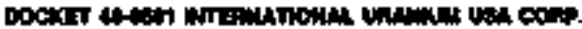

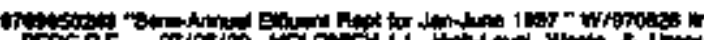

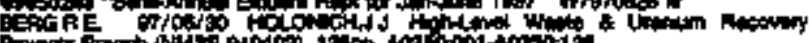

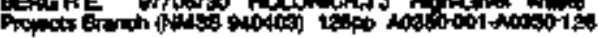

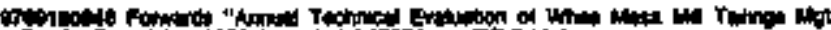

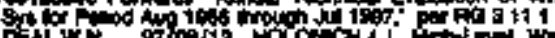

OAL

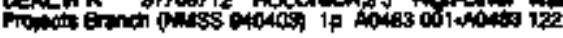

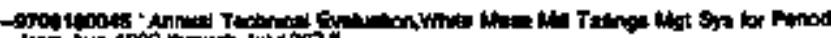

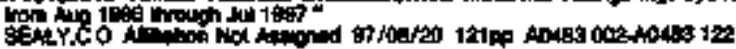

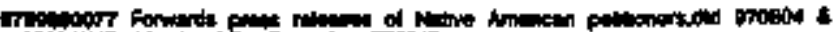

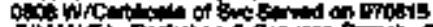

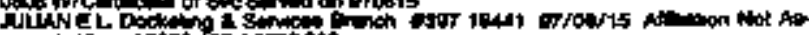

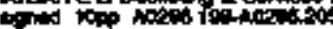




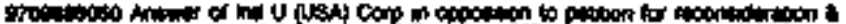

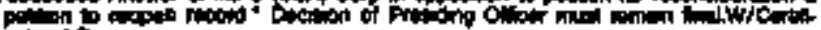
iㅓㅇ of 194 . $190000=0$

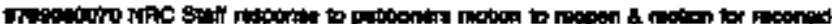

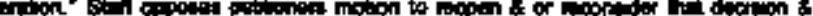

Tom,

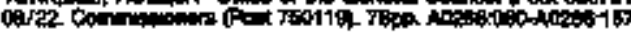

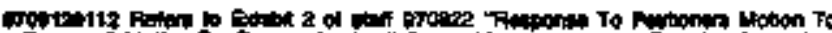

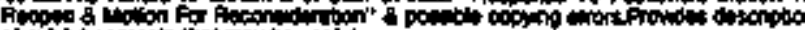

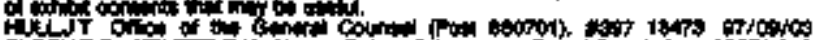

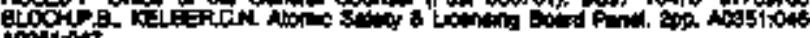
tosition.

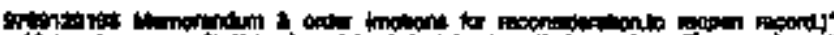

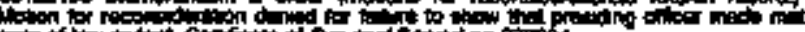

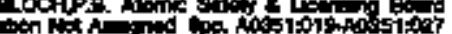

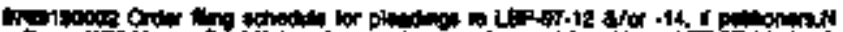

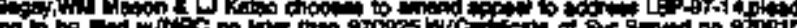

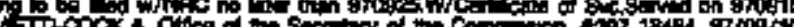
$L$ 工 AMar

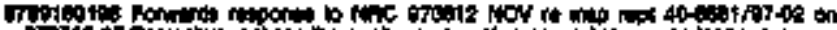

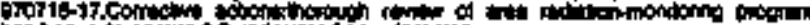

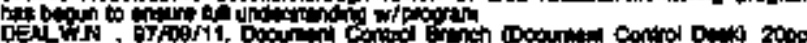

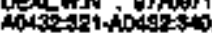

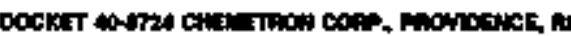

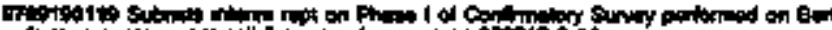

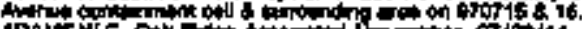

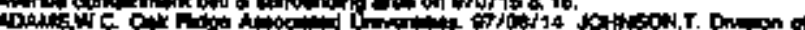

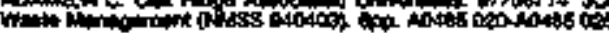

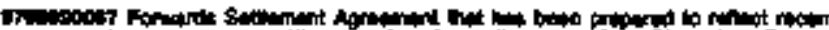

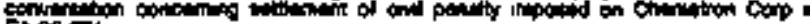
UA

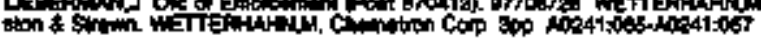

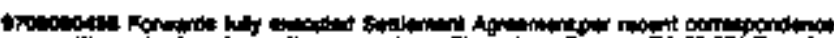

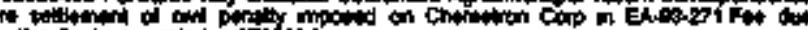

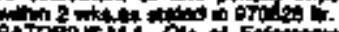

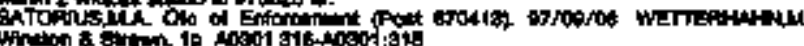

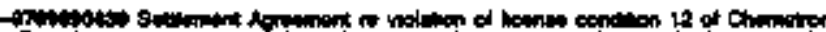

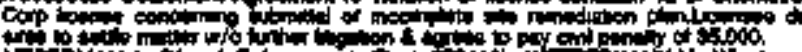

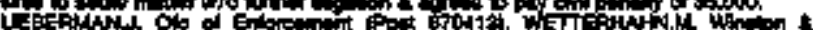

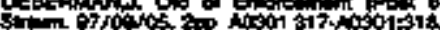

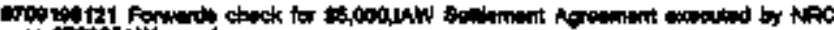

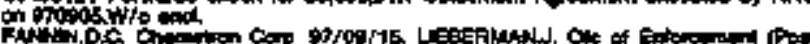

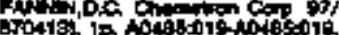

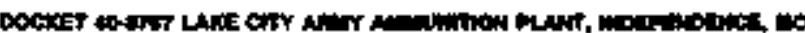

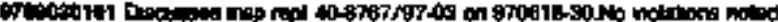

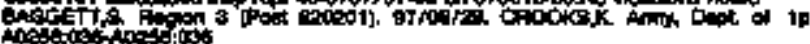

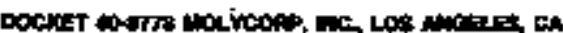

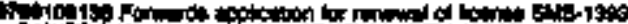

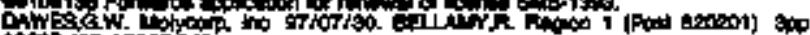
intorion.

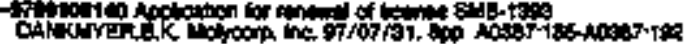

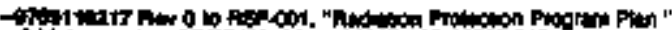

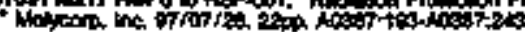

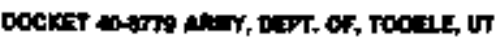

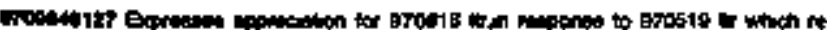

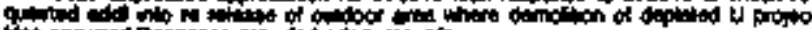

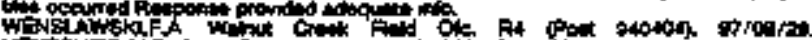

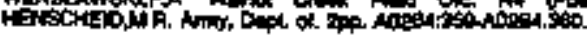

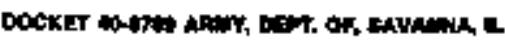

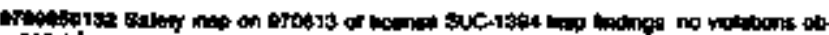

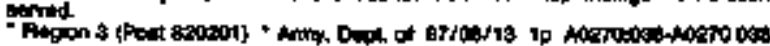

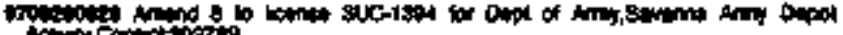

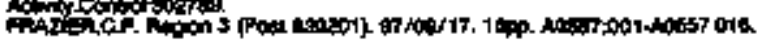

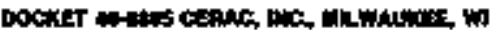

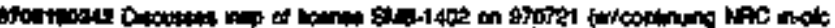

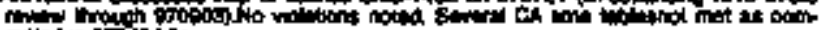
mitin in bristoin.

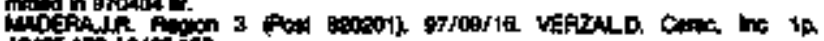

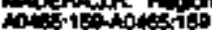

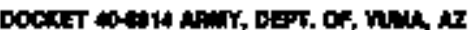

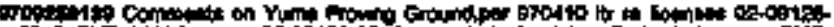

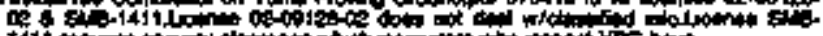

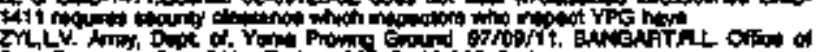

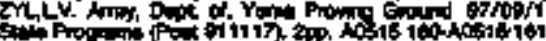

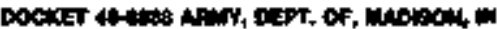

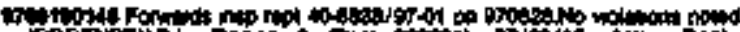

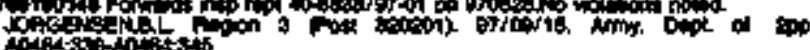

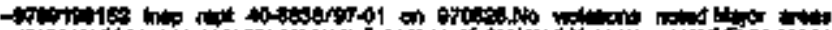

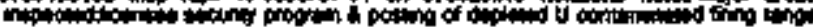

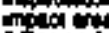

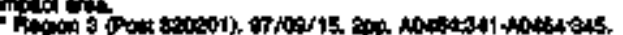

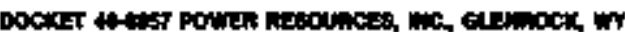

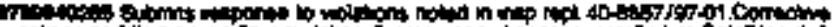

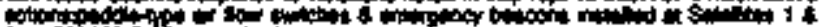
2 of

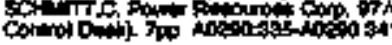

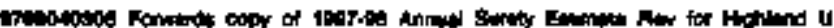

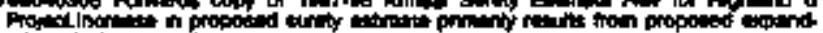

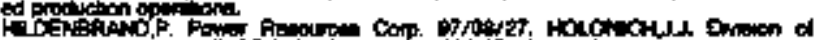

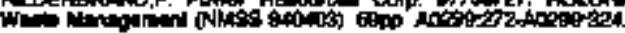

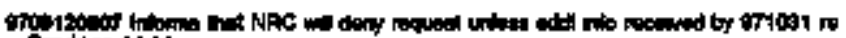

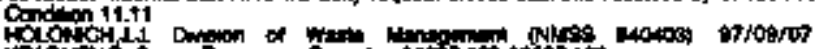

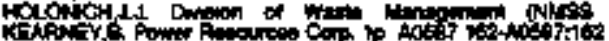

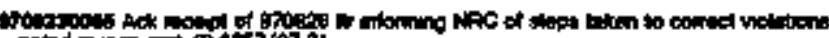

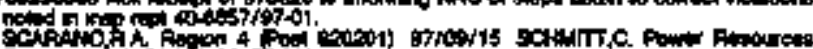

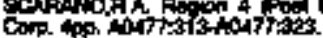

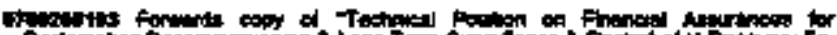

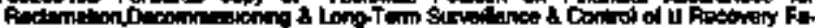

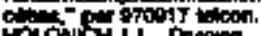

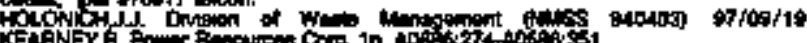

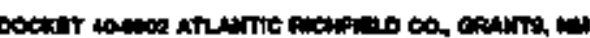

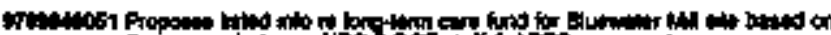

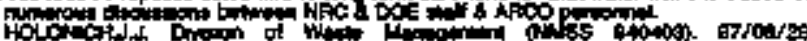

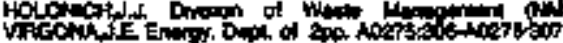

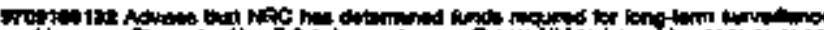

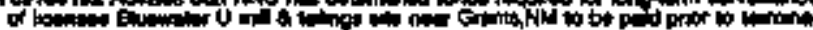

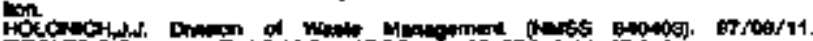

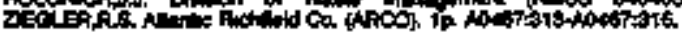

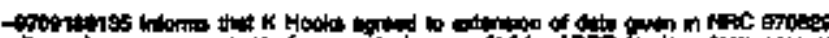

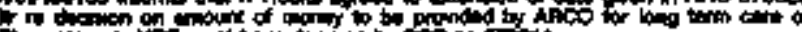

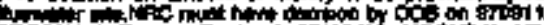

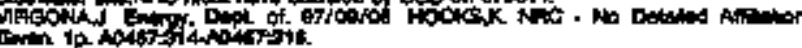

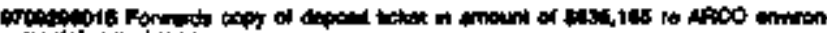

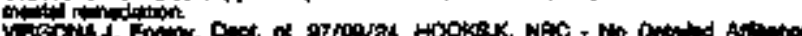

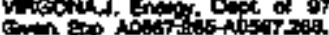

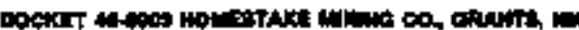

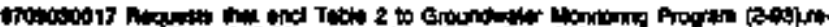

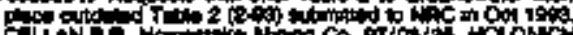

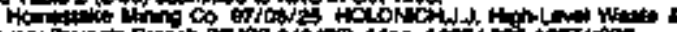

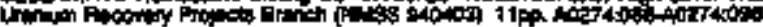

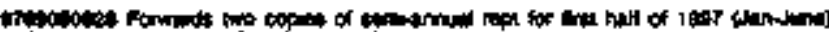

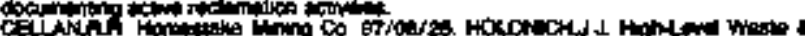

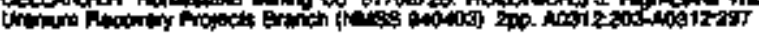

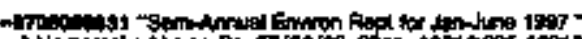

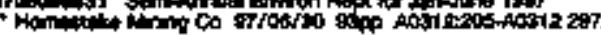




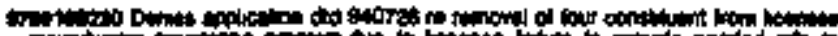

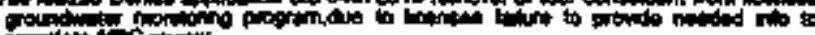

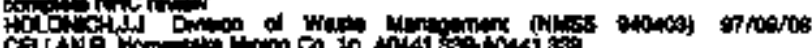
Cel

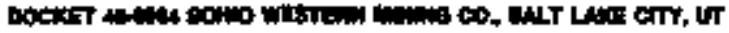

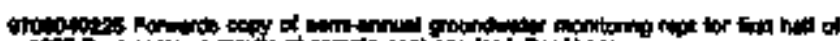

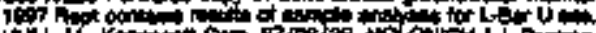

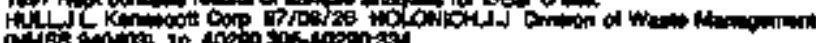
0 A

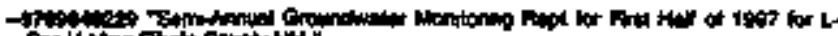

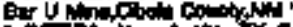

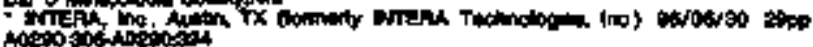

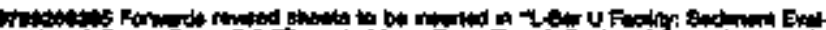

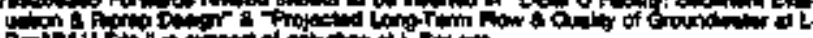
B r N

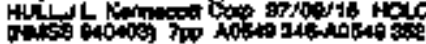

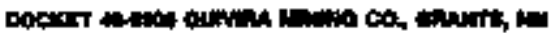

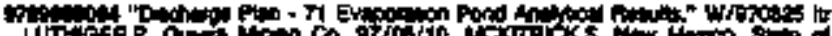
年

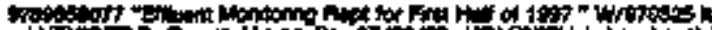

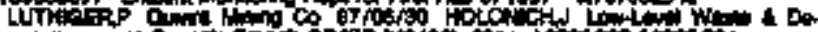

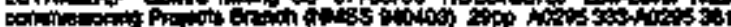

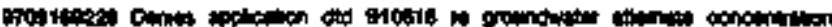

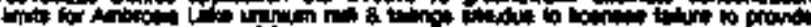

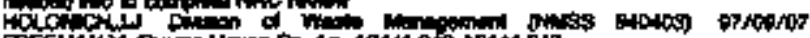

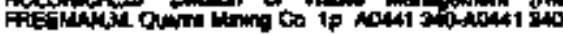

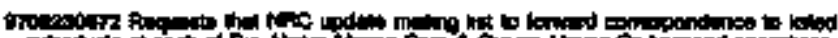

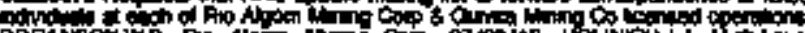

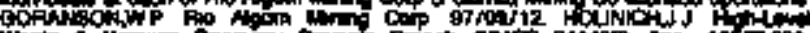

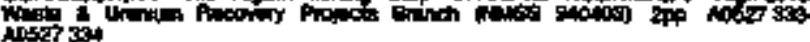

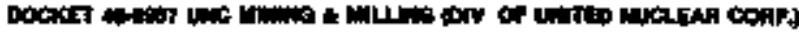

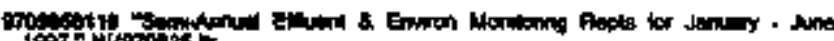
bit wrowes

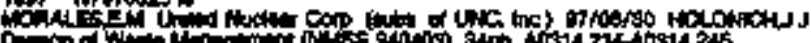

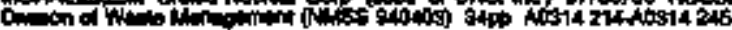

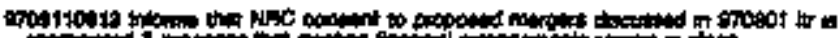

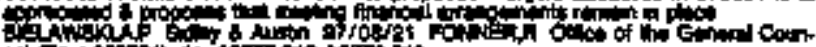

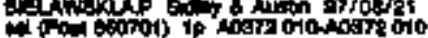

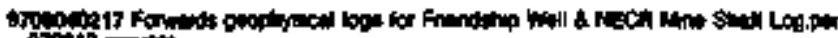

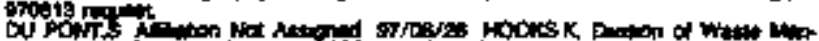

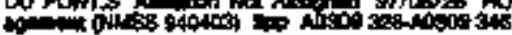

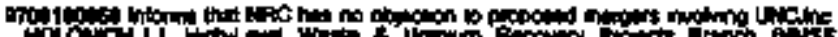

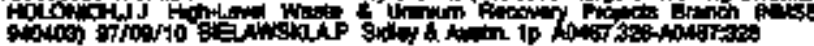

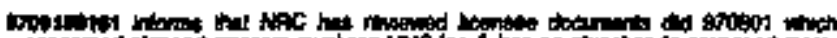

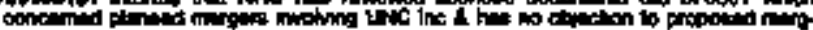

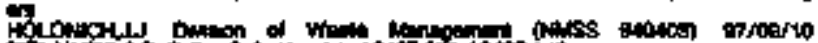

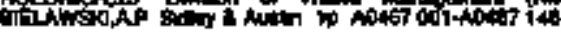

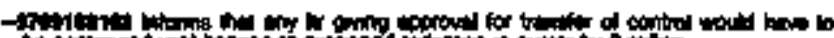

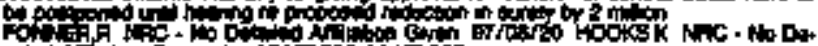

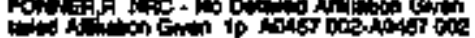

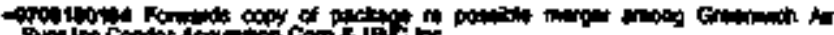

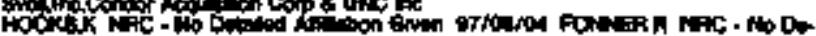

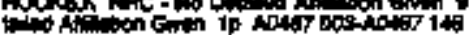

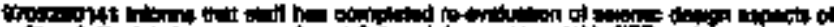

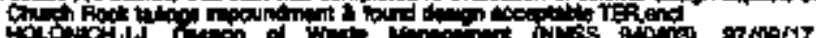

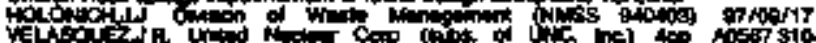

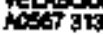

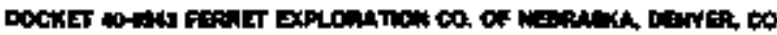

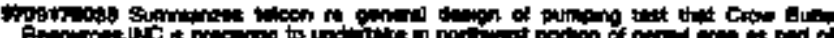

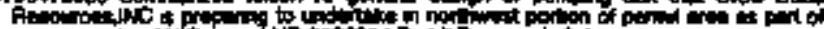

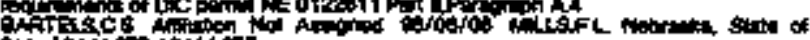

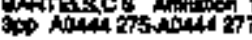

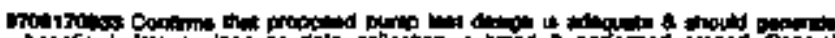

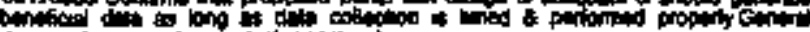

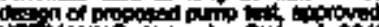

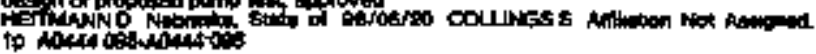

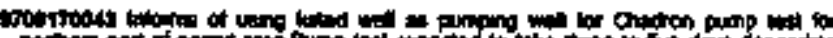

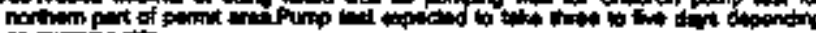
on pertition

COCf

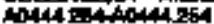

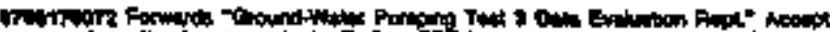

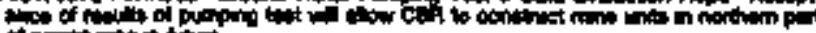
of

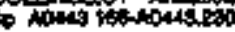

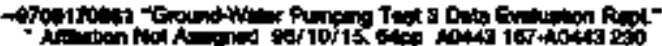

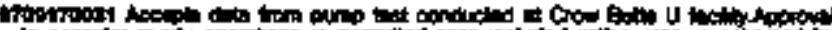

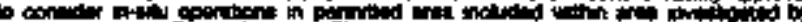
Ho

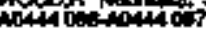

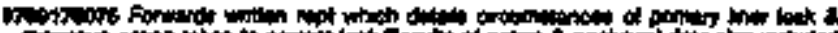

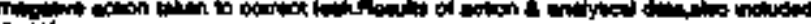

H thet

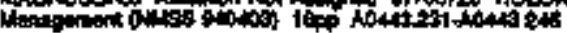

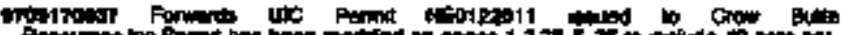

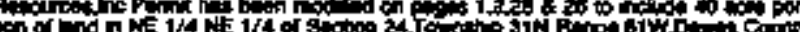
得

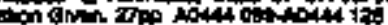

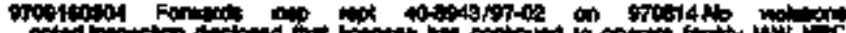

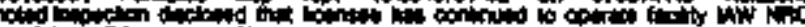

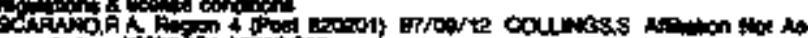

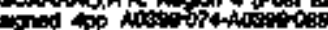

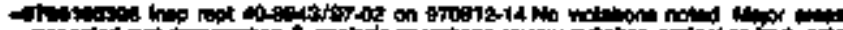

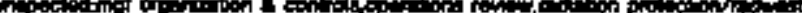

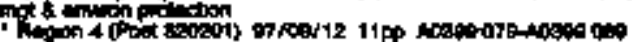

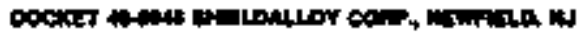

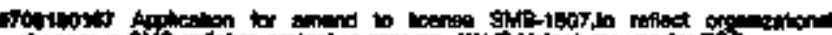

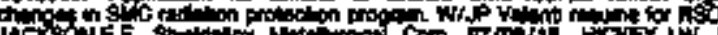

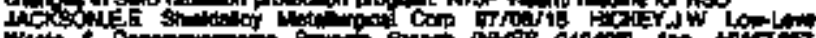

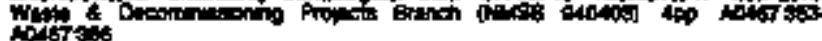

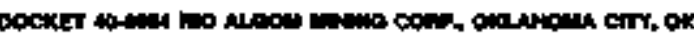

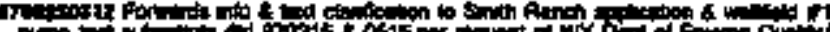

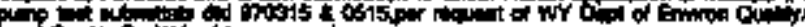

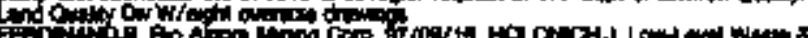

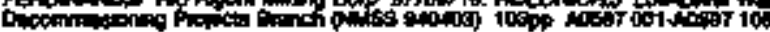

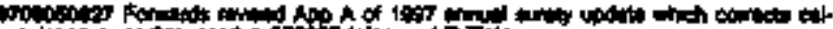

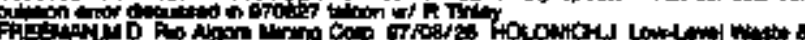

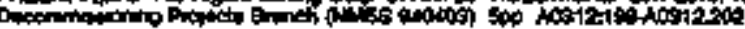

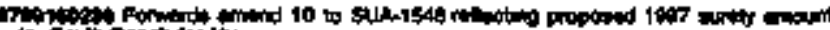

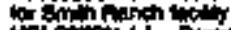

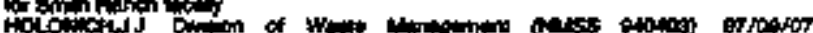

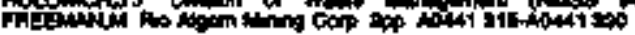

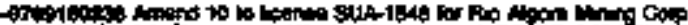

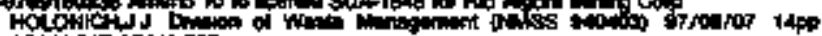
A041 $317+40441300$

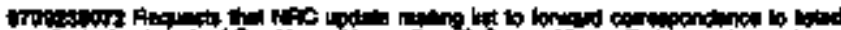

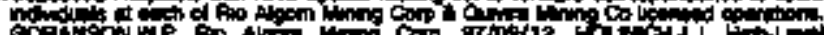

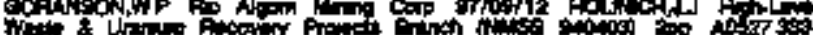
Hits

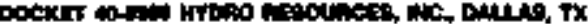

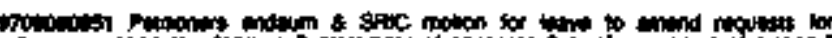

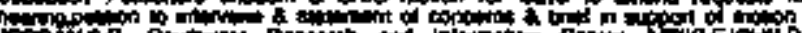

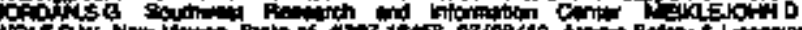

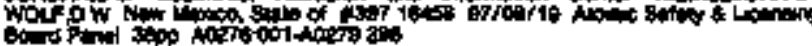

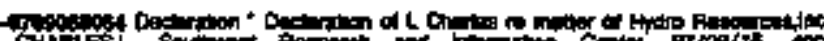

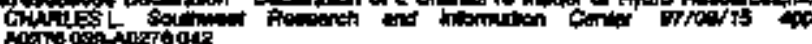

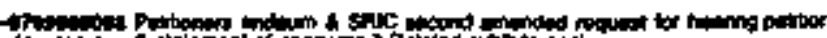

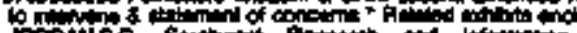

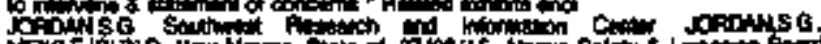

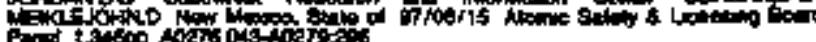




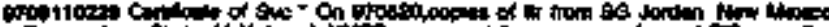

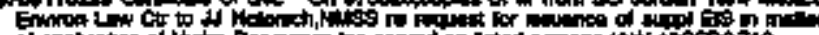

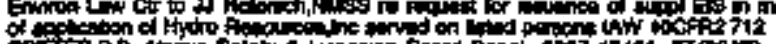

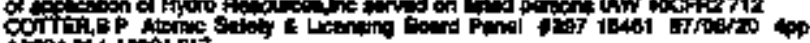

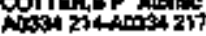

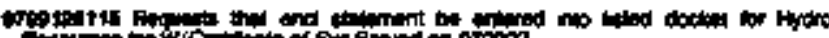

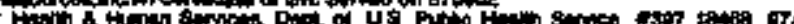

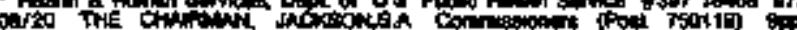

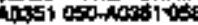

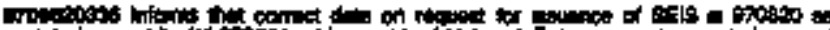

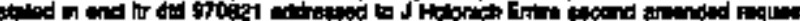

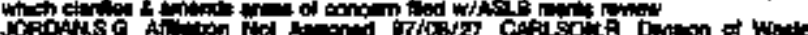

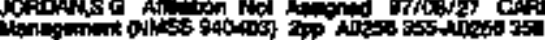

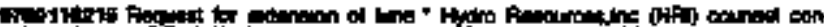

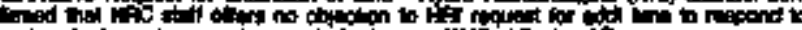

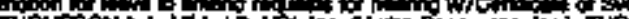

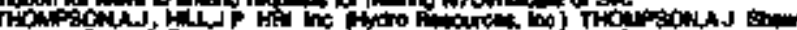

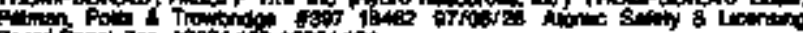

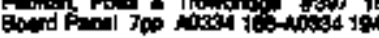

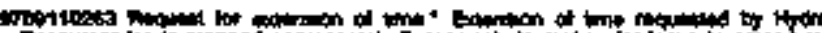

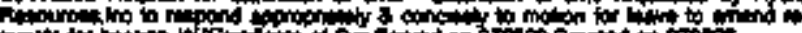

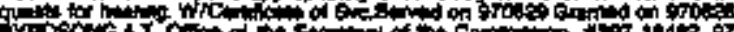

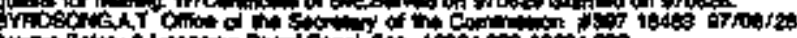

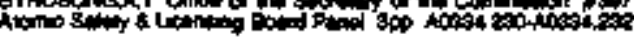

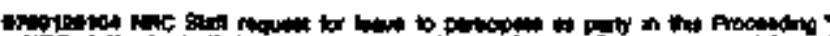

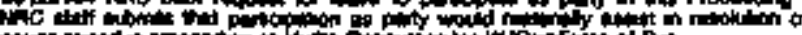

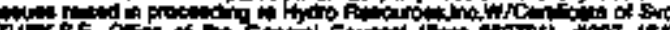

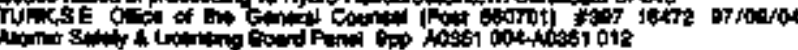

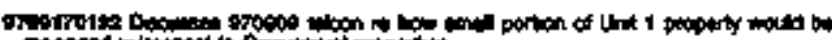

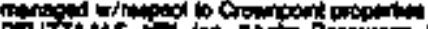

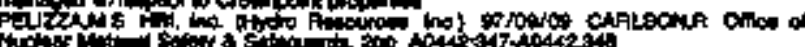

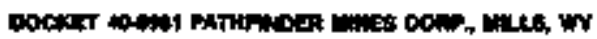

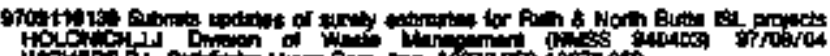

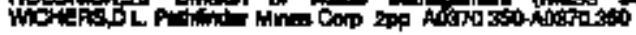

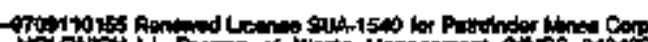

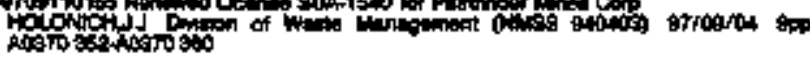

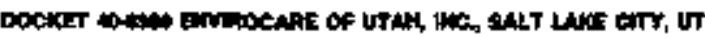

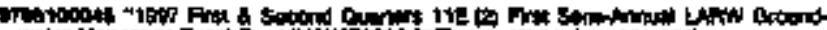

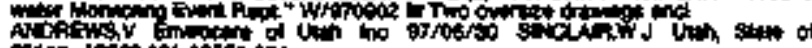

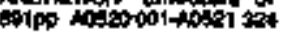

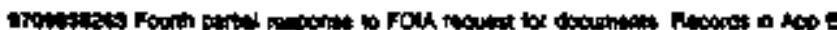

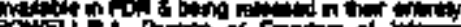

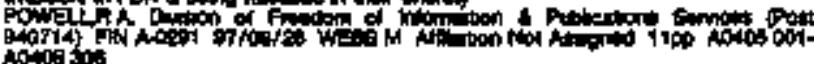

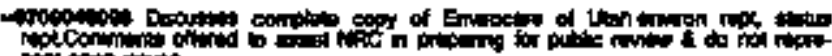

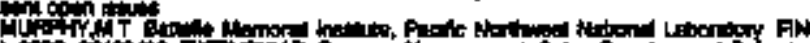

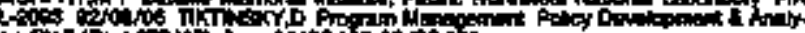

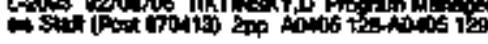

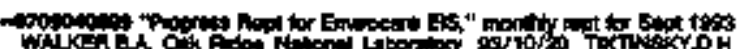

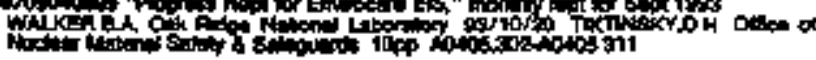

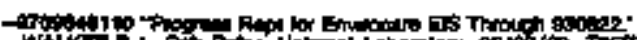

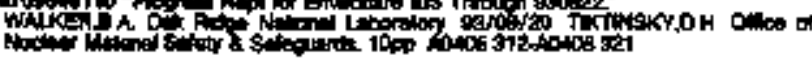

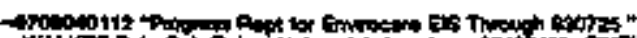

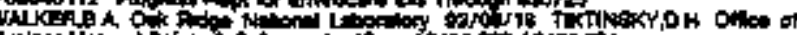

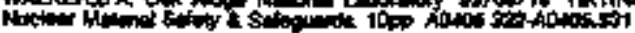

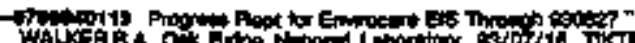

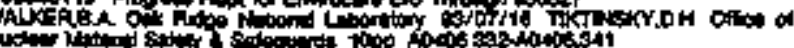

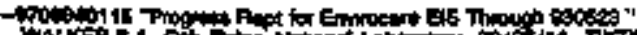

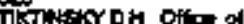

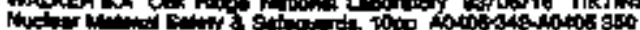

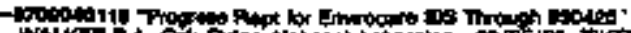

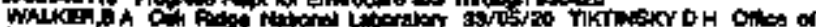

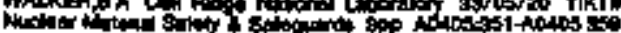

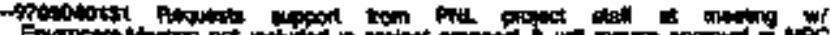

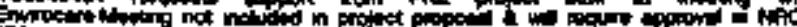

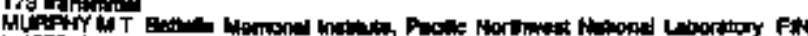

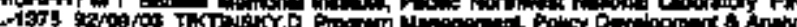

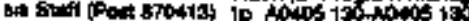

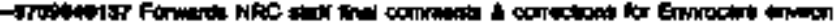

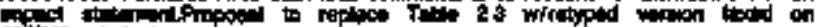

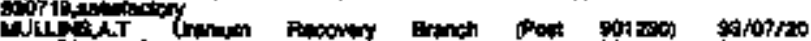

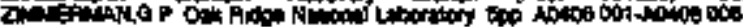

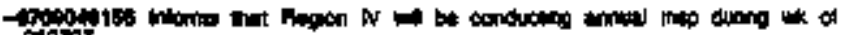
sor.t.

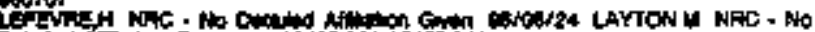

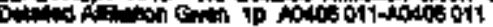

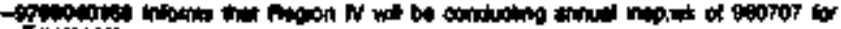

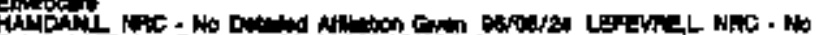

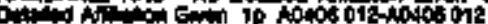

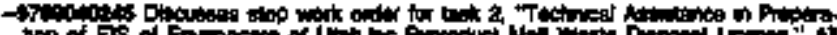

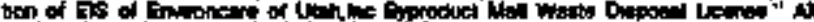

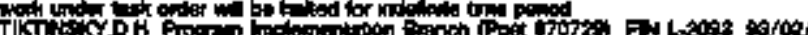

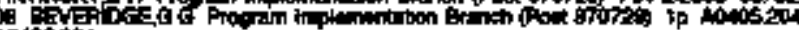
indosing

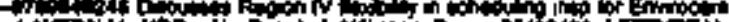

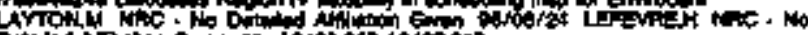

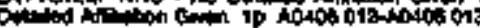

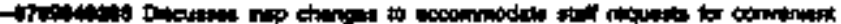

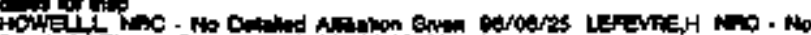

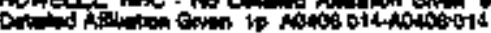

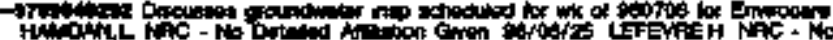

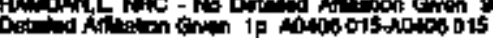

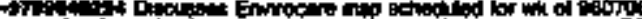
MLOW:

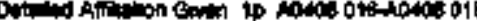

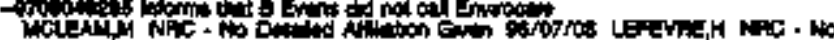

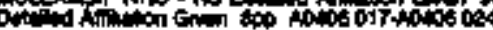

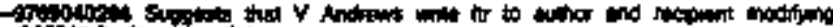

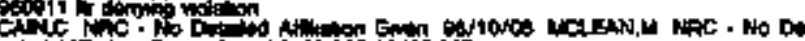

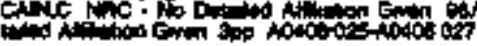

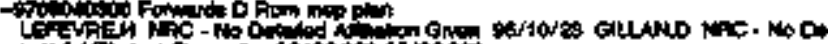

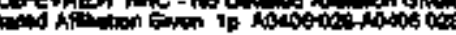

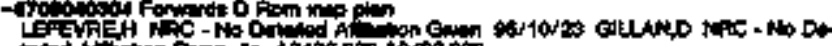

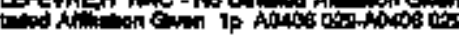

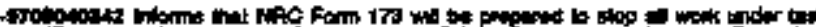

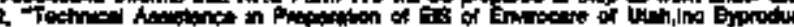

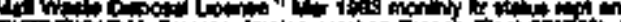

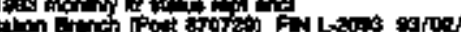

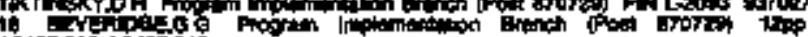

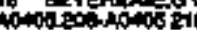

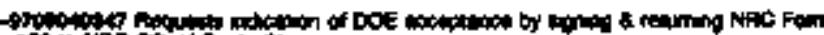

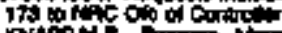

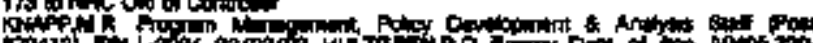

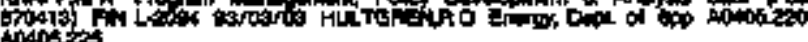

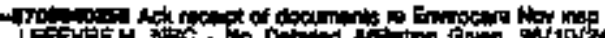

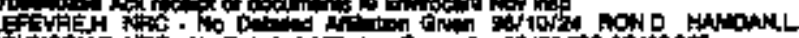

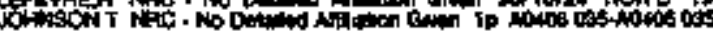

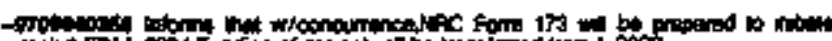

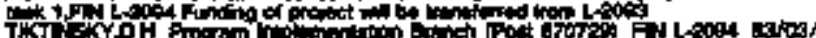
og of

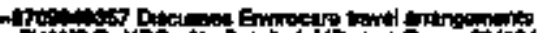

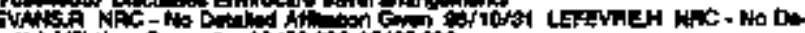
And A

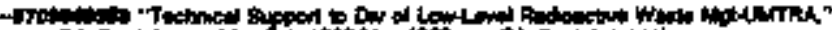

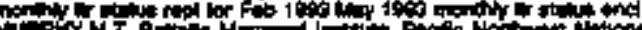

th

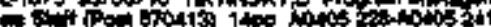

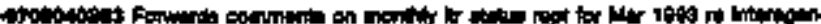

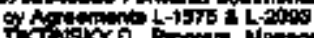

G

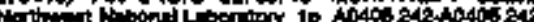

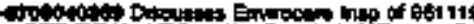

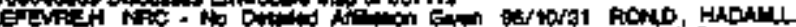

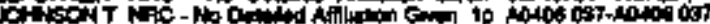

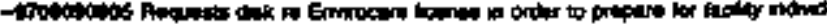

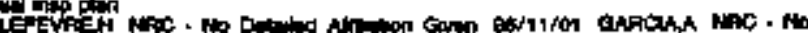

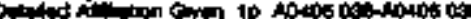




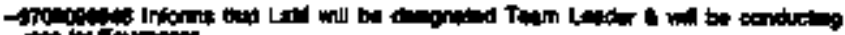

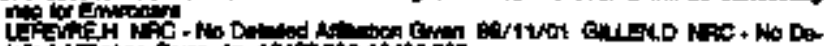

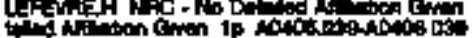

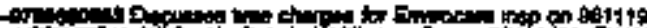

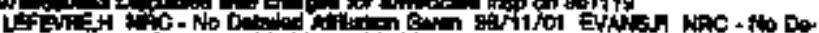

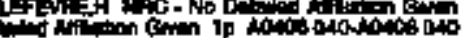

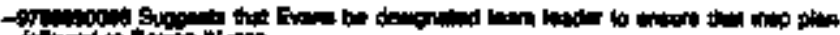

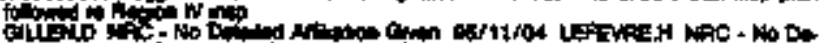

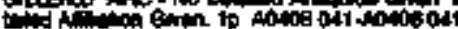

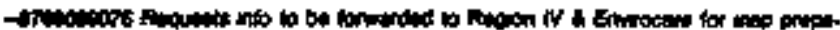

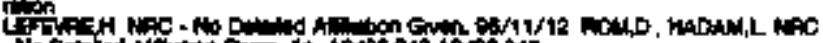

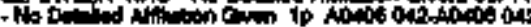

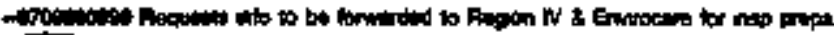

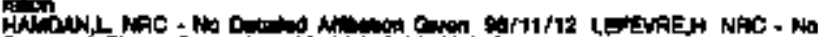

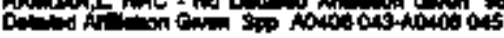

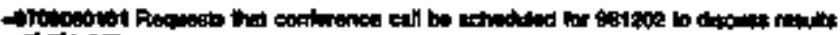

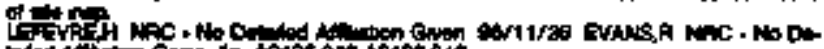

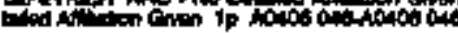

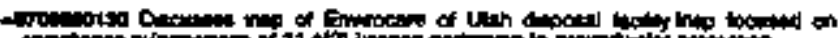

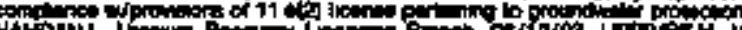

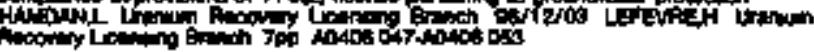

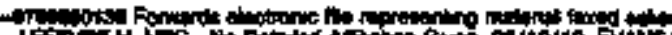

IE

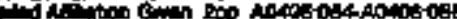

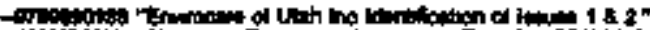

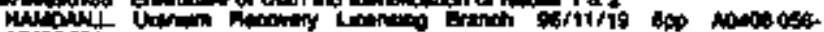
Antot od

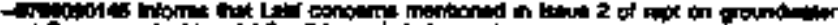

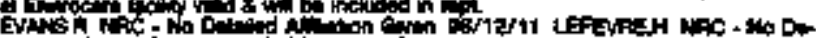

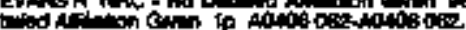

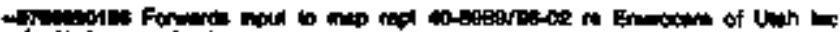

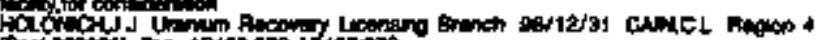

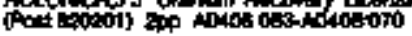

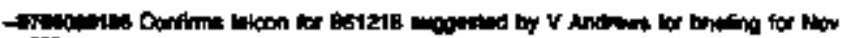

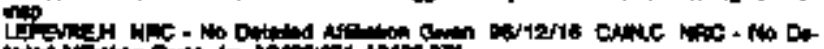

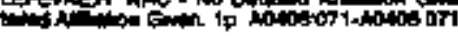

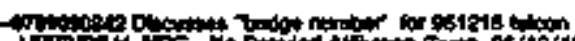

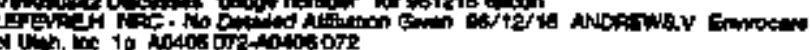

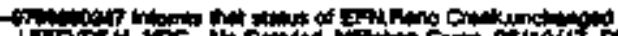
of

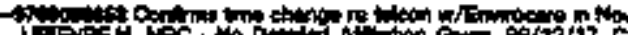

(1) Do

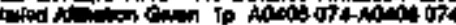

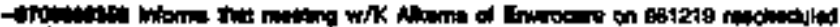

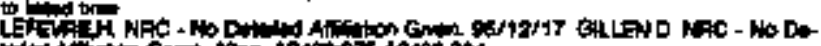

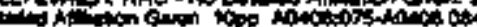

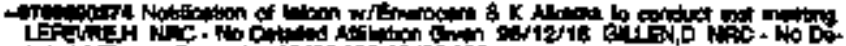

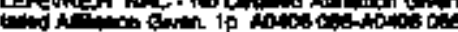

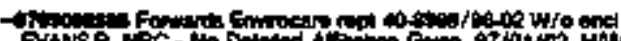

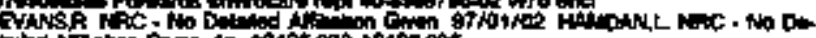

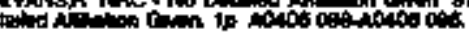

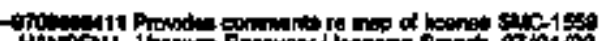

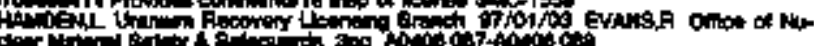

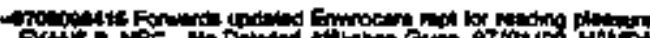

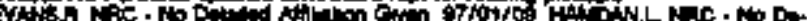

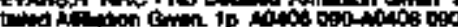

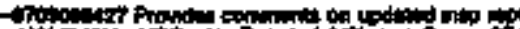

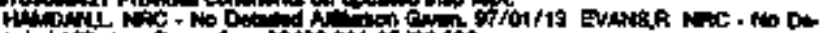

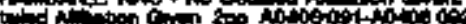

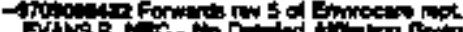
this

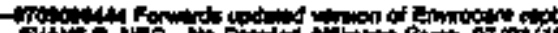

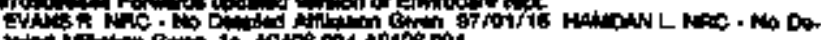

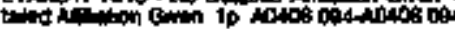

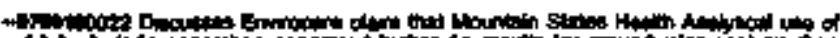

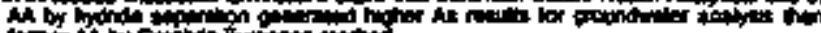

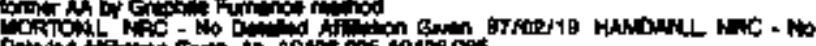

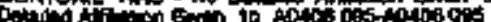

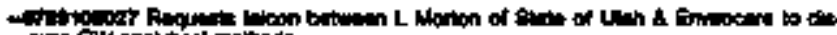

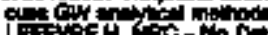

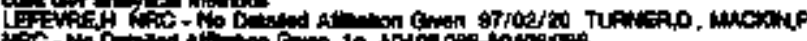

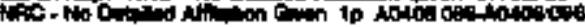

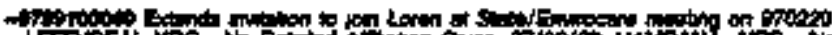

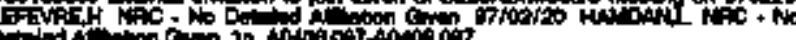

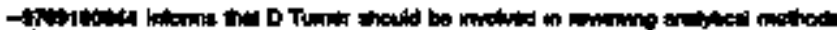

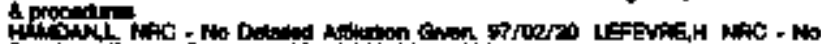

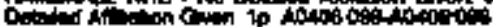

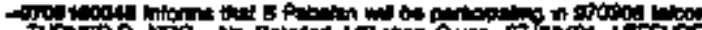

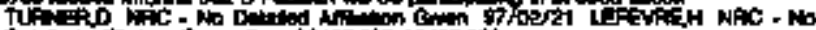

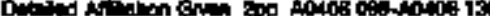

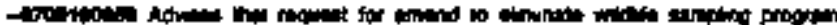

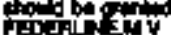

ic Nonto 10

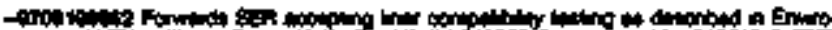

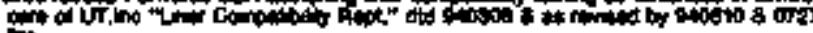

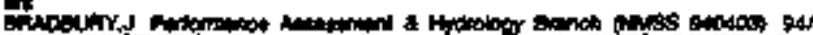

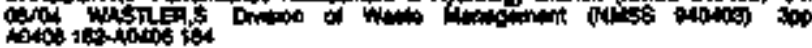

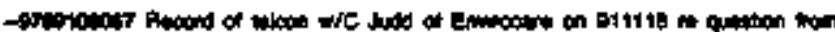

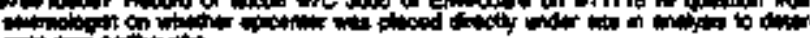

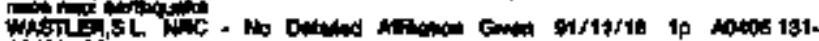
190.1900

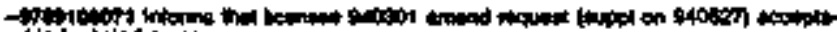
6.

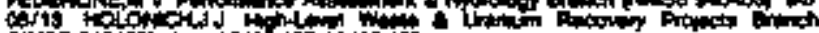

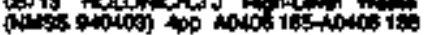

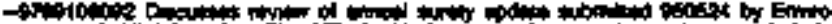

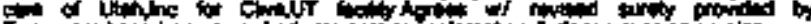

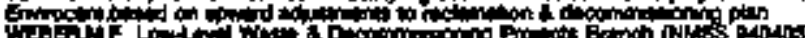

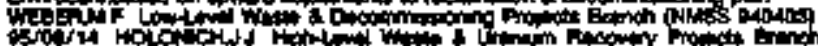

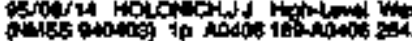

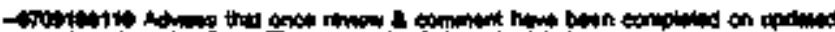

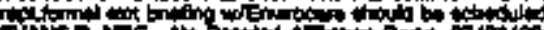

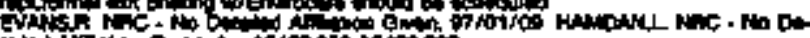

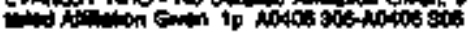

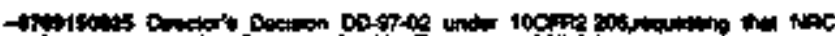

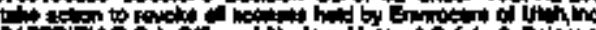

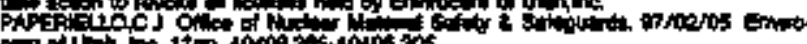

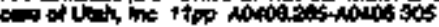

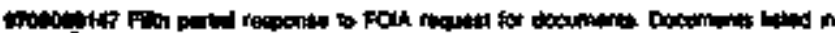

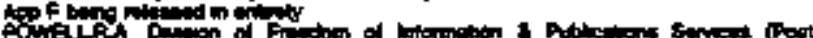

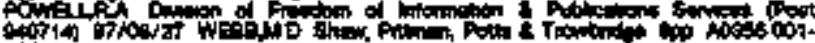
$\cos 10$

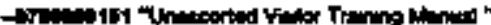

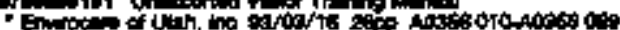

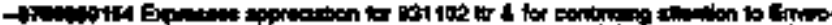

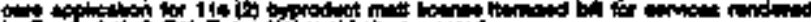

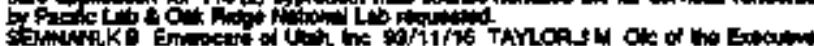

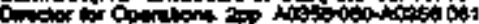

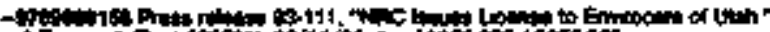

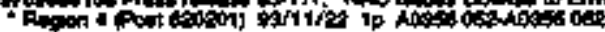

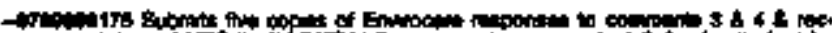

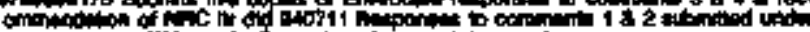

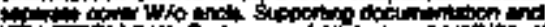

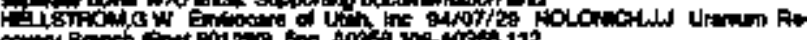

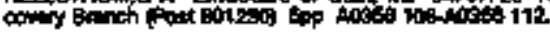

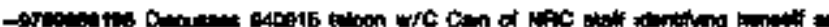

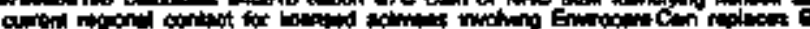

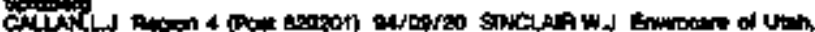
H.

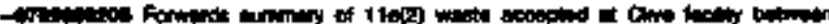

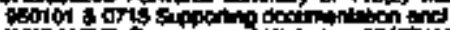

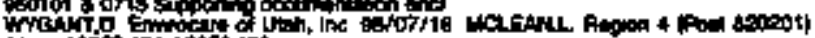

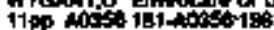

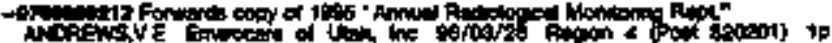

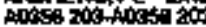

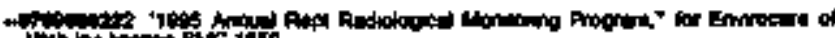

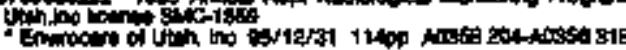

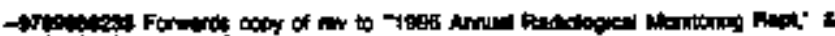

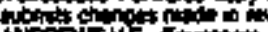

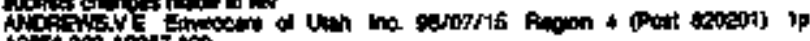

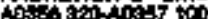




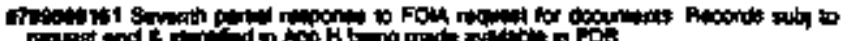

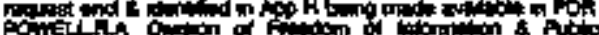

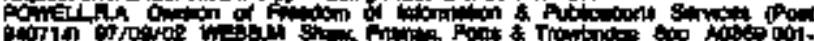

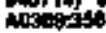

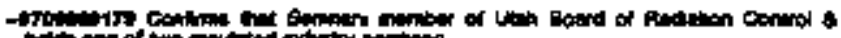

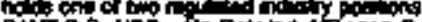

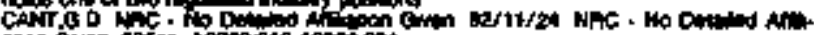

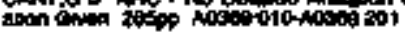

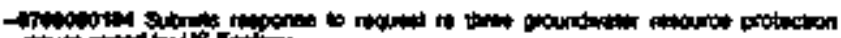

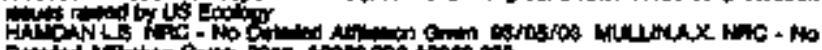

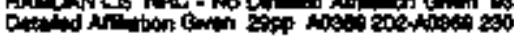

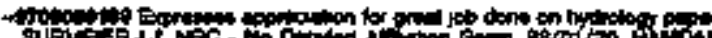

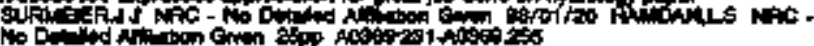

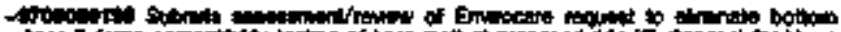

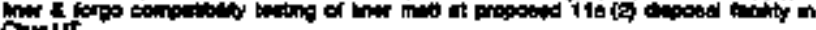
cintion

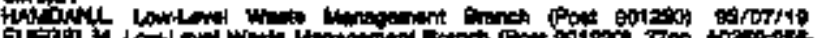

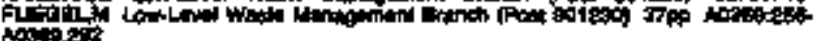

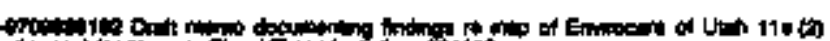

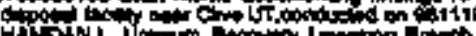

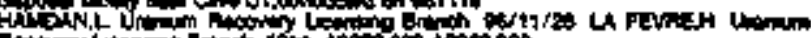

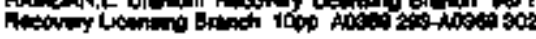

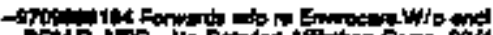

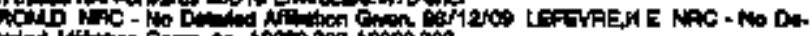
$03+\cos 000$

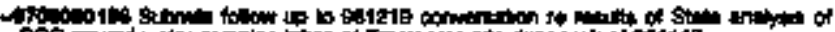

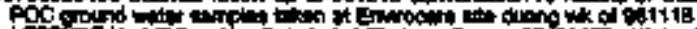

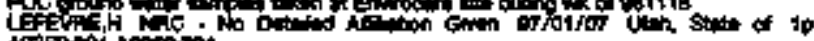

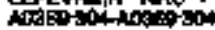

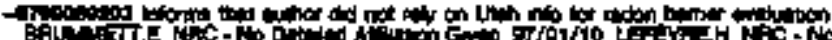

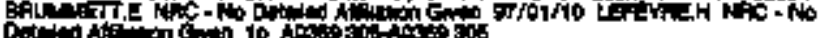

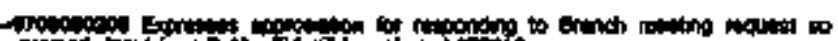

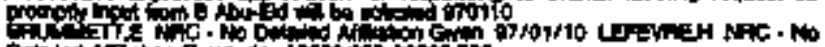

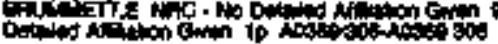

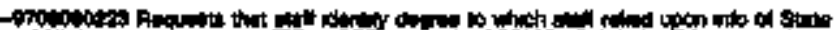

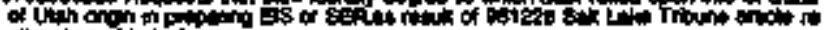

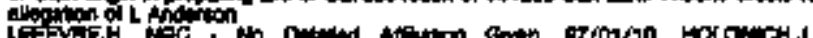

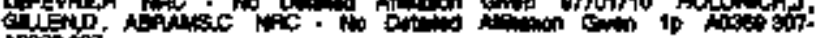
ADC

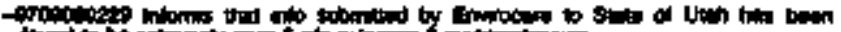

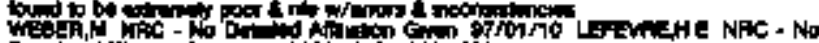

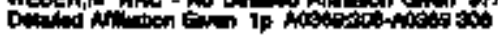

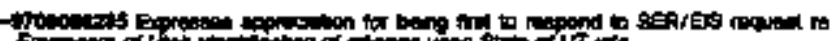

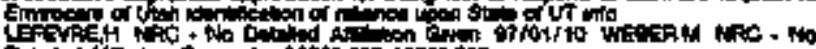

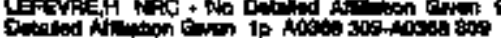

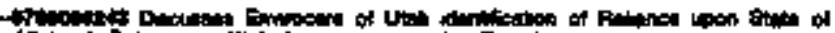

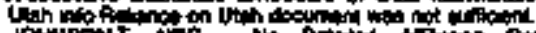

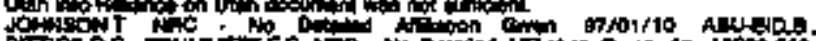

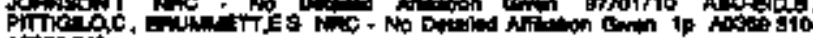

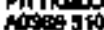

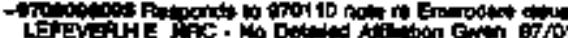

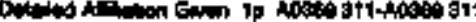

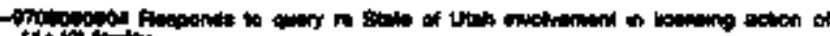
tiats the

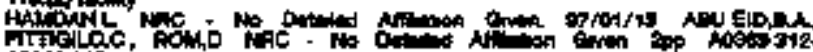
$\rightarrow 000$

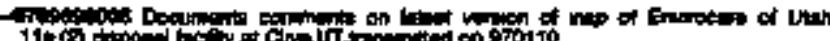

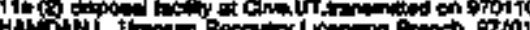

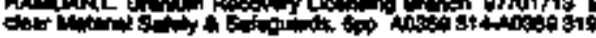

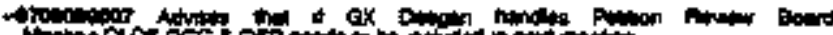

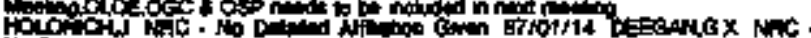

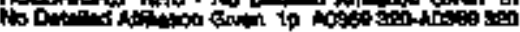

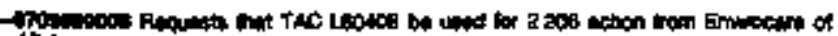

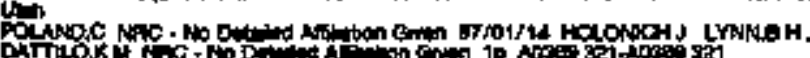

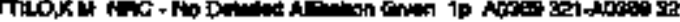

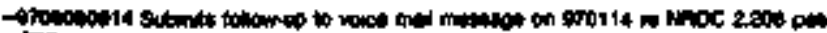

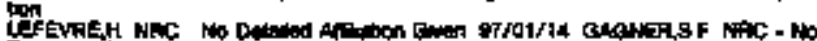

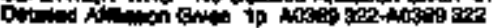

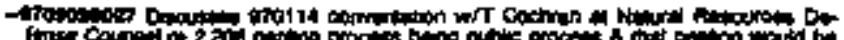

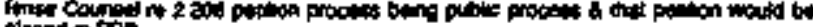

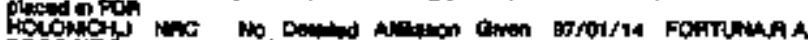

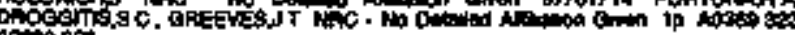

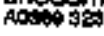

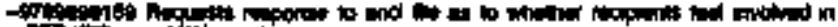
SuN or not ro not

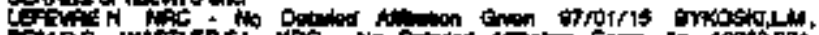

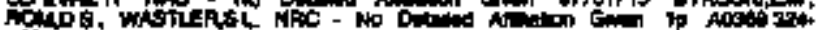
istom

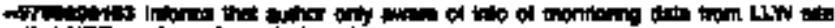

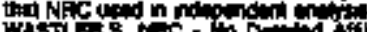

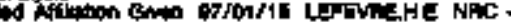

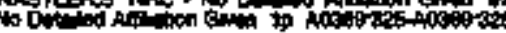

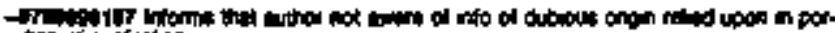

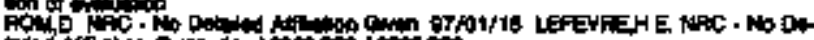

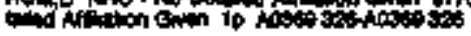

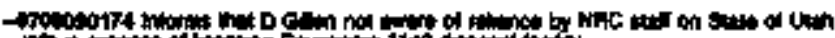

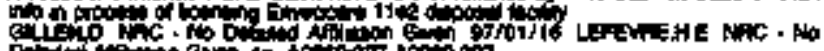
Dition

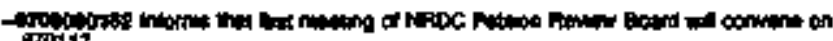
Wro: 17

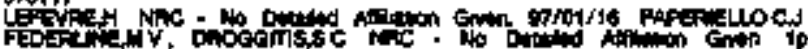
Hato

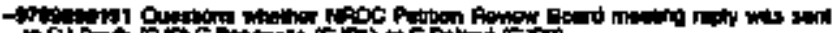

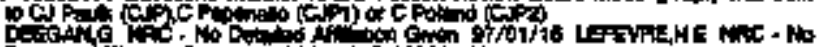

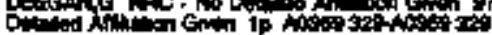

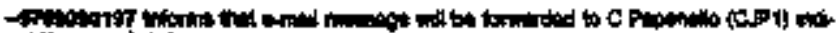
if

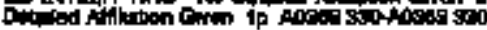

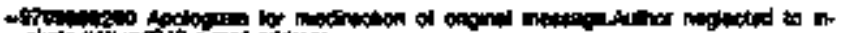

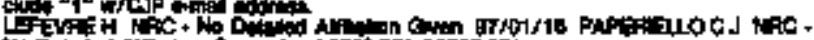

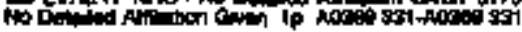

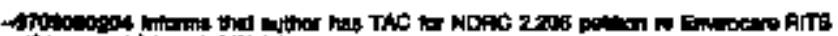

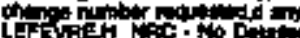

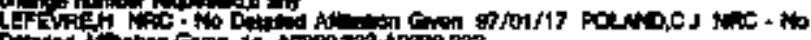

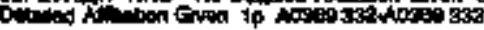

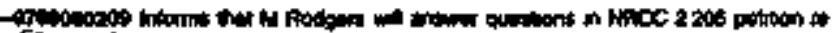

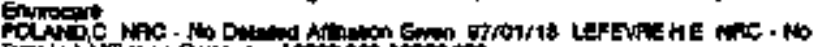

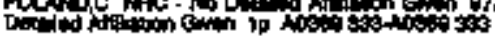

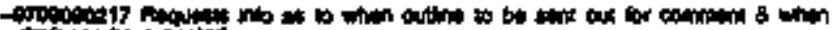

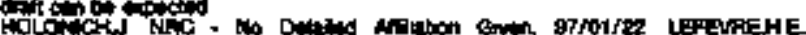

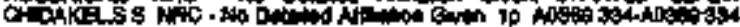

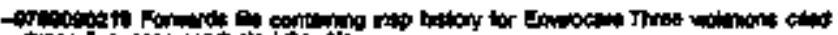

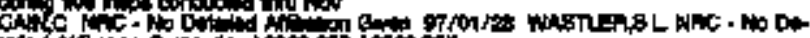

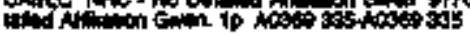

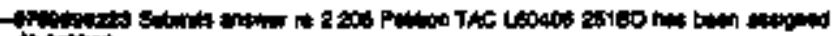

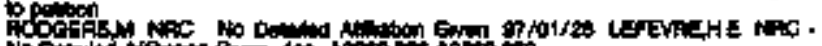

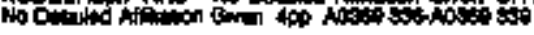

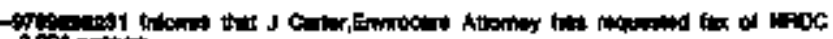
Le

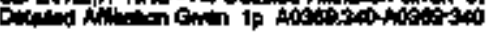

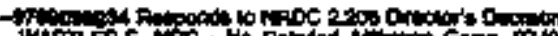

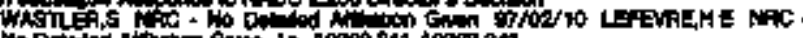

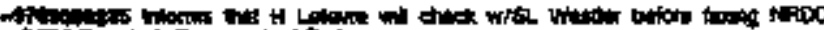

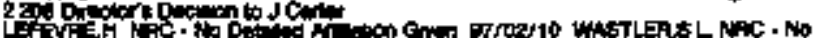

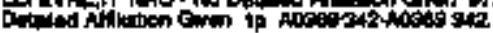

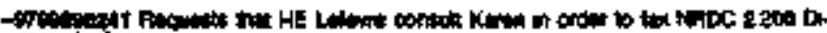

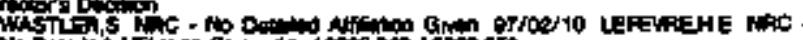
Tho of

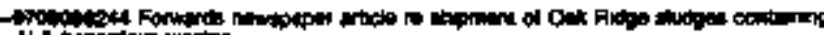
Y

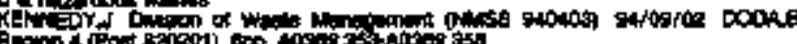
Amor 4 (Pod

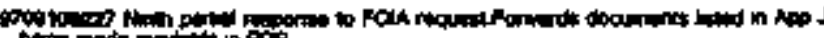

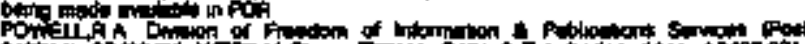

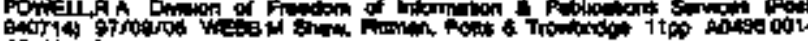
constis

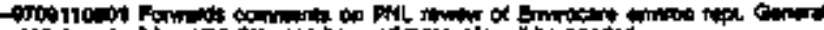

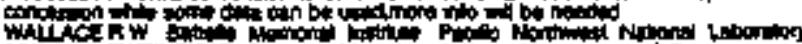

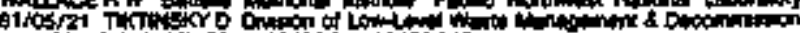

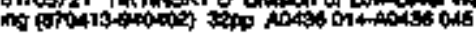

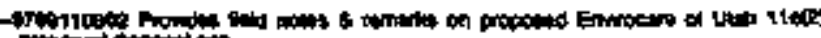

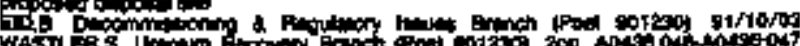

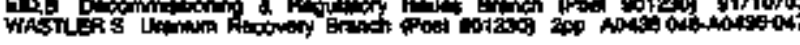




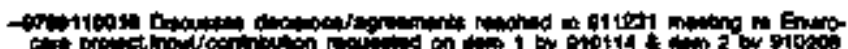

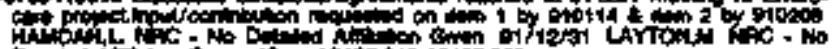

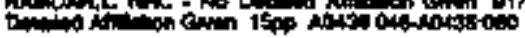

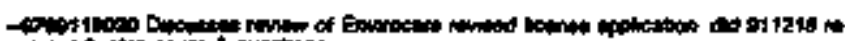

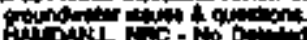

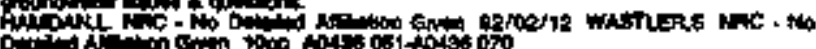

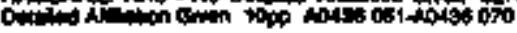

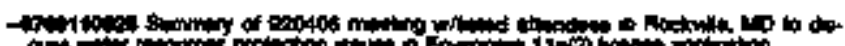

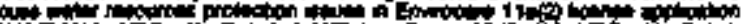

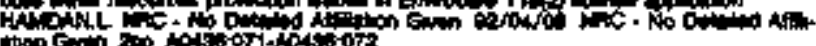
$\$ 07 \%$

- Py

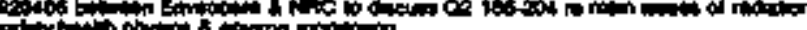

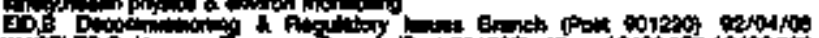
WA

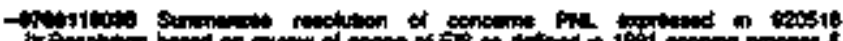

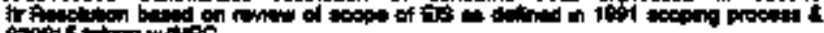
onsts

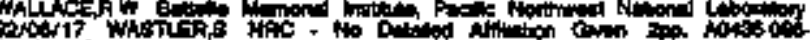
Apusach

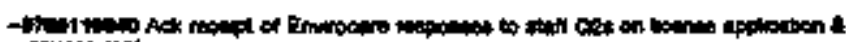

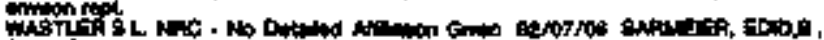

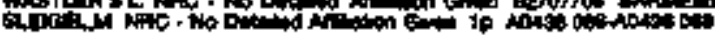

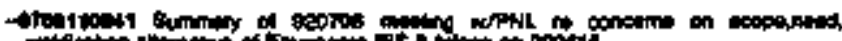

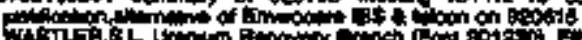

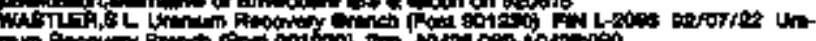

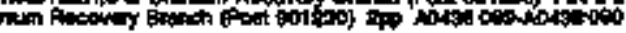

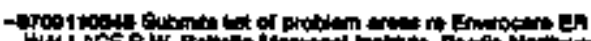

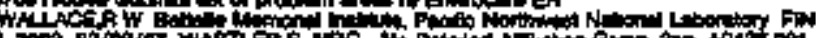

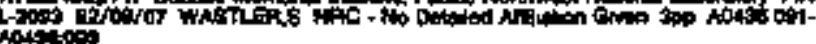

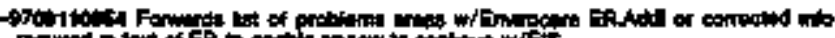

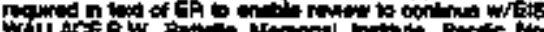

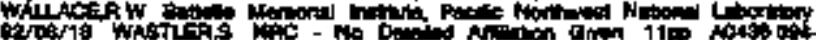
10.0.

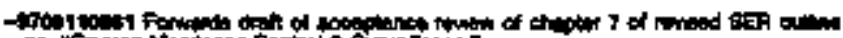

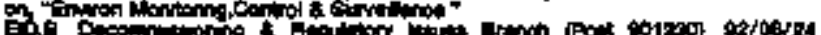
Whotu

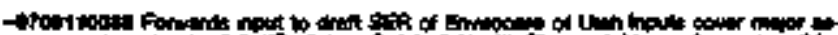

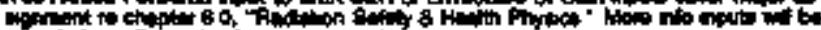

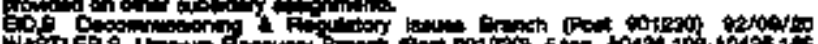
WAst a

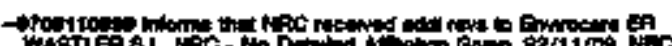

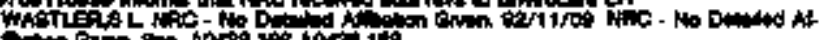

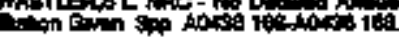

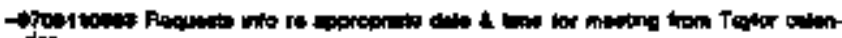

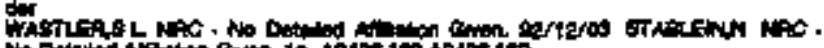

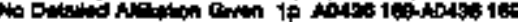

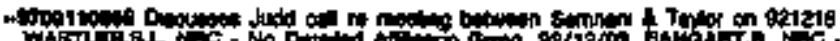

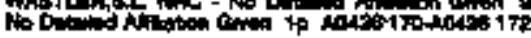

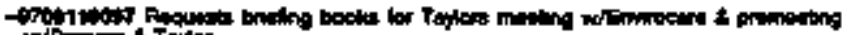

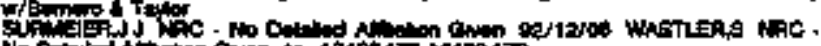

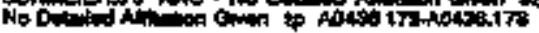

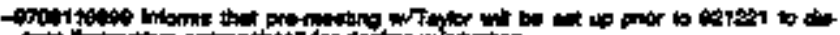

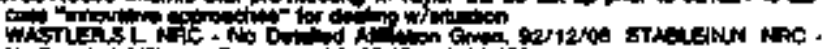

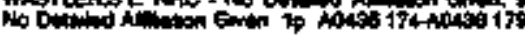

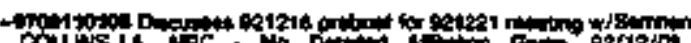

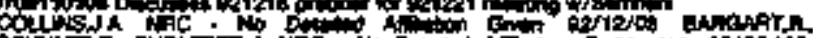

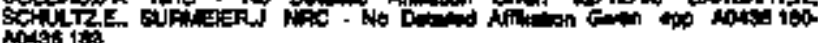

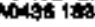

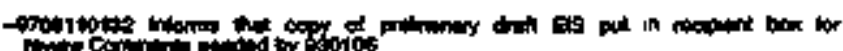

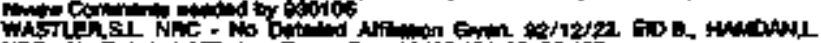

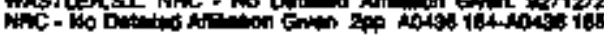

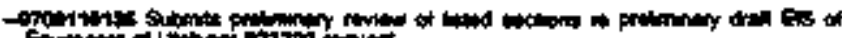

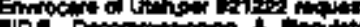

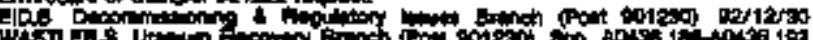

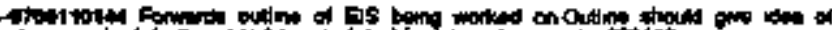

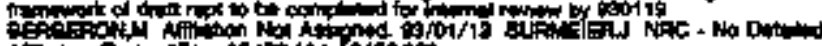

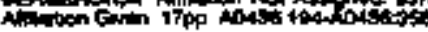

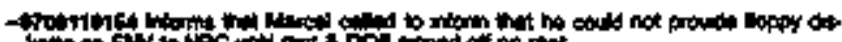

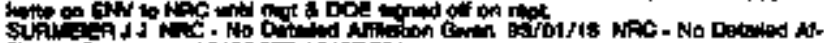

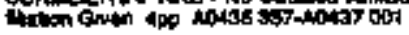

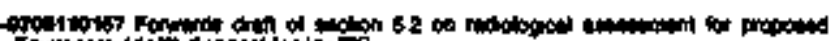

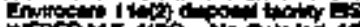

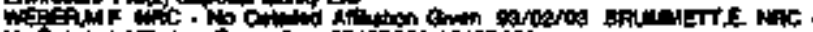

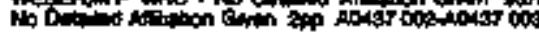

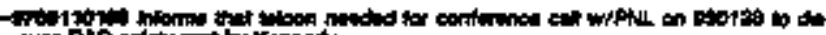

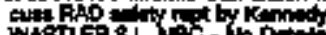

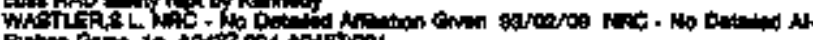

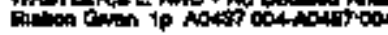

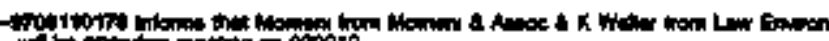

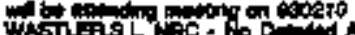

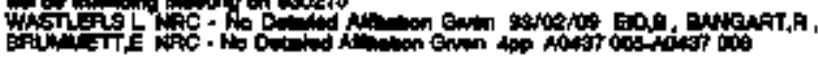

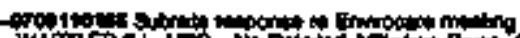

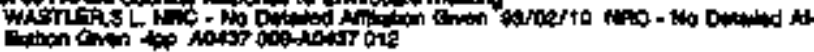

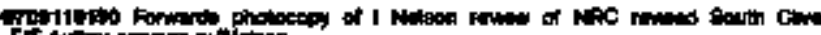

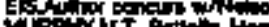

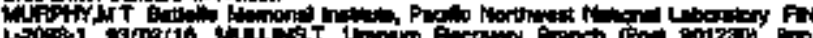

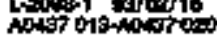

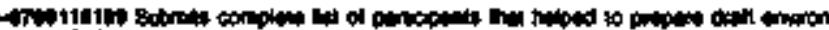

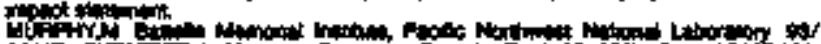

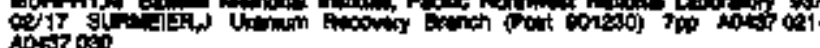

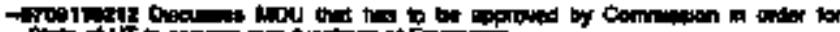

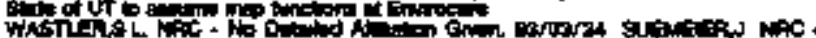

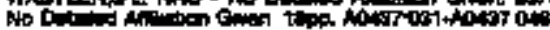

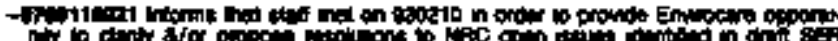

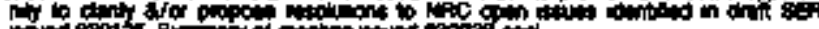

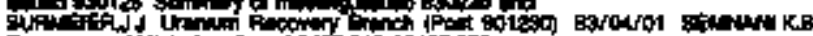

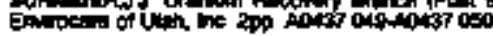

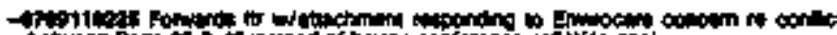

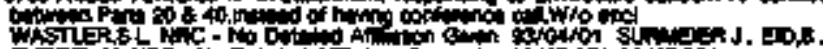
Fis

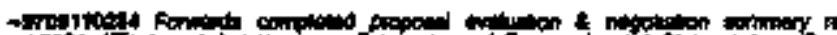
Look, "Thenc: A

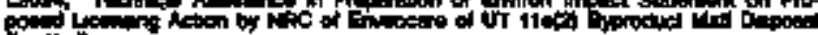
Par.t.

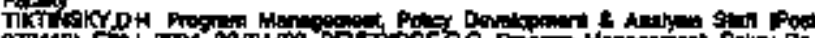

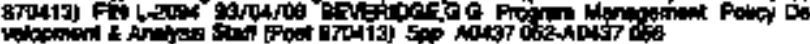

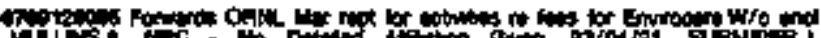

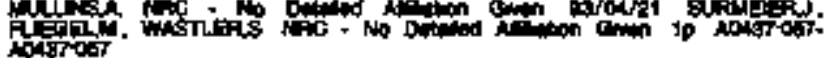

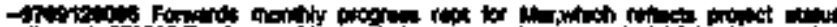

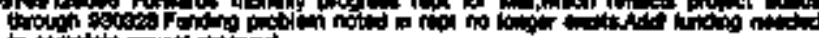

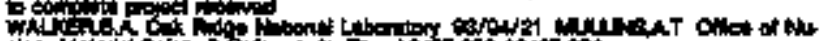

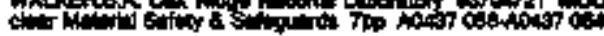

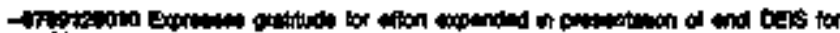

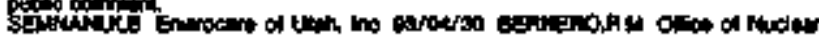

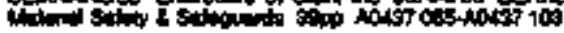

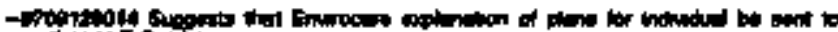

d

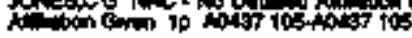

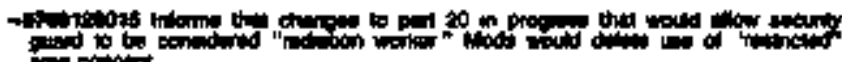
莦

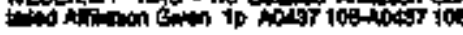

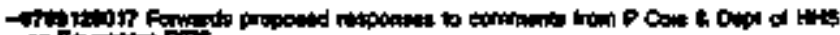
on Ef

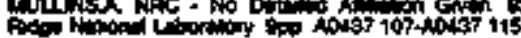

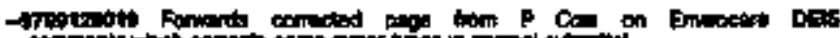

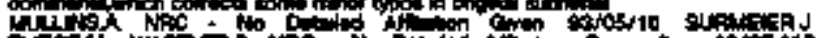

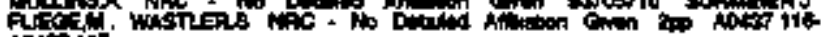
Natiti?

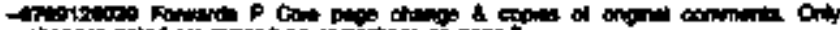

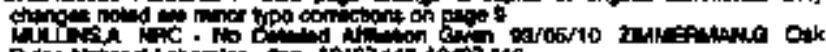

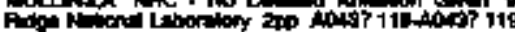

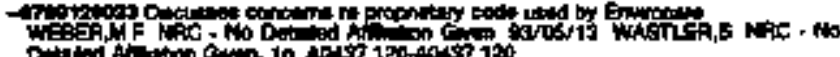

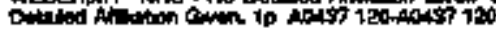

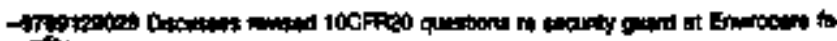

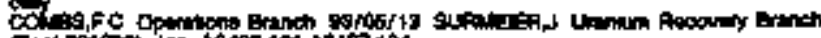

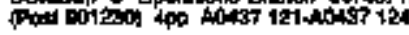




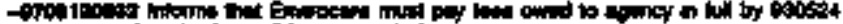

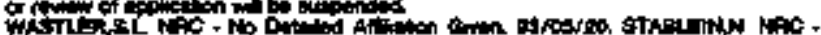

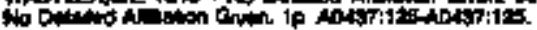

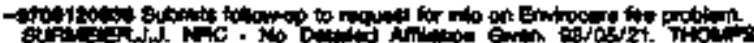
A

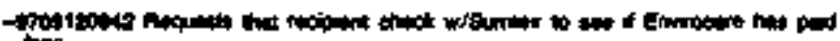

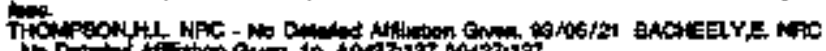

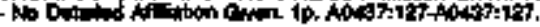

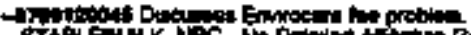

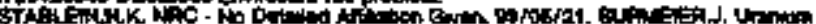

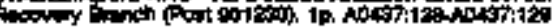

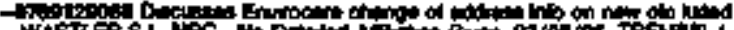

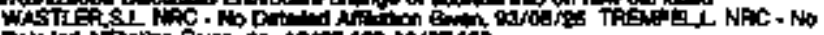

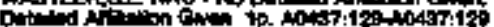

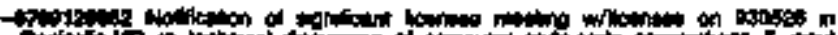

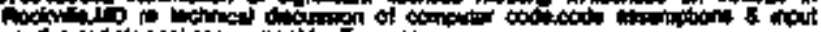
8tis

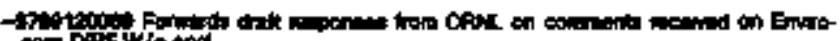

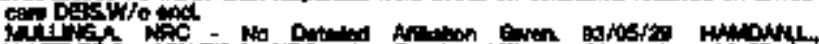

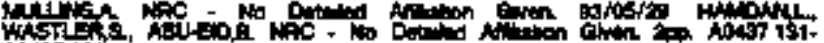
047132

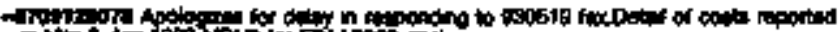

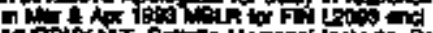

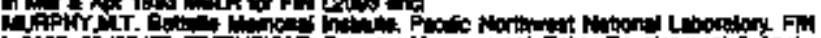

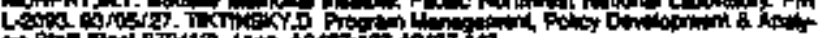

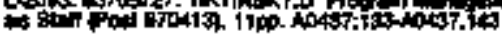

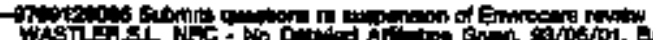

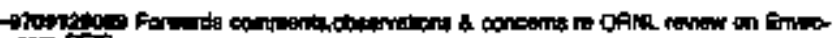
cotistion

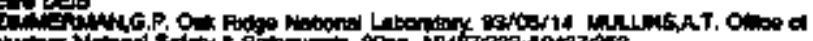

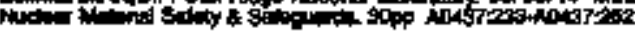

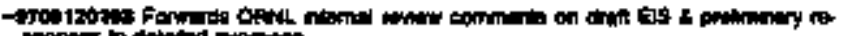

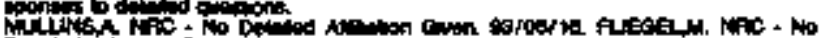

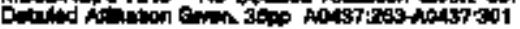

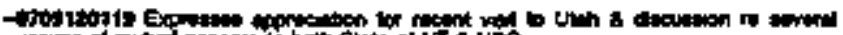

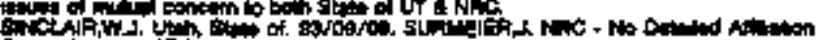

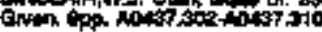

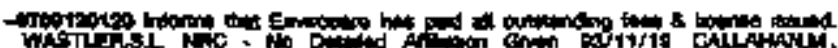

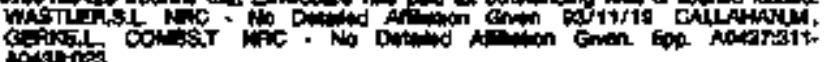
Aatate

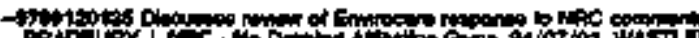

管

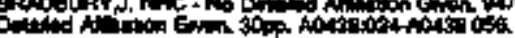

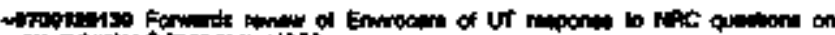

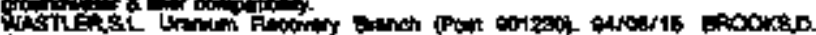

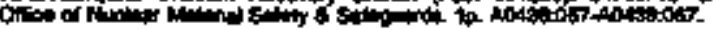

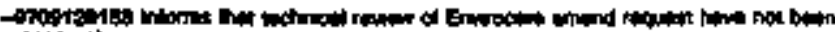

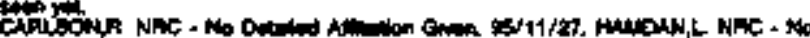

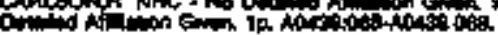

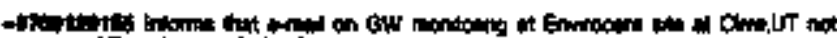

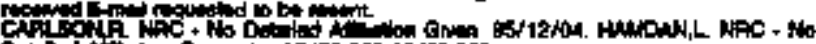

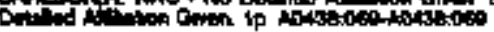

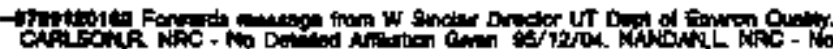

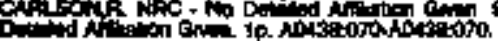

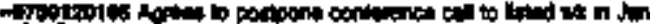

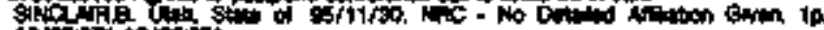

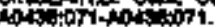

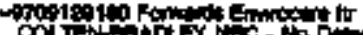

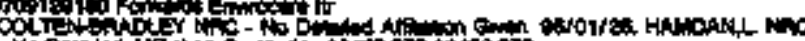

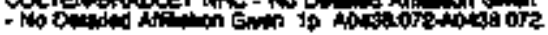

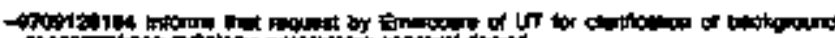

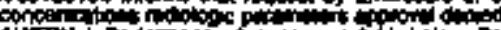

Allow

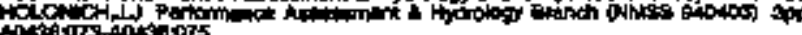

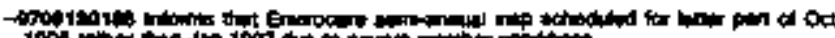

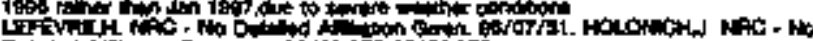

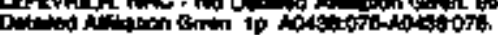

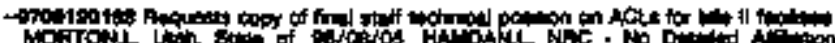

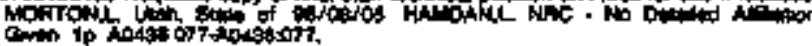

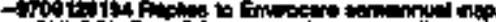

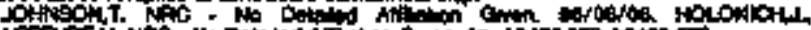

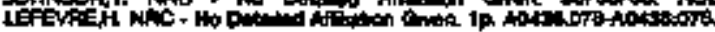

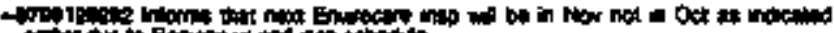

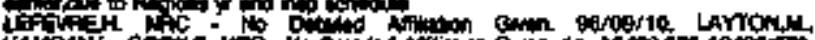

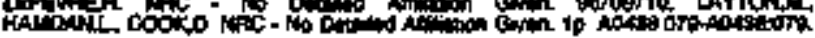

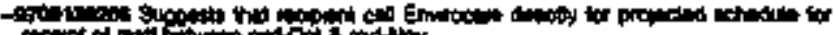

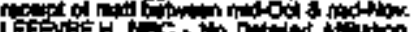

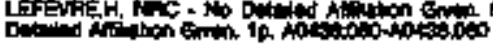

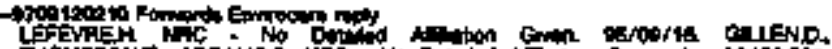

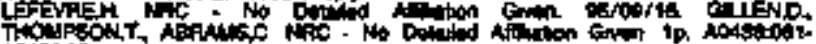
Aotstopic.

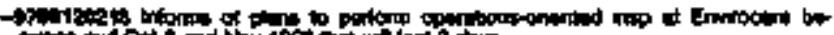

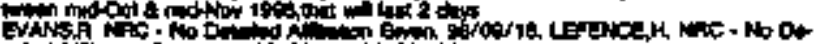

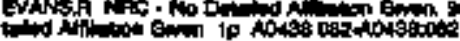

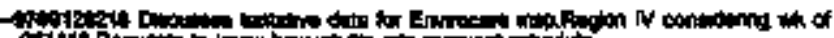

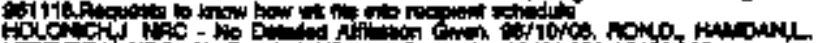
IE

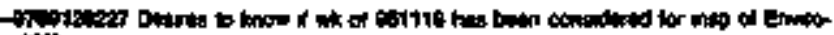

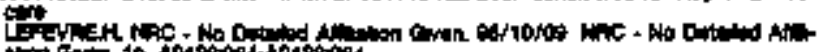

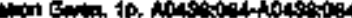

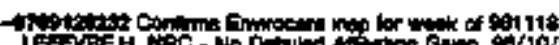

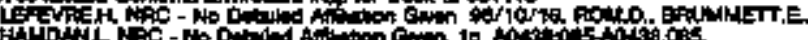

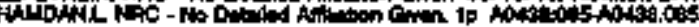

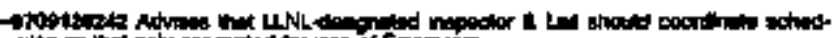

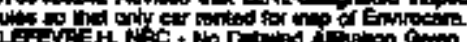

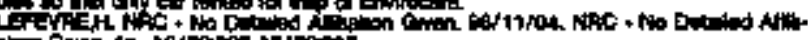

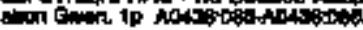

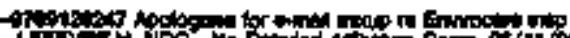

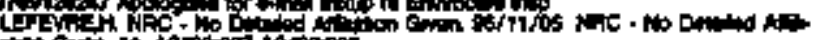

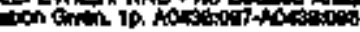

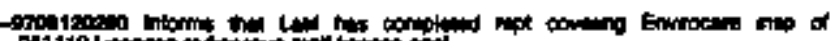

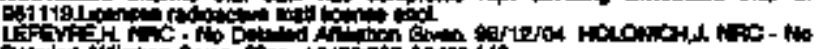

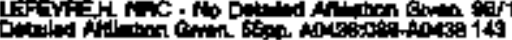

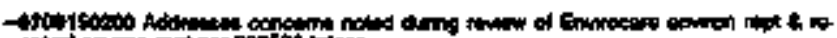

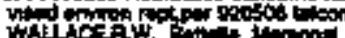

Wh

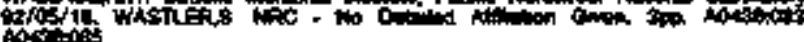

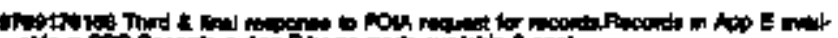

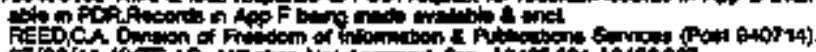
if

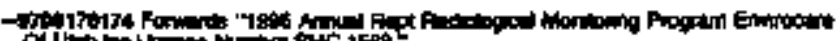
of U.

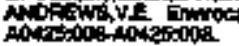

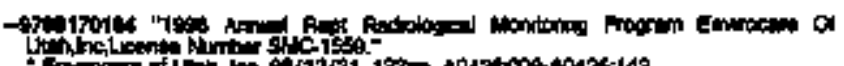

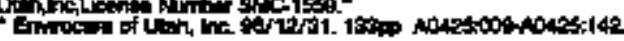

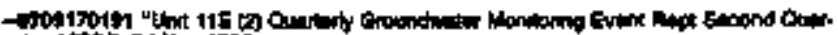
180 ad twow $100 \%$

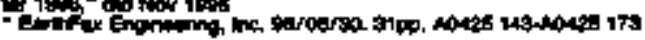

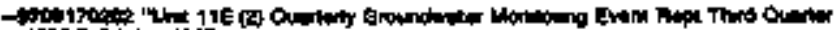

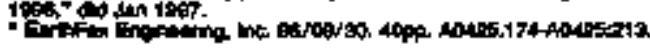

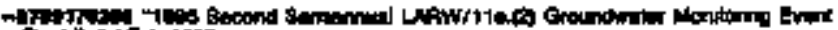

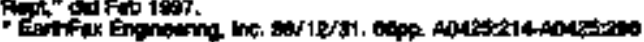

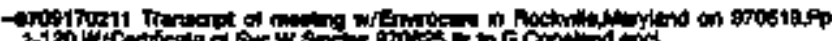

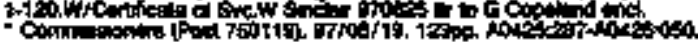

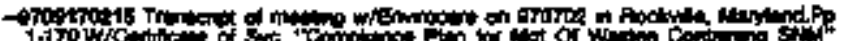
ond

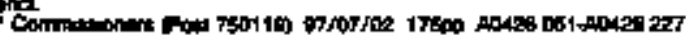

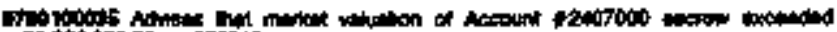

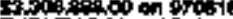

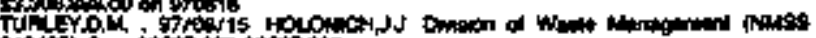

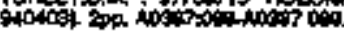

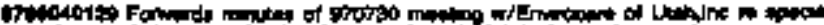

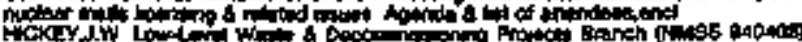
ol rowal 


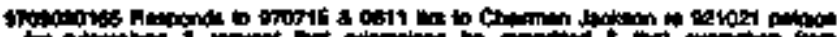

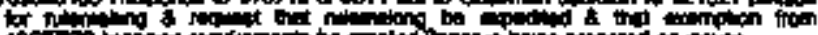

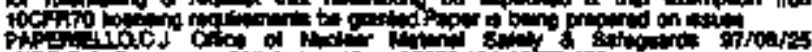

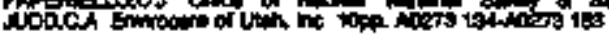

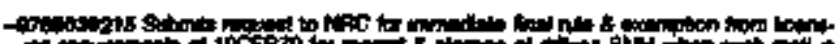

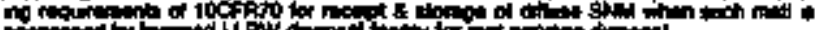

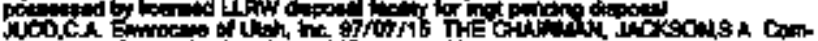

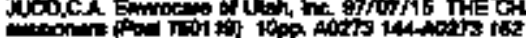

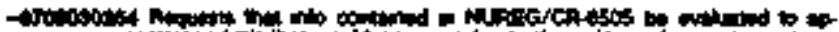

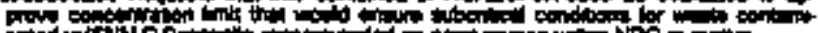

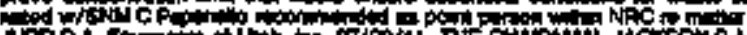

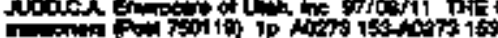

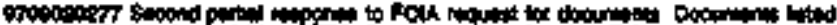

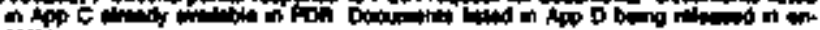
Tet

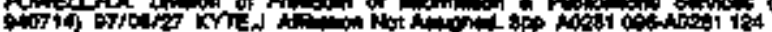

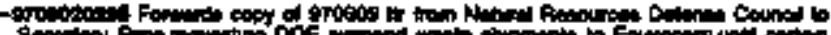

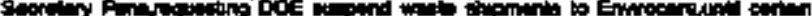

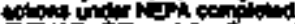

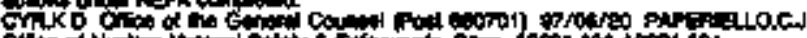

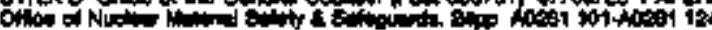

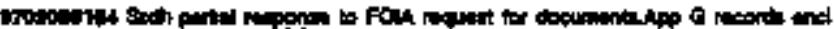

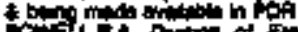

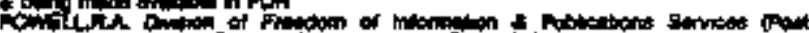

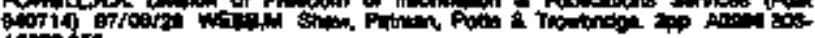
atos ix

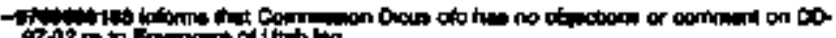

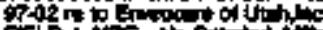

Filj J

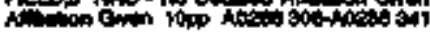

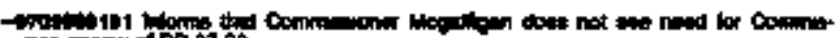
mon

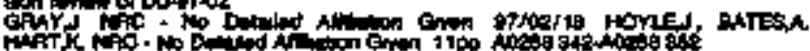

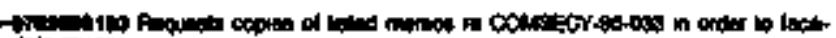

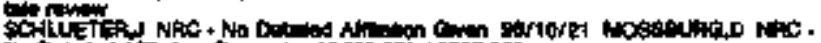

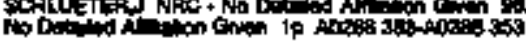

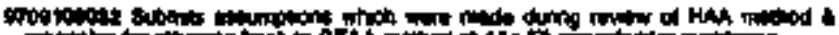

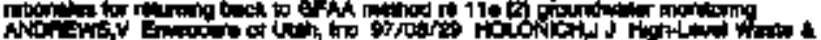

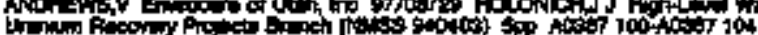

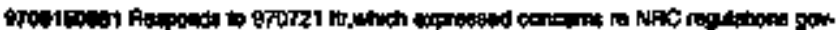

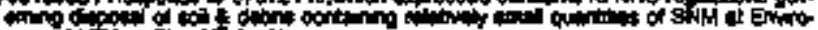

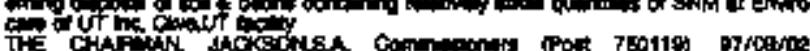

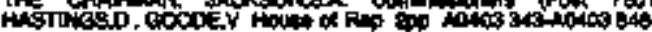

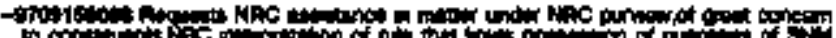

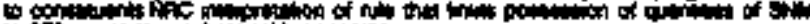

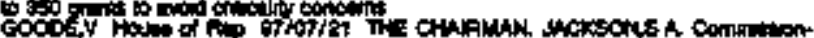

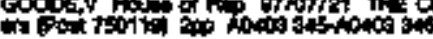

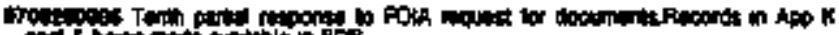

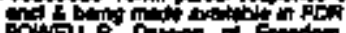

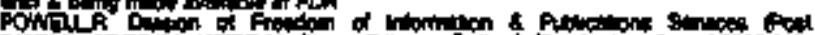
040714

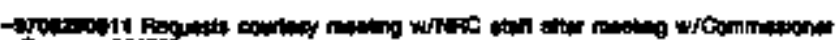

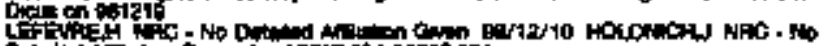

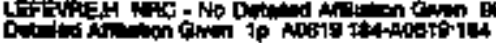

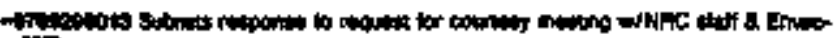

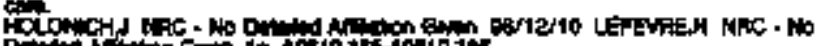

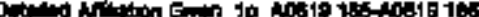

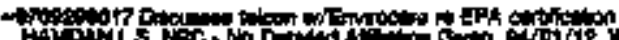

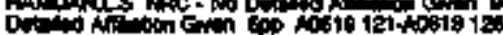

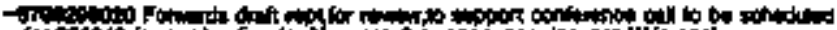

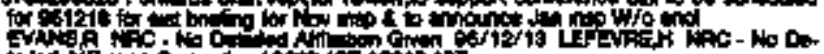
Find A

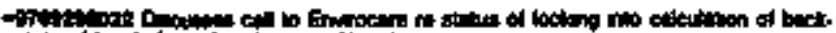

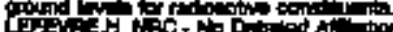

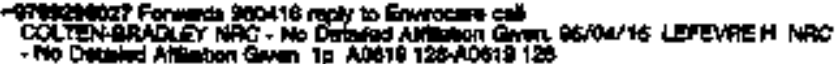

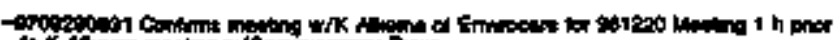

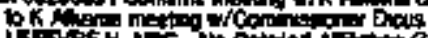

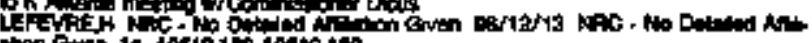

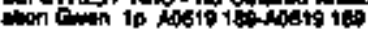

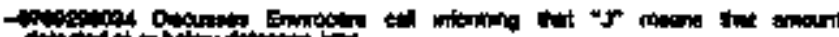

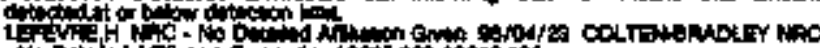

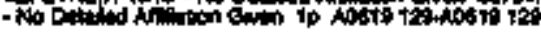

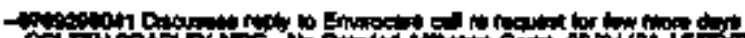

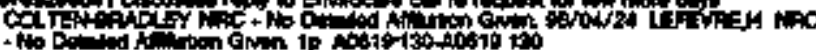

- if

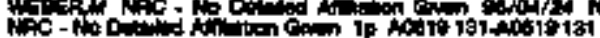

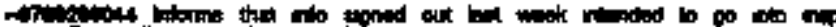

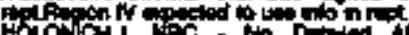

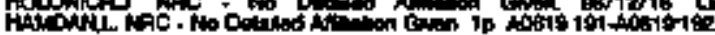

- How op

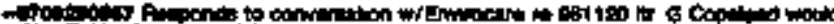

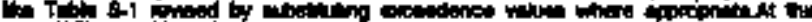

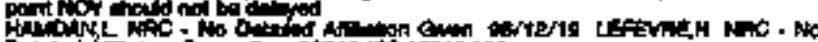

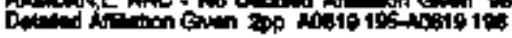

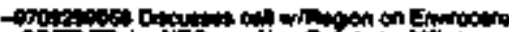

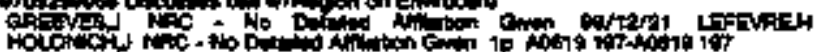

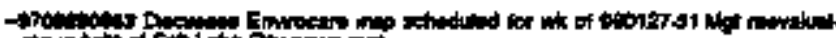

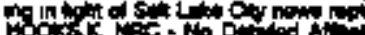

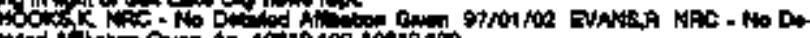

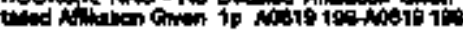

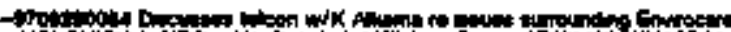

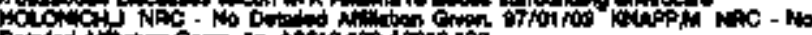

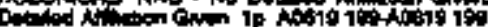

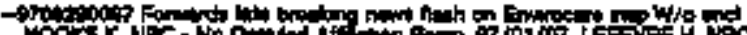

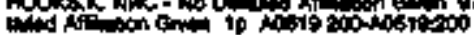

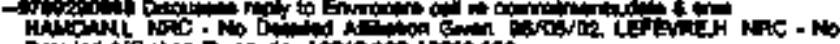

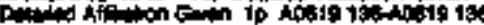

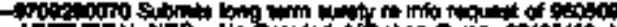

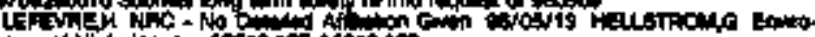

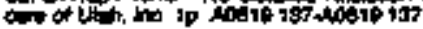

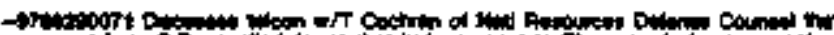

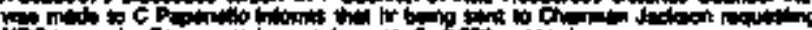

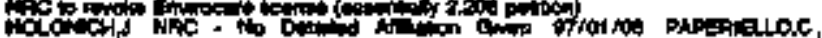

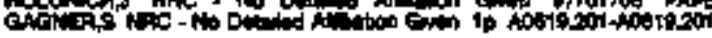

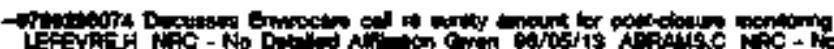

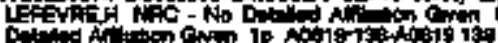

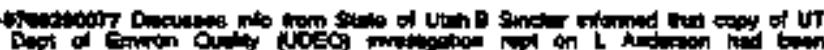

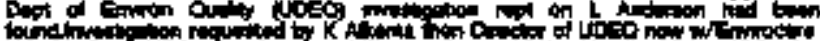

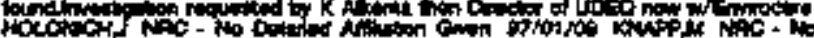

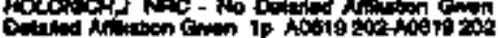

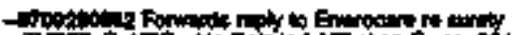

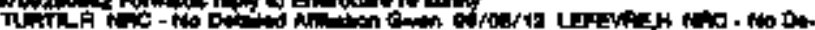

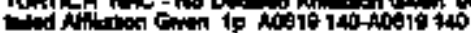

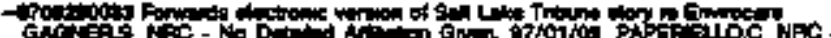
Go Dits

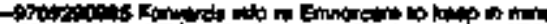
nch

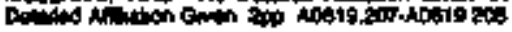

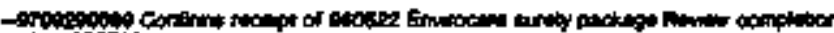

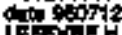

LCF

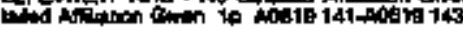

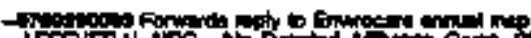

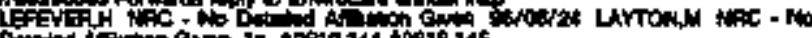

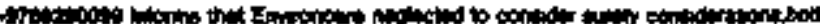

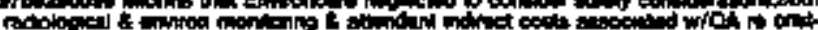

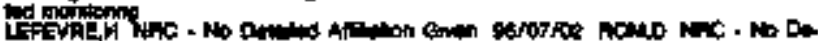

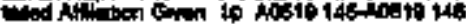

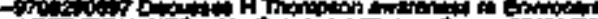

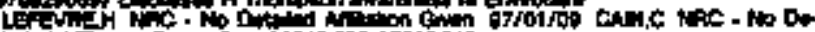

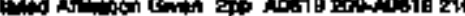

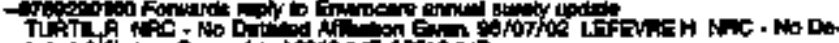

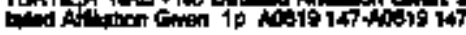

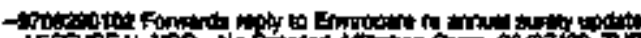

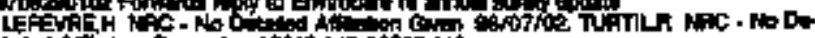

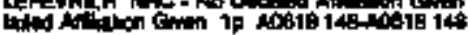




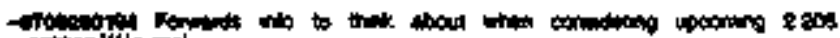

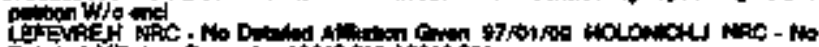

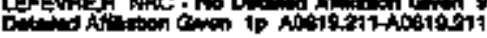

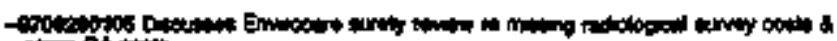

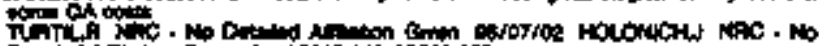

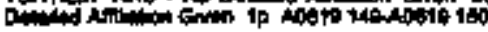

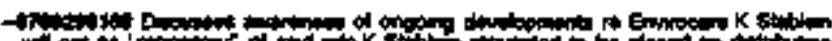

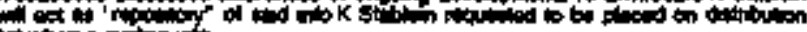

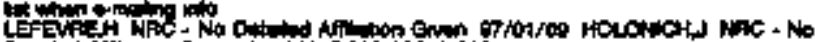

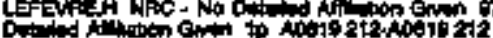

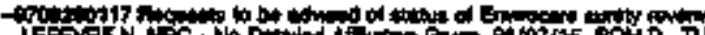

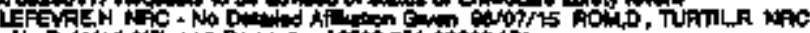

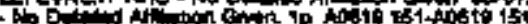

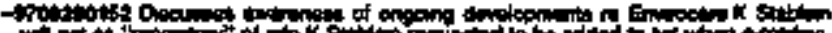

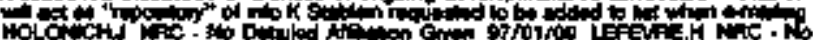

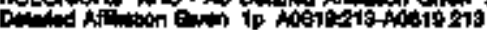

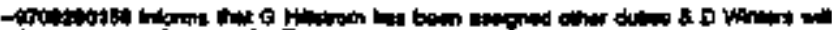

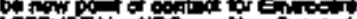

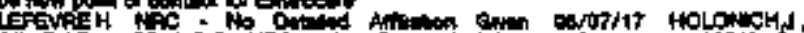

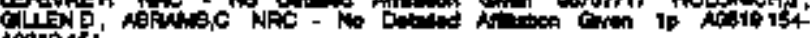
40019164

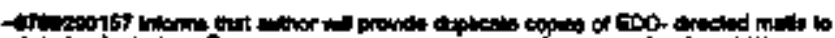
if

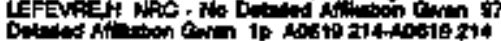

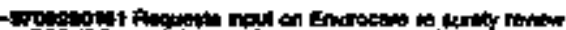
I

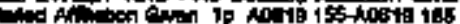

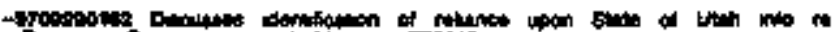

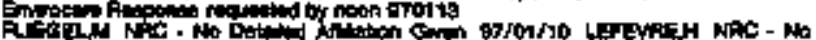

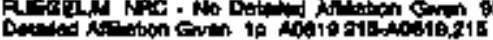

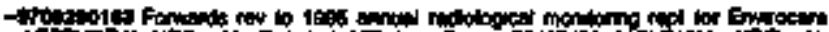

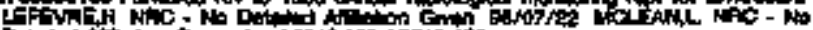

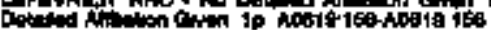

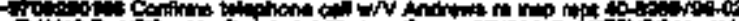

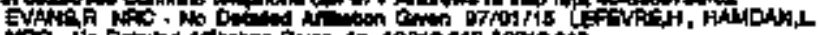

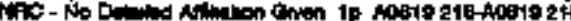

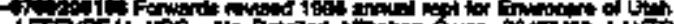

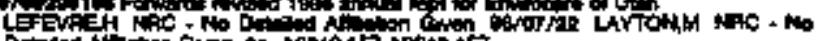

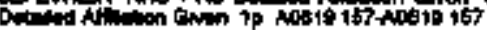

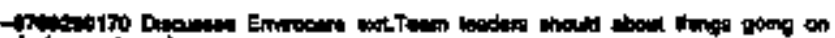

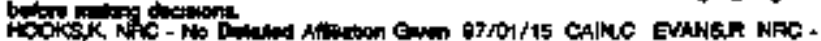

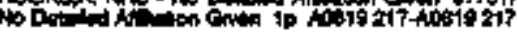

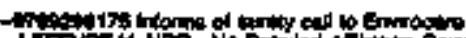

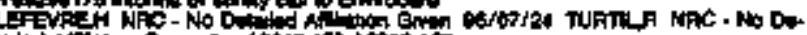

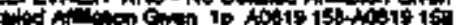

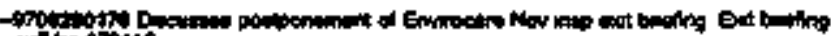
Tis

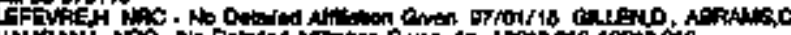

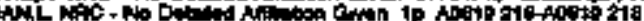

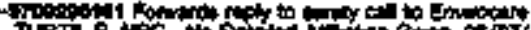

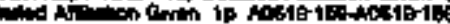

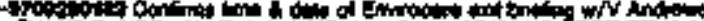

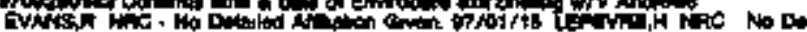

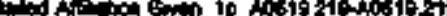

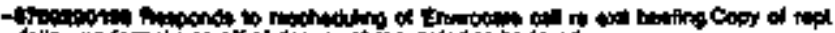

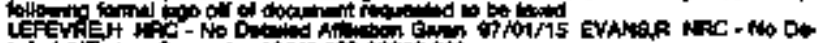

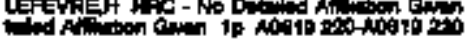

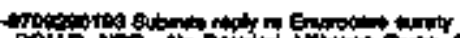

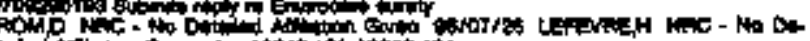

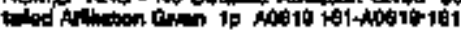

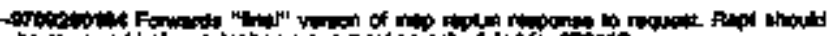

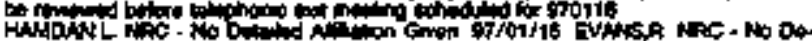

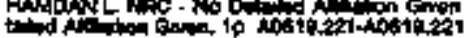

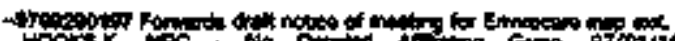

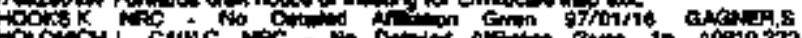
iosioges

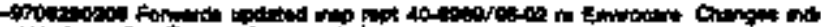

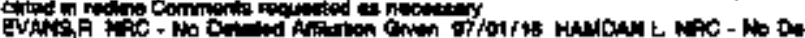

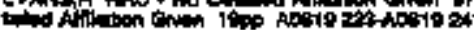

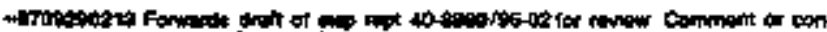

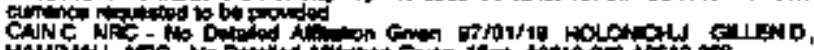

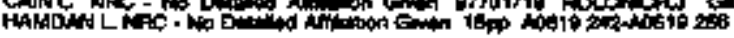

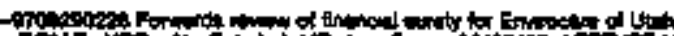

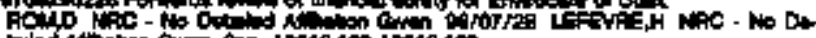

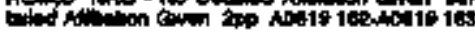

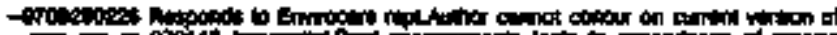

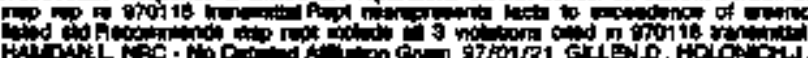

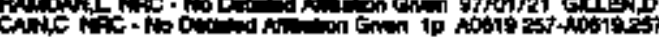

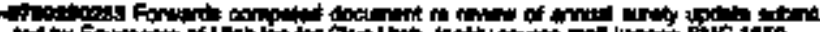

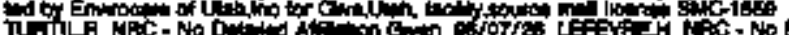

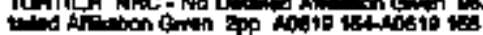

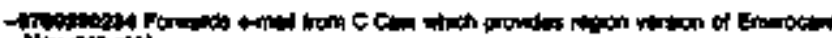

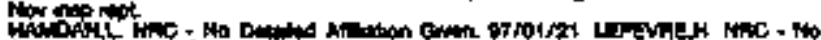

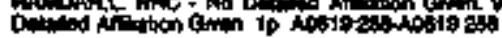

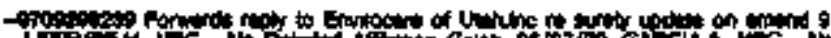

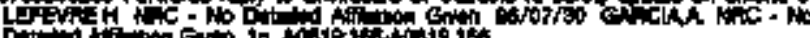

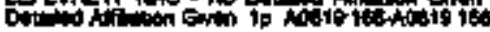

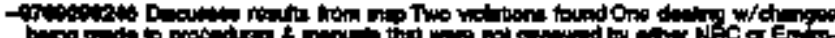

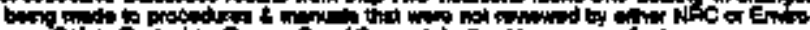

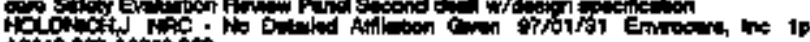

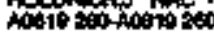

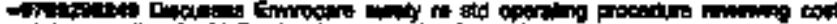
An

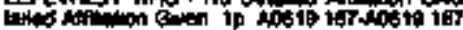

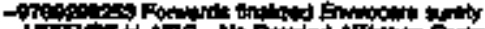

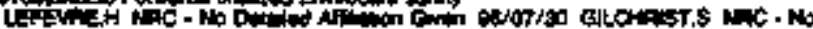

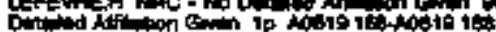

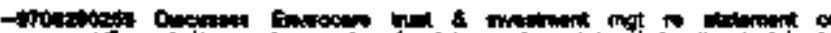

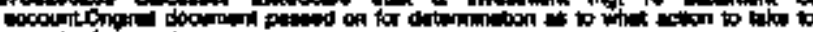

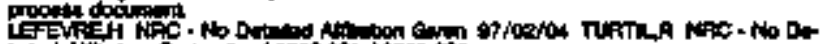

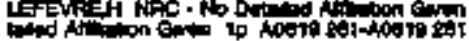

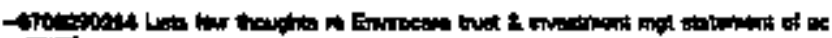

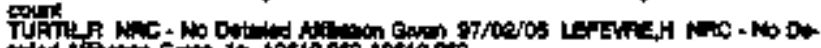
Hid Aifing

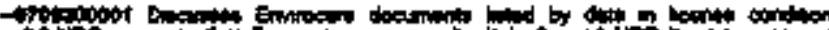

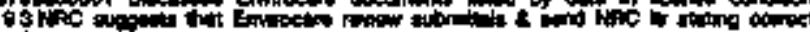

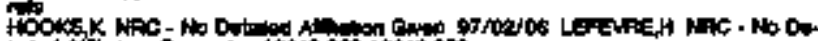

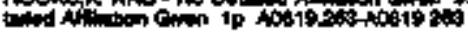

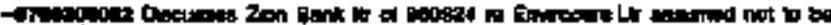

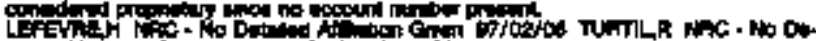

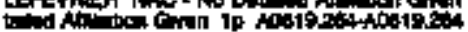

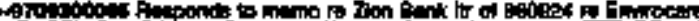

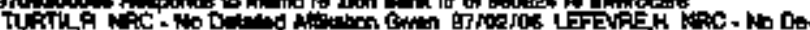

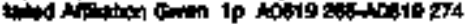

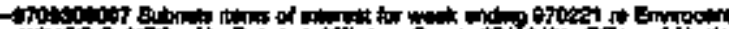

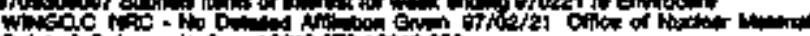

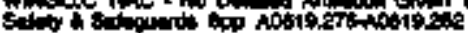

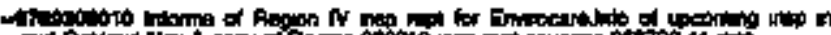

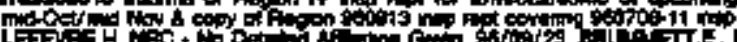

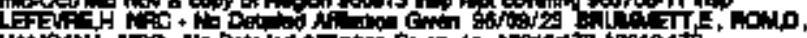

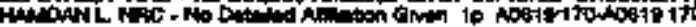

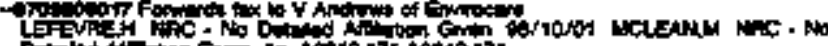

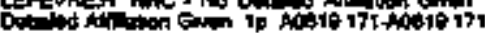

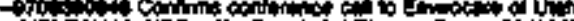

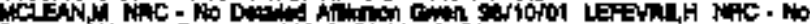

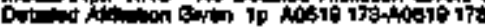

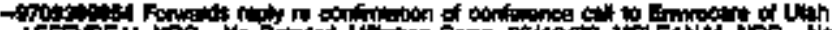
If

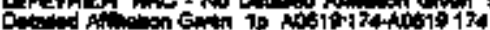

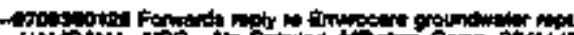

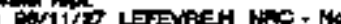

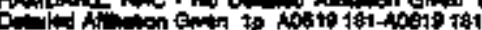

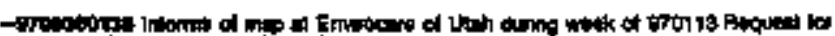

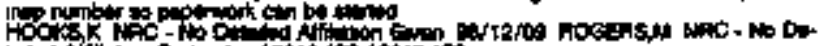

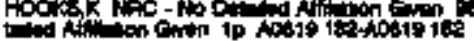

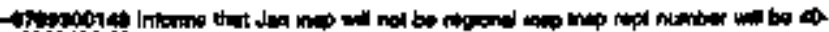

Hooks ith 


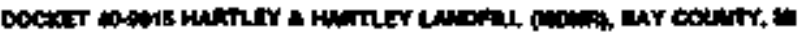

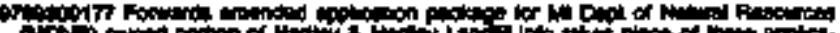

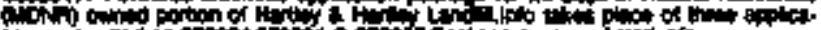

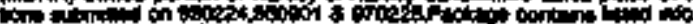

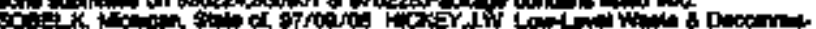

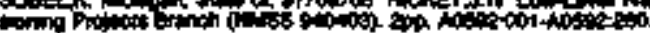

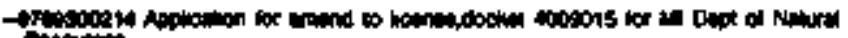

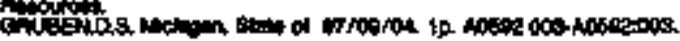

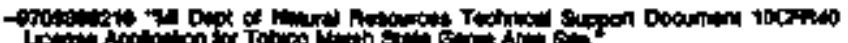

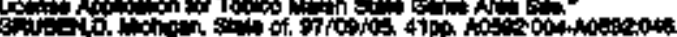

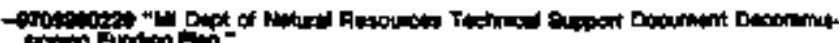

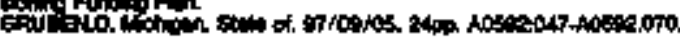

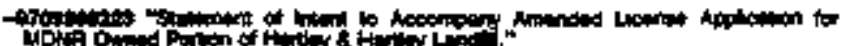

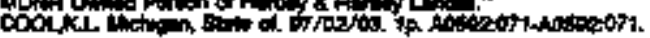

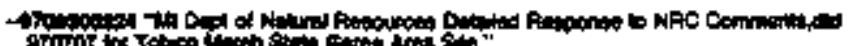

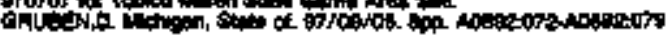

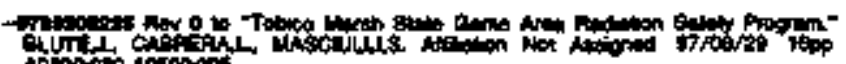

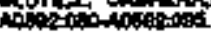

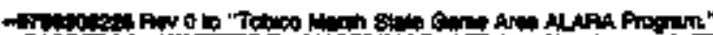

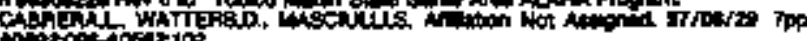

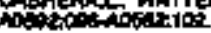

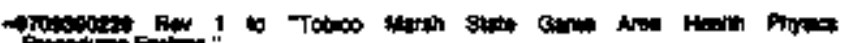

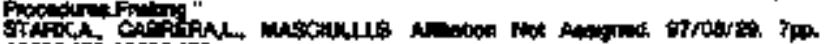

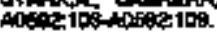

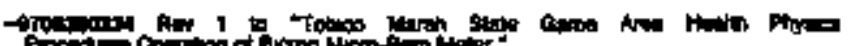

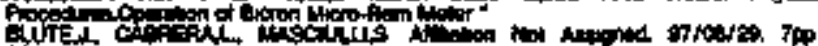

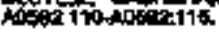

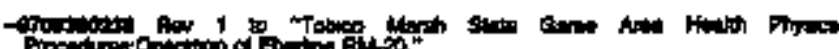

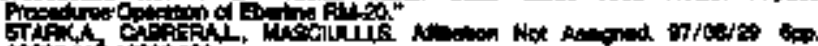

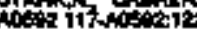

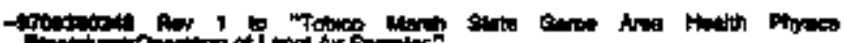

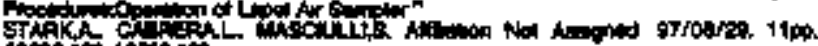

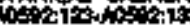

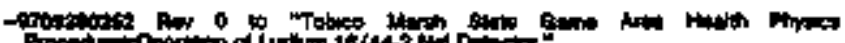

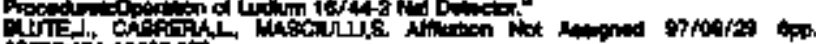

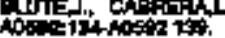

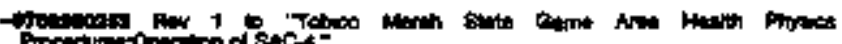

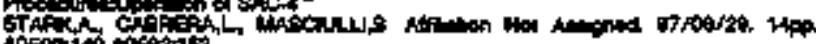

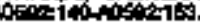

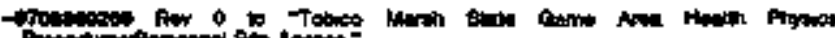

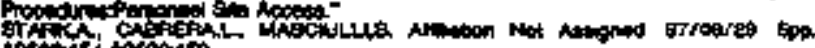

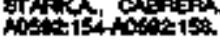

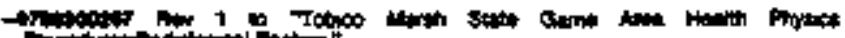

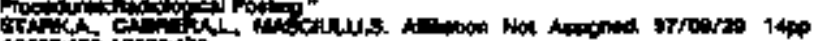

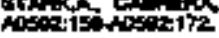

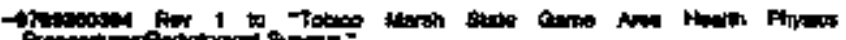

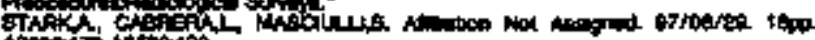

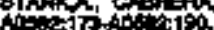

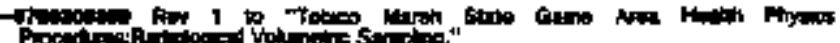

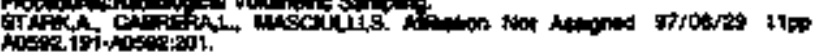

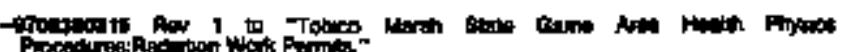

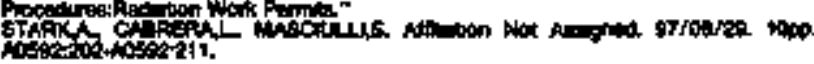

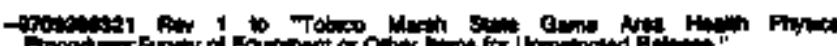
Sipod Sin

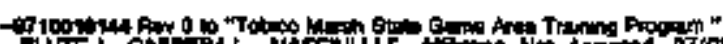
A

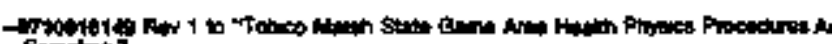

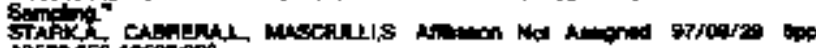

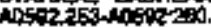

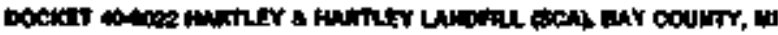

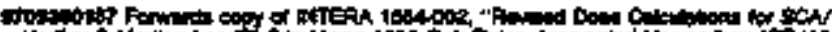

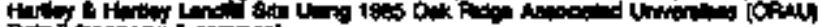

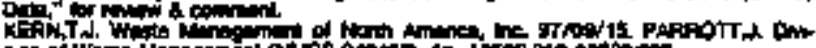

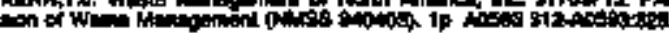

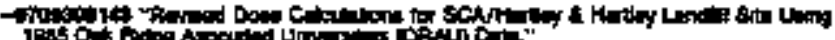

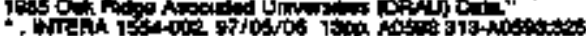

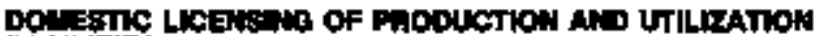 \\ Ficutitis}

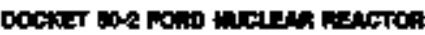

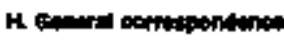

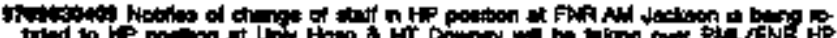

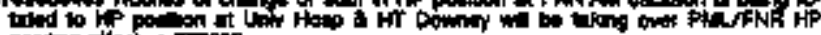

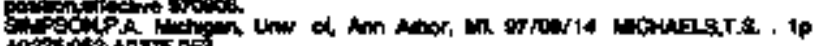

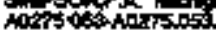

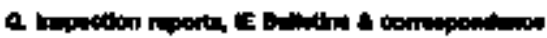

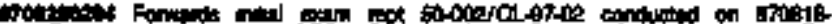

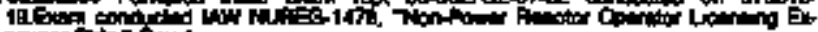

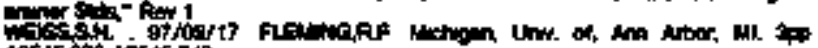

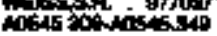

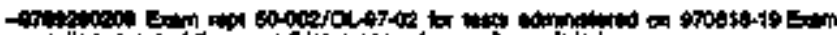

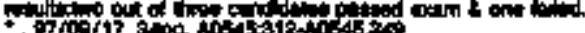

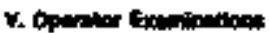

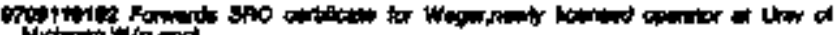

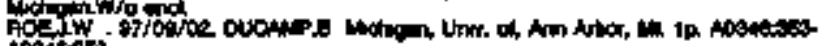

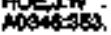

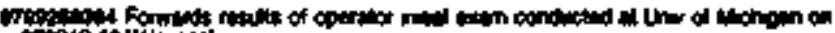

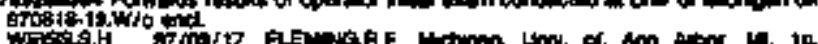

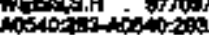

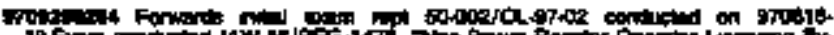

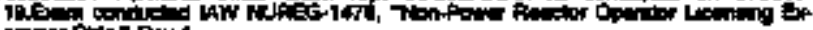
ariar

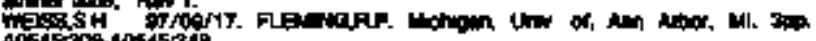

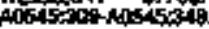

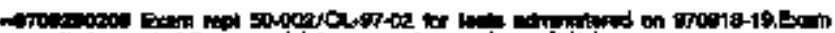

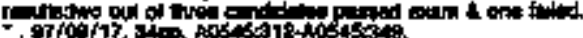

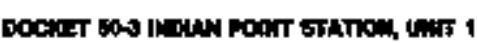

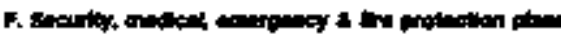

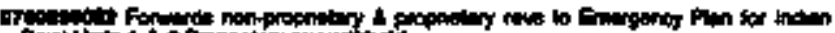

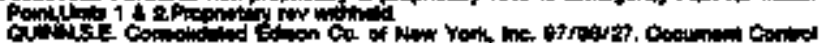

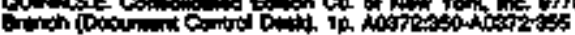

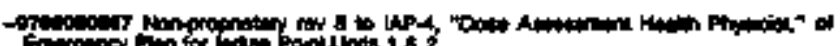

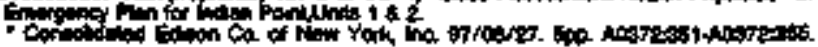

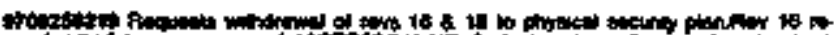

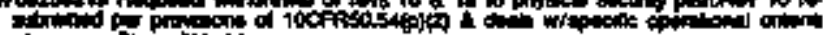
of thit

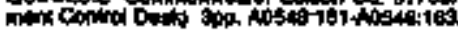

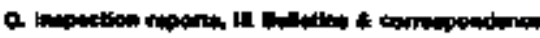

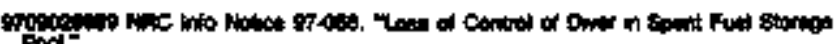

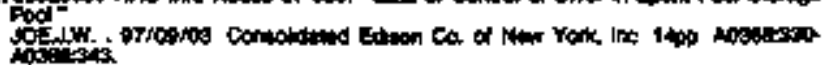

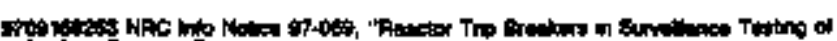

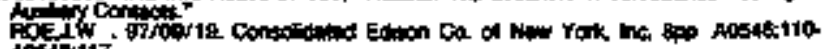
Alditit:

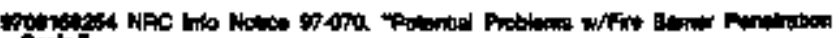

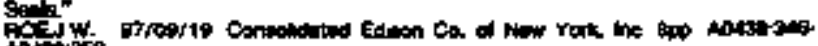
Pats. 


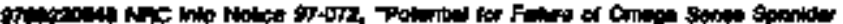

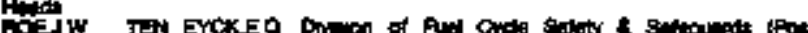

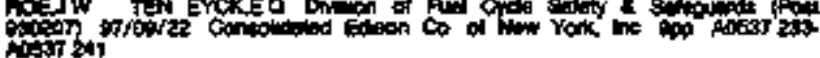

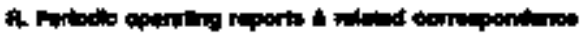

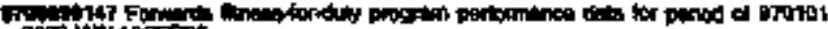

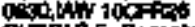

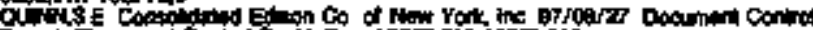

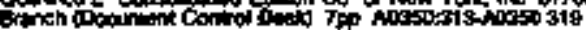

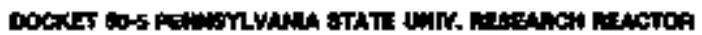

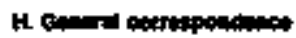

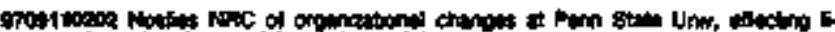

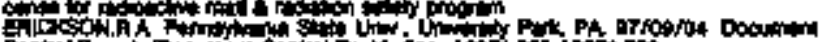

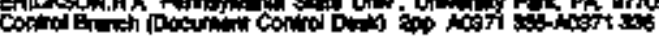

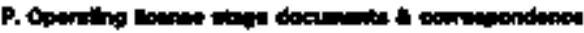

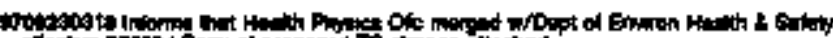

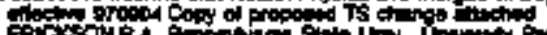

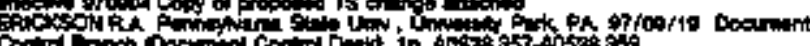

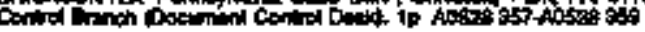

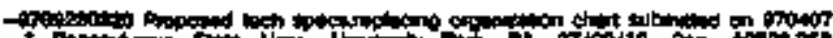

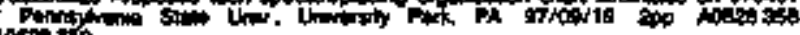

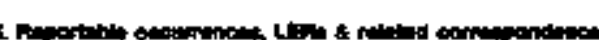

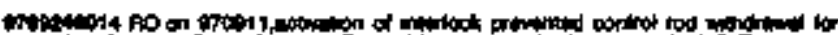

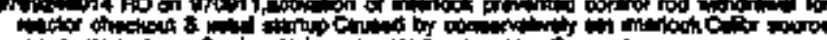

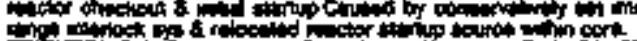

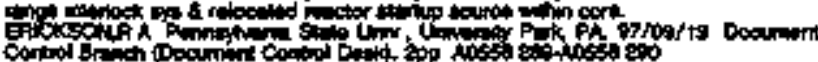

\section{Cpantor thention}

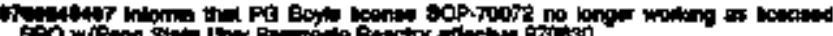

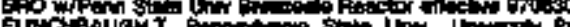

antion

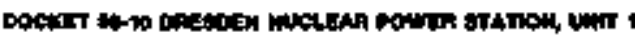

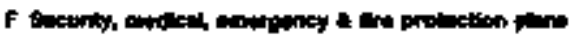

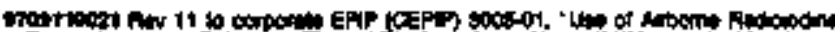

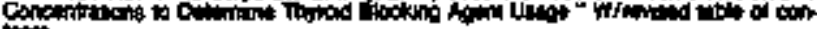
thition

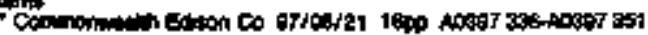

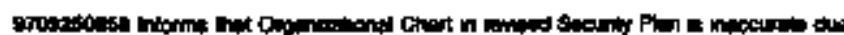

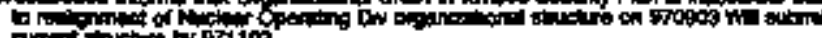

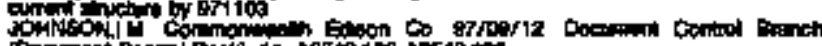

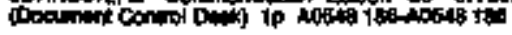

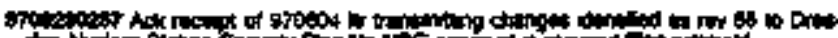

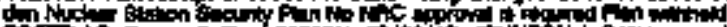

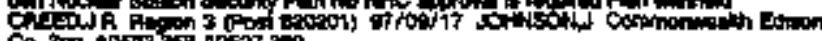
cong

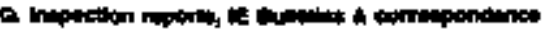

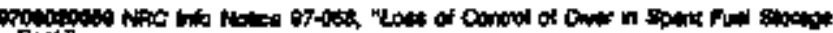

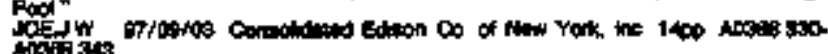

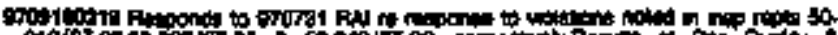

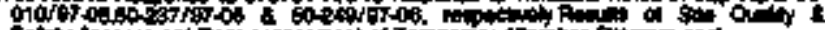

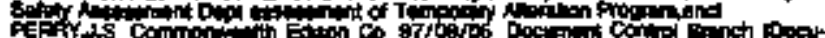

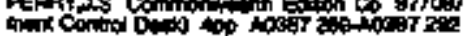

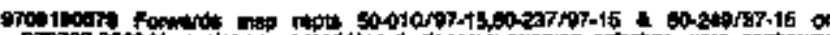

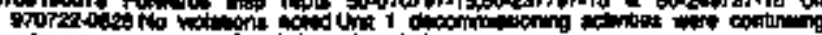

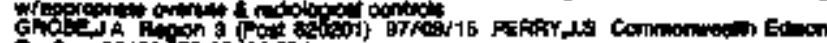

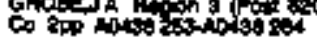

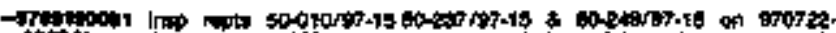

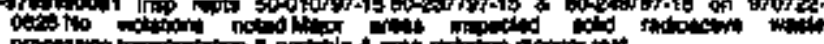

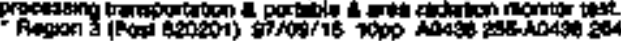

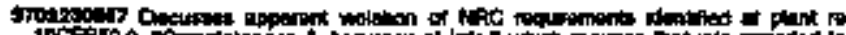

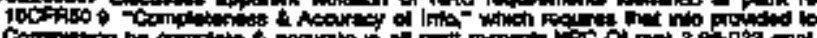

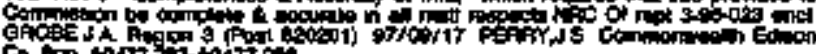

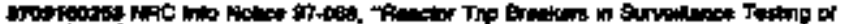

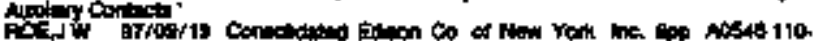
inste 117

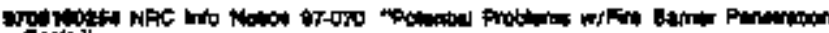

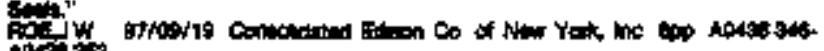

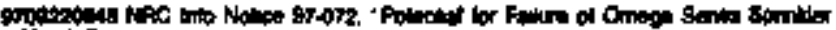

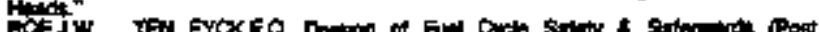

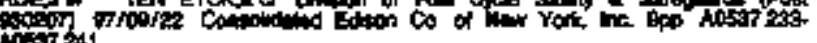

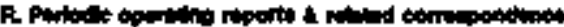

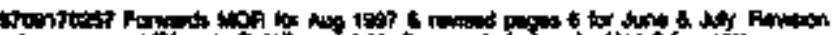

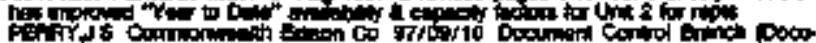

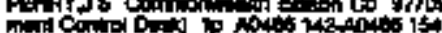

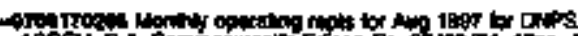

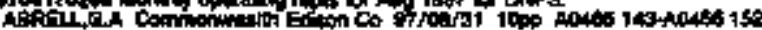

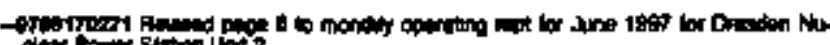

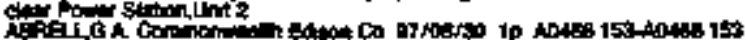

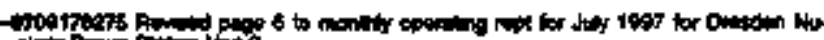

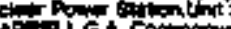

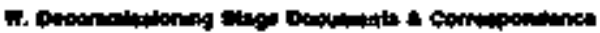

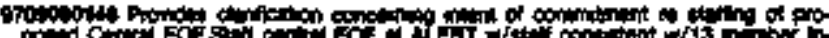

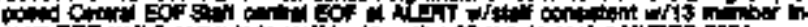

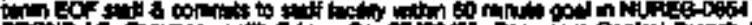
- Nons e C

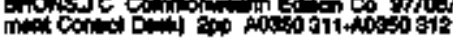

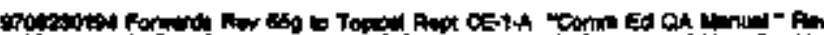

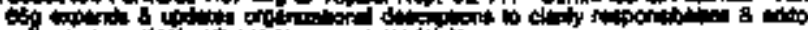

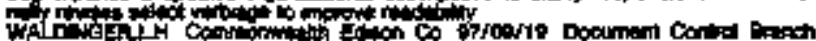

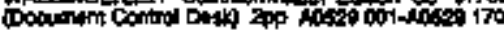

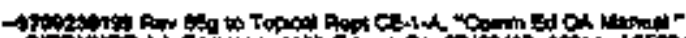

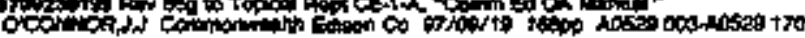

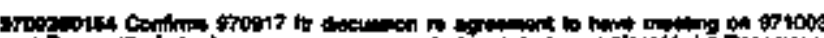

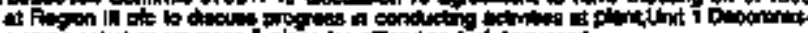
ron

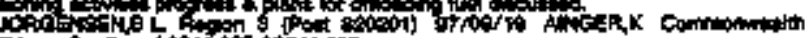

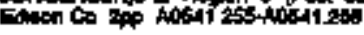

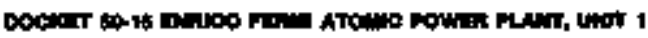

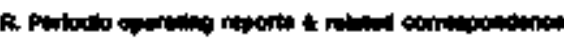

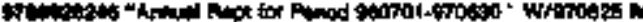

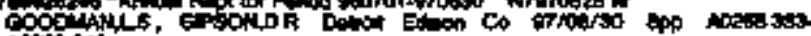
Aostono

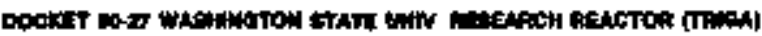

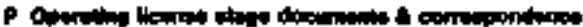

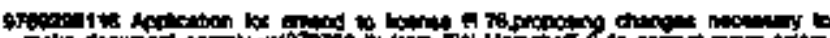

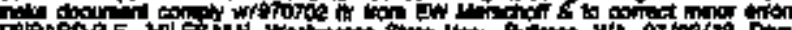

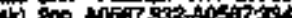

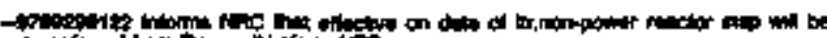

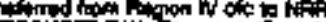

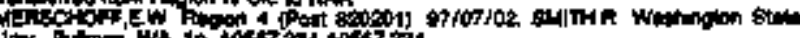

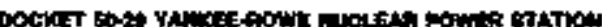

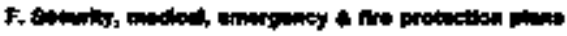

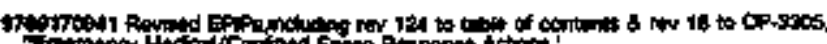

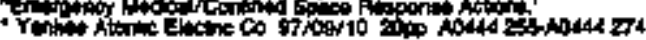

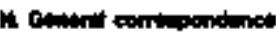

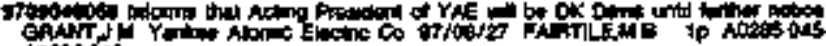
anows ons 


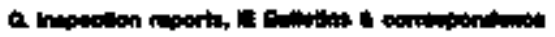

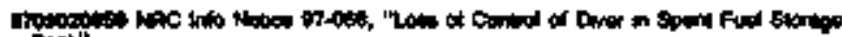

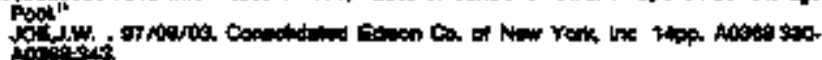

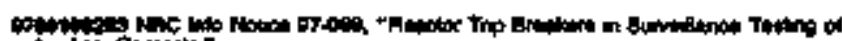

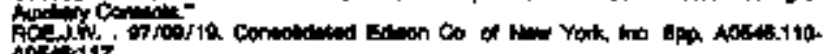

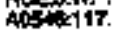

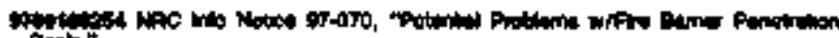

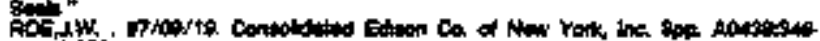
nosios:

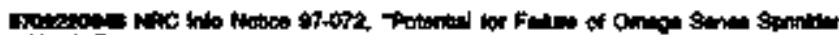

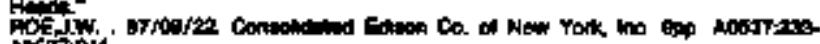

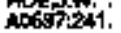

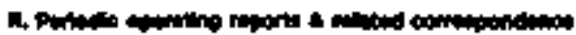

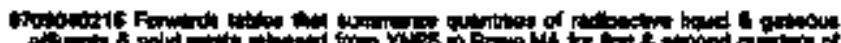

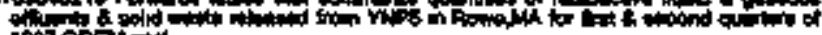
19p.opchet

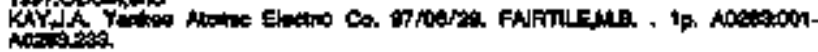

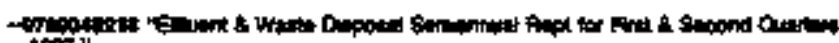

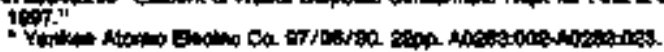

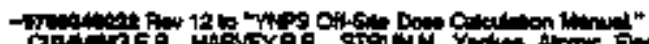

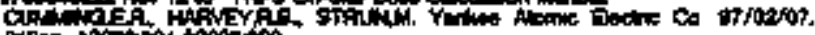

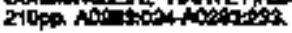

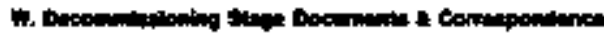

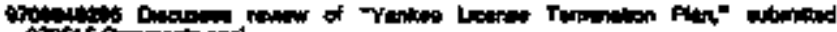

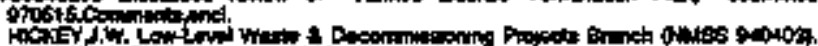

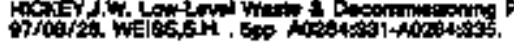

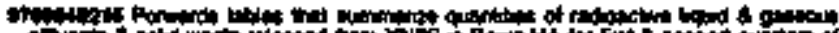

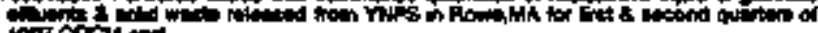

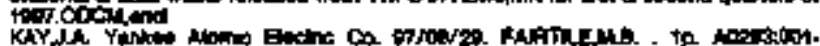

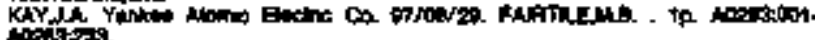

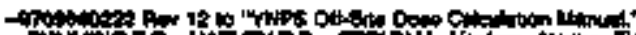

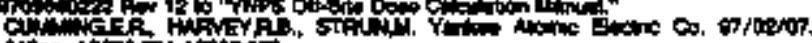

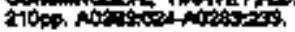

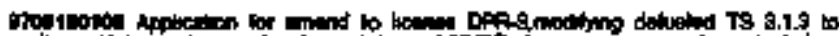

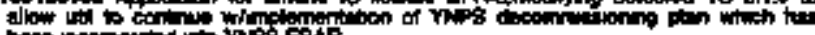
boin nowporting

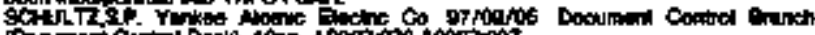

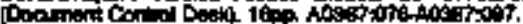

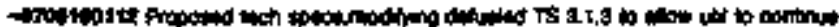

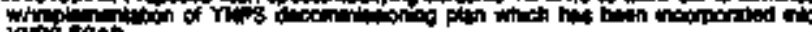
Pesila

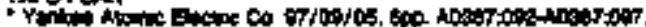

PIy 4050: 178

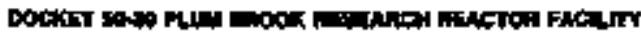

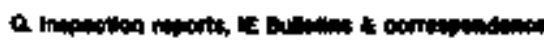

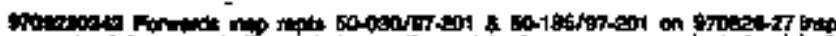

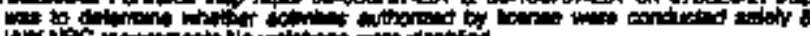

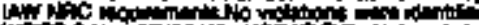

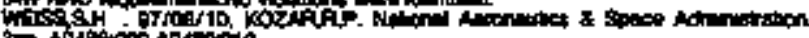

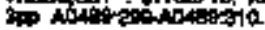

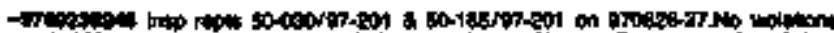

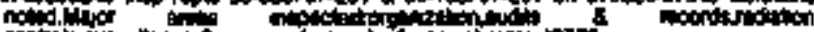

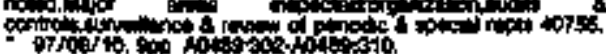

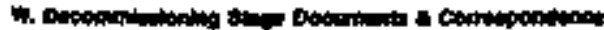

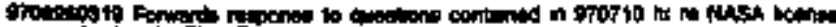

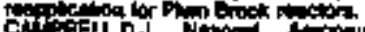

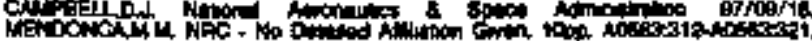

\section{Doctin Dore anmain}

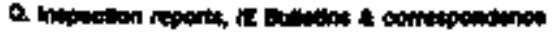

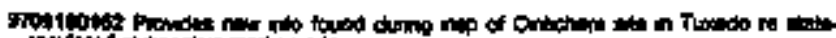

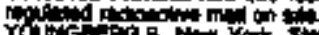

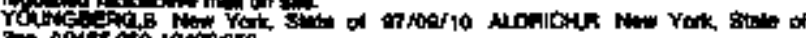

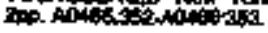

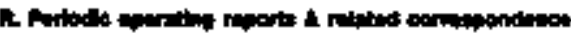

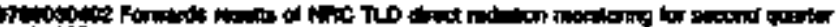

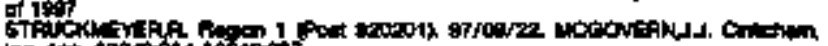

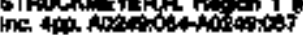

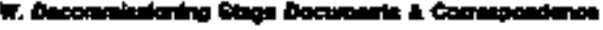

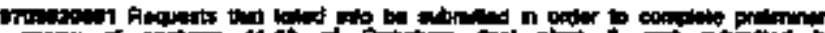

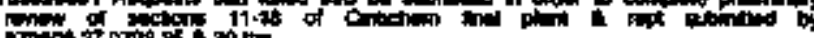

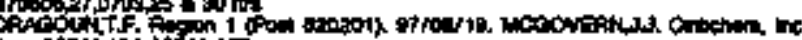

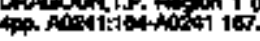

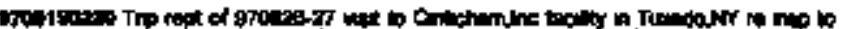

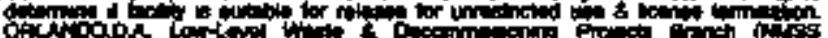

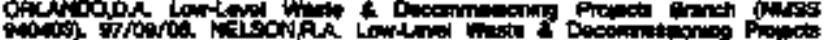

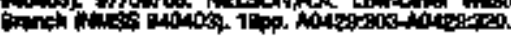

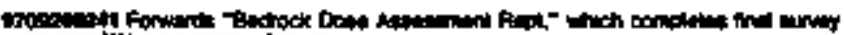

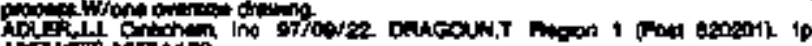

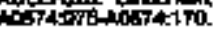

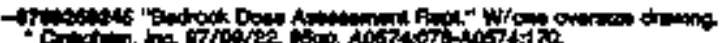

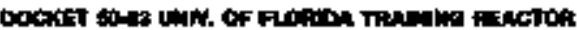

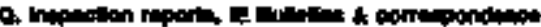

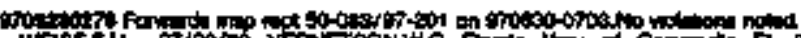

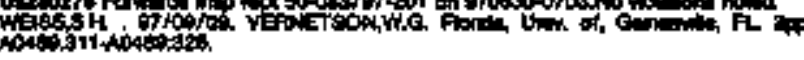

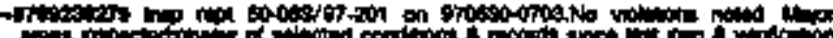

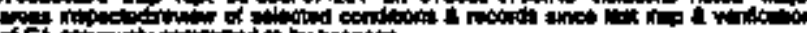

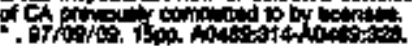

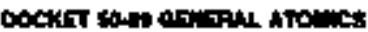

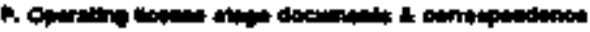

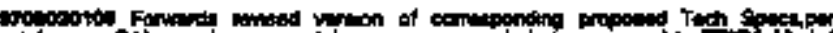

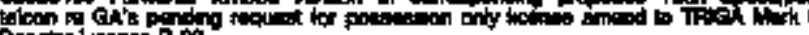

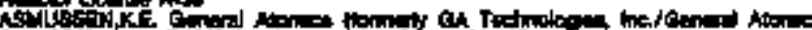
Co) gft

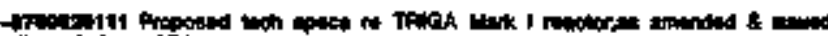

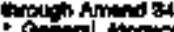
apo

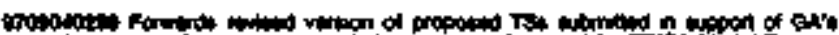

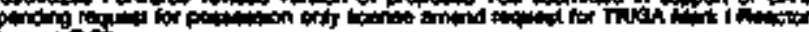

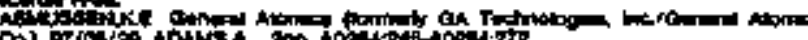

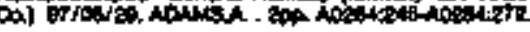

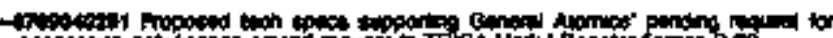

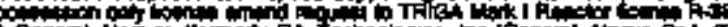

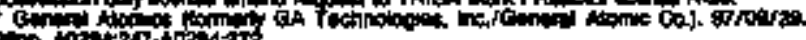

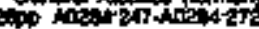

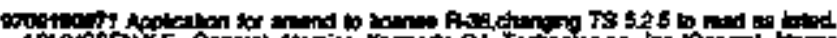

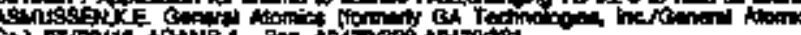

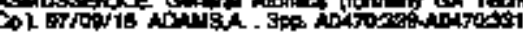

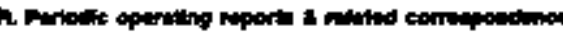

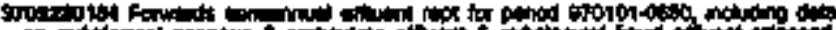

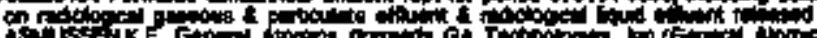

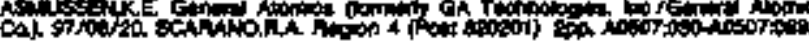

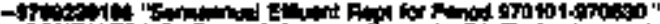

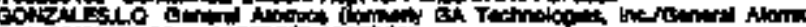

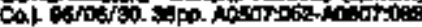

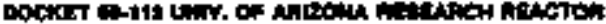

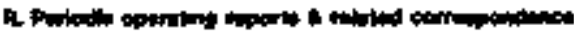

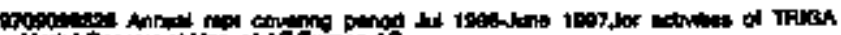

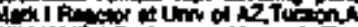

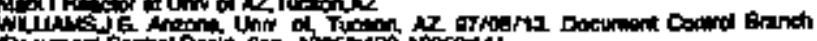

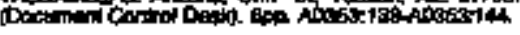




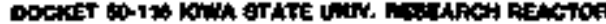

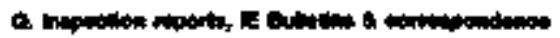

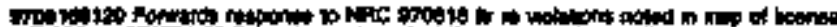

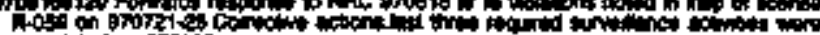

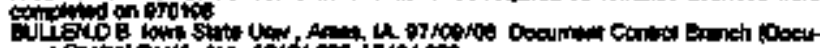

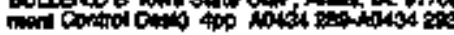

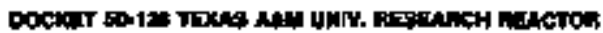

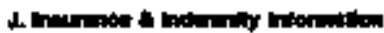

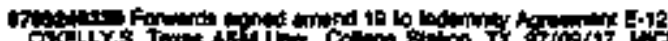

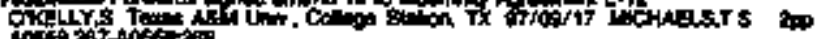

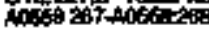

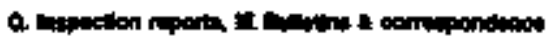

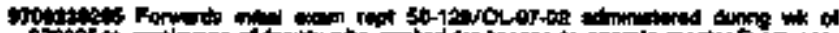

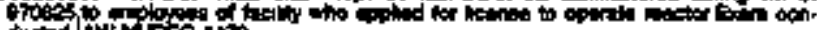

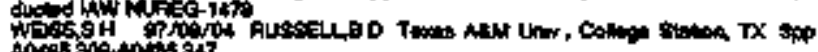

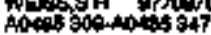

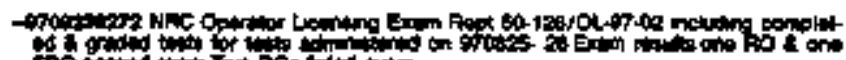

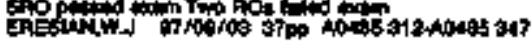

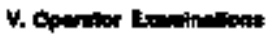

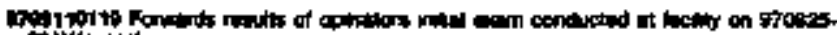

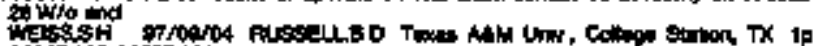

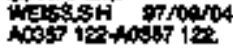

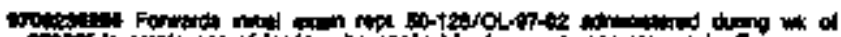

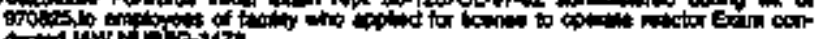

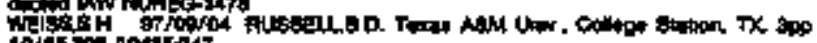

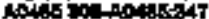

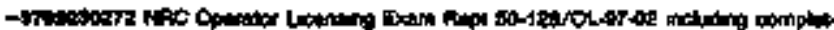

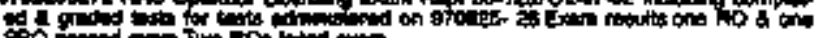

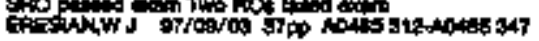

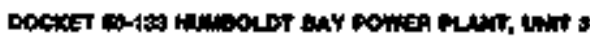

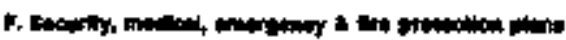

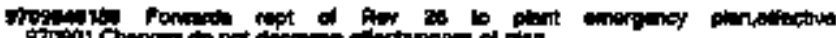

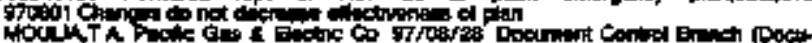

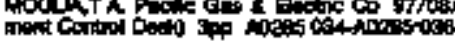

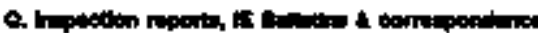

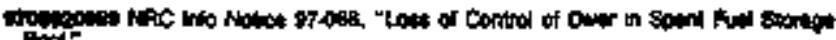

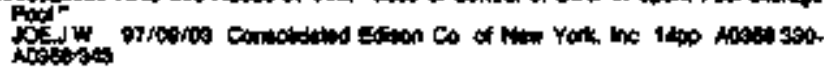

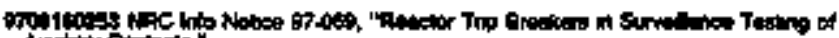

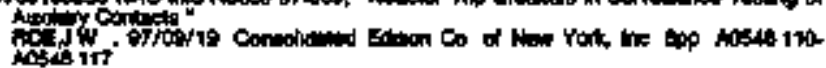

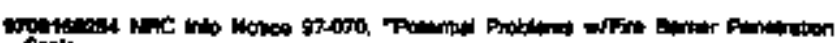

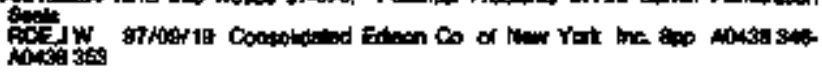

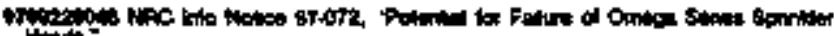

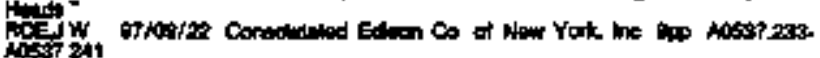

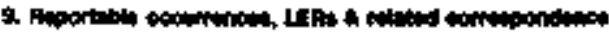

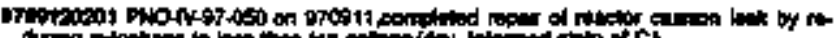

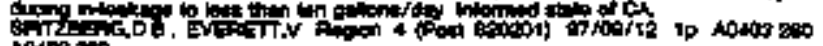
10403200

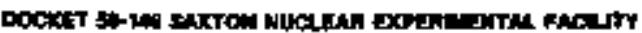

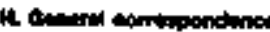

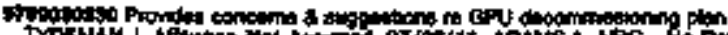

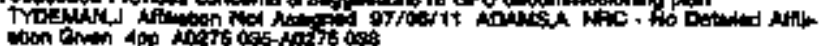

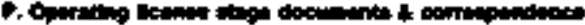

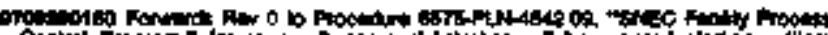

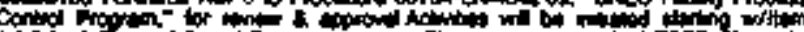

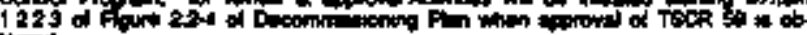

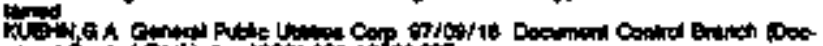

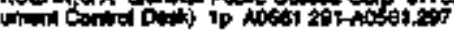

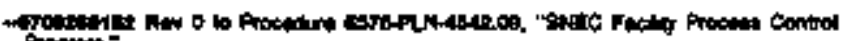

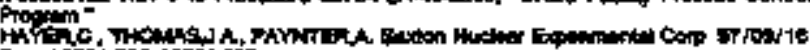

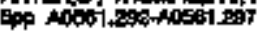

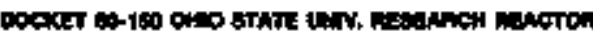

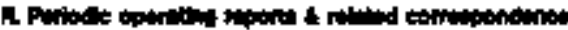

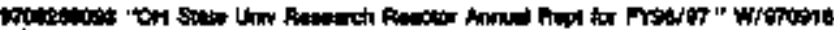

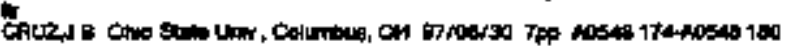

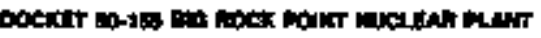

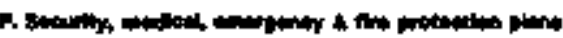

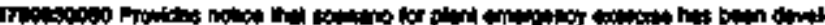

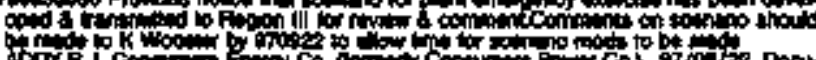

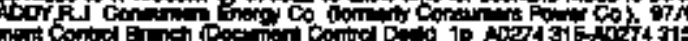

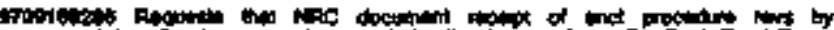

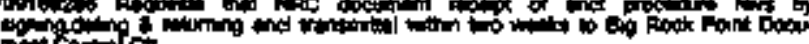

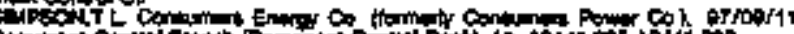

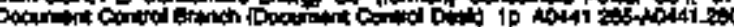

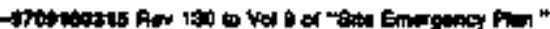

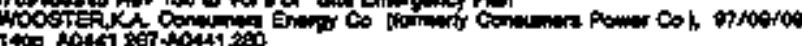

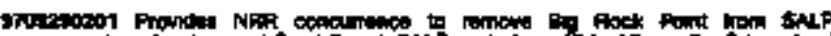

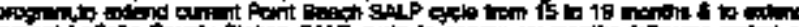

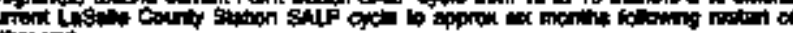

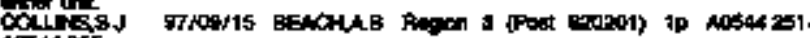

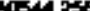

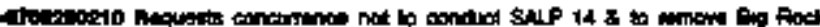

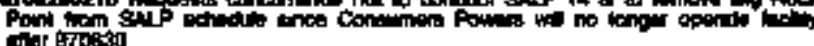

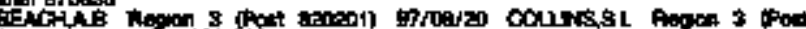

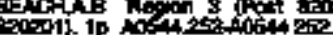

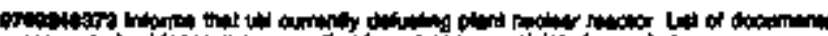

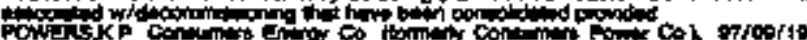

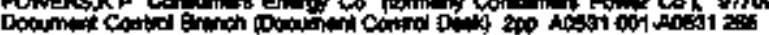

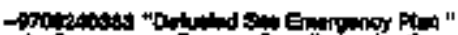

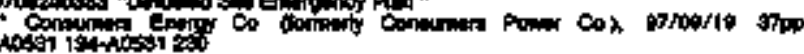

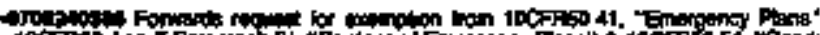
10 F For

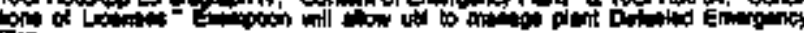

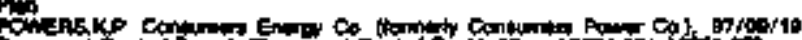

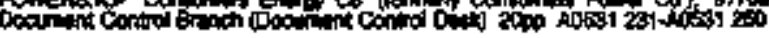

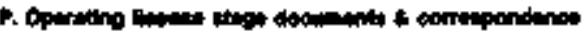

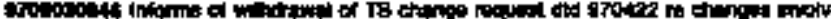

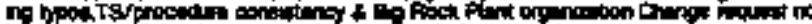

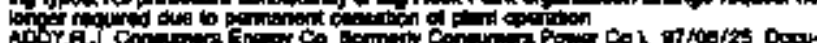

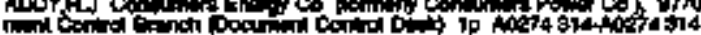

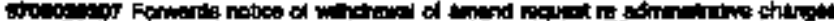

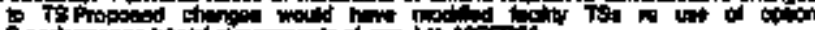

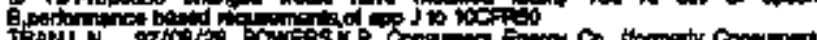

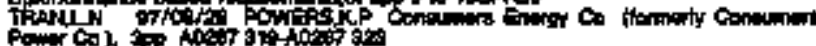

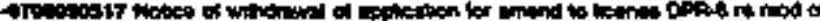

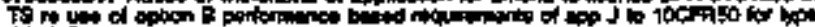

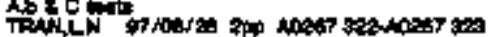

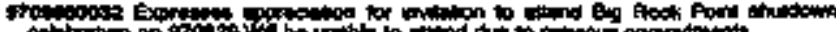

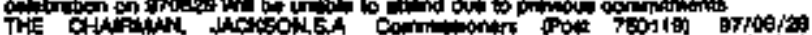
Jos. atos 


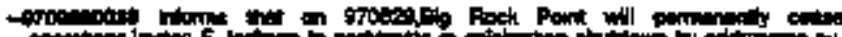

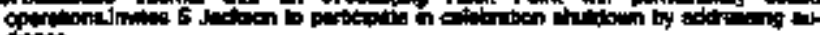
saming

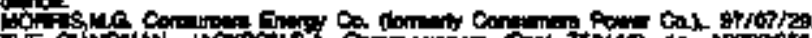

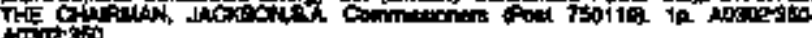

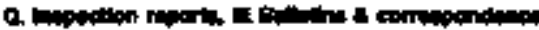

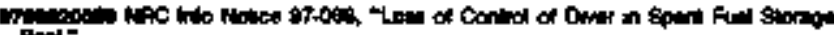

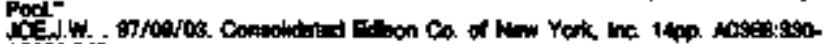

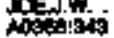

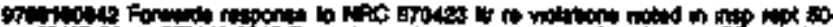

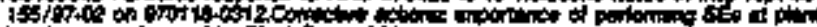

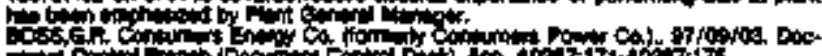
-

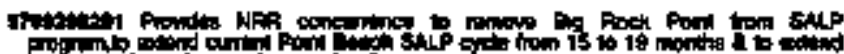

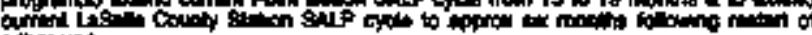
other unt

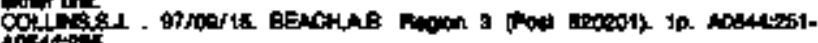
injestions

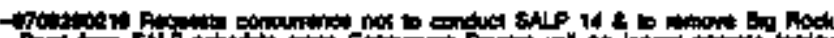

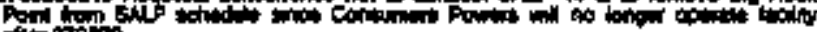

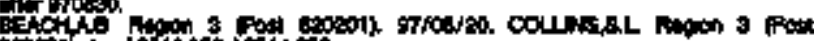

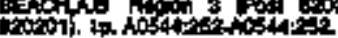

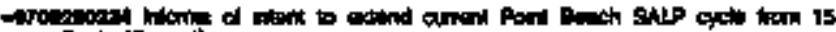

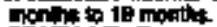

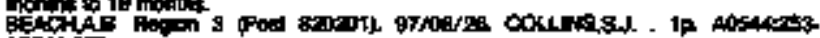
isinger.

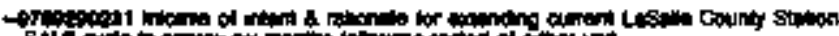

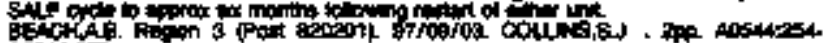
Bition

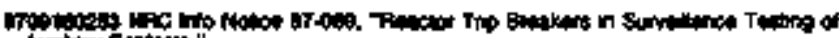

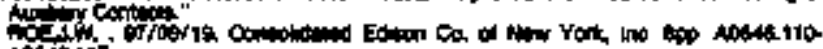
intitis?

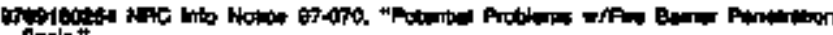

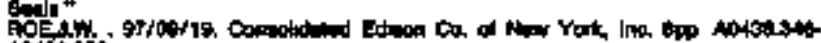

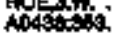

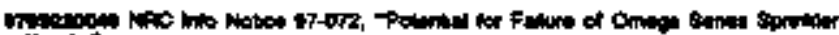

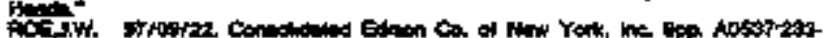
notions

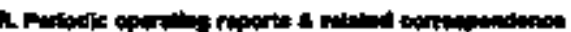

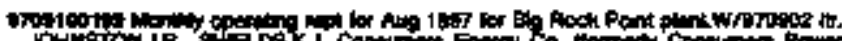

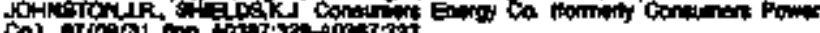

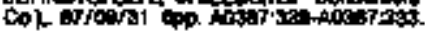

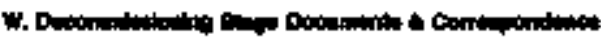

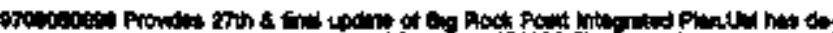

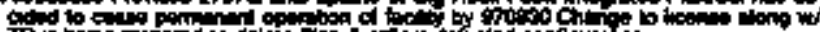

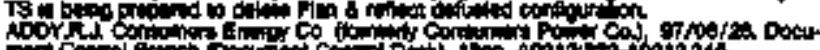

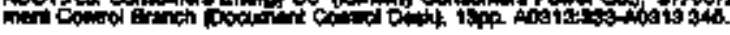

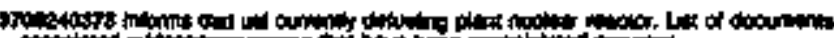
oching Dow jak

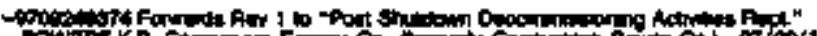

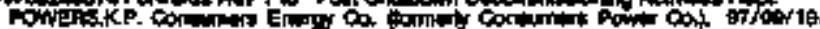

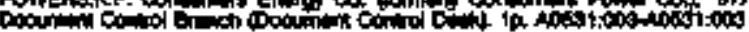

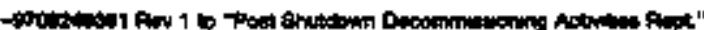

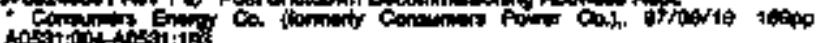

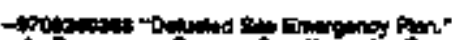

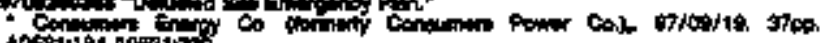

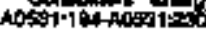

- o0,

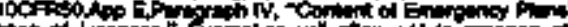

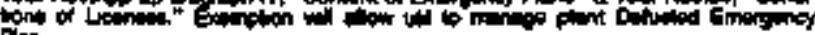

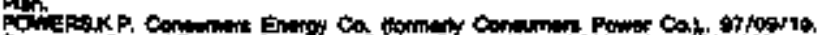

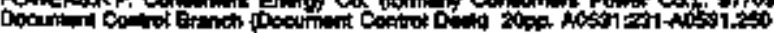

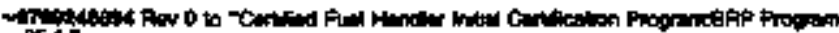

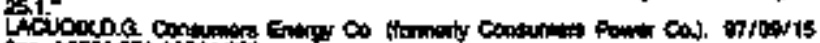

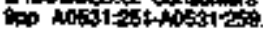

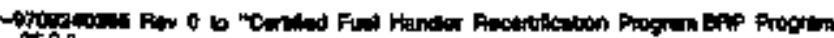

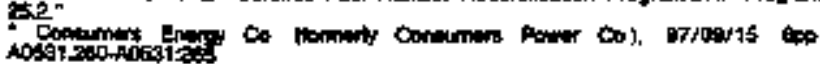

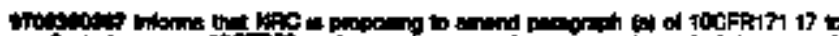

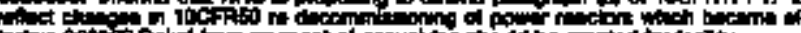

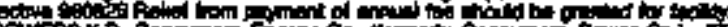

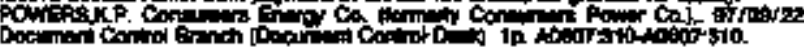

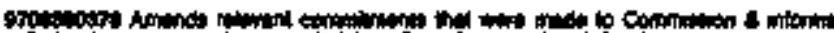

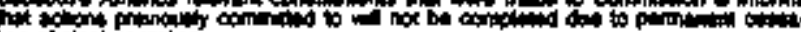
tin

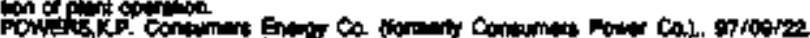

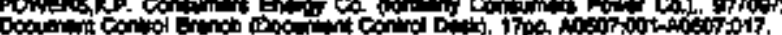

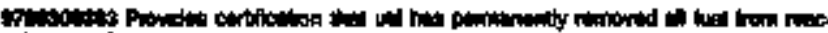

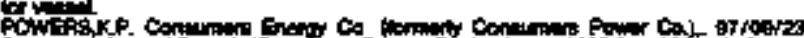

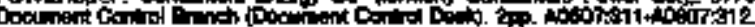

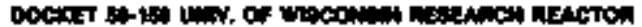

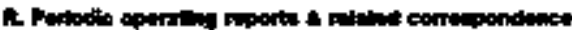

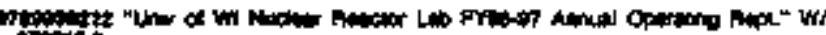
orions on

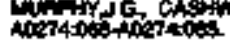

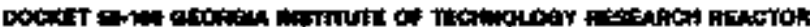

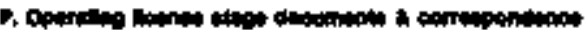

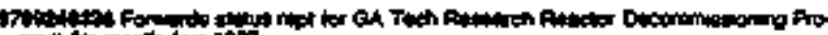

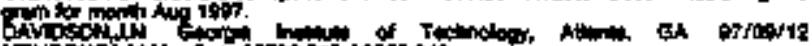

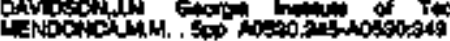

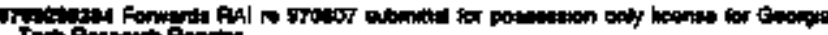

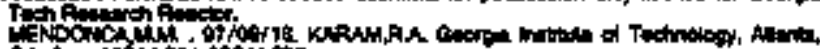
on.

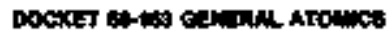

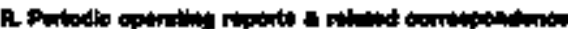

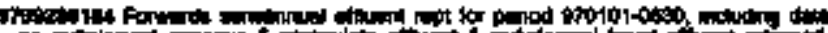

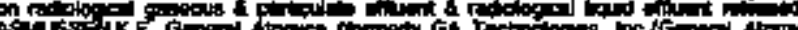

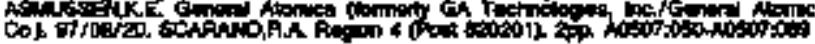

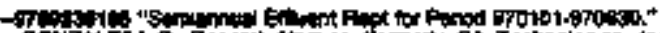

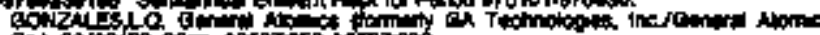

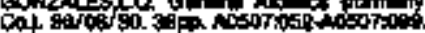

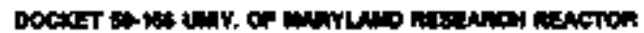

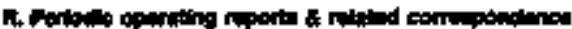

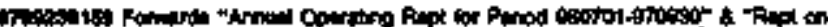

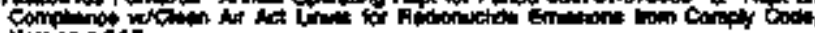
ition 1,

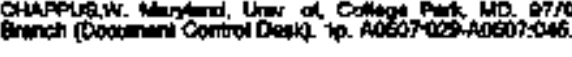

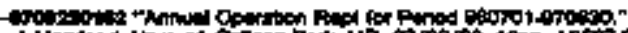

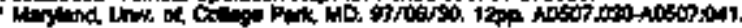

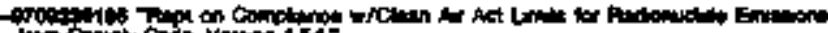

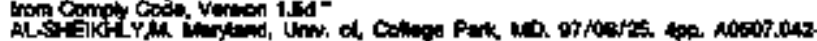
A00\%out.

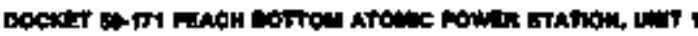

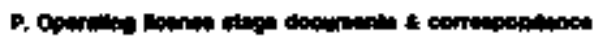

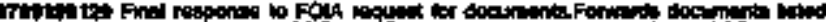

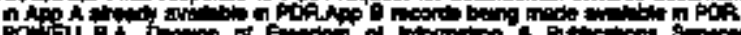

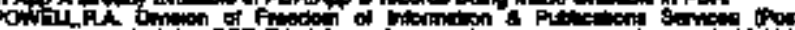

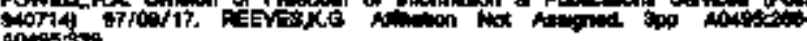
A04isis:

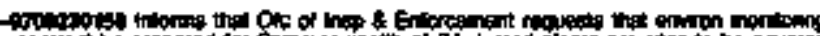

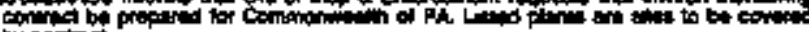

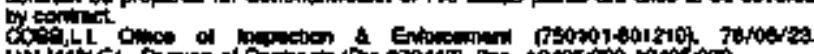

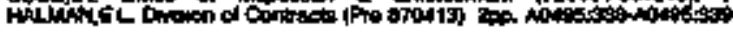

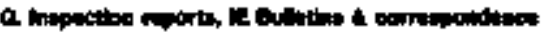

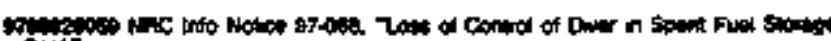

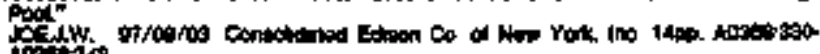
Actions 


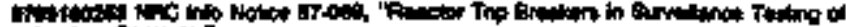

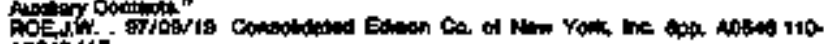
notiditio.

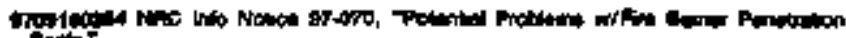

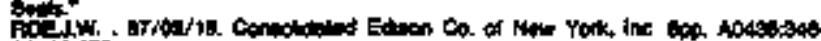
indisi.

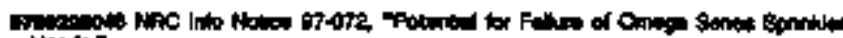

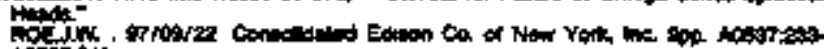
not.

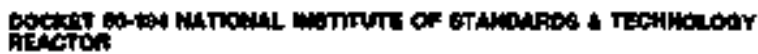

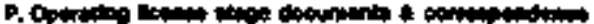

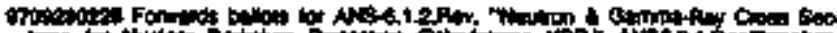

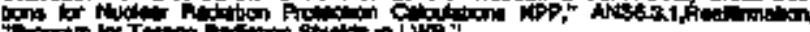
W

Wor

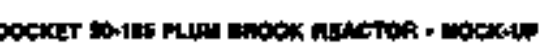

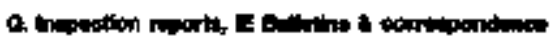

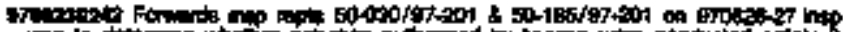

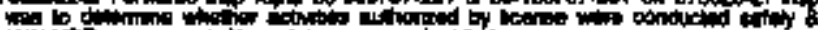

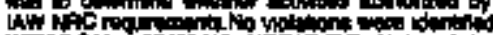

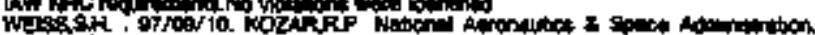

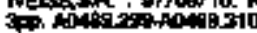

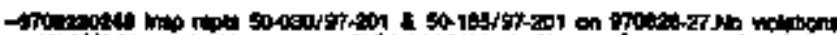

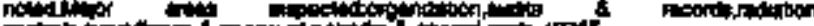

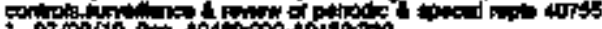

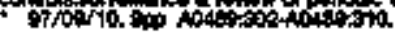

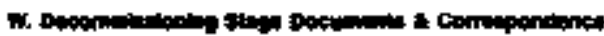

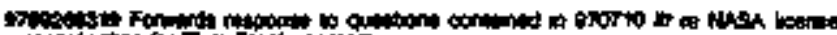

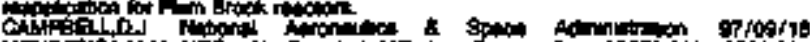

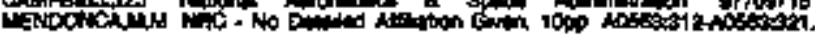

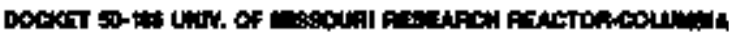

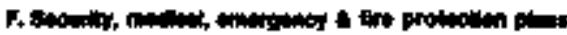

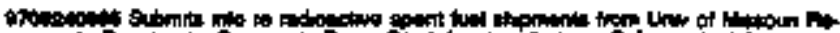

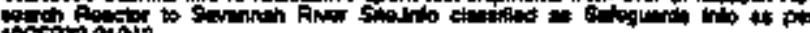
comition

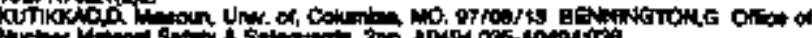

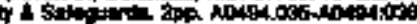

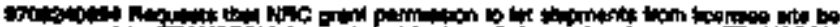

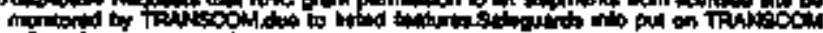
Finche

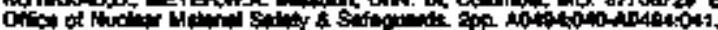

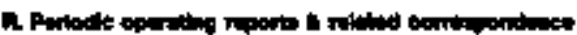

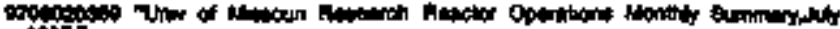
tós

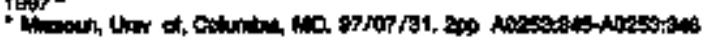

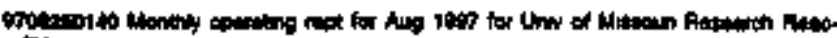
$+$

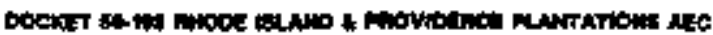

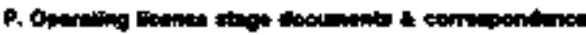

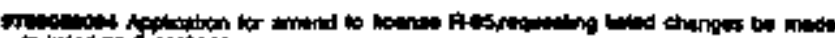

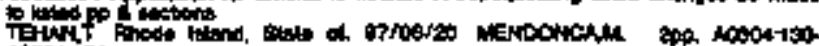
Torititis

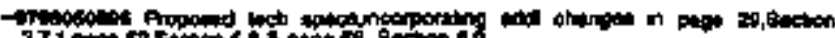

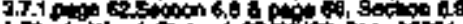

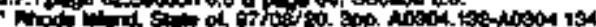

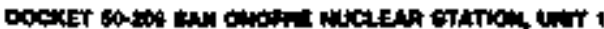

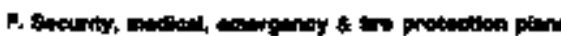

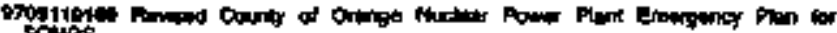
solios

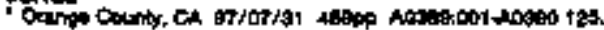

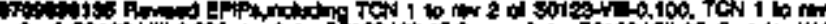
of sois कrong

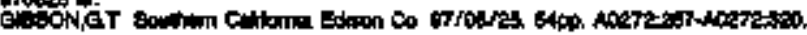

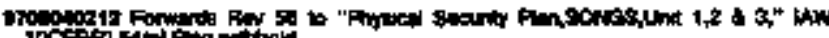

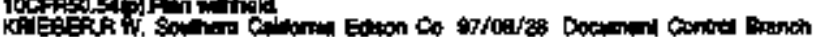

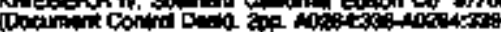

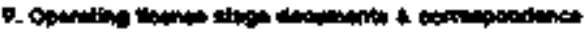

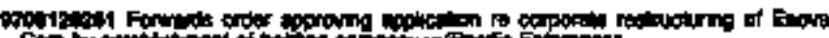

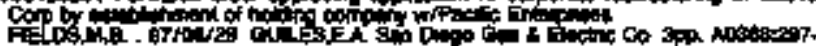
ation.

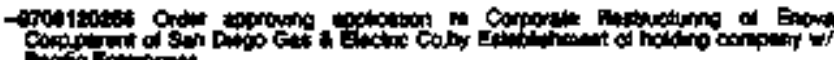

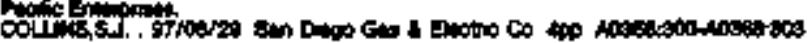

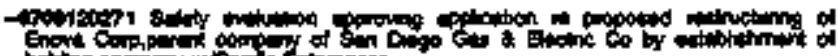

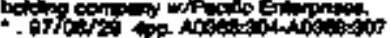

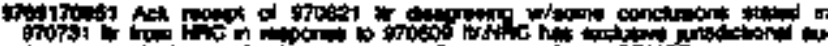

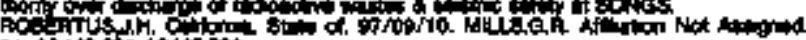

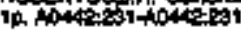

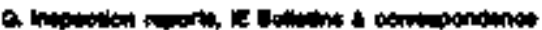

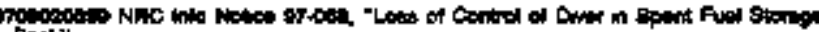

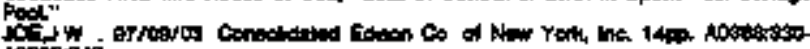
action.

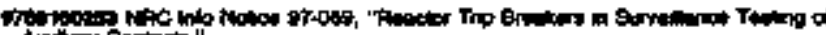

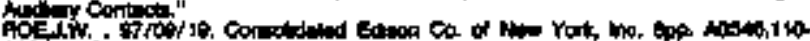

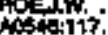

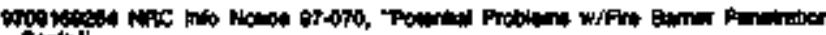

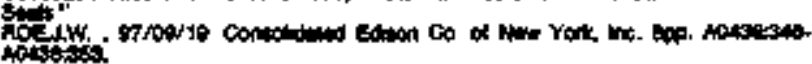

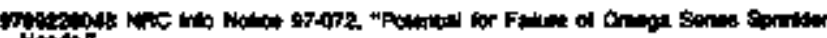

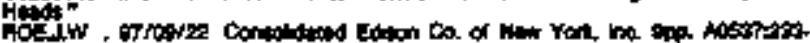
insositi.

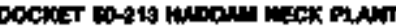

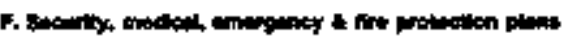

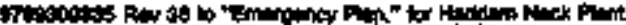

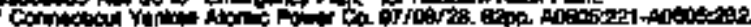

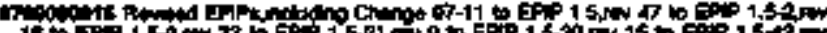

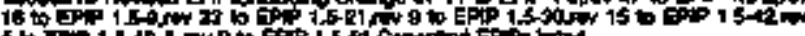

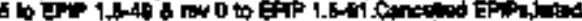

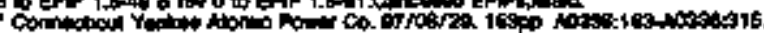

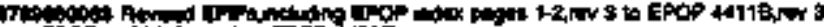

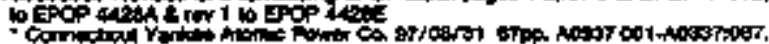

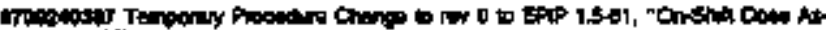

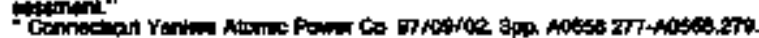

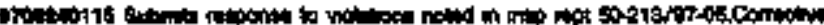

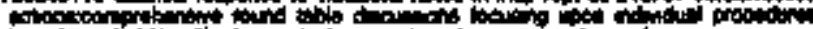

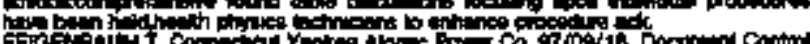

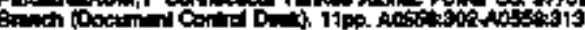

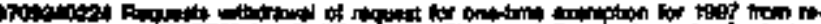

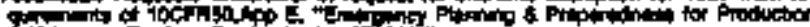

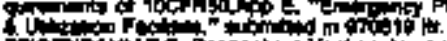

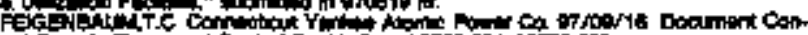

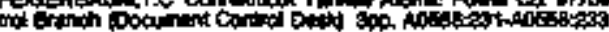

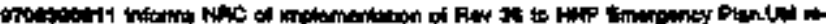

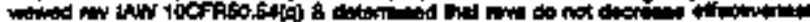
of Pin

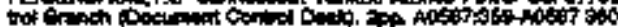

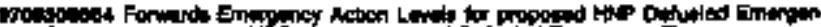

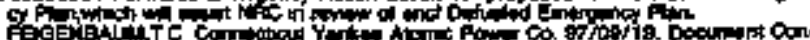

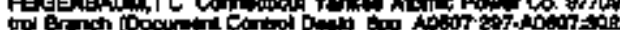




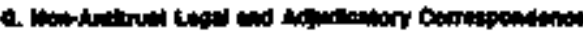

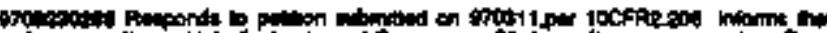

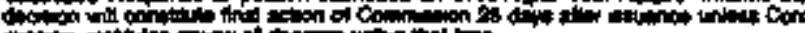

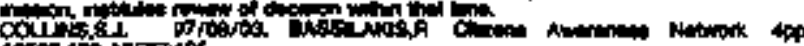

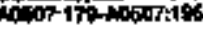

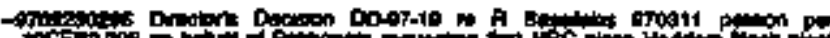

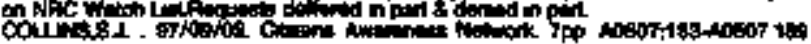

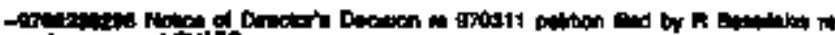

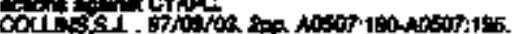

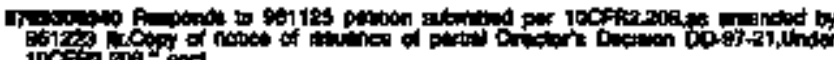

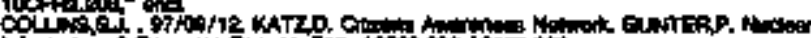

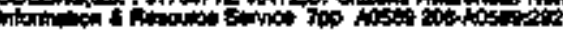

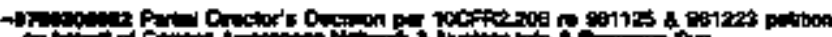
on tol of

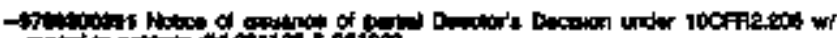

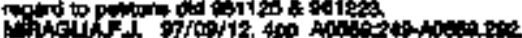

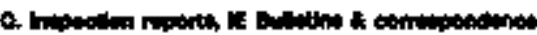

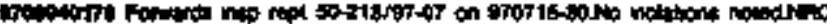

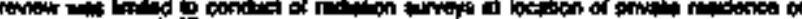

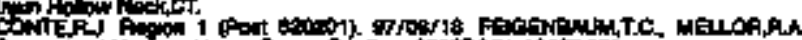

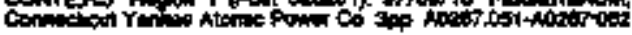

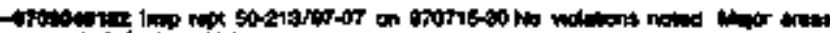

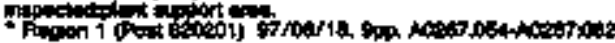

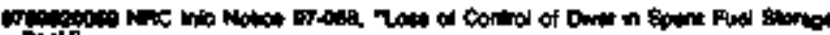

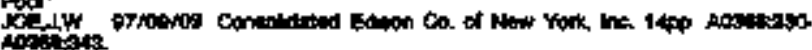

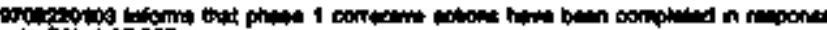

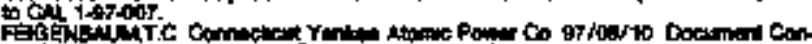

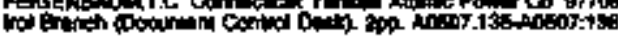

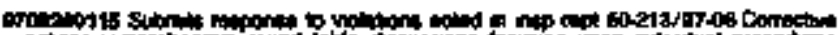

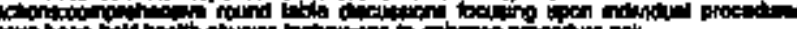

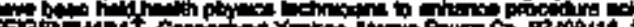

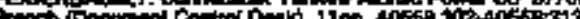

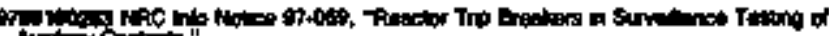
A Nositit

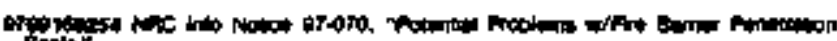

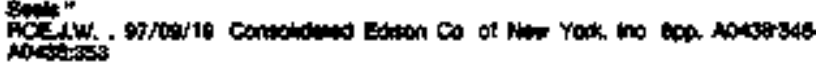

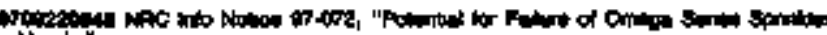

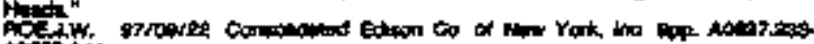

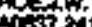

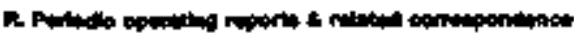

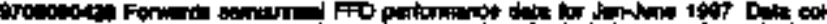

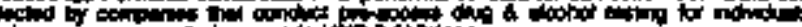

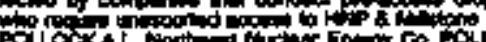

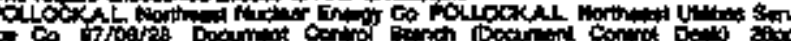

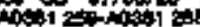

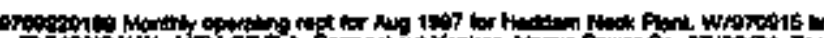

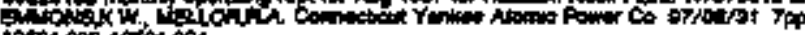
Aotol 200 intor 304 .

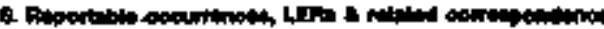

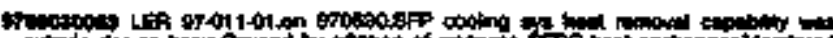

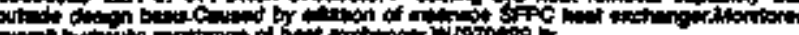

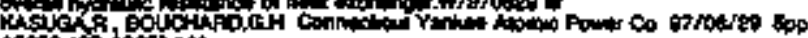

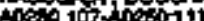

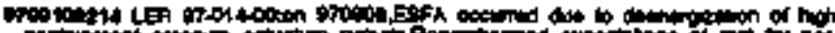

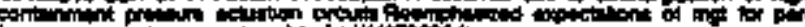

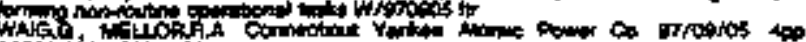

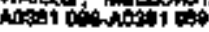

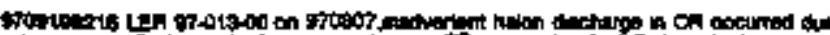

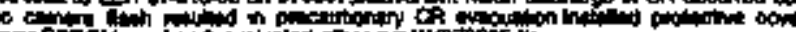

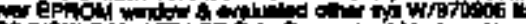

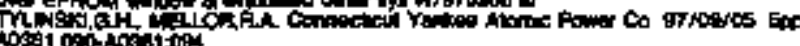

Thatic Fon

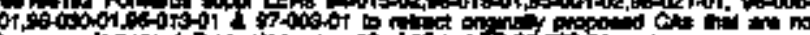

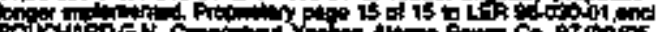

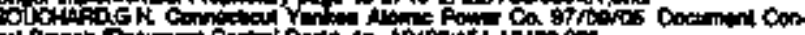

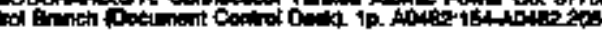

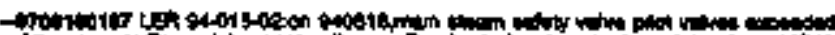

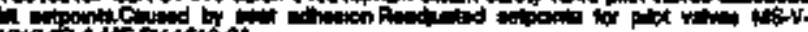

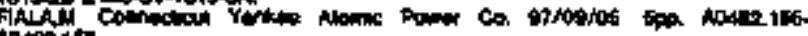

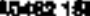

- WOH

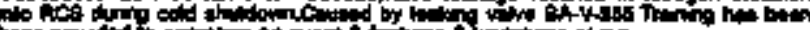

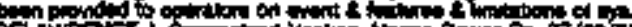

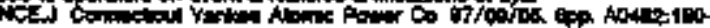

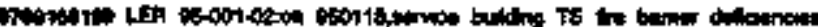

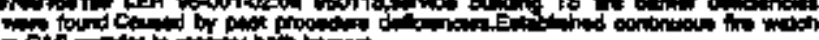

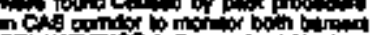

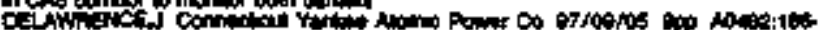

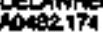

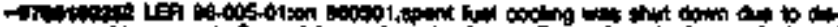

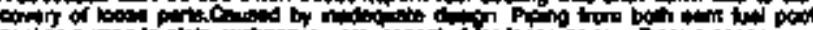

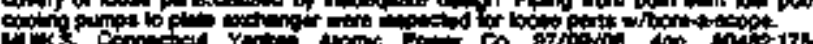

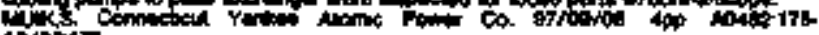
natein

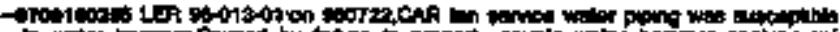

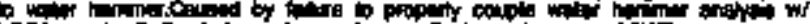

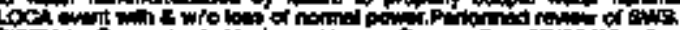

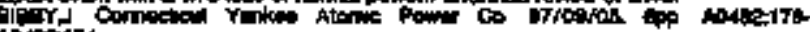
An:m1B

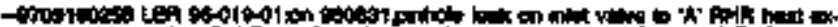

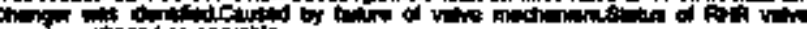

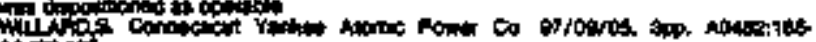
intis.

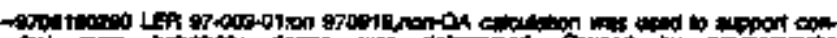

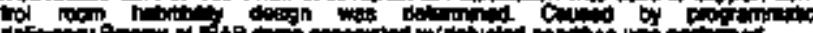

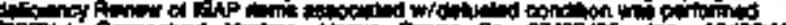

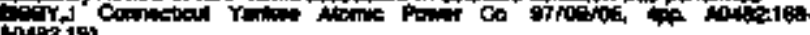
intin

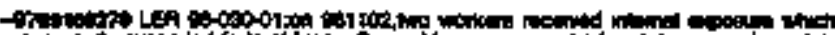

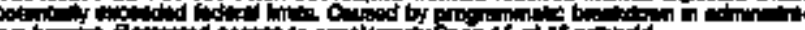

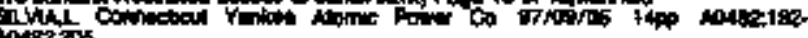

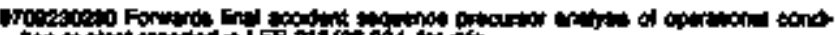

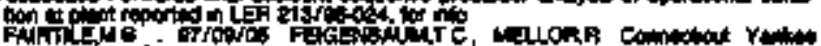
A

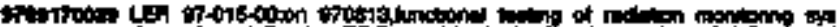

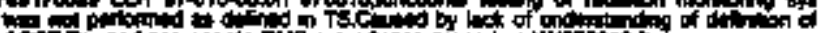

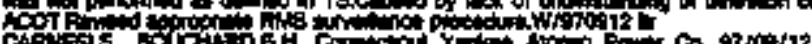

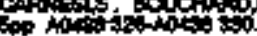

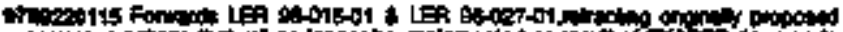

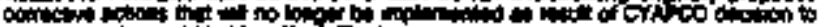

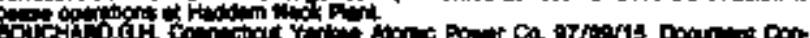

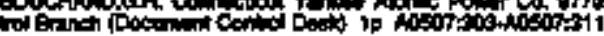

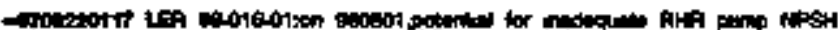

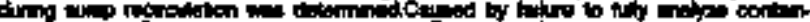

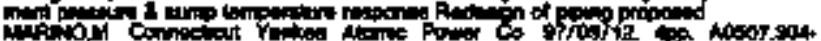
nocorion

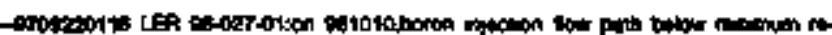

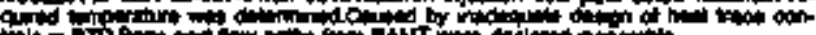

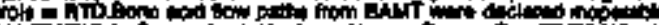

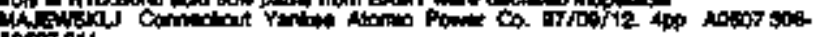
1000\%11

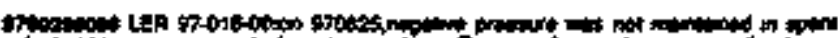

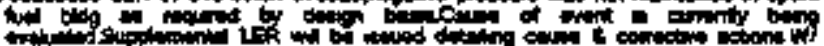
tiving

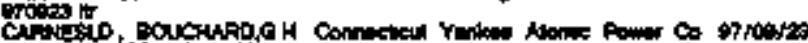
cring

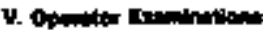

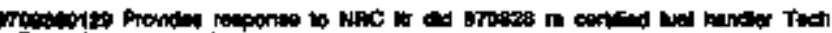

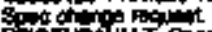

Pite

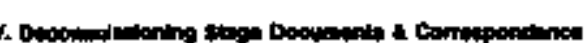

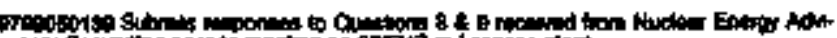

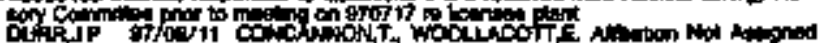

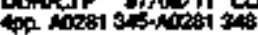




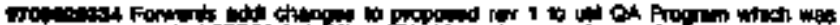

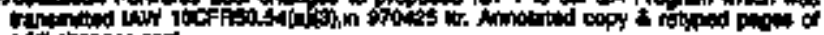

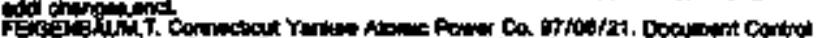

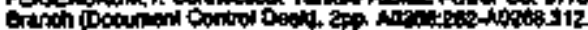

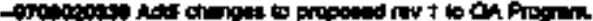

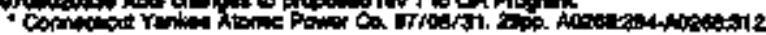

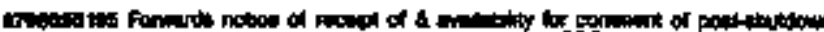

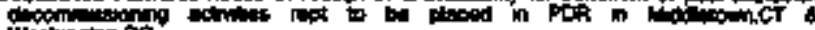

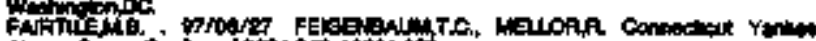

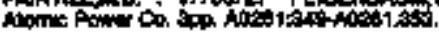

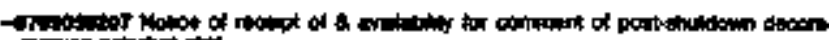

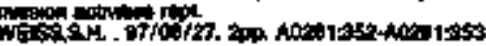

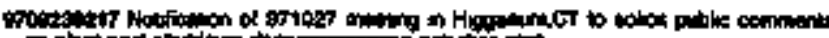

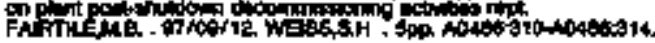

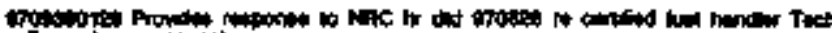

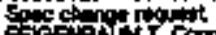

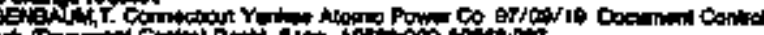
等

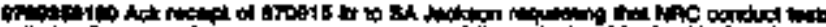

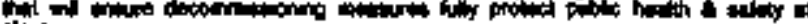

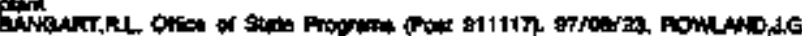

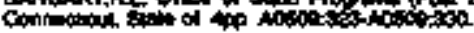

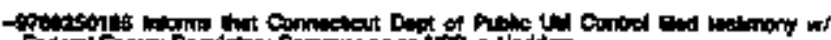

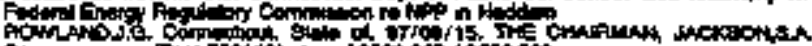

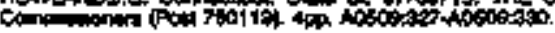

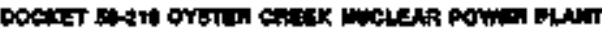

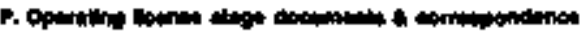

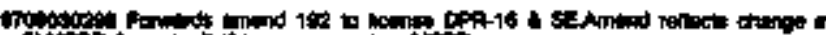

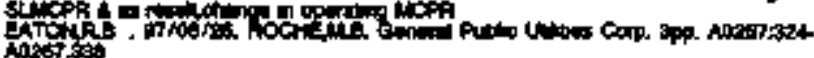

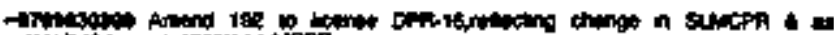

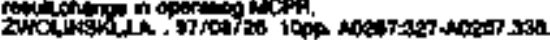

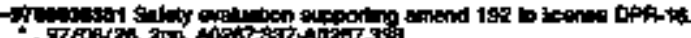

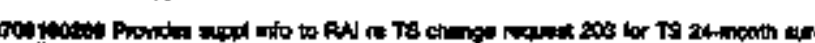

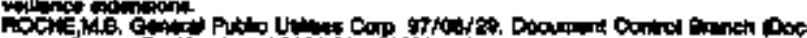

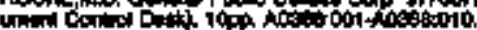

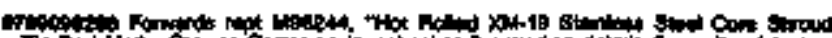

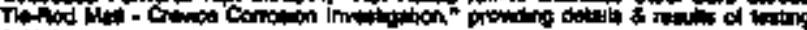

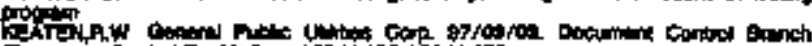

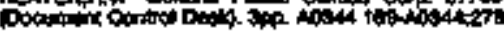

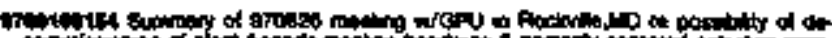

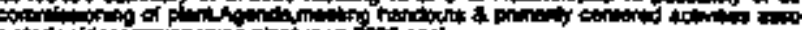

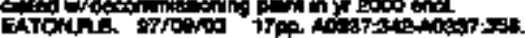

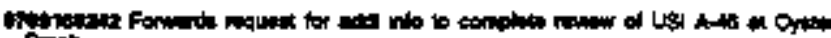

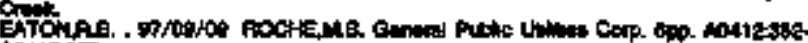
notizis?

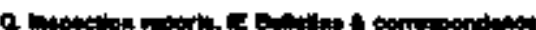

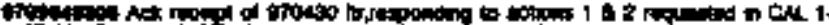

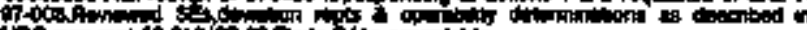

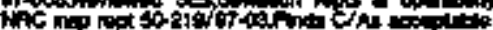
Hif coiph ât

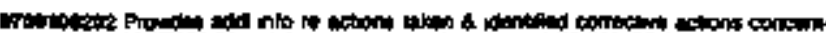

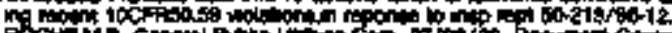

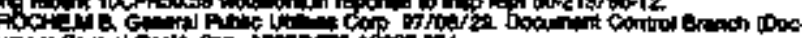

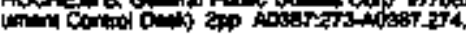

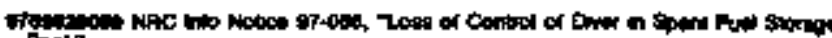

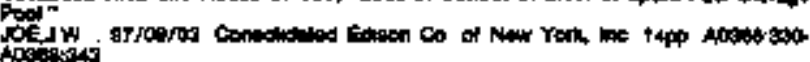

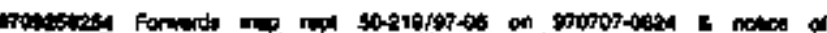

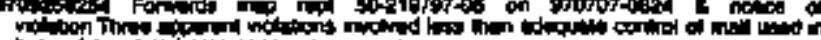

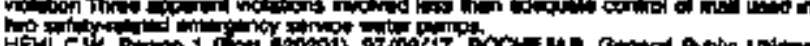

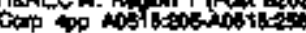

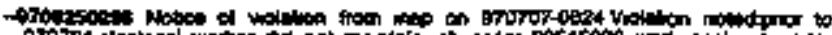

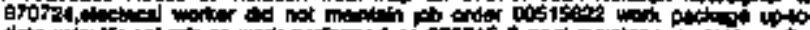

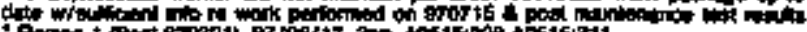

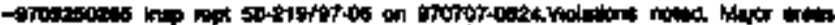

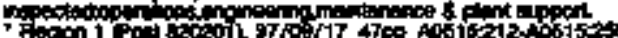

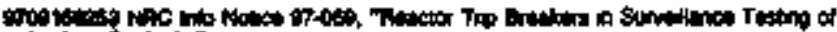

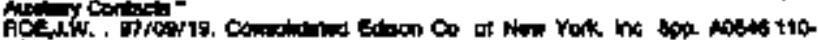
AOST:117.

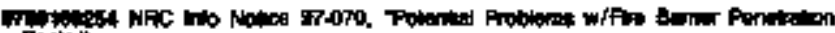

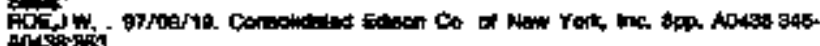

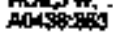

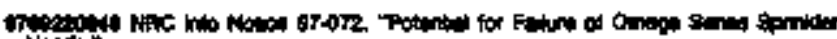

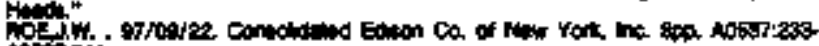
Aostinit

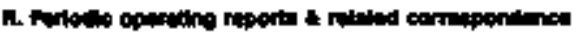

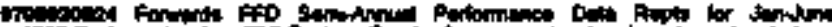

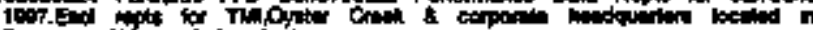

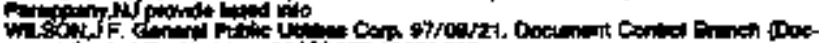

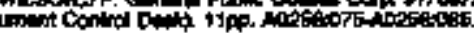

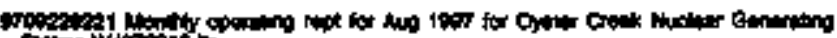

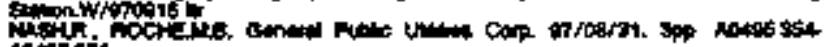
intions.

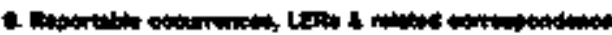

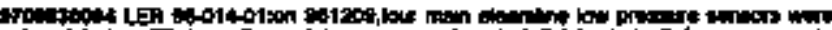

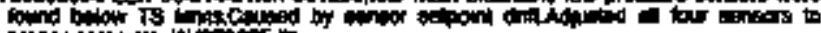

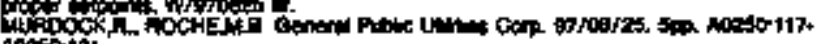
Antotion

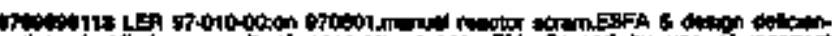

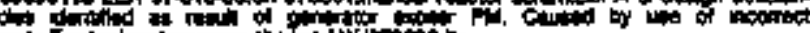

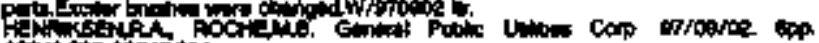

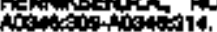

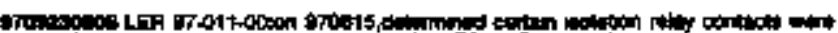

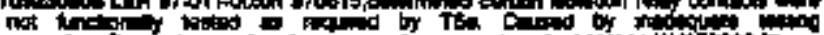

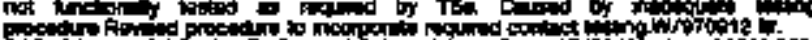

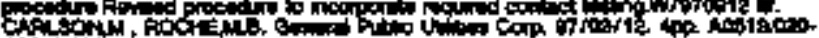
Aosisent

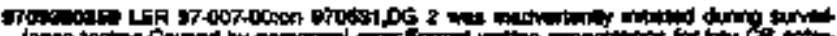

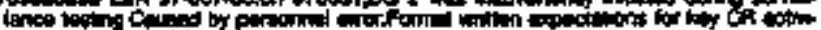

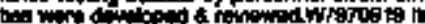

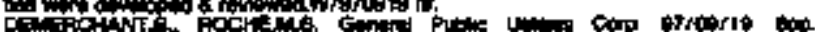

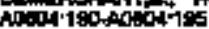

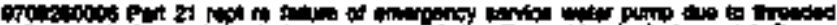

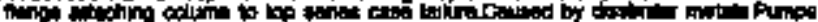

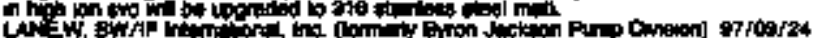

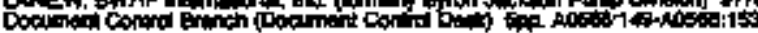

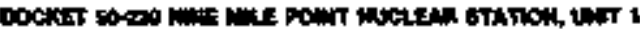

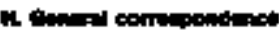

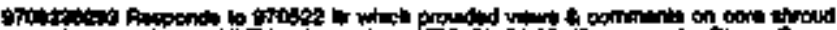

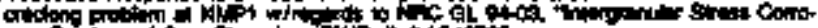
pon Alotion.

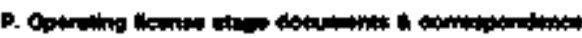

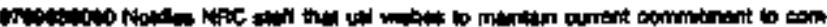

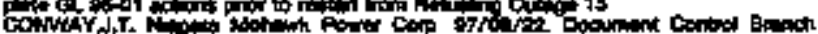
(D)

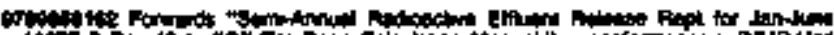

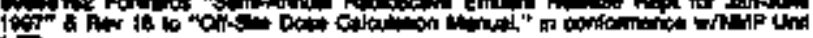

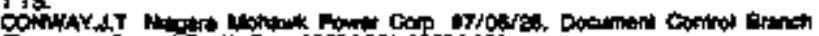

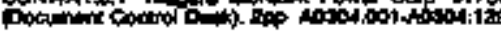

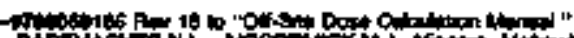

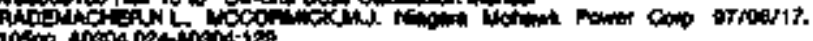

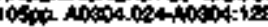

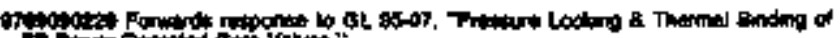

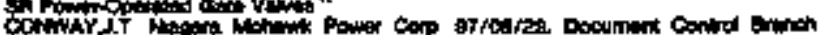

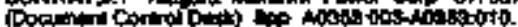

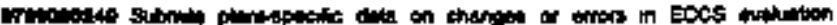

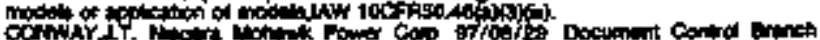

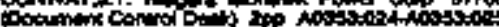




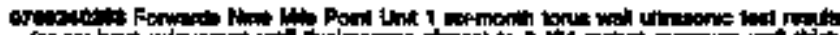

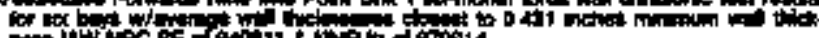

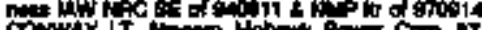

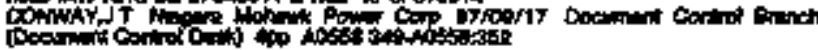

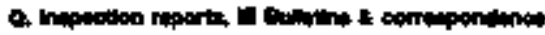

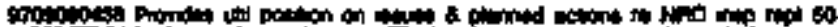

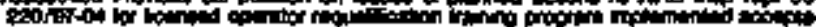

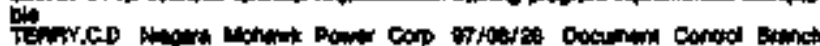

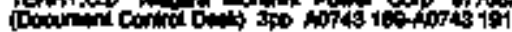

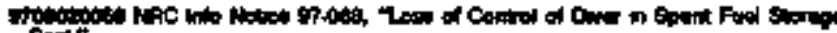

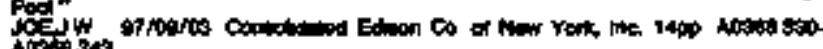

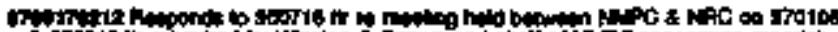

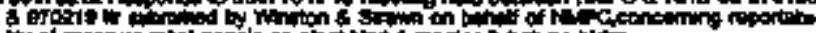

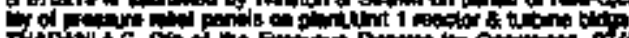

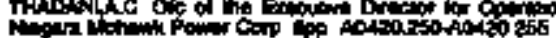

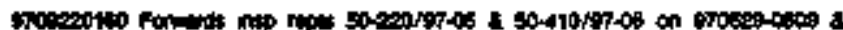

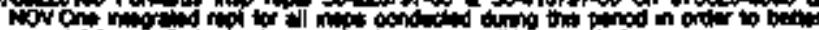

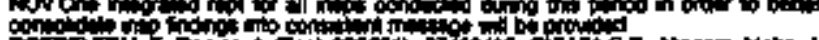

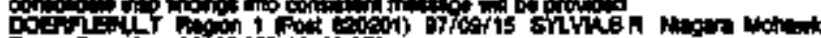

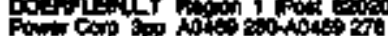

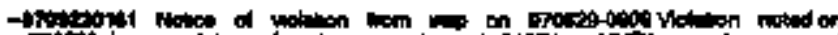

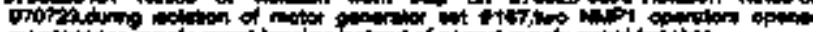
or

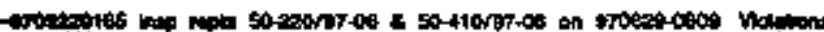

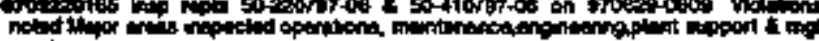

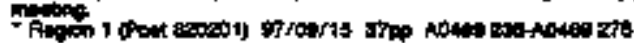

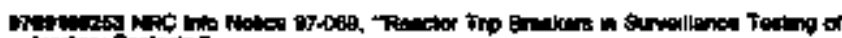

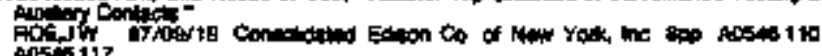

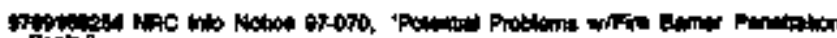

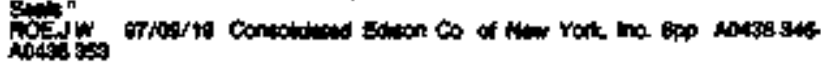

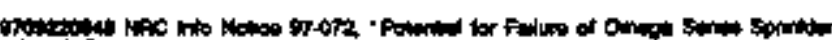

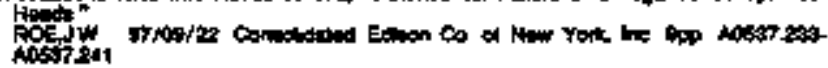

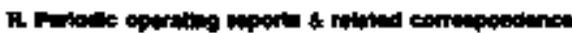

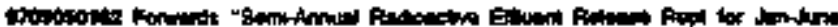

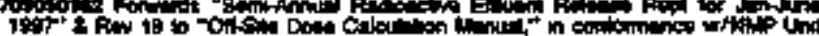

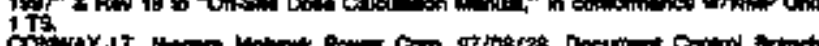

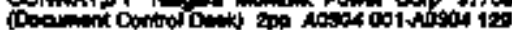

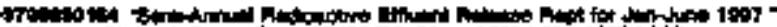

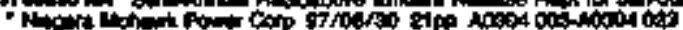

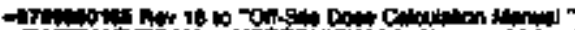

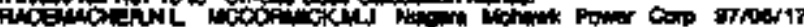

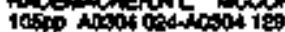

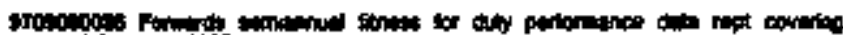

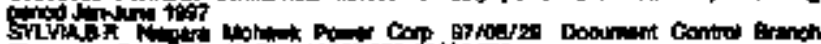

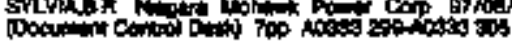

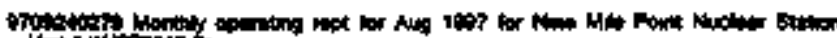

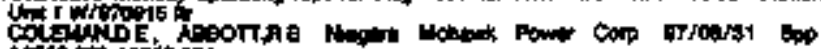
A

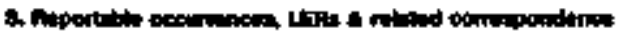

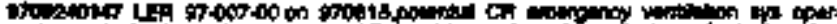

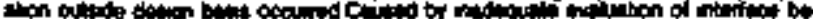

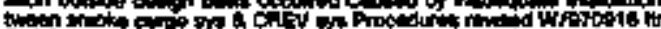

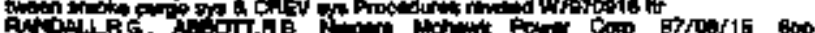

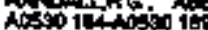

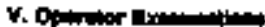

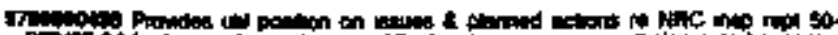

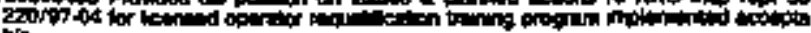

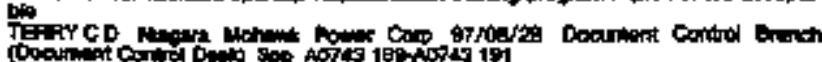

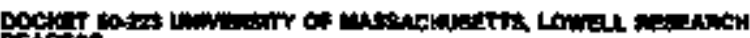
Fistor

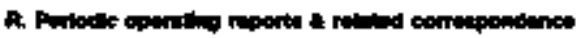

vitutiof

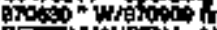

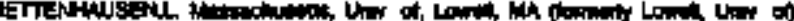

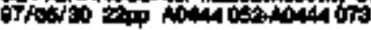

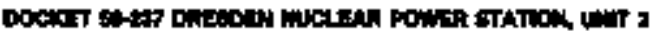

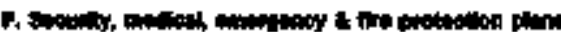

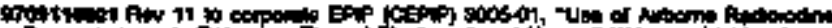

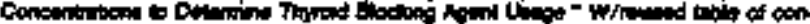

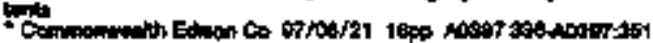

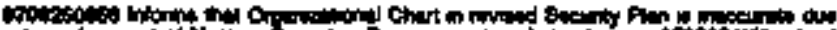

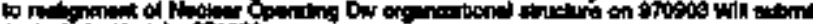

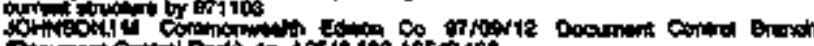

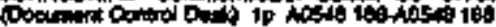

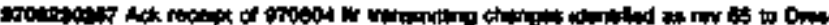

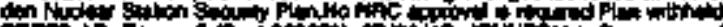
Co

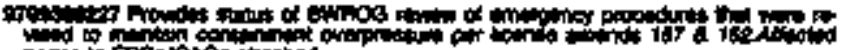

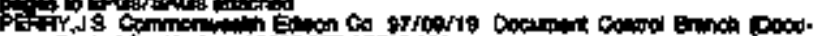

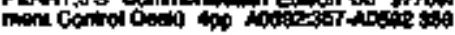

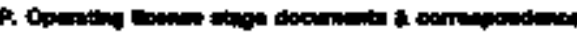

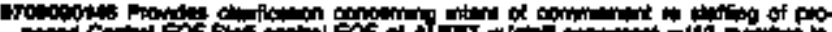

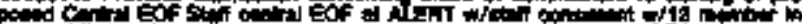

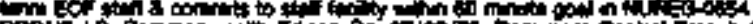

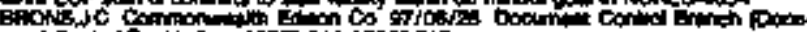

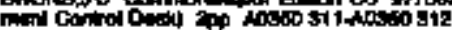

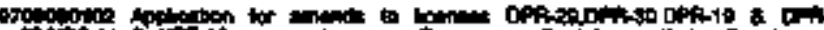

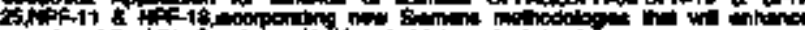

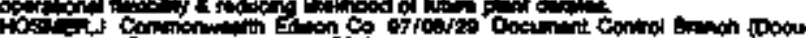

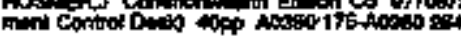

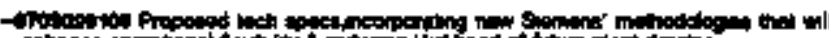

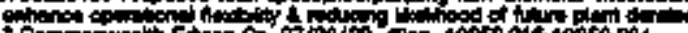

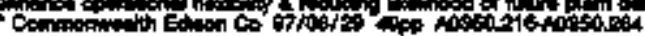

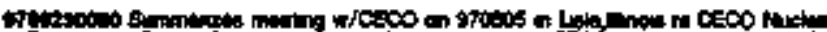

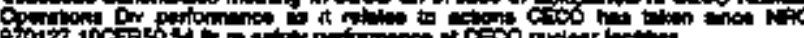
OPOT 10CF

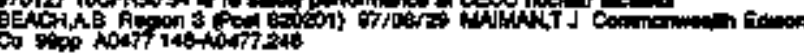

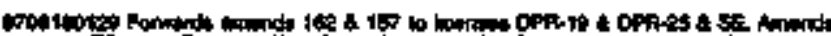

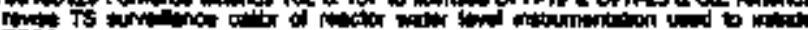
Exक

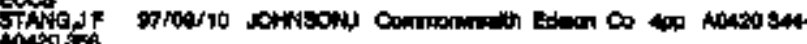

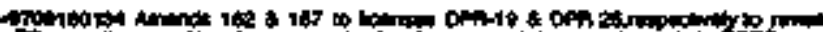

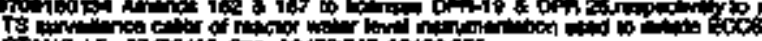

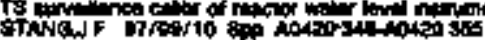

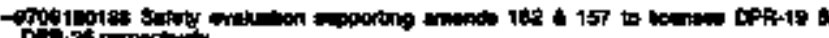

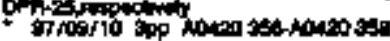

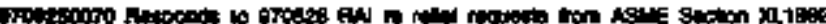

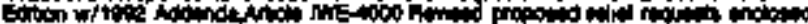

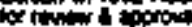

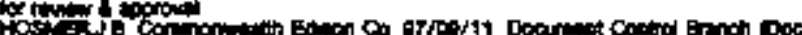

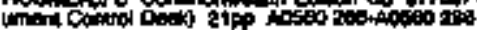

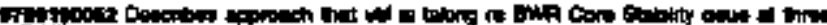

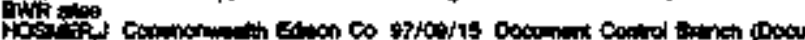

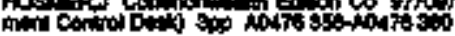

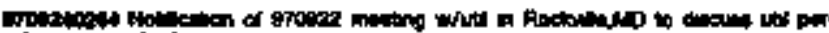

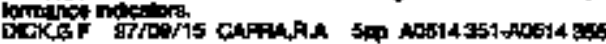

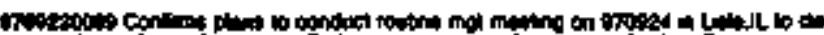

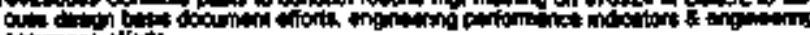

C:

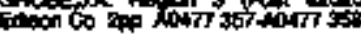

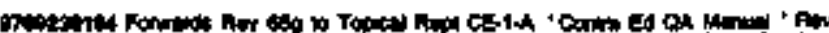

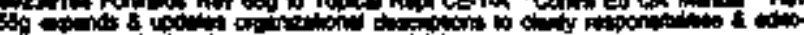

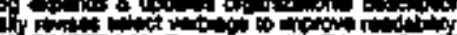

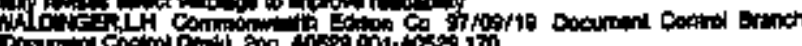




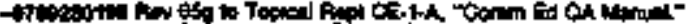

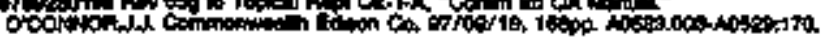

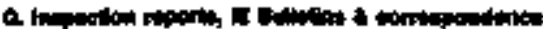

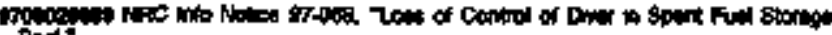
peatis Activis

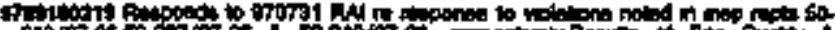

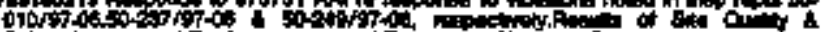

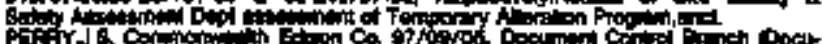

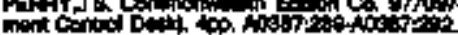

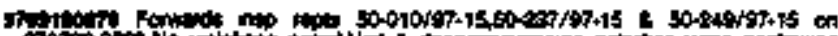

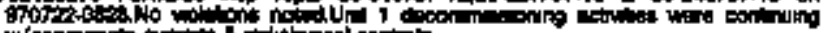

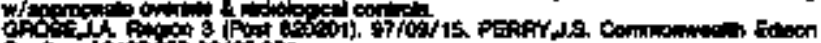

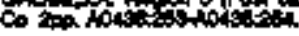

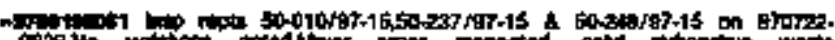

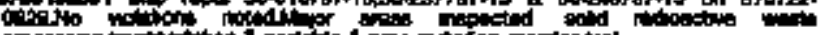

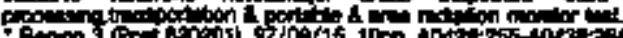

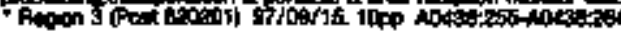

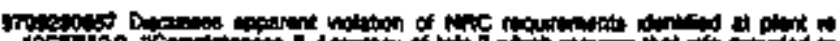

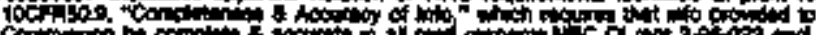

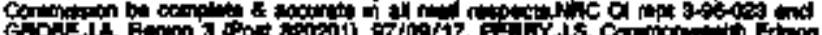

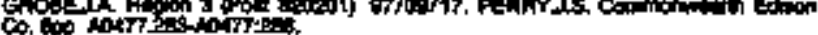

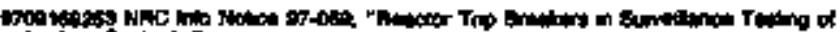

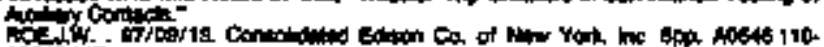
ingtis.

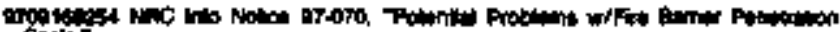

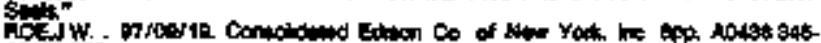
atistio

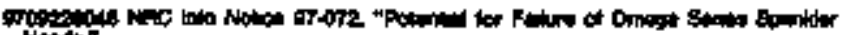

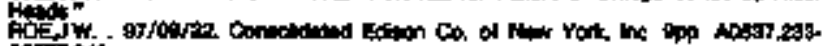

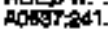

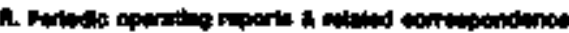

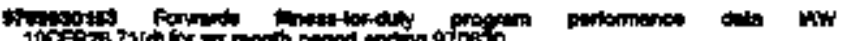

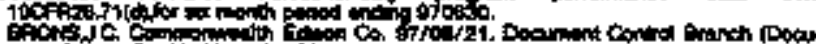

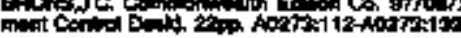

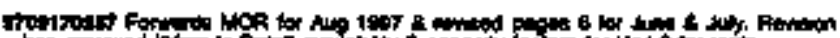

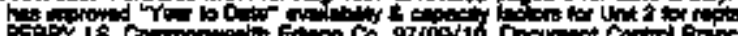

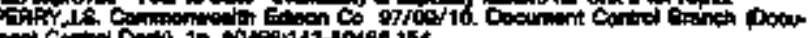

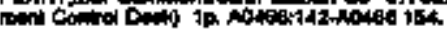

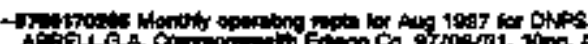

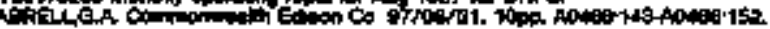

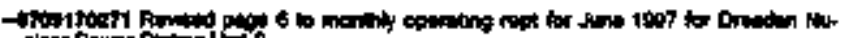

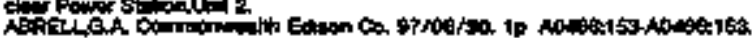

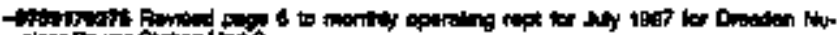

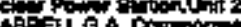

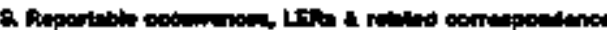

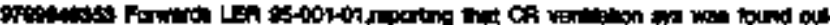

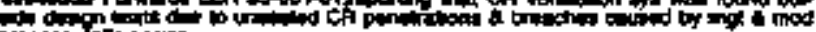

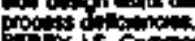

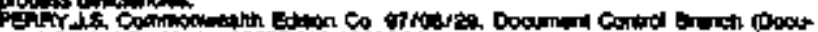

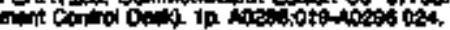

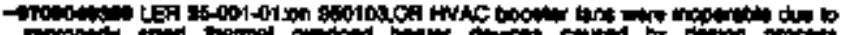

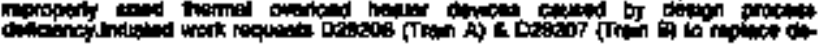

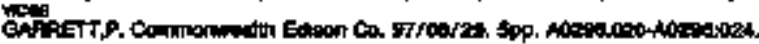

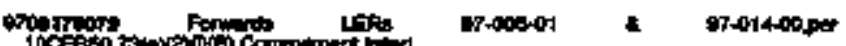
PCFif

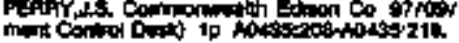

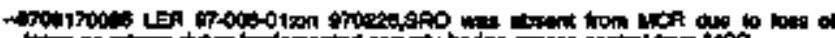

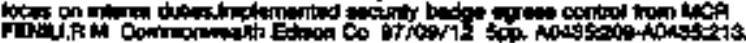

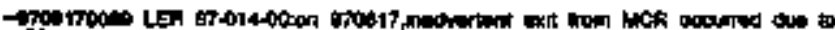

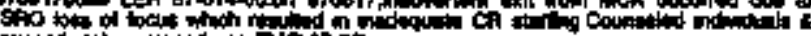

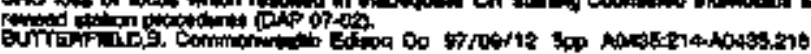

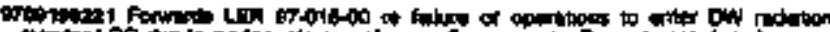

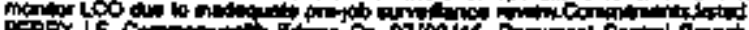

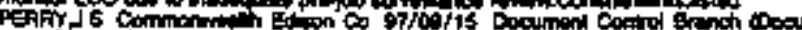

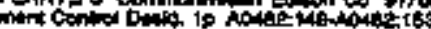

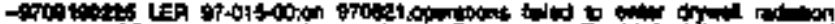

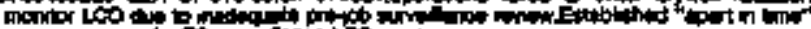

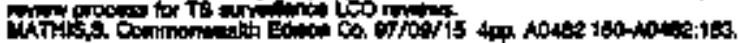

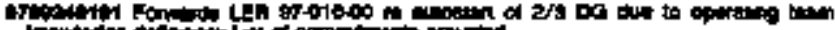

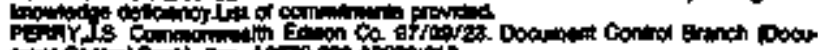
o.

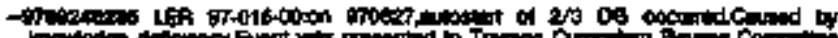

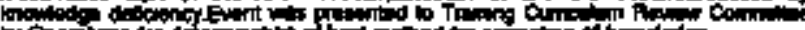

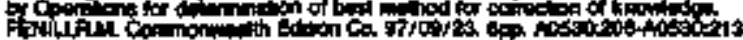

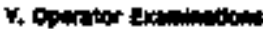

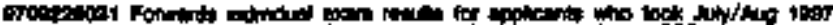

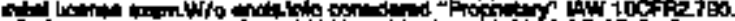

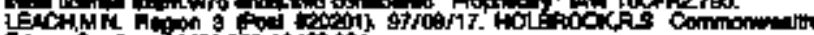

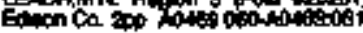

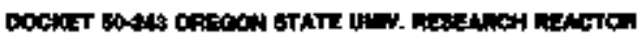

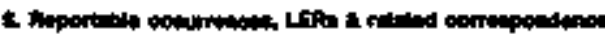

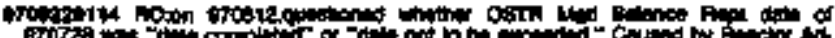

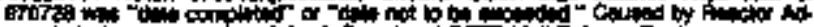
mopia

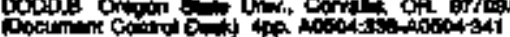

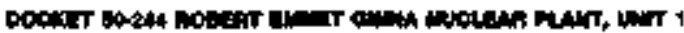

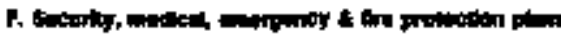

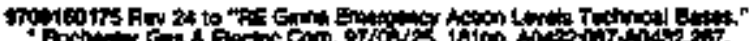

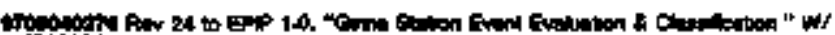

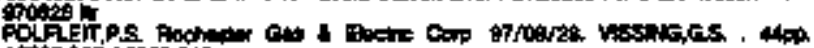
it500.002-19000045.

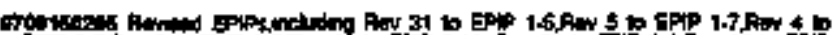

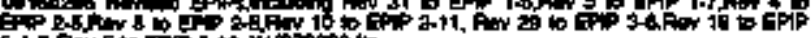
5is foy 5 w

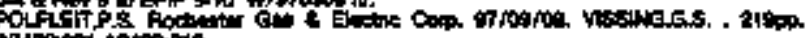

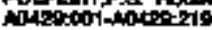

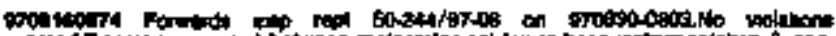

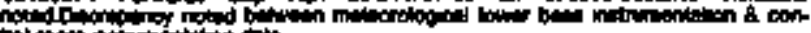

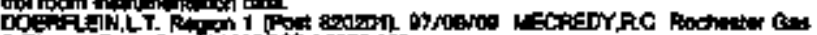
DO

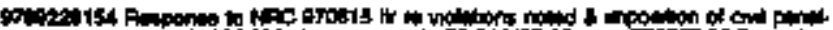

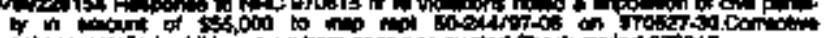

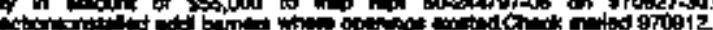
4.

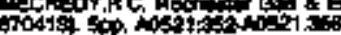

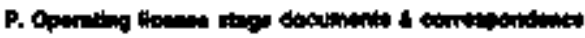

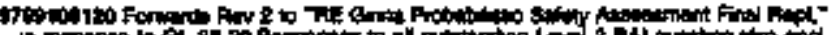

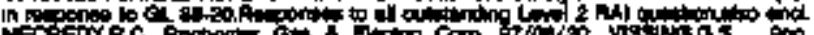

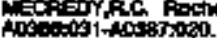

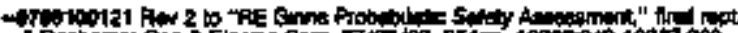

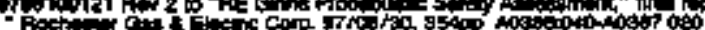

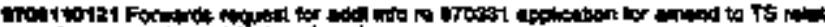

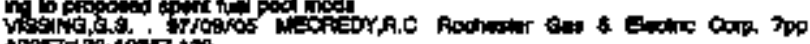

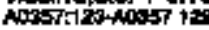

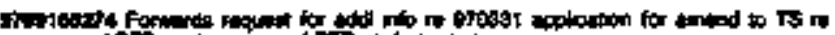

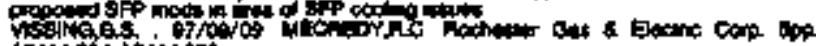

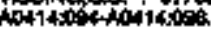

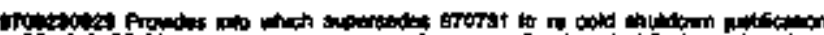

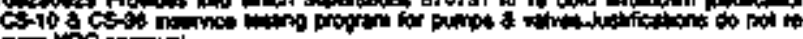
are

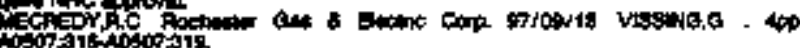

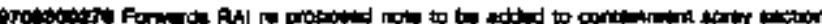

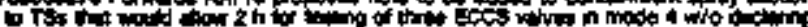

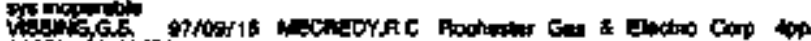

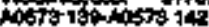

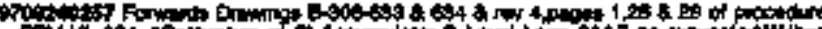

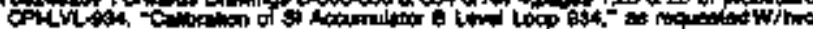

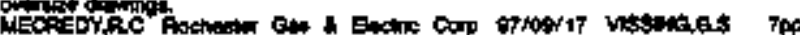

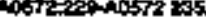




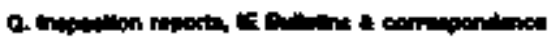

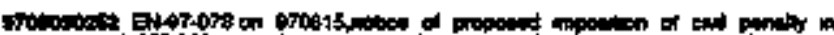

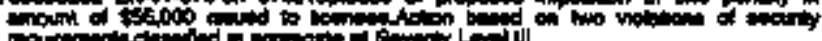

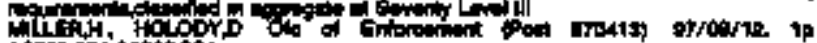

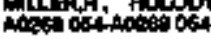

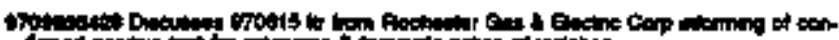

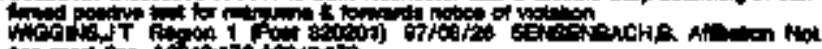

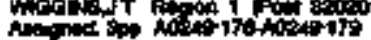

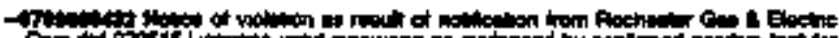

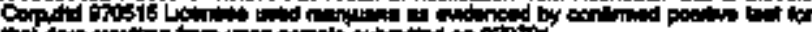

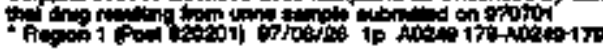

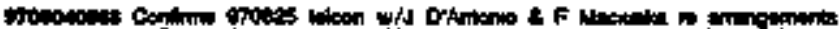

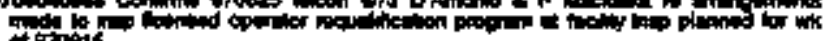

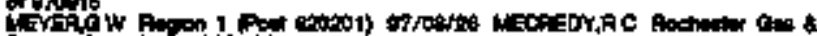

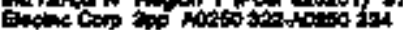

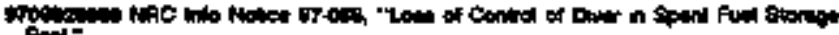
Pool -

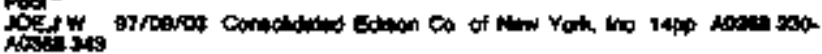

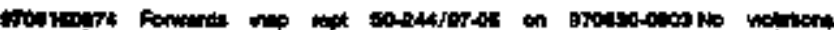

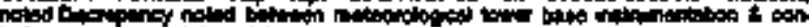

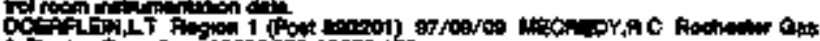
1 Evino

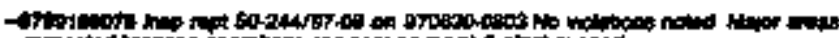

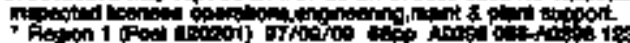

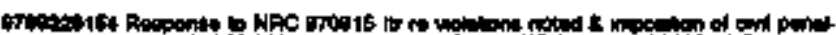

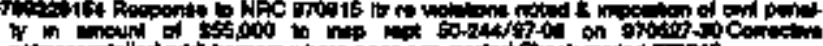

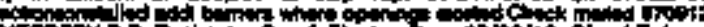

The

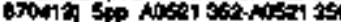

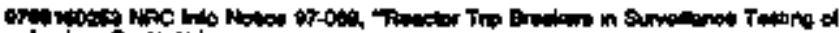

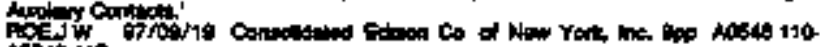

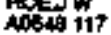

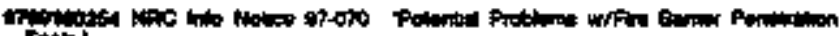

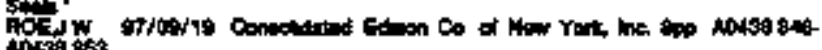

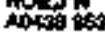

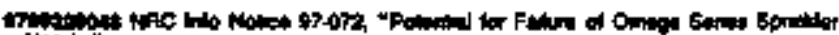

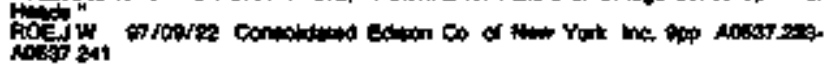

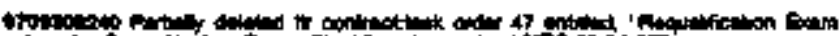

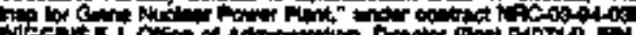

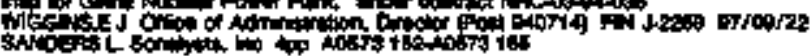

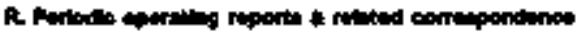

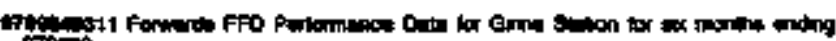
900

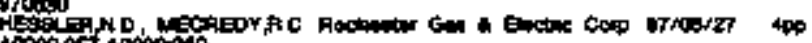
Atostation

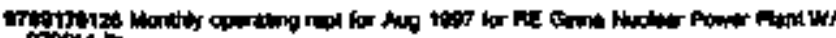

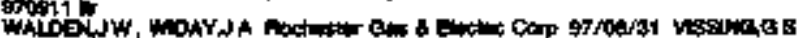

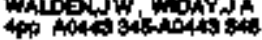

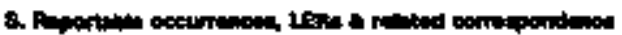

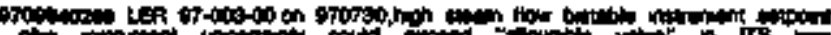

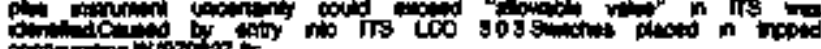

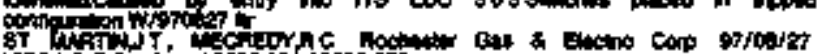

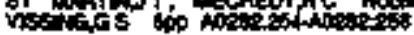

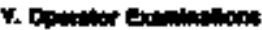

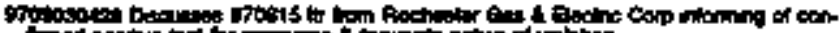

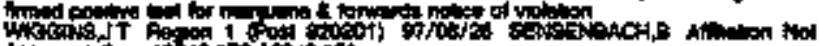

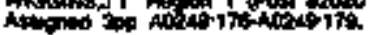

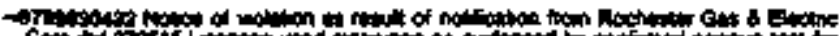

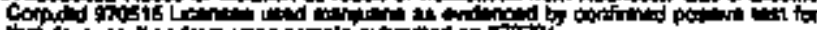

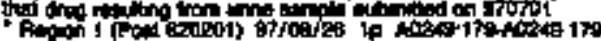

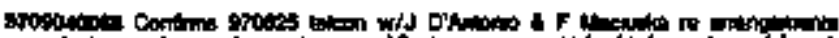

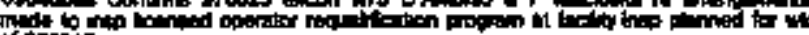
of bropts

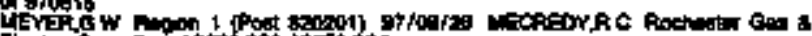

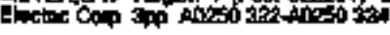

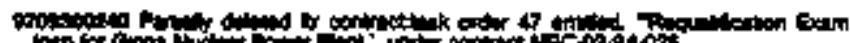

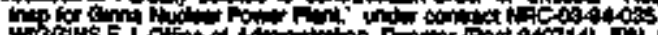
whotis

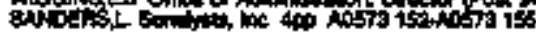

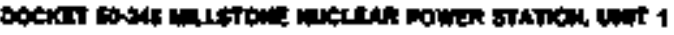

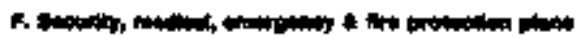

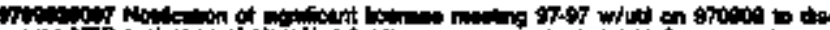

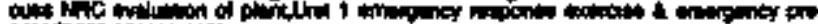

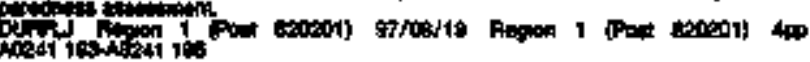

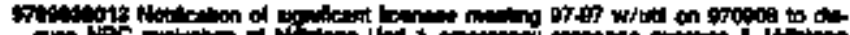

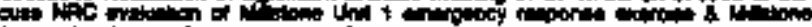

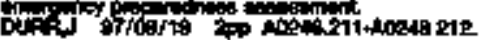

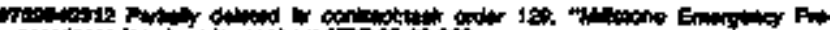

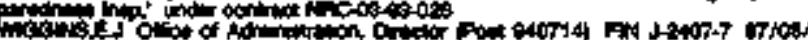

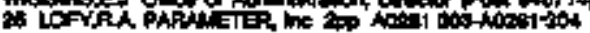

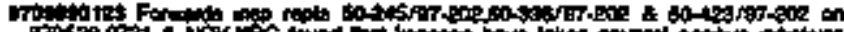

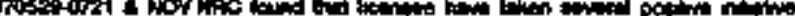

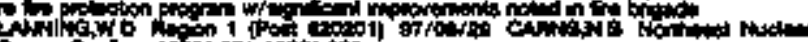

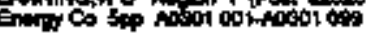

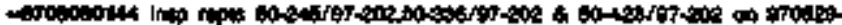

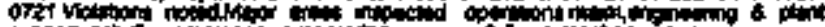

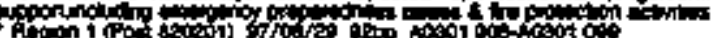

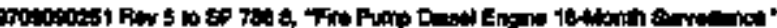

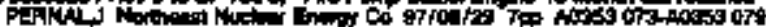

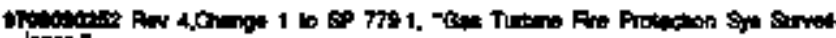

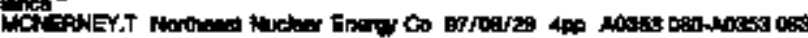

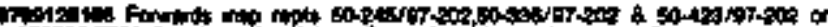

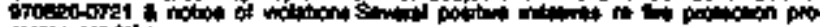

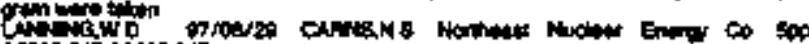

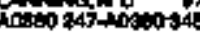

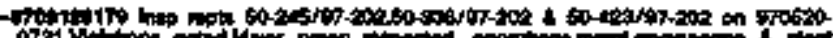
072

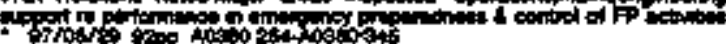

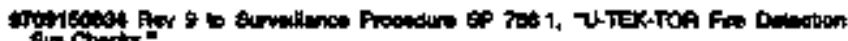

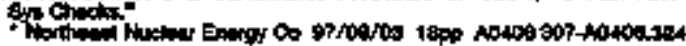

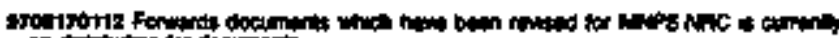

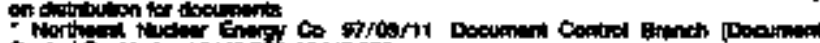

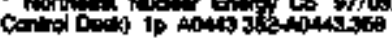

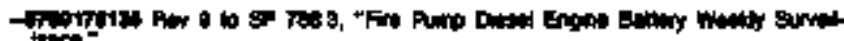

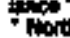

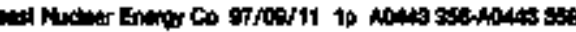

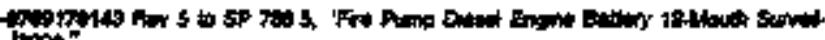

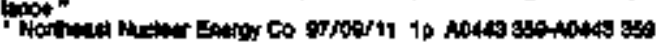

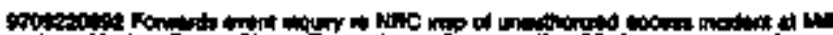

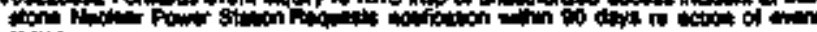

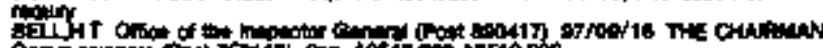

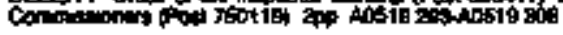

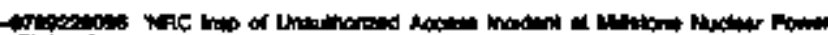

Sigen'

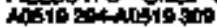

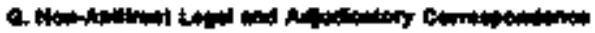

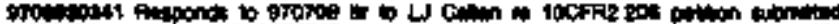

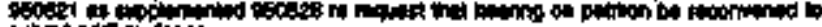

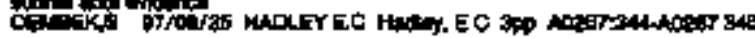

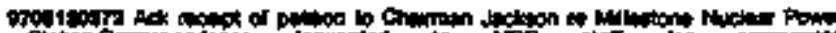

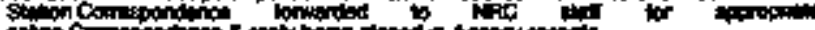

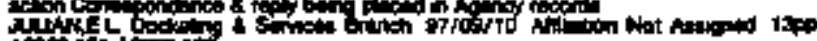
Astis is

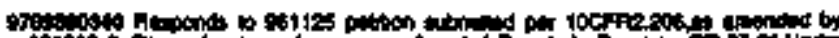

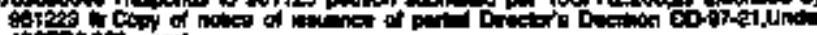

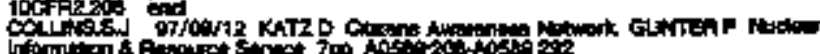

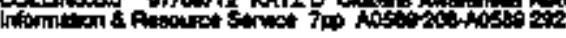




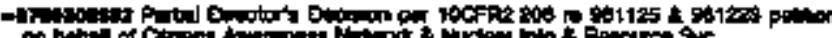

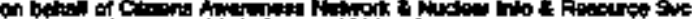

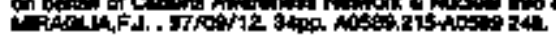

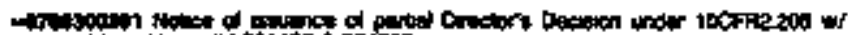

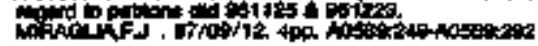

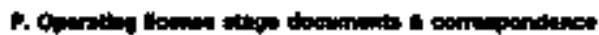

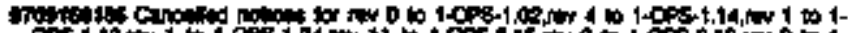

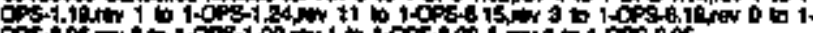

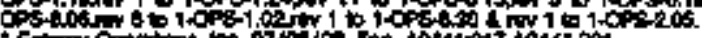

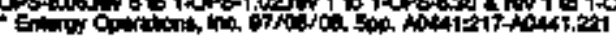

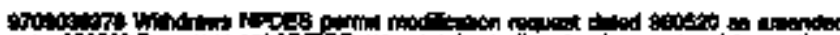

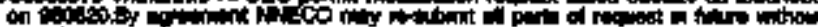

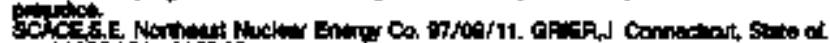
ip

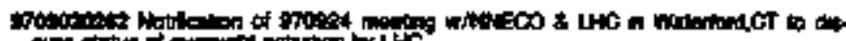

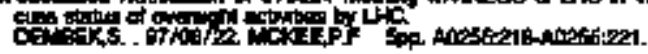

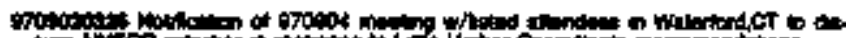

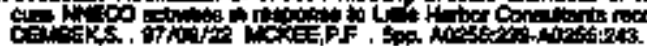

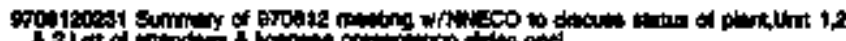

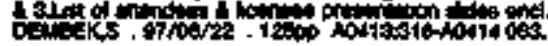

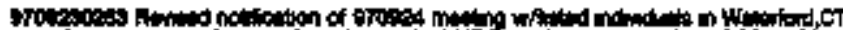
of

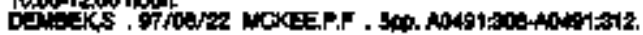

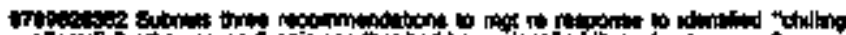

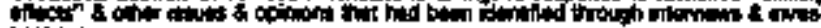

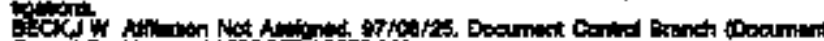

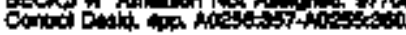

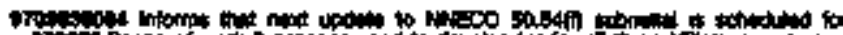

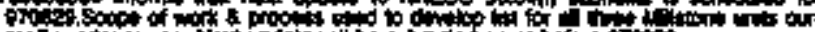

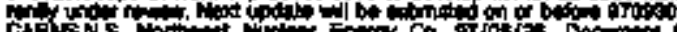

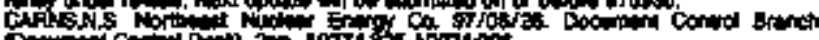

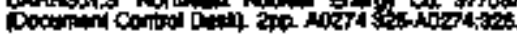

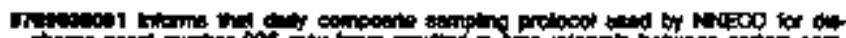

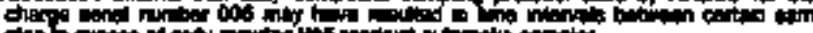

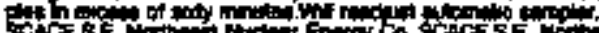

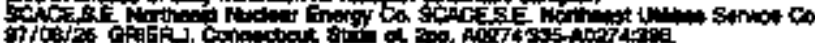

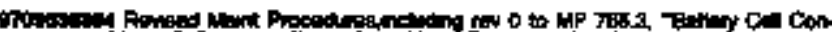

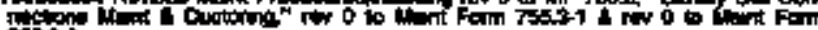
?t:

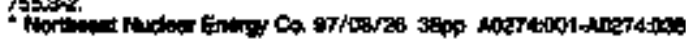

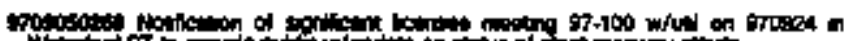

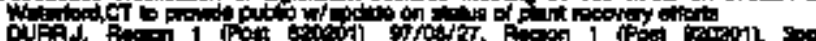

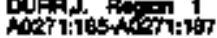

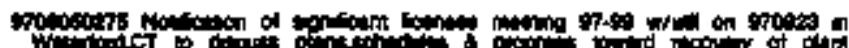
of

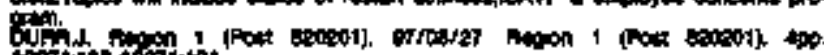

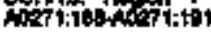

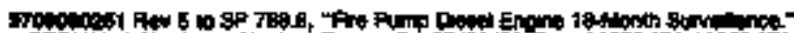

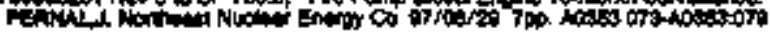

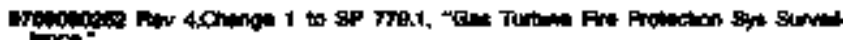

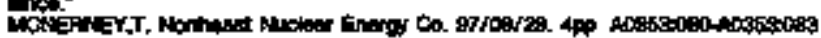

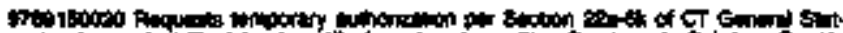

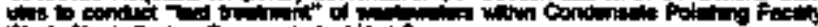

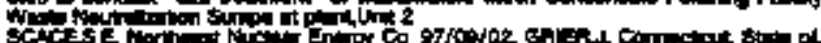
app. At

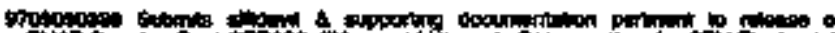

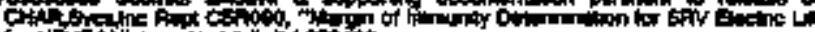

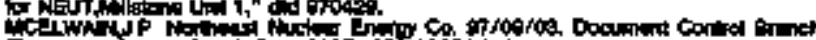

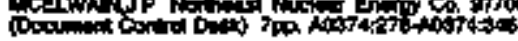

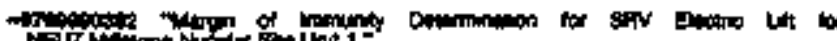
of

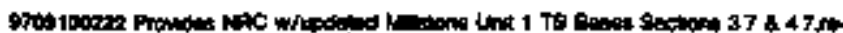

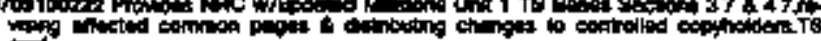

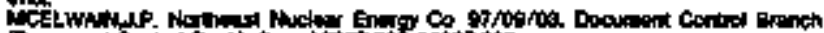

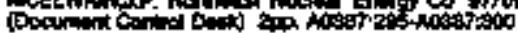

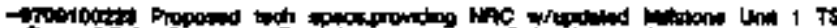

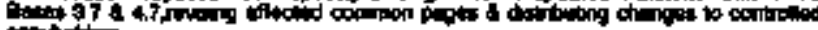

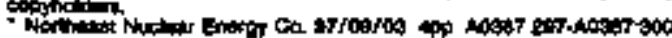

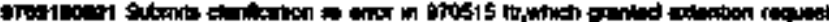

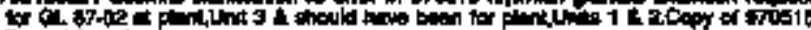

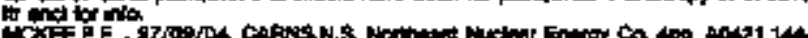
Notation.

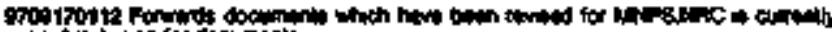

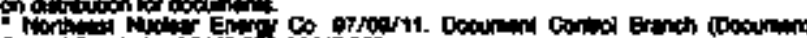

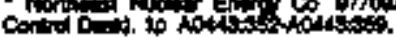

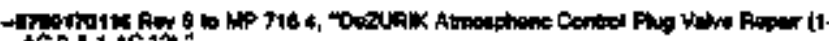

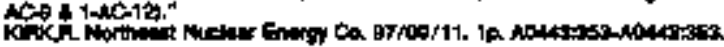

H H

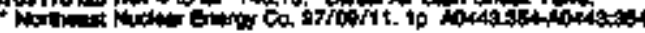

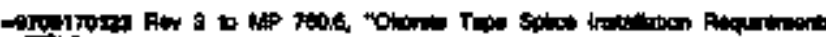

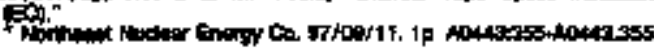

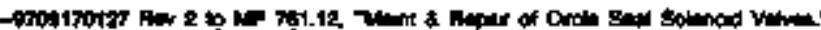

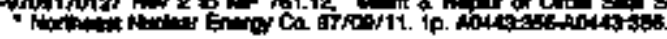

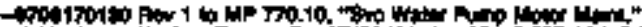

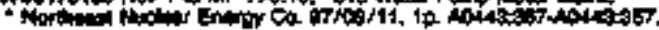

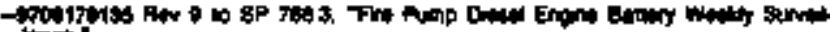

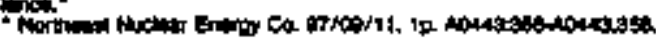

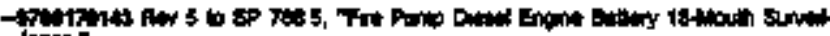

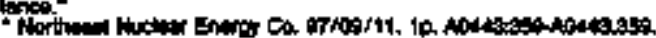

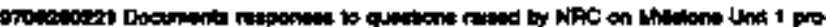

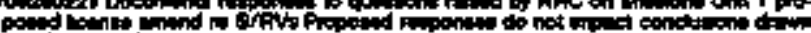

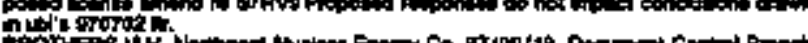

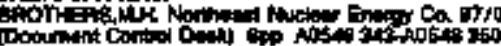

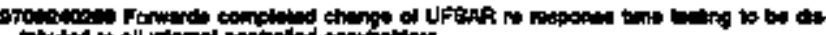

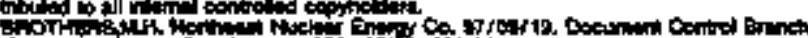

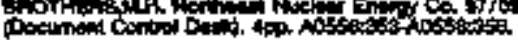

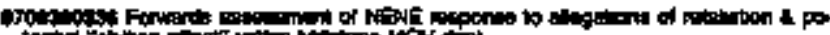

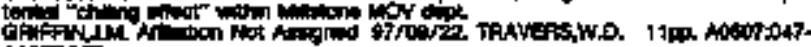
antions

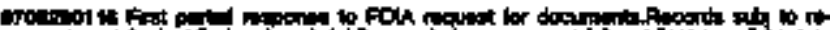

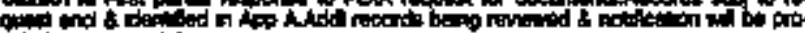

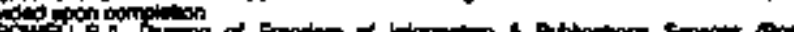

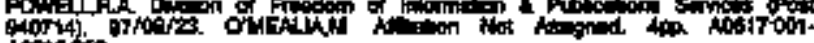
Montasse

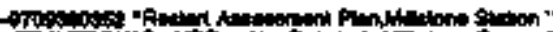

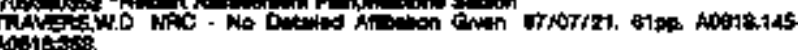

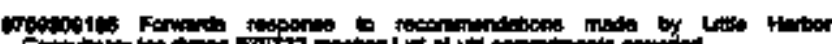

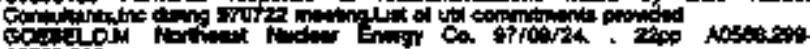

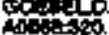

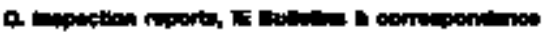

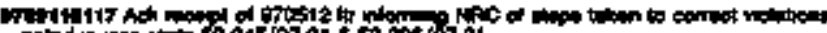
notid in mep phit 60-24

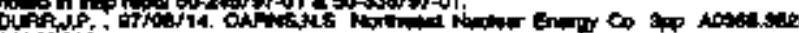
Aoctestis.

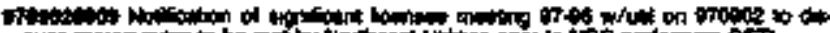

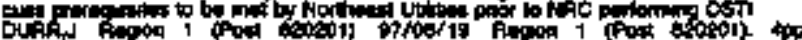

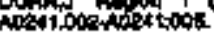

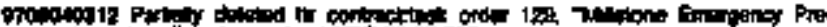

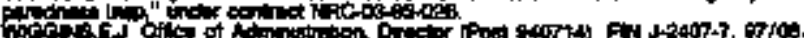

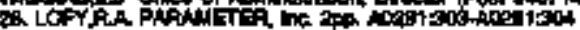

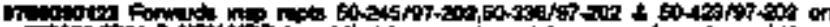

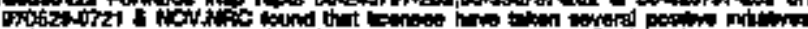

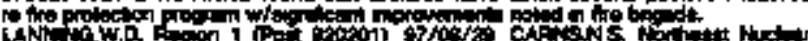
inniving,

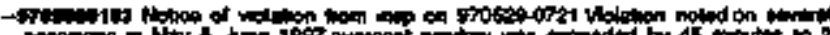

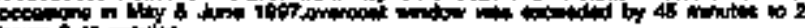
nols of th

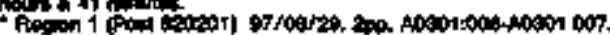

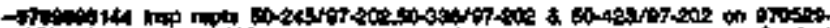

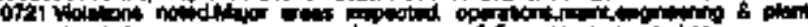

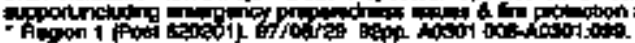




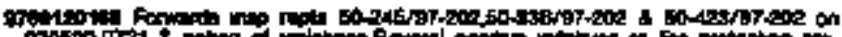

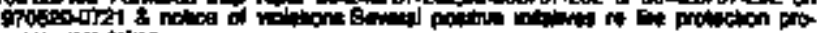

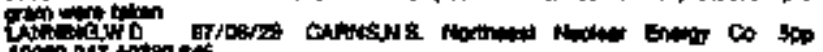

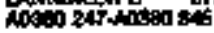

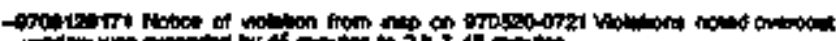

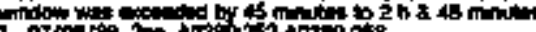

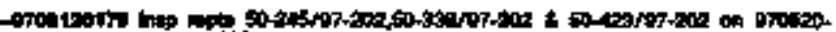

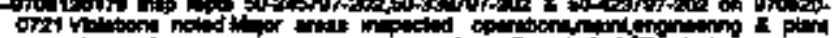

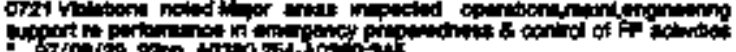

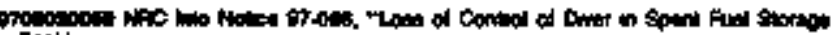

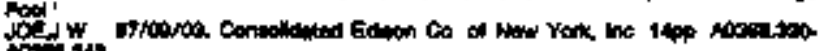

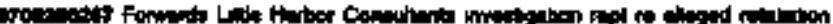

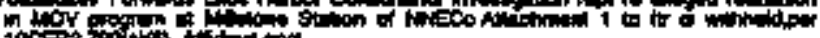

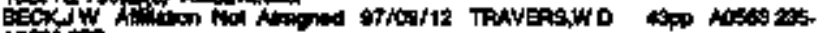
Antorit

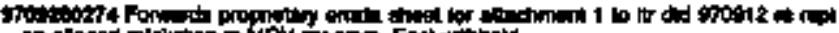

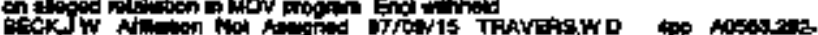
Noses

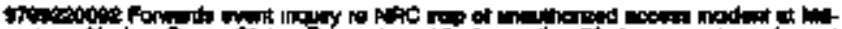

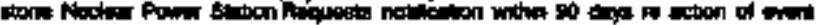

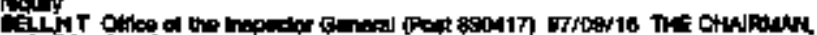

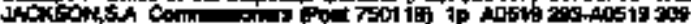

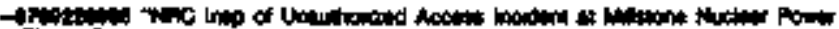

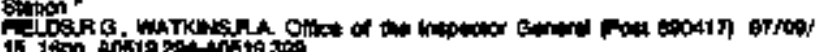

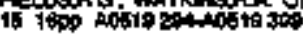

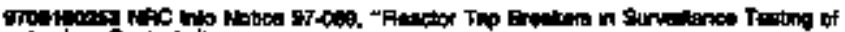
fos

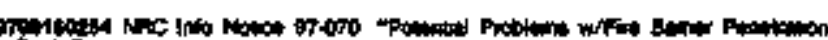

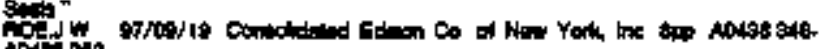
Aptosido

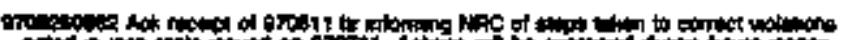

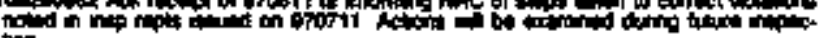

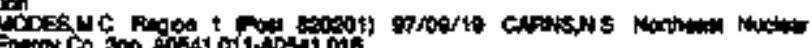

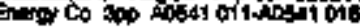

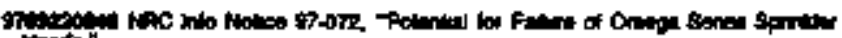

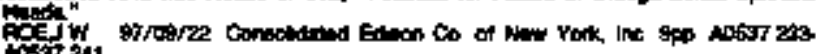

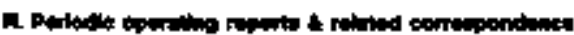

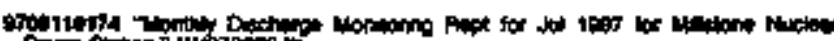

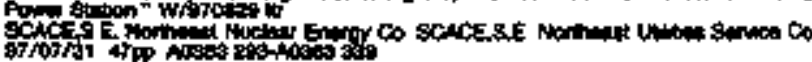

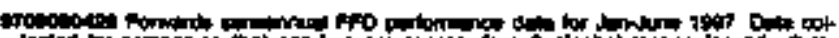

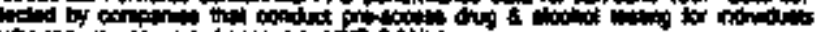

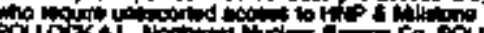

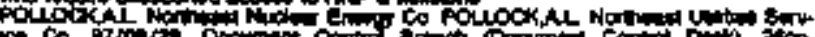

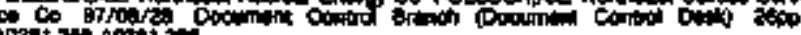

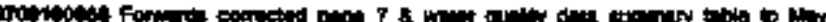

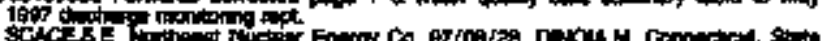
action

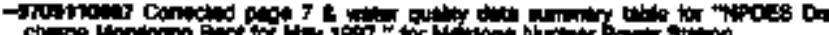

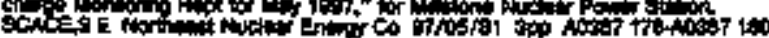

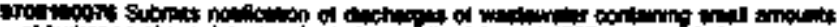

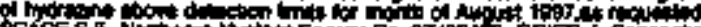

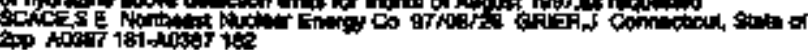

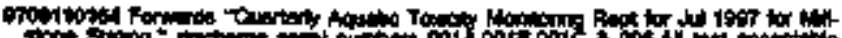

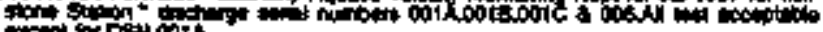

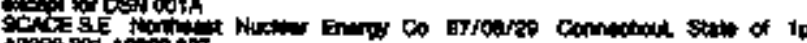
Nosos sor

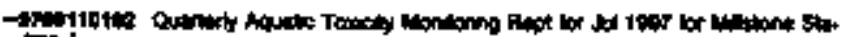

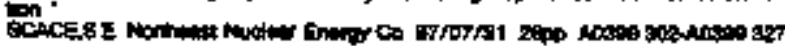

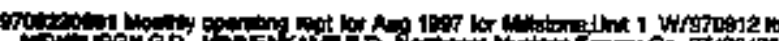

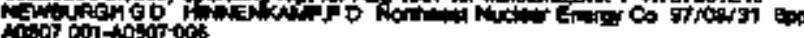

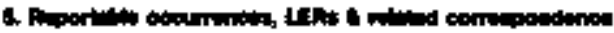

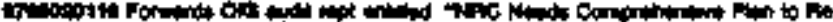

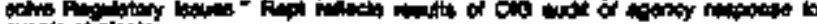

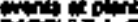

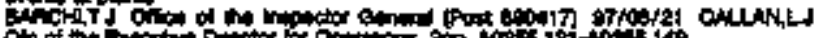

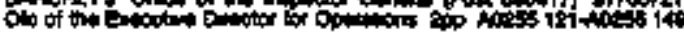

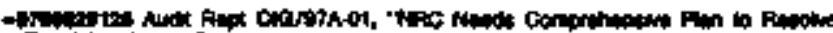

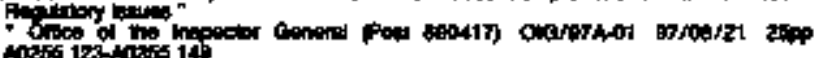

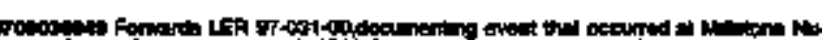

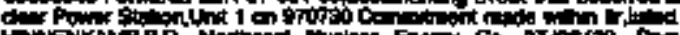

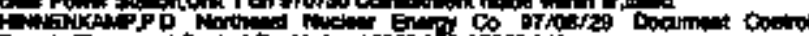

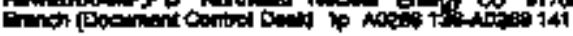

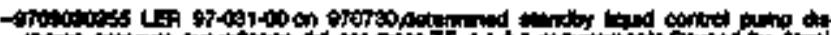

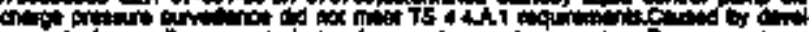

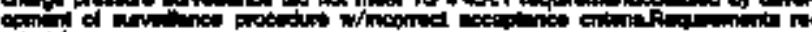
interd

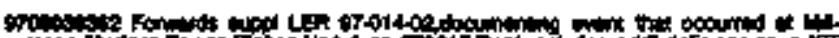

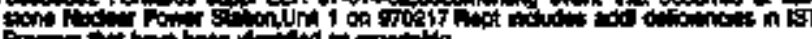

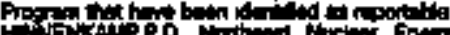

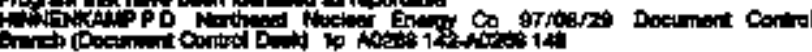

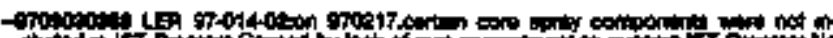

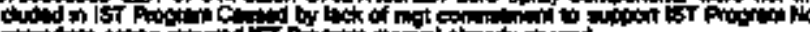

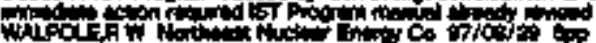

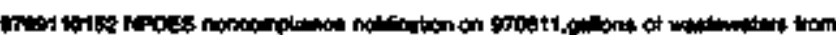

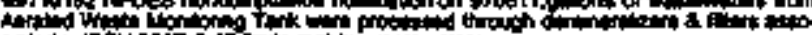

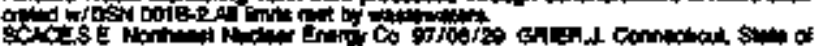

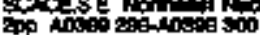

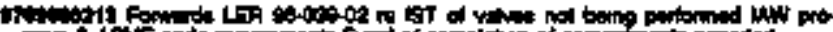

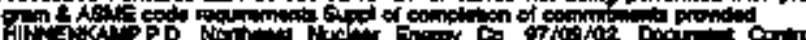

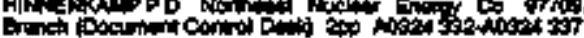

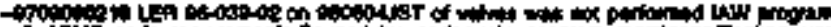

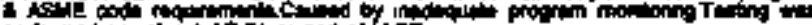

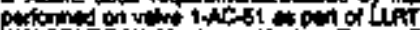

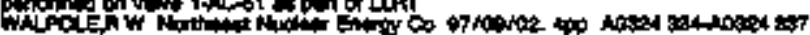

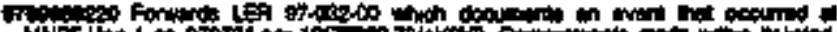

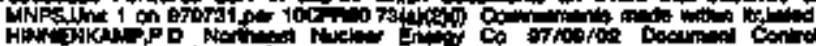

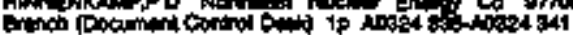

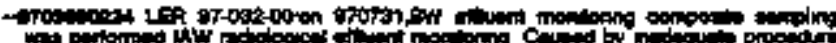

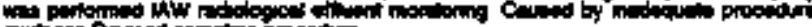

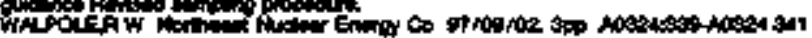

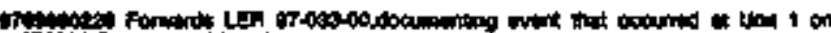

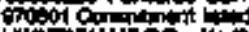

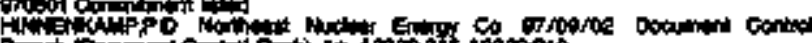

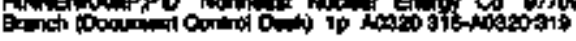

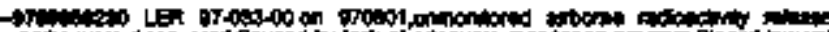

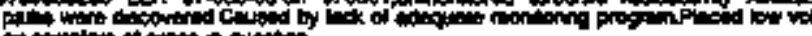

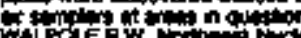

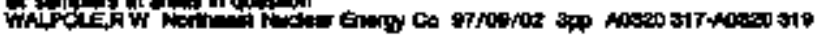

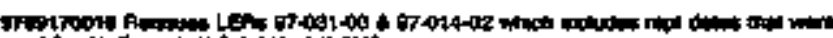

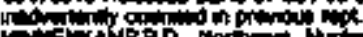

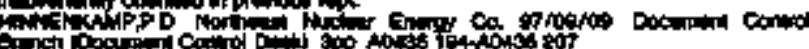

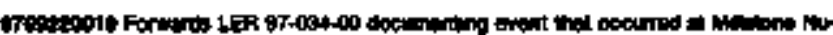

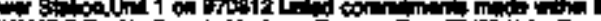

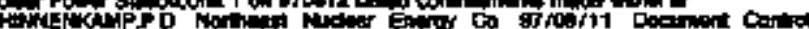

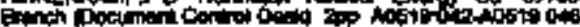

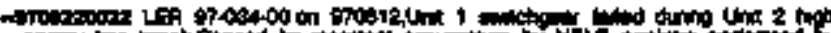

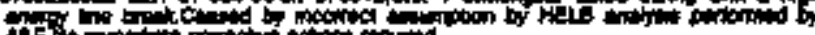

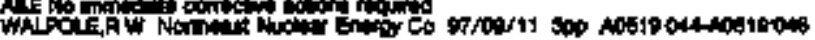

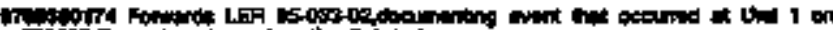

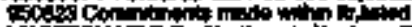

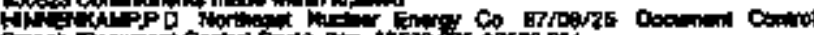

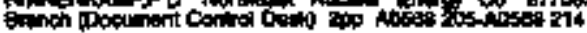

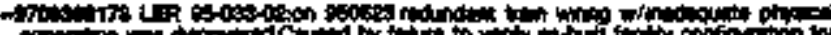

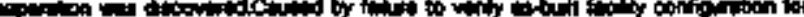

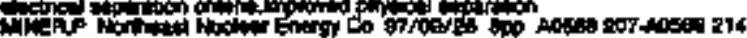

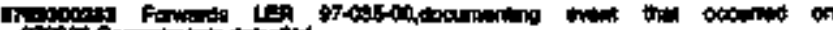
HWN

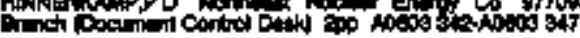

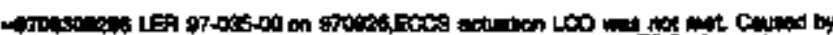

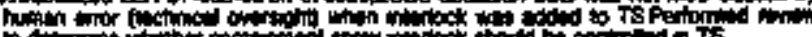

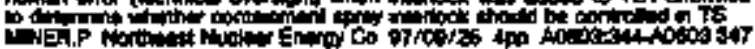




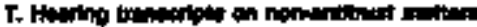

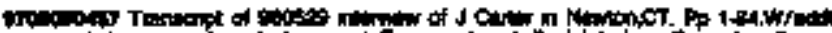

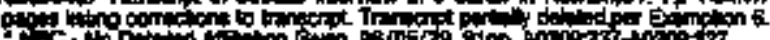

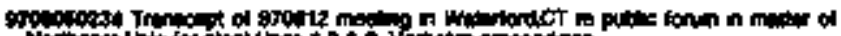

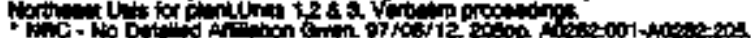

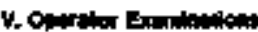

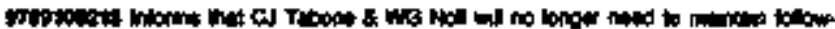

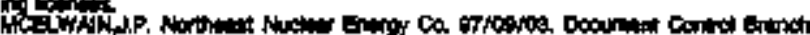

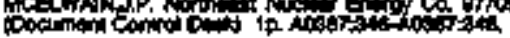

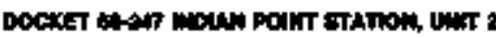

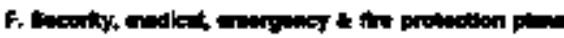

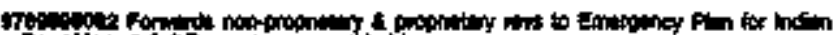

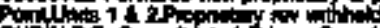

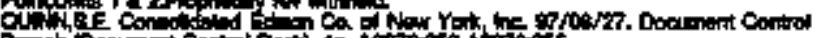

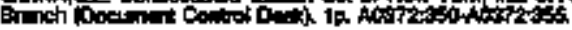

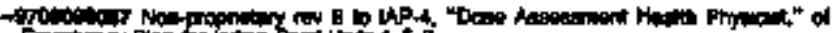

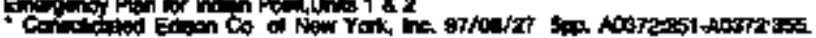

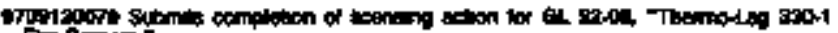
The curit:

OUWN

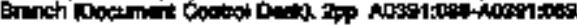

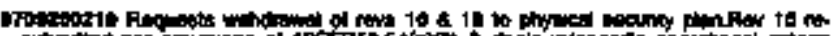

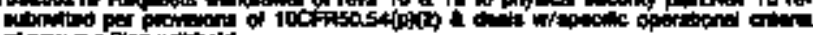

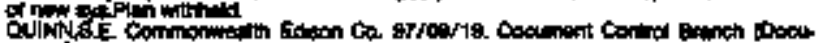

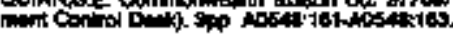

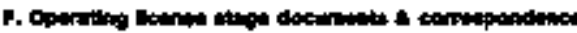

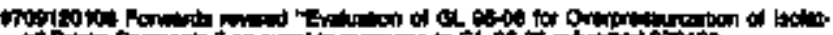
a P

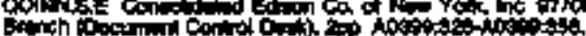

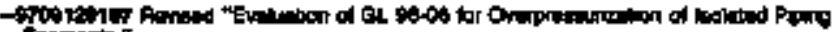

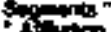

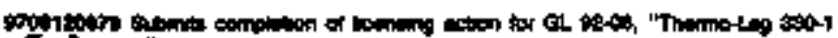

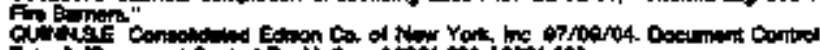

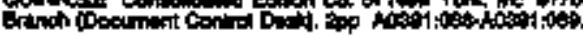

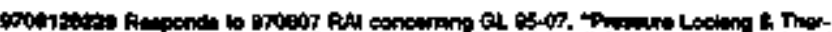

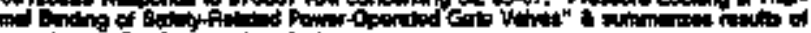

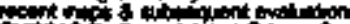

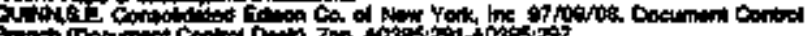

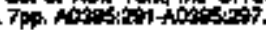

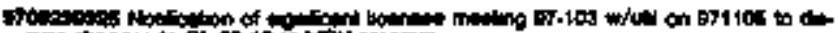

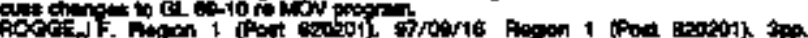

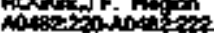

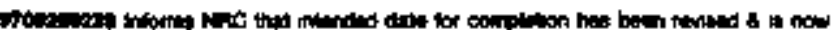

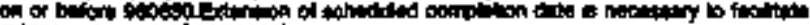

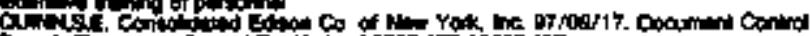

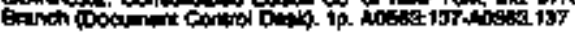

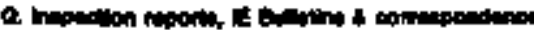

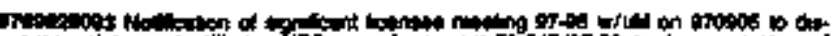

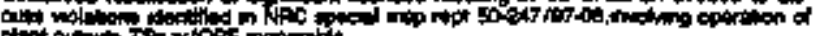

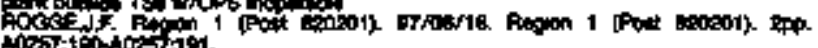

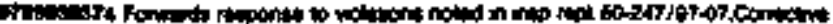

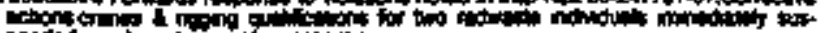

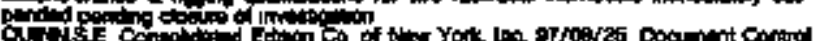

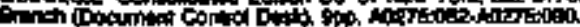

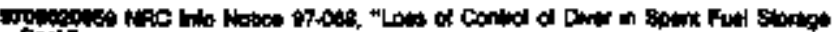

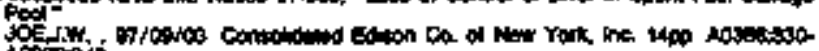
tos: 340

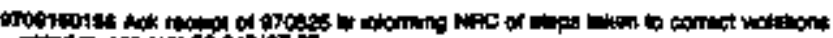

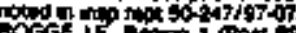

कo

-

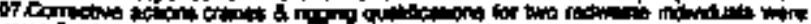
thine

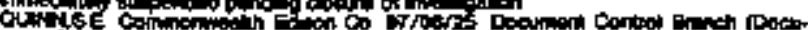

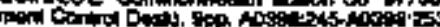

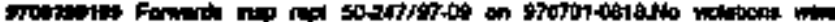

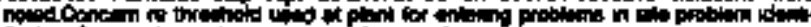

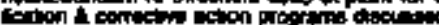

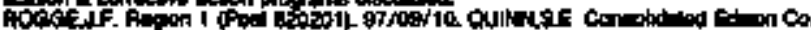
of

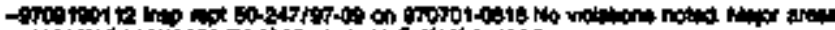

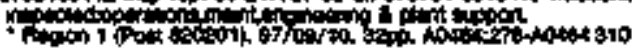

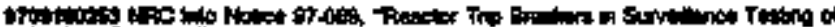

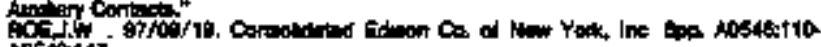

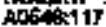

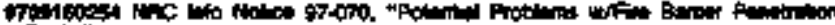

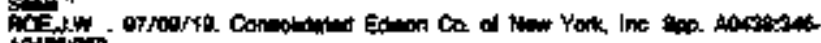
notion

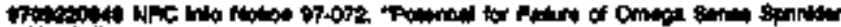

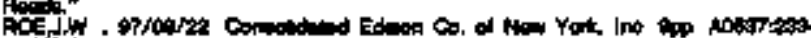
Aosititit.

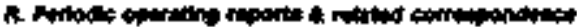

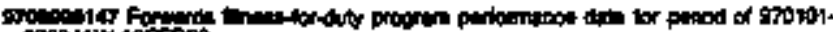

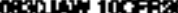

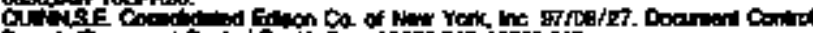

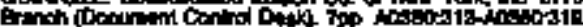

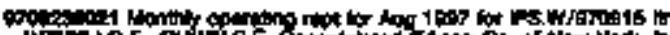

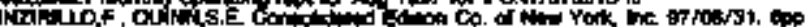

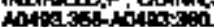

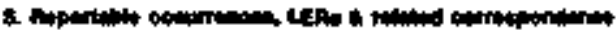

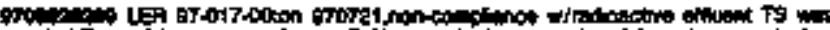

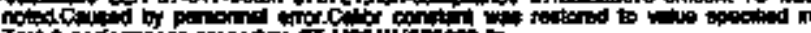

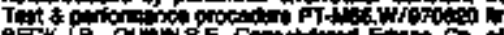

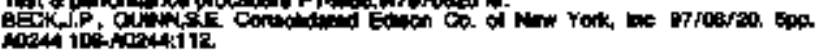

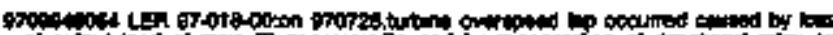

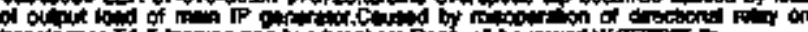

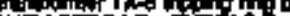

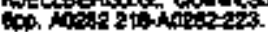

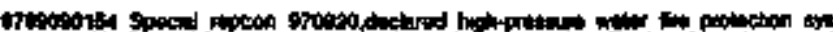

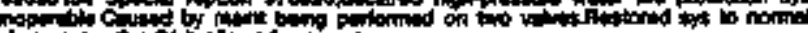

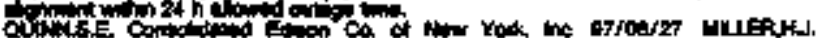

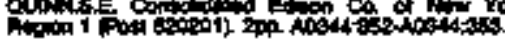

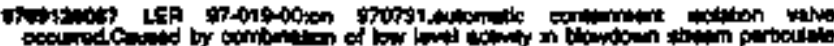

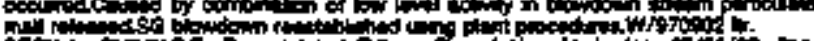

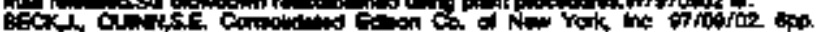
now0s

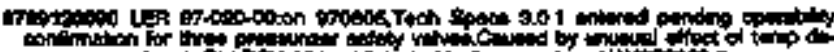

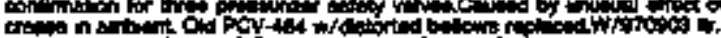

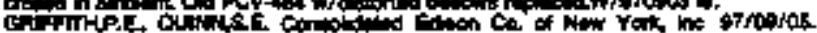

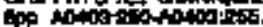

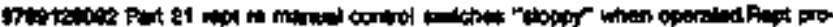

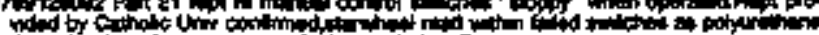

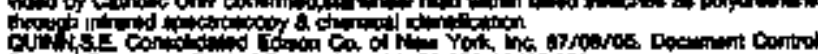

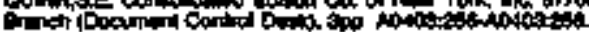

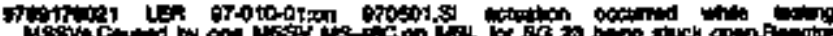

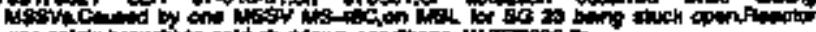

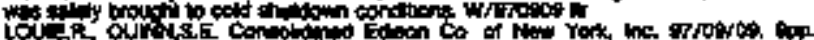

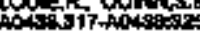

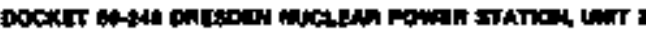

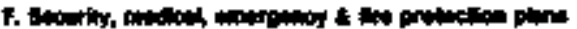

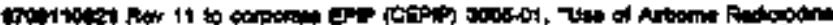

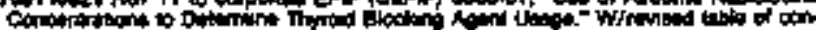

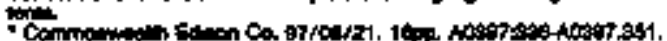

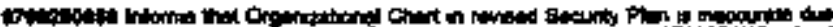

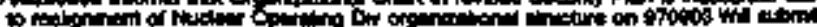

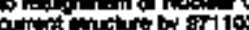

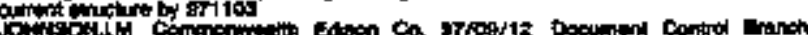

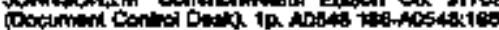

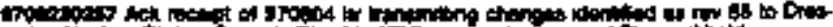

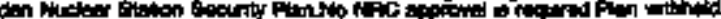

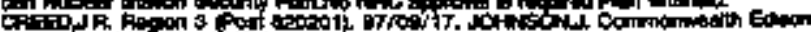

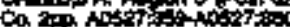




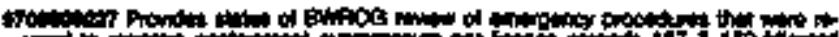

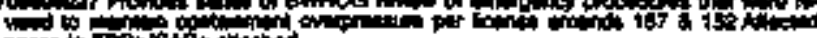

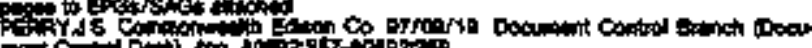

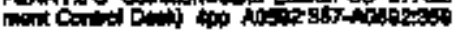

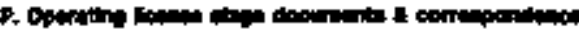

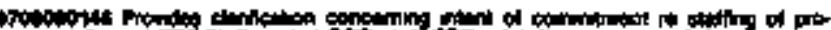

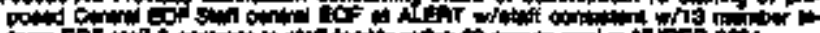

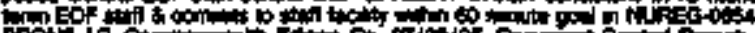

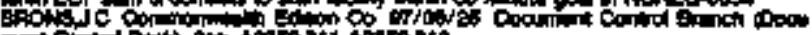

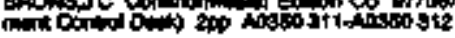

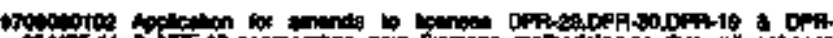

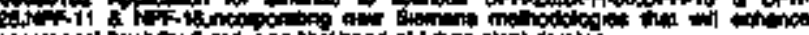

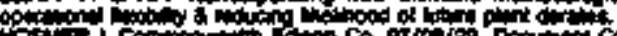

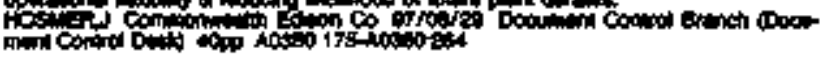

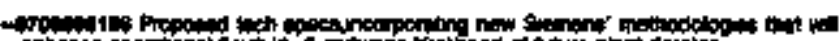

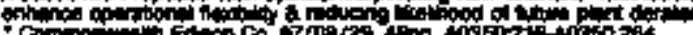

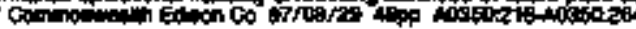

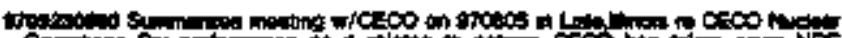

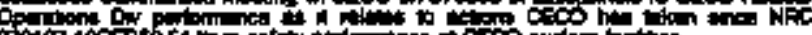

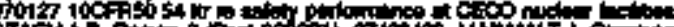

Mathon

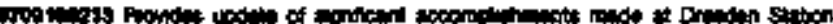

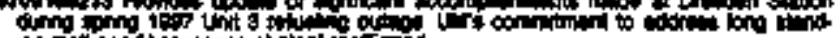

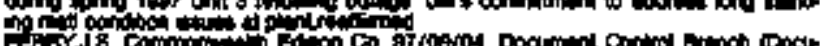

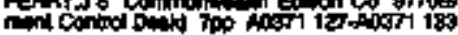

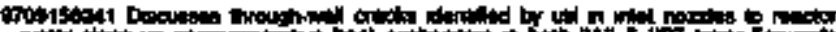

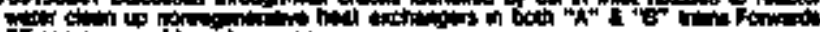

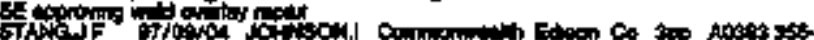
Hosto

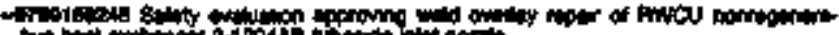

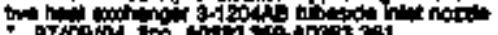

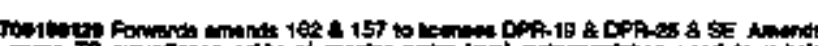

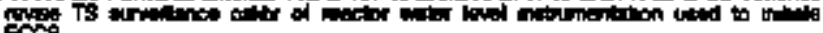

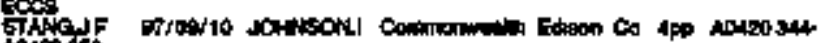
M40038

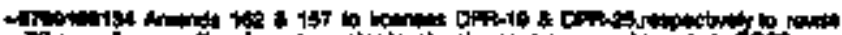
If

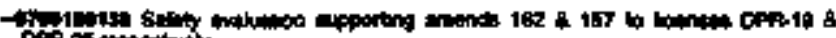

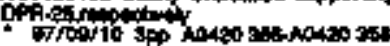

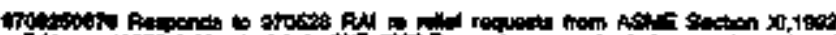

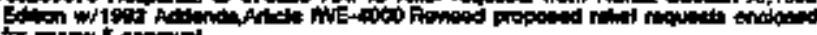
tonction

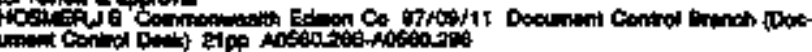

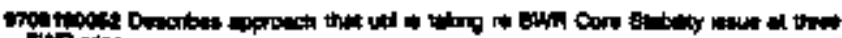

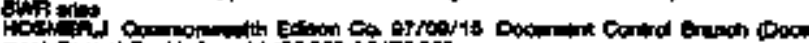

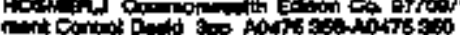

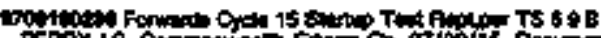

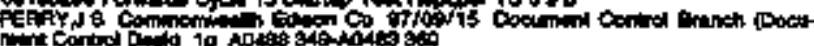

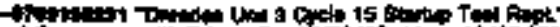

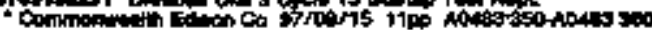

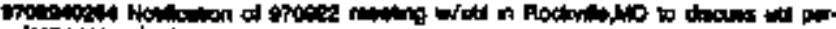

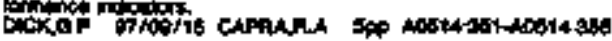

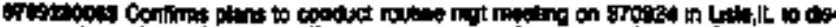

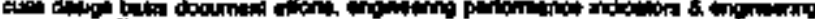

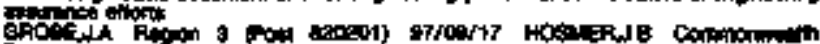

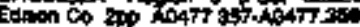

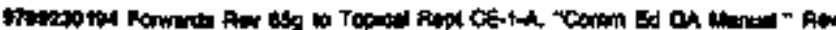

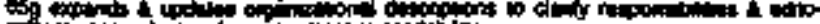

WA Dive

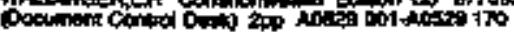

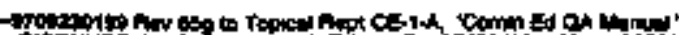

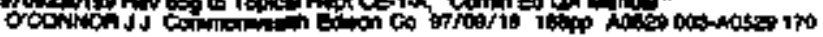

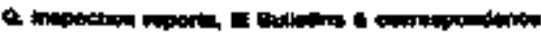

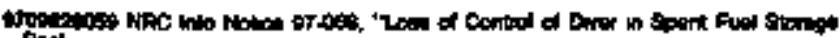

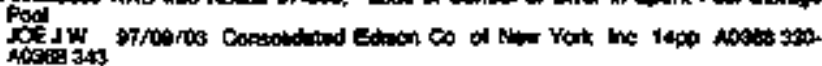

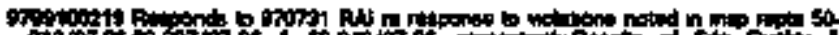

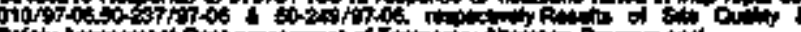

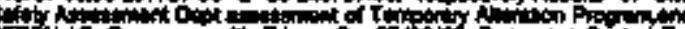

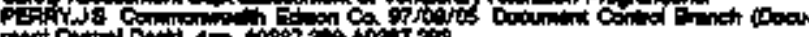

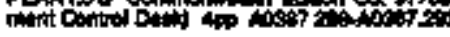

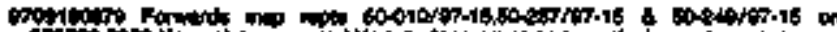

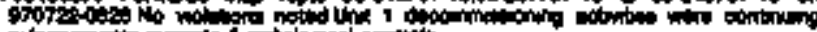

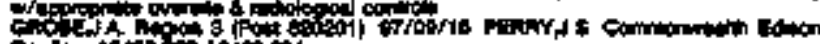

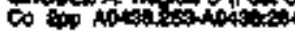

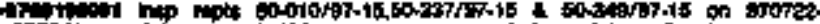

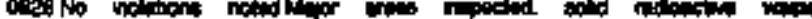

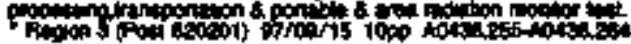

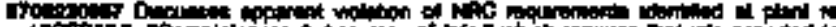

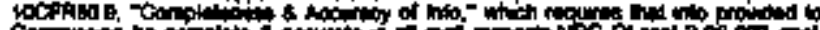

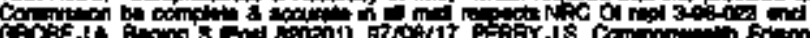

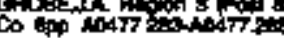

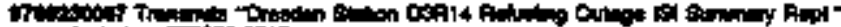

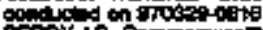

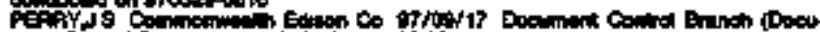

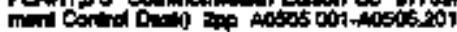

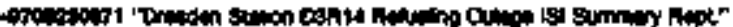

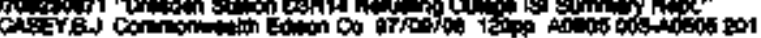

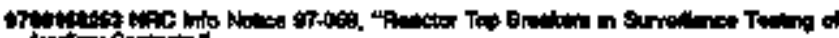

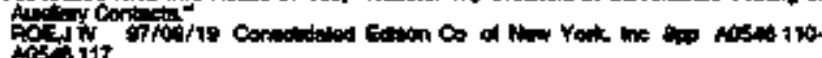

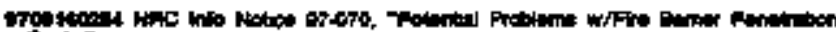

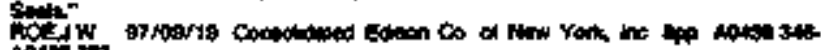
antin

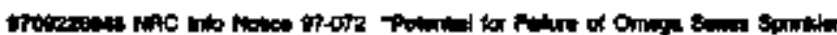

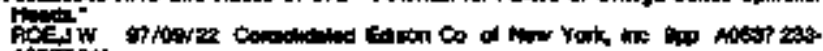
Nossinat

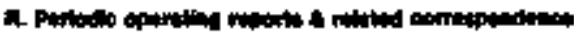

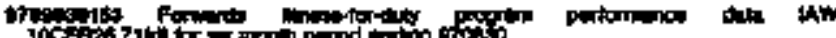

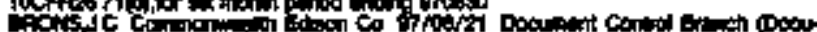
ment of

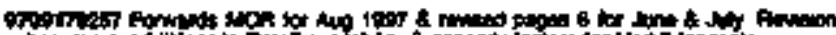

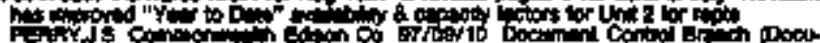

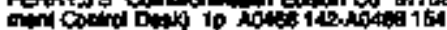

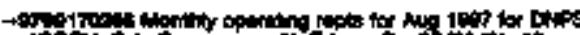

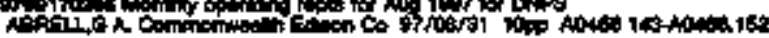

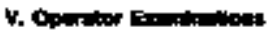

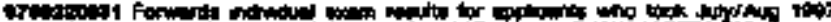

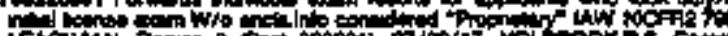

tshcitis Eath $C_{0}$ an

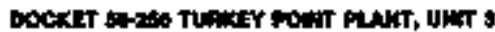

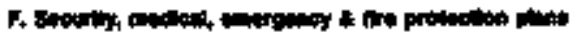

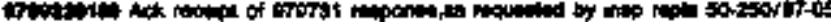

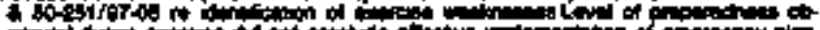

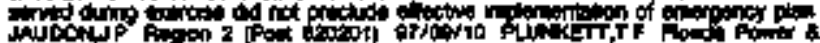

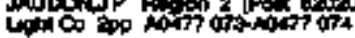

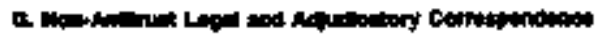

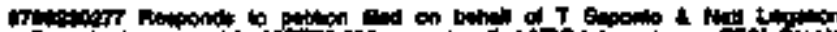

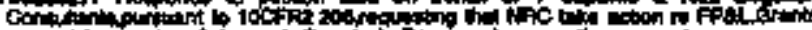

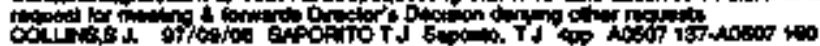

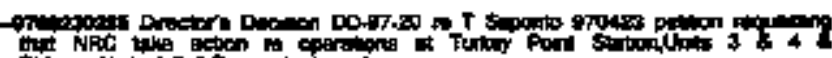

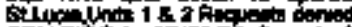

CoUf

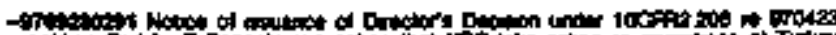

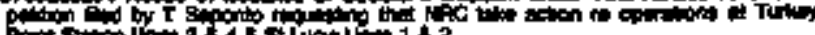

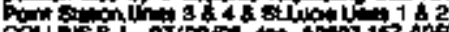

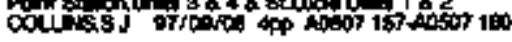




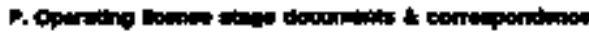

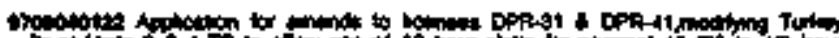

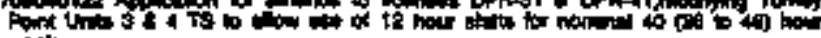

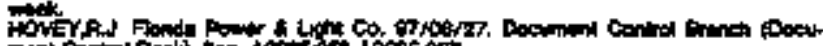

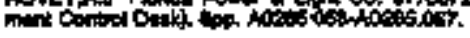

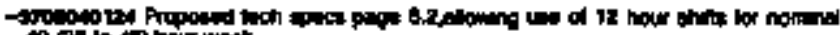

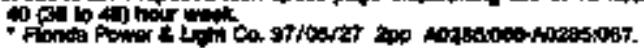

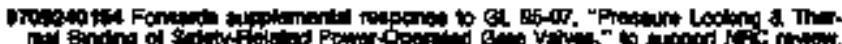

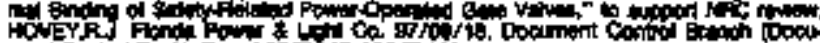

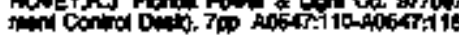

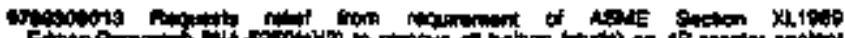

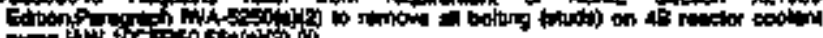
Pand

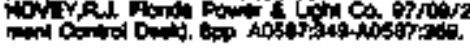

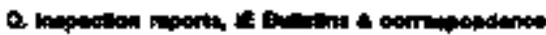

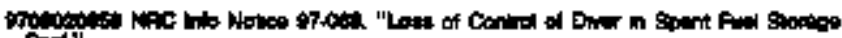

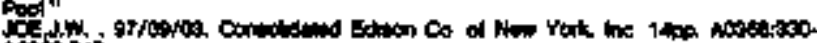

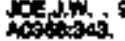

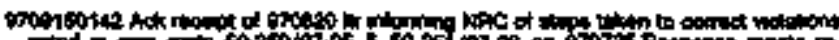

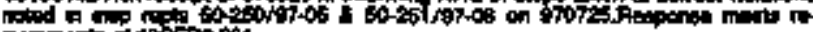

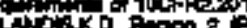

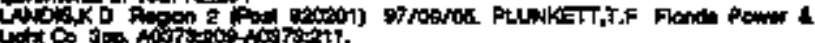

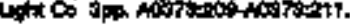

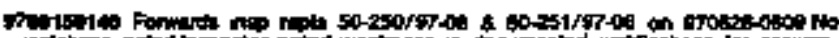

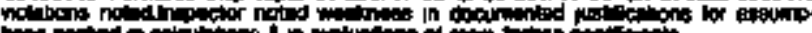

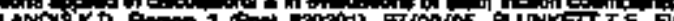
Freata Pown

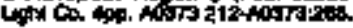

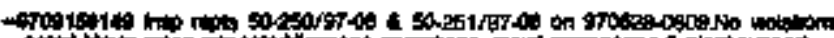

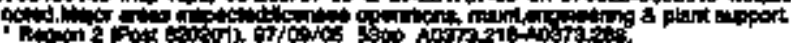

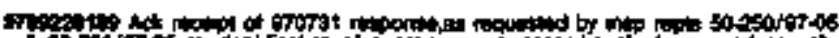

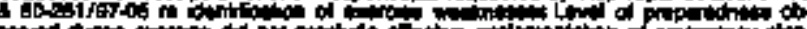

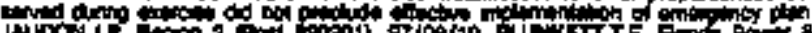

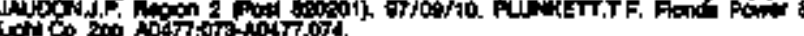
und

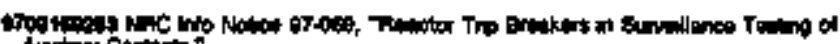

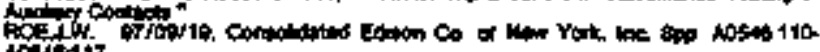
A. 1917

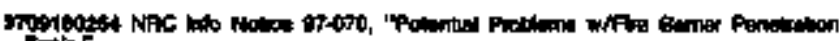

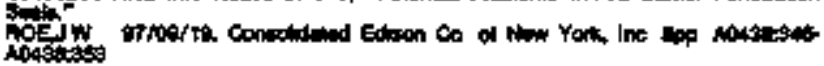

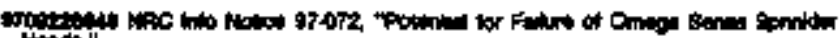

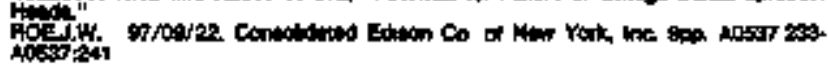

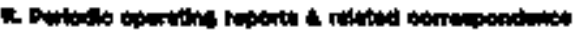

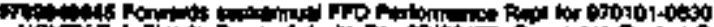

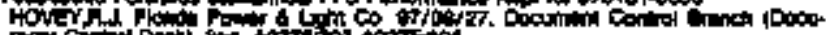

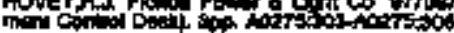

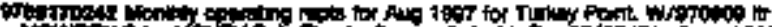

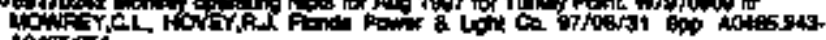

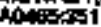

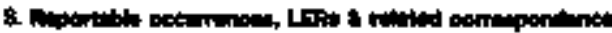

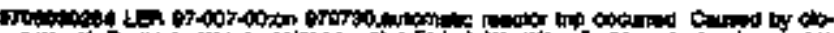

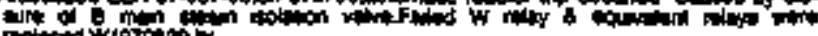
TH

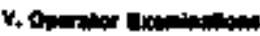

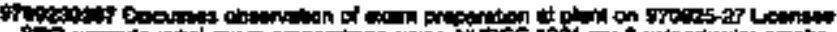

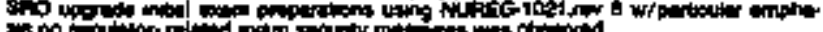

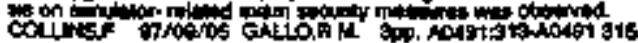

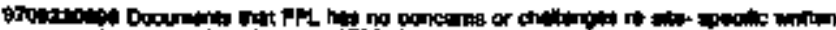

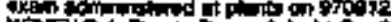

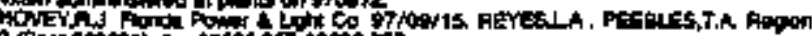

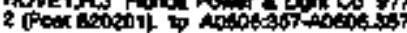

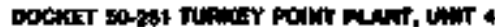

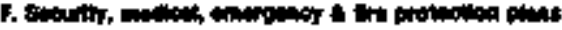

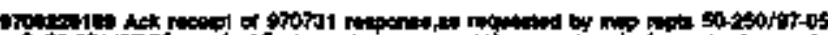

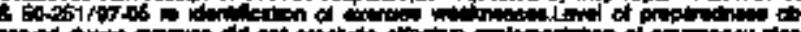

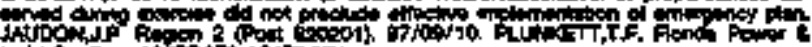

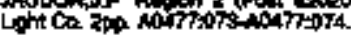

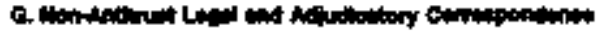

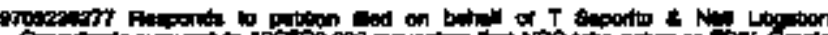

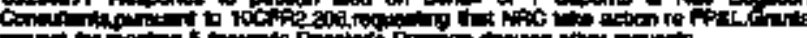

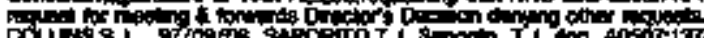

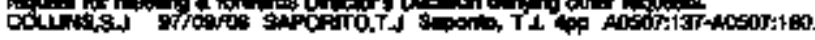

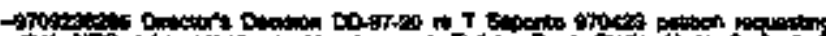

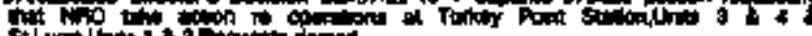

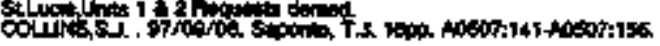

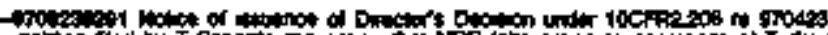

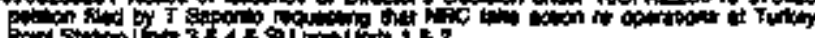

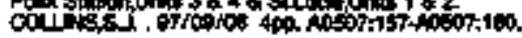

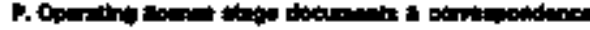

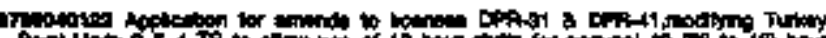

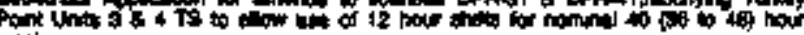

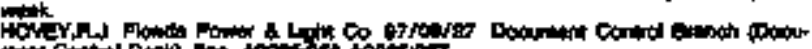

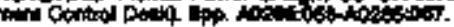

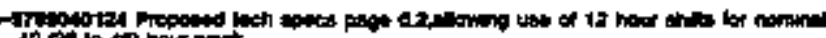

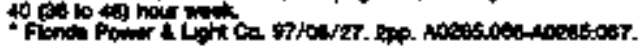

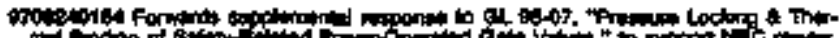

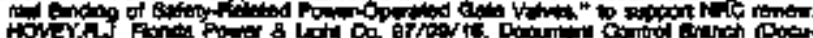

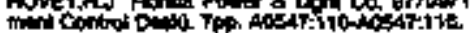

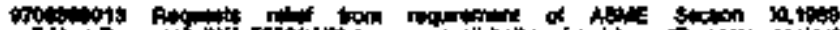

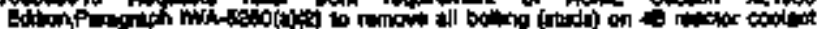

poh

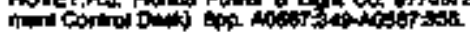

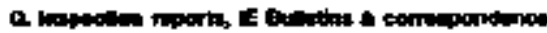

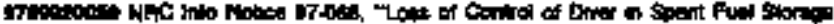
Rod" ation.

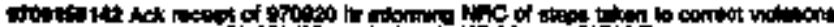

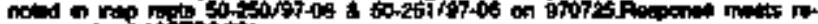

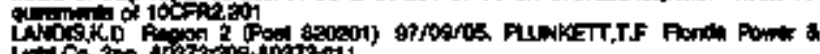

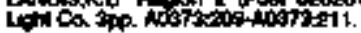

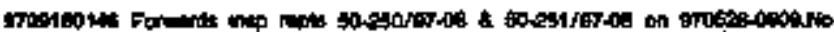

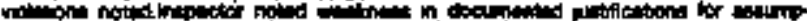

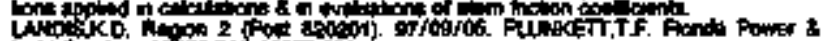

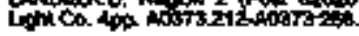

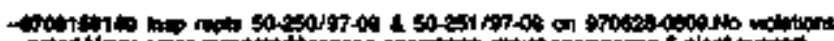

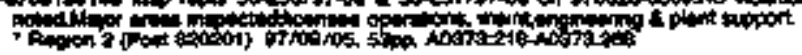

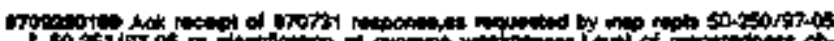

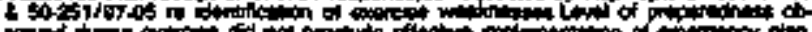

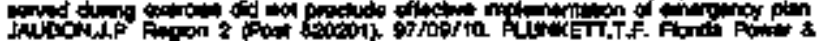

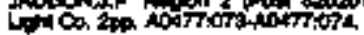

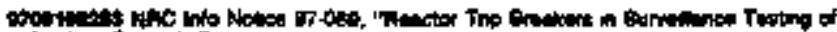

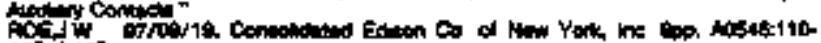
Notitit?.

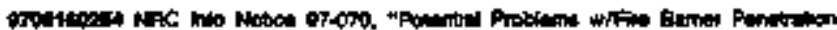

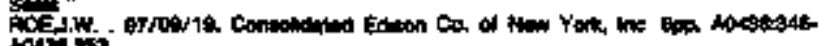
actisis.

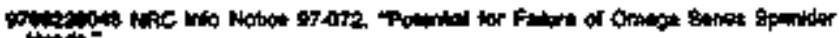

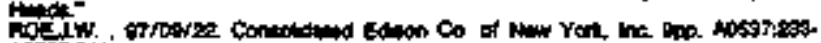

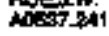

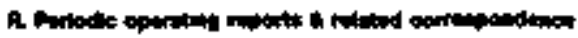

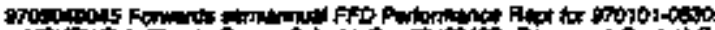

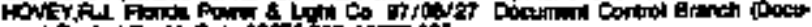

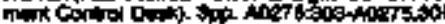




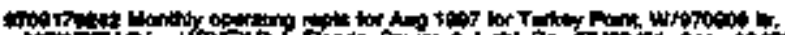

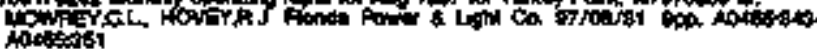

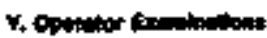

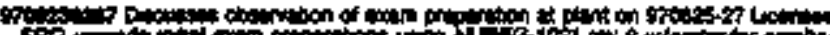

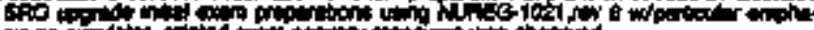
con

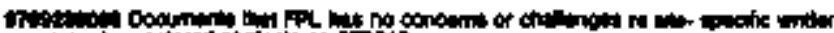

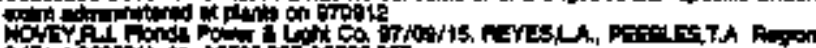

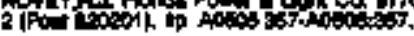

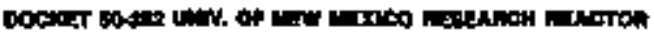

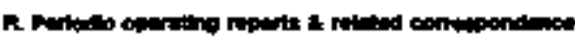

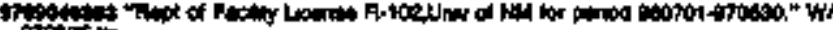
ortist

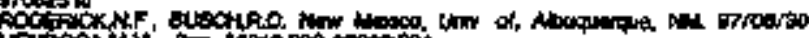

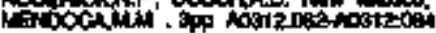

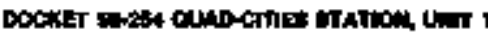

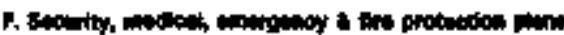

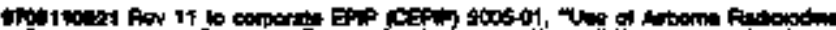

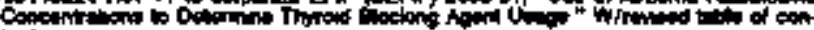

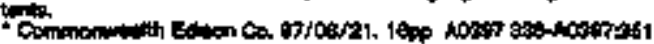

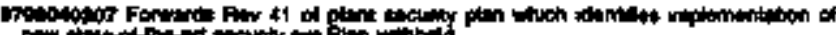

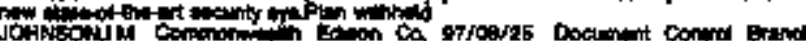

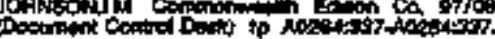

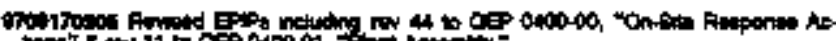

conter 11 to

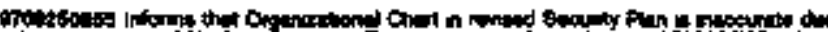

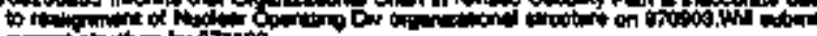

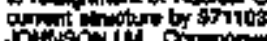

Dotho

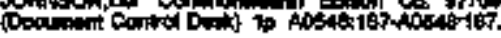

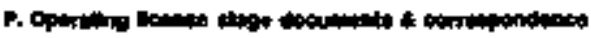

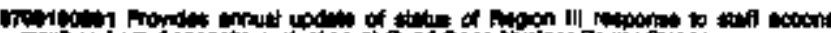

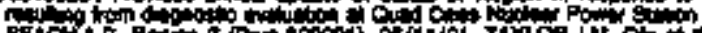

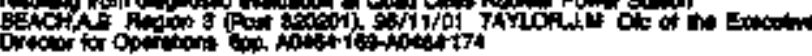

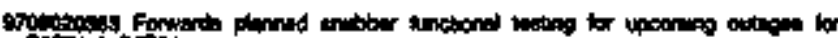

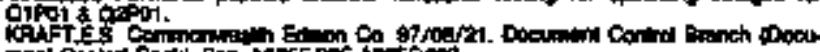

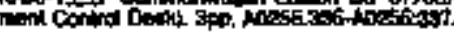

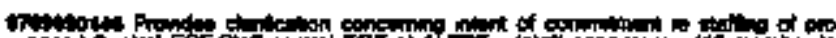

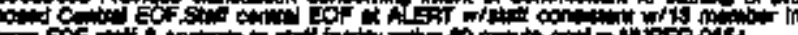

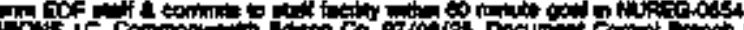

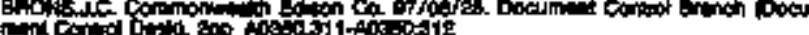

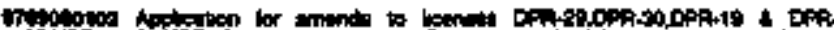

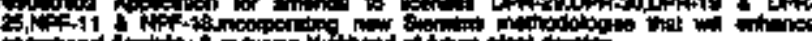

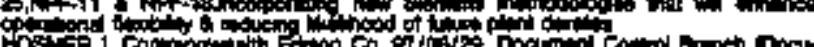

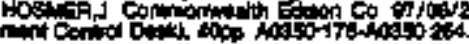

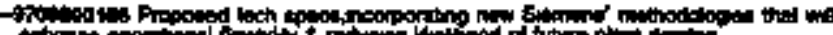

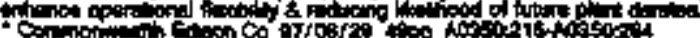

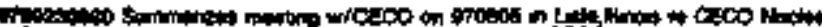

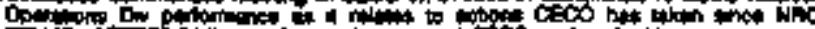

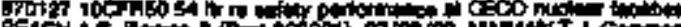

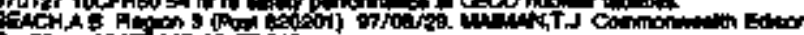

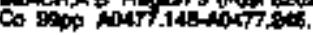

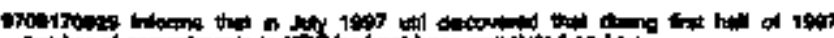

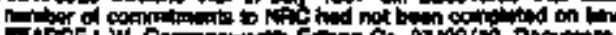

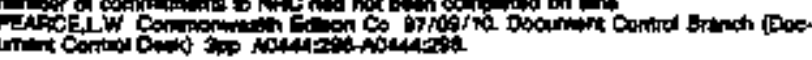

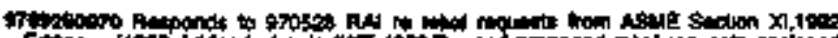

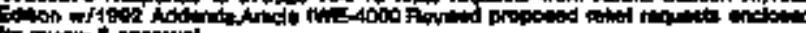
Hogry

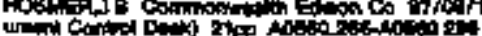

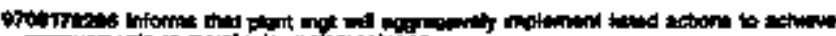

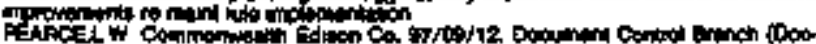

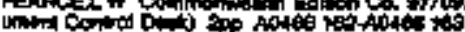

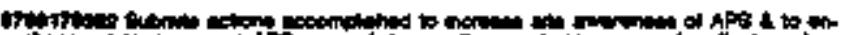

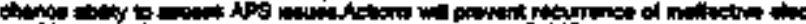

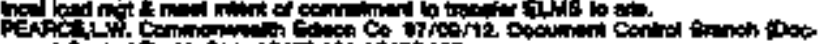

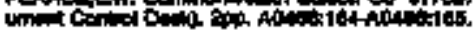

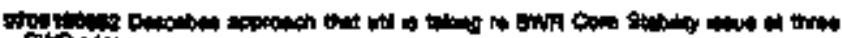

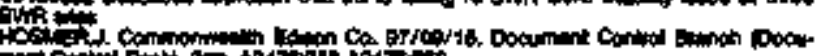

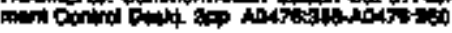

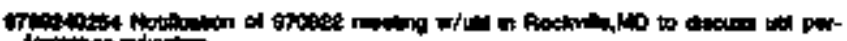

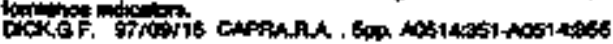

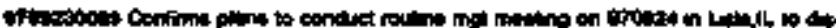

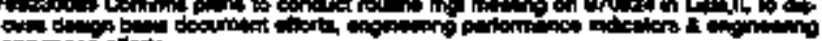

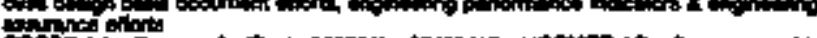

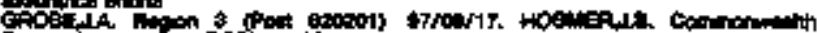

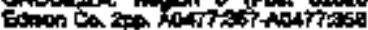

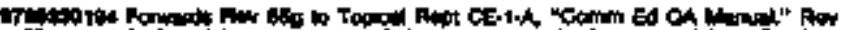

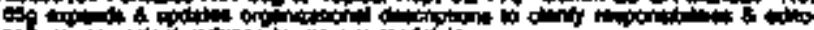

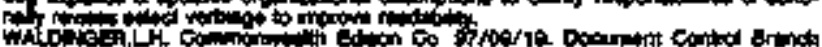

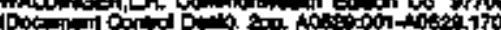

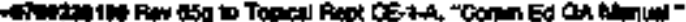

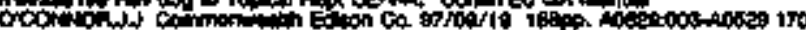

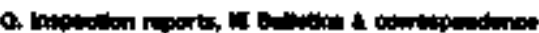

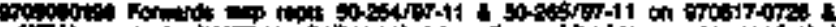

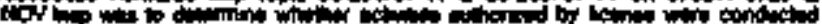

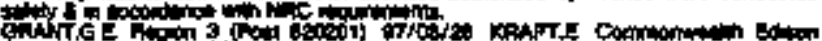

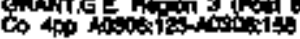

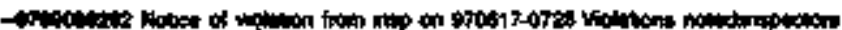

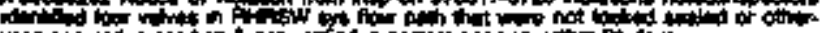

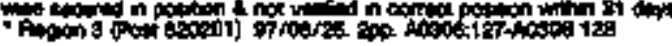

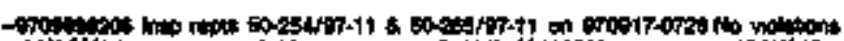

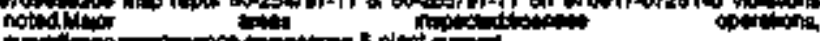

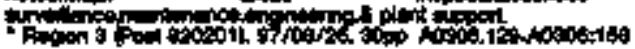

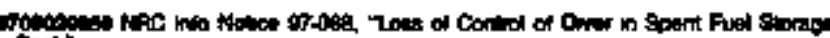

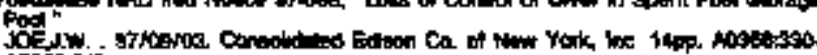

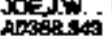

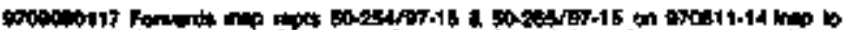

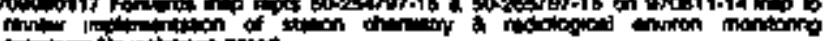

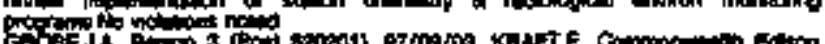

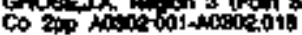

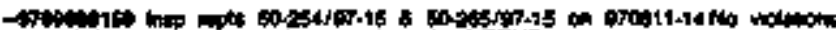

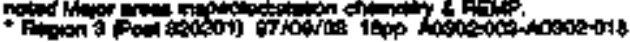

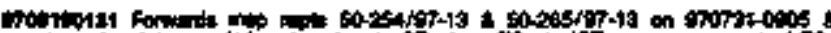

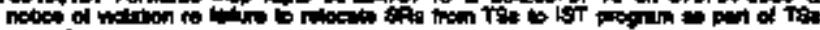

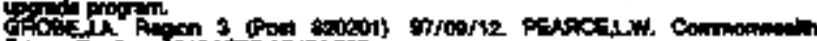

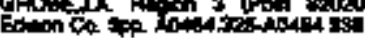

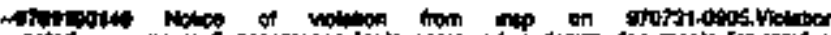

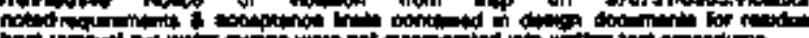

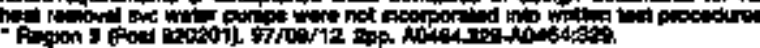

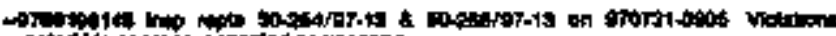

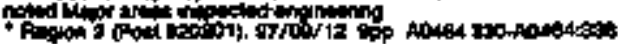

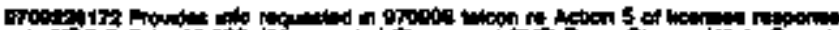

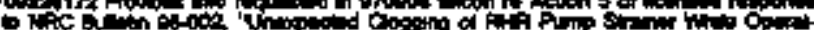

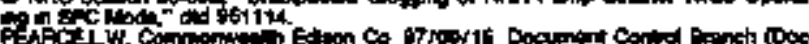

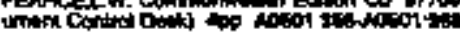

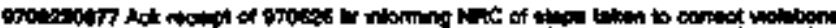

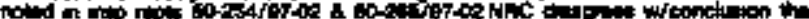

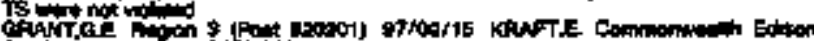

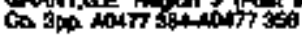

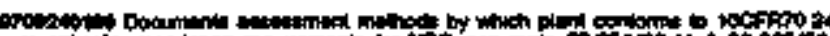

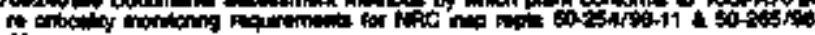

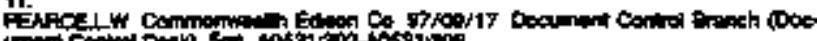

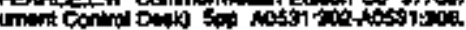

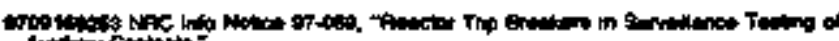

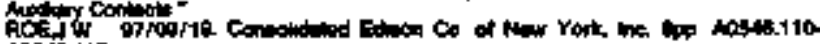
Aopers 117

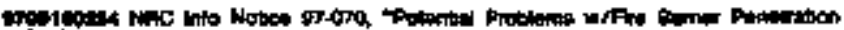

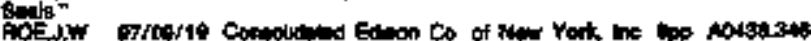
intors 


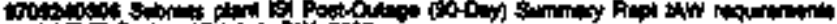

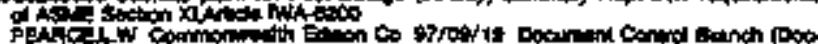

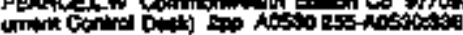

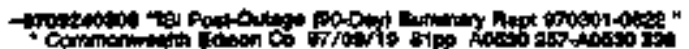

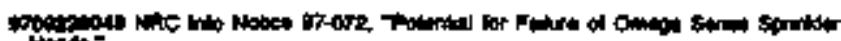

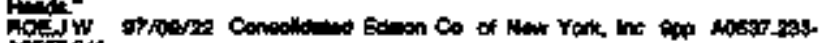

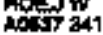

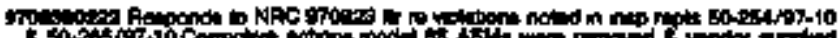

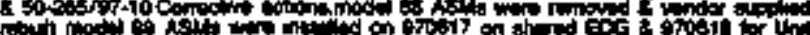
10.0.

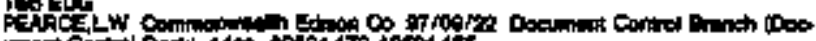

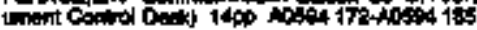

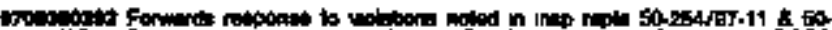

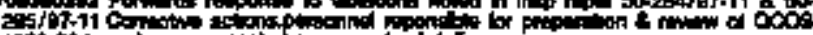
Fol of,

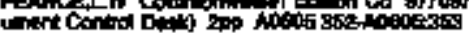

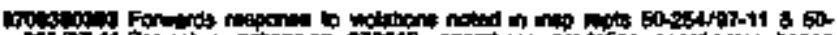

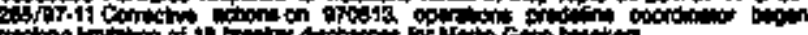

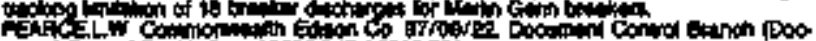

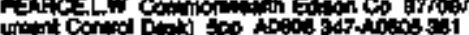

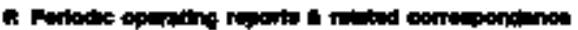

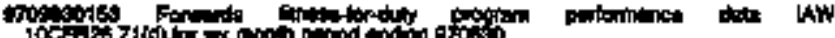

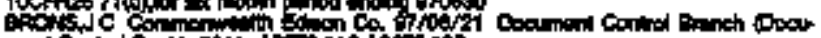

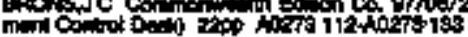

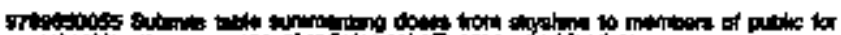

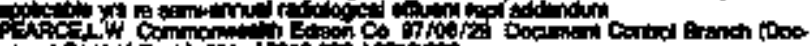

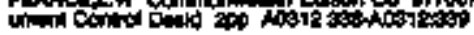

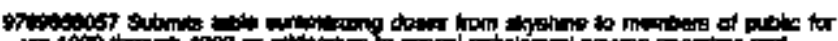

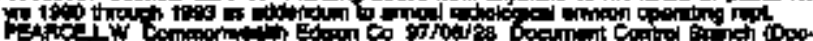

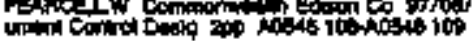

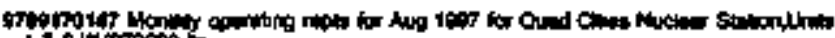

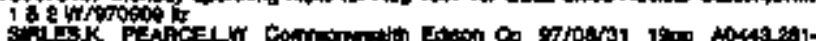
intion

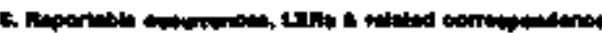

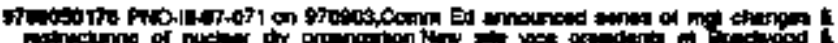

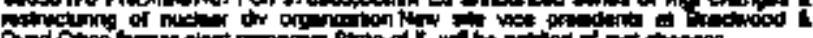

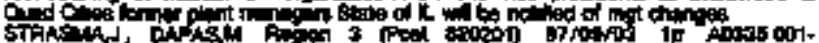
Anses 001

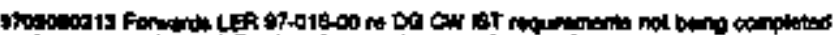

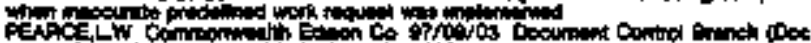

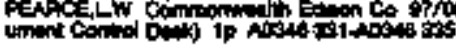

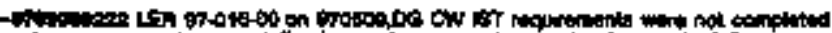

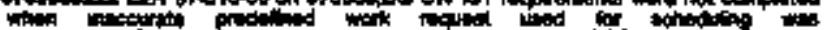

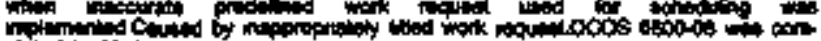

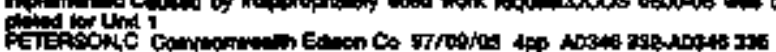

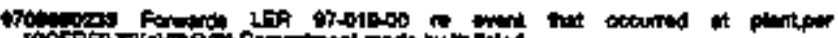
DCF

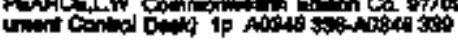

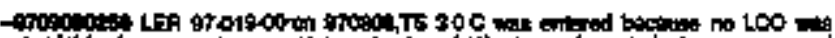

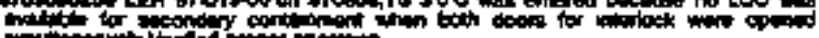

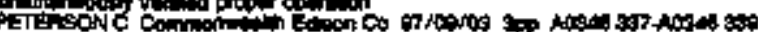

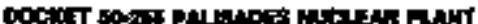

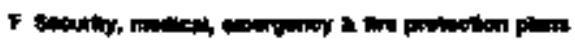

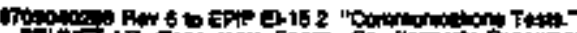

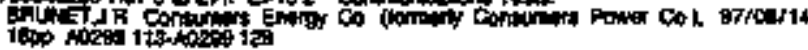

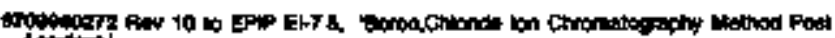
Actint

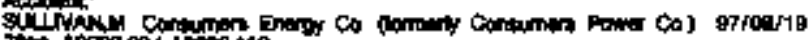

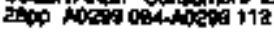

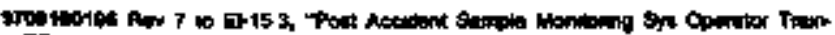

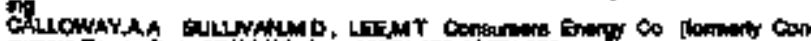

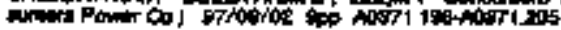

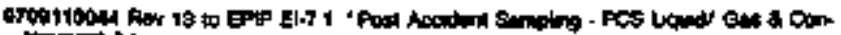

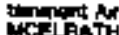

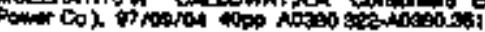

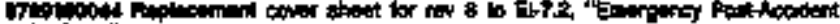

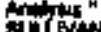

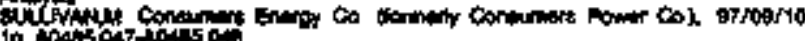

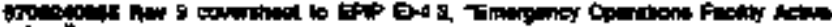

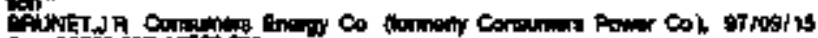

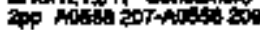

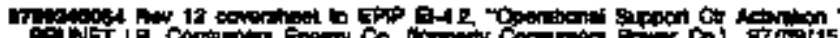

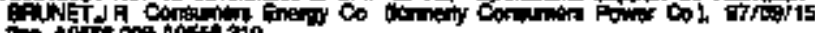

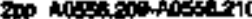

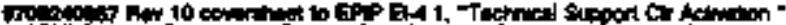

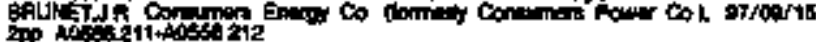

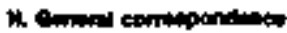

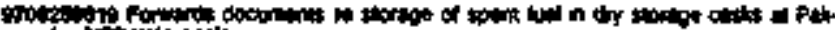
10

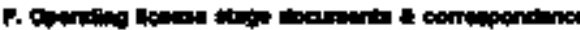

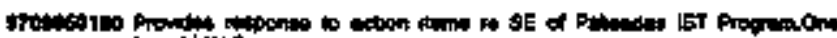
now

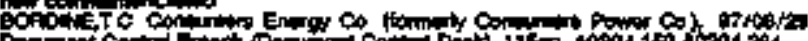

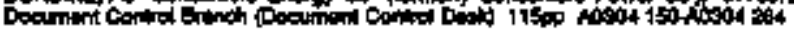

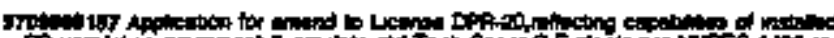

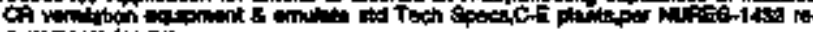
arming

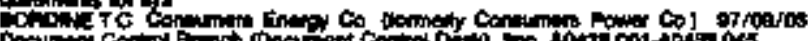

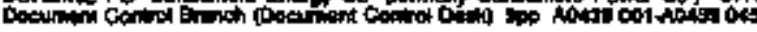

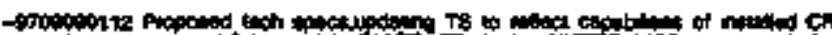

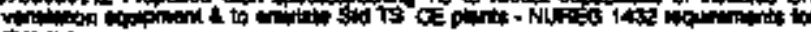

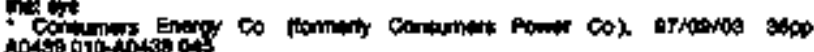

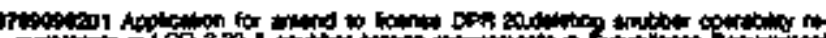

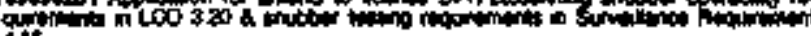

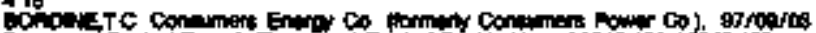

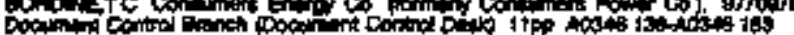

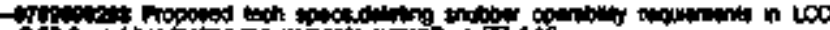

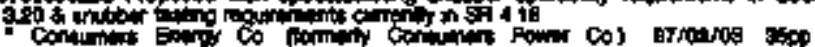
conting

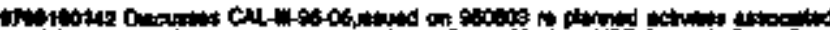

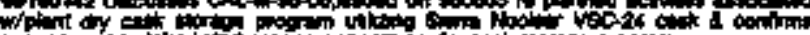

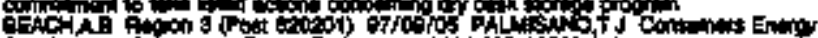

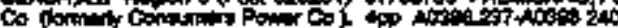

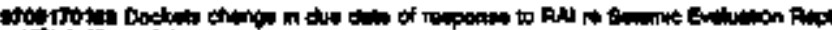

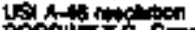

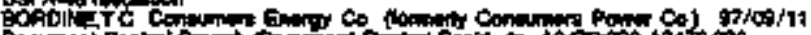

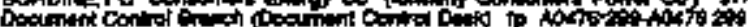

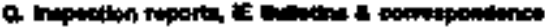

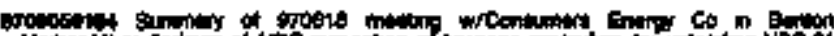

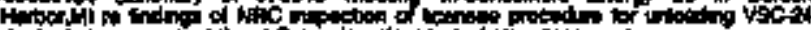
S.

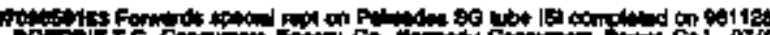

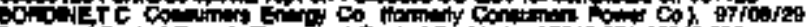

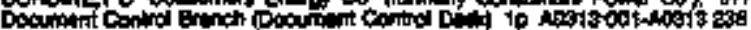

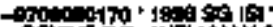

A A Pow Co $\mathrm{l}$,

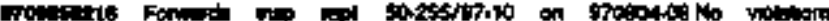

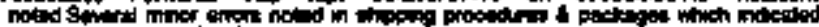

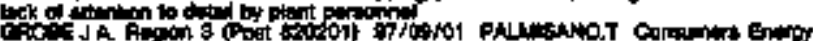

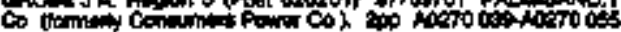

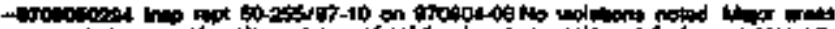

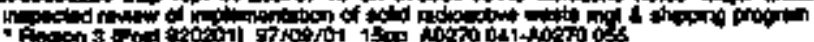

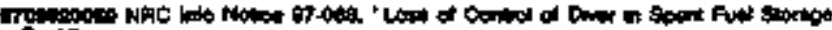

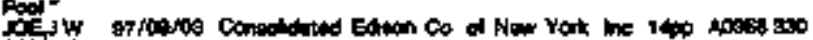
Adosis sas

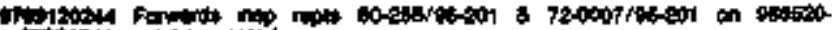

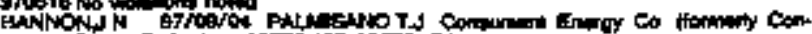

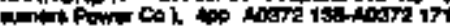




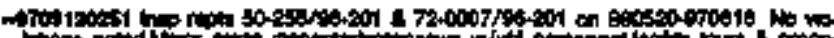

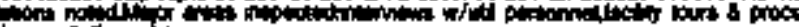

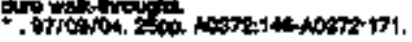

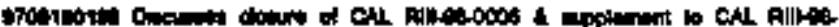

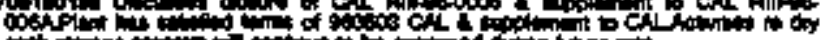

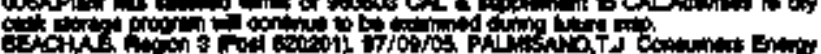

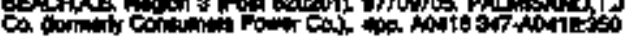

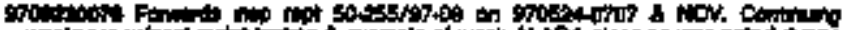

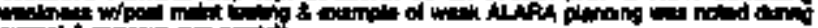

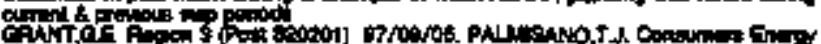

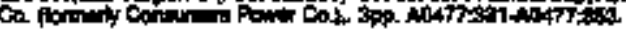

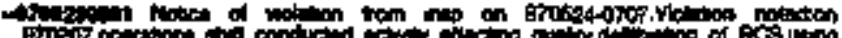

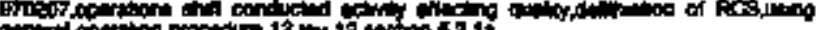

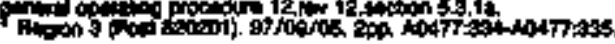

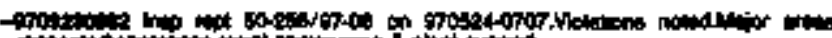

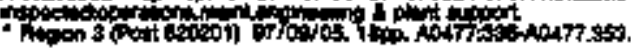

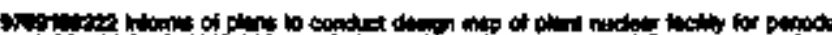

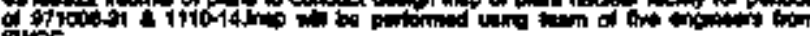

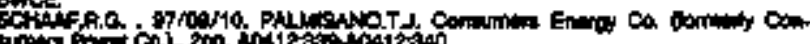

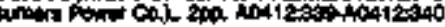

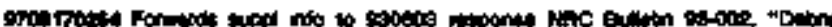

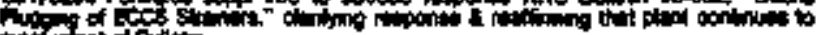

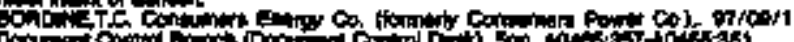

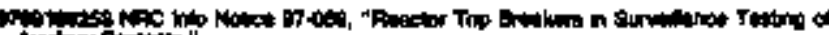

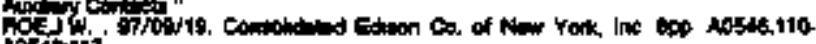
Hotiti?

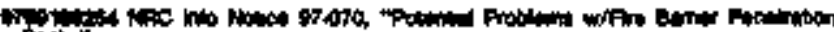

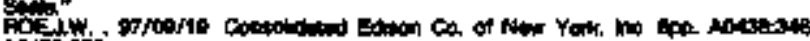

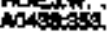

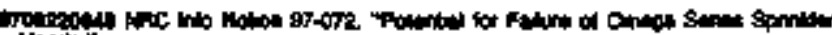

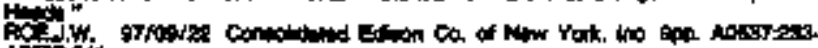
Ariogidit.

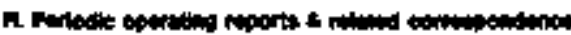

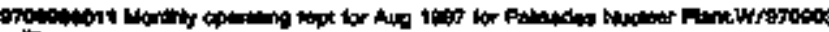

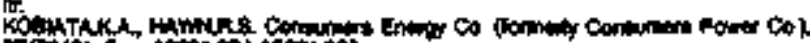

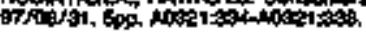

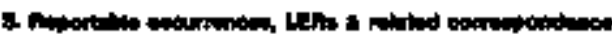

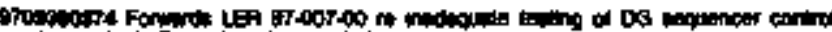

Pid.

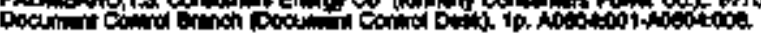

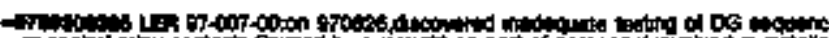

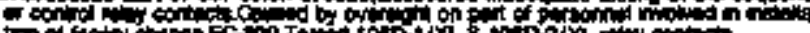

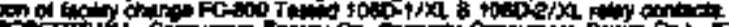

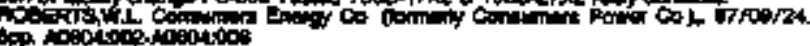

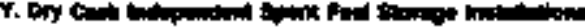

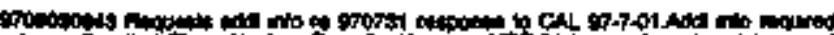

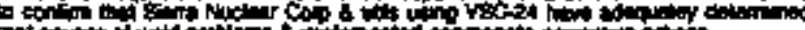

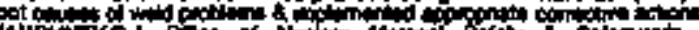

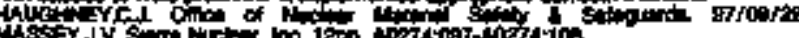

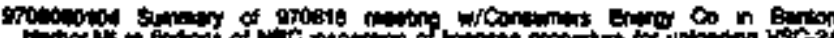

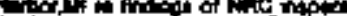

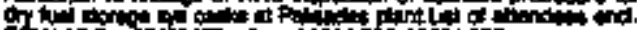

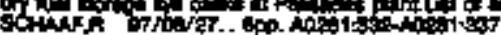

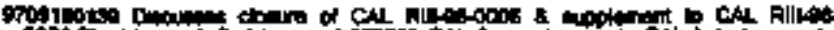

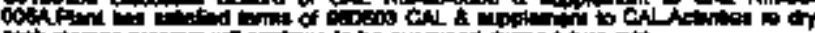

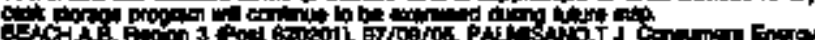

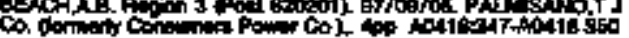

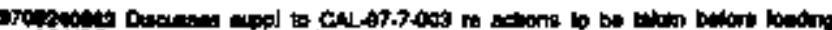

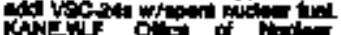
WN

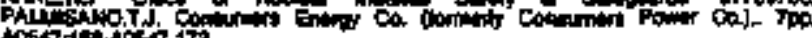

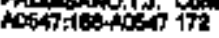

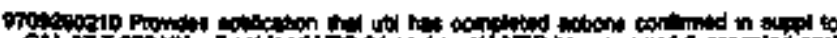

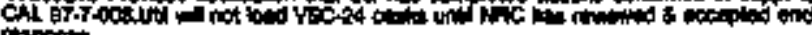

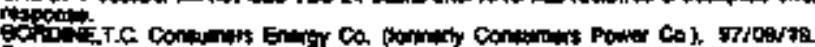

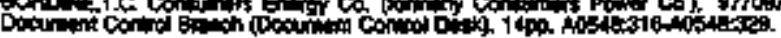

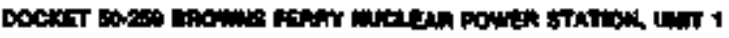

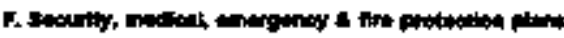

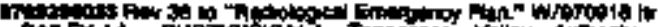

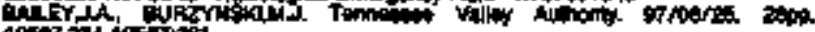

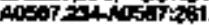

Orow

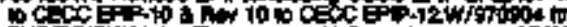

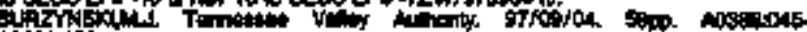
Aptos

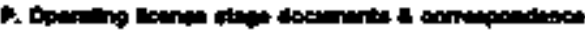

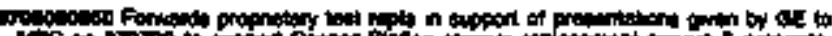

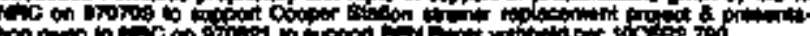

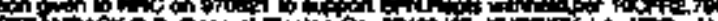

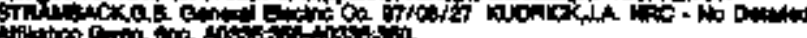

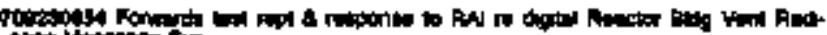

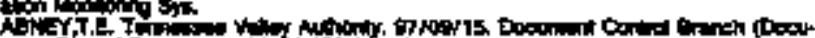

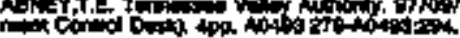

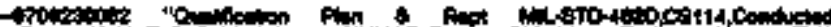

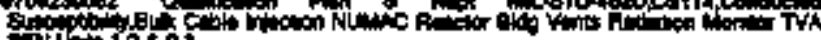
F. d:

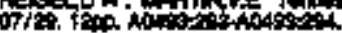

O.

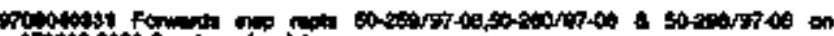

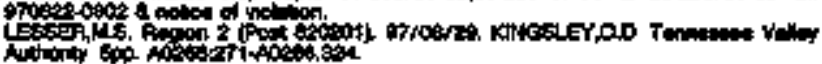

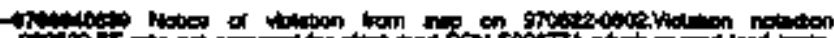

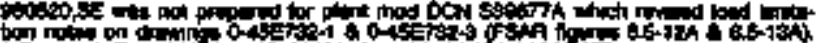

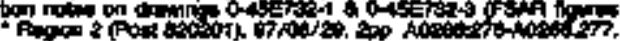

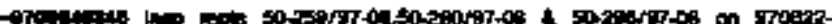

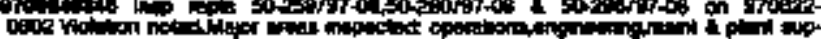

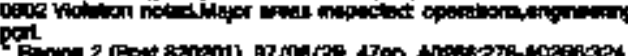

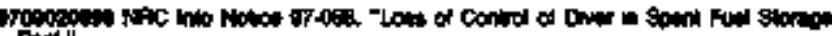

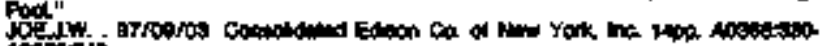

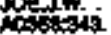

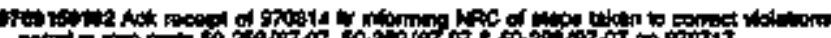

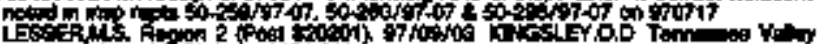

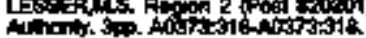

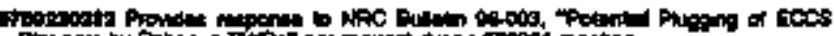

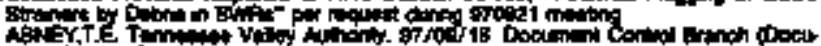

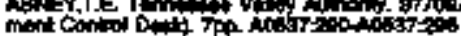

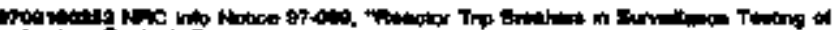

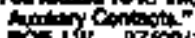
A0067117.

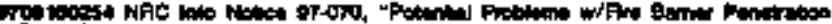

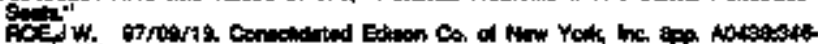
Notion

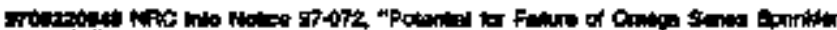

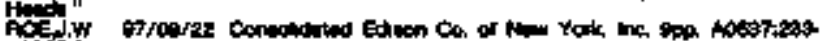
Asomat,

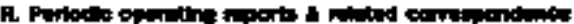

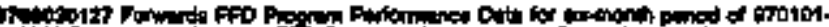

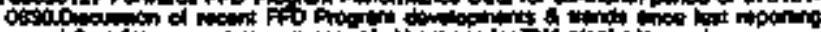

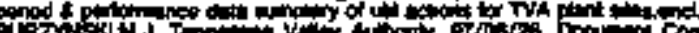

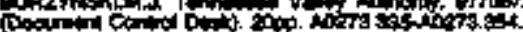

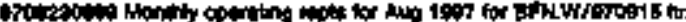

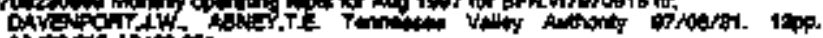

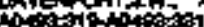

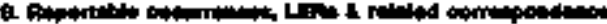

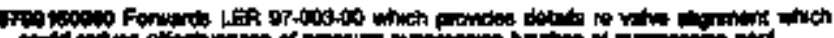

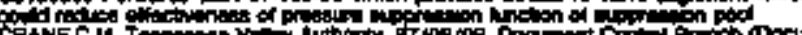

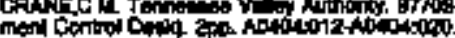




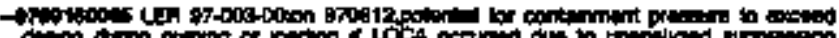

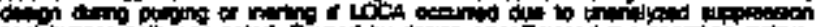

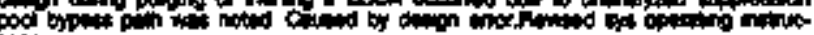
OE

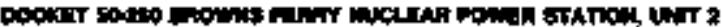

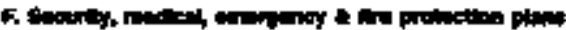

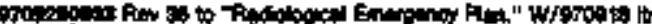

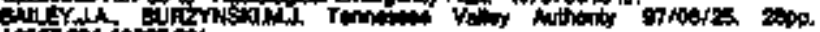
intestratiogit.

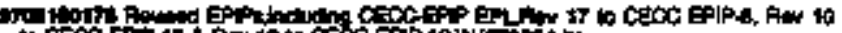
4 coc G Ansosios

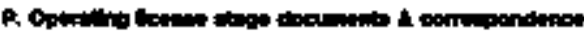

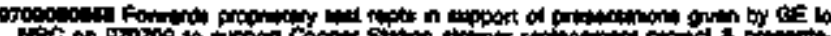

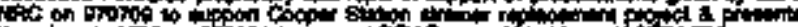

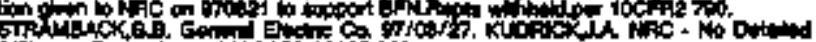

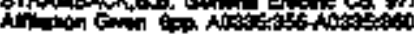

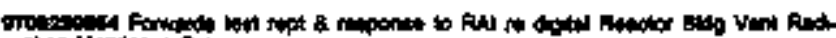
in Hontion st.

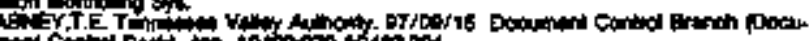

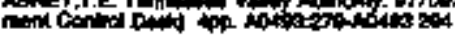

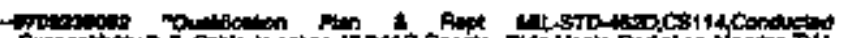

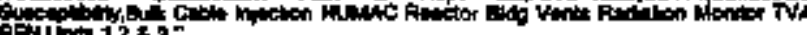

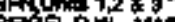
PEC

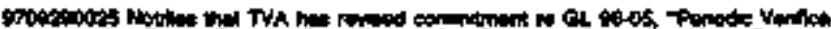

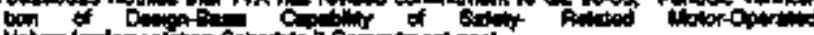

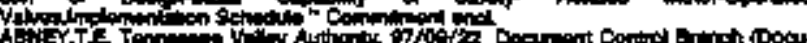

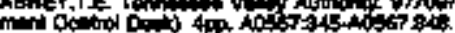

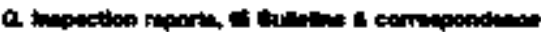

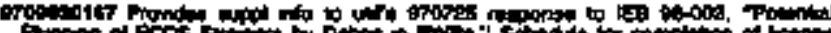

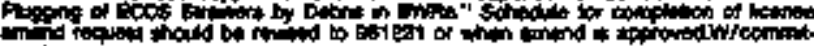
meith

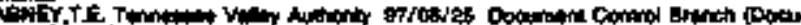

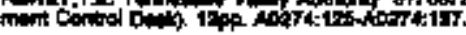

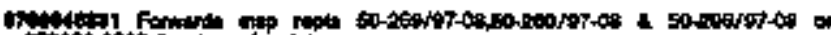

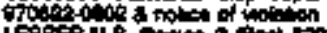

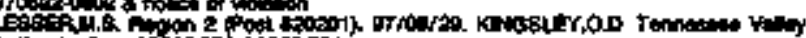

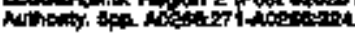

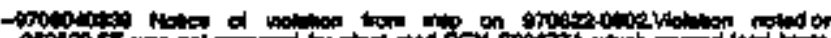

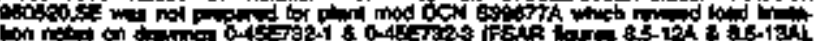

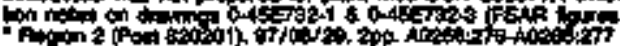

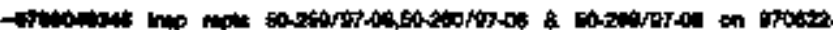

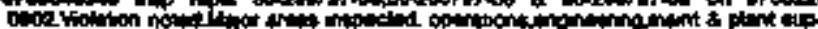

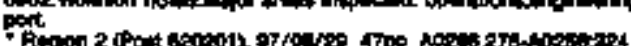

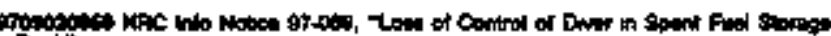

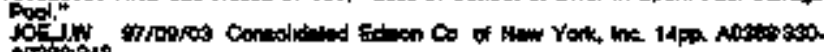

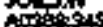

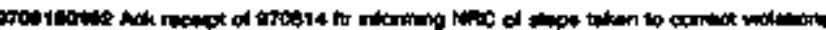

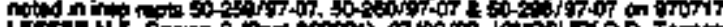
U.

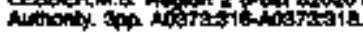

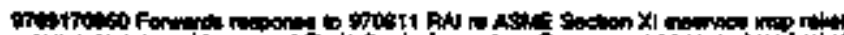

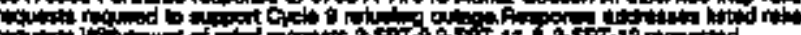

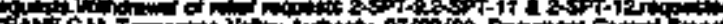

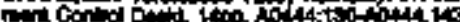

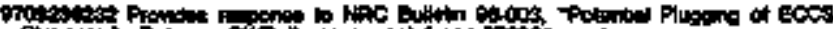

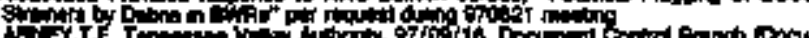

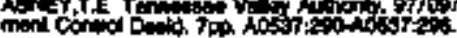

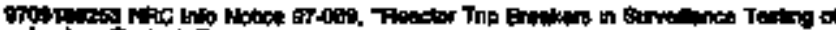
Ao

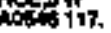

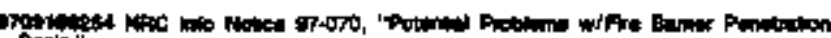

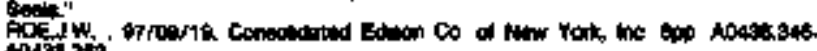
Alatis at

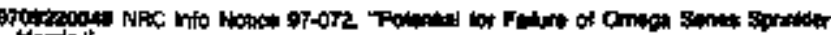

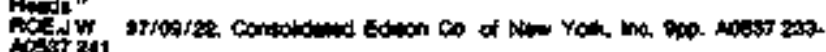
(1)

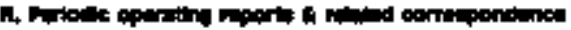

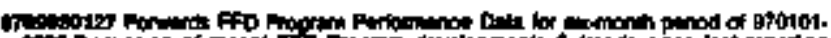

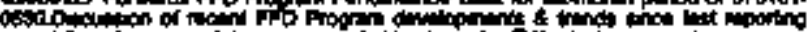

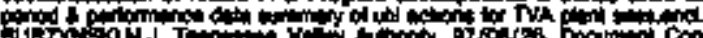

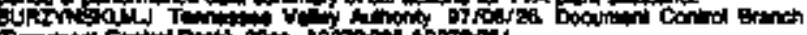

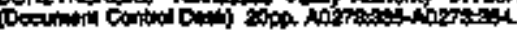

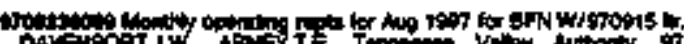

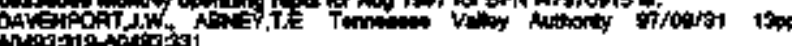

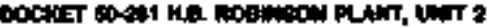

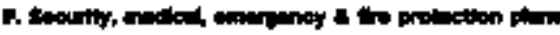

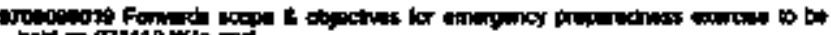

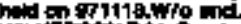

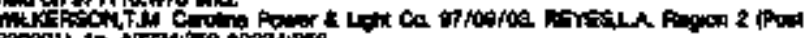

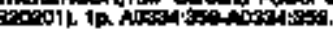

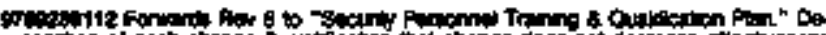

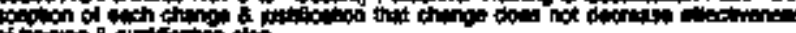

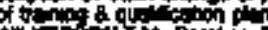

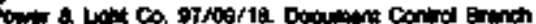

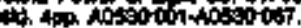

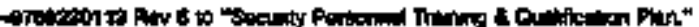

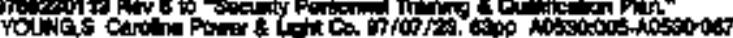

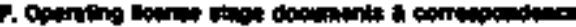

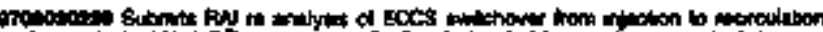

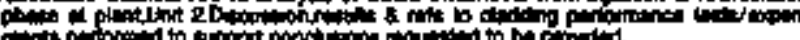

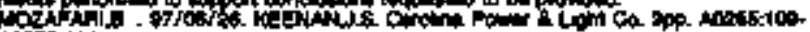
ing:T11.

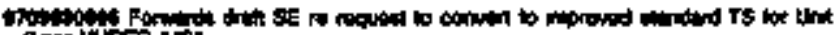

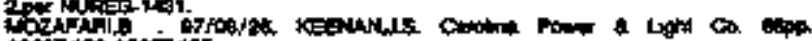

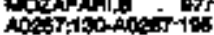

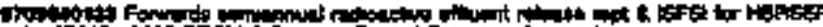

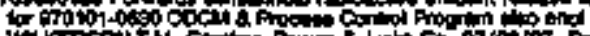

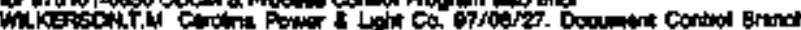

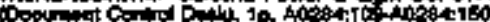

rot

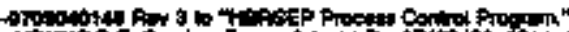

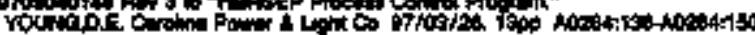

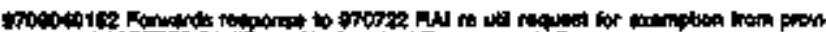

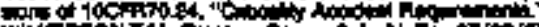

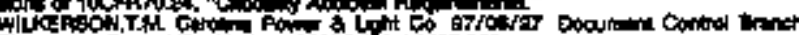

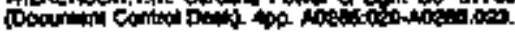

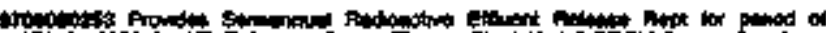

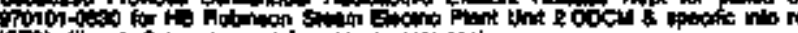

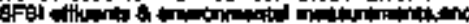

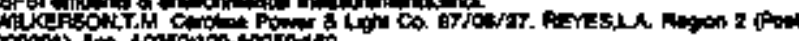

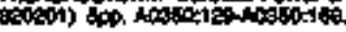

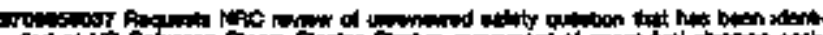

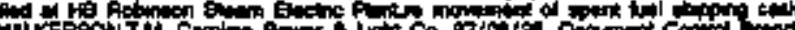

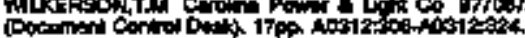

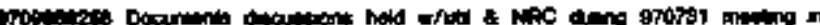

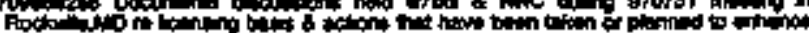

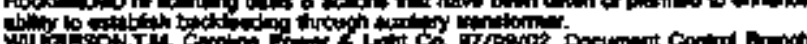

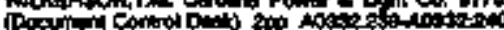

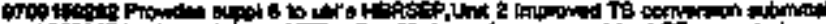

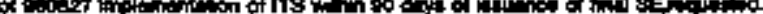

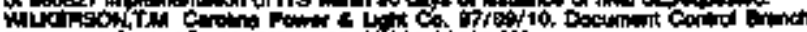

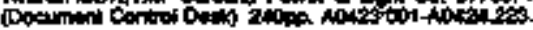

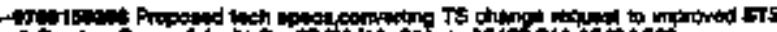

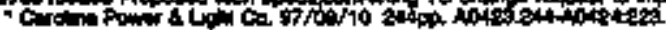

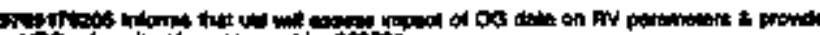

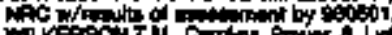

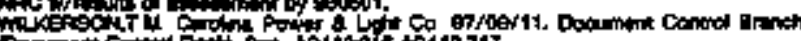

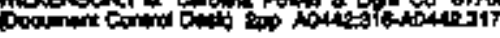

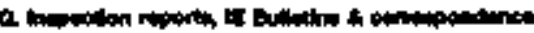

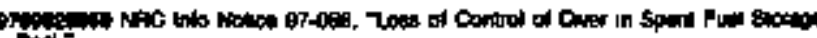

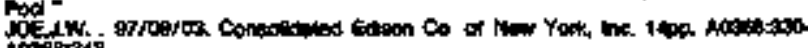
Antosis

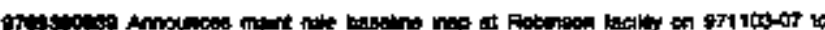

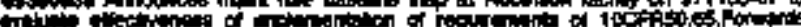

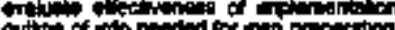

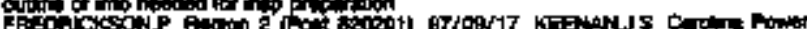

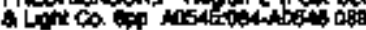




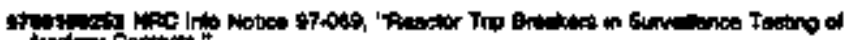

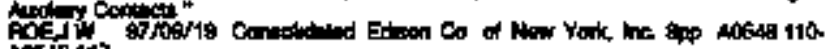
notust?

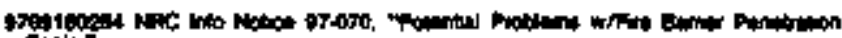

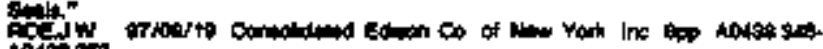
notion 392

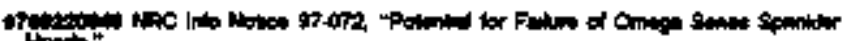

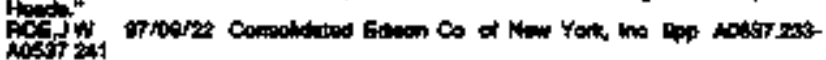

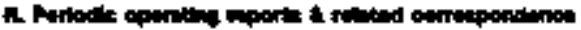

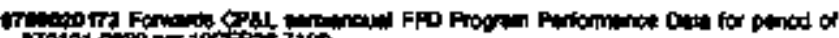
AlDoribsi

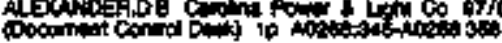

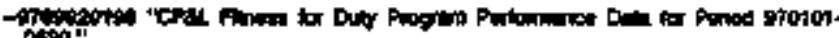

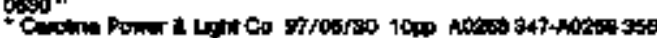

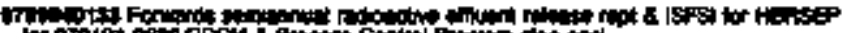

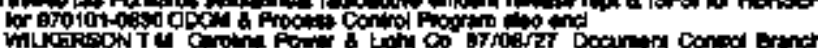

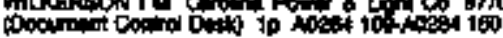

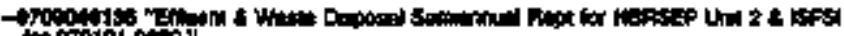

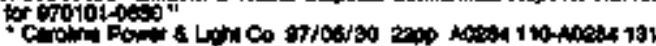

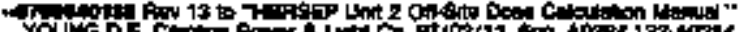

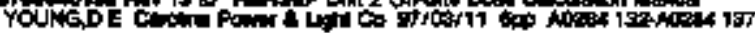

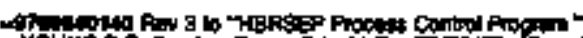

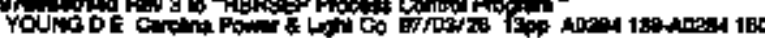

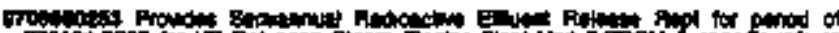

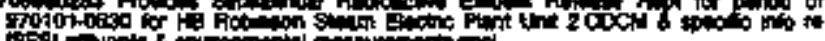
Wh

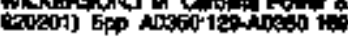

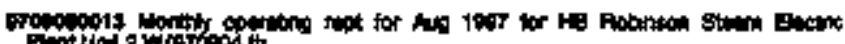

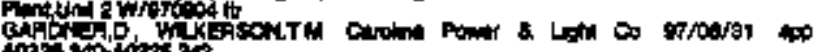

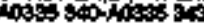

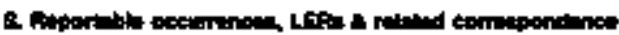

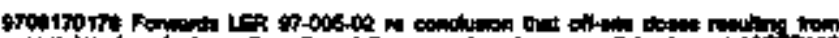

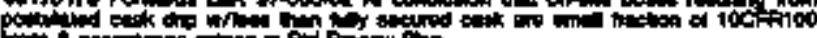

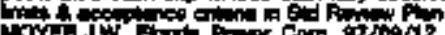

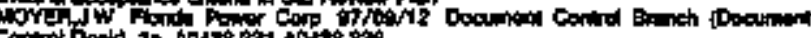

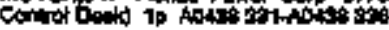

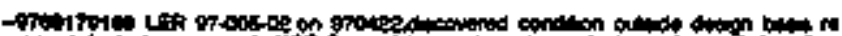

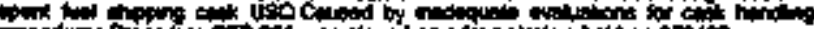

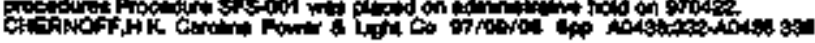

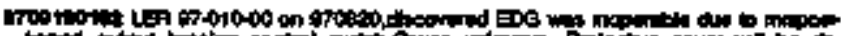

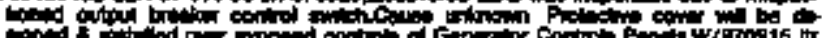

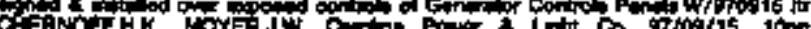

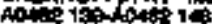

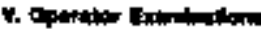

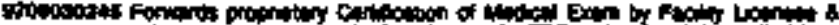

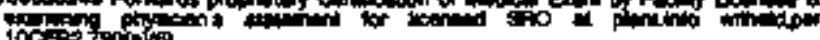

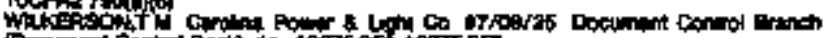

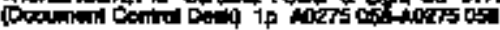

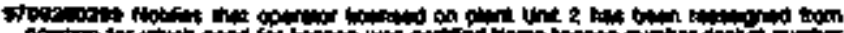

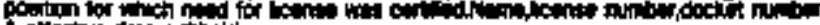

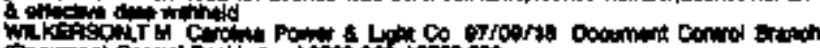

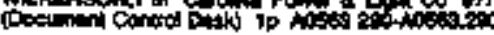

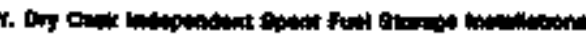

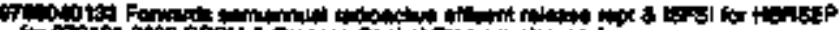

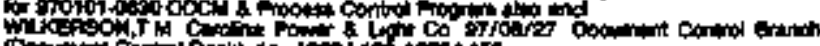

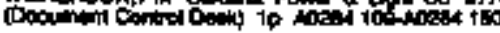

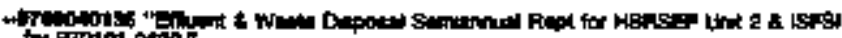

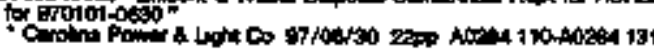

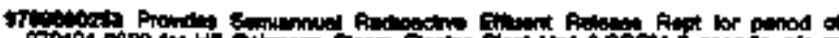

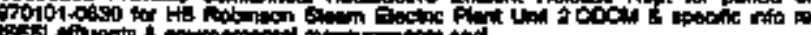

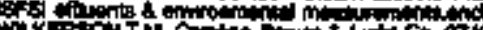

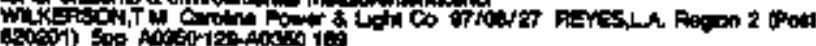

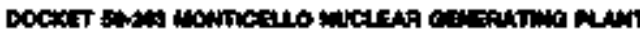

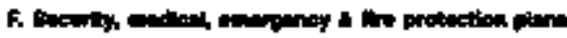

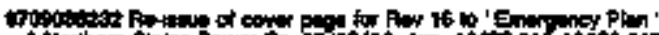

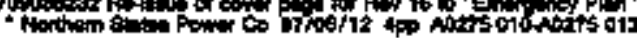

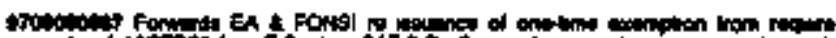

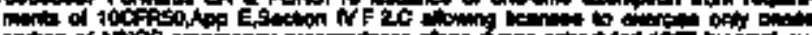

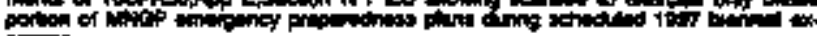

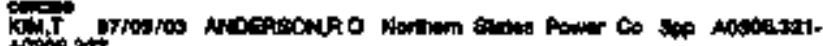
Antots

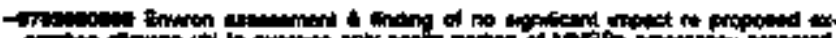

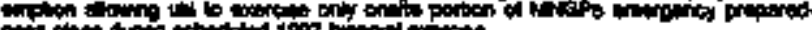

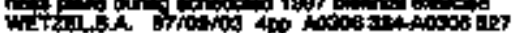

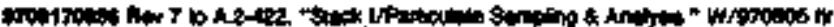

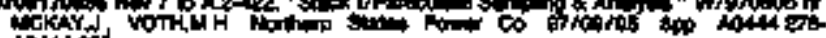
nim

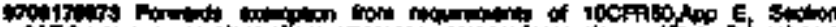

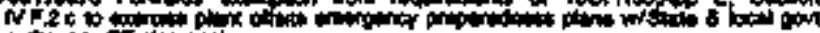

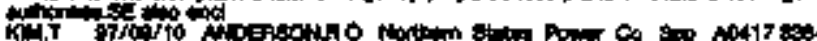
No117 30.

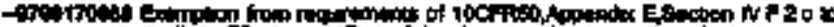

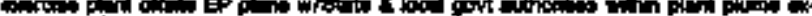

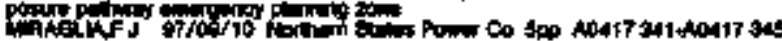

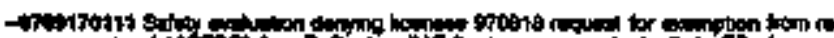

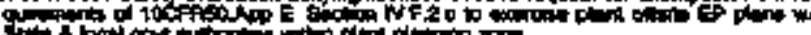

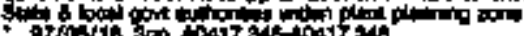

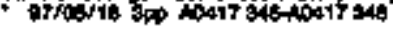

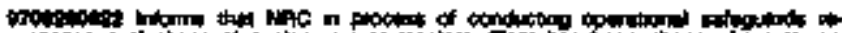

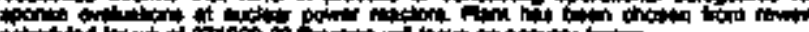

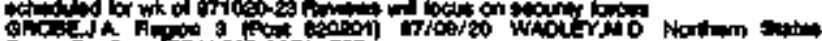

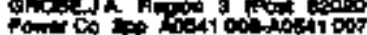

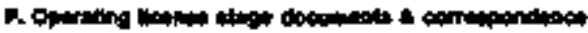

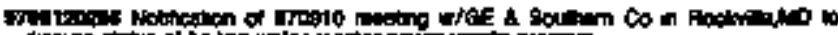

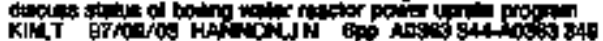

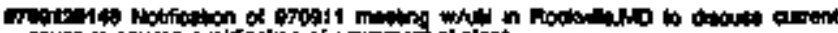

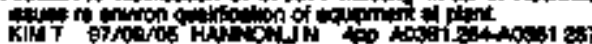

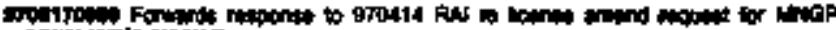

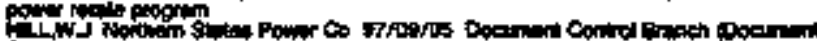

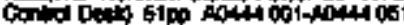

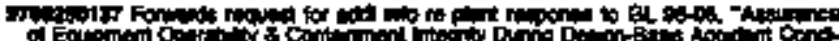

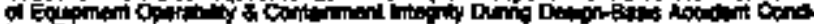

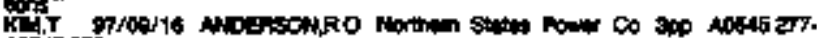
Motion

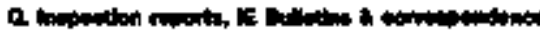

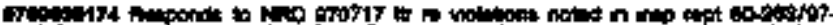

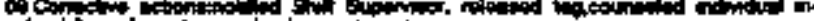

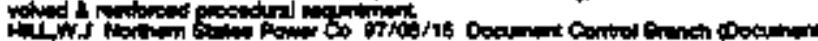

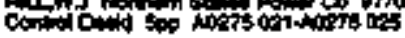

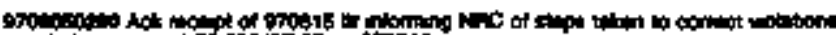

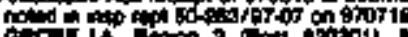

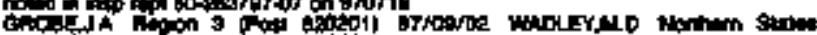

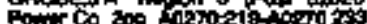

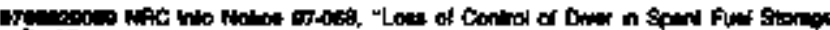

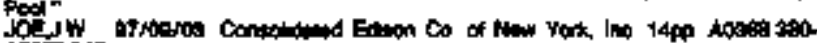

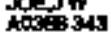

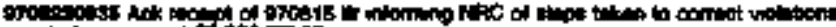

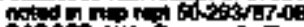

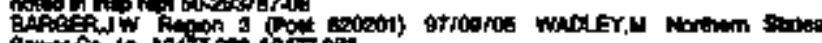

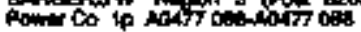

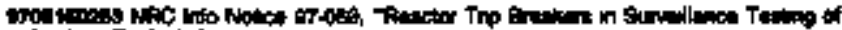

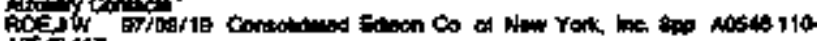
45.58 117

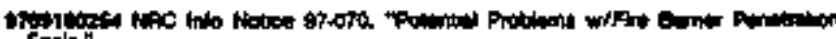

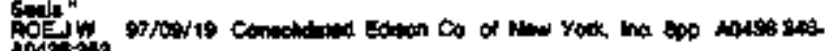
10450

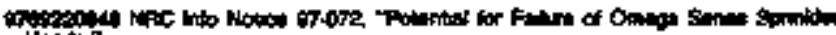

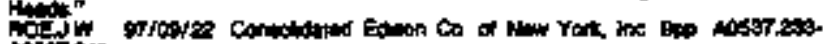
instias 


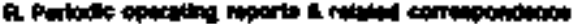

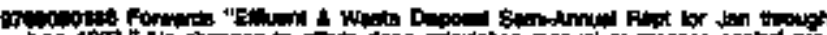

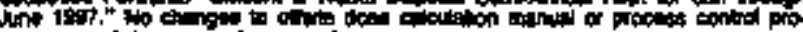

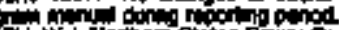

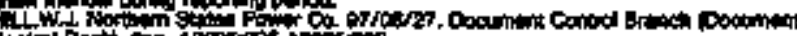

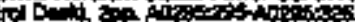

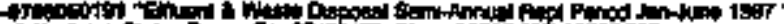

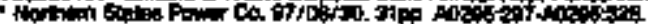

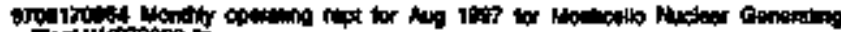

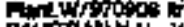

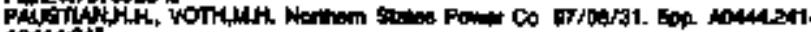
andivats

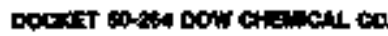

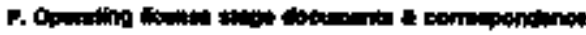

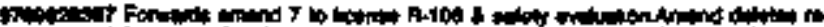

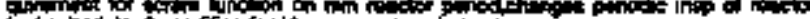

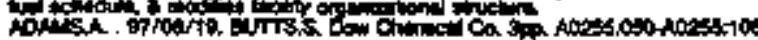

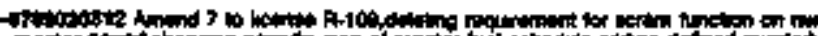

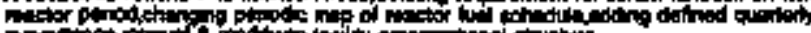

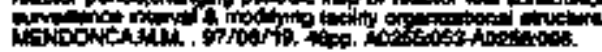

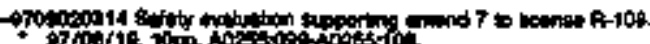

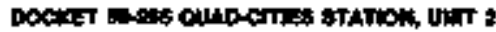

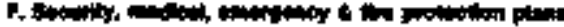

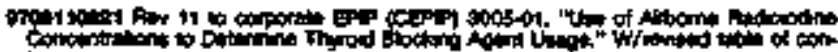

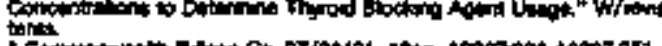

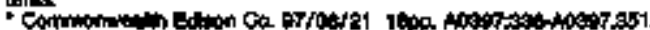

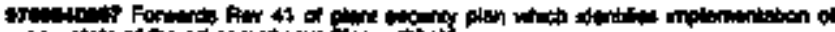

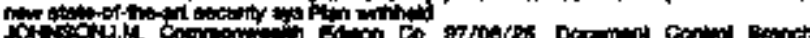

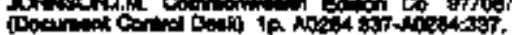

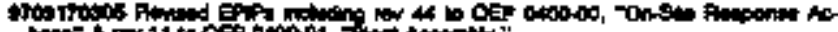

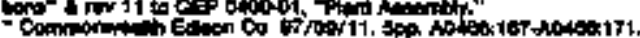

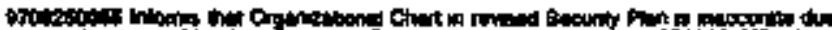

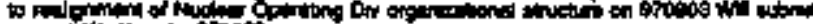

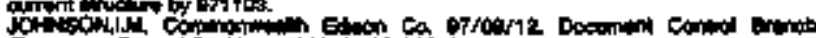

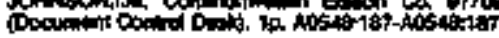

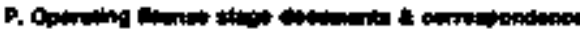

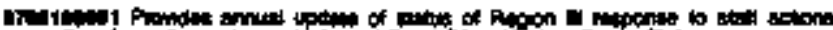

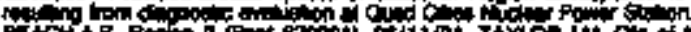

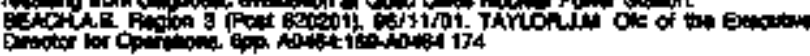

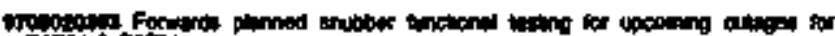
फ़िए copot.

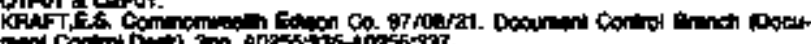

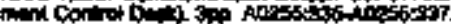

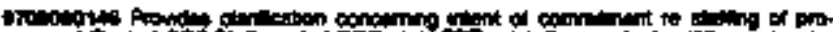

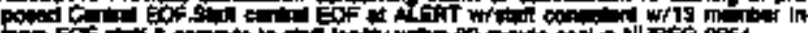

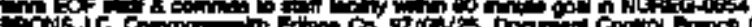

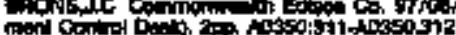

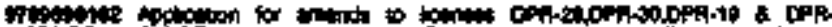

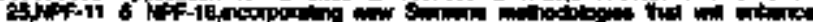

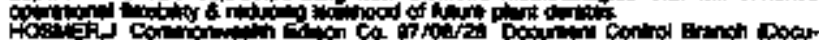

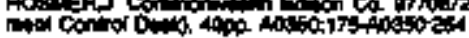

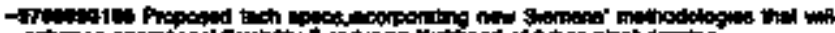

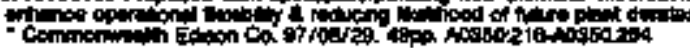

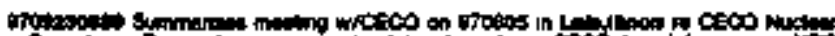

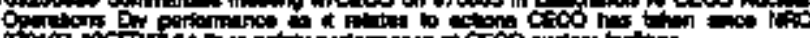
\$

0 or $77-206$.

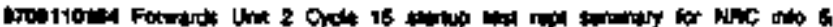

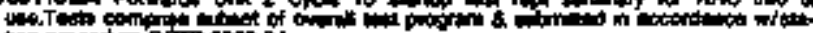

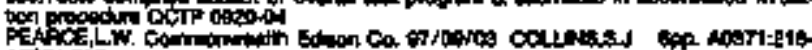
4

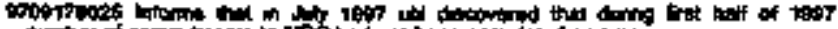

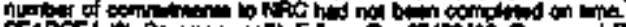

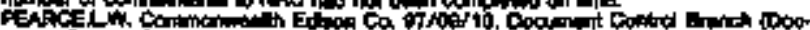

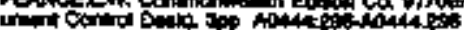

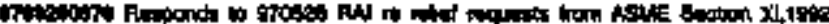

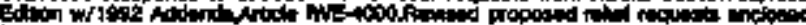

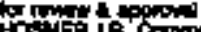

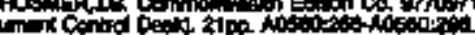

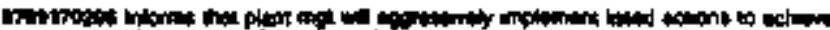

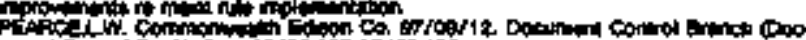

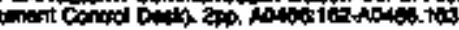

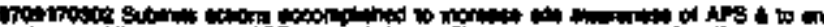

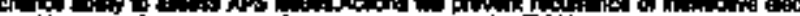

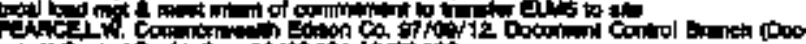

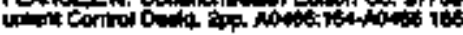

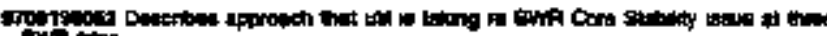
Fin

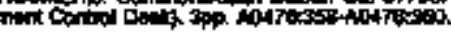

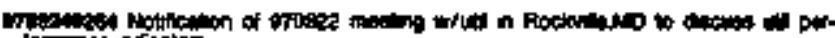

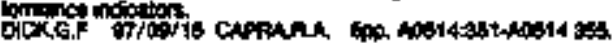

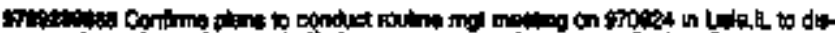

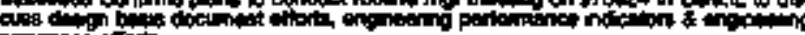

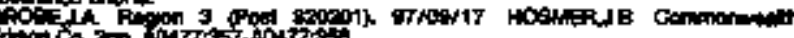

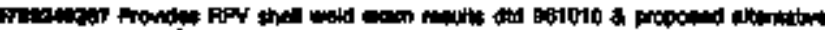

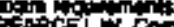

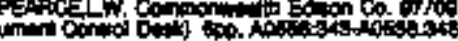

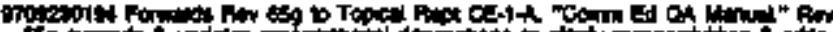

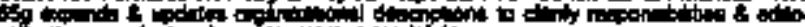

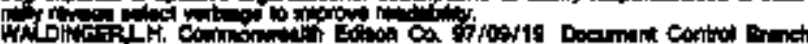

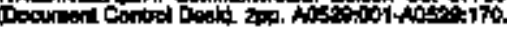

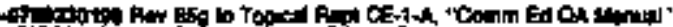

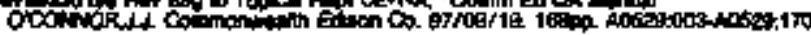

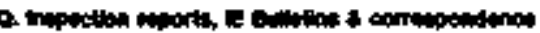

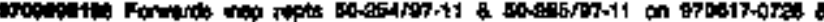

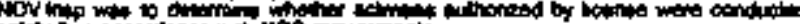

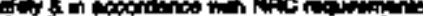

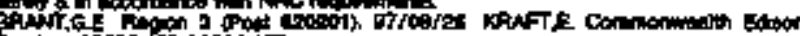

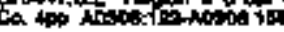

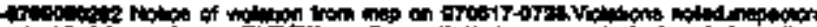

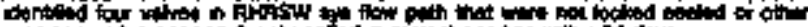

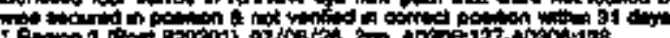

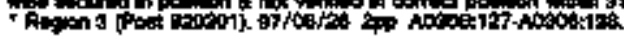

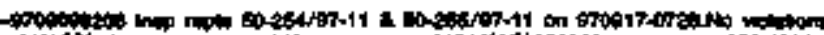

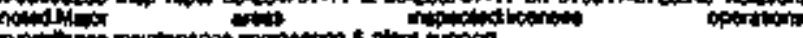

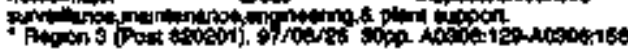

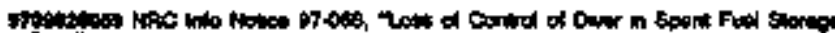

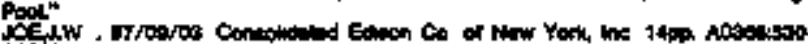

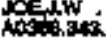

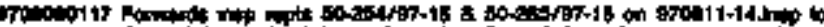

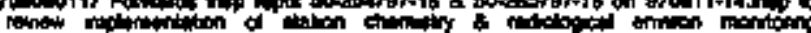

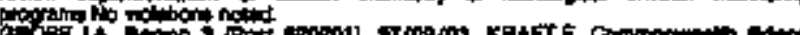

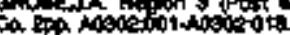

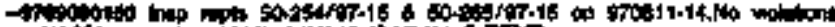

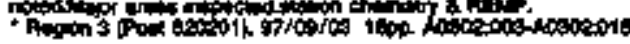

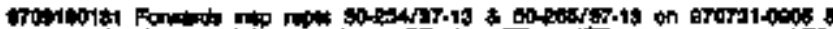

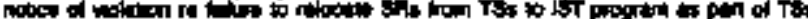

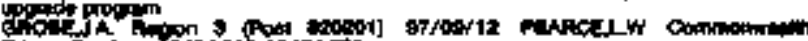

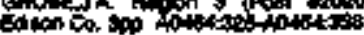

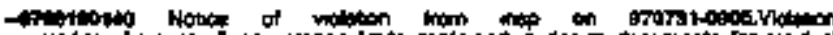

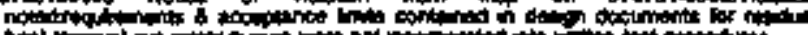

n

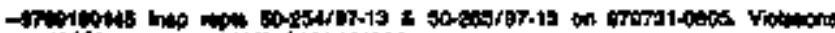

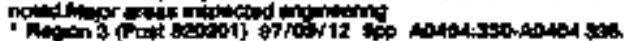

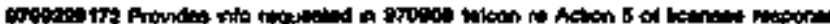

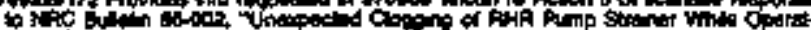

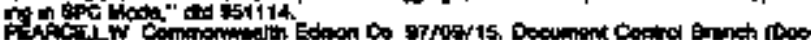

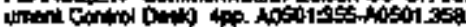

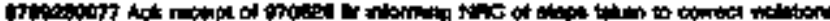

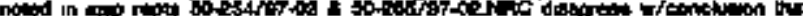

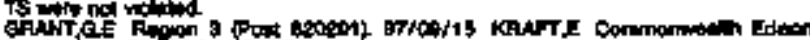
ka $3 \rightarrow 0$ a 


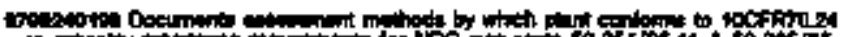

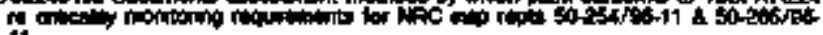

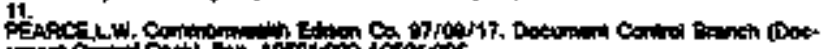

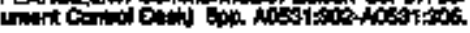

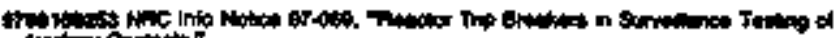
PCE AOSti1t:

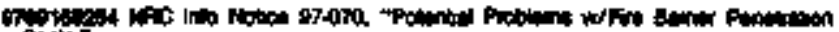

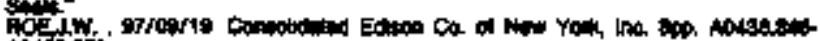
40,10460

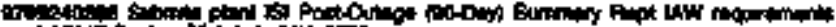

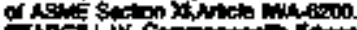

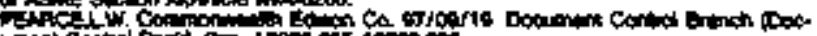

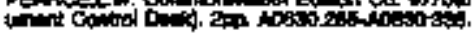

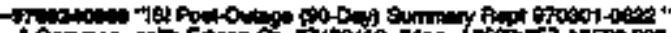

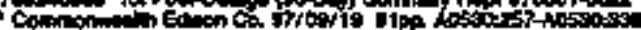

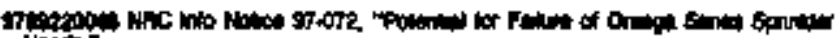

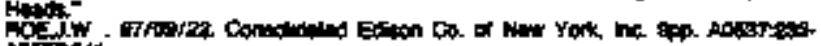
Anotiati.

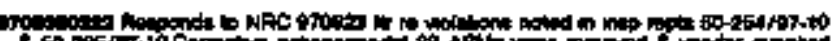

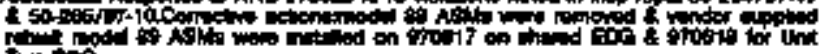
tos

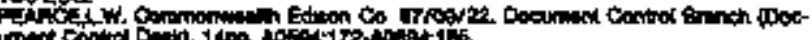

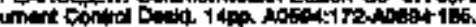

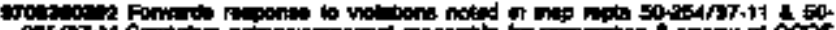

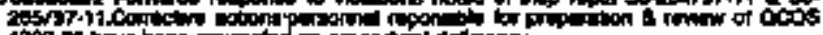
penact,

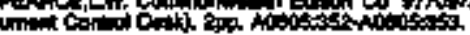

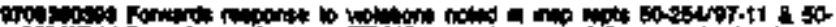

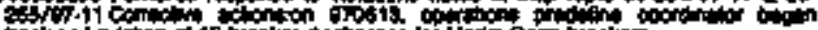
Péch

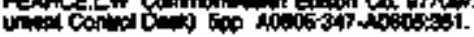

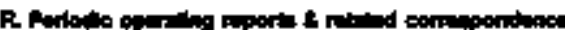

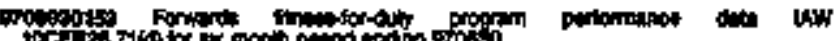

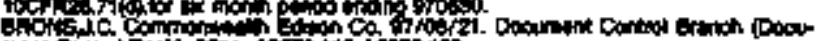

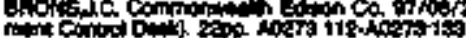

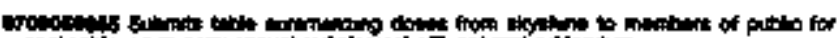

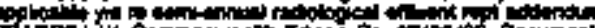

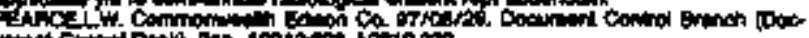

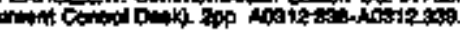

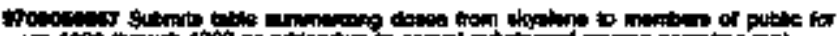

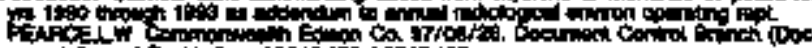

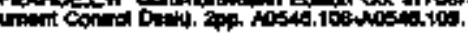

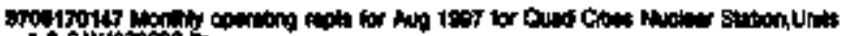

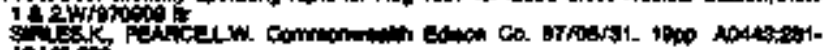
intion.

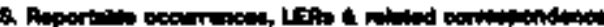

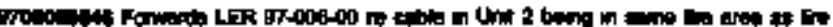

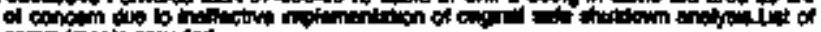

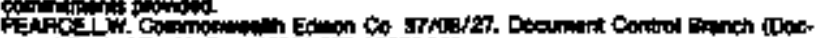

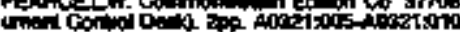

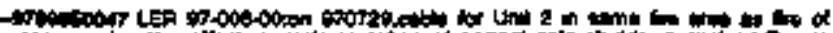

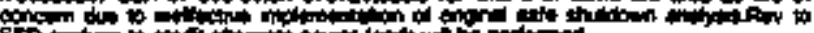

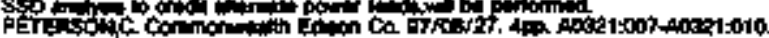

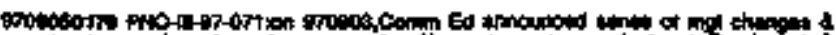

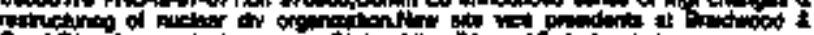

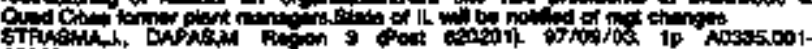

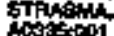

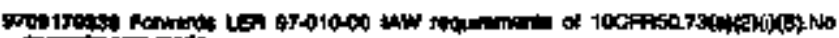
priping mada

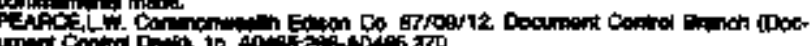

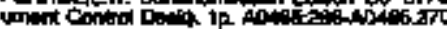

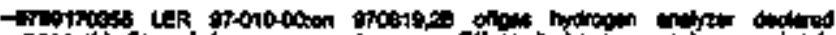

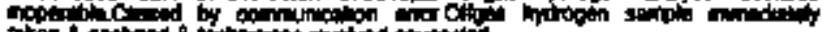

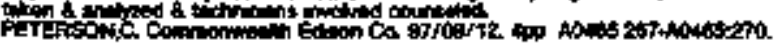

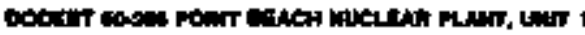

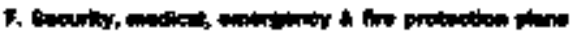

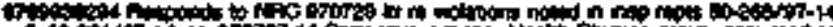

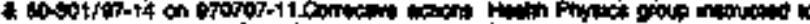

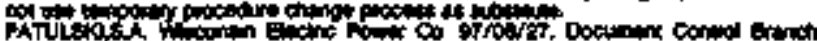

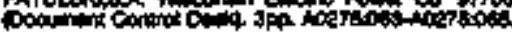

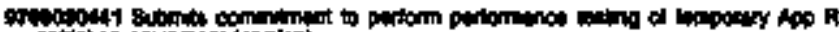

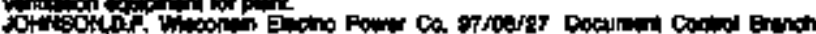

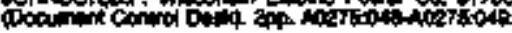

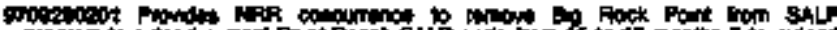

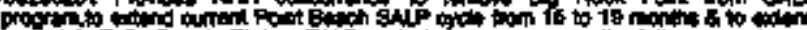

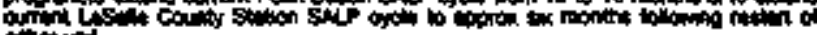

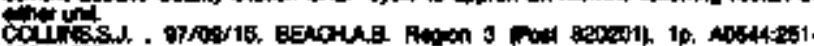
Antivis.

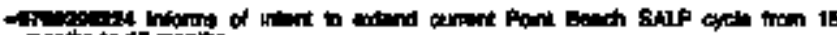

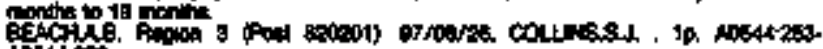
instion.

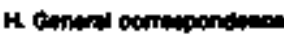

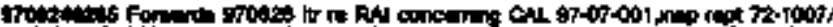

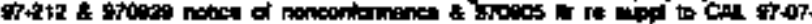
podwhend Aastat.

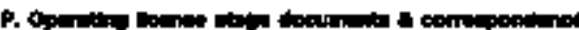

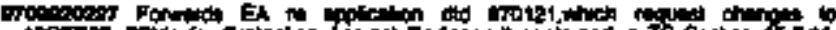

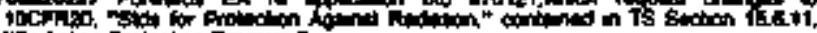

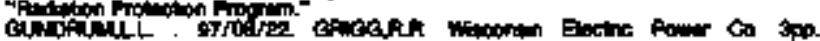

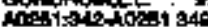

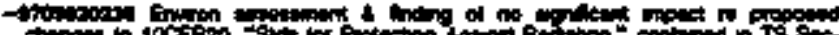

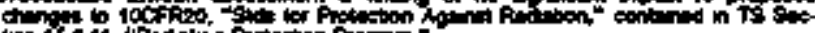

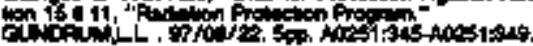

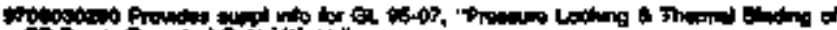

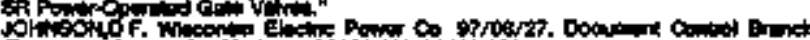

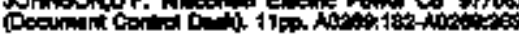

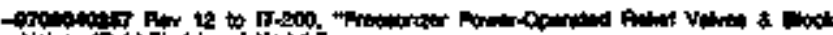

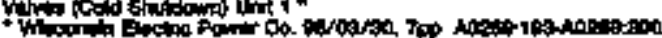

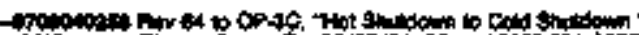

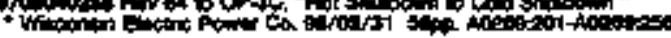

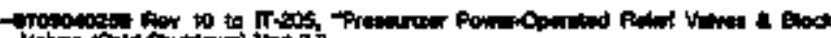

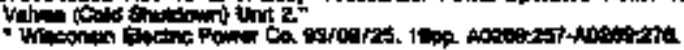

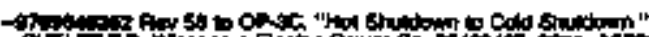

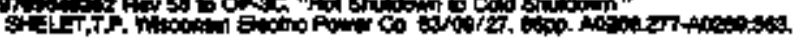

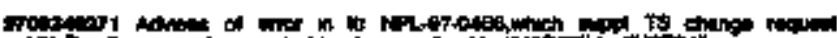

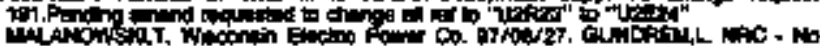

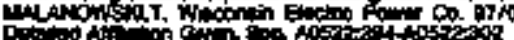

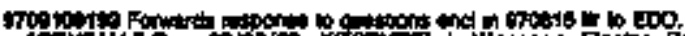

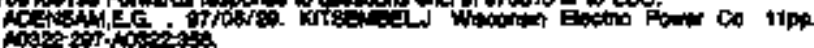

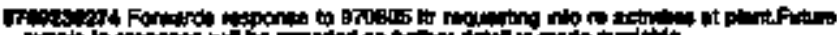

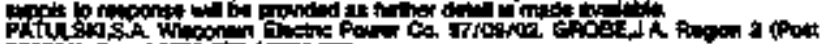

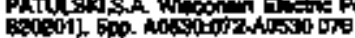

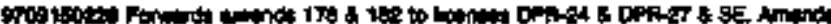

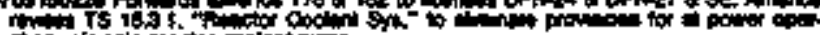

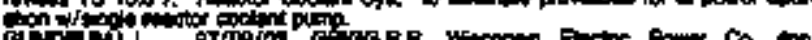

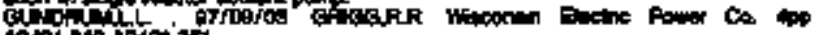

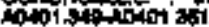

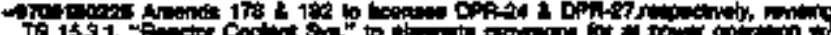

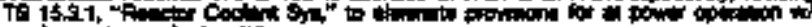

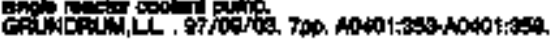

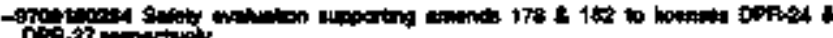

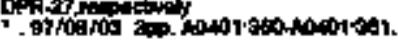

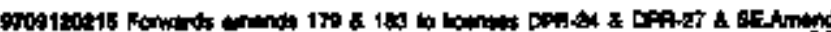
nites is LS

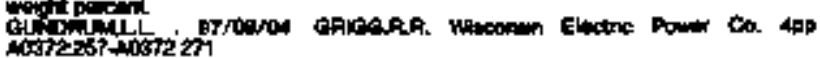




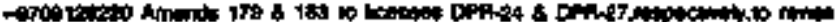

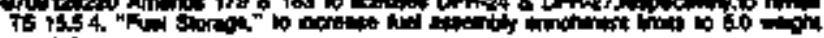

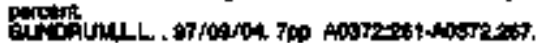

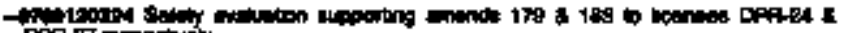
DPF-

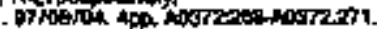

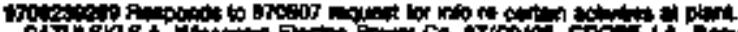

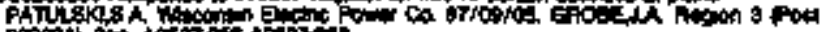

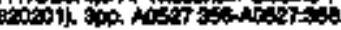

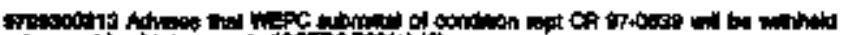

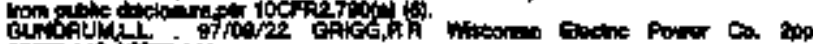

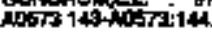

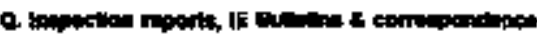

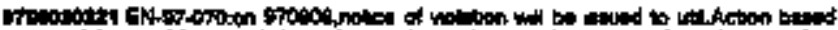

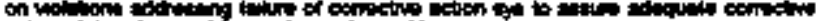

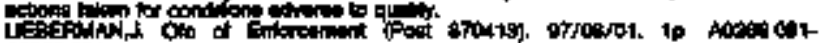
Acomososis.

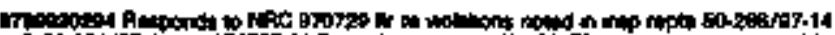

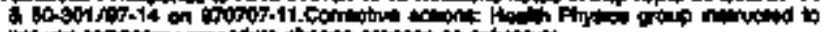

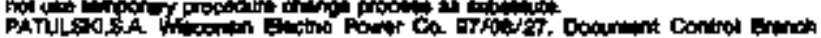

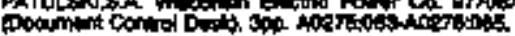

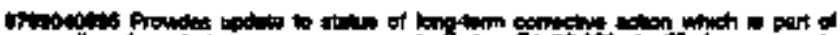

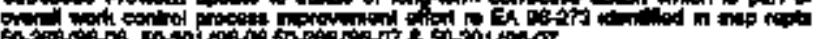

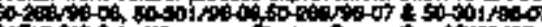

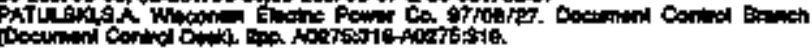

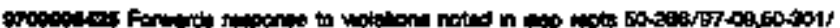

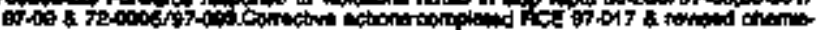

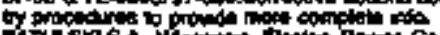

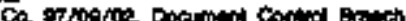

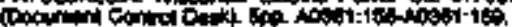

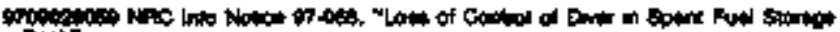

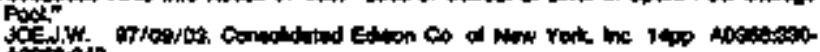

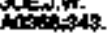

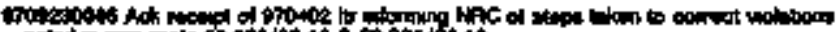

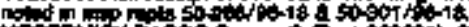

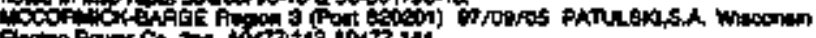

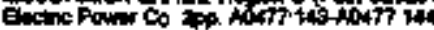

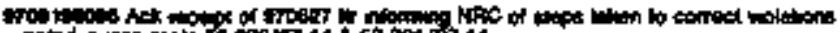

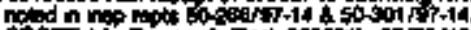

pich

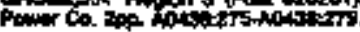

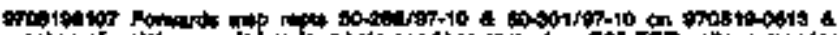

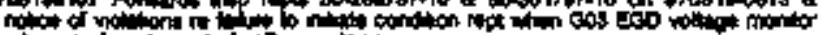

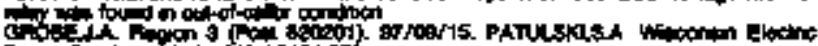

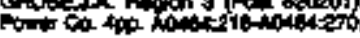

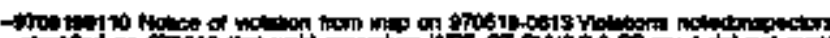

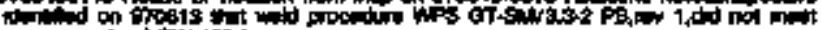

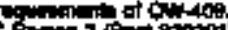

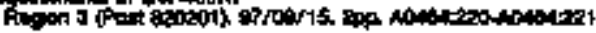

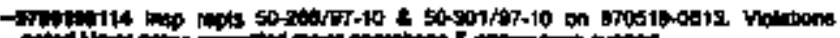

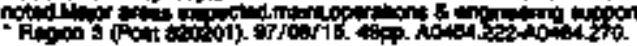

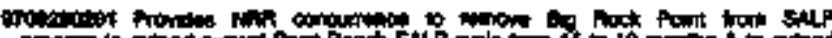

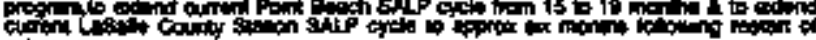

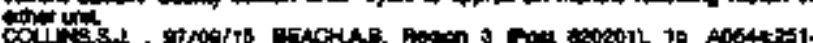
(x)

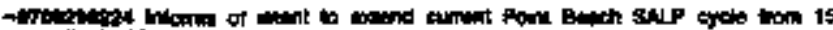

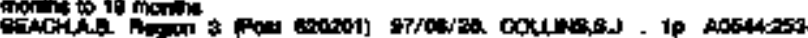
Nositios

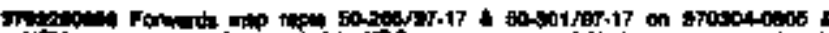

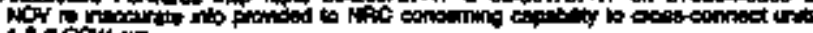

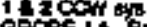

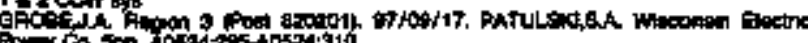

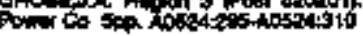

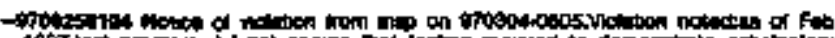

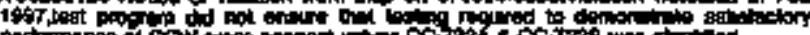

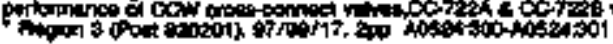

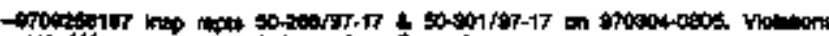

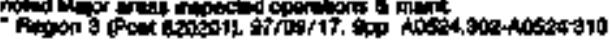

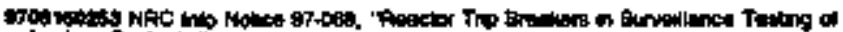
Anow

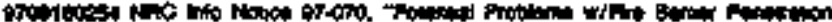

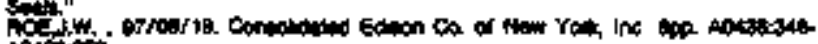
Notion

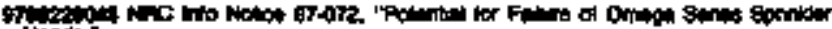

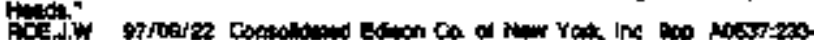
iactin.

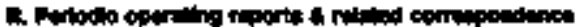

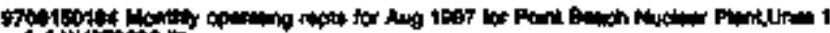
C. 2 Wropto

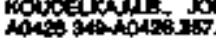

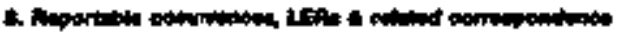

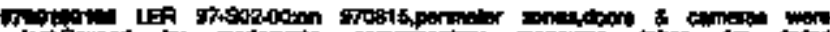

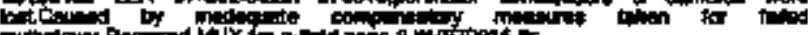

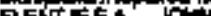

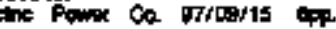

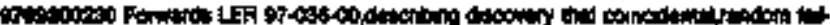

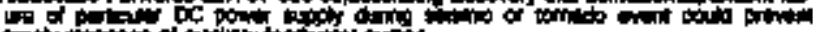

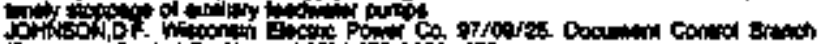

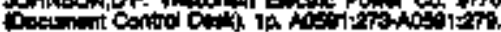

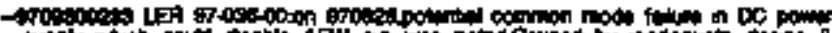

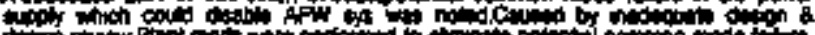

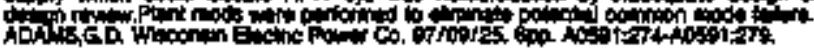

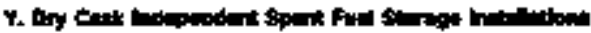

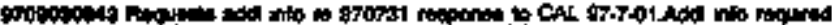

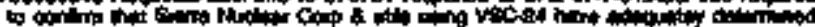

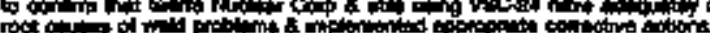

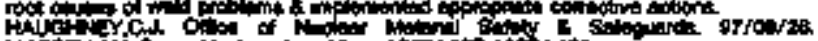

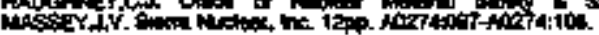

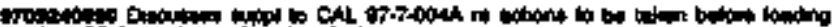

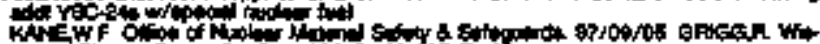

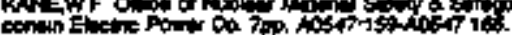

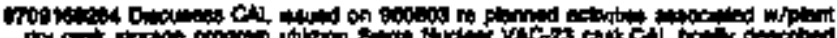

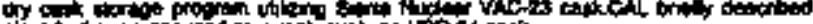

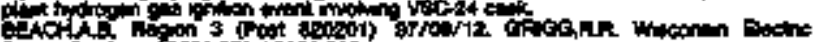

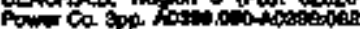

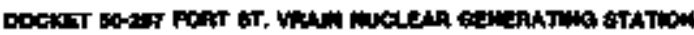

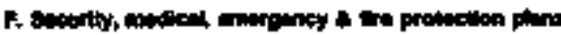

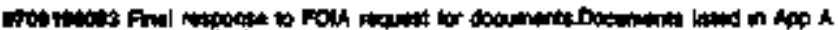

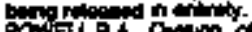

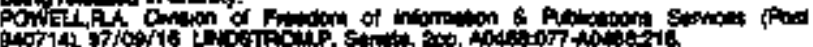

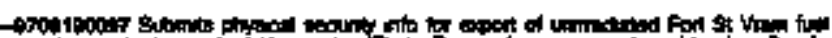

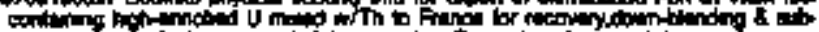

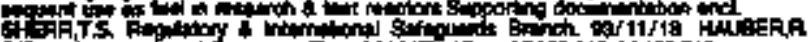

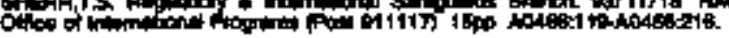

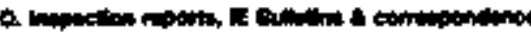

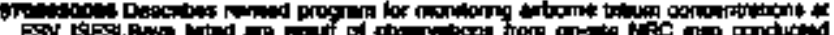

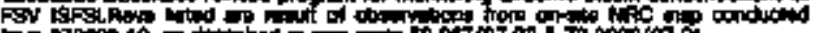

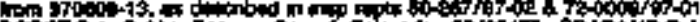

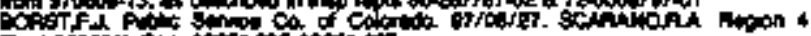

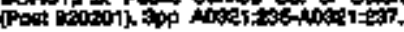

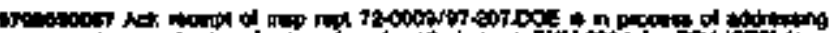

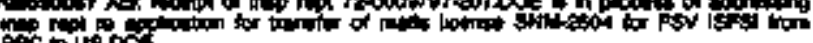
Pon to

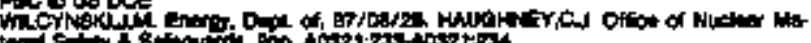

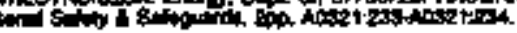

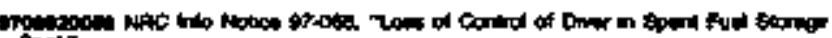
poed"

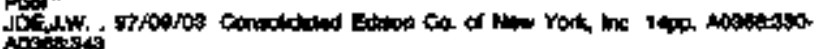

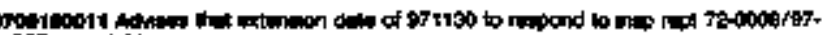

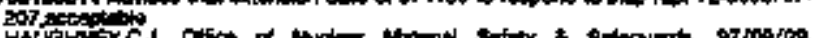

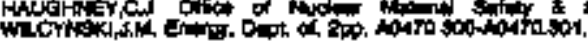

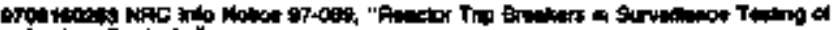

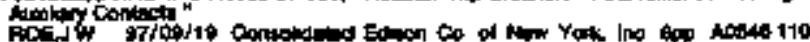
A0siditi 
ה

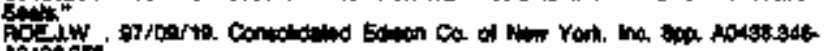
Anstias:

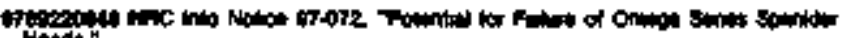

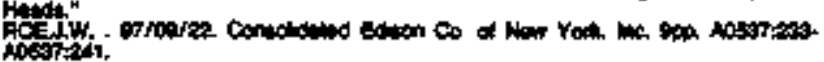

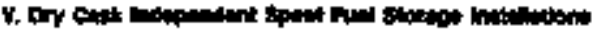

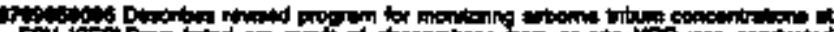

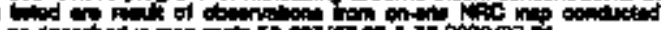

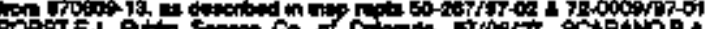

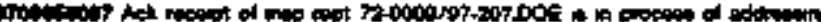

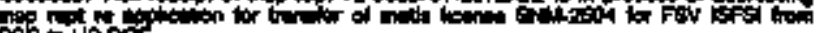

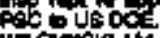

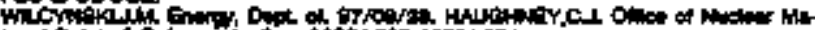

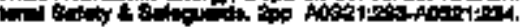

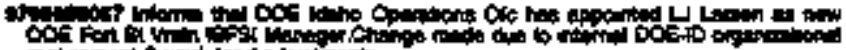

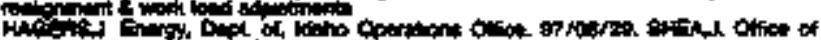

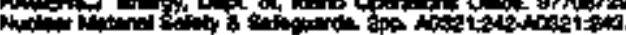

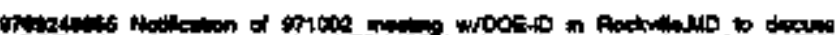

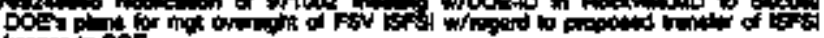

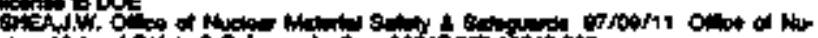

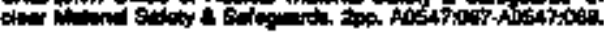

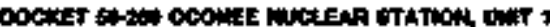

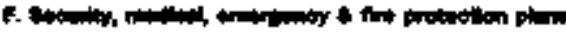

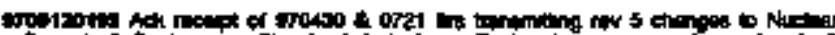

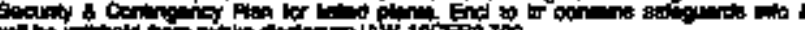

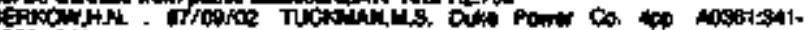

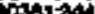

4. Francint intemention

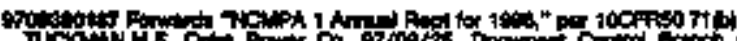

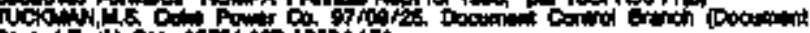

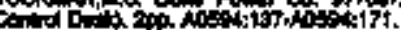

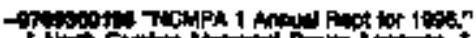

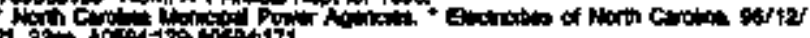

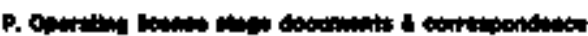

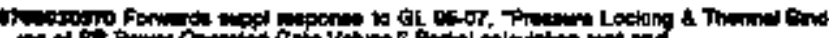

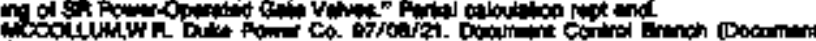

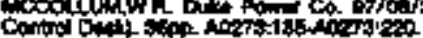

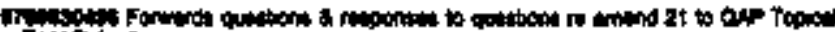

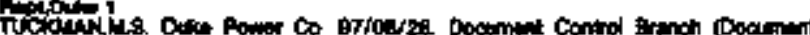

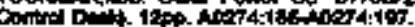

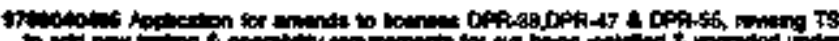

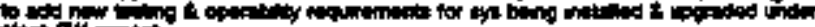

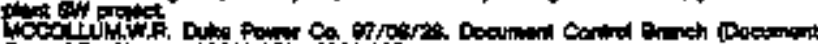

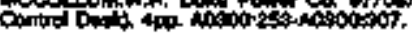

-

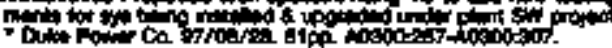

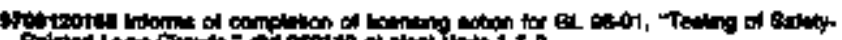

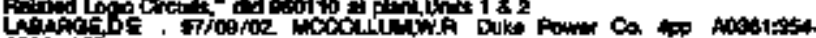
intist.

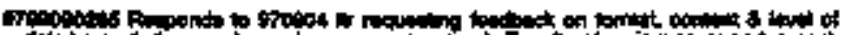

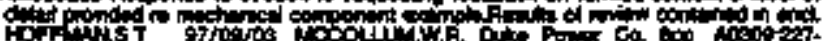
1000104

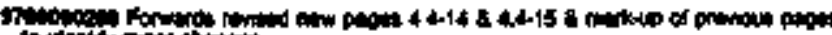

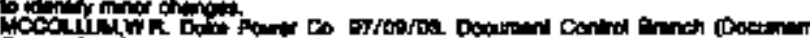

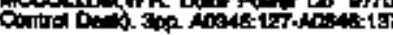

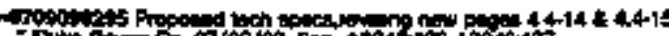

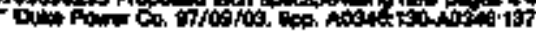

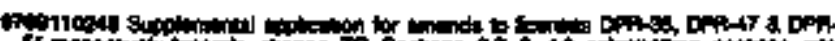

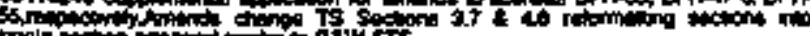

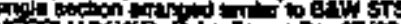
Cont D.

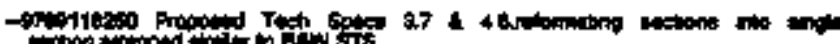

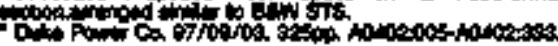

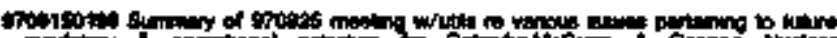

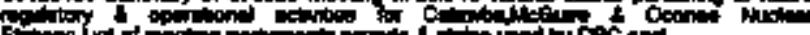

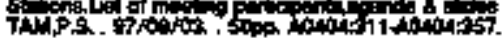

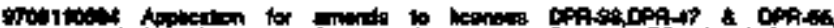

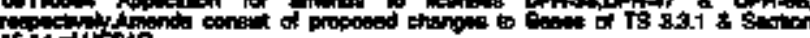
15.4 of $x=19$

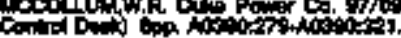

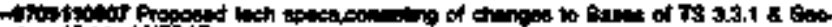

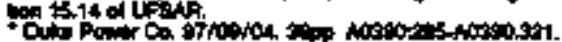

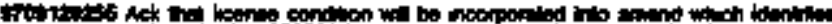

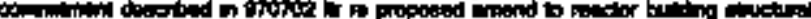

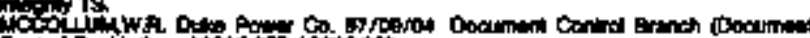

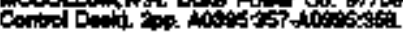

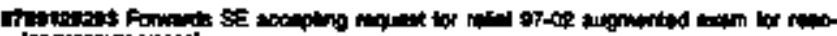

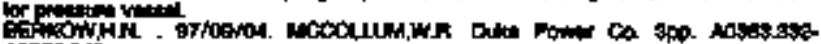
moskitsi3.

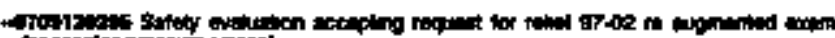

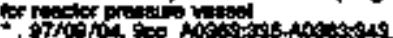

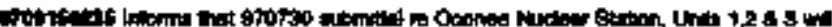

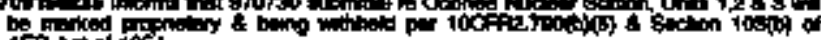

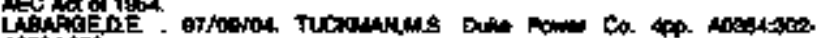
insolito

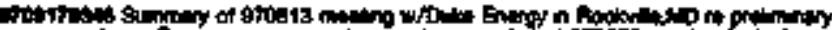

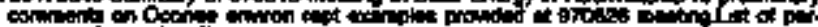

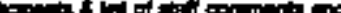

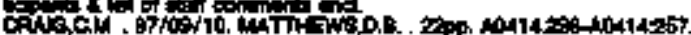

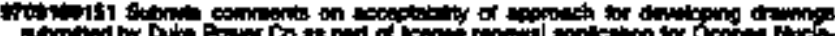

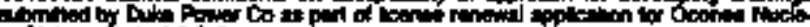

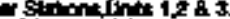

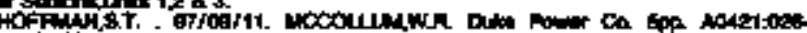
40421506 .

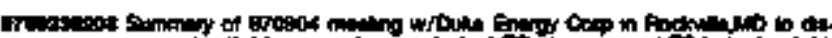

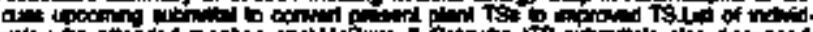

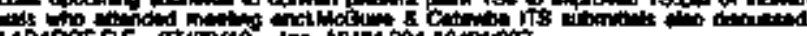

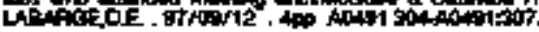

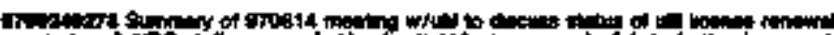

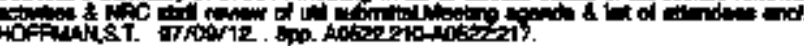

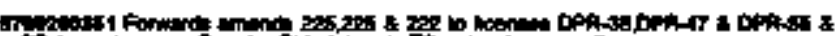

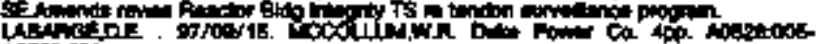
Lations

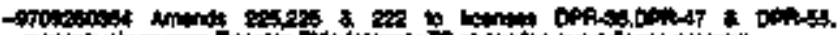

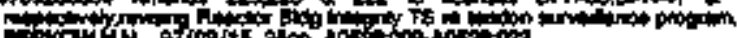

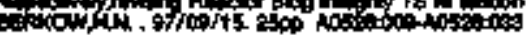

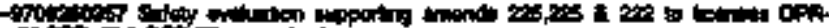

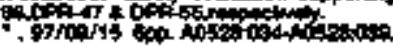

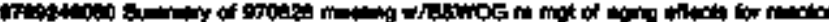

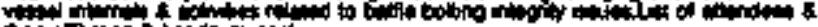

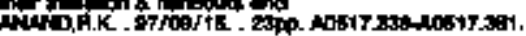

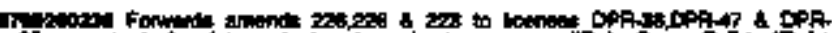

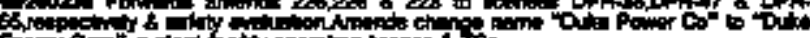

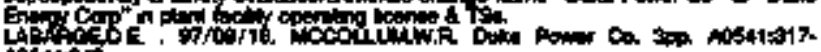
Antisid

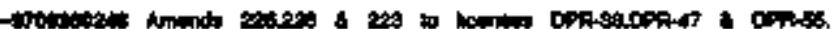

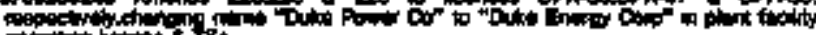

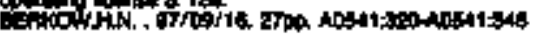

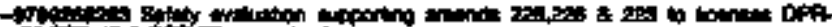

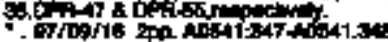

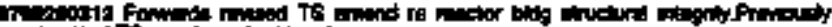

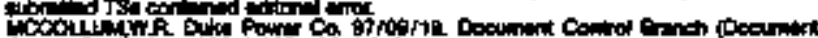

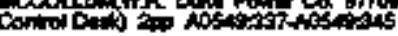

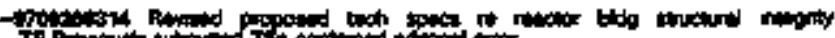

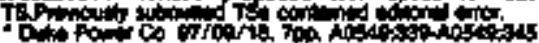

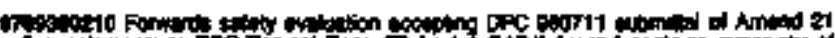

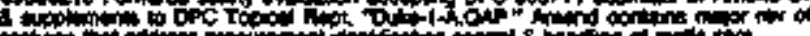

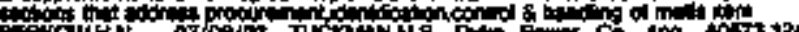
Aponatis 


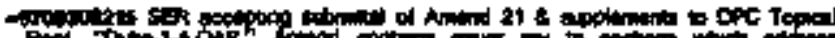

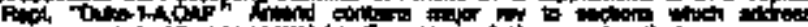

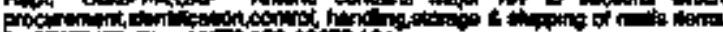

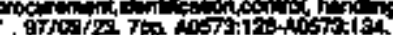

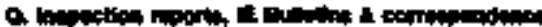

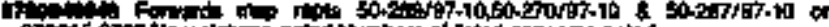

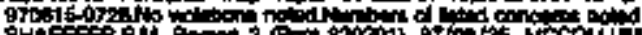

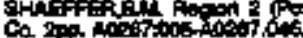

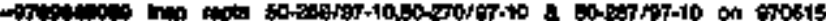

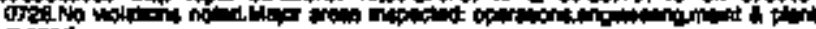

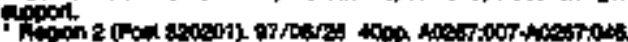

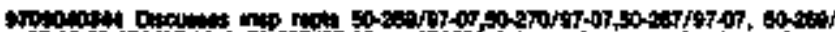

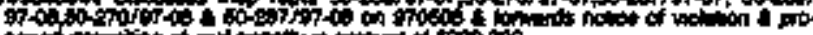

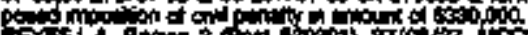
Pons

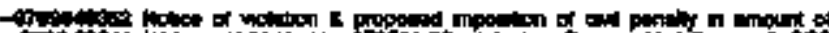

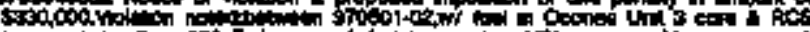

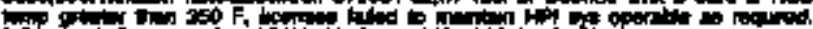

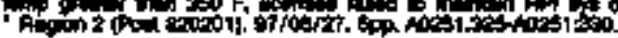

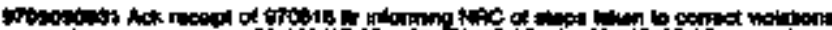

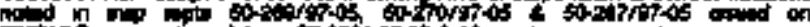

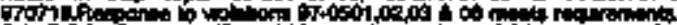

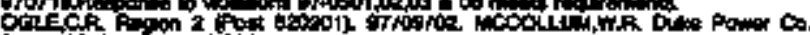

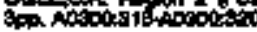

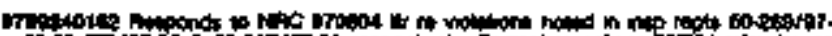

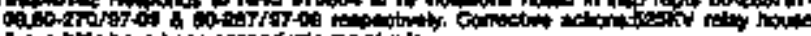

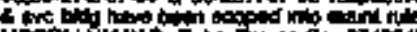

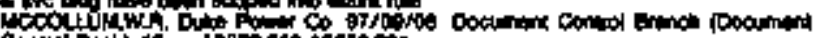

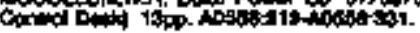

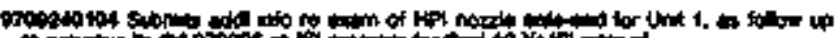

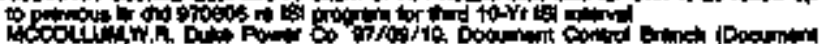

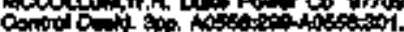

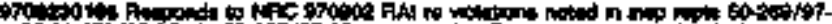

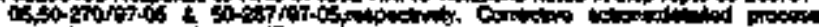

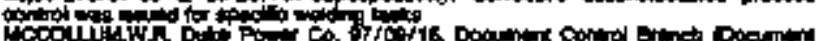

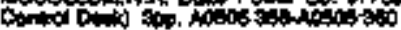

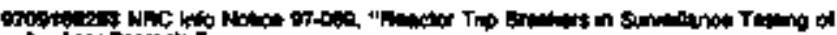

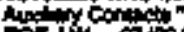

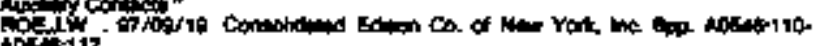
AOST17.

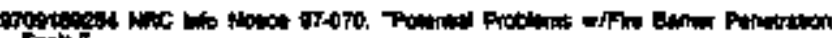

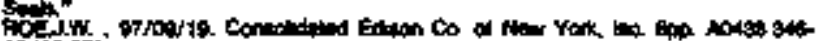
A

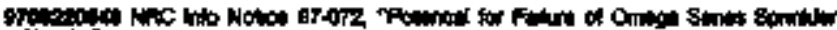

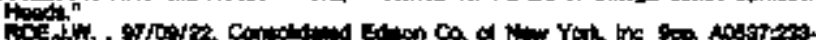
$\cos 241$.

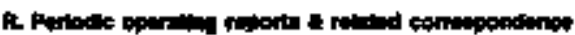

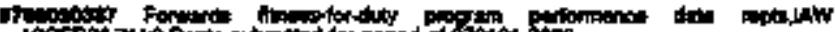

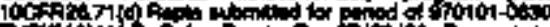

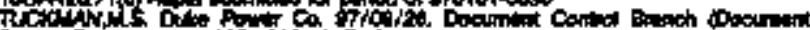

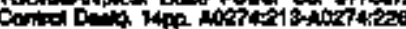

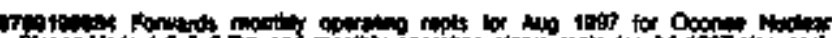
s.

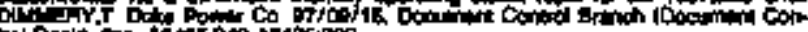

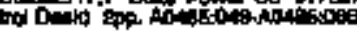

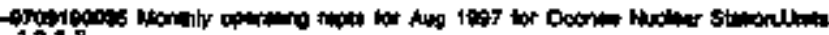
13 s.

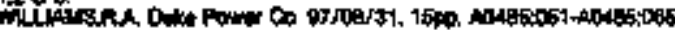

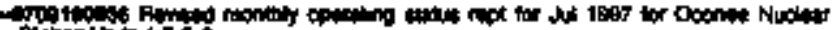

340 intor 12 a

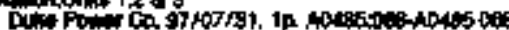

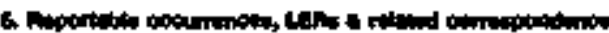

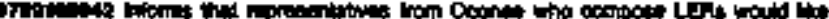

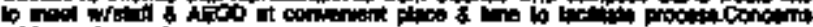

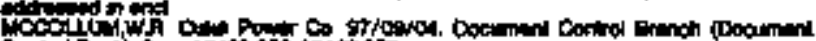

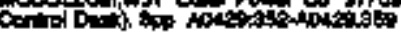

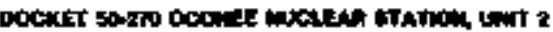

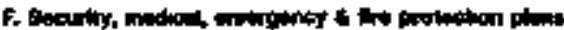

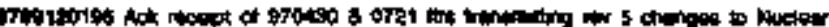

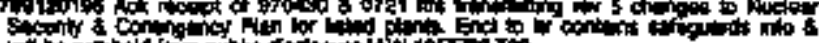

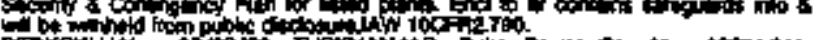
Acosed 3

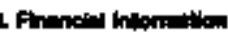

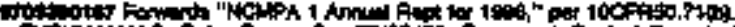

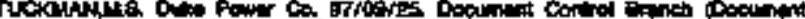

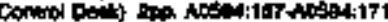

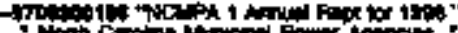

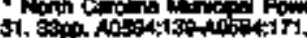

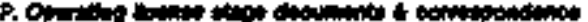

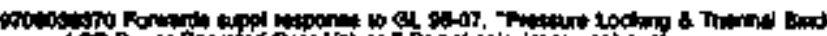

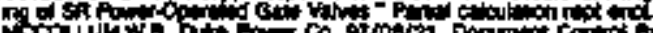

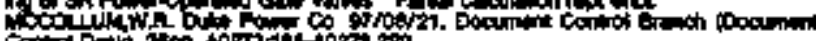

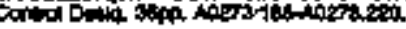

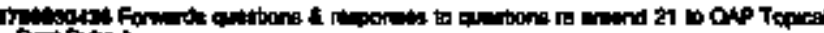
Pition

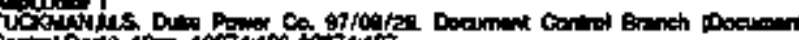

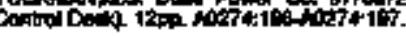

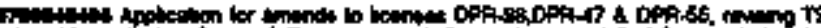

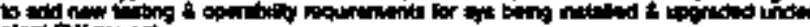

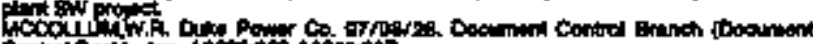

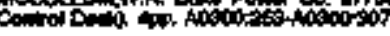

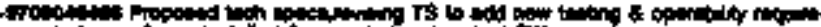

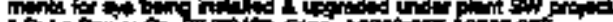

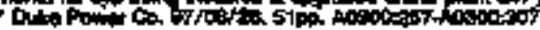

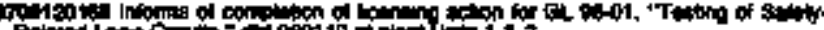

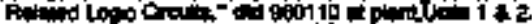

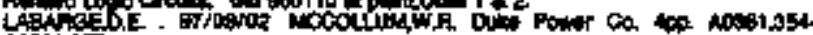
torist-35

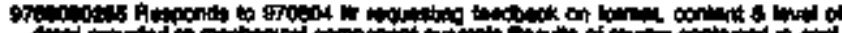

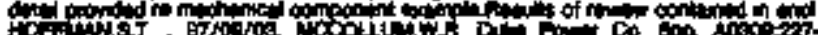
tion

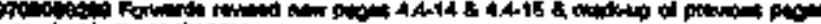

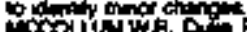

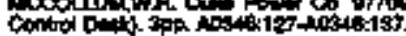

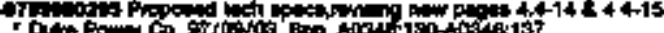

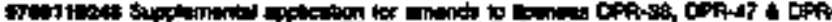

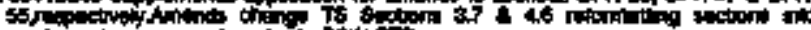

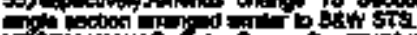

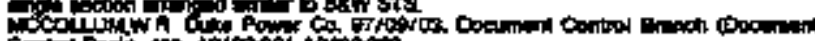

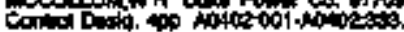

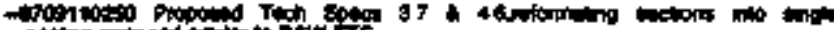

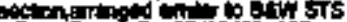

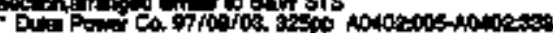

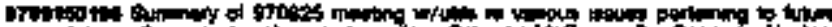

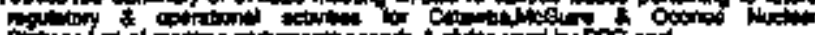

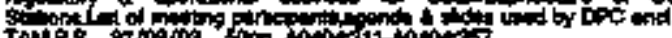

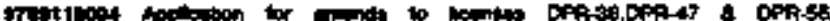

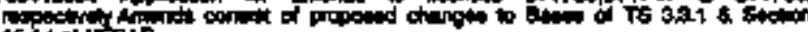

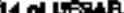

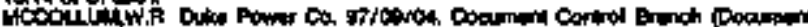

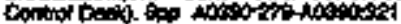

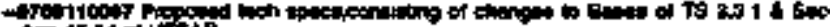

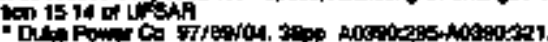

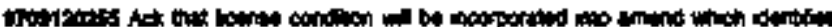

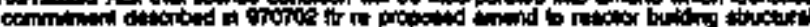
+

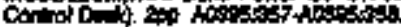

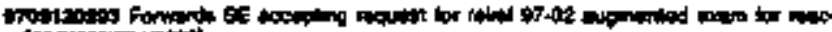

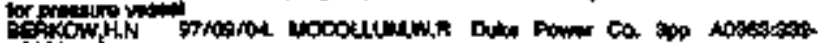
Nocosis.

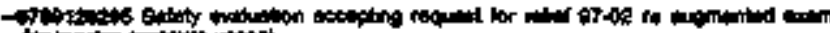

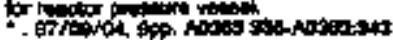

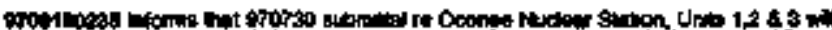

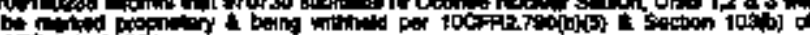

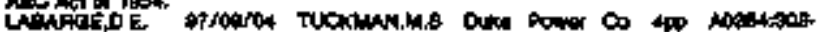
Arsid 305

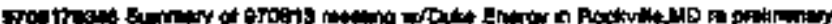

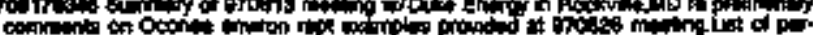

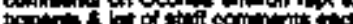

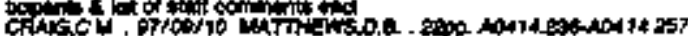




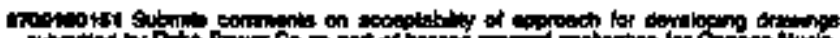

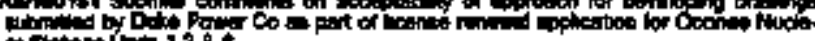

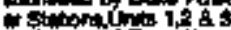

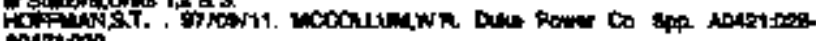
antioso.

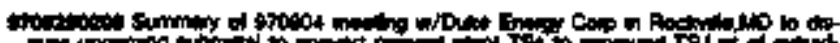

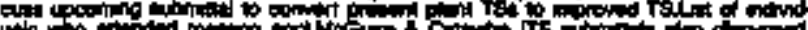

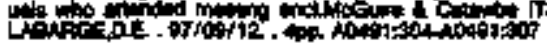

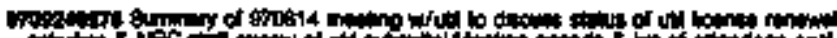

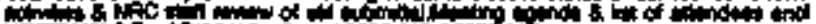

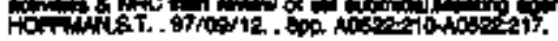

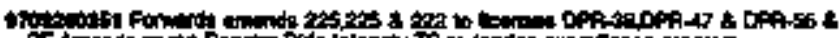

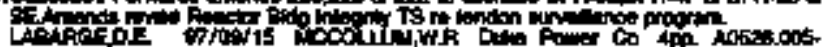
Atsing

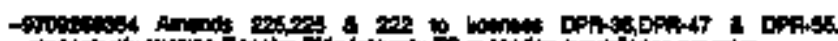

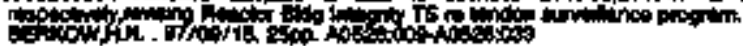

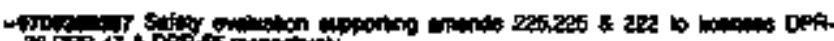

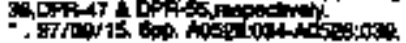

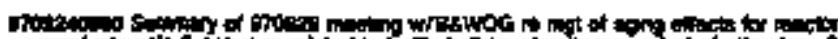

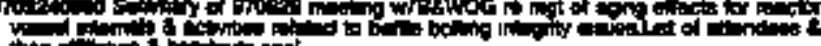

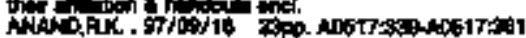

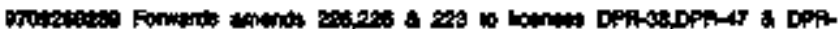

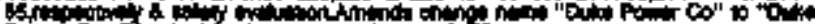

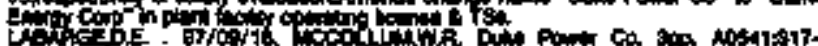
Acositisid

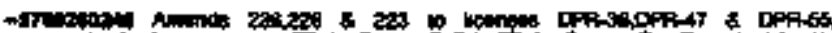

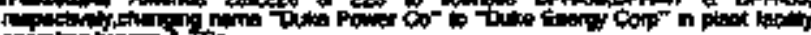

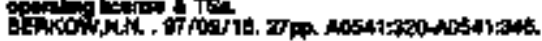

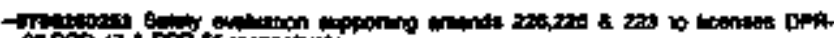

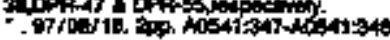

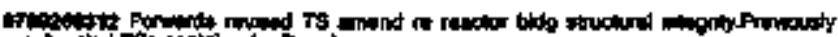

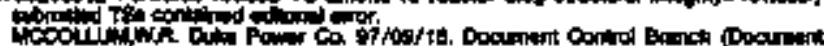

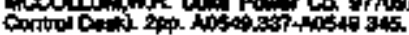

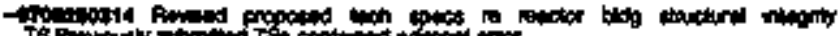

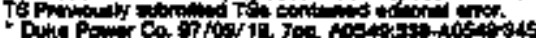

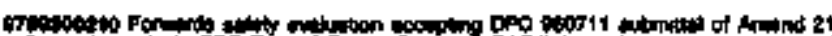

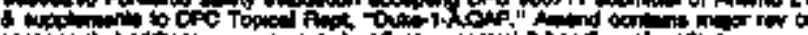

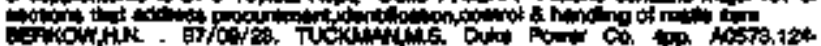
ingtion

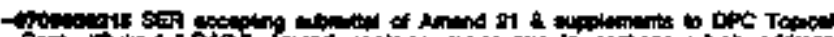

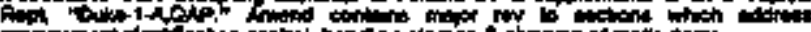

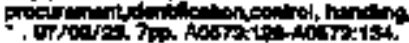

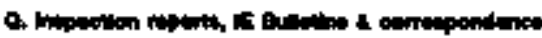

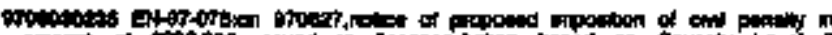

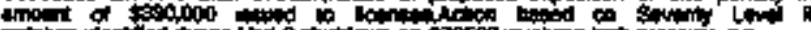

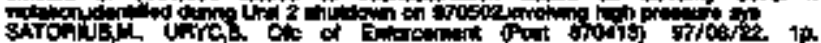
Afestom

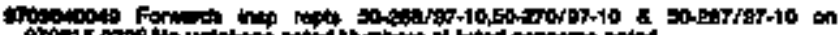

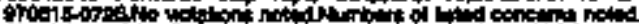

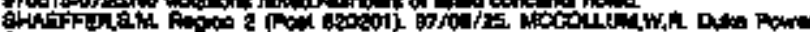

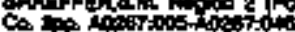

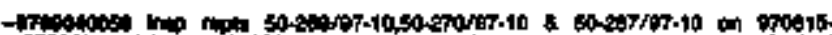

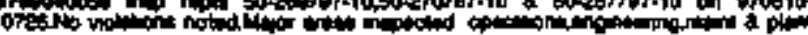

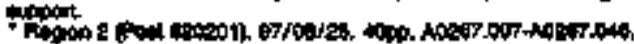

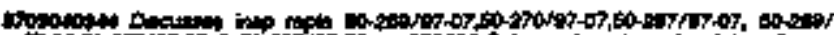

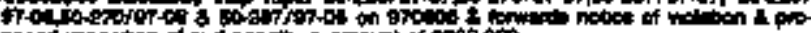

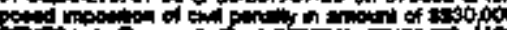

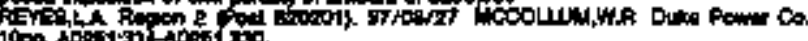

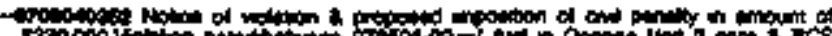

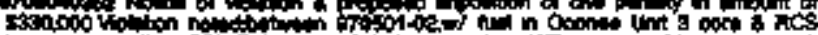

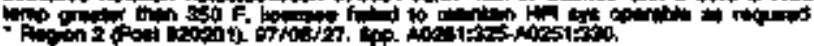

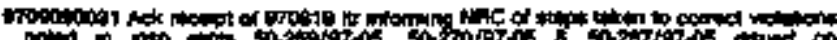

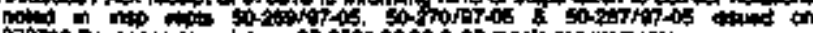

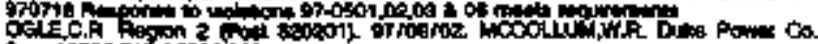

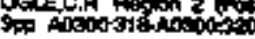

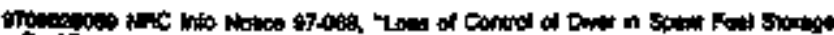

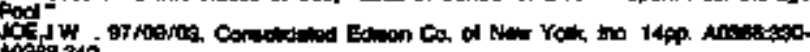
40

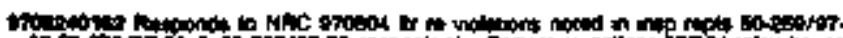

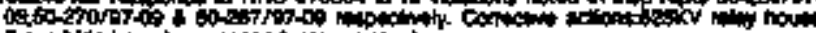

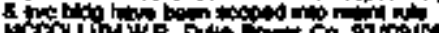

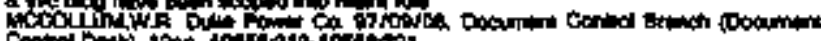

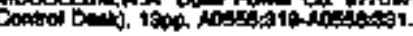

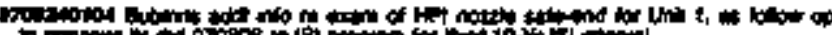

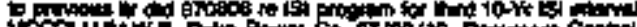

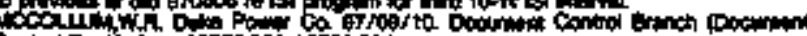

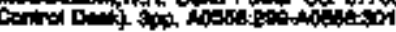

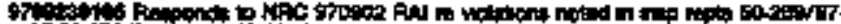
OSE0 2 mo

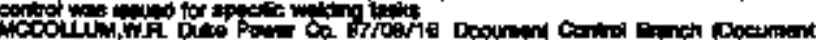

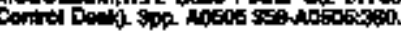

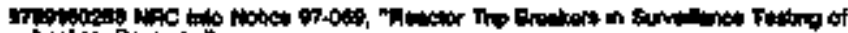

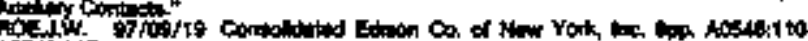

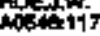

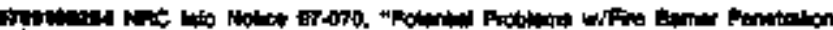

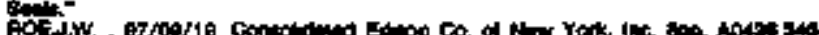
nototist.

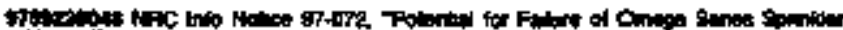

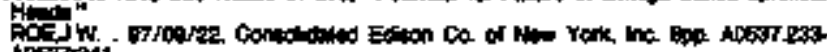
nosirini.

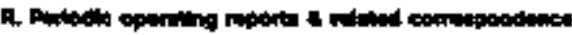

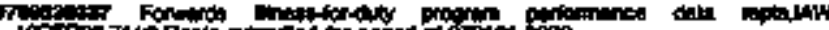
1000 UCA

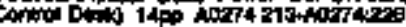

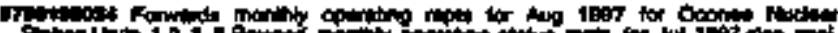

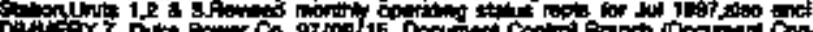

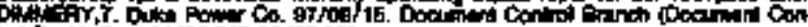

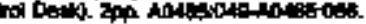

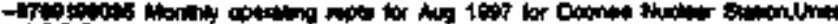

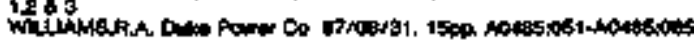

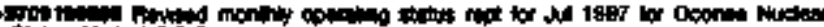

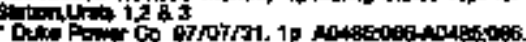

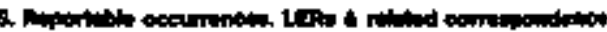

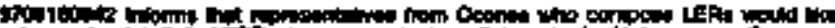

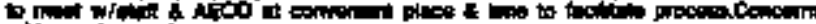

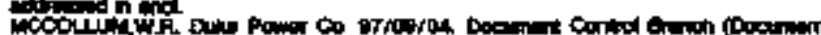

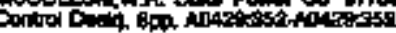

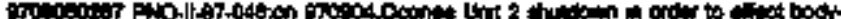

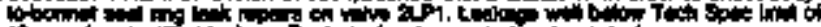

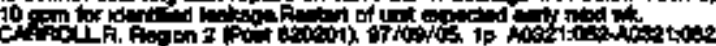

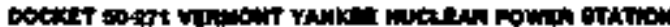

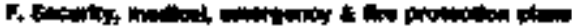

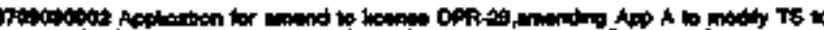

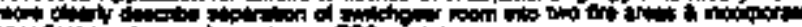

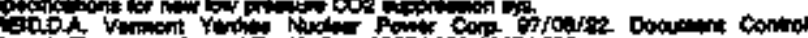

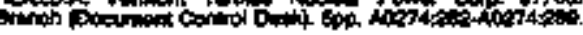

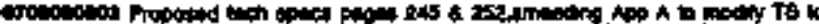

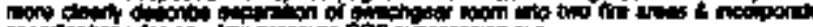

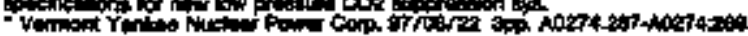

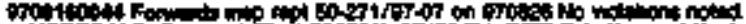

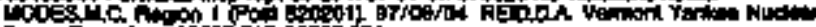

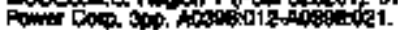

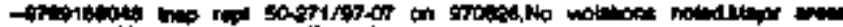

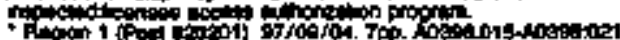

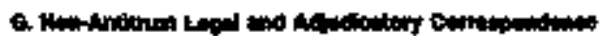

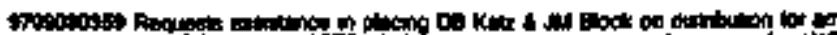

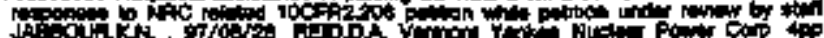

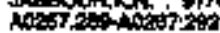




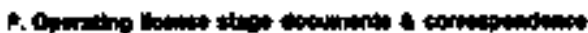

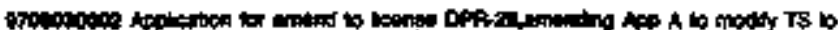

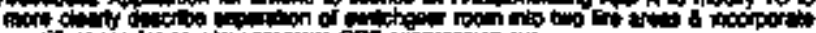

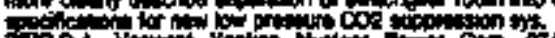

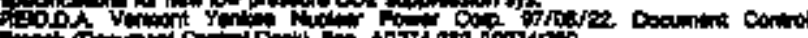

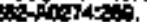

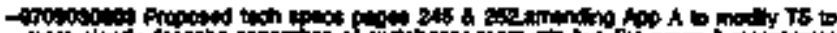

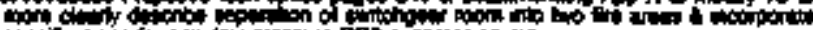
(n)

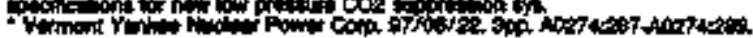

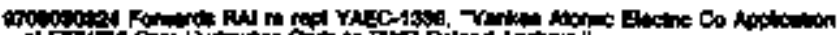
of Fint

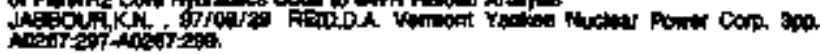

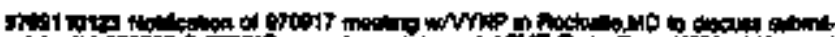

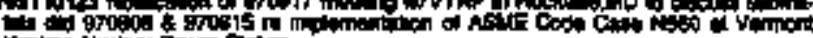

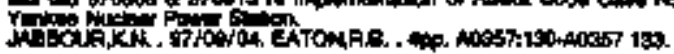

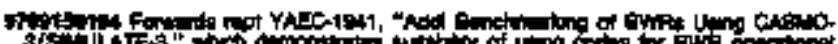

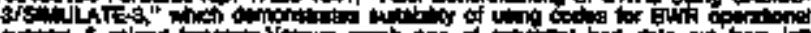

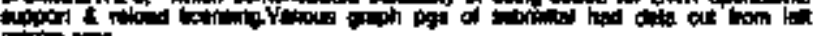
(1)

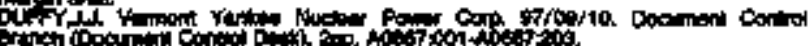

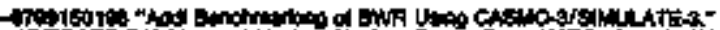

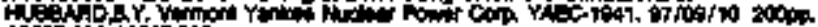

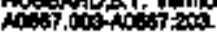

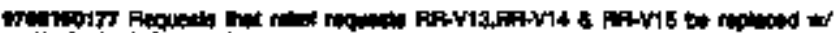

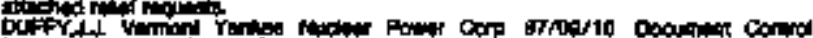

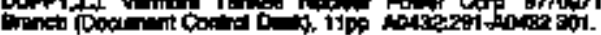

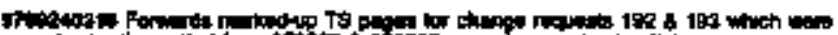

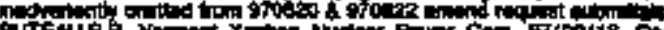

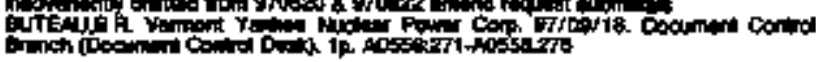

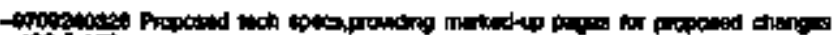
10\%

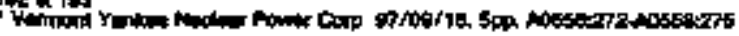

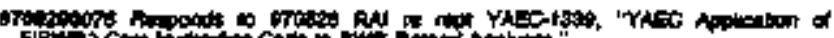

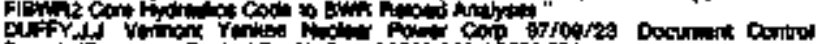

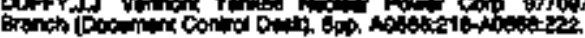

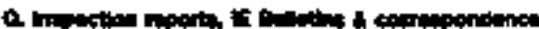

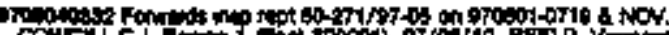

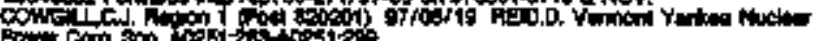

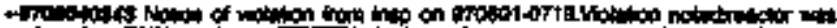

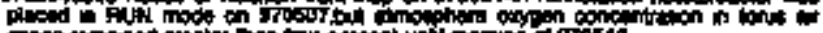

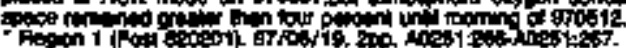

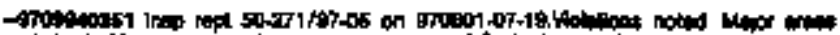

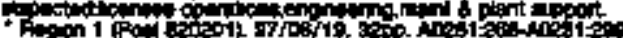

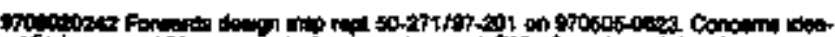

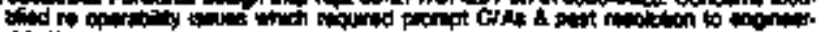

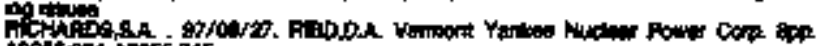

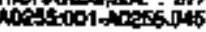

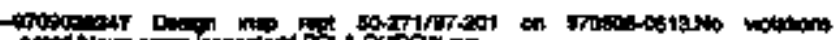

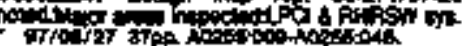

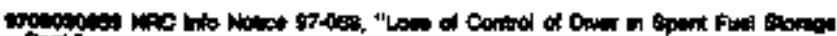

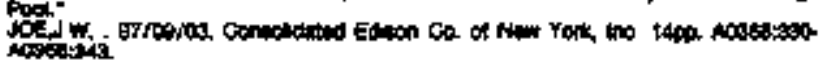

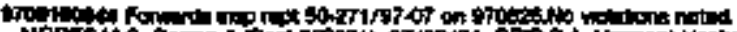

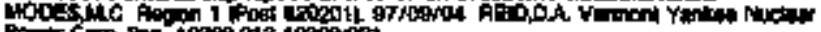

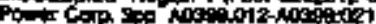

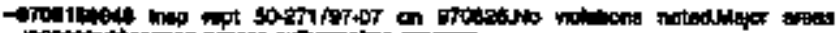

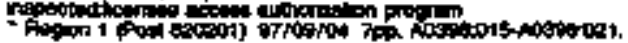

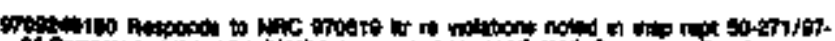

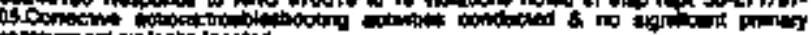

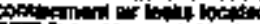

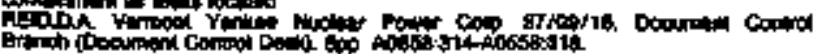

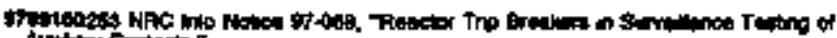

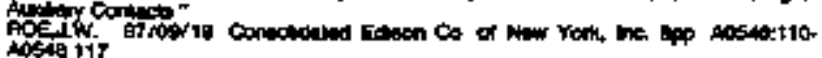

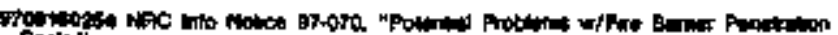

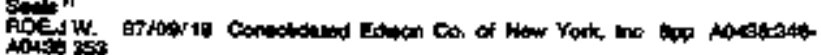

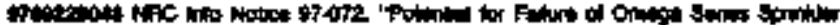

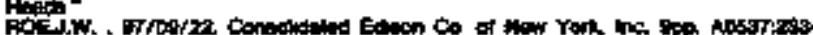
iositit.

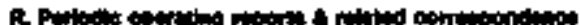

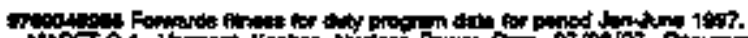

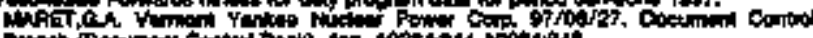

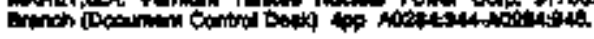

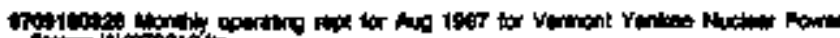

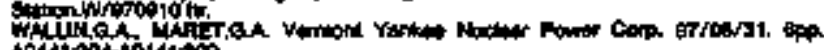

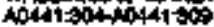

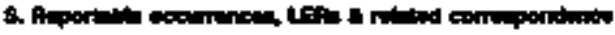

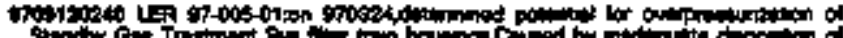

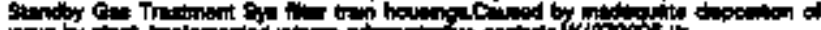

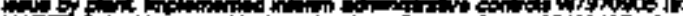

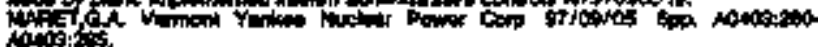

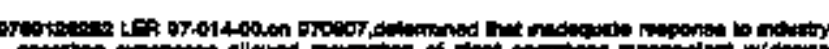

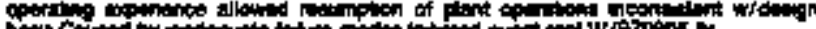

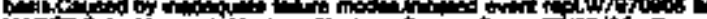

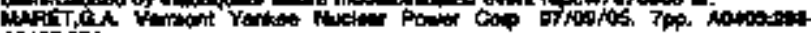
دow

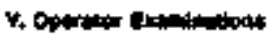

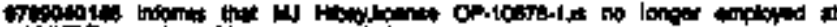

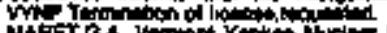

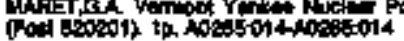

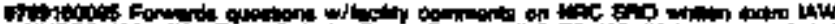

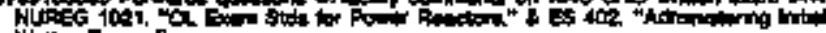

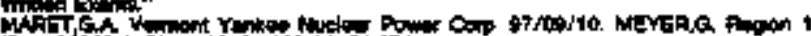
(A)

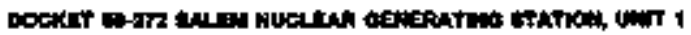

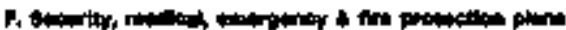

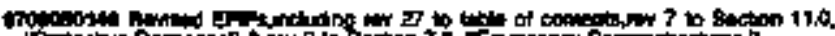

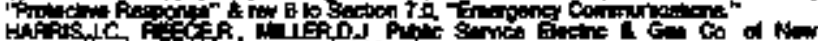

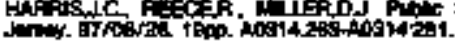

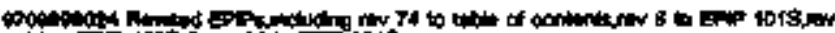

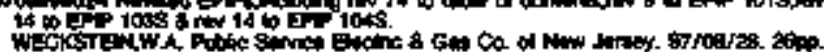

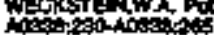

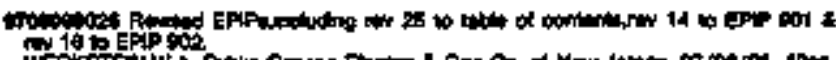
A 19 o

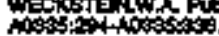

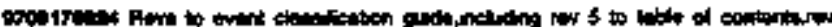

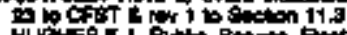
Aot4tor or

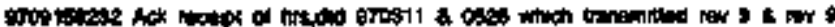

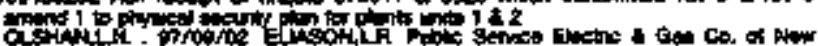

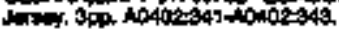

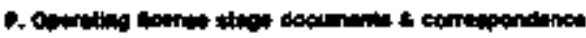

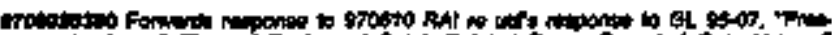

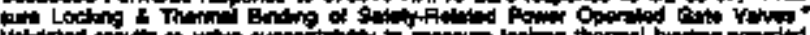

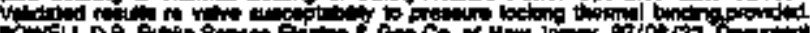

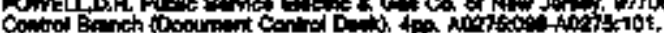

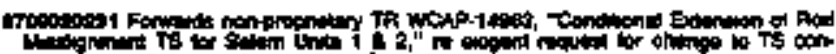

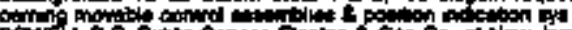

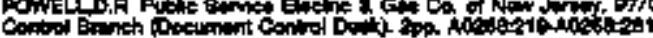

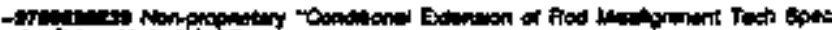

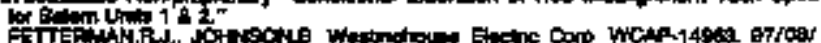

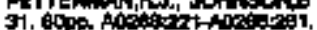

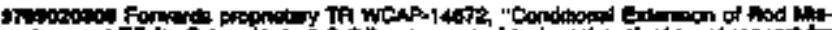

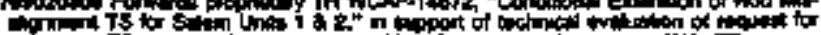

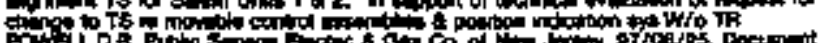

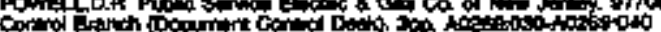




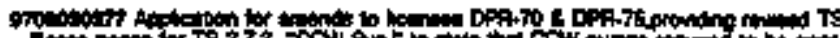

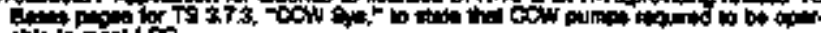

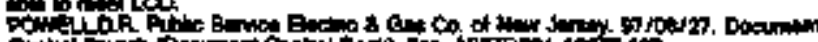

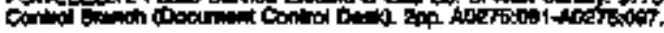

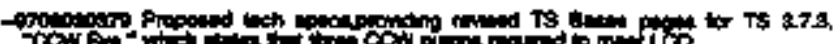

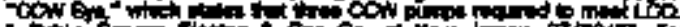

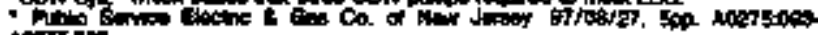
Aatron.

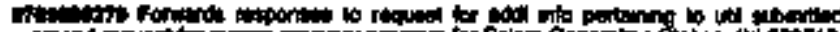

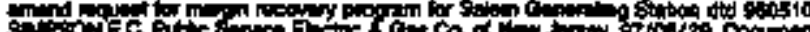

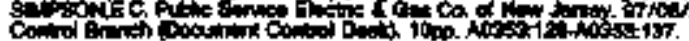

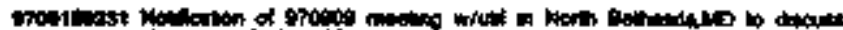

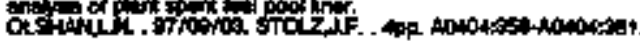

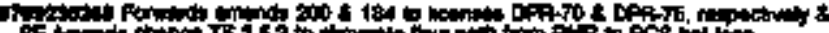

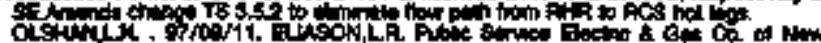

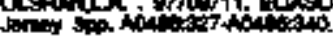

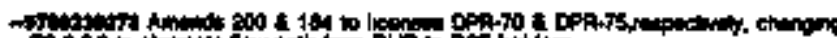

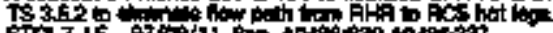

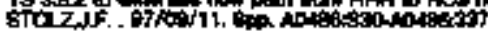

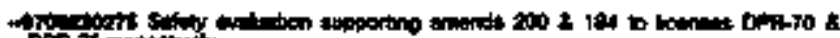

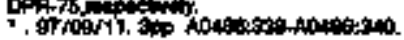

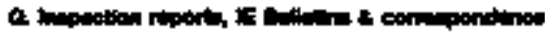

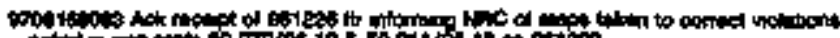

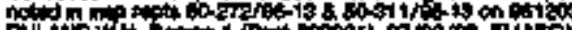

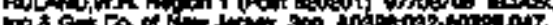

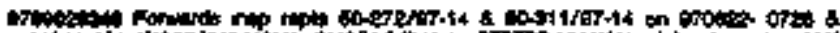

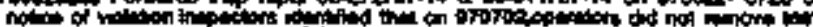

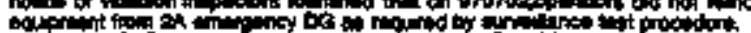

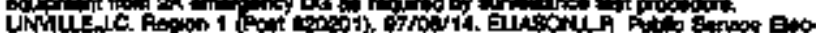

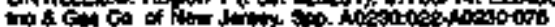

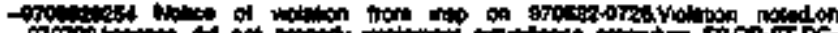

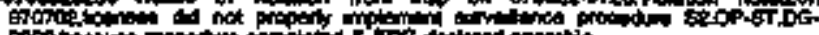

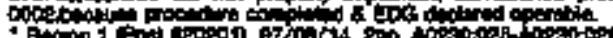

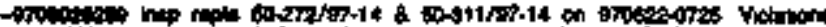

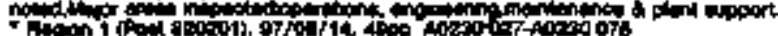

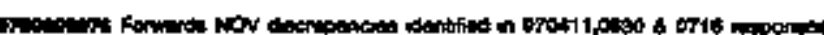

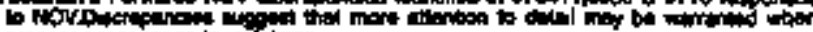

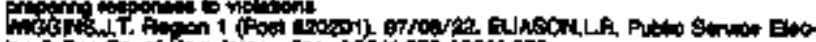

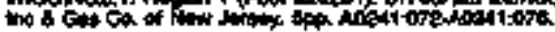

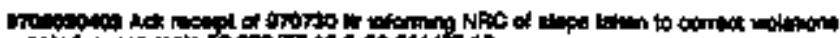

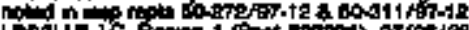

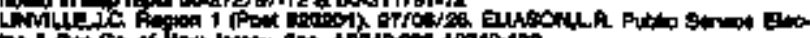

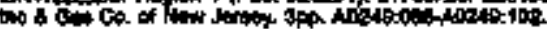

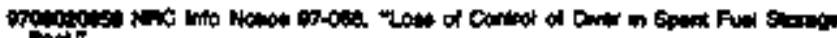

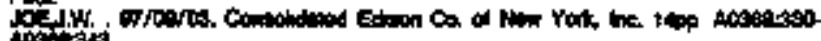

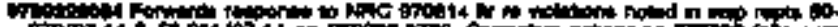

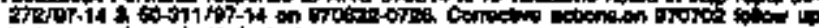

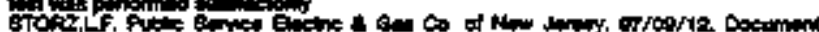

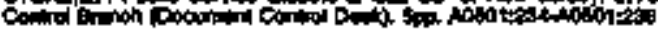

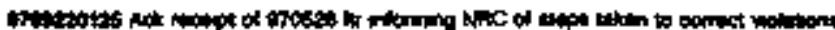

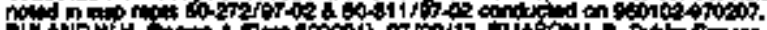

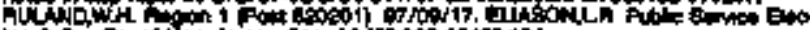

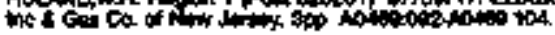

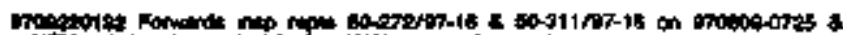

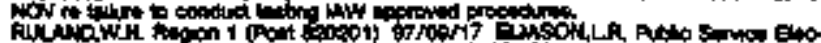

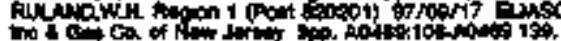

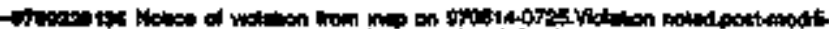

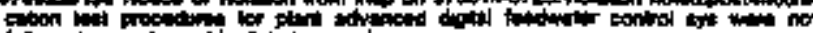

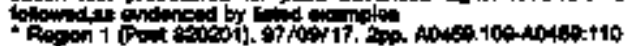

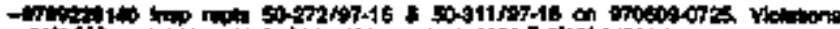

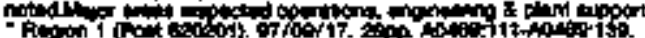

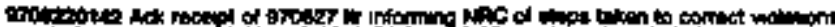

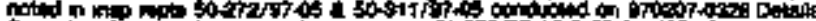

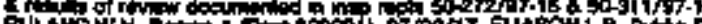

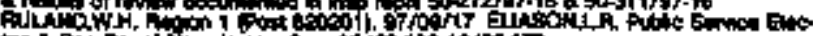

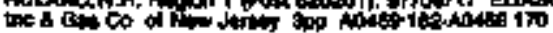

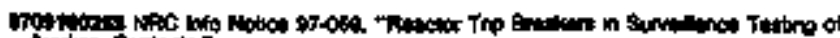

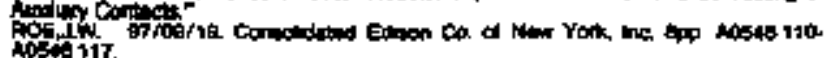

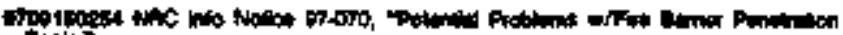

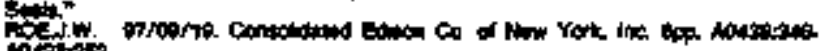
notion

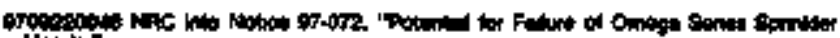

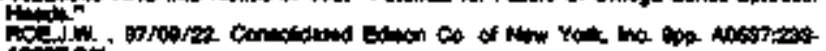

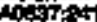

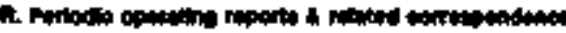

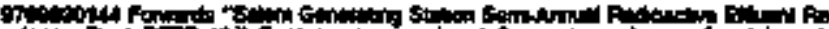

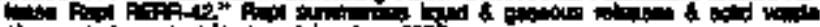

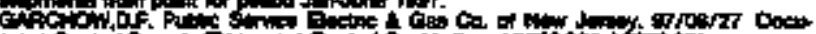

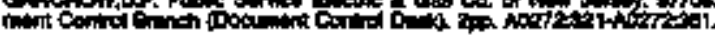

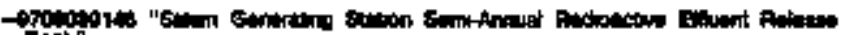

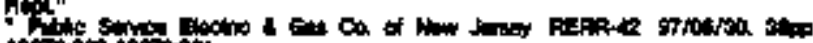

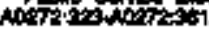

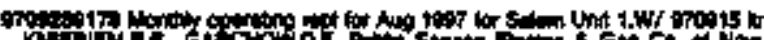
k.

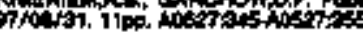

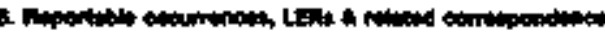

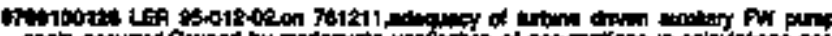

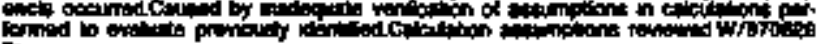

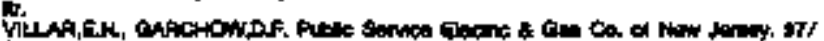
oltris.

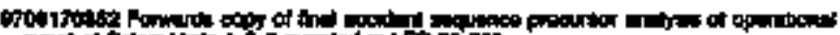

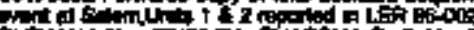

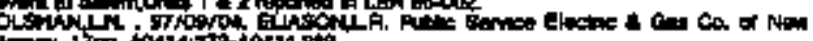

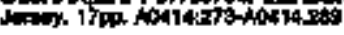

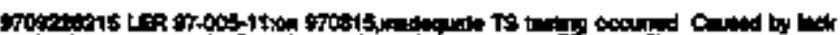

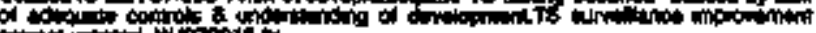

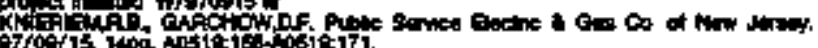

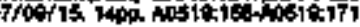

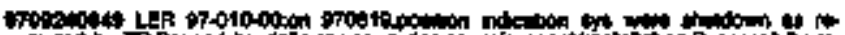

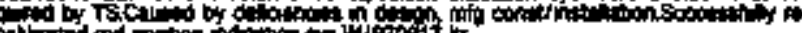

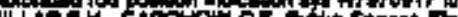

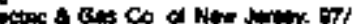
Dir.

Y. Dpotion Extrimion

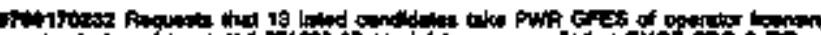

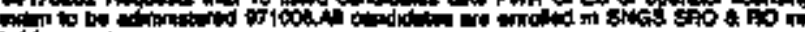

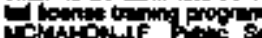

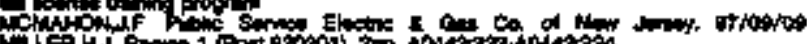

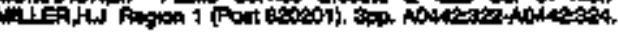

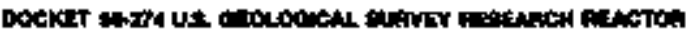

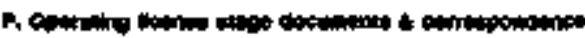

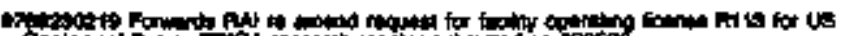

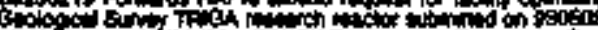

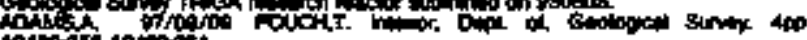

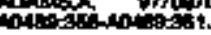

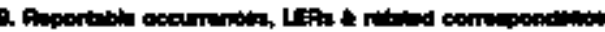

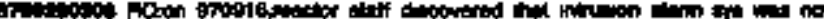

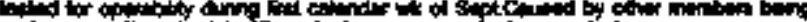

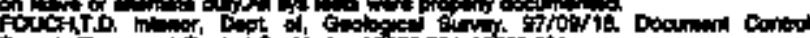

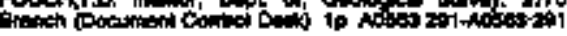

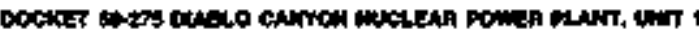

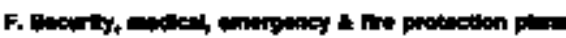

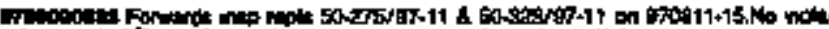

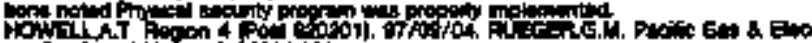

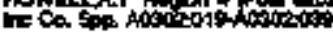

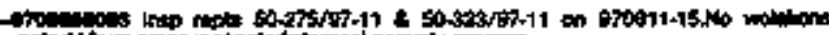

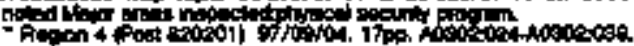

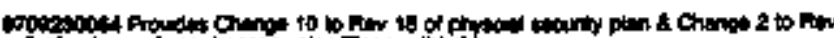

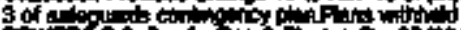

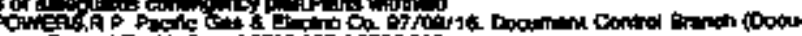

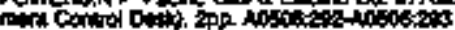




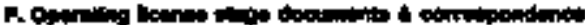

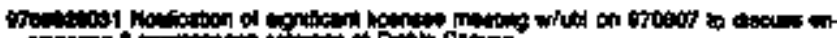

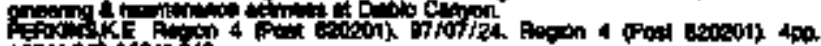

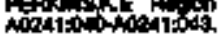

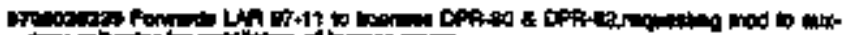

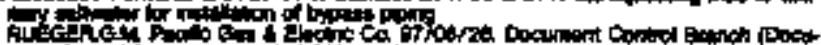

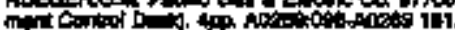

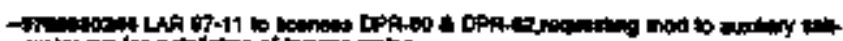

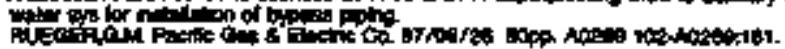

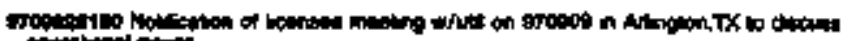

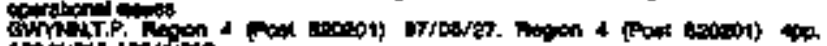

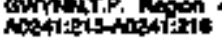

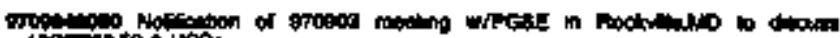

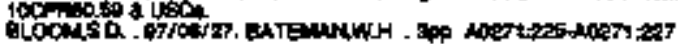

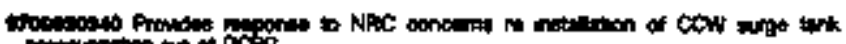

Pit

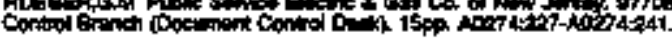

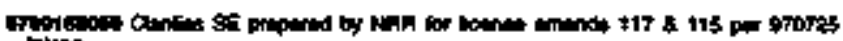

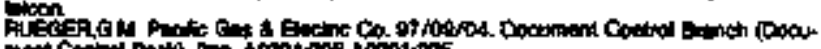

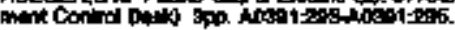

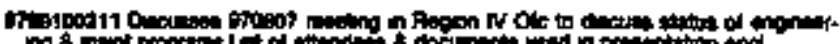

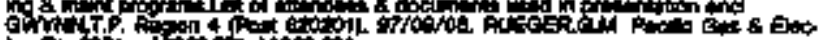

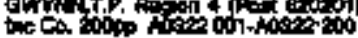

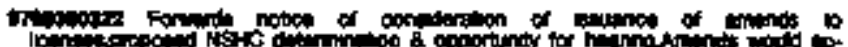

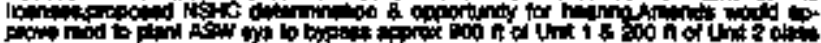

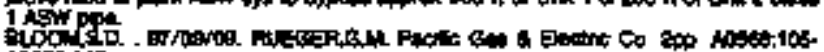
Apbigitio.

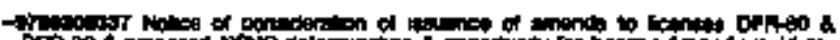

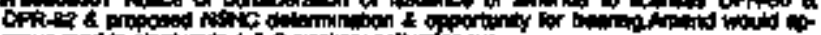

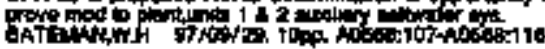

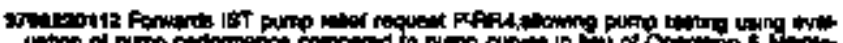

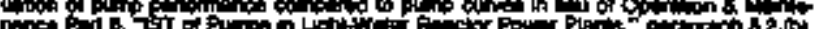

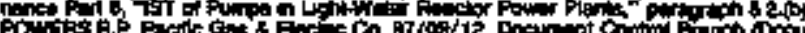

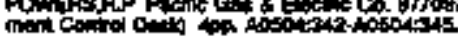

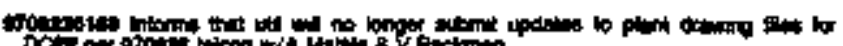

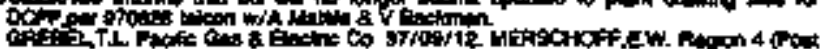

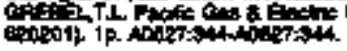

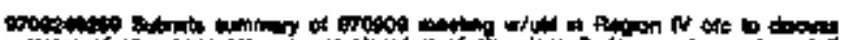

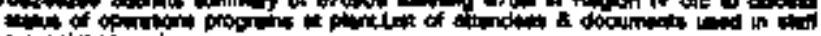

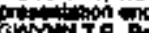

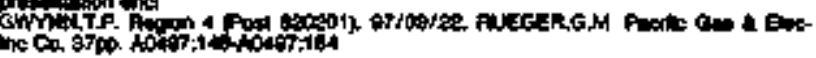

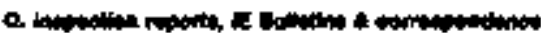

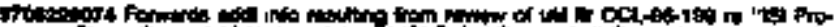

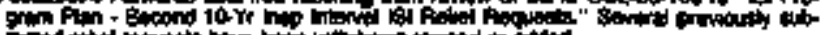

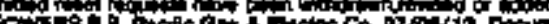

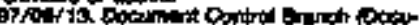

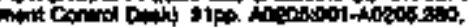

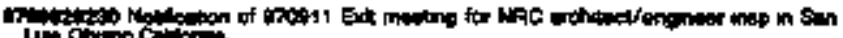

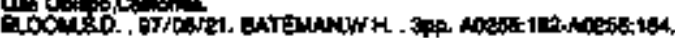

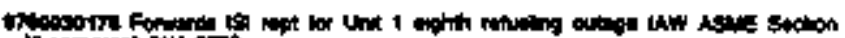

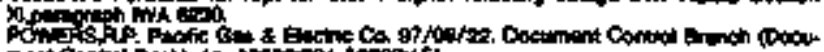

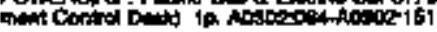

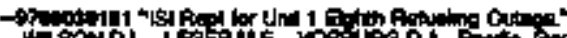

4how L LE⿱

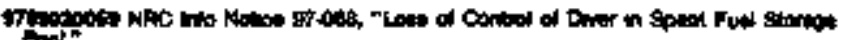

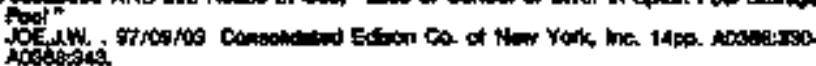

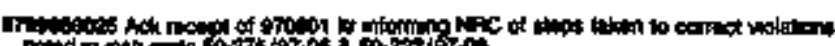

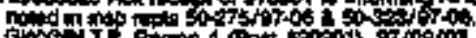

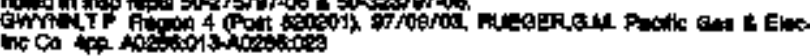

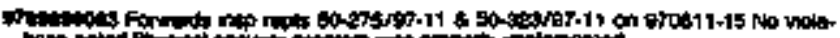

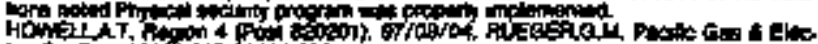

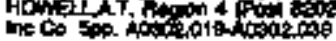

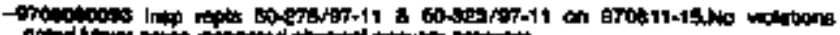

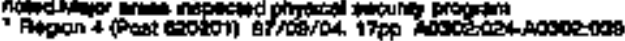

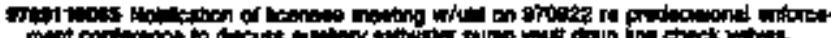

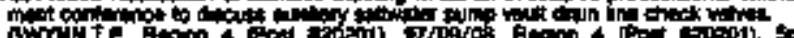

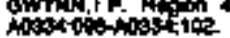

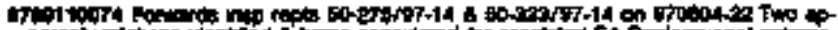

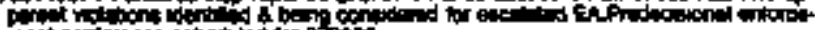

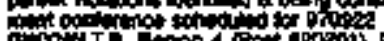

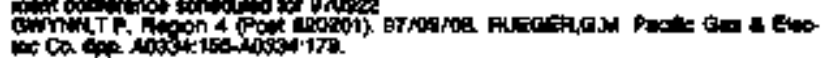

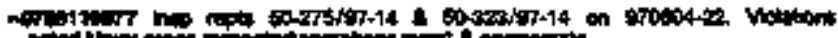

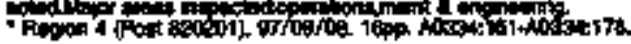

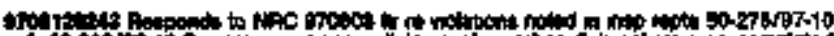

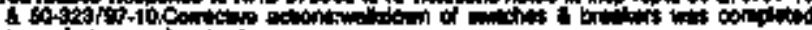

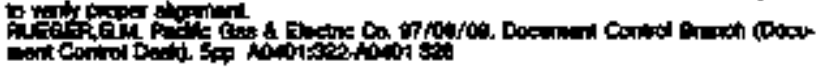

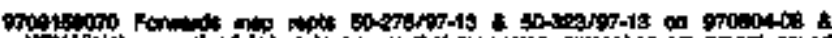

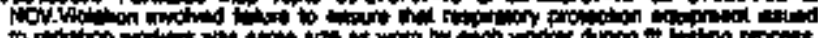

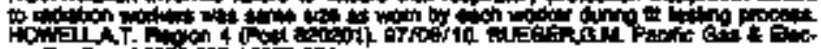

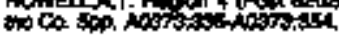

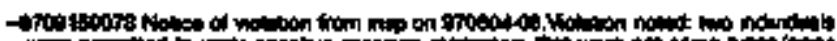

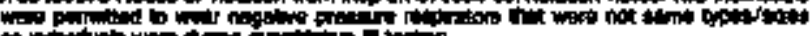

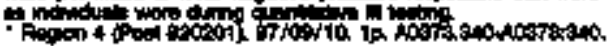

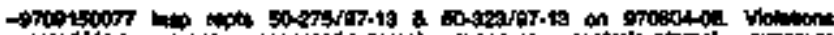

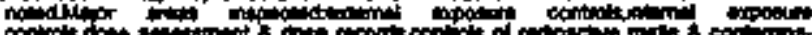

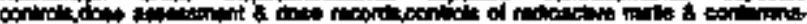
P.

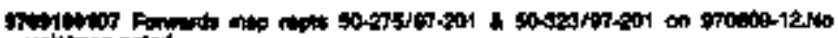

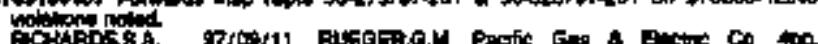

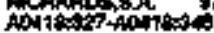

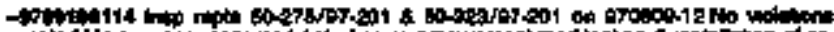

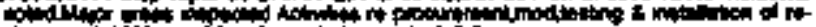

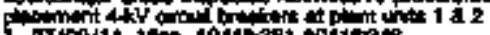

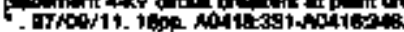

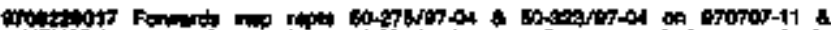

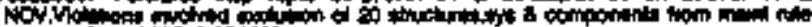
Fon

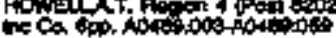

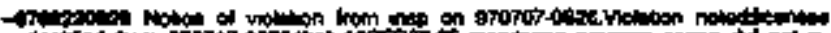

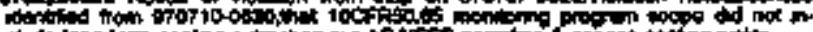

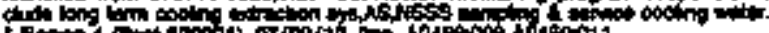

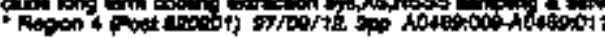

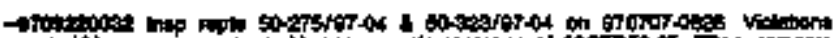

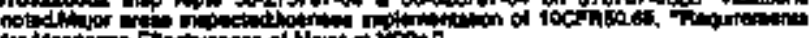

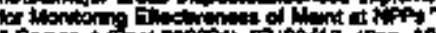

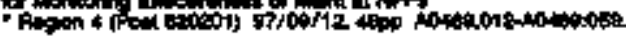

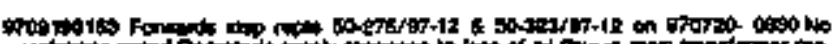

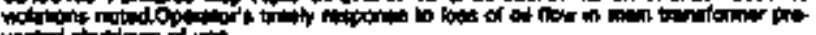

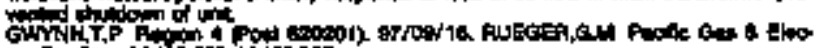

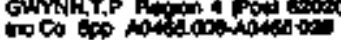

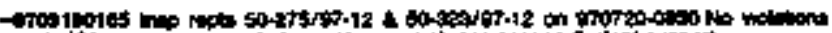

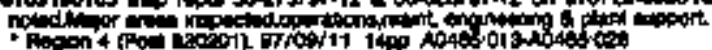

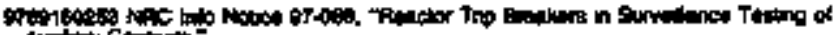

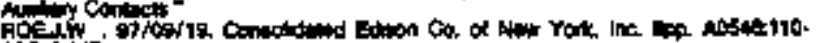

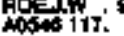

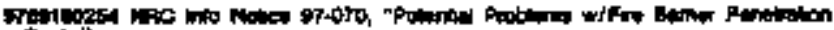

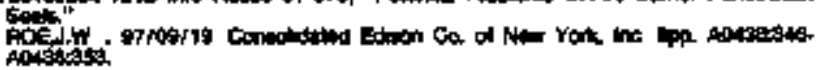

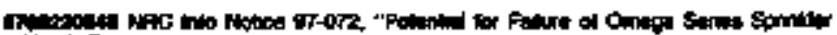
Hof 10065241.

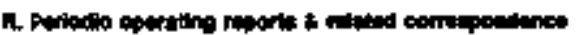

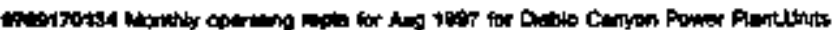

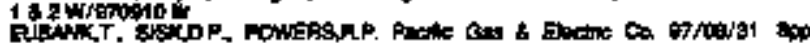

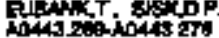

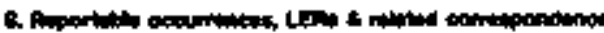

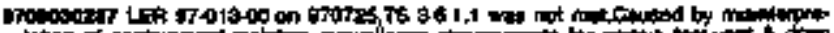

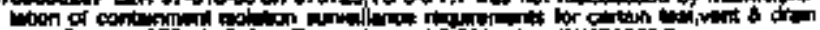

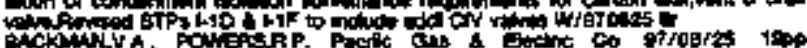

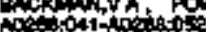




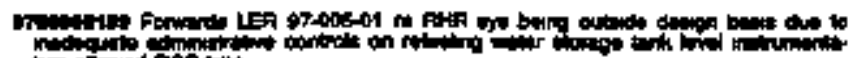

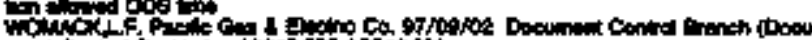

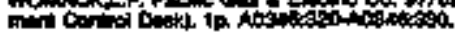

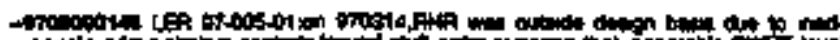

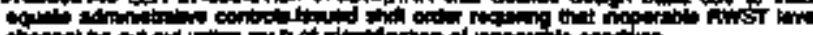

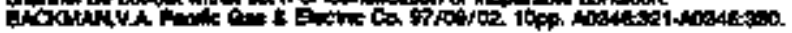

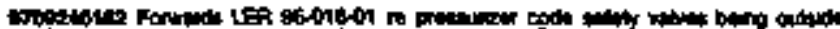

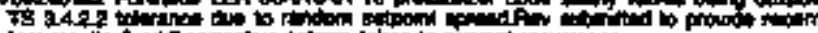

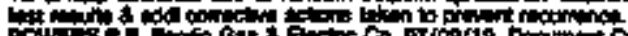

Doula

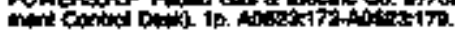

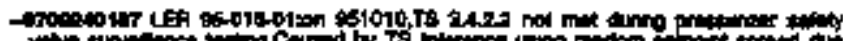

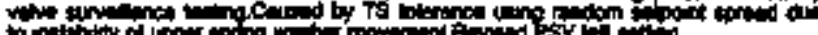

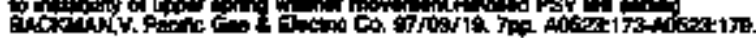

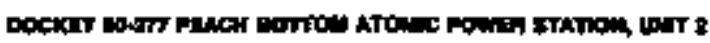

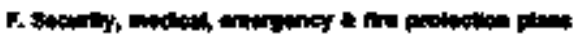

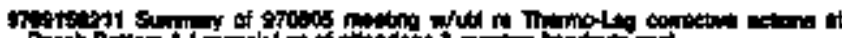

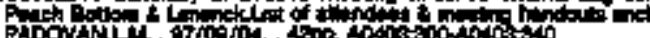

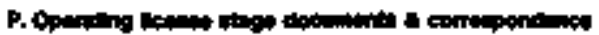

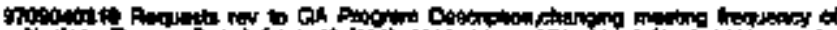

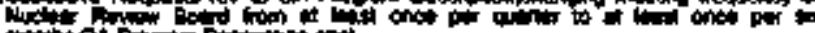

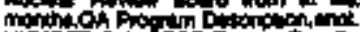
Pinth

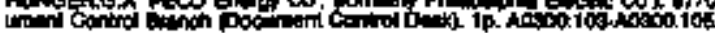

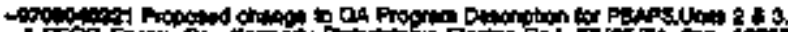

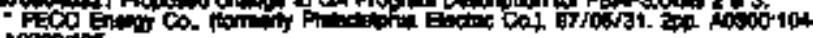
AOS00:00.

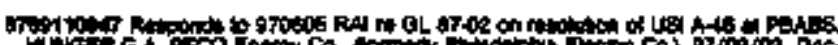

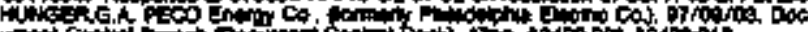

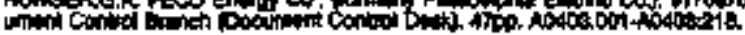

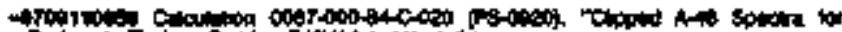

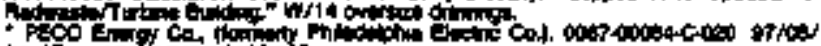

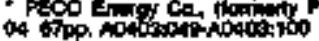

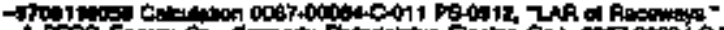

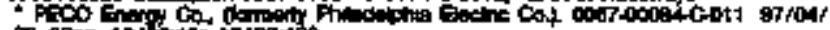
勿

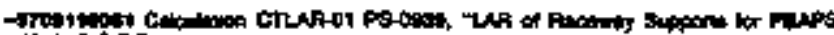
Untos 2 is

Pf

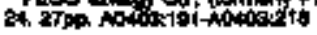

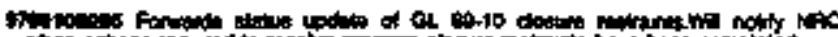

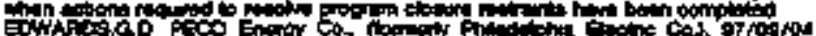

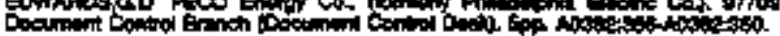

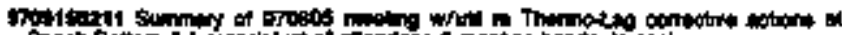

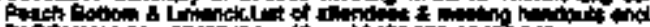

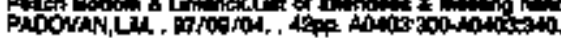

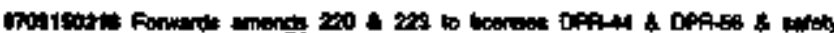

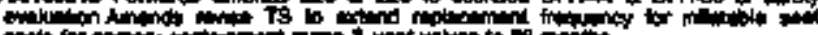

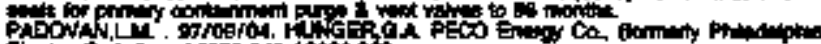

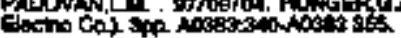

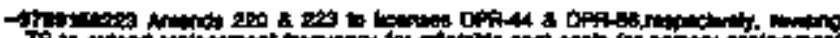

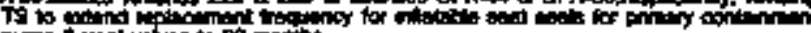

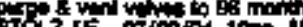

- Pros

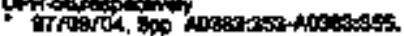

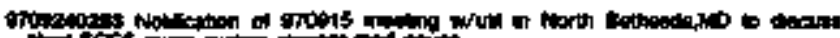

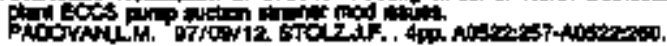

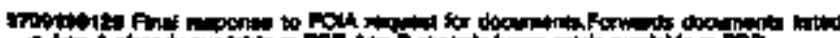

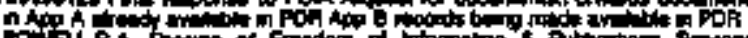

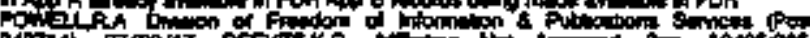

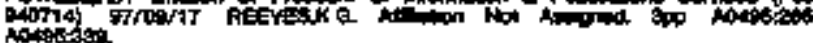

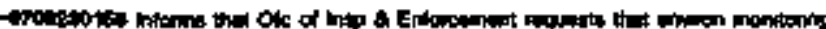

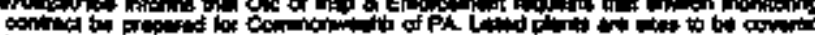

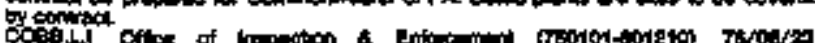

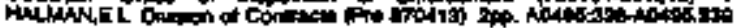

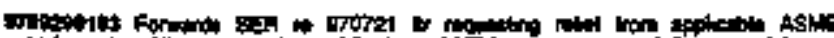

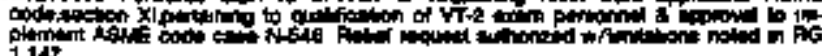
114

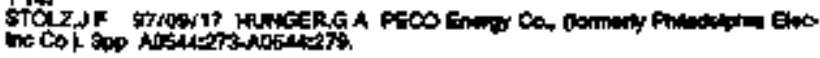

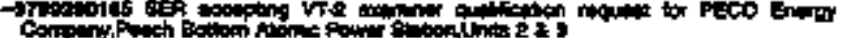
Comprif

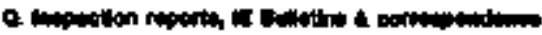

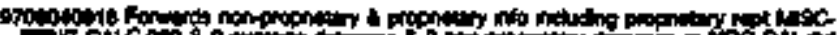

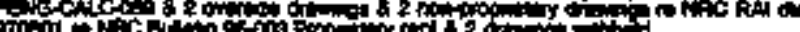

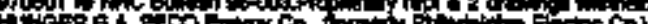

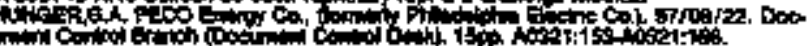

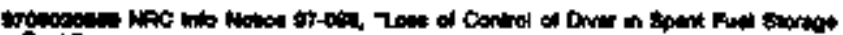
Podi:-

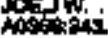

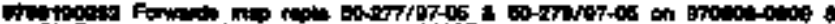

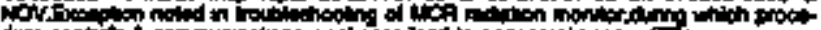

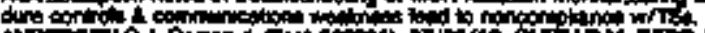

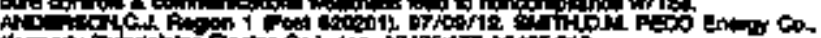

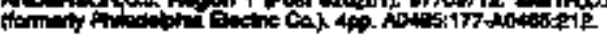

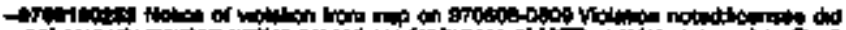

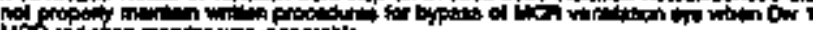

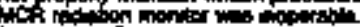

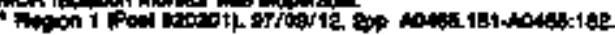

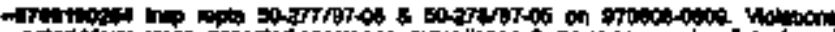

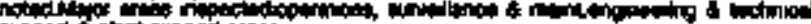

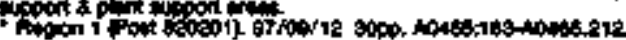

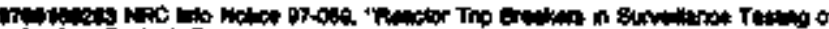

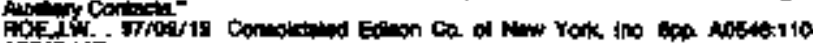
Aostiniti:

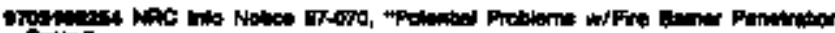

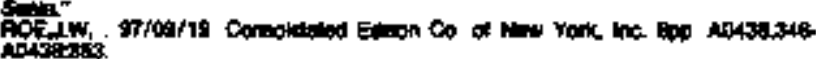
Monen

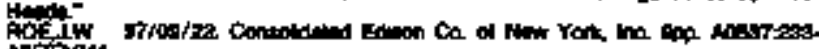
incisis

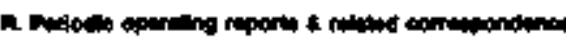

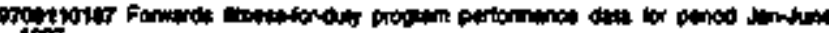

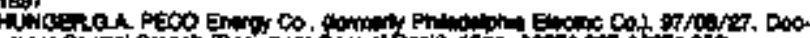

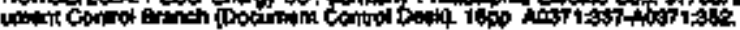

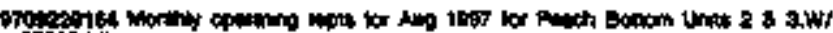
970 of 14

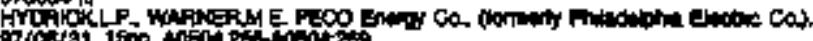

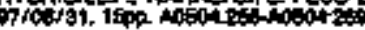

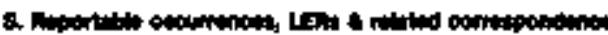

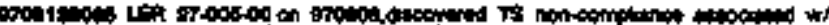

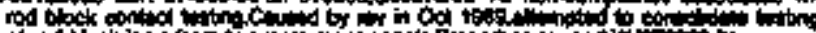

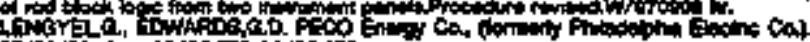

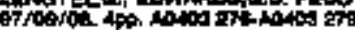

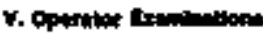

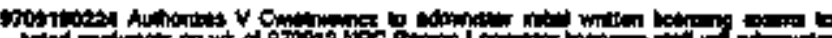

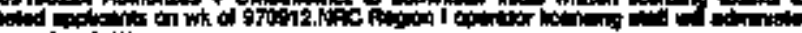

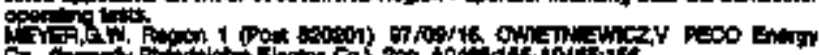

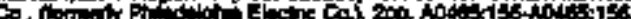

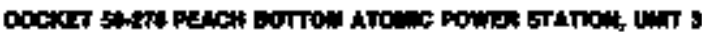

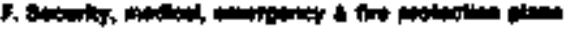

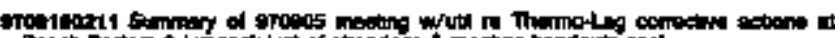

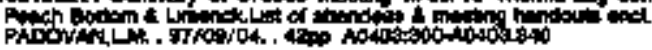

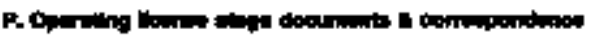

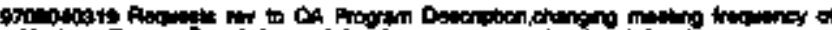

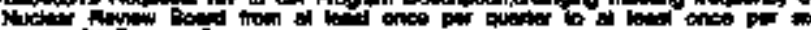

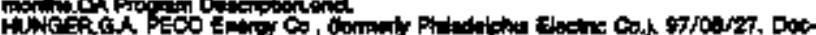

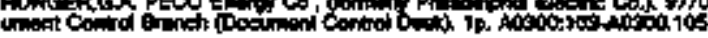

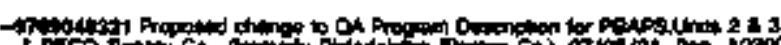

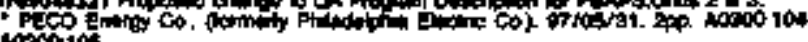
uscoitos 


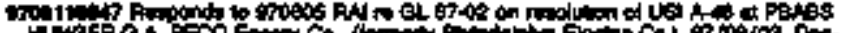

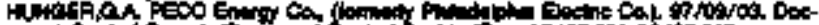

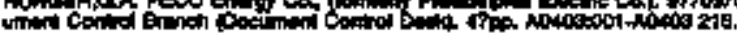

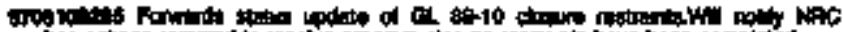

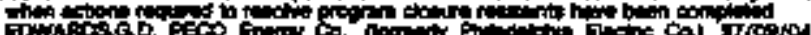

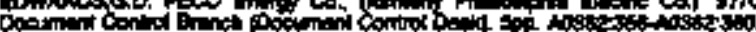

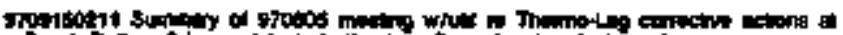

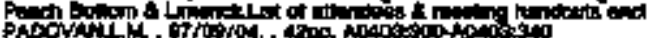

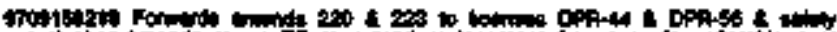

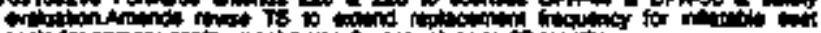

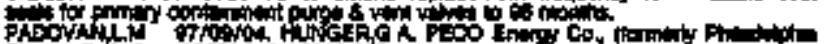

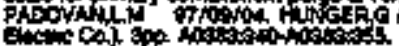

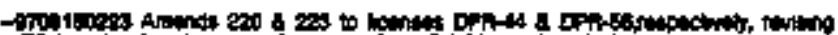

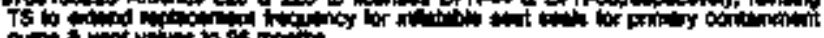

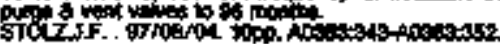

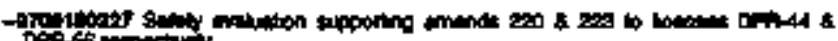

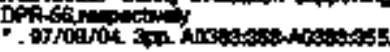

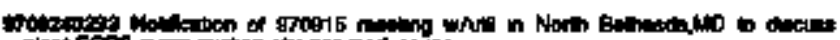

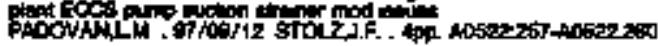

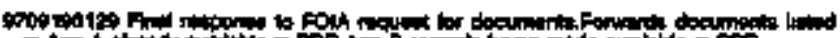

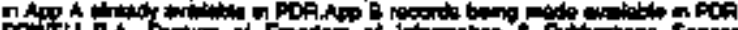

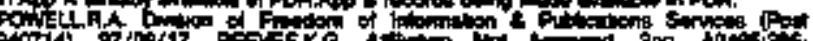

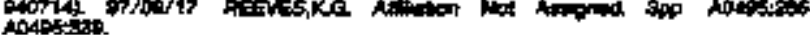

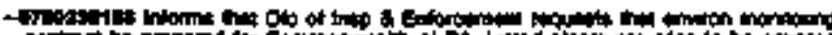

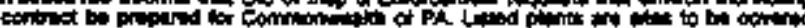

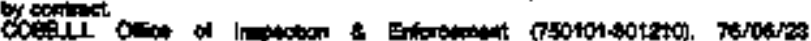

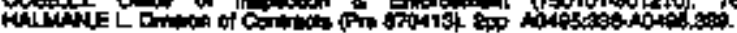

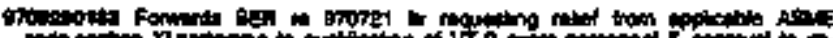

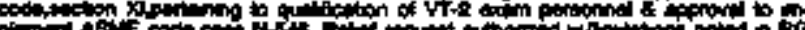

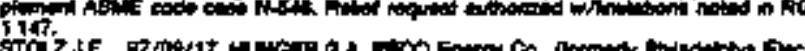

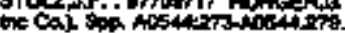

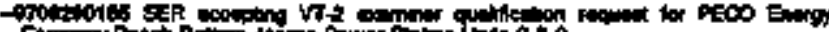

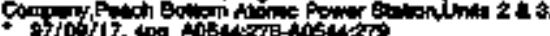

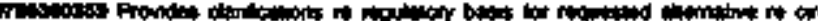

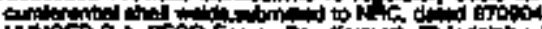

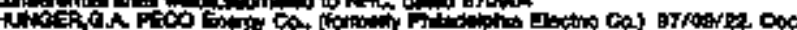

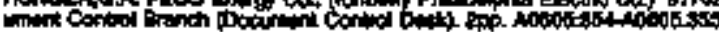

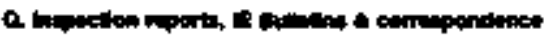

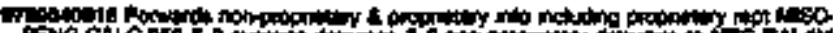

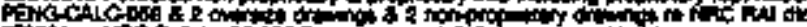

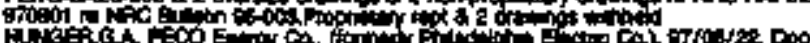

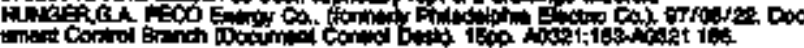

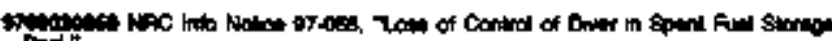

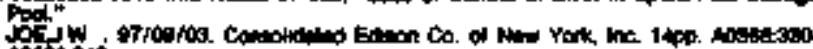
Axtosis

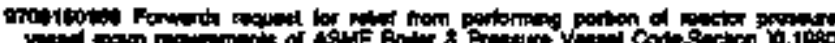

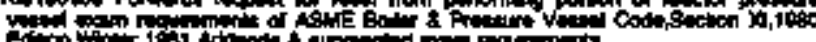

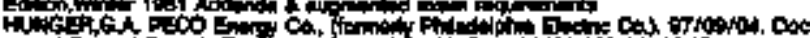

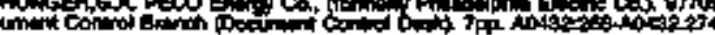

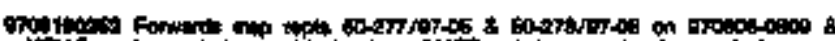

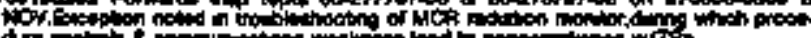

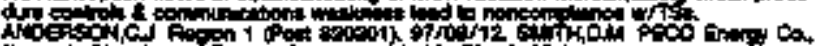

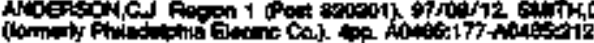

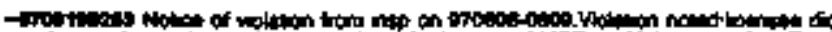

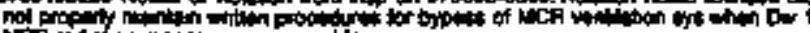

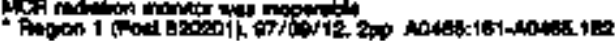

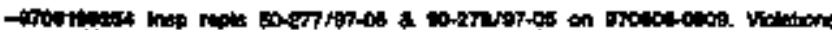

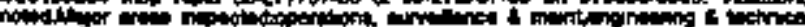

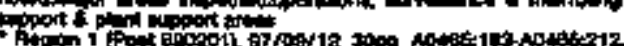

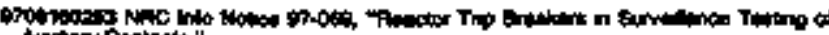

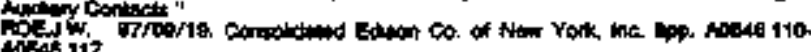

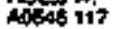

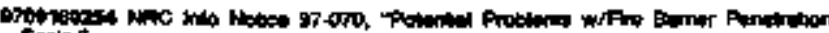

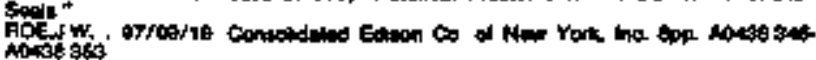

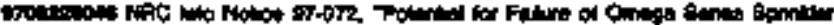

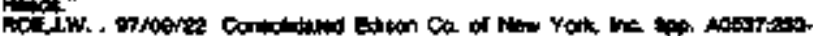

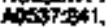

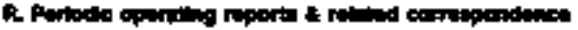

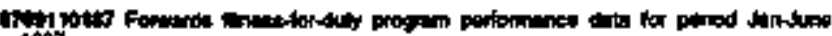

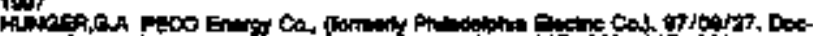

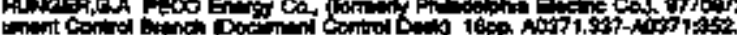

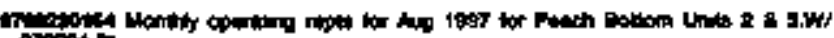

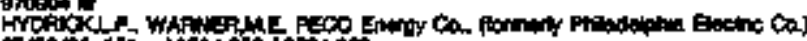

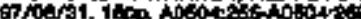

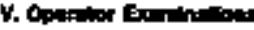

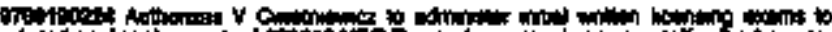

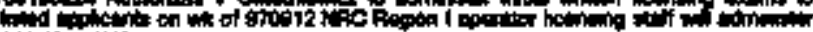

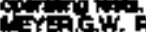

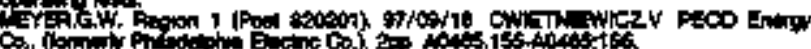

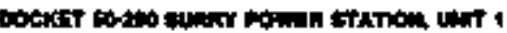

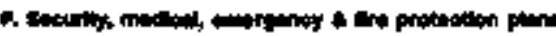

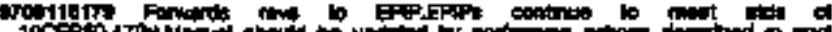

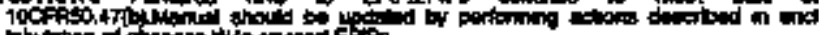

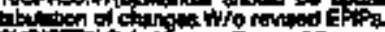

Cla

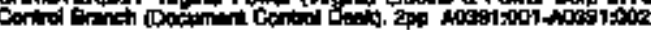

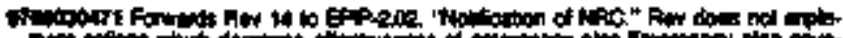

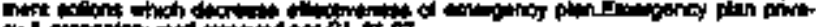

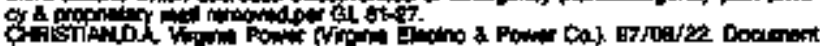

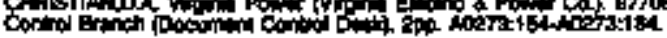

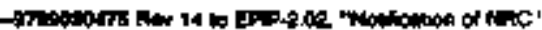

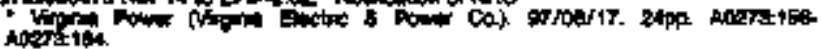

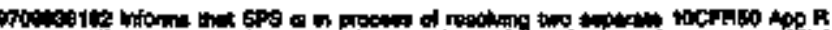

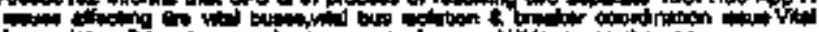

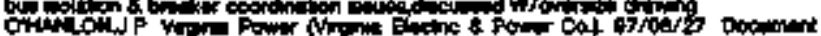

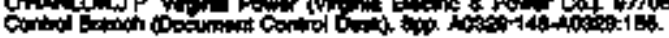

\$

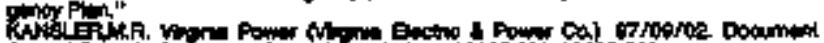

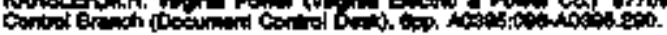

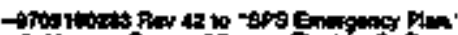

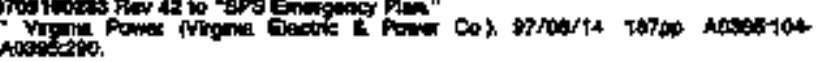

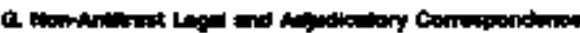

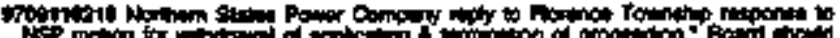

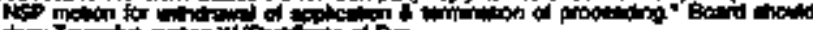

ôt Ton

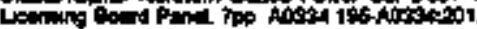

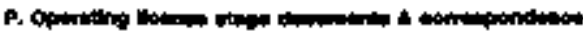

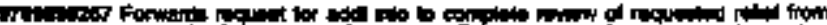
Fon

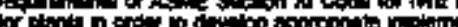

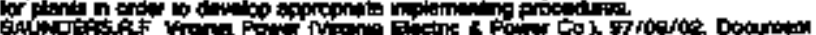

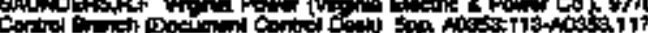

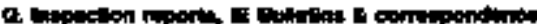

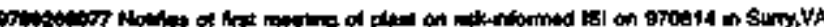

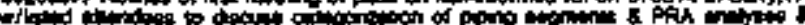

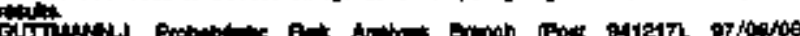

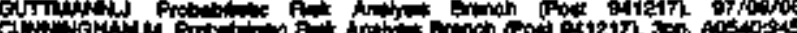
instast?

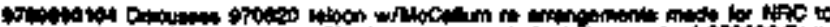

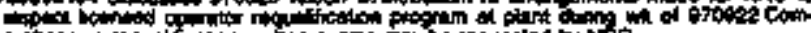

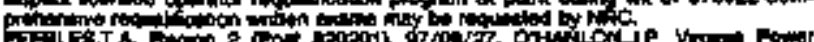

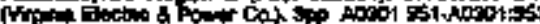

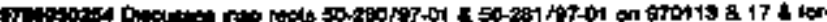

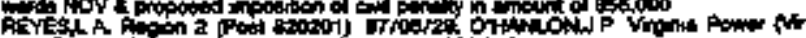

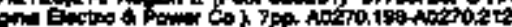




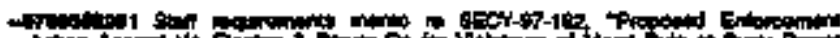

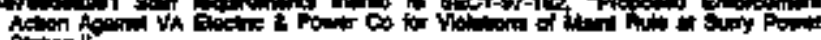

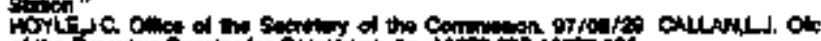

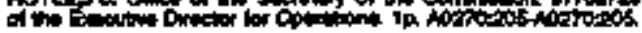

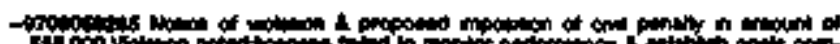

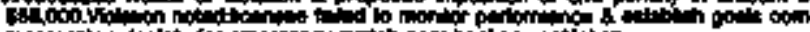

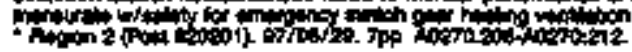

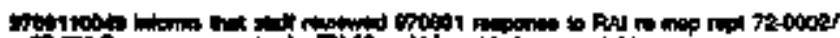

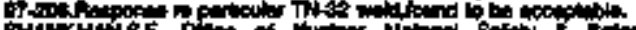

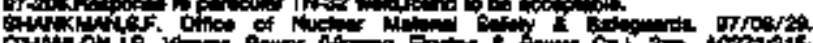
chetrityt.

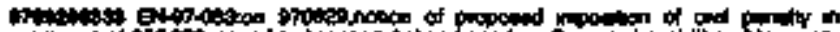

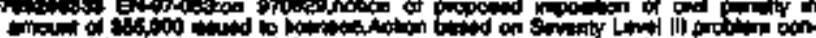

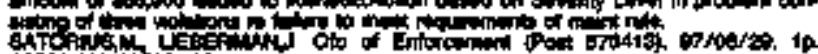
Aosons

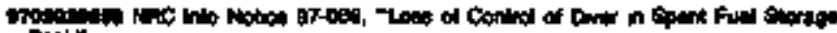

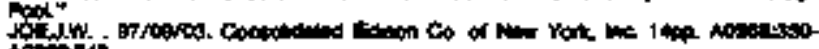
ATis:

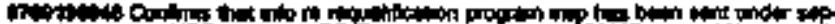

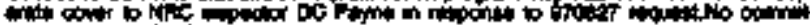

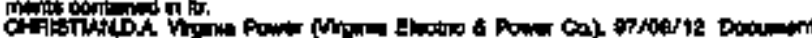

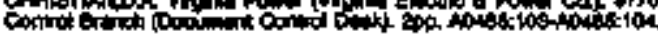

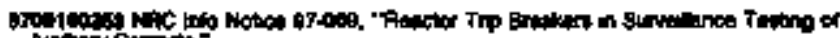

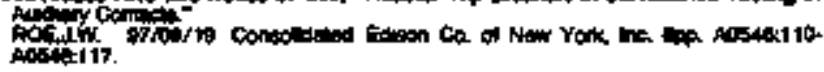

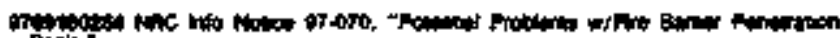

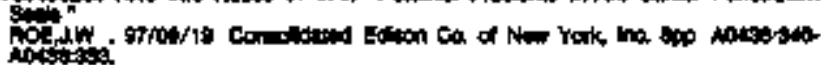

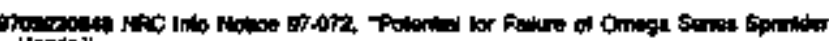

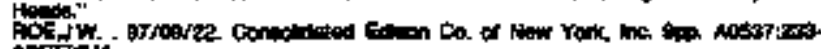
AOssinti1.

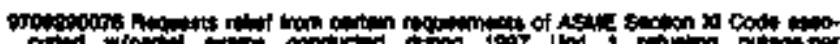

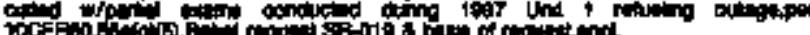

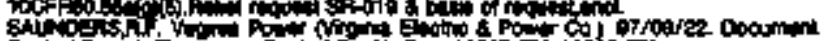

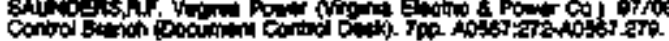

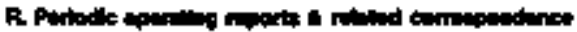

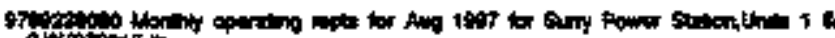

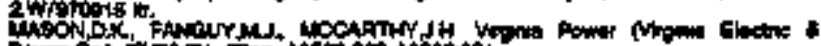
Pon Cox of

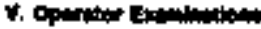

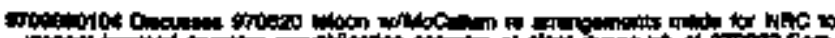

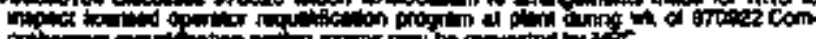

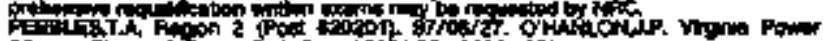
(Ving in

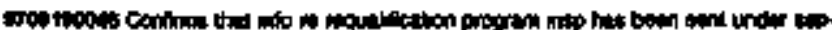

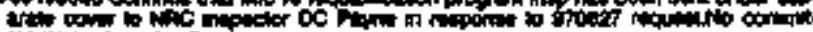
chistion

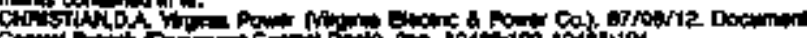

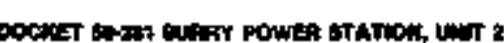

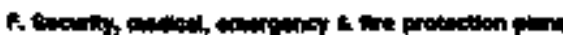

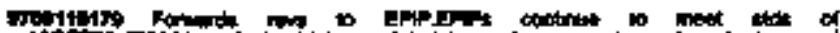

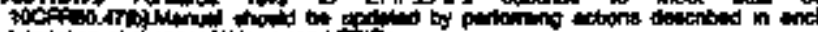

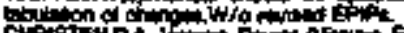

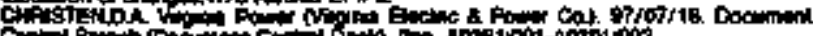

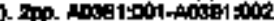

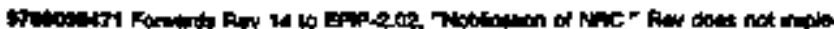

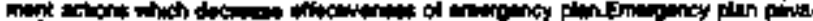
She

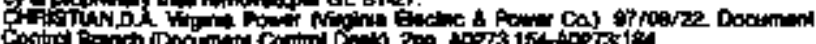

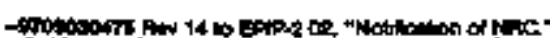

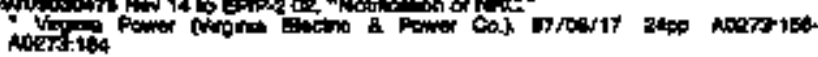

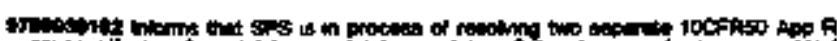

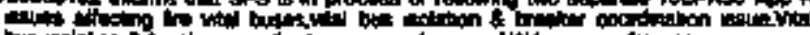

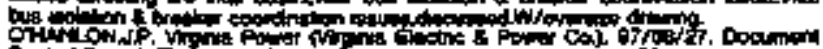

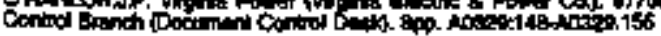

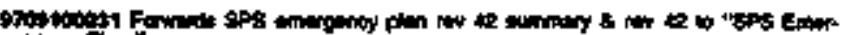
and $F$

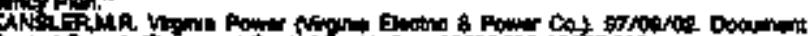

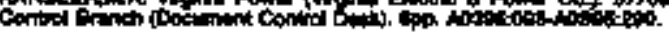

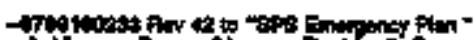

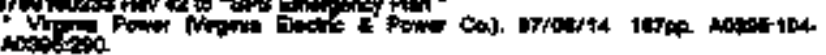

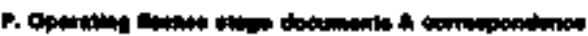

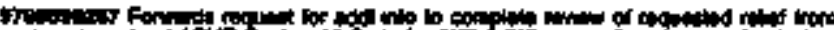

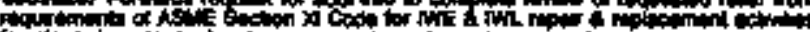

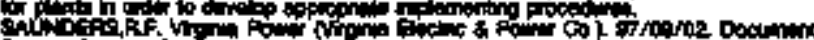

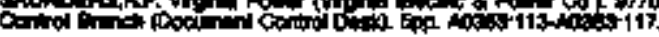

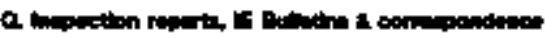

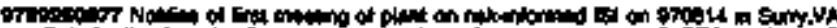

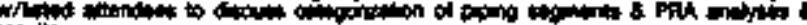
nition

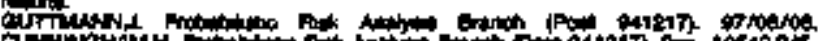

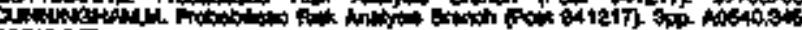
40.4037.

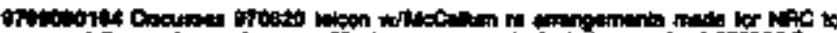

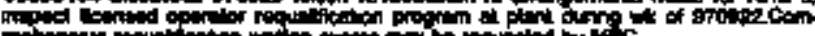

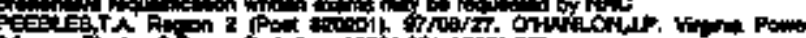

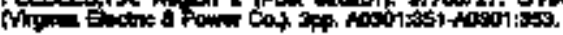

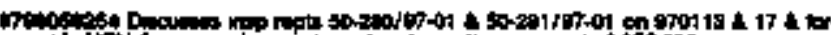

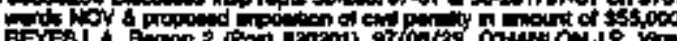

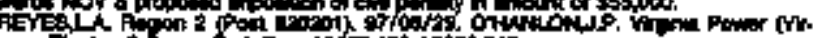

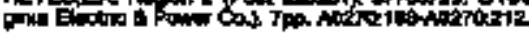

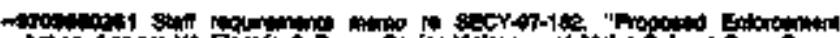

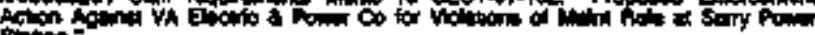

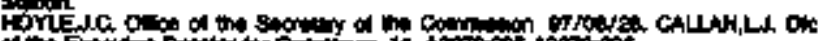

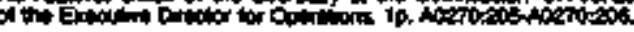

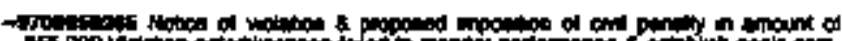

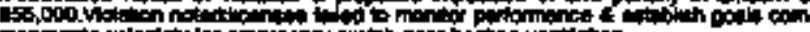

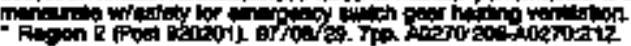

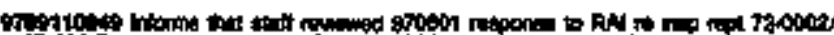
7.0.

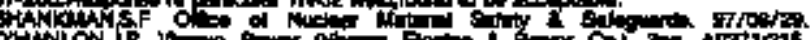
orition

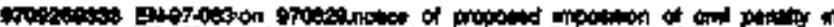

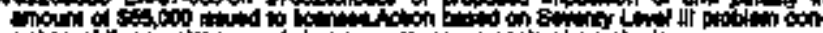

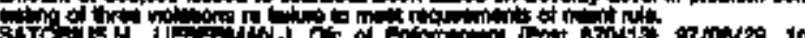

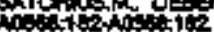

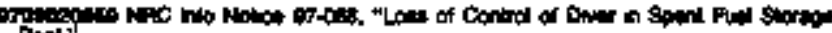
Aoct

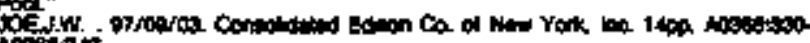
incisis

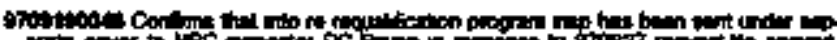

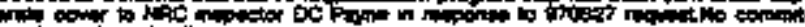

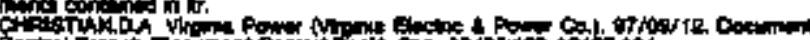

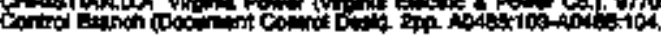

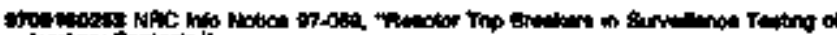

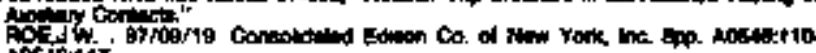
Nobit:117.

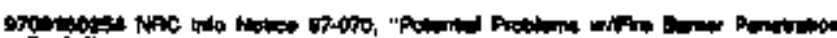

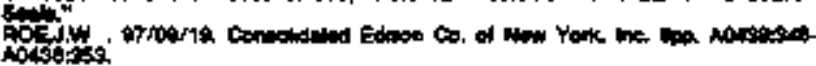

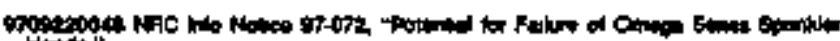

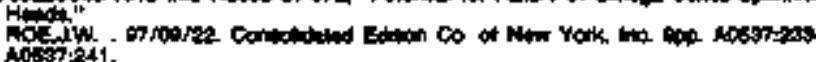

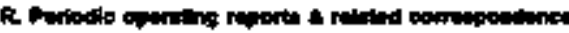

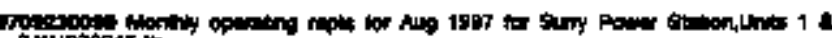

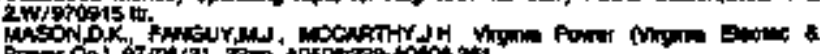

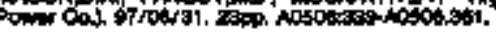

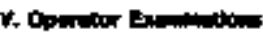

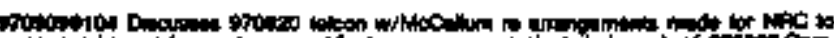

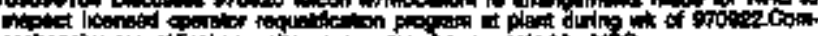

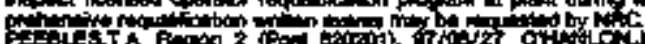

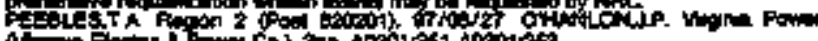

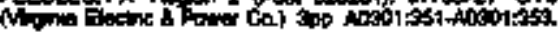




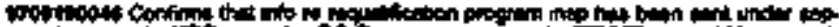

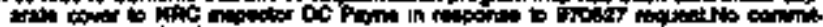

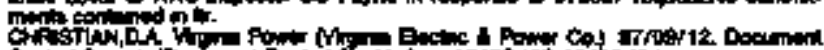

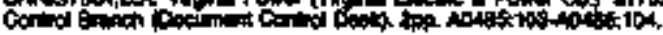

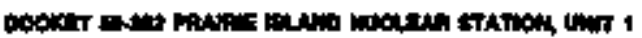

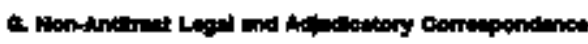

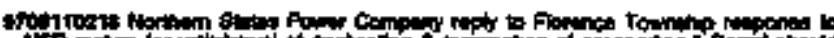

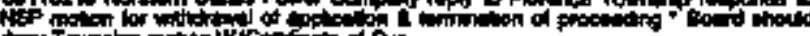

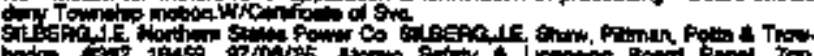

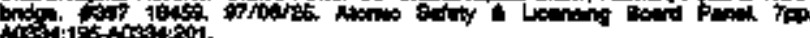

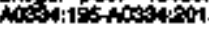

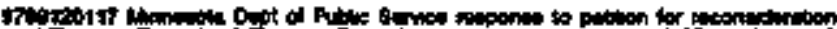

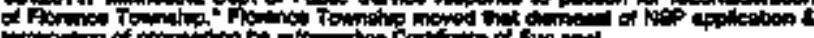
Vhectiof of

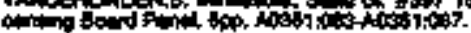

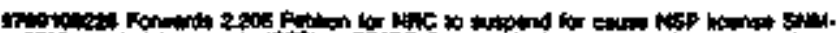

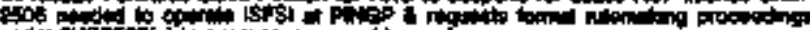

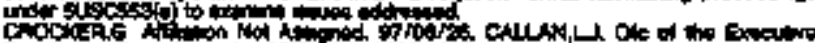

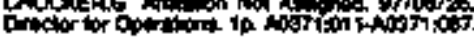

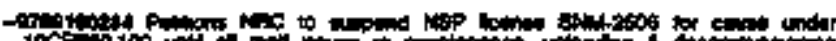

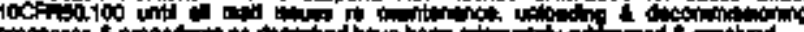

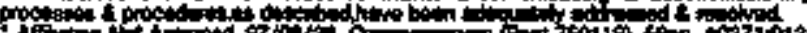

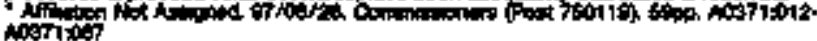

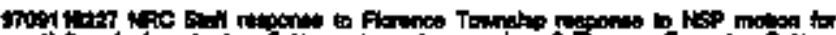

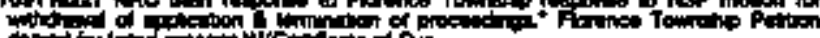

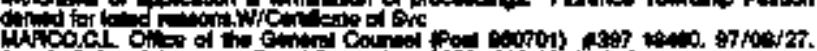

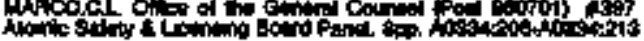

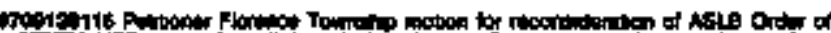

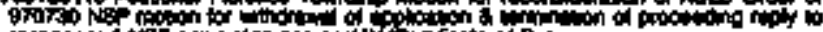

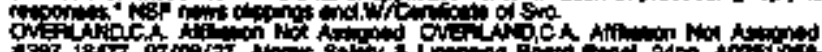

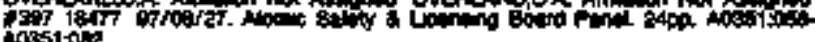
Masion:

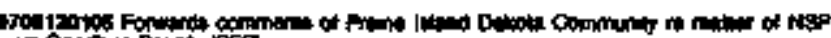

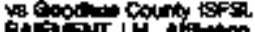

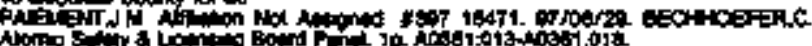
烈

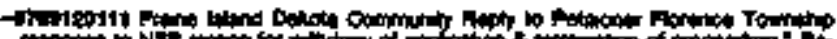

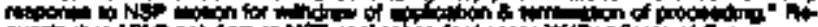

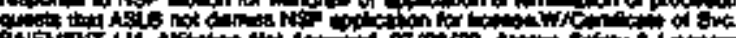

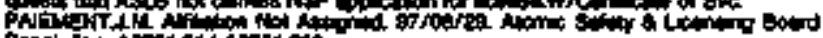

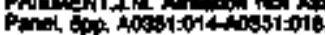

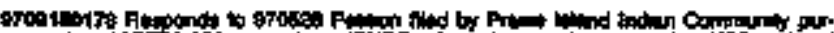

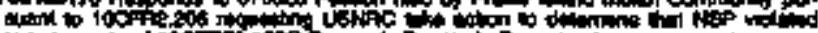

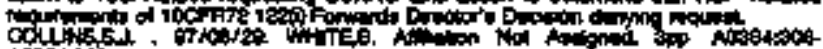

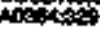

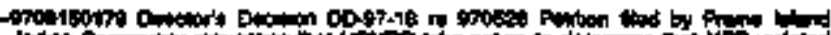

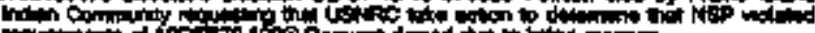

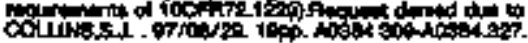

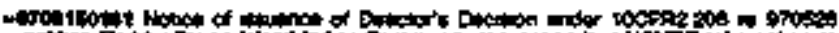

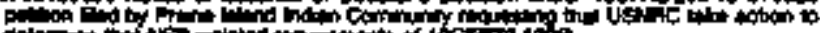

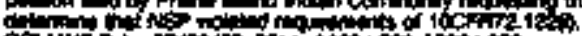

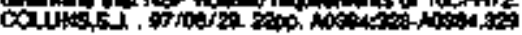

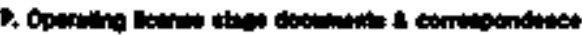

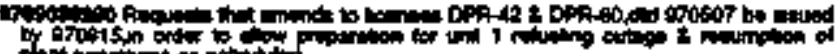

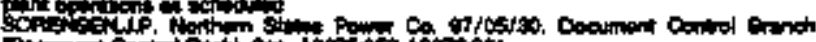

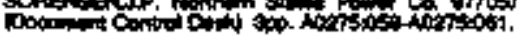

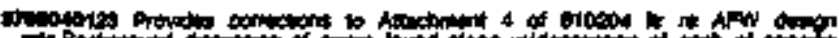

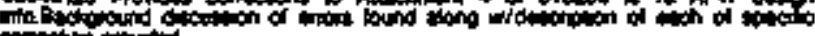

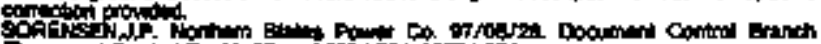

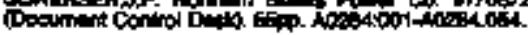

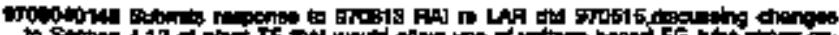

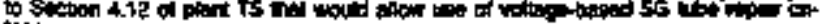

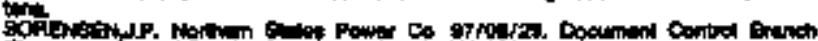

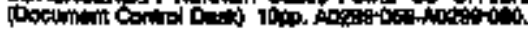

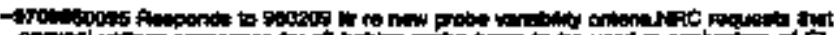

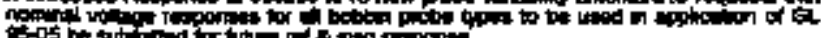

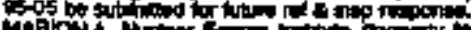

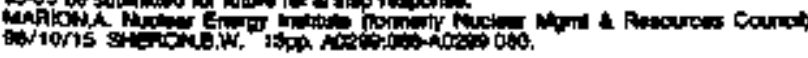

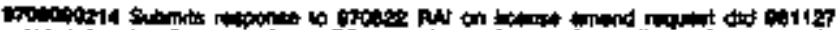

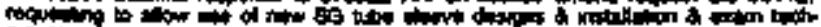

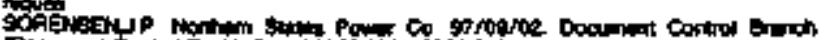

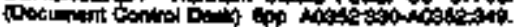

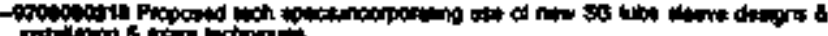

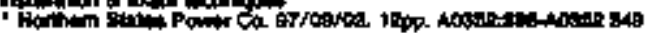

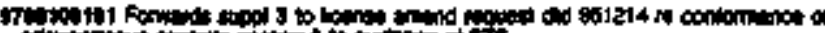

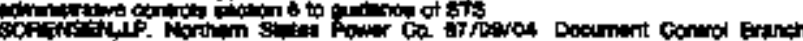

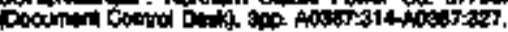

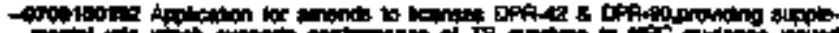

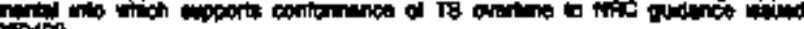

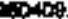

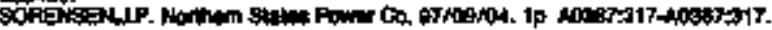

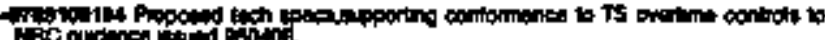

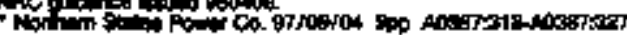

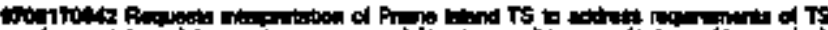

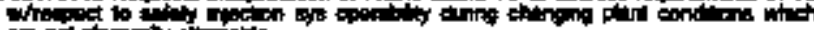

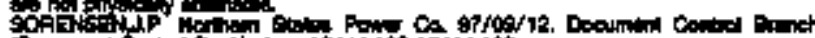

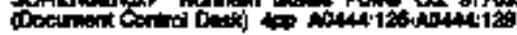

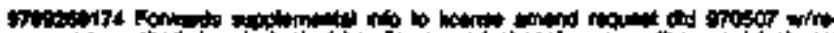

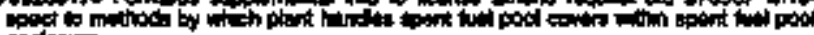
Anto:

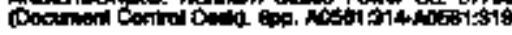

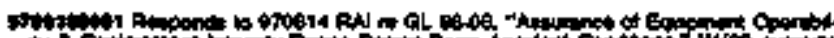

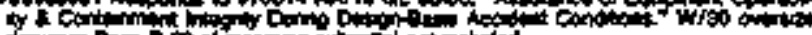

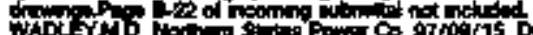

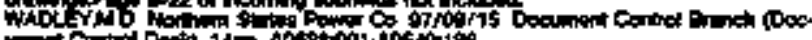

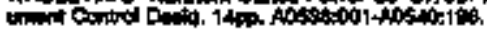

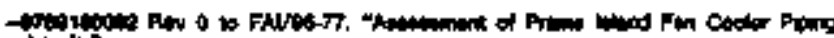

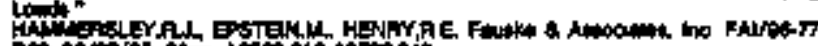

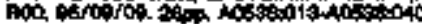

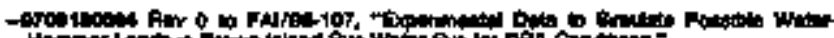

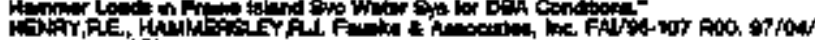

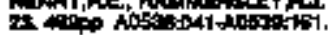

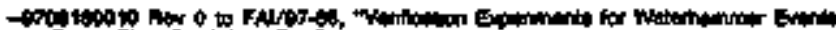

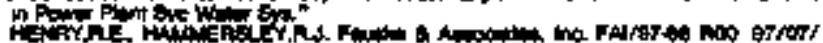

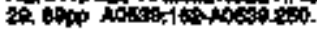

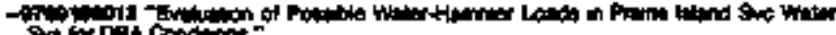

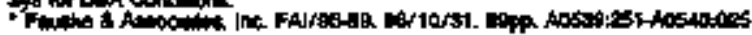

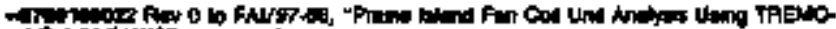

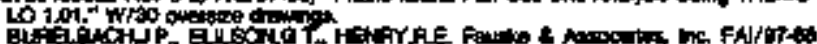

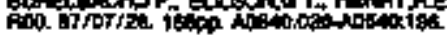

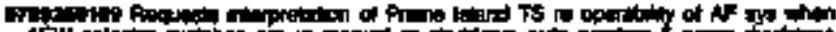

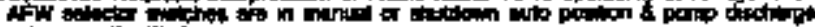

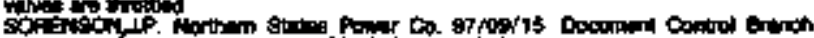

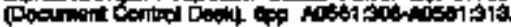

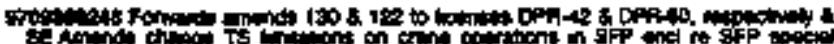

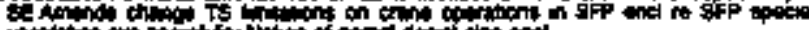

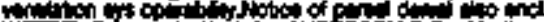

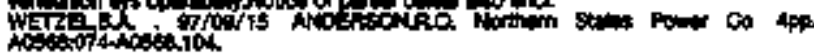

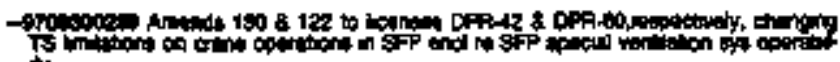

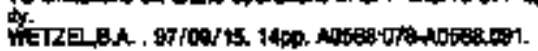

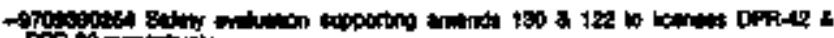

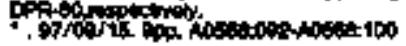

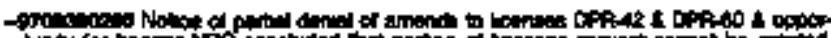

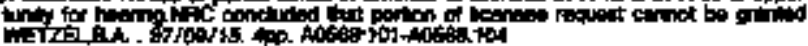

ip

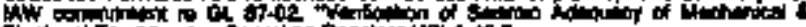

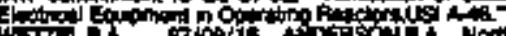

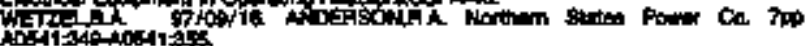

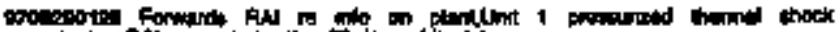

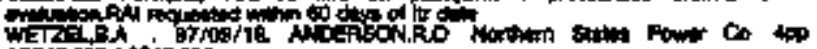

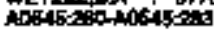

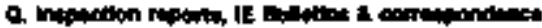

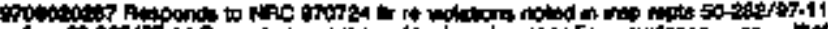

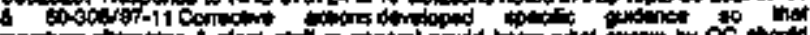

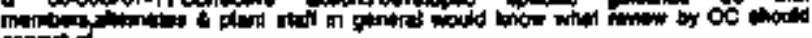

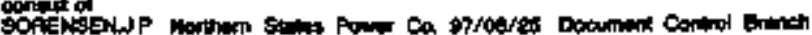

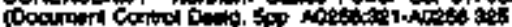




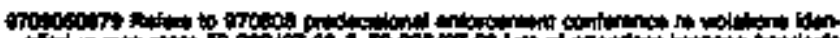

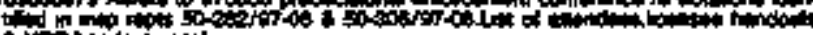

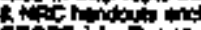

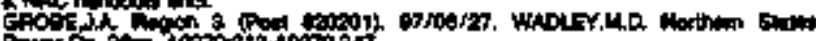

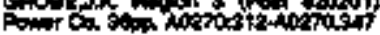

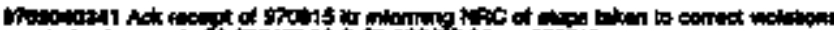

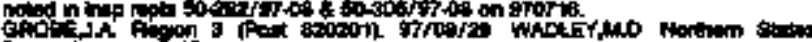

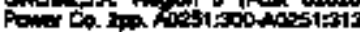

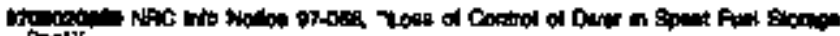

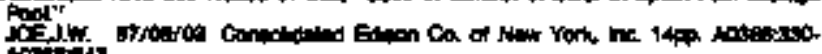
Arsistis

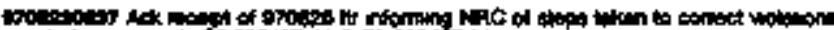

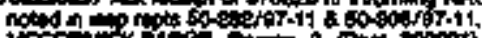

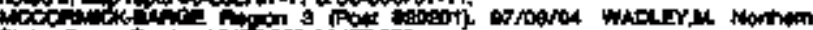

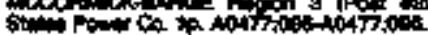

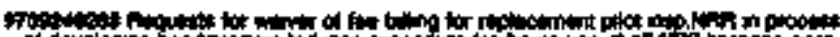

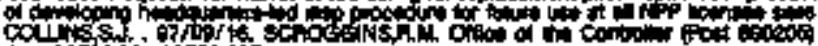

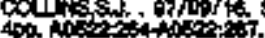

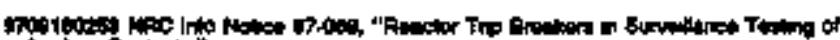
Ao

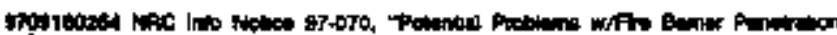

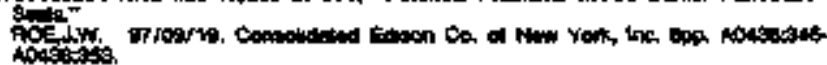

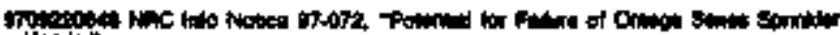

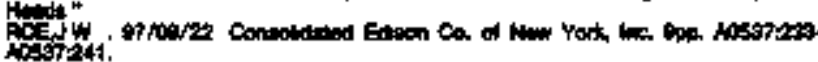

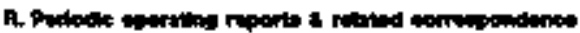

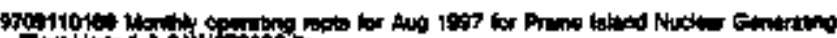

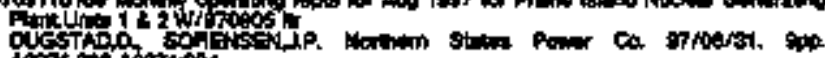

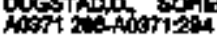

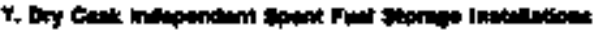

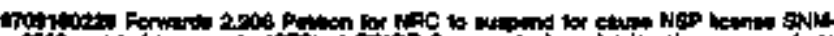

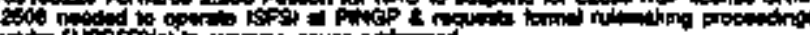

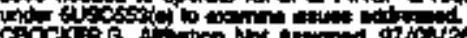

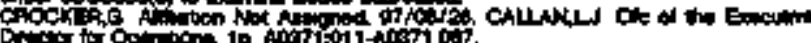

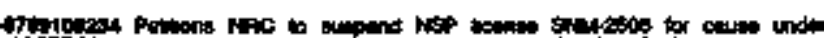

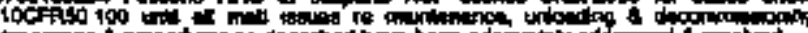

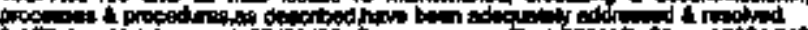

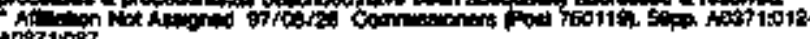
inos 1:00\%?

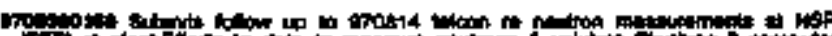

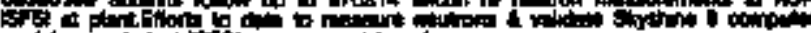

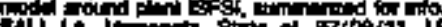

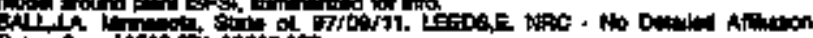

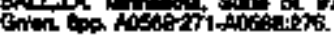

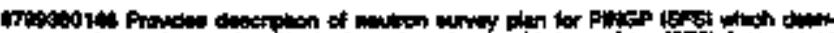

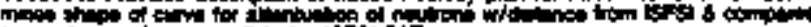

int on

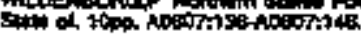

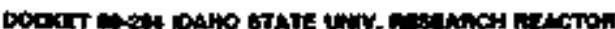

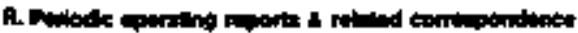

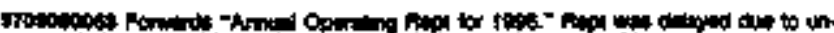

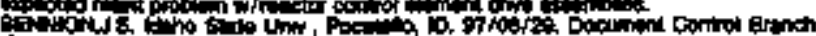

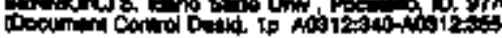

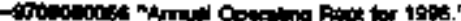

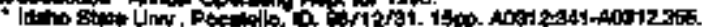

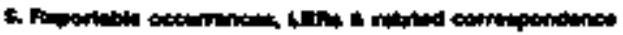

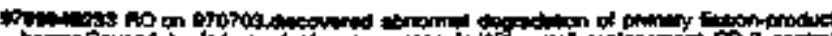

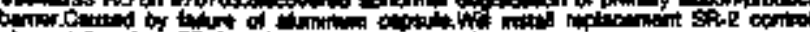

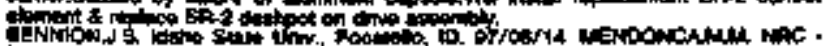

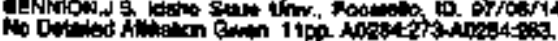

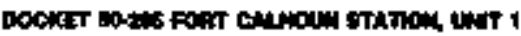

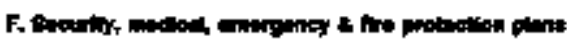

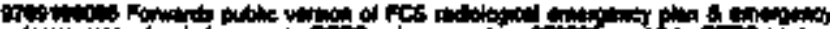

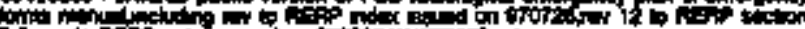
prew

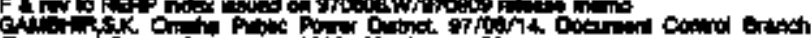

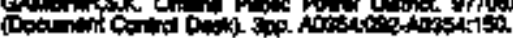

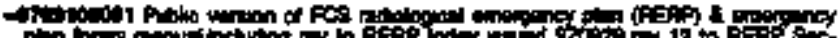

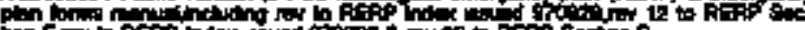

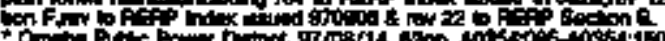

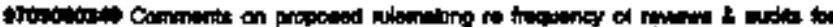

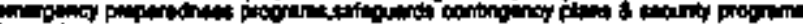

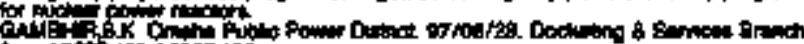

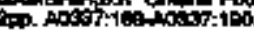

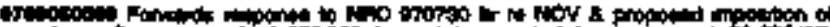

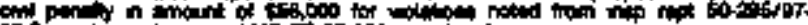

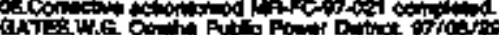

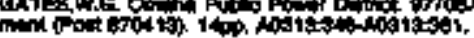

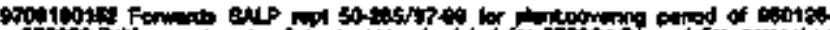

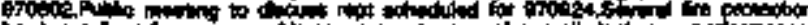

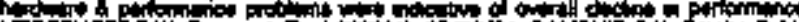
a

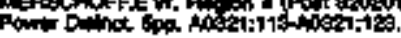

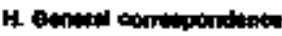

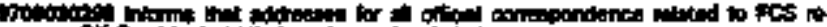

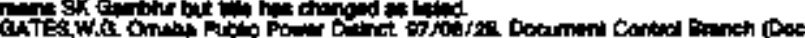

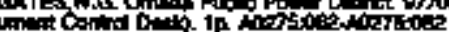

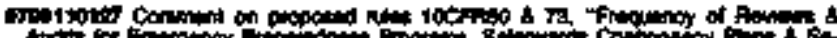

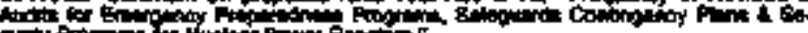

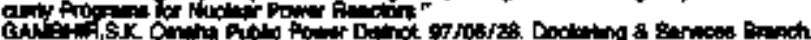

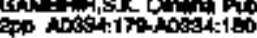

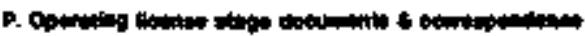

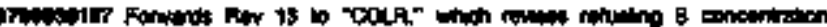

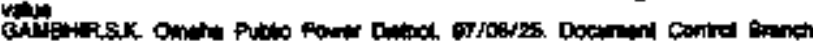

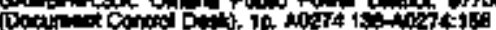

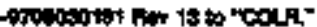

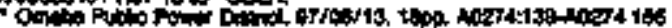

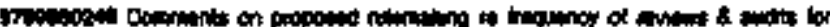

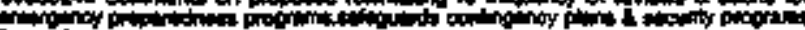
cinter

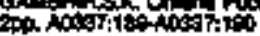

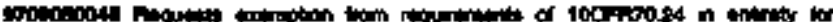

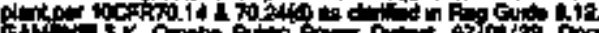

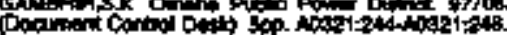

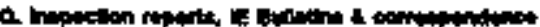

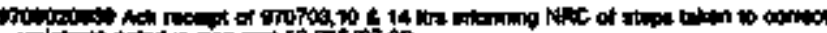

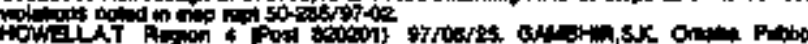

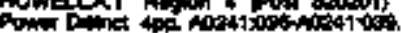

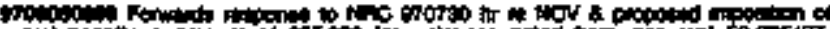

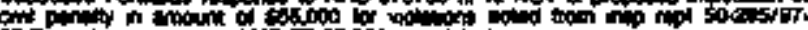

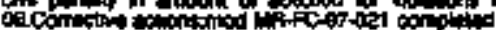

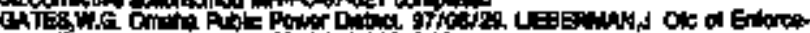

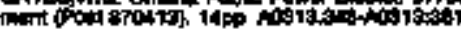

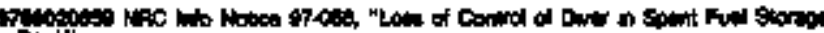

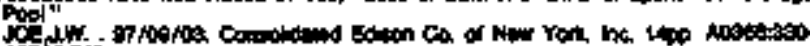
Antain

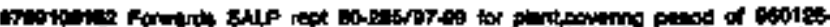

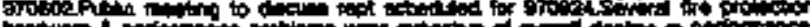

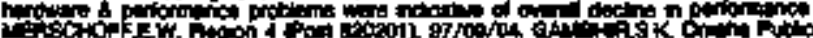

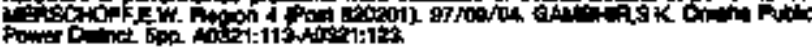

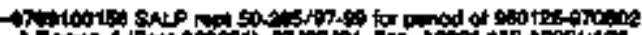

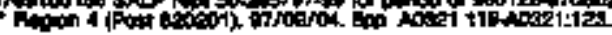

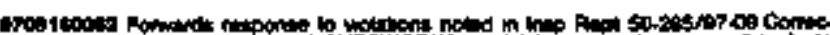

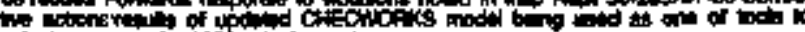

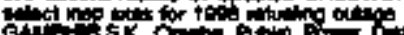

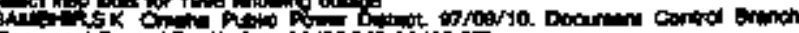

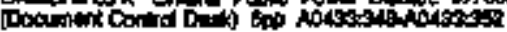




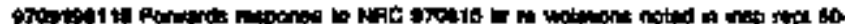

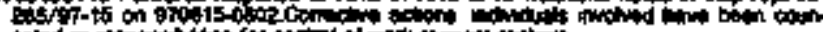

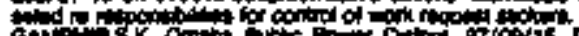

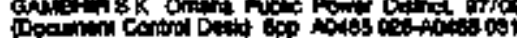

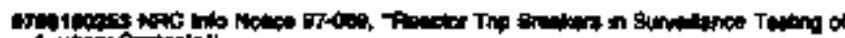

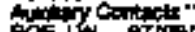
A0,

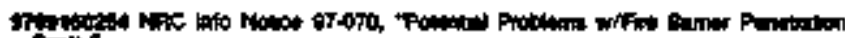

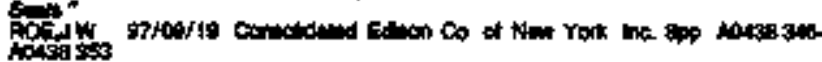

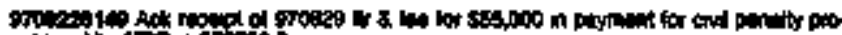

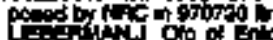

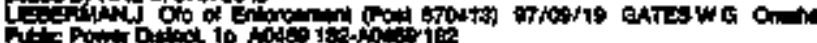

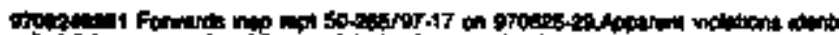

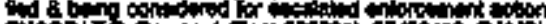

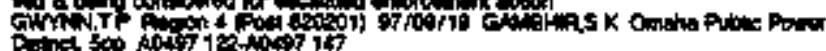

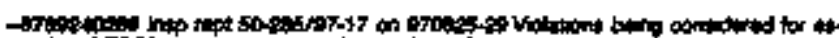

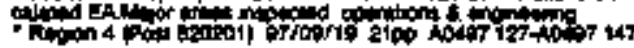

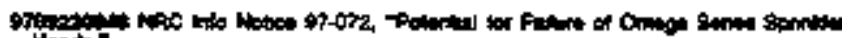

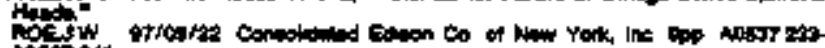
nostit

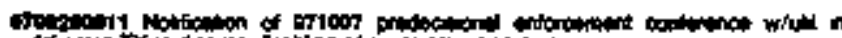

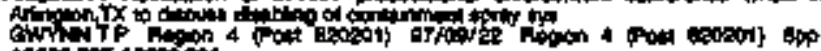

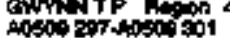

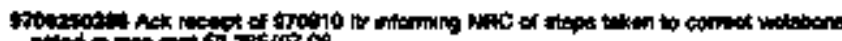

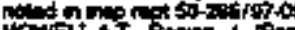

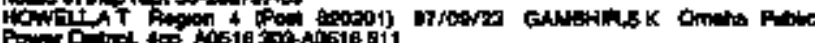

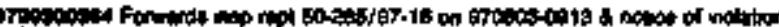

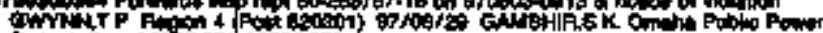

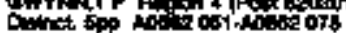

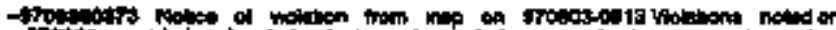

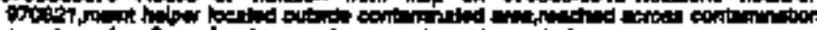

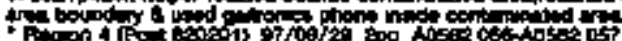

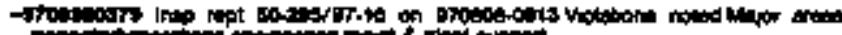

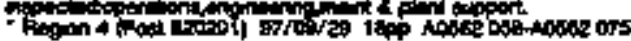

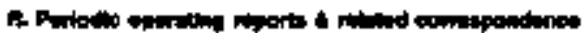

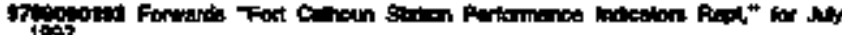

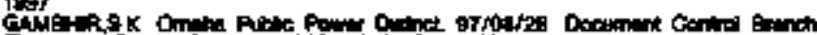

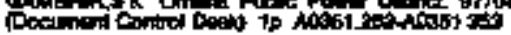

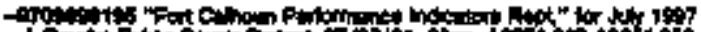

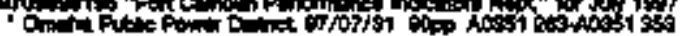

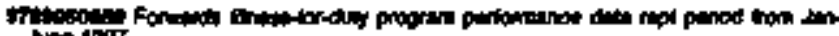

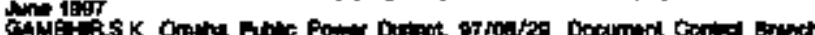

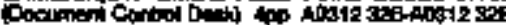

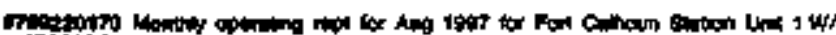
970012

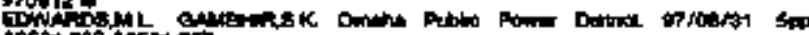

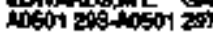

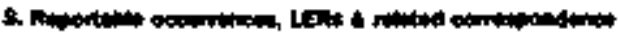

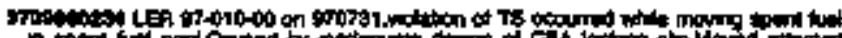

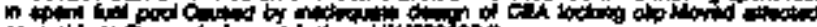

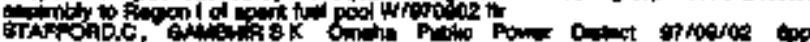

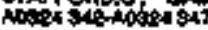

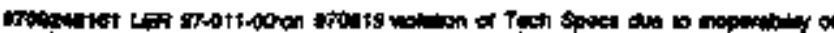

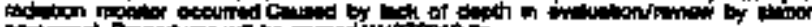

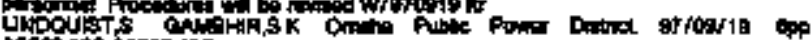

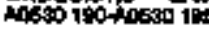

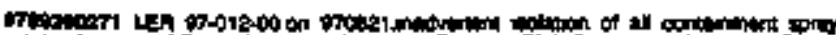

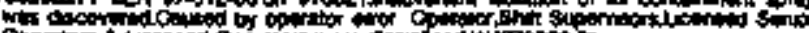

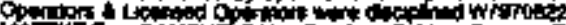

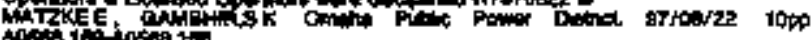

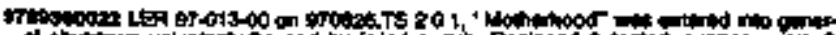

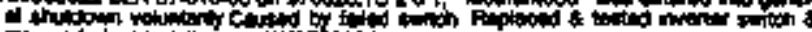

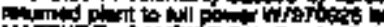

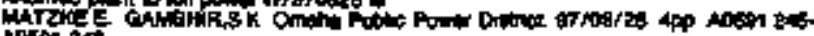
ADSol Ret

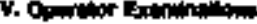

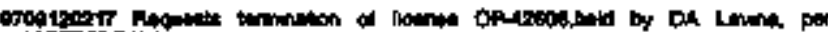

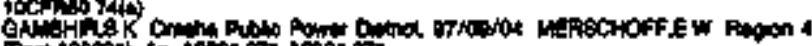

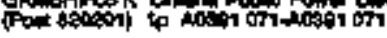

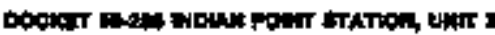

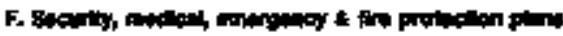

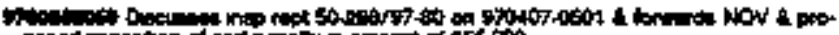

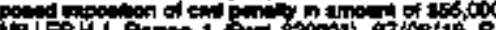

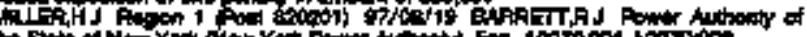

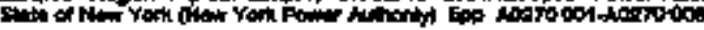

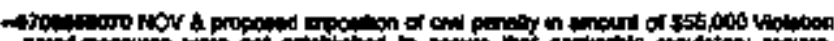

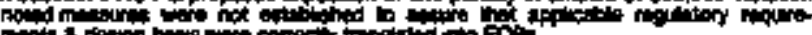

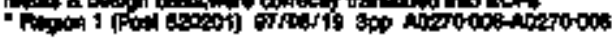

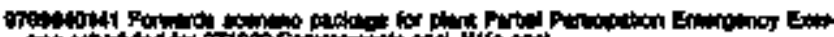

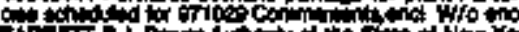

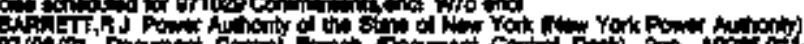

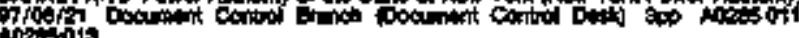
6015

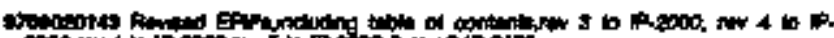

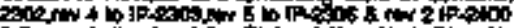

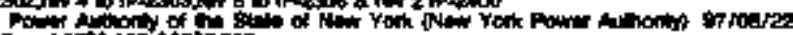

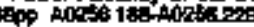

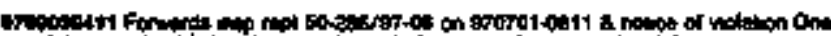

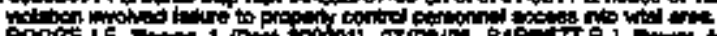

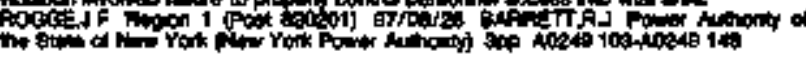

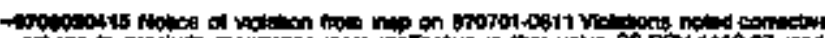

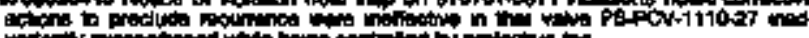

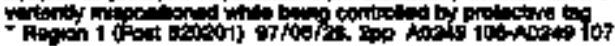

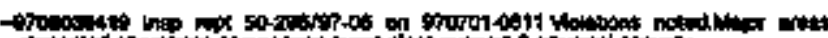
㽞

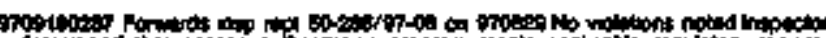

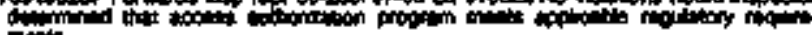

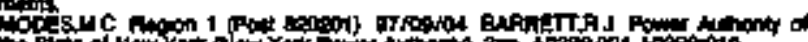

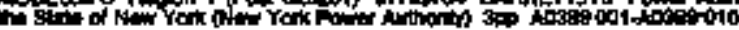

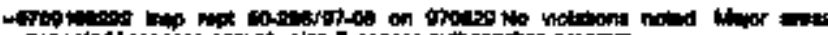

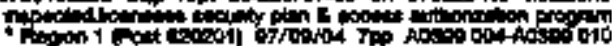

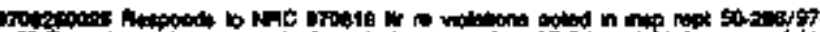

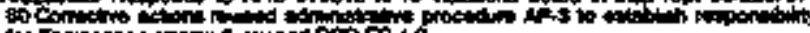

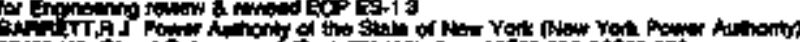

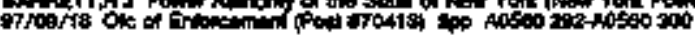

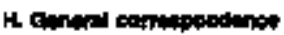

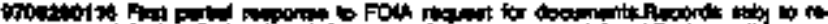

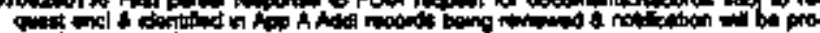

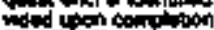

Pod Oy Now

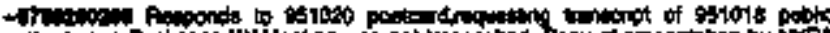

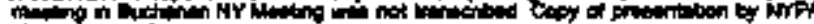
sintiond

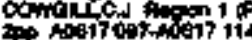

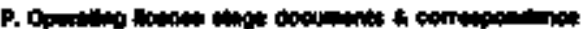

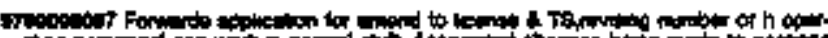

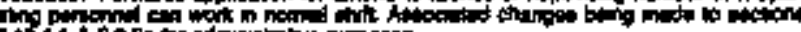

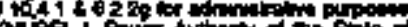

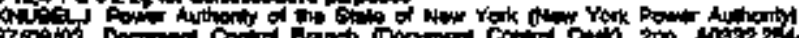
$\lim _{\cos }$

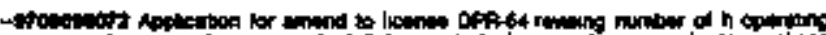

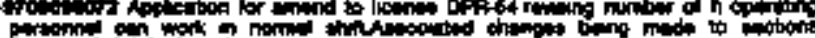

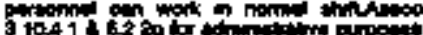

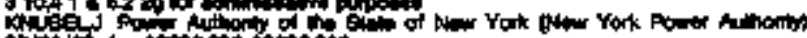
of

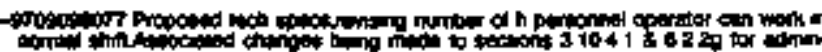

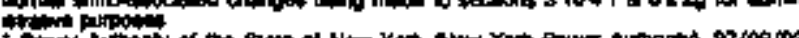

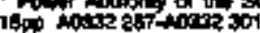




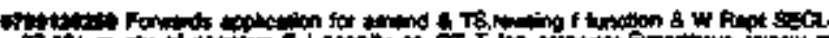

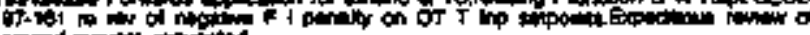

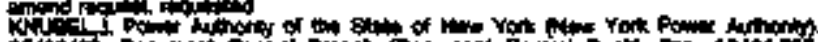

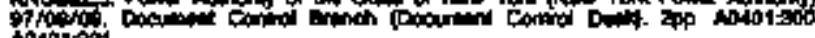
Nandist

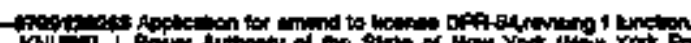

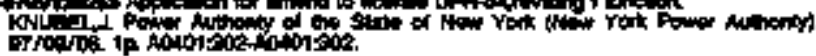

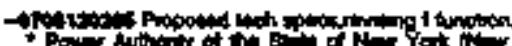

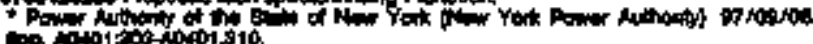

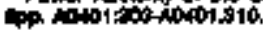

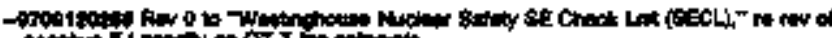

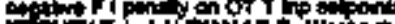

allow

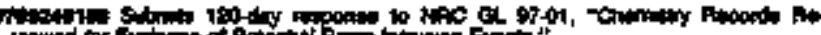

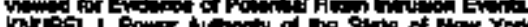

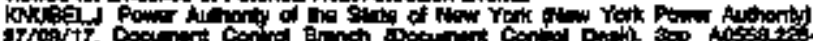
Aostion.

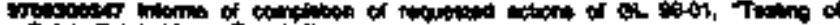

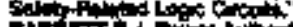

Fin

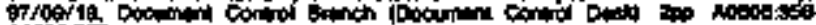

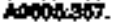

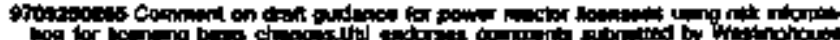

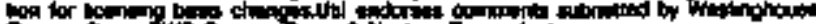

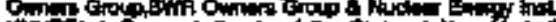

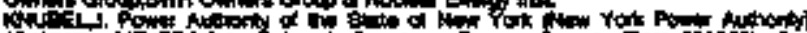

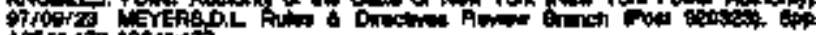

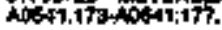

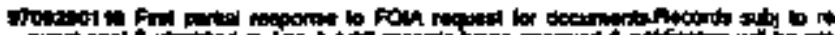

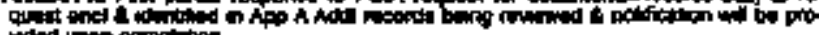

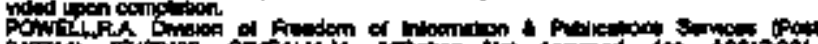

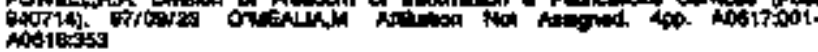

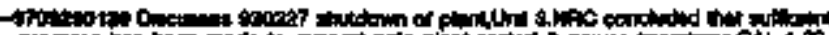

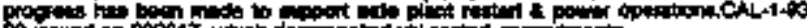
A

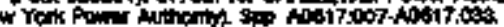

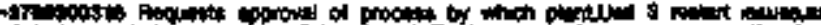

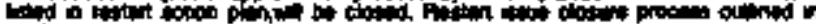

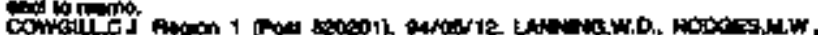

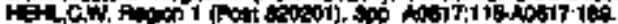

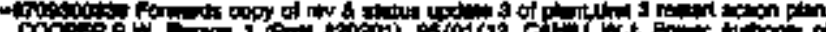

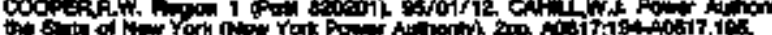

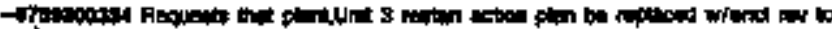

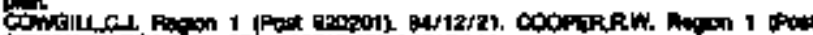

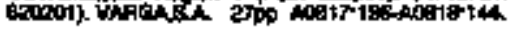

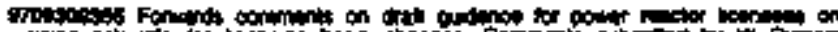

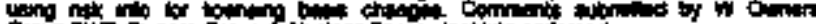

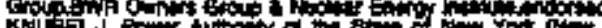

KNiL

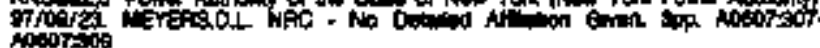

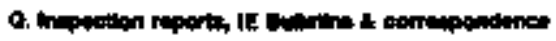

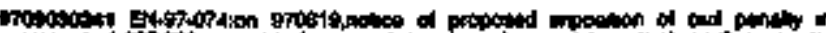

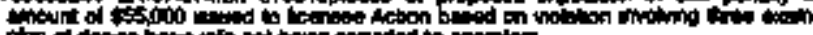

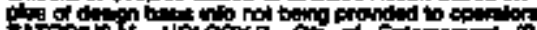

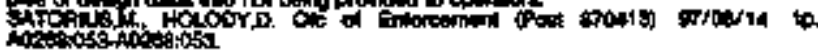

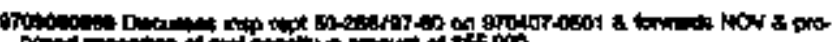

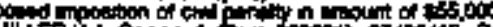

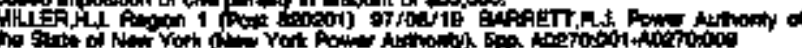

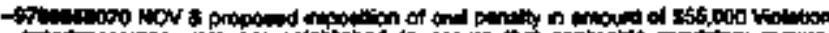

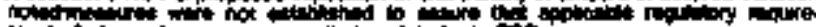

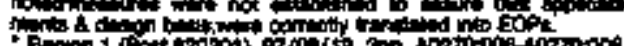

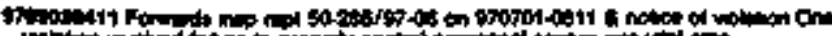

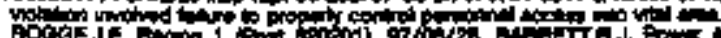
the

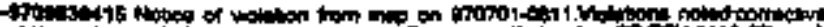

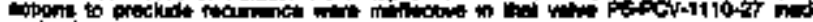

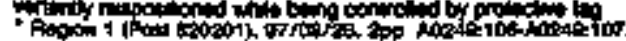

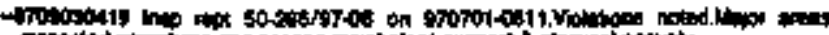

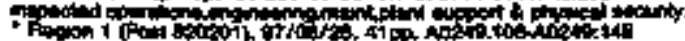

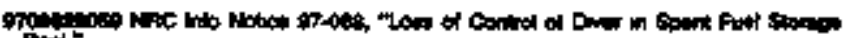

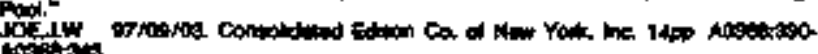
atosis

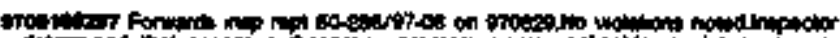

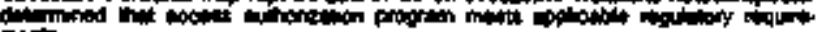

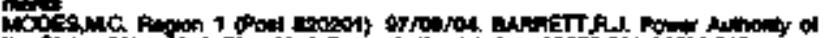

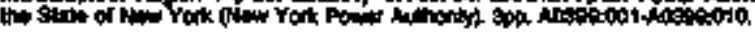

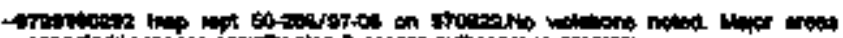

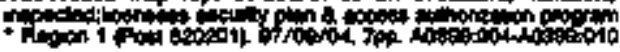

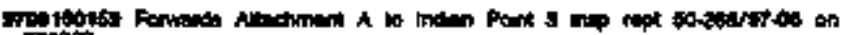
ropistis

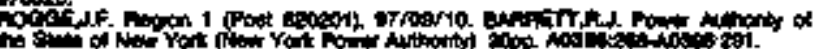

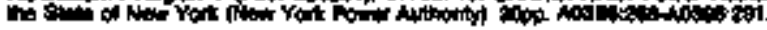

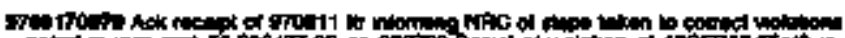

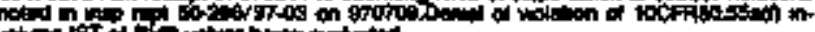
Q Wh of

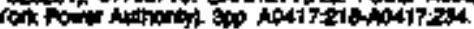

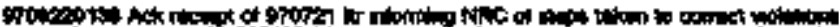

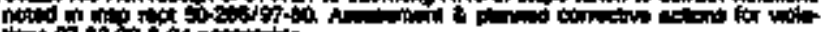

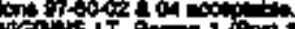

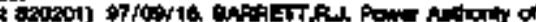

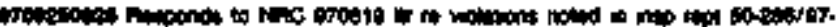

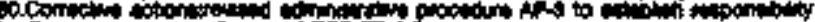
ce E

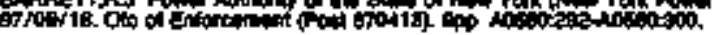

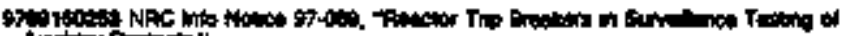

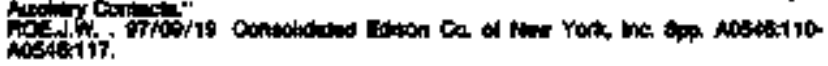

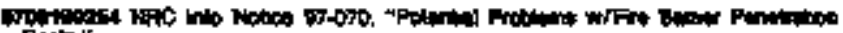

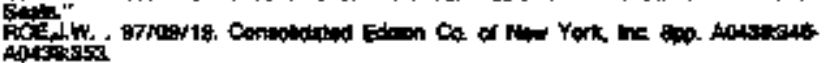

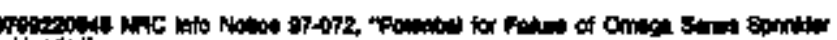

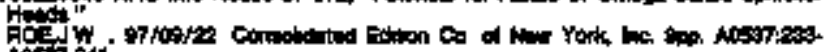

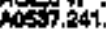

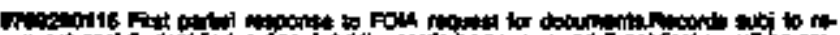

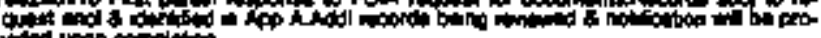

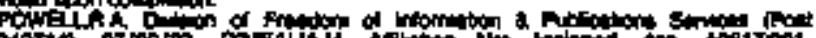

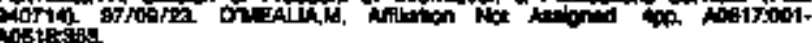

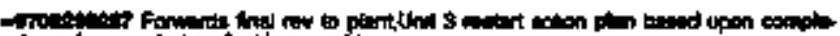

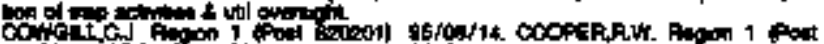

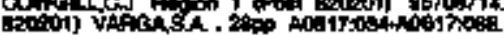

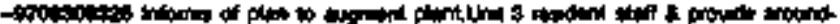

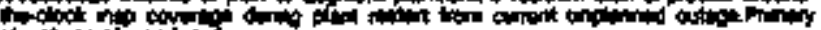

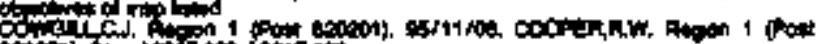

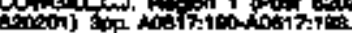

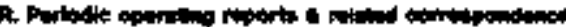

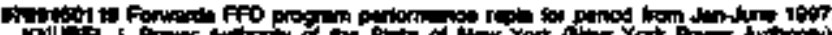
nTi

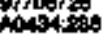

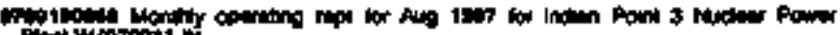
Pintworogit

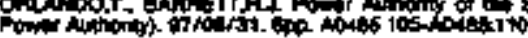

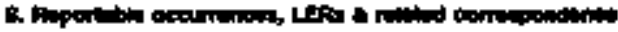

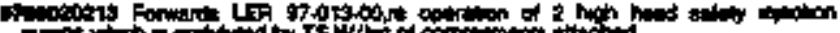

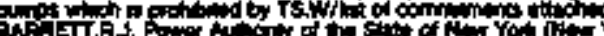

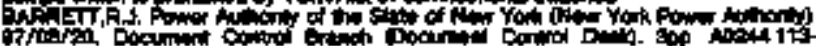

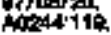

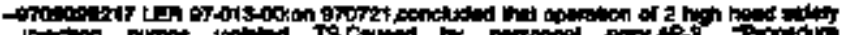

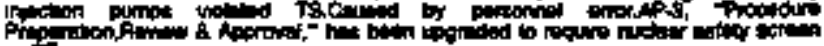
$x$ is

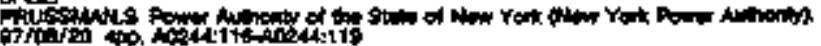

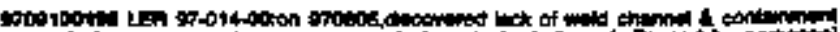

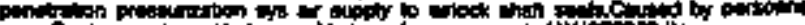

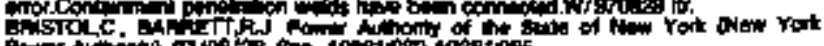

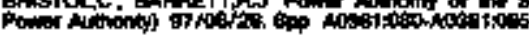




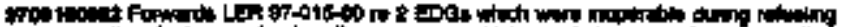

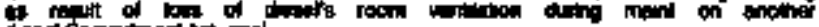

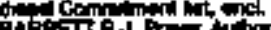

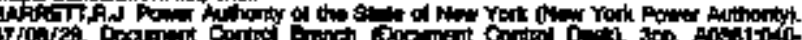

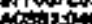

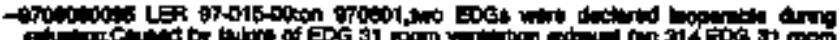

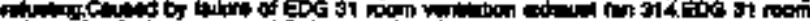

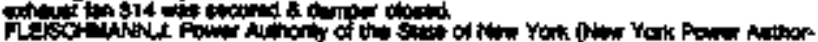

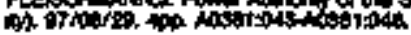

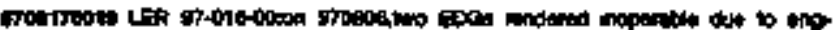

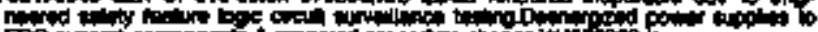

Fog.

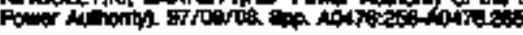

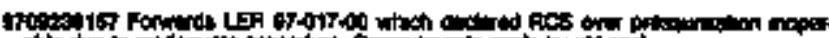

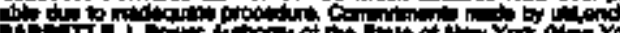

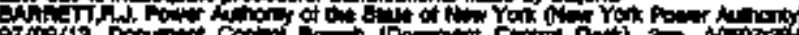

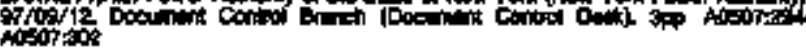

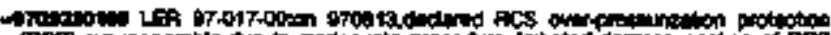

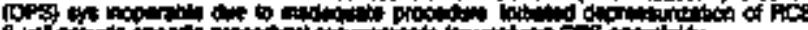

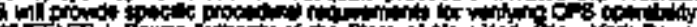
Tyon on

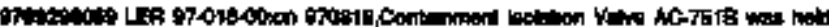

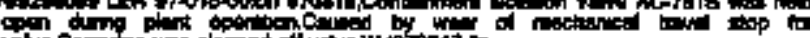

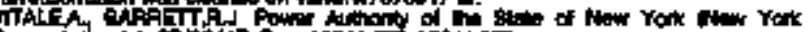

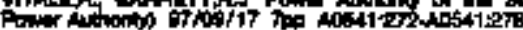

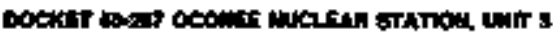

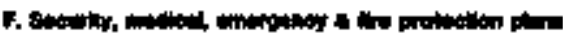

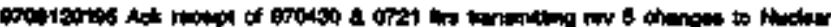

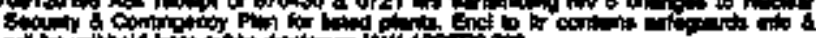

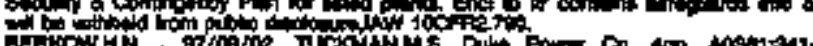

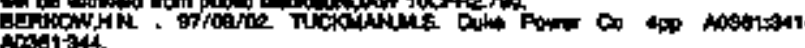

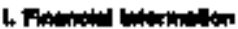

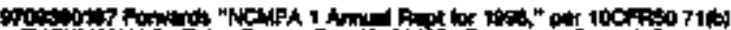

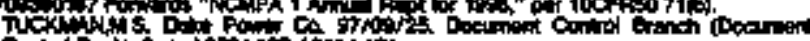

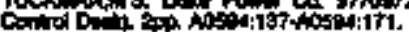

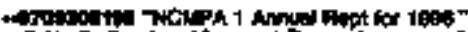

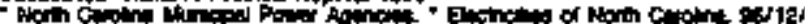

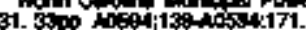

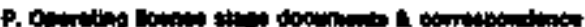

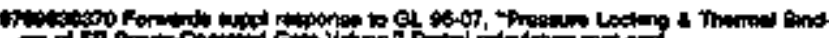

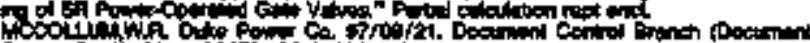

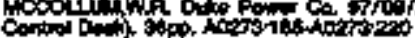

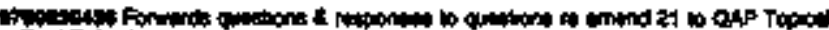

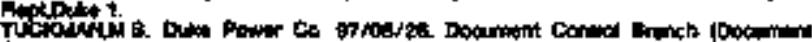

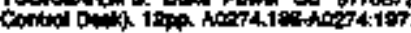

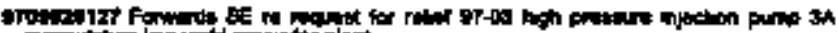

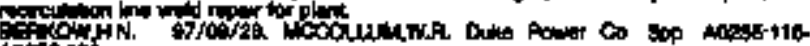
Antsition

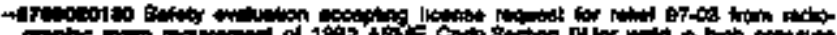

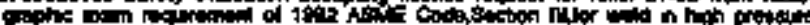

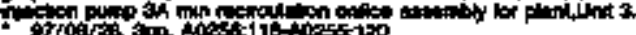

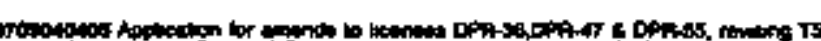

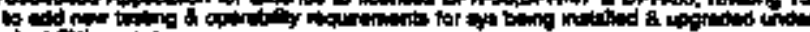

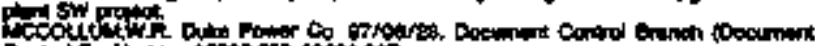

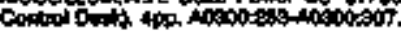

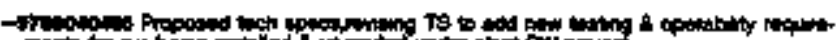

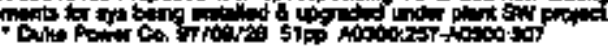

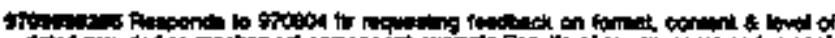

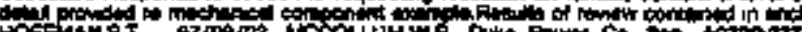
Nobstis

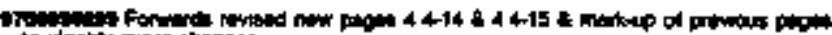

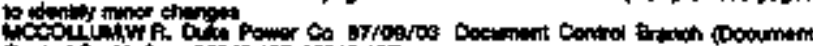

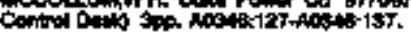

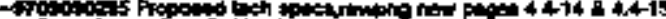

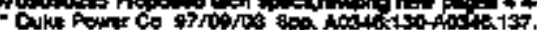

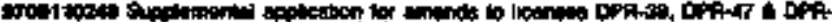

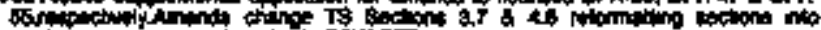

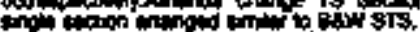

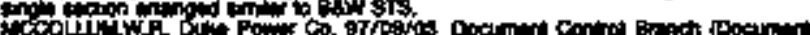

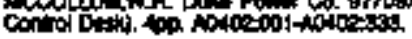

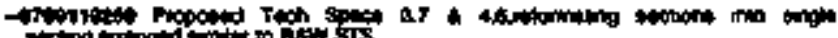
- Derit pon co of

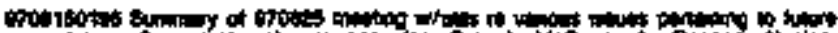

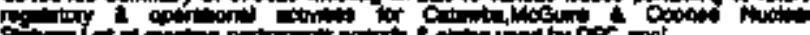

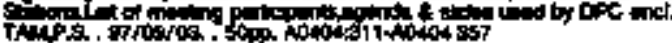

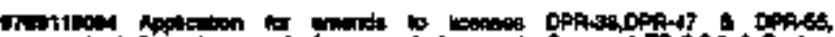
is 1514. UTBA

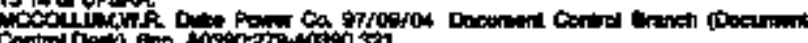

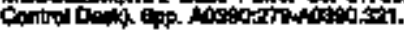

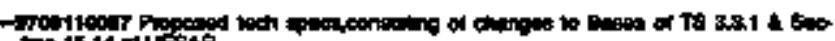

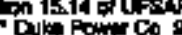

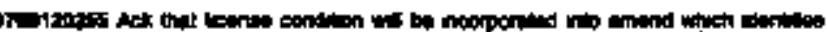

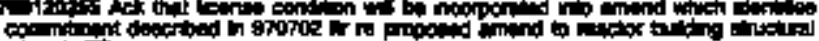

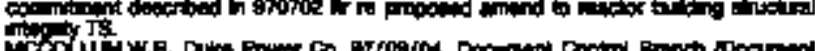

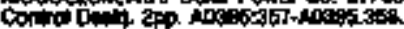

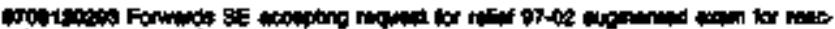

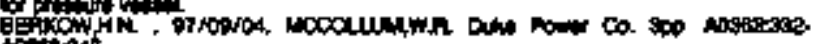
istonst

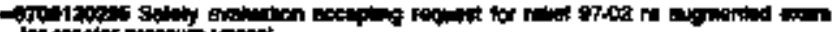

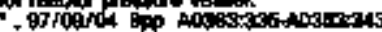

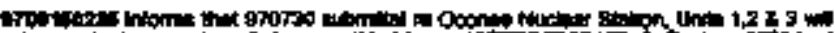

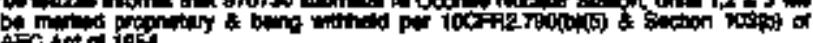

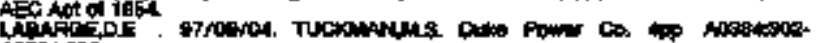
Ansoming

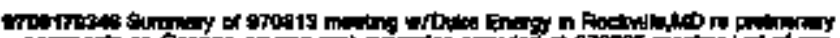

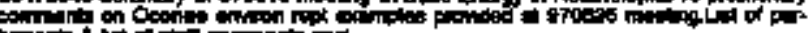

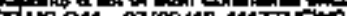

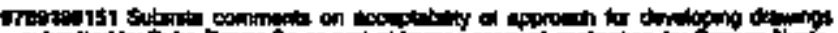

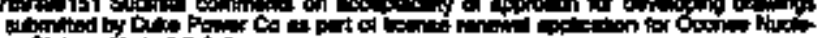

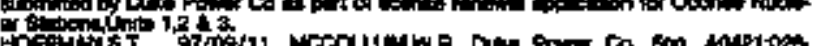
Aacertioso

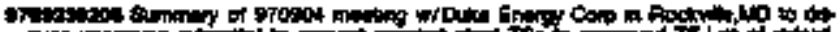

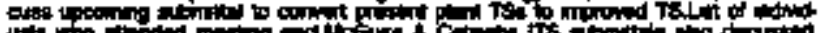

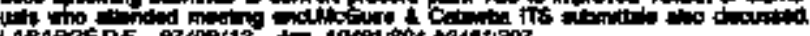

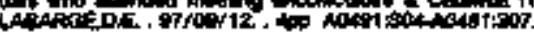

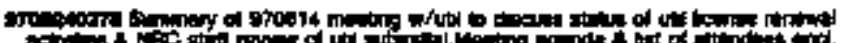

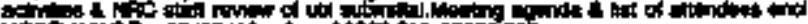

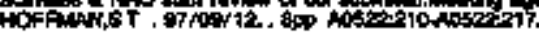

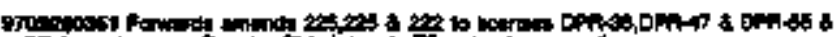

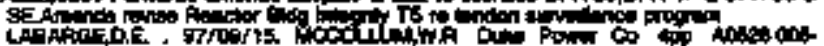

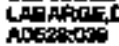

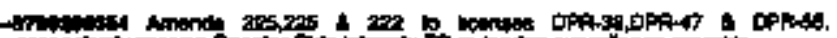

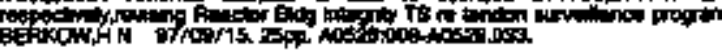

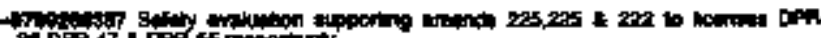
S.TPA

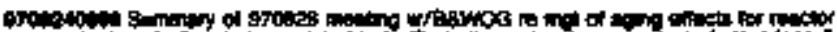

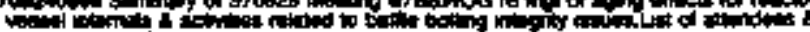

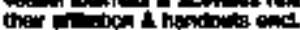

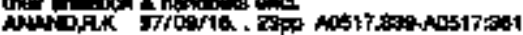

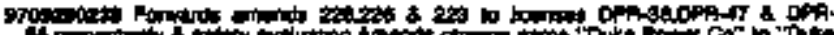

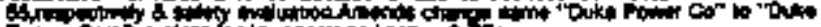
Gridus ins 1348

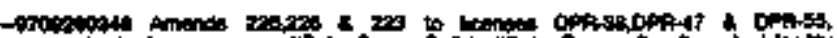

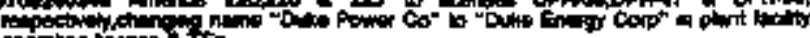

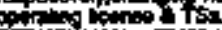

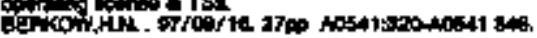

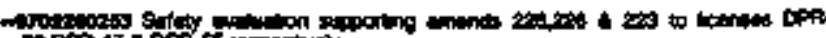

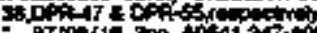

19:30.

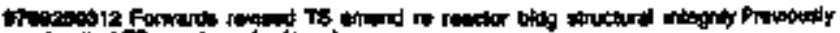

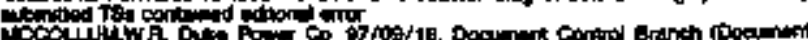

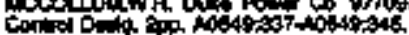

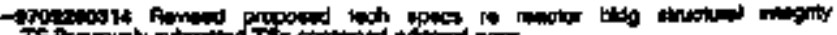

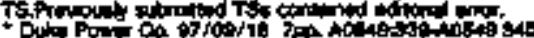




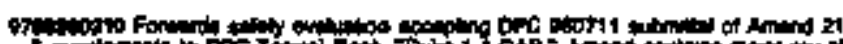

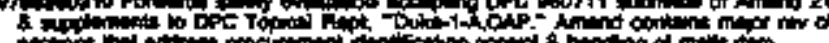

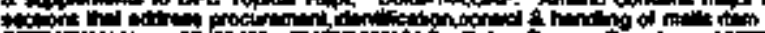

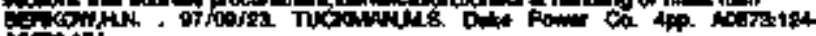
Aotion.

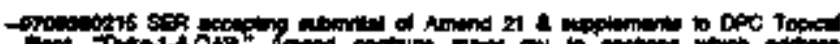

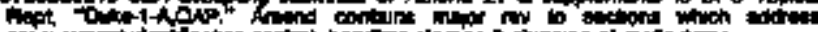

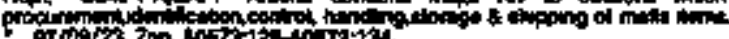

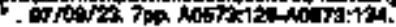

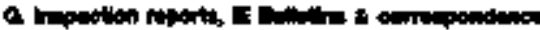

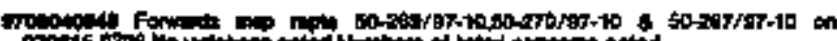

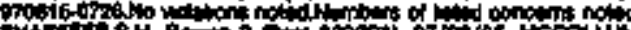

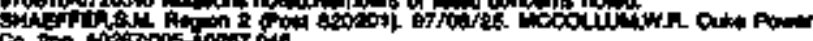
Co.

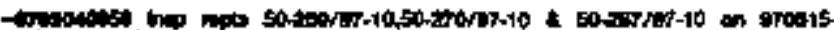

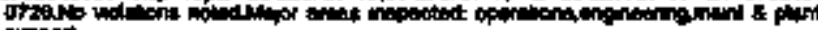

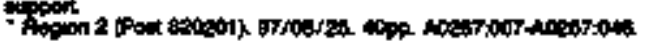

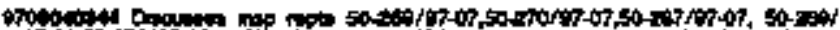

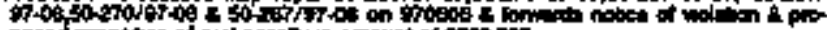

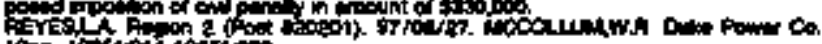

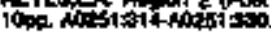

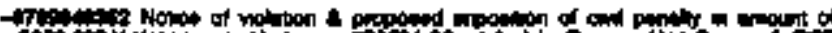

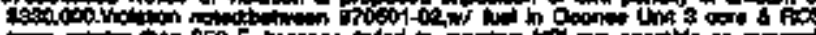

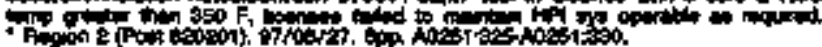

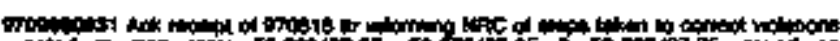

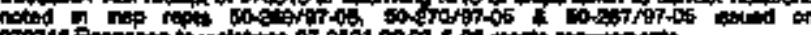

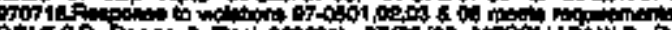

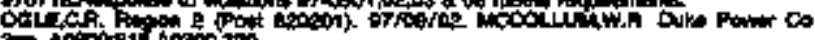

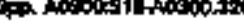

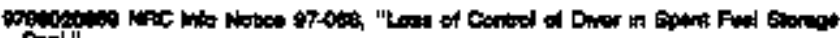

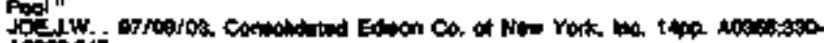

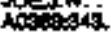

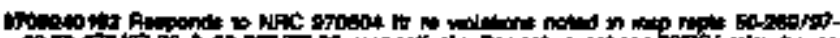

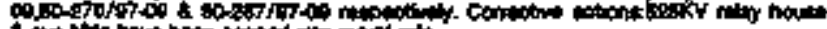
1

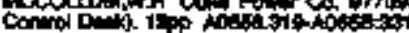

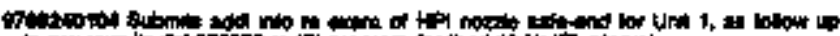

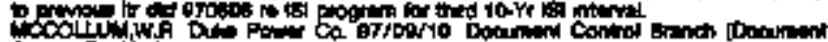

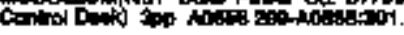

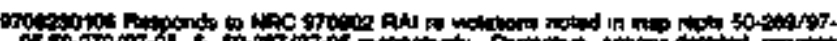
otgom

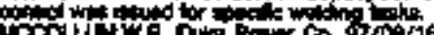

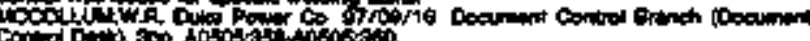

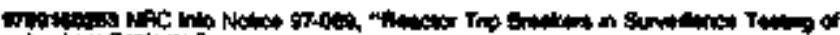

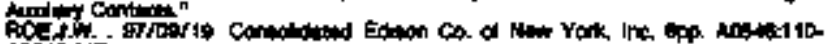
A0.4011\%.

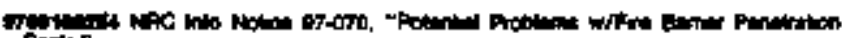

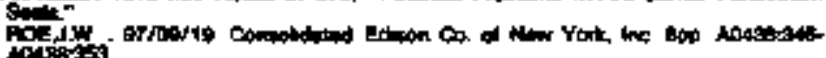

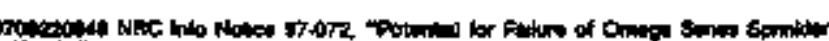

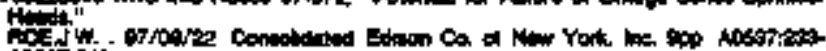

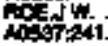

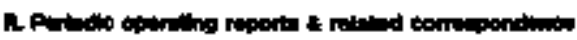

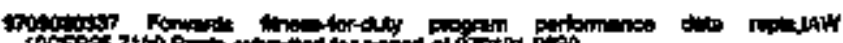

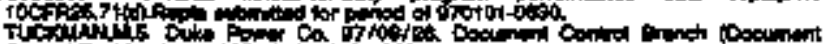

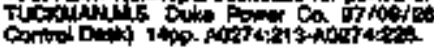

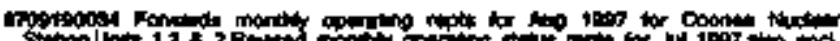

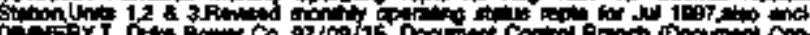

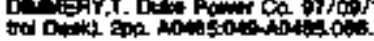

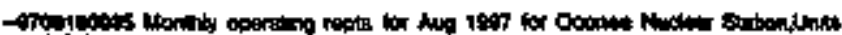

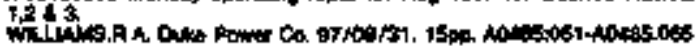

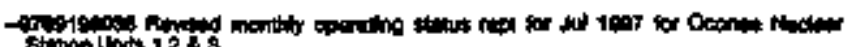

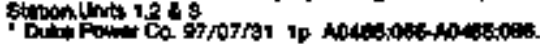

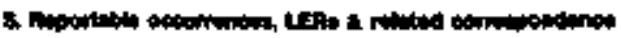

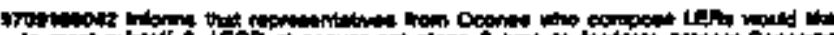

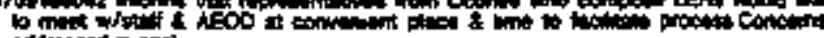

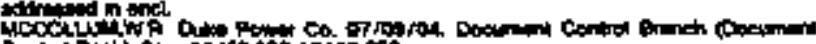

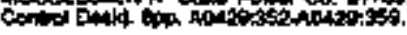

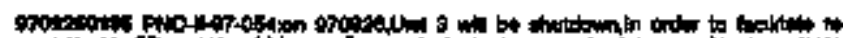

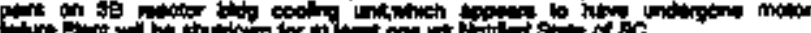

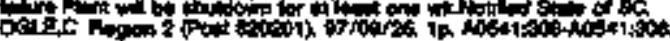

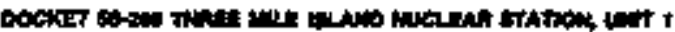

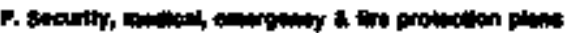

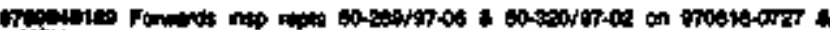

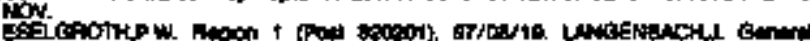

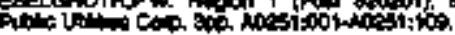

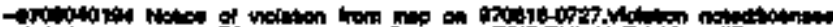

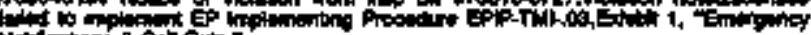

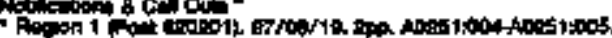

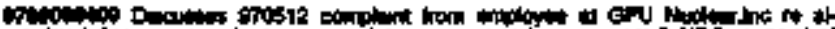

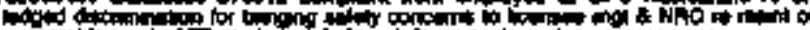

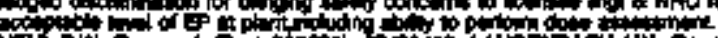

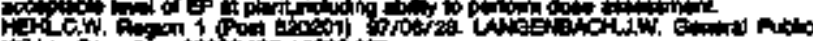

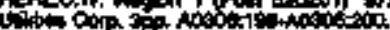

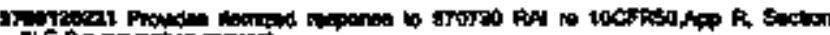
LA

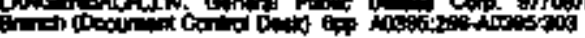

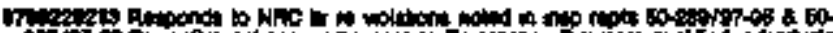

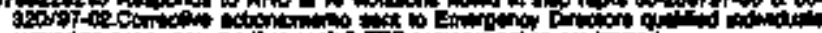

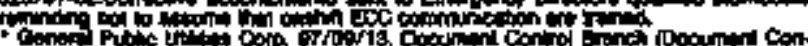

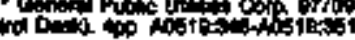

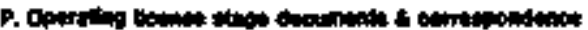

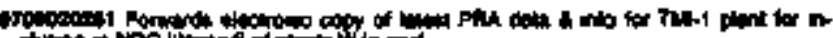

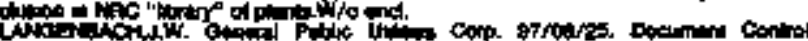

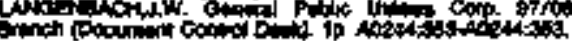

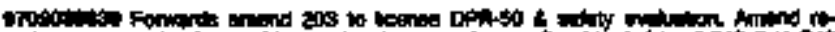

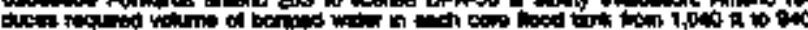

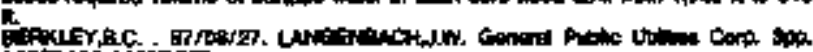

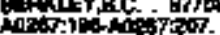

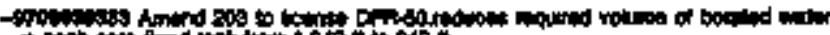

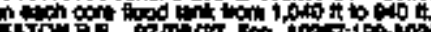

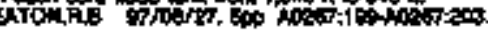

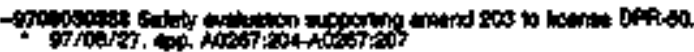

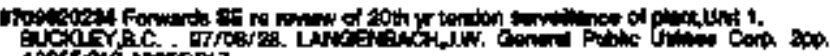

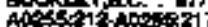

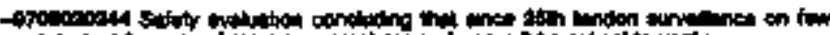

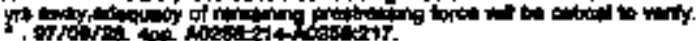

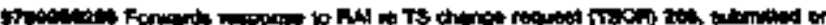

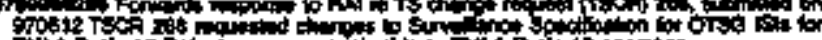
J

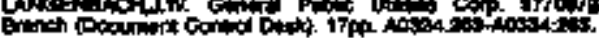

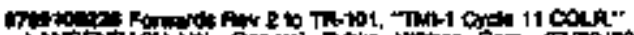

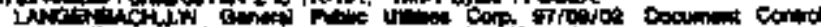

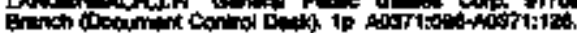

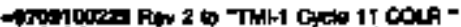

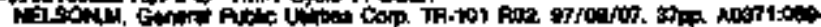
AOST1.12.

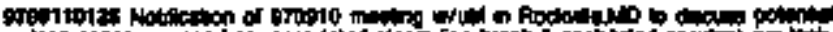

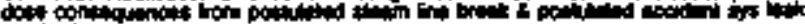

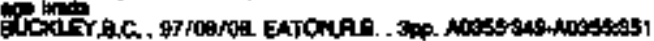

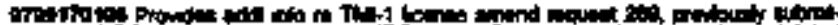

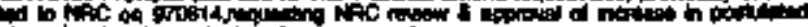

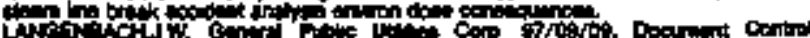

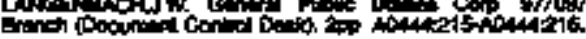

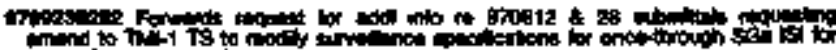

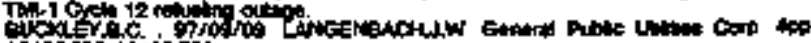

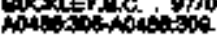

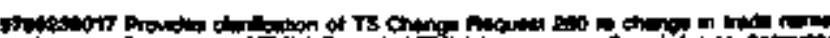

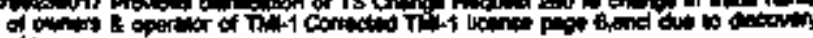

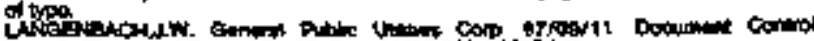

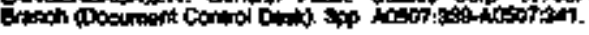




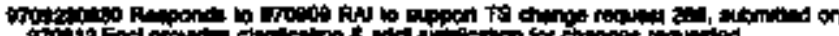

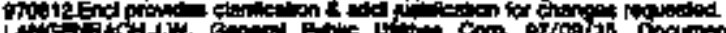

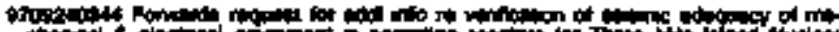

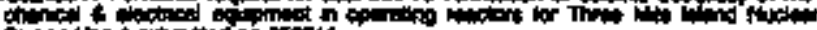

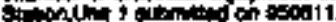

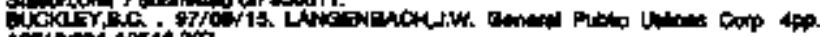

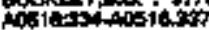

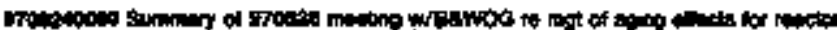

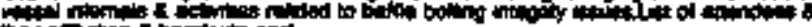

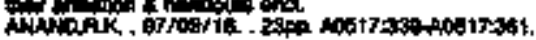

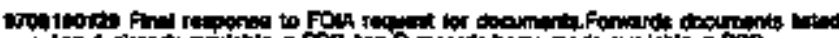

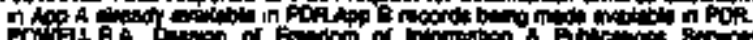

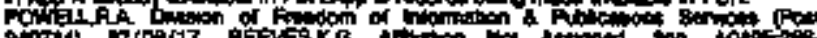

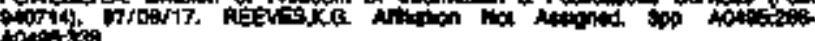

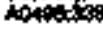

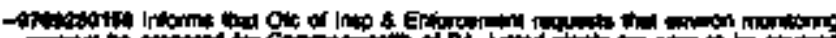

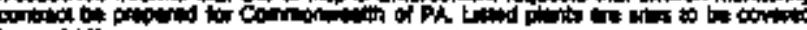

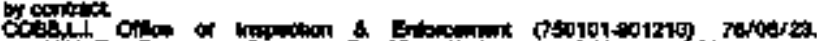

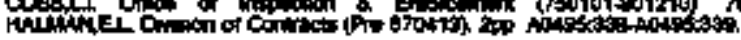

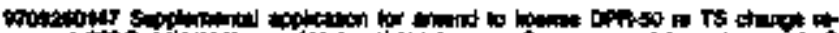

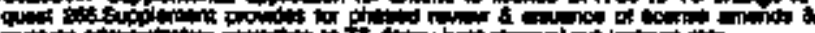

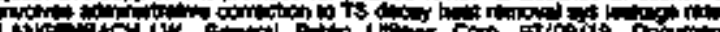

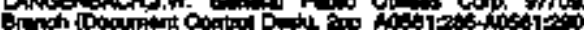

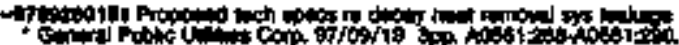

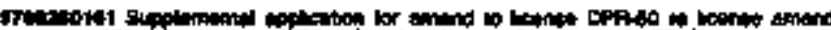

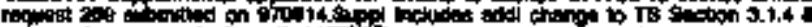

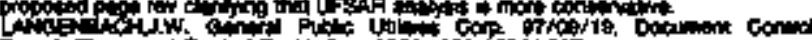

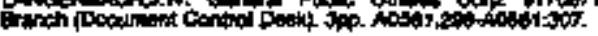

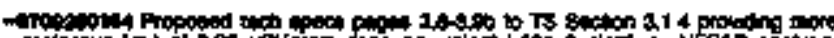

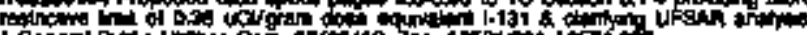

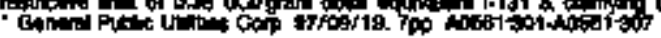

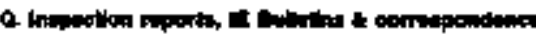

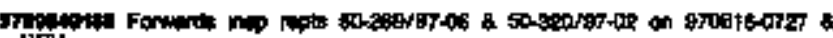
Not

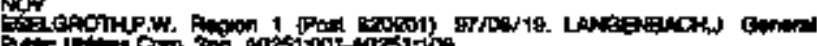

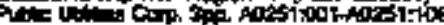

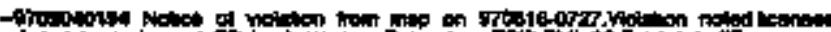

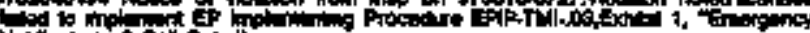

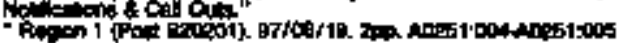

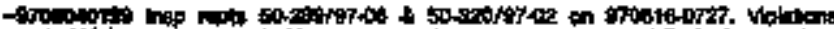

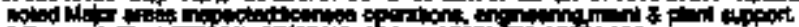

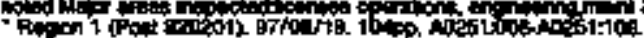

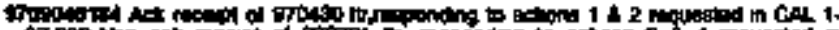

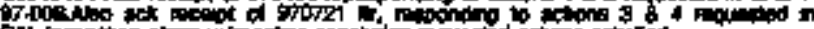

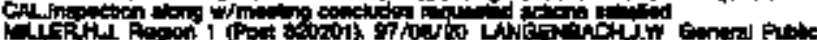

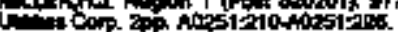

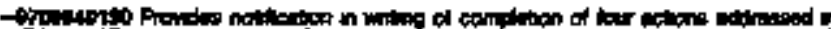

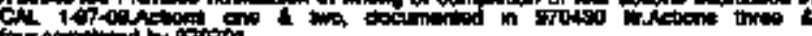

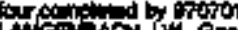

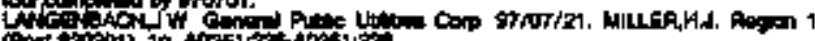

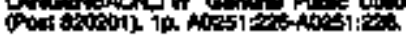

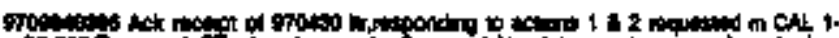

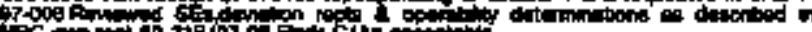

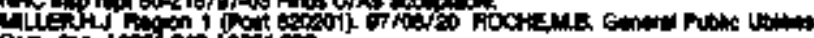

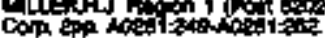

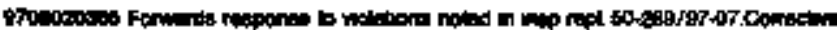

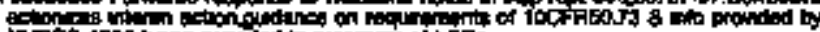

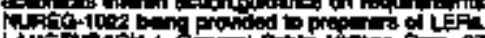

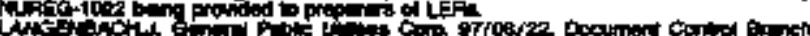

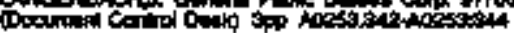

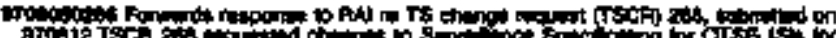

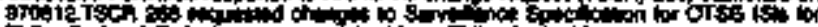

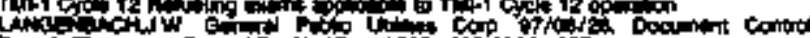

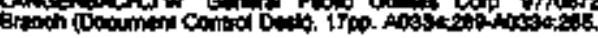

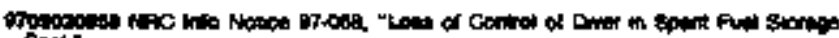

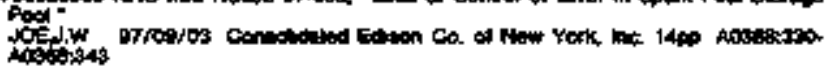

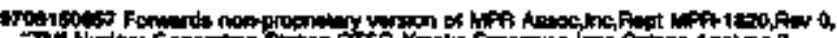

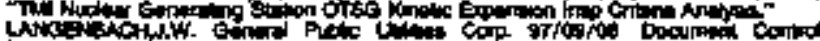

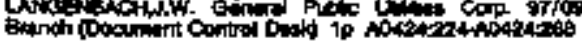

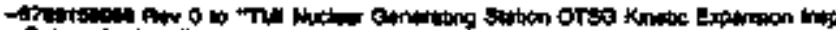

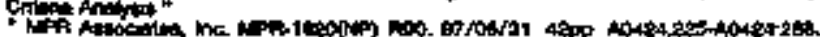

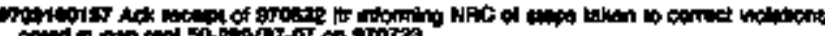

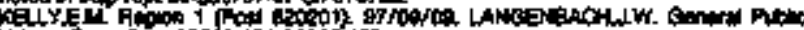

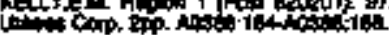

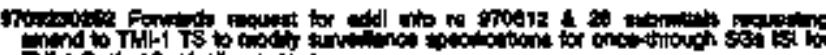

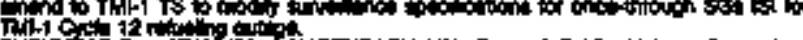

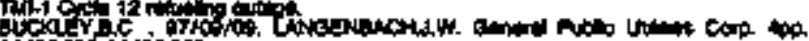

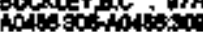

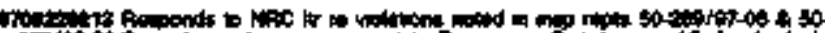

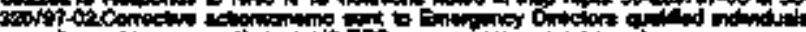

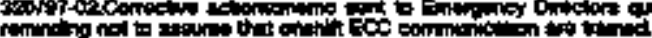

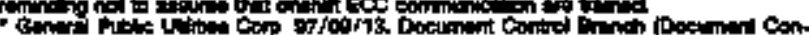

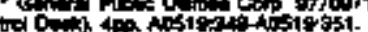

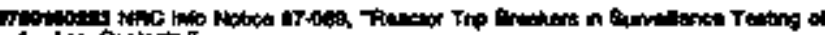

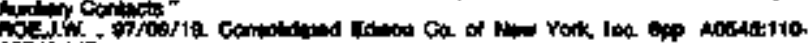
nititi.

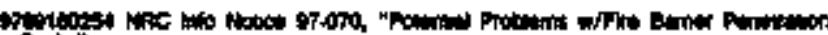

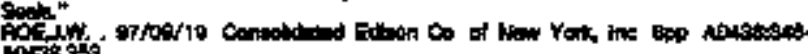
M0Fiste.

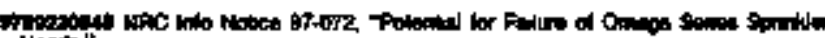

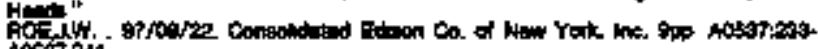
d000 24

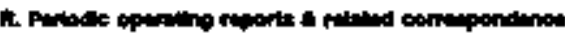

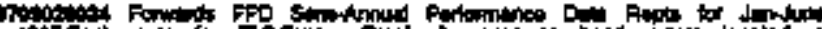

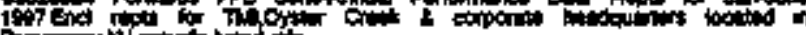

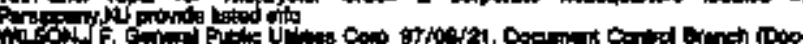

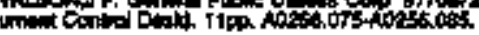

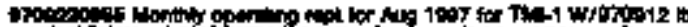

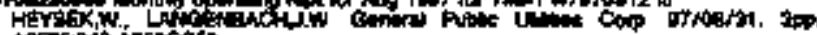

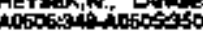

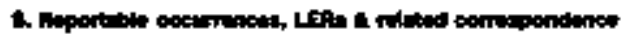

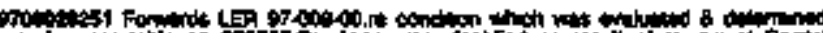

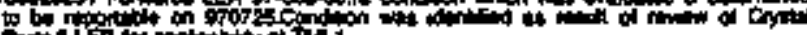

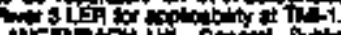

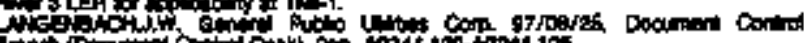

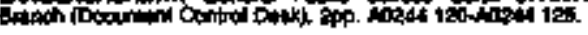

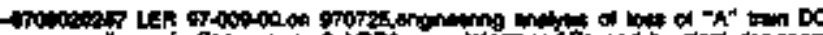

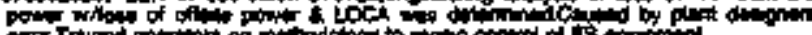

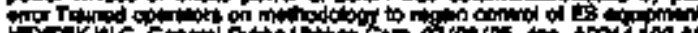

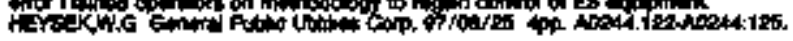

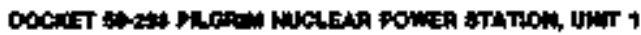

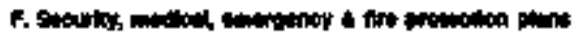

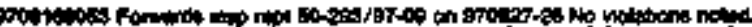

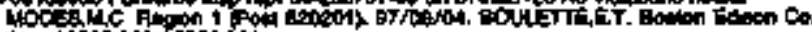

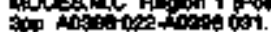

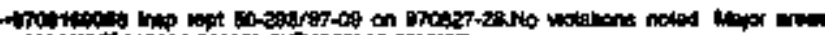

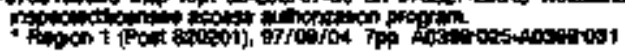

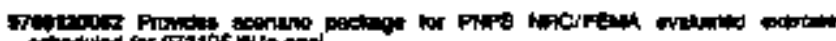

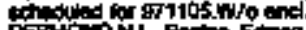

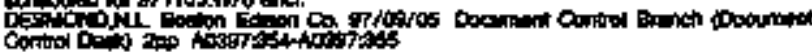

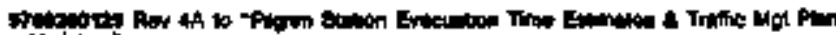

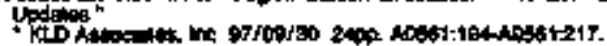

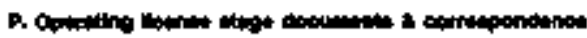

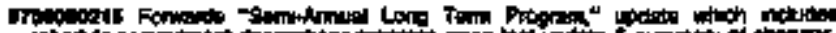

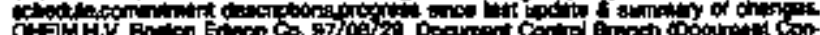

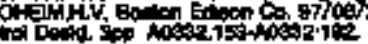

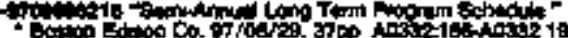

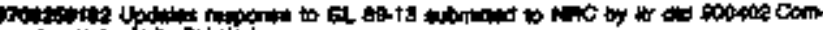

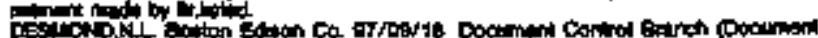

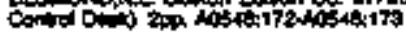

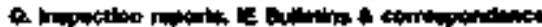




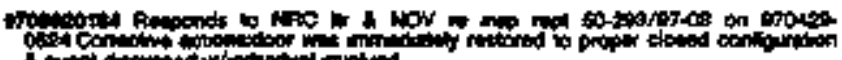

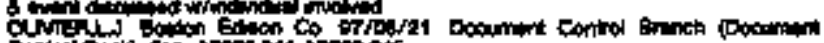

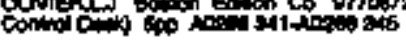

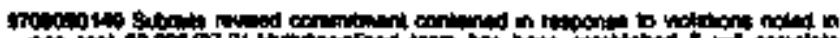

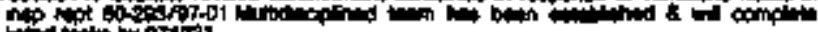

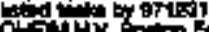

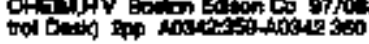

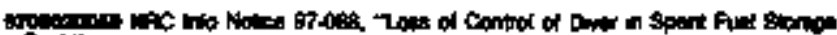

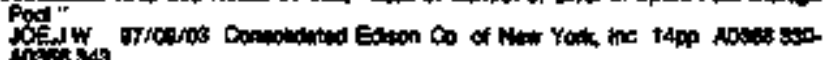

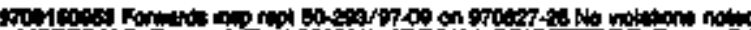

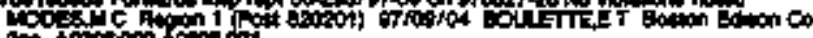

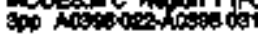

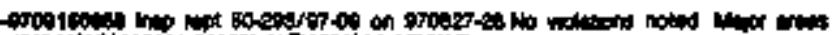

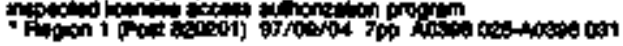

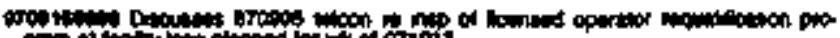

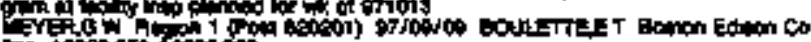
Fre

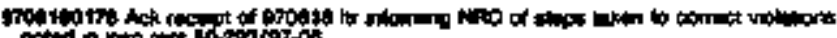

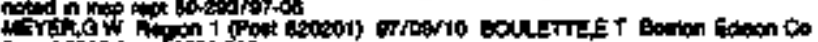

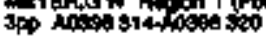

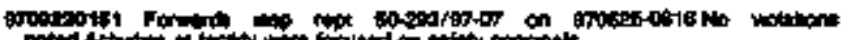

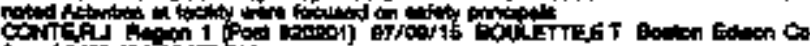

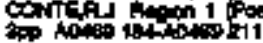

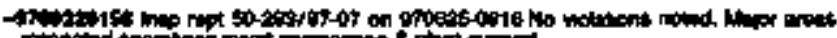

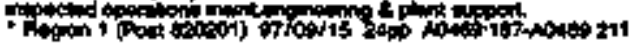

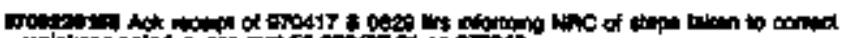

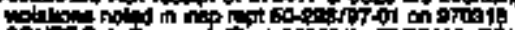

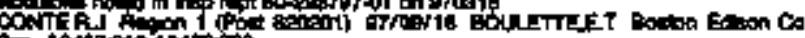

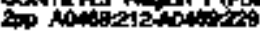

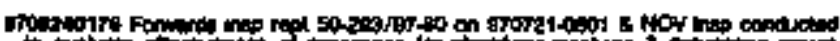

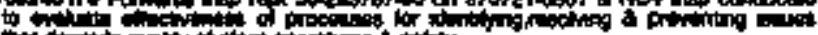

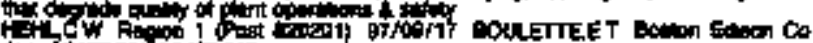
4 A

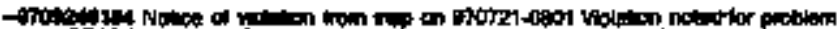

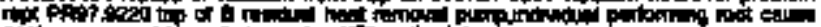

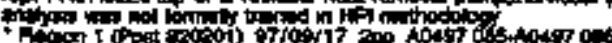

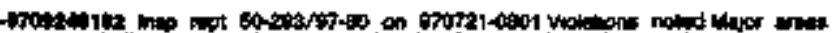

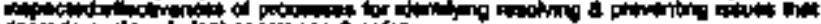

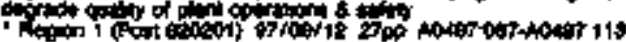

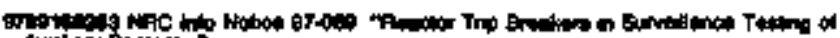

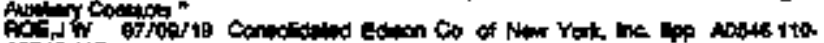
A05.17

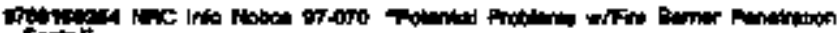

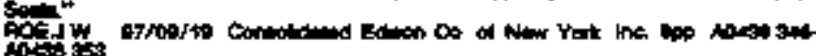

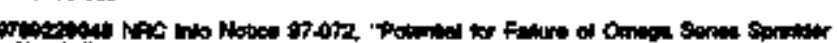

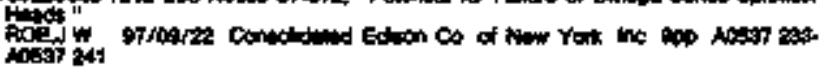

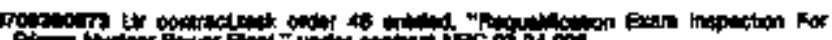

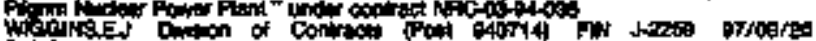

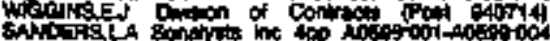

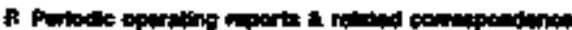

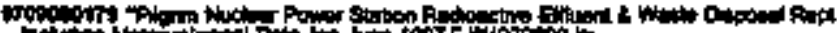

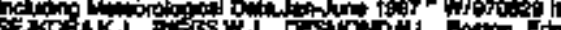

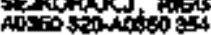

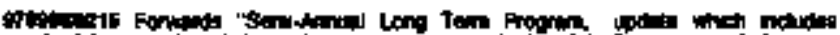

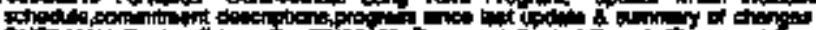

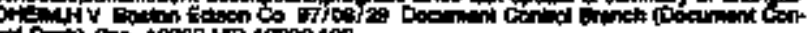

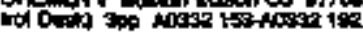

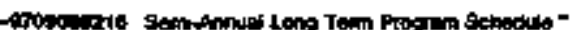

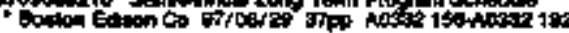

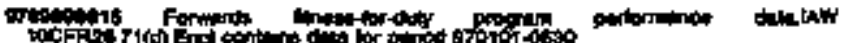

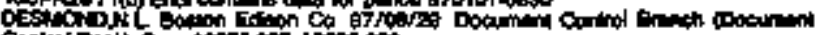

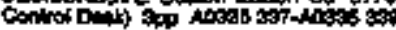

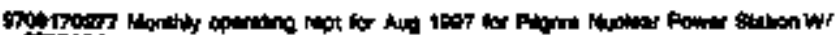

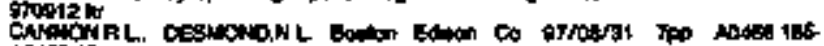
A014 151

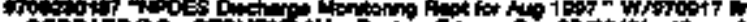

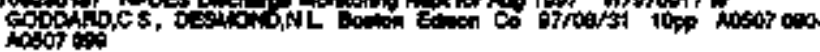

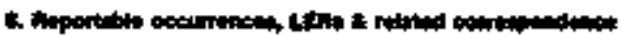

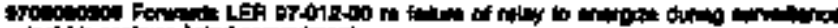

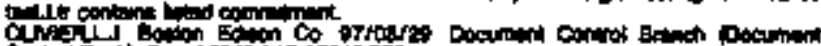

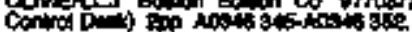

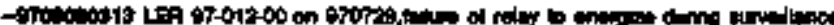

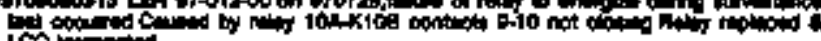

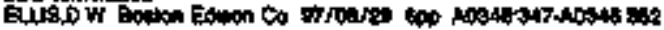

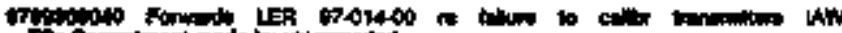

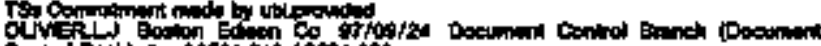

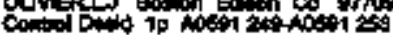

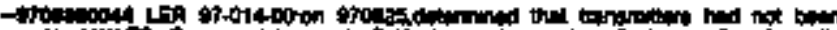

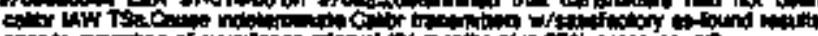

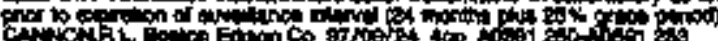

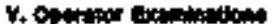

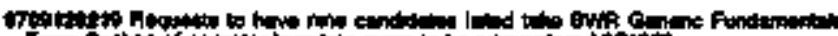

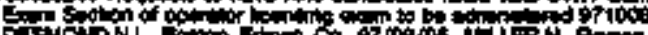

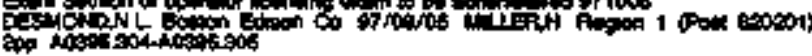

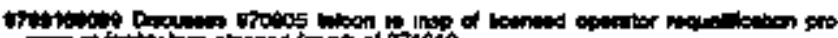

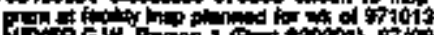

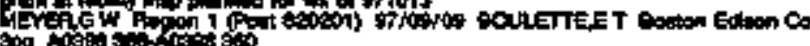

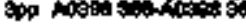

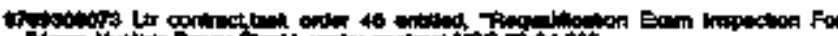

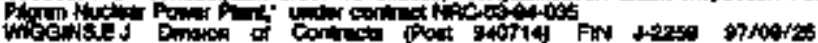

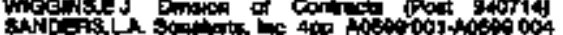

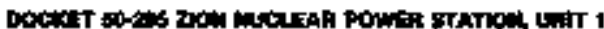

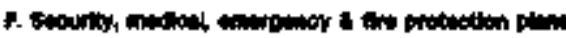

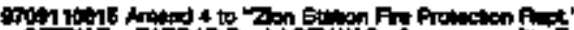

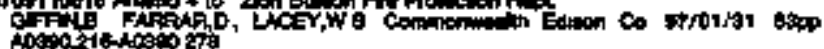

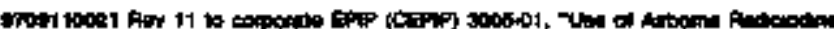

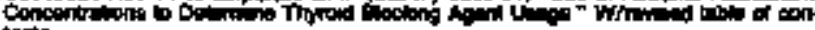

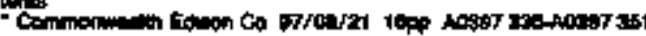

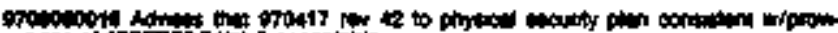

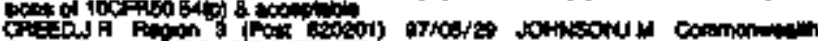

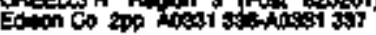

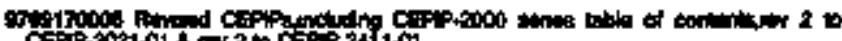

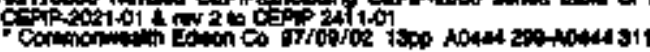

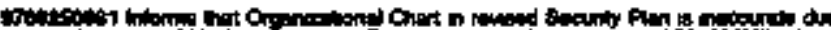

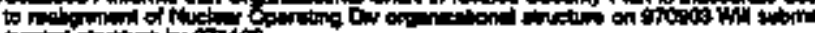

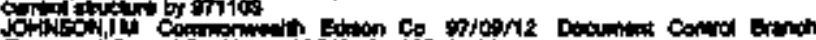

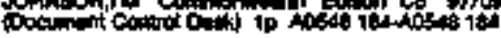

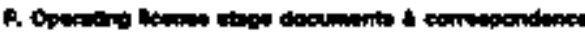

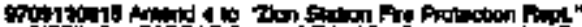

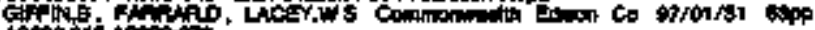
$\operatorname{cosec} 210 \cos 0=270$

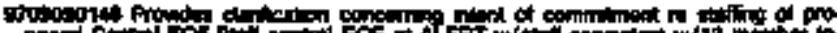

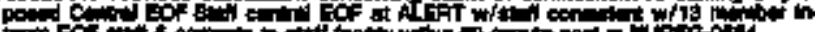

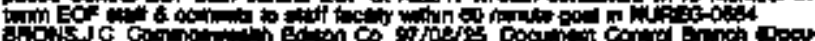

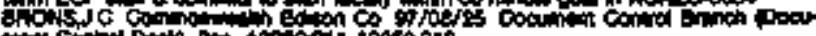

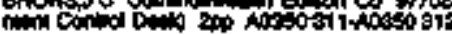

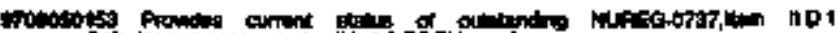

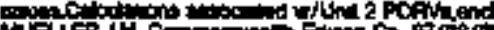

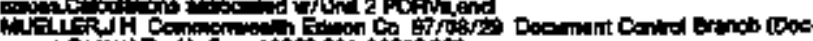

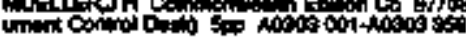

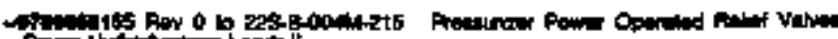

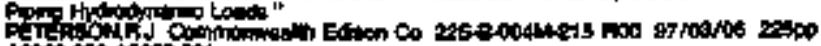
osisotion 201

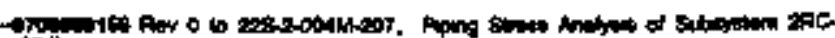

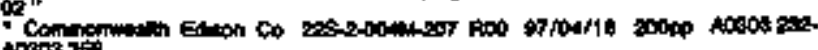
$\cos 03$ 


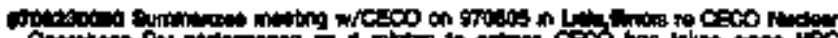

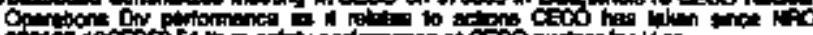

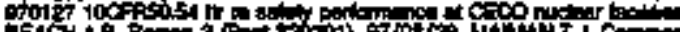

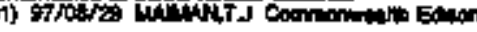

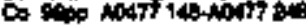

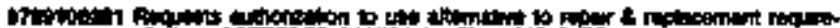

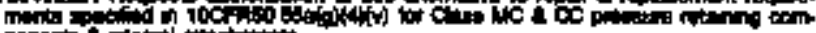

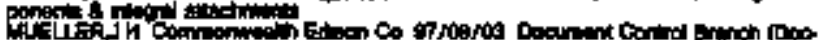

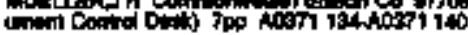

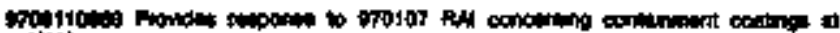

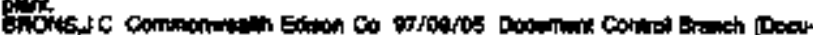

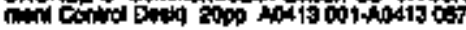

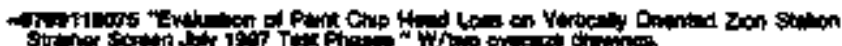

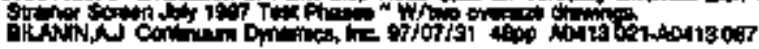

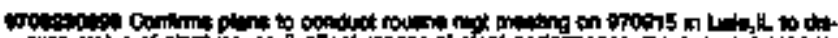

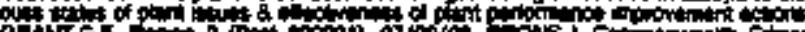

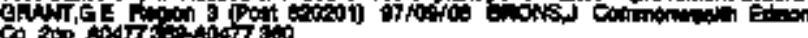

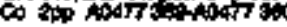

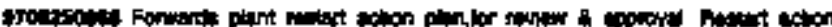

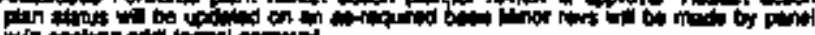

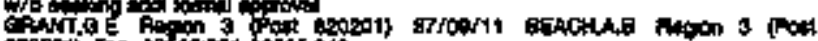

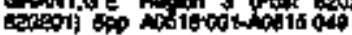

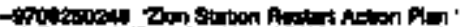

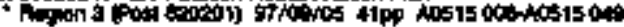

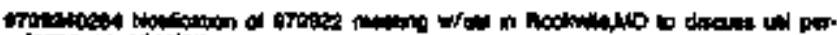

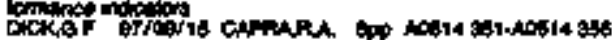

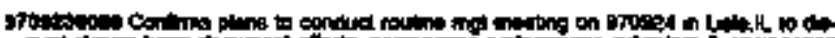

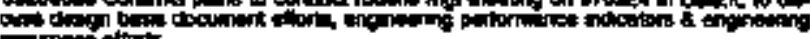

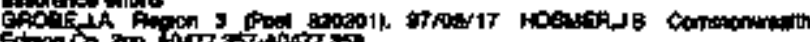

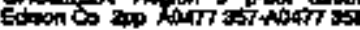

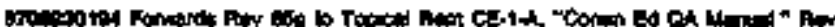

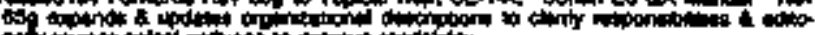

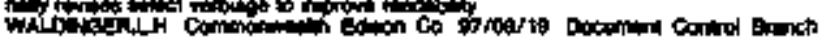

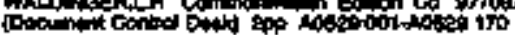

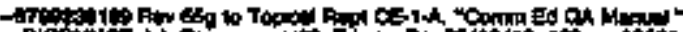

OCC

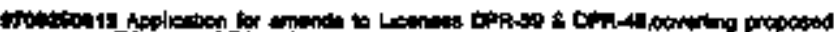

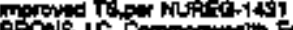

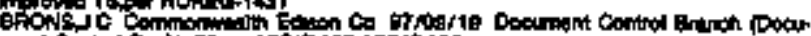

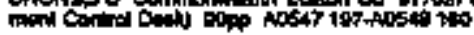

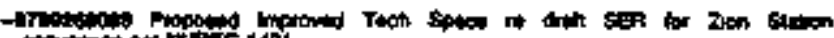

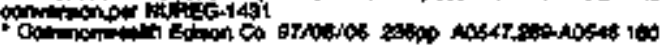

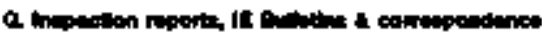

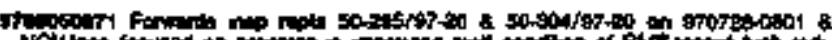

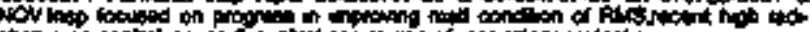

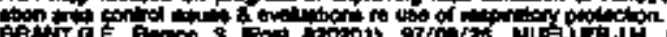

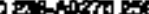

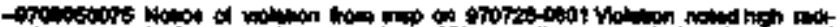

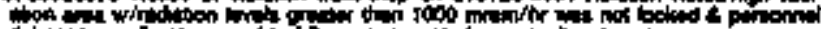

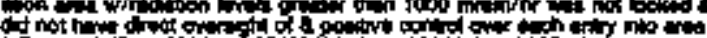

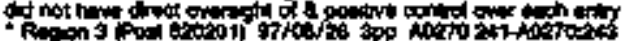

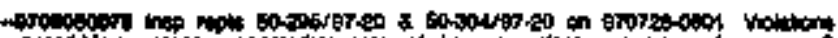

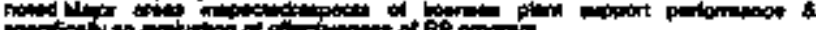

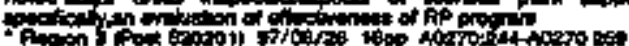

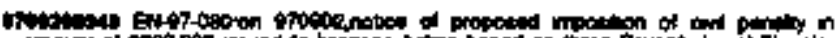

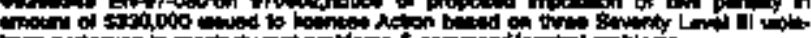

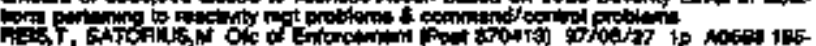

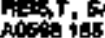

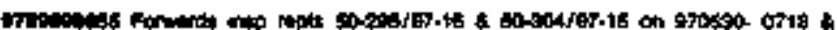

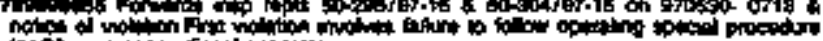

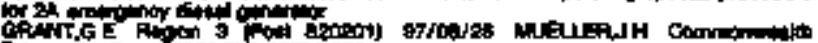

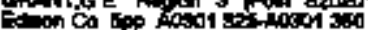

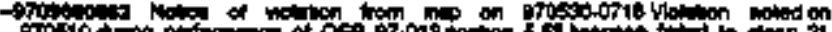

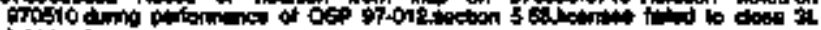

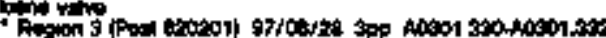

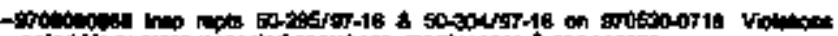

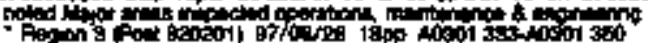

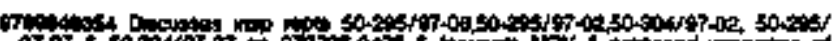

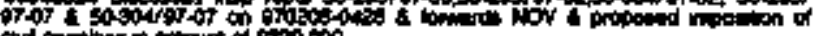

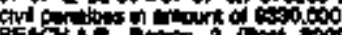

Eam

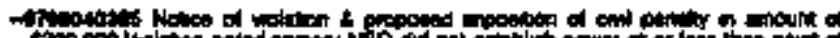

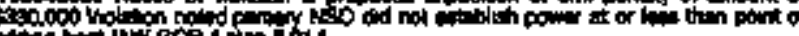

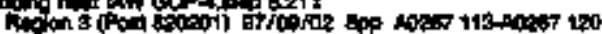

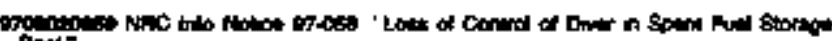

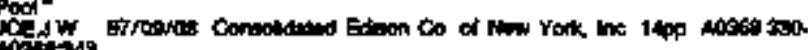

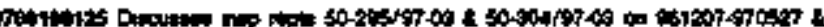

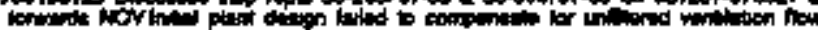

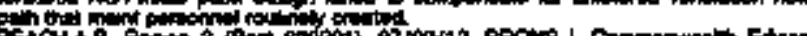

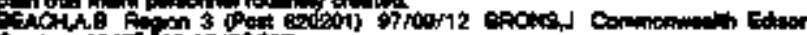

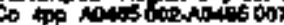

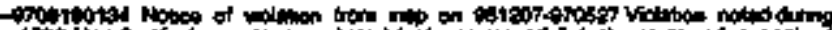

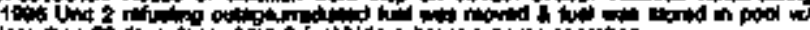

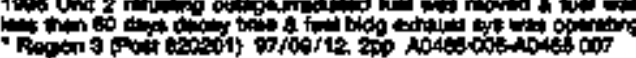

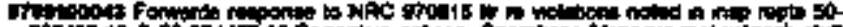

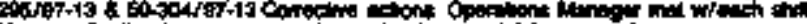

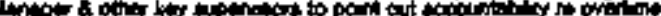

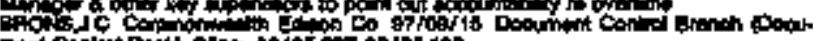

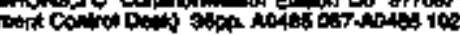

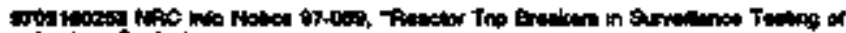

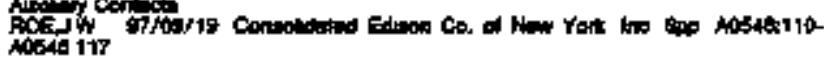

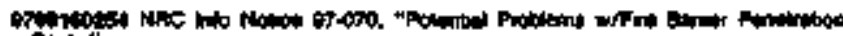

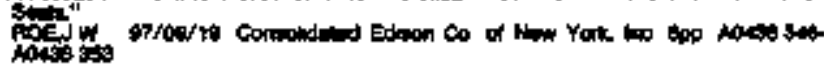

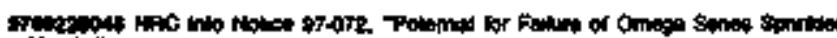

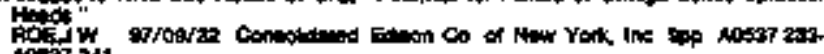
1009724

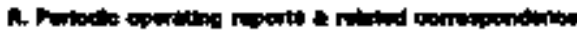

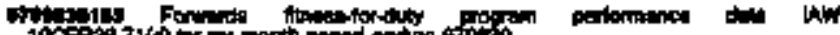

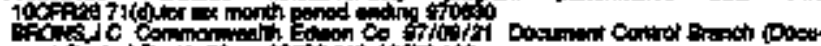

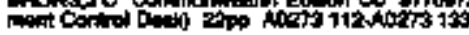

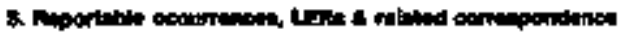

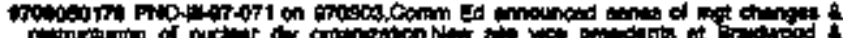

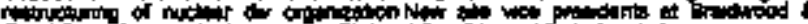

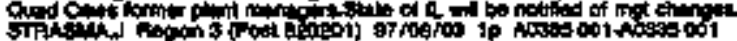

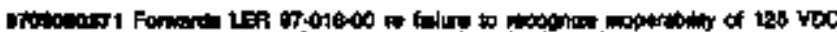

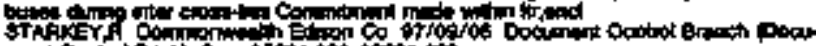

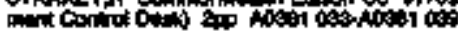

- Ar

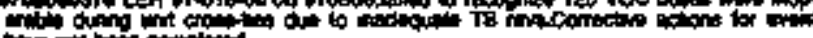

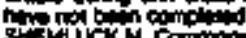

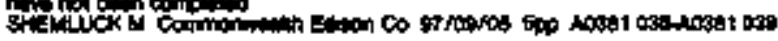

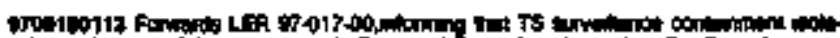

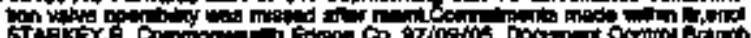

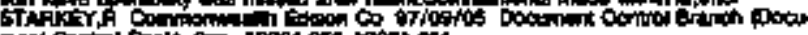

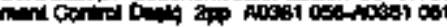

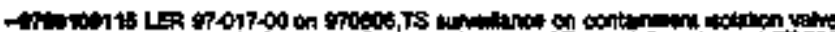

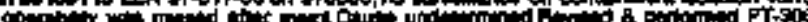

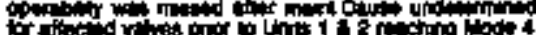

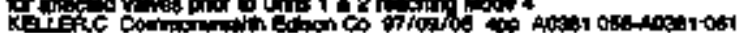

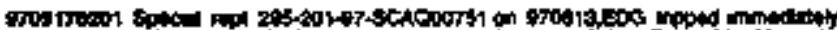

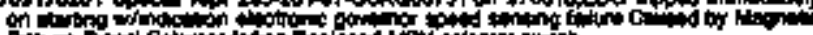

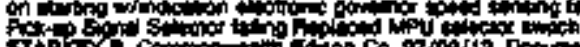

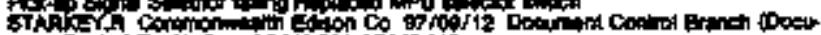

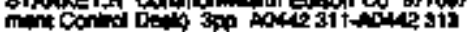

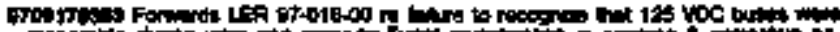

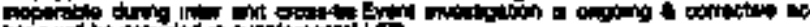

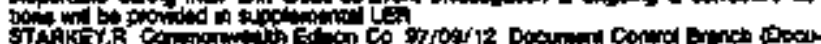

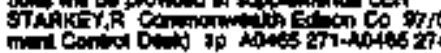

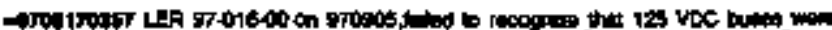

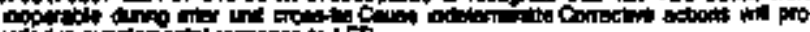

stidn

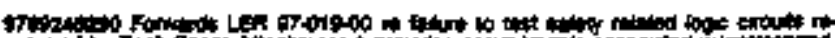

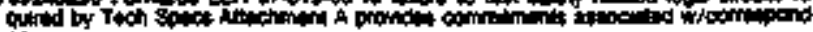

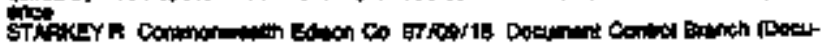

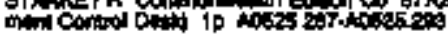

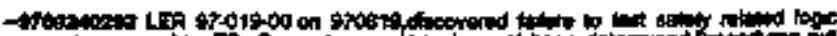

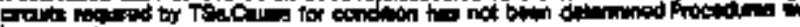

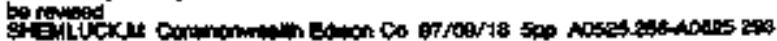




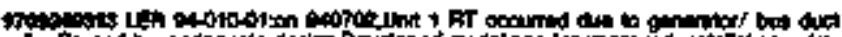

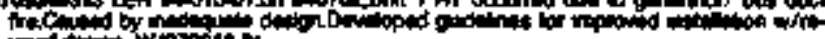

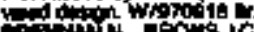

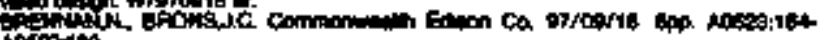
Atosistion

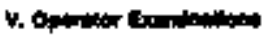

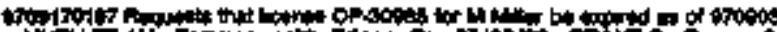

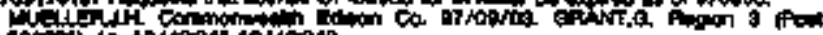

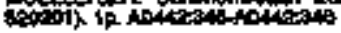

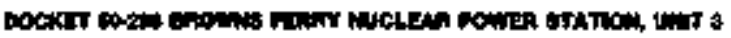

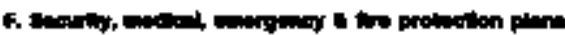

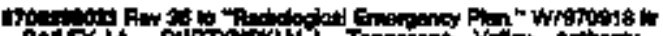

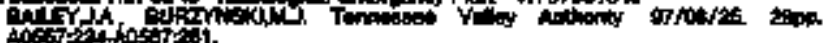

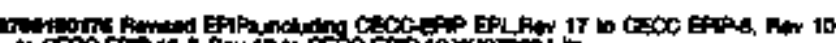

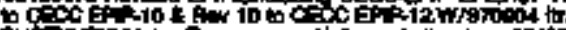

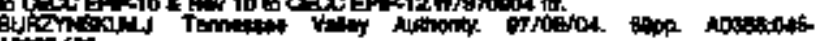
ingerts

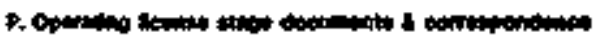

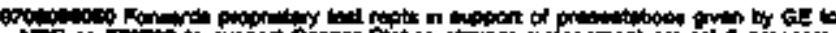

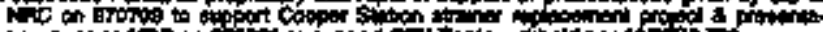

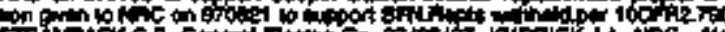

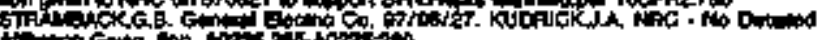
Ning

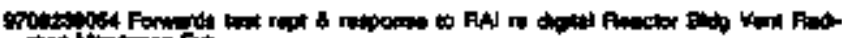

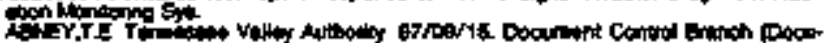

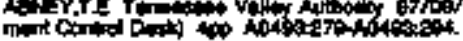

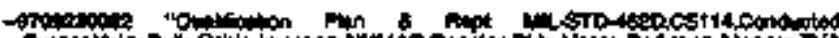

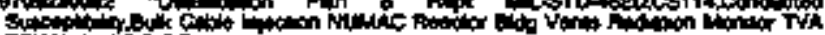

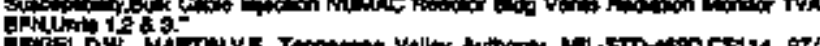

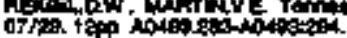

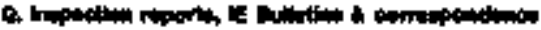

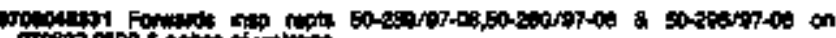

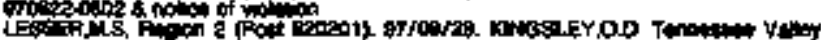

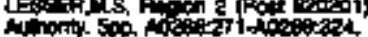

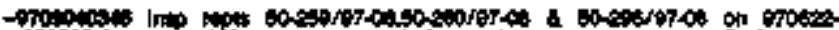

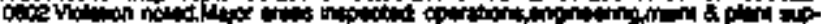

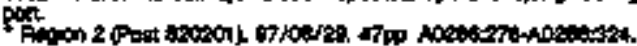

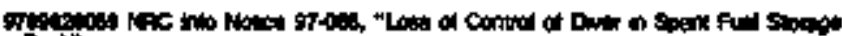

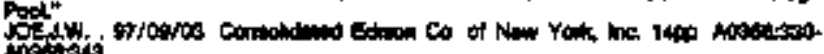

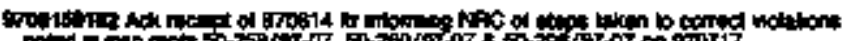

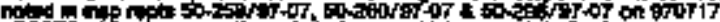

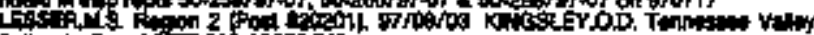

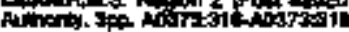

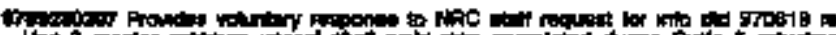

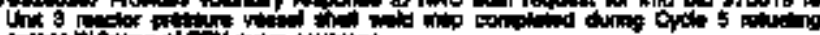
OF

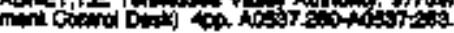

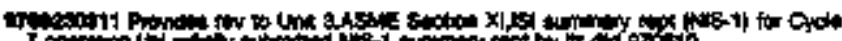

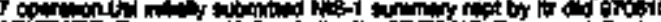

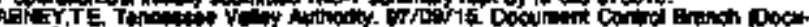

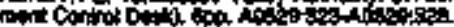

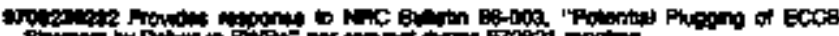

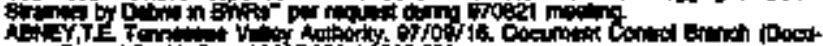

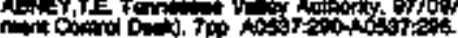

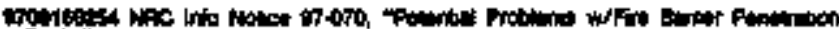

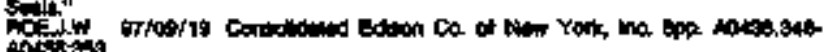
atsisto

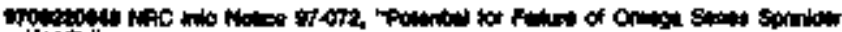

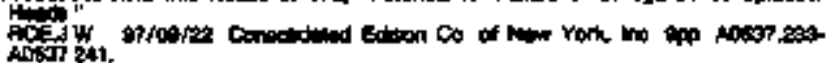

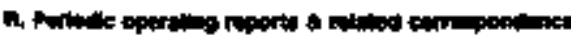

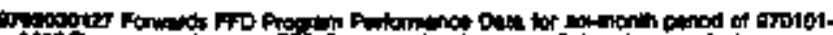

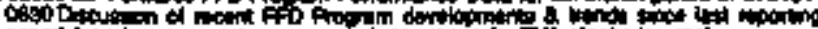

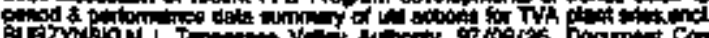

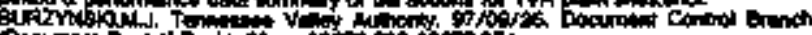

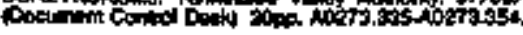

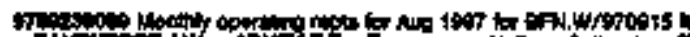
Dhe (1)

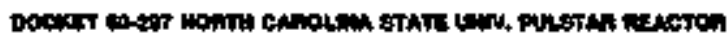

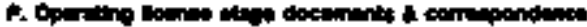

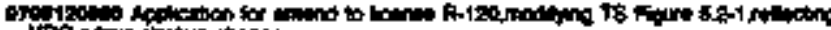

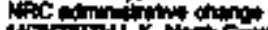

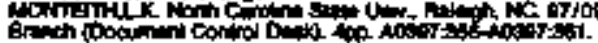

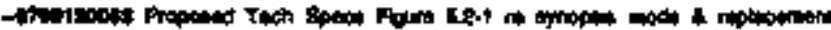

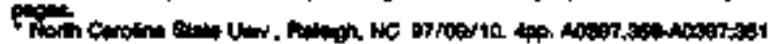

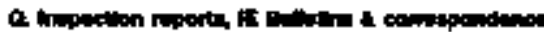

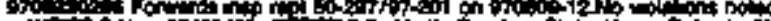

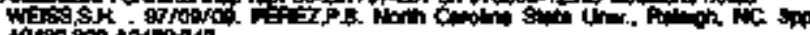

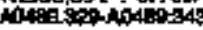

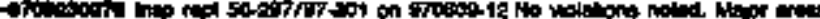

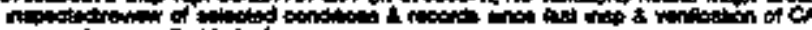

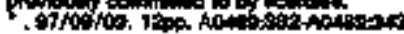

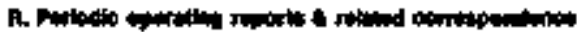

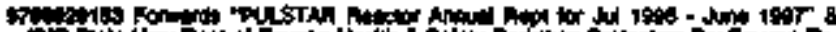

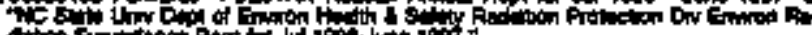

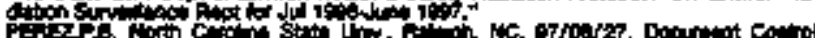

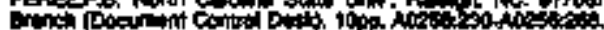

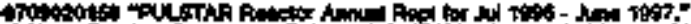

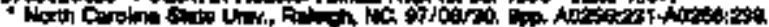

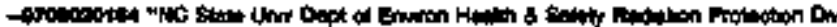

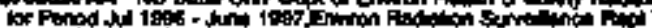

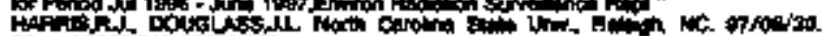

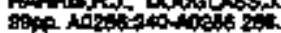

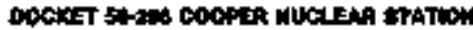

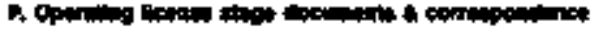

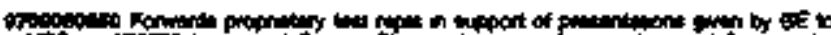

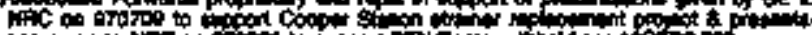

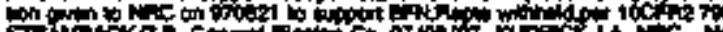

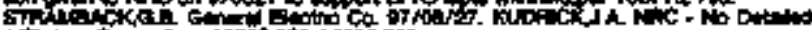

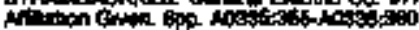

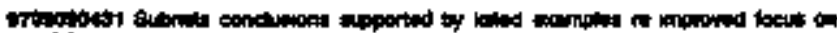

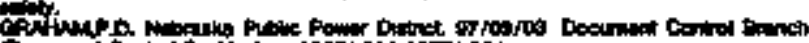

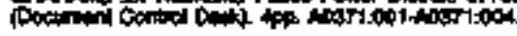

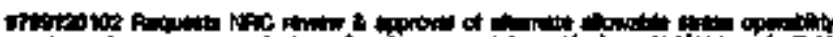

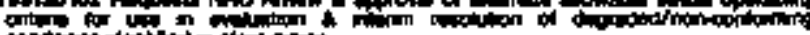

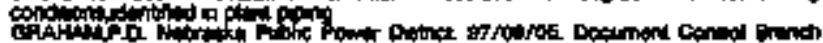

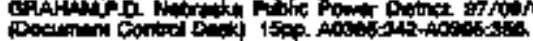

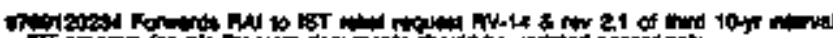

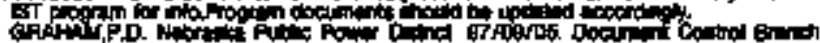

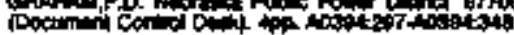

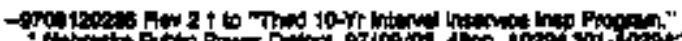

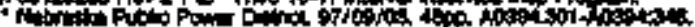

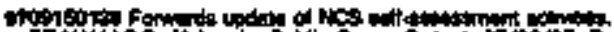

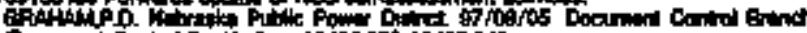

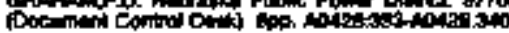

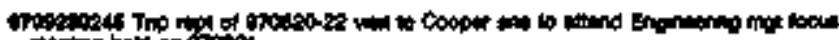

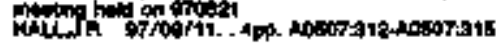

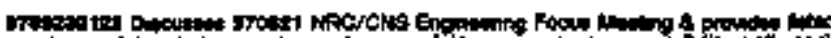

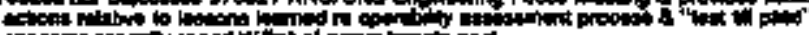

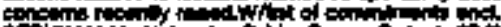

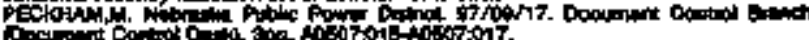

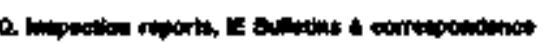

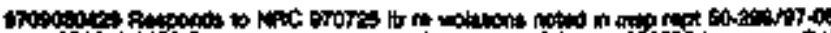

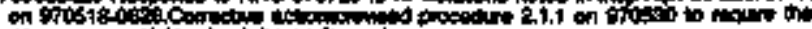

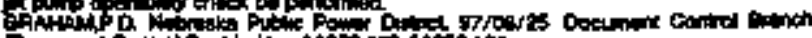

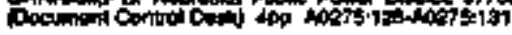




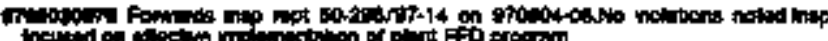

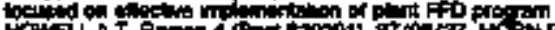

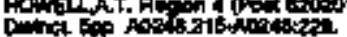

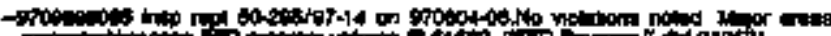

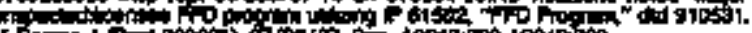

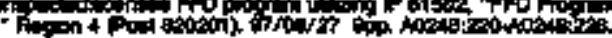

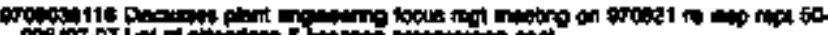

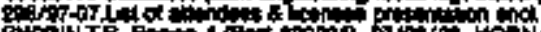

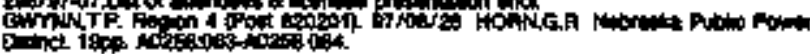

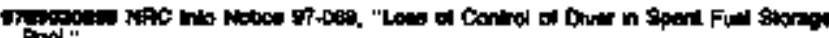

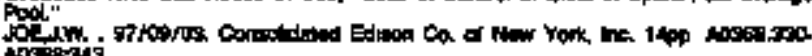
asiosis.

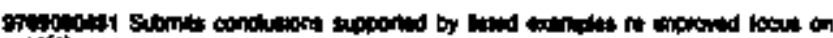

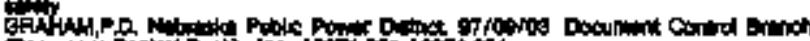

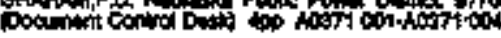

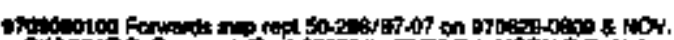

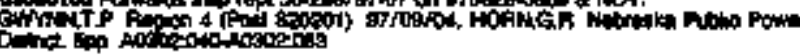

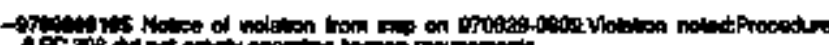

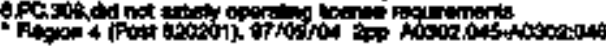

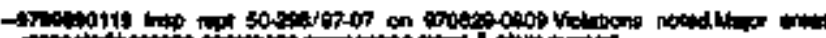

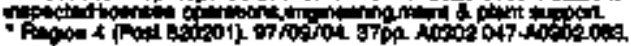

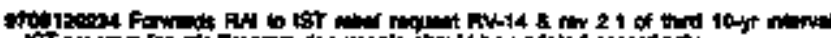

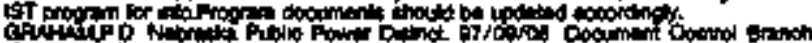

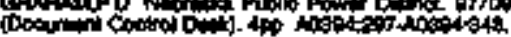

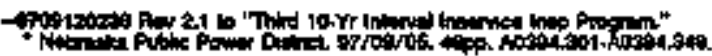

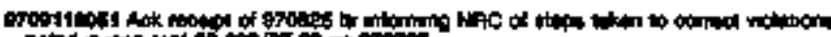

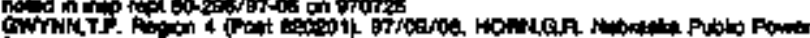

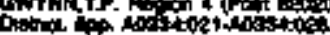

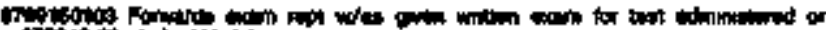

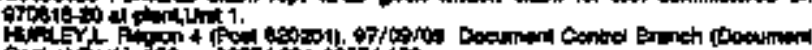

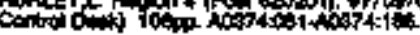

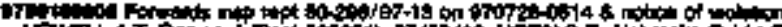

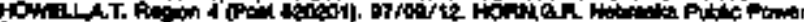

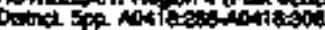

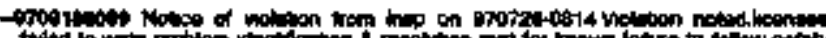

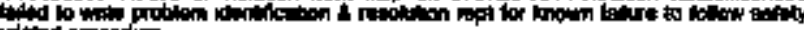
rintind proceder

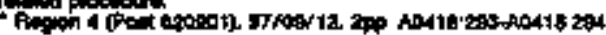

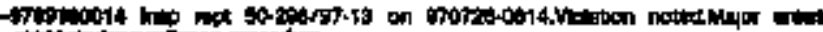

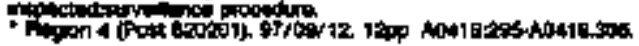

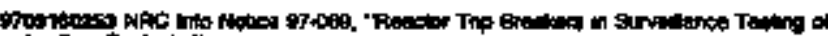
Pot

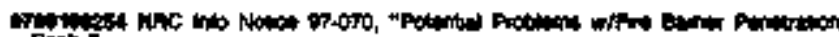

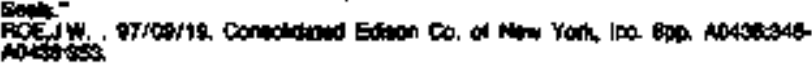

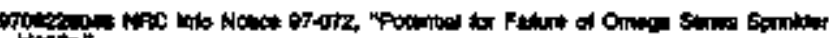

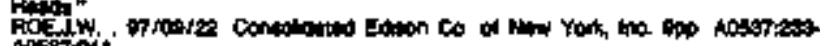
Aosstor.

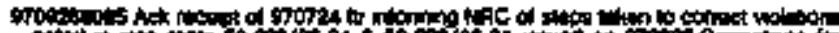

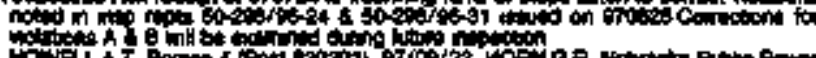

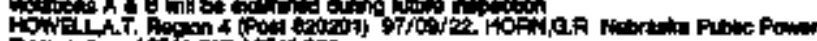

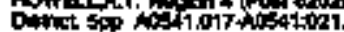

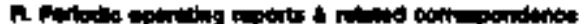

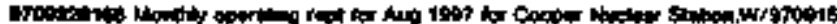

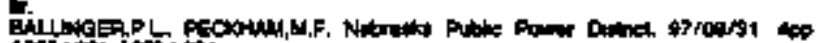

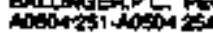

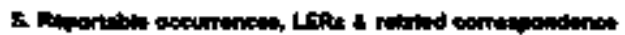

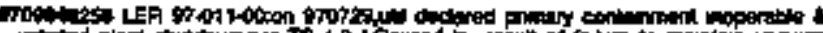

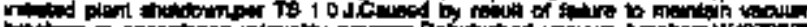

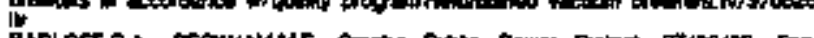

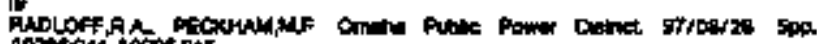

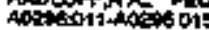

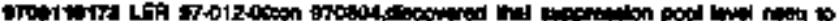

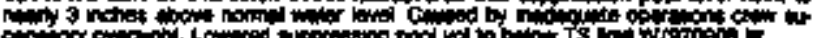

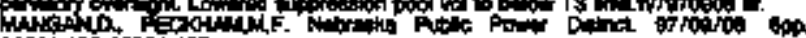
Aosti:102-1006t:107.

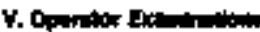

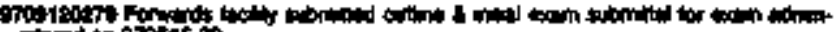

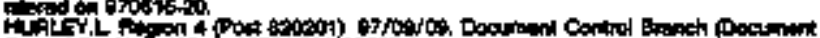

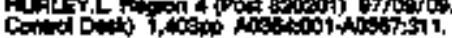

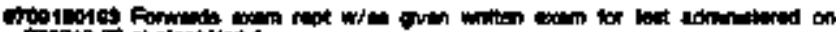

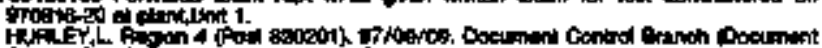

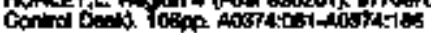

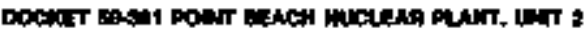

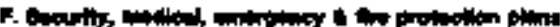

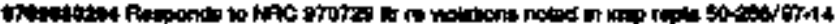
a so-

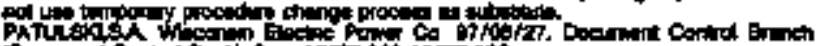

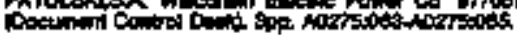

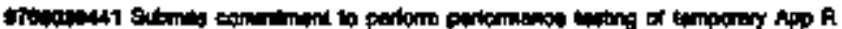

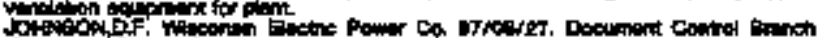

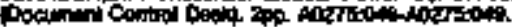

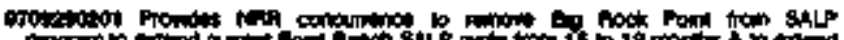

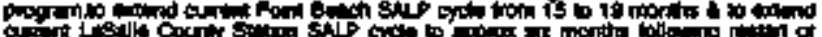

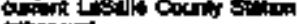
int und.

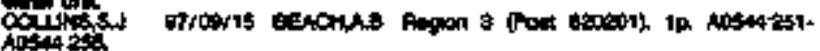

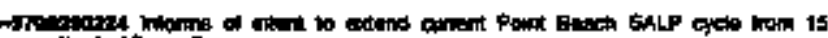

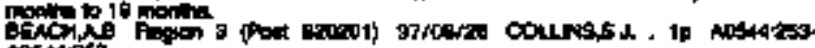
intersi

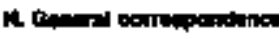

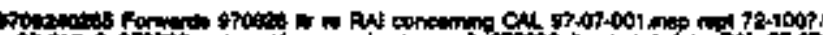

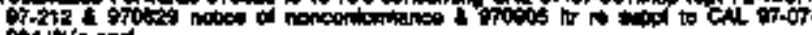
onditrond

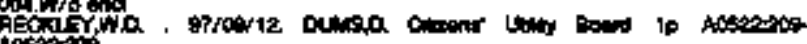
intosis.

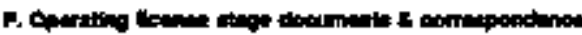

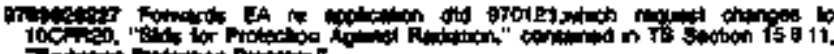

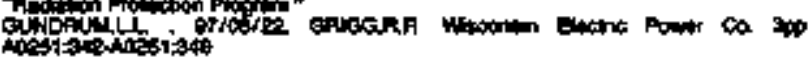

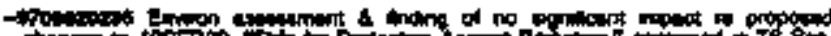

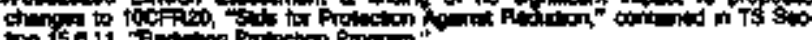

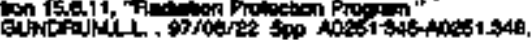

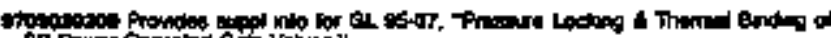

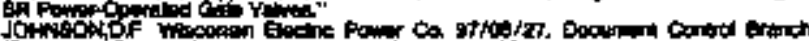

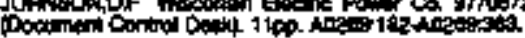

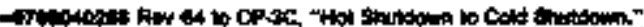

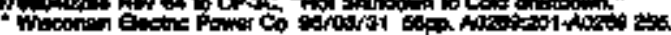

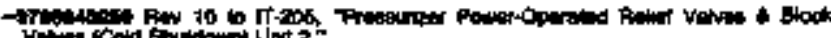

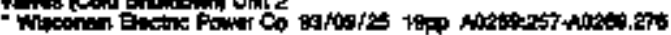

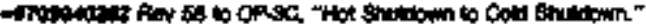

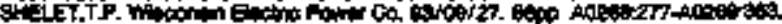

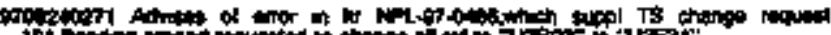

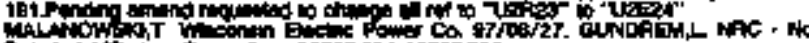

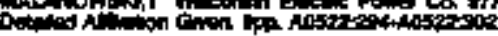

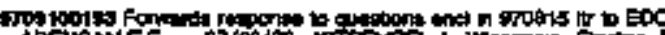

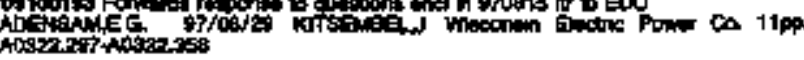

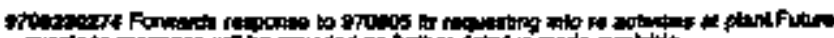

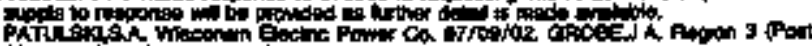

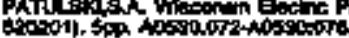

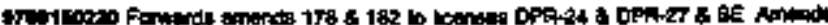

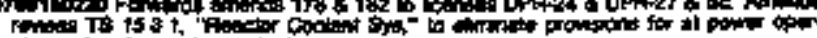

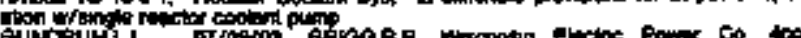

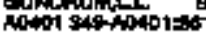




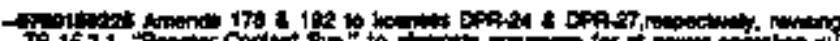

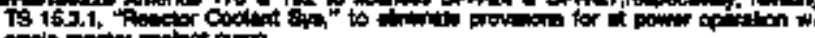

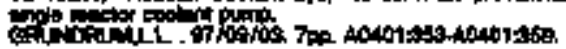

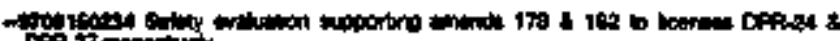

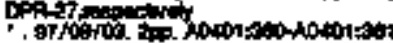

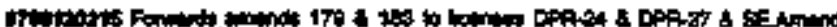

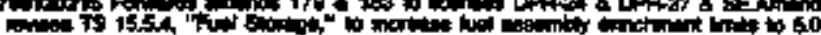

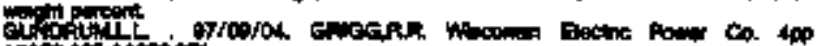

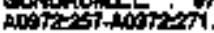

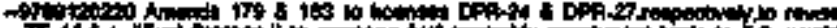

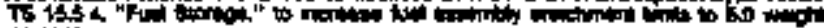

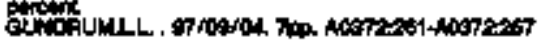

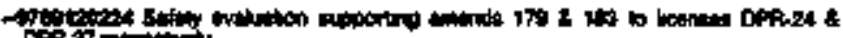

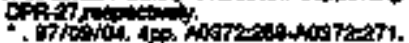

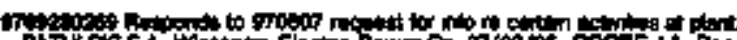

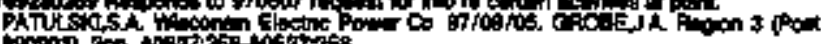

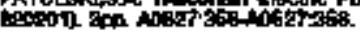

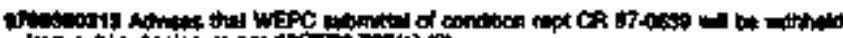
tomptim

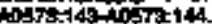

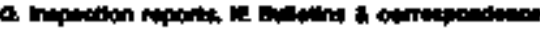

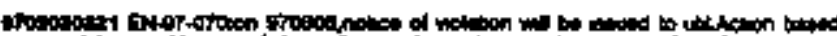

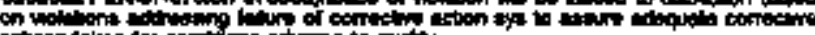

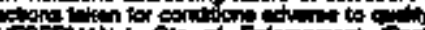

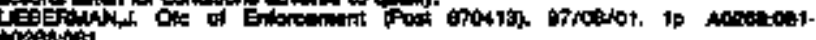
natest.

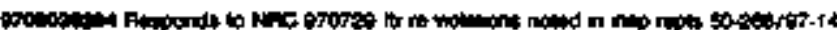

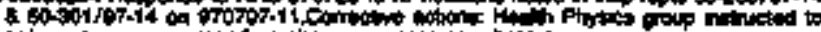

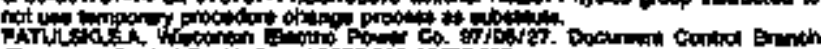

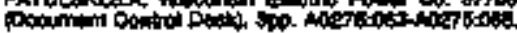

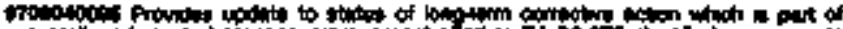

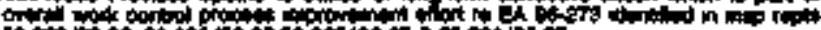

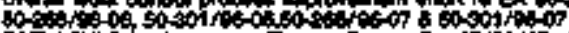

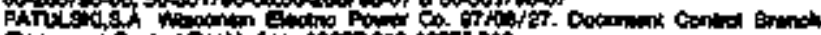

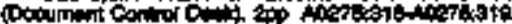

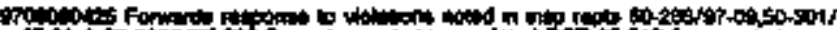

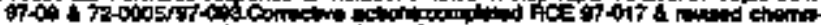

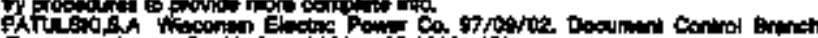

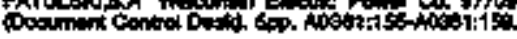

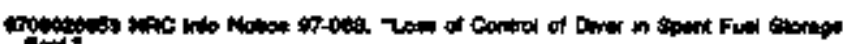

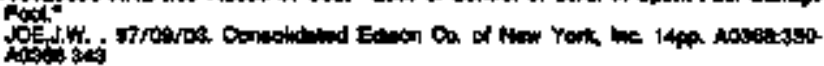

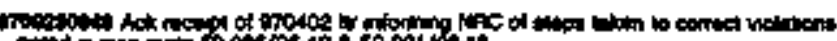

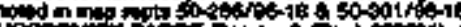

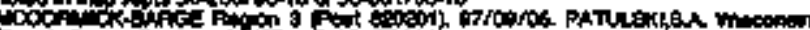

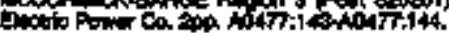

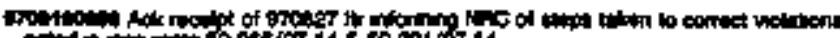

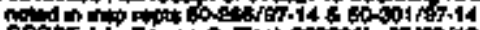

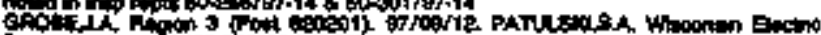

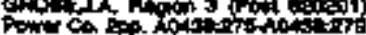

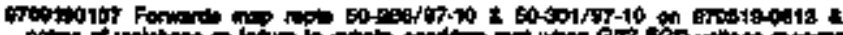

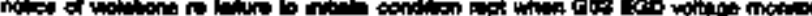

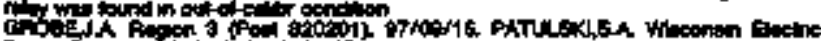

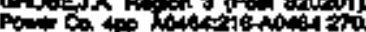

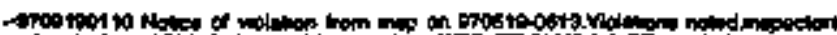

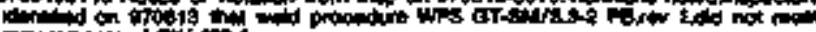

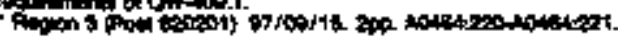

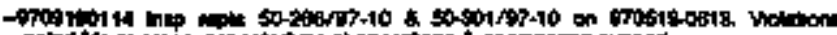

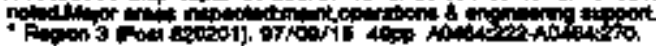

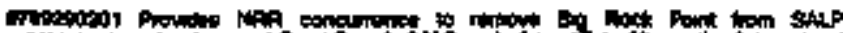

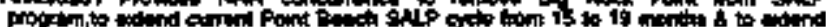

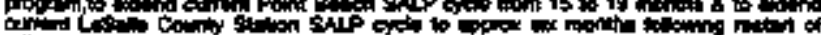

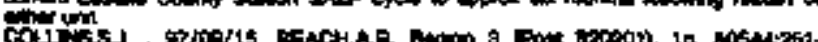
costitis

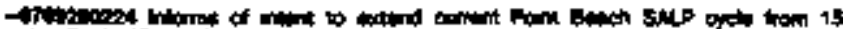

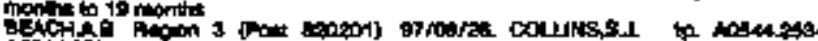
rositas

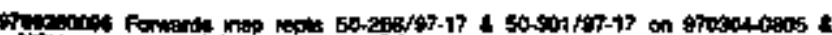

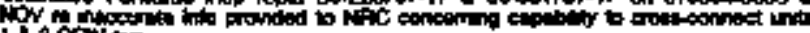
1820 ow the

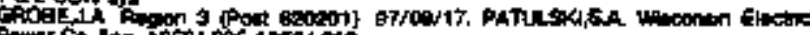

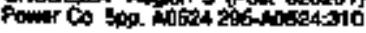

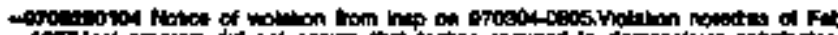
10\%

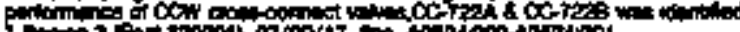

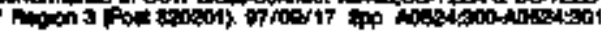

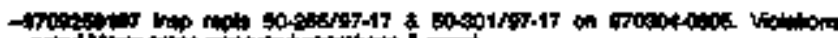

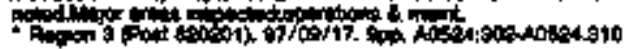

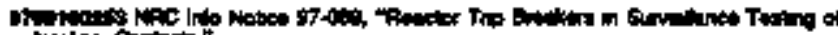

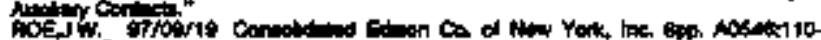
inomit.

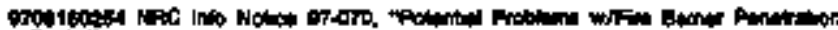

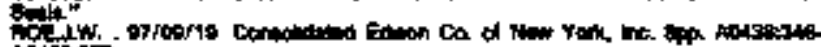
inting

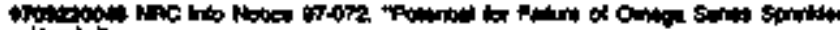

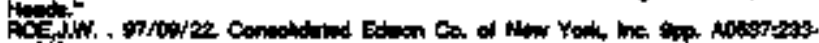
iximiti.

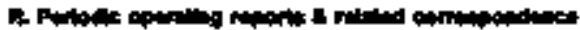

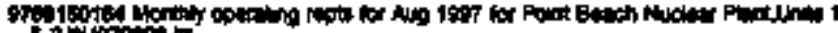

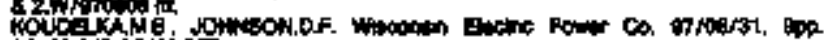

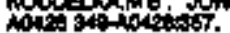

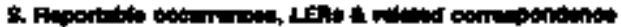

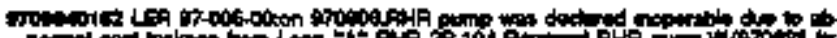

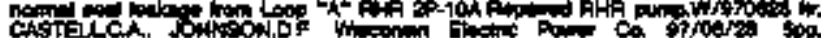

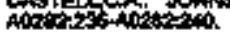

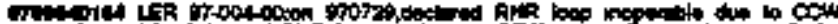

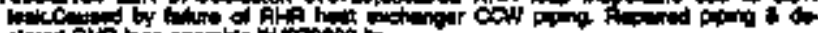

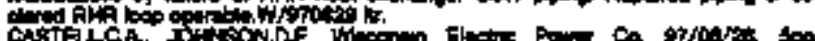
CAste LCA

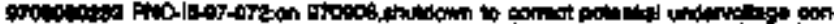

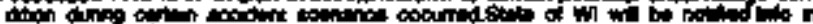

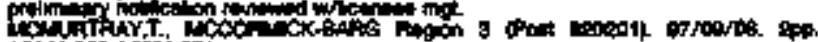

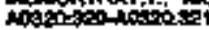

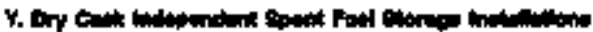

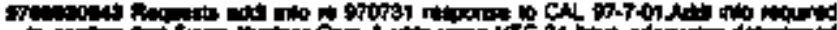

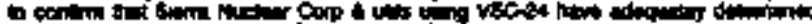

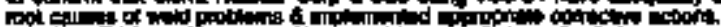

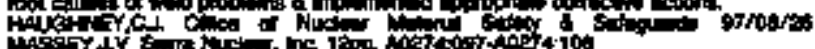

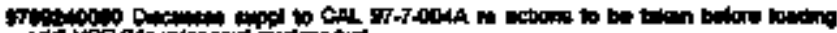

Kake

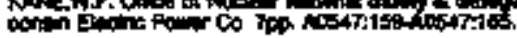

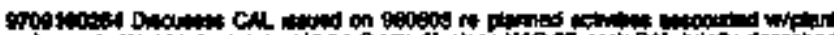

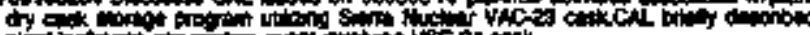

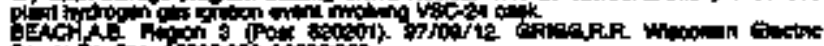

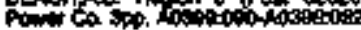

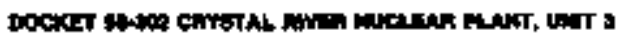

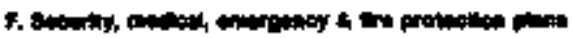

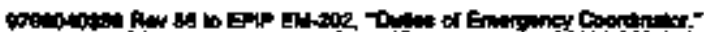

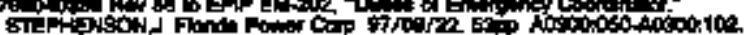

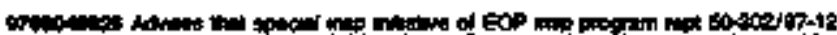

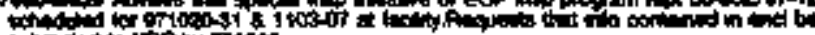

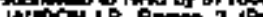

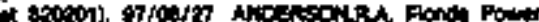
whengos

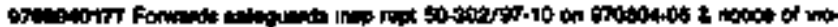

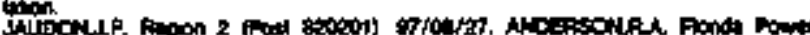

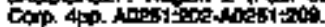

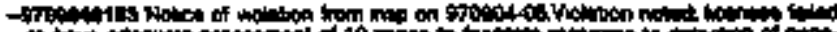

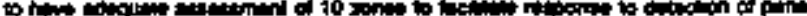

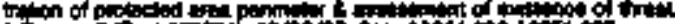

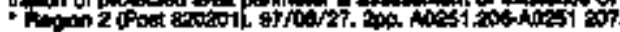

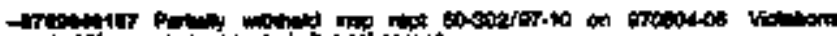

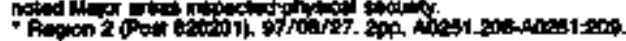

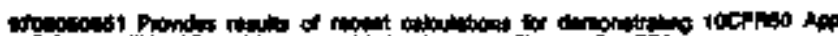
A

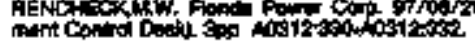




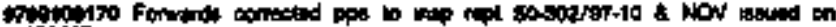

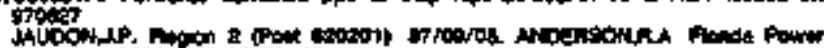

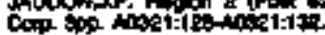

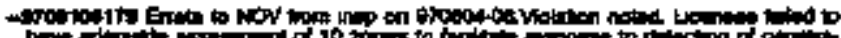

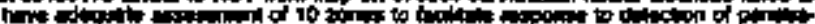

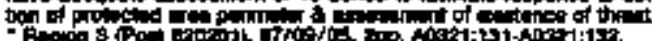

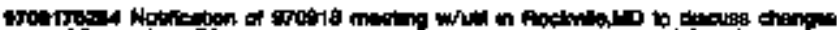

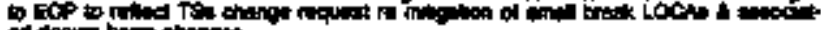

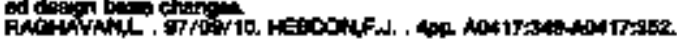

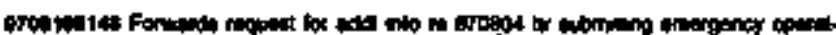

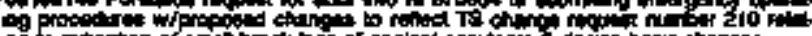

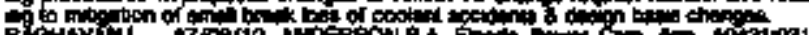

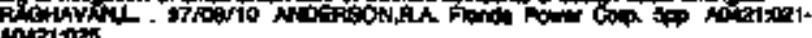
A0tist:tet.

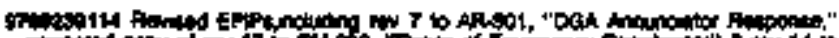

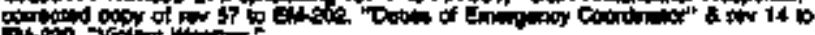

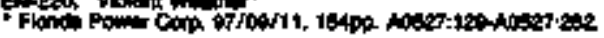

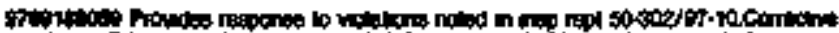

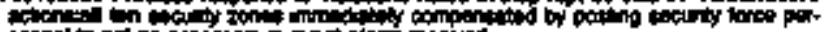

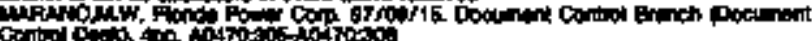

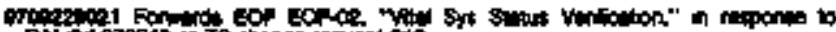

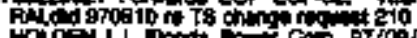

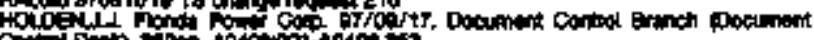

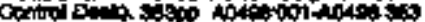

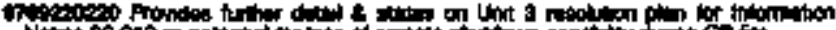

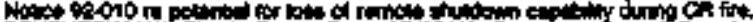

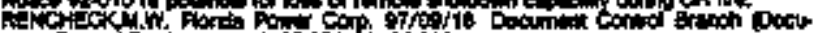

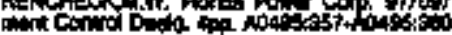

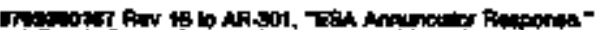

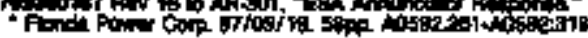

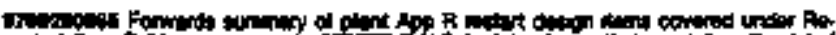

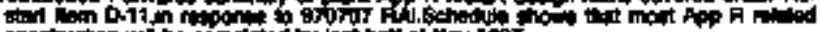
corn:

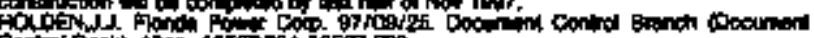

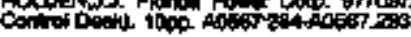

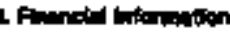

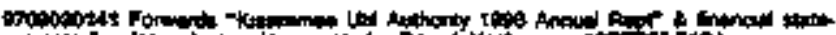

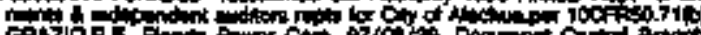

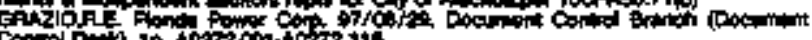

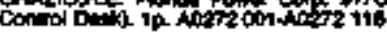

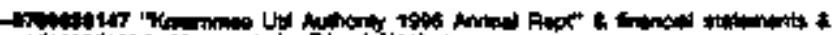

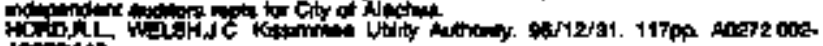
ingeith

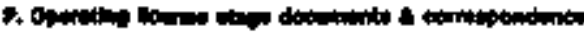

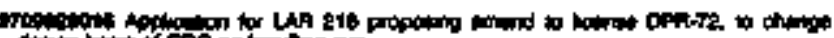
An

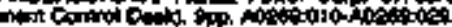

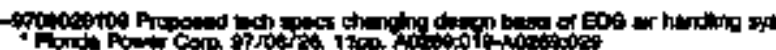

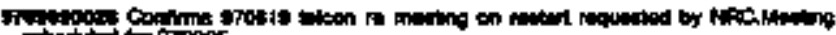

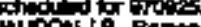

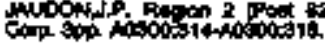

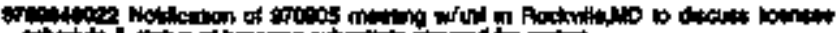

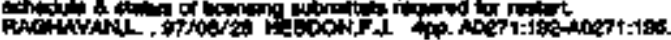

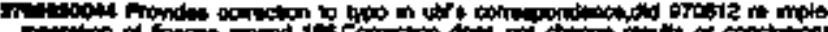

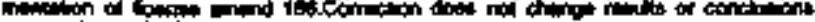

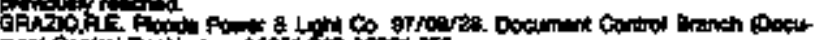

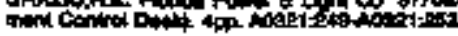

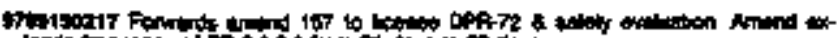

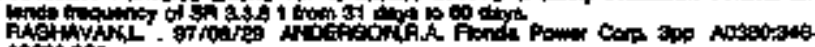

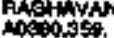

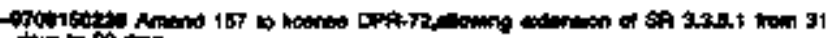

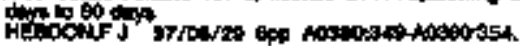

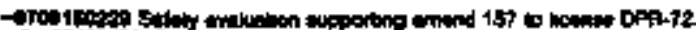

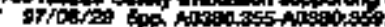

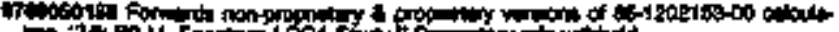

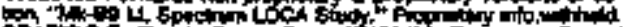

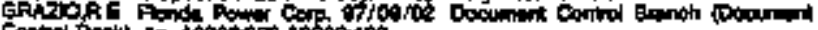

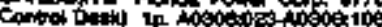

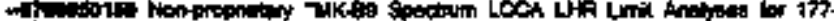

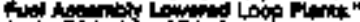

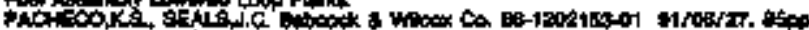

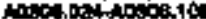

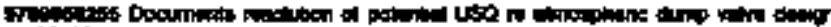

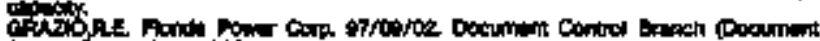

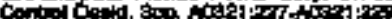

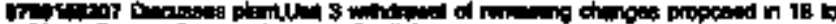

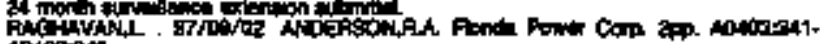
40iocise

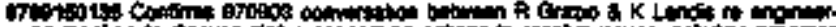

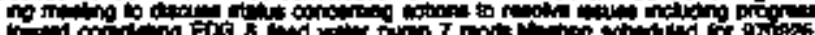

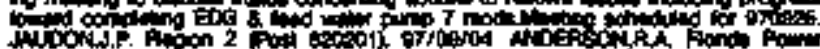

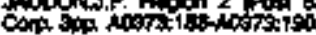

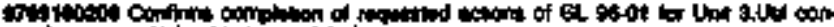

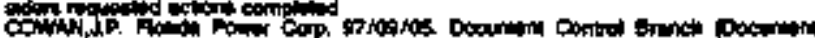

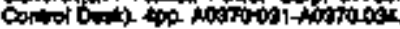

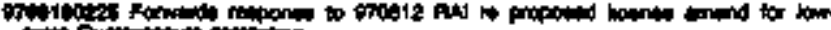

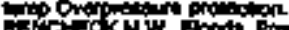

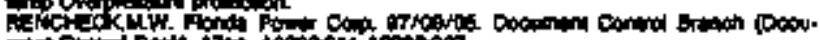

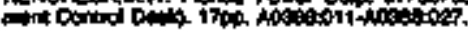

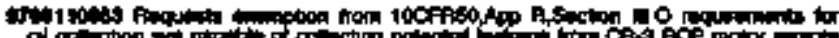

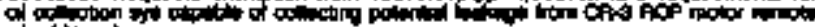

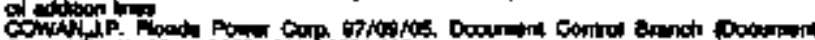

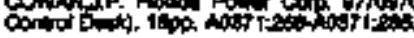

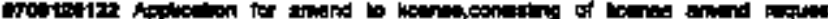

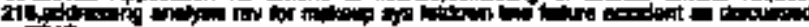

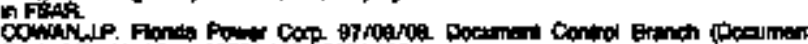

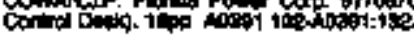

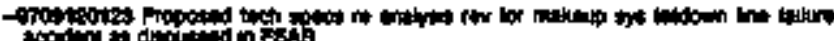

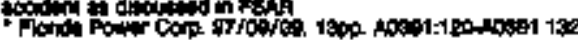

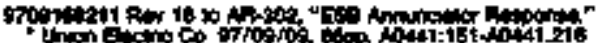

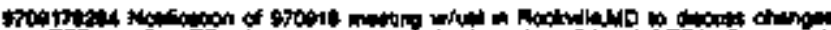

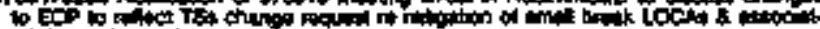

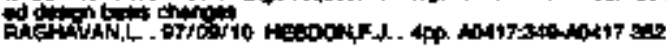

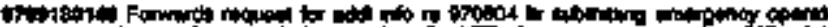

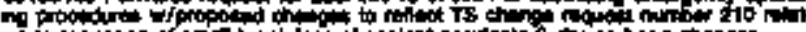

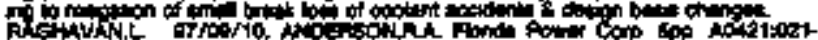
Antioge

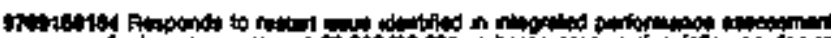

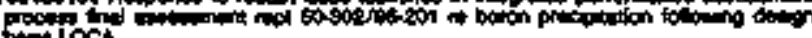
totitioc

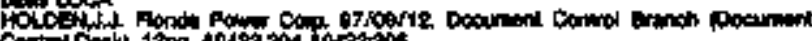

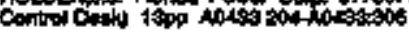

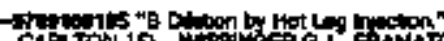

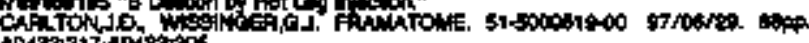
AD433217404030

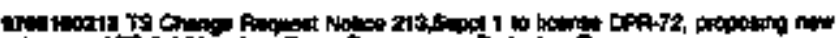

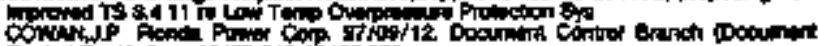

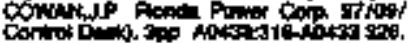

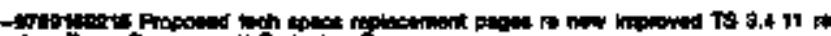

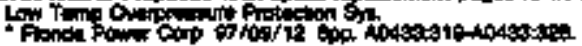

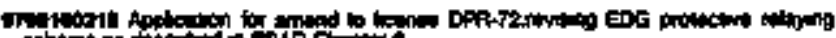

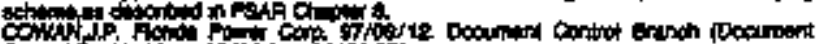

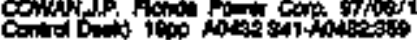

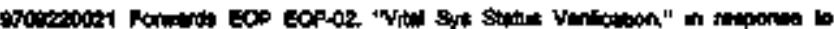

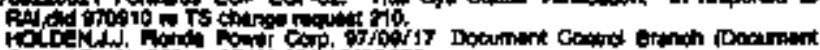

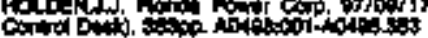

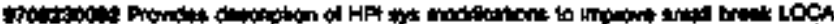

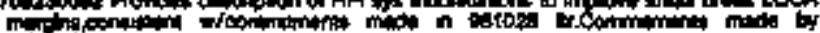

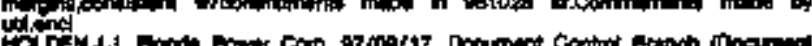

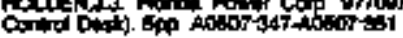

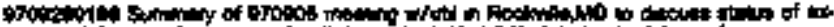

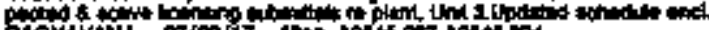

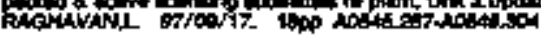

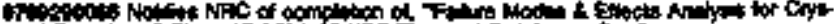

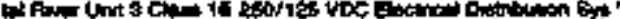

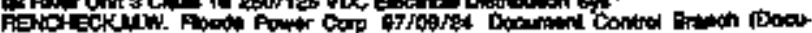

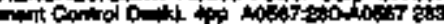




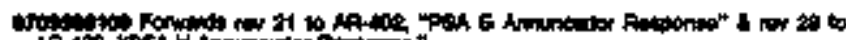
A A

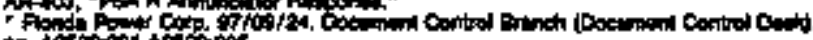

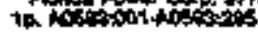

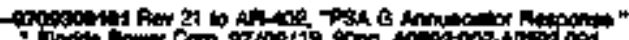

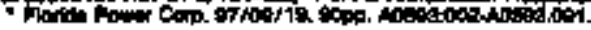

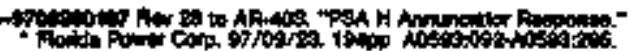

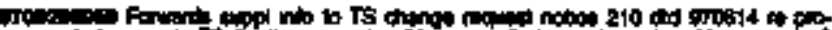

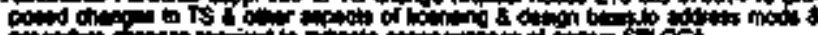

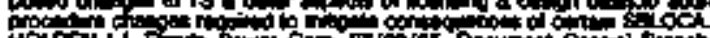

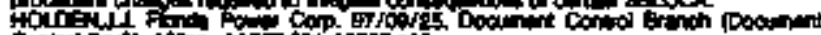

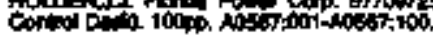

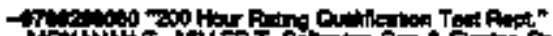

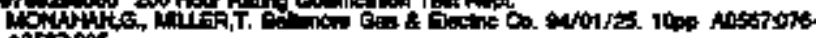
atorios

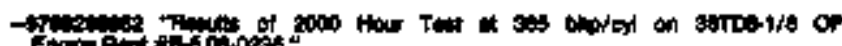
Co atosita.

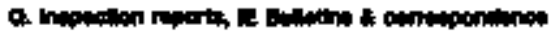

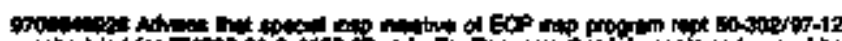

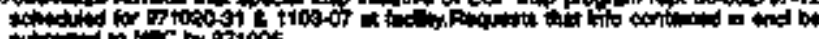

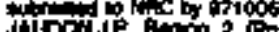

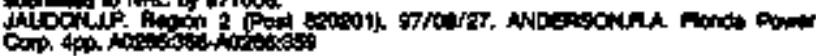

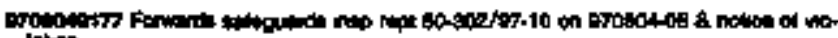

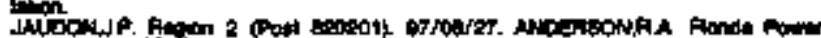

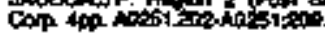

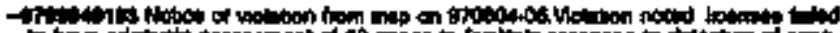

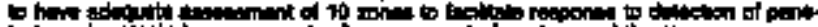

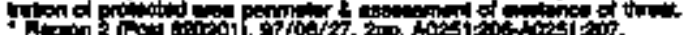

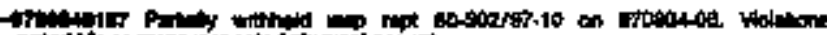

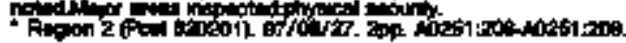

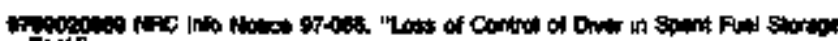

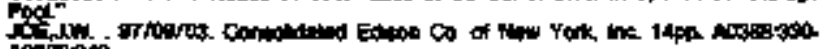

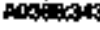

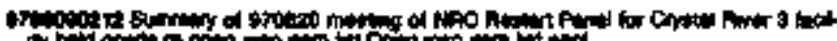

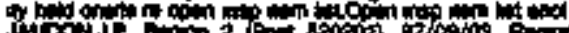

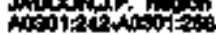

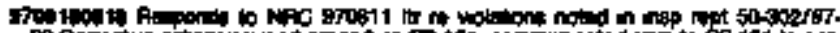

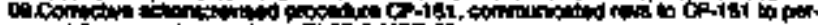

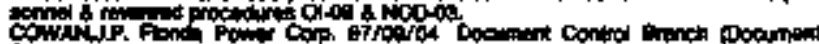

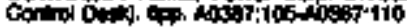

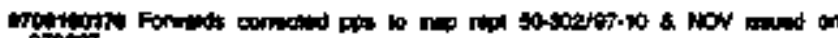
orot?

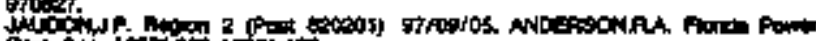

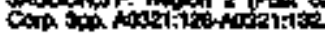

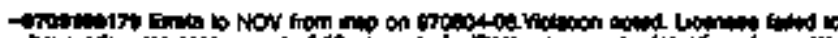
hat of

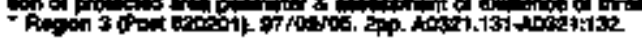

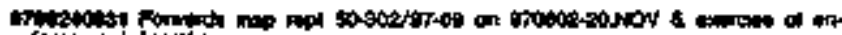

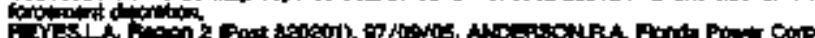

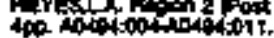

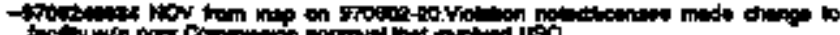

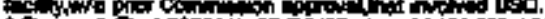

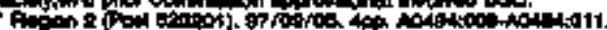

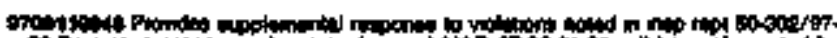

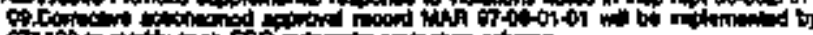

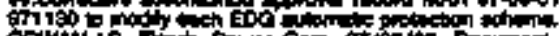

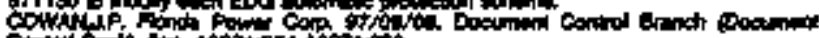

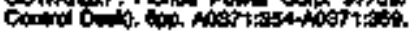

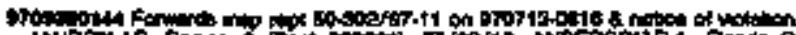

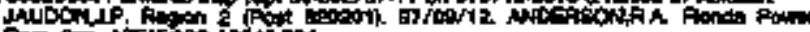
andition

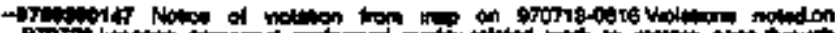

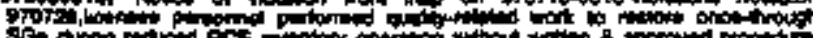

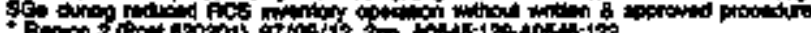

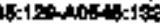

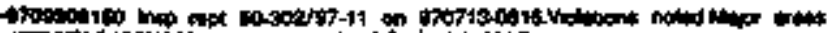

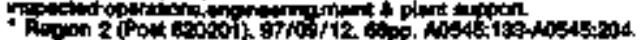

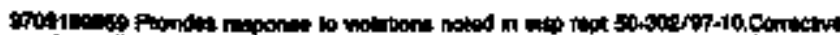

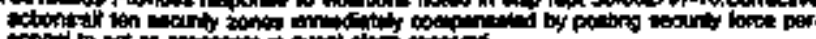

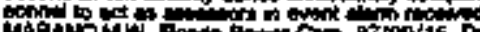

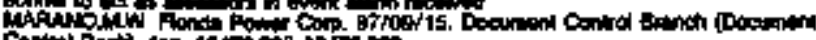

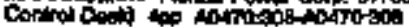

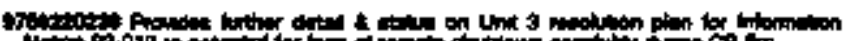

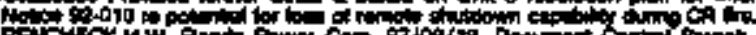

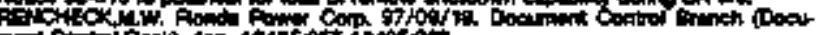

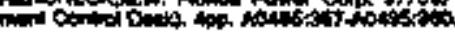

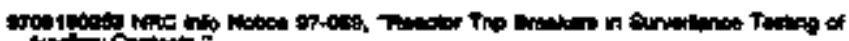
AOChy notistit?

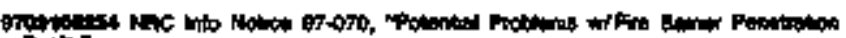

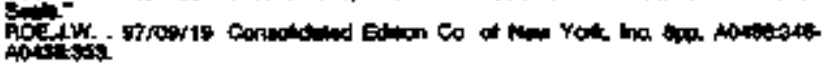

the

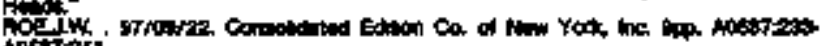
Aotizis.

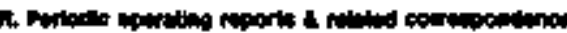

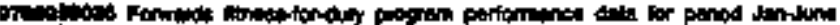

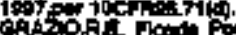

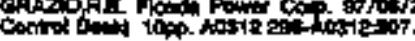

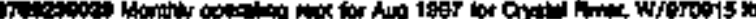

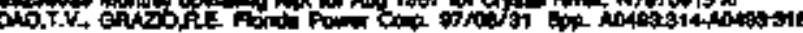

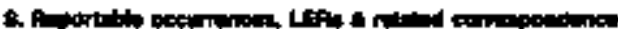

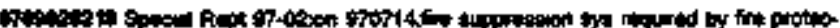

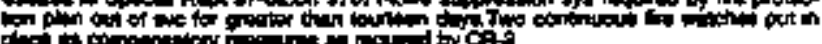

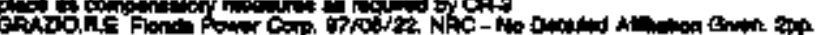

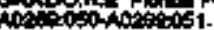

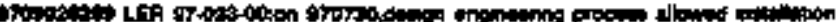

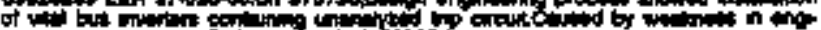

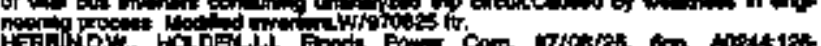
toostat.

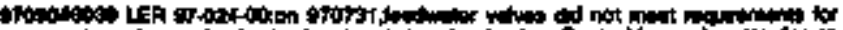

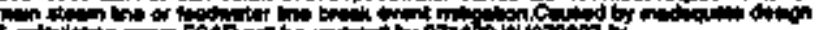
6.

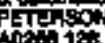

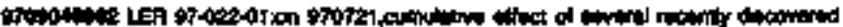

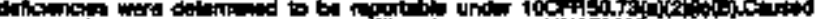

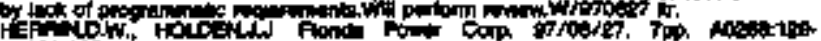
ADStis

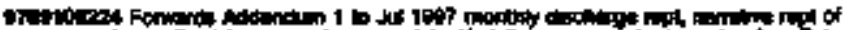

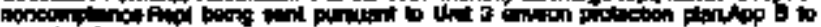

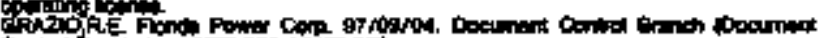

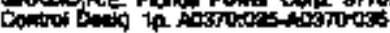

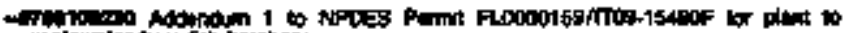

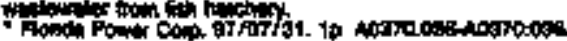

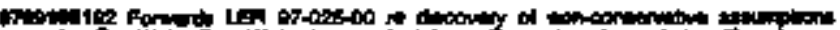
A7 in

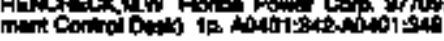

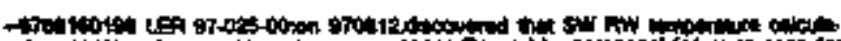

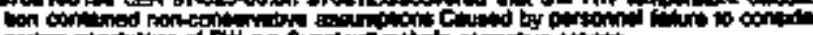

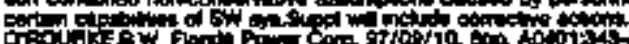

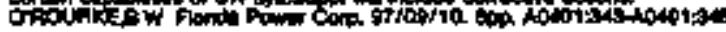

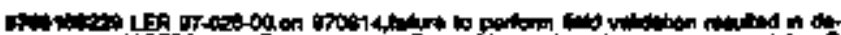

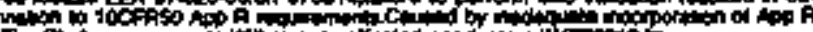

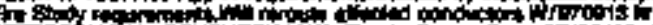

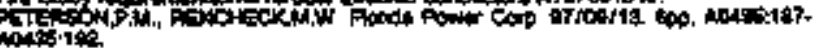

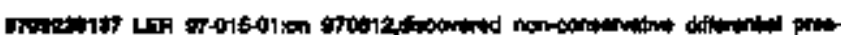

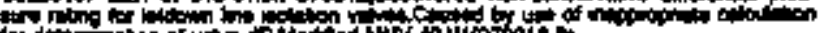

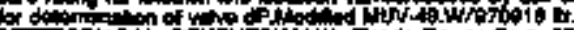

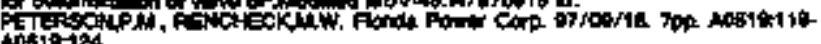

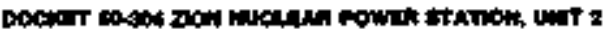

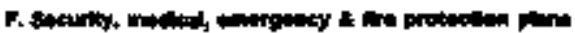

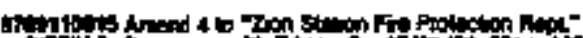

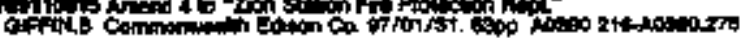

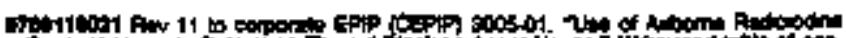

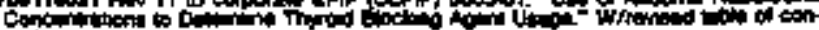

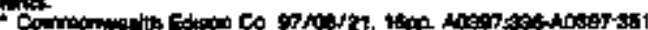




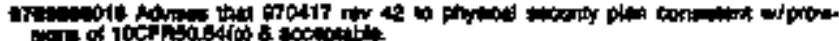
ic

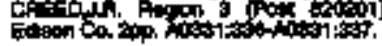

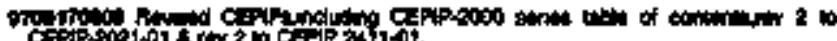

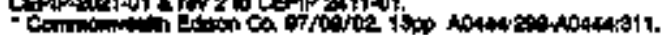

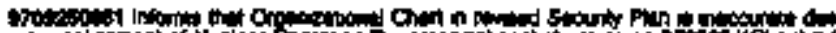

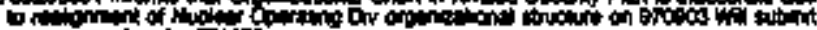

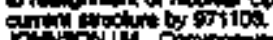

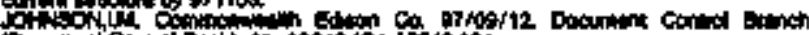

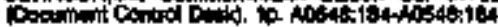

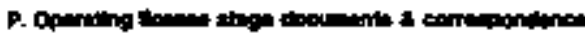

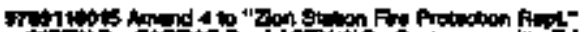

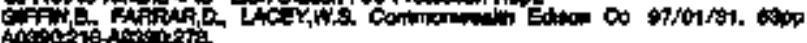

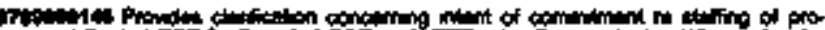

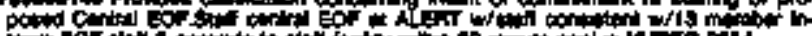
pon

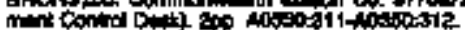

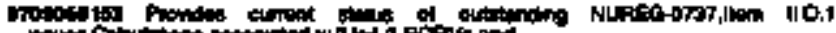

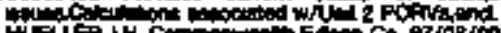
Inent Don

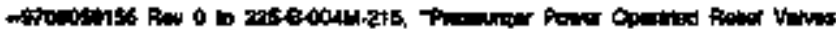
P-T. A

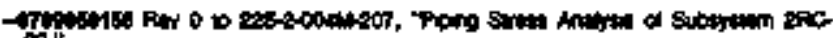

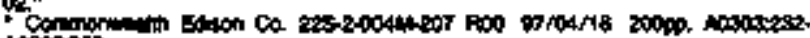
ustosito

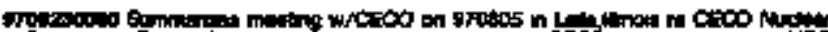

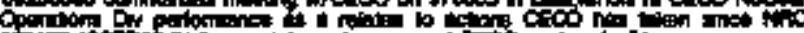
orter 10CF

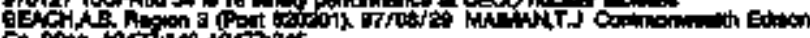
Cotation

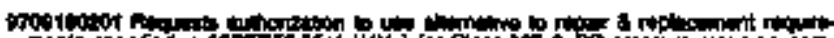

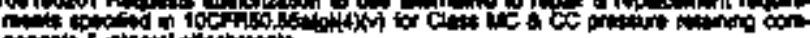

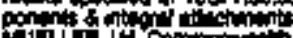

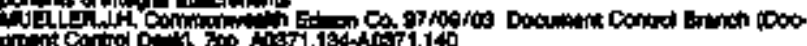

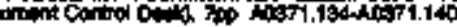

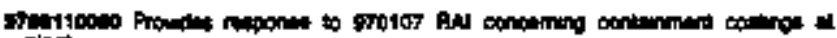
Din

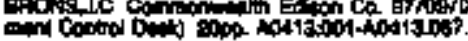

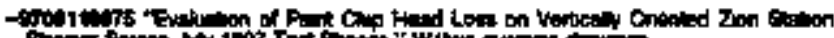

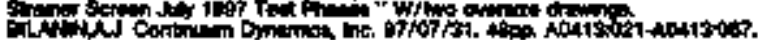

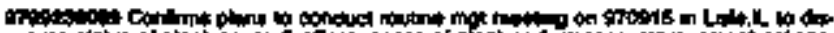

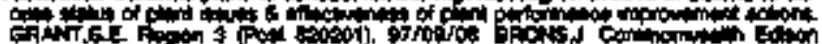

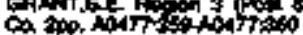

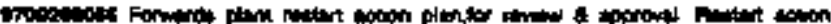

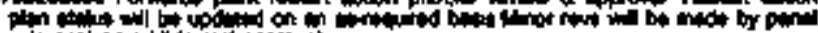
Wild

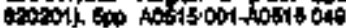

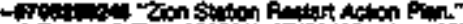

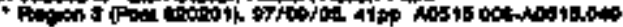

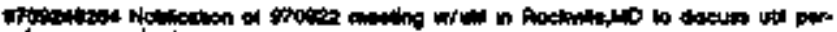

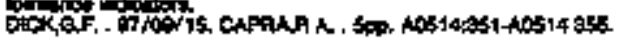

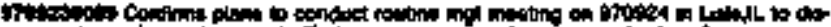

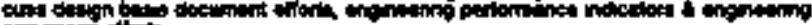

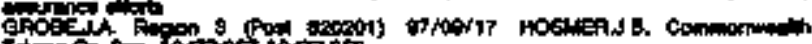

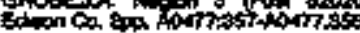

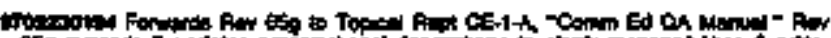

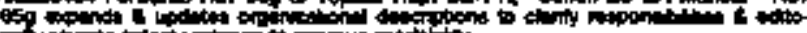

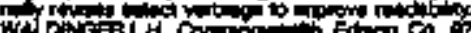

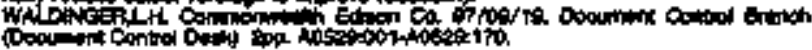

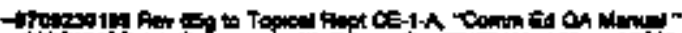

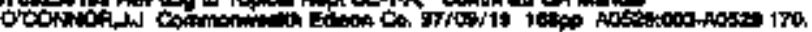

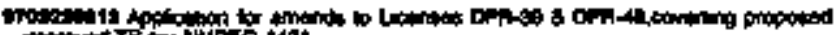

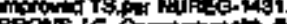

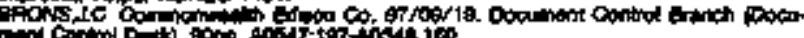

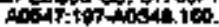

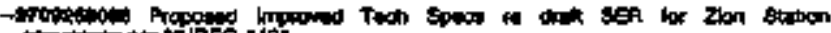

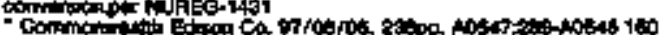

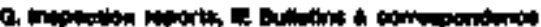

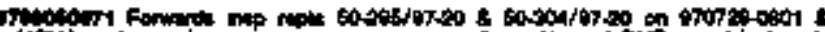

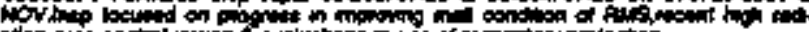

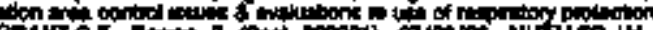

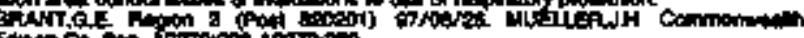

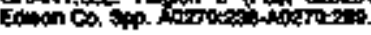

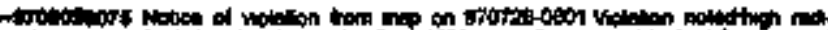

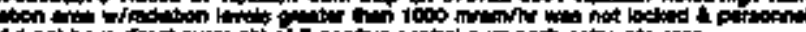

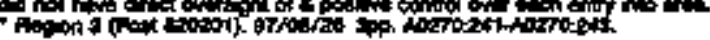

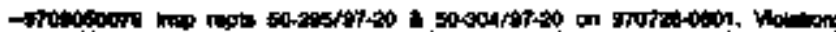

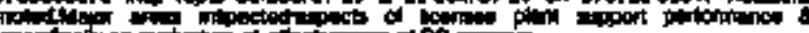

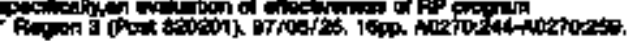

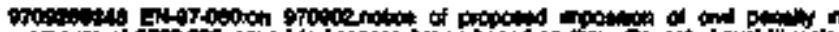

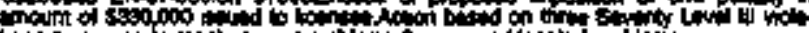

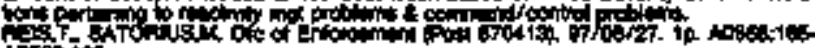

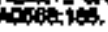

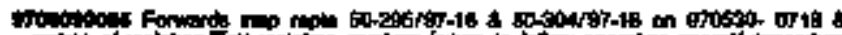

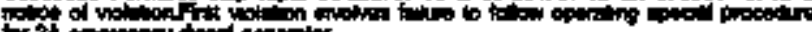

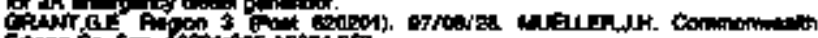

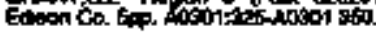

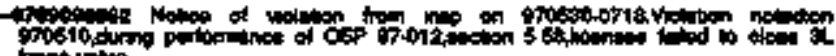

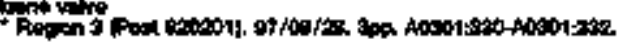

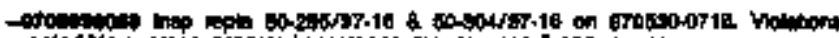

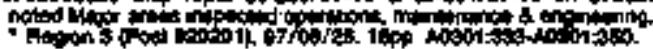

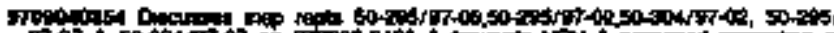

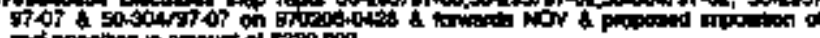

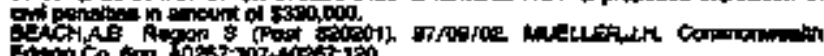

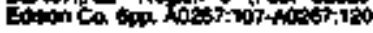

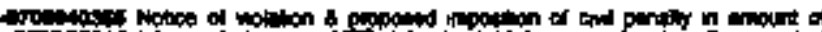

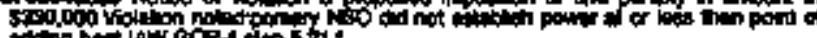

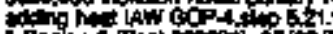

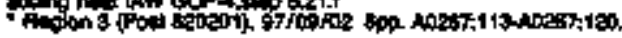

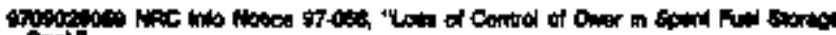

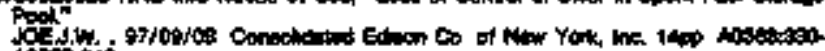

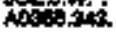

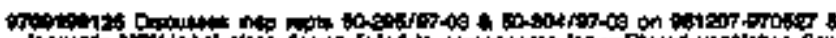

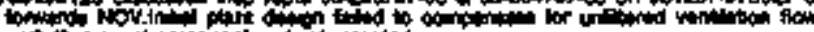

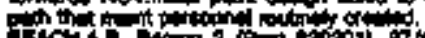

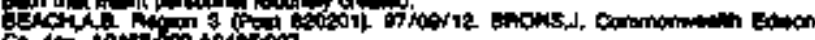

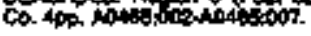

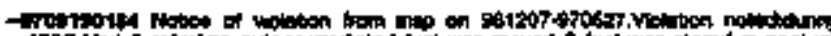

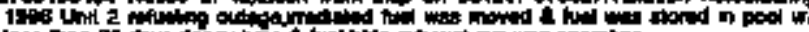

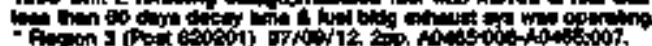

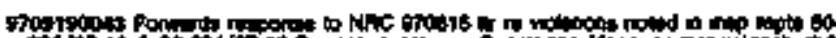

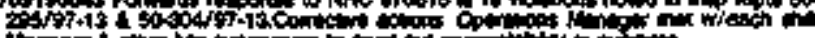

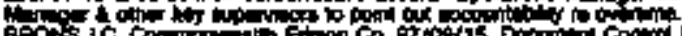

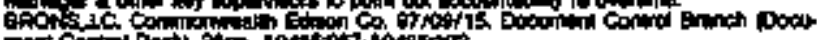

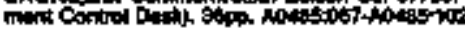

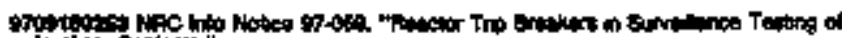

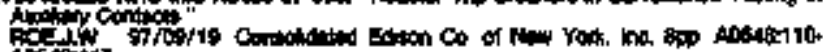
19.

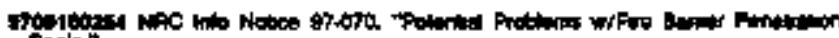

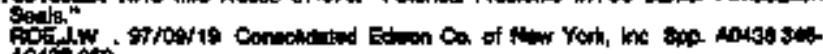
1040\%:

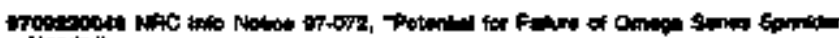

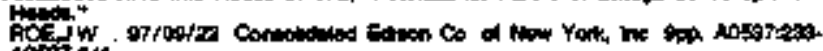
Actis 249 .

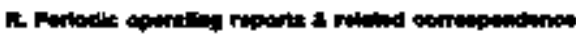

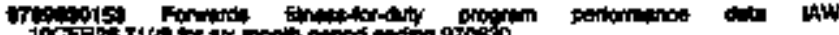

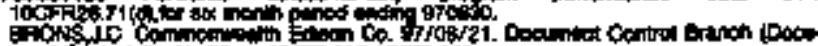

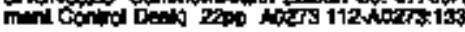

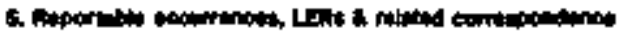

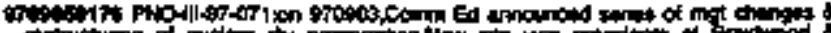

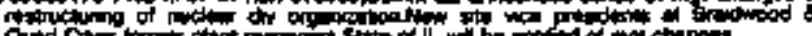

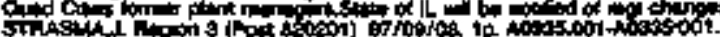




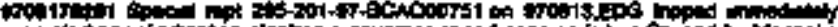

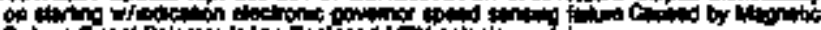

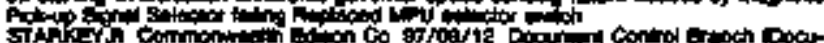

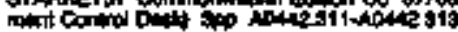

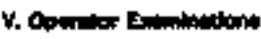

In

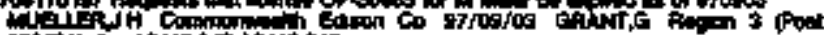

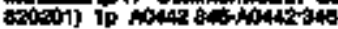

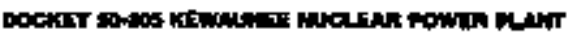

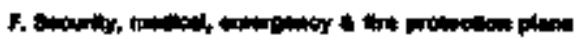

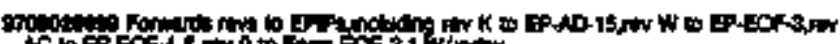
Ac loc

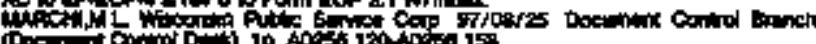

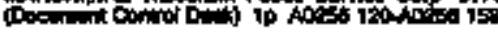

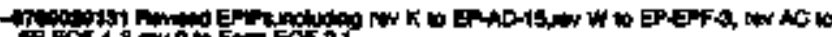

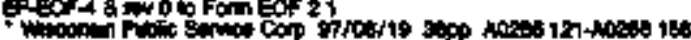

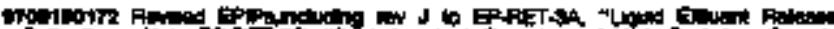

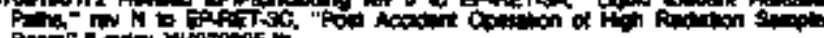

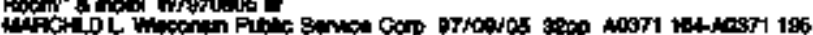

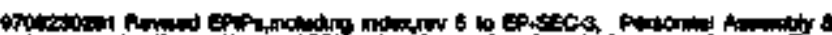
foum

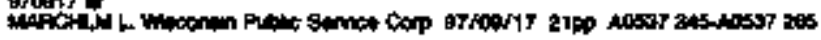

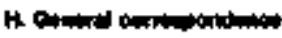

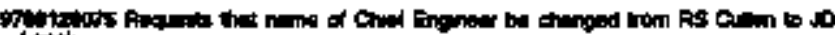
(1)

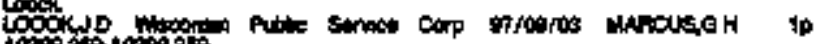

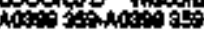

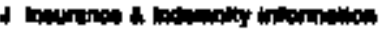

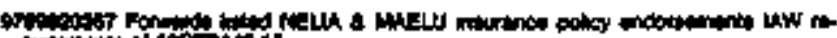

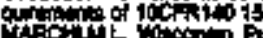

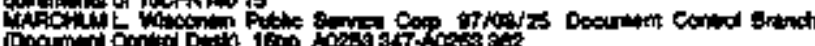

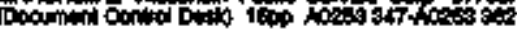

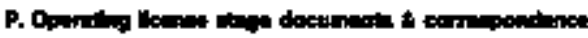

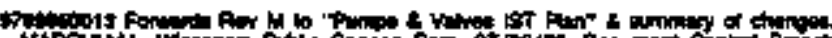

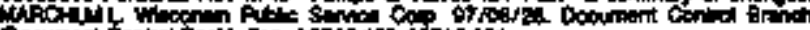

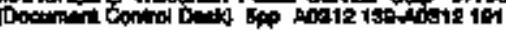

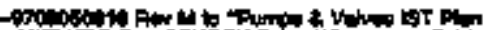

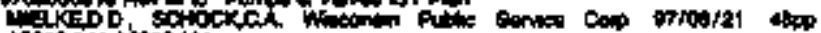

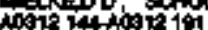

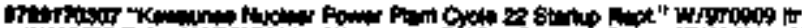

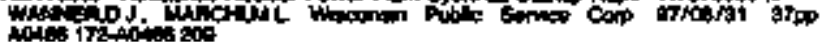

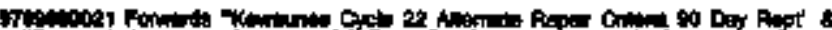

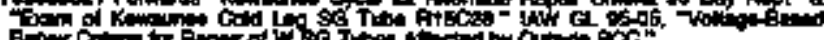
Fric

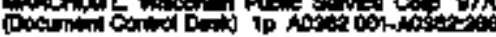

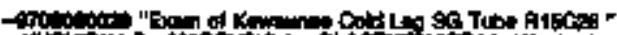

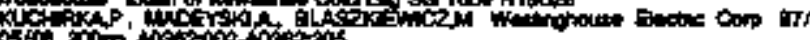

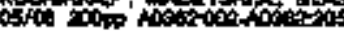

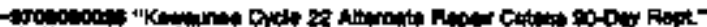

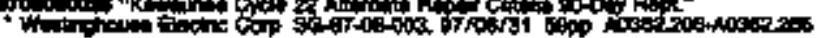

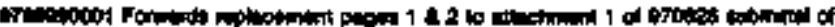
niv nof ist Pin

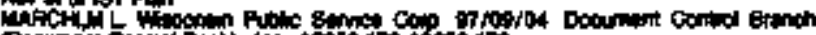

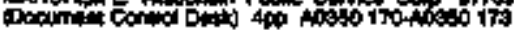

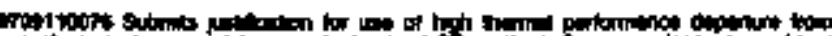

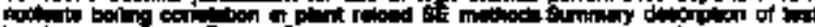
Which

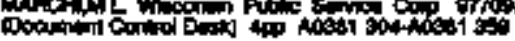

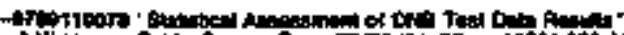

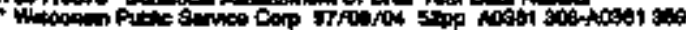

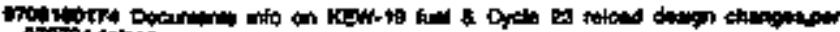

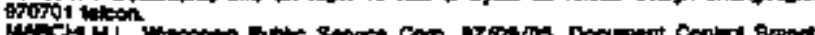

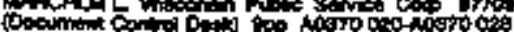

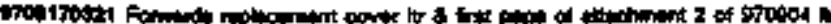

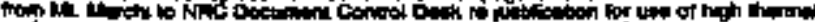

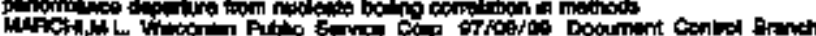

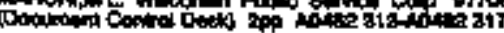

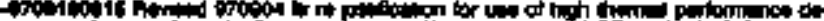

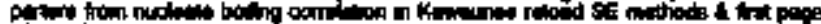

OA

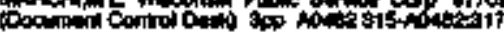

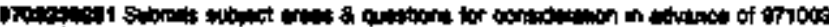

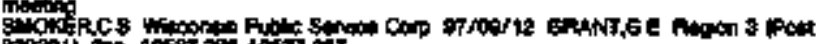

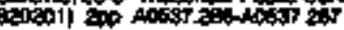

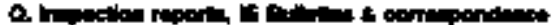

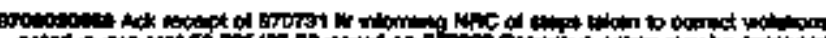

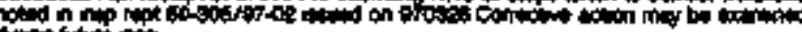
cint for to

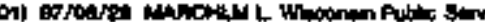

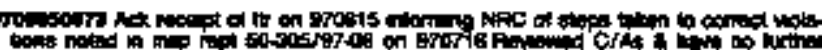

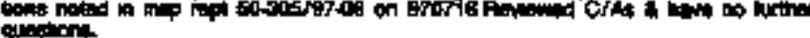

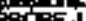

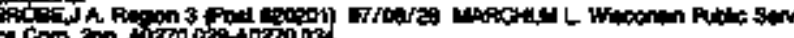

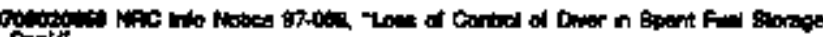

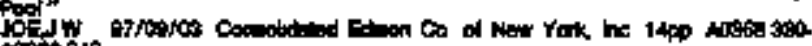

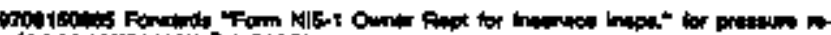

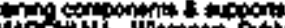

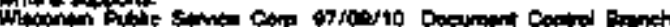

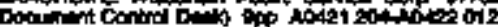

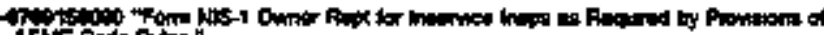

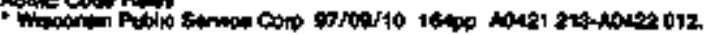

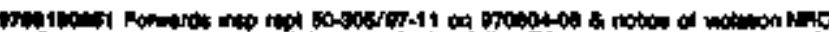

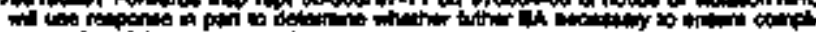

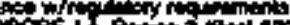

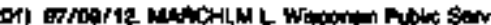

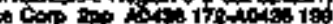

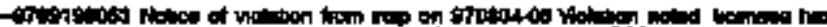

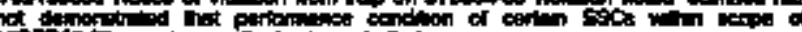

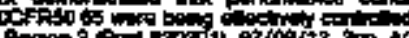

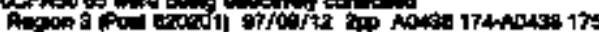

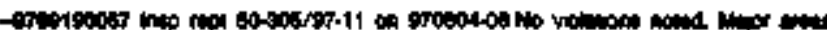

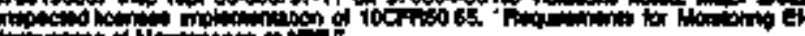

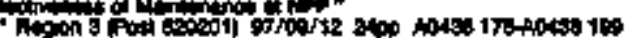

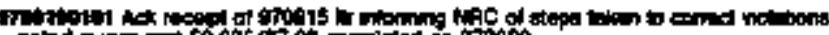

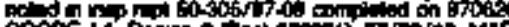

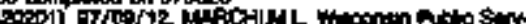

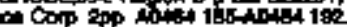

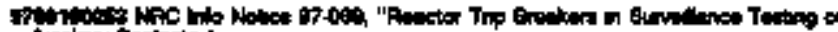

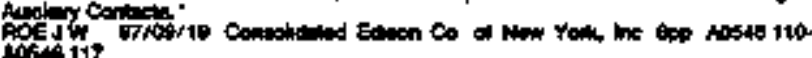

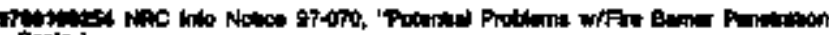

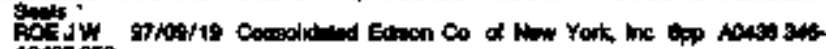
1043:

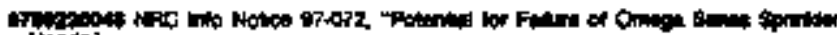

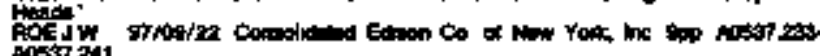

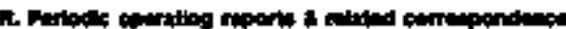

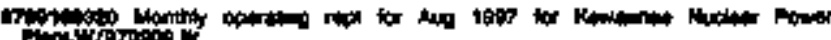

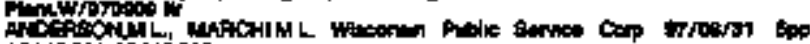

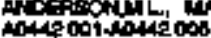

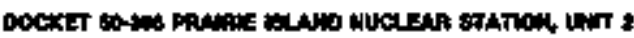

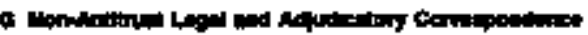

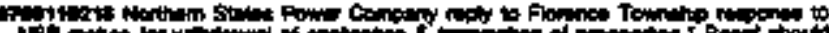

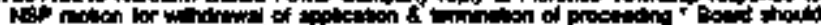

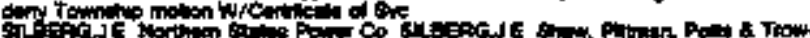

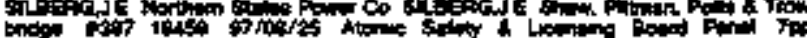

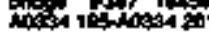

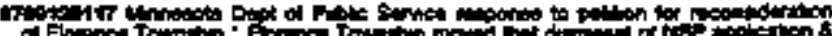

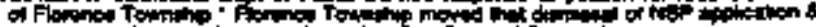

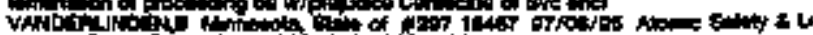

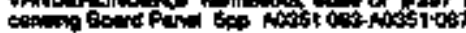




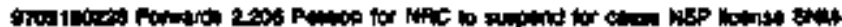

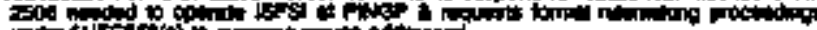

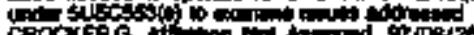

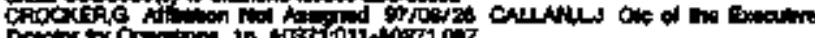
$11+40 \% 1007$

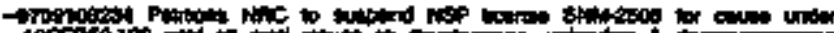

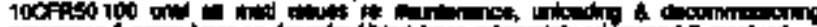

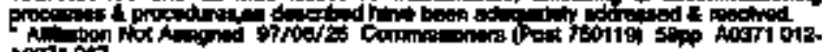
nosh 100 \%

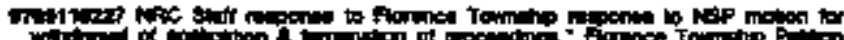

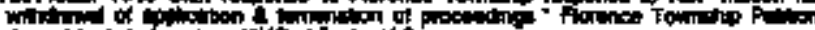

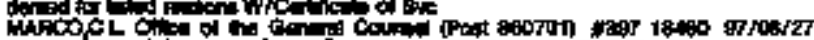

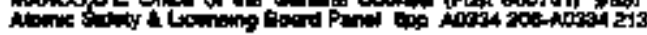

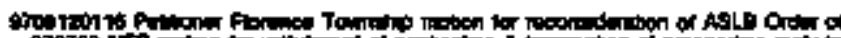

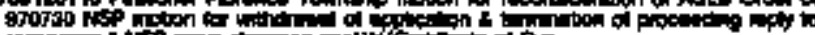

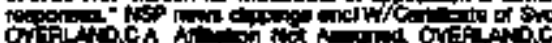

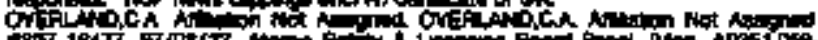

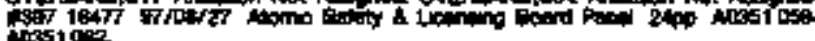

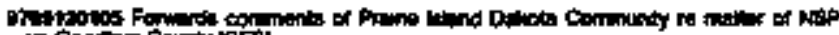

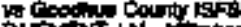

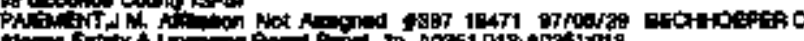

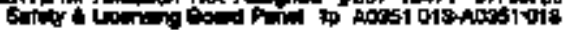

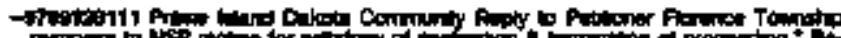

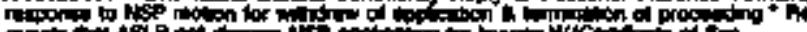

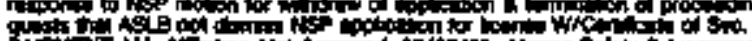

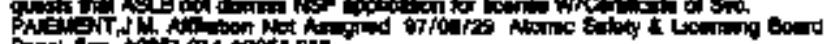

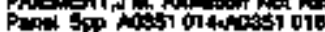

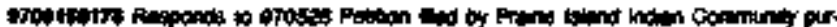

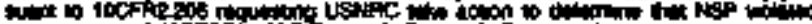

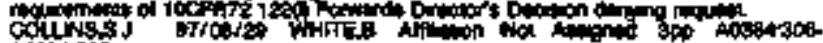
1000

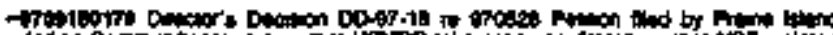

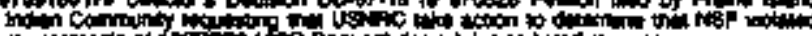

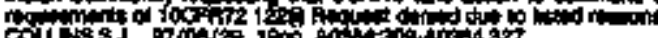

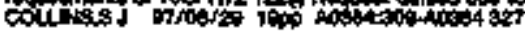

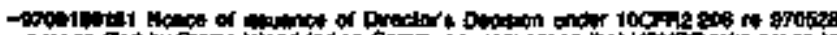

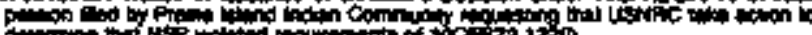
corit in

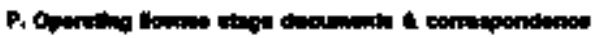

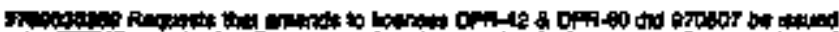

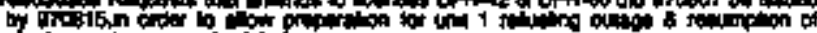

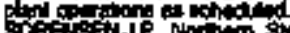

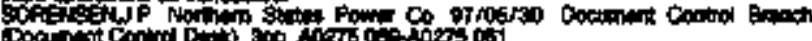

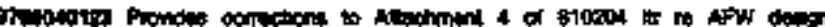

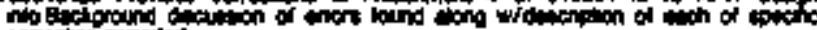

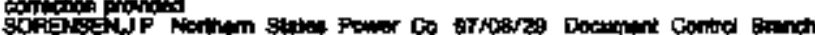

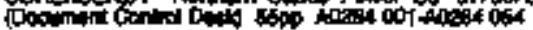

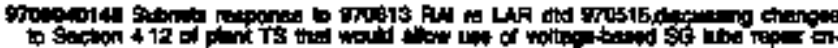

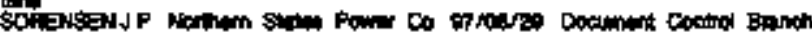

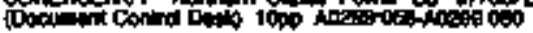

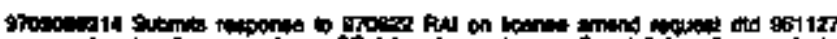

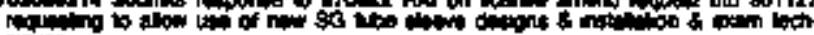

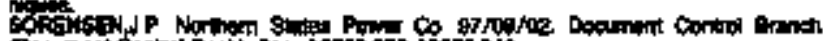

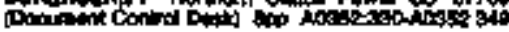

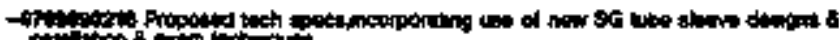

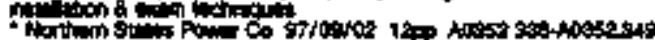

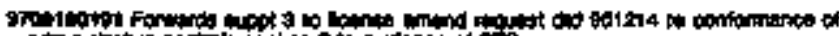

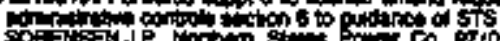

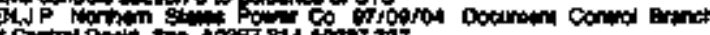

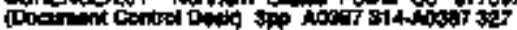

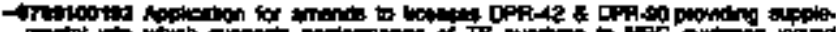

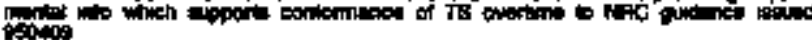

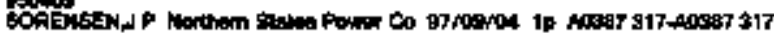

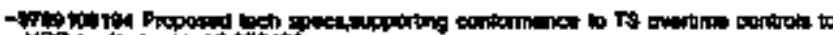

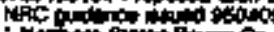

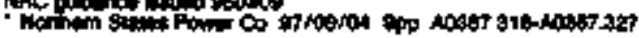

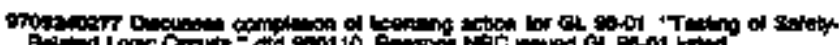

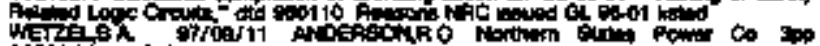

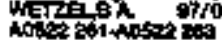

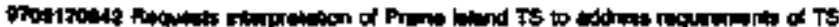

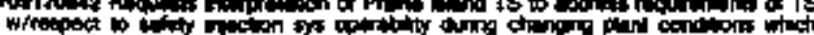
EOH

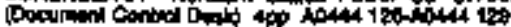

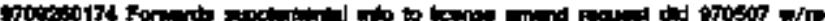

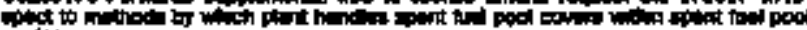

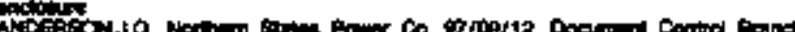

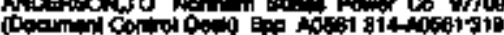

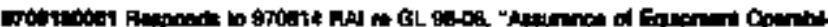

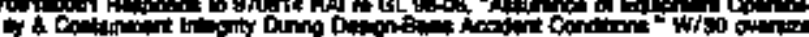

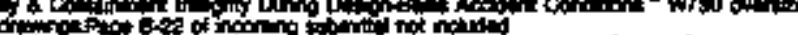

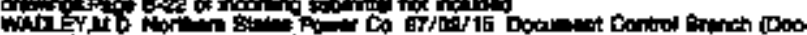

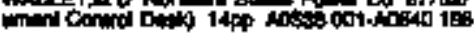

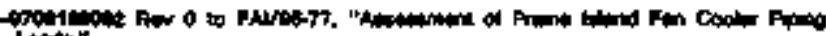

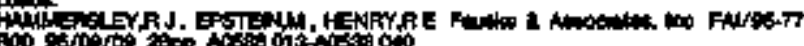

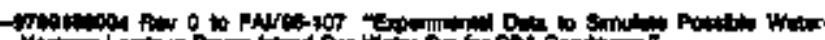

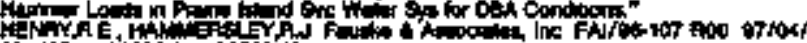
20 40.

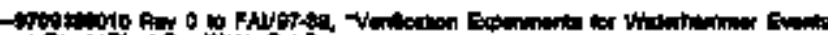
Fing

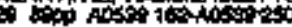

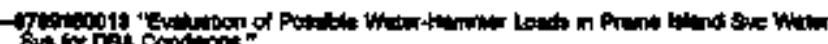

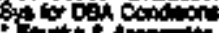

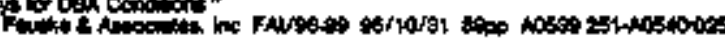

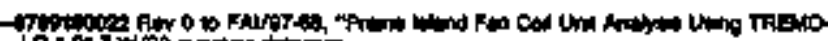

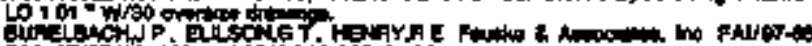
oto of

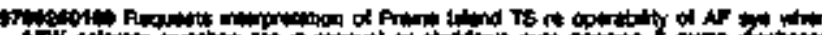

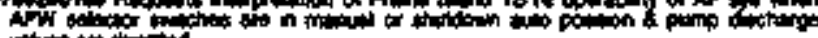

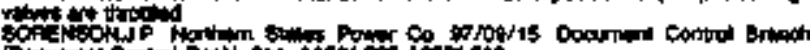

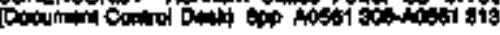

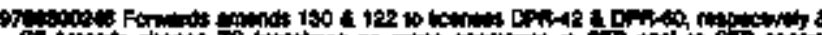

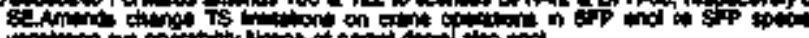

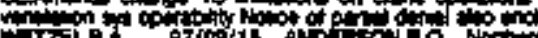

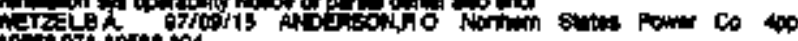

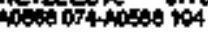

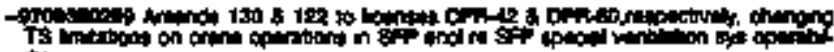

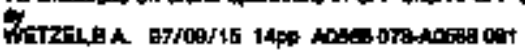

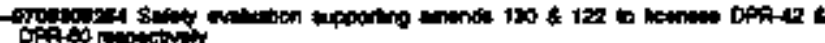

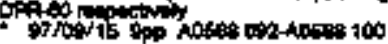

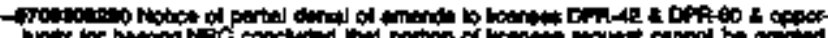

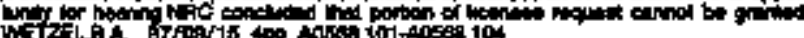

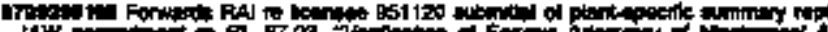

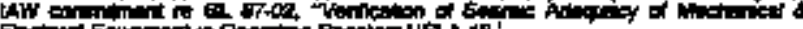

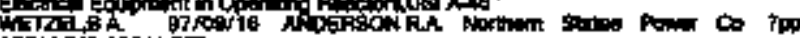

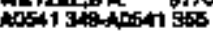

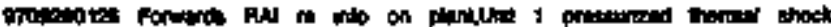

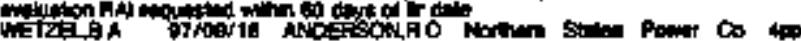

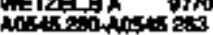

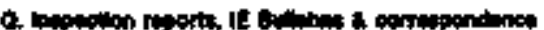

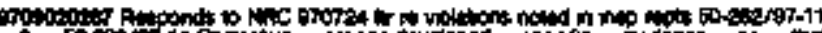
F0300\%-11 Go

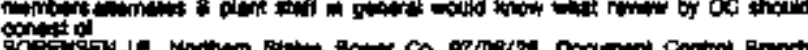

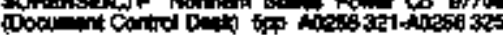

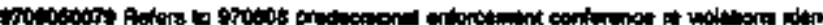

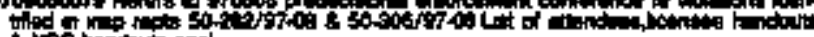

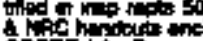

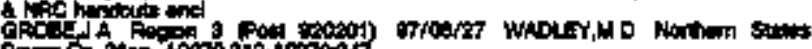

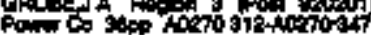

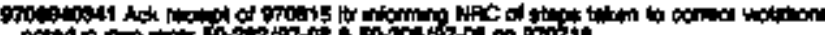

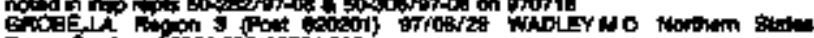

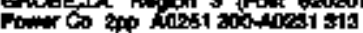

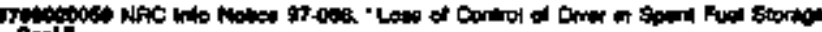

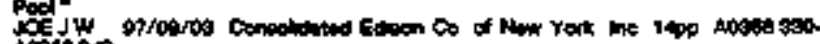
106\%

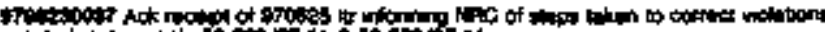

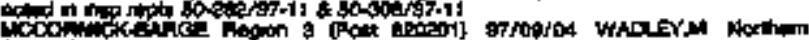

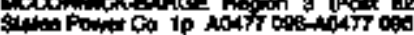




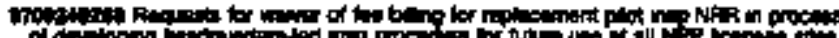

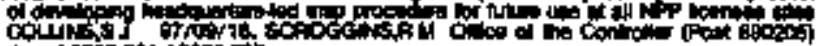

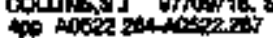

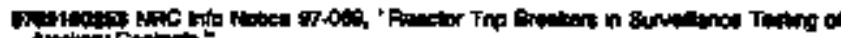

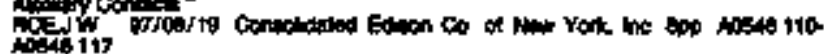

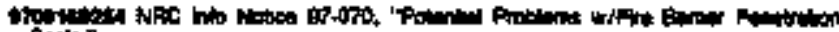

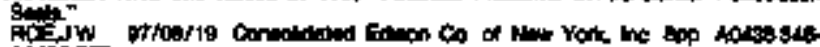
int.

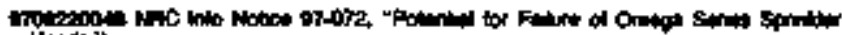

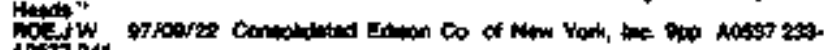

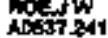

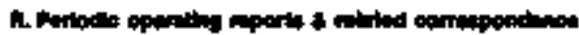

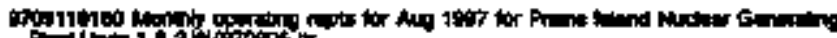

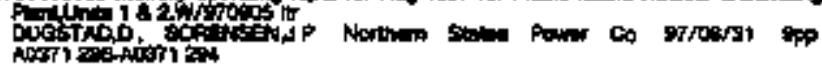

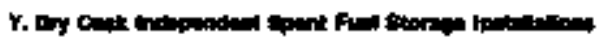

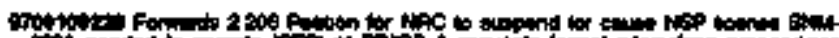

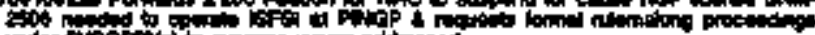

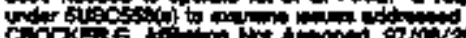

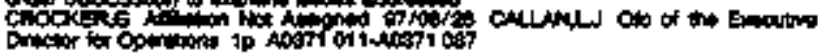

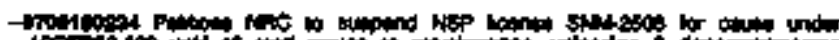

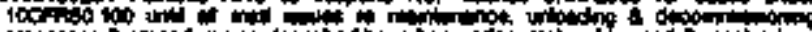

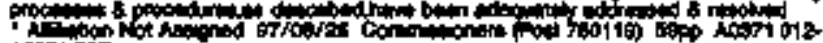
acomition

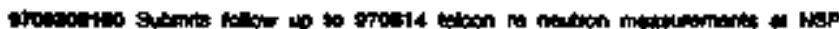

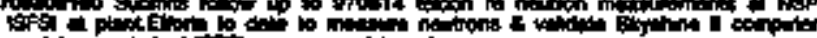

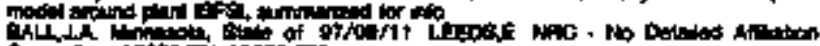

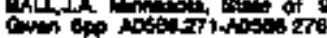

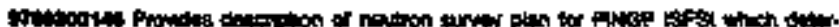

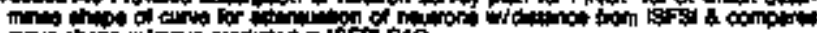

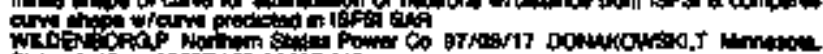
sto of

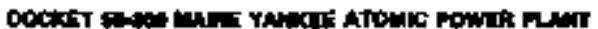

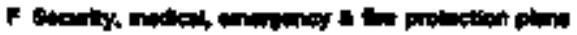

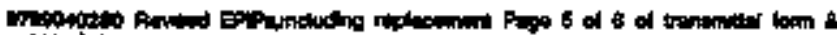

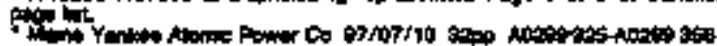

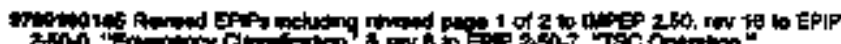

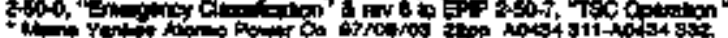

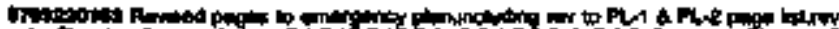

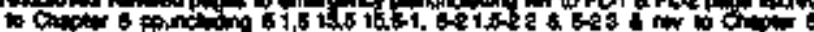

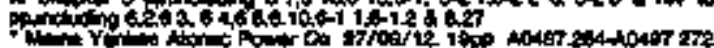

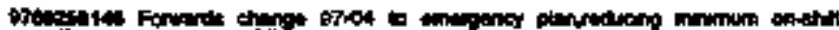
†

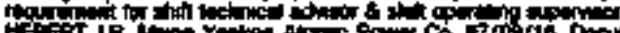

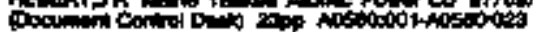

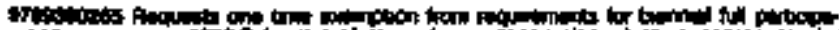

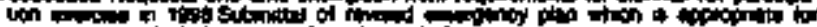

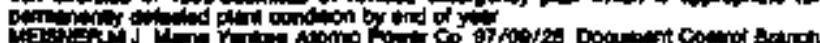

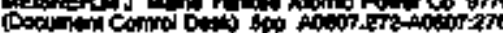

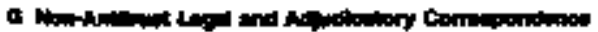

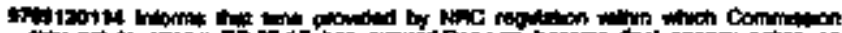

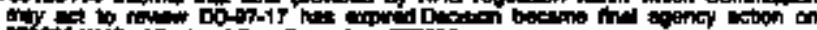

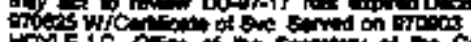

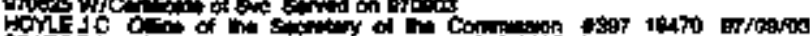

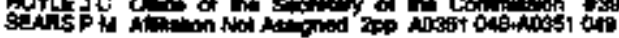

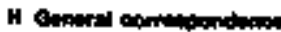

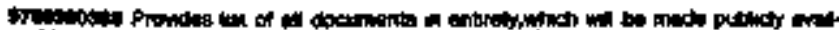

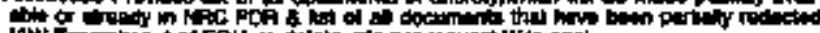

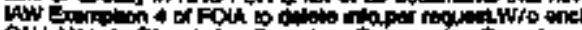

of

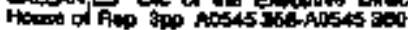

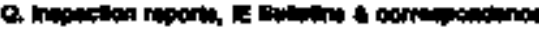

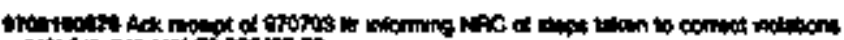

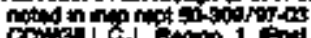

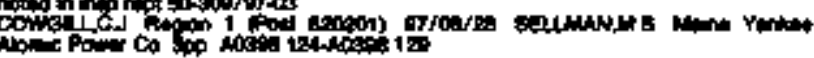

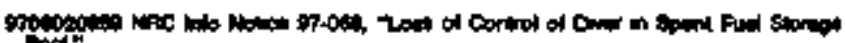

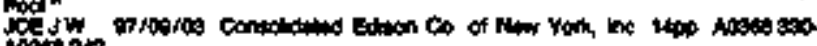
Notas

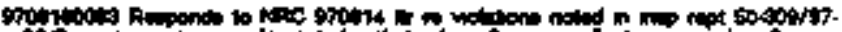

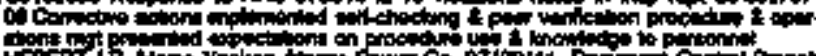

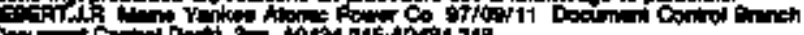

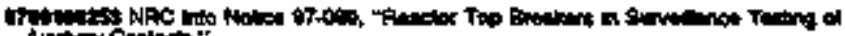
A

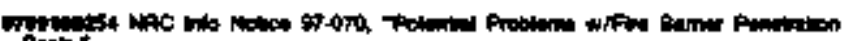

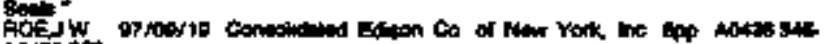
nomis 360

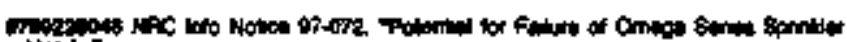

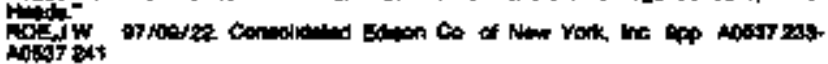

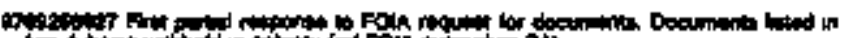

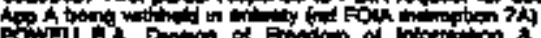
Bw

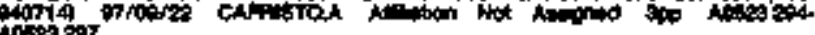

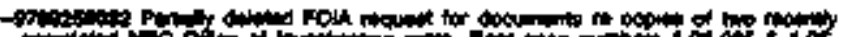

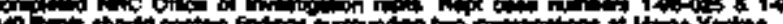

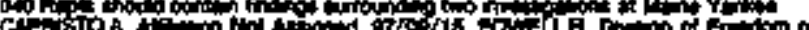

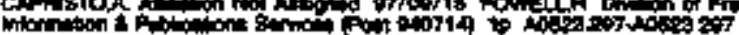

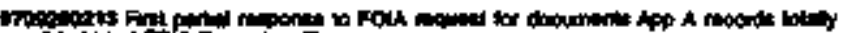

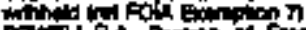

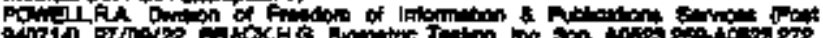

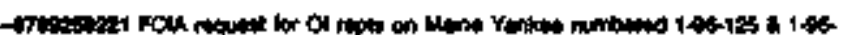

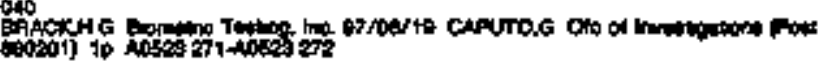

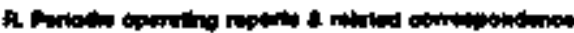

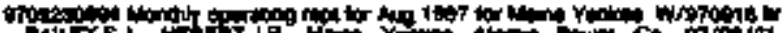

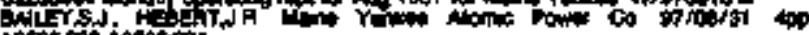

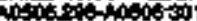

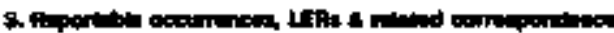

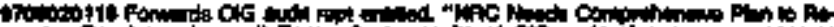

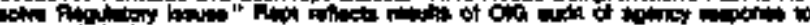

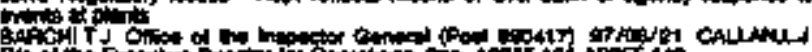

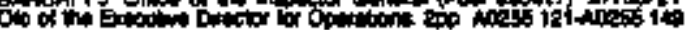

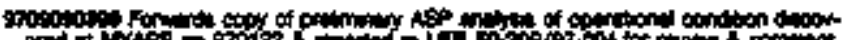

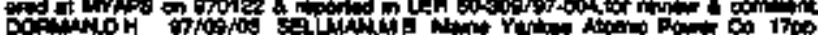

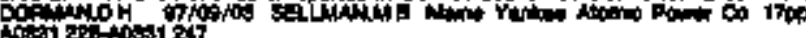

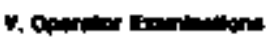

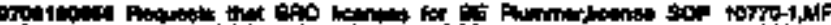

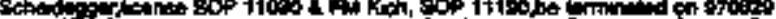

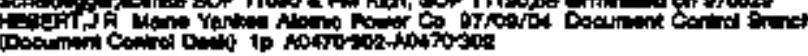

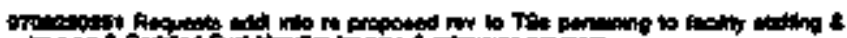

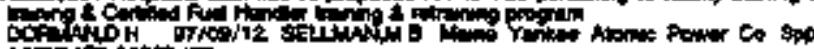
nosio $170+0000$ irs

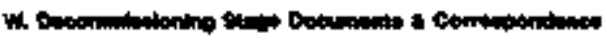

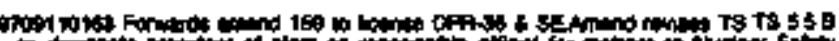

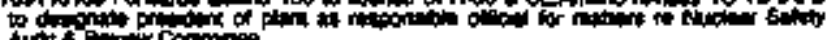

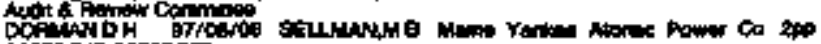

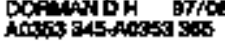

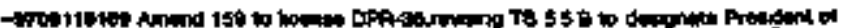
Hown compring

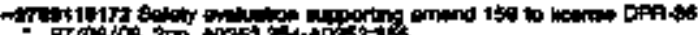




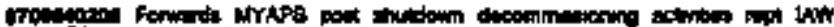

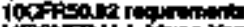

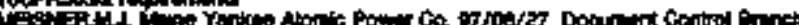

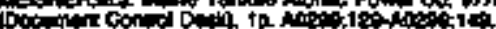

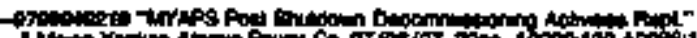

(1)

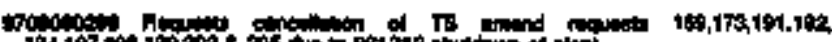

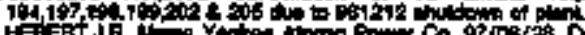

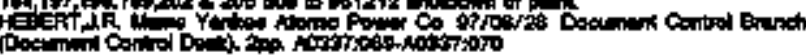

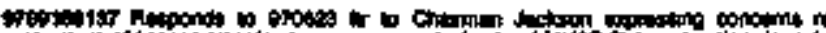

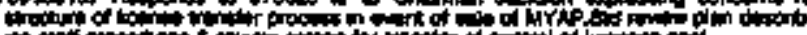

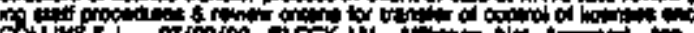

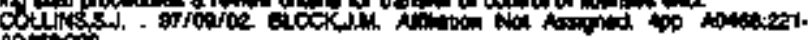
A A thos

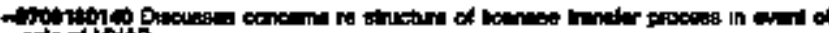

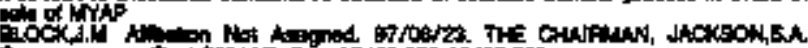

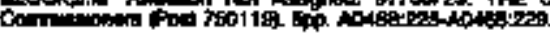

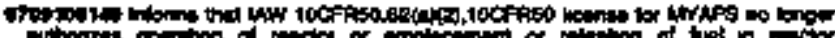

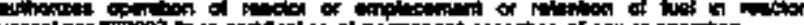

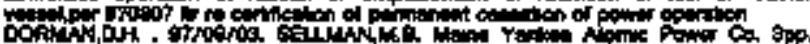

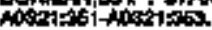

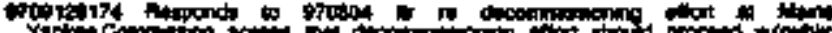

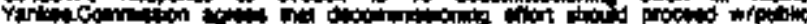

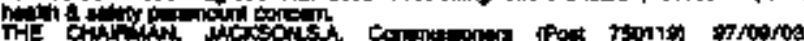

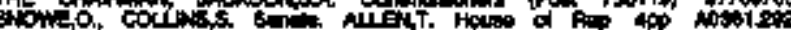
atosicts

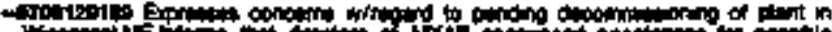

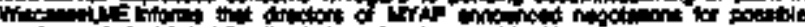

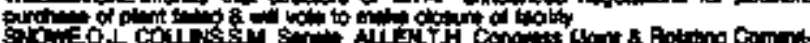
a

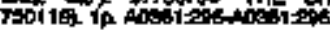

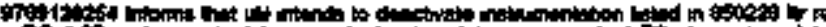

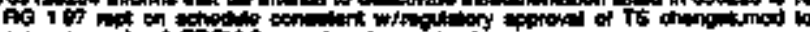
in

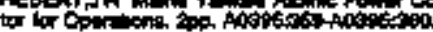

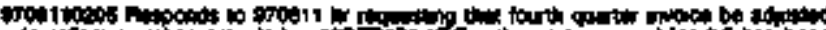

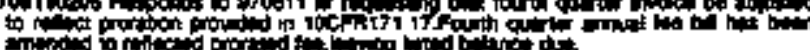

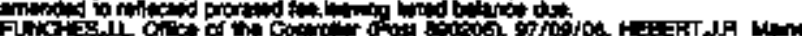
Yen

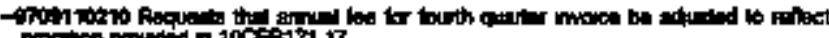

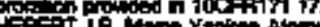

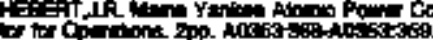

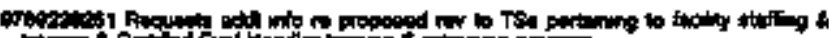

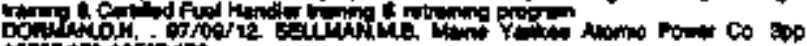

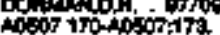

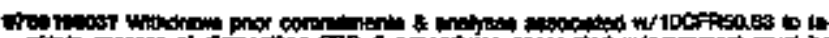

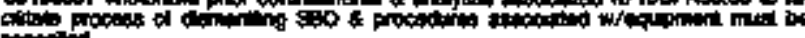
anconing

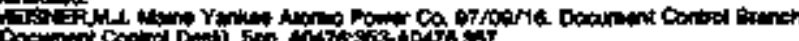

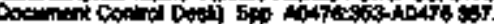

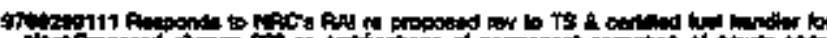

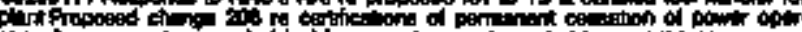

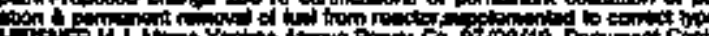

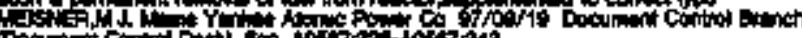

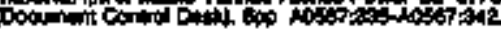

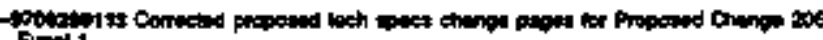
Eroil 1

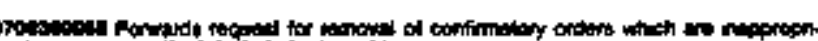

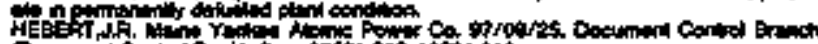

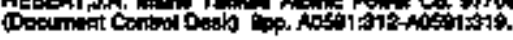

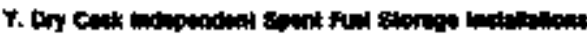

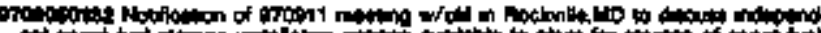

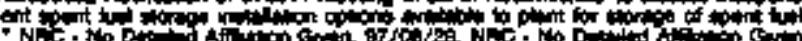
No.

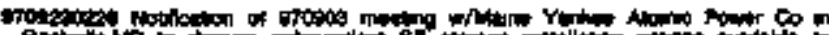
\$

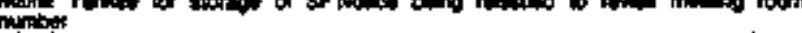

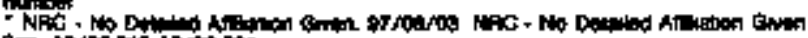

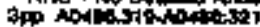

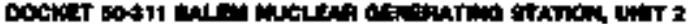

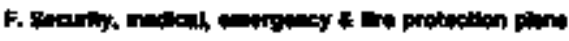

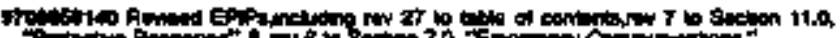

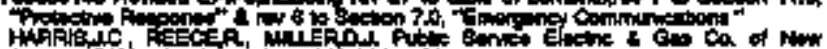

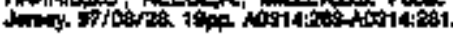

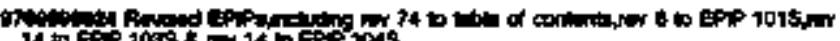

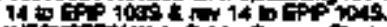

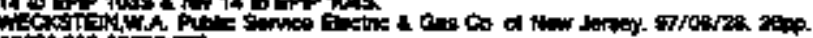

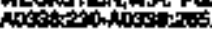

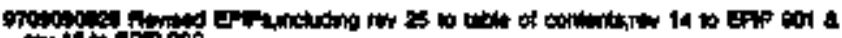

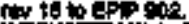

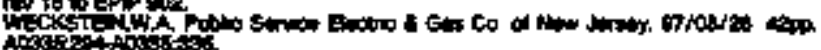

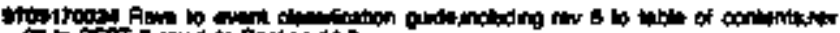

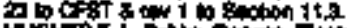

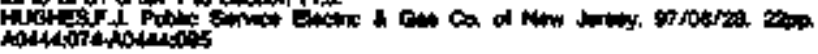

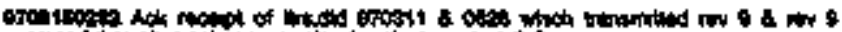

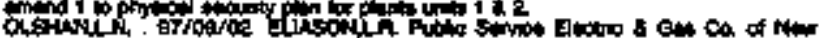

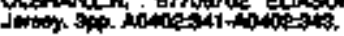

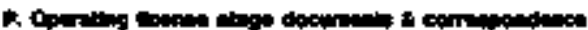

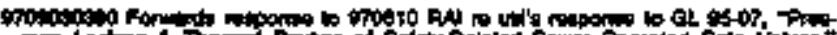

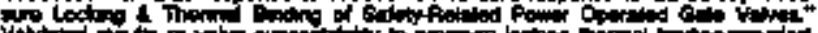

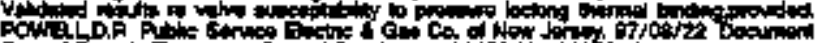

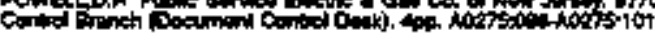

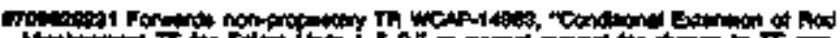

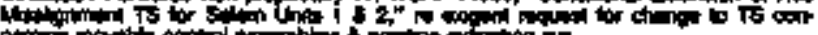

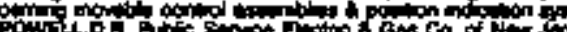
orjeyse Dotimen

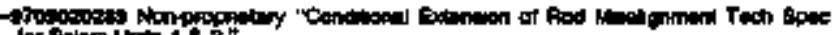

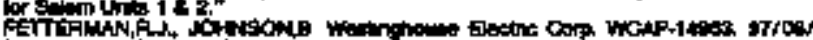

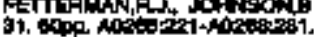

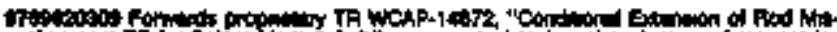

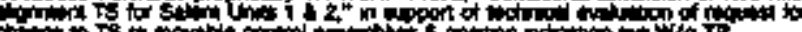

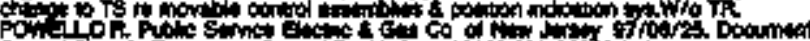

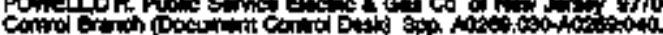

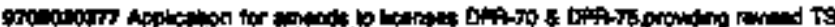

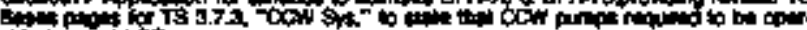
10

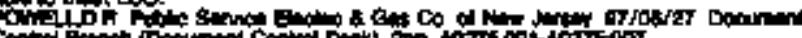

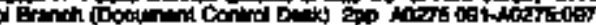

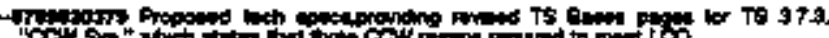

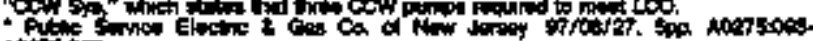
Atistion

Proviners For

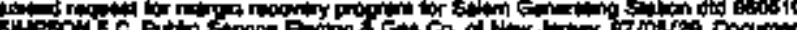
Coind :

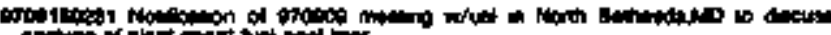

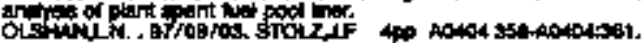

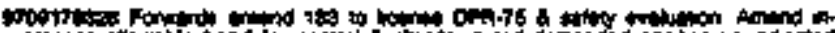

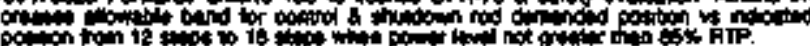

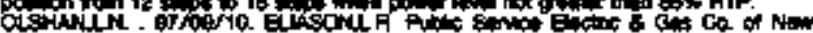

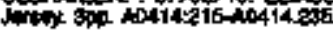

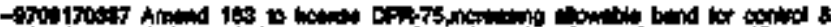

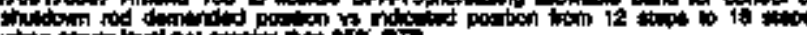

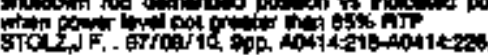

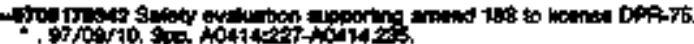

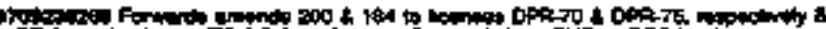

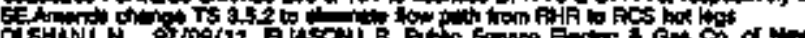

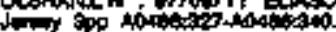

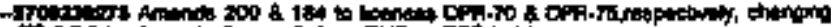

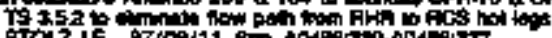

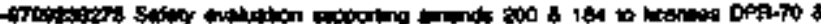

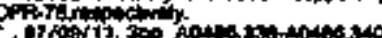




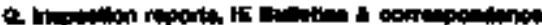

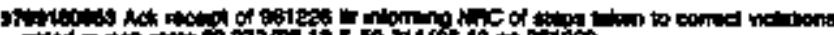

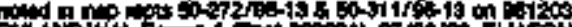

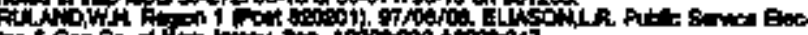

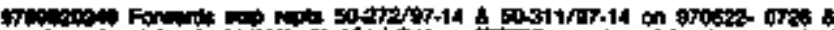

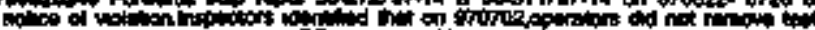

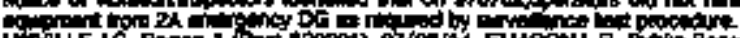

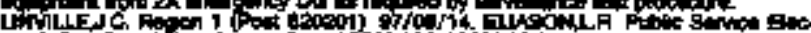

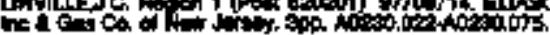

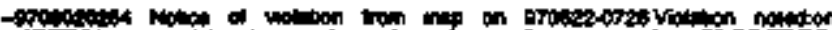

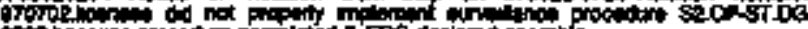

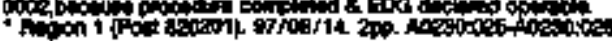

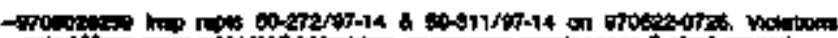

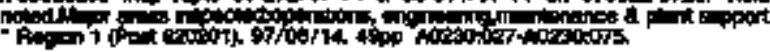

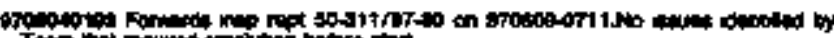

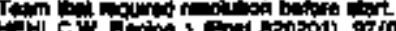

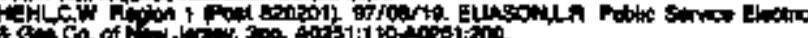

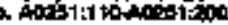

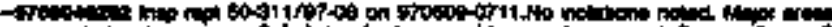

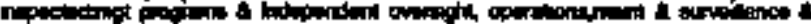
Ant

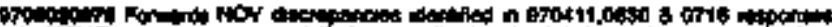

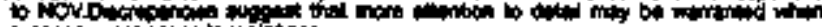

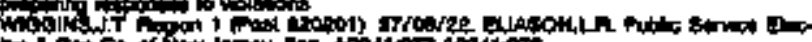

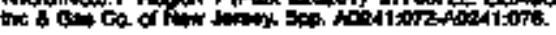

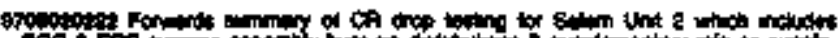

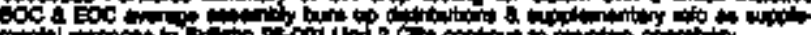

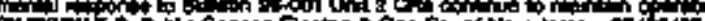

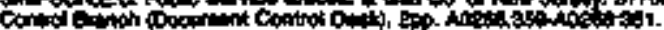

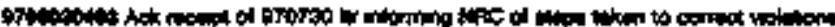

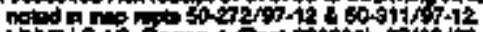

n'th por

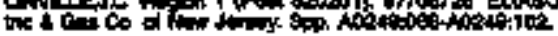

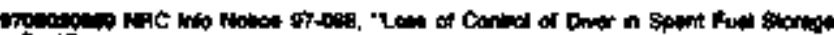
$\cos ^{\circ}$

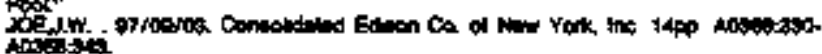

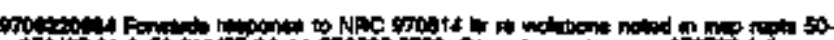

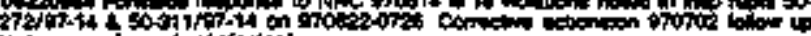

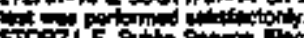

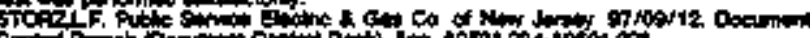

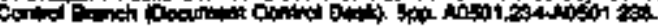

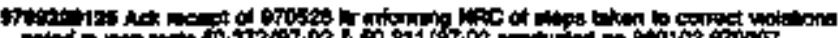

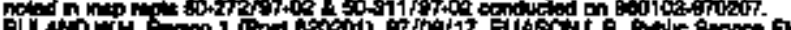

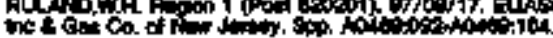

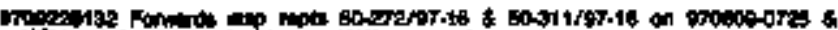

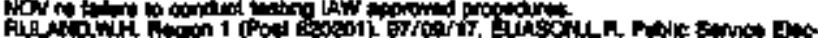

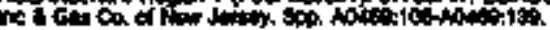

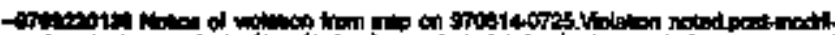

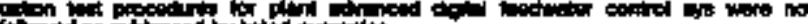

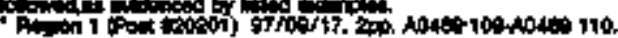

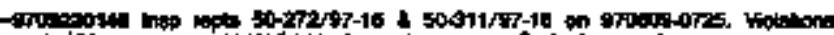

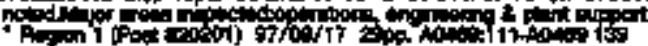

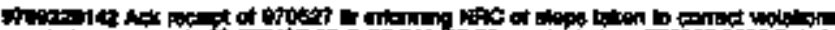

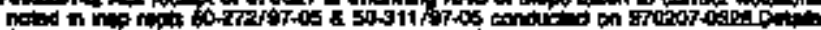

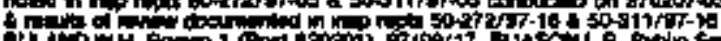
AUt

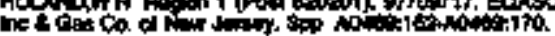

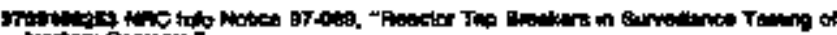

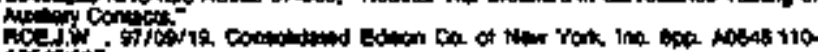
iostititis

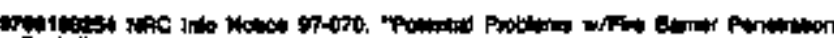

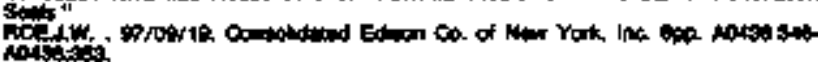

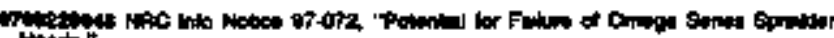

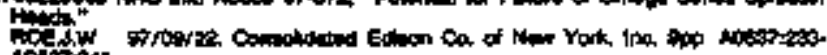
notion

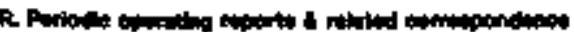

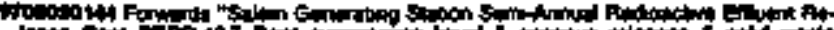

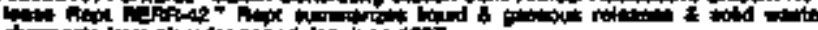

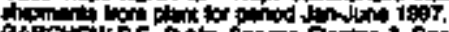

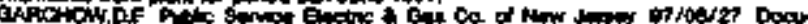

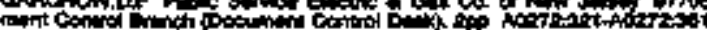

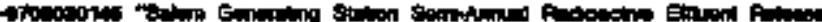

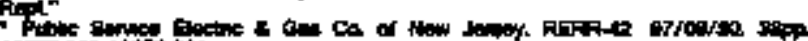

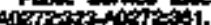

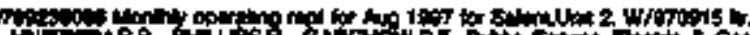

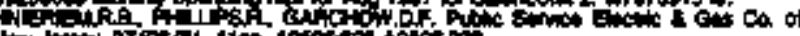

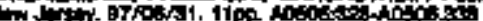

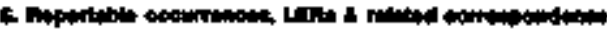

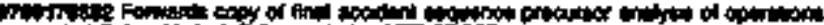
that 형

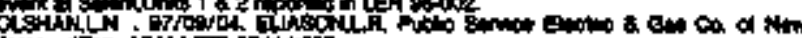

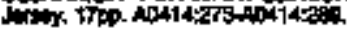

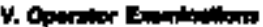

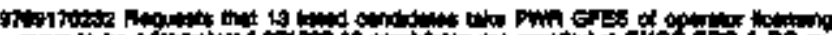

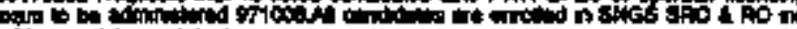

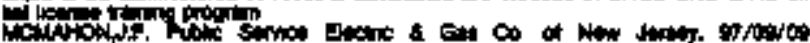

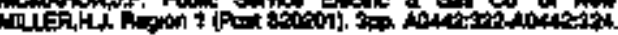

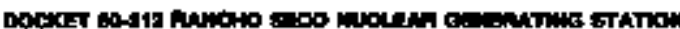

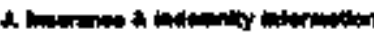

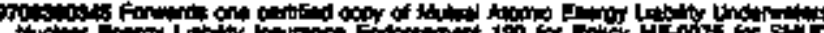

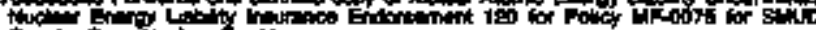

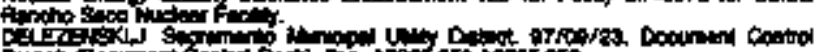

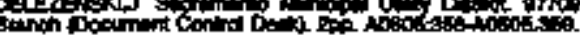

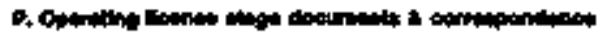

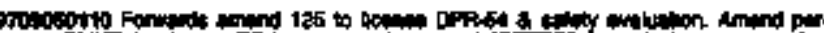

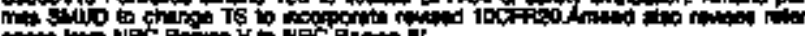

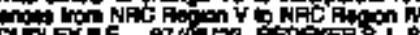

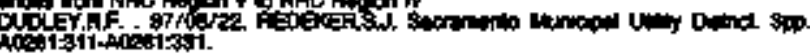

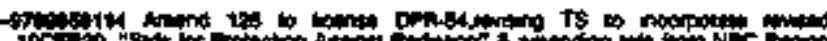

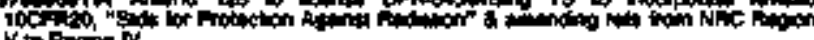
toping in

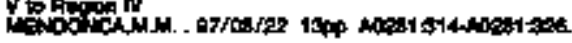

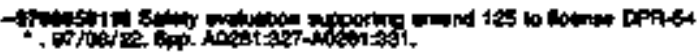

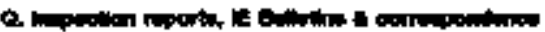

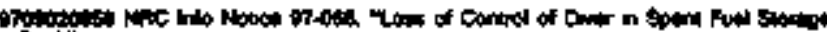

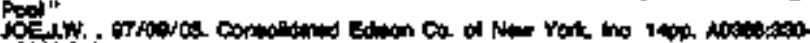
atsionis.

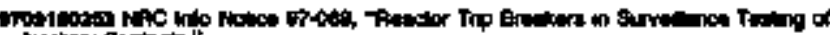

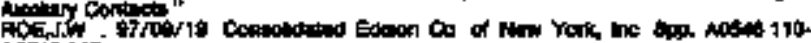
antertiz:

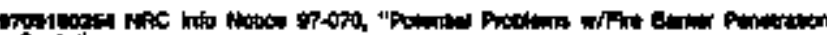

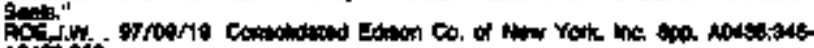
Notiosi.

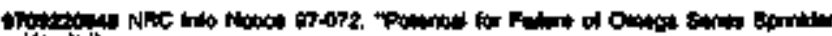

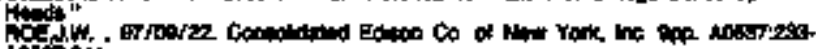
iostis

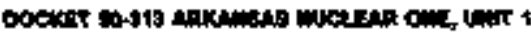

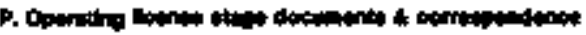

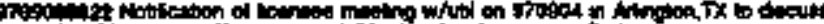

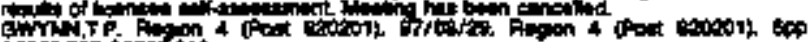

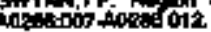

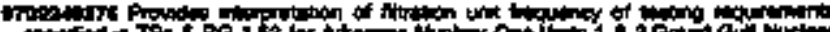

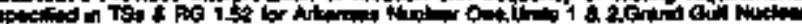

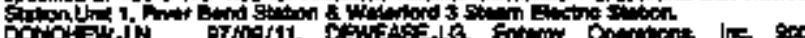
Dototions

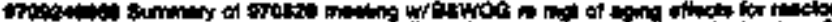

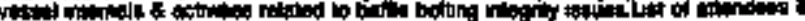

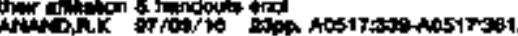




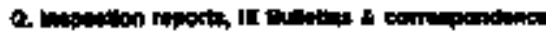

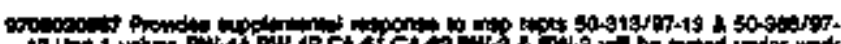

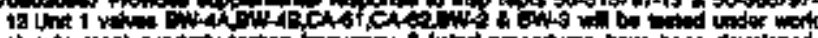

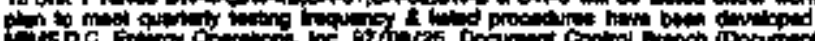

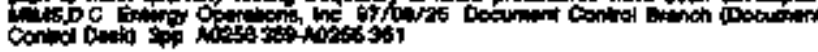

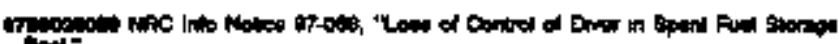

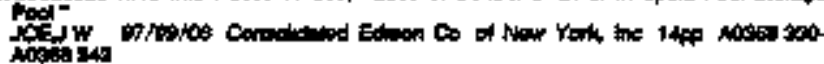

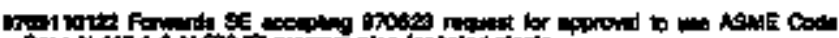

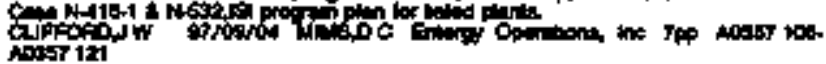

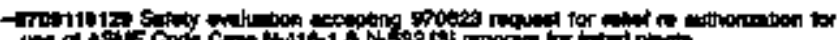
wat of A

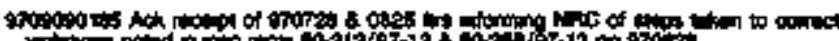

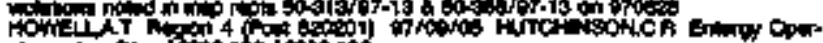

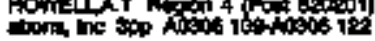

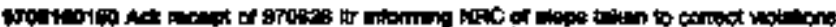

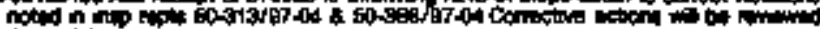

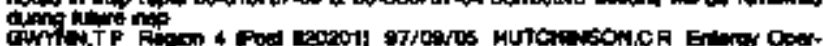

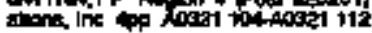

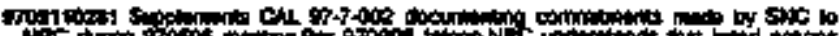

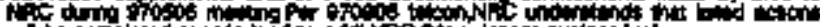

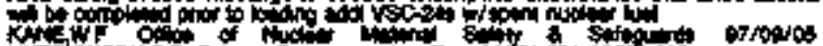

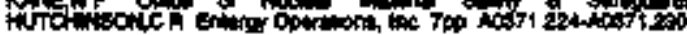

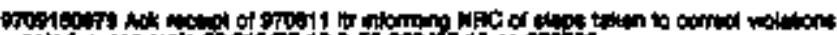

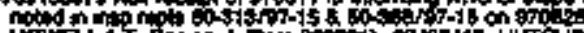

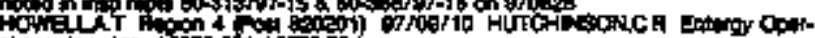

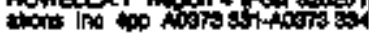

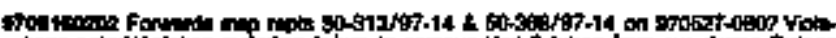

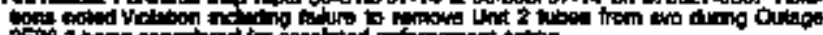

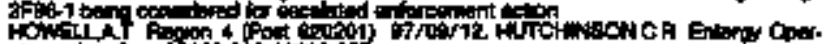

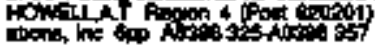

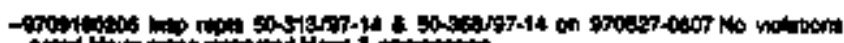

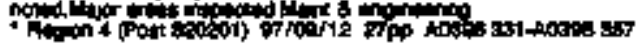

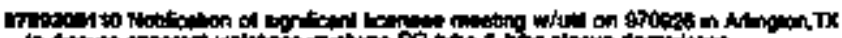

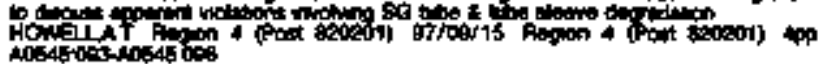

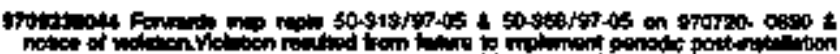

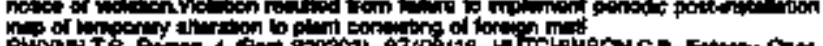

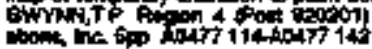

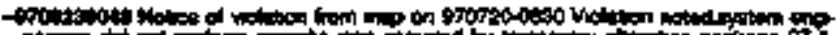

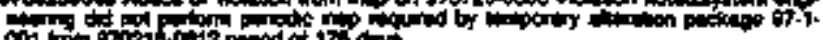

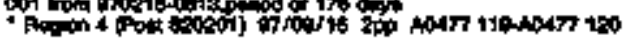

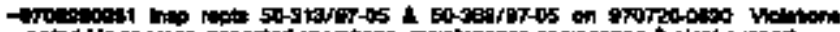

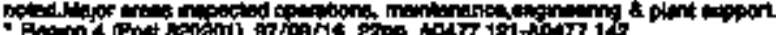

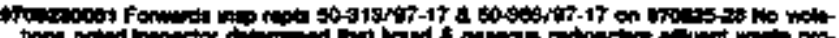

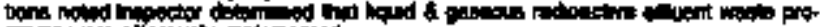
Fothe

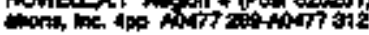

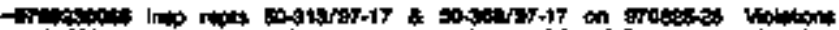

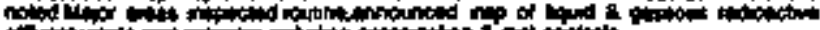

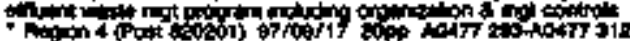

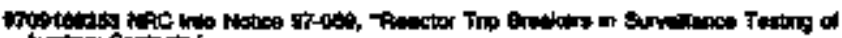

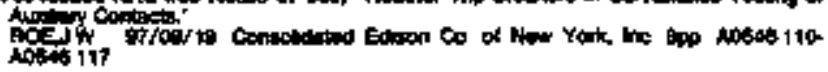

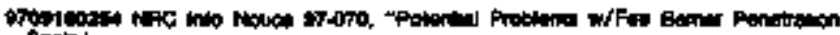

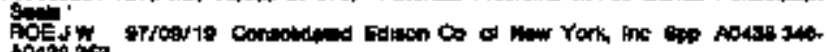
A0438 350

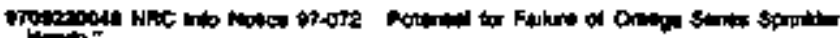

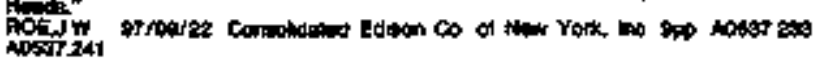

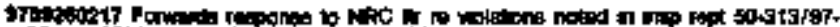

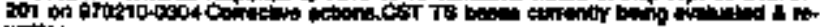

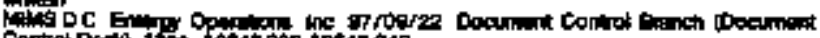

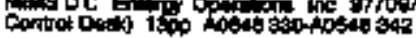

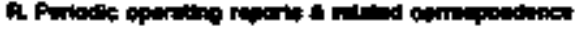

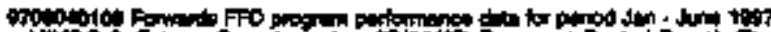

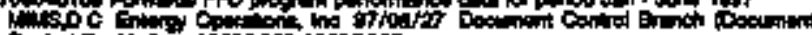

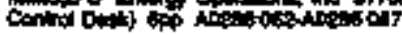

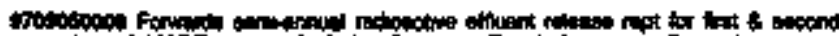

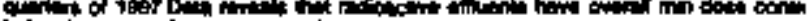

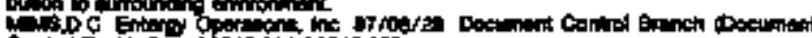

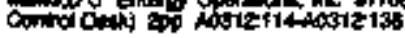

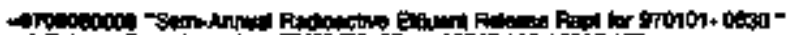

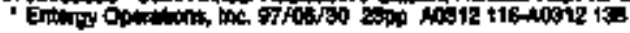

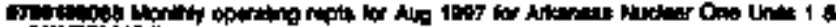
a

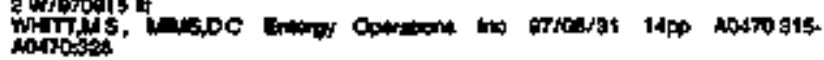

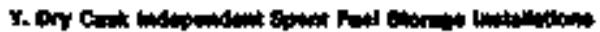

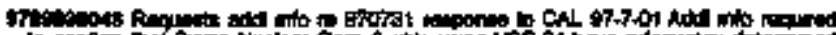

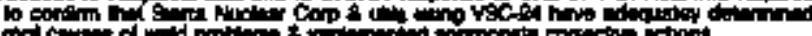

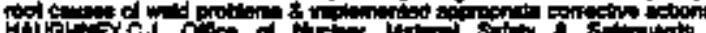

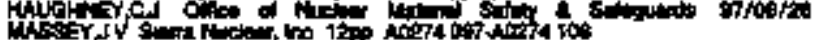

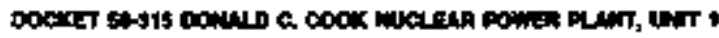

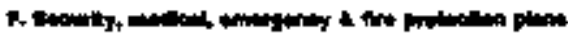

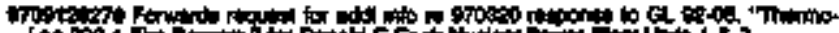

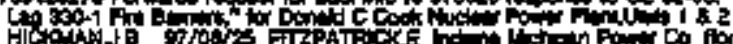
U.

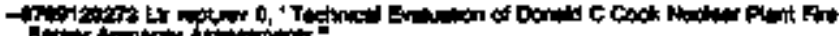

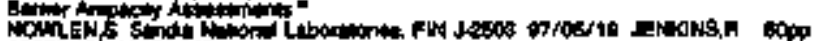

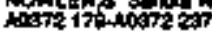

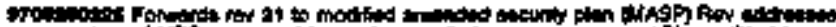

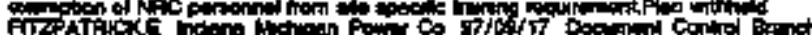

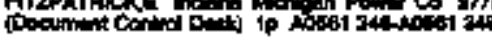

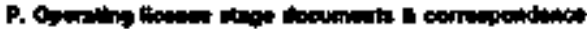

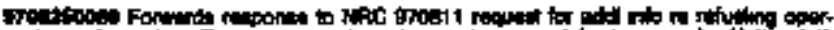

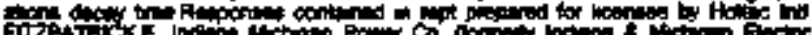

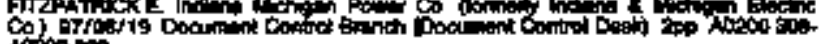
$\operatorname{tin} 000$

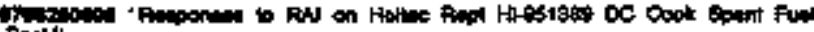
Pool

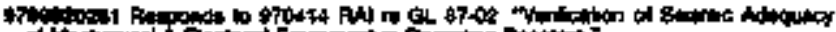

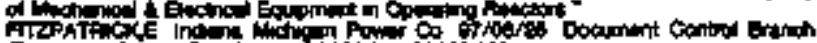

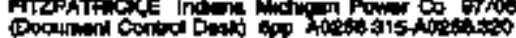

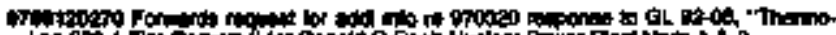

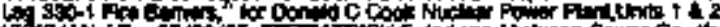

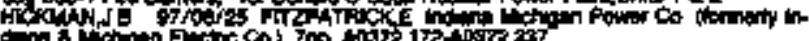

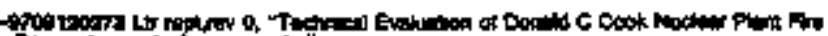

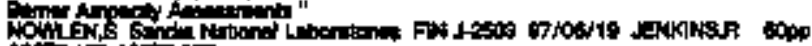

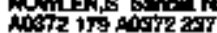

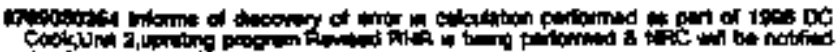

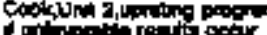

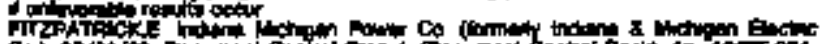

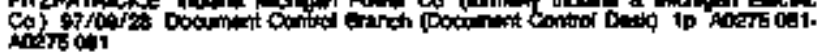

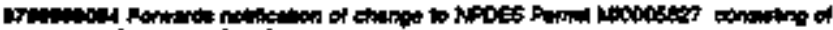

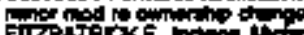

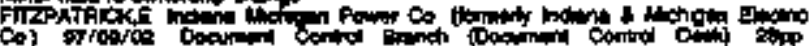
coison 12 hose

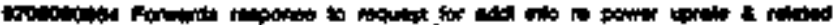

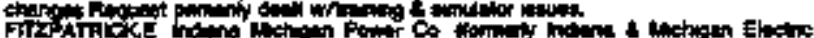
col of

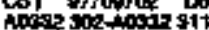

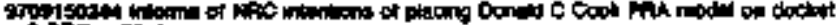

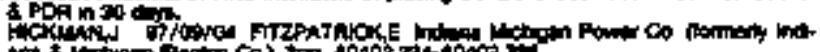

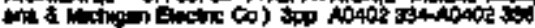




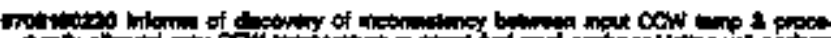

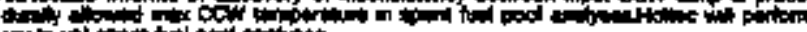

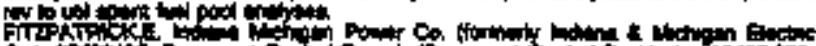

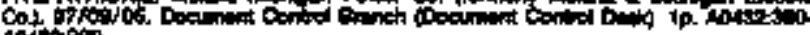
Aotions.

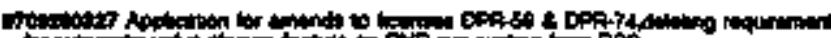

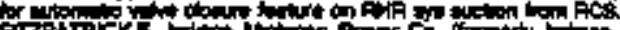
नाzo kT al 20097 D

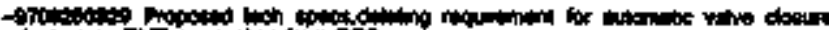

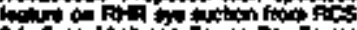

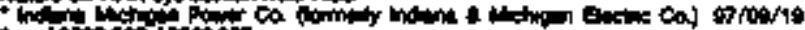

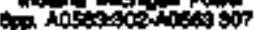

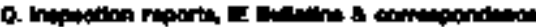

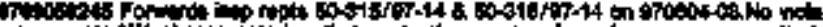

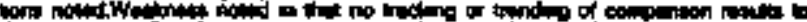

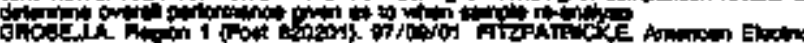

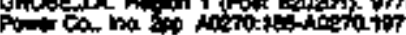

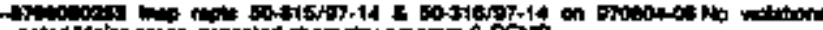

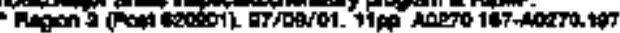

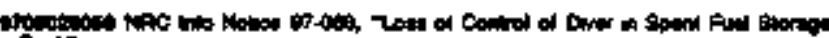

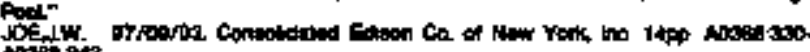
Aosians.

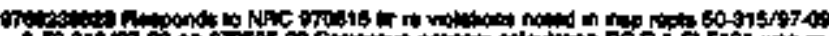

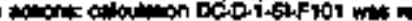

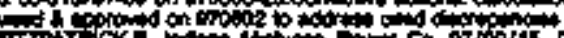

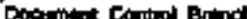

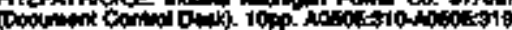

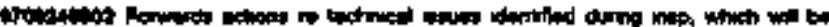

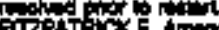

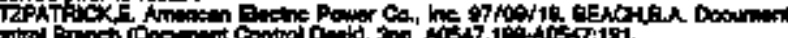

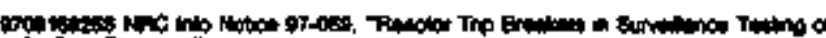

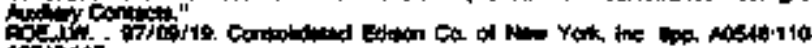
inotiotit.

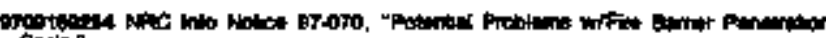
sentr

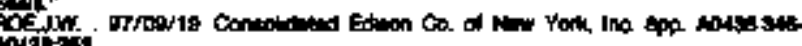
1043009

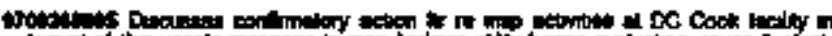

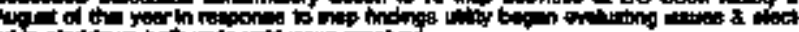

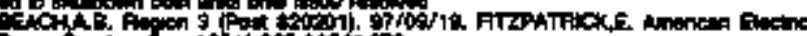

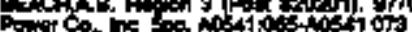

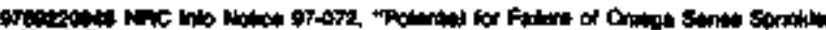

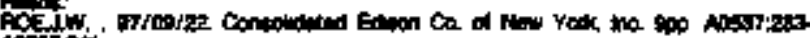
10:37.

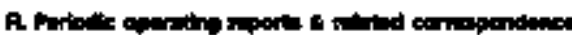

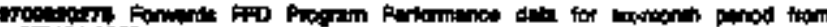
Contorso

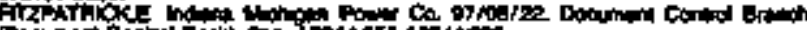

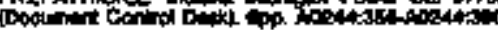

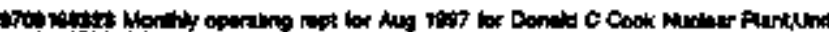

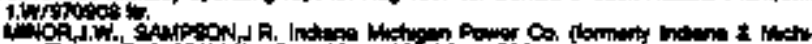
qn

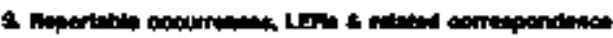

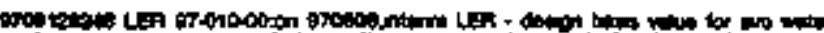

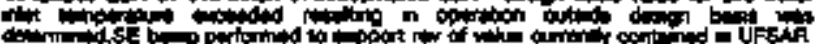

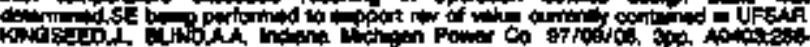
Kencipip.

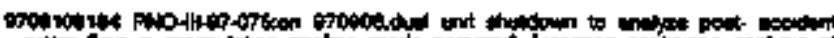

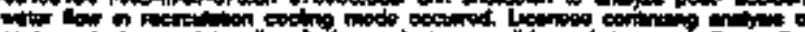

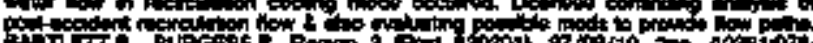

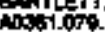

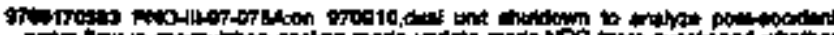

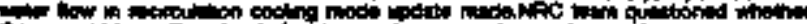

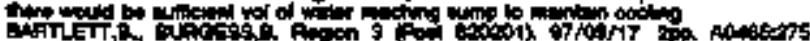
Apositit.

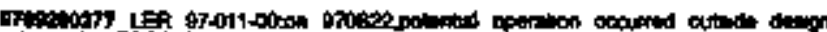

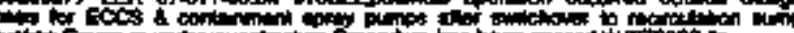

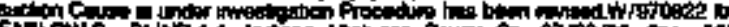

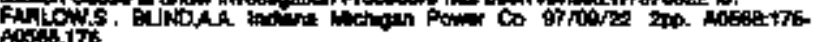

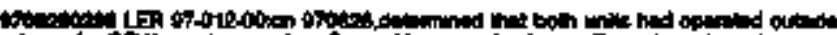

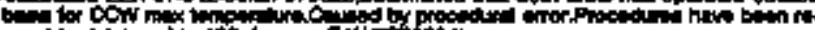
oli incositio.

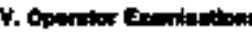

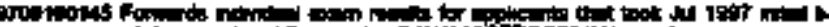

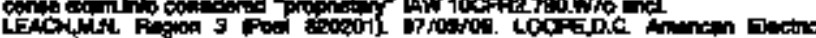

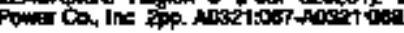

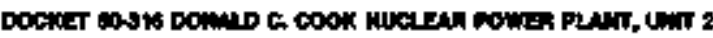

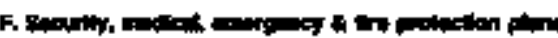

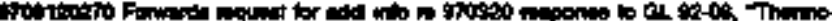

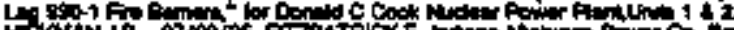

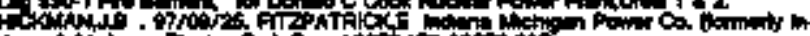

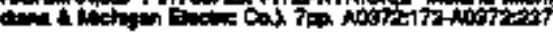

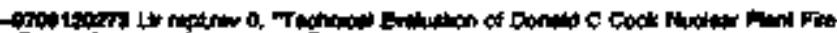

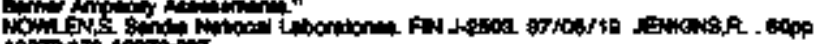

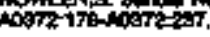

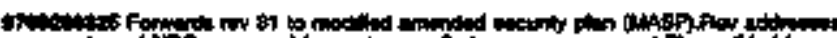
Fon

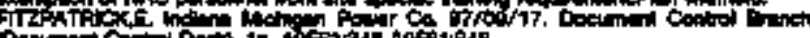

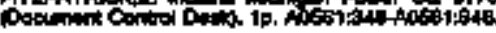

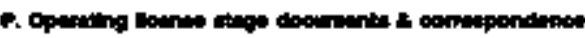

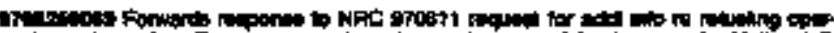
pront do Taj, sf tow Ad, sion.

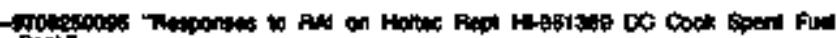

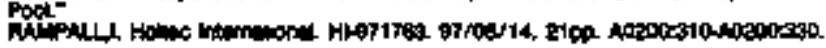

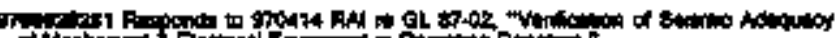

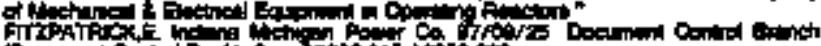

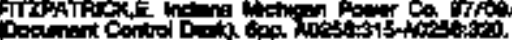

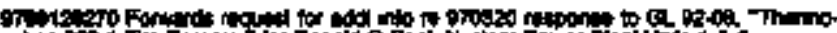

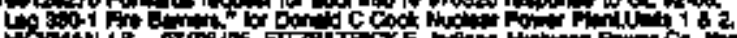

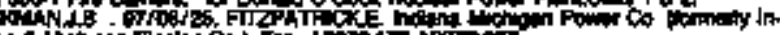

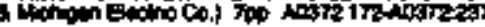

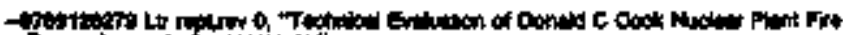

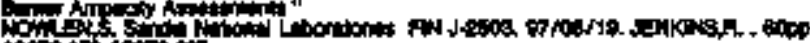
Nom 17 Hos

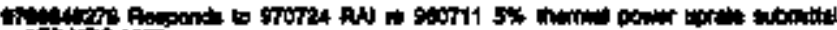
Apticin

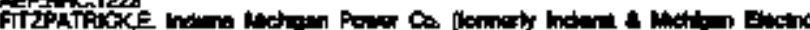

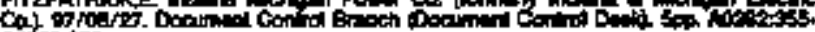
atipitis

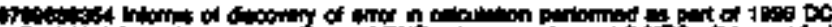

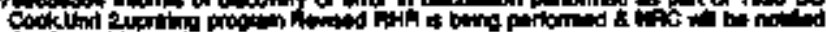

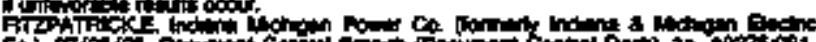

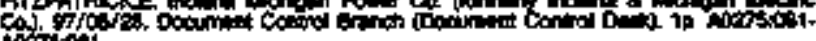
Axition.

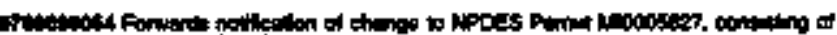

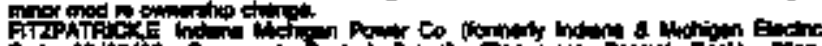

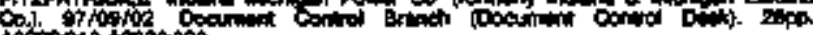

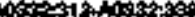

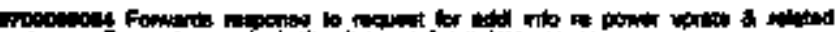

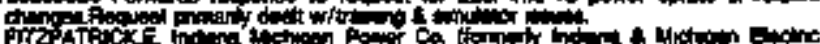

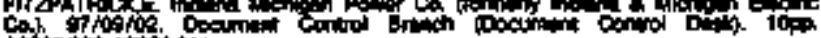

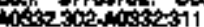

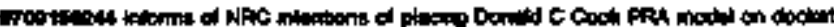

HCOA n jo dipe

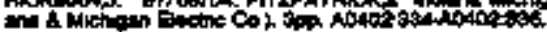

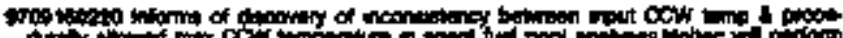

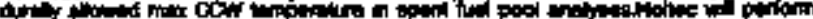

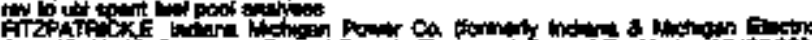

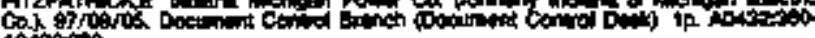
Aosion.

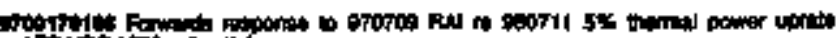

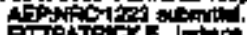

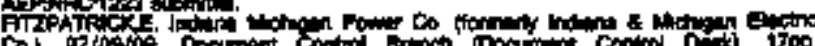

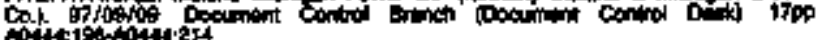




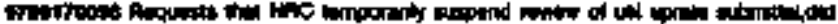

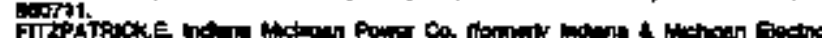

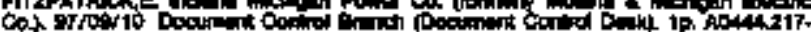

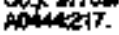

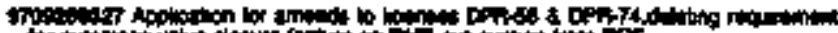

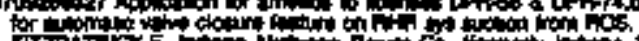

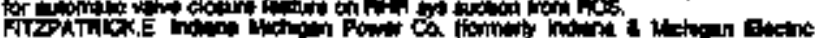

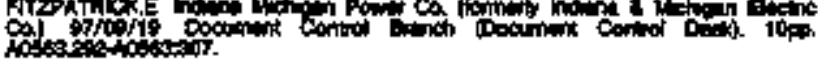

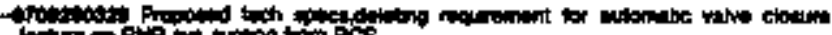

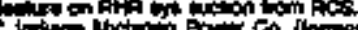

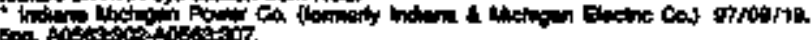

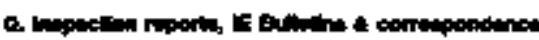

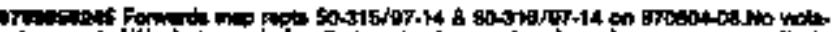

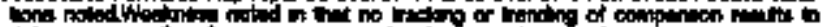
toce

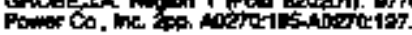

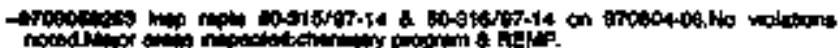

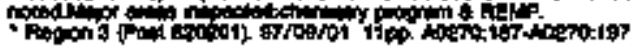

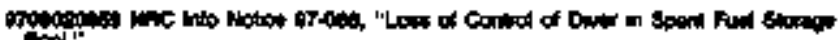

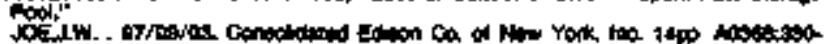
totisis

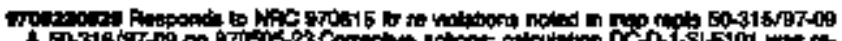

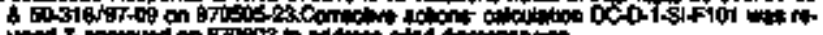

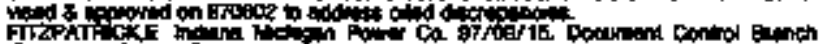

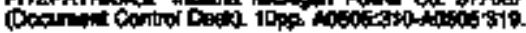

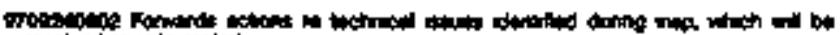

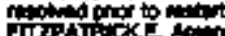

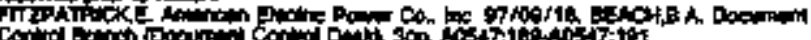
管

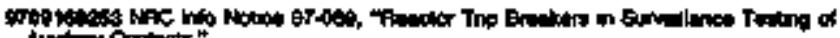

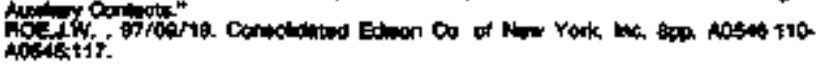

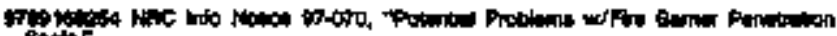
89.7\%

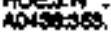

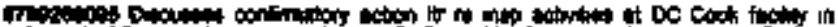

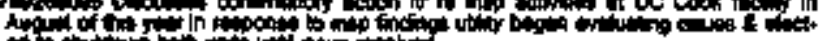

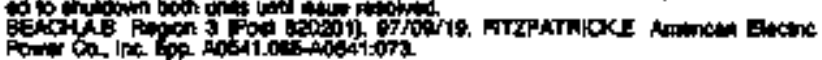

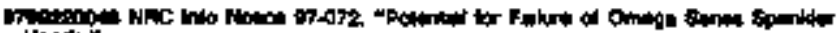

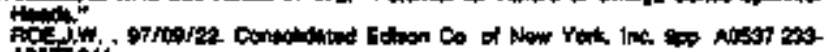
Axțin:

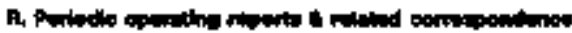

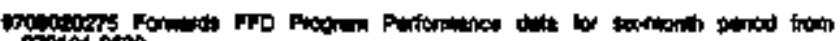

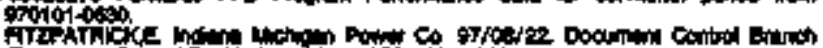

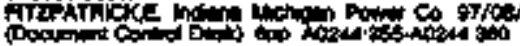

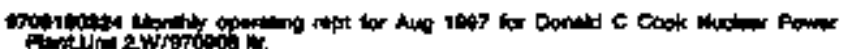

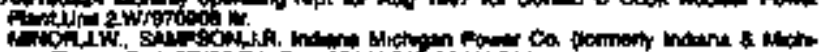

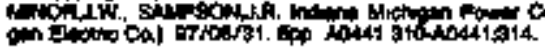

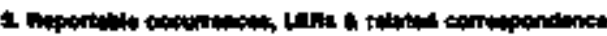

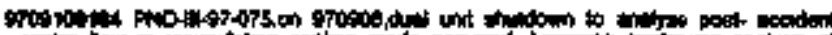

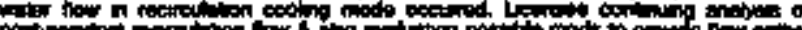

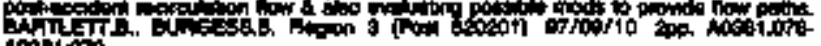
insion.

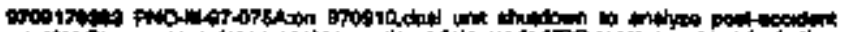

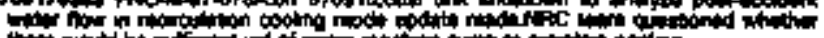

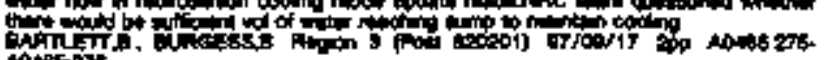
andints.

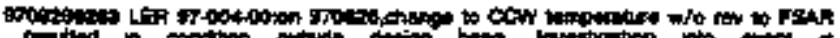

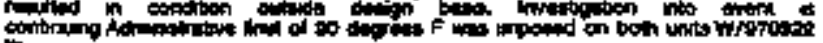

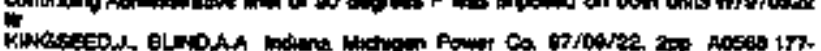
masa in

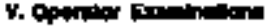

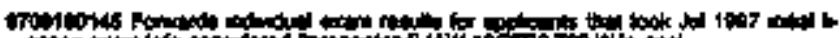
If

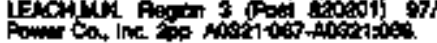

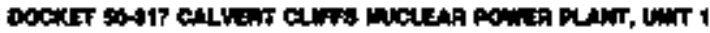

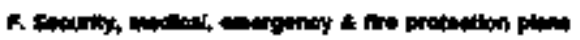

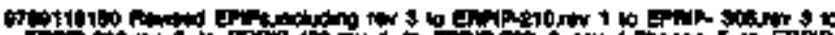

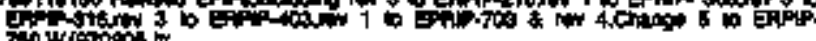

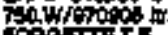

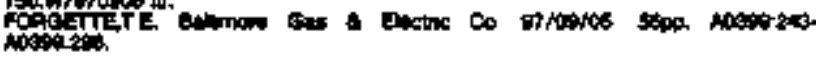

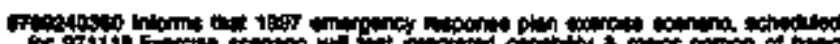

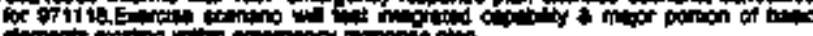

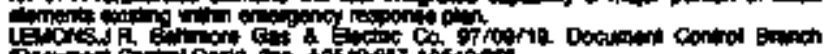

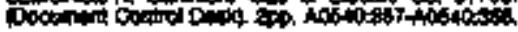

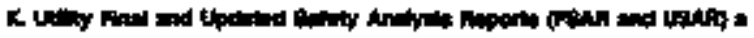

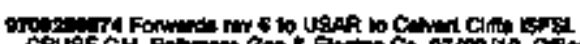

CPUS

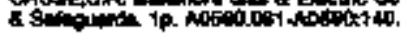

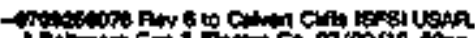

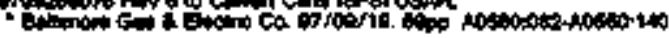

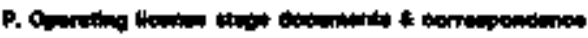

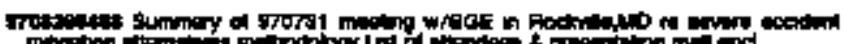

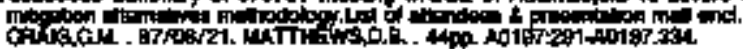

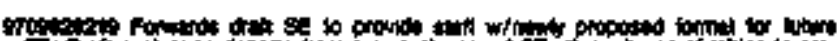

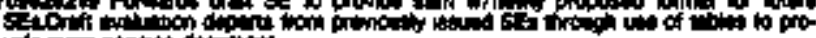

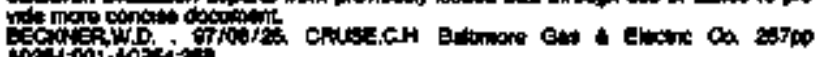

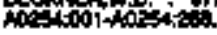

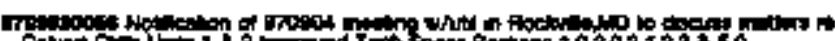

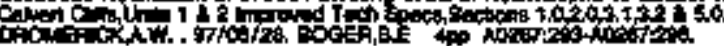

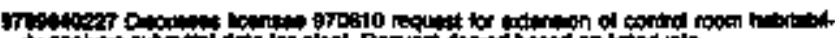
of ald

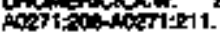

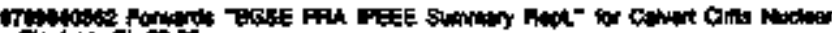

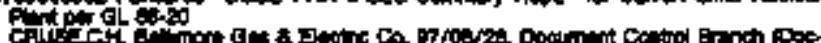

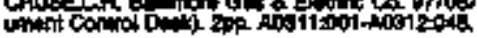

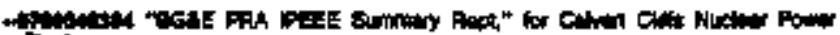

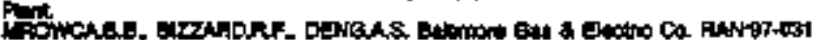

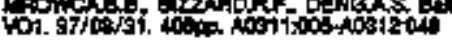

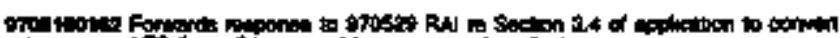

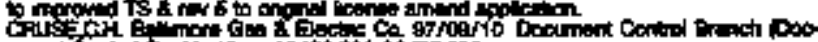

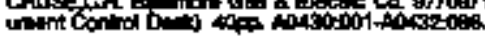

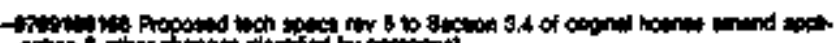

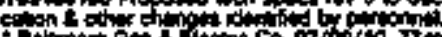

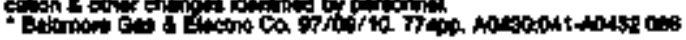

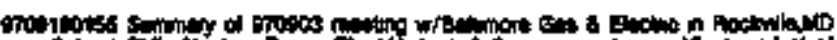

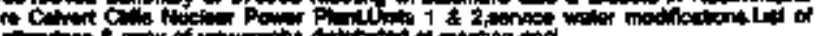

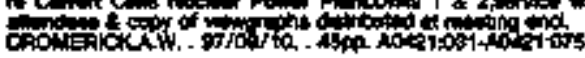

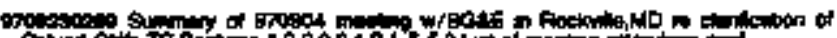

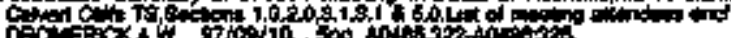

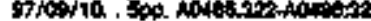

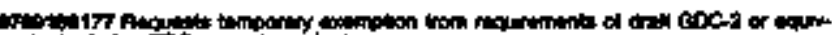

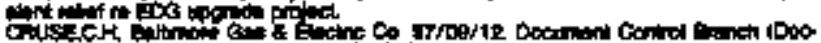

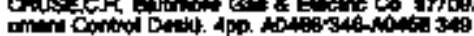

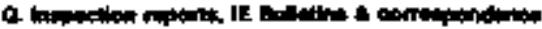

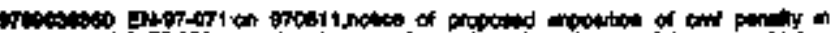

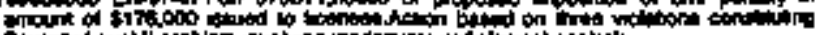

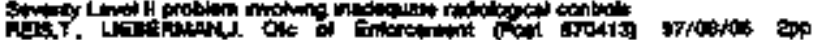

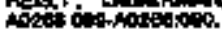

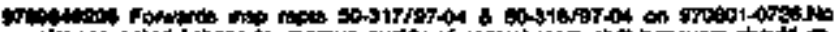

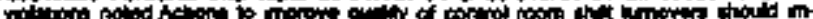

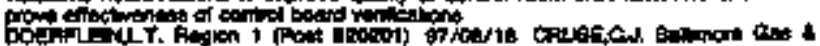

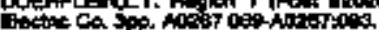




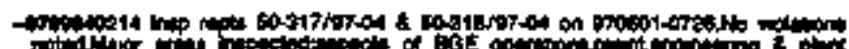

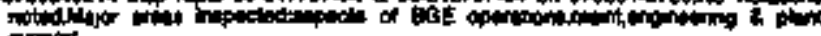

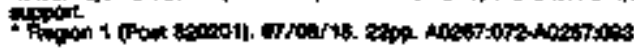

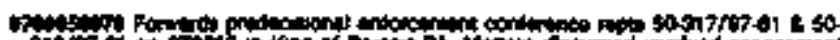
310)

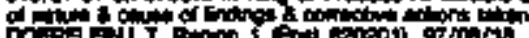

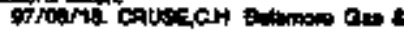
然

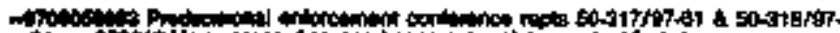

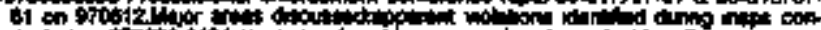

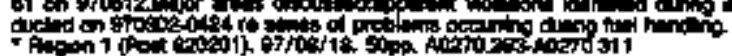

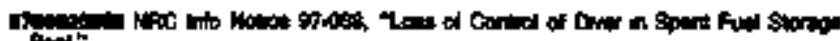
Anotion

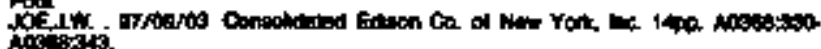

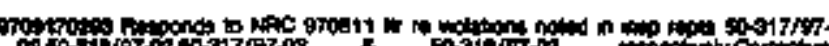

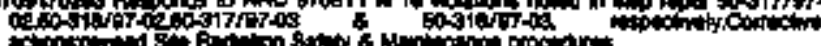

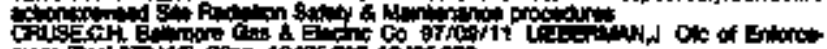

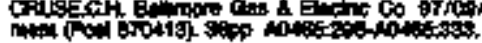

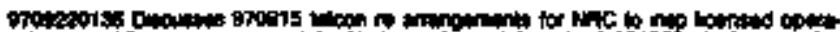

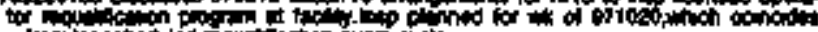

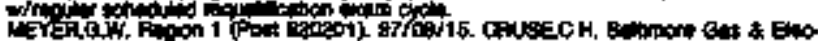
tec Co

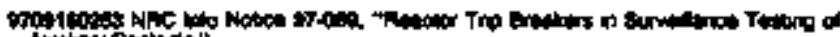
Abstit,

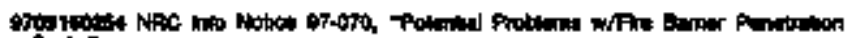

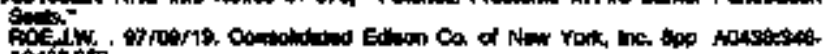
notistis:

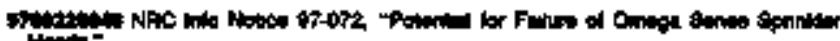

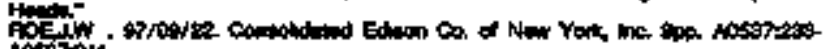

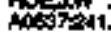

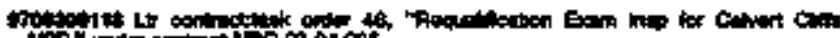

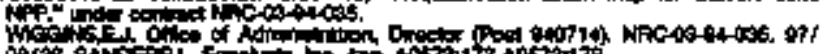

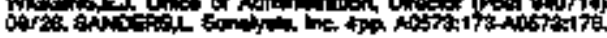

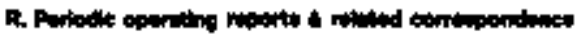

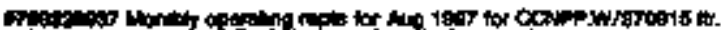

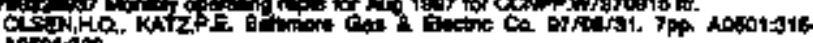

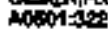

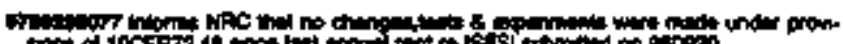

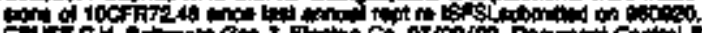

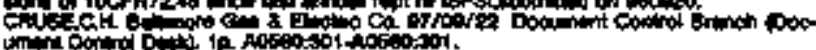

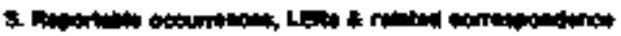

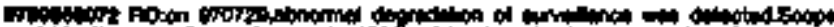

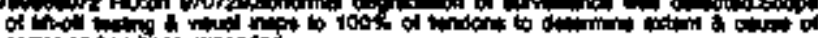

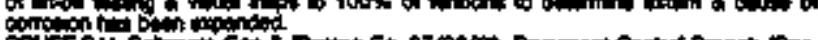

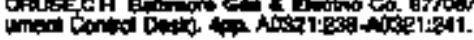

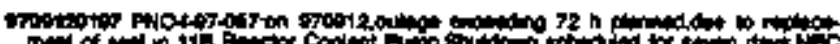

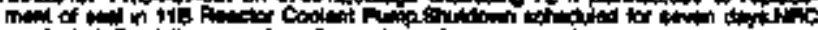

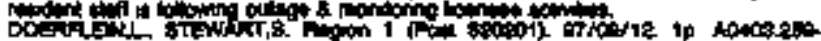
A0talon

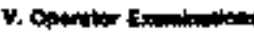

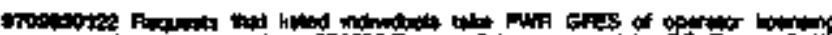

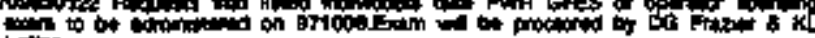

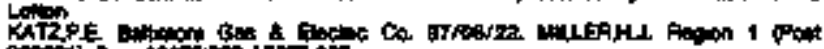

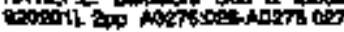

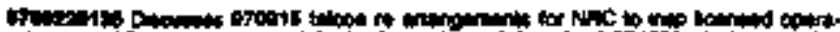

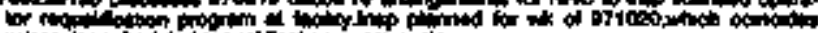

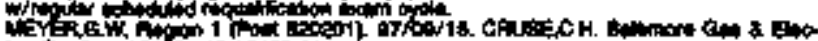

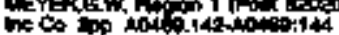

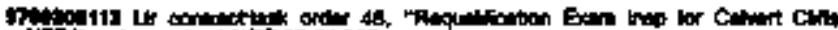

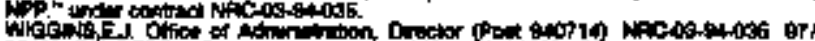

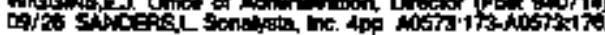

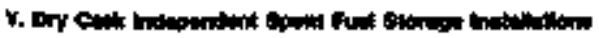

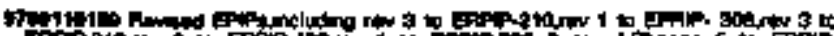

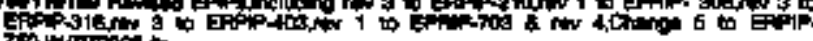
70 wirions to

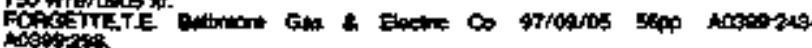

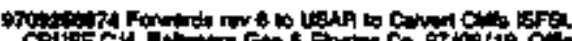

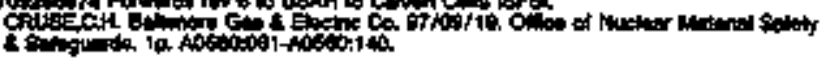

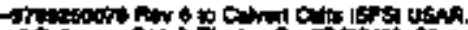

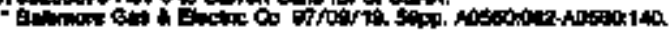

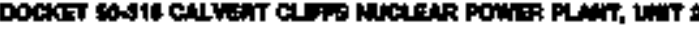

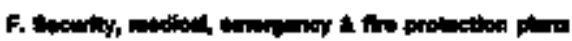

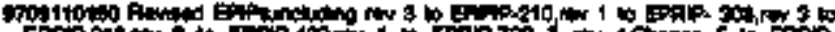

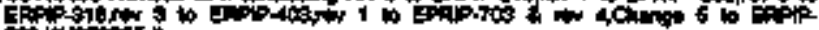

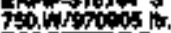

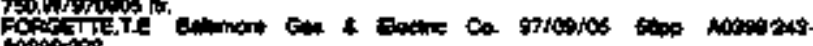
istos.

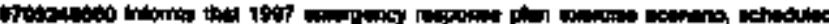

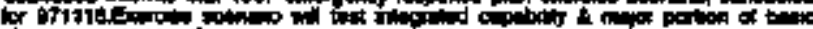

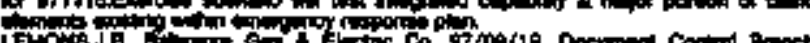
(1)

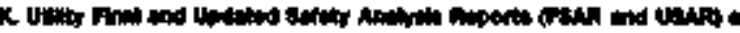

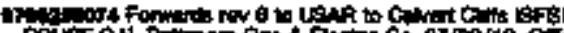

Calo

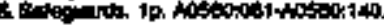

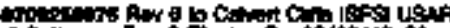

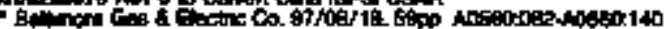

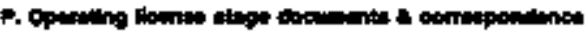

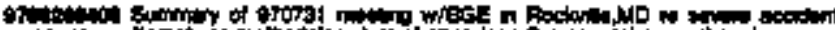

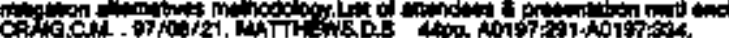

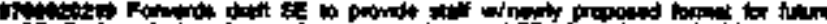

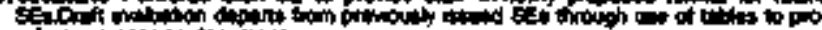

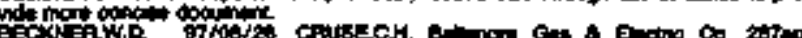

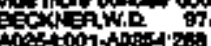

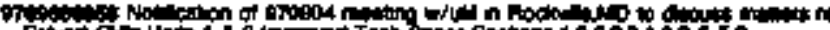

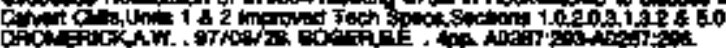

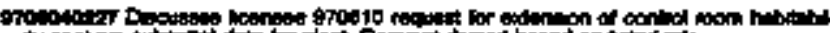

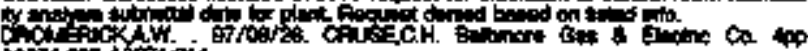
ancis

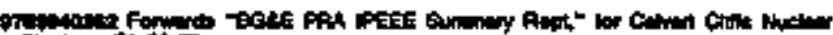

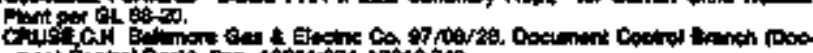
yilint

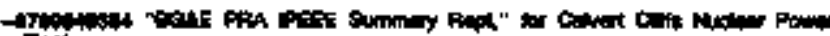

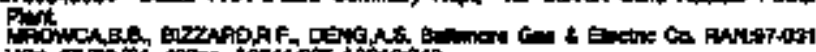

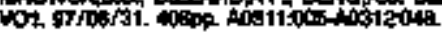

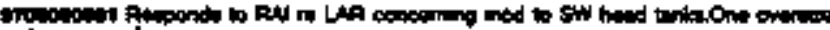
chistis ent

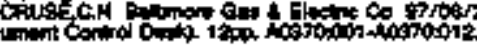

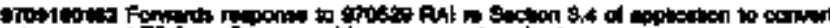

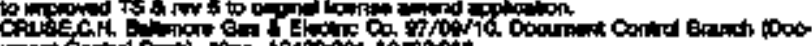

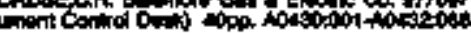

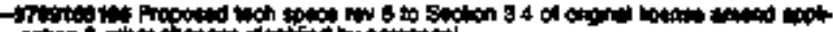

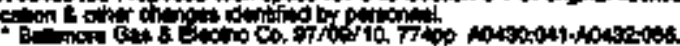

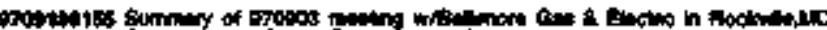

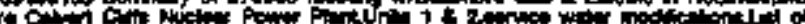
of

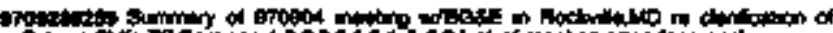

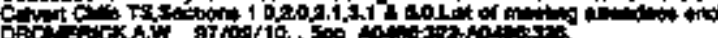

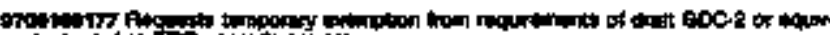

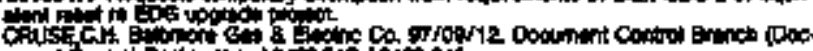

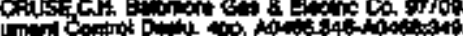

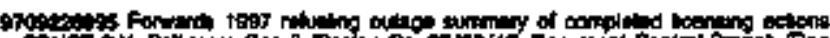

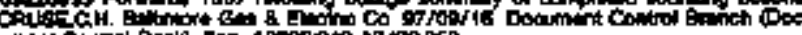

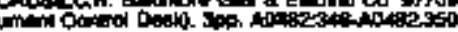




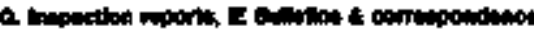

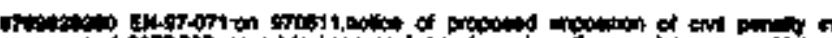

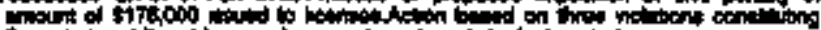

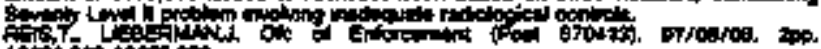

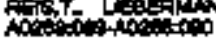

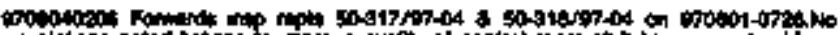

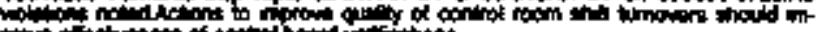

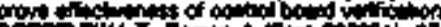

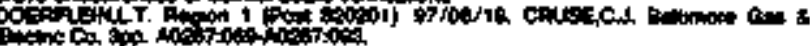

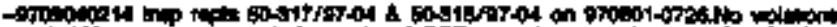

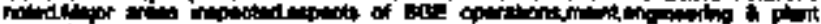
minger

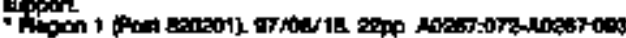

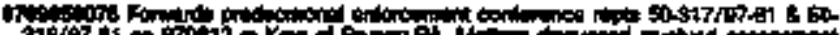

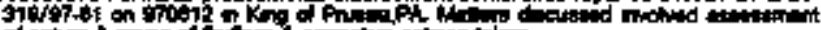

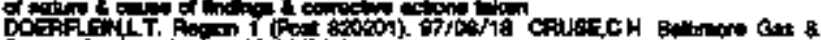

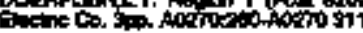

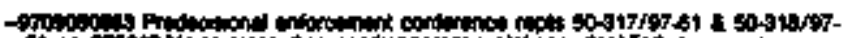

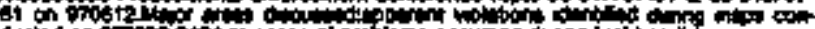

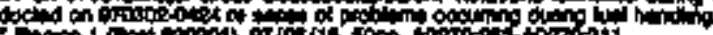

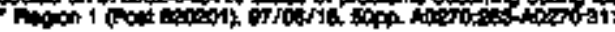

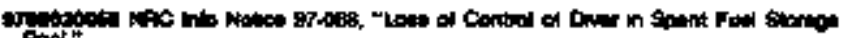

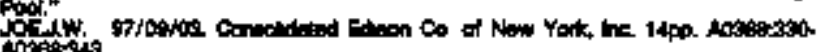

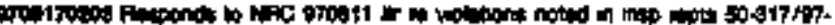

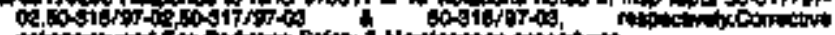

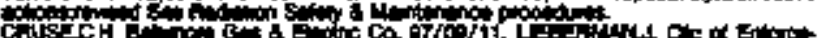

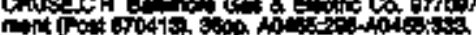

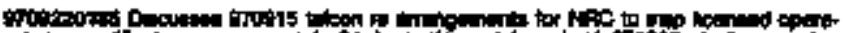

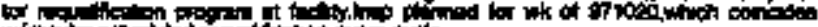

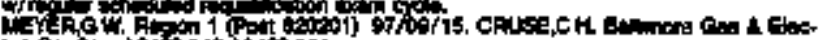

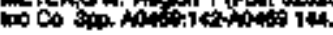

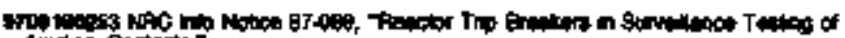

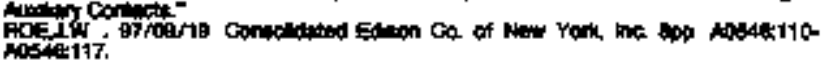

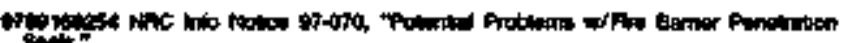

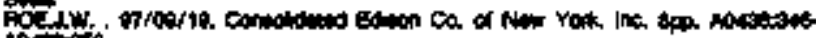

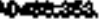

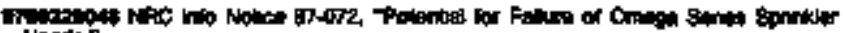

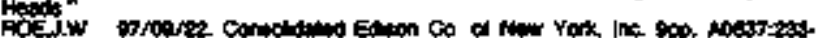

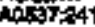

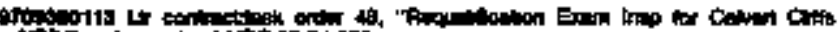

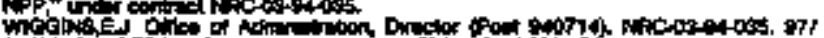

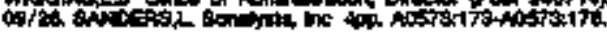

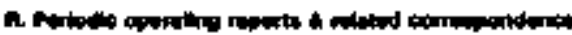

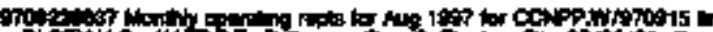
Oto $\cos 01.02$

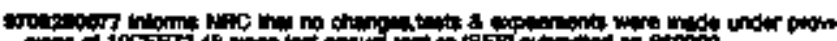

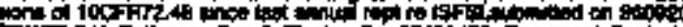

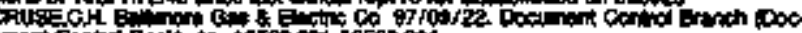

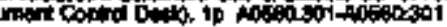

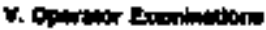

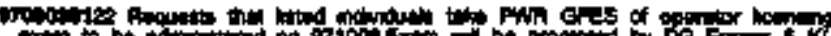

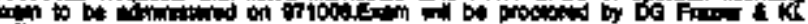

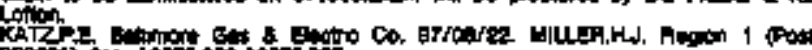
gacolit and

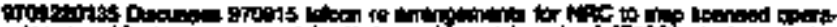

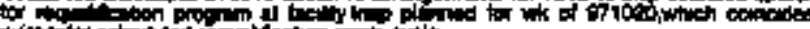

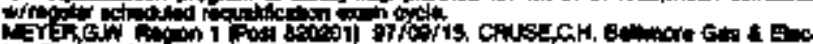

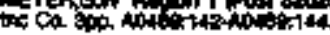

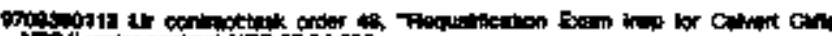

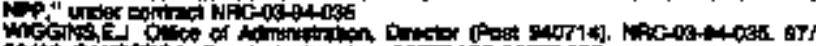

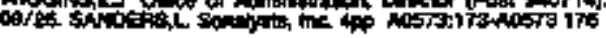

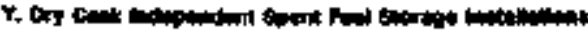

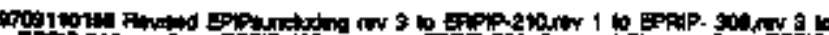

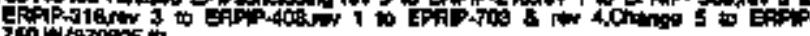

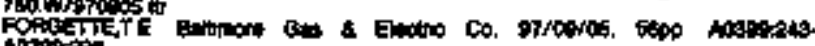
instats

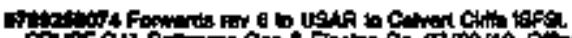

Citich of

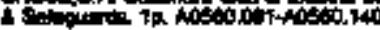

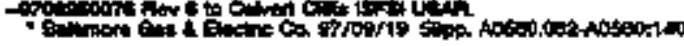

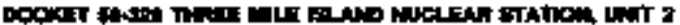

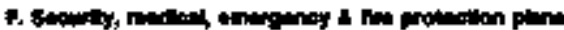

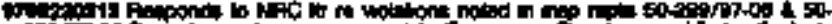

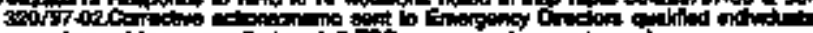

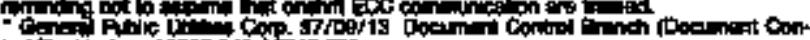
toil boik on

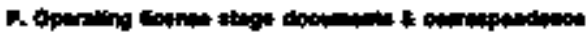

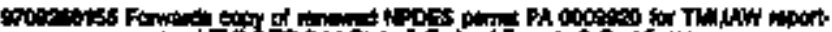

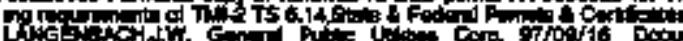

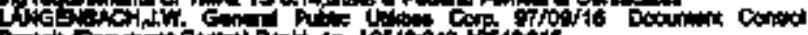

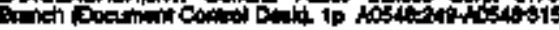

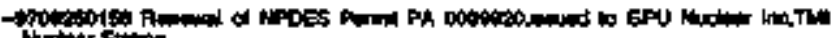

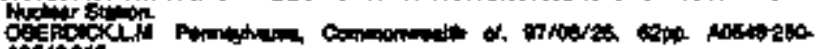
Anciotis.

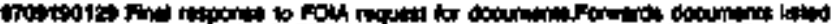

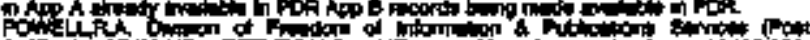

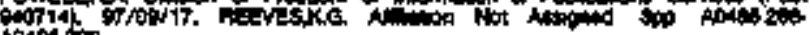

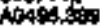

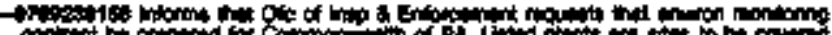

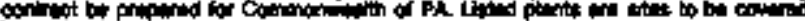

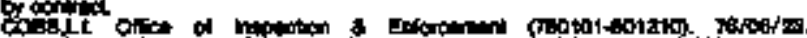

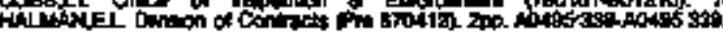

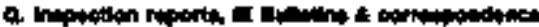

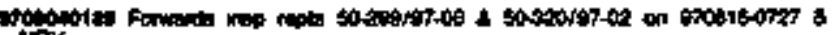

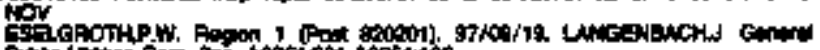

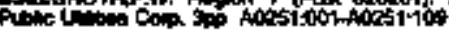

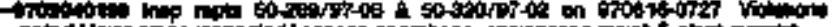

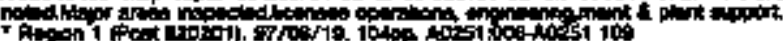

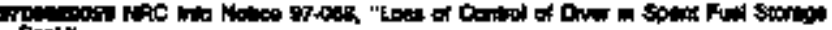

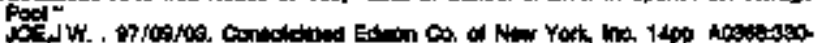
Aosidisis

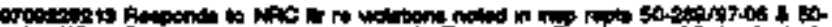

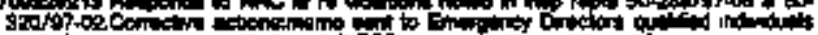

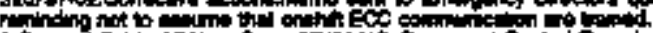

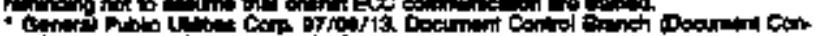
led boila

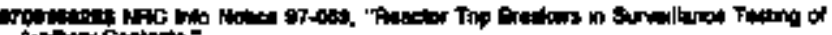

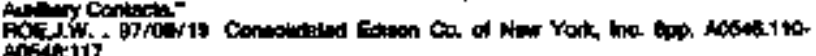

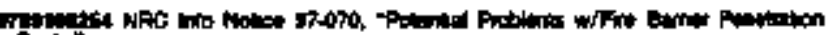

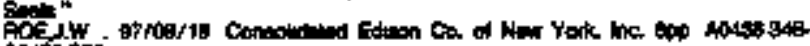
Aotsitise

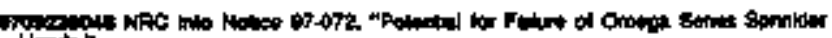

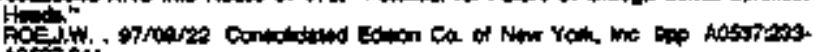
40.5\%24.

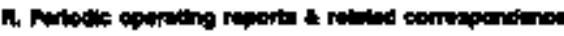

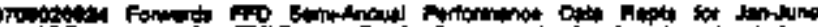

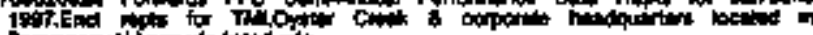

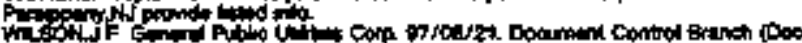

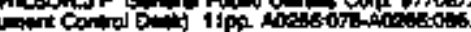

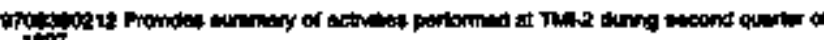

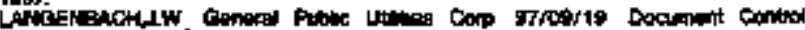

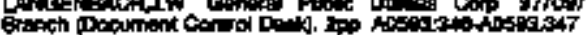

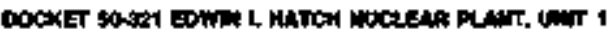




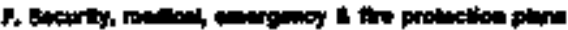

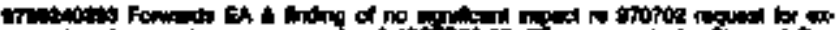

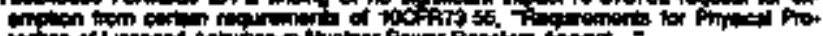

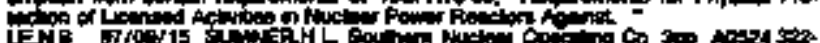
toing xis

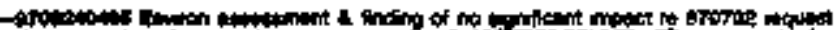

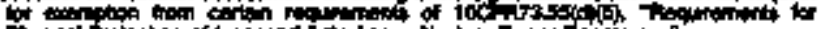
Pits

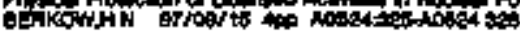

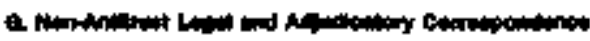

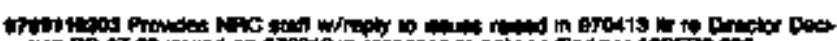

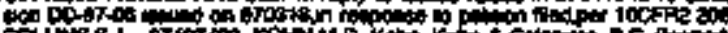

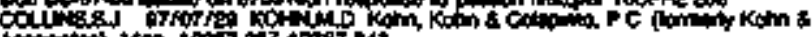

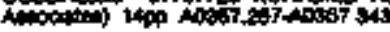

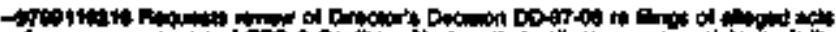

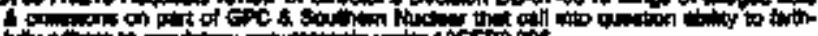

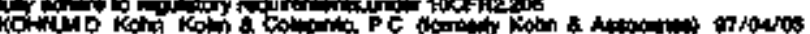

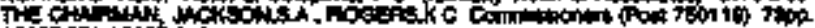

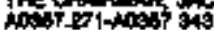

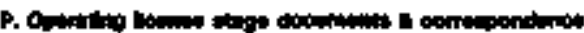

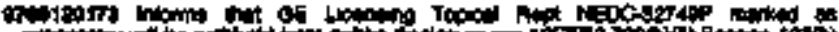

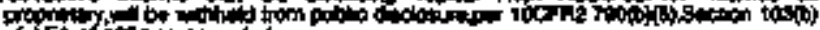

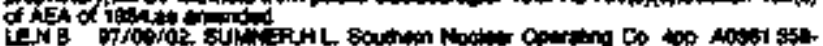
Alutiont

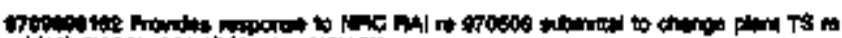

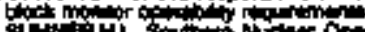

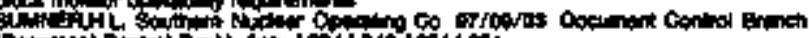

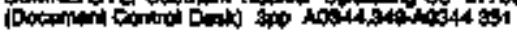

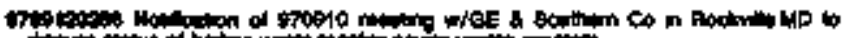

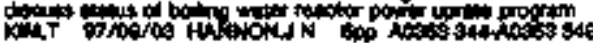

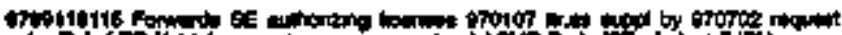

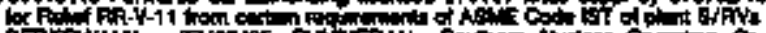
oration

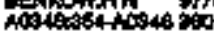

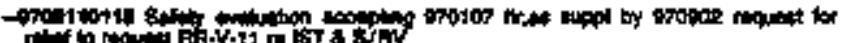

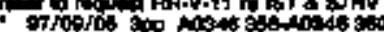

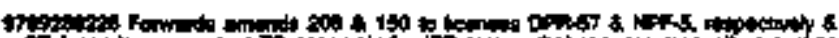

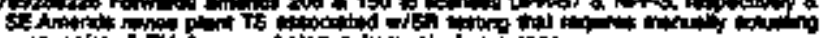

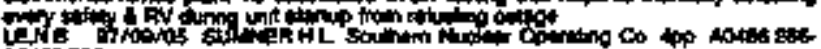
10060\%

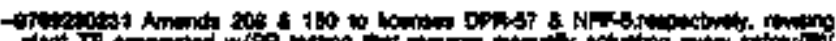

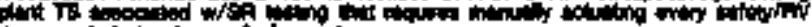

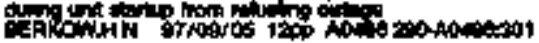

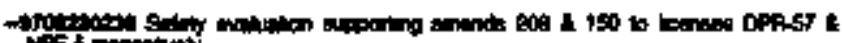

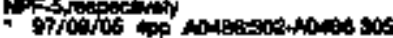

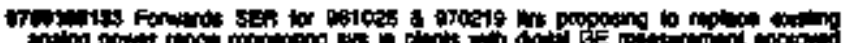

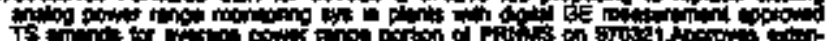

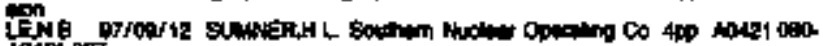
Aativi os

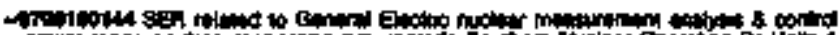

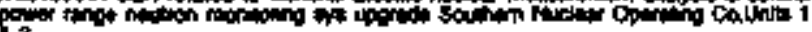

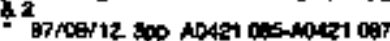

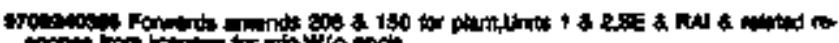

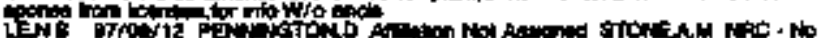

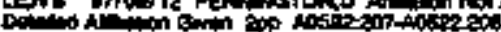

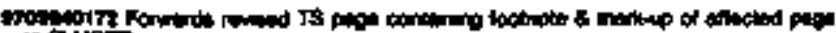

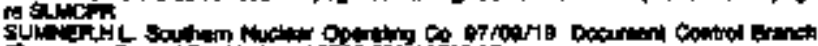

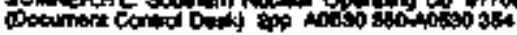

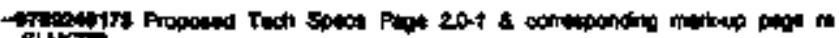
sulvin

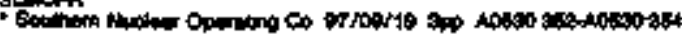

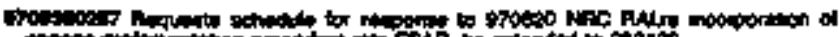

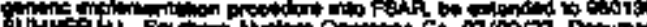

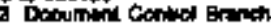

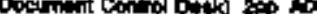

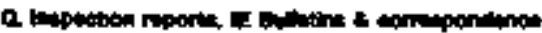

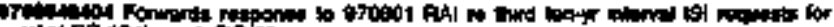

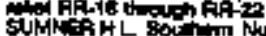

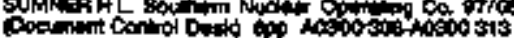

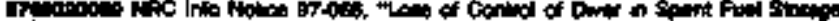

Aood

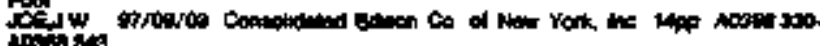

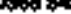

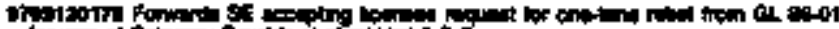

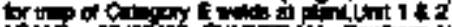

Uf A

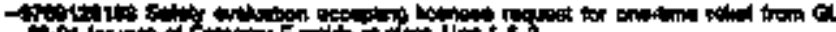

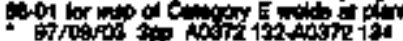

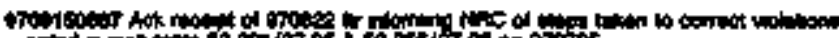

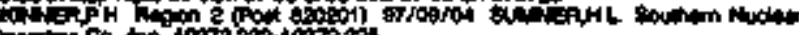

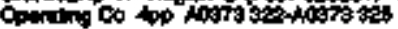

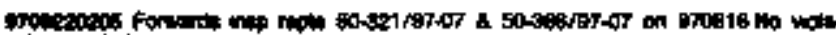

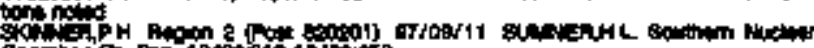

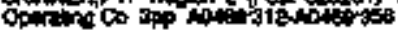

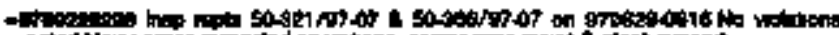

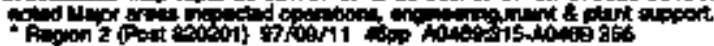

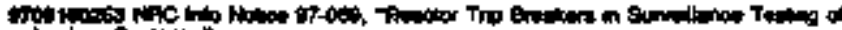

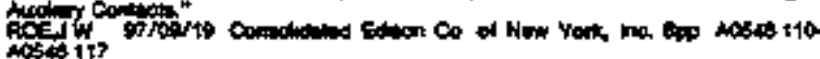

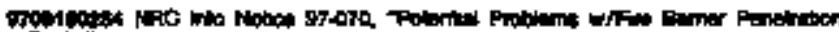

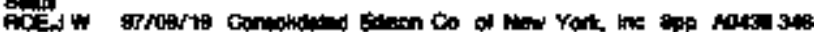
$\sin 305$

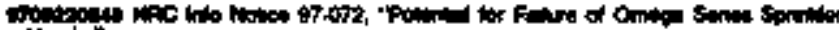

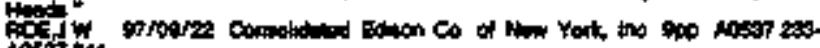
Nosist

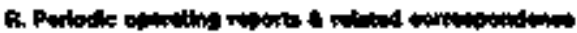

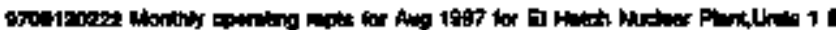

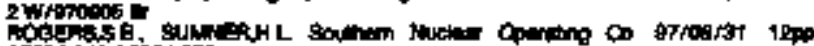

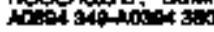

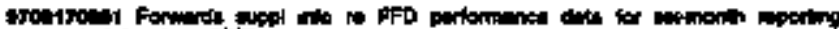

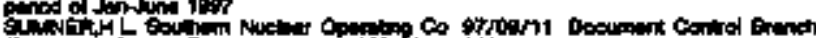

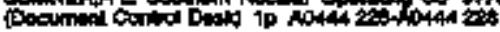

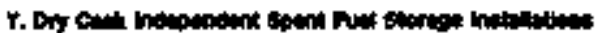

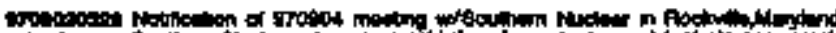

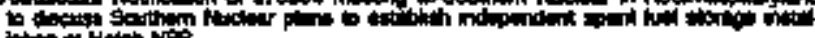

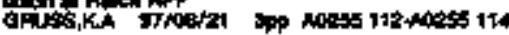

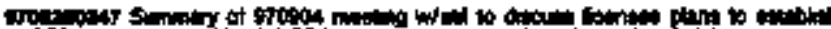

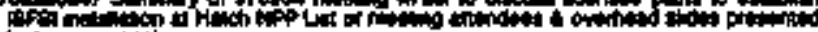

by

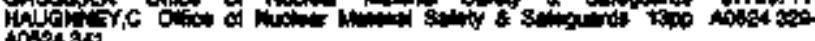
Nosit 34

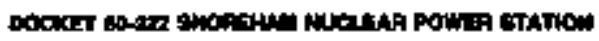

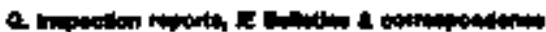

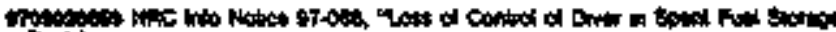

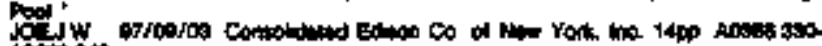
$\operatorname{Aos} \rightarrow 30$

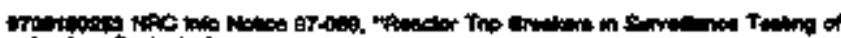

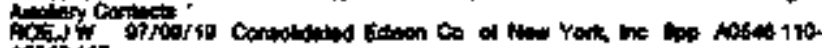
Aoft 117

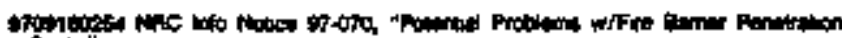

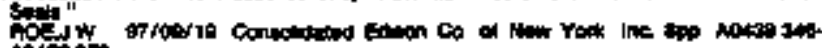
10453

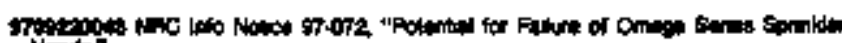

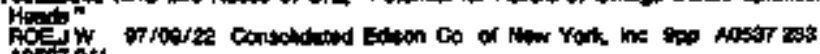
insis 24

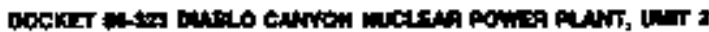




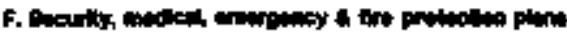

oH:

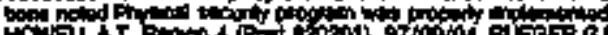

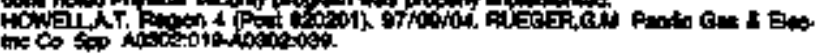

-

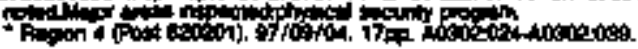

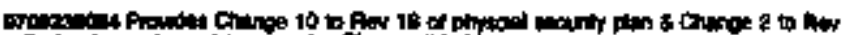

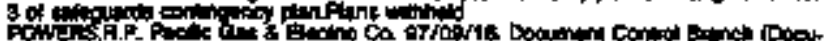

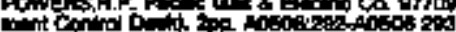

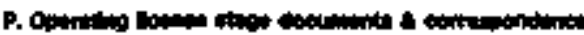

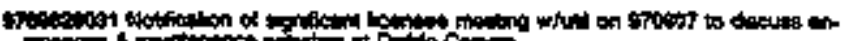

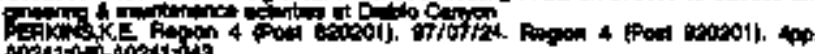

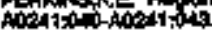

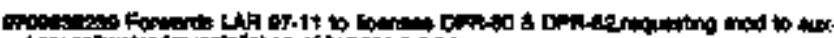
if is

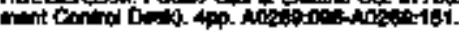

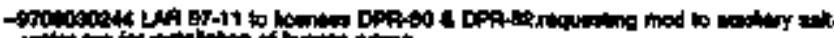
Futes

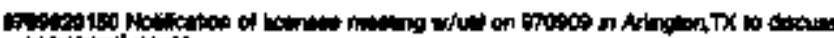

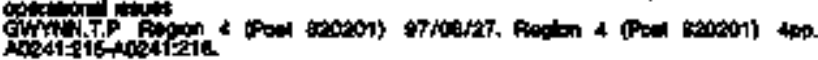

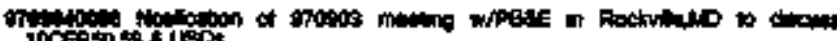

100\%

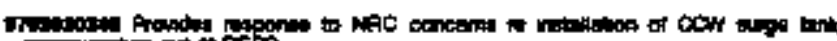

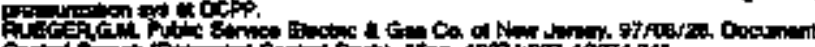

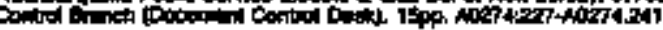

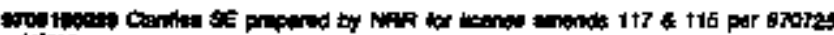
texp.

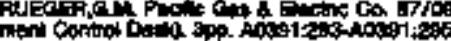

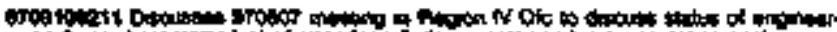
di

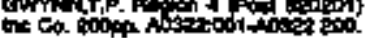

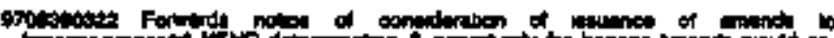

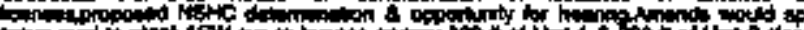

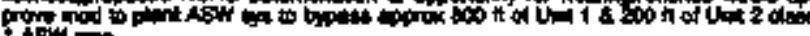

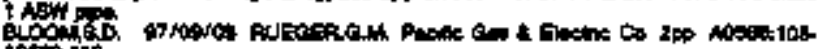
Astion

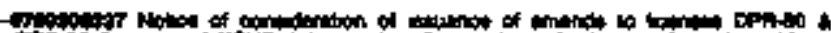

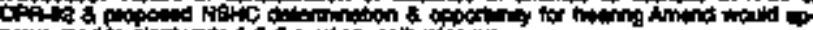

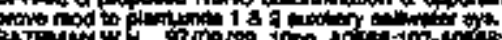

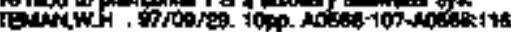

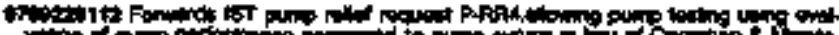

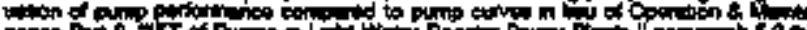

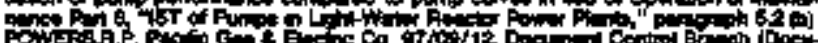

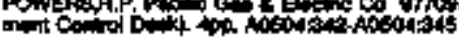

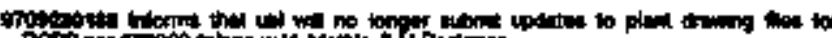

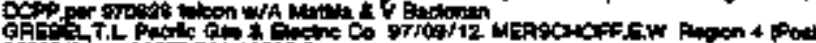

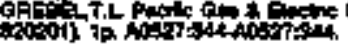

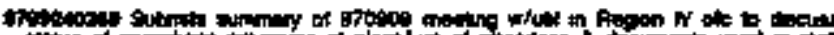

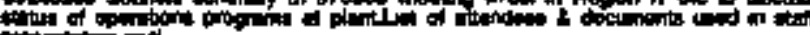

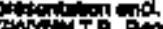

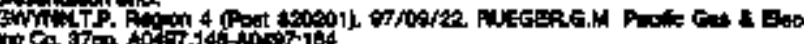

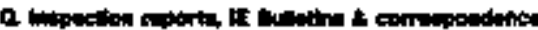

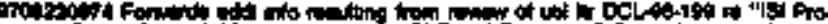

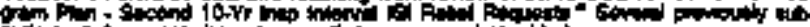

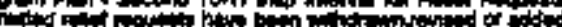

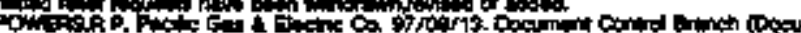

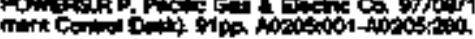

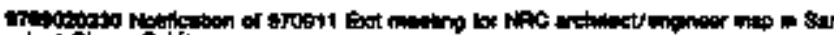

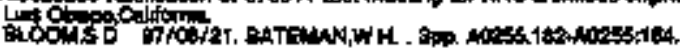

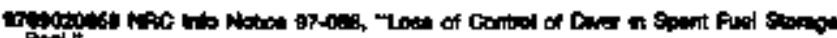

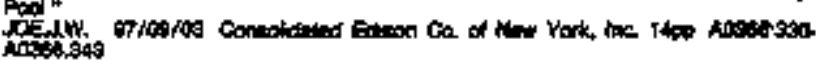

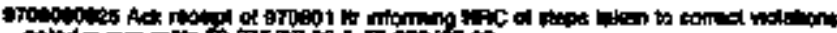

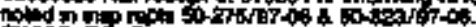

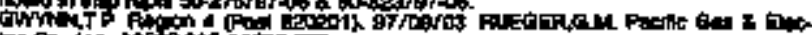

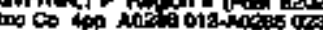

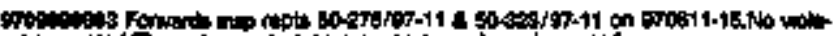

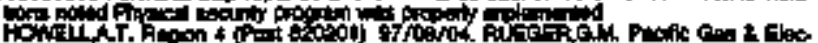
Fis

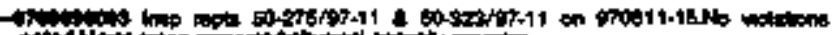

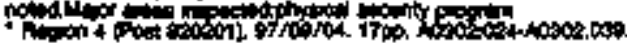

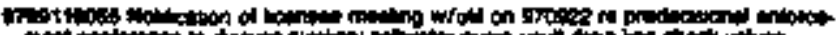

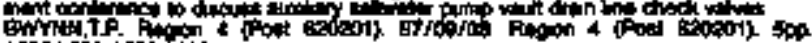

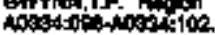

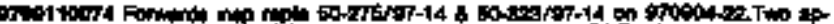

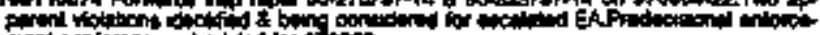

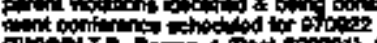

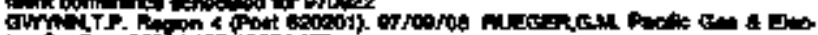

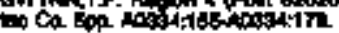

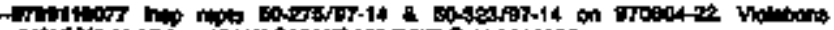

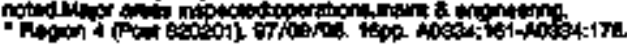

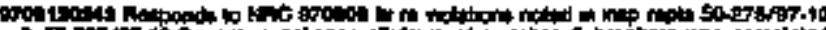

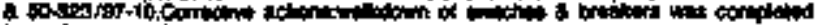

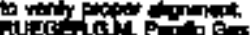

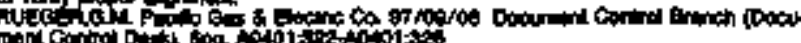

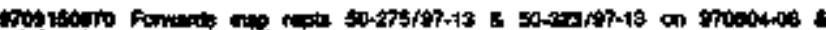

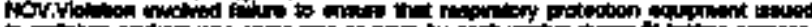

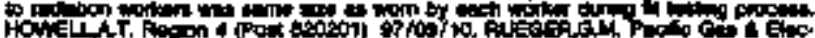

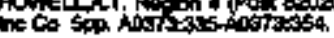

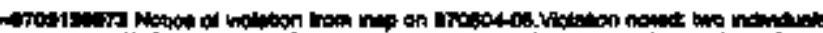

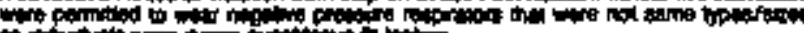

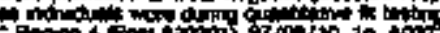

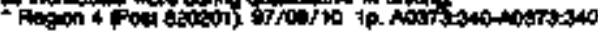

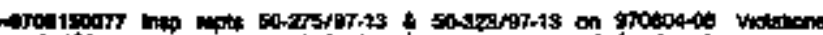

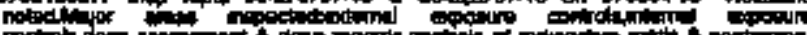

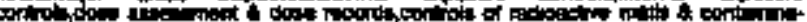
in

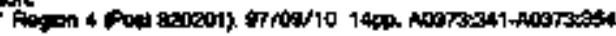

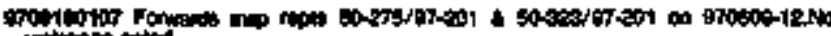

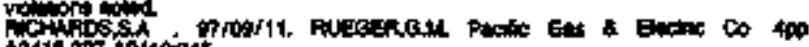

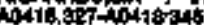

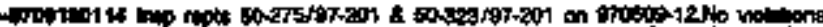

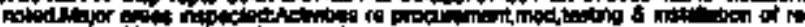

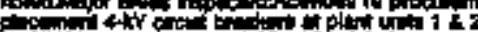

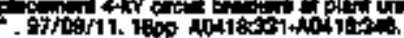

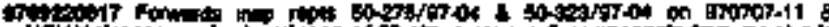

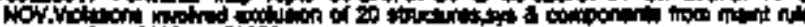

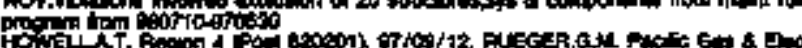
tr

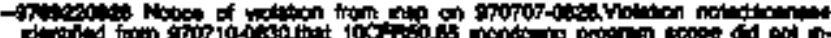

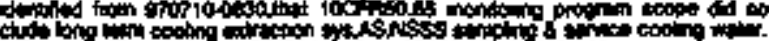

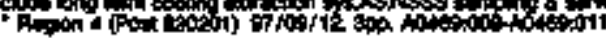

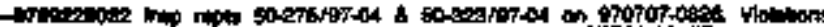

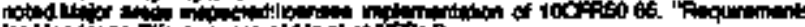

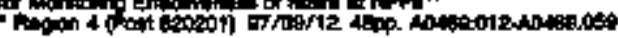

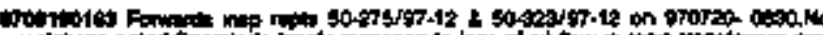

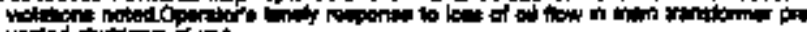

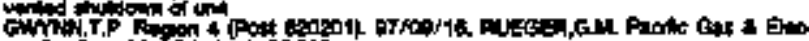

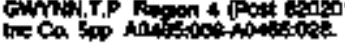

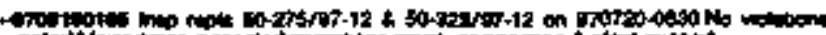

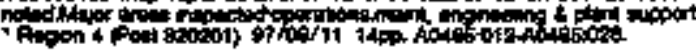

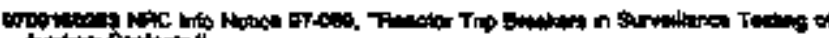

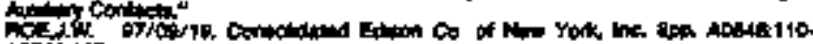

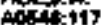

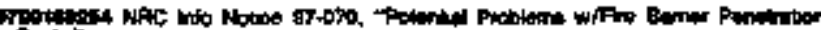

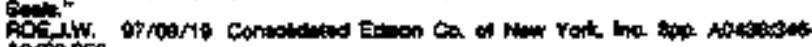
Anosios

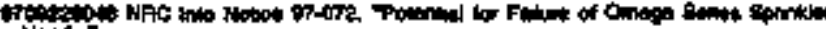

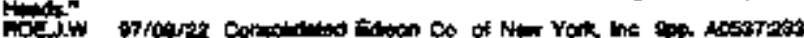
A0.

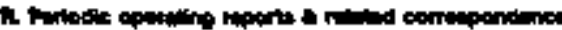

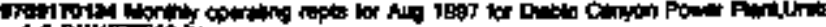

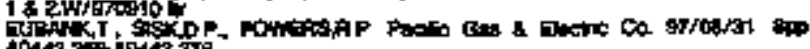




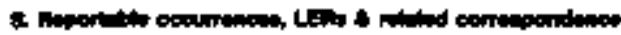

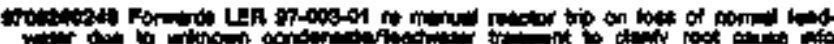

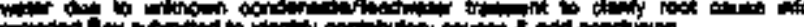
Fow

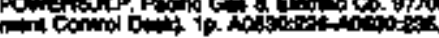

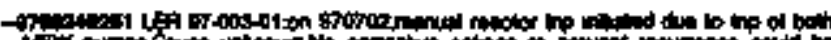

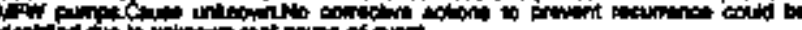
(a)

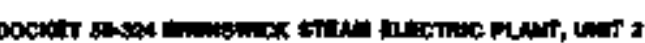

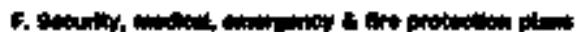

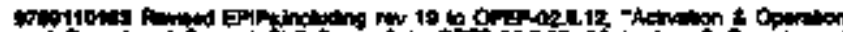

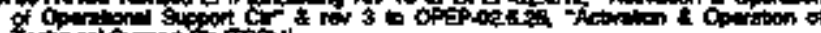

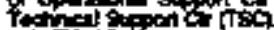

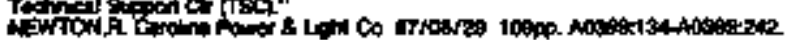

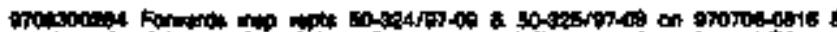

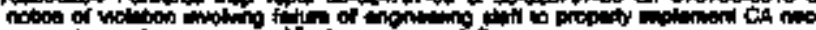

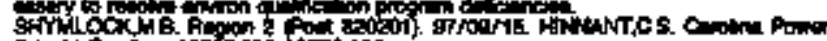

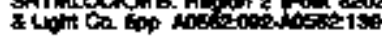

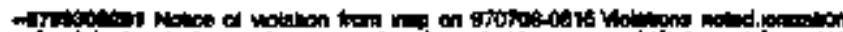

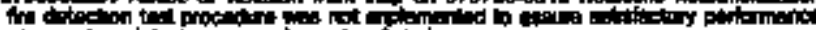

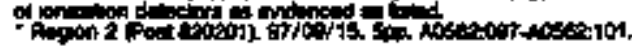

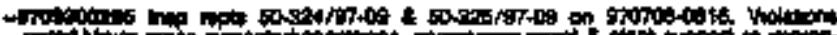

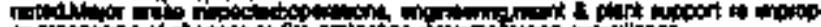

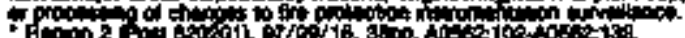

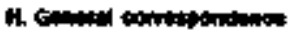

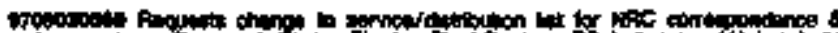

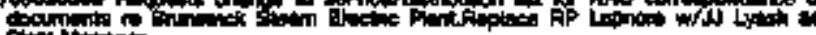

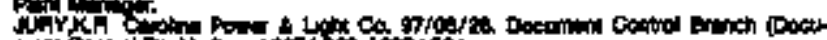

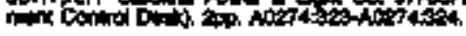

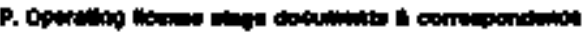

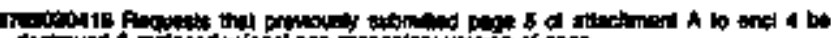

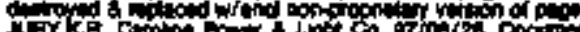

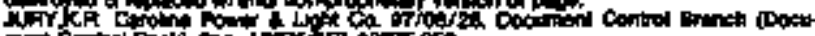

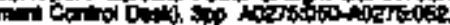

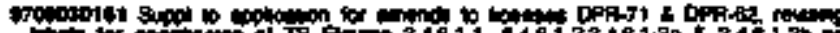

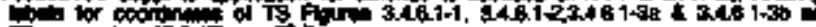

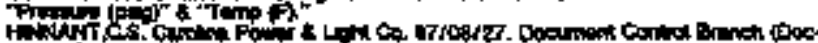

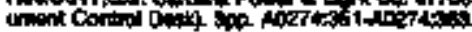

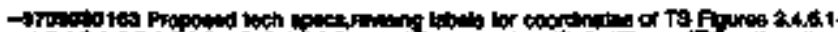

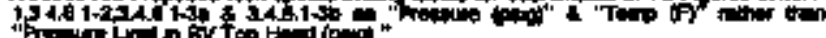

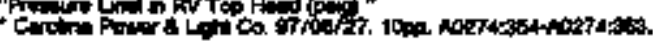

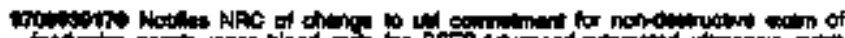

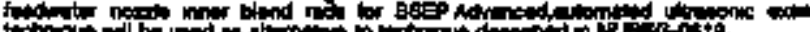

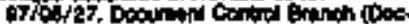

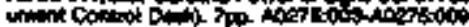

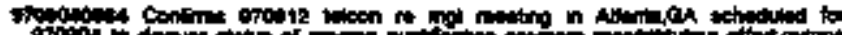

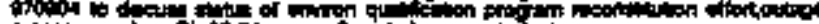

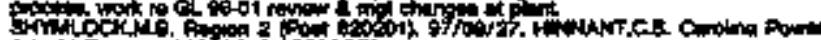

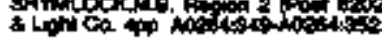

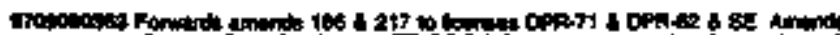

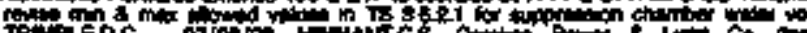

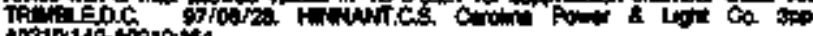

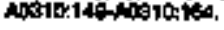

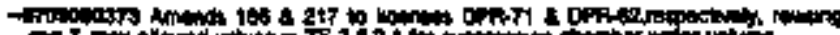
ED

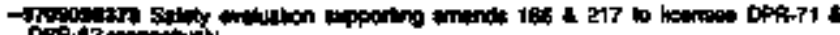

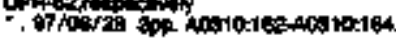

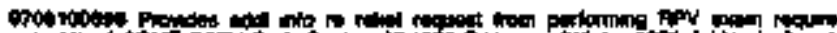

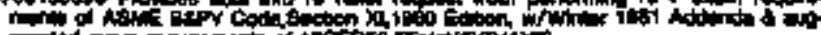

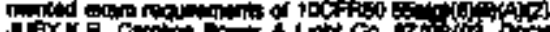

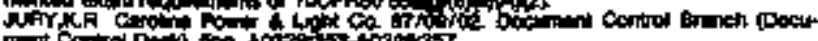

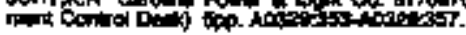

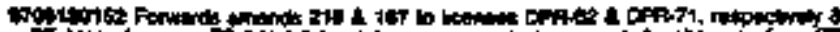

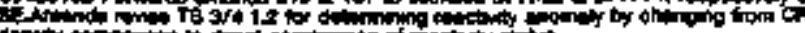

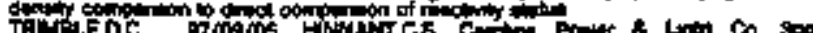

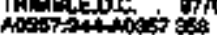

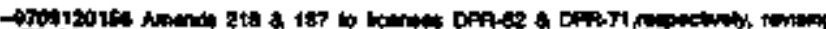

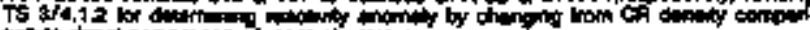

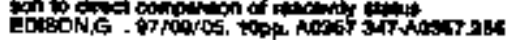

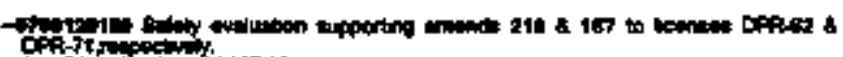

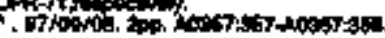

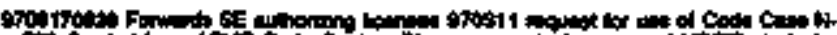

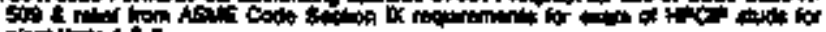

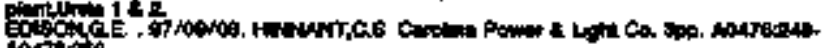
Anters

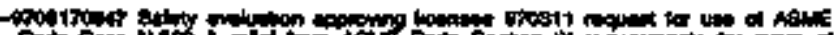

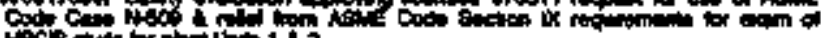

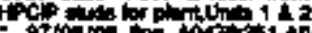

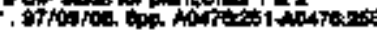

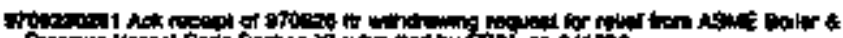

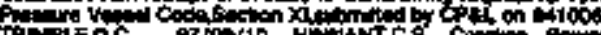

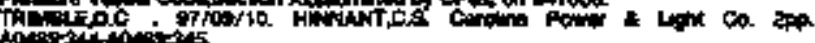

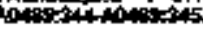

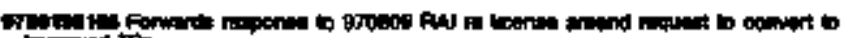

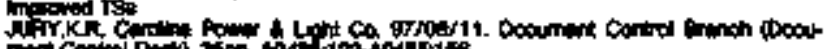

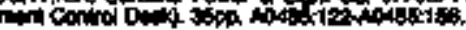

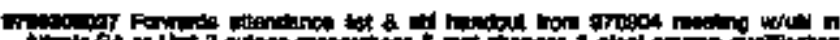

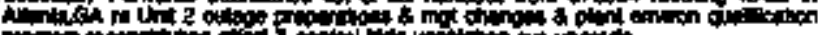

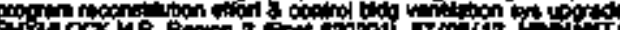

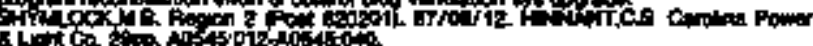

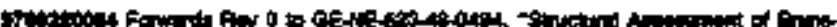

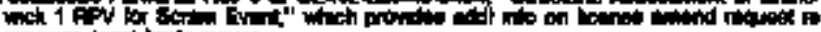

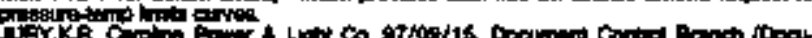

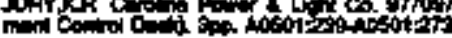

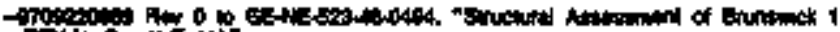

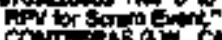

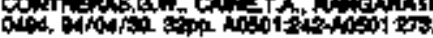

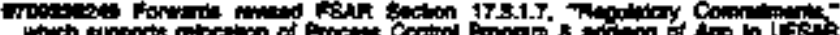

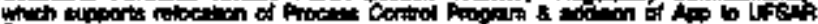

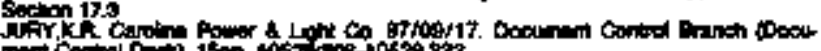

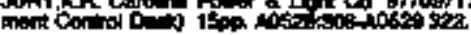

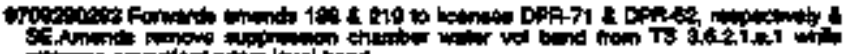

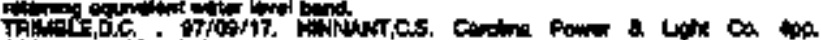

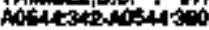

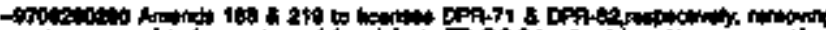

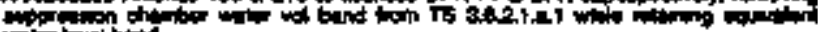

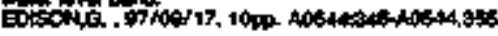

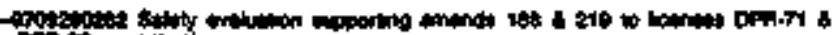

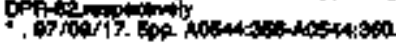

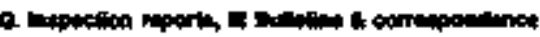

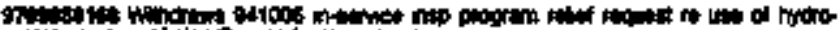
THY

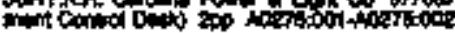

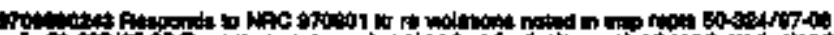

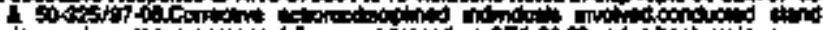

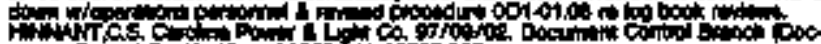

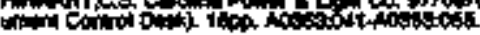

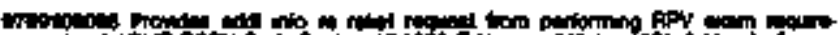

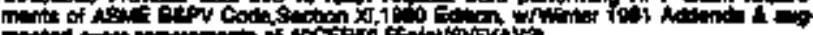

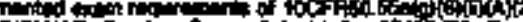

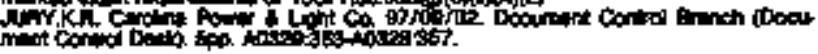

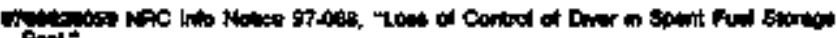

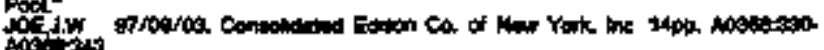

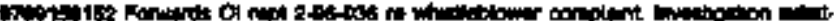

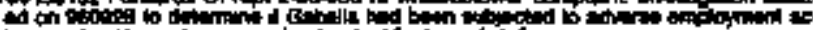

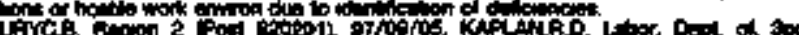

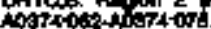

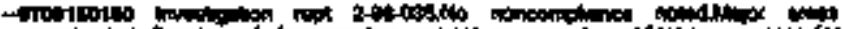

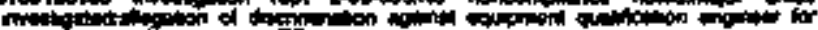

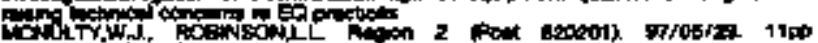

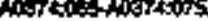

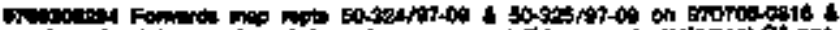

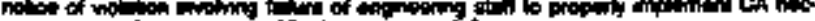

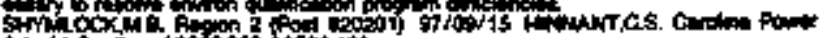

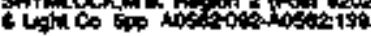




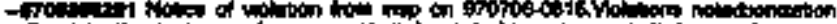

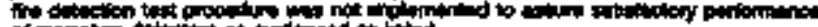

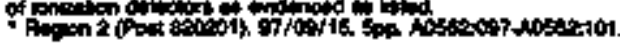

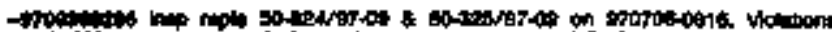

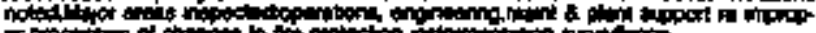

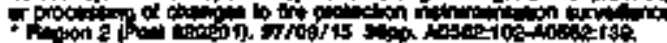

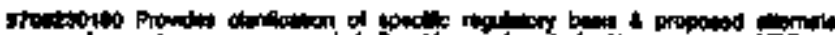

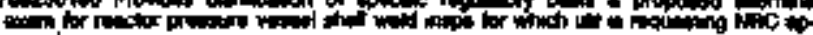

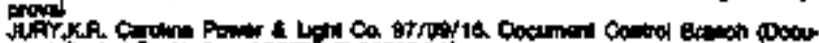

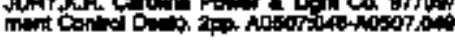

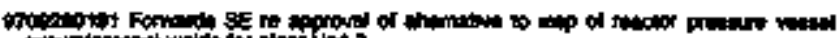

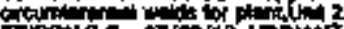

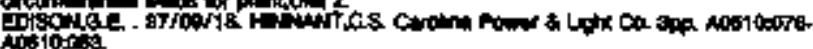
Atosion:

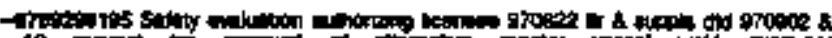

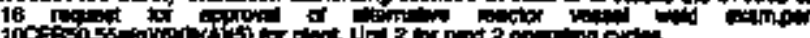

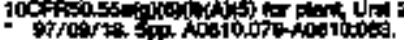

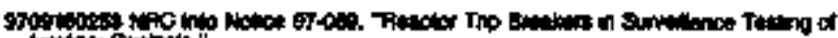

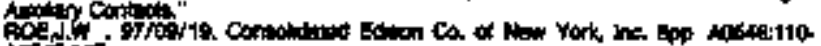
Potitis.

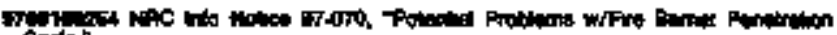
ofent

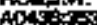

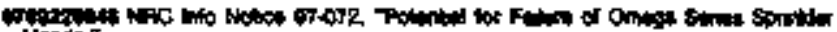

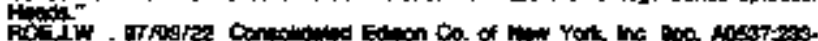
A05\%?:11

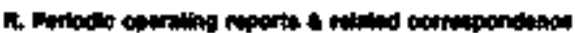

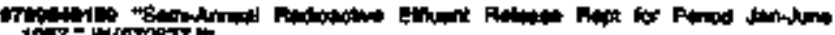

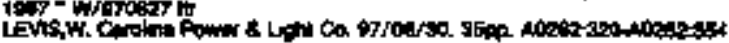

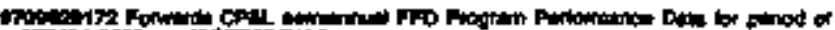

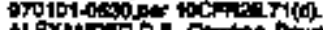

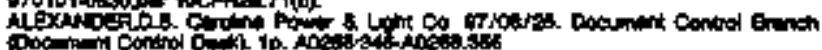

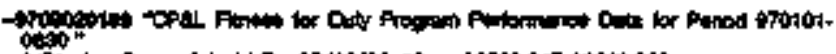

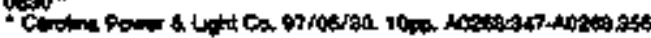

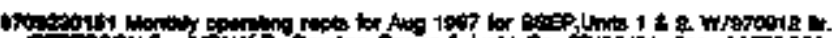

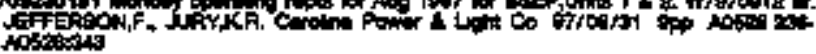

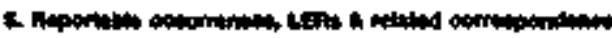

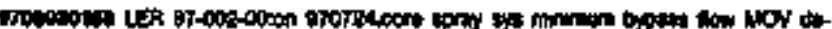

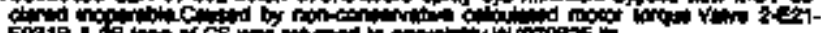

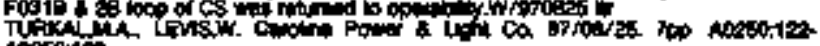
ifocitat

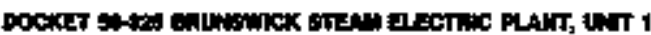

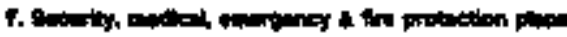

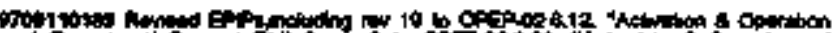

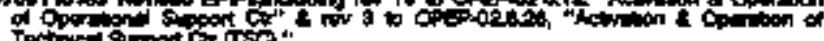

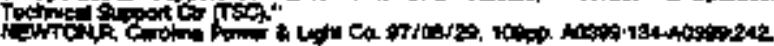

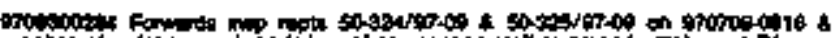

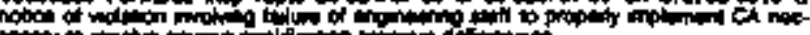

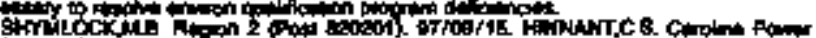

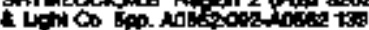

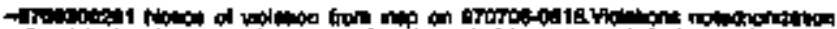

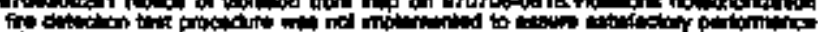

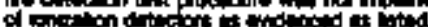

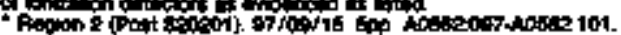

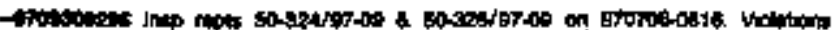

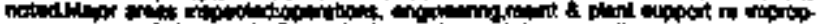

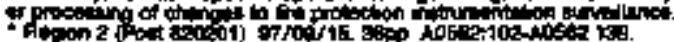

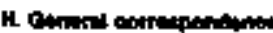

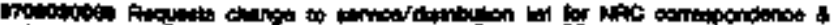

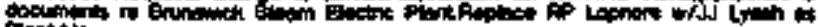
Plant thing.

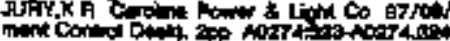

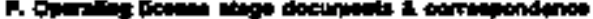

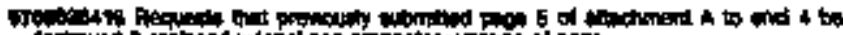

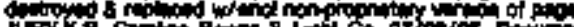

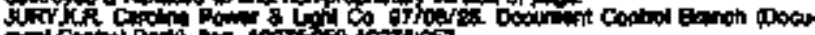

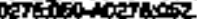

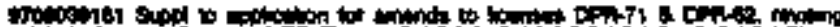

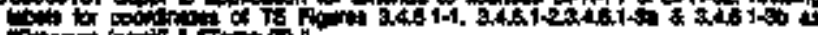
Pint

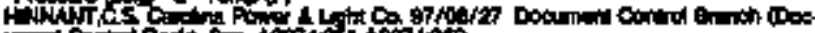

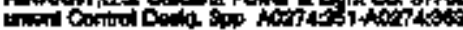

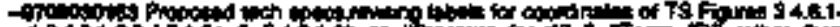

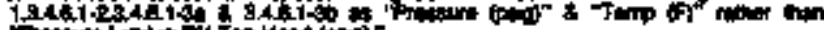

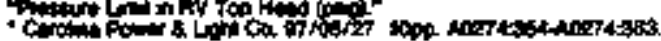

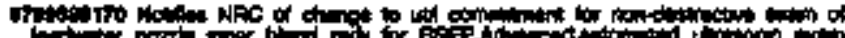

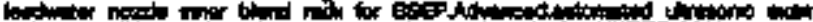

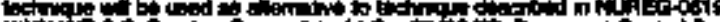
Hlintit C. 1.

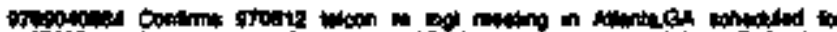

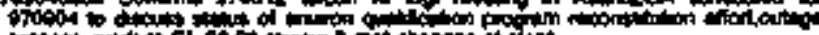

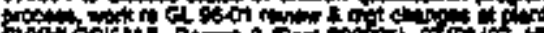

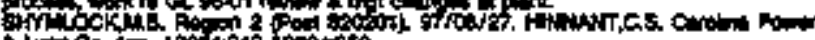

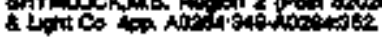

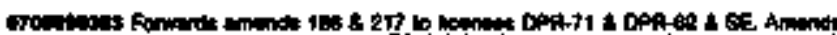

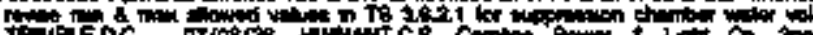

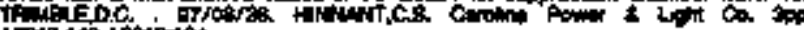
A

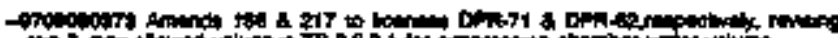

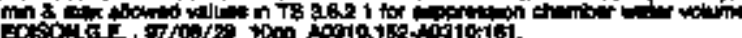

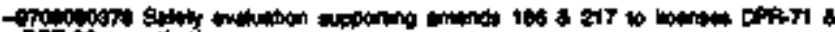

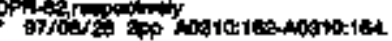

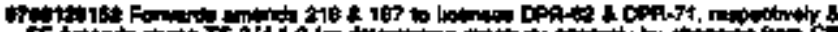

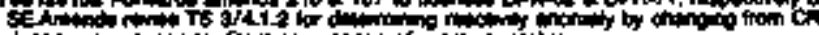

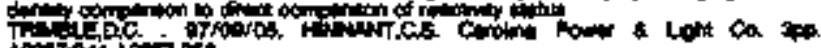

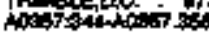

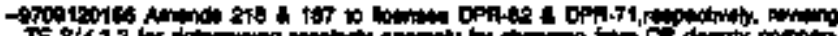

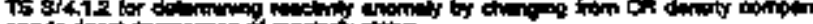

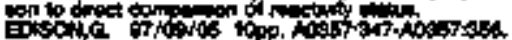

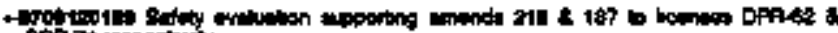

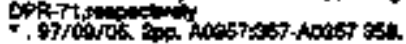

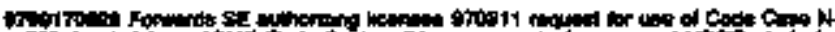

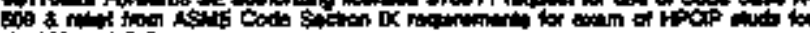

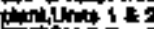

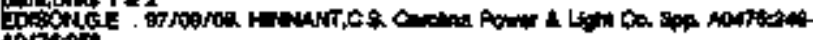
Aotroish

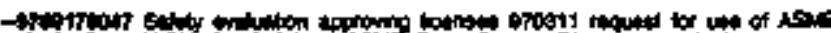

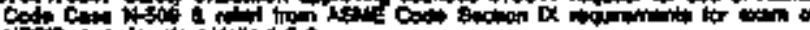

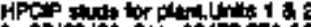

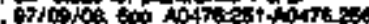

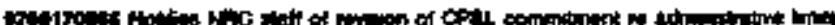

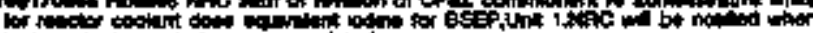

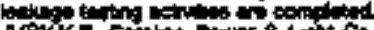

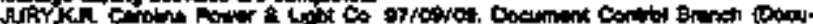

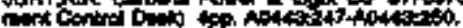

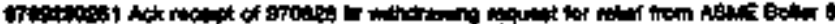

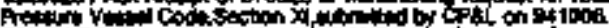

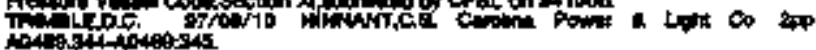

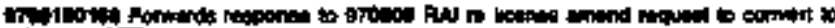

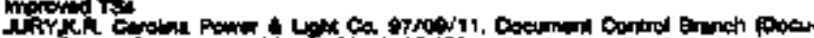

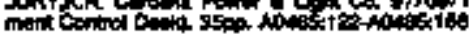

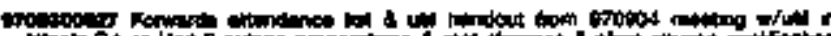

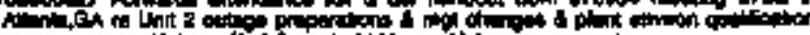

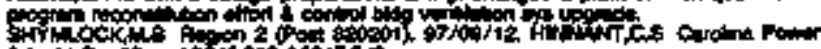

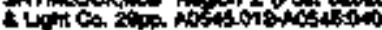

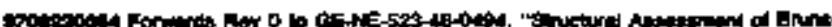

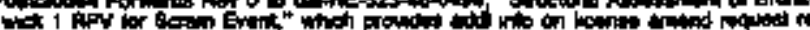

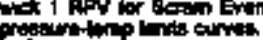

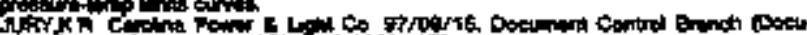

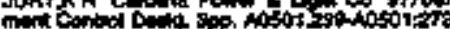

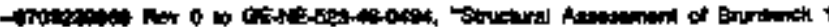
Apr tion so

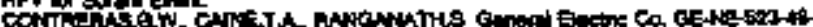

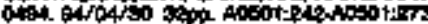

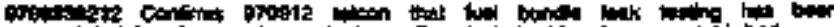
Difly

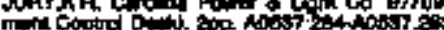




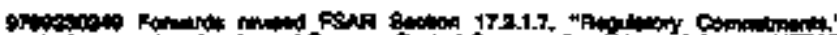

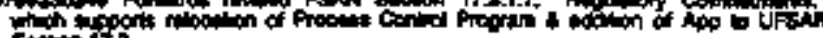
sting if

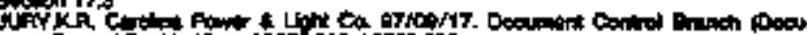

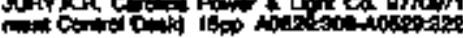
Pr

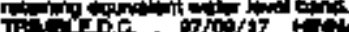

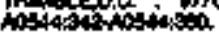

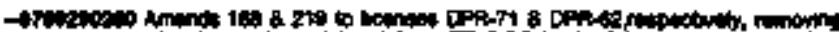

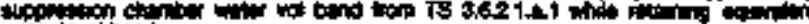

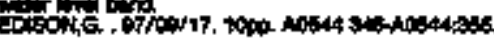

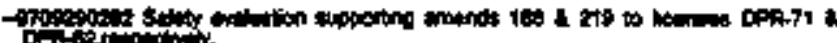

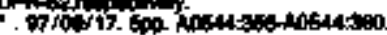

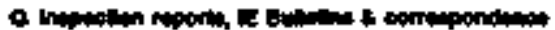

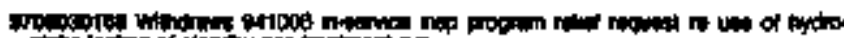

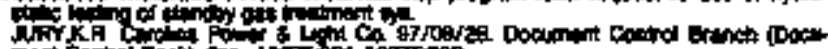

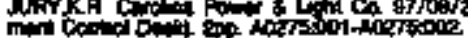

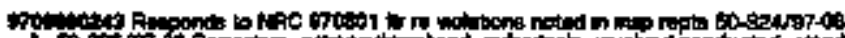

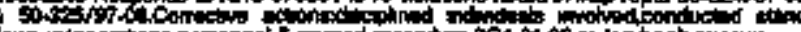

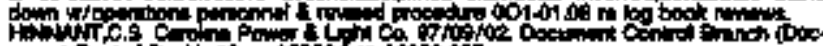

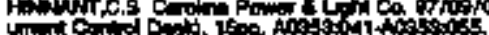

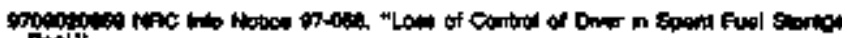
Pot

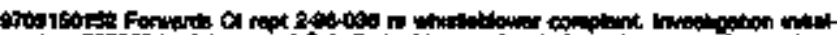

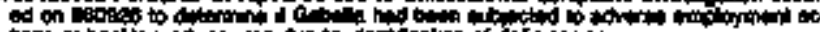

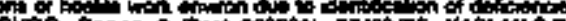

Latoot, Dent at, sip

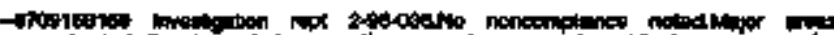

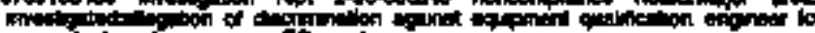

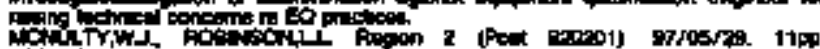

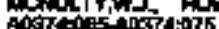

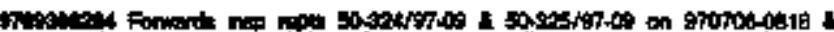

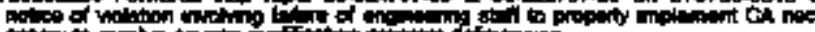

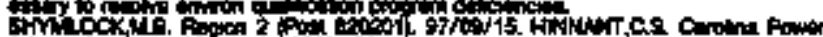
in

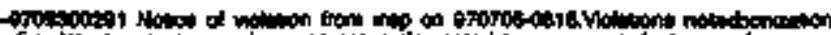

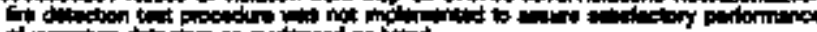

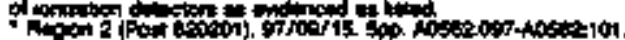

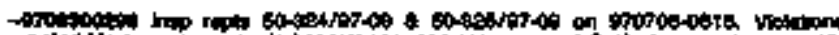

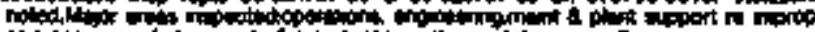

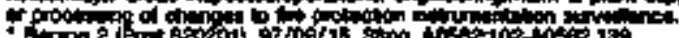

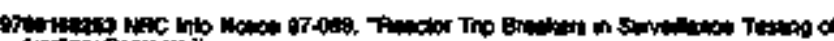

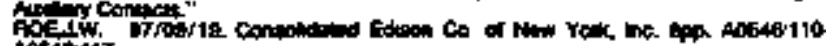
notorit 117

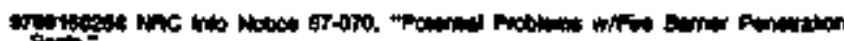

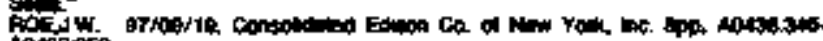
1003850

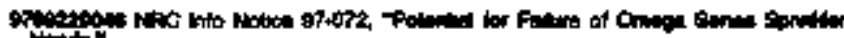

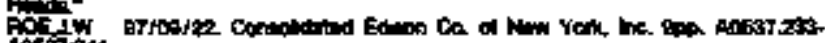
Antiont

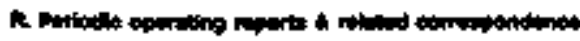

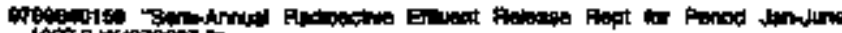

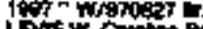

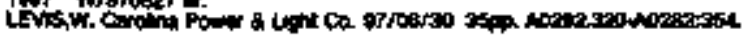

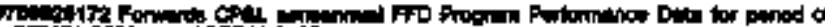

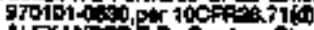

ALDGNGE

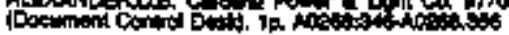

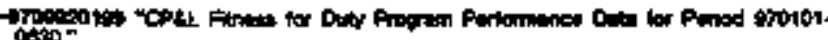

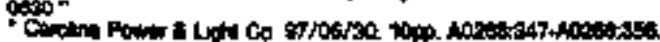

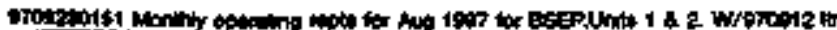
J

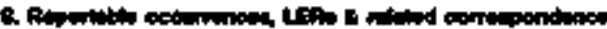

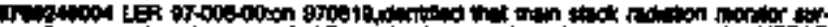

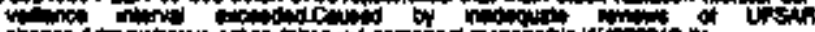

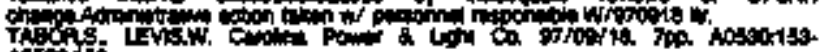
intos:

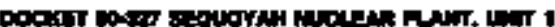

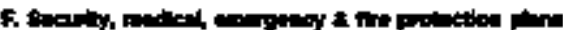

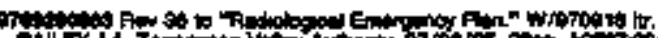

EN

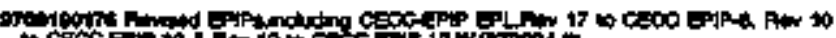

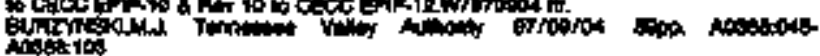

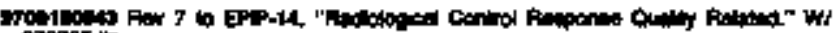
grogot to.

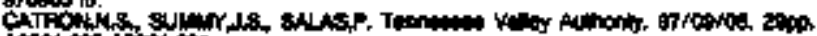

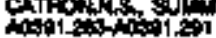

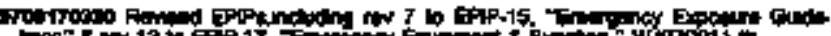

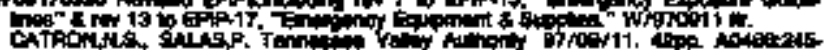
nowsint.

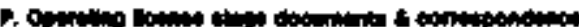

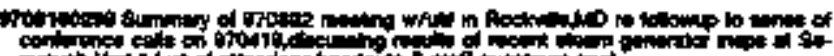

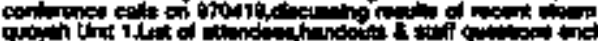
Q

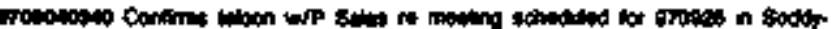

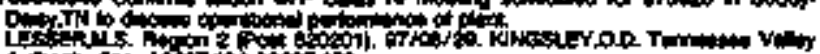
intion

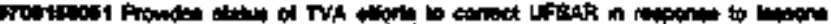

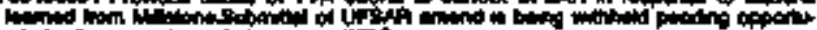

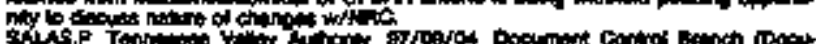

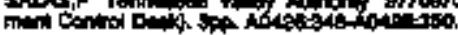

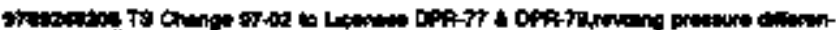

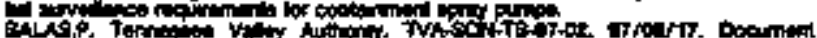

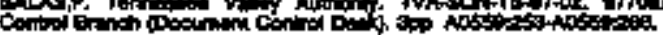

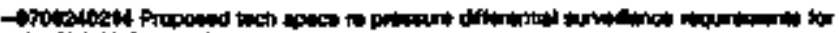

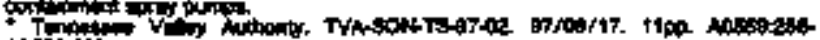
notion.

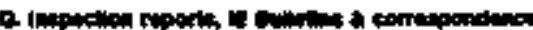

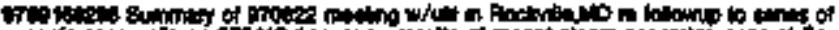

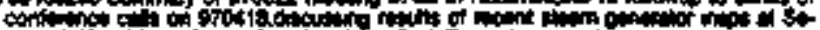

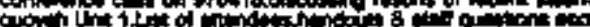

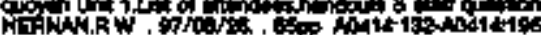

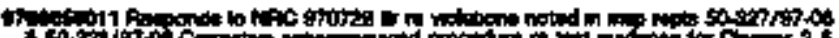
1 Sot

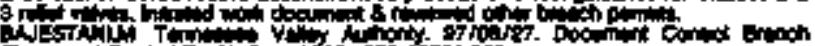

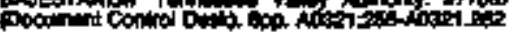

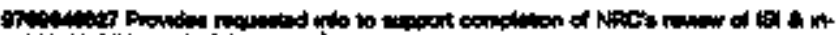

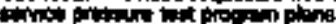

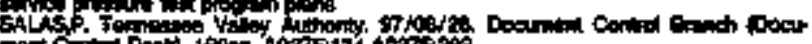

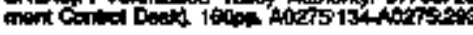

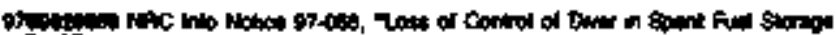
WE 10060 348

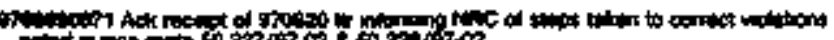
Hid n

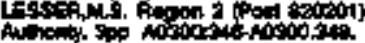

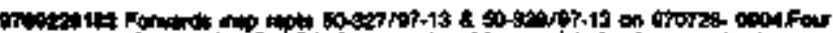

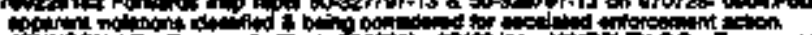

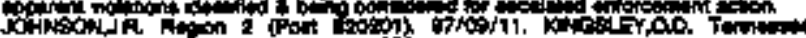

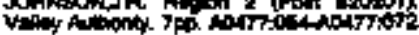

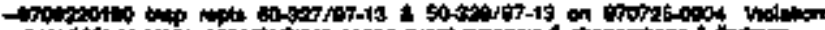

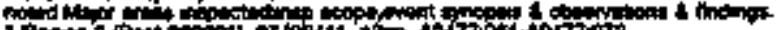

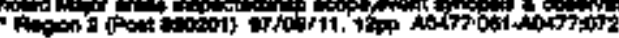

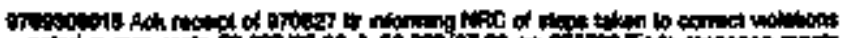

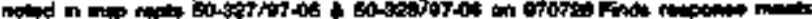

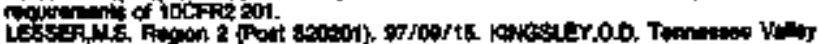

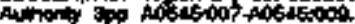




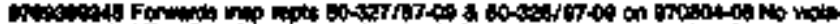

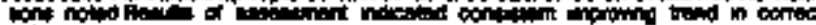
iton

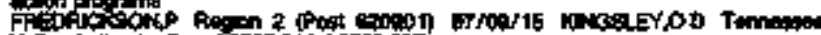

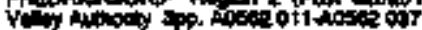

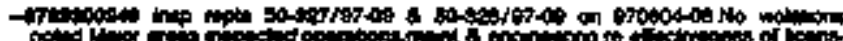

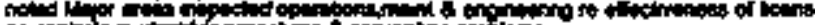

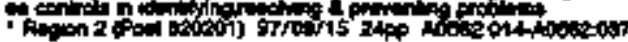

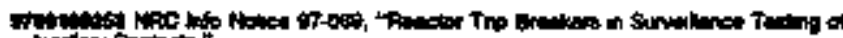

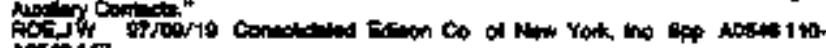
Ancis 1 t

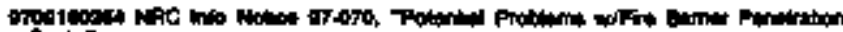

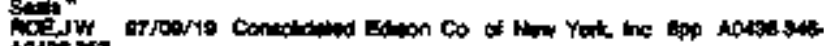
nations

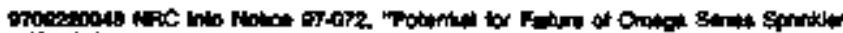

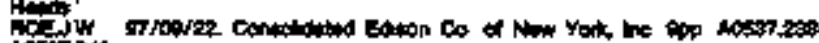
An-

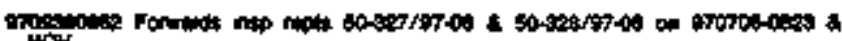

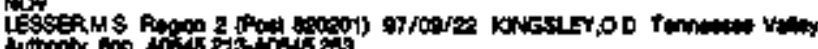

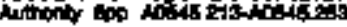

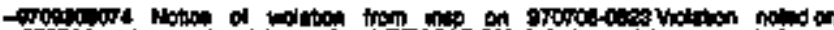

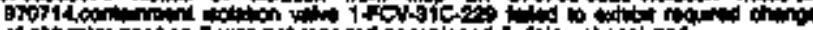

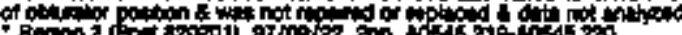

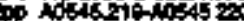

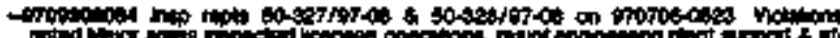

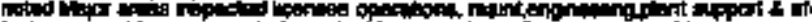

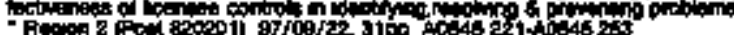

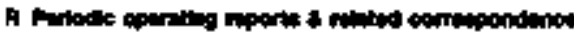

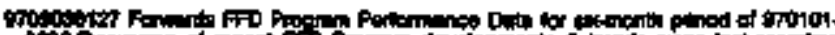

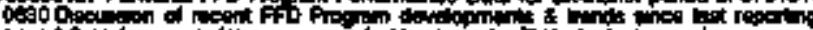

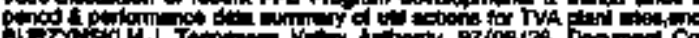

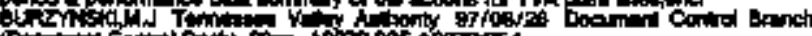

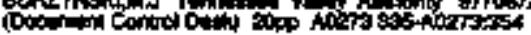

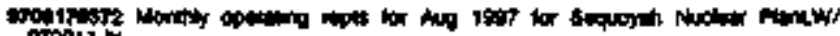
Tropis b.

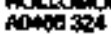

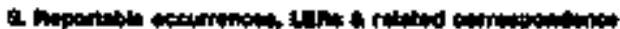

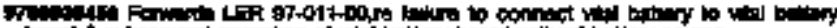
bo

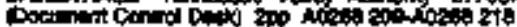

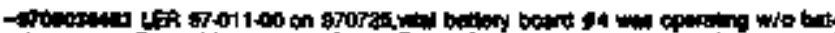

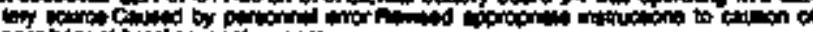

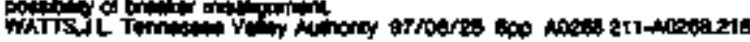

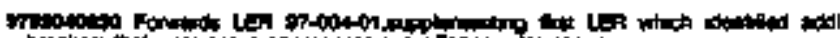

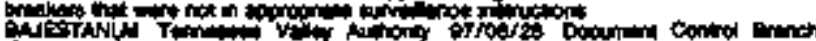

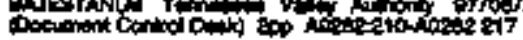

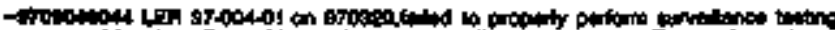

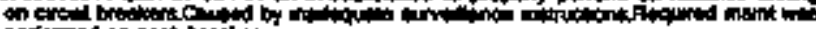

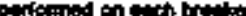

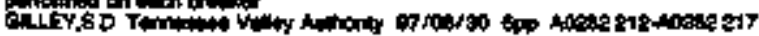

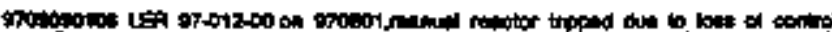

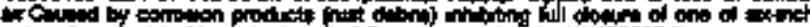

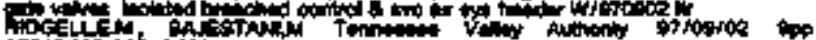

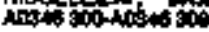

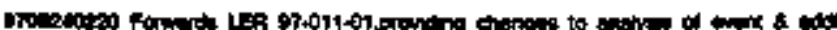

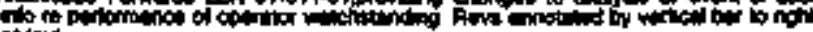

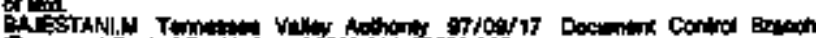

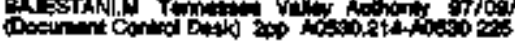

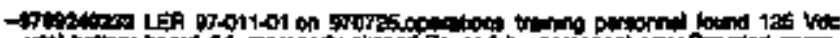

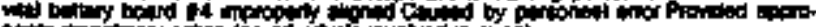

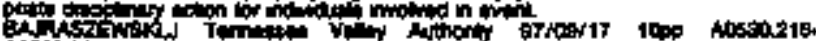
atsots

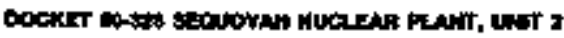

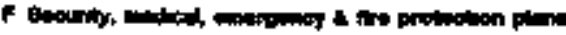

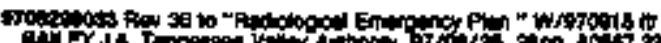

(1)

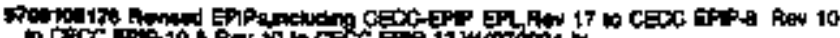

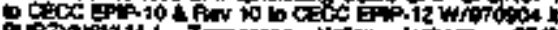

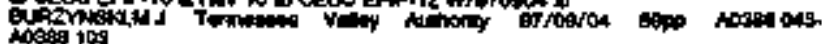

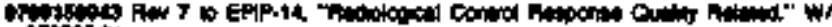
oropis tis

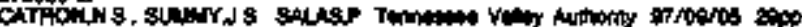

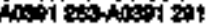

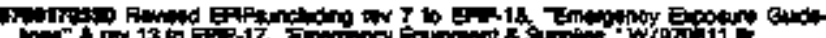

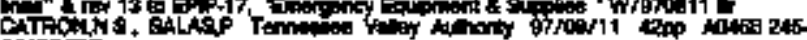
andigy

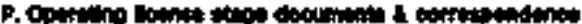

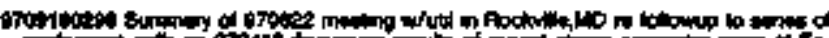

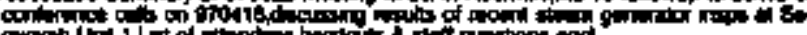

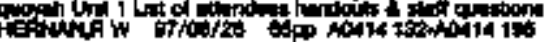

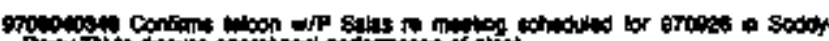

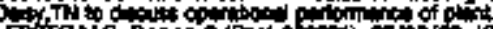

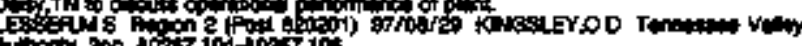

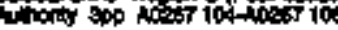

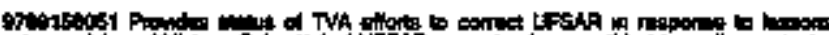

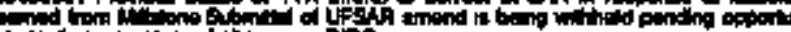

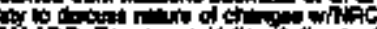

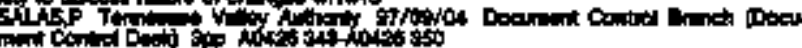

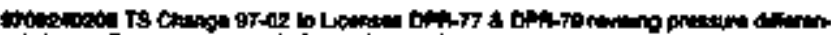

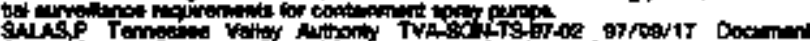

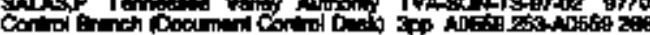

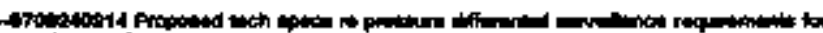

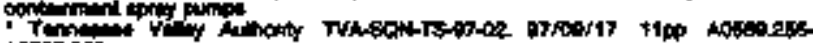
actionst

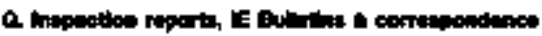

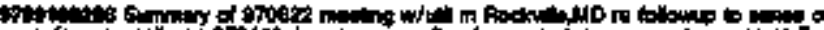

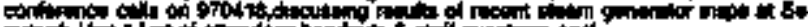

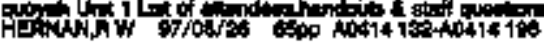

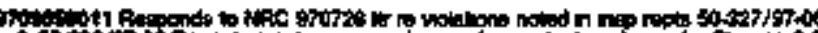

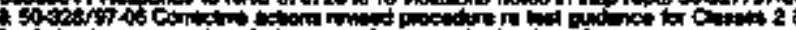

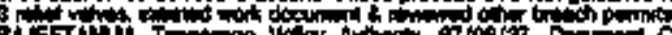

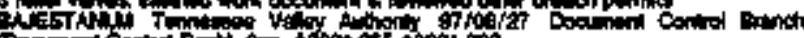

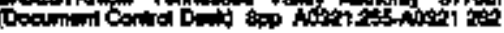

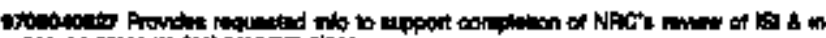

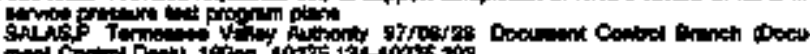

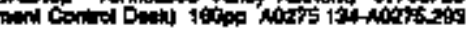

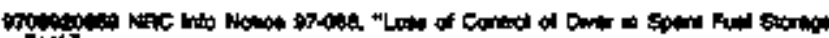
Foitix

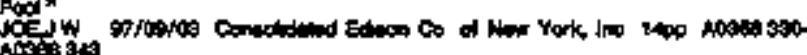
10030

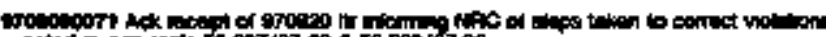

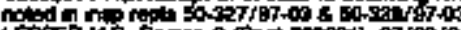

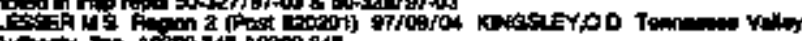

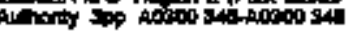

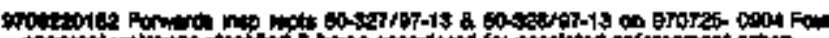

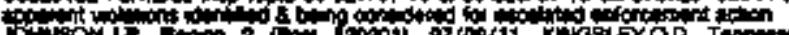

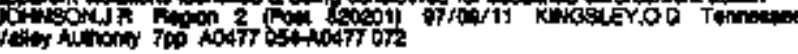

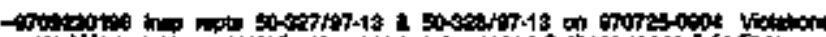

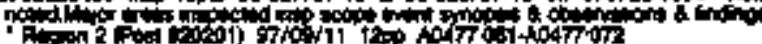

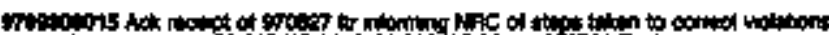
poted an nap li

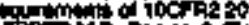

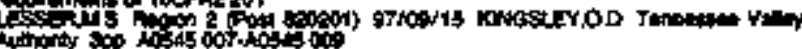

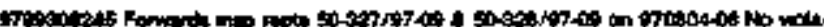

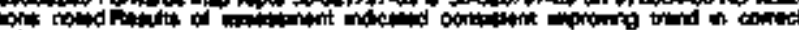

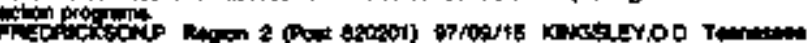

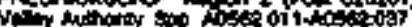

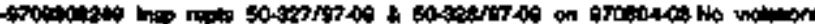

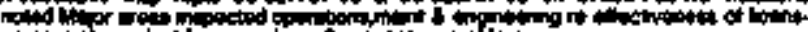

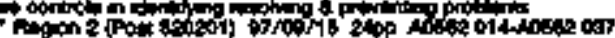

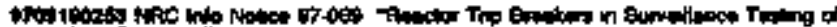

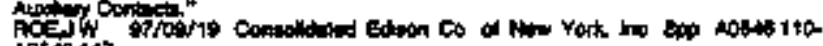
Ansis 11?

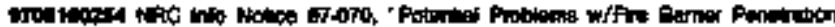

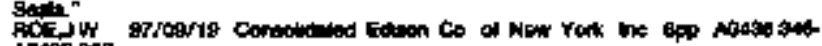
A 


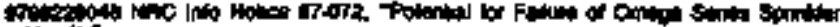

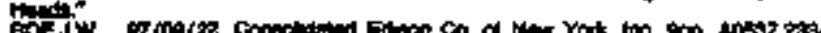
notist

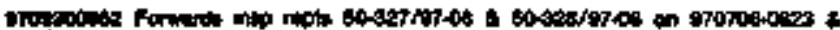

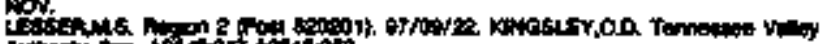

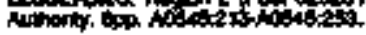

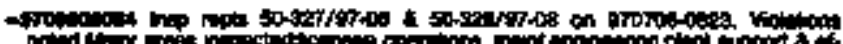

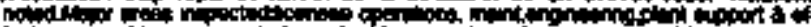

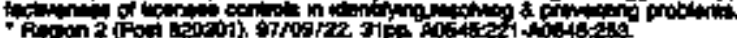

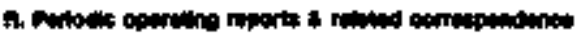

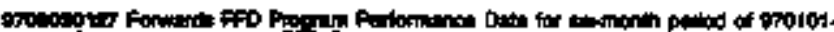

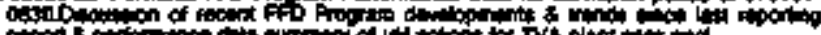

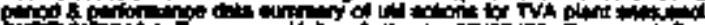

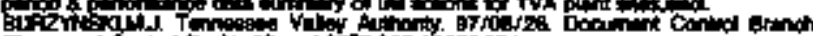

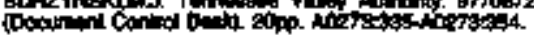

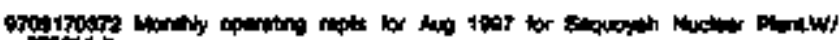

Fol' anditist.

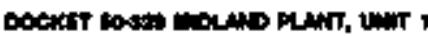

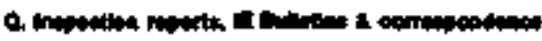

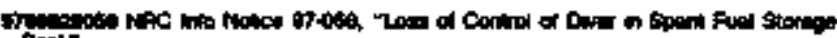
Poet-

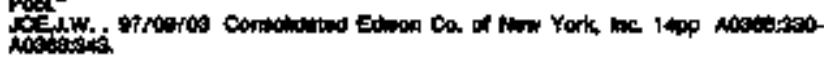

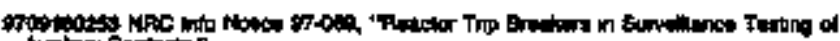
A

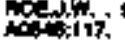

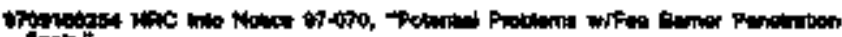

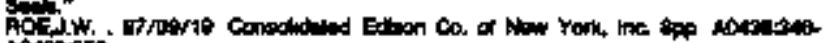
A0tofist:

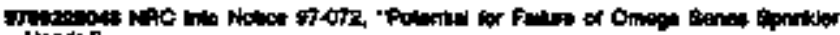

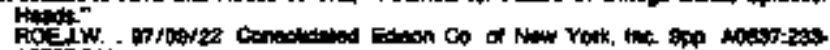
acosition.

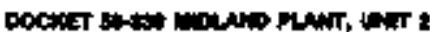

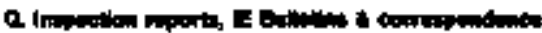

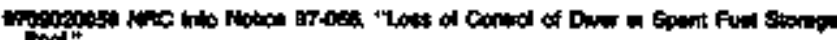

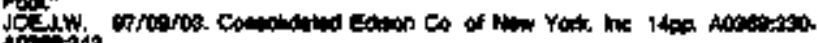

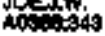

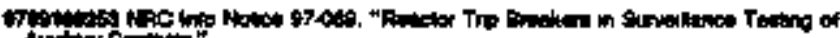

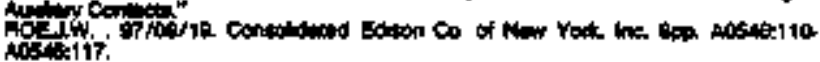

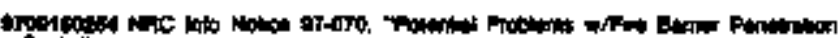

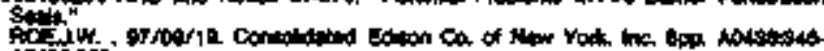
Atosing

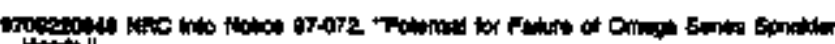

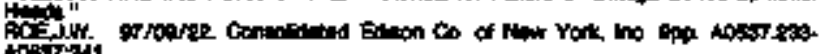

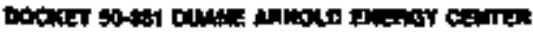

F. Foche

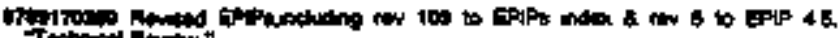

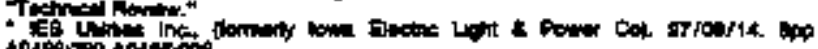
notecr.

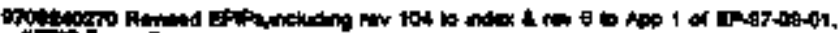

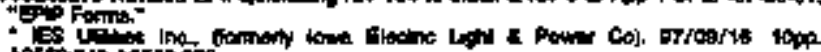

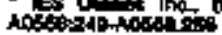

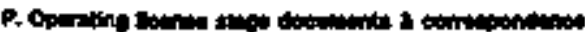

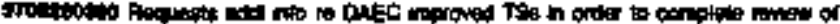

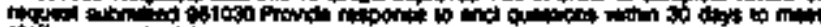

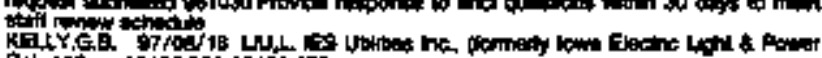

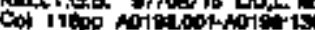

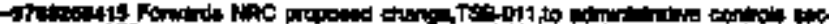

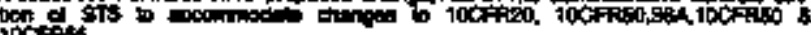

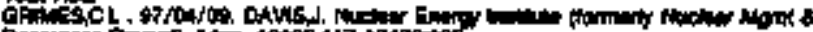

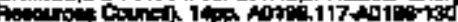

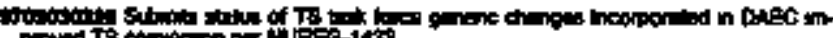

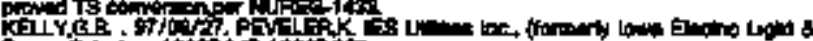

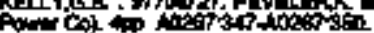

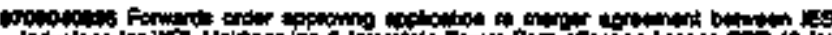

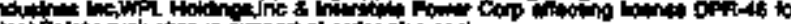

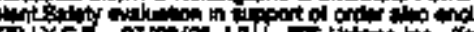

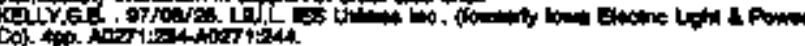

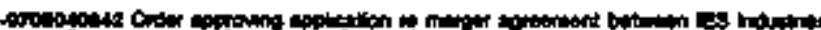

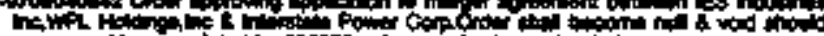

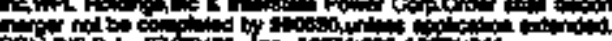
cotins

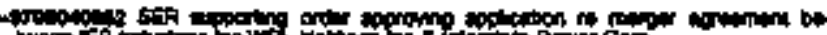

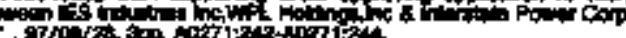

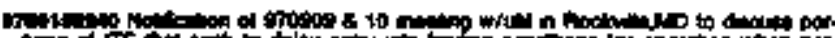

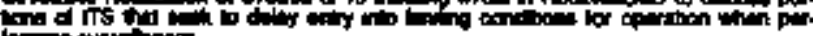

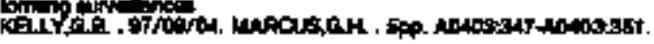

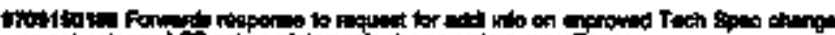

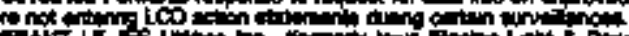

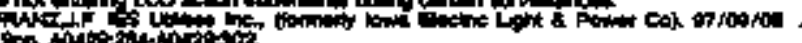

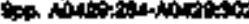

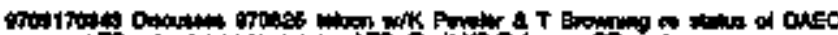

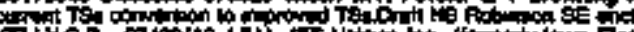

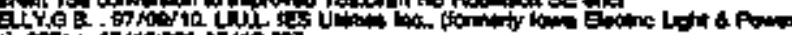

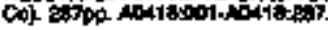

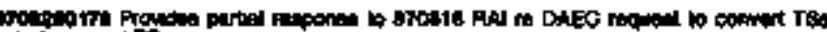

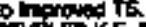

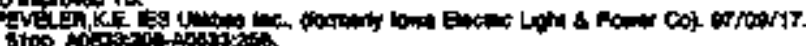

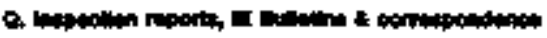

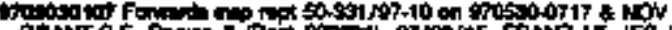

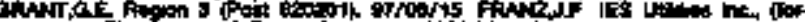

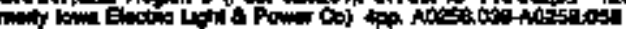

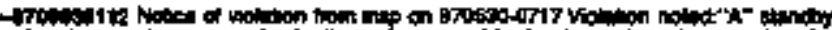
A

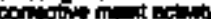

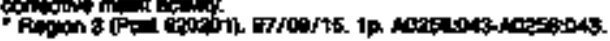

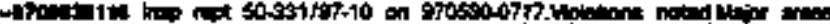

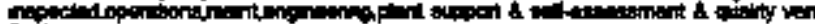

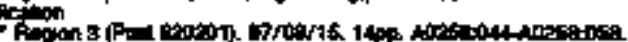

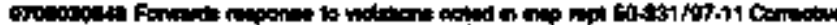

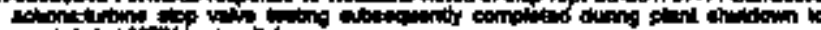

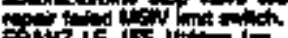

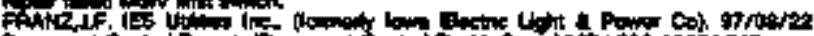

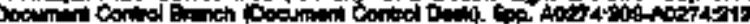

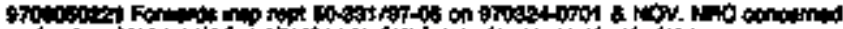

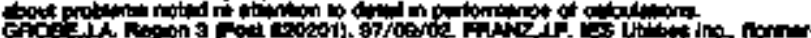

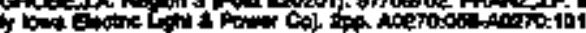

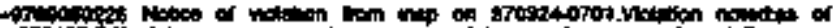

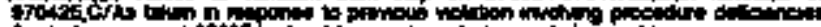

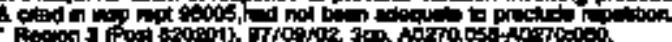

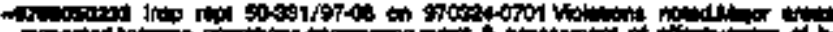

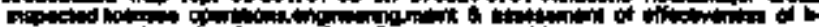

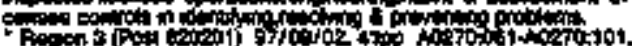

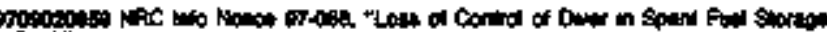

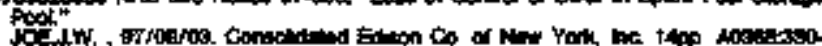

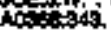

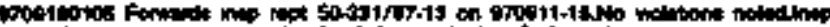

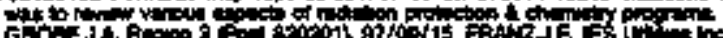

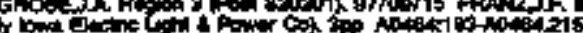

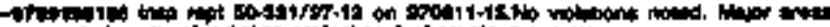

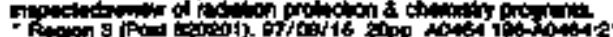

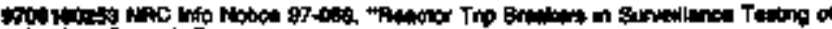

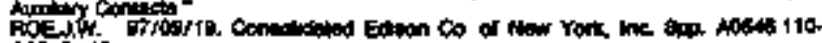
Ripti. 


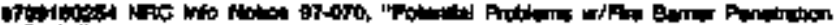

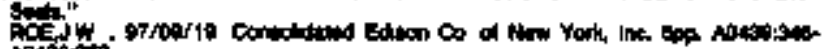
Notions

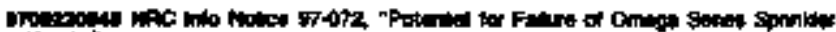

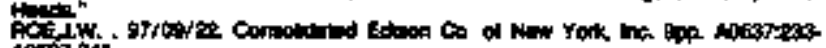
nosition

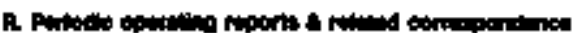

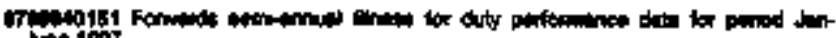
vito ion

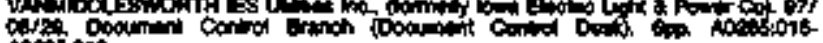
atopotio

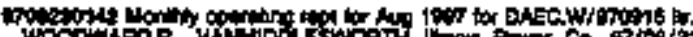

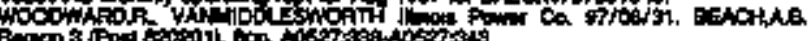

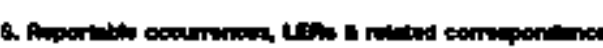

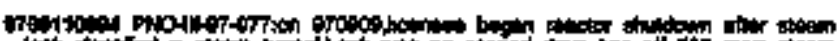

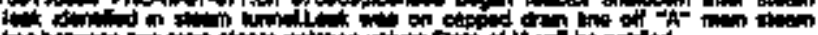
Dovis Dacistion.

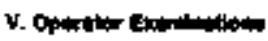

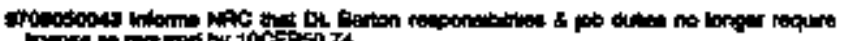
FAd

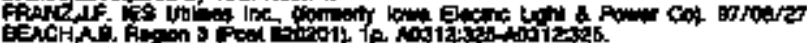

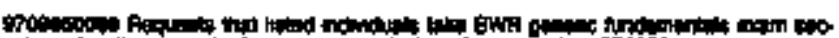

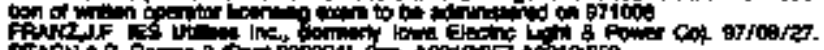

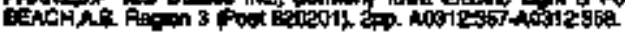

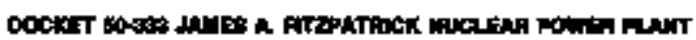

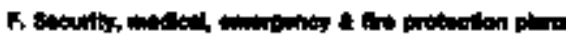

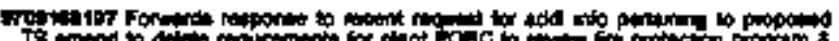

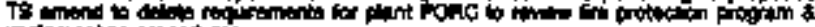

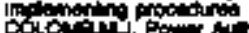

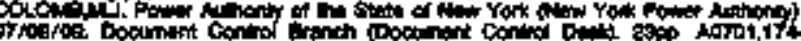
Aorolins:

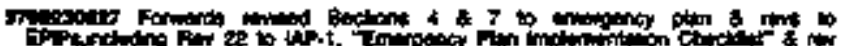

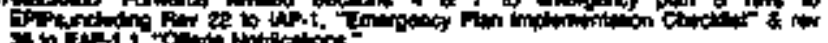

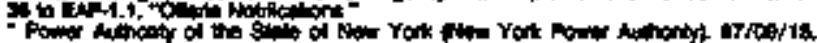

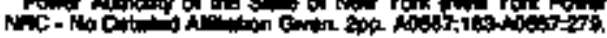

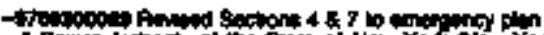

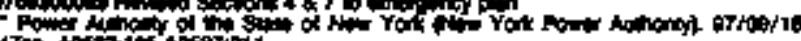

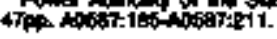

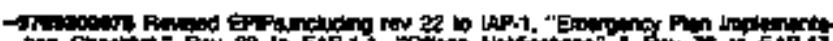

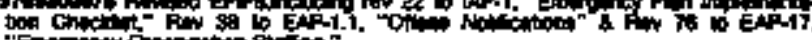

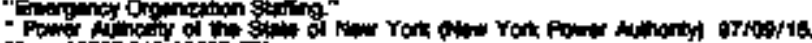

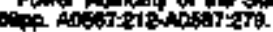

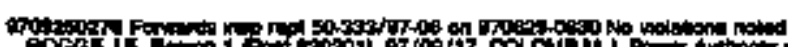

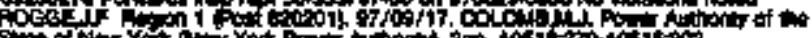
Stan of

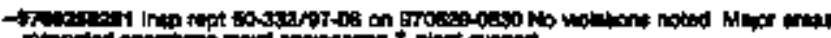

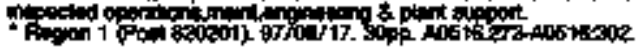

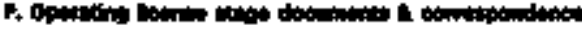

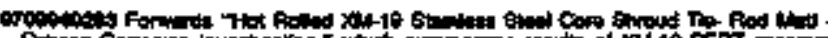

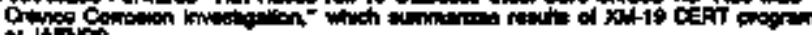

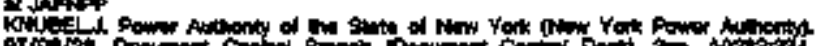

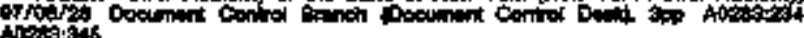
Nin:3:36

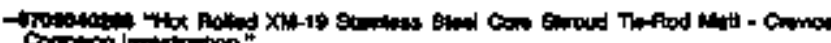

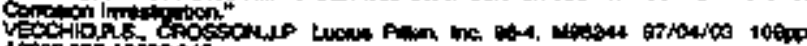

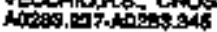

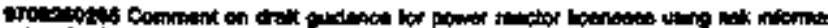

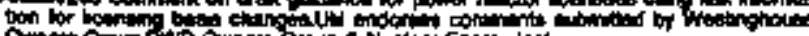

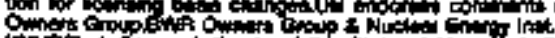

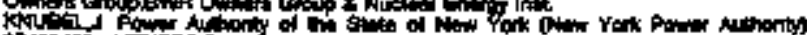

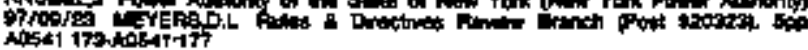

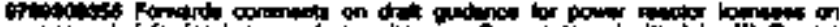

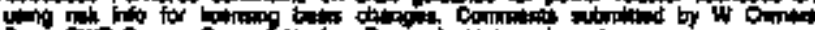

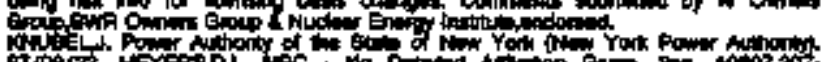

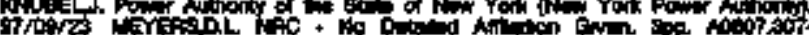
Aderiscos.

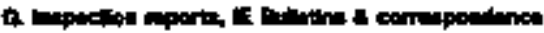

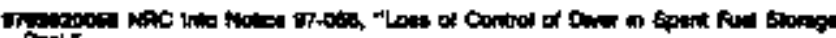

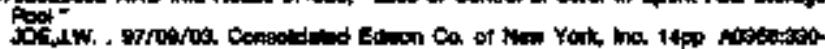
Axasis.

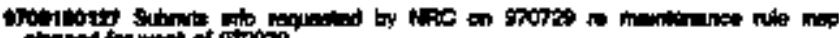

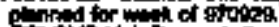

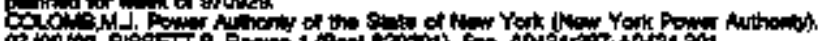

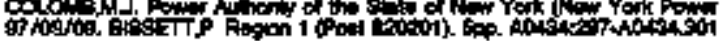

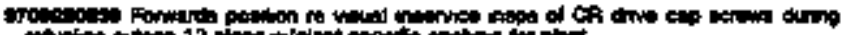

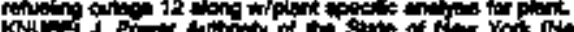

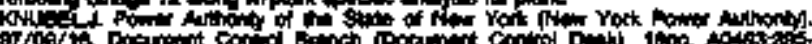

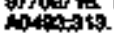

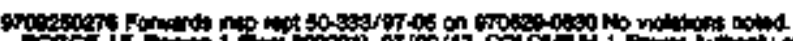

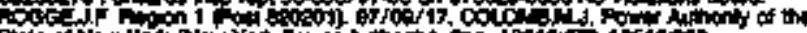

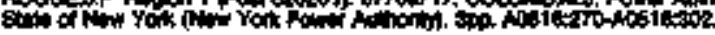

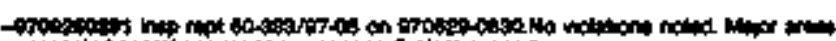

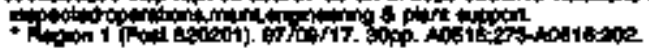

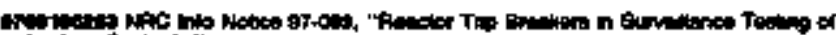

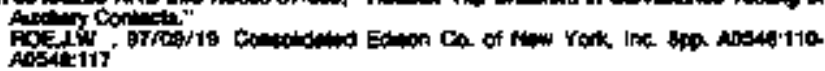

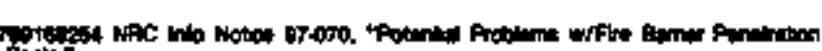

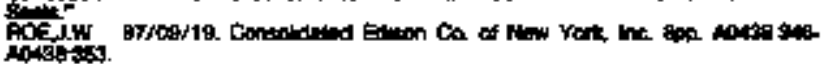

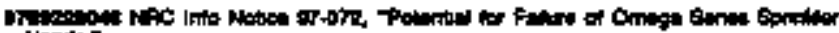

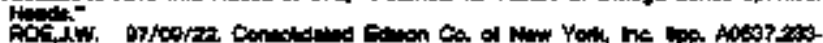
Aption:

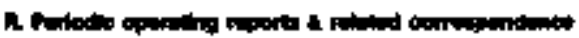

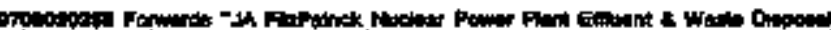

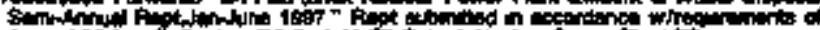

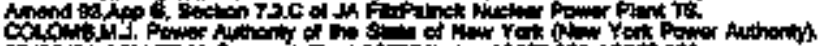

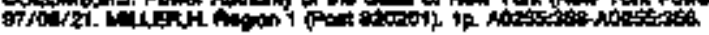

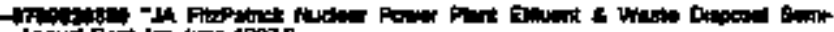

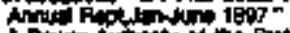

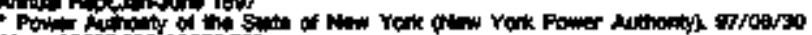
1.:ph, ind

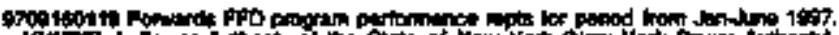
Fod 104040

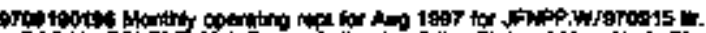

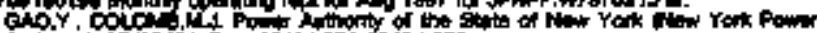

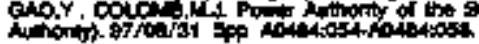

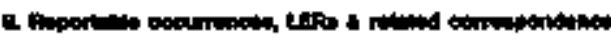

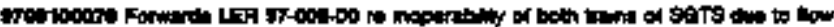

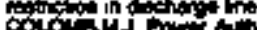

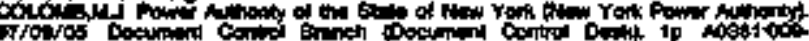
Nosistis

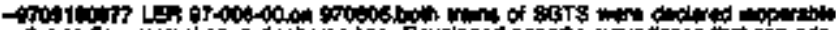

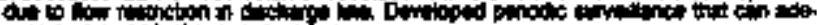

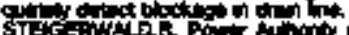

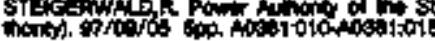

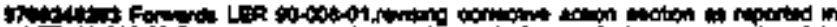

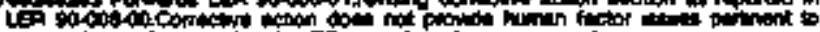

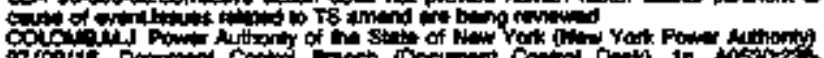

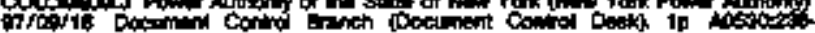

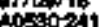

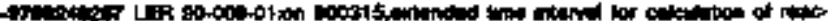

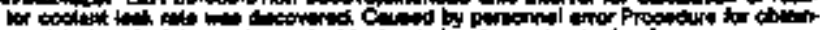

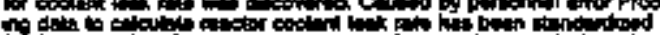

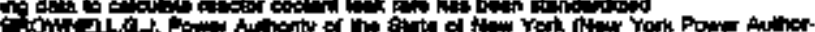

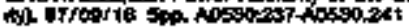




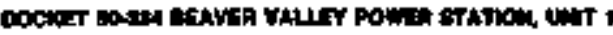

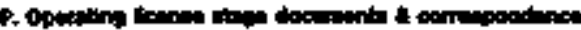

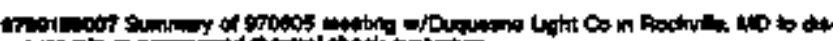

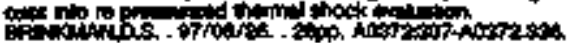

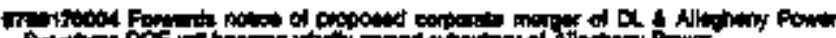

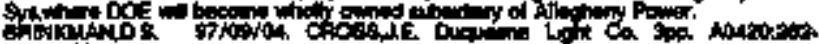
ind

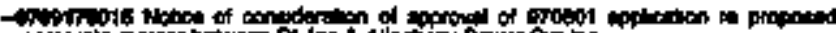

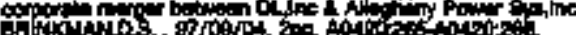

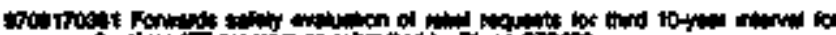

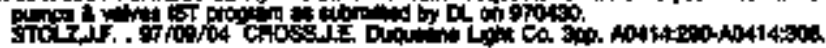

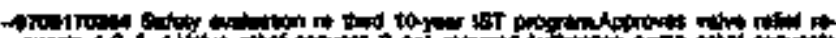

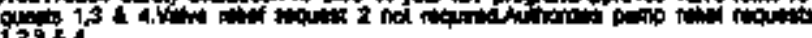
2954

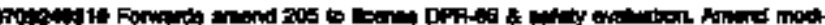

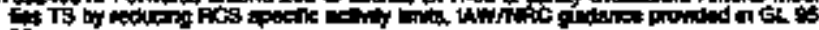

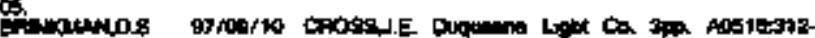
Atois

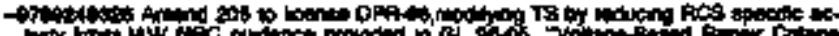

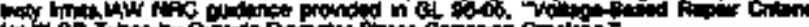

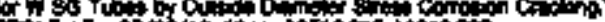

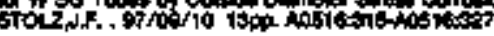

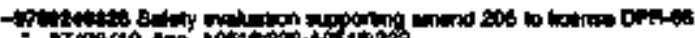

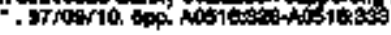

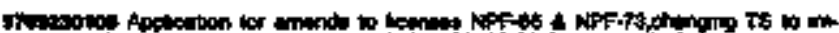

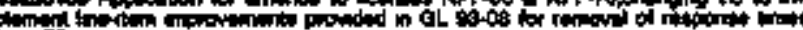
III T:

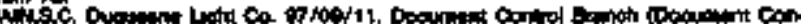

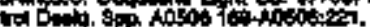

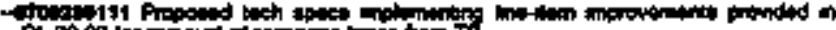

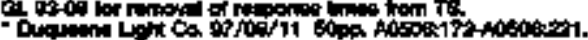

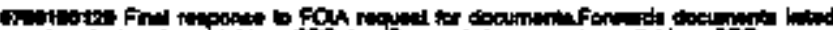

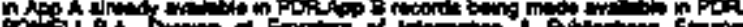

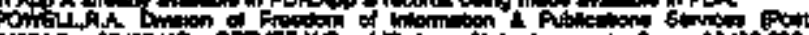

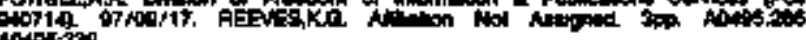
404050.

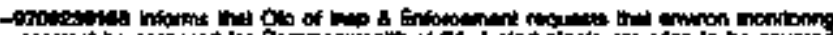

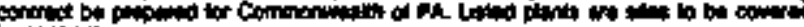

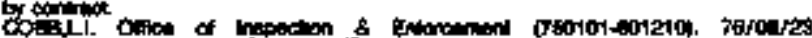

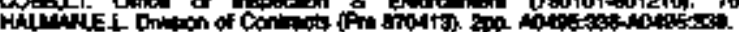

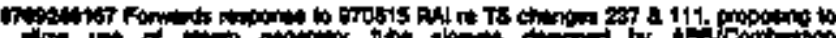

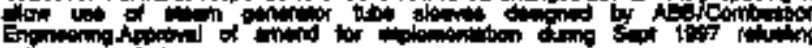

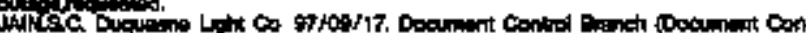

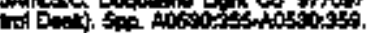

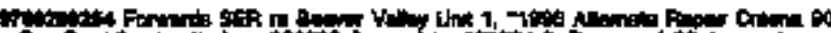

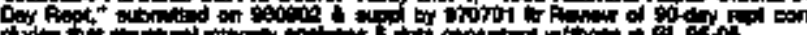

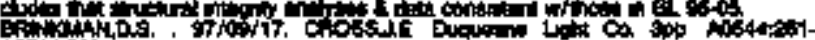
Alition

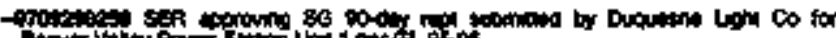

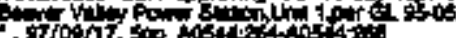

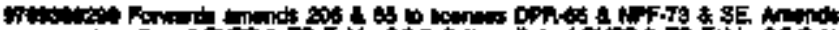

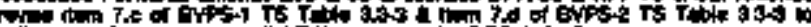

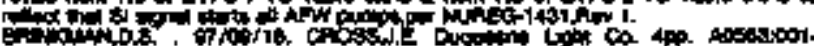
atosis.

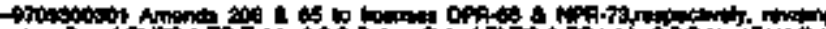

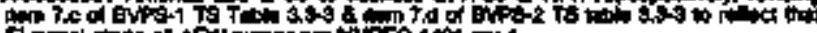

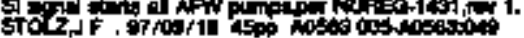

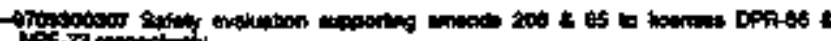

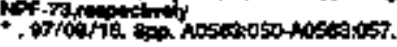

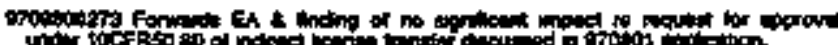

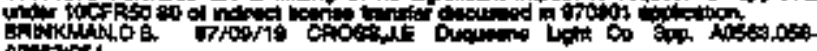
Alowatid

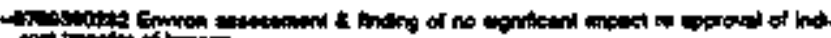

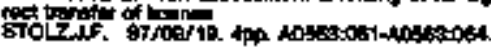

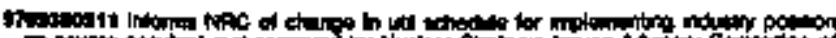

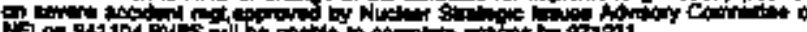

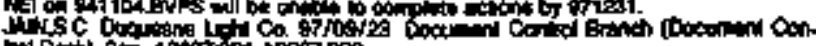
thet D.

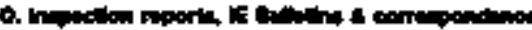

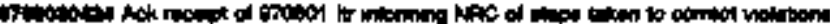

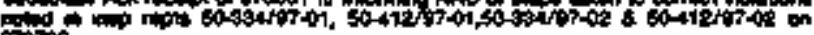
cotive. Es

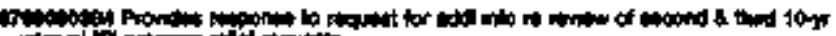

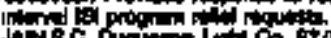

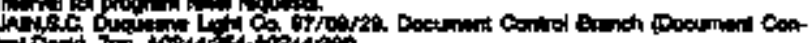

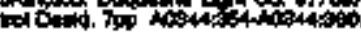

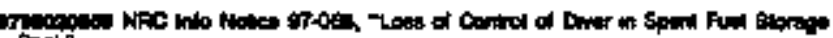
(a)

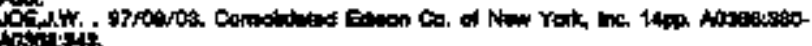

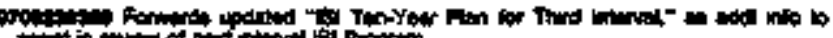

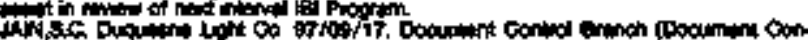

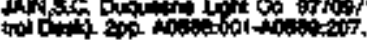

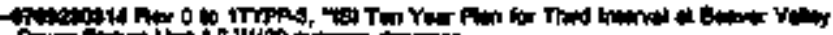

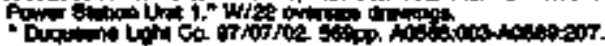

of

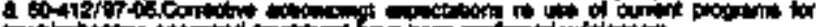

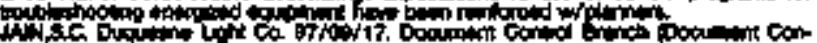

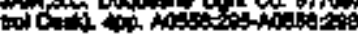

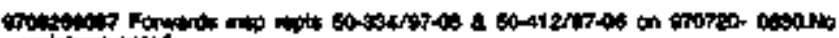

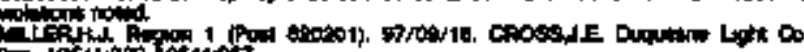

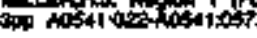

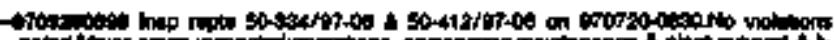

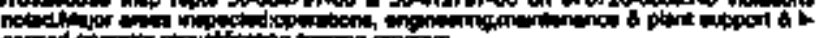

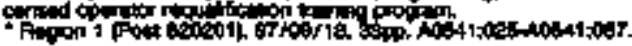

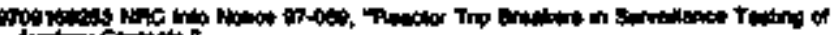

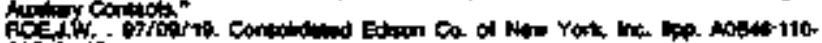
istatis.

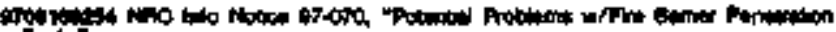

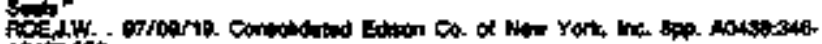
(1)

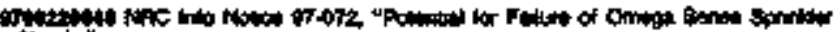

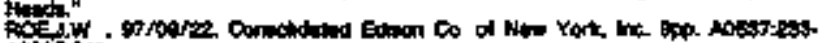
Atstivit.

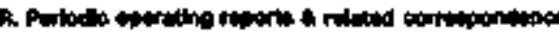

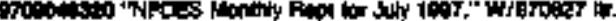

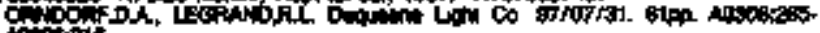
Antosicic

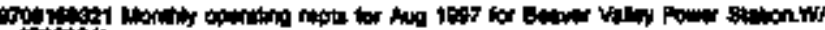

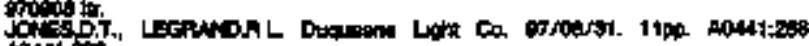
194120

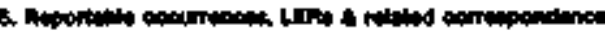

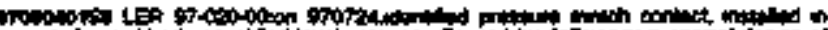

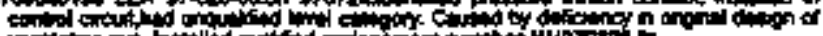

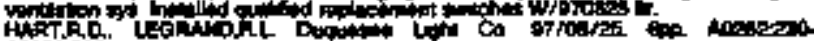

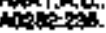

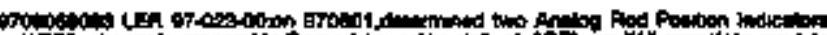

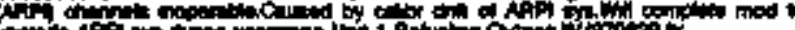

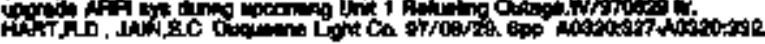

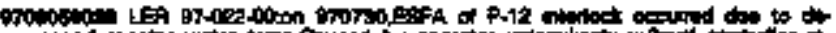

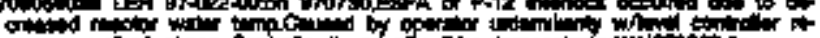

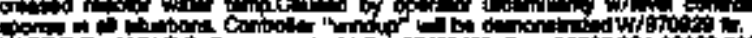

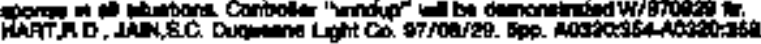

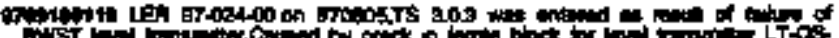

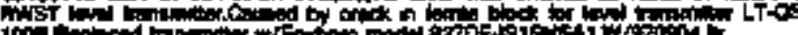

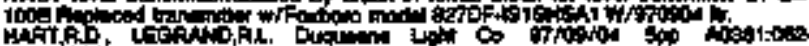
Nosation.

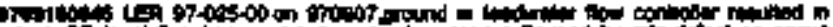

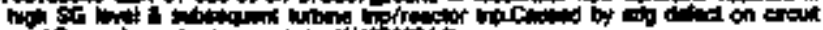

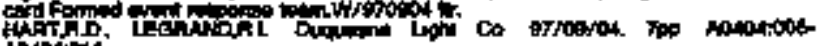
Arateris.

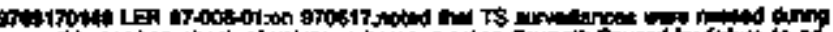

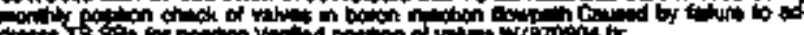

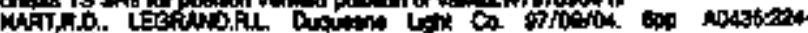
matif 


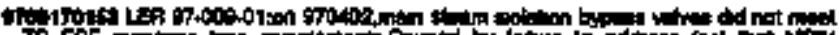

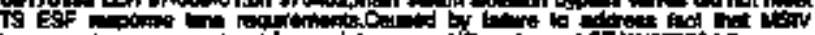

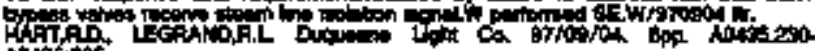
A

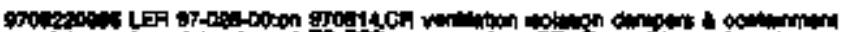

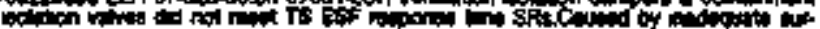

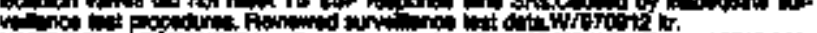

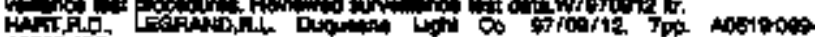
Mostion.

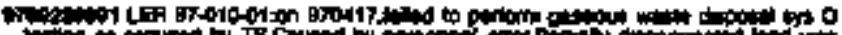

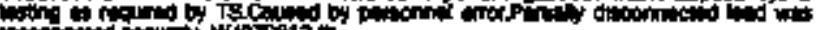

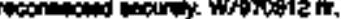

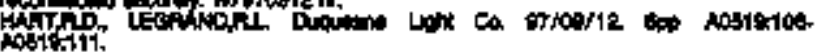

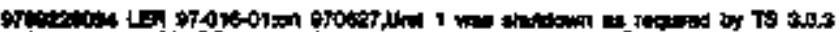

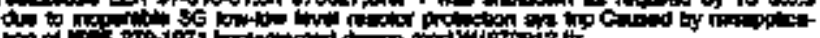
fon

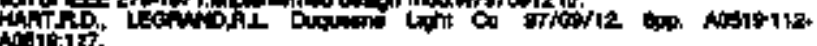
M0.10:17?

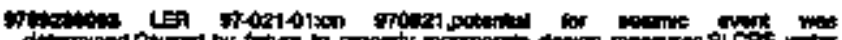

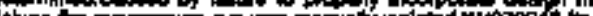

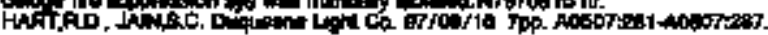

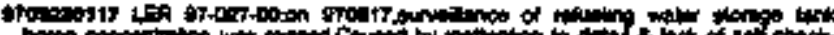

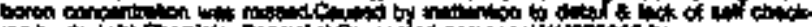

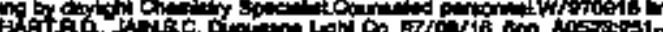

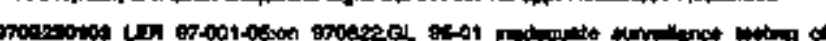

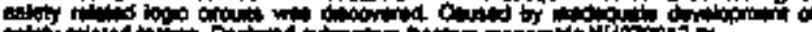

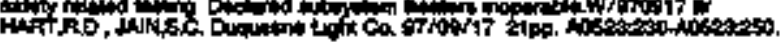

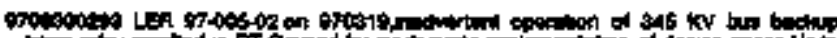

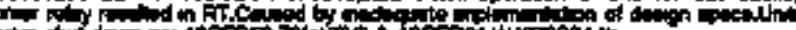

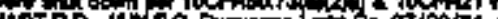

然

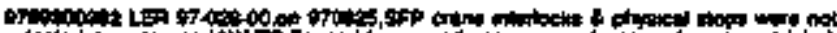

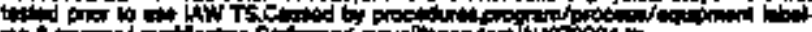

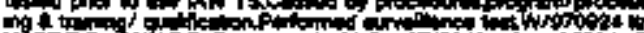

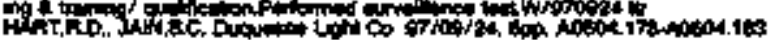

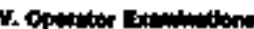

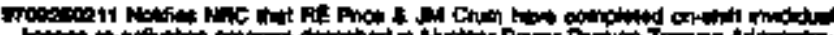

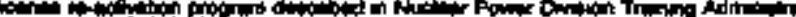

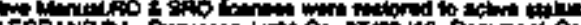

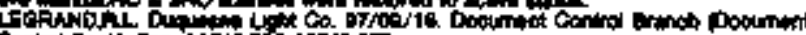

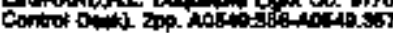

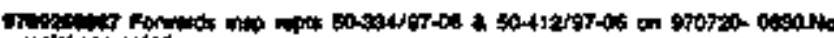
mingenting

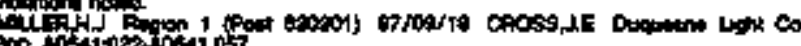

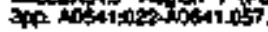

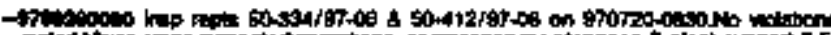

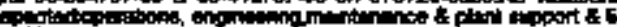

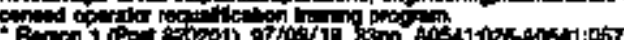

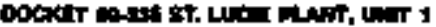

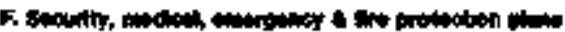

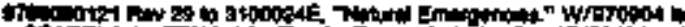

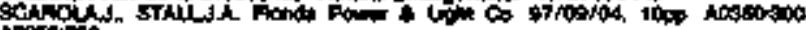
$\cos 00000$

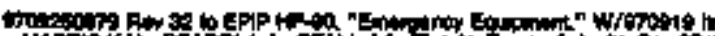

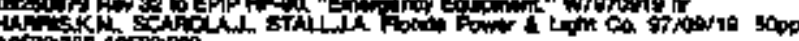

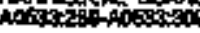

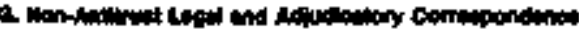

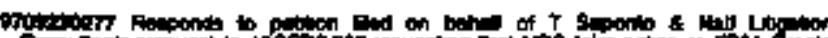

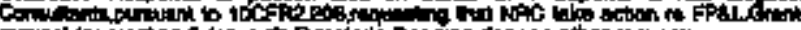

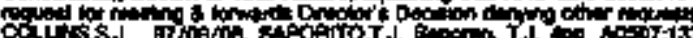

Coll th d D

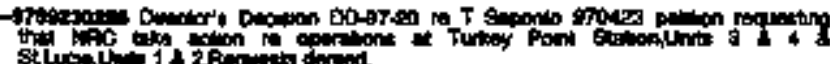

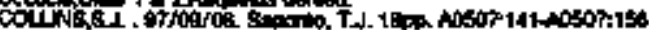

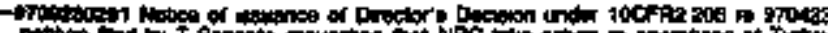

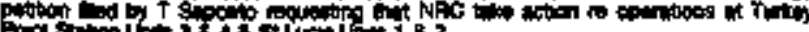

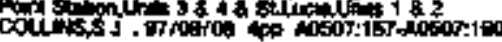

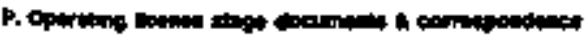

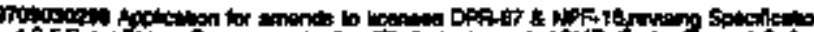

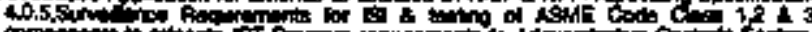

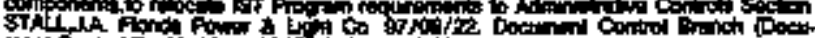

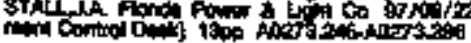

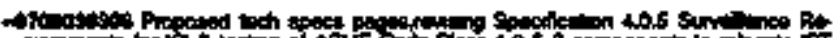
gen

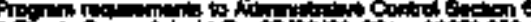

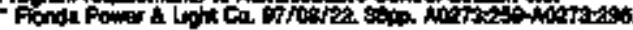

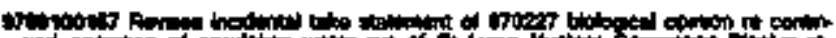

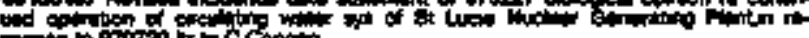

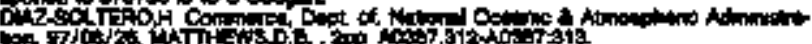
s

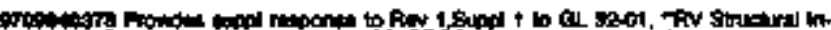

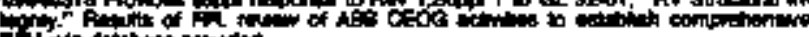
The 1

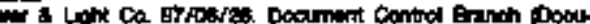

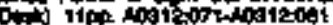

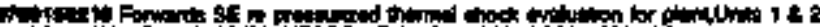

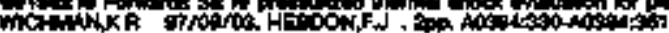

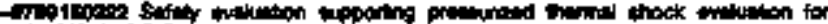
orisunt: 182

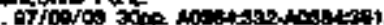

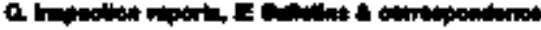

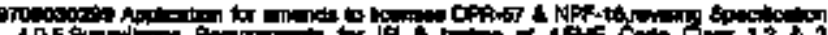

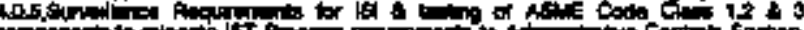

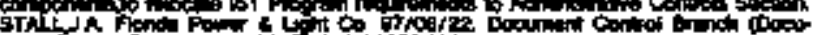

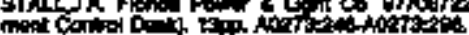

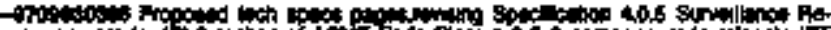

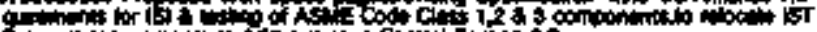

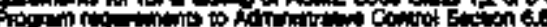

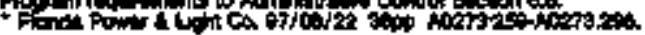

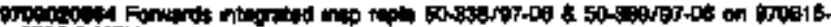
ortas now

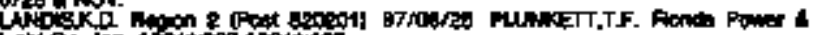

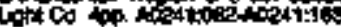

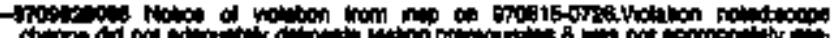

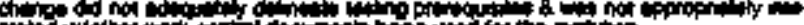

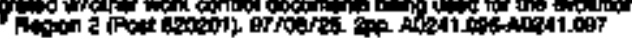

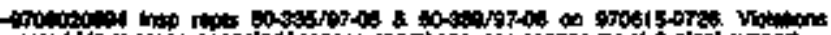

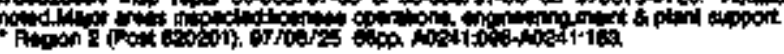

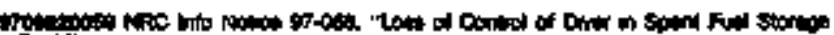

Pod

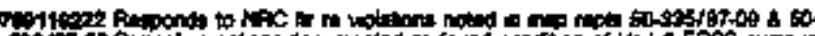

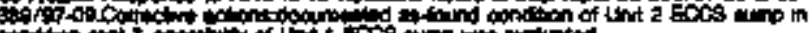

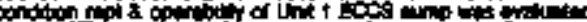

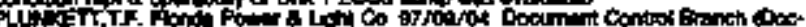

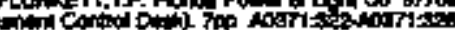

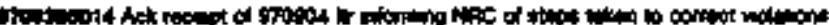

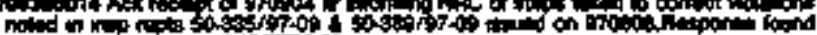

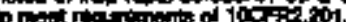

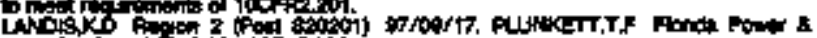

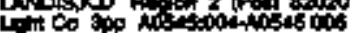

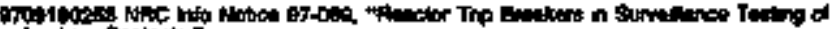

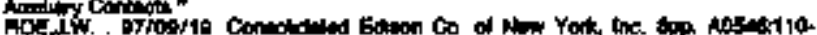
if 7 titis.

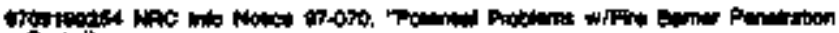

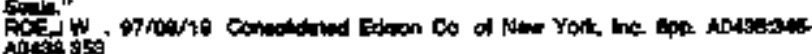

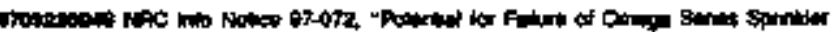

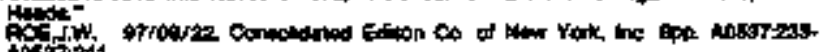
10:5

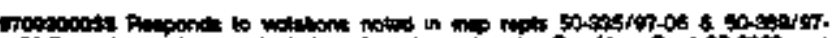

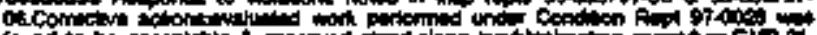

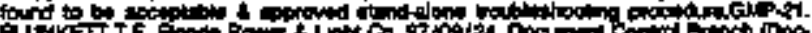

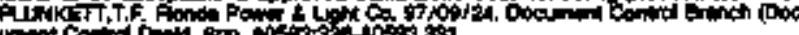

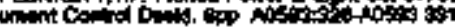

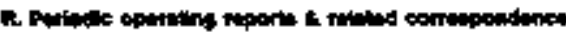

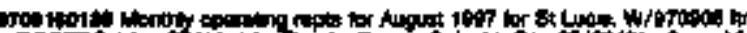

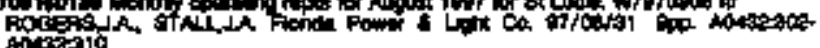

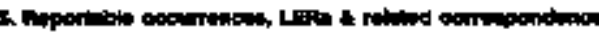

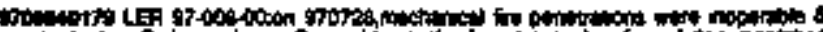

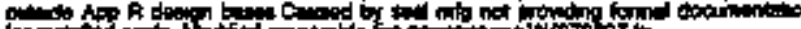

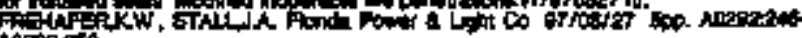
Aotar 20 


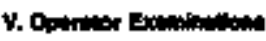

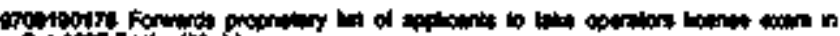

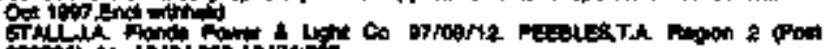

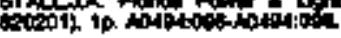

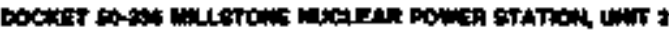

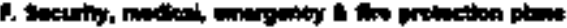

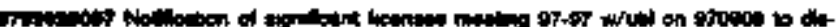

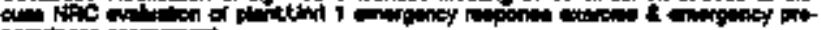

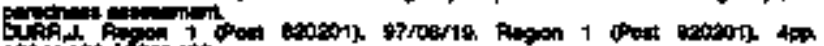

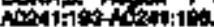

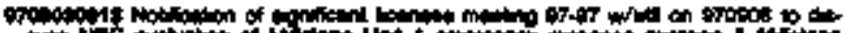

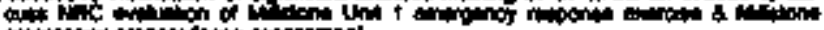

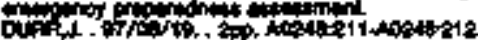

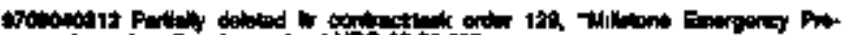

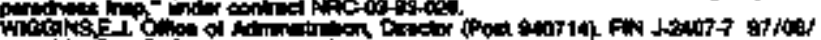

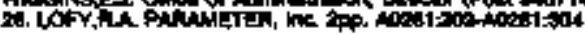

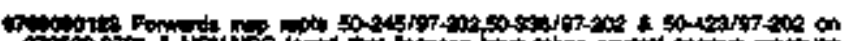

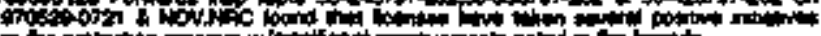

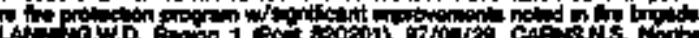

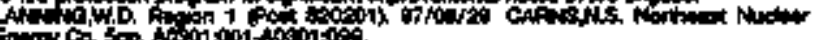

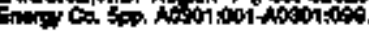

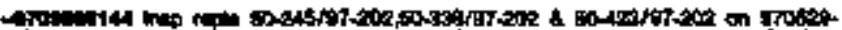

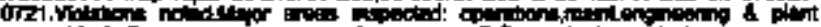

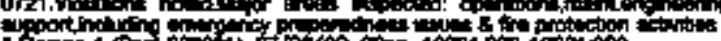

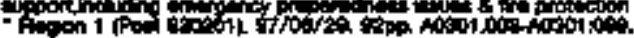

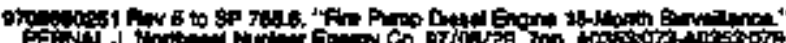

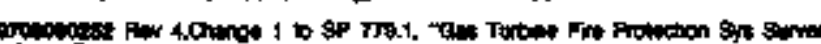

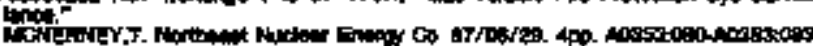

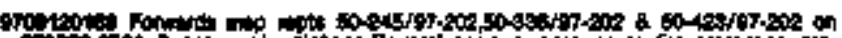

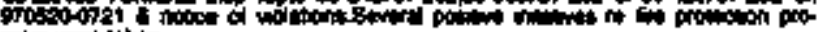

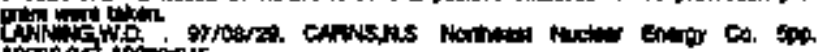

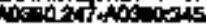

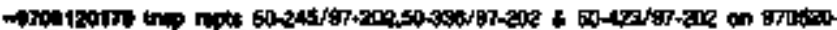

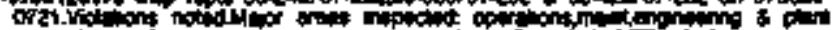
or a r.

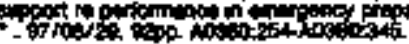

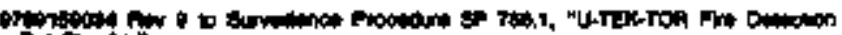

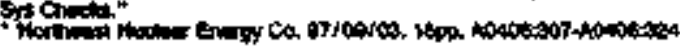

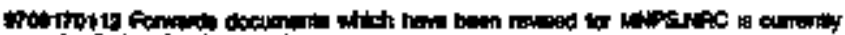

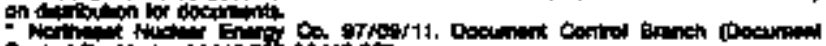

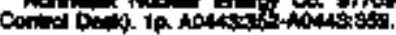

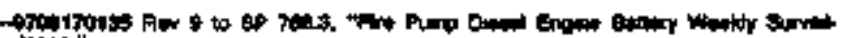

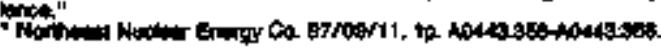

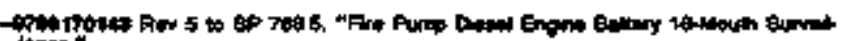

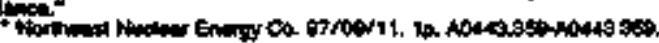

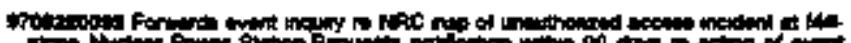

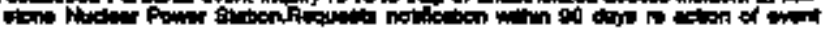

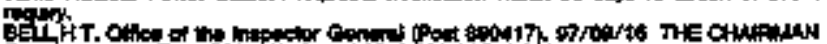

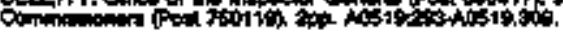

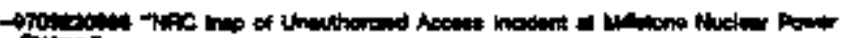

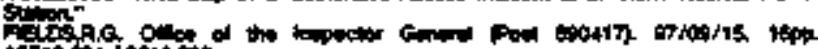

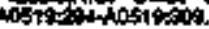

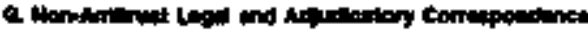

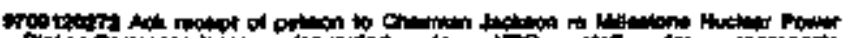

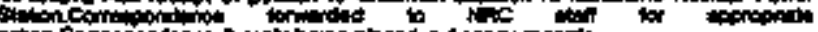

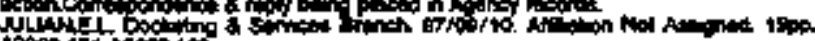
10.0.

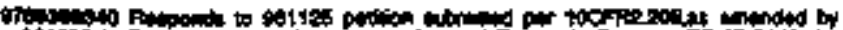

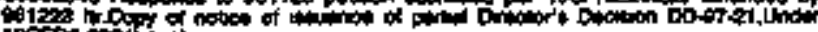

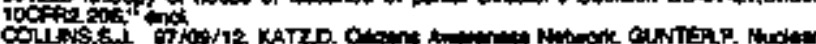

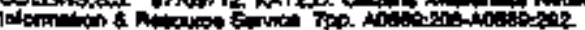

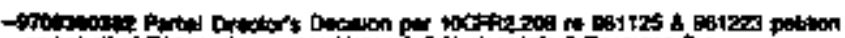

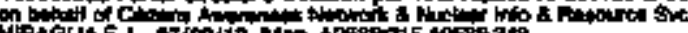

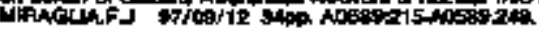

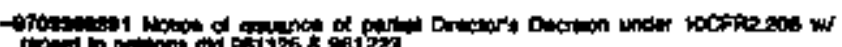

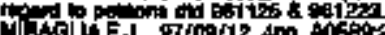

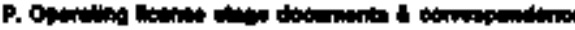

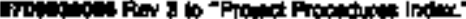

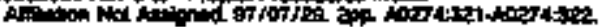

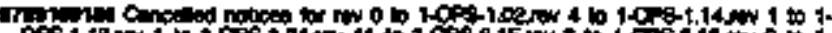

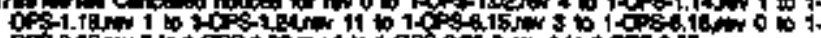
Opo

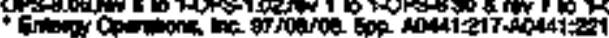

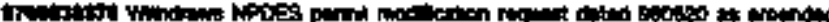

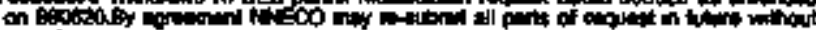

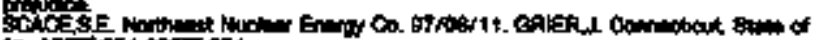

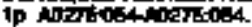

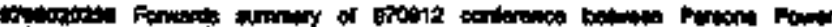

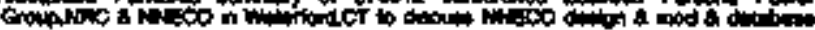

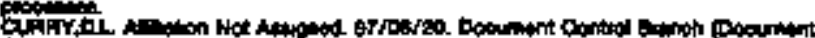

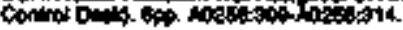

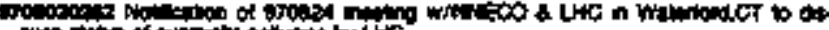

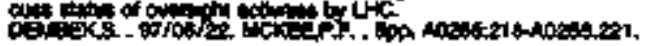

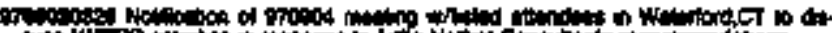

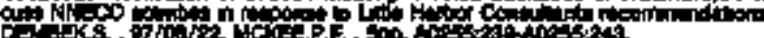

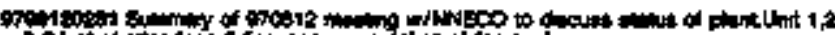

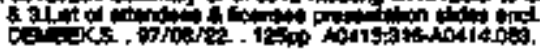

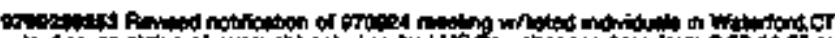

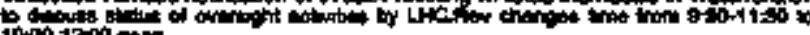

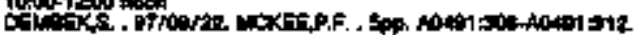

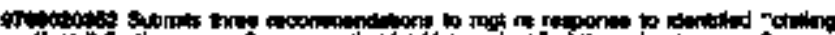

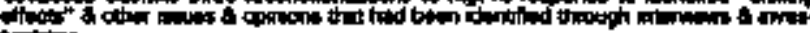

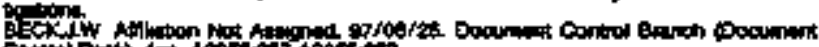

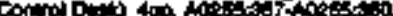

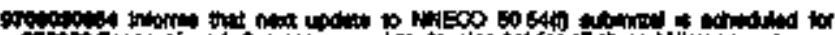

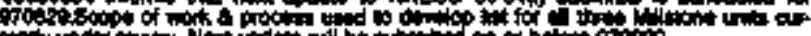

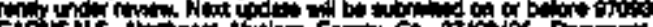

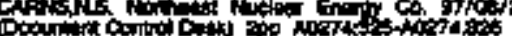

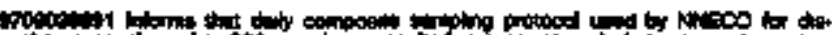

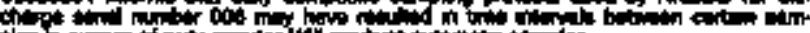

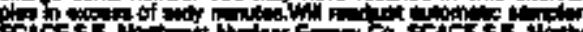

och.s.

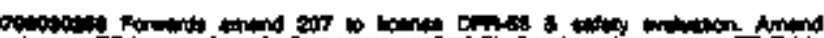

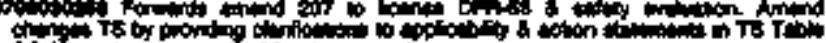

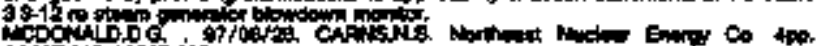
intion:

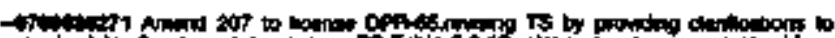

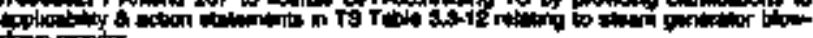

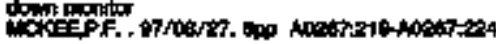

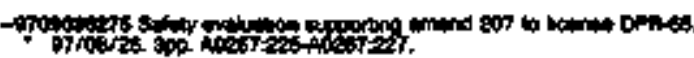

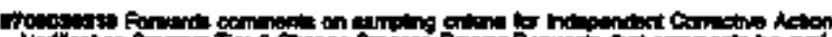

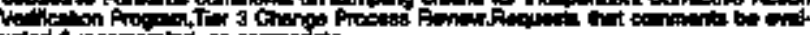

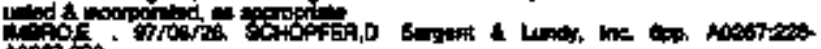
motes

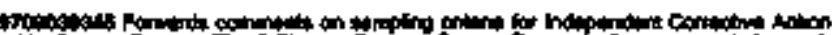

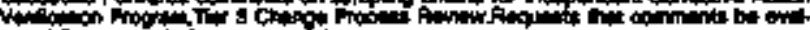

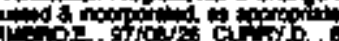

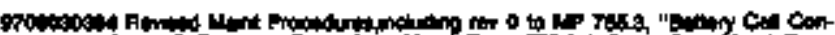

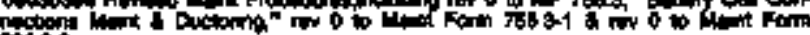
tots

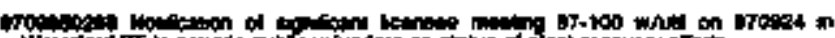

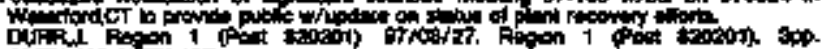

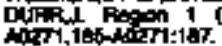

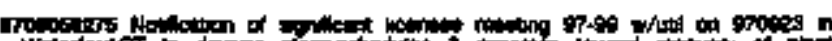

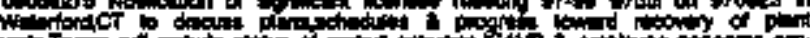

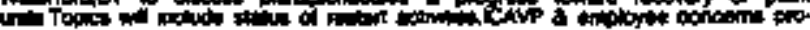

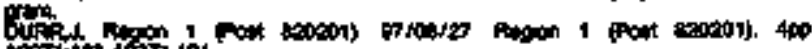

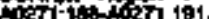

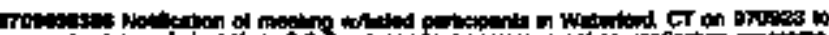

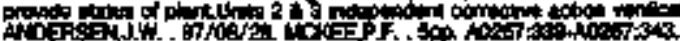




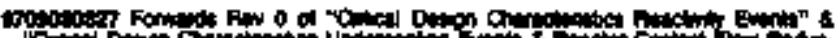

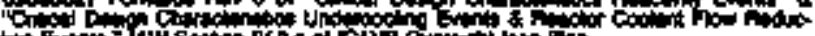

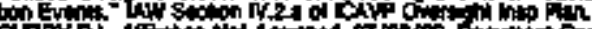

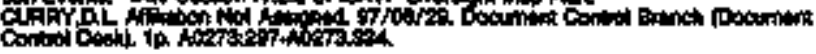

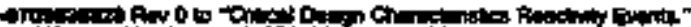

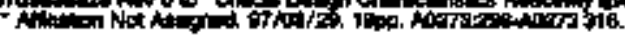

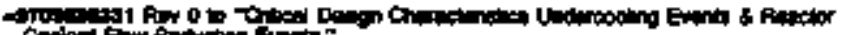

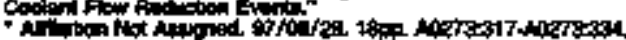

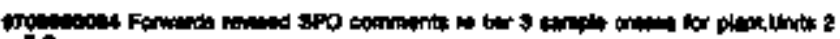
H Nonts.

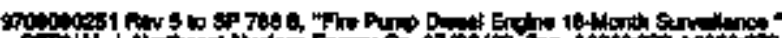

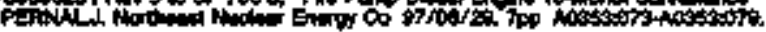

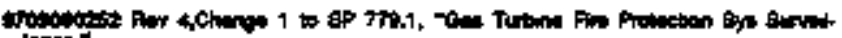

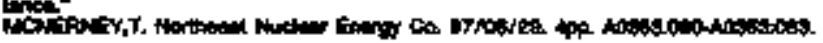

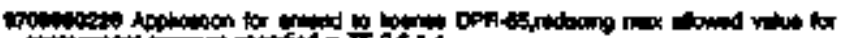

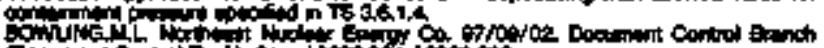

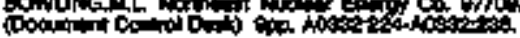

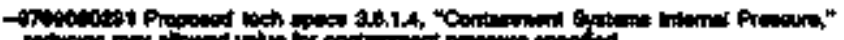

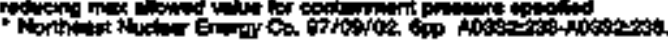

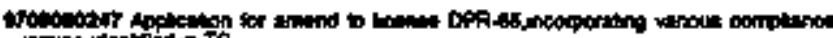

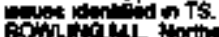

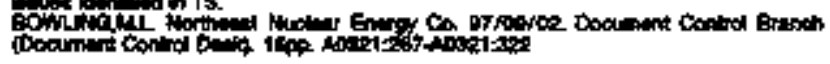

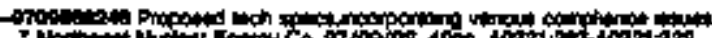

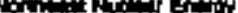

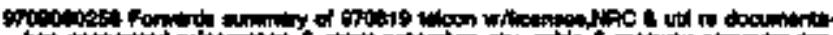

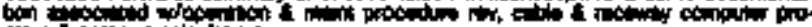
tots

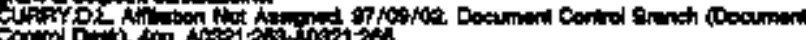

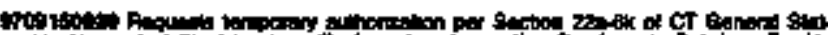

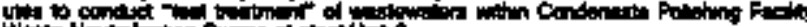

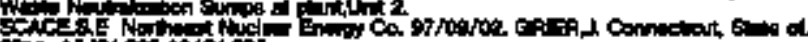

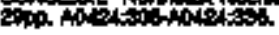

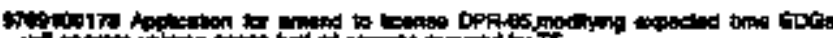
cold

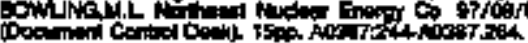

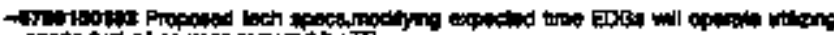

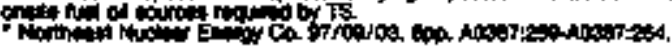

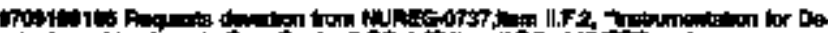

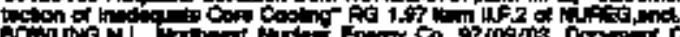

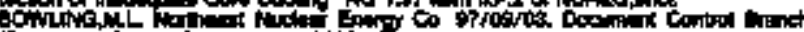

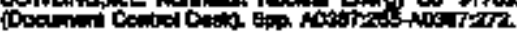

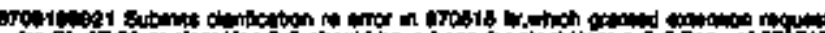

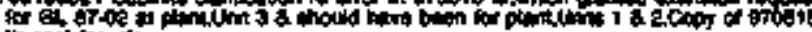

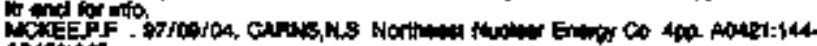
Nint:149.

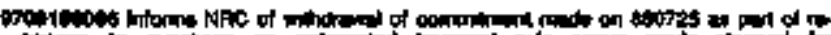

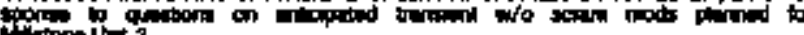

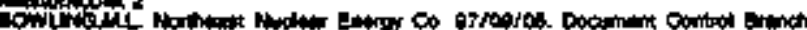

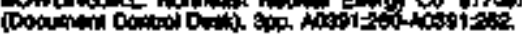

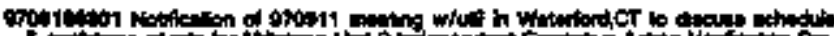

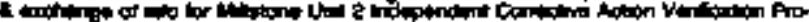

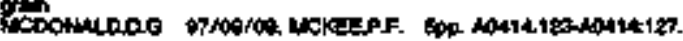

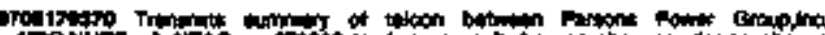

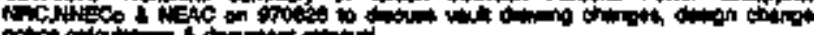

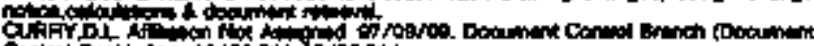

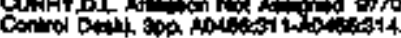

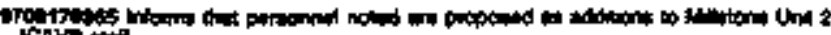
caverit.

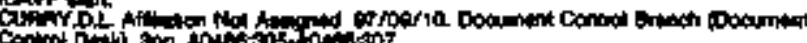

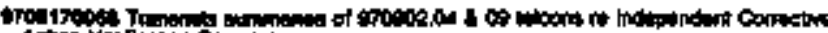

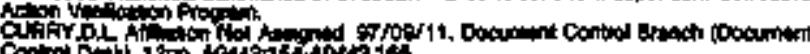

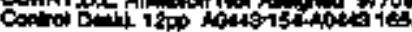

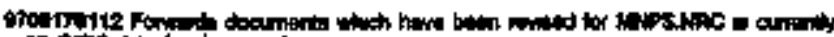

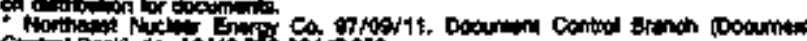

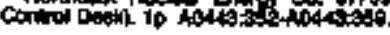

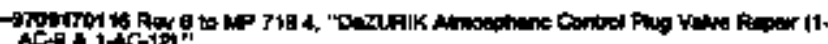

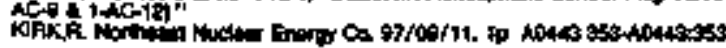

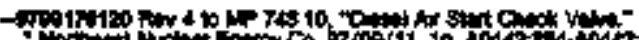

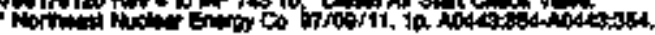

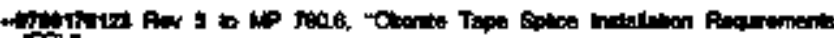
Epin

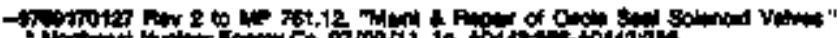

W

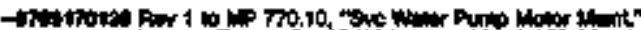

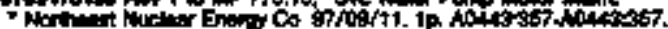

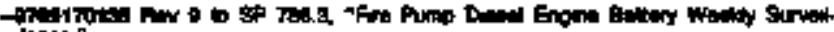

byon

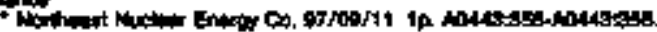

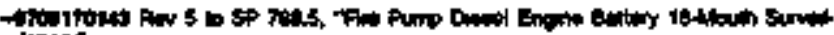
(n)

管

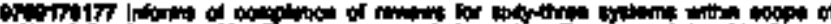

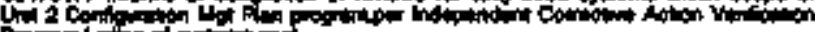

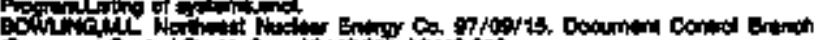

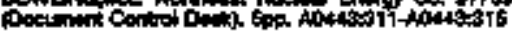

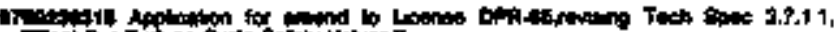

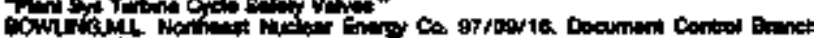

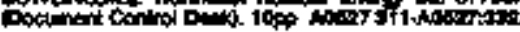

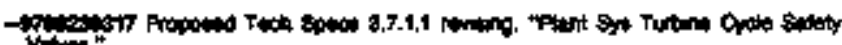

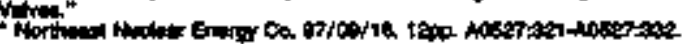

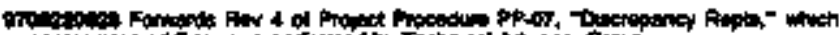

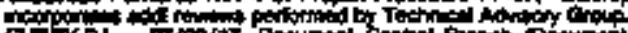

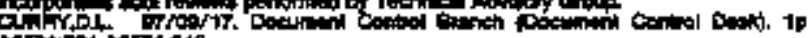
andotion

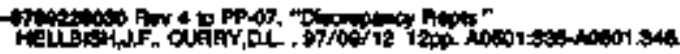

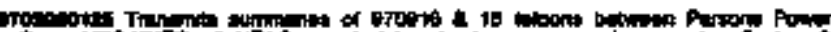

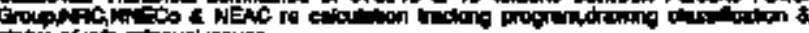

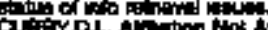

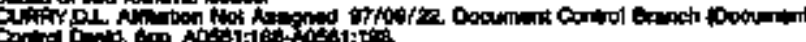

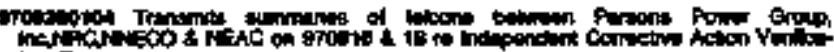

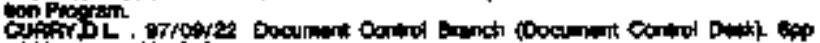

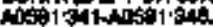

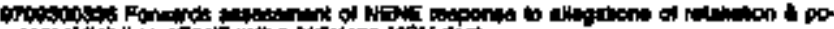

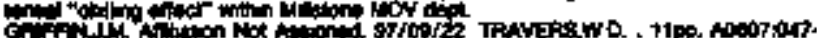
atsing

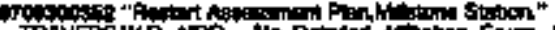

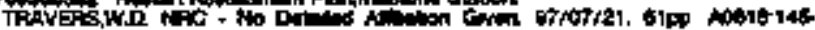
COA1EXss.

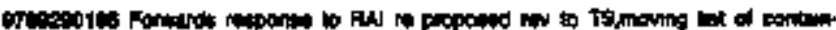

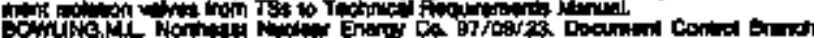

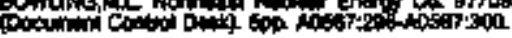

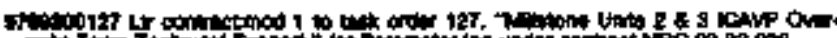

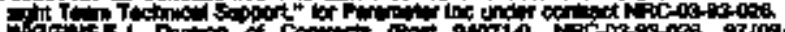

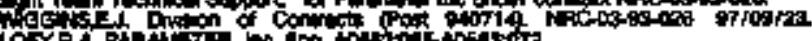

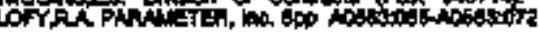

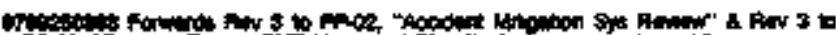

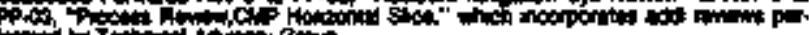

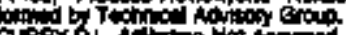

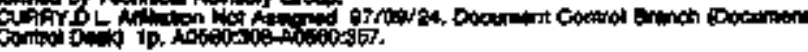

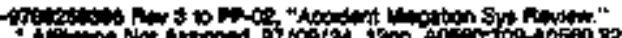

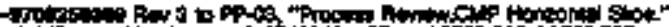

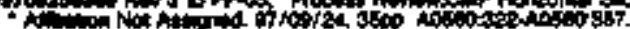

Th:

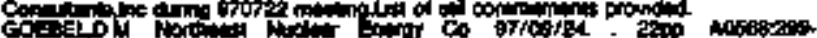

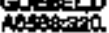

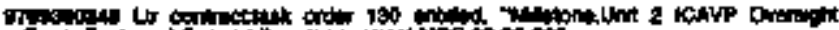

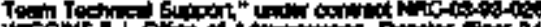

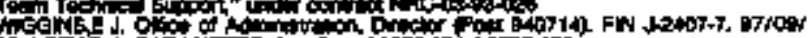

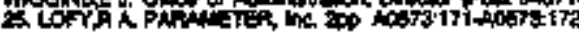

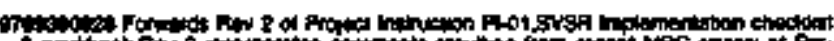

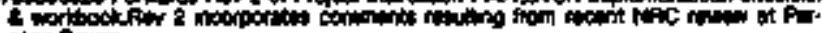

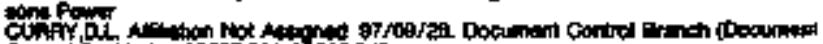

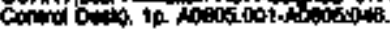

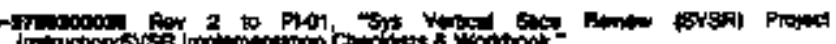

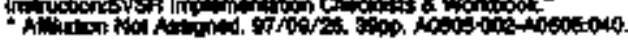




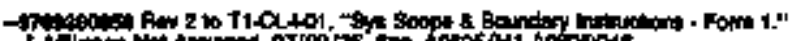

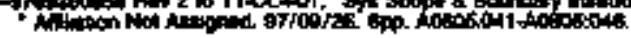

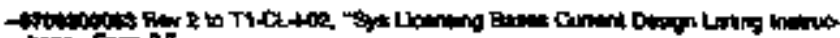

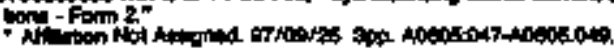

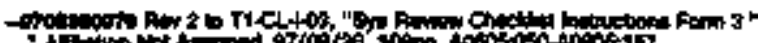

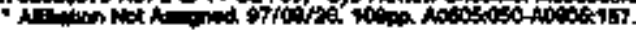

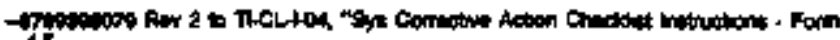

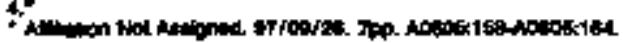

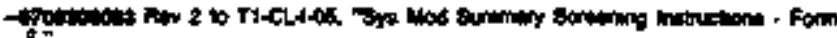

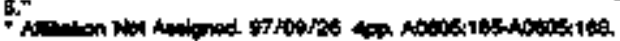

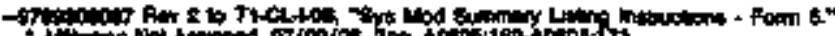

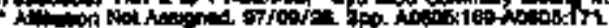

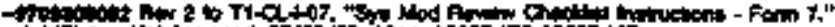

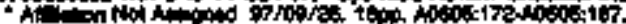

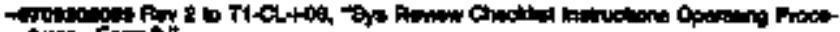

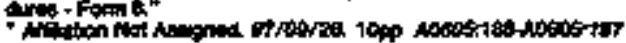

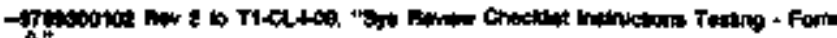

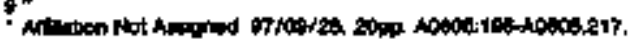

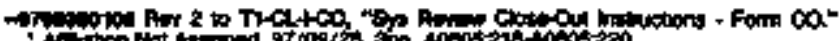

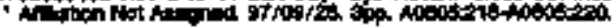

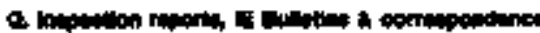

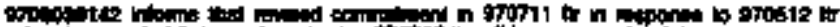

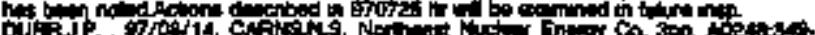
inderis.

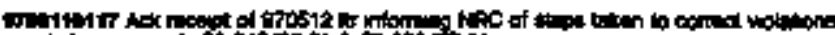

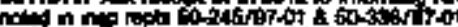

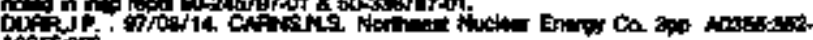
Notson.

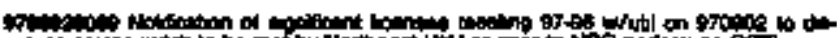

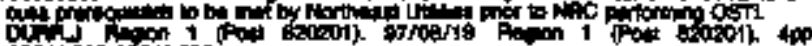
Dow1

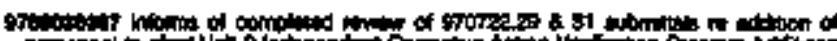

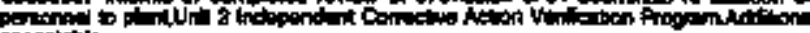

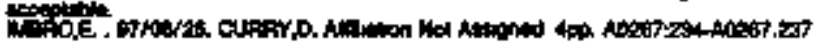

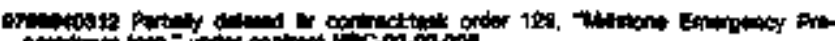

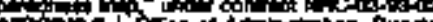

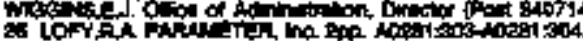

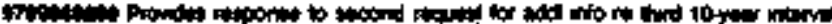

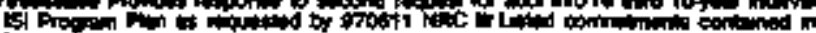

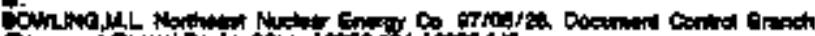

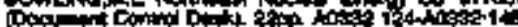

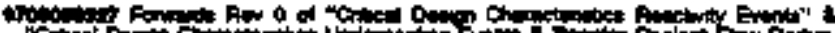

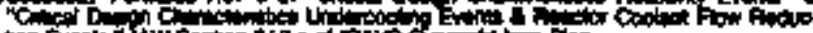

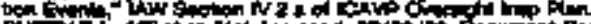

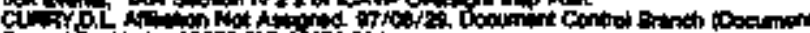

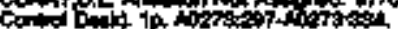

mocong Pon

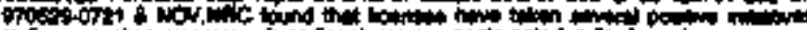

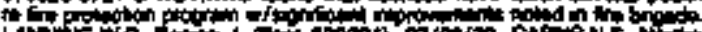

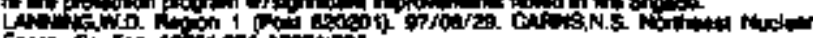

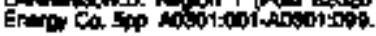

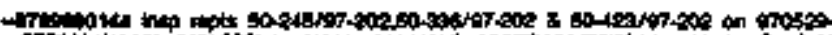

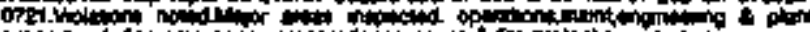

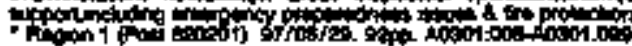

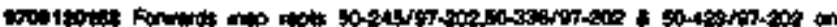

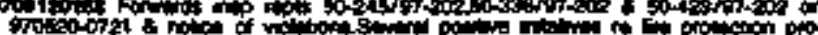

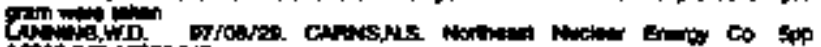
instion

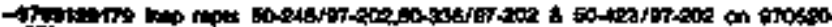

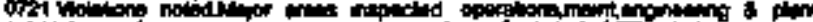

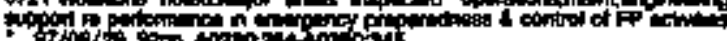

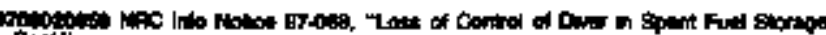
Piscidi

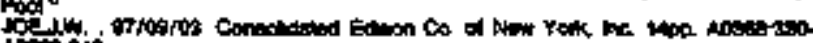

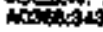

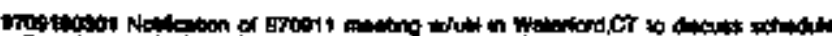

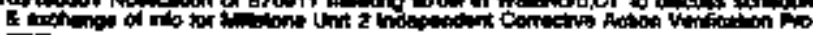

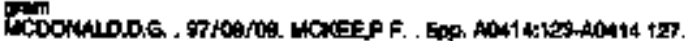

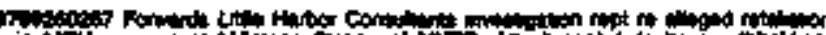

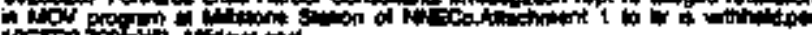

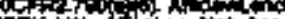
10.7\%

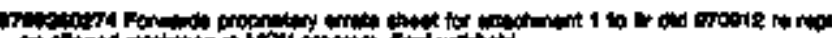

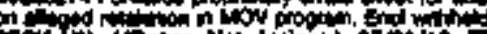

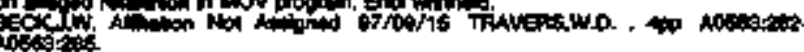

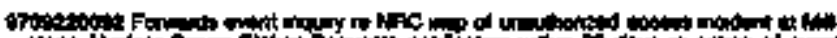

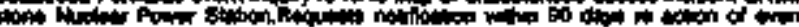

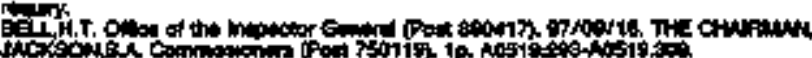

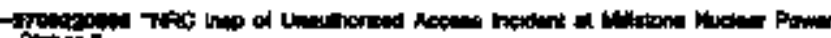

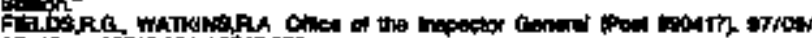

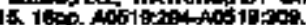

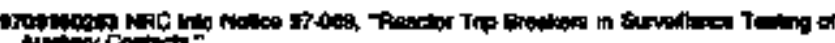

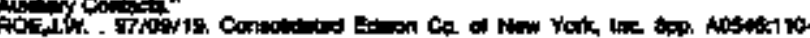
10601717.

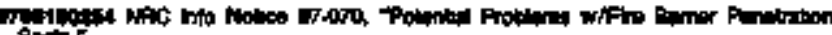

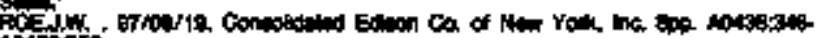
nitios.

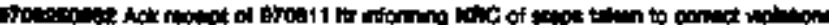

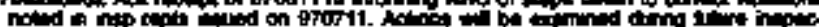

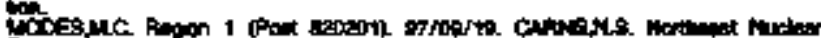

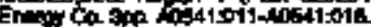

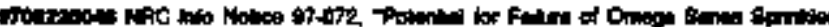

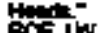
Actitit.

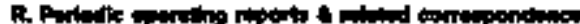

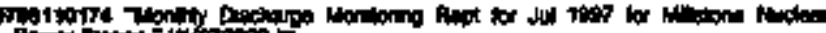

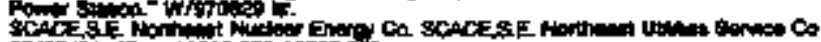
if

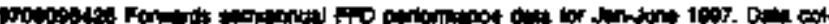
intof to of Wo

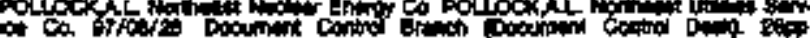

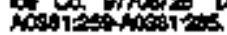

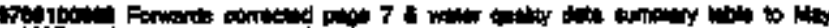

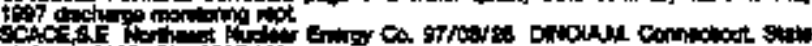

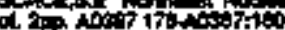

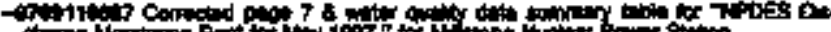

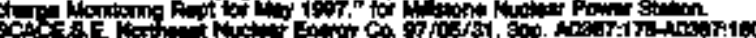

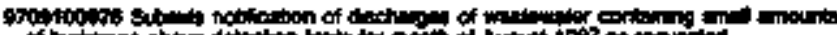

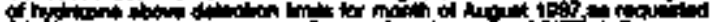
cetics.

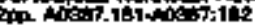

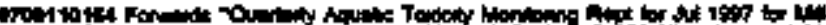

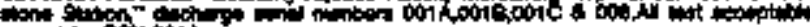

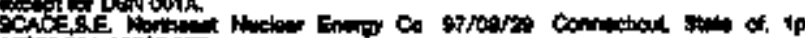
wing

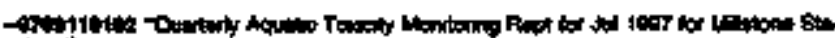

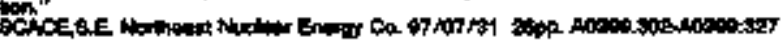

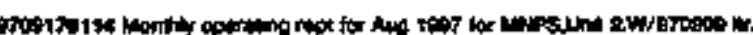

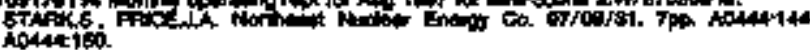

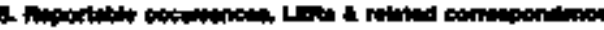

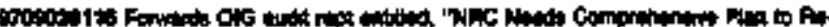

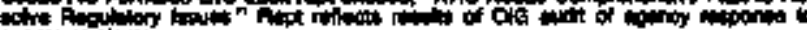

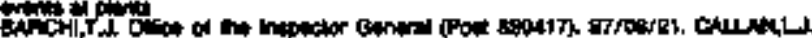

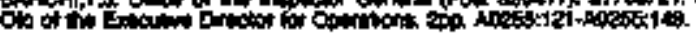

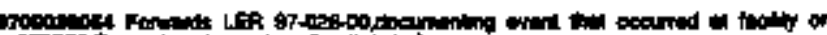

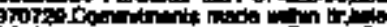

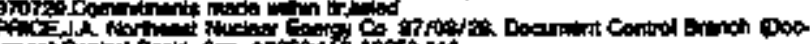

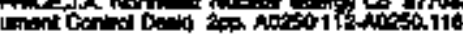

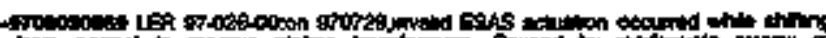

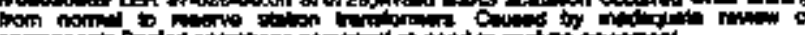

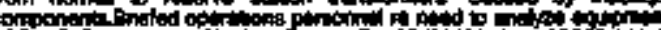

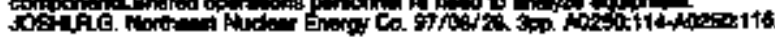




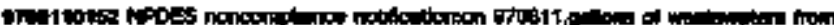

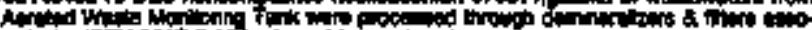

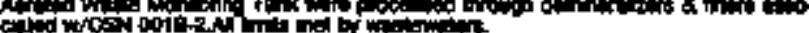

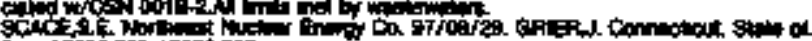

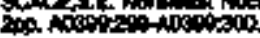

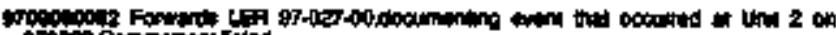

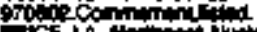

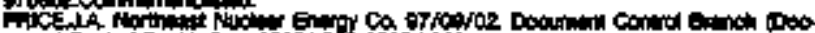

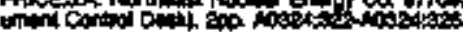

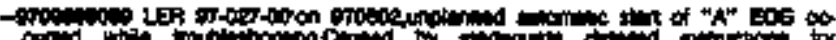

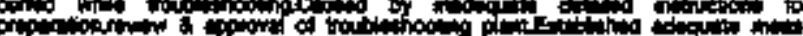

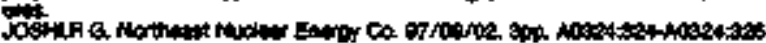

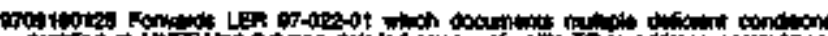

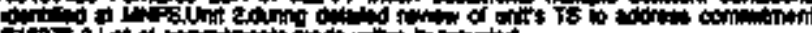

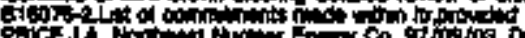

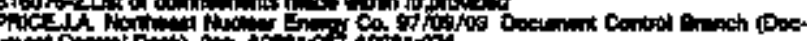

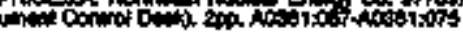

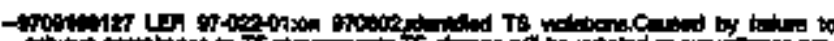

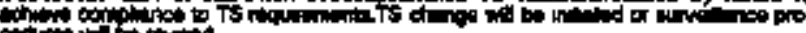

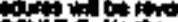

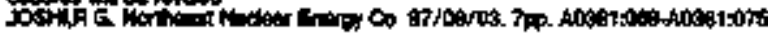

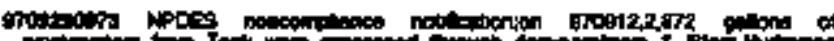

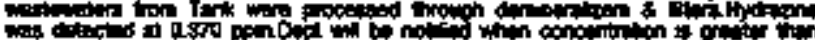

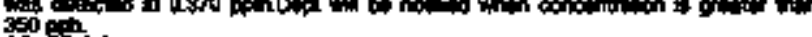
SCOC:

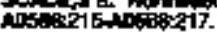

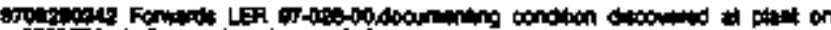
970 ice

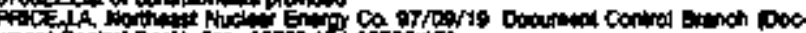

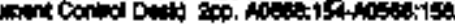

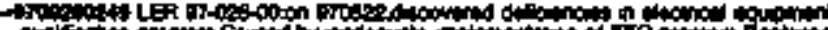

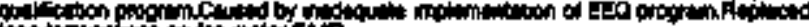

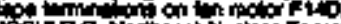

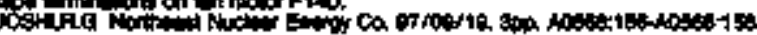

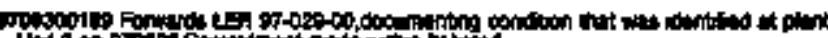

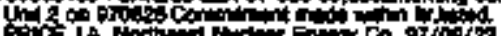

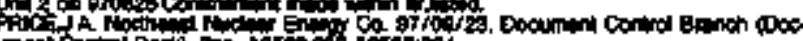

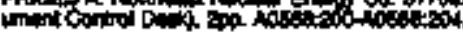

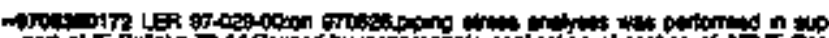

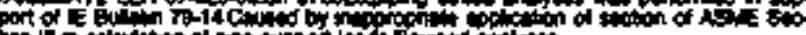

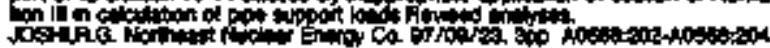

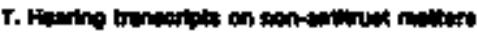

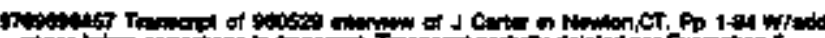

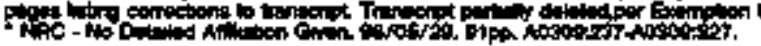

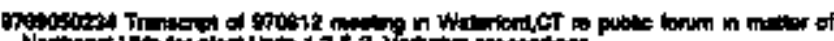

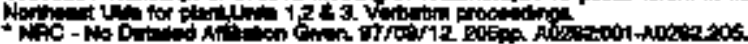

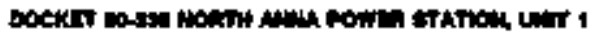

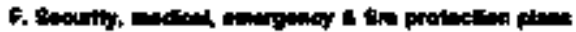

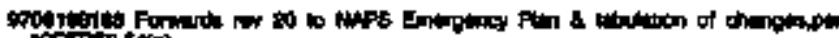

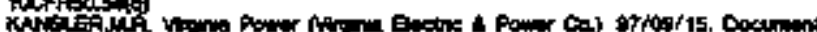

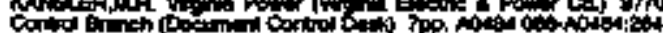

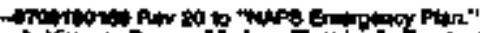

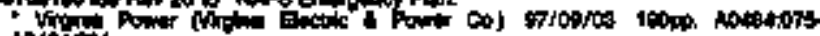
notition.

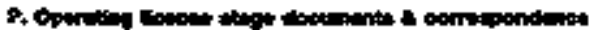

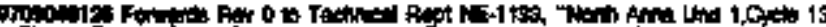

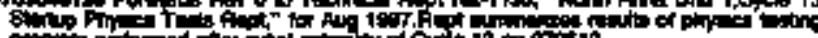

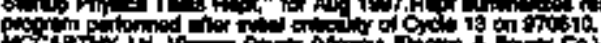

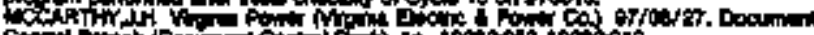

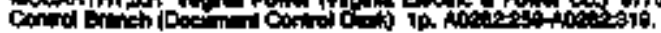

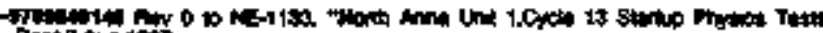
ingt, "Nint

(1)

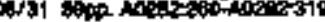

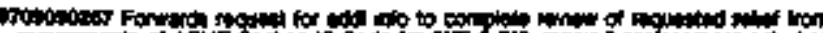

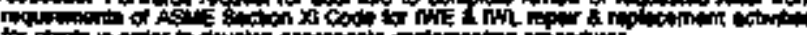

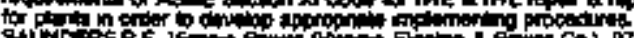

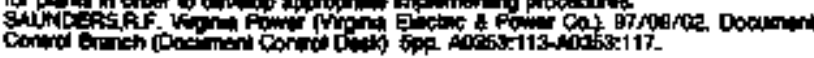

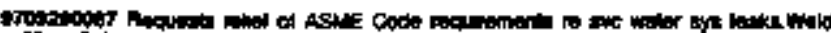

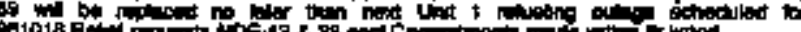

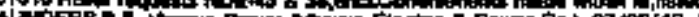

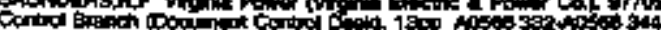

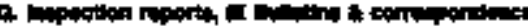

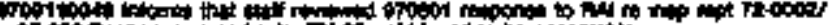

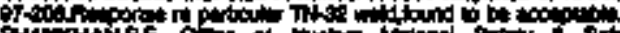

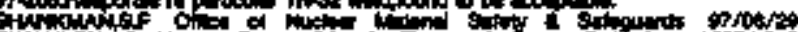

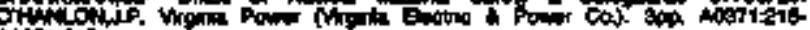
40379217 .

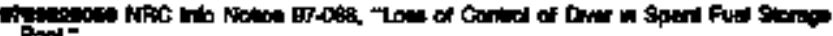

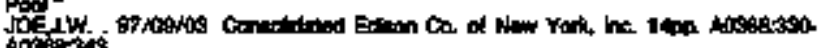

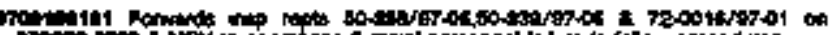

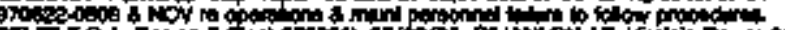
Q tone

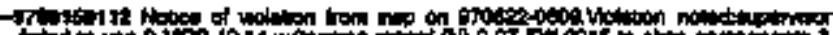

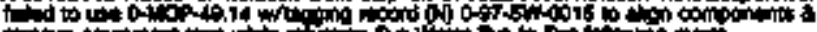

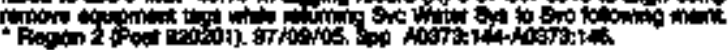

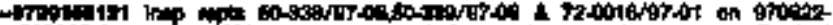

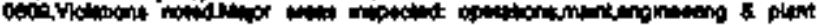

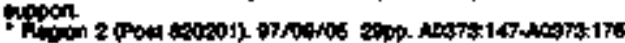

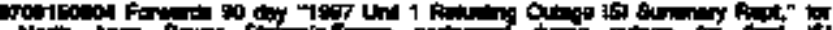

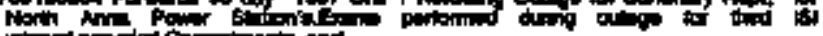

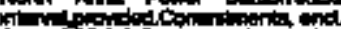

thinito

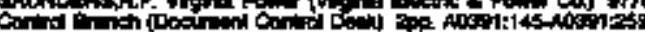

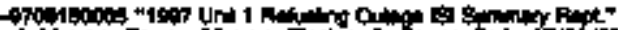

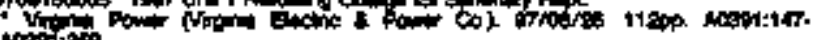

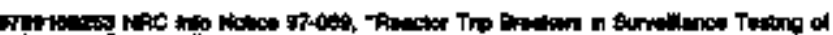

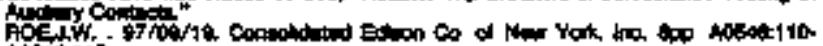
nosotit?

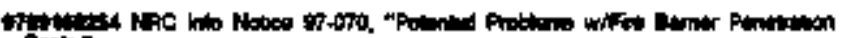

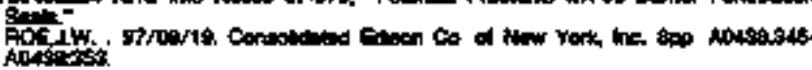

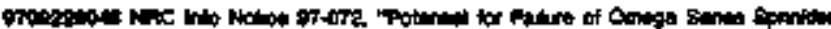

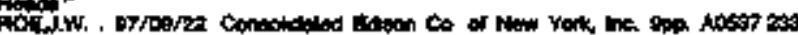
notist.

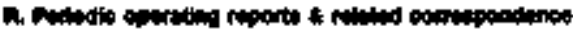

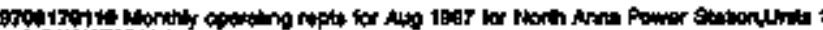

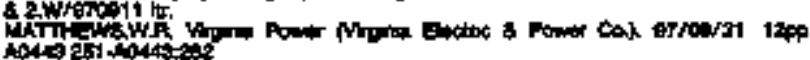

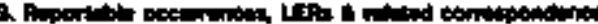

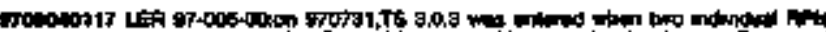

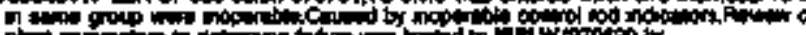

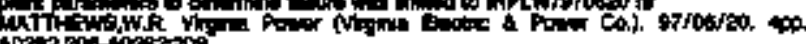

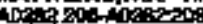

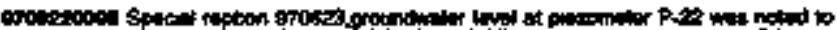

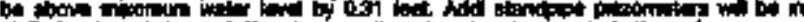
CNh

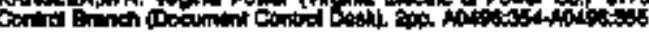

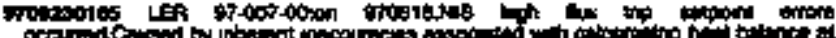

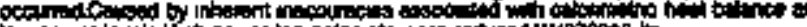
WA Tom

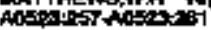

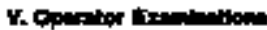

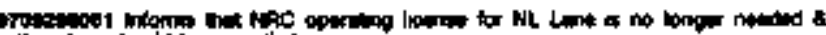

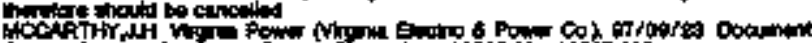

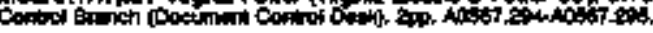

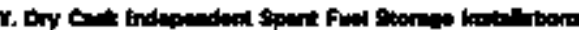

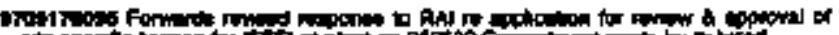

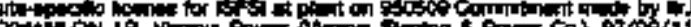

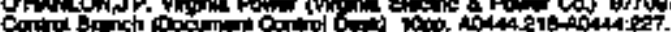




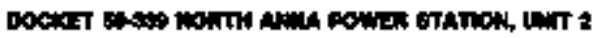

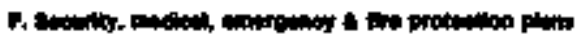

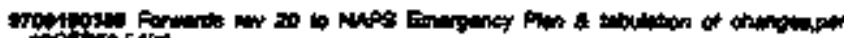
tocitiot 540

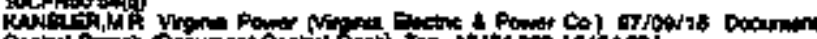

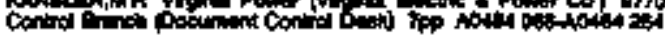

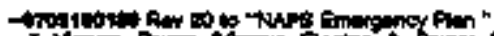

Vy

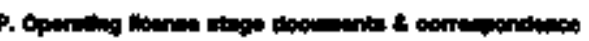

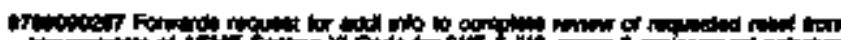

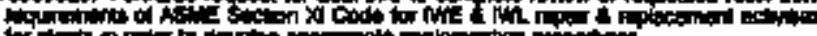

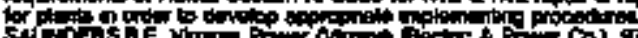

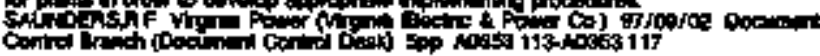

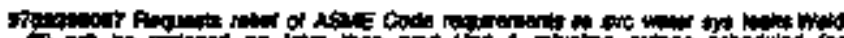

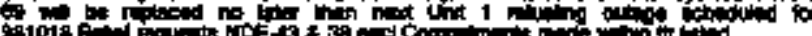

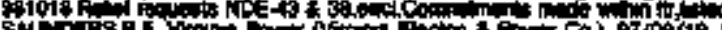

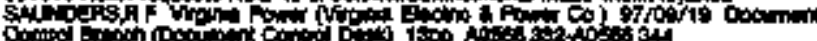

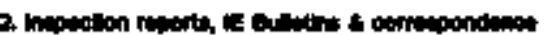

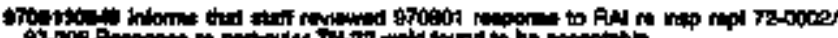

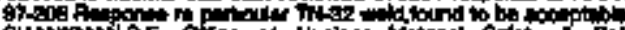

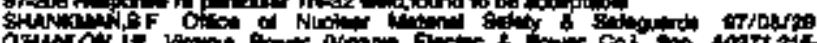
axilizit?

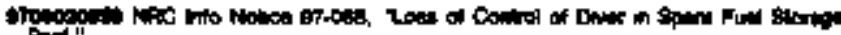

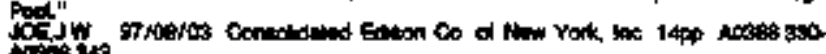
A.

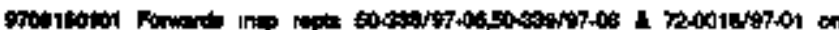
to

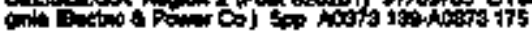

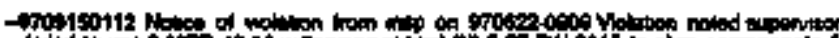
Hed to un of

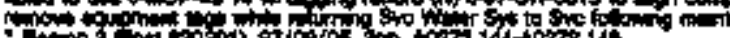

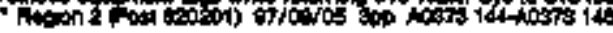

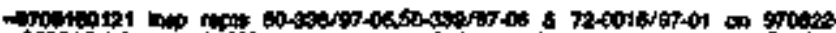

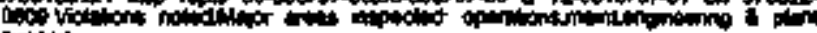
tiont

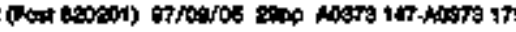

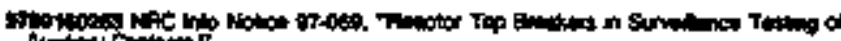

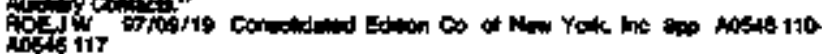

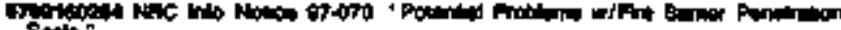

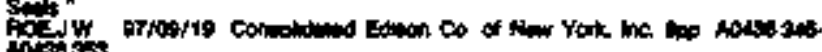

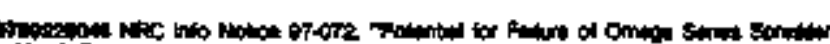

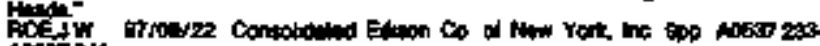
M.T.

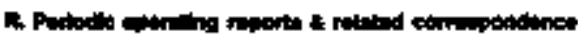

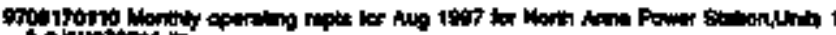

E

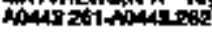

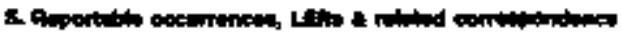

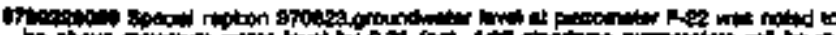

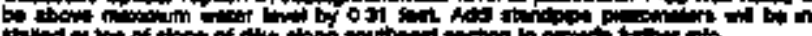

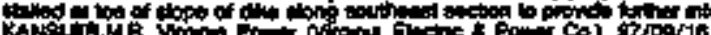

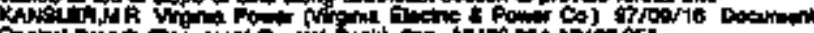

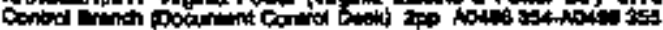

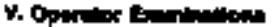

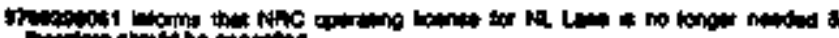

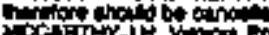

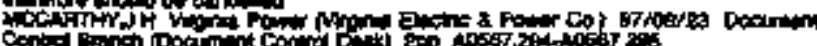

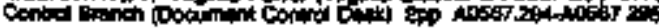

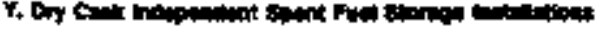

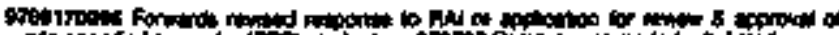

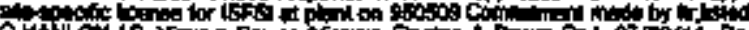

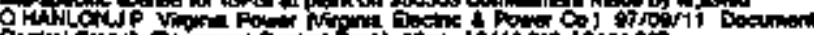

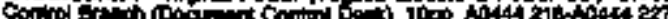

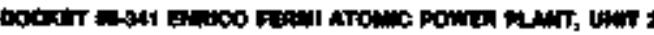

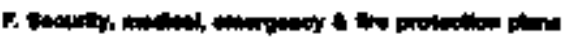

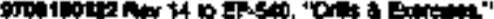

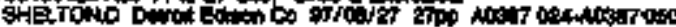

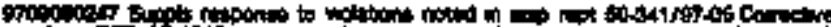

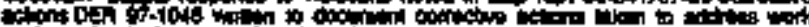

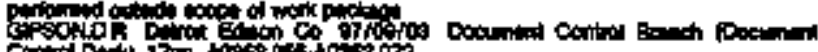

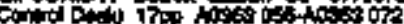

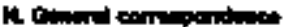

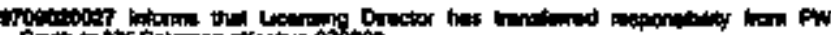

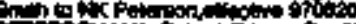

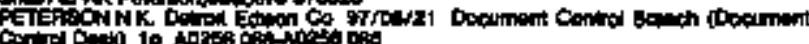

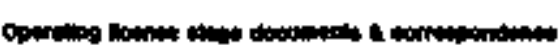

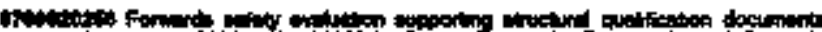

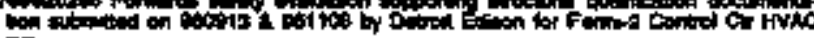

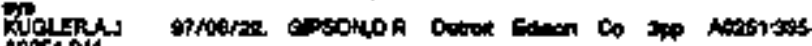
A

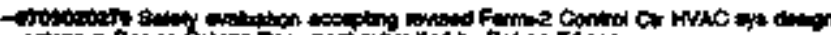

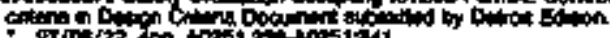

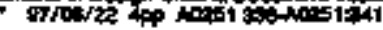

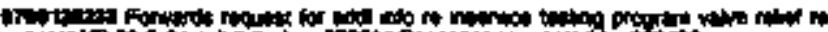

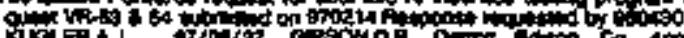

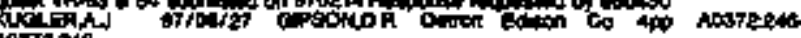
asm2 204:

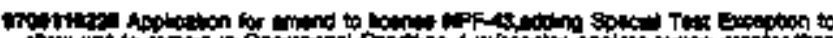

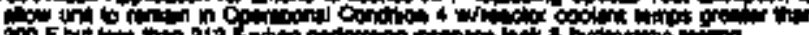

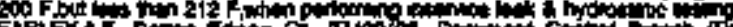

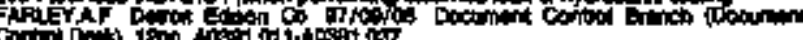

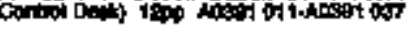

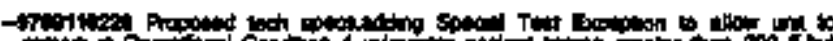

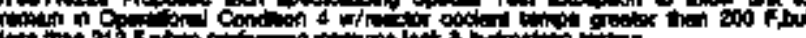

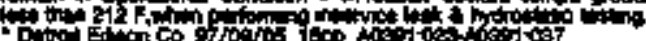

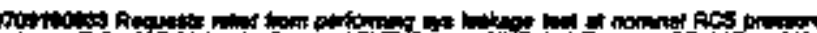

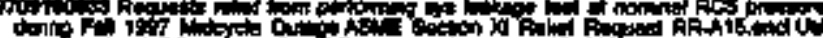

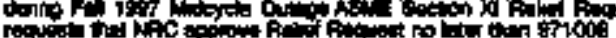

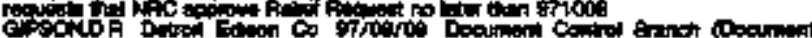

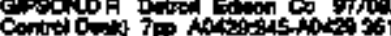

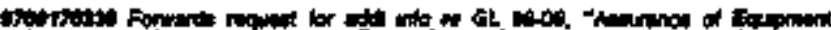

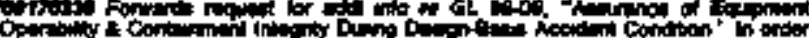

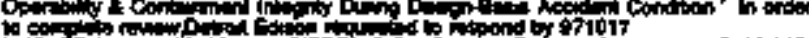

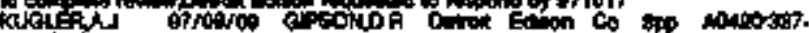
and

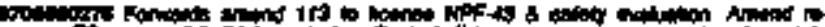

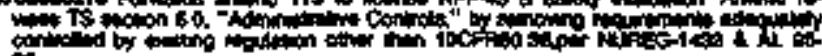

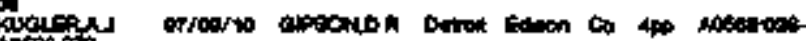
instion

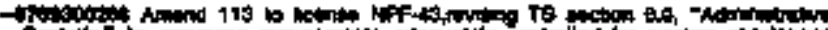

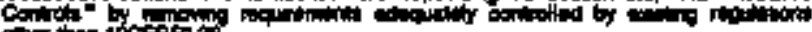

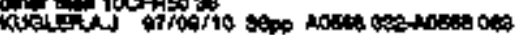

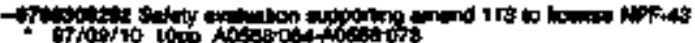

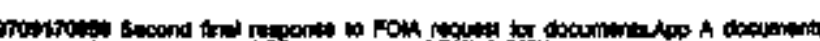
beng

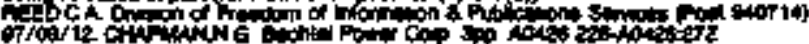

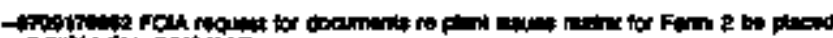

CPOA

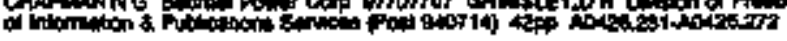

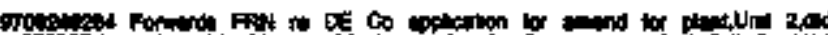

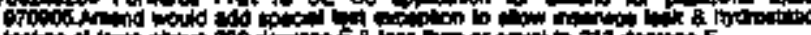
kLif AD:24313

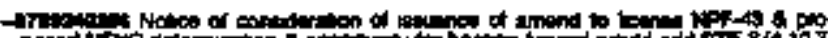

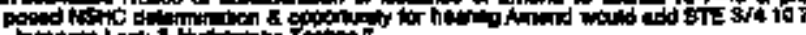

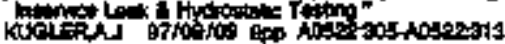

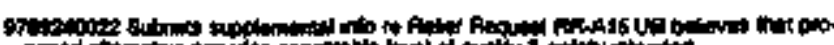

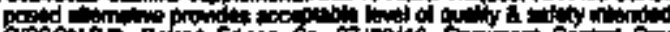

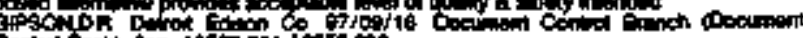

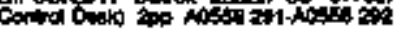




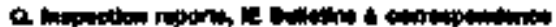

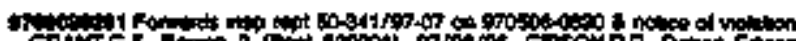

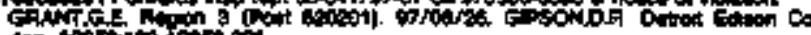

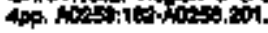

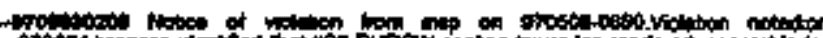

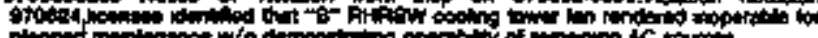

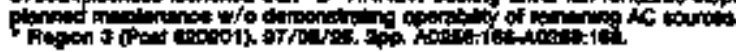

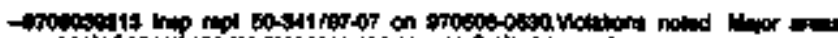

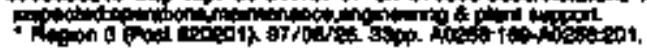

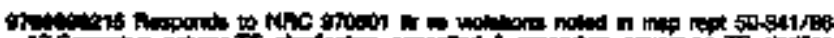

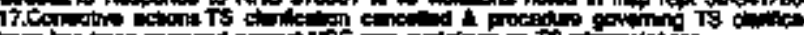

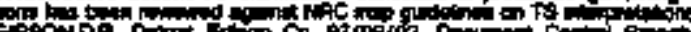

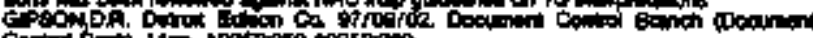

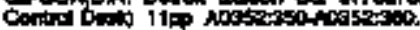

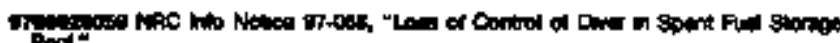

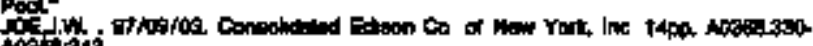
nostaras.

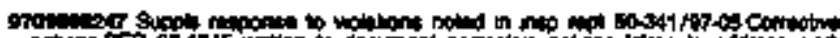

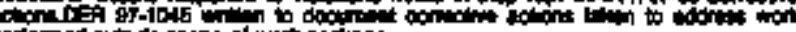
rod

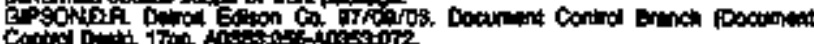

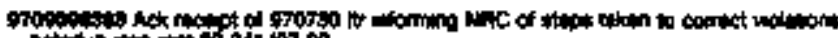

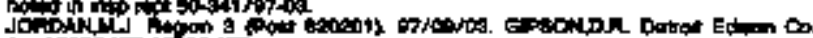

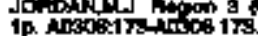

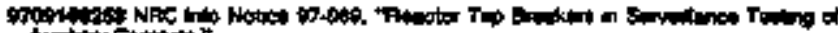

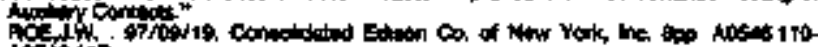
it: 17

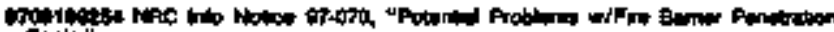

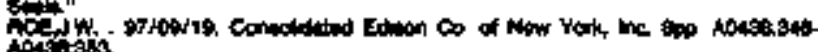
notion

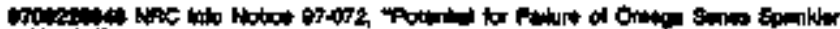

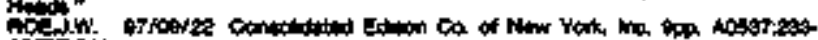
7.ton.

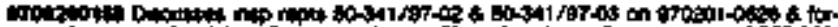

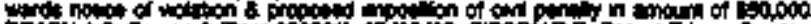

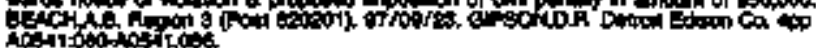

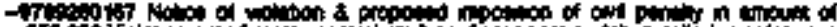

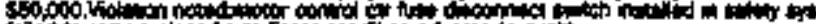

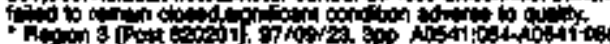

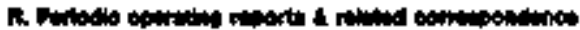

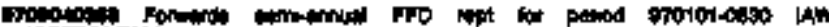

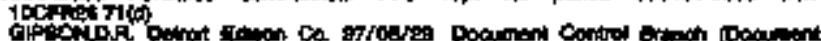

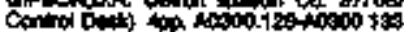

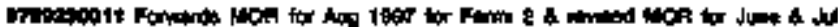

Peir. Cincin

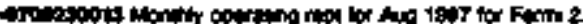

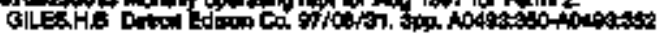

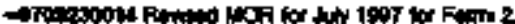

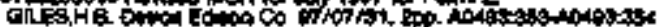

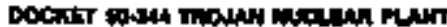

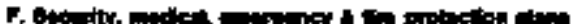

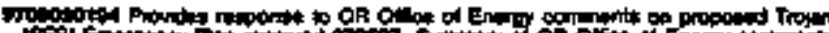

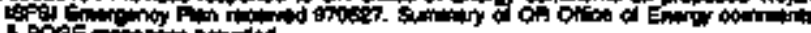

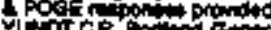

Plot

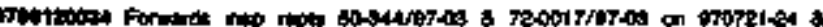

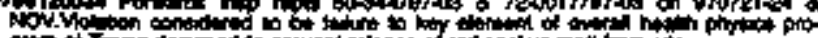

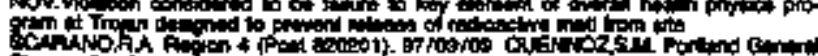

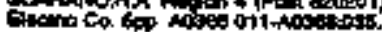

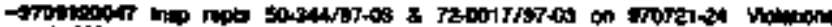

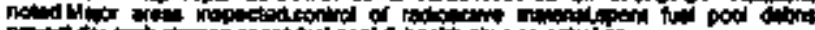

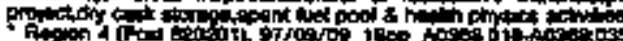

1. In

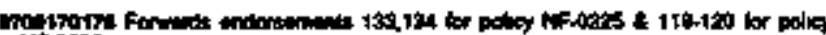

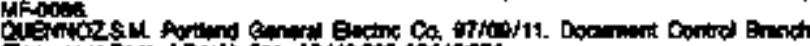

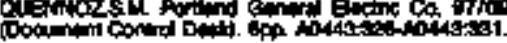

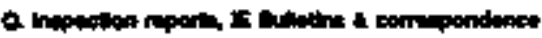

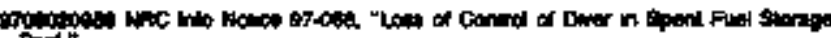
Pod

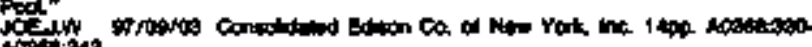
sition.

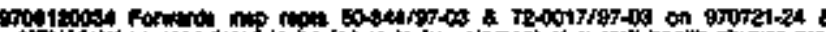

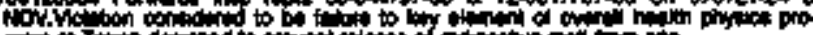

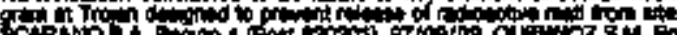

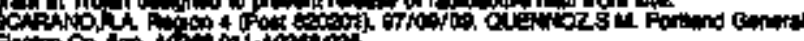

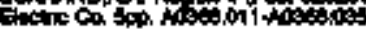

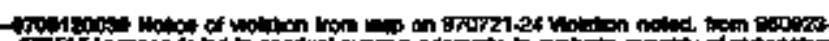

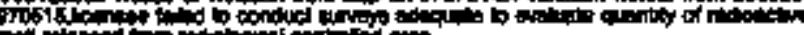

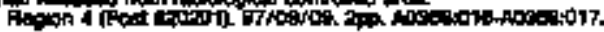

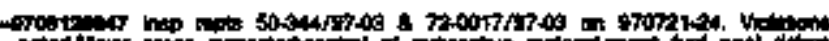

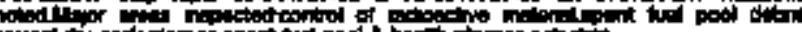

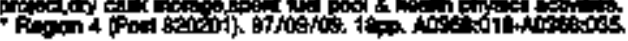

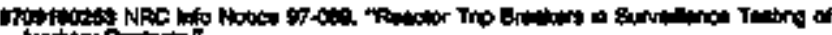

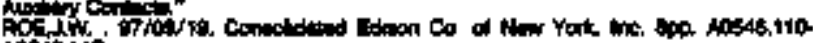
athent?

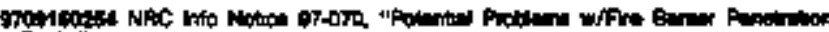

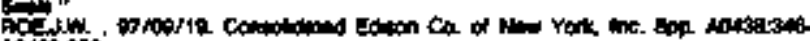
4040.

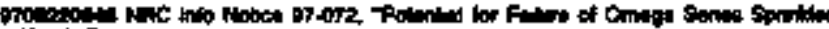

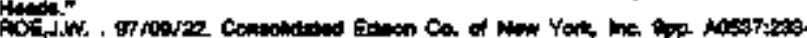
No6roty:

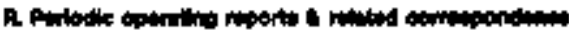

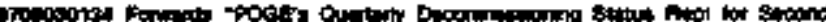

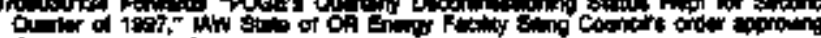
teocrin: onn pien

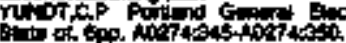

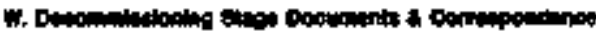

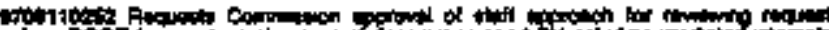

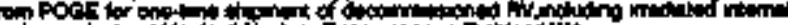

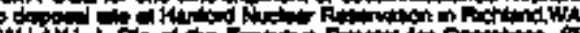

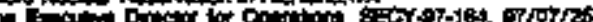

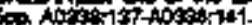

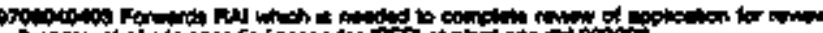

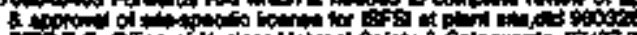

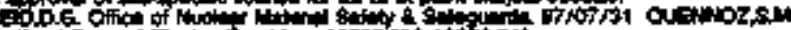

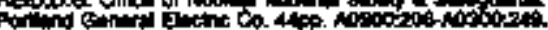

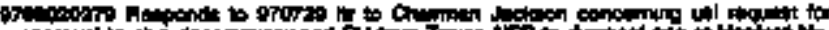

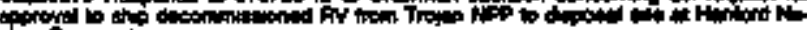

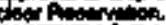

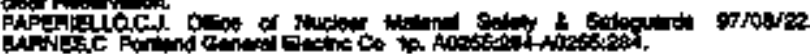

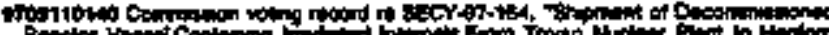

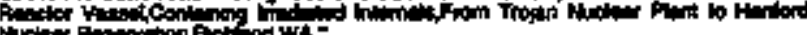

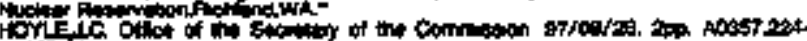
10057.200

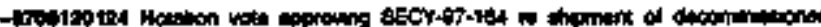

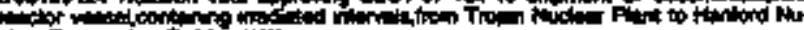

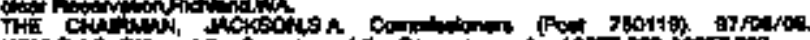

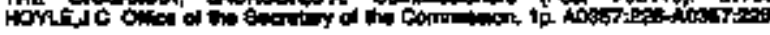

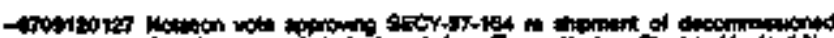

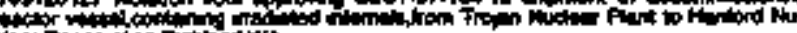

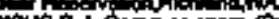

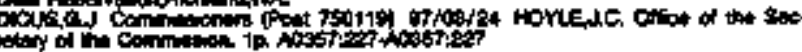

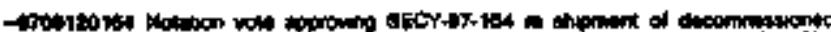

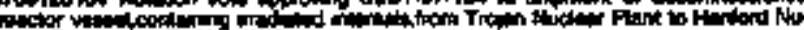

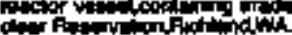

ortid by

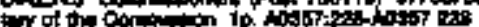

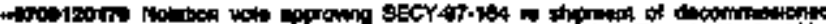

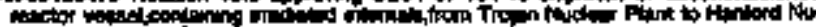

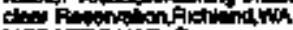

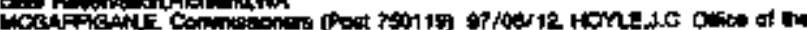

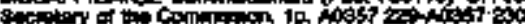




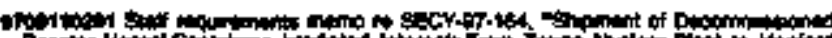

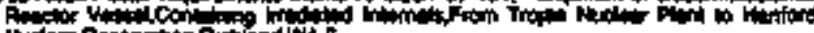

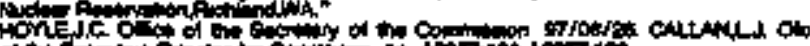

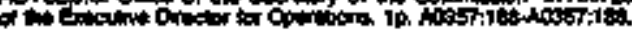

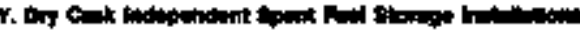

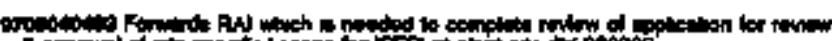

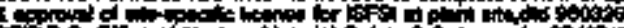

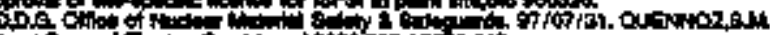

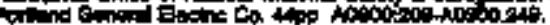

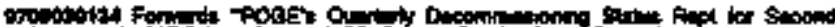

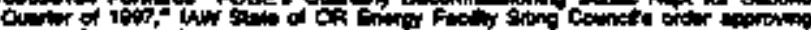
decoming

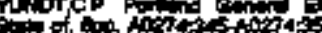

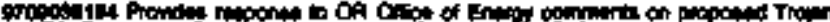

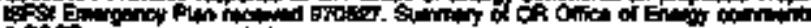

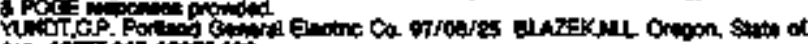

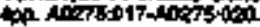

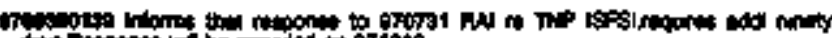

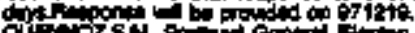

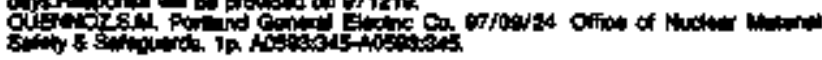

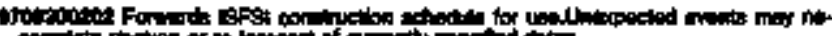

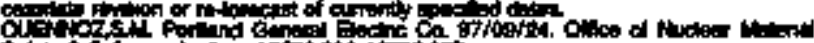

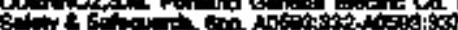

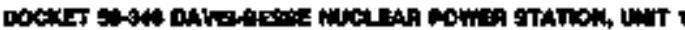

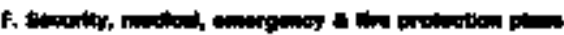

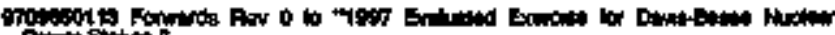

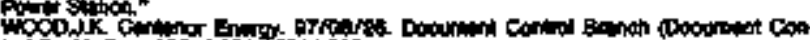

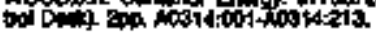

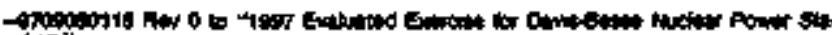

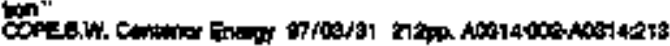

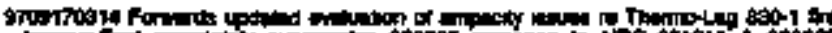

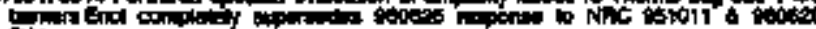
Fintorition

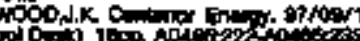

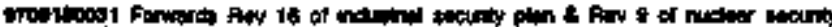

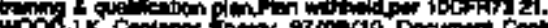

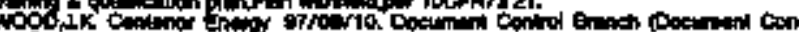

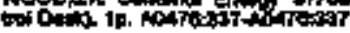

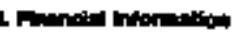

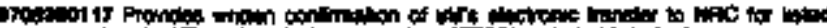

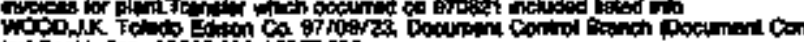

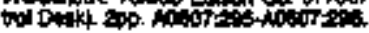

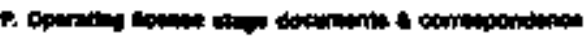

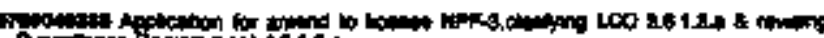

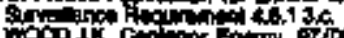

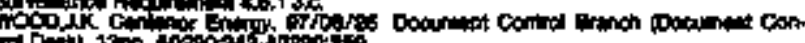

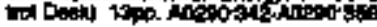

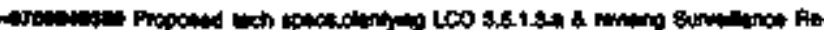
and

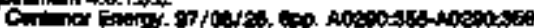

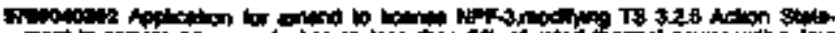

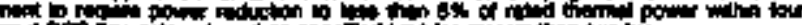

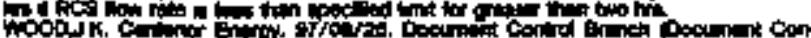

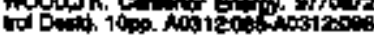

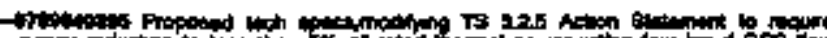

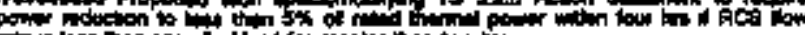

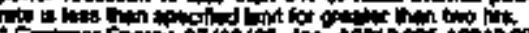

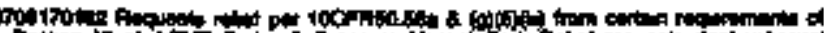

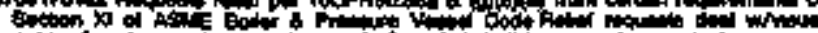

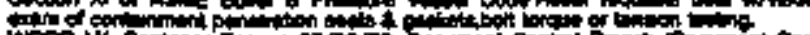

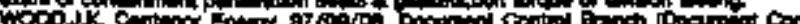

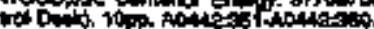

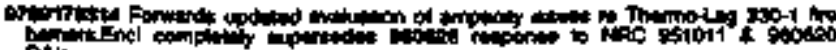

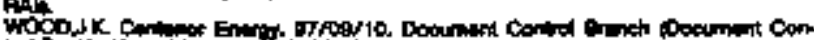

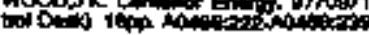

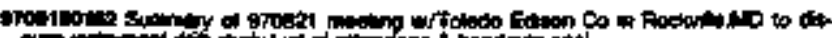

A

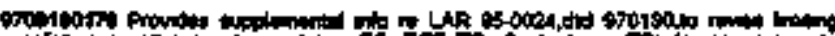

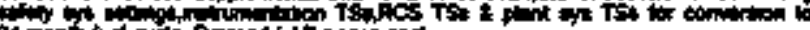

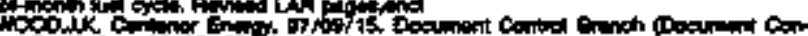

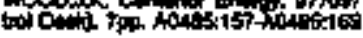

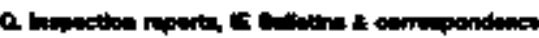

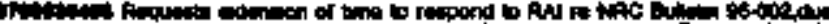

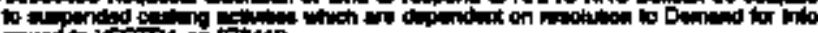

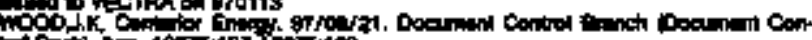

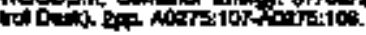

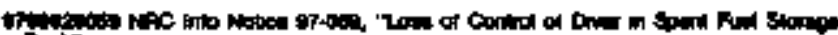
Poct.

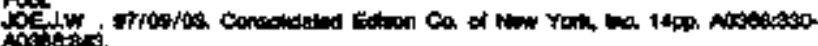

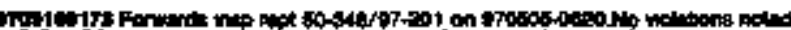

Dow rowas,

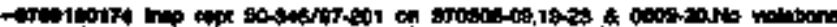

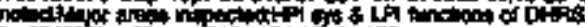

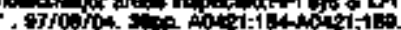

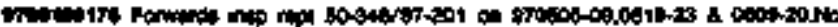

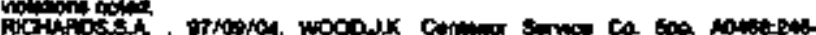
Aptition

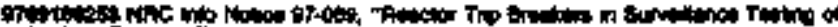

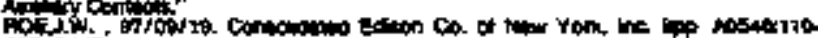
Aositis?

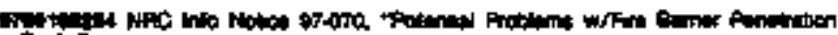
POE Aositis:

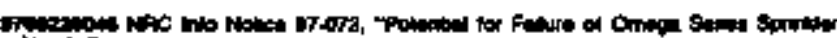

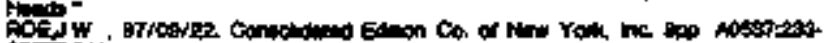
Arstint:

It ri-

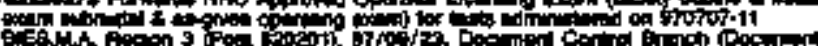

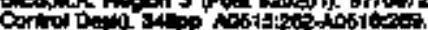

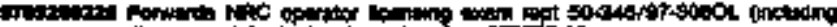

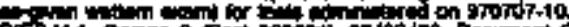

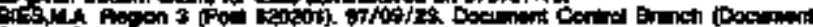

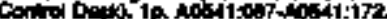

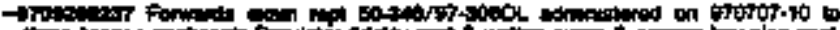

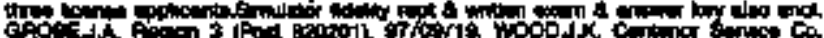

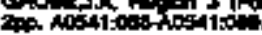

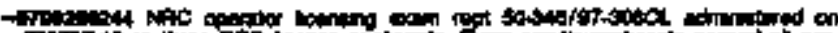

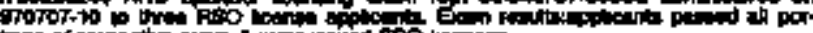

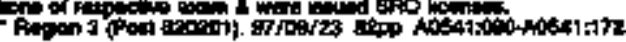

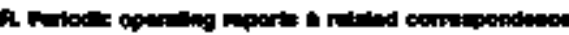

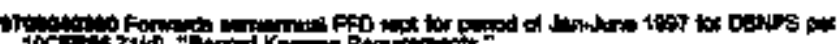
TCC Tod

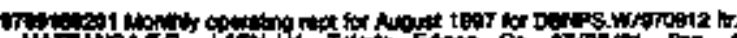

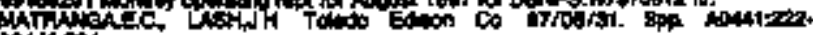
inititat

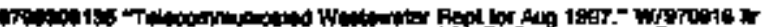

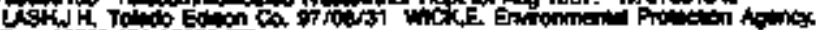
an 100 in

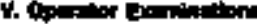

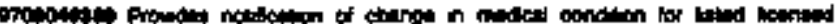

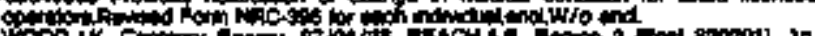

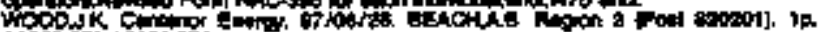
isto

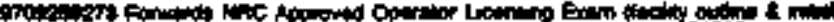

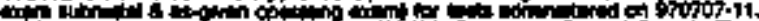

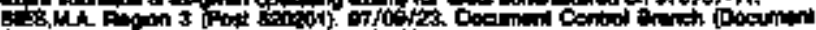

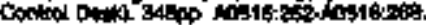

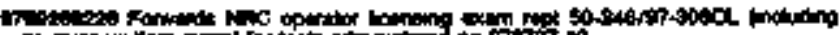

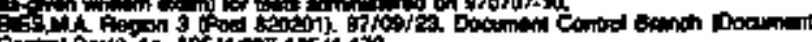

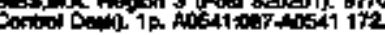

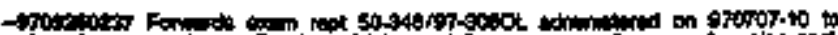

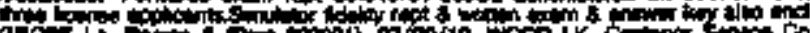

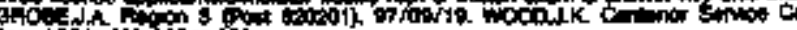

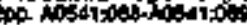




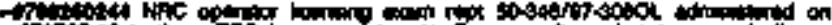

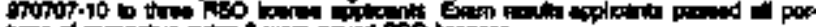

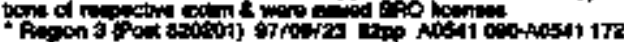

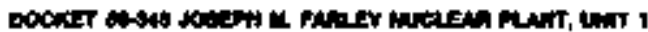

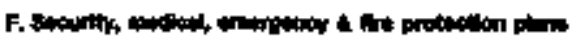

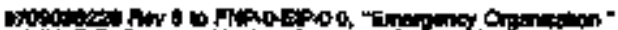

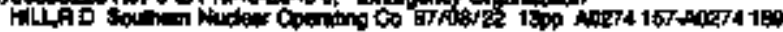

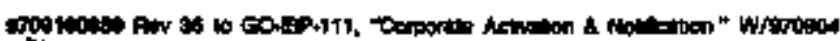

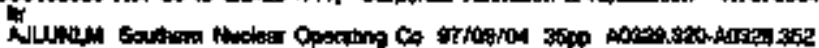

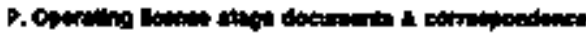

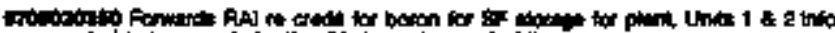

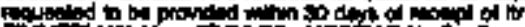

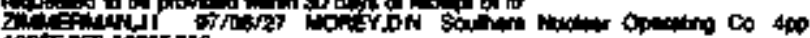

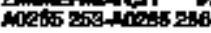

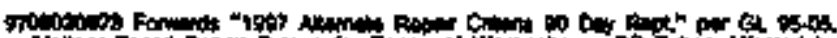

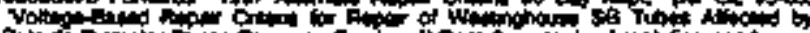

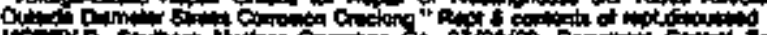

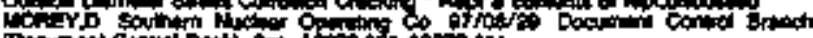

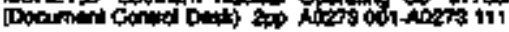

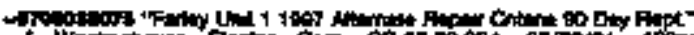

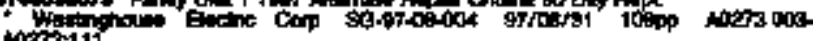

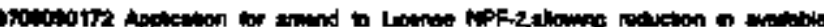

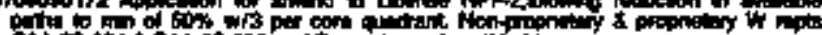

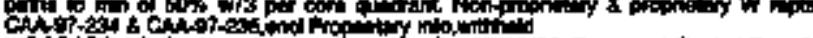

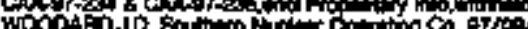

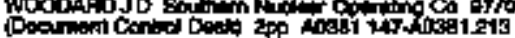

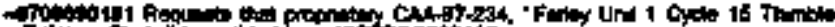

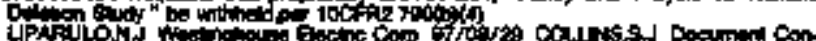

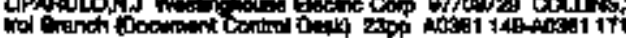

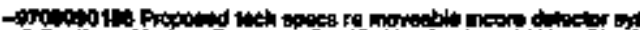

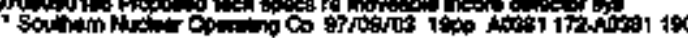

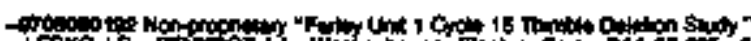

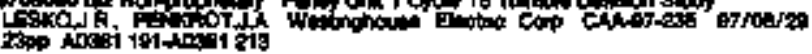

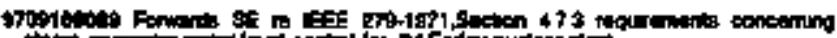

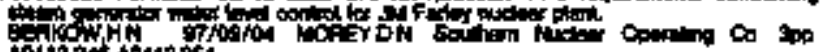
tont2

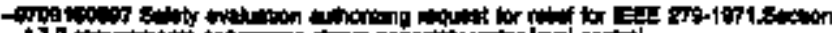

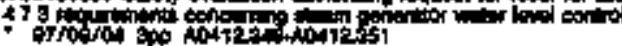

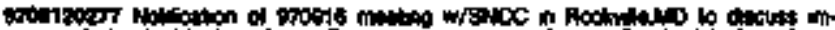

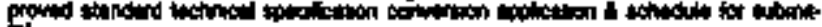

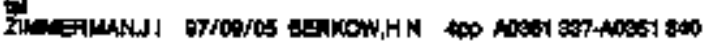

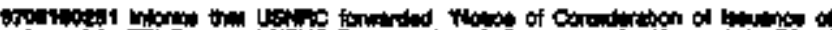

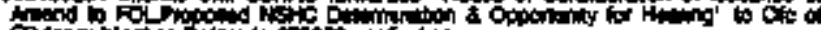

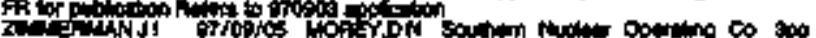

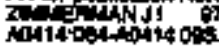

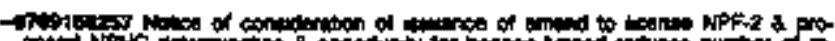

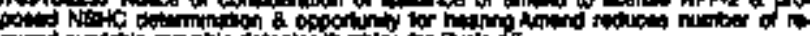

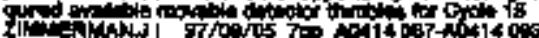

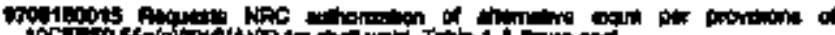

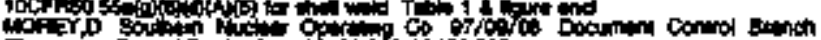

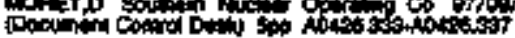

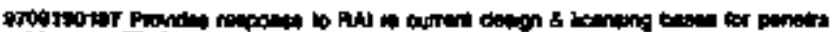

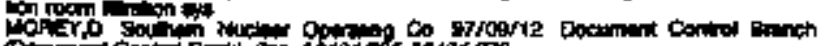

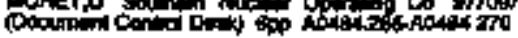

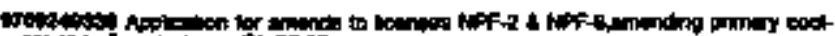

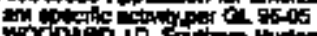

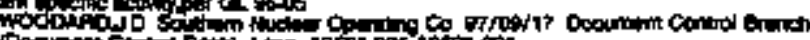

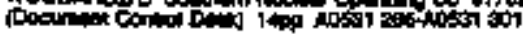

- Py

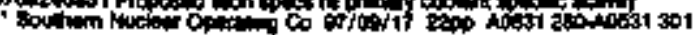

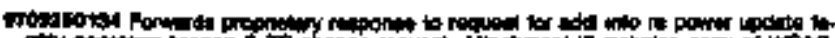

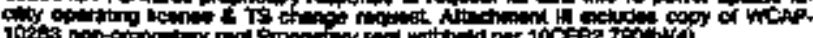

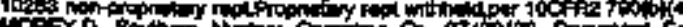

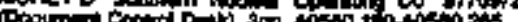

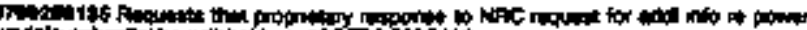

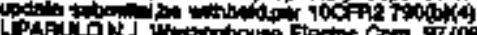

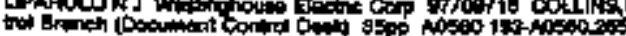

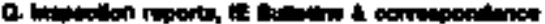

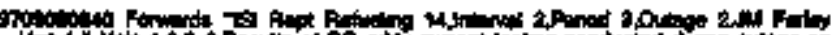

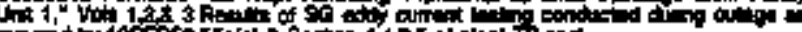

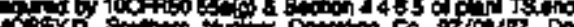

Can

"

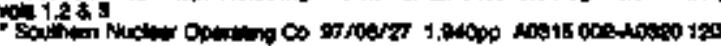

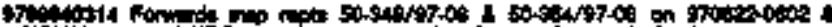

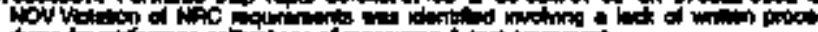

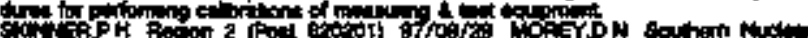

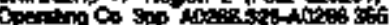

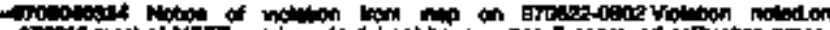

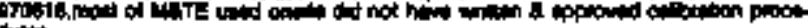

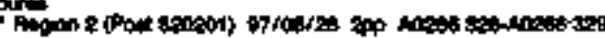

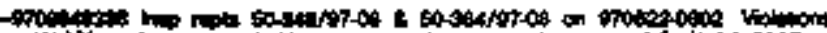

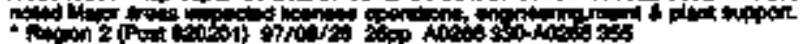

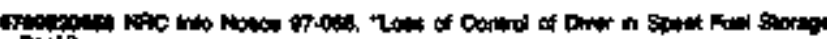

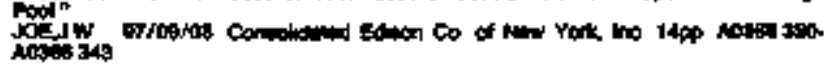

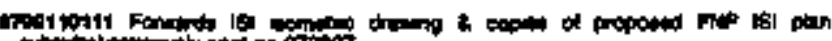

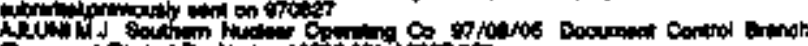

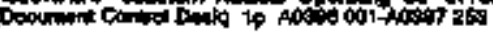

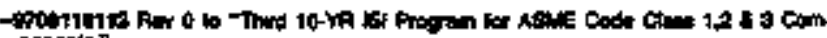

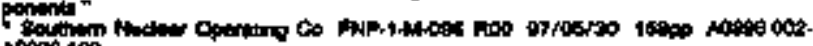
notist 160

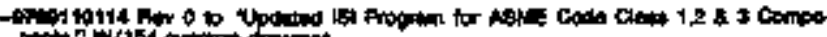

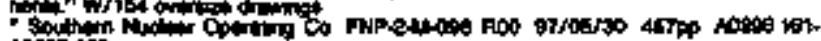

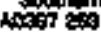

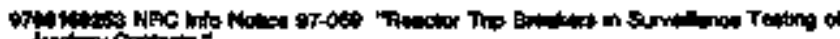
Fon 4int: 17

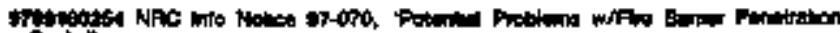

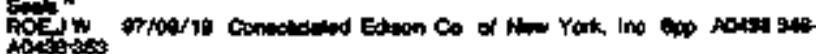

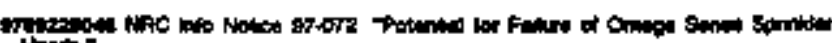

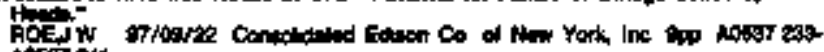
Aosit 241

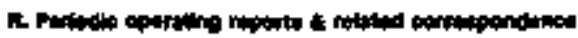

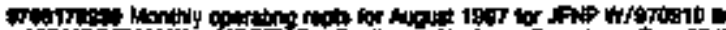

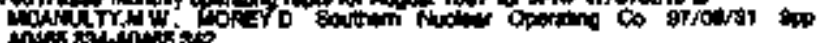

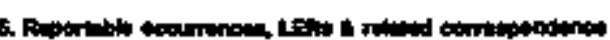

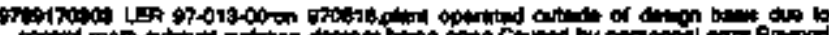

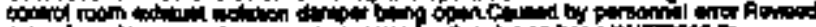

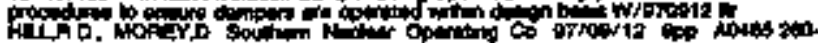
10.6.

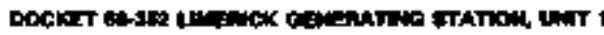

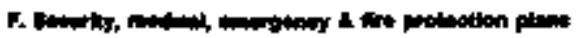

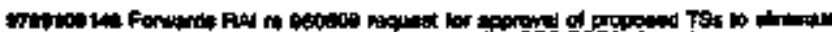

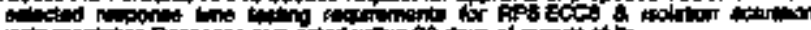

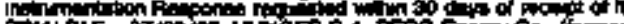

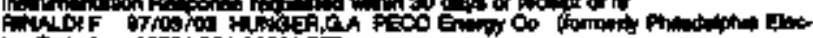

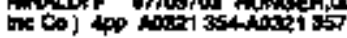

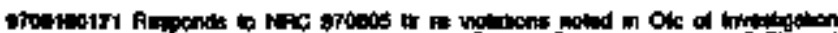

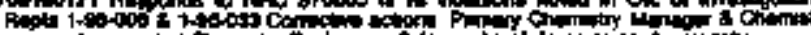

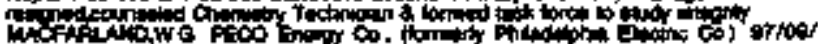

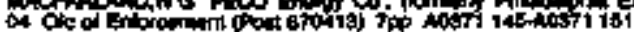

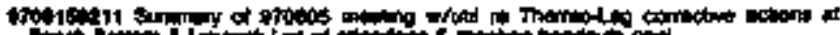

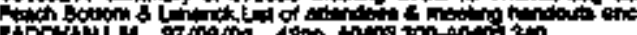

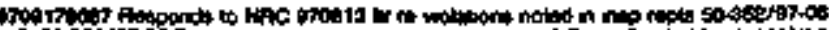

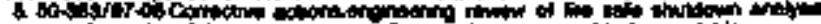

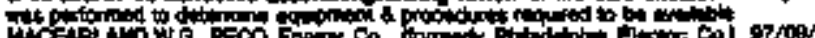

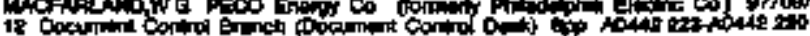




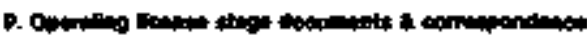

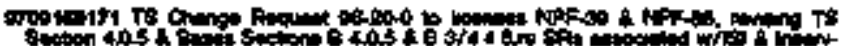

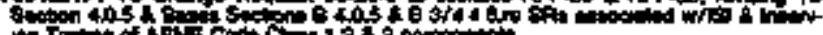

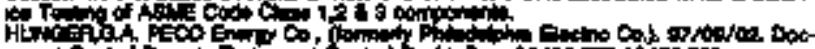

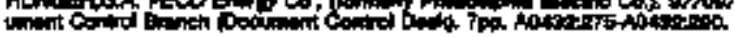

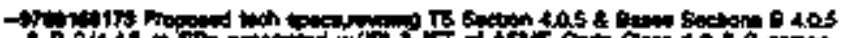

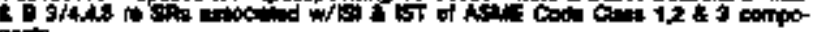

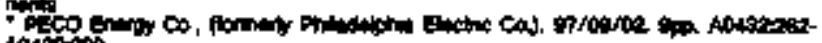
Notionso

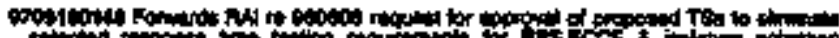

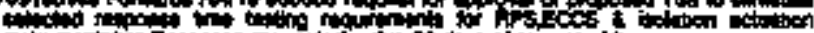

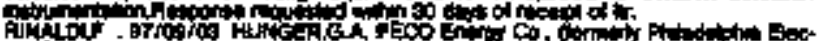

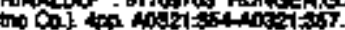

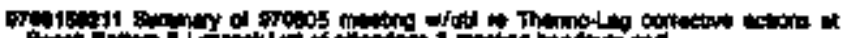

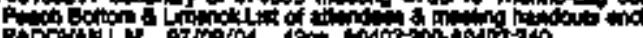

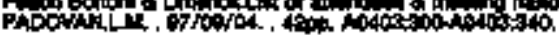

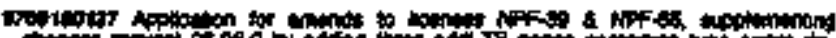

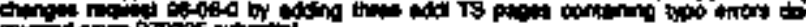

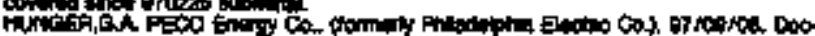

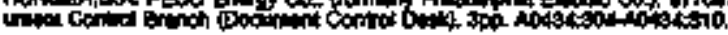

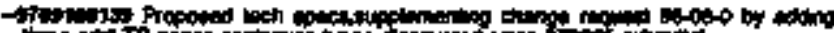

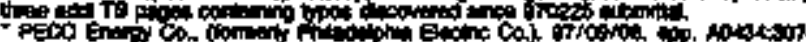
notisto.

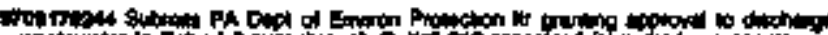

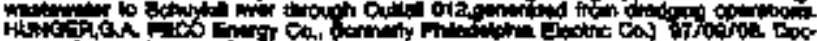

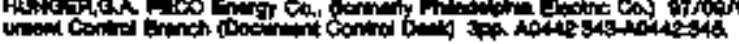

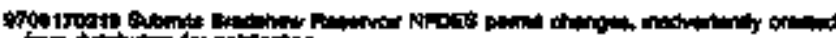

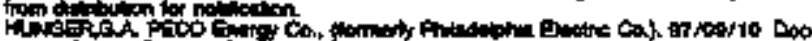

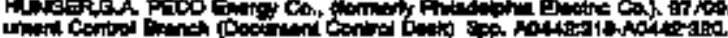

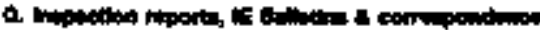

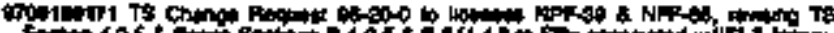

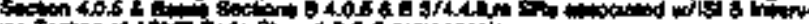

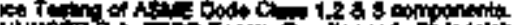

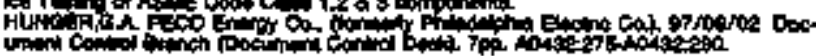

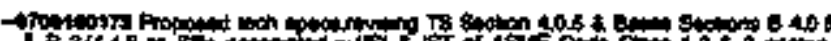

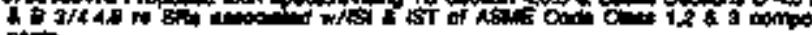

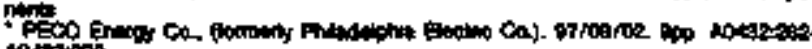
$100 \mathrm{sic}=0$

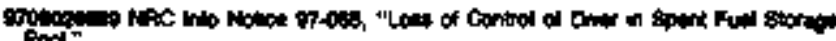
Pod A0sinsis:

Fon

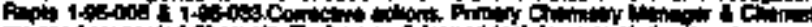

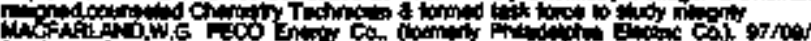

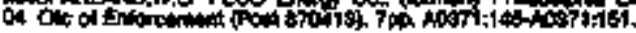

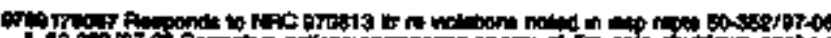

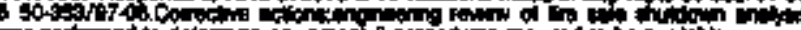

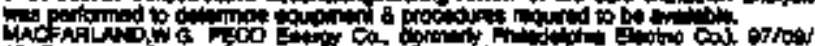

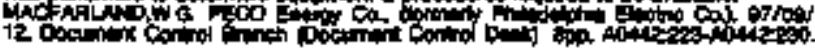

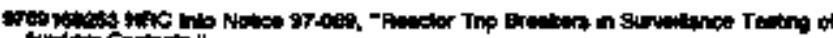

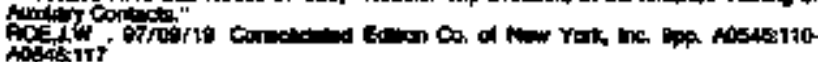

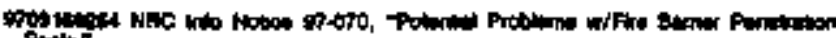

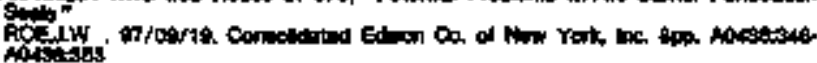

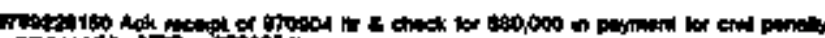

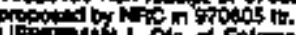

Foc

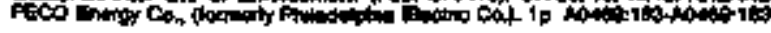

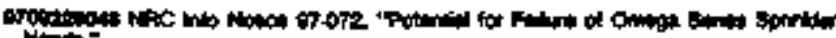

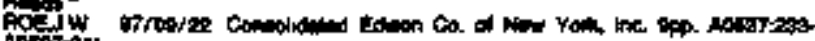

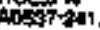

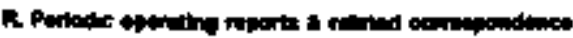

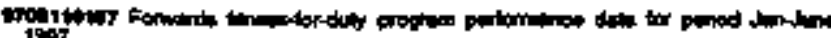

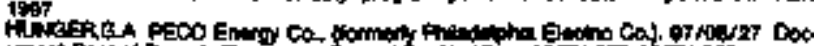

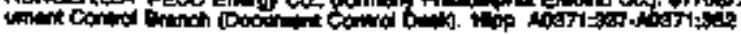

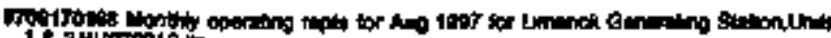

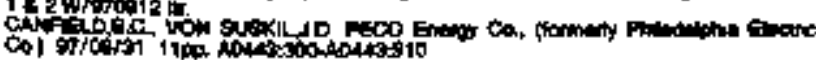

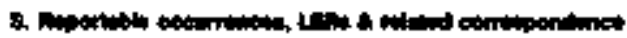

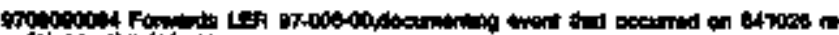

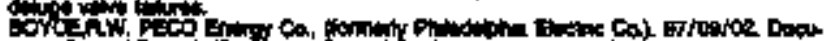

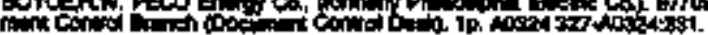

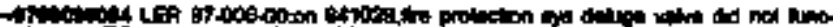

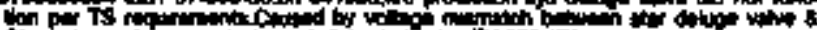

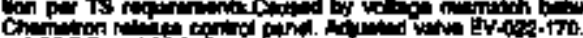

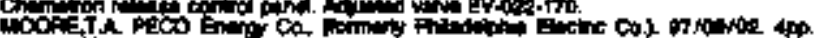

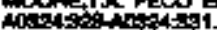

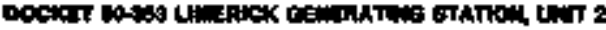

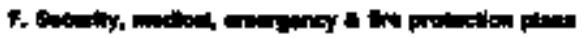

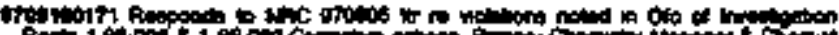

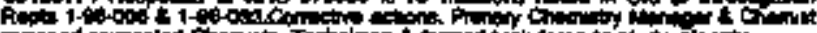

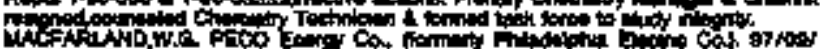

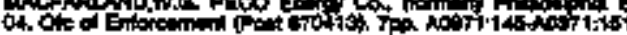

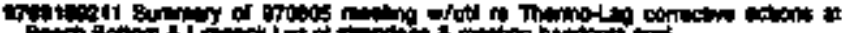

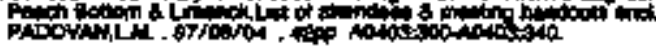

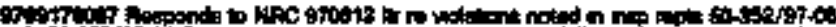
A 80 or

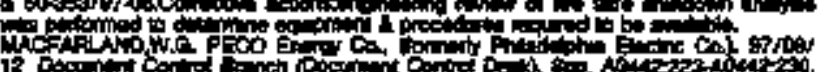

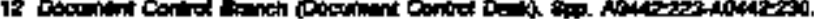

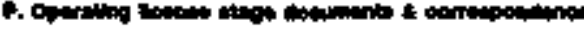

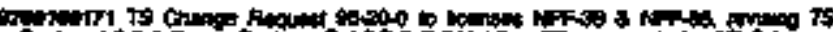

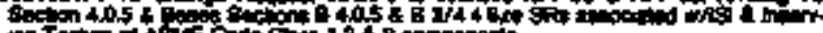

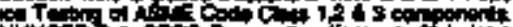

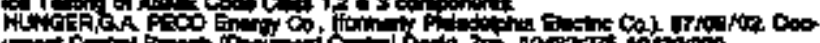

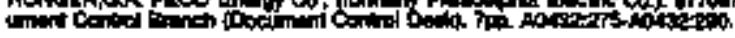

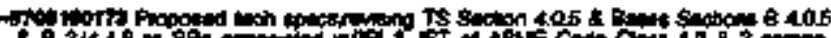

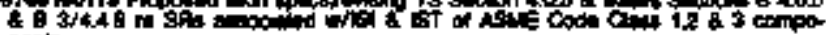

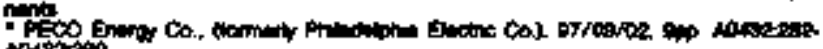
notion.

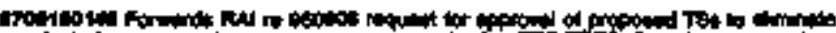

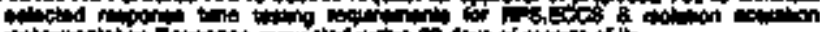

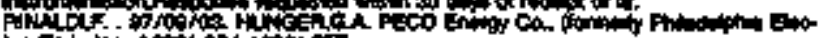
tont 0 1

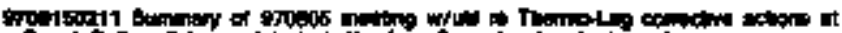

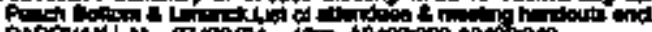

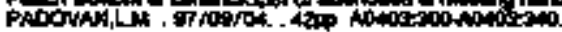

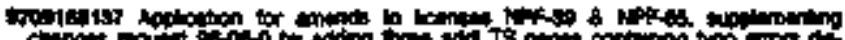

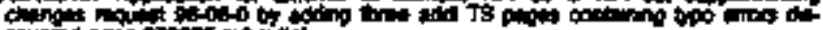
coting

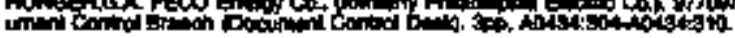

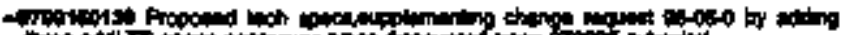

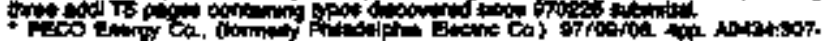
atgite.

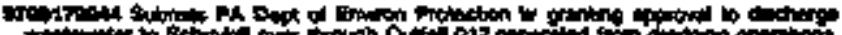

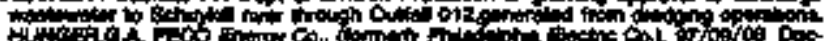

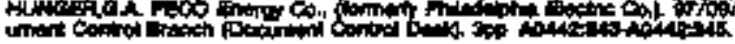

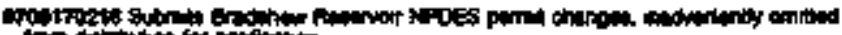

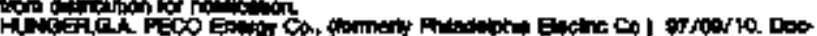

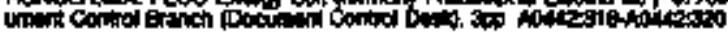

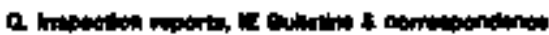

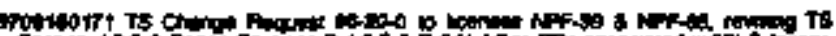

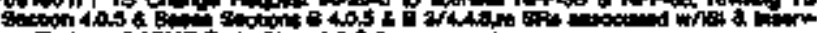

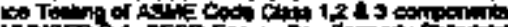

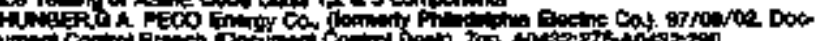

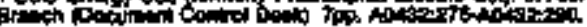

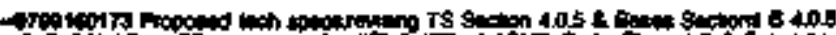

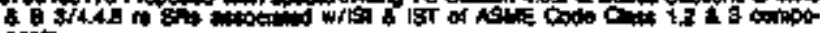

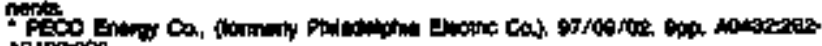

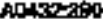

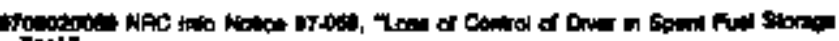

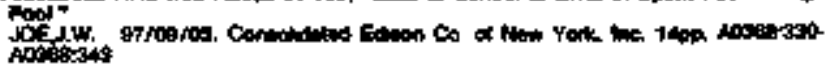




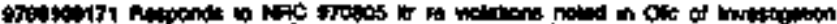

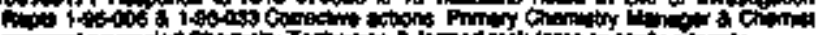

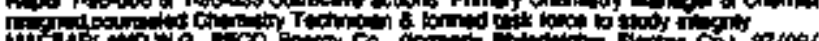

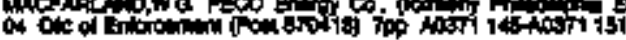

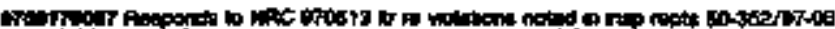

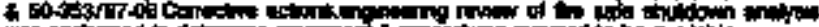

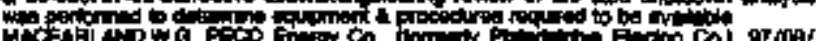

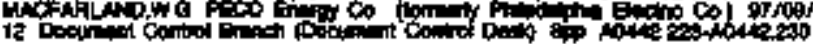

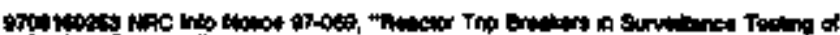

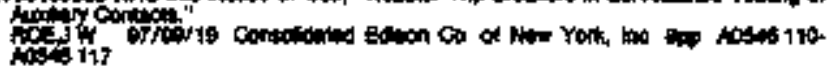

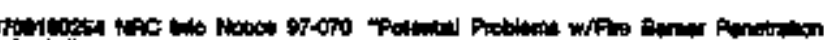

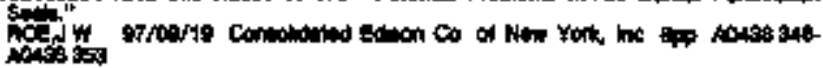

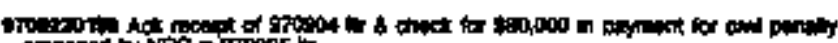

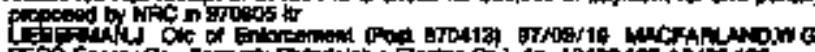

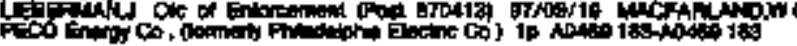

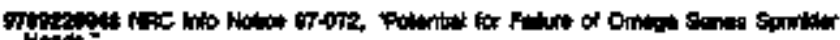

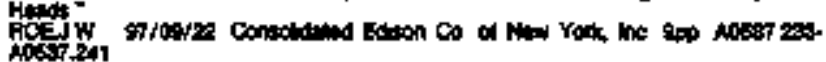

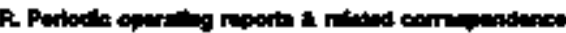

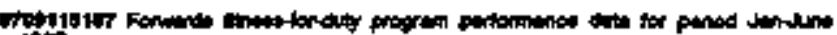

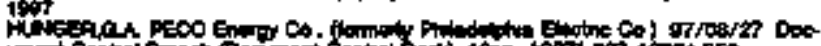

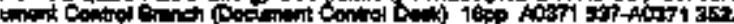

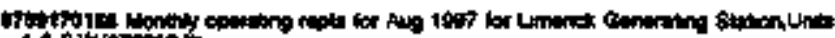

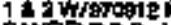

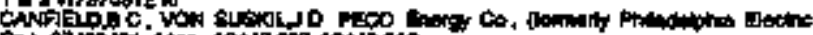

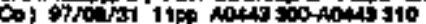

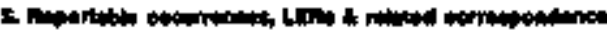

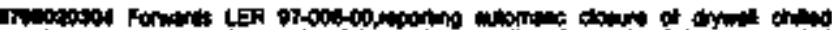

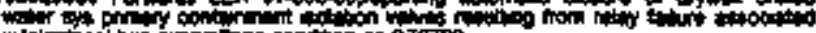

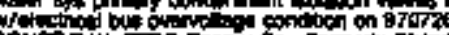

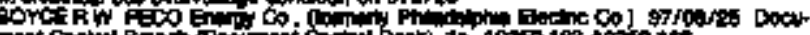

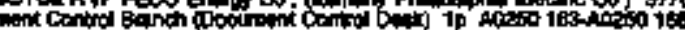

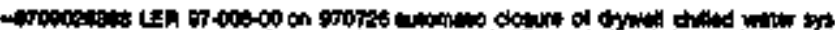

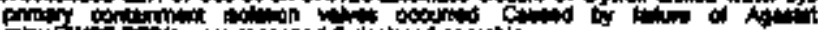

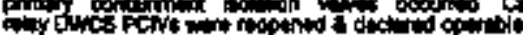

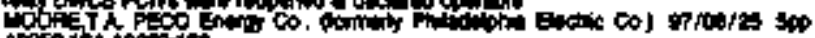

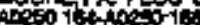

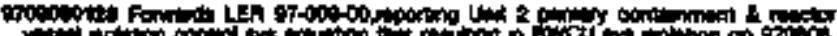

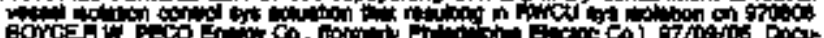

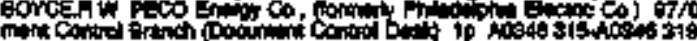

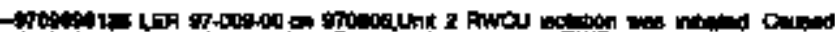

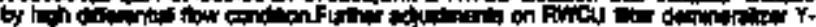

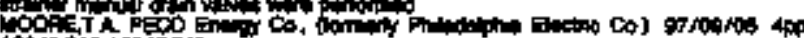

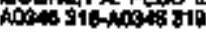

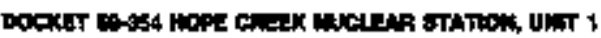

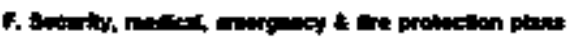

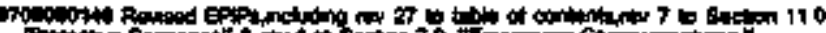

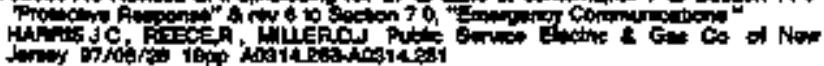

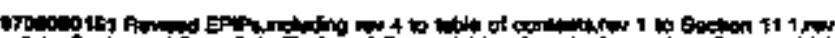

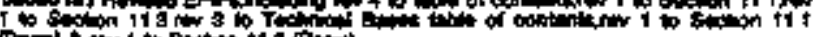

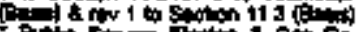

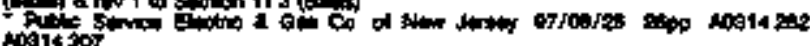

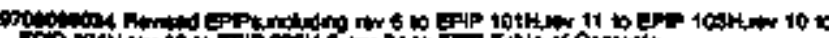

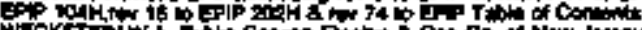

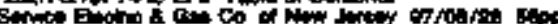
4392000034245

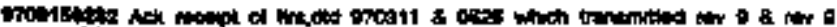

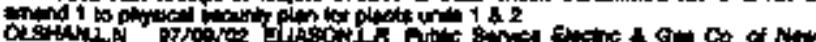

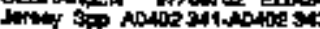

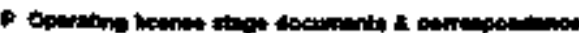

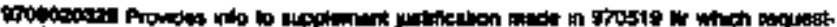

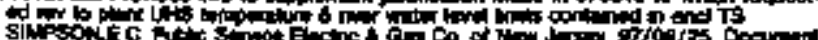

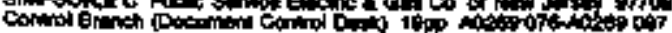

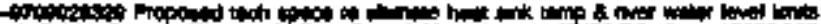

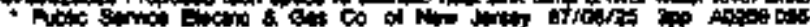
notion

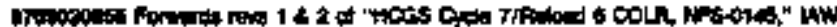
Istopis 10

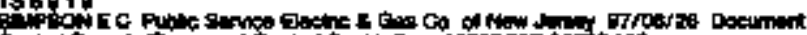

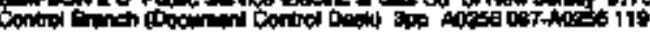

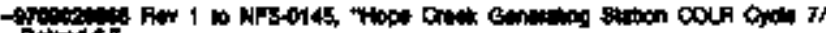

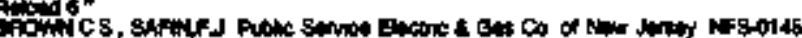

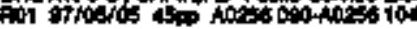

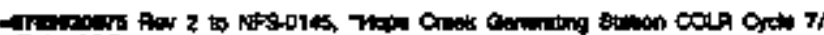

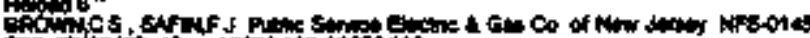

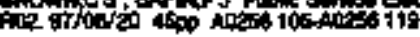

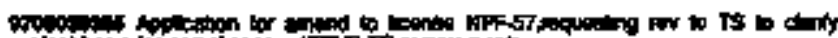

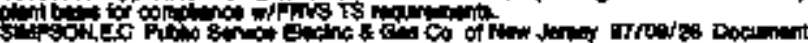

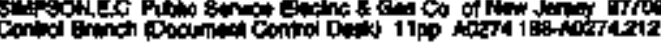

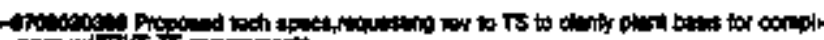

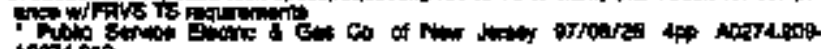
Aotions

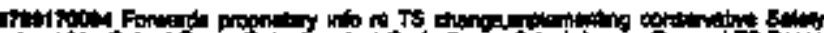

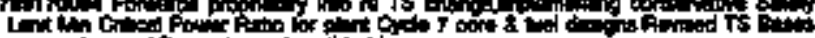

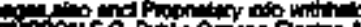

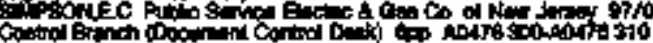

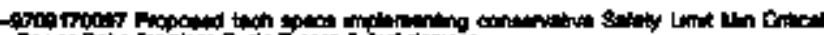

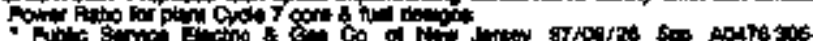
Notrisitio

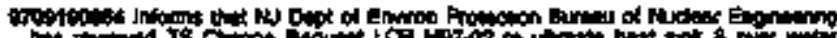

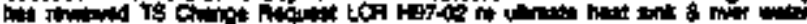

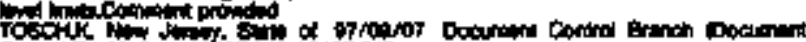

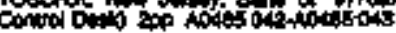

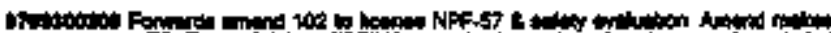

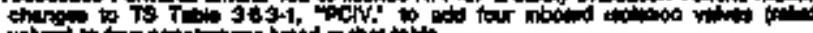

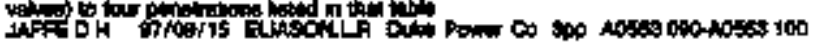

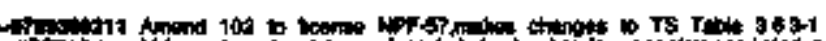

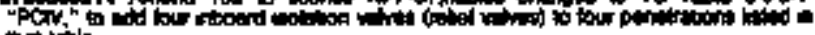

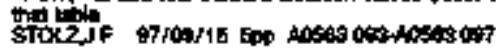

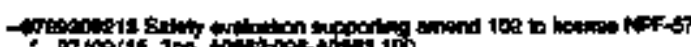

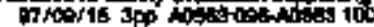

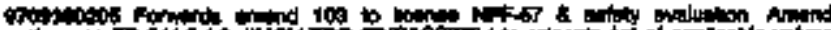

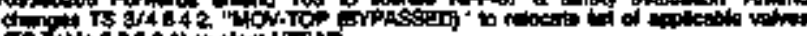

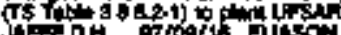

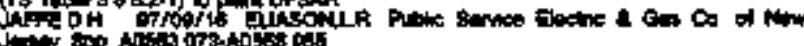

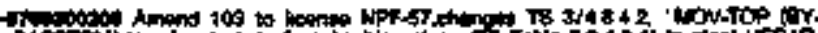

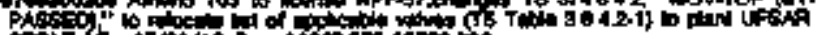

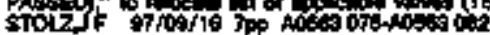

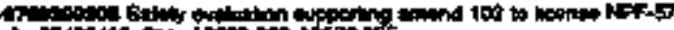

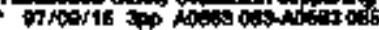

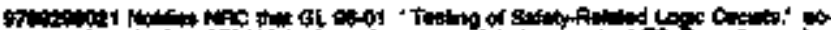

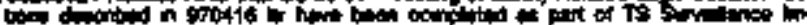

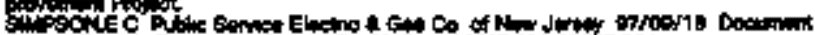

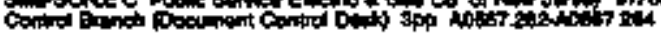

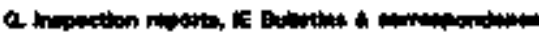

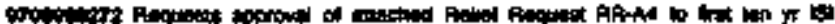

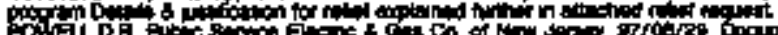

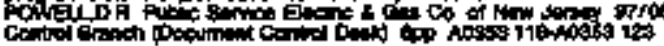

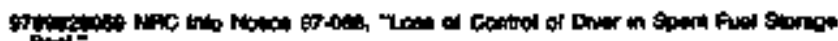

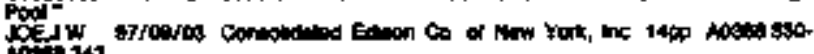
1010

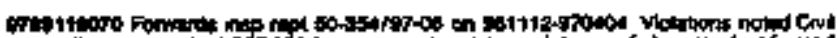

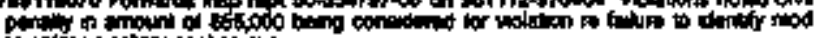

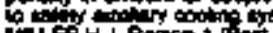

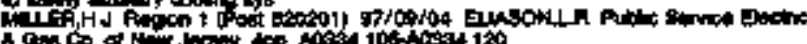

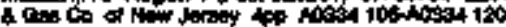

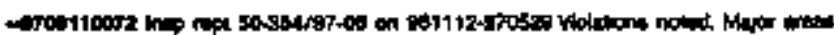

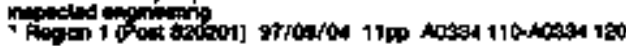

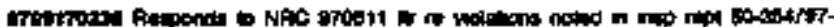

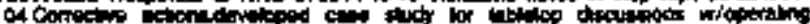

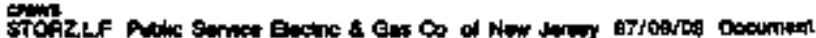

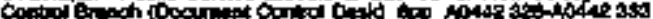




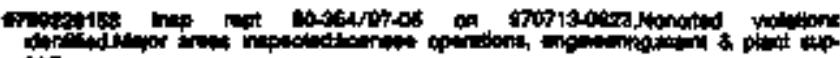

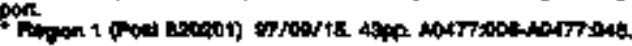

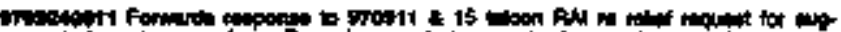

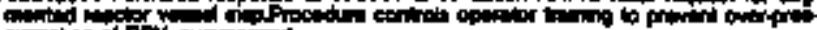

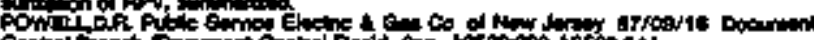

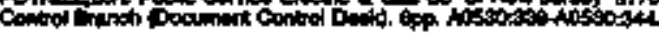

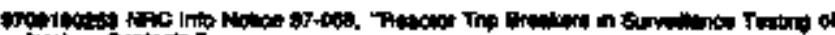

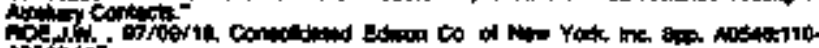

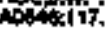

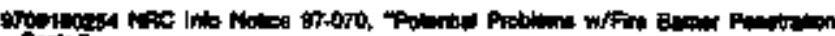
Fo: A

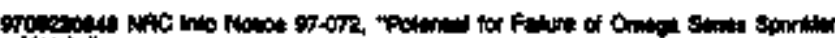

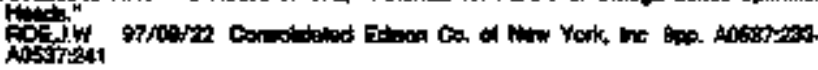

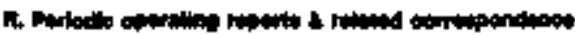

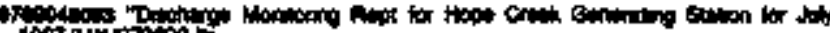
$1+7$ -

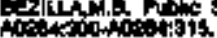

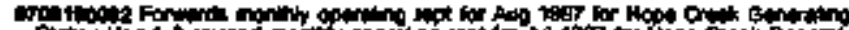

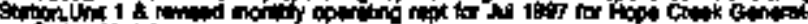
aristint 1

B⿺辶寸

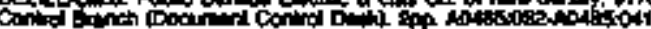

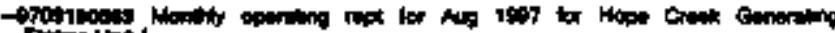

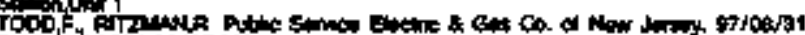

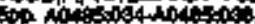

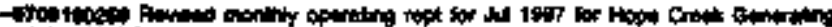

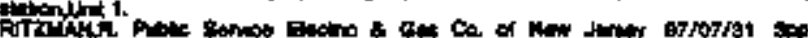

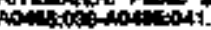

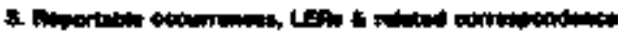

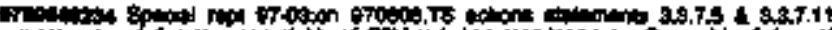

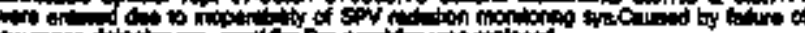

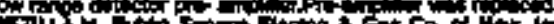

(1)

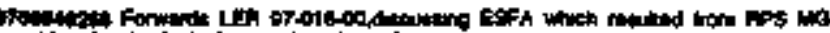

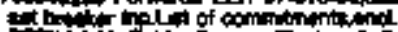

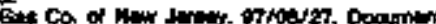

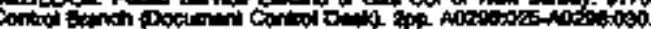

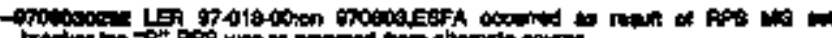

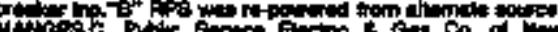
Cop

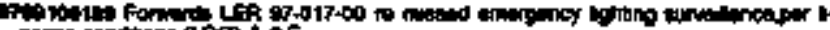

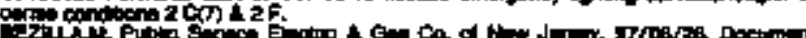

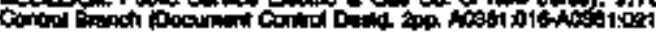

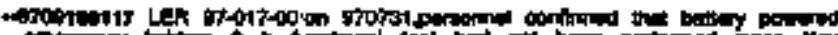

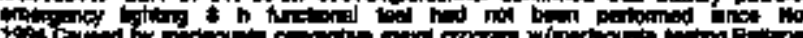

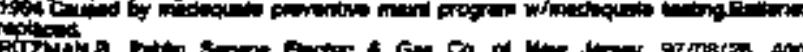

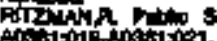

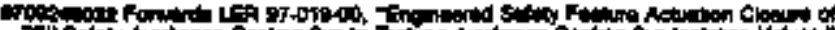

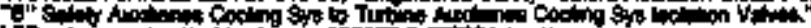

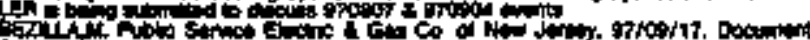

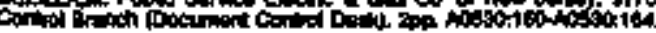

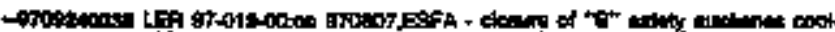

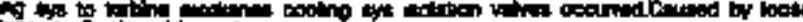

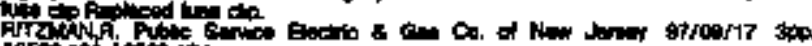
Aositivinistitid

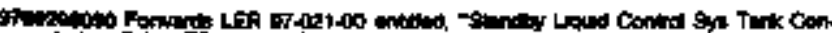

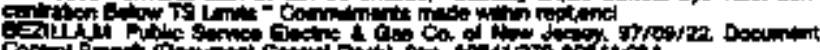

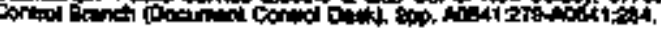

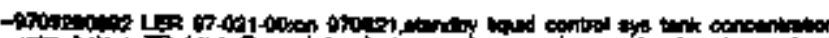

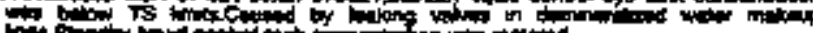

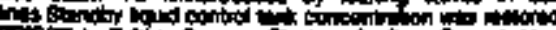

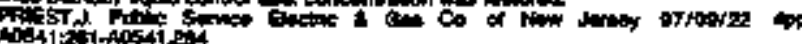

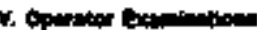

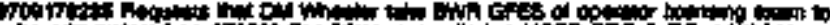

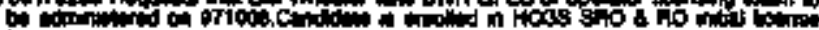

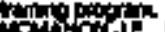

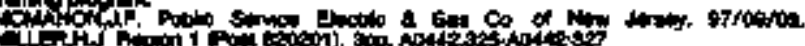

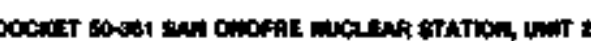

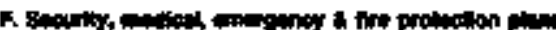

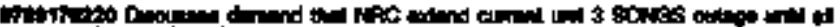

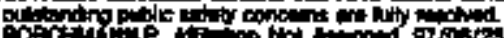

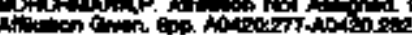

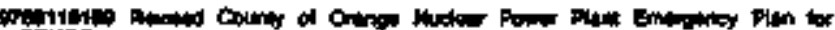

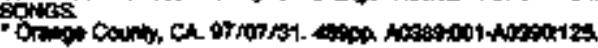

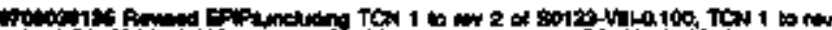

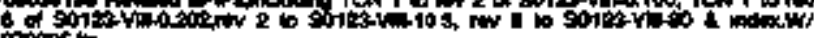
oftestr.

Cofow,

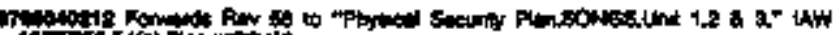

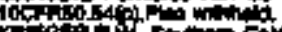

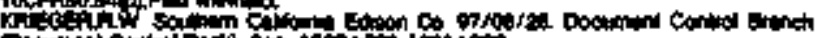

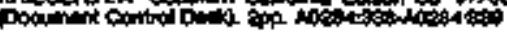

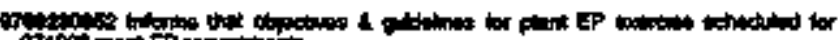

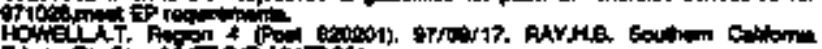

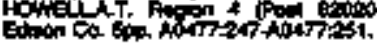

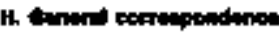

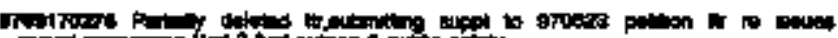

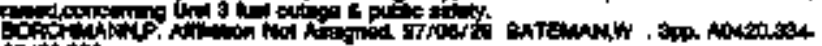

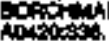

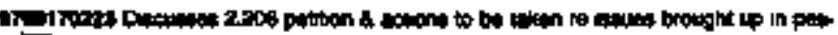
in

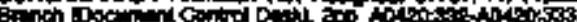

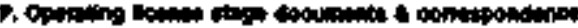

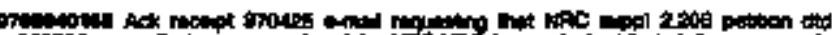

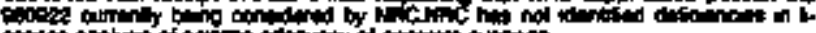

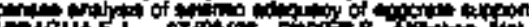

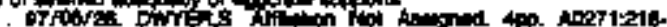
$10271-24$

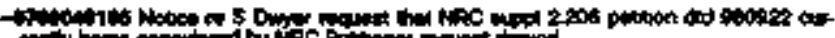

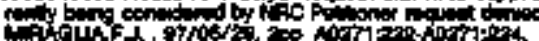

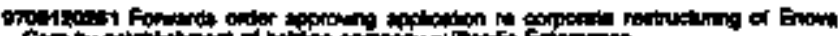

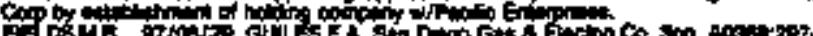
iosion.

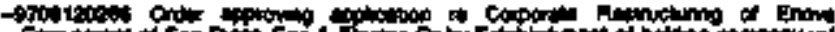

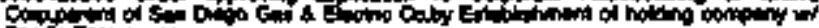

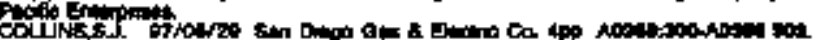

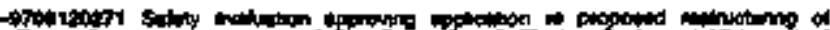

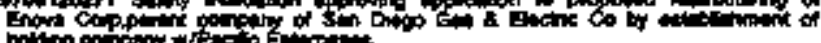

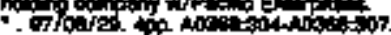

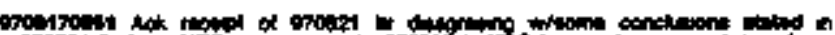

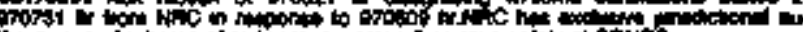

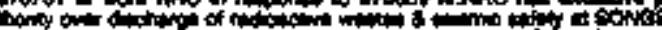

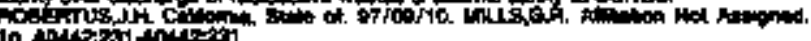

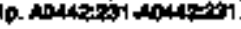

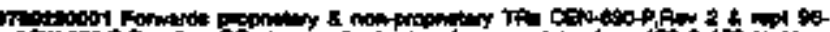

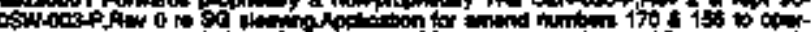

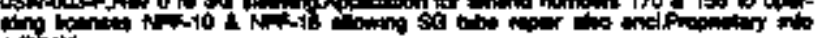

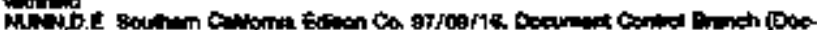

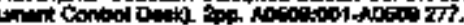

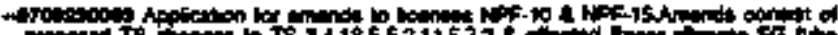

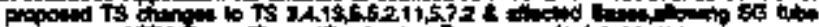
AOH.

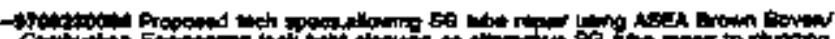

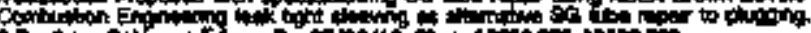

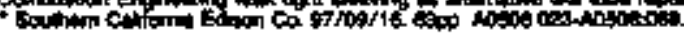

-

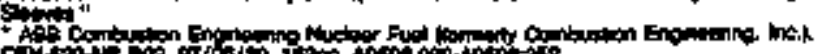

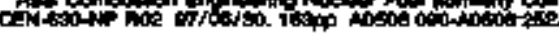




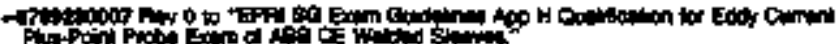

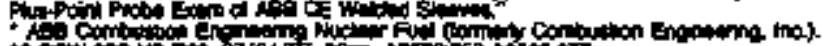

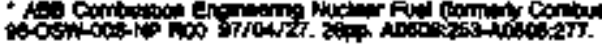

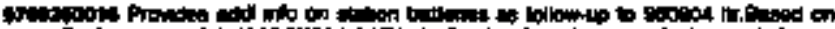

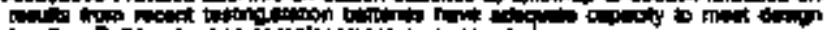

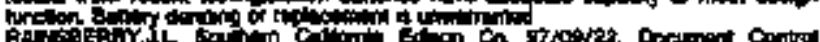

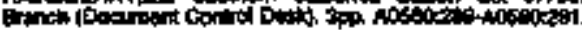

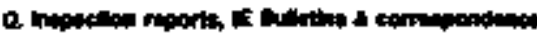

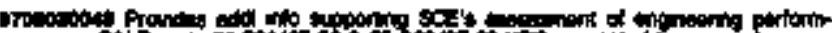

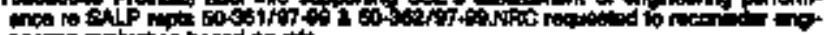

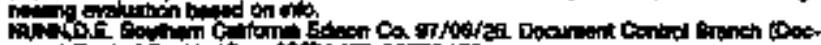

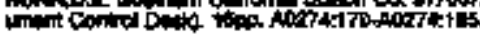

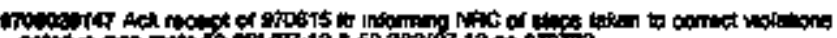

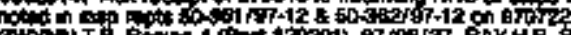

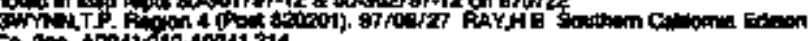
$\cos \sin 4021212-40241214$

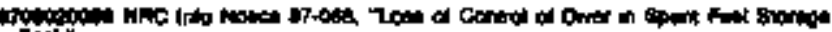

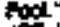

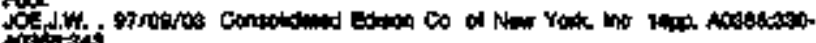

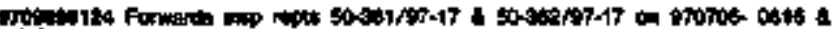
now.

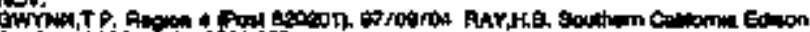
Co.

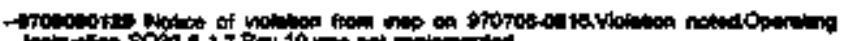

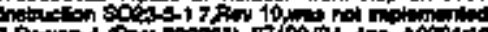

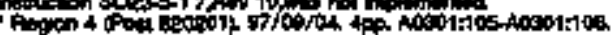

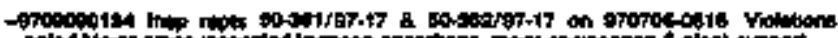

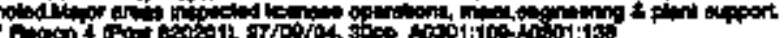

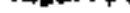

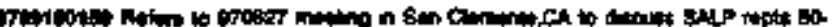

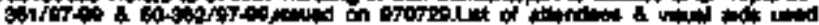
cint

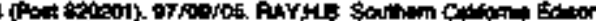

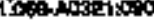

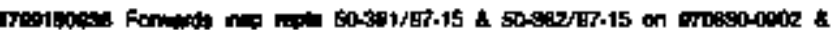
MOF PT

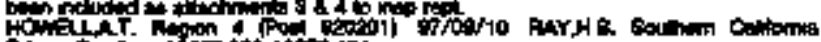

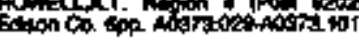

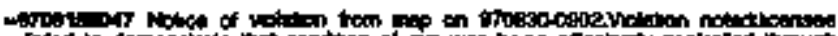

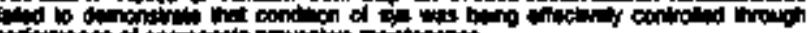
Popin 4 for

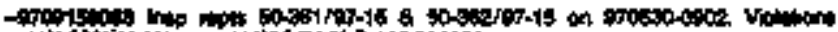

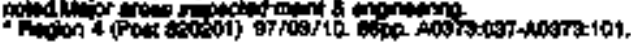

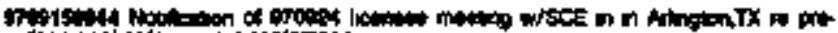

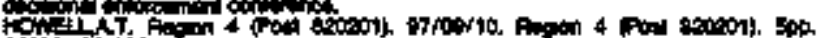

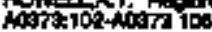

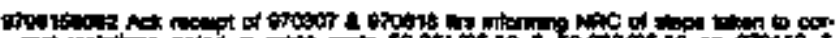

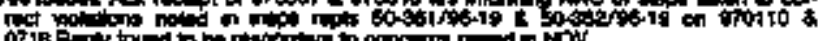

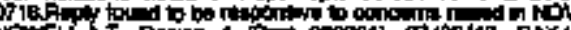

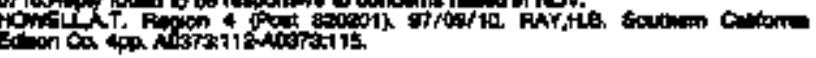

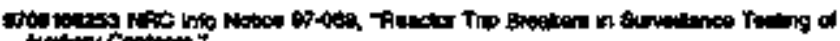

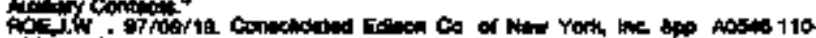
A0sitit:

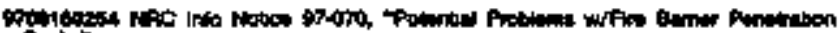

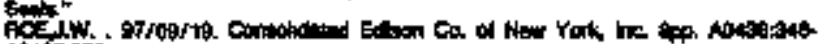
Mot395:

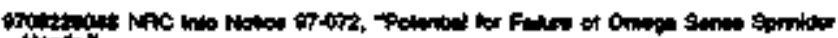

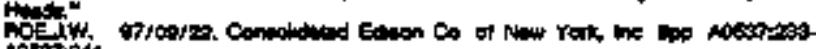

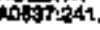

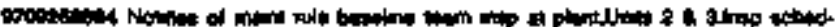

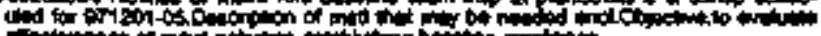

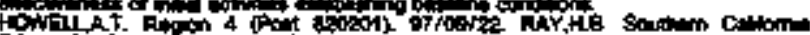

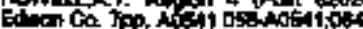

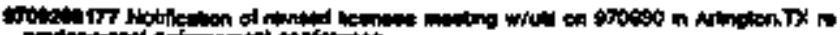

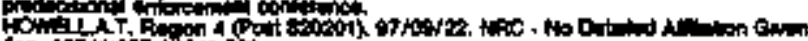

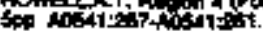

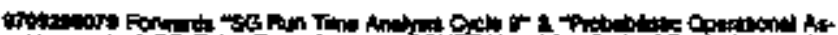

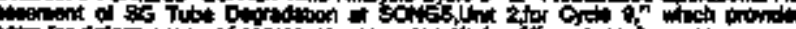

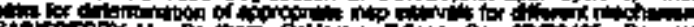

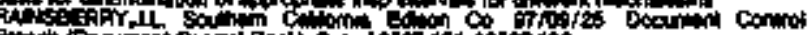

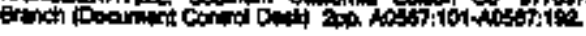

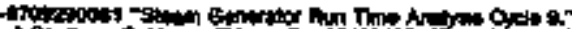

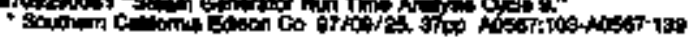

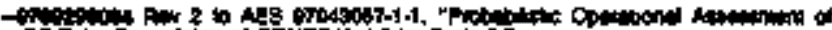

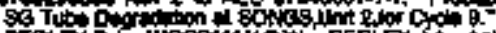

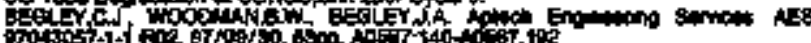

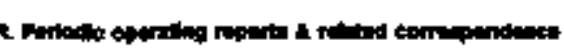

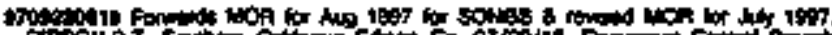

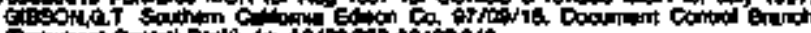

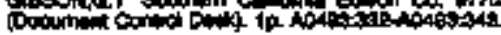

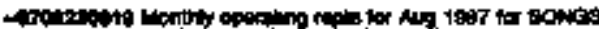

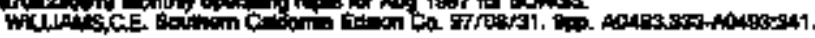

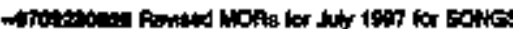

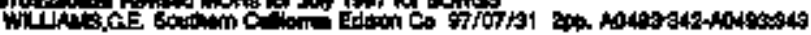

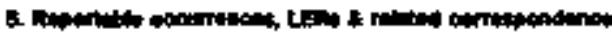

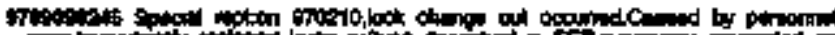

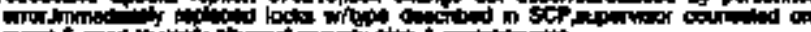

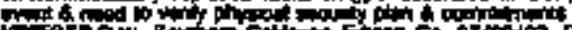

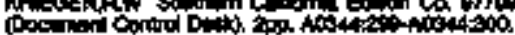

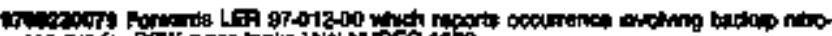

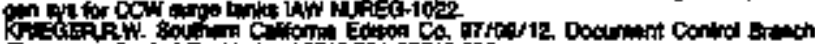

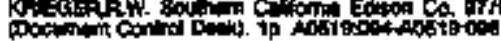

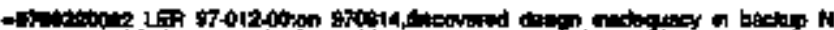

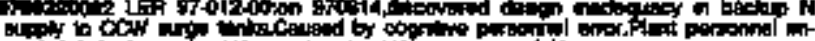

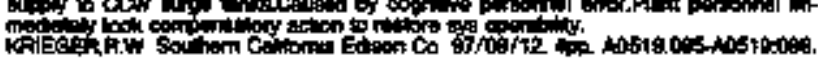

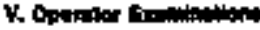

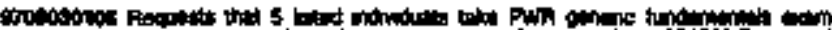

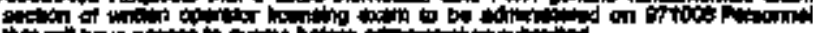

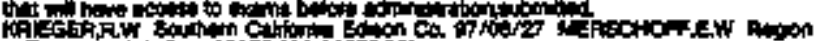
4 (4)

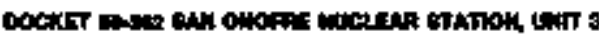

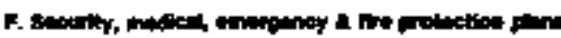

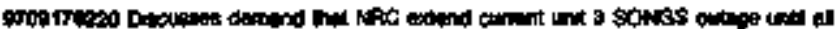

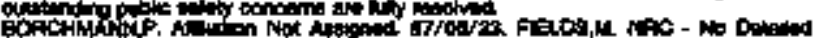

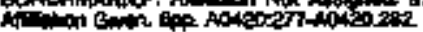

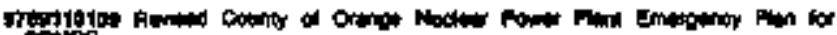

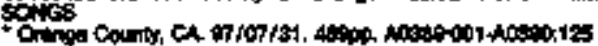

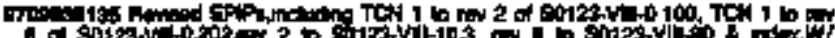

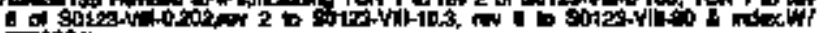
Erises b

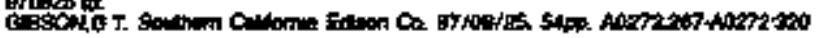

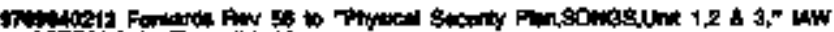

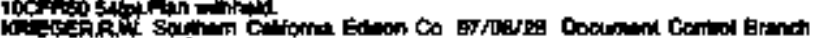

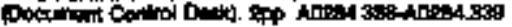

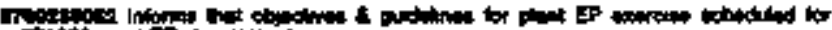

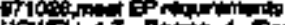

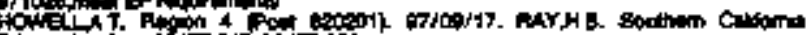

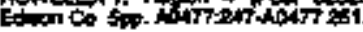

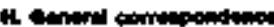

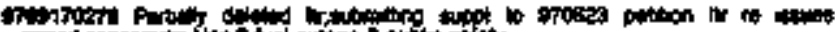

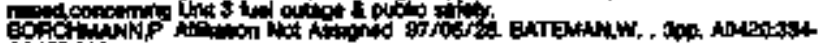
Aorots

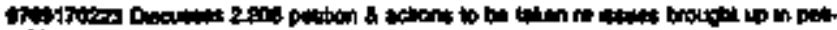

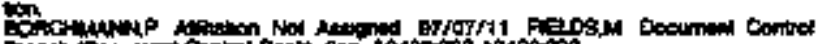

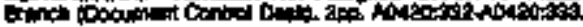

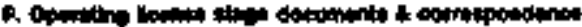

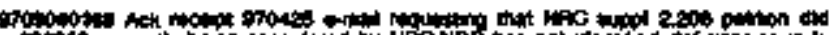

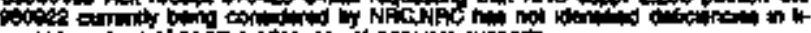

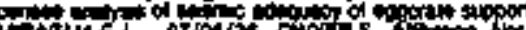

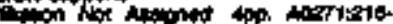

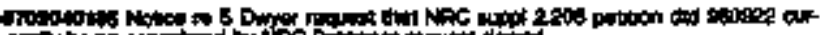

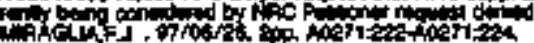




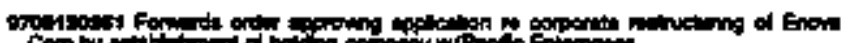

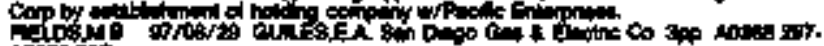

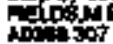

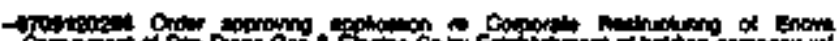

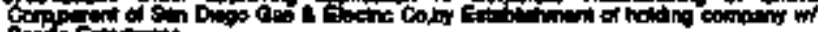

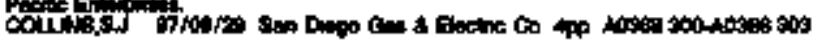

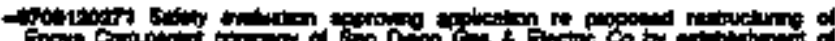

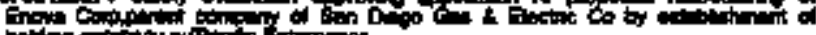

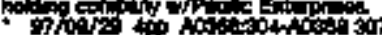

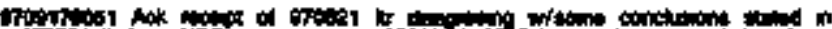

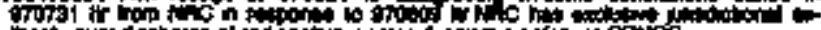

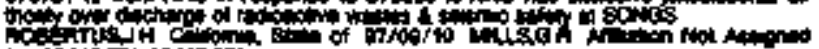

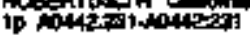

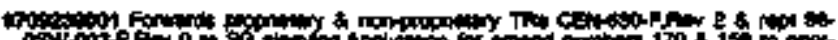

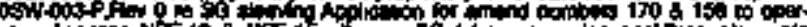
年

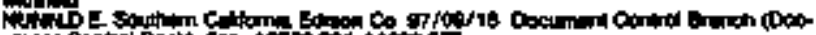

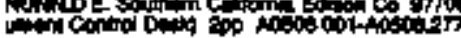

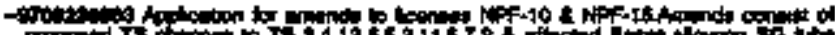

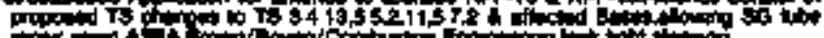
Now

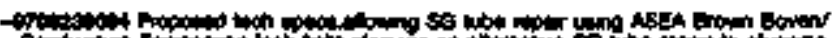

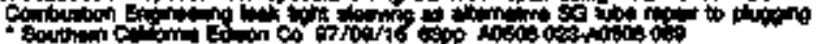

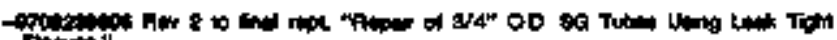

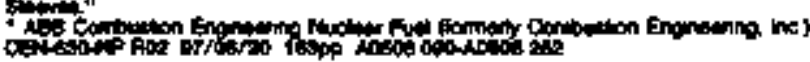

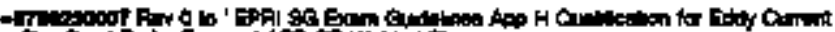

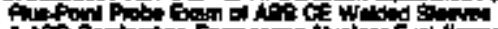

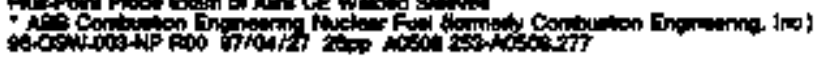

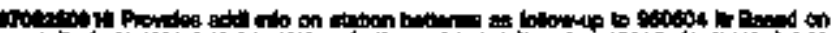

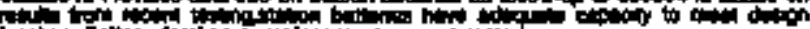
All

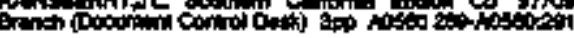

a.

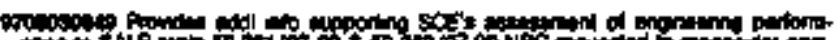

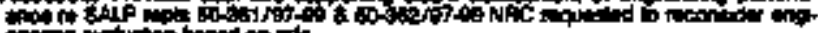

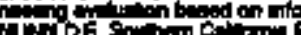

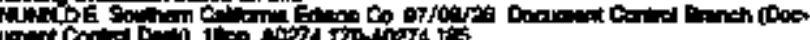
4 towary to

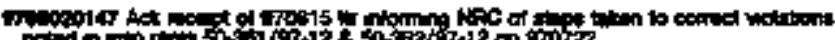

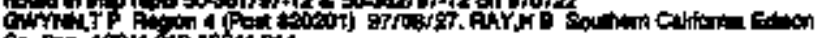

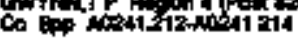

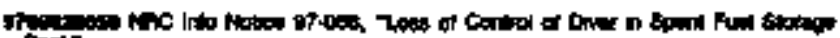

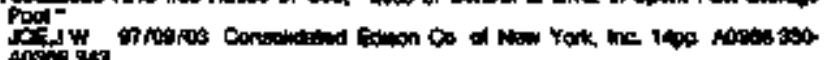
A0Eod 500

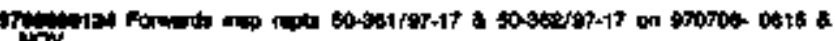

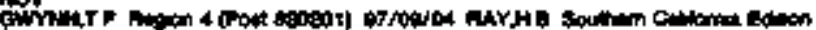
Con

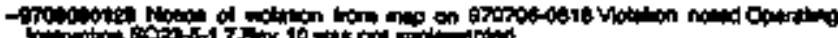

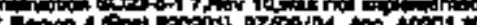

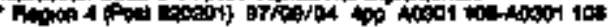

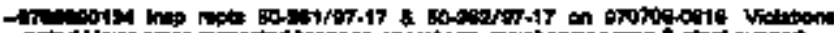

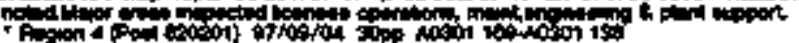

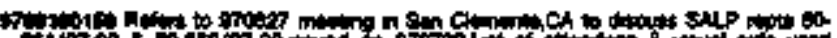

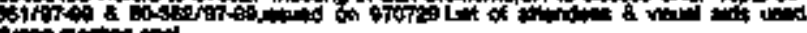

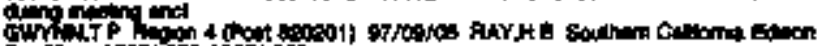
Co 220 ing

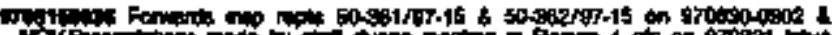

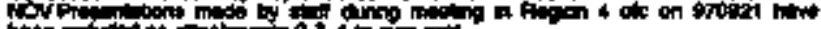

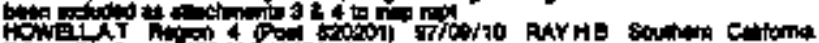

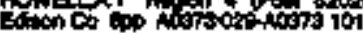

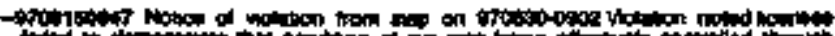

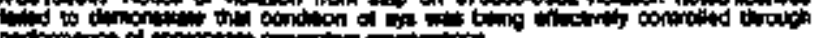

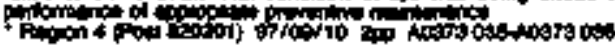

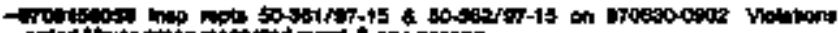

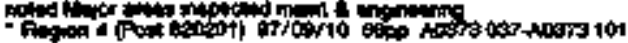

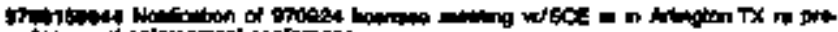

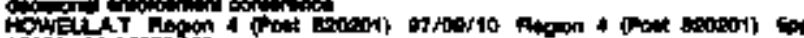

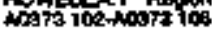

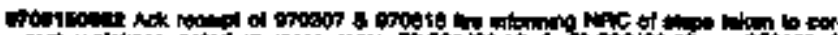

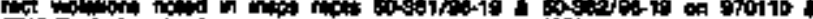

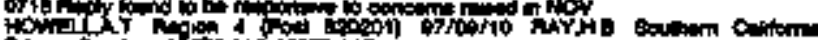

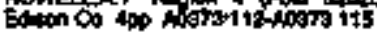

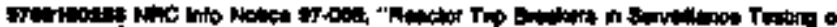

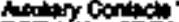

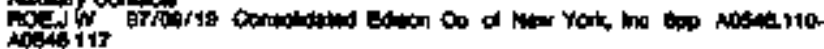

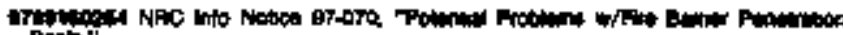

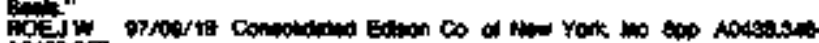

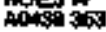

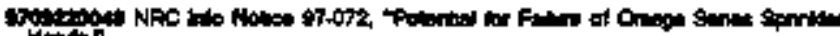

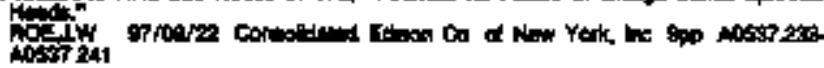

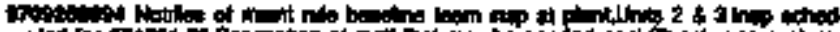

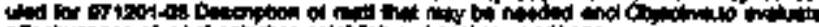

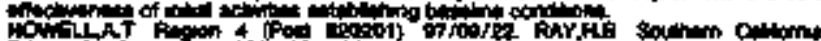

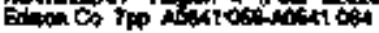

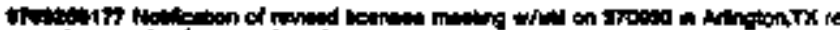

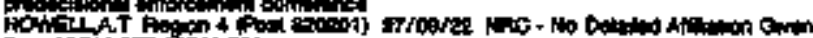

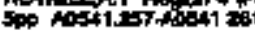

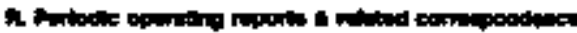

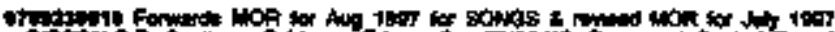

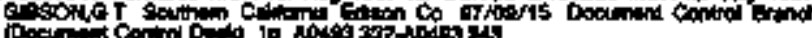

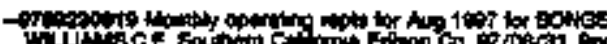

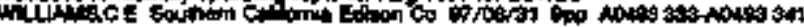

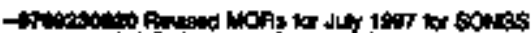

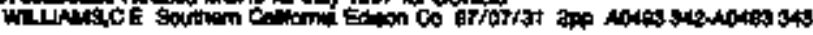

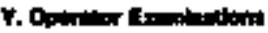

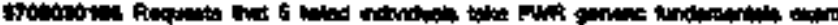

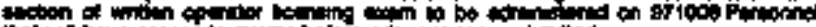

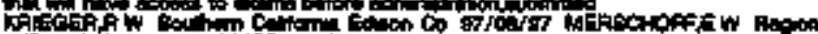

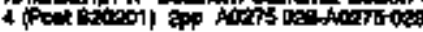

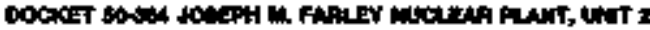

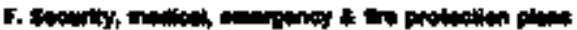

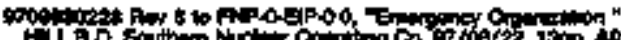

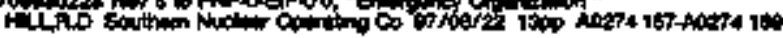

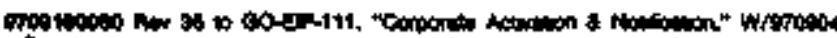

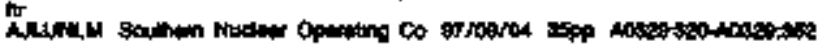

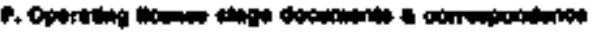

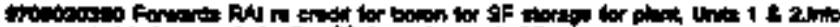

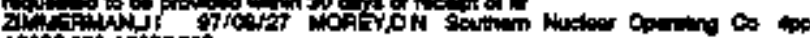
A

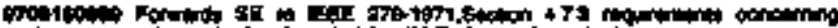

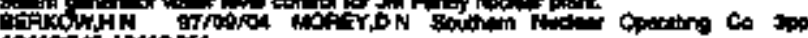

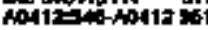

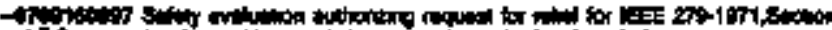

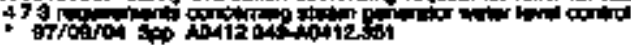

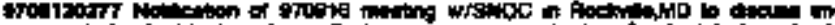
prowed :

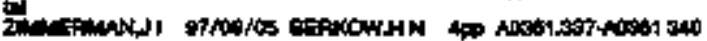

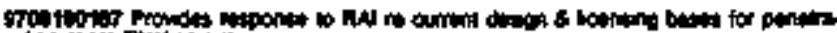

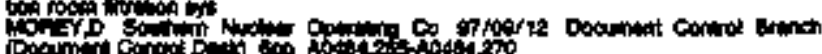

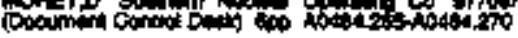

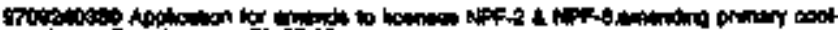

Whoth

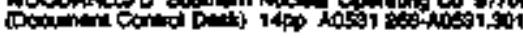

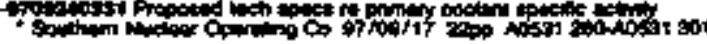




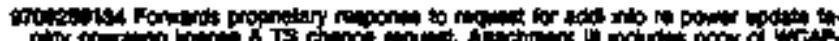

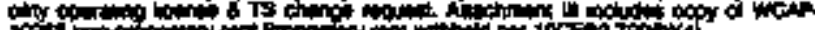

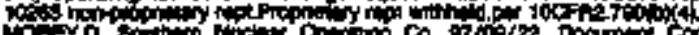

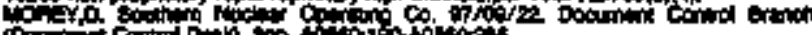

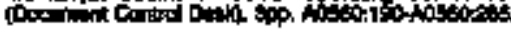

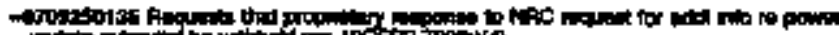

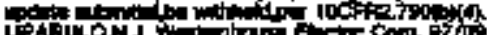

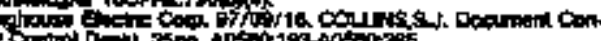

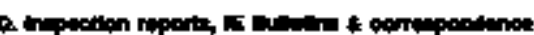

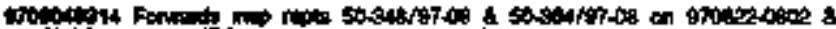

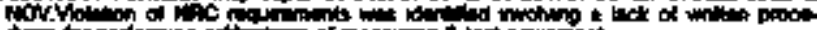

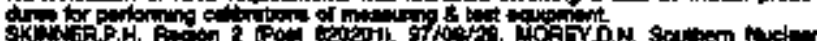

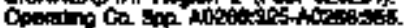

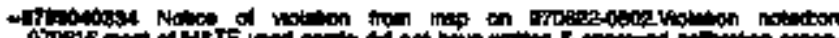

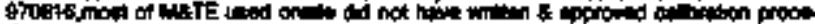

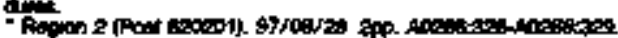

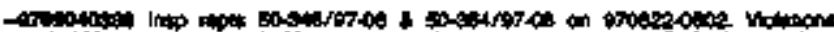

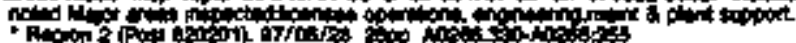

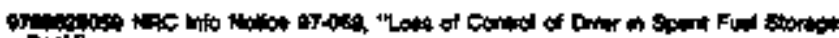

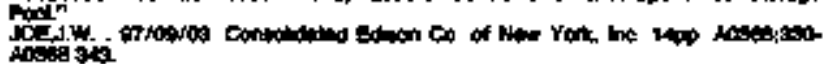

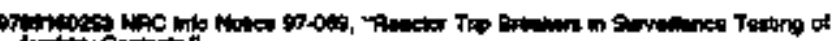
Hof J iostit?

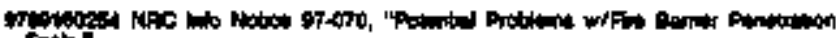

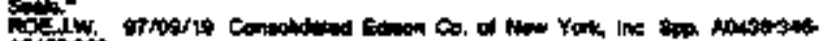

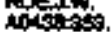

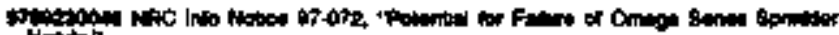

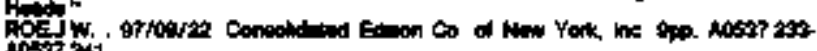
into

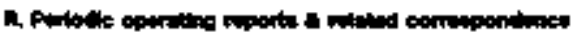

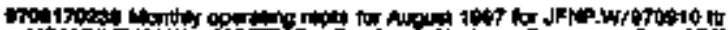

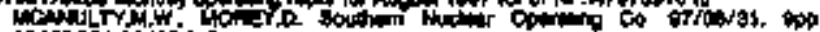

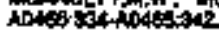

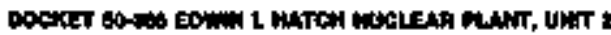

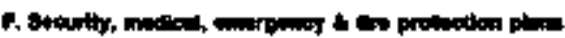

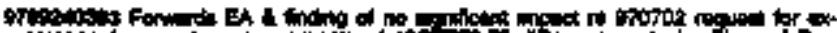

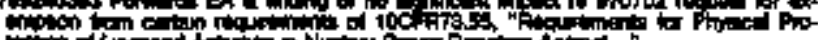
I.

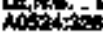

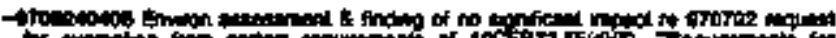

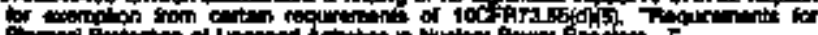

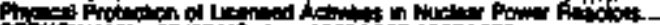

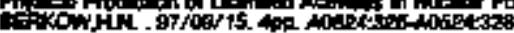

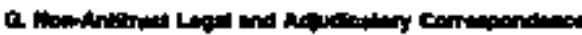

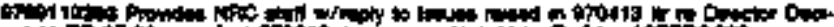

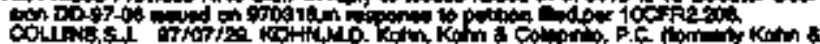

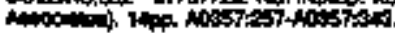

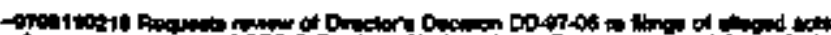
a om

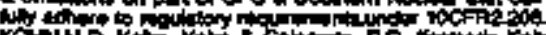

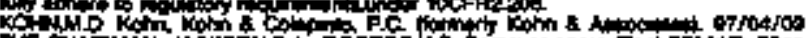

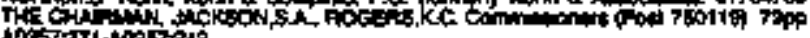

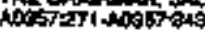

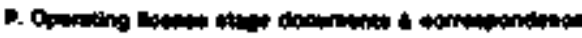

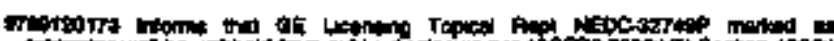

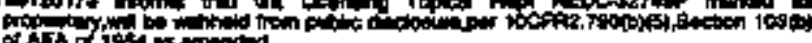
of $17 \lambda$ or it

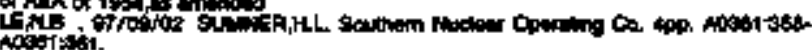

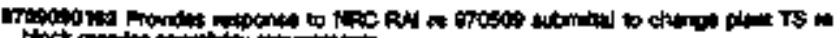

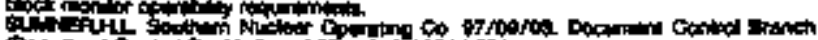

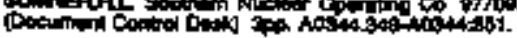

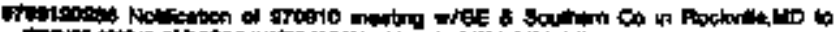

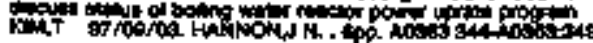

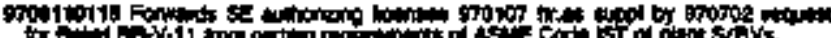

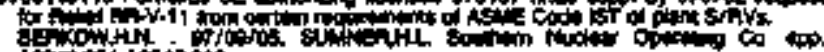

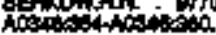

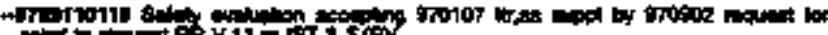
ring to

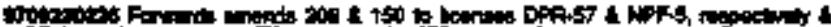

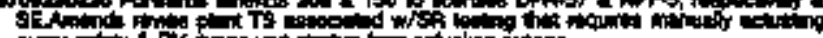

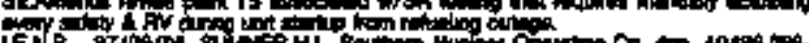

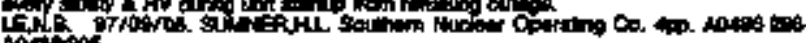
anteros.

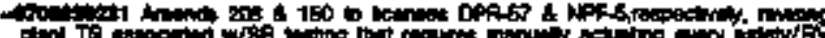

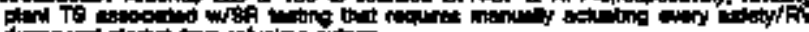

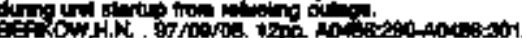

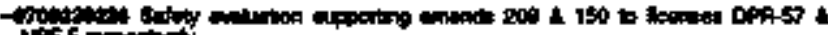

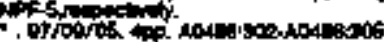

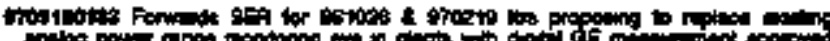

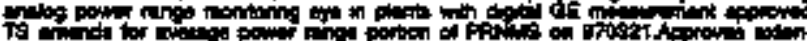

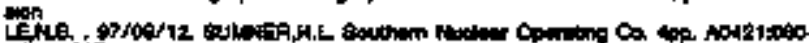
Not?isis?.

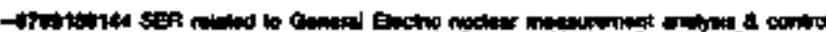

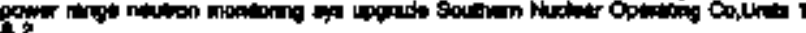
. Itrost12.

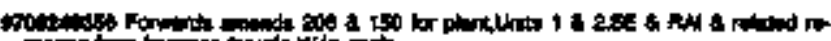

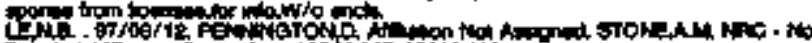

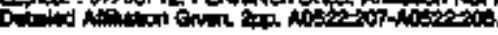

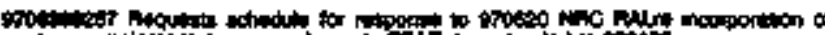

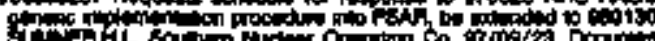

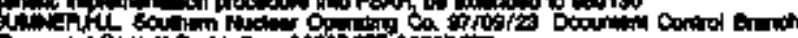

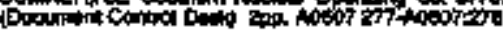

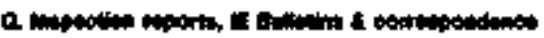

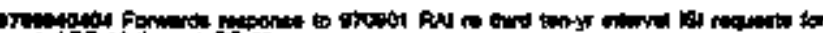

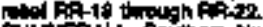

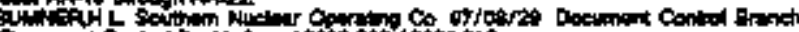

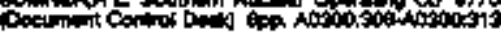

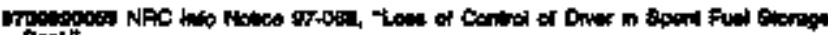
pood

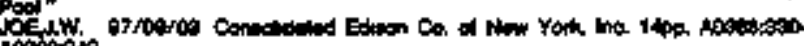
atomis

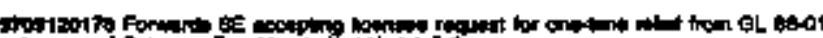

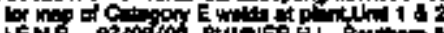

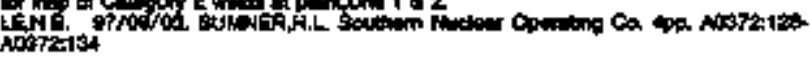

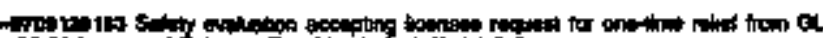

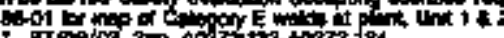

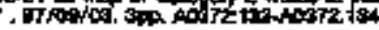

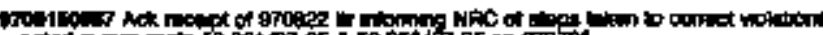

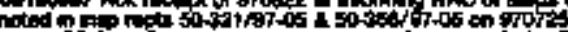

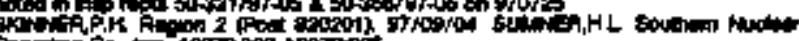

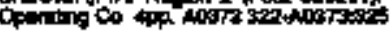

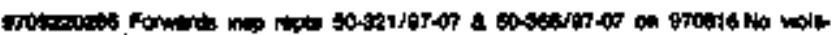

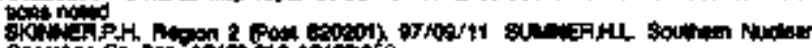

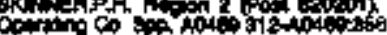

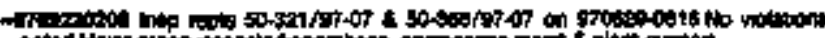

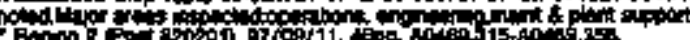

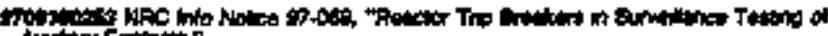

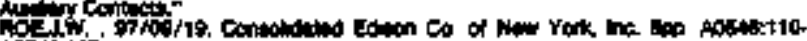
astitit.

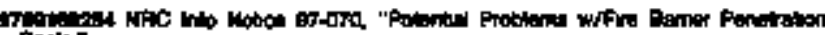

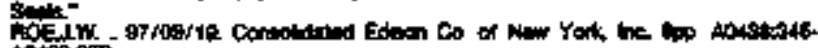

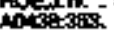

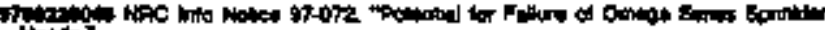

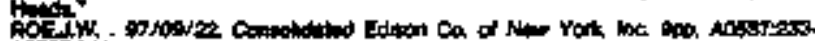
An: 241

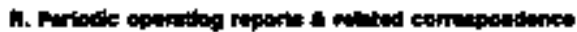

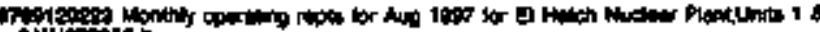

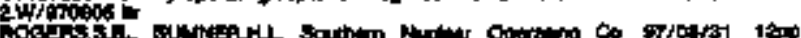

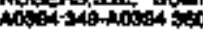




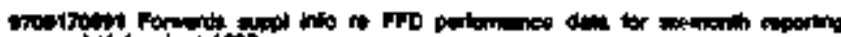

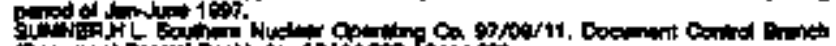

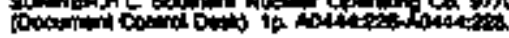

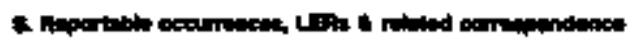

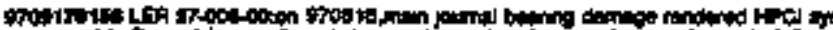

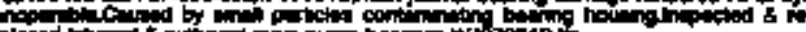

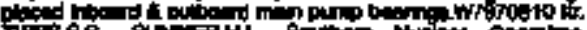

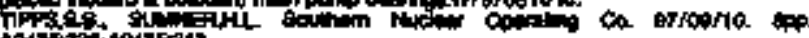

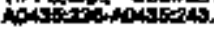

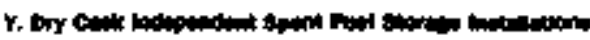

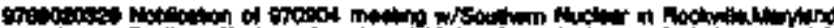

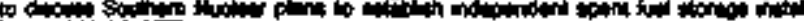

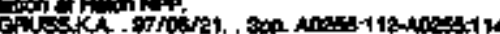

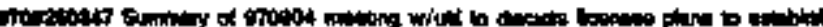

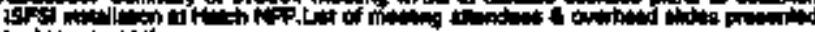

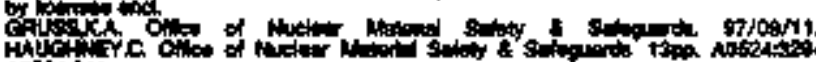
istatiat.

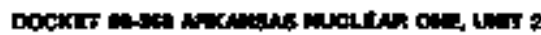

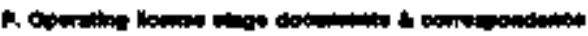

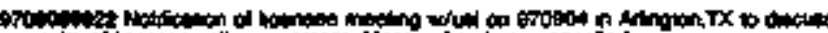

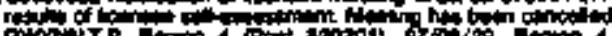

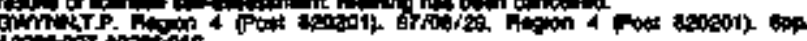

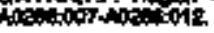

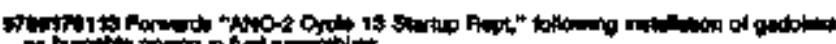

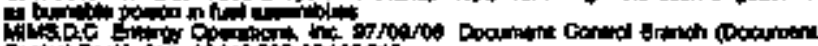

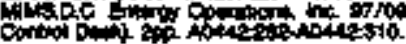

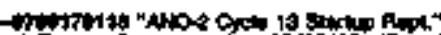

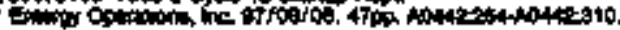

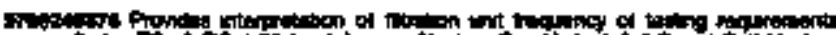

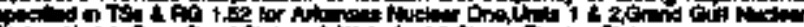

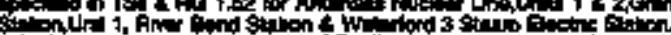

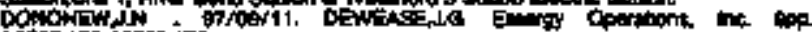

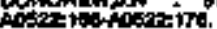

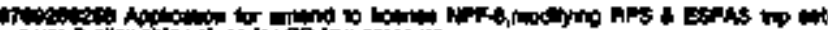

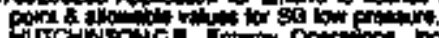

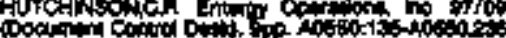

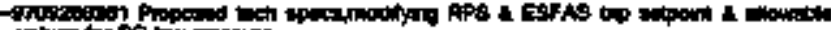

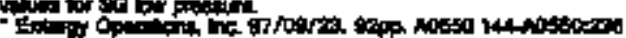

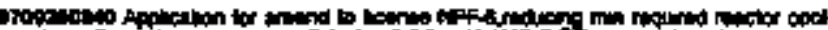

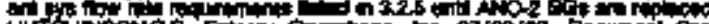
HUtC H o

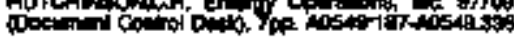

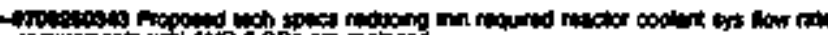

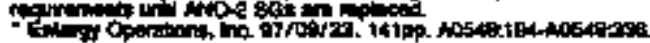

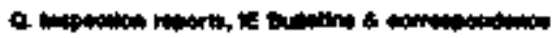

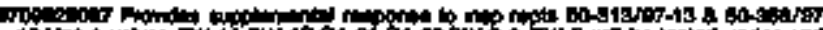

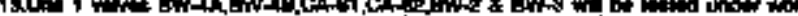

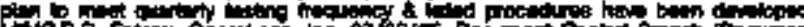

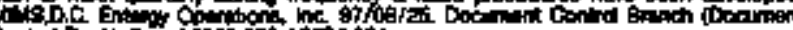

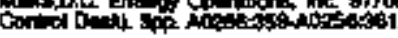

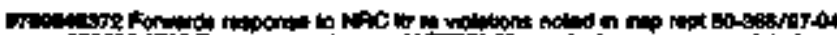

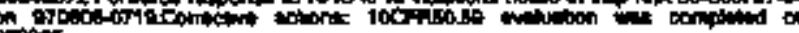
(a)

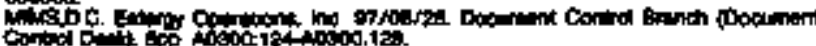

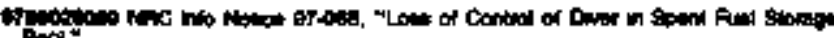

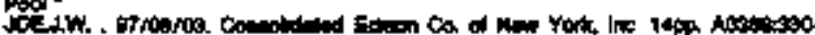

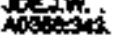

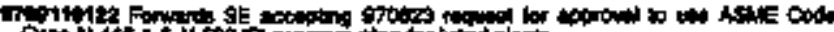

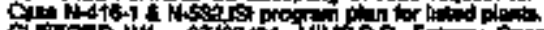

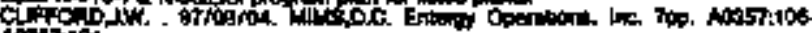
105;121.

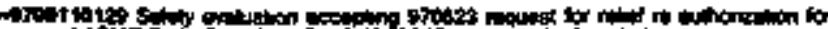

toof of

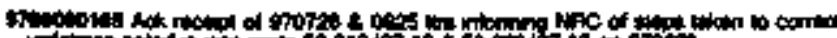

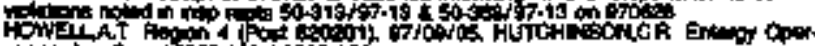

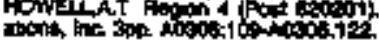

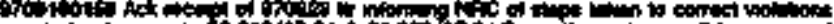

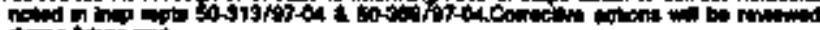

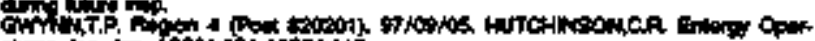

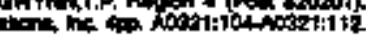

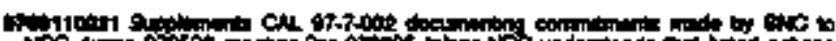
NAC ung o

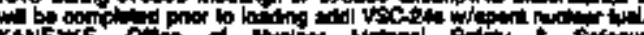
KN

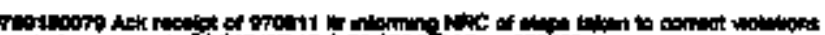

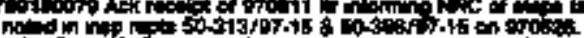

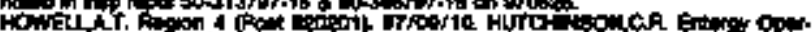

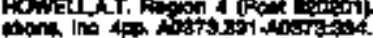

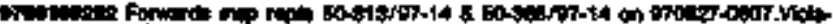

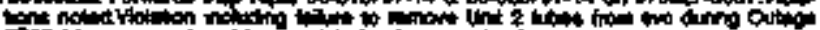

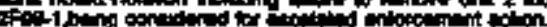

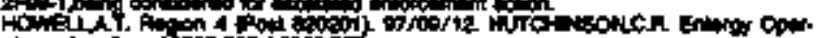

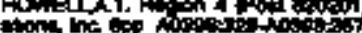

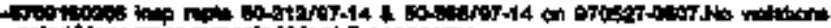

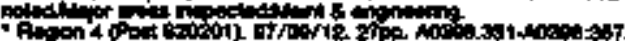

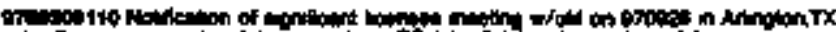
Fow

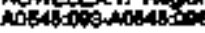

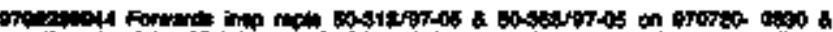

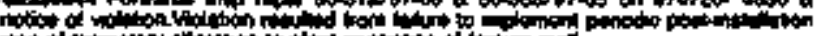

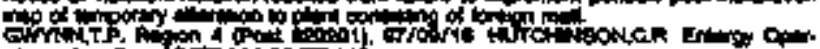

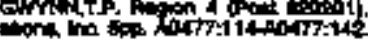

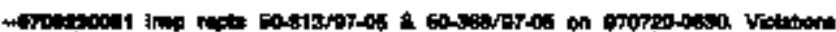

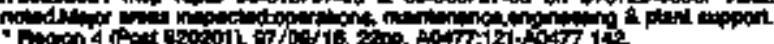

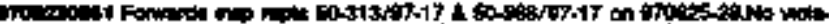

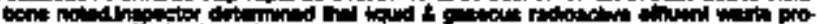

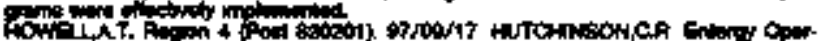
$\operatorname{con} 10$.

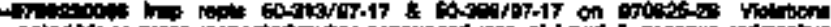

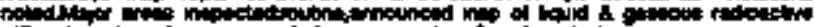

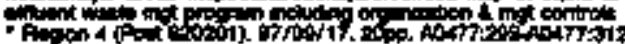

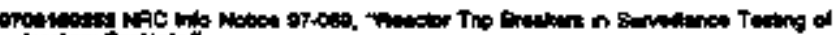

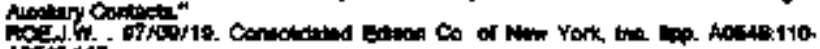
ind $177^{-}$

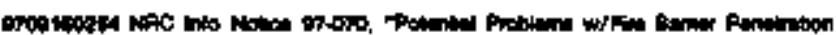

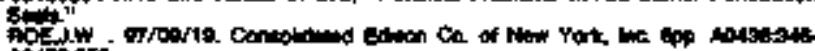
Hotion

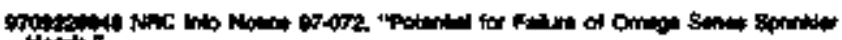

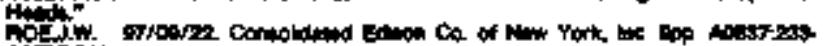
notorat

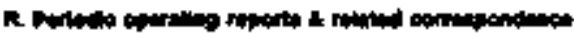

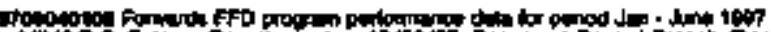

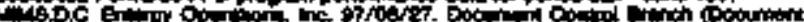

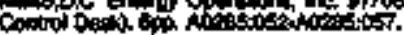

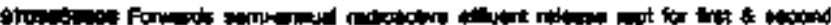

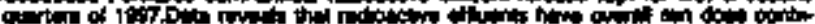

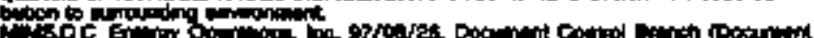

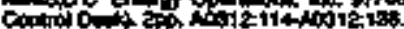

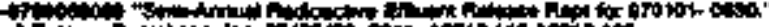

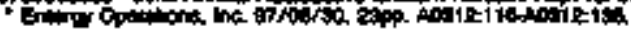

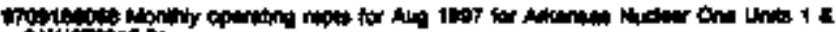

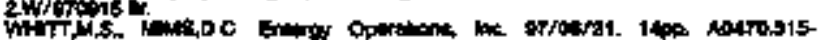
notrotin

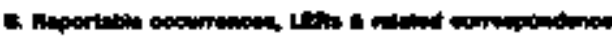

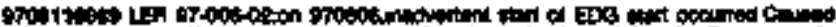

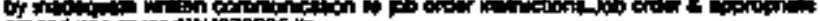

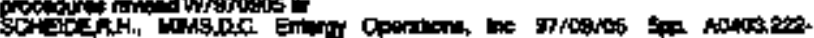

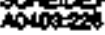

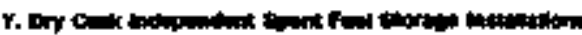

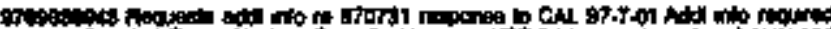

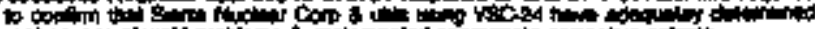

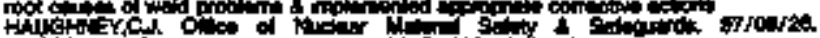

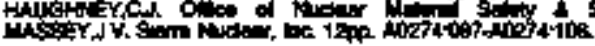




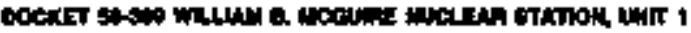

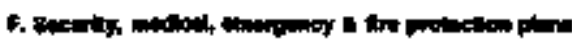

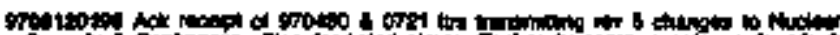

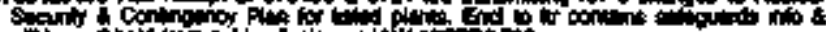

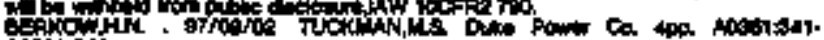
istist

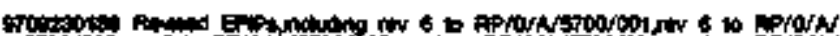

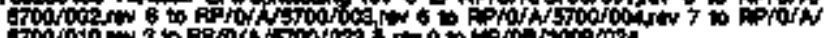

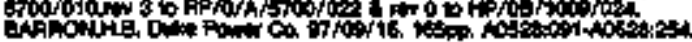

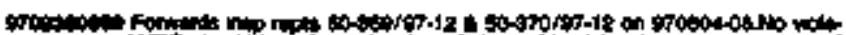

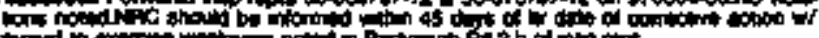

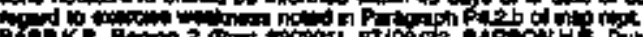

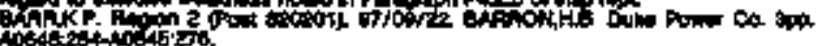

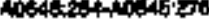

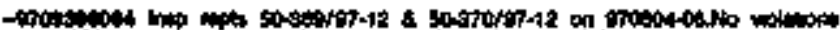

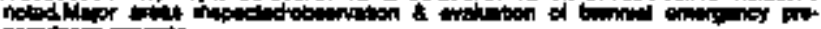

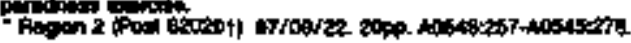

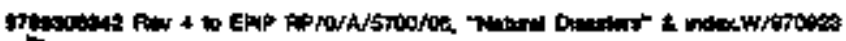

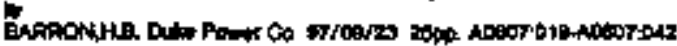

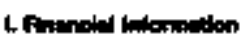

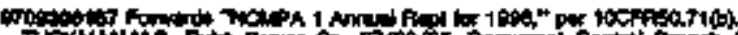

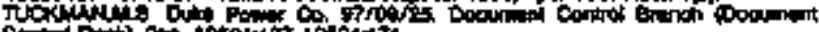

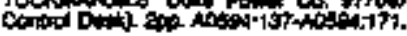

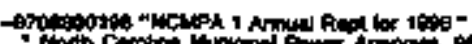

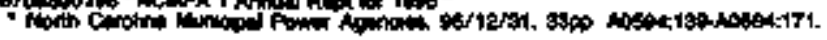

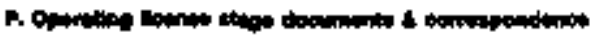

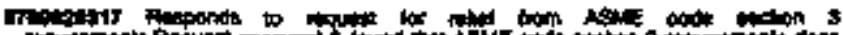

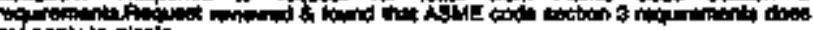

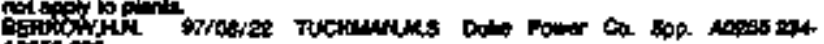

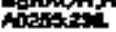

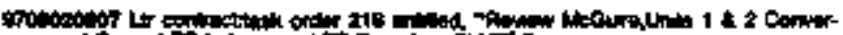

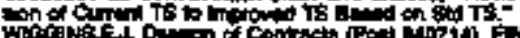

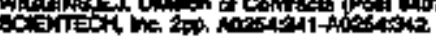

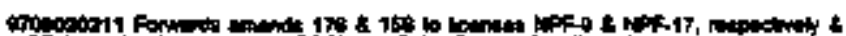

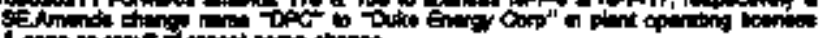

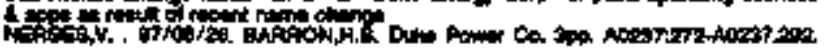

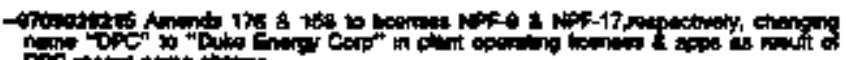

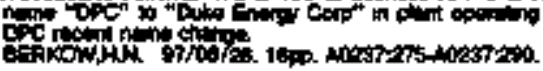

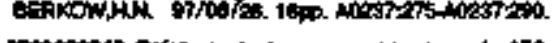

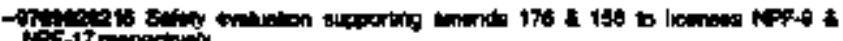

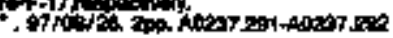

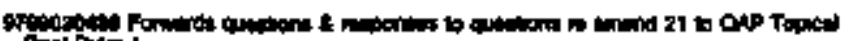

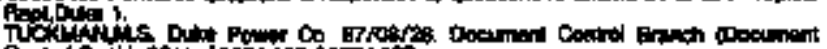

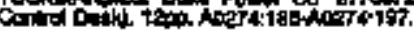

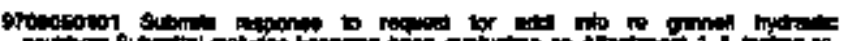

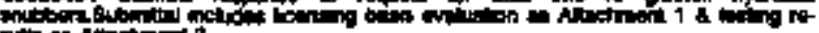
ENin

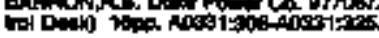

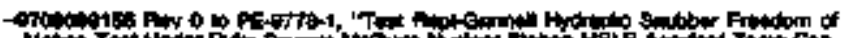

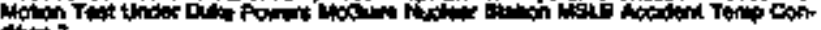

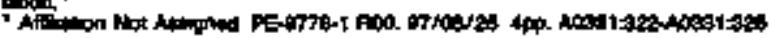

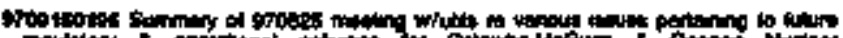

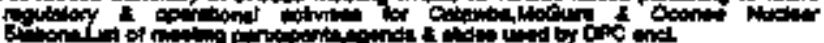

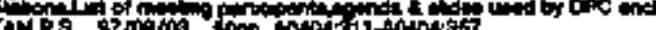

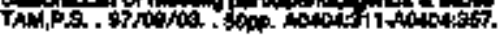

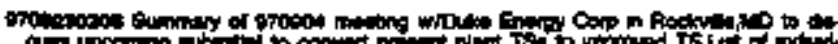

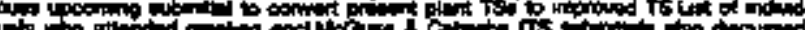

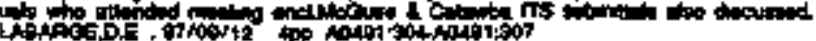

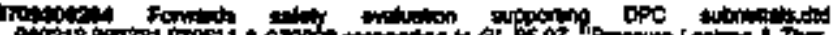
Hot 1 is

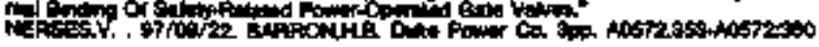

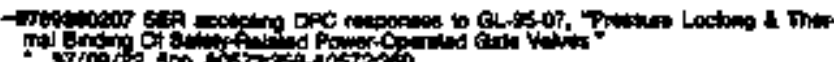

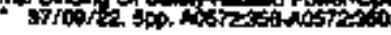

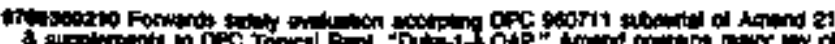

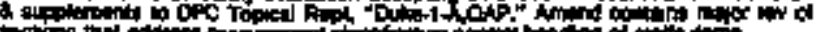
A nits

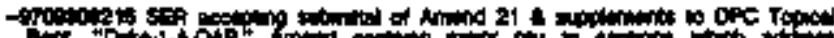

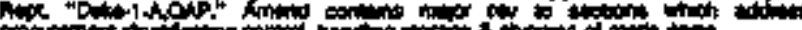

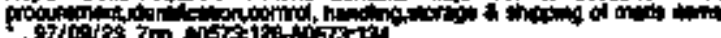

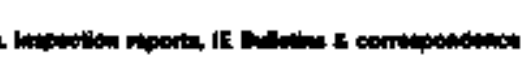

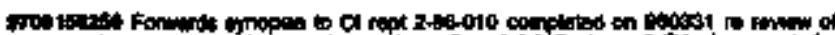

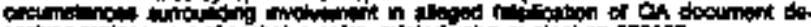

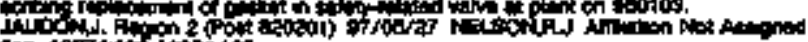

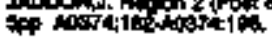

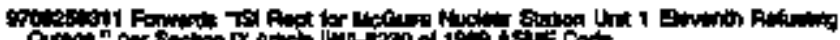

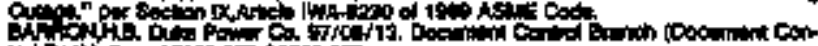

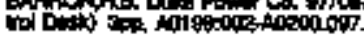

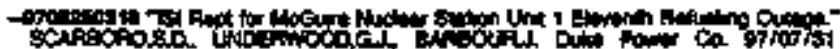

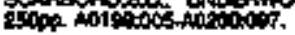

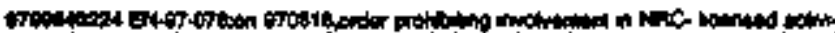

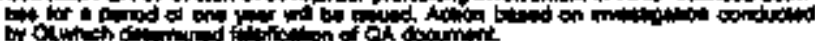

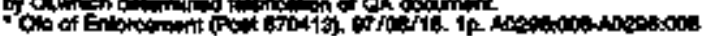

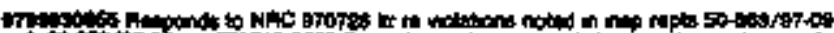

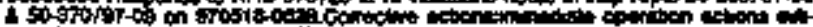

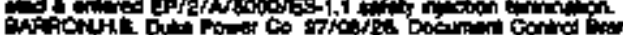

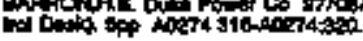

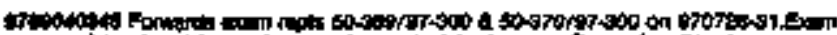

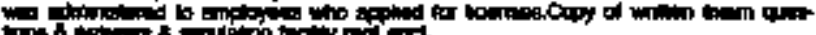
pheis

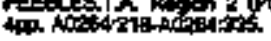

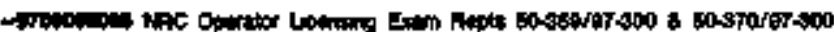

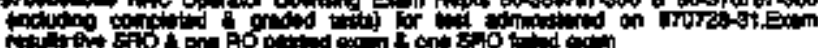

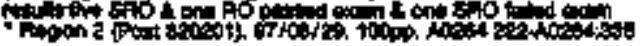

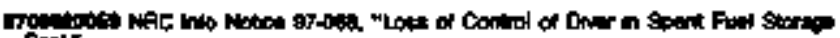
Poot" ition

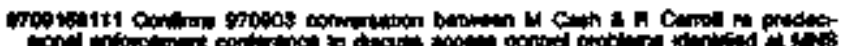

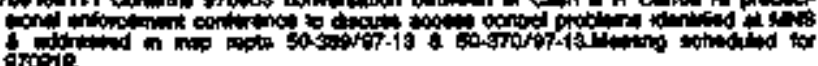
artore

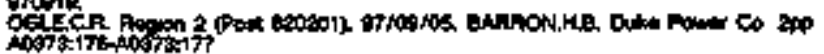

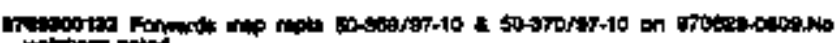

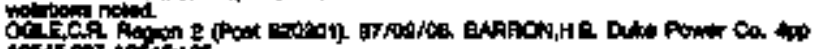
axas of 7 -

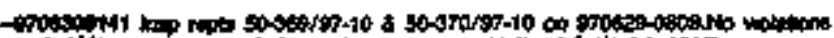

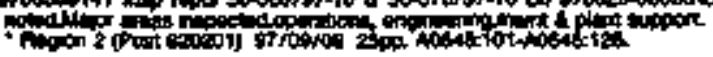

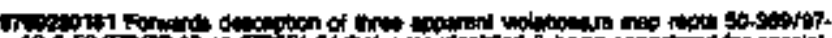

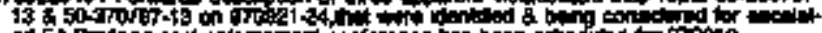

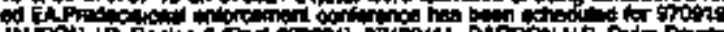

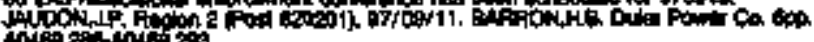

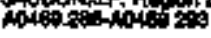

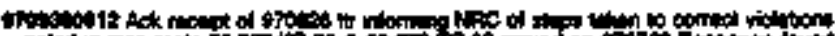

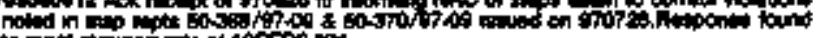

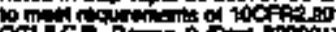

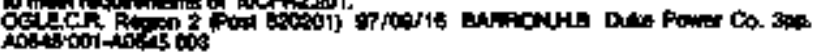

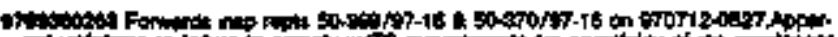

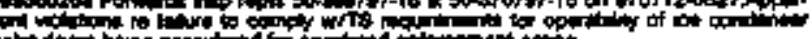

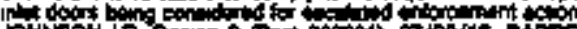

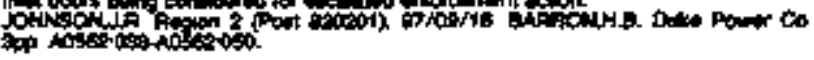

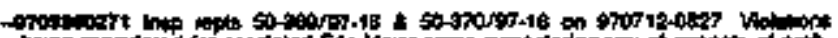

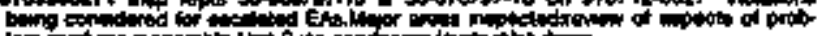

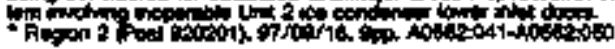

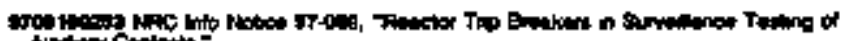

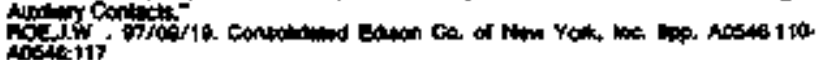

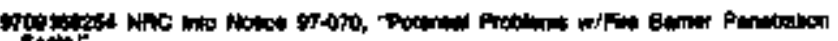

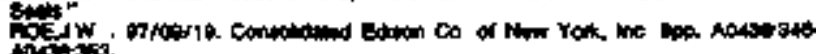
apdosis.

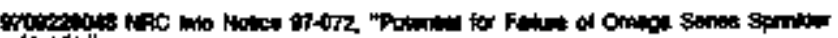

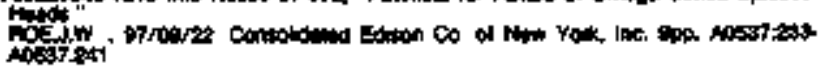




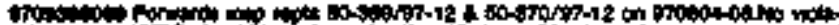

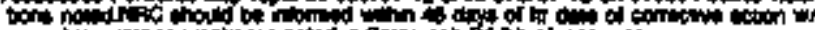
Ty d to

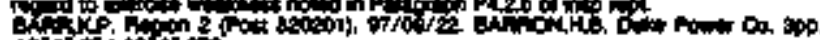

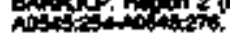

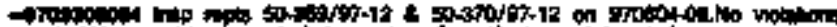

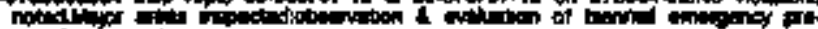

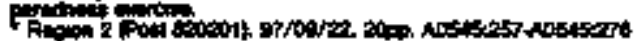

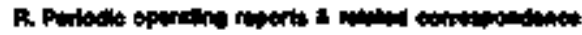

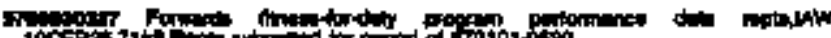

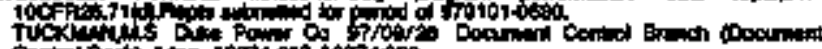

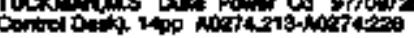

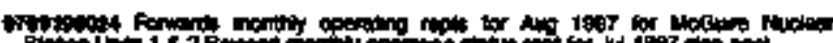

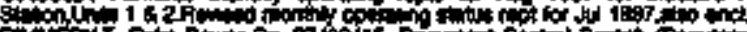

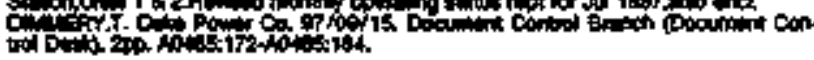

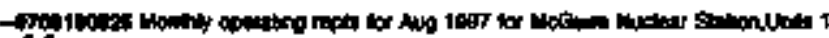
곤

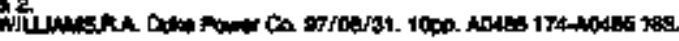

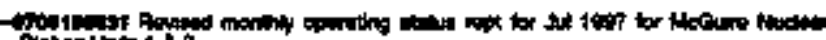

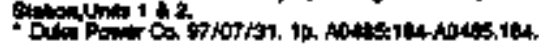

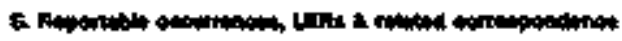

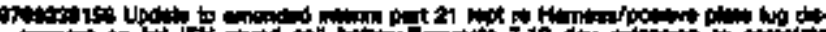

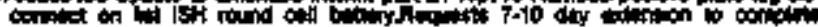

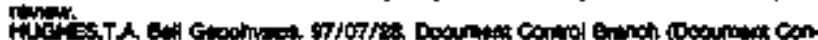

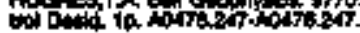

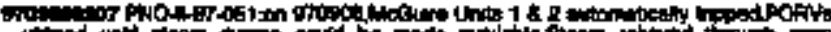

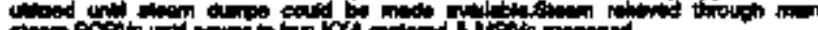

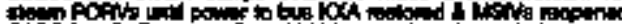

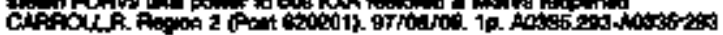

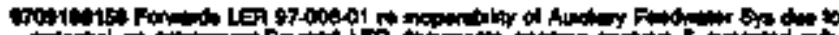

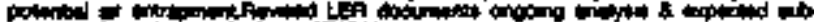

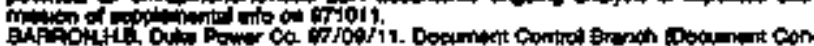
tol D.m.

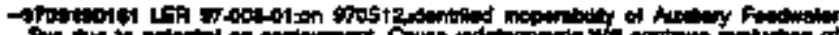

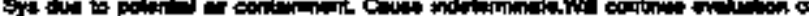

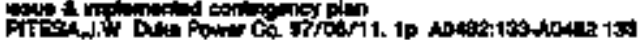

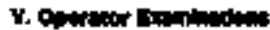

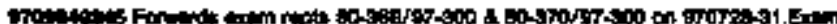

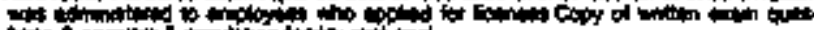
Ptes

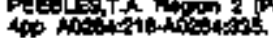

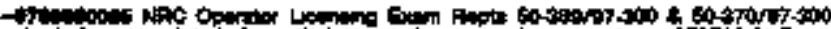

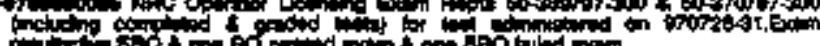

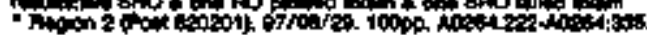

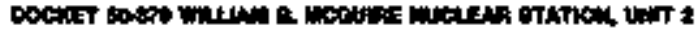

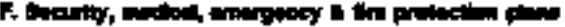

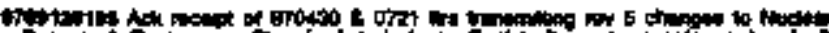

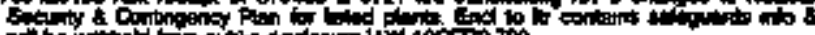

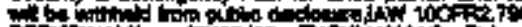

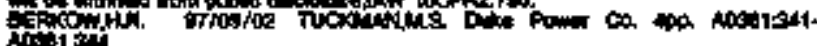

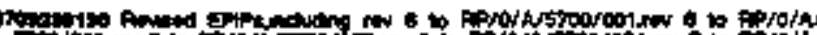

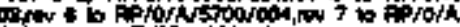

(1)

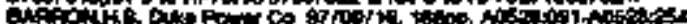

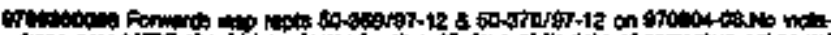

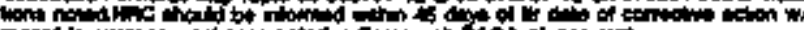

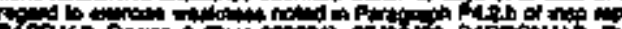

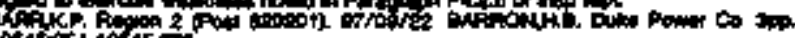

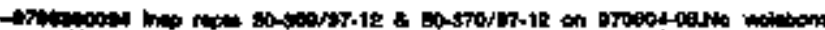

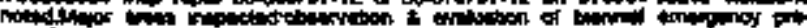

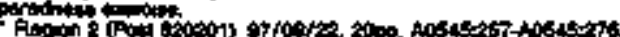

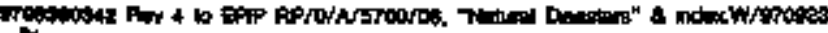

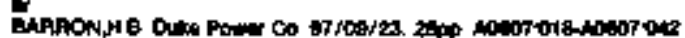

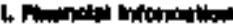

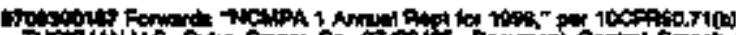

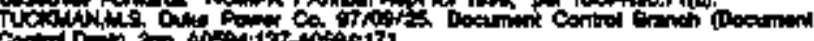

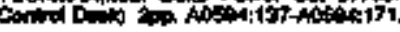

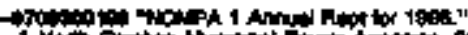

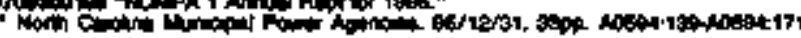

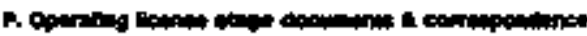

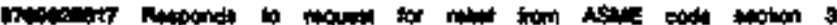

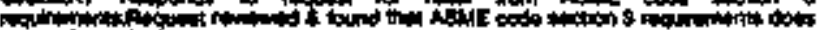

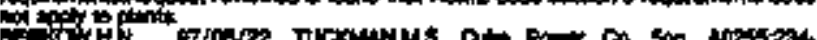
ition

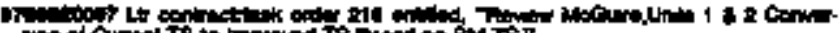

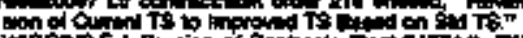

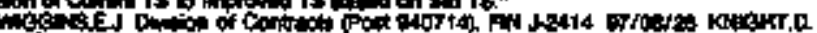

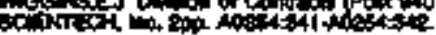

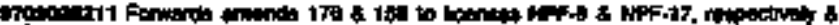

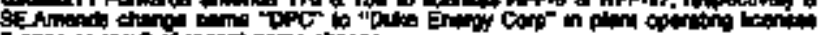

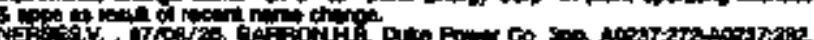

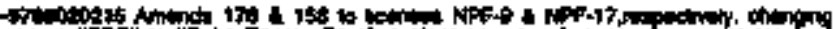

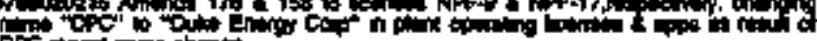

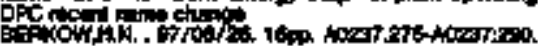

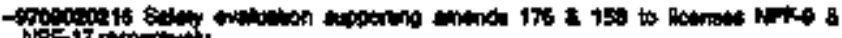

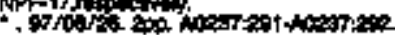

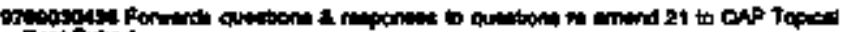

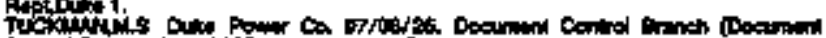

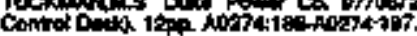

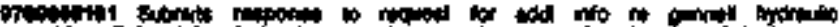

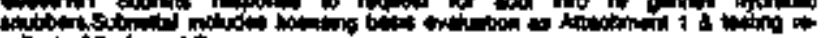

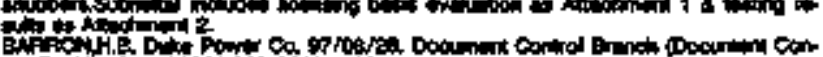

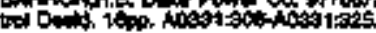

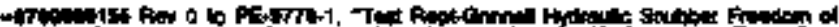

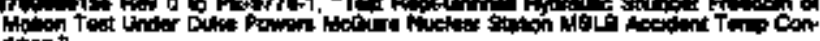

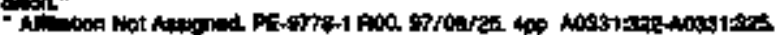

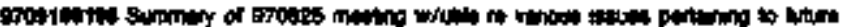

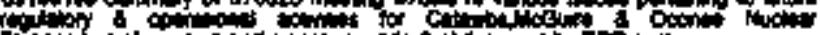

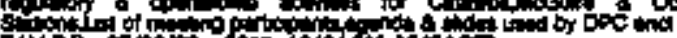

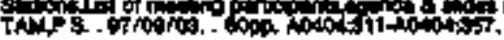

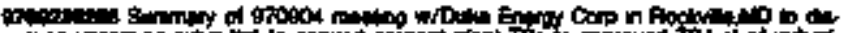

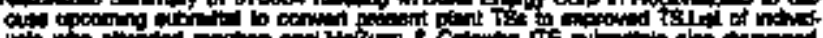

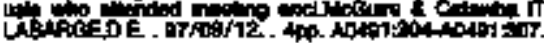

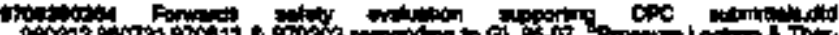

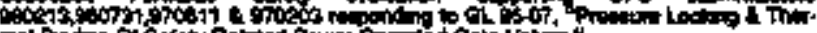

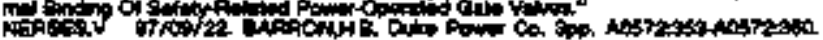

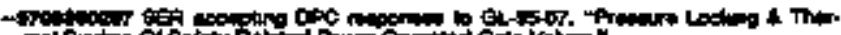

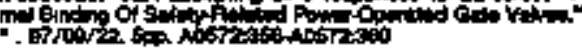

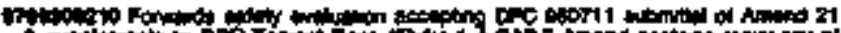

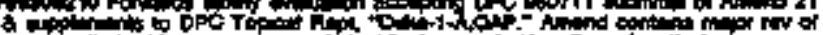

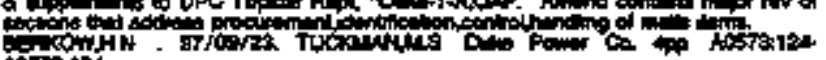
itotiats

-

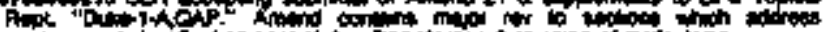

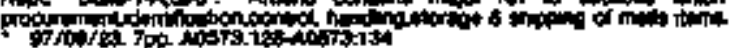

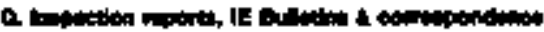

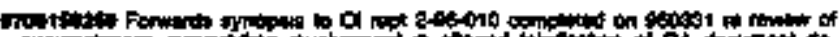

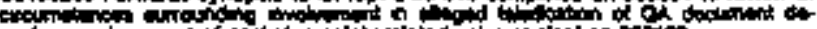

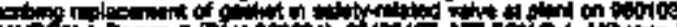

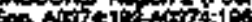

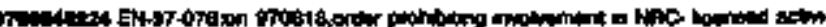

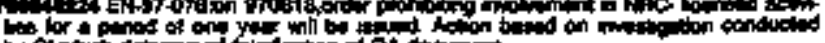

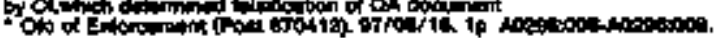

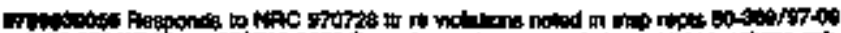

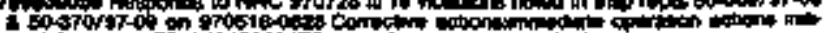

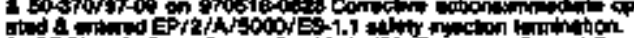

-

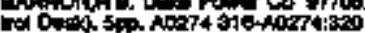




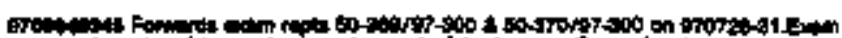

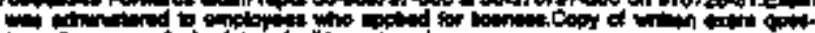

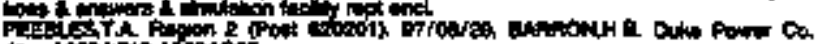
40. Ning

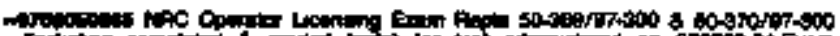

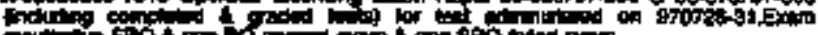

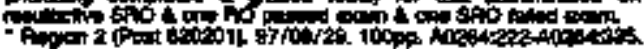

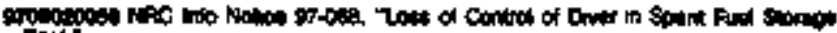

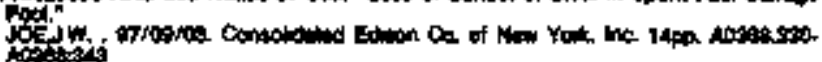

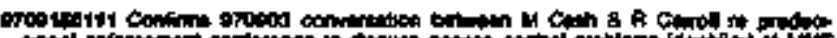

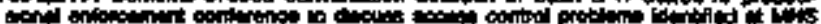

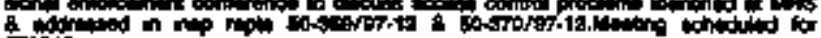
groplo

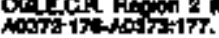

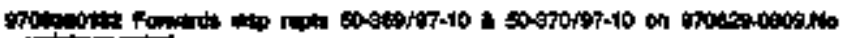

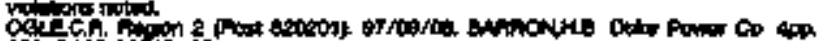

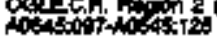

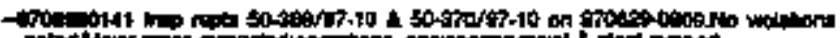

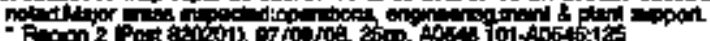

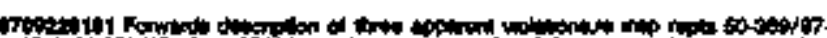

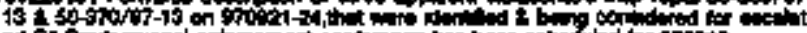

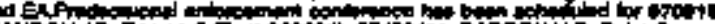

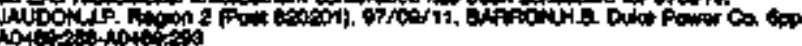

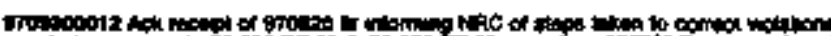

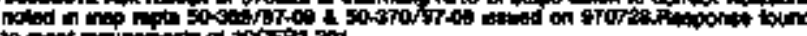

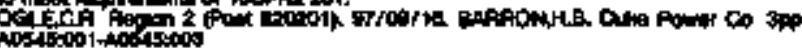

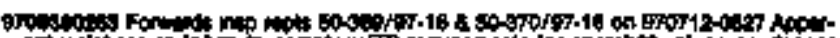

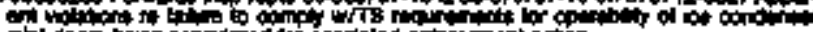

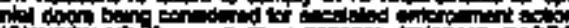

Whis

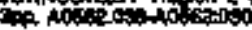

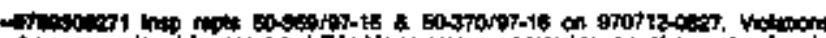

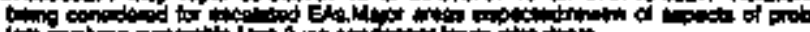

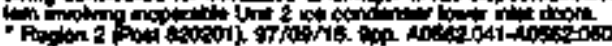

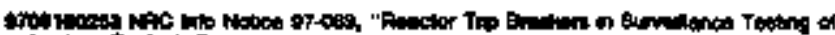

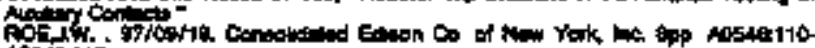
A

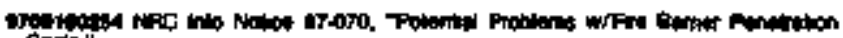

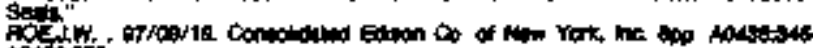

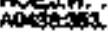

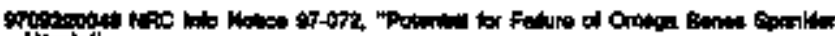

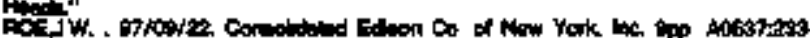
Acosizit.

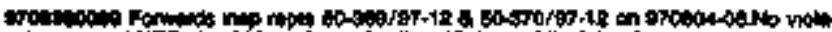

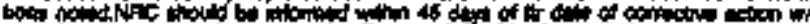

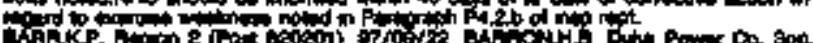

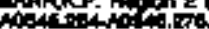

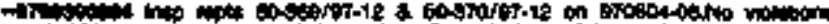
netarin

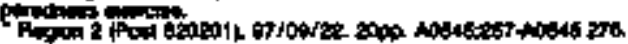

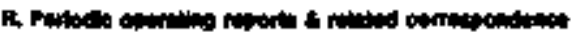

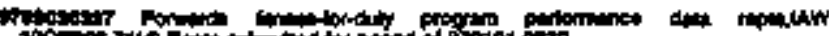

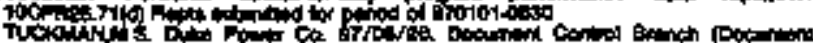

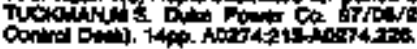

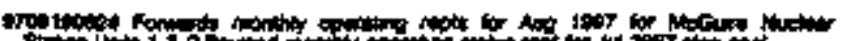

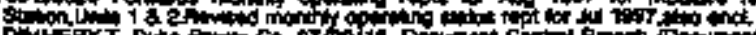

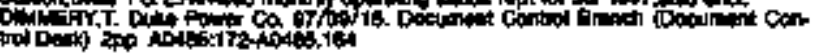

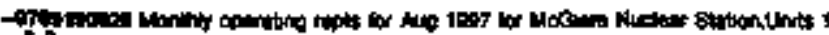
of

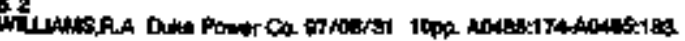

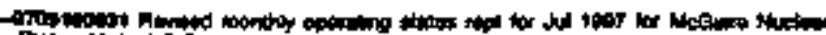

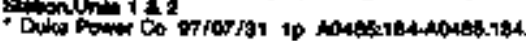

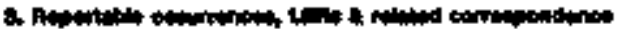

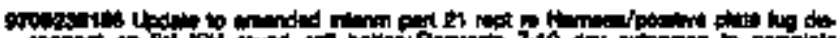

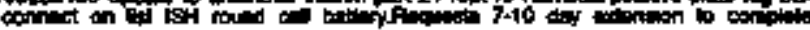

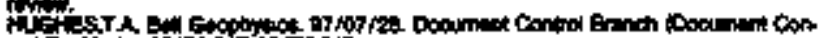

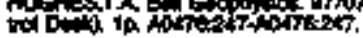

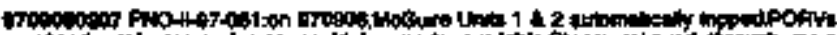

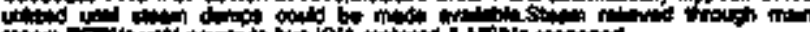

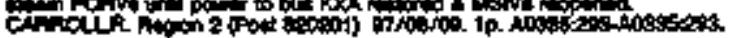

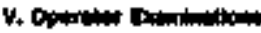

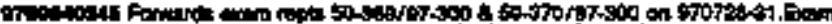

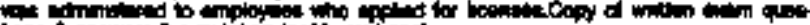

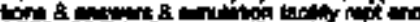

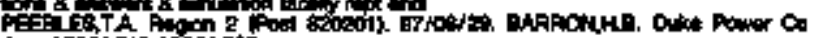

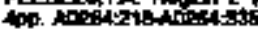

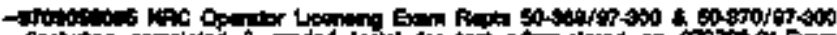

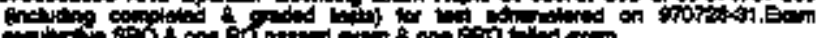

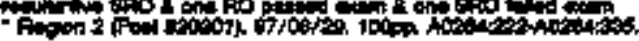

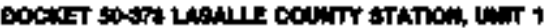

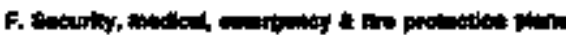

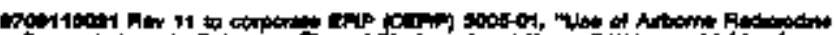

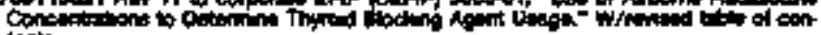
inter.

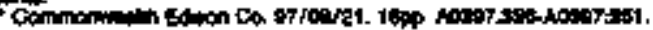

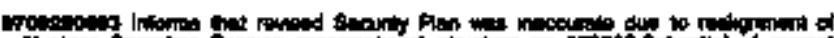

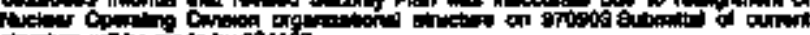

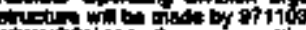

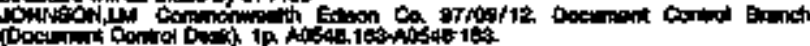

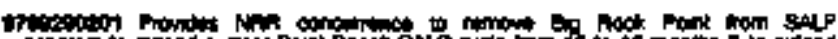

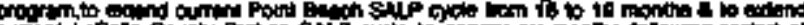

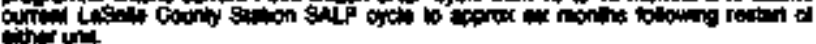
(n)

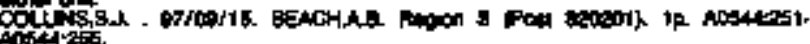

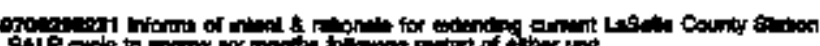

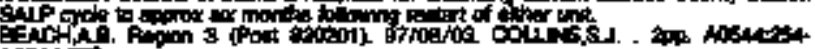
Anotition

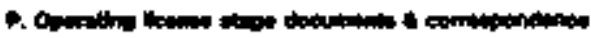

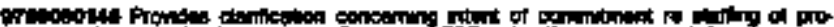

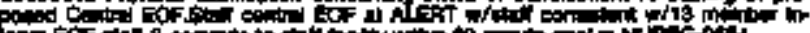

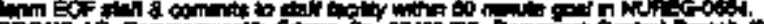
arows

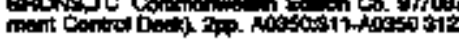

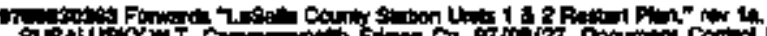

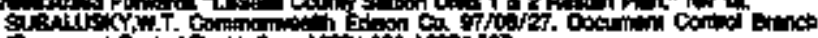

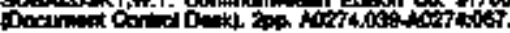

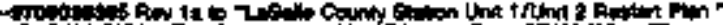
sudit tory, CorT. 1027\%0.7.

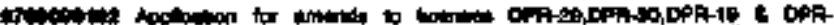

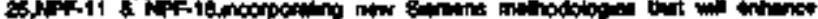

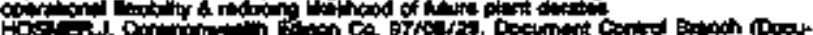
Hoj

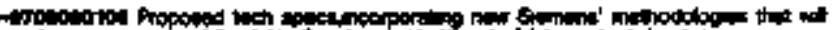

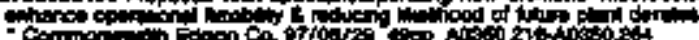

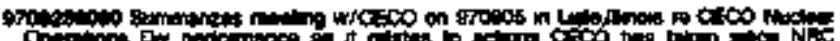

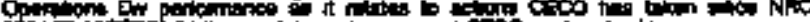

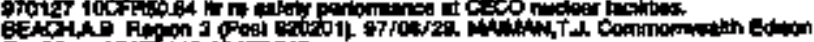

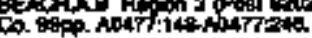

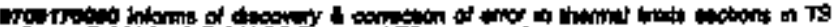

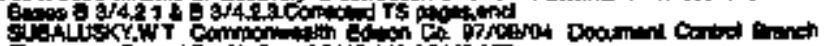

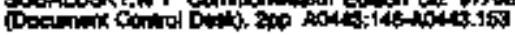

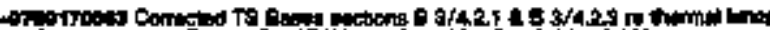

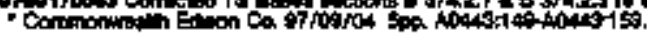

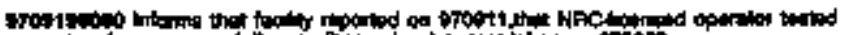

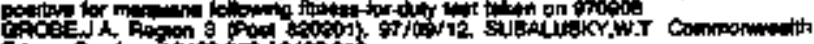

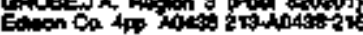




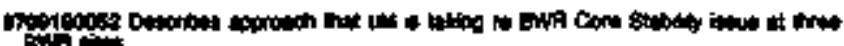

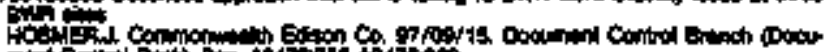

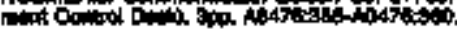

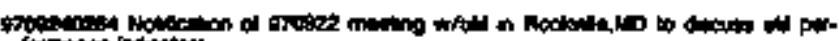

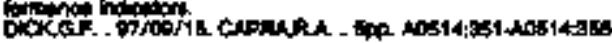

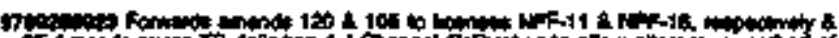

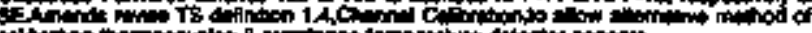

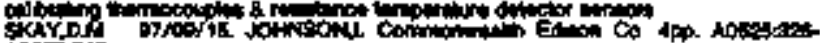
itcipens

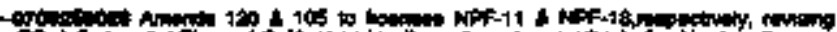

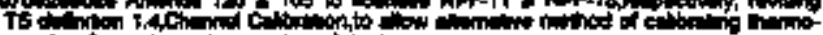

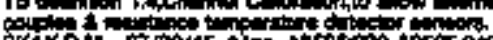

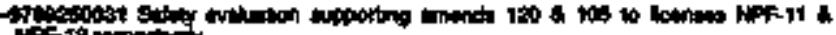

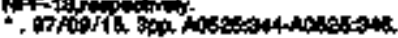

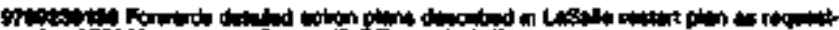

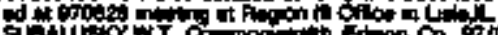

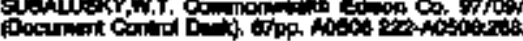

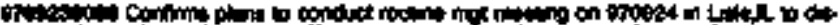

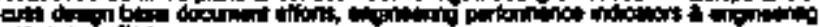

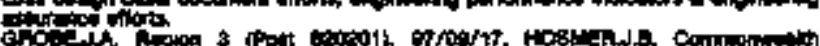

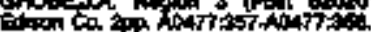

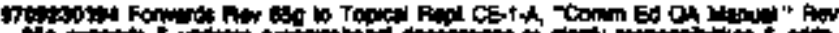

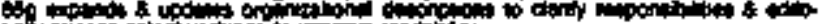

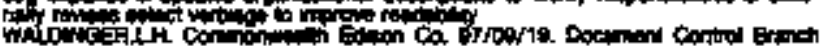

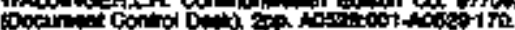

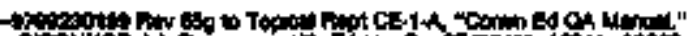

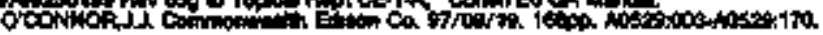

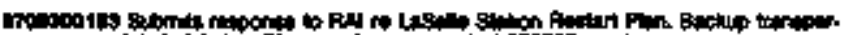
술

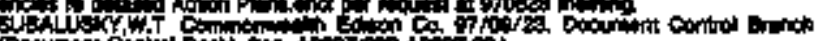

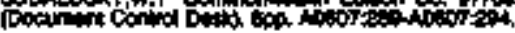

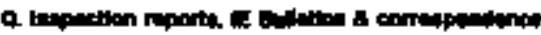

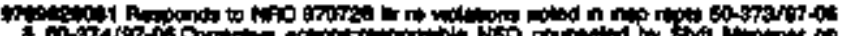

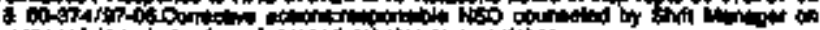

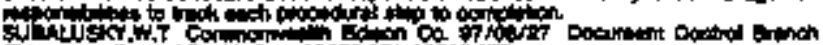

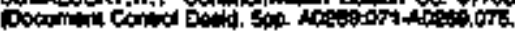

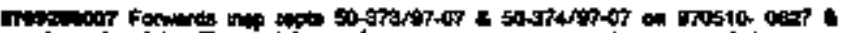

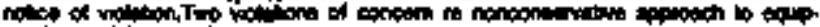
L

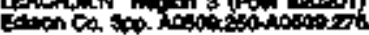

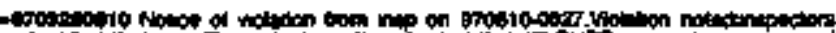

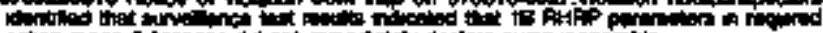

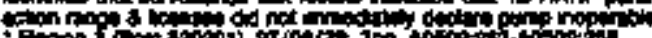

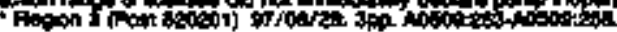

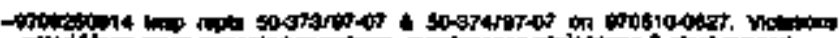

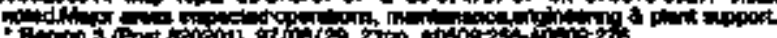

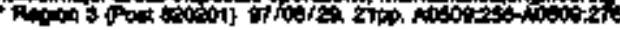

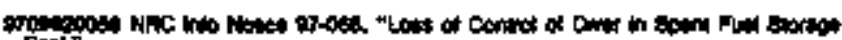
Poet

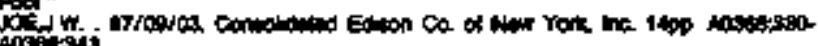

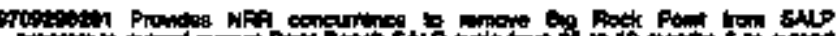

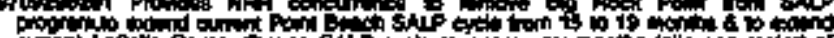

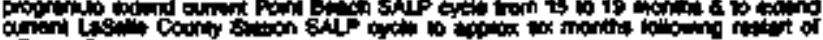

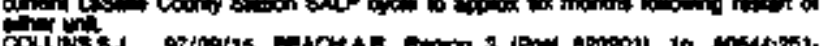

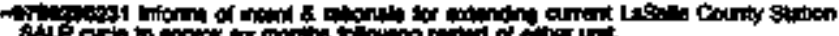

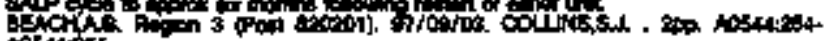
aris

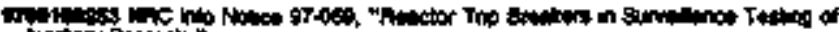

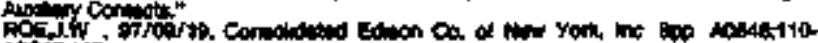
A

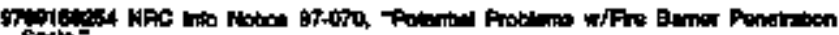
FOF ADAST 353

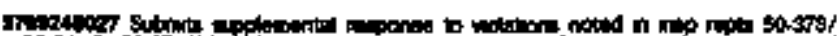

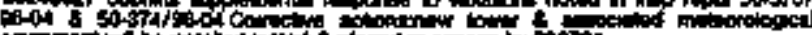

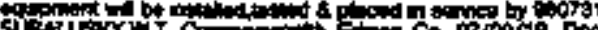

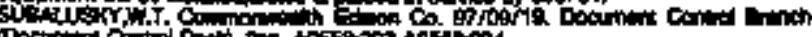

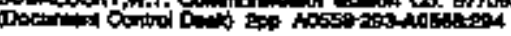

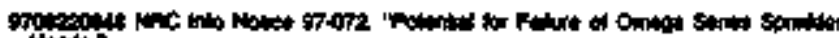

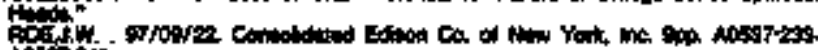
Piosinit.

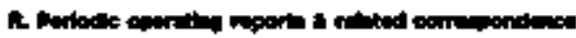

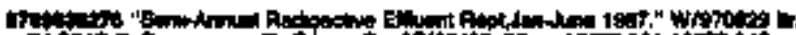

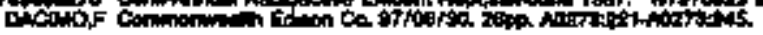

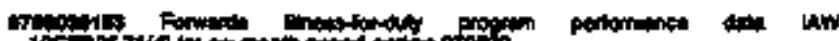

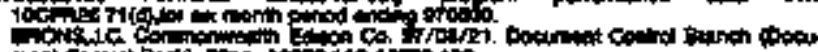

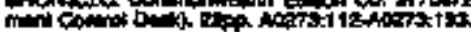

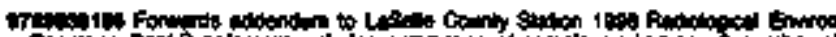

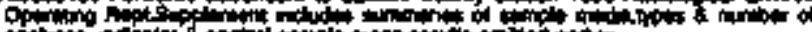

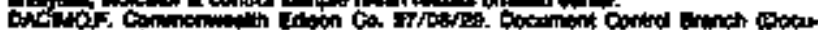

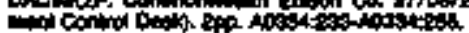

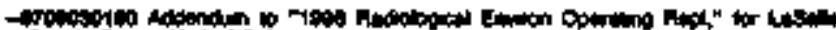

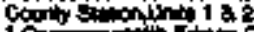

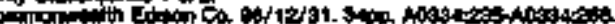

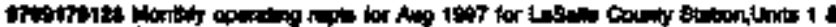

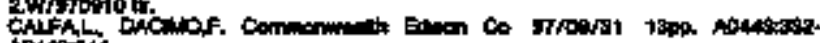

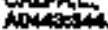

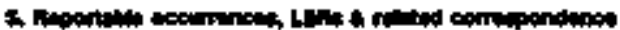

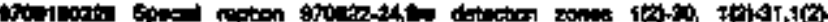

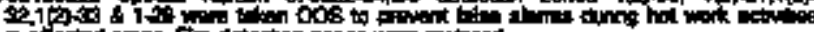

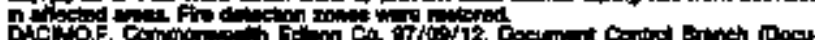

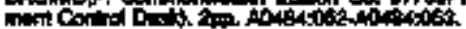

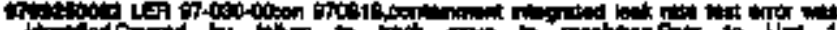

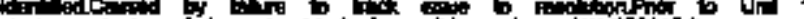

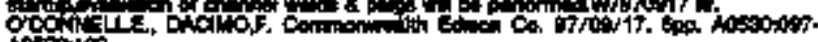
atosicto

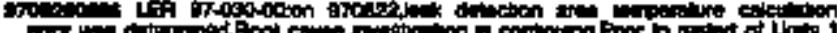

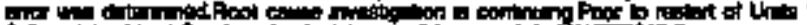

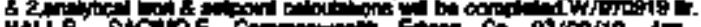

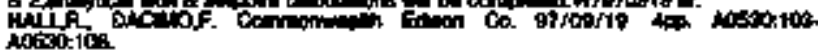

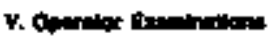

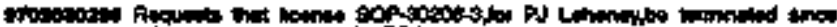

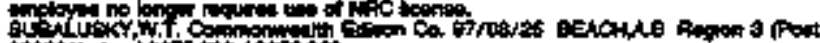

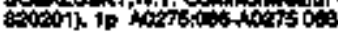

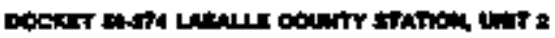

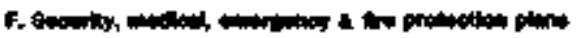

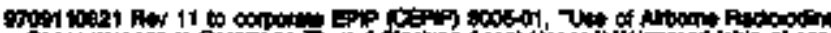

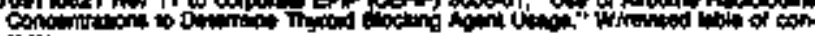

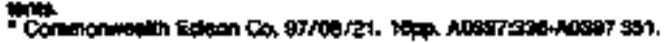

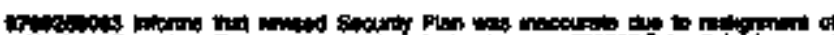

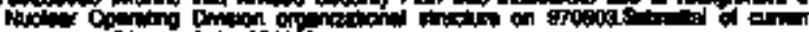

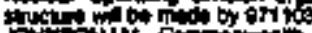

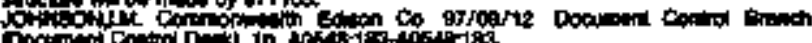

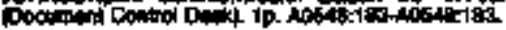

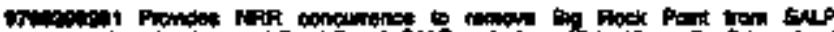

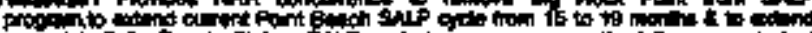

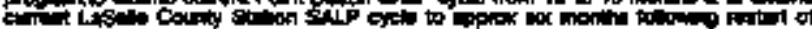
(1)

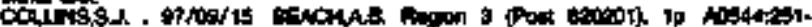

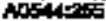

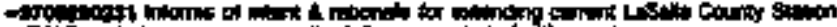

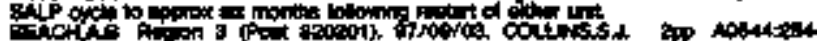
nosidents.

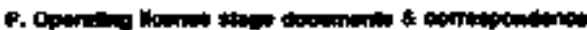

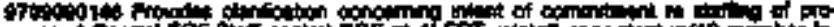

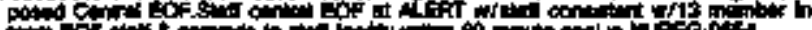

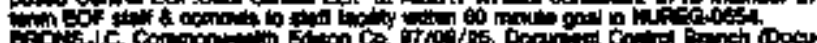

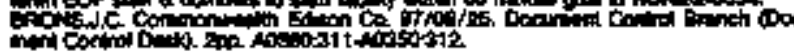

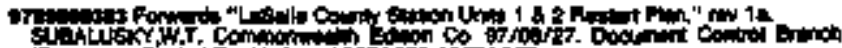

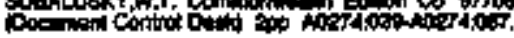

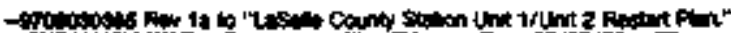

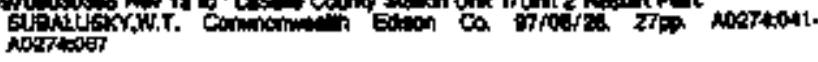




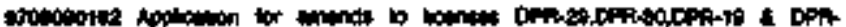

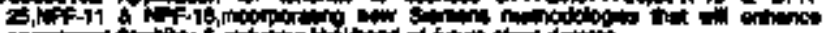

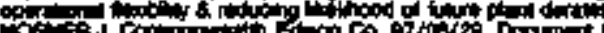

(t)

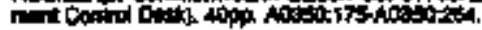

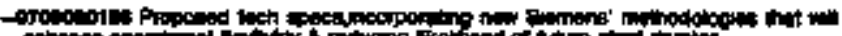

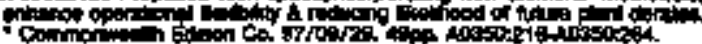

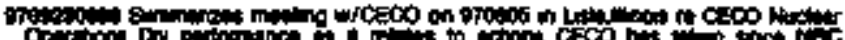

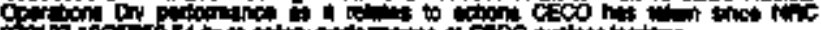

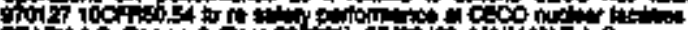

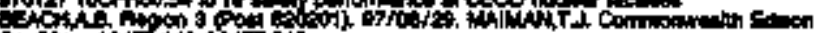

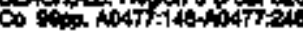

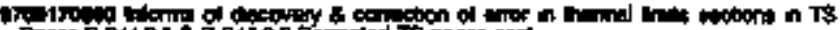

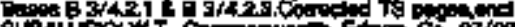

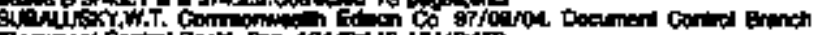

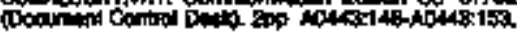

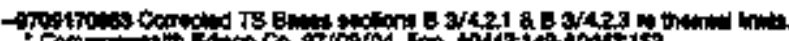

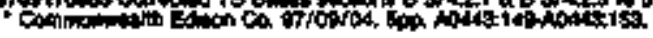

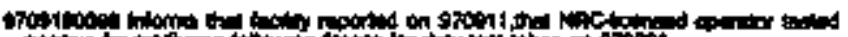

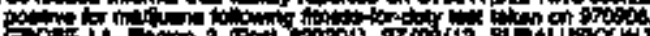

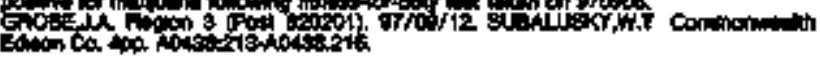

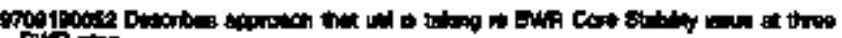
等

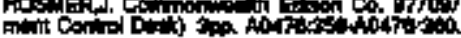

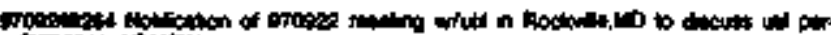

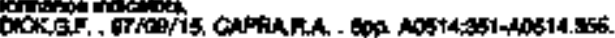

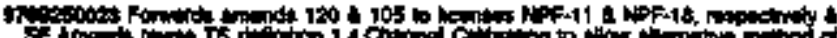

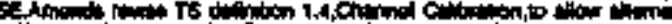

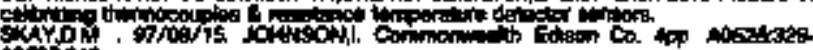
$00 x^{3}$ sit.

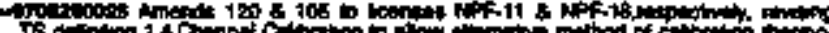

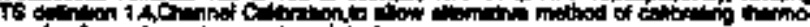
Dorto

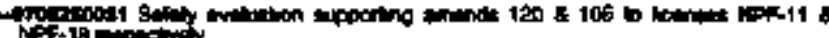

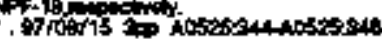

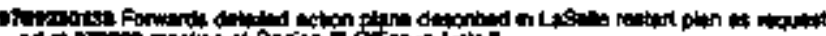

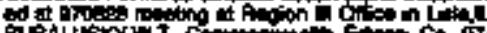

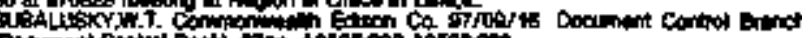

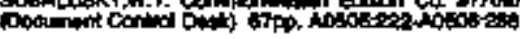

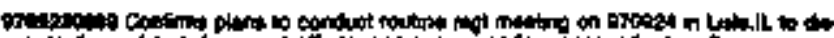

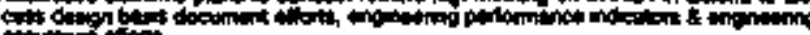

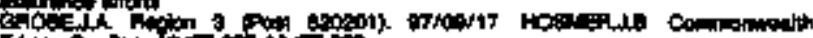

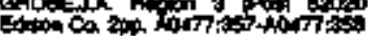

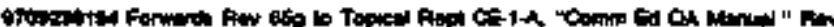

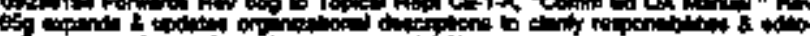

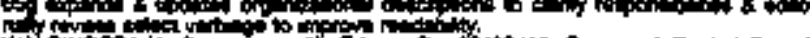

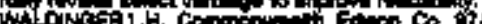

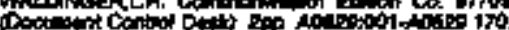

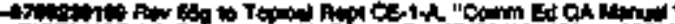

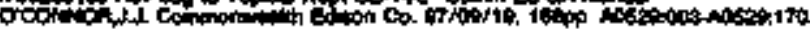

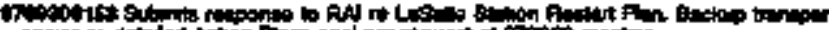

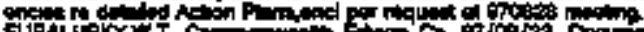

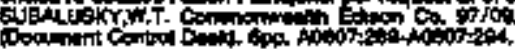

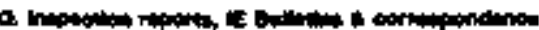

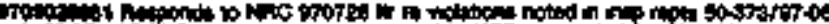

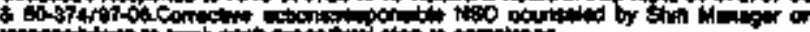

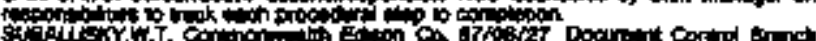

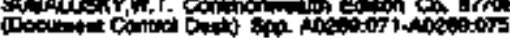

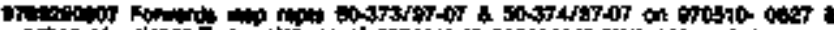

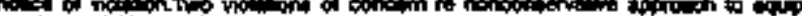

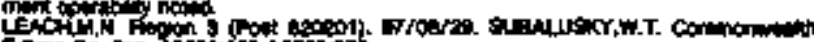

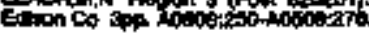

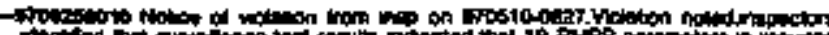

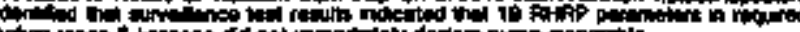

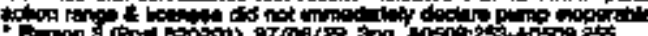

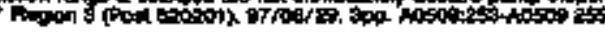

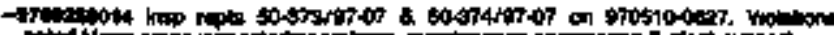

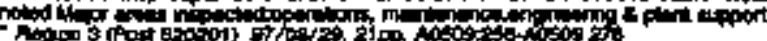

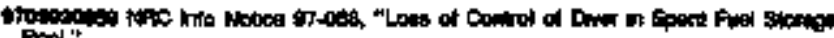
Pow" "10

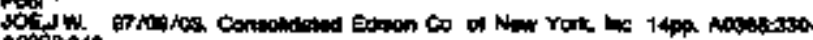

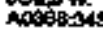

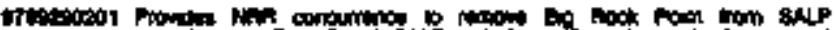

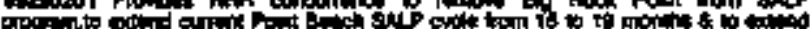

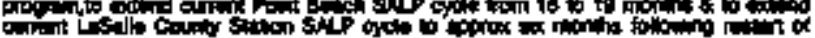

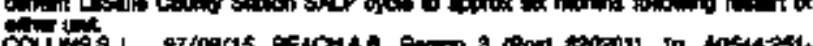

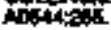

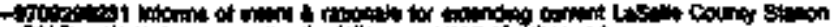

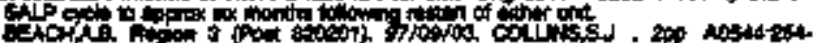
Nomptos.

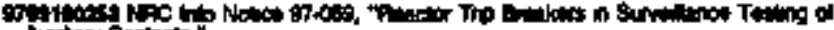

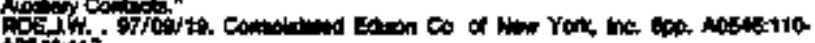
Nositit:

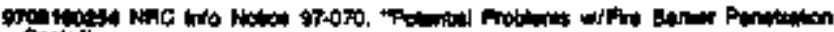

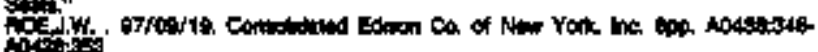

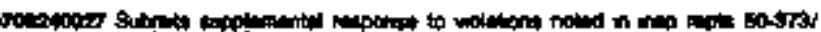

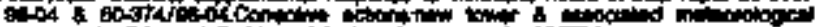

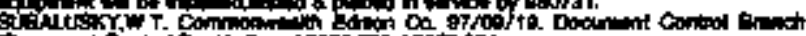

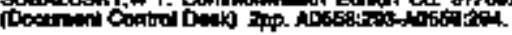

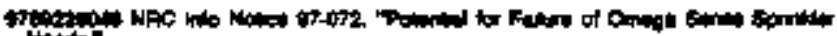

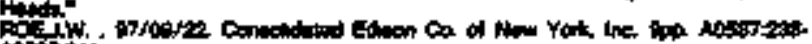

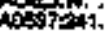

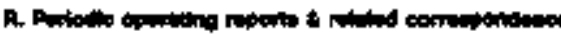

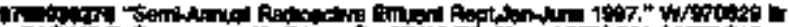

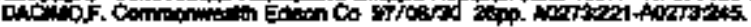

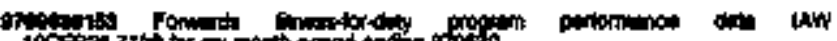

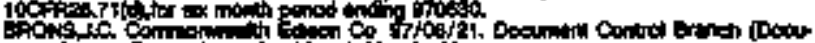

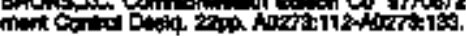

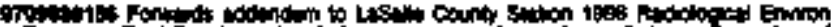

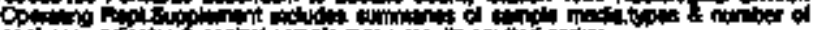

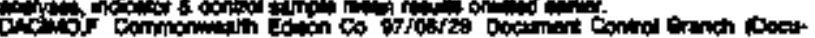

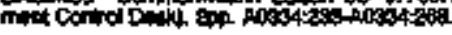

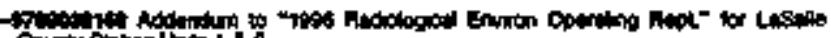

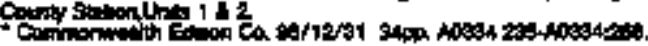

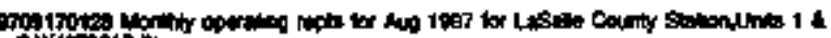

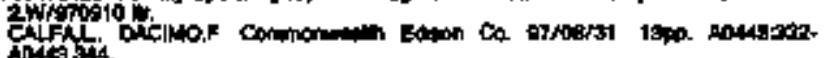

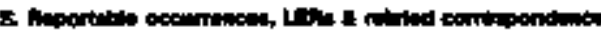

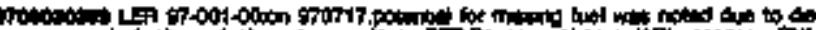

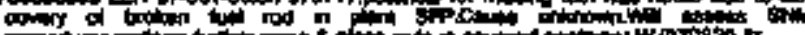

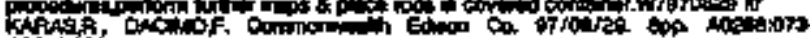
Notion

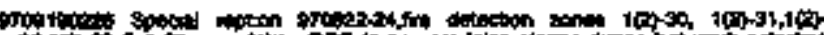

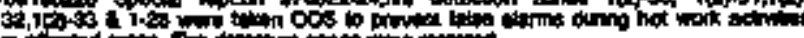

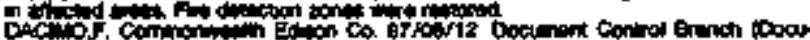

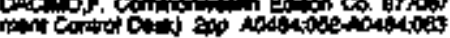

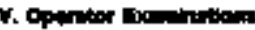

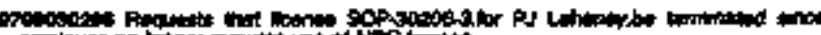

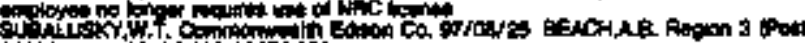

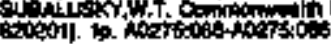

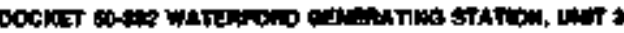

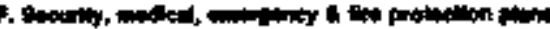

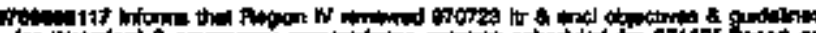

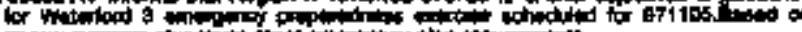

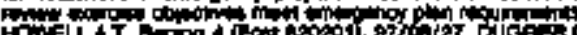

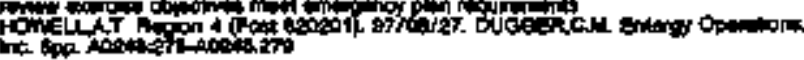

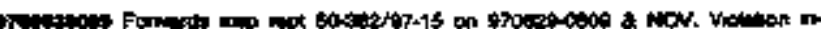

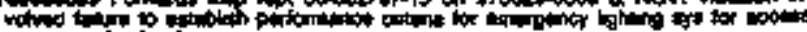

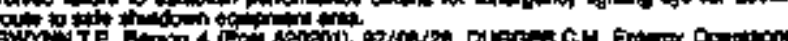

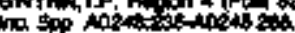

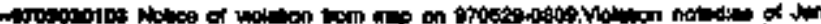

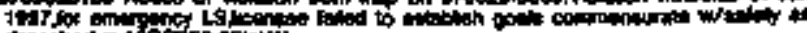

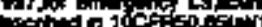

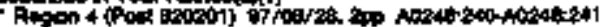




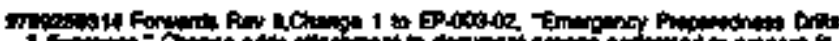

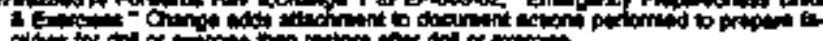

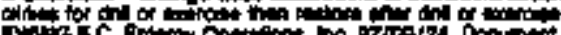

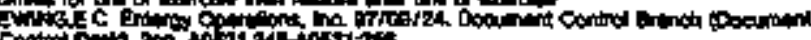

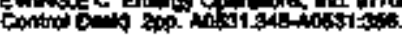

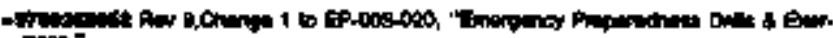

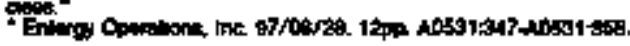

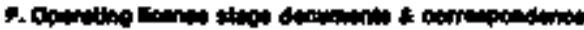

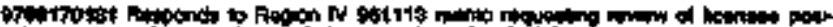

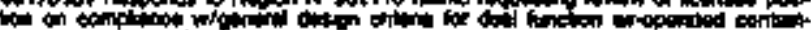

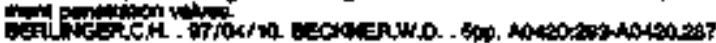

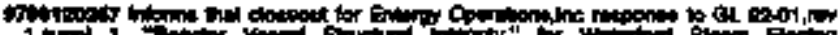

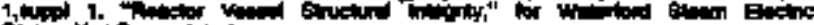

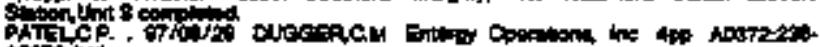

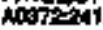

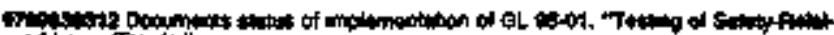

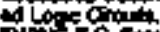

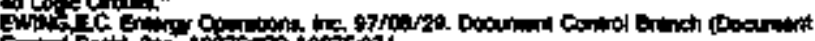

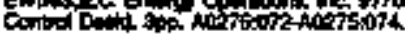

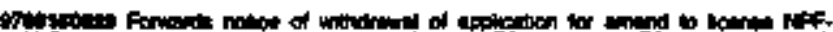
3.

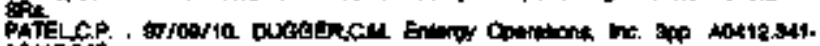
nortats.

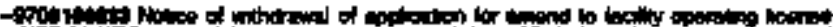

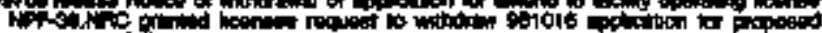

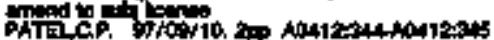

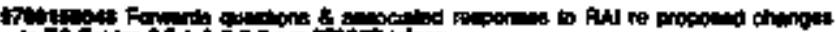

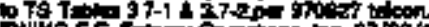

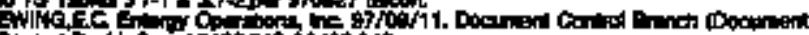

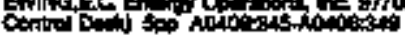

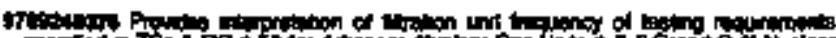

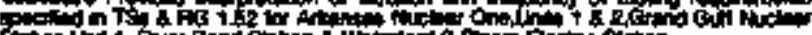

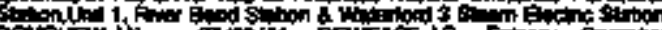
ocipit

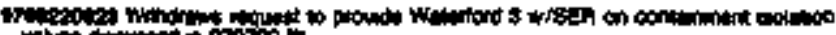

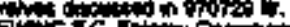

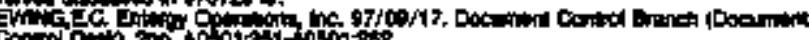

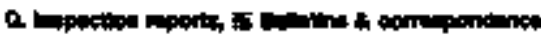

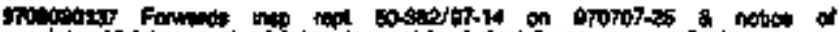

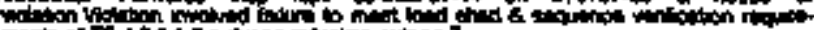

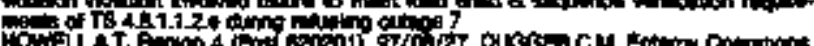

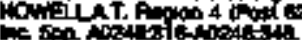

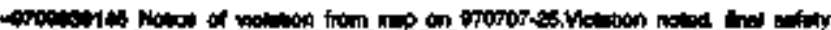

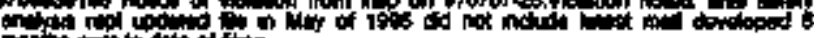

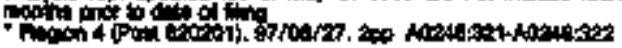

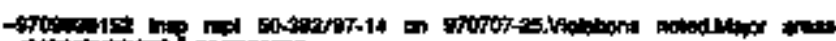

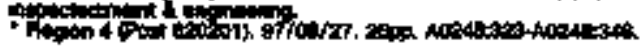

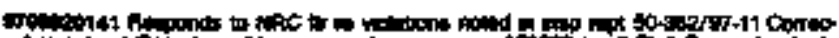

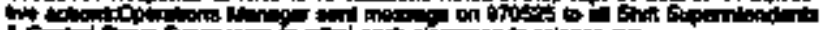

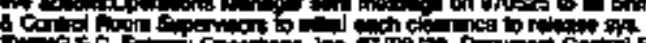

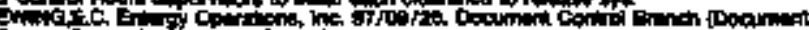

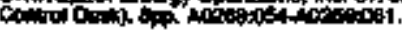

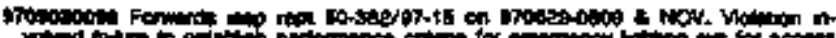

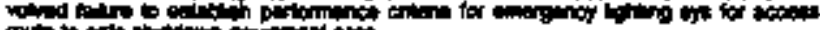

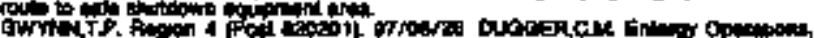

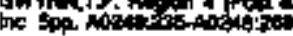

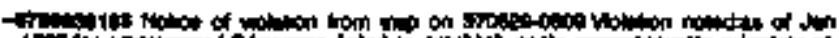

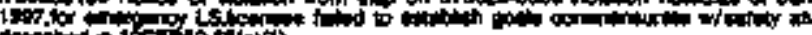

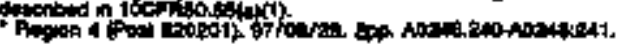

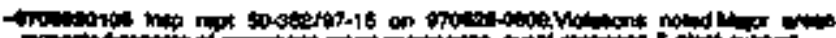

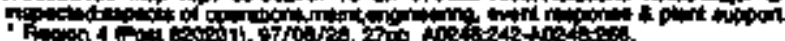

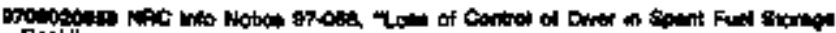

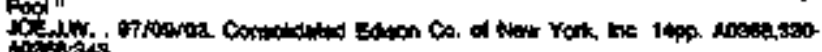

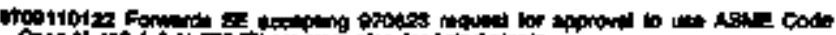

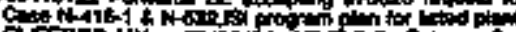

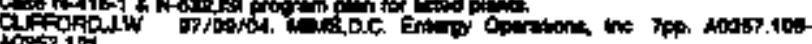
124

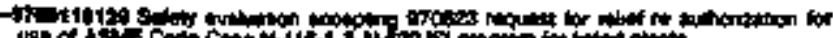

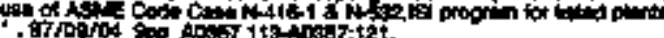

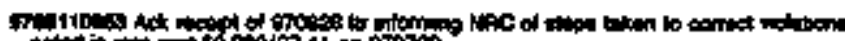
nection

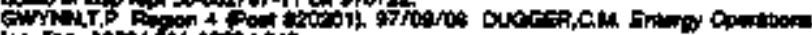

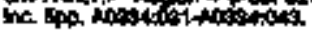

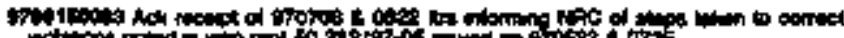

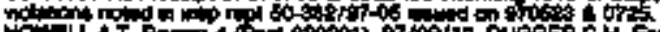

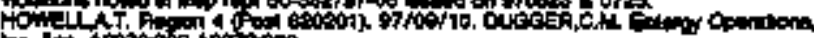

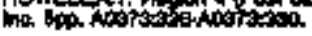

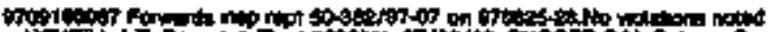

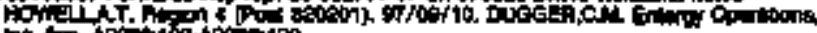

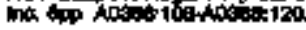

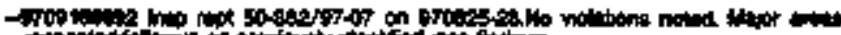

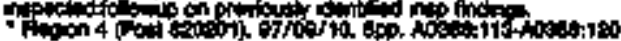

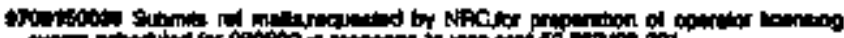

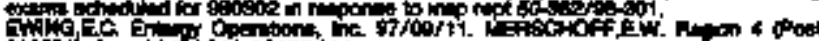

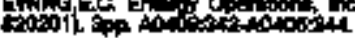

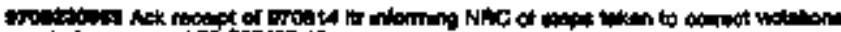

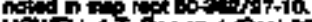

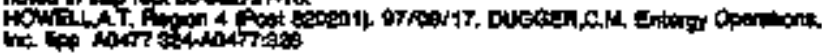

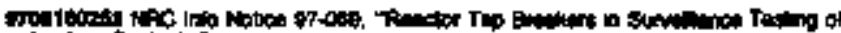

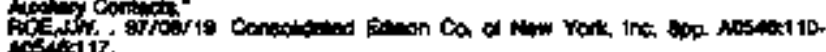
istititi.

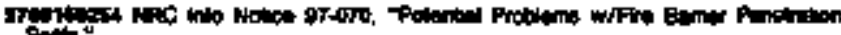

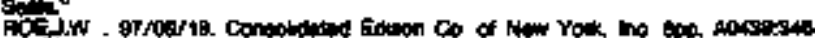

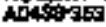

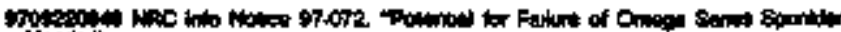

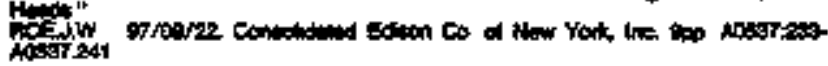

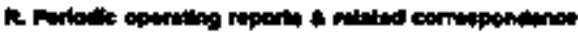

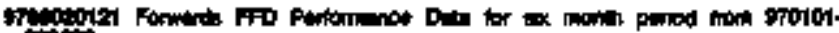

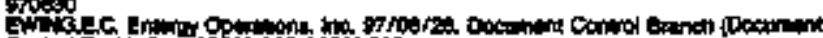

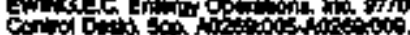

ITod

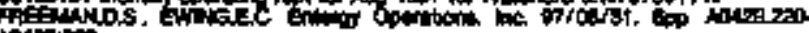
intorati.

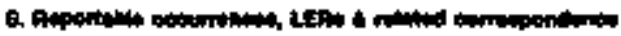

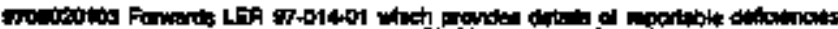

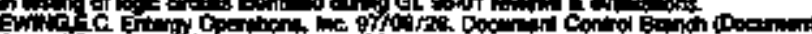

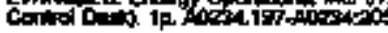

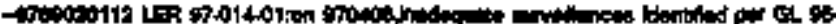

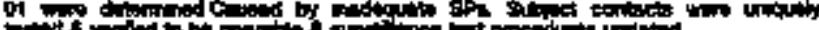

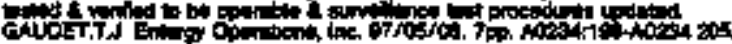

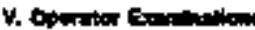

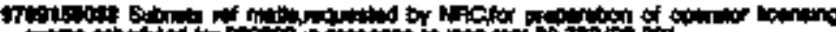

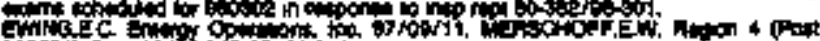
-

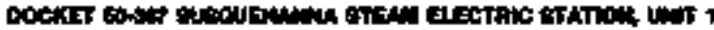

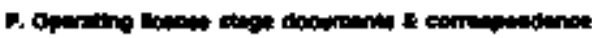

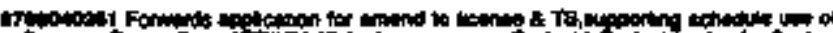

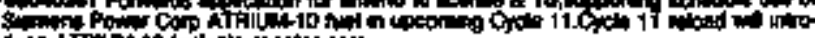

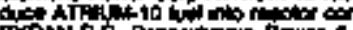

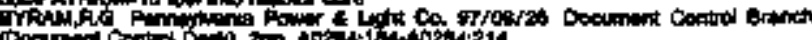

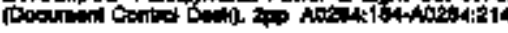

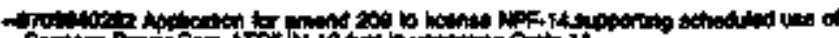

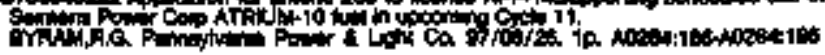

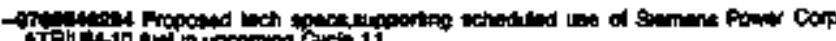

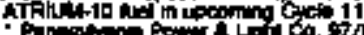

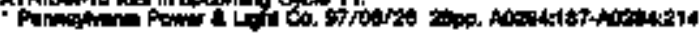

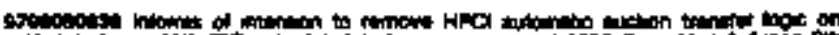

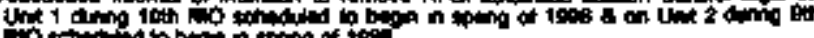

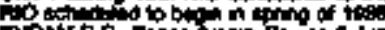

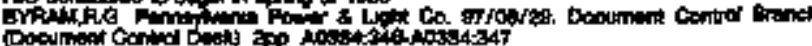




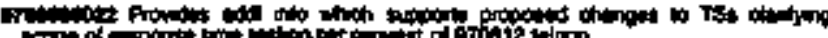

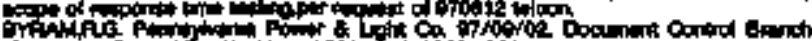

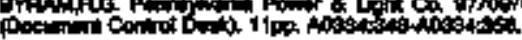

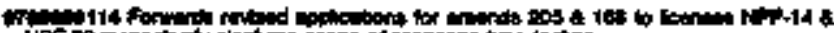

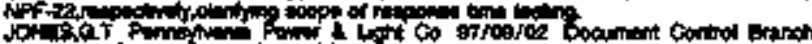

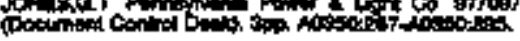

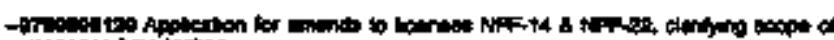

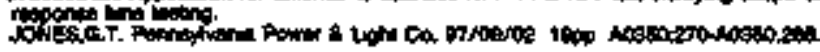

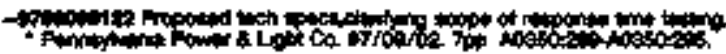

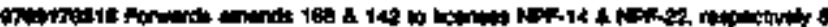

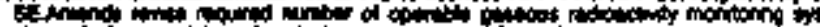

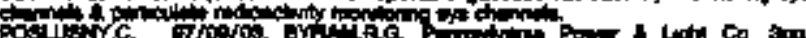
Hif 400 -

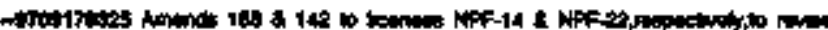

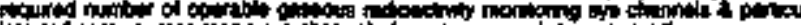

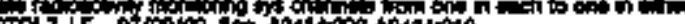

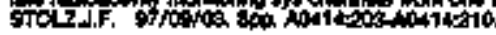

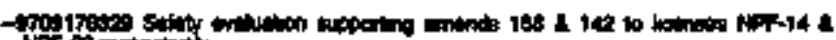
Nothering

97 TOW

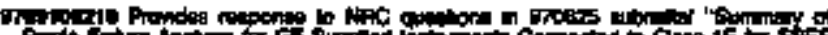

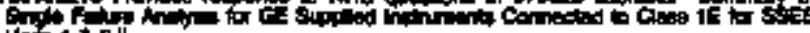

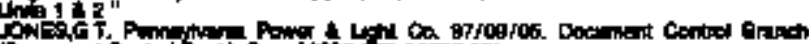

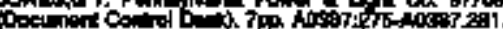

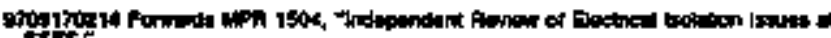

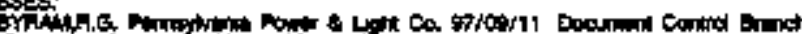

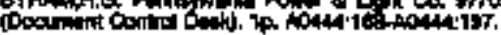

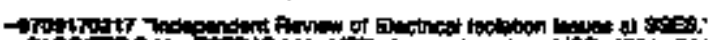

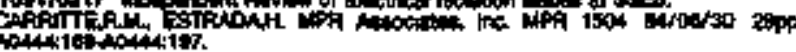

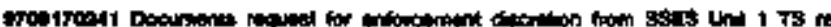

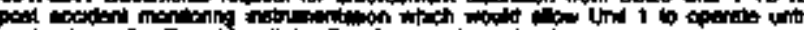

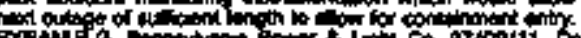

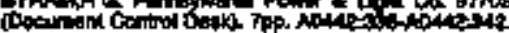

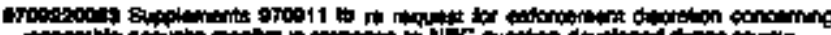

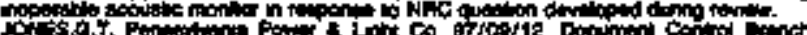

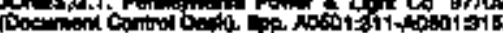

-

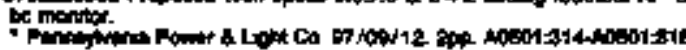

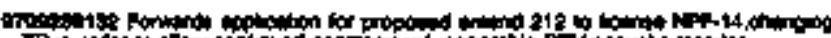

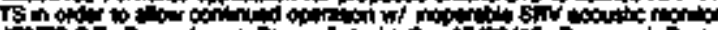

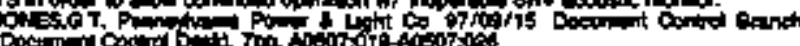

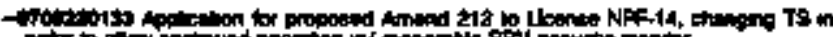

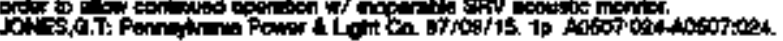

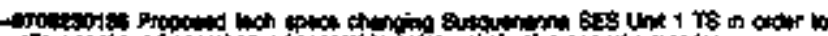

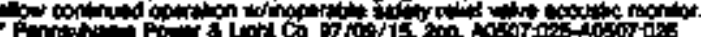

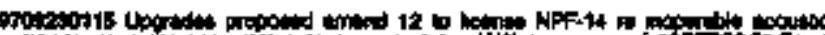

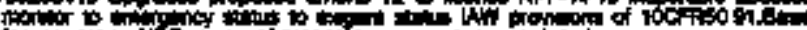

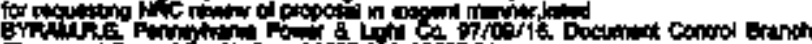

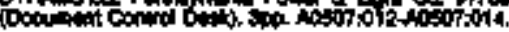

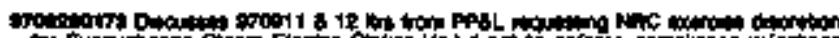

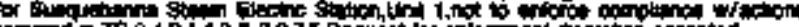

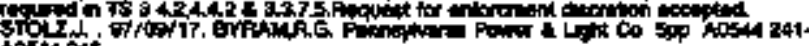
10.4.

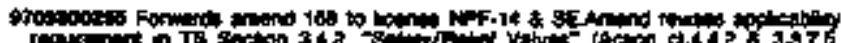

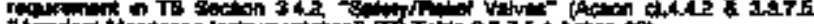

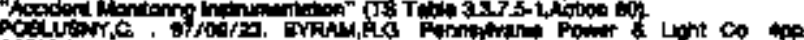
$100001-40 \sin 15$

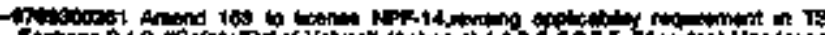

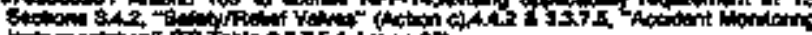

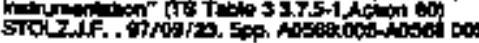

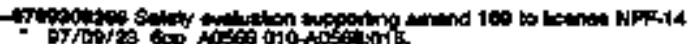

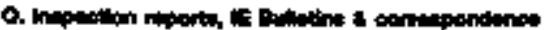

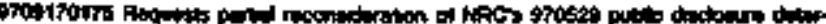

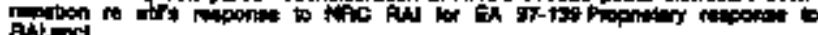

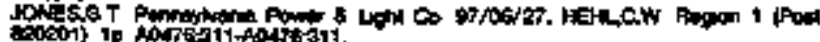

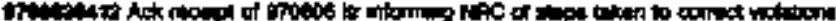

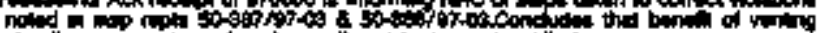

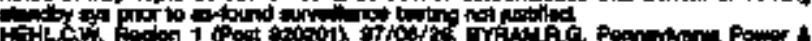

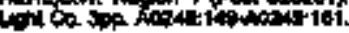

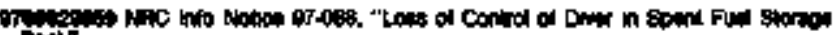

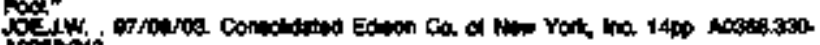
Antoing

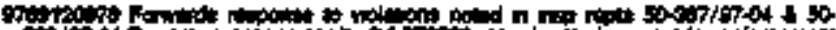

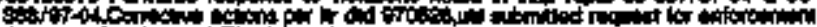

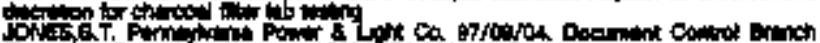

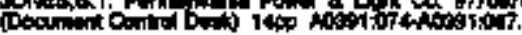

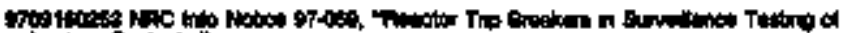
Bo 1051117.

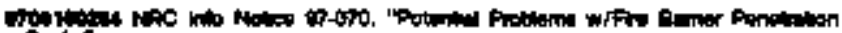

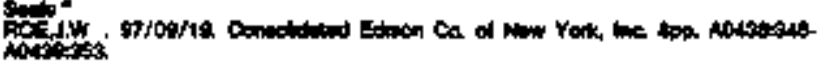

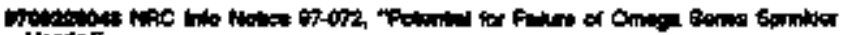

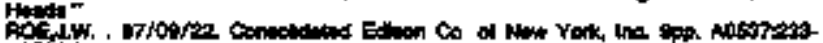
nostititi:

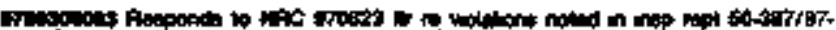

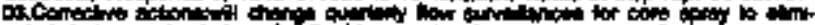

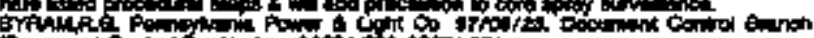

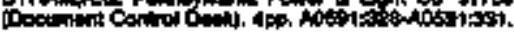

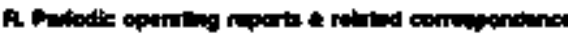

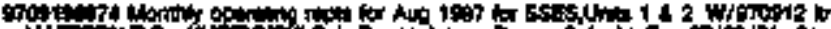

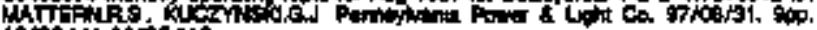

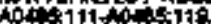

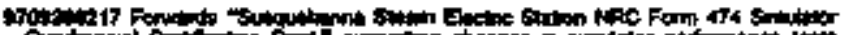

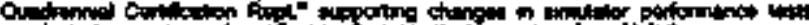

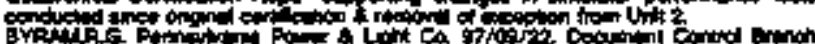

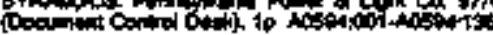

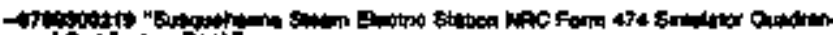

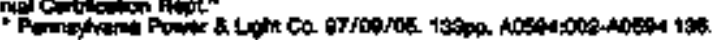

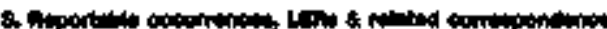

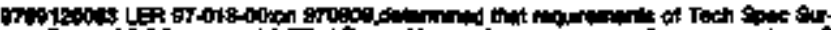

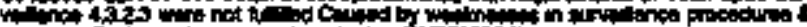

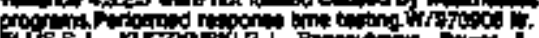

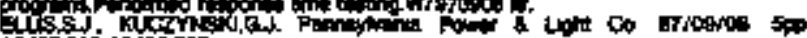
intosimation

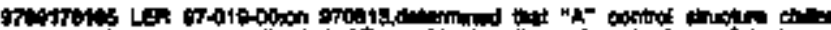

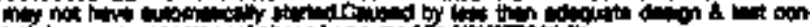

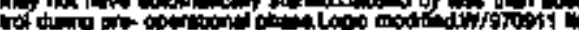
ilis

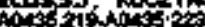

\section{Opintor Go.imberione}

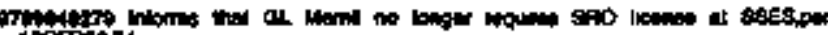

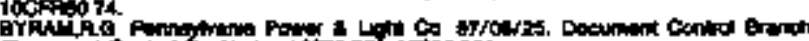

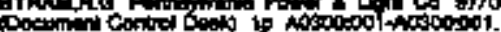

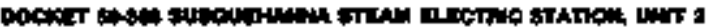

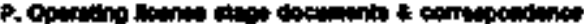

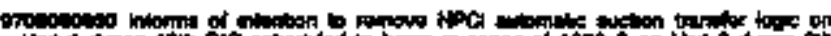

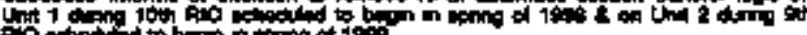
it

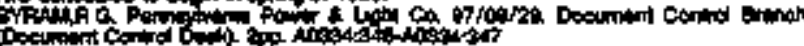

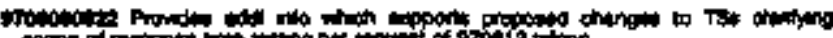

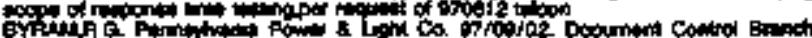

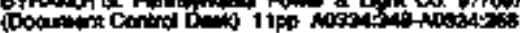

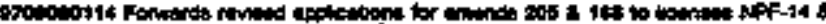

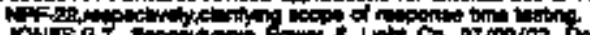

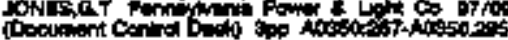

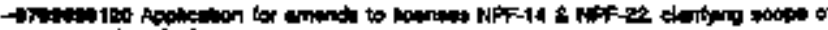

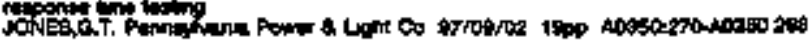




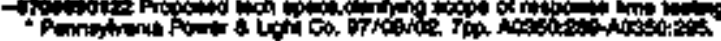

Ir:

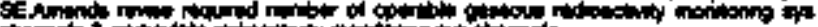

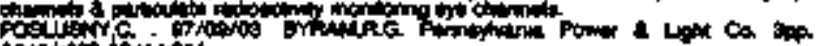

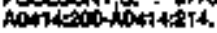

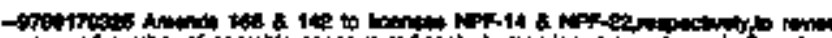

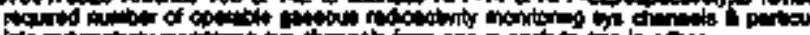

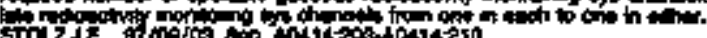

STOL2 J7. G

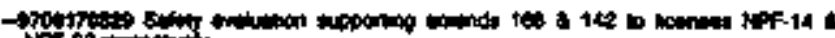

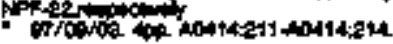

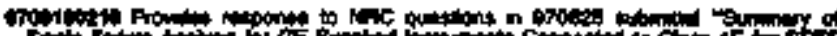

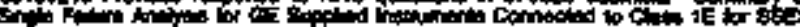

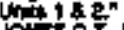

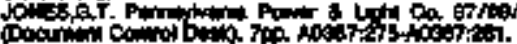

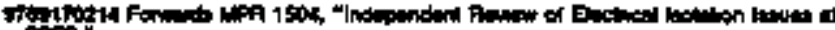

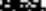

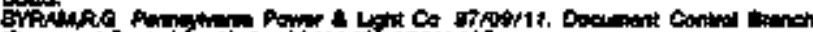

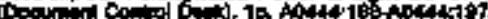

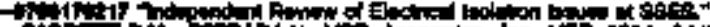

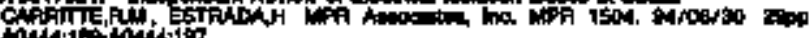

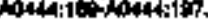

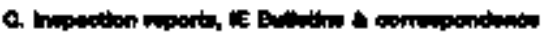

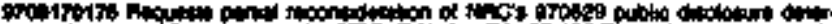

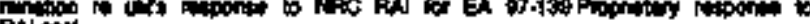
Rulinist

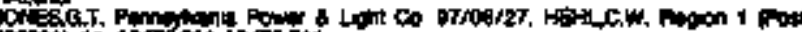

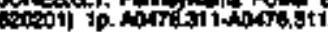

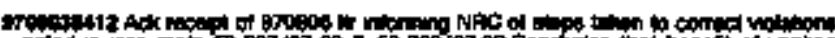

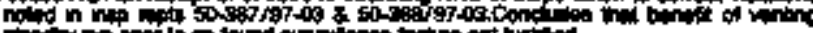

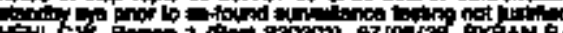

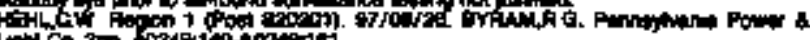

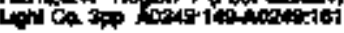

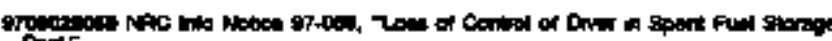

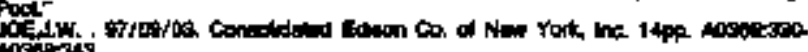

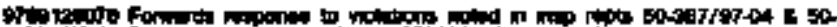

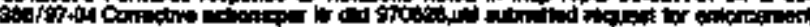

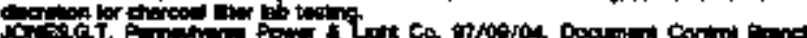

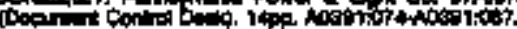

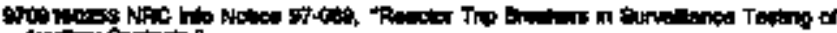

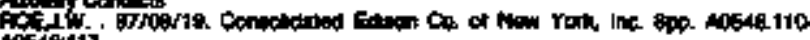
ASA4117.

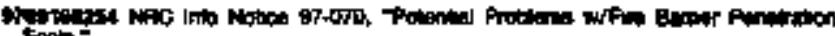

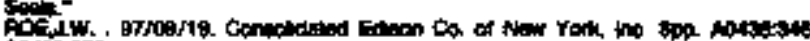

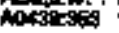

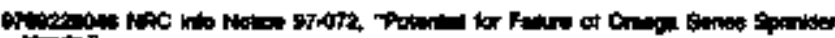

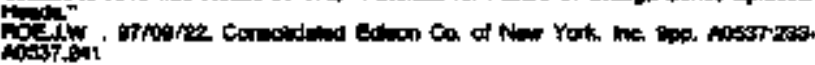

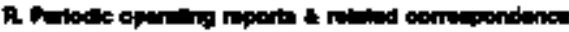

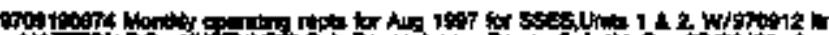

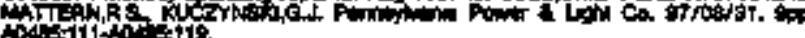

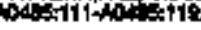

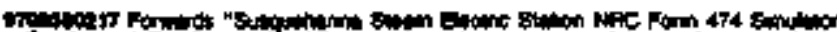

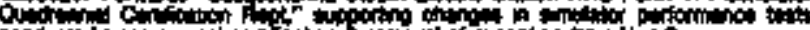
anduct inc of

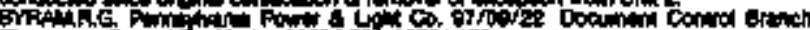

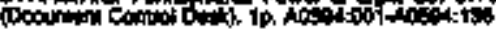

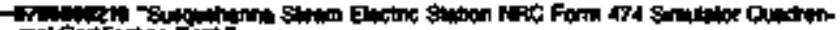

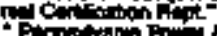

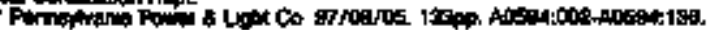

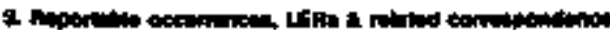

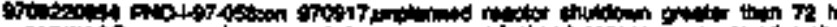

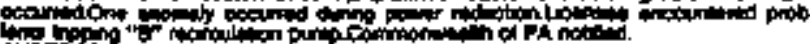

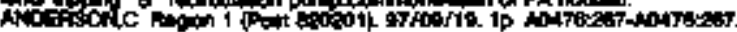

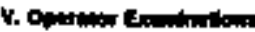

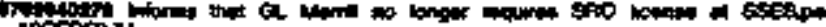
100 casor.

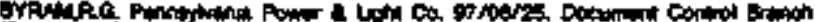

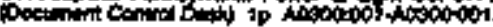

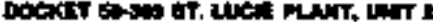

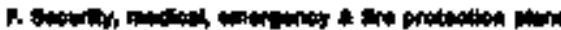

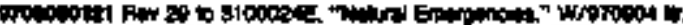

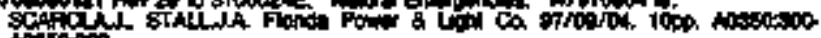
insolio.

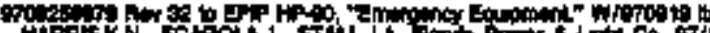

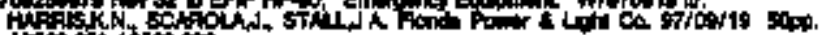

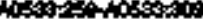

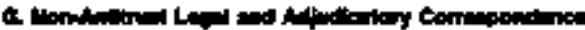

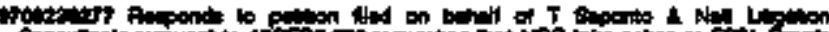

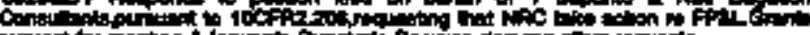

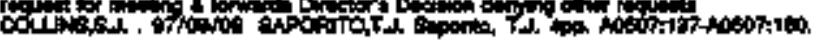

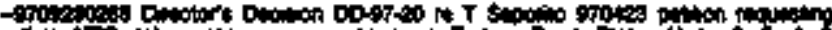

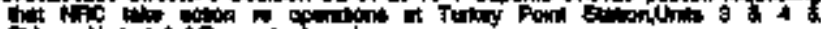

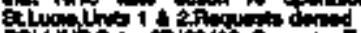

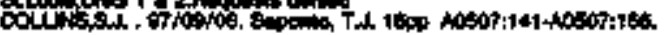

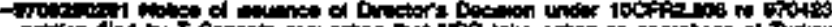

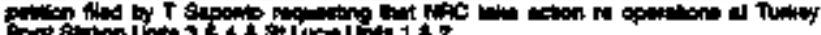

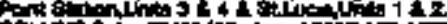

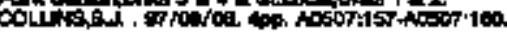

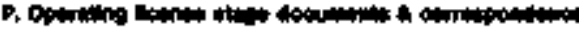

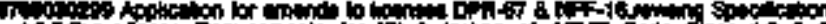

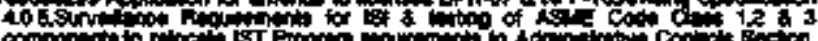

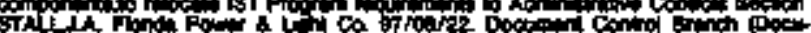

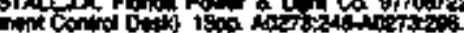

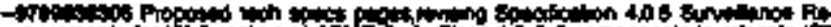

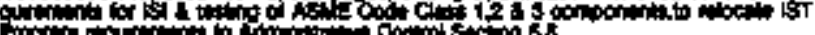

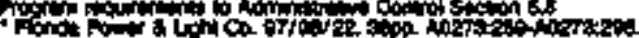

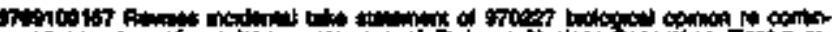

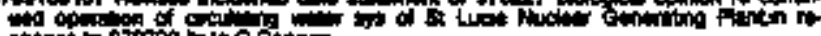

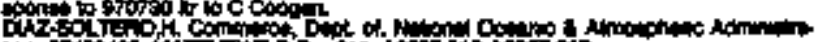

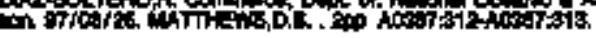

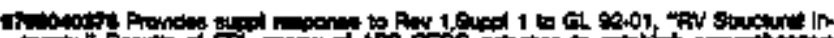

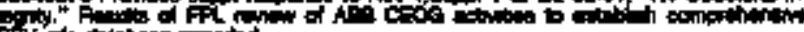
辛y

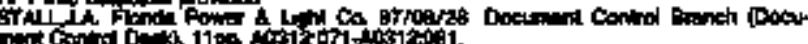

If 1 F

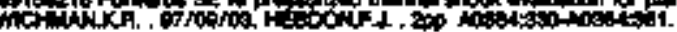

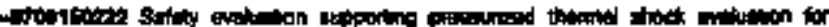
Plit:

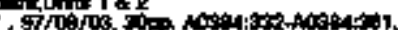

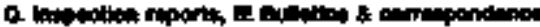

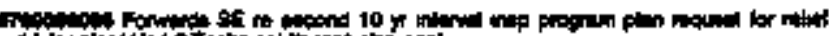

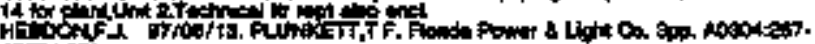
Anstion

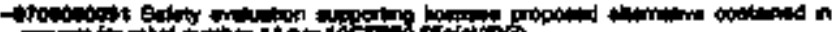

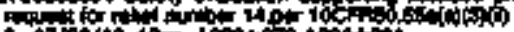

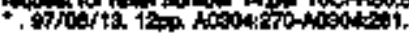

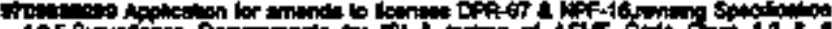

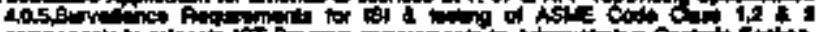

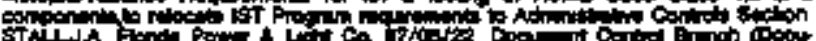

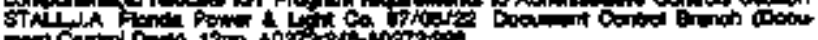

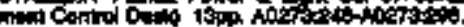

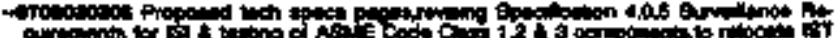

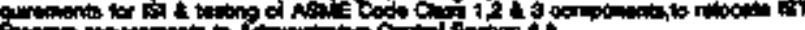

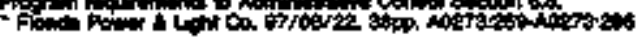

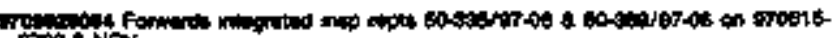
ortant

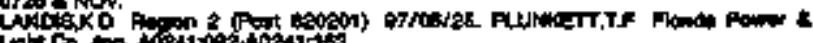

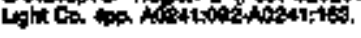

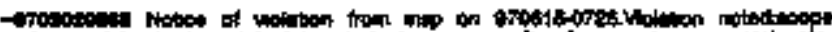

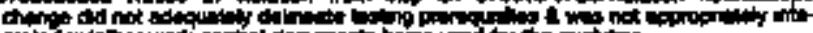

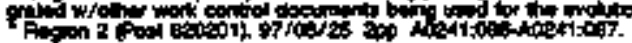

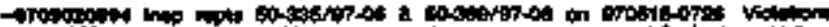

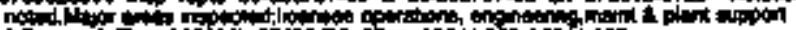

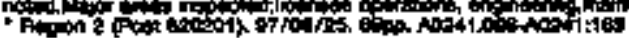

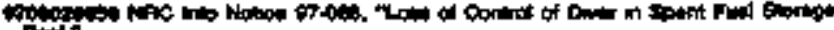
Put

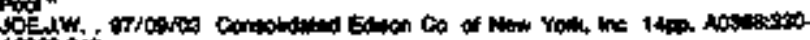
Actoris 


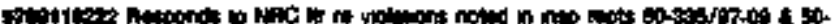

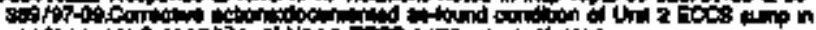

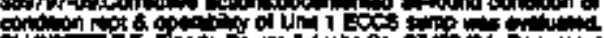

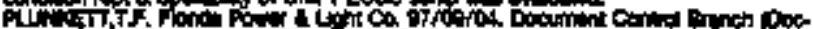

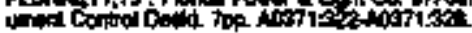

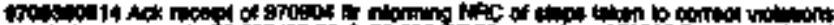

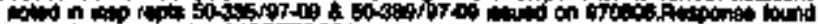
0 on

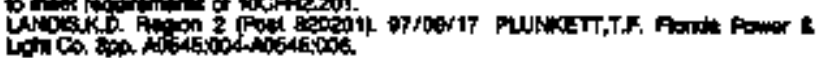

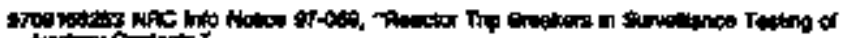

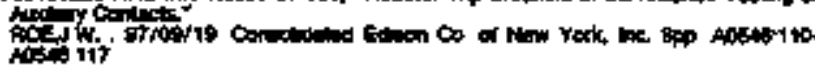

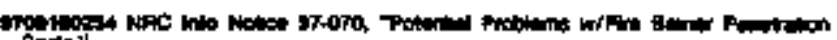

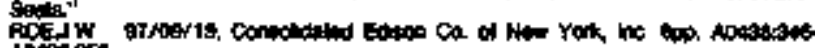

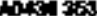

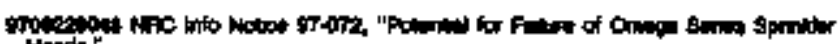

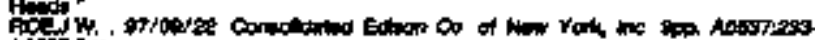
AOST-B 61 .

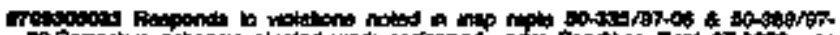

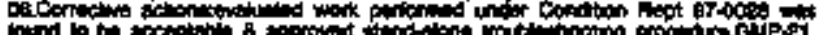

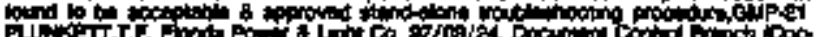

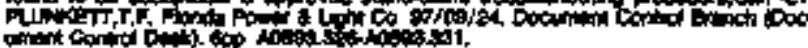

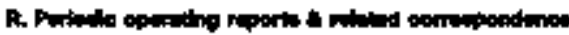

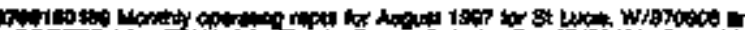

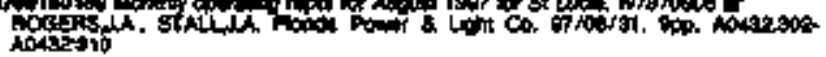

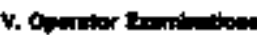

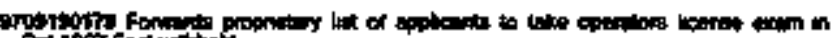

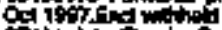

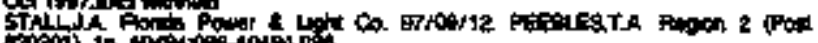

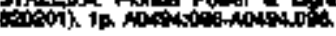

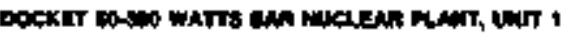

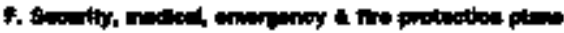

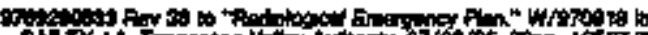

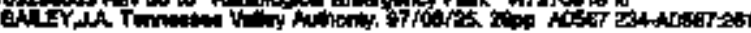

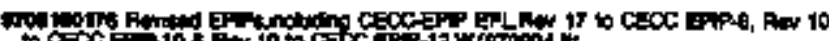
gule

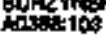

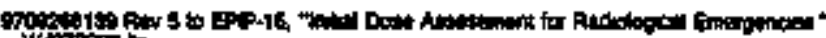

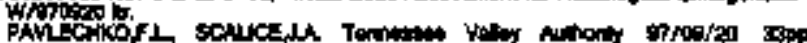

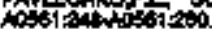

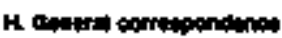

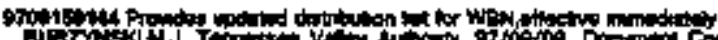
Drich

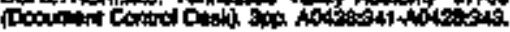

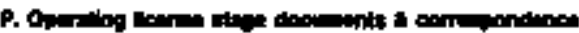

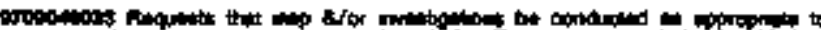

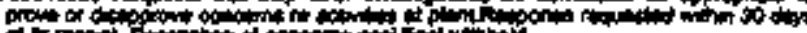

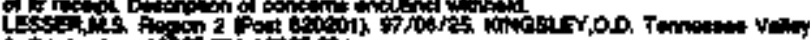

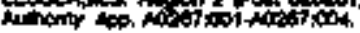

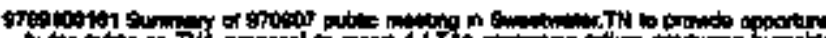

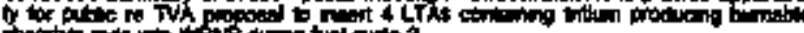

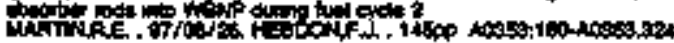

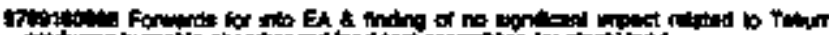

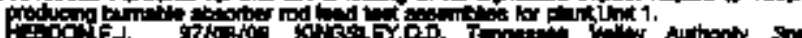

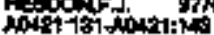

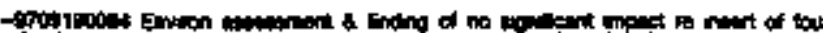

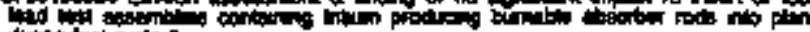

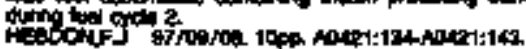

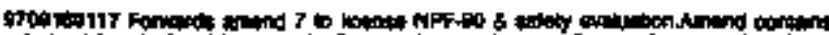

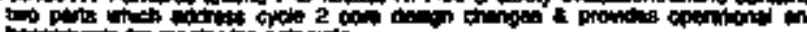

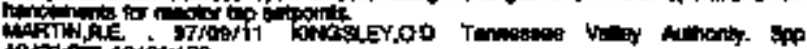

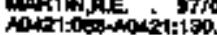

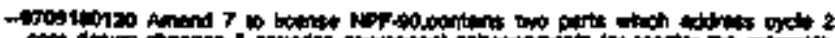

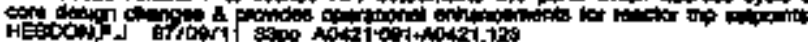

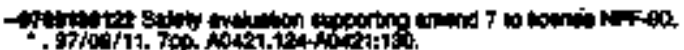

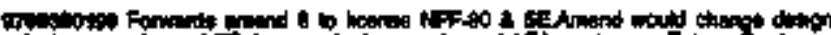

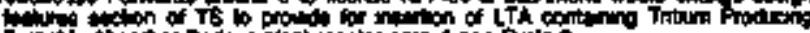

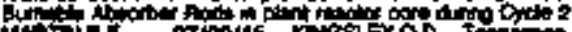
Vatay Nutwory

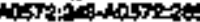

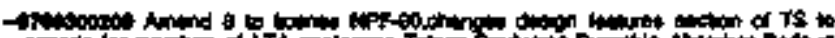

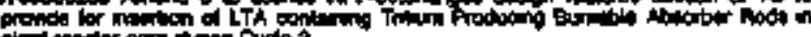

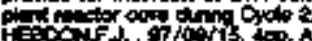

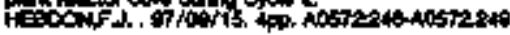

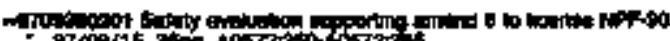

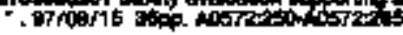

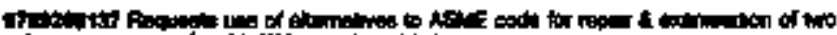

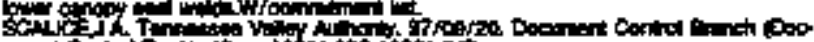

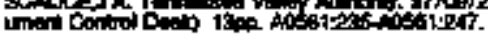

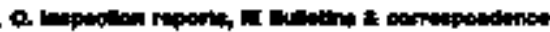

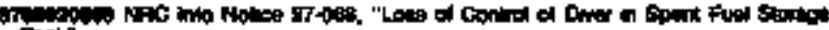

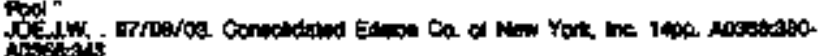

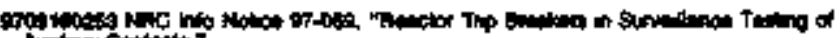

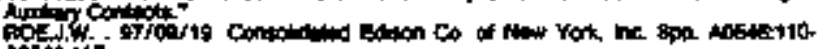
fix.

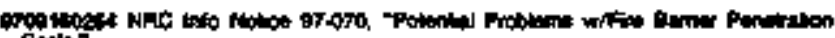

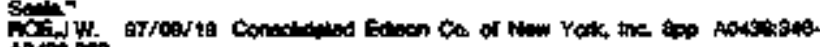
Andsing

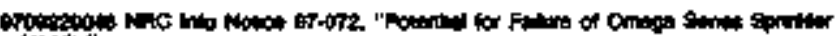

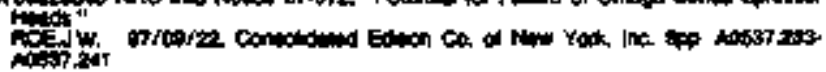

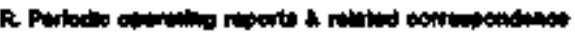

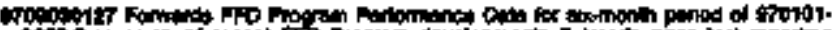

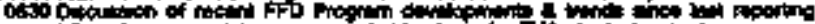

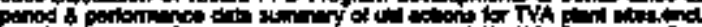

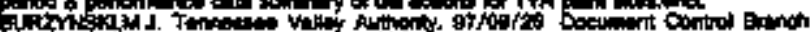

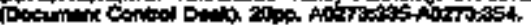

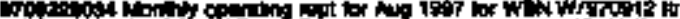

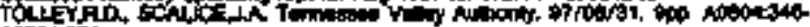
100061

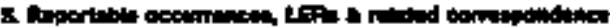

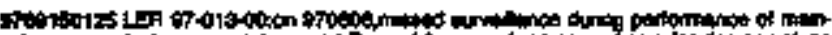

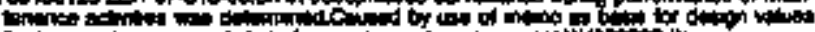

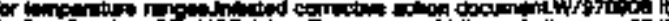
1000

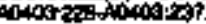

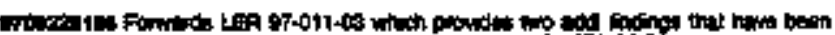

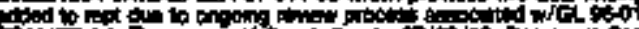
S:

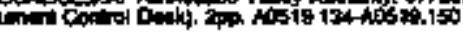

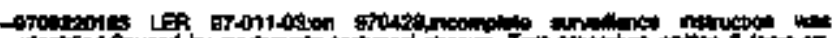

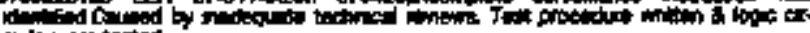

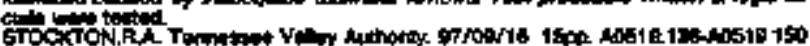

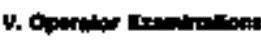

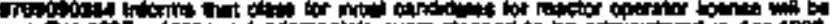

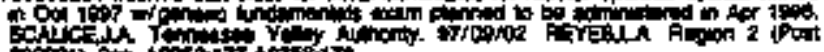
-

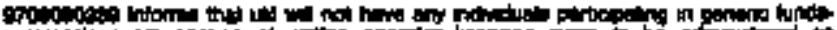

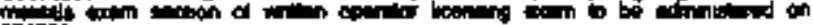

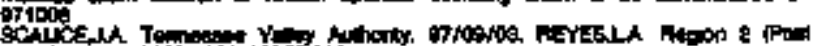

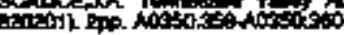

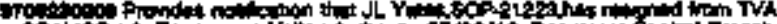

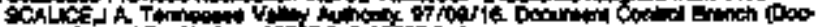

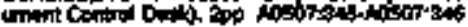

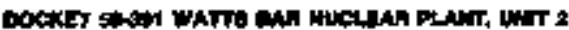

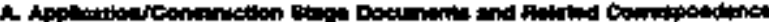




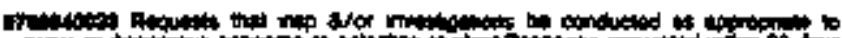

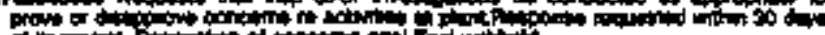
of to

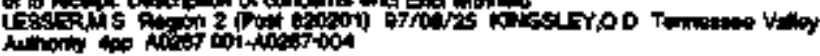

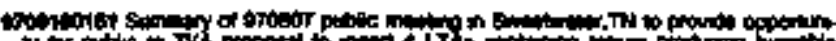

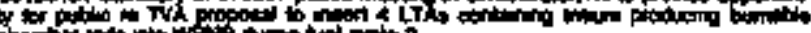

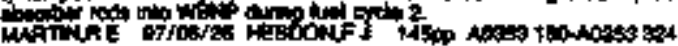

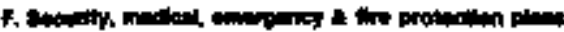

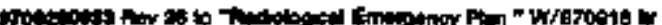

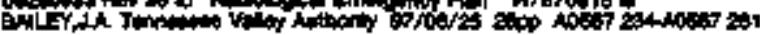

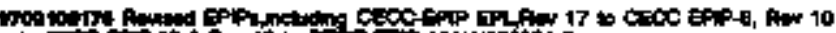

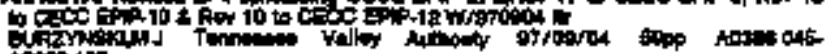
Hosic 100

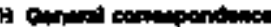

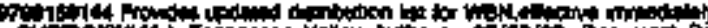

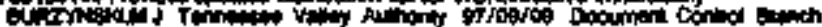

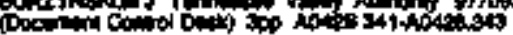

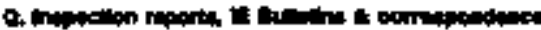

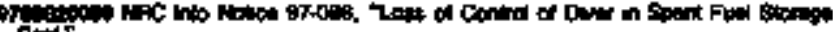
Fo: ind

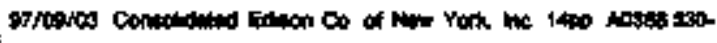

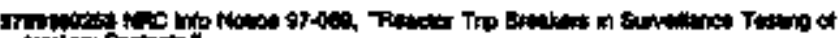

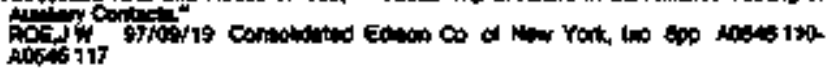

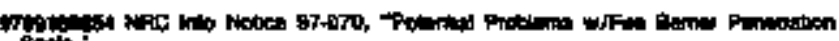

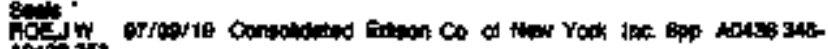

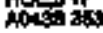

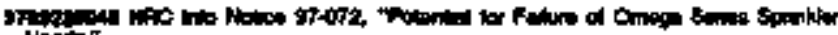

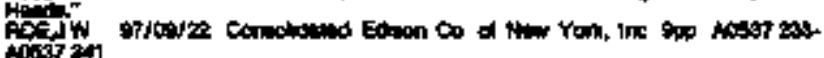

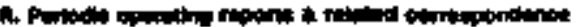

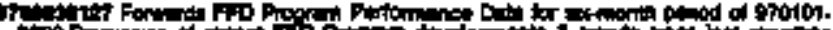

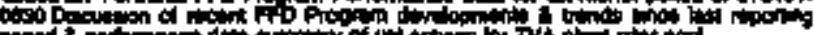
Peing Doon

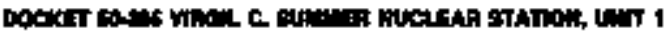

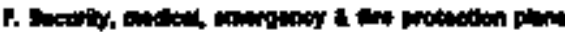

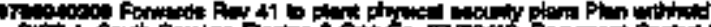

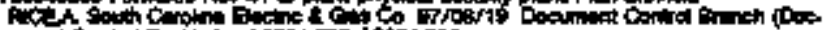

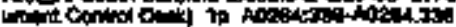

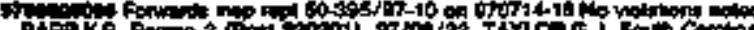

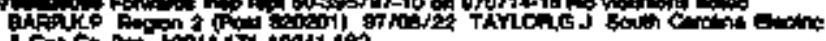

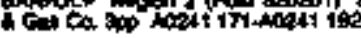

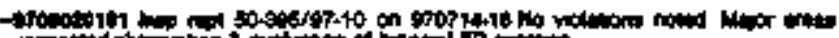

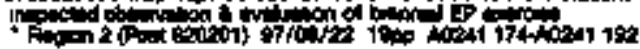

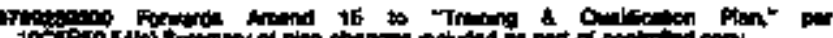

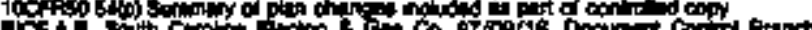

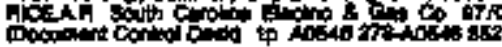

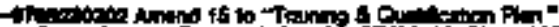

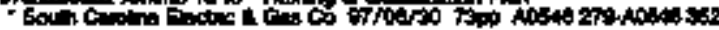

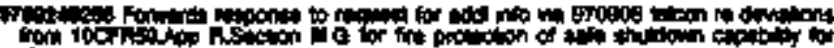

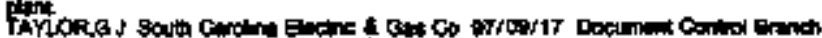

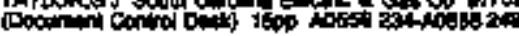

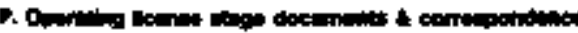

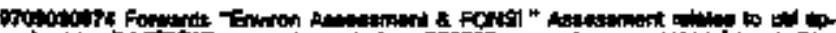

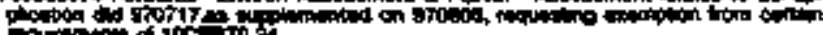

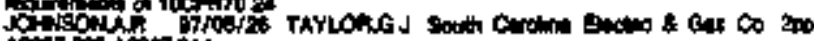

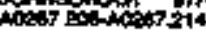

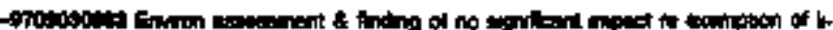

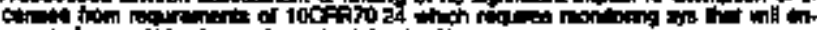

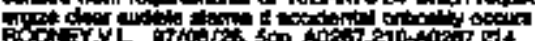

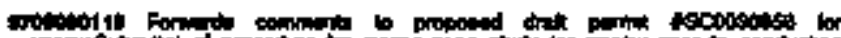

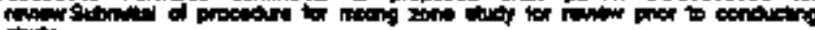

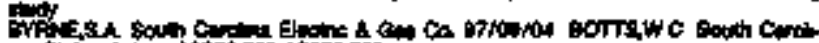

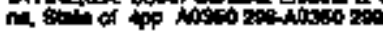

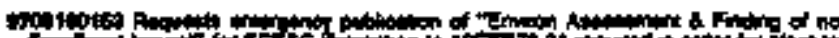

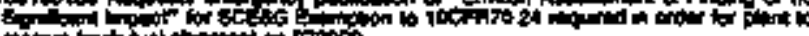

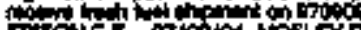

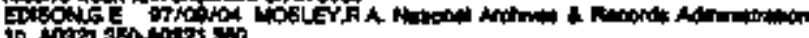

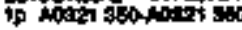

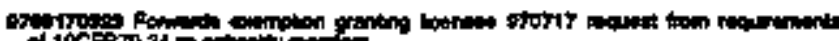
of 10CF

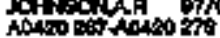

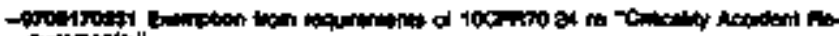

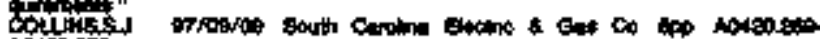
(a)

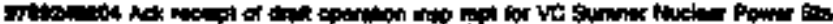

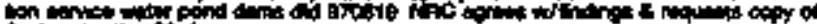

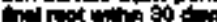

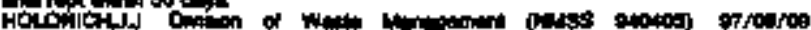

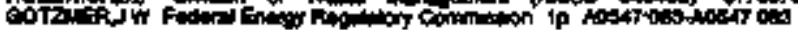

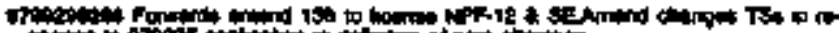

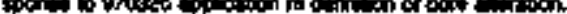

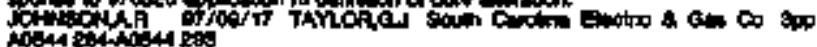

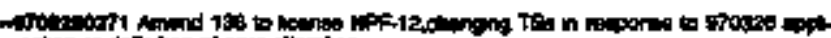

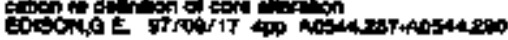

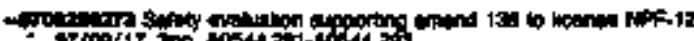

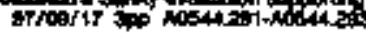

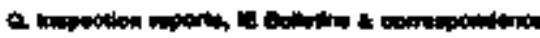

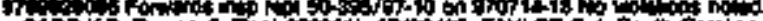

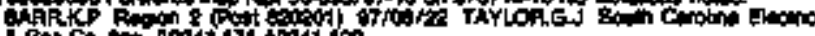

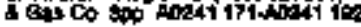

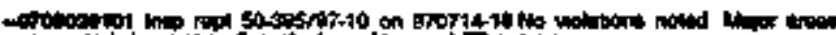

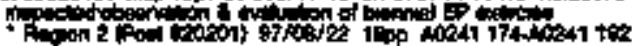

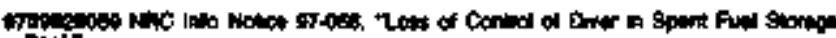

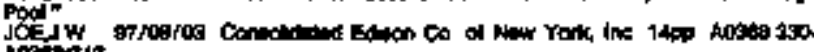
iotiats

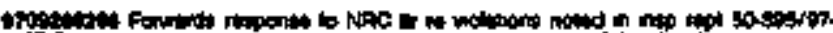

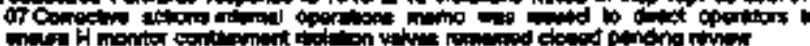

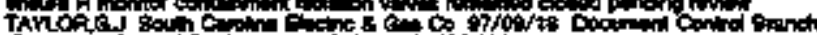

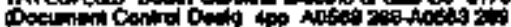

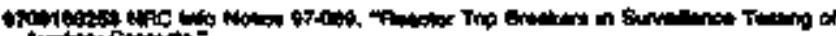

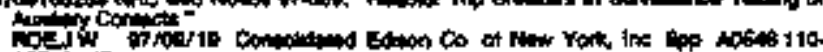
100117

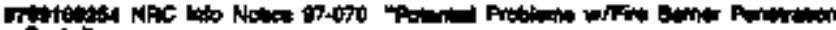

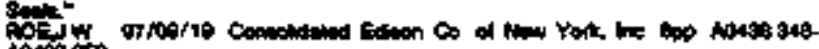
A0.0. is

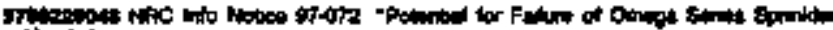

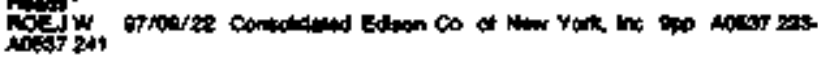

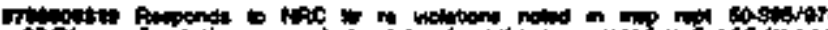

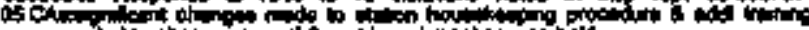

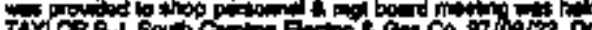

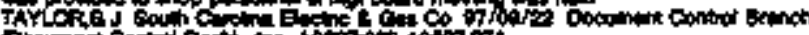

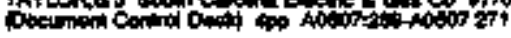

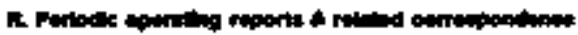

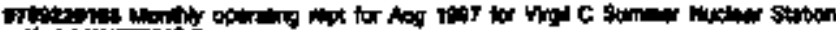

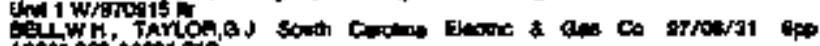

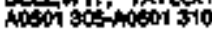

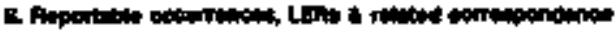

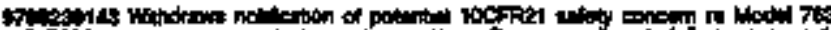

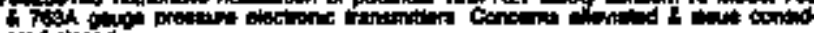

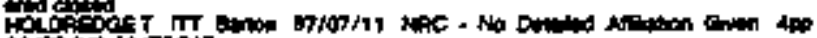

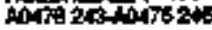

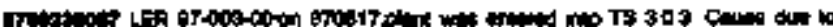

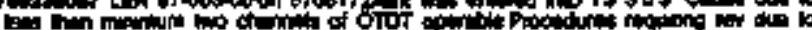

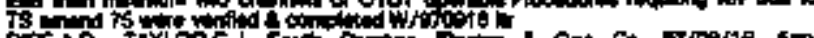

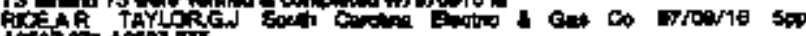

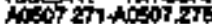


Y. Opander formination

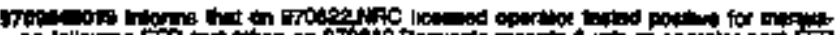

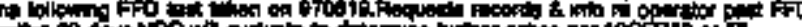
Hit 30 of thoch

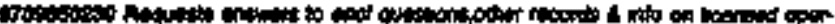

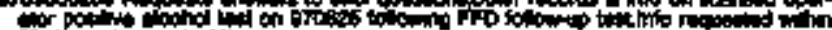

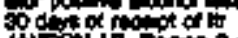

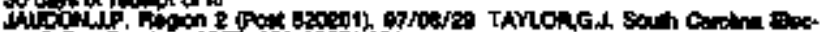

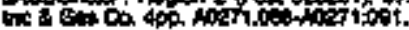

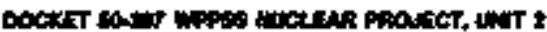

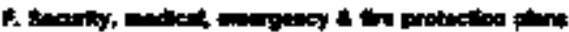

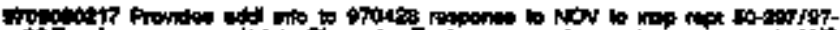

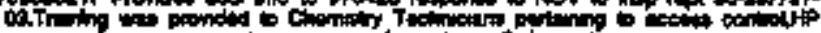

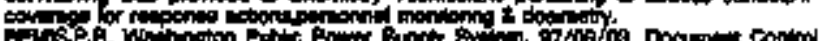

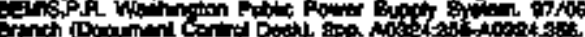

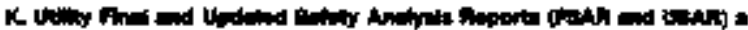

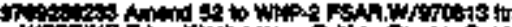

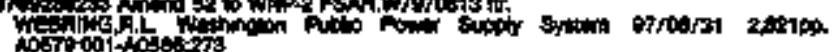

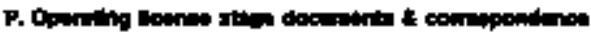

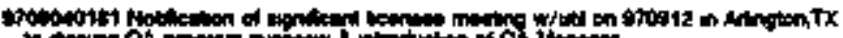

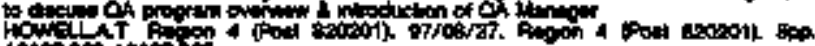

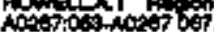

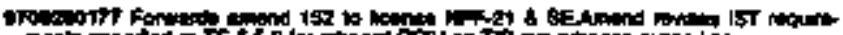

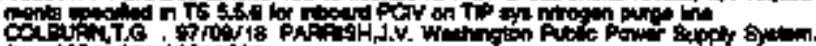

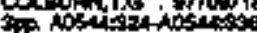

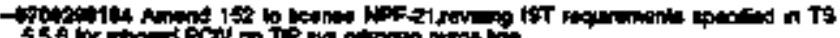

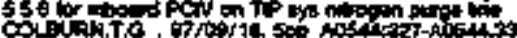

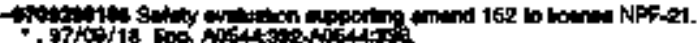

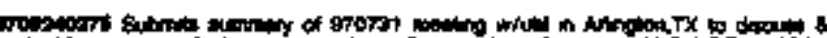

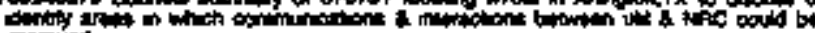

mitivind

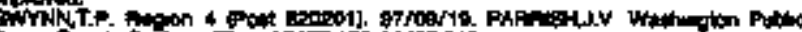

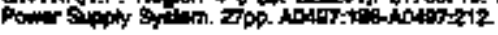

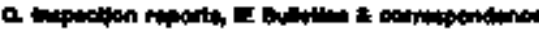

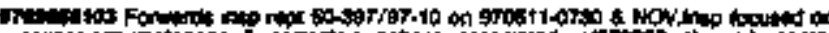

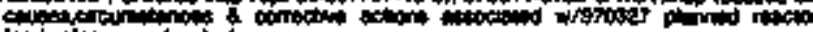

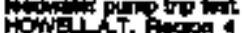

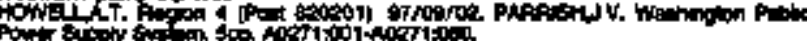

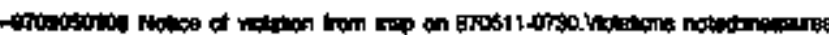

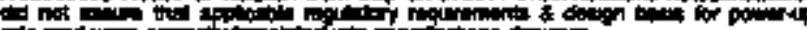

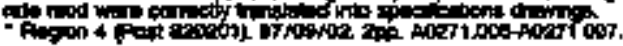

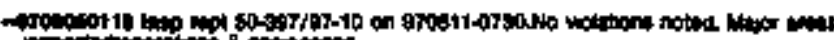

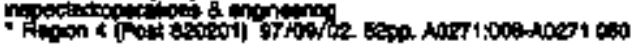

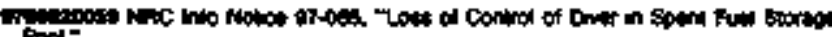

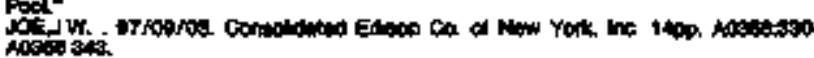

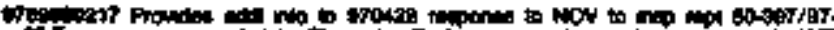

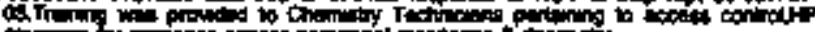

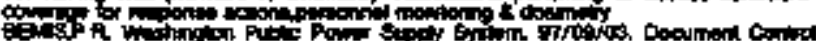

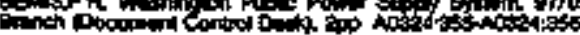

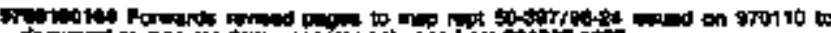

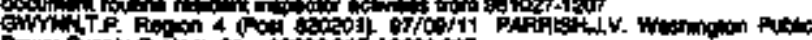

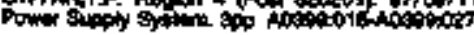

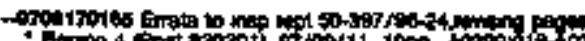

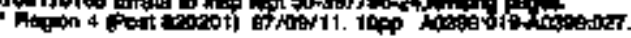

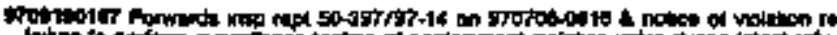

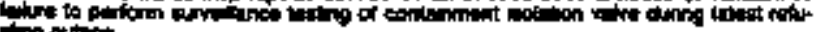
aterte

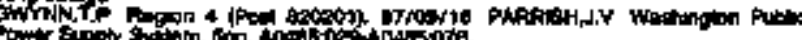

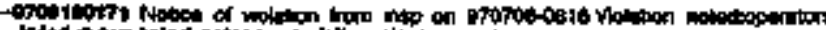

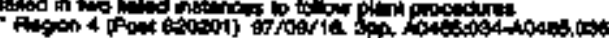

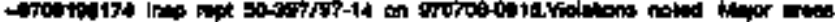

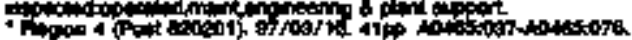

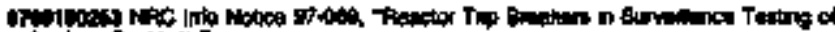

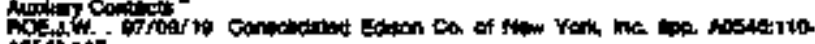
10.74:117,

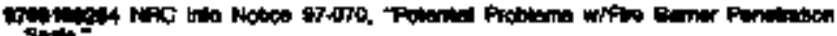

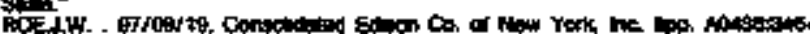
Notions

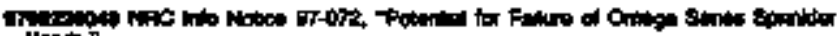

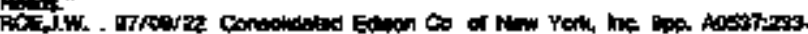
astivit.

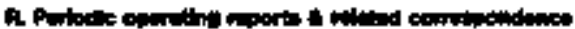

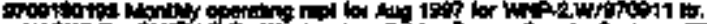

Kustole

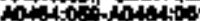

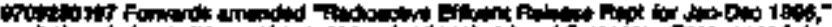

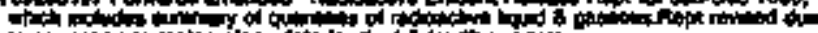

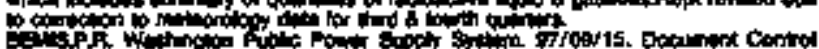

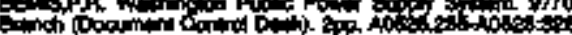

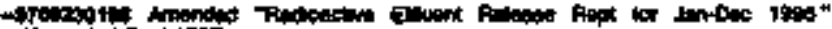

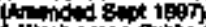

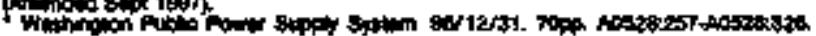

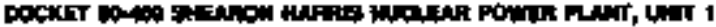

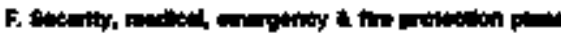

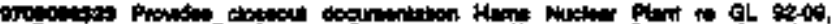

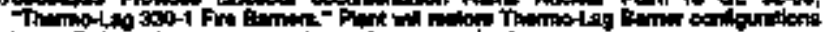

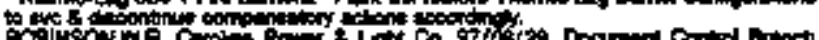

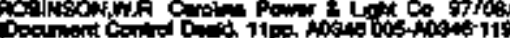

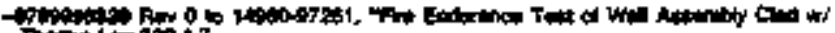

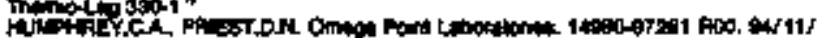

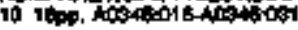

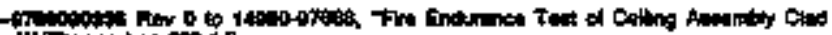

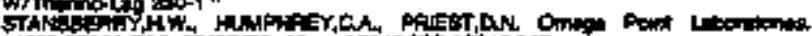

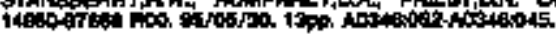

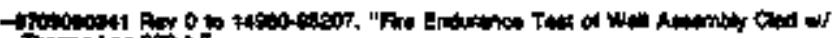

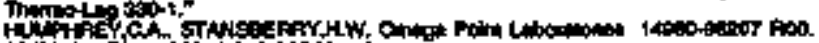

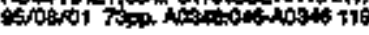

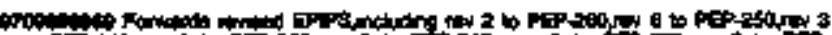

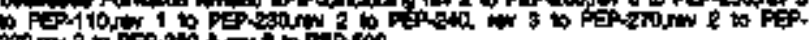

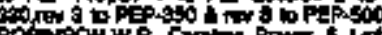
Ro:

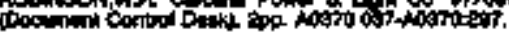

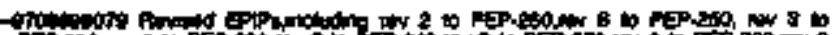

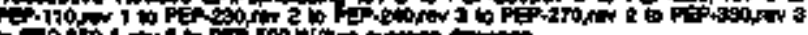

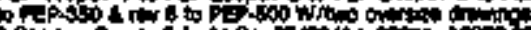

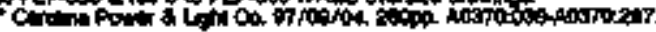

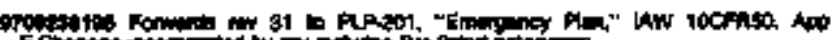

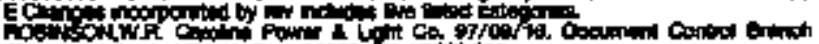

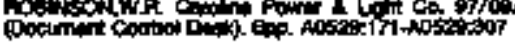

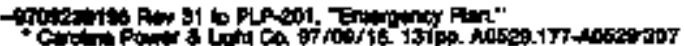

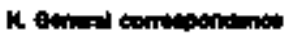

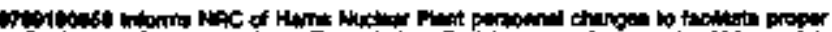

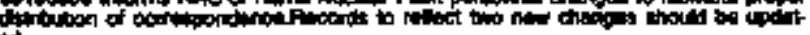

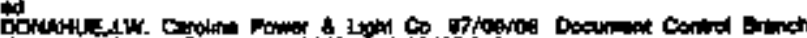

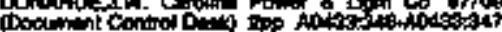

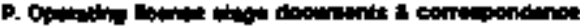

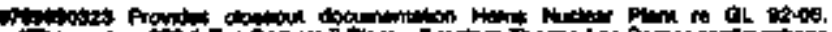

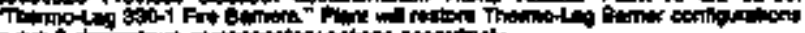

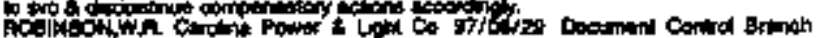

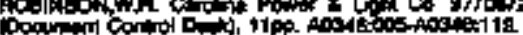

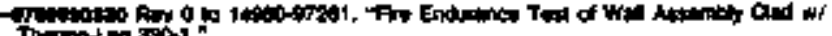

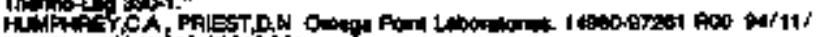

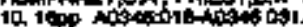




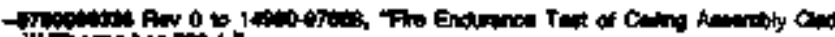

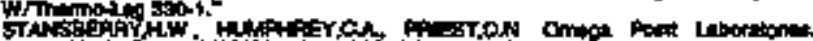

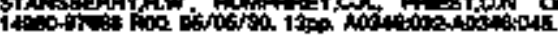

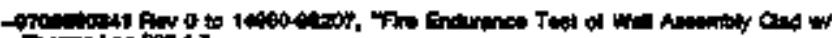

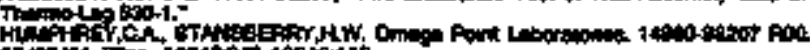

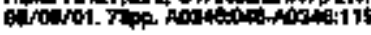

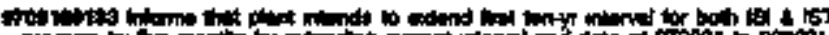

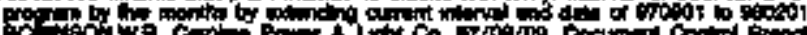

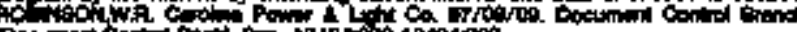

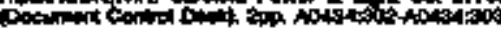

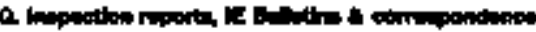

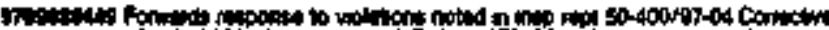

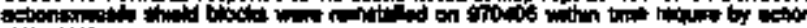

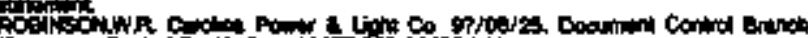

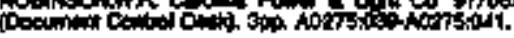

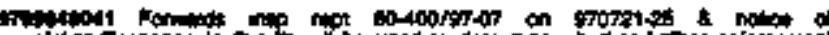

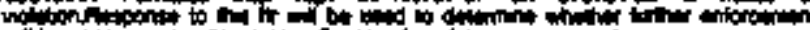

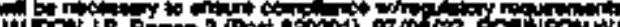

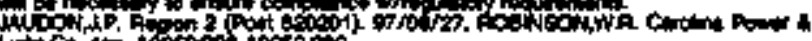

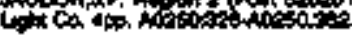

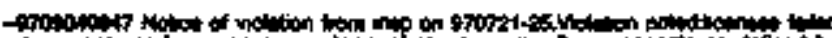

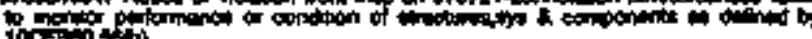
Toc-

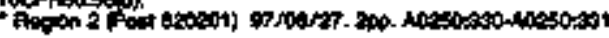

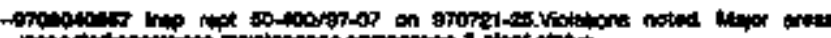

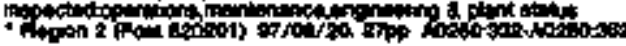

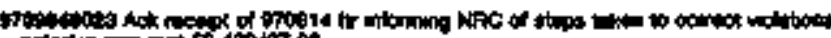

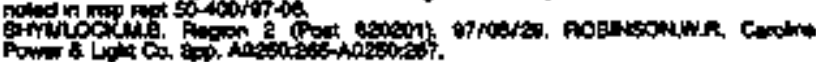

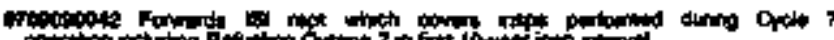

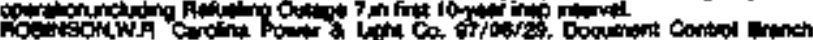

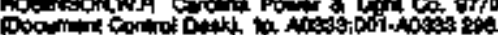

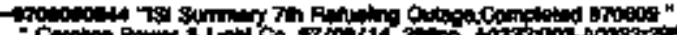

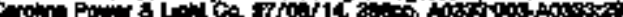

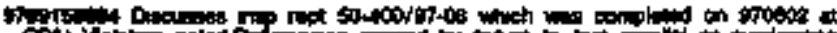

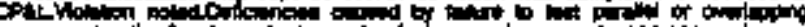

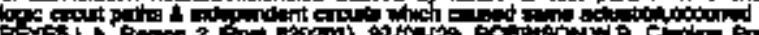

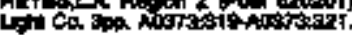

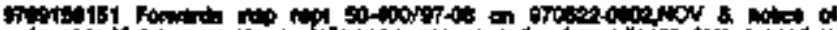

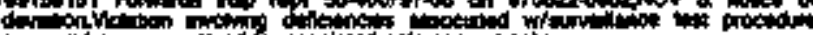

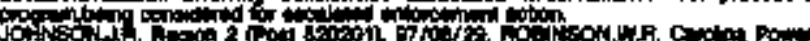

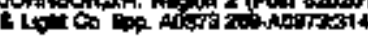

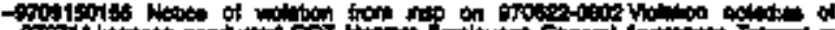

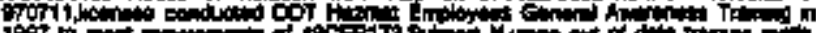

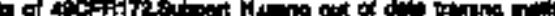

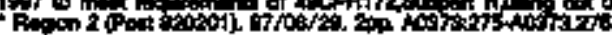

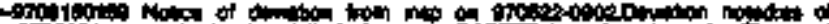

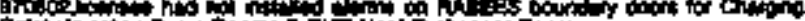

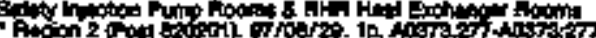

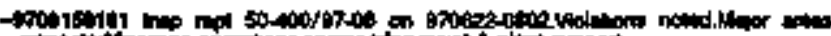

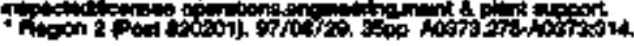

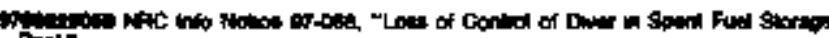
Poof

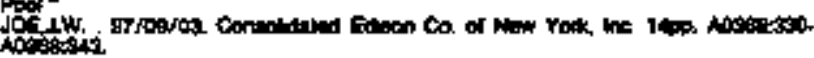

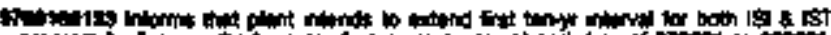

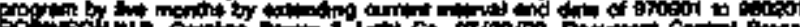

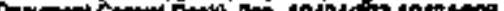

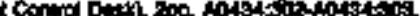

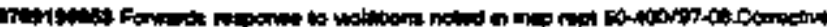

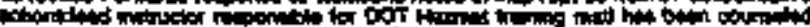

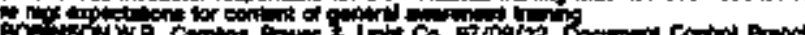

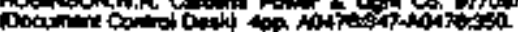

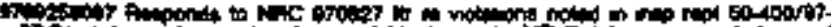

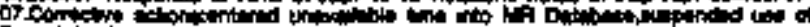

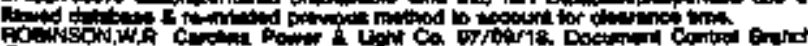

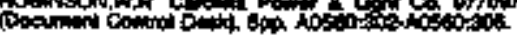

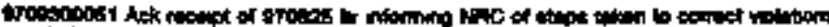

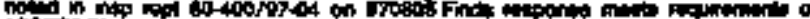

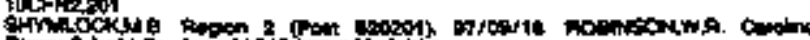

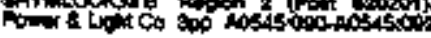

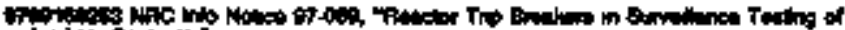

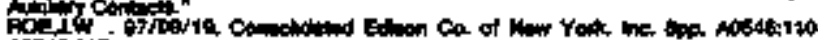
A0site1ti,

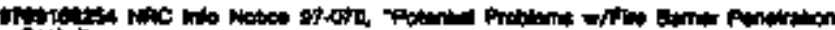

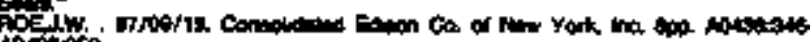
A0

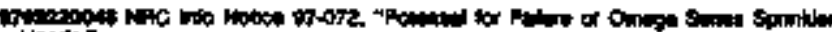

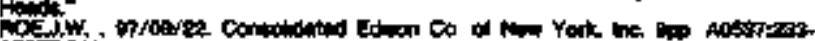
Nostrit:

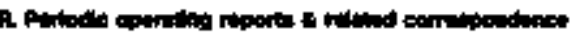

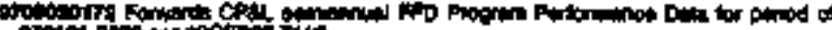

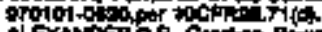

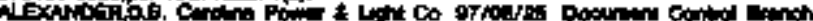

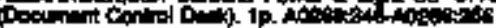

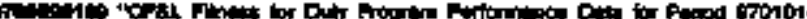
$\log _{0}$

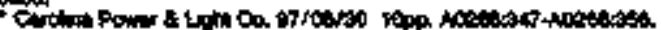

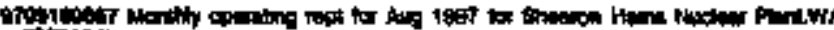

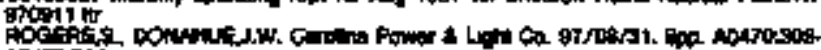
Nonosid

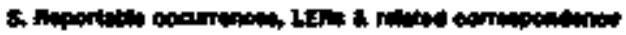

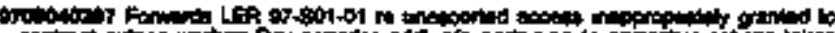

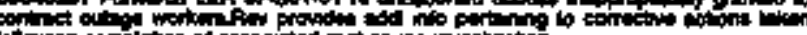

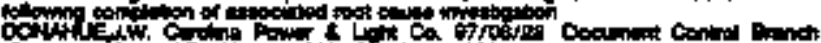

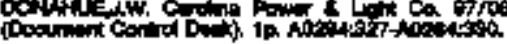

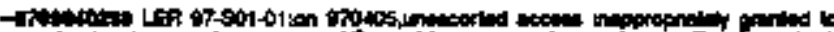

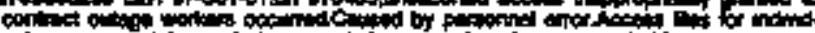

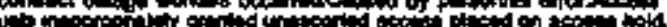

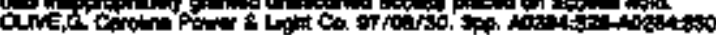

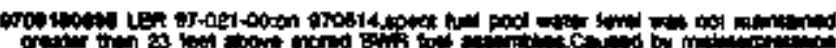

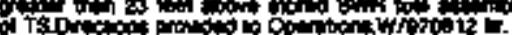
o Ts. Aartiot

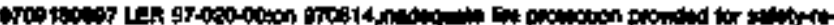

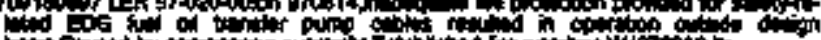

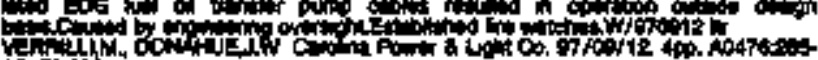
1040.0.

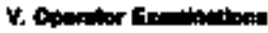

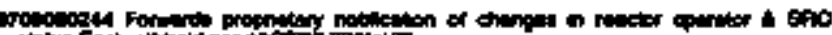

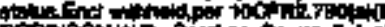

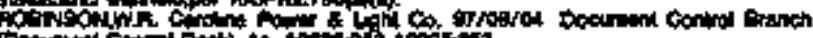

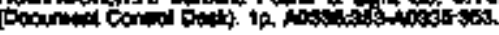

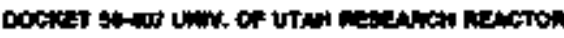

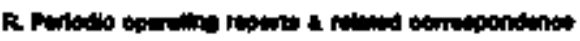

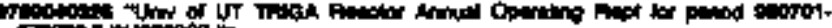

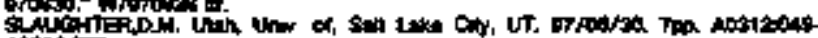
10012505 .

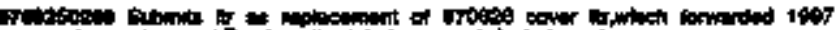

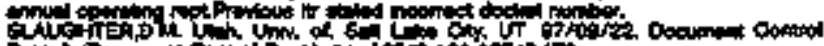

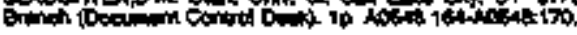

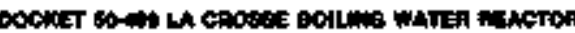

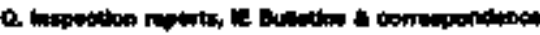

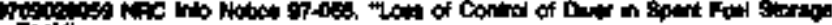

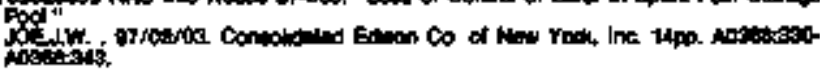

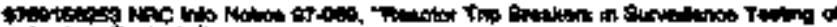

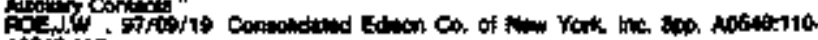
iosititi:

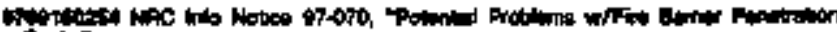

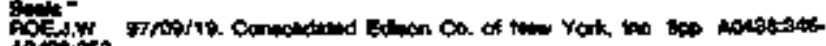
10.759 


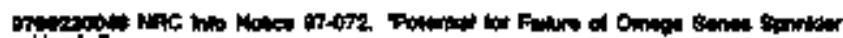

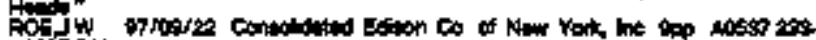
A 0552

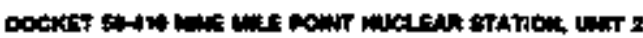

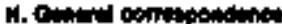

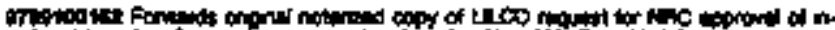

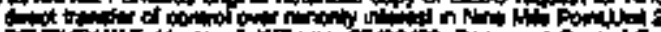

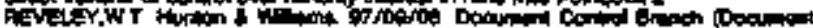

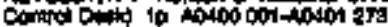

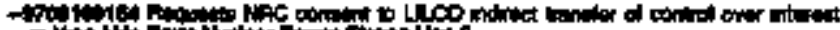

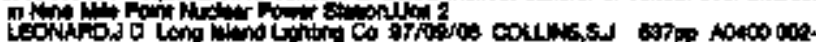
A0.101 278

1 Frition

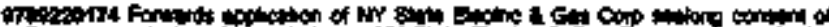

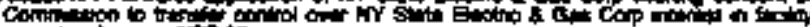

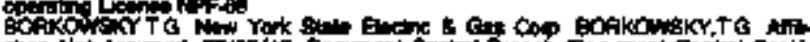

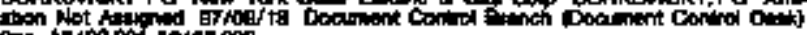

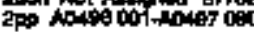

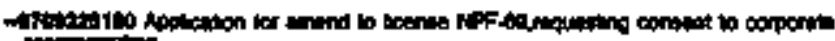

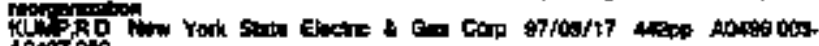

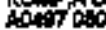

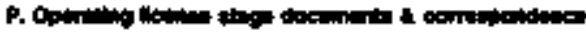

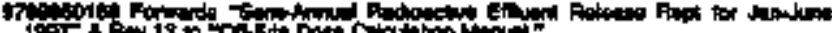

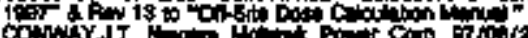

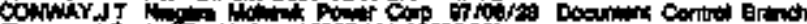

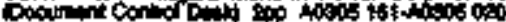

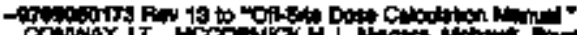

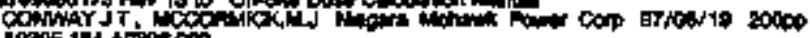

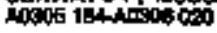

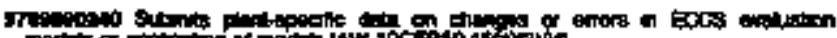

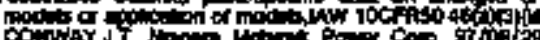

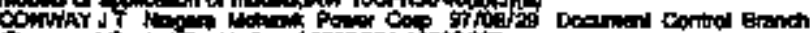

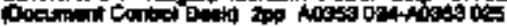

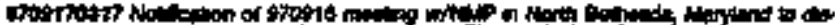

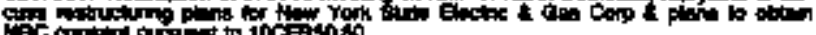

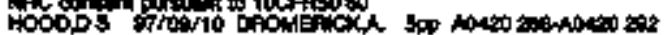

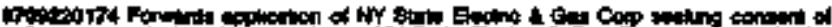

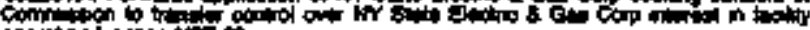

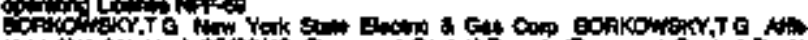

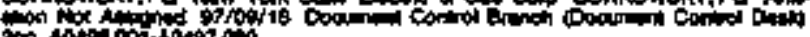

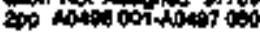

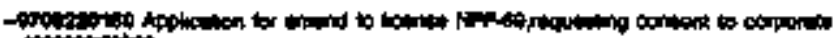

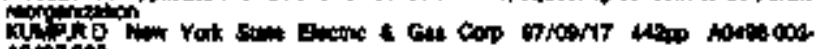

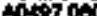

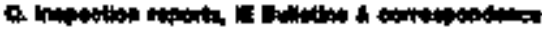

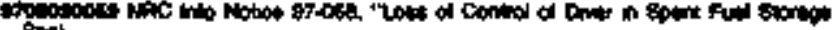

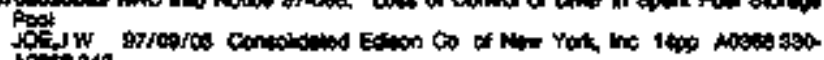

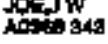

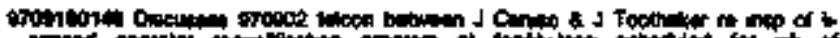

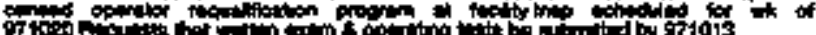

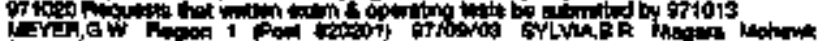

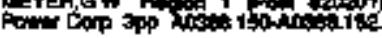

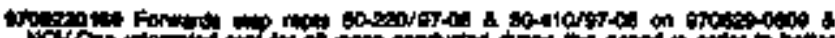

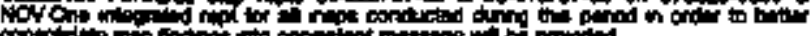

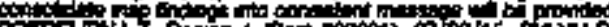

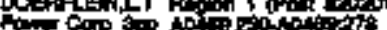

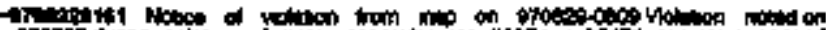

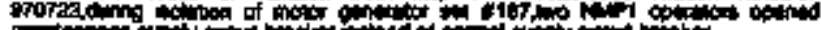

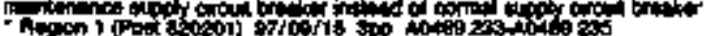

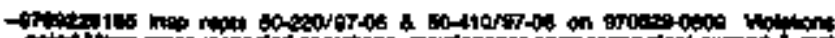

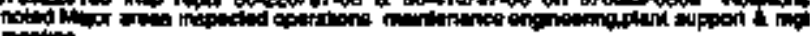

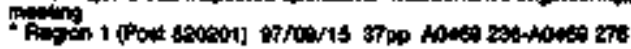

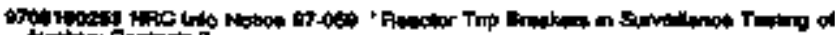

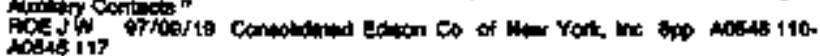

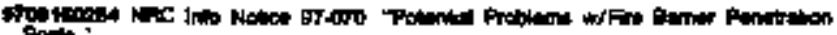

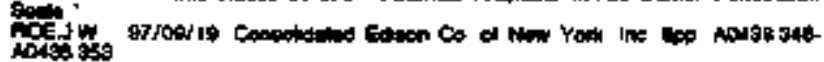

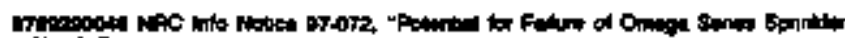

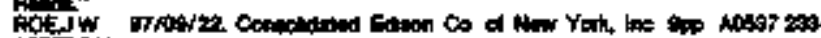
iocis?

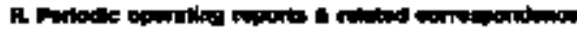

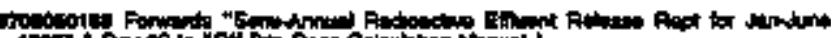

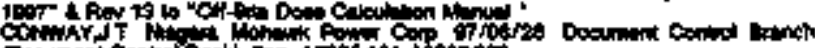

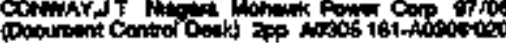

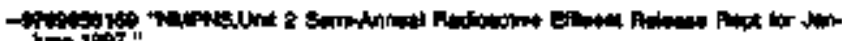

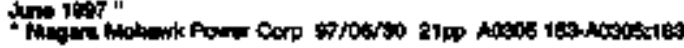

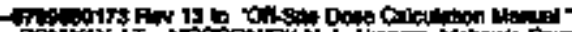

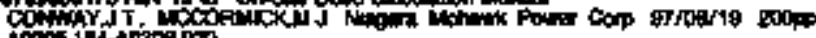

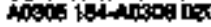

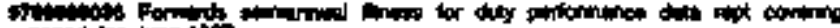

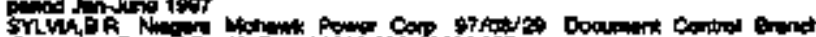

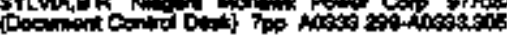

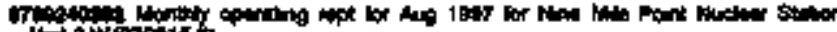
Vid 2 whoroms

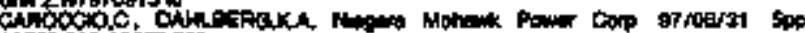
10\%

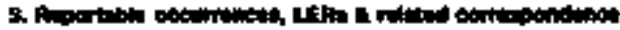

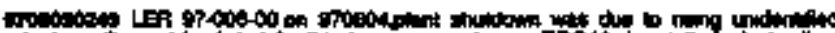

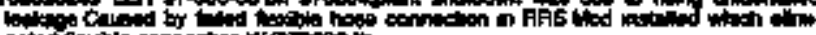

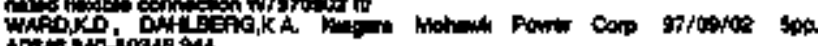
incos 240.103499

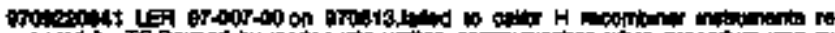

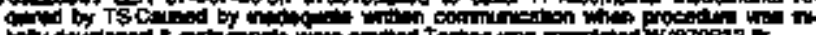

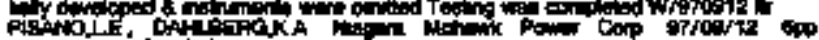

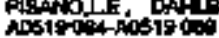

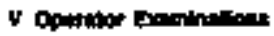

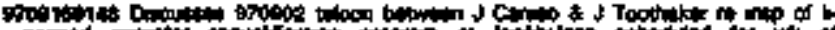

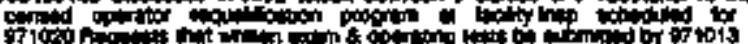

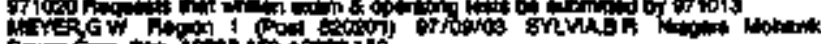

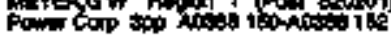

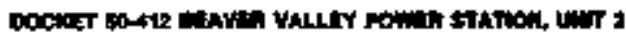

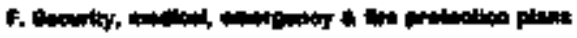

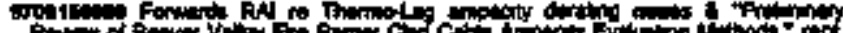

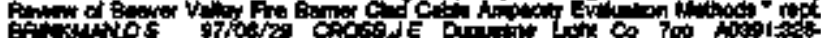
Anstion

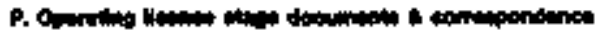

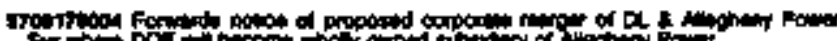

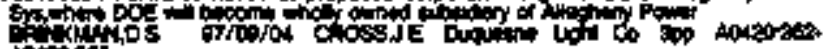
thoto

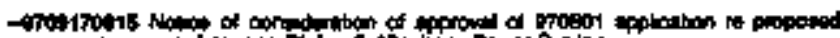

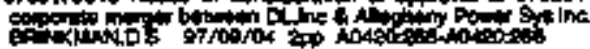

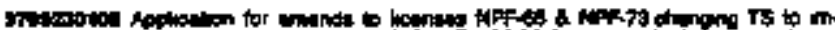

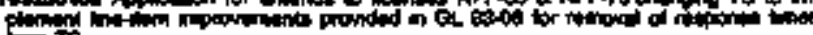
in 7

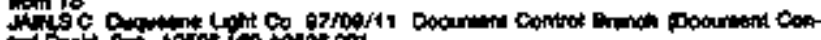

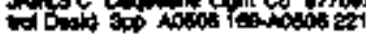

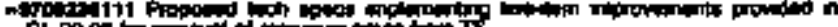

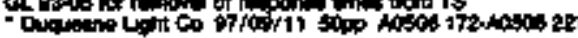

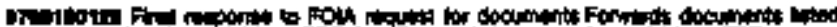

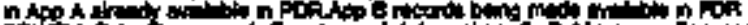

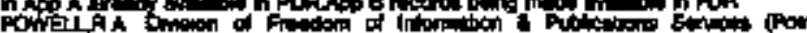

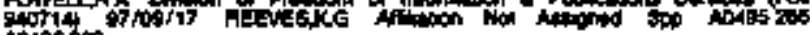
Aotos 30

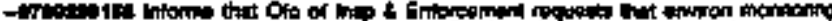

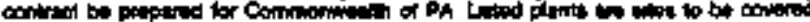

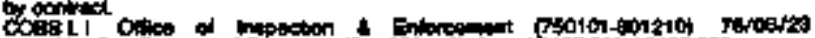

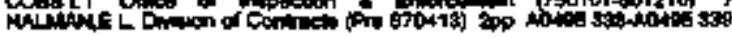




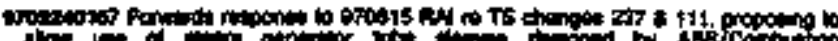

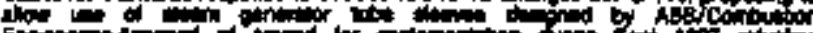

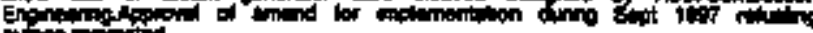

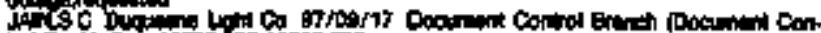

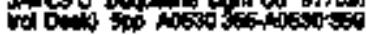

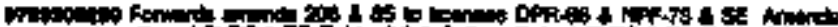

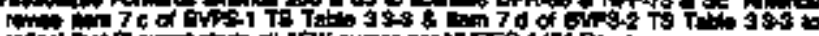

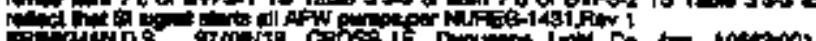

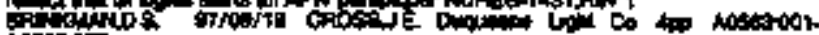
Ancisiog

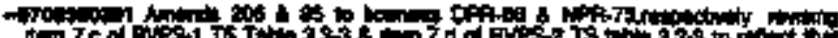
tin 76 od Dh

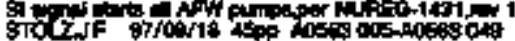

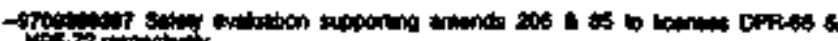

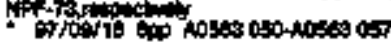

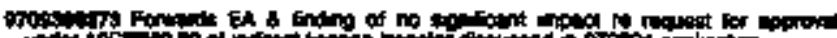

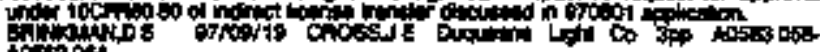

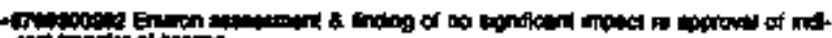

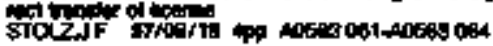

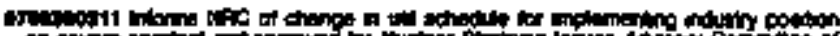

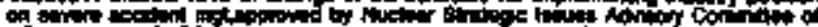

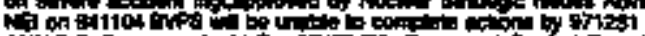

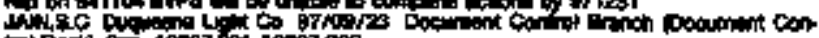

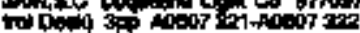

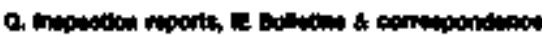

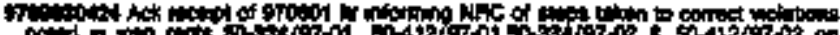

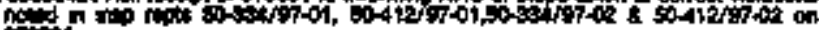
grotos

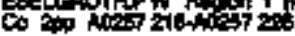

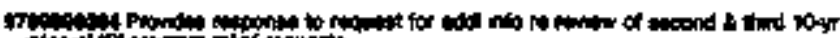

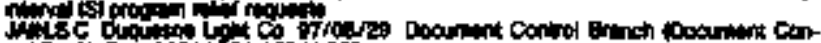

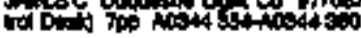

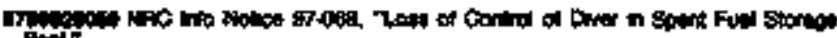

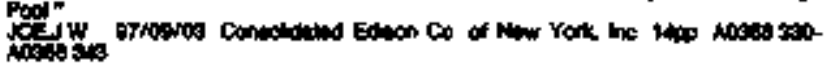

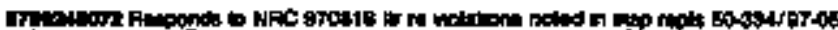

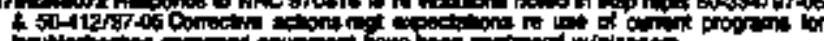

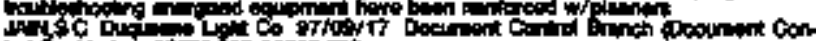

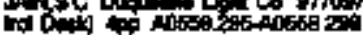

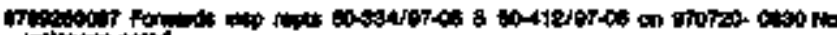

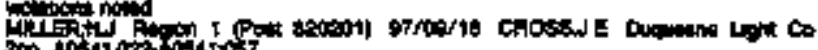

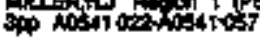

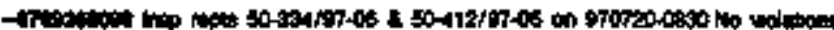

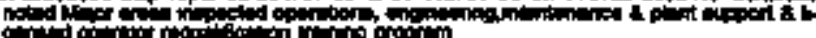

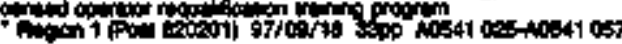

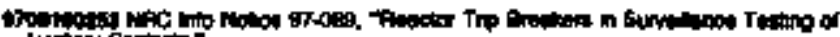

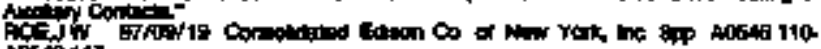
Mosin:13

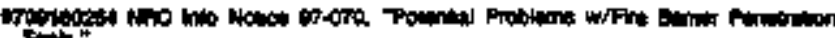

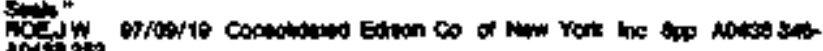
10.5.,

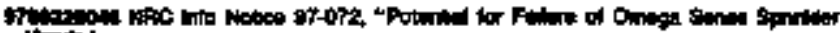

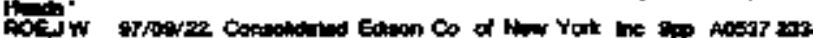
notions

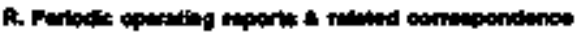

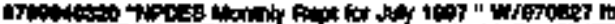

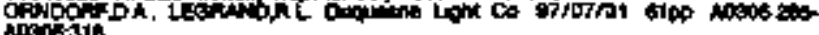
nocolosic

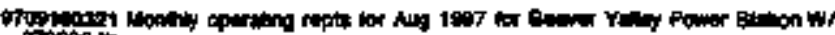

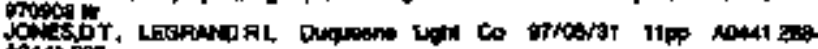
1044201

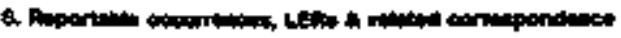

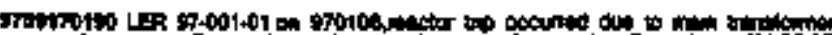

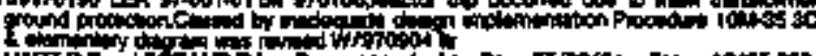

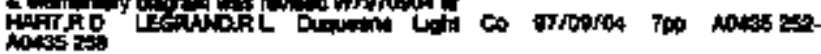

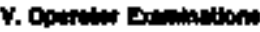

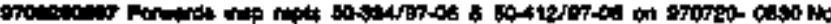

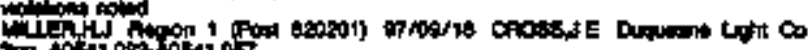

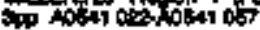

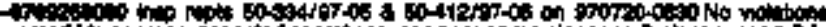

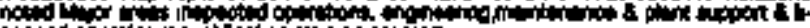

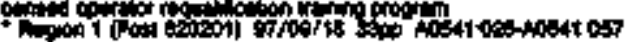

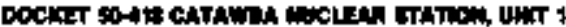

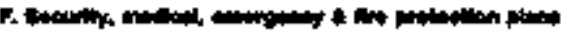

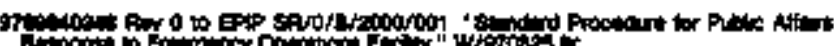

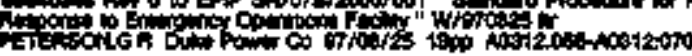

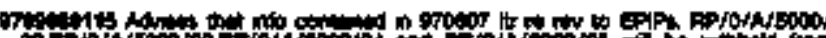
of

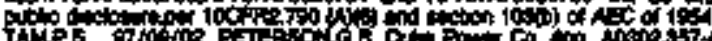

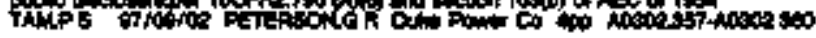

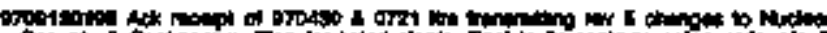

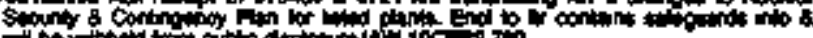

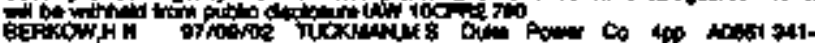
(a) 134

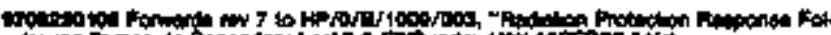

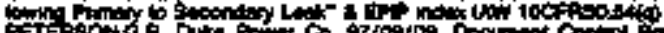

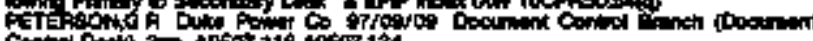

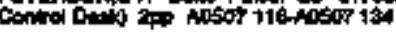

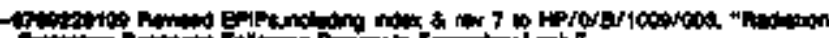

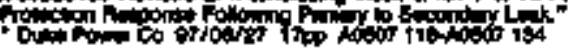

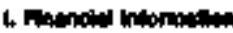

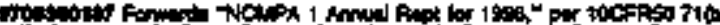

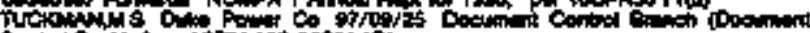

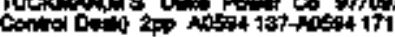

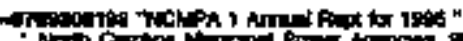

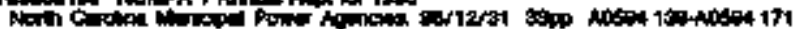

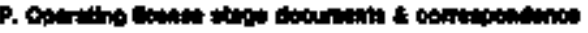

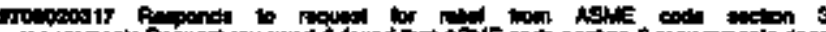

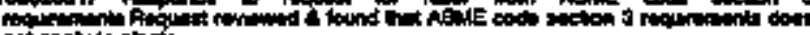

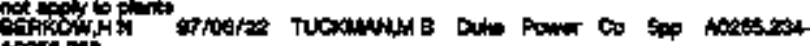
tones 20

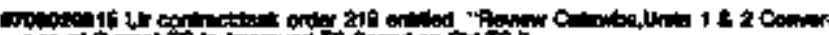

Win of

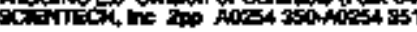

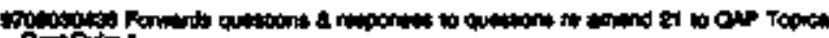

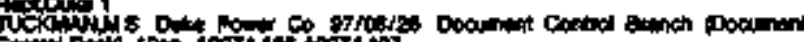

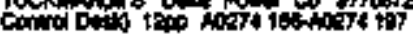

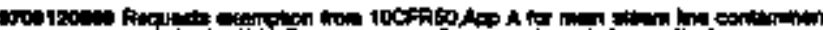

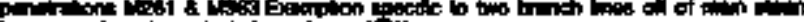

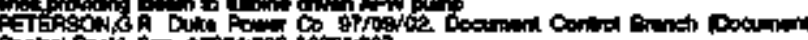

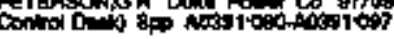

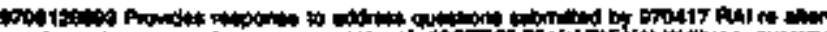

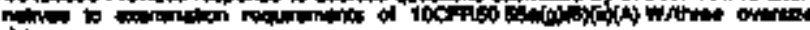

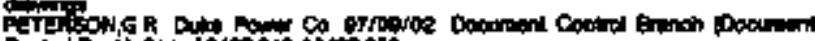

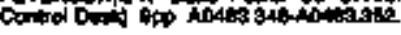

Fos

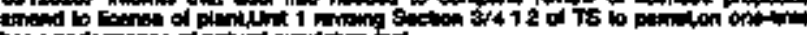

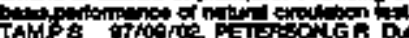

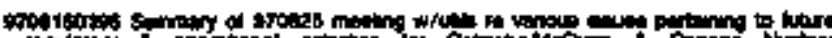

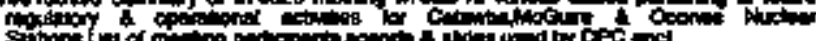

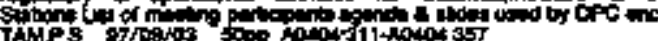

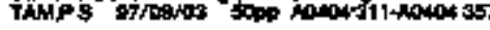

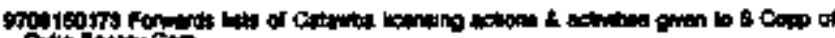

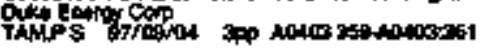

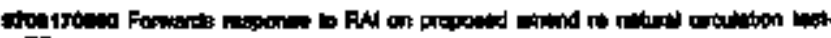

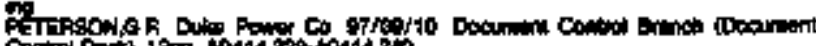

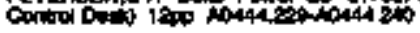




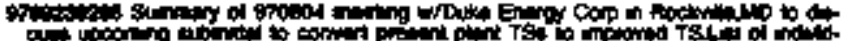

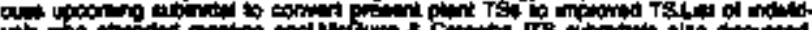
LAtive

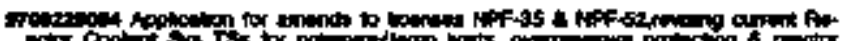

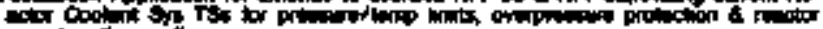

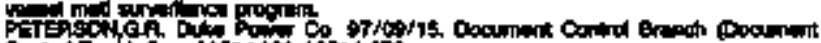

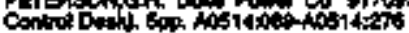

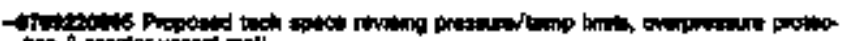

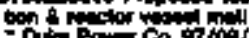

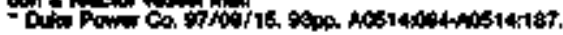

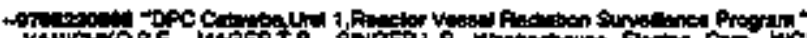

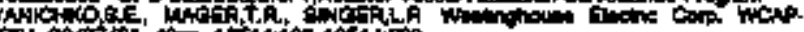

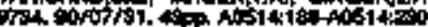

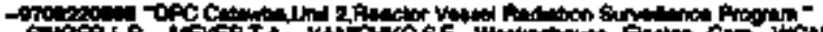

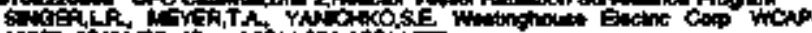

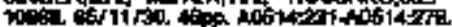

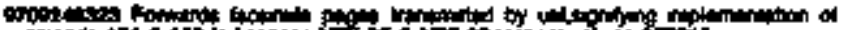

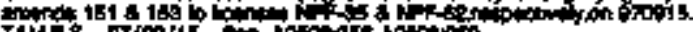

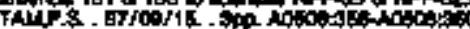

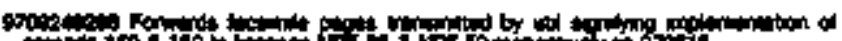

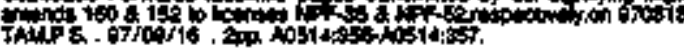

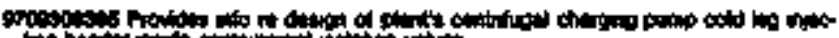

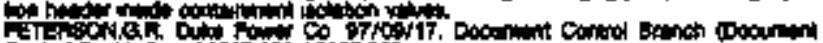

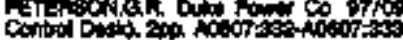

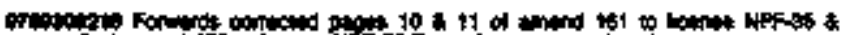

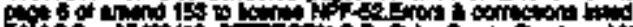

TAtho

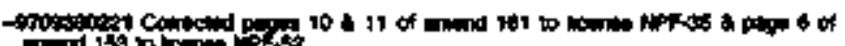

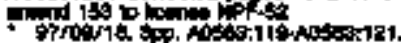

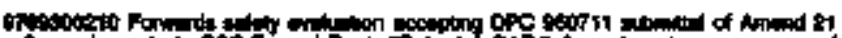

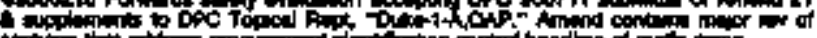

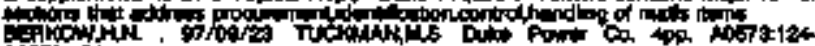
$\lim _{0 \rightarrow 3}$

- *7w

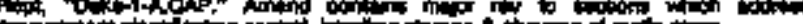

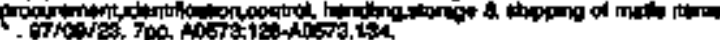

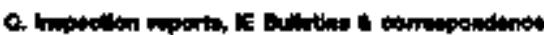

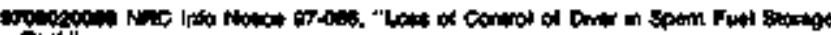

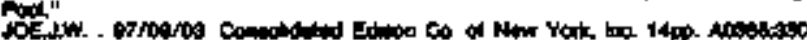

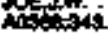

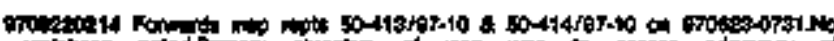

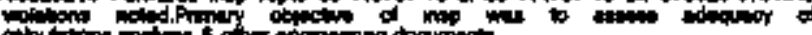

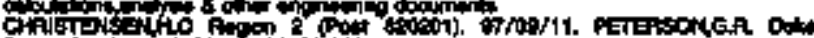

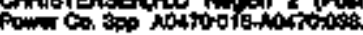

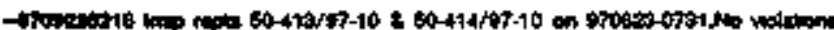

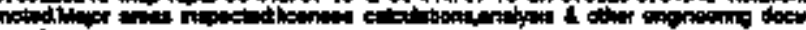

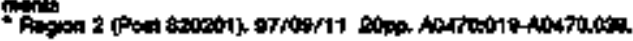

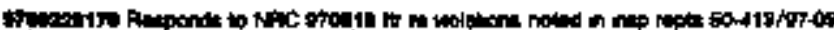

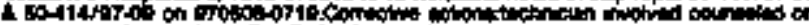

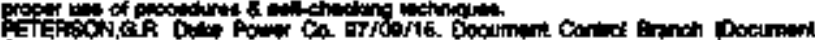

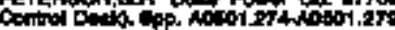

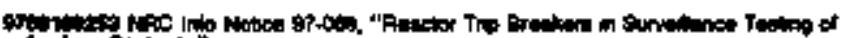
ping instit:

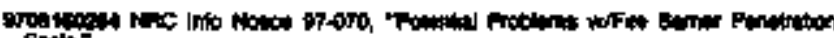

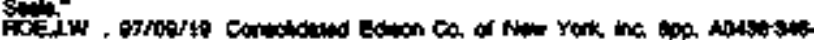

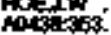

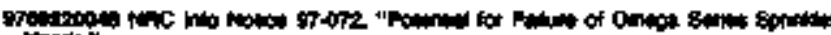

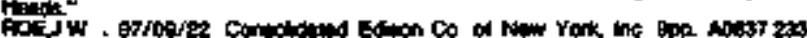
AOSTitit

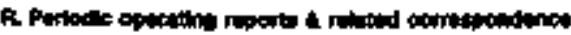

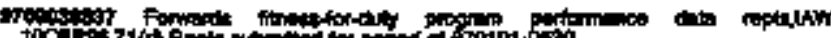

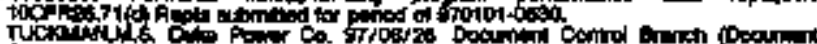

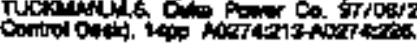

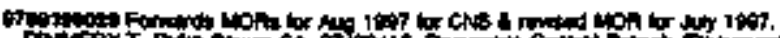
of.

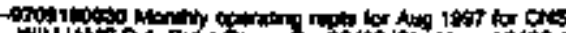

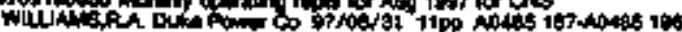

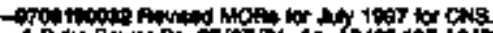

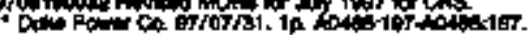

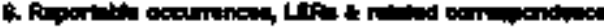

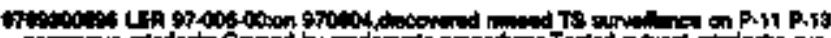

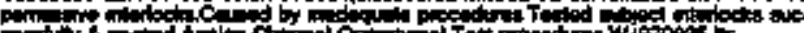

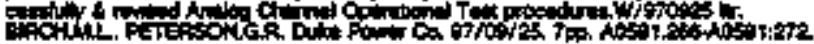

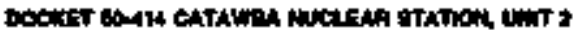

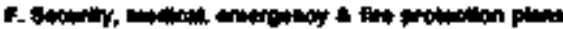

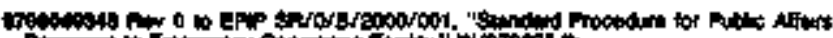

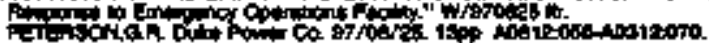

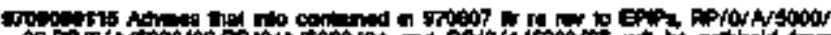

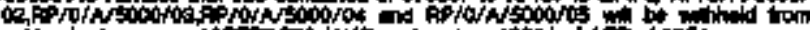

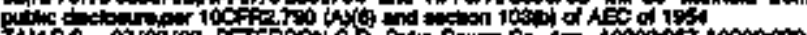

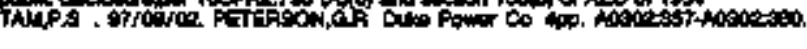

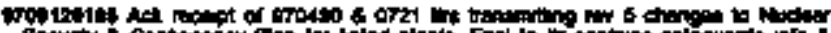

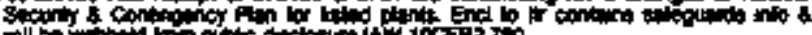

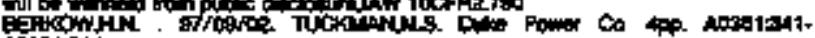
mosolitit

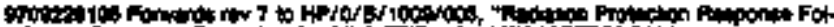

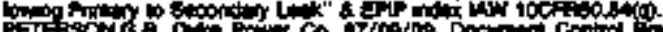

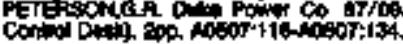

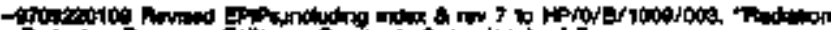

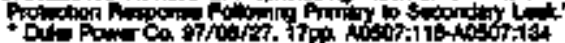

L Fiance tom tom

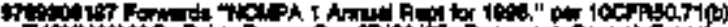

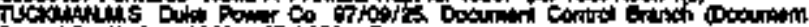

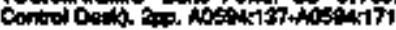

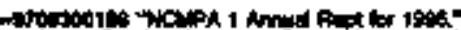

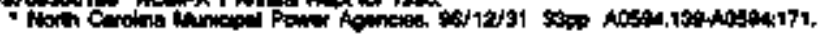

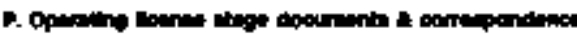

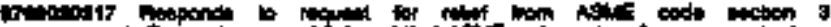

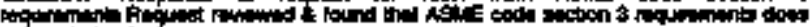

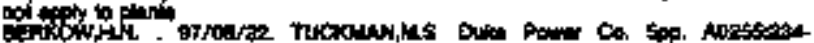
incistix

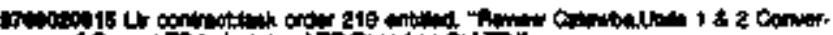
con of C C

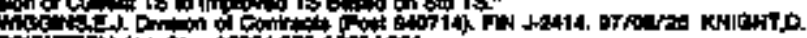

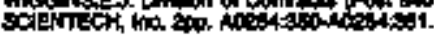

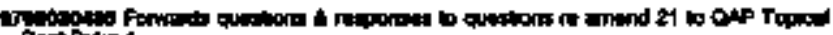

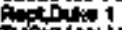

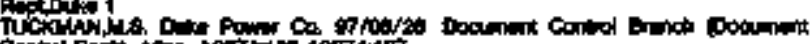

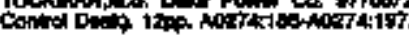

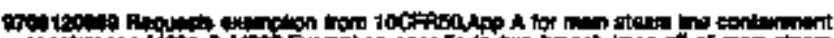

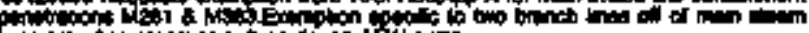

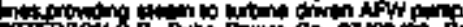

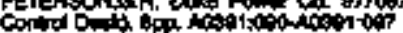

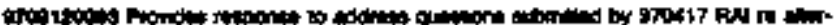

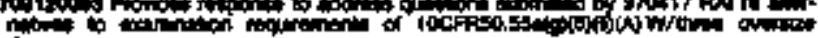
drimo

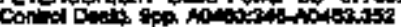

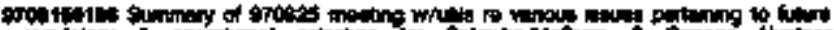

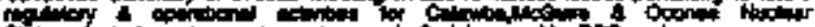

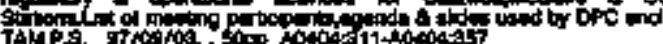

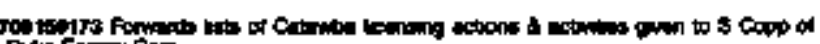

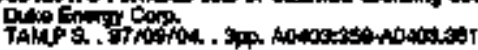

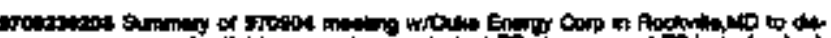

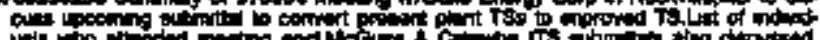

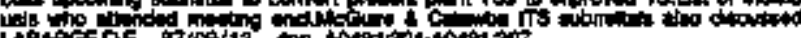

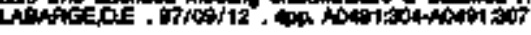

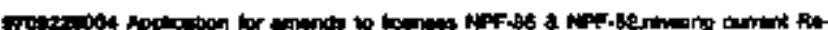

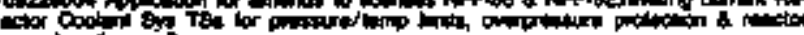

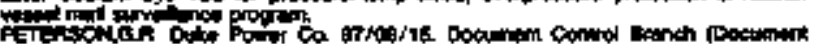

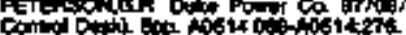

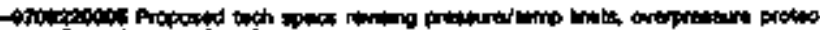

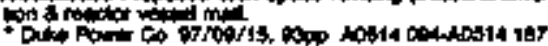




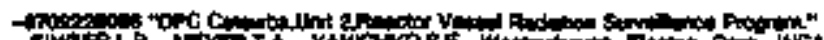

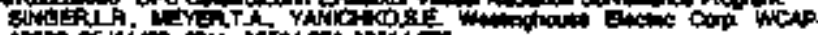

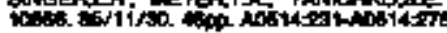

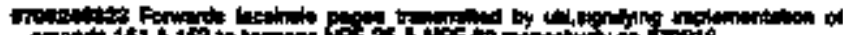

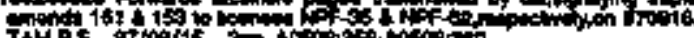

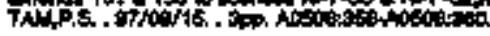

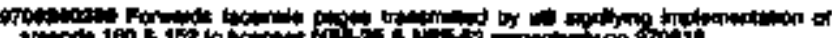

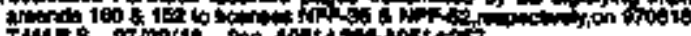

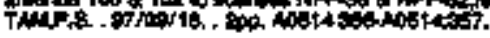

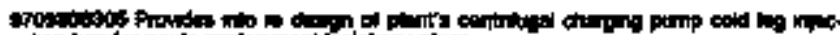

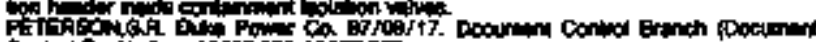

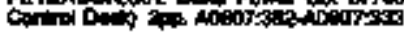

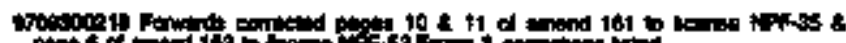

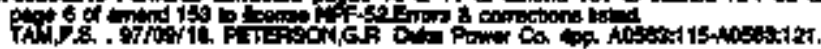

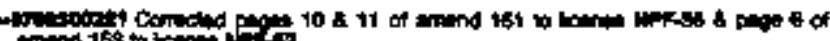

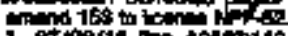

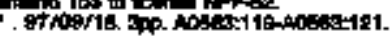

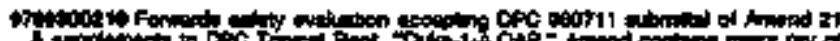

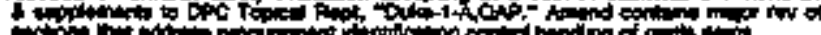

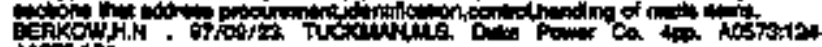
Aotoriat

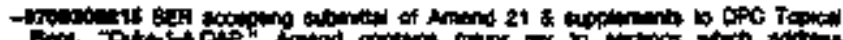

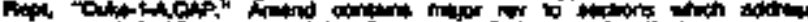
procerementanch

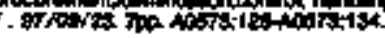

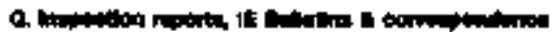

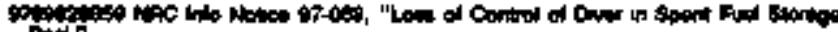

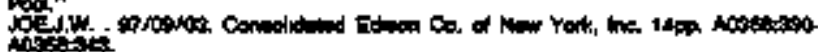

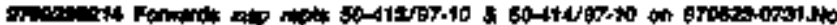

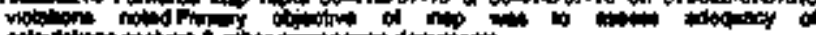

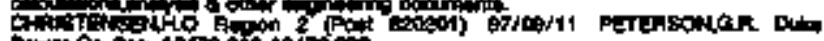

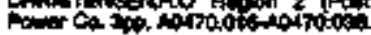

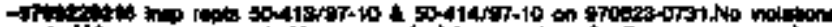

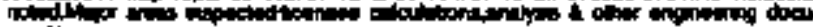

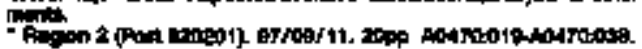

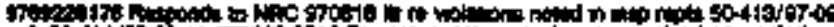

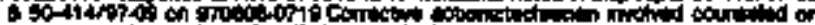

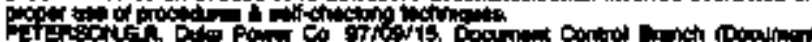

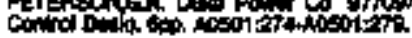

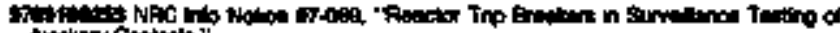

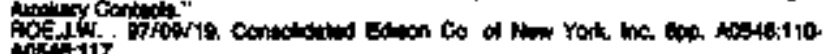

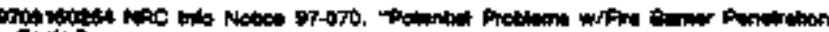

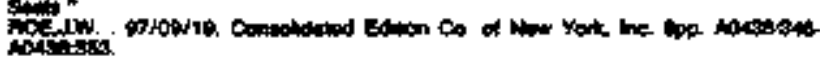

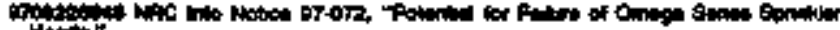

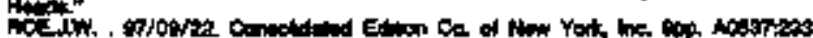
$\operatorname{lom}_{0 \rightarrow 1}$

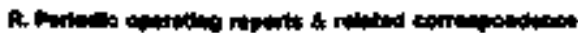

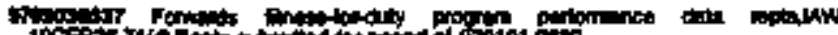

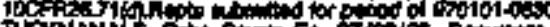

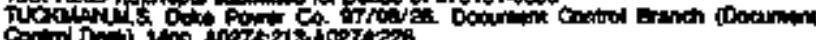

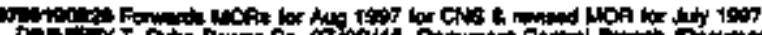

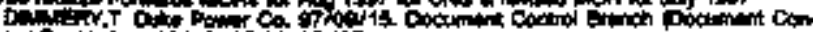

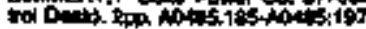

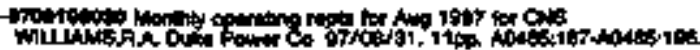

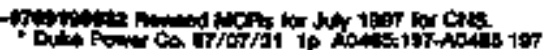

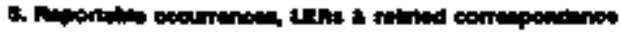

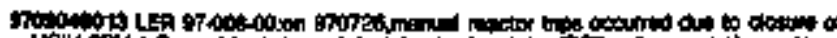

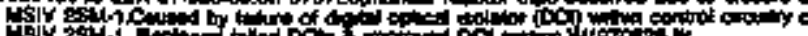

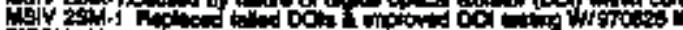

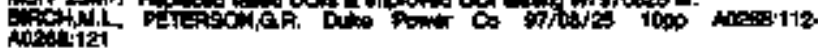

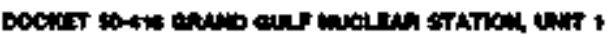

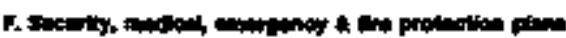

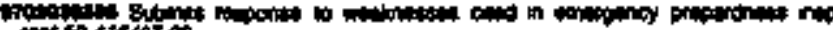
rot $50016 / 970$

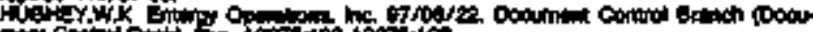

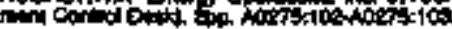

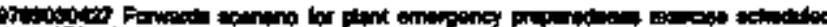

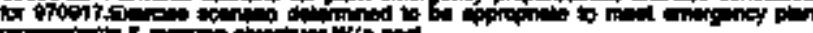

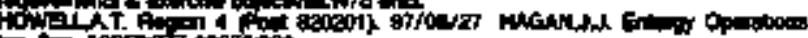

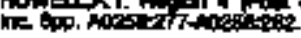

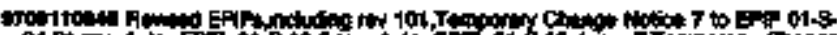
$0421,4+4$ to

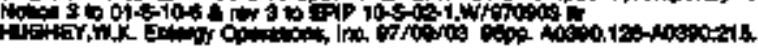

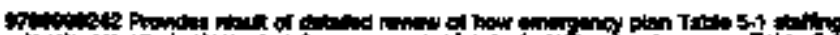

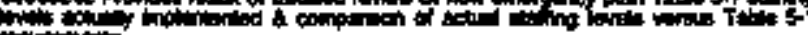

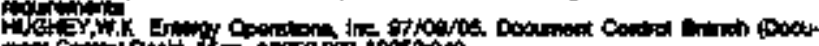

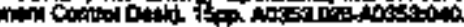

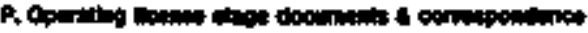

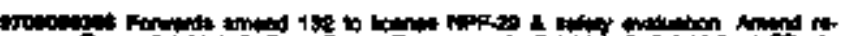

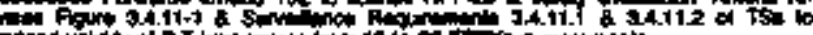

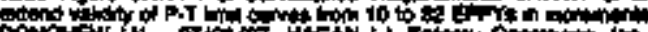

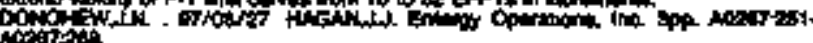
cotestate

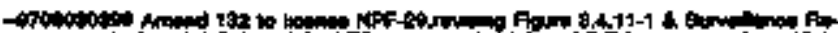

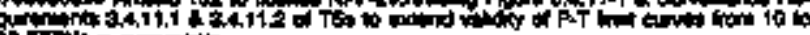
Dow

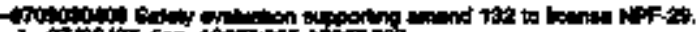

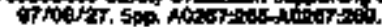

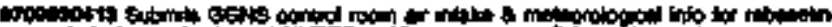

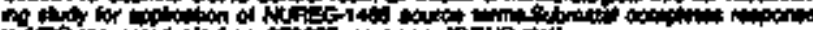

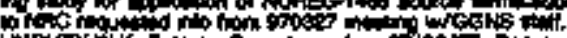

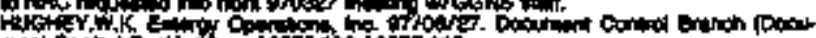

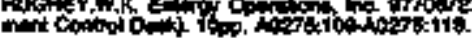

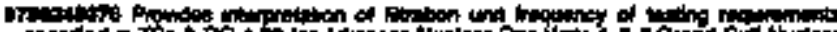

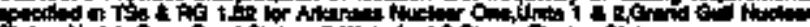

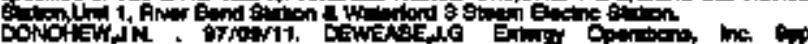

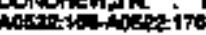

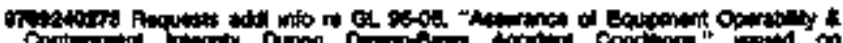

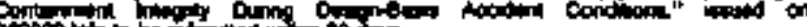

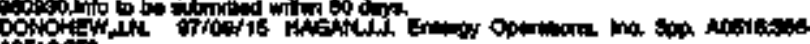
Aosicion.

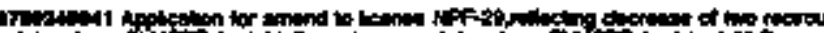

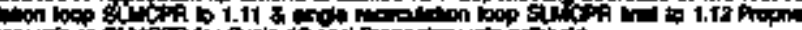

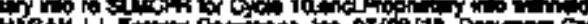

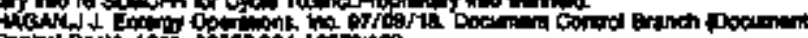

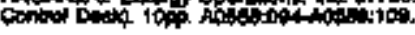

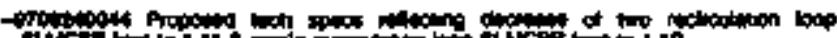

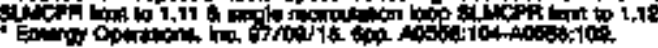

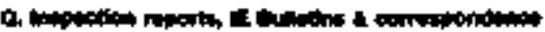

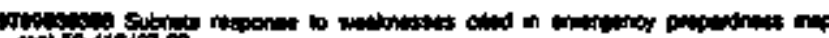

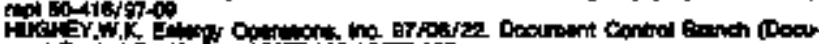
ond tom,

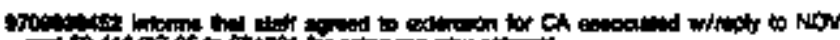

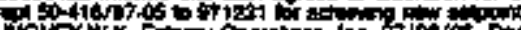

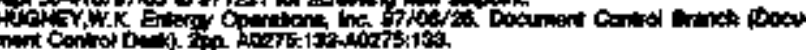

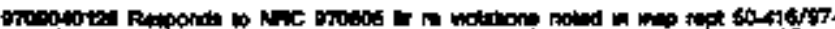

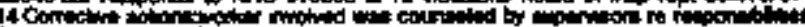

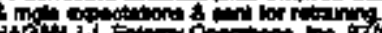

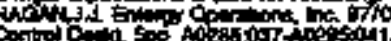

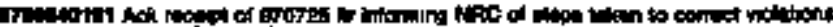

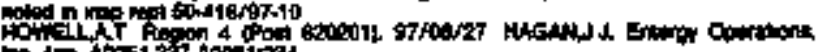

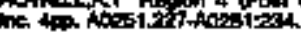

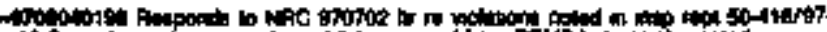

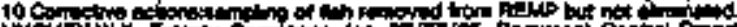

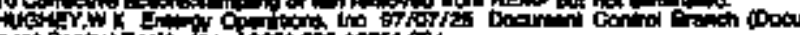

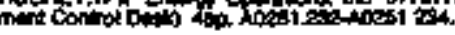

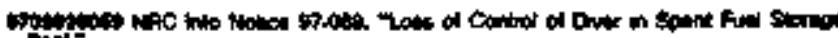

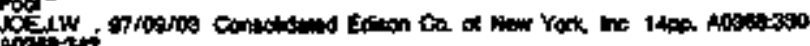
tionetis 


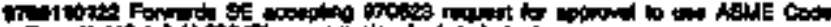

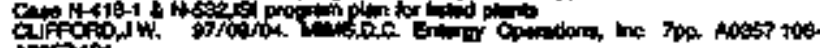
instivit.

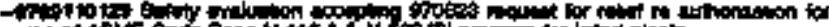

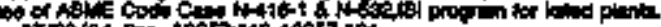

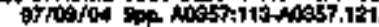

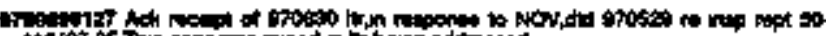

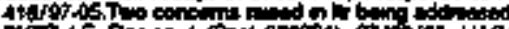

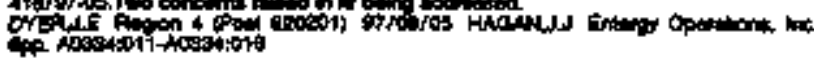

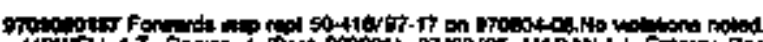

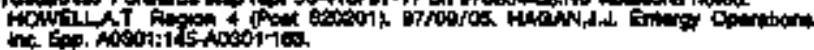

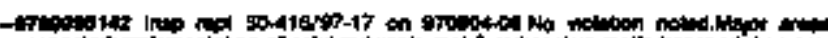

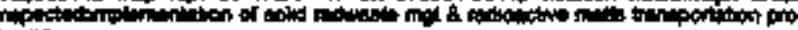
Finter

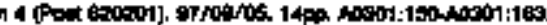

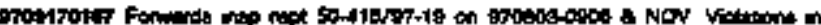

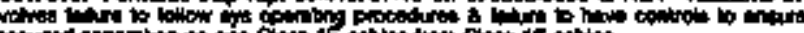

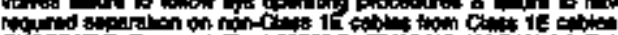

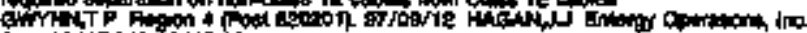

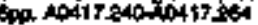

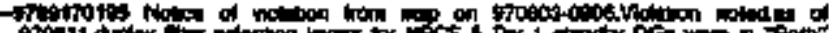

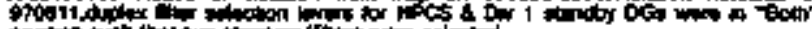

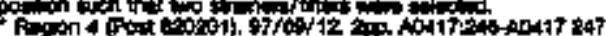

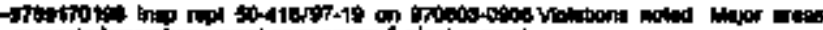

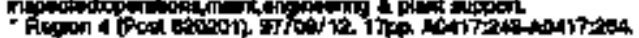

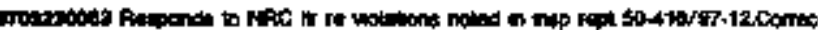

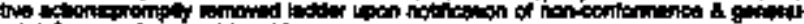

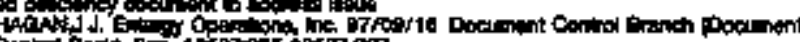

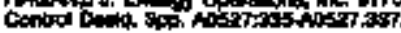

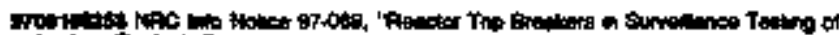
Fo mitis.

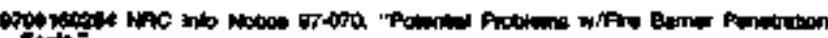

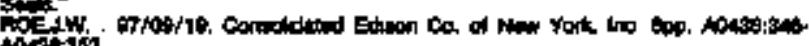

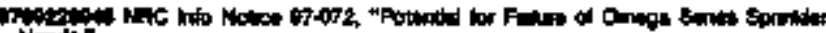

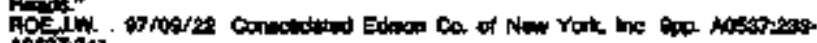
intoriay.

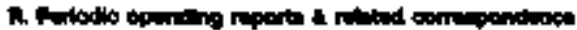

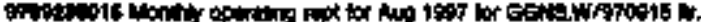

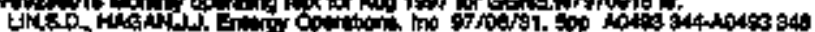

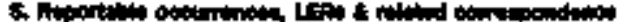

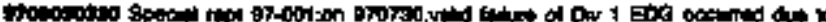

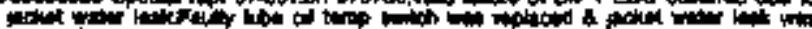

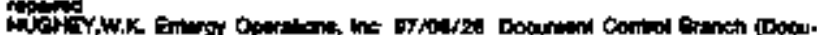

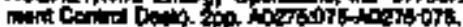

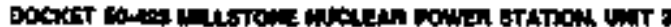

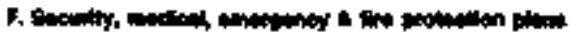

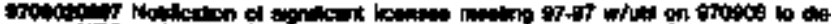

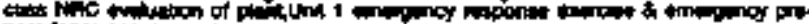

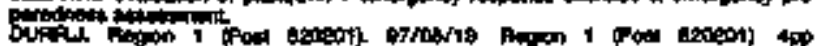

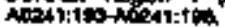

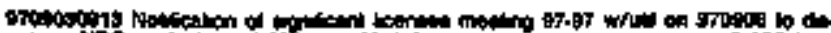

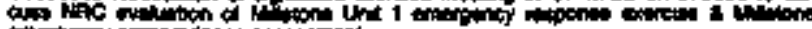

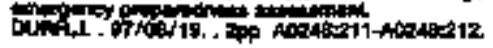

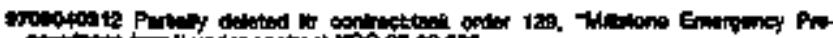

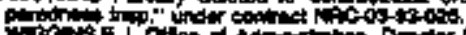

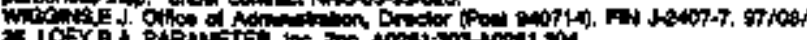

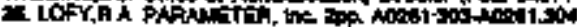

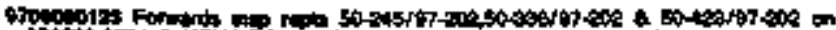

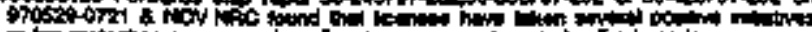

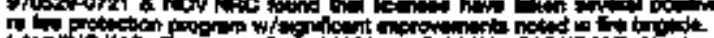

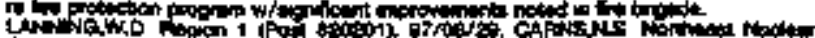

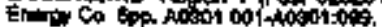

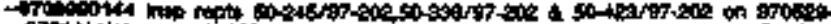

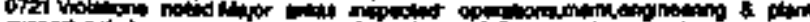

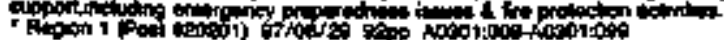

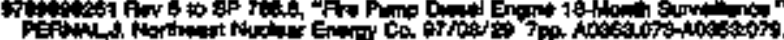

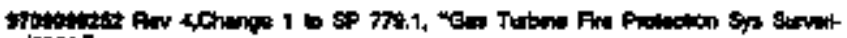

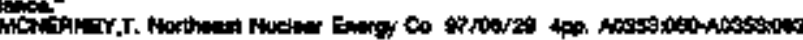

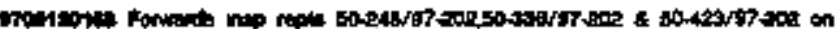

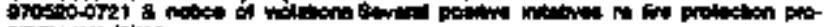

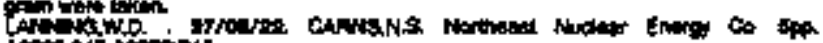

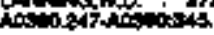

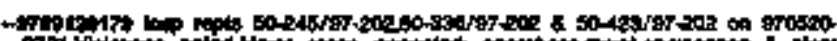

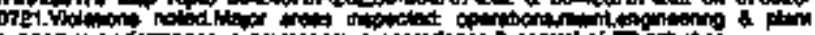

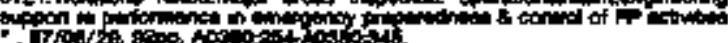

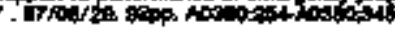

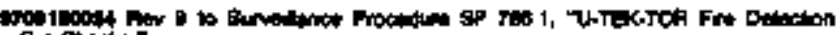

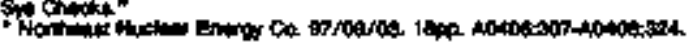

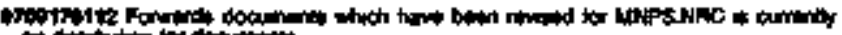

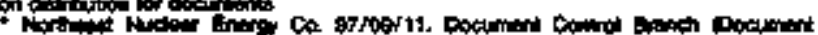

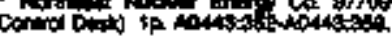

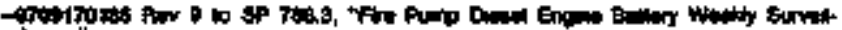

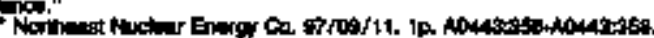

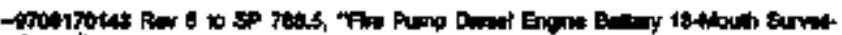
- teater.

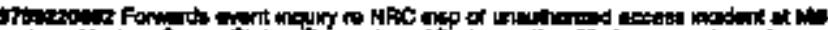

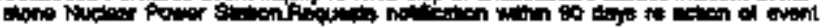

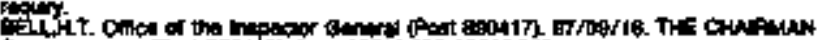

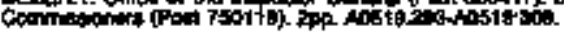

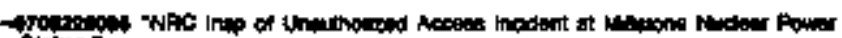

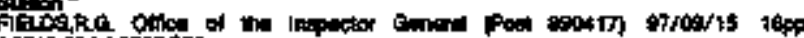

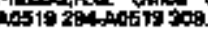

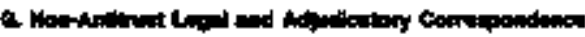

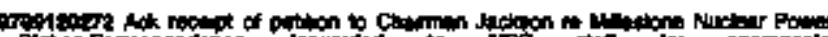

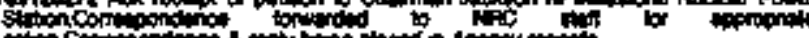

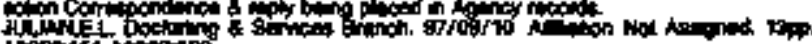

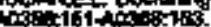

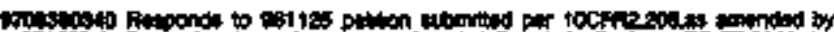

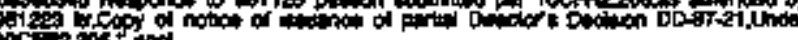

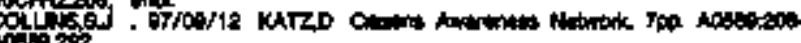
actores

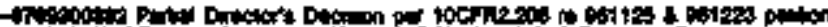

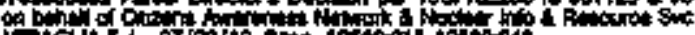
a

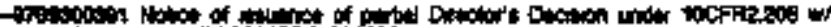

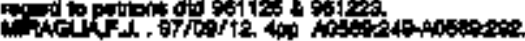

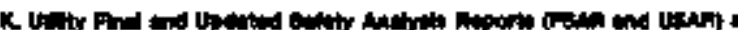

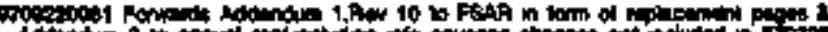

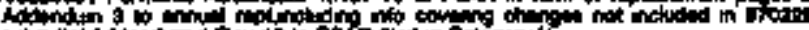

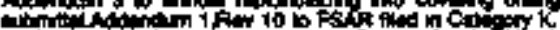

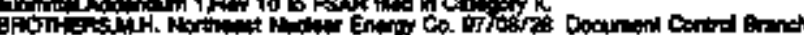

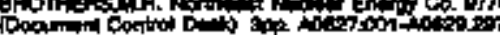

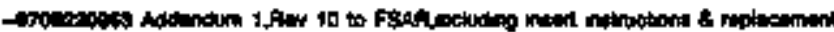

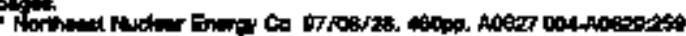

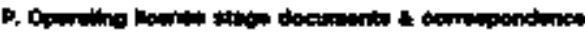

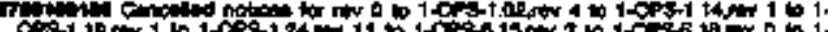

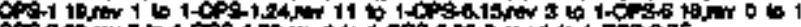

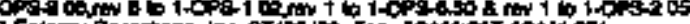

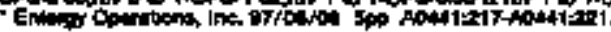

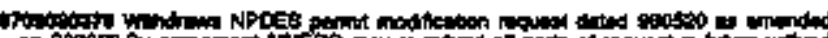

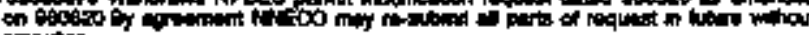

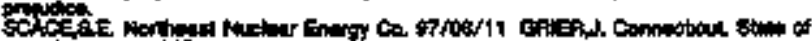

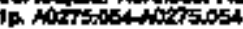

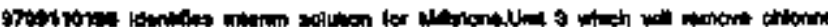

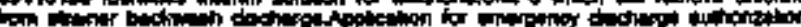

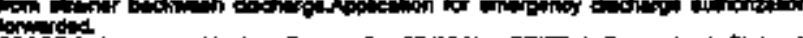

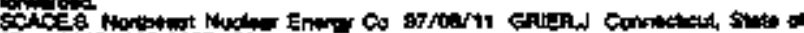
Top A

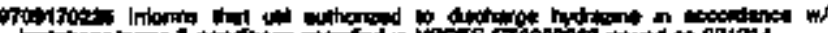

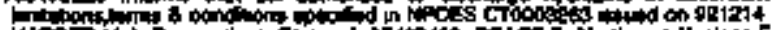

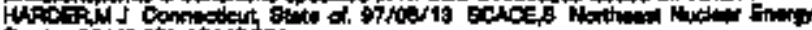

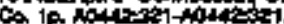




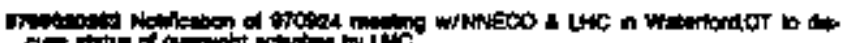
ciche

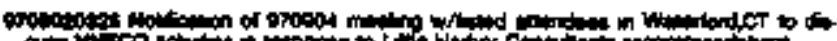

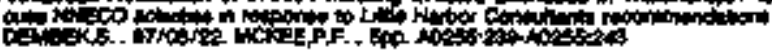

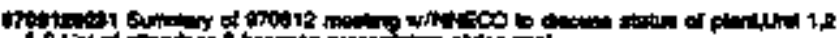
D.

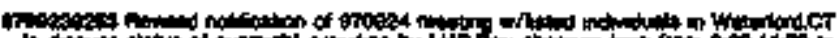

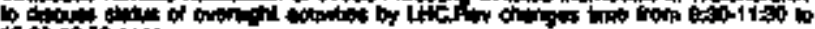

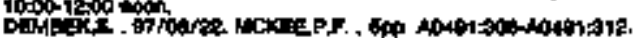

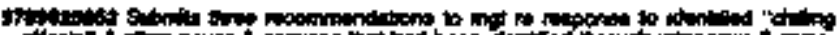

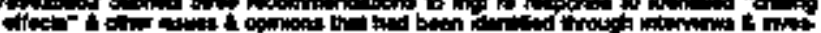

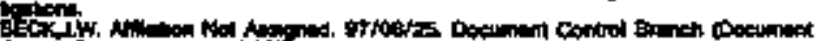

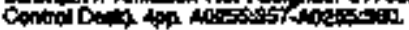

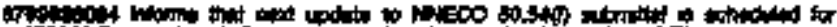

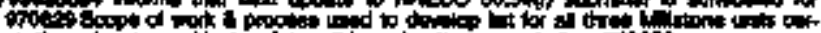

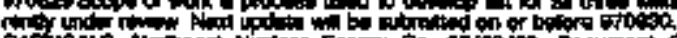

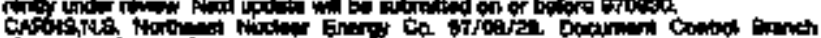

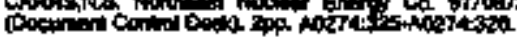

Wy

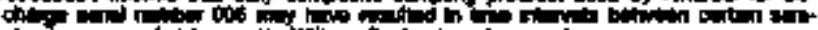

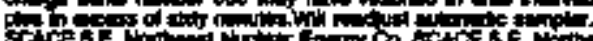

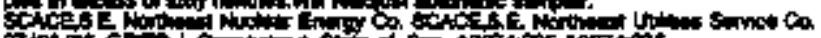
of $h_{0}$.

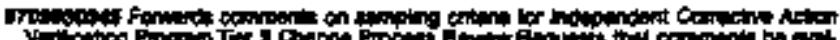

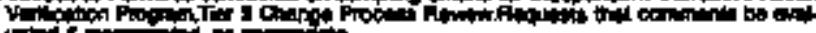

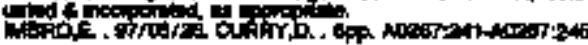

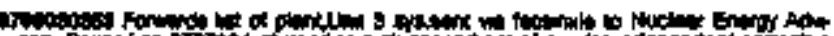

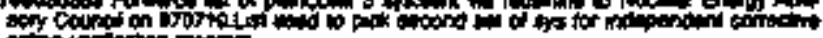

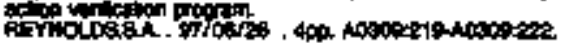

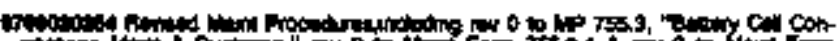

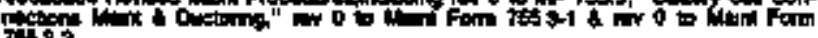
The

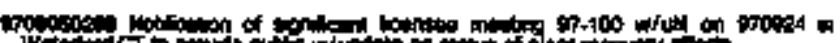
ou

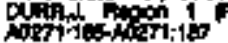

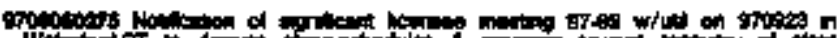

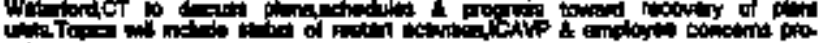

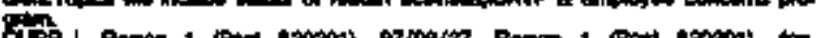

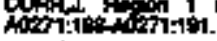

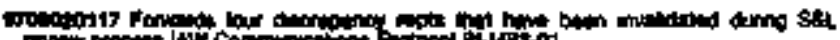

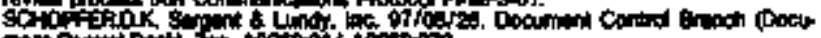

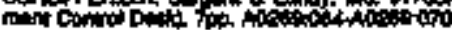

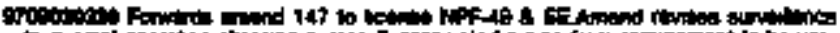

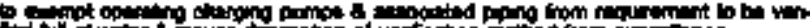

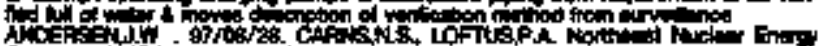

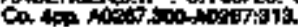

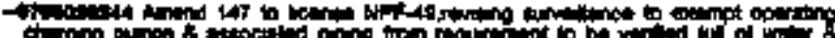

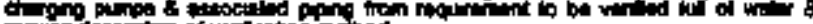

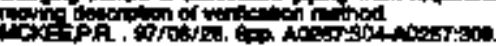

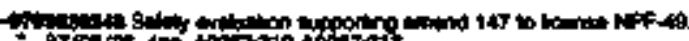

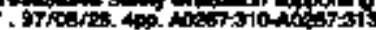

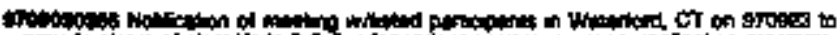

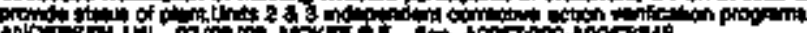
A

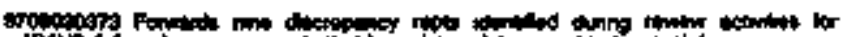

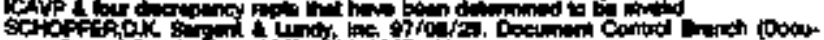

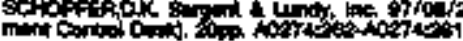

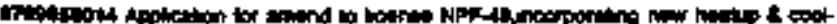

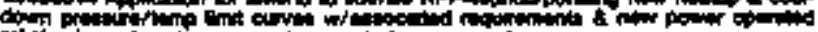

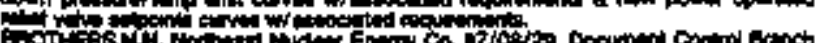
Docom con

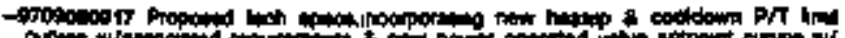

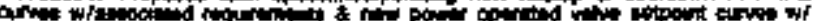

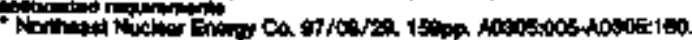

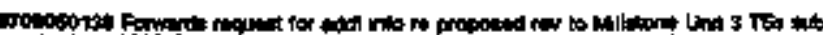

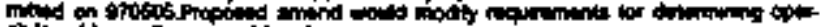

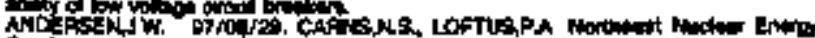

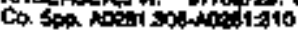

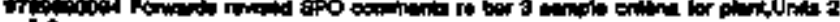

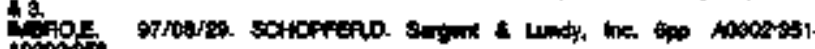
rosions.

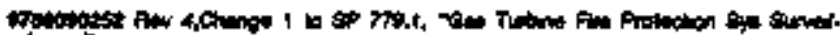

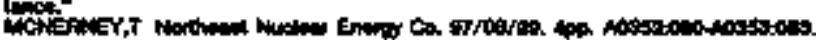

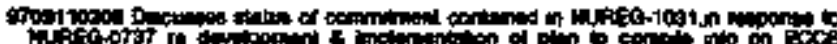

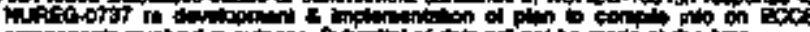

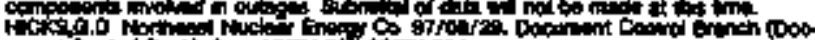

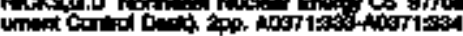

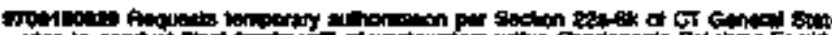

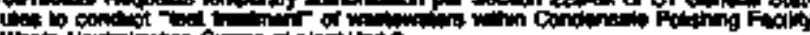

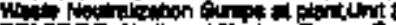

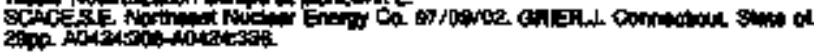

s70

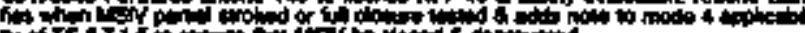

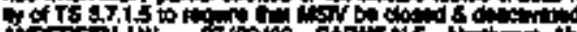

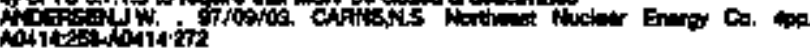

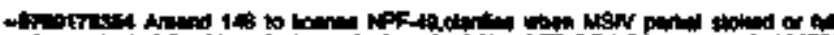

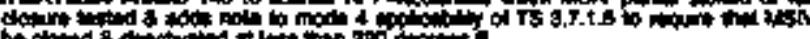

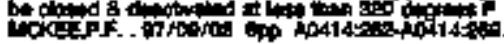

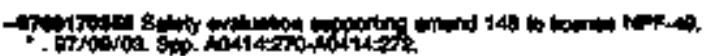

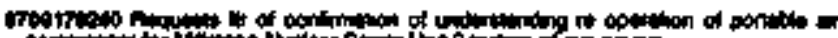

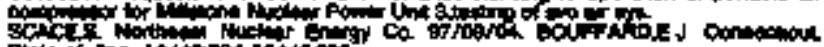

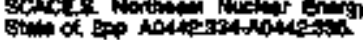

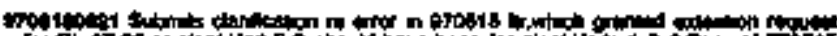

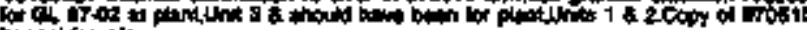

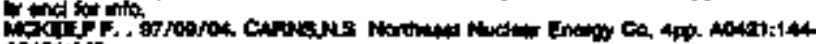
colitis.

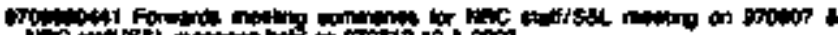

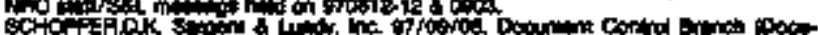

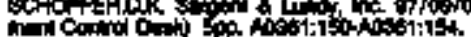

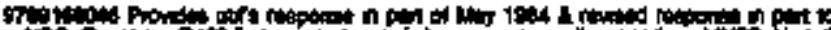

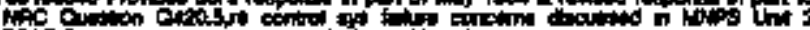

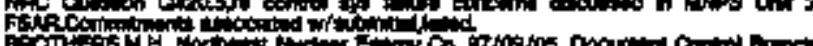

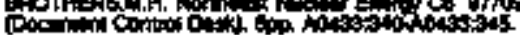

sTht

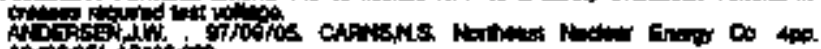

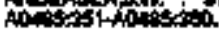

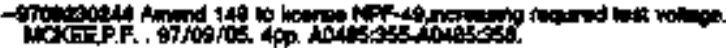

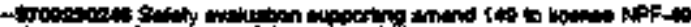

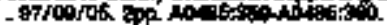

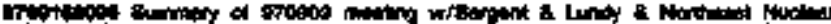

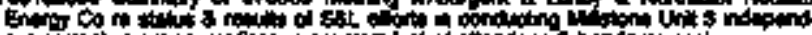

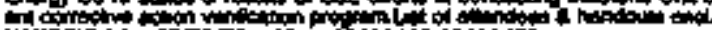
Whos

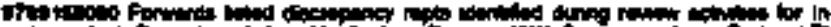

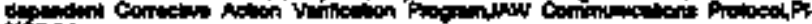
101

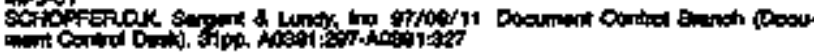

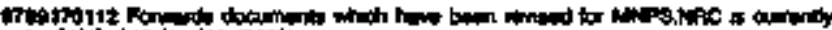

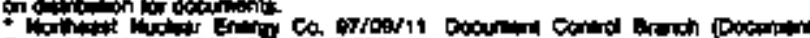

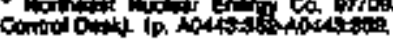

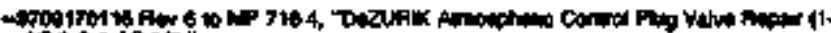

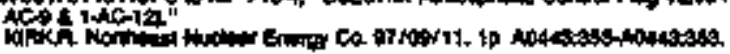

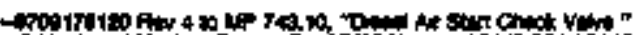

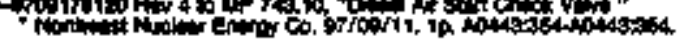

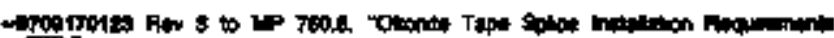

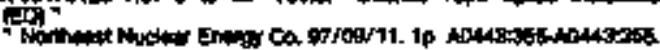

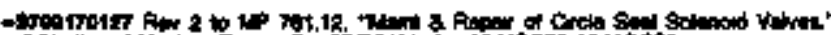

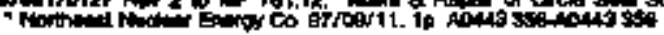

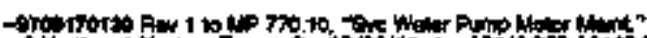

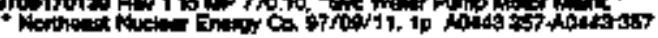

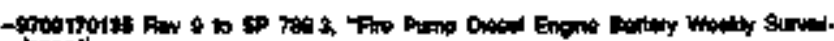

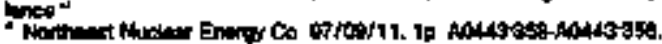




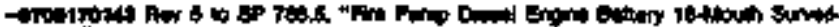

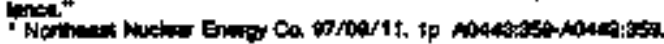

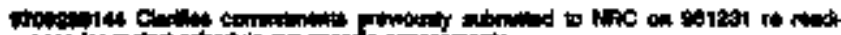

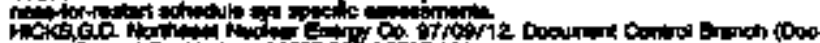

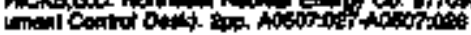

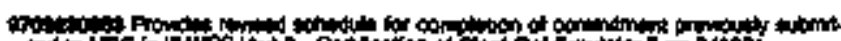

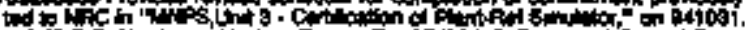

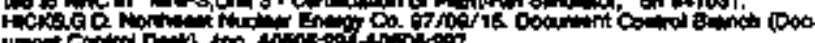

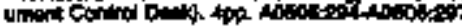

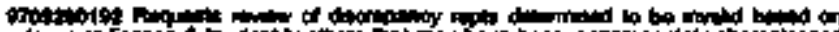

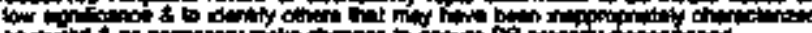

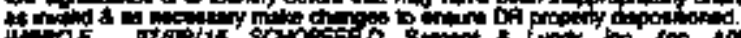
mitize

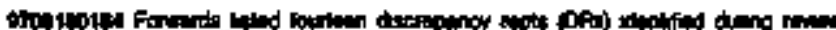

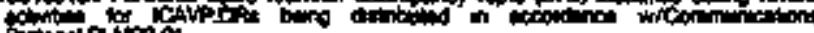

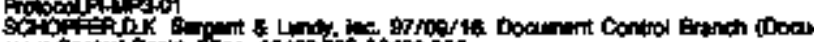

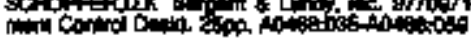

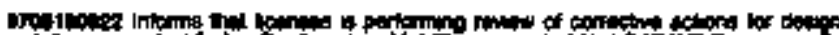

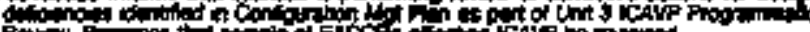

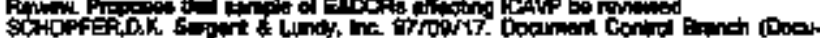

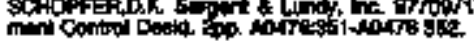

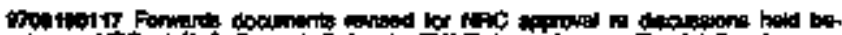

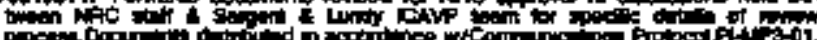

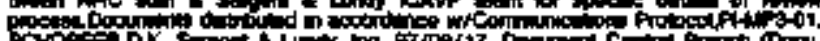

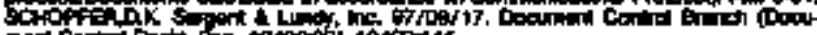

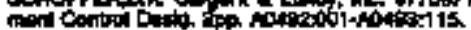

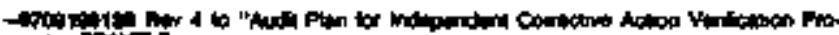

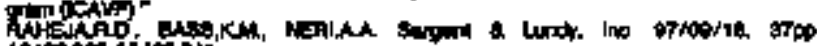

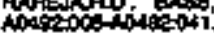

-

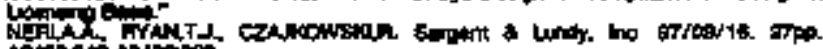

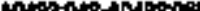

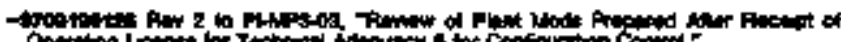

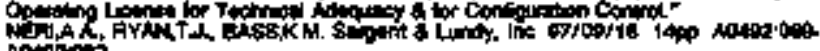
Aotians

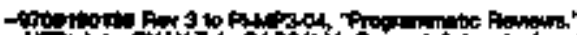

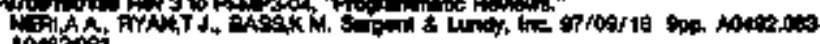
Nomzaoi.

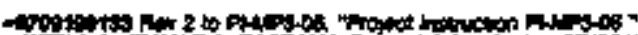

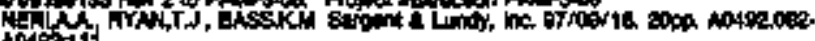
Anotratis.

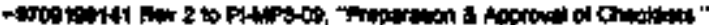

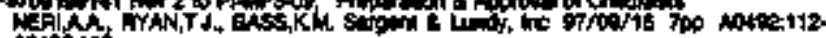
A0402+10.

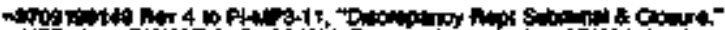

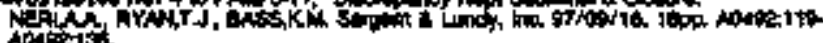

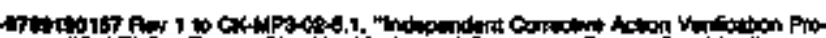

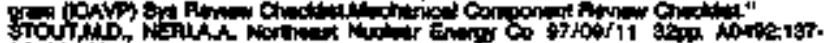
sotion.

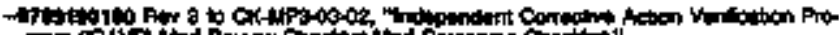

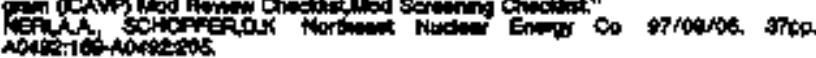

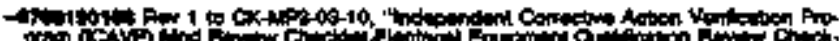

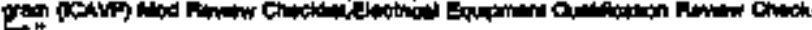

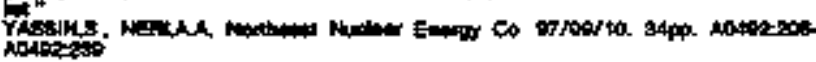

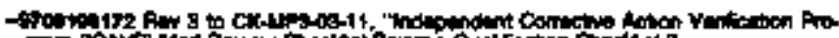

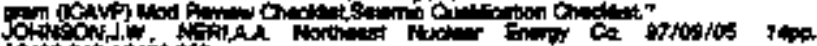

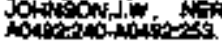

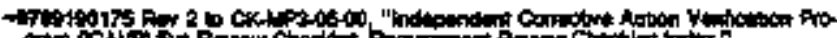

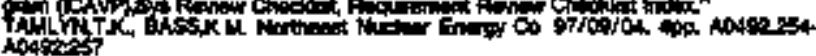

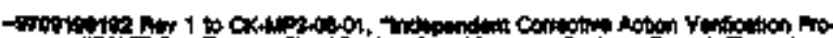

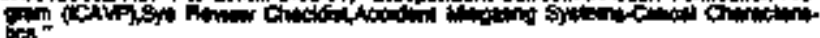

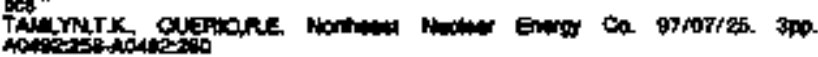

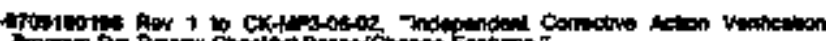

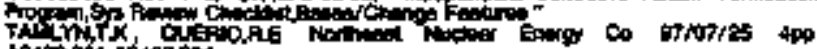

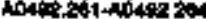

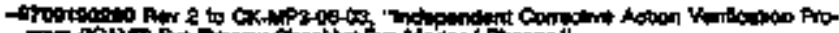

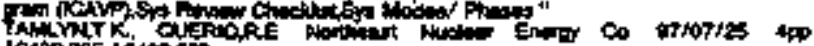

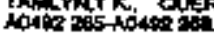

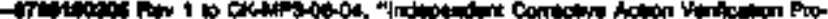

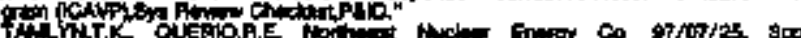
(1)

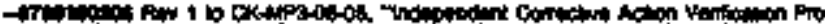

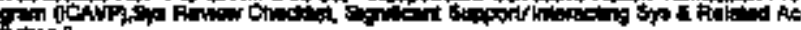

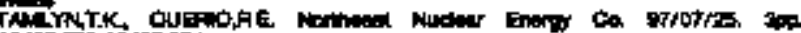

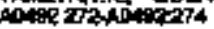

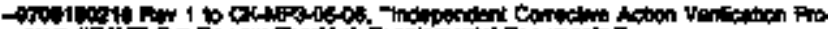

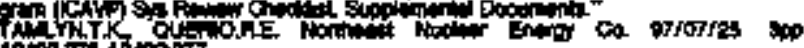

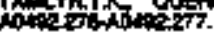

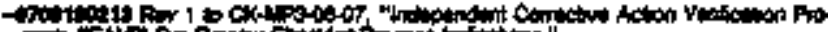
Fin

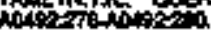

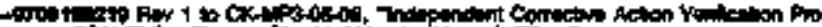

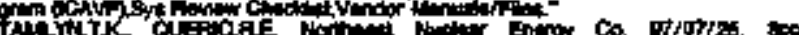
ater at tom

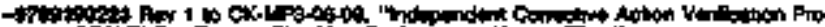

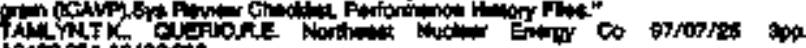

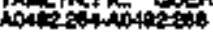

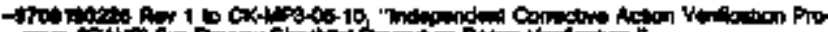

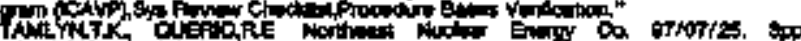

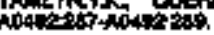

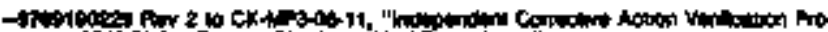

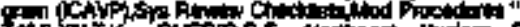

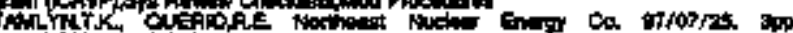

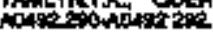

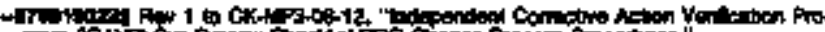

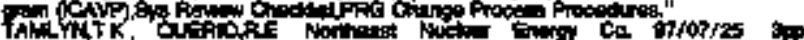

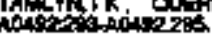

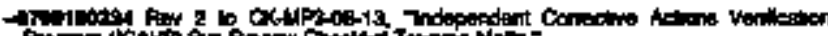

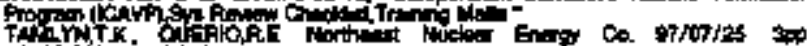
(n)

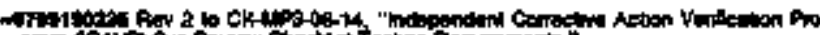

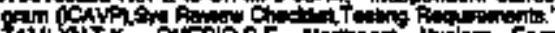
A AL A0.

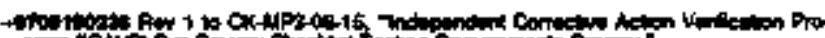

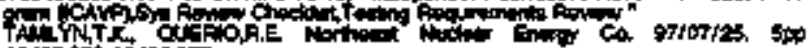

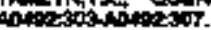

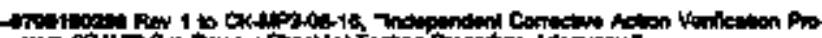

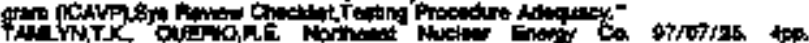

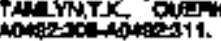

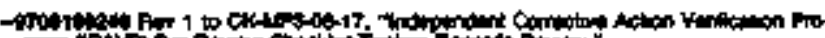

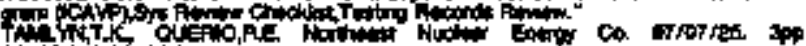
(1)

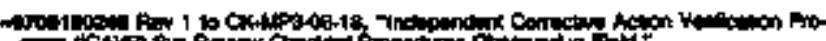

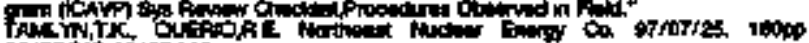

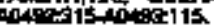

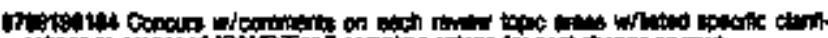

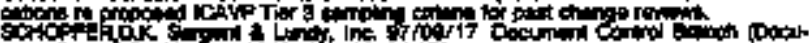

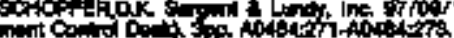

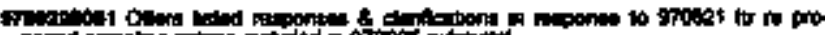

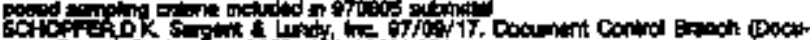

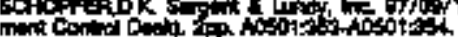

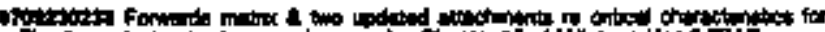

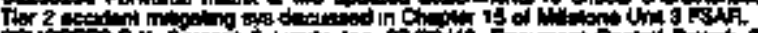

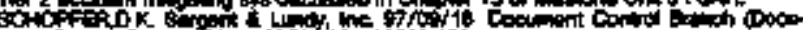

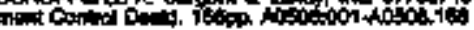

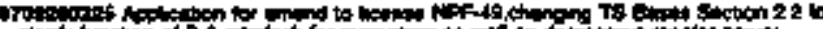

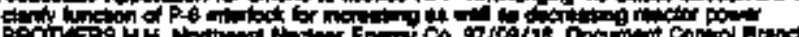

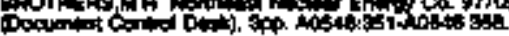

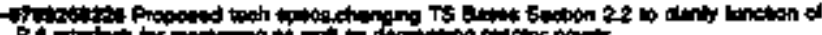

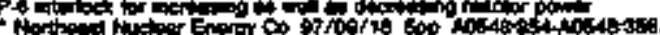

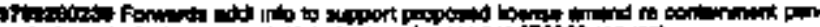

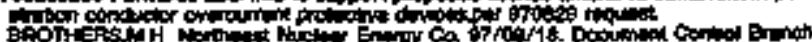

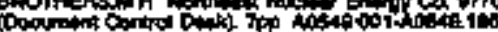




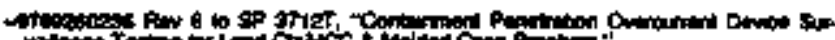

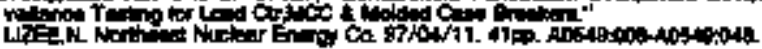

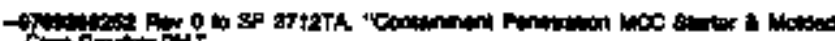
U.

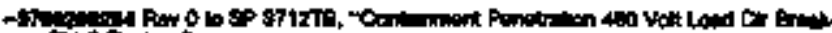

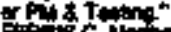

等

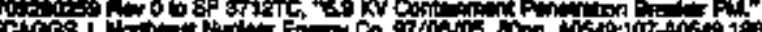

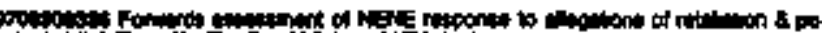

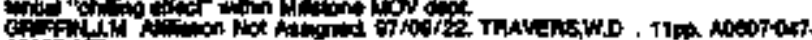
antorits.

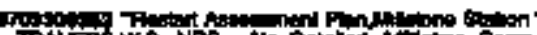

TRA antitis

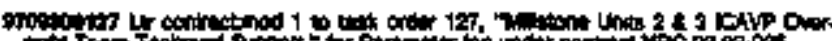

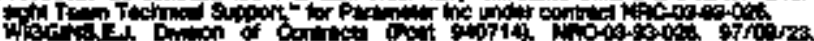

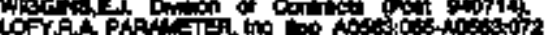

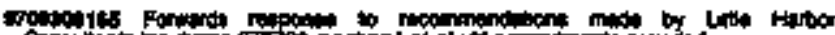

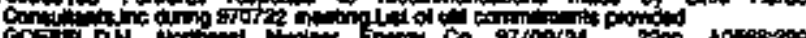

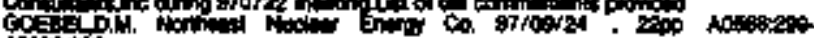

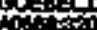

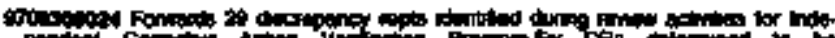

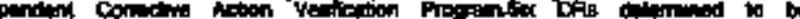

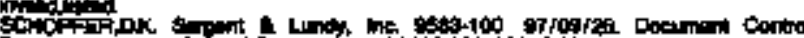

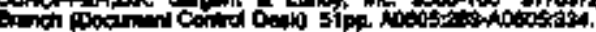

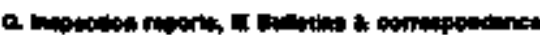

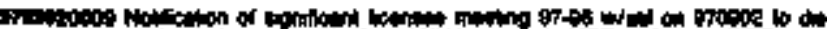

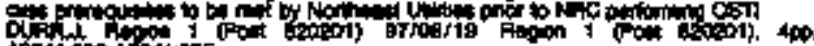

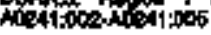

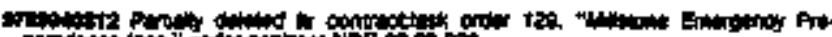

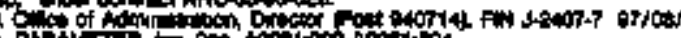
Wh

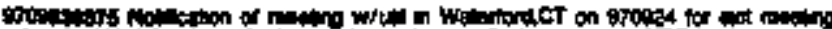

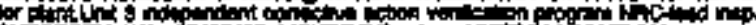

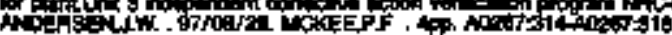

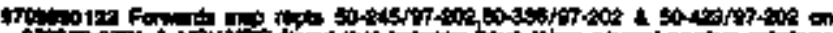

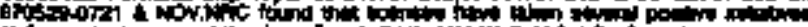

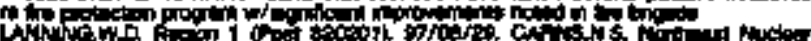

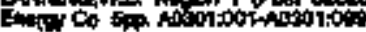

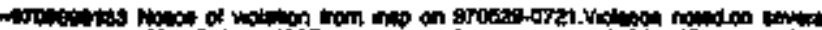

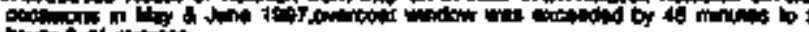

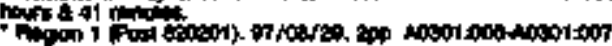

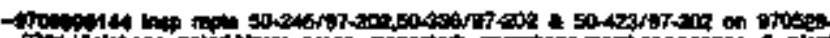

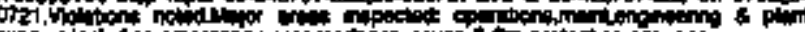

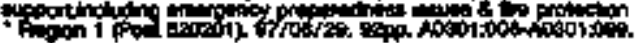

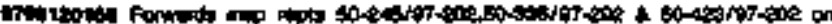

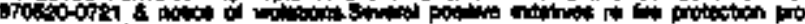

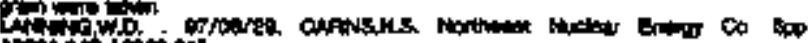

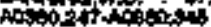

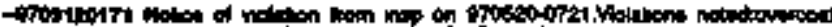

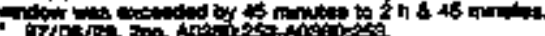

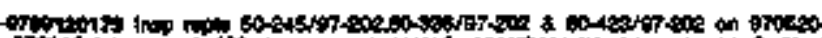

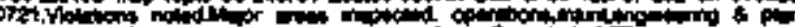

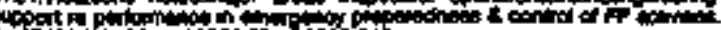

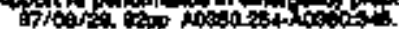

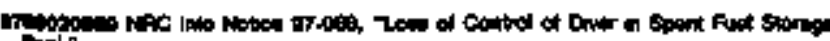

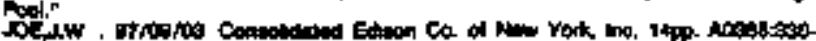

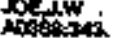

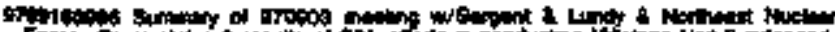

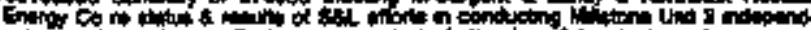

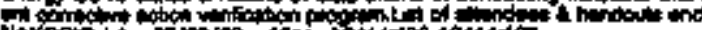

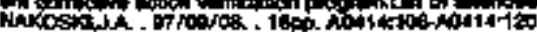

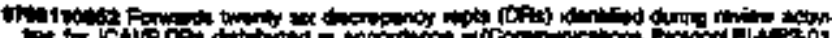

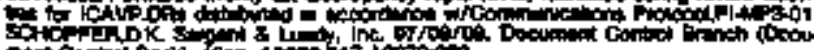

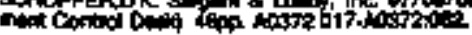

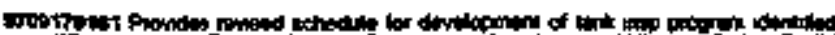

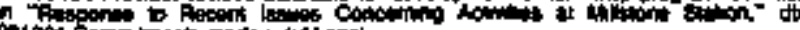

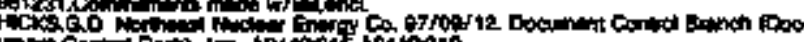

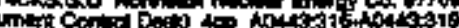

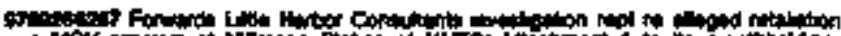

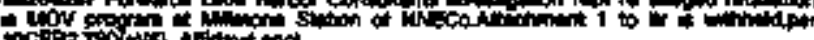

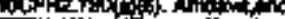

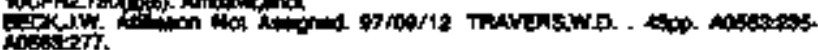

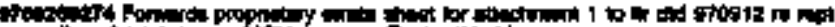

on itotion.

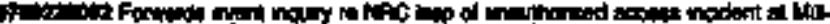

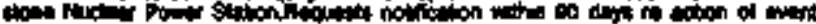

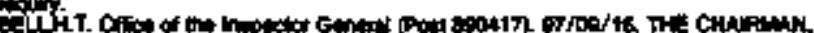

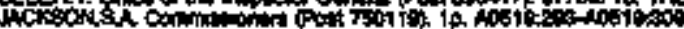

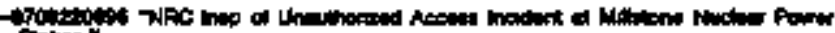

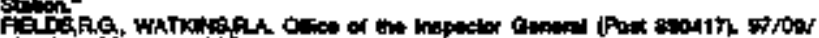

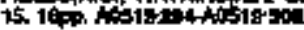

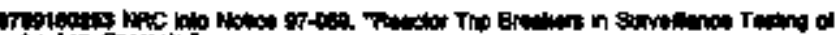
Pó. Antis.

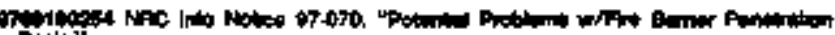

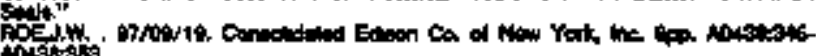

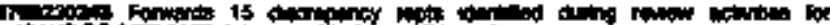

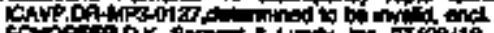

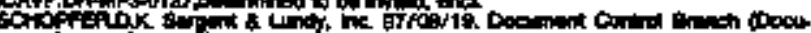

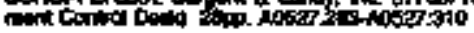

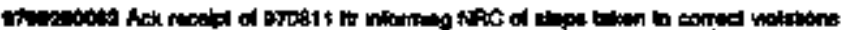

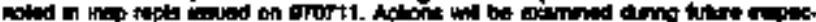
pon

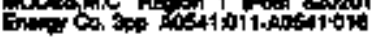

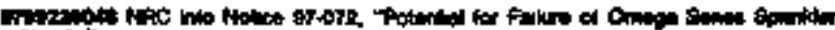

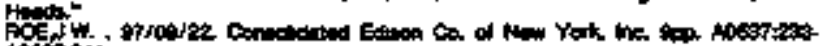
nosisian:

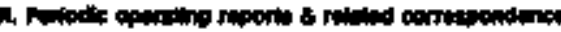

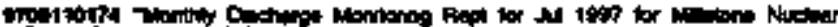

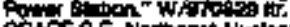

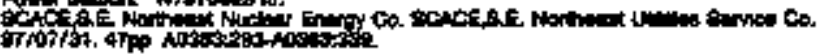

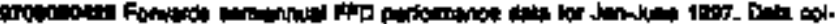

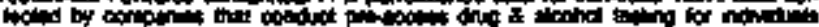

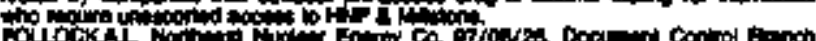

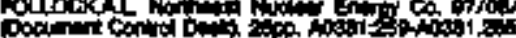

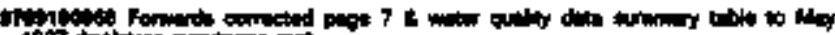

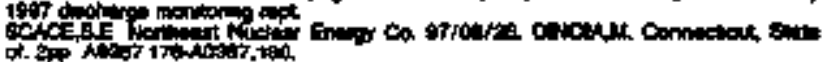

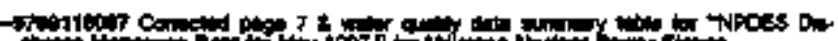
sing

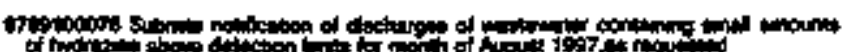

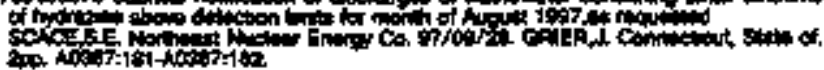

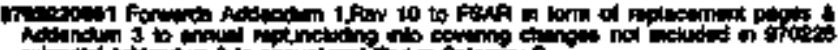

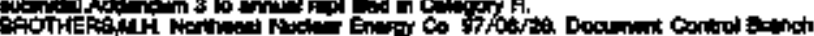

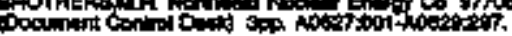

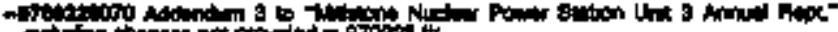

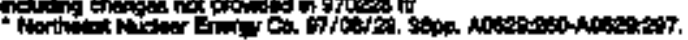

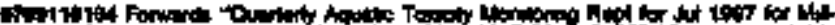

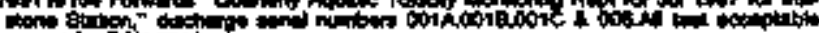

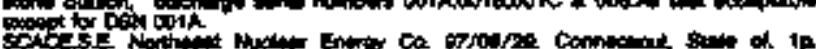

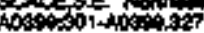

Arob1101: '

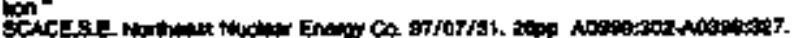

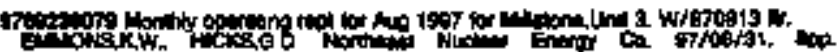

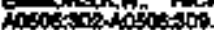




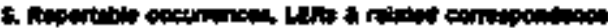

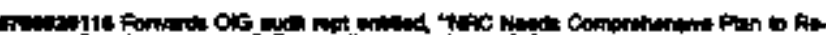

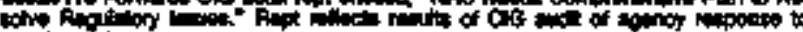
(1)

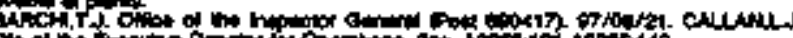

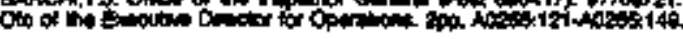

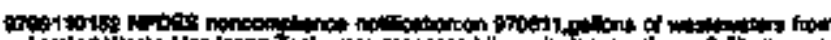

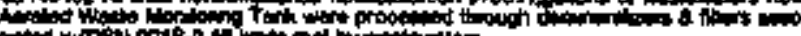

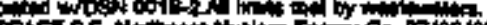

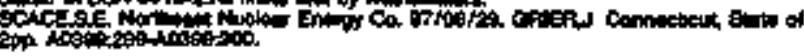

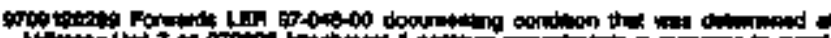

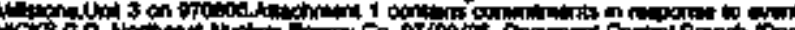

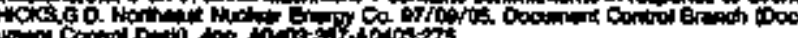

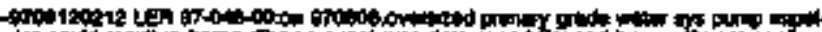

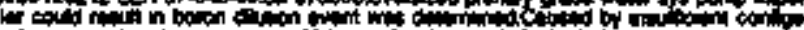

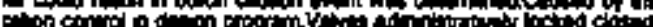

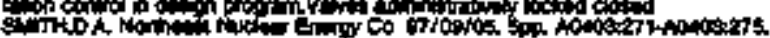

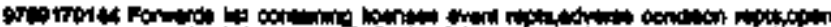

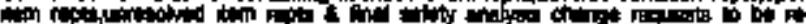

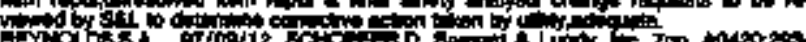
A.t.

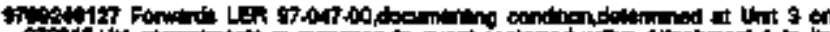

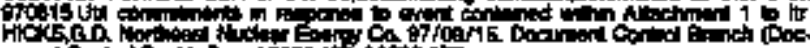

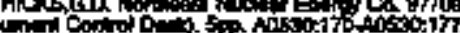

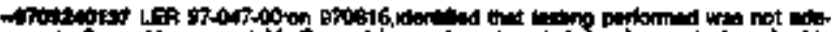

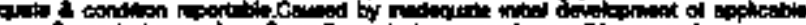

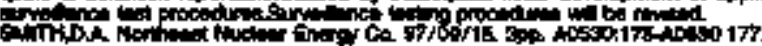

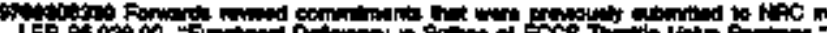
If

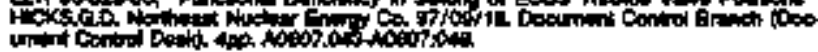

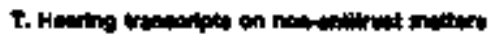

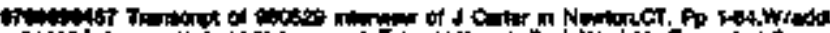

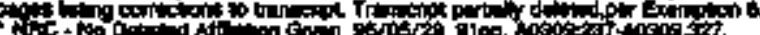

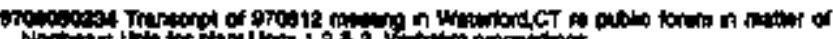

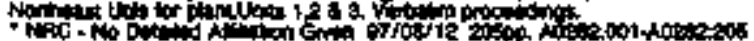

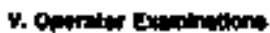

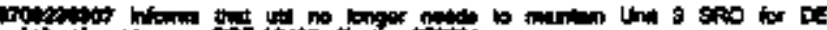
An

baO'

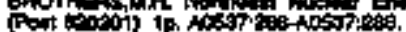

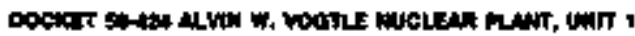

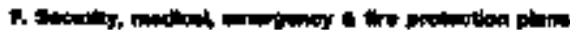

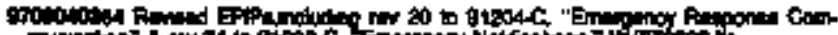

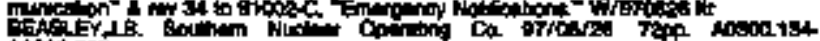
1000000

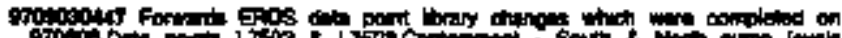

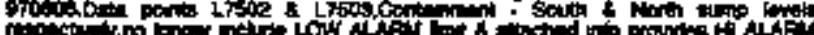

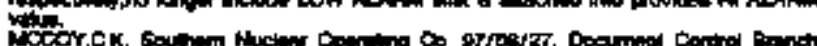

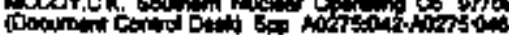

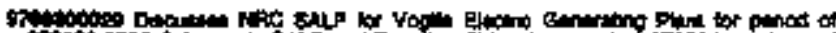

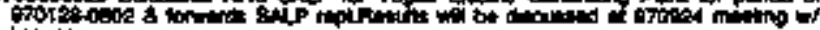

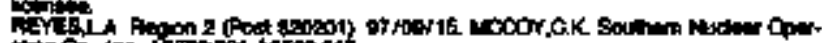

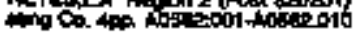

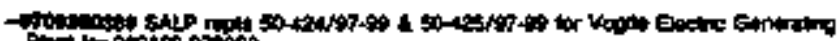

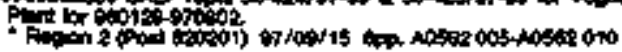

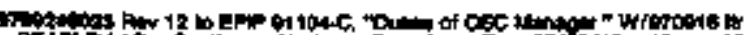

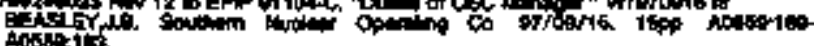

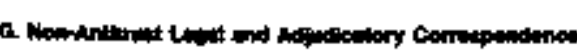

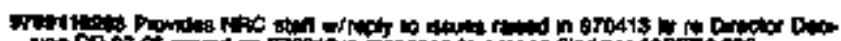

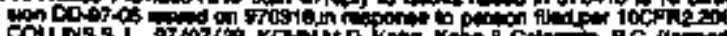

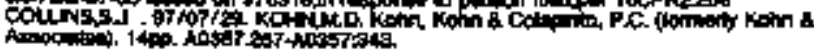

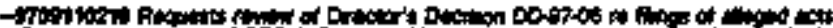

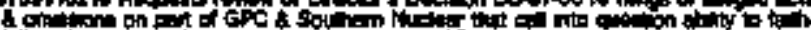

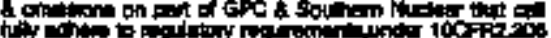

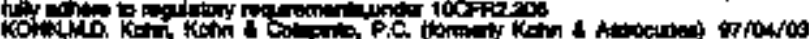

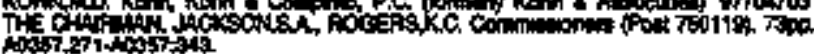

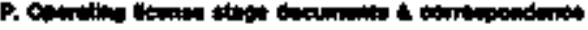

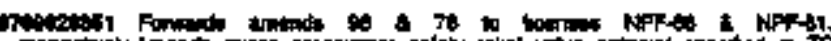

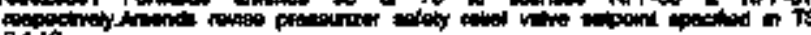

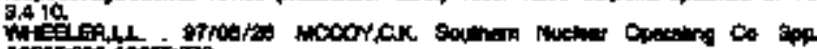

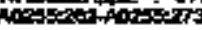

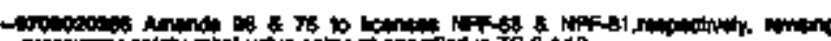

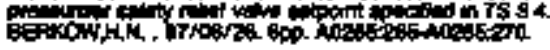

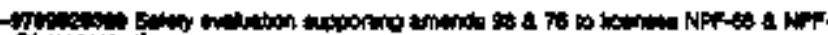

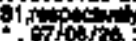

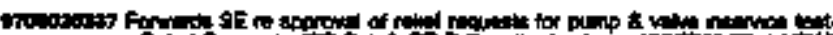

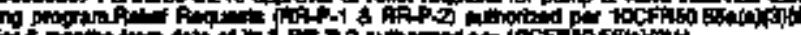

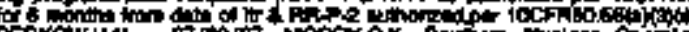

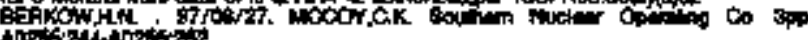

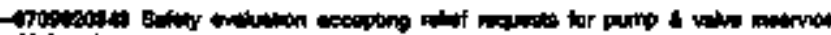

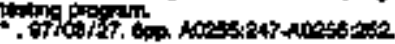

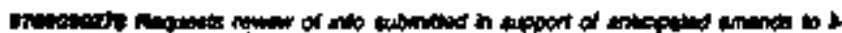

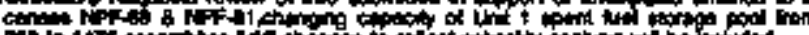
Dxall.

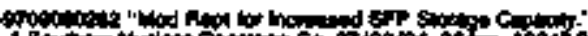

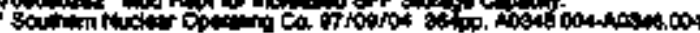

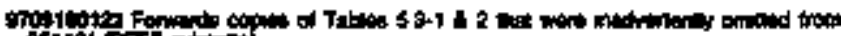

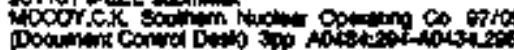

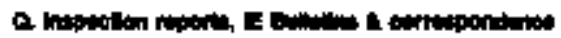

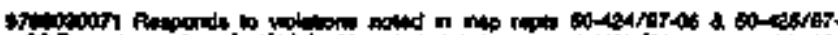

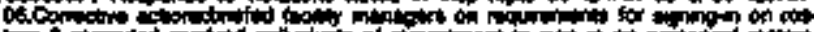

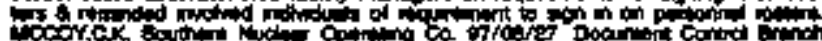

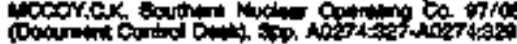

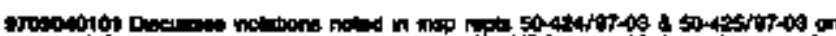

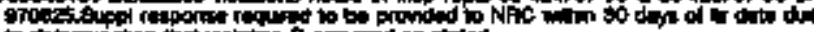

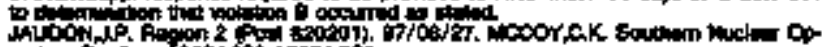

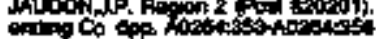

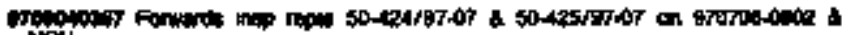

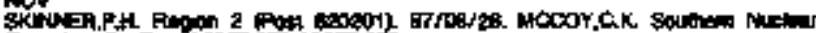

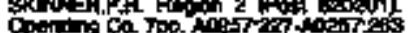

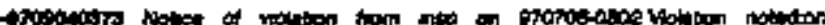

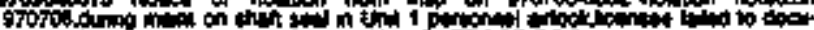

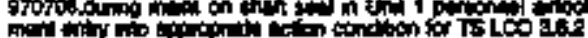

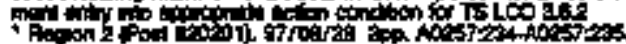

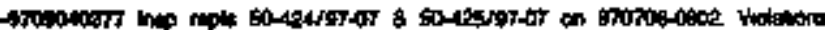

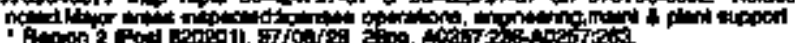

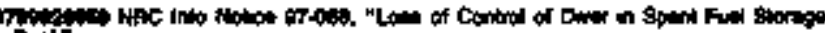

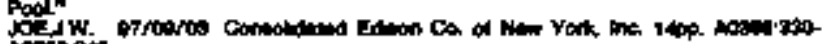
Mosionis

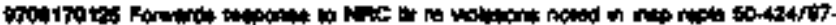

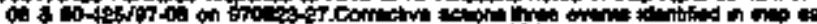

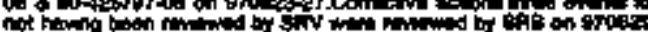

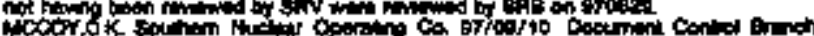

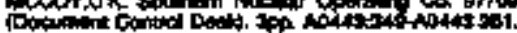

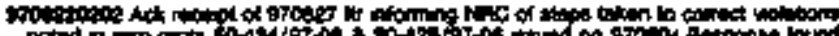

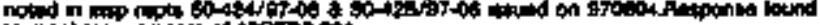
by

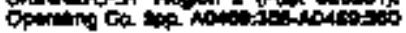

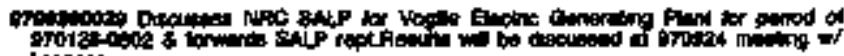

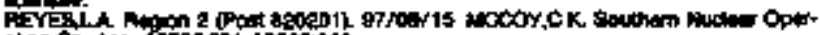

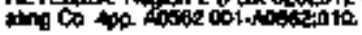

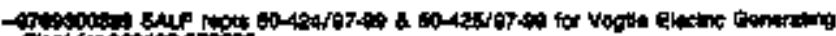

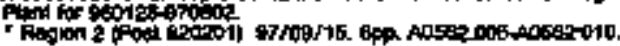




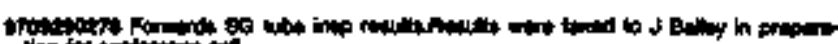

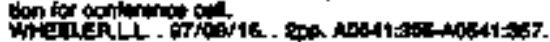

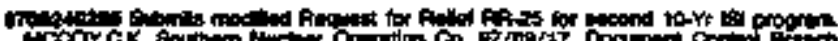

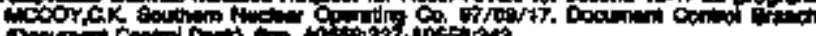

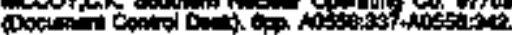

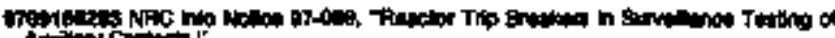
Fob,

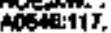

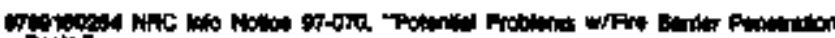

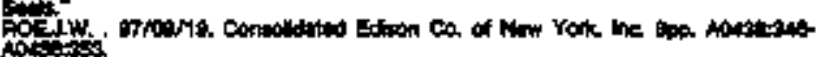

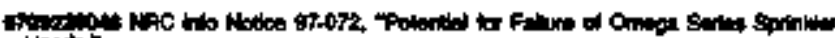

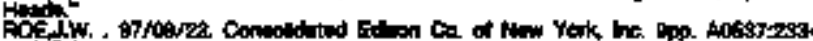

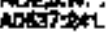

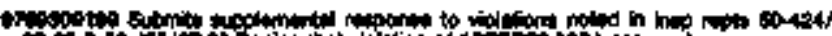

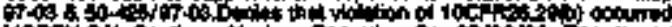

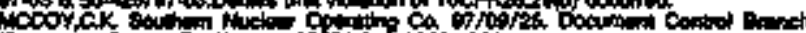

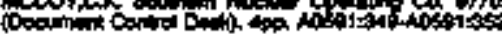

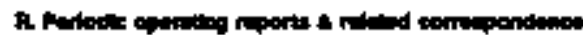

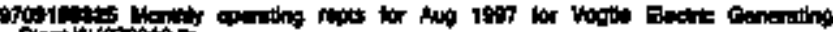

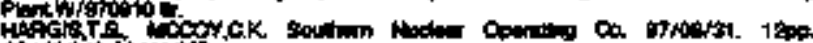

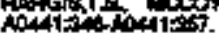

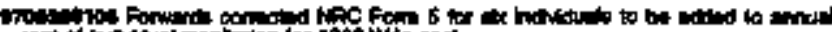

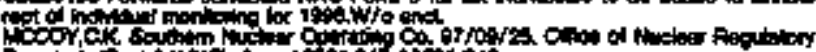

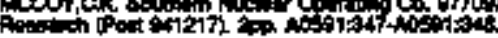

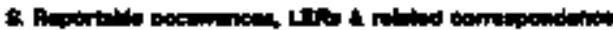

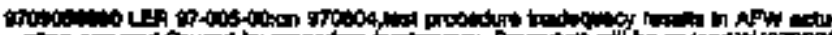

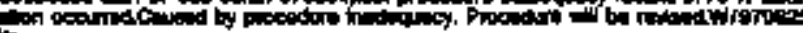

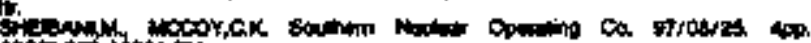
ithotiony

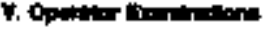

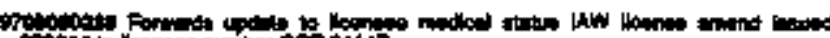

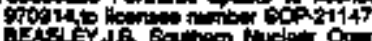

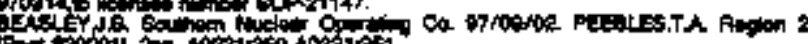

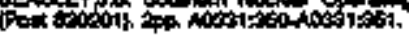

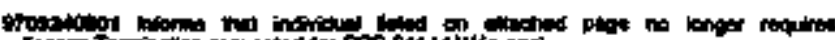

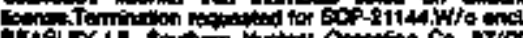

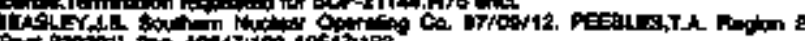

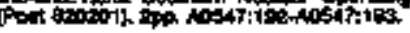

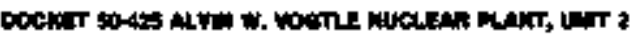

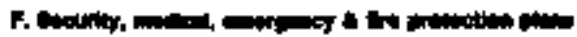

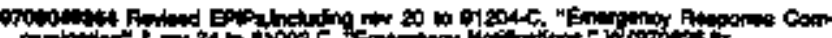

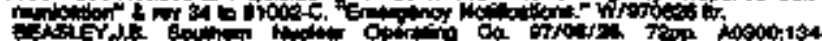
ingorits

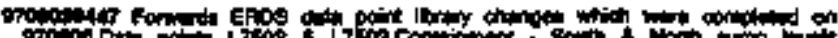

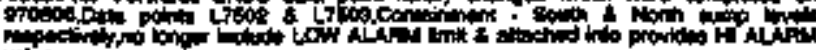
min.

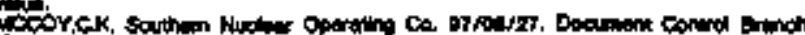

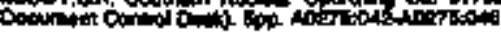

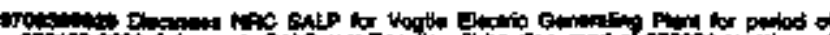

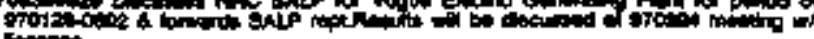

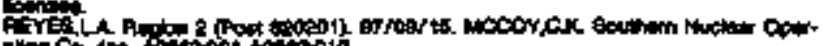
and cos 4p.

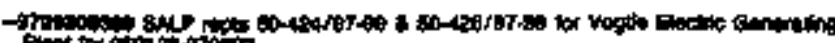

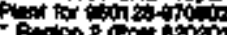

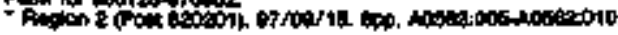

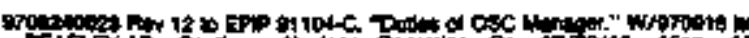

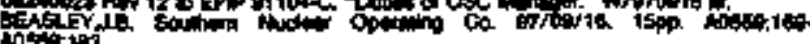

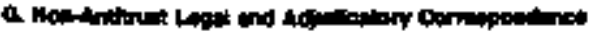

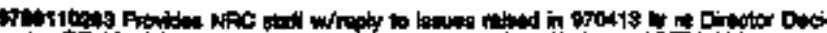

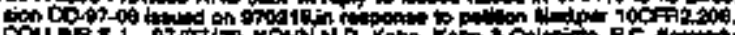

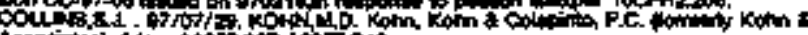

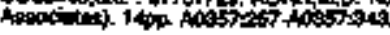

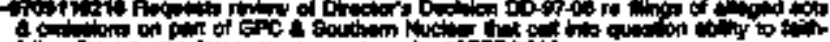

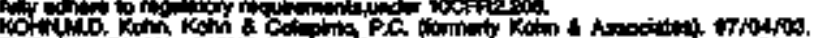

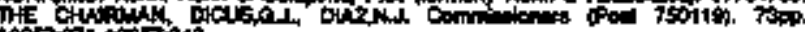

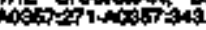

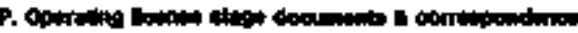

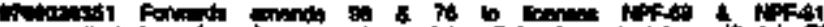

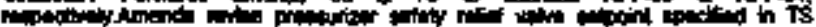

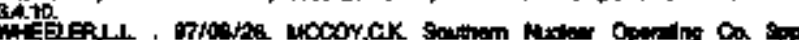

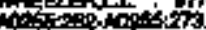

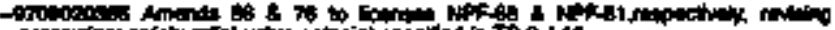

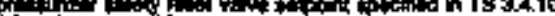

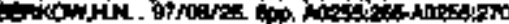

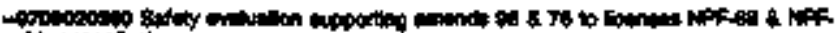

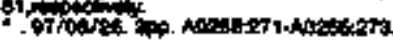

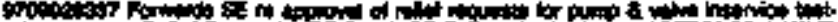

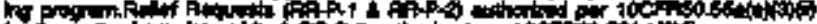

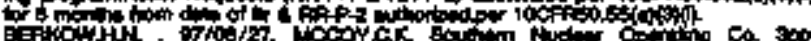

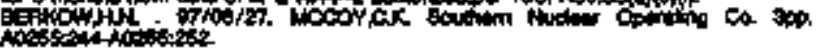

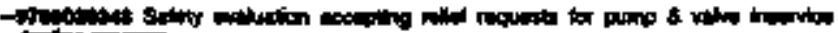

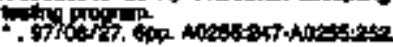

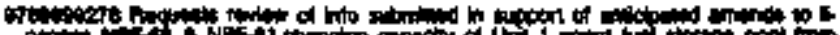

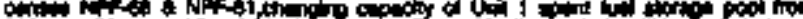

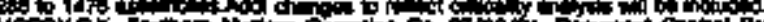

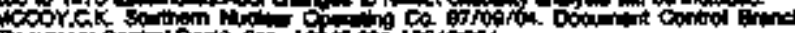

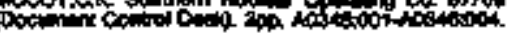

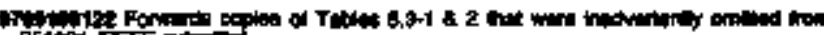

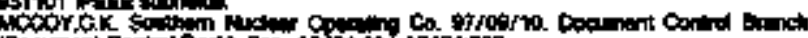

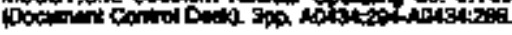

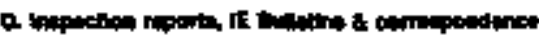

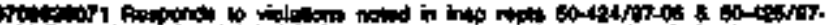

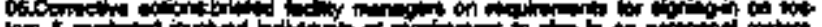

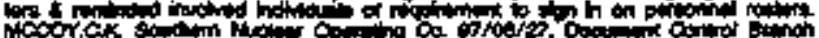

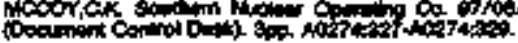

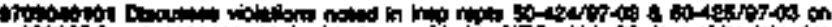

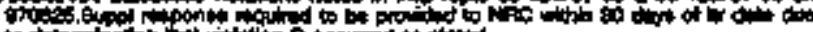

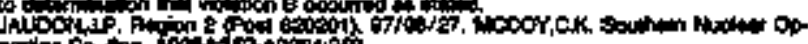

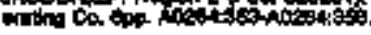

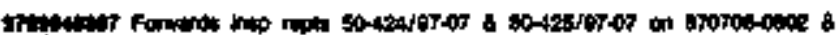

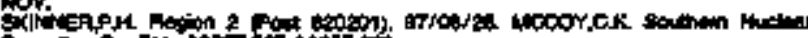

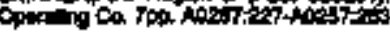

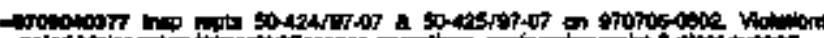

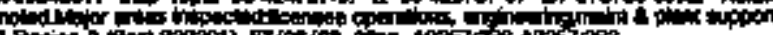

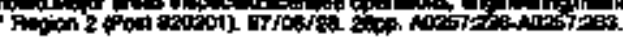

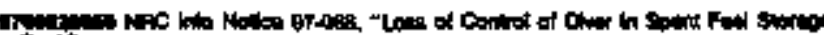

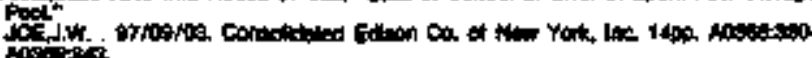

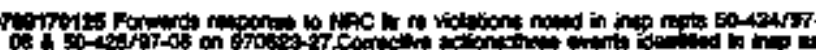
o.

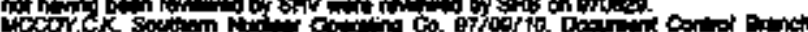

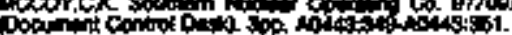

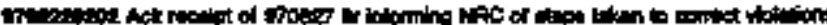

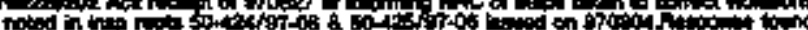

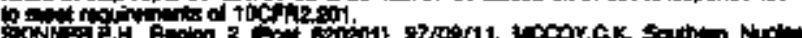

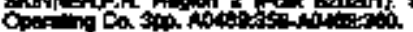

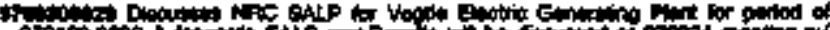

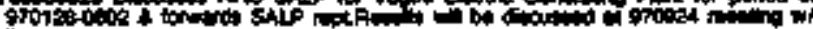

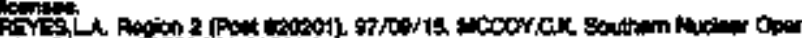

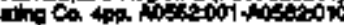

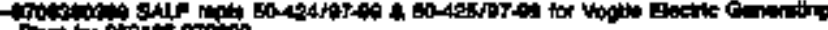

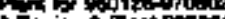

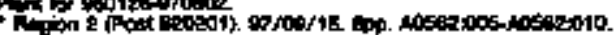

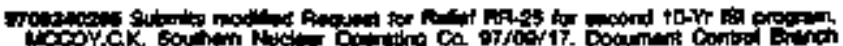

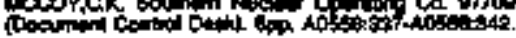

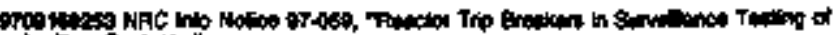

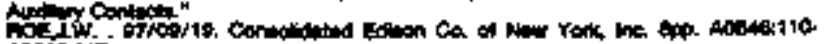
indfir. 


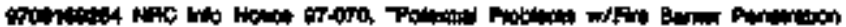
$8+1$ Roctiv.

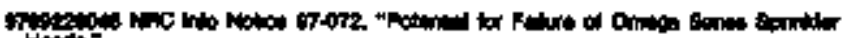

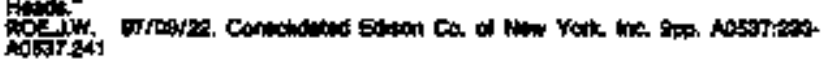

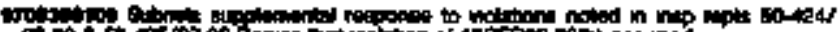

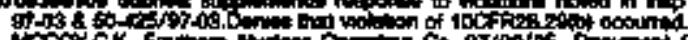

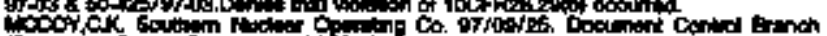

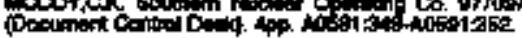

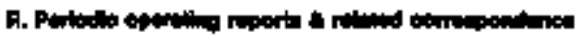

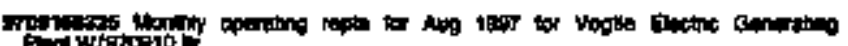

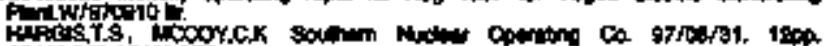

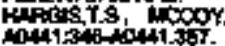

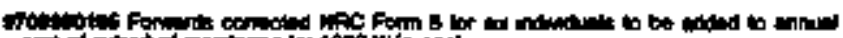

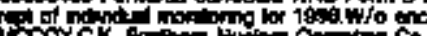

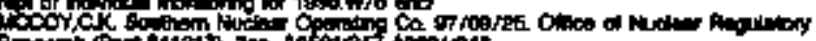

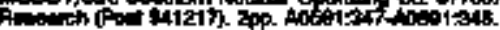

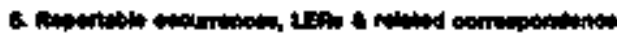

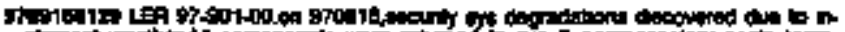

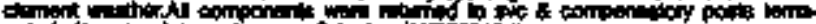

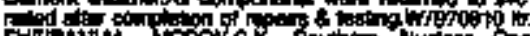

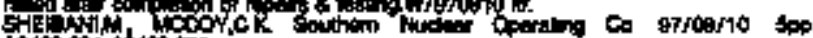

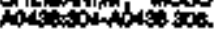

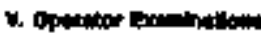

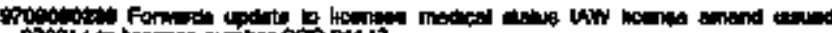

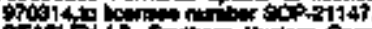

Q 0.4030135:

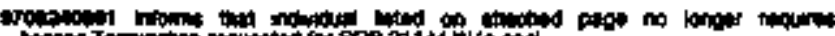

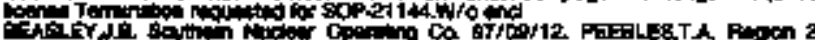

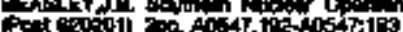

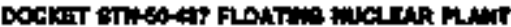

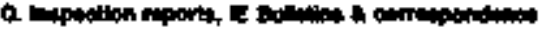

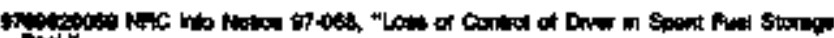

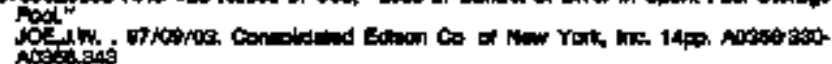

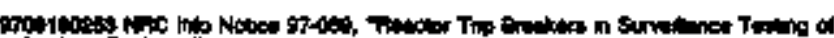

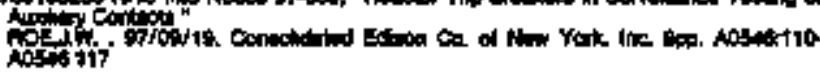

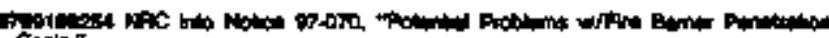
soisti"

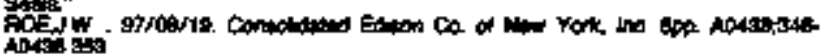

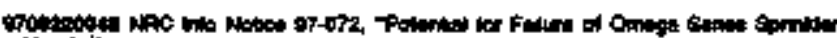

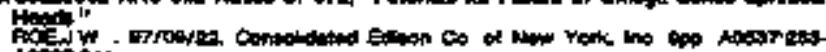
Alosition

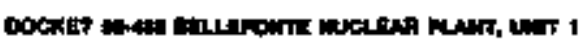

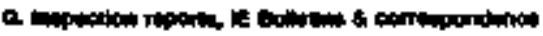

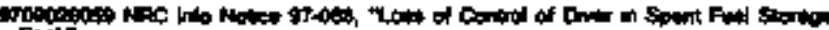

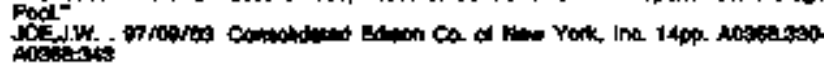

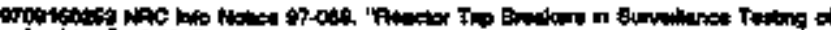

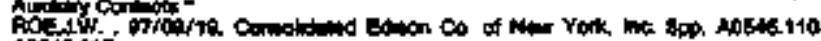

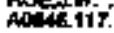

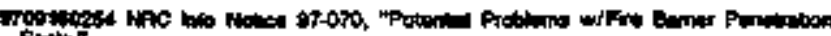

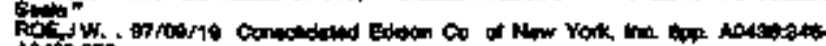
A 0 4. 35.

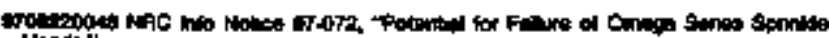

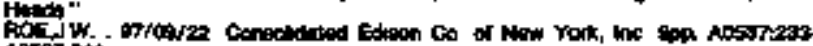
Ansosit 241 .

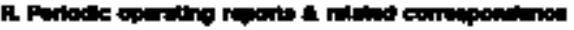

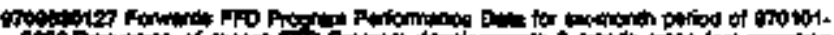

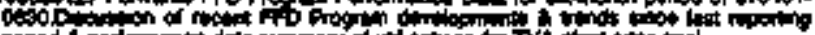

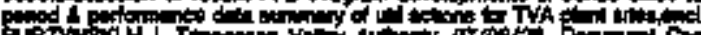

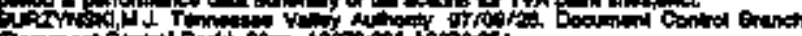

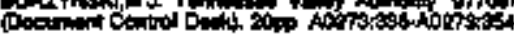

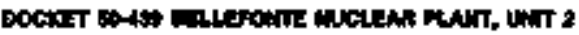

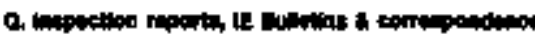

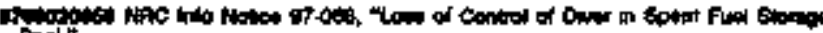
Peod.".

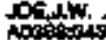

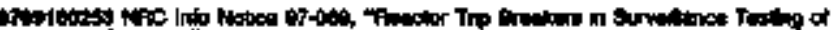

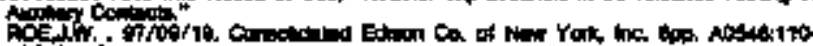
Astitis:

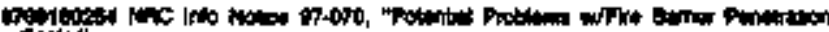

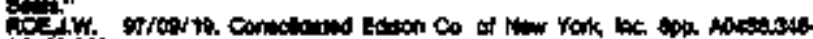
10.0.6.

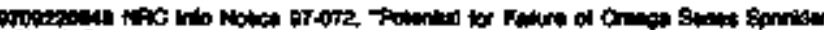
Hectic ictint.

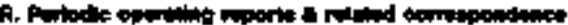

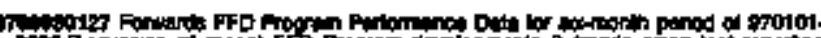

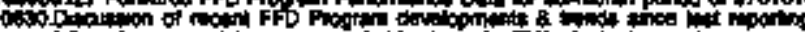

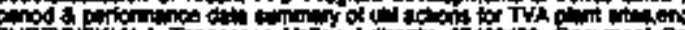

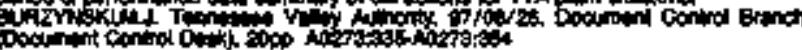

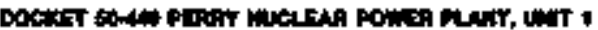

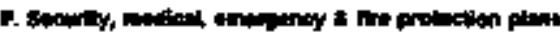

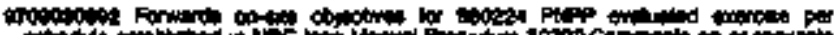

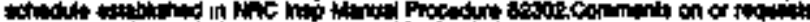

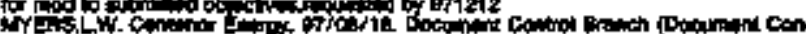
Dol ontion.

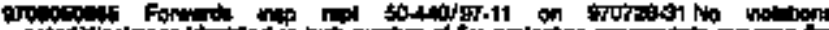

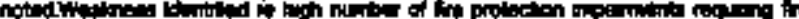
chich

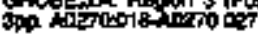

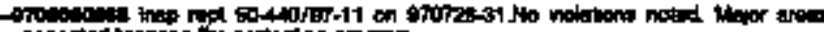

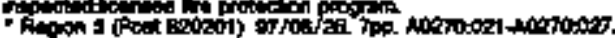

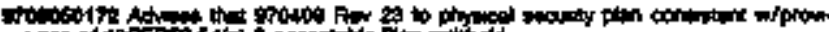

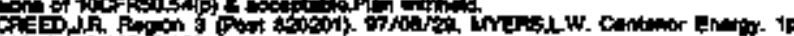

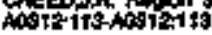

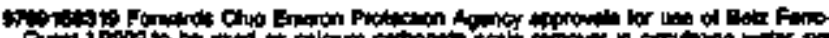

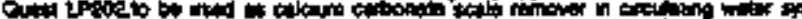

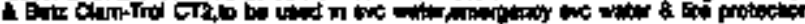
FE

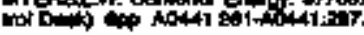

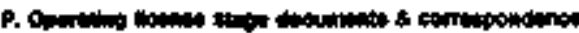

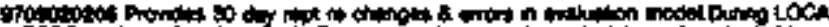
COCS -

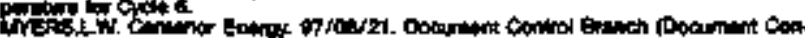

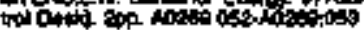

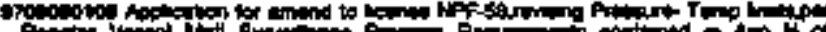

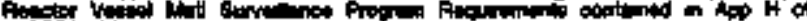

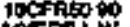

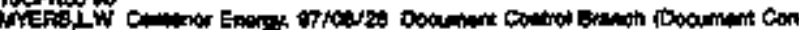

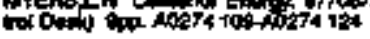

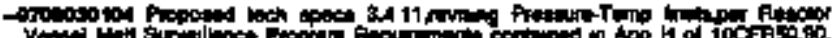

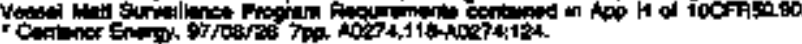

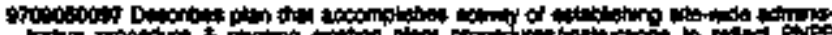

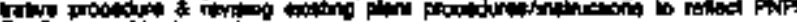
in

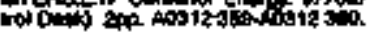




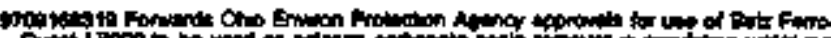

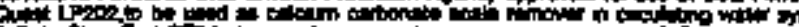

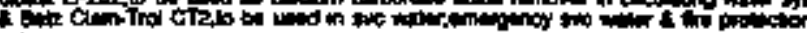

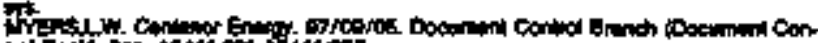

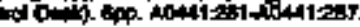

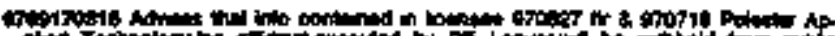

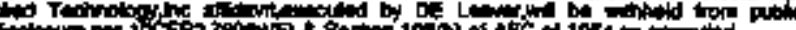

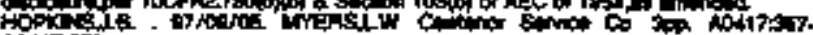

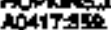

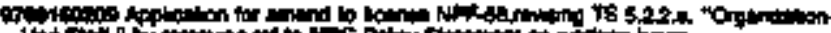

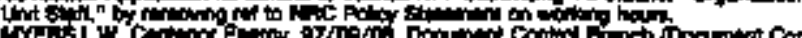

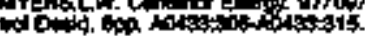

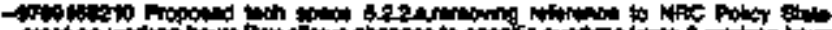

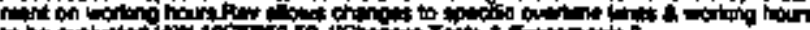

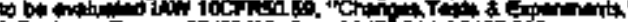

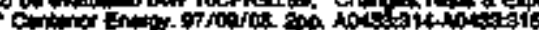

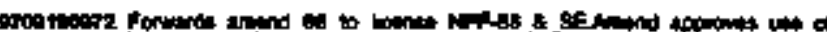

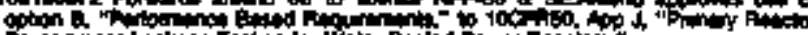

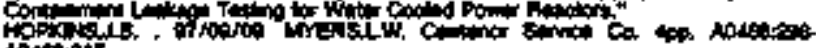

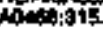

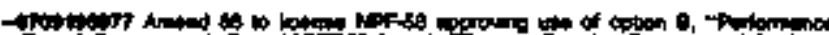

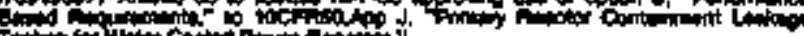

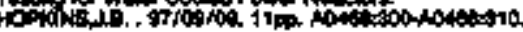

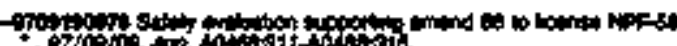

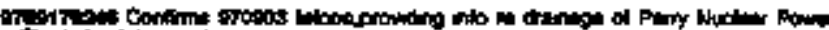

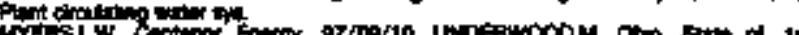
Motion

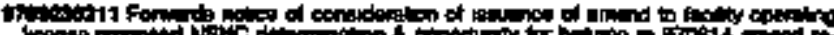

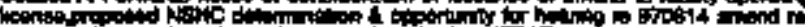

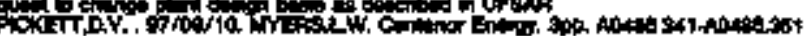

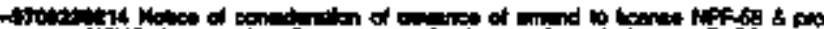

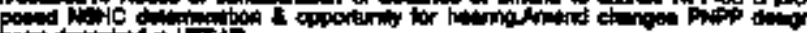

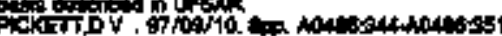

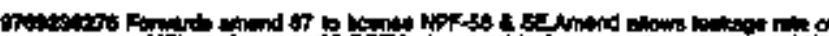
on or now to of the or exil to 100 bet

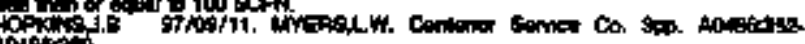
Nortatos

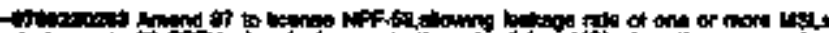
to bete t

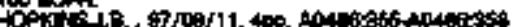

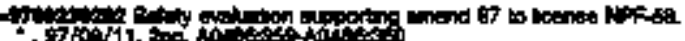

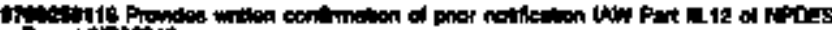
Pint toonto

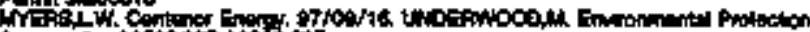

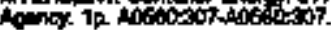

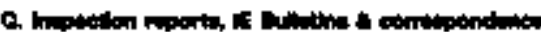

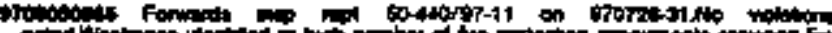

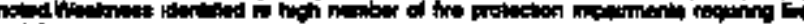

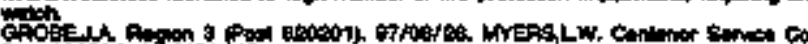

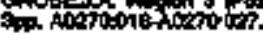

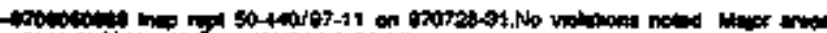

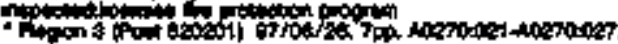

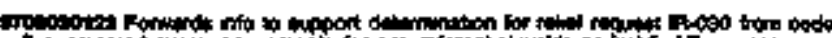

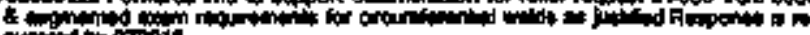

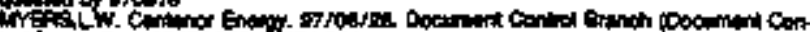

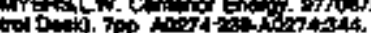

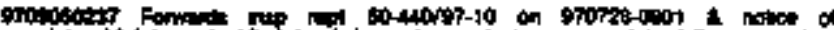

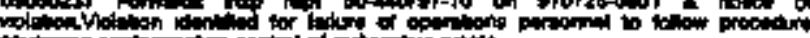

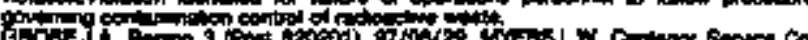

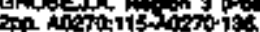

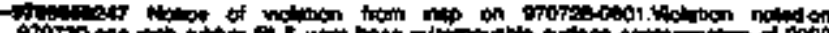

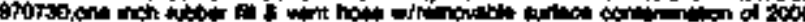

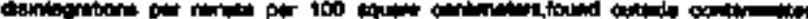

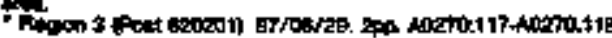

-

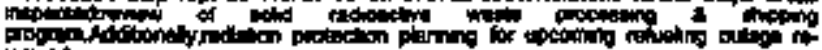

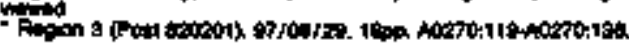

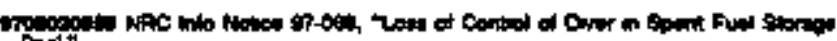
$\sin$

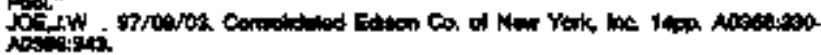

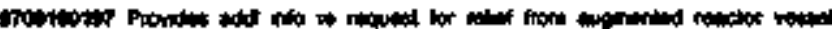

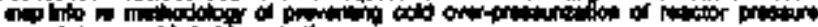

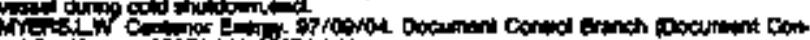

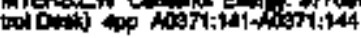

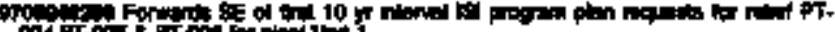

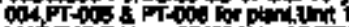

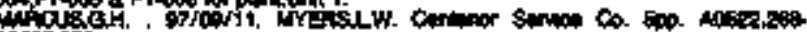
Nitezo.

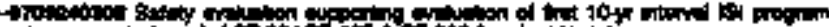

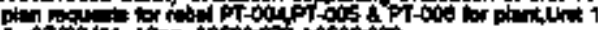

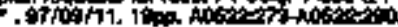

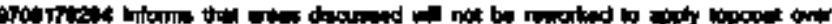

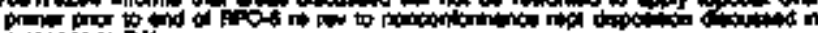
Dim

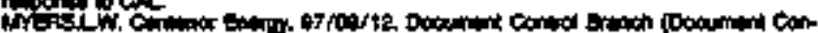

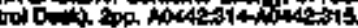

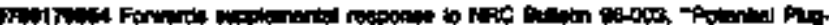

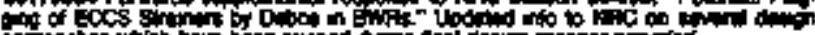

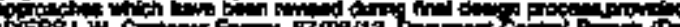

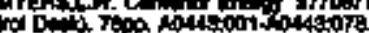

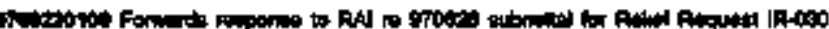

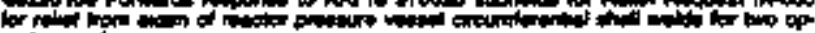

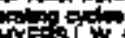

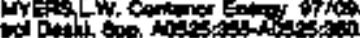

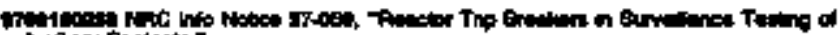

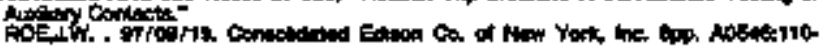
Nositi17.

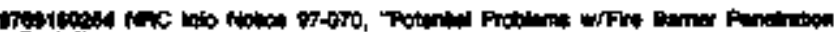

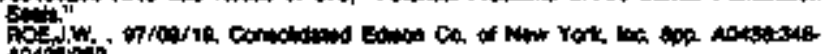

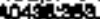

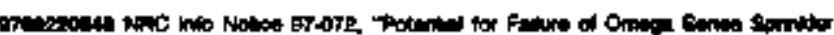

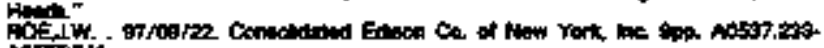

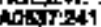

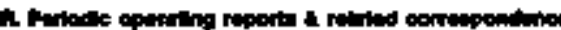

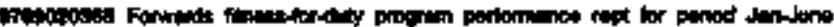

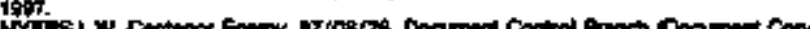

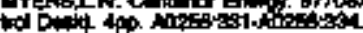

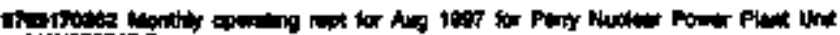

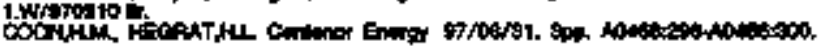

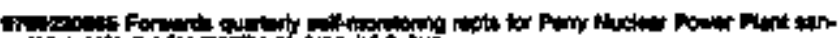

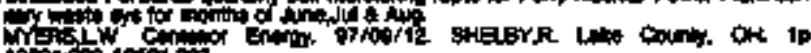
AOP

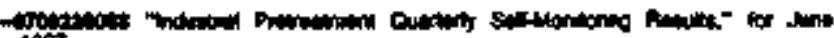

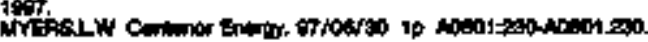

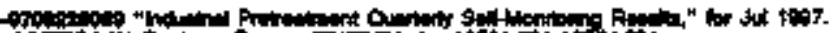

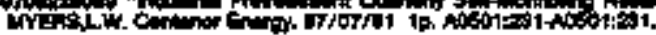

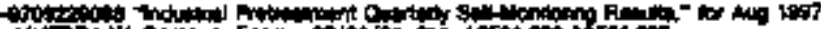

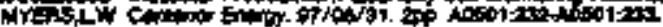

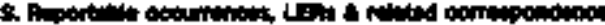

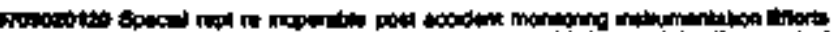

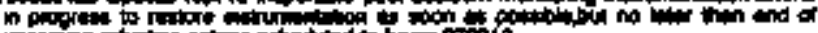

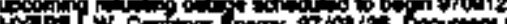

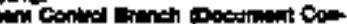

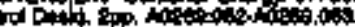

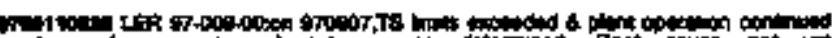

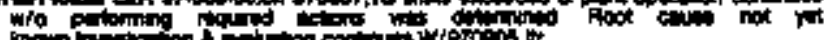

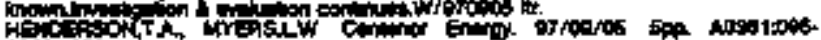
Hition

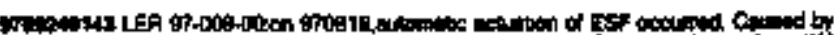

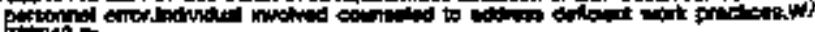

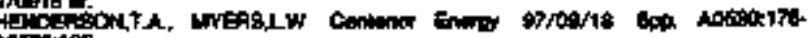
A050:180. 


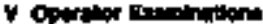

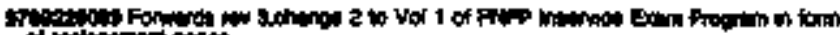

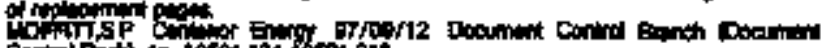

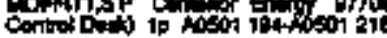

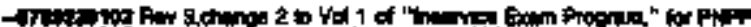

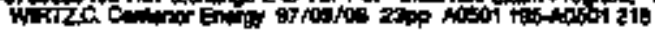

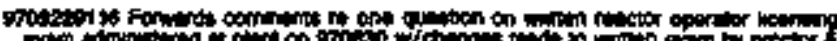

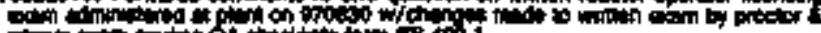

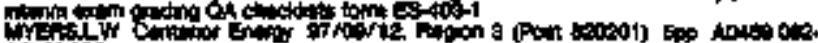
Aomitos

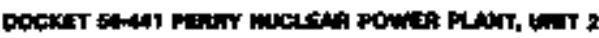

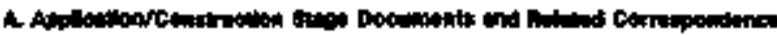

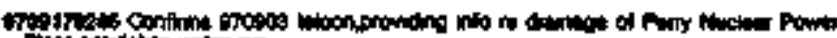

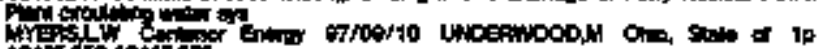

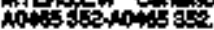

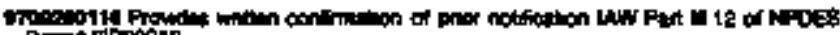
int $30001 \%$

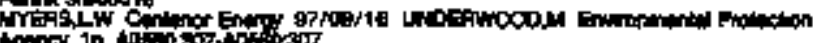

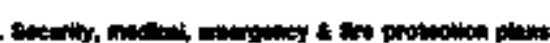

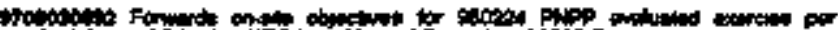

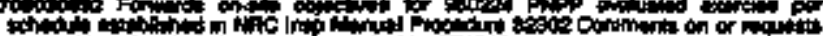

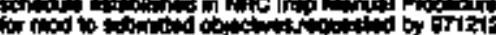

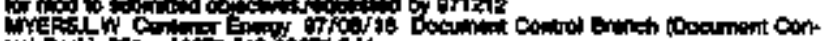

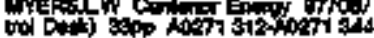

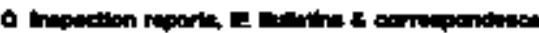

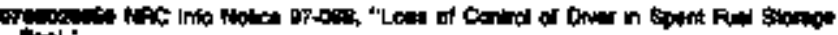

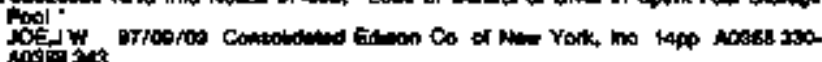

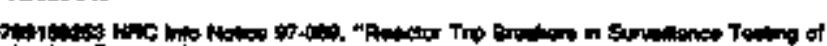

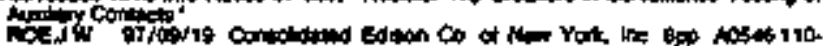
10:0117

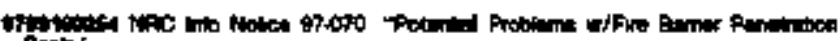

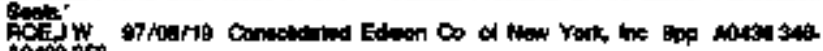
A04so 160

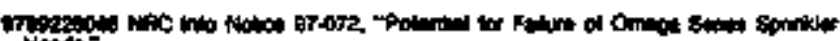

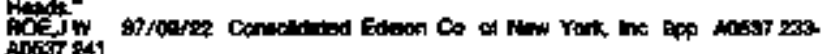

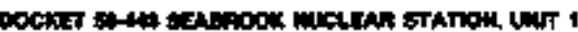

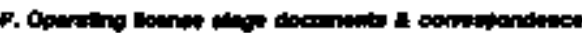

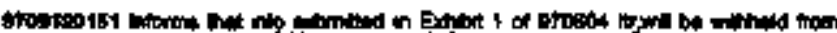

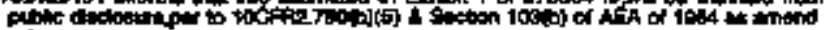

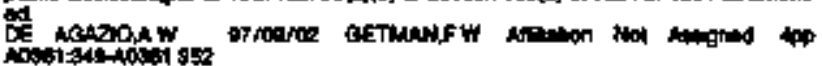

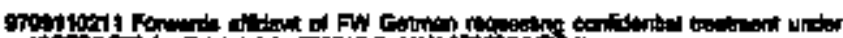

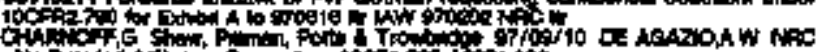

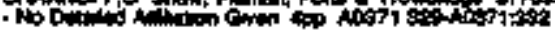

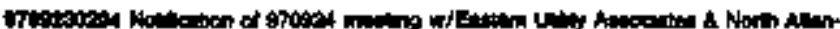

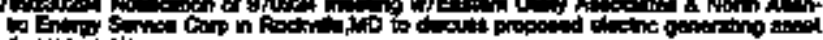

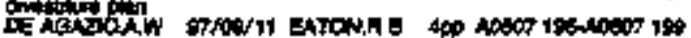

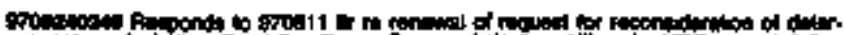

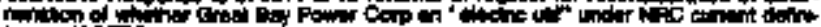
con locFinoz

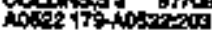

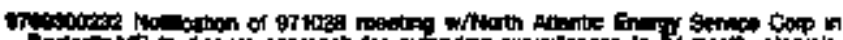

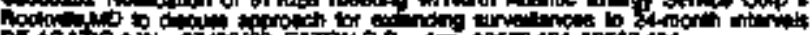

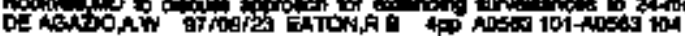

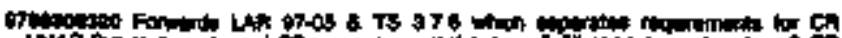

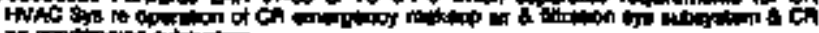

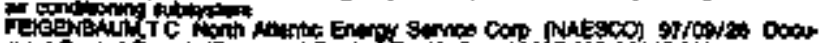

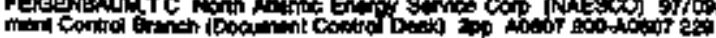

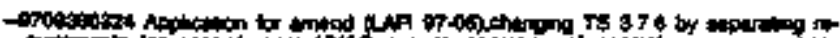

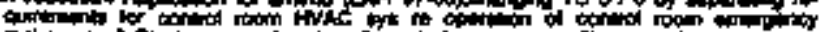

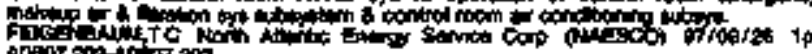

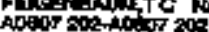

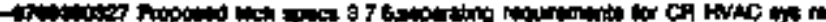

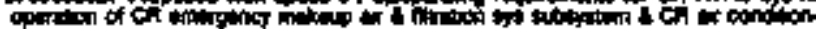

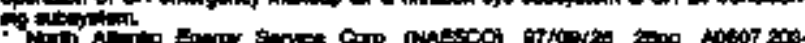
notion 20.

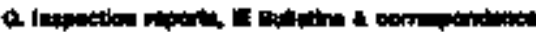

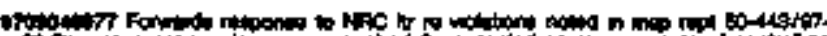

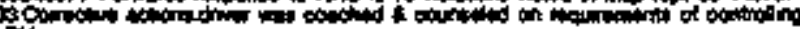

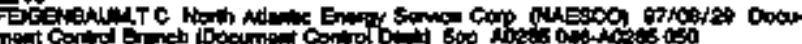

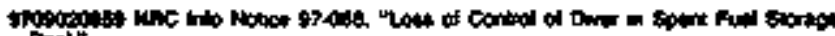
Pool" Nis...

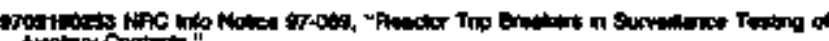

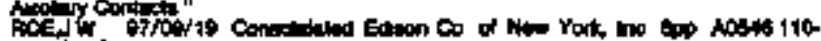
iasis it?

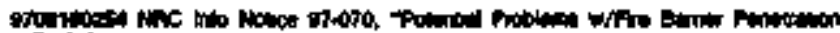

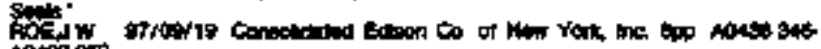
$\ln =0$

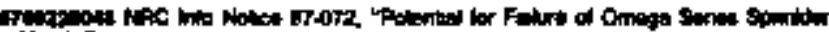

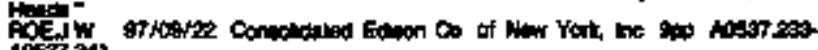
Aiston

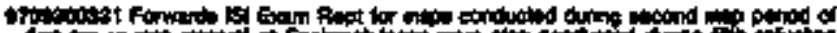

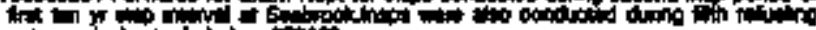

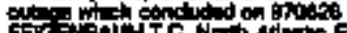

Frow

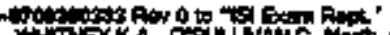

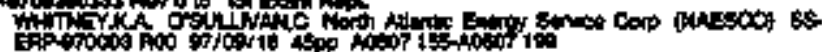

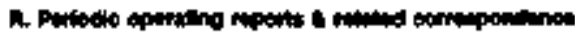

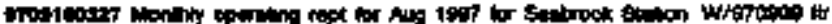

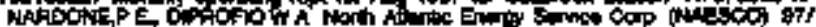

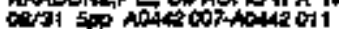

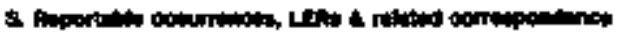

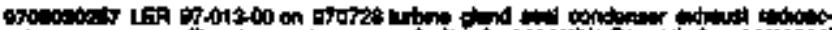

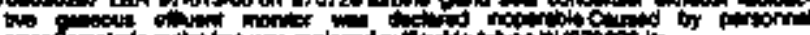

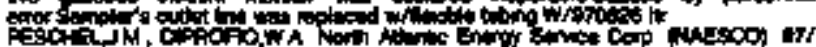
cillow

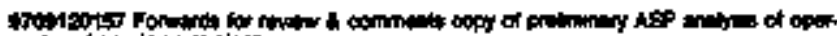

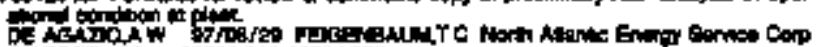

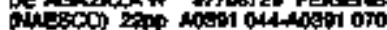

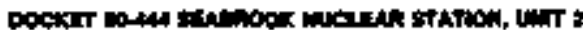

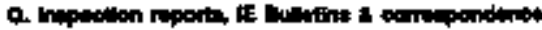

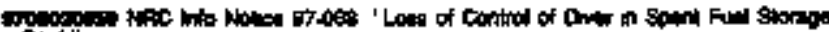

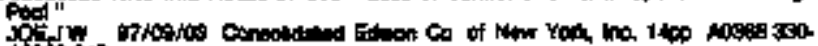

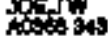

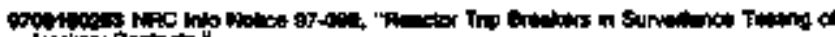

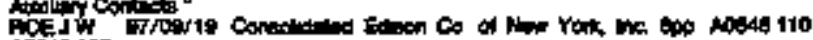
$\lim _{0.117}$

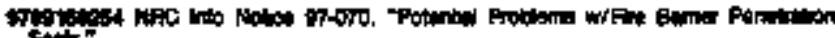

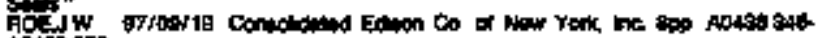
$10+303$

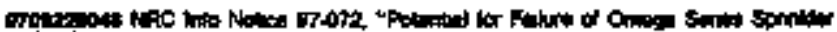

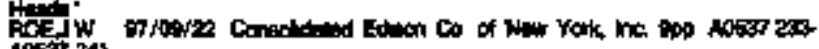
A0sort 24t

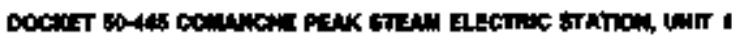

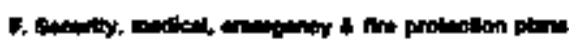

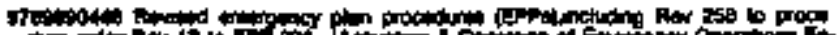

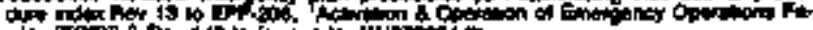

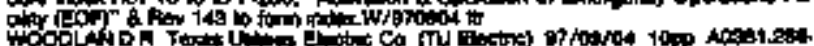
Aoth 19 


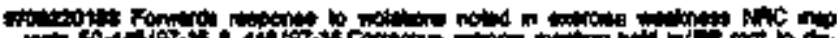

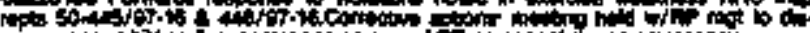
For

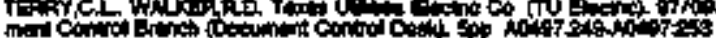

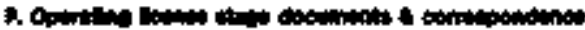

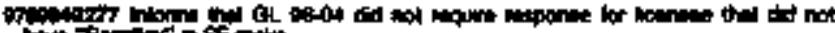

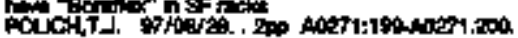

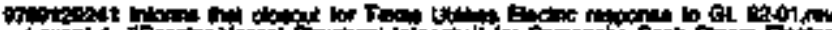

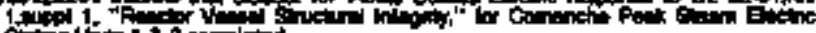

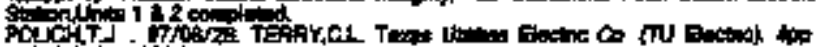

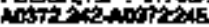

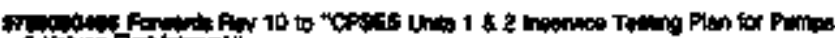

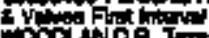

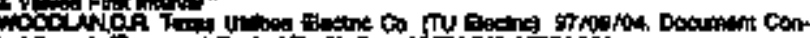

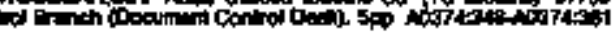

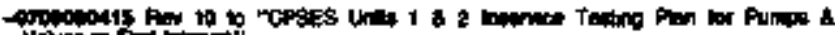

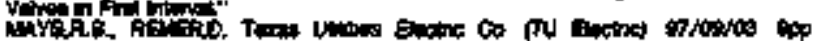

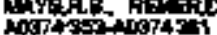

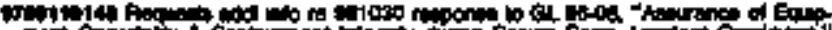

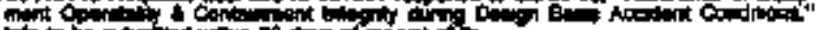

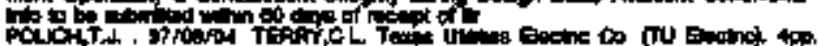

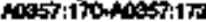

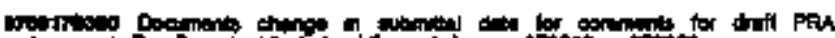

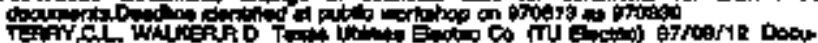
1

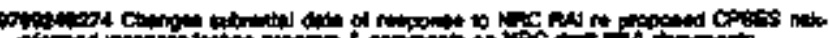

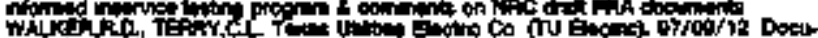

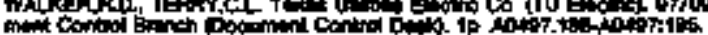

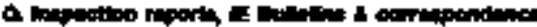

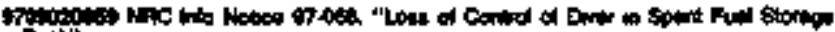

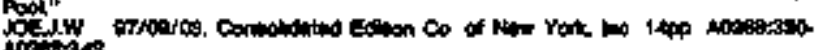

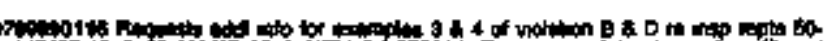

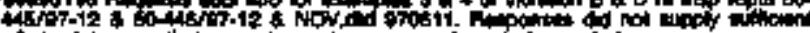

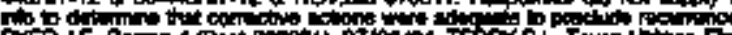

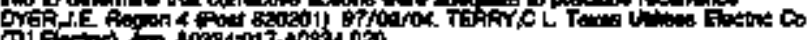

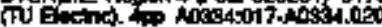

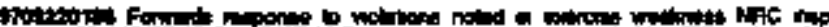

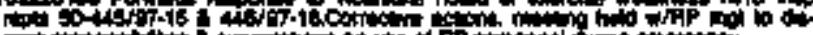
5it

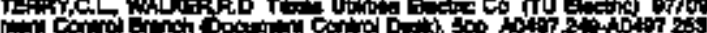

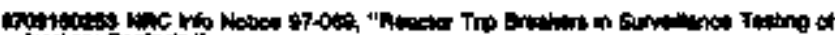

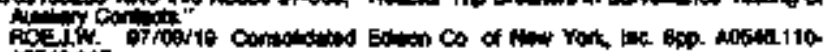
10.40117.

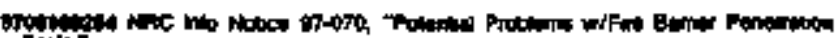

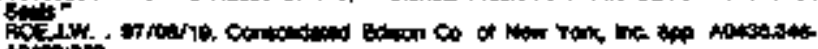
intivise.

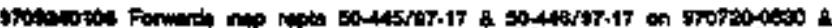

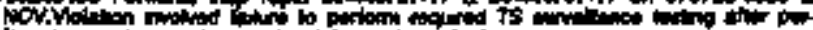

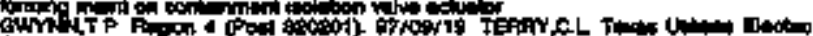

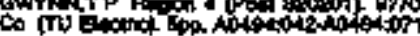

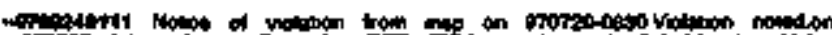

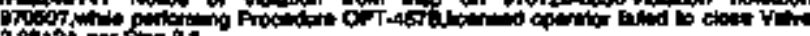

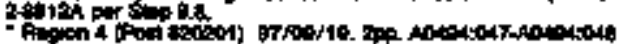

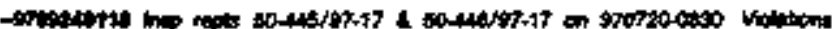

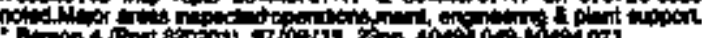

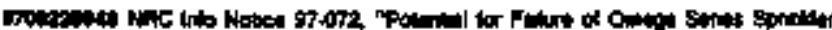

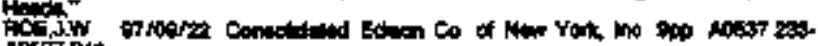
$\cos 3724$.

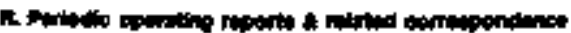

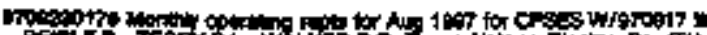

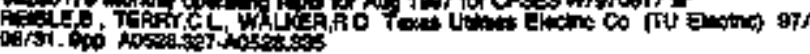

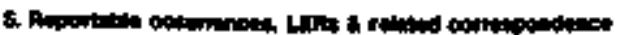

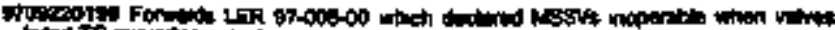

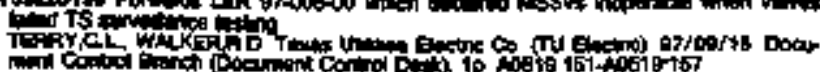

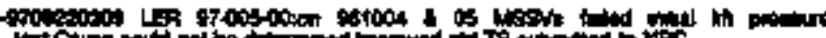

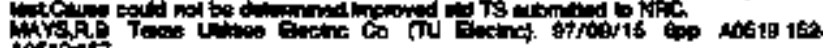
tosionts

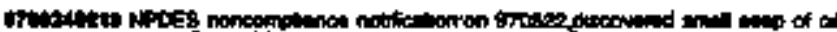

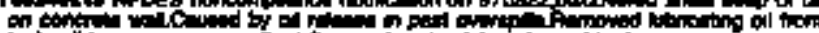

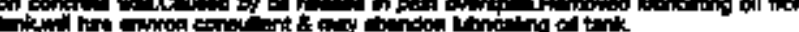

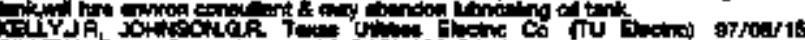

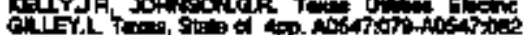

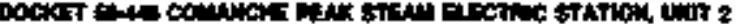

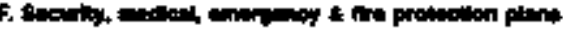

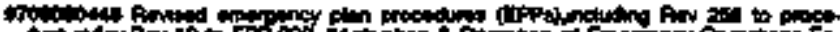

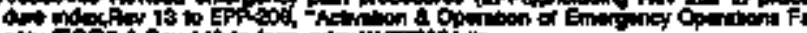

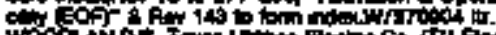
Atotise

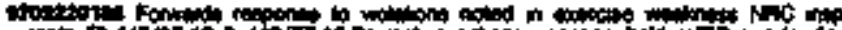

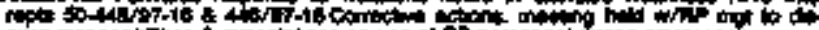

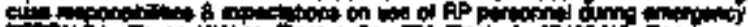

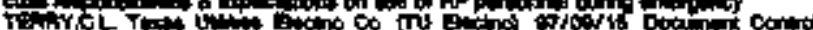

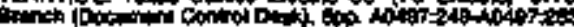

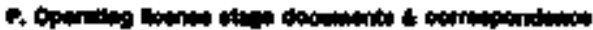

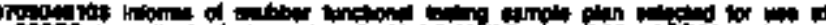
Co

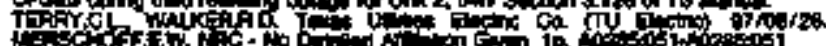

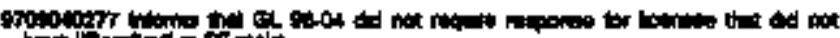

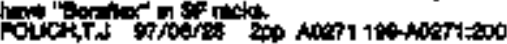

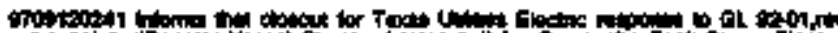

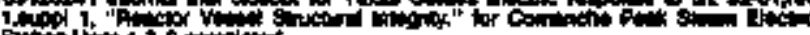

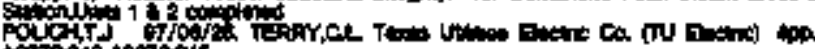

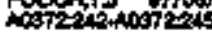

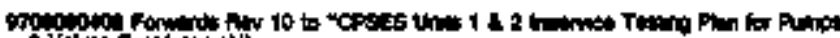

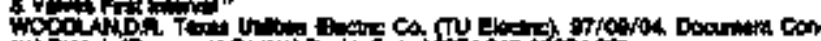

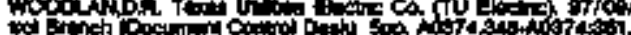

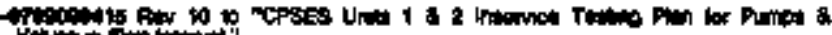

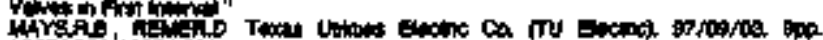
A

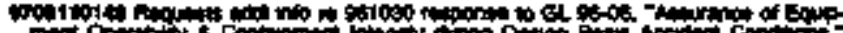

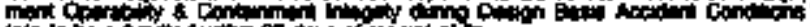

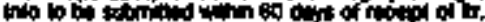

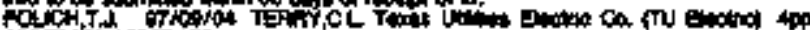
A

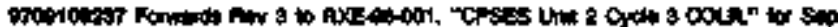

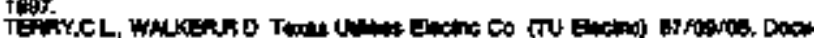

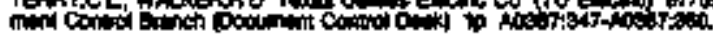

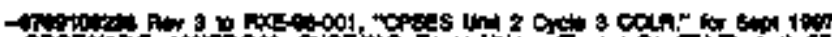
of

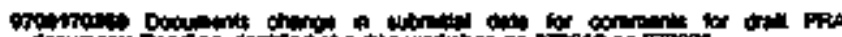

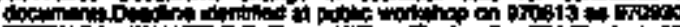

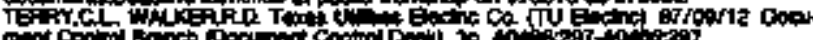

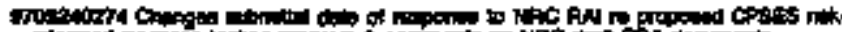

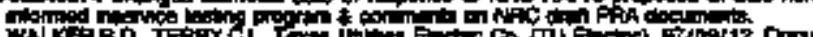

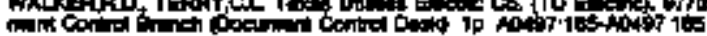

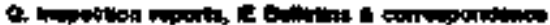

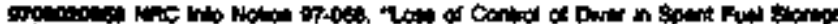

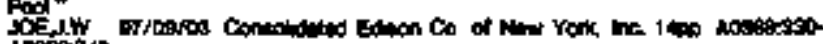
Antas

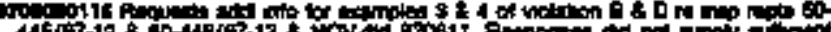

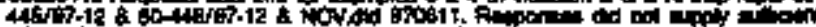

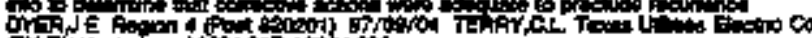

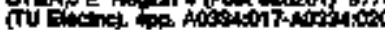

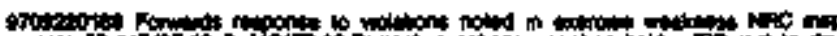

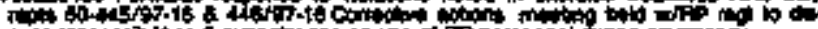

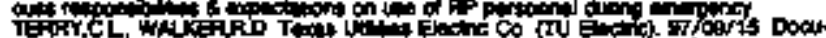

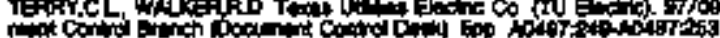

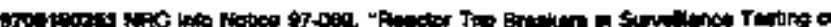

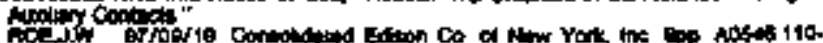
inction 


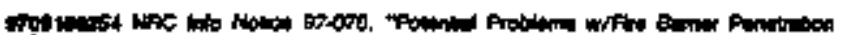

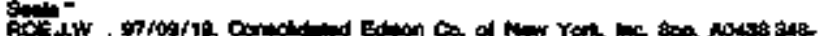
$\cos =0$

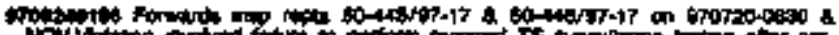

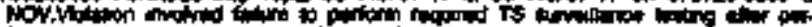

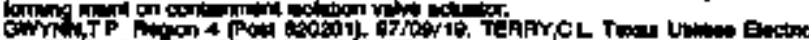

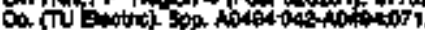

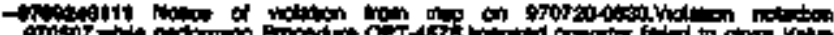

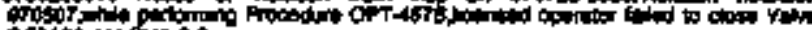

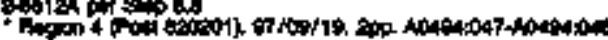

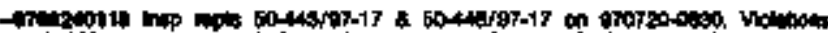

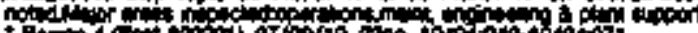

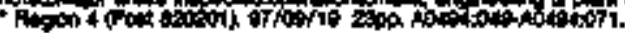

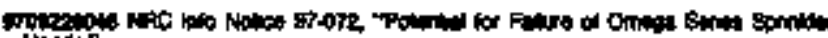

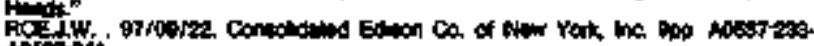
antitit.

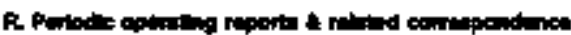

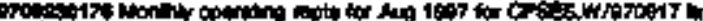

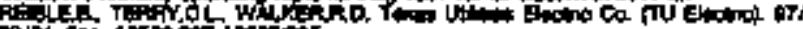

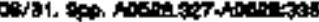

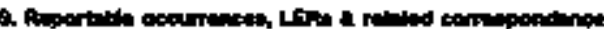

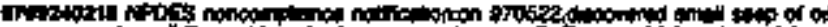

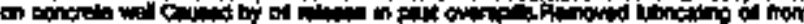

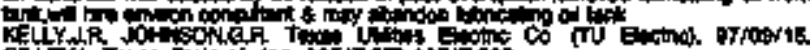

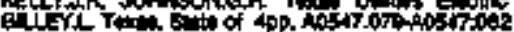

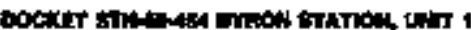

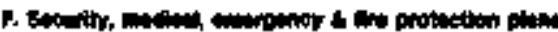

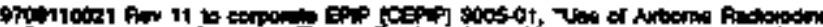

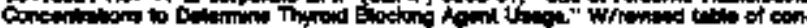

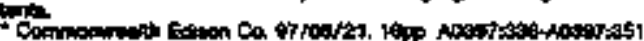

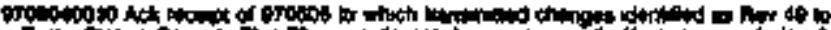

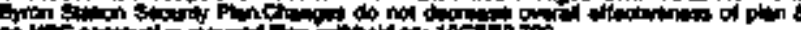

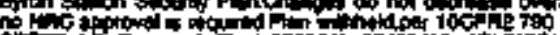

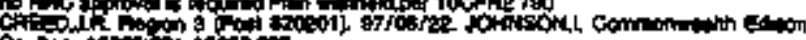

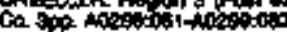

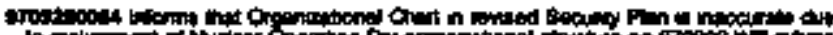

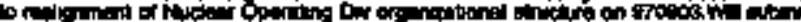

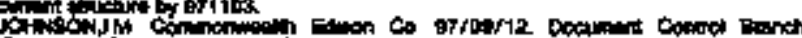

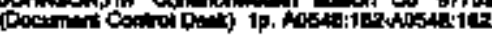

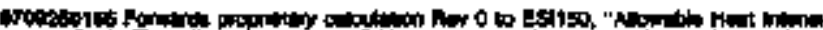

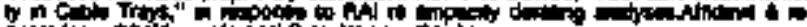

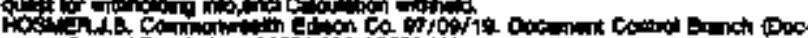

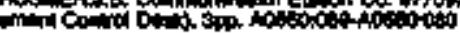

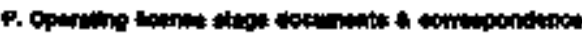

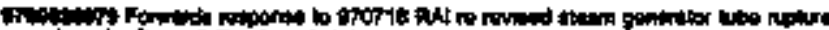

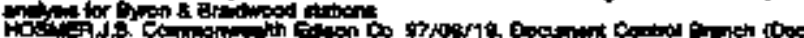

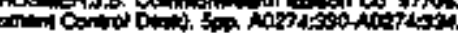

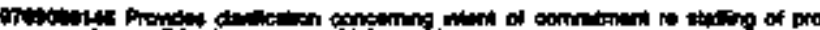

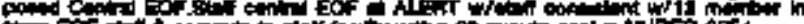

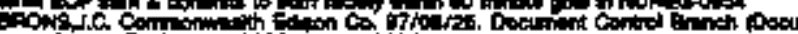

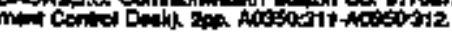

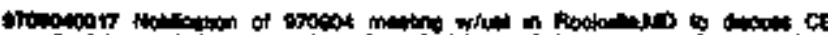

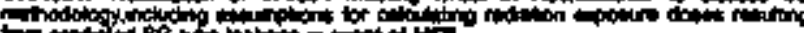

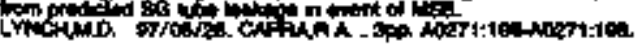

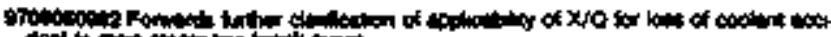

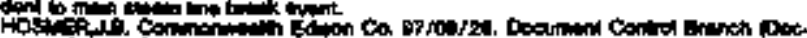

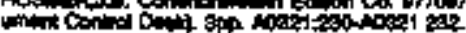

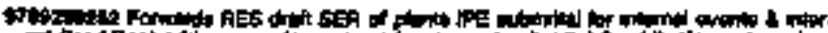

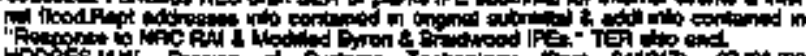

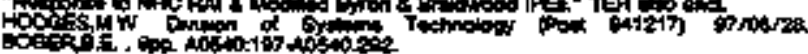

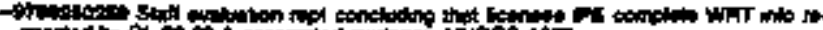

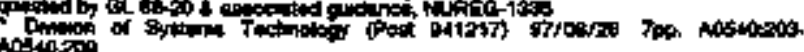

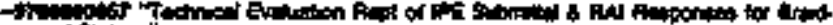

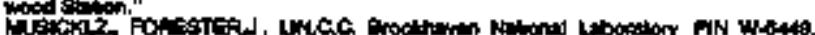

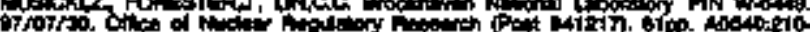

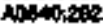

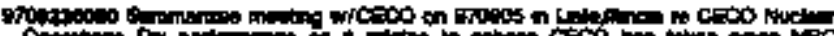

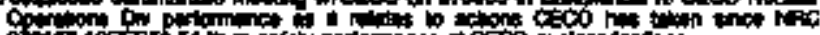

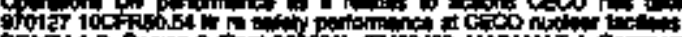

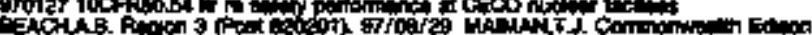

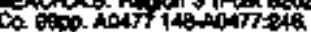

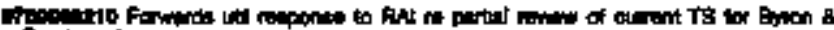

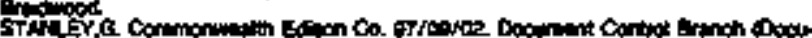

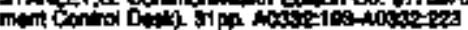

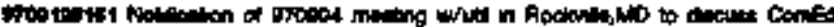

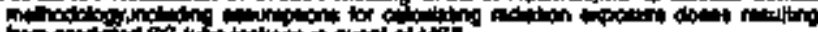

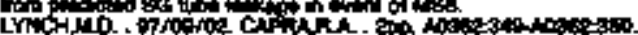

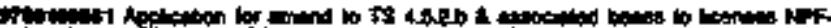

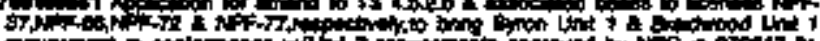

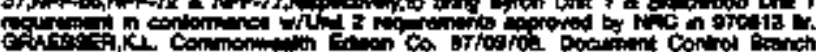

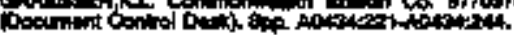

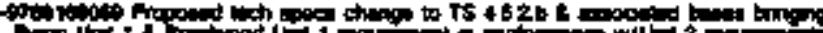

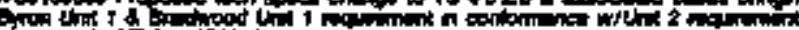

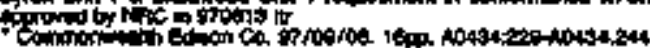

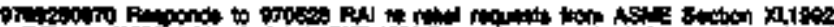

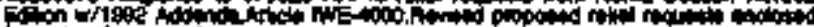

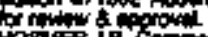

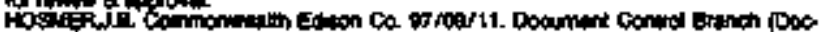

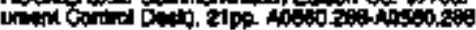

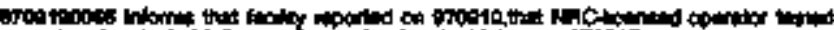

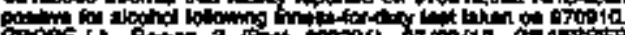

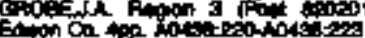

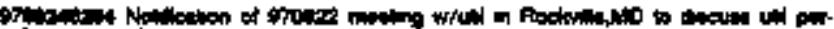

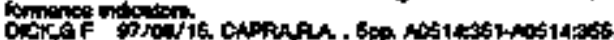

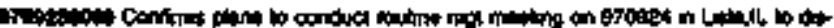

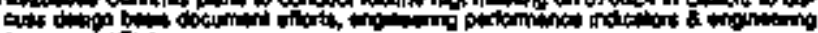

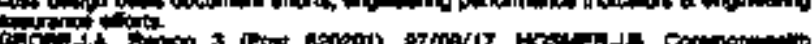
iding to

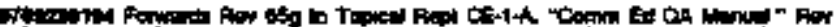

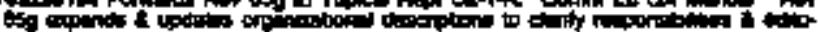

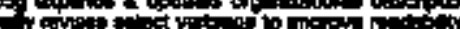

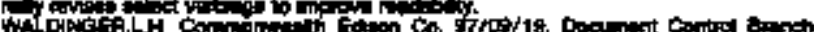

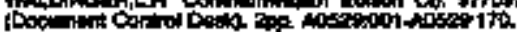

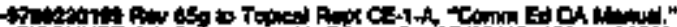

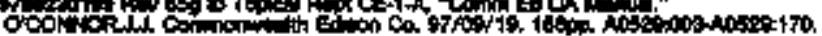

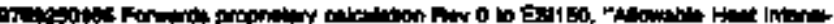

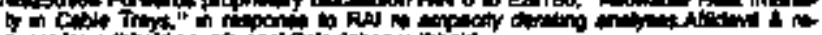

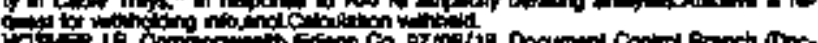

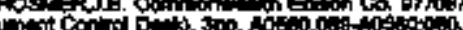

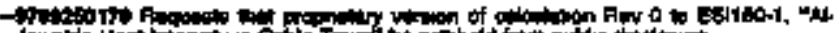

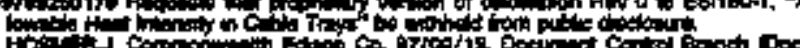

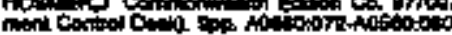

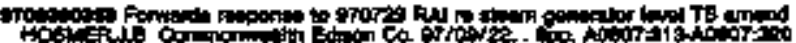

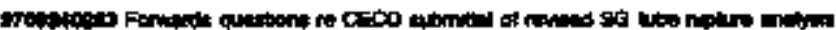

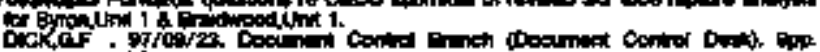

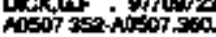

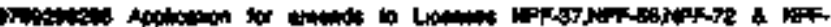

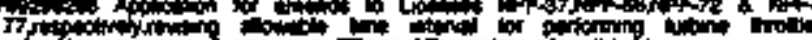

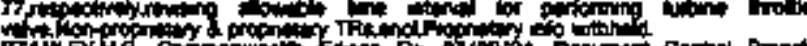

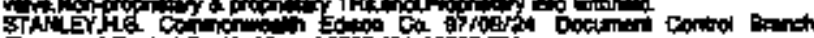

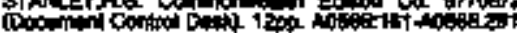

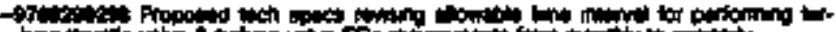

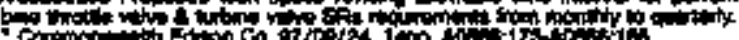

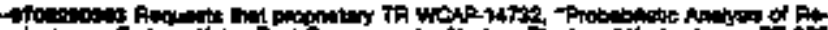

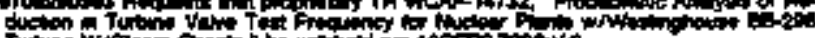

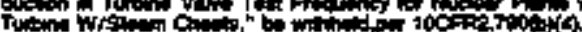

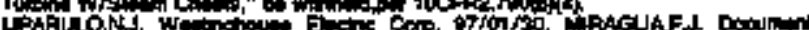

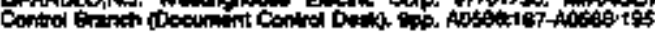

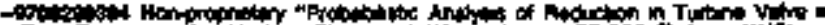

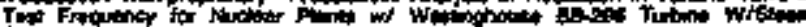

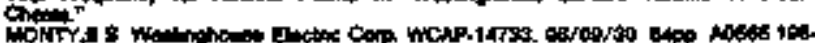
nowest. 


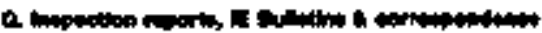

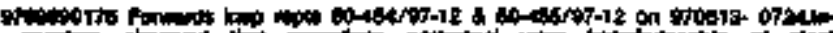

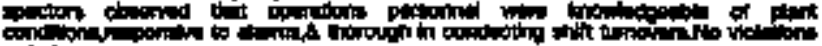
weind

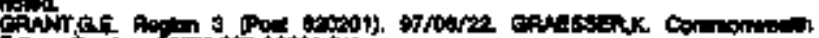

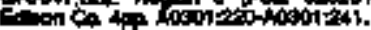

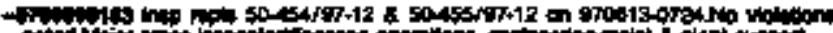

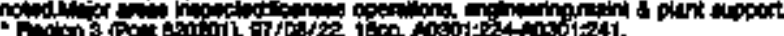

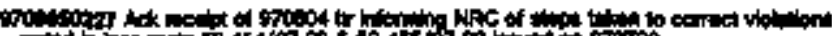

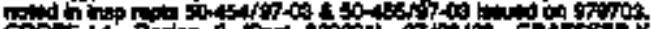

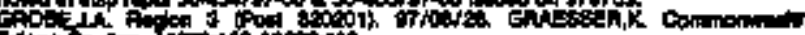

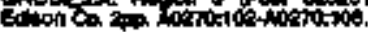

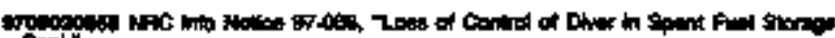

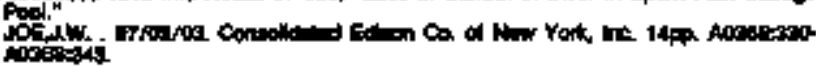

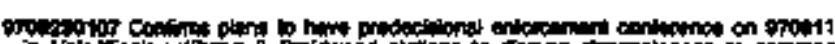

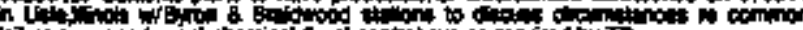

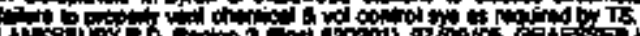

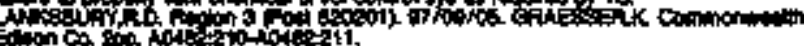

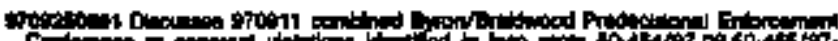

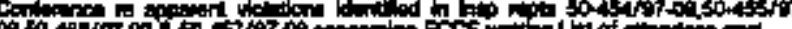

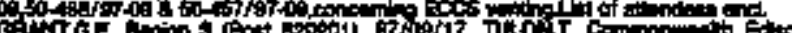
Co. 20.9.

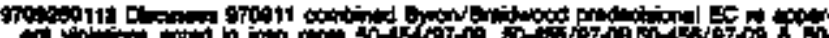

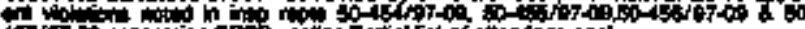

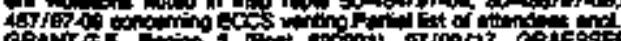
chan Ge

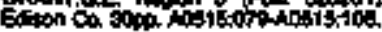

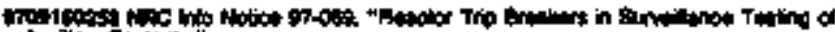

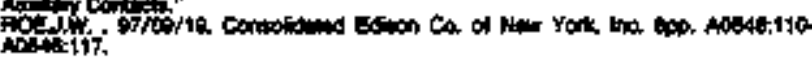

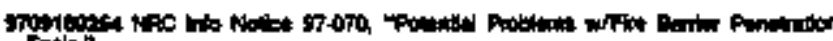

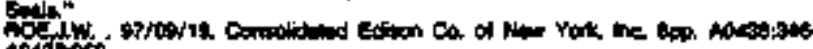
Noditsos.

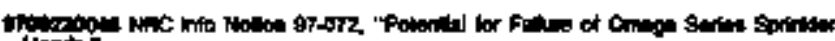

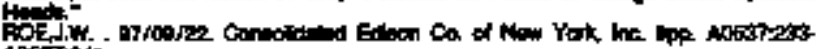
Moshoti.

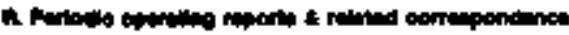

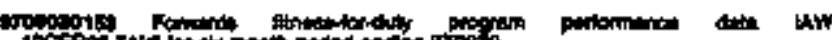

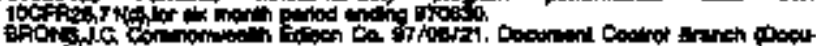

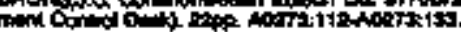

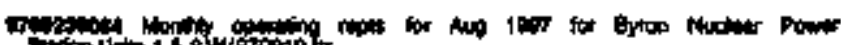

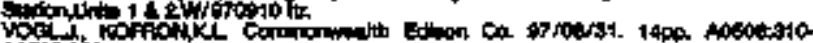

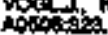

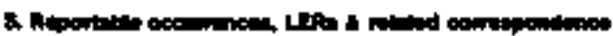

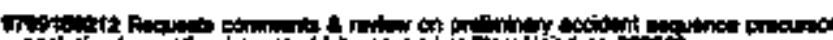

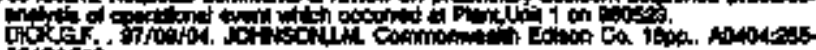
Dofotis:

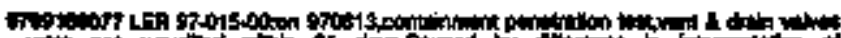

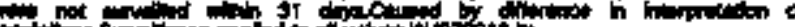
c. inotionst.

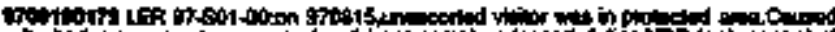

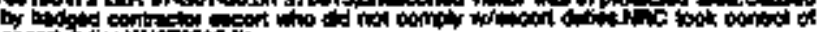

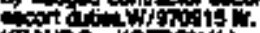

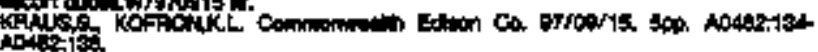

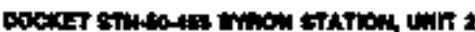

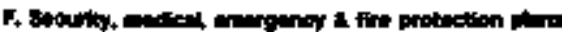

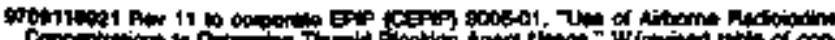

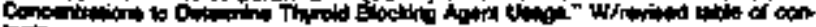
Con

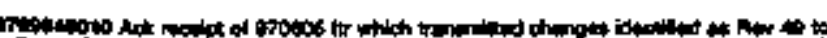

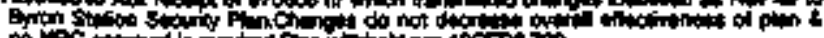

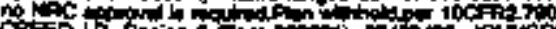

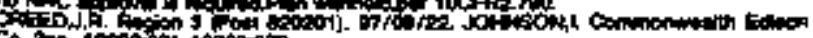

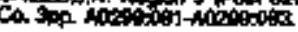

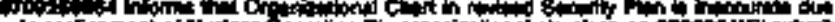

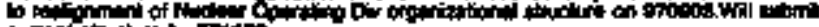

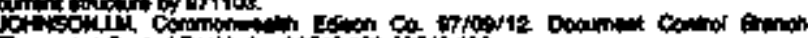

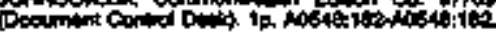

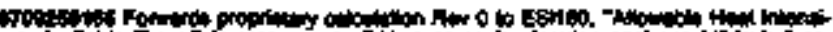

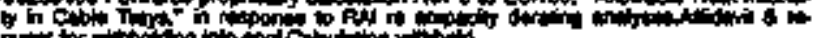

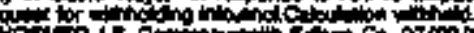

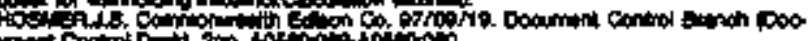

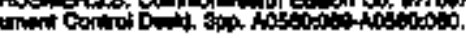

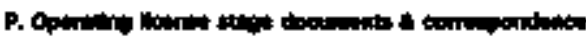

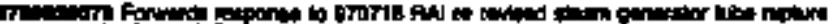

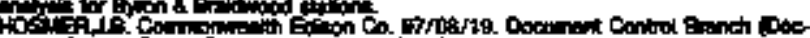

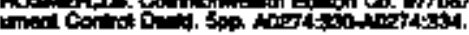

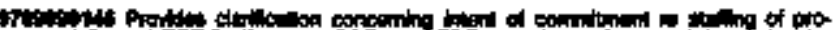

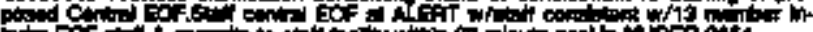

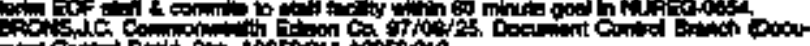

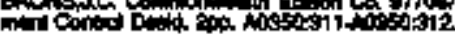

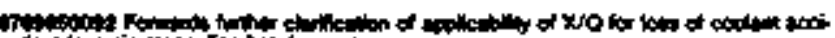

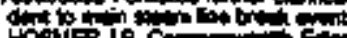

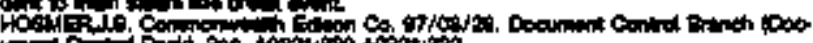

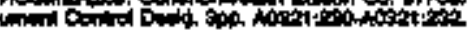

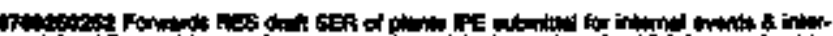

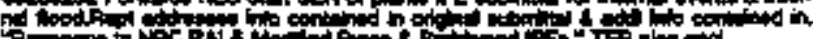

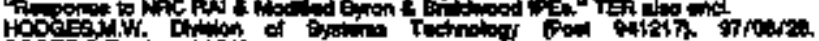

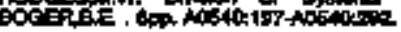

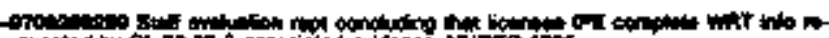

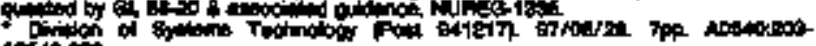
ionition

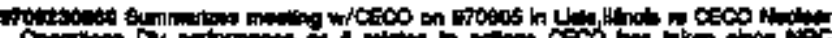
Oponten

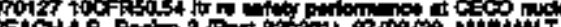

Co wipe

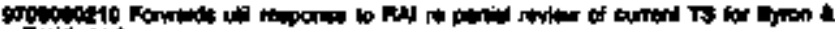

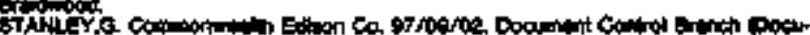

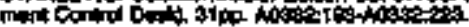

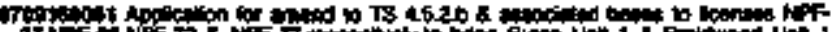

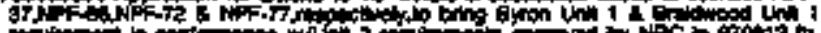

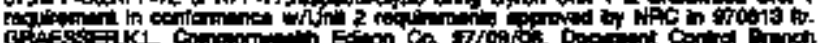

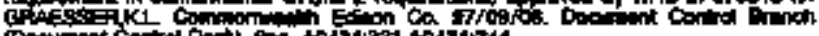

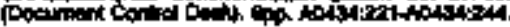

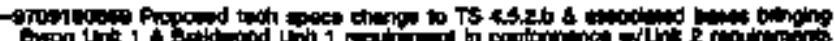

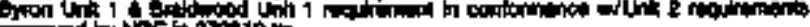

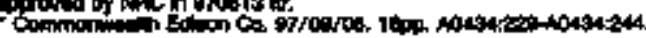

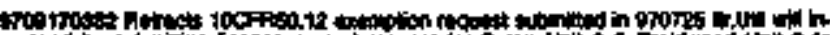

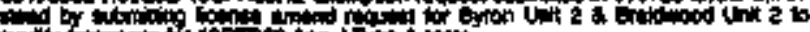
hod"

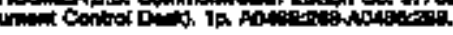

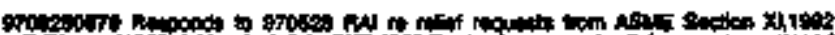

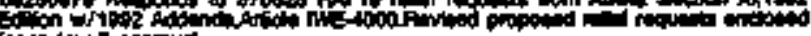

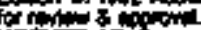

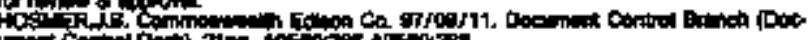

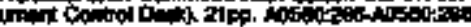

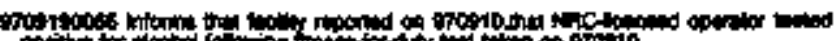

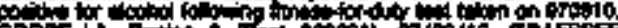

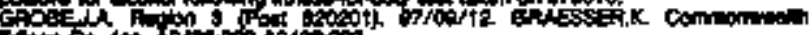

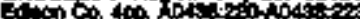

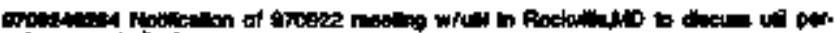

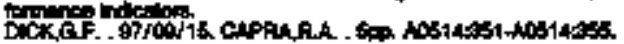

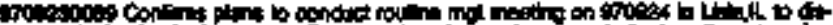

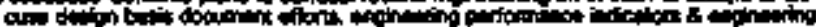

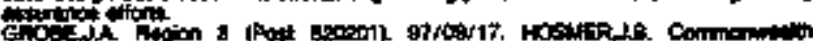

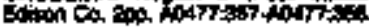

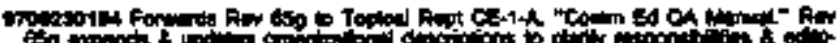

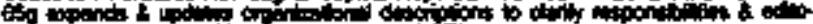

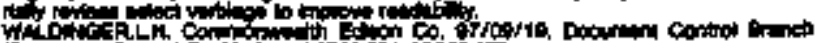

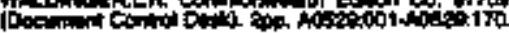

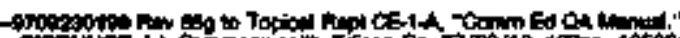

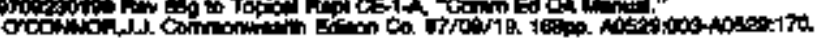

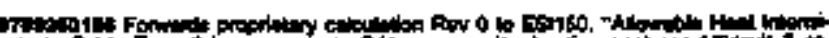

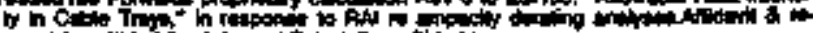

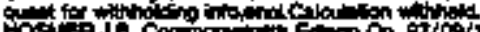

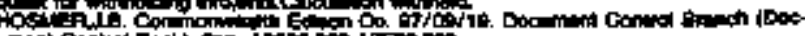

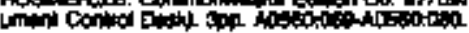




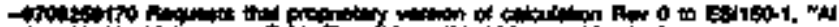

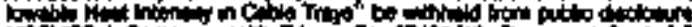

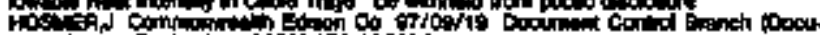

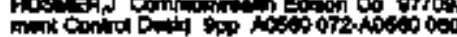

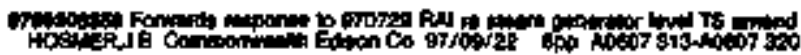

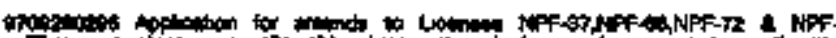

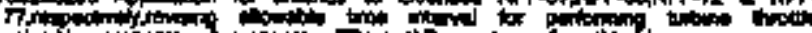

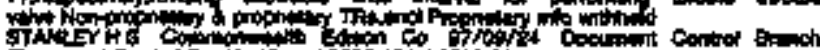

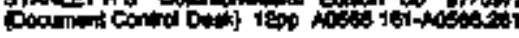

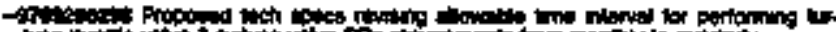

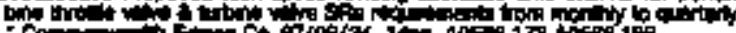

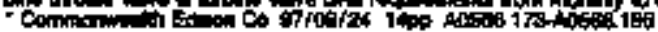

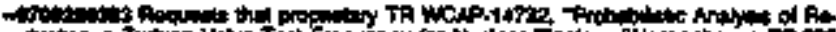

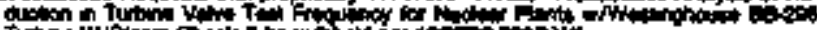

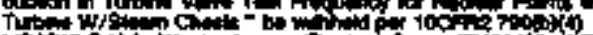

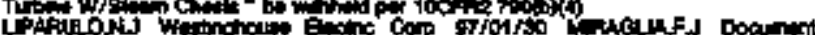

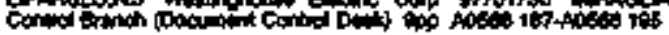

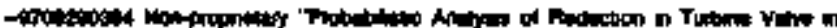

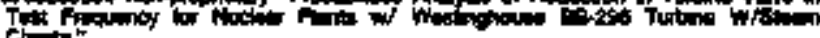

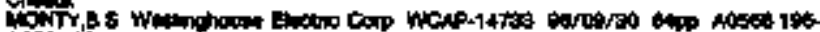
Achos

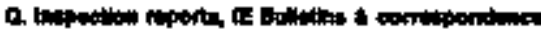

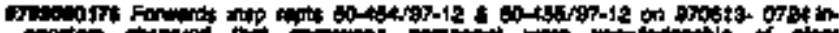

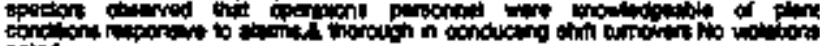
noind

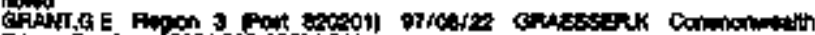

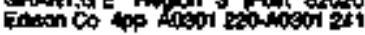

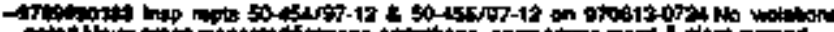

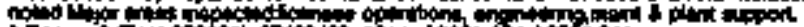

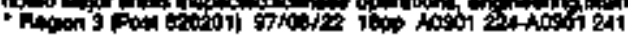

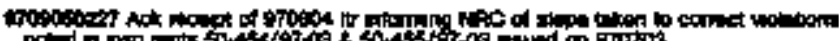

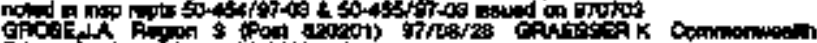

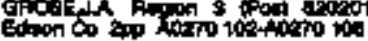

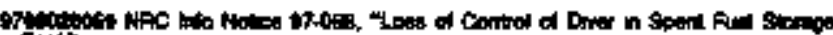

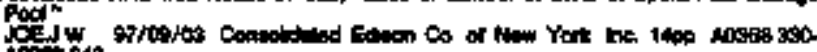
AT. 849

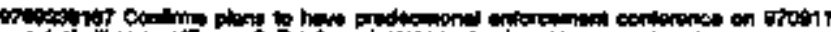

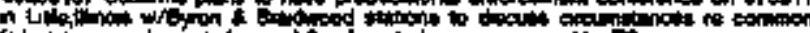

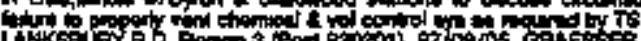
UAKKSOU

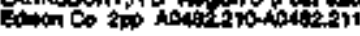

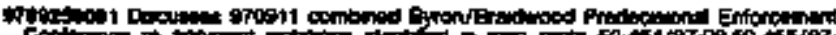

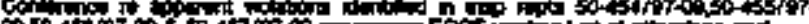

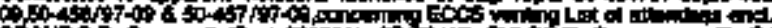

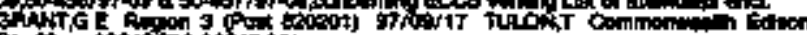
con 60 in

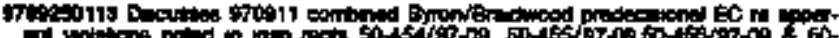

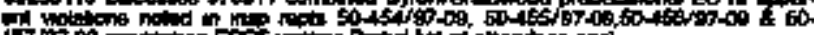
of

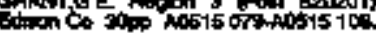

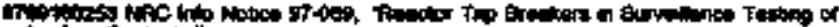

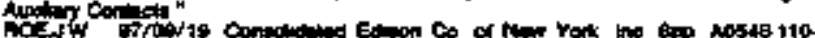
notion

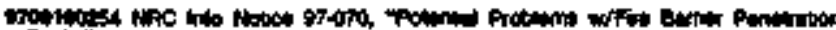

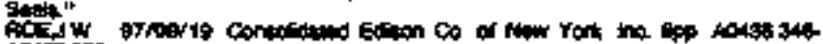

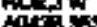

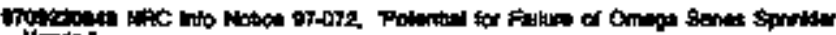

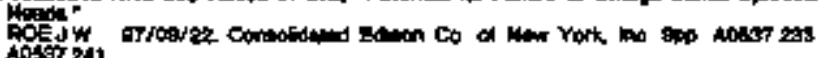

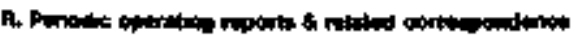

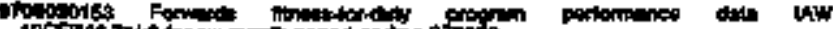

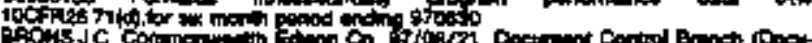

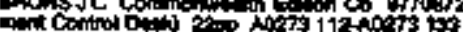

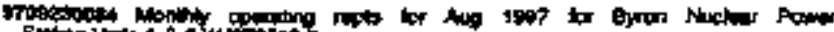

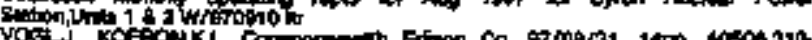

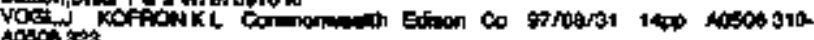

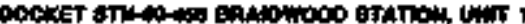

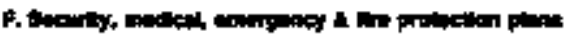

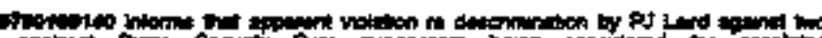

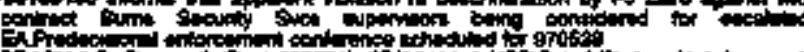

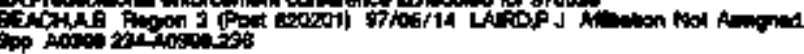

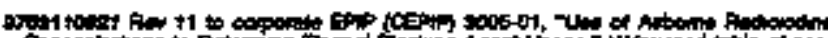

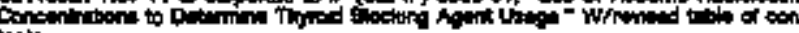
tion

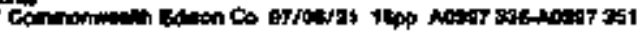

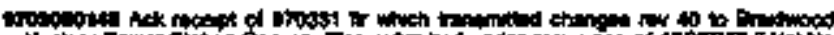

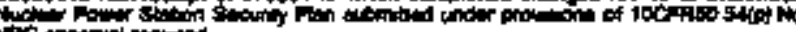

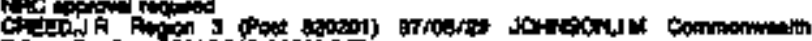

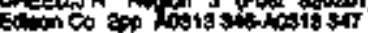

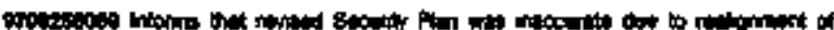

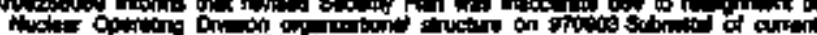

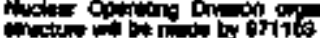

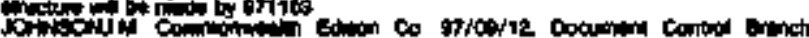

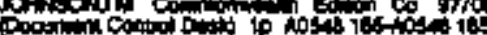

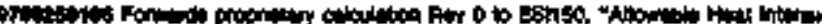

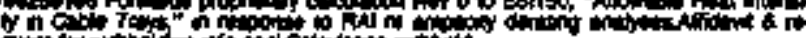

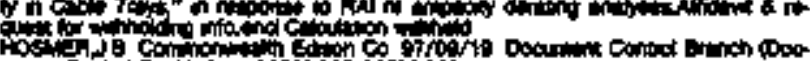
Q

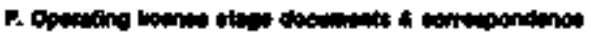

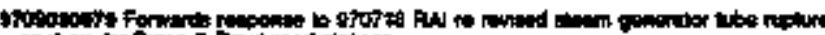

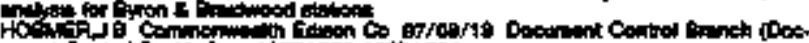

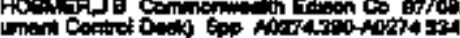

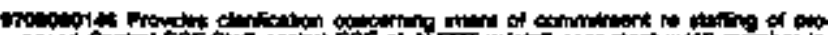

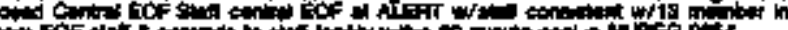

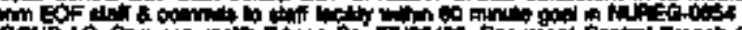

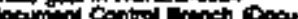

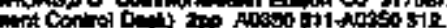

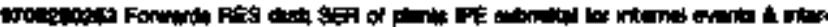

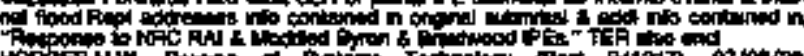

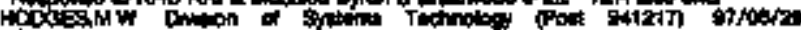

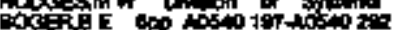

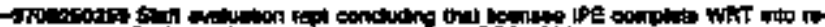

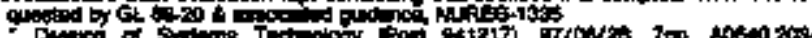
Ansingen

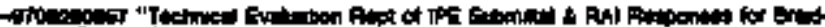

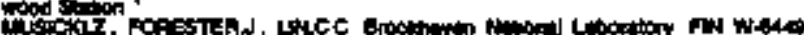
of 104079

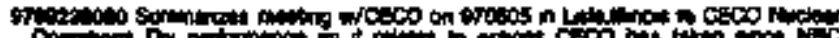

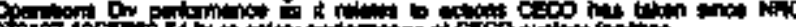
oflis 10c

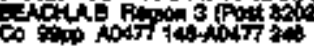

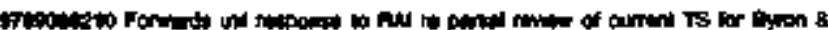

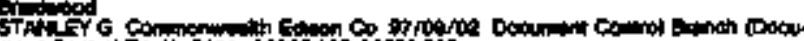

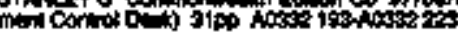

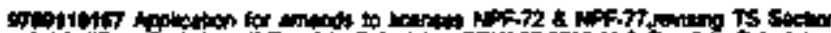

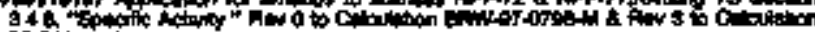

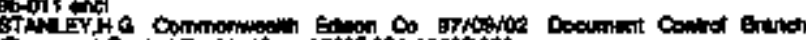

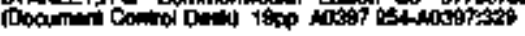

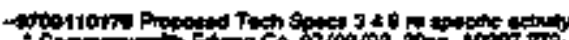

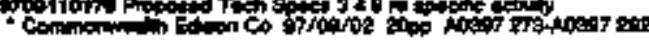

-

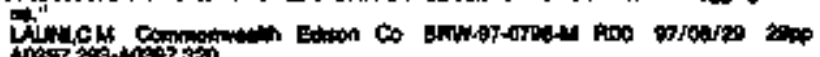

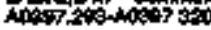

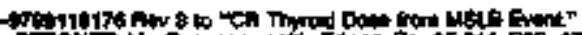

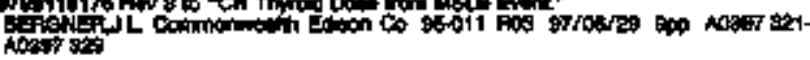

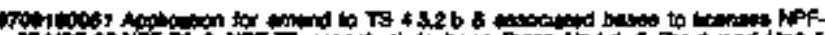

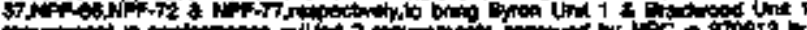

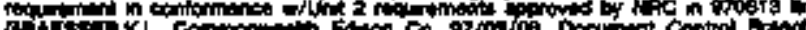

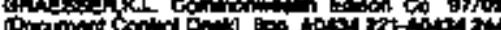

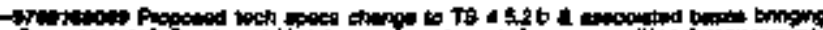

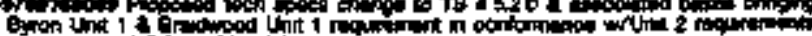

Frowed by toc in ghosis to 


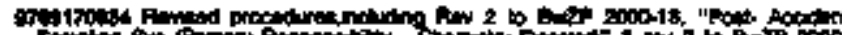

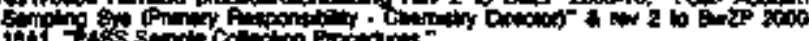

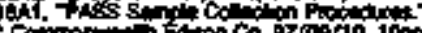

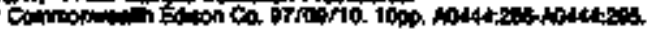

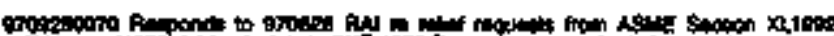

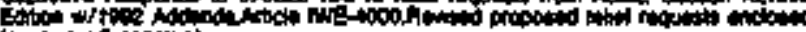

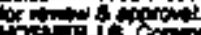

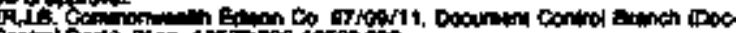
等

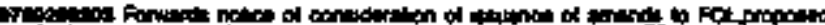

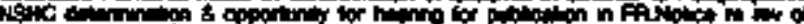

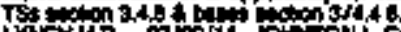
totist

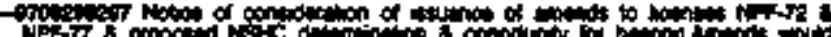

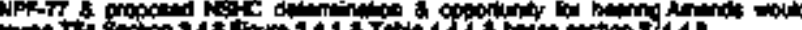

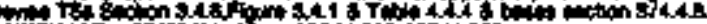

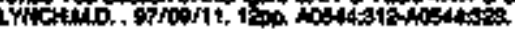

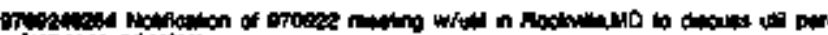

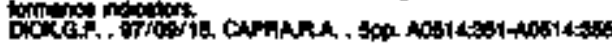

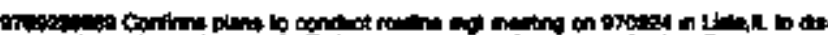

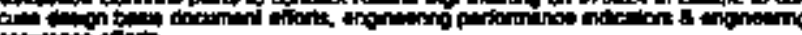

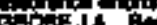

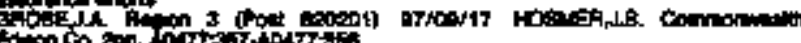

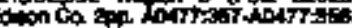

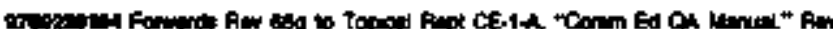

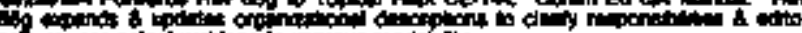

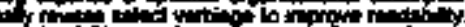

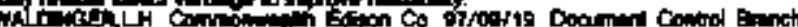

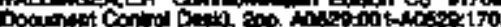

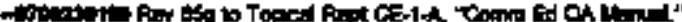

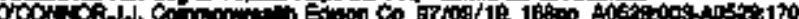

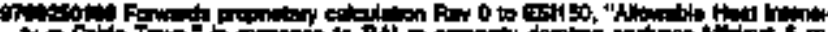

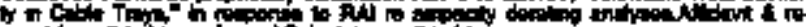

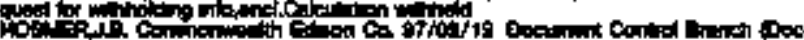

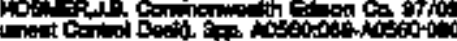

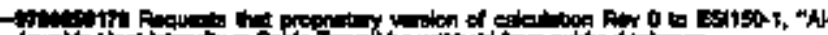
Ho in

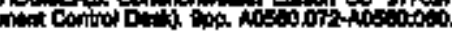

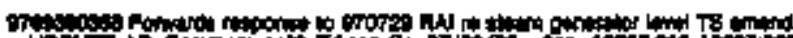

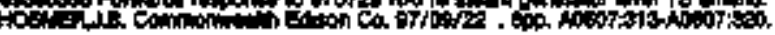

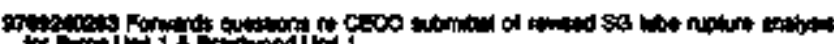

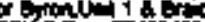

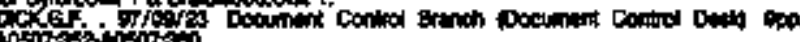

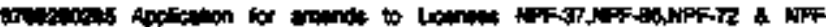

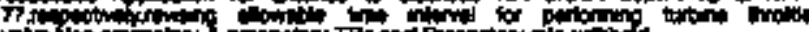

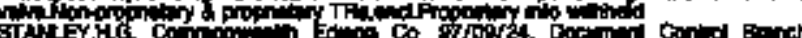

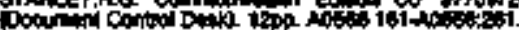

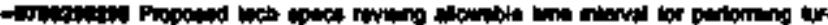

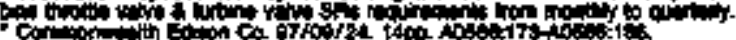

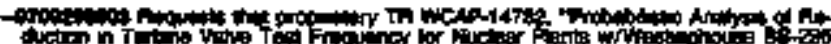

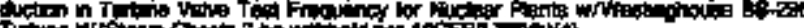

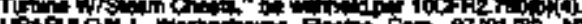

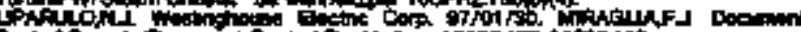

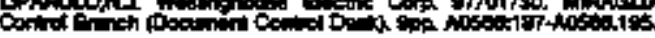

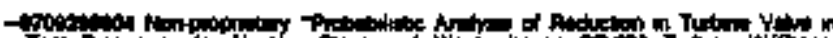

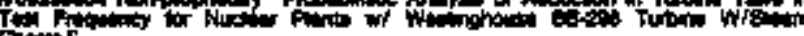

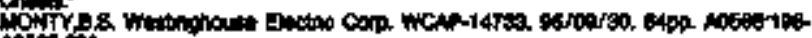
not.

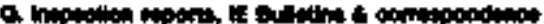

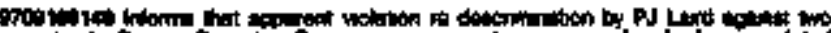

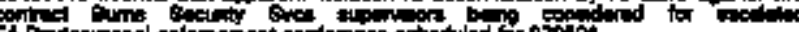

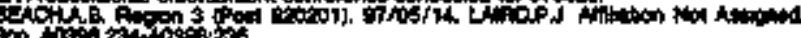

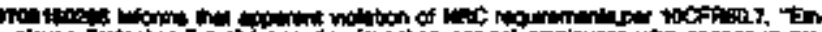

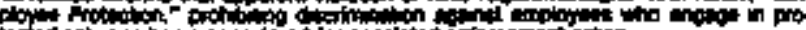

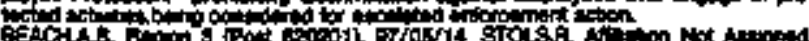

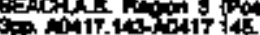

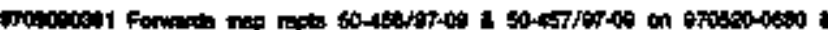

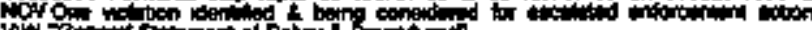
AN

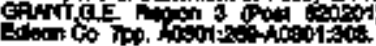

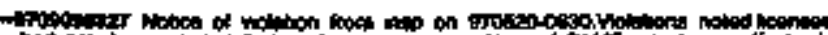

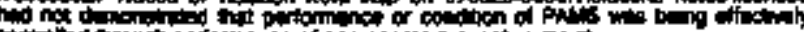

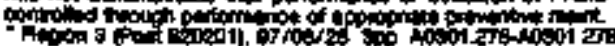

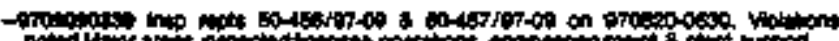

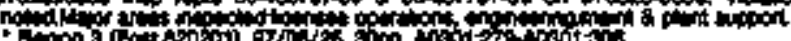

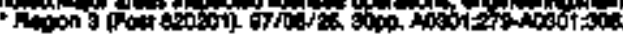

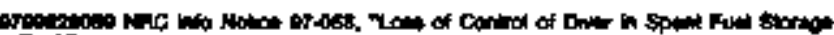
nod

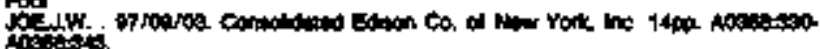

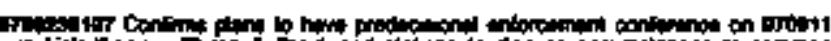

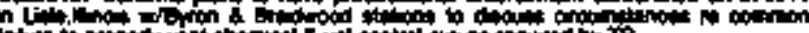

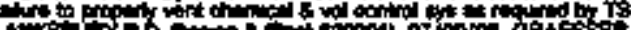

Ak Commennom

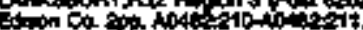

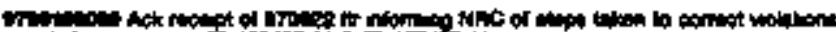

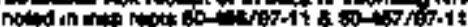

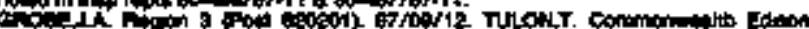

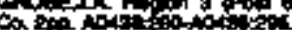

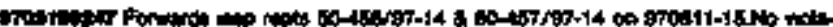

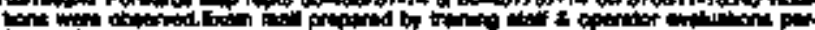

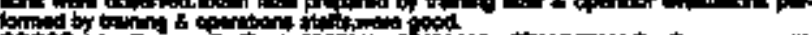

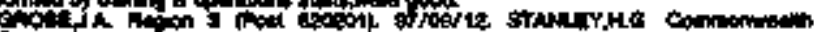

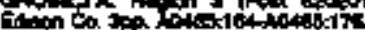

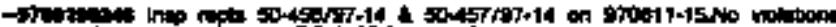

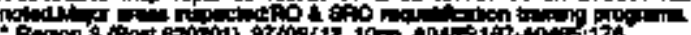

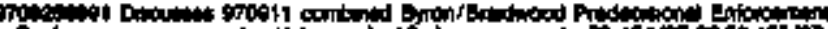

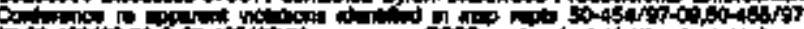

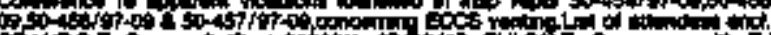

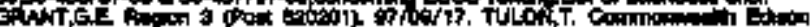

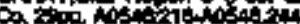

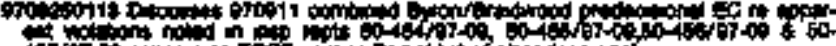

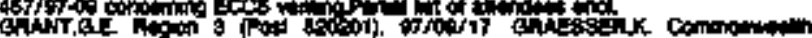

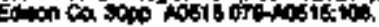

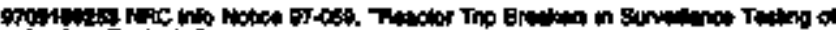

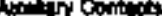
Fic

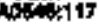

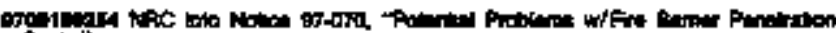
A

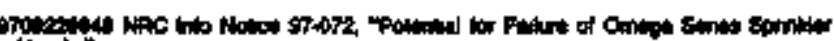

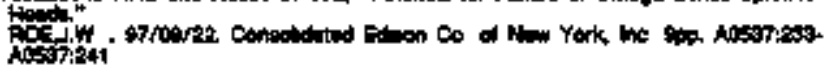

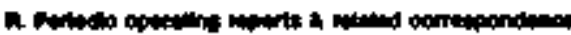

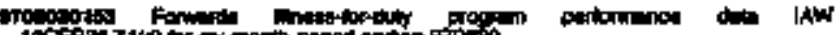

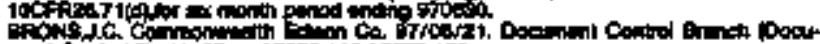

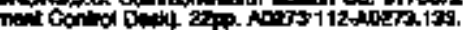

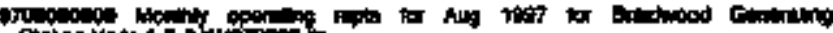

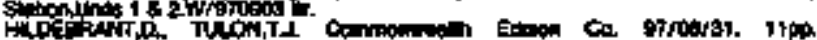

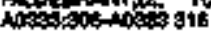

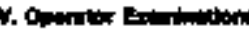

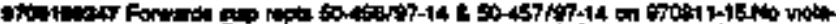

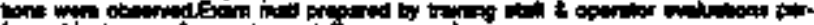

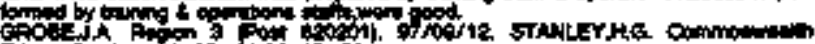

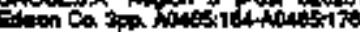

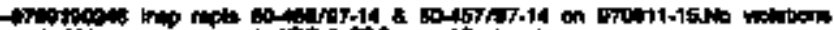

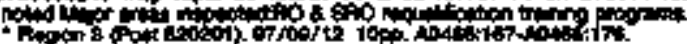

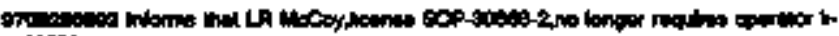

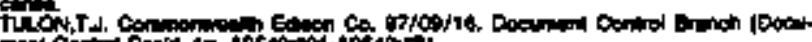

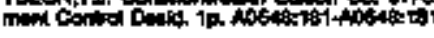

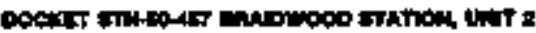

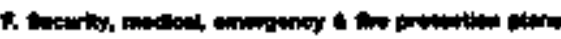

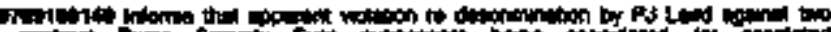

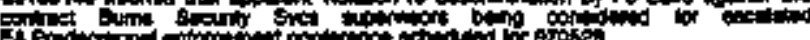

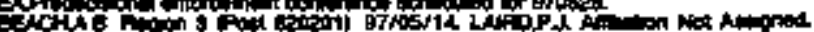

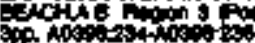

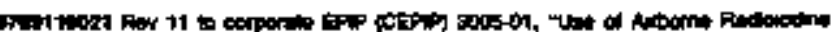

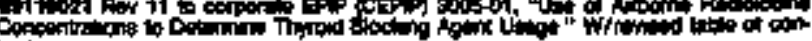

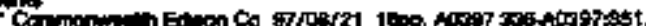




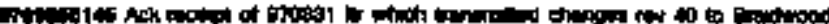

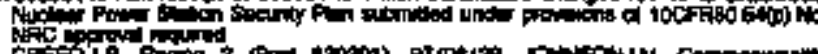

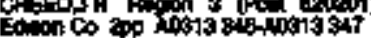

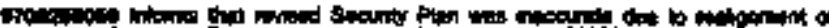

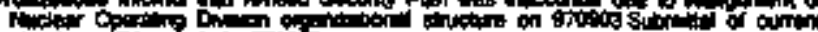

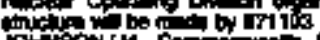

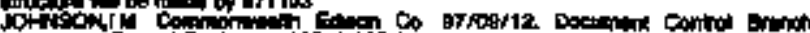

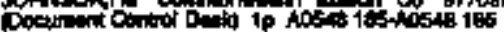

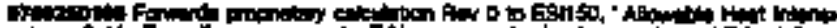

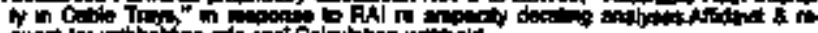

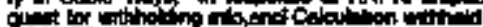

Oot

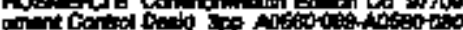

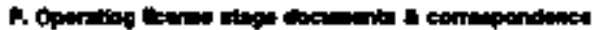

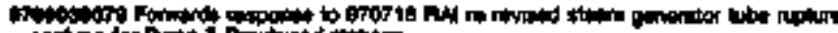

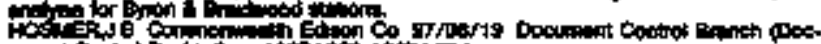

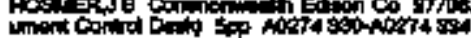

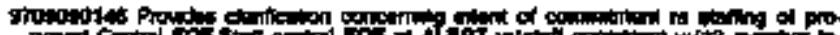

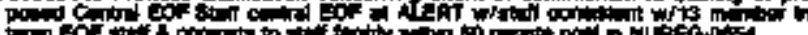

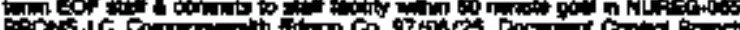

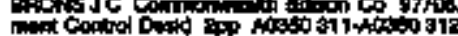

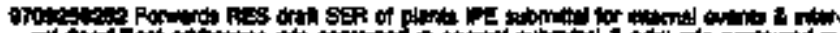

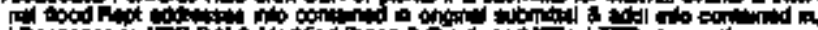

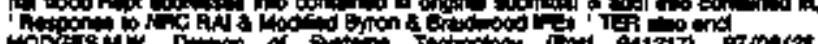

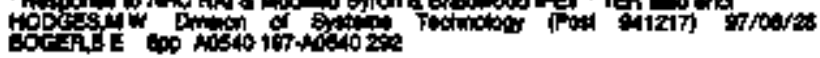

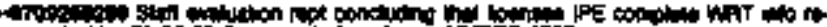

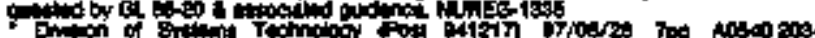
istongs

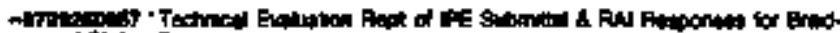
Food batin"

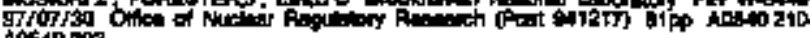
d0640290

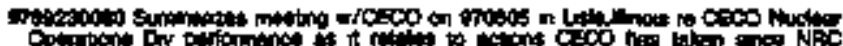

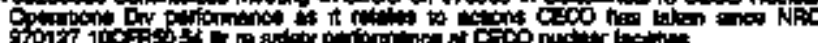

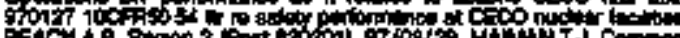

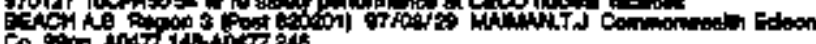

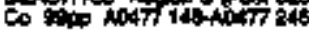

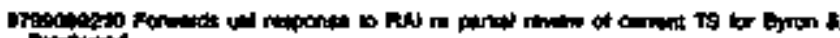
Bitiod

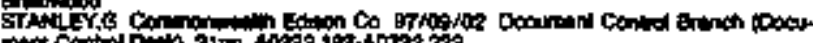

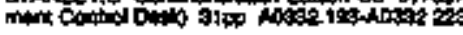

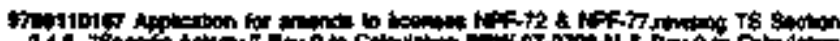

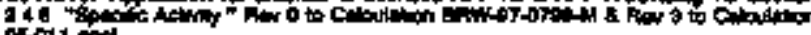

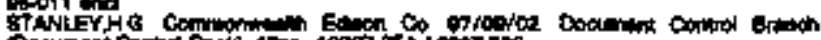

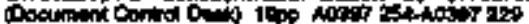

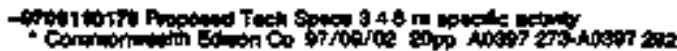

-

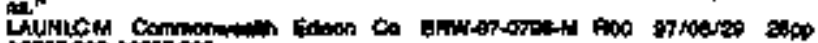
now 2 itom

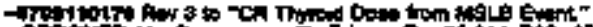

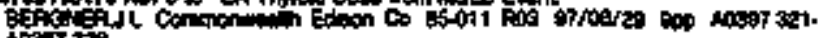
Atipt

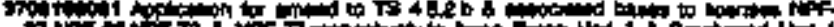

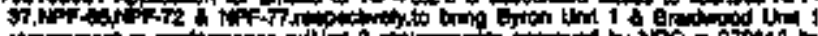

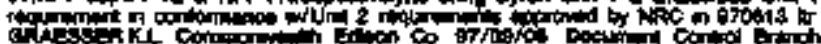

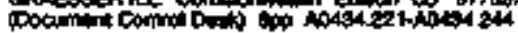

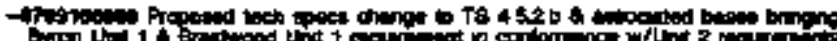

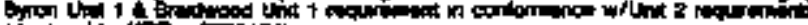

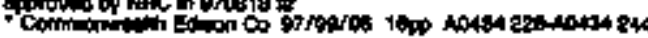

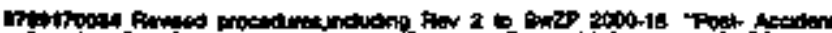

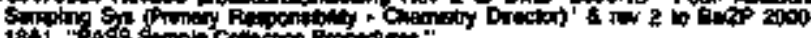

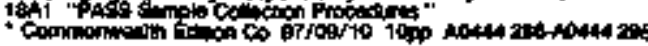

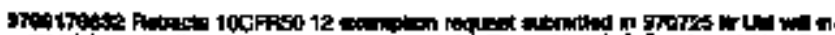

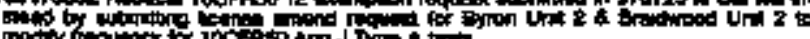

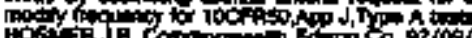

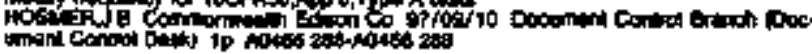

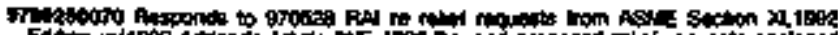

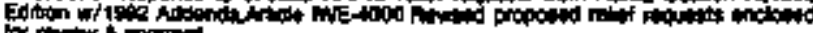

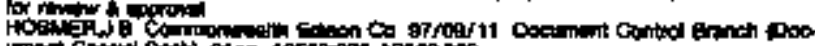

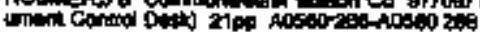

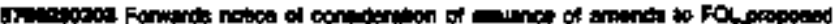

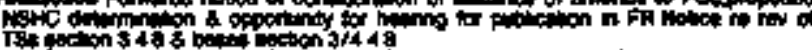
1. timation

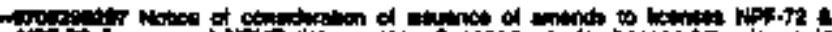

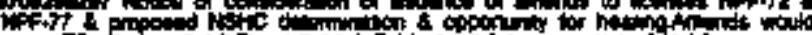

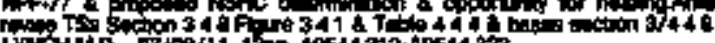

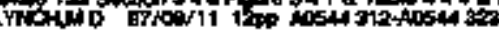

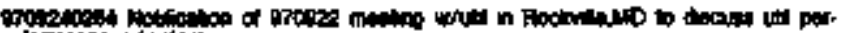

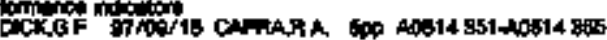

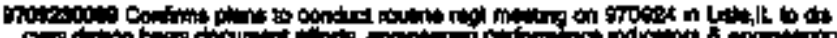

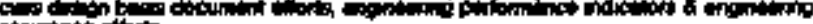

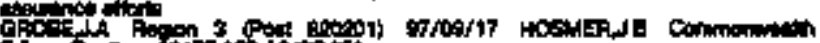

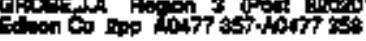

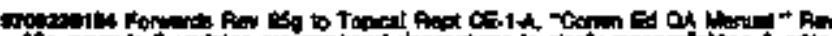

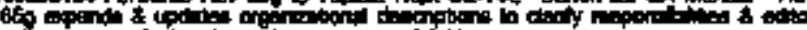

Hint

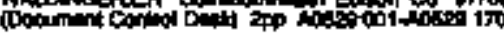

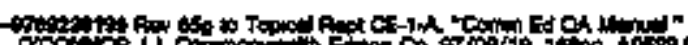

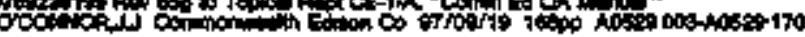

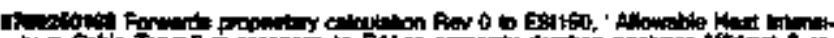

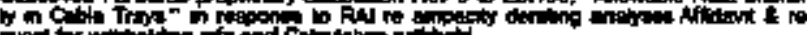

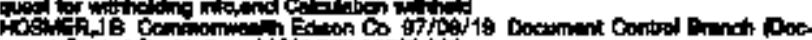
W

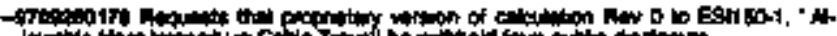

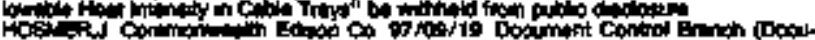

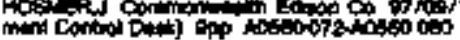

17is

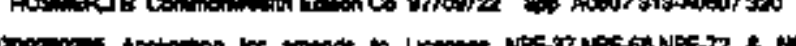

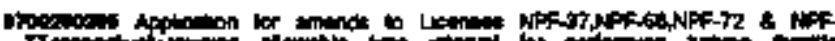

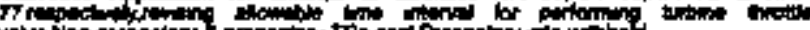

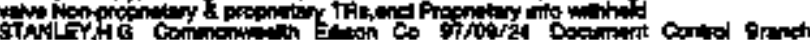

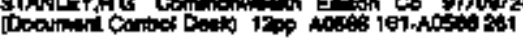

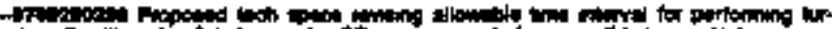

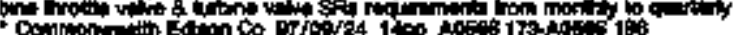

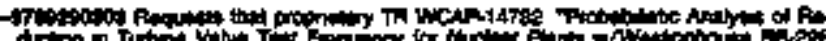

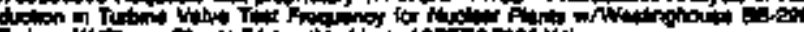

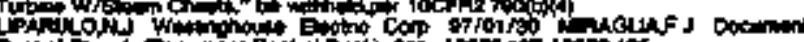

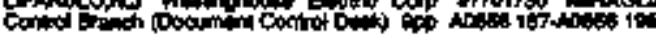

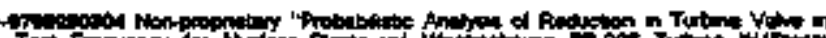

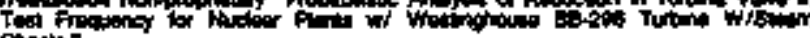

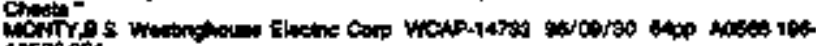

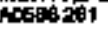

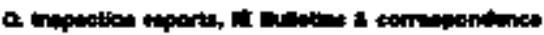

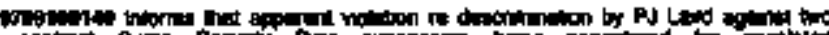

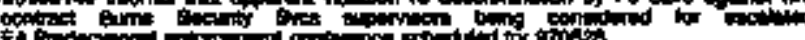

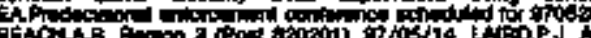

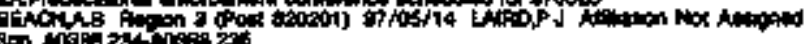

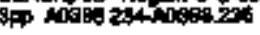

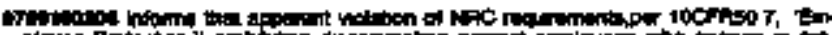

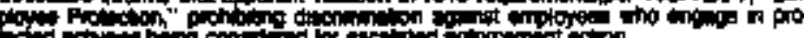

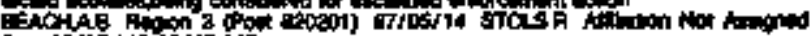

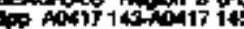

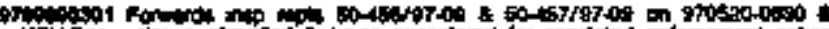

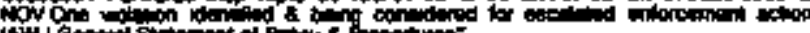

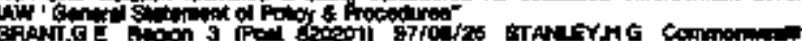

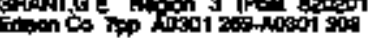

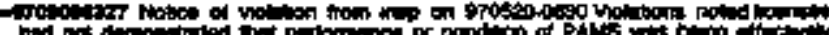

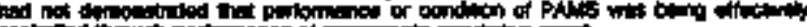

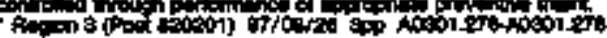

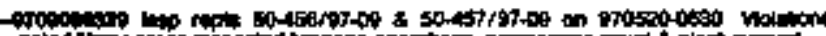

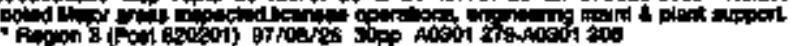

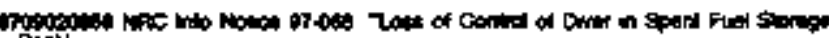

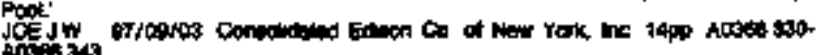
40

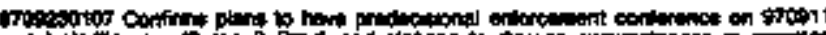

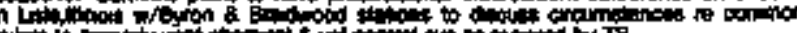

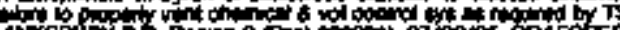

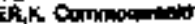




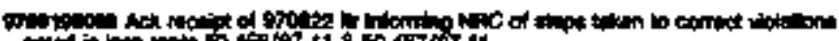

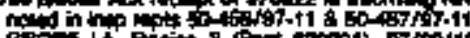

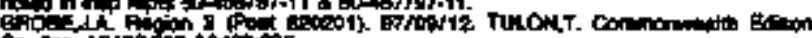

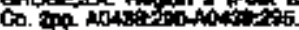

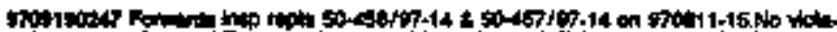

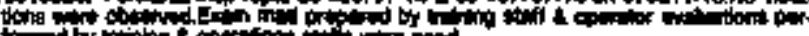

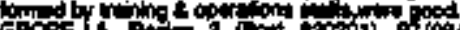

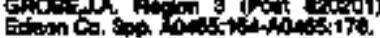

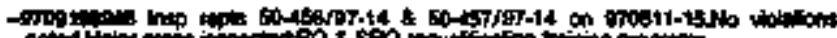

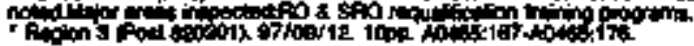

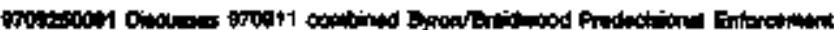

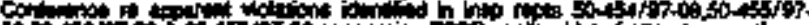

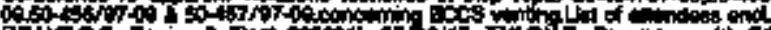

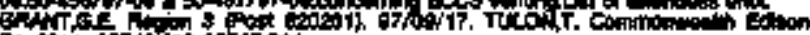

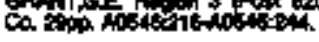

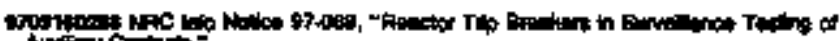

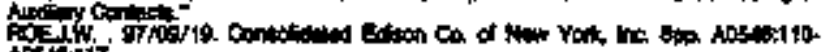
Axtititi.

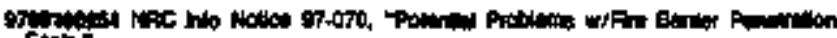

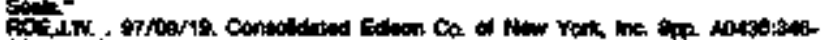
atiosis

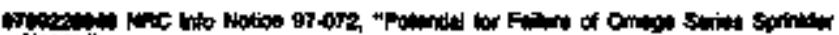

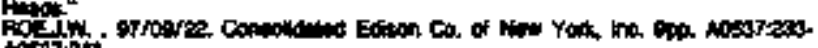
atsitisi.

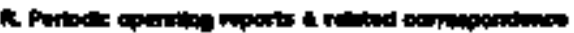

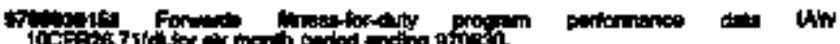

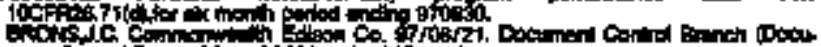

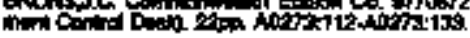

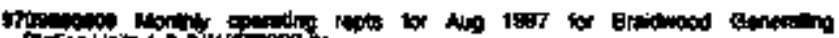
His

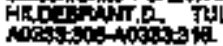

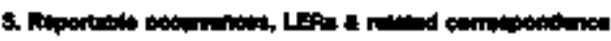

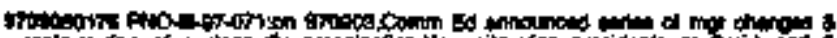

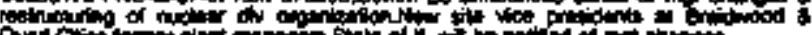

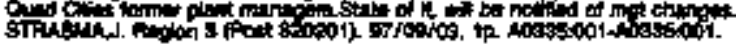

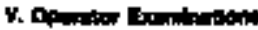

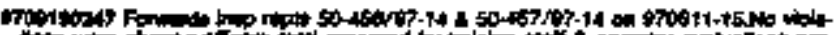

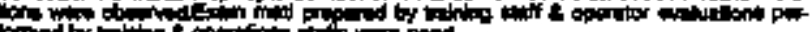

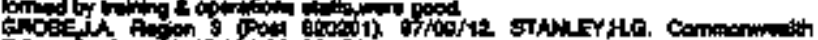

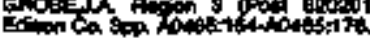

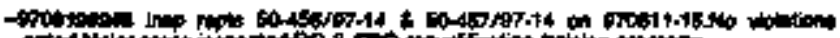

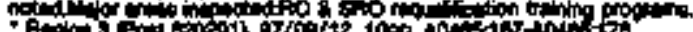

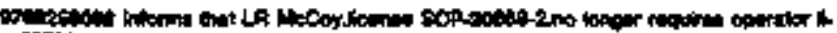

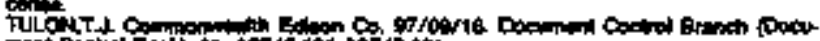

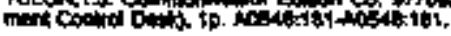

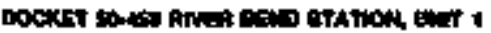

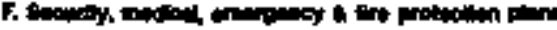

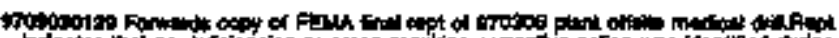

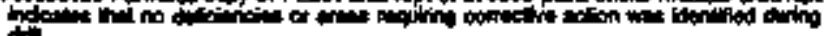

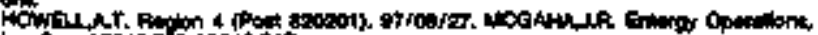

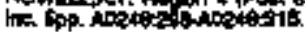

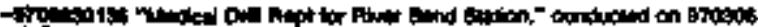

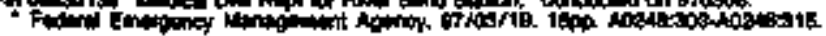

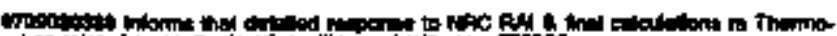

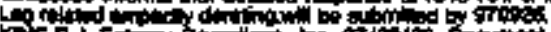

1. Contor Do

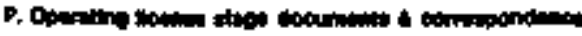

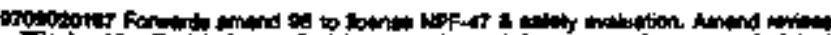

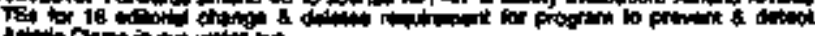

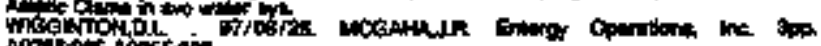

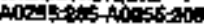

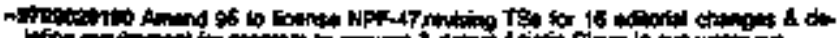

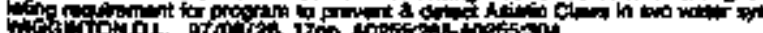

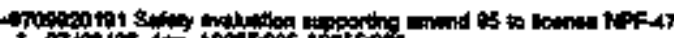
+

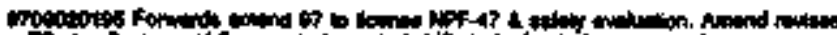
TS: b

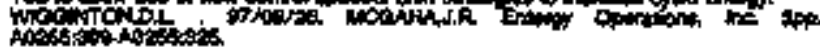

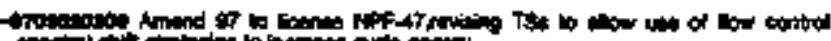

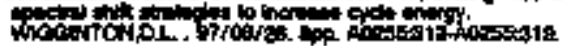

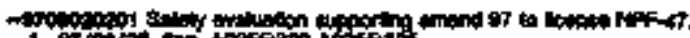

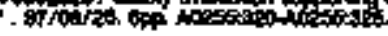

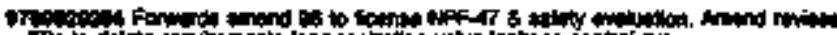

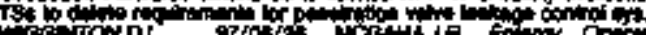

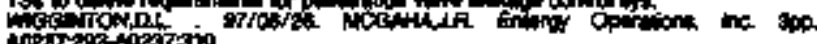

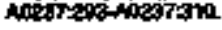

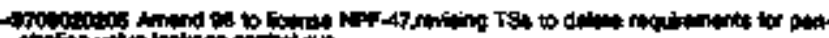

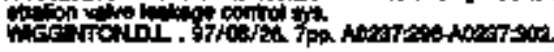

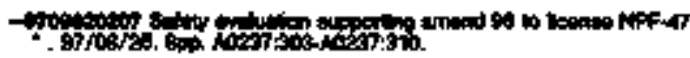

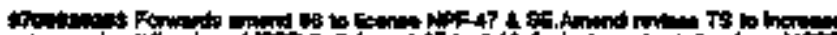

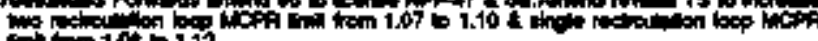

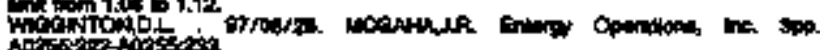

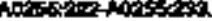

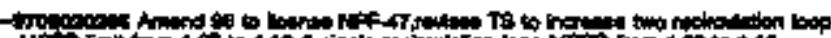

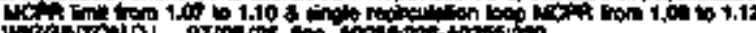

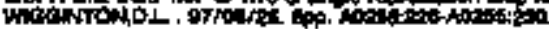

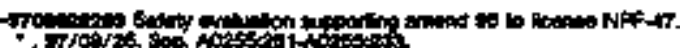

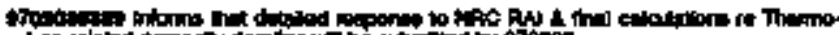

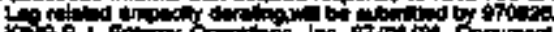

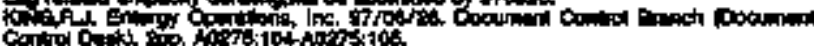

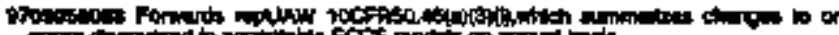

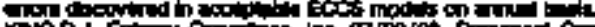

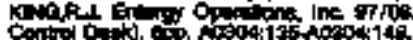

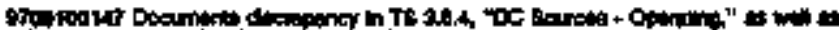

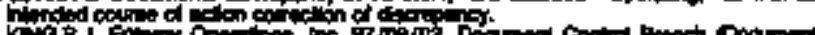

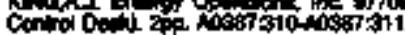

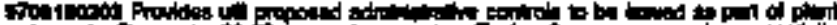

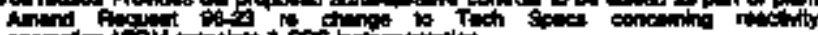

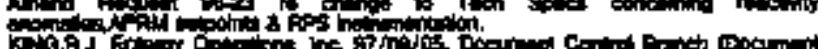
Corto D G

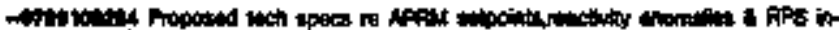

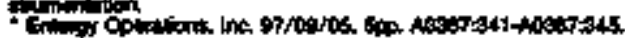

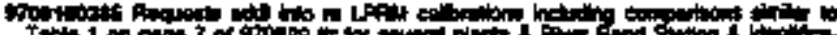

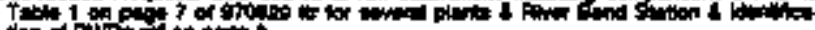

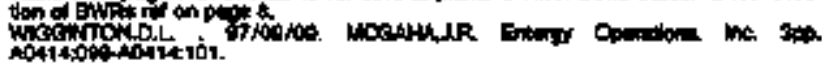

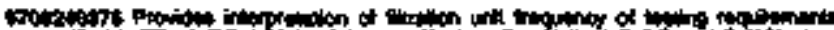

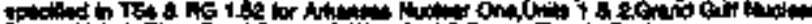

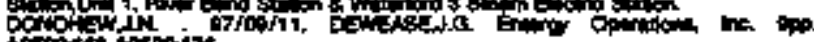

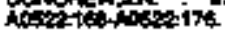

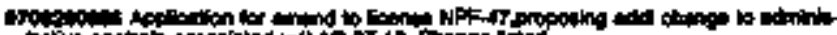

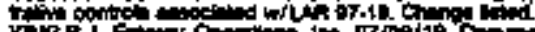

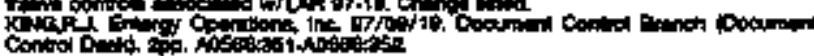

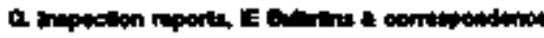

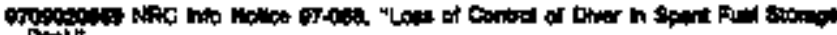

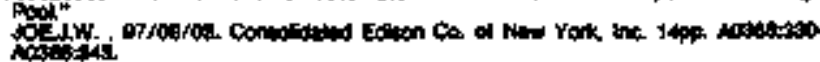

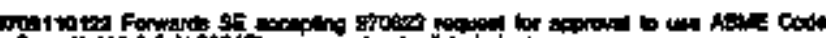

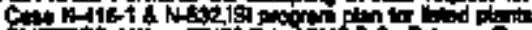

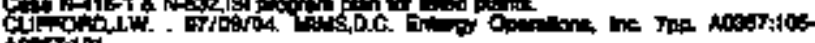
ator:124,

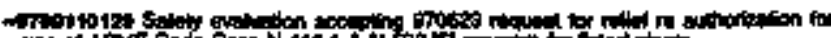

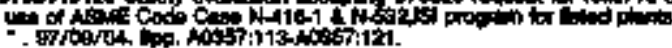

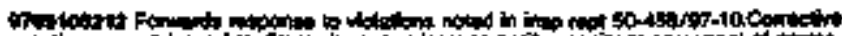

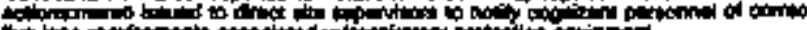

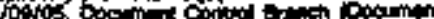

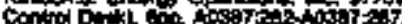




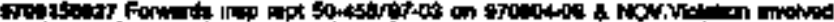

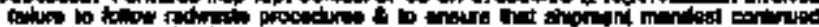

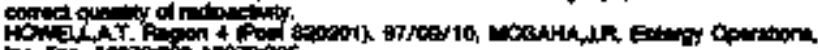

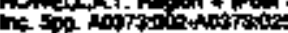

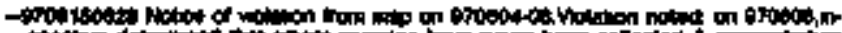

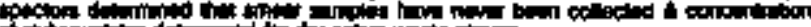

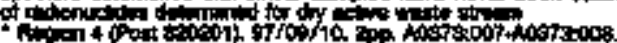

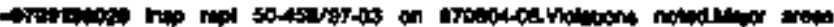

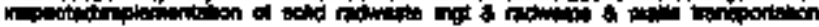
Dorithe

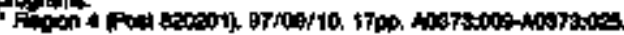

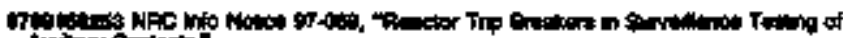

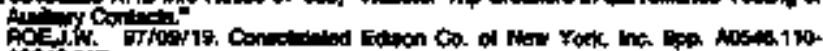
A0sitit.

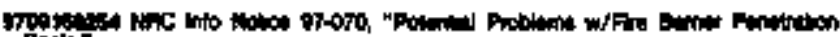

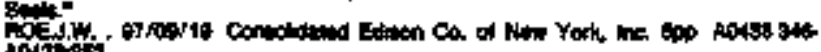

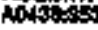

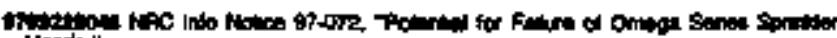

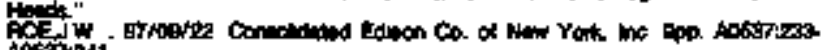

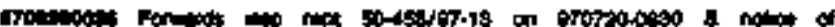

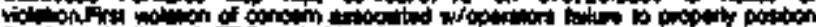

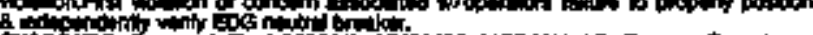
Gint th. P.

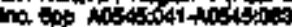

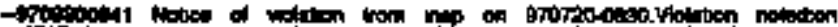

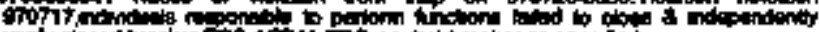

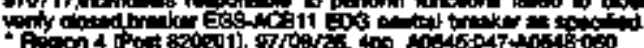

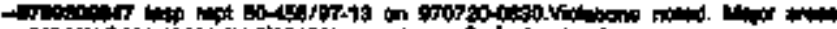

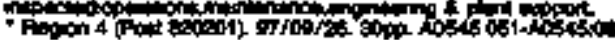

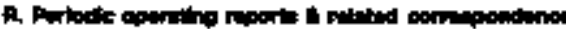

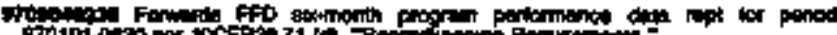
MING

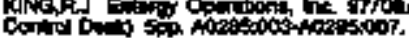

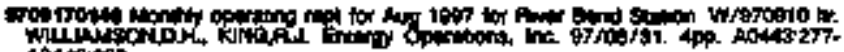
Mactosio

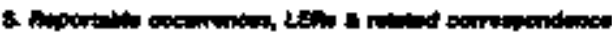

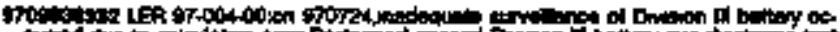

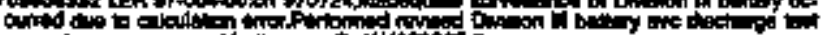

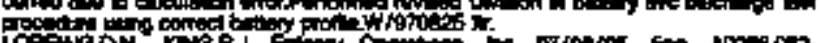

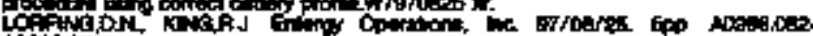
Lotiving

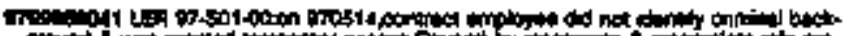

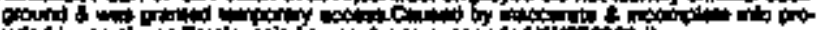

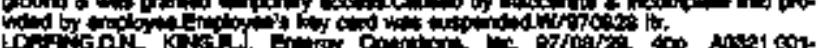
inteition,

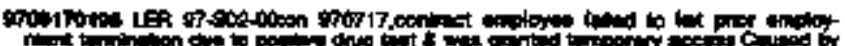

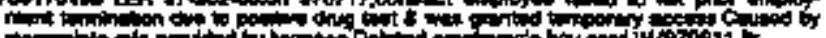

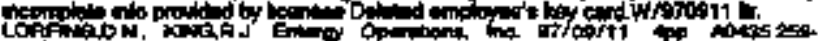
int: 407

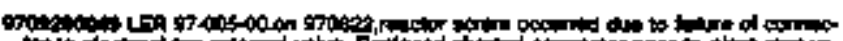

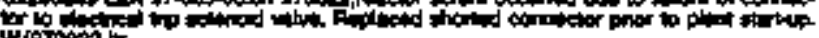

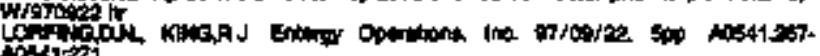

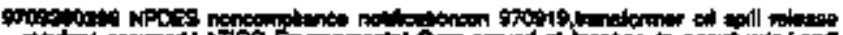

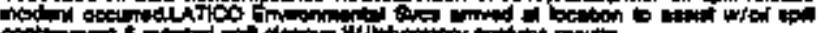

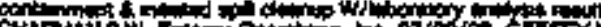

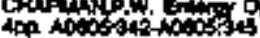

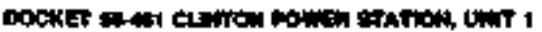

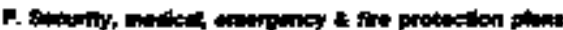

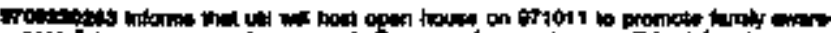

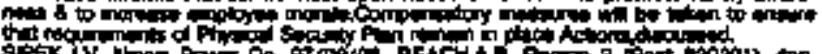

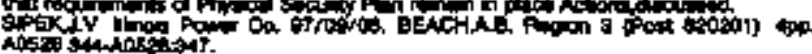

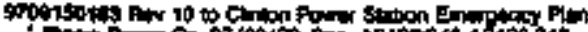

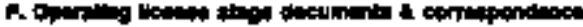

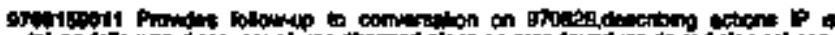

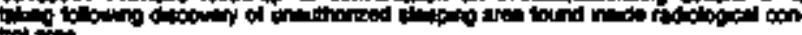

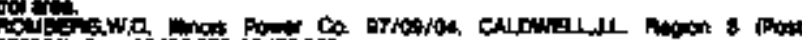
onit.

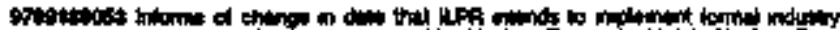

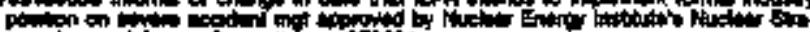

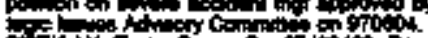

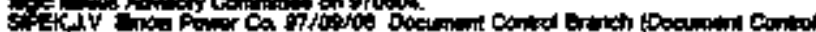

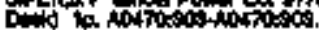

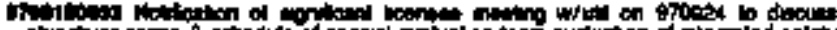

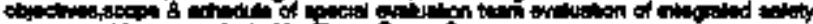

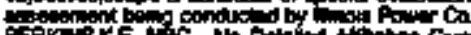

Figkn

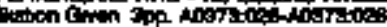

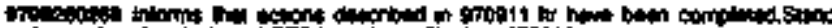

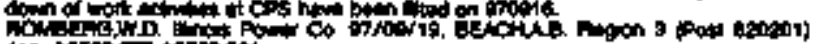

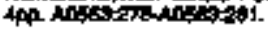

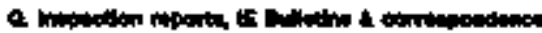

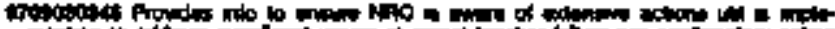

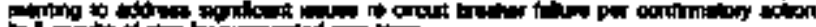

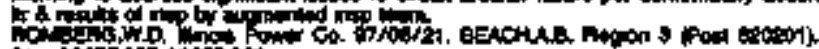

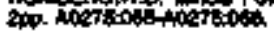

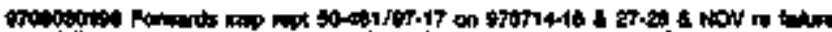

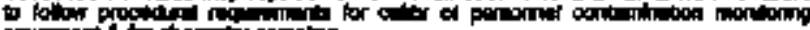

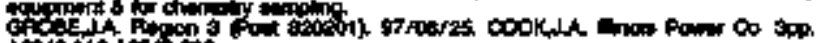

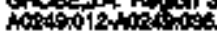

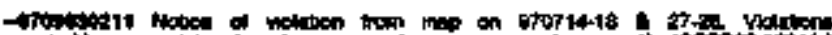

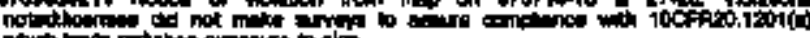

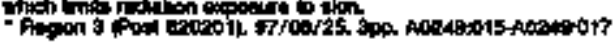

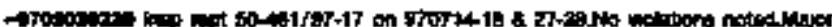

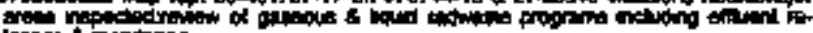

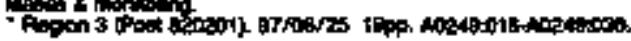

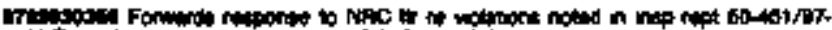

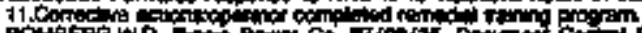

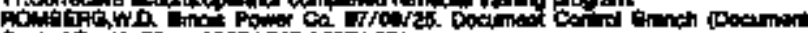

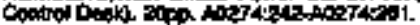

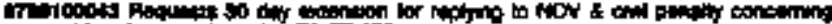

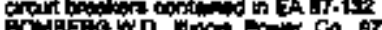

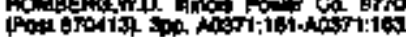

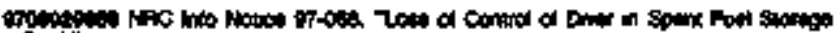
Pod

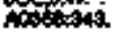

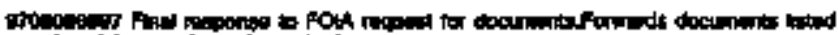

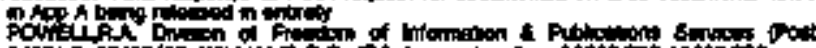

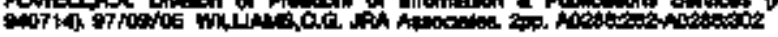

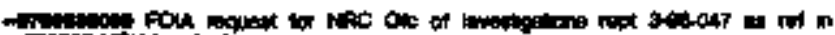

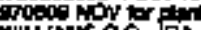

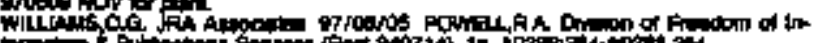

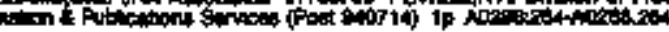

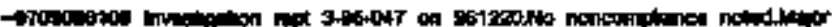

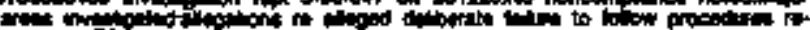
ay aption

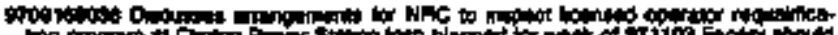
br 1

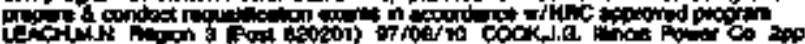

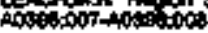

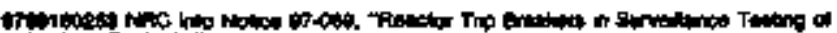

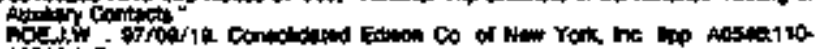

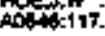

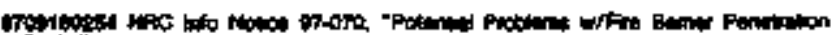

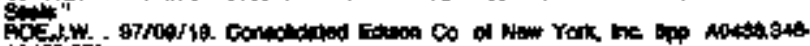
abotisa

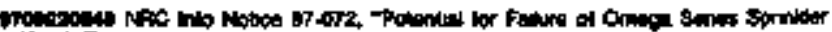

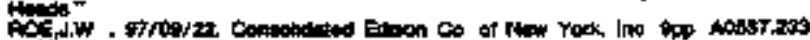
Astinget.

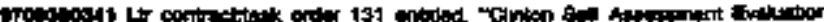

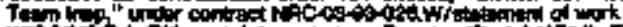
is Wh Lof 


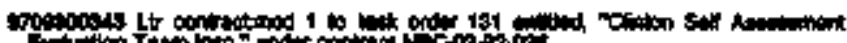
Whet the tory

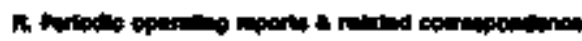

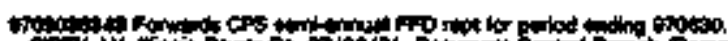

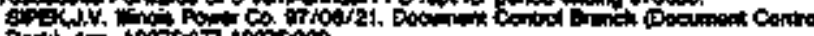

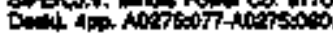

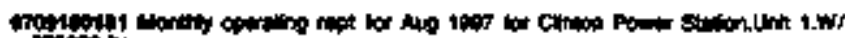

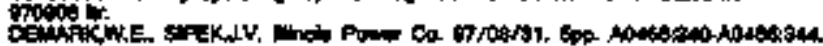

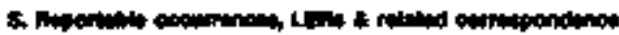

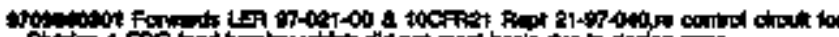

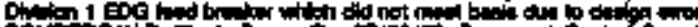

Pow

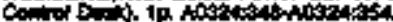

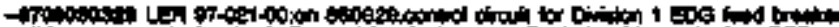

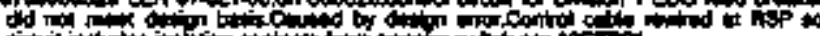

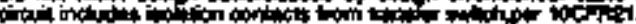

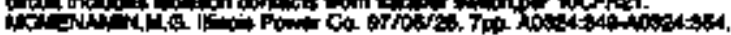

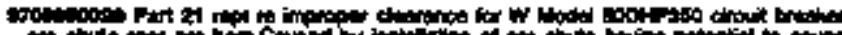

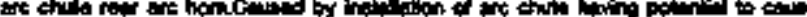

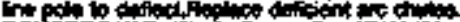

Pol

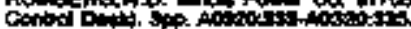

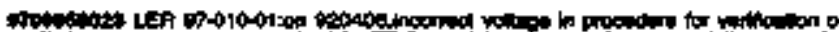

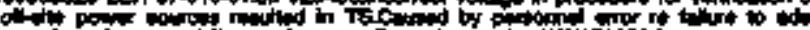

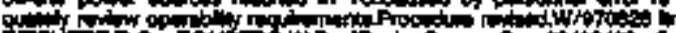

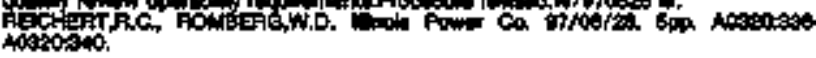

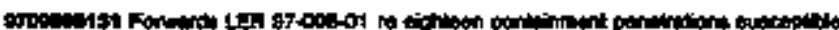

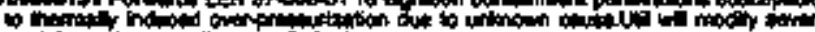

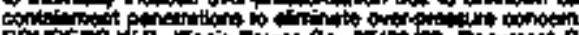

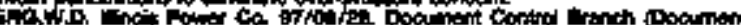

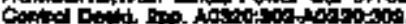

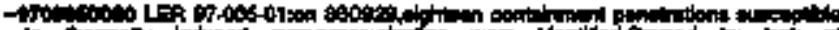

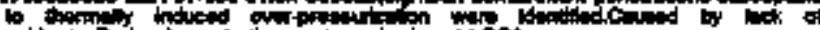

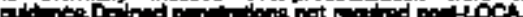

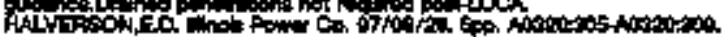

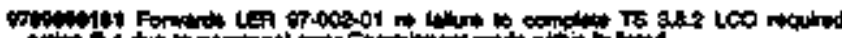

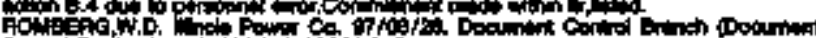

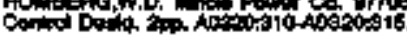

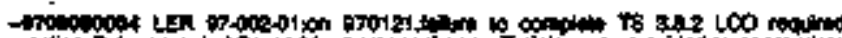

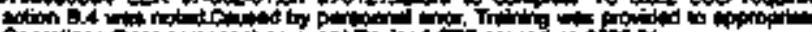

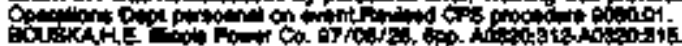

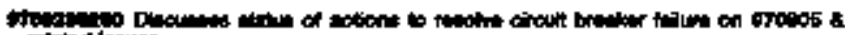

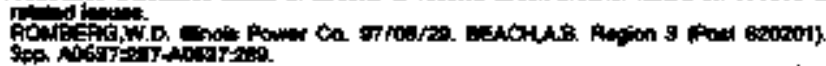

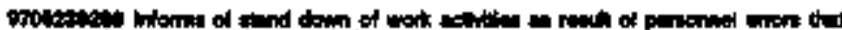

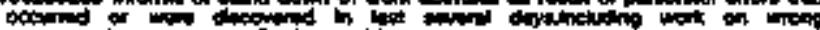

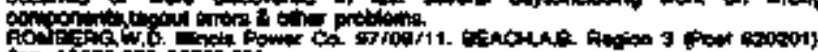

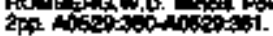

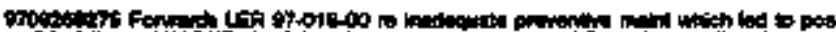

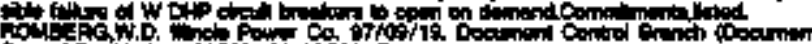

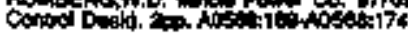

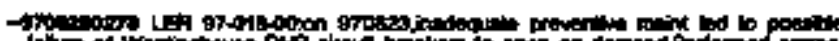

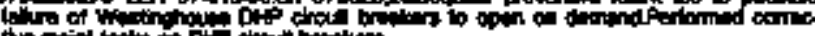

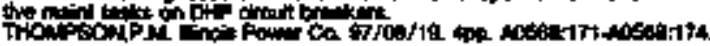

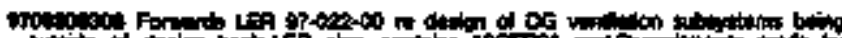

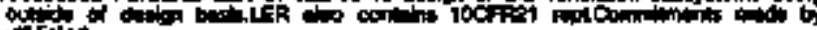

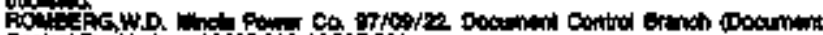

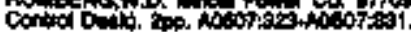

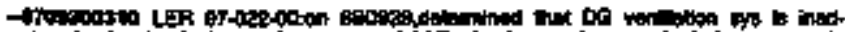

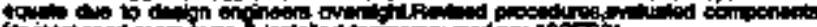

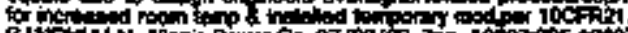

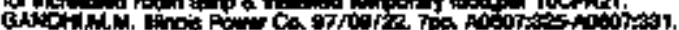

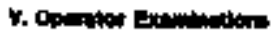

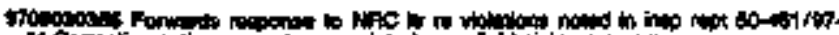

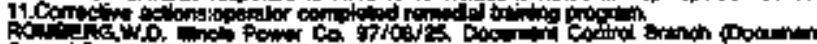

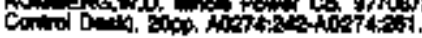

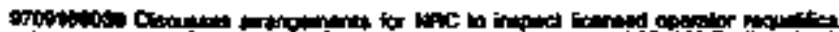

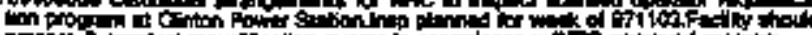

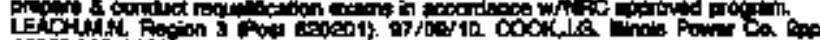

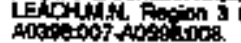

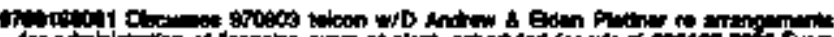

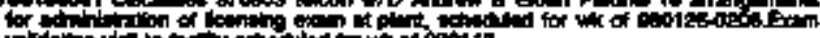

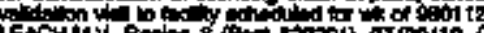

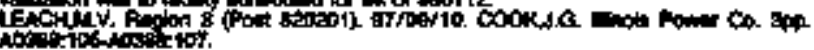

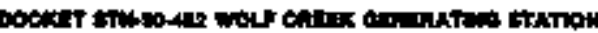

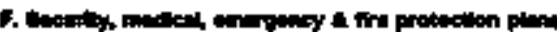

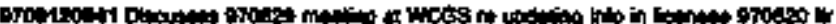

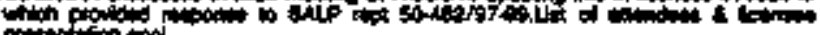

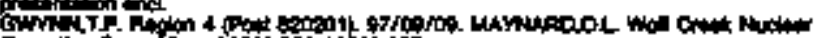

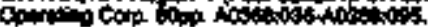

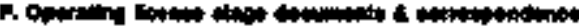

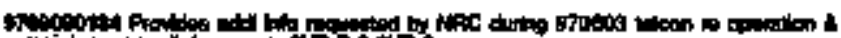

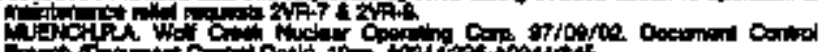

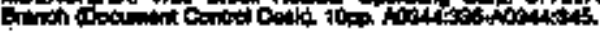

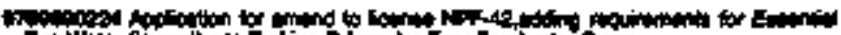

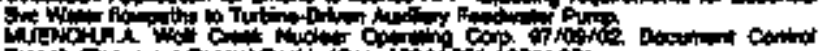

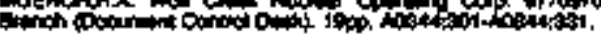

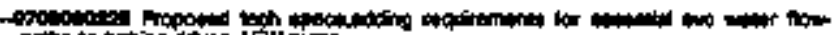

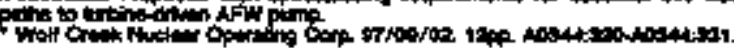

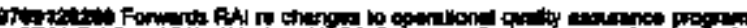

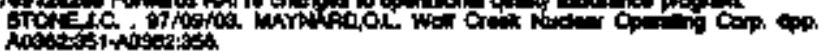

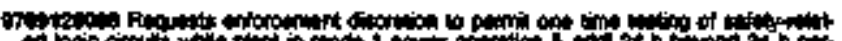

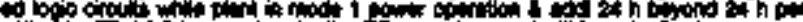
What

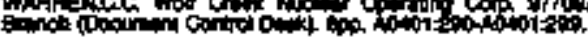

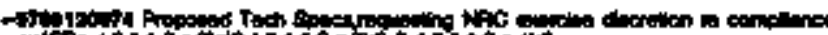

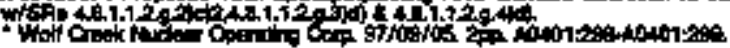

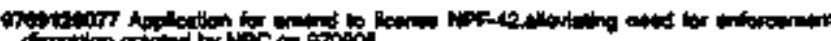

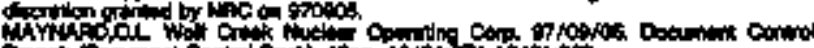

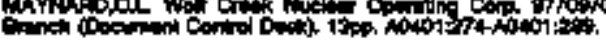

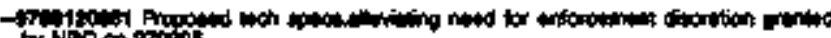

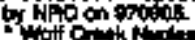

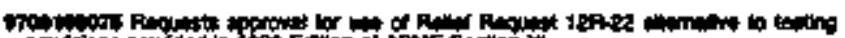

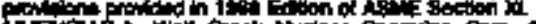

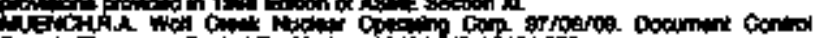

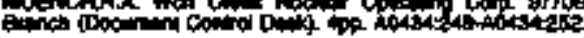

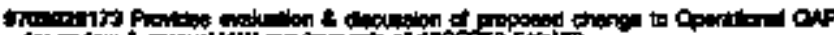

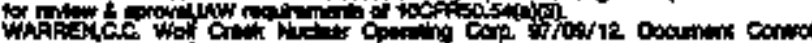

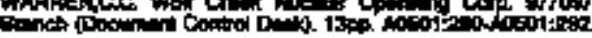

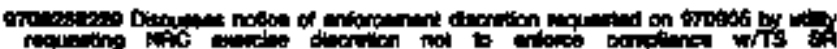

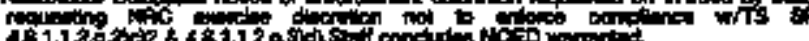

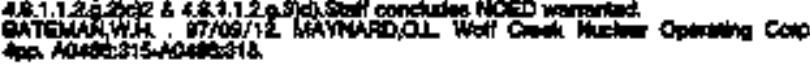

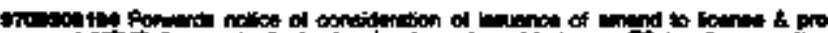

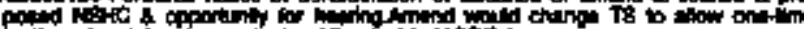

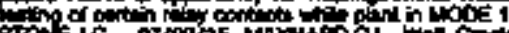

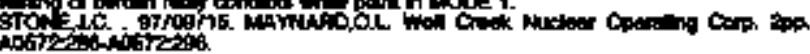

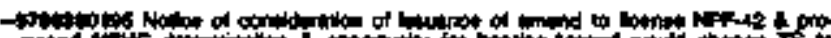

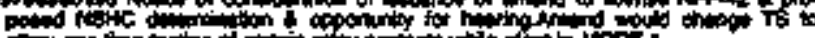

EN on

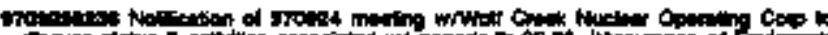

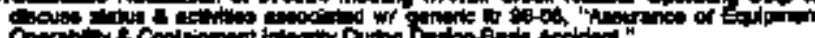

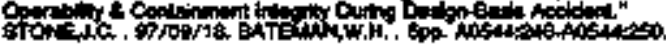

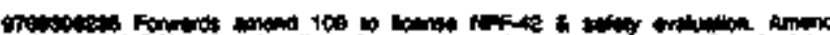

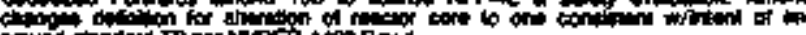

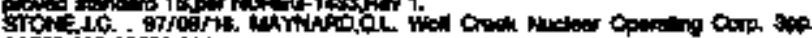
notet 106mingtild.

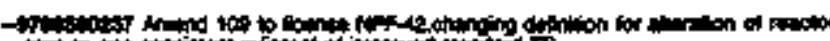
sto to

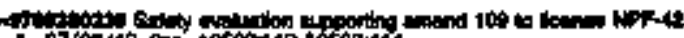

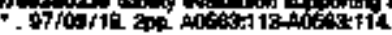




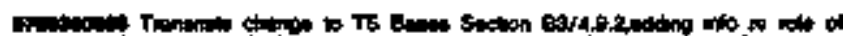

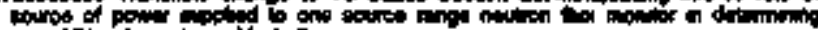

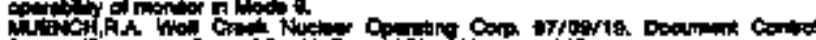
pach

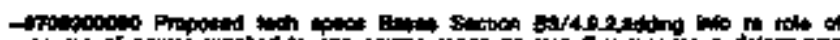

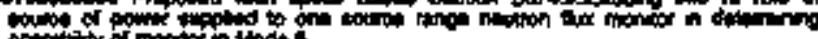

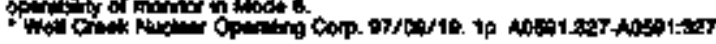

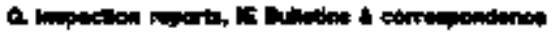

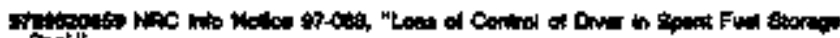

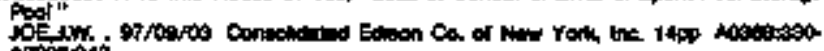

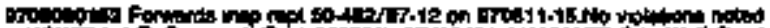
Hoth

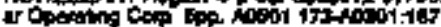

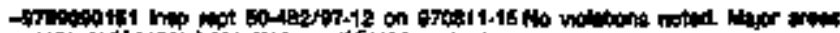

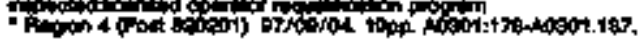

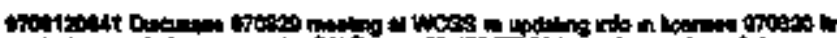

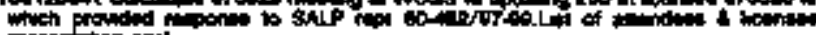

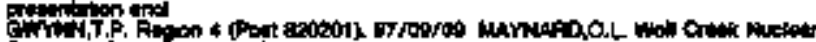

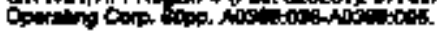

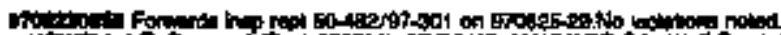

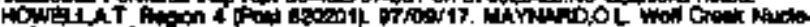

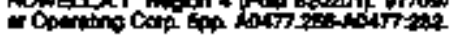

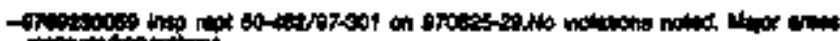

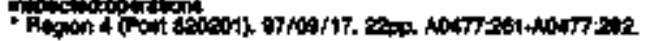

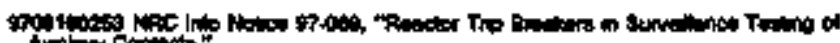

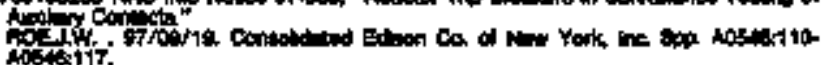

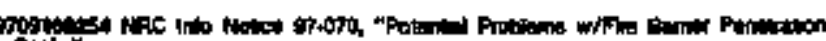

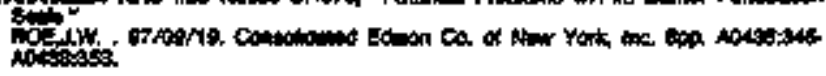

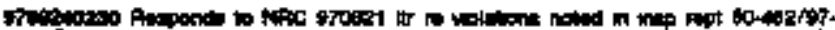

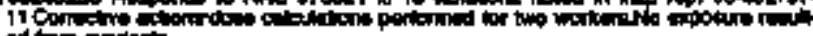

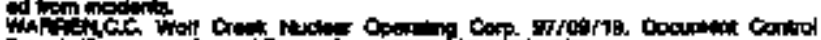

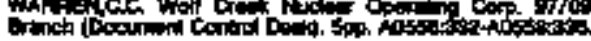

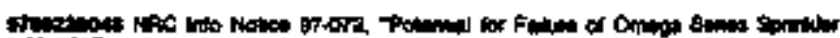

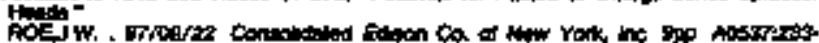
nosis?

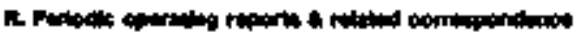

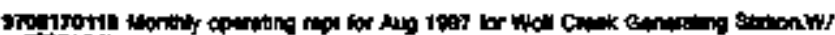

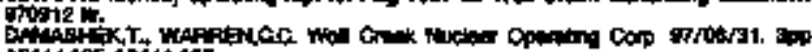

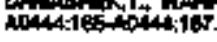

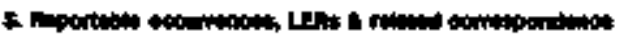

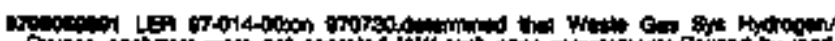

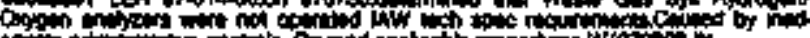

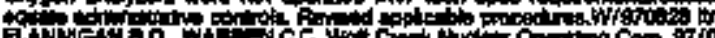
Fitw

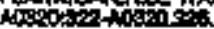

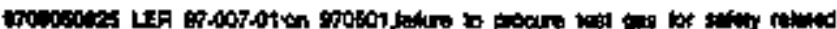

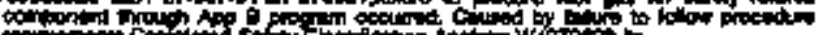

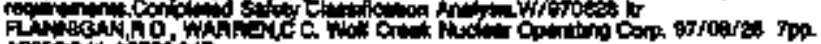

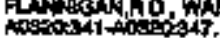

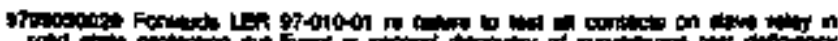

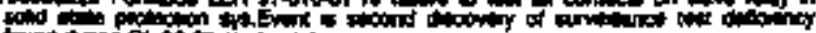

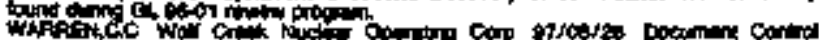

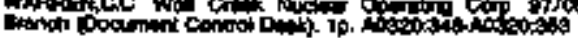

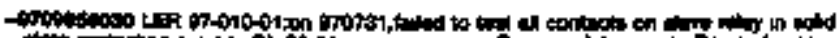

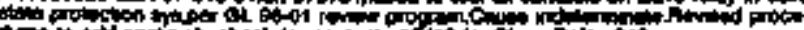

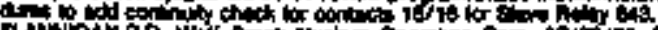

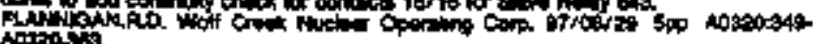

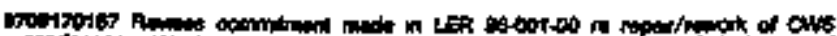

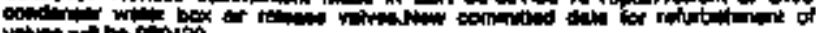

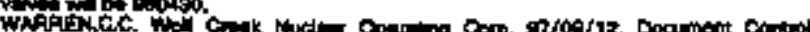

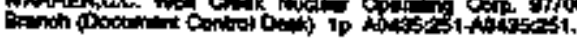

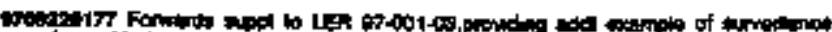

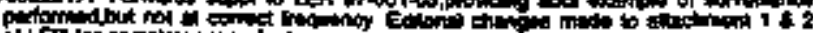

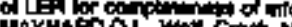

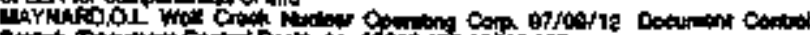

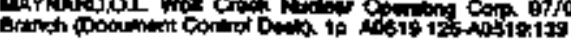

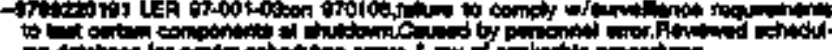

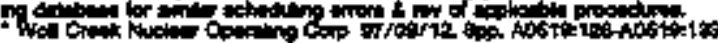

wopurico left of

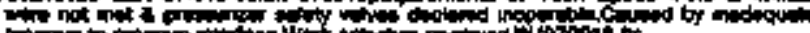

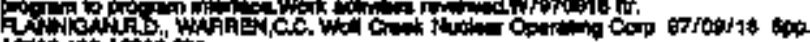

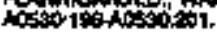

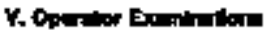

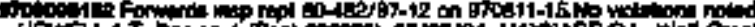

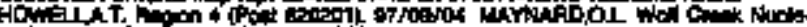

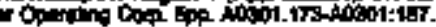

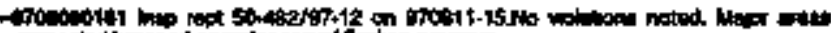

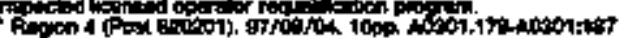

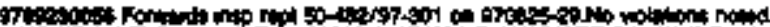

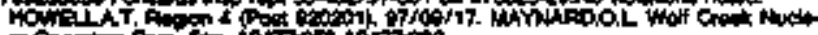

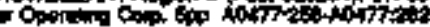

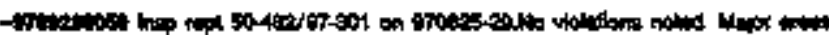

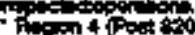

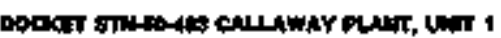

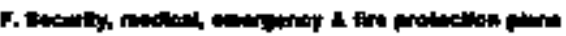

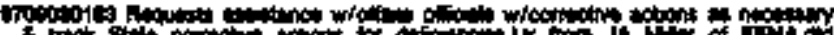

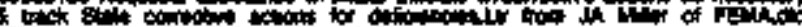

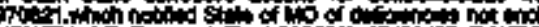

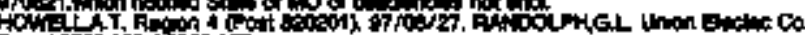

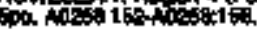

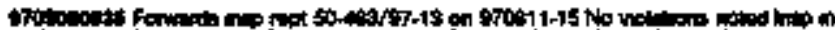

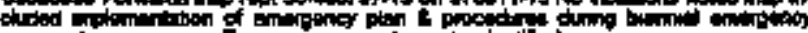

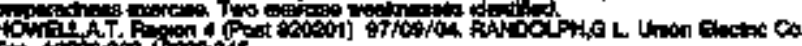

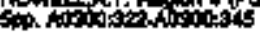

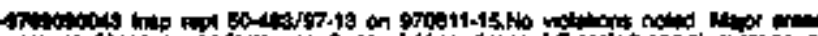

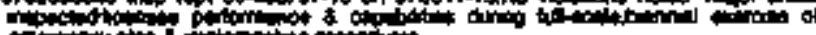

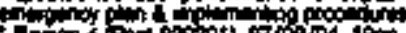

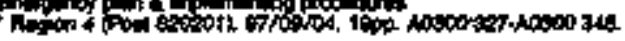

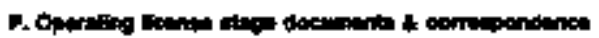

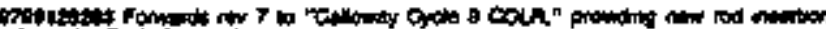

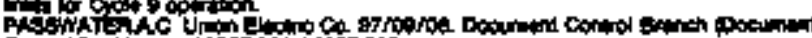

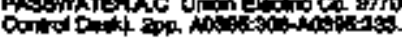

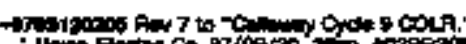

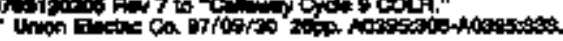

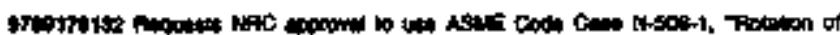

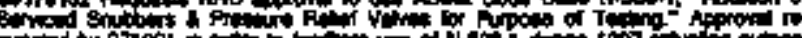

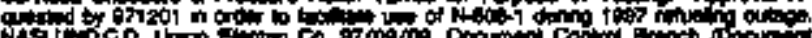

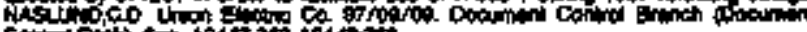

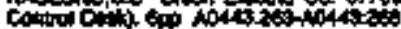

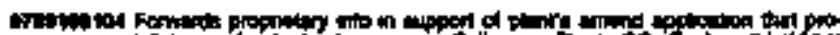

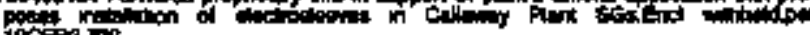

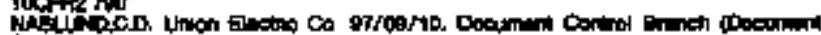

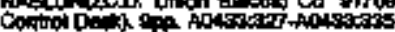

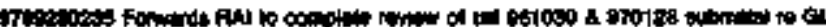

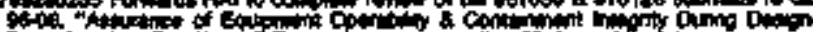

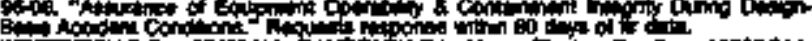

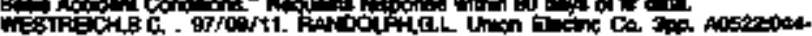

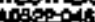

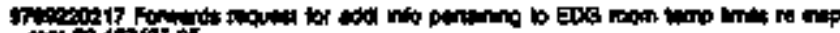

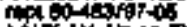

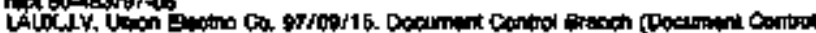

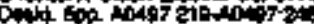

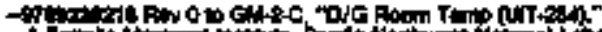

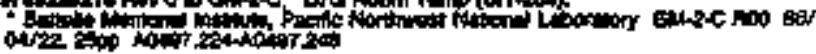

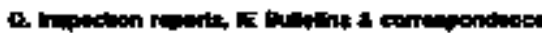

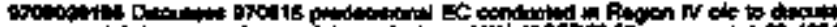

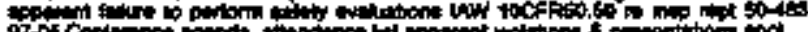

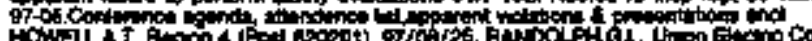

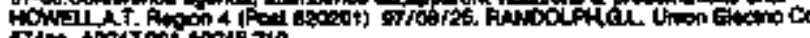

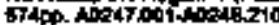

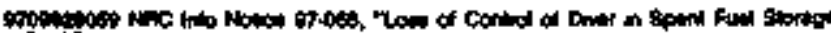

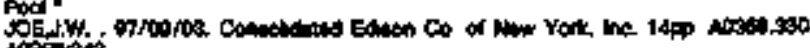

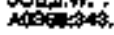




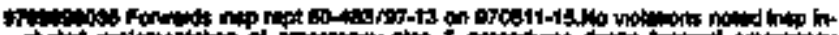

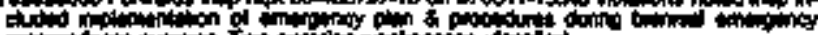
Fon

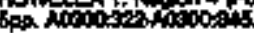

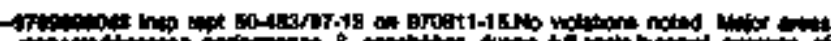

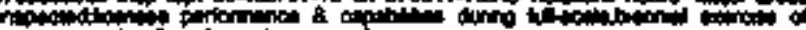

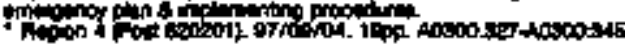

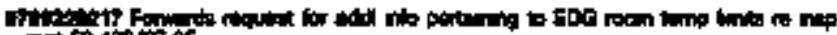

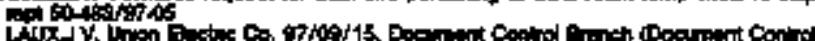

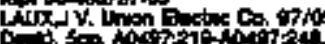

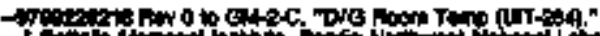

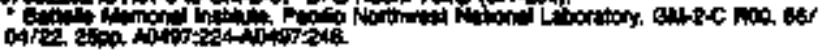

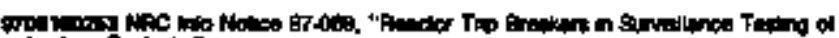

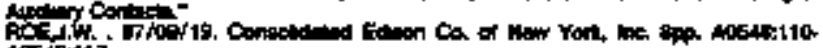
Alowitit.

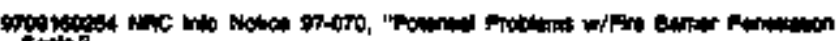

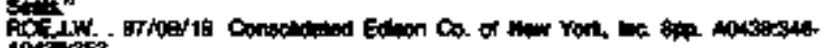

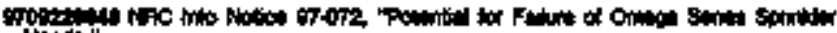

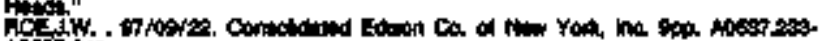

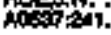

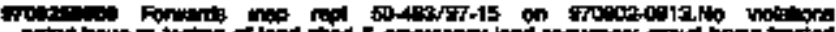

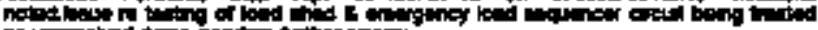

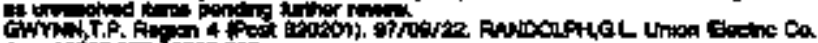

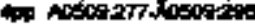

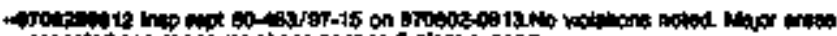

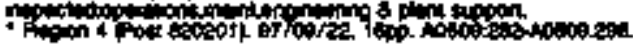

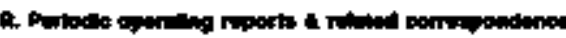

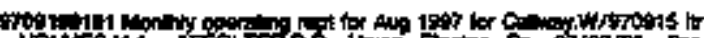

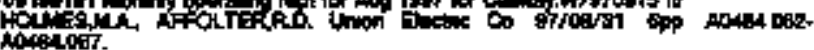

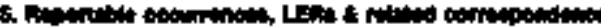

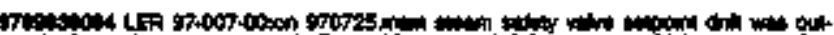

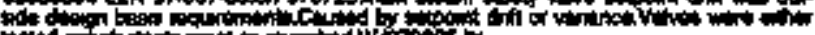

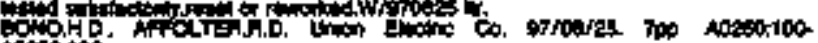
100.106.

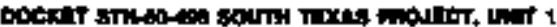

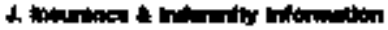

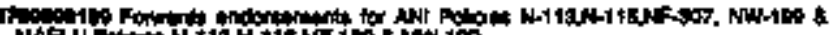

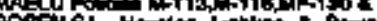

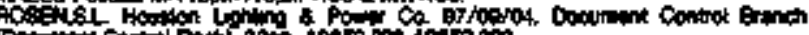

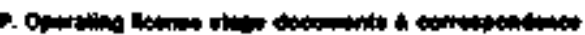

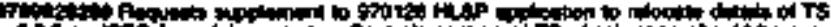

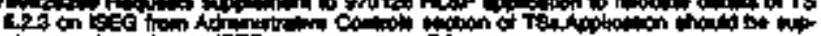

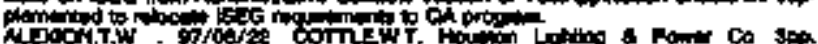

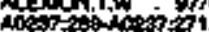

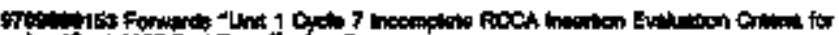

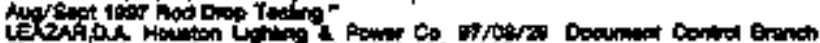

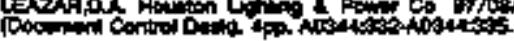

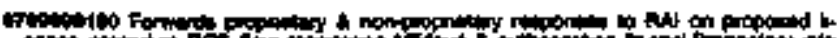

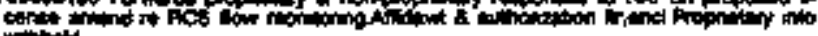

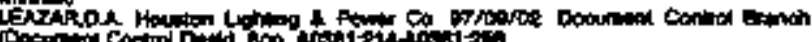

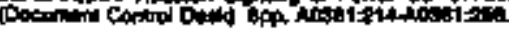

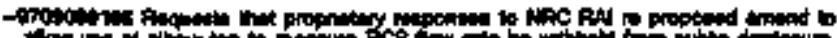

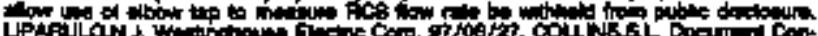

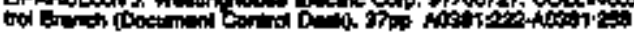

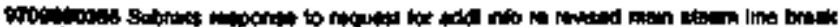

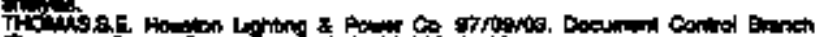

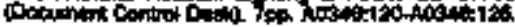

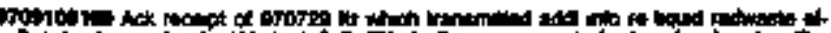

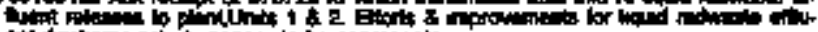

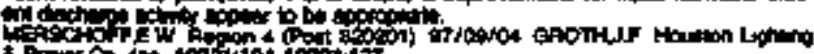

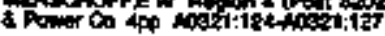

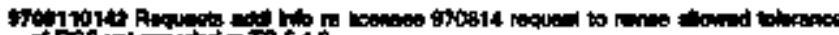
of Biss vol

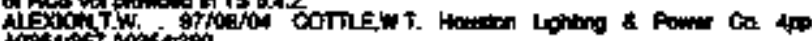

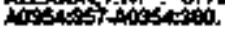

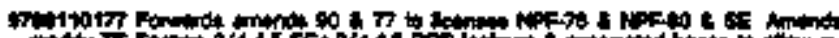

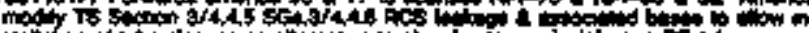

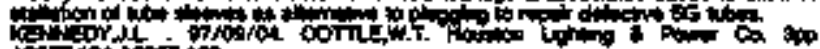

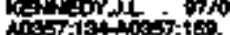

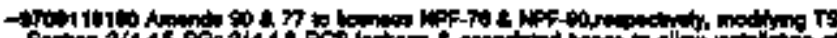

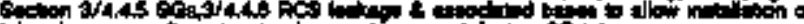

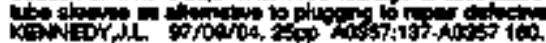

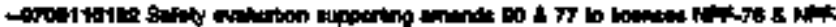

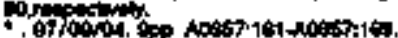

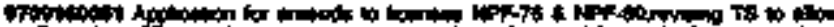

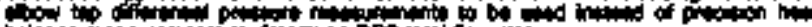

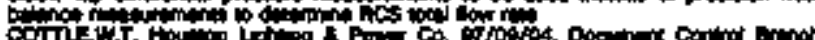

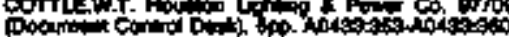

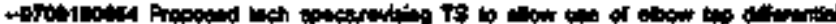

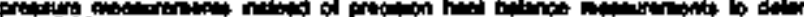

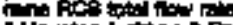

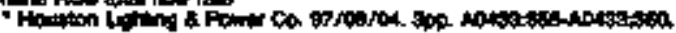

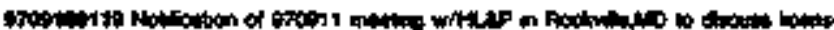

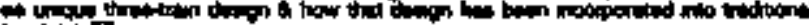

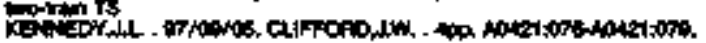

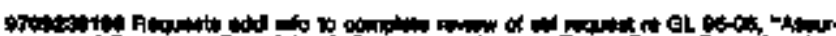

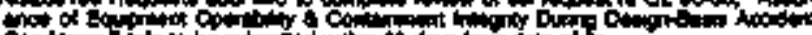

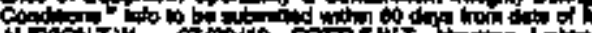

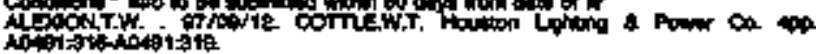

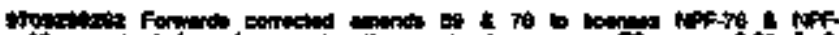

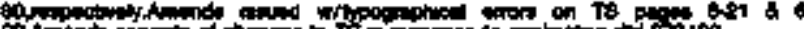

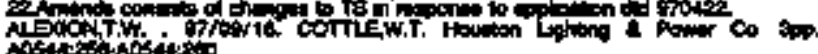

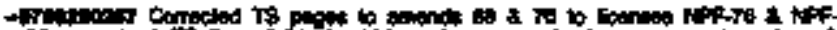

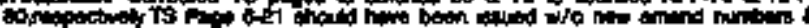

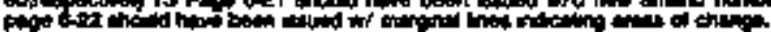

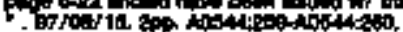

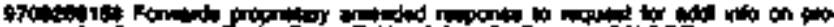

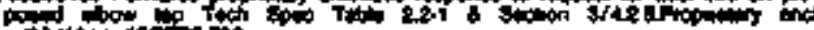

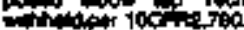

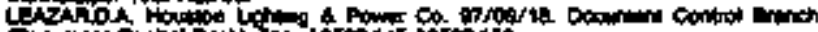
(D)

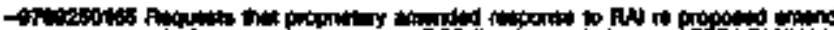

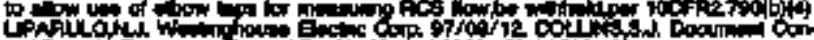

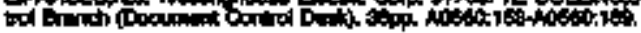

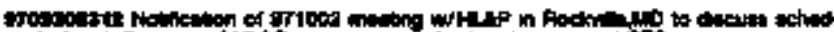

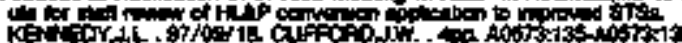

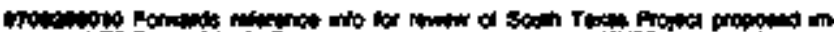

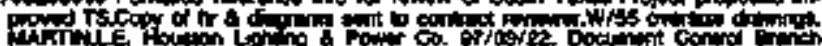

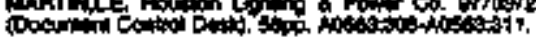

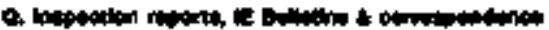

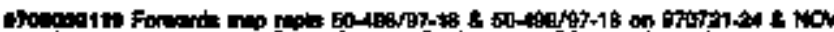

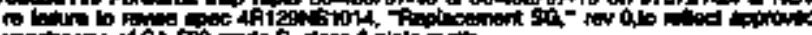

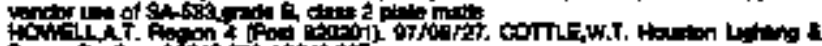

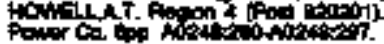

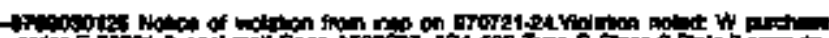

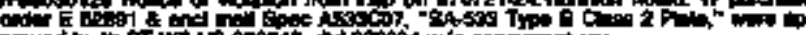

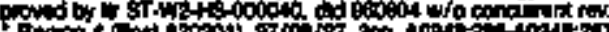

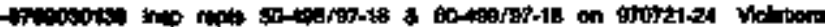

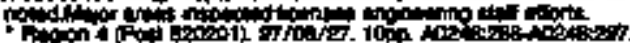

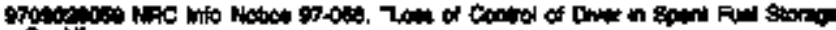

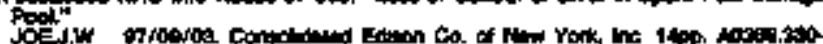
Not:

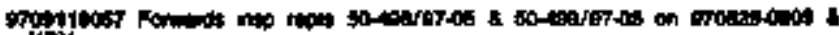

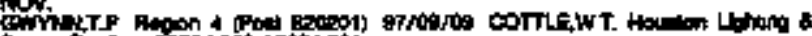

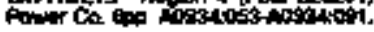

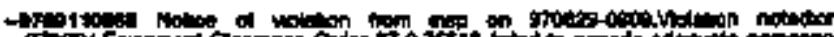

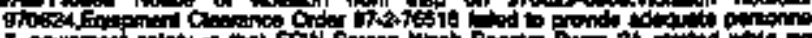

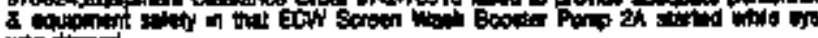

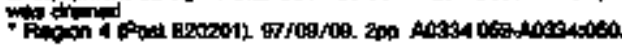




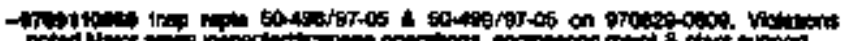

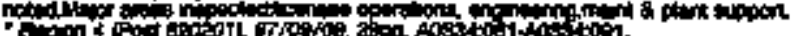

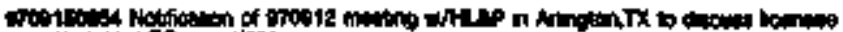

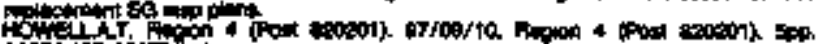

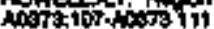

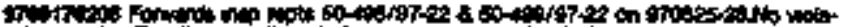

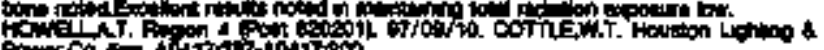

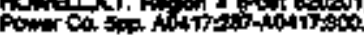

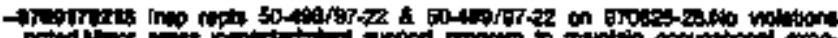

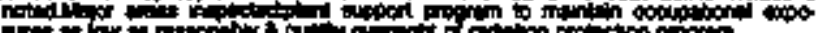

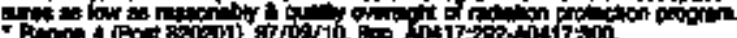

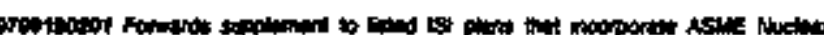

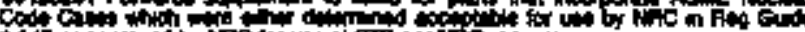

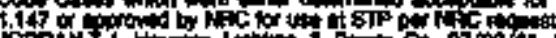

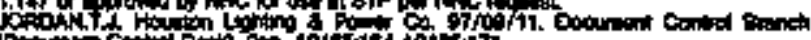

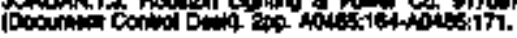

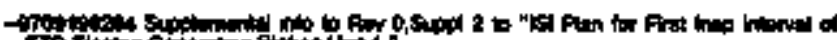

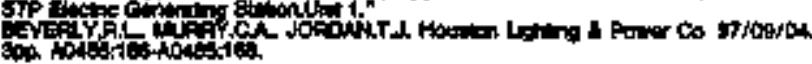

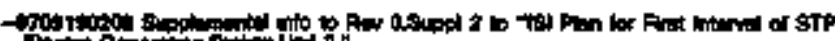

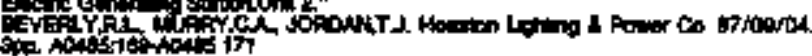

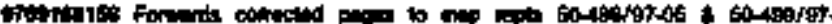

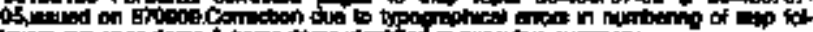
Gin

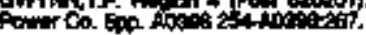

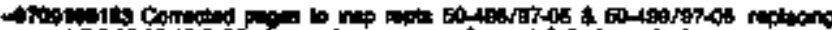

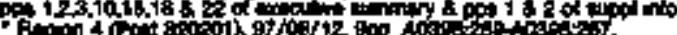

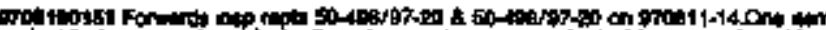

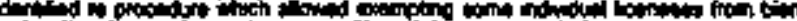

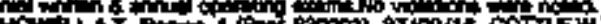

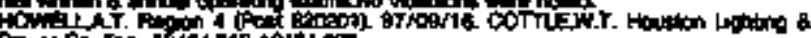

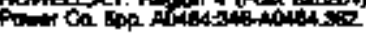

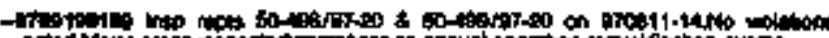

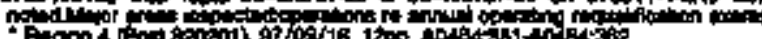

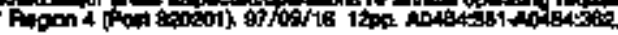

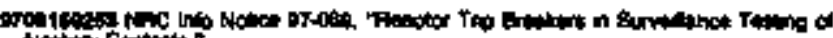

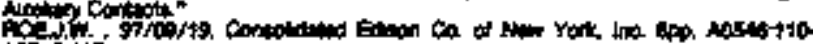
Ap.

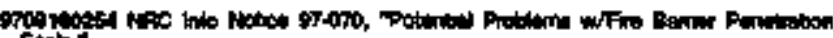

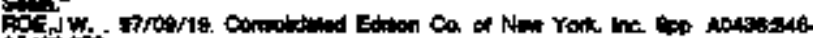
Antis.

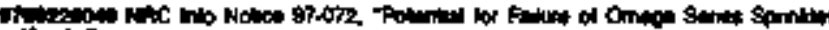
toit" 10.0교.

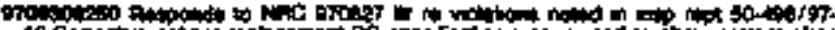

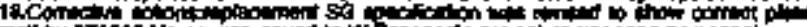

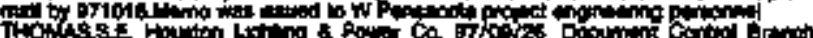

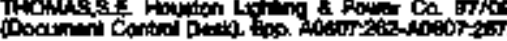

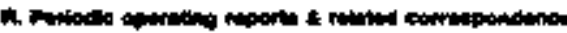

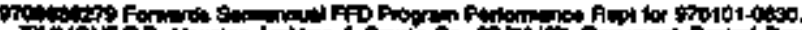

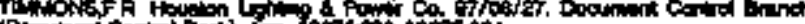

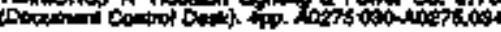

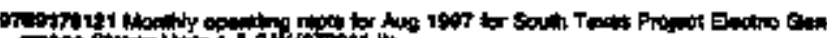

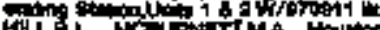

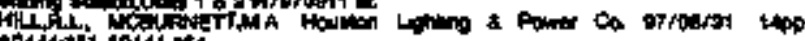

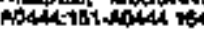

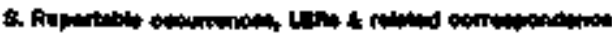

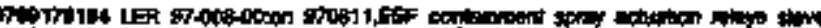

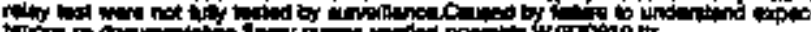

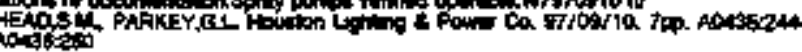

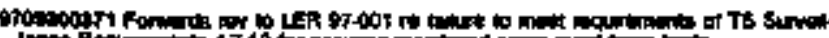

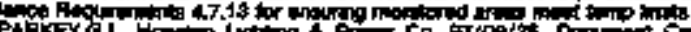

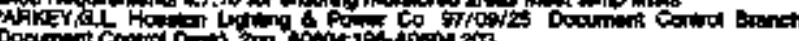
-

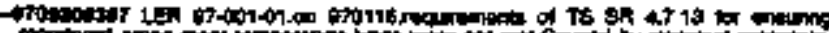

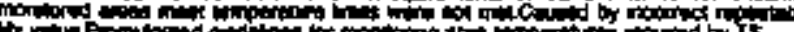
Cit

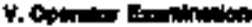

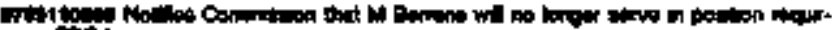

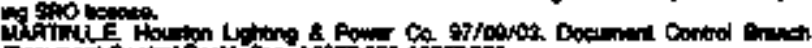

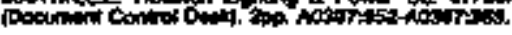

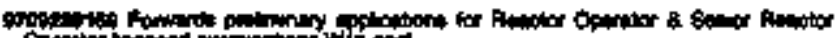

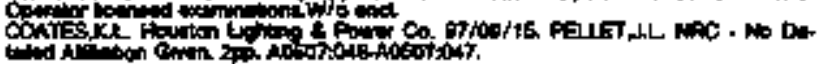

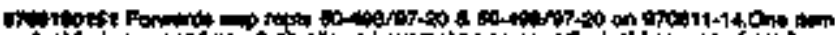

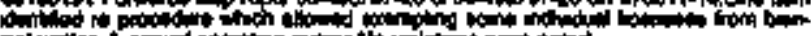

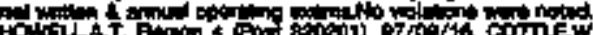

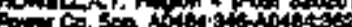

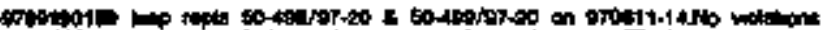

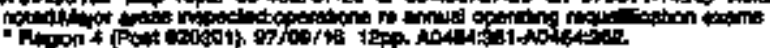

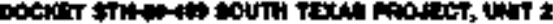

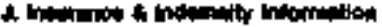

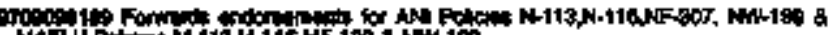

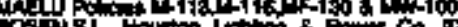

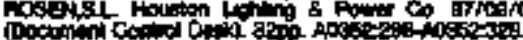

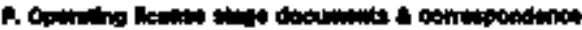

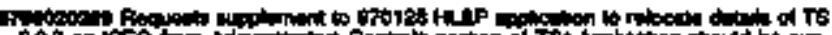

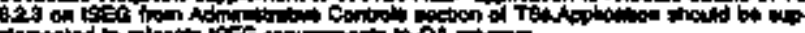

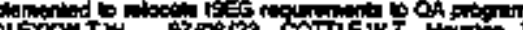

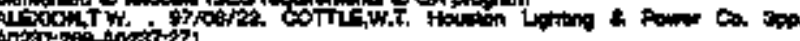

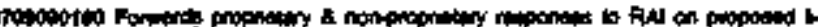

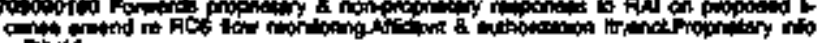

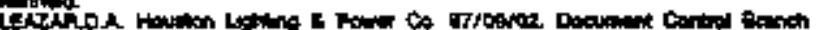

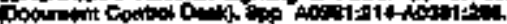

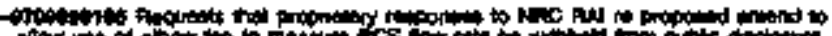
for

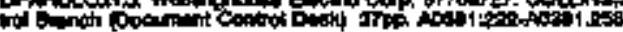

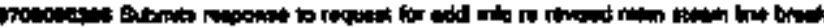

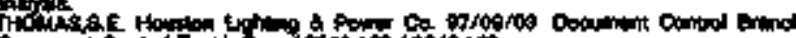

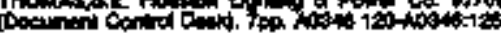

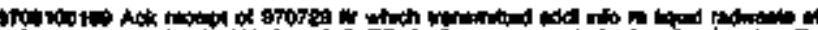

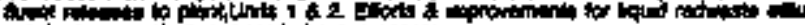

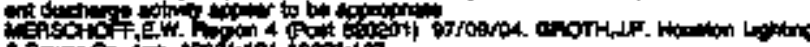

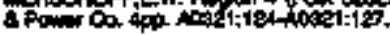

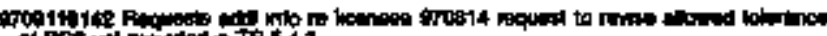

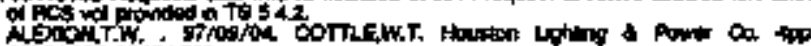
A

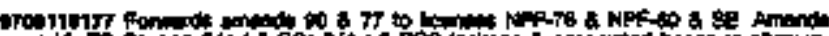

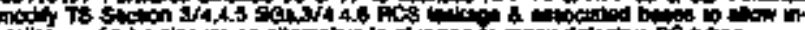

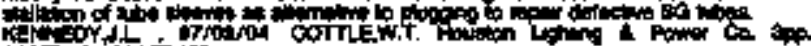

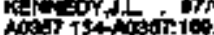

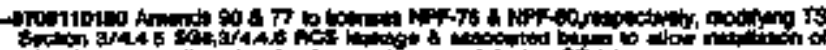

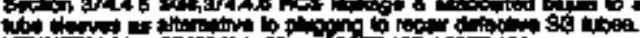

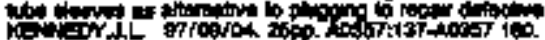

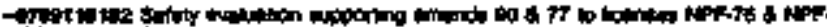

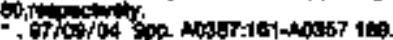

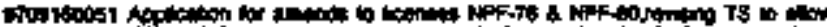

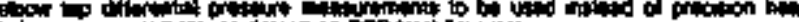

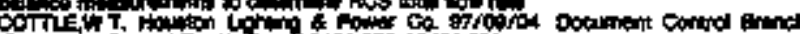

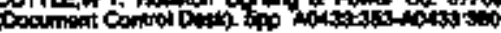

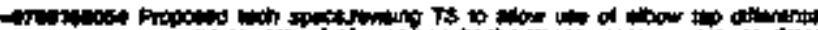
Fie

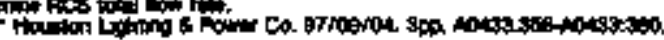

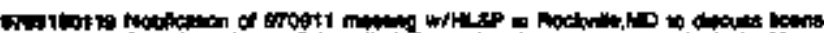

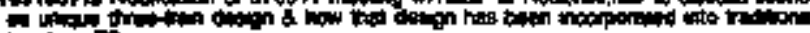

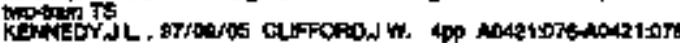

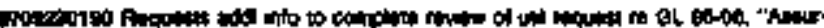

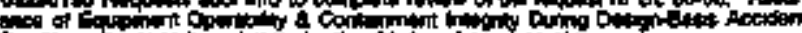

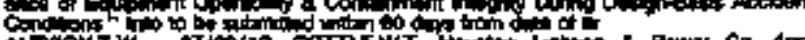

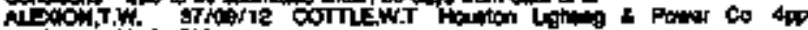

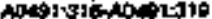




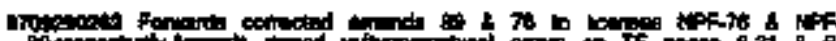

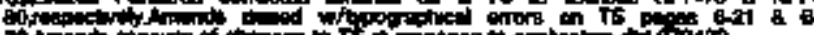

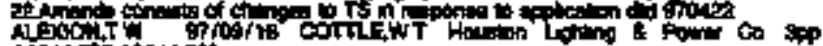

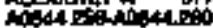

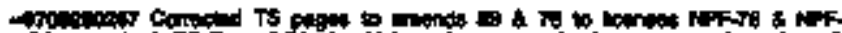

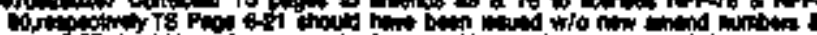

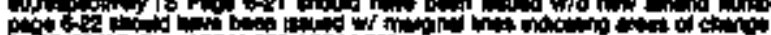

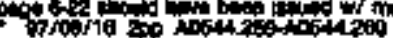

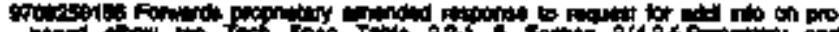

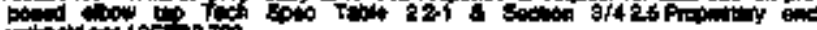
mition of $10 \mathrm{c}$ ine 7 \%

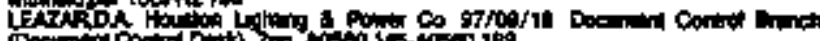

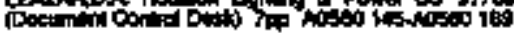

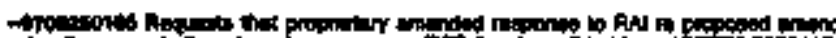

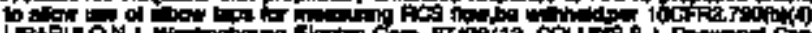

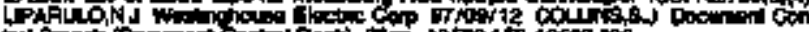

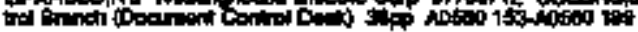

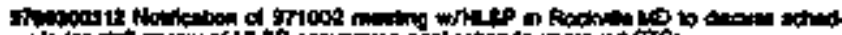

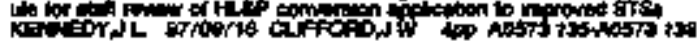

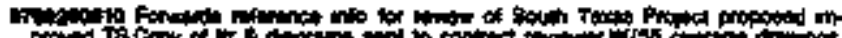

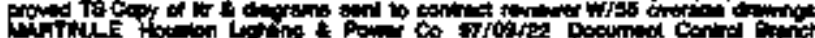

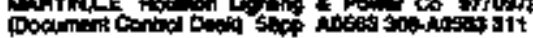

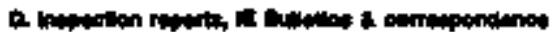

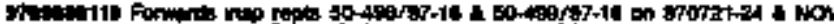

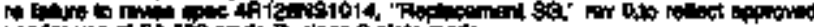

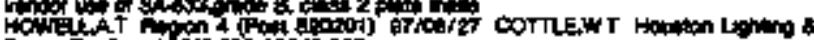

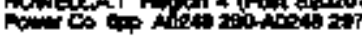

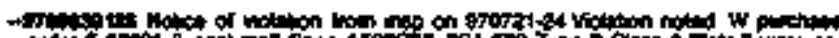

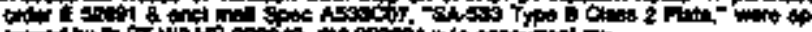

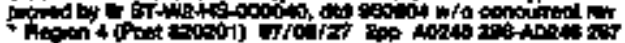

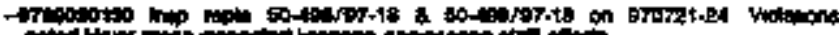

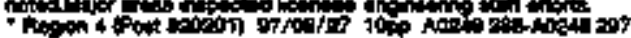

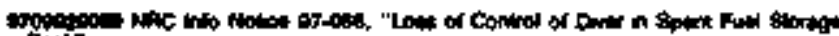
Poct"

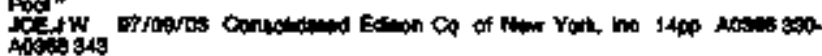

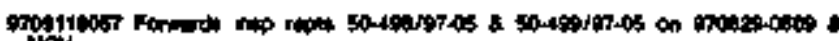
mo.

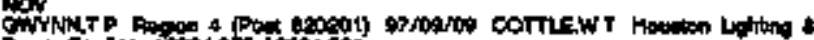

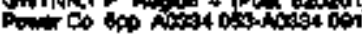

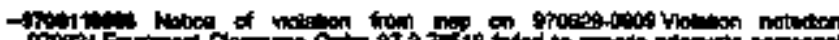
9rocel -

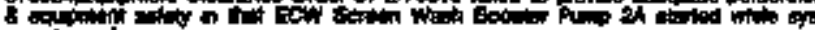

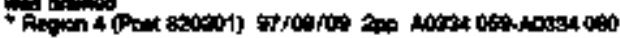

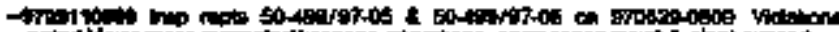

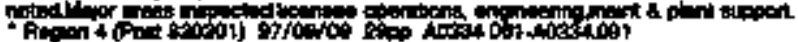

shom

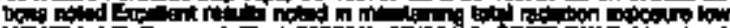

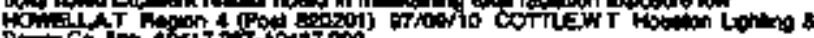

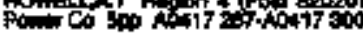

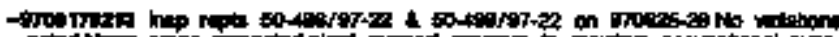

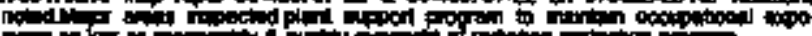

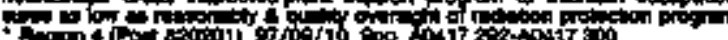

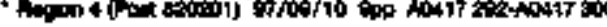

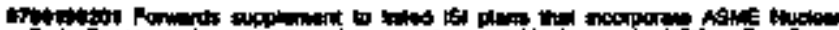

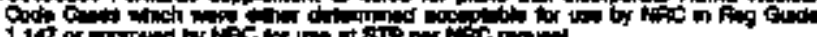

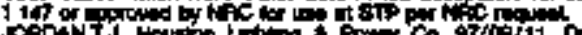

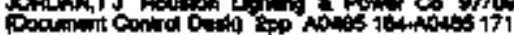

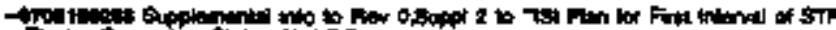

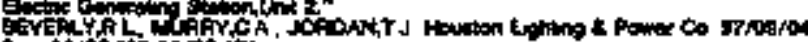
舟

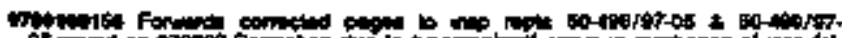

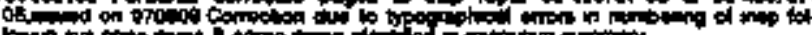

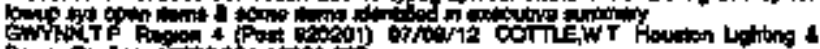

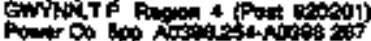

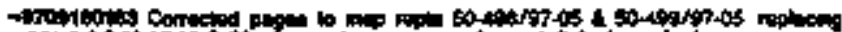

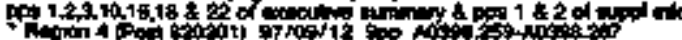

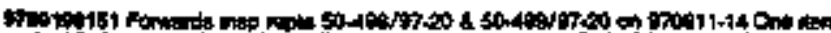

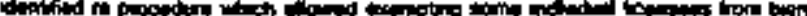

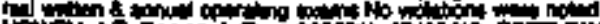

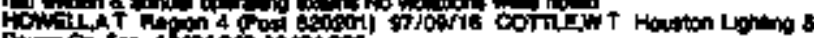

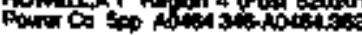

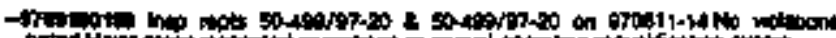

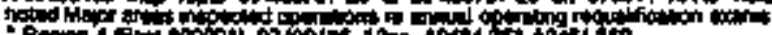

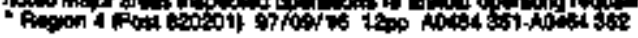

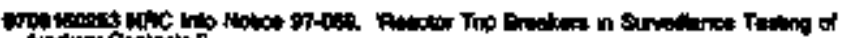

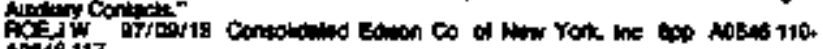
Ais: 197

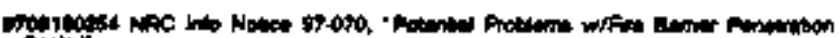

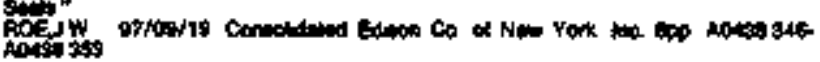

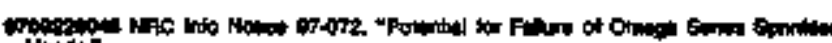

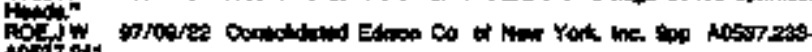

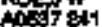

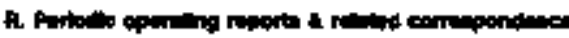

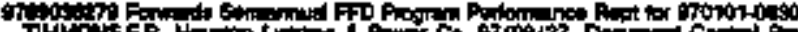

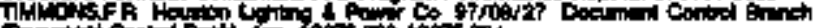

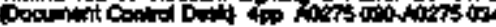

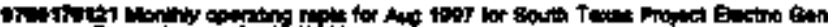
Gith stuch,

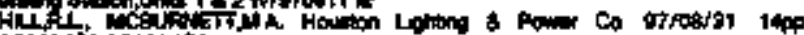

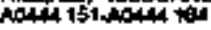

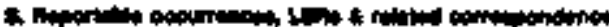

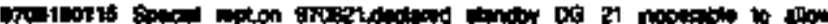

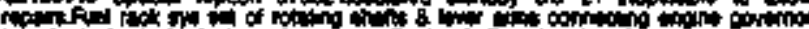

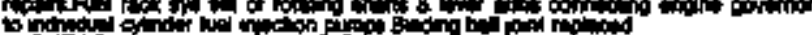

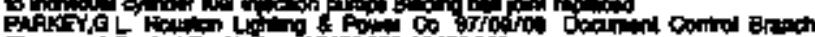

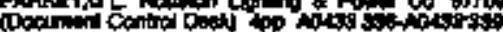

Y. Oparion Exominations

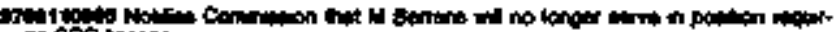

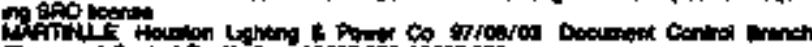

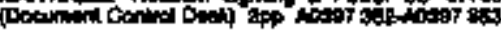

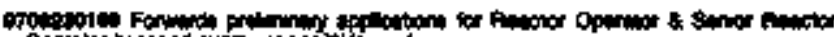

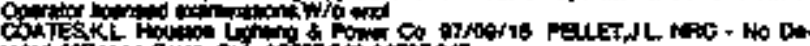

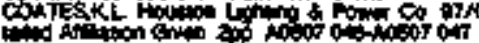

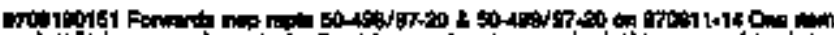

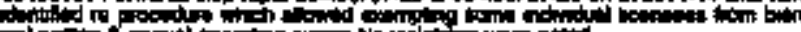
-

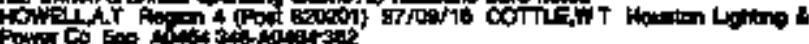

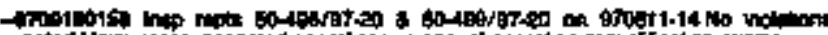

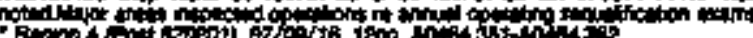

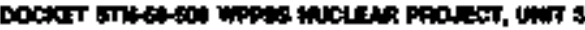

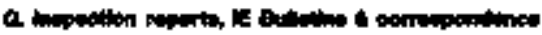

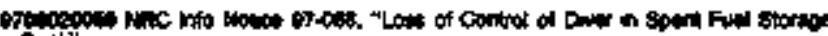

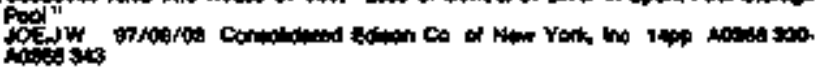

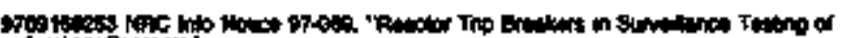

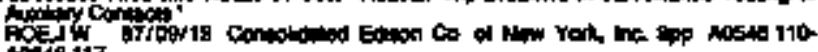
1048 117

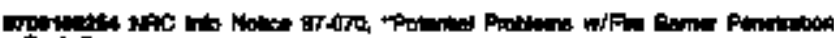

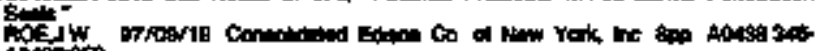
atom

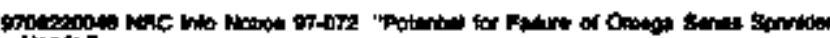

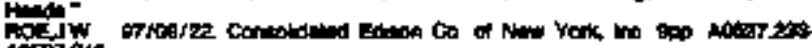
10637241

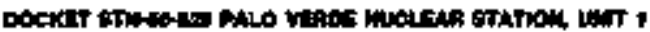

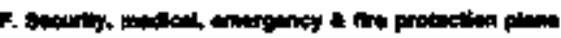

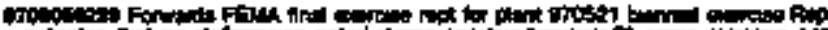

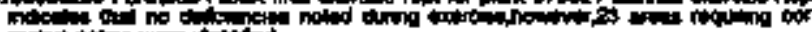
Fity

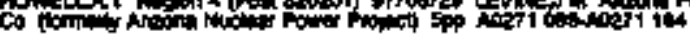

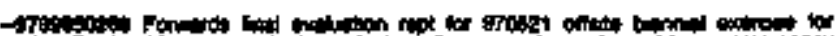

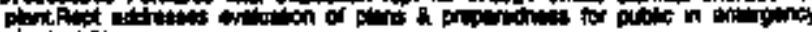

Pitlind

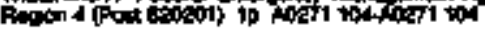




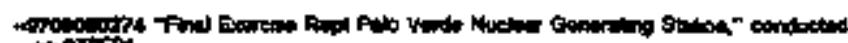

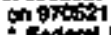

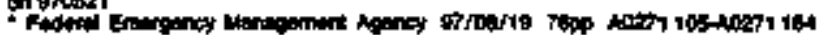

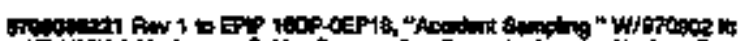

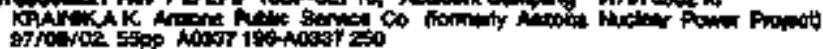

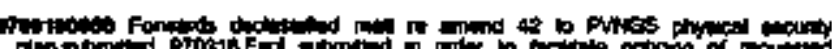

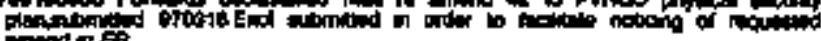

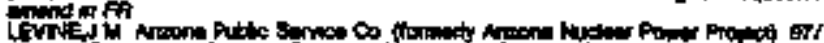

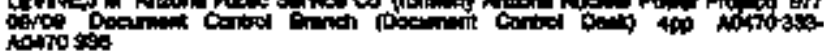

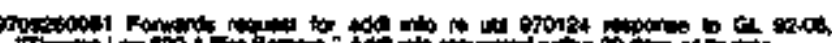
Thin Thowhs,

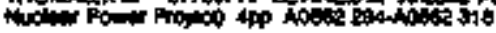

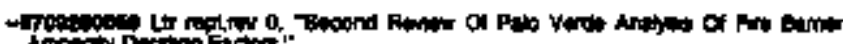

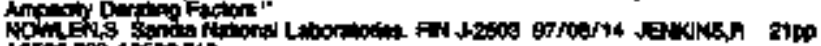

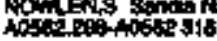

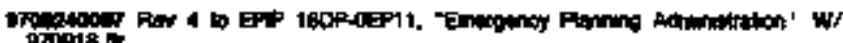
9 ,

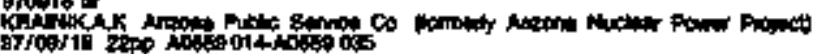

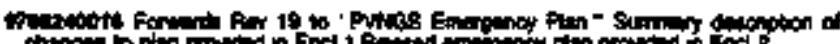

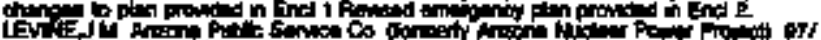

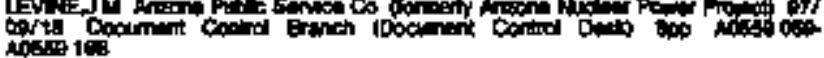

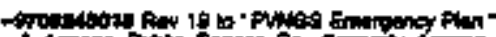

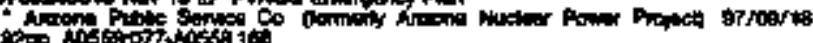

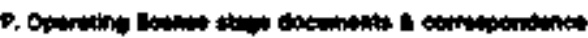

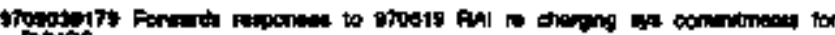

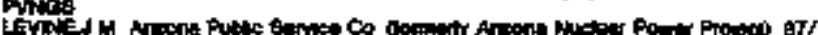

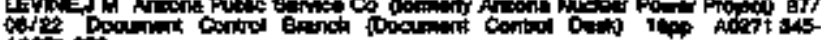
tion

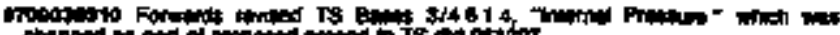

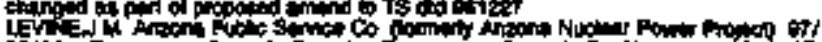

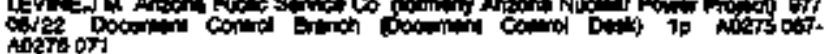

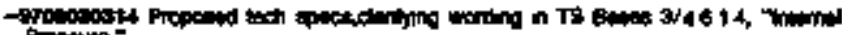

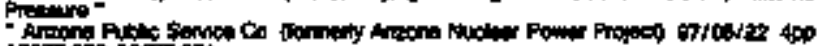

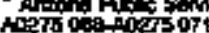

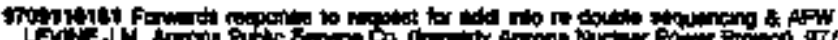

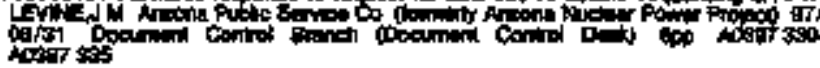

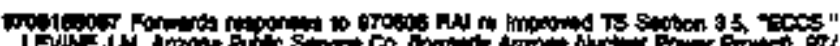

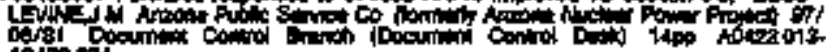
4042 a

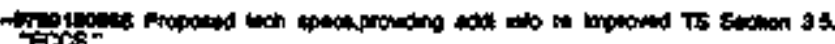
tracs

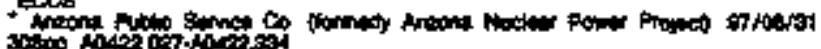

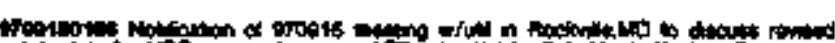

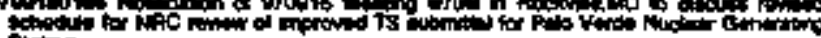

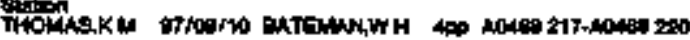

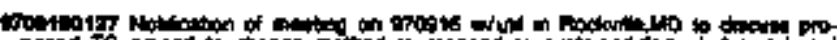

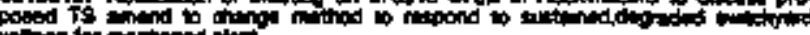

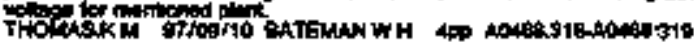

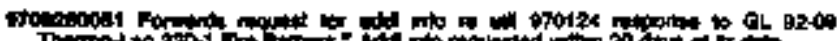

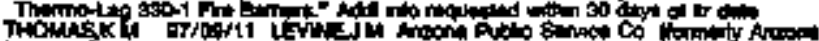

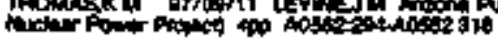

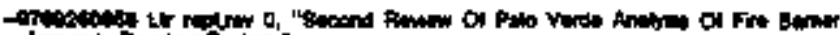

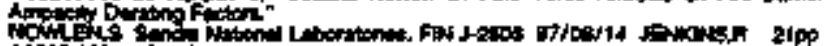

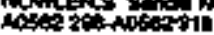

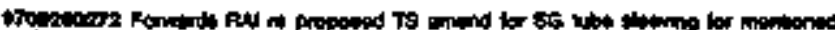

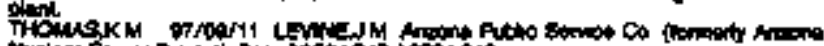

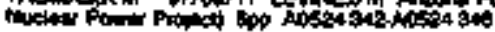

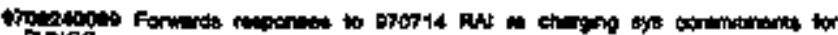
PYNas

I

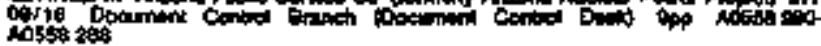

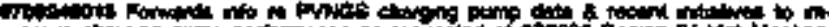

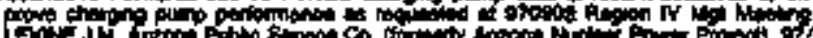

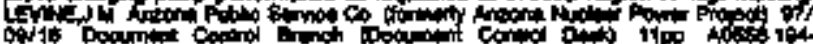
Hosis

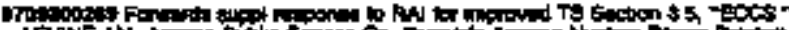

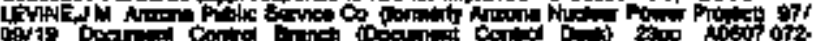
nogurout

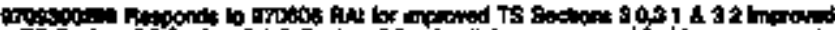
TS Salon 30 of

OHe EC intorio

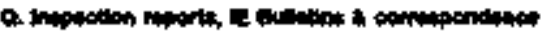

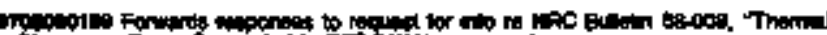

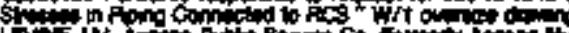

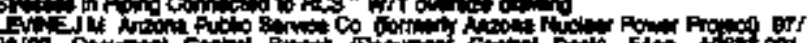

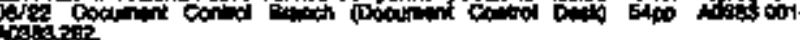
10303212

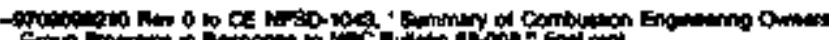

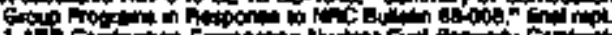

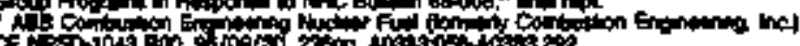

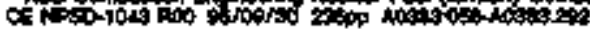

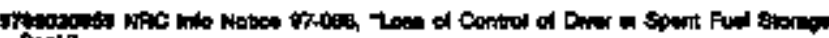
策

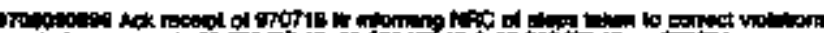

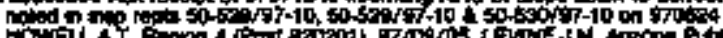

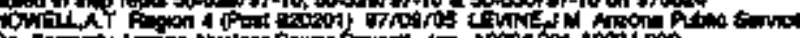

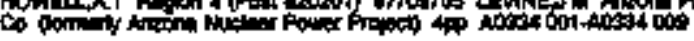

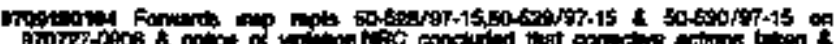

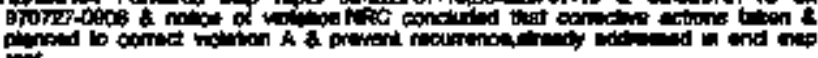

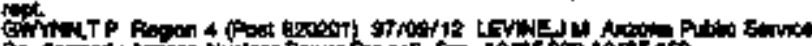

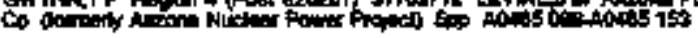

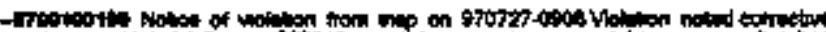
tolions in

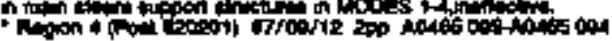

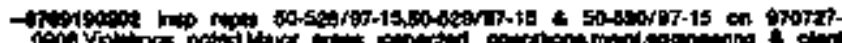

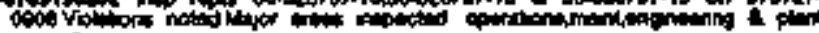

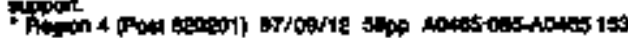

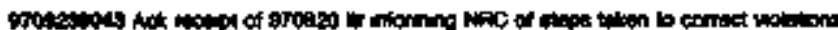

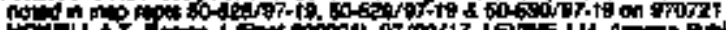
Col-L

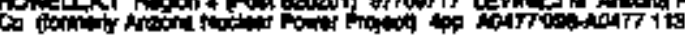

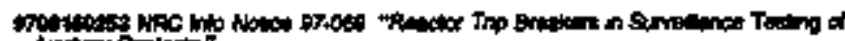

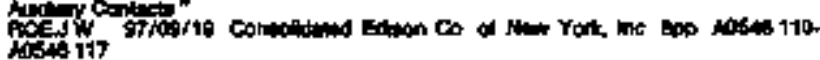

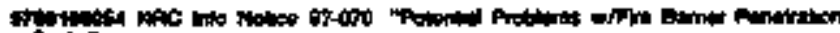

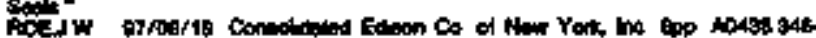
Aith

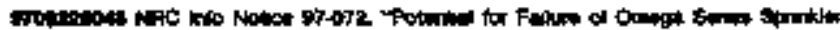

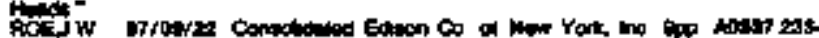
100972

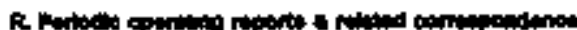

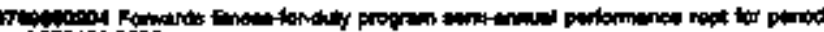

Kriflik A K A

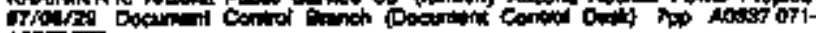
or

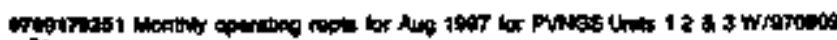

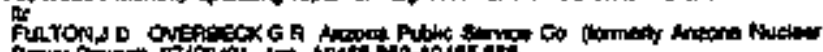

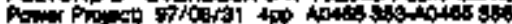

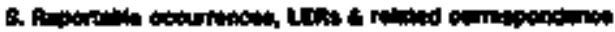

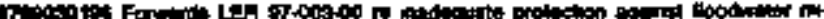

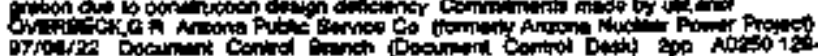

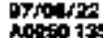




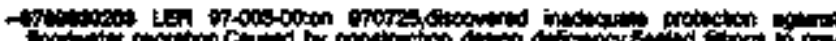

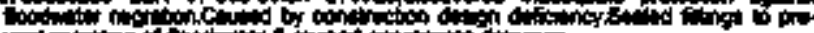

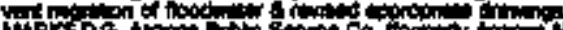

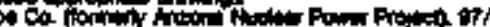

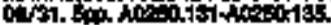

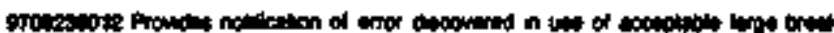

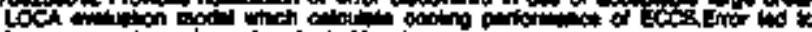

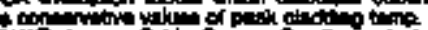

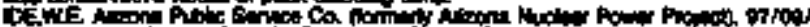

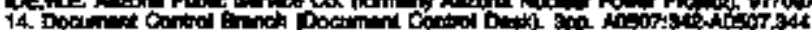

Doche som

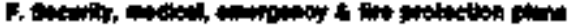

DH:

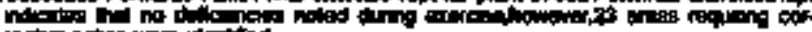

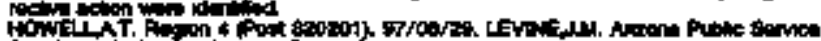

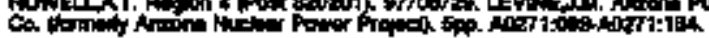

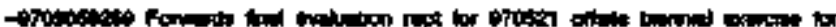

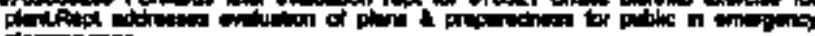

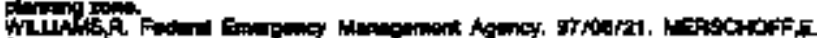

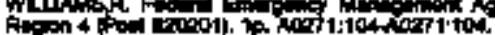

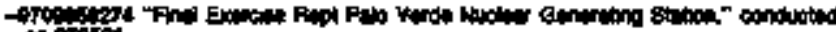

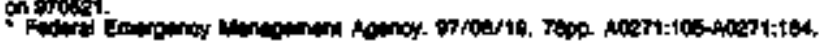

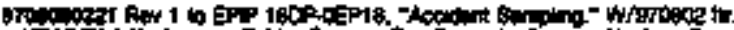

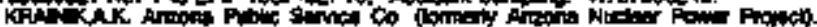

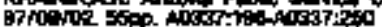

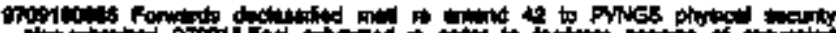

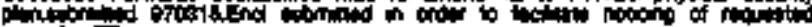

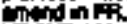

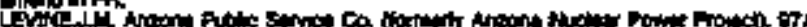

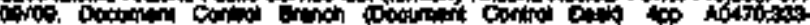
1970 309

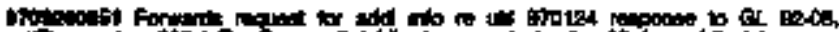

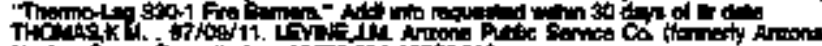

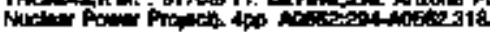

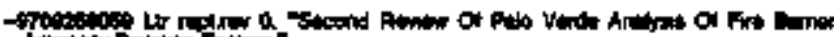
Ninction

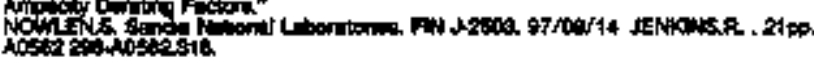

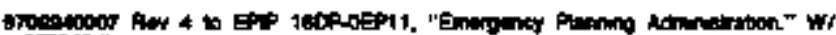
molit.

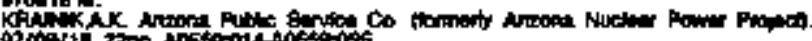

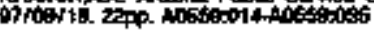

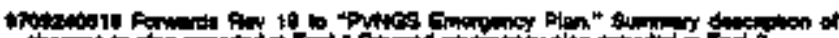

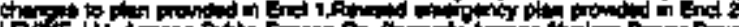

IV $\lim _{10}$

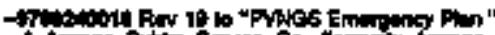

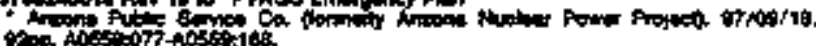

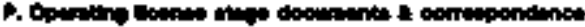

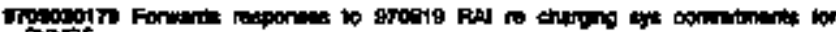

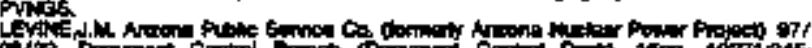

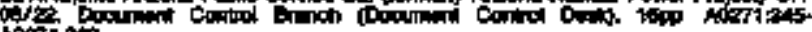

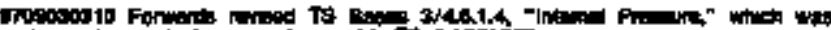

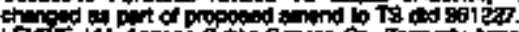

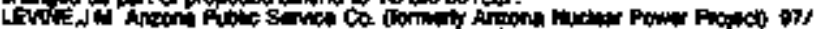

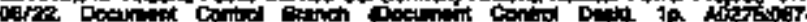
Ants the

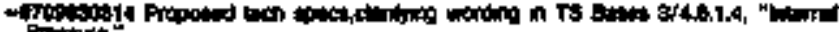

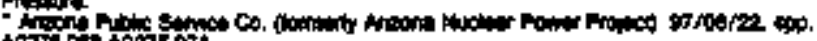

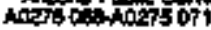

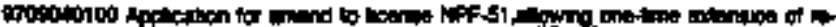

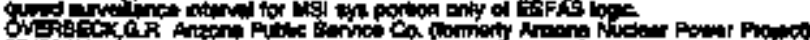

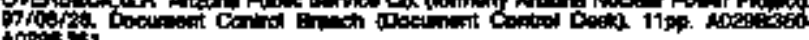
notions

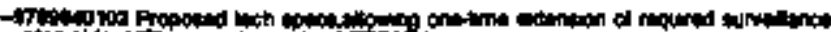

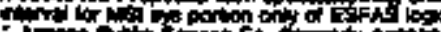

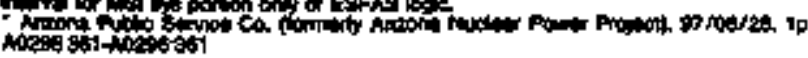

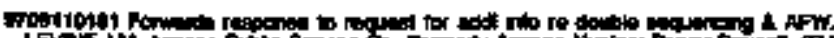

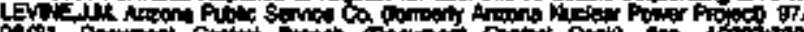

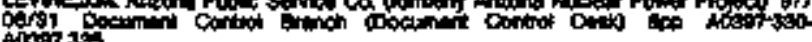

O7. L

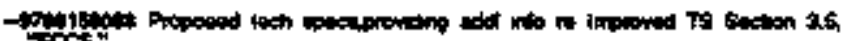
2005

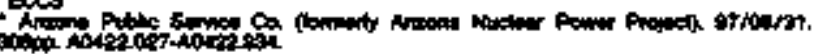

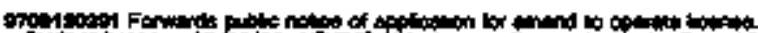

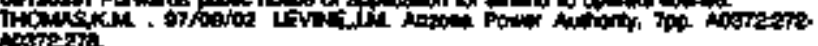

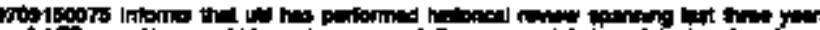

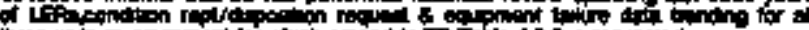

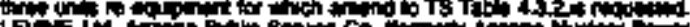

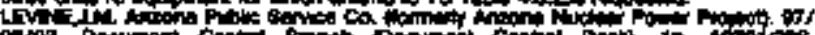

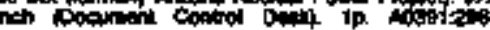
aspitan

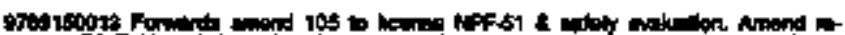

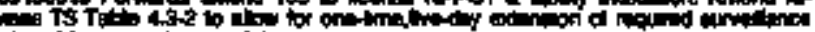

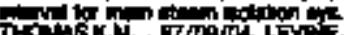

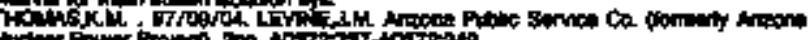

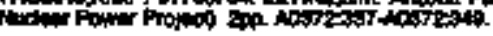

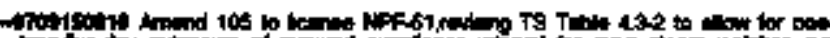

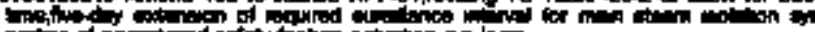

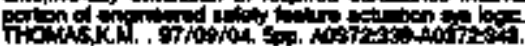

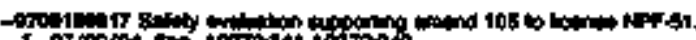

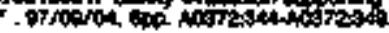

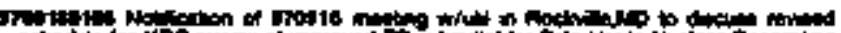

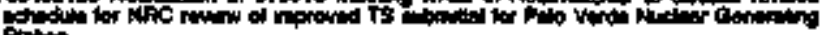

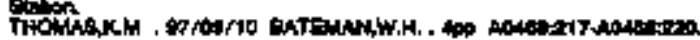

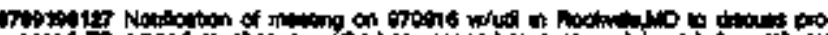

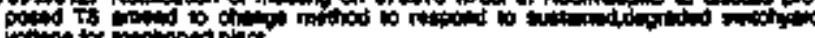

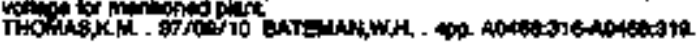

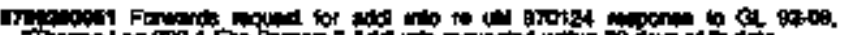
fhe

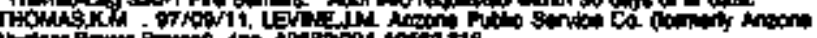

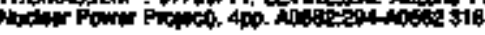

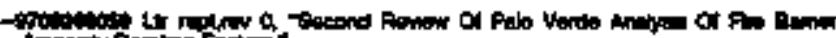

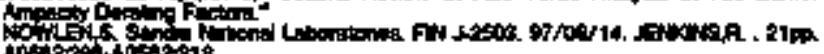

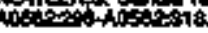

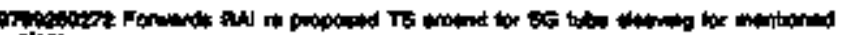

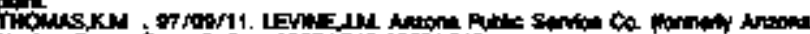

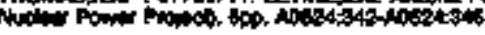

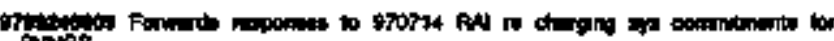
prives.

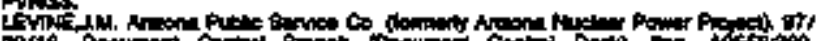

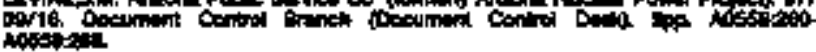

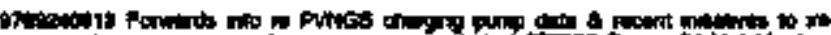

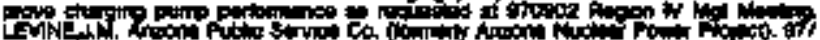

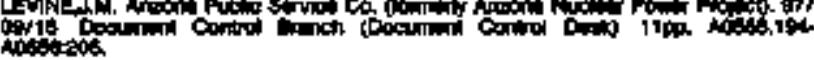

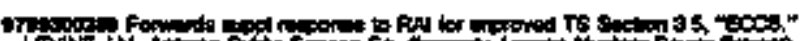

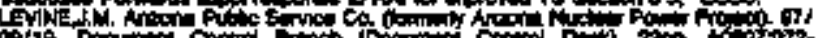

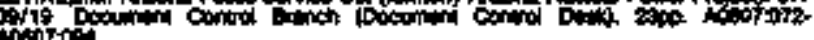

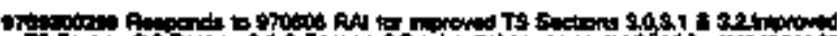

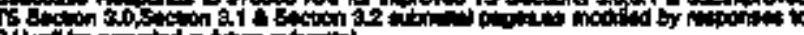
A

ow notorets

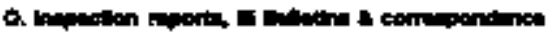

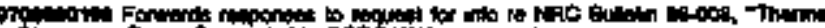

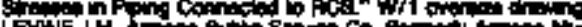

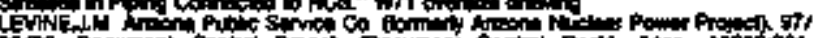

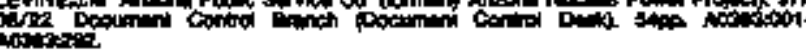

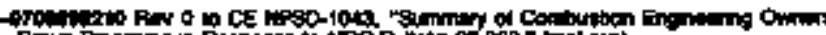

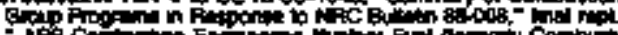

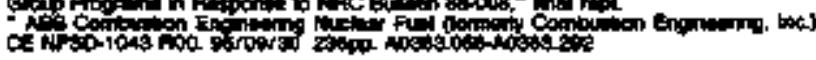

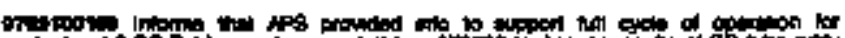

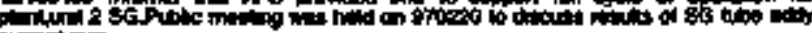

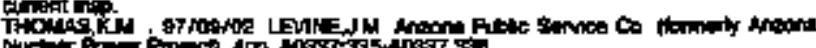

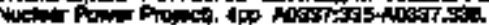




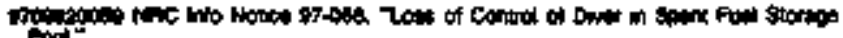

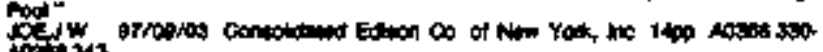
40

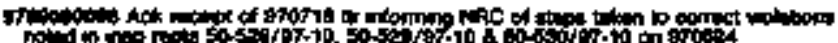

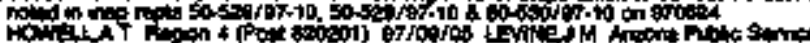

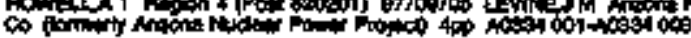

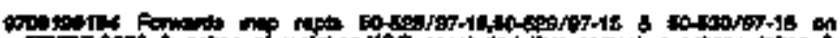

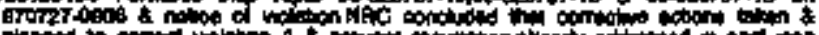

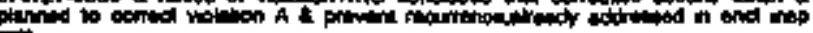

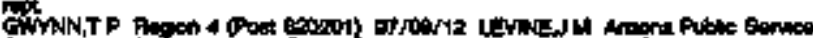

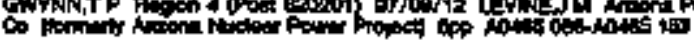

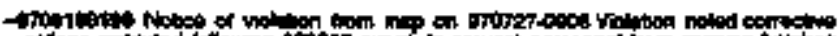

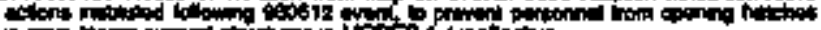

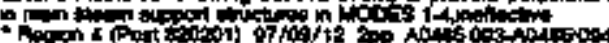

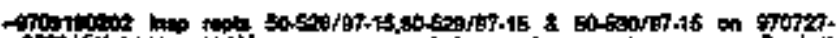

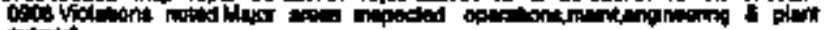

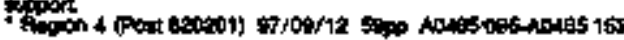

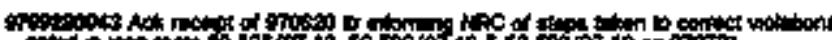

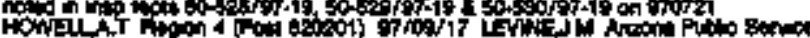

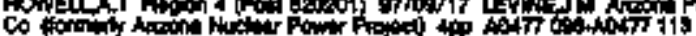

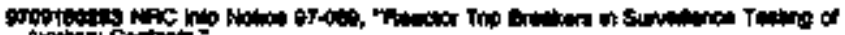

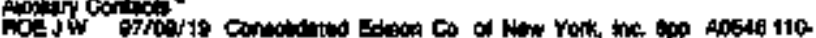
10.0. 117

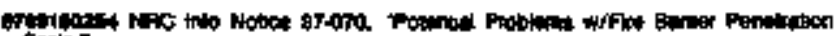

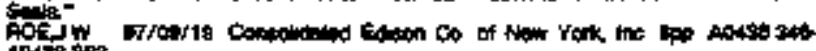
AD4:S

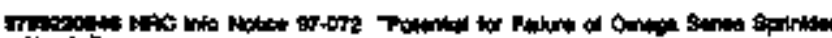

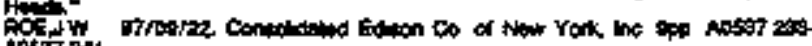

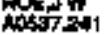

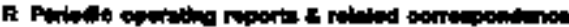

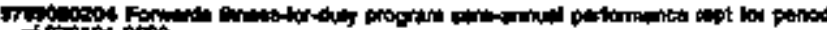
$0+0101-0.50$

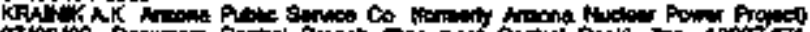

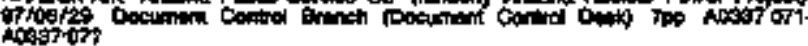

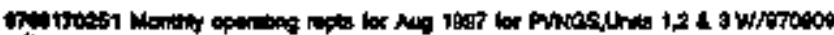

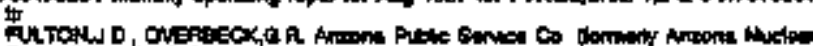

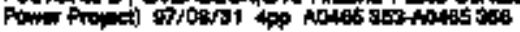

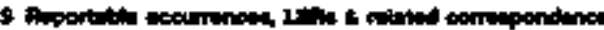

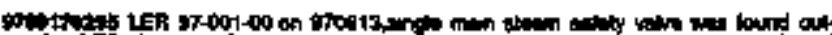

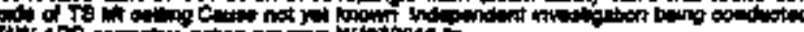

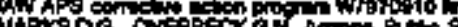

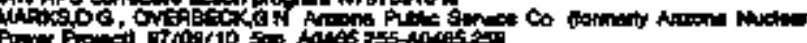

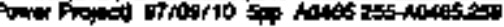

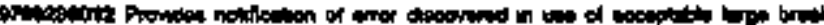

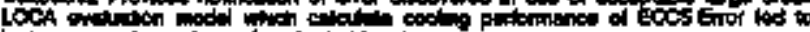

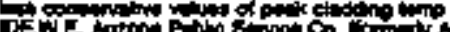

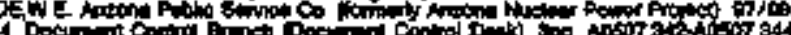

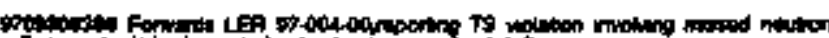

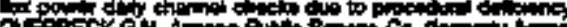

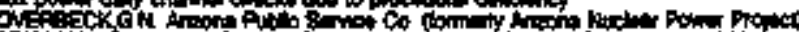

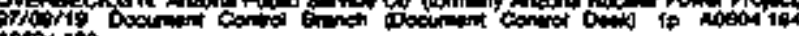
Noso4 1 to

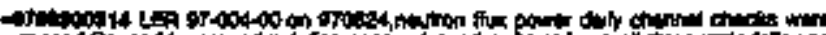

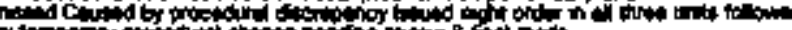

作

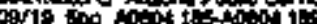

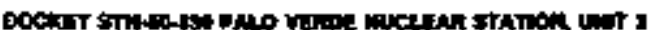

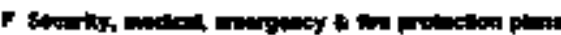

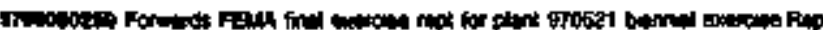

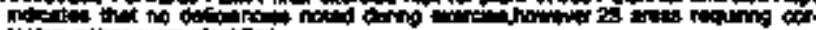

Hon

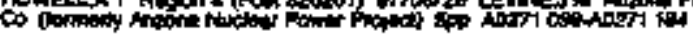

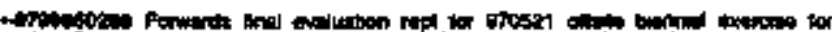

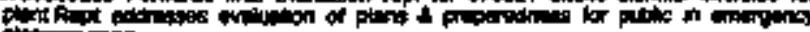

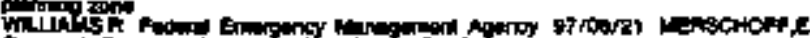
Arogen 4 (f)

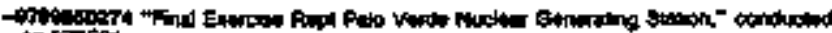

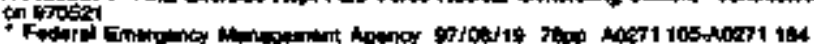

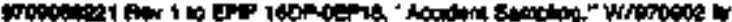

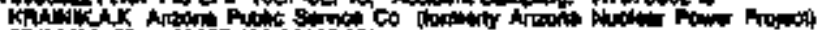

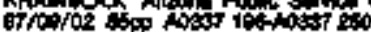

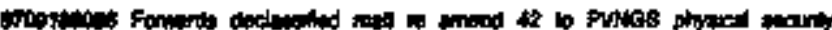

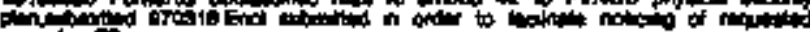

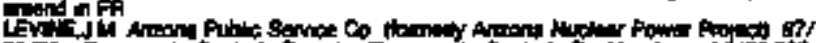

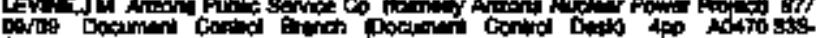
A04 303

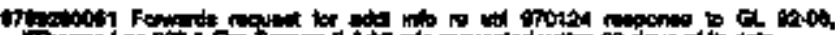

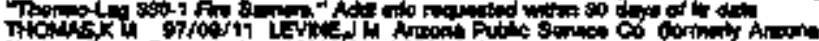

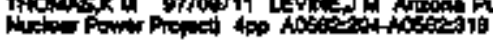

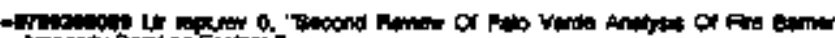
Aroin

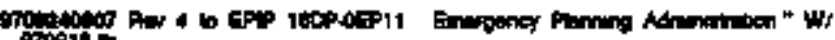
Tion

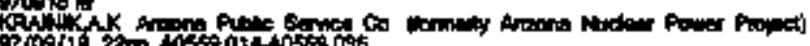

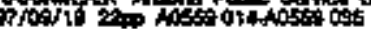

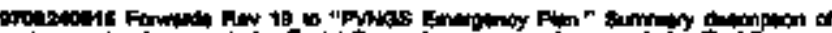

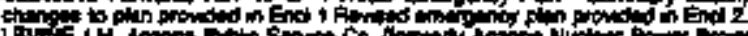

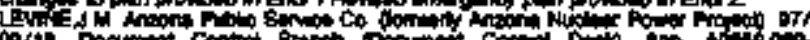

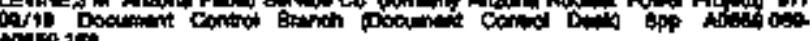
Ades 180

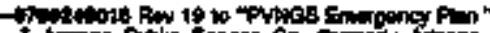

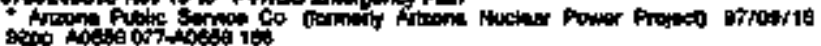

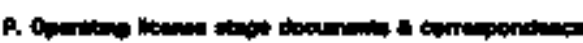

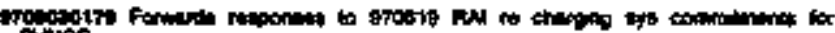

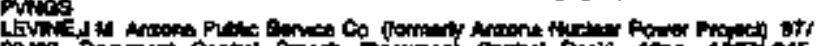
inger 300

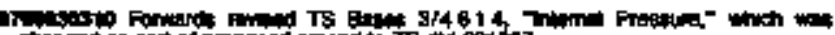

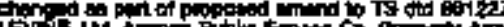

Win arem ort

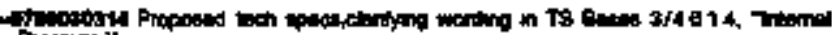

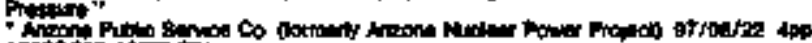

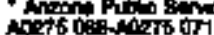

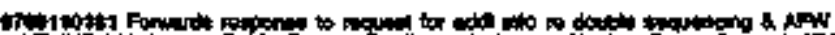

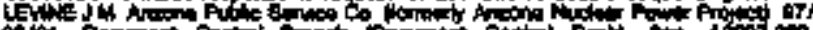

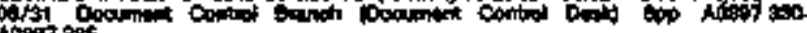
tho os

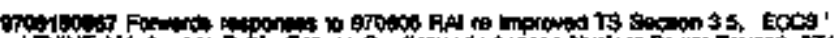

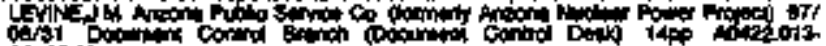
0.191

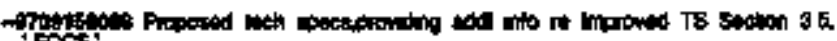
EDes

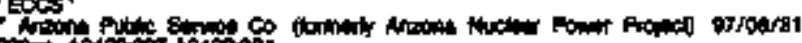

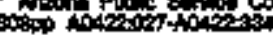

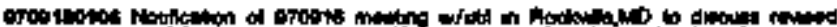

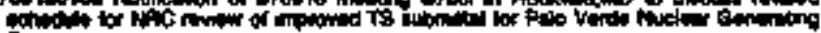

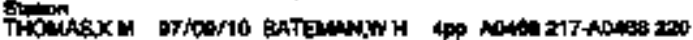

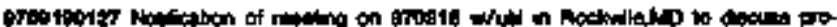

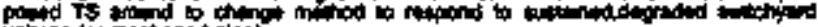

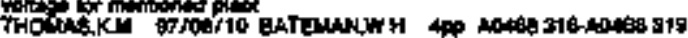

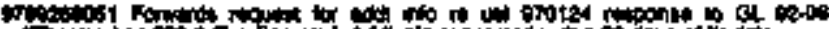

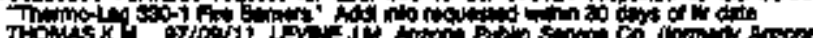

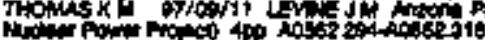

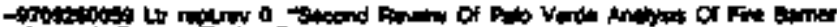

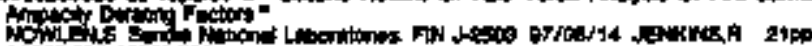

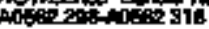

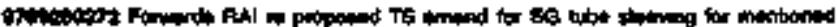

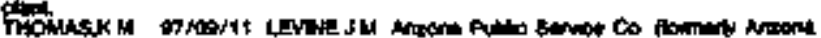

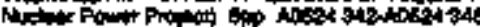

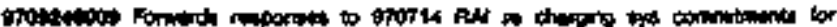
Prives

ly

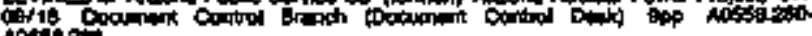
notio a 


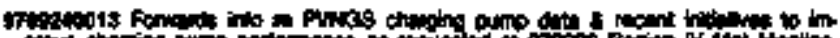

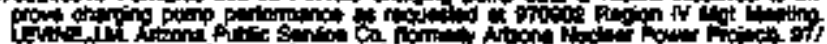

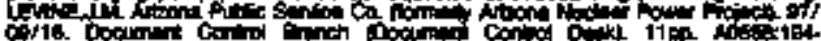
Q18.

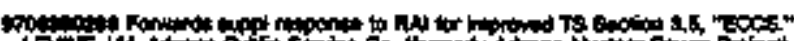

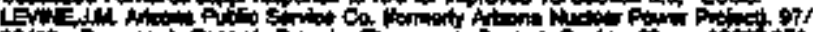

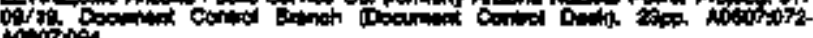

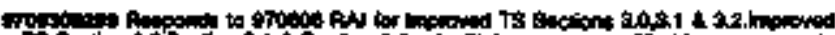

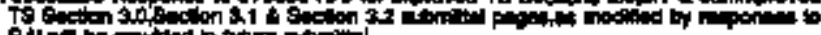

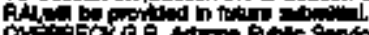

OY

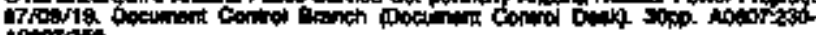
40000 ind

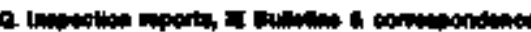

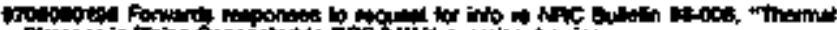

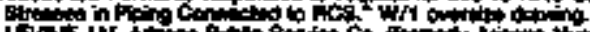

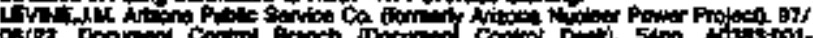
(1)

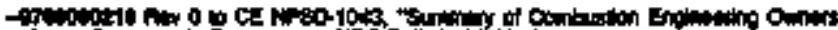

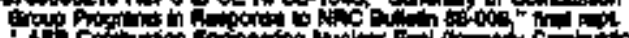

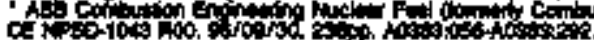

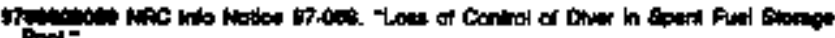

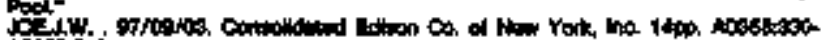
antivis.

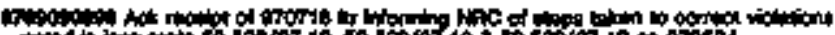

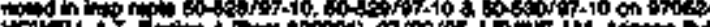

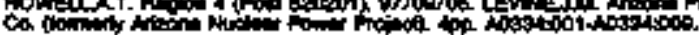

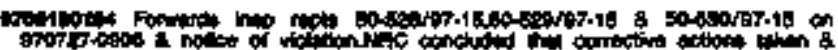

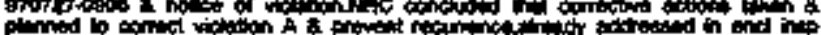

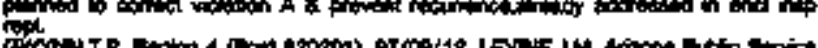

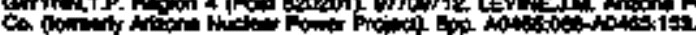

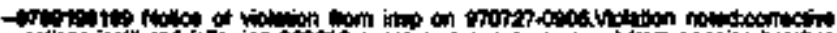

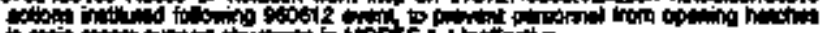

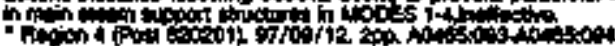

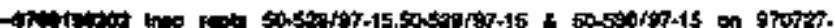

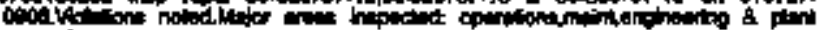

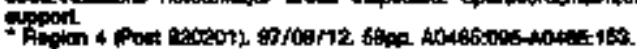

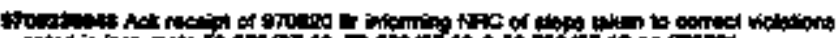

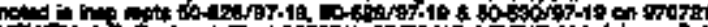

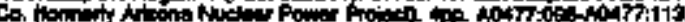

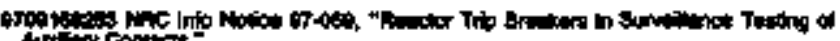

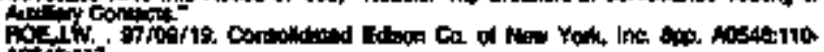
Aititivit.

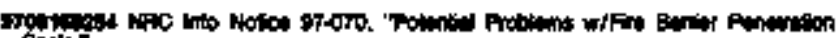

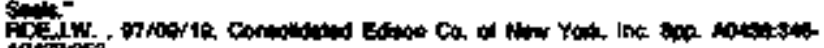
Natios;

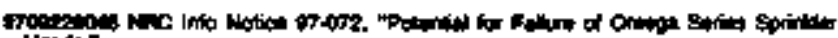

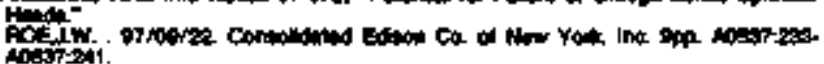

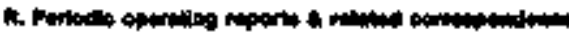

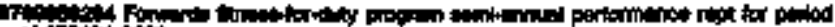
of 0 intotion

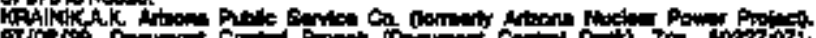

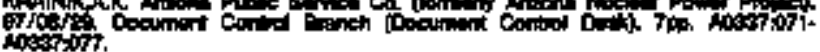

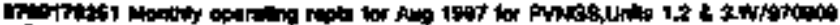

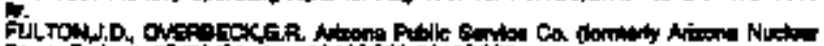

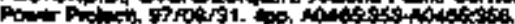

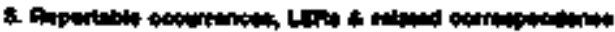

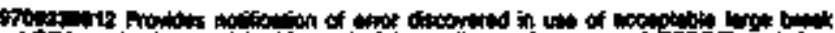

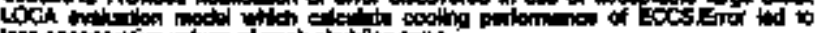

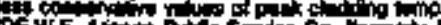

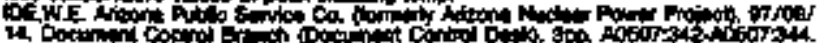

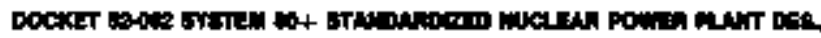

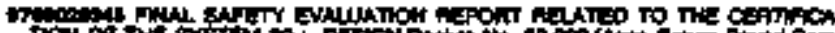

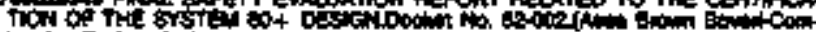

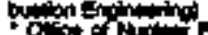
ato

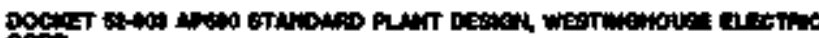
cotin.

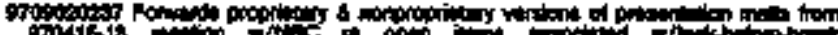

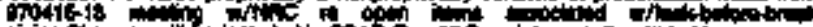

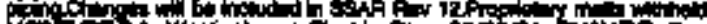

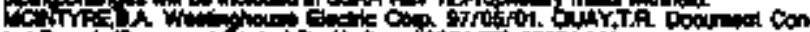

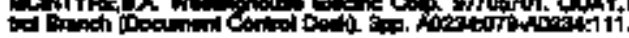

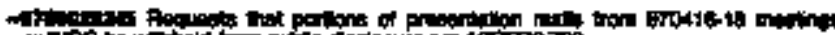

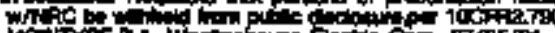

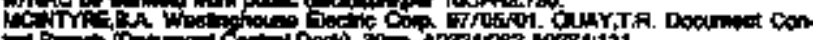

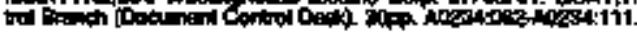

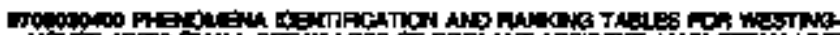

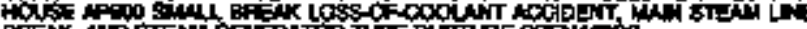

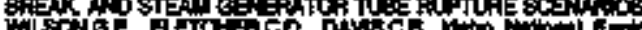

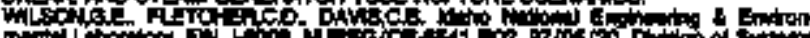
Drition of sycint

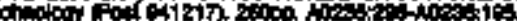

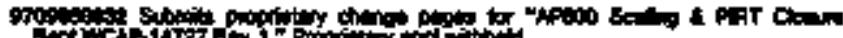

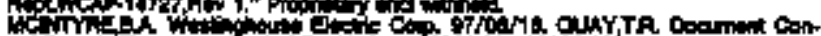

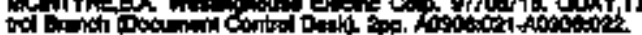

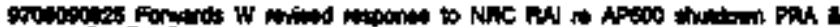
itcin Foothy

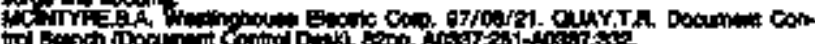

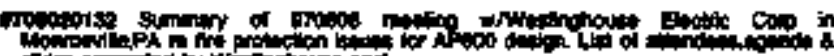

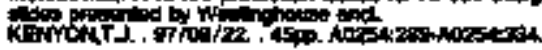

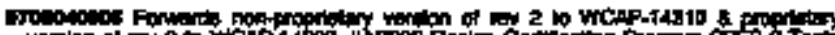

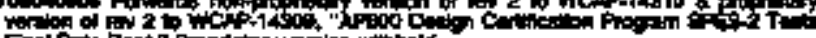

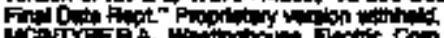

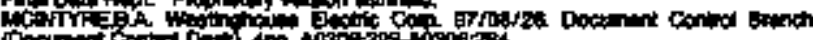

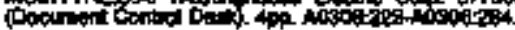

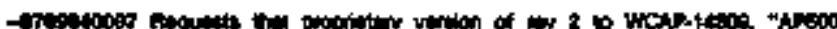

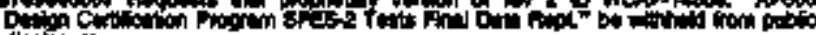

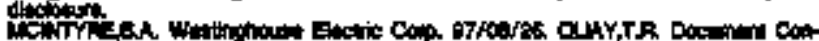

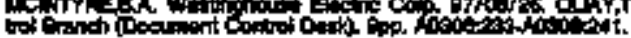

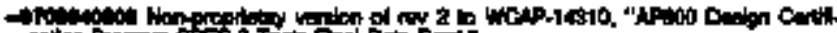

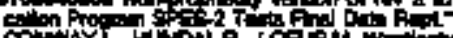

Colar.

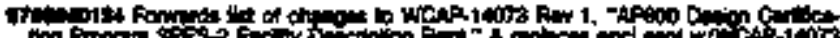
boa Pror: in wotos.

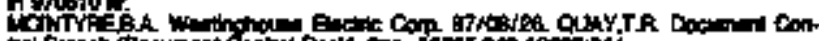

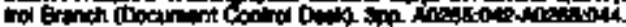

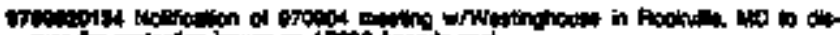
gese

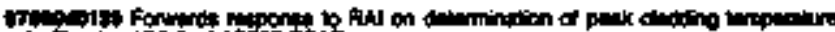

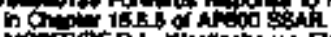

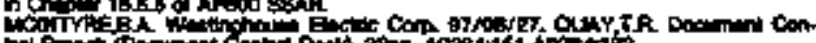

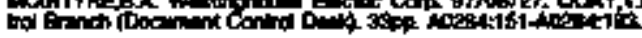

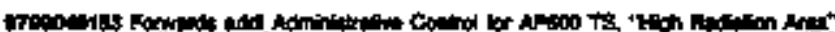

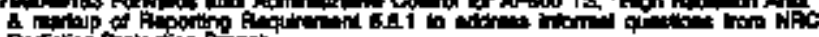

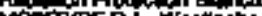

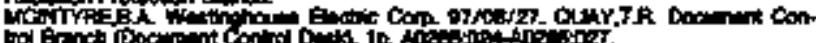

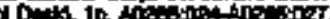

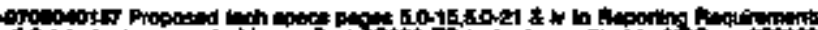

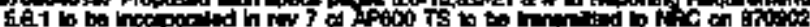

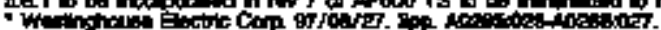

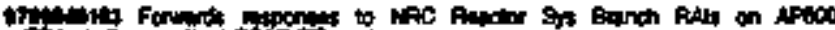

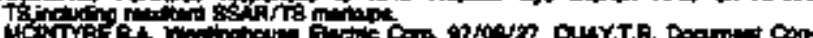

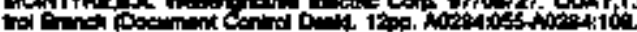

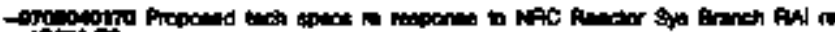

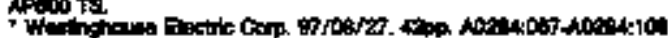

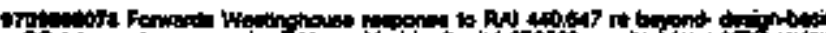

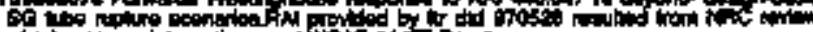

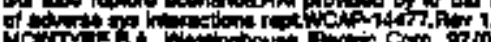

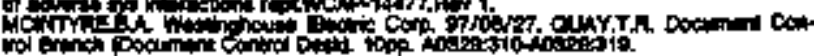

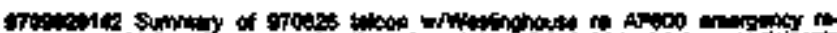

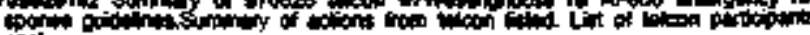

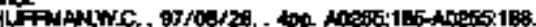




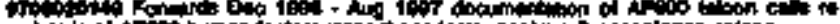

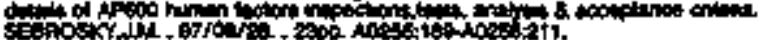

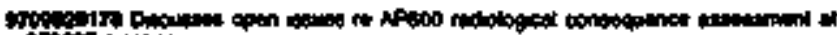

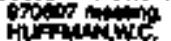

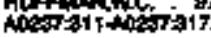

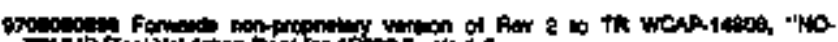

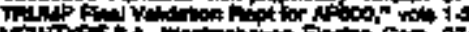

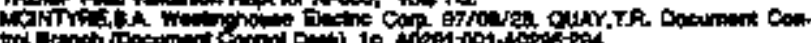

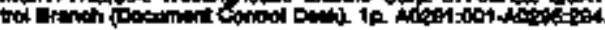

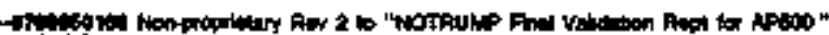

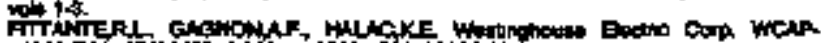

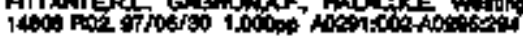

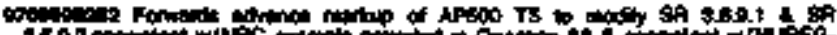

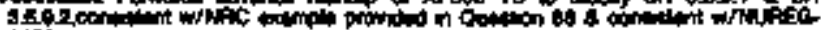
testo

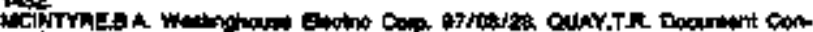

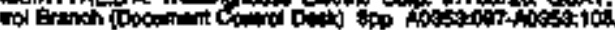

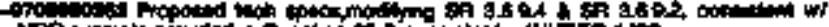

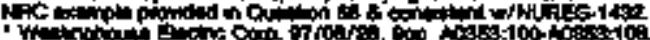

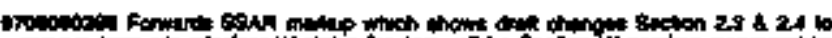

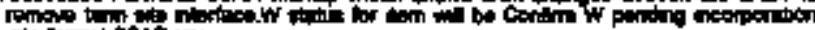

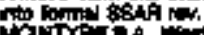

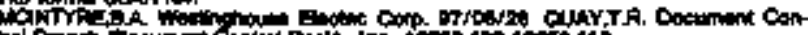

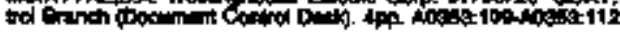

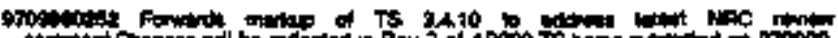

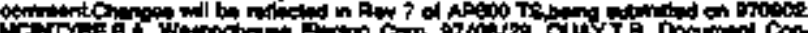

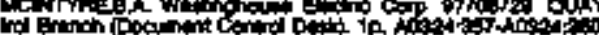

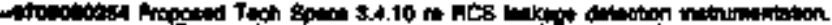

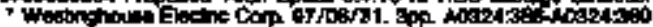

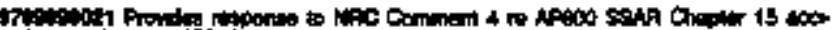

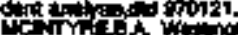

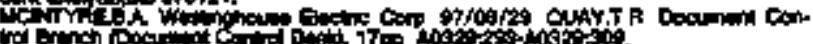

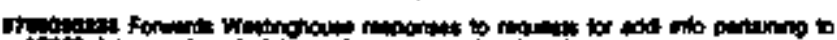

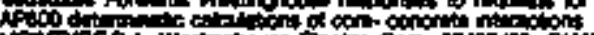

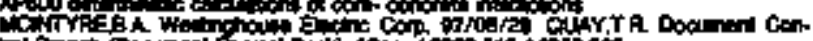

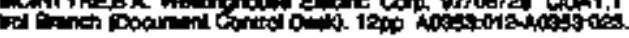

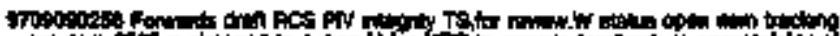

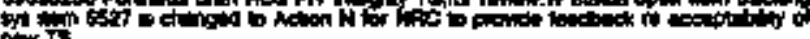

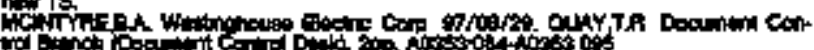

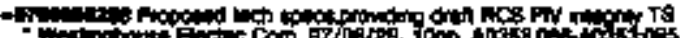

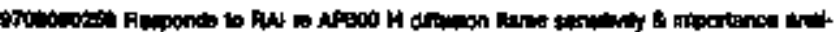

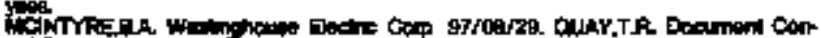

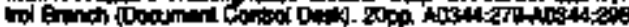

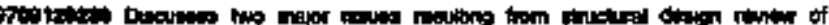

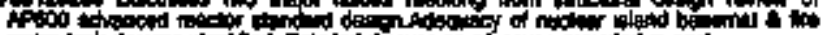

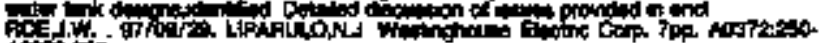

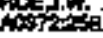

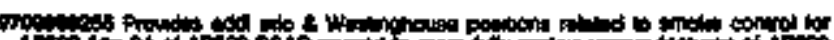

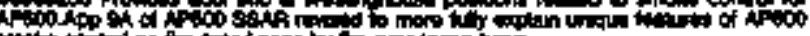

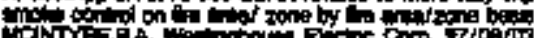

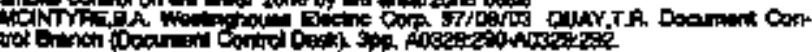

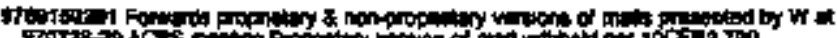

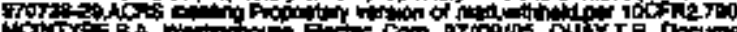

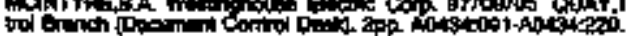

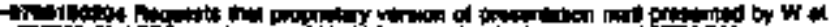

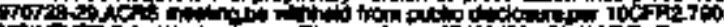

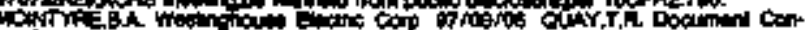

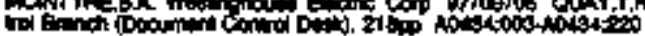

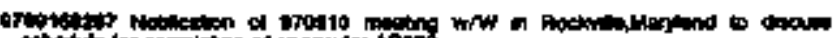

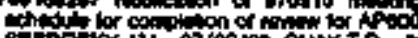

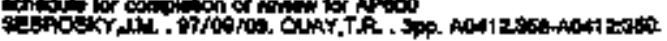

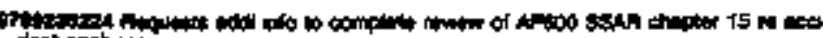

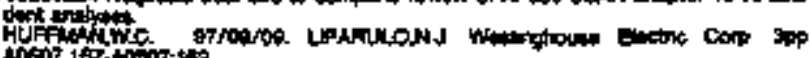

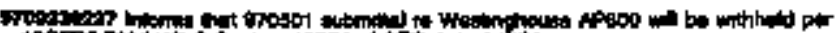

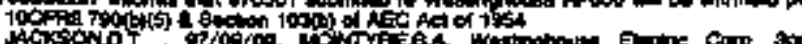

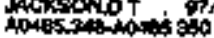

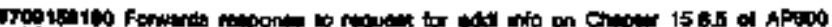

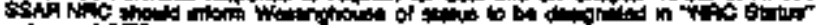

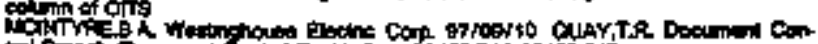

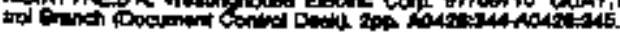

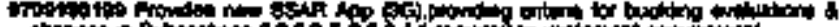

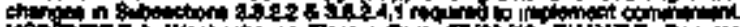

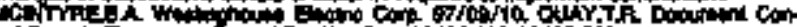

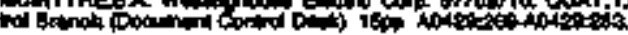

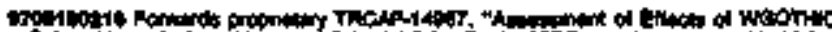

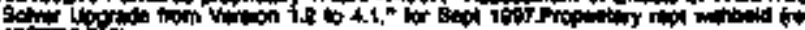

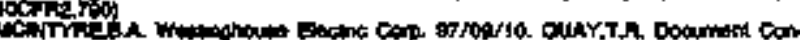

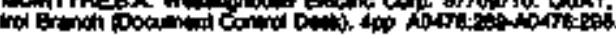

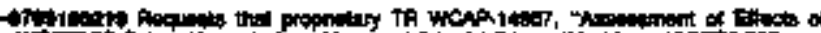

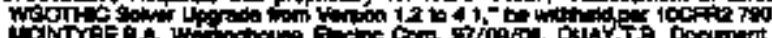

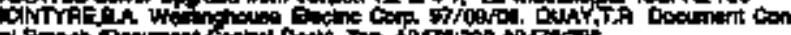

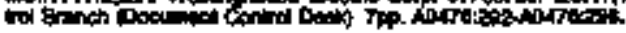

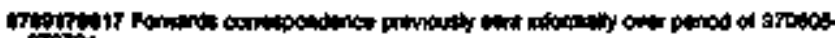
9ropit

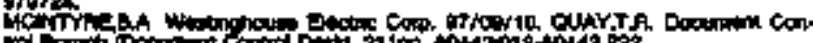

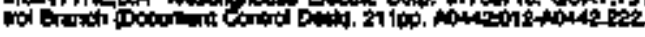

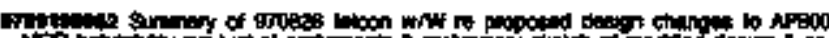

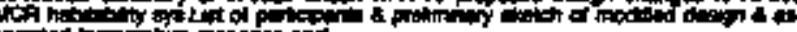

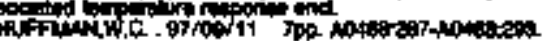

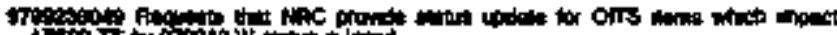

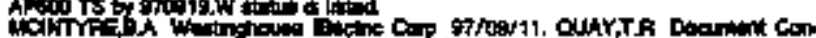

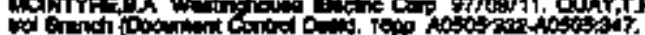

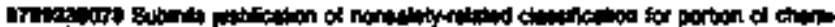

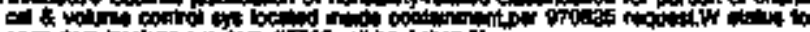

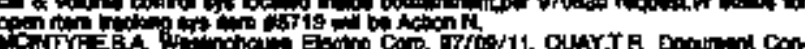

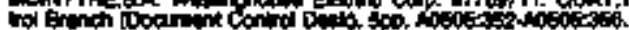

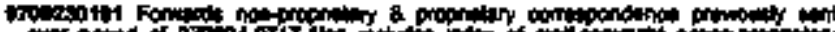

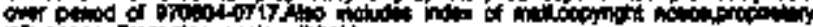

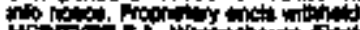

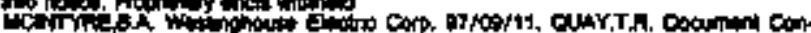

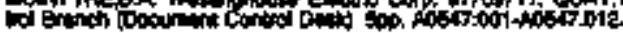

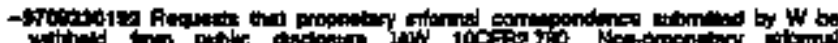

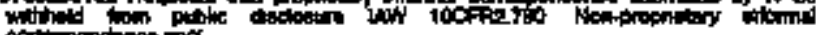

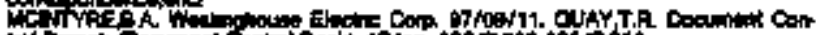

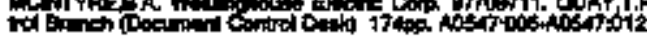

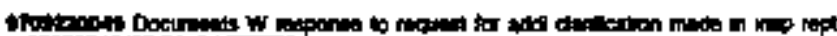

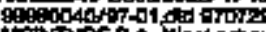

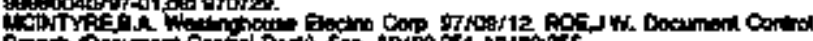

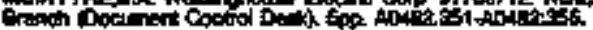

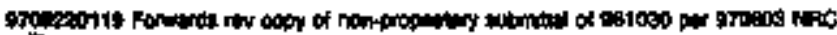

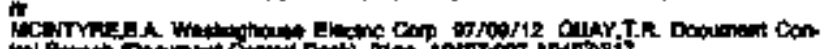

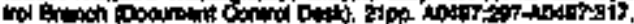

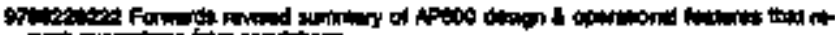

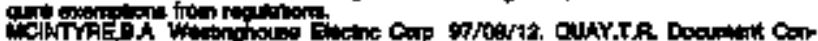

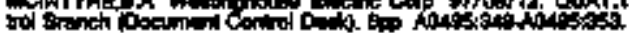

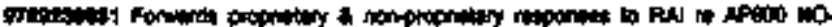

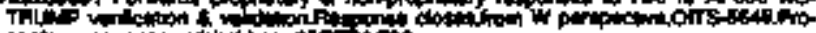

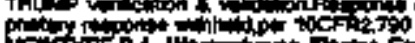

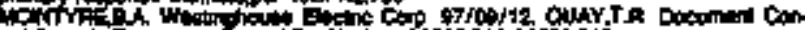

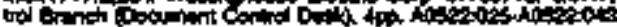

- Ton:

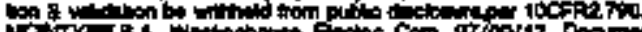

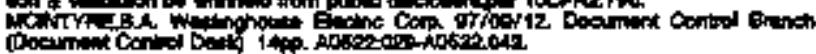

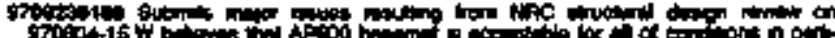

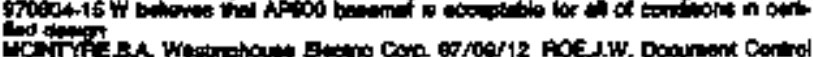

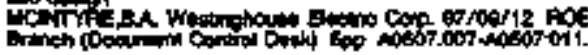

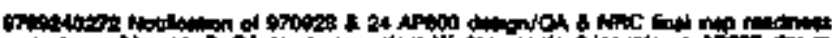

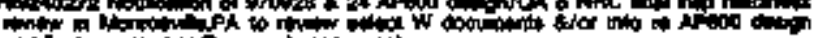

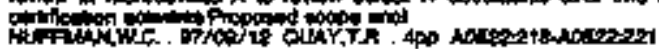

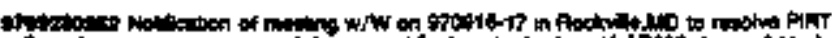

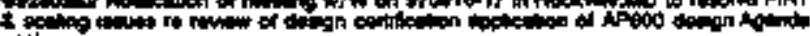

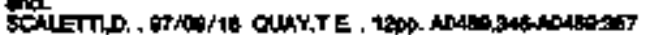

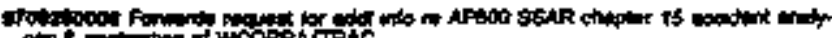

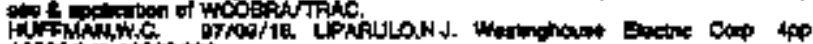
iosoming

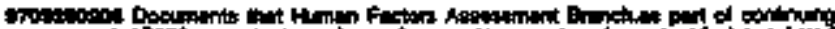

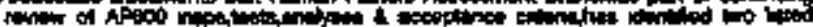

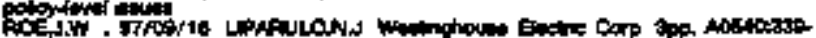
$\cos 40 \sin$

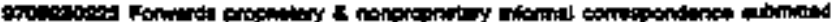

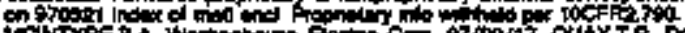

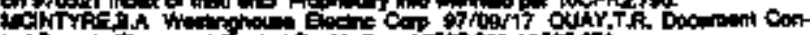

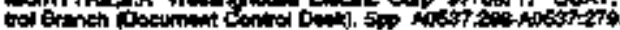




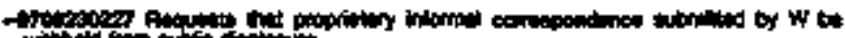

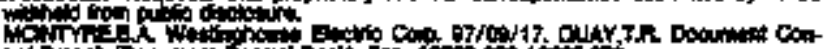

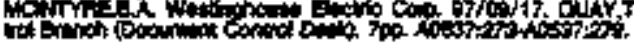

IF

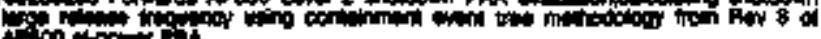

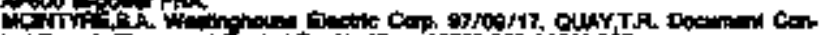

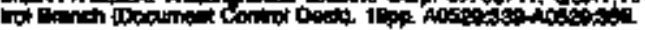

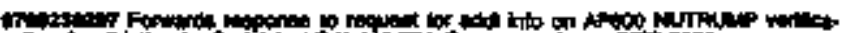

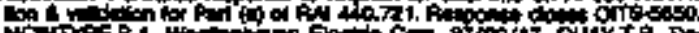

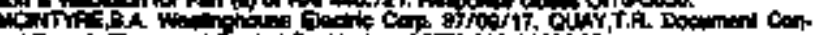

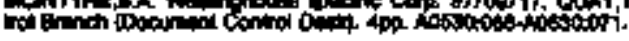

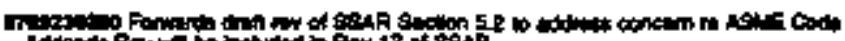

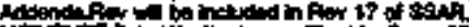

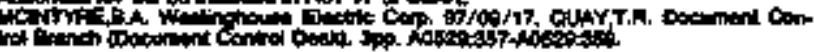

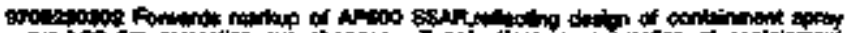

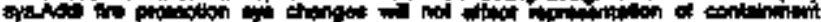

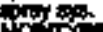

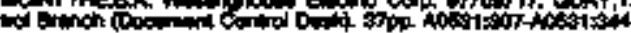

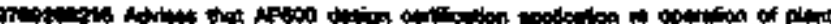

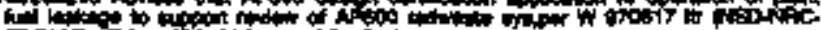

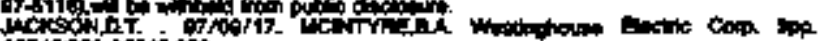

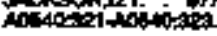

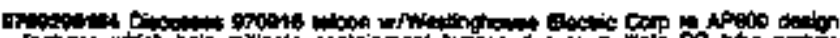

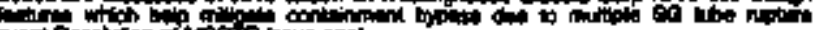

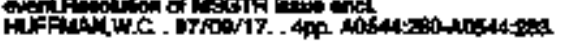

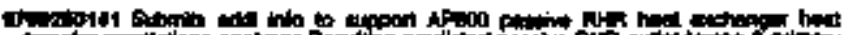

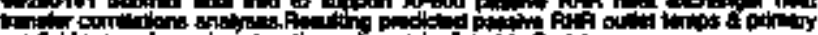

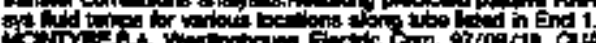

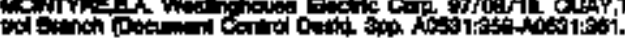

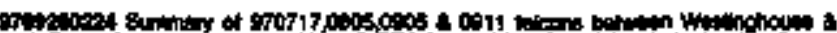

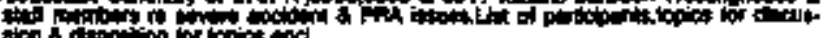

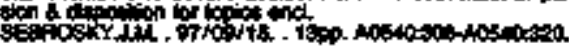

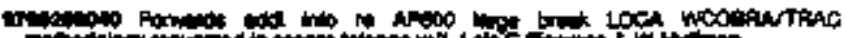

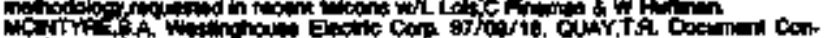

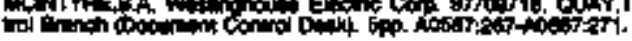

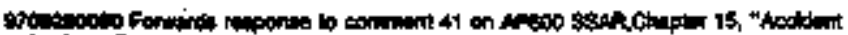

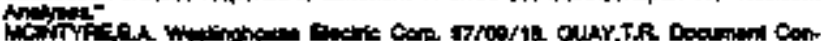

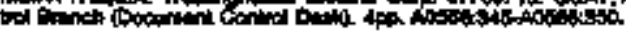

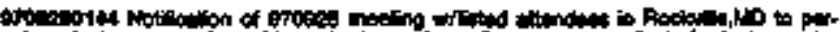

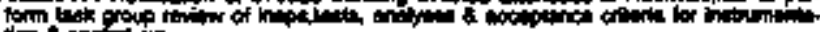

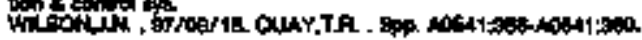

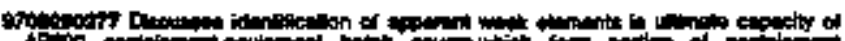

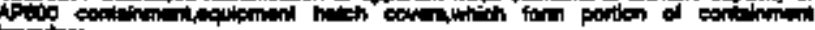

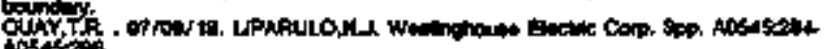

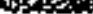

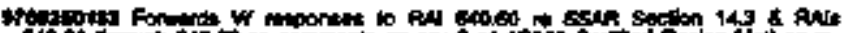

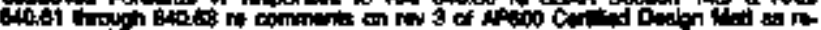
Fitivith

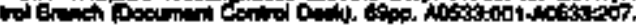

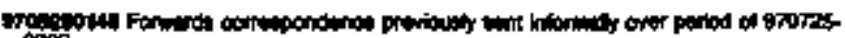

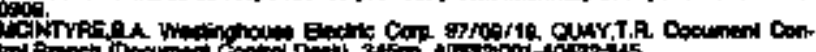

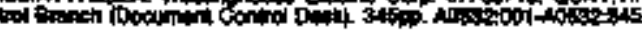

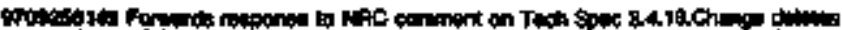

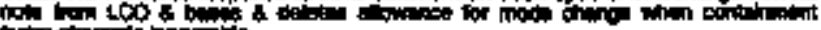

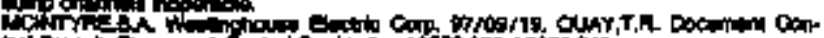

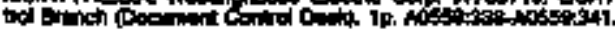

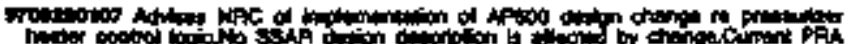

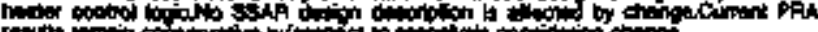

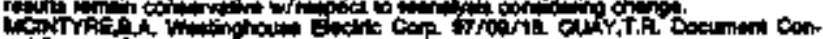

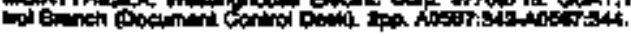

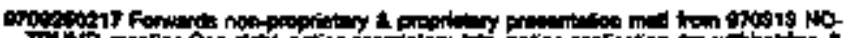

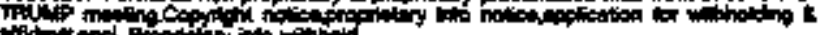

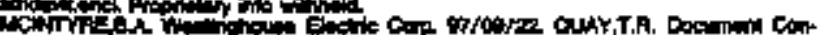

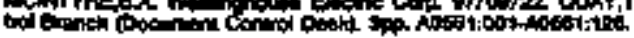

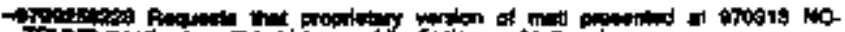

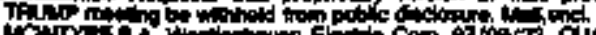

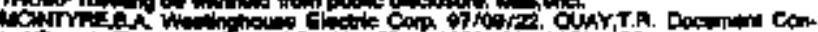

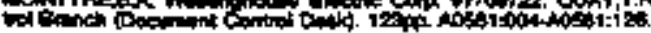

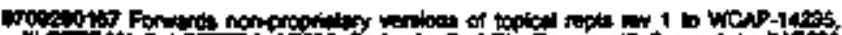

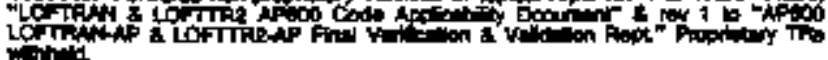
Hing

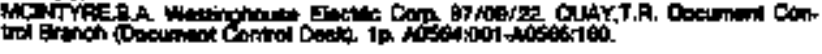

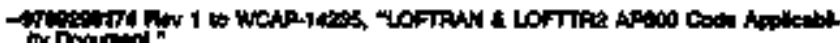

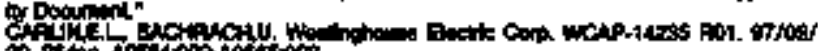

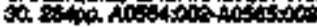

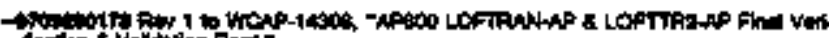

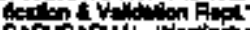

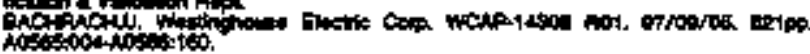

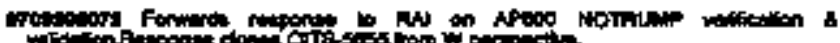

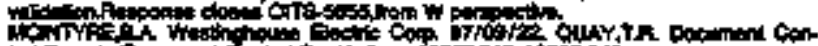

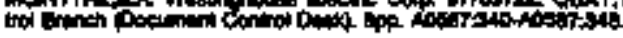

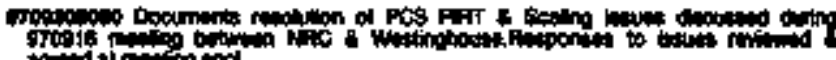

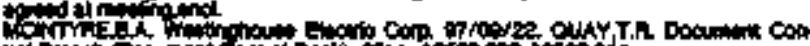

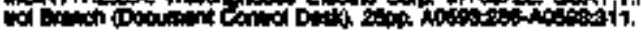

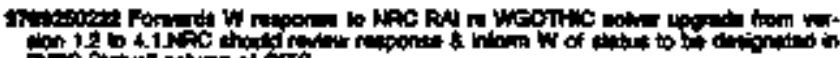

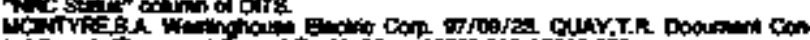

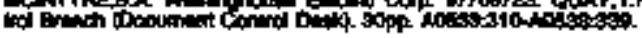

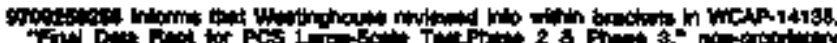

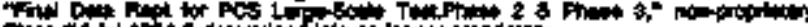

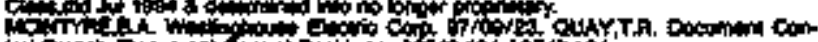

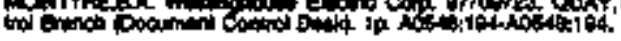

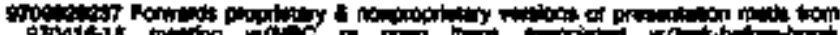

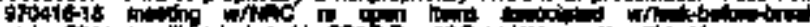

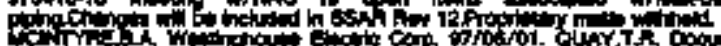

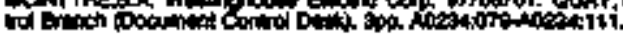

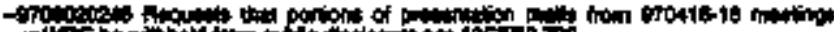
Whe bet

Lor.

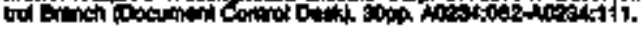

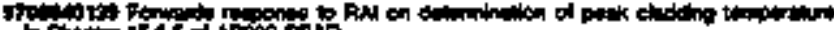

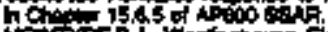

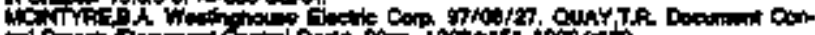

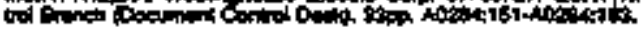

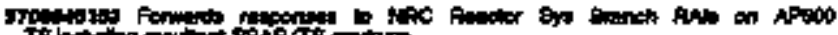

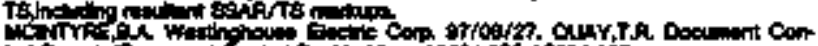

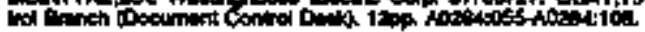

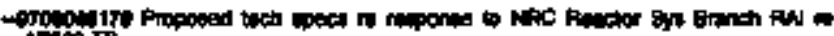

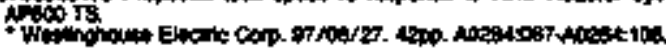

由)

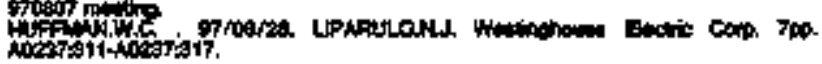

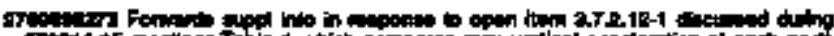

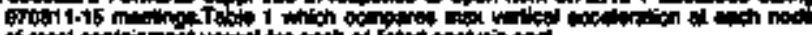
of Whet Can

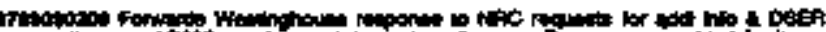

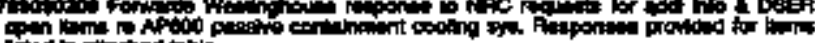

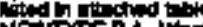

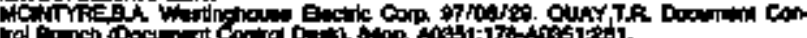

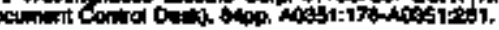

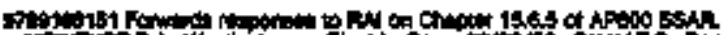

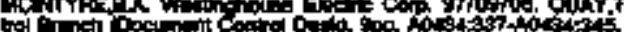

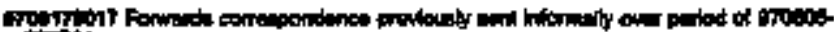

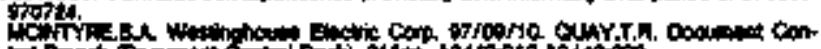

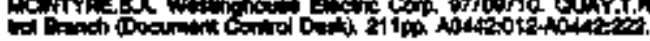

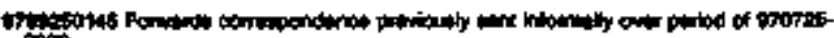

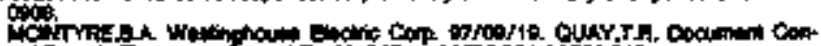

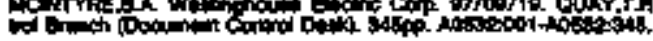

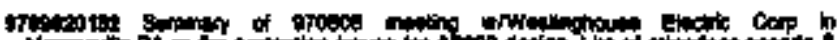

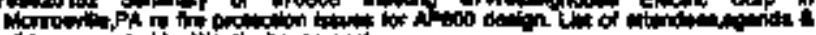

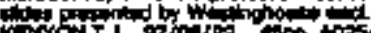

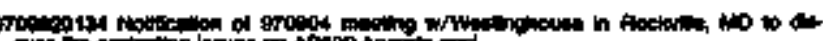

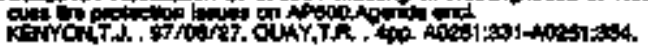

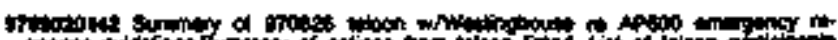

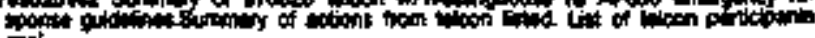

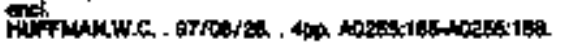




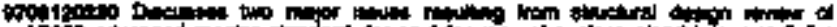

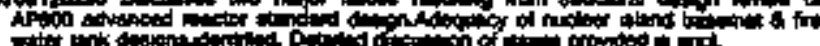

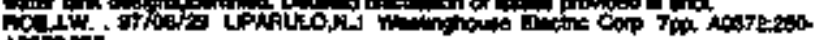

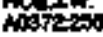

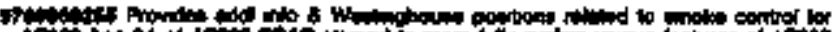

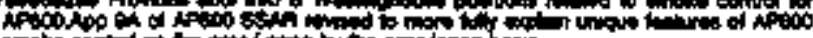

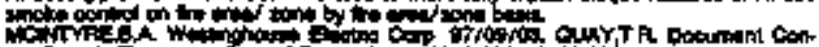

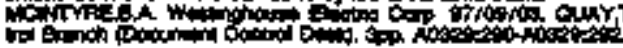

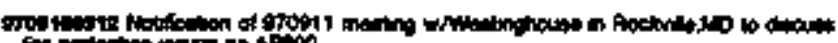

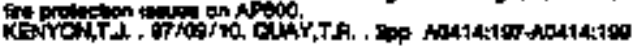

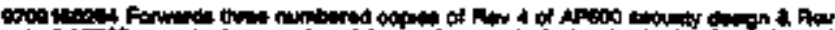

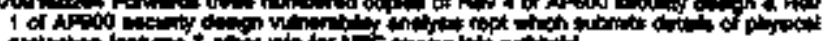

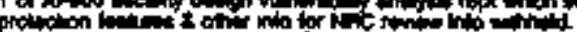

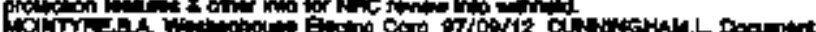

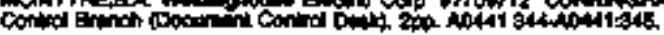

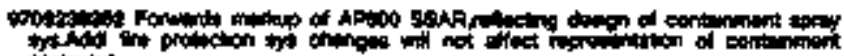

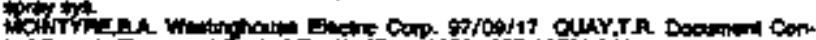

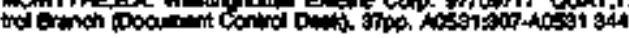

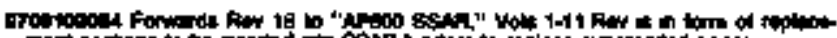

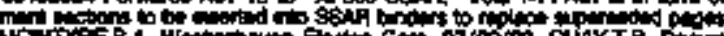

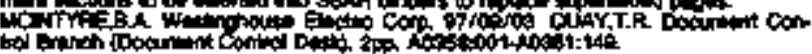

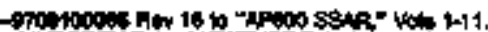

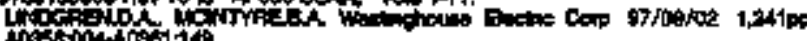

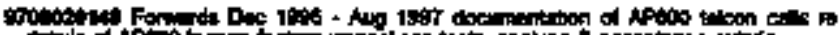

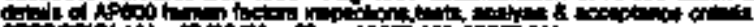

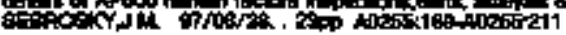

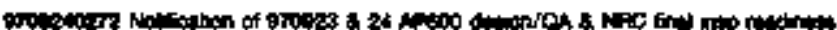

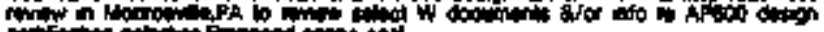

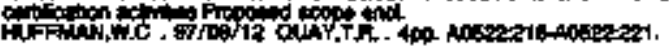

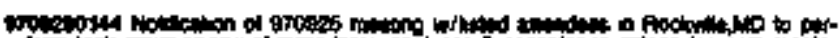

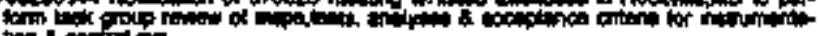

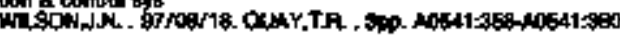

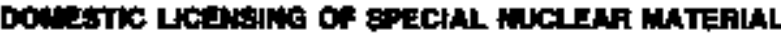

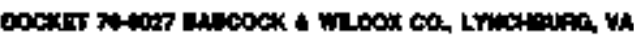

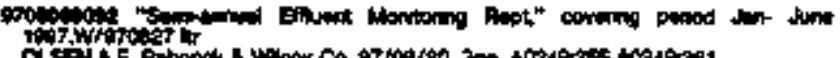

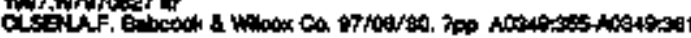

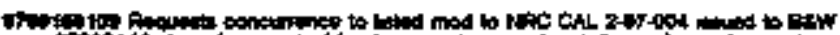

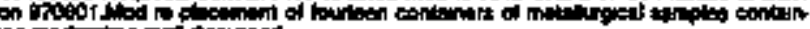

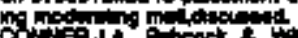

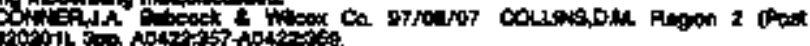

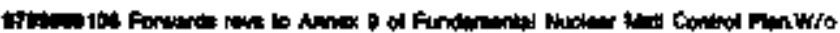
chet

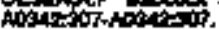

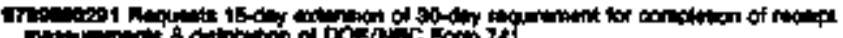

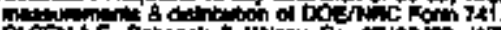

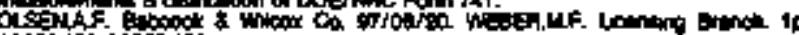

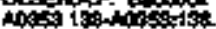

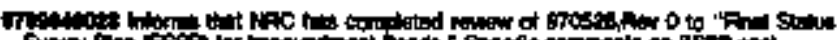

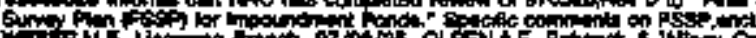

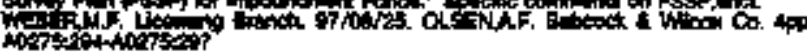

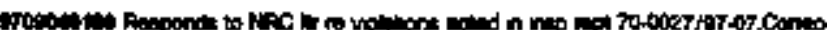

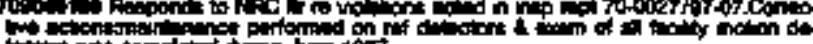

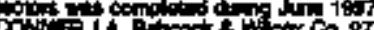

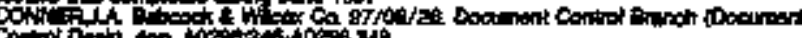

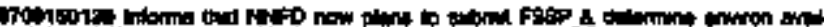

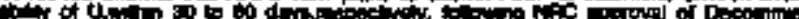

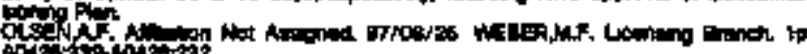
A

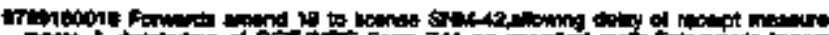

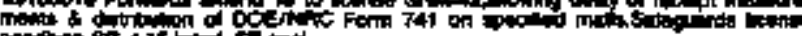

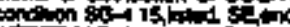

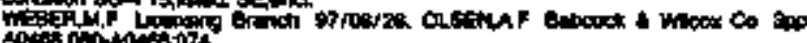

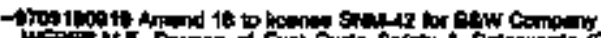

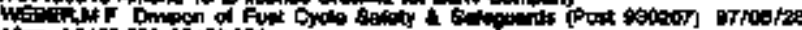
10T Nowescons

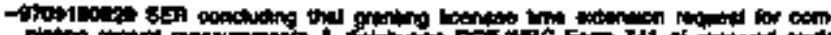

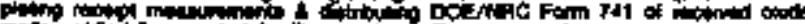

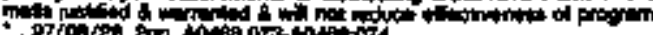

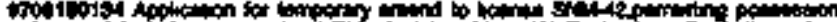

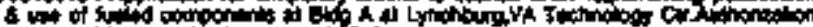
of

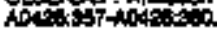

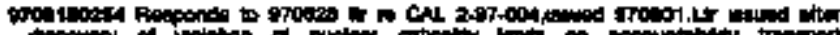

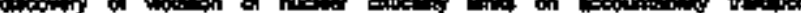

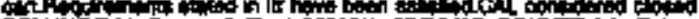

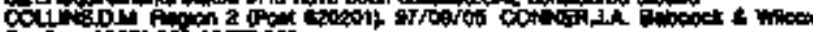

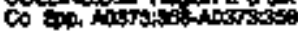

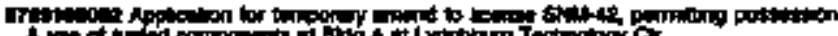

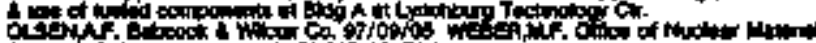

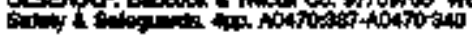

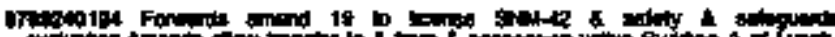

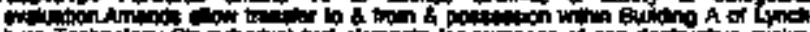

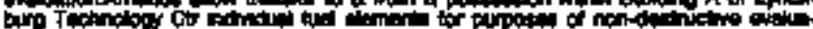
in

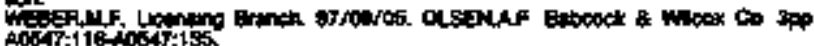

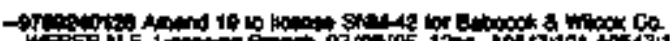
(1)

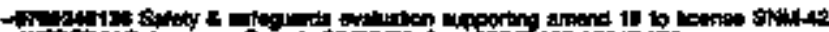

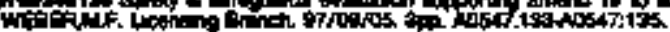

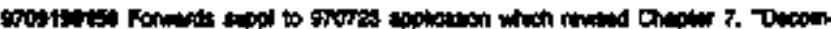

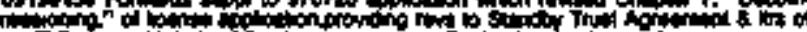

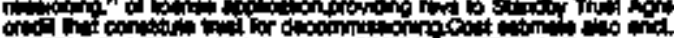

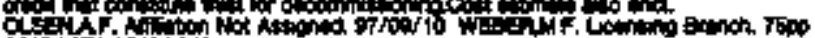

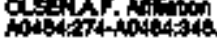

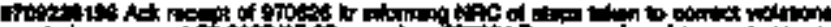

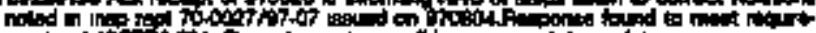

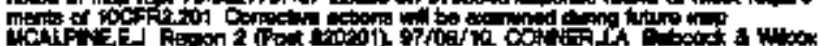

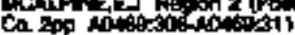

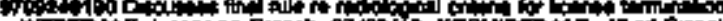

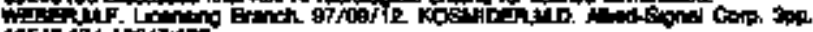

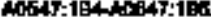

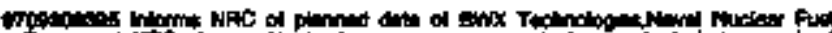

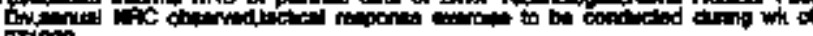

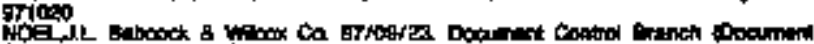

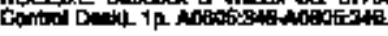

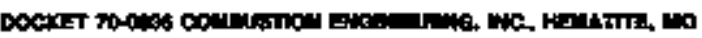

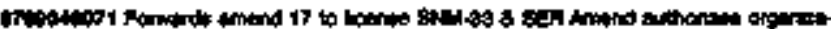

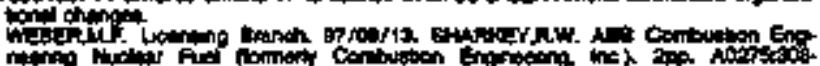
nation

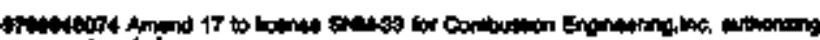

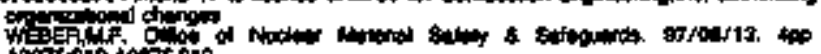
A

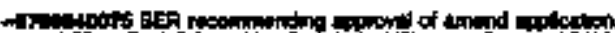

Wh Antsits

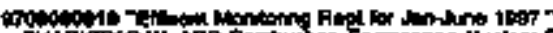

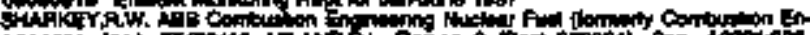
grotion

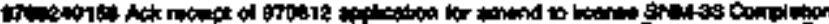

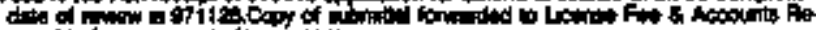

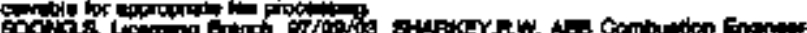

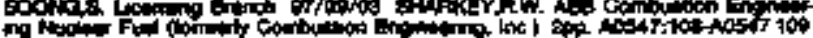

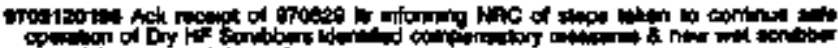

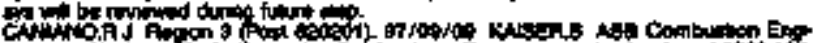

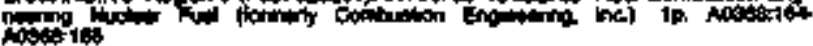

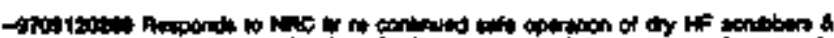

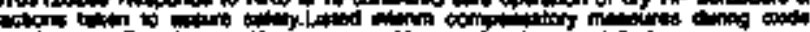

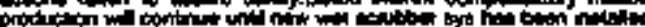

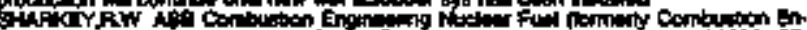

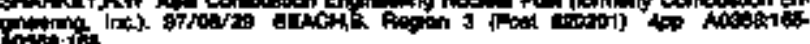

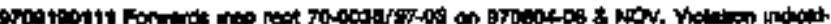

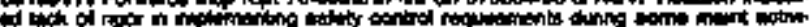

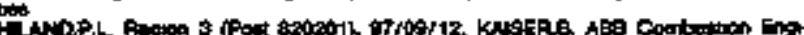

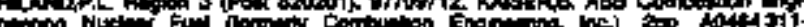
nomistist

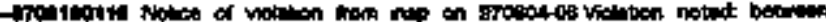

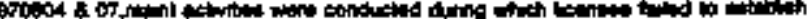

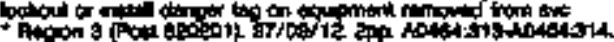




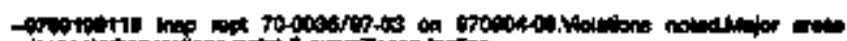

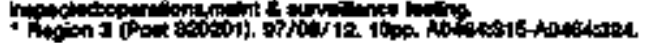

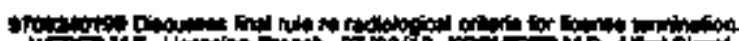

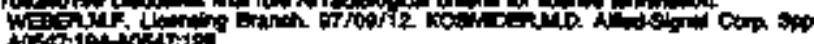

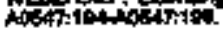

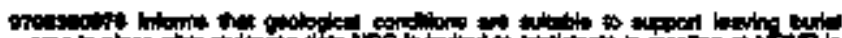

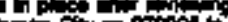

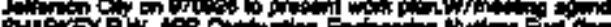

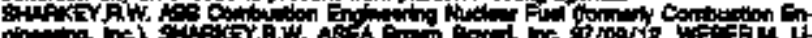

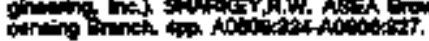

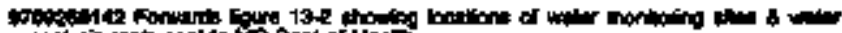

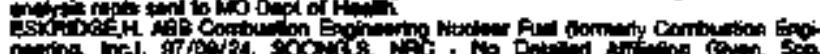

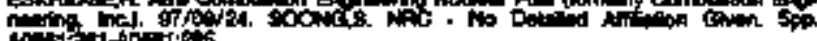

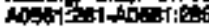

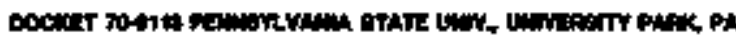

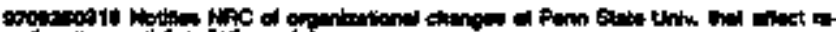

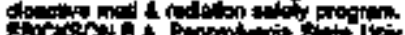

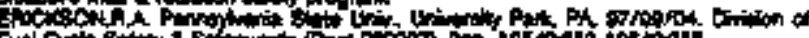

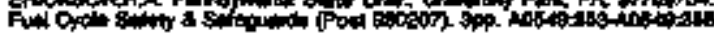

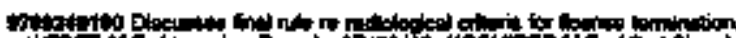

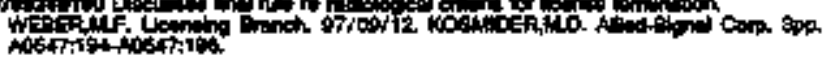

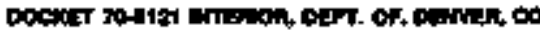

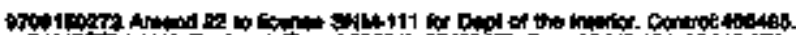

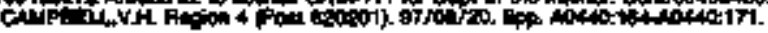

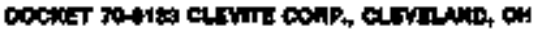

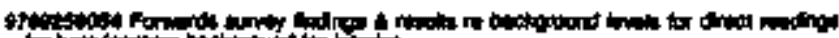

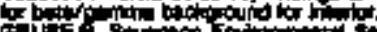

Fites

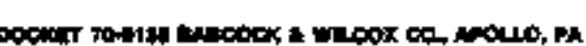

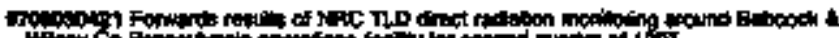

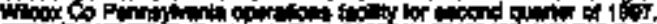

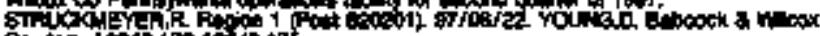

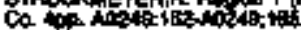

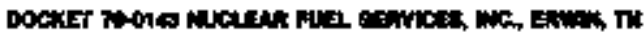

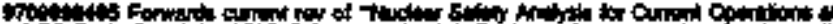

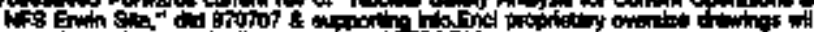

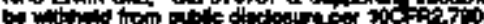

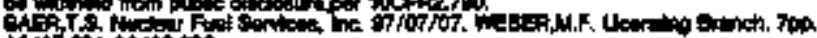

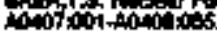

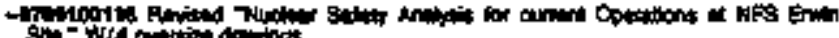

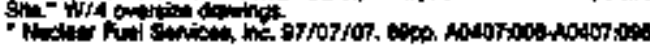

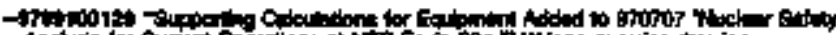

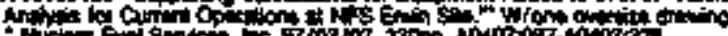

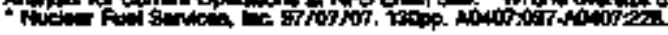

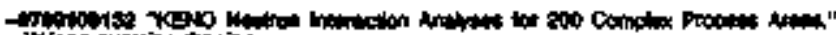

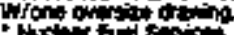

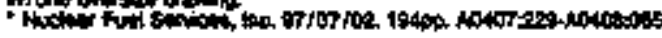

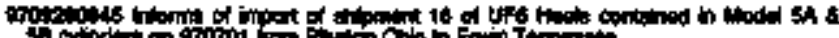
puth

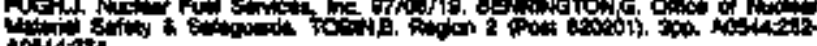

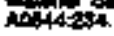

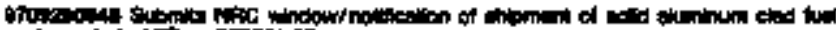

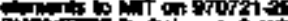

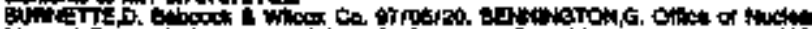

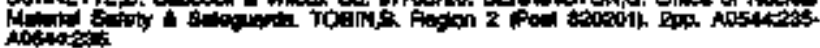

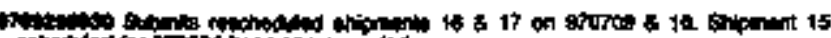

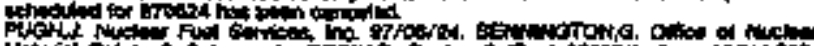

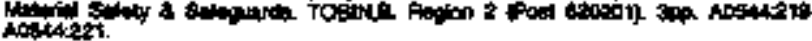

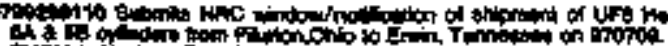

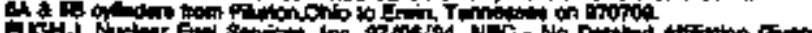

apos

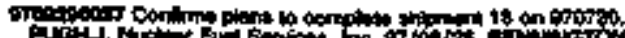

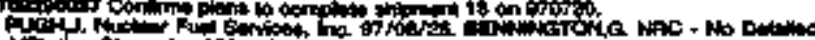

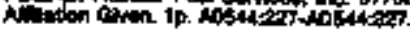

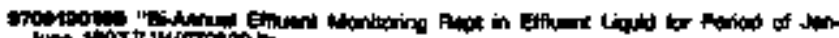

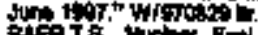

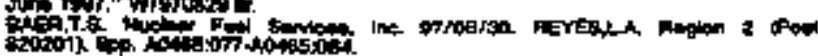

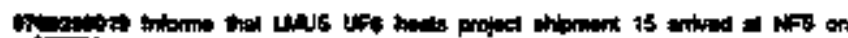

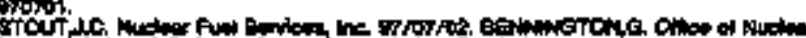

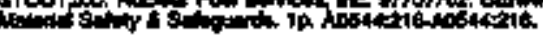

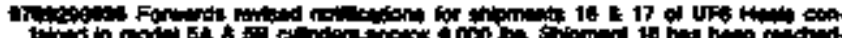

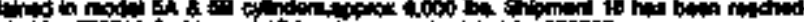

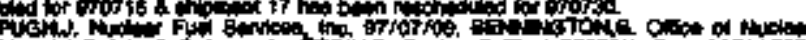

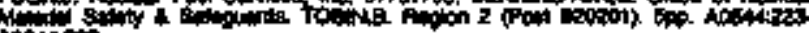
Nosidis

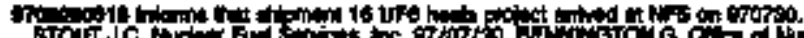

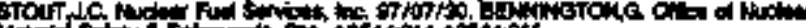

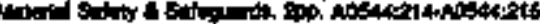

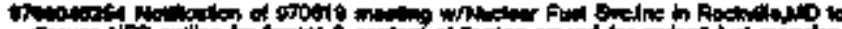

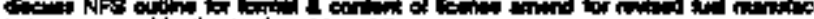

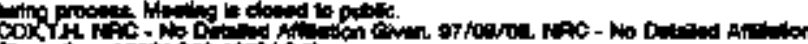

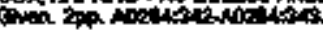

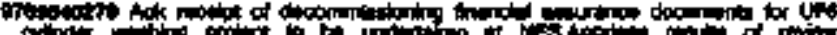

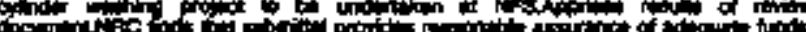
oc Nivis

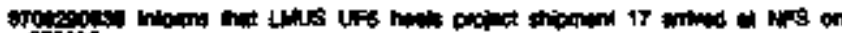
STour

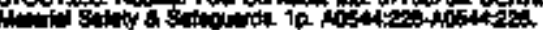

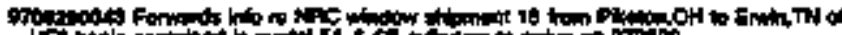

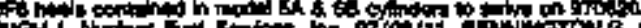

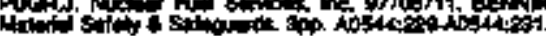

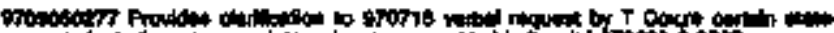

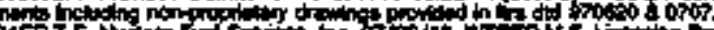
action

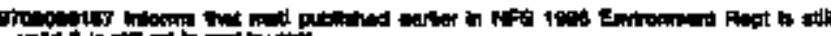

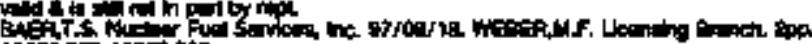
ing

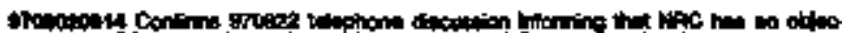

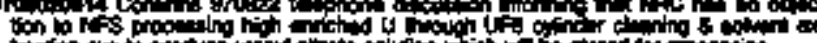

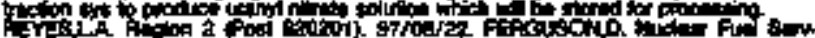

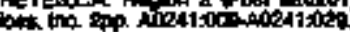

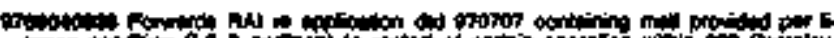

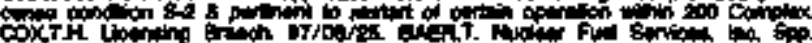

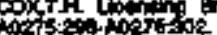

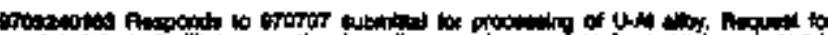

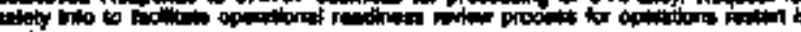

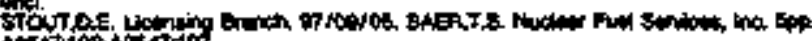
Mentiositistitor.

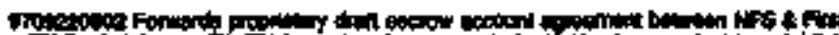

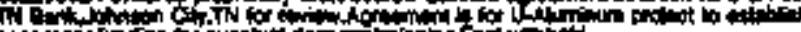

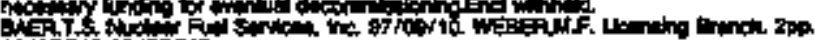

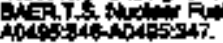

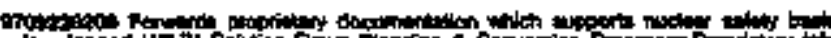

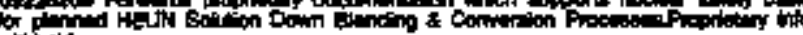

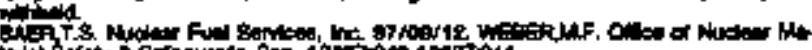

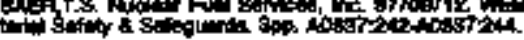

I H

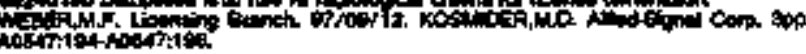

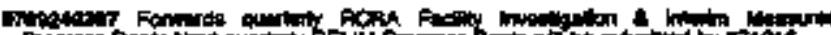

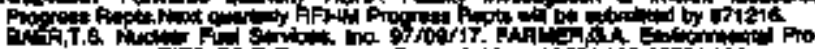

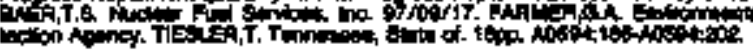

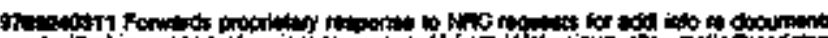

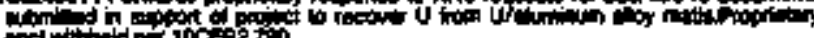

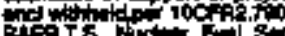

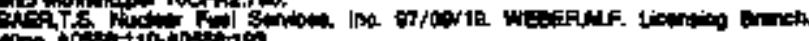

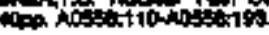

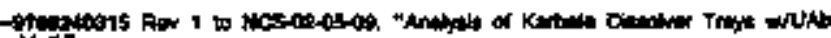

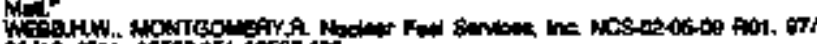

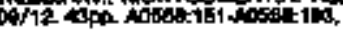

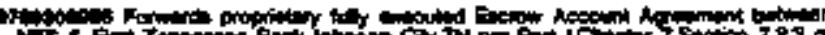

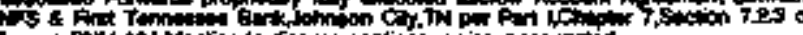

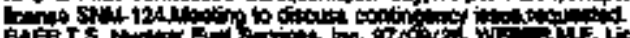

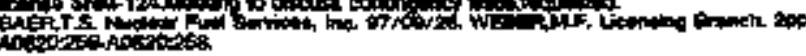




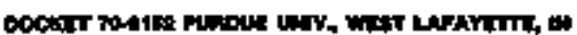

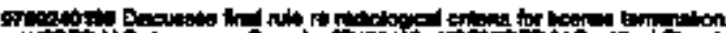

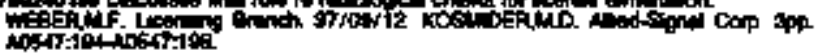

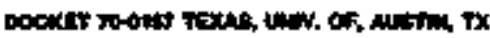

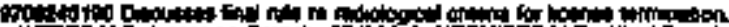

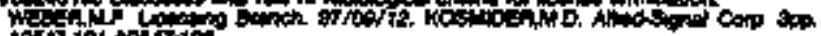

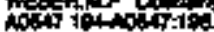

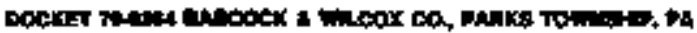

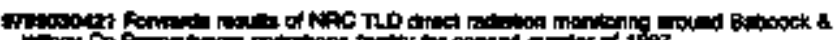
I

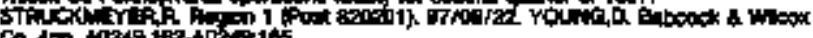

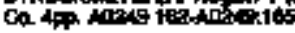

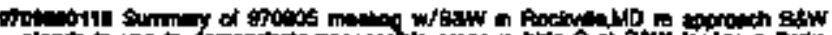

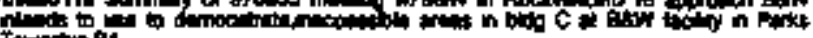
Tominger

CFilk

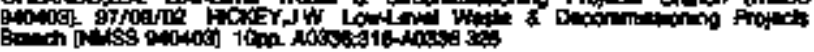

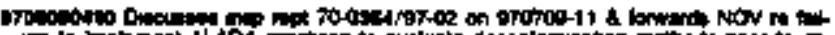

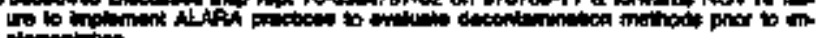

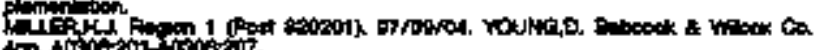

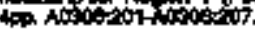

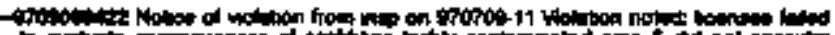

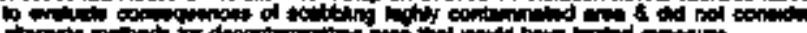

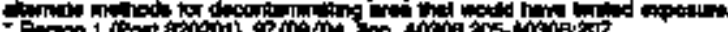

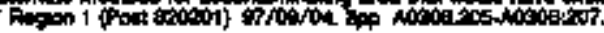

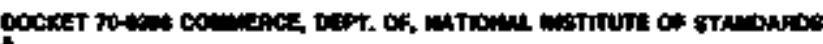

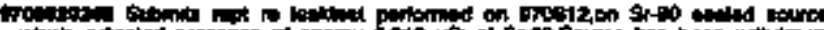

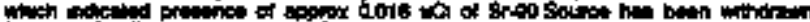

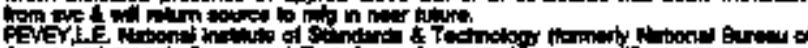

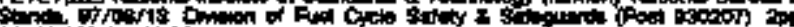
Aochositation

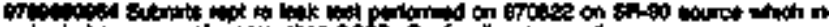

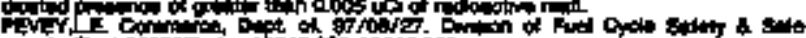

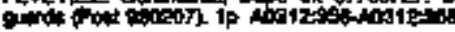

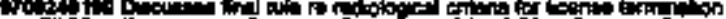
if Nostricumastivis.

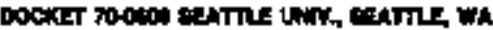

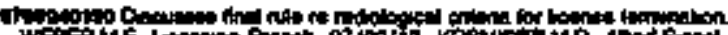

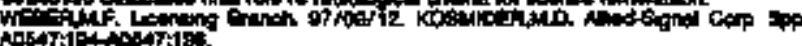

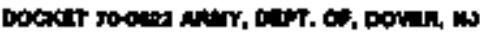

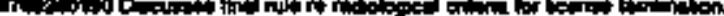

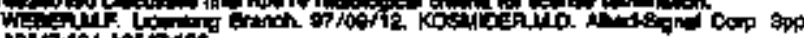

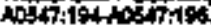

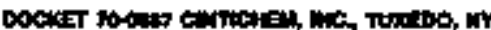

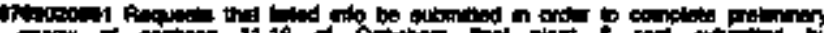

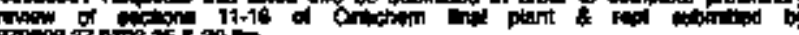
Gow

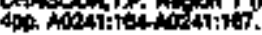

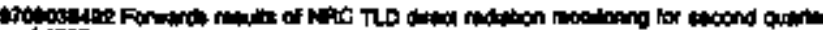

S1B9y

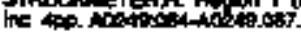

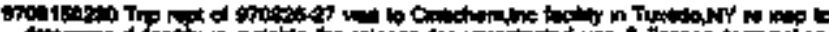

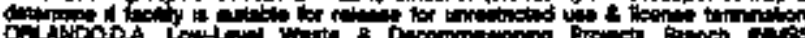

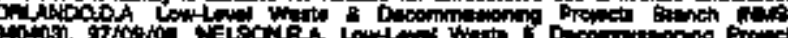

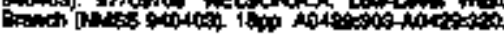

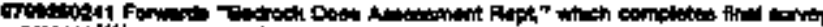

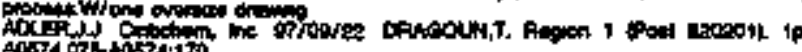

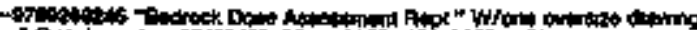

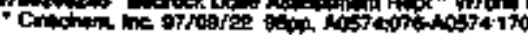

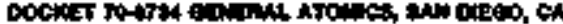

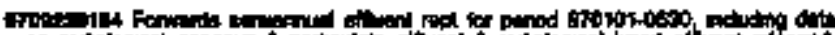

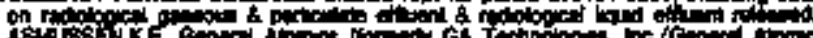

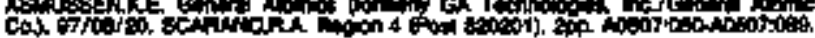

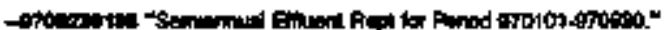

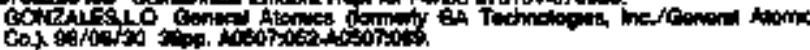

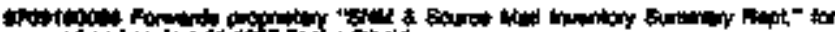
Fold

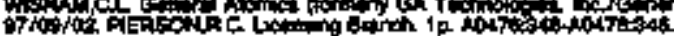

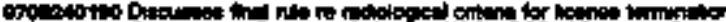

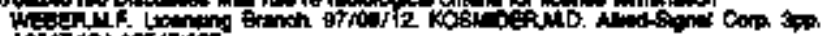

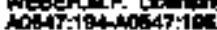

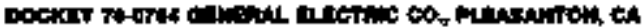

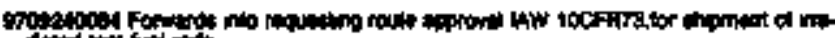

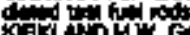

CFll LAD JHW. F

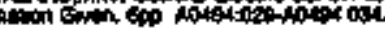

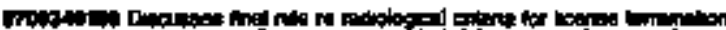

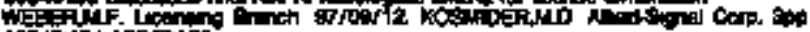
A07

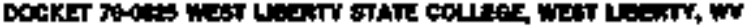

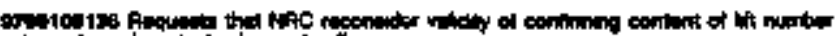
HR

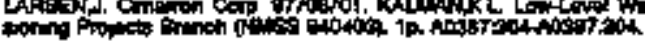

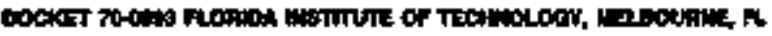

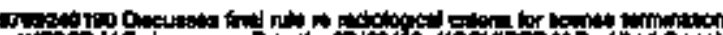

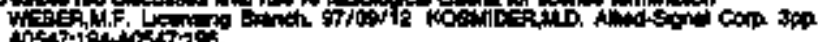

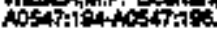

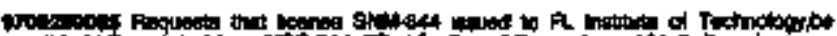

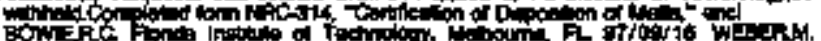

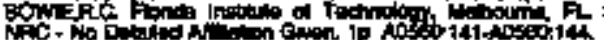

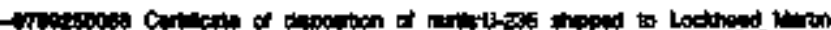

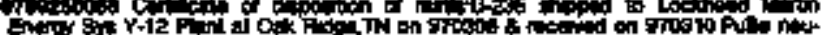

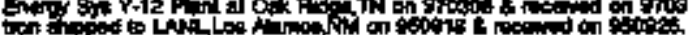

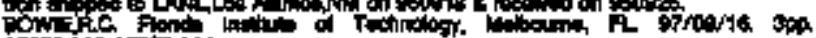
and $142+100010$

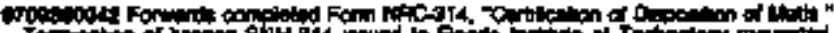

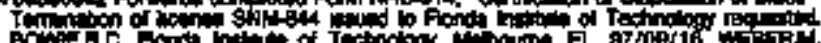

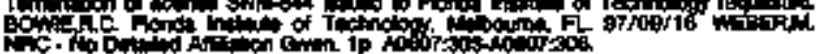

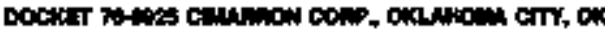

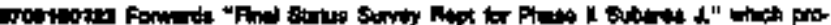

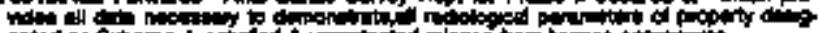

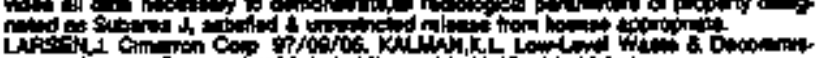

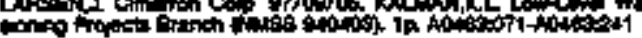

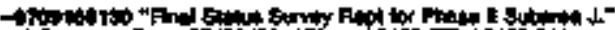

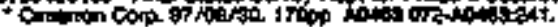

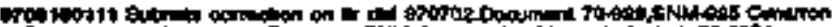

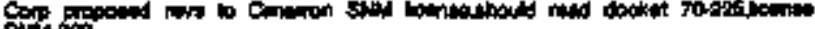
S

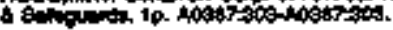

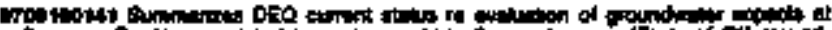

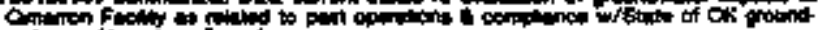

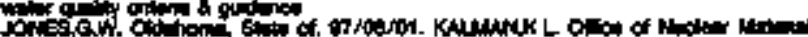

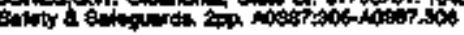

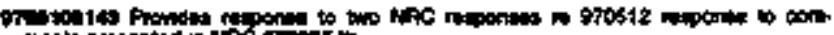

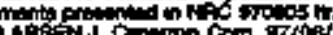

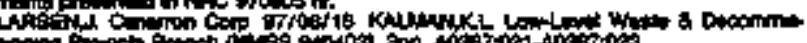

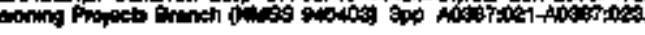

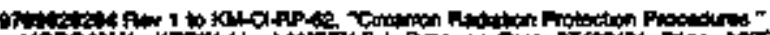

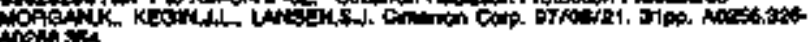

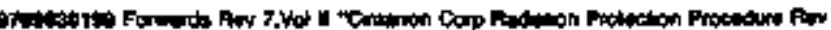

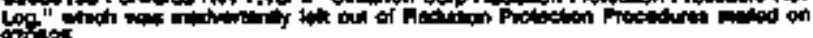
(ant incistions

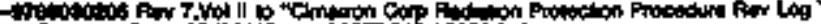

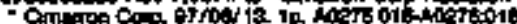


wogens14 Fon

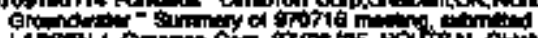

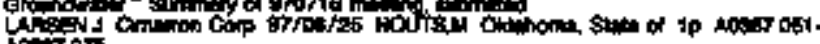
An: on

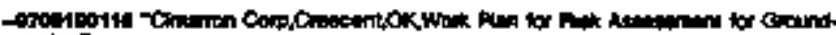

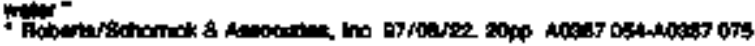

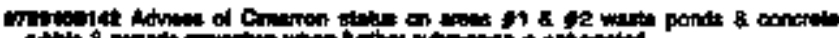

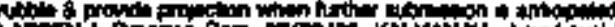

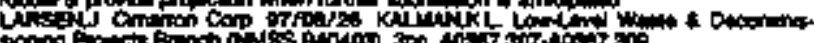

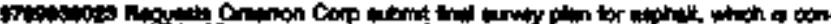

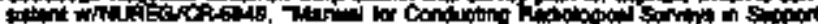

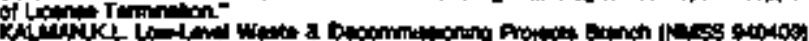

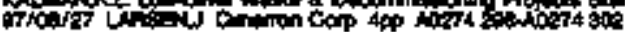

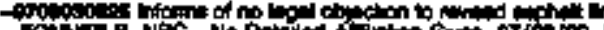

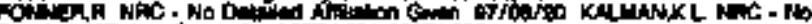

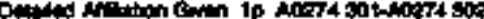

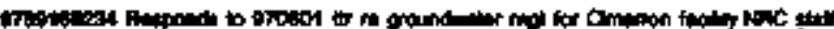

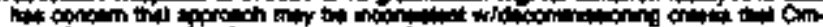

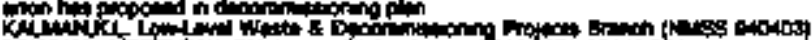

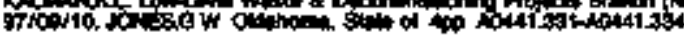

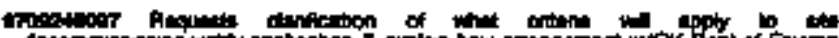

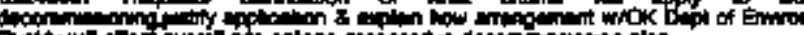

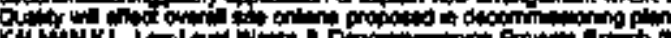

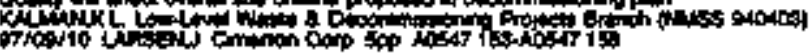

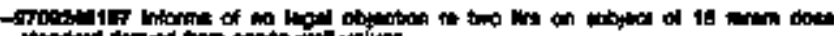

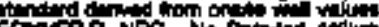

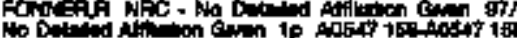

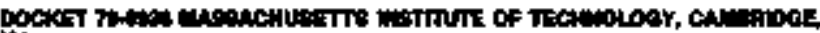

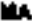

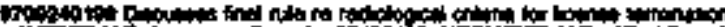

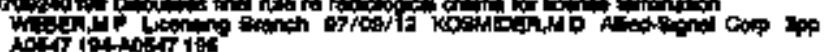

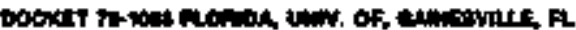

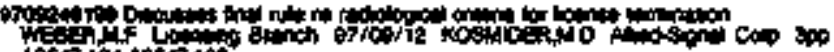

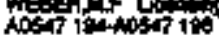

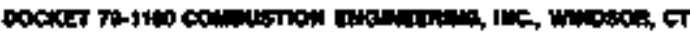

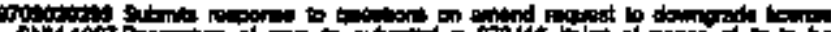

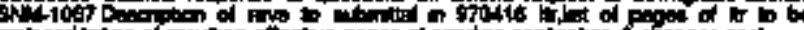

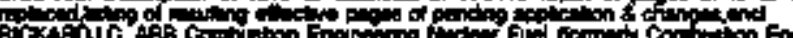

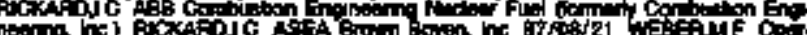

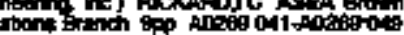

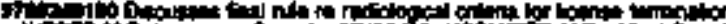

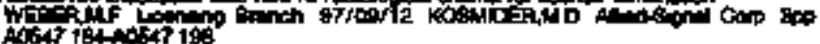

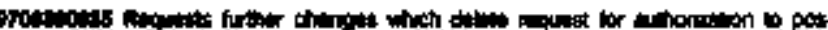

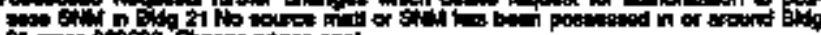

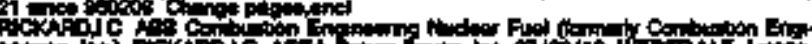

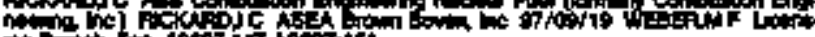
on

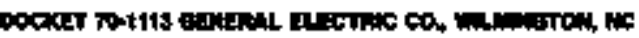

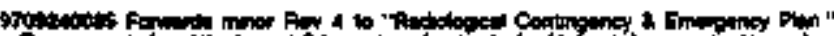

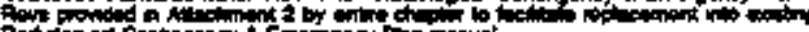

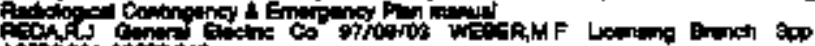

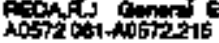

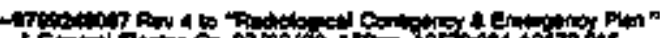

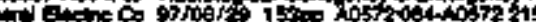

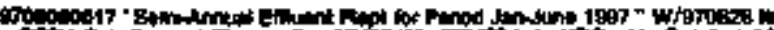

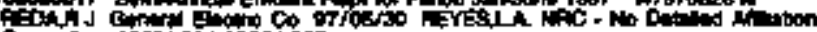

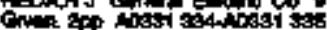

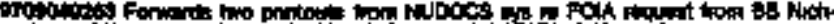

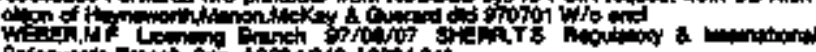

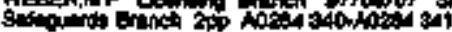

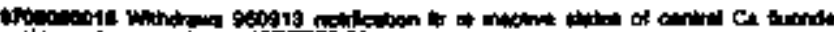

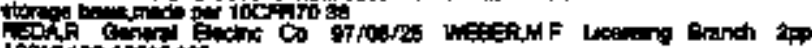

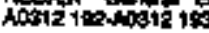

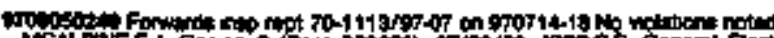

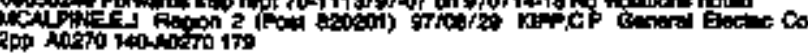

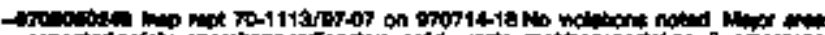

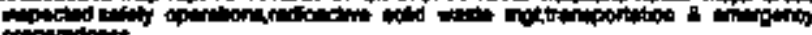

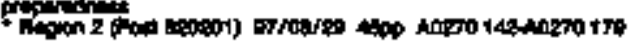

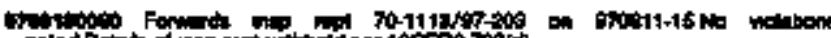

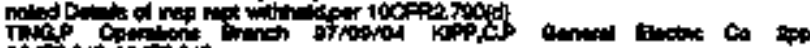
Hont

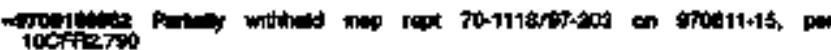

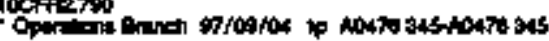

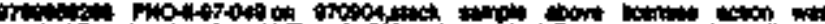

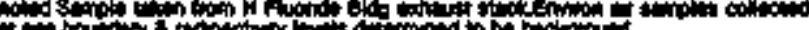

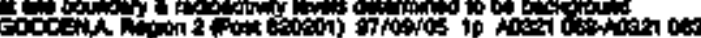

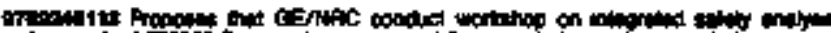

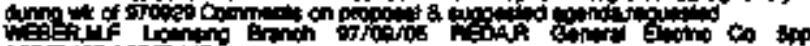

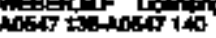

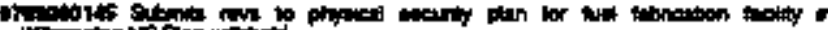

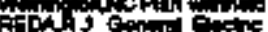

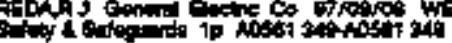

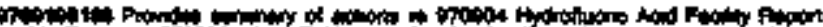

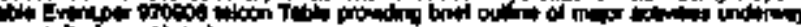
chof tomisositotes of

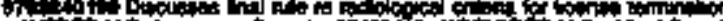

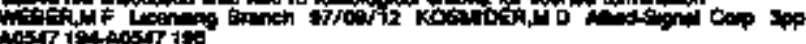

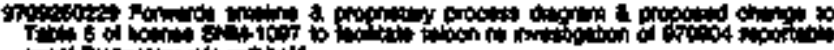

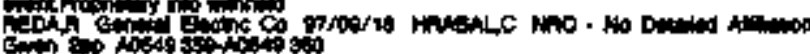

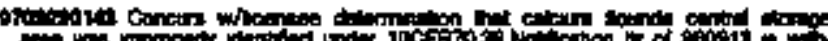

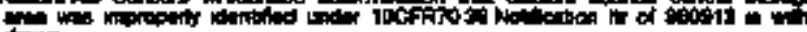

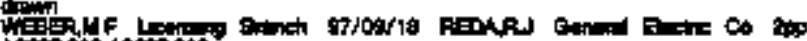

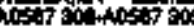

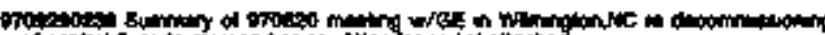
of

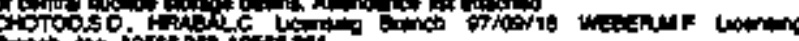

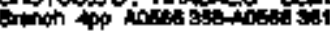

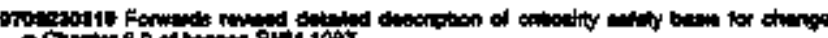

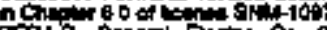

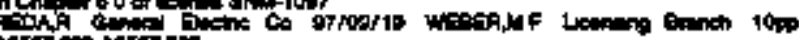

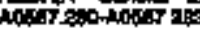

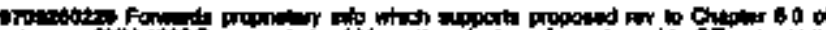

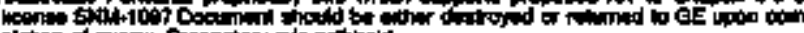

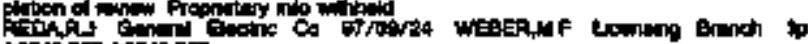
nosto sionotion

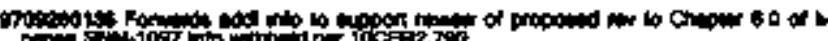

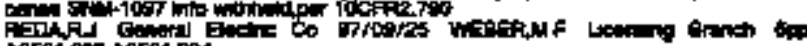
Hocki2s

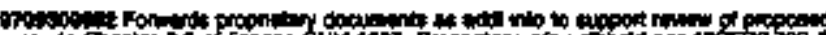

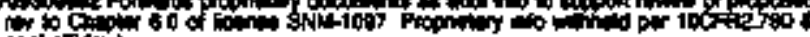

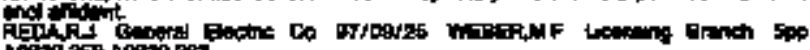

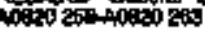

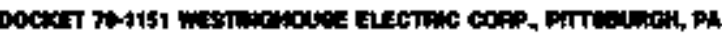

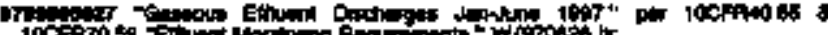

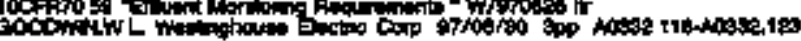

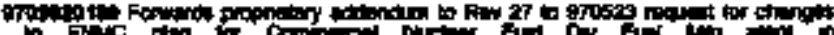

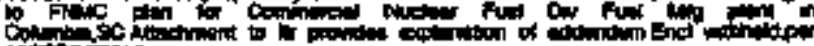

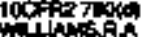

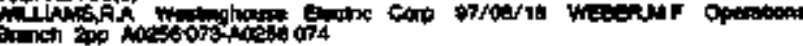

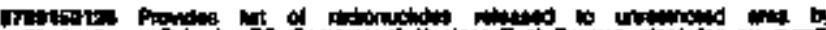

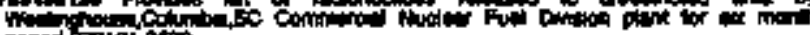
penod

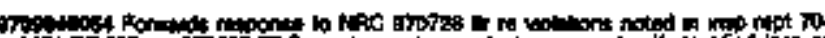

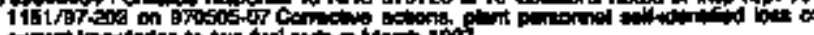

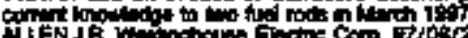

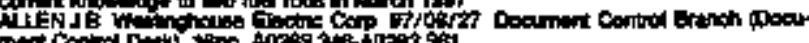

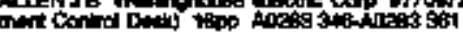




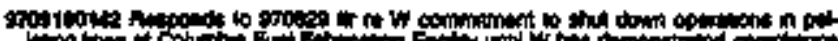

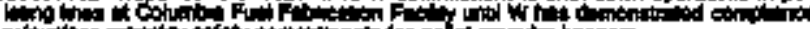

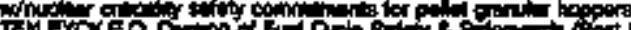

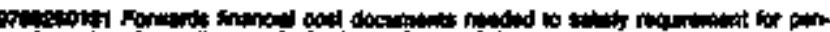

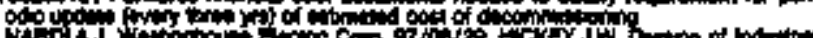

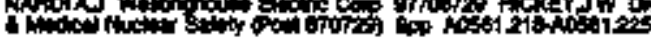

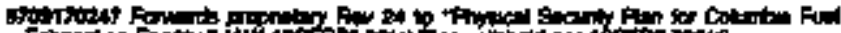

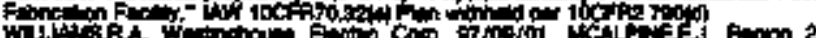

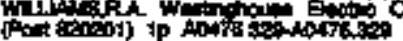

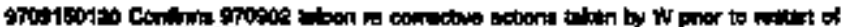

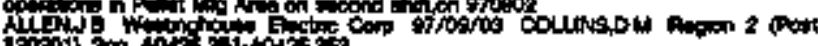

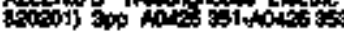

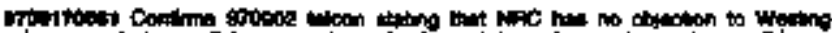
hol.

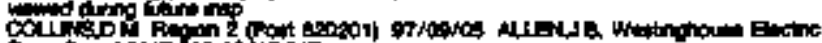

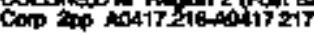

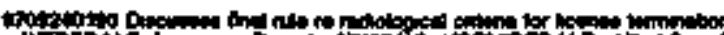

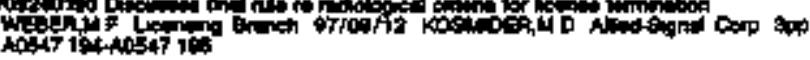

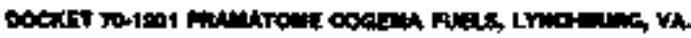

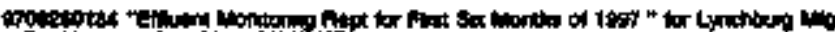

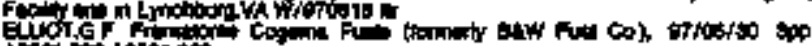

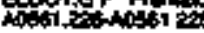

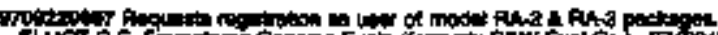

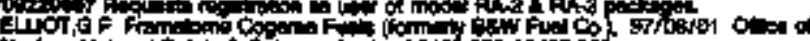

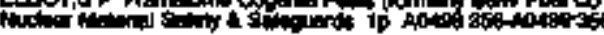

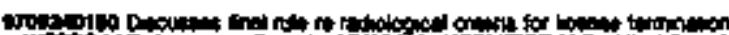

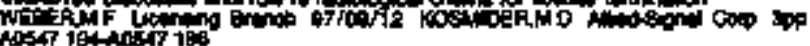

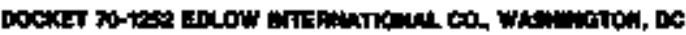

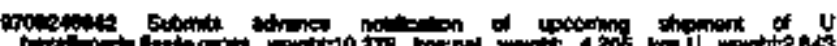

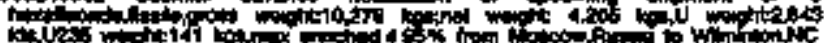

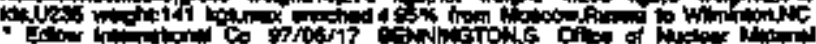

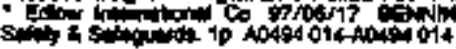

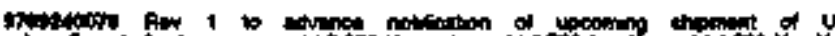

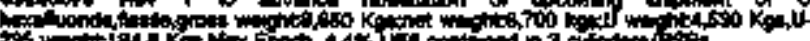

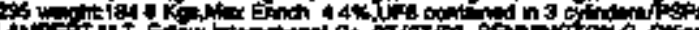

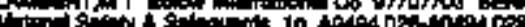

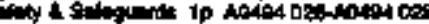

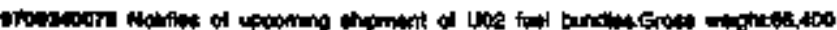

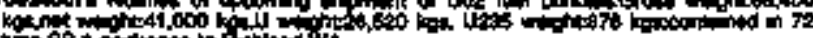

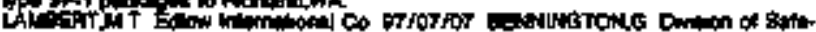

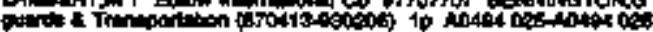

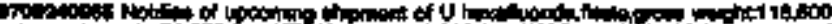

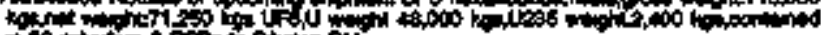

Lit

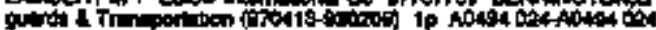

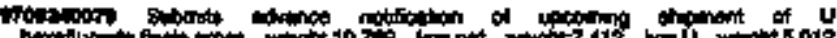

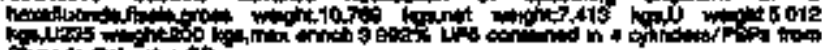

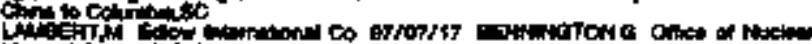

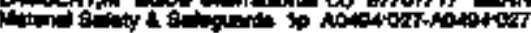

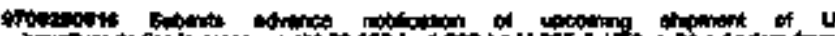

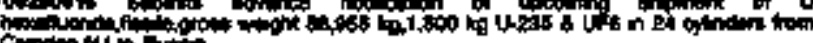

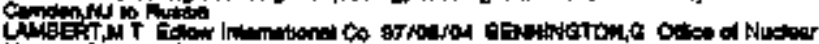

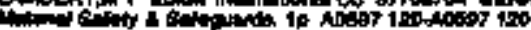

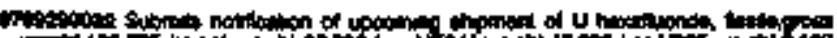

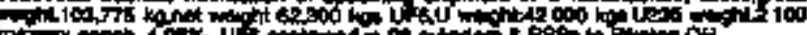

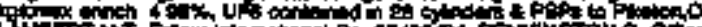

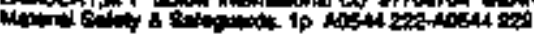

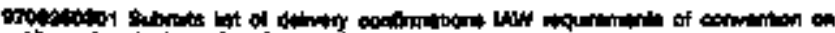

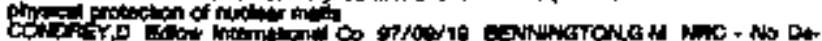

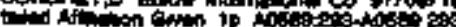

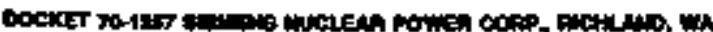

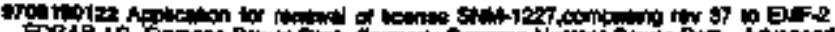

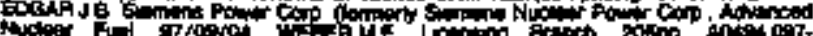
Hotion

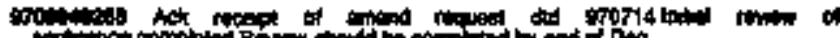

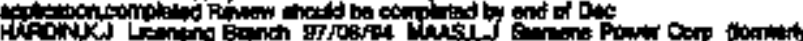

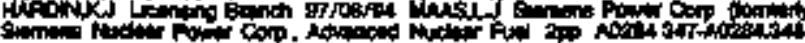

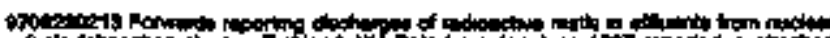

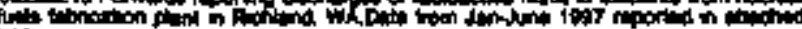

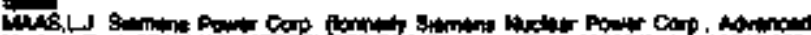

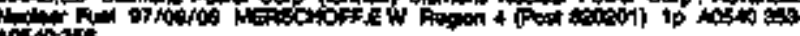

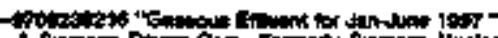

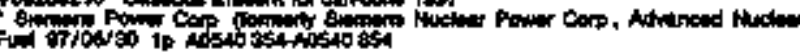

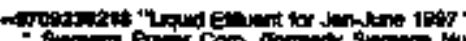

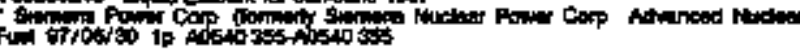

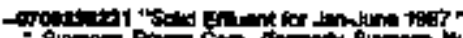

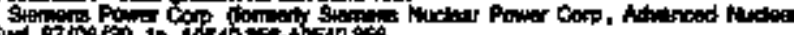

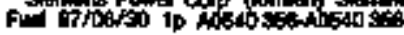

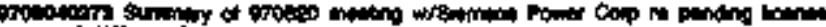

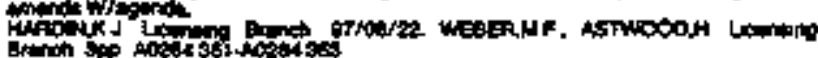

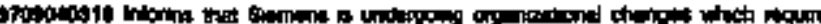

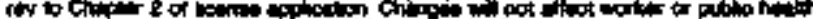

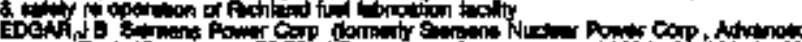

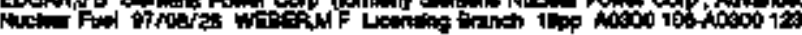

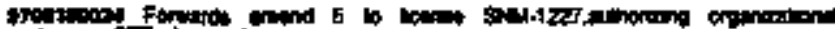
The

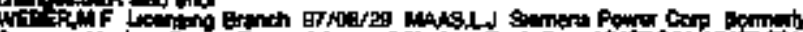

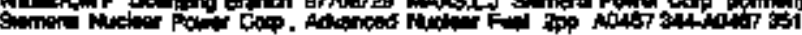

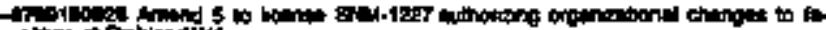
civen ot phiandim

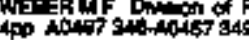

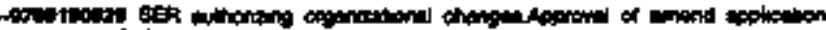

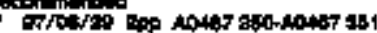

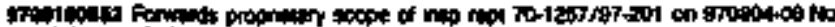
netwonit noted

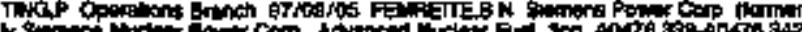

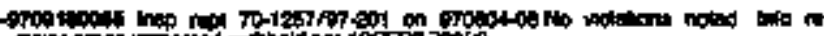

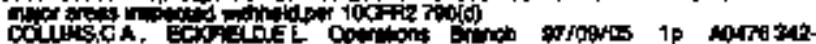
distion

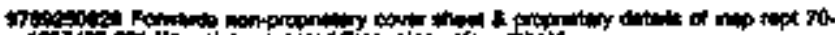

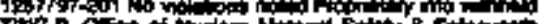

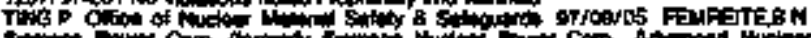

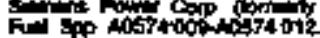

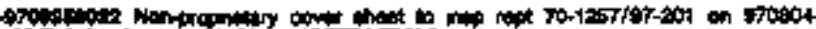

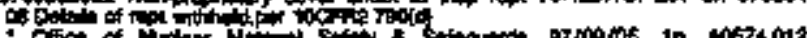

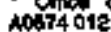

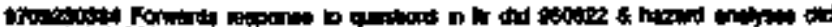
$070 \sin 10 \cos$

COSO CDGNN

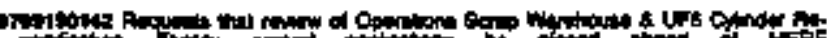

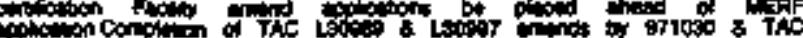

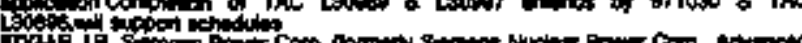

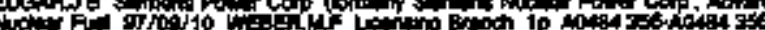

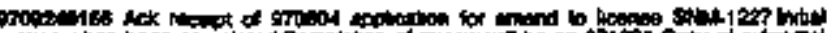

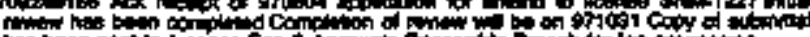

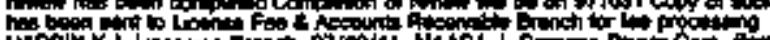

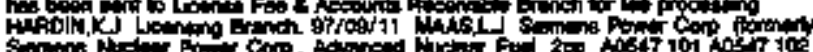

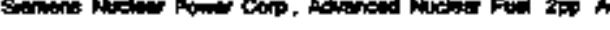

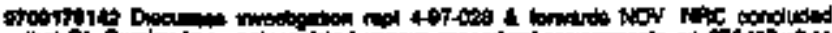

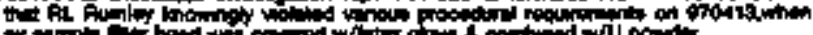

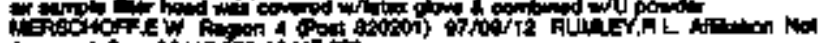

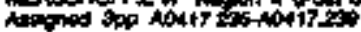

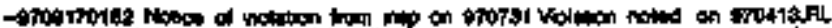

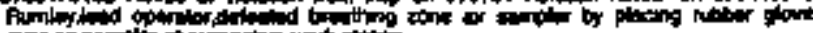

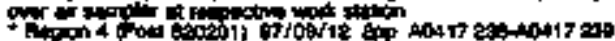

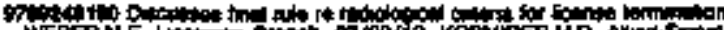

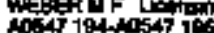




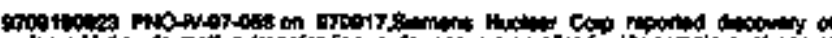

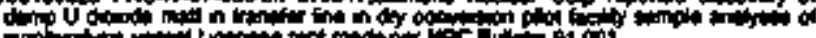

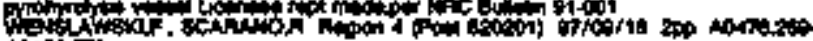
A

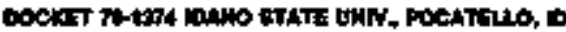

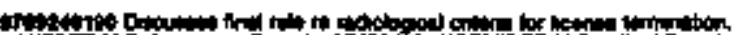

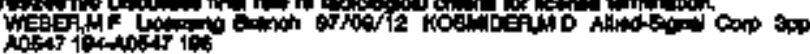

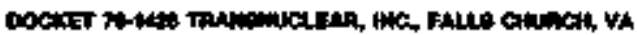

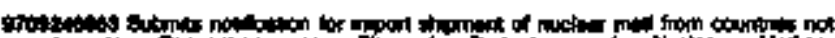

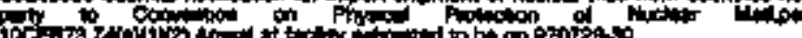

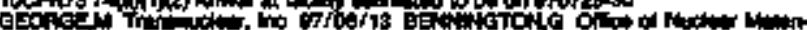

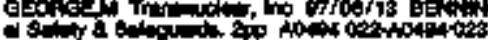

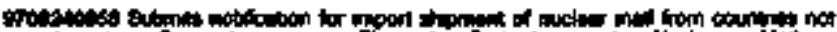

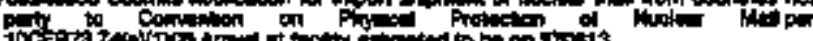

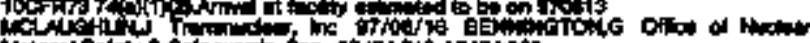

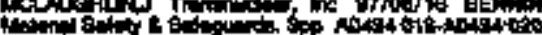

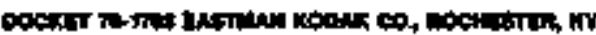

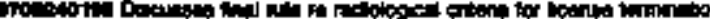

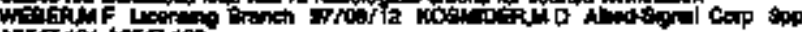
ADST 194 Hos 190

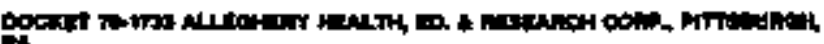
Pl

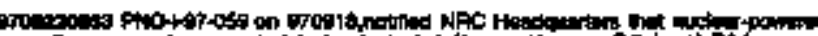

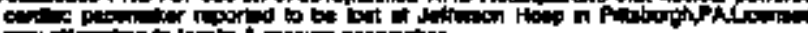

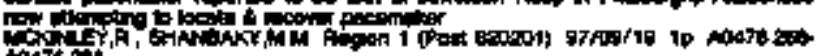
147620

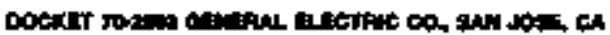

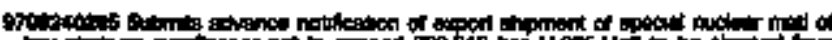

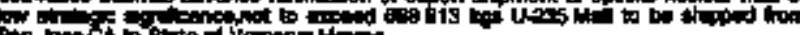
in

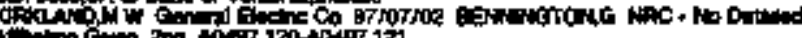

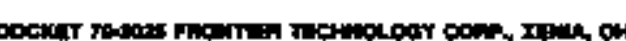

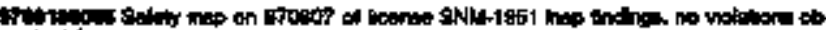

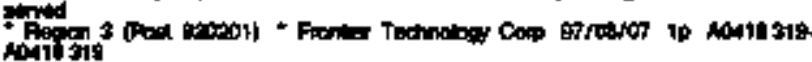

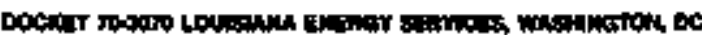

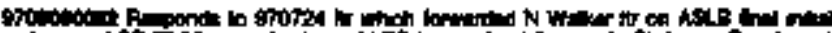

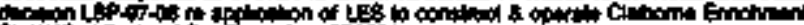

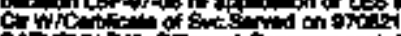

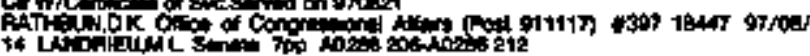

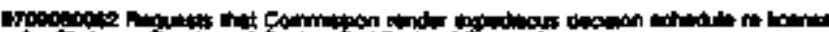

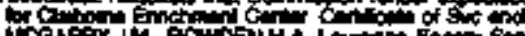

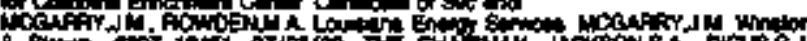

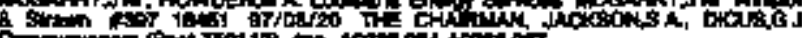

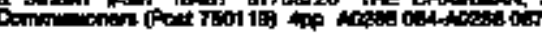

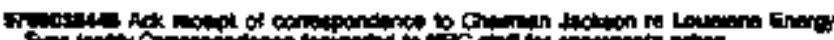

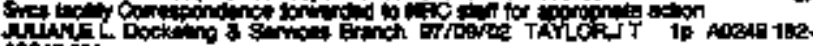

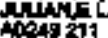

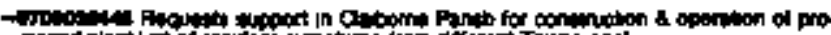

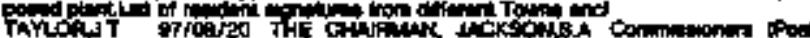

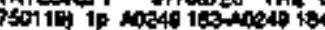

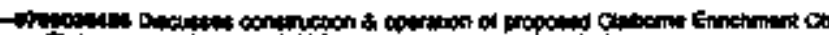

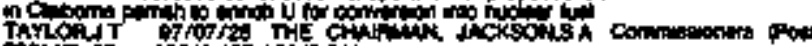

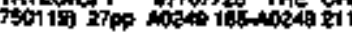

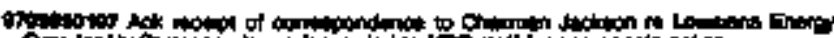

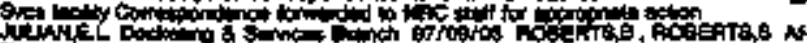
Alent

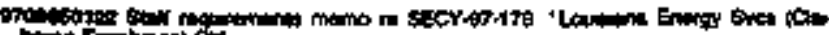

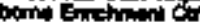

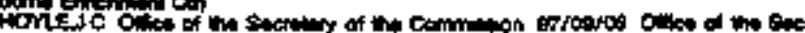

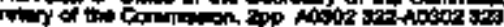

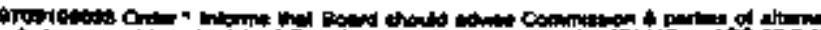

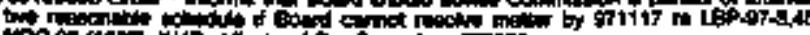

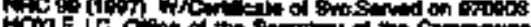
win

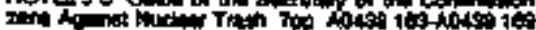

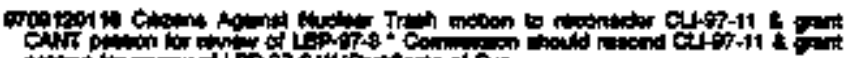

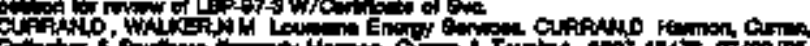

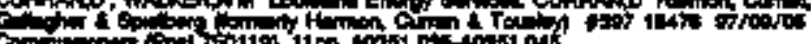

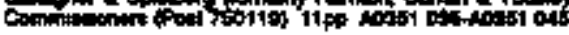

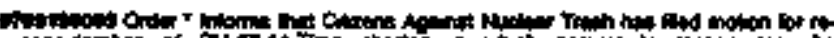

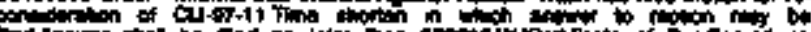

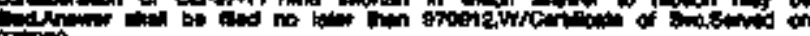

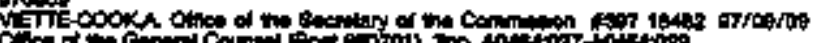

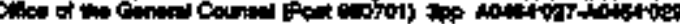

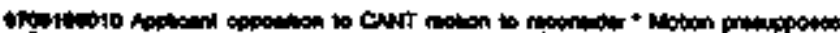

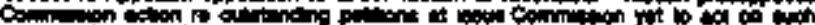

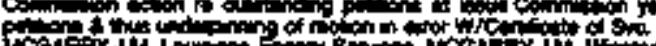

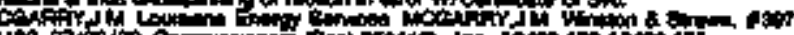

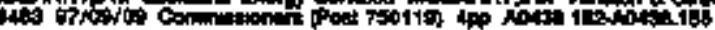

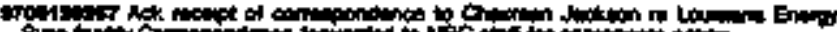

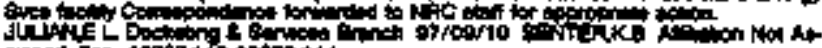

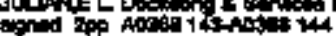

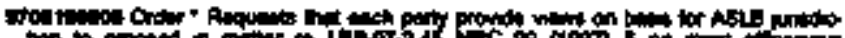

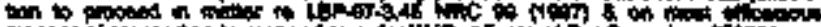

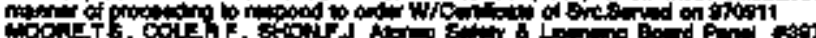

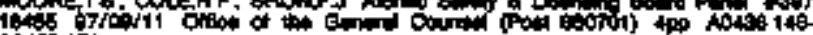
104\$: 1

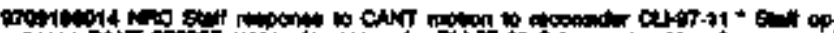

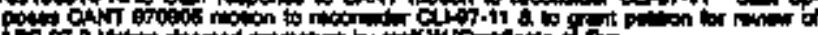

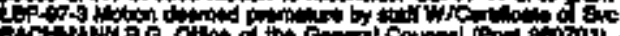
ie Con

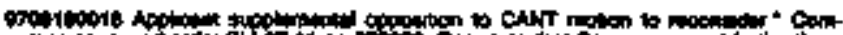

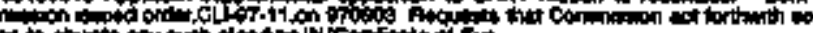

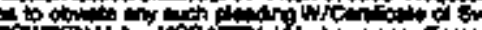

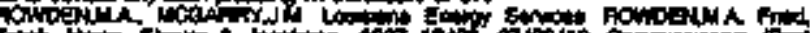
int

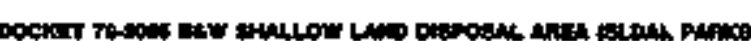

Jining,

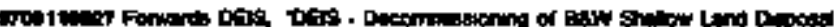

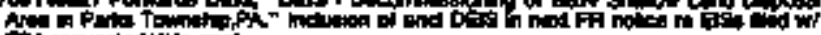
ron

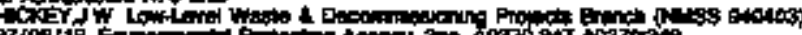

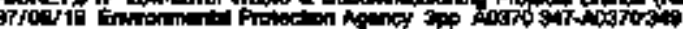

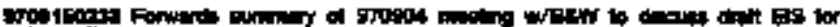

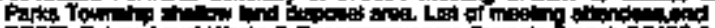

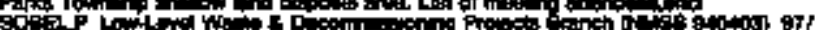
oovo

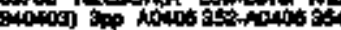

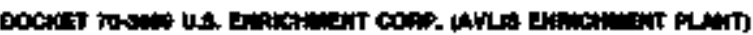

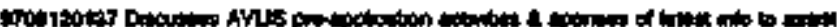

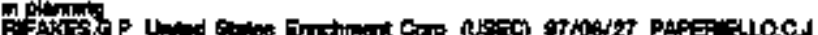

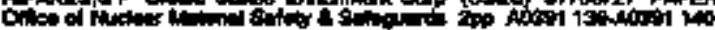

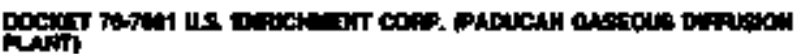

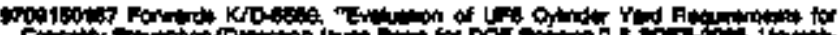

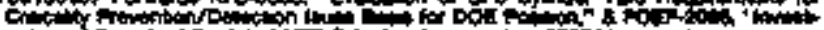

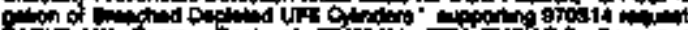

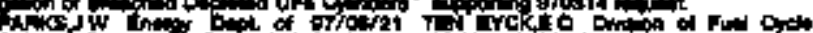

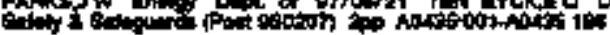

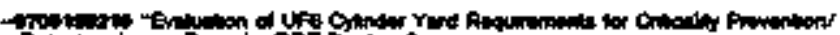
Rotipton A

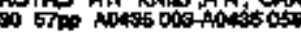

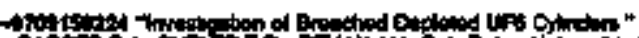

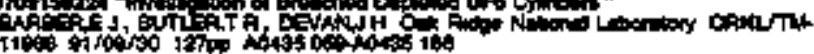

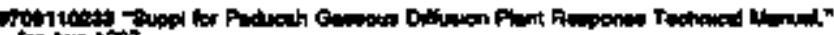

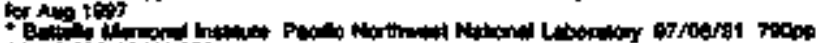

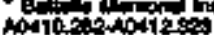

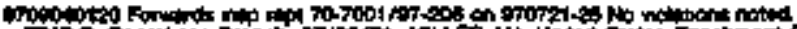

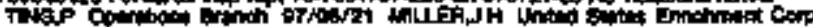

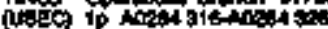

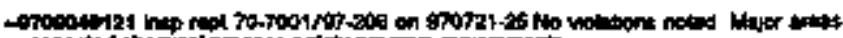

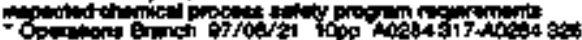

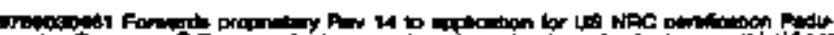

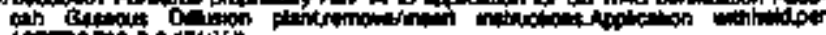
1067270017060

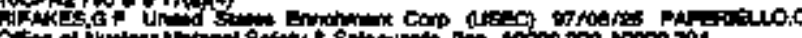

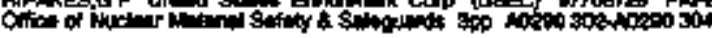




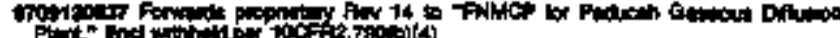

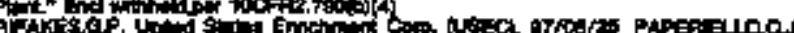

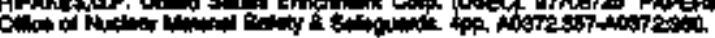

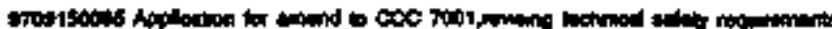

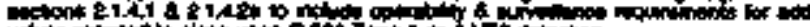

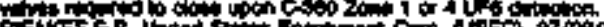

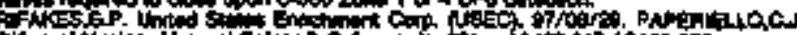

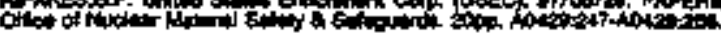

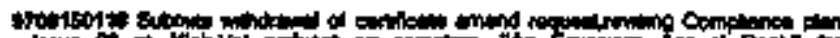

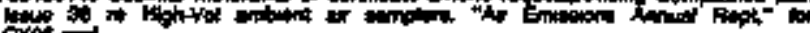

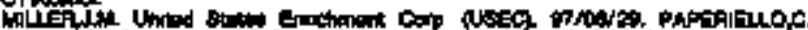

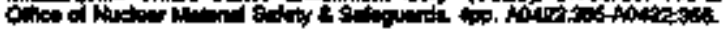

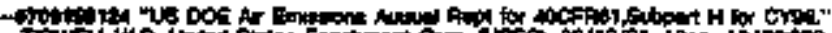

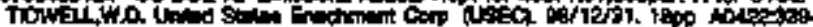
Aatosis

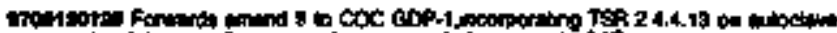

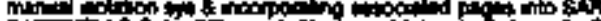
PA Mlle

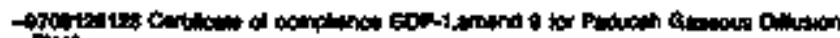
Phich

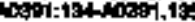

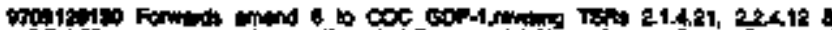

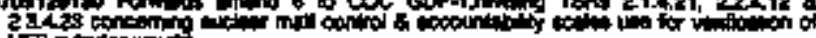

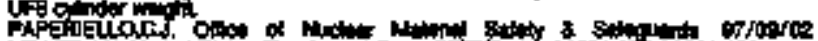
A

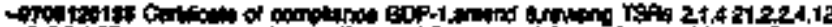

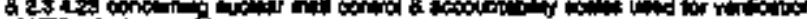

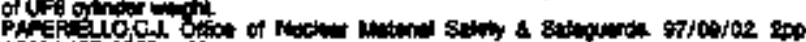

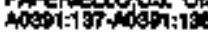

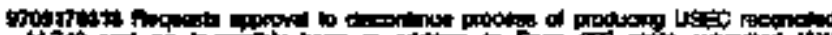

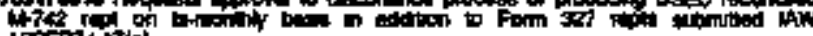

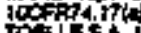

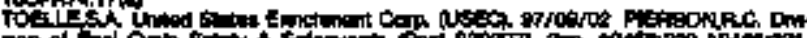

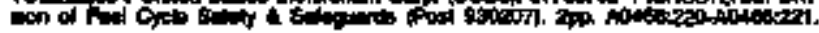

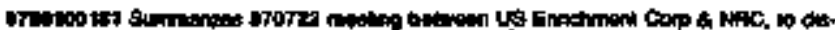

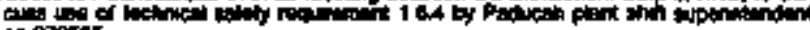

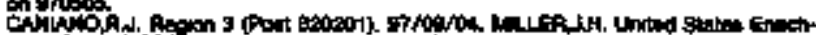

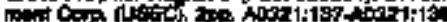

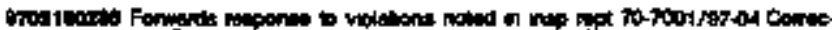

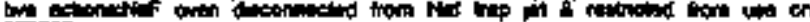

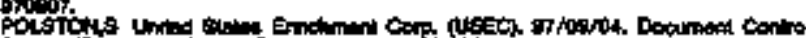

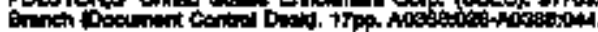

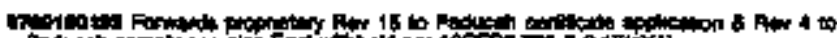

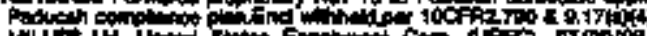

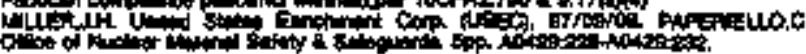

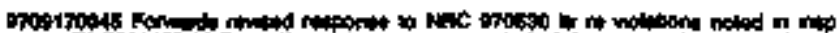

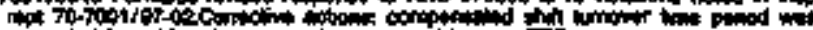

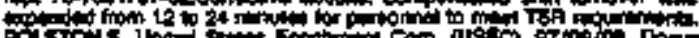

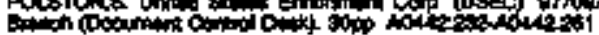

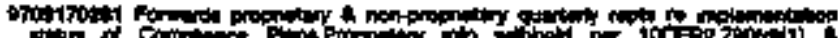

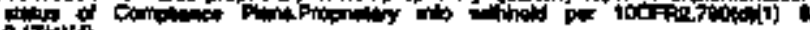

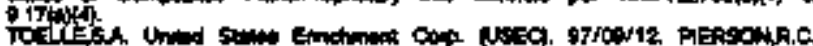

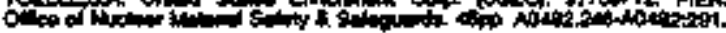

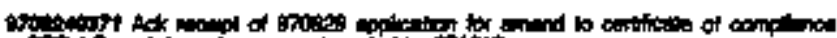

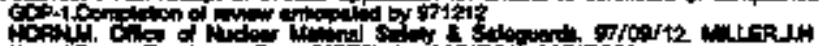

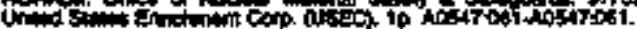

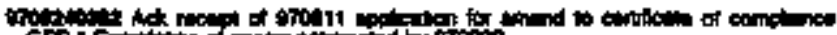

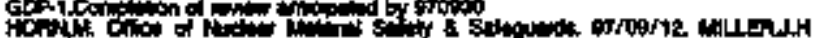

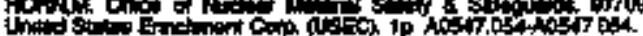

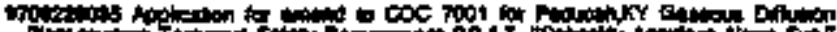

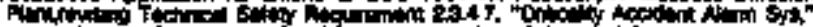

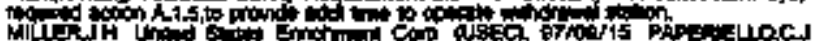

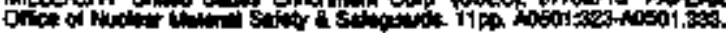

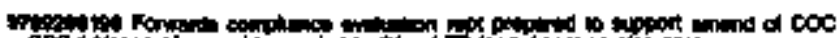

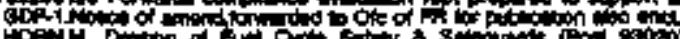

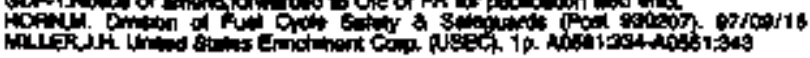

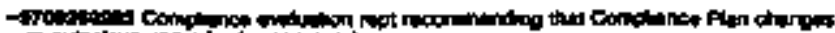

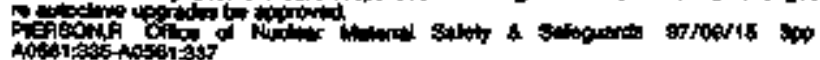

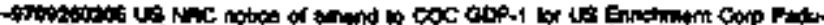

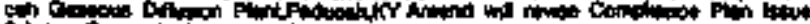

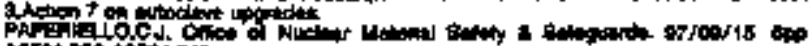

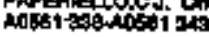

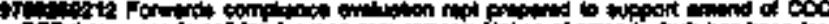

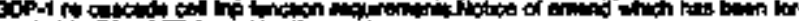

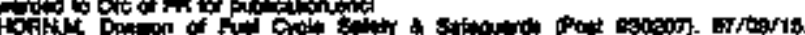

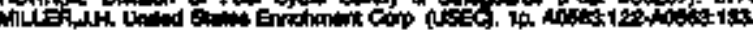

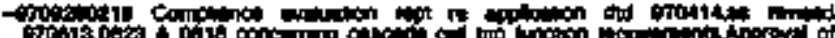

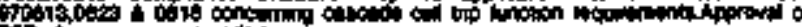

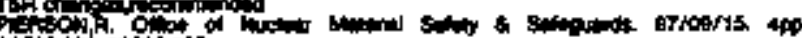

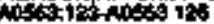

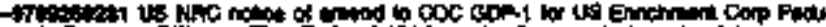

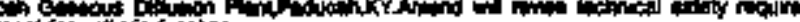

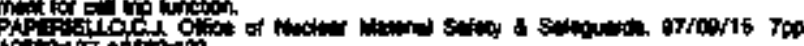

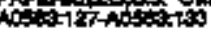

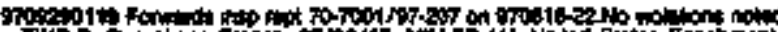

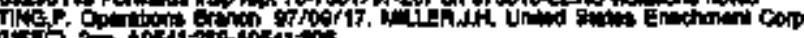
ister

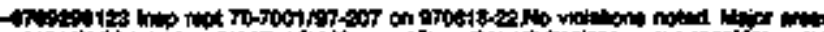

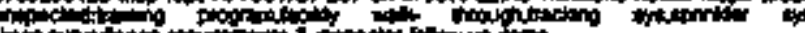

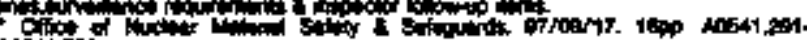
$\cot _{0 \rightarrow 100}$

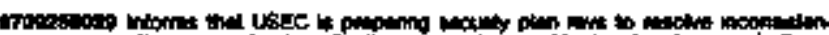

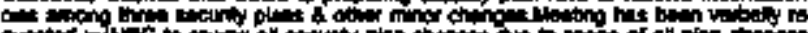

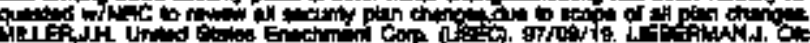

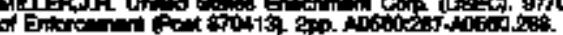

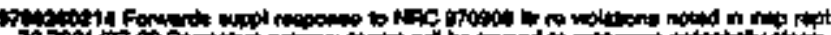
(1) Cod

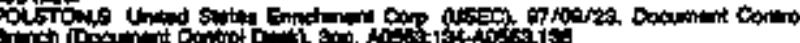

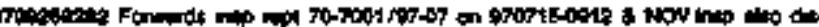

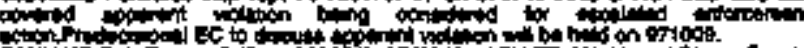
Chiliko,

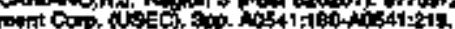

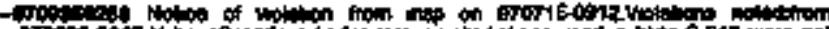

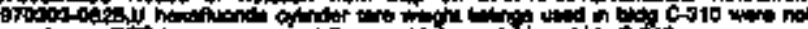

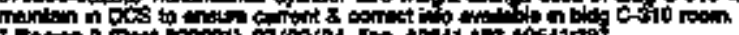

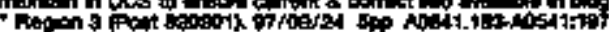

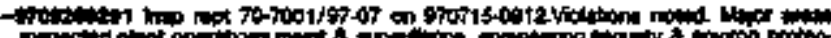

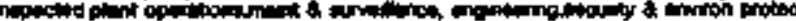

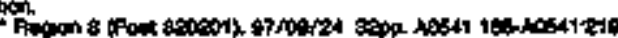

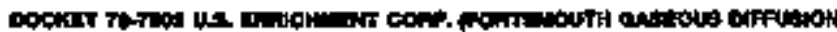

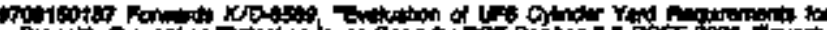

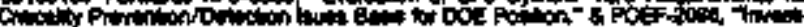

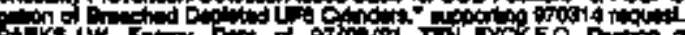

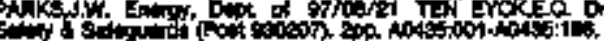

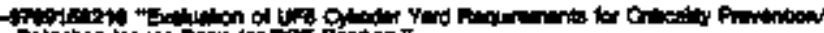

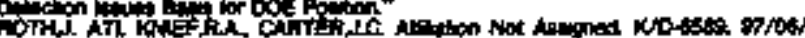

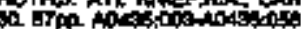

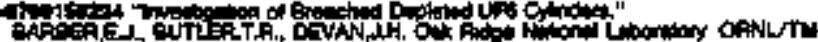

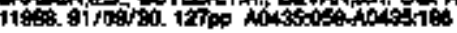

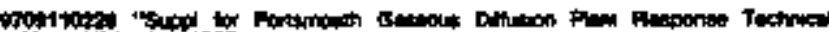

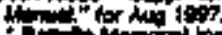

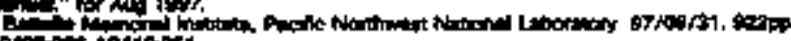

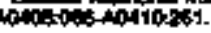

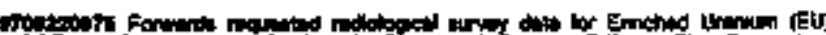

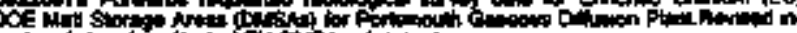

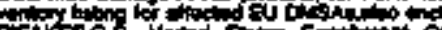

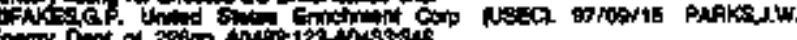

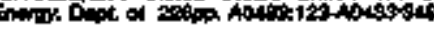

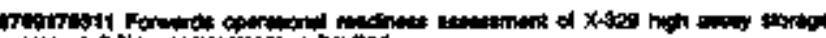

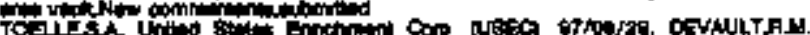

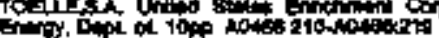

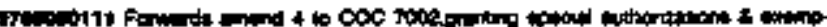

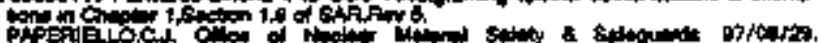

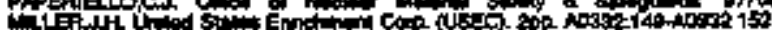

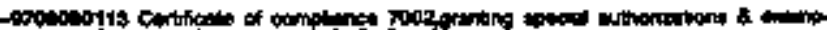

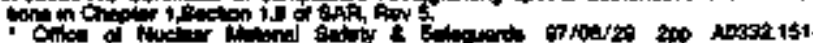

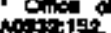

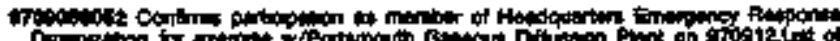

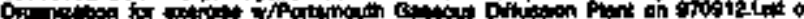

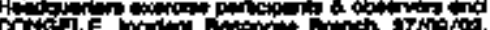




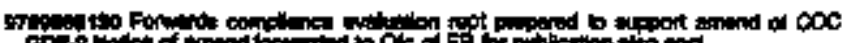

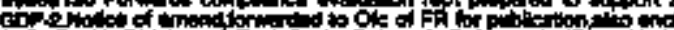

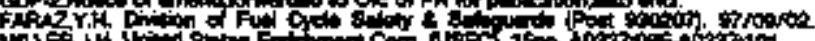

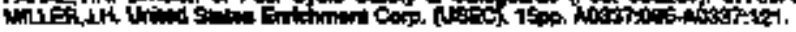

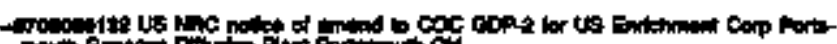

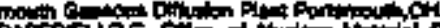

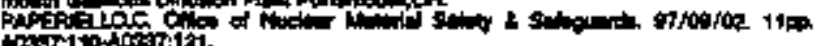

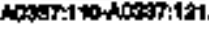

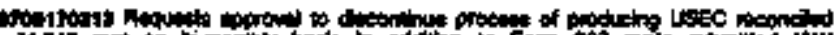
474

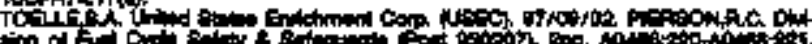

17.0.

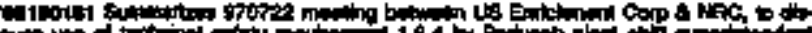

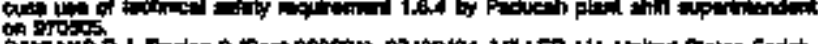

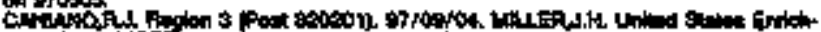

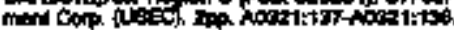

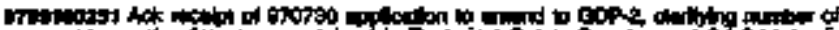

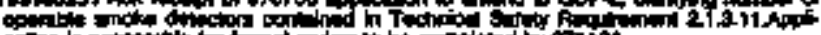

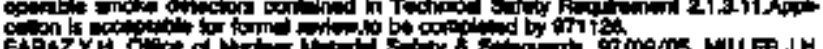

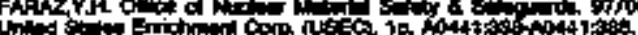

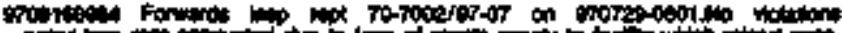

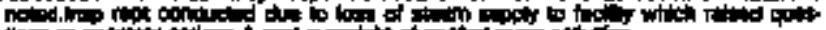

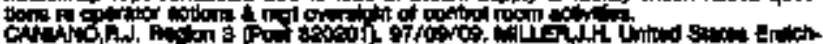

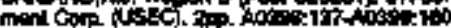

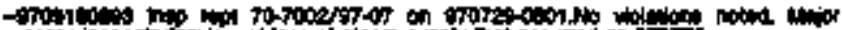

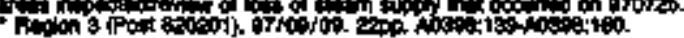

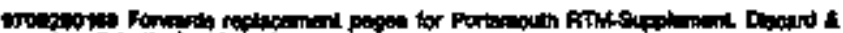

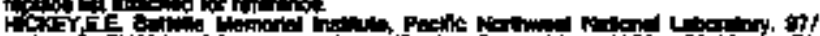

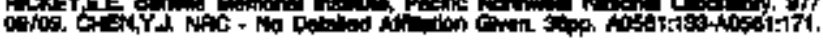

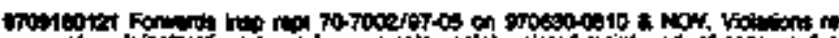

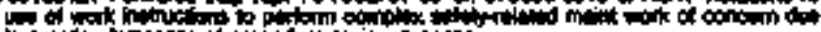

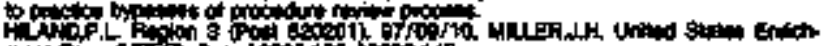
intid

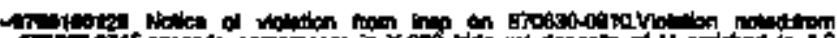

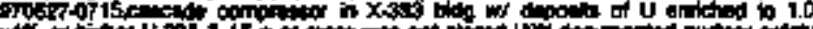

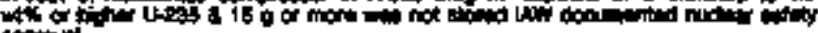

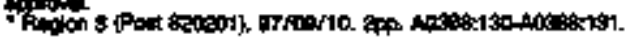

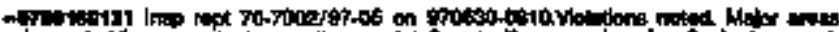

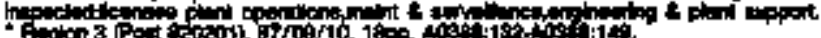

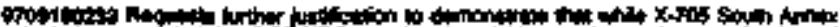

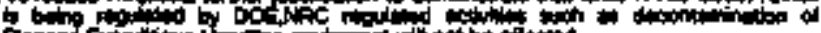

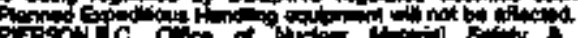

4.

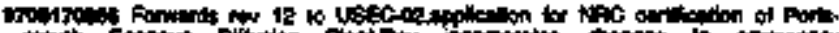

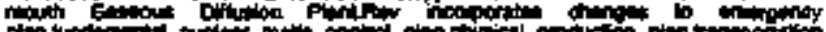

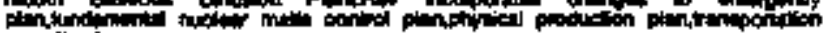

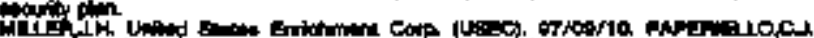

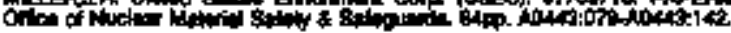

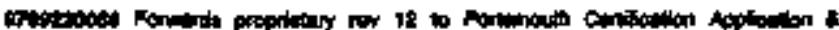

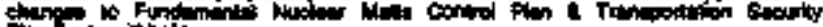

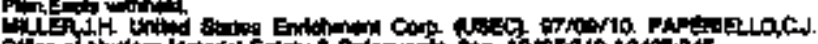

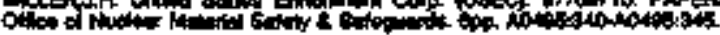

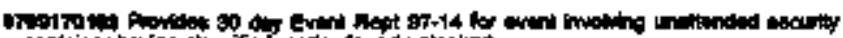

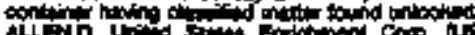

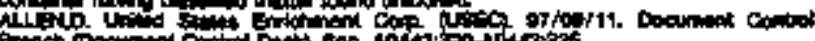

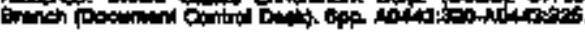

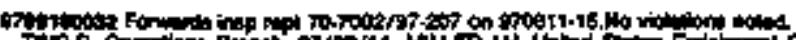

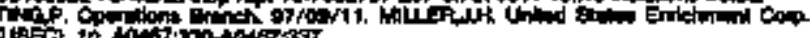

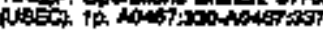

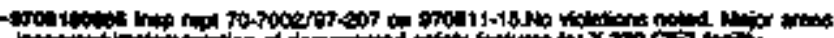

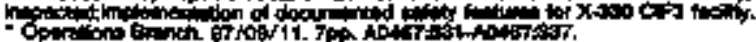

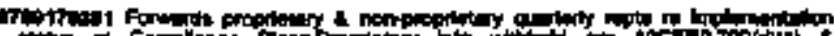

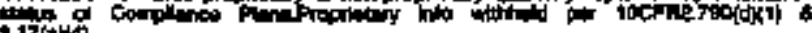

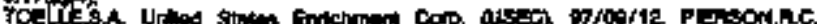

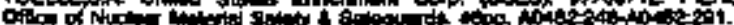

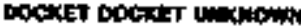

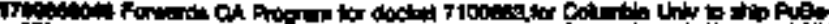

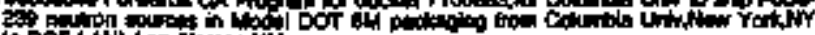

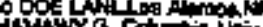

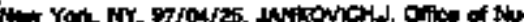

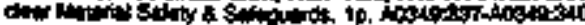

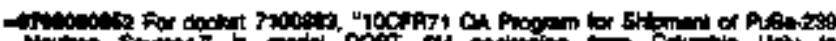

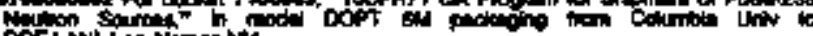

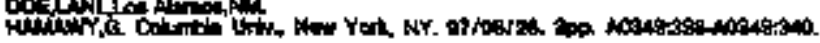

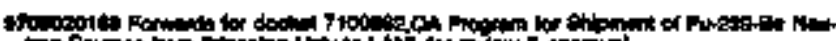

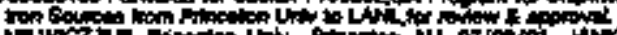

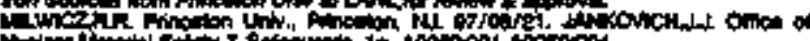

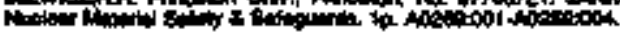

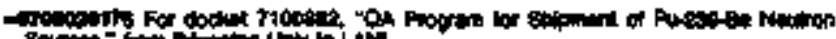

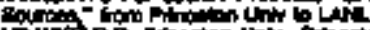

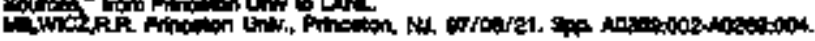

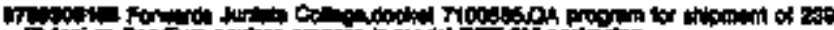
L

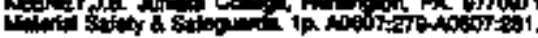

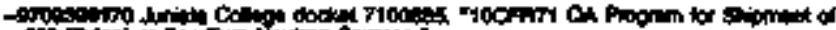

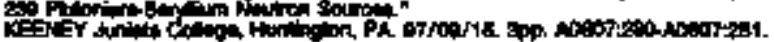

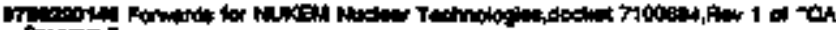

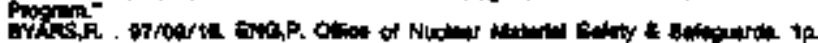

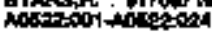

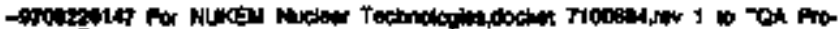

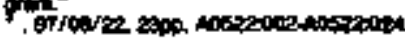

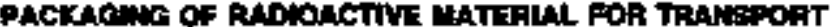

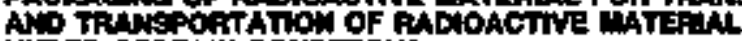

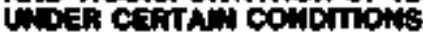

\section{bocket meas niwroo ous}

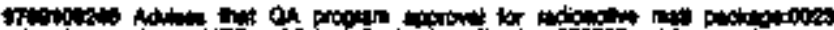

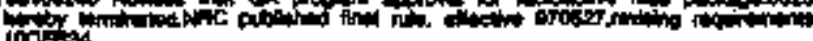

10070

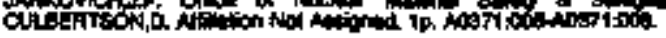

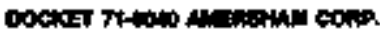

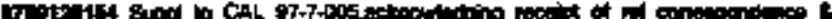

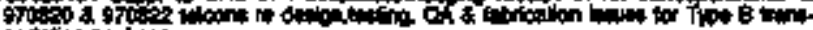

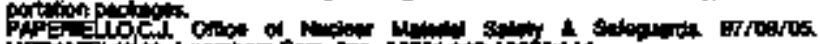

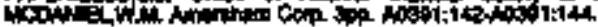

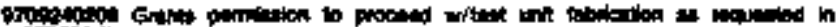

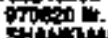

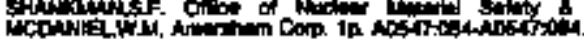

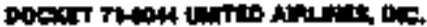

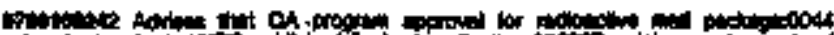

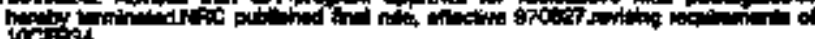

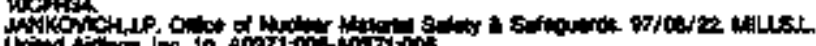

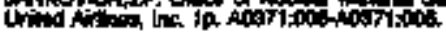

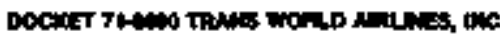

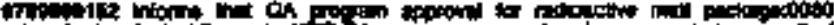

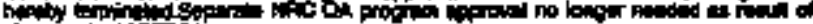

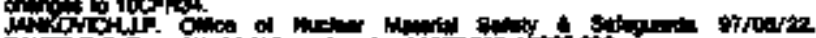

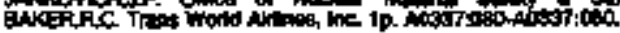

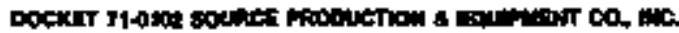

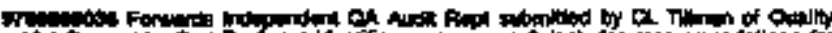

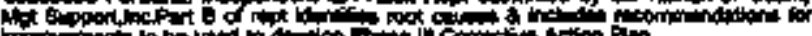
Dex

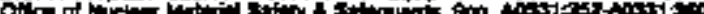

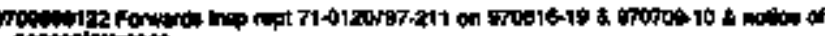

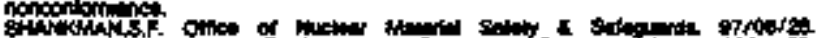

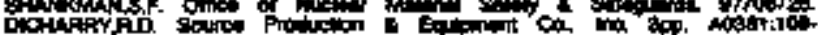
1058:144.

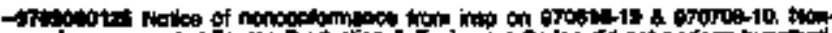

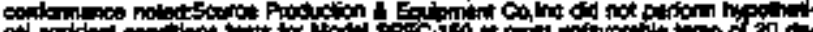

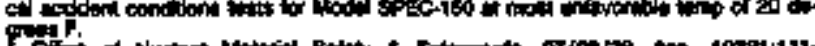

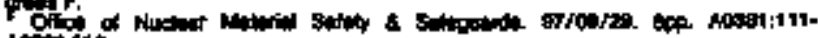
Ansti118. 


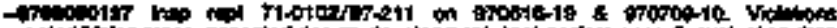

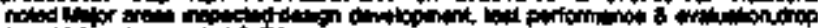

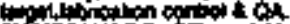

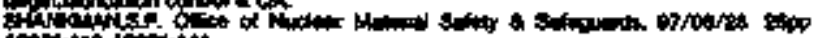

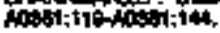

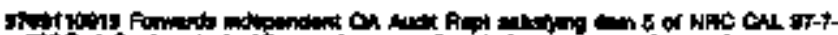

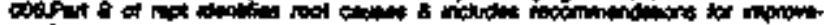

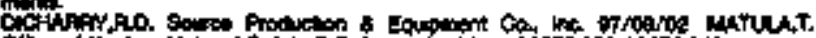

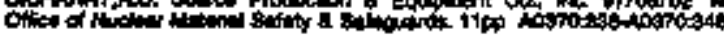

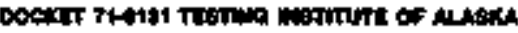

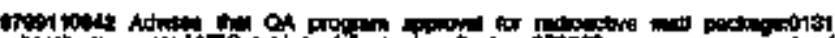

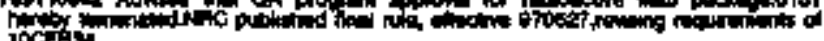
$10 \mathrm{cos}$.

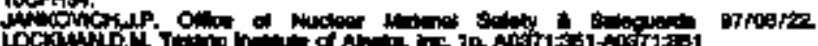

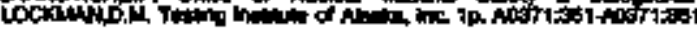

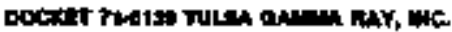

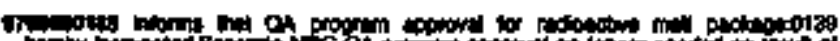

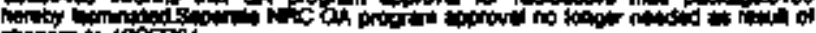

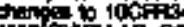

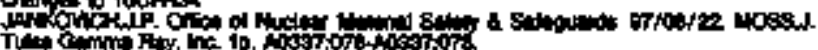

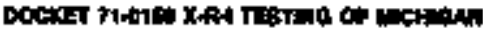

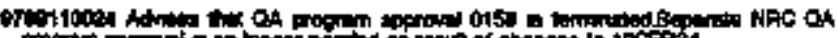

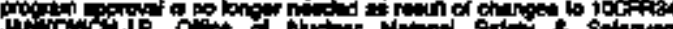

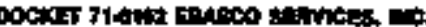

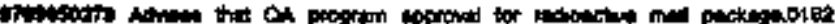

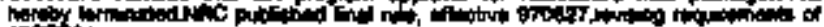
100 (1)

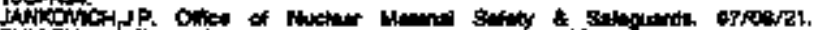

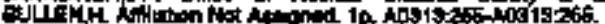

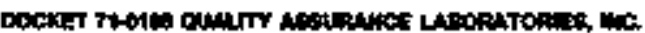

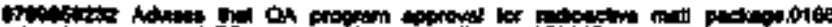

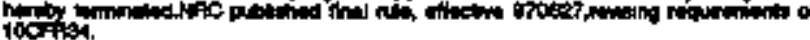

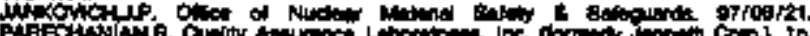

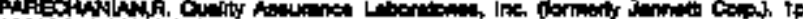

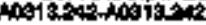

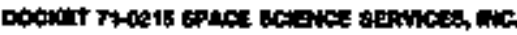

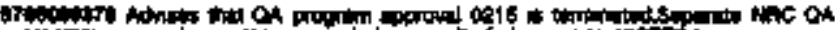

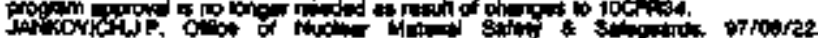

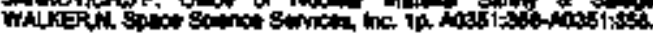

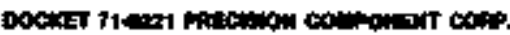

S7.

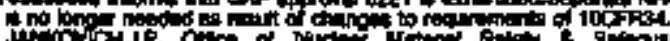

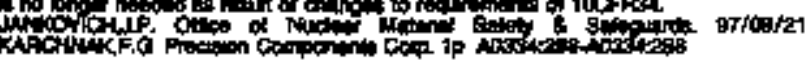

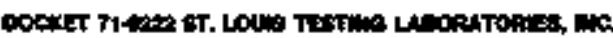

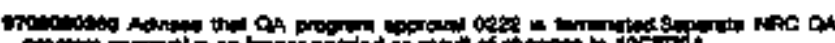

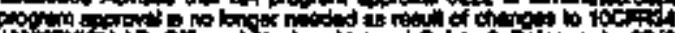

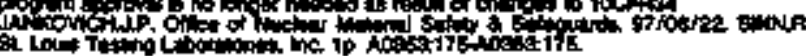

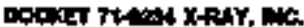

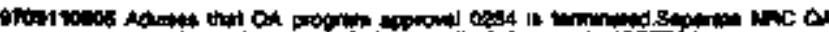

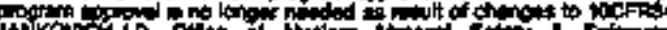

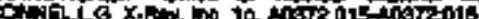

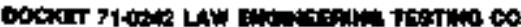

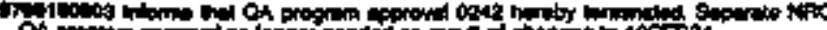
OA proph Q

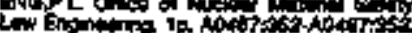

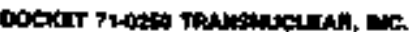

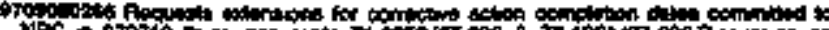

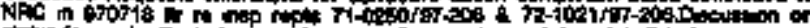

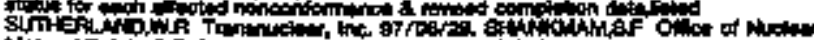

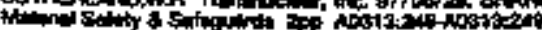

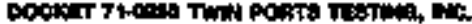

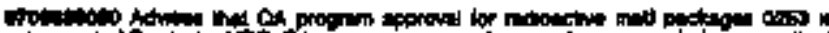

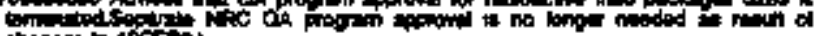

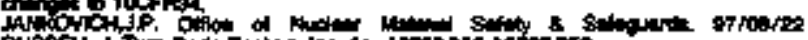

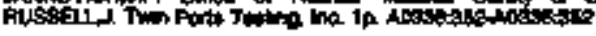

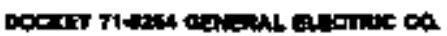

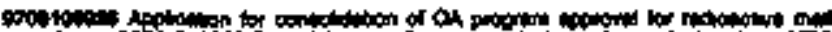

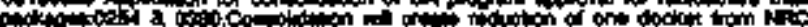

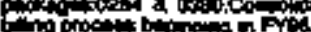

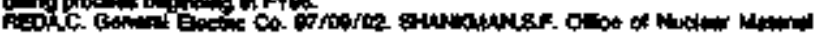

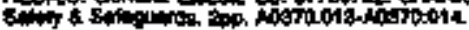

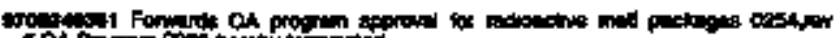

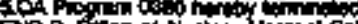

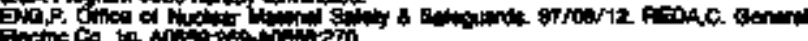

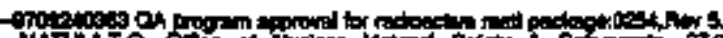

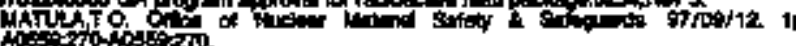

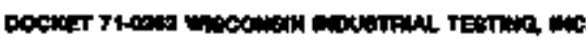

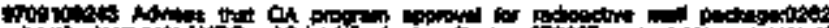

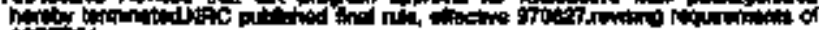
toction.

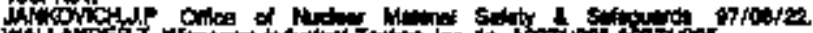

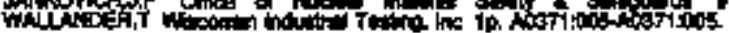

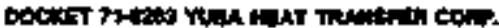

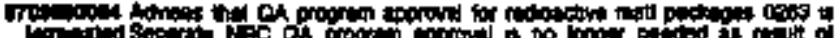

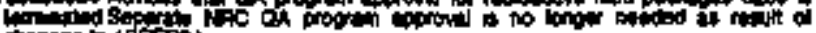

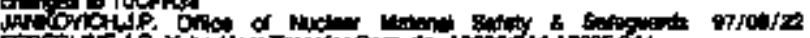

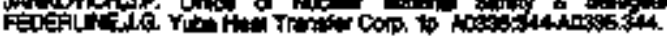

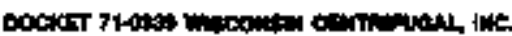

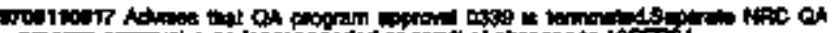

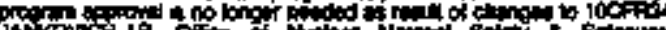

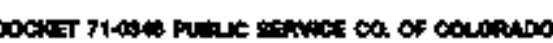

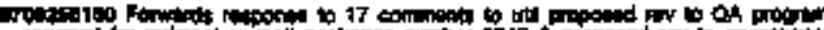

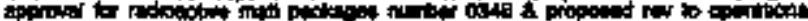

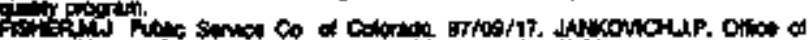

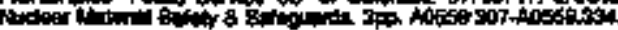

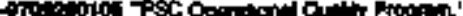

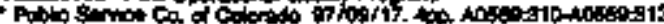

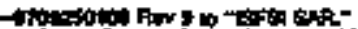

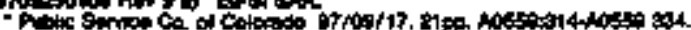

Docket rupin Gegine dectinc on

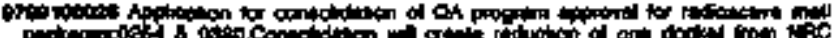

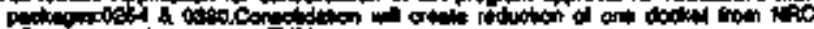

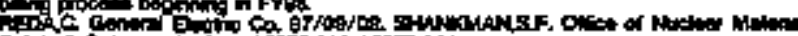

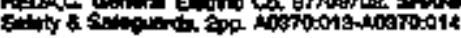

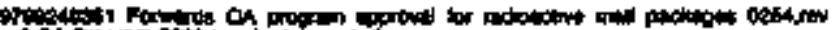

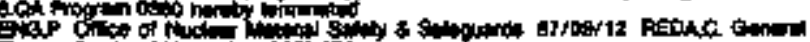

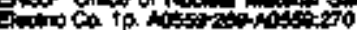

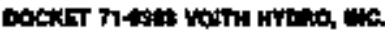

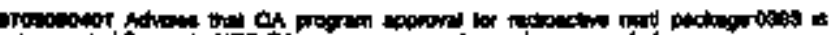

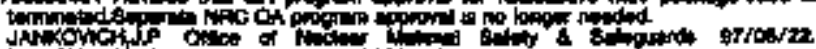

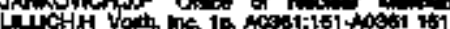

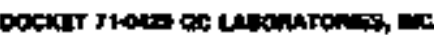

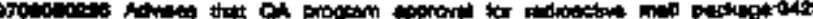

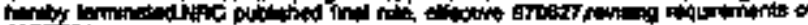
$100 \times 12$

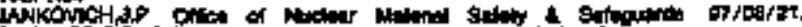

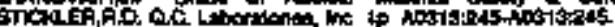




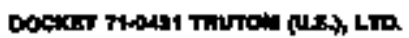

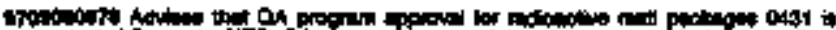

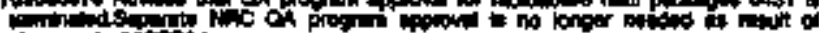

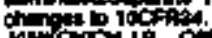

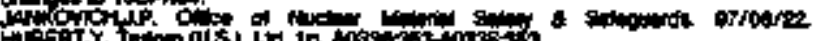

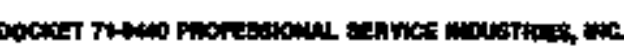

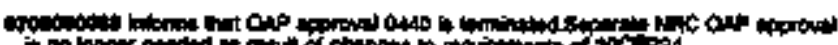

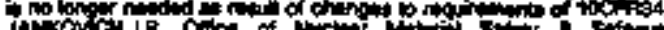

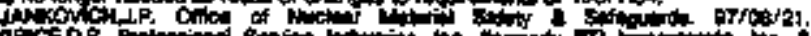

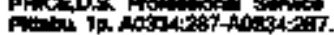

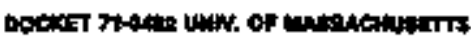

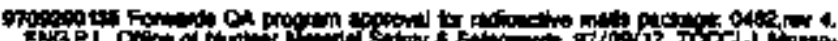

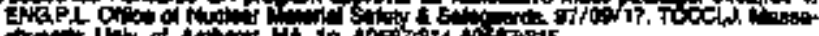
of

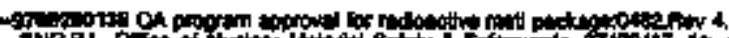

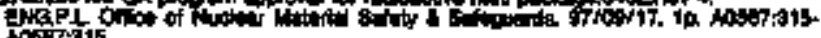

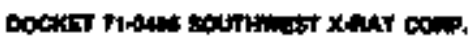

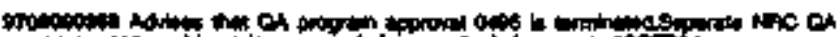

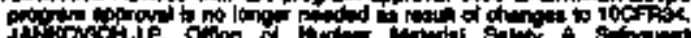

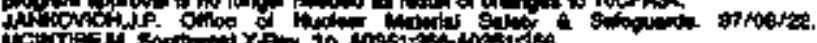

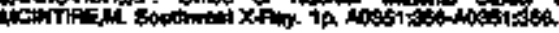

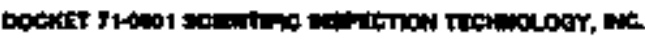

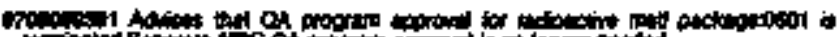

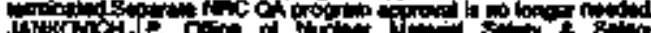
WWY DW W

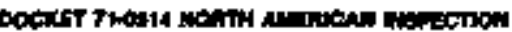

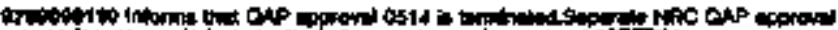

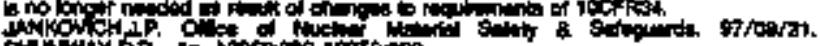

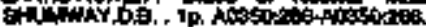

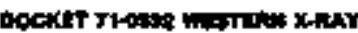

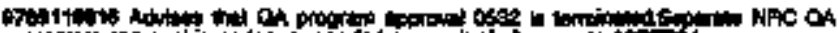

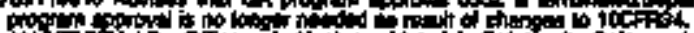

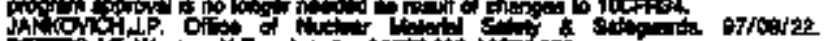

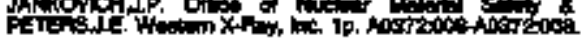

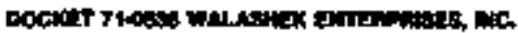

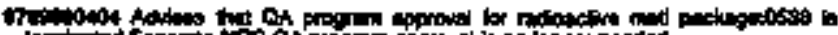

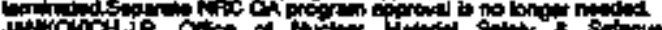

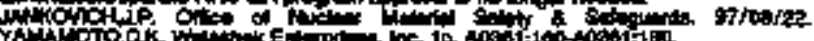

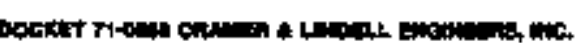

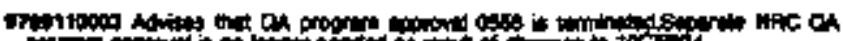

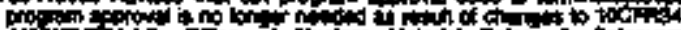
An

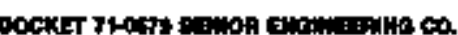

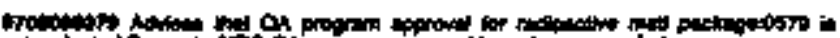

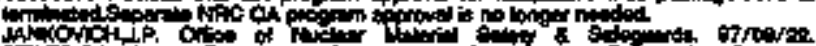
STus

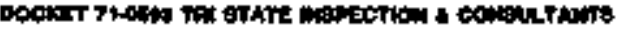

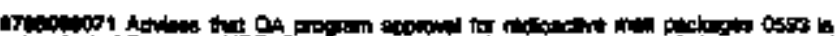
in

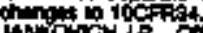

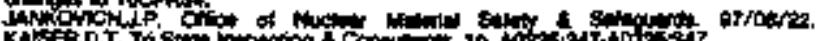

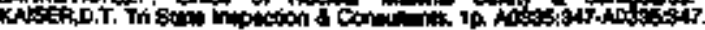

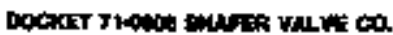

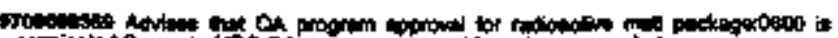

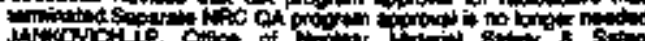

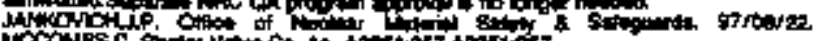

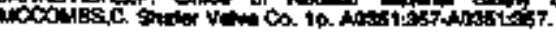

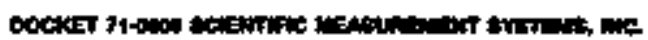

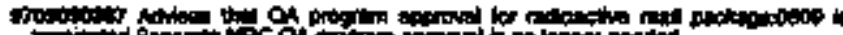

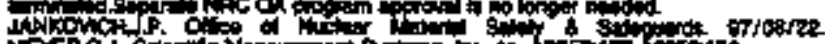

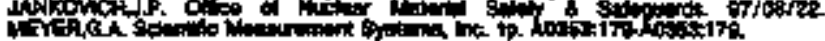

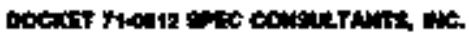

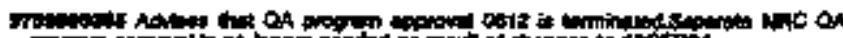

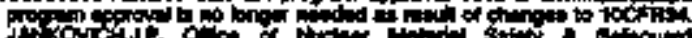
HAC L

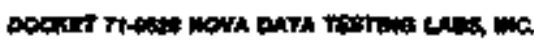

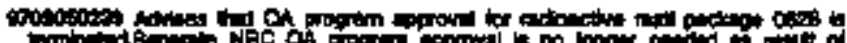

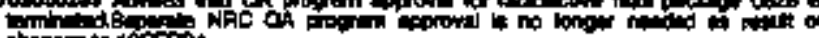

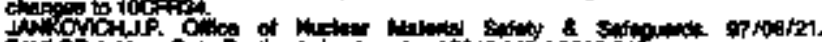
TAY OA

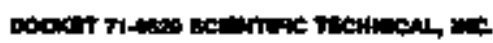

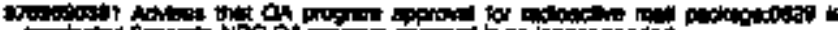
In

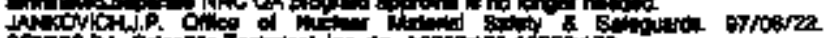

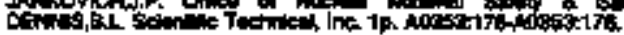

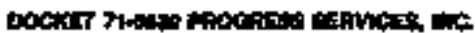

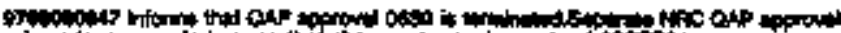

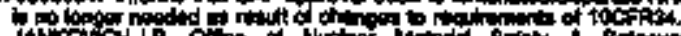

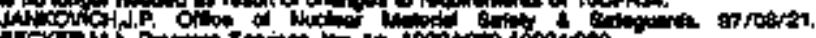

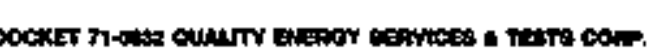

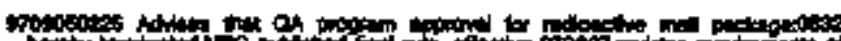

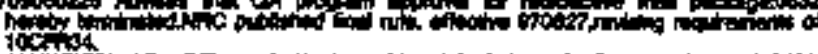

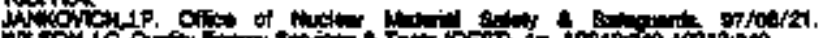

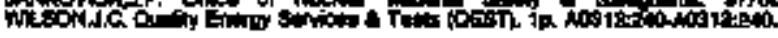

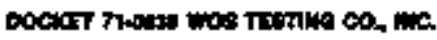

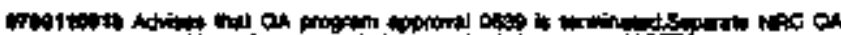

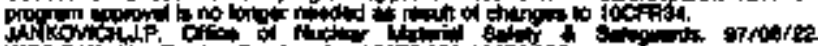
Wo:

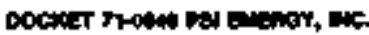

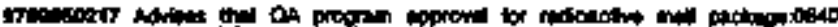

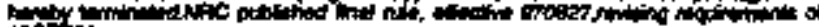

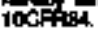

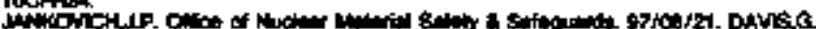

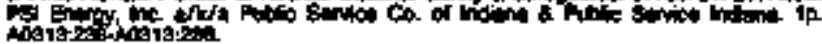

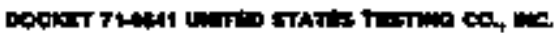

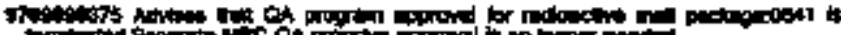

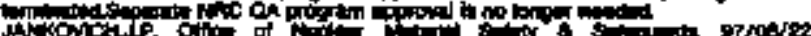

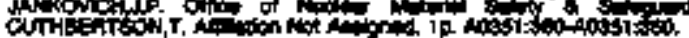

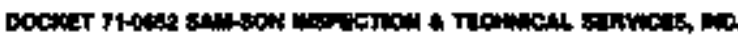

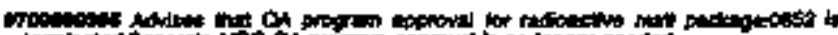

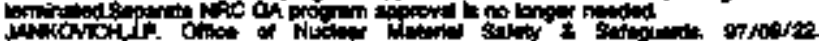

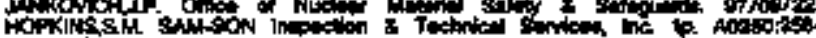
notoos.

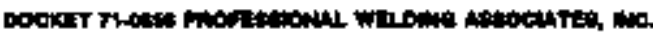

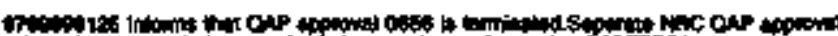

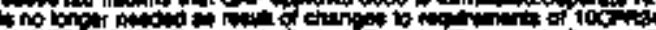

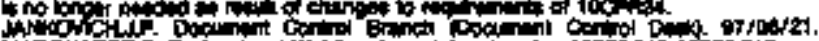

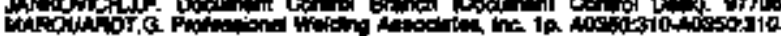

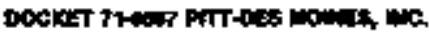

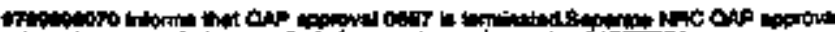

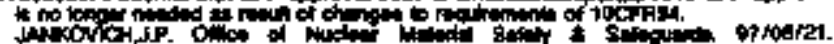

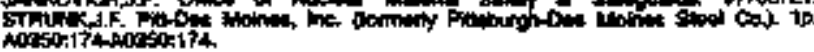




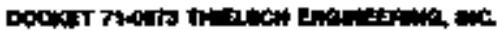

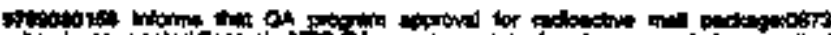

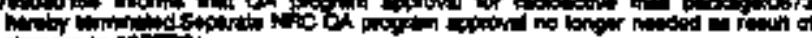

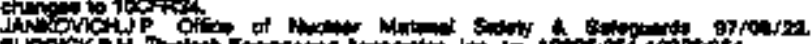

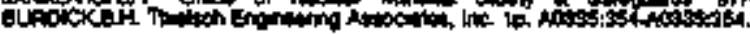

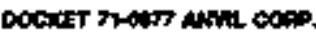

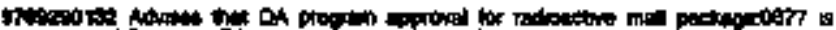

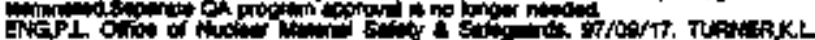

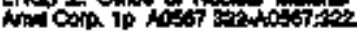

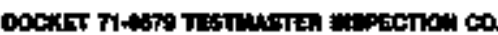

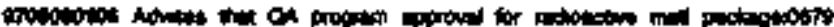

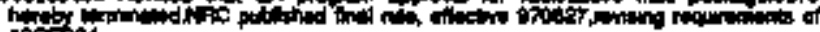

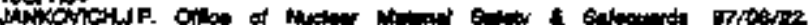

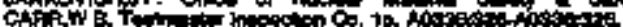

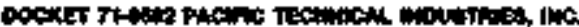

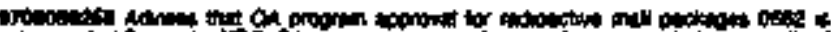

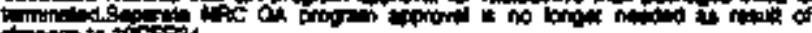
chis

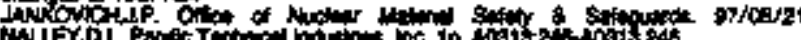

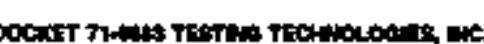

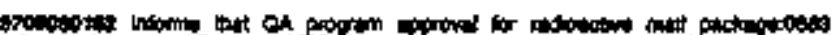

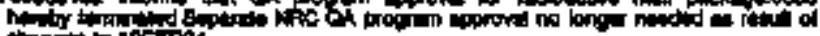

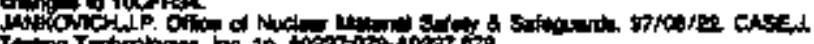

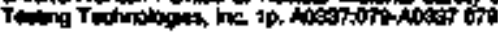

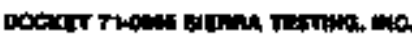

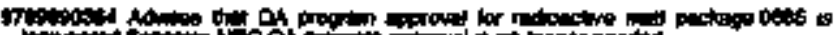
Hintids

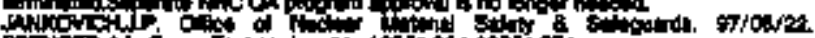

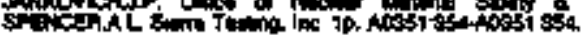

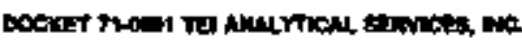

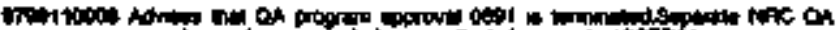
PR

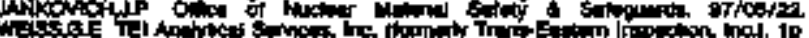

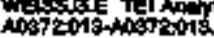

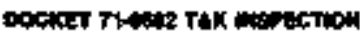

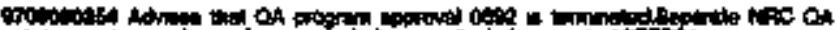

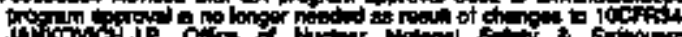

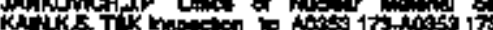

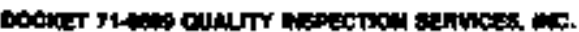

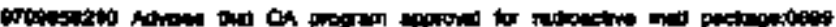

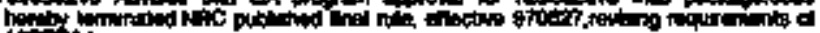

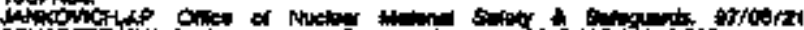

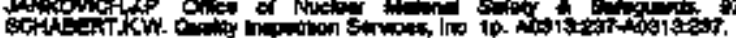

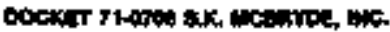

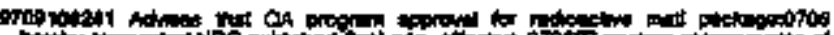

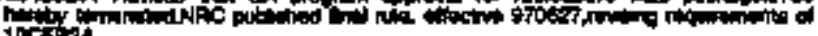

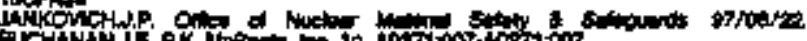

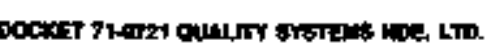

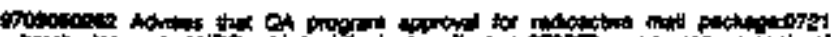

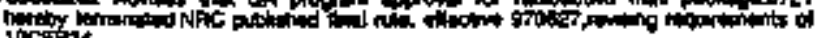

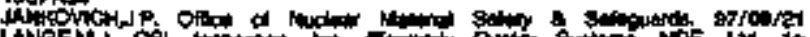
Hate

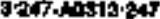

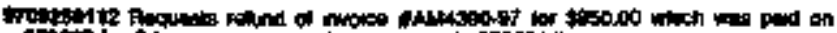

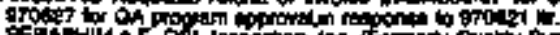

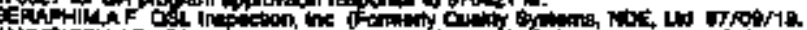

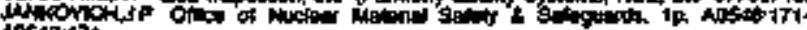
atose'1?

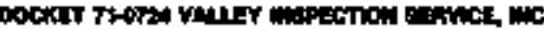

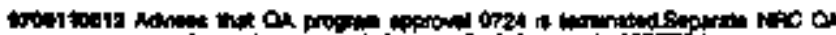

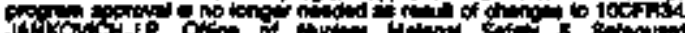

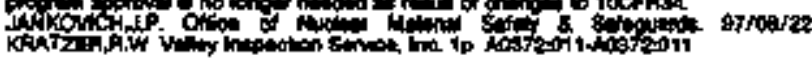

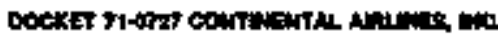

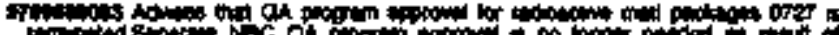

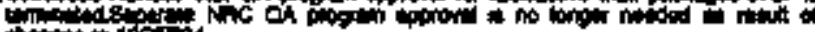

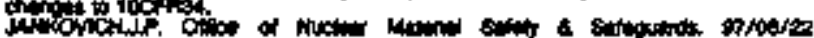

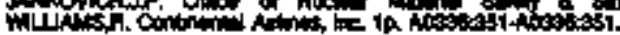

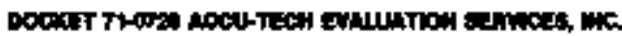

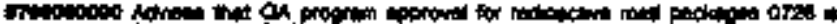

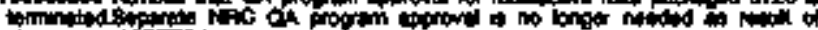

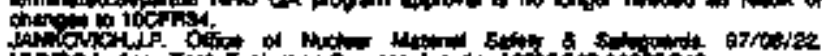

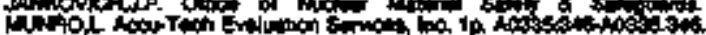

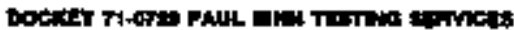

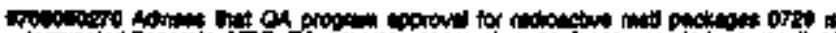

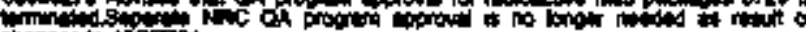

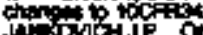

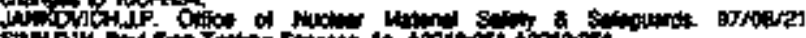

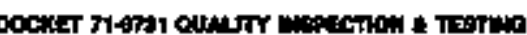

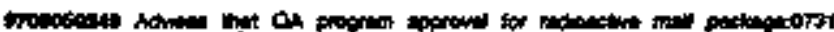

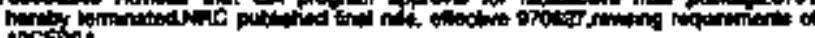

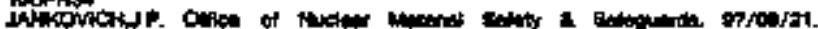

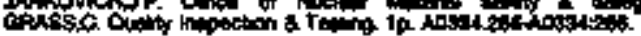

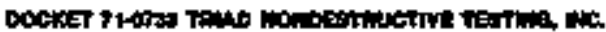

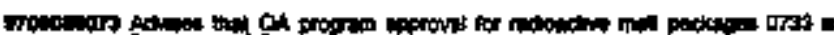

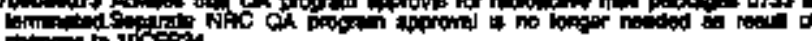

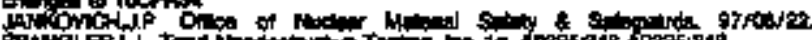

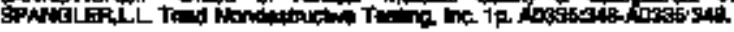

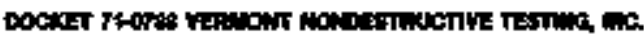

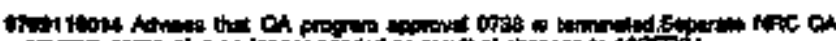

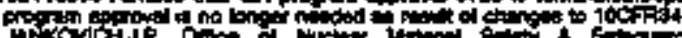

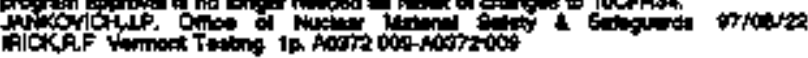

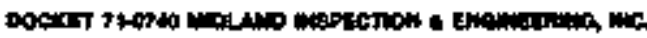

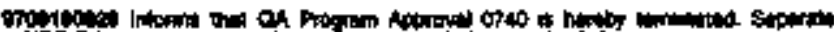

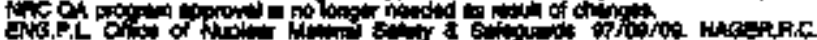

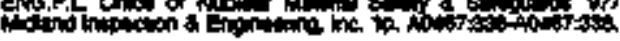

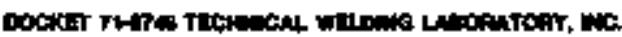

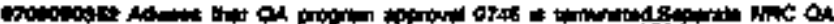

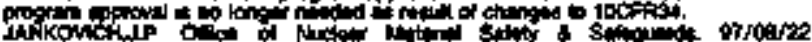

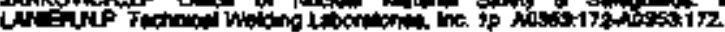

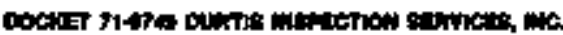

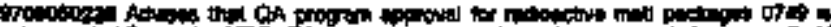

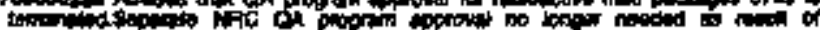

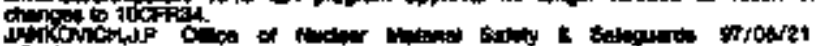

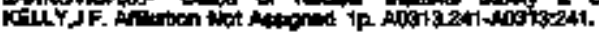

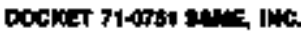

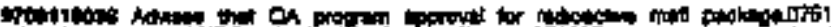

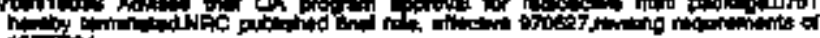
$100+34$

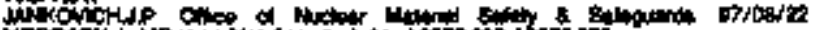

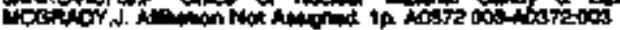

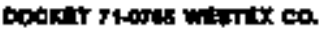

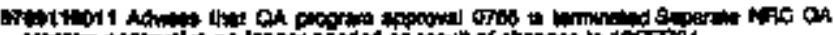

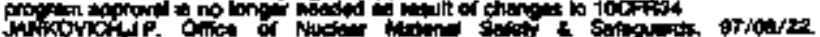

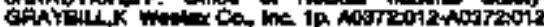




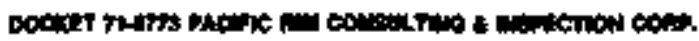

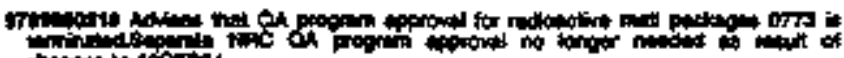

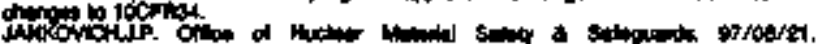

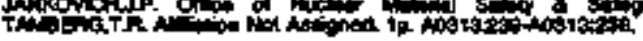

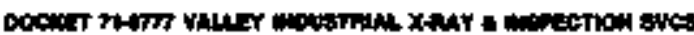

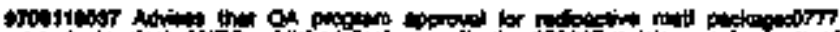

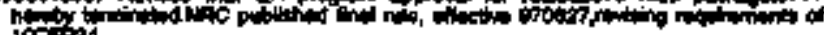

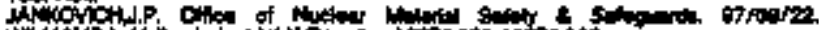

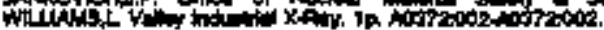

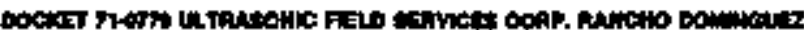

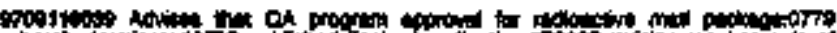

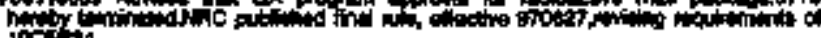

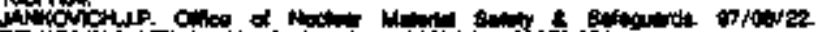

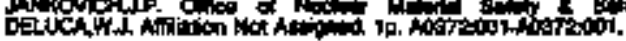

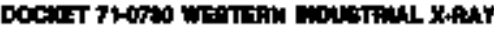

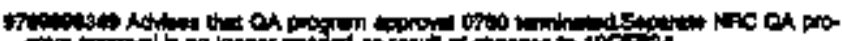

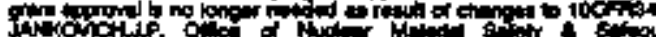

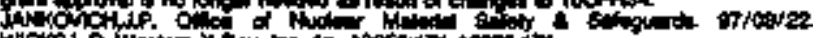

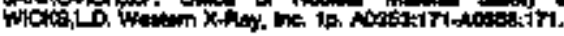

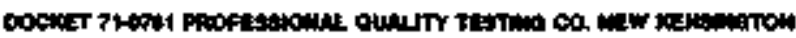

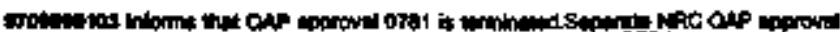

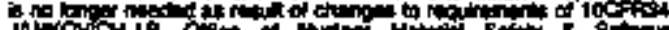

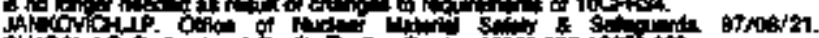

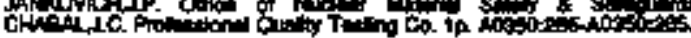

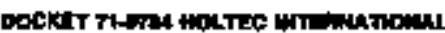

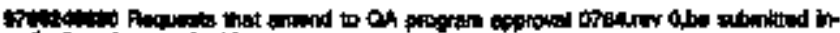

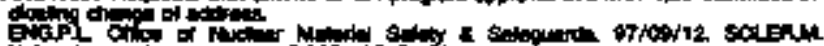

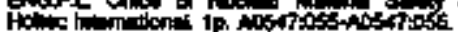

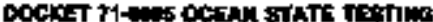

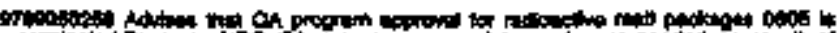

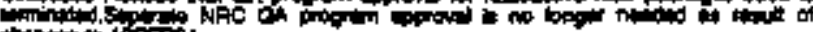

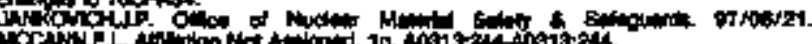

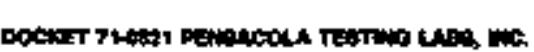

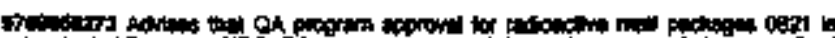

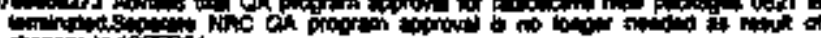

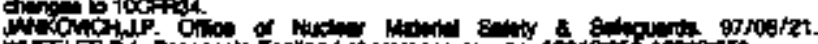

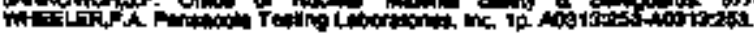

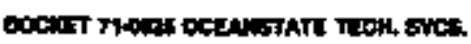

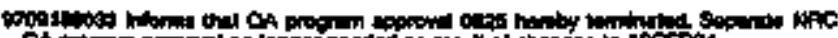

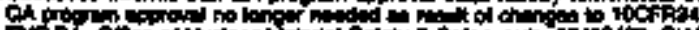

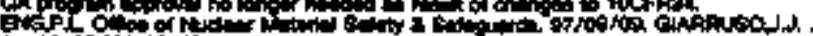
ip. Anotion

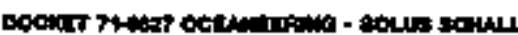

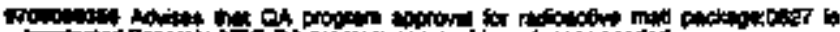

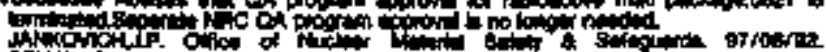

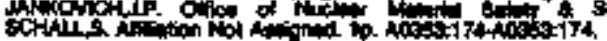

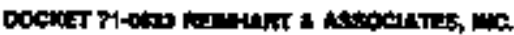

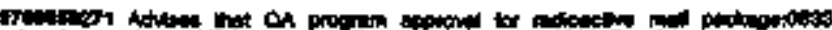

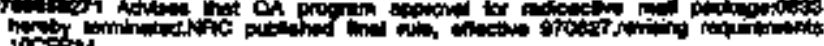

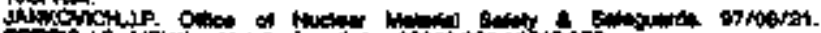

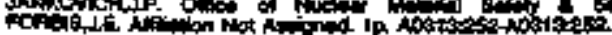

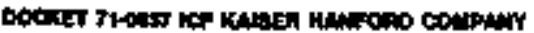

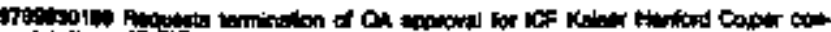

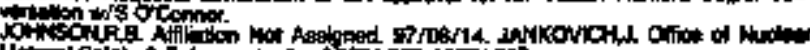

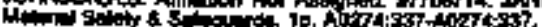

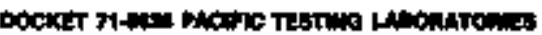

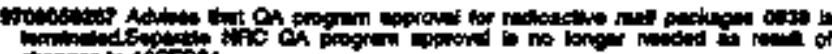

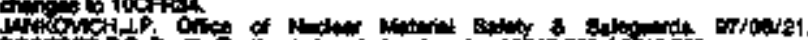

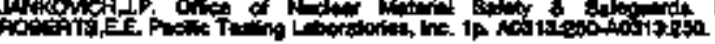

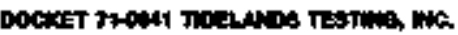

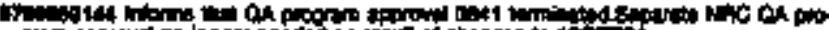

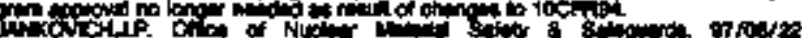

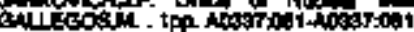

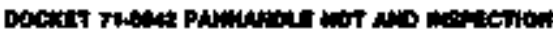

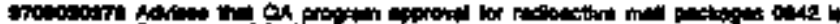

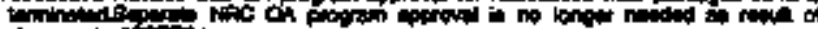

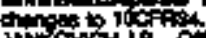

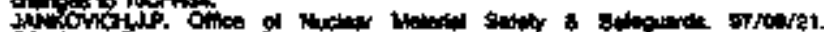

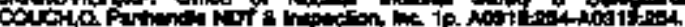

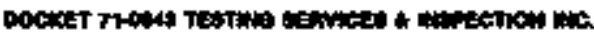

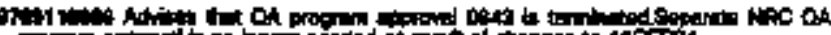

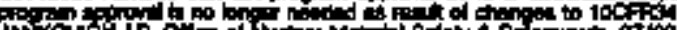

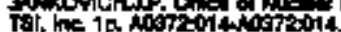

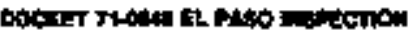

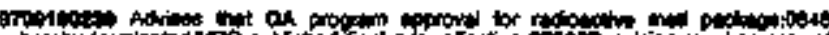

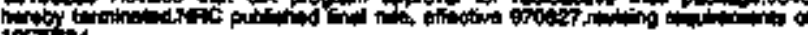

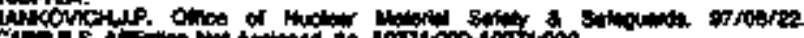

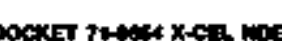

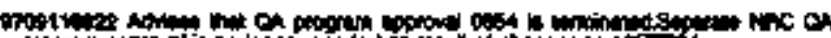

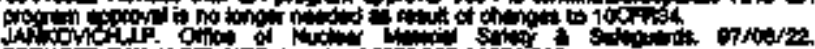

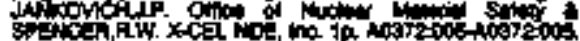

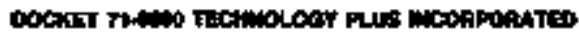

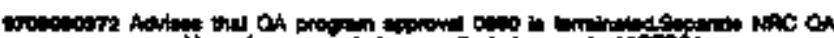

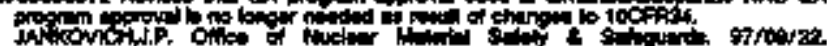

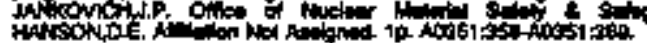

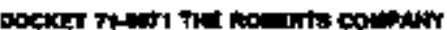

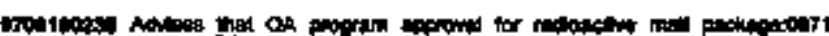

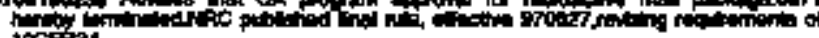

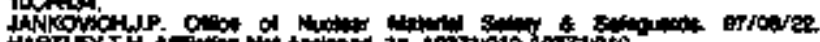

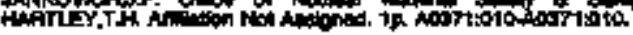

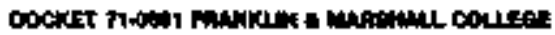

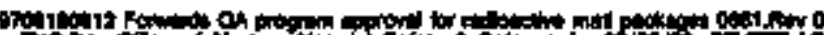

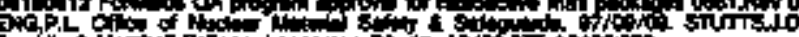

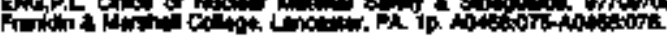

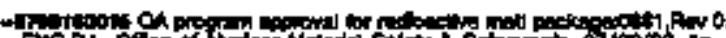

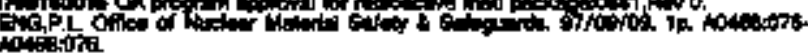

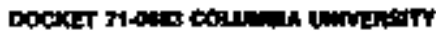

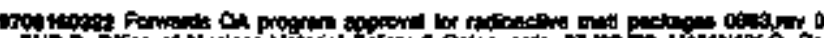

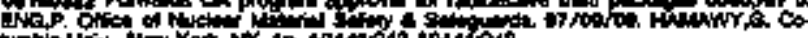

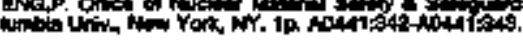

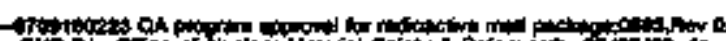

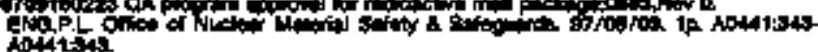

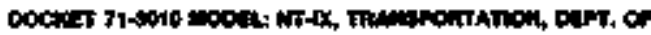

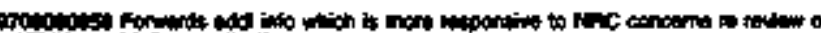

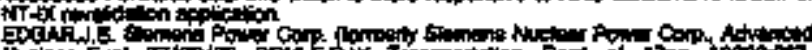

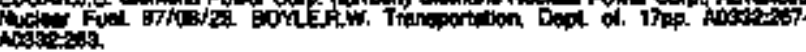

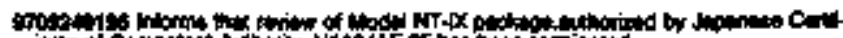

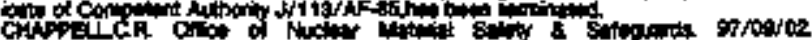

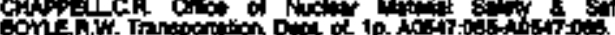




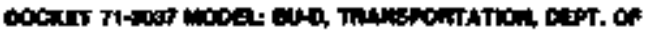

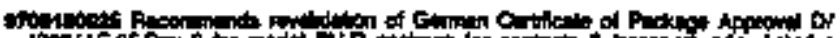

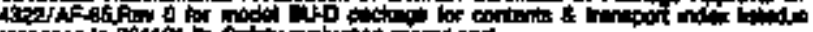

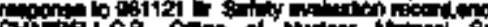

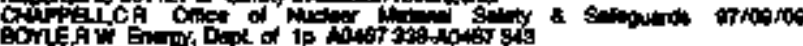

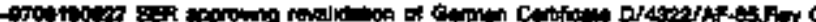

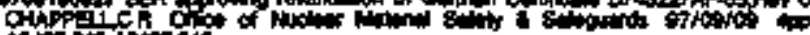

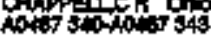

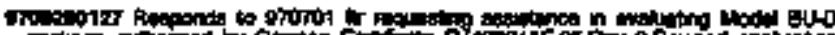

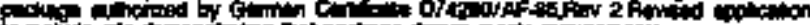

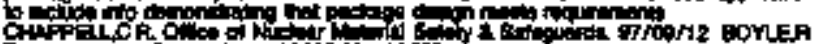

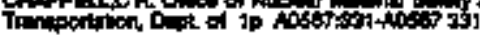

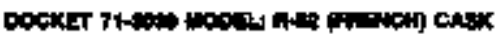

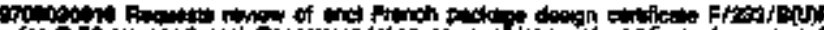

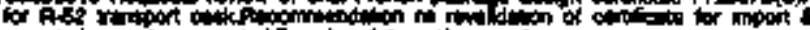

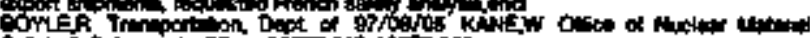

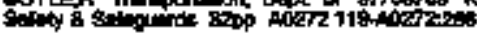

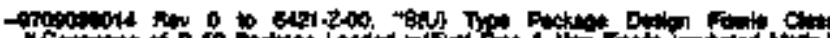

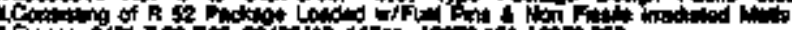

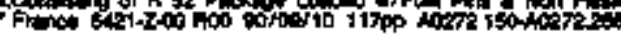

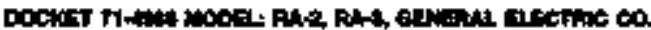

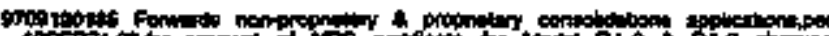

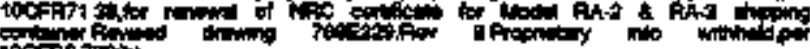

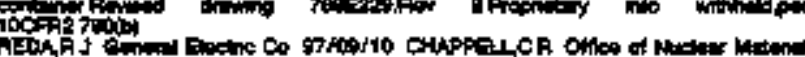

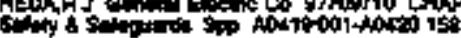

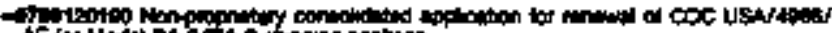

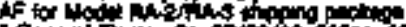

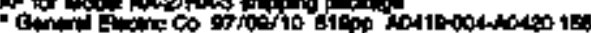

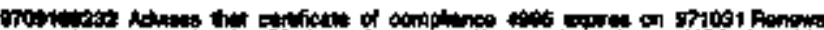

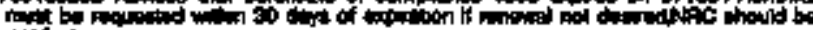

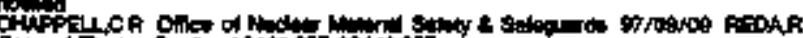

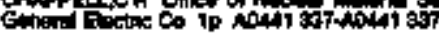

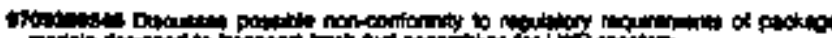

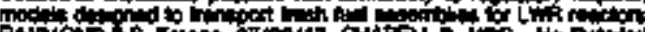

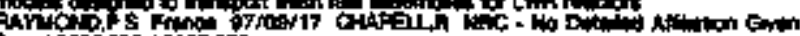

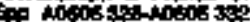

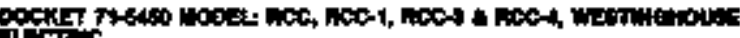

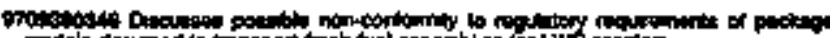

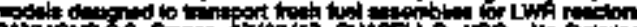

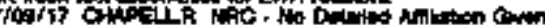

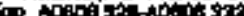

Dbthe 71-

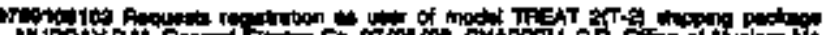

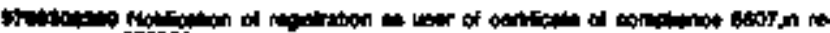

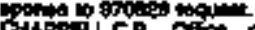

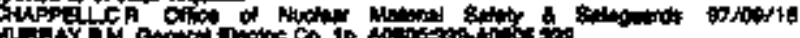

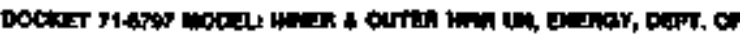

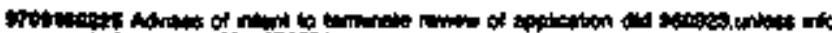

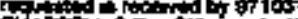

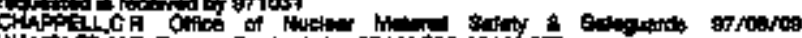

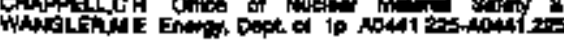

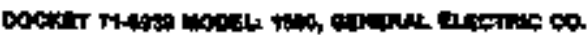

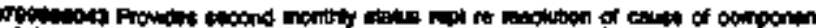

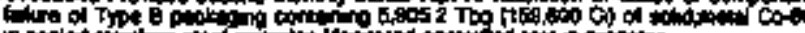

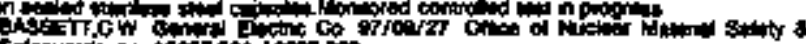

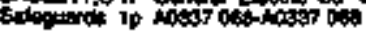

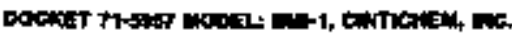

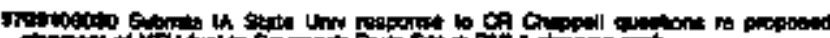

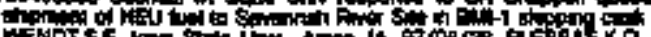

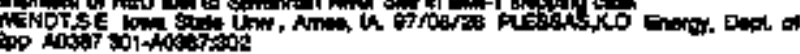

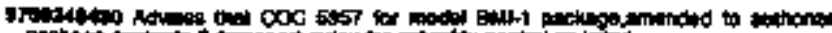

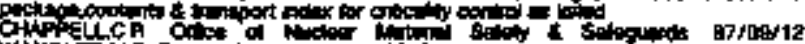

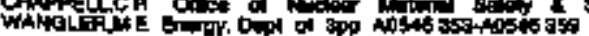

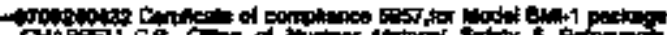

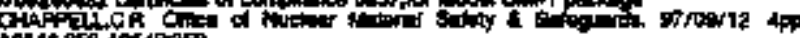

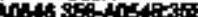

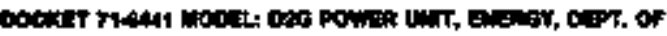

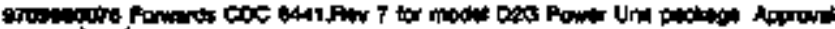

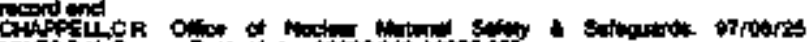

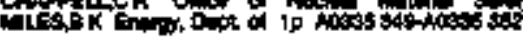

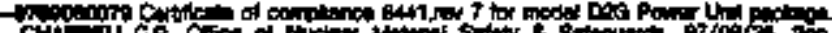

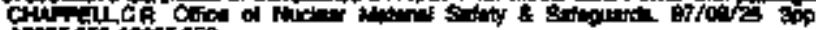

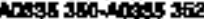

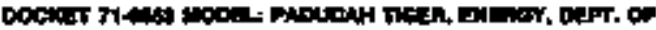

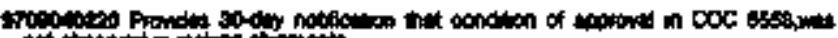

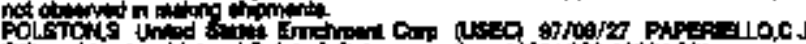

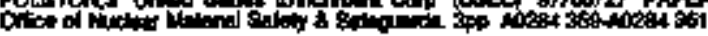

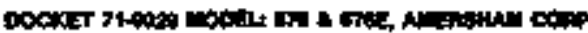

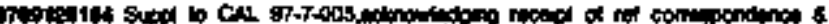

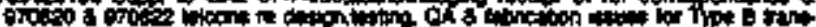

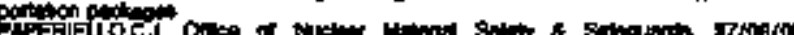

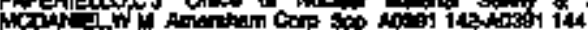

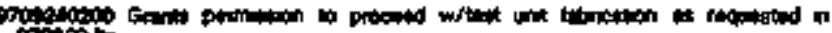

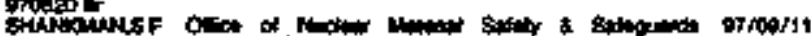

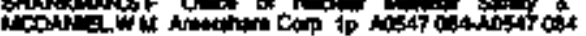

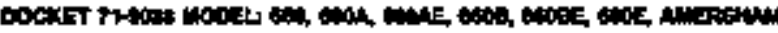

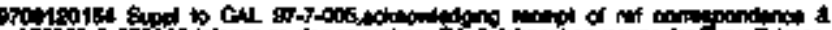

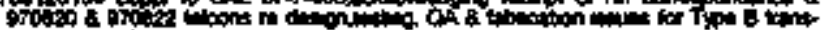

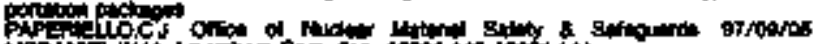

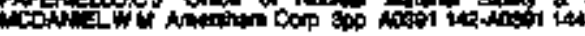

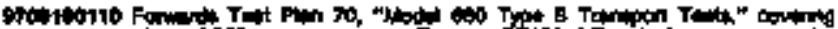

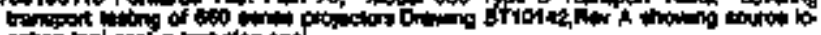

Find Soldit s.

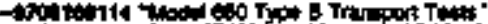

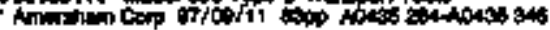

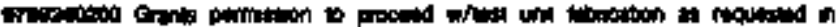

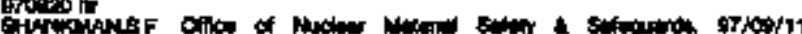

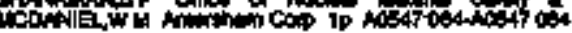

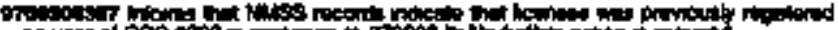

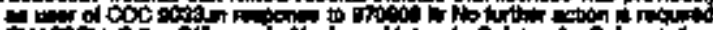

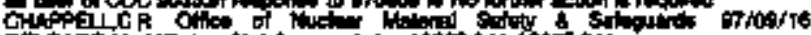

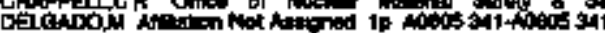

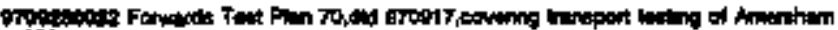

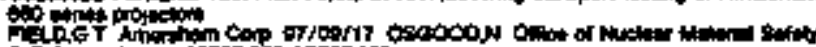

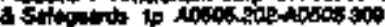

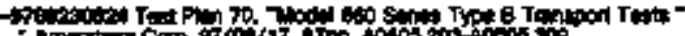

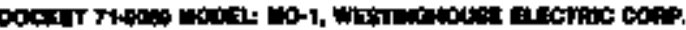

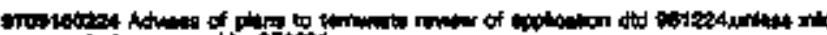

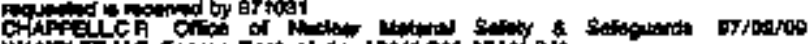

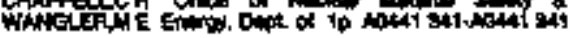

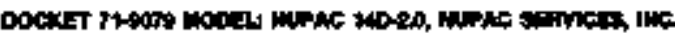

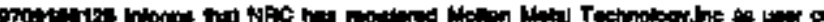

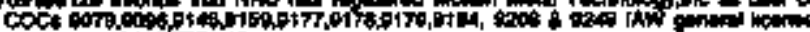

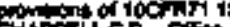

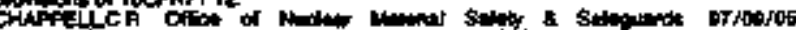

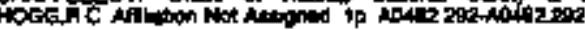

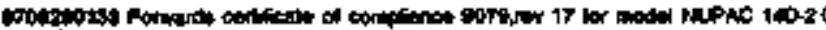

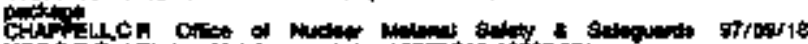

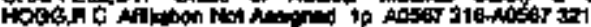

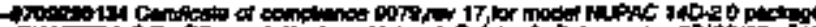

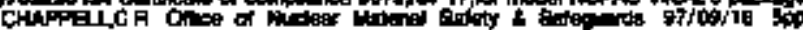
A0E87 317 ALA67 321 


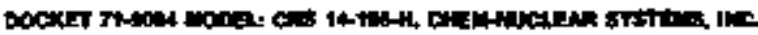

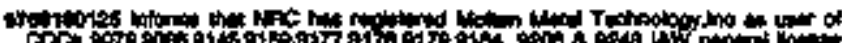

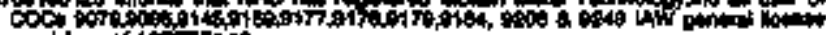

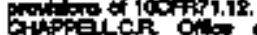

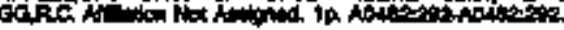

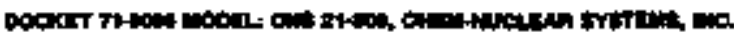

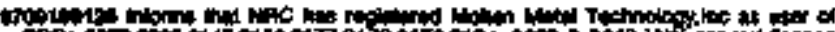

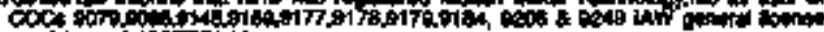

Cotint

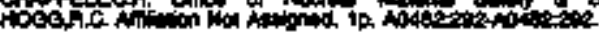

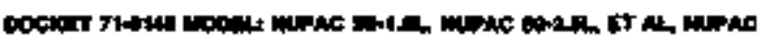

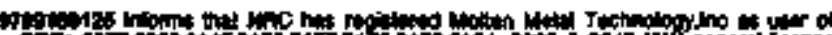

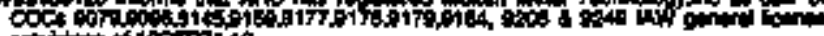

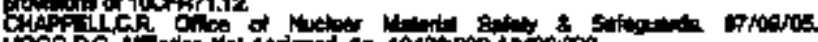

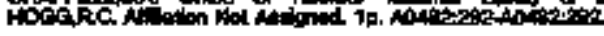

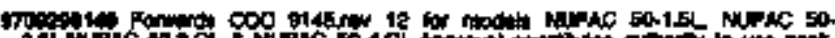

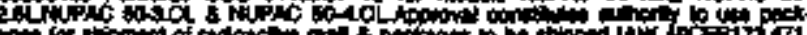

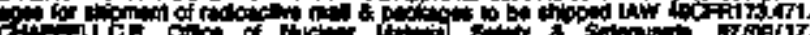

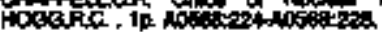

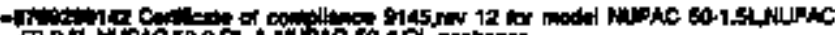

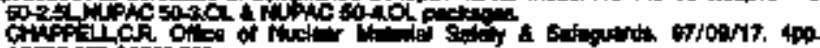

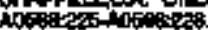

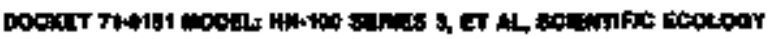

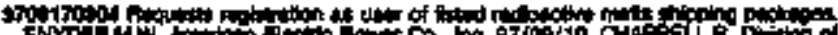

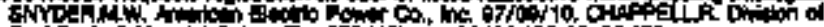

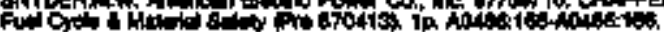

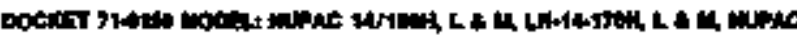

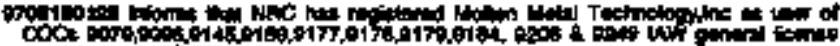

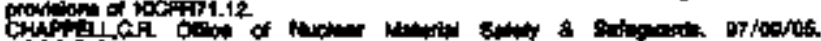

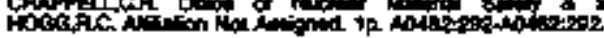

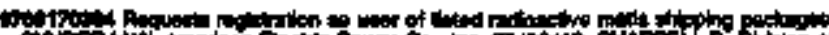

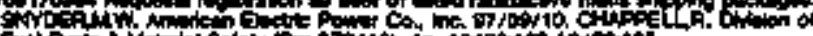

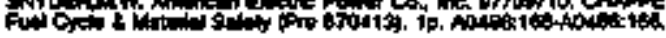

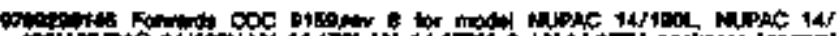

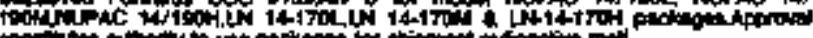
and

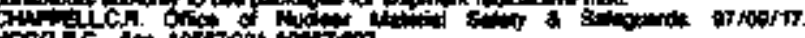

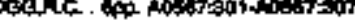

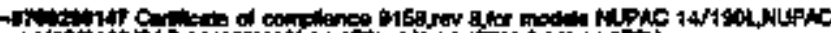

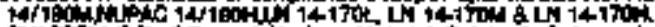

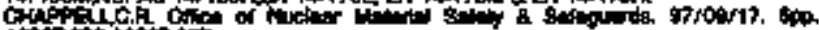

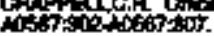

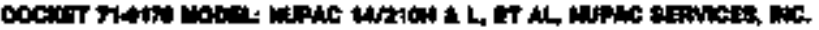

If

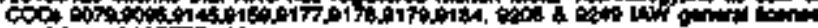

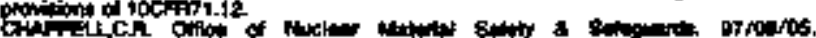

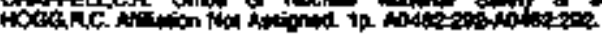

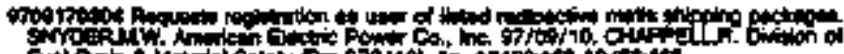

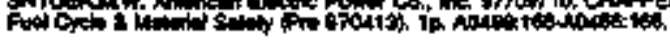

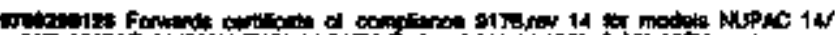

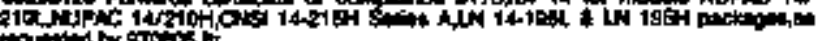

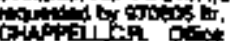

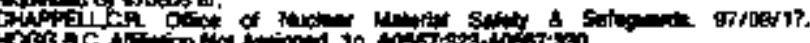

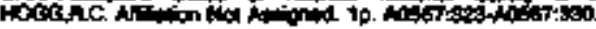

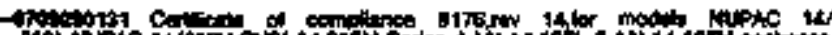

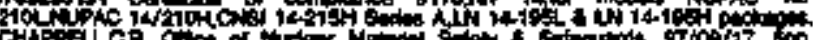

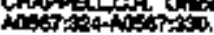

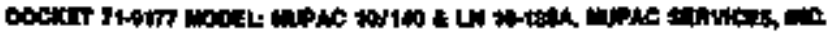

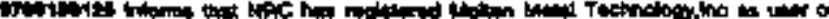

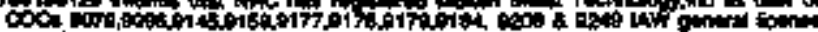

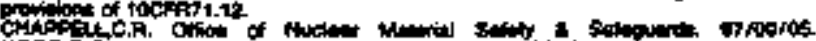

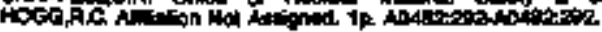

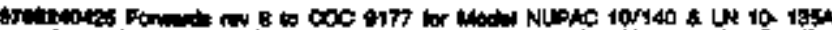

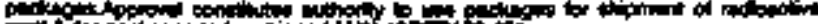

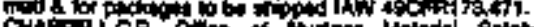

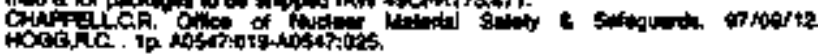

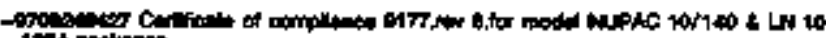

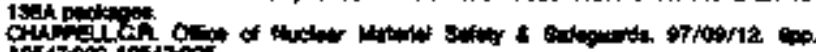

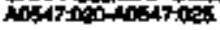

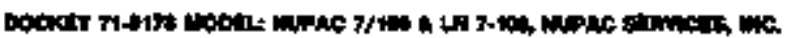

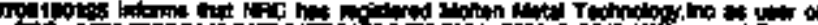

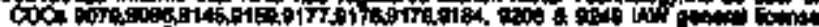

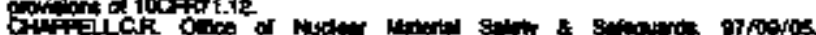

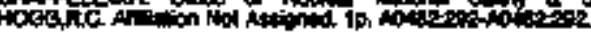

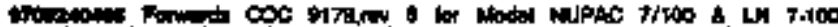

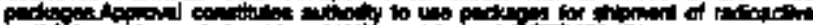

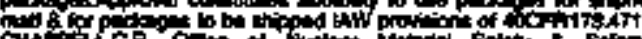

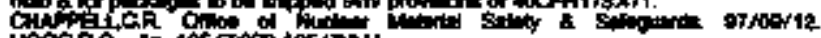

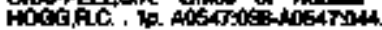

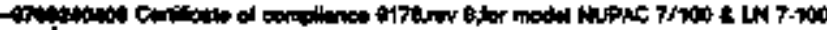

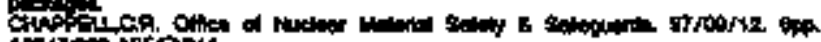

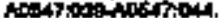

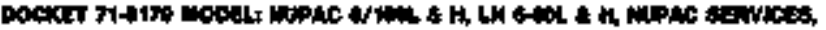

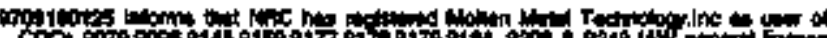

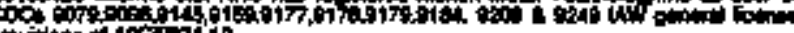

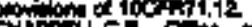

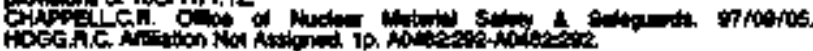

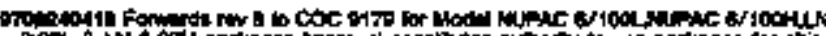

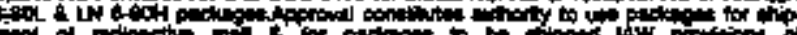

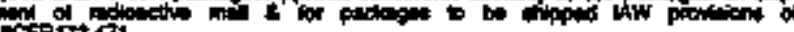

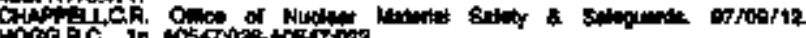

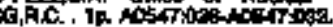

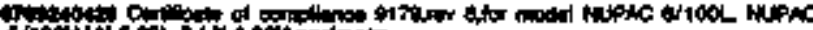

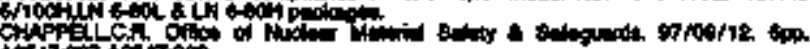

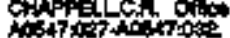

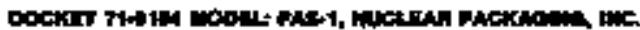

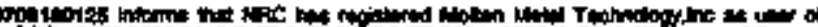

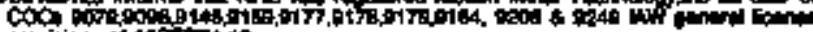

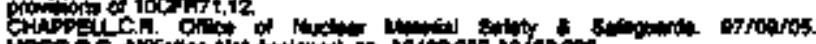

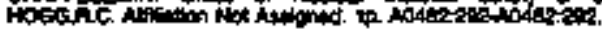

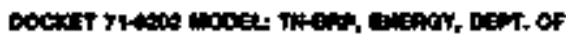

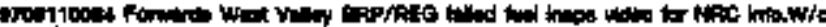

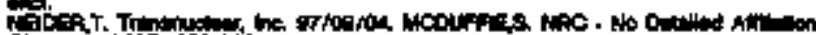

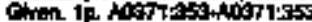

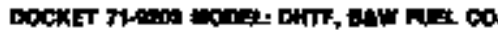

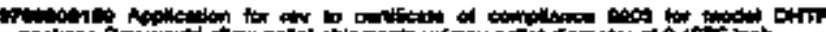

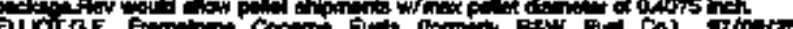

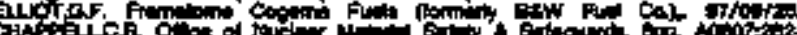
Aistion.

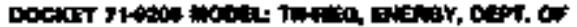

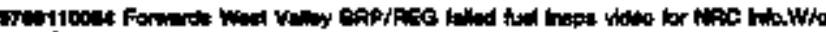
Cot

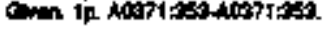

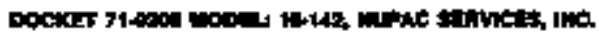

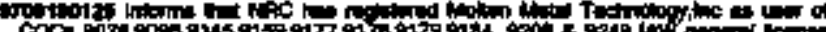

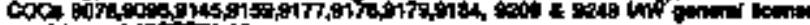

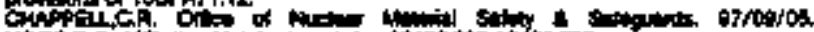

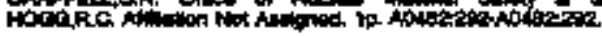

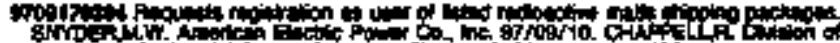

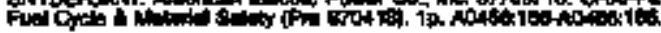

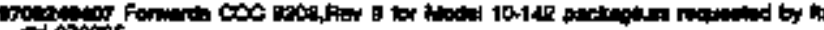

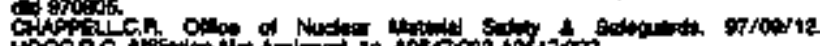

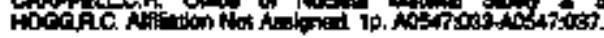

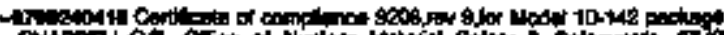

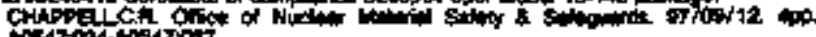

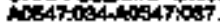




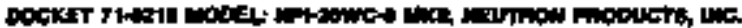

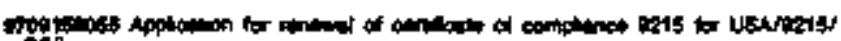

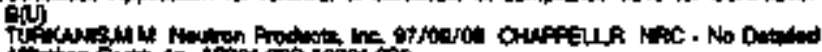

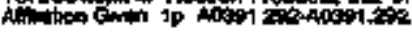

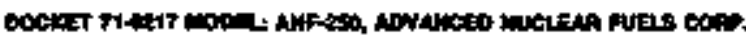

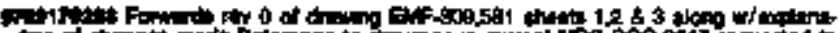

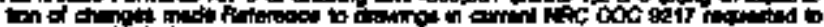

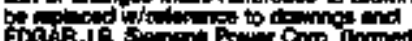

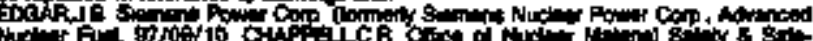

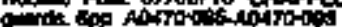

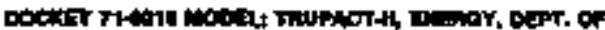

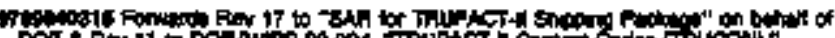

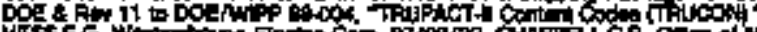

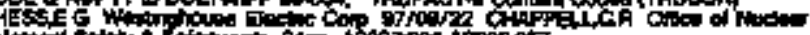

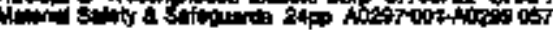

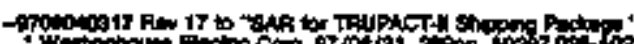

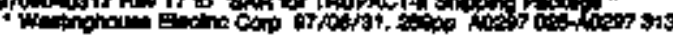

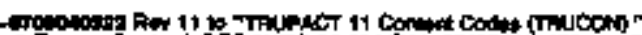

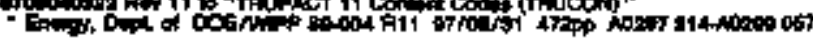

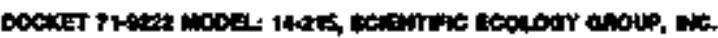

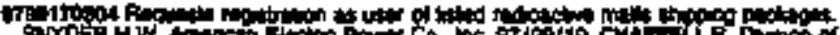

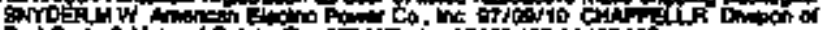

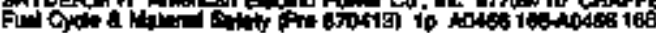

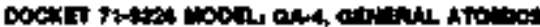

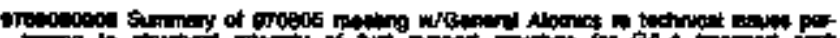

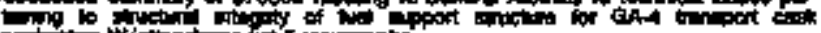

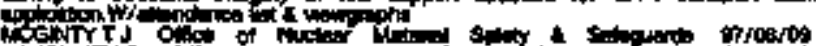

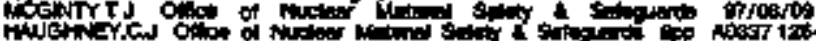
10.0.

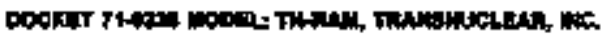

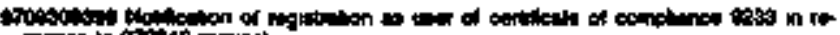

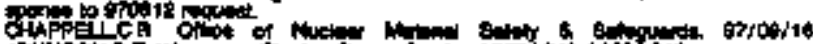
Dof

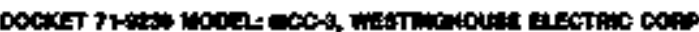

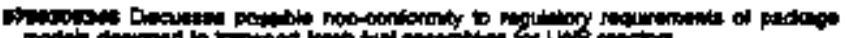

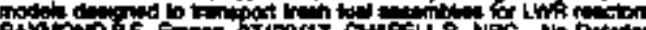
top towes so notos

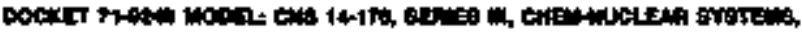

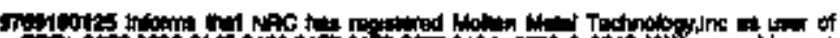

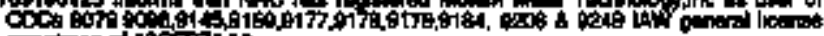

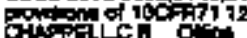

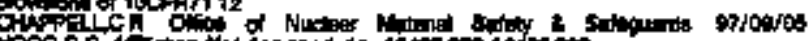

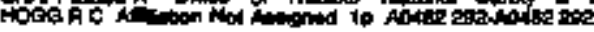

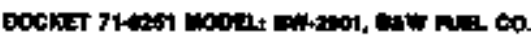

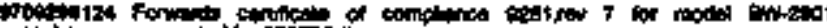

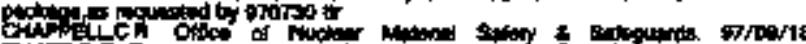

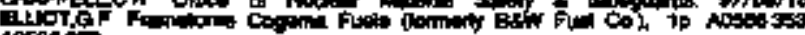
AOSAd \$क्ष

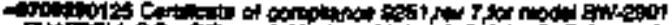

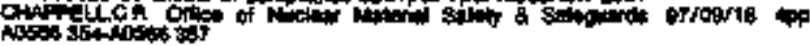

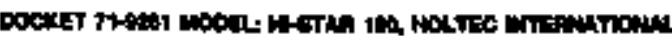

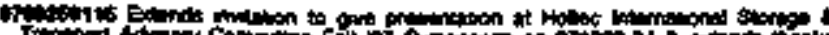

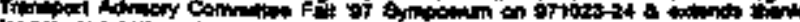

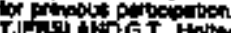

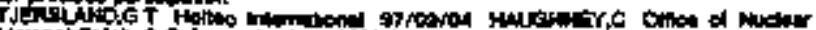

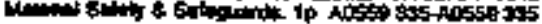

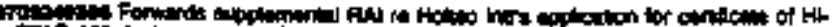

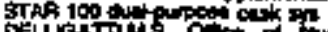

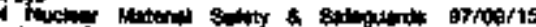

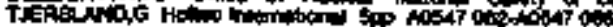

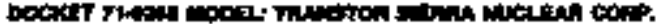

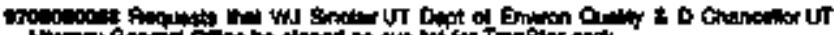

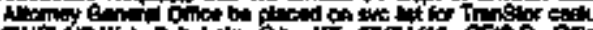

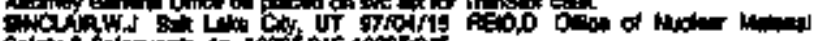

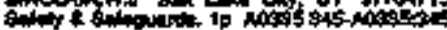

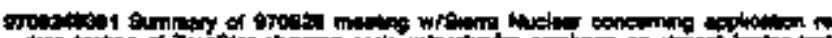

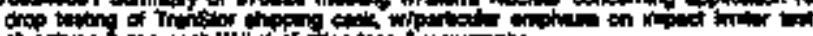

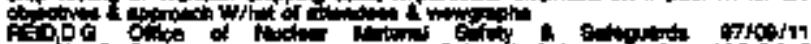

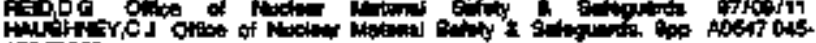
inction of

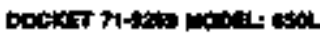

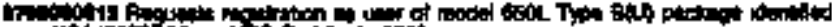

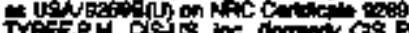

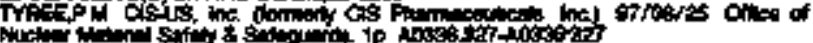

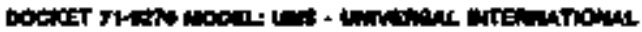

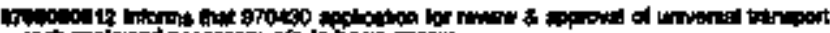

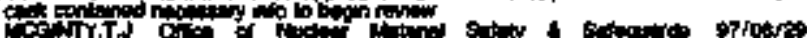
ThE

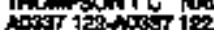

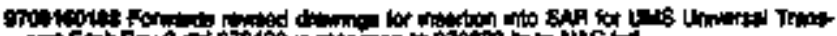

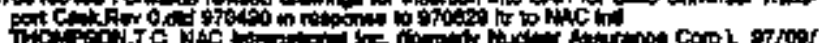

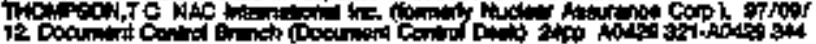

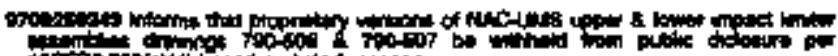

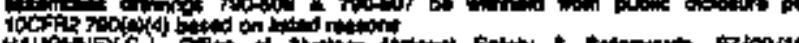

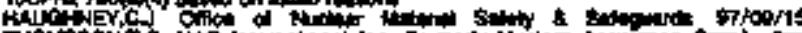

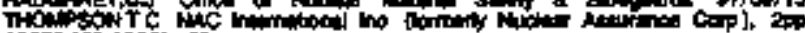

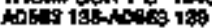

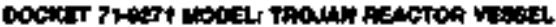

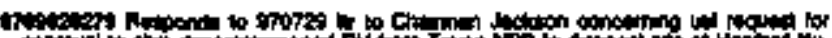

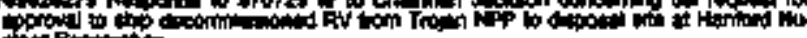

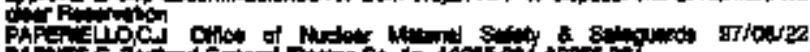

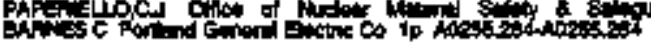

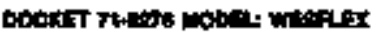

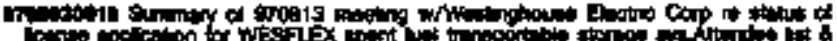

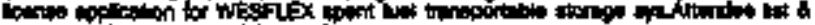

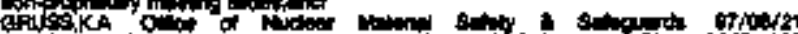
Filk ANT 131

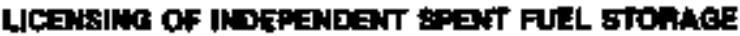

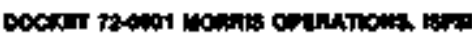

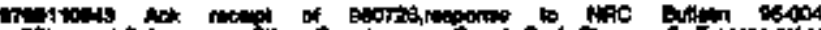

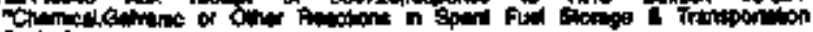

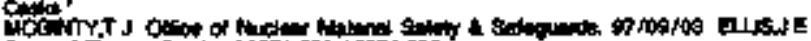

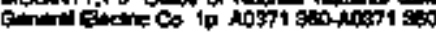

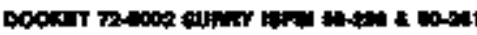

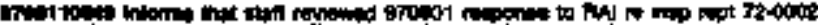

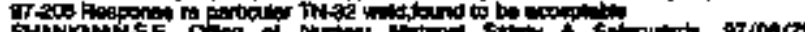

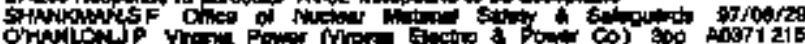
atiot 217

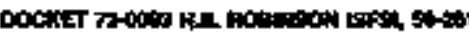

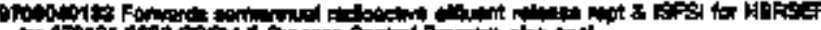

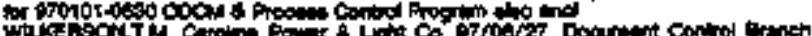

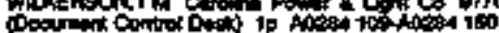

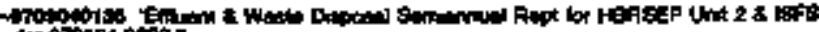

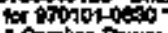

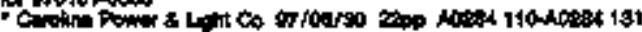

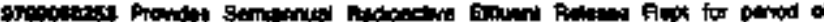

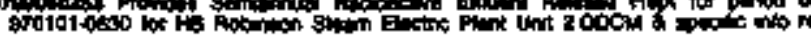
is

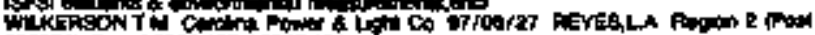

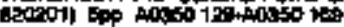




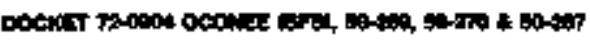

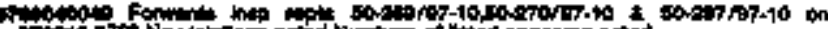
of

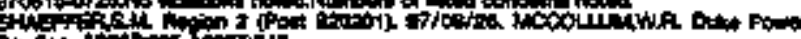

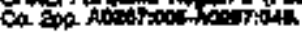

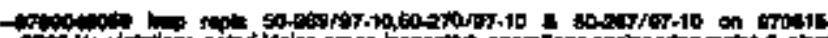

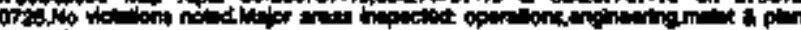

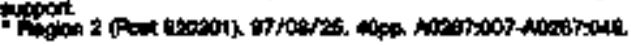

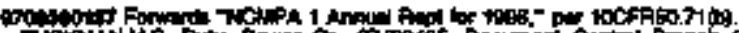

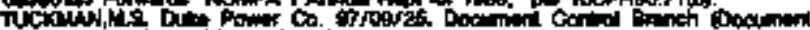

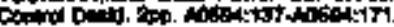

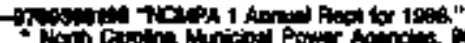

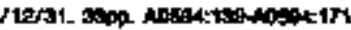

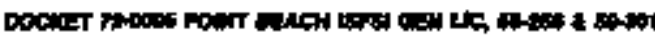

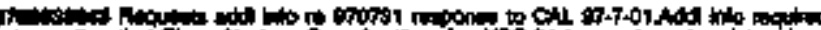

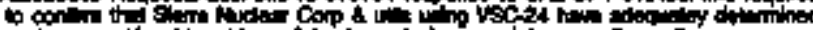

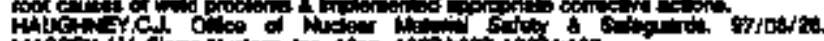

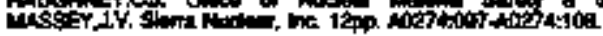

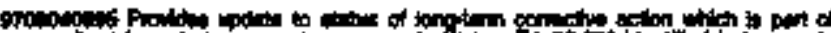

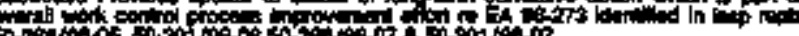

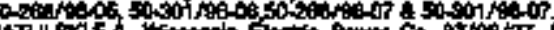

ATULSA

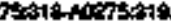

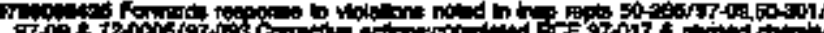

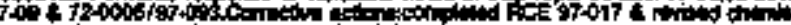

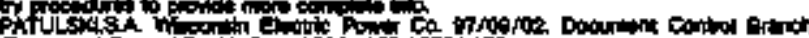

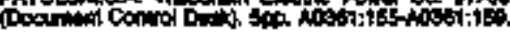

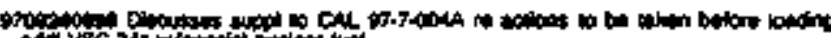

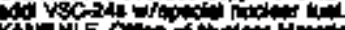

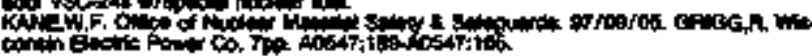

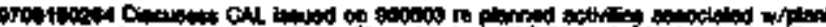

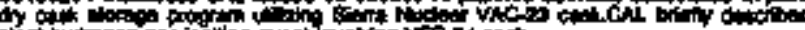

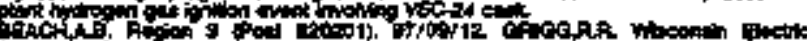

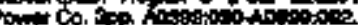

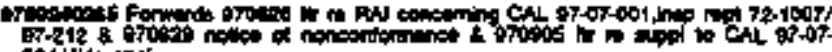

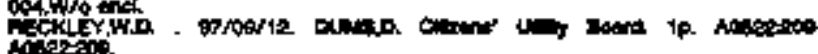
A

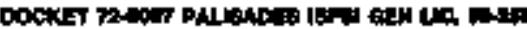

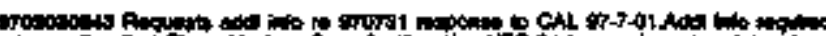

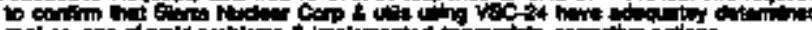

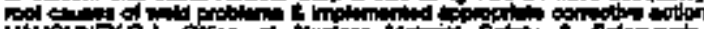

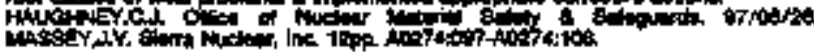

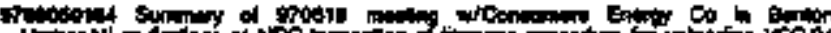

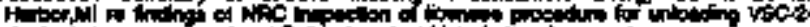

sylut

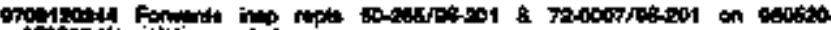

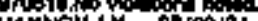

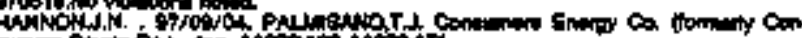

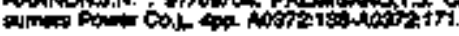

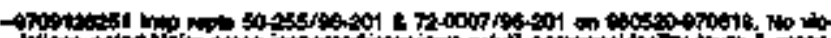

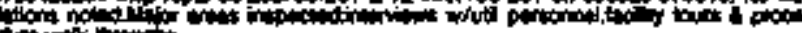

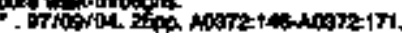

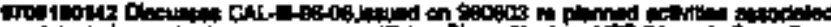

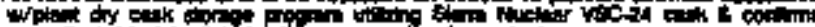

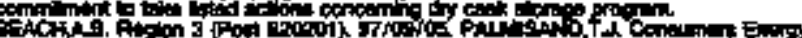

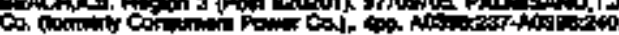

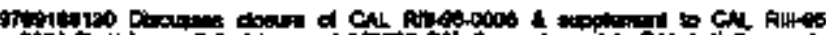

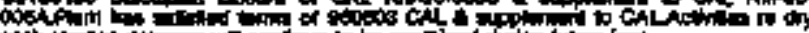

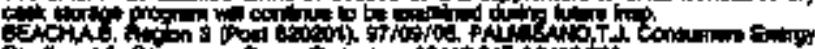

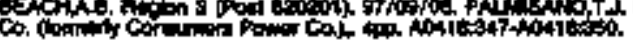

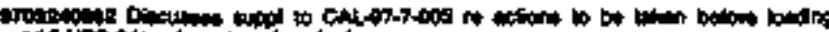
act vete 24, of

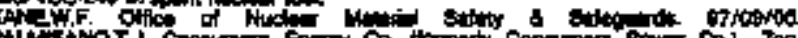

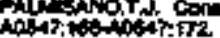

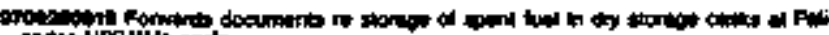

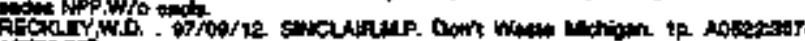
Nitionst.

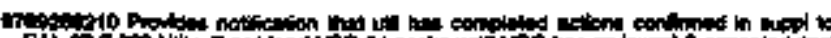

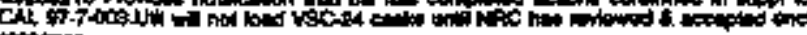

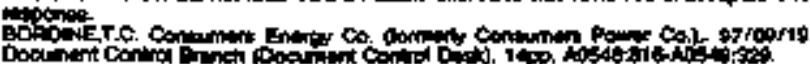

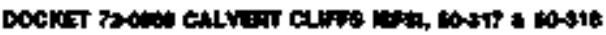

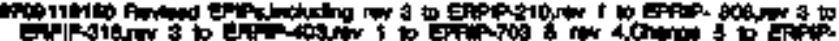

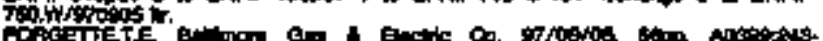
(n)

in:

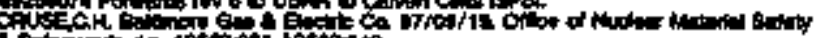

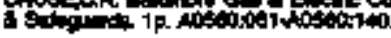

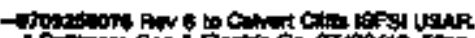

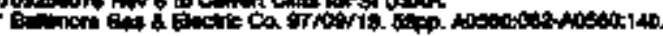

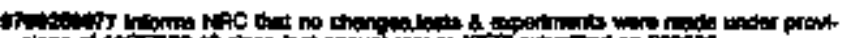

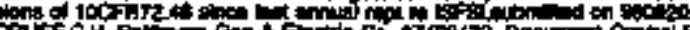

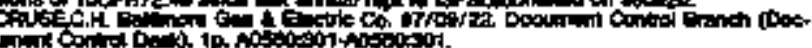

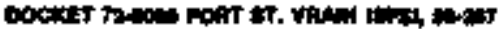

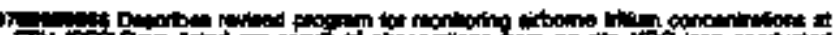

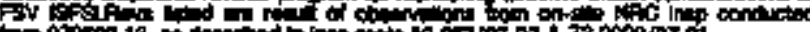

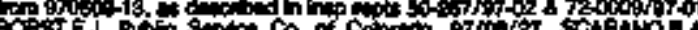

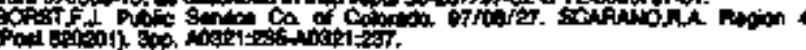

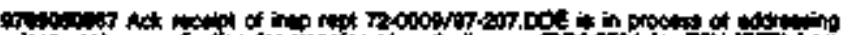
lis no

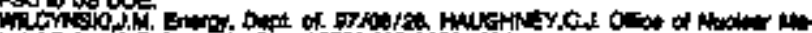

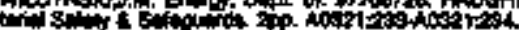

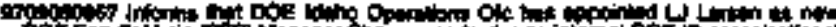
Co

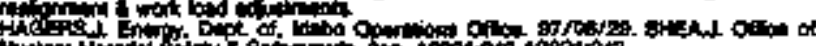

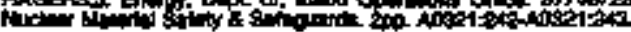

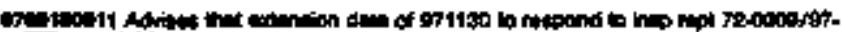

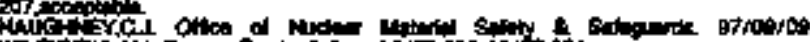

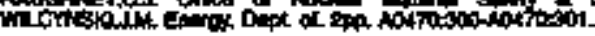

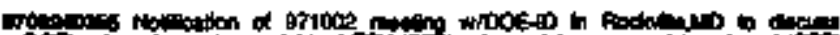

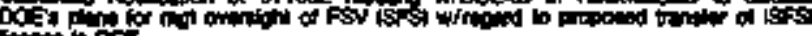

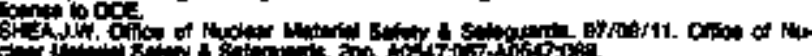

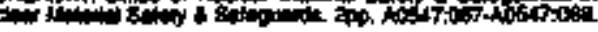

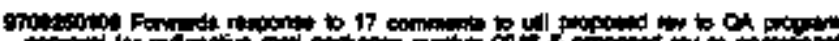

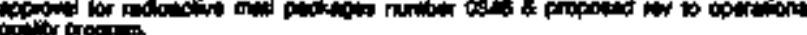

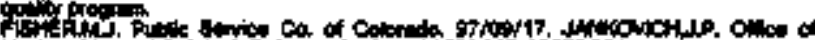

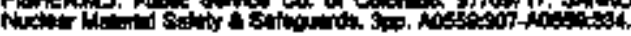

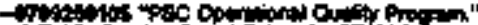

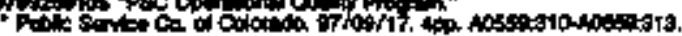

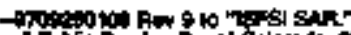

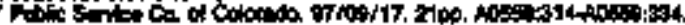

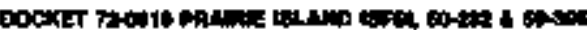

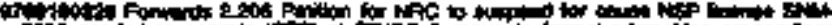

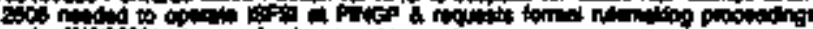

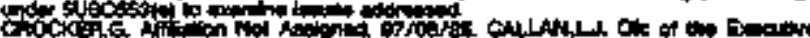

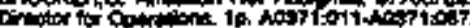

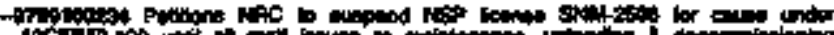

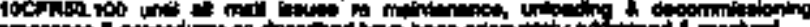

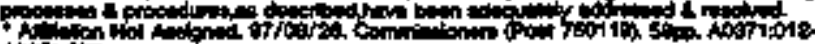
A

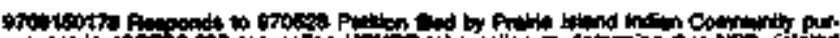

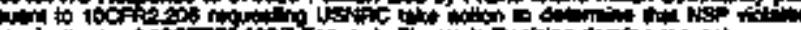

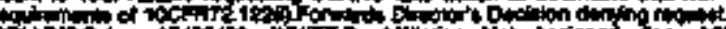

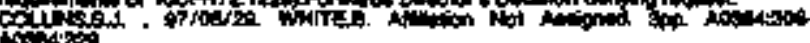

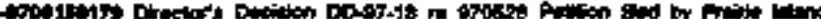

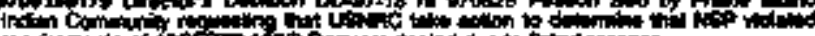

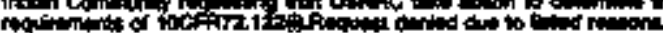

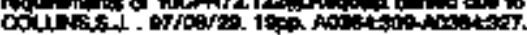

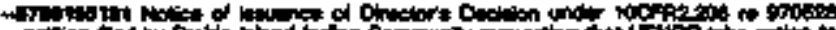

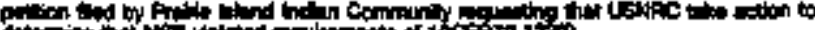

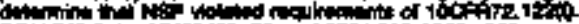

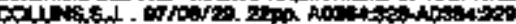

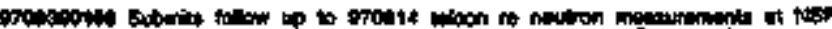
IF

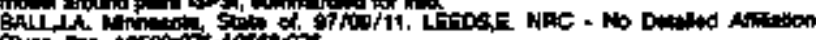

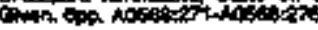

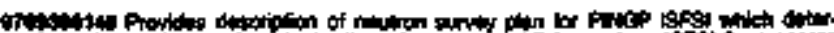

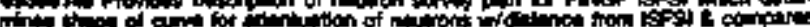

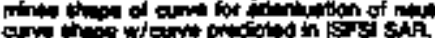

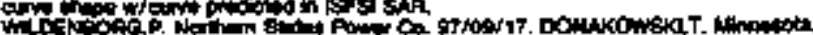

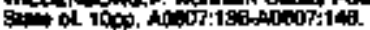




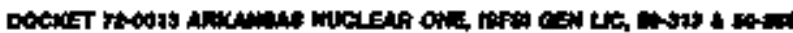

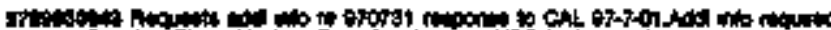

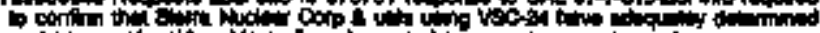

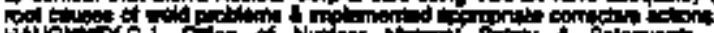

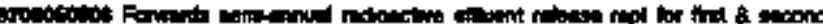

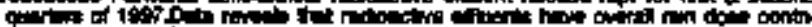

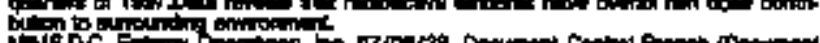

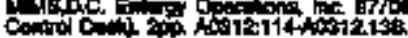

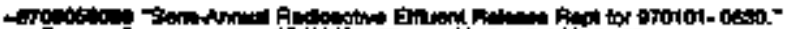

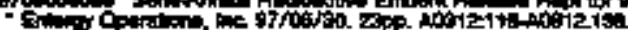

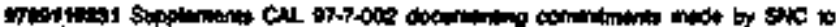

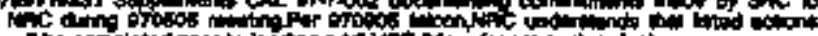

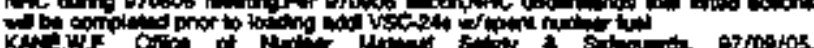

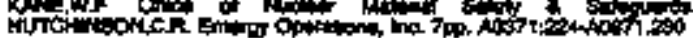

otrosios.

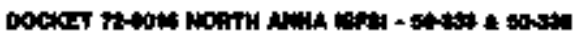

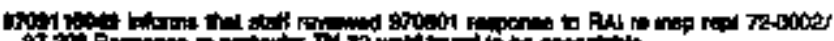

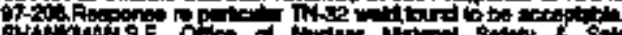

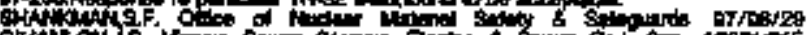
ortion

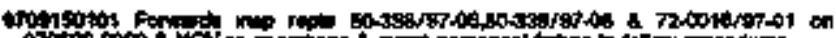
解

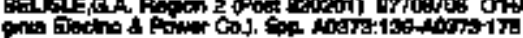

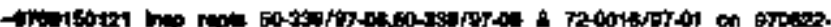

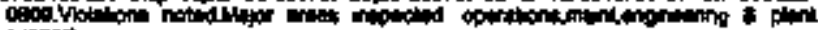

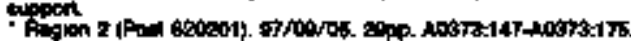

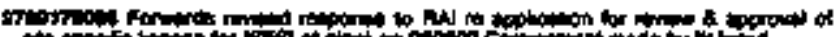

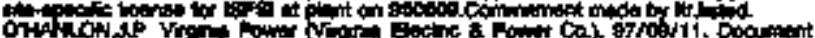

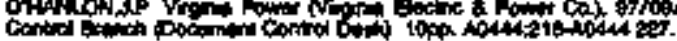

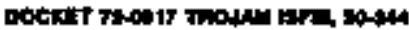

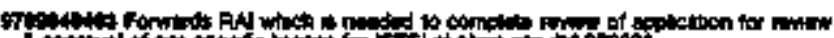

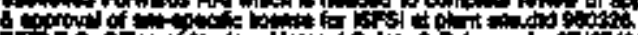

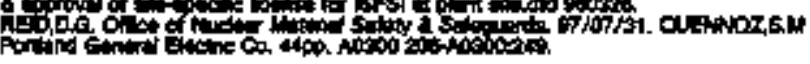

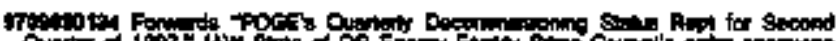

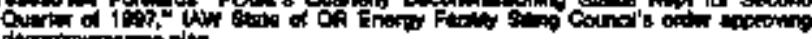

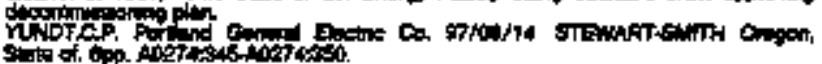

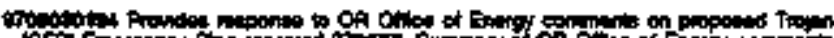

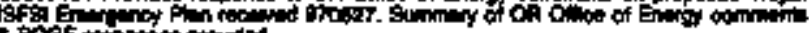

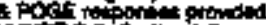

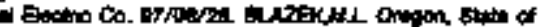
7 incistato

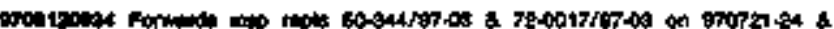

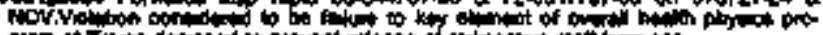

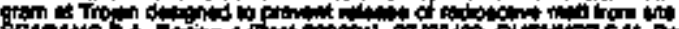

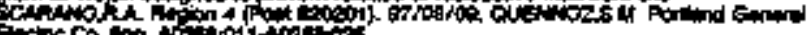

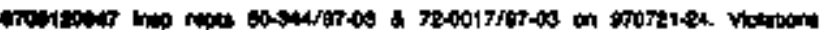

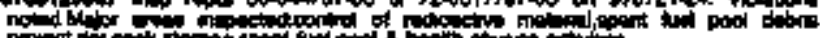

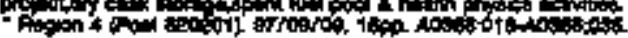

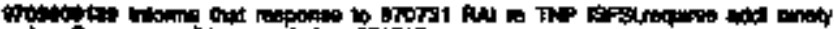

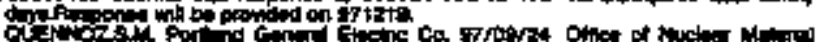

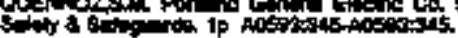

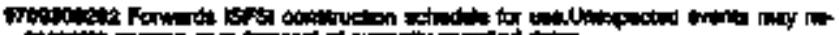

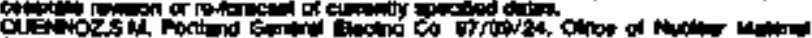

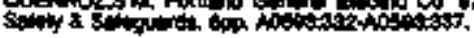

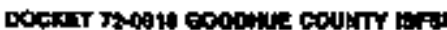

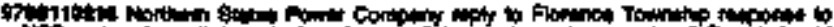

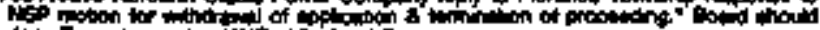

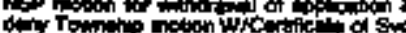

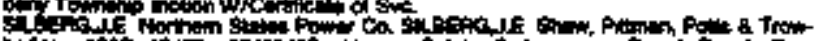

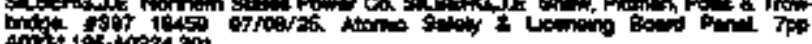

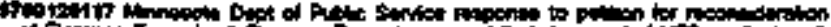

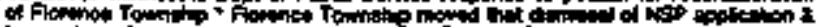

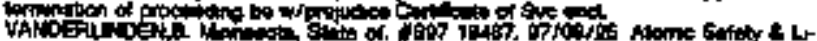

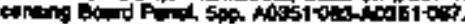

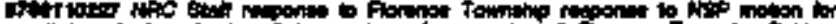

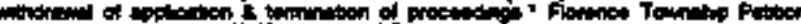

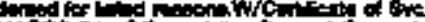

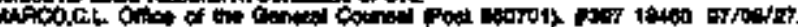

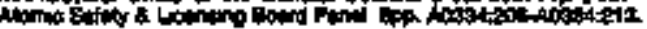

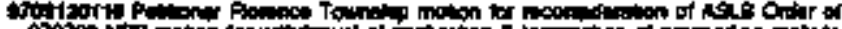

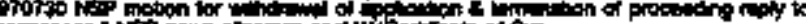

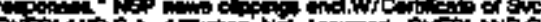

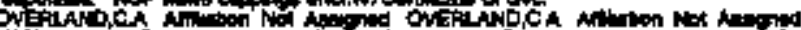

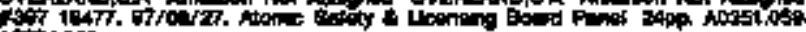
Atotion?

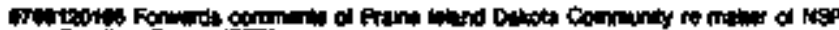

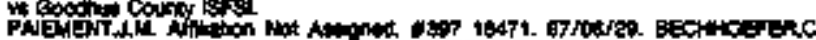

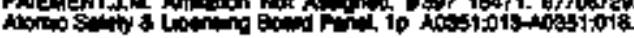

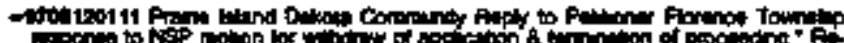

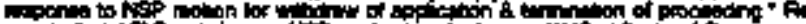

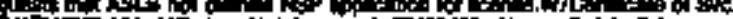
wi

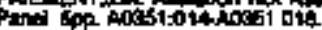

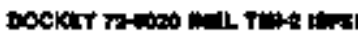

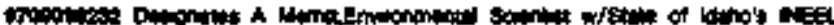

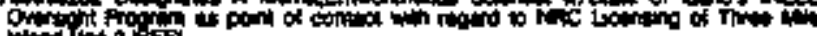

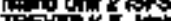

and io

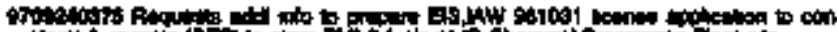

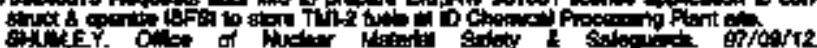

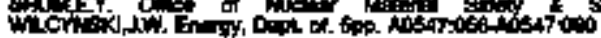

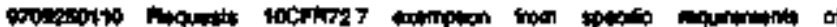

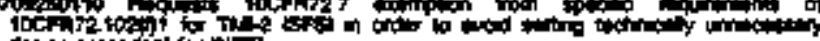
dinction

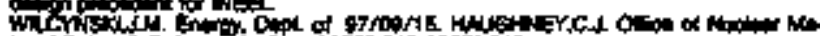

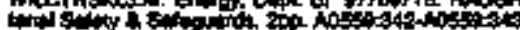

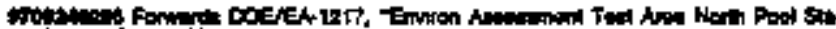

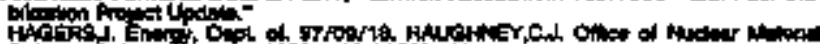

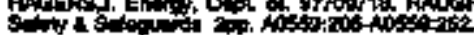

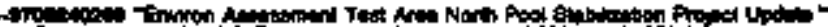

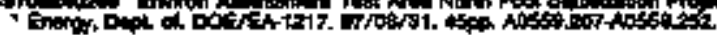

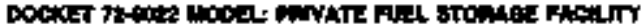

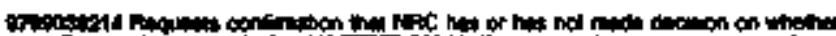

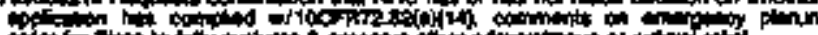

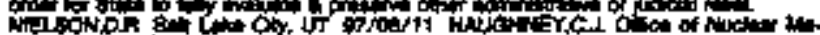

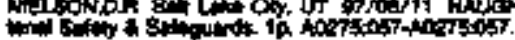

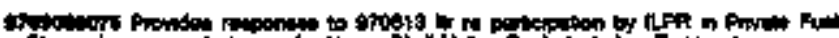

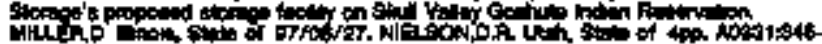
norition

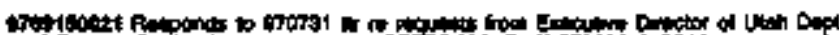

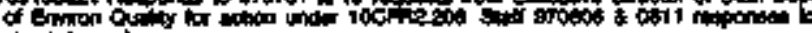

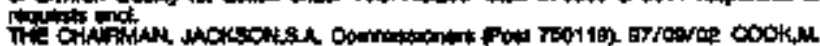

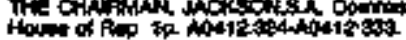

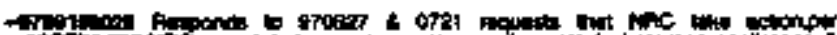

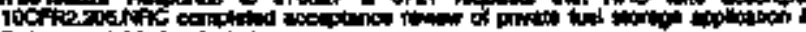

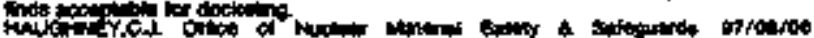

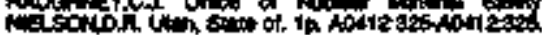

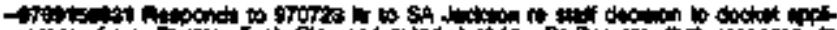

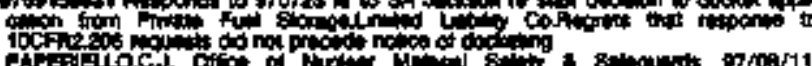

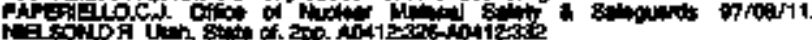

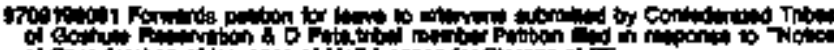

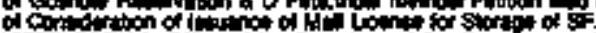
OH

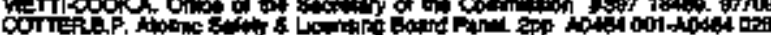

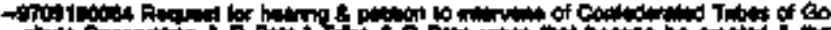

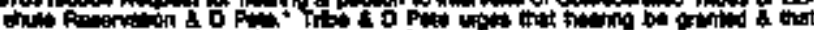

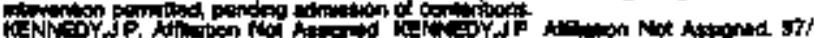

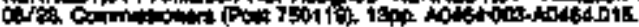

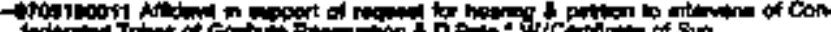

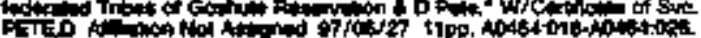

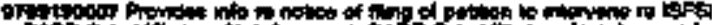

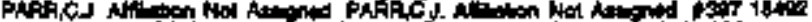

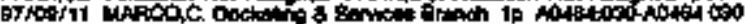




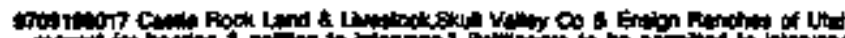

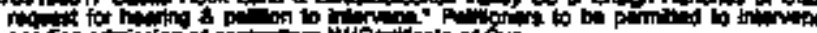

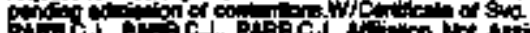

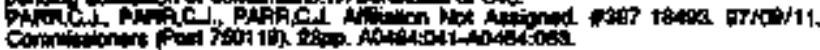

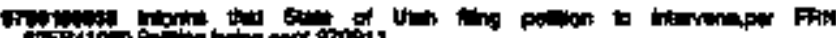

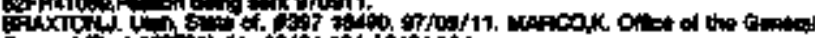

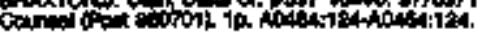

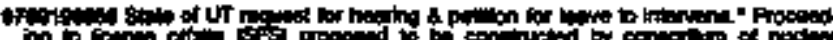
in to rener off

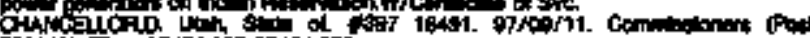

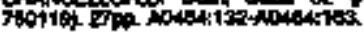

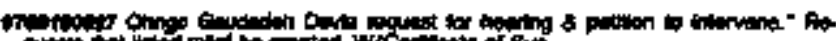

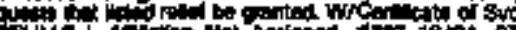

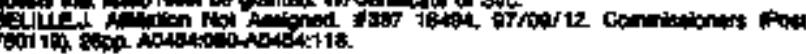

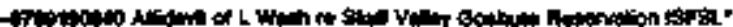

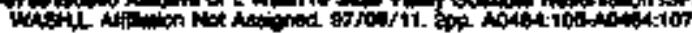

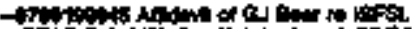

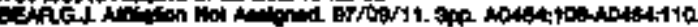

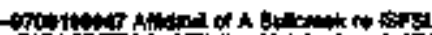

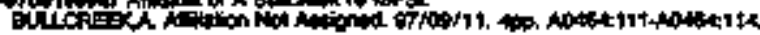

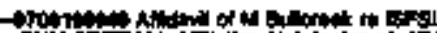

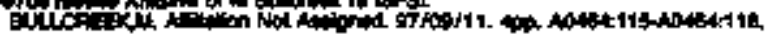

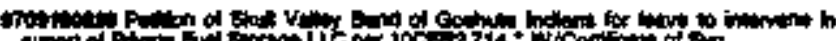

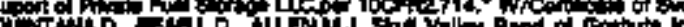

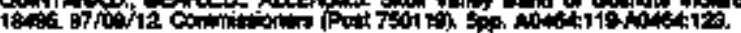

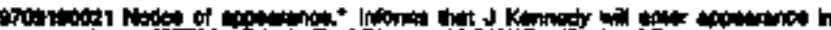

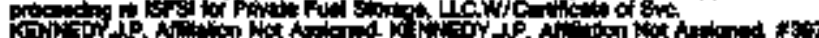

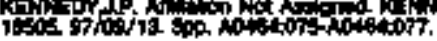

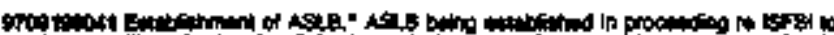

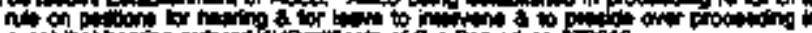

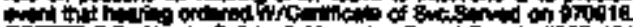
CorTy

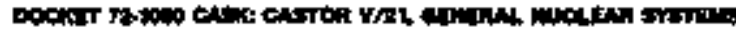

I0

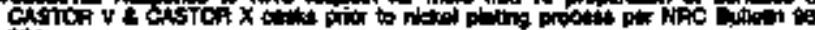

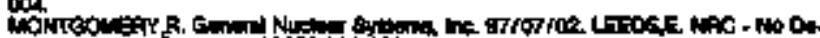
wiod him

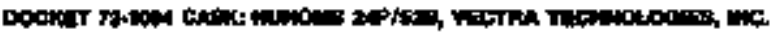

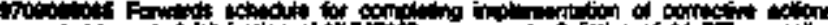

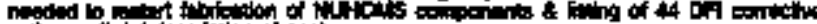

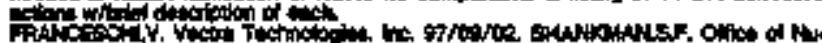

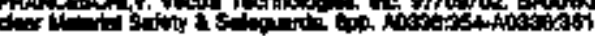

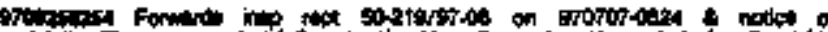

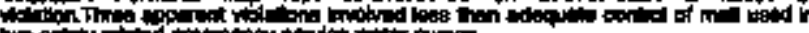

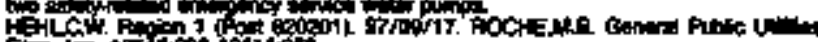

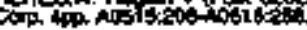

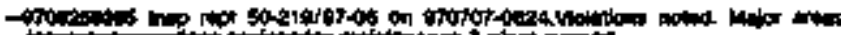

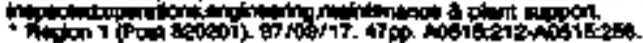

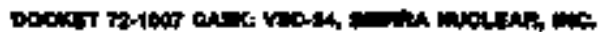

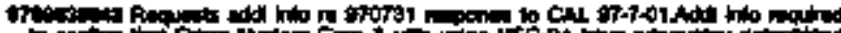

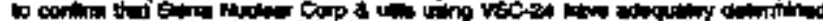

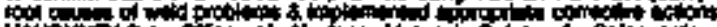
4 H AtSE:T,

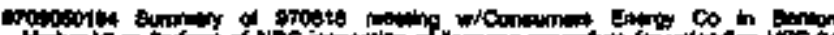

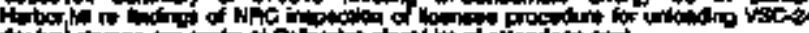

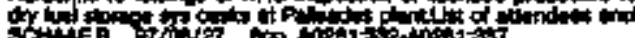

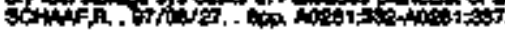

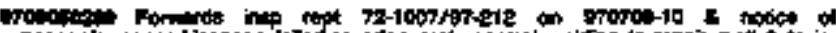

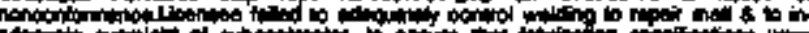

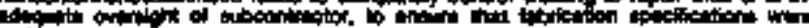

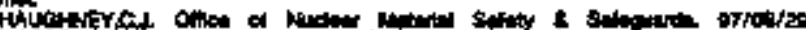

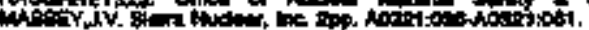

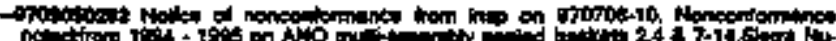

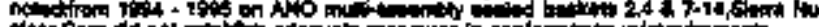

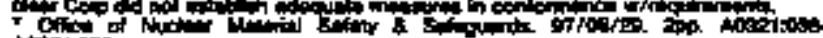

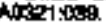

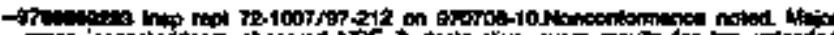

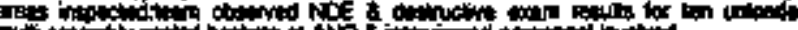

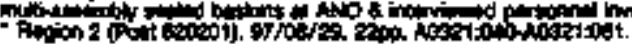

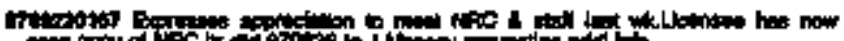

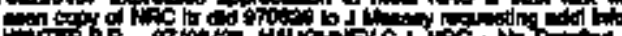

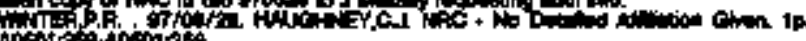
tostristasonst.

of

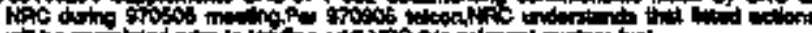

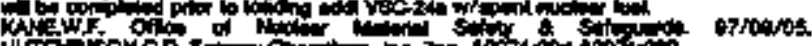

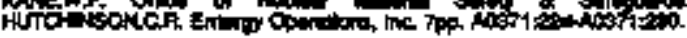

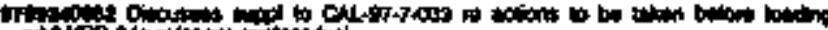

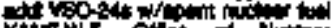

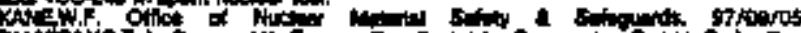
BNI 10567160+4047:972

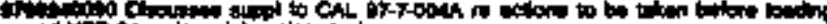

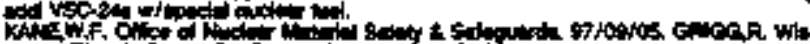

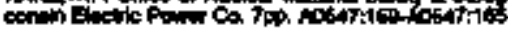

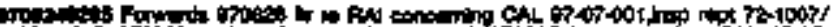

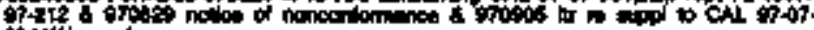

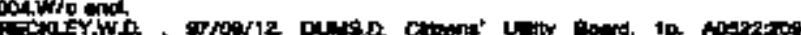
isto

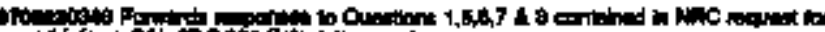

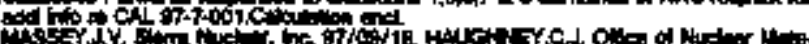

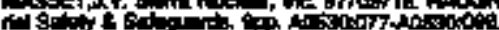

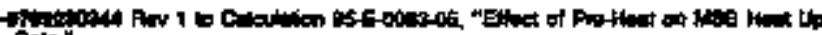

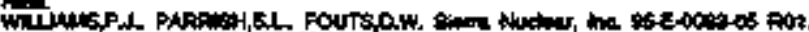

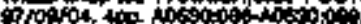

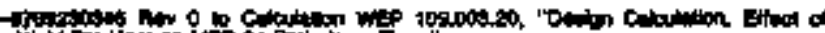

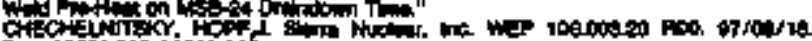
Con

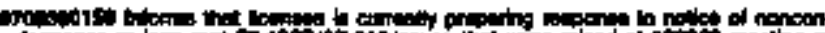

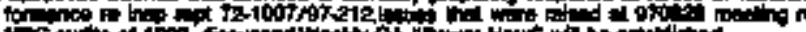
the

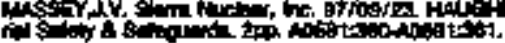

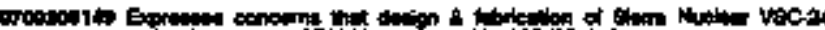

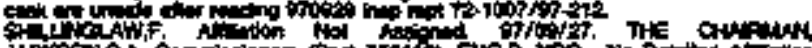

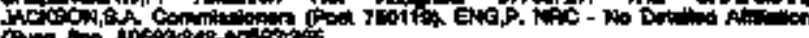

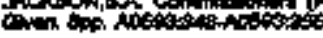

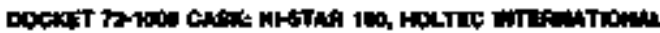

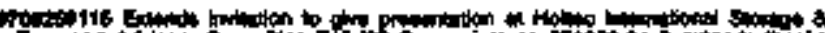

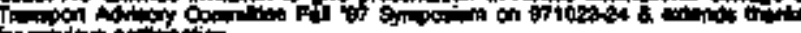

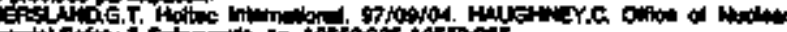

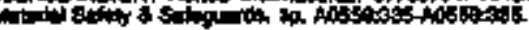

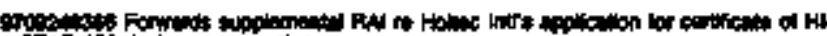

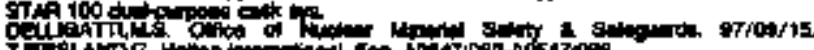

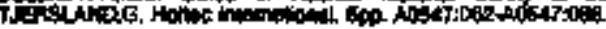

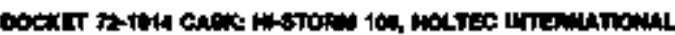

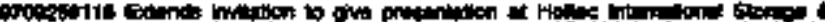

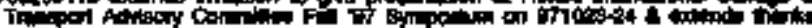

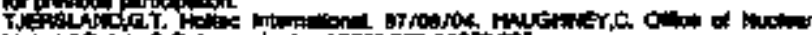

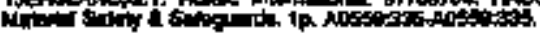

$\sin$

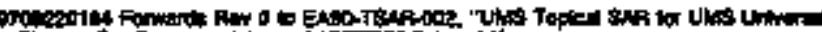

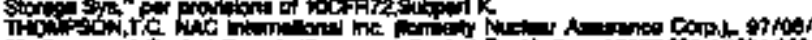

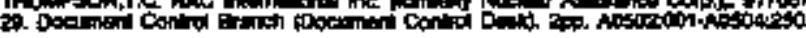

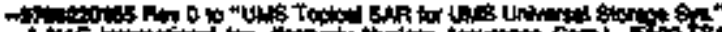

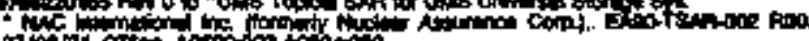

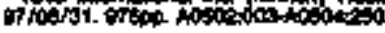

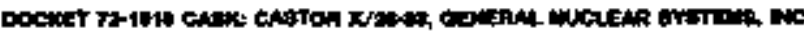

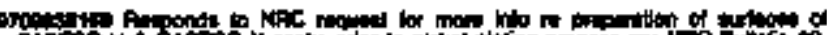
C4s OPd t. 


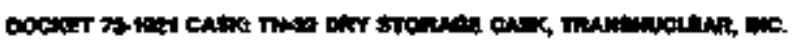

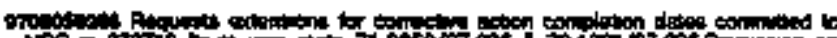

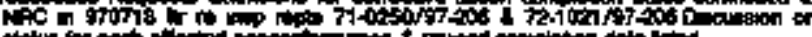

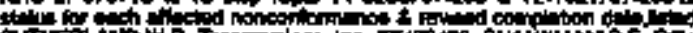
צuTh

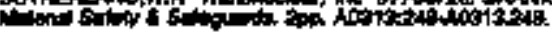

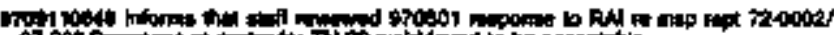

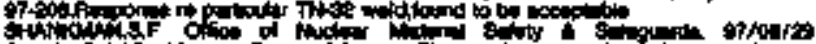

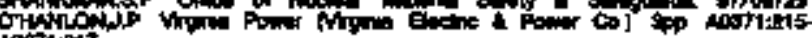
A0sia:217.

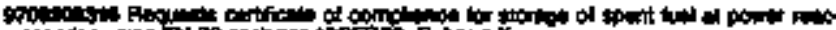

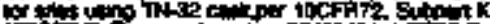

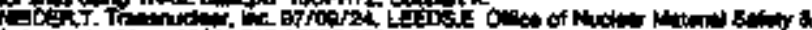

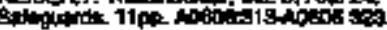

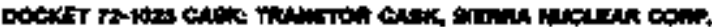

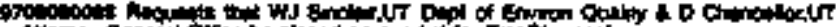

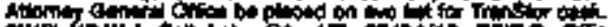

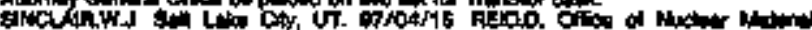

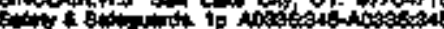

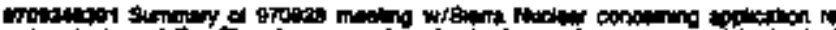

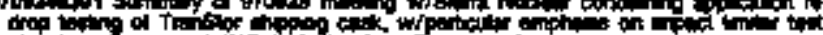

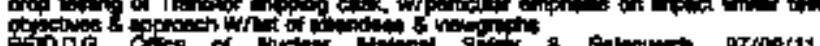
inus ADSA ot

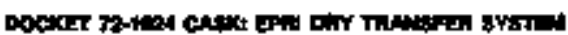

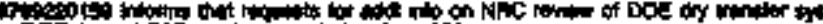

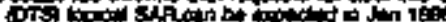

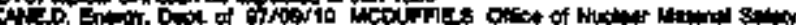

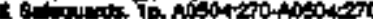

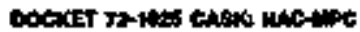

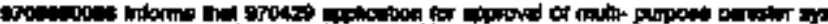

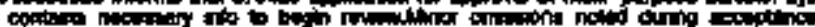

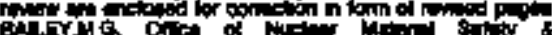

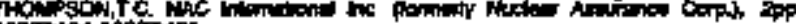
A035:134 Hids:19s.

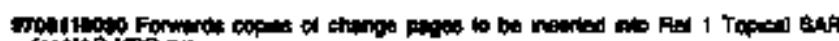

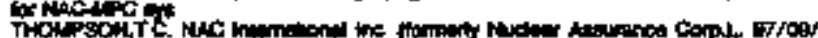

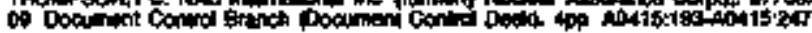

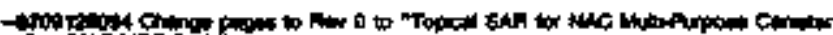

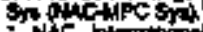

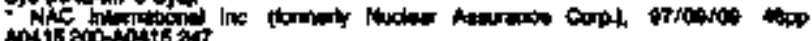

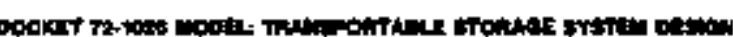

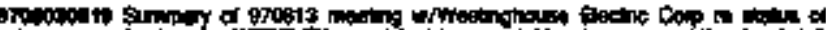

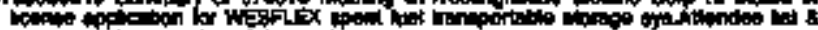

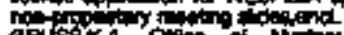

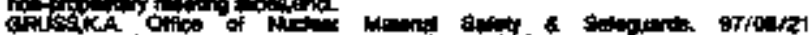

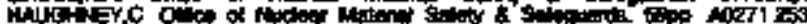
Ahertal 


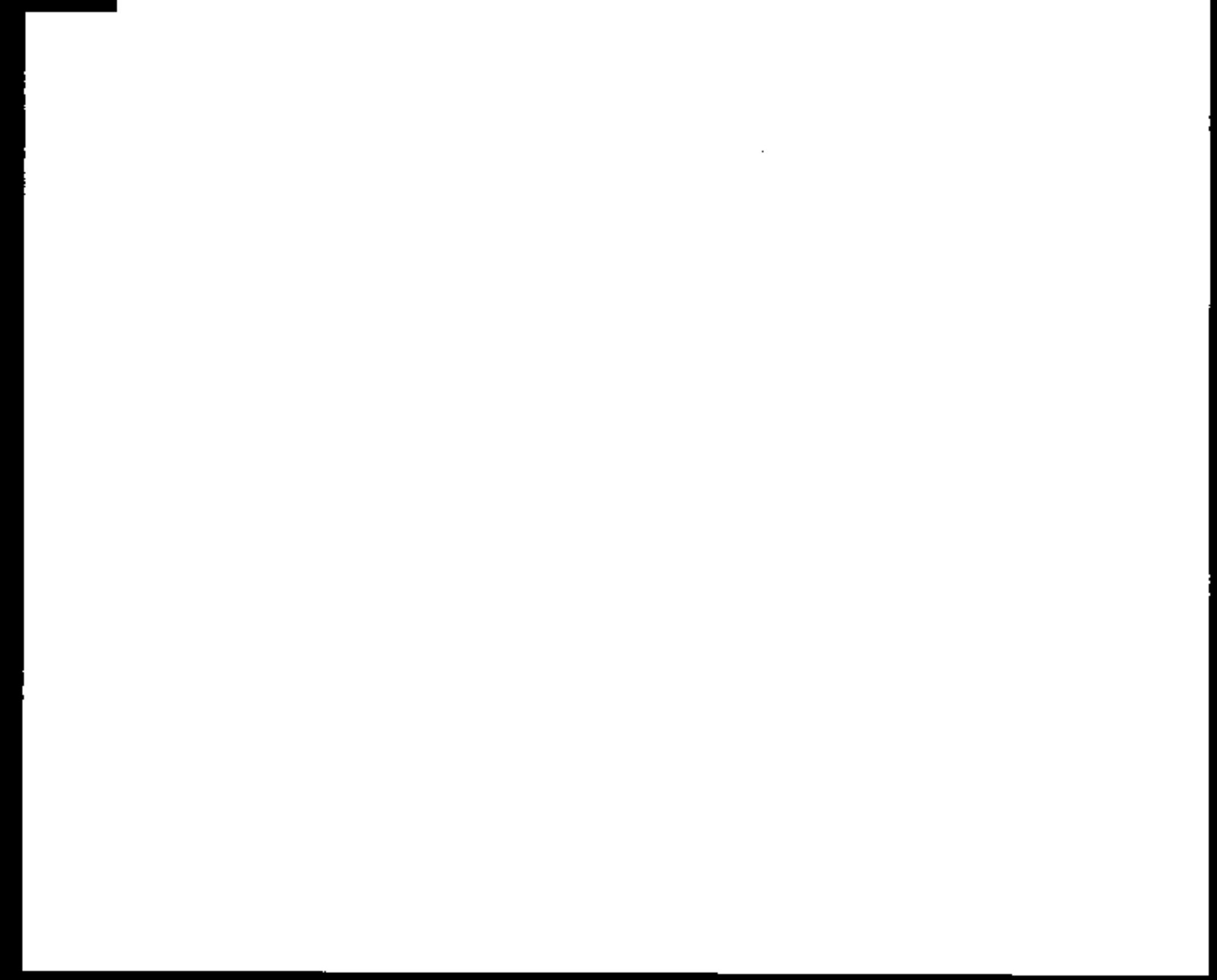




\section{NONDOCKETED ITEMS}

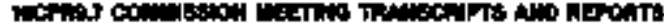

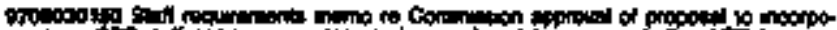

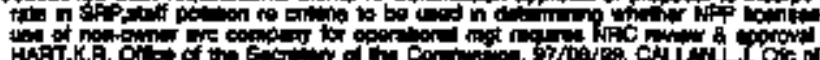

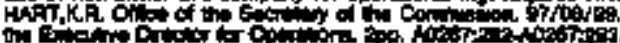

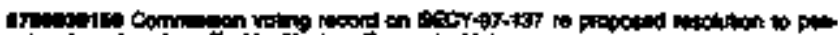

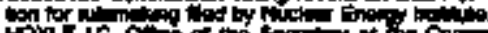

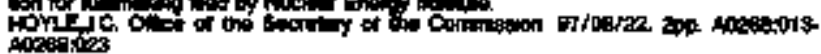

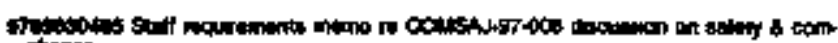
FOY

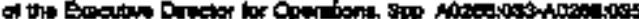

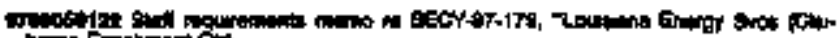

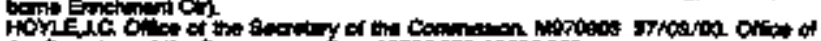

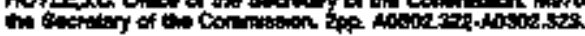

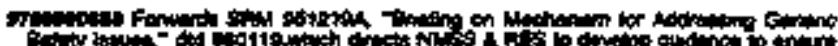

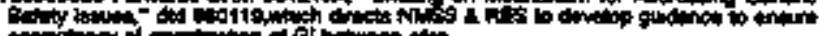
con cith

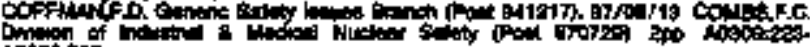
100000

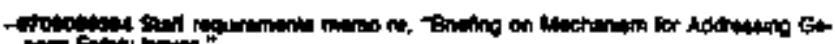

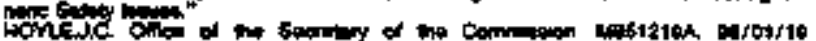

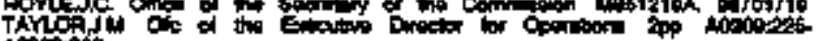

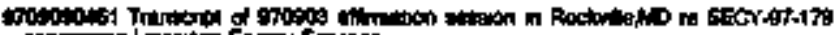

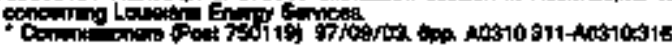

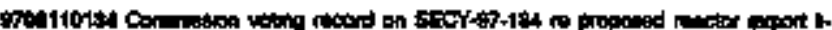

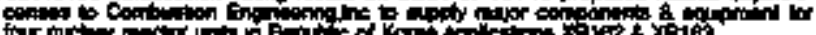

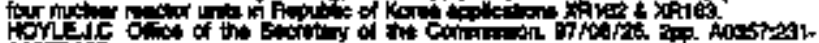
astoritis

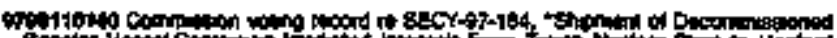

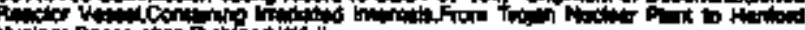

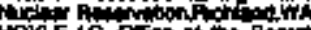

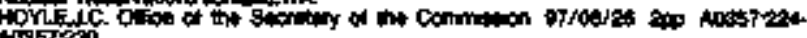

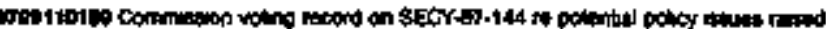

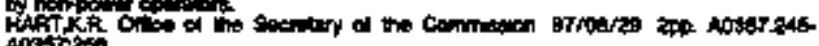
Aostritis

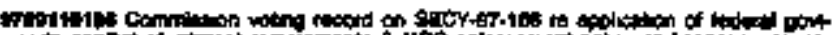

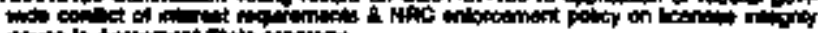

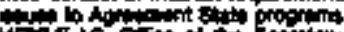

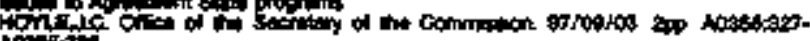
Aosolist.

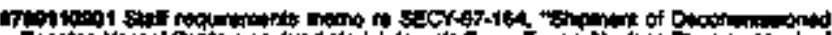

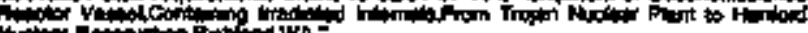
tom

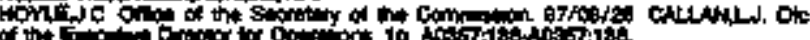

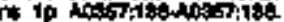

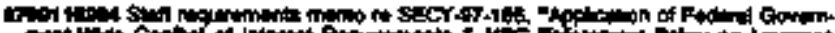

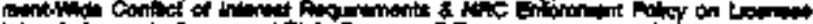

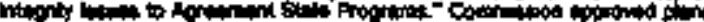

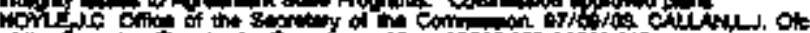

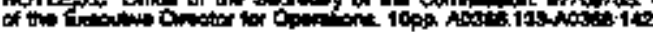

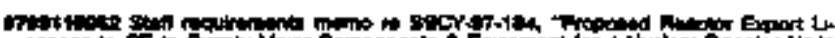

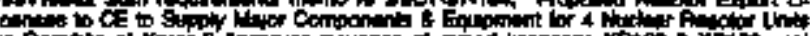

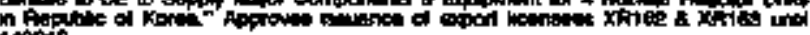

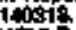

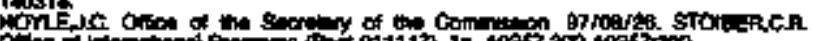

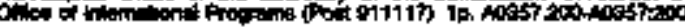

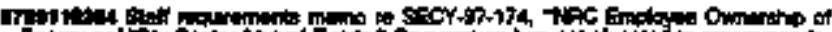

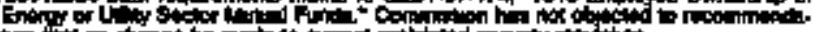

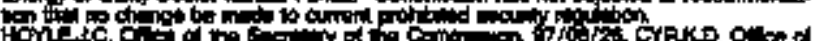

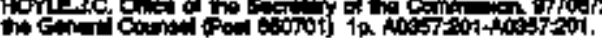

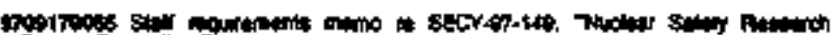

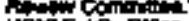

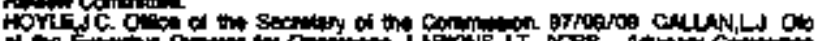

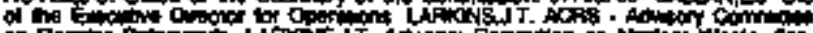

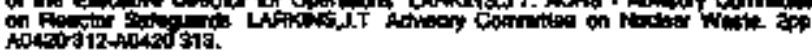

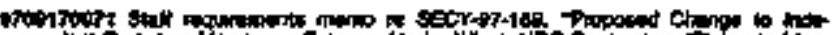

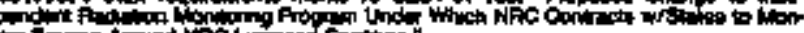

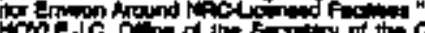

of $\rightarrow$ hot

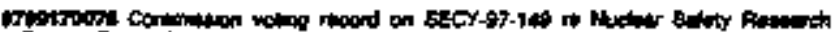

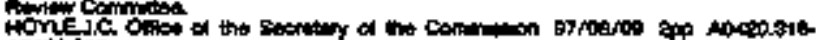
nOA20032.

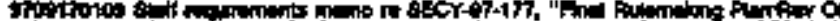

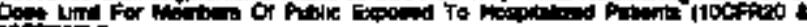

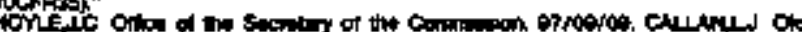

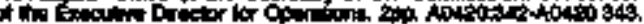

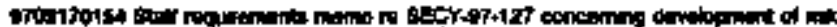

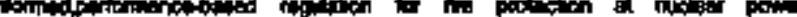

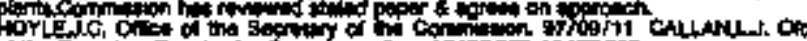

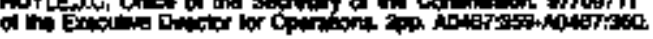

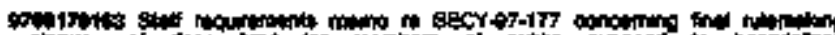

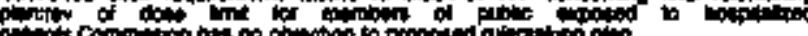

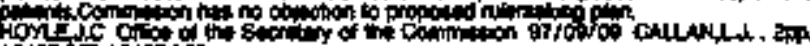
(10)

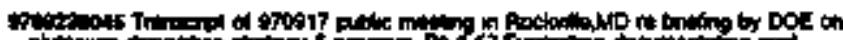

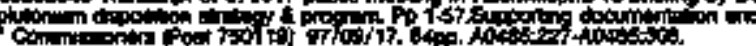

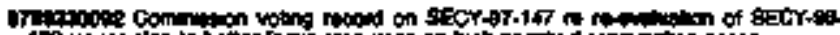

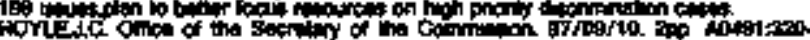

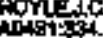

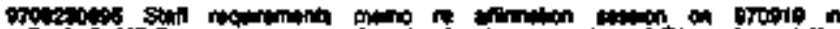

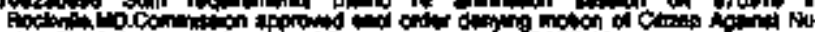

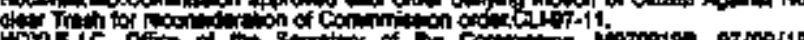

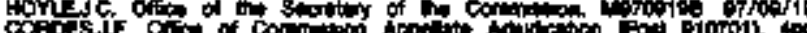
This

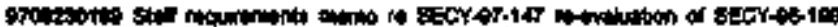

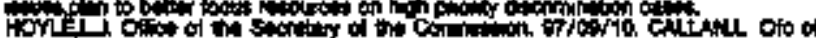

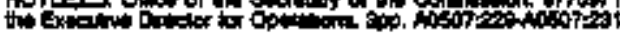

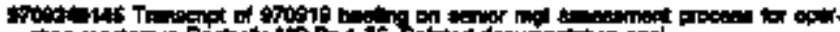

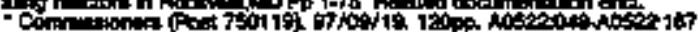

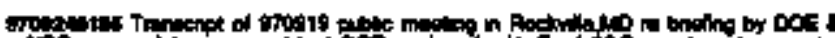

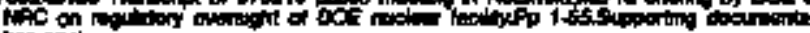
tom the

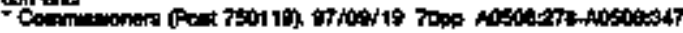

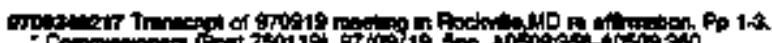

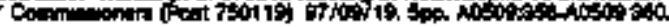

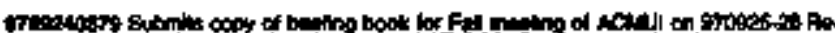

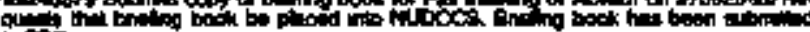

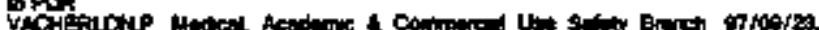

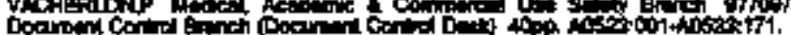

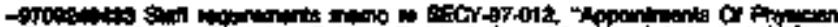

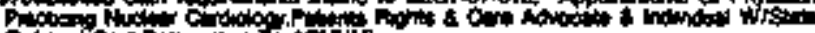

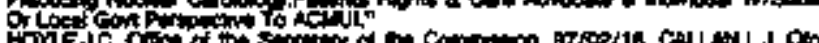

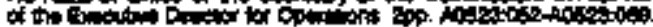

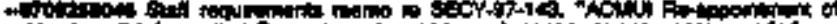

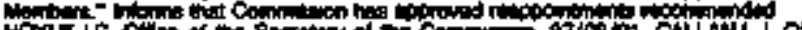

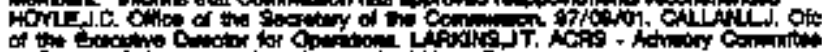

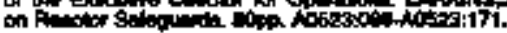

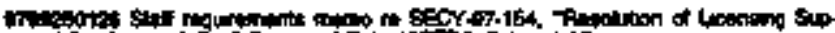

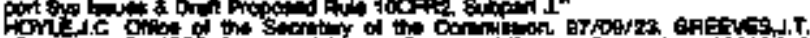

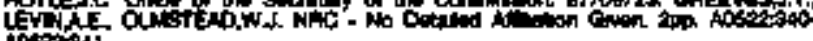
itosest

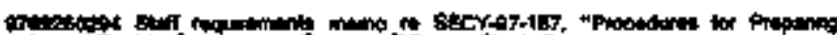

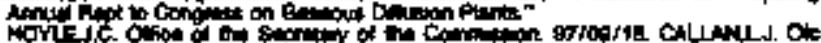

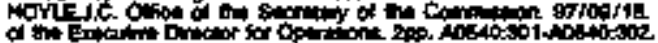

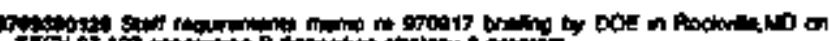

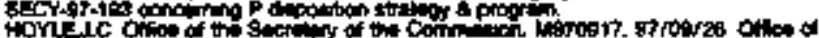

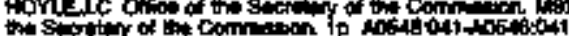

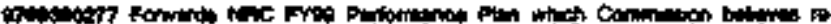

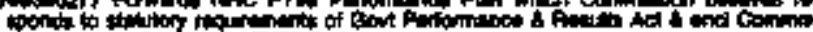

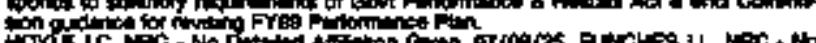

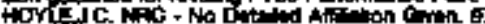

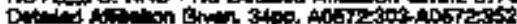

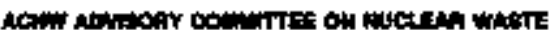

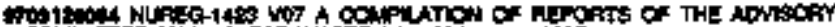

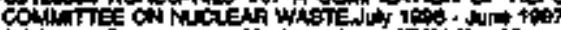

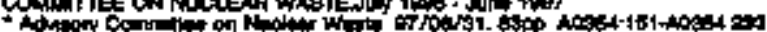

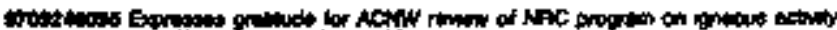

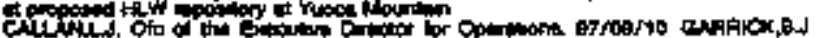
indirary $\alpha$. 


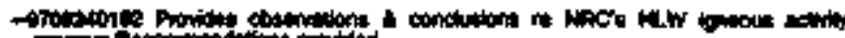

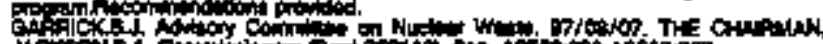

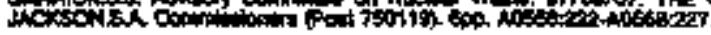

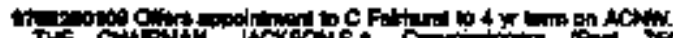

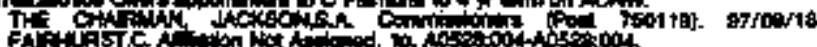

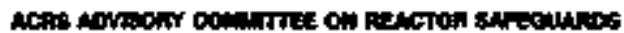

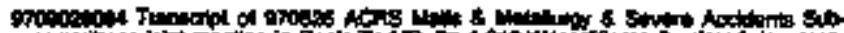

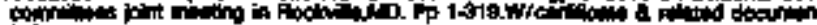

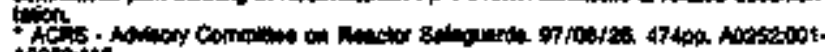
ANotit.

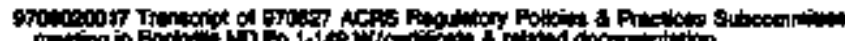

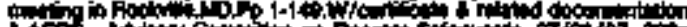

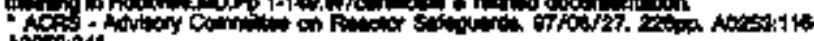
Alotist.

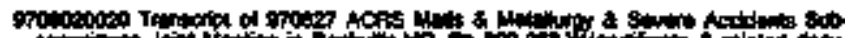

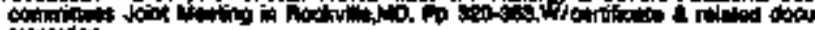

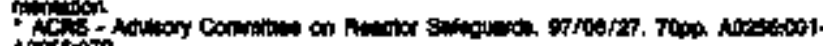
Ations

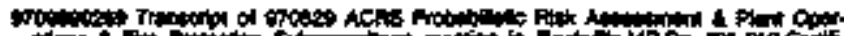

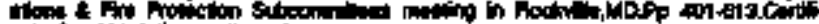

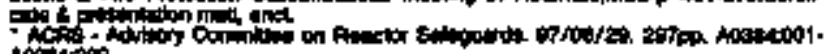

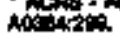

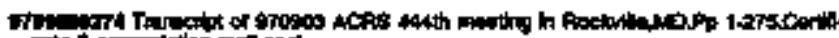

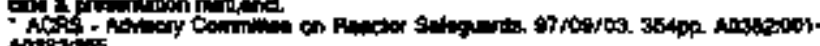
nation.

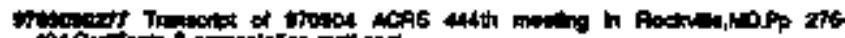

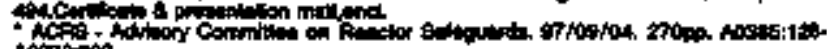
notation

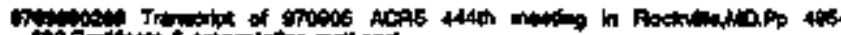

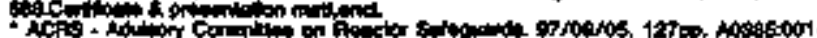
itotizt.

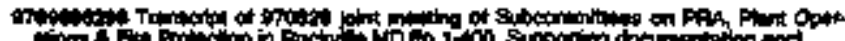

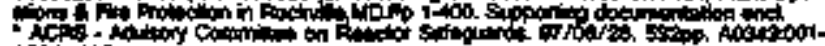
nosution

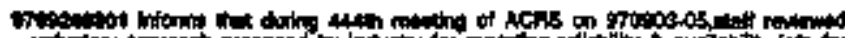

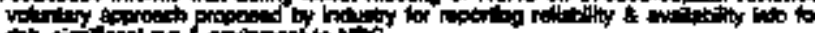

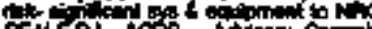

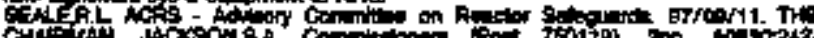

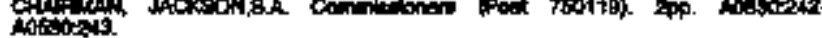

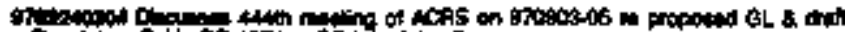

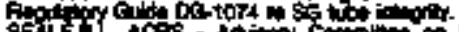

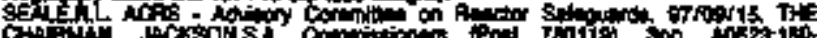
Antatide

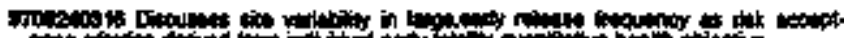

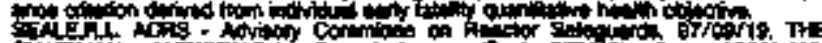

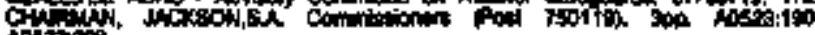

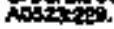

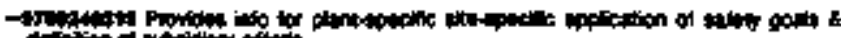

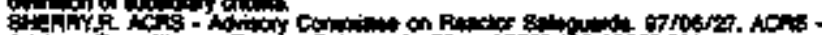

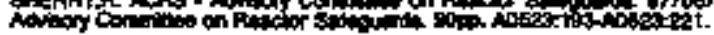

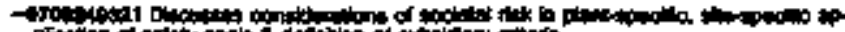

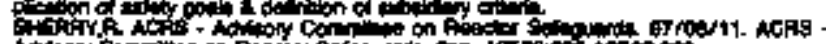

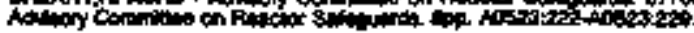

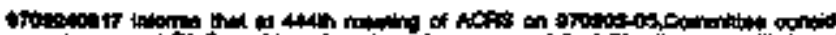

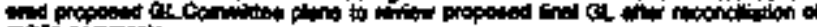

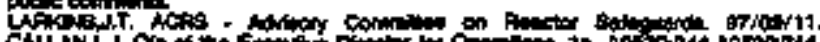

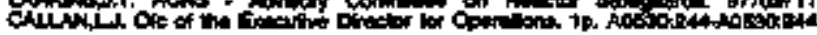

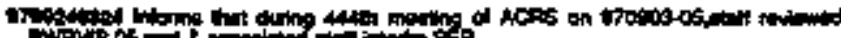

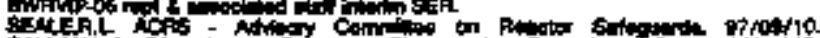

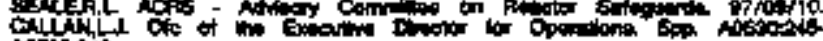
antorate

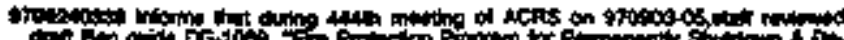

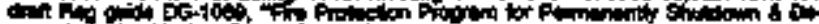

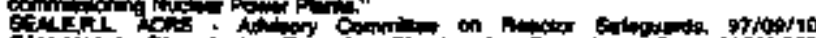

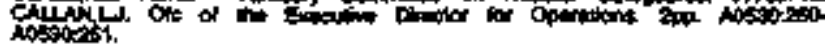

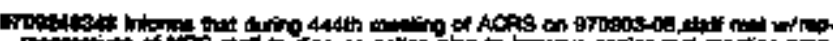

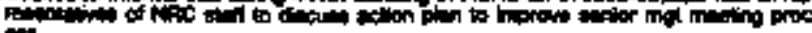

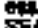

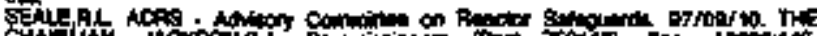

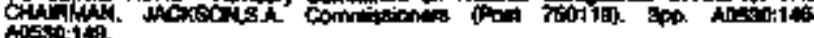

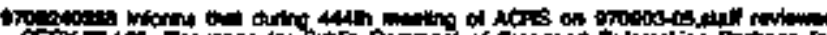

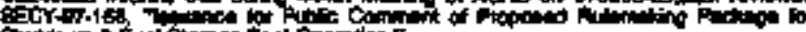

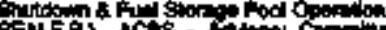

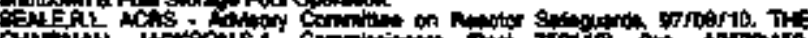

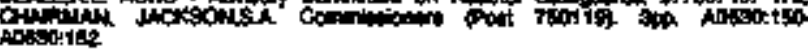

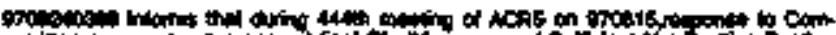

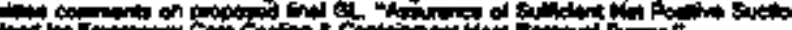

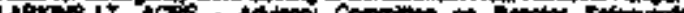

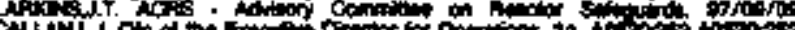

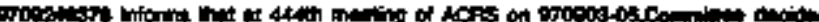

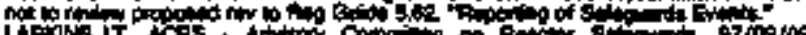

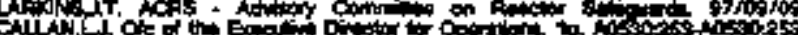

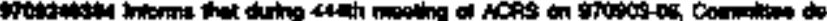

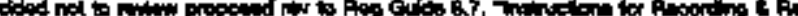

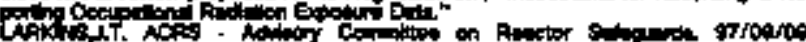

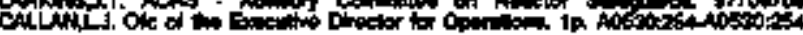

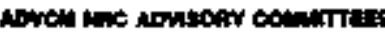

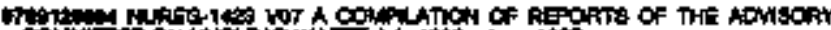

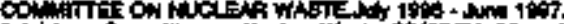

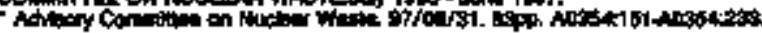

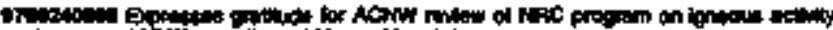

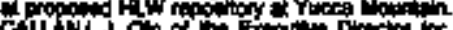

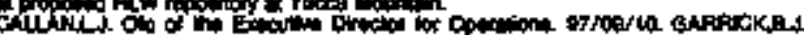

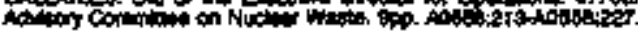

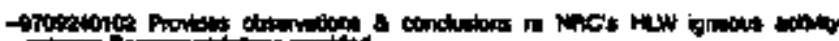

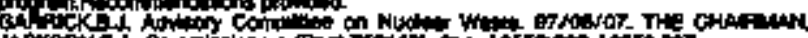

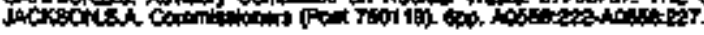

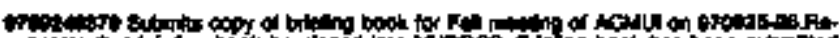

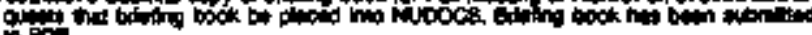

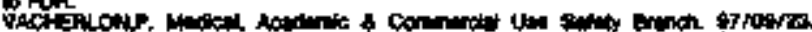

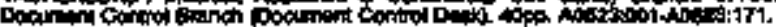

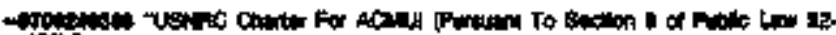
4 arescel

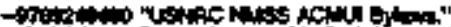

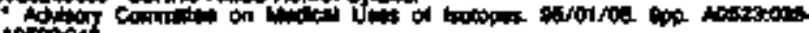
notron.

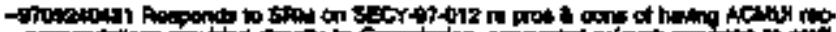

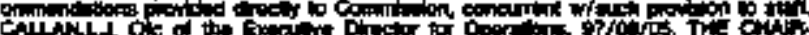

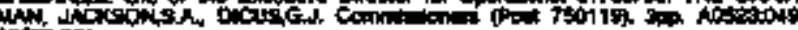
Aotrexist.

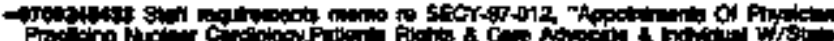

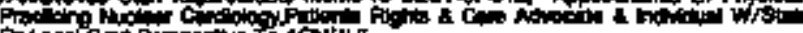

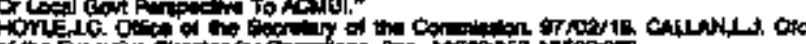

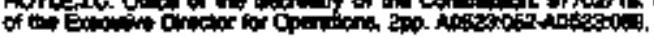

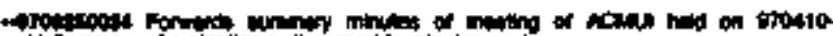

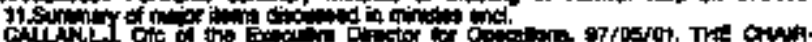

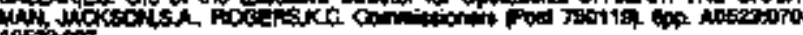
atomot.

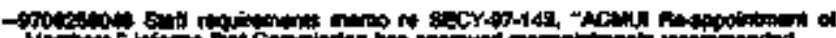

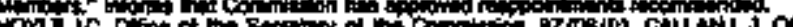

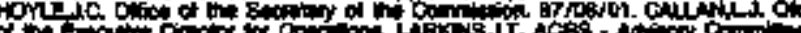

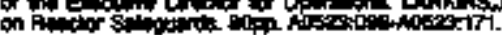

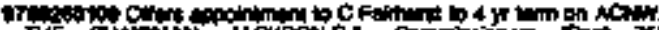

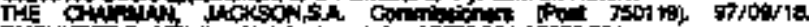

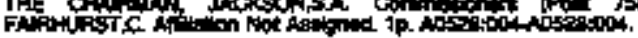

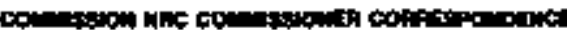

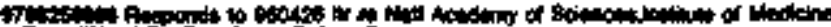

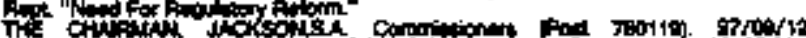

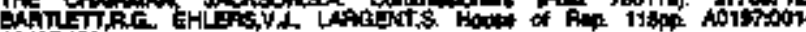
A0107172.

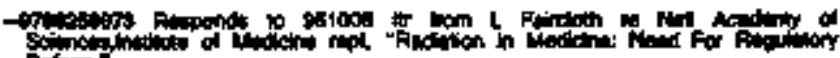

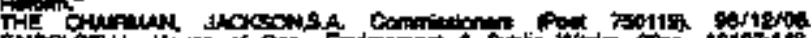

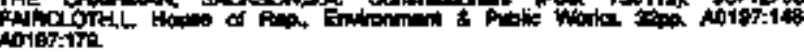

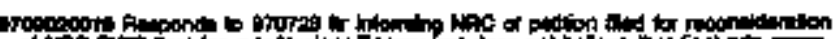

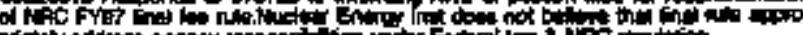

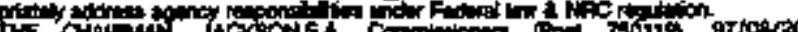

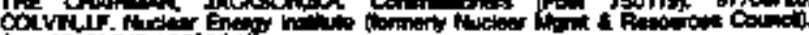

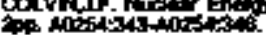




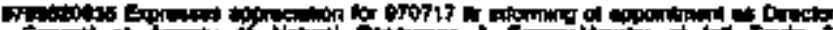

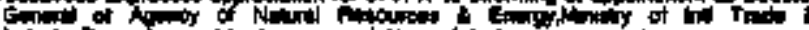

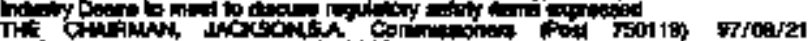

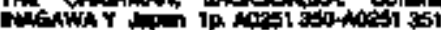

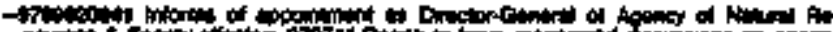

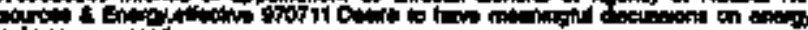

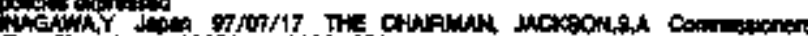

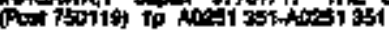

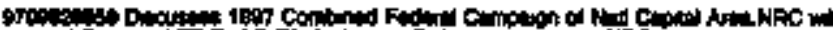

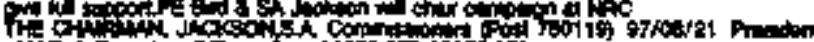

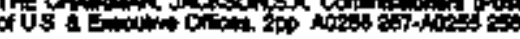

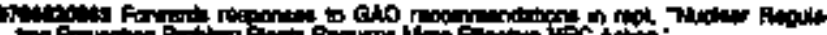

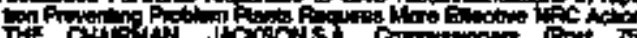

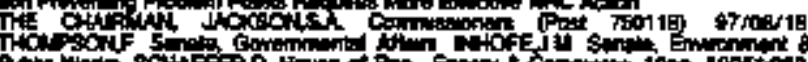

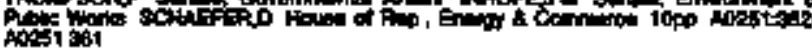

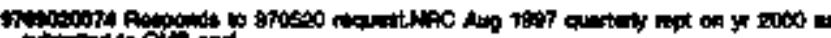

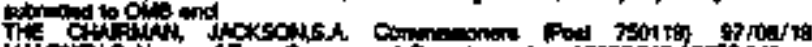
Nhor

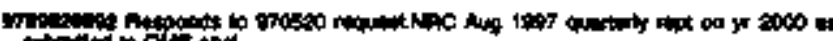

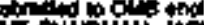

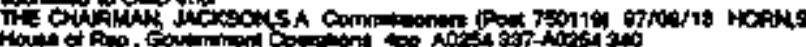

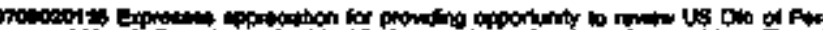

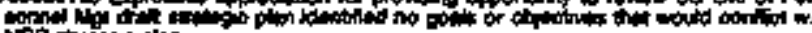

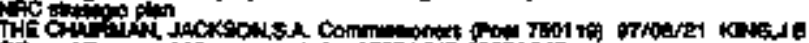

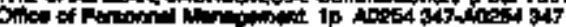

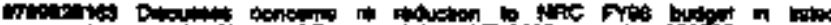

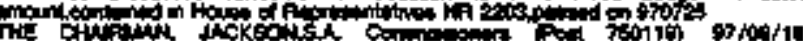

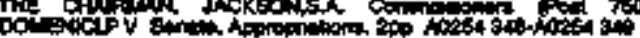

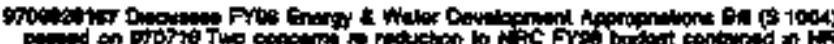

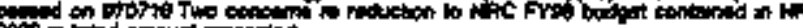

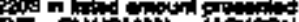

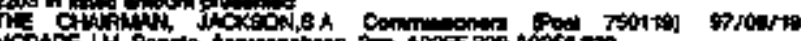

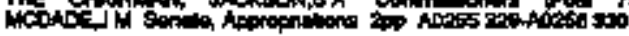

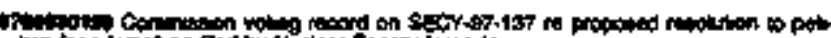

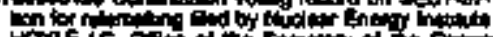

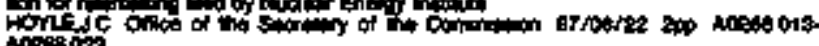
$\cos 000$

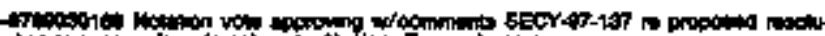

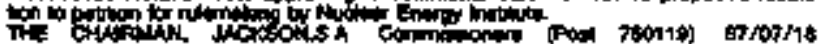

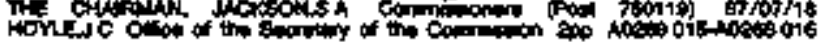

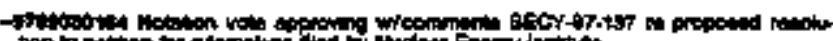
Cop

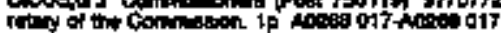

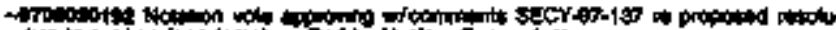

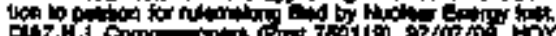

tar or com

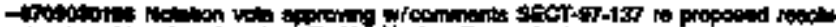

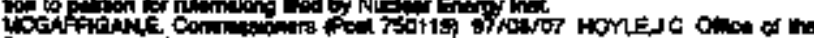

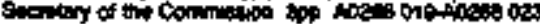

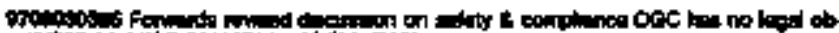

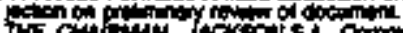

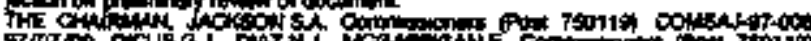

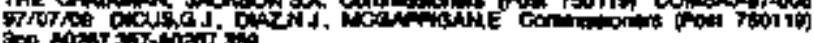

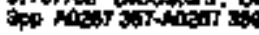

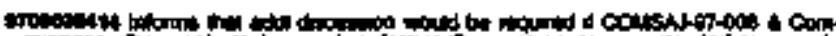

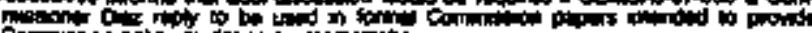

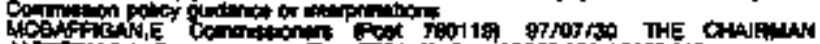

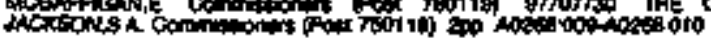

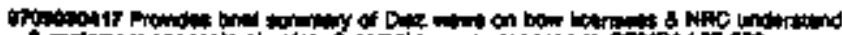

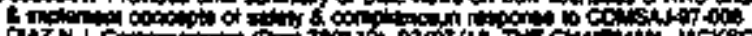
oction 190

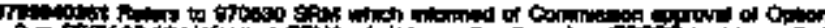

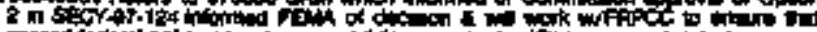

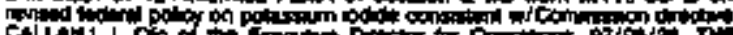

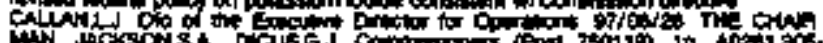
Ath

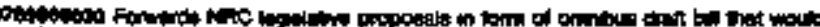

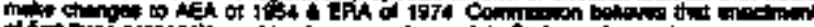
帛

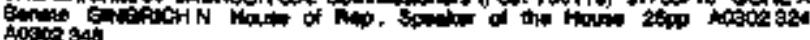

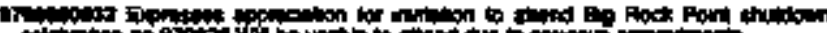
TE of

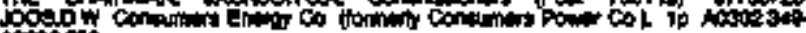

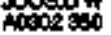

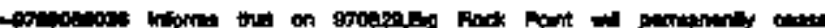

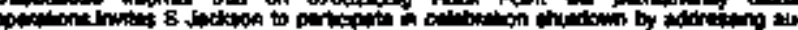

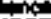

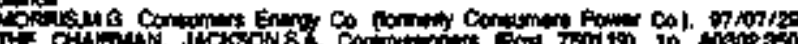
intog

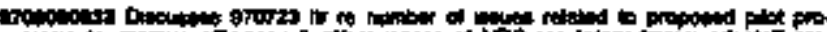

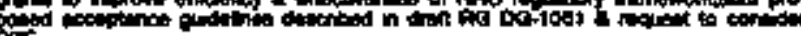

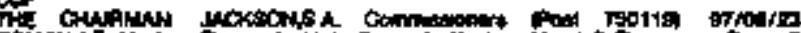

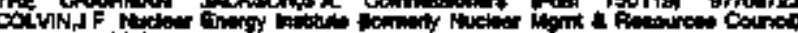

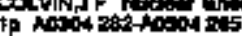

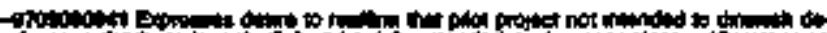

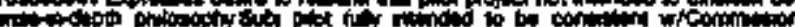
Pin

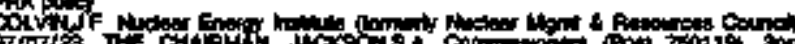

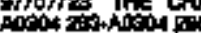

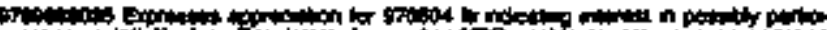

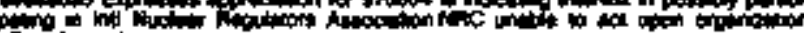

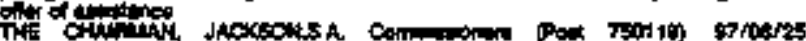
Thino

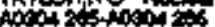

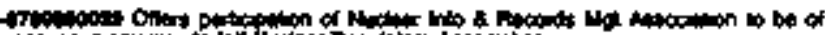

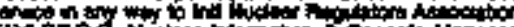

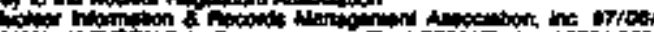

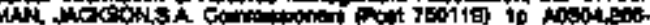

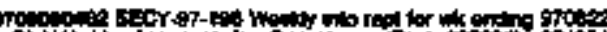
o

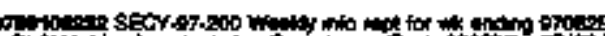

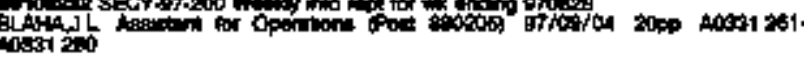

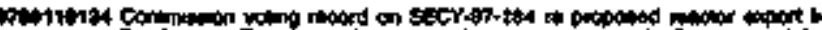

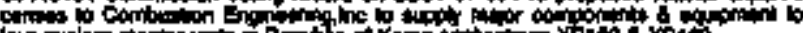

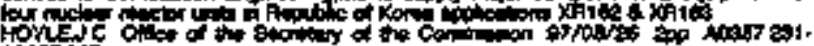
insis 20

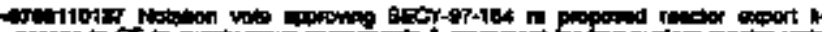

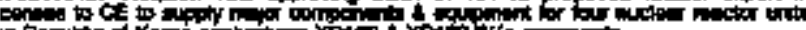

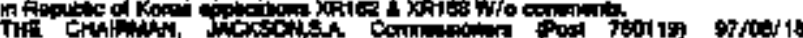

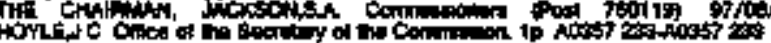

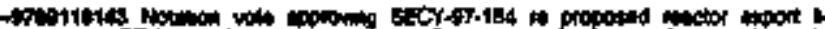

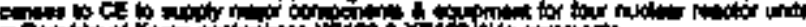

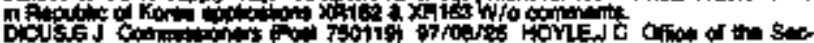

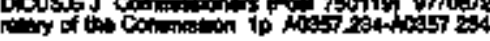

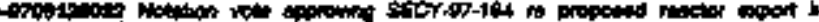

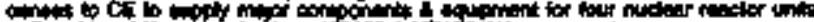
havist of Kor

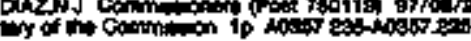

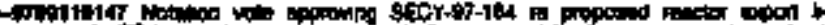

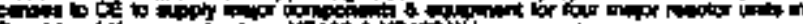

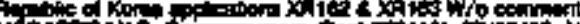

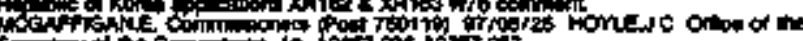

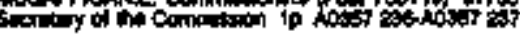

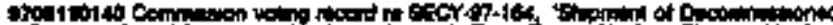

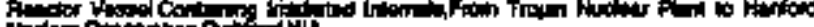

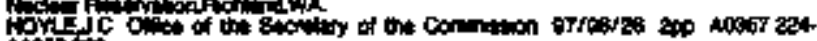
100520

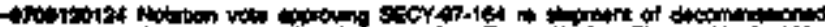

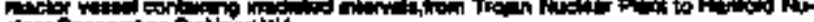

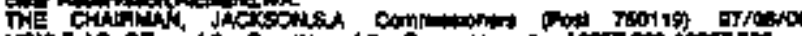

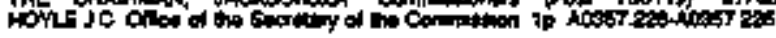

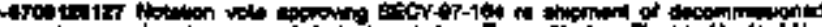

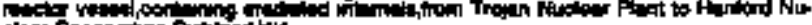

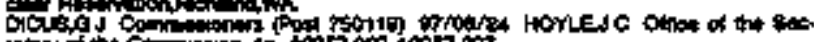

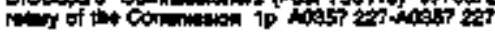

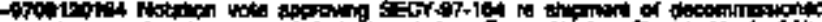

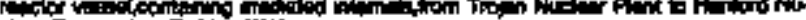

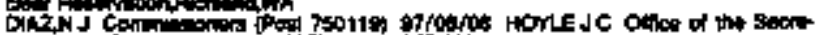

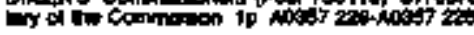




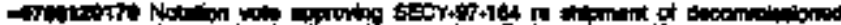

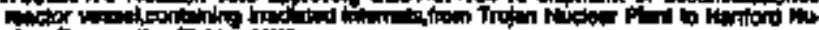

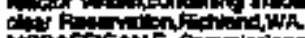

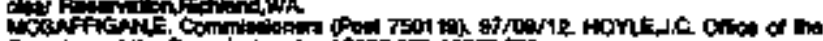

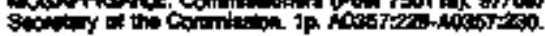

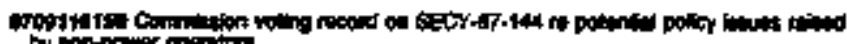

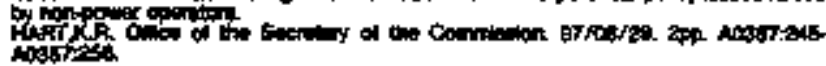

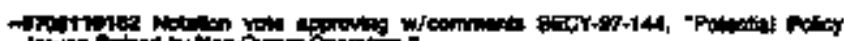

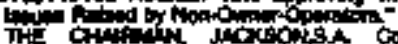

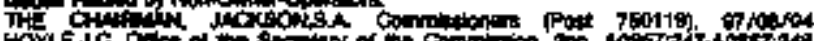

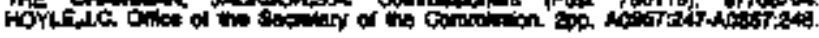

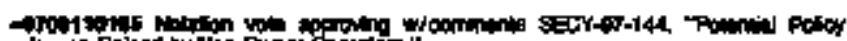

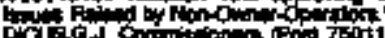

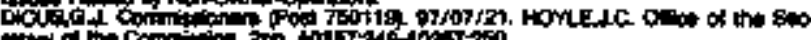

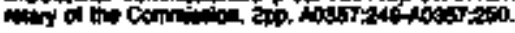

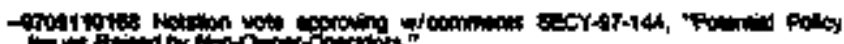

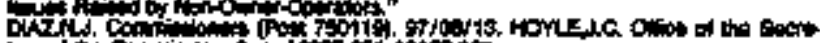

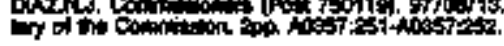

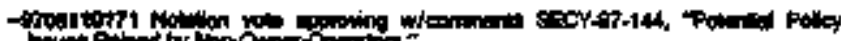

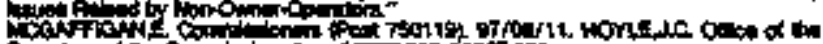

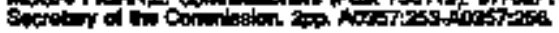

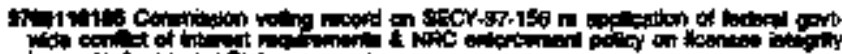

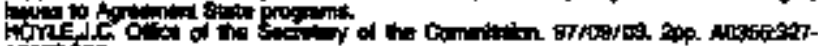
Anstista

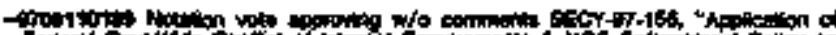

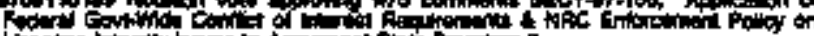

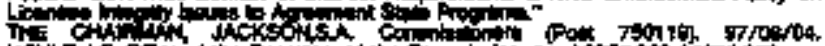

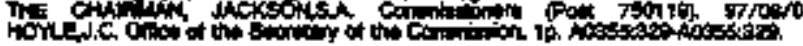

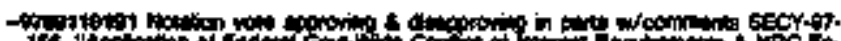

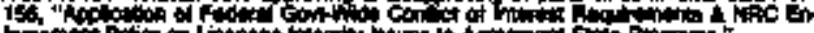

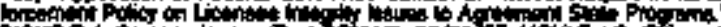

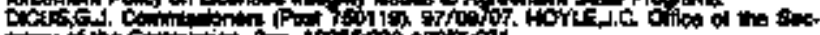

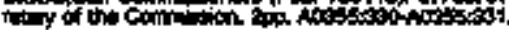

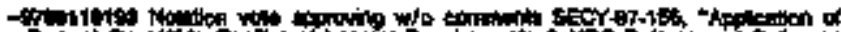

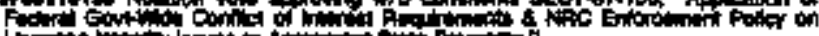

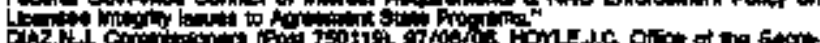

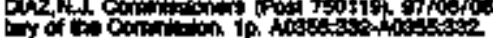

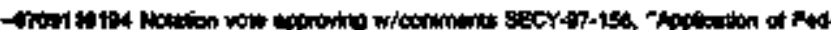

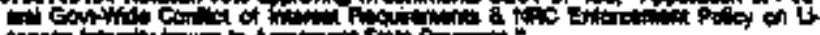

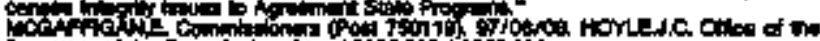

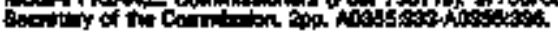

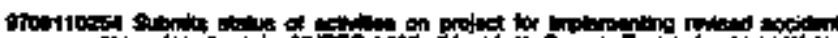

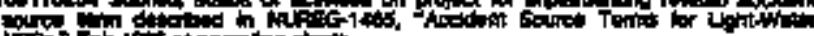

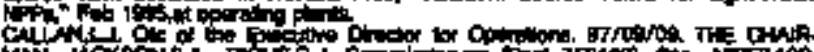

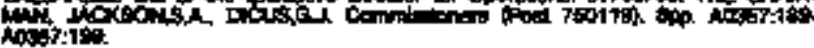

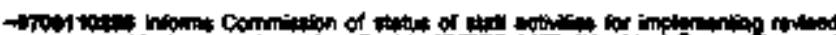

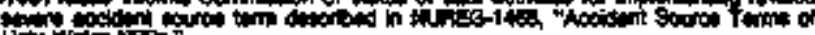

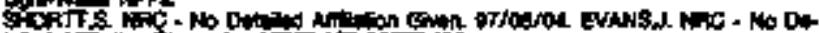

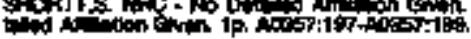

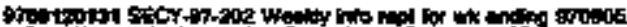

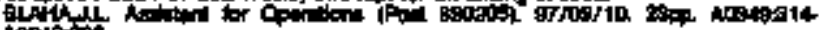
moris

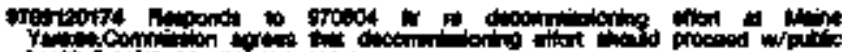

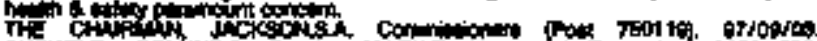

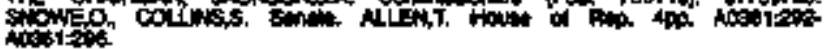

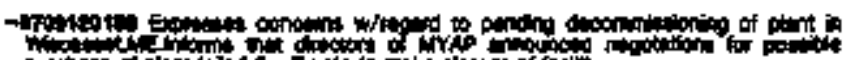

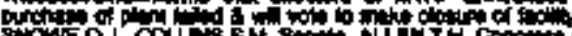

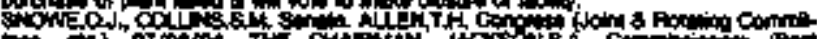

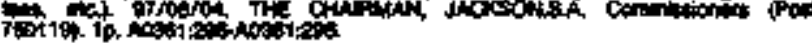

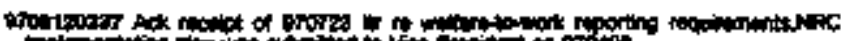

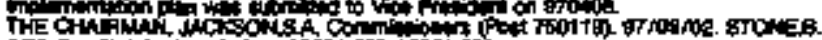

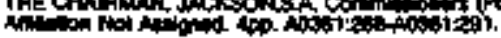

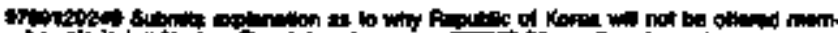

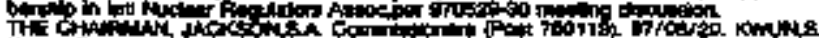

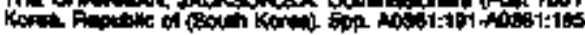

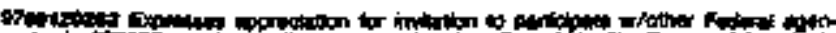

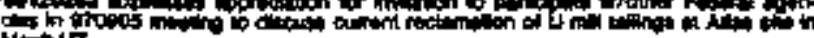
Hoing.

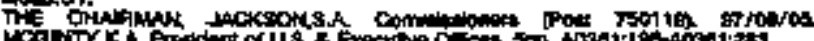

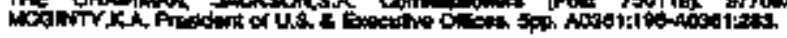

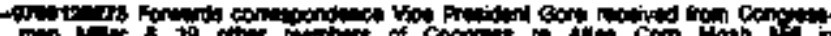

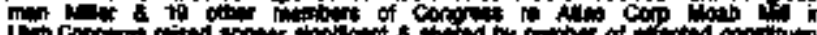

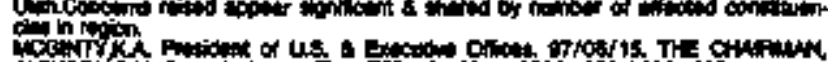

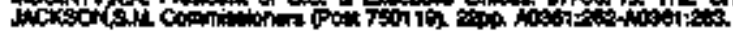

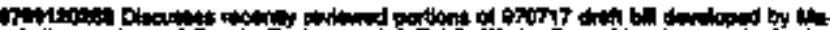

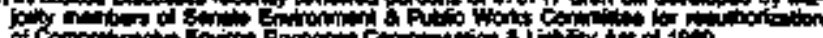

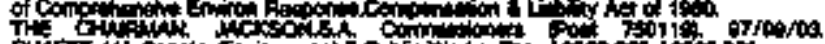

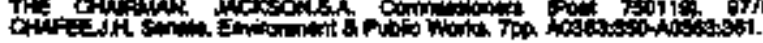

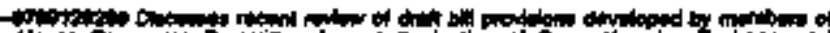

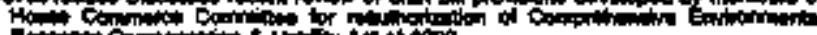

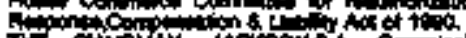

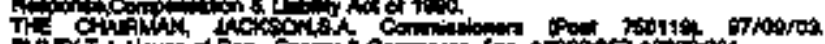

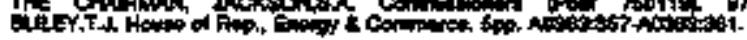

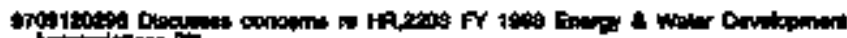

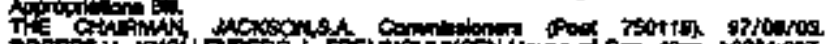
Toco itositis.

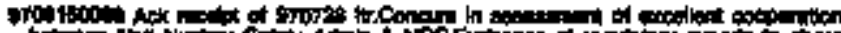

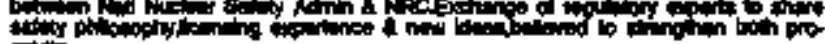

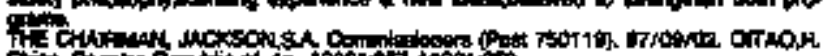

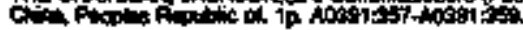

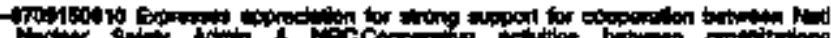

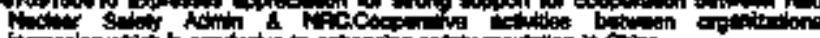

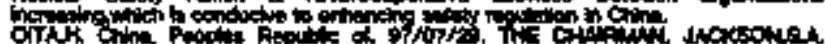
Can

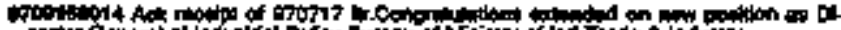

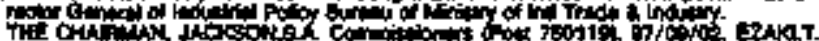

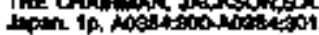

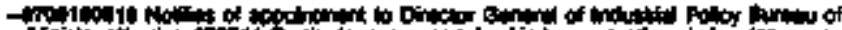

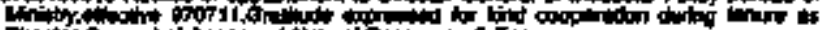

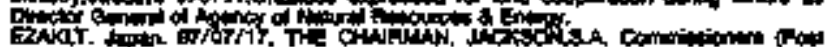

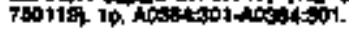

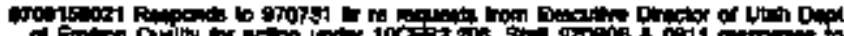

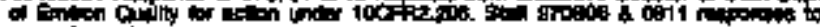

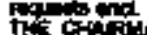

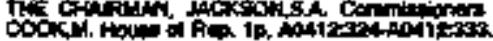

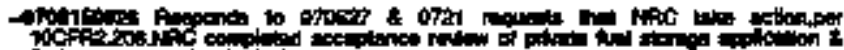

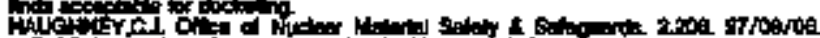

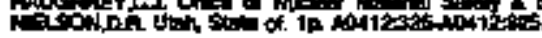

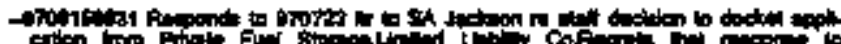

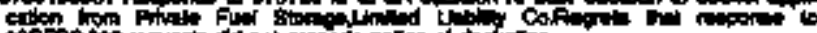

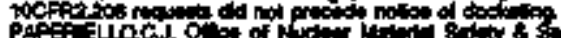

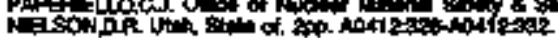

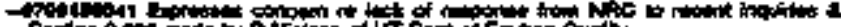

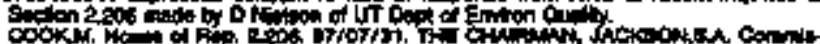

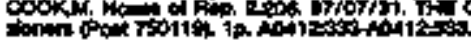

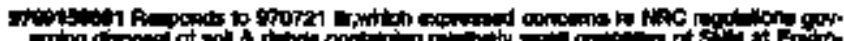

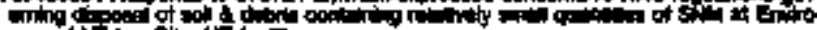

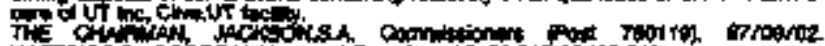

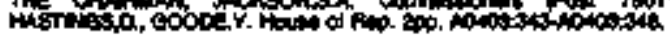

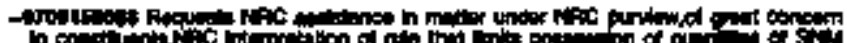

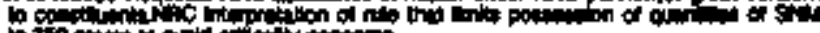

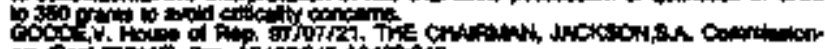

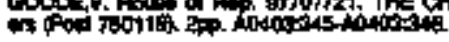

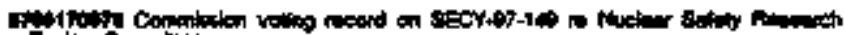

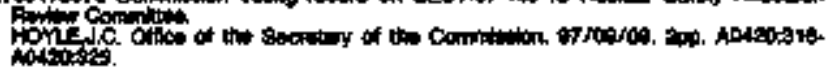

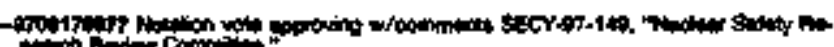

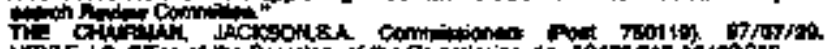

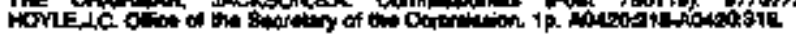

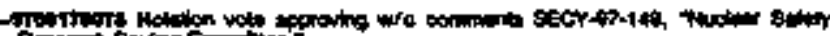

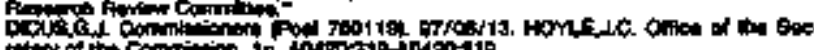

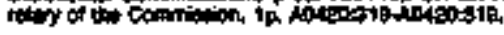

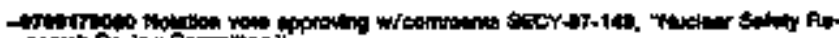

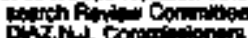

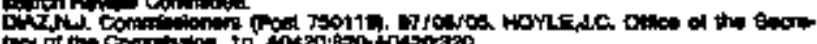

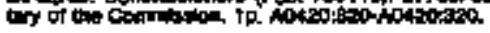




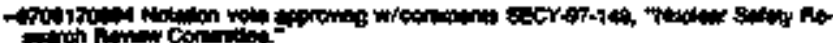

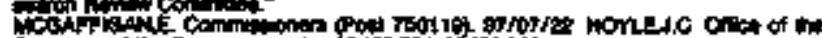

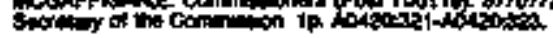

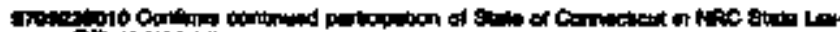

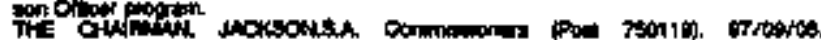

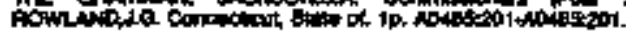

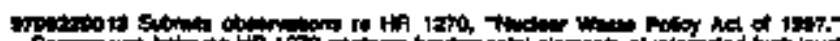

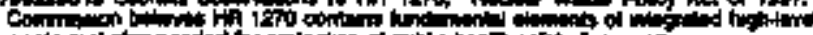

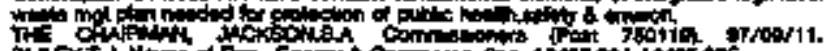

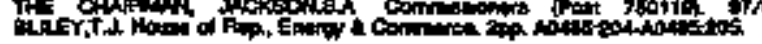

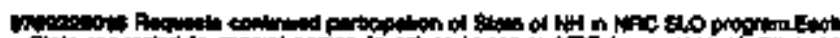

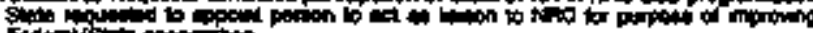
Roprifstion.

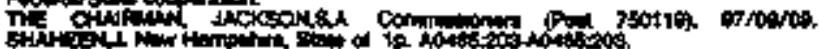

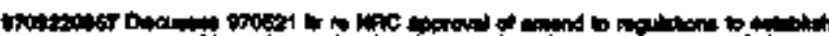

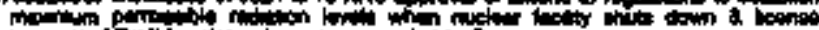

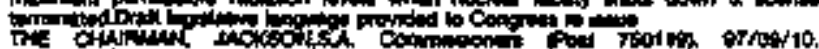

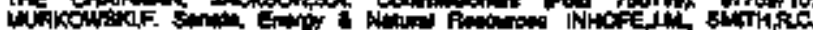

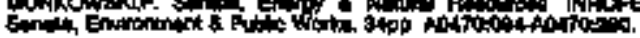

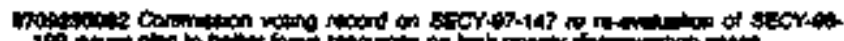

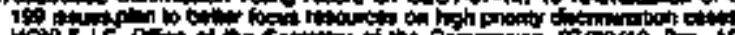
Hoy

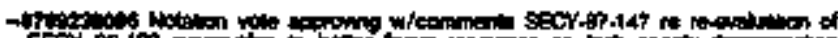

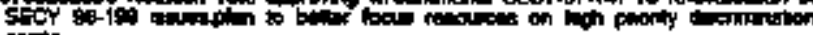

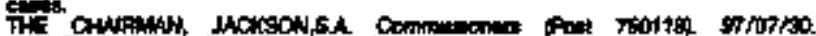

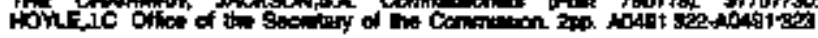

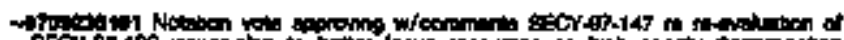

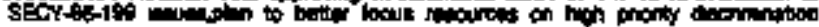

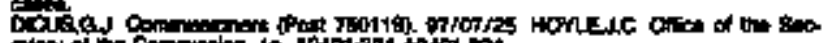

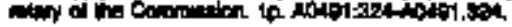

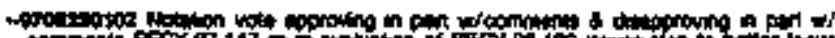

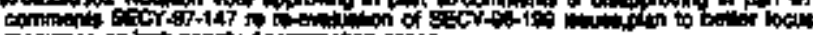

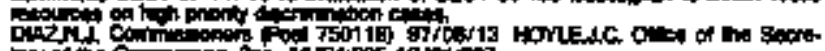

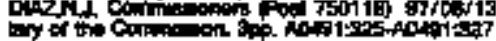

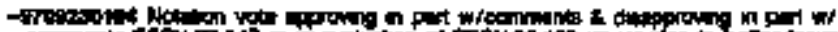
tor.

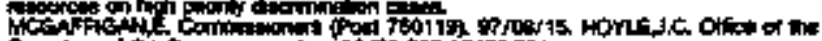

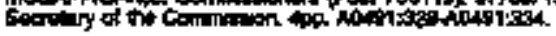

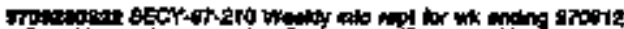

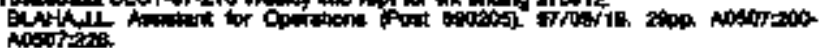

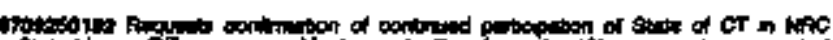

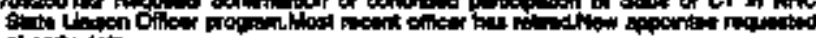

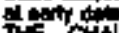

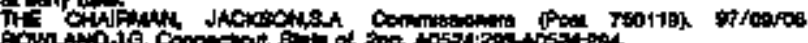

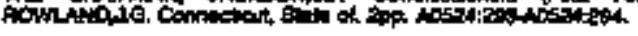

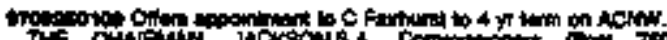

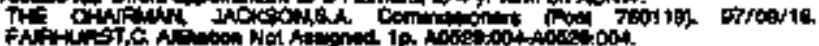

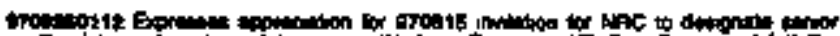

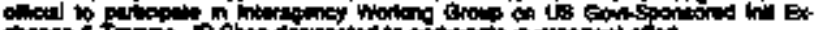

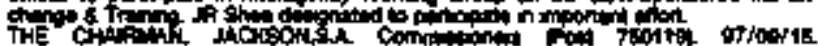

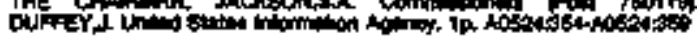

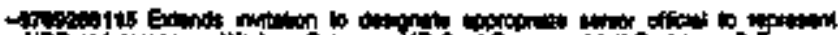

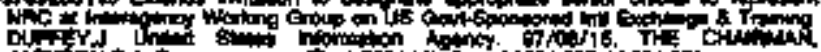

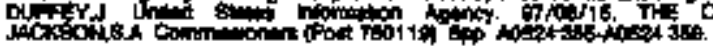

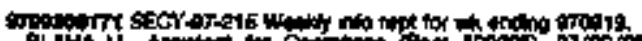

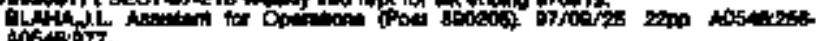

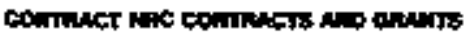

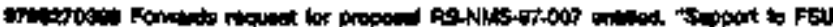

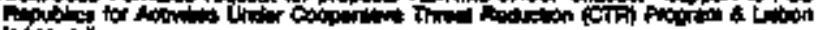
ithe

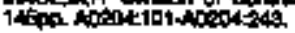

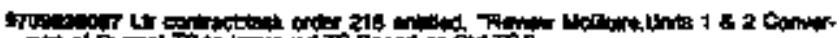

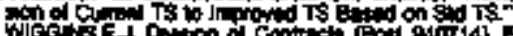

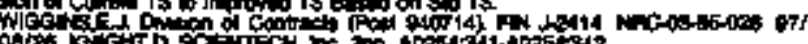

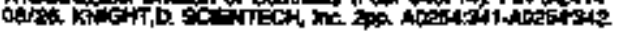

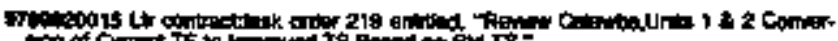

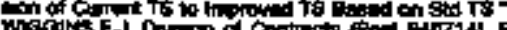

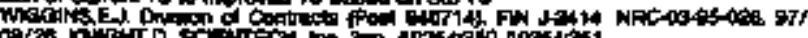

yonarsosis.

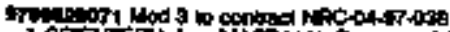

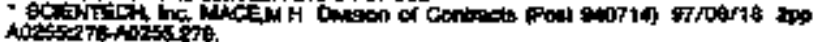

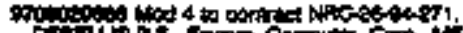

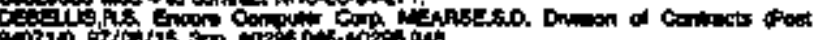
$040-1020$ ind

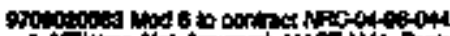

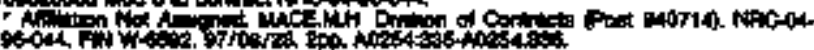

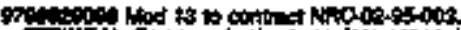

II) A

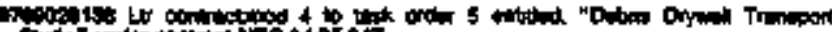

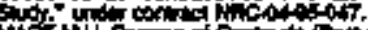

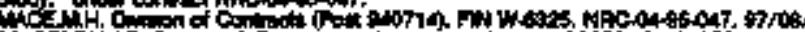

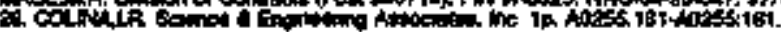

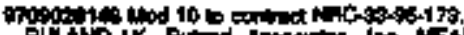

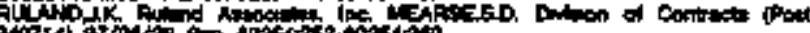

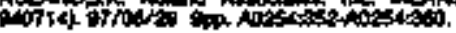

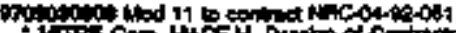

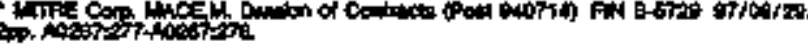

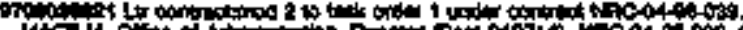

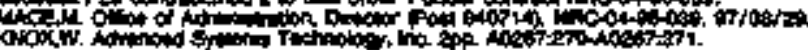

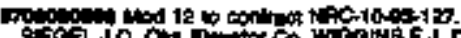

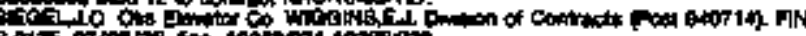

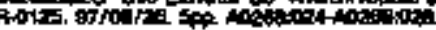

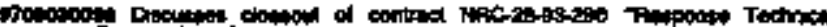

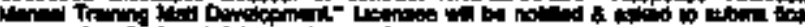

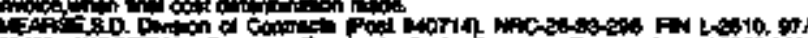

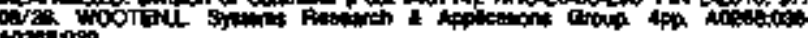
natios.

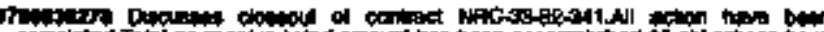

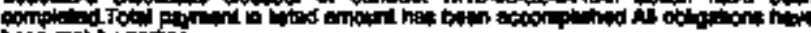

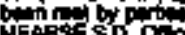

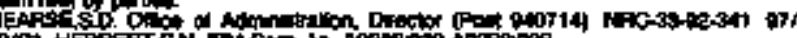

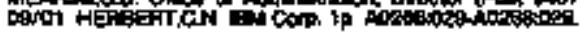

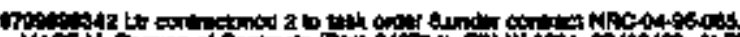

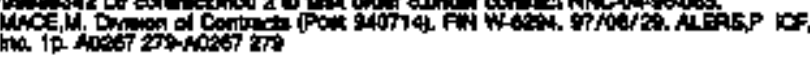

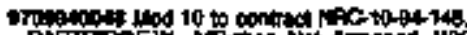

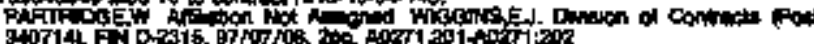

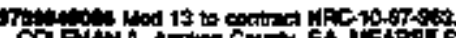

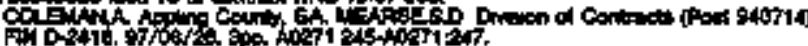

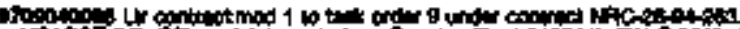

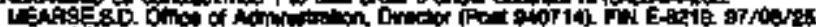

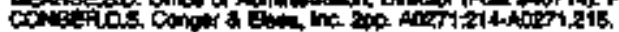

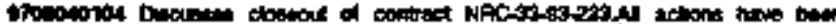

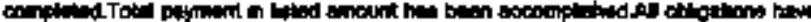

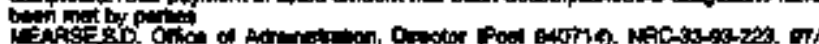

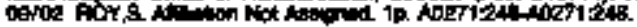

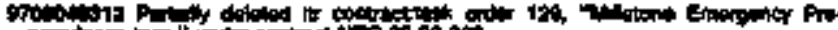

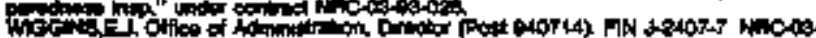

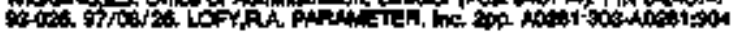

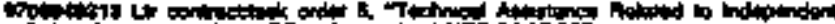

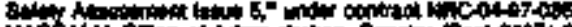

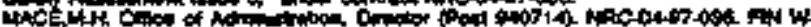

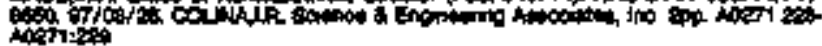

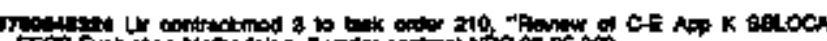

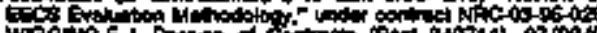

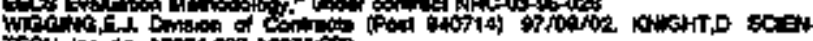

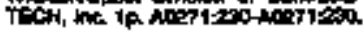

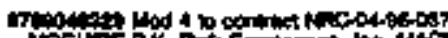

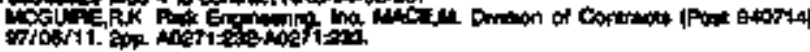

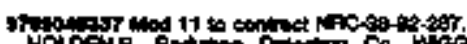

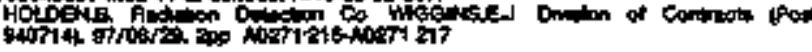

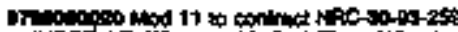

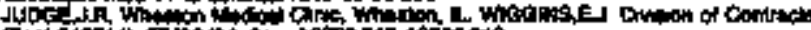
(Foli 140 id)

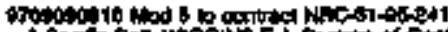

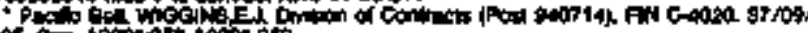
axpis

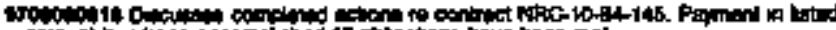

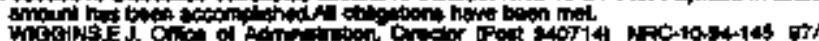

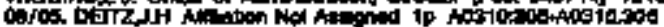




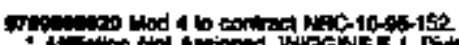

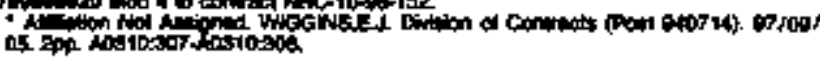

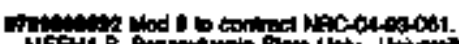

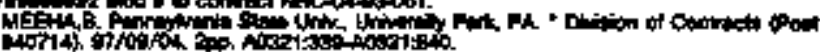

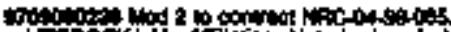

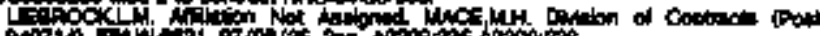

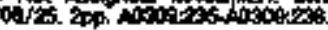

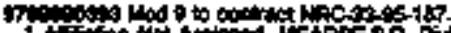

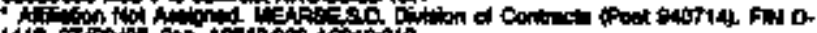
1419. का

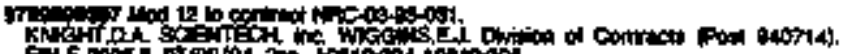

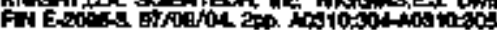

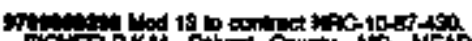

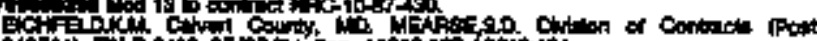

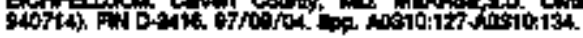

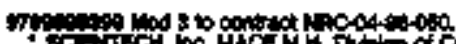

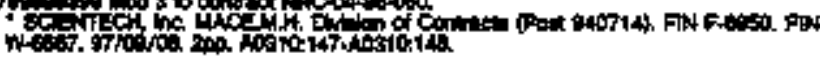

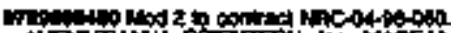
Ad

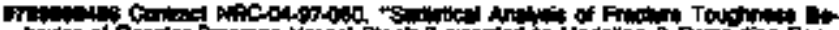

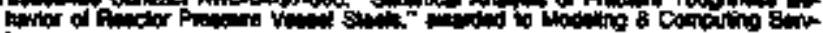

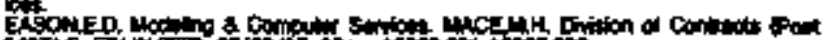

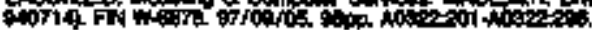

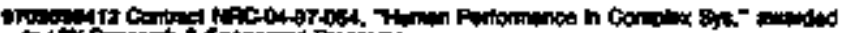

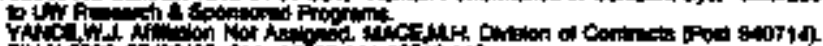
F $\mathrm{K}$ K-

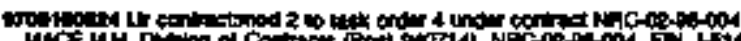

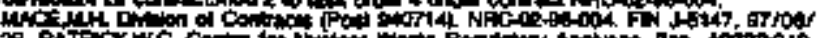

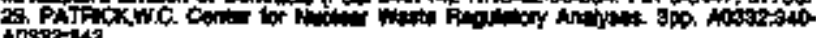

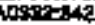

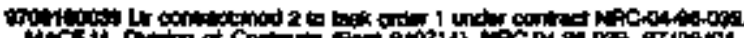

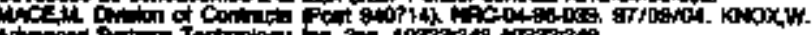

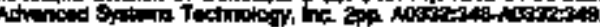

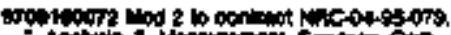

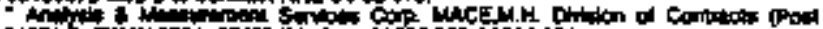

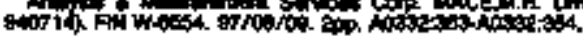

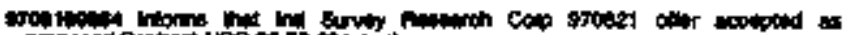

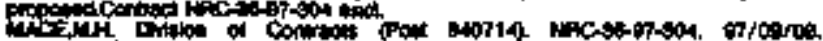

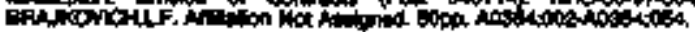

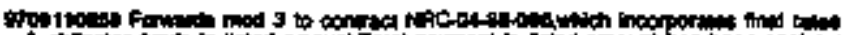

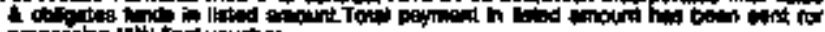

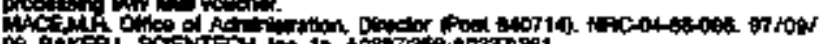
of. ENA

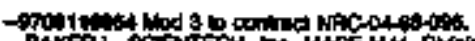

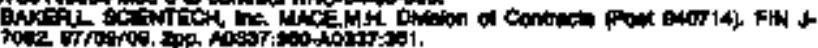

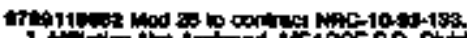

4. tription.

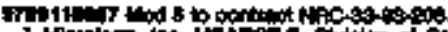

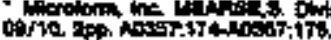

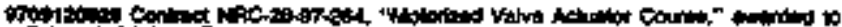

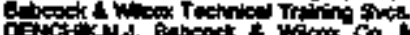

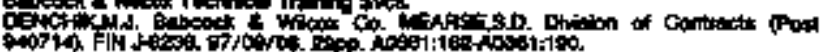

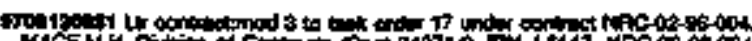

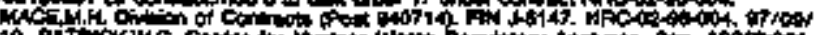
10. PA

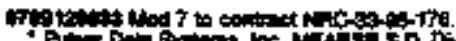

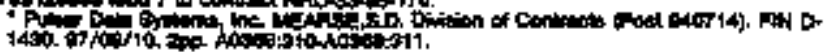

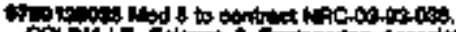

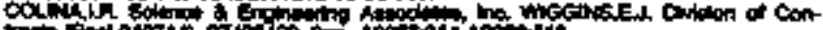

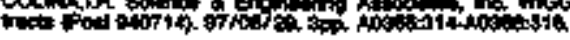

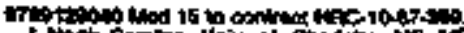

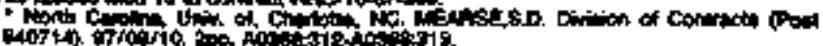

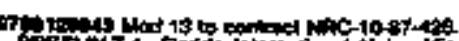

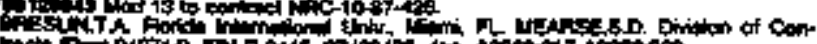

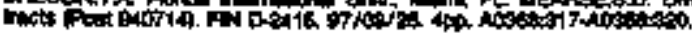

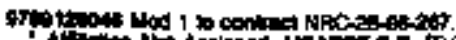

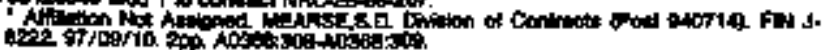

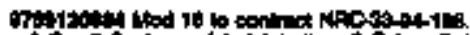

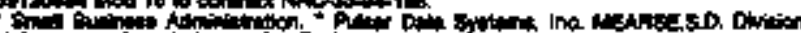

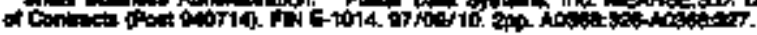

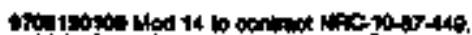

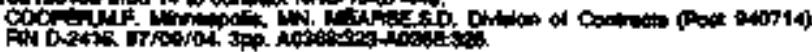

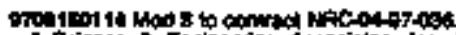

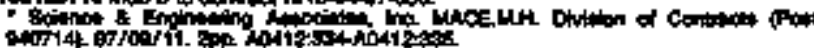

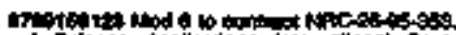

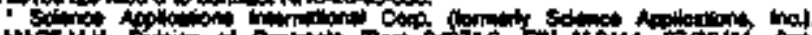

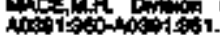

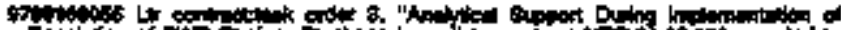

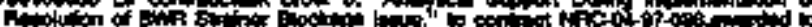

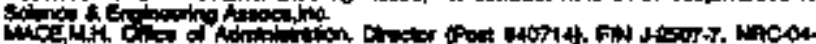

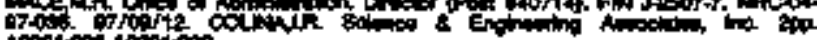

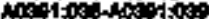

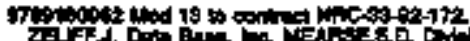

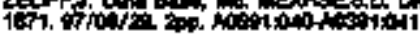

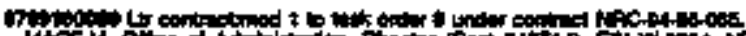

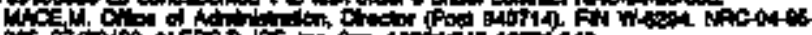

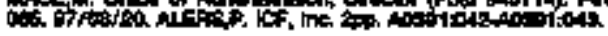

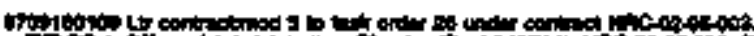

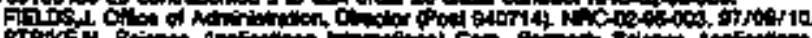

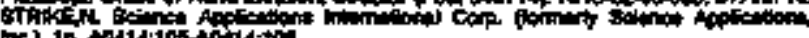

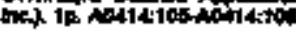

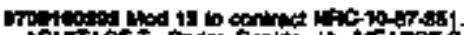

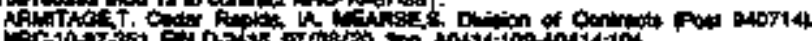

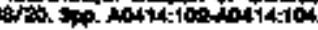

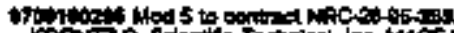

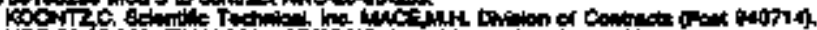
1.

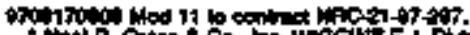

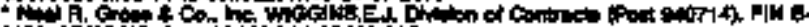

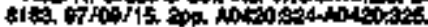

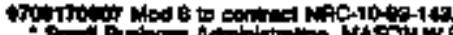

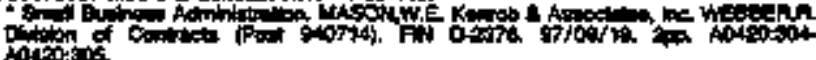

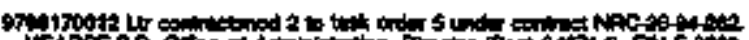

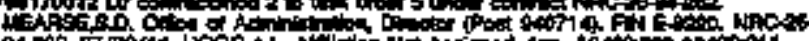

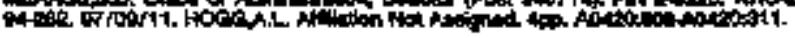

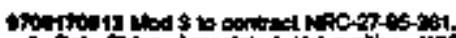

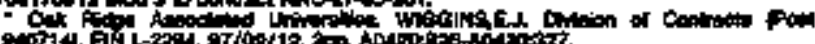

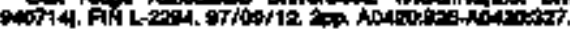

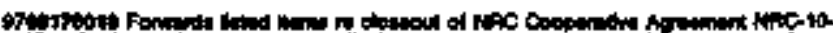

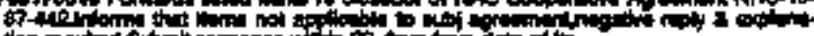

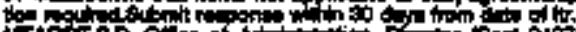

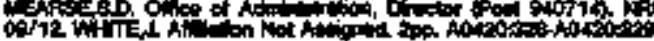

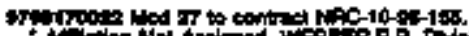

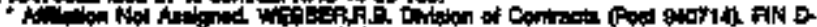

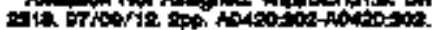

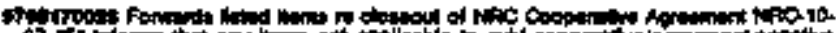

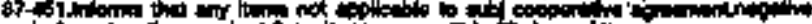

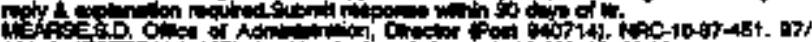

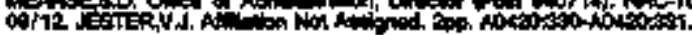

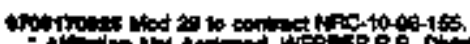

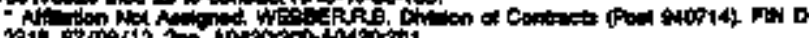

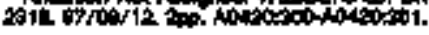

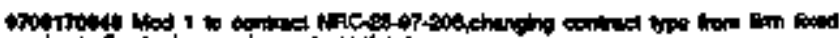

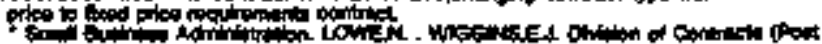

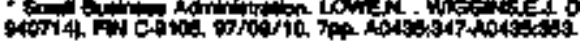

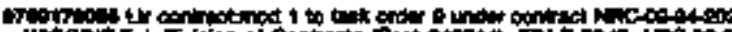

Whe

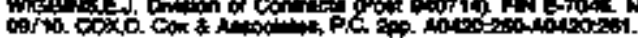

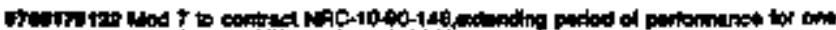

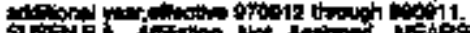

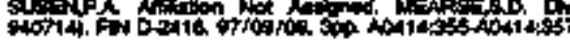

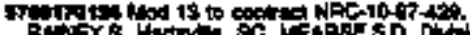

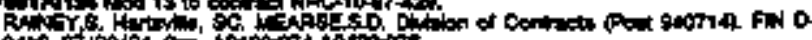

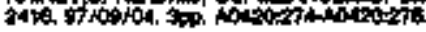

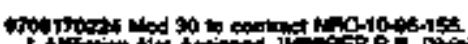

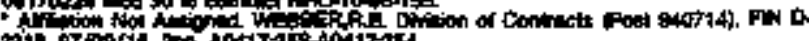

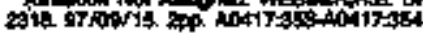

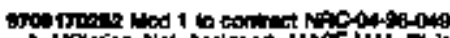
or ie 


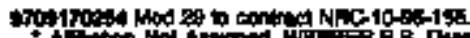

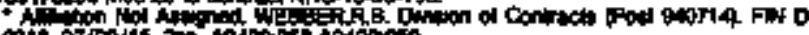

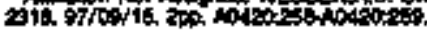

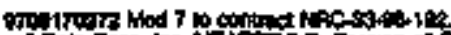
Dona

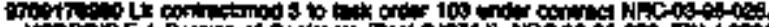
HOF

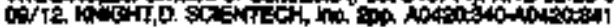

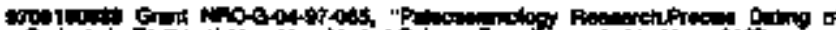

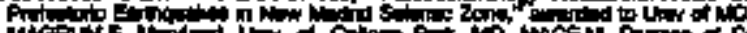

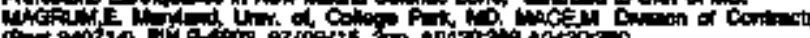

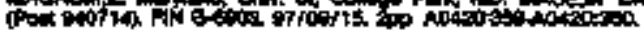

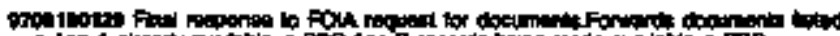

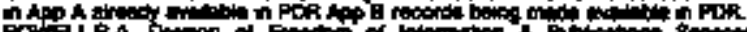

ain it

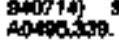

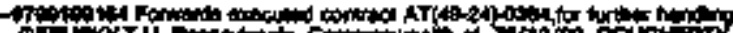

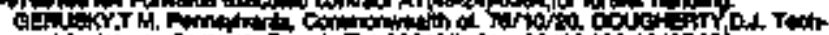

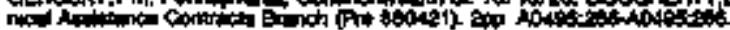

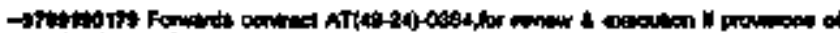
contationd work

cello

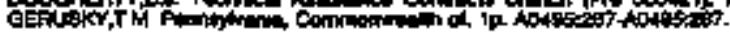

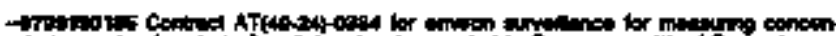

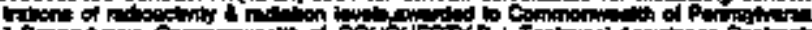

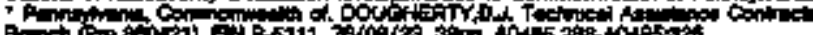

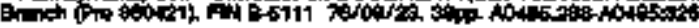

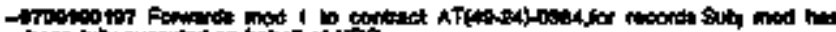

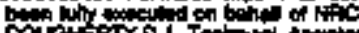

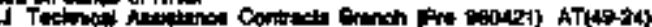
ation

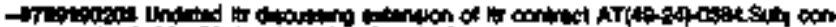

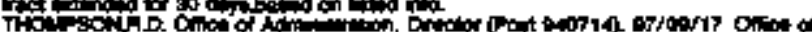

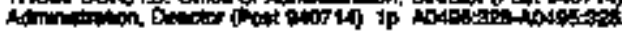

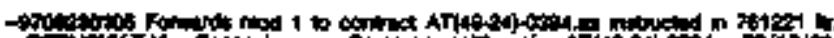

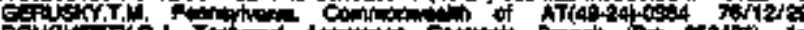

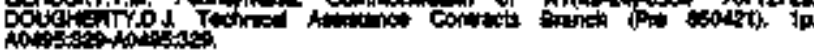

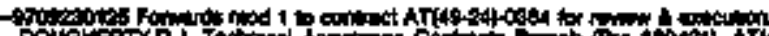

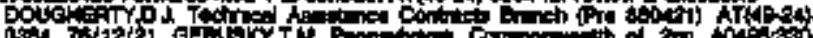

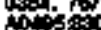

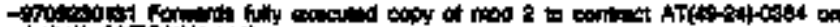

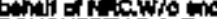

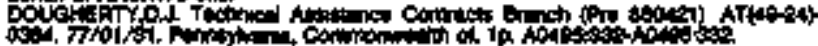

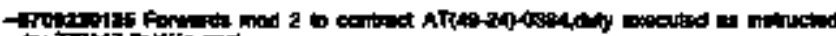

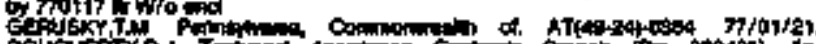

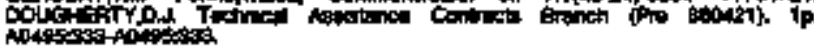

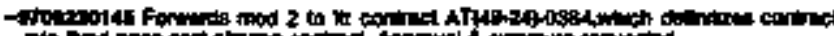

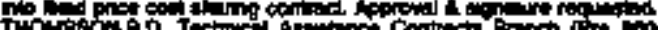

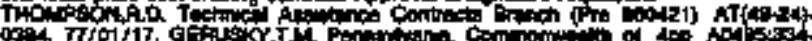
istion

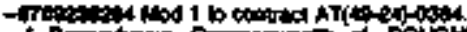

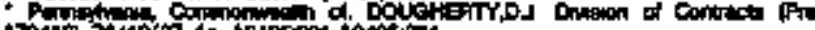

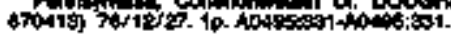

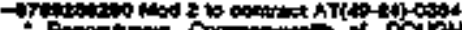

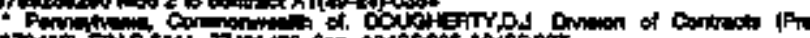

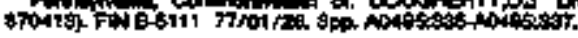

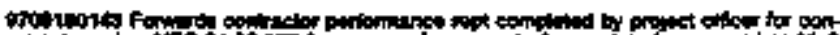

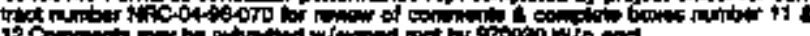

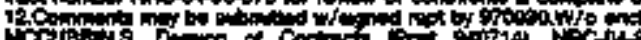

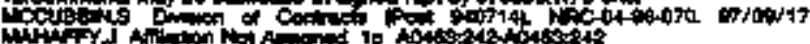

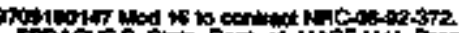

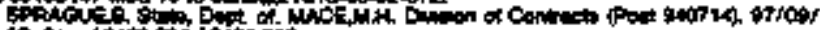

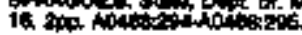

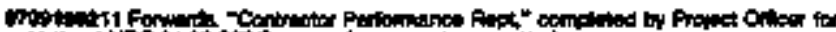

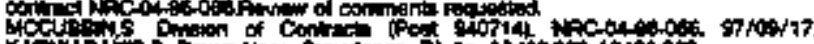

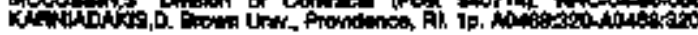

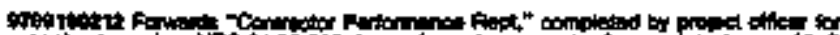

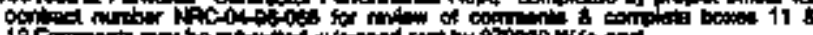
120.

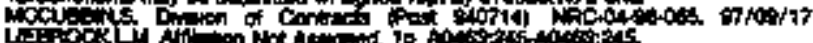

atsons.

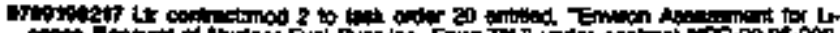

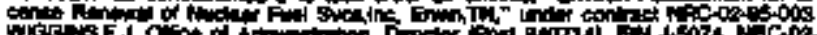
T50

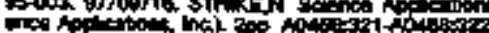

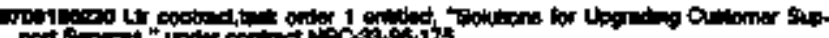
pent

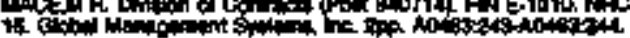

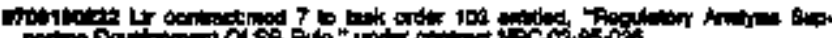

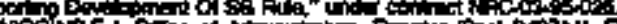
Recin

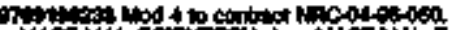

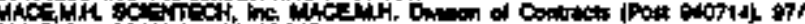

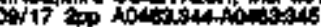

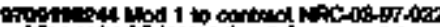

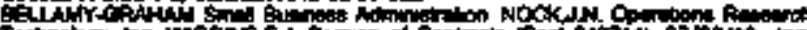

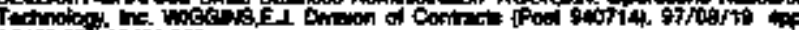

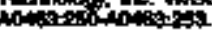

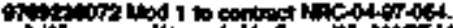

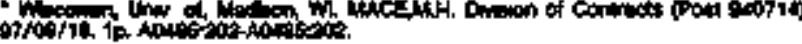

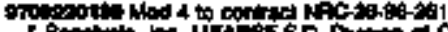

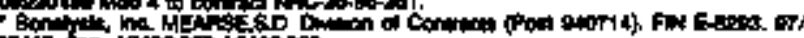

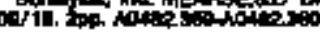

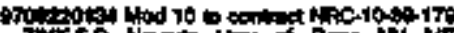
a

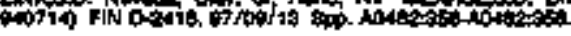

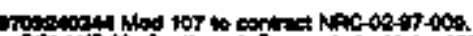

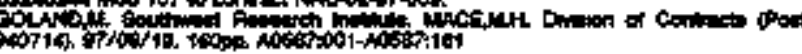

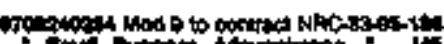

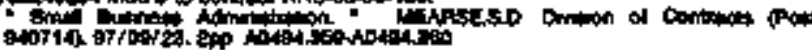

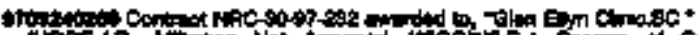
Lock

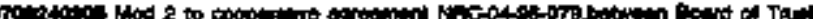

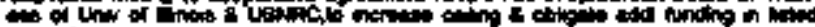

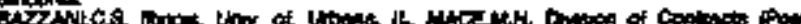

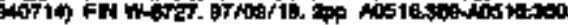

wow

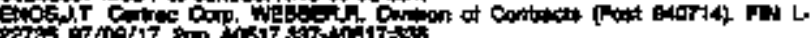

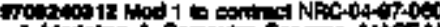

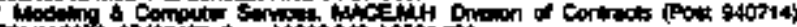

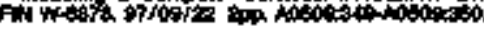

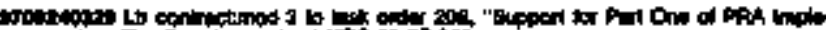
moth

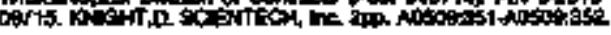

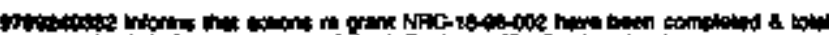
F

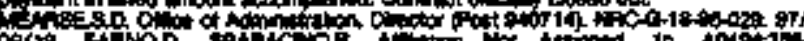

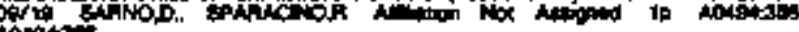

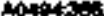

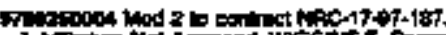

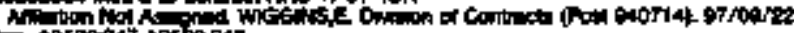

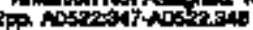

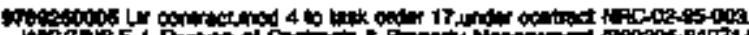

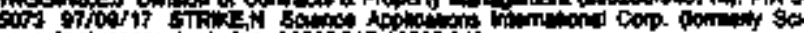

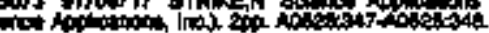

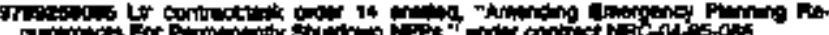

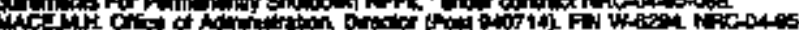
oth of

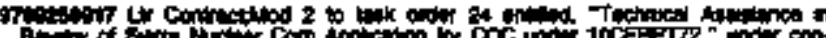

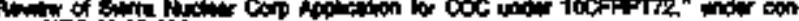

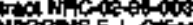

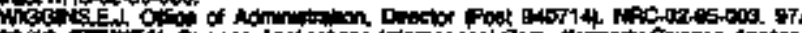

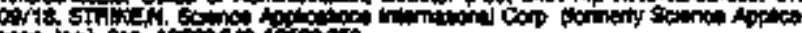

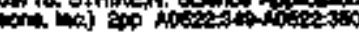

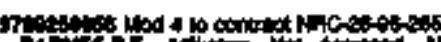

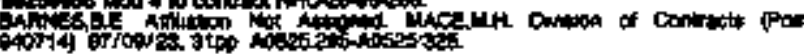

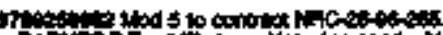

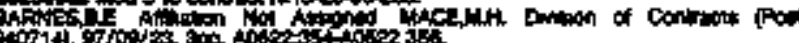
(4) 14 .

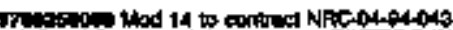

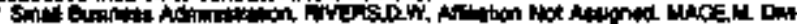

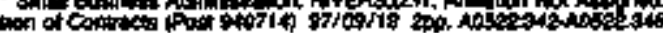

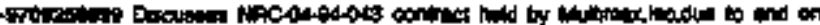

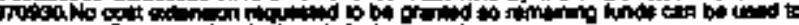

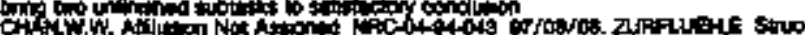

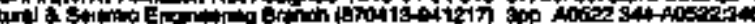




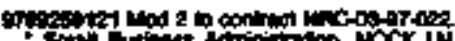

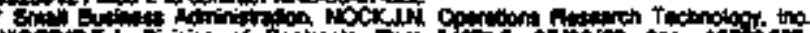

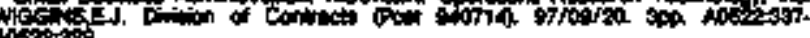

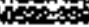

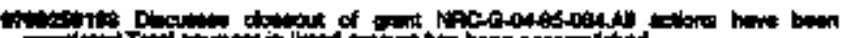

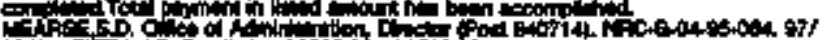

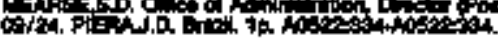

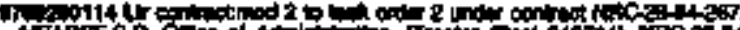

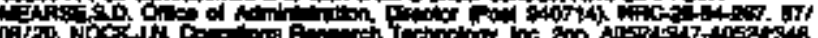

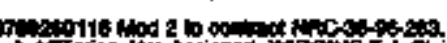

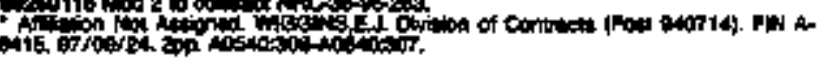

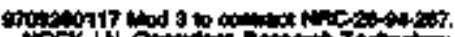

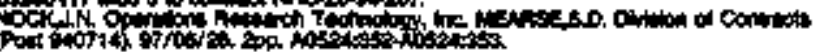

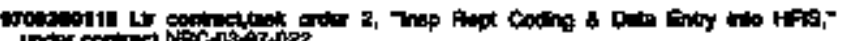

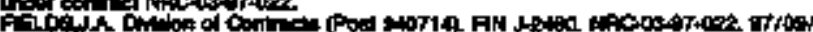

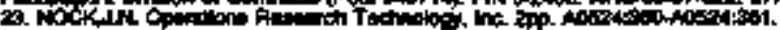

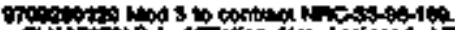

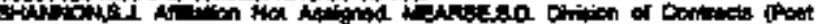

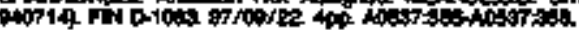

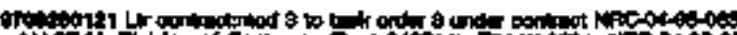

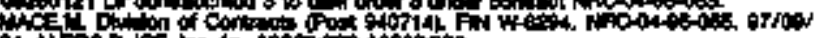

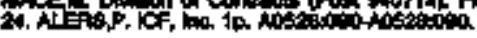

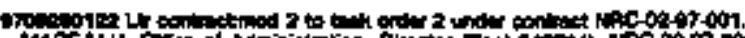

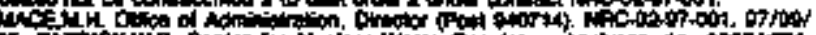

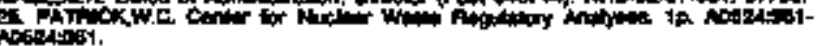

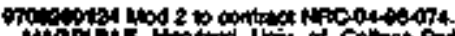
Peiti on

of

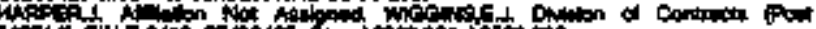

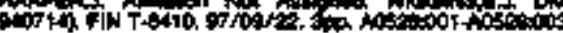

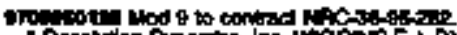

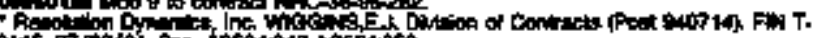

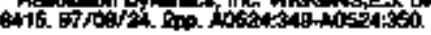

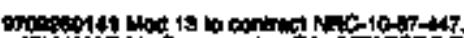

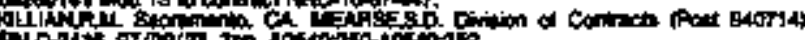

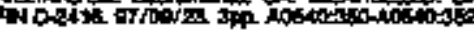

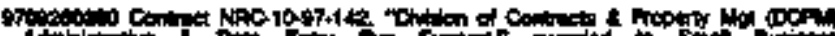

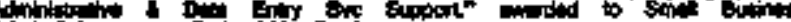

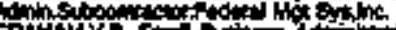

Fithy

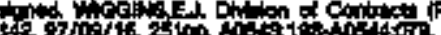

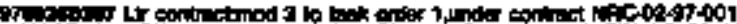

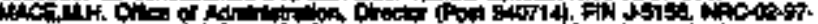

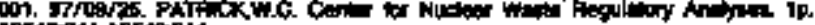

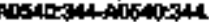

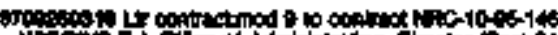

Het

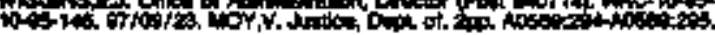

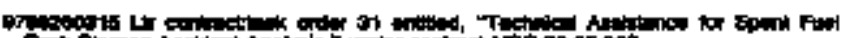

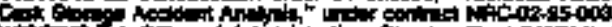

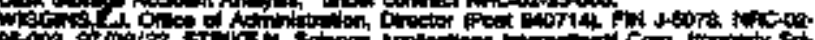

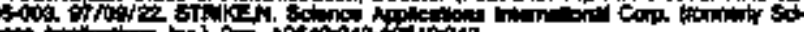

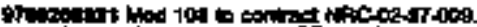

Af

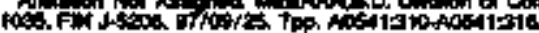

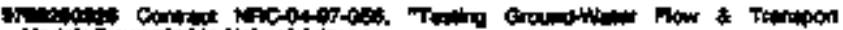

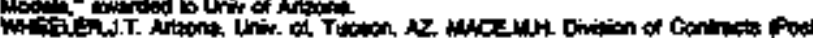

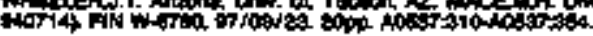

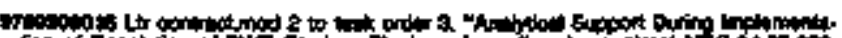

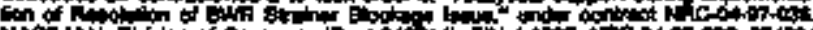

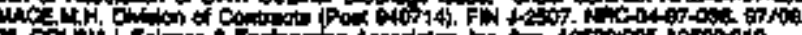

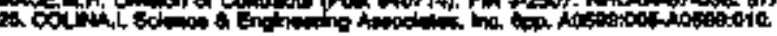

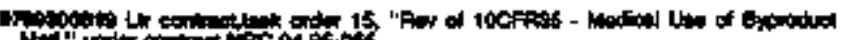

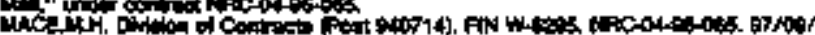

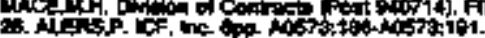

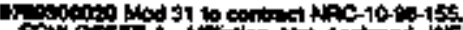

20

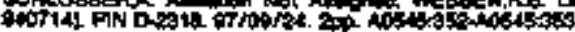

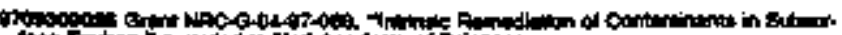

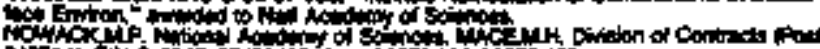

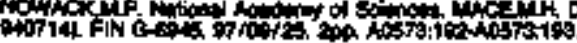

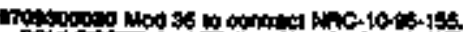

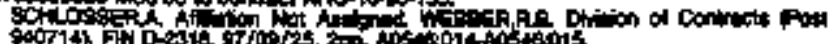

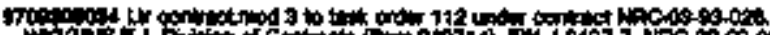
He

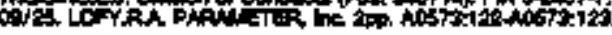

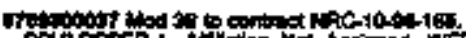

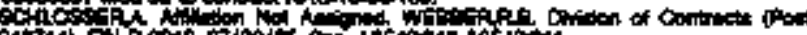

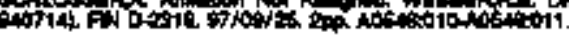

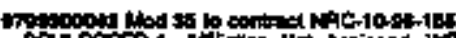

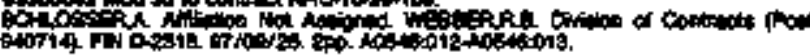

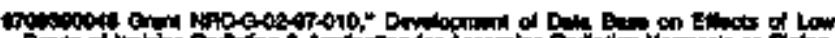

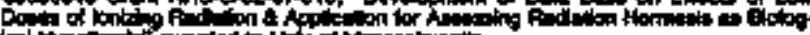

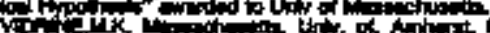

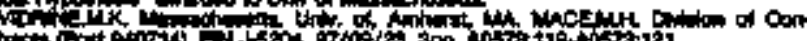

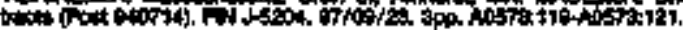

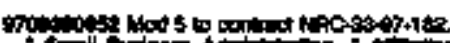

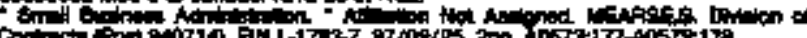

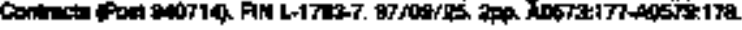

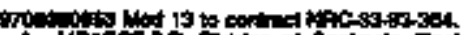

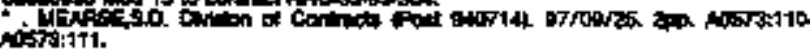

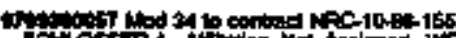

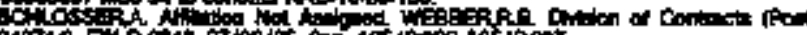

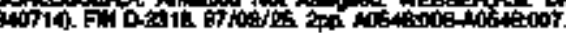

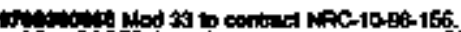

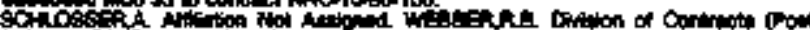

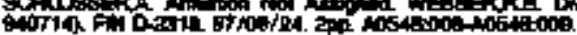

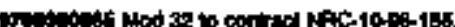
Sh Tos

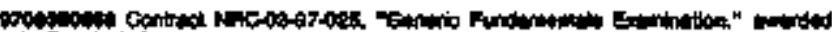

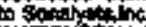

Whan

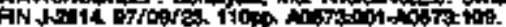

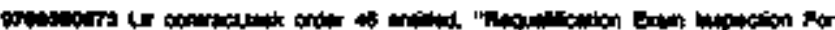

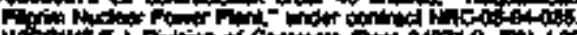

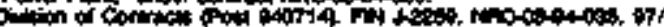
swio

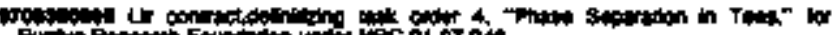
Mace

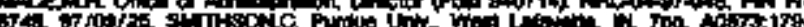
Anstis

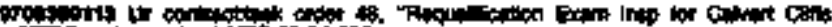

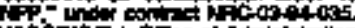

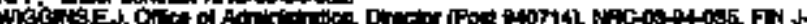

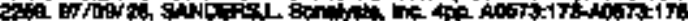

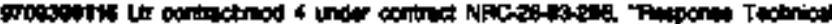

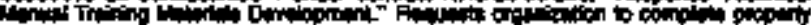

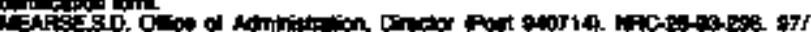

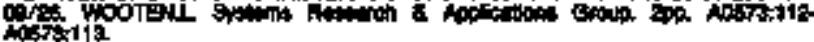

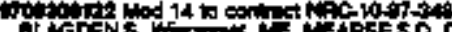

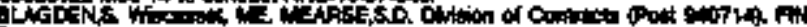

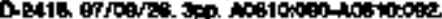

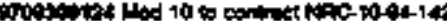

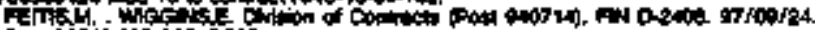

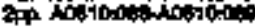

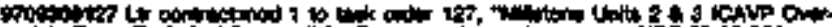

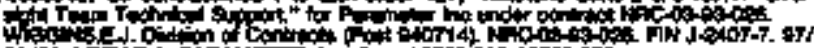

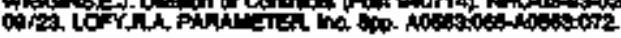

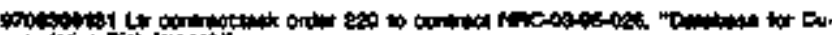

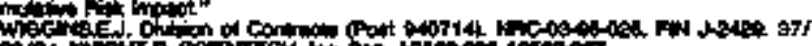

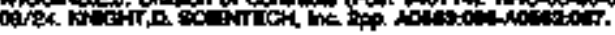

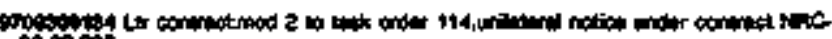

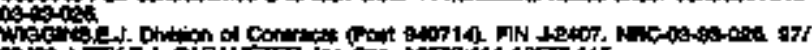

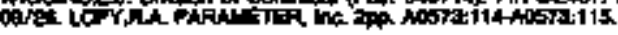

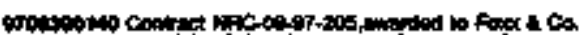
R.

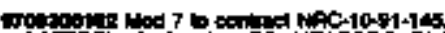

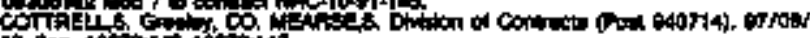

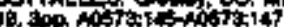

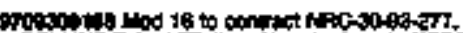

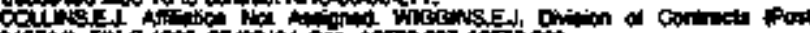

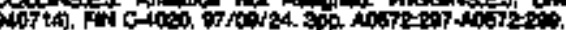

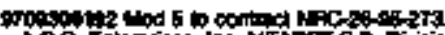

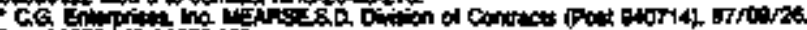
con 


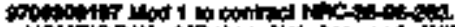

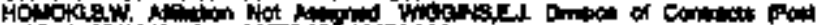

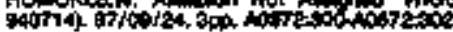

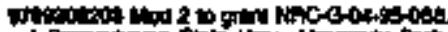

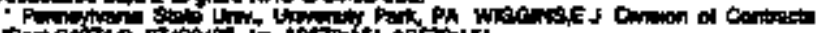
toos of

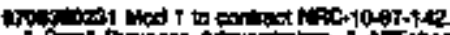

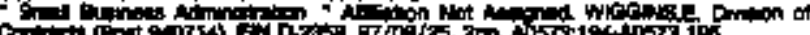

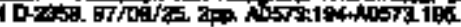

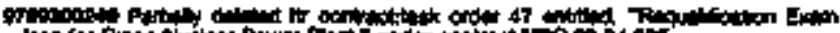

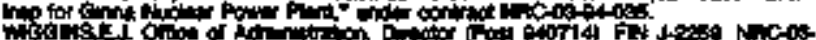

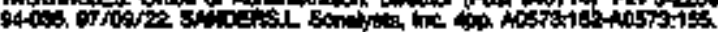

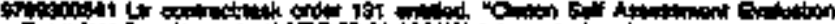

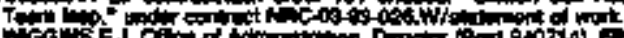

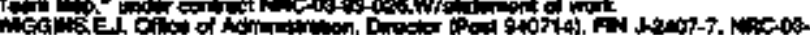

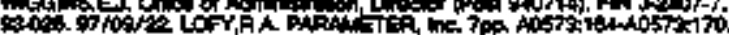

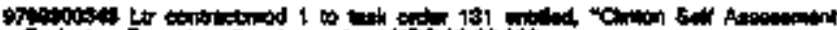

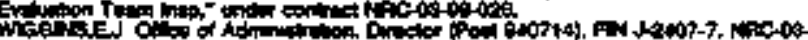
opos of

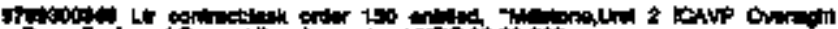

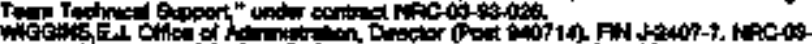

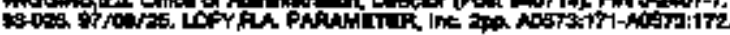

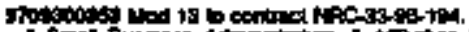

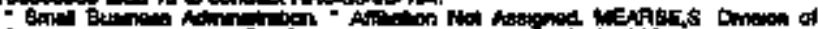

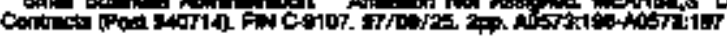

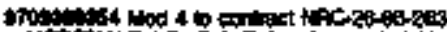

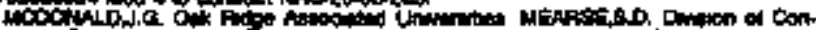

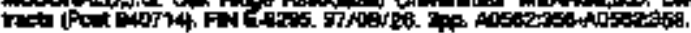

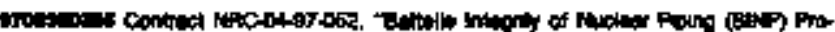

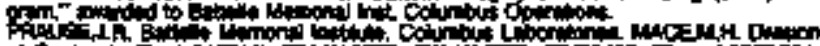

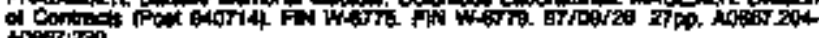
Adoedtron.

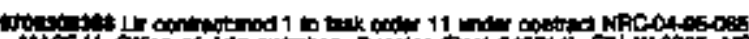

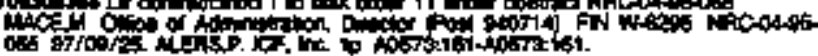

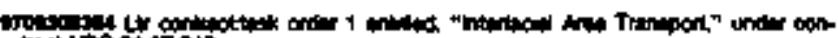
tractictions

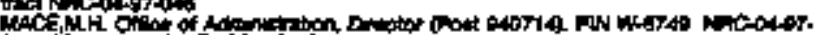

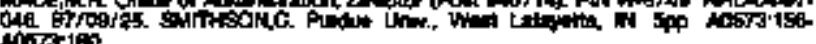

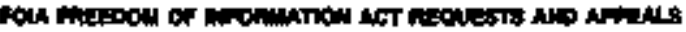

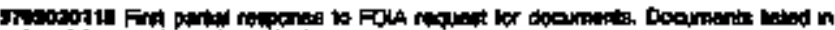

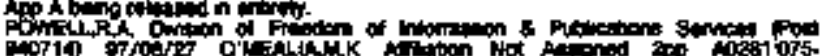

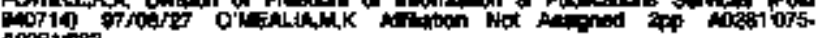
$\operatorname{arcosing}$

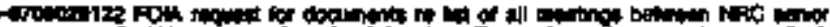

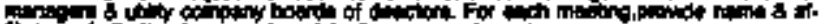
ot

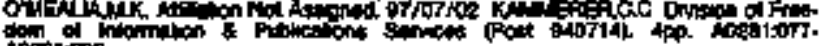

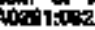

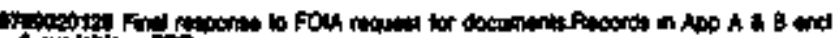

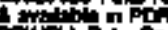

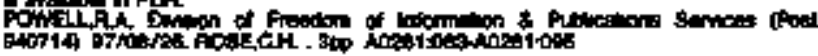

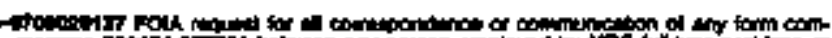

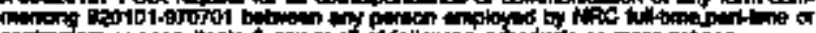

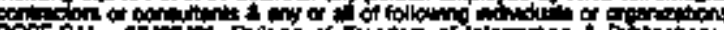

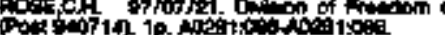

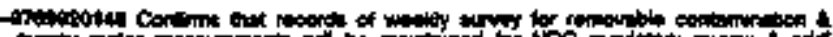

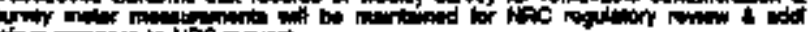
Uen

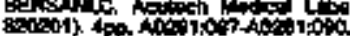

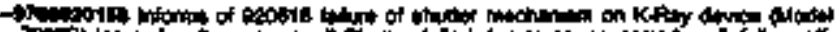

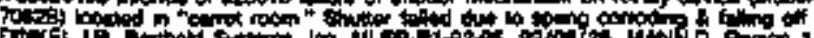
(1)

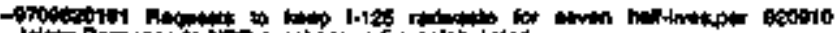

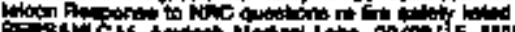

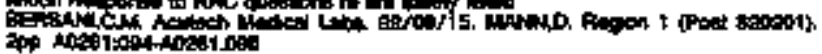

部

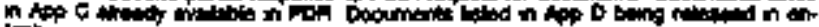

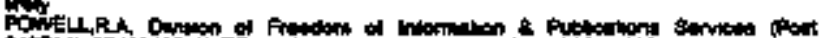

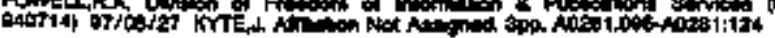

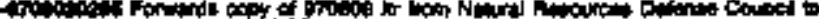

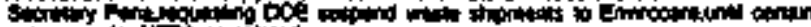

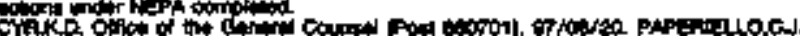

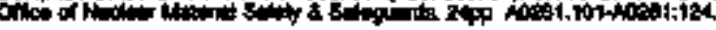

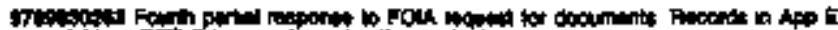

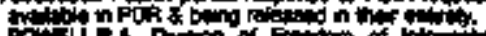

Pon

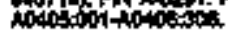

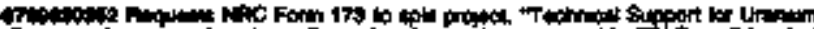

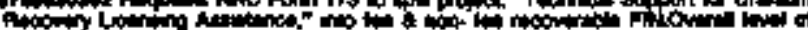

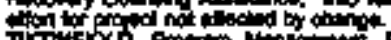

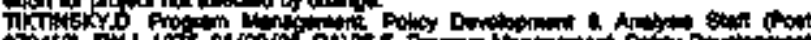

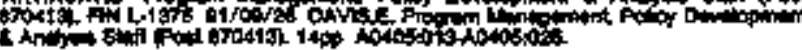

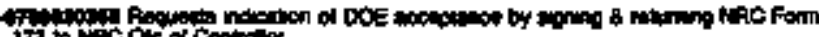

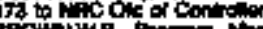

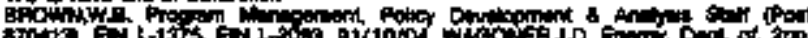

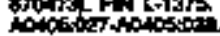

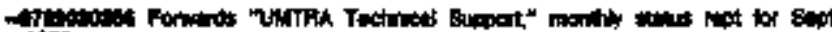

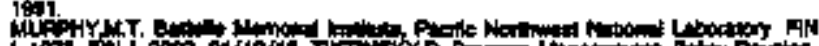

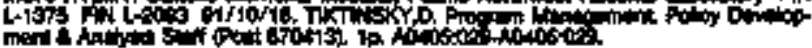

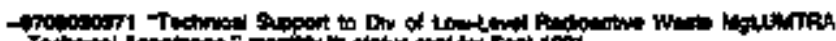

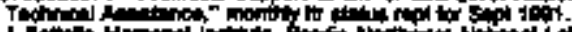

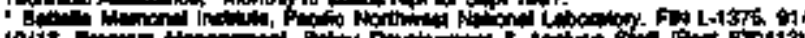

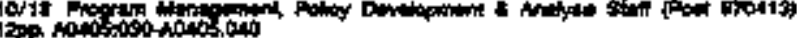

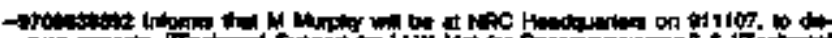

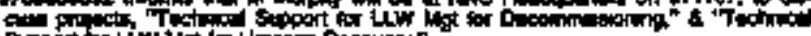

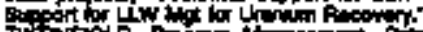

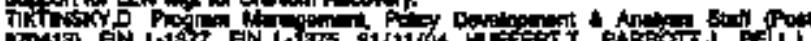

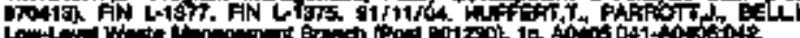

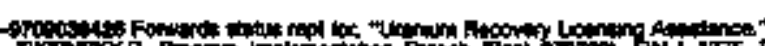

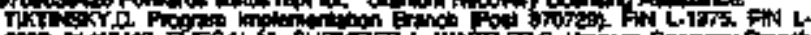
pes of

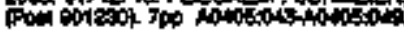

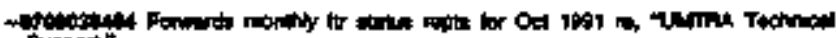

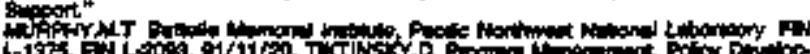

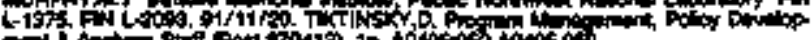

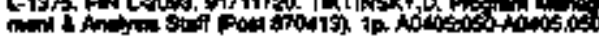

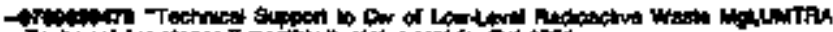

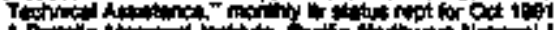

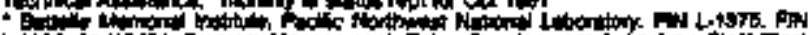

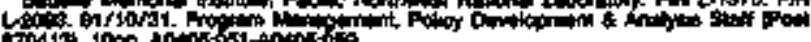

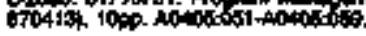

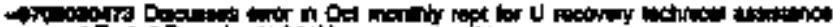

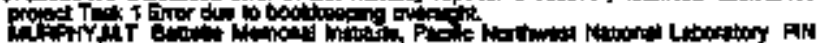

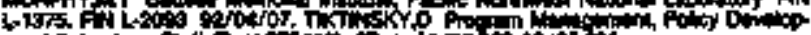

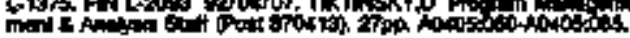

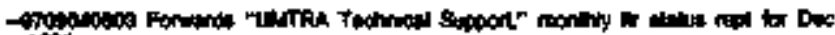
101

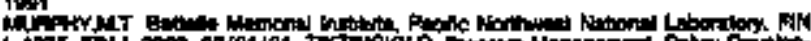

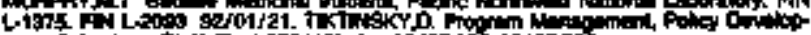

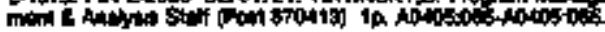

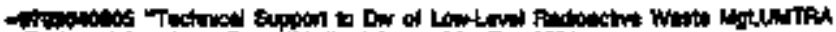

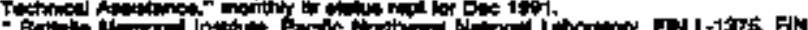

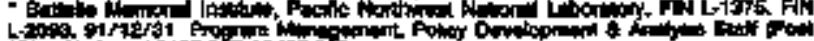

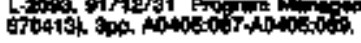

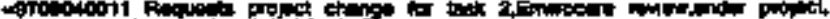

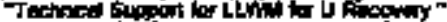

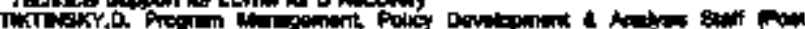

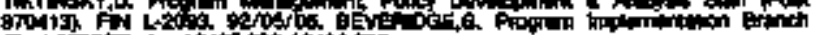

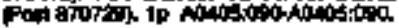

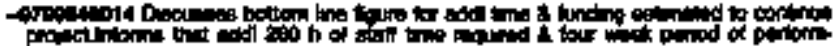

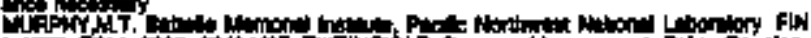

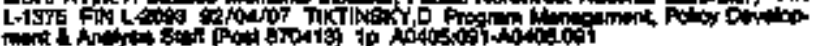

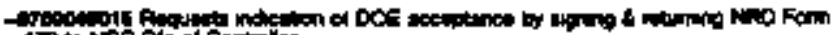

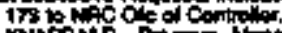

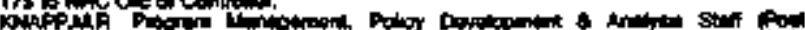

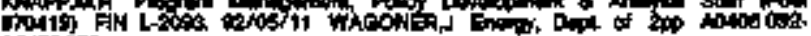
nodostas

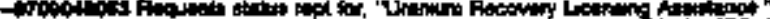

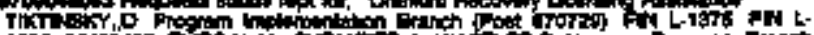

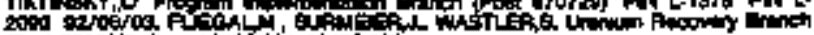

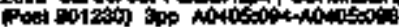




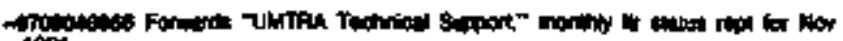
100 .

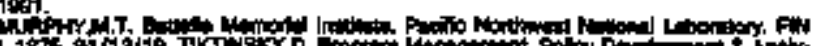

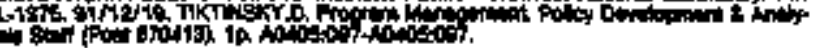

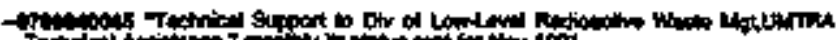

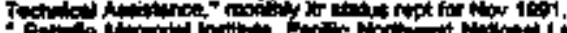

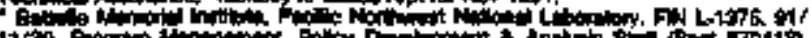

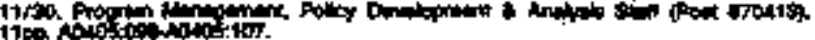

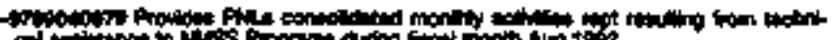

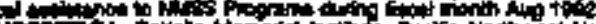

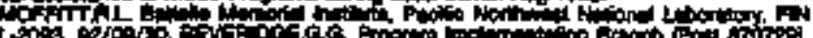

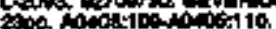

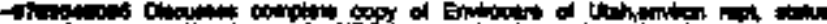

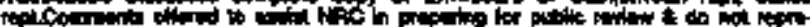

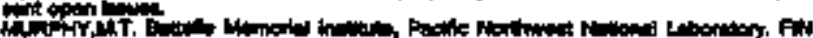

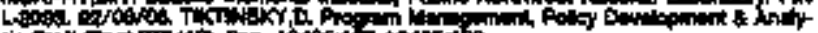

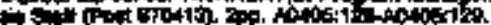

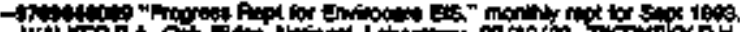

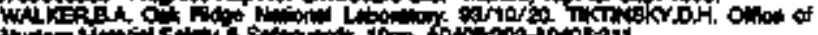

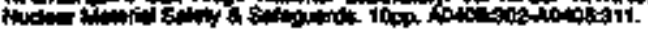

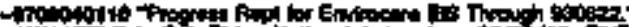

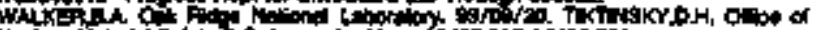

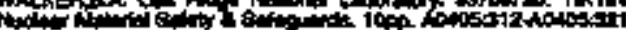

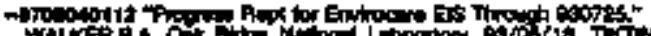

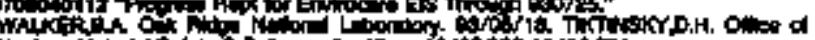

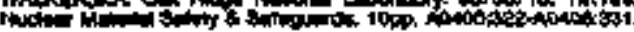

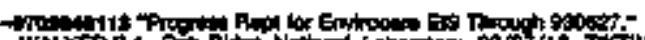

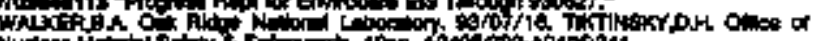

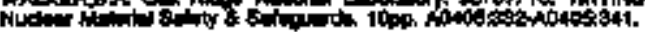

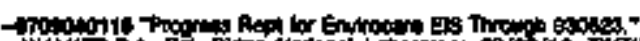
Nutur

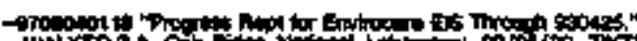

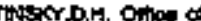

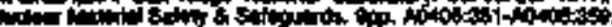

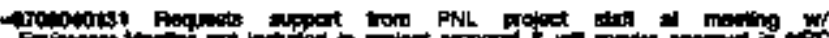

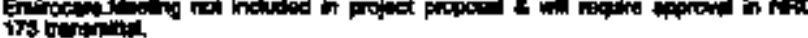

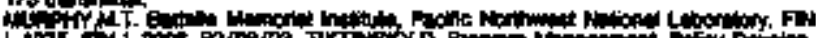

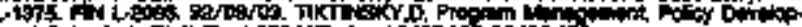

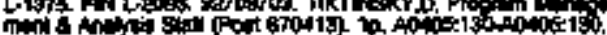

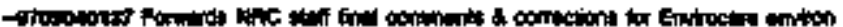

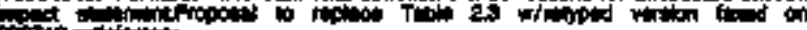

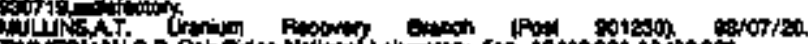

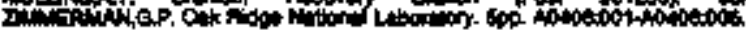

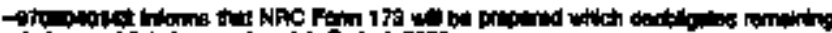

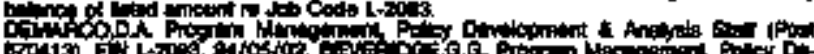

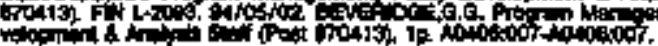

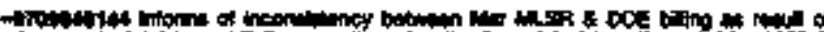

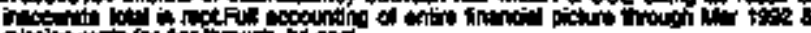

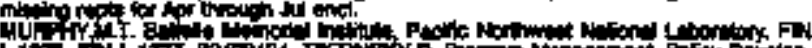

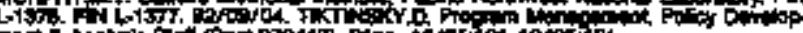

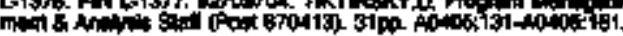

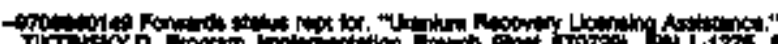

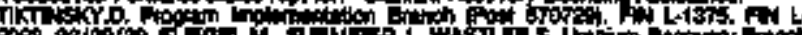

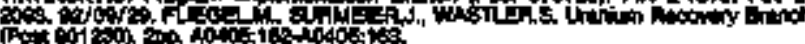

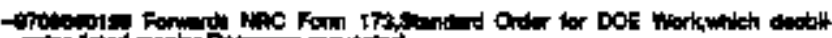

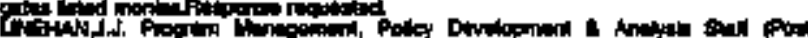

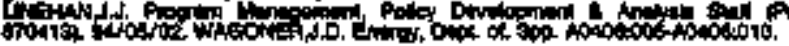

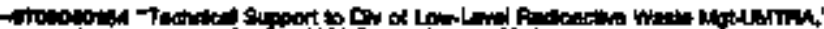
Fonly

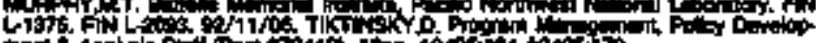

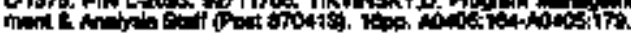

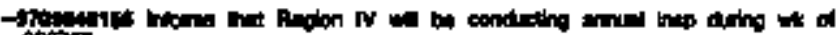

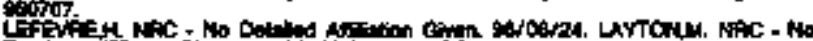

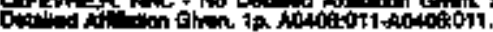

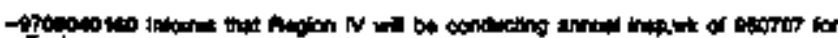

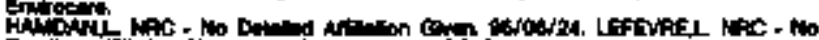

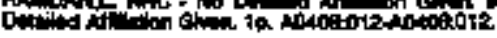

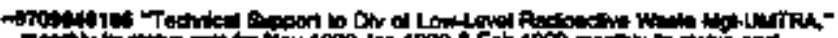

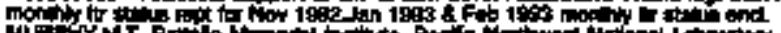
Mif

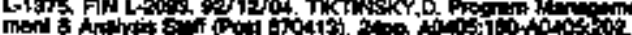

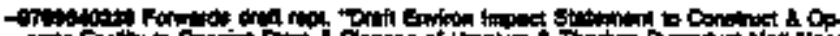

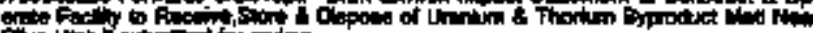

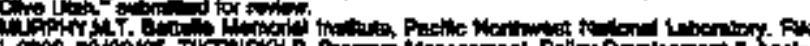

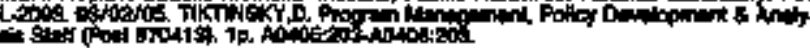

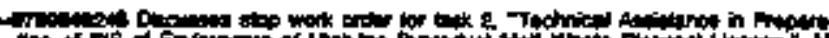

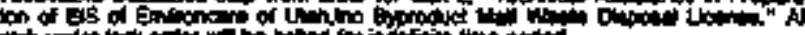

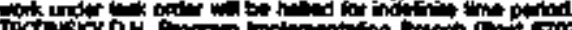

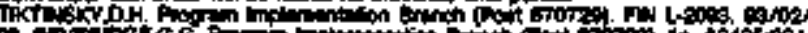
con:

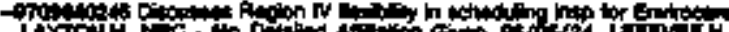

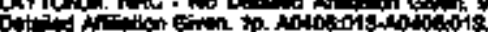

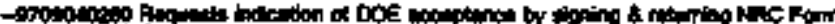

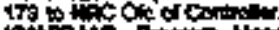

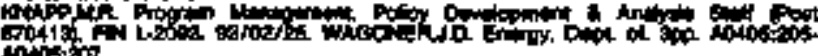
ionstion

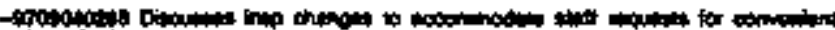

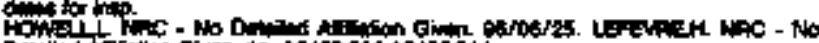

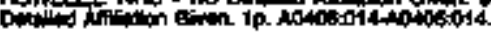

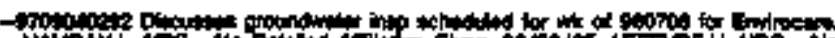

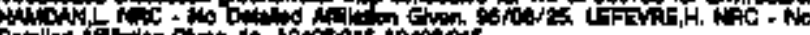

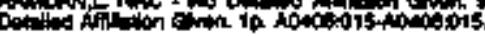

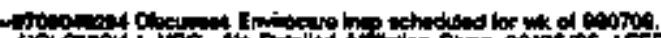

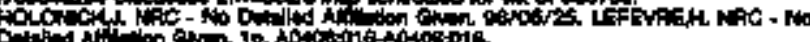

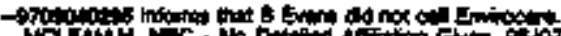

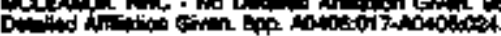

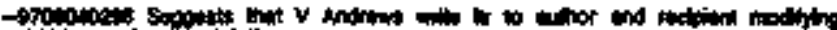

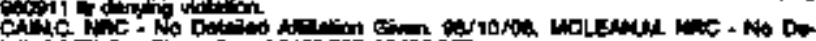

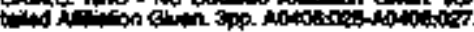

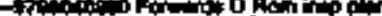

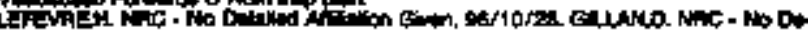

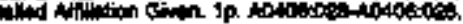

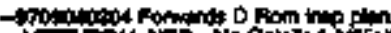

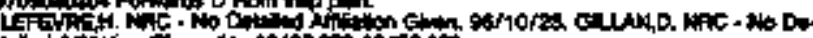

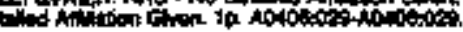

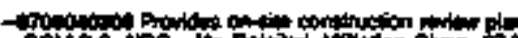

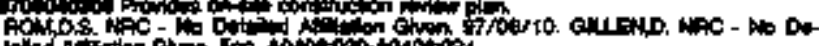

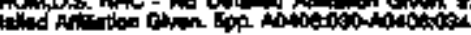

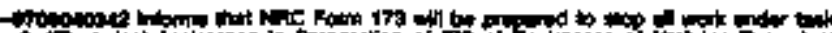

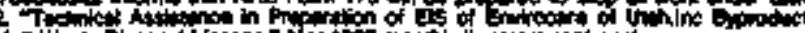

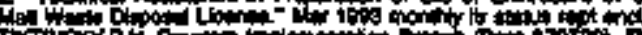

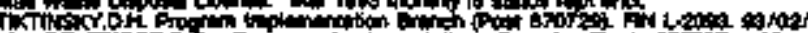

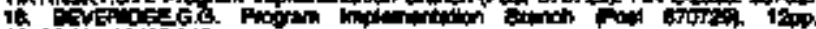

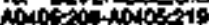

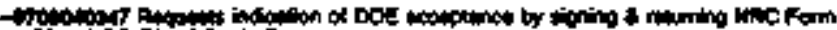

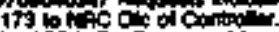

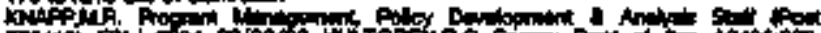

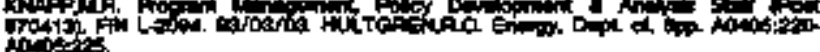

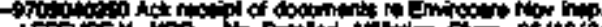

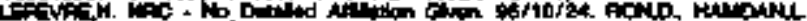

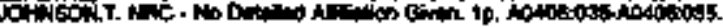

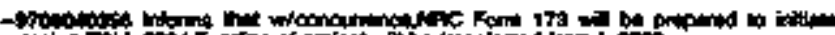

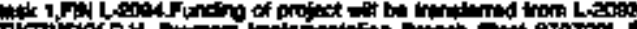

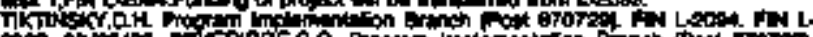

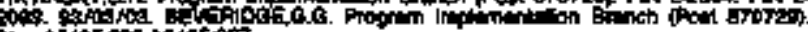

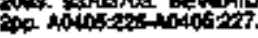

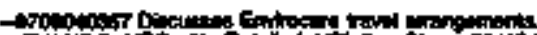

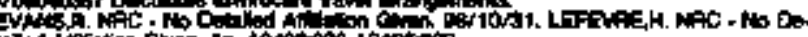
tonosos.

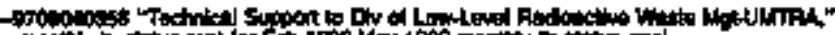

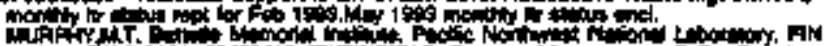

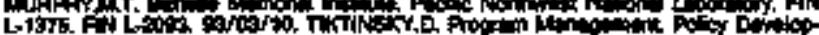

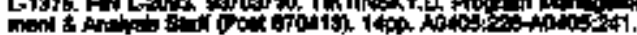

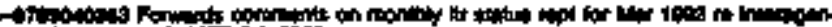
C.

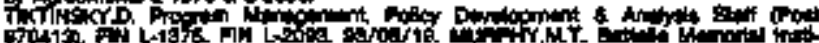

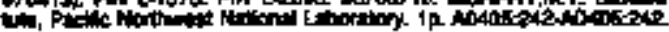

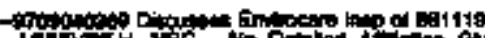

U.P

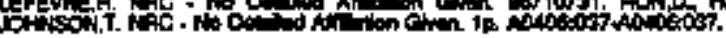

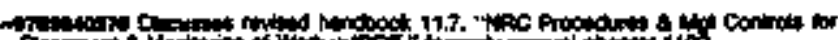

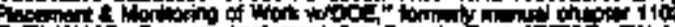

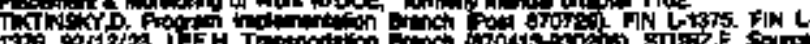

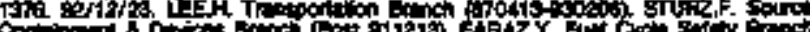

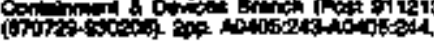




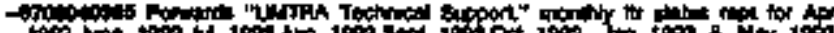

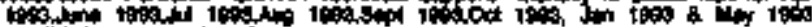

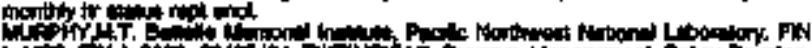

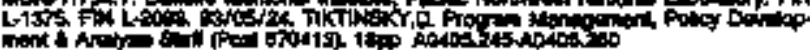

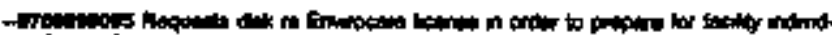

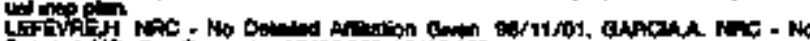

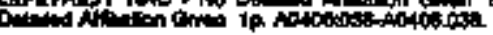

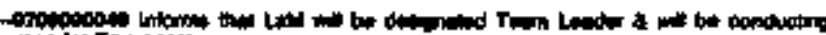

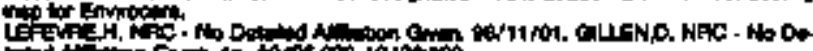

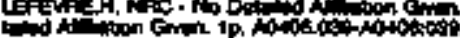

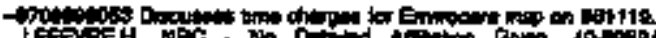

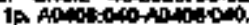

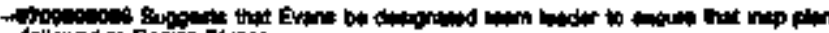
allow

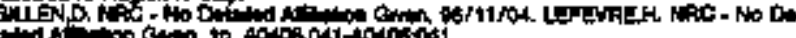

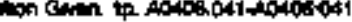

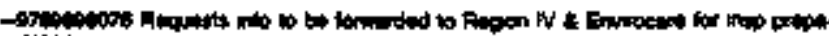

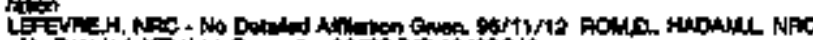

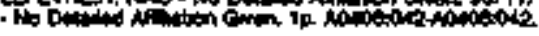

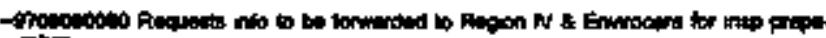

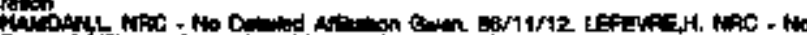

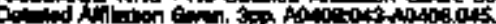

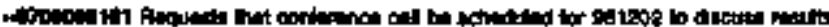
If

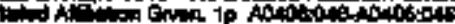

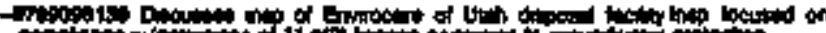

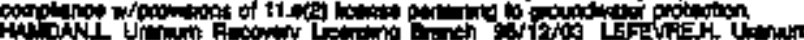

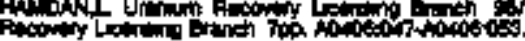

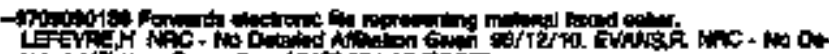

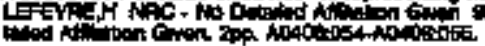

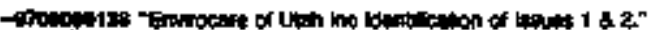

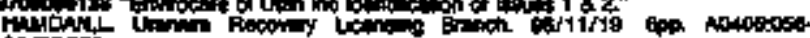

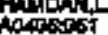

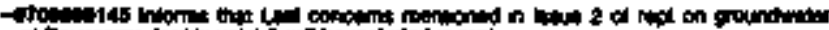

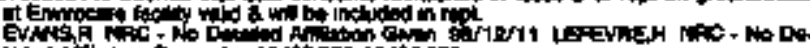

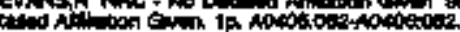

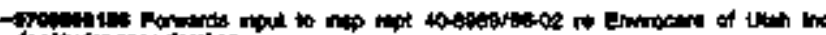

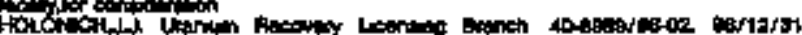

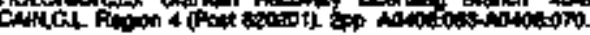

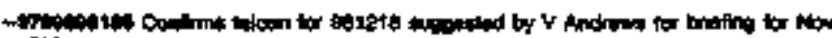

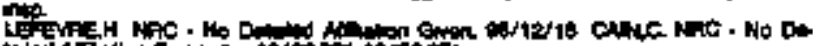

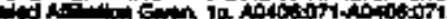

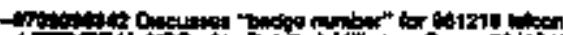

Fin

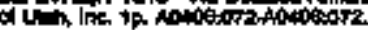

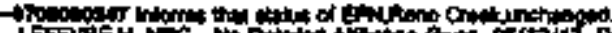

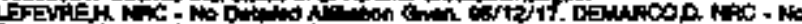

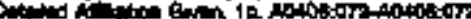

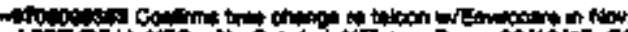

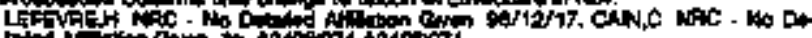

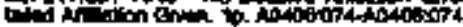

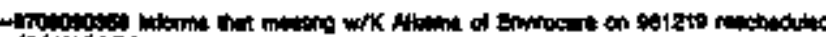

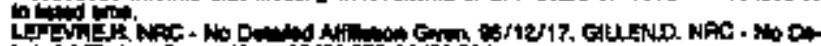

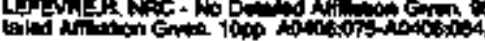

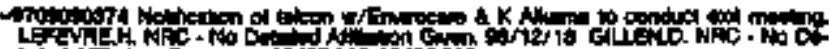

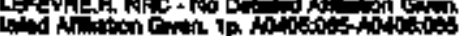

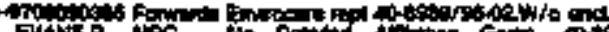

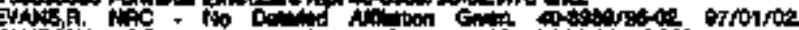

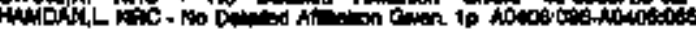

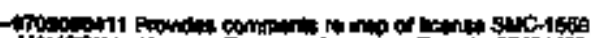

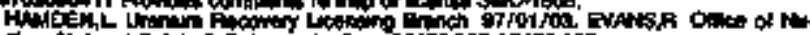

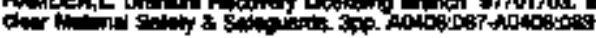

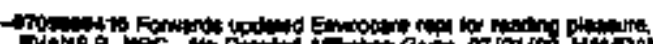

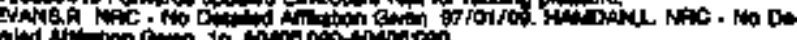

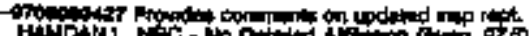

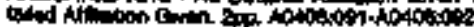

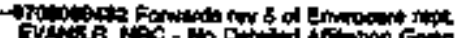

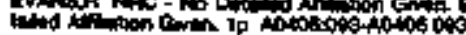

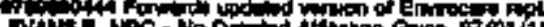

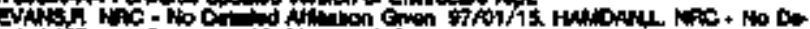

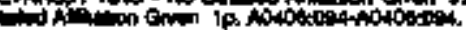

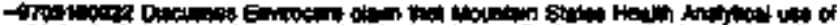

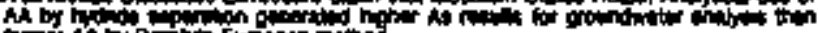

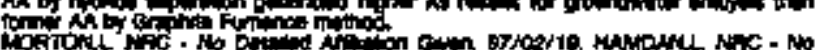

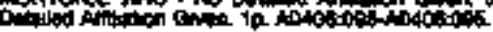

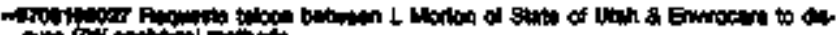

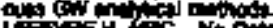

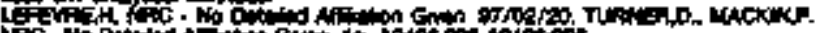

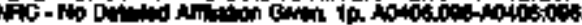

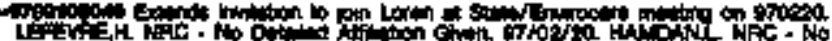

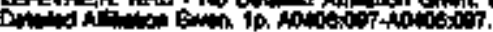

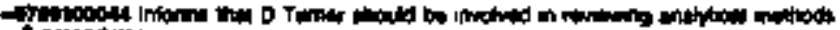

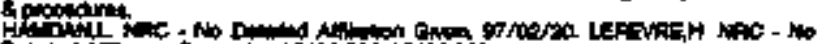

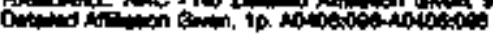

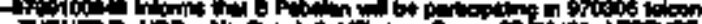

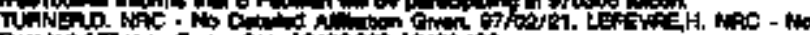

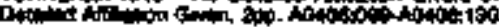

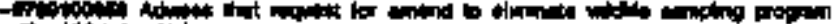

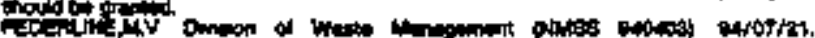

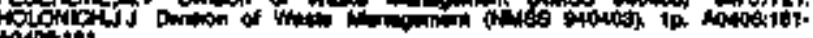
Motitit.

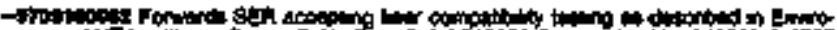
oare of UT,

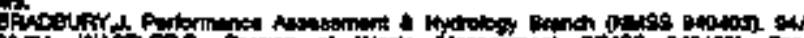

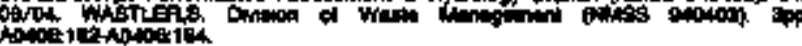

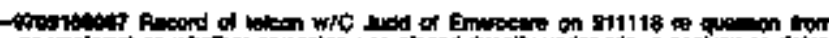

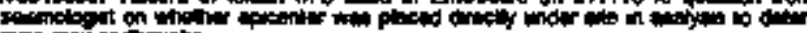

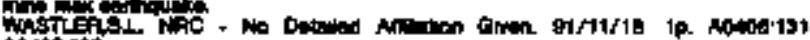
1000. 700 .

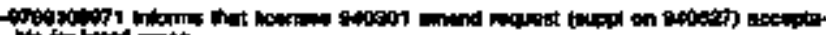

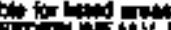

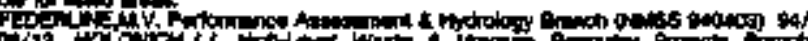

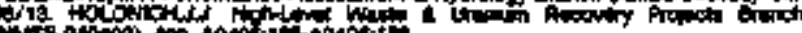

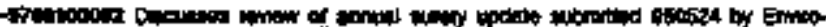

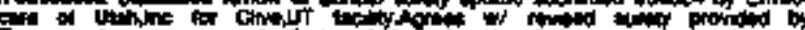

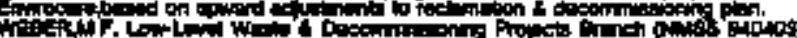

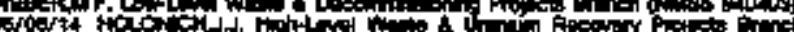

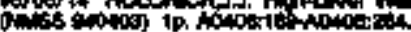

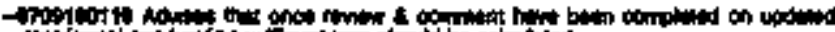

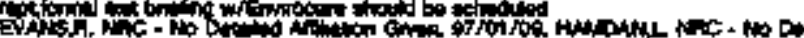

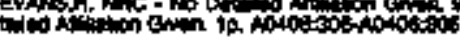

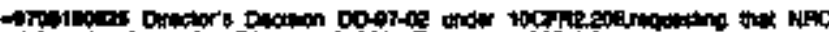

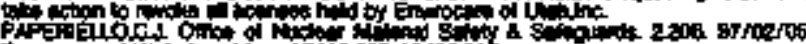

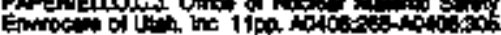

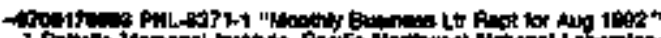

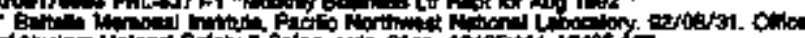

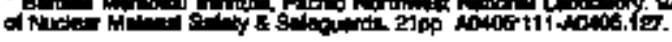

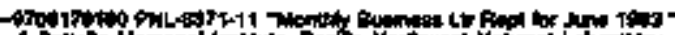

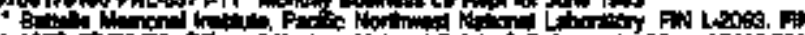

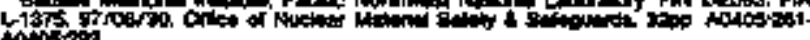
$10+00 \%$

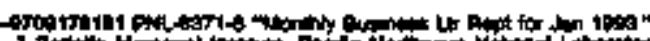

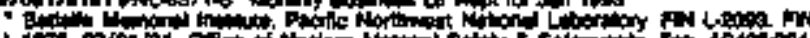

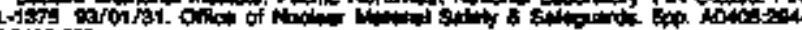

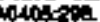

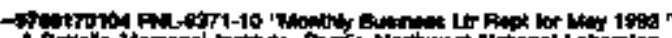

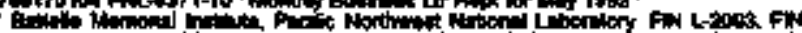

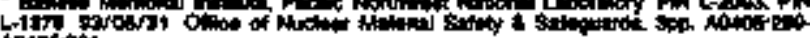
scint.

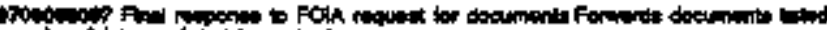
nA

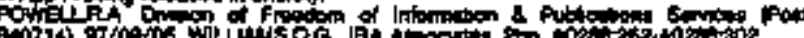

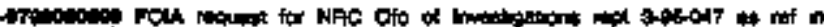
orotos Noy for elint

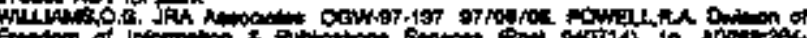
ation.

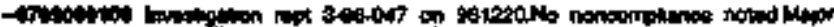

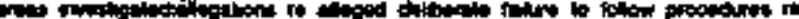

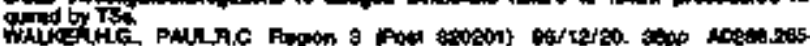

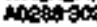




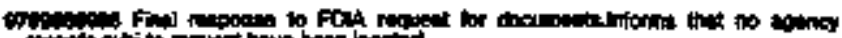

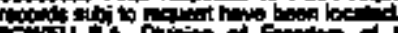

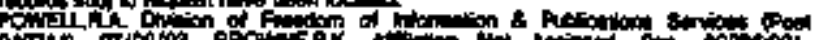

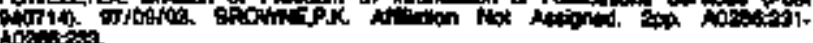
lotions

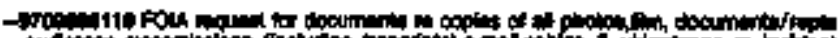

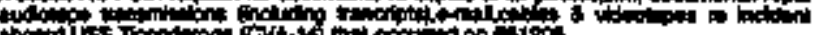

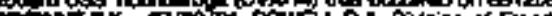

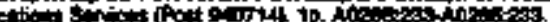

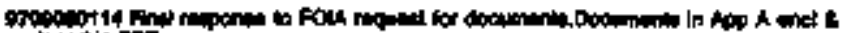

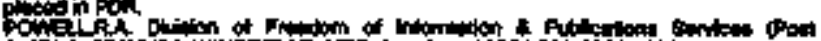

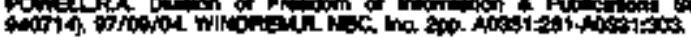

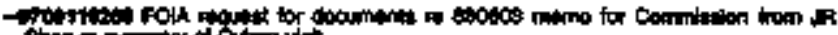

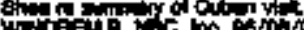

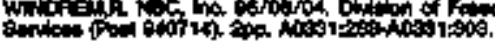

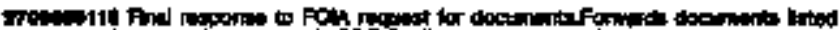

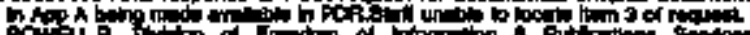
on

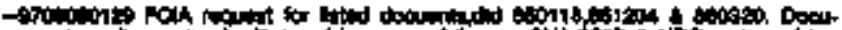

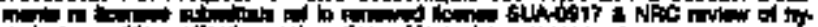

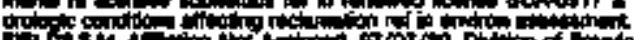

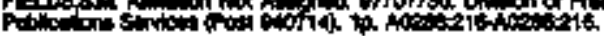

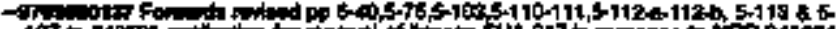

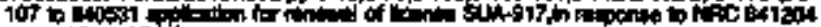

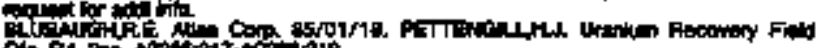

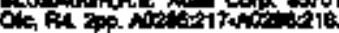

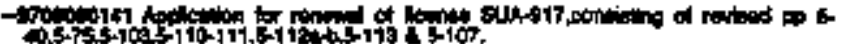

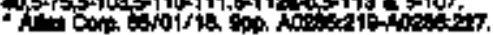

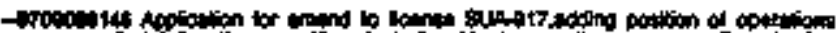

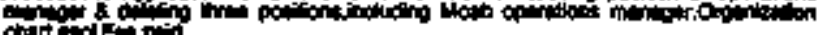

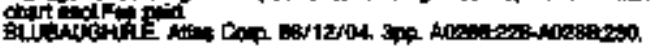

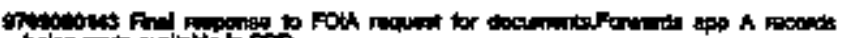

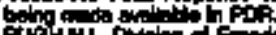

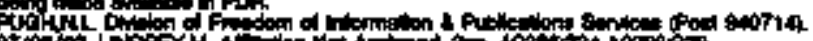

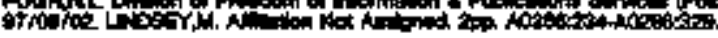

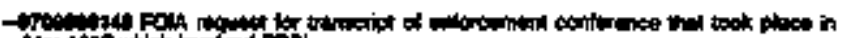

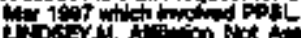

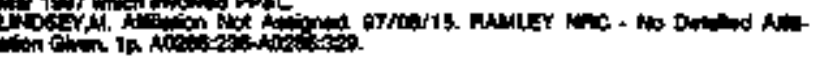

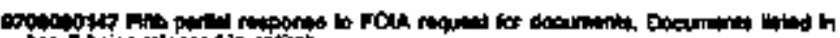

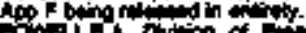

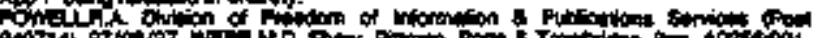

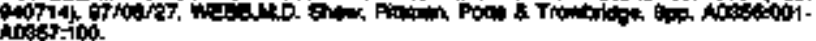

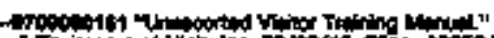

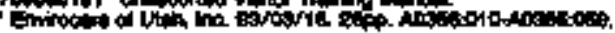

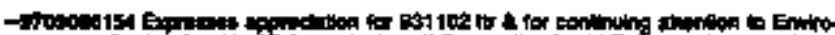

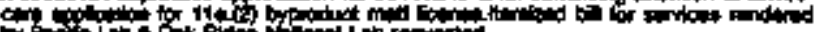

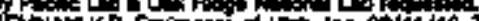

ginN

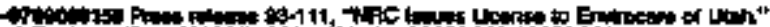

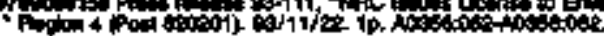

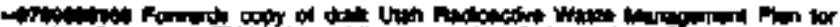

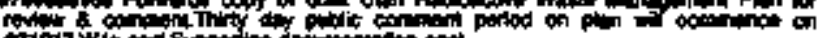

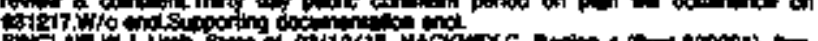

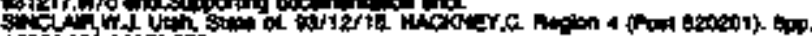

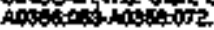

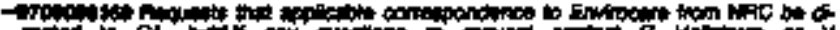

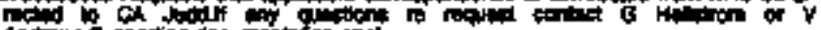

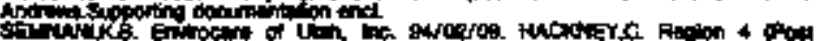
S

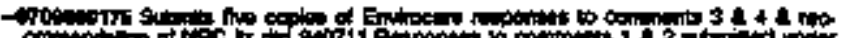

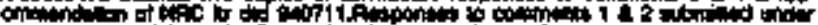

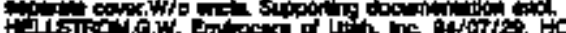

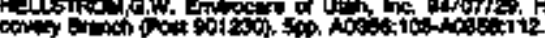

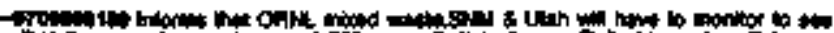

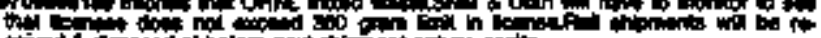

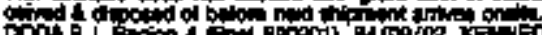
Fogit.j.

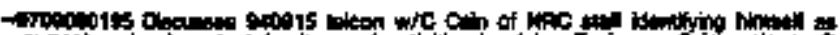

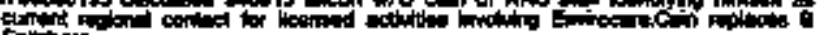

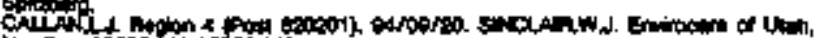

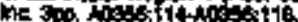

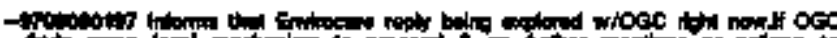

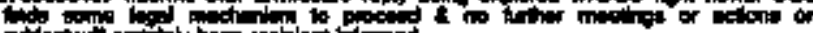

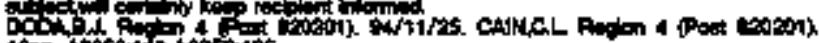

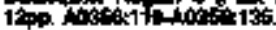

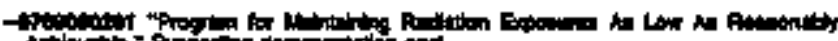

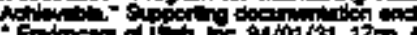

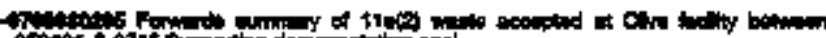

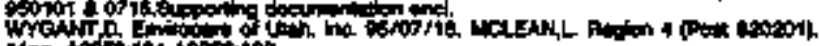

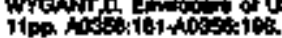

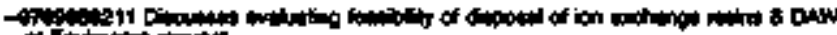

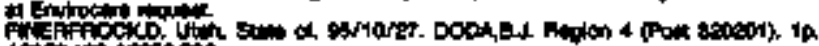
ind

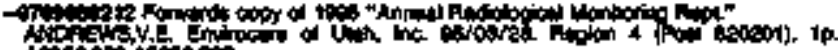

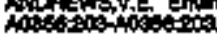

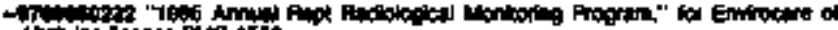

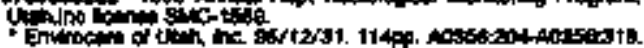

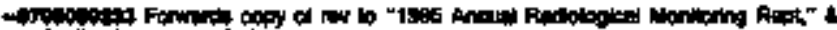

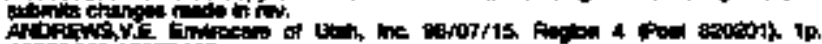

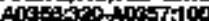

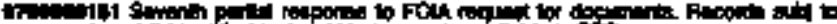

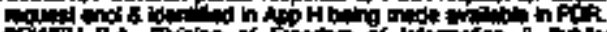

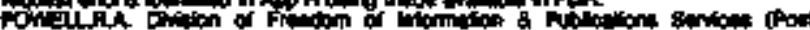

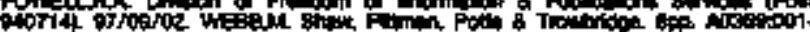
1009068.

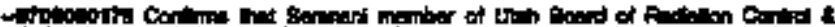

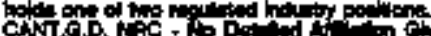

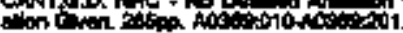

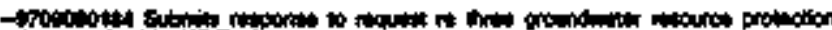

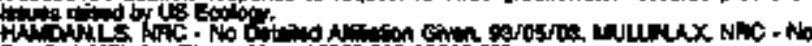

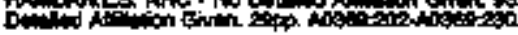

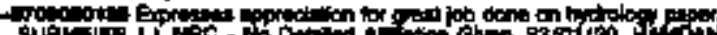
SuAp

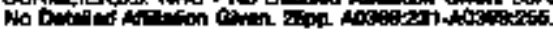

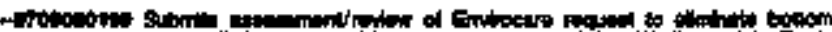

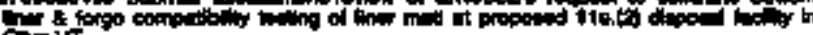

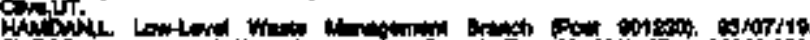

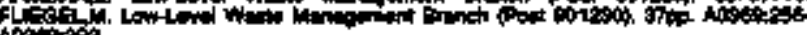
A $00.0 \%$

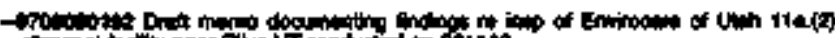

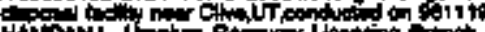

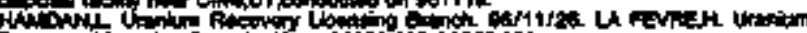

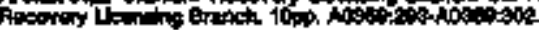

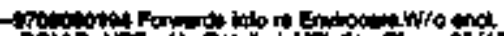

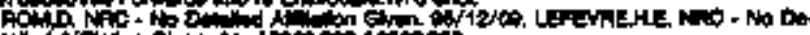
Af

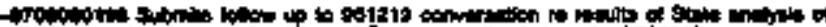

U

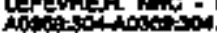

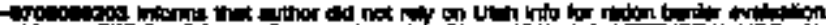

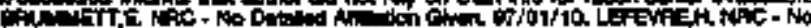

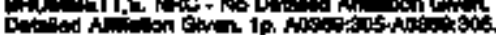

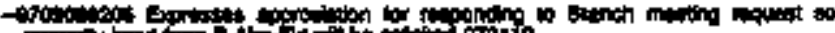

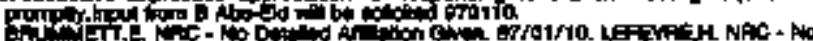

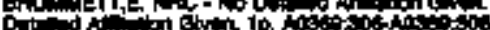

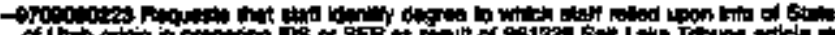

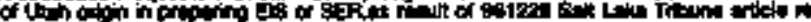

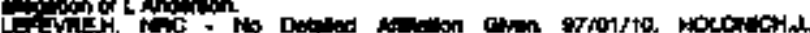

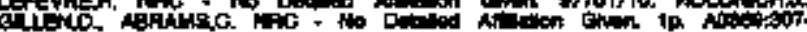
Anstions.

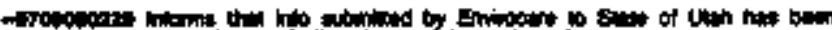

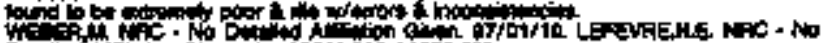

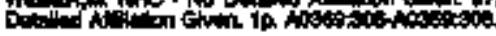

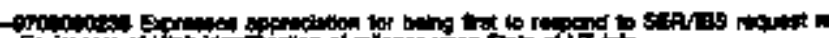

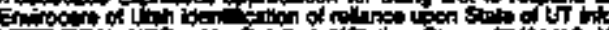

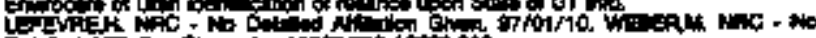

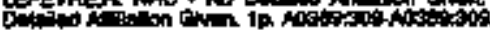

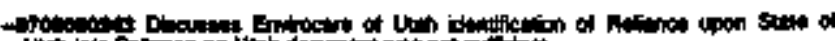

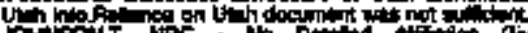

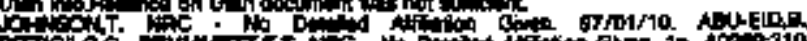
Auts 
4

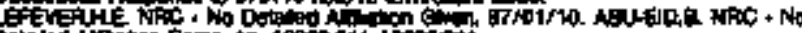

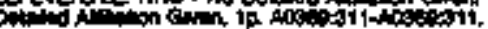

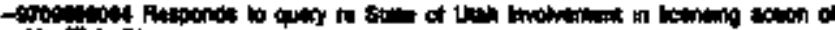

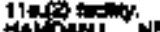

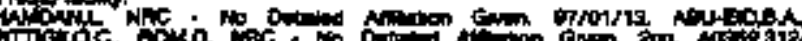

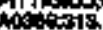

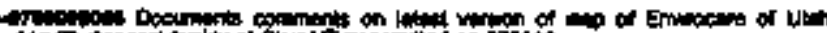

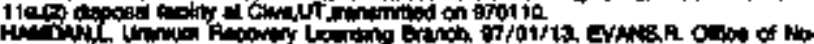

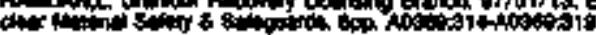

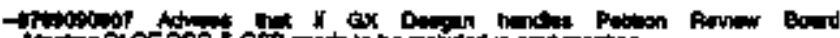

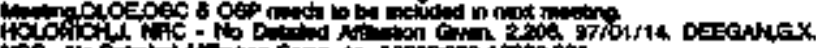

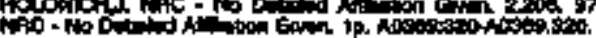

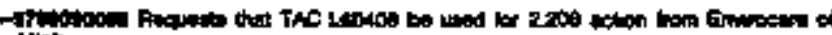

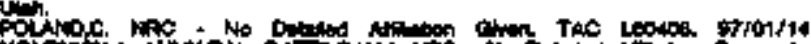

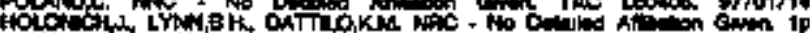

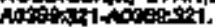

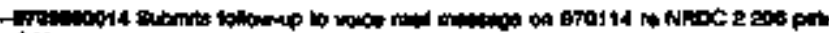

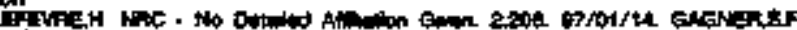

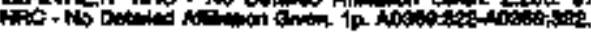

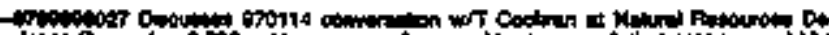

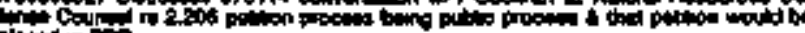
Ping

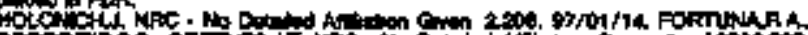

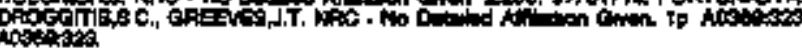

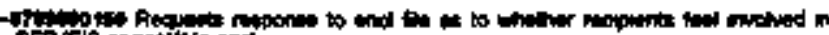
SAacks or netifio and

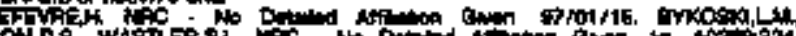
10.4.

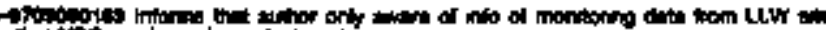

WhSThes

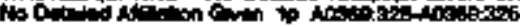

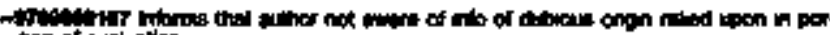

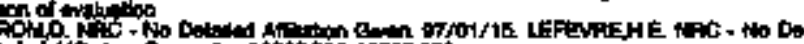

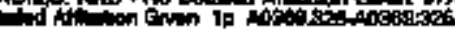

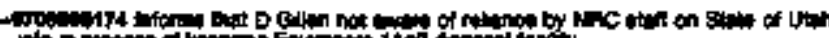

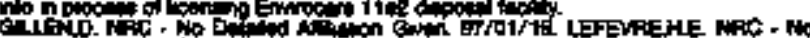

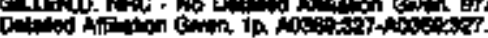

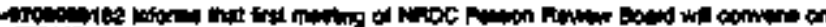
Brati?

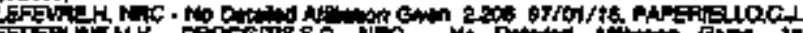

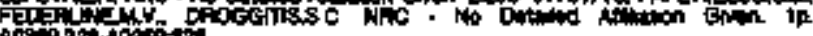

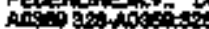

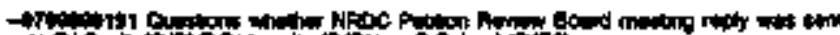

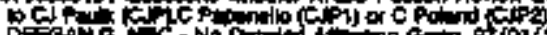

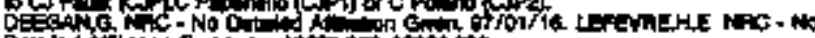

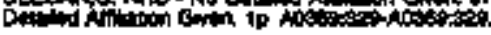

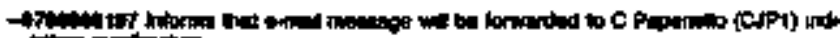

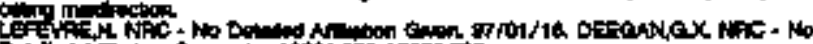

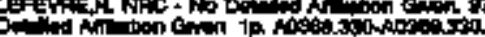

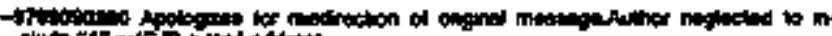

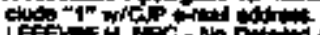

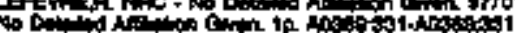

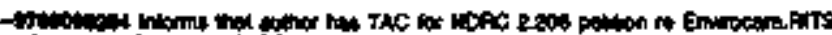

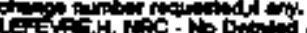

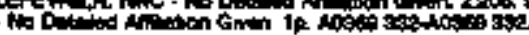

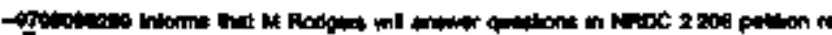

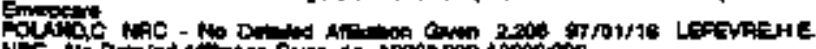

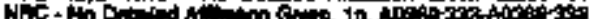

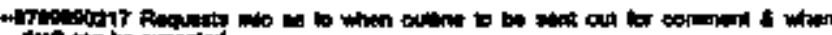

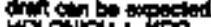

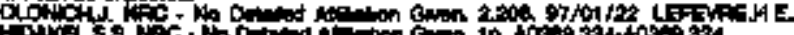

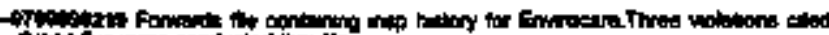

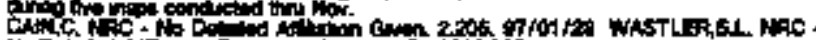

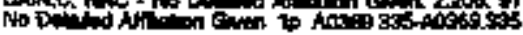

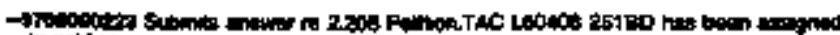
$\tan$

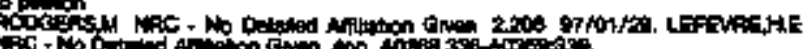

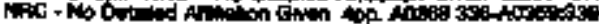

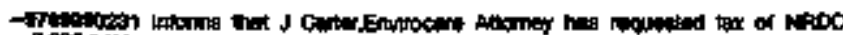
L

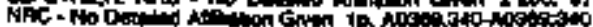

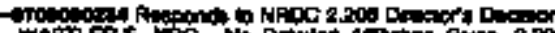

Whis

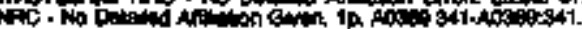

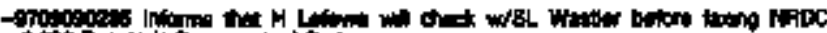

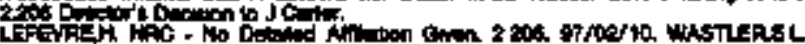

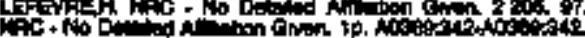

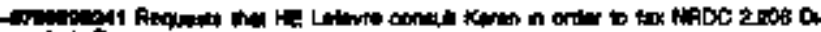

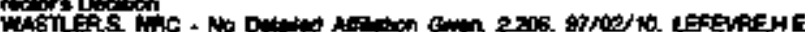

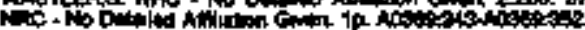

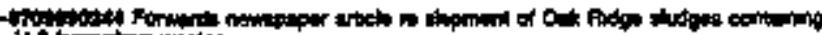

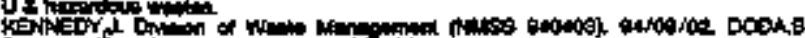

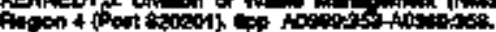

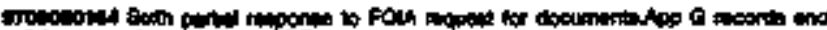

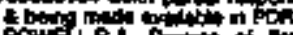

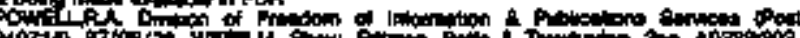

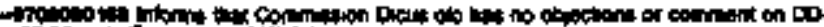

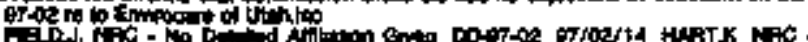

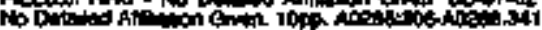

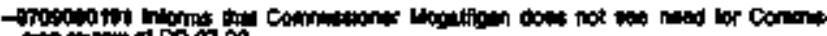

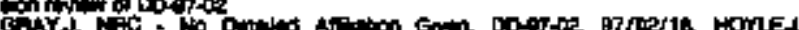

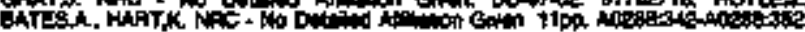

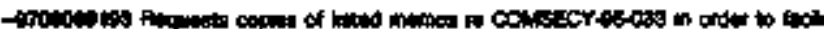

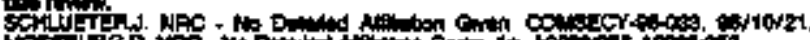

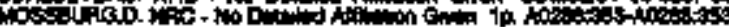

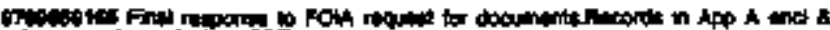

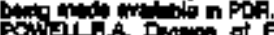

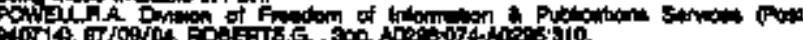

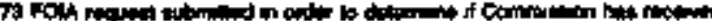

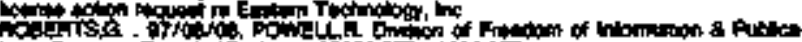

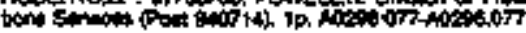

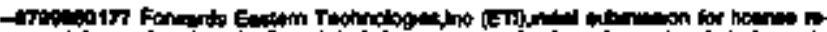

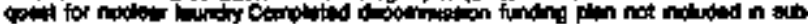

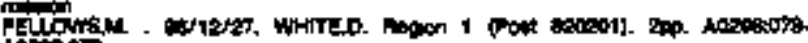
ation

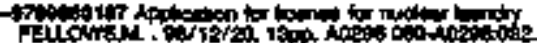

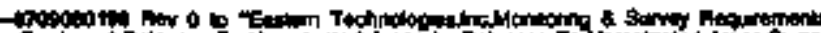

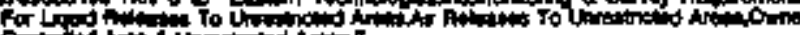

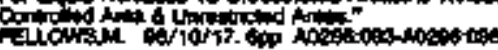

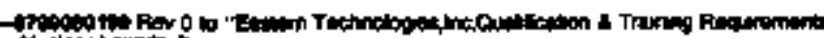

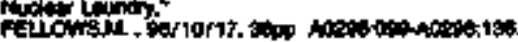

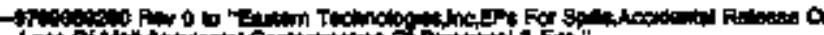

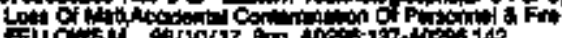

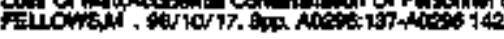

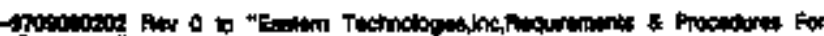

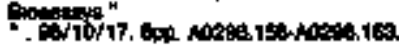

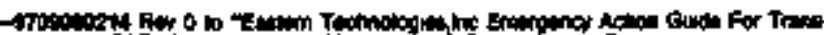

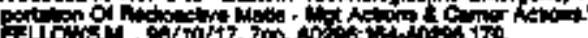

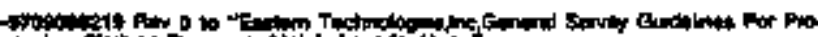

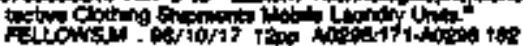

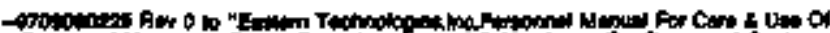

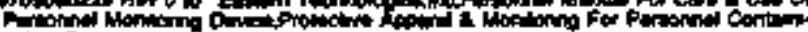
fition"

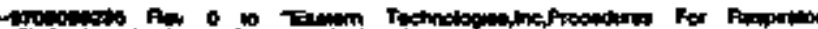
Ftow:

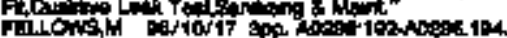

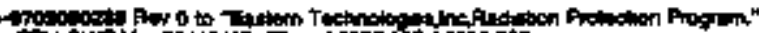

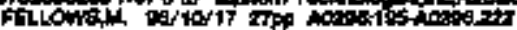

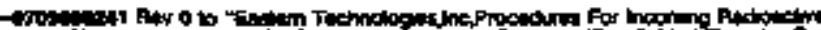

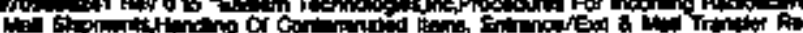

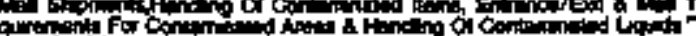

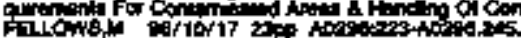

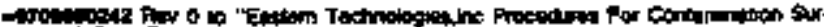

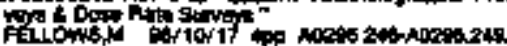




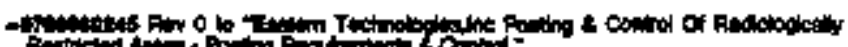

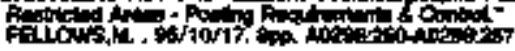

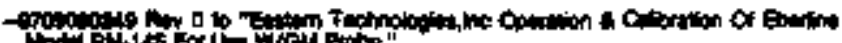

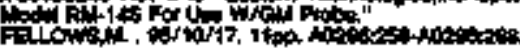

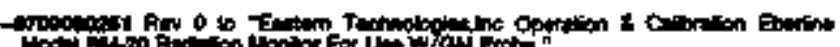

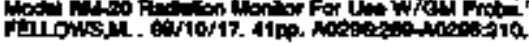

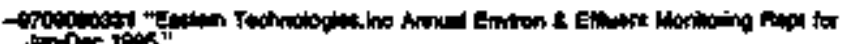

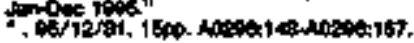

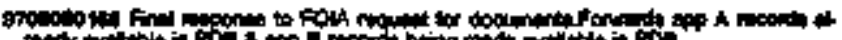

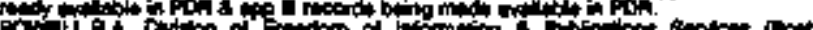

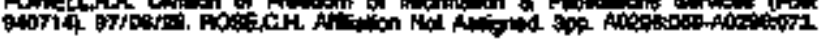

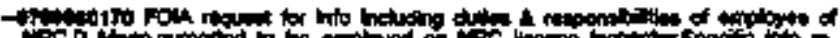

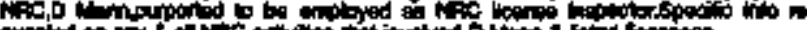

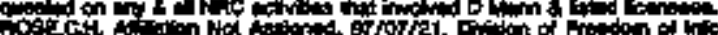

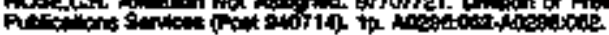

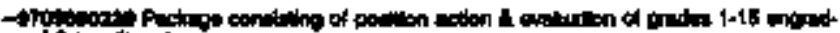

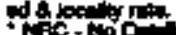

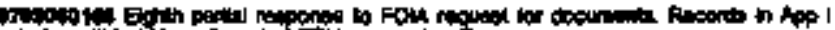

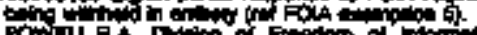

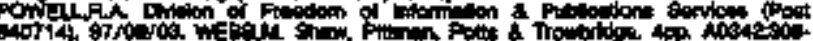
Ansizis.

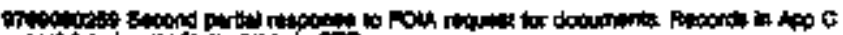

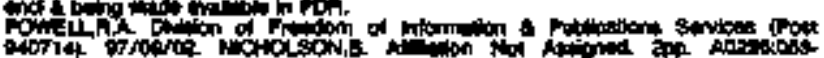
iogotisin

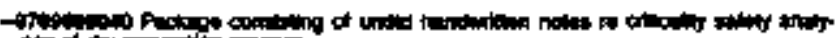

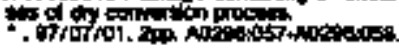

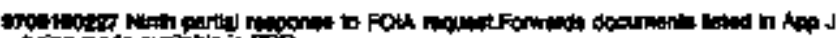

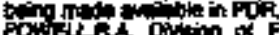

Pow

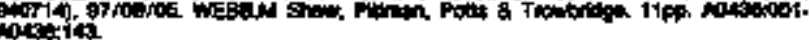

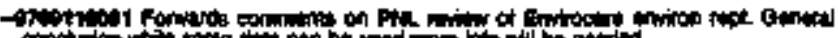

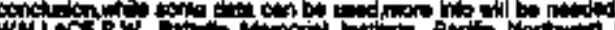

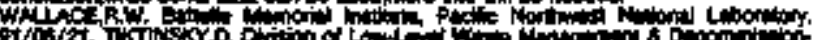

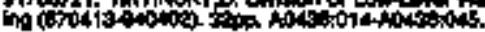

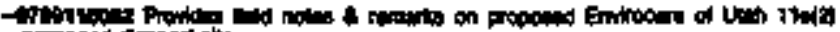

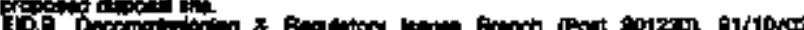

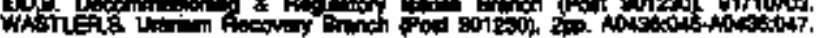

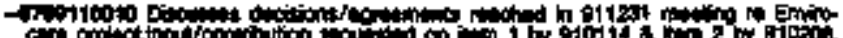

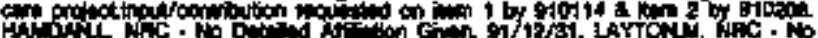

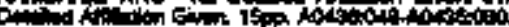

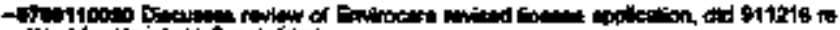

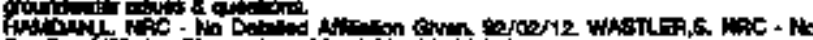

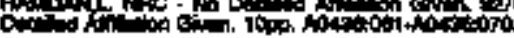

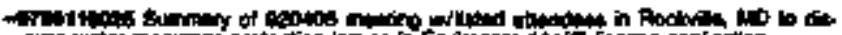

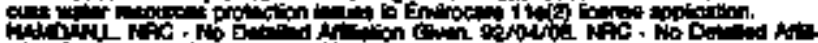

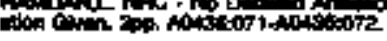

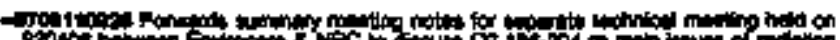

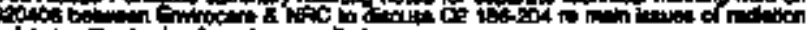

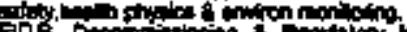

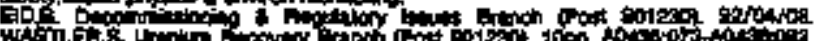

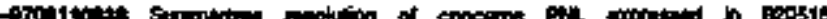

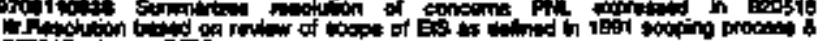

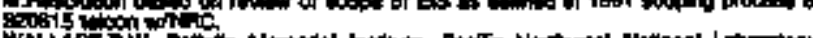

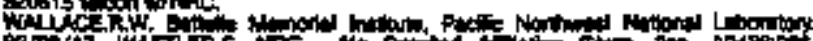

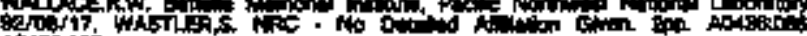
axton.

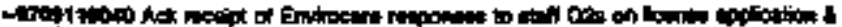
whinger.

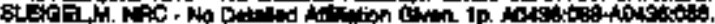

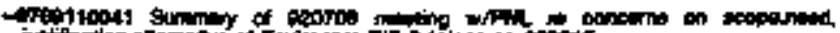

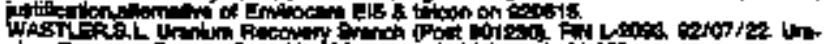

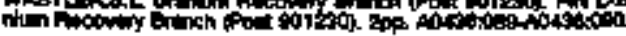

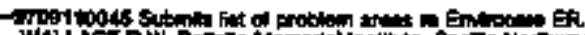

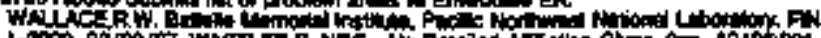

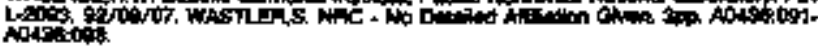

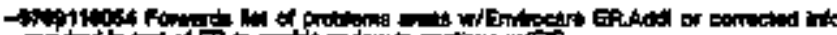

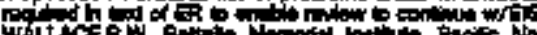

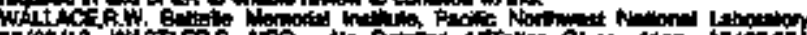
grody itom

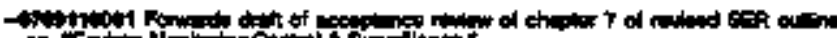

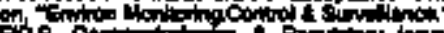

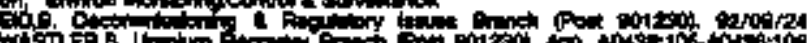

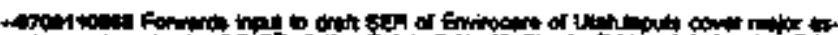

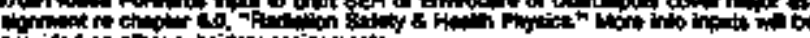

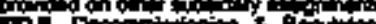

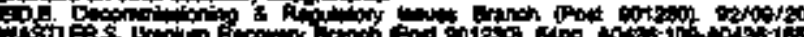

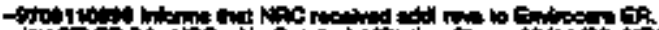

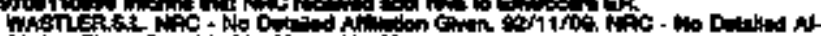

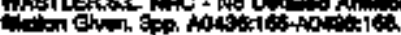

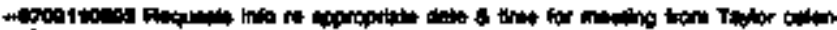

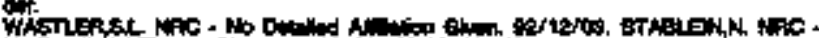

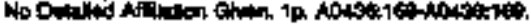

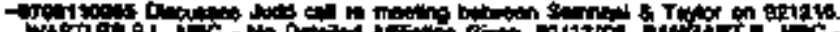

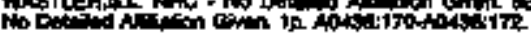

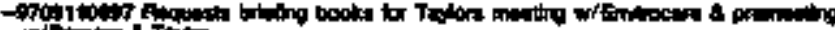

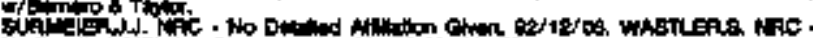

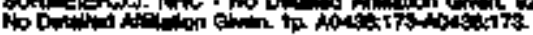

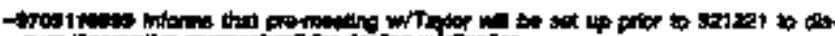

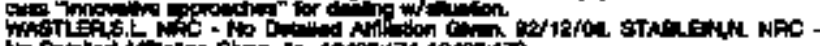

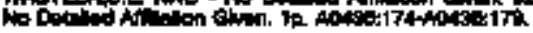

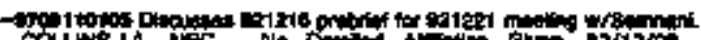

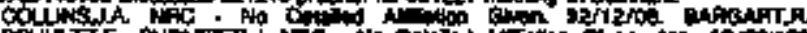

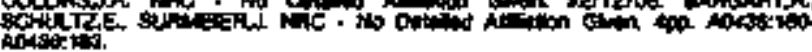

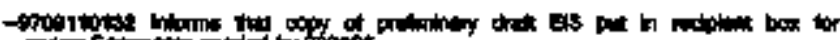

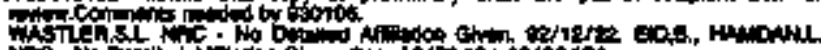

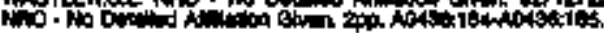

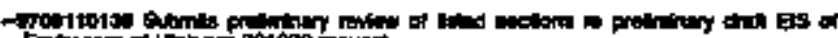

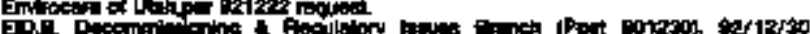

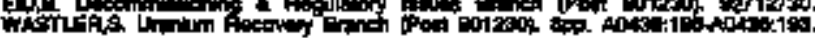

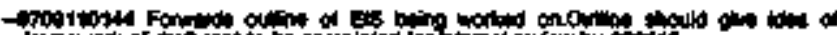

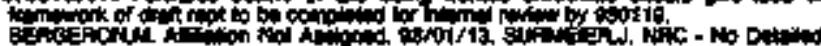

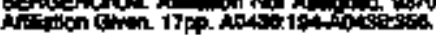

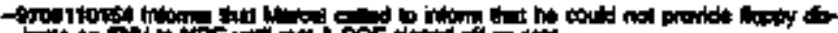

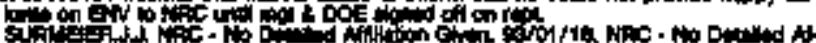

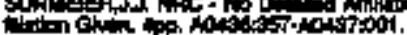

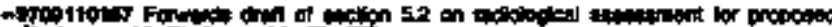

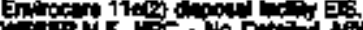

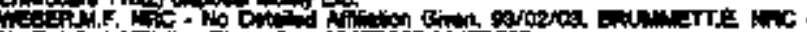

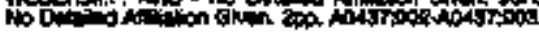

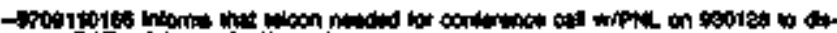

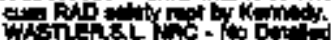

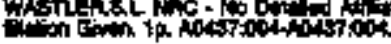

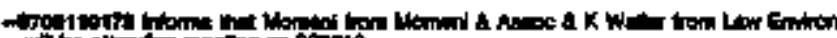

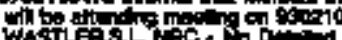

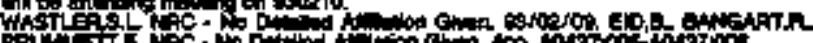

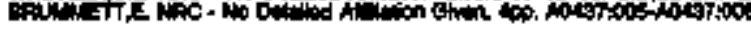

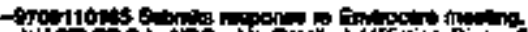

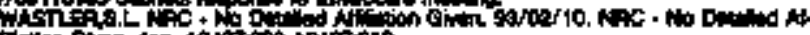

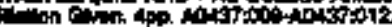

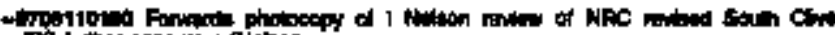

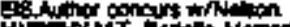

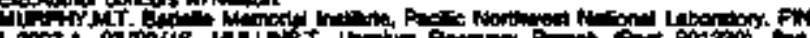
10437s ois

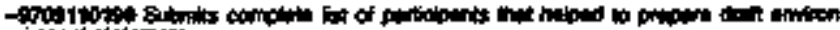

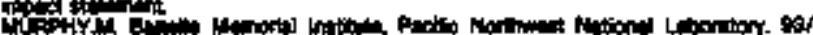

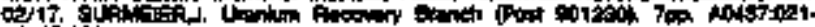

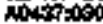

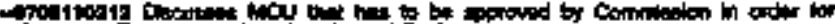

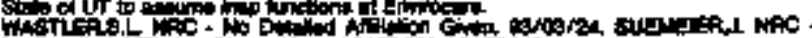

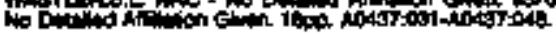

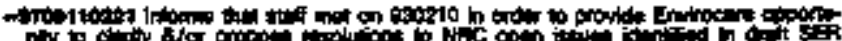

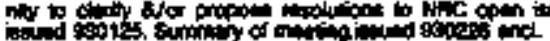

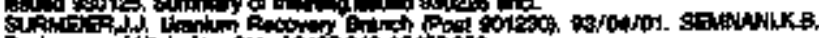

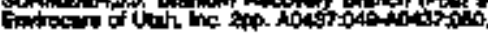




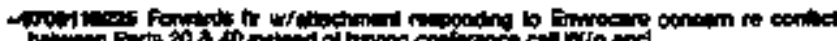

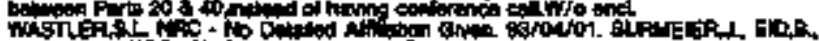

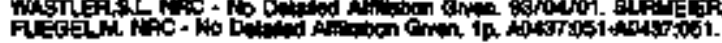

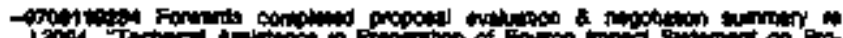

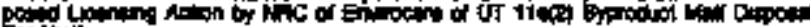
PH:

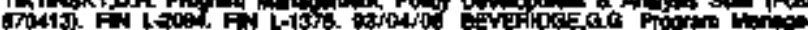

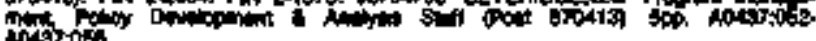

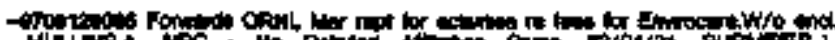

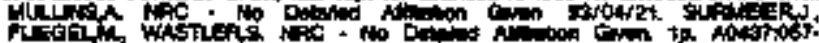

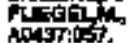

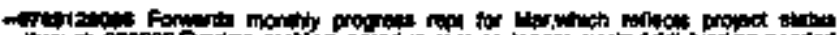

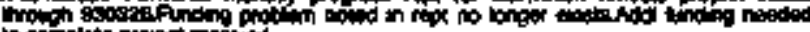

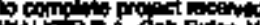

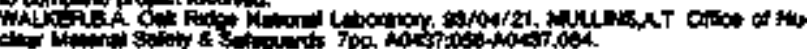

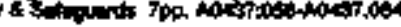

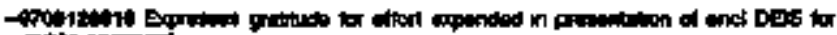

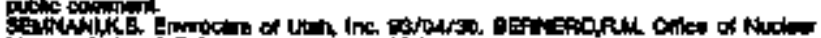

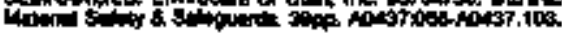

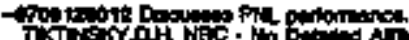

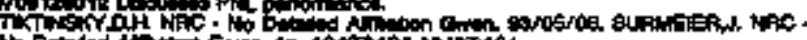

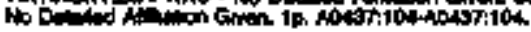

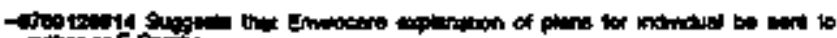

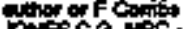

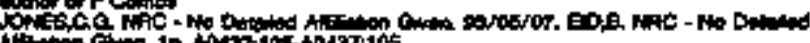

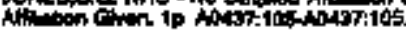

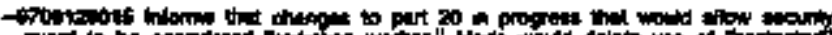

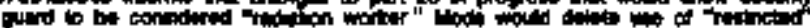

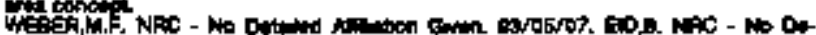

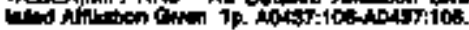

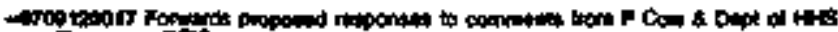
on Finmotic CEes

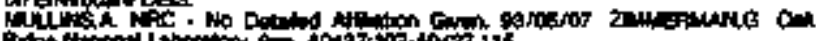

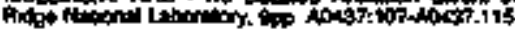

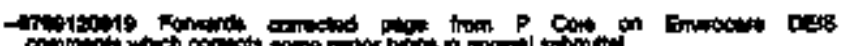

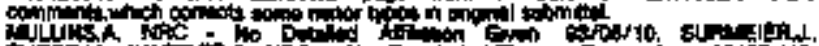

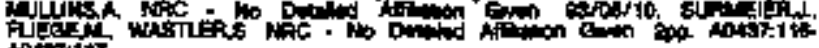
absis:it?

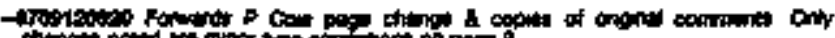

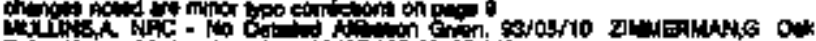

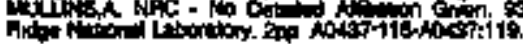

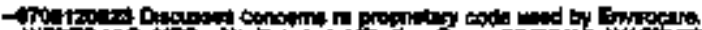

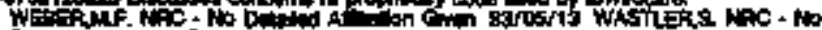

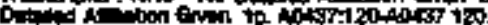

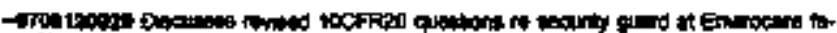

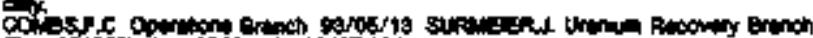

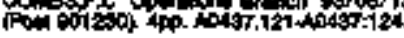

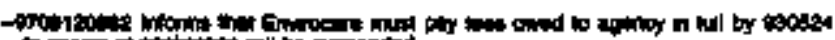
Whint of Wo DT

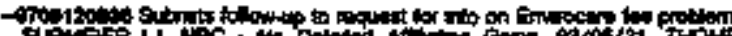
E NHC - Ko Dot

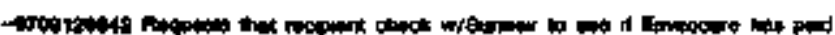

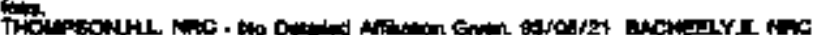

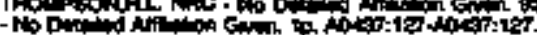

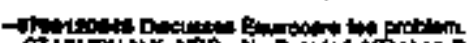

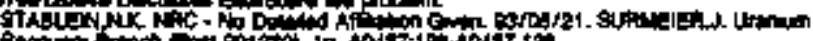

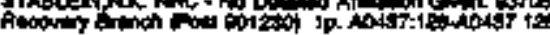

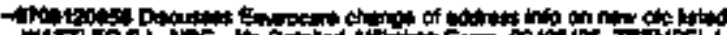

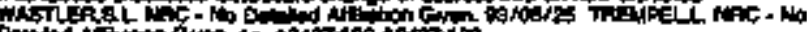

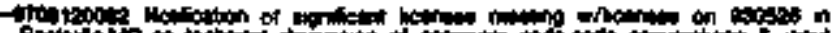

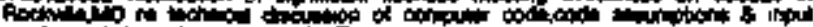

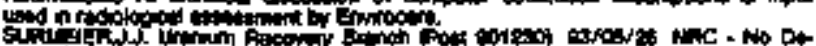

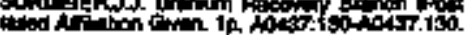

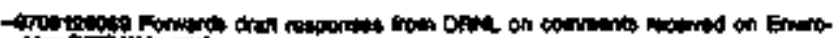

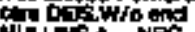

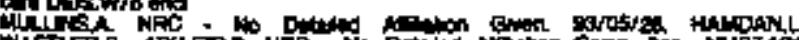

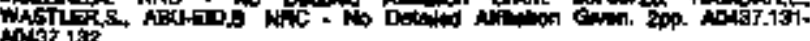

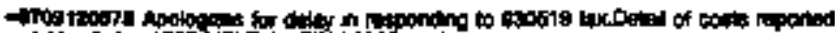

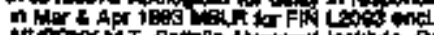

Hifrir Fip

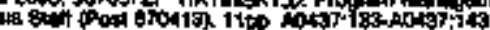

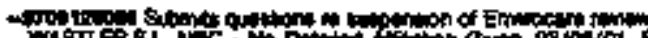
GMTARTR MAC

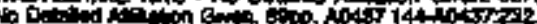

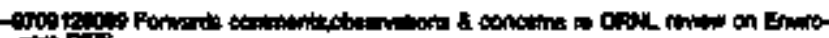

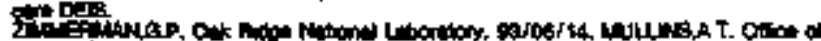

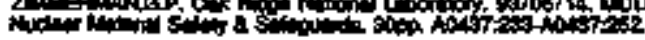

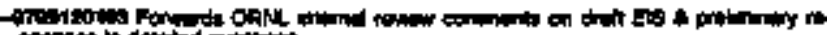

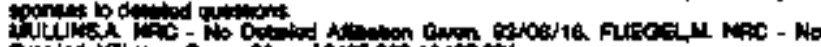

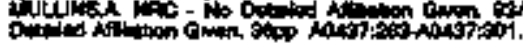

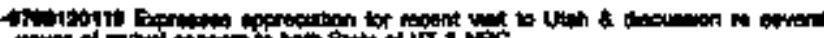

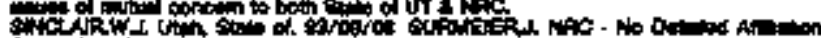

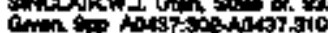

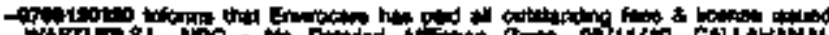

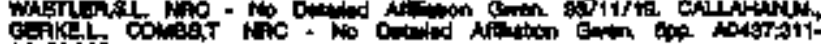

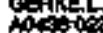

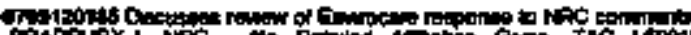

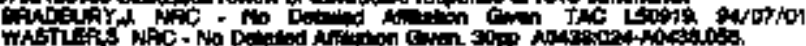

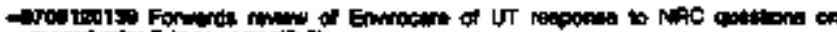

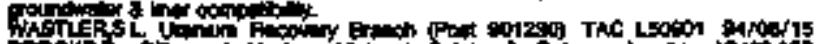

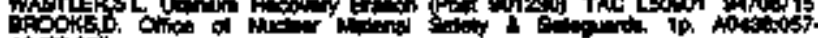

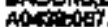

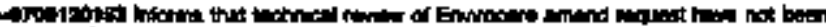

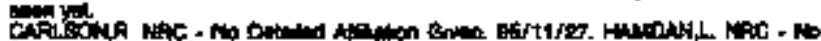

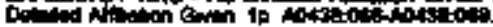

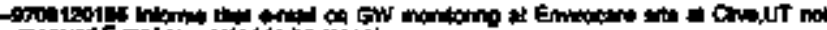

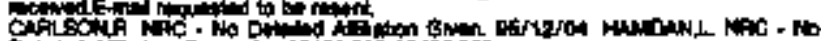

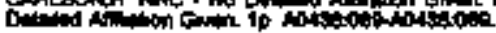

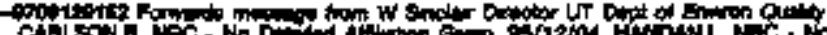

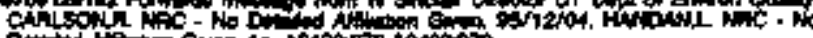

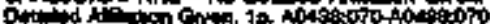

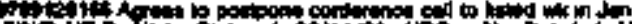

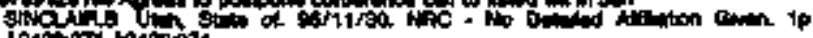
costortinationi.

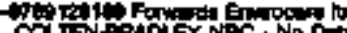

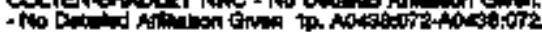

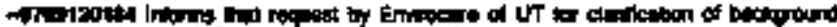

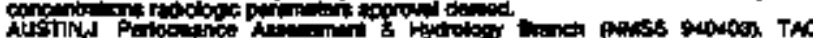

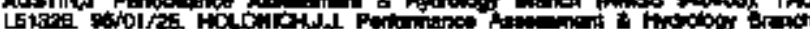

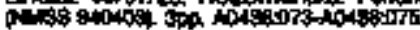

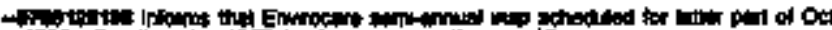

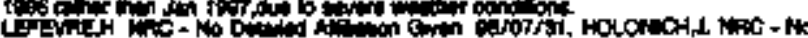

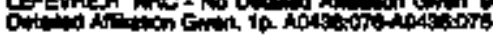

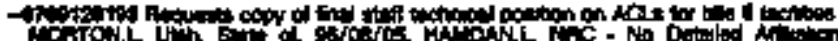

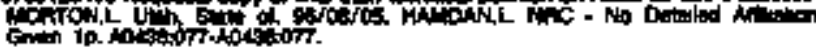

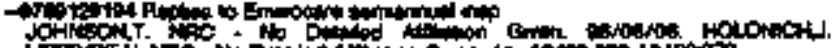
i

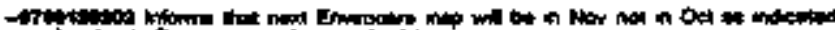

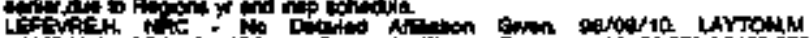

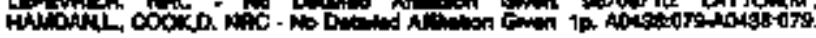

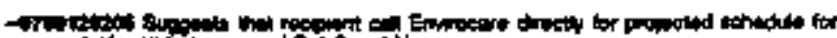

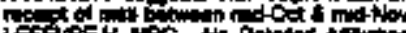

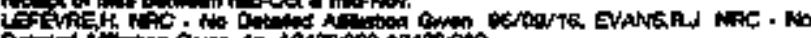

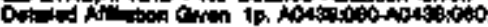

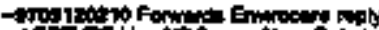

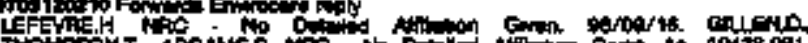

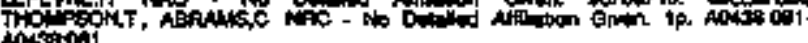

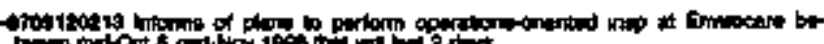

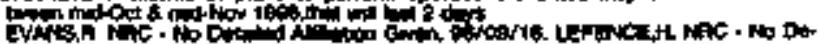

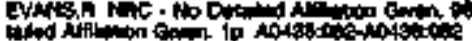

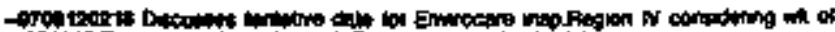

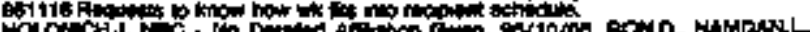

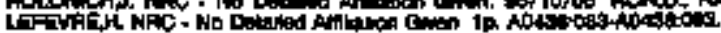

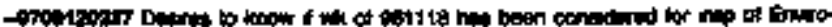
Ut

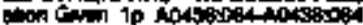




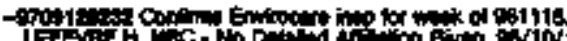

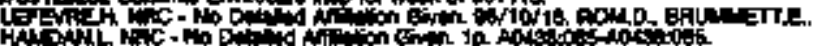

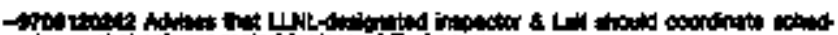

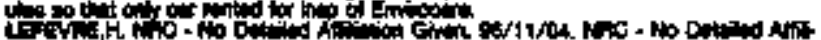

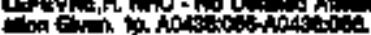

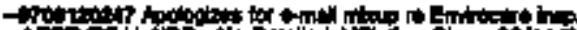

Q H

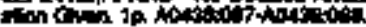

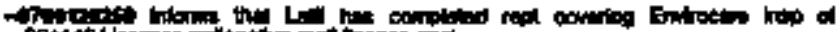
$8911+10$ la

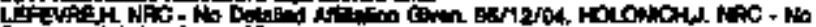

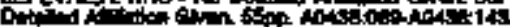

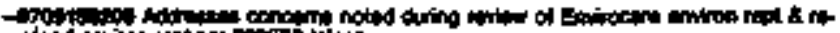

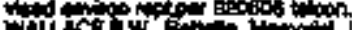

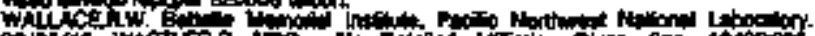

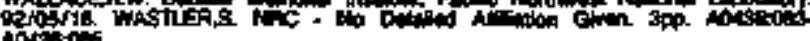
Notiones

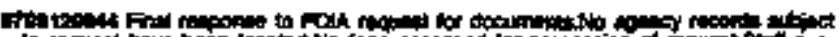

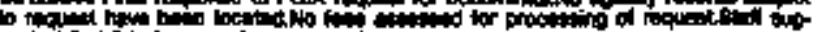
8.

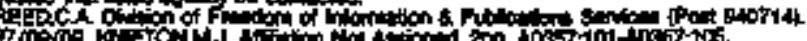

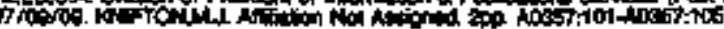

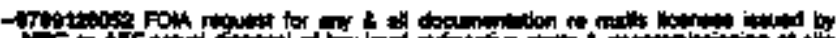
C. N M.

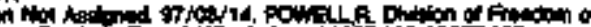

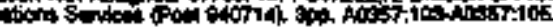

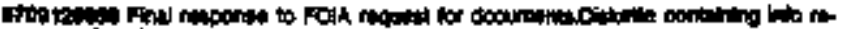

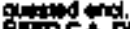

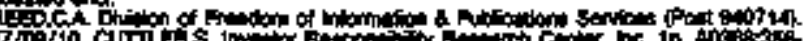
troter

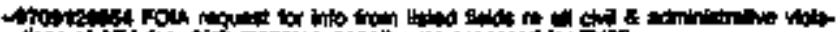
bont of A

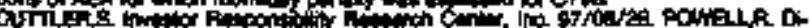

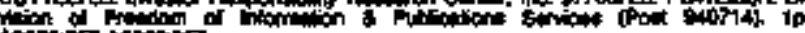

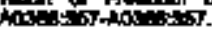

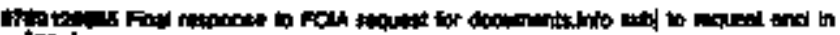
An 1

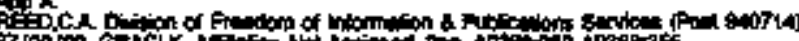

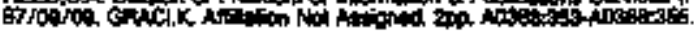

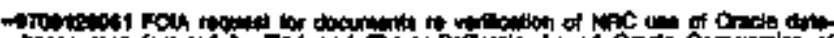

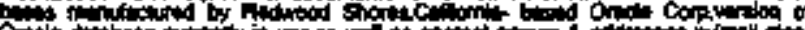

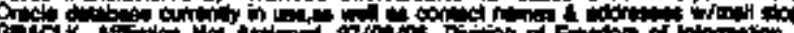

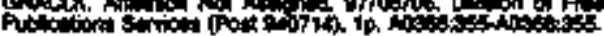

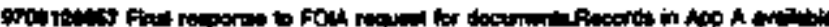

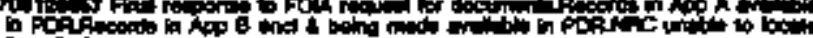

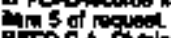

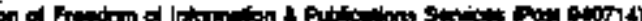

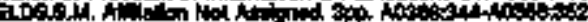

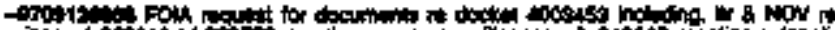

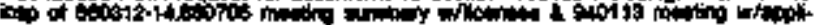

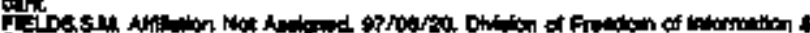

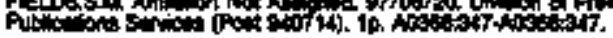

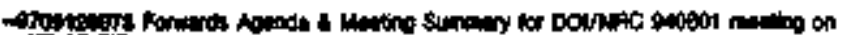

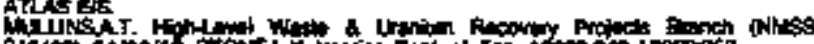

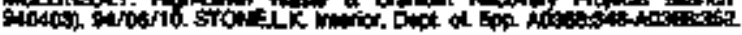

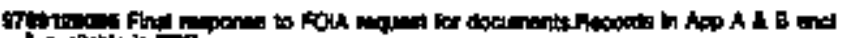

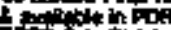

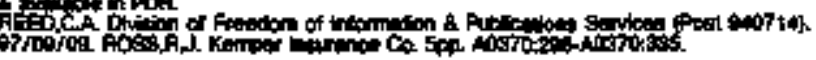

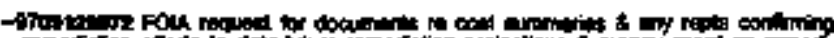

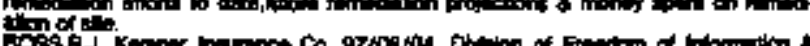

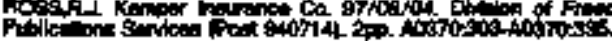

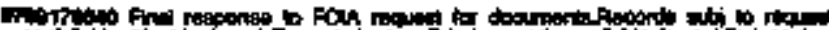

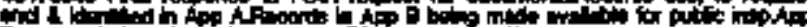

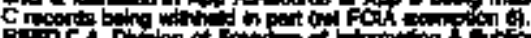

F

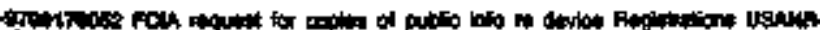

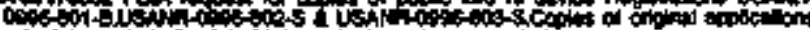

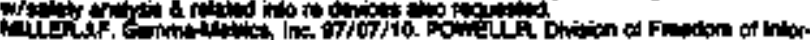

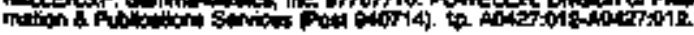

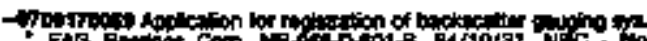

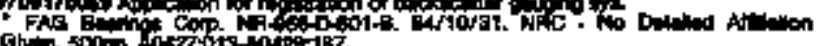

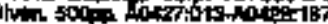

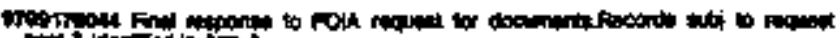

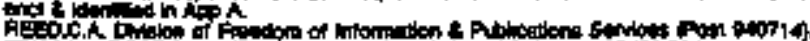

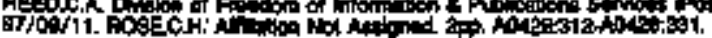

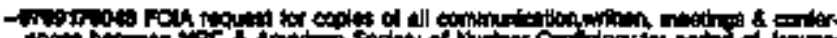

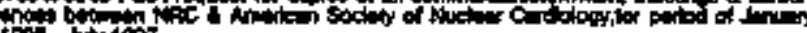

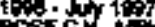

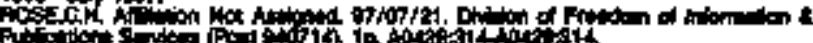

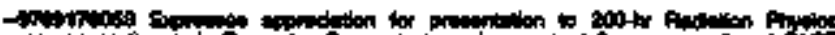

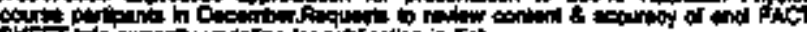

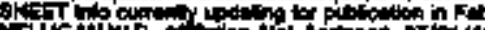

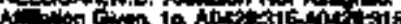

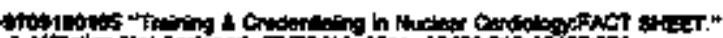

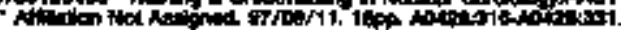

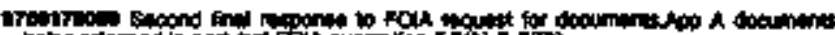

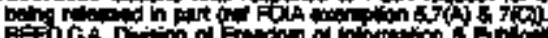

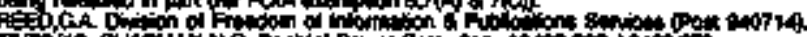

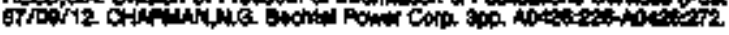

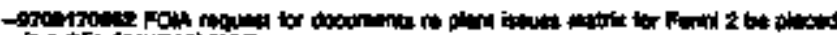

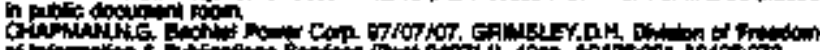

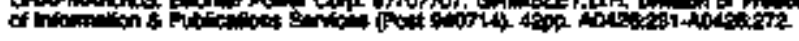

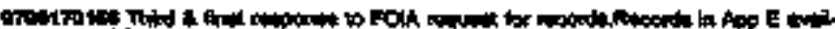

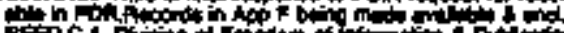

Pe

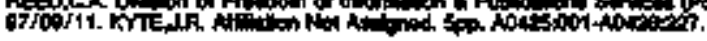

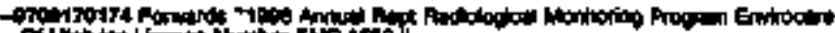

Fof

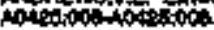

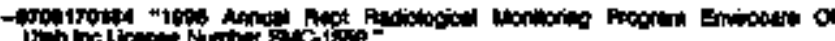

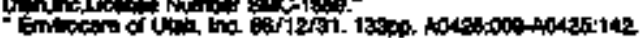

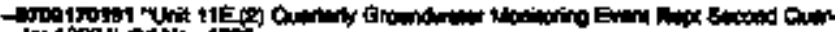

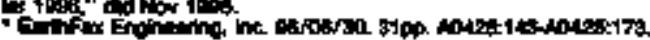

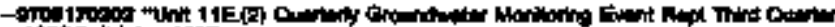

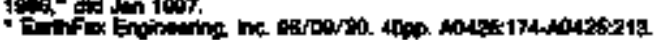

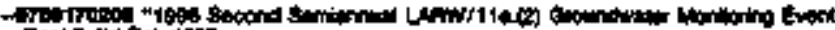

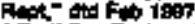

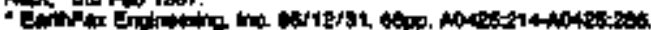

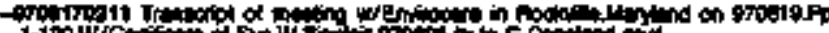

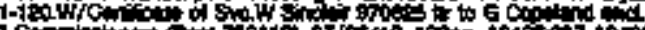

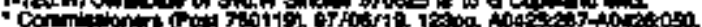

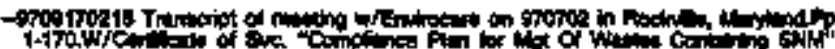

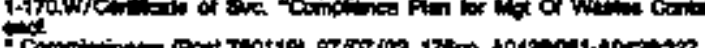

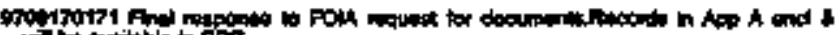

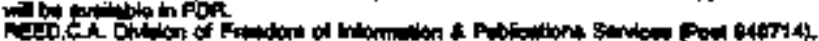

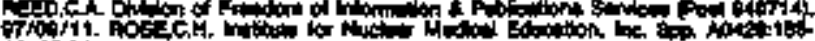
Nontisil.

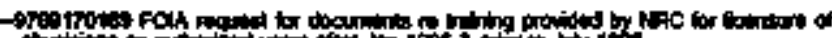

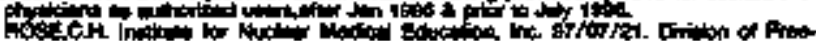

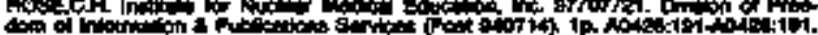

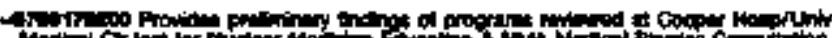

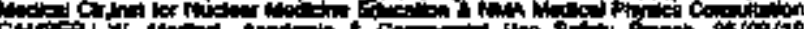

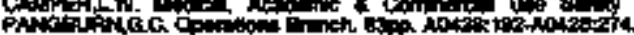

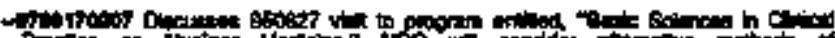

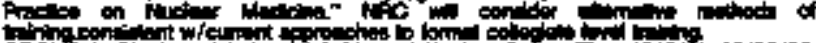

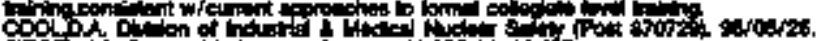
Bed

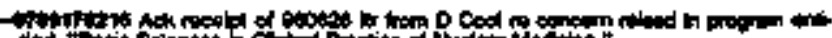

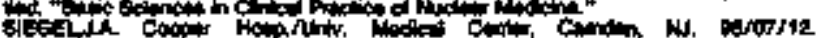
F

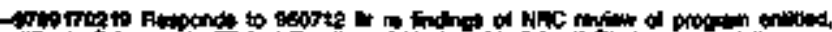

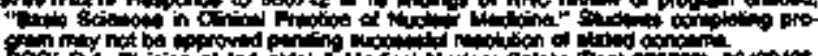

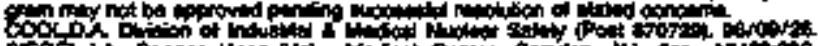
sit.

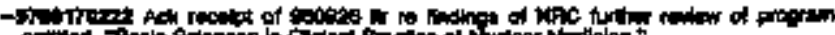

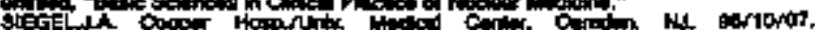

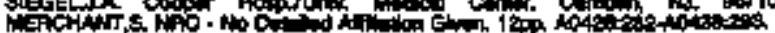

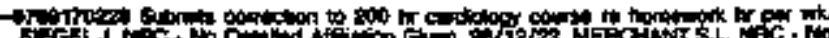
OFich 


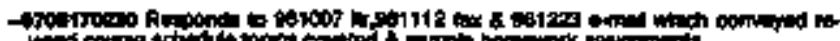

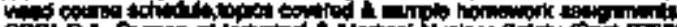

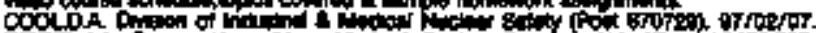

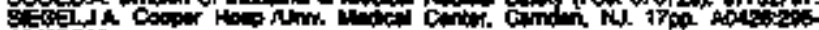

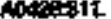

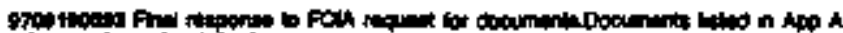

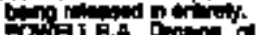

Fin

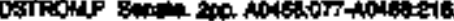

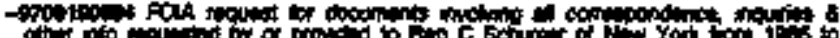

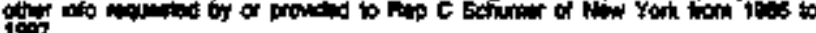

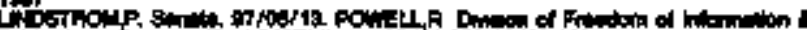

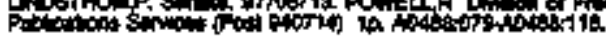

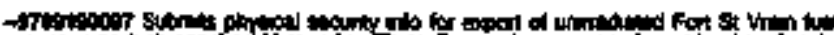

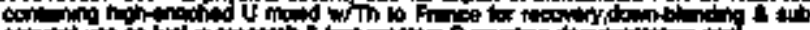

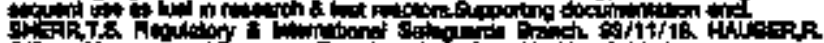

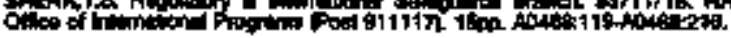

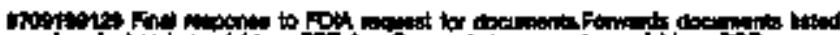

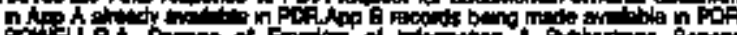

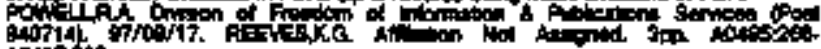
ater

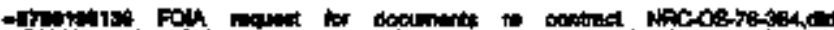

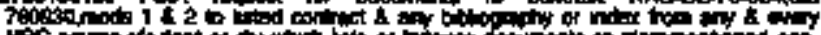

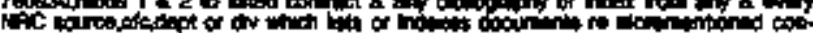

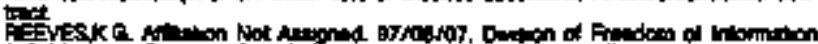

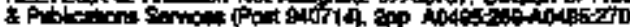

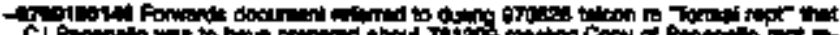

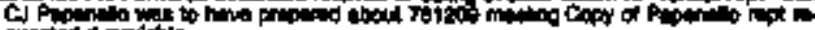

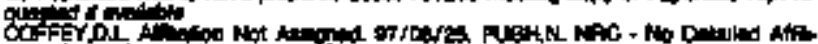

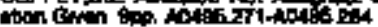

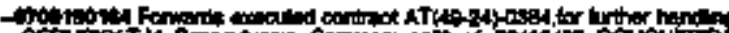

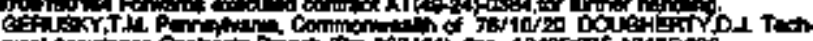

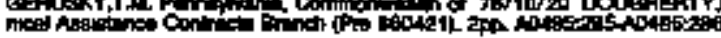

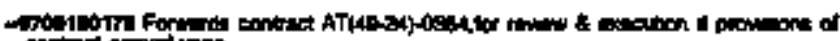
Doftä

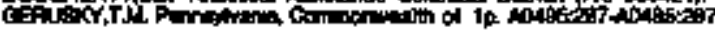

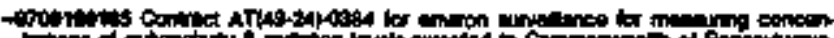

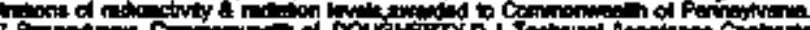

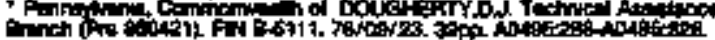

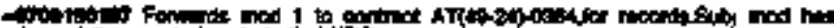

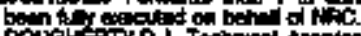

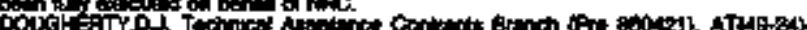

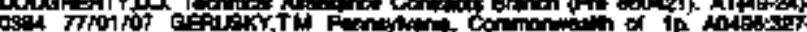
ithest

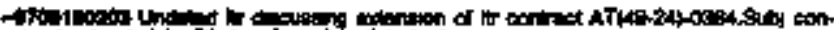

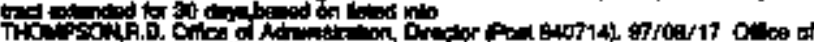

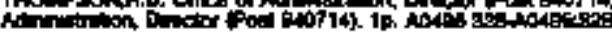

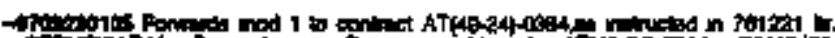

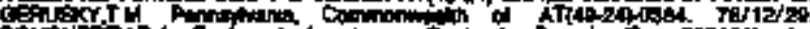
oof

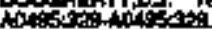

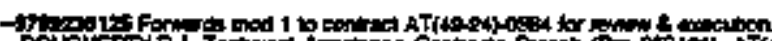

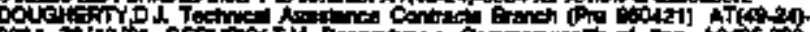

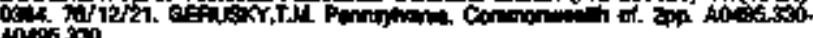

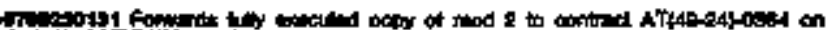

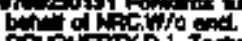

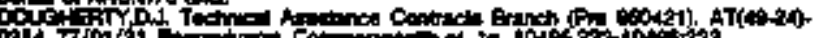

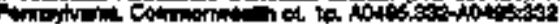

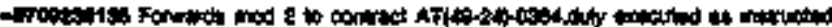

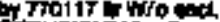

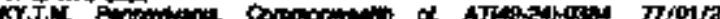

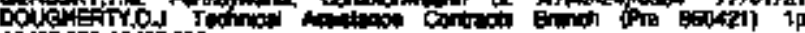

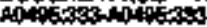

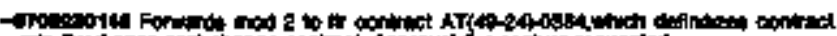

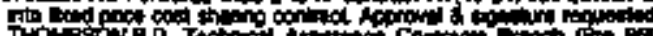

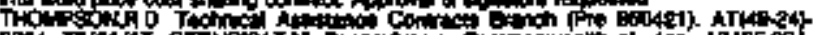

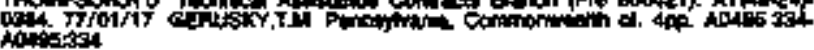

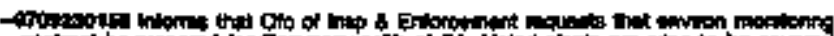

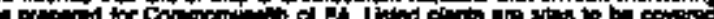

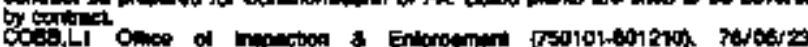

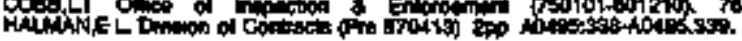

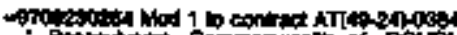

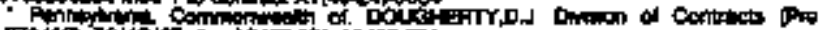

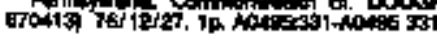

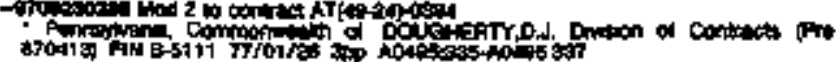

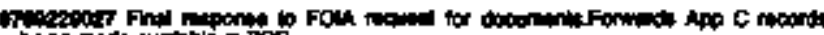

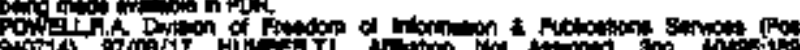

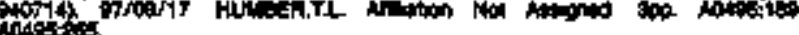

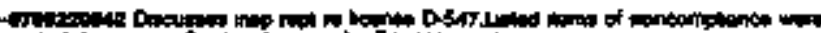

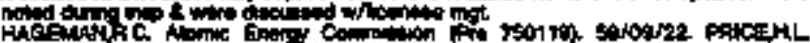

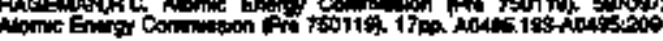

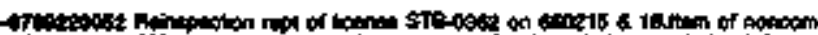

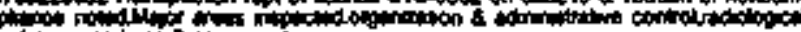

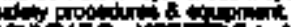

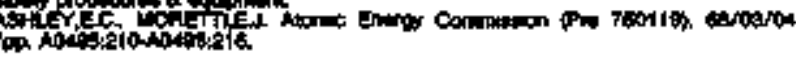

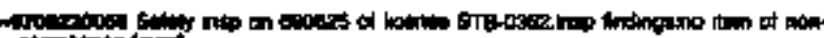

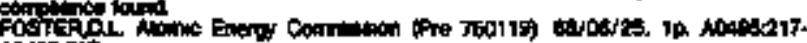
10406017.

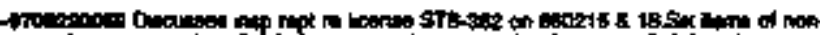

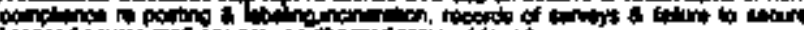

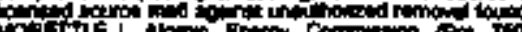

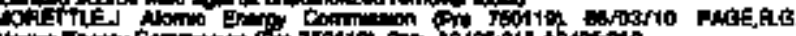

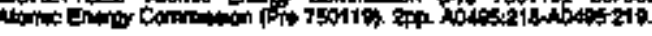

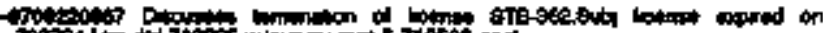

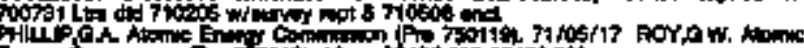

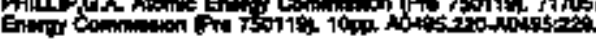

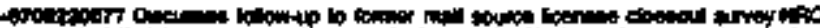

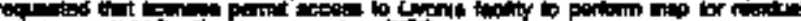

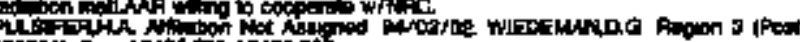

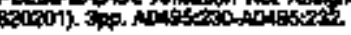

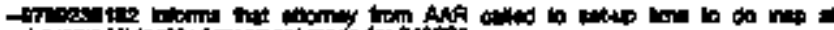

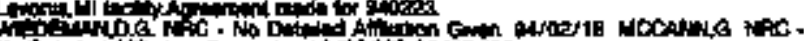

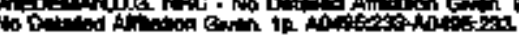

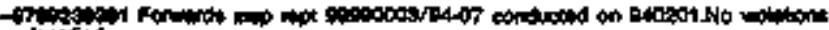

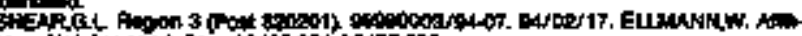

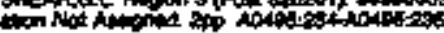

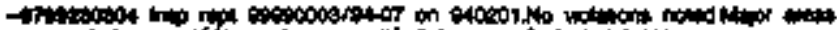

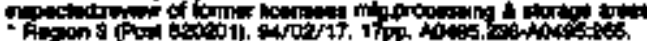

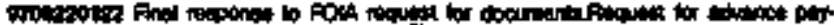

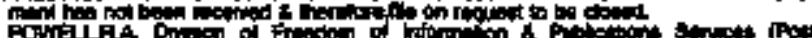

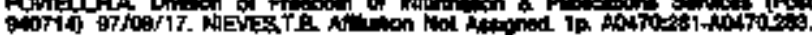

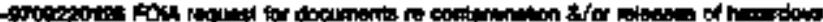

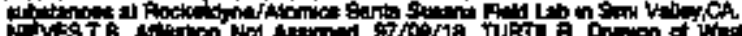

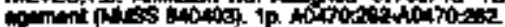

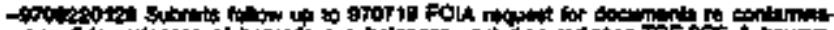

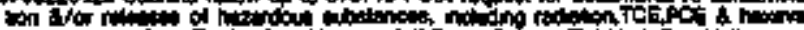

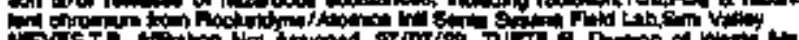

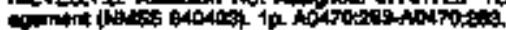

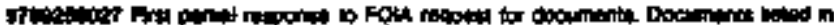

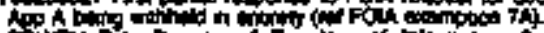

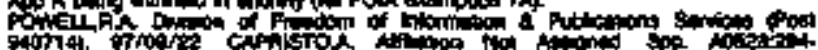
actsict.

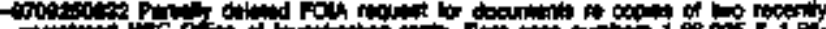

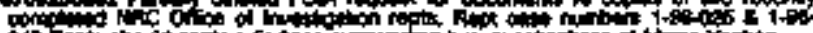

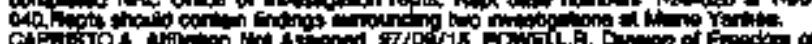

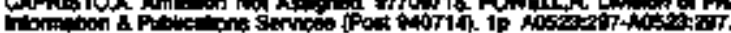

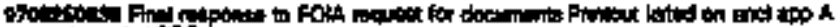
Thathin at GOA.

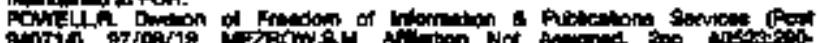

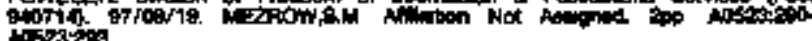

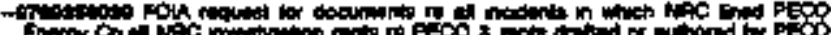

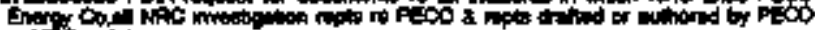

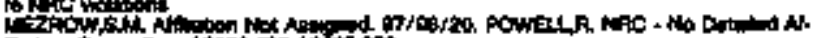

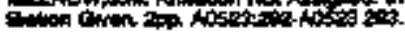

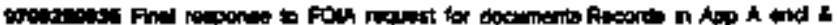
araichio in FOA

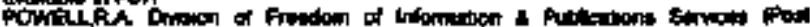

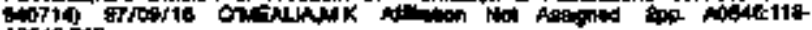
ADS6R21:

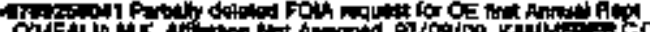

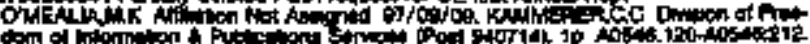




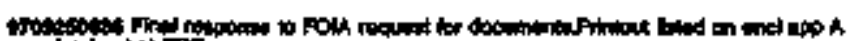

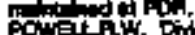
PCw Actosition

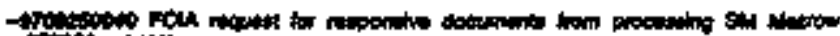

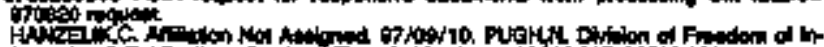

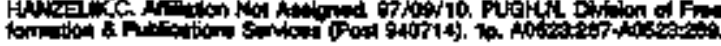

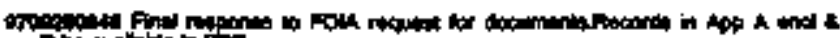
abe of

POM

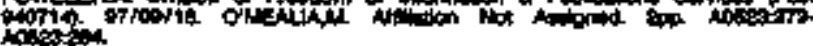

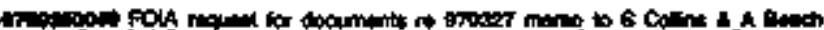
then $\mathrm{J}$ fiot $\mathrm{J}$.

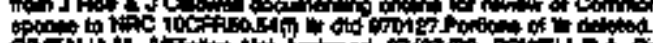

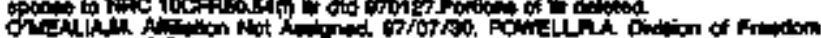

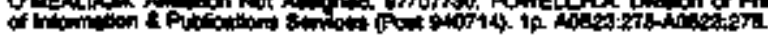

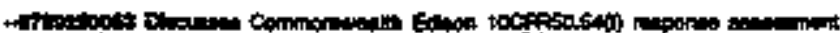

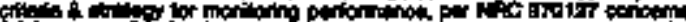

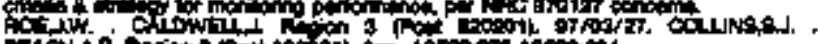

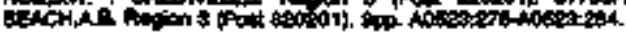

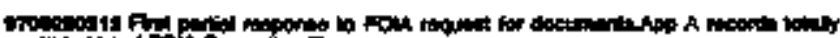

Pow

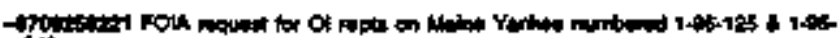
onto

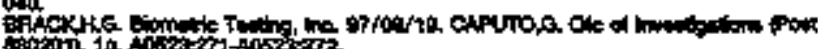

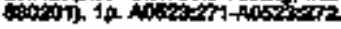

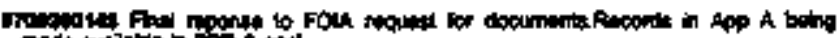

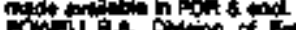

An

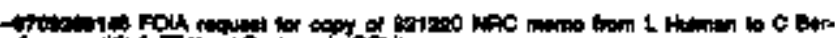

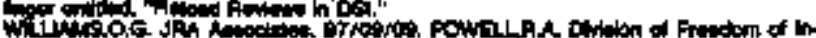

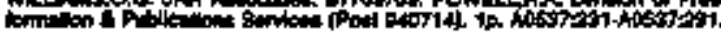

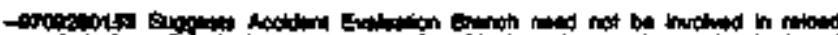

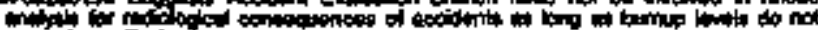

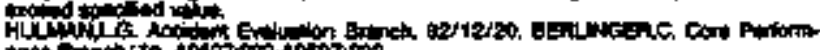

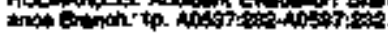

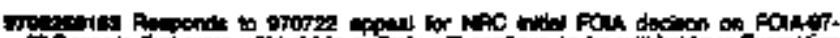

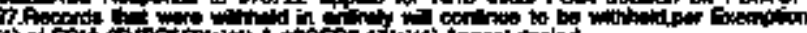

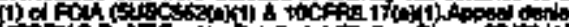

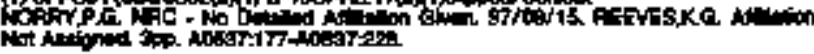

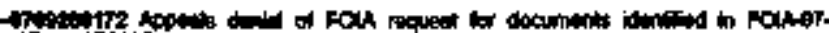

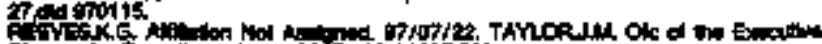

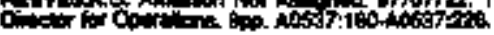

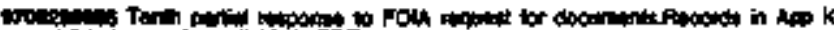

Ponj

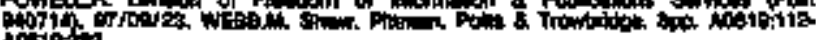
notole

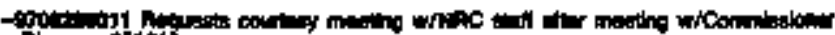
If

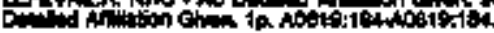

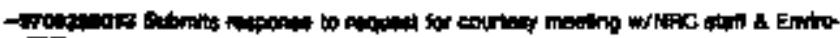
Fictor

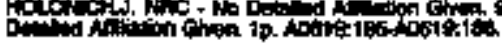

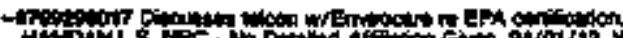

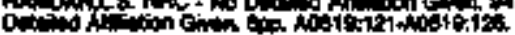

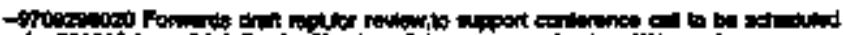

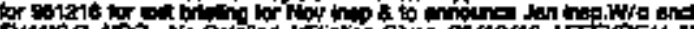

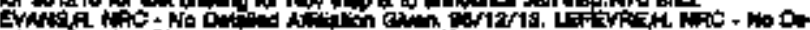

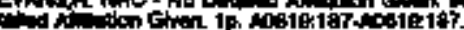

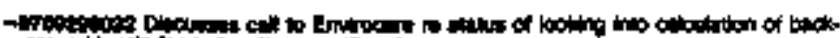

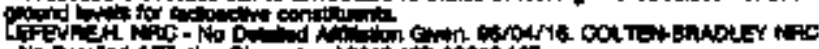

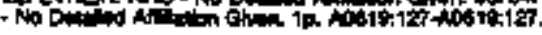

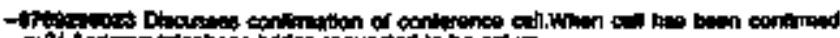
WN hid

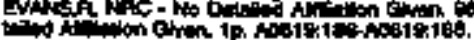

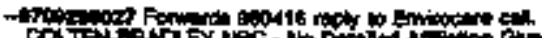

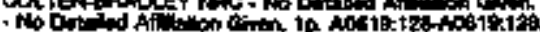

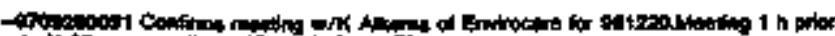

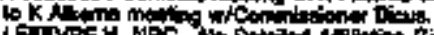

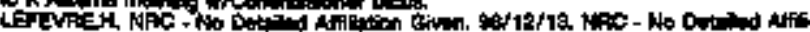

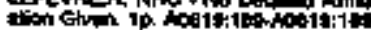

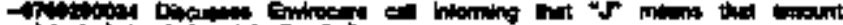

U

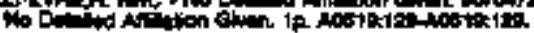

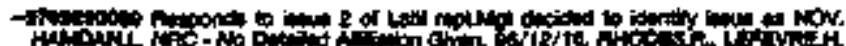

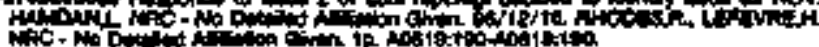

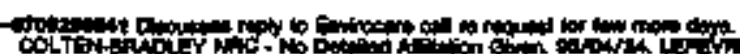

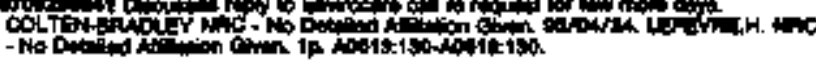

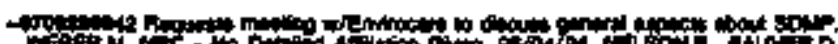

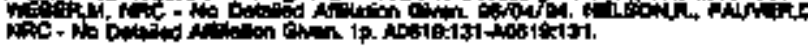

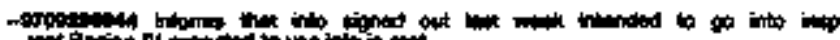

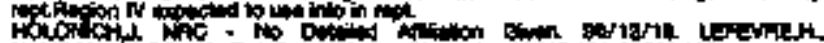

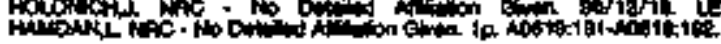

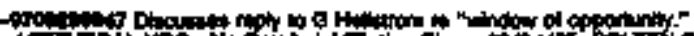

L

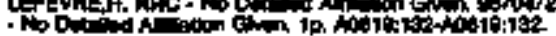

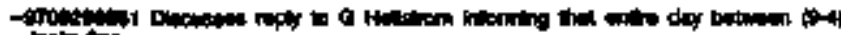

colit the

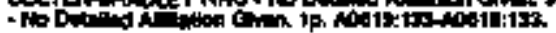

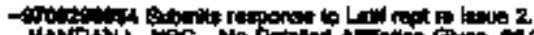

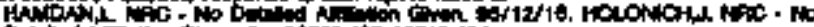

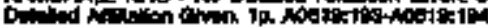

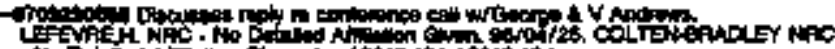
- Tho Dotid A

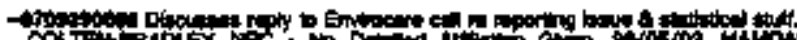

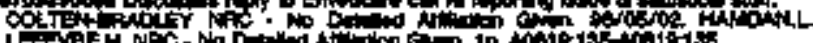

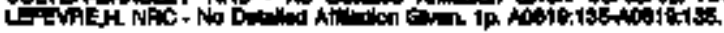

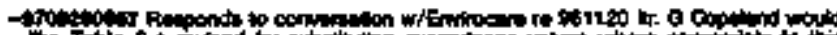

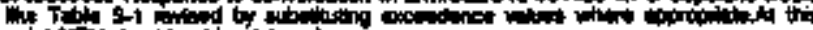

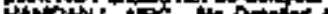

Darien

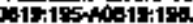

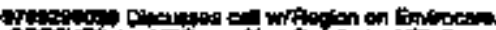

a

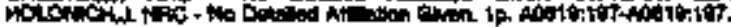

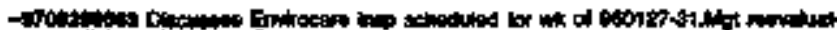

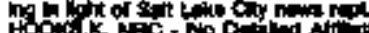

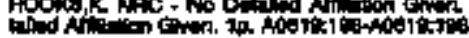

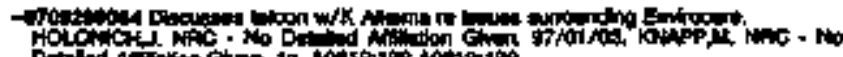

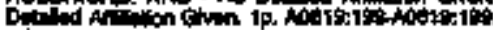

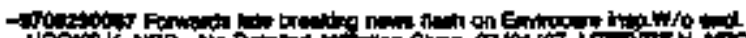

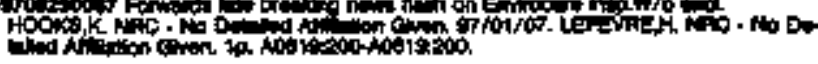

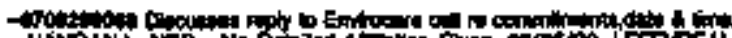

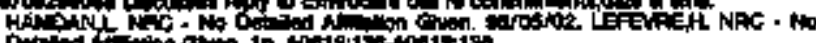

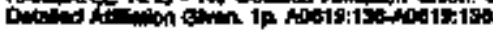

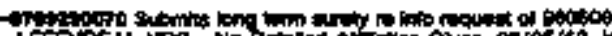

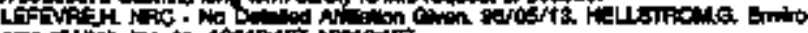

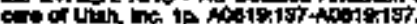

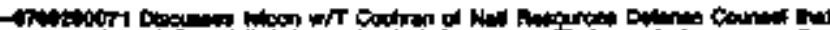

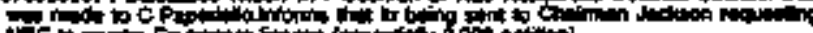

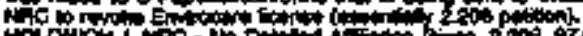

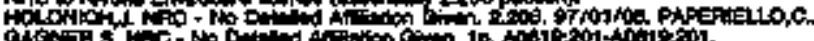

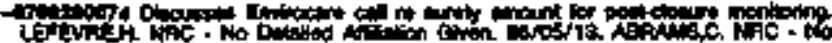

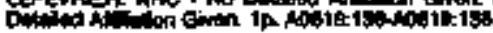

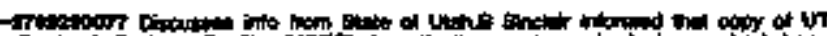

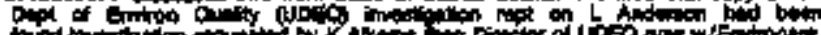

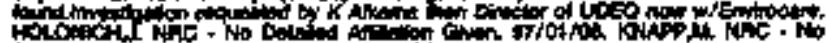

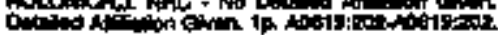

- MT:

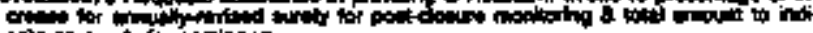

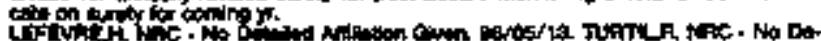

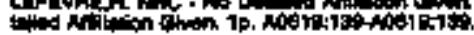

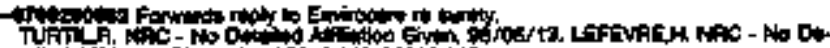

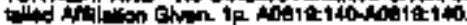

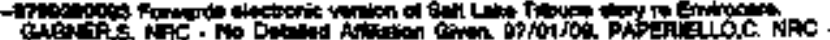

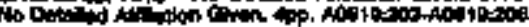

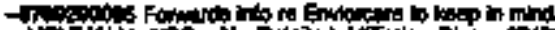

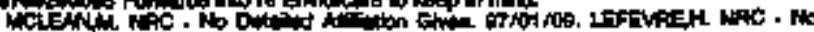

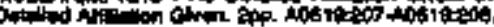




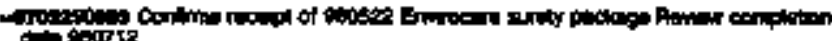

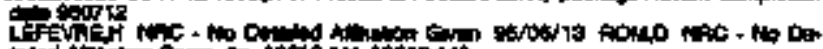

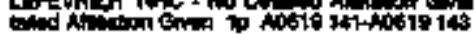

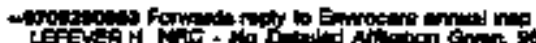

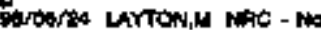

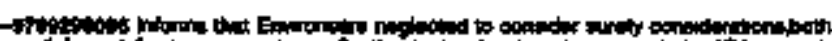

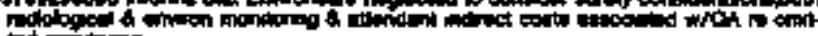

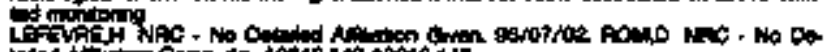

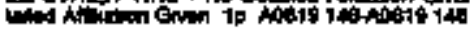

[r.

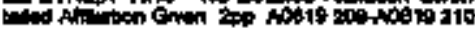

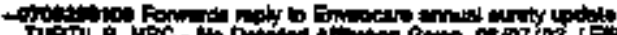

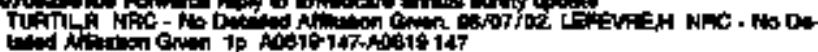

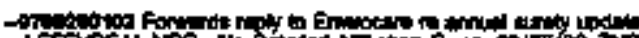

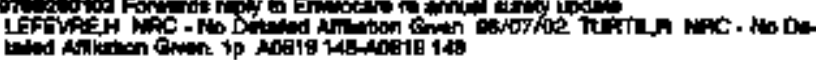

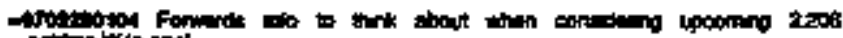

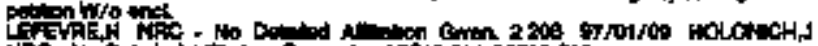

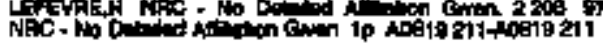

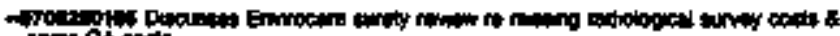

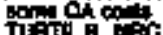

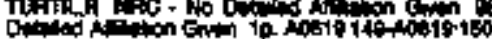

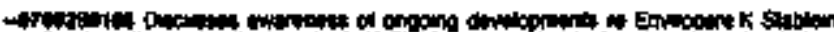

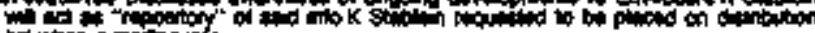

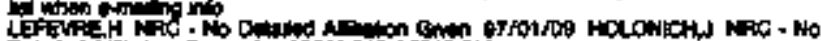

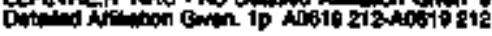

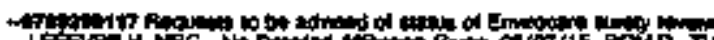
action

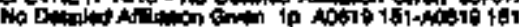

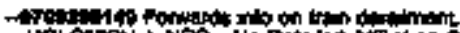

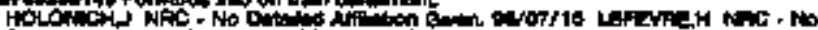

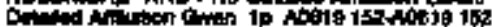

-Ty Hot

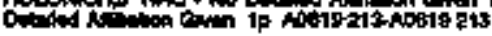

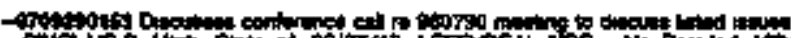

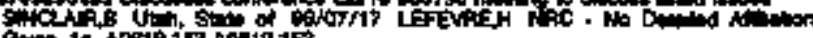

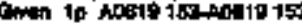

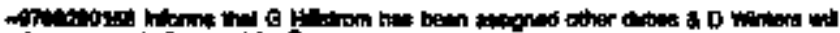

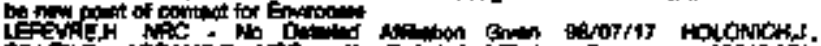

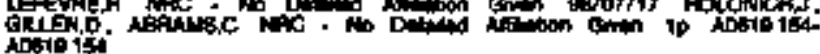

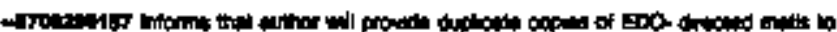

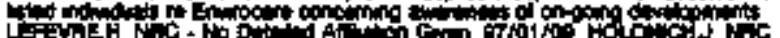

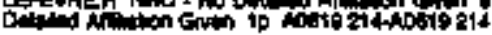

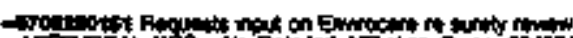
UFP F ide

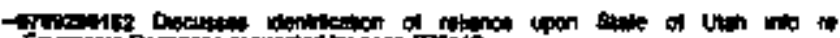

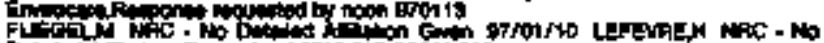

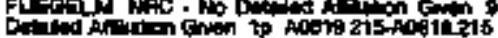

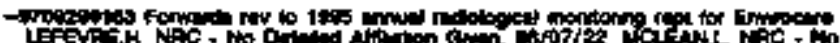

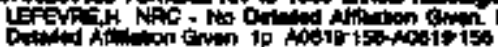

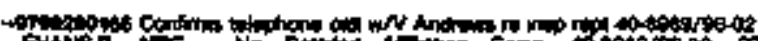

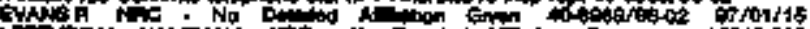

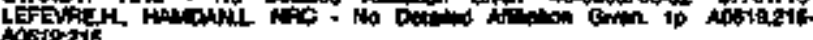

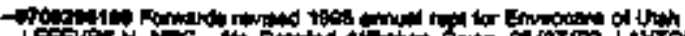

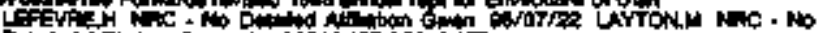

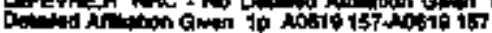

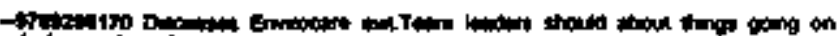

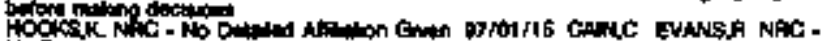

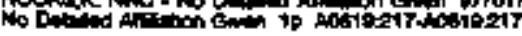

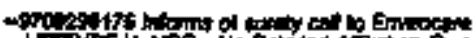

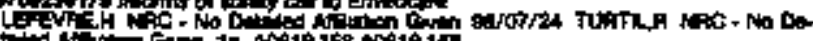

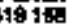

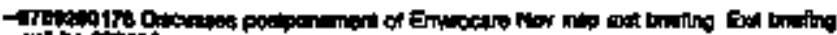

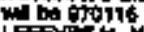

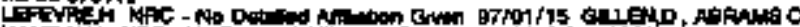

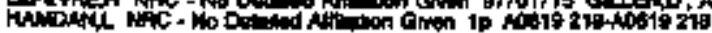

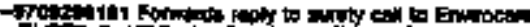

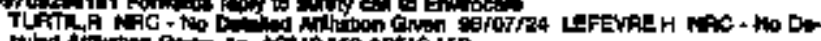

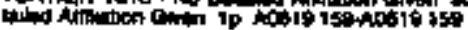

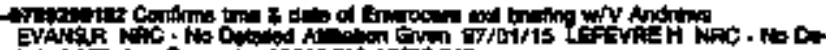

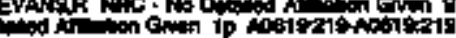

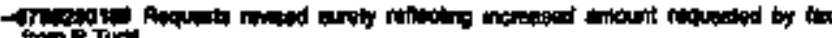

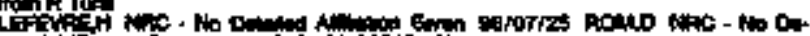

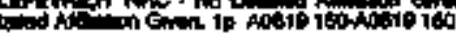

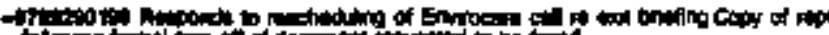

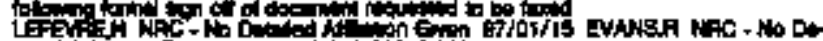

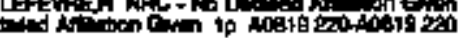

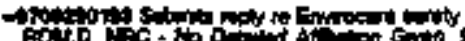

Alo

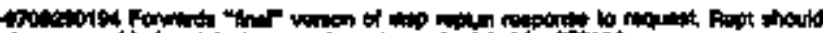

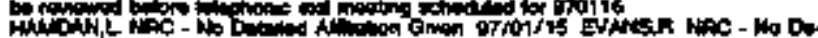

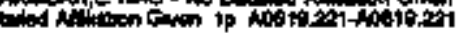

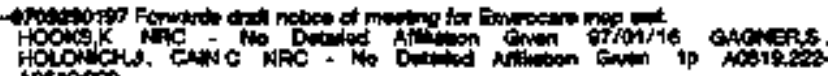
Aotiots

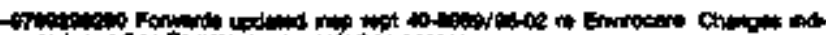

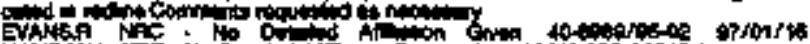
FWM

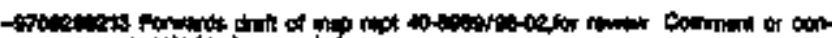

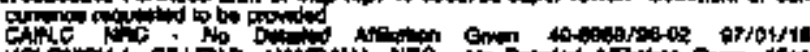

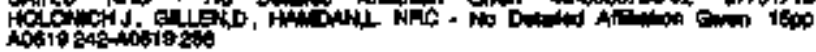

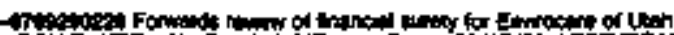

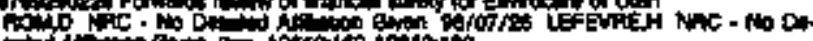

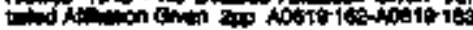

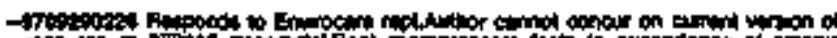

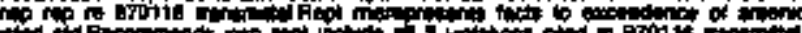

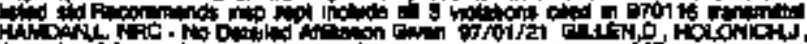

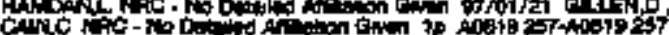

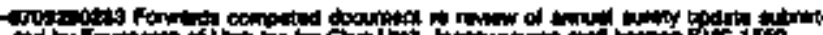
Ju定

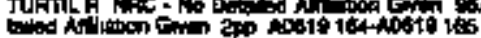

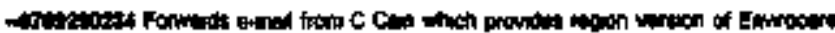

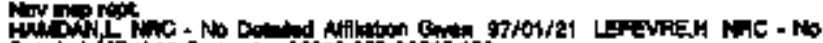
Dotedy

ifl

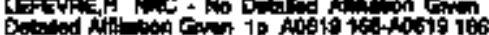

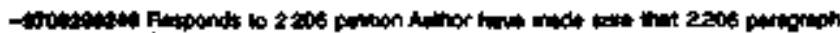

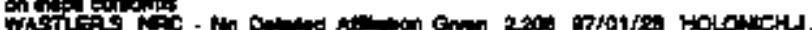

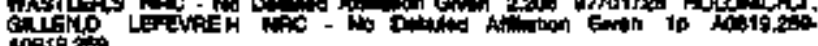

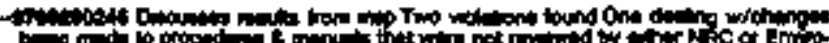

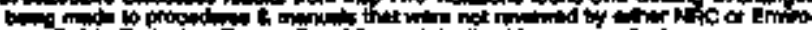

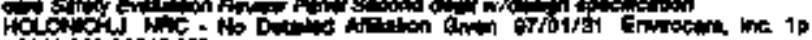

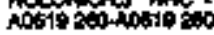

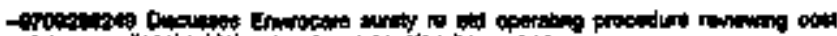
UE

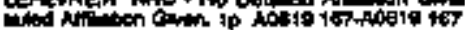

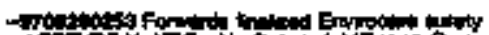

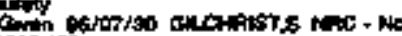

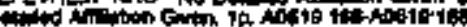

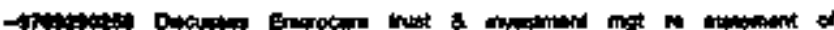

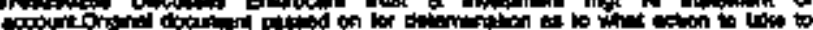

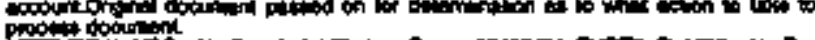

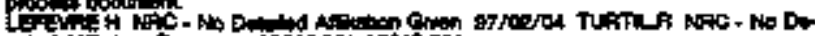

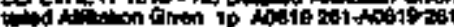

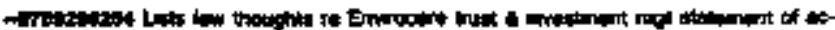

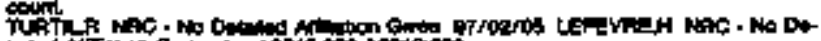

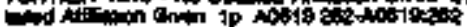

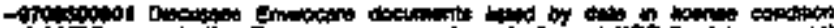

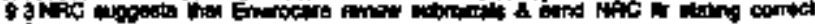

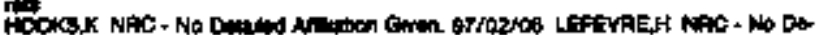

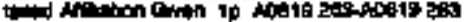

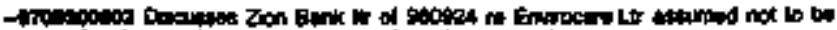

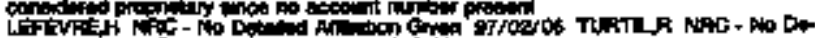

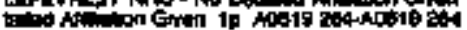




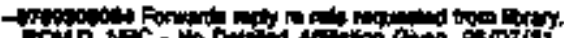

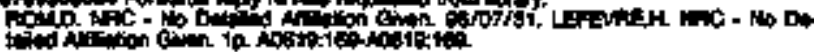

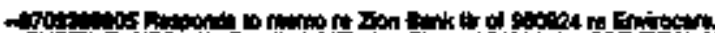

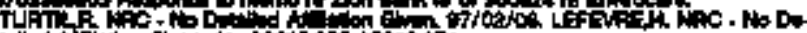

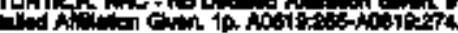

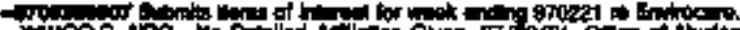

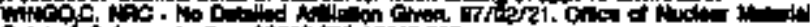

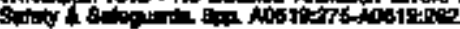

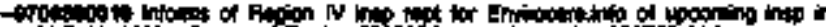

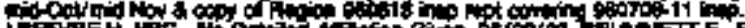
(1)

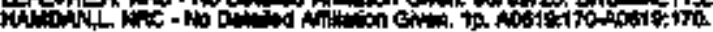

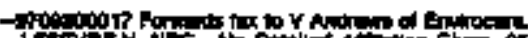

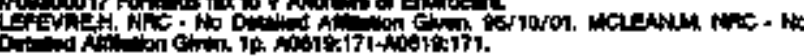

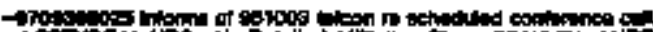

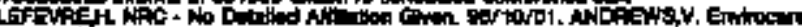

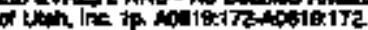

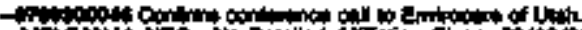

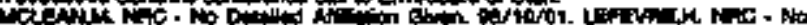

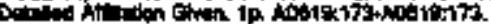

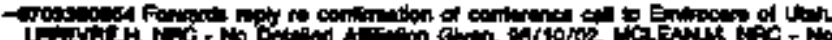

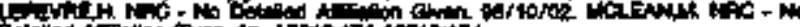

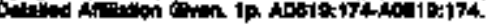

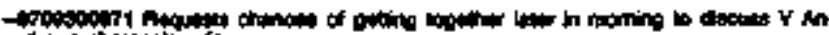

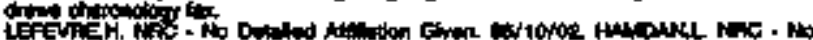

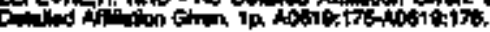

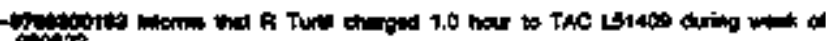
etrots

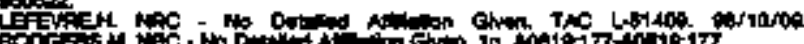
政

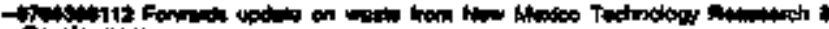

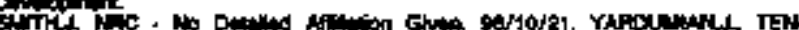

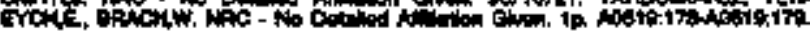

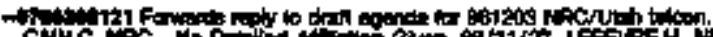

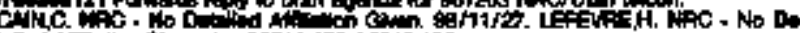

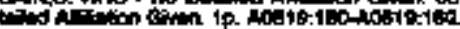

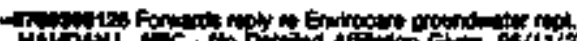

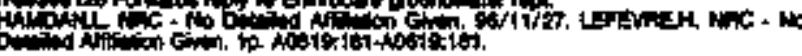

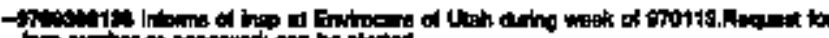

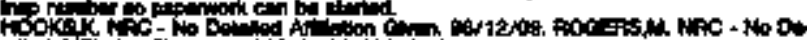
and

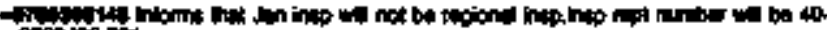

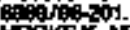

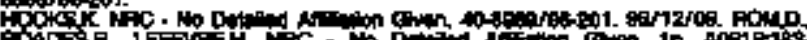

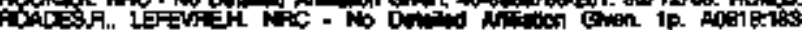
Find

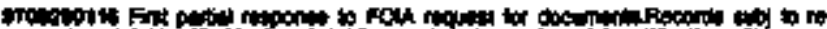

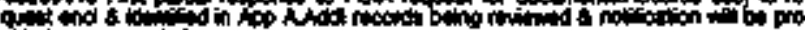

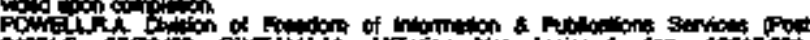

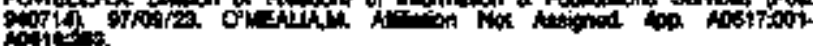

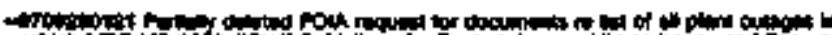

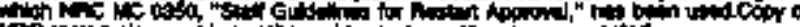

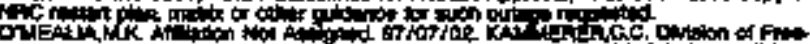

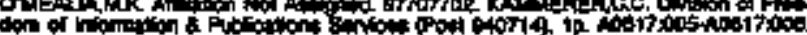

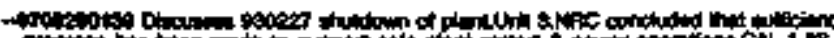

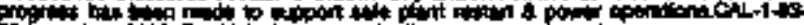

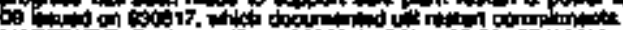

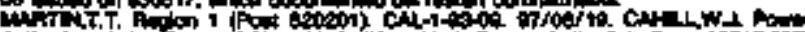

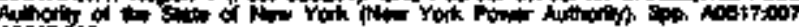
Anditions

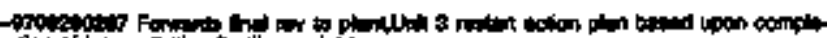

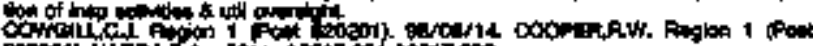

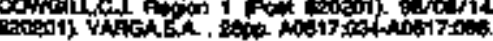

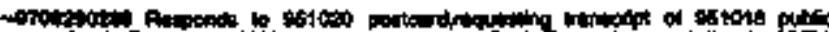

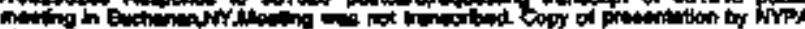
11 inting and

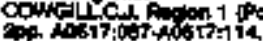

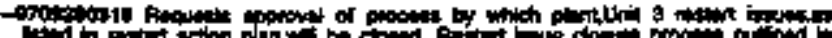

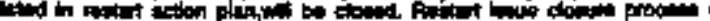

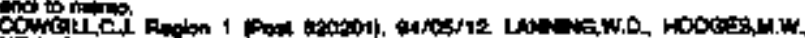

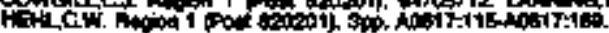

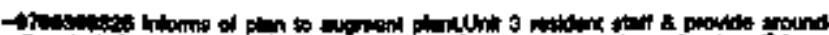

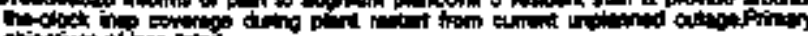

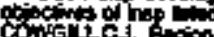

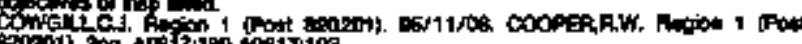

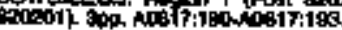

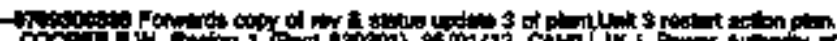

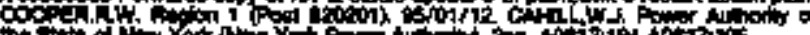

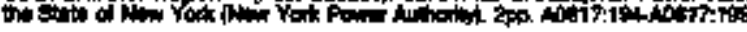

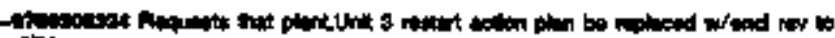

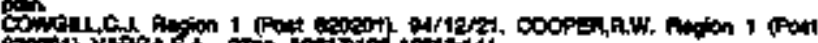

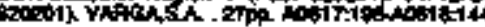

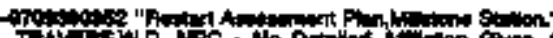

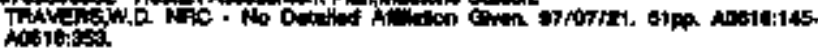

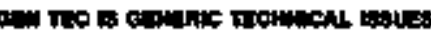

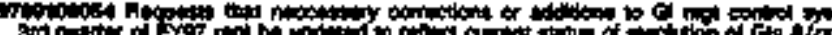

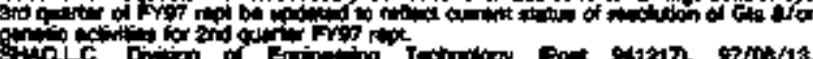

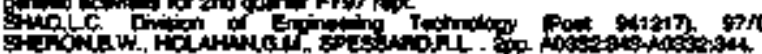

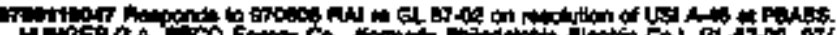

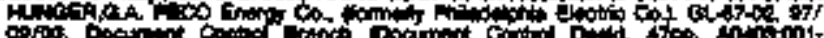
Aonotite

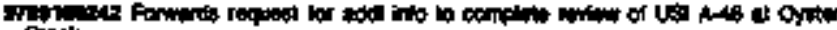

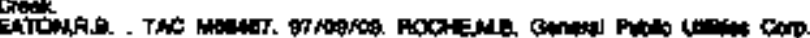

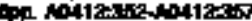

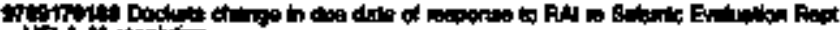

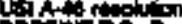

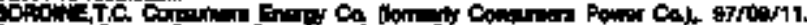

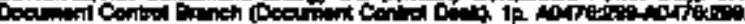

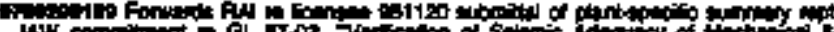

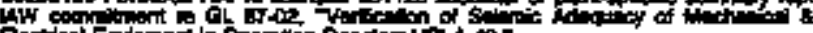

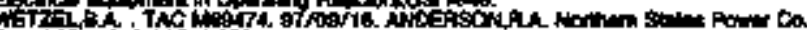

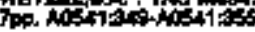

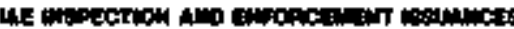

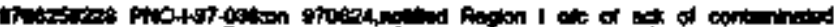

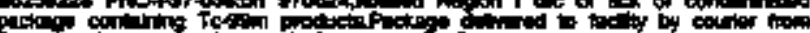

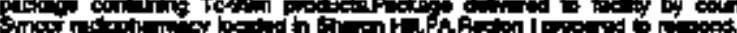

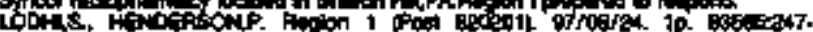
Boted 247 .

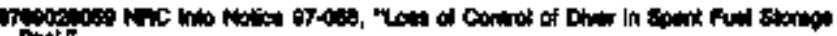

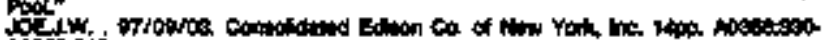
Atowis

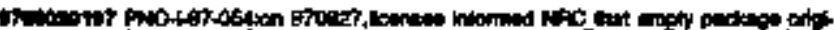

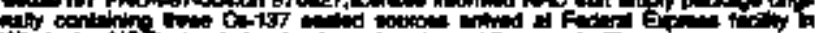

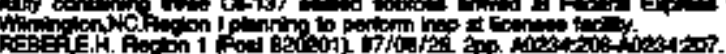

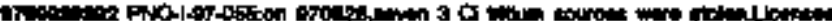

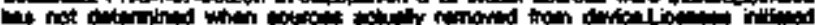
oning A060

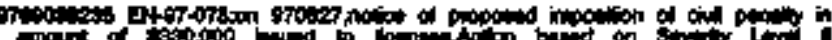
oncin of

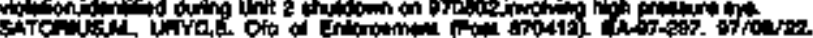
ip.

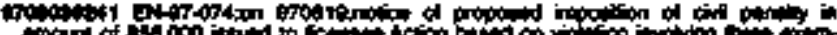

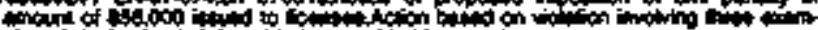

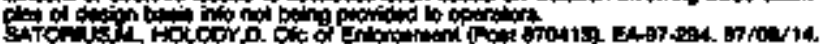

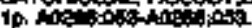

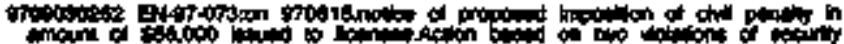

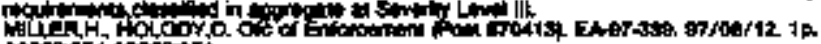

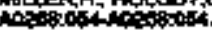

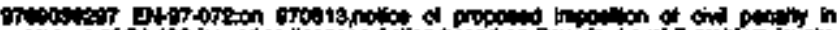

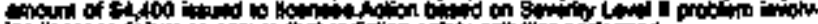

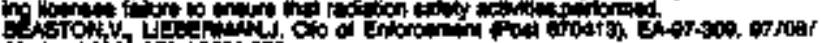

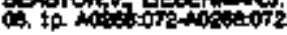

If

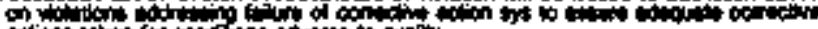

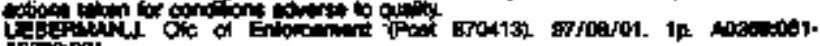

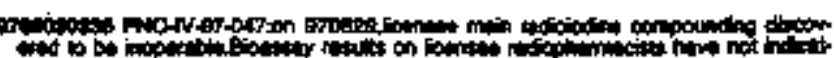

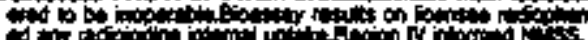

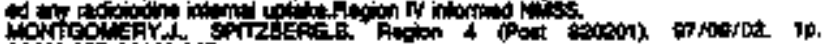
ration 


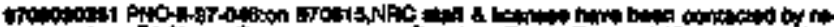

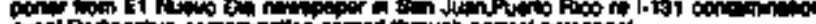

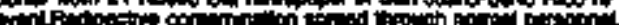

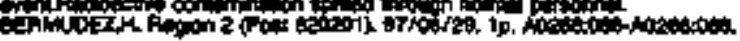

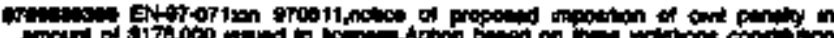

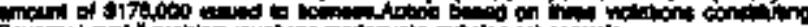
s.

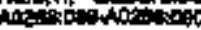

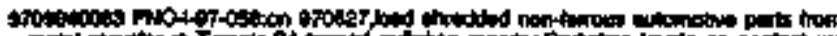

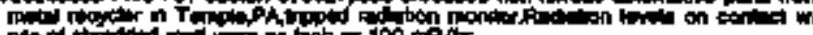

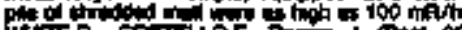

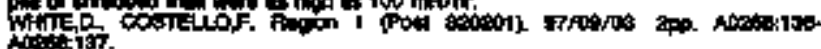

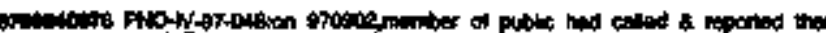

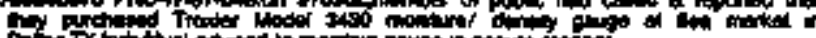
(1)

nogtint.

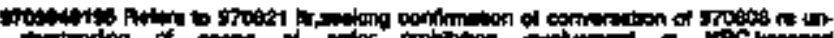

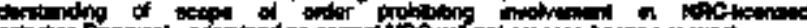

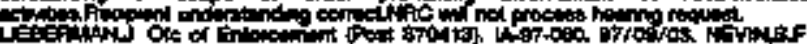

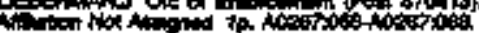

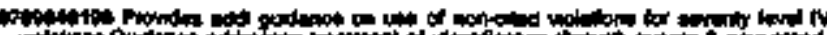

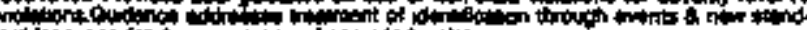

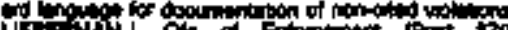

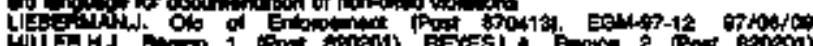

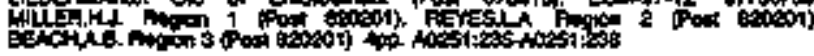

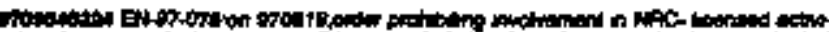

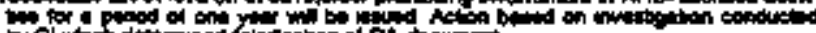

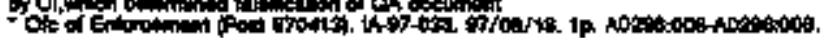

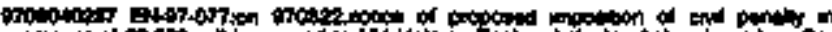

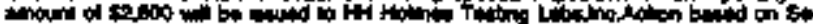

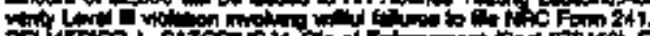

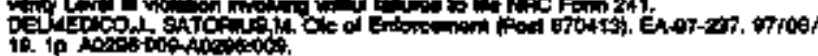

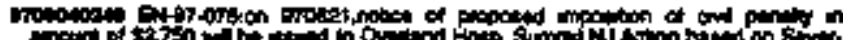

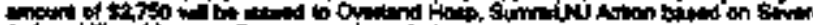

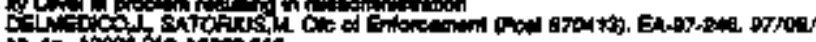

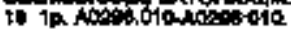

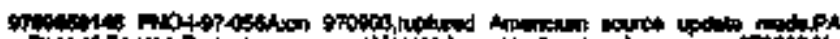

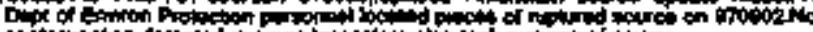

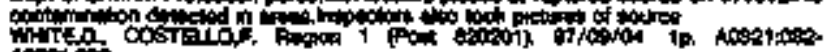
10.ton

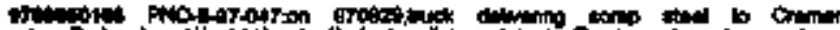

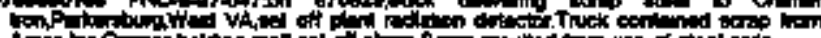

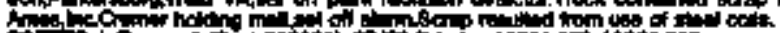

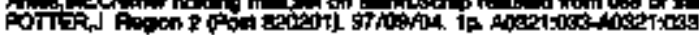

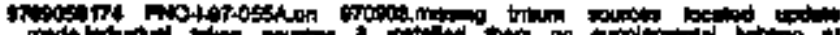

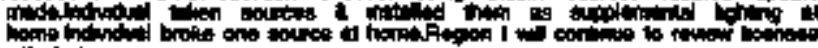

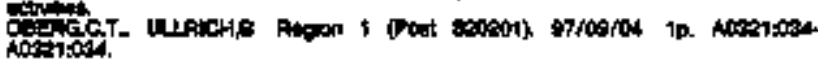

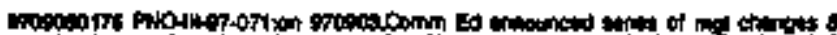

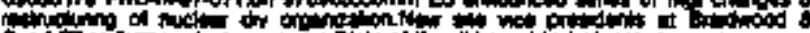

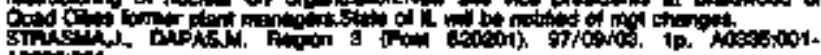

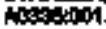

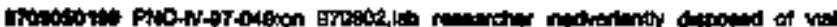

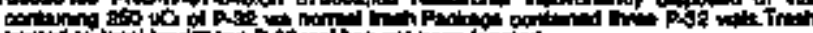

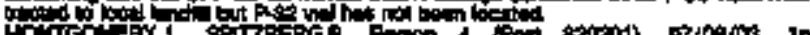
ins

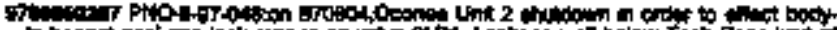

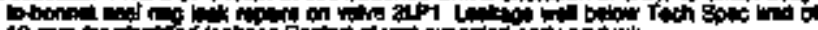

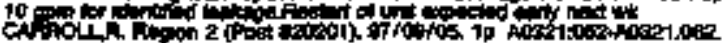

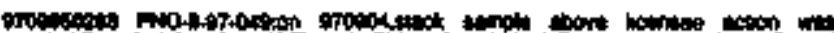

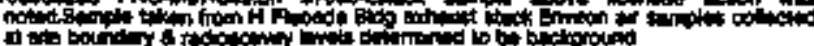

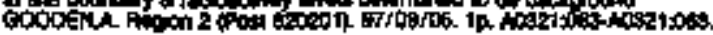

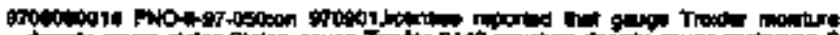

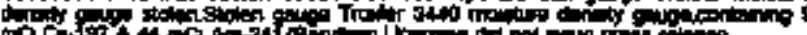
Thof

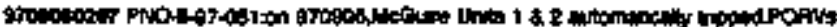

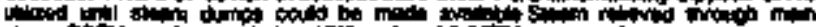

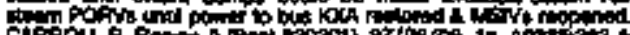

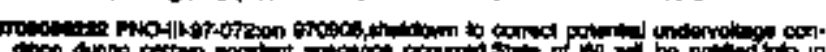

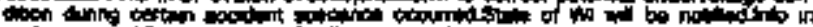

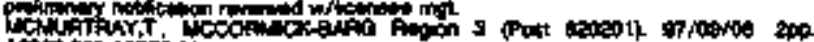

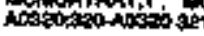

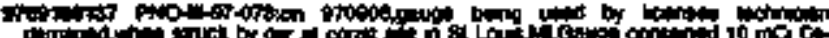

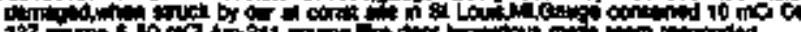

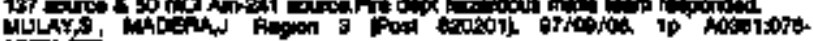
ADST

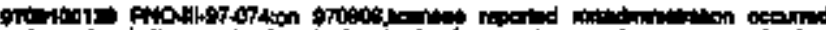

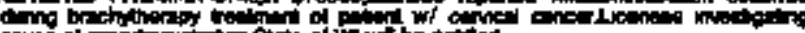

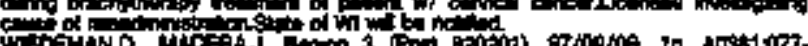
Antositor

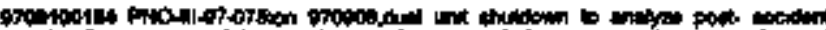

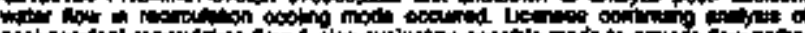

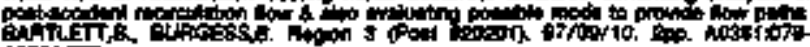
Nomet:or

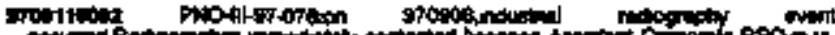

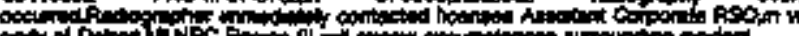

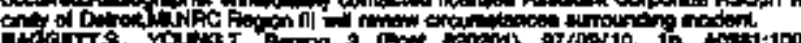
Andition

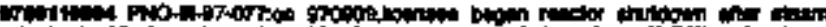

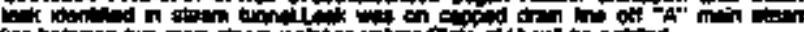
DUPCwis, Acti:10i.

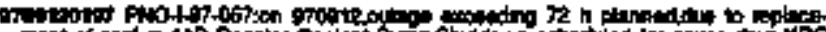

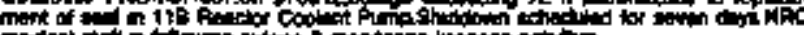

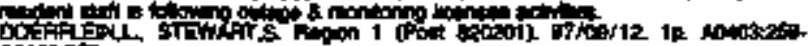

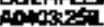

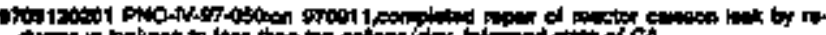
sFtzMatoseso.

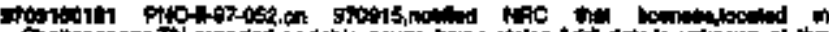

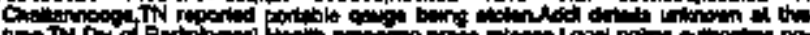

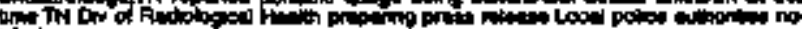

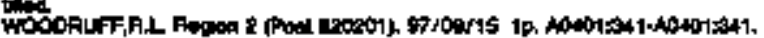

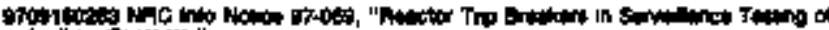
for. instivit

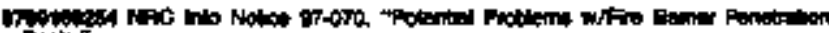

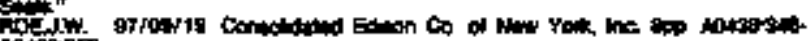
10

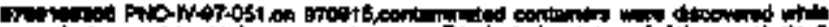

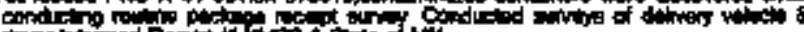

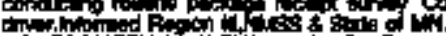

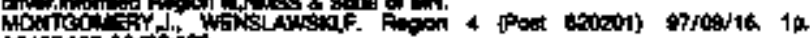

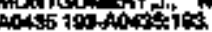

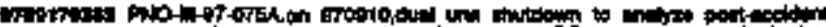

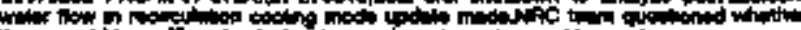

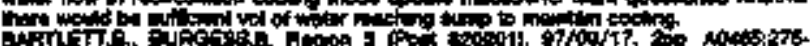
naterore

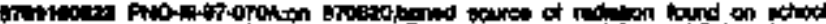

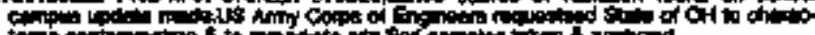
tares ent

L

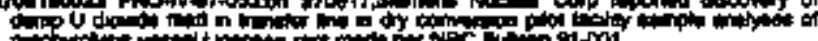

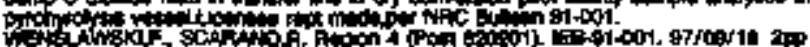
No4

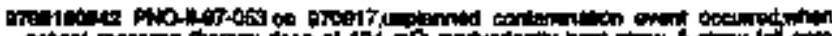

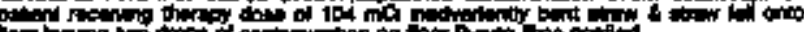

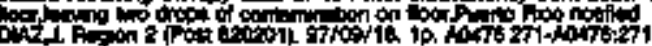

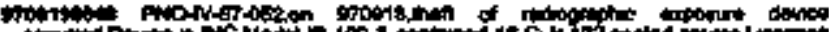

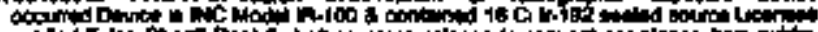

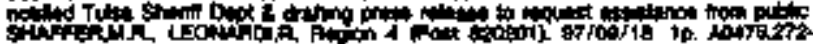
intrition

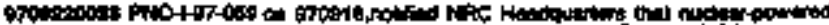

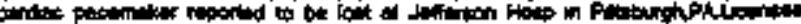

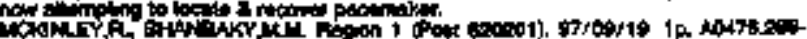

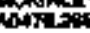

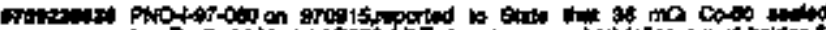

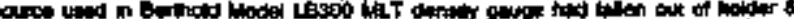

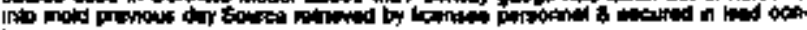

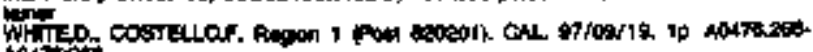
104 


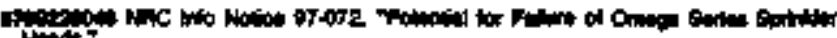

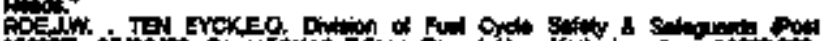

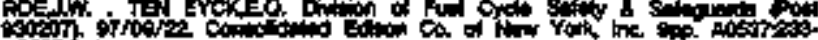

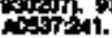

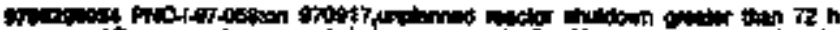

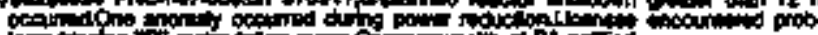

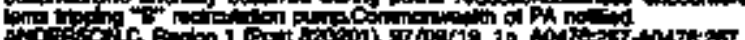

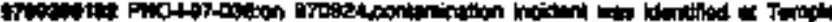

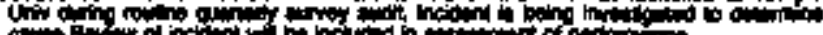

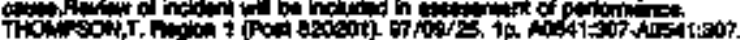

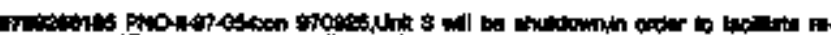

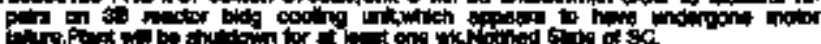

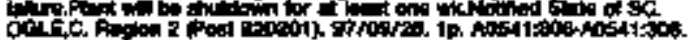

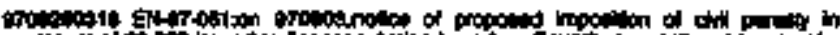

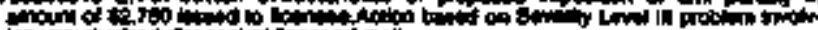

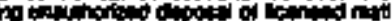

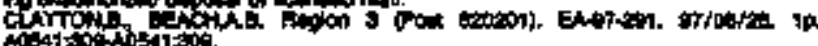

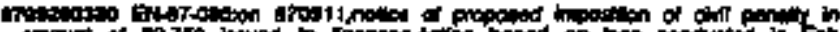

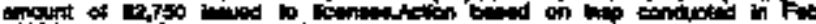

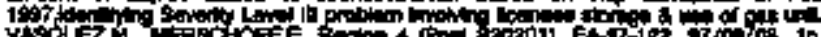
A06

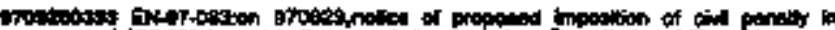

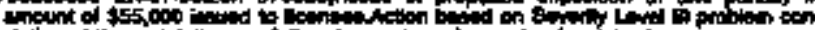

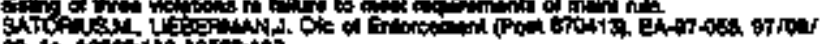

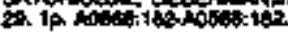

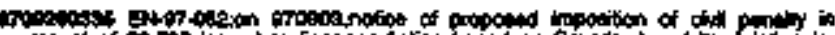

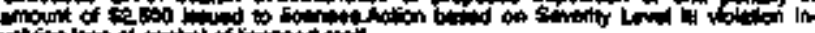

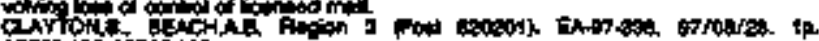

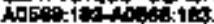

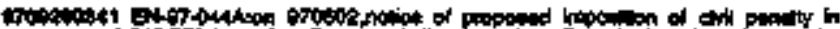

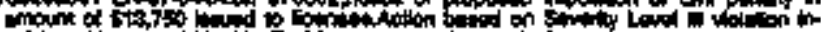

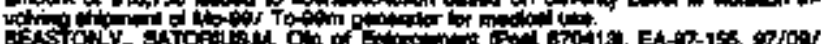

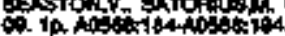

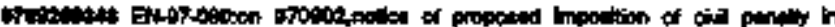

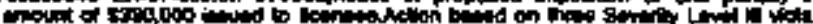
A Nostions

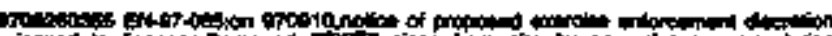

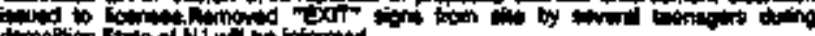
a

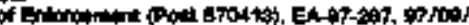

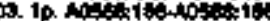

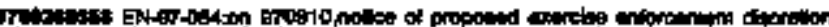

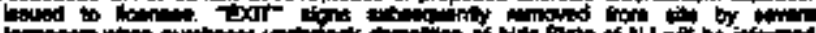

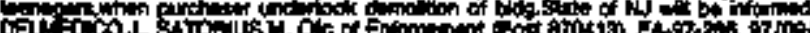

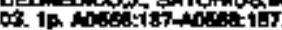

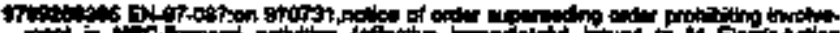

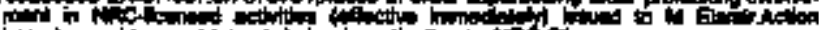

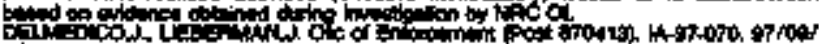

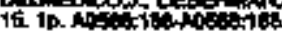

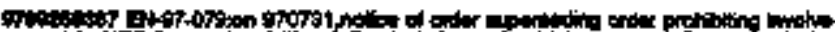

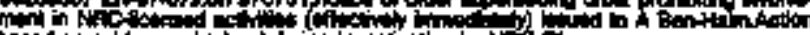

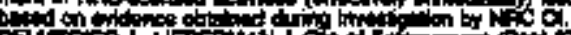
27. 1p. An

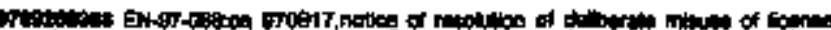

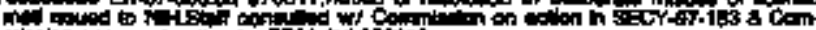

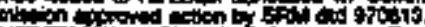

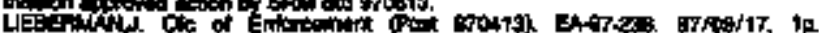
not

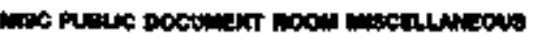

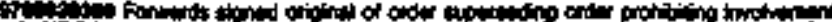

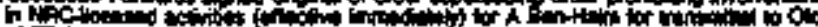

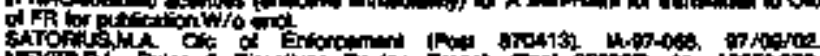

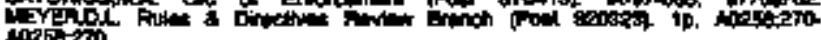

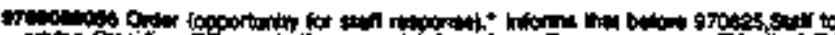

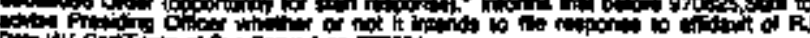

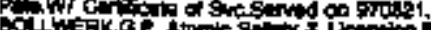

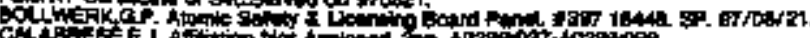

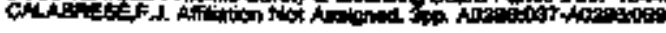

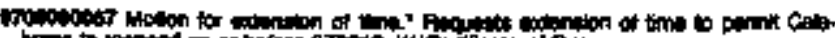

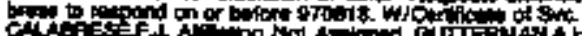

Det

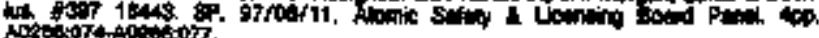

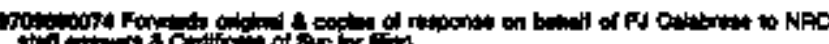
of

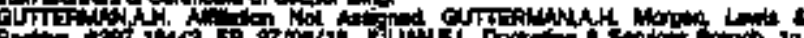

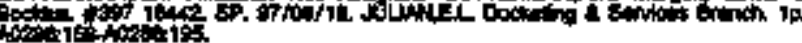

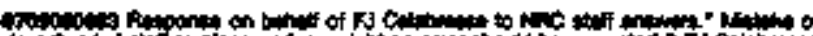

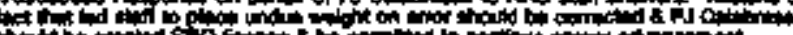

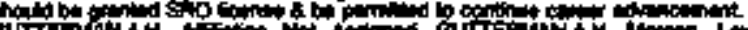
(I) ofonts.

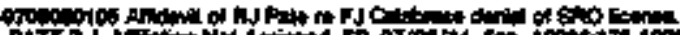

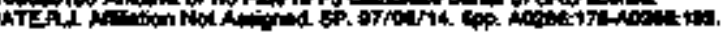

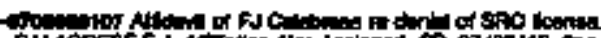

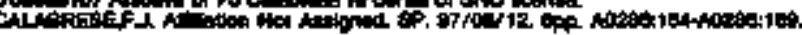

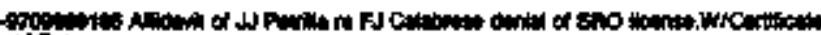
여요요

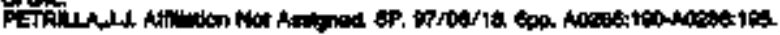

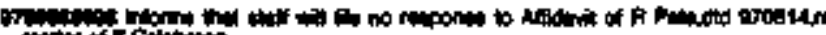

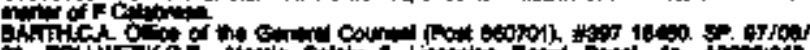

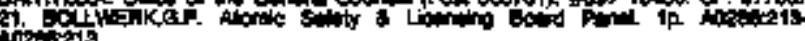

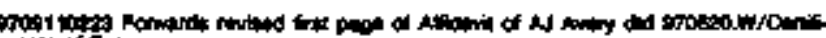

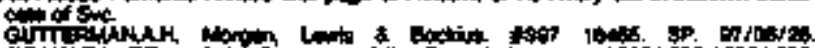

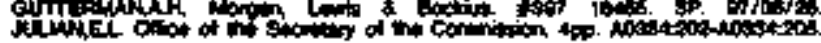

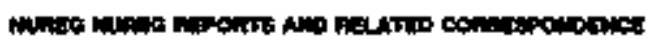

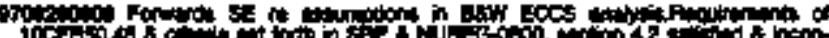

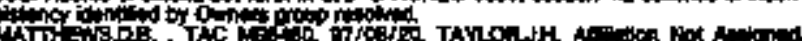

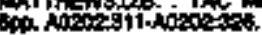

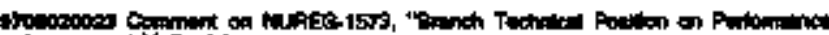

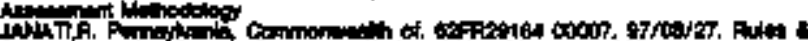

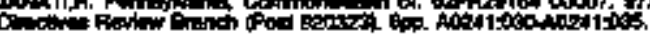

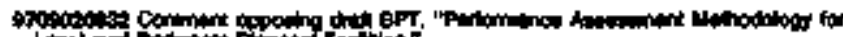

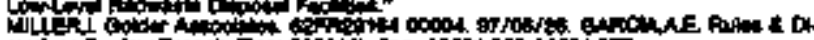

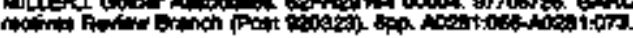

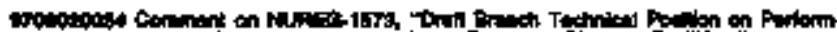

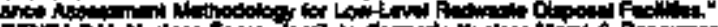

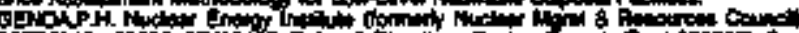

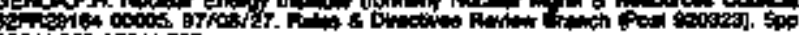
Antrinsentiont toss.

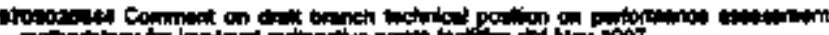

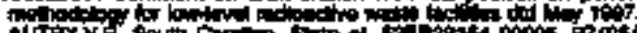

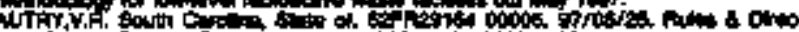

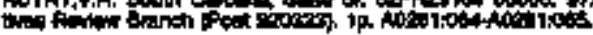

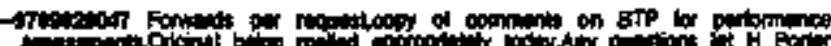

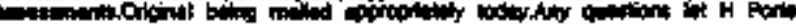

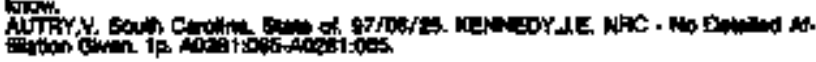

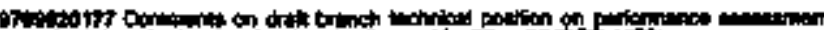

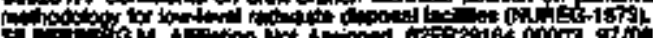

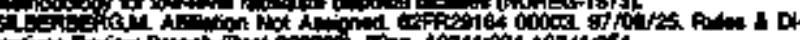

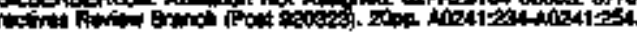

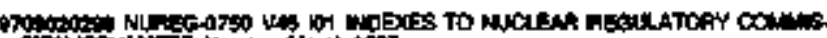

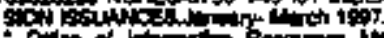

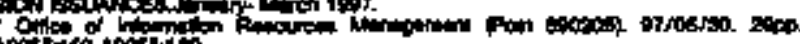
An

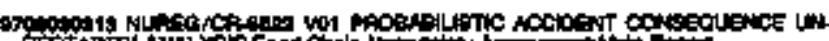

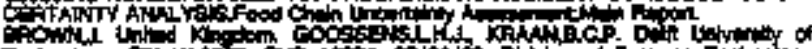

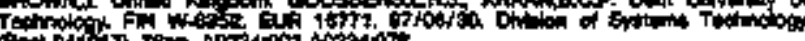

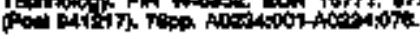

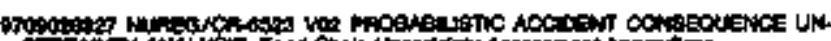
C

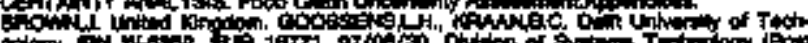

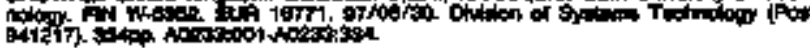

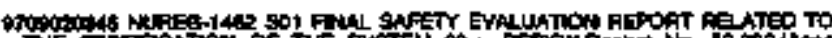

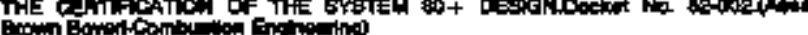

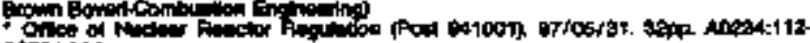
notionstat.

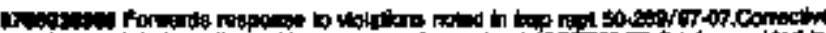

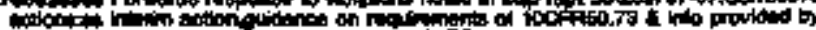

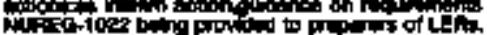

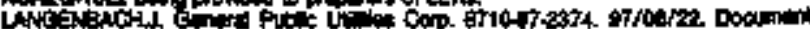

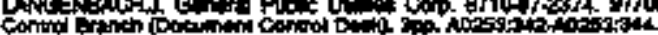




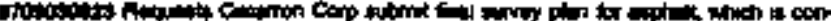

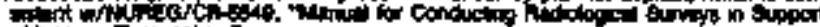

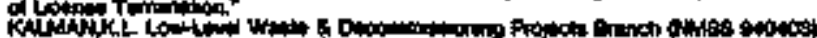

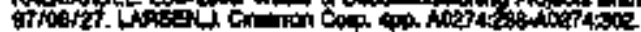

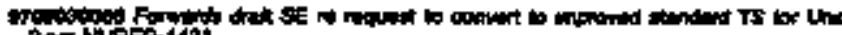

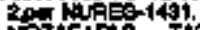

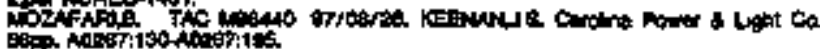

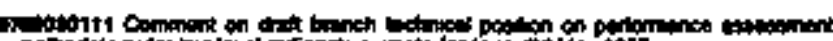

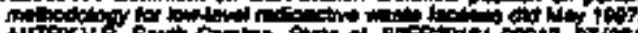

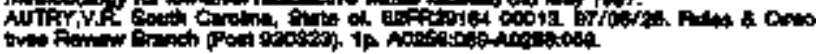

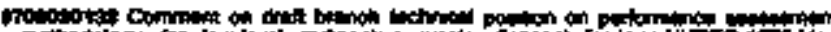

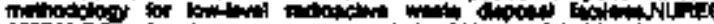

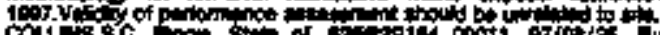

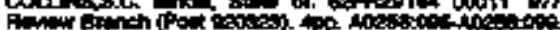

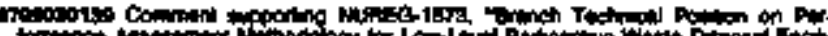

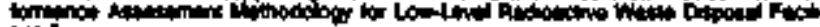

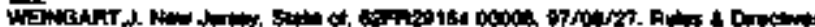

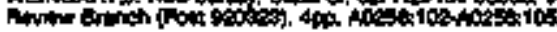

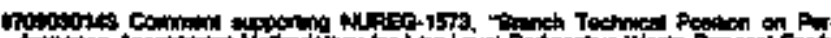

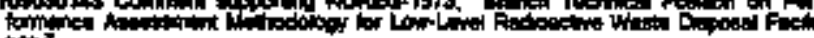

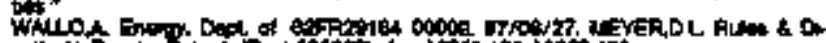

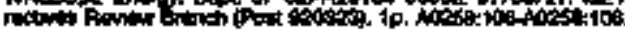

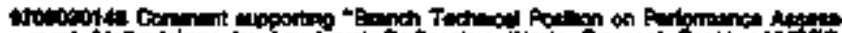

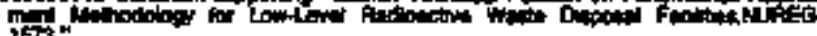

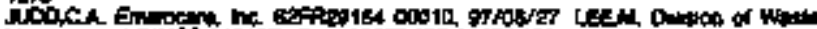

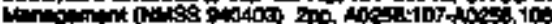

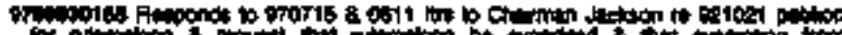

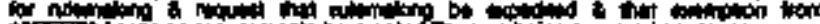

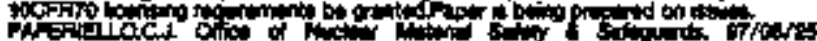

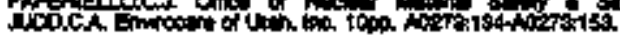

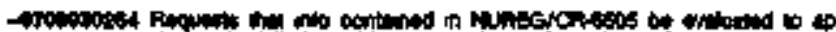

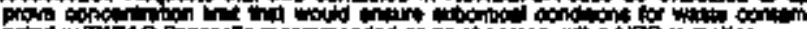

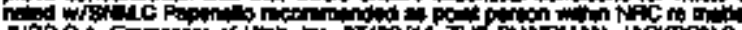

Jotace Emocen of

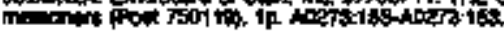

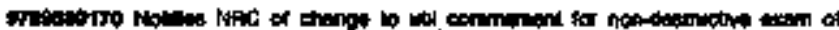

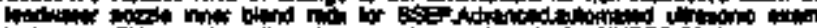

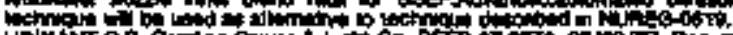

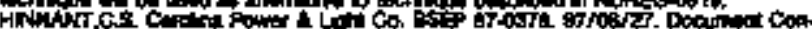

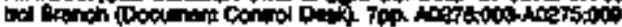

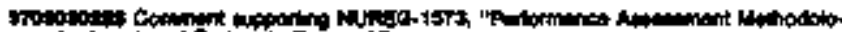

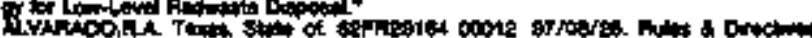

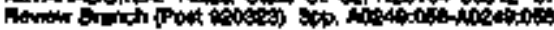

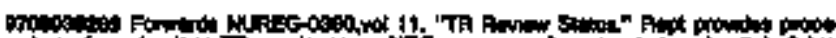

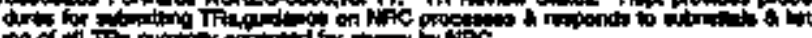

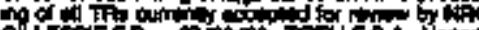

chict

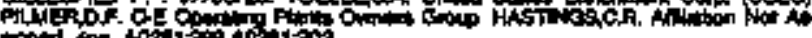

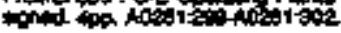

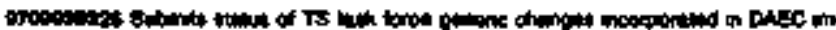

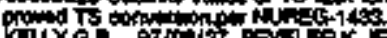

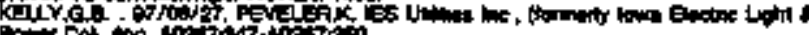

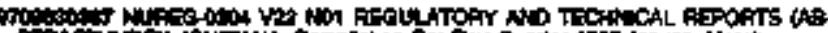

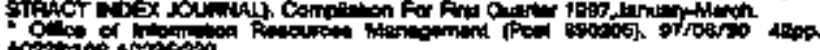

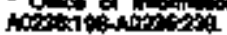

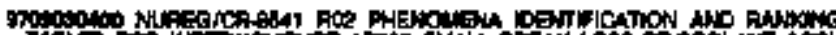

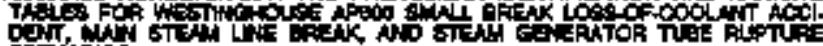
octirition

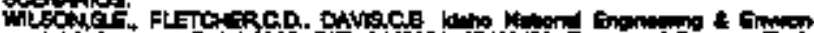

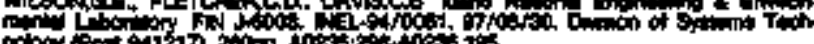

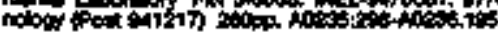

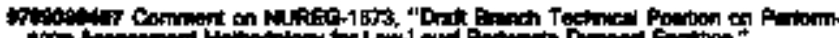
E- A

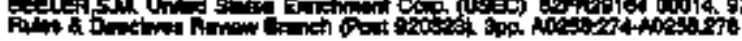

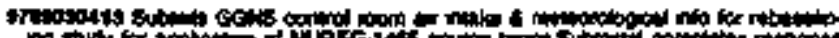

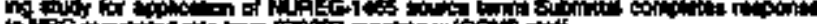

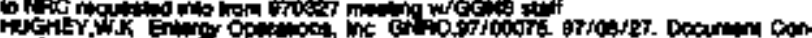

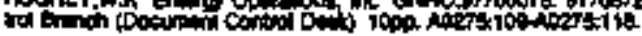

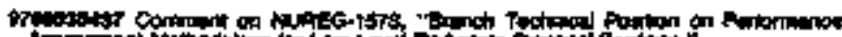

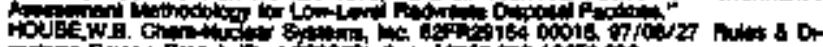

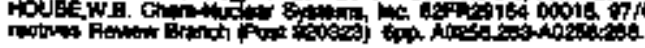

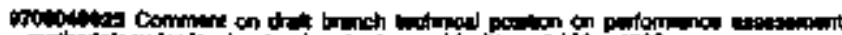

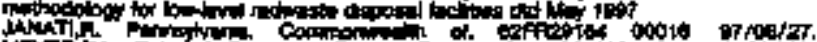

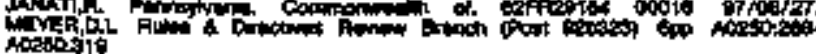

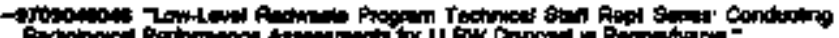

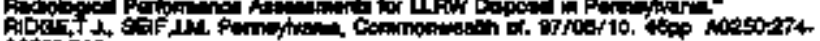
Anstist.

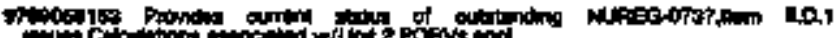

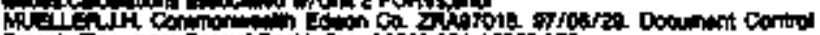

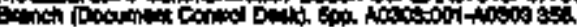

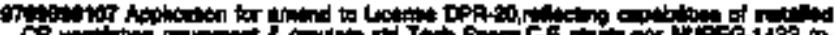

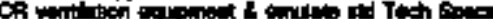

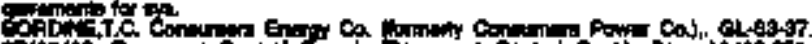

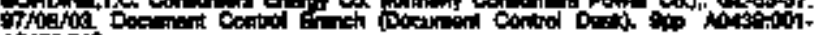
thengitis

- in

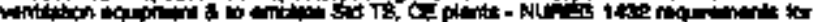

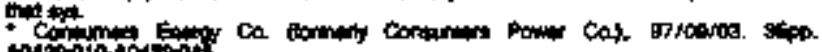

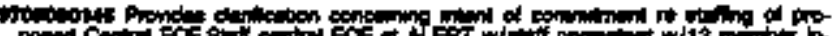

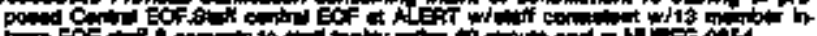

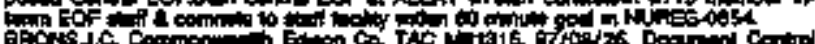

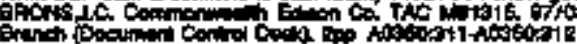

mowapes Fon

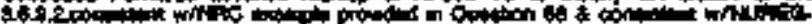

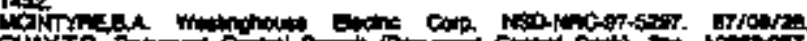

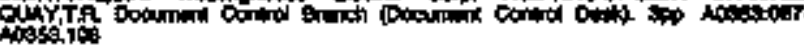

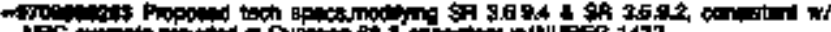

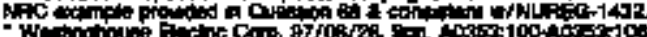

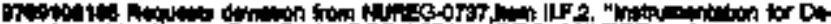

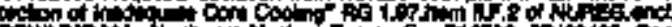

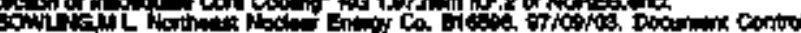

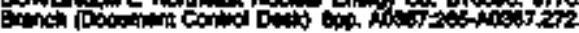

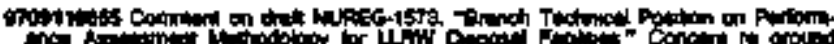

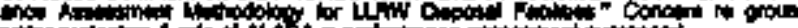

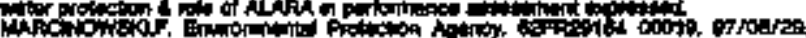
minc

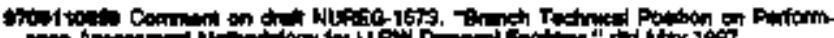

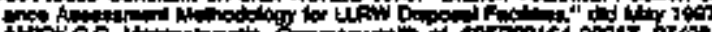

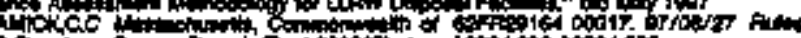

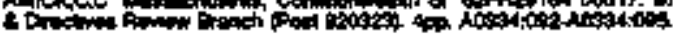

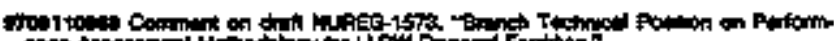

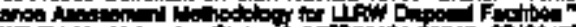

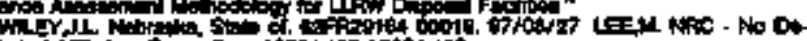

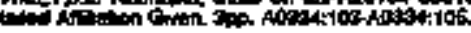

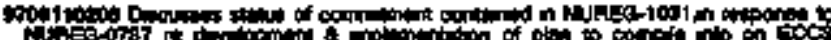

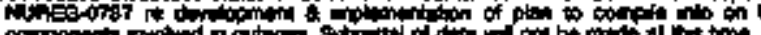

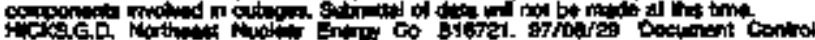

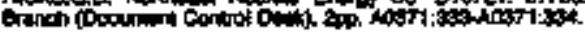

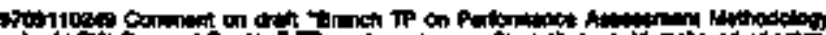

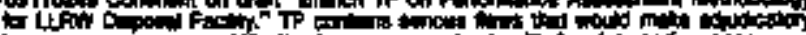

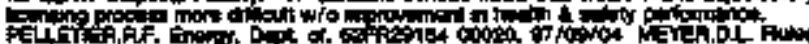

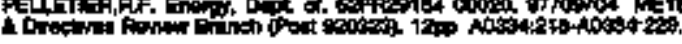

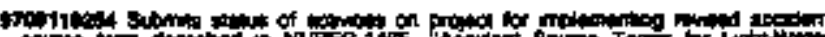

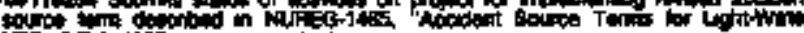

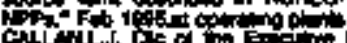

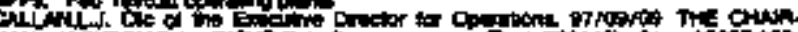

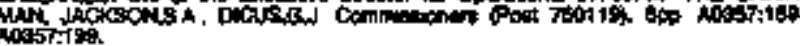

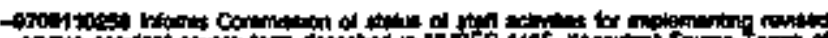

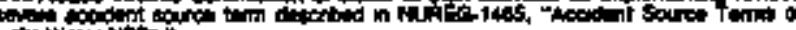

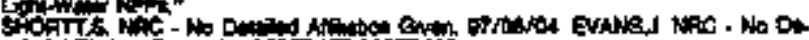
the dif

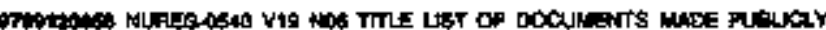

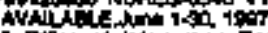

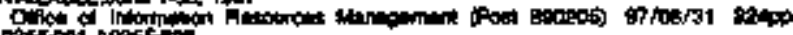

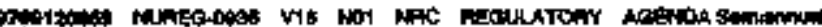

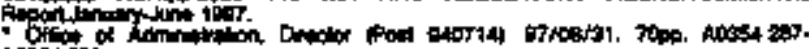
ansisento

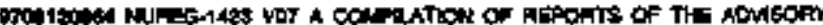

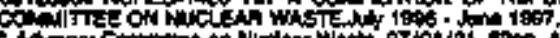

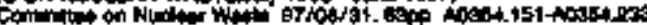

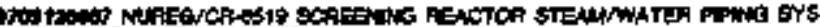
B

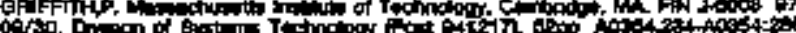




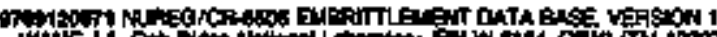

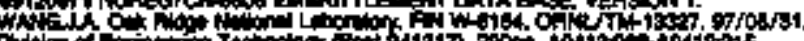

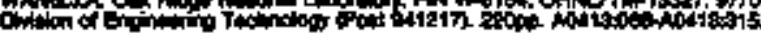

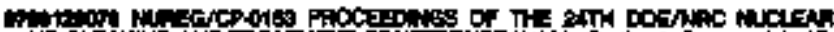

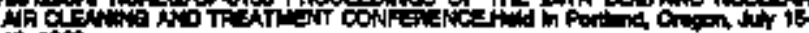

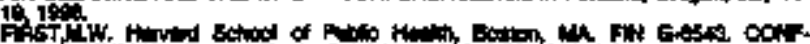

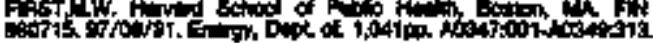

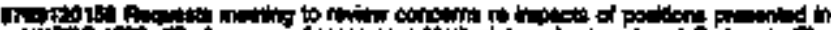

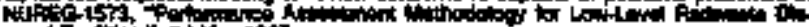

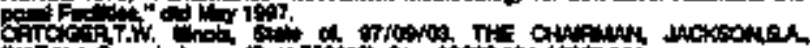
OH

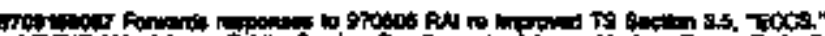

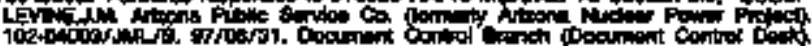

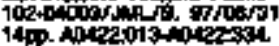

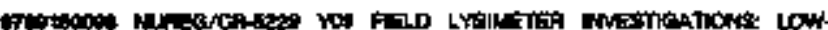

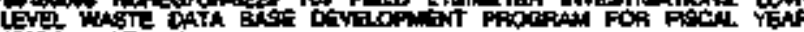

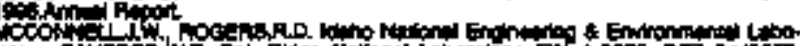
OFt

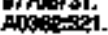

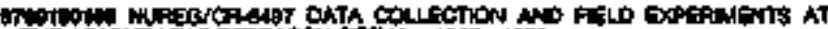

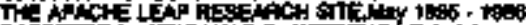

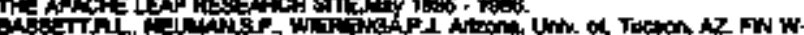

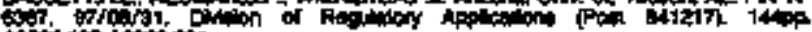

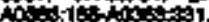

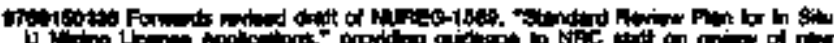

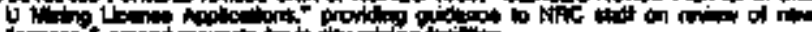

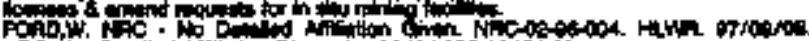

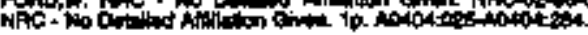

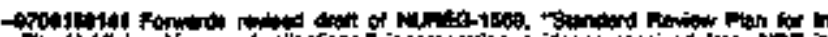

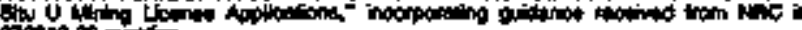

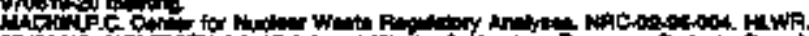

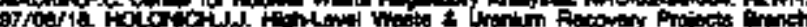

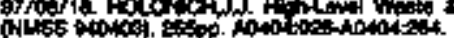

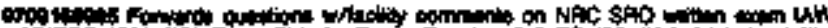

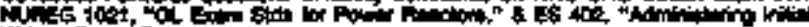

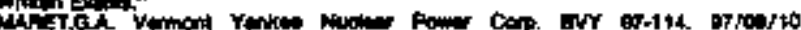

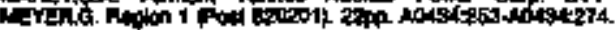

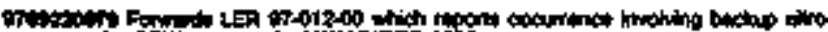

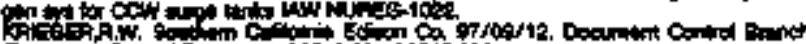

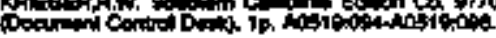

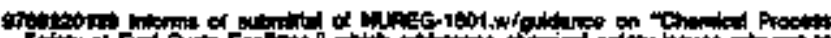

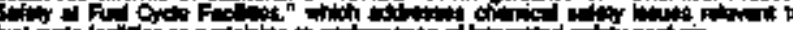

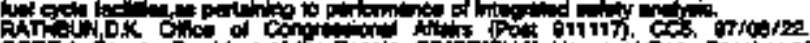

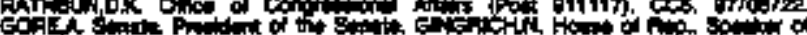

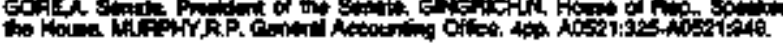

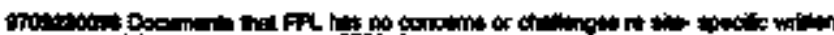

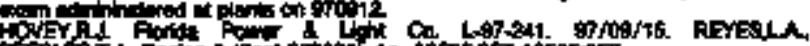

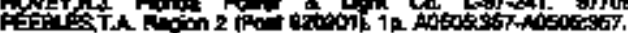

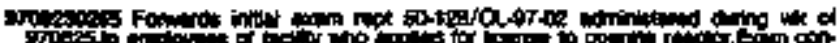

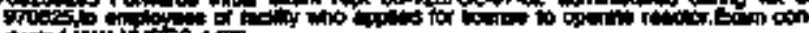

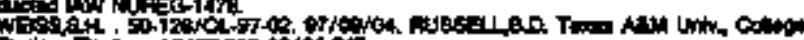

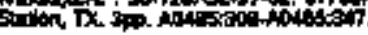

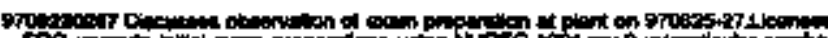

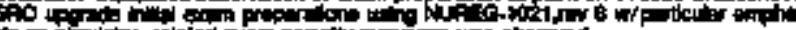

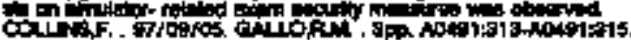

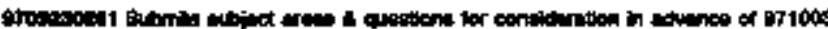

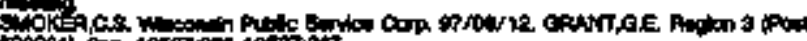

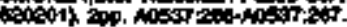

Or:

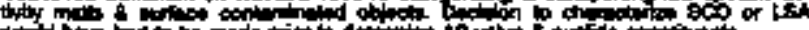

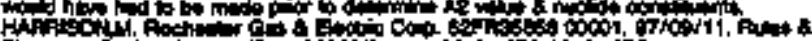

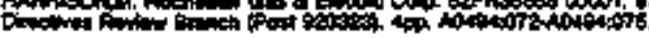

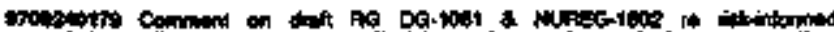

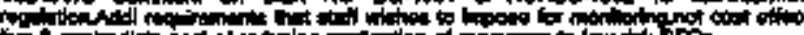

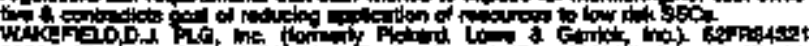

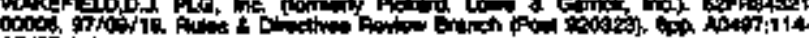
ODAFititi

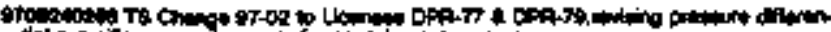

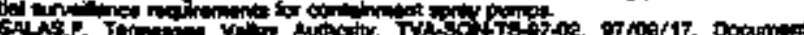

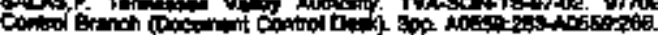

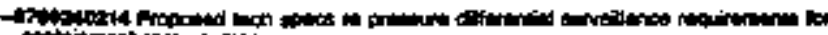

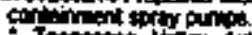

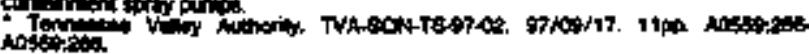

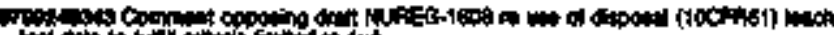

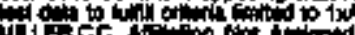

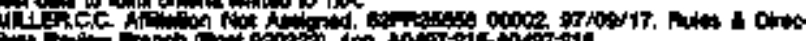

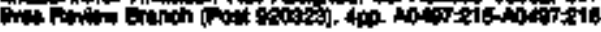

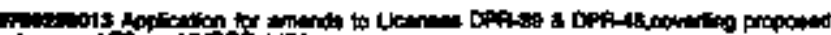

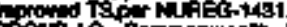

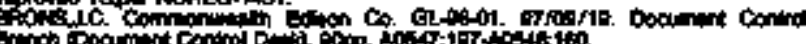

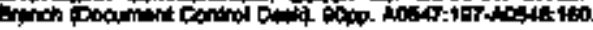

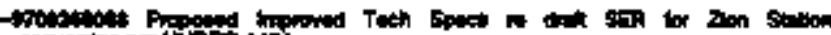

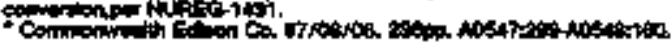

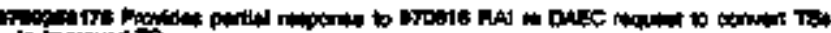
lotapowitis

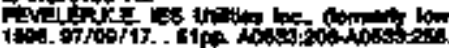

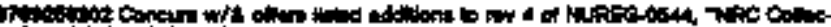
bint 연

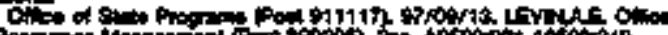

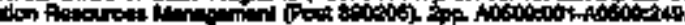

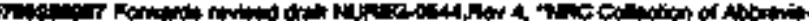

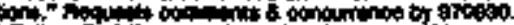

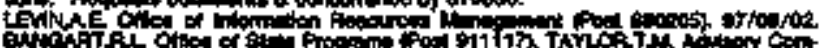

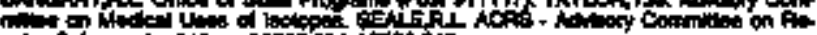

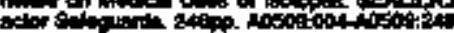

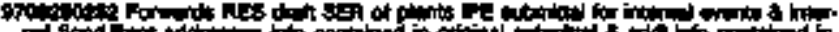

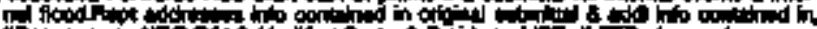

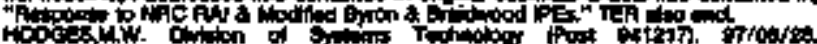

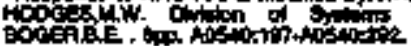

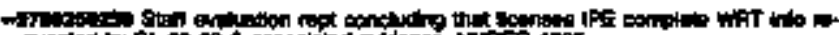

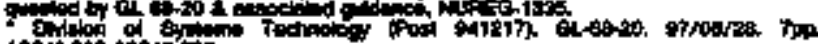

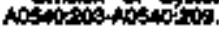

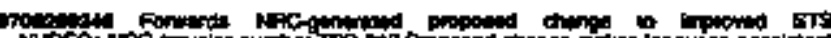

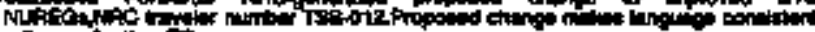

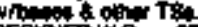

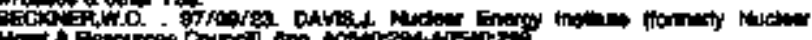

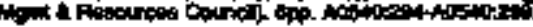

on

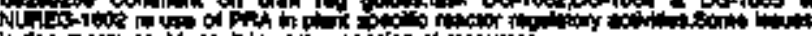

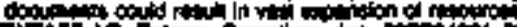

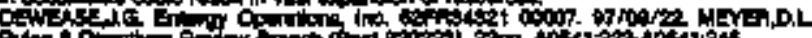
等

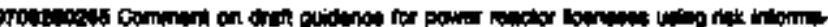

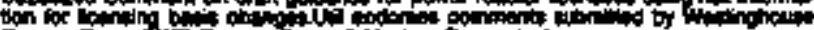

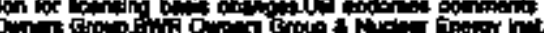

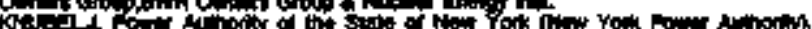
os Q

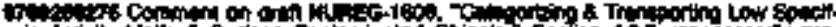

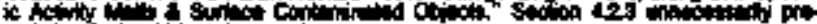

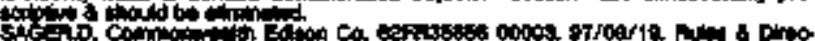

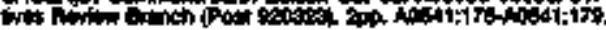

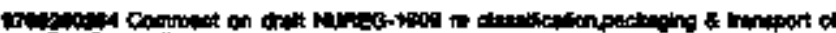

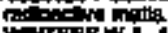

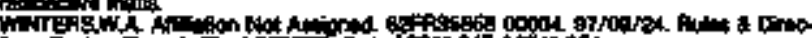

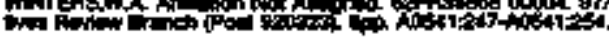

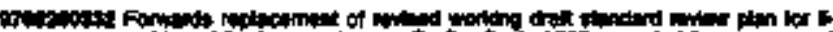

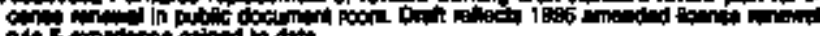

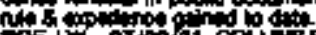

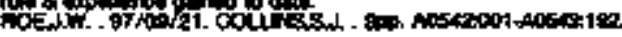

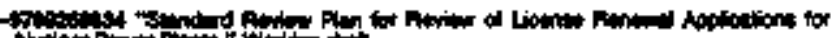

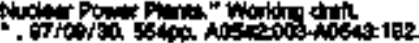

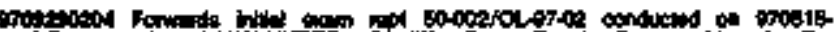

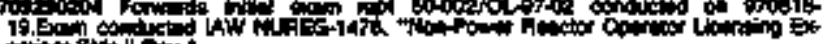

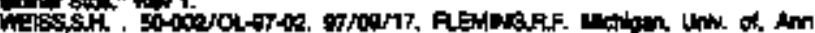

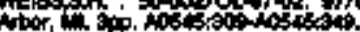

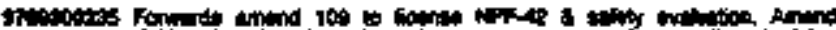

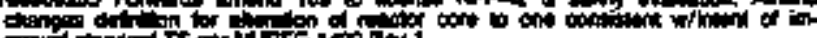

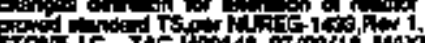

sin is

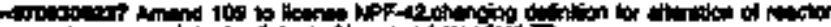

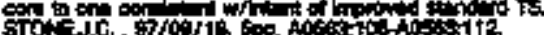

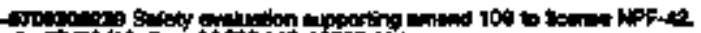
- .

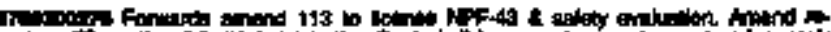

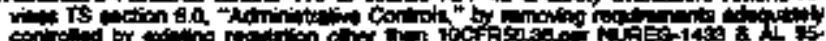

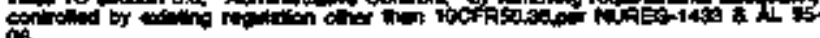

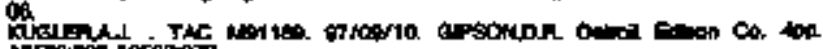

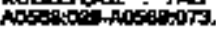




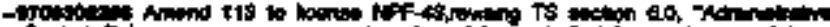

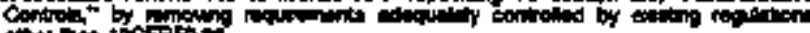

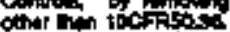

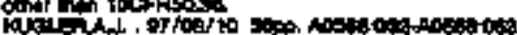

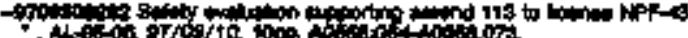

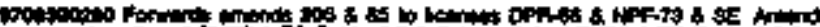

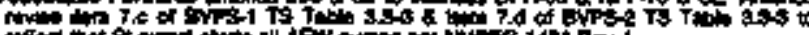

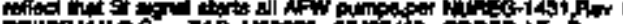

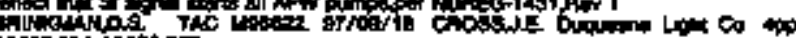

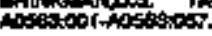

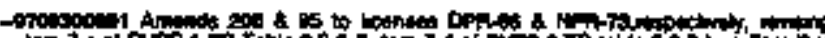

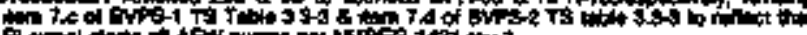

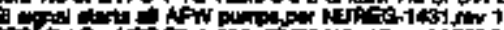

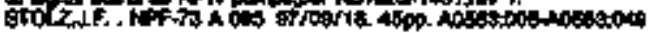

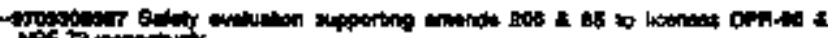

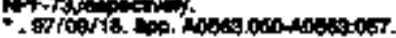

ond ofmurentions

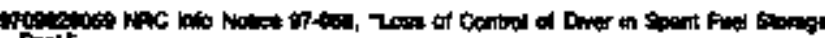

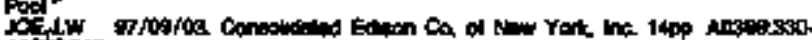
astisto

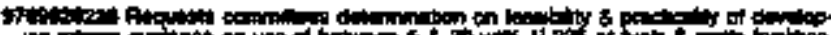

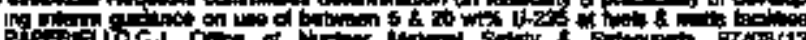

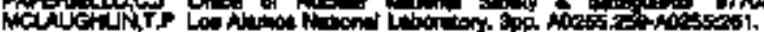

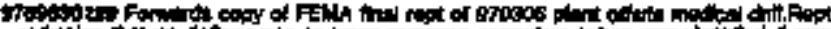

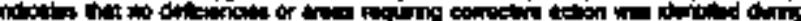

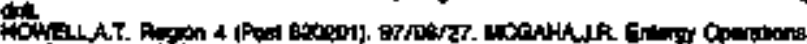

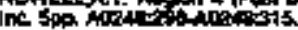

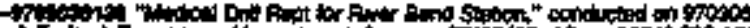

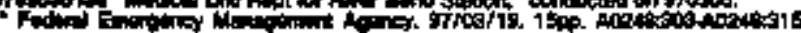

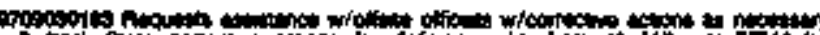

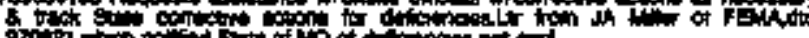

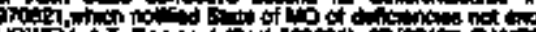

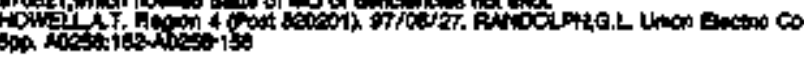

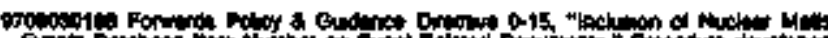

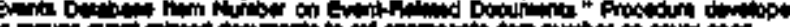

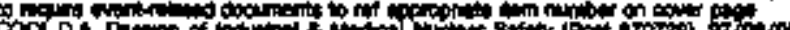

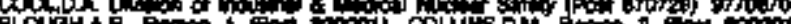

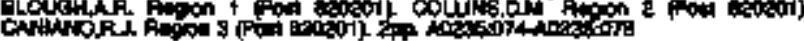

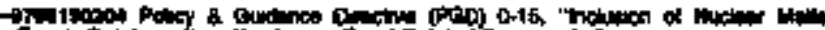

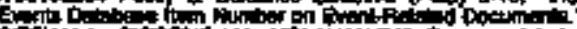

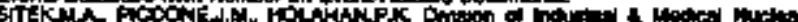

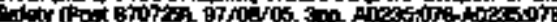

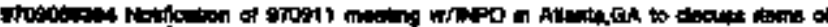

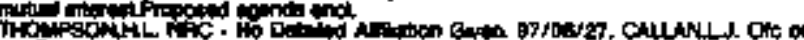

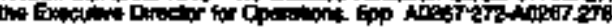

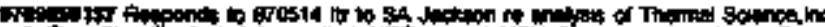

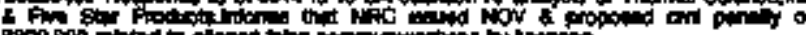

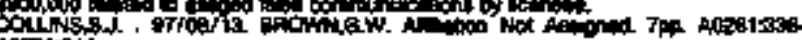

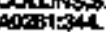

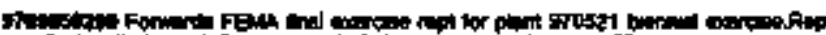

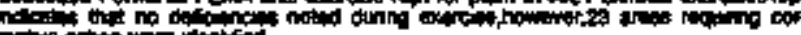

Co

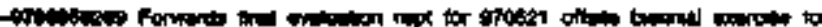

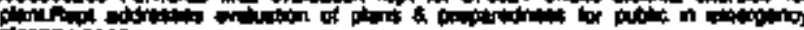

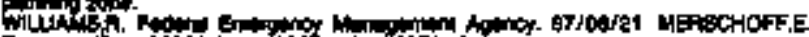

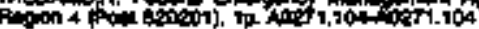

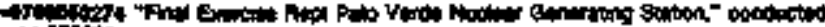
on $\cos$.

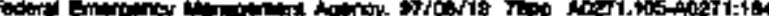

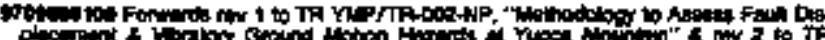

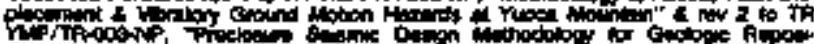

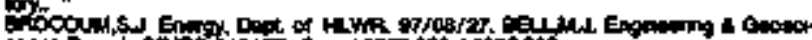

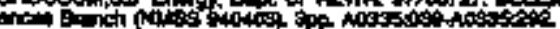

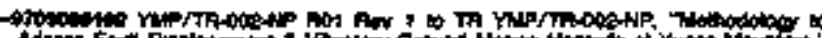

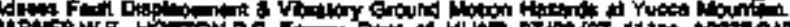

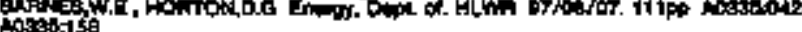

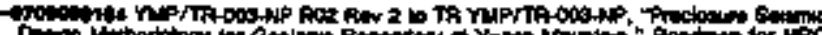

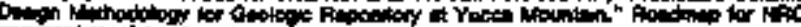

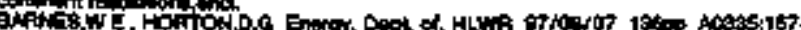
nosstais

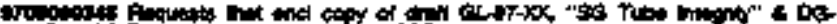

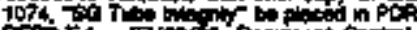

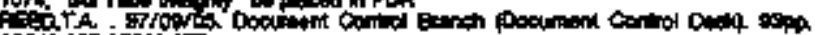

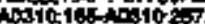

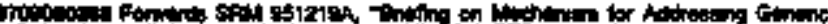

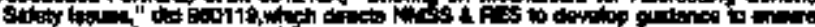
cot inct

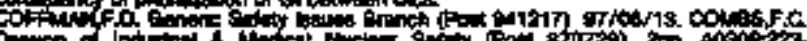
Anotion

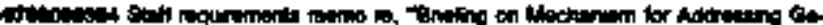

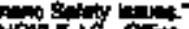

XYy tictors

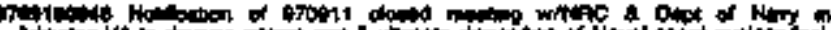

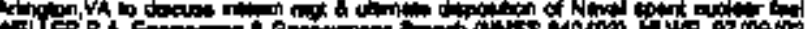

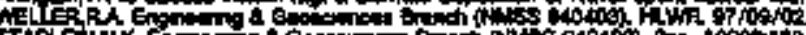
sise:

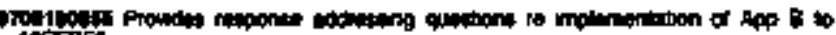

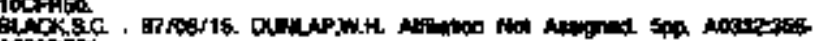
intorit.

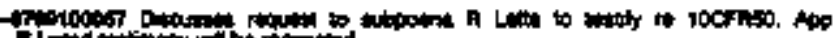

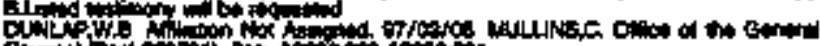

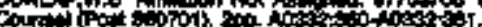

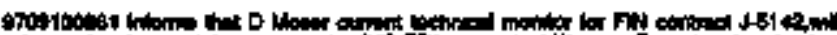

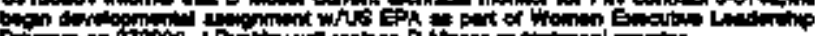

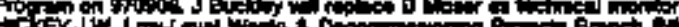

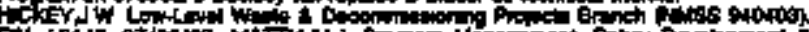

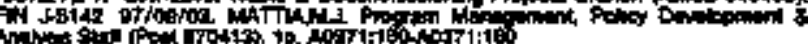

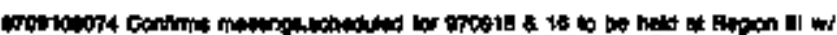

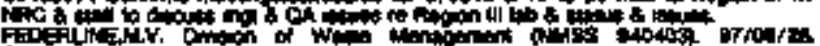
Fin:

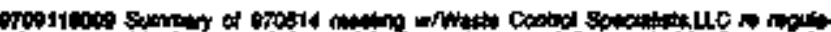

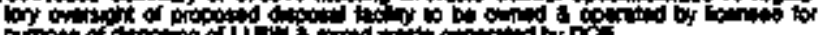

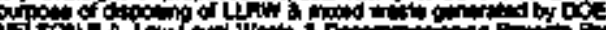

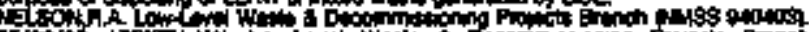

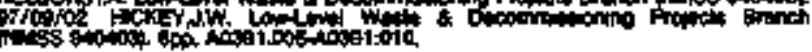

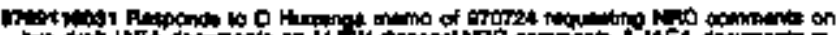

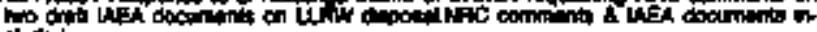
thenction

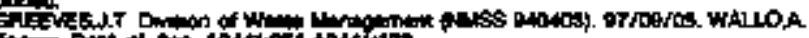

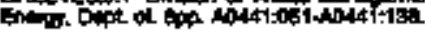

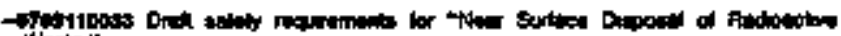

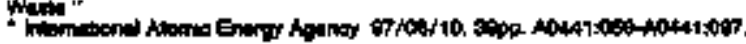

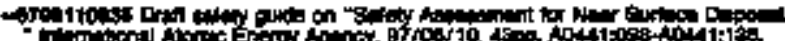

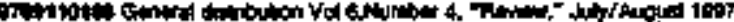

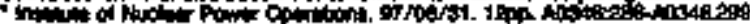

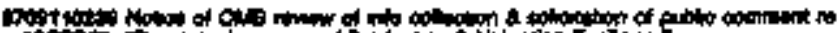
I0CF

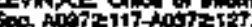

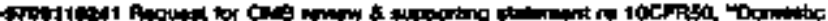

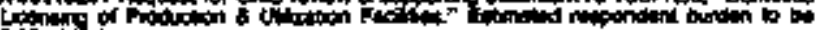

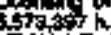

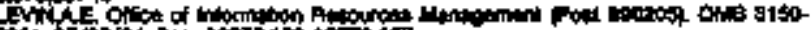
Di1 G700\%

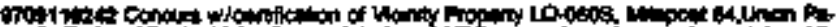

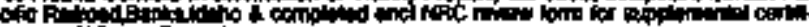

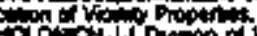

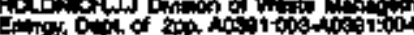

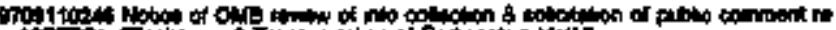

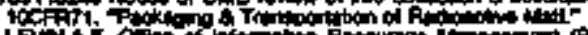

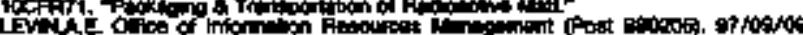

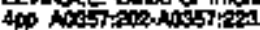

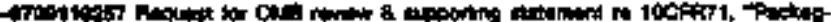

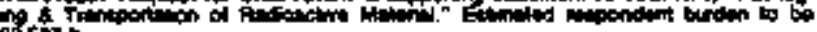

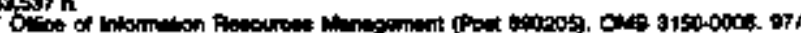

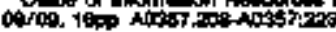

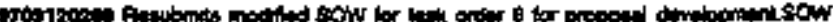

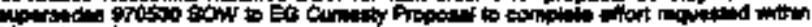

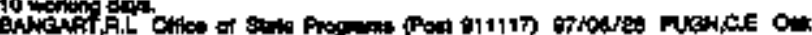

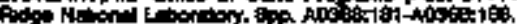




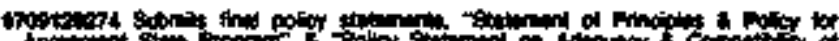

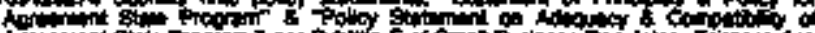

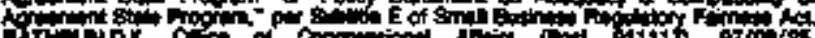

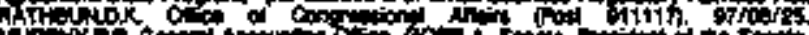

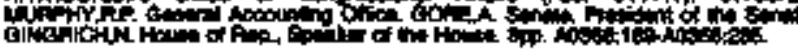

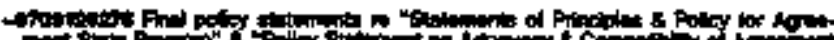

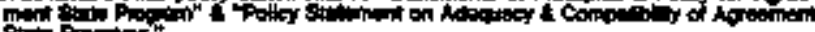

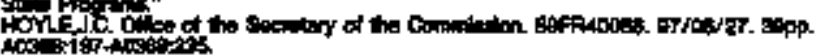

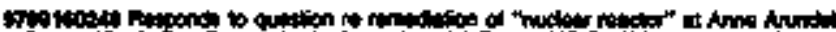

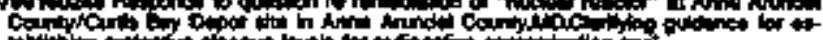

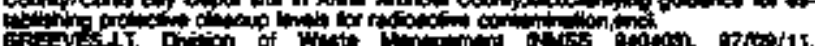

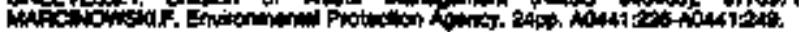

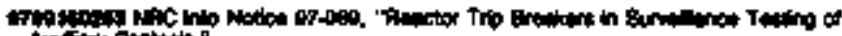

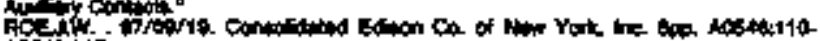
Aostatit?

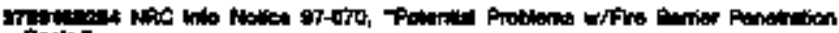

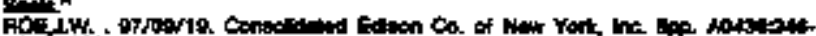
Aocicoses

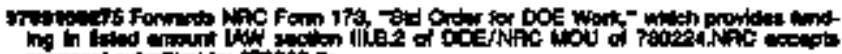

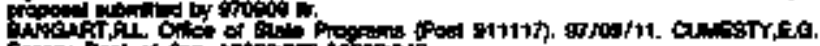

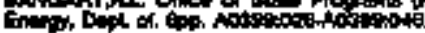

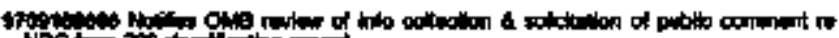

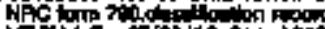

Low

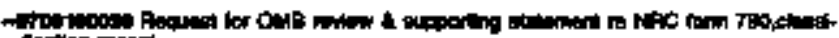

7.

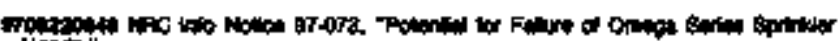
BC:

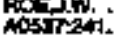

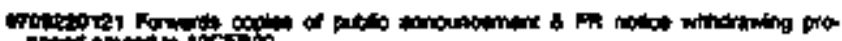

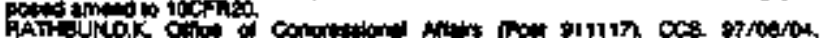

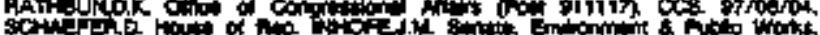

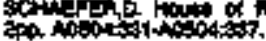

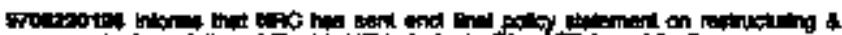

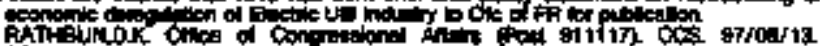

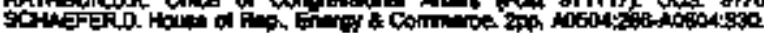

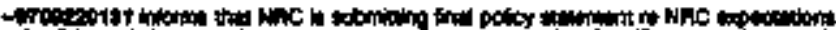

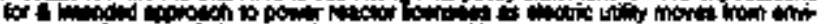
ofith

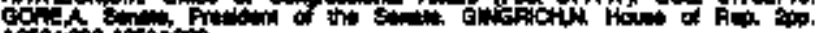
000400000

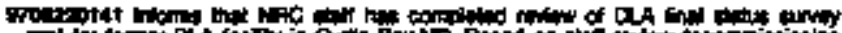

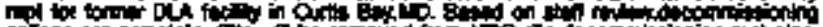

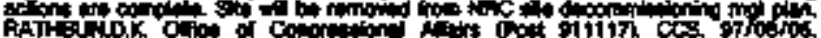
AATH

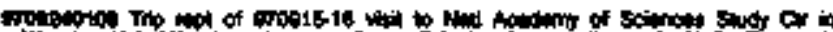

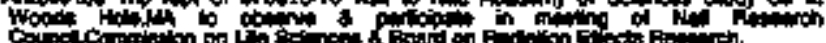

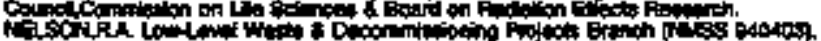

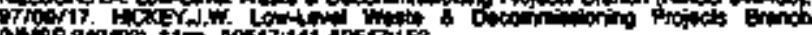

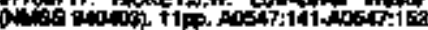

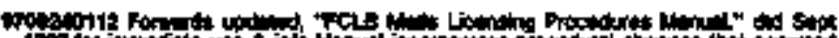

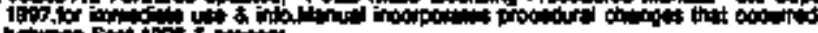

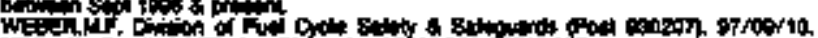

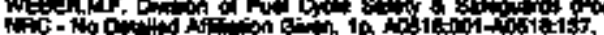

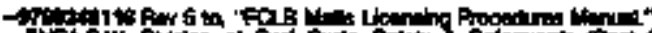

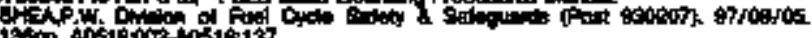

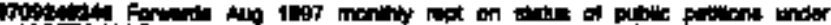

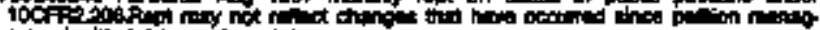

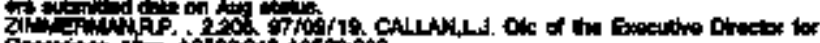

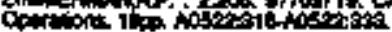

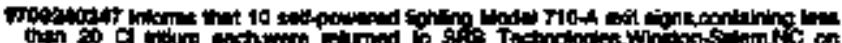

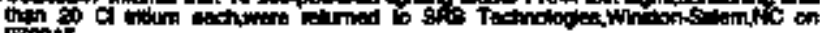
Eroets.

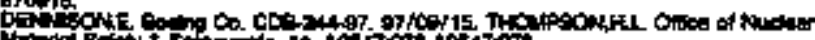

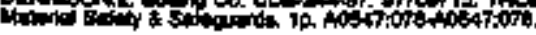

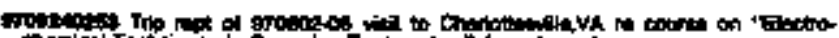

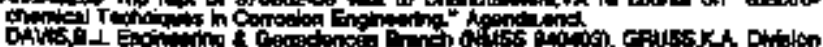

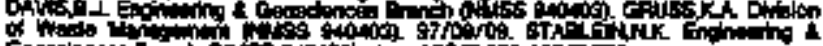

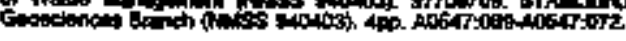

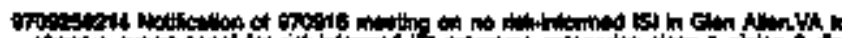

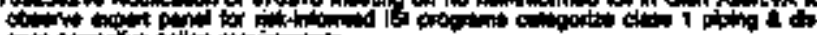

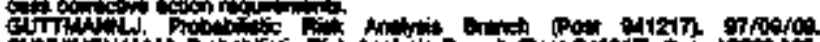

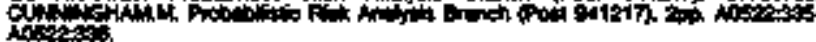

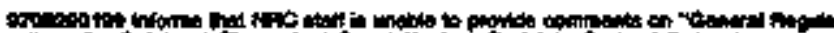

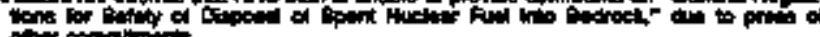

of

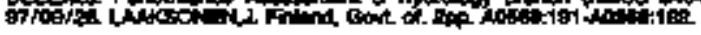

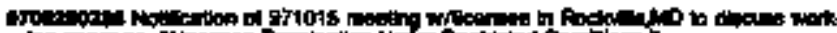

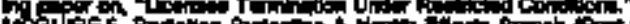

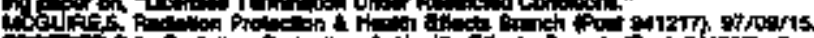

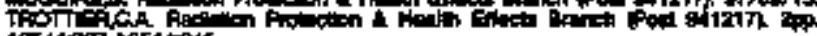

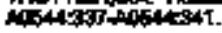

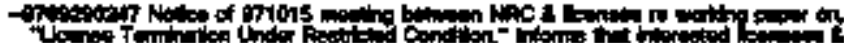
Il dipon

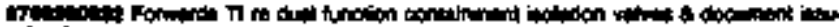

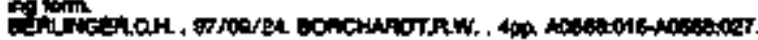

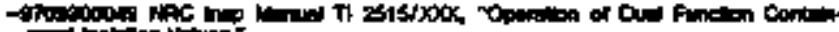

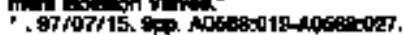

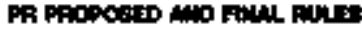

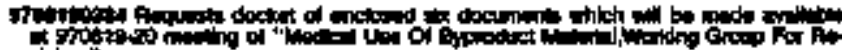

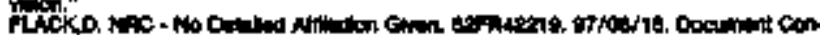

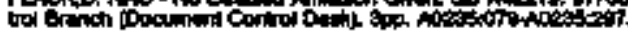

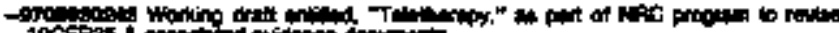

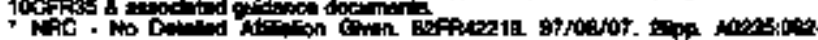
notion.

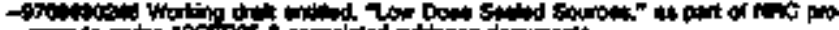

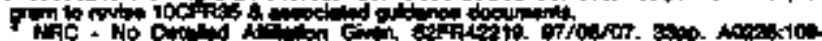
nes:140.

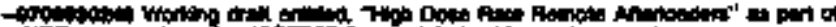

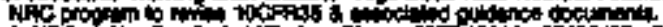

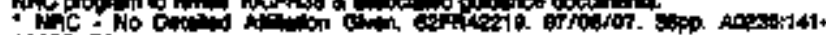
notion

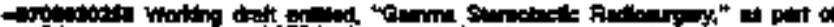

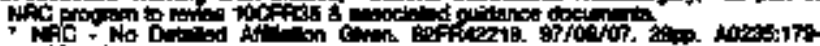
nokstistit.

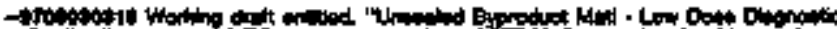

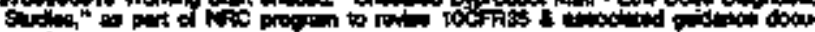

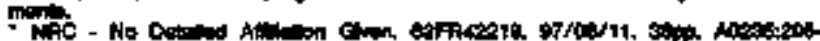
notristis.

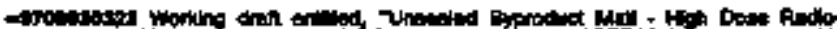

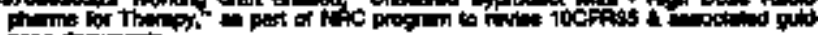

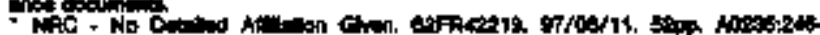
nozpoter.

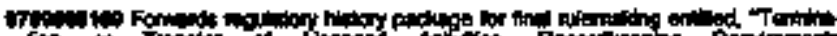

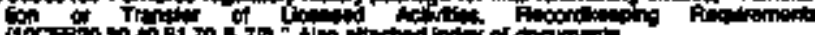

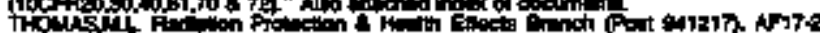

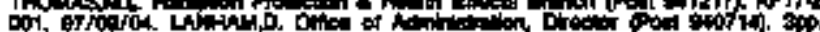

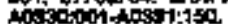

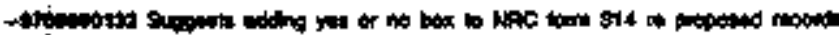

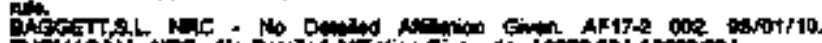

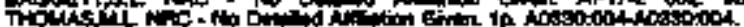

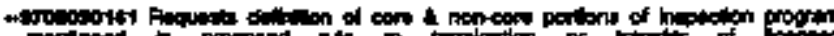

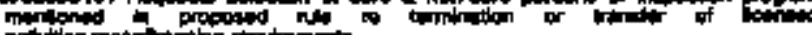

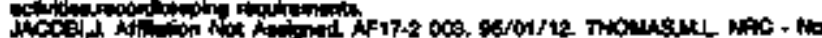

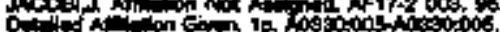

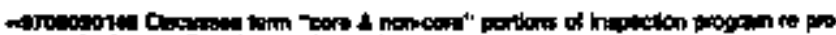

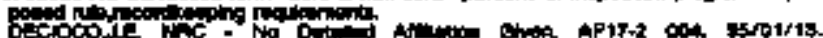

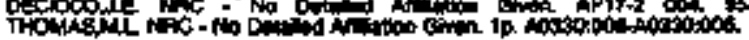

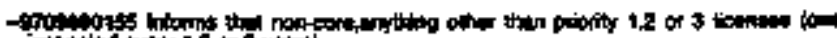

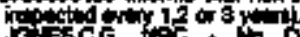

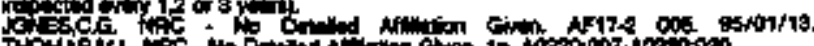

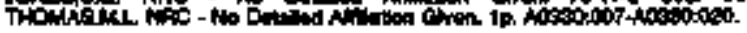




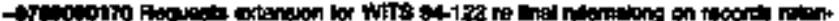

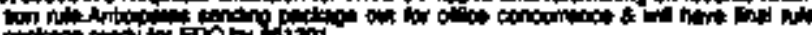

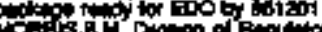

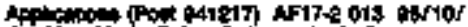

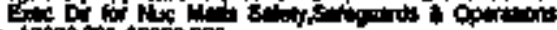

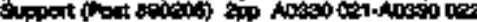

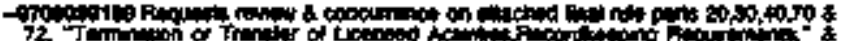

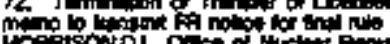

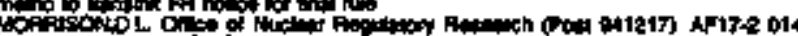

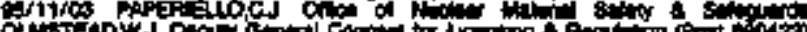

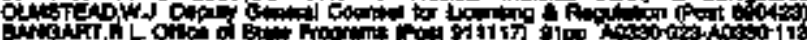

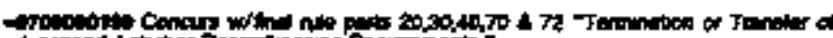

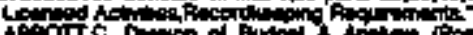

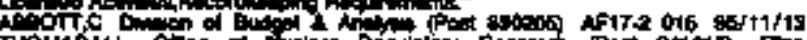

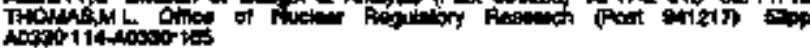

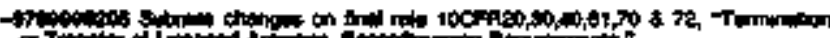

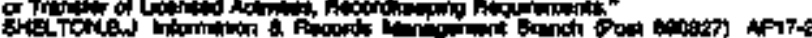

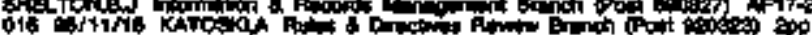

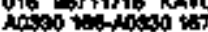

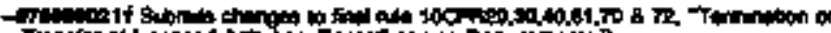

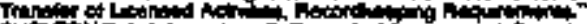

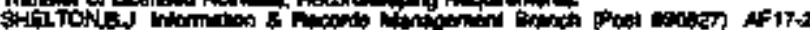

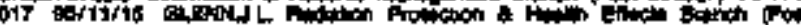

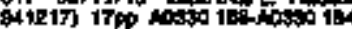

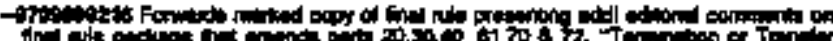

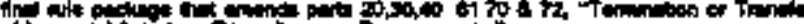

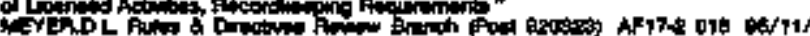
18 贷

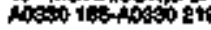

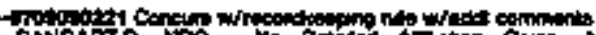

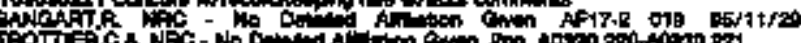

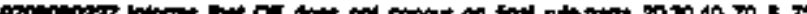

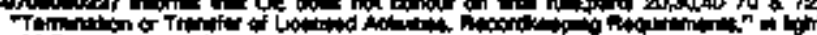
of wôf

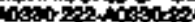

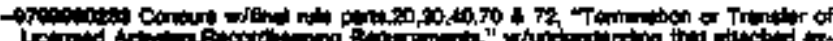

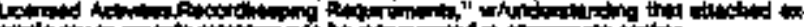

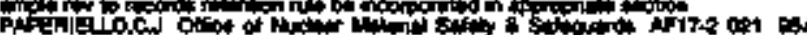

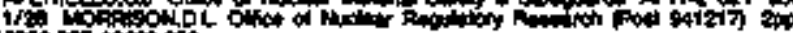

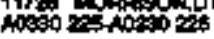

- Ho

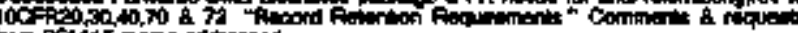

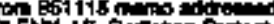

等 等

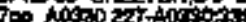

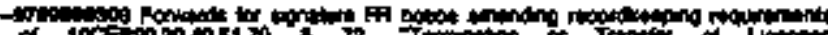

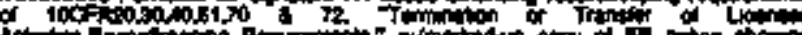

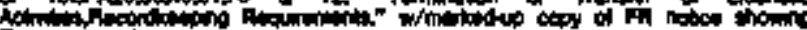

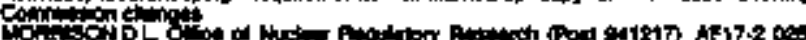
wolfo TAY Aosto

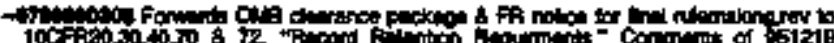

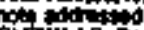

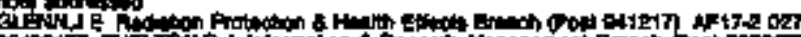

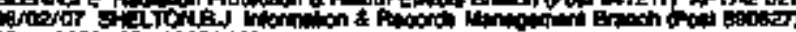

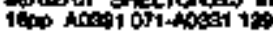

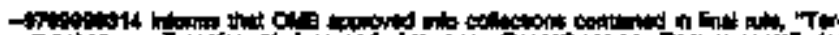

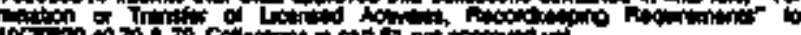

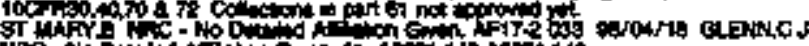

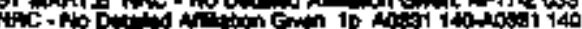

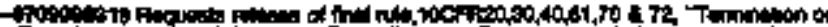

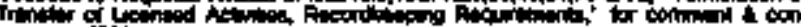

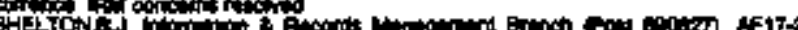

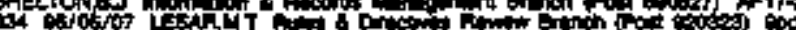
Acosi 141 14005 100

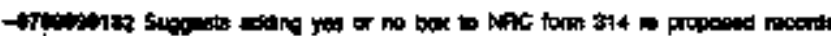

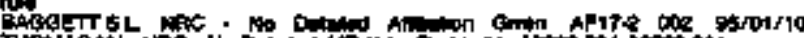

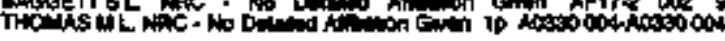

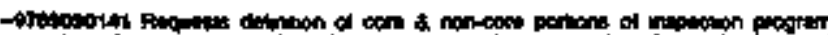

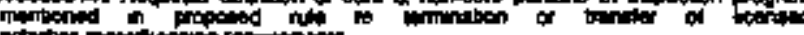

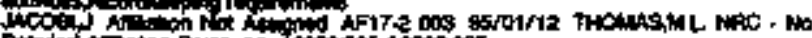

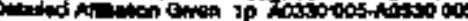

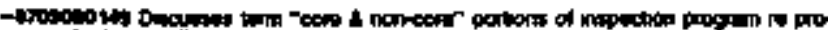

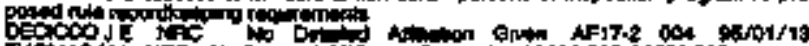

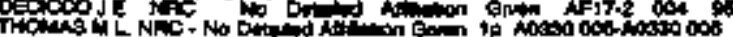

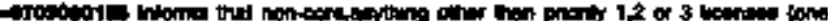

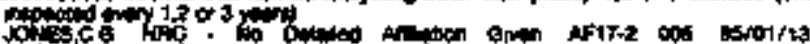

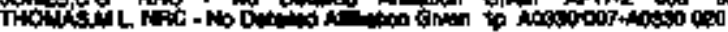

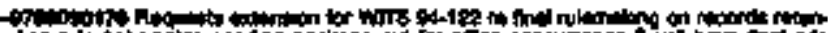

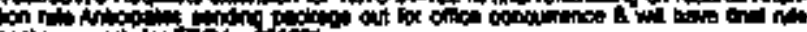

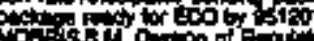

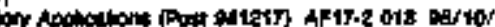

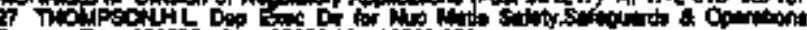

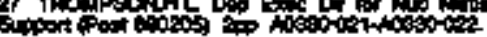

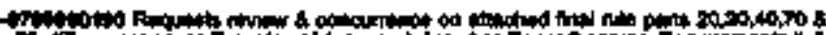

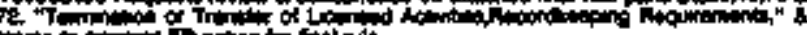

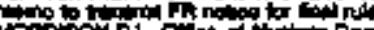

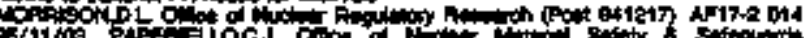

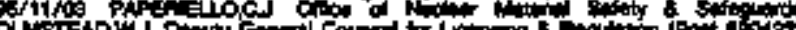

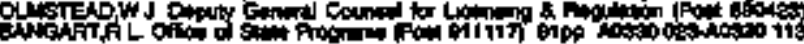

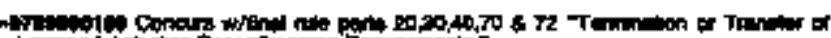

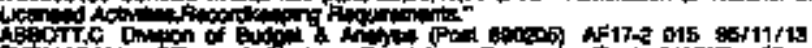
170

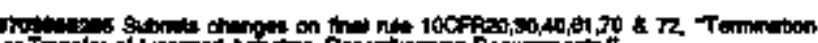

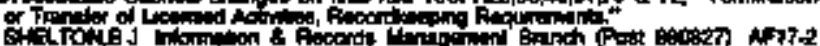

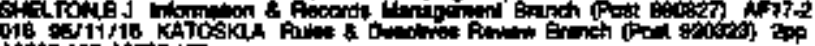
(16)

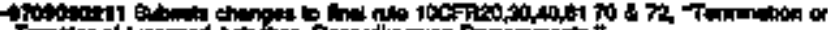

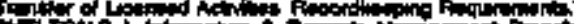

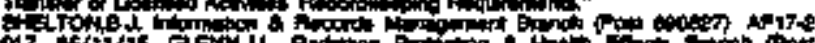

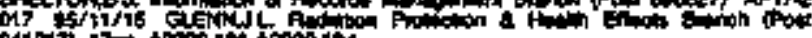

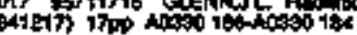

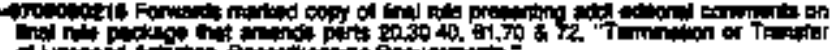

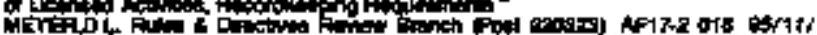

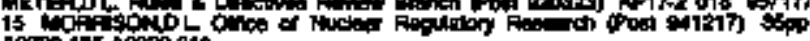

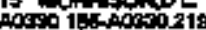

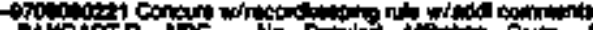

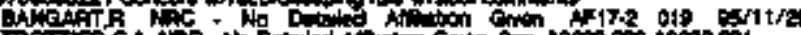
TOÓT丨

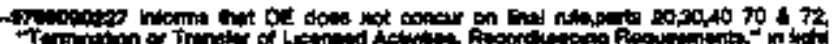

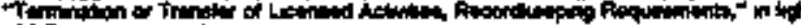
of

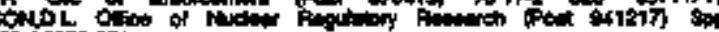
10000 ecencoso 224

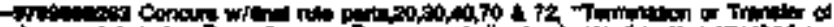

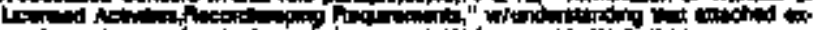

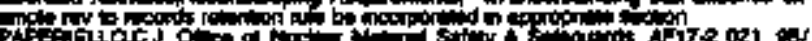
Nhicict of

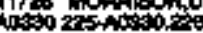

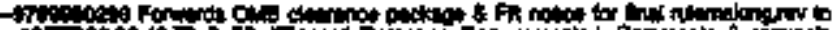

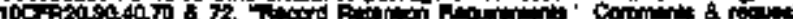

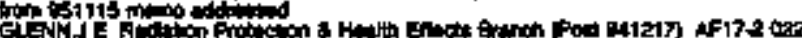

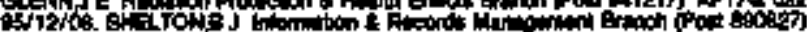

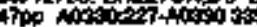

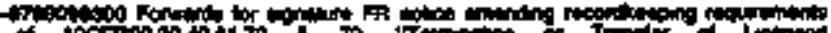

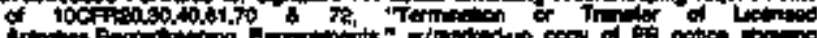

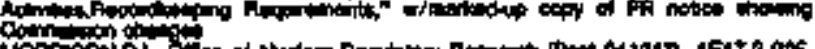

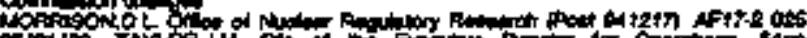

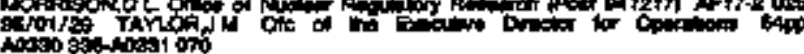

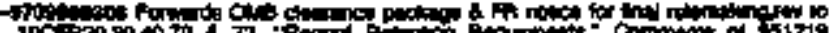

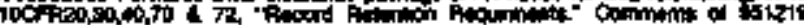

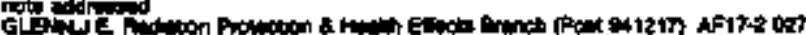

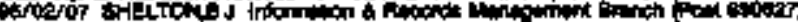

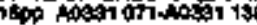

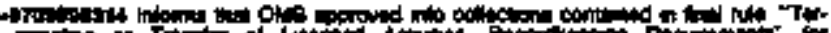

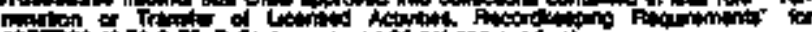

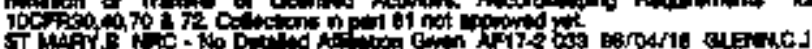
Ric-Fo

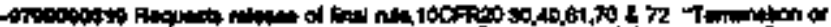

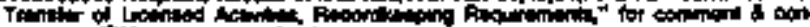

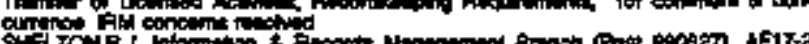

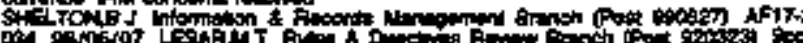

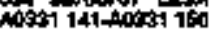

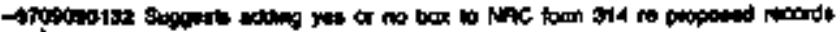

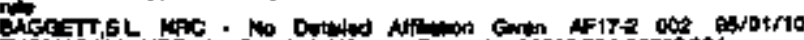

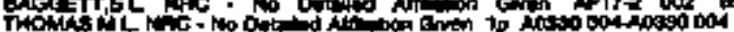




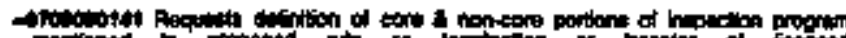

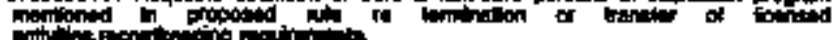

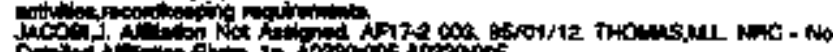

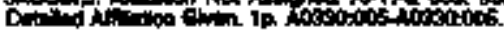

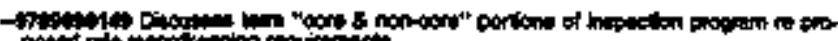

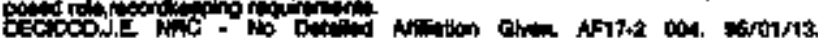

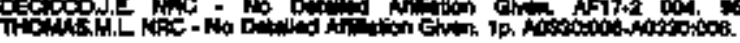

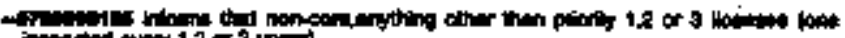

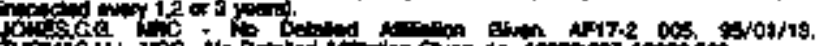
titis

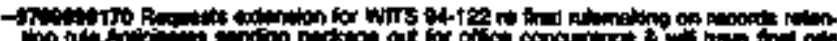

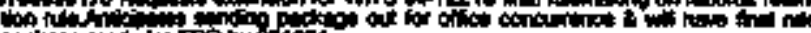

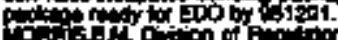

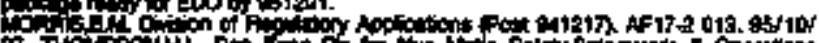

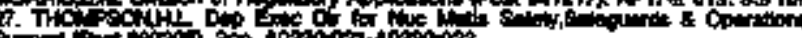

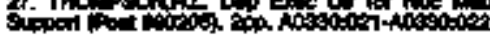

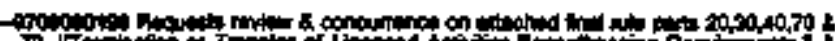

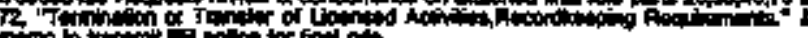

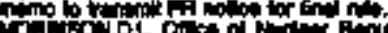

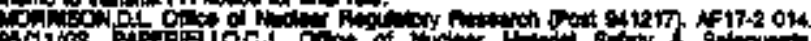

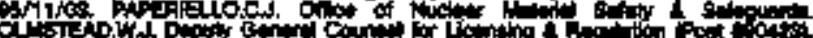

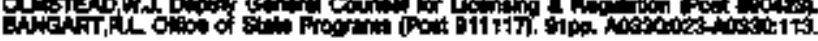

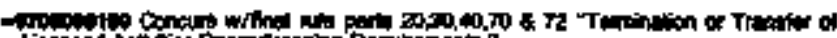

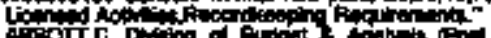

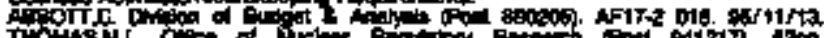

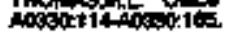

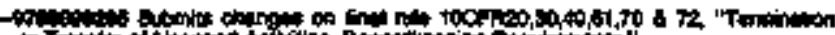

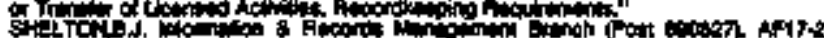

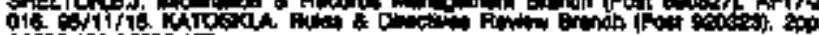

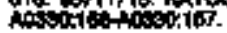

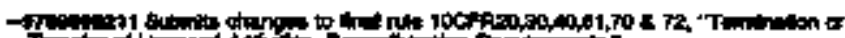
Th

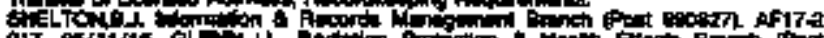

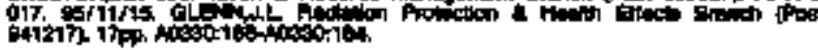

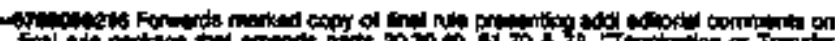

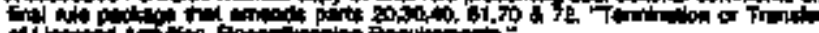
of 1 by of a 1.

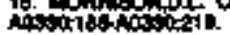

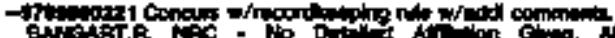

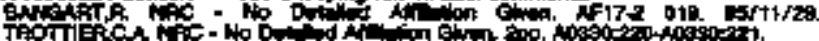

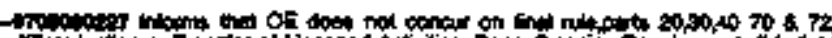

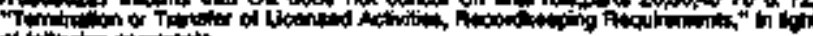

of

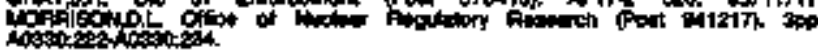

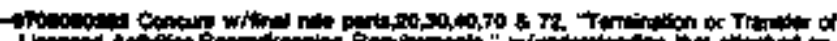

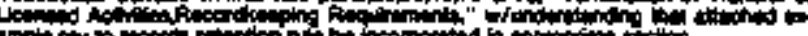

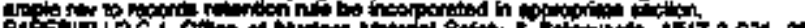

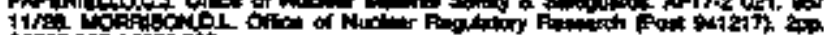

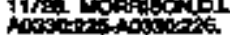

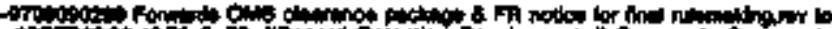
toc

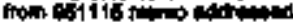

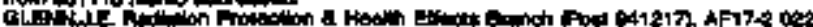

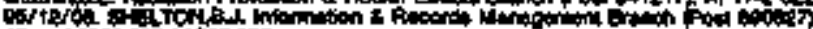

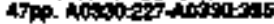

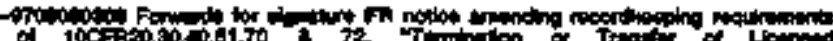

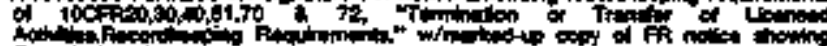

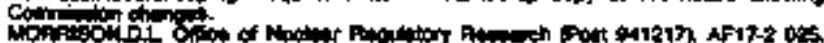

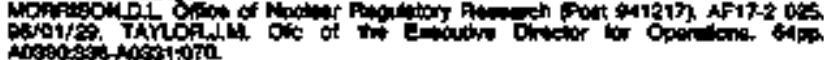

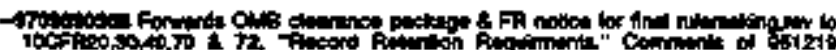

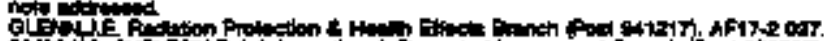

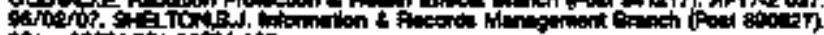

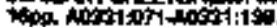

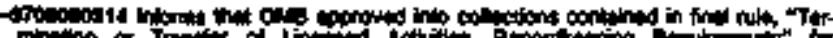

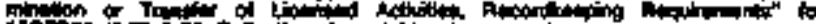

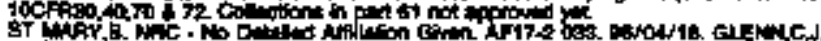

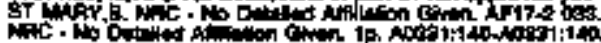

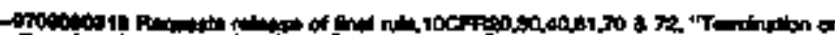

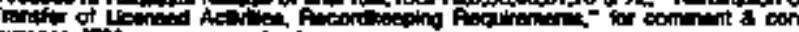

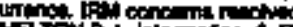

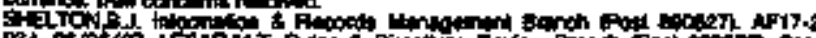

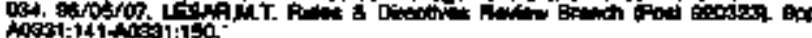

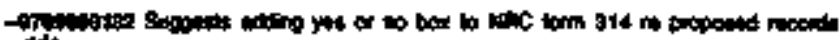

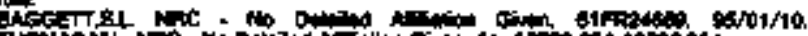

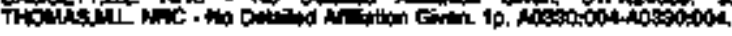

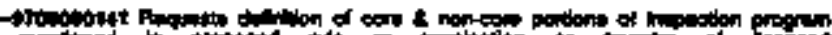

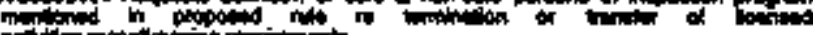

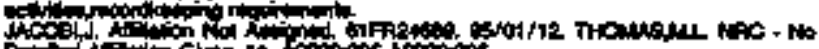

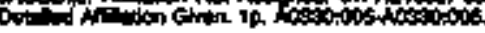

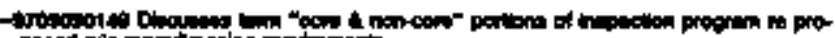

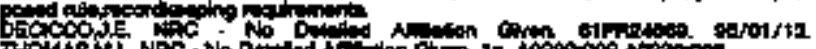

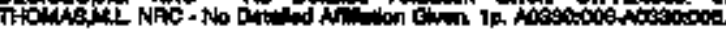

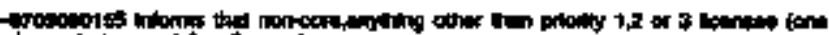

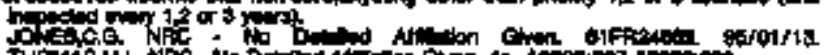

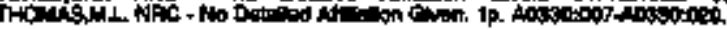

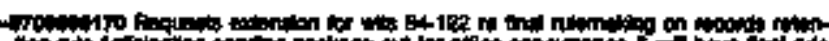

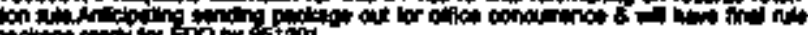

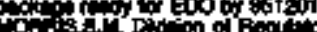

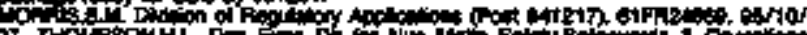

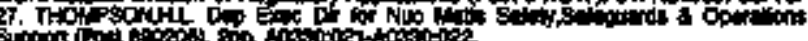

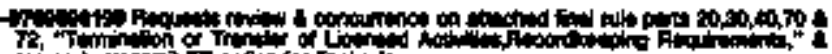

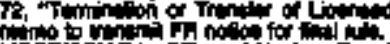

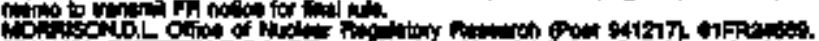

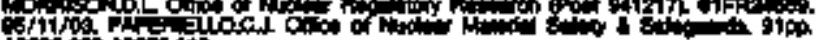

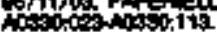

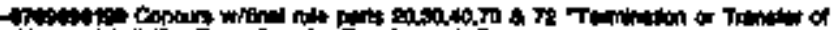

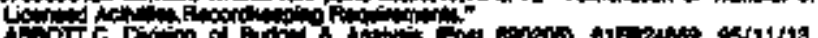

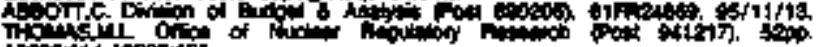

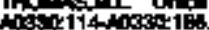

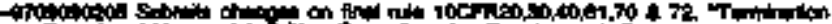

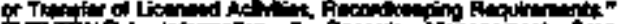

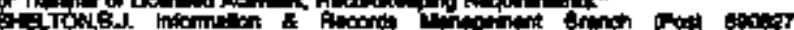

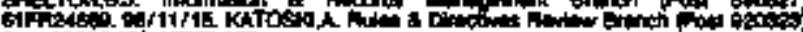

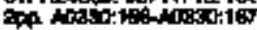

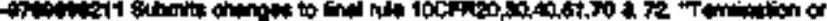

git:Tow

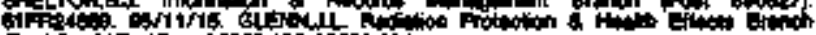

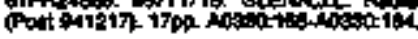

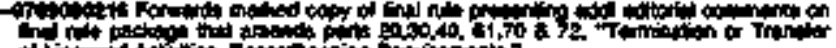

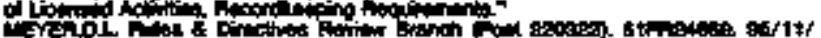

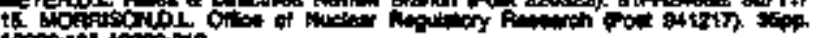

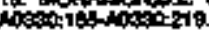

M

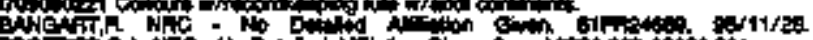
TAOTI

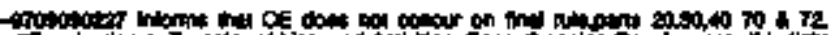

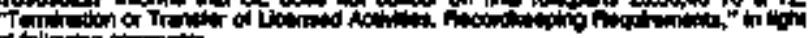

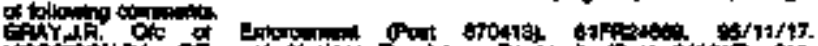

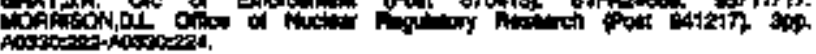

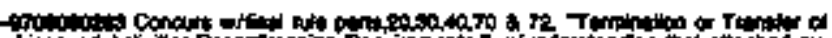

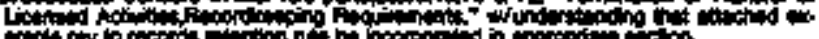

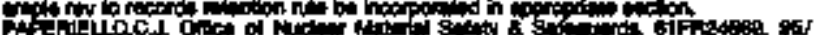
11: 1180

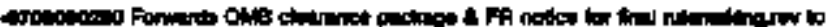
10<

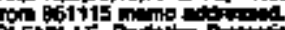

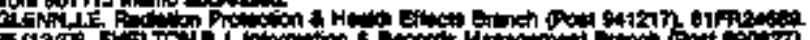

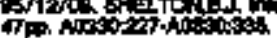

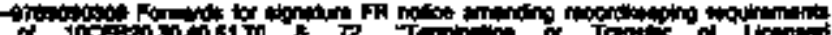

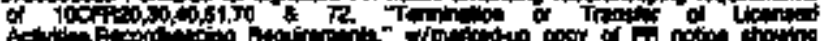

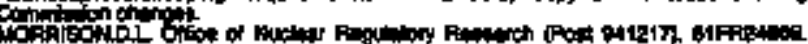

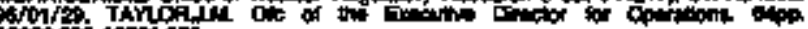

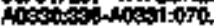

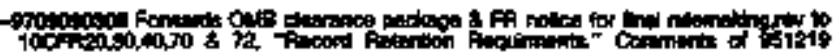

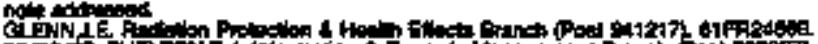

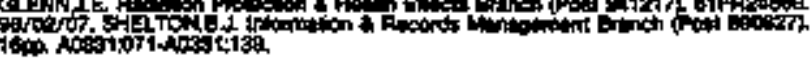

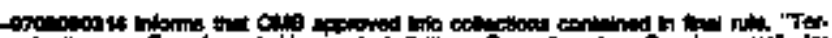
of

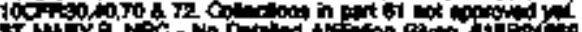




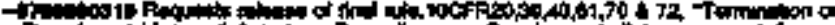

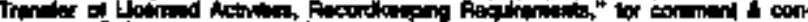

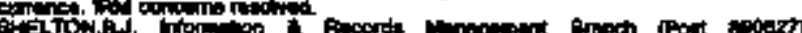

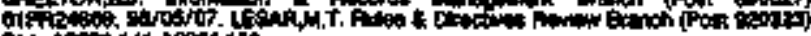

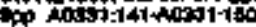

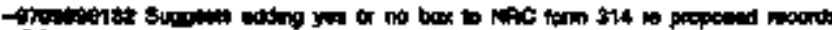

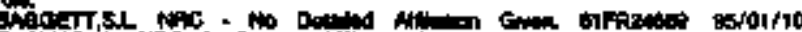

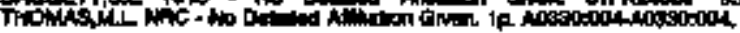

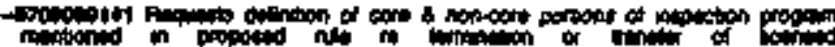
Wow

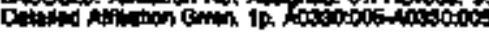

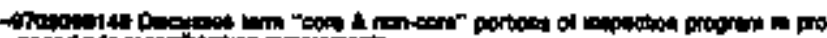

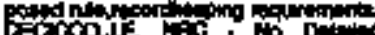

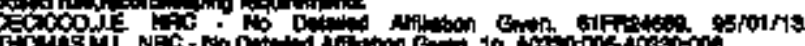
THCW

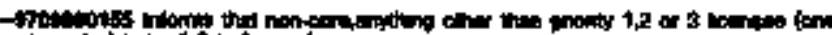

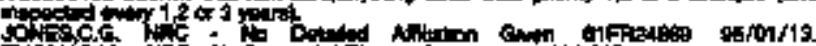

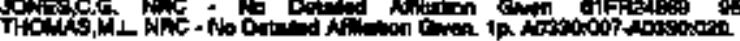

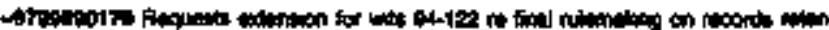

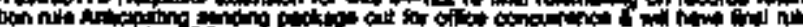

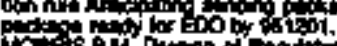

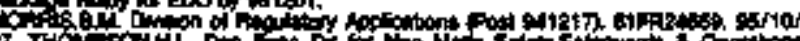

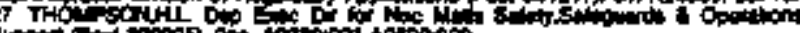

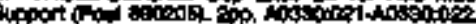

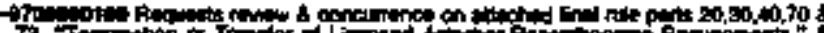

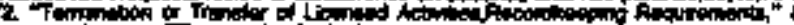
wo

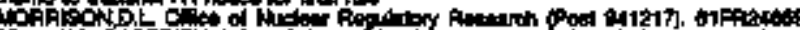

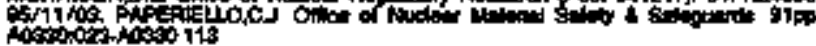

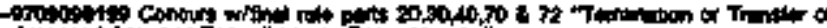

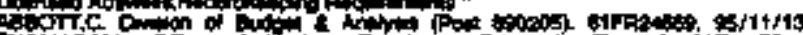

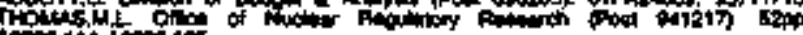

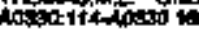

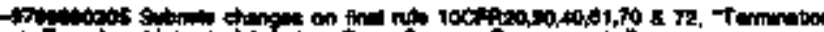

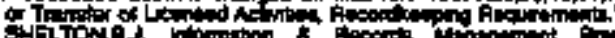

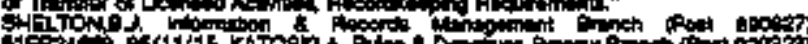

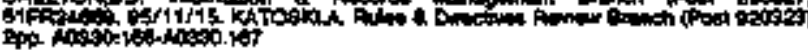

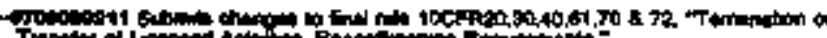

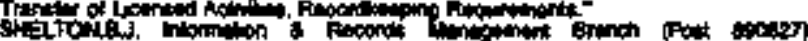

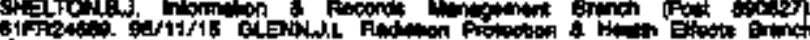

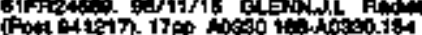

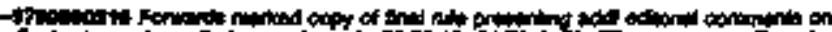

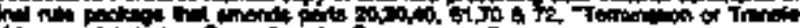

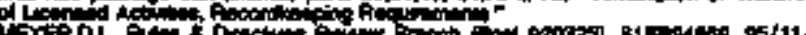
is

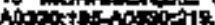

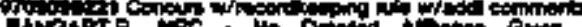

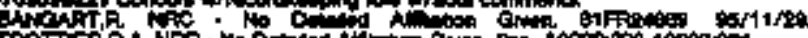

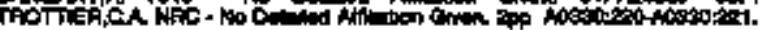

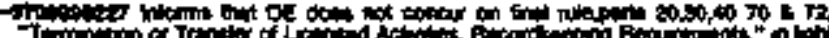

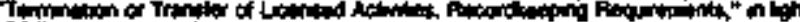

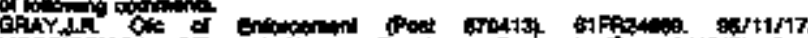

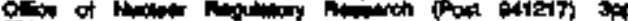

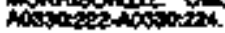

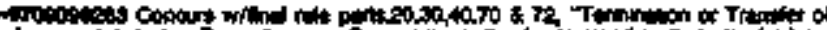

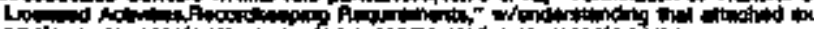

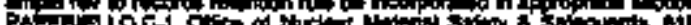

PA J 1914

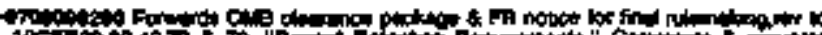

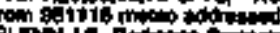

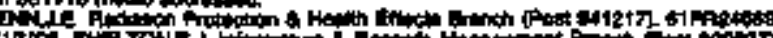

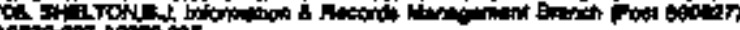

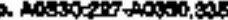

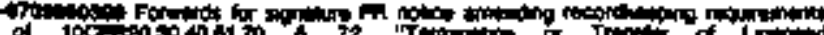

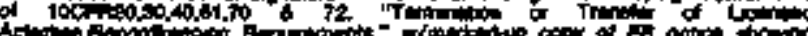

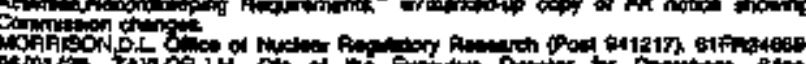

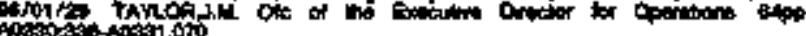

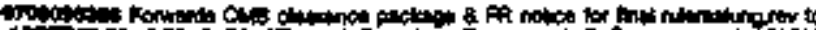

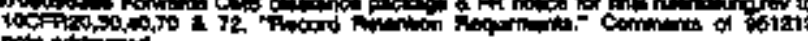

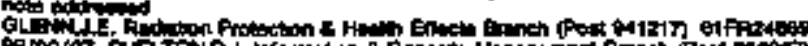

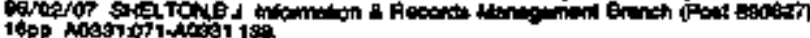

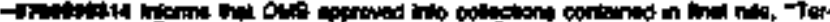

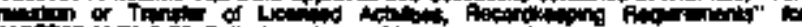

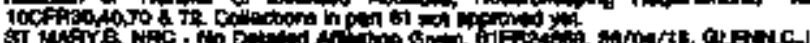

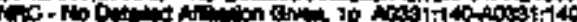

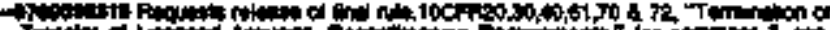

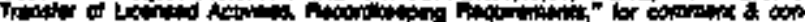

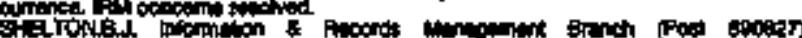

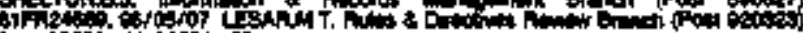

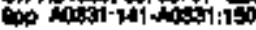

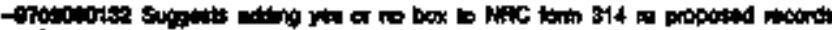

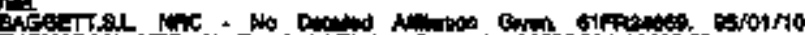

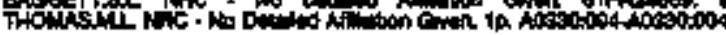

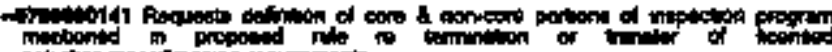

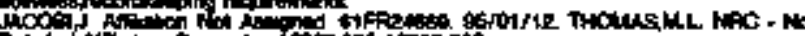

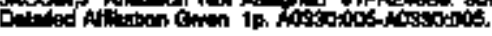

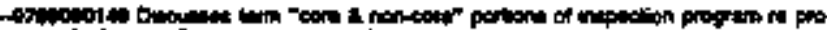

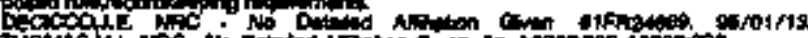

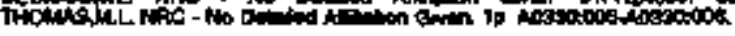

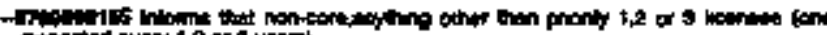

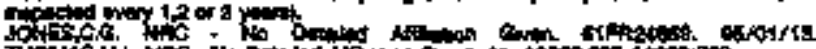

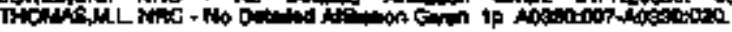

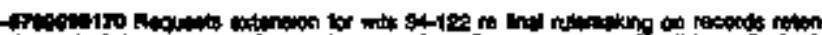

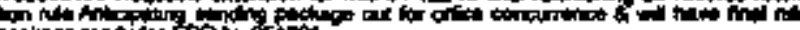

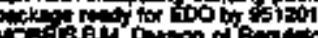

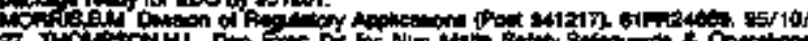

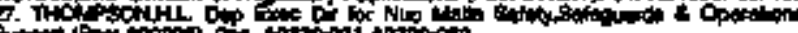
等

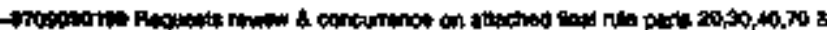

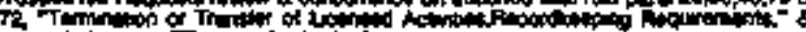

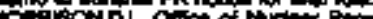

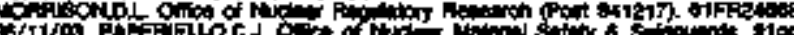

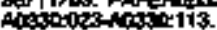

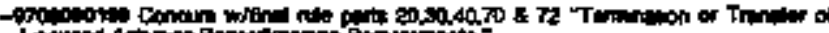

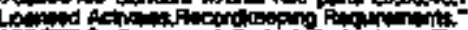

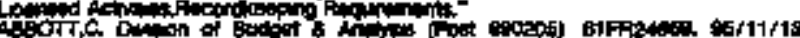
To

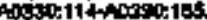

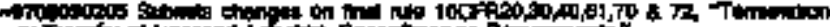

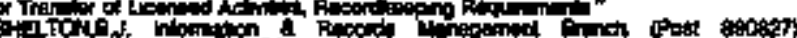

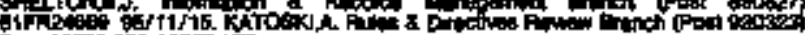

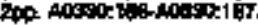

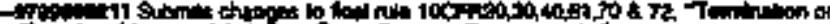

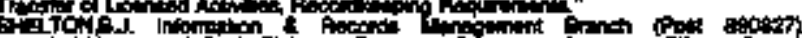

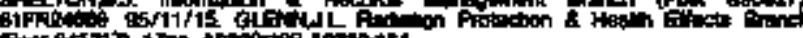

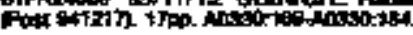

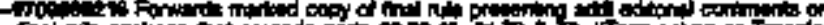

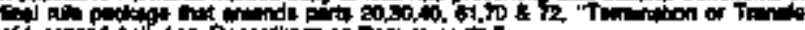

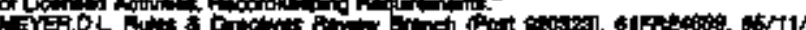
FY

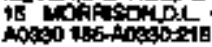

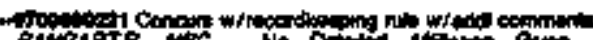

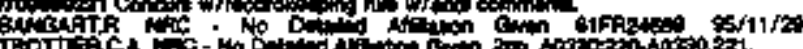

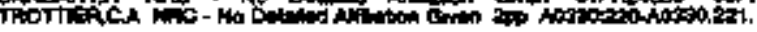

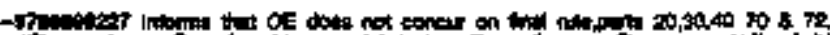

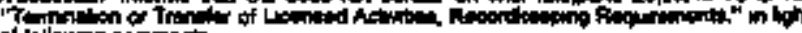

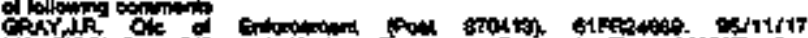

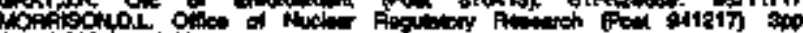

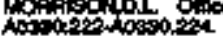

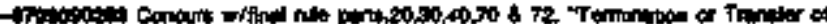

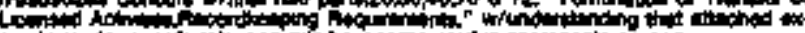

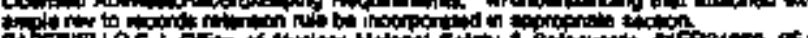

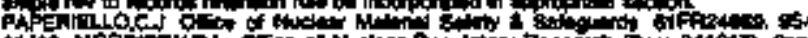

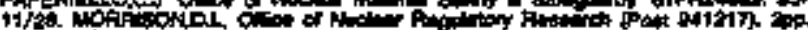

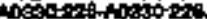

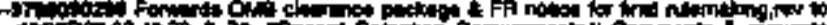

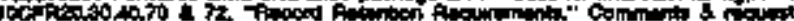

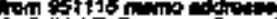

a

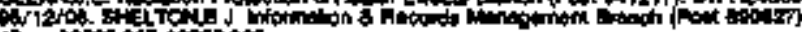

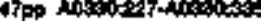

47w

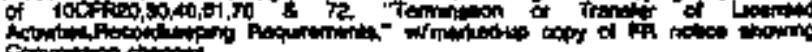

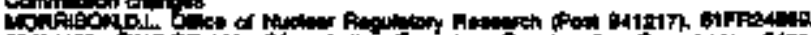

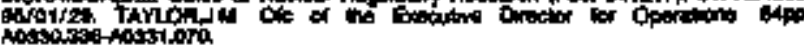


100. not. tivition

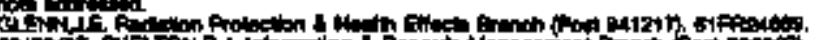

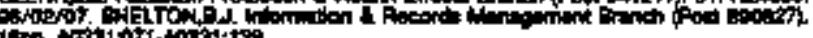

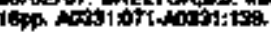

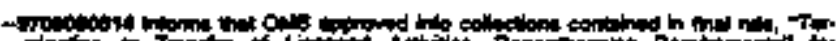

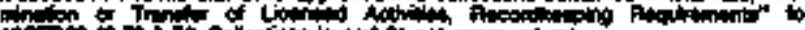

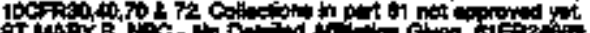

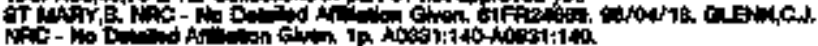

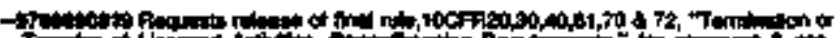

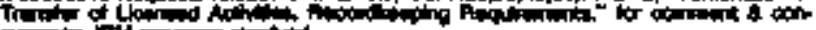

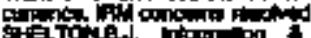

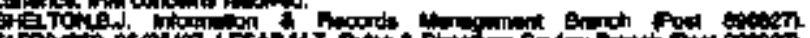

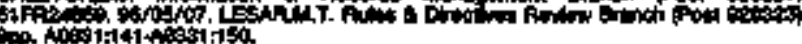

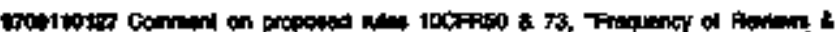

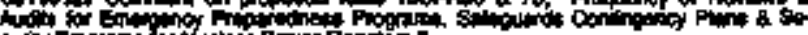

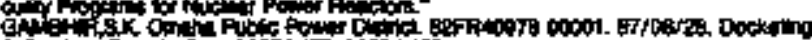

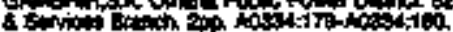

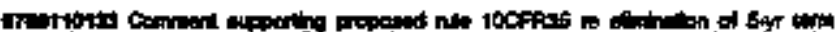

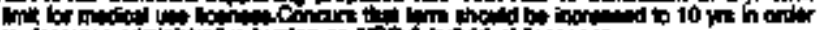

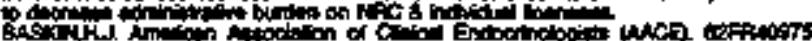

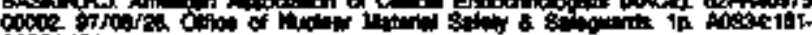

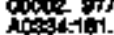

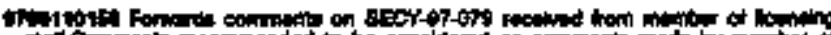

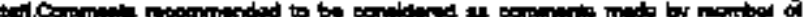

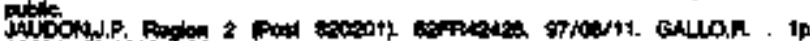
toto 4 ,

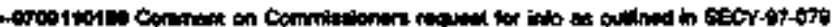

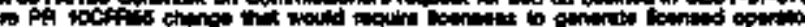

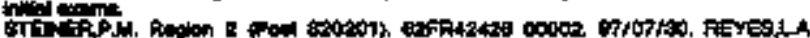

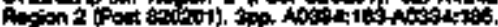

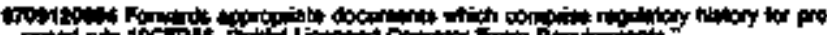

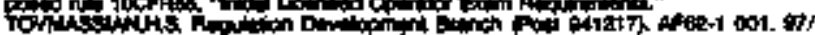

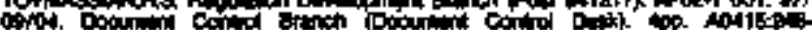
Alition.

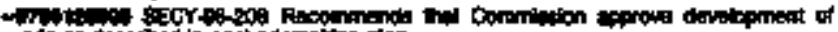

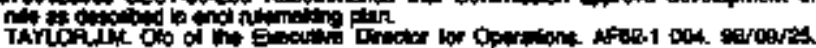

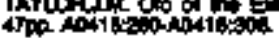

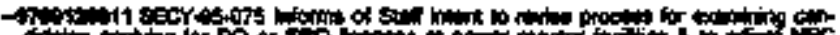

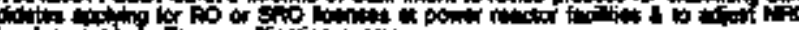

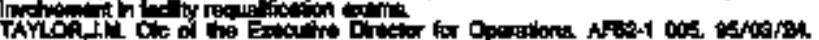

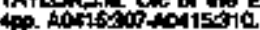

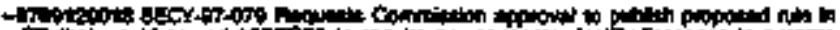

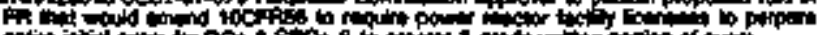

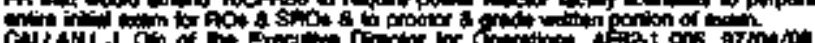

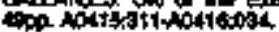

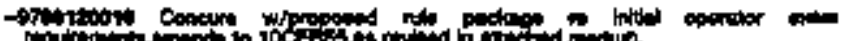

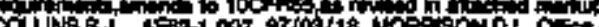
COLNB

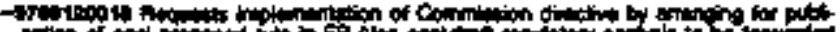

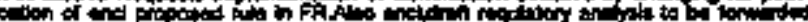

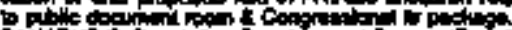

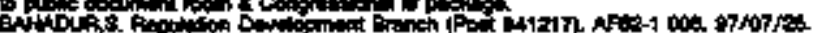

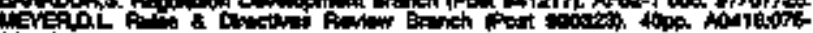
rontisid.

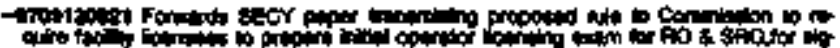

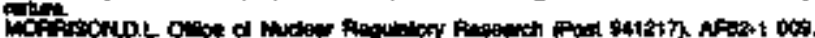

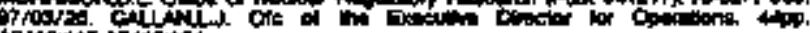

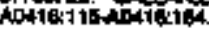

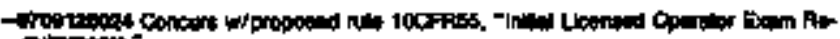

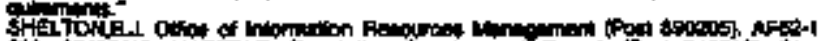

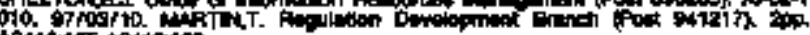

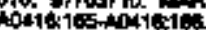

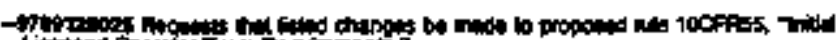

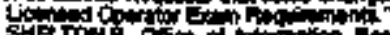

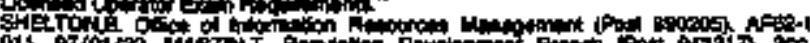

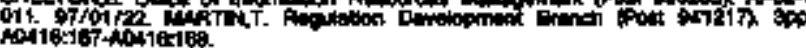

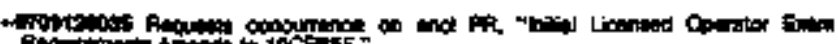

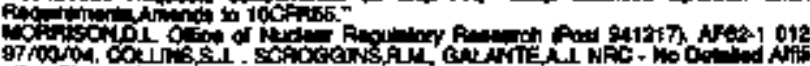

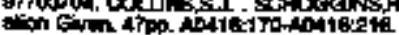

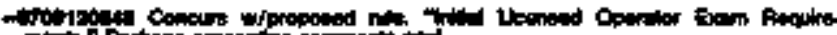

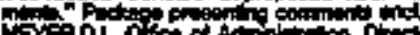

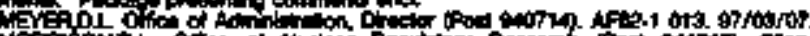

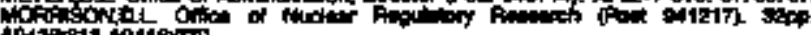

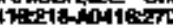

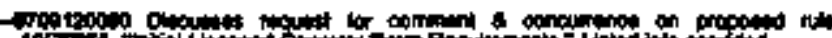

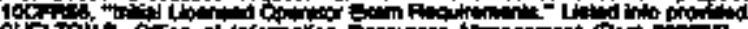

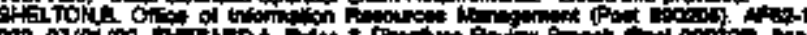

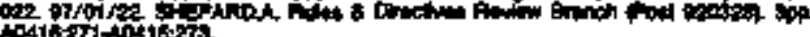

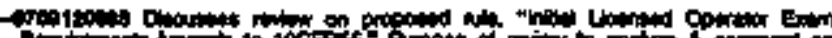

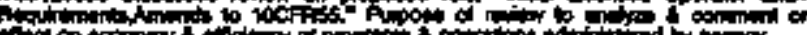

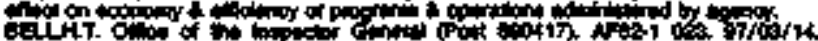

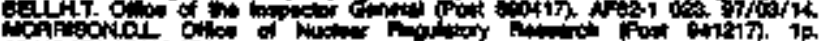
Nitiog

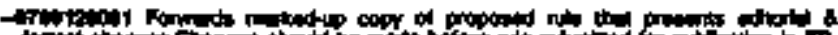

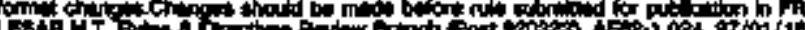

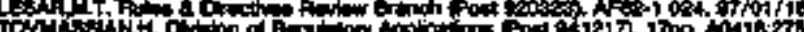
401609.

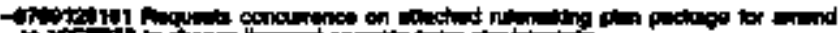

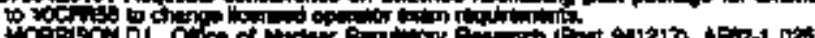

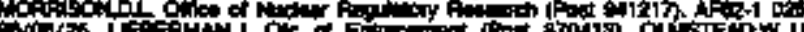

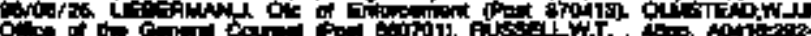
Antoris.

Hividi

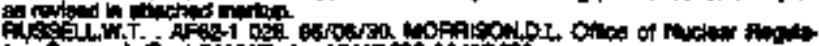
tor $\mathrm{F}$.

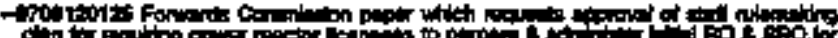

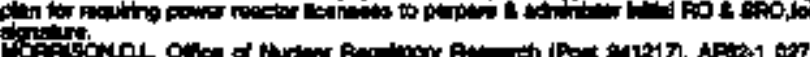

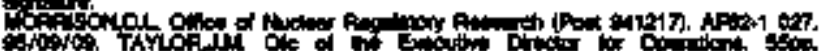

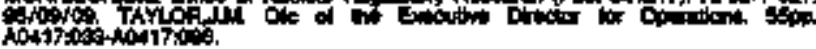

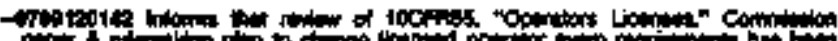

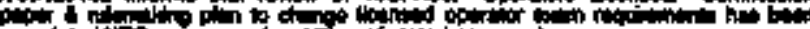

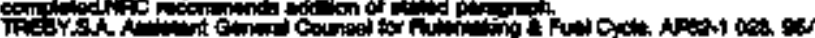

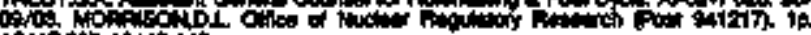
Alithes-404t?:140

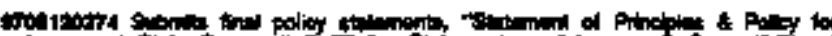

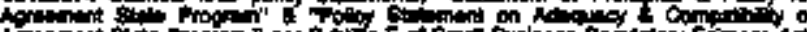

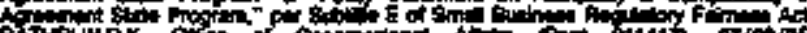

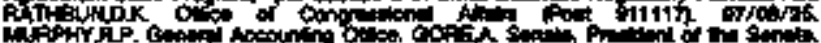

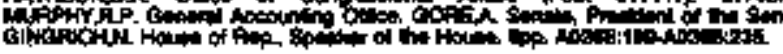

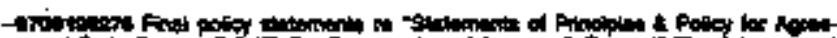

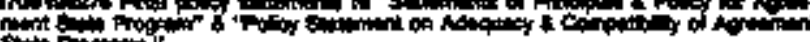

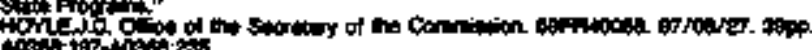

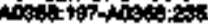

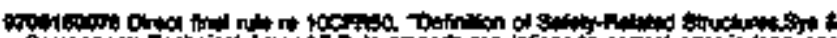

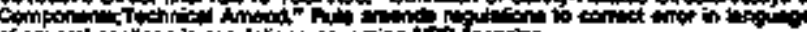

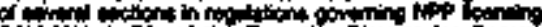

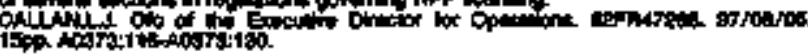

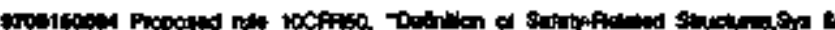

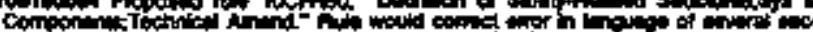

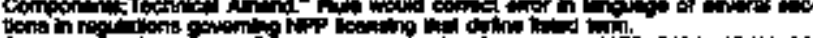

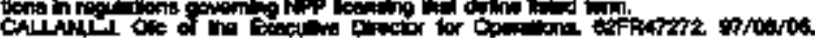

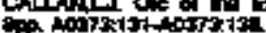

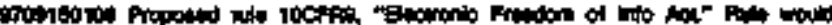

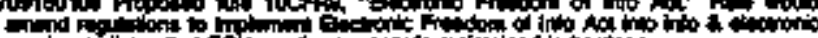

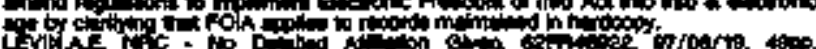

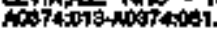

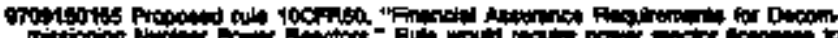

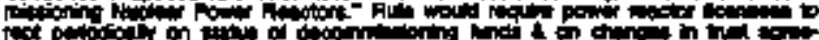

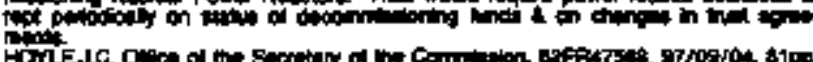

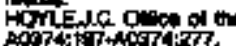

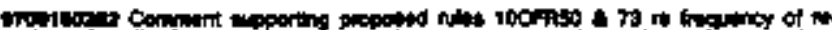

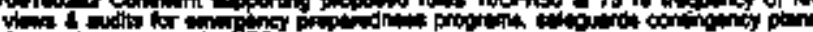

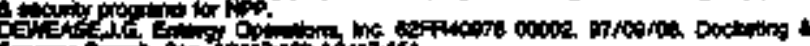

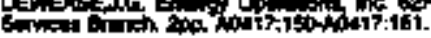

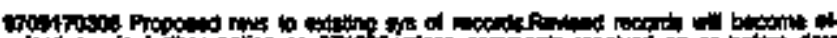

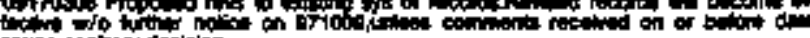

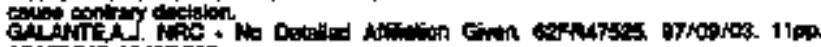

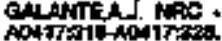

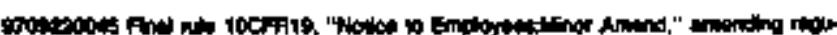

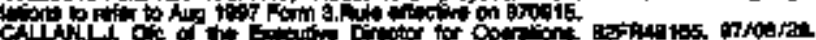

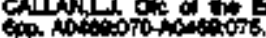




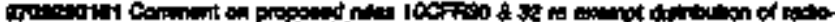

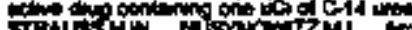

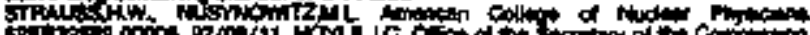

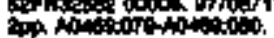

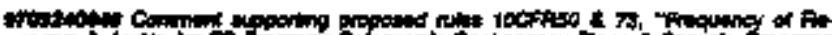

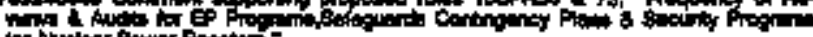

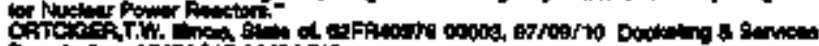

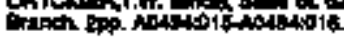

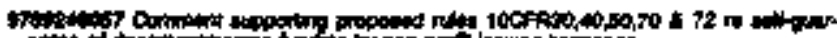

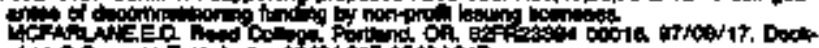

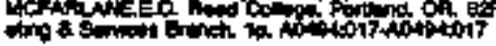

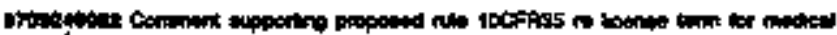

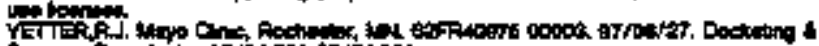

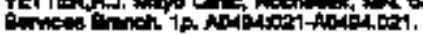

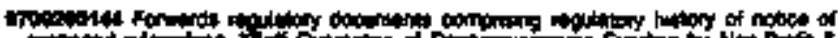

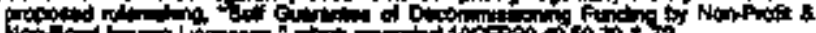

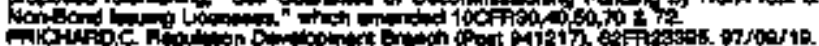

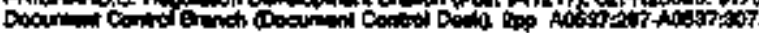

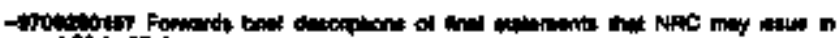

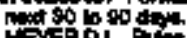

hiva

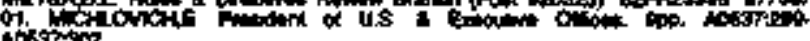
Absistor.

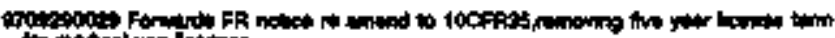

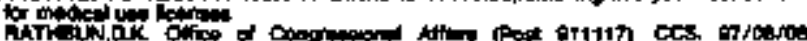

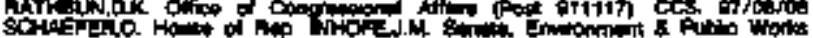

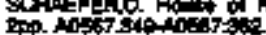

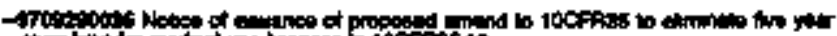

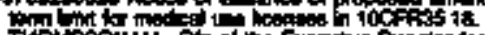

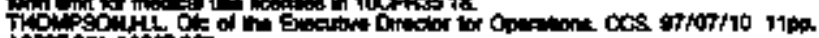

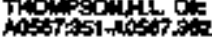

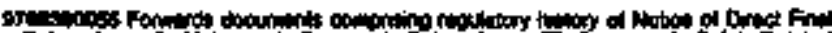

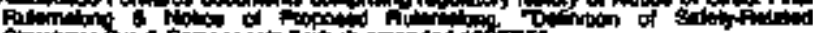

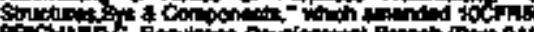

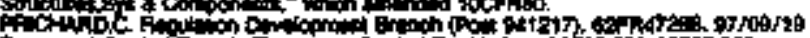

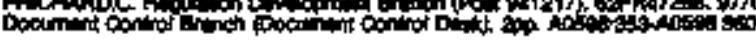

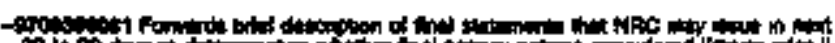

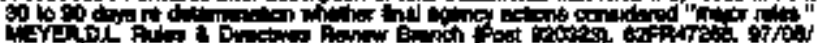

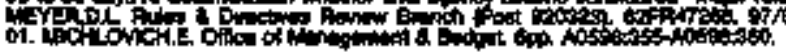

PAOS PAOPFert

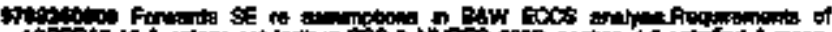
106-

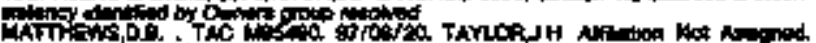

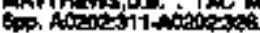

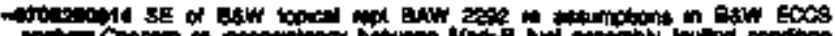

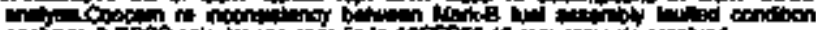

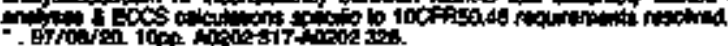

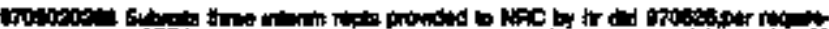

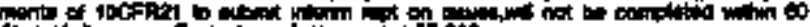

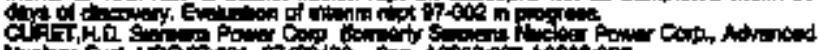

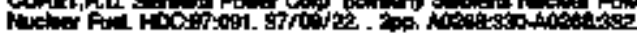

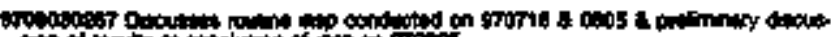
iches of

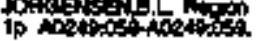

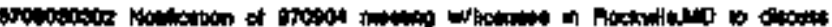

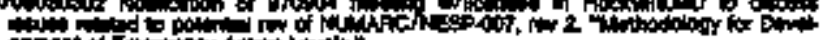

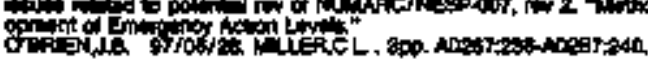

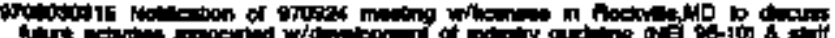

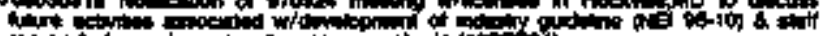

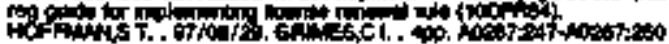

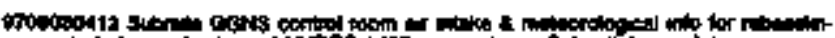

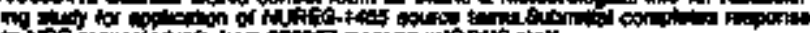

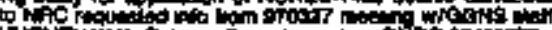

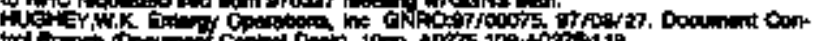

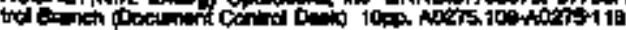

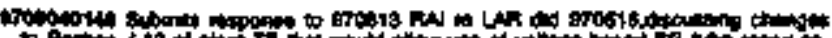

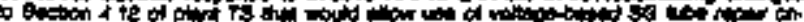

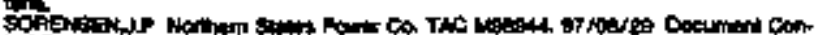

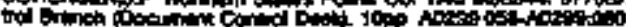

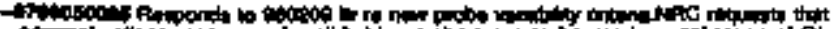

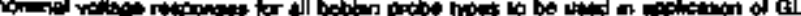

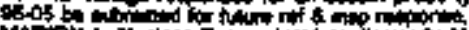

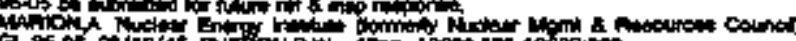
G

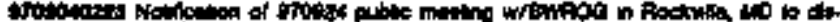

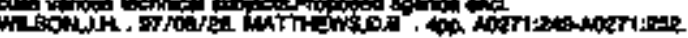

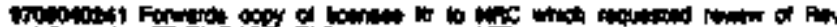

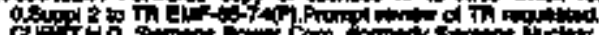

Cint

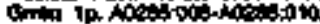

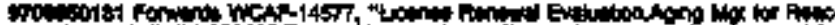

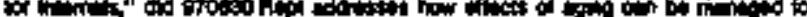

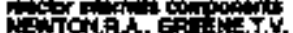

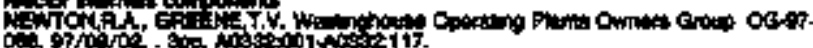

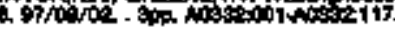

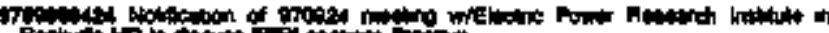

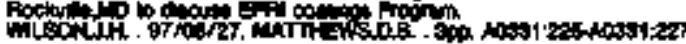

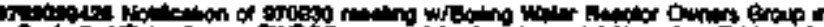

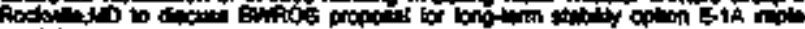

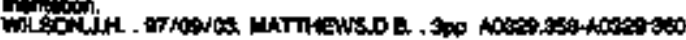

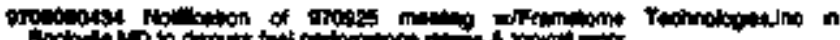

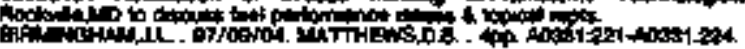

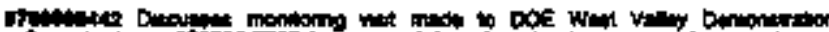

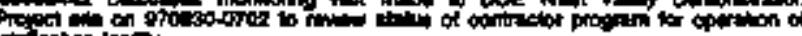
Iivicion me-

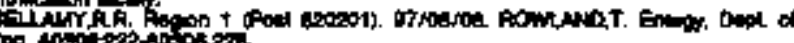

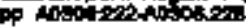

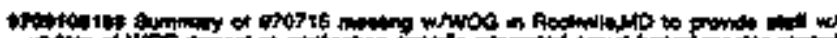

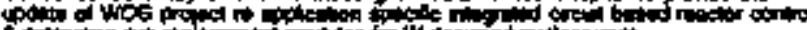

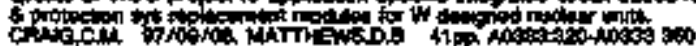

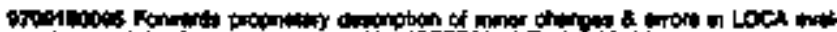

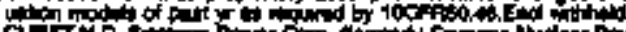

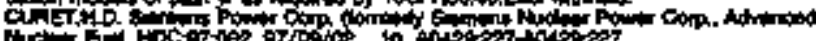

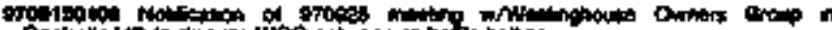

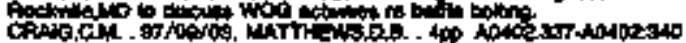

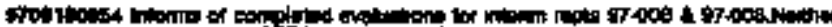

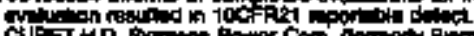

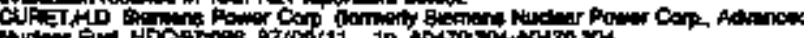

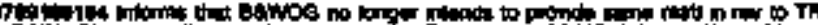

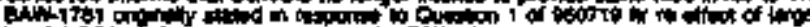

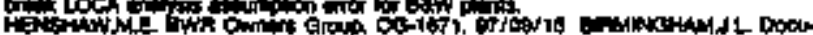

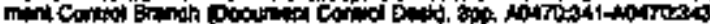

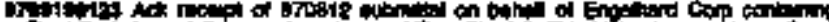

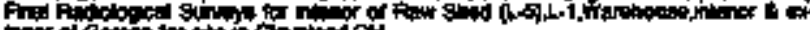

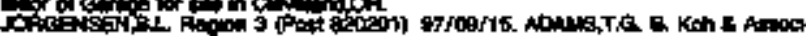

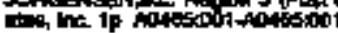

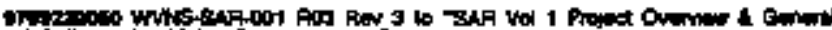

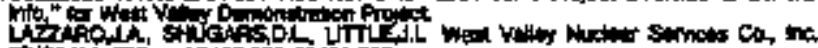

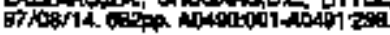

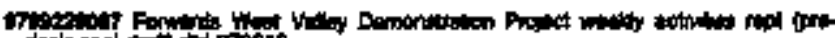

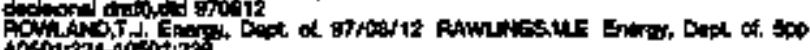

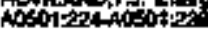

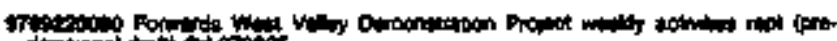

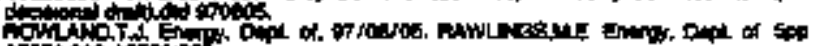

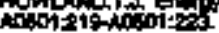

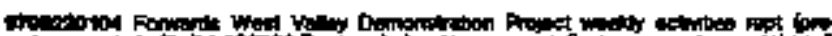

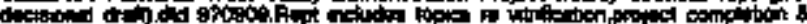

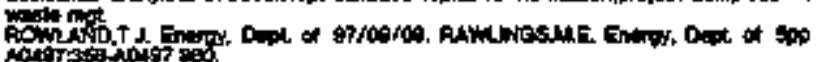

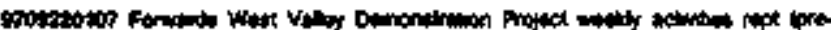

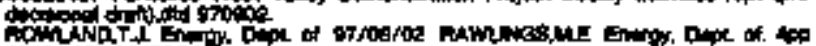
sin

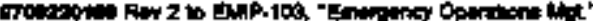

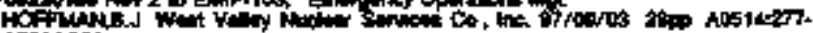
10514306

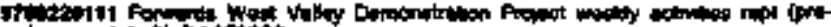

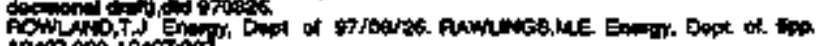
ADen

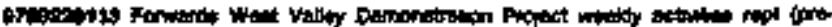

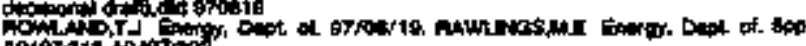

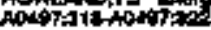




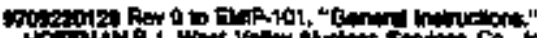

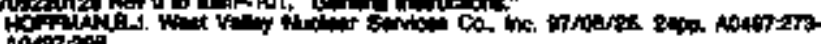
A0miatia

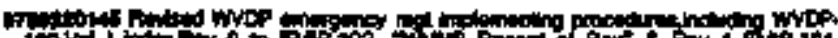

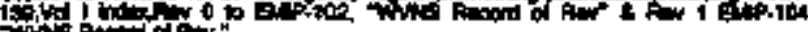

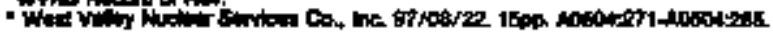

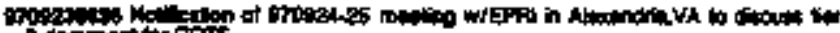

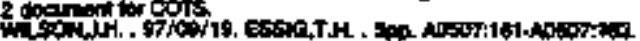

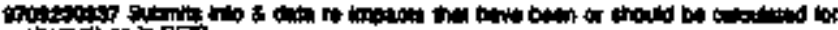

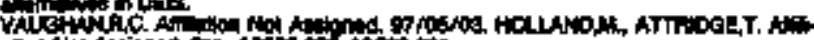

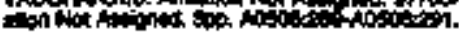

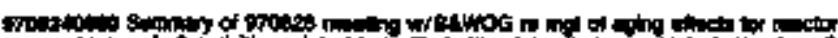

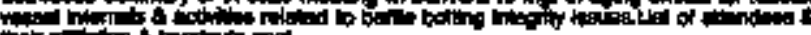

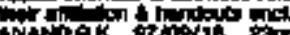

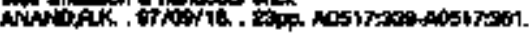

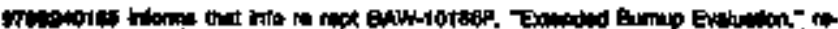

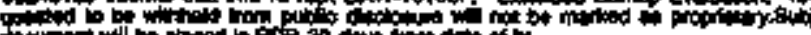

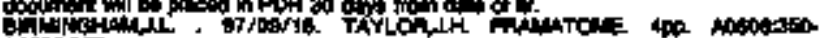

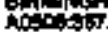

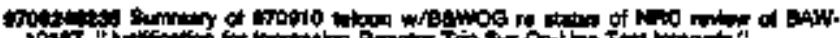

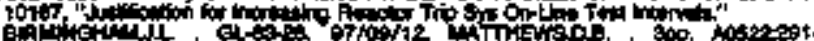
$\lim _{0 \rightarrow 0}$

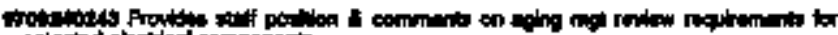

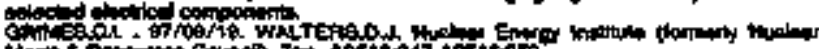

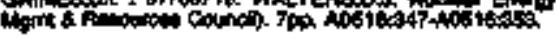

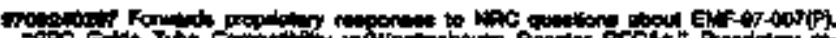

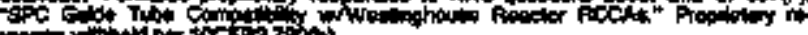
octer

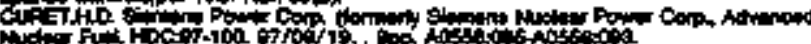

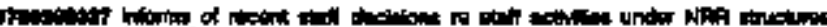

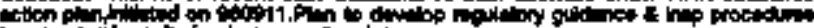

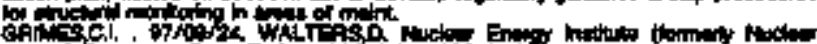

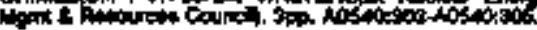

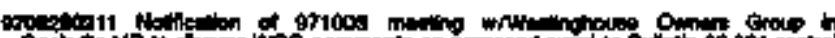

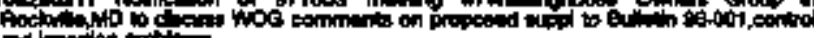

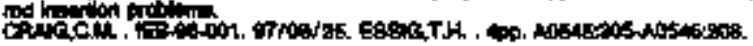

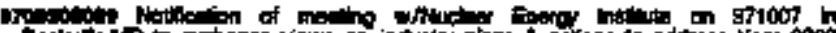

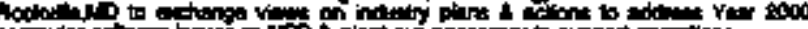

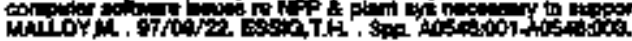

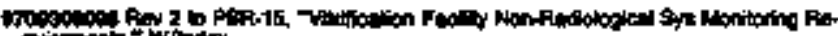

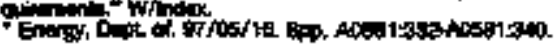

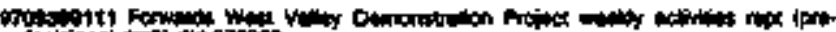

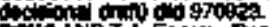

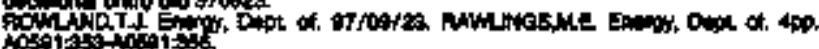

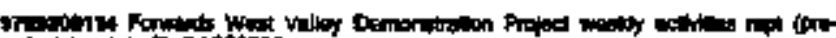

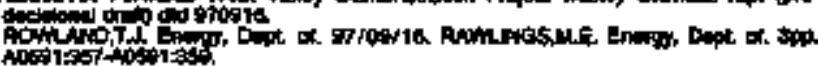

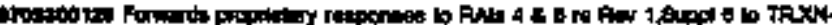

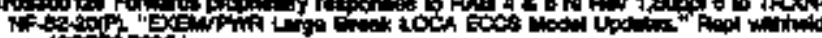

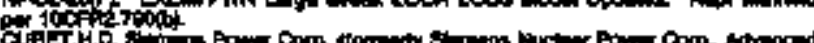

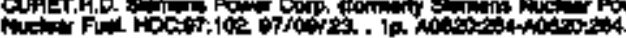

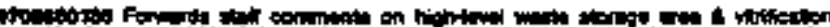

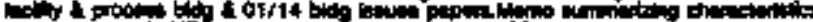

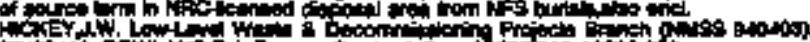

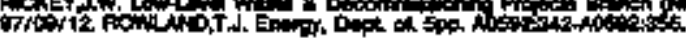

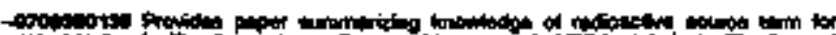

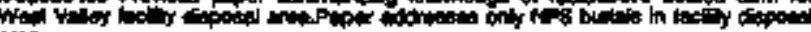

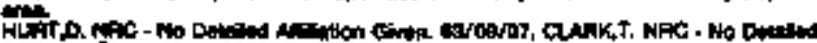
Anting

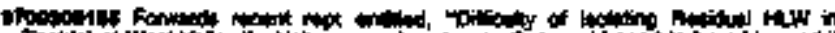

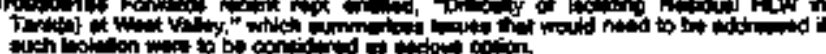

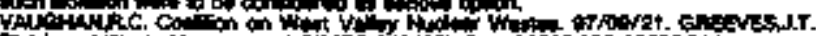

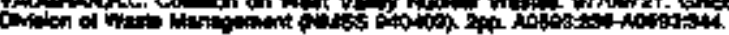

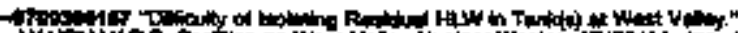

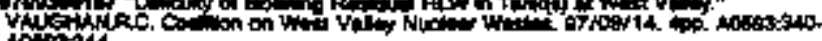
An:abie

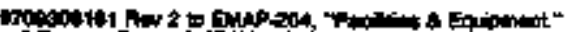

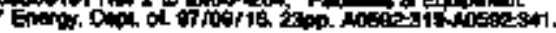

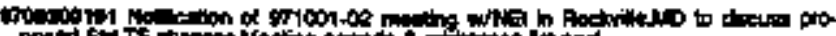

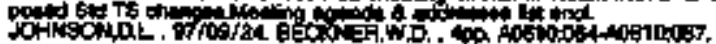

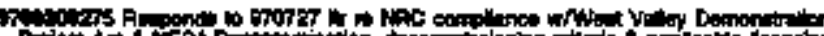

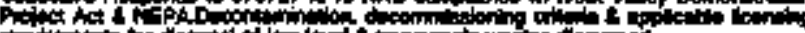

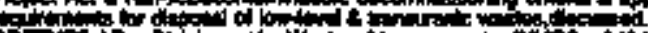
of

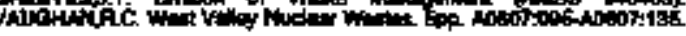

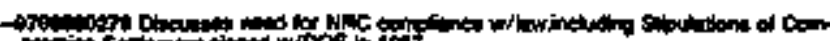
rot

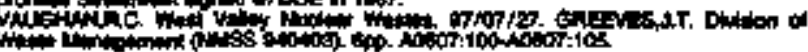

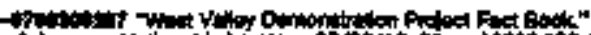

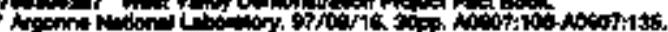

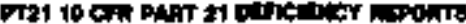

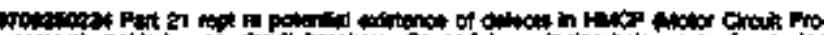

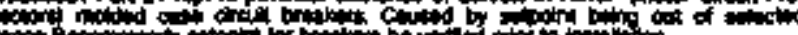

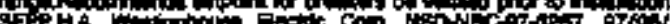

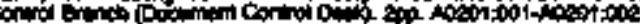

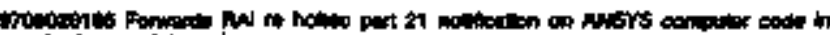

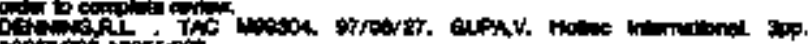

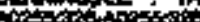

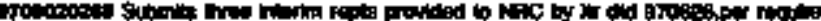

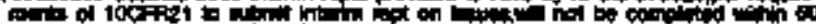

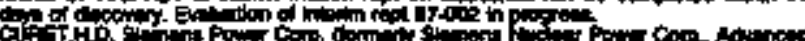

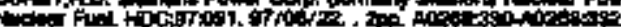

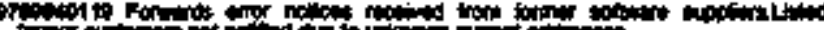

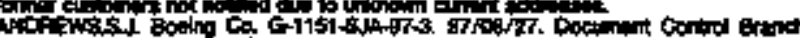

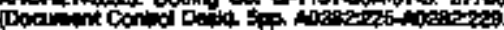

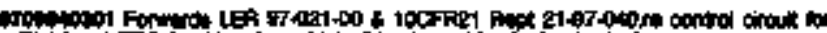

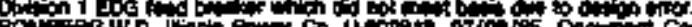

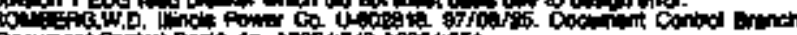

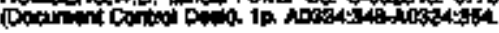

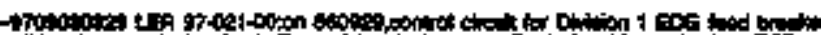

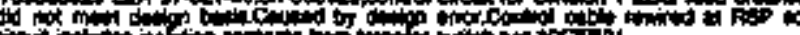

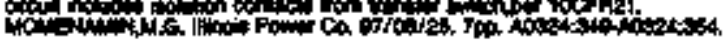

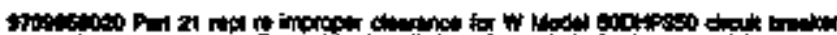

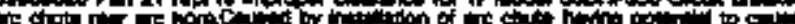

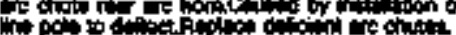

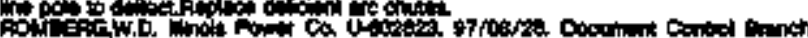

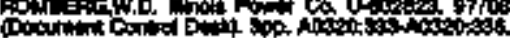

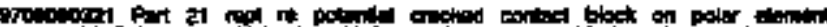

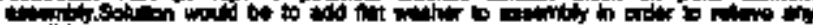

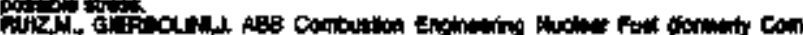

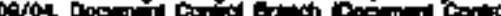

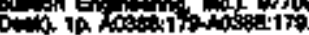

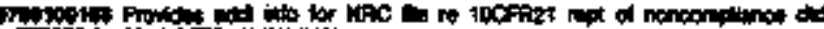

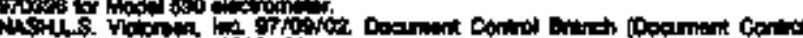

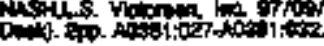

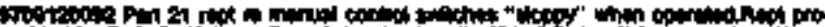

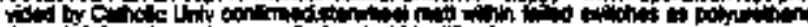

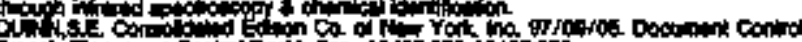

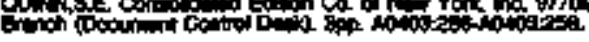

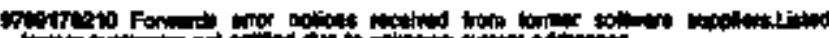

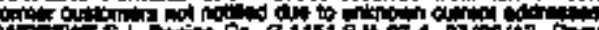
Thons.

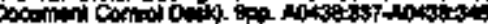

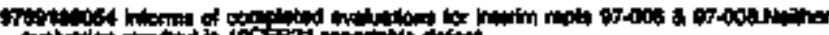

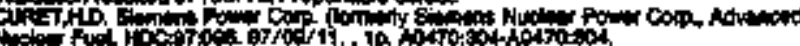

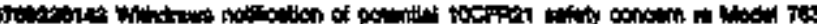

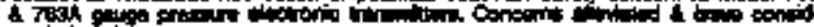

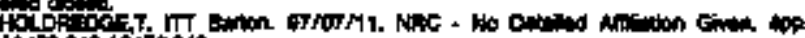

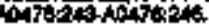

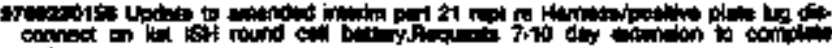

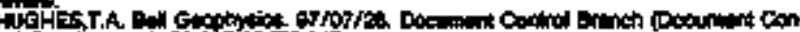

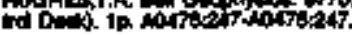

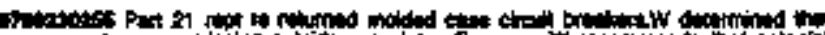

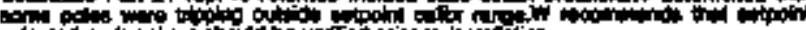

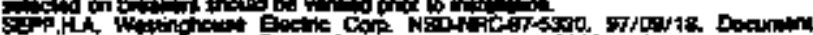

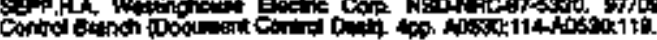

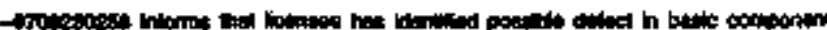

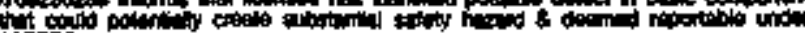

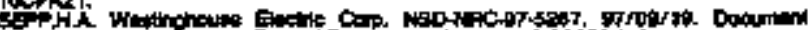

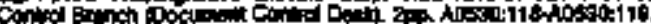




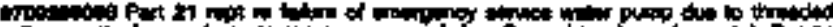

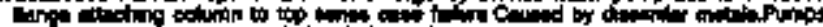

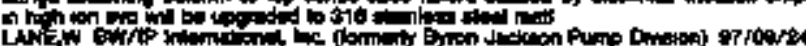

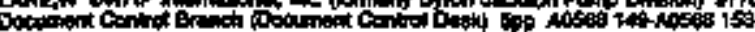

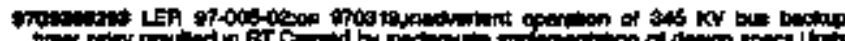

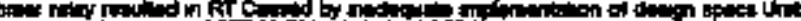

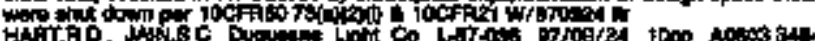

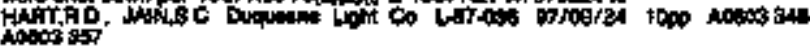

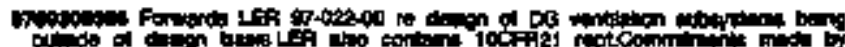
titing

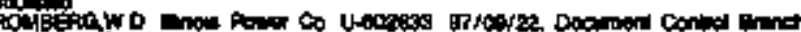

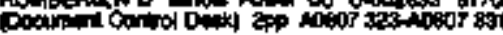

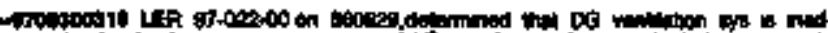

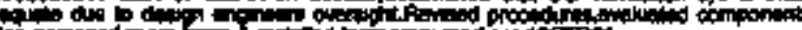

Banilim

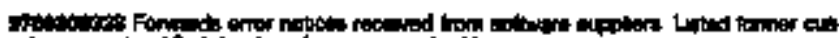

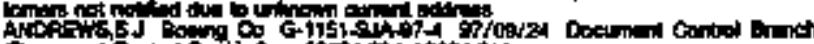

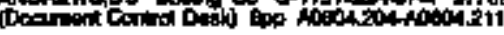

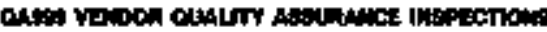

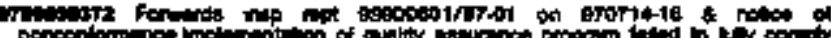

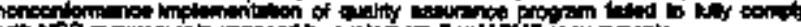

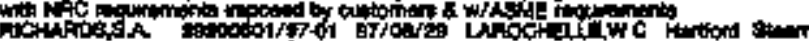

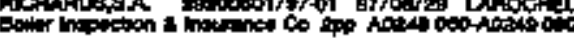

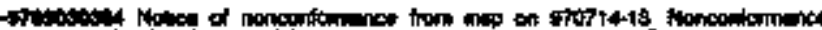

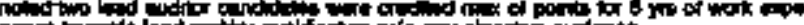

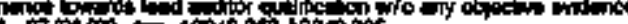

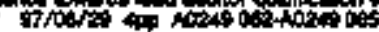

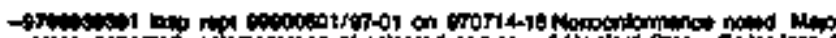

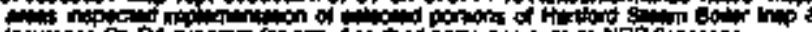

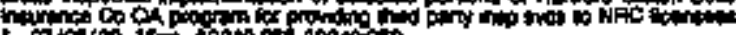

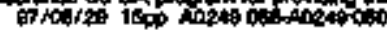

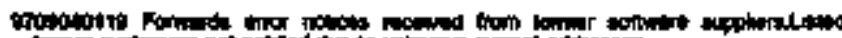

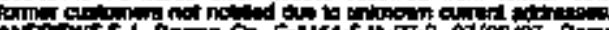

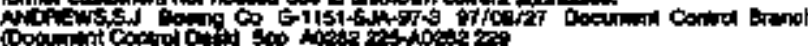

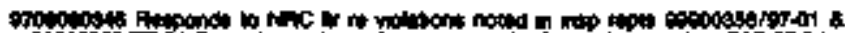

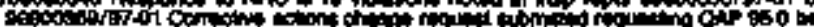
nitid to do

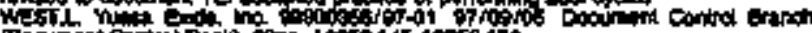

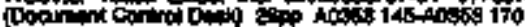

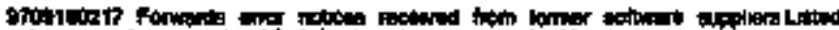

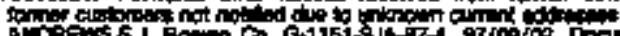

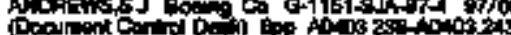

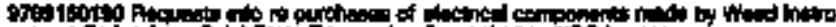

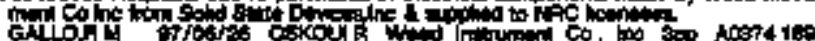
iostis

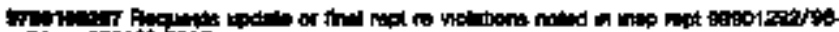

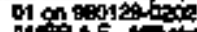

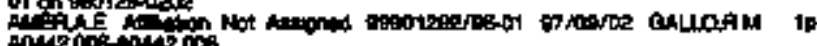

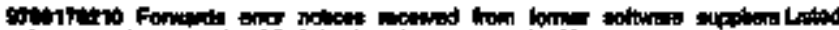

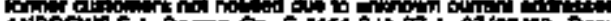

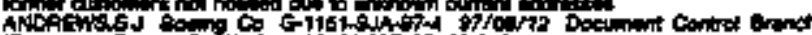

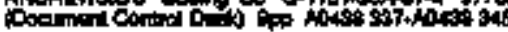

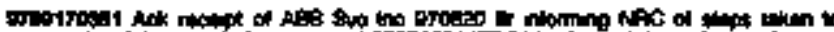

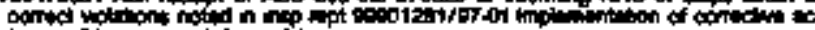
Find of

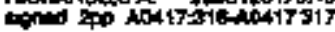

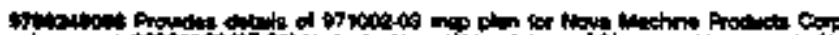

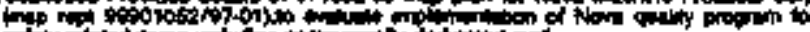

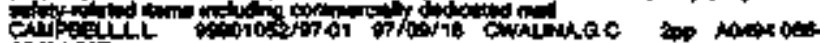
rotor on

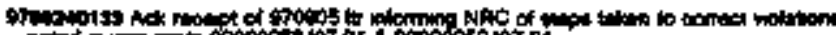

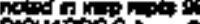

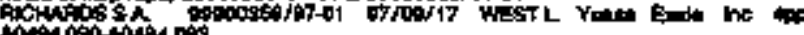

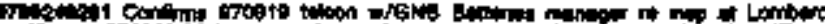

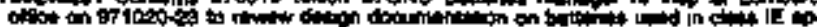

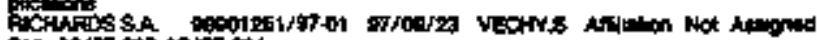

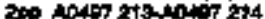

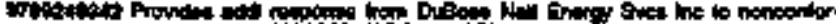

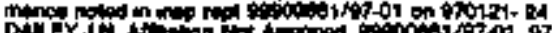

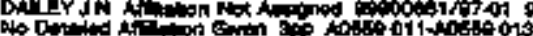

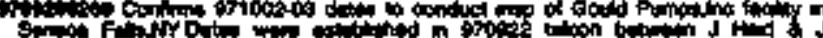

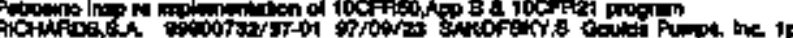

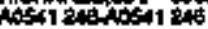

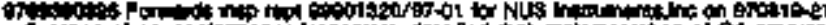

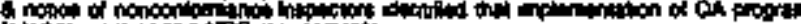

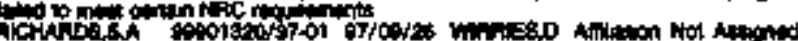

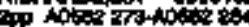

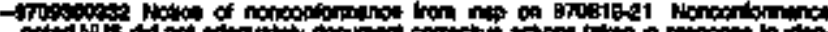

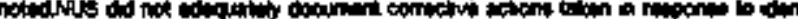

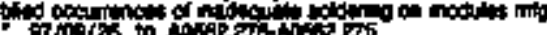

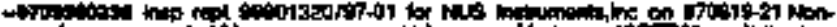

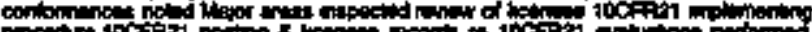

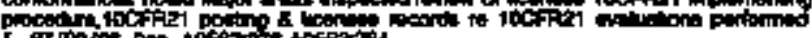

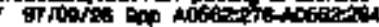

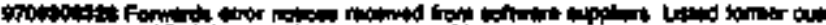

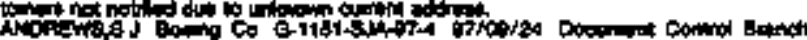

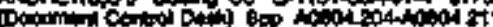

bitre

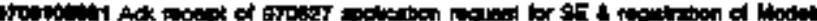

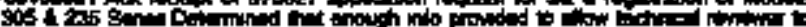

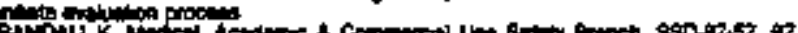

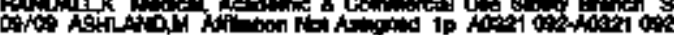

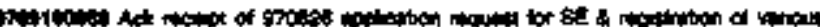

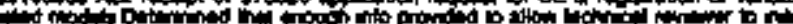

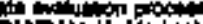

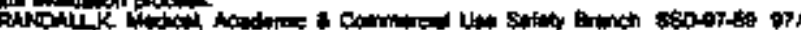

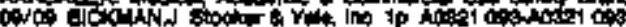

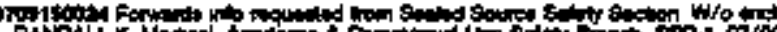

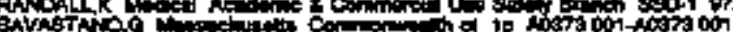

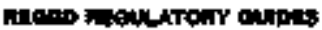

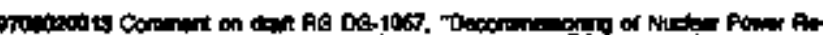

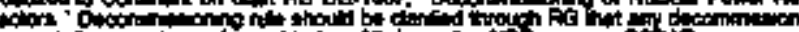

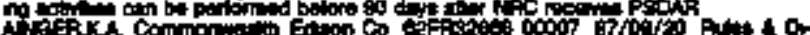

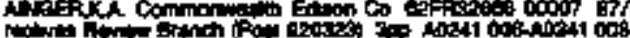

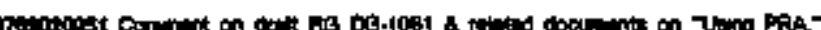

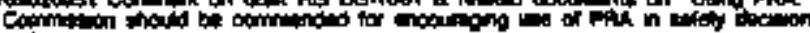

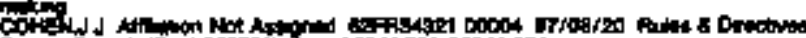

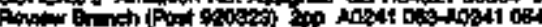

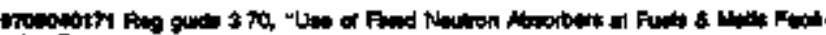

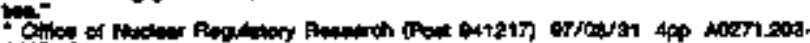
Antion

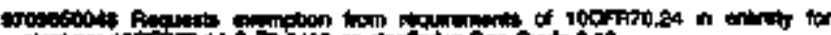

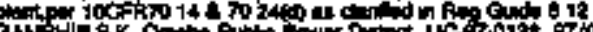
Dooumat can

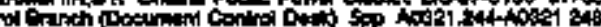

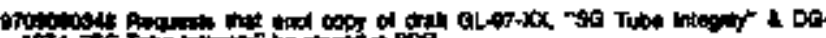

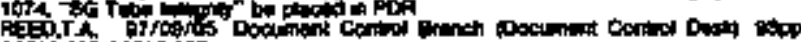
action

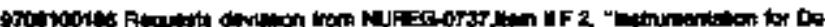

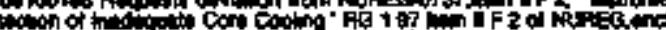

Con

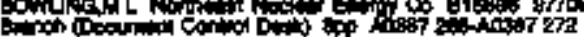

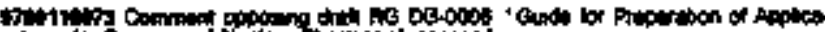

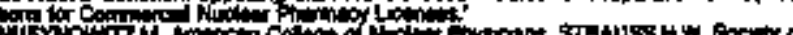

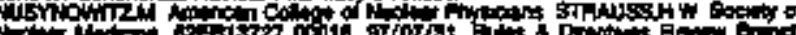

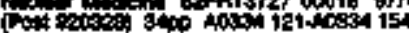

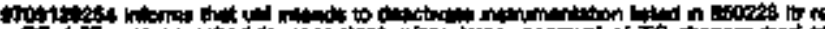

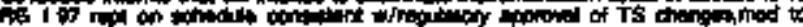
Fit.

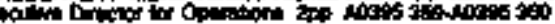

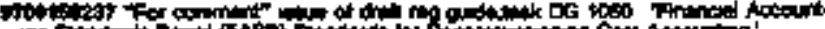

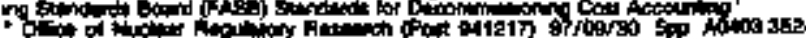
A0nosis

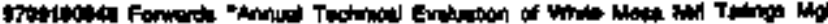

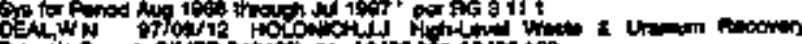

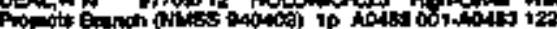




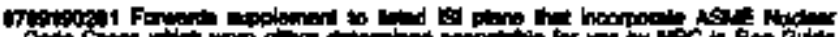

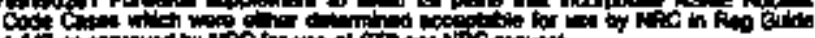

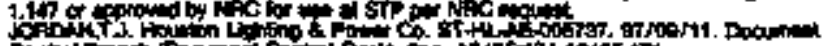

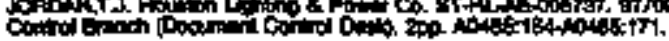

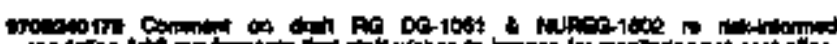

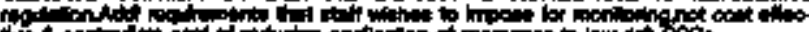

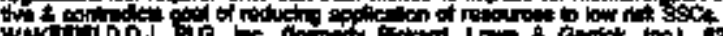

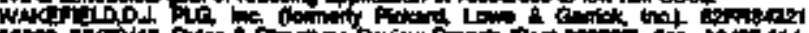

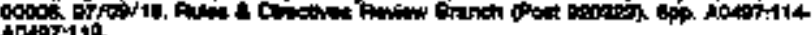

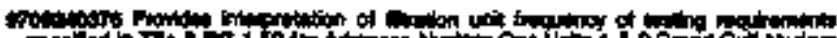

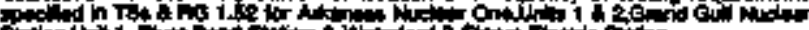

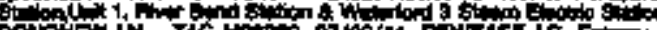

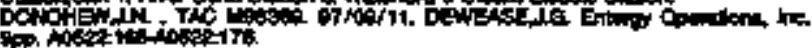

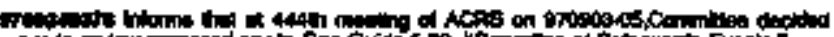

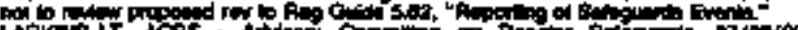

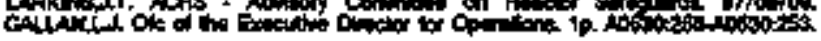

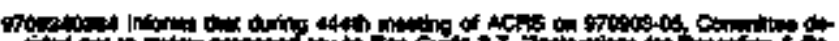

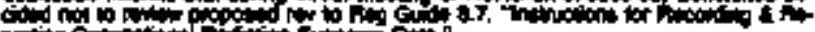

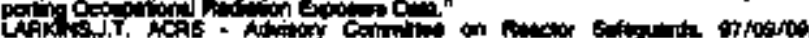

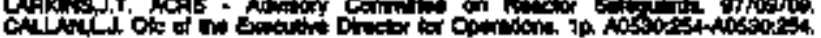

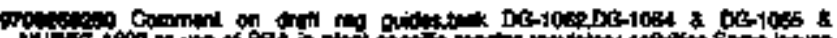

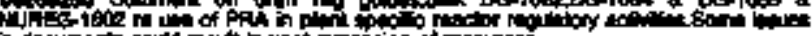

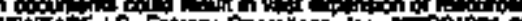

Din to

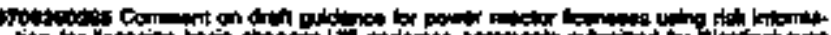

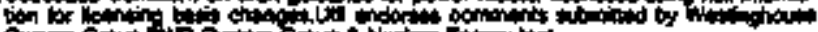

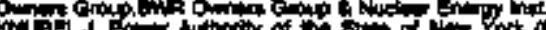

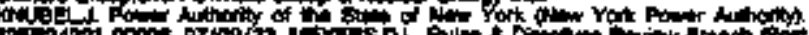
oristis.

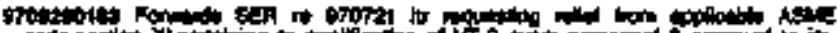

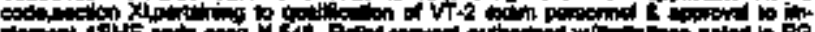

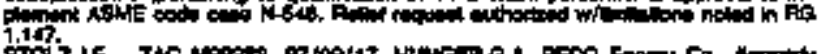

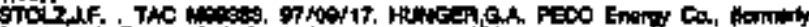

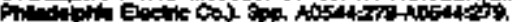

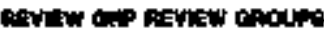

sho:

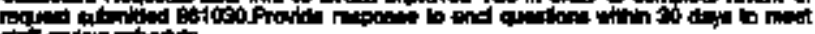

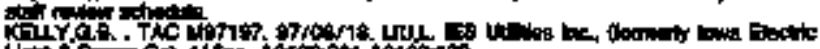

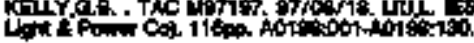

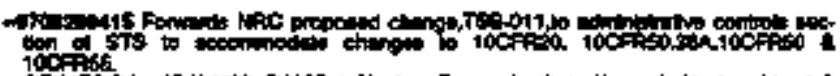

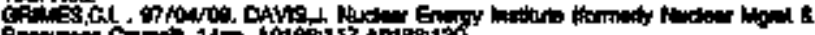

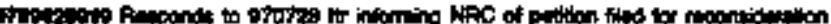

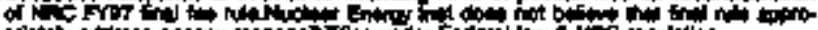

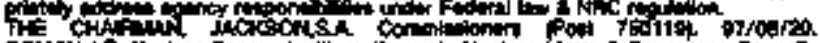

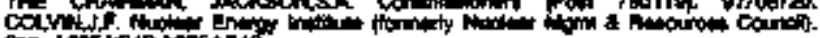

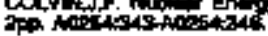

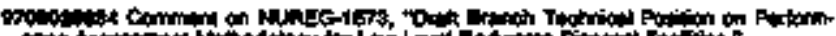

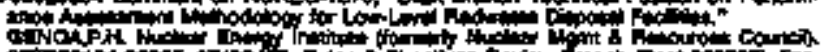

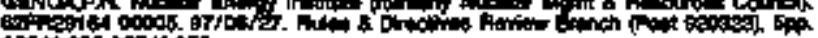

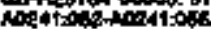

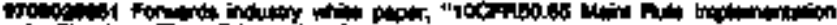

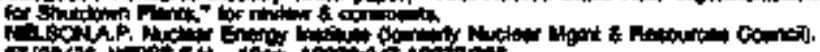

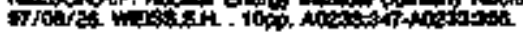

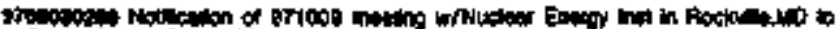
Tre⿻三丨 A

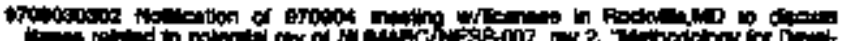

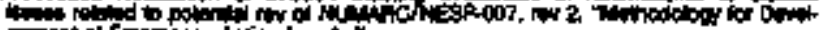

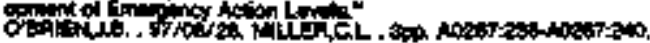

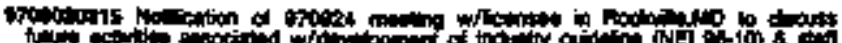

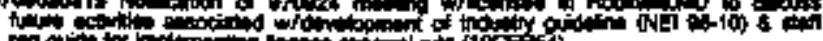

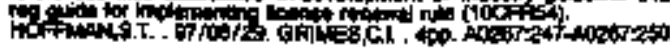

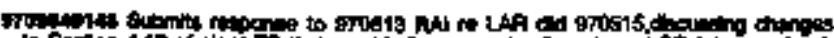

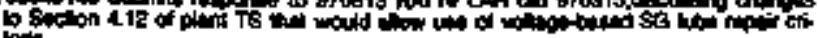

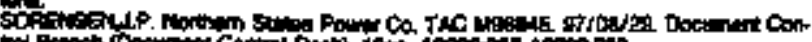

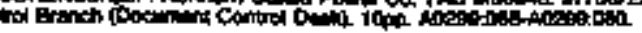

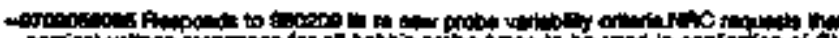

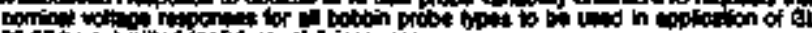

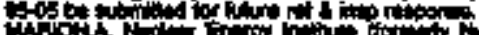

Whol Now

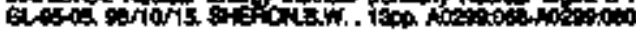

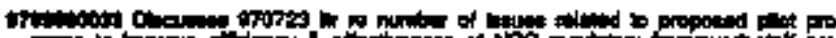

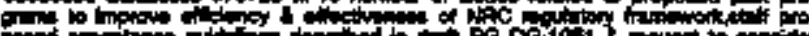

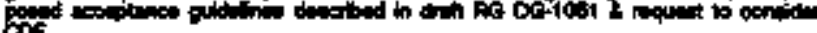

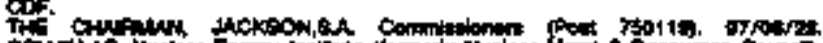

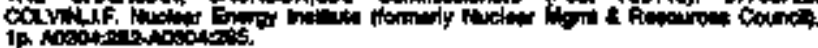

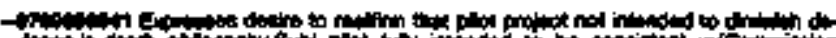

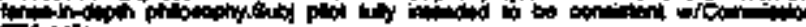
ofloction

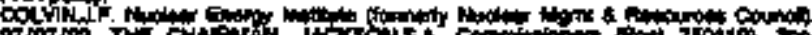
$900 \%$ The GN

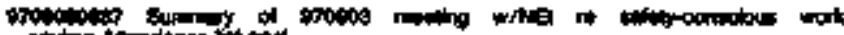

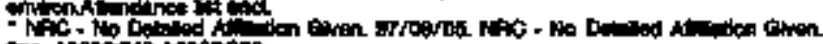

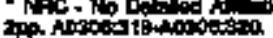

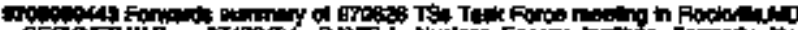

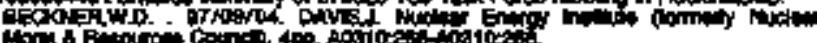

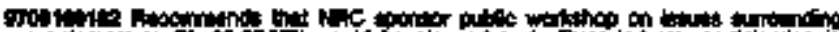

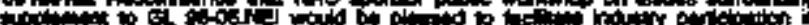
toth

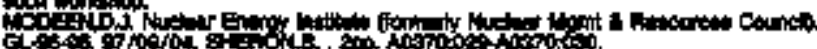

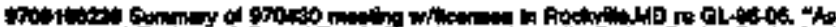
trice of Eq

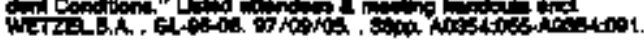

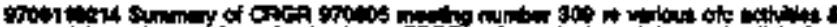

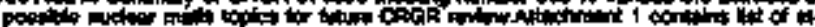

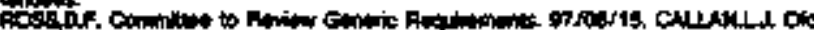

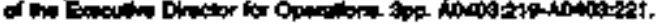

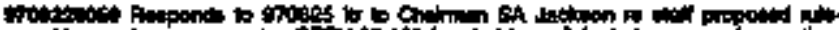

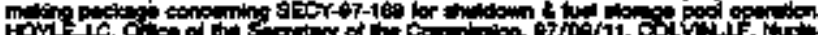

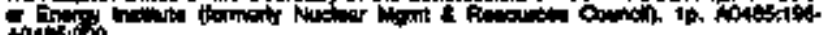
Aarsidio

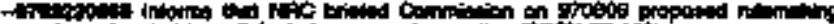

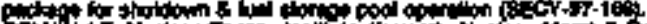

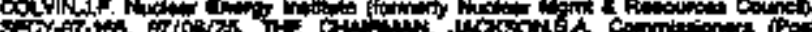

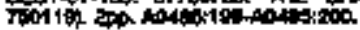

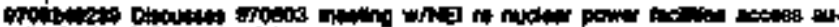
cit

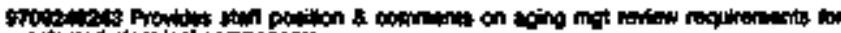

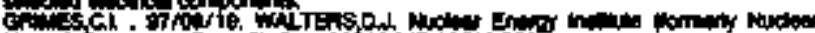

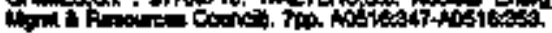

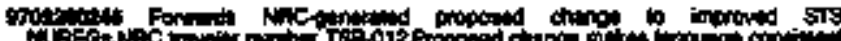
WHEC

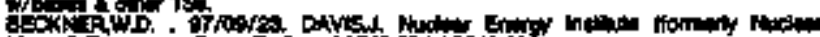

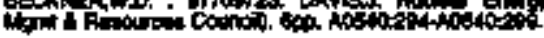

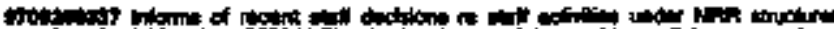

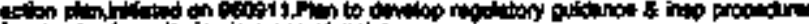

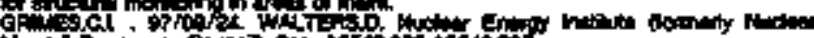

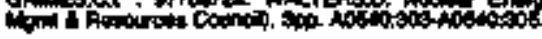

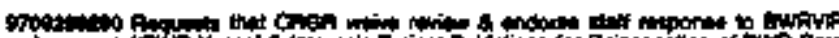

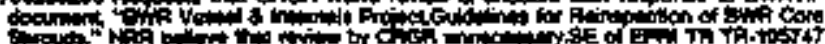
tincour.

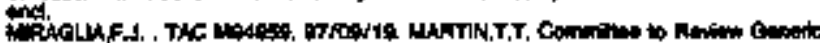

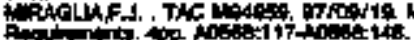

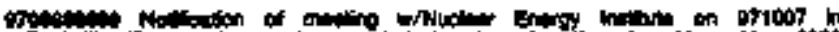

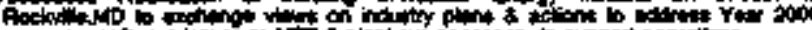

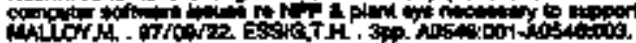

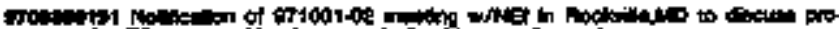

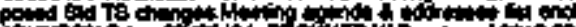

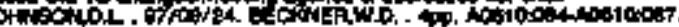

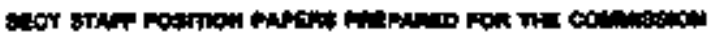

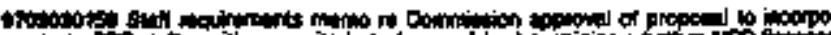

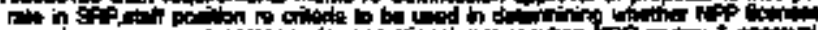

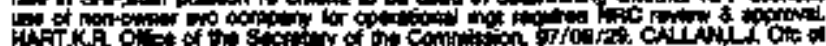

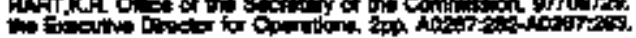




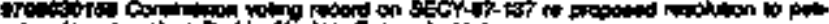

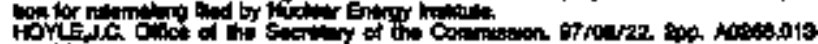
ingent

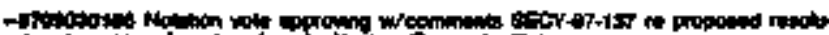

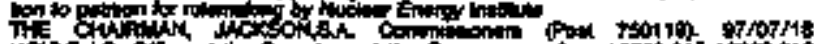

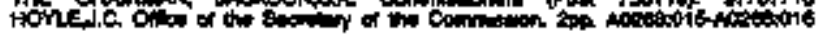

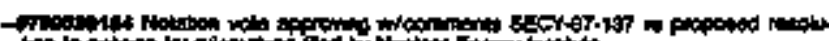

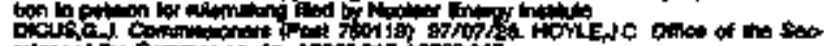

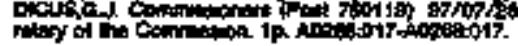

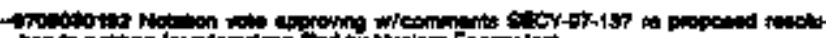

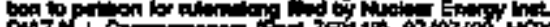

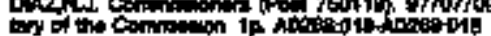

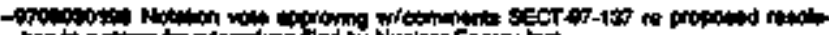

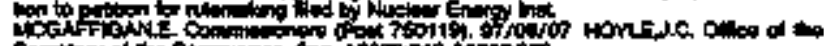

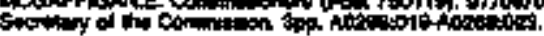

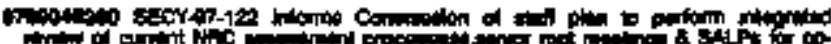

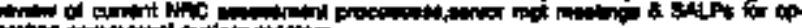

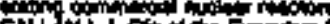
indinto

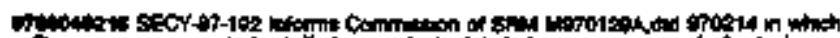

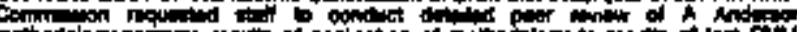

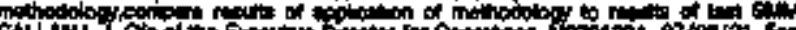

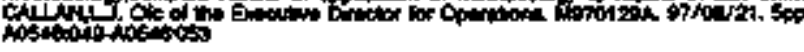

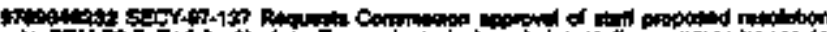

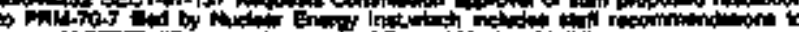

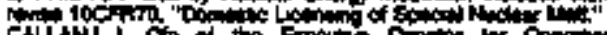

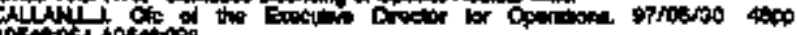

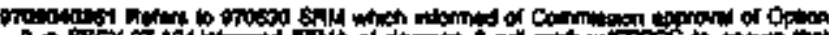

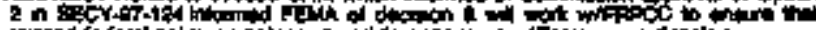

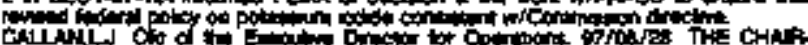

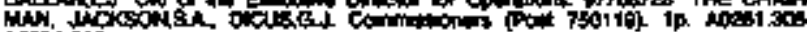

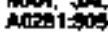

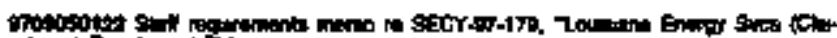

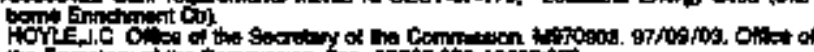

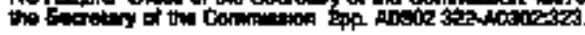

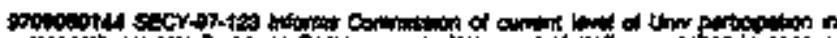

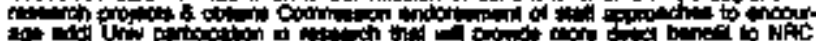

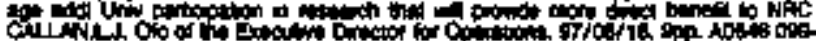
Arstion.

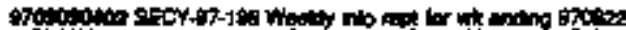

ofthell introxide

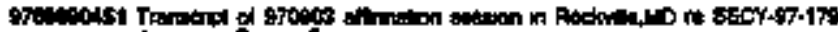

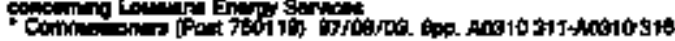

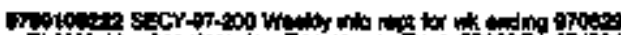

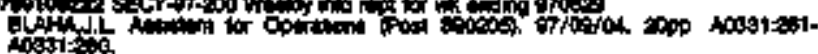

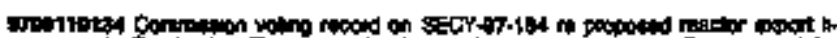

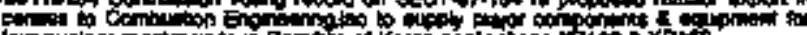

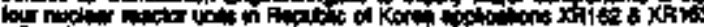

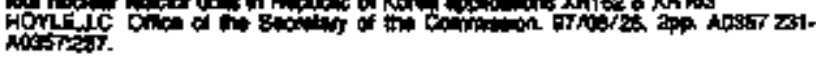

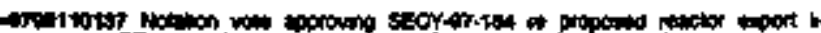

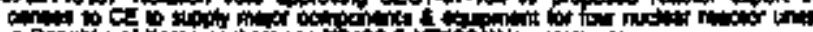

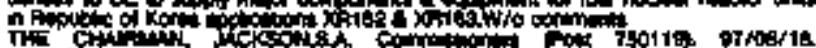

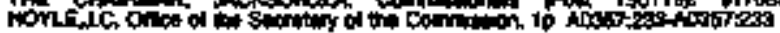

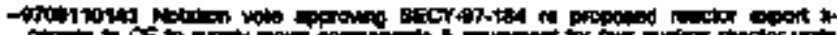

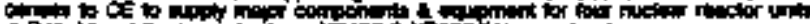

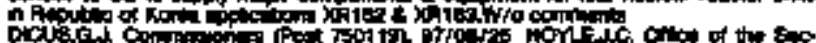

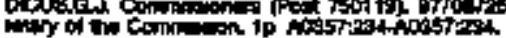

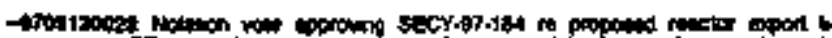

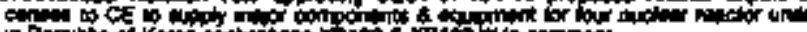
ID

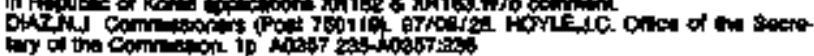

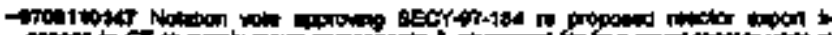

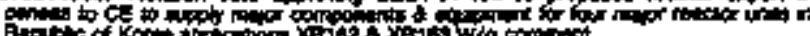

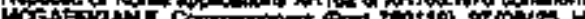

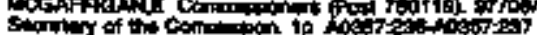

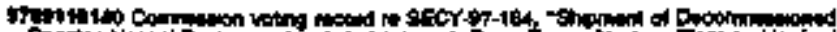

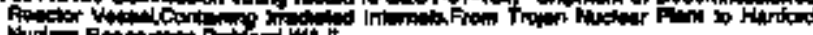

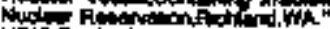

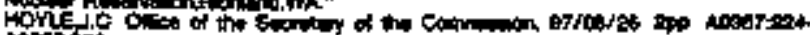
nosit? 200 .

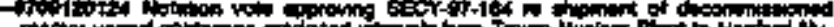

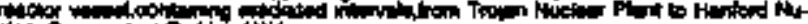

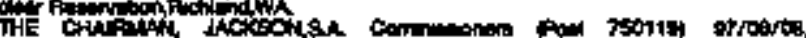

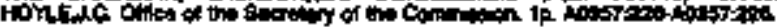

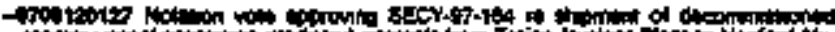

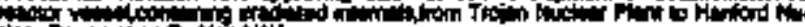
The

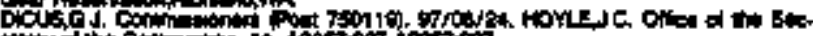

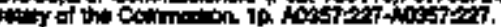

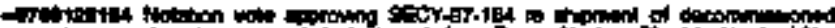

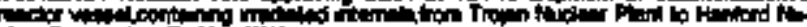

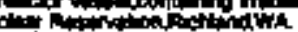

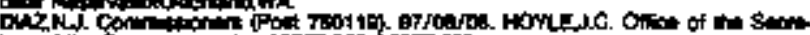

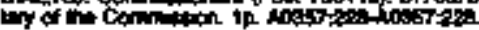

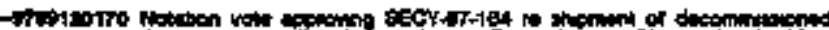

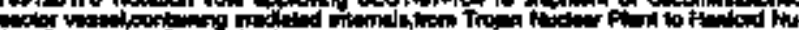

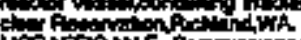

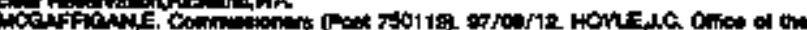

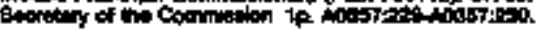

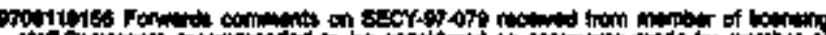

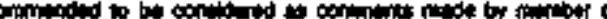

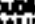

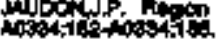

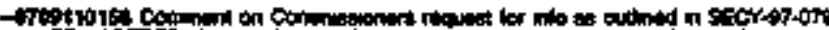

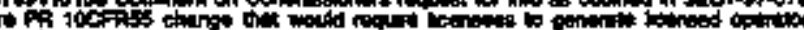

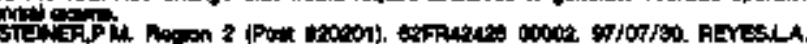

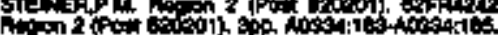

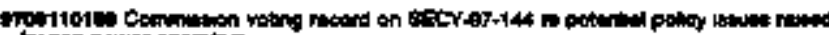

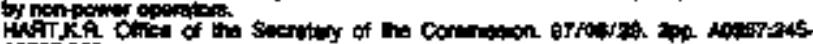
Anstion

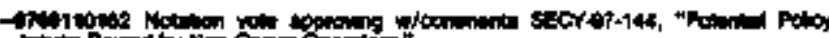

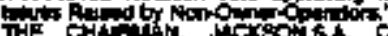

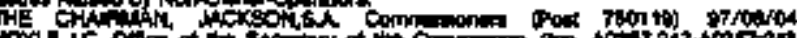

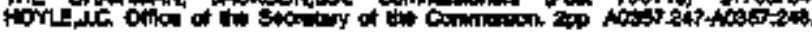

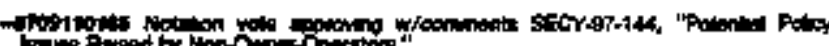

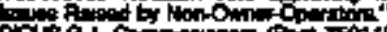

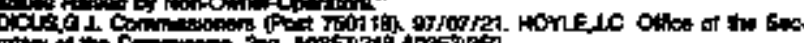

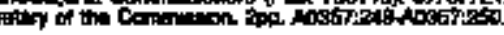

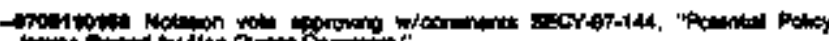

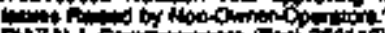

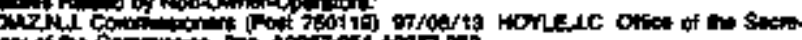

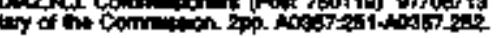

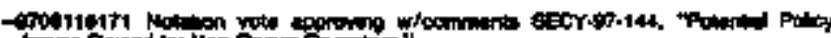

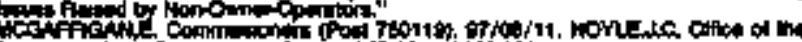

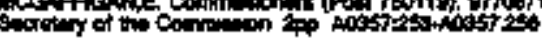

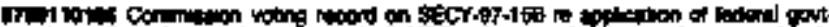

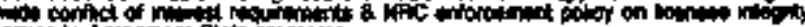

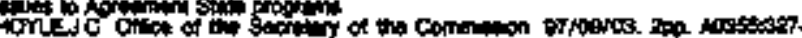

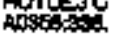

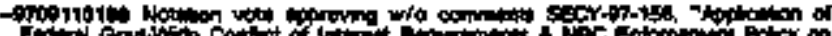

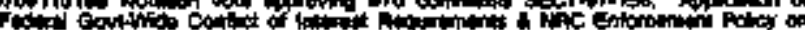

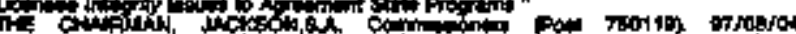
Hofl $=\mathrm{d} C$ G

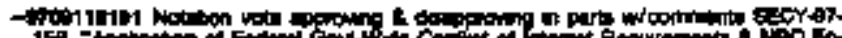

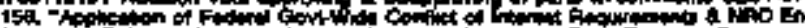

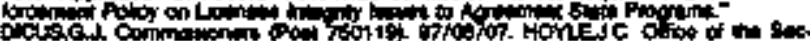

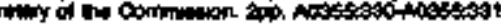

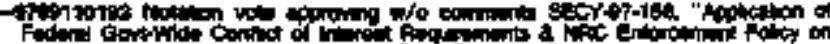

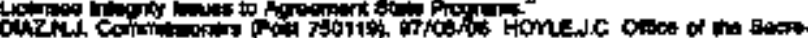

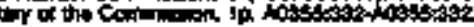

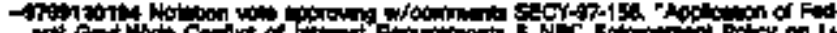

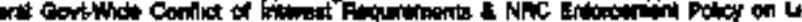

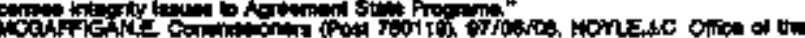

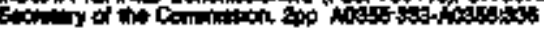

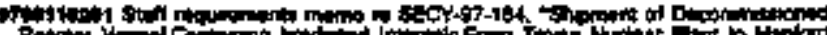

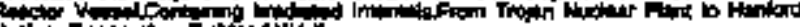

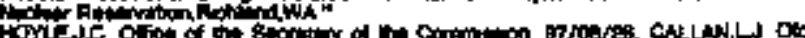

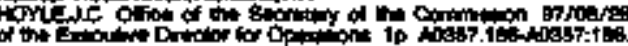

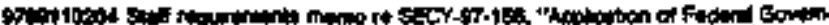

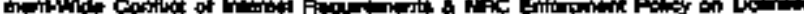

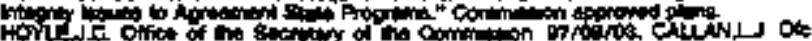

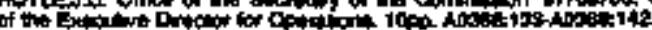




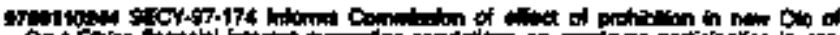

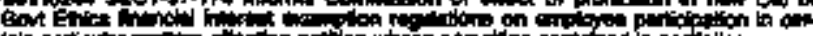

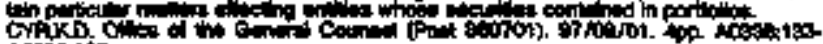
Crikd $\mathrm{C}$

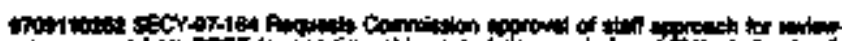

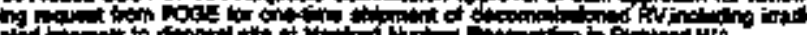

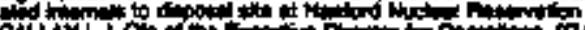

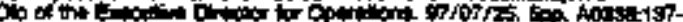

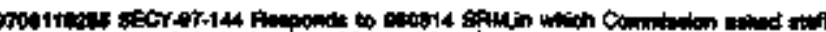
to

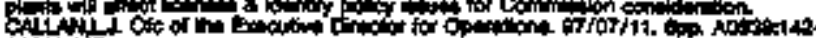
A0s

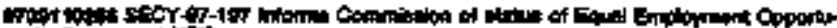

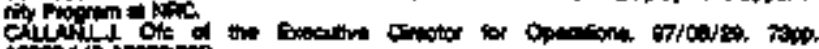

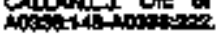

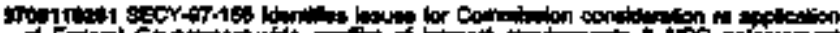

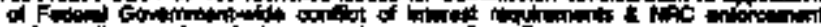

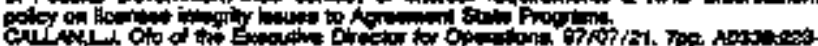
insos.

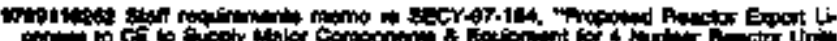

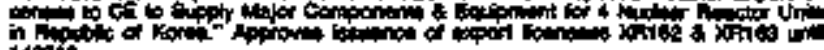

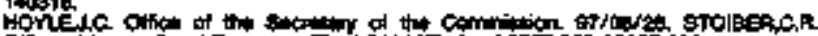

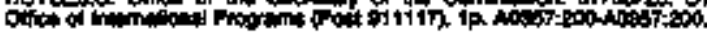

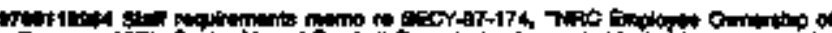

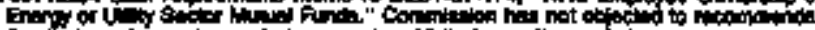

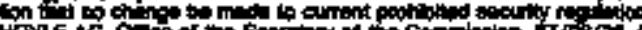

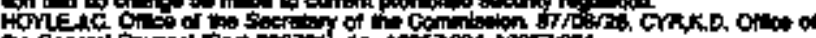

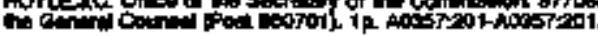

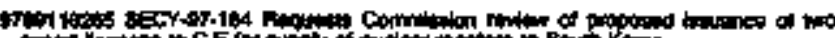

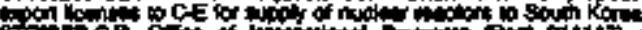

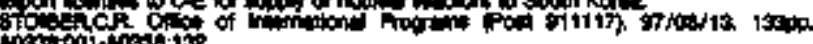

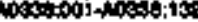

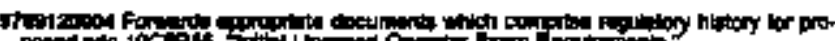

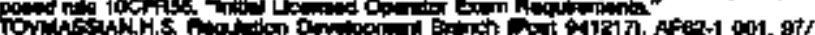

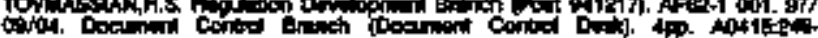
1017\%10.

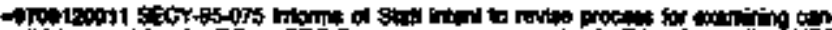

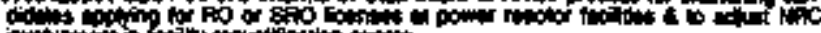
TAy ow.

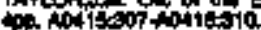

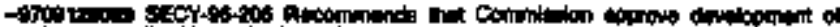

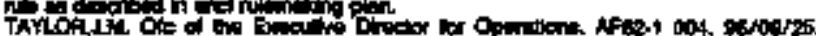

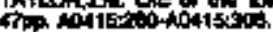

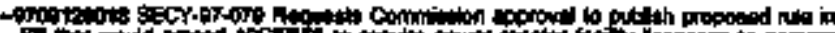

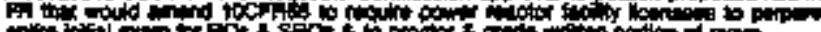

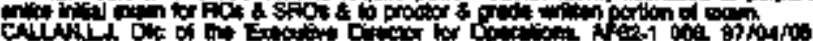

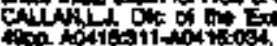

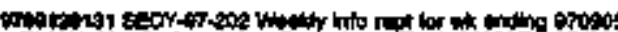

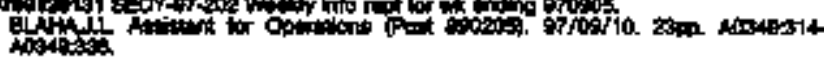

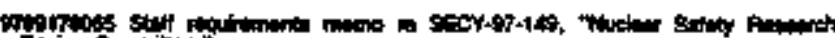

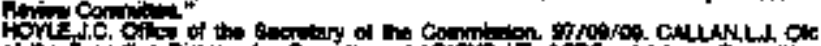

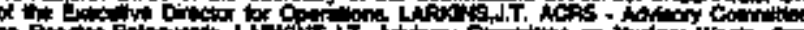

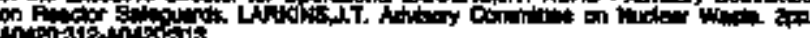

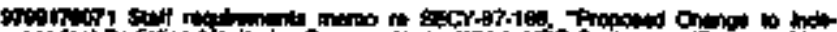

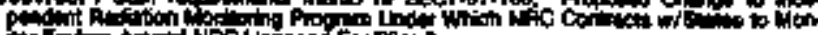
or pron now

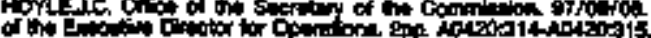

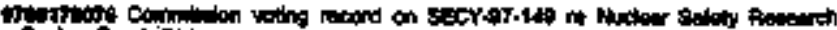

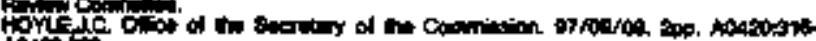
10istes.

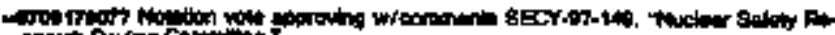

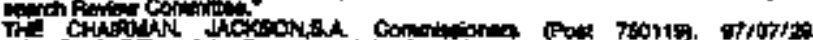

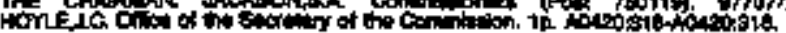

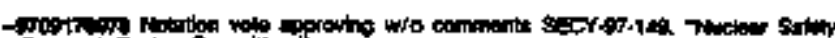
cictis

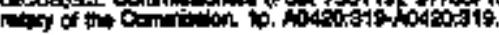

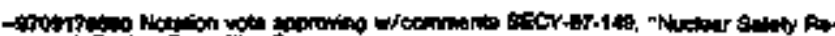

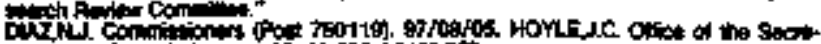

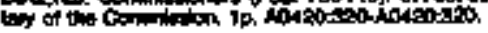

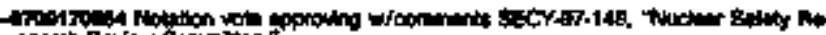
:

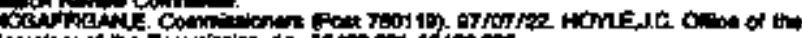

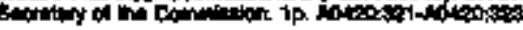

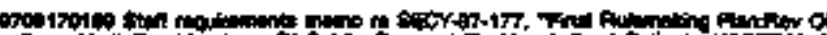

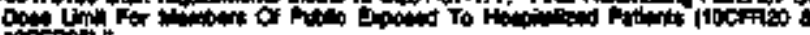

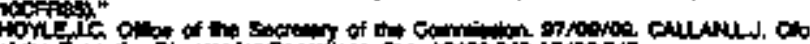

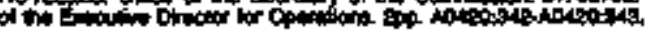

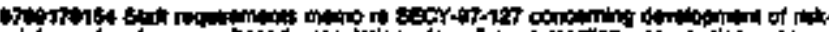

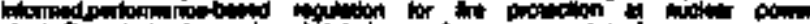

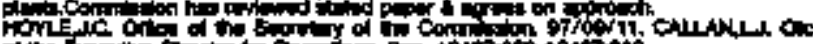

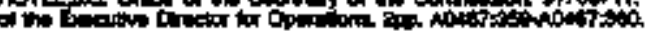

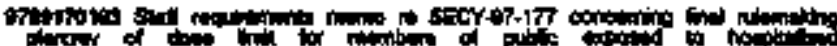

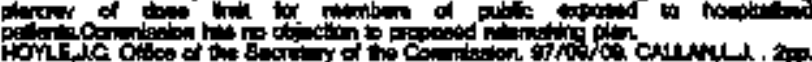

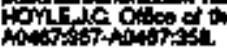

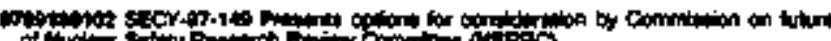

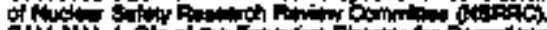

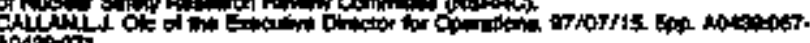
Notsint.

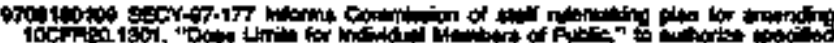

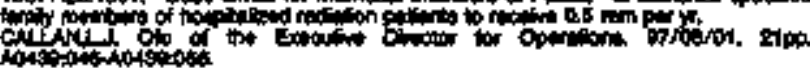

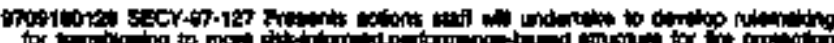

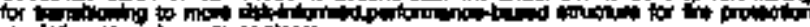

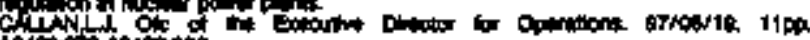

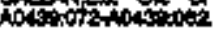

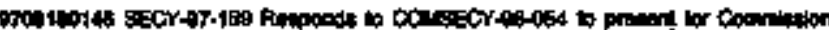

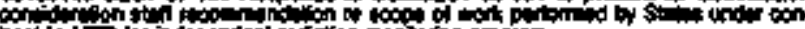

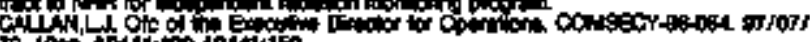

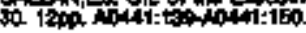

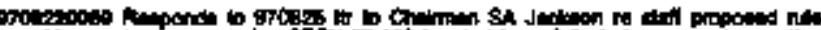

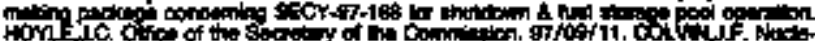

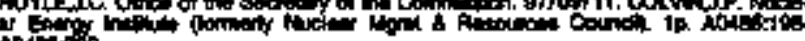
Notestio.

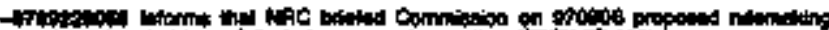

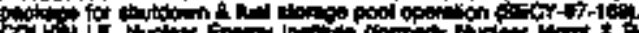

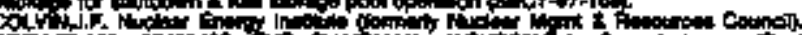

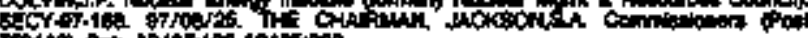

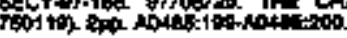

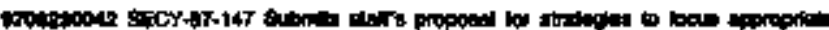

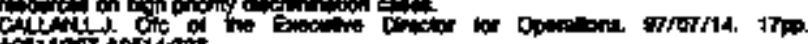

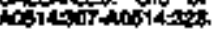

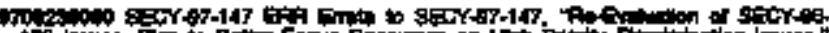

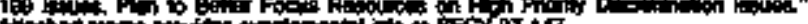

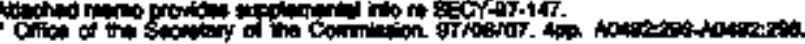

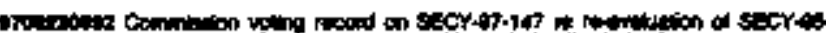

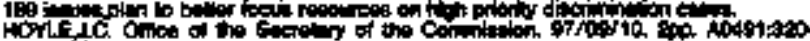
nontsist.

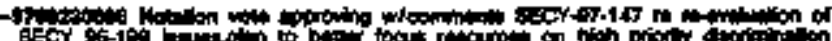

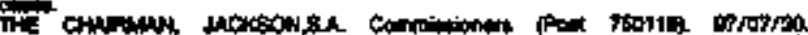

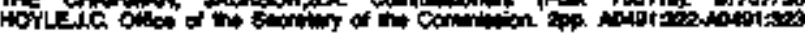

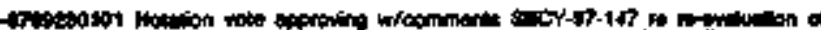

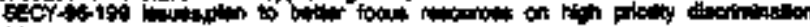

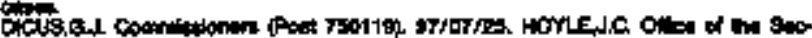

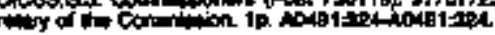

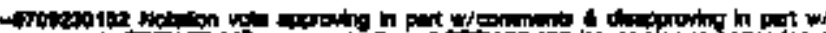

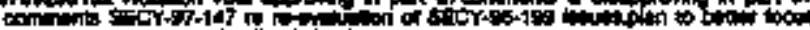

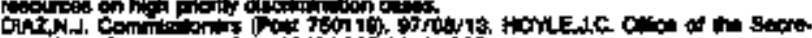

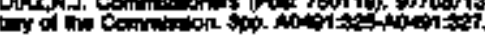

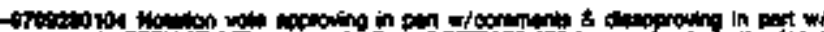

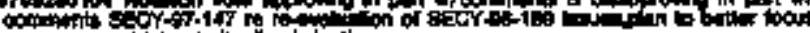

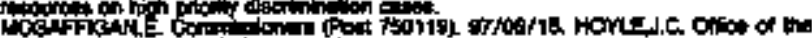

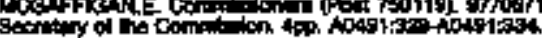

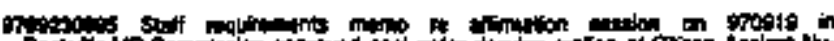

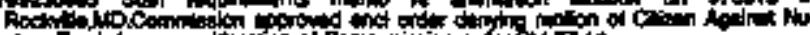

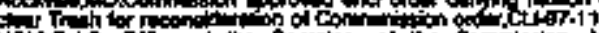

The

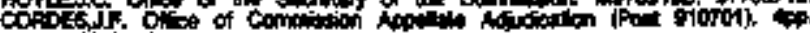
And 130 की 


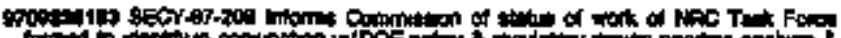

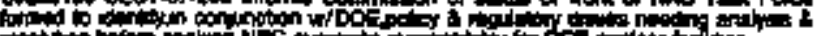

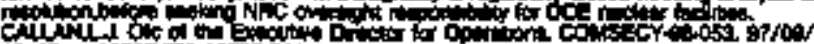

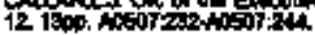

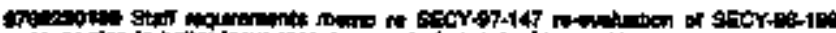

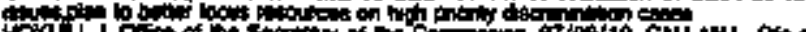

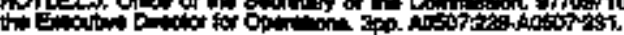

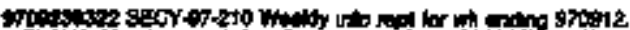

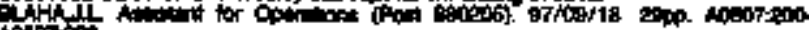
Aropition

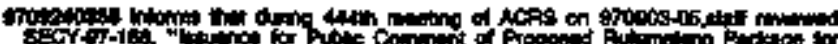

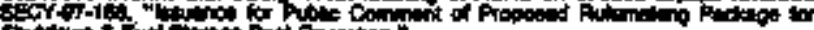

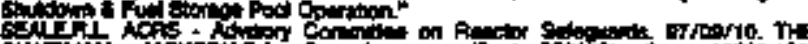

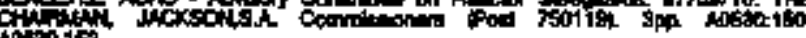
atom

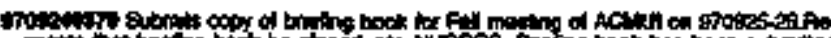

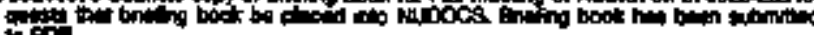

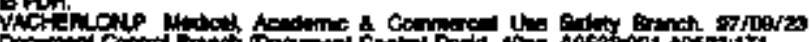

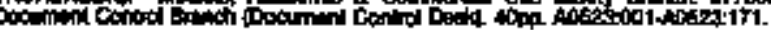

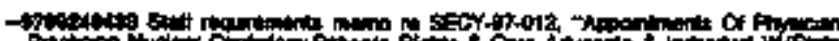

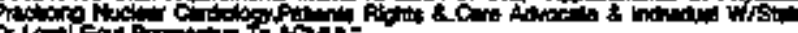
or Lo

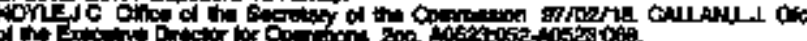

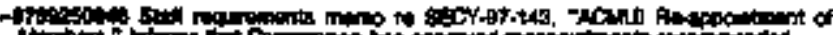

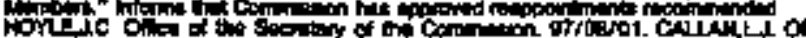

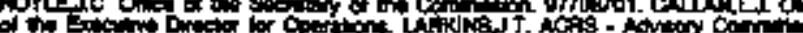

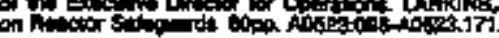

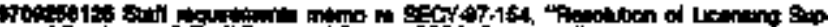

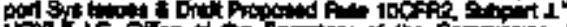

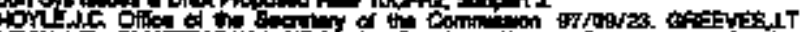

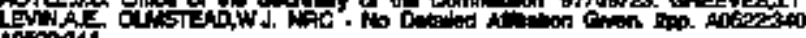
A06estit,

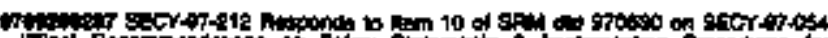

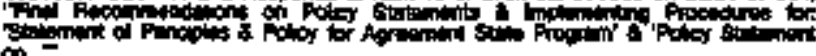

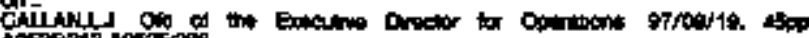

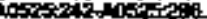

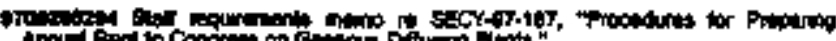

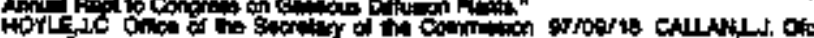

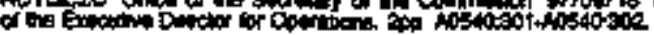

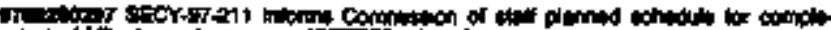

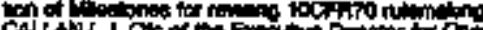

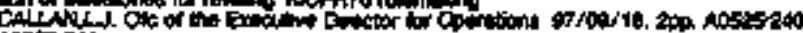
astisit.

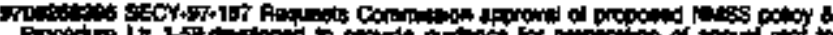

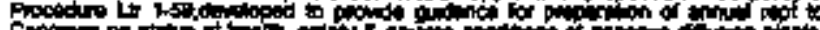

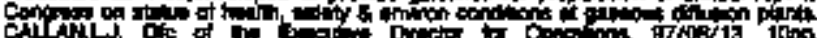
AnL

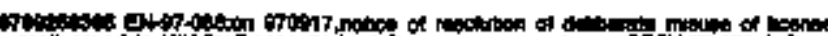

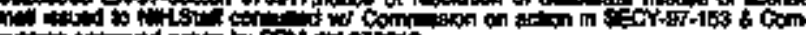

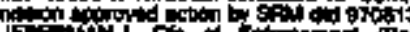

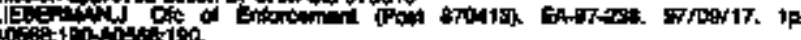

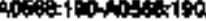

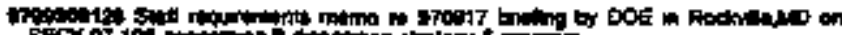

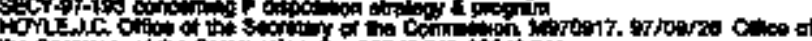

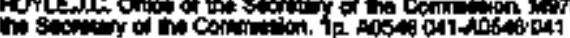

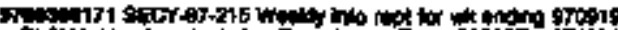

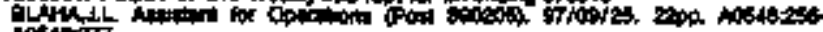
nostegrt

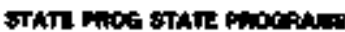

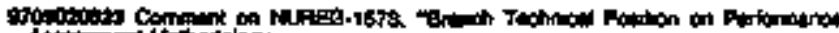

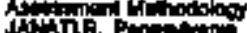

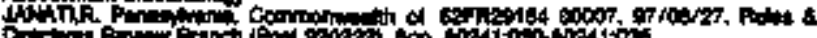

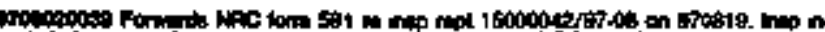

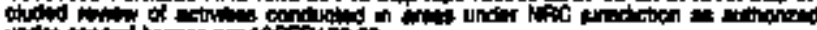

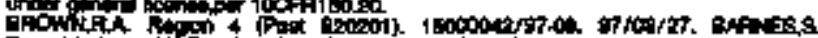

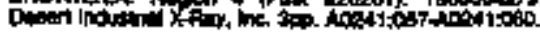

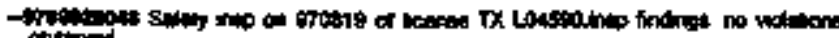

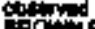

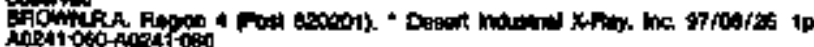

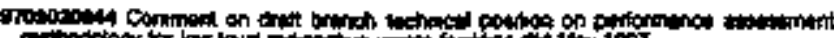

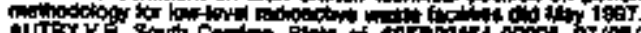

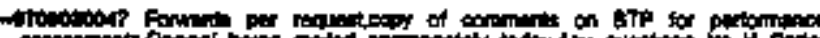

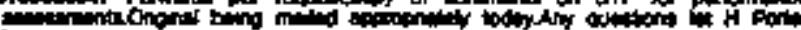

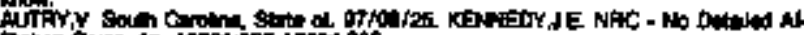

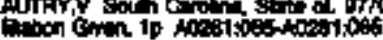

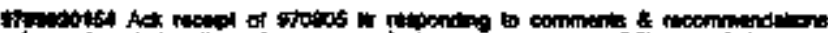

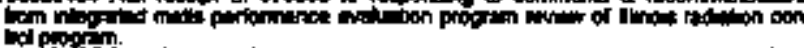

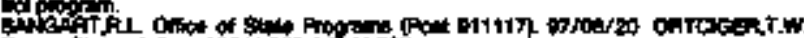

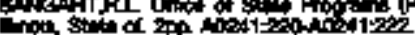

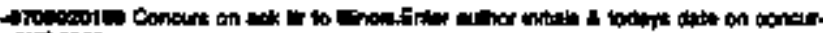

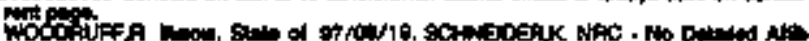

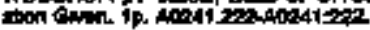

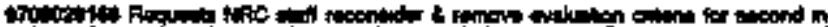

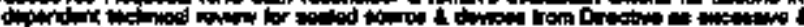

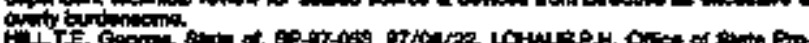

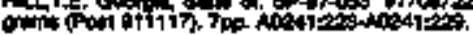

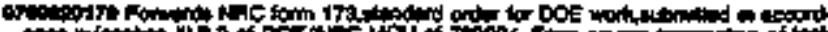

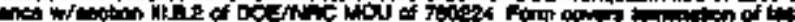

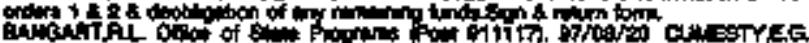

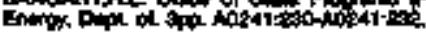

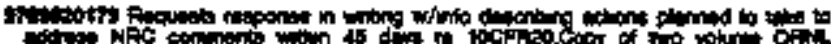

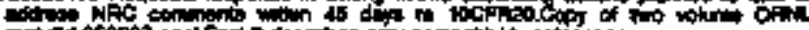

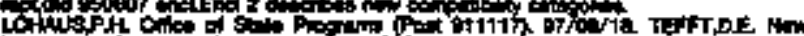

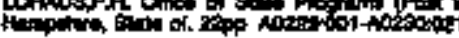

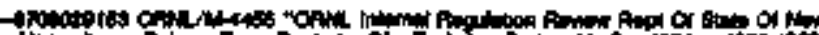

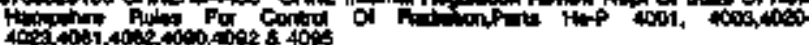
KU.

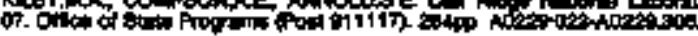

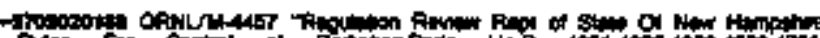

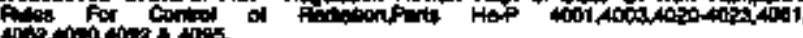

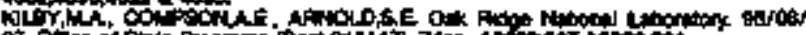

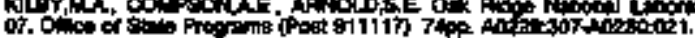

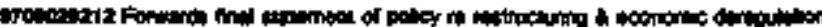

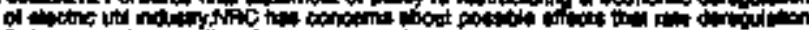

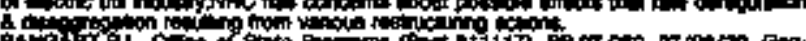

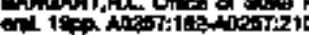

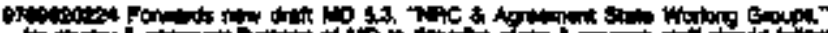

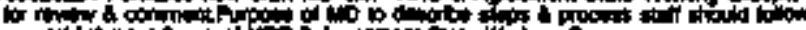
a

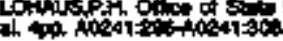

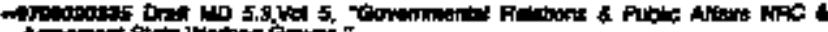

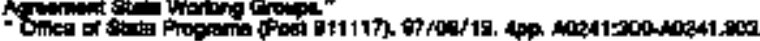

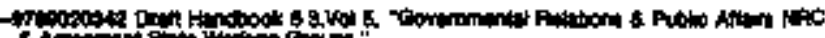

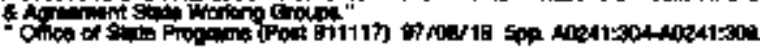

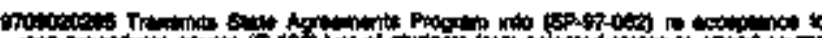

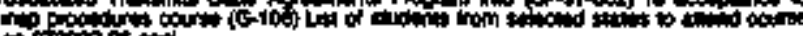

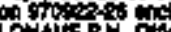

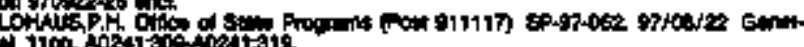

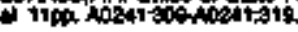

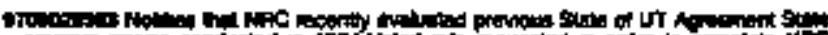

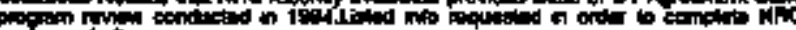
and

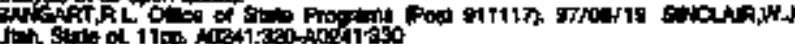

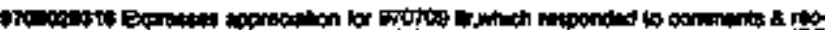

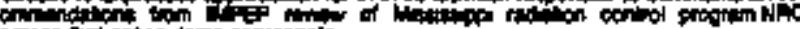

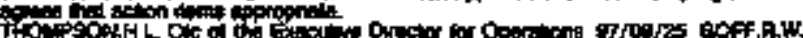

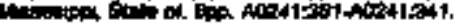

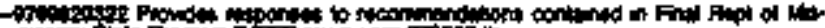

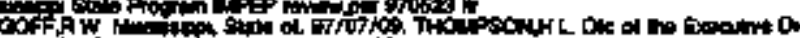

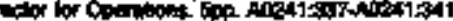

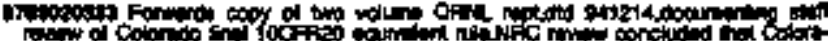

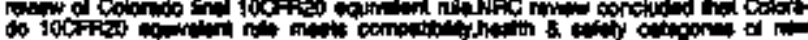

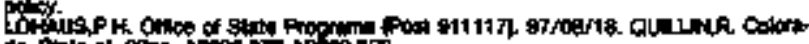

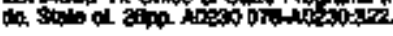

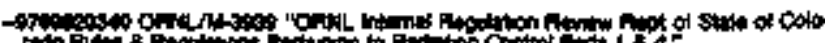

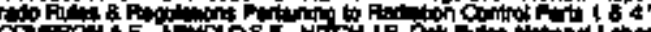

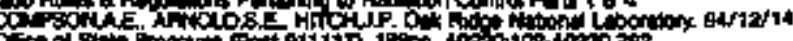

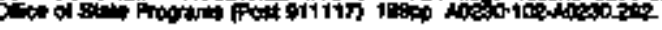

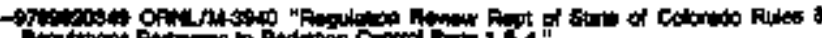

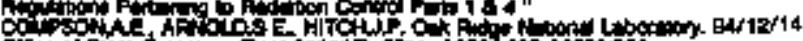

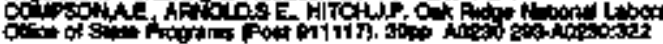




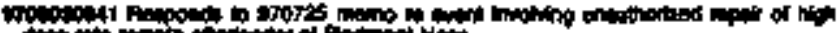

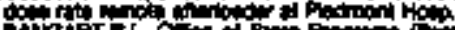
IN

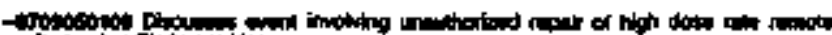

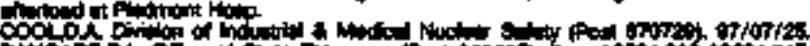
ENGMA,

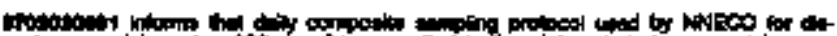

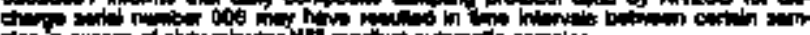

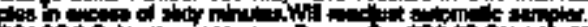

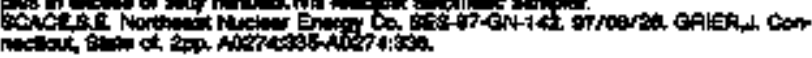

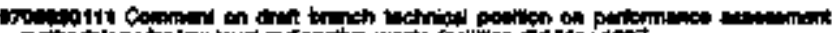

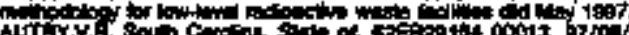

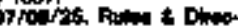

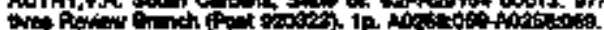

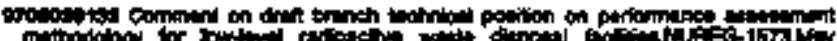

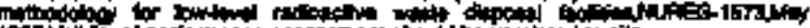

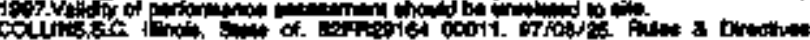

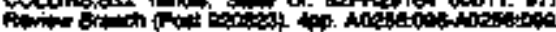

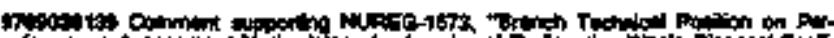

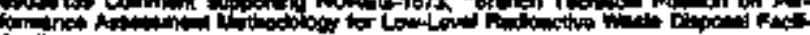
(1)

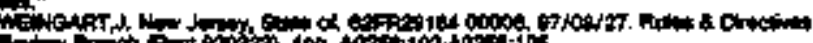

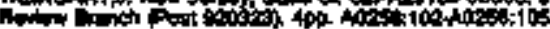

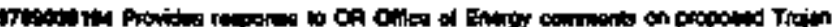

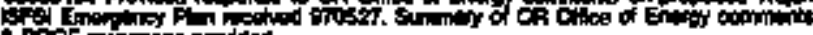

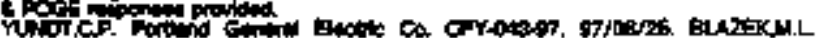

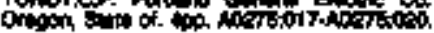

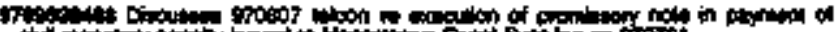

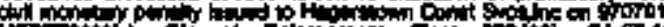

C.t.

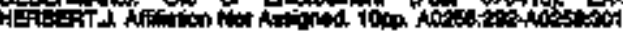

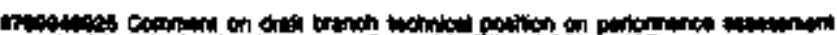

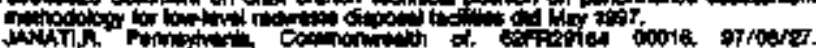

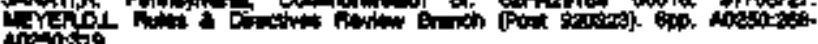

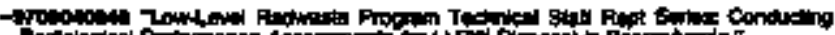

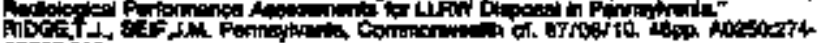
noxisio.

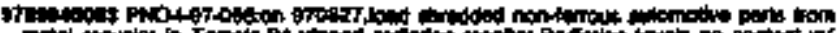

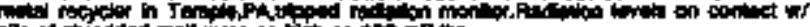

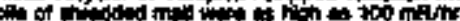

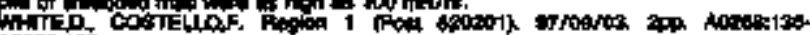

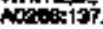

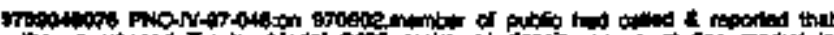

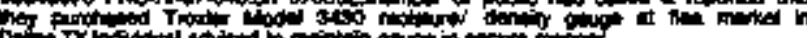

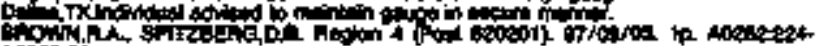
rotito

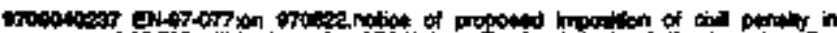

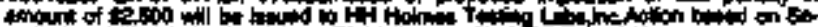

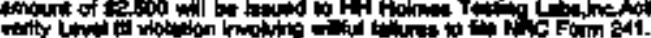

D.

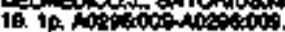

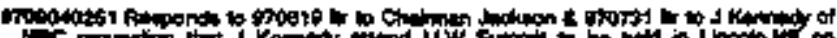

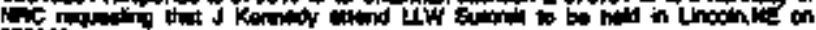
omate

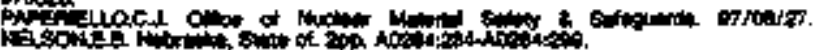

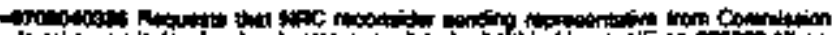

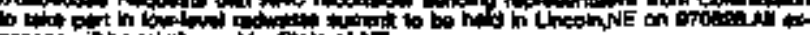

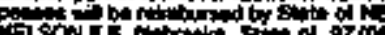

kison,

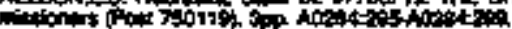

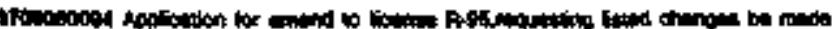

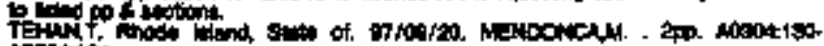
Aovitisi,

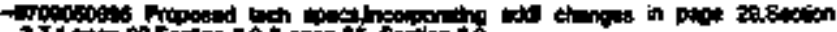

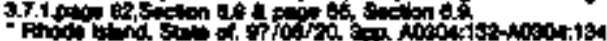

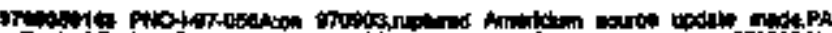

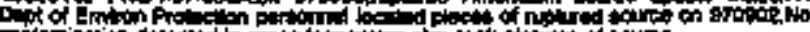
Fon $10021=0$

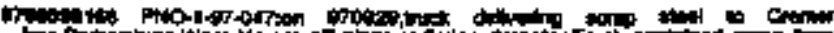

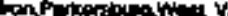
A

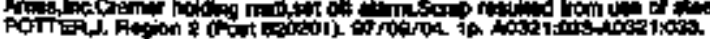

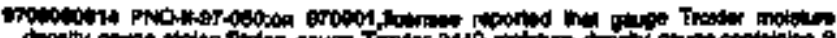

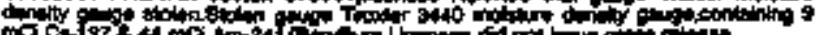

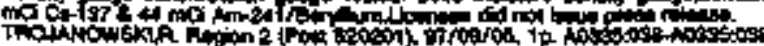

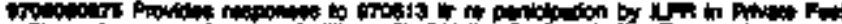

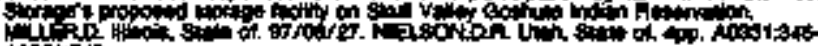
atistip.

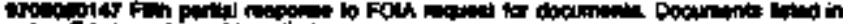

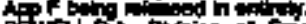

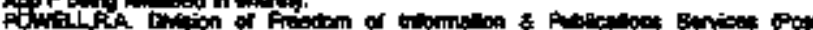

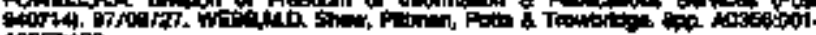
Angorido.

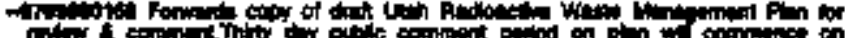

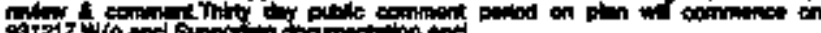

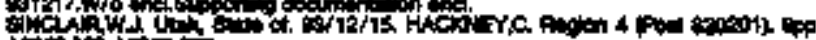

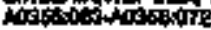

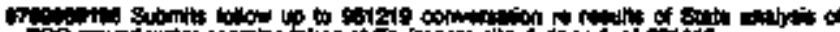

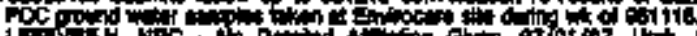
I

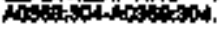

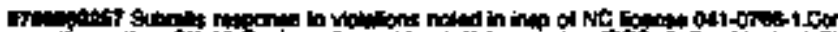

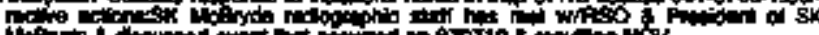

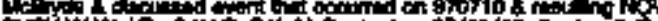

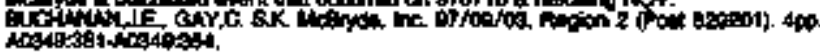

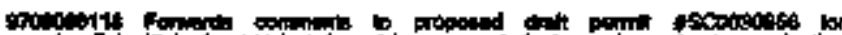

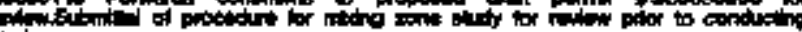

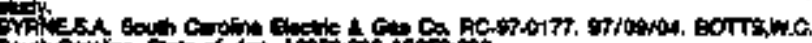

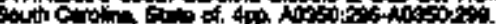

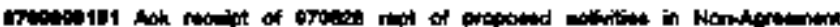

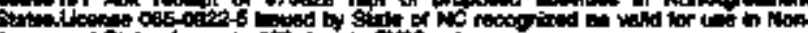

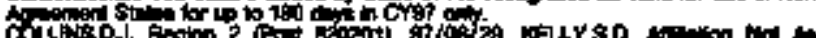

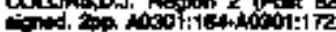

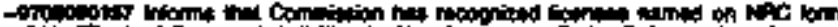

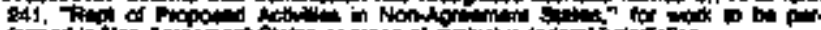

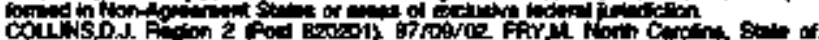

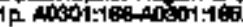

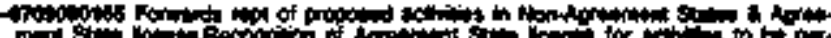
monl of

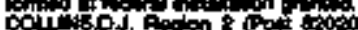

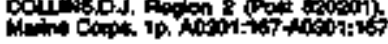

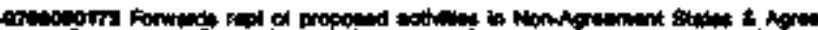

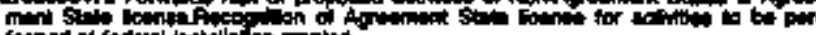

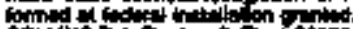

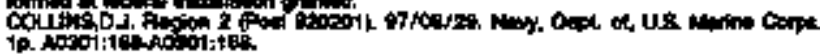

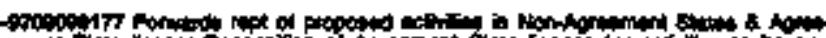
The

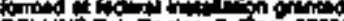

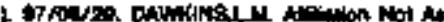

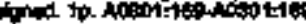

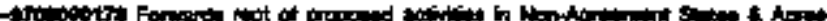

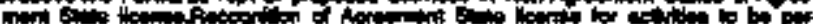

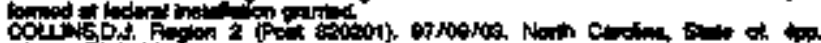

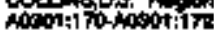

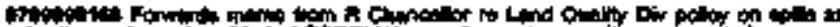

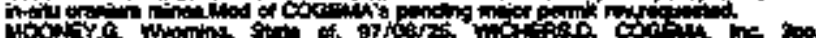

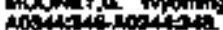

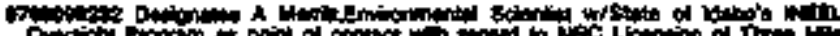

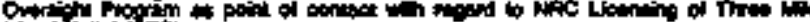

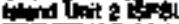

This the

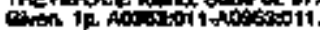

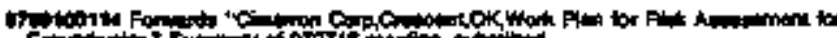

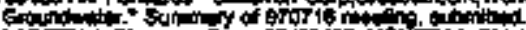

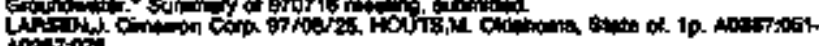

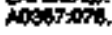

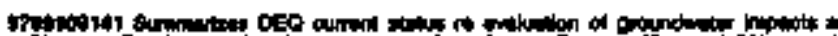

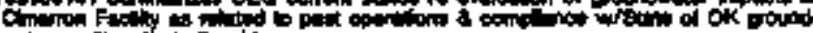

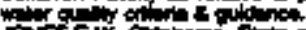

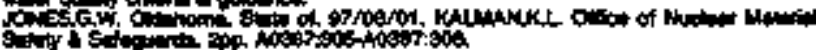

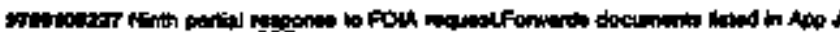

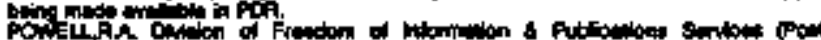

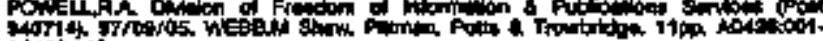

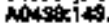

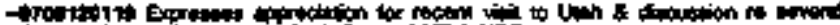

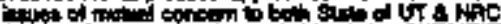

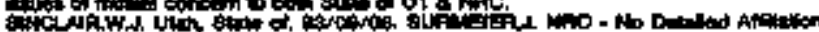

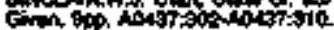

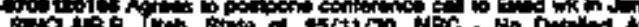

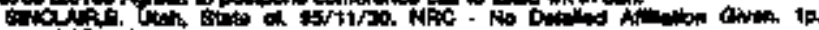

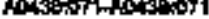




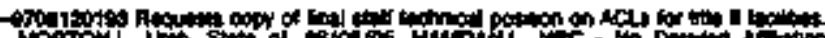

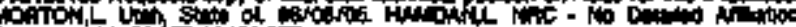

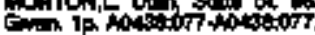

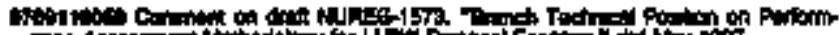

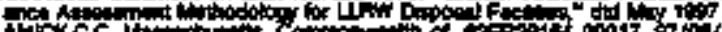
7Norza, Fum

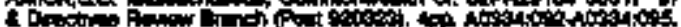

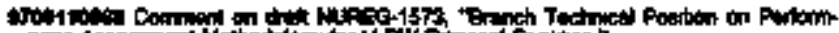
Wit A

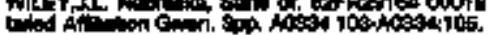

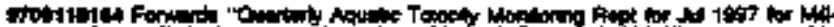

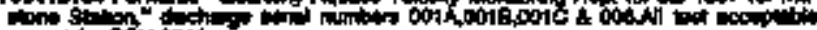

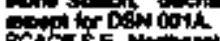

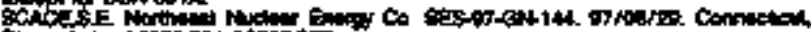

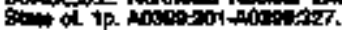

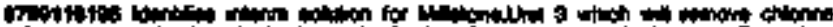

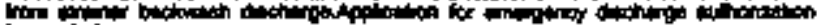

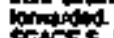

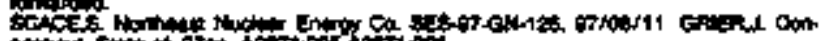

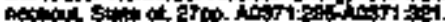

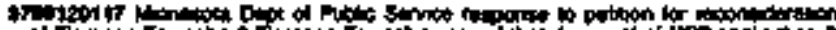

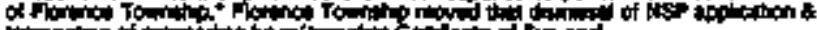
vitowe of

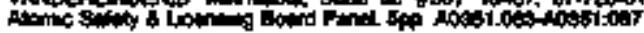

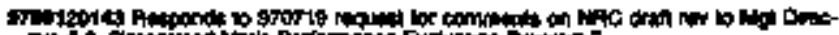

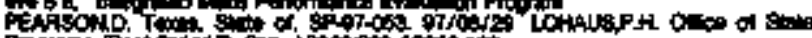

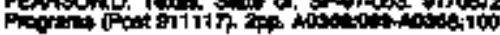

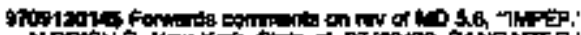

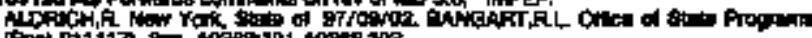

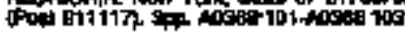

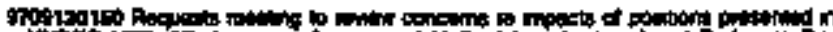

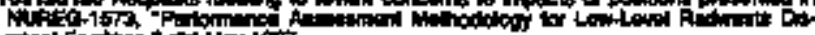

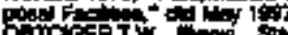

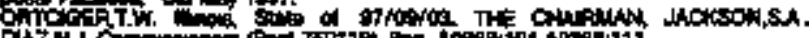

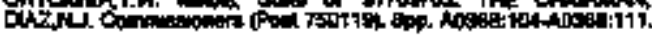

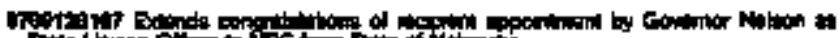
Fithon

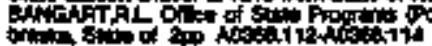

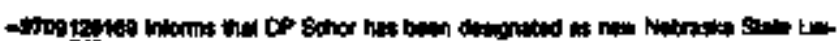

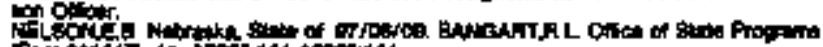

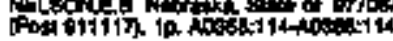

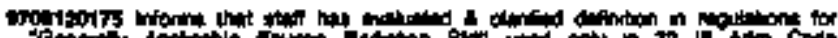

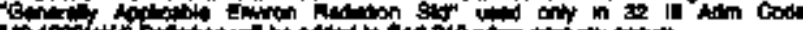
30.125.4.

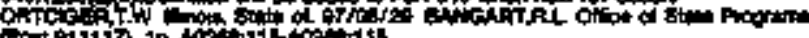

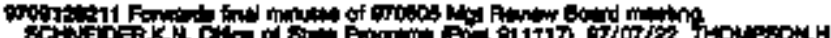

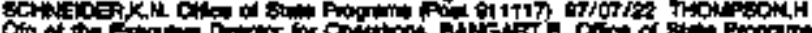

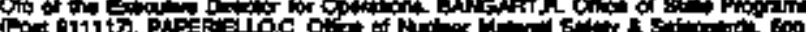

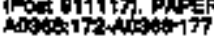

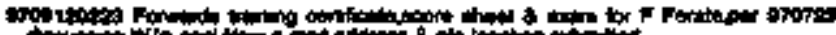

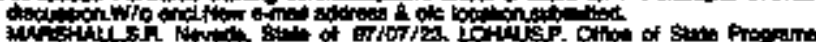

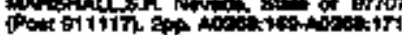

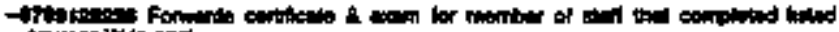
ogingation and

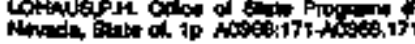

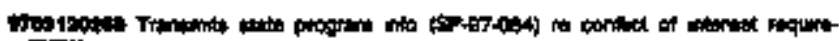

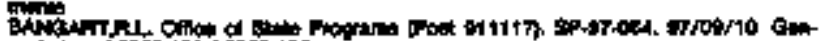

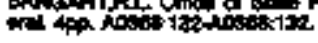

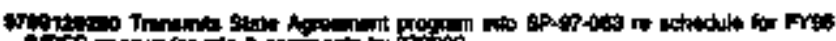

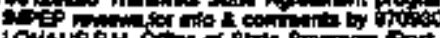

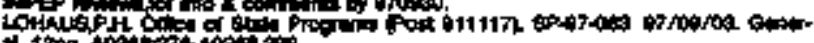

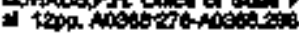

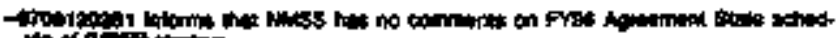

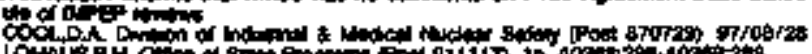

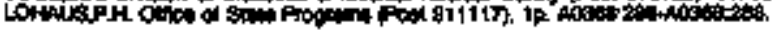

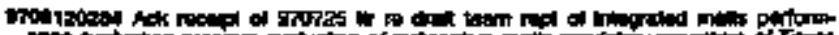

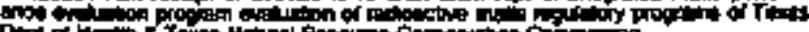
Poif of

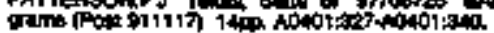

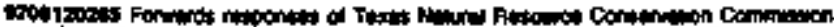

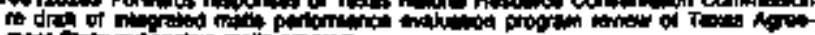

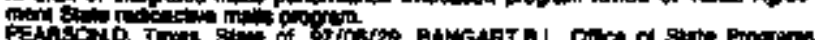

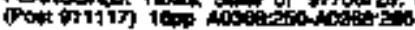

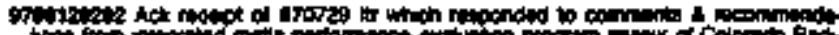

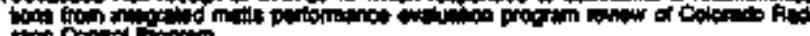

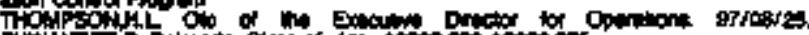

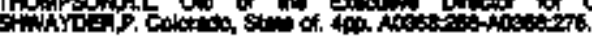

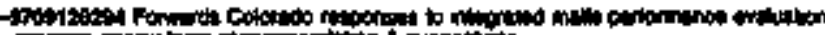

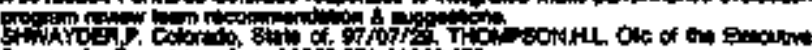

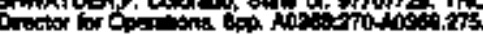

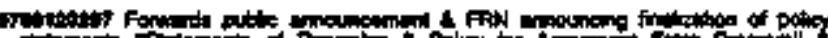

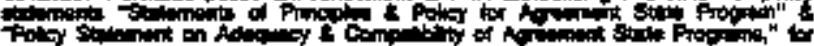

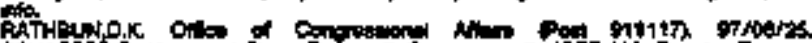

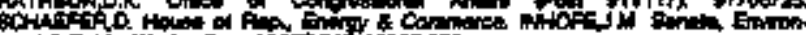

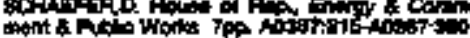

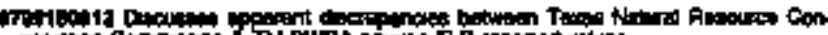

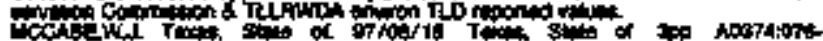
intistiont.

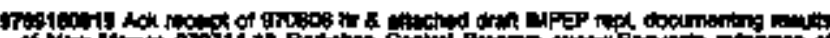

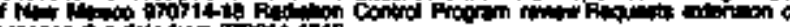

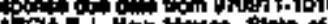

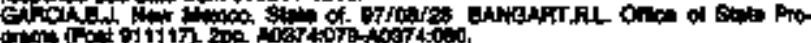

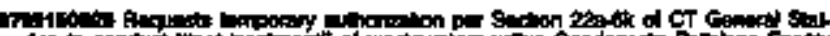

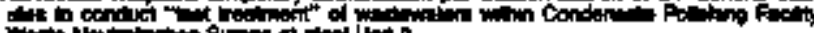

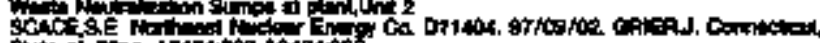

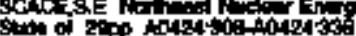

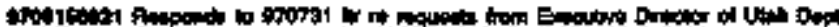

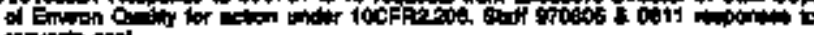

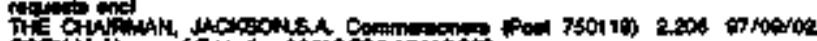

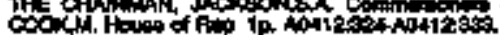

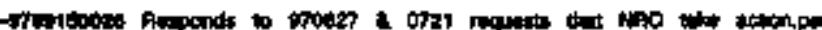

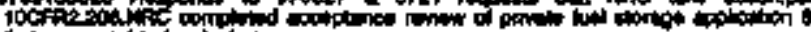

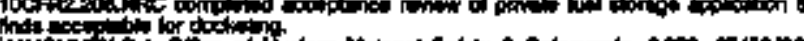

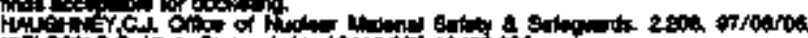

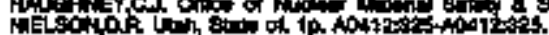

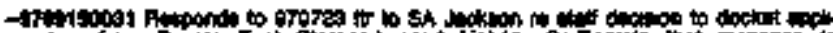

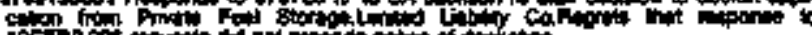
PC

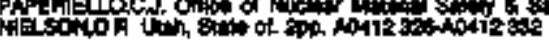

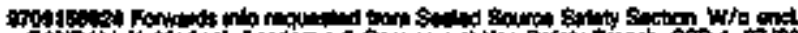

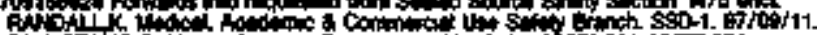
Shlost

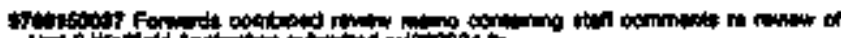

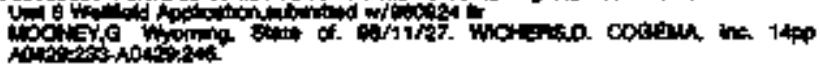

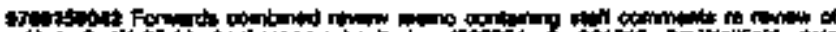

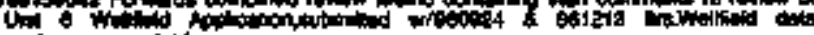

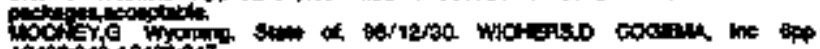

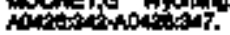

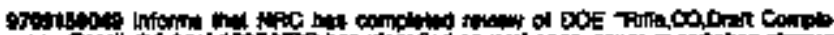

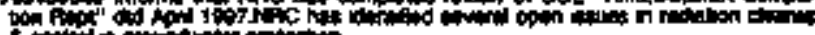

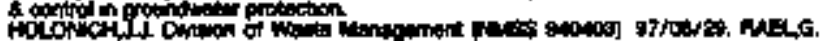

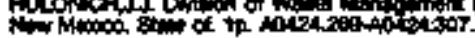

If

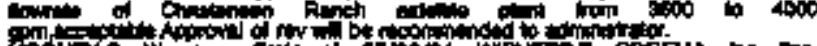

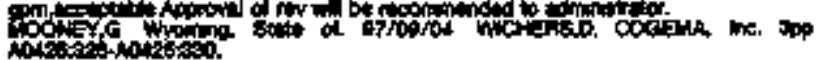

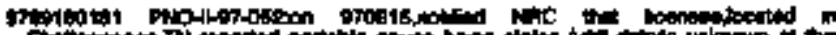

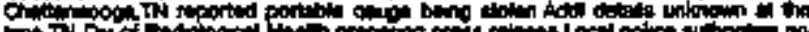

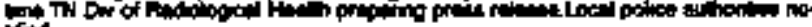

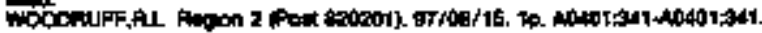

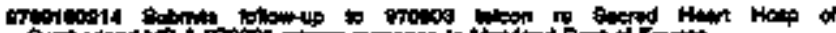

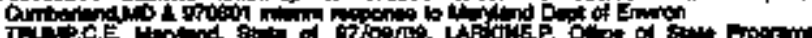

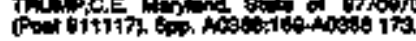

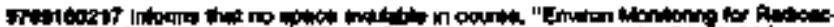

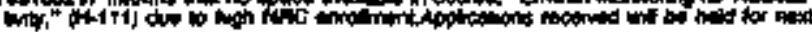

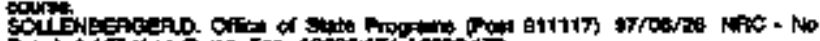

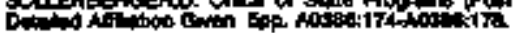

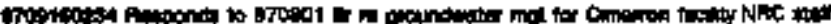

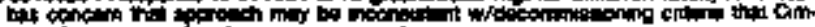

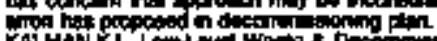

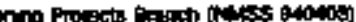

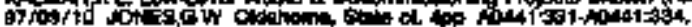




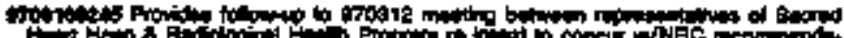

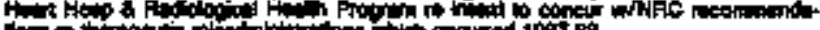

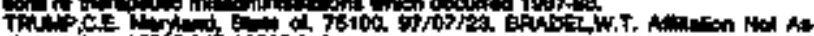

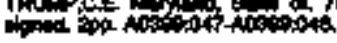

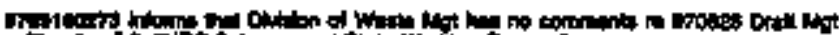

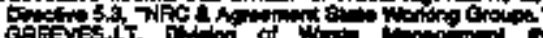

GA

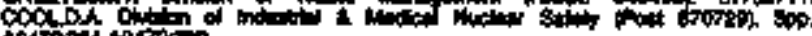

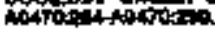

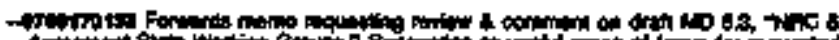

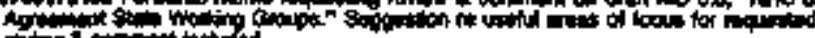

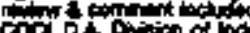

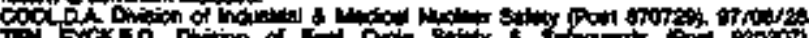

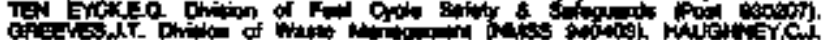

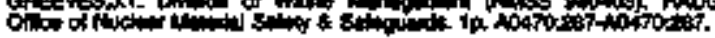

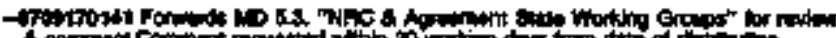

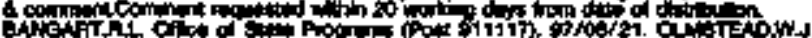
anc

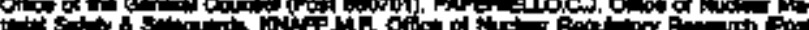

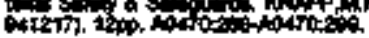

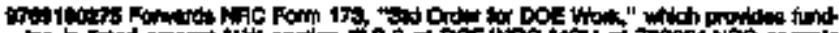

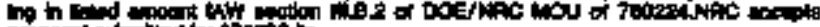

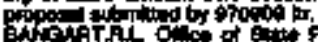

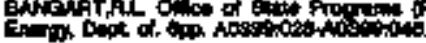

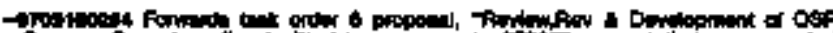

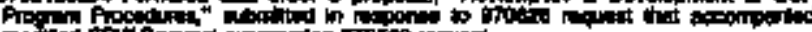

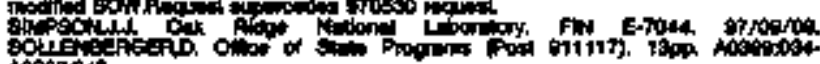

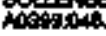

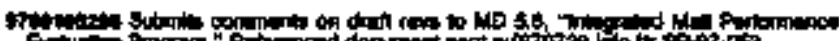

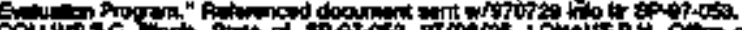
A

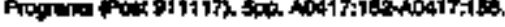

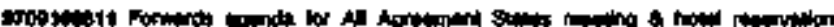

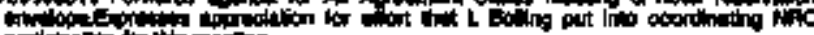

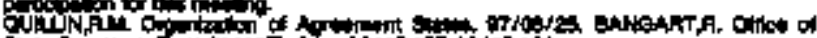

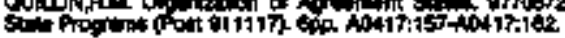

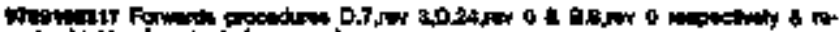

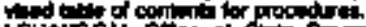

totils

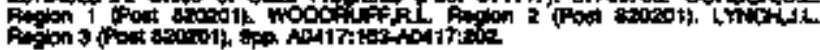

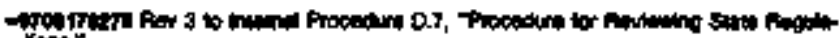

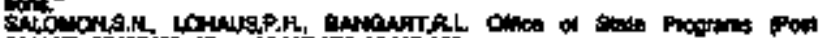

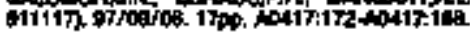

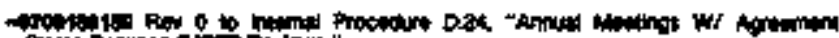

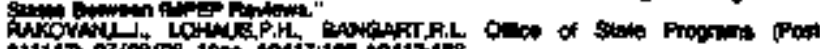

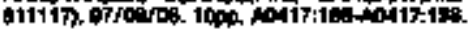

- Fow

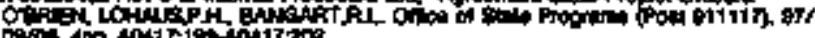

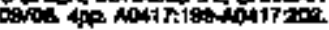

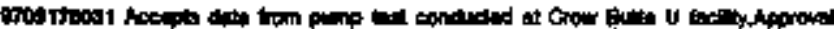

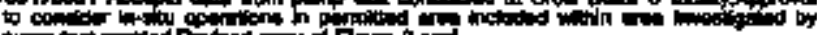

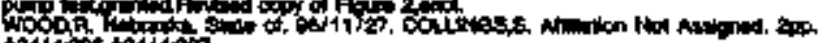
Apt40\%

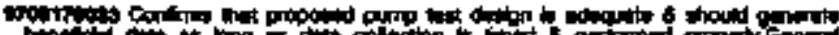

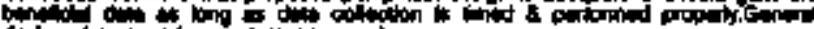

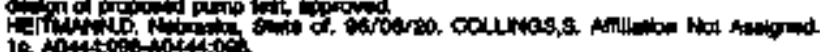

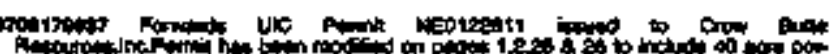

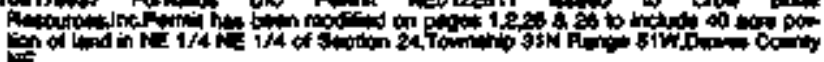

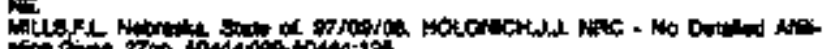

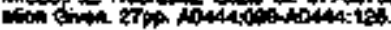

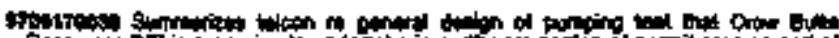

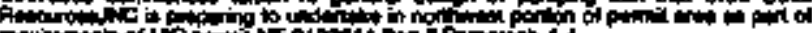

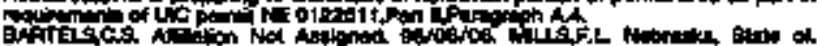

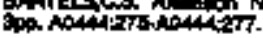

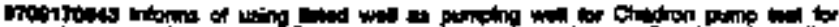

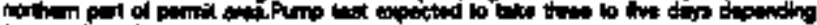

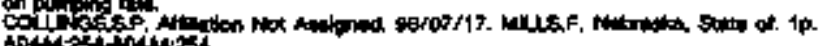

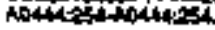

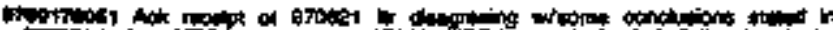

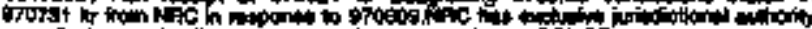
a

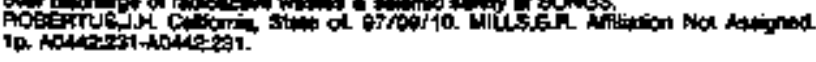

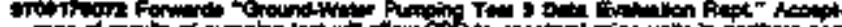

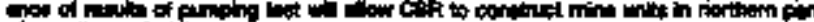

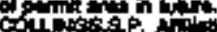

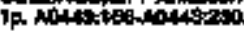

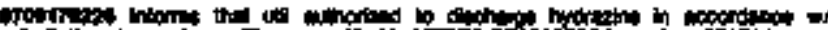

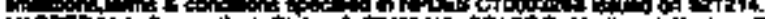

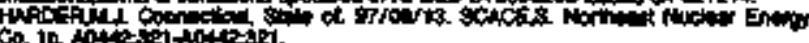

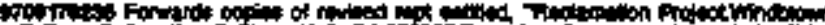

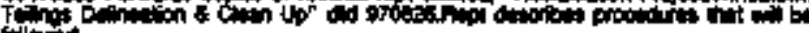

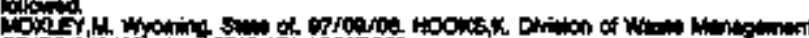
6.

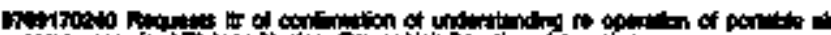

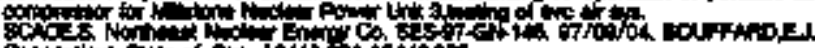

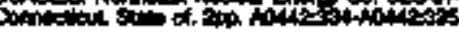

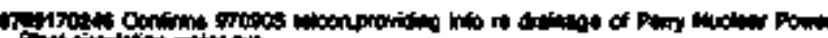

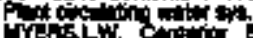

Yy

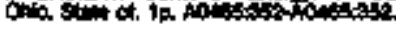

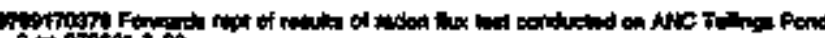
intopit

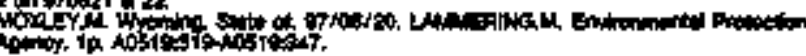

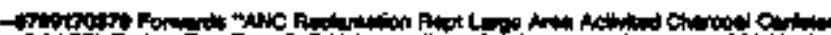

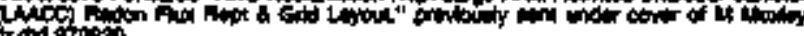

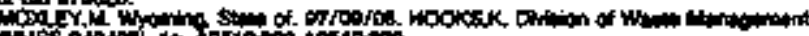

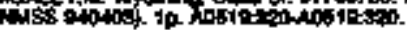

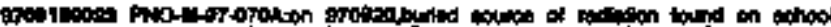

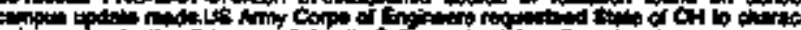

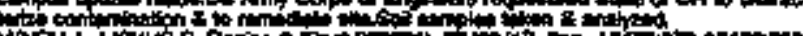

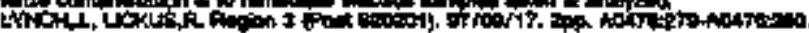

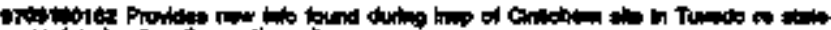

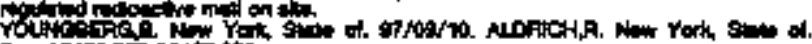

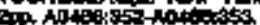

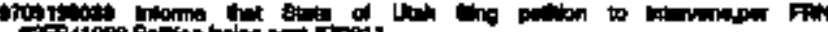

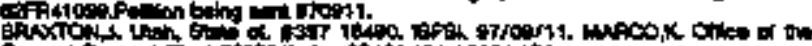

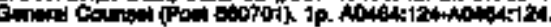

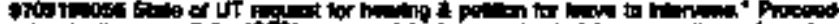

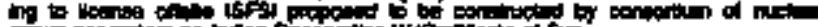

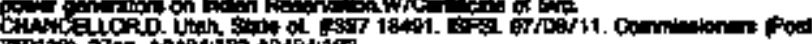
Fo'

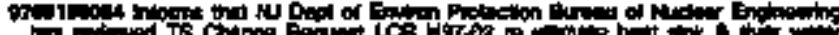

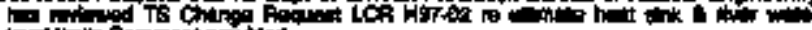

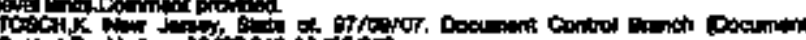

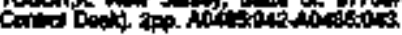

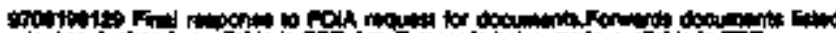
in A $^{\circ} \mathrm{A}$. ow

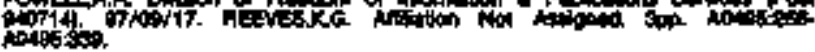

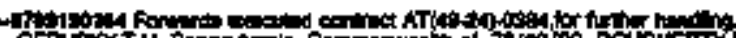
G

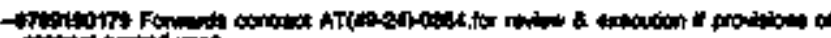
ơ

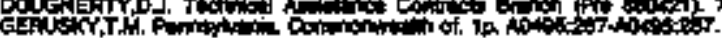

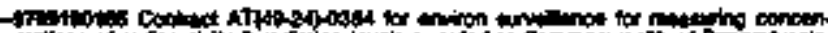

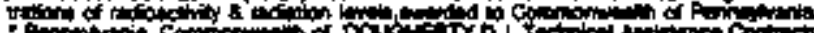

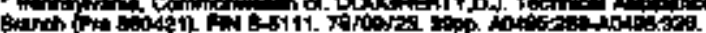

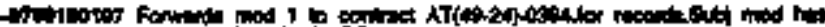

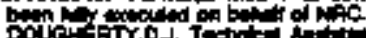

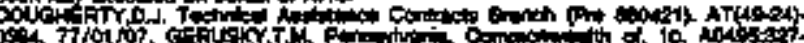
Aoussor.

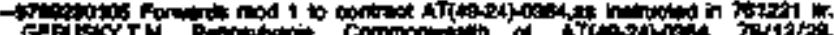

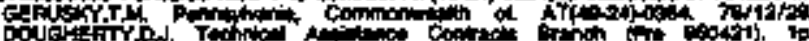

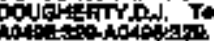

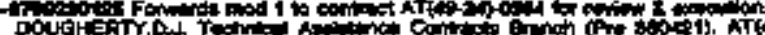

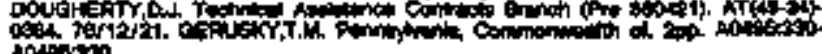
romition.

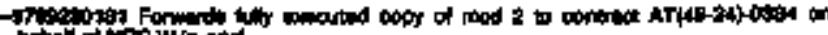

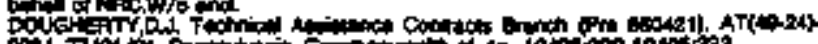

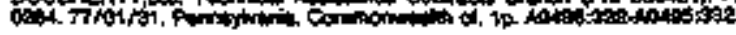




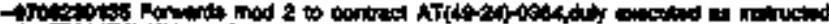

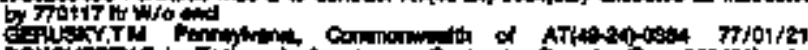

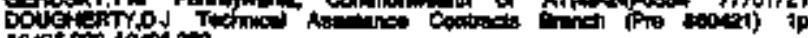

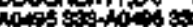

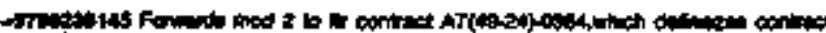

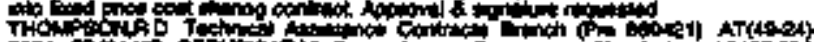

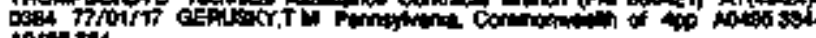
Alatis sild

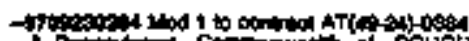

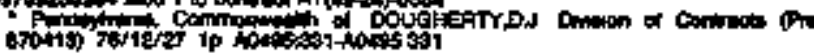

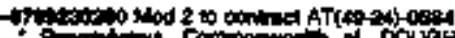

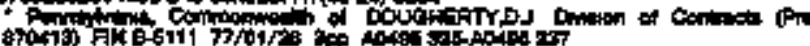

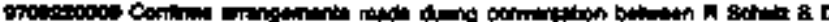

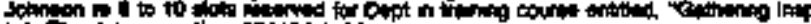

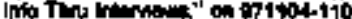

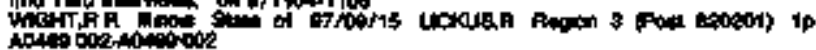

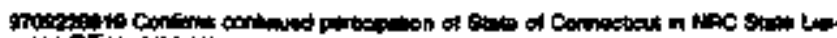

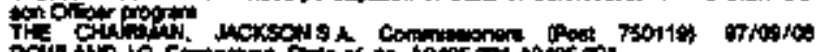

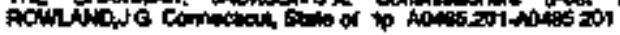

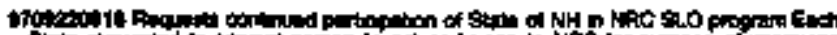

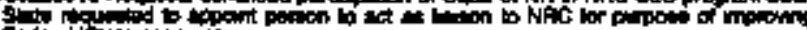

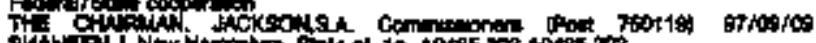

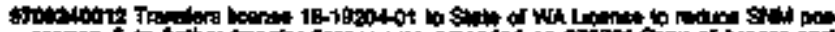

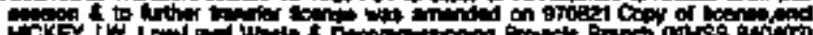

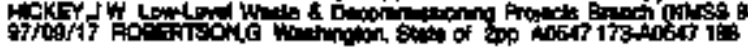

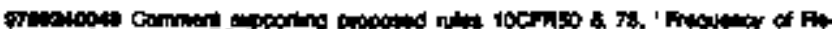

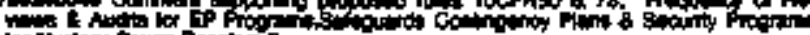

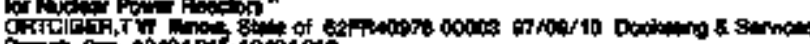

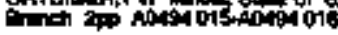

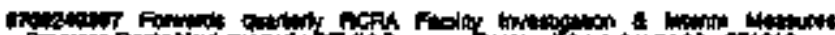

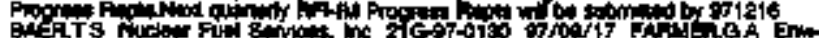

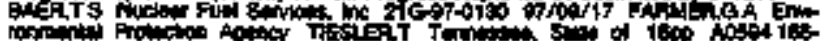
mintinges

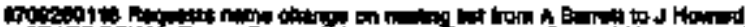

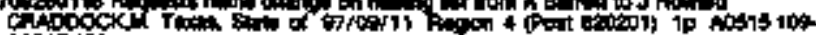
intsis

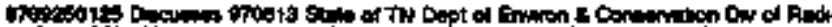

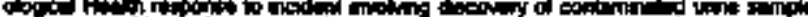

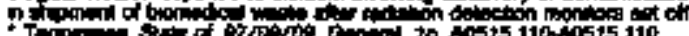

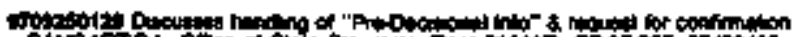

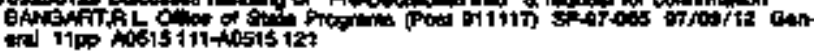

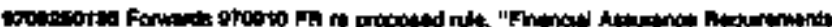

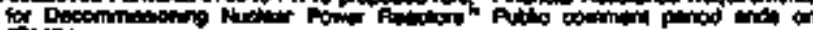

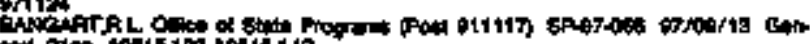

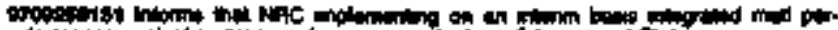

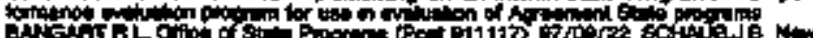

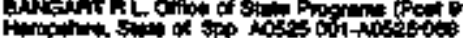

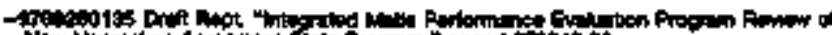

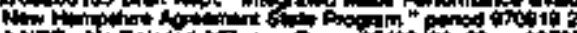

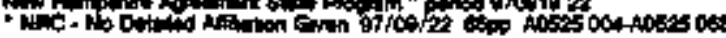

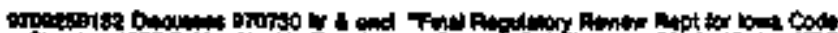

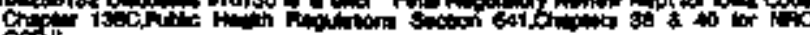

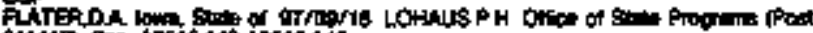
91117? $\sin$ A.

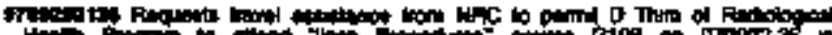

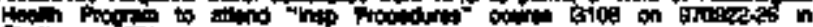
Chaturgatis

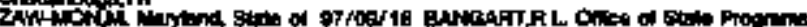

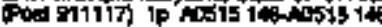

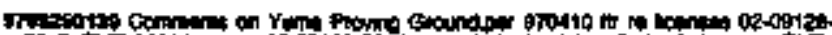

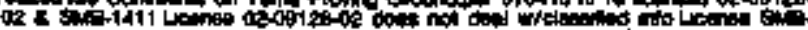

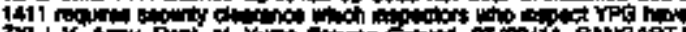

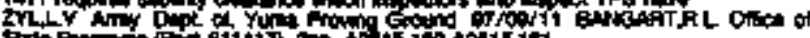

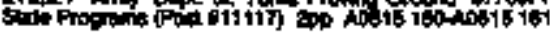

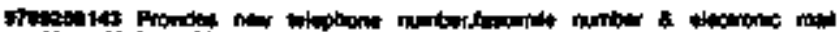

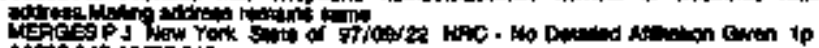

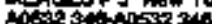

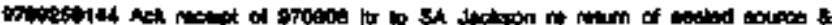

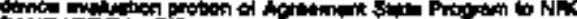

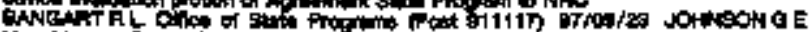

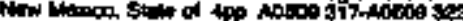

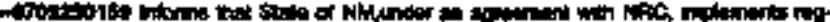

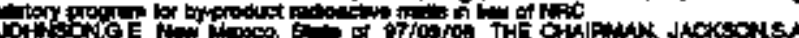

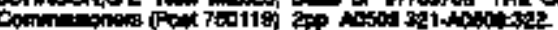

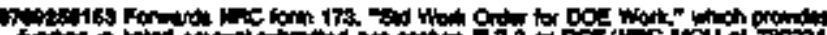

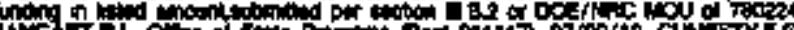
ont ind

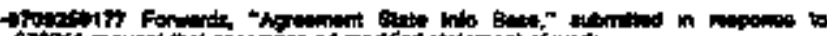

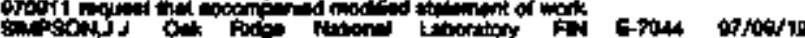

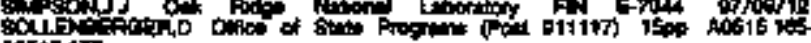
40.1日 178

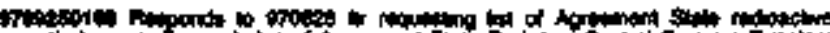

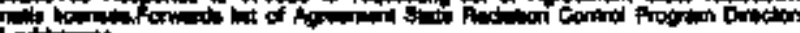

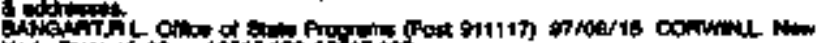
row

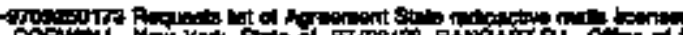

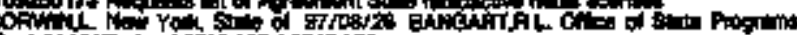
Pont ज्ञा117 के

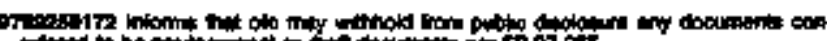

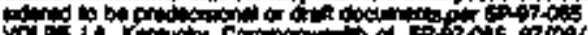

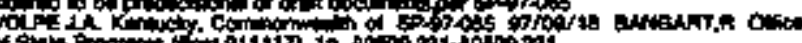

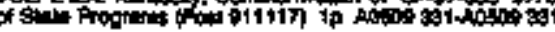

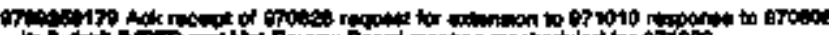

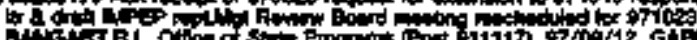

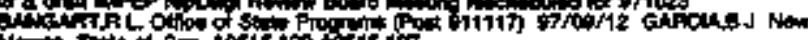

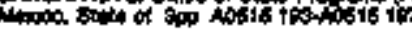

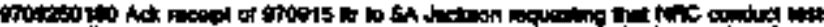

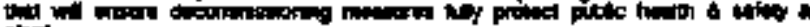

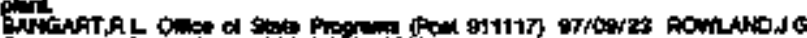

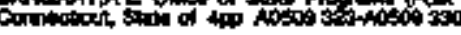

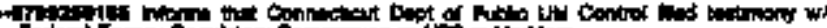

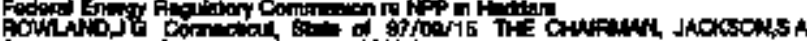

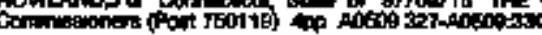

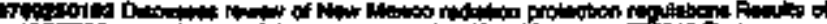

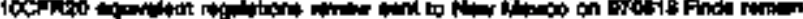

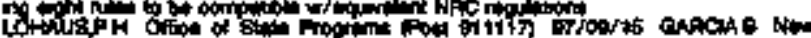

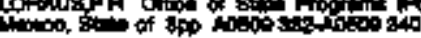

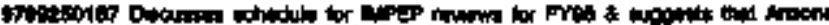

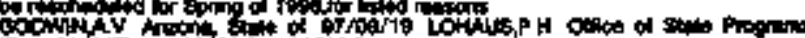

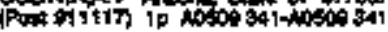

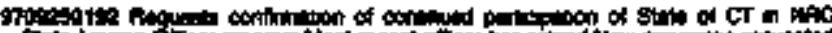
H.

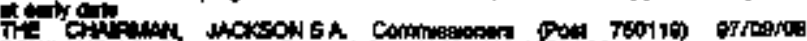

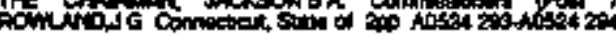

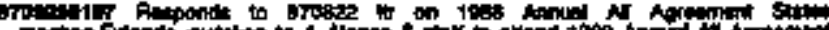

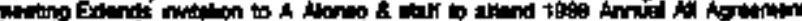

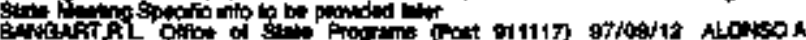

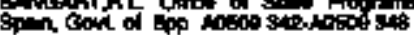

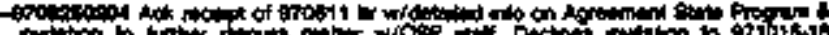

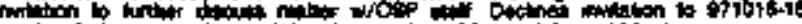

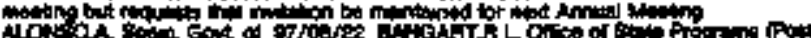

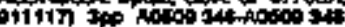

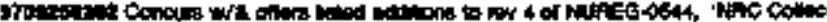

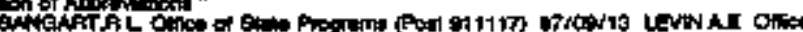

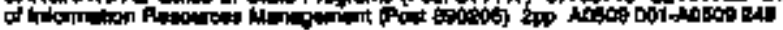

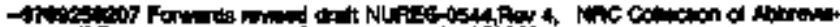

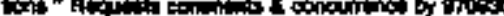

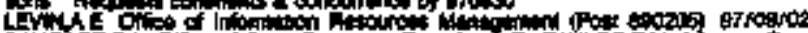

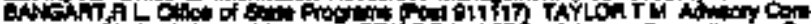

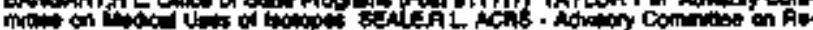

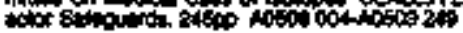

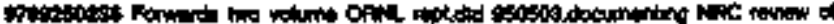

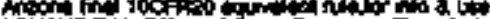

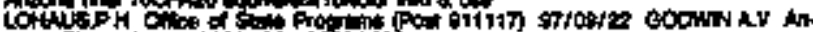

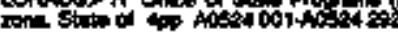

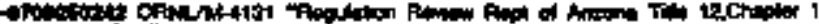
Axtrion 1 to 4

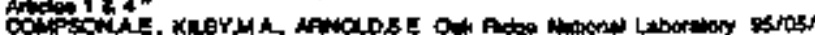

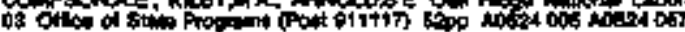

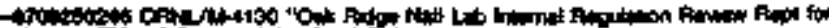

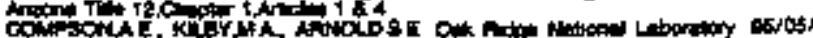

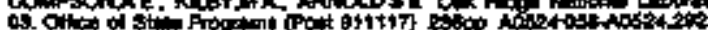




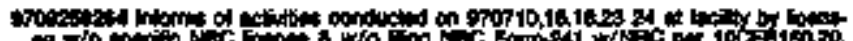

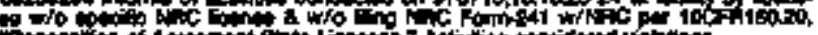

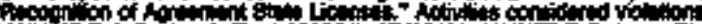

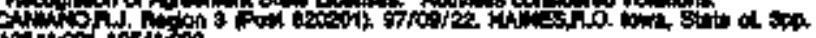
nitiond

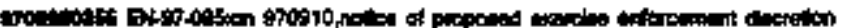

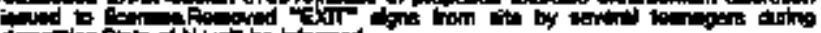

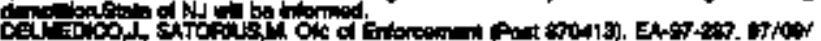

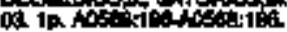

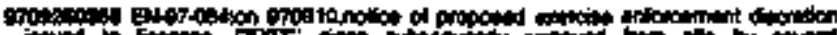

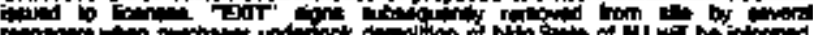
ond

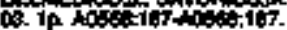

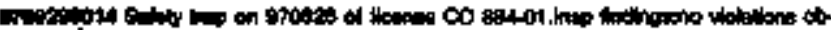

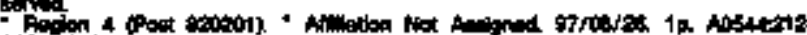
istition

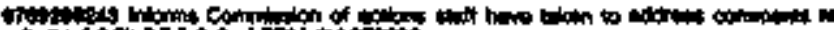

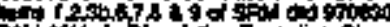

Coll

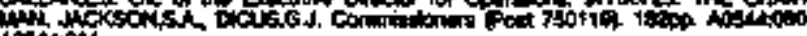
Ansteititi.

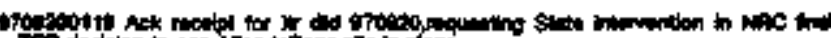

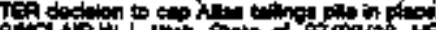

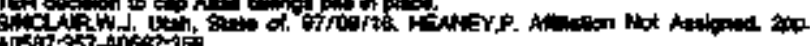

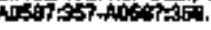

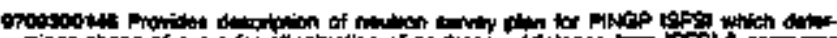

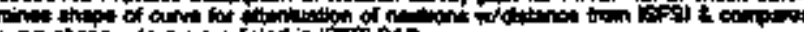

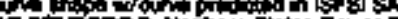

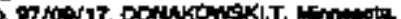
on:14

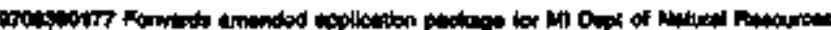

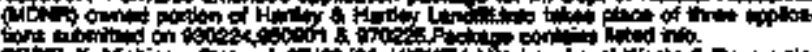

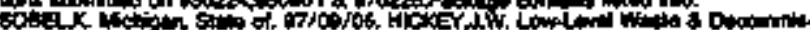

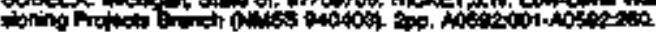

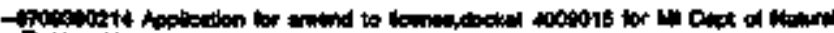

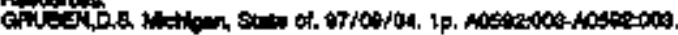

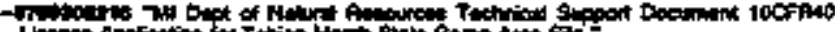

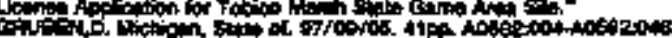

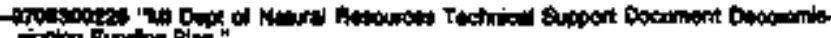

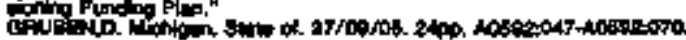

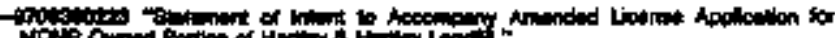

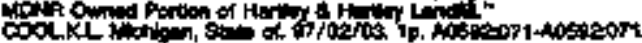

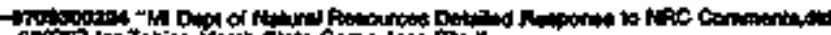

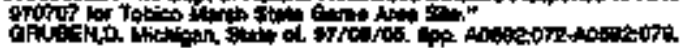

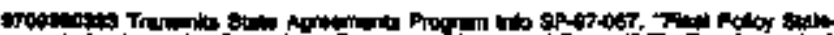

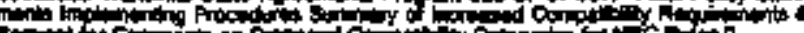

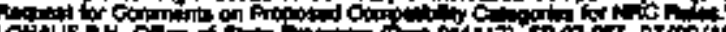

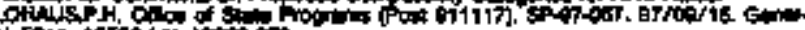

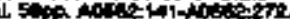

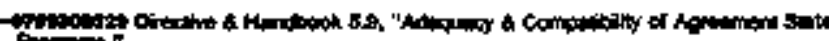

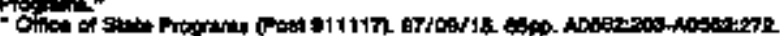

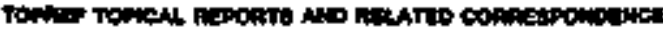

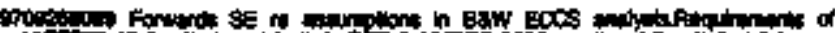

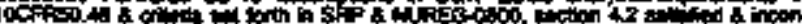

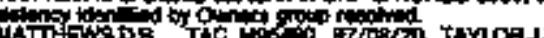

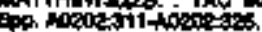

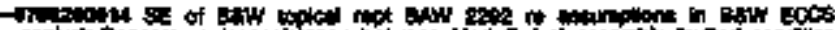

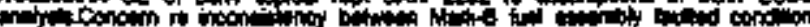

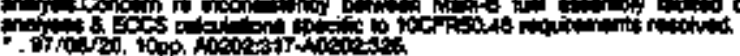

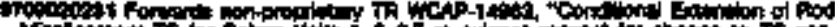

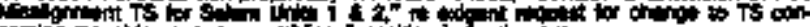

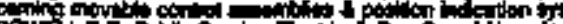

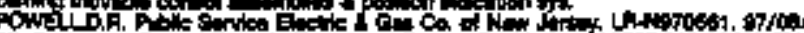

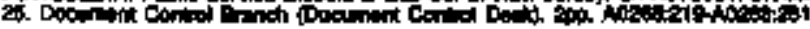

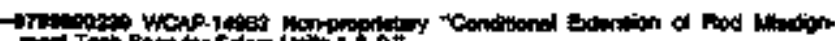
"

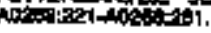

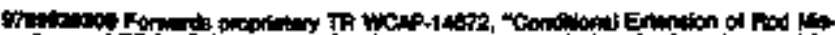

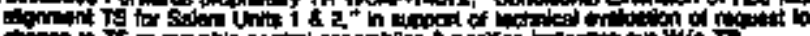
Dow

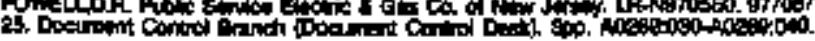

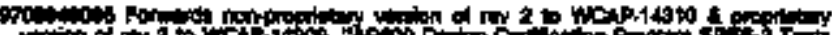

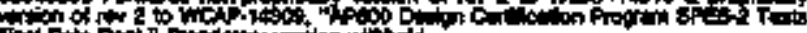

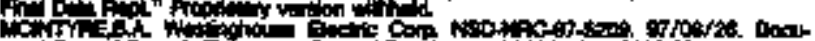

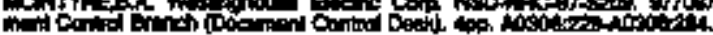

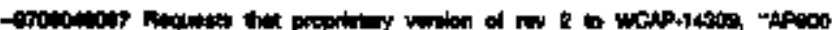

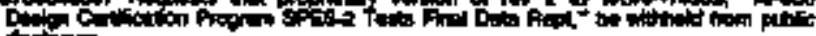

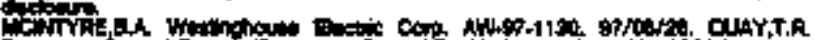
Docill

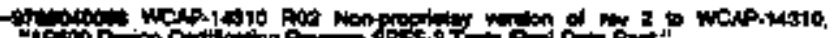
corith L L

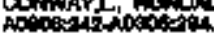

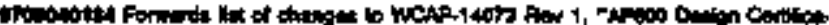

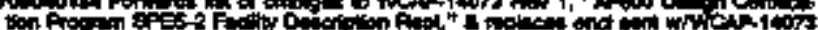
h

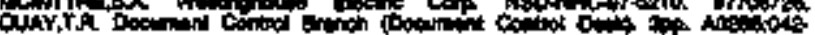
Antopits

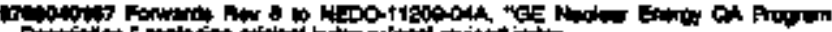

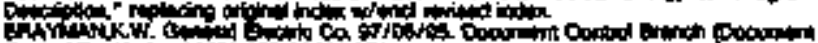

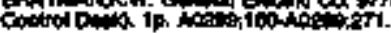

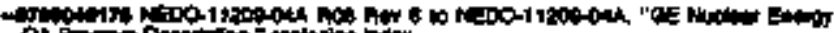

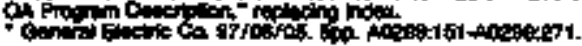

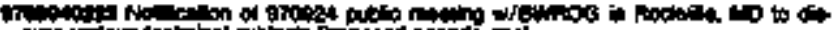

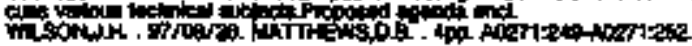

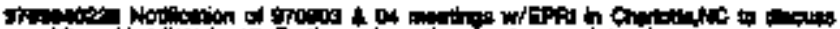

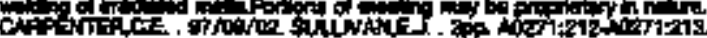

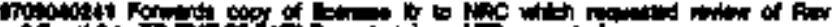

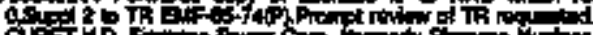

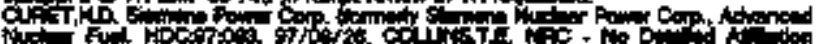

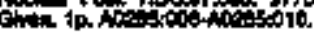

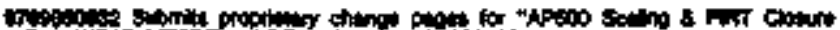

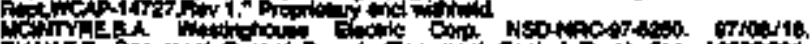

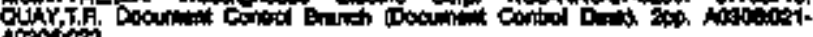
Astion.

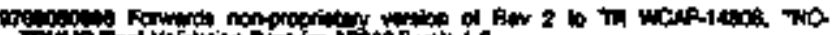

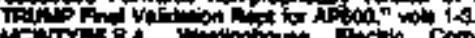

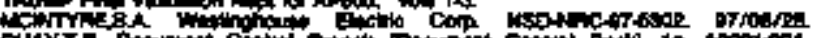

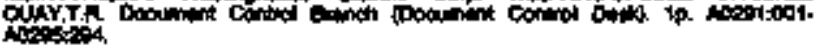

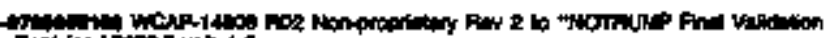

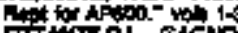

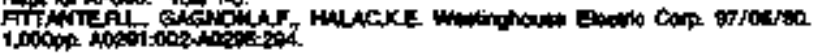

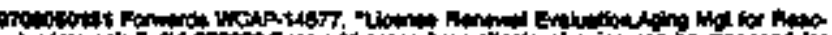

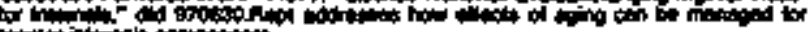

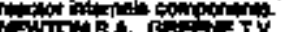

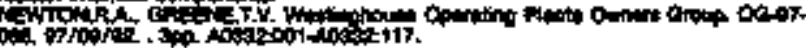

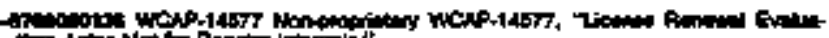

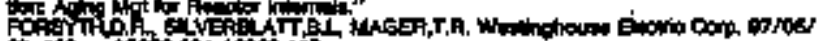
औ.

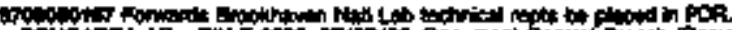

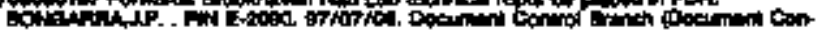

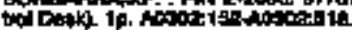

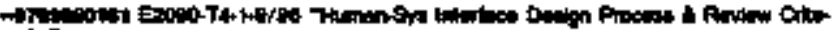

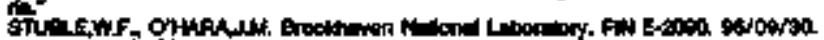

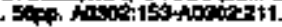

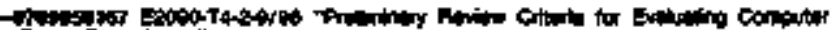

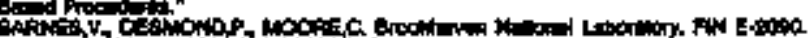

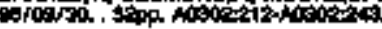

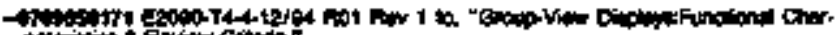

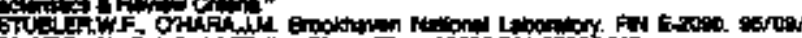

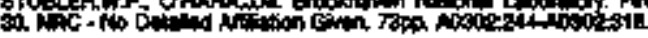

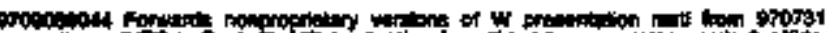

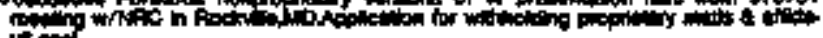

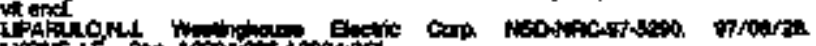

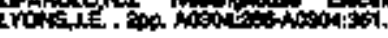

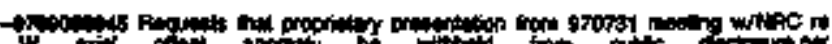

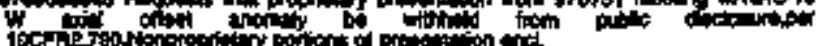

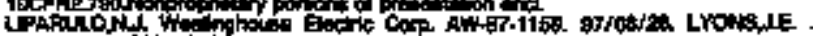
70 An phis 


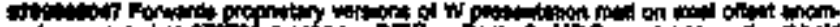

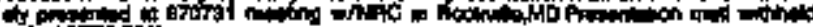

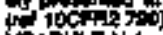

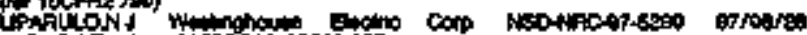

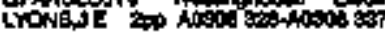

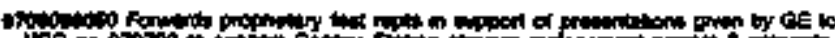

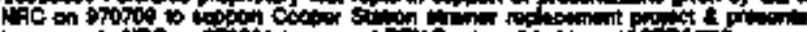

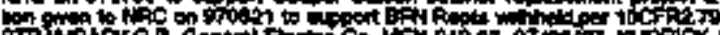

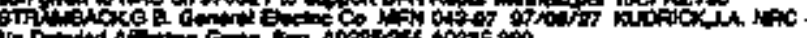

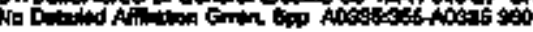

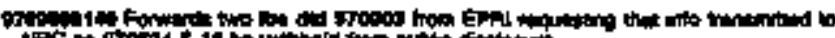

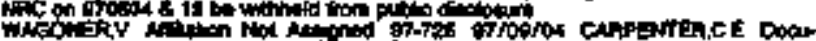

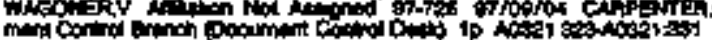

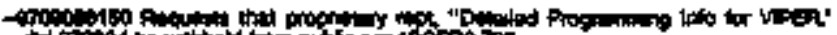
Fon

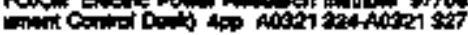

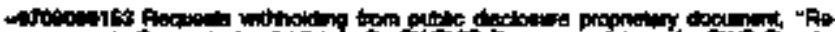

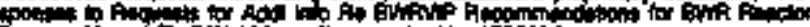

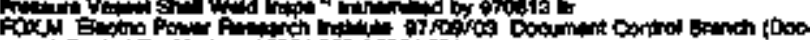

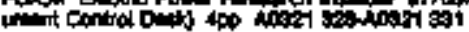

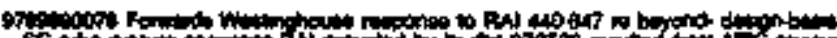

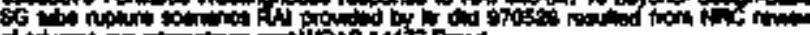

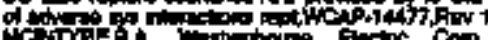

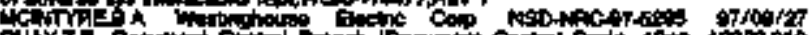

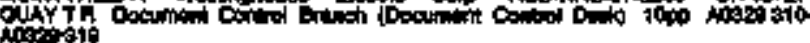

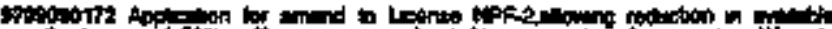

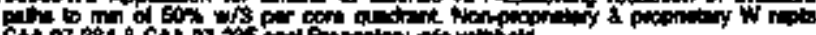
Cuter mo do

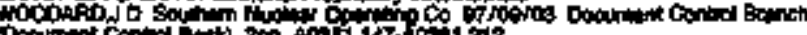

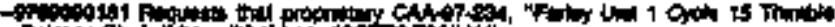

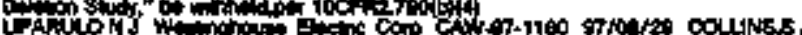

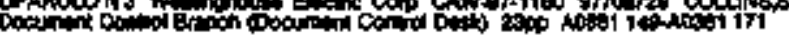

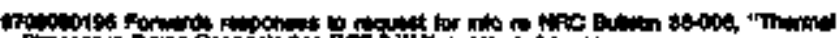

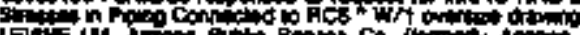

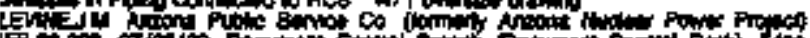

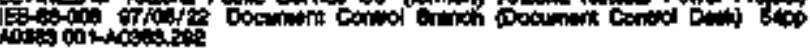

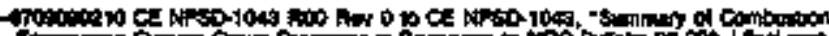

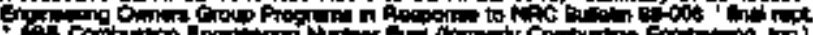

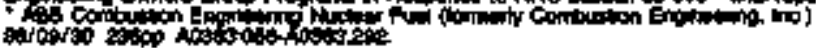

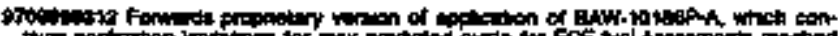

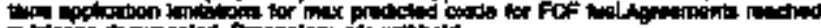

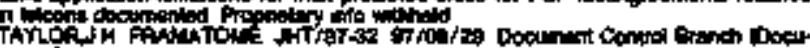

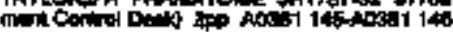

Truposts

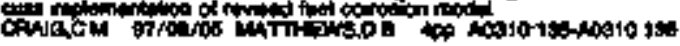

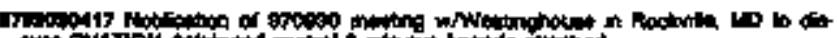

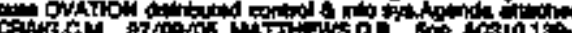

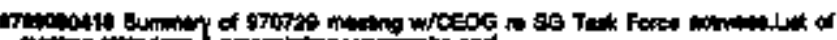

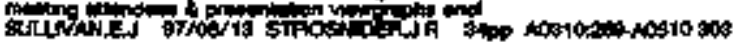

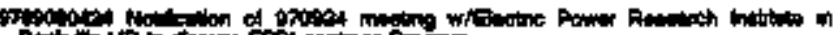

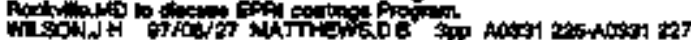

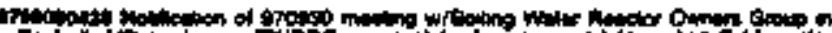

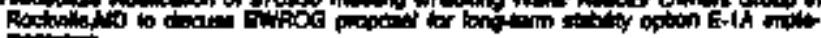

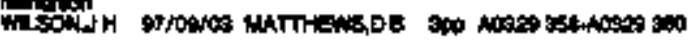

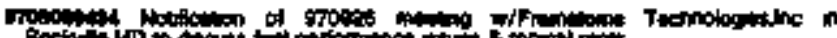
Fochis

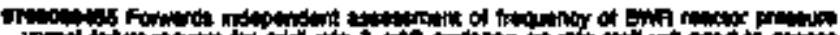

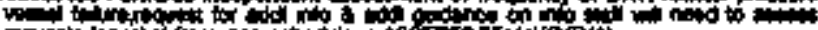

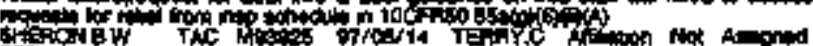

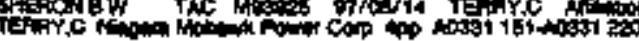

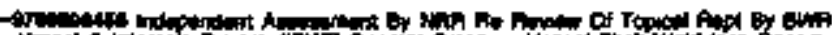

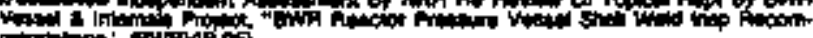
(1)

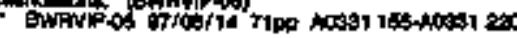

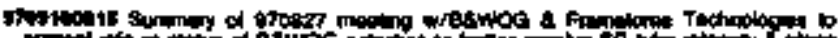

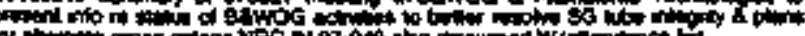

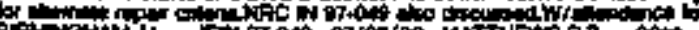

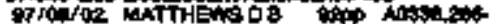

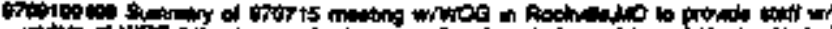

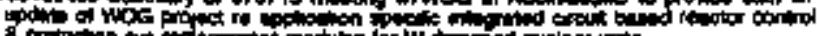

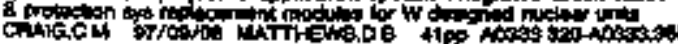

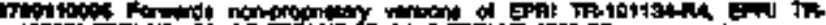

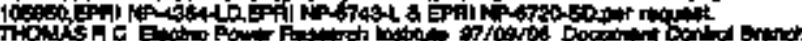

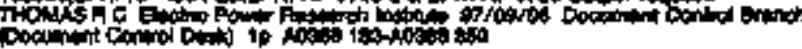

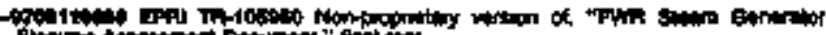

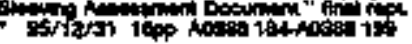

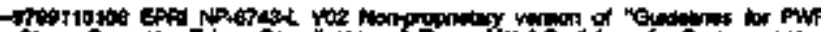

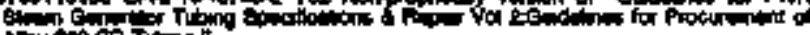

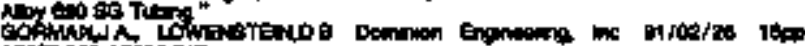

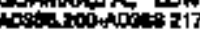

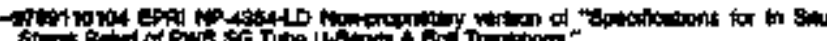

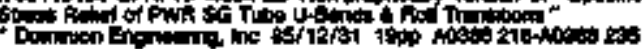

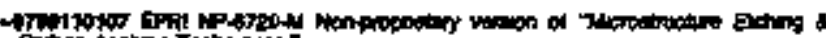

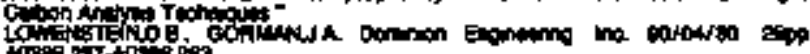

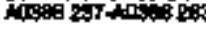

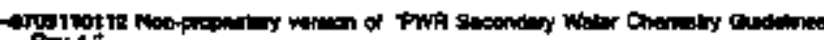

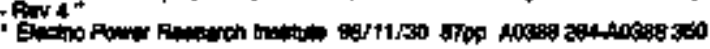

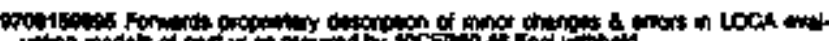

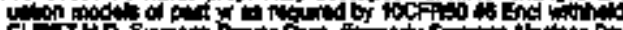

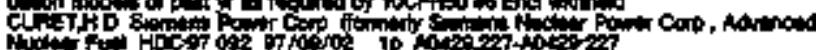

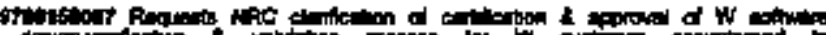

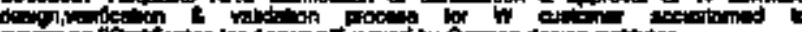

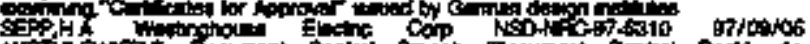

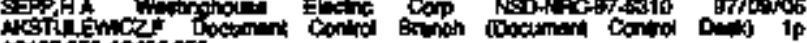
Alos 0

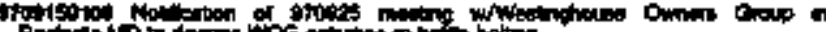
cats

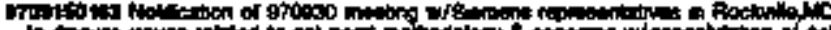

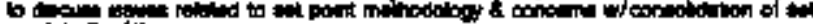

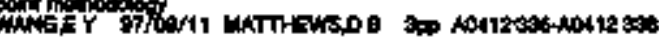

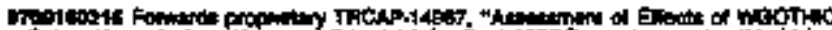

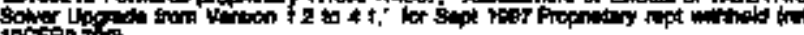

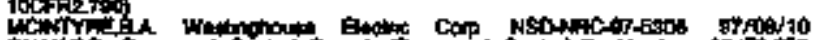

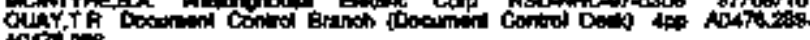
Misto:

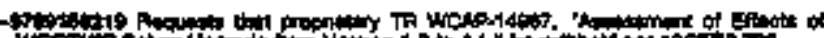

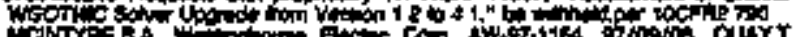

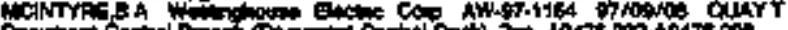

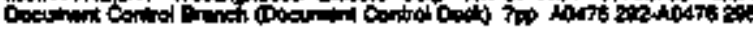

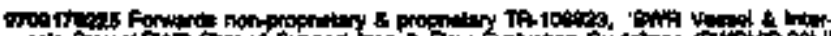

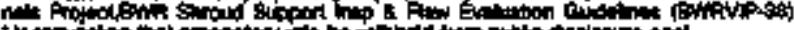

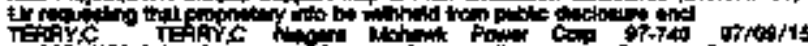
CNApH

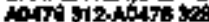

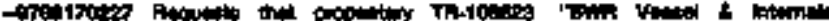

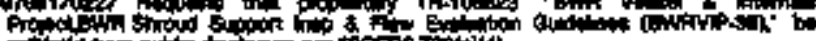

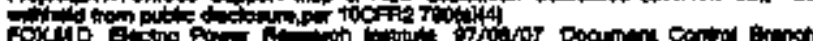

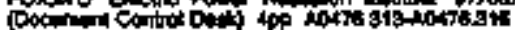

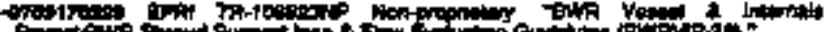

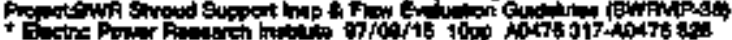

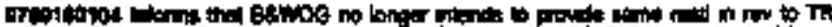

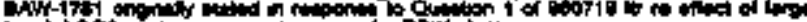

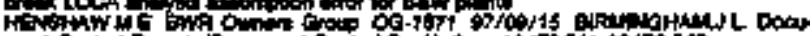

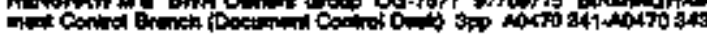

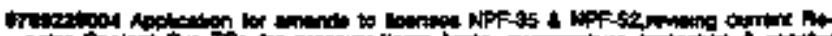

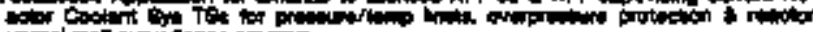

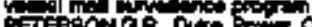

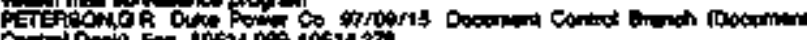

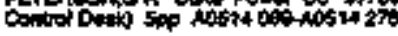

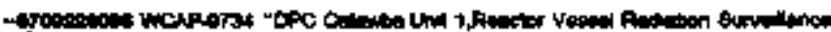

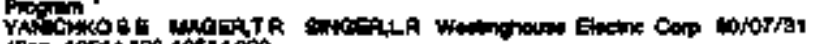

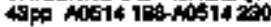

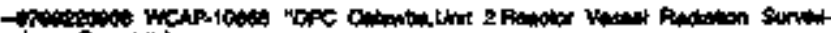

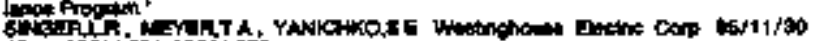
toph

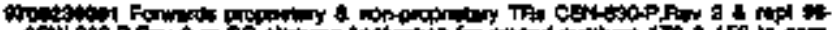

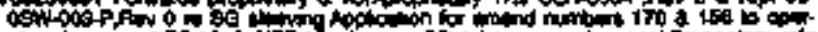

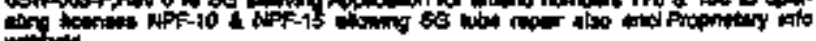

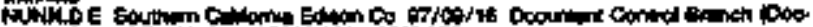

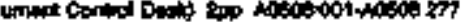




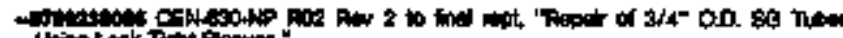

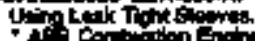

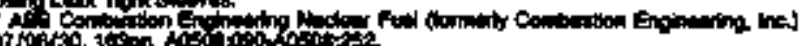

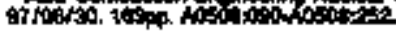

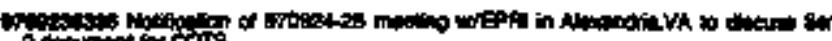

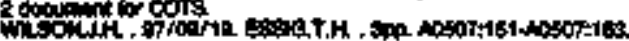

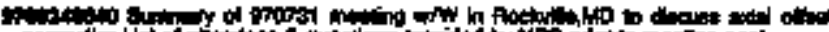
corich of

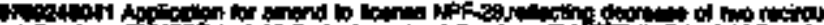

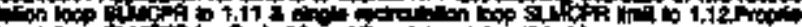

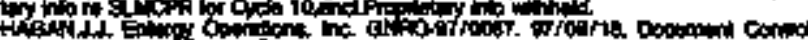

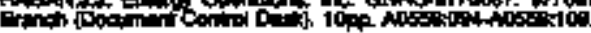

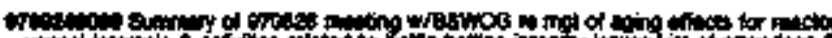

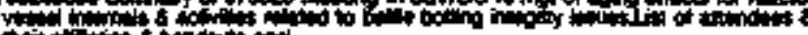

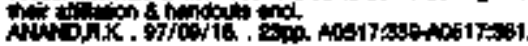

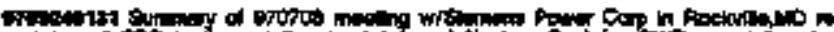

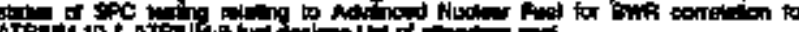

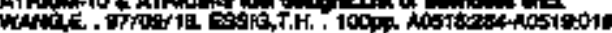

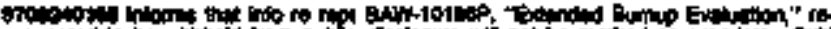

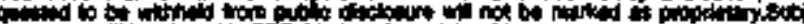

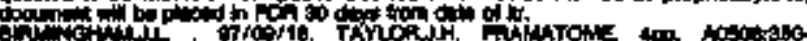
A

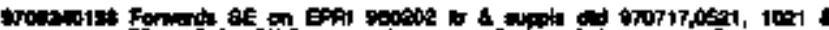

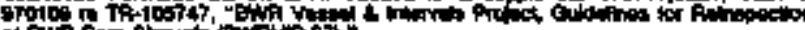
a tha

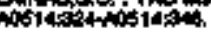

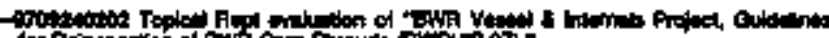

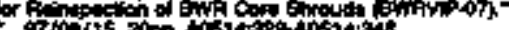

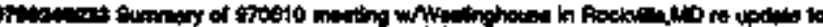

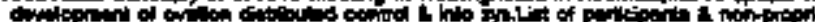

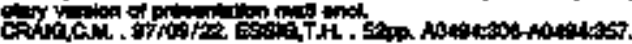

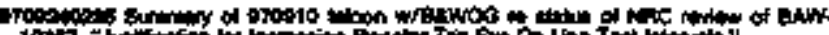

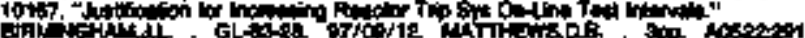

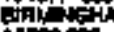
inotiat

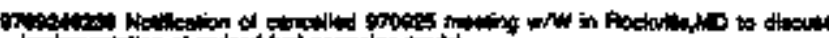

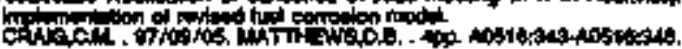

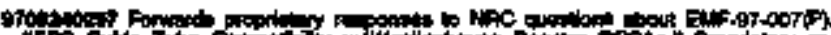

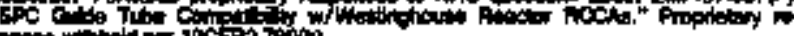

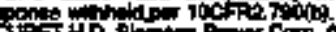

Jin

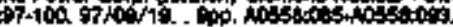

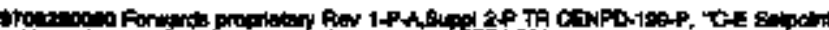

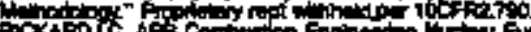

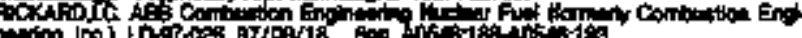

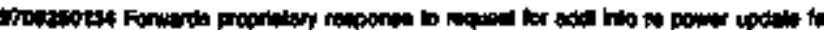

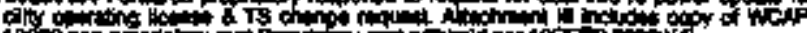

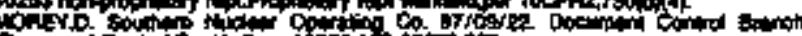

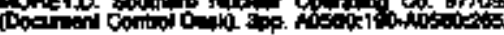

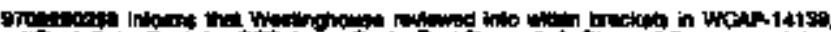

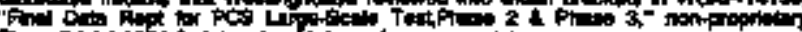

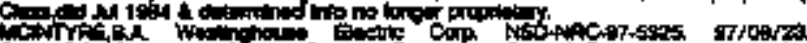

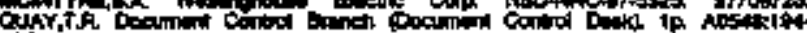
Astiotion.

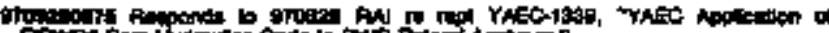

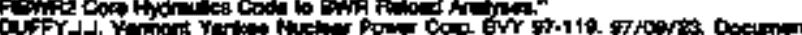

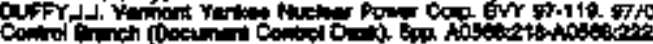

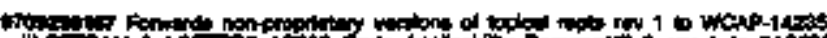

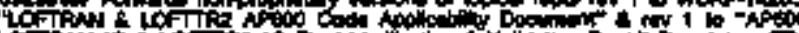

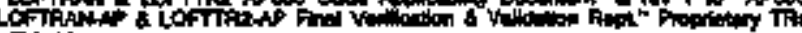
Cintid

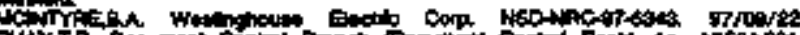

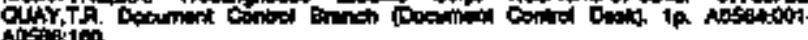

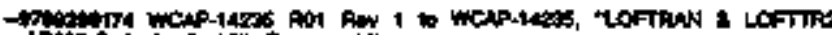

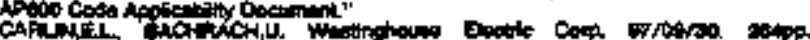
intert tos anction

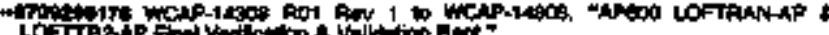

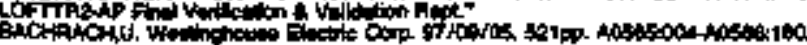

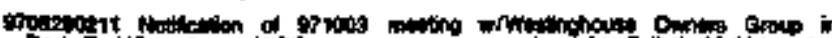

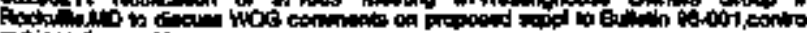

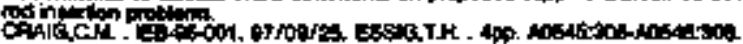

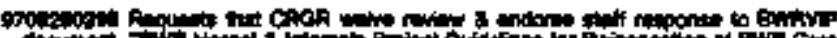
gonem,

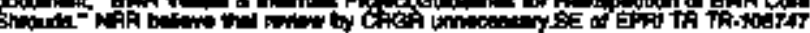

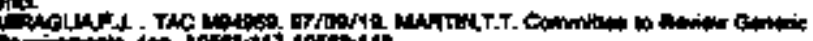

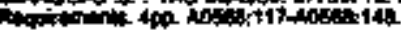

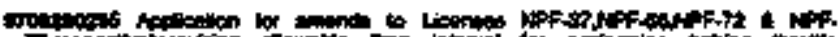

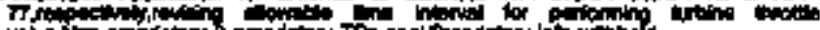

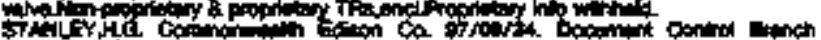

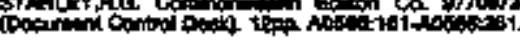

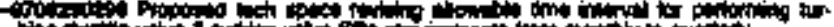

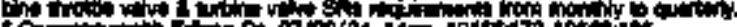

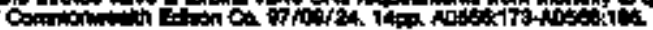

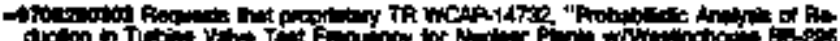

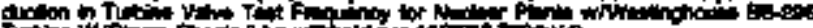

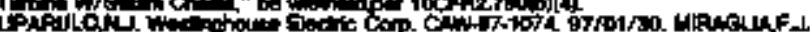

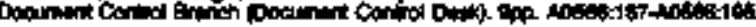

- Fot

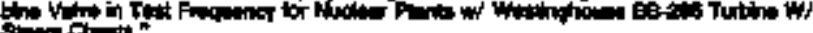

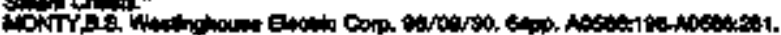

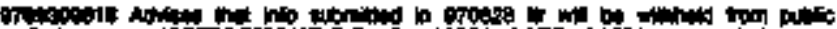

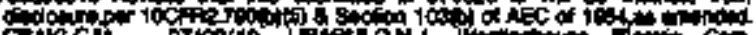

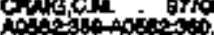

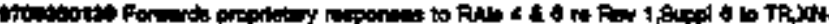

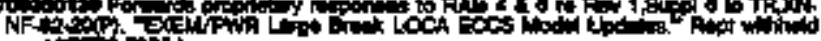

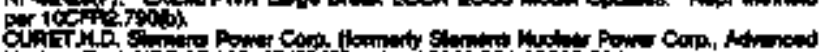

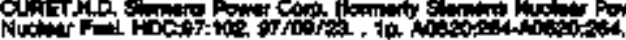

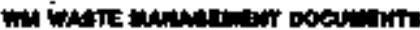

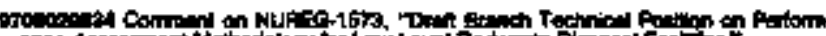

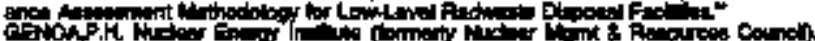

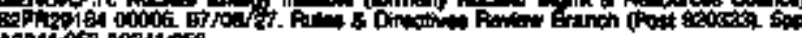

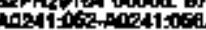

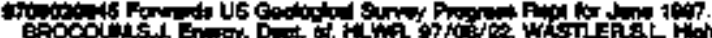

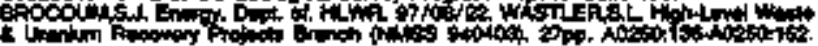

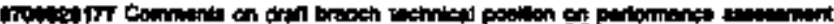

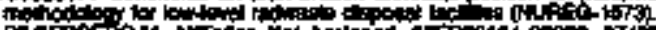

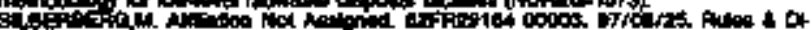

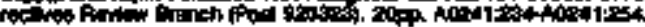

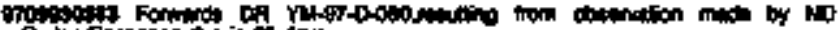

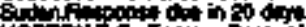

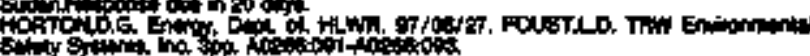

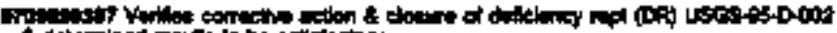

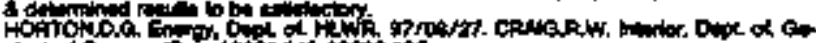

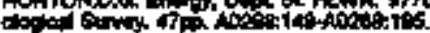

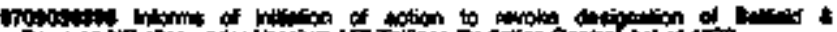

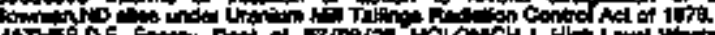

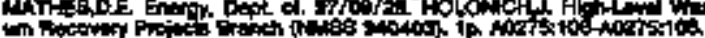

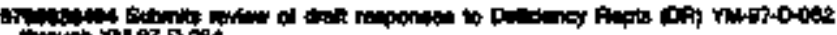

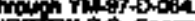

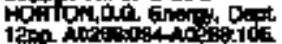

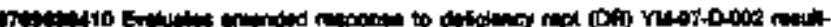

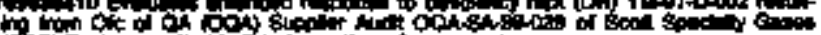

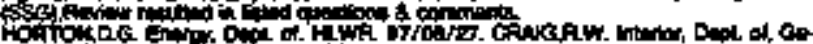

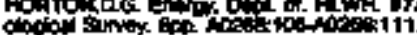

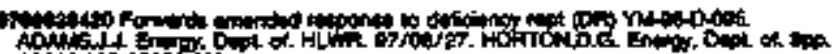
A

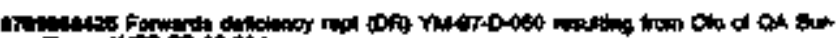

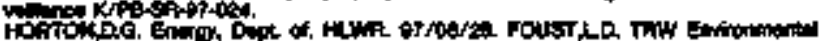

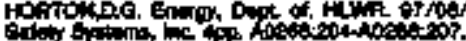

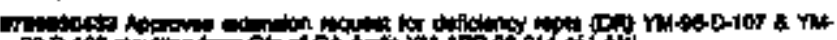

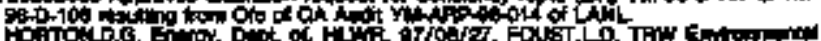

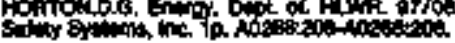

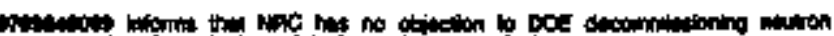

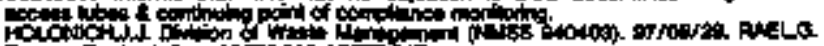

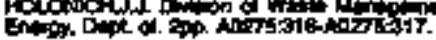




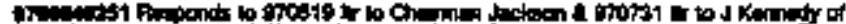

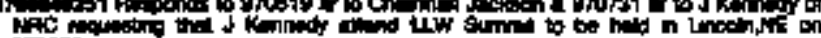
artions

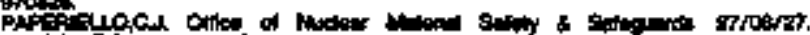
4⿴囗大

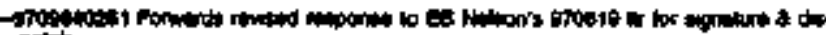

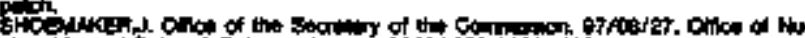
d.

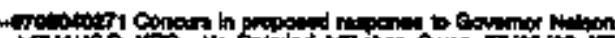

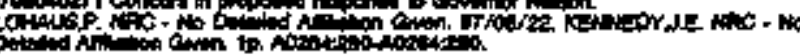

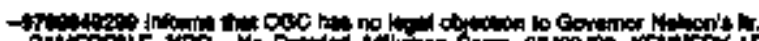

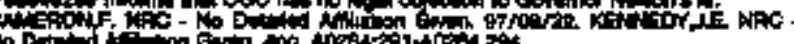

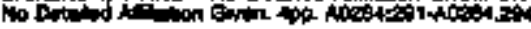

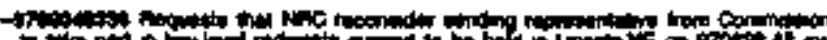

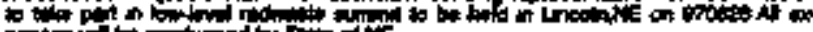

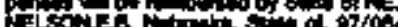

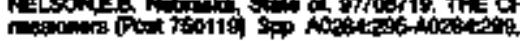

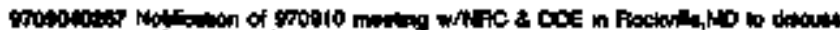

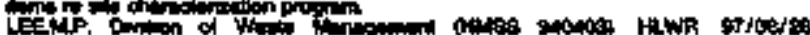

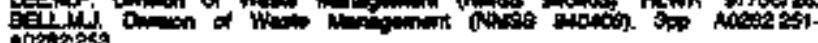

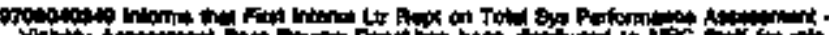

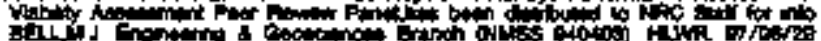

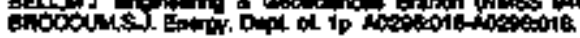

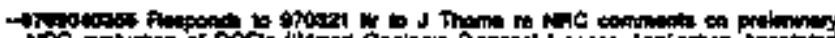

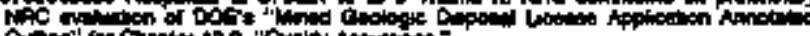

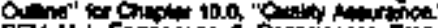

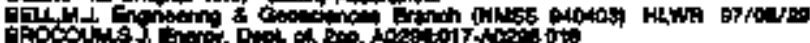

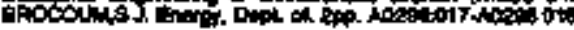

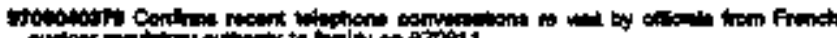

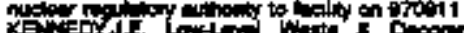

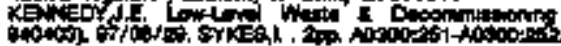

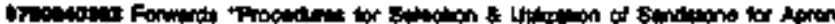

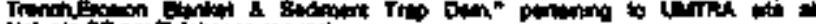

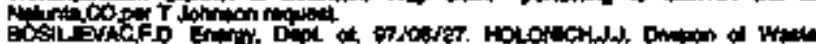

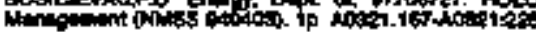

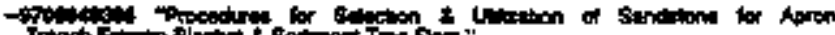
Thixh

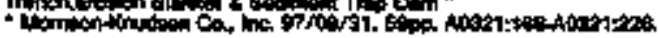

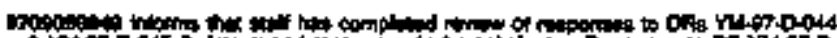

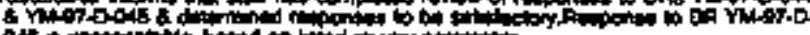

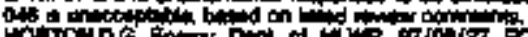

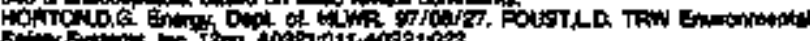

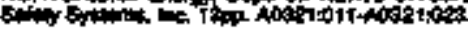

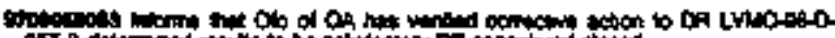

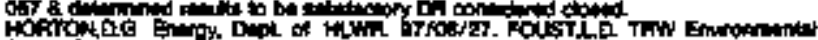

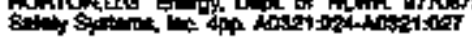

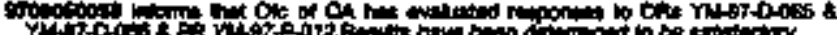

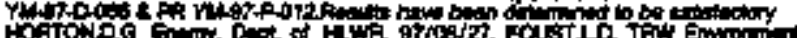

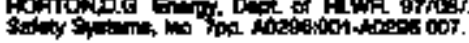

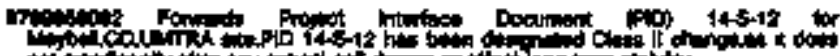
mog

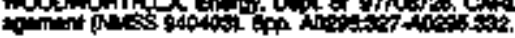

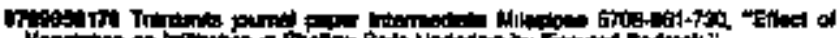

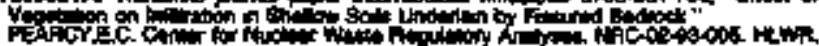

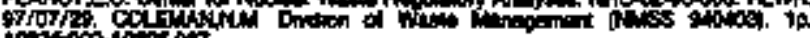

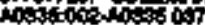

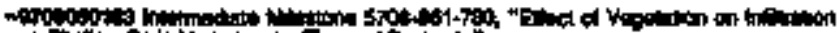

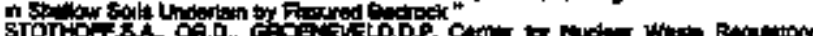
sToriol

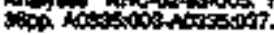

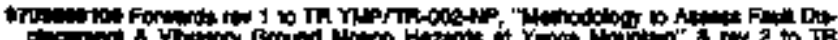

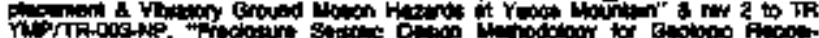

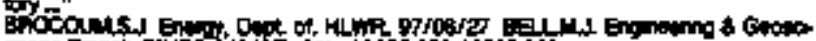

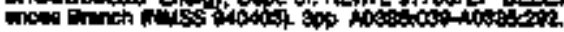

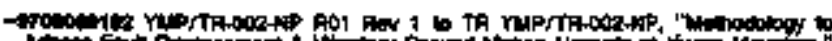

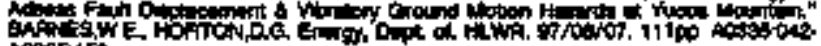
roostitis

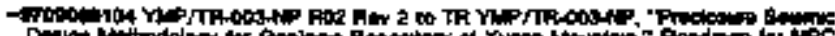

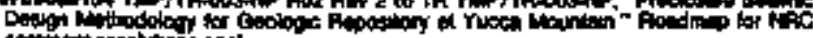

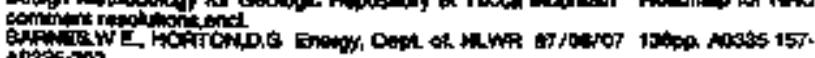
Nhats 202

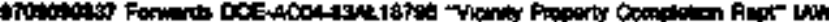

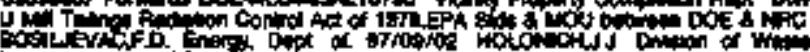

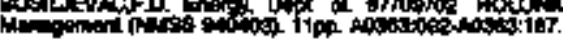

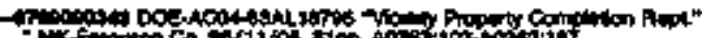

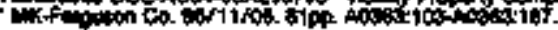

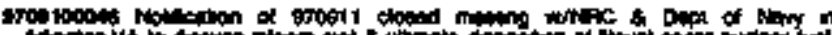

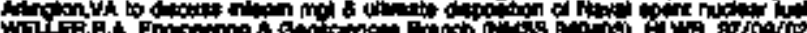

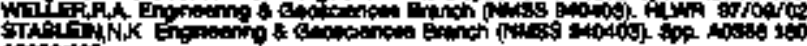
iosicto

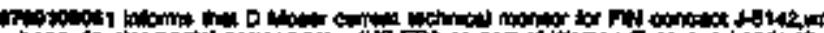

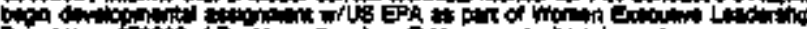

Fik

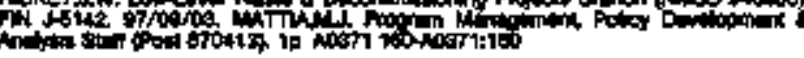

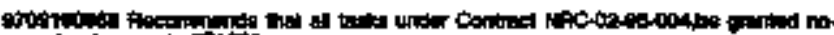

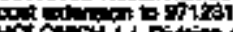

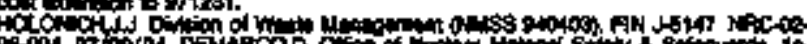

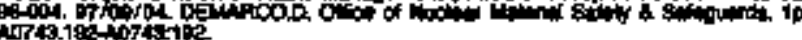

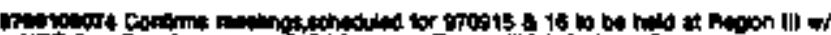

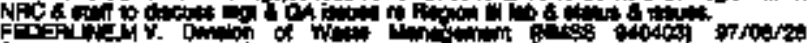

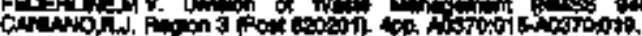

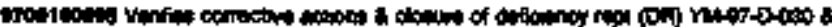

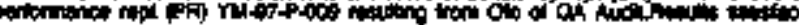

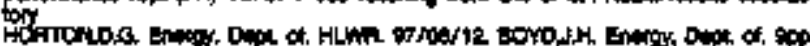

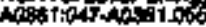

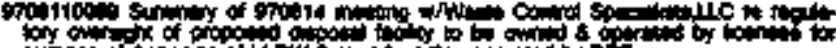

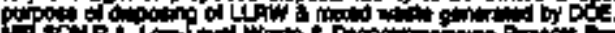

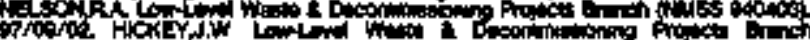

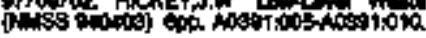

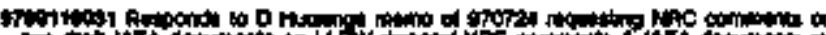

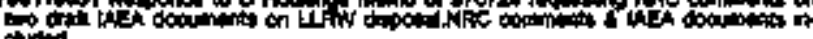
clicin

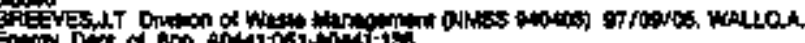

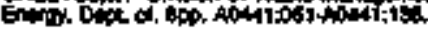

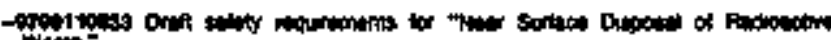

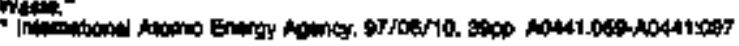

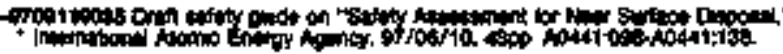

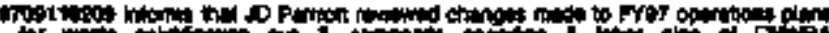

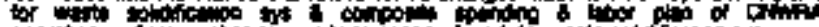

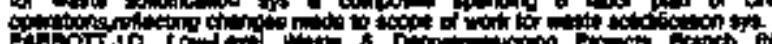

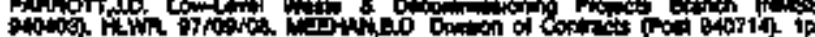

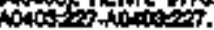

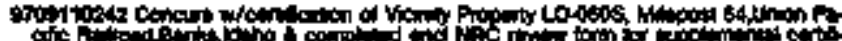

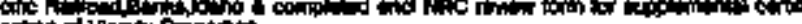

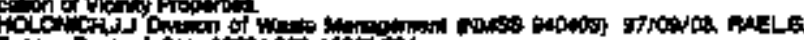

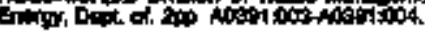

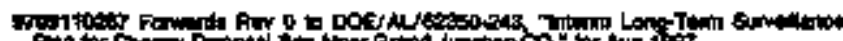

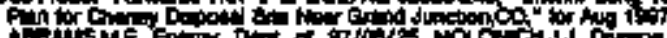

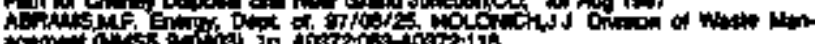

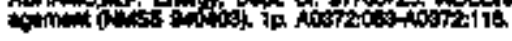

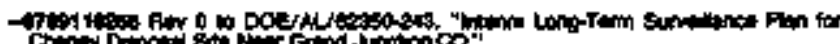

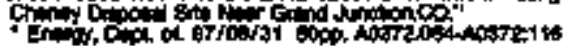

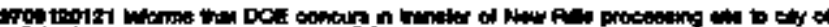

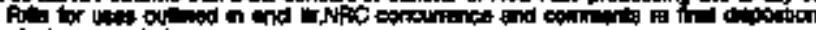

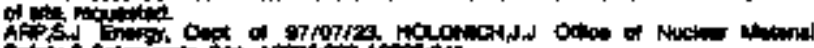

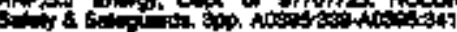

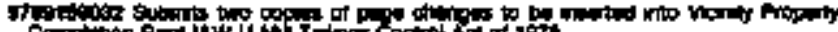

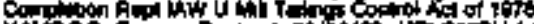

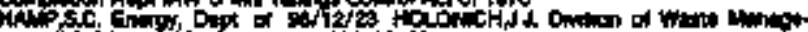

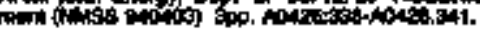

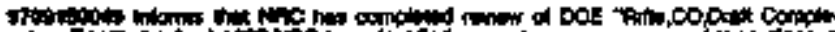

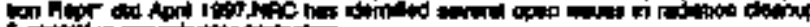

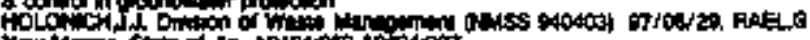

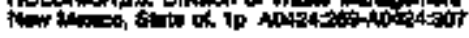

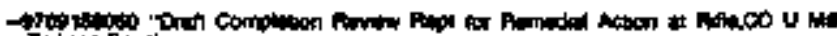

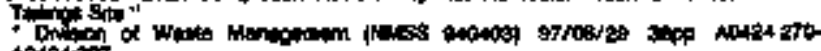
notovion.

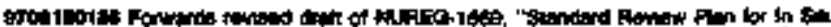

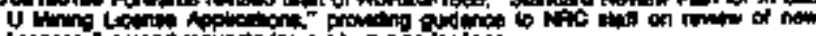

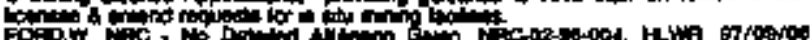

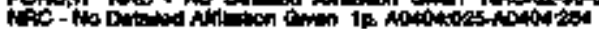




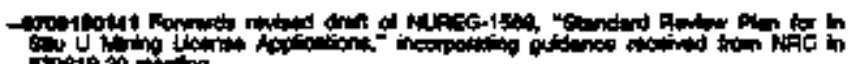

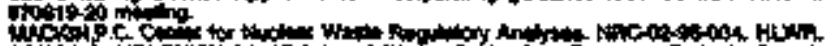

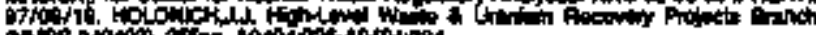

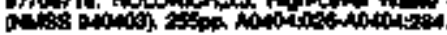

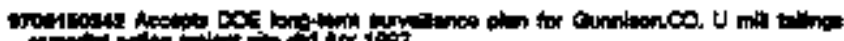

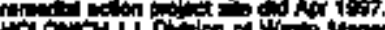

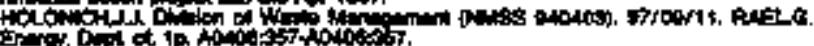

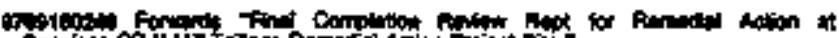

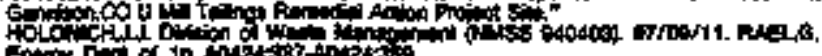

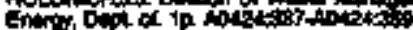

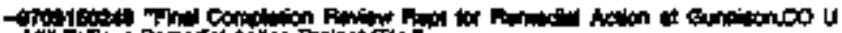

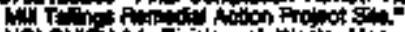

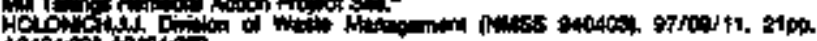

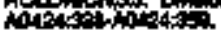

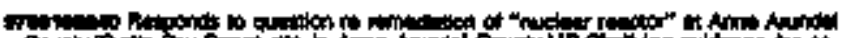

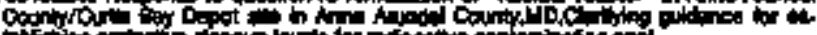

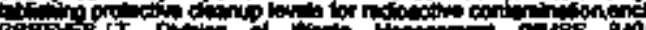

C5:

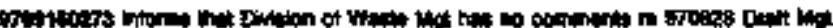

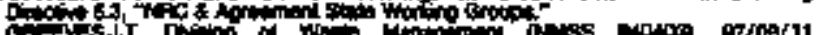

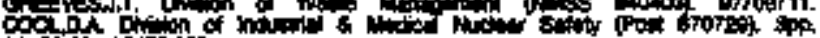
a

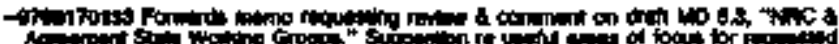

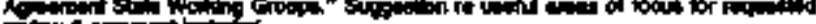
(1)

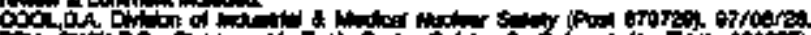

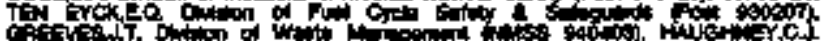

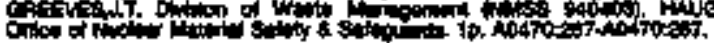

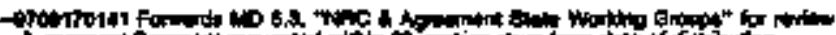

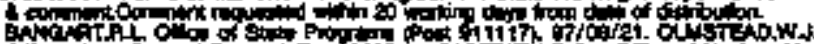
onter of the $\mathrm{E}$.

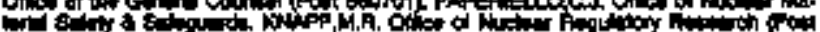

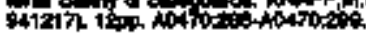

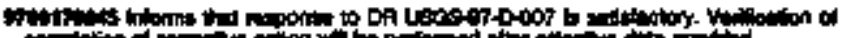

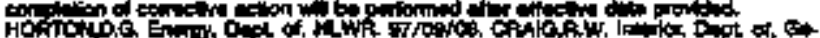

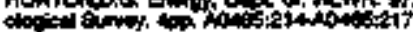

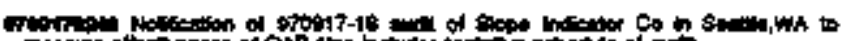

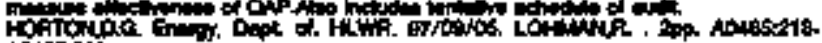
Notimatio

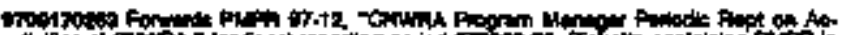

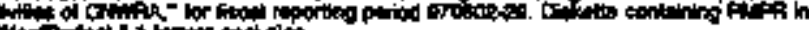

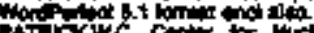

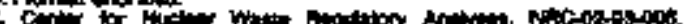

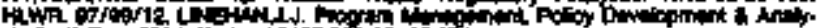

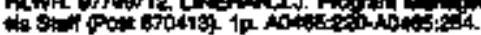

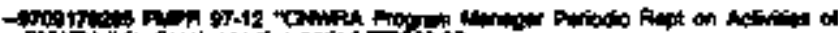

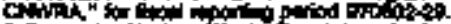

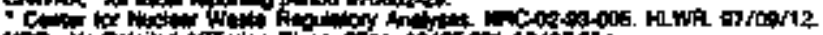

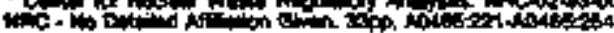

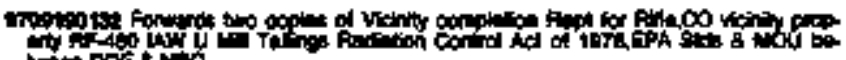

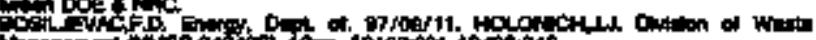

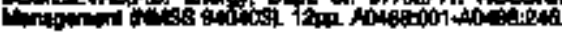

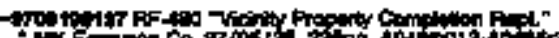

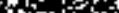

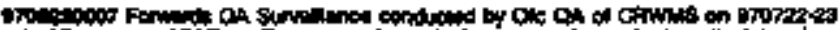

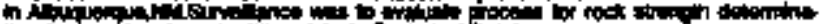

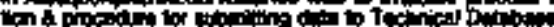

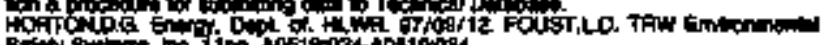

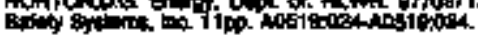

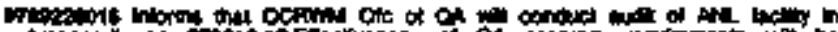

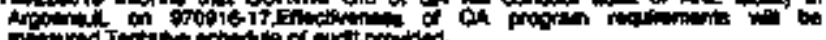

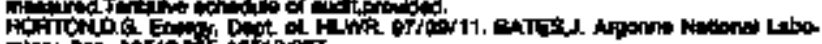

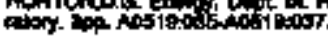

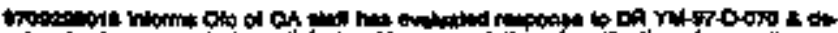

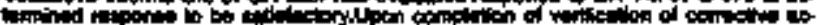

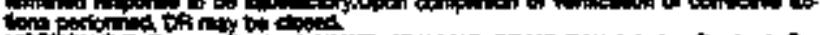

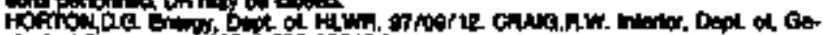

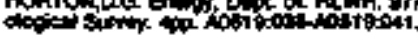

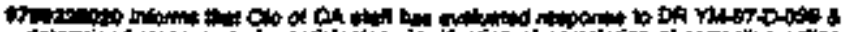

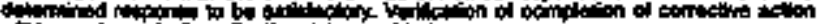

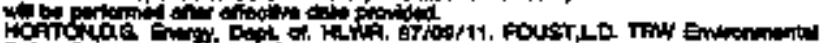

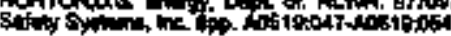

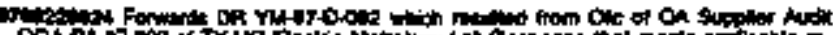

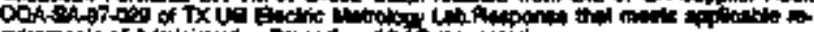

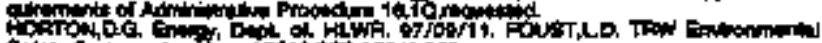

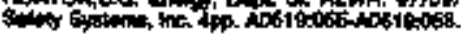

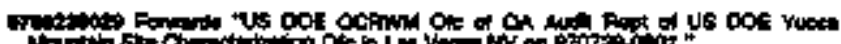

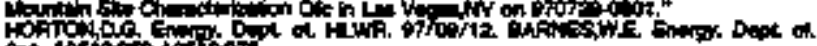

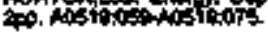

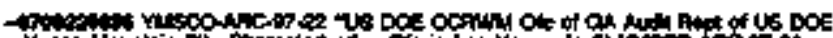

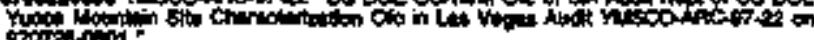

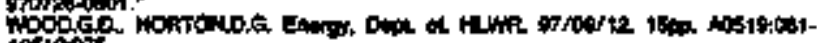
A0:

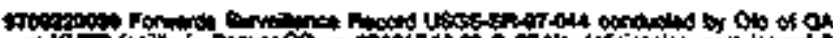
at Y

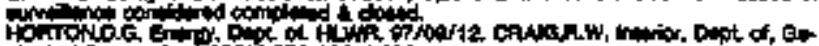

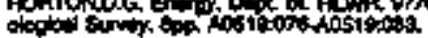

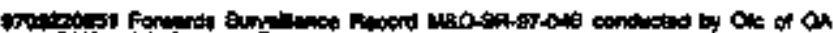

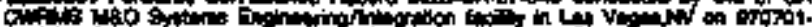

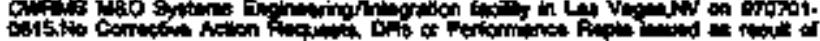

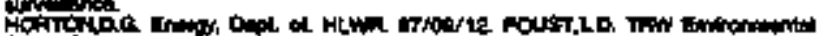

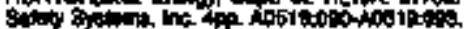

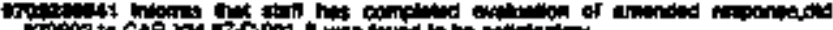

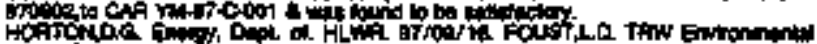

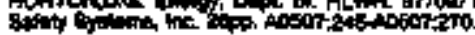

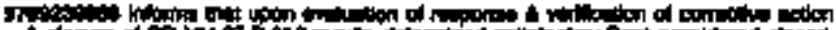

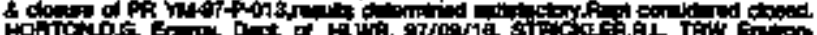

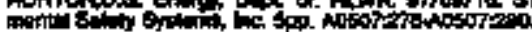

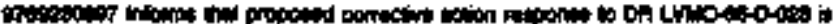

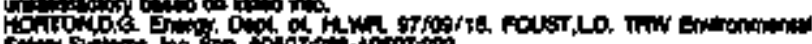

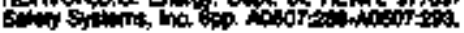

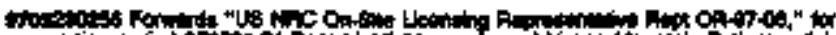

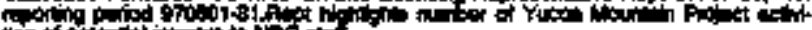
of of

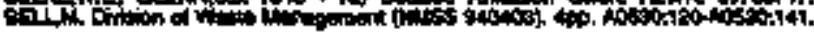

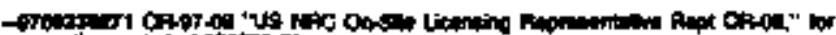

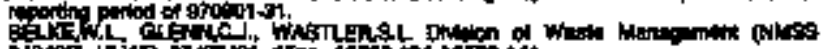

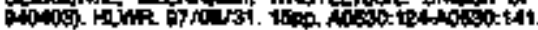

का

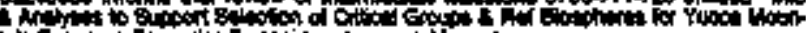

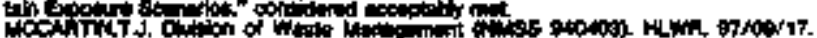

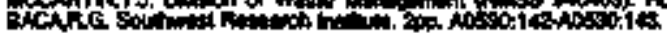

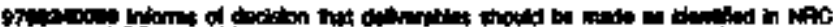

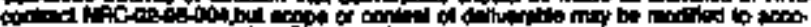

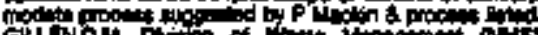

G

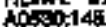

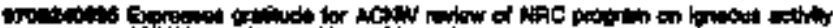

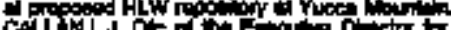

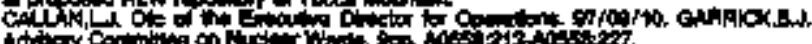
等

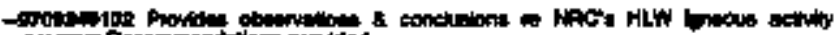

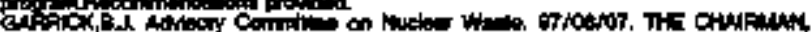

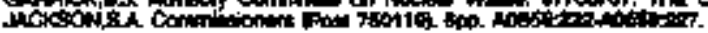

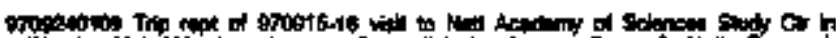

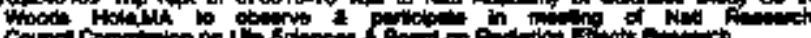

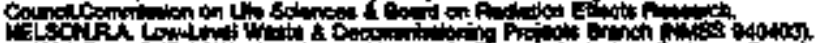

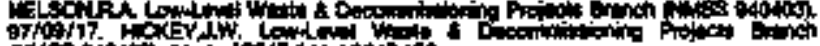

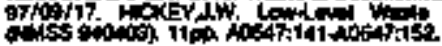

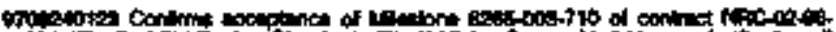

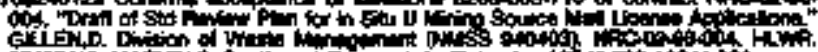

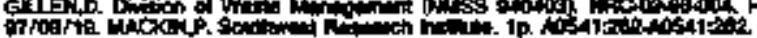

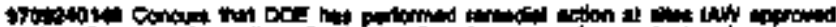

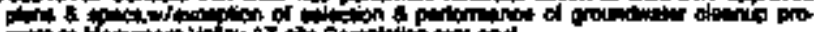
Din

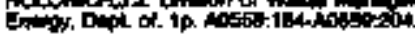

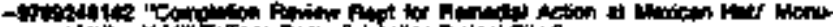

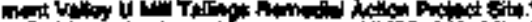

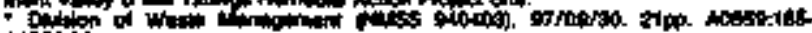
AOC:

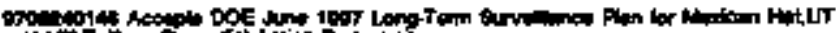

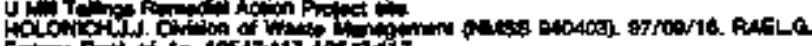

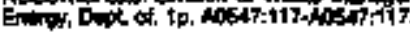

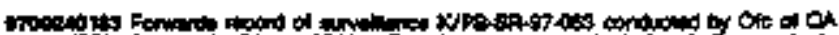

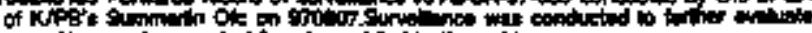

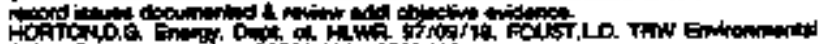

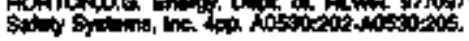




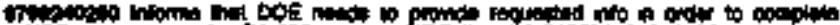

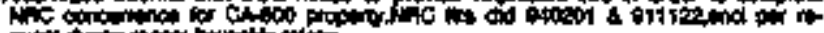

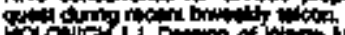

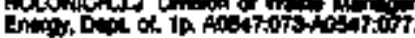

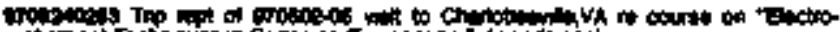

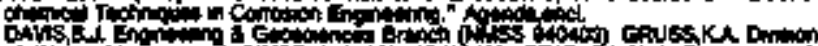

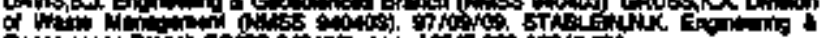

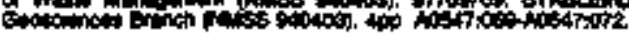

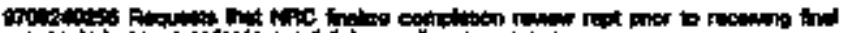

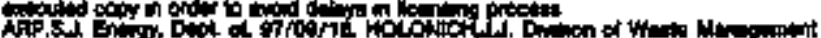

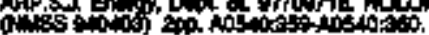

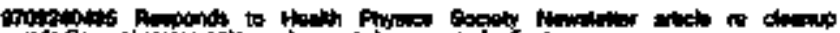

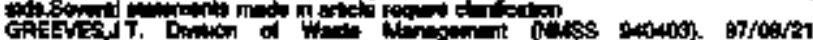
AOEb

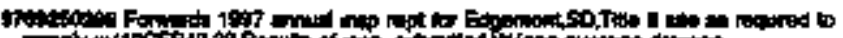

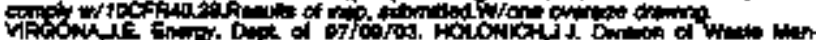

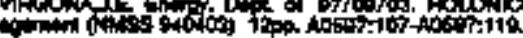

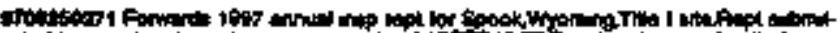

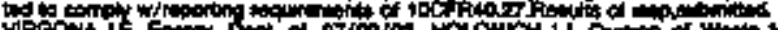

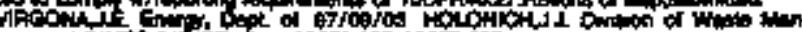

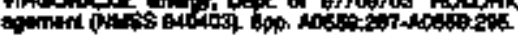

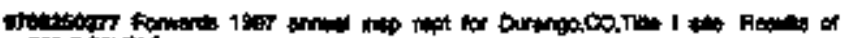

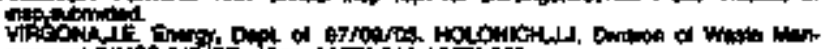

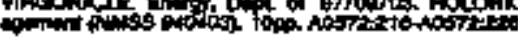

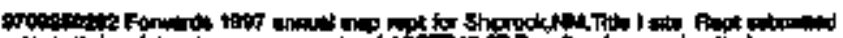

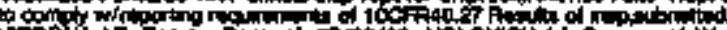

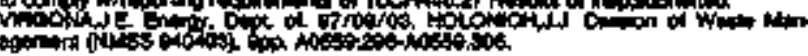

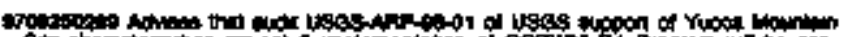

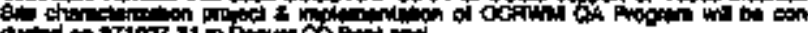

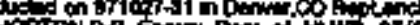

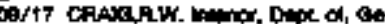
todictomo 118

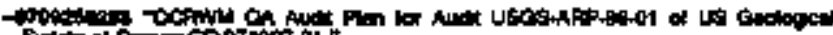

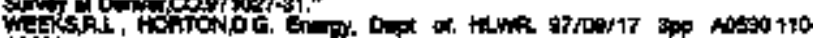
Aotolita.

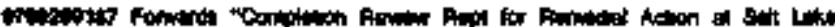

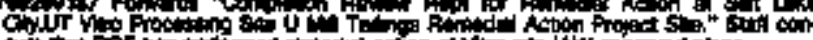
a

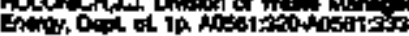

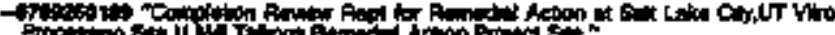

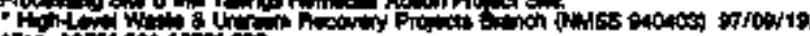

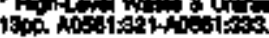

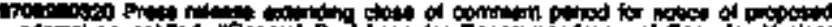

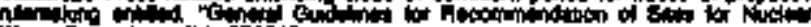

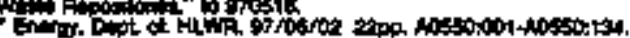

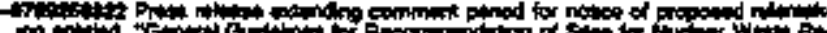

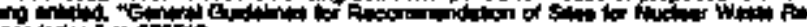

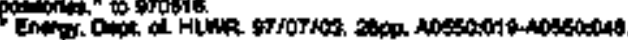

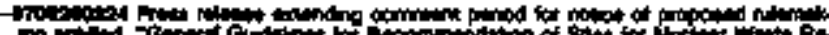

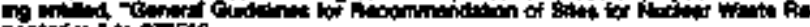

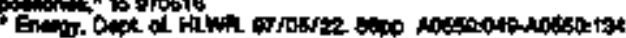

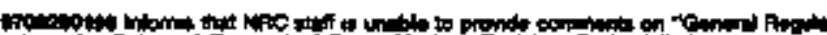

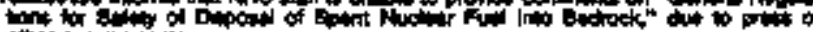

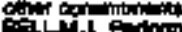

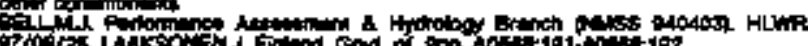

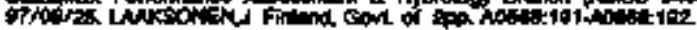

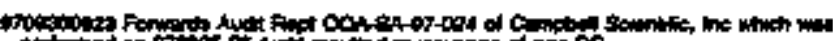

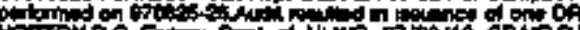

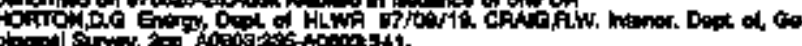

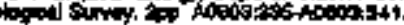

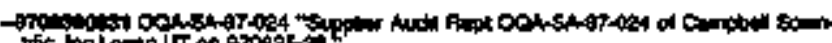

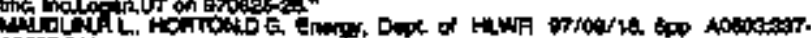
Apeotioni.

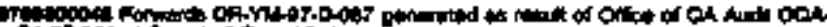

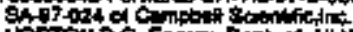

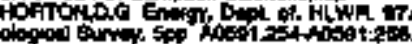

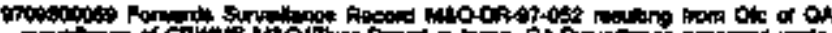

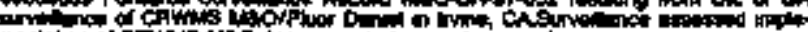

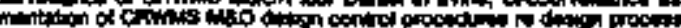

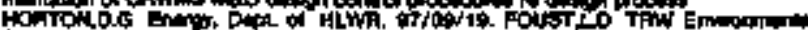

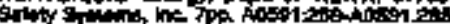

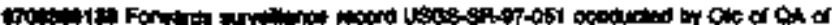

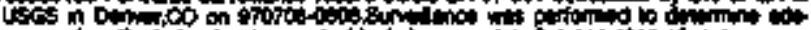

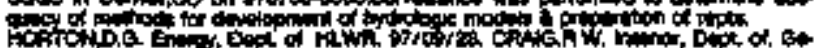

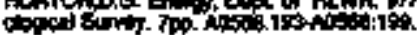

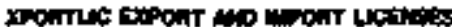

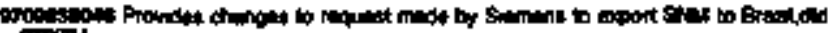

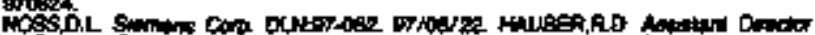

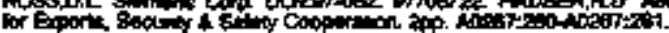

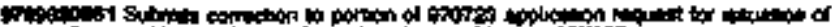

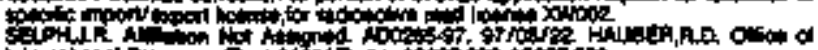

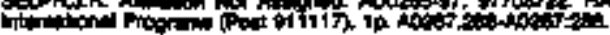

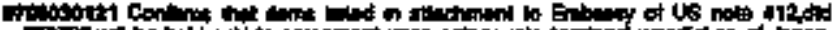

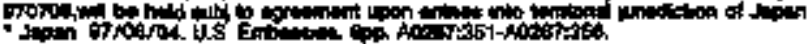

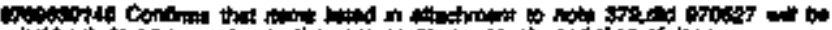

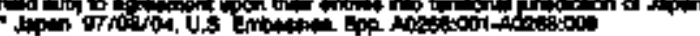

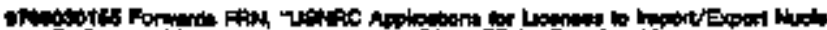

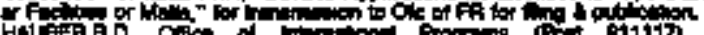

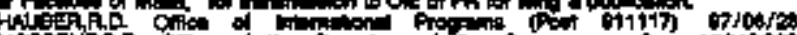

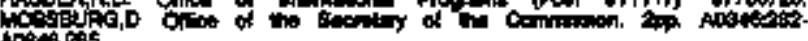
nowitos

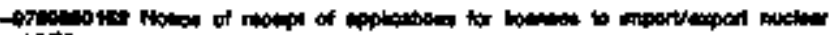

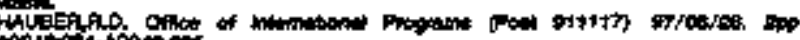

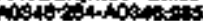

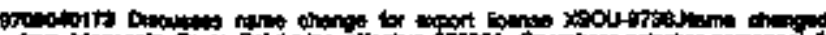

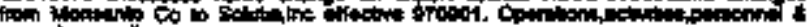

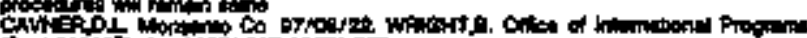

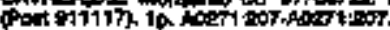

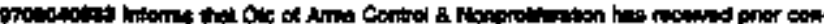

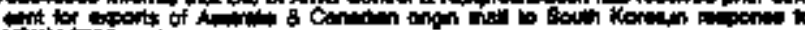

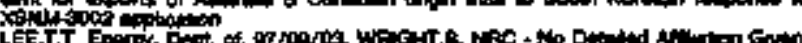
LEE,T,T Eneopy Dept of

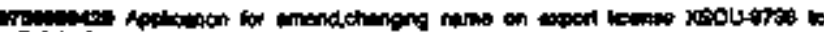

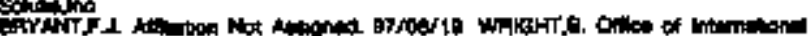

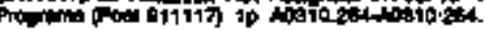

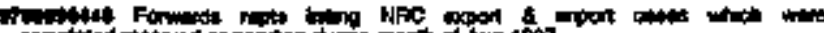

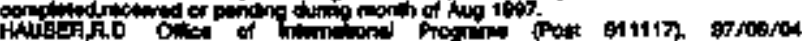
Del

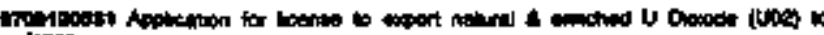

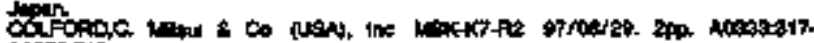
ictesitis

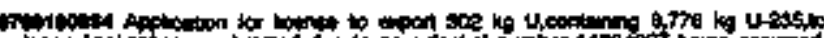

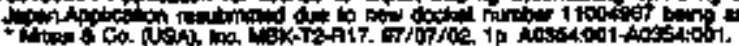

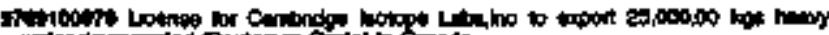

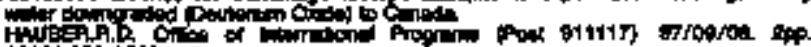

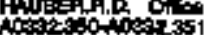

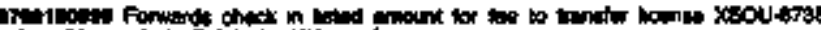

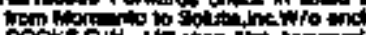

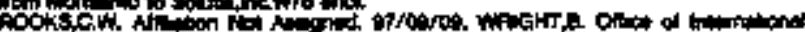

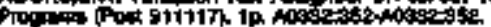

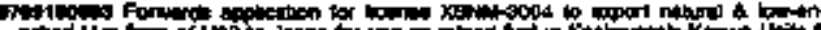

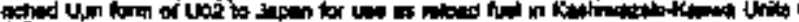

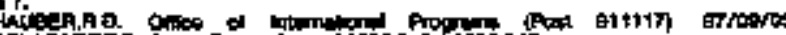

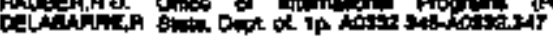

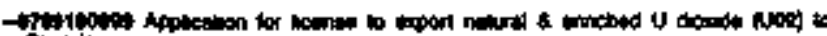

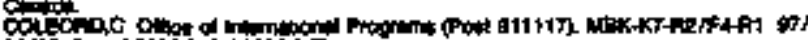

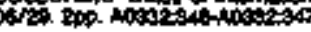

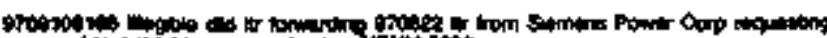

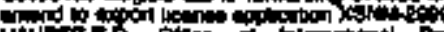

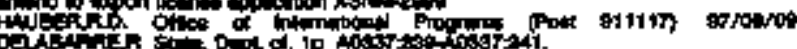

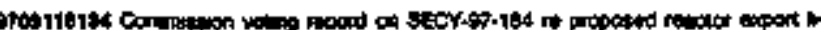

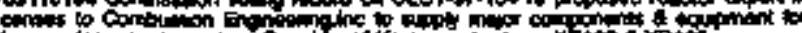

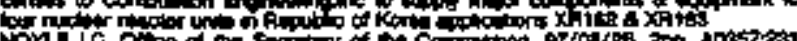
absitit

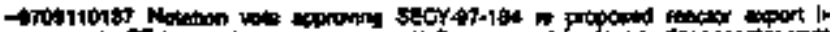

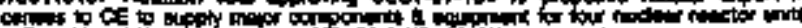

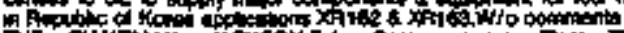

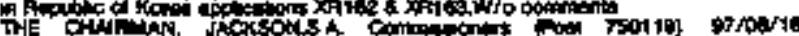

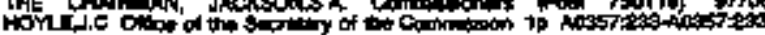




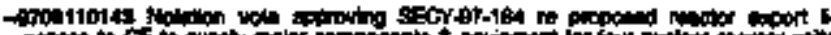

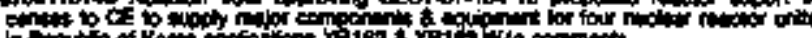
I n

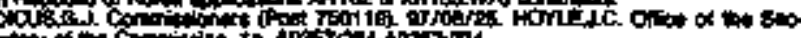

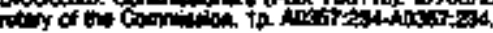

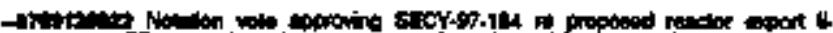

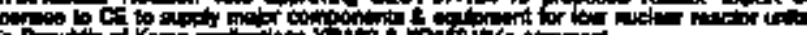
of

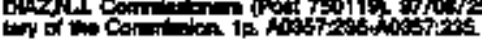

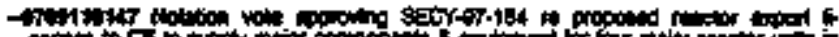

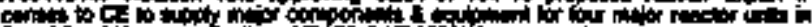
A

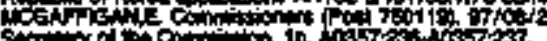

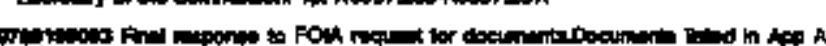

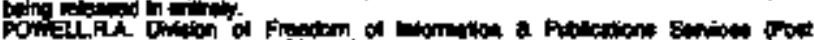

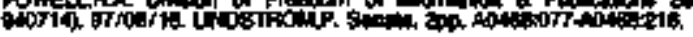

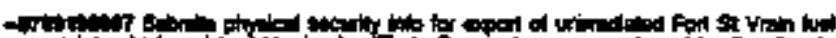

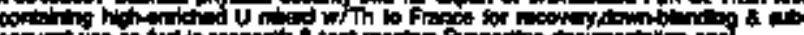

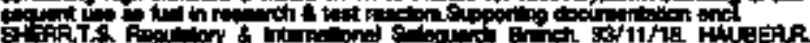

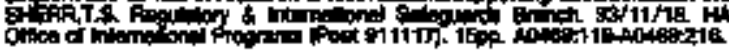

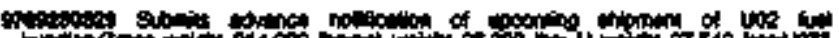

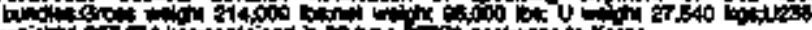

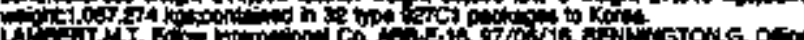

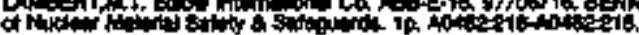

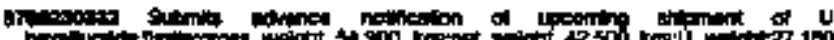

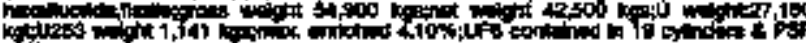
bicis

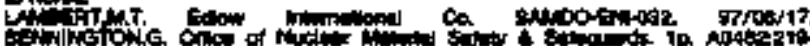

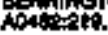

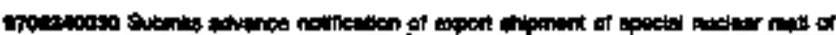

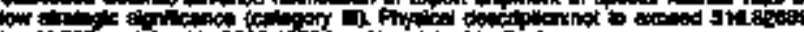

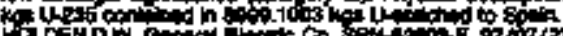

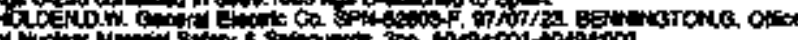

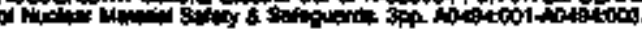

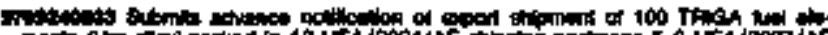

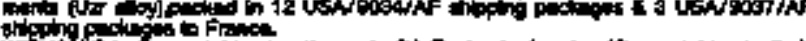

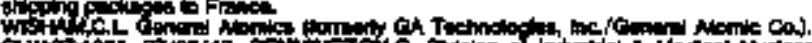

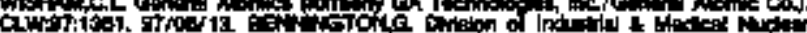

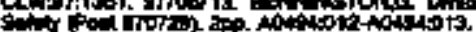

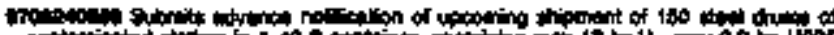

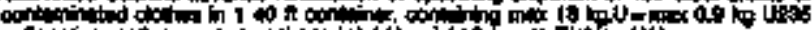

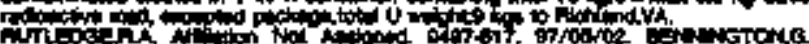
Ot:

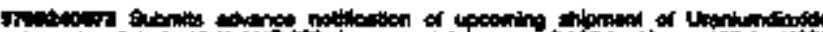

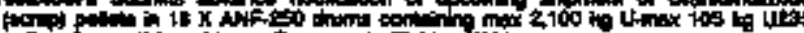

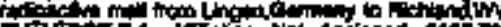

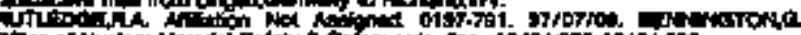

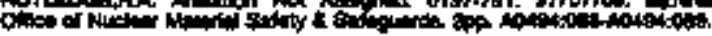

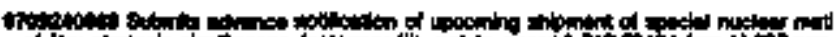

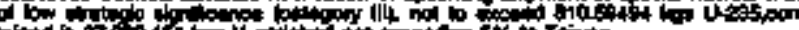

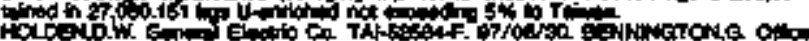

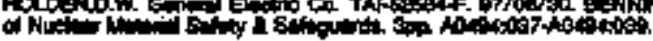

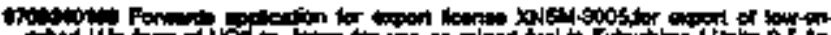

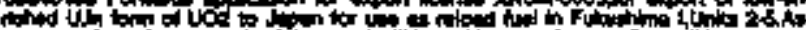

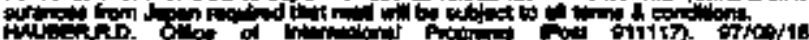
Da

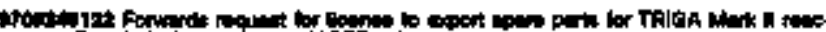

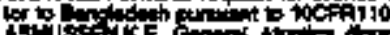

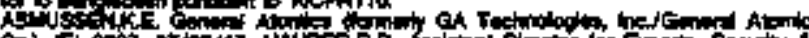

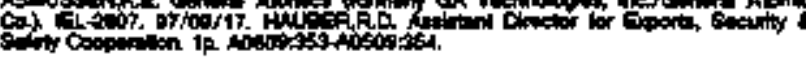

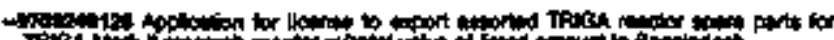

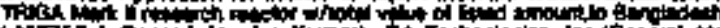

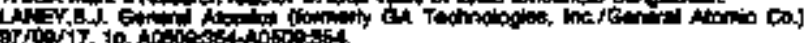

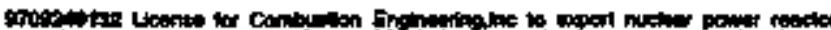

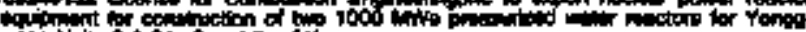

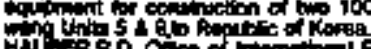
10.

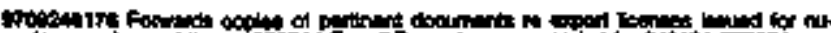

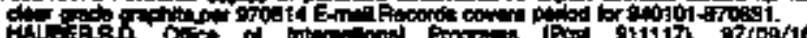
STAN

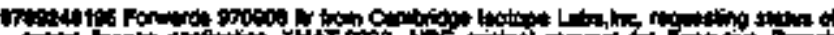

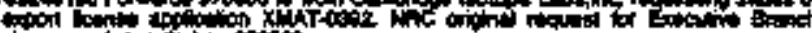

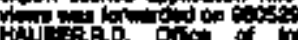

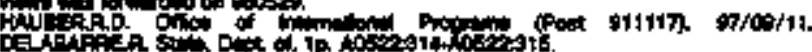

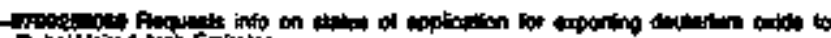

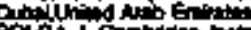

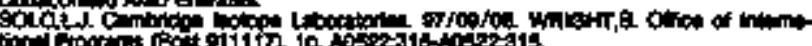

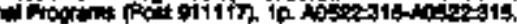

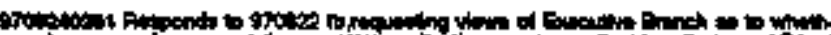

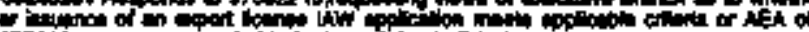

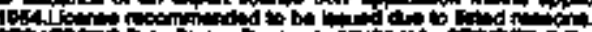

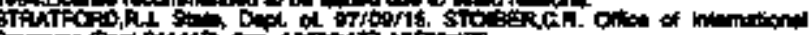

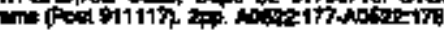

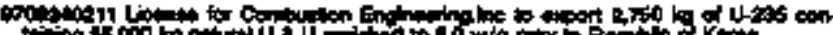
Hef

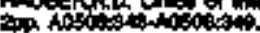

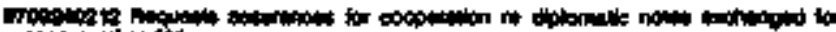

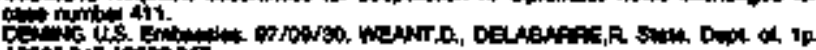

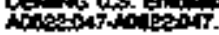

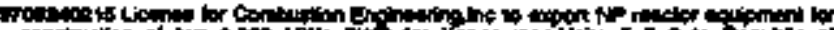

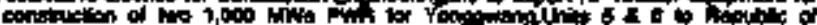

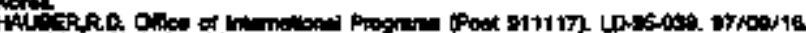

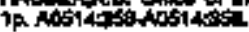

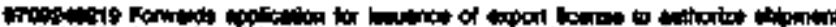

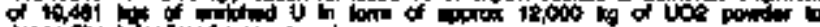

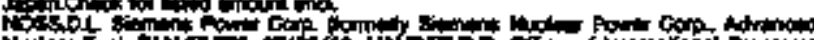

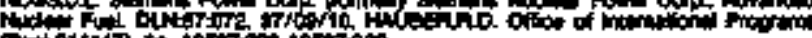

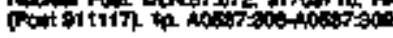

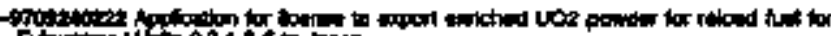
A

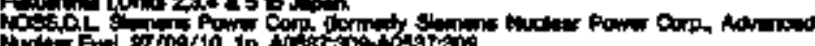

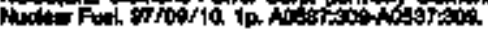

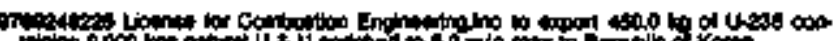

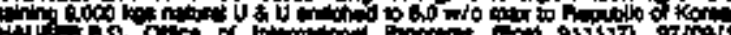

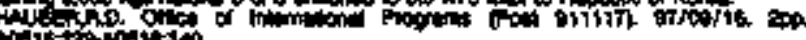

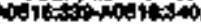

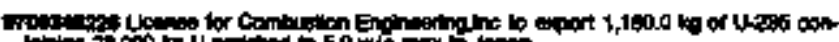

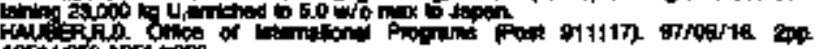

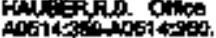

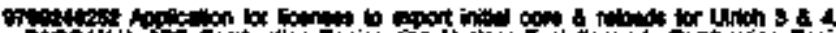

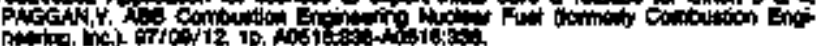

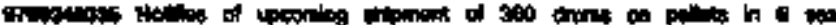

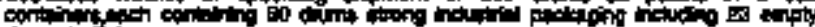

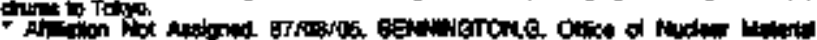

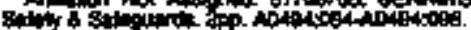

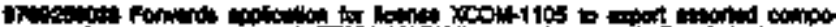

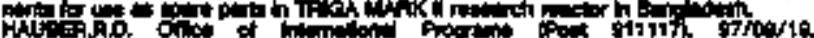

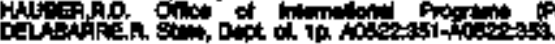

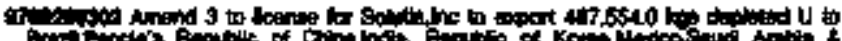

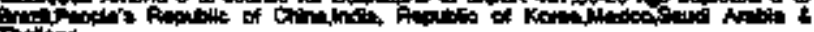

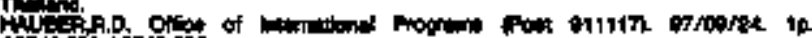

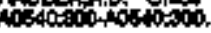

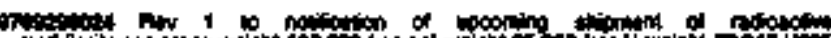

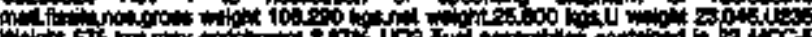

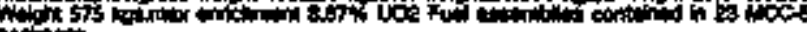

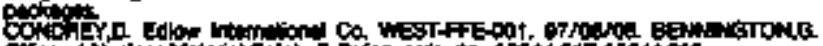

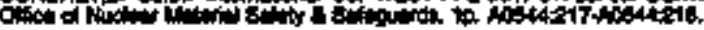

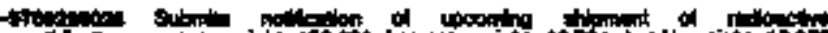
The

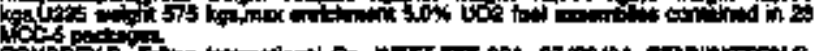

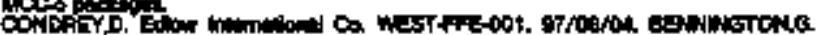

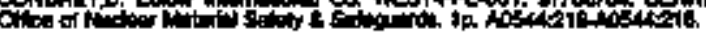

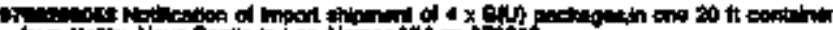

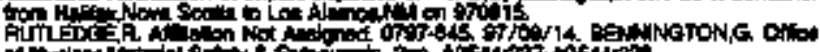

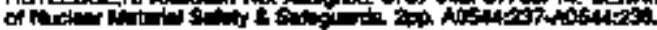

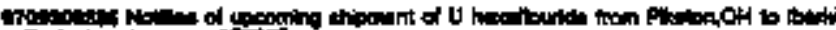

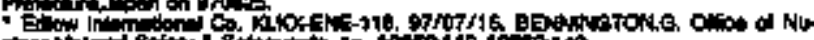

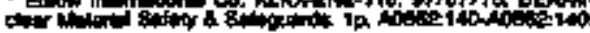




\section{PERSONAL AUTHOA INDEX}

The subject category column in this index gives the docket number and the symbol for the category of information within that docket; for example, 50-320 P. The docket subject categories are defined in Apperi. dix A.

Subject categories for nondocketed ftems are also eritered in this column by symbol; for exampla, ACRS These categories are defined in Appendix 8 and listed in the Contents.
An asterisk following the accession number th this irxdex ind"cates that the document is an enclosure to another document. To obtein the sccession number of the princtpal document under which the encioesure is listed, refer to the index entitled "Cross Reference of Enclosumes to Principal Documents."

\begin{tabular}{|c|c|c|c|c|}
\hline Alminan & 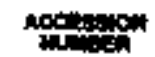 & 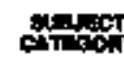 & & AUNHOA \\
\hline 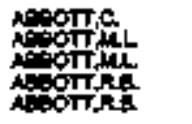 & 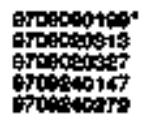 & 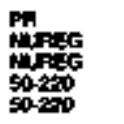 & $\mathbf{8}$ & 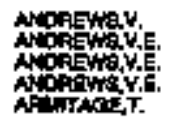 \\
\hline 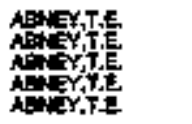 & 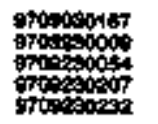 & 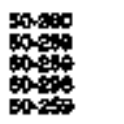 & $\begin{array}{l}0 \\
\text { h } \\
p \\
0 \\
0\end{array}$ & 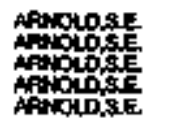 \\
\hline 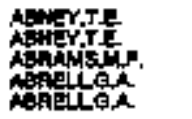 & 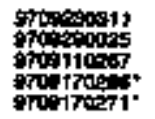 & $\begin{array}{l}50-290 \\
50-100 \\
5=10 \\
50-10\end{array}$ & $\begin{array}{l}Q \\
P \\
\text { A } \\
\text { A }\end{array}$ & 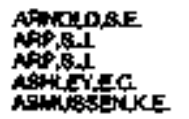 \\
\hline 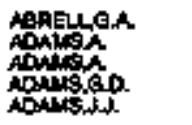 & 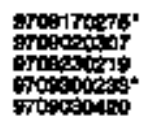 & $\begin{array}{l}60-19 \\
6004 \\
00404 \\
004\end{array}$ & $\begin{array}{l}n \\
\text { p } \\
\text { s }\end{array}$ & 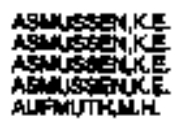 \\
\hline 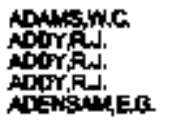 & 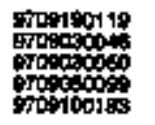 & 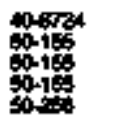 & w & 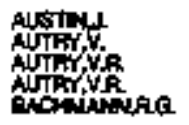 \\
\hline 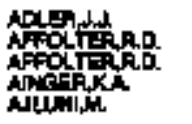 & 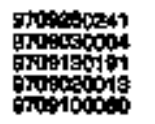 & 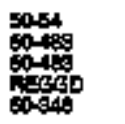 & $\begin{array}{l}\text { w } \\
\text { s } \\
\text { f }\end{array}$ & 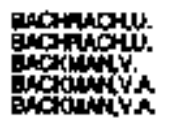 \\
\hline 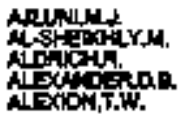 & 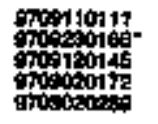 & 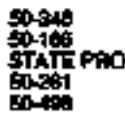 & $\begin{array}{l}0 \\
\text { R } \\
\text { h } \\
\text { D }\end{array}$ & 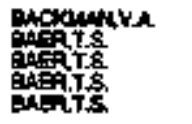 \\
\hline 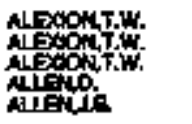 & 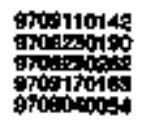 & 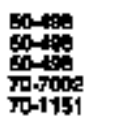 & p & 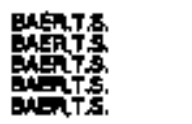 \\
\hline 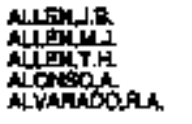 & 970015019 & 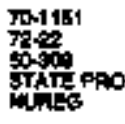 & W & 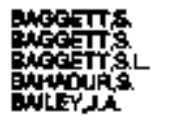 \\
\hline 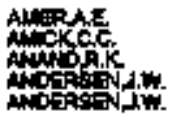 & 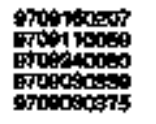 & 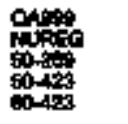 & p & 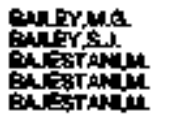 \\
\hline 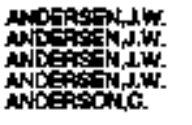 & 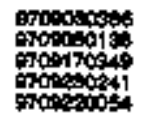 & 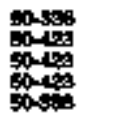 & $\begin{array}{l}\mathbf{p} \\
\mathbf{p} \\
\mathbf{p} \\
\mathbf{p} \\
\mathbf{8}\end{array}$ & 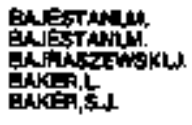 \\
\hline 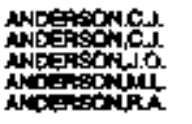 & 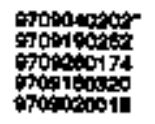 & 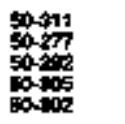 & 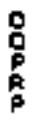 & 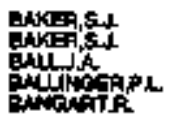 \\
\hline 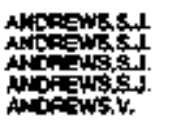 & 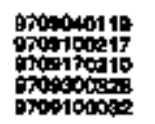 & 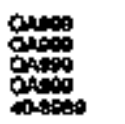 & & 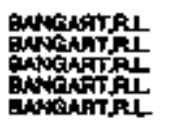 \\
\hline
\end{tabular}

\begin{tabular}{|c|c|c|c|}
\hline 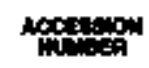 & \multicolumn{2}{|c|}{ 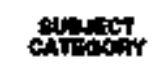 } & muthot \\
\hline 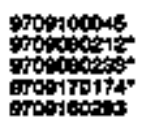 & T: & & 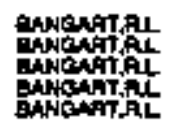 \\
\hline 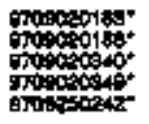 & 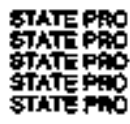 & & 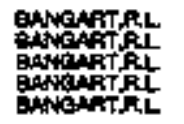 \\
\hline 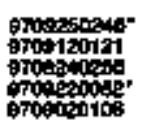 & 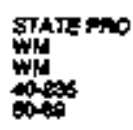 & 09 & 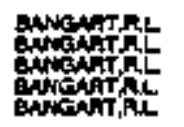 \\
\hline 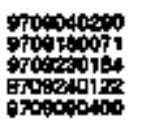 & 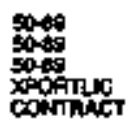 & p & 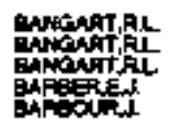 \\
\hline 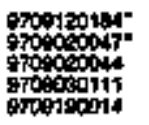 & 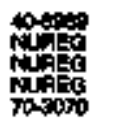 & & 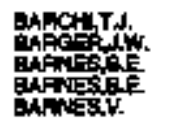 \\
\hline 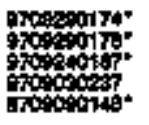 & 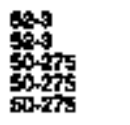 & $\begin{array}{l}6 \\
5 \\
8\end{array}$ & 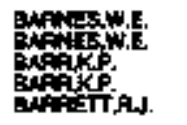 \\
\hline 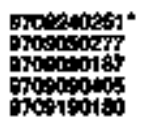 & 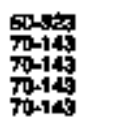 & $s$ & 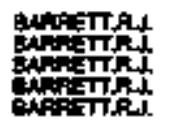 \\
\hline 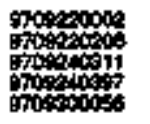 & $\begin{array}{l}70-149 \\
70-149 \\
70-149 \\
70-143\end{array}$ & & 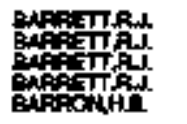 \\
\hline 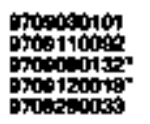 & 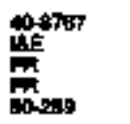 & $\mathbf{F}$ & 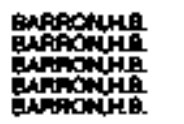 \\
\hline 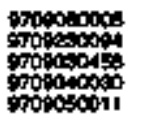 & 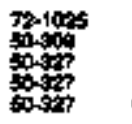 & $\begin{array}{l}\text { D } \\
5 \\
5 \\
0\end{array}$ & 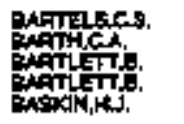 \\
\hline 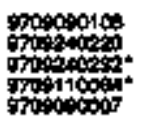 & 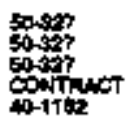 & \$ & 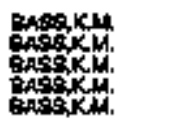 \\
\hline 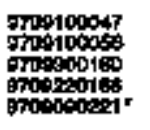 & 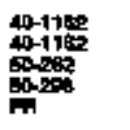 & $\mathbf{Y}$ & 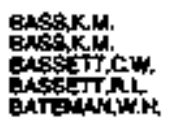 \\
\hline poogons & 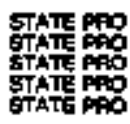 & & 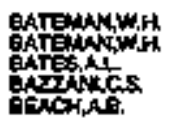 \\
\hline
\end{tabular}

\begin{tabular}{|c|c|}
\hline 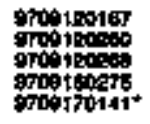 & 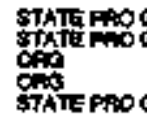 \\
\hline 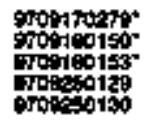 & 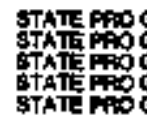 \\
\hline 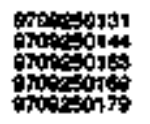 & 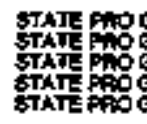 \\
\hline 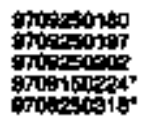 & 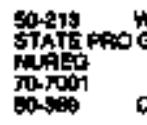 \\
\hline 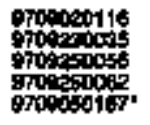 & 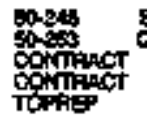 \\
\hline 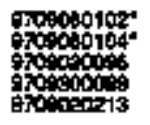 & 证 \\
\hline 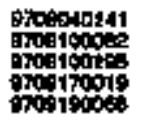 & 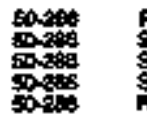 \\
\hline 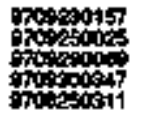 & 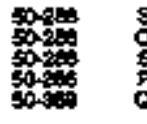 \\
\hline 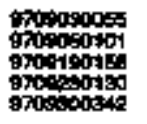 & 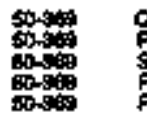 \\
\hline 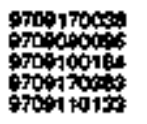 & 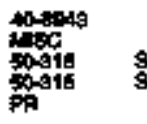 \\
\hline 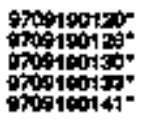 & 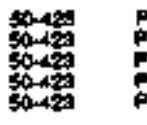 \\
\hline 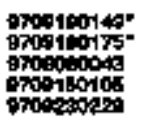 & 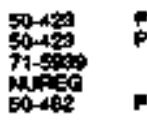 \\
\hline 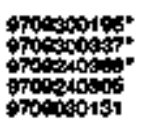 & 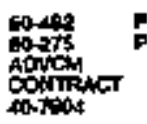 \\
\hline
\end{tabular}




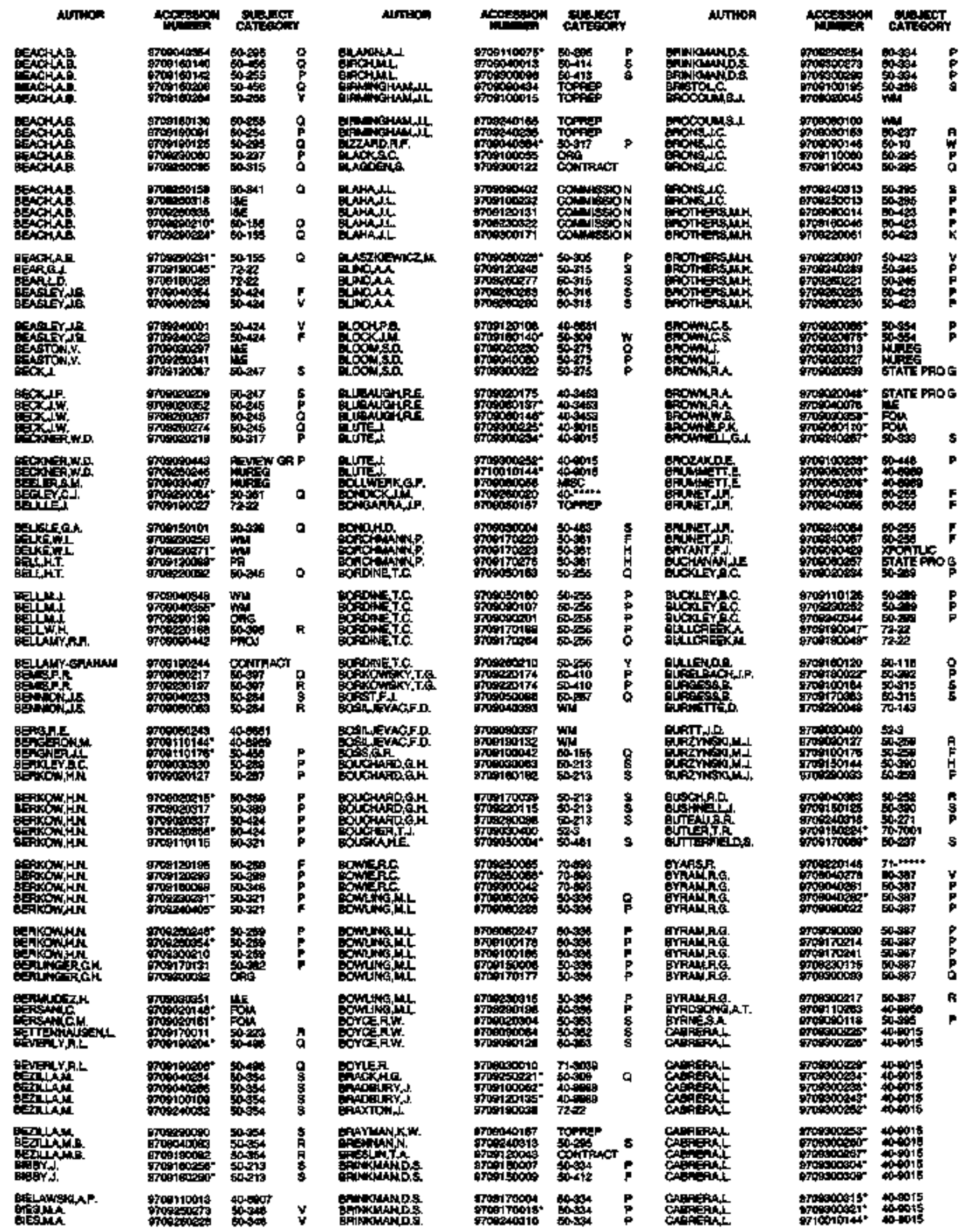




\begin{tabular}{|c|c|c|c|c|c|c|c|c|c|c|c|}
\hline AUTmos & 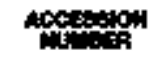 & 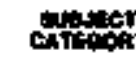 & & Mmopr & 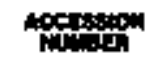 & ơlect & & MTw & 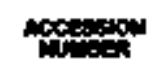 & civipury & \\
\hline 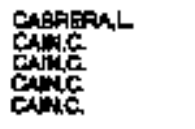 & 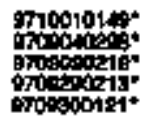 & 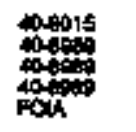 & & 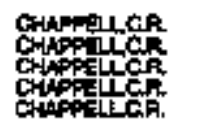 & 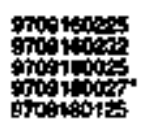 & 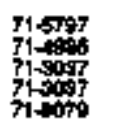 & & 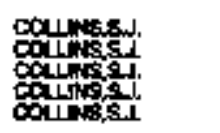 & 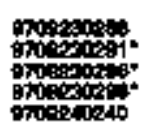 & 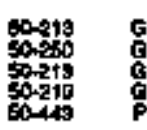 & $\begin{array}{l}\text { G } \\
\text { G } \\
\text { G } \\
\text { G } \\
\mathbf{p}\end{array}$ \\
\hline 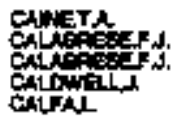 & 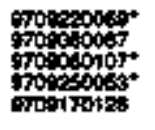 & 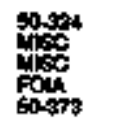 & $\mathbf{A}$ & 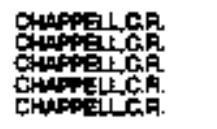 & 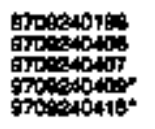 & $\begin{array}{l}710010 \\
7+70 \\
7 \rightarrow+70 \\
7 \rightarrow+\infty\end{array}$ & & 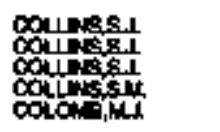 & 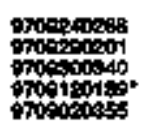 & 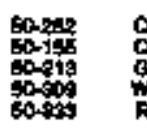 & $\begin{array}{l}\stackrel{8}{0} \\
\text { g } \\
\text { H }\end{array}$ \\
\hline 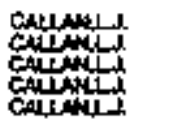 & 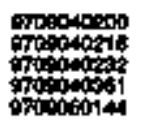 & 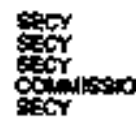 & & 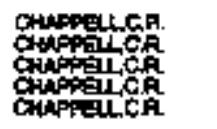 & 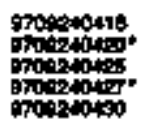 & 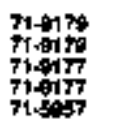 & & 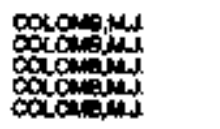 & 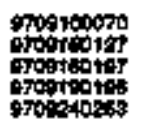 & 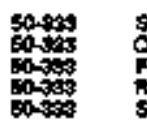 & 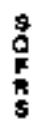 \\
\hline . & 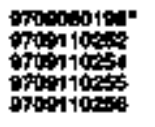 & 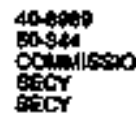 & ON & 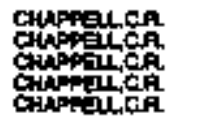 & 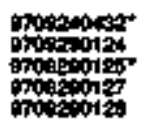 & 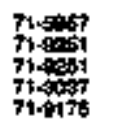 & & 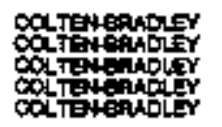 & 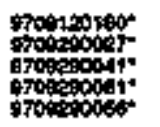 & 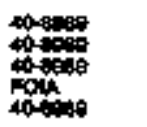 & \\
\hline 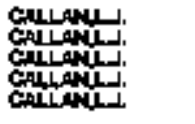 & 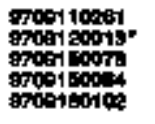 & 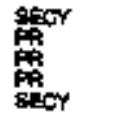 & & 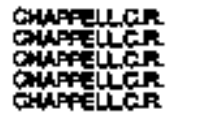 & 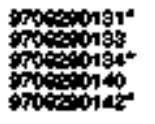 & 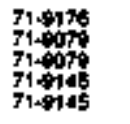 & & 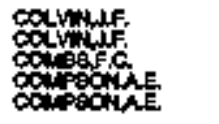 & 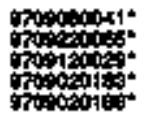 & 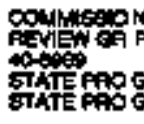 & \\
\hline 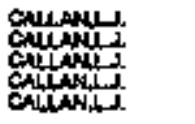 & 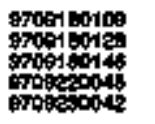 & 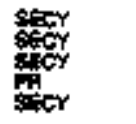 & & 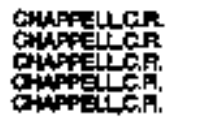 & 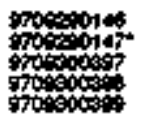 & 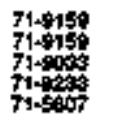 & & 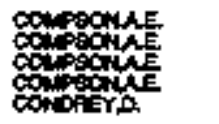 & 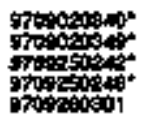 & 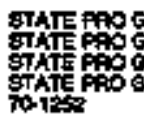 & \\
\hline 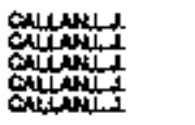 & 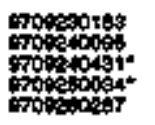 & sold & & 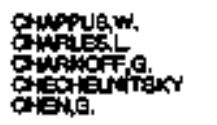 & 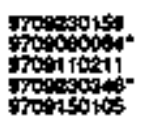 & 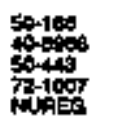 & $\begin{array}{l}\text { A } \\
P\end{array}$ & 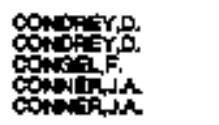 & 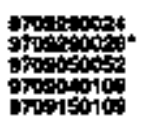 & 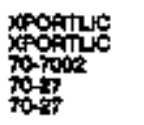 & \\
\hline 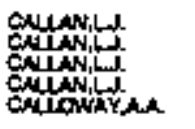 & prepeser & 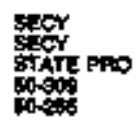 & to & 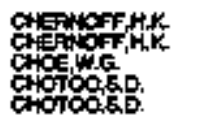 & 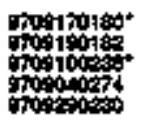 & 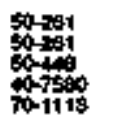 & 管 & 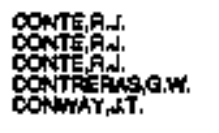 & 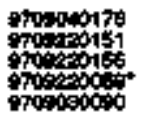 & 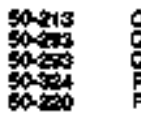 & \\
\hline 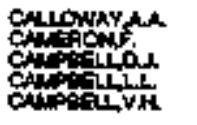 & 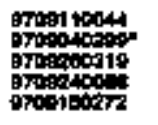 & 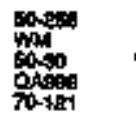 & $w$ & 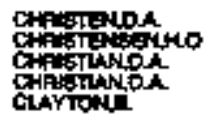 & 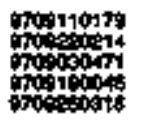 & 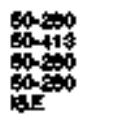 & $\begin{array}{l}f \\
9 \\
q \\
0\end{array}$ & 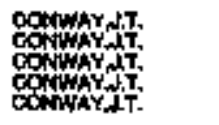 & 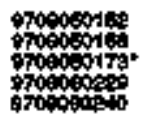 & 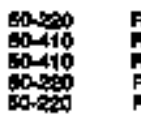 & \\
\hline 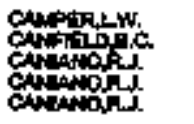 & 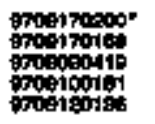 & 然 & n & 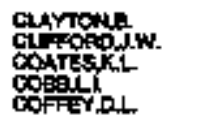 & 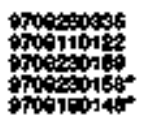 & Poin & $\begin{array}{c}q \\
\text { p }\end{array}$ & 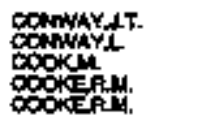 & 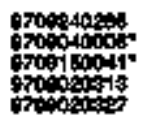 & 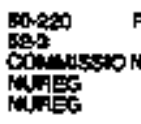 & \\
\hline 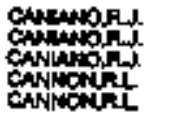 & 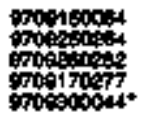 & 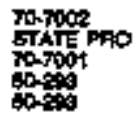 & ค & 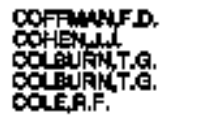 & 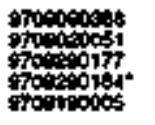 & 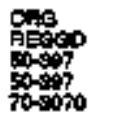 & $\mathbf{P}$ & 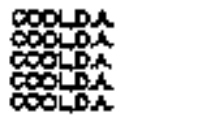 & grosing & 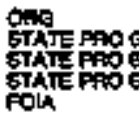 & \\
\hline 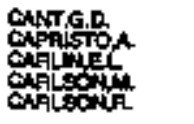 & 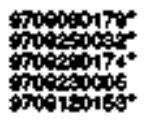 & 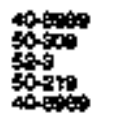 & $\begin{array}{l}0 \\
9\end{array}$ & 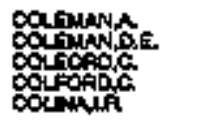 & 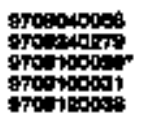 & 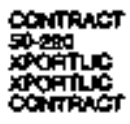 & R & 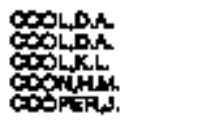 & 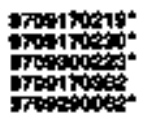 & rolk & \\
\hline 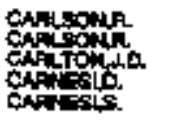 & 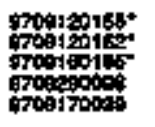 & 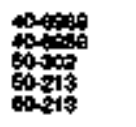 & ह & 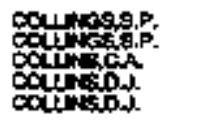 & 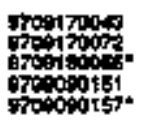 & 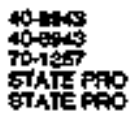 & & 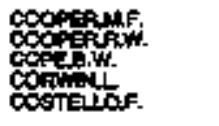 & 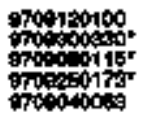 & 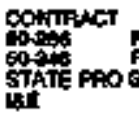 & \\
\hline 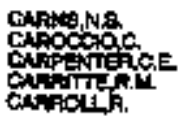 & 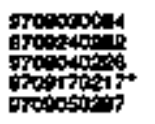 & 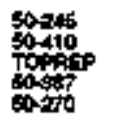 & $\begin{array}{l}p \\
b \\
B\end{array}$ & 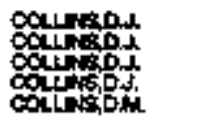 & 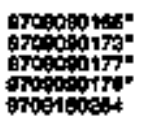 & 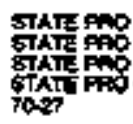 & & 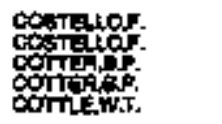 & 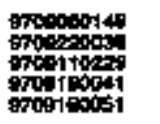 & 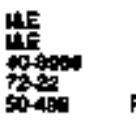 & \\
\hline 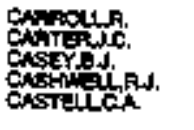 & 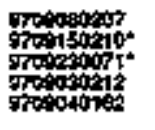 & 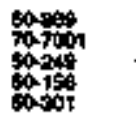 & 昌 & 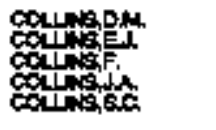 & 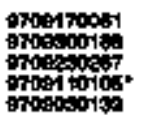 & 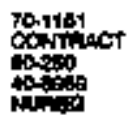 & $v$ & 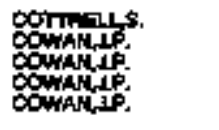 & 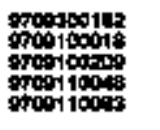 & 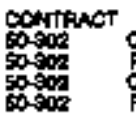 & \\
\hline 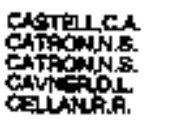 & 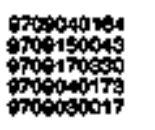 & 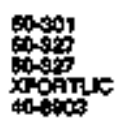 & $\begin{array}{l}5 \\
F \\
F\end{array}$ & 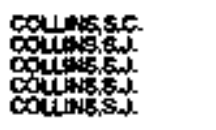 & 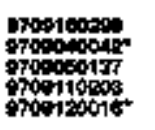 & 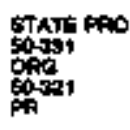 & p & 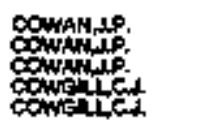 & 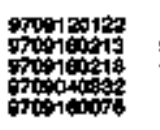 & 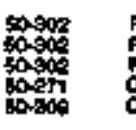 & p. \\
\hline 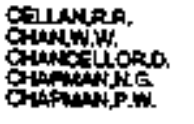 & 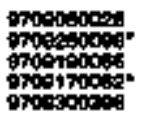 & 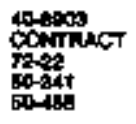 & $\stackrel{8}{8}$ & 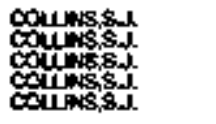 & 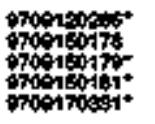 & $\log _{0 \rightarrow 0}$ & 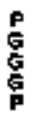 & 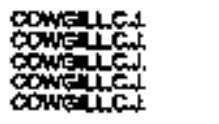 & orectosit: & 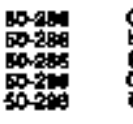 & $\begin{array}{l}\mathrm{g} \\
\mathrm{p} \\
\mathrm{p} \\
\mathrm{p}\end{array}$ \\
\hline 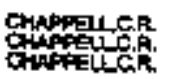 & oroposors & 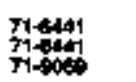 & & counss. & 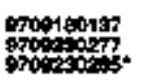 & 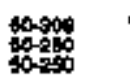 & $\frac{w}{a}$ & 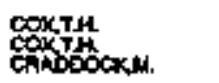 & 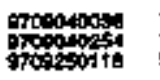 & 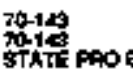 & \\
\hline
\end{tabular}




\begin{tabular}{|c|c|c|c|c|c|c|c|c|c|c|c|}
\hline AUTHOA & 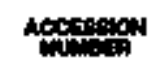 & 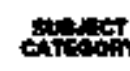 & & Antrion & 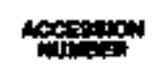 & 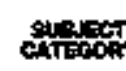 & & Aunces & A & 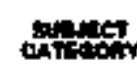 & \\
\hline 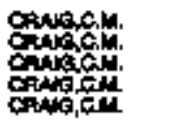 & 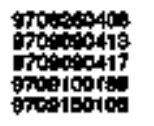 & 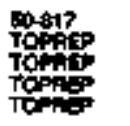 & م & 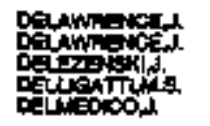 & 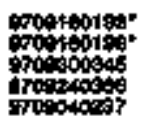 & 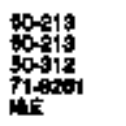 & j్ & 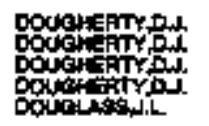 & 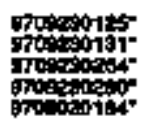 & 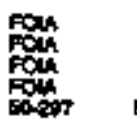 & A \\
\hline 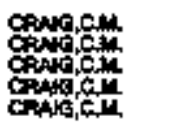 & 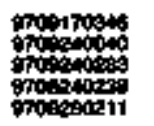 & 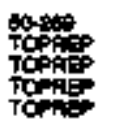 & P & 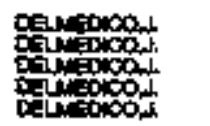 & 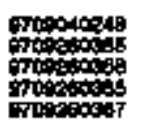 & 喿 & & meouxti & 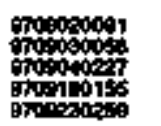 & 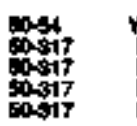 & p \\
\hline 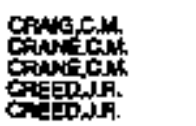 & 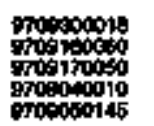 & 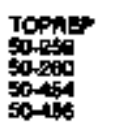 & 点 & 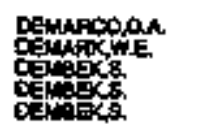 & 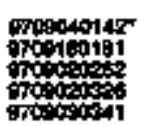 & 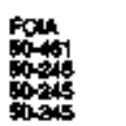 & p & 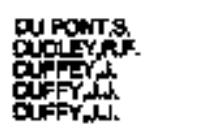 & 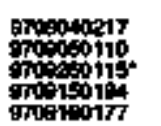 & 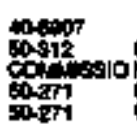 & of \\
\hline 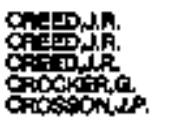 & 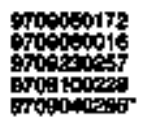 & 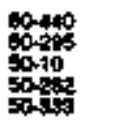 & 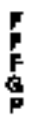 & 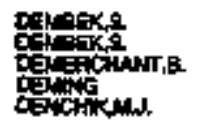 & 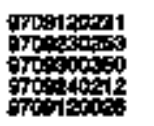 & 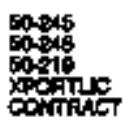 & $\begin{array}{l}\mathrm{p} \\
\mathrm{p} \\
\mathrm{s}\end{array}$ & 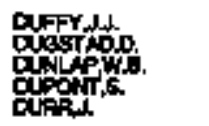 & 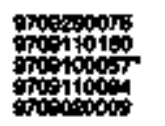 & 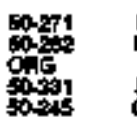 & $\begin{array}{l}P \\
R \\
5 \\
G\end{array}$ \\
\hline 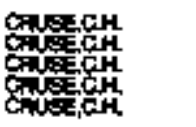 & 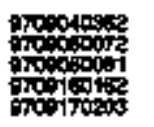 & 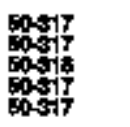 & 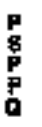 & 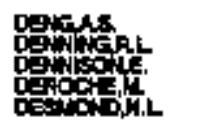 & 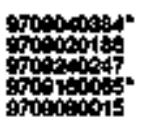 & 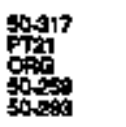 & ด̆ & 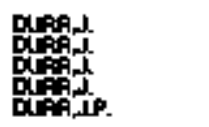 & 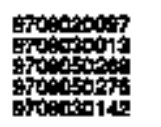 & 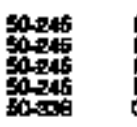 & $\begin{array}{l}\mathbf{F} \\
\mathbf{p} \\
\mathbf{p} \\
0\end{array}$ \\
\hline 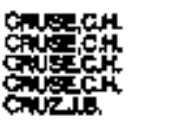 & 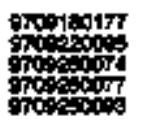 & 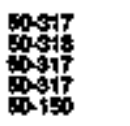 & $\frac{p}{k}$ & 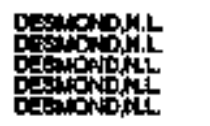 & 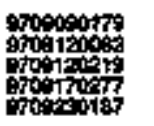 & 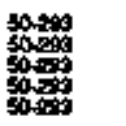 & $\begin{array}{l}\text { R } \\
\text { E } \\
\text { B } \\
\text { R }\end{array}$ & 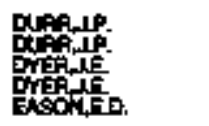 & 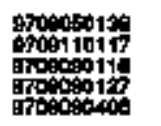 & 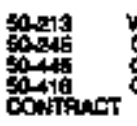 & $\begin{array}{l}\text { w } \\
0 \\
0\end{array}$ \\
\hline 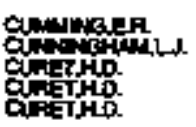 & 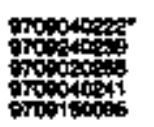 & 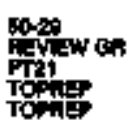 & $\mathbf{p}$ & 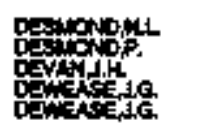 & 和 & 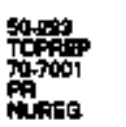 & p & 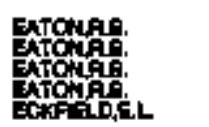 & 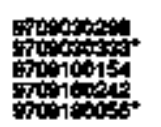 & 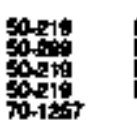 & p \\
\hline 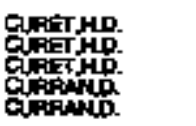 & 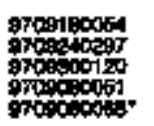 & 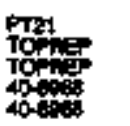 & & 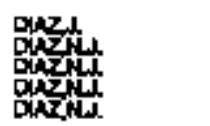 & moner & $\begin{array}{l}45 \\
105\end{array}$ & & 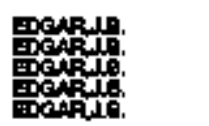 & 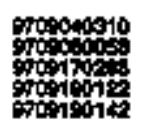 & 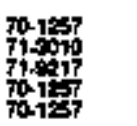 & \\
\hline 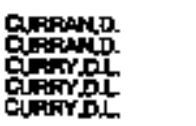 & 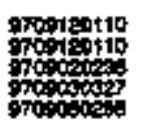 & 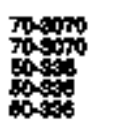 & ? & 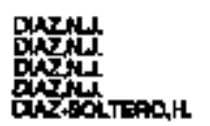 & 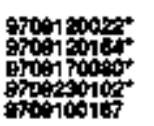 & 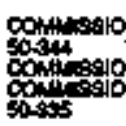 & & 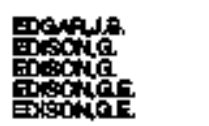 & 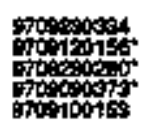 & 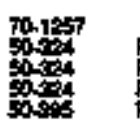 & $p$ \\
\hline 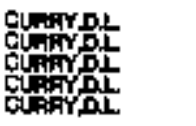 & 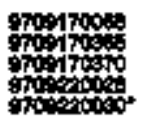 & 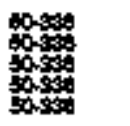 & P & 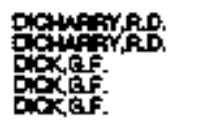 & 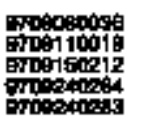 & 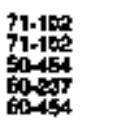 & $\frac{8}{6}$ & 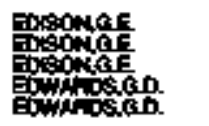 & 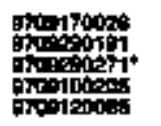 & 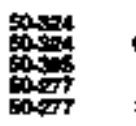 & $\begin{array}{l}\text { p } \\
\text { p } \\
\text { p } \\
\text { s } \\
\text { s }\end{array}$ \\
\hline 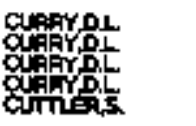 & 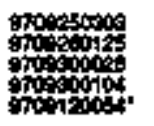 & 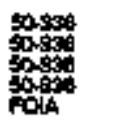 & $p$ & 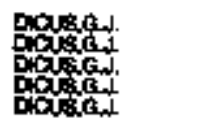 & 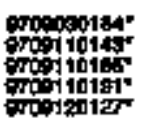 & consopos & & 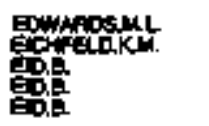 & 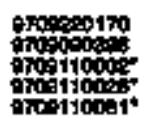 & 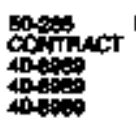 & $r^{n}$ \\
\hline 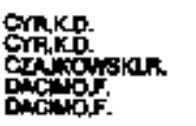 & 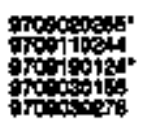 & 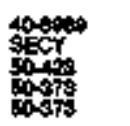 & 8 & 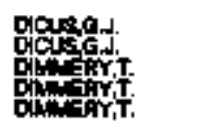 & 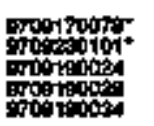 & 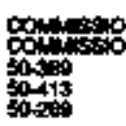 & ON & 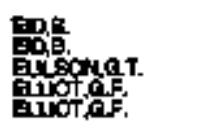 & 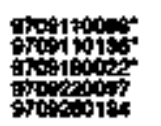 & 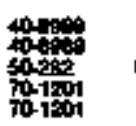 & p \\
\hline 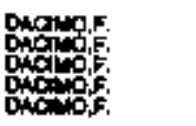 & 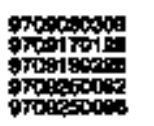 & 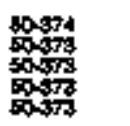 & 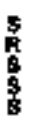 & 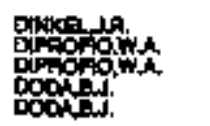 & 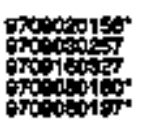 & 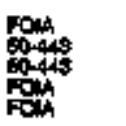 & $\mathbf{B}$ & 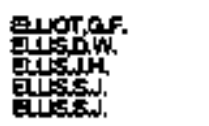 & 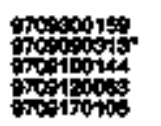 & 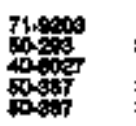 & $\begin{array}{l}s \\
s \\
s\end{array}$ \\
\hline 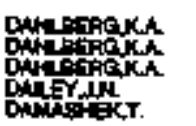 & 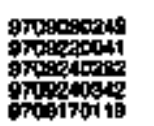 & 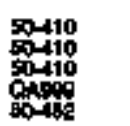 & $\stackrel{8}{8}$ & 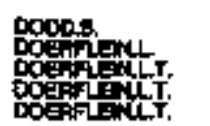 & 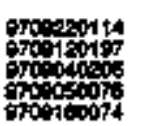 & 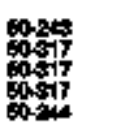 & $\begin{array}{l}8 \\
8 \\
8 \\
0\end{array}$ & 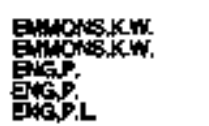 & 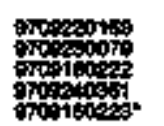 & 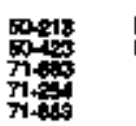 & R \\
\hline 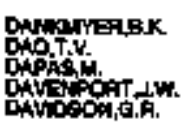 & 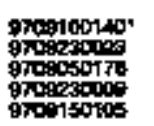 & 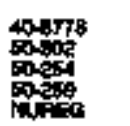 & 疍 & 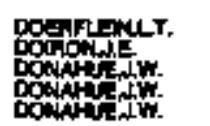 & 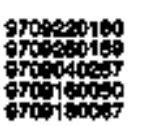 & 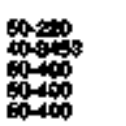 & 0 & 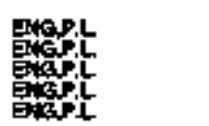 & 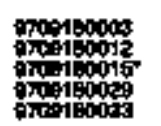 & 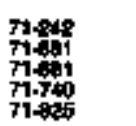 & \\
\hline 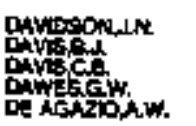 & 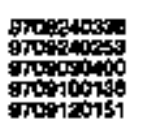 & 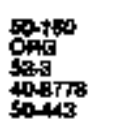 & $\mathbf{P}$ & 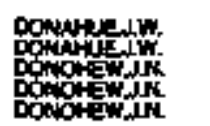 & 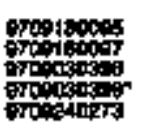 & $\begin{array}{l}50-490 \\
50-40 \\
50416 \\
50416 \\
50418\end{array}$ & $\$$ & 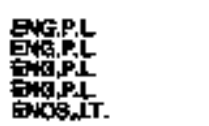 & 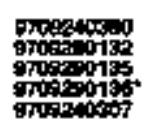 & 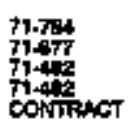 & T \\
\hline 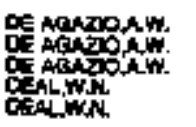 & 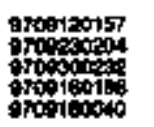 & 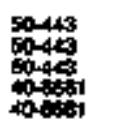 & $\mathbf{8}$ & 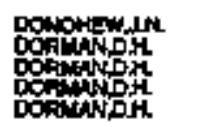 & 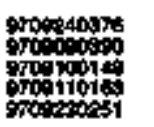 & 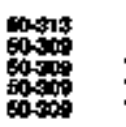 & w & 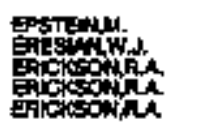 & 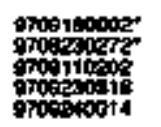 & 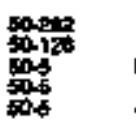 & $\begin{array}{l}P \\
y \\
H \\
\text { s }\end{array}$ \\
\hline 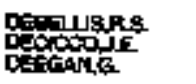 & 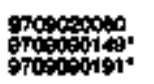 & Tomimat & & 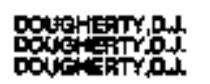 & 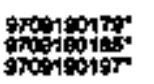 & rous & & 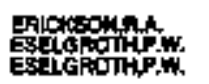 & 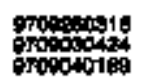 & 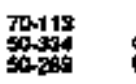 & 8 \\
\hline
\end{tabular}




\begin{tabular}{|c|c|c|c|c|c|c|c|c|c|c|c|}
\hline Allmoin & 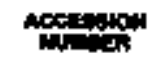 & cover & & AUTHCA & MCtsing & chivecr & & Amica & AGezaly & citheoris & \\
\hline 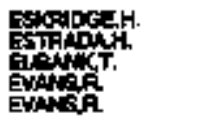 & 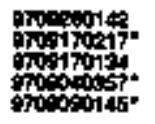 & 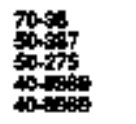 & P & 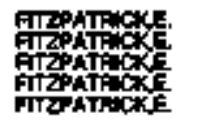 & 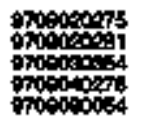 & 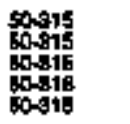 & $\begin{array}{l}\text { p } \\
\text { p } \\
\text { p } \\
\text { p }\end{array}$ & 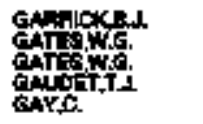 & 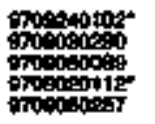 & 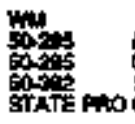 & 竞 \\
\hline 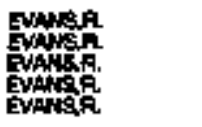 & 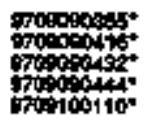 & 舟 & & 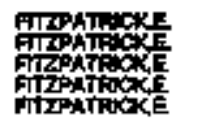 & 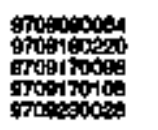 & 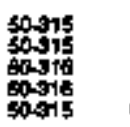 & $\begin{array}{l}p \\
p \\
p \\
p \\
q\end{array}$ & 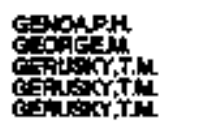 & 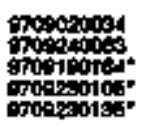 & 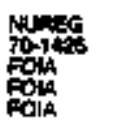 & \\
\hline 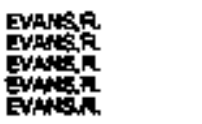 & 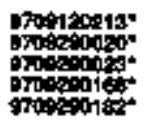 & 舟 & & 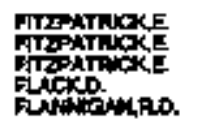 & 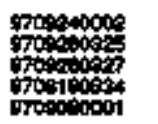 & coass & $\begin{array}{l}0 \\
k \\
0 \\
5\end{array}$ & 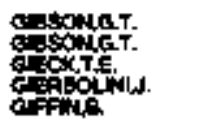 & 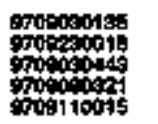 & 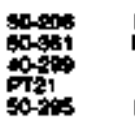 & $\mathbf{r}$ \\
\hline 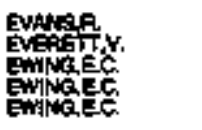 & 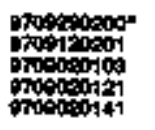 & 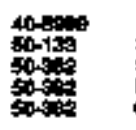 & 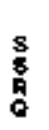 & 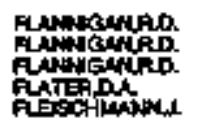 & 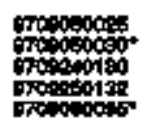 & 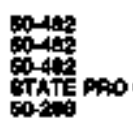 & $\begin{array}{l}\mathbf{g} \\
8 \\
\frac{5}{5} \\
g \\
g\end{array}$ & 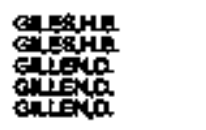 & 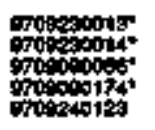 & 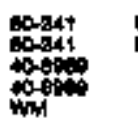 & $\mathrm{R}$ \\
\hline 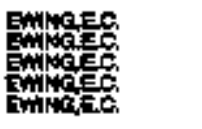 & 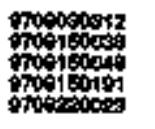 & 象此 & $\begin{array}{l}p \\
p \\
p \\
p \\
p\end{array}$ & 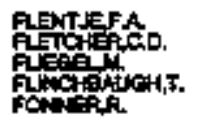 & 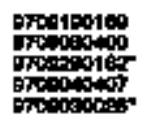 & 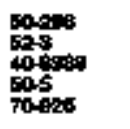 & $s$ & 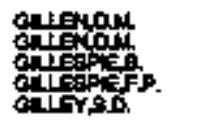 & 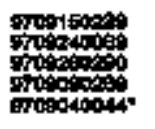 & 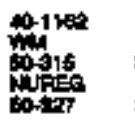 & $\begin{array}{l}5 \\
s\end{array}$ \\
\hline 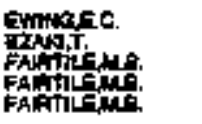 & 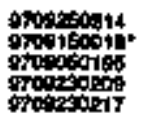 & 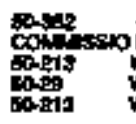 & $\underset{w}{w}$ & 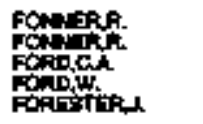 & 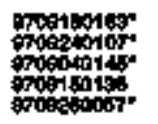 & 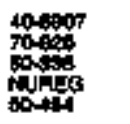 & P & 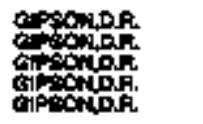 & 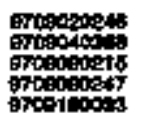 & 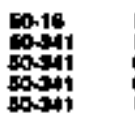 & 高 \\
\hline 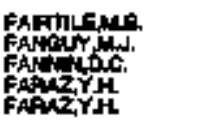 & 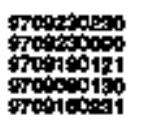 & 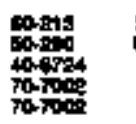 & 咅 & 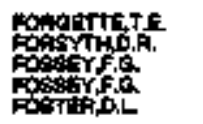 & 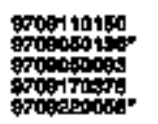 & 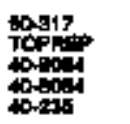 & $\mathbf{F}$ & 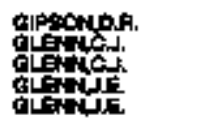 & 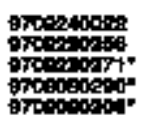 & 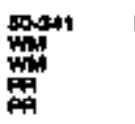 & $p$ \\
\hline 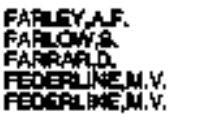 & 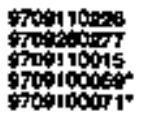 & 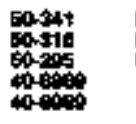 & 点 & 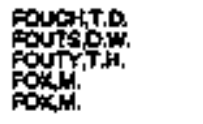 & 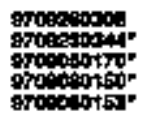 & 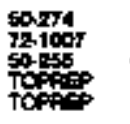 & $\begin{array}{l}0 \\
0\end{array}$ & 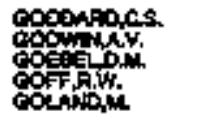 & 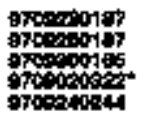 & 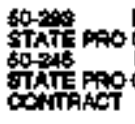 & $\begin{array}{l}1 \\
0.0 \\
0.0 \\
0 \\
0\end{array}$ \\
\hline 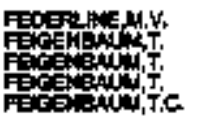 & 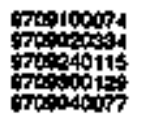 & 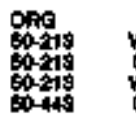 & $\begin{array}{l}\text { wo } \\
\text { 总 } \\
\text { o }\end{array}$ & 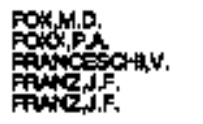 & 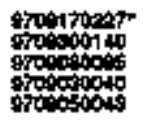 & 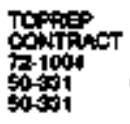 & $\stackrel{q}{v}$ & 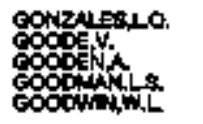 & 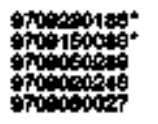 & 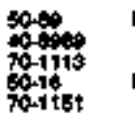 & F \\
\hline 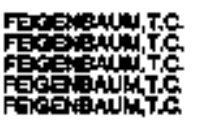 & 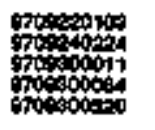 & 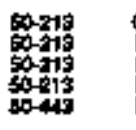 & $\begin{array}{l}q \\
F \\
F \\
F\end{array}$ & 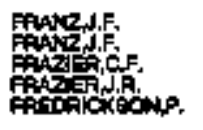 & 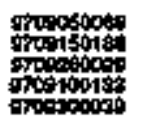 & 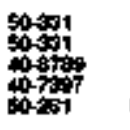 & $\stackrel{v}{p}$ & 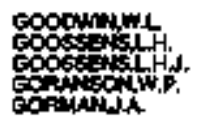 & 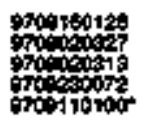 & notion & \\
\hline 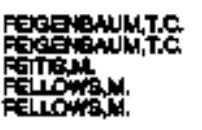 & 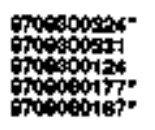 & 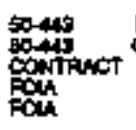 & 8 & 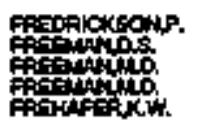 & 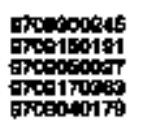 & 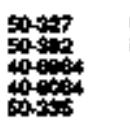 & $\begin{array}{c}\mathrm{G} \\
\mathrm{s}\end{array}$ & 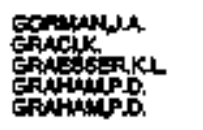 & 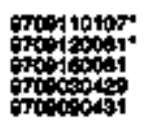 & Toris & $\stackrel{P}{\mathbf{p}}$ \\
\hline 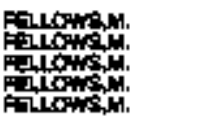 & 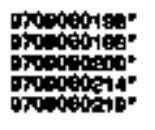 & Rak & & 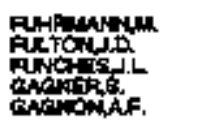 & 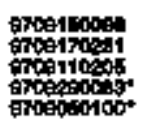 & 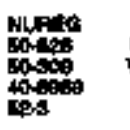 & $\stackrel{R}{W}$ & 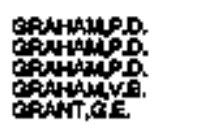 & 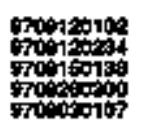 & solat & $\begin{array}{l}\mathbf{p} \\
\mathbf{P} \\
\mathbf{p} \\
\mathbf{p}\end{array}$ \\
\hline 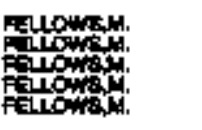 & oropoes: & rouk & & 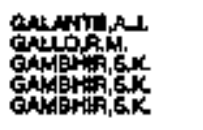 & 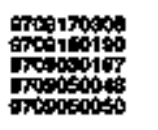 & 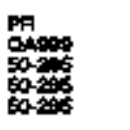 & $\begin{array}{l}p \\
p\end{array}$ & 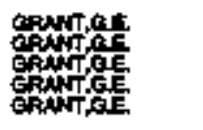 & 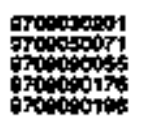 & कot & $\begin{array}{l}0 \\
0 \\
0 \\
0 \\
0\end{array}$ \\
\hline 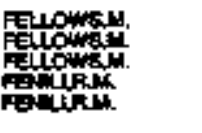 & 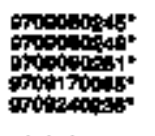 & 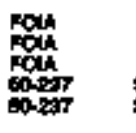 & $\$$ & 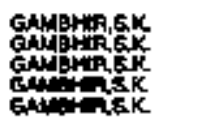 & 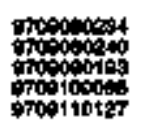 & sos & $\stackrel{5}{\mathbf{p}}$ & 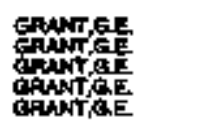 & 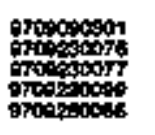 & 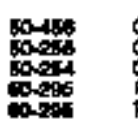 & $\begin{array}{l}o \\
o \\
0 \\
0 \\
p\end{array}$ \\
\hline 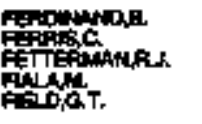 & 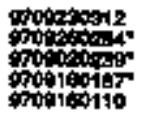 & 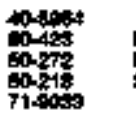 & $\begin{array}{l}\mathbf{P} \\
\mathbf{p}\end{array}$ & 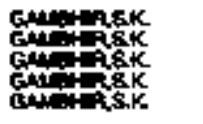 & 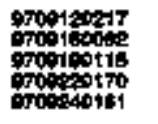 & coses & & 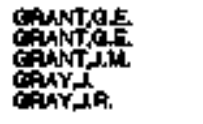 & 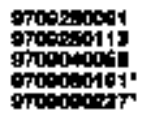 & 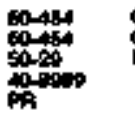 & 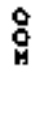 \\
\hline 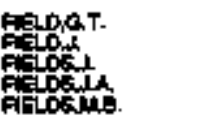 & 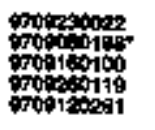 & 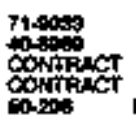 & 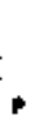 & 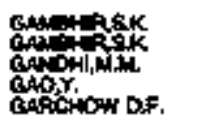 & 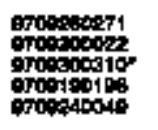 & $\begin{array}{l}60-255 \\
60-75 \\
50-41 \\
50-333 \\
60-712\end{array}$ & & 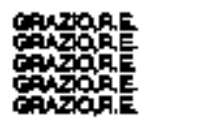 & 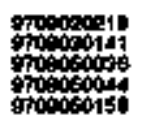 & sotog & $\begin{array}{l}\mathbf{5} \\
\mathbf{1} \\
\mathbf{p}\end{array}$ \\
\hline 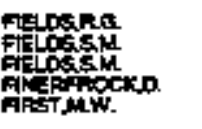 & 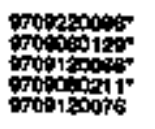 & 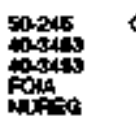 & 0 & 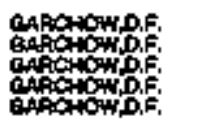 & 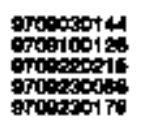 & 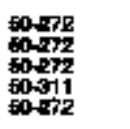 & คิ & 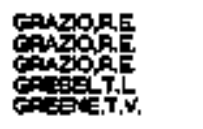 & 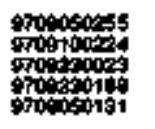 & 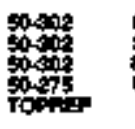 & 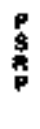 \\
\hline che & prosono & 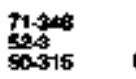 & $p$ & 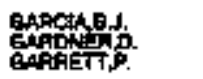 & 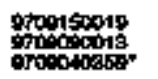 & 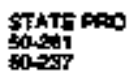 & & 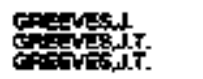 & 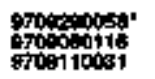 & 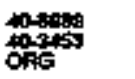 & \\
\hline
\end{tabular}




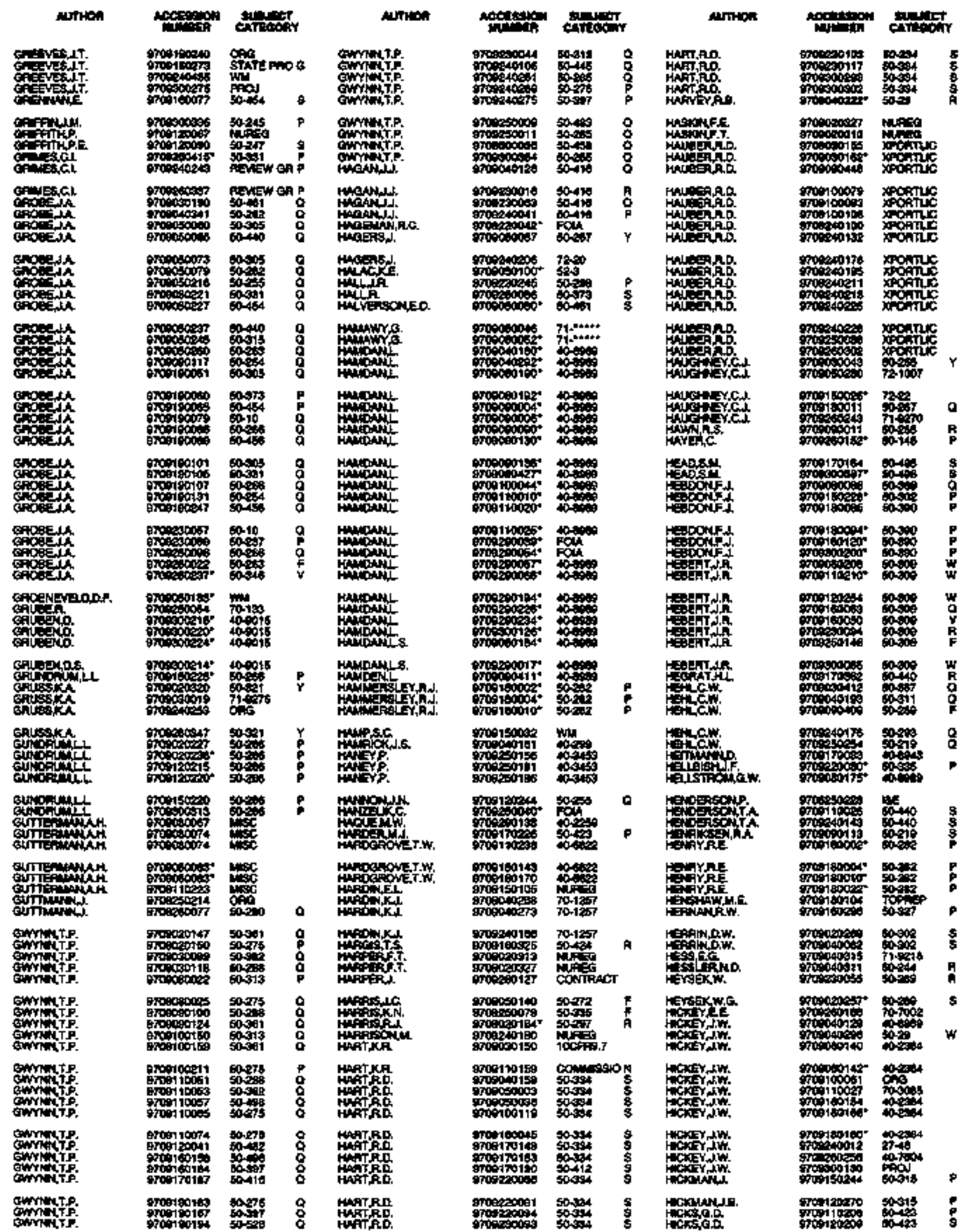




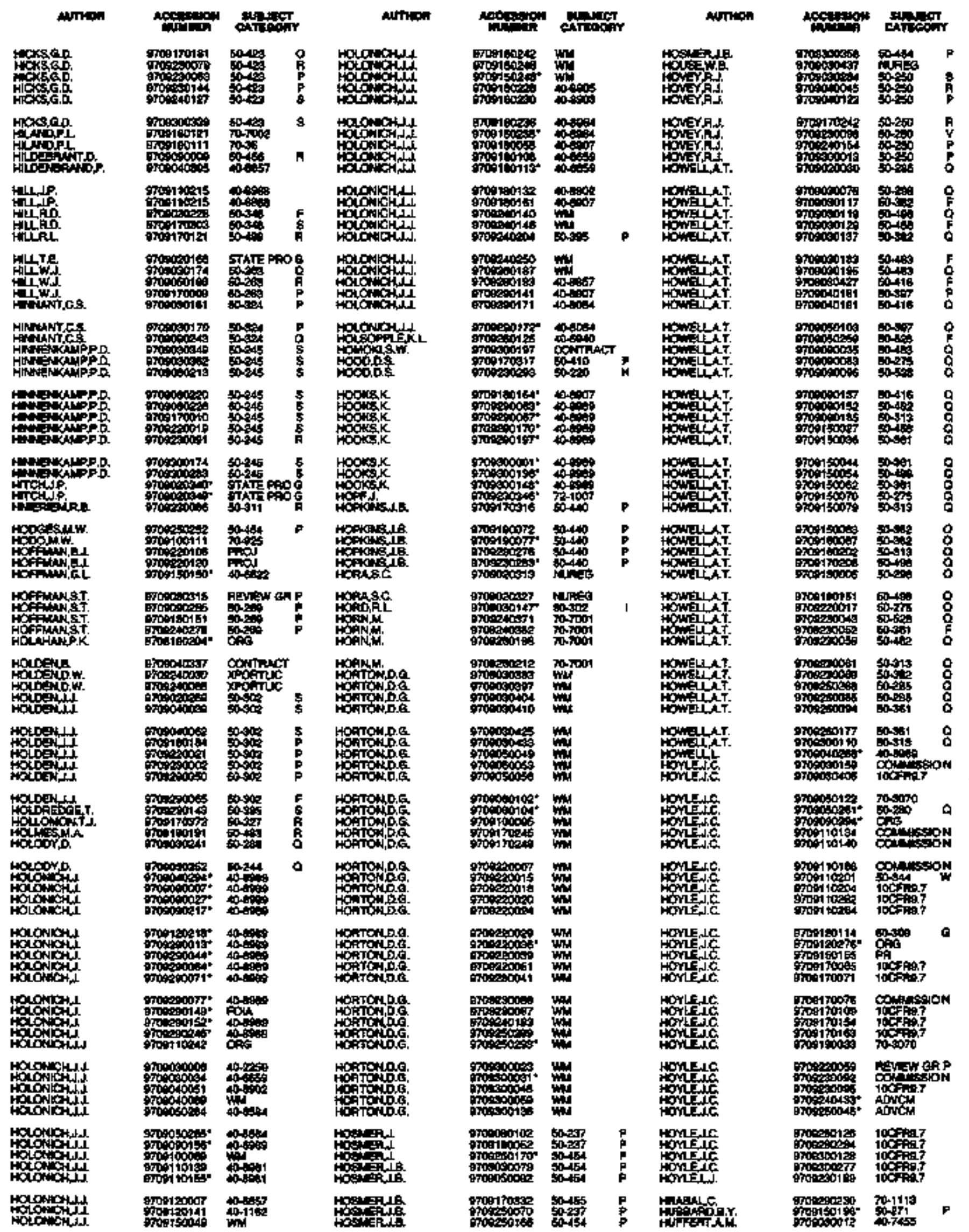




\begin{tabular}{|c|c|c|c|c|c|c|c|c|c|c|}
\hline annes & 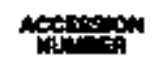 & \multicolumn{2}{|c|}{ 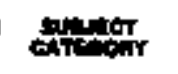 } & almion & 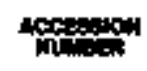 & \multicolumn{2}{|c|}{ congect } & MTTWOA & 10cestion & 4⿻ \\
\hline 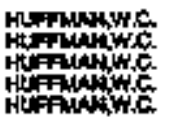 & 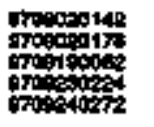 & \multicolumn{2}{|l|}{ 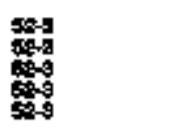 } & 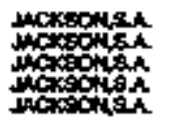 & 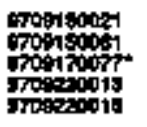 & \multicolumn{2}{|c|}{ N } & 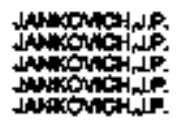 & 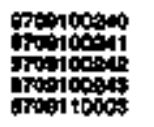 & 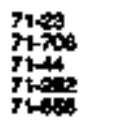 \\
\hline 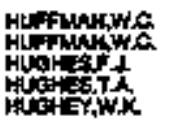 & 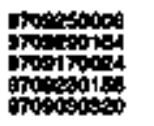 & 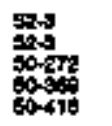 & 8 & 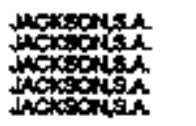 & 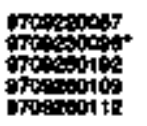 & \multicolumn{2}{|c|}{ 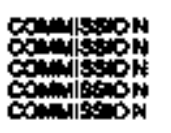 } & 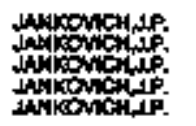 & 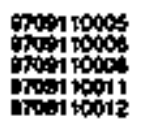 & 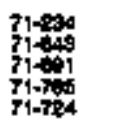 \\
\hline 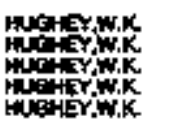 & 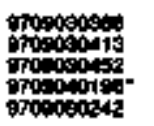 & 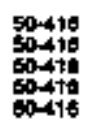 & 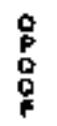 & 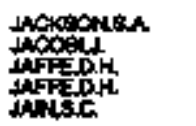 & 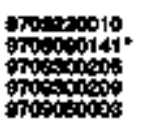 & \multicolumn{2}{|c|}{ 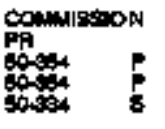 } & 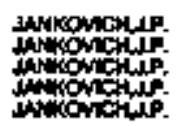 & 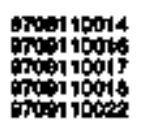 & 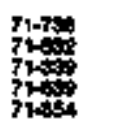 \\
\hline 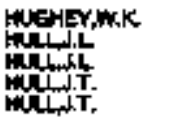 & 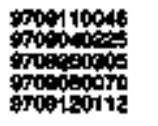 & 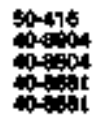 & $F$ & 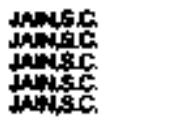 & 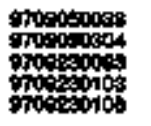 & 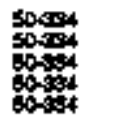 & 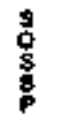 & 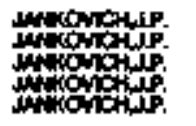 & 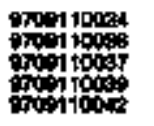 & 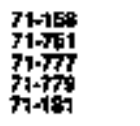 \\
\hline 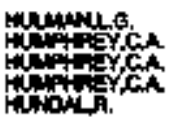 & 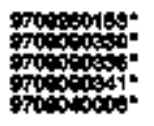 & 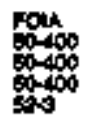 & : & 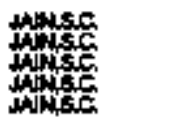 & 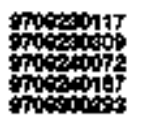 & 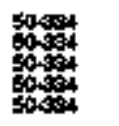 & $\frac{8}{8}$ & 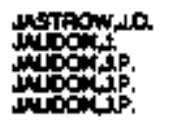 & 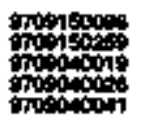 & 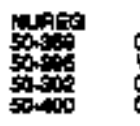 \\
\hline 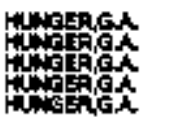 & 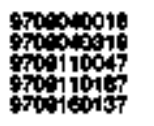 & 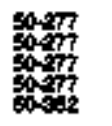 & 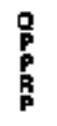 & 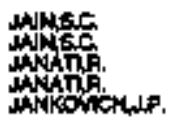 & 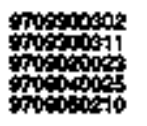 & 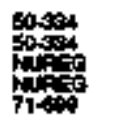 & $\$$ & 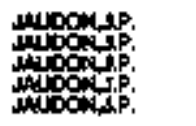 & 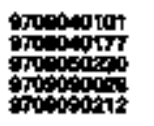 & 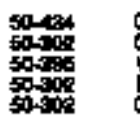 \\
\hline 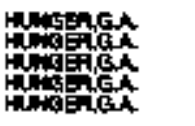 & 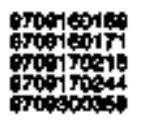 & 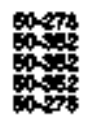 & ? & 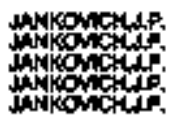 & 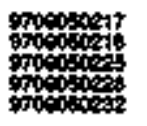 & 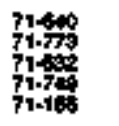 & & 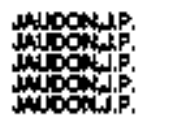 & 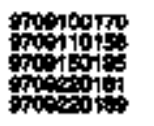 & 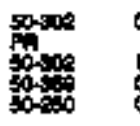 \\
\hline 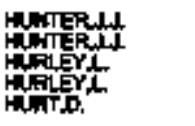 & 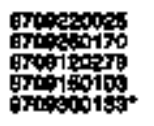 & 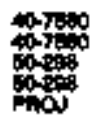 & $\checkmark$ & 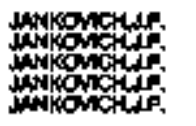 & 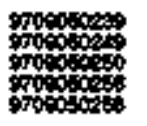 & 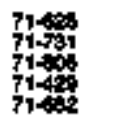 & & 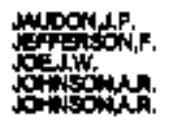 & 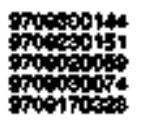 & 0 \\
\hline 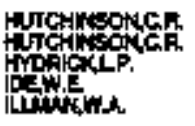 & 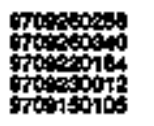 & 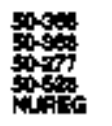 & $\begin{array}{l}\text { P } \\
\text { P } \\
\text { R } \\
\text { S }\end{array}$ & 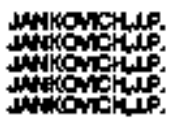 & 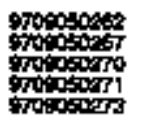 & 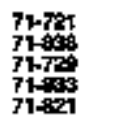 & & 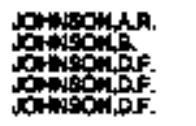 & 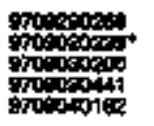 & 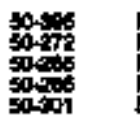 \\
\hline 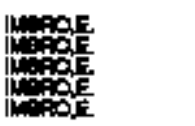 & 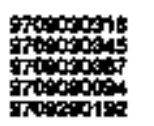 & 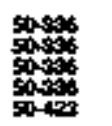 & $\frac{1}{p}$ & 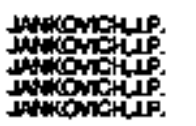 & 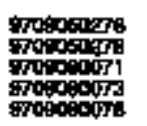 & 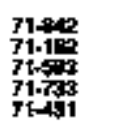 & & 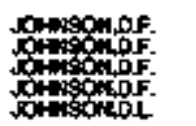 & 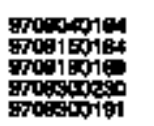 & 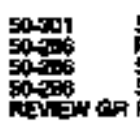 \\
\hline 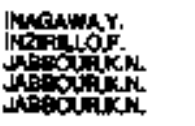 & 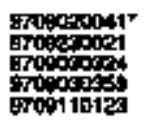 & 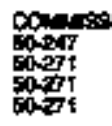 & $\begin{array}{r}\text { On } \\
\text { P } \\
\text { P } \\
\text { P }\end{array}$ & 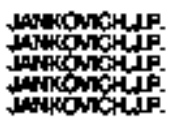 & 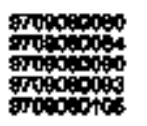 & 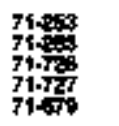 & & 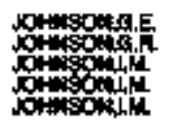 & 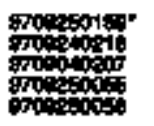 & 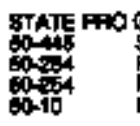 \\
\hline 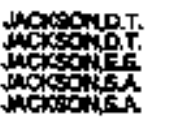 & 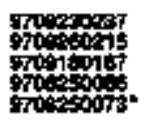 & 㹂 & & 政 & 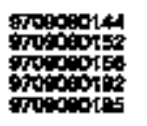 & 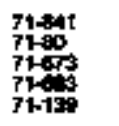 & & $\frac{1}{104}$ & 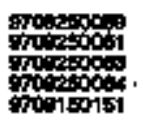 & 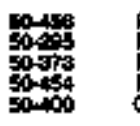 \\
\hline 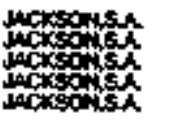 & 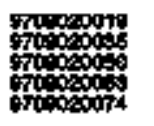 & 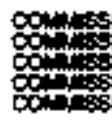 & & 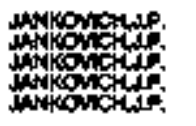 & 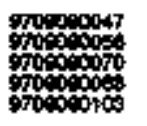 & 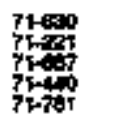 & & 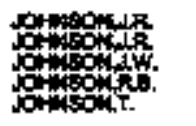 & 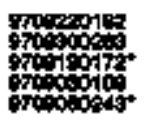 & 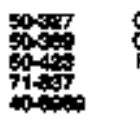 \\
\hline 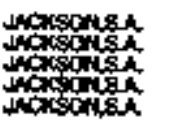 & 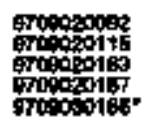 & 皮 & & 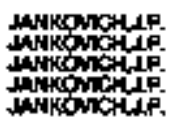 & 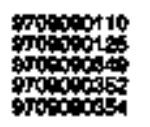 & $\begin{array}{l}71,414 \\
77,96 \\
71,700 \\
71,702\end{array}$ & & 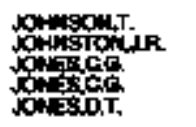 & 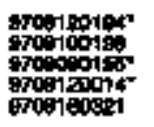 & 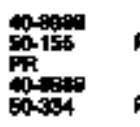 \\
\hline 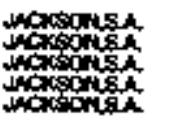 & 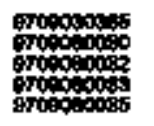 & 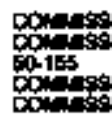 & & 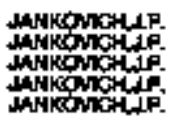 & 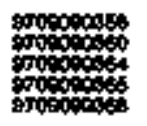 & 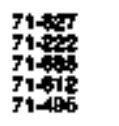 & & 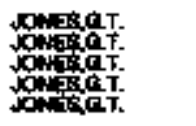 & 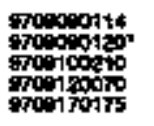 & 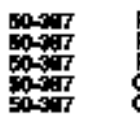 \\
\hline 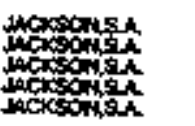 & 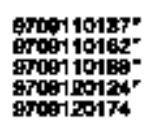 & 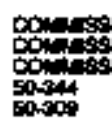 & & 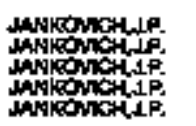 & 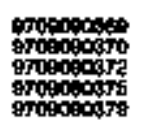 & 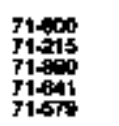 & & 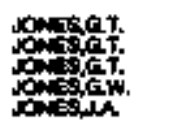 & 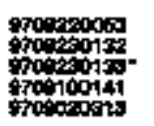 & 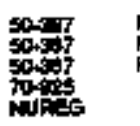 \\
\hline 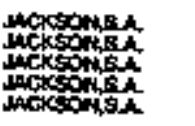 & 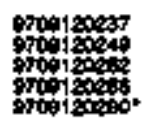 & 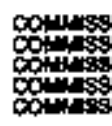 & & 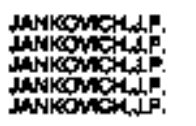 & 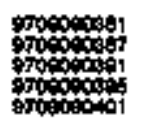 & 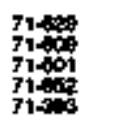 & & 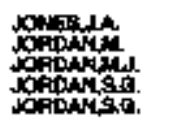 & 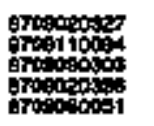 & 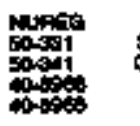 \\
\hline 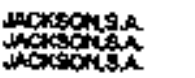 & 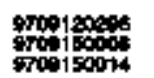 & $\begin{array}{l}c 015 s \\
\infty \\
\infty\end{array}$ & & 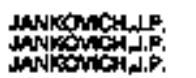 & 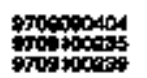 & 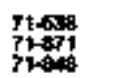 & & 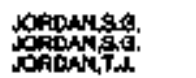 & 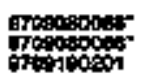 & 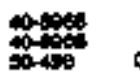 \\
\hline
\end{tabular}




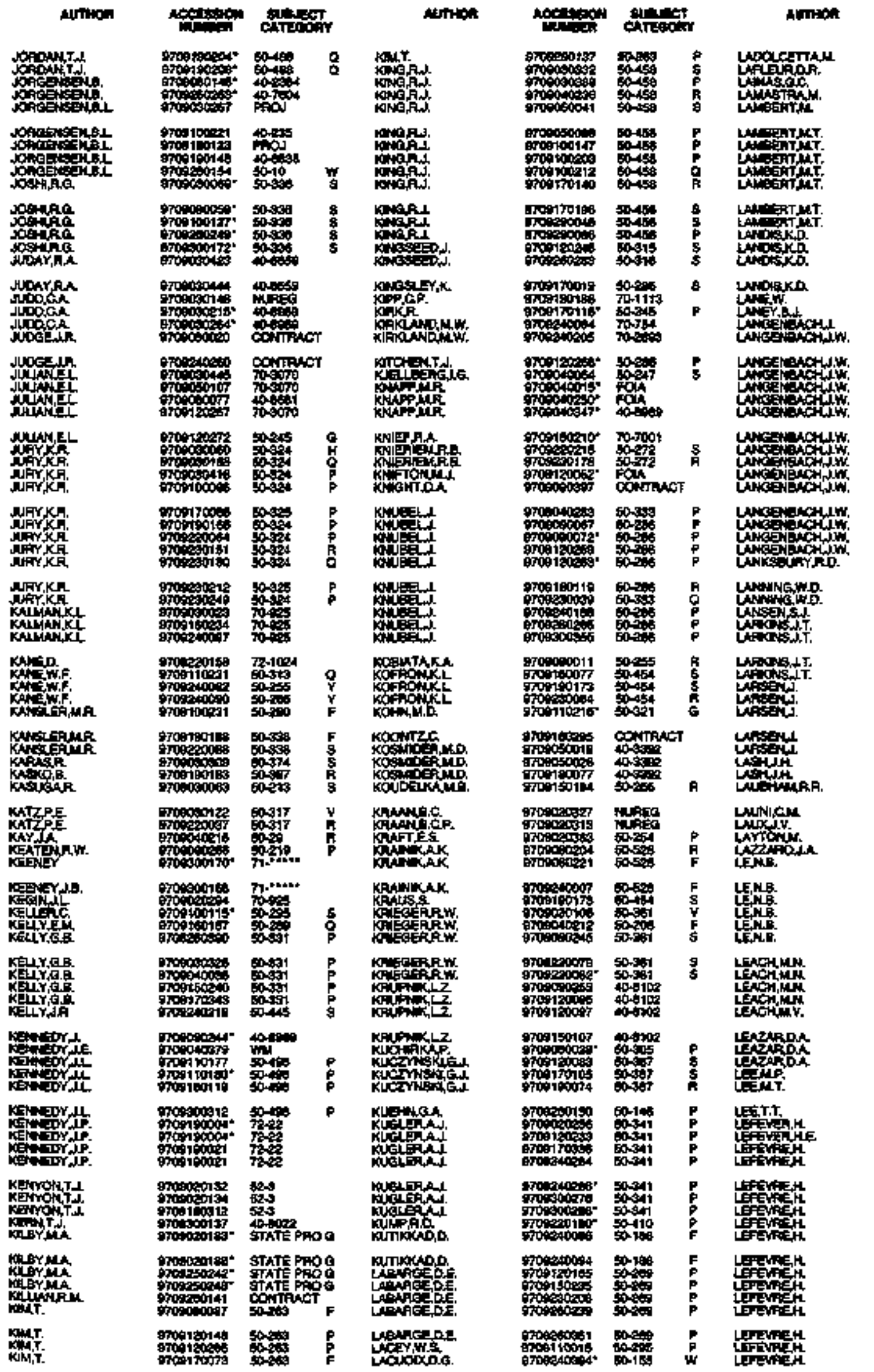

\begin{tabular}{|c|c|c|}
\hline 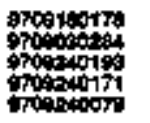 & 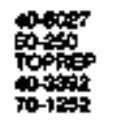 & $s$ \\
\hline 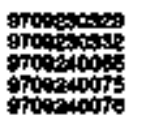 & 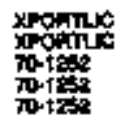 & \\
\hline 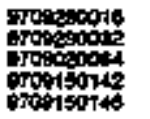 & 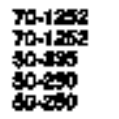 & ధి \\
\hline 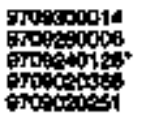 & 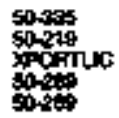 & $\begin{array}{l}0 \\
8 \\
0\end{array}$ \\
\hline 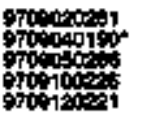 & & $\stackrel{p}{p}$ \\
\hline 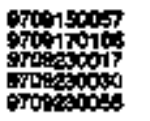 & & $\begin{array}{l}q \\
p \\
p \\
p \\
\text { p }\end{array}$ \\
\hline 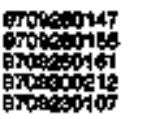 & & $\stackrel{p}{\frac{P}{9}}$ \\
\hline 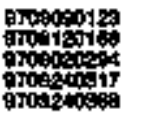 & 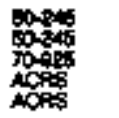 & $g$ \\
\hline 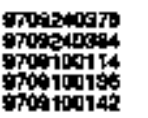 & 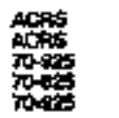 & \\
\hline 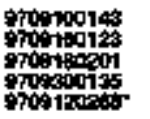 & 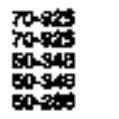 & $\begin{array}{l}\mathbf{R} \\
\underset{\mathbf{R}}{\mathbf{p}}\end{array}$ \\
\hline 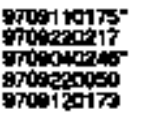 & 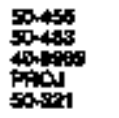 & p \\
\hline 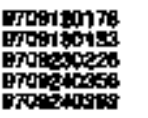 & 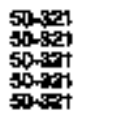 & $\begin{array}{l}\text { p } \\
p \\
p \\
p \\
p\end{array}$ \\
\hline 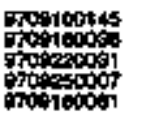 & $\begin{array}{l}50 \\
50 \\
00 \\
00\end{array}$ & $\stackrel{y}{q}$ \\
\hline 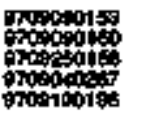 & & $\begin{array}{l}p \\
p \\
p\end{array}$ \\
\hline 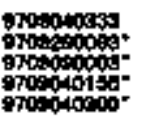 & 8 & \\
\hline 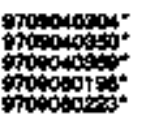 & & \\
\hline 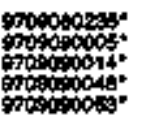 & & \\
\hline ophosp:- & & \\
\hline
\end{tabular}


218 PERSONAL AUTHOR MDEX

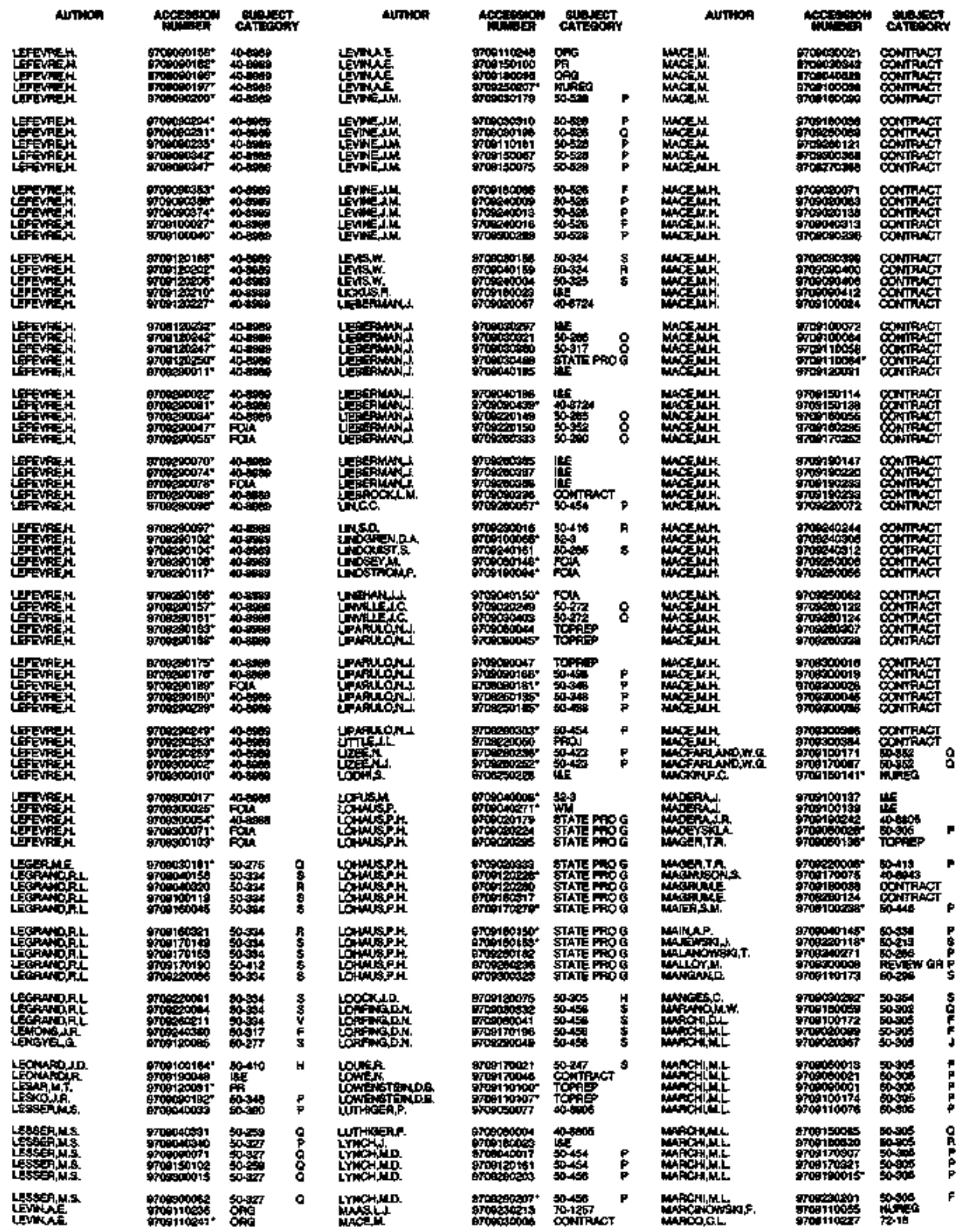




\begin{tabular}{|c|c|c|c|c|c|c|c|c|c|c|}
\hline \multirow{2}{*}{ 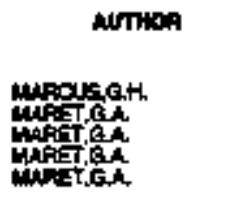 } & \multirow{2}{*}{ 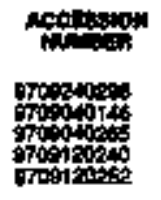 } & \multicolumn{2}{|c|}{ aypestry } & \multirow{2}{*}{ 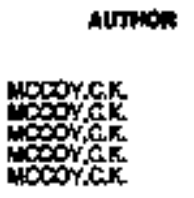 } & \multirow{2}{*}{ 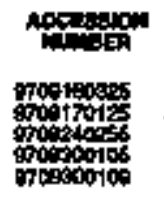 } & 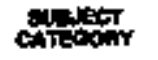 & \multirow{2}{*}{ 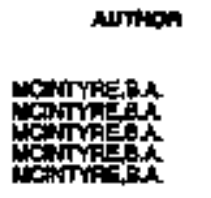 } & \multirow{2}{*}{ 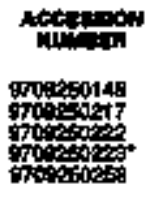 } & \multicolumn{2}{|l|}{ ז'T } \\
\hline & & 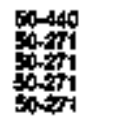 & $\begin{array}{l}9 \\
\qquad \\
8 \\
8 \\
3\end{array}$ & & & 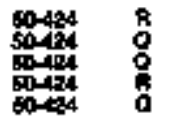 & & & 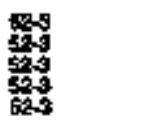 & \\
\hline 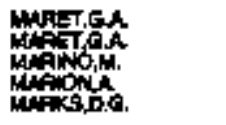 & 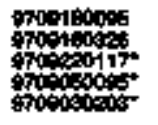 & 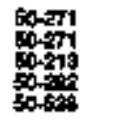 & $\begin{array}{l}v \\
\text { V } \\
8 \\
\mathbf{s} \\
\mathbf{s}\end{array}$ & 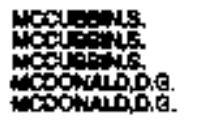 & 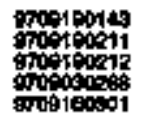 & 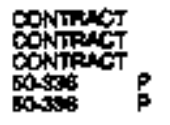 & 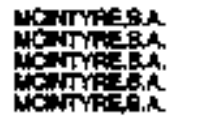 & 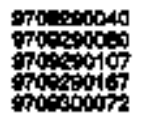 & 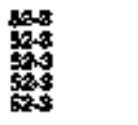 & \\
\hline 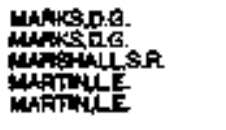 & 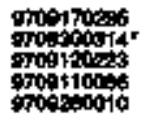 & 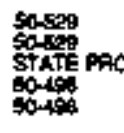 & s.s & 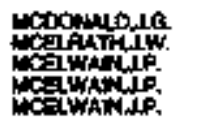 & 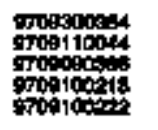 & 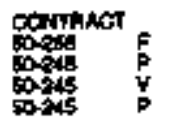 & 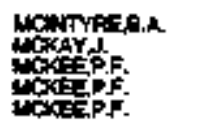 & 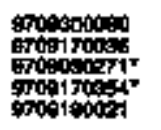 & 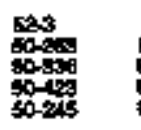 & p \\
\hline 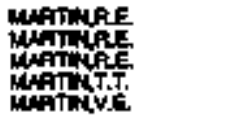 & 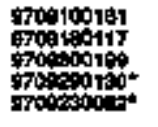 & 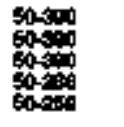 & p & 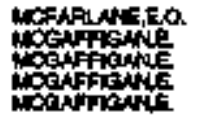 & 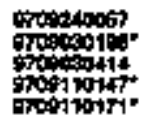 & 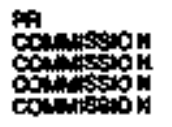 & 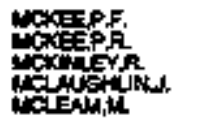 & 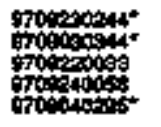 & 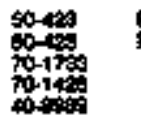 & $\rho$ \\
\hline 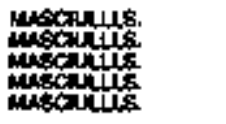 & 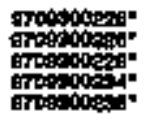 & $\begin{array}{l}400015 \\
400015 \\
400015 \\
400015\end{array}$ & & 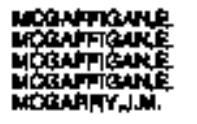 & 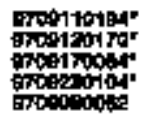 & 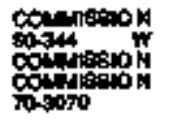 & 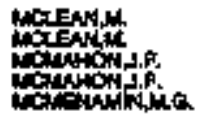 & 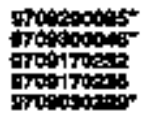 & 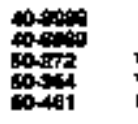 & $\begin{array}{l}Y \\
\mathbf{Z}\end{array}$ \\
\hline 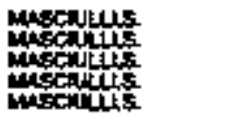 & 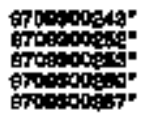 & 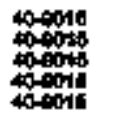 & & 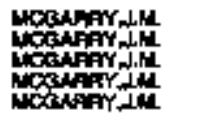 & 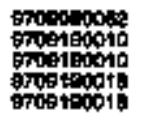 & 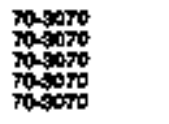 & 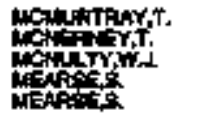 & 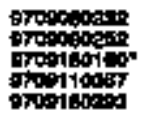 & 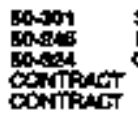 & 8 \\
\hline 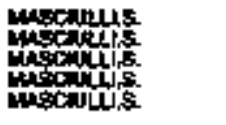 & 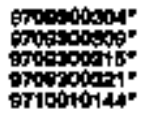 & $\begin{array}{l}400015 \\
40,0016 \\
400015 \\
400015\end{array}$ & & 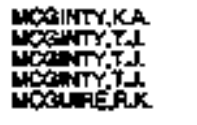 & 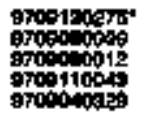 & 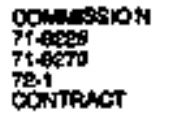 & 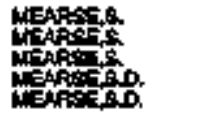 & 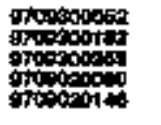 & 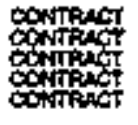 & \\
\hline 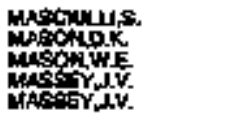 & 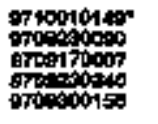 & 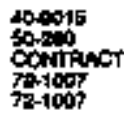 & ค & 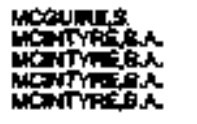 & 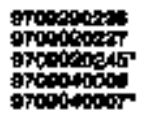 & 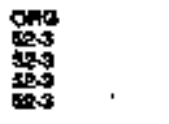 & 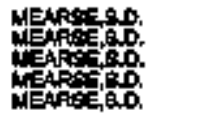 & 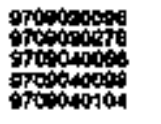 & 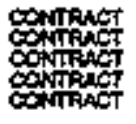 & \\
\hline 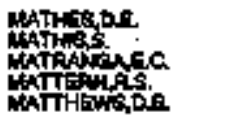 & 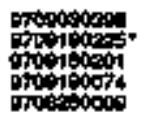 & 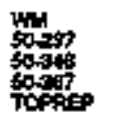 & 急 & 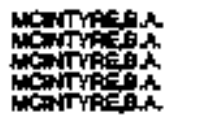 & 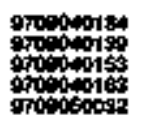 & $\begin{array}{l}509 \\
503 \\
503 \\
503\end{array}$ & 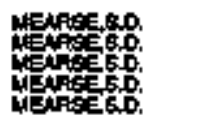 & 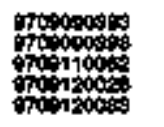 & 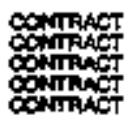 & \\
\hline 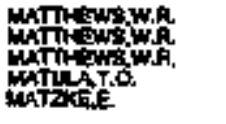 & 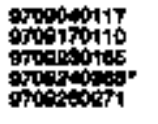 & 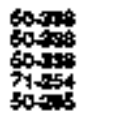 & $\begin{array}{l}\stackrel{8}{A} \\
\text { s } \\
s\end{array}$ & 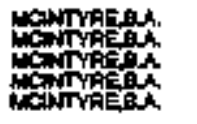 & 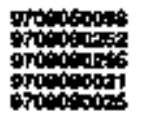 & 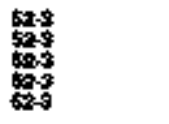 & 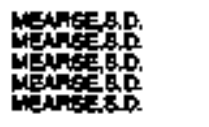 & 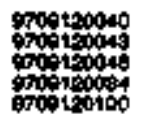 & 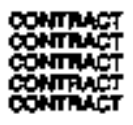 & \\
\hline 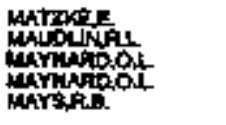 & 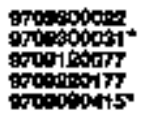 & 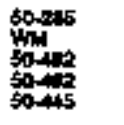 & 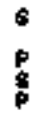 & 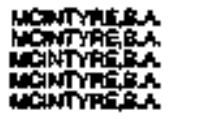 & 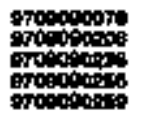 & 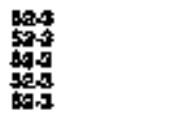 & 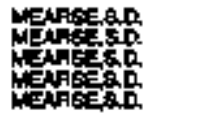 & 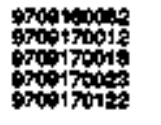 & 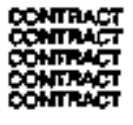 & \\
\hline marsere & 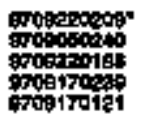 & 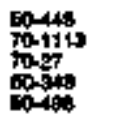 & n̂ & 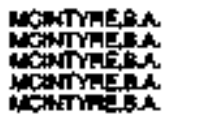 & 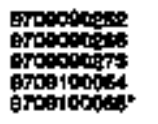 & $\frac{108}{608}$ & 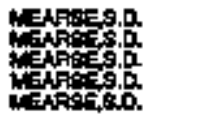 & 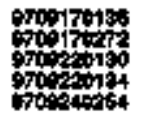 & 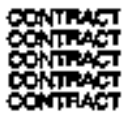 & \\
\hline 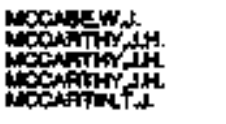 & 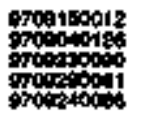 & 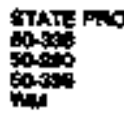 & $\begin{array}{l}5 \\
\mathbf{p} \\
\mathbf{p}\end{array}$ & 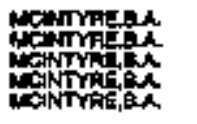 & 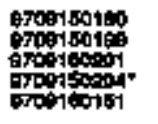 & $\begin{array}{l}103 \\
63\end{array}$ & 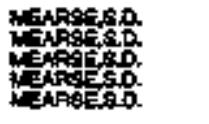 & 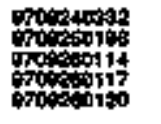 & 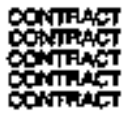 & \\
\hline 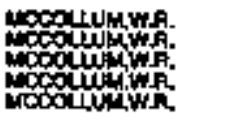 & 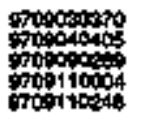 & 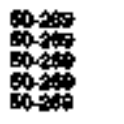 & $\stackrel{p}{p}$ & 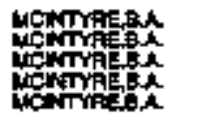 & Dropon & 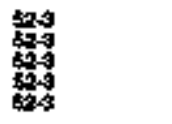 & 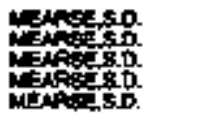 & 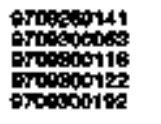 & 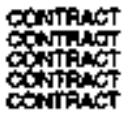 & \\
\hline 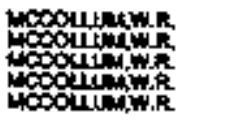 & 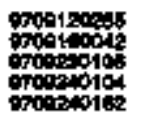 & 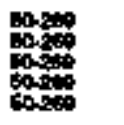 & $\begin{array}{l}\mathbf{p} \\
8 \\
8 \\
0\end{array}$ & 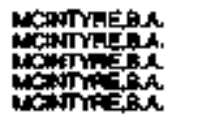 & 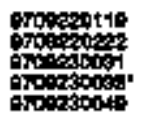 & 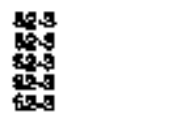 & 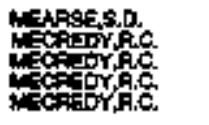 & 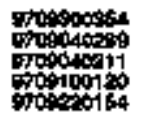 & conmpact & $\begin{array}{l}5 \\
5 \\
0 \\
0 \\
0\end{array}$ \\
\hline 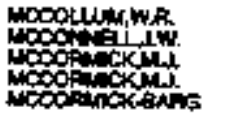 & 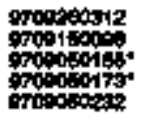 & 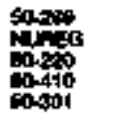 & $p$ & 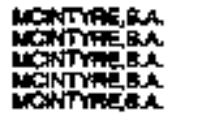 & 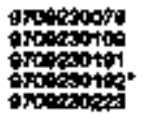 & $\begin{array}{l}52-3 \\
025 \\
063 \\
023\end{array}$ & 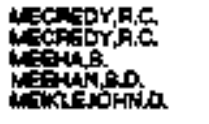 & 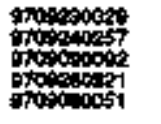 & 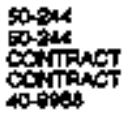 & p \\
\hline 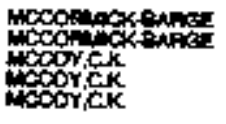 & 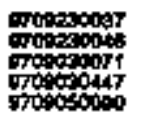 & 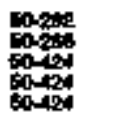 & $\begin{array}{l}\frac{\alpha}{9} \\
\text { o } \\
8\end{array}$ & 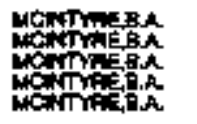 & 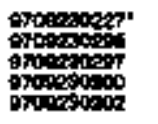 & 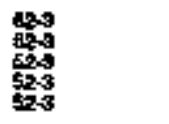 & miscon & 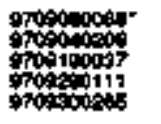 & 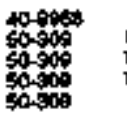 & 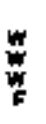 \\
\hline 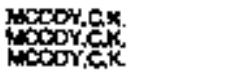 & 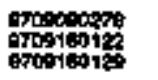 & 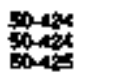 & $p_{5}^{p}$ & Hentractis & chospors & 率行 & 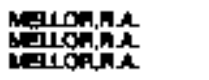 & 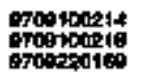 & 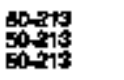 & $\begin{array}{l}\mathbf{s} \\
5 \\
\text { p }\end{array}$ \\
\hline
\end{tabular}




\begin{tabular}{|c|c|c|c|c|c|c|c|c|c|c|c|}
\hline alrom & "4 & 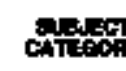 & & ANTHOM & Mecsons & sing & & Mring & 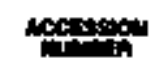 & 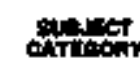 & \\
\hline 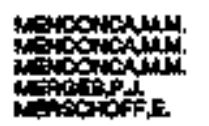 & 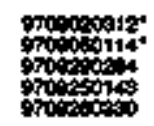 & 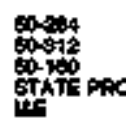 & 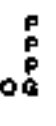 & 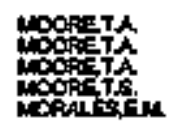 & 酐 & 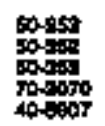 & 8 & 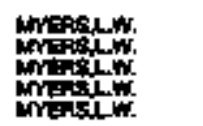 & 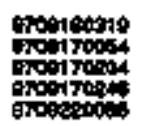 & 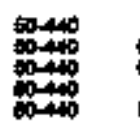 & $\begin{array}{l}p \\
8 \\
8 \\
p\end{array}$ \\
\hline 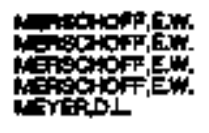 & 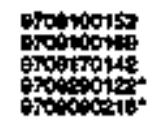 & 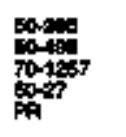 & $\begin{array}{l}\circ \\
P\end{array}$ & 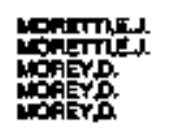 & 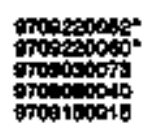 & 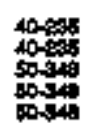 & $\stackrel{8}{\circ}$ & 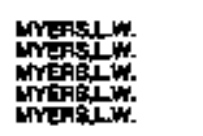 & 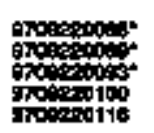 & $\begin{array}{l}50-400 \\
60.40 \\
50+40 \\
50+10 \\
50+10\end{array}$ & \\
\hline 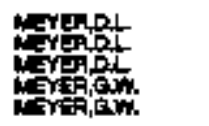 & 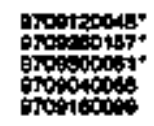 & 曹 & 8 & 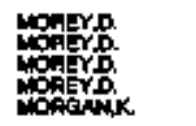 & 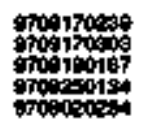 & 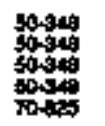 & $\begin{array}{l}\text { म } \\
\text { S } \\
8\end{array}$ & 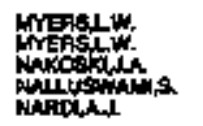 & 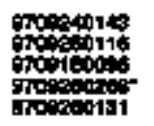 & 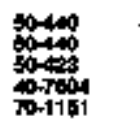 & s \\
\hline 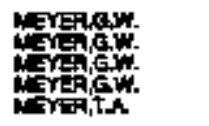 & 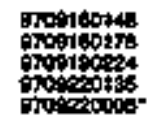 & 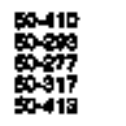 & $\begin{array}{l}y \\
8 \\
8 \\
0\end{array}$ & 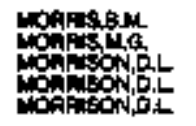 & 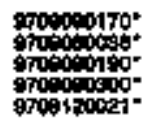 & 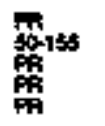 & $D$ & 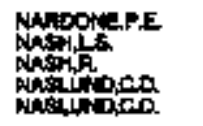 & 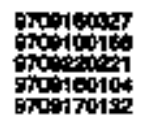 & 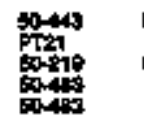 & $\mathbf{R}$ \\
\hline 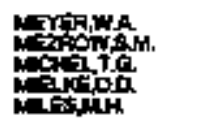 & 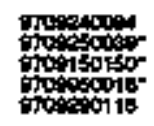 & 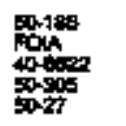 & p & 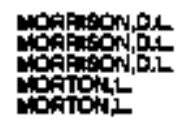 & 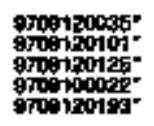 & 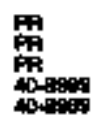 & & 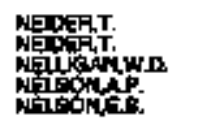 & 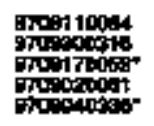 & 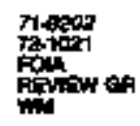 & \\
\hline 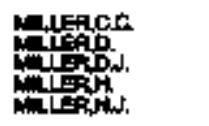 & 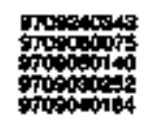 & 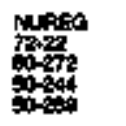 & $\stackrel{8}{8}$ & 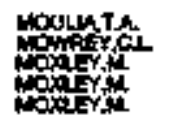 & 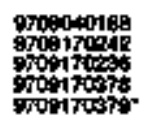 & 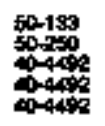 & $\mathbf{F}$ & 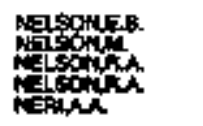 & 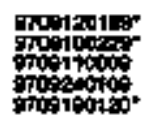 & 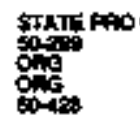 & $\stackrel{o g}{p}$ \\
\hline 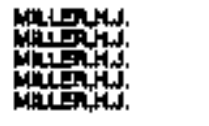 & 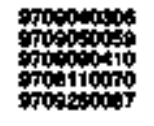 & 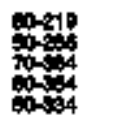 & $\begin{array}{l}8 \\
8\end{array}$ & 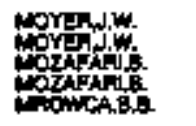 & 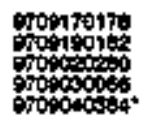 & 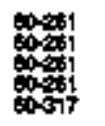 & 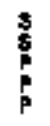 & 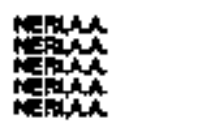 & 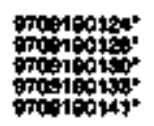 & 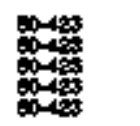 & 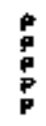 \\
\hline 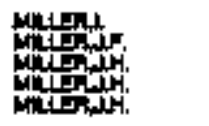 & 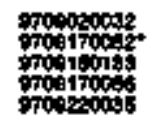 & 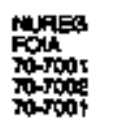 & & 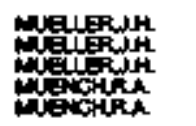 & 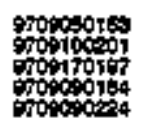 & 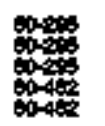 & 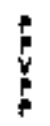 & 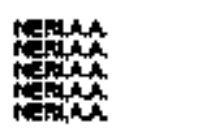 & 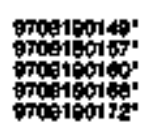 & 69y & $\stackrel{p}{?}$ \\
\hline 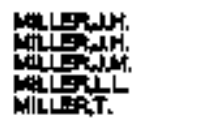 & 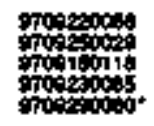 & 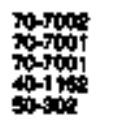 & 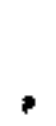 & 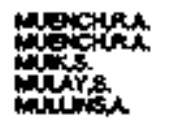 & 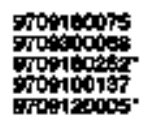 & 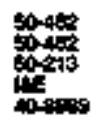 & p & 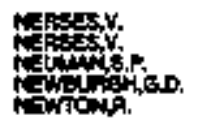 & 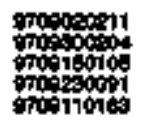 & 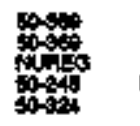 & $\underset{\mathbf{F}}{\mathbf{R}}$ \\
\hline 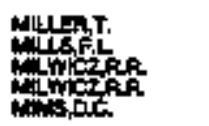 & 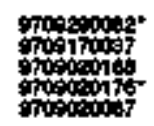 & 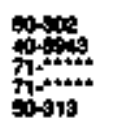 & + & must & 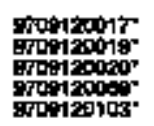 & 象 & & 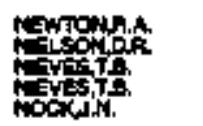 & 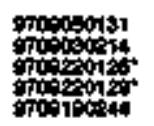 & 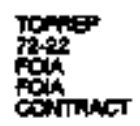 & \\
\hline 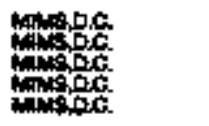 & 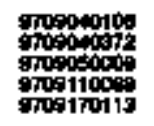 & 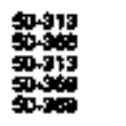 & 章 & 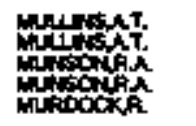 & 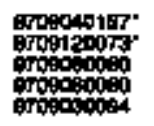 & 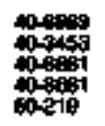 & g & 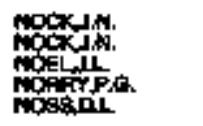 & 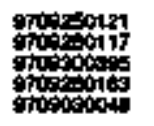 & 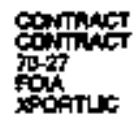 & \\
\hline tion. & 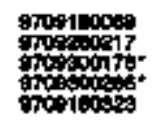 & 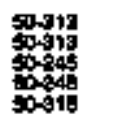 & 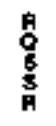 & 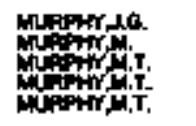 & 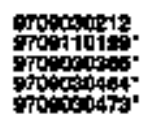 & 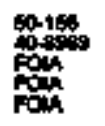 & म & 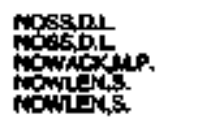 & 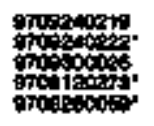 & 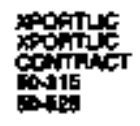 & p \\
\hline thondwi & 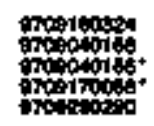 & 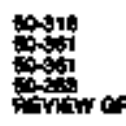 & 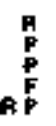 & mpoprint. & 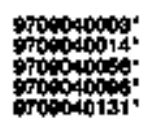 & 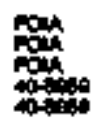 & & Musuce & 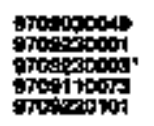 & 舟 & $\frac{0}{p}$ \\
\hline 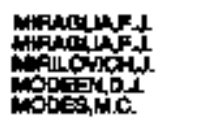 & 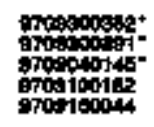 & 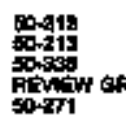 & 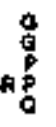 & 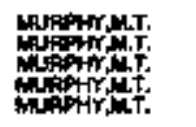 & 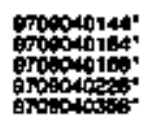 & 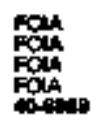 & & 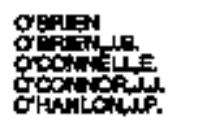 & 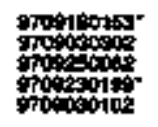 & 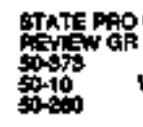 & 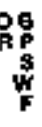 \\
\hline 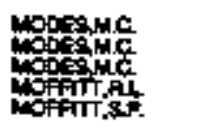 & 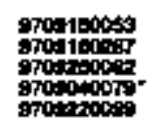 & 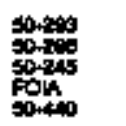 & $g$ & 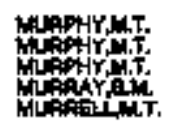 & 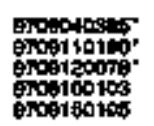 & 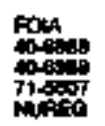 & & 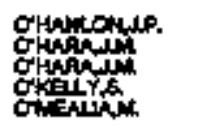 & 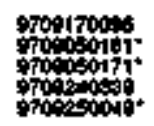 & 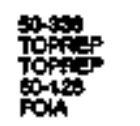 & $r$ \\
\hline 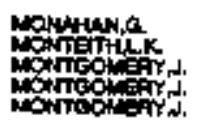 & 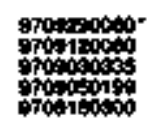 & 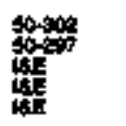 & ; & 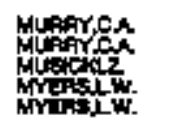 & 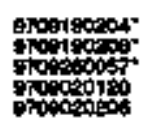 & 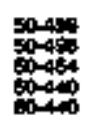 & $\begin{array}{l}0 \\
8 \\
8\end{array}$ & 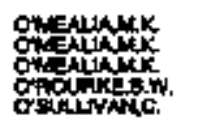 & 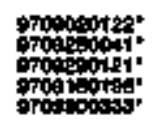 & 我 & ร్ \\
\hline 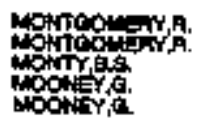 & 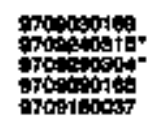 & 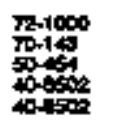 & p & 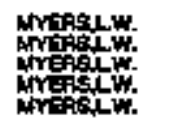 & 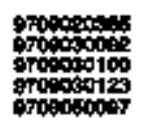 & $\begin{array}{l}60-40 \\
50+40 \\
50+40 \\
50+40 \\
50+40\end{array}$ & $\begin{array}{l}0 \\
+ \\
0 \\
0\end{array}$ & 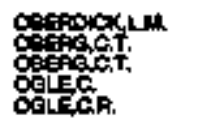 & 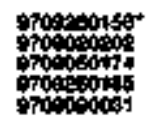 & 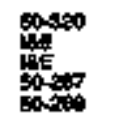 & $P$ \\
\hline$x_{0 \rightarrow 0}=x$ & 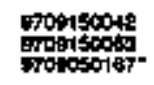 & 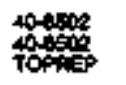 & & 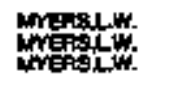 & 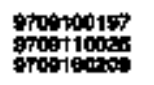 & $\begin{array}{l}50900 \\
50+400 \\
50-400\end{array}$ & $\frac{8}{5}$ & 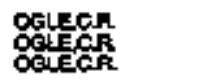 & 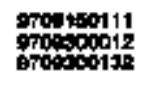 & sasp & g \\
\hline
\end{tabular}




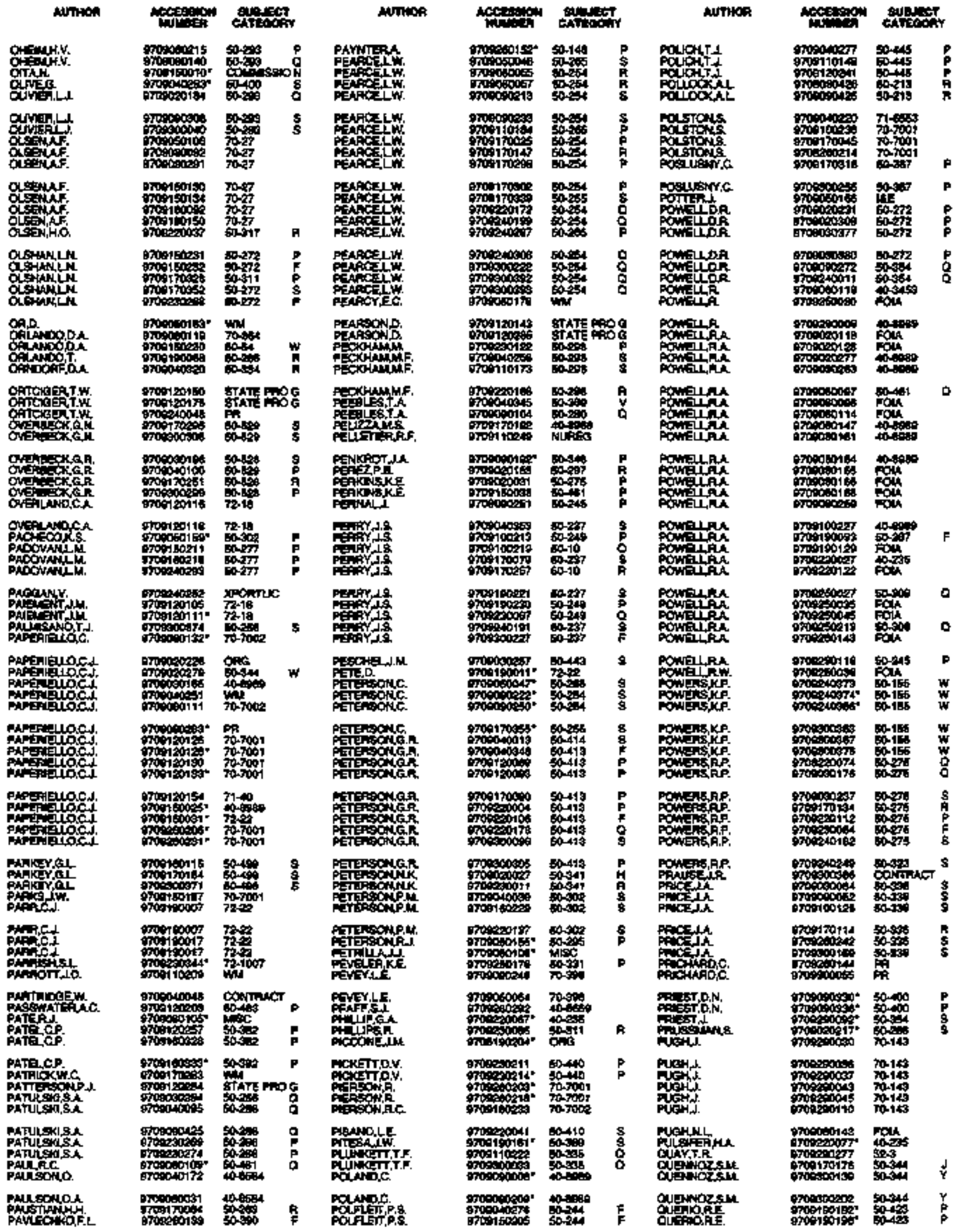




\begin{tabular}{|c|c|c|c|c|c|c|c|c|c|c|c|}
\hline AUTHON & in & 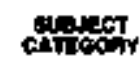 & & MTHOM & A & 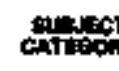 & & Aurntoph & stom & Strivest & \\
\hline 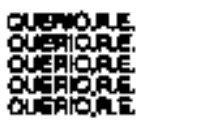 & 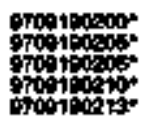 & 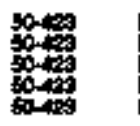 & p & 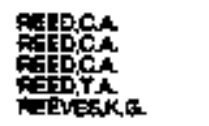 & 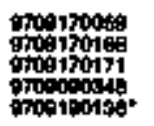 & 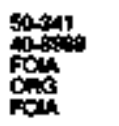 & p & 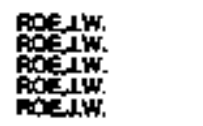 & 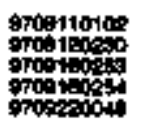 & 幽 & $\begin{array}{l}v \\
8 \\
8\end{array}$ \\
\hline 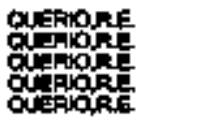 & 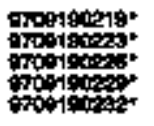 & 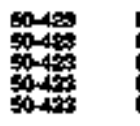 & p & 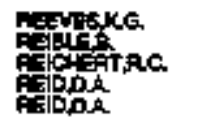 & 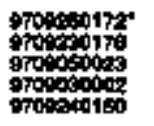 & 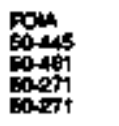 & $\begin{array}{l}\text { R } \\
8 \\
8 \\
0\end{array}$ & 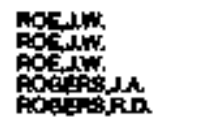 & 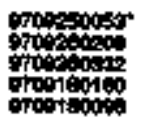 & 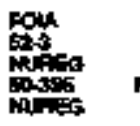 & n \\
\hline 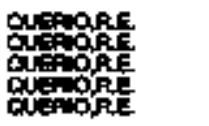 & 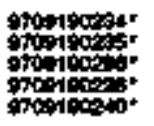 & 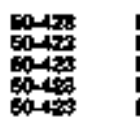 & & 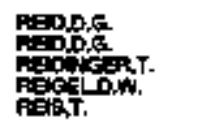 & 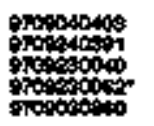 & 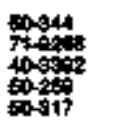 & b & 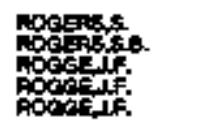 & 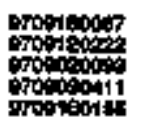 & 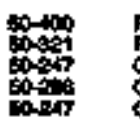 & $\begin{array}{l}\text { R } \\
\text { : } \\
8 \\
8\end{array}$ \\
\hline 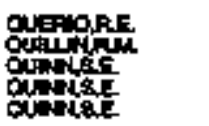 & 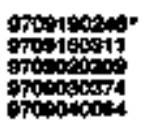 & 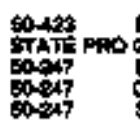 & 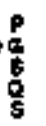 & 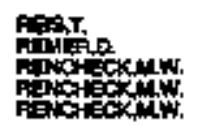 & 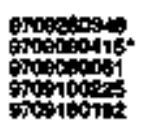 & 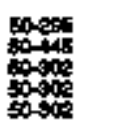 & $\begin{array}{l}9 \\
8 \\
8 \\
8\end{array}$ & 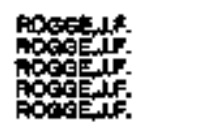 & 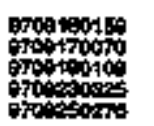 & 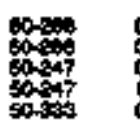 & $\begin{array}{l}0 \\
0 \\
0 \\
0\end{array}$ \\
\hline 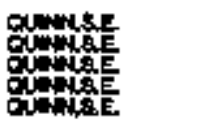 & 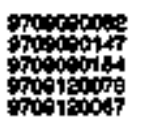 & 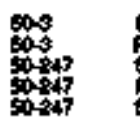 & $\begin{array}{l}6 \\
8 \\
8 \\
8 \\
8\end{array}$ & 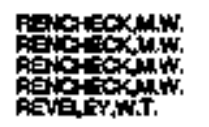 & 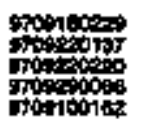 & 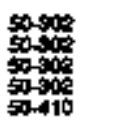 & 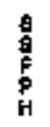 & 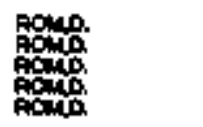 & 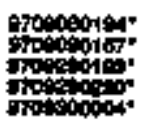 & 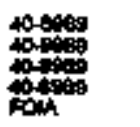 & \\
\hline 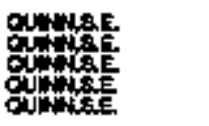 & 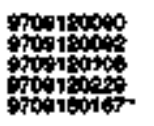 & 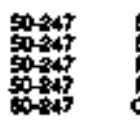 & 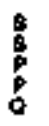 & 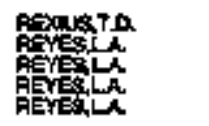 & 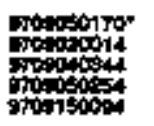 & 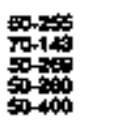 & g. & 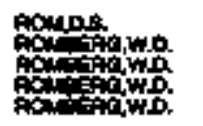 & 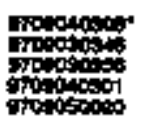 & 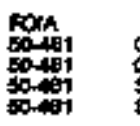 & $\begin{array}{l}a \\
0 \\
b \\
3\end{array}$ \\
\hline 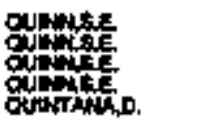 & 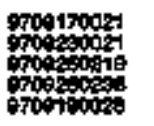 & 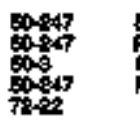 & $\begin{array}{l}5 \\
\qquad \\
\\
\end{array}$ & 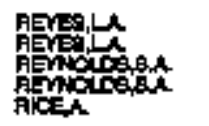 & 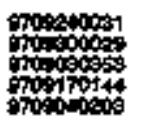 & 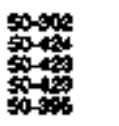 & $\begin{array}{l}\text { g } \\
\text { p } \\
\text { g }\end{array}$ & 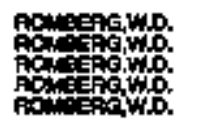 & 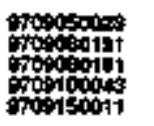 & 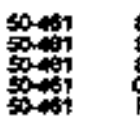 & $\begin{array}{l}8 \\
8 \\
8 \\
0 \\
\end{array}$ \\
\hline 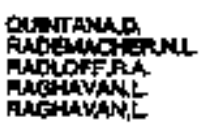 & 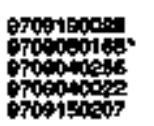 & 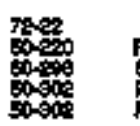 & म & 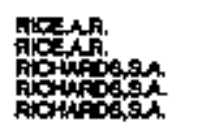 & 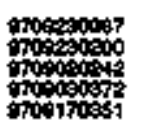 & 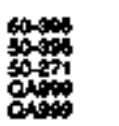 & g & 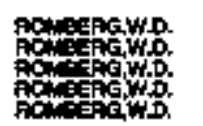 & 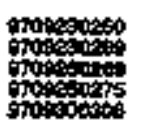 & 我 & $\begin{array}{l}8 \\
8 \\
8 \\
8 \\
8 \\
8\end{array}$ \\
\hline 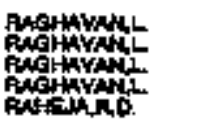 & 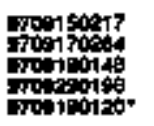 & 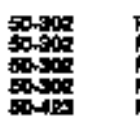 & p & 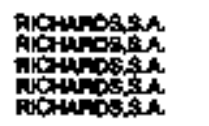 & 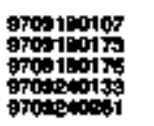 & 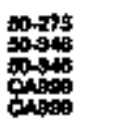 & $\stackrel{8}{8}$ & 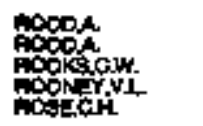 & 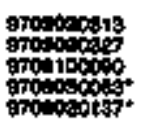 & $\frac{\operatorname{mag}}{400}$ & $=$ \\
\hline 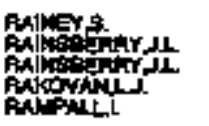 & 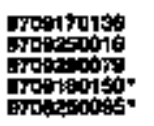 & 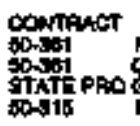 & : & 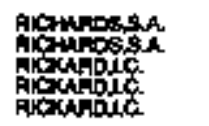 & 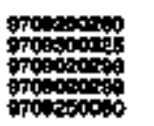 & 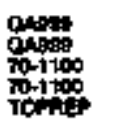 & & 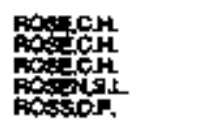 & 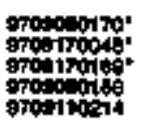 & mente & F \\
\hline ponguk & 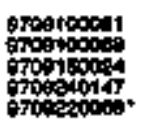 & 然 & $s$ & 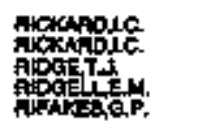 & 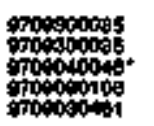 & 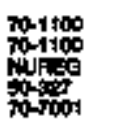 & 5 & 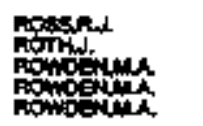 & 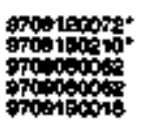 & 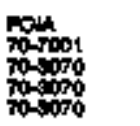 & \\
\hline 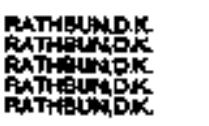 & 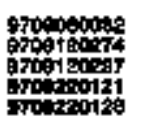 & 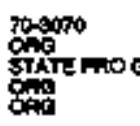 & & 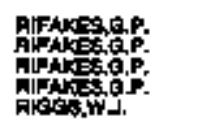 & 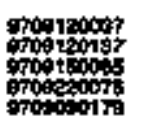 & 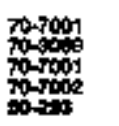 & n & 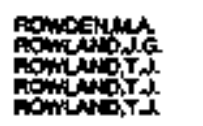 & 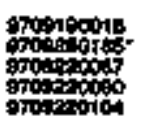 & 舟 & $w$ \\
\hline 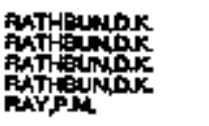 & 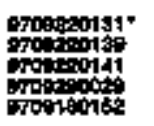 & 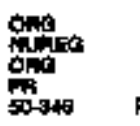 & $P$ & 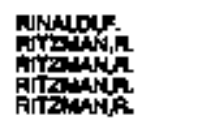 & 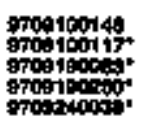 & 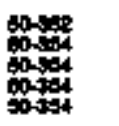 & $\begin{array}{l}\mathbf{p} \\
\mathbf{s}_{\mathbf{5}} \\
\mathbf{n} \\
\mathbf{8}\end{array}$ & 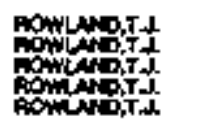 & 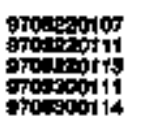 & 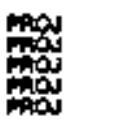 & \\
\hline 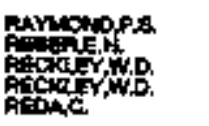 & 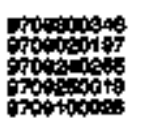 & 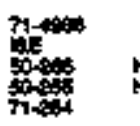 & H & 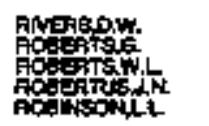 & 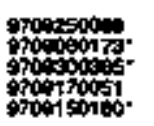 & 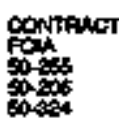 & s. & 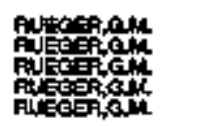 & 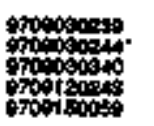 & 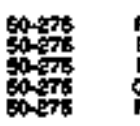 & p \\
\hline 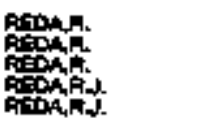 & 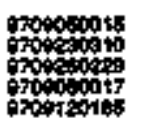 & 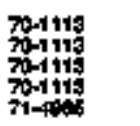 & & 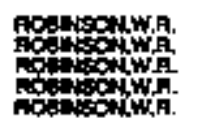 & 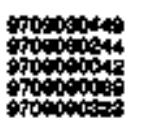 & 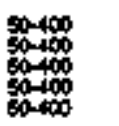 & $\frac{g}{y}$ & 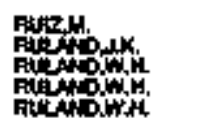 & 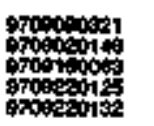 & 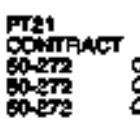 & 8 \\
\hline 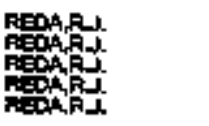 & 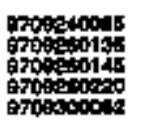 & 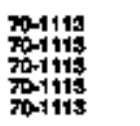 & & 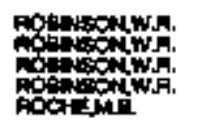 & 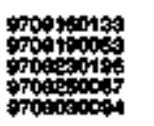 & 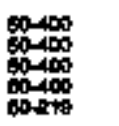 & $\begin{array}{l}p \\
9 \\
8 \\
8 \\
8\end{array}$ & 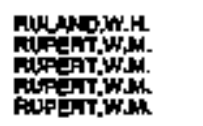 & 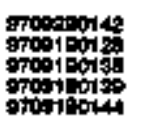 & 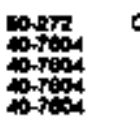 & 0 \\
\hline 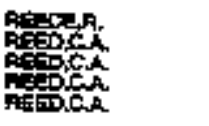 & 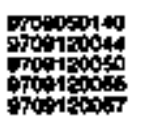 & 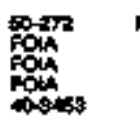 & $F$ & 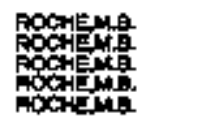 & 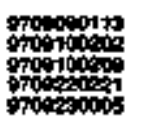 & 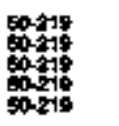 & $\begin{array}{l}8 \\
8 \\
0 \\
8 \\
8 \\
5\end{array}$ & 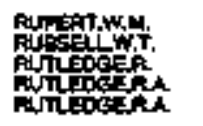 & 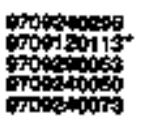 & 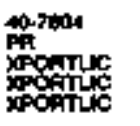 & \\
\hline 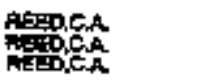 & 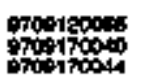 & roa & & 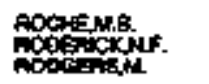 & 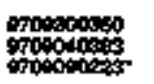 & carese & 务 & 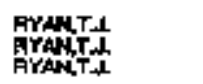 & oronserses & 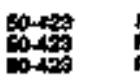 & 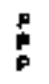 \\
\hline
\end{tabular}




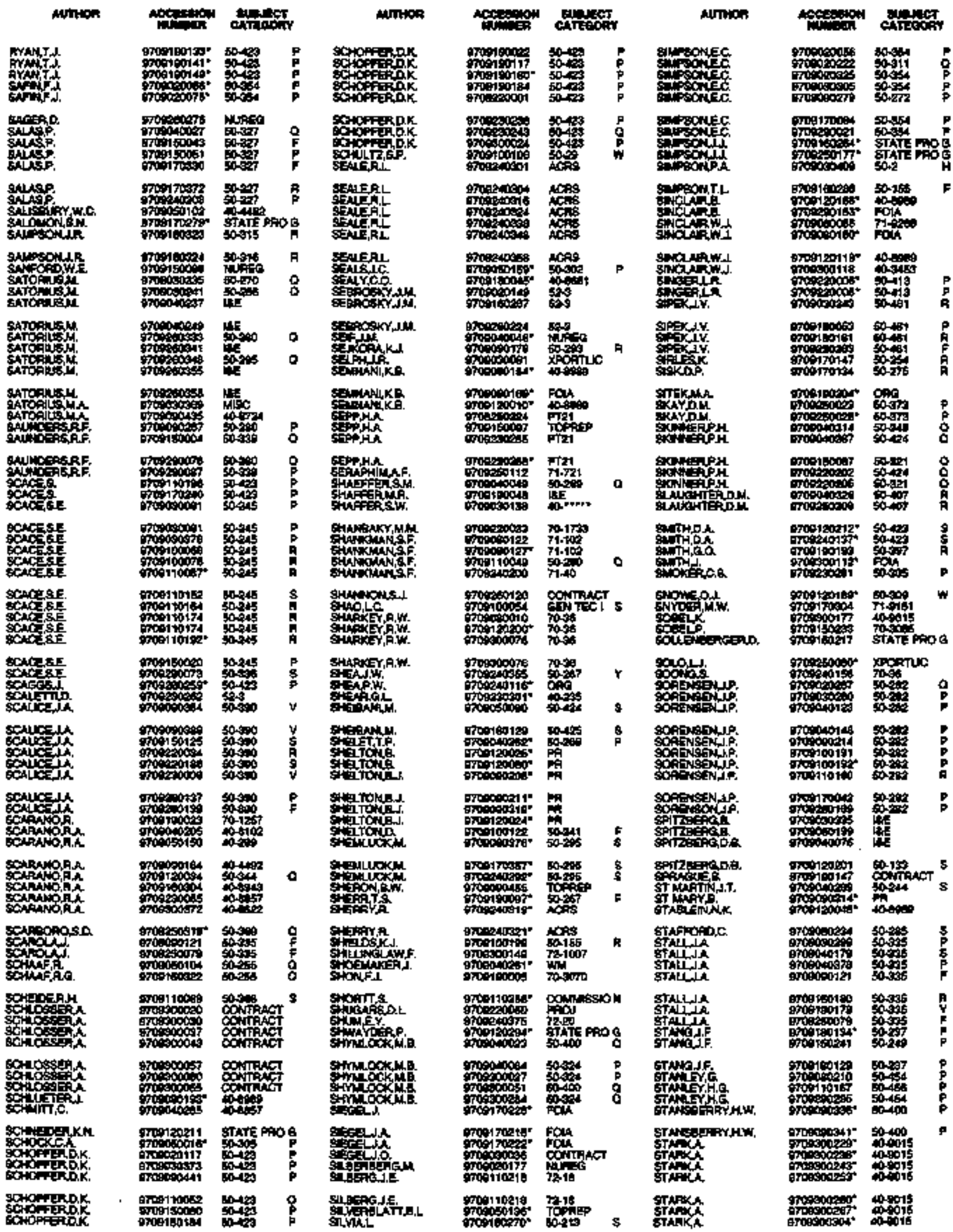




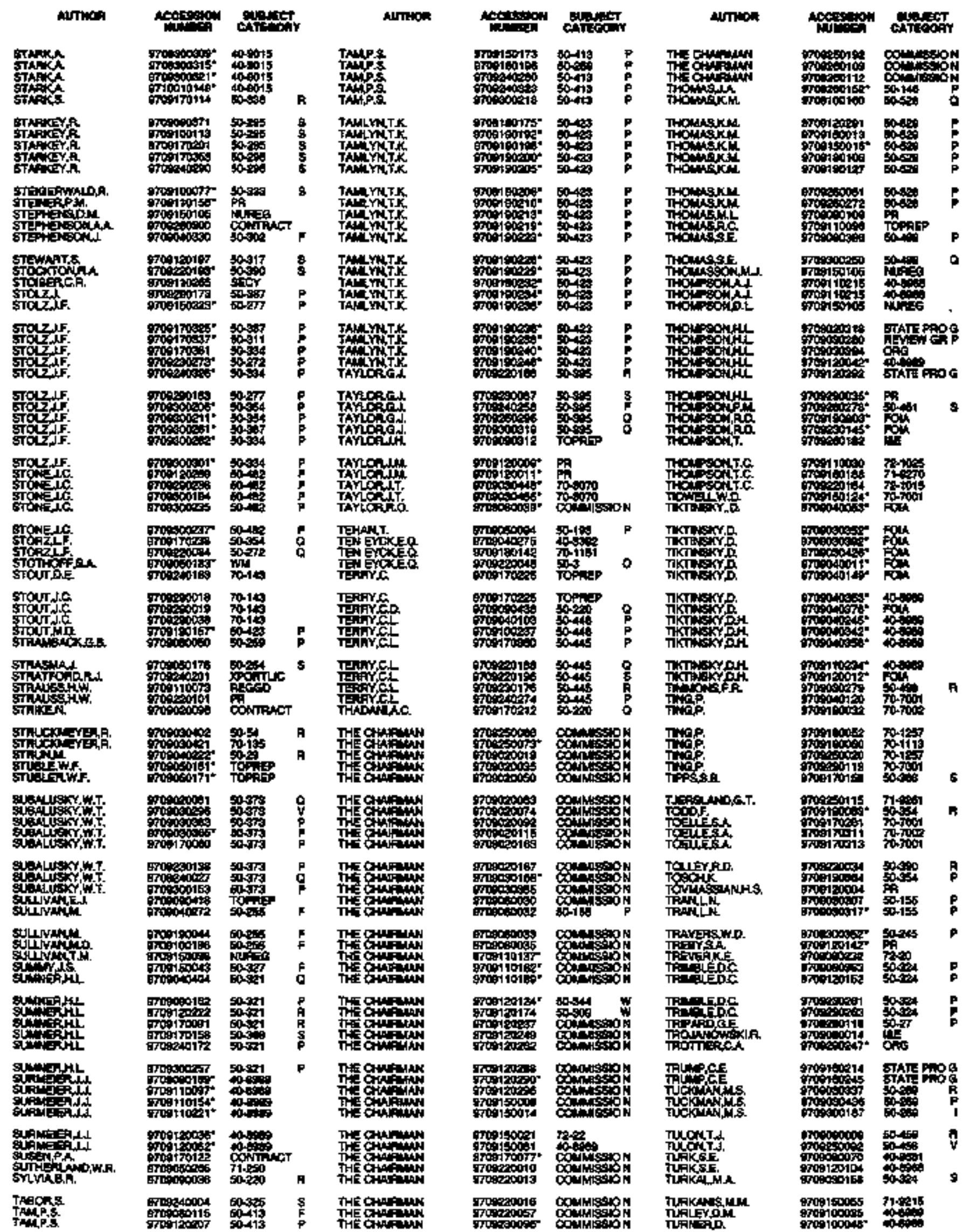




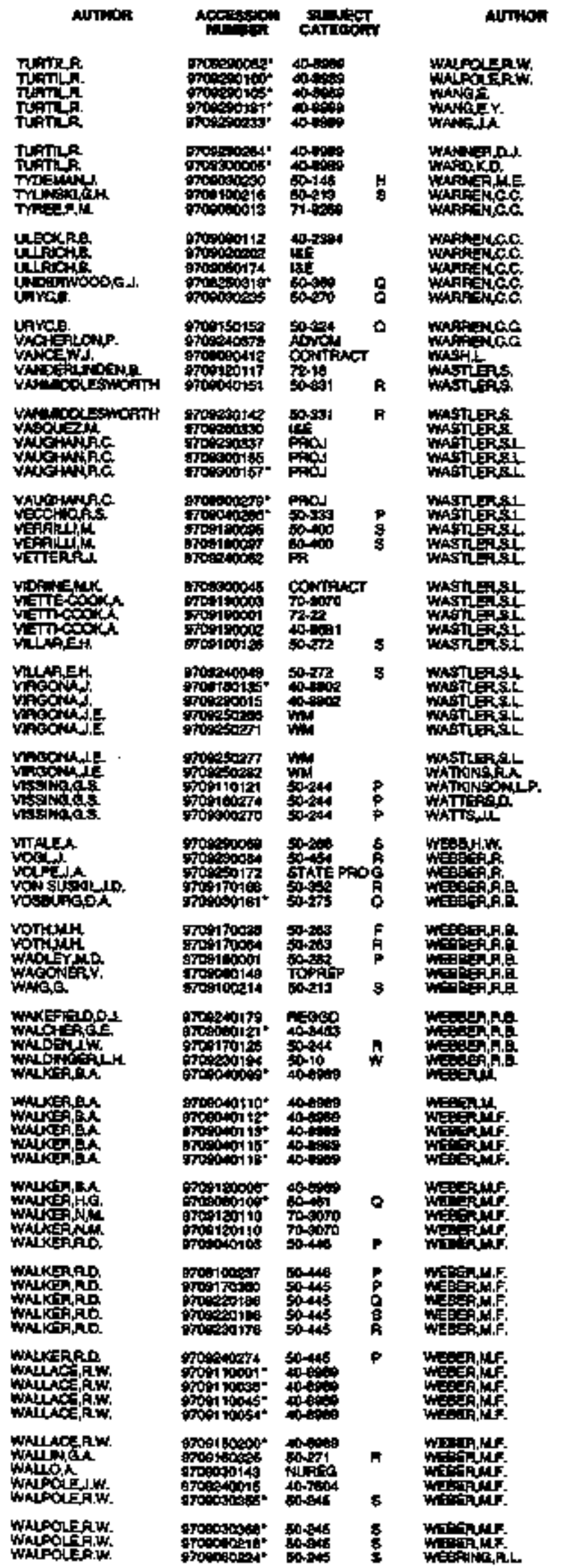

\begin{tabular}{|c|c|c|c|}
\hline 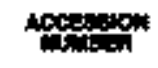 & 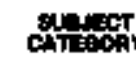 & & Антюсн \\
\hline 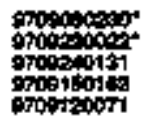 & 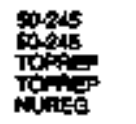 & 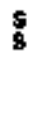 & 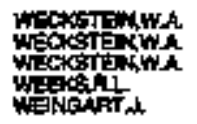 \\
\hline 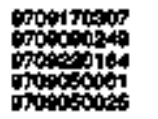 & 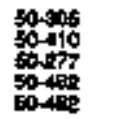 & $\begin{array}{l}8 \\
8 \\
8 \\
8\end{array}$ & 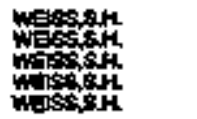 \\
\hline 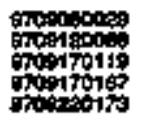 & $\begin{array}{l}60-462 \\
0-462 \\
50-482 \\
50-40 \\
50-40\end{array}$ & $\begin{array}{l}5 \\
\mathbf{p} \\
\mathbf{p} \\
\mathbf{p} \\
\mathbf{p}\end{array}$ & 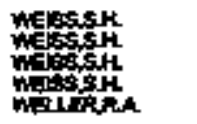 \\
\hline 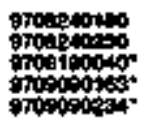 & 我 & $\$$ & 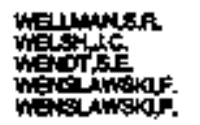 \\
\hline 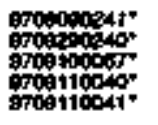 & 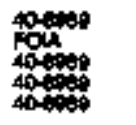 & & 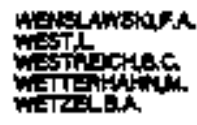 \\
\hline 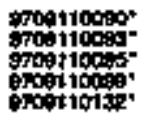 & 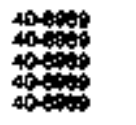 & & 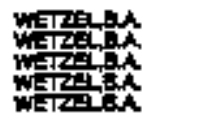 \\
\hline 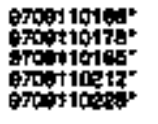 & 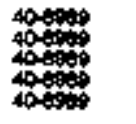 & & 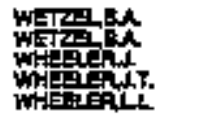 \\
\hline 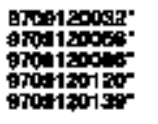 & 我 & & 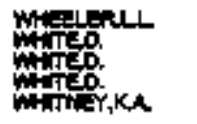 \\
\hline 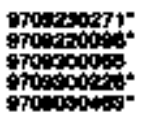 & 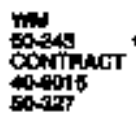 & $\begin{array}{l}0 \\
\mathrm{~s}\end{array}$ & 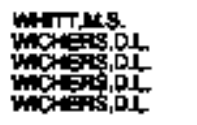 \\
\hline 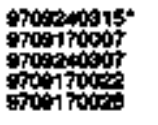 & 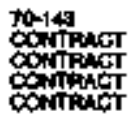 & & 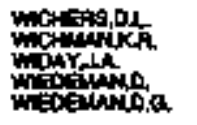 \\
\hline 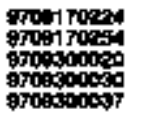 & 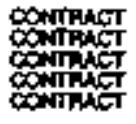 & & 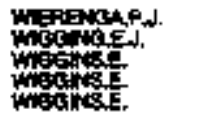 \\
\hline 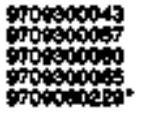 & 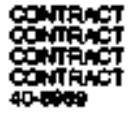 & & 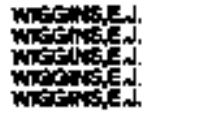 \\
\hline 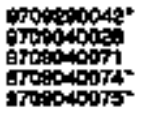 & $\frac{409}{700}$ & & 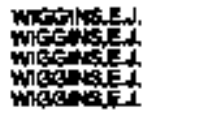 \\
\hline 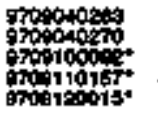 & 㔔1143 & & 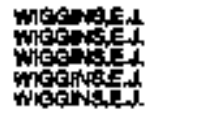 \\
\hline 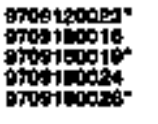 & 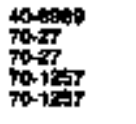 & & 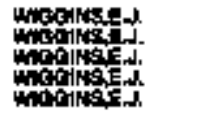 \\
\hline 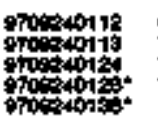 & 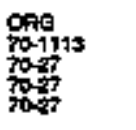 & & . \\
\hline 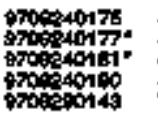 & 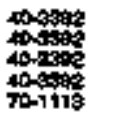 & & 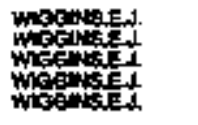 \\
\hline 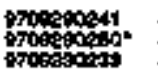 & $\begin{array}{l}40.7 \% 8 \\
0.7+\infty\end{array}$ & * & 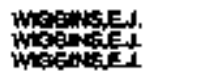 \\
\hline
\end{tabular}

Accosen civist

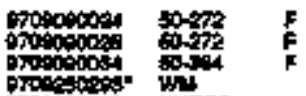

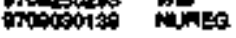

prosocor sogis

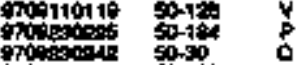

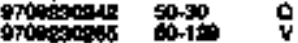

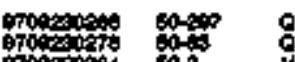

trootion

0700000170.00000

0000000 i

(1)

s7oponour

tom

Dor

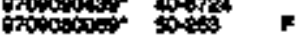

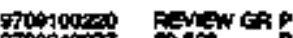

T00,

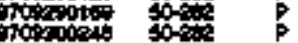

pogones: socas p

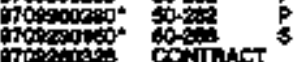

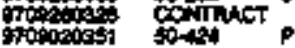

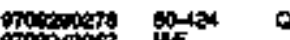

\%00001\%

Thositiona 9

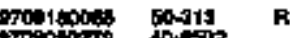

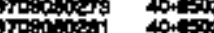

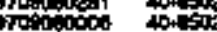

Tropocos 40

Frogeors1 400002

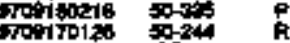

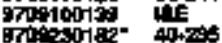

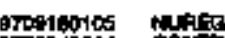

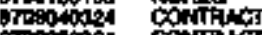

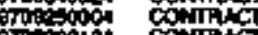

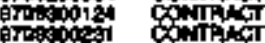

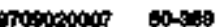

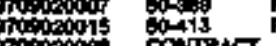

Thos contret

${ }_{10}$

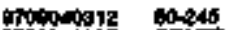

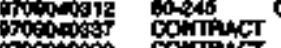

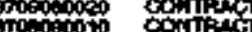

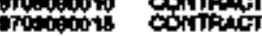

g70000009 corminct

Dotertoos.

G00

sropinopes contract

roposes conmets

Fin

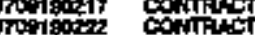

moricoes4 conmact

Nonesos contruc

Neavos

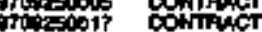

mostons contract

To on

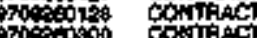

moososisto CxwTsacy

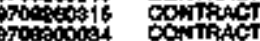




\begin{tabular}{|c|c|c|c|c|c|c|c|c|c|c|}
\hline Nmnon & A & andestict & & Allmion & Accecien & 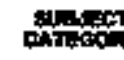 & & Al/miton & 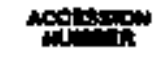 & 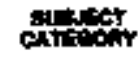 \\
\hline 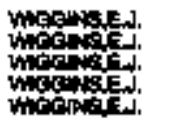 & 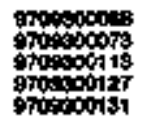 & 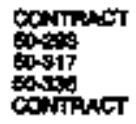 & $\begin{array}{l}y \\
y\end{array}$ & 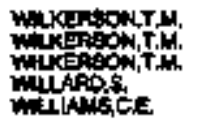 & 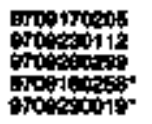 & 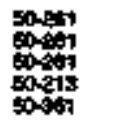 & $\begin{array}{l}\mathbf{p} \\
\mathbf{F} \\
\mathbf{8} \\
\mathbf{8}\end{array}$ & 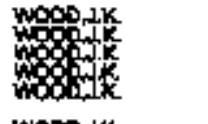 & 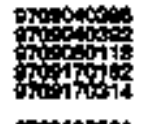 & 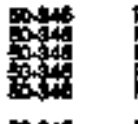 \\
\hline 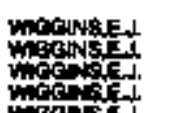 & 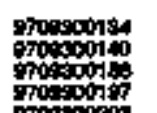 & 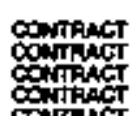 & & 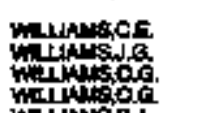 & 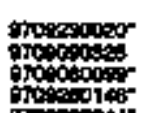 & 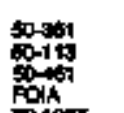 & h & 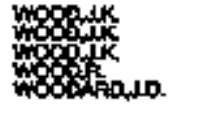 & 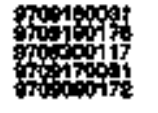 & 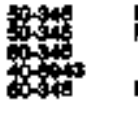 \\
\hline 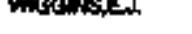 & ropasoens & COWHAMT & & $\mathrm{n}=\mathrm{N}$ & Eropes & $72 \cdot 100$ & & & & \\
\hline Woposict & 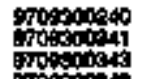 & $\begin{array}{l}0.244 \\
0.41 \\
0.494\end{array}$ & 8 & 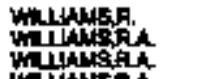 & 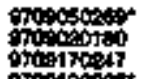 & 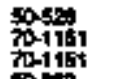 & $\boldsymbol{F}$ & 䃕 & & \\
\hline Trets & 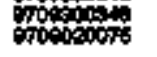 & 506 & $\mathbf{p}$ & Whingsin & Troposto & $50-13$ & h & & 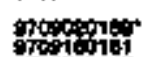 & \\
\hline mescinst. & 20000009 & $50-244$ & : & 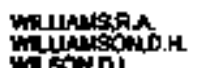 & porichos. & 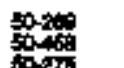 & ค & & & \\
\hline Hor TON & oposolsos & 50150 & $\mathbf{p}$ & 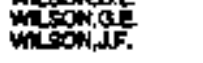 & 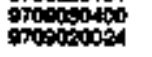 & 50 & F & & & \\
\hline Whesentontes & gromosocos & 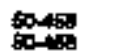 & $\stackrel{p}{p}$ & mostur & 700004 & Topep & & & & \\
\hline 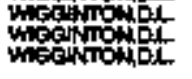 & 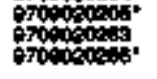 & tont & P & Hotith & 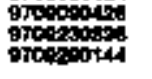 & 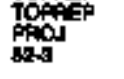 & & & 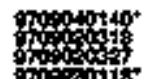 & 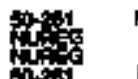 \\
\hline Wentintor & proosuch & 络45 & p & Whongun & $20011000 \%$ & Fonk & & & & \\
\hline . & oropoct & 象象 & 0 & 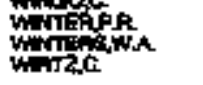 & 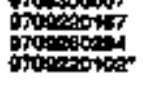 & totion & $\mathbf{v}$ & in & 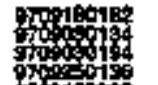 & \\
\hline mity & 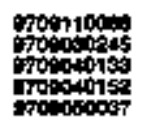 & 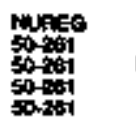 & $\begin{array}{l}y \\
\text { p } \\
\text { p }\end{array}$ & 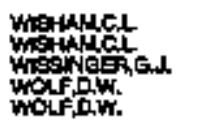 & 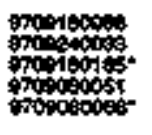 & 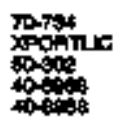 & $\mathbf{P}$ & 䣏: & 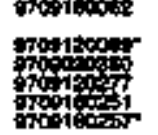 & 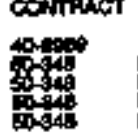 \\
\hline 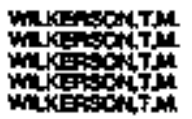 & 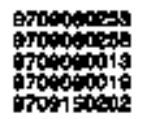 & sol & $\begin{array}{l}\mathbf{p} \\
\mathbf{p} \\
\mathbf{f} \\
\mathbf{p}\end{array}$ & 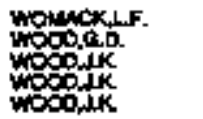 & 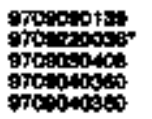 & 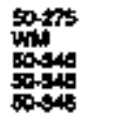 & 8 & 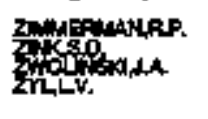 & 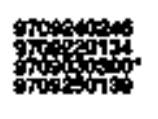 & 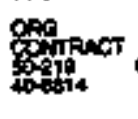 \\
\hline
\end{tabular}




\section{CORPORATE SOURCE INDEX}

The subject category column in this index gives the docket number and the symbol for the category of Informatlon within that docket; for exampla,50-232 P. The docket subject categories are defined in Appendix A.

Subject categories for nondocketed items are tiso entered in this column by symbol; for example, ACRS.

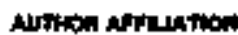

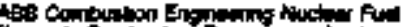

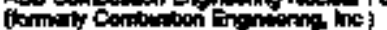

MgEA Brom Bowher, ke

AII Prominaral Cokp

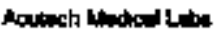

Anoterou Cop

Anences Abocation of Onusy

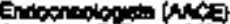

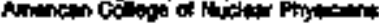

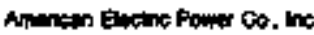

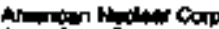

AUn.

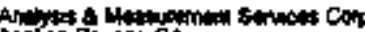

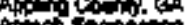

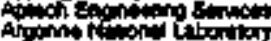

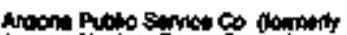

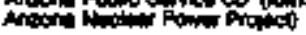

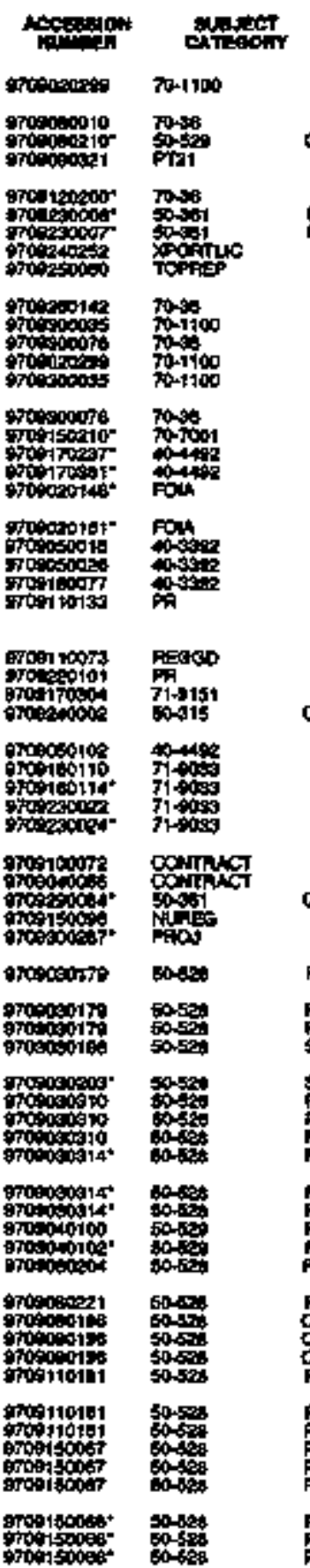

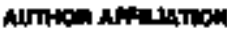

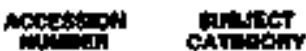

proptsition

oroditions

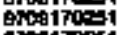

sodinues

mastoon

moascost

oxpanocor

orocolotion

opontopos

500000

$5 x+2009$

prosentos

sropesools

stooswoils

(1)

toto

orosanopis:

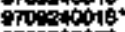

970 siones

9700000:-2

$9 \mathrm{POCNOOP}=$

970000099

mopocats

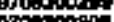

oropoinsta

Arwich Sitw of

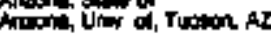

propey

Toiviar

ropis 10

ordontos

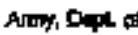

Afry, Doper of, Yum Prowag Ground

Q

gonesot3a

Atotions

axpopipis?

stocentas"

solsto

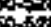

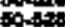

$0-40$

50-60:

the

$+\infty$

sitio

50

stit.

$+4$

sosid

50-60.

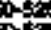

安

S06

50

o-

sos.

$50+52$

ERE?:

E0-5

Ea-5:

and

50-609

STATE RAO

bo-113

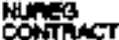

$40-10$

40691

$40-345$

$40-345$

$40-3450$

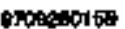

900200

most:012

mosis

(1)

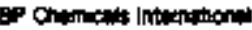

Ixo919014

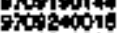

orotions

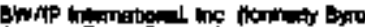

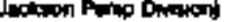

sma dationg

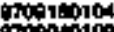

70s0soido.

propopipa

$40-3<5$

40

40.700

40.7504

$40-7004$

idion

क्षेत्र

독

TCyins

T0.2

intor

970000021

grooracose

rich

ropropes

$70-7$

conitine

rom

70-14

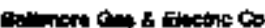

97000035

ponosola

tro

Poonto

170

reopenosey. 9700000 roostod

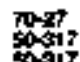

sol?

$50-317$

50ㄴㄱㄴ 


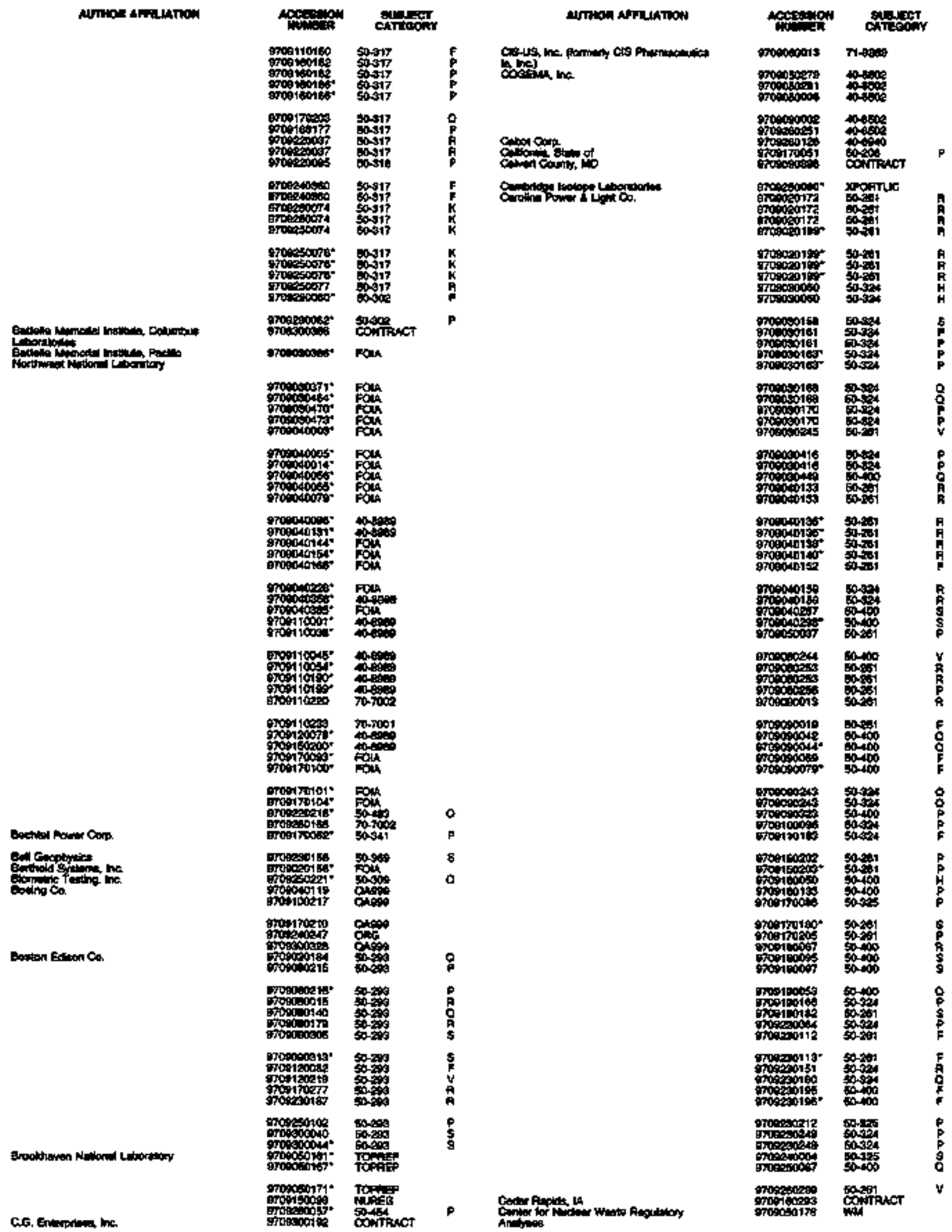




\begin{tabular}{|c|c|c|c|}
\hline 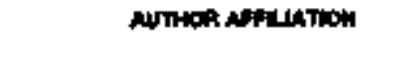 & 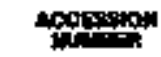 & chition & \\
\hline \multirow[t]{9}{*}{ Cortaror then } & 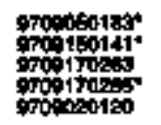 & (1) & s \\
\hline & 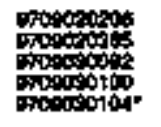 & $\begin{array}{l}50-40 \\
50+40 \\
5 \alpha+40 \\
50+40 \\
50-40\end{array}$ & P \\
\hline & 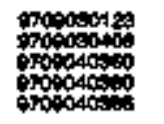 & 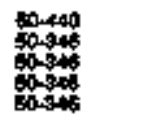 & $\begin{array}{l}9 \\
G \\
\mathbf{R} \\
\mathbf{P}\end{array}$ \\
\hline & 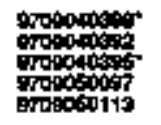 & $\begin{array}{l}50-448 \\
50-348 \\
00-346 \\
50-440 \\
00-46\end{array}$ & $\begin{array}{l}p \\
? \\
? \\
? \\
?\end{array}$ \\
\hline & 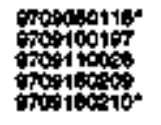 & $\begin{array}{c}00340 \\
0-40 \\
0-40 \\
0 \rightarrow 40 \\
0-40\end{array}$ & $\begin{array}{l}\mathbf{5} \\
0 \\
5 \\
p \\
p\end{array}$ \\
\hline & 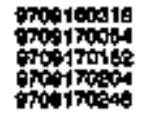 & 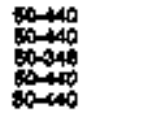 & $\begin{array}{l}8 \\
0 \\
0 \\
p\end{array}$ \\
\hline & 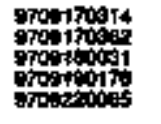 & $\begin{array}{l}50.340 \\
50.440 \\
50.340 \\
50.40 \\
50.40\end{array}$ & $\begin{array}{l}\stackrel{p}{P} \\
\stackrel{P}{F} \\
\stackrel{p}{\mathbf{p}}\end{array}$ \\
\hline & 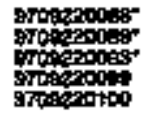 & $\begin{array}{l}50-40 \\
50-40 \\
50-40 \\
50-40 \\
50-40\end{array}$ & $\begin{array}{l}\mathbf{R} \\
\mathbf{R} \\
\mathbf{t} \\
\mathbf{6}\end{array}$ \\
\hline & 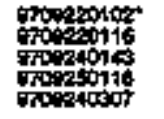 & 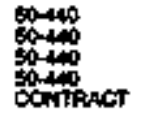 & $\begin{array}{l}\mathrm{y} \\
\mathrm{y} \\
\mathbf{s}\end{array}$ \\
\hline \multirow[t]{2}{*}{ 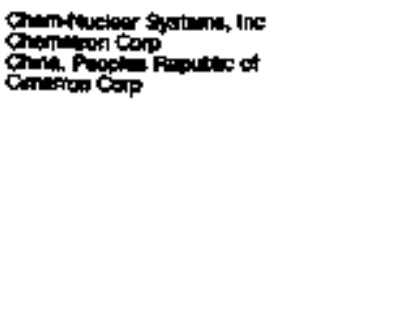 } & 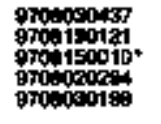 & 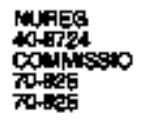 & $\mathbf{N}$ \\
\hline & 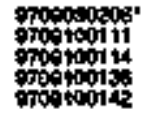 & 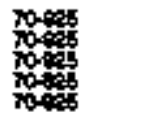 & \\
\hline Conlantwin ine & 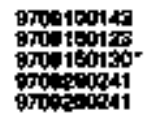 & 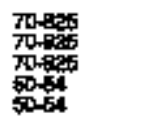 & $\underset{w}{w}$ \\
\hline 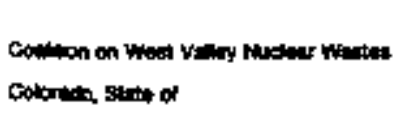 & 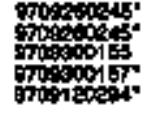 & 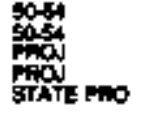 & w \\
\hline 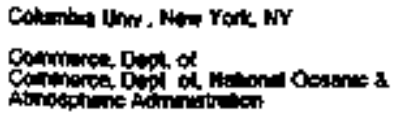 & 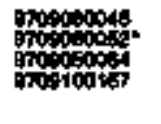 & 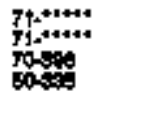 & D \\
\hline \multirow[t]{4}{*}{ 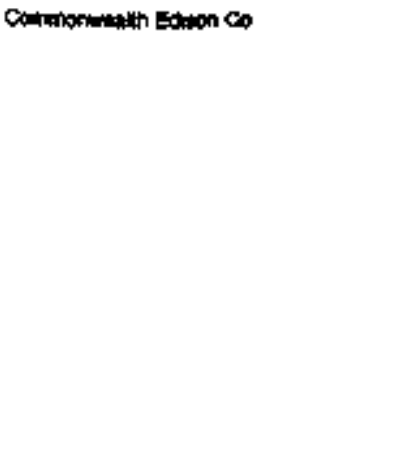 } & 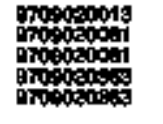 & 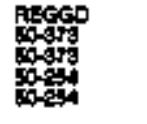 & $\begin{array}{l}9 \\
g \\
p\end{array}$ \\
\hline & 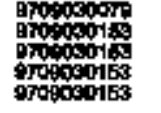 & 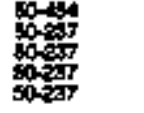 & $\begin{array}{l}\mathbf{F} \\
\mathbf{R} \\
\mathbf{R} \\
\mathbf{R}\end{array}$ \\
\hline & 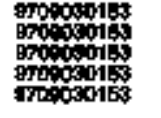 & 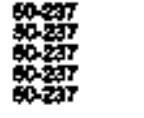 & $\frac{\mathbf{n}}{\mathbf{n}}$ \\
\hline & 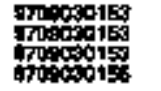 & 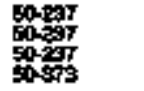 & $\begin{array}{l}\mathbf{R} \\
\mathbf{R} \\
\mathbf{R} \\
\mathbf{R}\end{array}$ \\
\hline
\end{tabular}

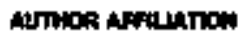

100

iver

50.5

orocostes bo-

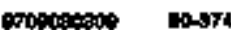

octor

pos.

acom

meanary

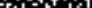

970004040

000000

orotionst

rrososos

Totosos

orosect

rrotosiones

$9 \operatorname{mocos} 1 \mathrm{~s}$

$0001 \%$

$001 \%$

To60\%

sropesooce

grogeopos

troperces

oreposofor

onsosolos

spotopiog.

Thorios:

Donos.

Troosonos

40

moovents

0700014

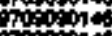

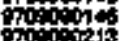

.

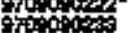

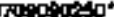

40.0.

009100113

8 성 100115.

Eros 100ect

Frog 10003

snoposes

Notot

ow 100

oxp1003

Droo1socon

opot rose

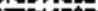

GJor 1002

aros inas

ordent

Frogit 100

groprtioges

100

Tर्व 1016

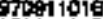

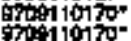

50110175-

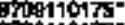

gog191:

tosionto

Pror 101:4

prog10001

suscosos

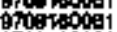

9rontoder

groptaboez-

7ronecos:

7 70900

50에

tors

$50-204$

$00-25$

so-ese

on

ofot

sonos

sones

mats

$00-44$

so-4se

50.

5om

50 287

coaㅛ

쇼

sow

oxir

60.

sots

60-75

6-10

$50-10$

我10

So-10

Stored

50-3연

tis

502

socs

苏

S0-40

so-20

solo

tor

<-10

$50-70$

$50-10$

50.10

SA-10

50-10

案电

5010

sangs

sols

pits

sods:

son

50.4

6.25

$\$ 45$

50

506

70-4 4

$50-4$

sonat 


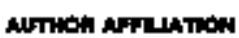

\begin{tabular}{|c|c|}
\hline 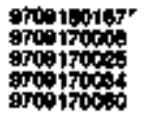 & 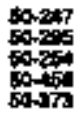 \\
\hline 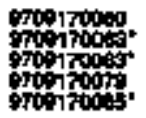 & 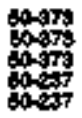 \\
\hline 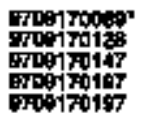 & 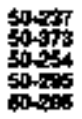 \\
\hline 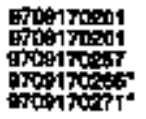 & 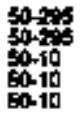 \\
\hline 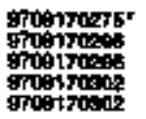 & 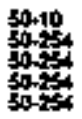 \\
\hline 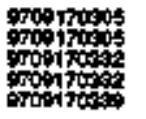 & 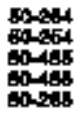 \\
\hline 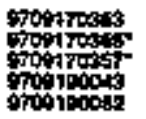 & 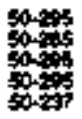 \\
\hline 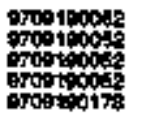 & 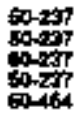 \\
\hline oropotas & 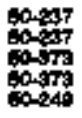 \\
\hline 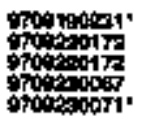 & 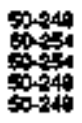 \\
\hline 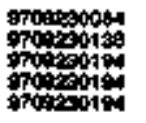 & 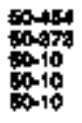 \\
\hline 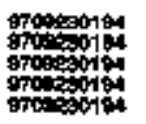 & $\begin{array}{l}50-10 \\
50-10 \\
5-10 \\
50-10 \\
5-10\end{array}$ \\
\hline 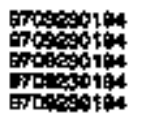 & $\begin{array}{l}50-10 \\
50-10 \\
60-10 \\
50-10 \\
50-10\end{array}$ \\
\hline 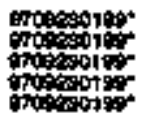 & $\begin{array}{l}50-10 \\
60-10 \\
00-10 \\
00-10 \\
00-10\end{array}$ \\
\hline 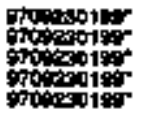 & $\begin{array}{l}60-10 \\
60-10 \\
00-10 \\
0-10 \\
0-10\end{array}$ \\
\hline 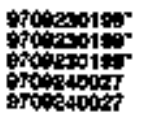 & 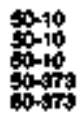 \\
\hline 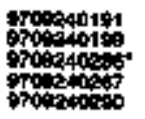 & 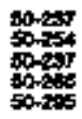 \\
\hline & 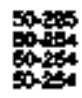 \\
\hline
\end{tabular}

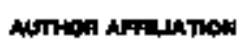

\section{臬}

p

\section{西}

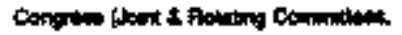

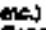

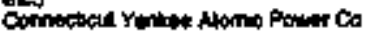

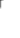$$
w
$$$$
\text { }
$$$$
\underset{w}{w}
$$$$
\text { r }
$$$$
\text { w }
$$$$
\text { w }
$$$$
\text { 湈 }
$$

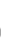$$
\text { : }
$$

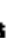

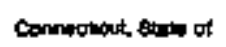

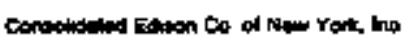

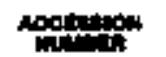

ctovictict

9 opounst3

900000013

$\operatorname{mog} 0000$

croosons.

9000,000

woospon

9roos 0001

ronososos

Fonton

700

\%od

970000000

$00+00$

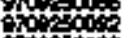

Bxocosos

9roosoys:

Tox

Fotion.

Trasor

mogectios:

sog.

irootrong

Hotwo

mopareas

ropertes

croparats

orotoras

mosecoes.

moses.

pos

orotims

grosonses

970000

row

of

Then

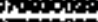

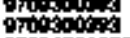

proolactes?

sors

pos

0005

60-254

co-10

sto.

50

605

5037

5037

$50+\frac{15}{50}$

10000

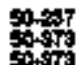

tons

Don

$50-44$

304

Sorfy

50-19

const

50

5

Do- 6

Mers

50 tod

601464

ind

soss

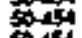

s.44

sts

cos

Dast

cong

Dons

const

0054

80.300

spopososes

Froobor s

mogoedots

gropiporit

9700 popite

gronetsot.

ancoreotis

repotio

prospese

troptotets

oromerop:

rop

$+4$

(100010

Dropen 17

4.

otow

tot

reocoust

sotow

ostotion?

00004

Grooknow:

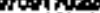

moowos?

9az)

$+2$

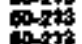

P.7.

tow

stata

t大+ta

为幽

我年1

402-21:

S4-1:

Dris

50 궁

\$p.219

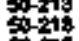

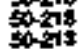

s0ㅕㄴ

ros

cots

0.27\%

50

50219

6024

oropounse

priptose

700000

sopat?

so 


\begin{tabular}{|c|c|c|c|}
\hline 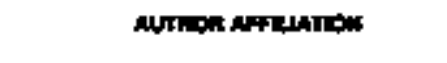 & 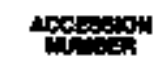 & anto & \\
\hline & 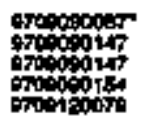 & $\begin{array}{l}503 \\
503 \\
503 \\
0067 \\
00247\end{array}$ & \\
\hline & 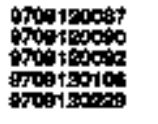 & 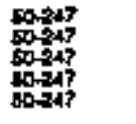 & \\
\hline 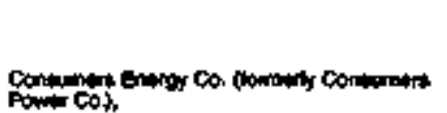 & 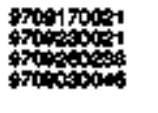 & 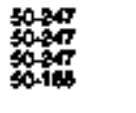 & \\
\hline - & 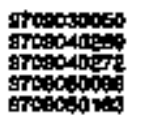 & 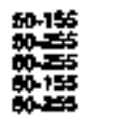 & \\
\hline & 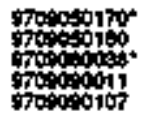 & 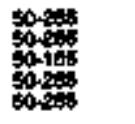 & \\
\hline & 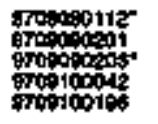 & 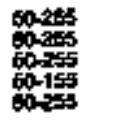 & \\
\hline & 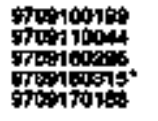 & 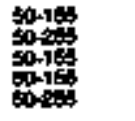 & \\
\hline & 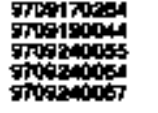 & 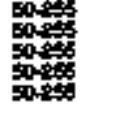 & \\
\hline & 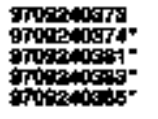 & 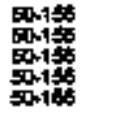 & \\
\hline & 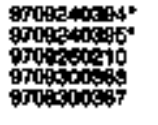 & 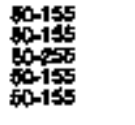 & \\
\hline 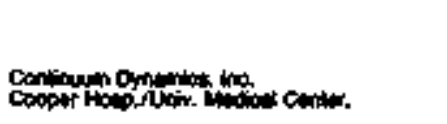 & 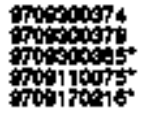 & 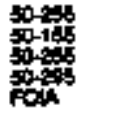 & \\
\hline 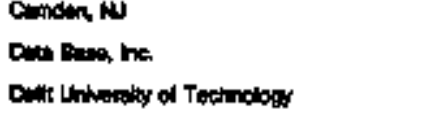 & 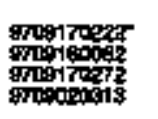 & 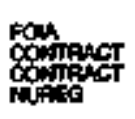 & \\
\hline 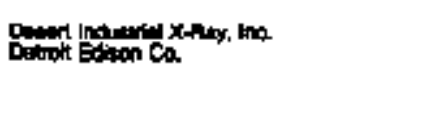 & 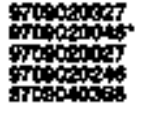 & 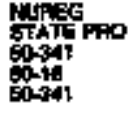 & \\
\hline & 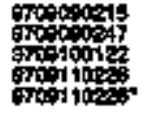 & 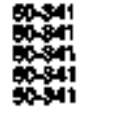 & \\
\hline & 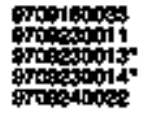 & 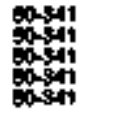 & \\
\hline 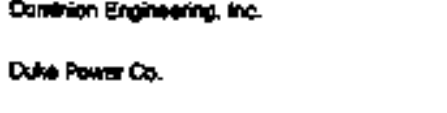 & 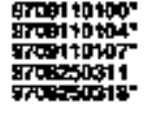 & 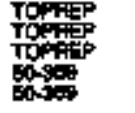 & \\
\hline & 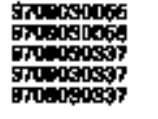 & 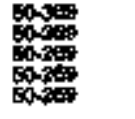 & \\
\hline & 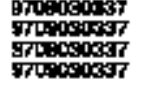 & $\begin{array}{l}50207 \\
502 \\
502 \\
502\end{array}$ & \\
\hline
\end{tabular}

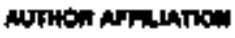

17cision

Path

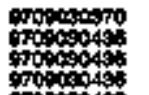

ortotions

croogentes

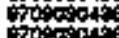

roments

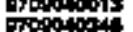

07000034

oraconos

4900040.

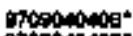

0700

901010

gropeses

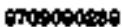

nopeces:

moenocis.

ondionst

Tosi10004

9700110004

07:

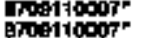

ononiouse

Finimis

sinis

ontloeto

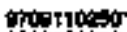

ror

190

का

Frodians

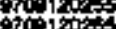

ototo

propoto

Frondrow

oforotrowa

prespoes.

erorexe

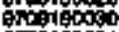

sprigxop:

Exorexpex

oremecoses:

on 100 s.

sinions.

onfors

poopacose

(j)

1rogero

gripratos.

propecosta

9700 सetr

90 PePs 010

Brisis or 30

Broas

9700240462

900 pos

9700 chas

970000034

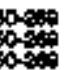

sot.

10.

tons

sita

$00-1$

$\infty=1$

0-413

cor

sot

00

$50-209$

5

s.

$\infty$

sato

\$i⿻

.

安的

50.90

5.t.

$50-209$

00209

ton

som

ste

1.

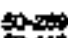

solf

\$1

\$4-1

soses

stis

sol.

60-19

E000

5041

sor.s.

50413

50-413

5

$60-1$

50

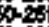

$\rightarrow$

solitis

50-413

50.4

\$ant

5-19

50-13

041

som.

=0

50and

sat

.

son

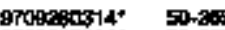

970000314050

9 ros0008s 0.41

9 ropoonty

eroseoses

$50.4+3$

orossoct 42

gropay

का:

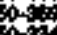

50

$50-304$

$\operatorname{socoses}$

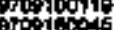

co-3sa

cons

50

$50-334$

\section{$p$
$p$
$p$
$p$
$p$
$p$
$p$
$p$
$p$}




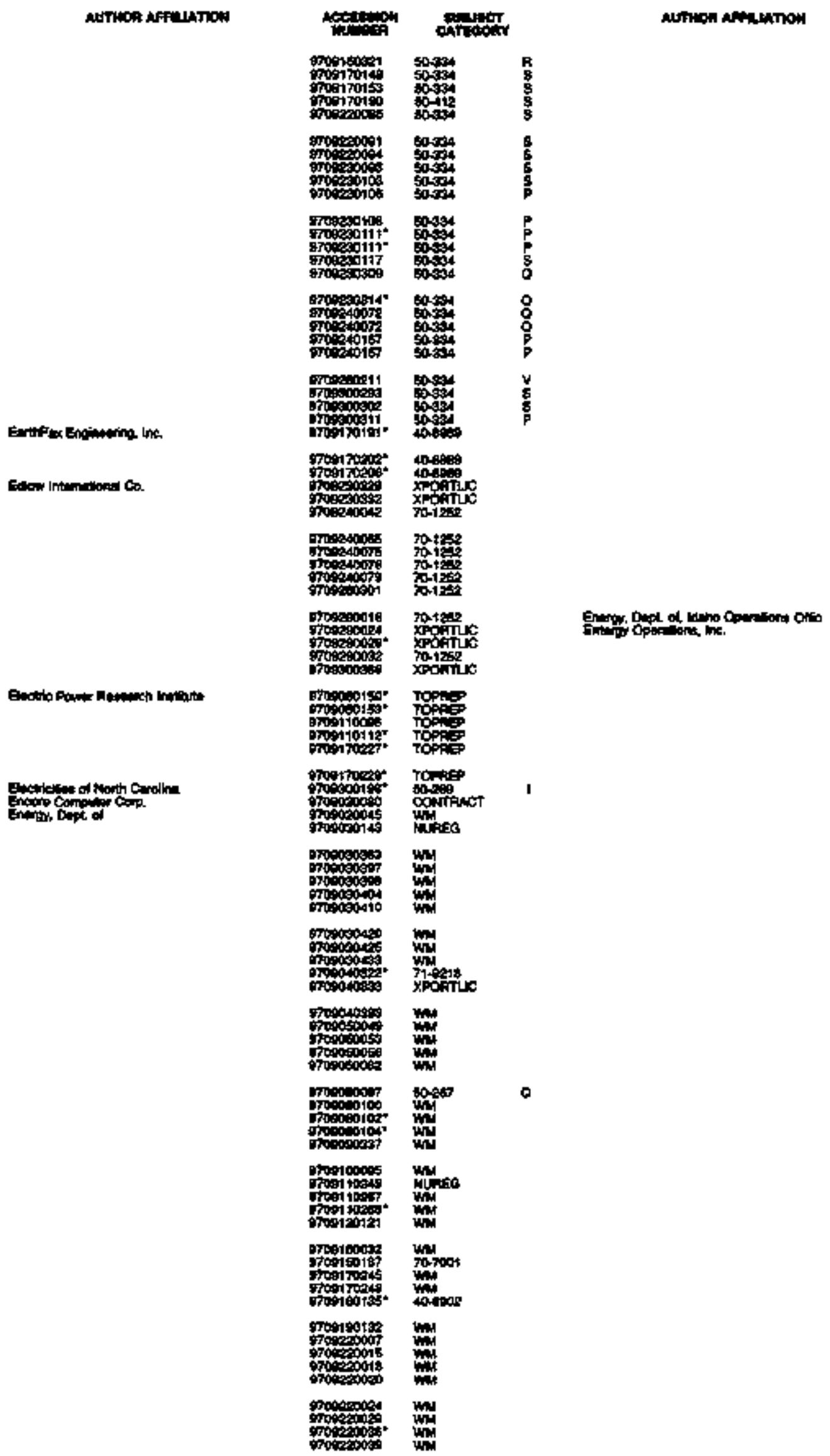

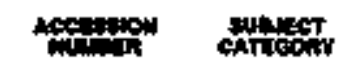

oroesposi

oxtopion

roproul1

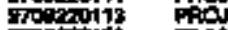

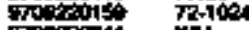

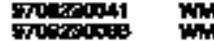

proseanogy

poctos

0 ox $\quad 70$

कrot in

sncesos to

igron

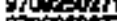

(rosions

700

.

7roespoes.

in

Tiscos.

7.n.

Brogecors

0000003

ordoge0004

orrosocosos wh

mopsooses fond

propony

optocis

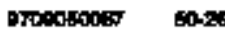

Brocosoor $60-31$

Grobeanios sos

Broxvin

oxtoconem

ortoresolst

Propesayt

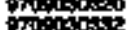

orisosis

sosese

intis

010

orososect

200001

$\ldots$

aro

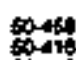

todi.

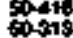

oroanc120

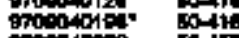

moences

700040372504

ondopoce

50000

700000

970000000

50

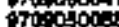

osis

50 sis

$80-40$

Drococoner

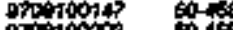

ont

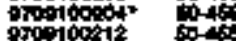

9 9090 1004

ondrom

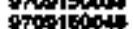

ofom

wats

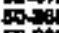

Brit

急宛

oroored 1 \%

oporgote

is

ond

co:16

sion.

sos.

700170140 to-40

9701701\%

Thosot

00016

spest

thas

o-4

coure

s70020041 Dest

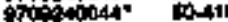

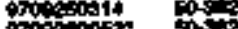

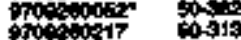

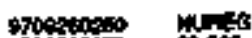

Hoteoc:

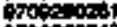

sastest

ints 


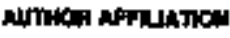

Entrocert 에내, in

Eatocare, no

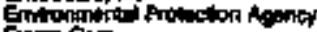

Eorn CAP

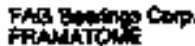

Firiond, the

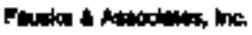

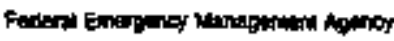

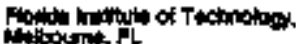

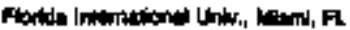

Fortila Pawer \& Lotk Ca.

Forida Pawer Core

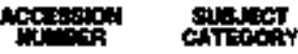

oropios"

mopoceses. wowes

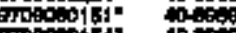

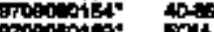

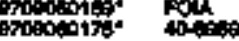

Fropogar: For

(1)

wot.

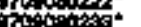

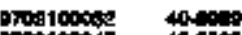

posionos 40.8.

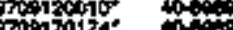

(1)

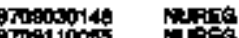

9ros 10ch

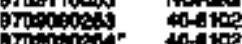

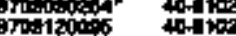

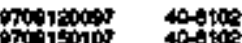

andion

institorts

Poin

groegroos

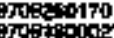

roptocose

mortsonto

propocts

wip.

rotos.

artion.

orvescoost

orveroos

9 onsatoct

9700120049

proposers

$9700 \mathrm{cos}$

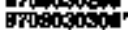

Briveososon

Proposs

wions

wond

Actom

ropuras:

BNo305Ts

0700004

spococort

$9700110 \%$

orotioger

9700017019

9rotisons.

roterator

cruproces

opictor.

moto

oritome1:

orusousto

Poosoys

700 .

ivenog

170

chomen

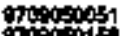

pitos

7optoor:

rotionso

proptaress

700 poes

orotoreso

$40-7500$

40.7580

stan

s0a

c0020.9

cost

bods

osto

theses

rotes

CowitinteT

Bo. 20

50

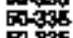

자요

50요잉

to

50에

thene

Antors

toms

bogst

cons

tor

tost

s0he

soses

502010

solat

敌-270

rats

$\rightarrow \infty$

sospos

soriog

bos

tode

bote

Doros

50000

Woo

xote

coroc

coros

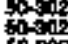

$\sin$

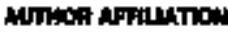

$p$
8
8

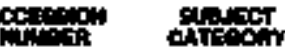

propioces sos

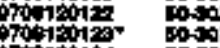

povition 50402

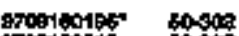

opoos

ropisazs

Toaroter

sroprotr:

retion

Potoptor

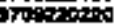

oreseapose

(a)

(t)

trons

meananos

rotoposo

400030.

ritoporis?

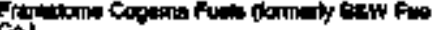
Calh

\section{Fraxe}

onotorat?

prosing

oritosiso.

$\log _{0 \rightarrow 0}$

50302

sop:

5000

$\operatorname{son}$

$\operatorname{son} 2$

6rop?

$\sin$

sonite

$\operatorname{sos} 0$

coton

ofor

$\sin$

conth

70-1201

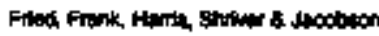

Brosopose

groposocis

900010004

9rograosis

Frowior Toundogy Corp

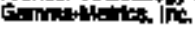

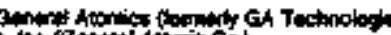

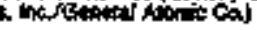

orocosinos

moseraris

Tocosis:

700001

870018001

70.19000

roresoris

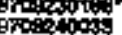

somatsons

Powatis:

Ther

artolotis

gropon?

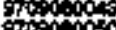

50000

1000100

Todzons:

gropranso

moevipas

groesecose

mopecoso

Socons

ros

$5002007^{*}$

7130 \%

$70-4006$

70.970

70

rom

sous

sto

sog

son

$0+34$

50

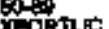

090004025

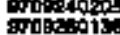

For

wootitas

wooderes

xpentuch

Tcoing

70-1113

7.1119

我

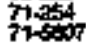

Tr- +400

ritestos

जol1

50.119

yecarulc

Tots

70119.13.

70,

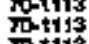

oxposogs

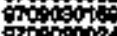

grototacest

ortacts

soovent

Puotens.

pos

20+113

$70-1113$

\%

牙我

s.

so

0-21

需娄

roopen 13

100

$\$ 0100$

Foloto.

Thoplotis:

sroproceser

popdzar

Spos soos

sor 15

borts

ofats

cors

50 . 


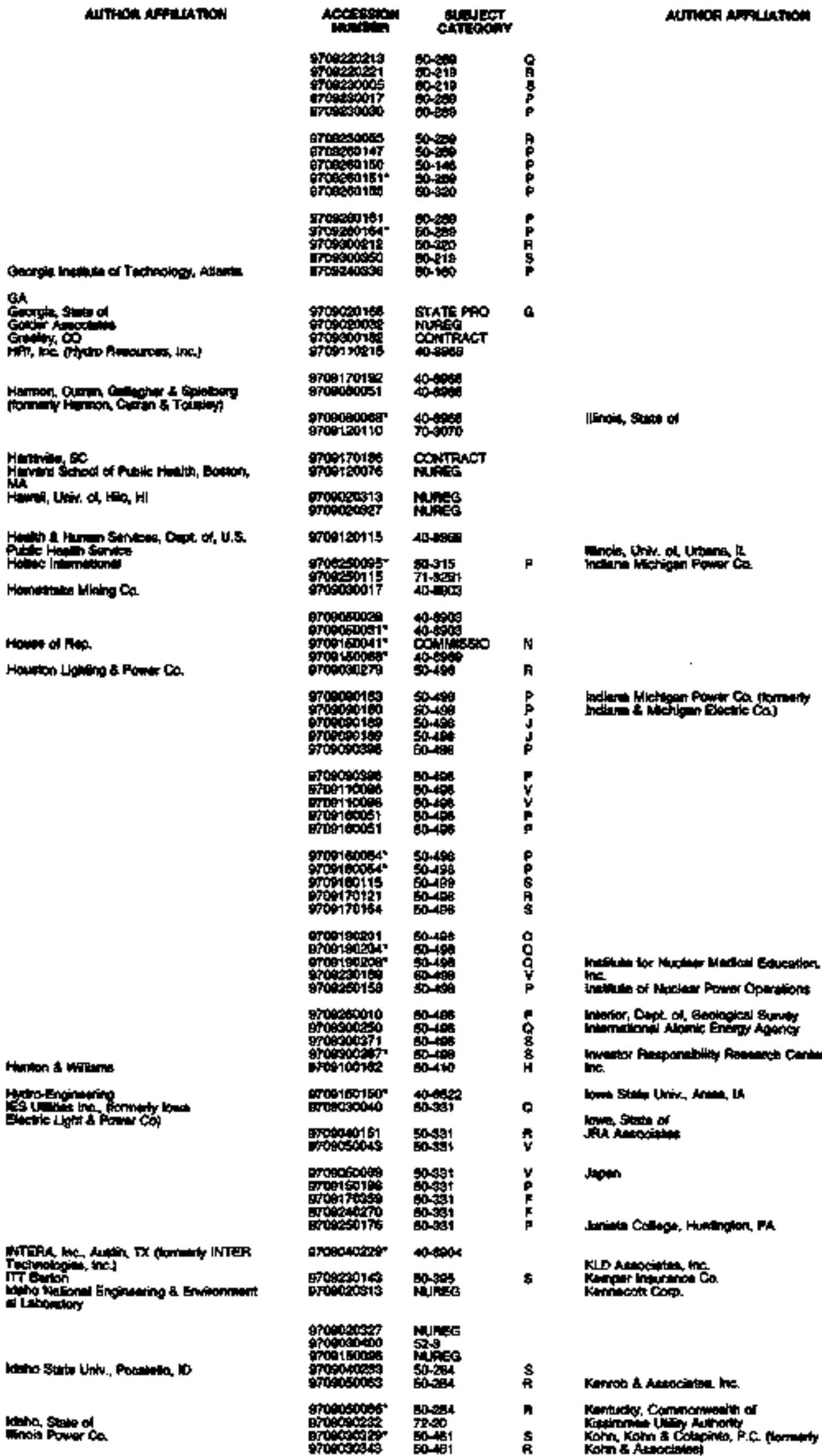

\begin{tabular}{|c|c|}
\hline 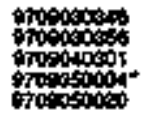 & 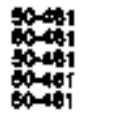 \\
\hline 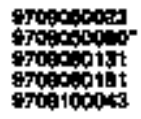 & $\begin{array}{l}504 y \\
5044 y \\
50-461 \\
50-45 \\
50-40\end{array}$ \\
\hline spodsont & 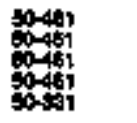 \\
\hline 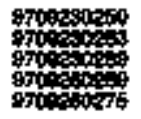 & $\begin{array}{l}50-41 \\
50-41 \\
50-41 \\
50-41 \\
50-41\end{array}$ \\
\hline 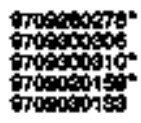 & 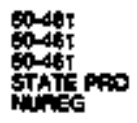 \\
\hline 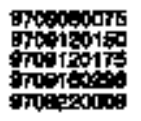 & 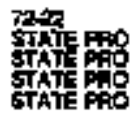 \\
\hline
\end{tabular}

groesaposa

970 - 190.

Propolats

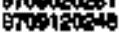

PA

CONERAT

50315

50315

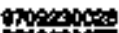

ofort

onctos

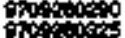

groread

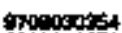

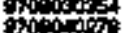

\%roto

opotions

Fiontes

retion

Protestes

posporat

mog 700 \%

800170108

oroticiset

tropancer

propose

froct1018

5015

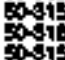

oxs:

50315

sors

Bowis

E0315

cosis

$\rightarrow 0$

solits

sto

整田

$60-915$

$50-315$

sons

rot

gropartsos

OAR

on-2h

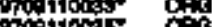

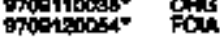

970910000

gropio180

(1)

oropoporta

gronand1"

gototiot

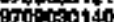

goto 10018 .

grososis:

oracosory

17000012a

Sroverate

gropodirz

gropoons?

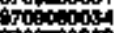

20

roweoso

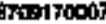

거의

stivis man o

son

coningois $\quad N$

inonitu

conthon Contoss

74.men

Bonges

Fit

40 tod

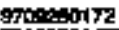

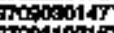

\section{4}

$40-4$

coritivet

STARE 10

oro 


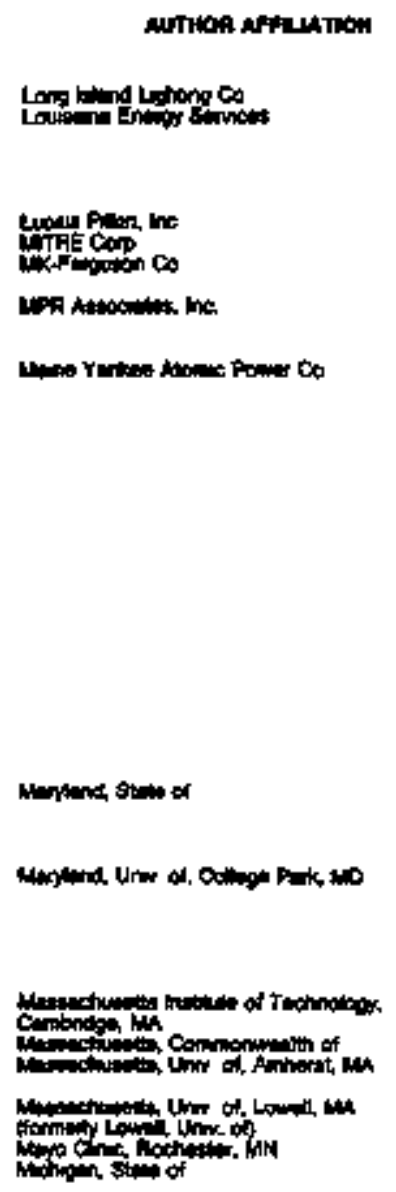

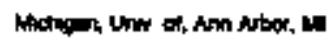

Mertorian ine

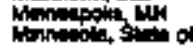

Mimenoper stabe of

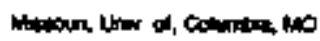

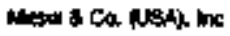

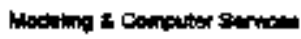

Motsom be

Mantoin 6

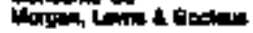

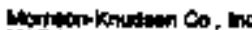

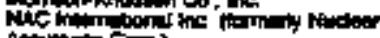

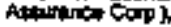

$\operatorname{lic}$ ing

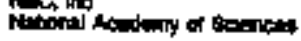

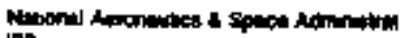

Ion

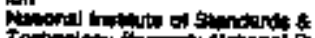

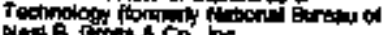

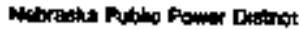

\begin{tabular}{|c|c|c|c|}
\hline 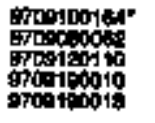 & 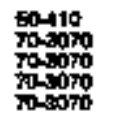 & H & \\
\hline 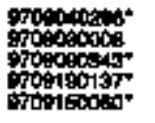 & cominger & $\theta$ & Nabsilke Stato of \\
\hline 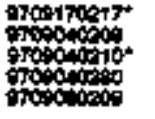 & 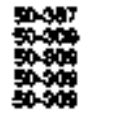 & $\underset{w}{w}$ & 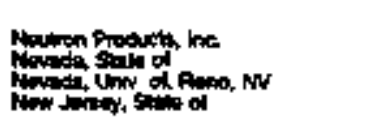 \\
\hline 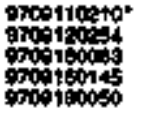 & 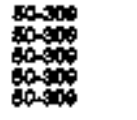 & W & 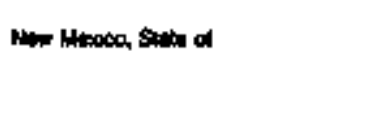 \\
\hline $\cos _{0 \rightarrow 000}$ & sotion & $\underset{\underset{W}{W}}{\underset{F}{\boldsymbol{F}}}$ & 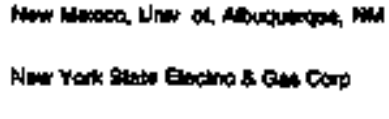 \\
\hline 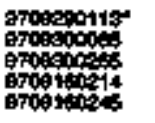 & 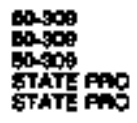 & $\underset{G}{W}$ & 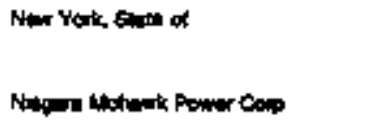 \\
\hline 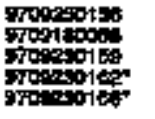 & 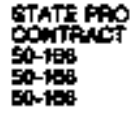 & $\begin{array}{l}\mathbf{G} \\
\mathbf{n} \\
\mathbf{n}\end{array}$ & \\
\hline popectos & Tomplat & & \\
\hline 9rosilooses & Moner & & \\
\hline$\$ 700370011$ & so- 200 & A & \\
\hline oroestones & pions & & \\
\hline 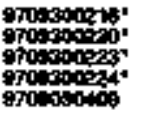 & 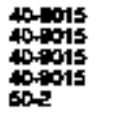 & H & Noth \\
\hline rot now & GowTRMT & & (ntsecos) \\
\hline
\end{tabular}

Foor

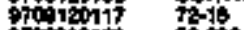

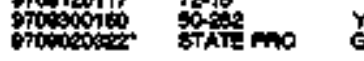

stanomas 50-100

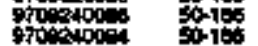

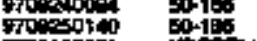

का001000

ipofuc

$9709+0003$

oroento4a.

mogrodis

Fondo014

groor t0:31"

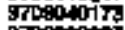

Brisocioct?

Fopoond

xofictuc

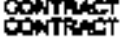

40.97s

40-7ra

40878:

isc

证安

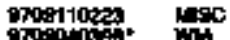

97011000 72-1085

stortro0s4 72-1005

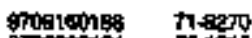

intaciol 4 ritons

oporois: 721015

970s110008\% Fold

9roestosto 50,0

soponetio

50,00

s7agh roos

COATRAET

Oroctives

aroontot

gropitents

socta

$50-298$

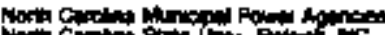

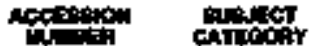

grograces

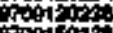

tortson

groesolits

Dreancis:

Potilopos.

7 .

mitrocs

spoon roos?

orentects

570012003

onoteds

arosustas

srosotion

97000004

9rosisoos

50

onochersis

poposest

propicias

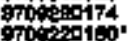

9row安14

inof

9ropacyis.

970 solis

poposora:

Topostiga

groports.

prosolote

Tromosonis

40

onotitión

970000240

07000

970000\%

oroofroens

orostionat

modsis

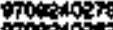

potongto

ortionat?

gionocourt

stoptits

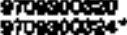

ensoposers

$8 \pi \times 00093$

ragcoses.

6ropolos

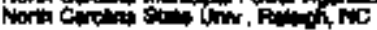

rocosang:

का

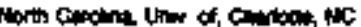

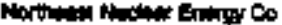

9700000004

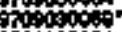

20004

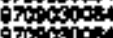

$\operatorname{mosecos}$

$\rightarrow 00000$

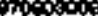

oposcost.

moworage

poctostos

octisent

700

orroses?

Pooson

s.to 40 +

Foogist

Dot

int sits

50

6ris

und

Nivis

sing DFo

0 -

내요

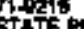

STIE Pa

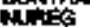

$50-45$

\%.t.

Tht

otisto

Nung:日e

$\infty$

sonto

STATE BAO

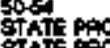

sidit $\mathrm{PAO}$

sotery

otis

$0-20$

6-610

$50+10$

sacto

然管

然

50-200

$50+410$

50-ars.

S0-410

oges

tow

40-10

60

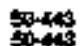

tom

504

$50-49$

3048

1048

50 as

s007

㔔焉

करा

cosis

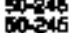

Dop

soas

sos

torats

bats

50245

ons

60.45

00.245

50.25

504

6

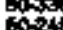

$\stackrel{p}{p}$

6

P

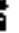

g

今

,

R

3020

$50-25$ 
Mmkin Mrftentist

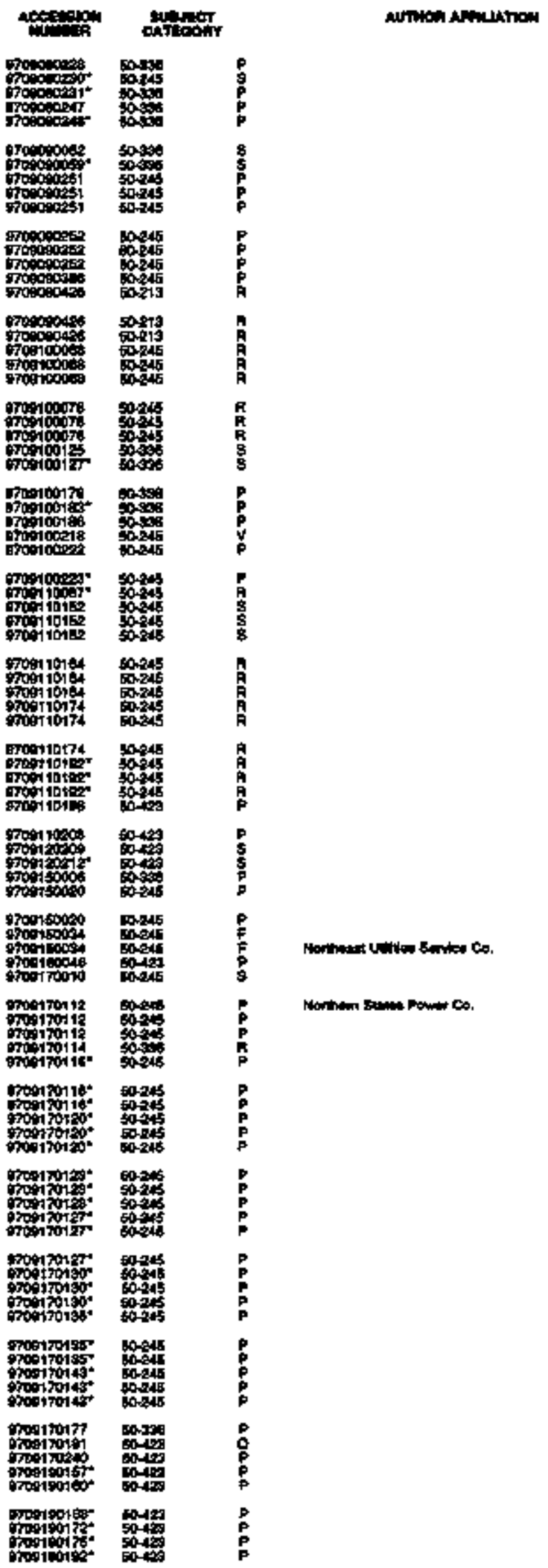

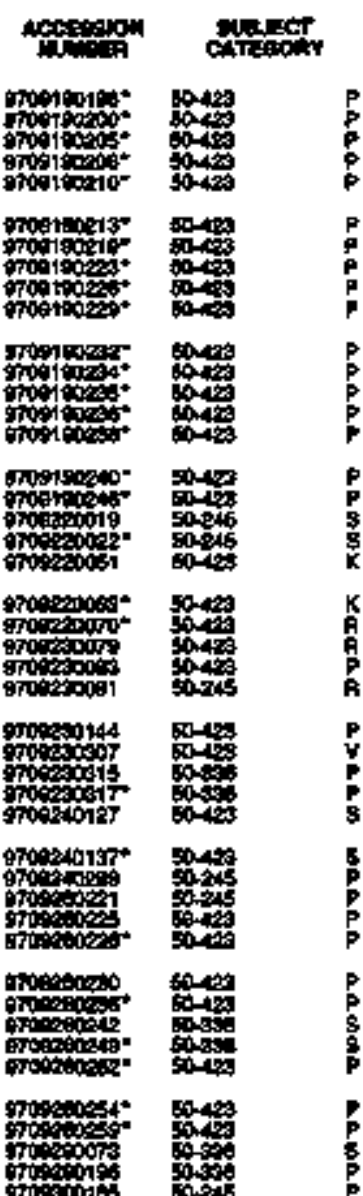

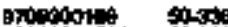

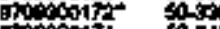

(a)

of tom

orpoeonas:

97000003

$5 \sin x$

froptont 4

groporang

97000

shon

orototo

Troperars

Foneros

(1)

(1)

Troposina

cropeores.

7000014

stom

syont.

sportiond

ophomit

onotome.

togicont:

(1)

prosicosis

orositert

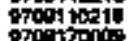

prog

$\rightarrow \infty$

rogitioge

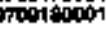

orosiston

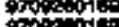

67000017

sonte

sow

sit.

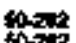

tis

5-1.

couss

50

to.t.

co.25

6 연

toses 


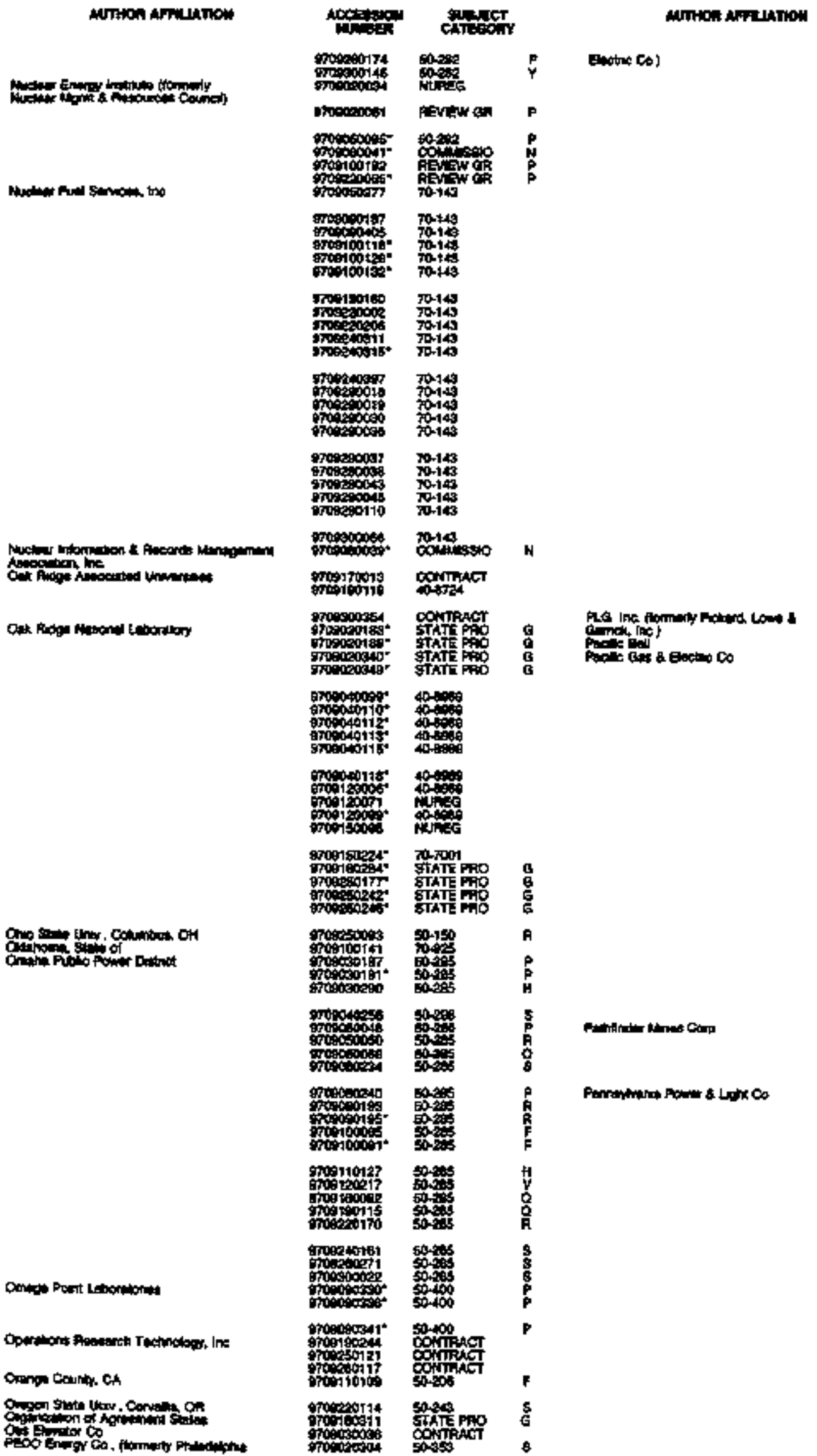

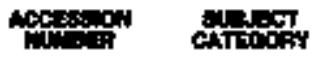

\begin{tabular}{|c|c|}
\hline 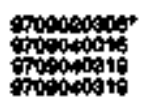 & 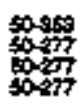 \\
\hline 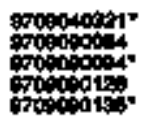 & 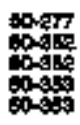 \\
\hline 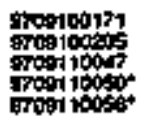 & 然然 \\
\hline 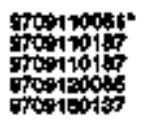 & 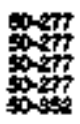 \\
\hline 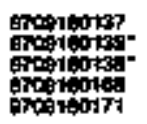 & 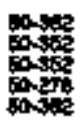 \\
\hline 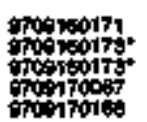 & 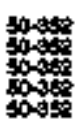 \\
\hline
\end{tabular}

orogrogig

970170044

9ropiroment

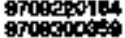

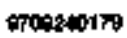

orocosono $\sin x$

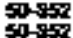

sols

$50-56$

50 क्त

n:TED

CONIPACT

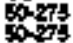

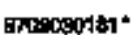

Bntecosols

WN0000

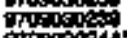

thososin.

Brososocen:

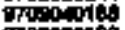

970000013

7000010.

s.t.5

s.25

sis

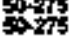

s.275

0130

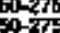

$50+25$

970950059

poontili

rosery 12

stots

$60-275$

(t)-275

groosarit:s

Procentits

oroctats

orotion

$50-275$

50275

50 it

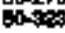

pooruas: 1

rooritis:

7ronisus

shise

$40+3$

0 er

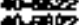

orootsolfo

\section{0-3e7}

oroposeres

ropoter

970000002

$0 \times 0000002$

20000

$70+114$

ropocolán

roe000120.

07000122

0040125

orotocarto

sroraport

cor 700

670970

poris

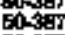

Bastar

60-35i

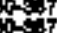

Gen

Dones

$90-5$

soles

Dongty

$50-5$

505

cos

0 tr

So-s

grogr rag

Tras 


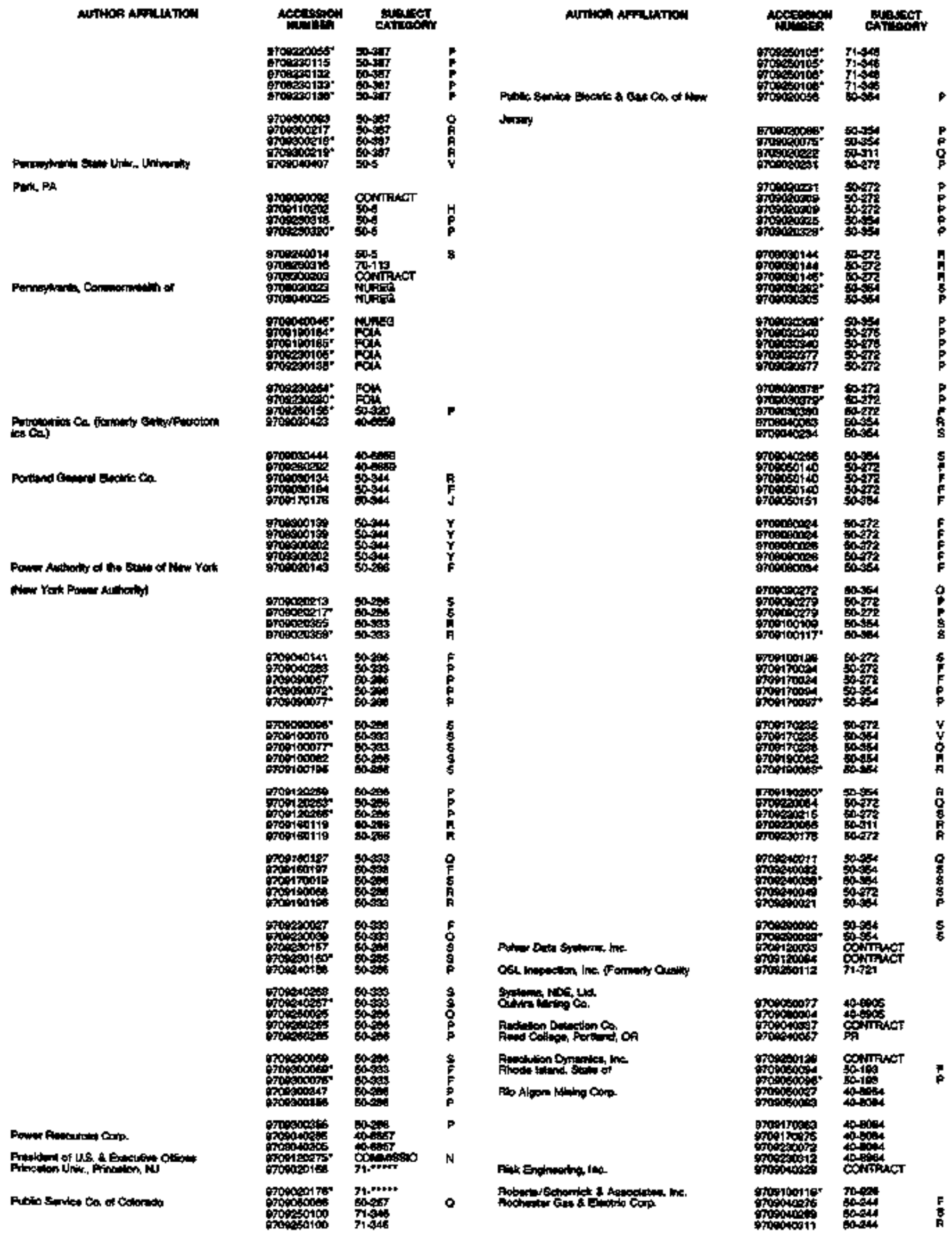


Authos Armuthtion

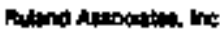
sk Hetion in

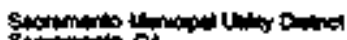

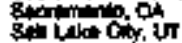

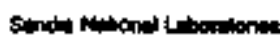

Saroent 8 Lund. Ino

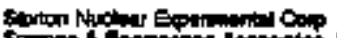

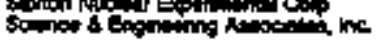

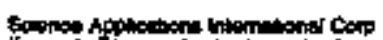

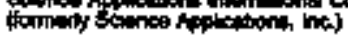

Scanter to the

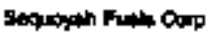

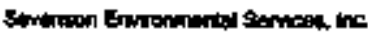

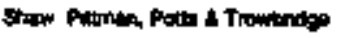

Shophens Buller, the

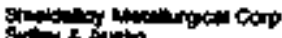
oning

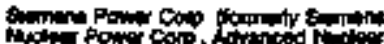

Eming Noxtor, tor

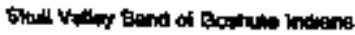

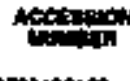

suenct

oproporat so-24

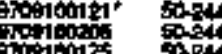

peis:ons

omocans

roper

inotits

omosonest

rivenow

roedecos

onotocostos

oporitodes.

Fingers

ropopsts

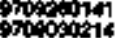

oreopeses

atomots

gropososer

Dinetors.

roepeost?

postos

(1)

portond

gepoces

tor

otoibotat

6rot80125"

cons.

7rootion.

$0700+90140$

6rooto0194

ropespos

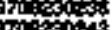

prostons

proptanter

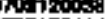

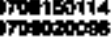

07015012.

propara

709010

roption

propacore

propasone

pontosit

9700110z'

Brosiioets

omerengo

opostos

9rotionsto

aropotation

JToso4041

50000

spacosos.

Trostsotad

orogr 7uza

Foricos

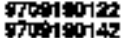

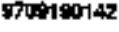

Monsat 13

rostoger

7000025

Trotios

JFane M0Z19.

Troweoper

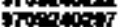

$7 \cos 0120$

9700000

Bringocin"

popesos.

9700a015s

contrans

oft?

poin

T1- eaj

14.

onsts

otes

60-4

6-4i密

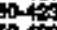

Borix

$60-4 \pi$

\$-40

s-its

to-4es

5040

sto

$50-4$

x-40

to-

1.4.

tond

60-429

50-14\%

CONTIRACT

Cortitat

cotrintat

contruct

ONiRH

60000\%

Fon

40.7604

$70-13$

$50-44$

72.10

40 or

411162

atitio

XPofertic

คT)

TCPR:

7atas?

TCryep

File

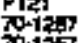

\%12

70 1며이

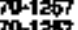

p-12क

xorulc

xporTh

Tivic

T2100\%

rop

$30+\infty$

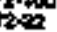

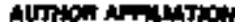

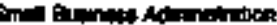

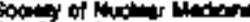

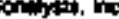

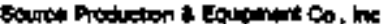

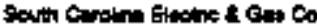

Bowh Croine, Sthe of

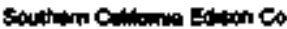

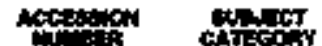

spitioo

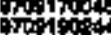

intives

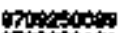

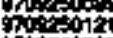

extagotion

900000

9ropocosis

97000

motoras

porow

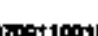

prot 10018

rootonit:

97002010:

Contin

Oonit

onter

CONTRACT

ONying

contale

CONTACT

coptrict

Proso.

ofymer

71-102

$7+100$

conses

polas

Dousts

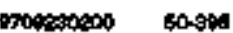

moetarar borta

rownos sols

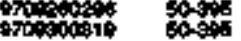

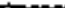

make

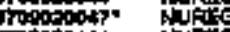

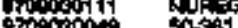

50.54

ranosol

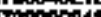

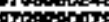

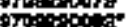

cropescos:

Fruesocos.

ertolitas.

(1)

$\operatorname{ros} 11$

oposzopor

ron 200 or

sposecosis

9ropeopors

9740001

740 is:

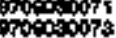

raposons:

Tropout

Fropopost

ropescos.

50-59

6009

Bo-

1

Dot

sarst

60\%

10.51

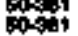

50-ง91

$50 .+1$

sonst

sosit

00-31

oris

0

$30-34$

solus

$50-40$

$50-424$

5044

oroposent

intopios

The

poctions

Troctots:

Treaconts

Do:

Toropas

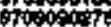

0.01

0.45

orsa

torse

50341

$+0,4$

70078

rog

60017013

sourd

$8+2$

solit.

5060

M0011014:

polot

molor

Tos

gropiote

ardipare

700104

tris

The

ropirote

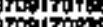

7 Tota

grongas

sorase

sor 


\begin{tabular}{|c|c|c|c|c|c|c|c|}
\hline \multirow[t]{3}{*}{ AUTHCA AFTukTIN } & \multirow{2}{*}{ 然 } & \multicolumn{2}{|c|}{ 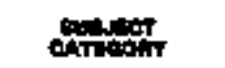 } & \multirow[t]{2}{*}{ 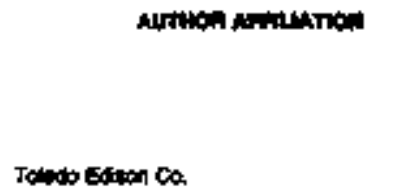 } & \multirow{2}{*}{ 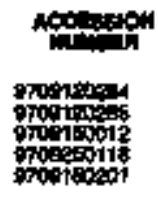 } & \multicolumn{2}{|c|}{ 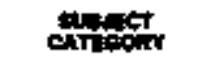 } \\
\hline & & $\begin{array}{l}50424 \\
50044 \\
50344 \\
50,44\end{array}$ & $\begin{array}{l}q \\
p \\
p \\
p\end{array}$ & & & 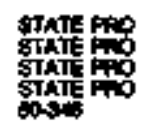 & $\begin{array}{l}0 \\
6 \\
6 \\
6 \\
6\end{array}$ \\
\hline & 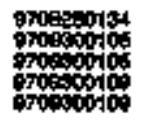 & 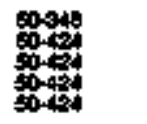 & $\begin{array}{l}\mathbf{p} \\
\mathbf{R} \\
\mathbf{R} \\
\mathrm{O} \\
\mathbf{0}\end{array}$ & Tnenenedes, ine & 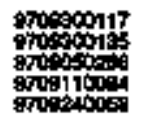 & 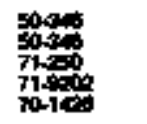 & $\begin{array}{l}1 \\
\mathbf{R}\end{array}$ \\
\hline 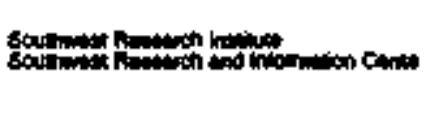 & 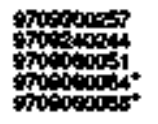 & 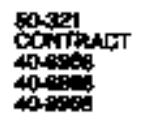 & $\mathbf{P}$ & 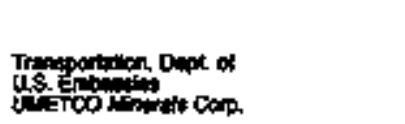 & 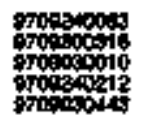 & 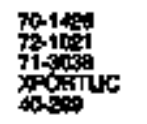 & \\
\hline \multirow[t]{10}{*}{ Soming gon of } & 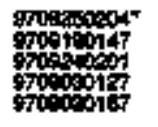 & 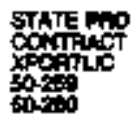 & 贵 & \multirow[t]{2}{*}{ Inlow fiectie Co. } & 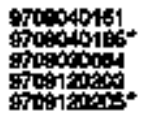 & 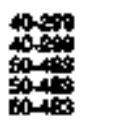 & $\stackrel{9}{p}$ \\
\hline & 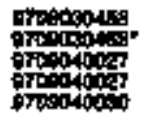 & 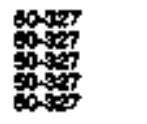 & $\begin{array}{l}\text { s } \\
\text { ğ } \\
\text { है }\end{array}$ & & 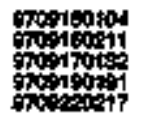 & 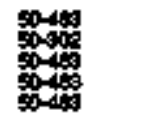 & ? \\
\hline & 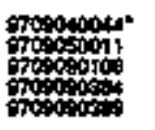 & 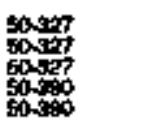 & 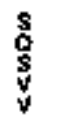 & 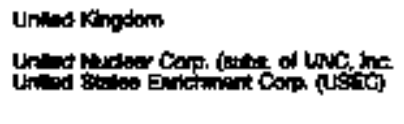 & 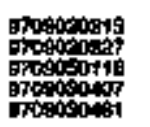 & 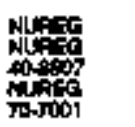 & \\
\hline & 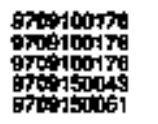 & 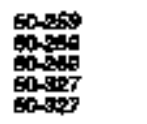 & $\begin{array}{l}\boldsymbol{F} \\
\mathbf{F} \\
\mathbf{F} \\
\boldsymbol{f}\end{array}$ & & 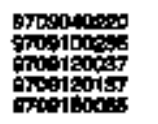 & 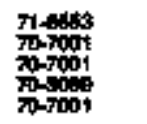 & \\
\hline & 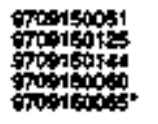 & $\begin{array}{l}50 x \\
5000 \\
50+0 \\
50+0\end{array}$ & $\begin{array}{l}9 \\
9 \\
4 \\
8 \\
5\end{array}$ & & 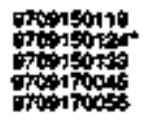 & 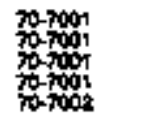 & \\
\hline & 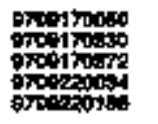 & 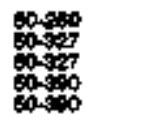 & $\begin{array}{l}0 \\
\mathbf{k} \\
\mathbf{R} \\
\mathbf{R}\end{array}$ & & 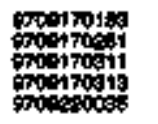 & 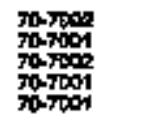 & \\
\hline & 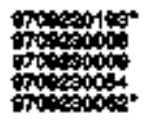 & $\begin{array}{l}50-60 \\
5000 \\
50000 \\
50250\end{array}$ & 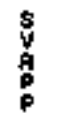 & 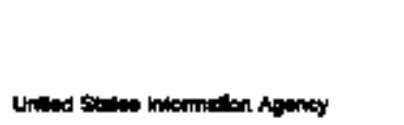 & 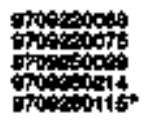 & 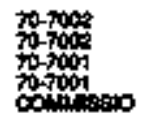 & N \\
\hline & 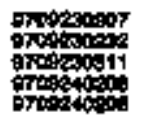 & 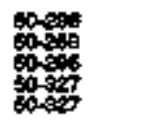 & $\begin{array}{l}0 \\
0 \\
0 \\
0 \\
8\end{array}$ & Vith san of & 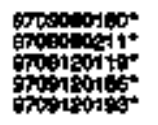 & ron & \\
\hline & 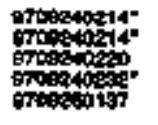 & 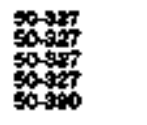 & $\begin{array}{l}\text { p } \\
\text { : } \\
8\end{array}$ & 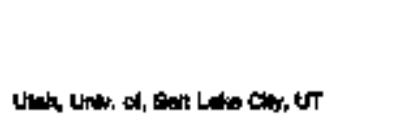 & 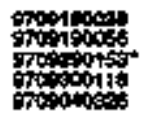 & $\underbrace{700 x}_{0 \rightarrow \infty}$ & \\
\hline & 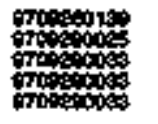 & 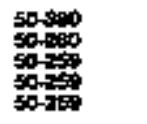 & $\begin{array}{l}F \\
F \\
F \\
F\end{array}$ & 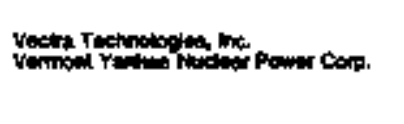 & 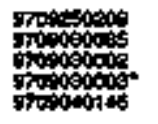 & 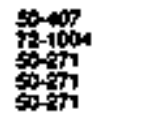 & $\begin{array}{l}\text { n } \\
\text { p } \\
\text { p }\end{array}$ \\
\hline \multirow[t]{5}{*}{ Tomacen } & 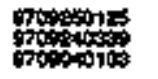 & 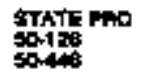 & $\stackrel{9}{p}$ & & \multirow{2}{*}{ 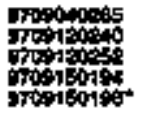 } & \multirow{2}{*}{ 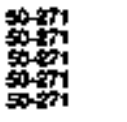 } & $\begin{array}{l}\text { R } \\
\text { के } \\
8\end{array}$ \\
\hline & oreaposid & $50-445$ & $\boldsymbol{p}$ & & & & क \\
\hline & 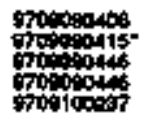 & 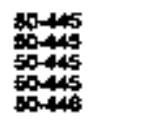 & $\begin{array}{l}p \\
F \\
F\end{array}$ & & 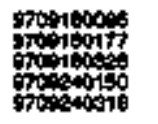 & 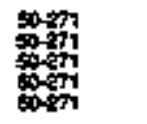 & $\begin{array}{l}\mathbf{y} \\
\mathbf{p} \\
\mathbf{p} \\
\mathbf{p}\end{array}$ \\
\hline & 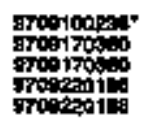 & 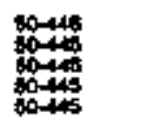 & $\begin{array}{l}p \\
p \\
p \\
g\end{array}$ & \multirow[t]{3}{*}{ 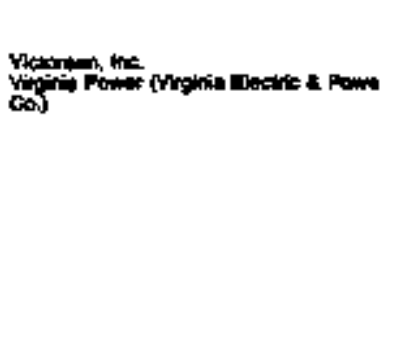 } & 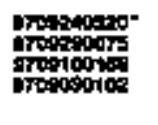 & 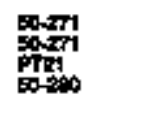 & $\begin{array}{l}\rho \\
F\end{array}$ \\
\hline & 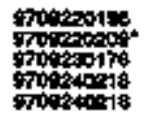 & 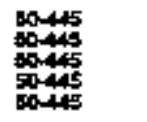 & $\begin{array}{l}\text { 量 } \\
\text { 量 } \\
3\end{array}$ & & 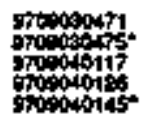 & 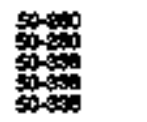 & $\begin{array}{l}\text { E } \\
\text { F } \\
8 \\
\text { P } \\
\text { P }\end{array}$ \\
\hline Thenes state of & 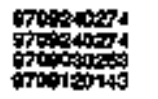 & 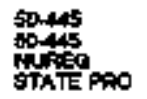 & 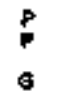 & & 舟 & 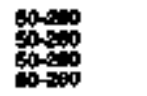 & $\begin{array}{l}\mathrm{P} \\
\mathrm{p} \\
\mathrm{p}\end{array}$ \\
\hline
\end{tabular}




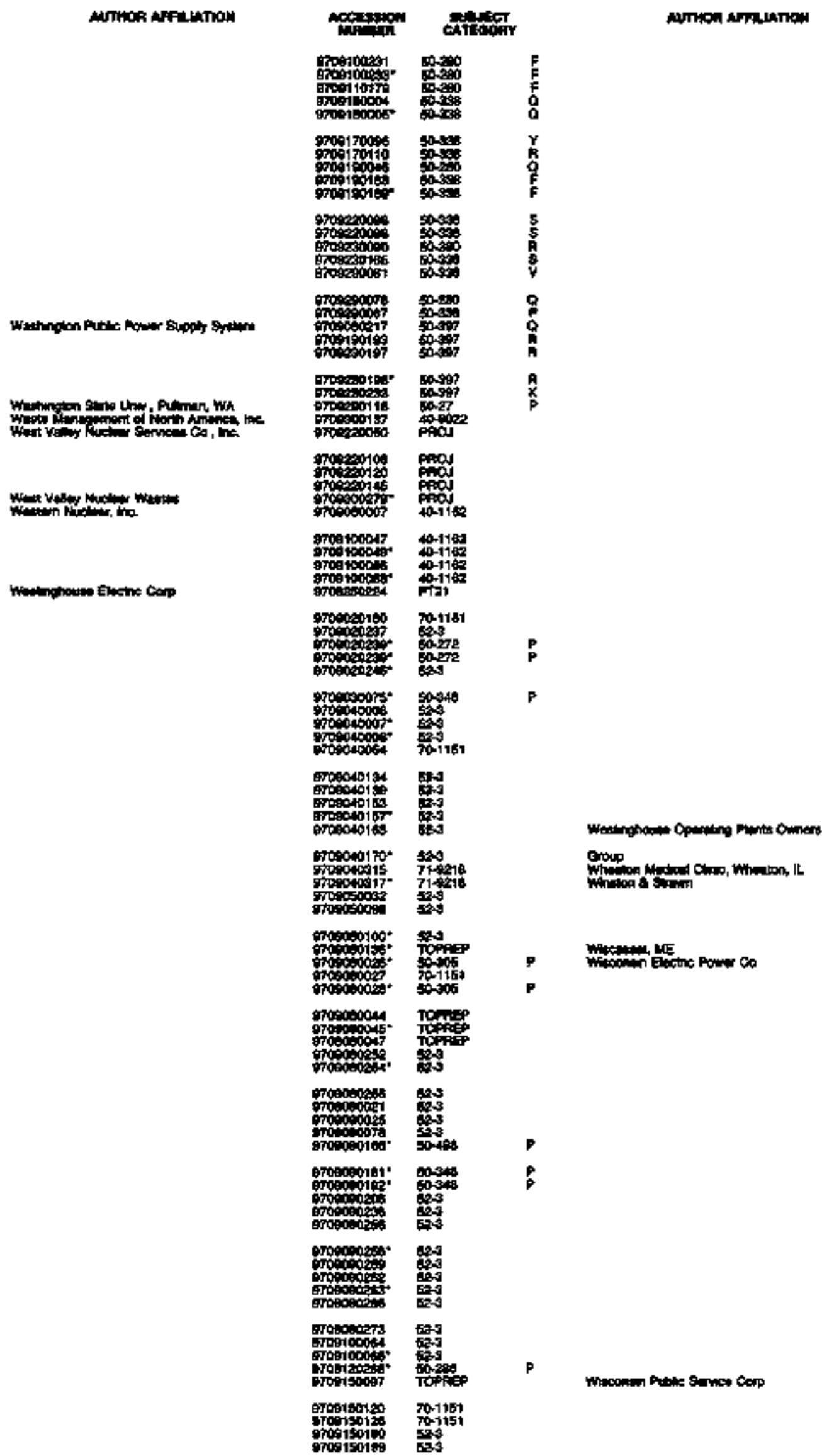


CORPORATE SOURCE IMEX

MUno: 4rinton

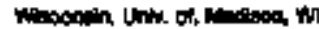

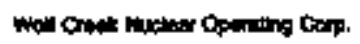

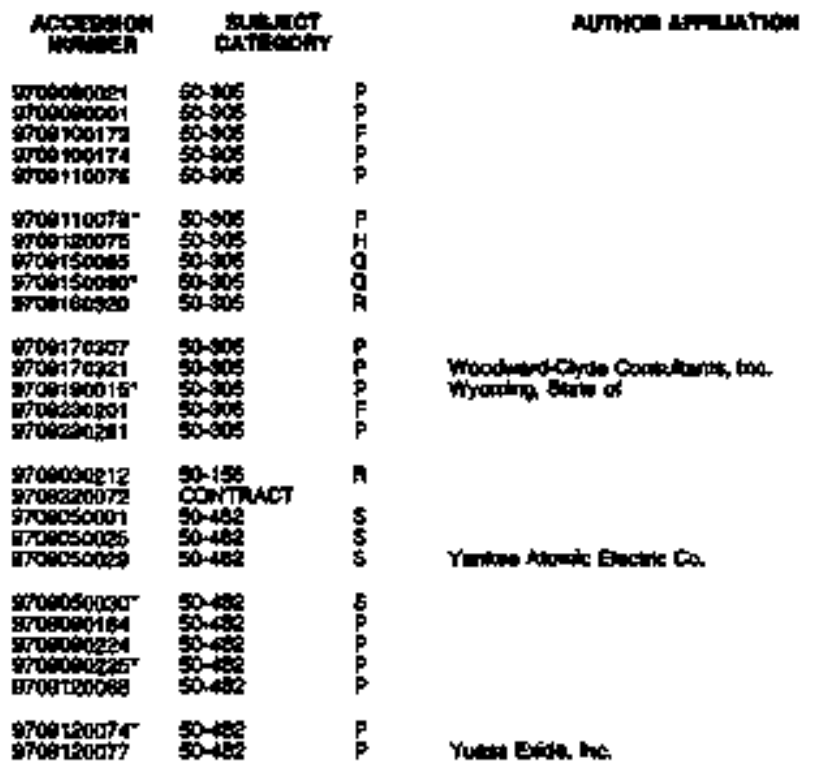

\begin{tabular}{|c|c|}
\hline 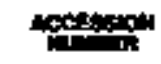 & 慗 \\
\hline 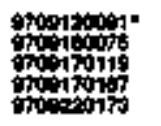 & 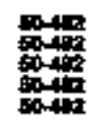 \\
\hline 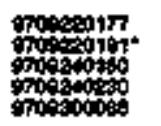 & 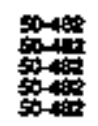 \\
\hline 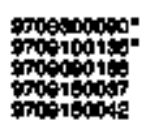 & 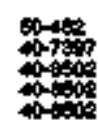 \\
\hline 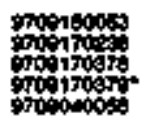 & 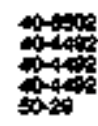 \\
\hline 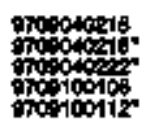 & 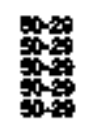 \\
\hline operpous & 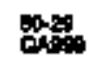 \\
\hline
\end{tabular}




\section{REPORT NUMBER INDEX}

The subject category colvinn in this index gives the docket number and the symbol for the category of information with in that docket; for example,50-233 P. The docket stbject categories are defined in Appendib A.

Subfect categories for nondocketed litems are also entered in this column by symbol; for example, ACRS.

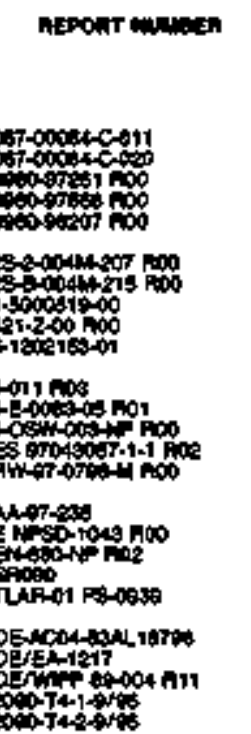

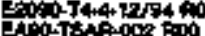

PRAl

Fin

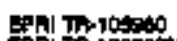

GRI TRID:Th:

FAlfor 107 R00

FAltion R

ENArores 500

CAliopis tion

FNP.1 1 ToS RDO

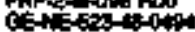

c496 $\mathrm{Am}$

Herites

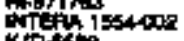

rops.

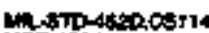

MPA 7 104

MFitson 800

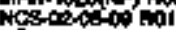

NE-1 33000

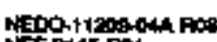

1.t

Wo-140 Pot

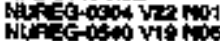

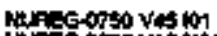

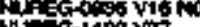

Mhactis

Nithingerois

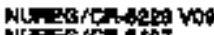

NLF Fsichlotit?

NIL

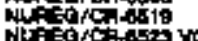

Neficarch exes vos

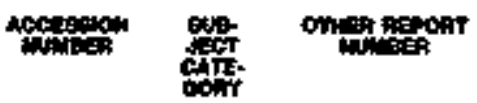

\begin{tabular}{|c|c|c|c|}
\hline 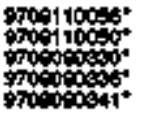 & 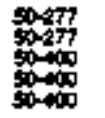 & $\begin{array}{l}p \\
0 \\
0 \\
p \\
p\end{array}$ & 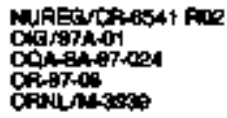 \\
\hline propion: & 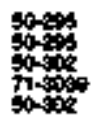 & $\begin{array}{l}\mathbf{p} \\
\mathbf{p} \\
\mathbf{p}\end{array}$ & 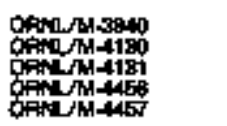 \\
\hline opolis: & 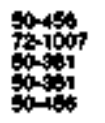 & $\begin{array}{l}p \\
q \\
p\end{array}$ & 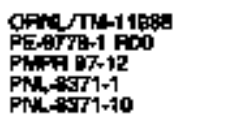 \\
\hline crocolo: & 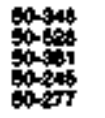 & $\begin{array}{l}P \\
q \\
P \\
P\end{array}$ & 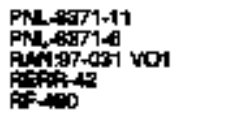 \\
\hline 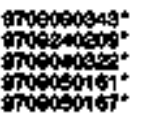 & 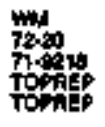 & & 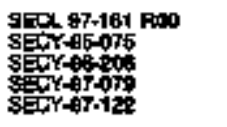 \\
\hline 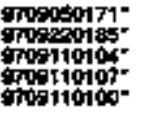 & 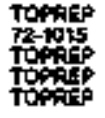 & & 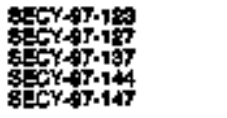 \\
\hline 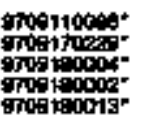 & 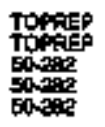 & 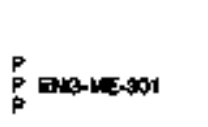 & 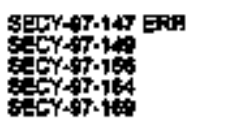 \\
\hline 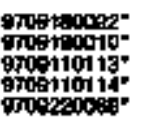 & 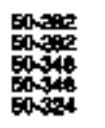 & 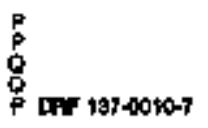 & 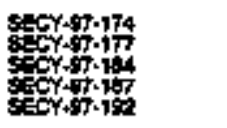 \\
\hline 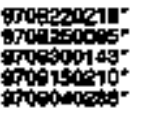 & 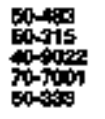 & $\stackrel{9}{P}$ & 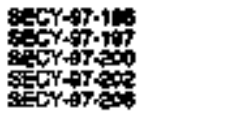 \\
\hline 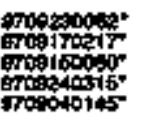 & $\begin{array}{l}00+96 \\
50-7 \\
50-209 \\
70-143 \\
50-500\end{array}$ & 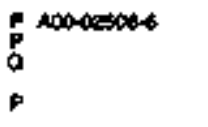 & 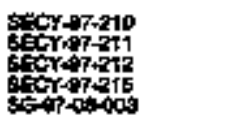 \\
\hline 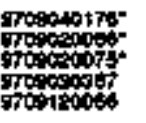 & $\cos _{0 \rightarrow 0}$ & $\mathbf{P}$ & 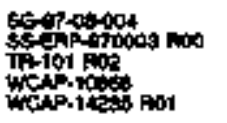 \\
\hline 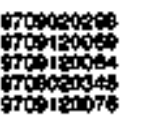 & 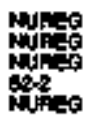 & Comisactis & 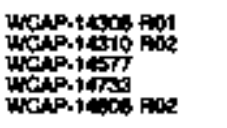 \\
\hline 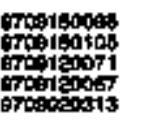 & Mng & 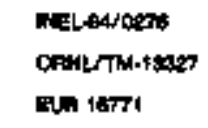 & 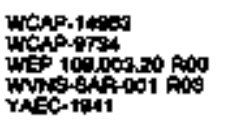 \\
\hline oncicases & MUREs & Еงด 16771 & Mertholesto AO1 \\
\hline
\end{tabular}

These categories are defined in Appendix $\mathrm{B}$ and Itsted in the Contents. An asterisk following the accession number in this index indicates that the docut ment is an enclosure to another document. To obtatn the accession number of the principal document under which the enclosure is listed, rofor to the index entitted "Cross Reference of Enclosures to Ptincipal Documents."

\begin{tabular}{|c|c|c|c|c|}
\hline 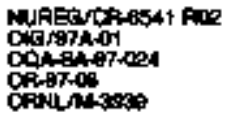 & 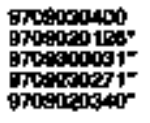 & 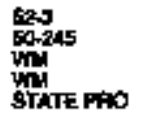 & 8 & 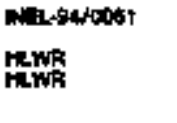 \\
\hline 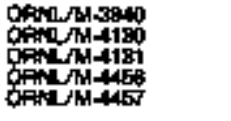 & 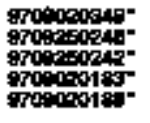 & 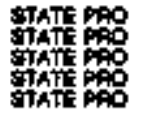 & $\begin{array}{l}5 \\
\frac{6}{5} \\
5 \\
5 \\
5 \\
5\end{array}$ & \\
\hline 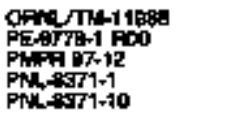 & 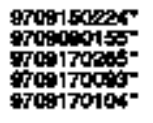 & $\begin{array}{l}\text { mond } \\
\text { soses } \\
\text { poun } \\
\text { poun }\end{array}$ & P & 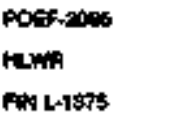 \\
\hline 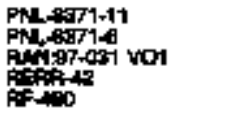 & 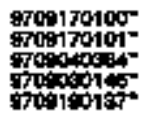 & 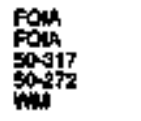 & $\boldsymbol{p}$ & 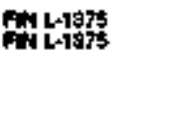 \\
\hline 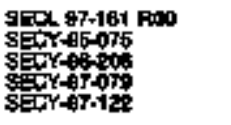 & 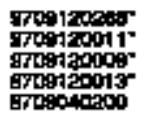 & 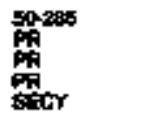 & $\varphi$ & 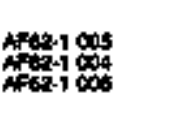 \\
\hline 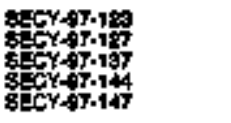 & 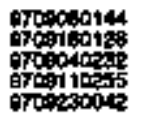 & 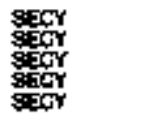 & & \\
\hline 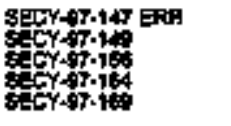 & 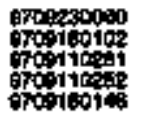 & 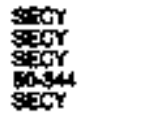 & $\mathbf{w}$ & Consecteroos \\
\hline 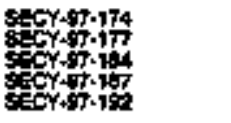 & 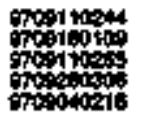 & 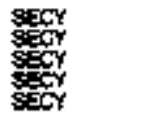 & & 4prot294 \\
\hline 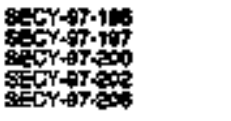 & 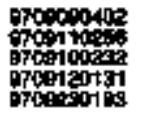 & 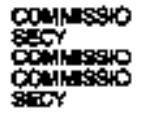 & $\begin{array}{l}\text { N } \\
\text { N } \\
\text { N }\end{array}$ & constecrasoss \\
\hline 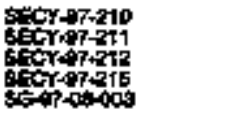 & 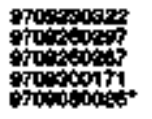 & 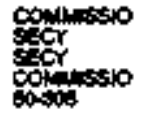 & $\stackrel{\mathbf{w}}{\mathbf{w}}$ & \\
\hline 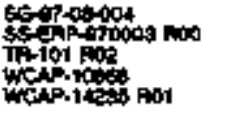 & 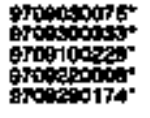 & 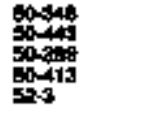 & $\underset{p}{p}$ & \\
\hline 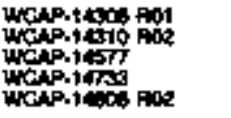 & 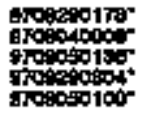 & 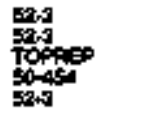 & $\mathbf{P}$ & \\
\hline 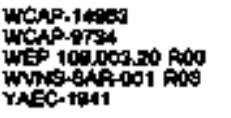 & 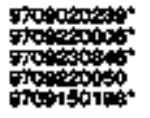 & 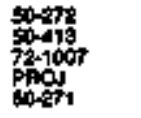 & $\stackrel{p}{p}$ & \\
\hline 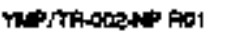 & 9rosonoter" & whit & & rewa \\
\hline
\end{tabular}


244

REPORT NUAMEER MNDX

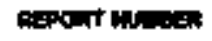

YMPTTROOSAP Fite actariton intitin

대알

OTHEA RPQMT

ofir

9700000104" WH intig

R:ost wates

Mt释

Yusco AAC 07

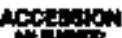
Atimis

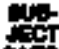
cois orocenses" mis

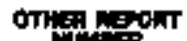
NTtis 14พ⿴囗十 


\section{CROSS REFERENCE OF ENCLOSURES TO PRINCIPAL DOCUNENT}

Item listed as enclosures are those indicated by an asterisk following the accession number in the indexes. The second column gives the accession number of the principal document under which the enclosure Is listed. Knowing the accession number of the principal document the date issued,and the catepory enables locating the title listing of the enclosure.

The subject category column in this index gives the docket aumber and the symbol for the categron of information within that docket; for example, 50-320P. The docket subject categortes are defined in Appenthx A.

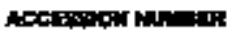

\begin{tabular}{|c|c|c|c|}
\hline 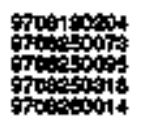 & 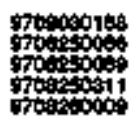 & 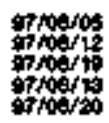 & 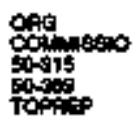 \\
\hline 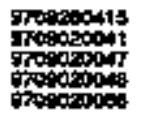 & 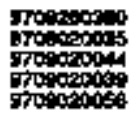 & 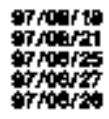 & 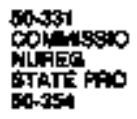 \\
\hline 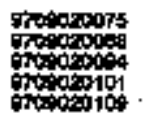 & 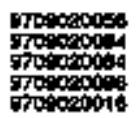 & 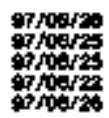 & $\begin{array}{l}0.354 \\
50-355 \\
50-305 \\
50-300\end{array}$ \\
\hline 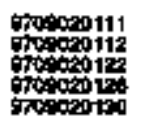 & 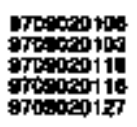 & 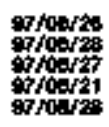 & 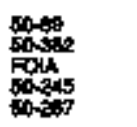 \\
\hline 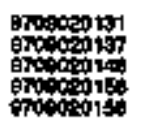 & 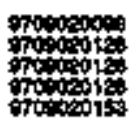 & 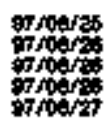 & 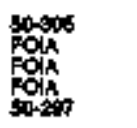 \\
\hline 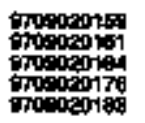 & 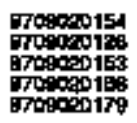 & 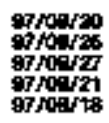 & 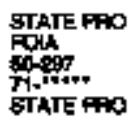 \\
\hline 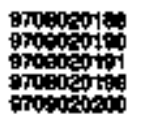 & 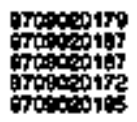 & 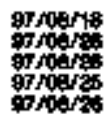 & 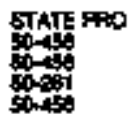 \\
\hline 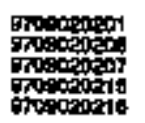 & 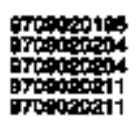 & 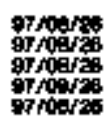 & \\
\hline 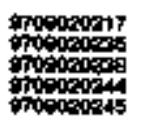 & 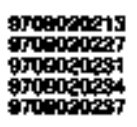 & 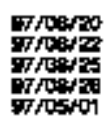 & 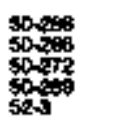 \\
\hline 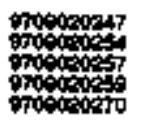 & 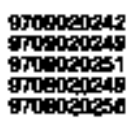 & 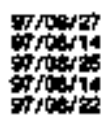 & 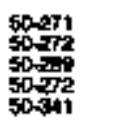 \\
\hline 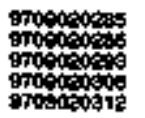 & 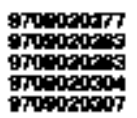 & 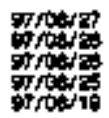 & 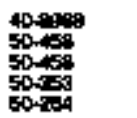 \\
\hline 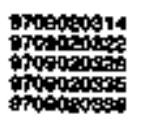 & 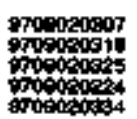 & 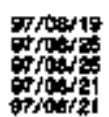 & 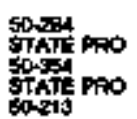 \\
\hline
\end{tabular}

Subject categories for nondocketed titems are also entered in this column by symbol; for example, ACAS. These categories are defined in Appendk B and listed in the Contents.

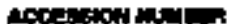

\begin{tabular}{|c|c|}
\hline 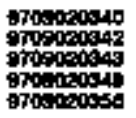 & \\
\hline 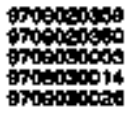 & \\
\hline 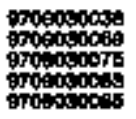 & ' \\
\hline 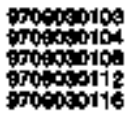 & \\
\hline 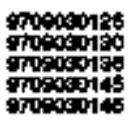 & \\
\hline 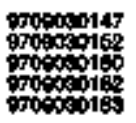 & \\
\hline 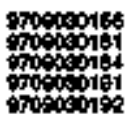 & \\
\hline ocoses & \\
\hline 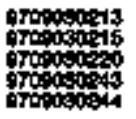 & \\
\hline 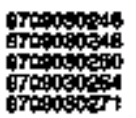 & \\
\hline 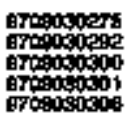 & \\
\hline 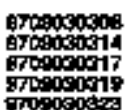 & \\
\hline
\end{tabular}

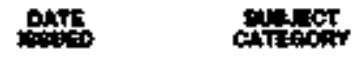

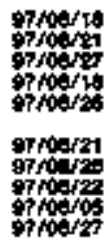

sinows

oroes

rrowat

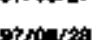

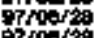

oprow

arowis

grroarz

orfoeraz

orpourat

orpourz

growes

Mrow

grroare

9proousto

83rouraz

orroarz2

grooras

oroarar

groerze

8710122

자막

Hrows

97708\%

ond

970010

oroot 18

isoert

Pot

intors

等0

pow

Fo요

on 1002

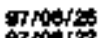

9700

150et

由rote

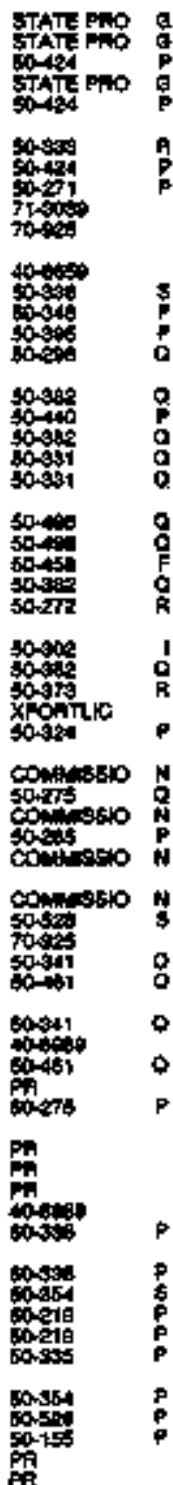




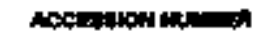

\begin{tabular}{|c|c|}
\hline$\frac{20003}{3}$ & 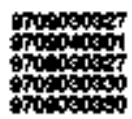 \\
\hline 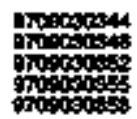 & 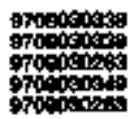 \\
\hline 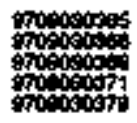 & 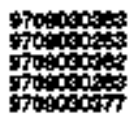 \\
\hline 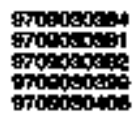 & 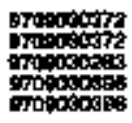 \\
\hline octs & 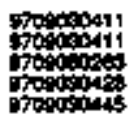 \\
\hline 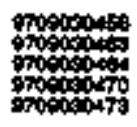 & 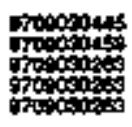 \\
\hline 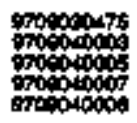 & 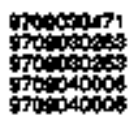 \\
\hline 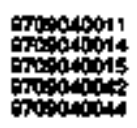 & 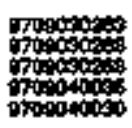 \\
\hline 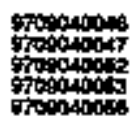 & 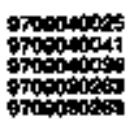 \\
\hline 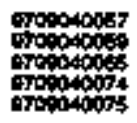 & 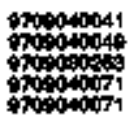 \\
\hline 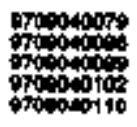 & 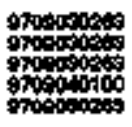 \\
\hline 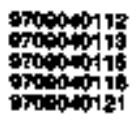 & 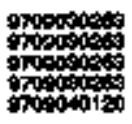 \\
\hline 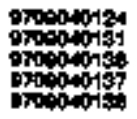 & 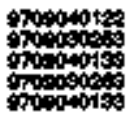 \\
\hline 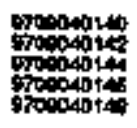 & 9ropentas \\
\hline 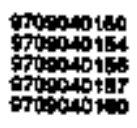 & 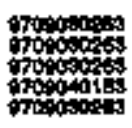 \\
\hline 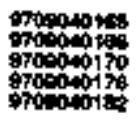 & 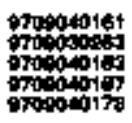 \\
\hline 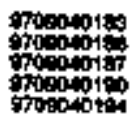 & 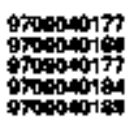 \\
\hline 6roposo19a & groould \\
\hline
\end{tabular}

necd

\begin{tabular}{|c|c|c|c|}
\hline 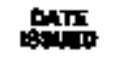 & chnte & & 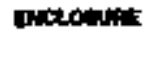 \\
\hline 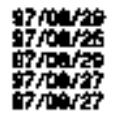 & 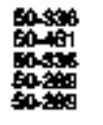 & $\begin{array}{l}\text { P } \\
\text { p } \\
\text { ? }\end{array}$ & 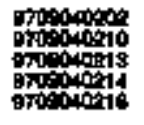 \\
\hline 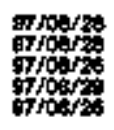 & 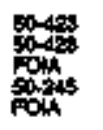 & P & 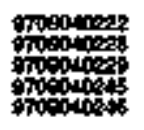 \\
\hline 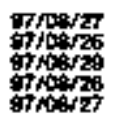 & 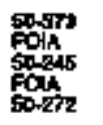 & $\begin{array}{l}s \\
p\end{array}$ & 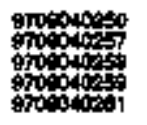 \\
\hline 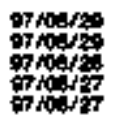 & 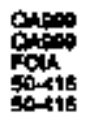 & p & 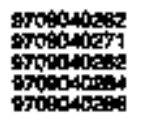 \\
\hline 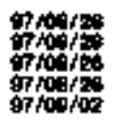 & 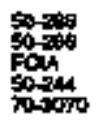 & 8 & 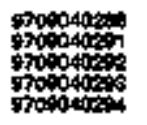 \\
\hline 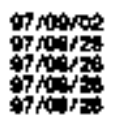 & 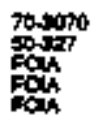 & $\mathbf{s}$ & 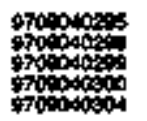 \\
\hline
\end{tabular}

grtoter

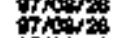

ition

然rod

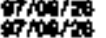

or

orjoarz

oropta

orotas

orotis

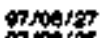

कrots

spotis

roer 1

oris

9000

का

prave

कrotict

spever

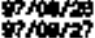

家lowat?

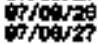

कroter

or

propts

orroparas

rrots

optain

onsta.

970

orradits

stopis

$90 \mathrm{cos}$

orroats

orroditas

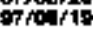

orforar

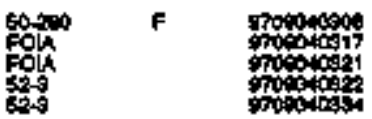

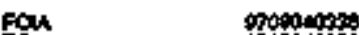

Fot

50.31 : P

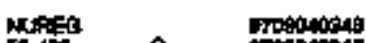

$00-400$ o

Fol

rou

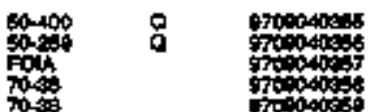

Fon

$\operatorname{lom}_{0 \rightarrow 0}$

tot.

4 두요

0

to

xartor

60.50

$00 \%$

00

60 -

sow

pots.

rat

rouk

con.

tos

.

tapos

Foth

5

sotis

sorson

5031

sion

0009

5040
Doctint

Grosonicas

Foopes

sonerose

moncets

omeopogis

7400

700

roposnes:

rrovescon

rogosopen

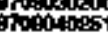

orropososen

pocons

prons

Proptof

posucosogs

7000

7roc0soces

Tocosose

100000

toto

poocosos

Fochlots

prontosts

Prisoness

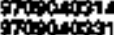

Protion

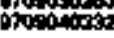

oporatiogry

Fococon

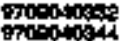

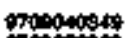

70000

70000

smoonecton

7romeris

orotos

stomonas

8700000

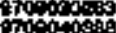

monoto

mopousese

00009

mopois

Profotols

Dusososut

rif

rato

Prososots

proposopes

7ropolet

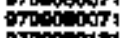

monerost

.

070

800000

snoosoros

Thospon

$\$ 700011$

Crotosito

socosonos
만단

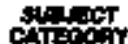

Expoestie

Shoers

ordour

Grow

g70018

orto

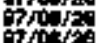

orosat

G)

97\%0s

grober

orpoy

970y?

Not

काtow

rotaze

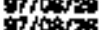

$4 / 08 / 28$

onderst

4t/ds:

Tris

trosz

0 cor

sfos

orom

卉

gratz?

Prous

7 or

ortosio

orotan

orous

grour

orrosiz

prays

protex

prot

of hert

proses

Fow

点

proder

ofots

orots

ofow

070043

rooy

$\rightarrow$ Hod

orropers

prota

70

67

P

orros

growas

prots

or

9700 hes

ortostas

$9700 / 2$

inders

ofotion

9 ropos

$\mathrm{OHOH}^{2}$

orom

कीNA

spopoes sorst

sosio

mis

$\rightarrow$

tax

Por

$40 \mathrm{H}+1$

ron

poras

Dowes

nit

sowes:

sols

50.57

30

400

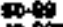

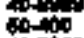

40 fect

4⿻丷요

4 4.

40

Fou

$71+1$

D

50345

tw

8034

50 .

Eotit

50-289

40

Bow

b0-60

wid

40

to

tar

40

40

entid

cond

fous.

opst

4at

sow

00091

0000

6ont

$80-40$

ates

Ba네

$80-40$

sater.

box

cont

0

-10

s0\%

牙?

sost

so-3i?

0

Q 


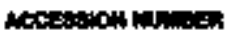

\begin{tabular}{|c|c|}
\hline 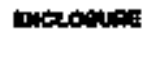 & ofoction \\
\hline 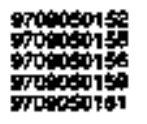 & 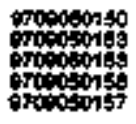 \\
\hline 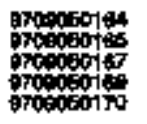 & 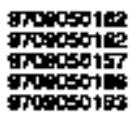 \\
\hline proponst & 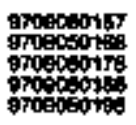 \\
\hline 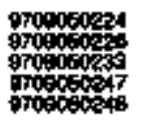 & 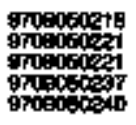 \\
\hline 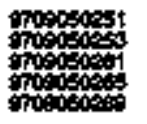 & 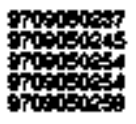 \\
\hline 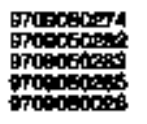 & 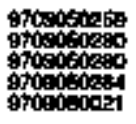 \\
\hline mopocosis & 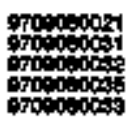 \\
\hline 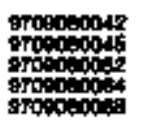 & 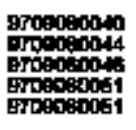 \\
\hline mopoporg & oropopops \\
\hline 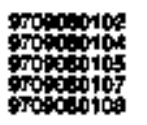 & 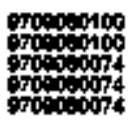 \\
\hline 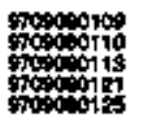 & spopos? \\
\hline 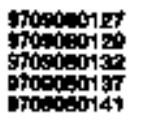 & 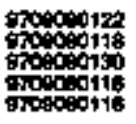 \\
\hline 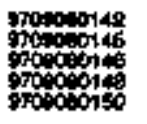 & 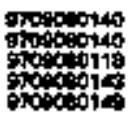 \\
\hline oropecis & 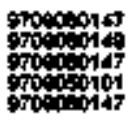 \\
\hline 90 & 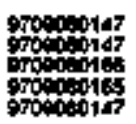 \\
\hline 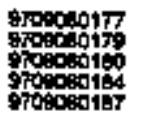 & 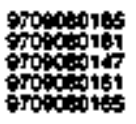 \\
\hline 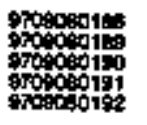 & 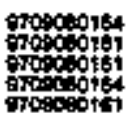 \\
\hline 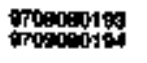 & Prosotitat \\
\hline
\end{tabular}

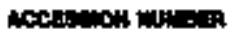

\begin{tabular}{|c|c|c|c|c|c|c|c|}
\hline th & apect & & ENCLOEURE & 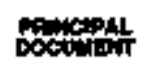 & pants & \multicolumn{2}{|c|}{ coflecters } \\
\hline 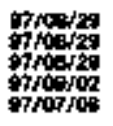 & 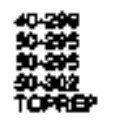 & ? & 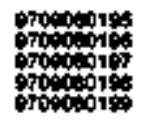 & 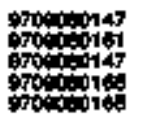 & 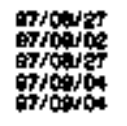 & 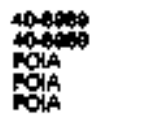 & \\
\hline 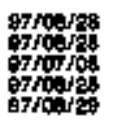 & 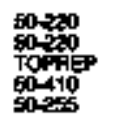 & $\stackrel{\mathbf{R}}{\mathbf{Q}}$ & 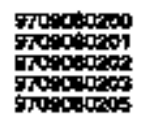 & 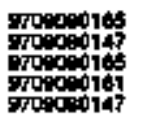 & 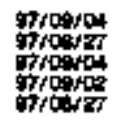 & 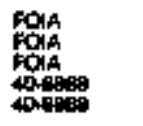 & \\
\hline 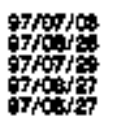 & 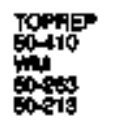 & w & 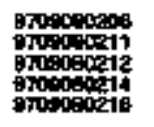 & 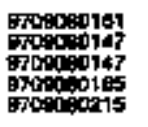 & 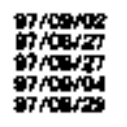 & 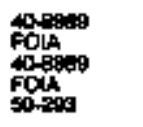 & p \\
\hline 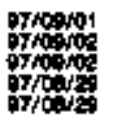 & 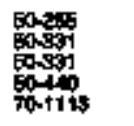 & $\begin{array}{l}8 \\
8 \\
8\end{array}$ & 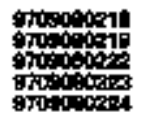 & 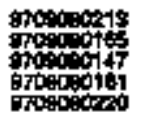 & 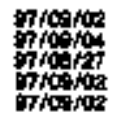 & 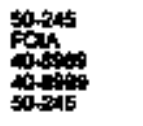 & s \\
\hline 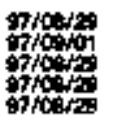 & 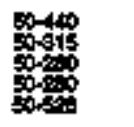 & 8 & 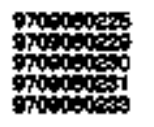 & 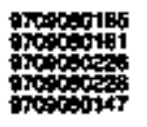 & 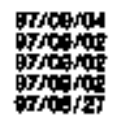 & 然 & 8 \\
\hline 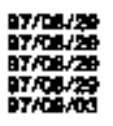 & 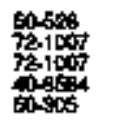 & $\mathbf{F}$ & 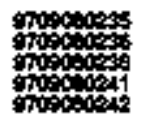 & 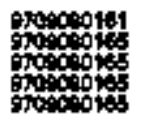 & 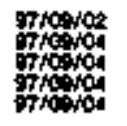 & 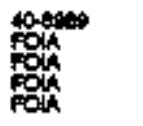 & \\
\hline 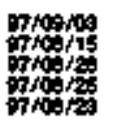 & 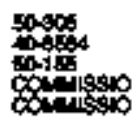 & W & 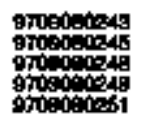 & 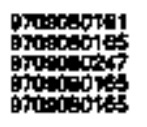 & 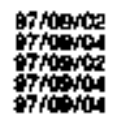 & 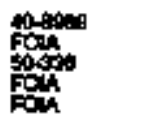 & p \\
\hline 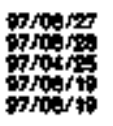 & 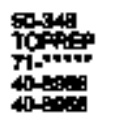 & 0 & 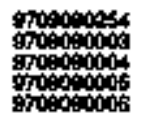 & 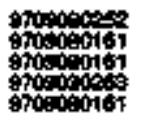 & 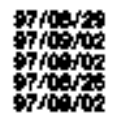 & 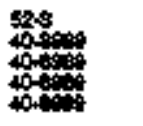 & \\
\hline 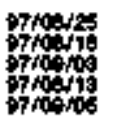 & 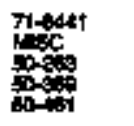 & g & 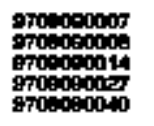 & 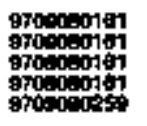 & 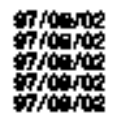 & 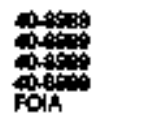 & \\
\hline 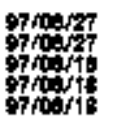 & 㟧 & & 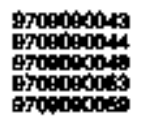 & 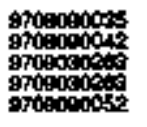 & 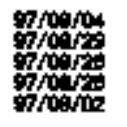 & 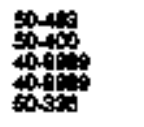 & 8 \\
\hline 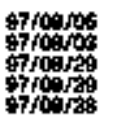 & 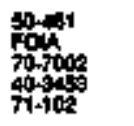 & 0 & 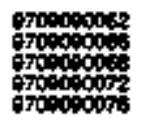 & 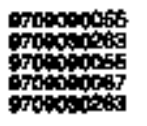 & 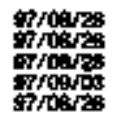 & 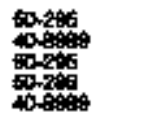 & $\begin{array}{l}\circ \\
0\end{array}$ \\
\hline 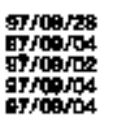 & 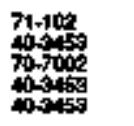 & & 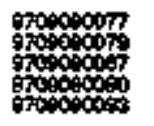 & 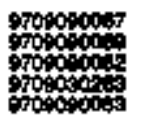 & 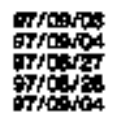 & 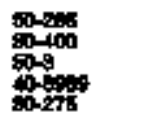 & $\begin{array}{l}p \\
\mathbf{f} \\
\mathbf{q}\end{array}$ \\
\hline 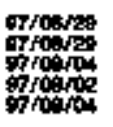 & 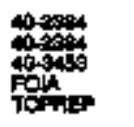 & & 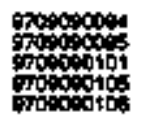 & 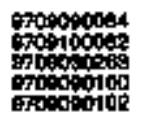 & 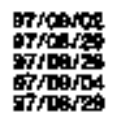 & 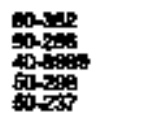 & $\begin{array}{l}8 \\
8 \\
0 \\
8\end{array}$ \\
\hline 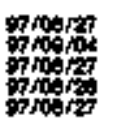 & 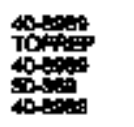 & $\mathbf{p}$ & 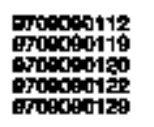 & 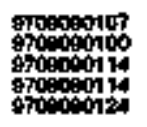 & 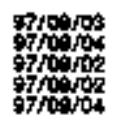 & 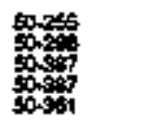 & $\begin{array}{l}p \\
0 \\
\vdots \\
0\end{array}$ \\
\hline 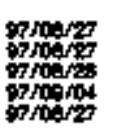 & son & & 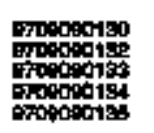 & 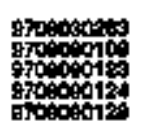 & 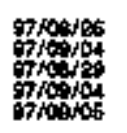 & 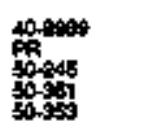 & $\stackrel{0}{8}$ \\
\hline 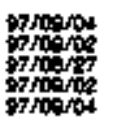 & 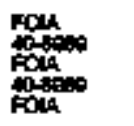 & & $\frac{1}{1000013}$ & 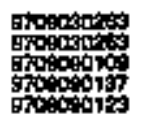 & 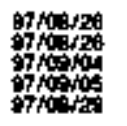 & 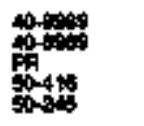 & 8 \\
\hline 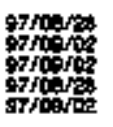 & 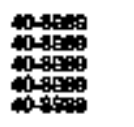 & & 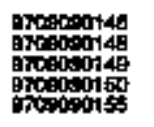 & 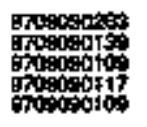 & 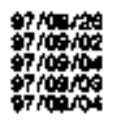 & 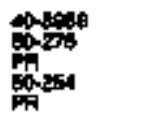 & 0 \\
\hline 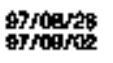 & 40.089 & & 90000158 & 9700000050 & $9700 / 28$ & 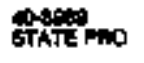 & g \\
\hline
\end{tabular}


AOC:

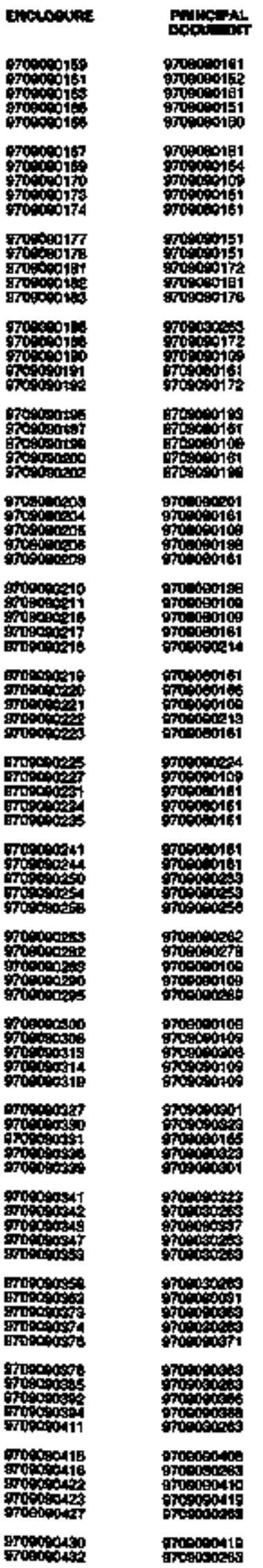

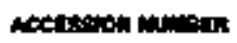

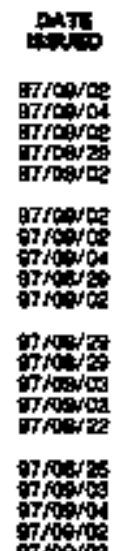

\begin{tabular}{|c|c|c|c|}
\hline 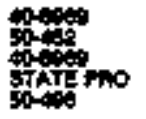 & $\frac{0}{p}$ & 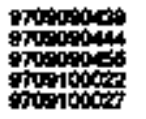 & 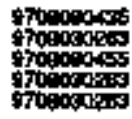 \\
\hline 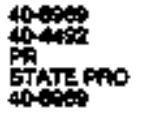 & a & 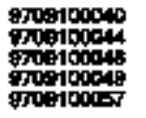 & 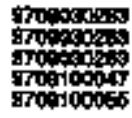 \\
\hline 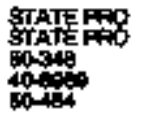 & $\begin{array}{l}6 \\
0 \\
0 \\
0\end{array}$ & 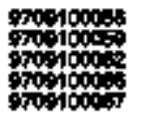 & 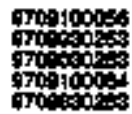 \\
\hline
\end{tabular}

growates

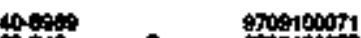

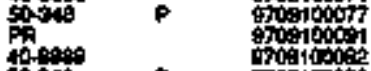

कroovos

oner

oftorion

ofotoc

orota.

ortos

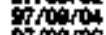

oftas

broters

审rotiol

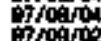

Errotros

prronine

prrowa

Br roporos

of rodur

Erreosap

aritosto

Frepora

oryoras

7 tort

orpor

or for

oftoges

Broerd

groout

sprograd

orrot

oftey

ortoot

orrouzs:

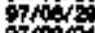

orpopo

orroter

orpers

Tros

wris

orfaras

proaris

Drowes

wrost

Eroara

Erfoaras

grropos

eropie

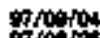

oforo

grota

91000

orroers g7000000\%

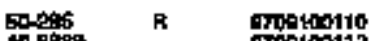

Fiseg grophyis

tome o

Bond P P

40-8969

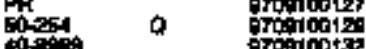

50-5e日 0 oropots

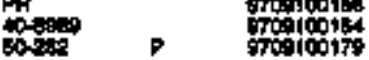

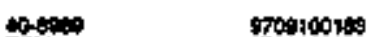

pon

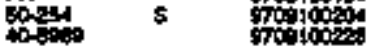

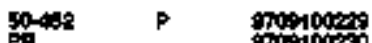

400\%

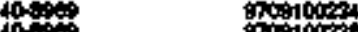

4000\%

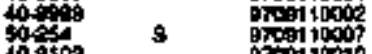

40 f 402 \&

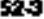

tora

is

Ban.

蒠

tom

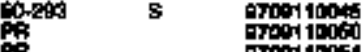

contor

solio $p$

0.45 ghertho

40.

itim

grositoors

novitoon

gropioges

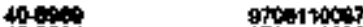

$40 .-4$

Tos

tore $P$ ofsonto

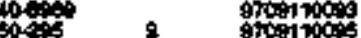

sosan $P$ orstront

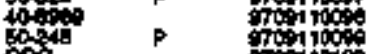

Cof

505

tont

pit?

ores vo10

orrag 1010

orois tais 13

$40-909$

40.7604

701ำ
50\%
700000

groposation

risposion

0ritiontis

Expionts

ropoonis

(n)

970000

oropotat

aropots

anconot

provonte

poinon

Norot

arinoter

Propores:

trotoxis

orotions

sporopen

towooes?

Foodtoog

ortorover

9monoces?

opopoger:

poitoosi

prosionet

monover?

9 monocest

orowoper

groptoos?

proes rot?

ragition

groes 1005

어엉

2001100\%

r.

rog $100 \%$

bogiontes

ond 100 e

otoloter

soprones?

motipos

owition.

oros 1100 क

orotanes?

asis

rop11011

g्रु0* 10115

grodid

\section{ormonos}

\%

of

prosest

BDAB

PRen

EThat15

orropes

8 row

Froto

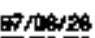

Pragos

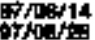

groence

Proparas

0

$\theta$

orion

Fors

9ropos

Prorfor

970013

Fromo

gropos

or poeros

Thoo

ordot.

$\rightarrow 0000$

ryora

70

70

Thowo

Froes

0 tow

ond

$07 / 00 / 05$

groaros

Frow

Whons

970000

9700

8rpolot

Protios

orposes

900

7000

srowo

srosot

sine

frow

ofrose

7rome

Fro

Fropos

ornowas

orforats

irroests

ofronas

Growos

Aropo

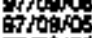

FIOWNOS

ginotos

chity

4007

0.

H.

onter

40.00

oflice

40-1169

400

索

cotos

sing

H.

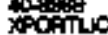

40 ope

00

70

E00sid

70143

0004

Fits

\%

$40-7307$

50 ㄱ:

$50-100$

bases

000

$\infty+1$

마.

sorat

0

S0-44.

40

th

낭

4

coss

40 의

40.40

40

sto

sor $27 \pi$

cotprant

5040

are

实迹

$\infty$

50.4.

4

40.6.

40.is:

Tof

0

Tomiti?

topentat

Top

然

stos

50 为 $\quad P$ 


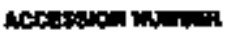

\begin{tabular}{|c|c|c|c|c|c|}
\hline anciogun & ofoctitit & 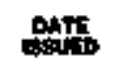 & cAnts & & GWLOAUA: \\
\hline 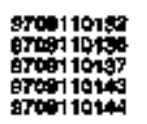 & 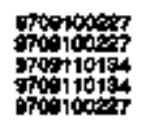 & 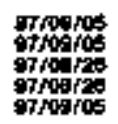 & 舟 & H & 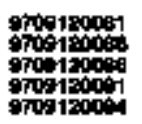 \\
\hline 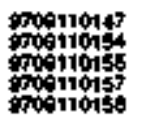 & 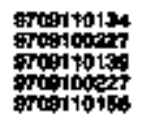 & 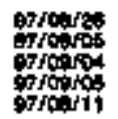 & 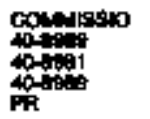 & M & 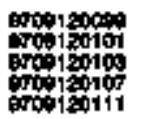 \\
\hline 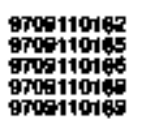 & 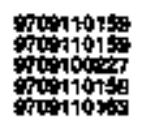 & 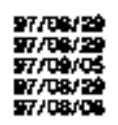 & 然 & y & 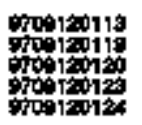 \\
\hline 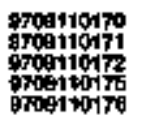 & 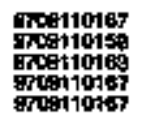 & 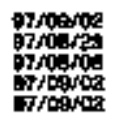 & 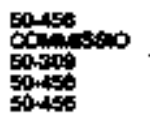 & $p$ & 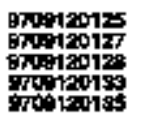 \\
\hline 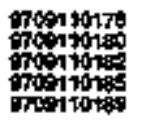 & 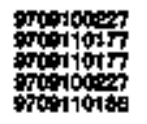 & 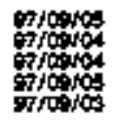 & & p & 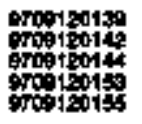 \\
\hline 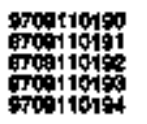 & 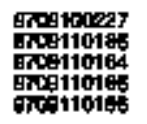 & 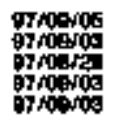 & 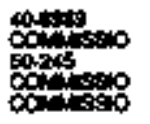 & $\begin{array}{l}4 \\
8\end{array}$ & 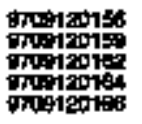 \\
\hline 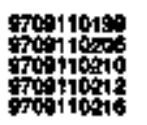 & 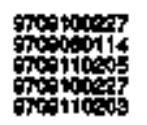 & 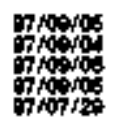 & 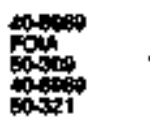 & $w$ & 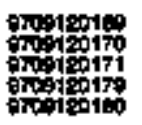 \\
\hline 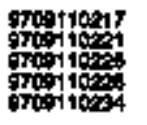 & 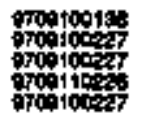 & 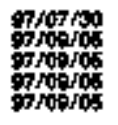 & 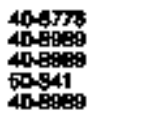 & P & 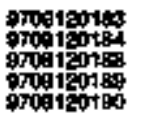 \\
\hline 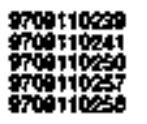 & 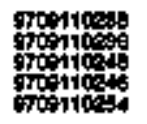 & $\begin{array}{l}\text { propos } \\
\text { prowos } \\
\text { prowos }\end{array}$ & 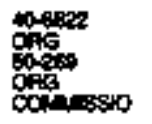 & $\begin{array}{l}\mathbf{P} \\
\mathbf{N}\end{array}$ & 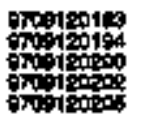 \\
\hline 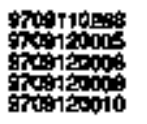 & 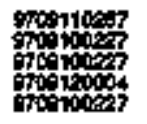 & 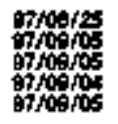 & 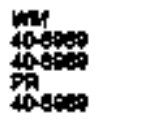 & & 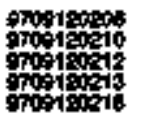 \\
\hline 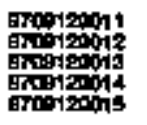 & 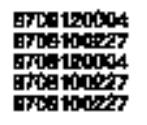 & 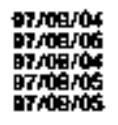 & 翼 & & 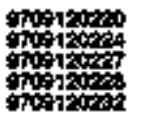 \\
\hline 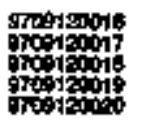 & 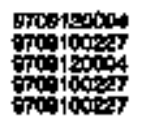 & 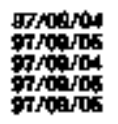 & 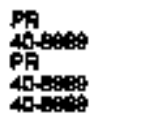 & & 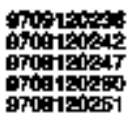 \\
\hline 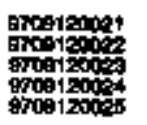 & 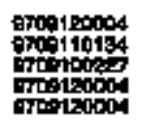 & 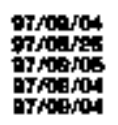 & parsos & H & 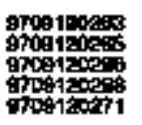 \\
\hline 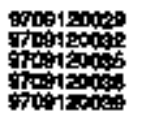 & 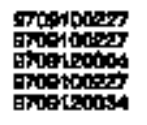 & 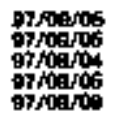 & 急 & 0 & 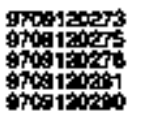 \\
\hline 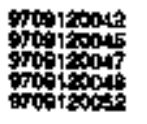 & 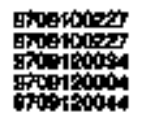 & 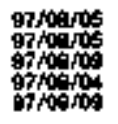 & 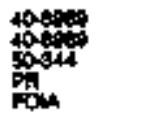 & 0 & 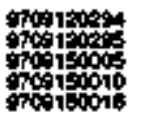 \\
\hline 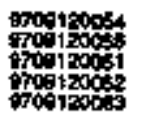 & 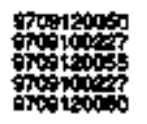 & 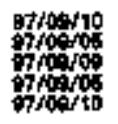 & 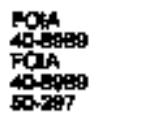 & $p$ & 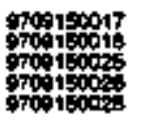 \\
\hline 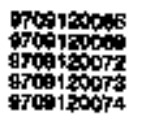 & 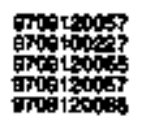 & 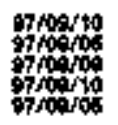 & $\begin{array}{l}40,0453 \\
006858 \\
604453 \\
009483\end{array}$ & $p$ & 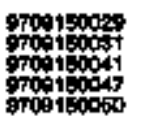 \\
\hline 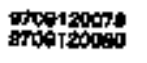 & Forpoer & 9yooros & 40.989 & & 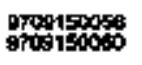 \\
\hline
\end{tabular}

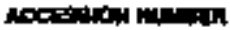

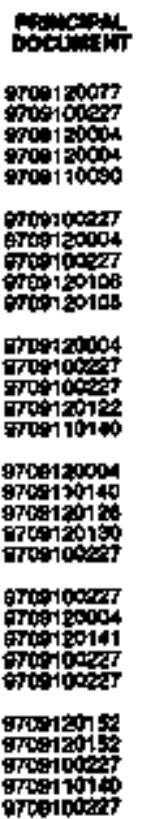

새곤

otrow os

moaro

Dropos

of

970905

97090

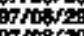

$97 / 08 / 29$

ofion

가에

ond

Trowar

$\min$

Thoos

oftows

97 roves

770 ara

$9 \mathrm{gr} 09 \mathrm{9}$

yroous

How

7.

Hot

oromanir

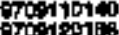

ortotsoles

Doeraotre

9rotiogers

9rostors

oroorizits

arcorocar

xpon

propigation

orcerocpa

(20030

trotoz

prosens

(1)

然 $100 \%$

sponpeses

970900027

groetcous

97061002s?

90012005

onctoos

ming

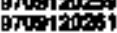

Bropizusc

sock

ond

कot

pooraen

ros 1000

\%001500

70015001s:

900150014

9709050003

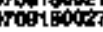

orop15000

Fo915002

90001.005

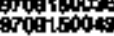

gordsong
9709010

grosiz

yrosters

Mrowos

orapos

g

Finotos

Thiot 10

grosos

grogye

Tho

grrow

gryosicos

Troovo

grow

onpos

$7 / 000$

$7 \mathrm{NON}_{0}$

0 rowos

7 ow

090000

gropos

Tromen

rosio

ato

(1)

7.

rotion

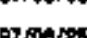

1,000

aptoro

mpor

propes

2700

7ifosp

onrost 19

g7fos 70

97 rowa

or 000

irfots

garoos 70

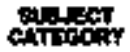

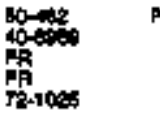

tonst

a

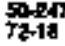

Pn.

40

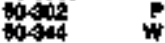

\%

oxidy

7070

40-8099

岸

$40-1182$

40 a

Eos

tot

ont w

STATE PAO

5034

30045

sons

bogr

dof

00

popes

sise

tots

(20000

tons

sto

artes

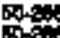

(9)

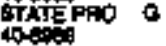

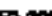

40

40

40

4has

D.t.

solo

casys $P$

突皮

STAEF⿱

GT실 PRO 9

ojons

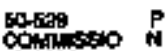

in

onde

soust 0

Cotusson

cotips

50301 


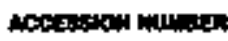

\begin{tabular}{|c|c|c|}
\hline 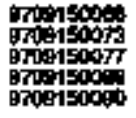 & 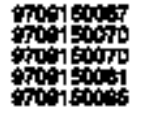 & 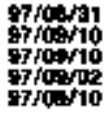 \\
\hline 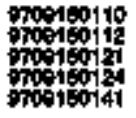 & 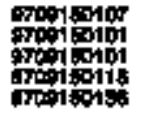 & 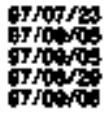 \\
\hline 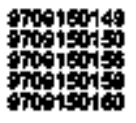 & 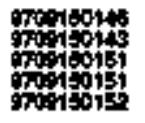 & 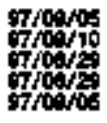 \\
\hline 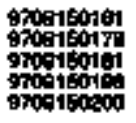 & 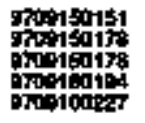 & 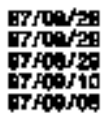 \\
\hline 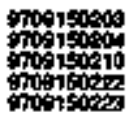 & 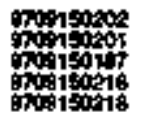 & 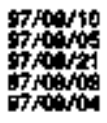 \\
\hline 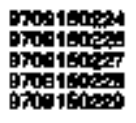 & 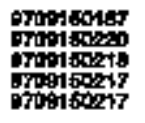 & 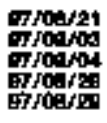 \\
\hline 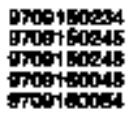 & 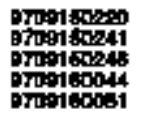 & 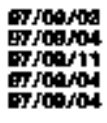 \\
\hline 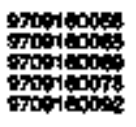 & 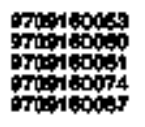 & 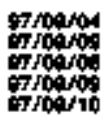 \\
\hline 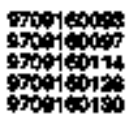 & 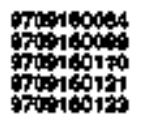 & 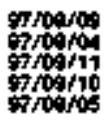 \\
\hline 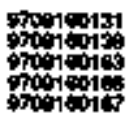 & 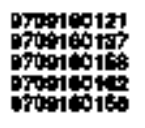 & 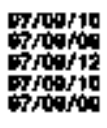 \\
\hline 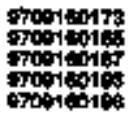 & 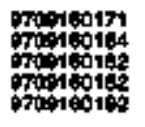 & 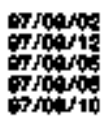 \\
\hline 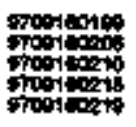 & 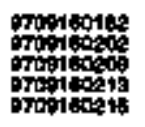 & 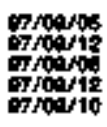 \\
\hline 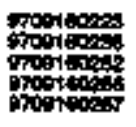 & 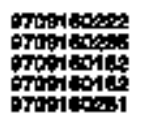 & 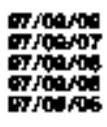 \\
\hline 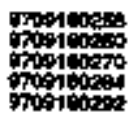 & 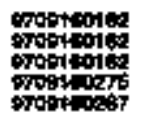 & 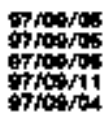 \\
\hline 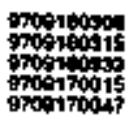 & 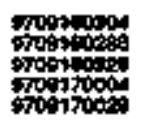 & 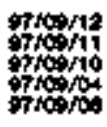 \\
\hline 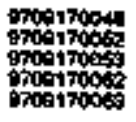 & 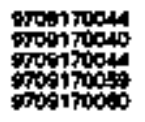 & 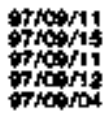 \\
\hline 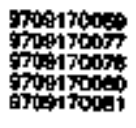 & 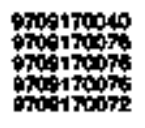 & 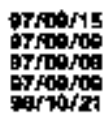 \\
\hline spopropes & 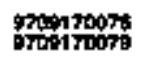 & $\begin{array}{l}\text { grospos } \\
\text { Trtosti2 }\end{array}$ \\
\hline
\end{tabular}

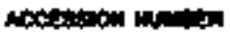

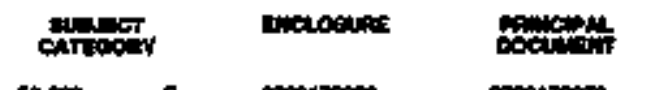

phre

tritiot

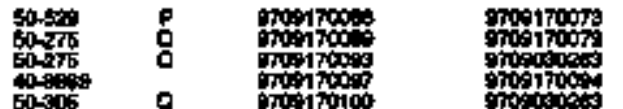

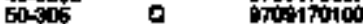

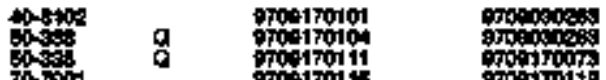

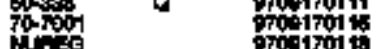

oropingits

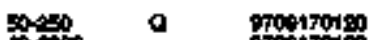

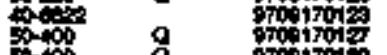

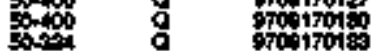

EO-wo

$\stackrel{g}{\mathrm{~g}}$

Popro-18s

tosiroit

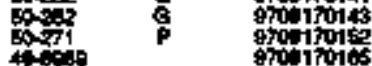

440

ropiroits

sots

30.7001

sart

70. 1001

rame

Ea-27\%

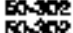

son:

SO-2

ind

So-271

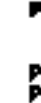

70017047

700170184

posipon

970 1 0195

gropints

sposinesos

stipinats

spopinats

\$popine

spoptow

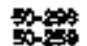

5014

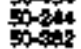

prodsues

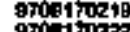

9700tioges

oroditoes

ro-rone prostrees.

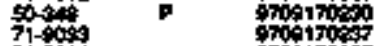

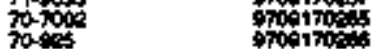

masoor

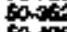

const

$\stackrel{8}{0}$

soginat

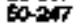

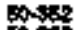

然急

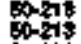

sotios

proptrots

Foitsila

mor.112

ofotitis

gencisist

somitedt

poingones:

moviromic.

orotrof?

shot Toss

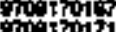

porion

-rostrola

toperoin

Dor

atorion

groverozut

gitor 1017

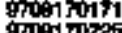

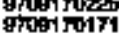

grter rom

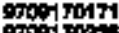

oropras:

orot The

sood res?

was

oto

pointore

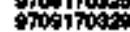

grovroces

7ing.

For

onov rosed

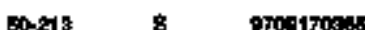

ह0-15

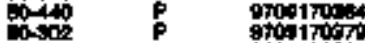

5

otopindsi

71 ias

s7oo1000

E0.215 8 97019000

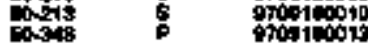

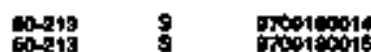

60-13
sints

Bome日s

4ons

sis of

ofistions

aripane.

Wision

oritas

movions

Fela

fin

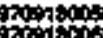

axtion

orotecos.

roth

Conisos:

Comisisto $N$

Cowiss

dxp9:8011

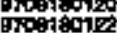

oxporats

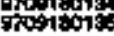

combstion of
Fror rose

incorocis

otom

groprood

Hor

spridoto

For

spontopos

pantons

propicic

Drontoos

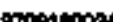

mopisole

poripos

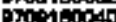

prosiboces

Finos:

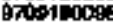

97001010:

grosiono

popipan

poing

onconos

popigists ortorio

Fint

phas.

intar.

orropas

ortopio

orionis

nowat

300

orit

srosing

growi

grjoa/11

grjoa/t

proper

orotis

growit

oxpog/ 12

的ori1

ond

stoost1

monto

optodit

orrosit

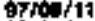

070

grodis

ormodis

7001

prosios

ondos

ortor

Fin

1000

ortosnos

orropos

Frow

Frow

on

orroolos

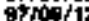

ortosto

然保

Trom/16

0001

0 tront

ofosit

7 row

Fropo

Fop

जowits

o7jes:

ofrow

ofsy

oropis

croarse

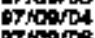

7 roogt

FroOrit

9700

7 rovi

PoN11

opdotiv

oryosen ofts

fout

For

Rola

optos?

olis:

P

obits

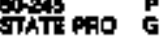

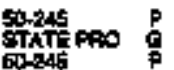

6x-4:

colver?

कotid

Forton

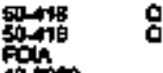

की

Fon.

iptis

tont

Dosio

for

Tates

Ttpos:

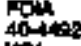

into

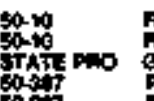

sons

隹

sis

st?

60-4

tor

ind

60-20

要然

sol

牙部

so-sos

7 \%

So-

T2-12.7

거운

70.427

4006

T0-1207

70-1113

연:

opts:

som

con

po- 39

媐密

g

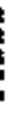

p

p

\section{?}

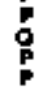

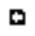




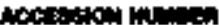

\begin{tabular}{|c|c|c|c|c|c|}
\hline 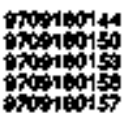 & 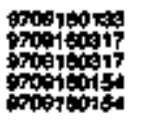 & 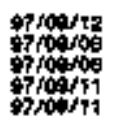 & 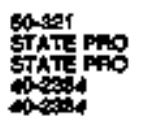 & $\begin{array}{l}\text { f } \\
\mathbf{6} \\
\mathbf{6}\end{array}$ & 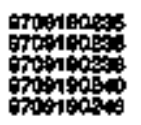 \\
\hline 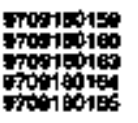 & 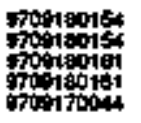 & 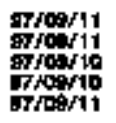 & & & 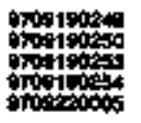 \\
\hline 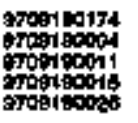 & 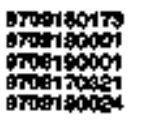 & 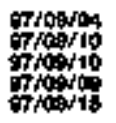 & $\frac{5004}{70-27}$ & p & $\log _{000}$ \\
\hline 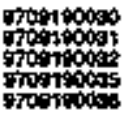 & 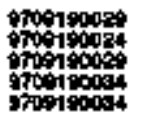 & 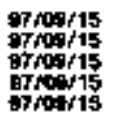 & & $\begin{array}{l}\mathbf{P} \\
\mathbf{R} \\
\mathbf{R} \\
\text { R } \\
\text { P }\end{array}$ & 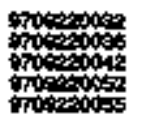 \\
\hline 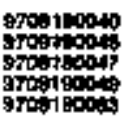 & 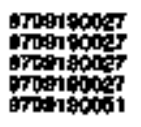 & 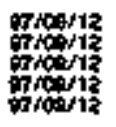 & $\begin{array}{l}\frac{72-27}{72-22} \\
\frac{72-72}{7-72} \\
50-605\end{array}$ & 0 & 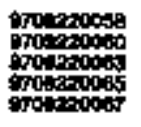 \\
\hline 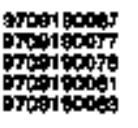 & mosisood & 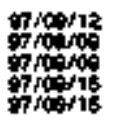 & & $\begin{array}{l}\text { g } \\
\text { p } \\
\text { 욤 }\end{array}$ & 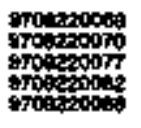 \\
\hline 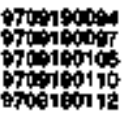 & speraos & 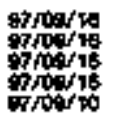 & 4 & م & 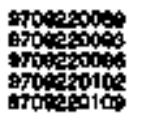 \\
\hline 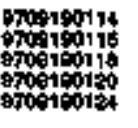 & 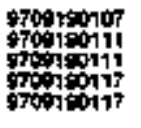 & 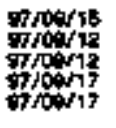 & & $\stackrel{q}{\mathbf{p}}$ & 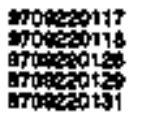 \\
\hline 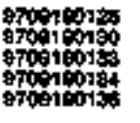 & 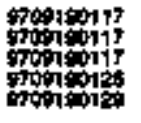 & 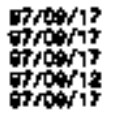 & & $\underset{p}{p}$ & 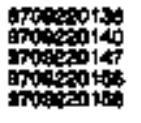 \\
\hline 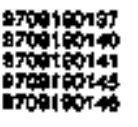 & 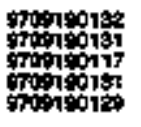 & 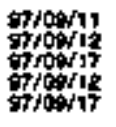 & 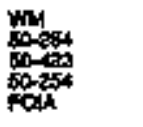 & 古 & 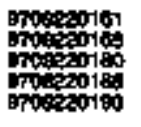 \\
\hline 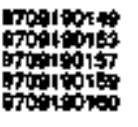 & 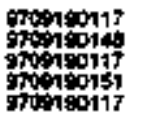 & 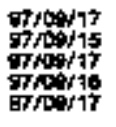 & 象 & $\begin{array}{l}\mathbf{p} \\
\mathbf{p} \\
\mathbf{p}\end{array}$ & 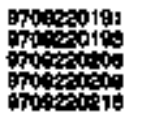 \\
\hline 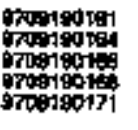 & 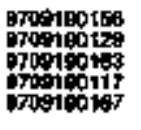 & 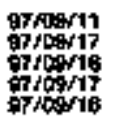 & 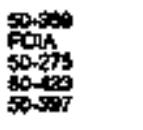 & $\begin{array}{l}8 \\
0 \\
0\end{array}$ & 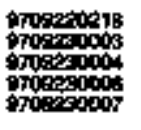 \\
\hline 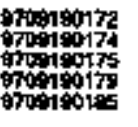 & 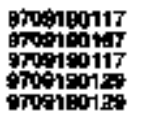 & 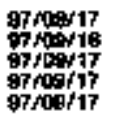 & so-dis & $\stackrel{P}{\dot{P}}$ & 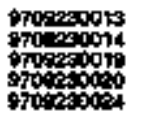 \\
\hline 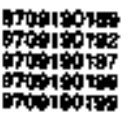 & 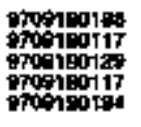 & 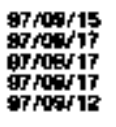 & & $\begin{array}{l}5 \\
5 \\
0\end{array}$ & 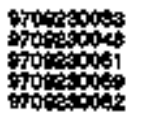 \\
\hline 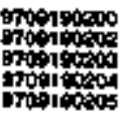 & 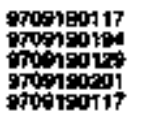 & 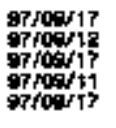 & & $\begin{array}{l}p \\
9 \\
9\end{array}$ & 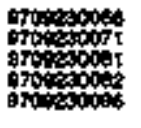 \\
\hline 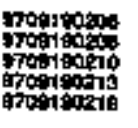 & 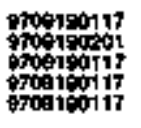 & 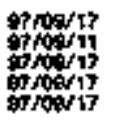 & 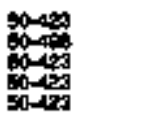 & $\begin{array}{l}P \\
Q \\
p \\
p\end{array}$ & 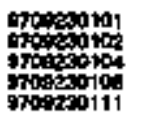 \\
\hline 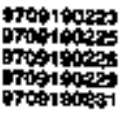 & 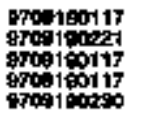 & 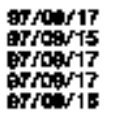 & 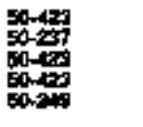 & $\begin{array}{c}p \\
5 \\
p \\
8\end{array}$ & 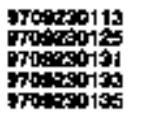 \\
\hline 9709009 & 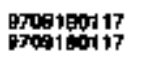 & $\begin{array}{l}\text { orroar17 } \\
\text { arrodit }\end{array}$ & $50-40$ & $p$ & 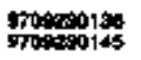 \\
\hline
\end{tabular}

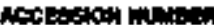

\begin{tabular}{|c|c|c|c|}
\hline 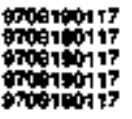 & 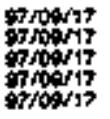 & 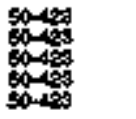 & $\begin{array}{l}\mathbf{p} \\
\mathbf{p} \\
\mathbf{p}\end{array}$ \\
\hline 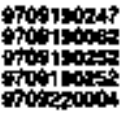 & 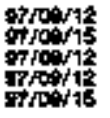 & $\begin{array}{l}50-460 \\
50-54 \\
50-77 \\
50,77 \\
50-413\end{array}$ & 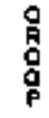 \\
\hline 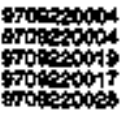 & 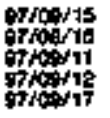 & 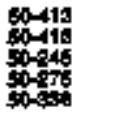 & $\begin{array}{l}\mathbf{p} \\
\mathbf{5} \\
0 \\
0\end{array}$ \\
\hline 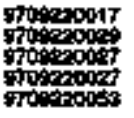 & 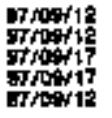 & 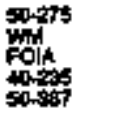 & $p$ \\
\hline 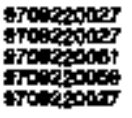 & 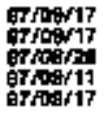 & 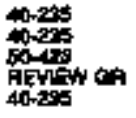 & $\frac{K}{p}$ \\
\hline 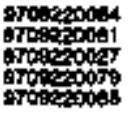 & 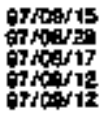 & 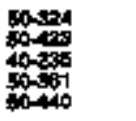 & $\mathbf{S}$ \\
\hline$\frac{170000}{600000}$ & 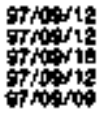 & 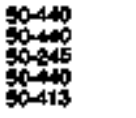 & $\begin{array}{l}\mathbf{P} \\
\mathbf{n} \\
\stackrel{\mathrm{Z}}{\mathbf{F}} \\
\mathbf{F}\end{array}$ \\
\hline 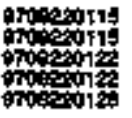 & 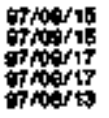 & satis & 乌 \\
\hline 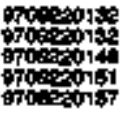 & 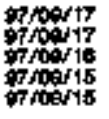 & 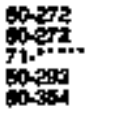 & 음 \\
\hline 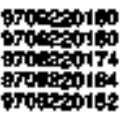 & 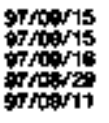 & 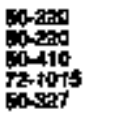 & $\begin{array}{l}0 \\
\frac{0}{p} \\
0\end{array}$ \\
\hline 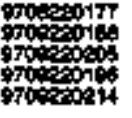 & 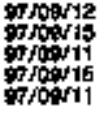 & 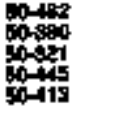 & $\begin{array}{l}5 \\
5 \\
0 \\
6 \\
0\end{array}$ \\
\hline 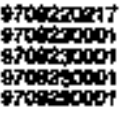 & 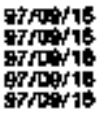 & 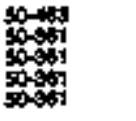 & $\begin{array}{l}o \\
p \\
p \\
p \\
p\end{array}$ \\
\hline 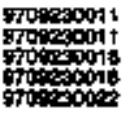 & 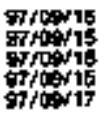 & 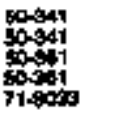 & $\begin{array}{c}\text { ด } \\
\text { ค } \\
\text { ค̆ } \\
\text { ค } \\
\text { ค }\end{array}$ \\
\hline 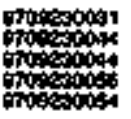 & 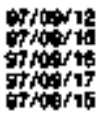 & 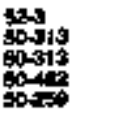 & 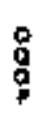 \\
\hline 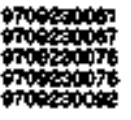 & 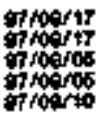 & 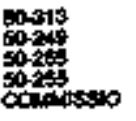 & $\frac{9}{0}$ \\
\hline 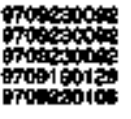 & 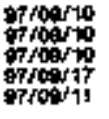 & 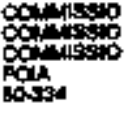 & 樒 \\
\hline 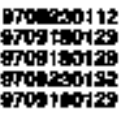 & 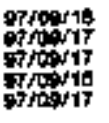 & 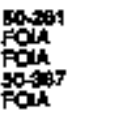 & $P$ \\
\hline 8000018 & orrogurs & $\begin{array}{l}\text { romis? } \\
\text { Roin }\end{array}$ & $\mathbf{P}$ \\
\hline
\end{tabular}




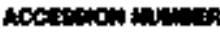

\begin{tabular}{|c|c|c|}
\hline 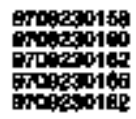 & & 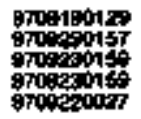 \\
\hline 9rapays & & 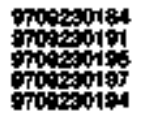 \\
\hline 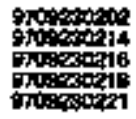 & & 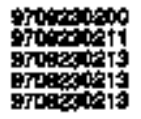 \\
\hline 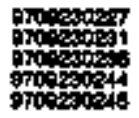 & & 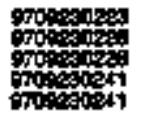 \\
\hline 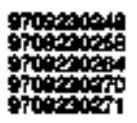 & & 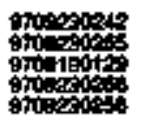 \\
\hline 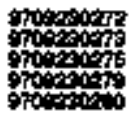 & & 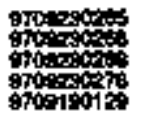 \\
\hline 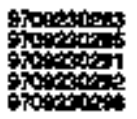 & & 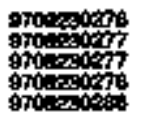 \\
\hline 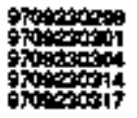 & ' & 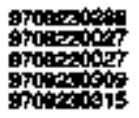 \\
\hline 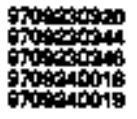 & & 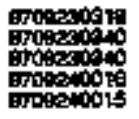 \\
\hline 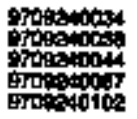 & & 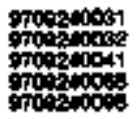 \\
\hline 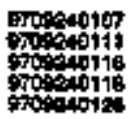 & & 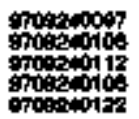 \\
\hline 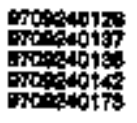 & & 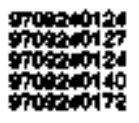 \\
\hline 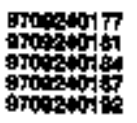 & & 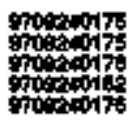 \\
\hline 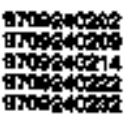 & & tonsos \\
\hline 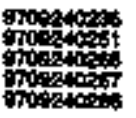 & & aring \\
\hline 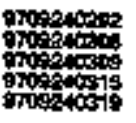 & & 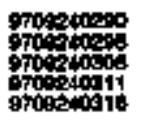 \\
\hline 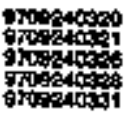 & & 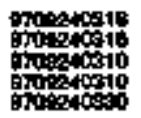 \\
\hline grocactoss & & 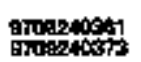 \\
\hline
\end{tabular}

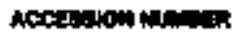

\begin{tabular}{|c|c|c|c|c|}
\hline 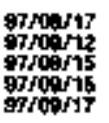 & 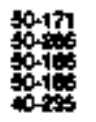 & $\begin{array}{l}\mathbf{s} \\
\frac{8}{n} \\
\frac{1}{n}\end{array}$ & 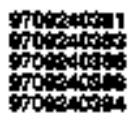 & 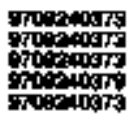 \\
\hline 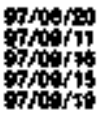 & 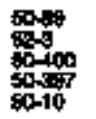 & $\begin{array}{l}\mathbf{R} \\
\mathbf{F} \\
\mathbf{R}\end{array}$ & 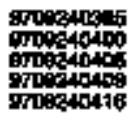 & 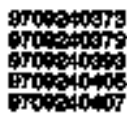 \\
\hline 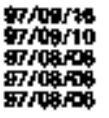 & $\frac{505}{70-15}$ & $\underset{F}{F}$ & 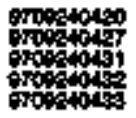 & preacotis \\
\hline 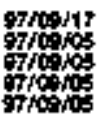 & 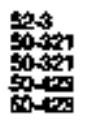 & $\begin{array}{l}\mathbf{p} \\
\mathbf{p} \\
\mathbf{p}\end{array}$ & 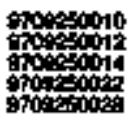 & 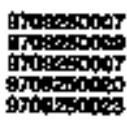 \\
\hline 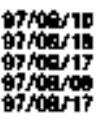 & 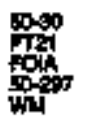 & 0 & 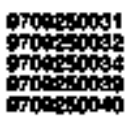 & 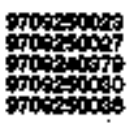 \\
\hline 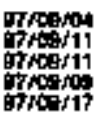 & 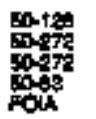 & $\begin{array}{l}\mathbf{p} \\
\mathbf{p} \\
\mathbf{p}\end{array}$ & 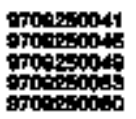 & 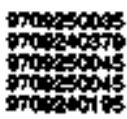 \\
\hline
\end{tabular}

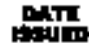

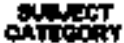

oprosis

70019

700

जow

57Now1:

770002

Emow 1

$07 / 00612$

orropite

ofores

7 ood

ntroara

Troeraz

growes

prows

ow

pon

9roto

prosts

70

7 ow

stopecos

oy/0g/41

1700

g7hoorit

gyroaros

groant

groert

orosto

9709

6ridis

\%7/00/19

propos

propsts

7 ropos

sroesto

970019

\%

sroent

970005

7 tonos

stow1

nowose

of

oftowis

onders

inopis?

ontion

oroers

ofor

$0 \sin ^{\circ}$

gyroess

9709

ofoep

sints

贺胫

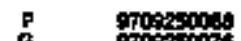

然

20045

的果

Ditopito

orrogrts moto

optontod

ontionts

\$213 $B$ groptor

tis

Dow 010

W0

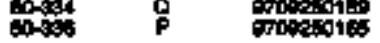

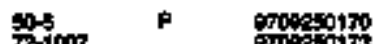

72-100

ose F F

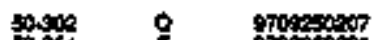

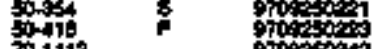

this

70

무요

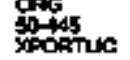

ifucosont

oroosanes

70-?

究

s stoposess

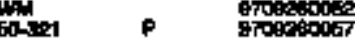

$40-309$

40,0

0000

siones

Tom:

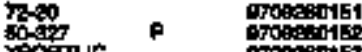

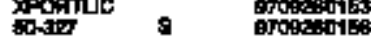

$\sin$

she

St.31

2700004

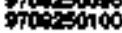

oncosocos

$70 \rightarrow+0$

ortions

oropsonce

Hom

oforing

oroesonos

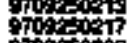

goto 0 se

stivinase

Propontost

gopos

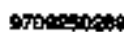

prosectos

procitas 14

由roposi

Evionsors

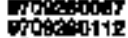

由rogerota1

rogespr4s

9rocesols

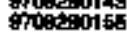

Stooes0144

ofogis

$50 \%$ \% 0101

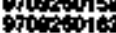

Fropone7

sons

stis

垈果

soms

60.34

$50-348$

Titast movis

770

700

7row

prowit

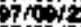

कrowa

9001

7roov.

7000

orroerta

proovis

nions

91002

groevis

orropit

gropit

$0700 / 17$

71007

ind

grogras

s7oms

7/00\%

7ron:

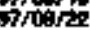

scon

列

Pris

onotit

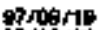

onts

개

groph

ayoortip

Froots

groot

orroars

moors

orions

onto

gropir

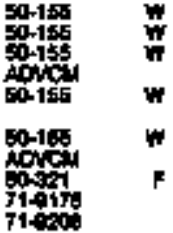

71 in

ADts

ADici

cosas

50.75

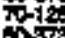

cons?

A

ow

rom.

애

Fon

XpontrL

constiver *

7

sone 0

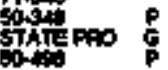

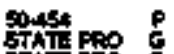

空能F

जिसाए PAO है

MAT?

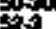

STMTEPOO

borar

sonst

ond

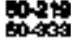

nat

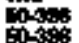

poste

$0-154$

$50-609$

50.75

coinsos

pouk

Bong

50-145

$\operatorname{lin}_{0 \rightarrow 0}$

A

40 oss

50

con

ind

$70-7001$

$70-700$

50-101

rowon

0

Eato

28

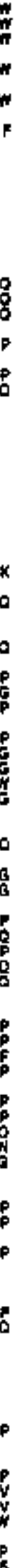




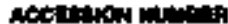

\begin{tabular}{|c|c|c|c|c|c|}
\hline 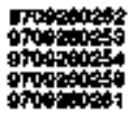 & 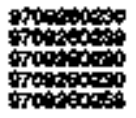 & 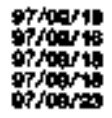 & 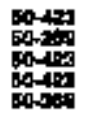 & $\stackrel{p}{p}$ & 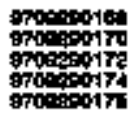 \\
\hline 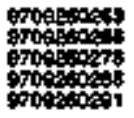 & ortopes & 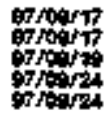 & 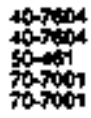 & 5 & 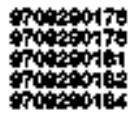 \\
\hline 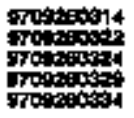 & 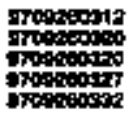 & 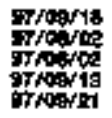 & 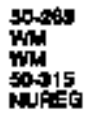 & $p$ & 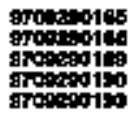 \\
\hline 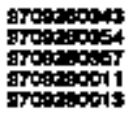 & 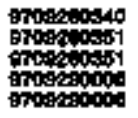 & 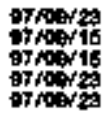 & 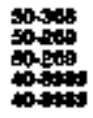 & $\begin{array}{l}p \\
\stackrel{p}{p} \\
p\end{array}$ & 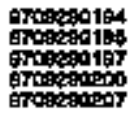 \\
\hline 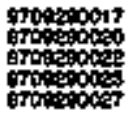 & 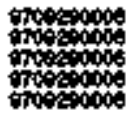 & 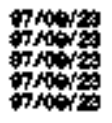 & & & 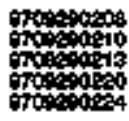 \\
\hline 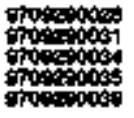 & 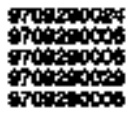 & 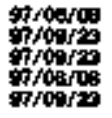 & 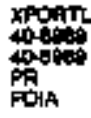 & & 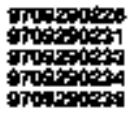 \\
\hline 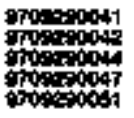 & 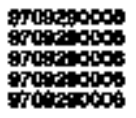 & 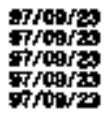 & 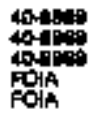 & & 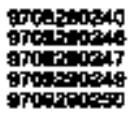 \\
\hline 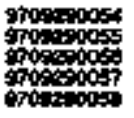 & 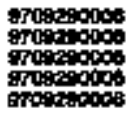 & 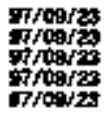 & 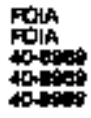 & & 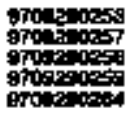 \\
\hline 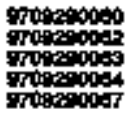 & 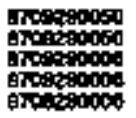 & 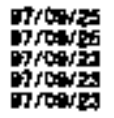 & & p & 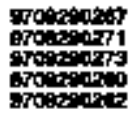 \\
\hline 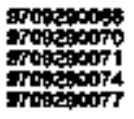 & 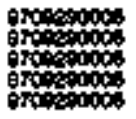 & 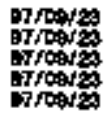 & & & 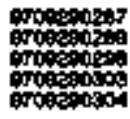 \\
\hline 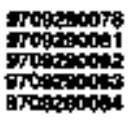 & 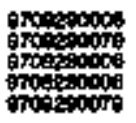 & 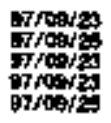 & $\begin{array}{l}\text { FO } \\
60\end{array}$ & 0 & 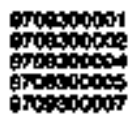 \\
\hline 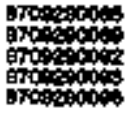 & 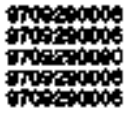 & 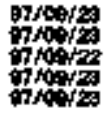 & & 5 & 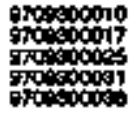 \\
\hline 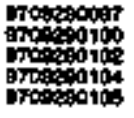 & 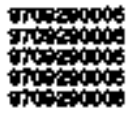 & 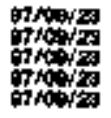 & & & 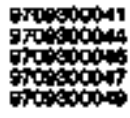 \\
\hline 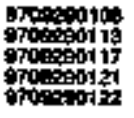 & 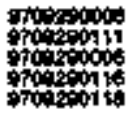 & 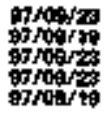 & 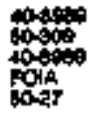 & w & 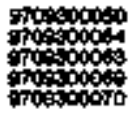 \\
\hline 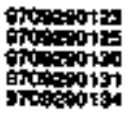 & 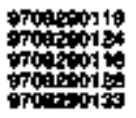 & 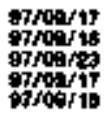 & 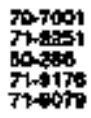 & $\mathbf{F}$ & 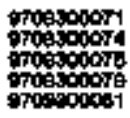 \\
\hline 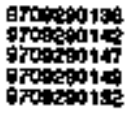 & 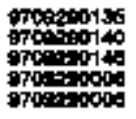 & 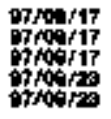 & 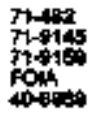 & & 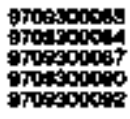 \\
\hline 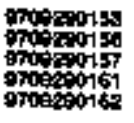 & 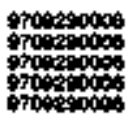 & 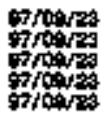 & 8. & & 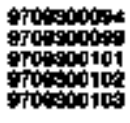 \\
\hline 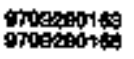 & $\begin{array}{l}970 \% \text { oposs } \\
\text { sropsopos }\end{array}$ & कraps & $40-4$ & & 970000107 \\
\hline
\end{tabular}

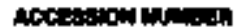

\begin{tabular}{|c|c|c|}
\hline 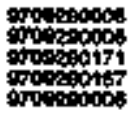 & 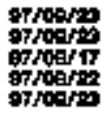 & 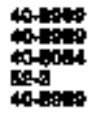 \\
\hline 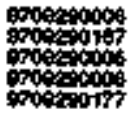 & 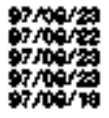 & \\
\hline 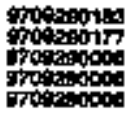 & 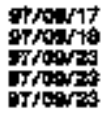 & \\
\hline 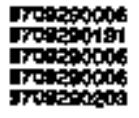 & 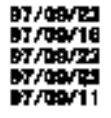 & \\
\hline 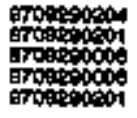 & 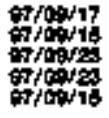 & \\
\hline 等 & 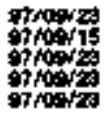 & \\
\hline 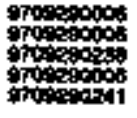 & 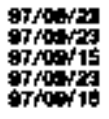 & \\
\hline 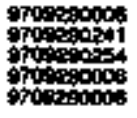 & 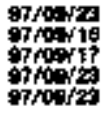 & \\
\hline 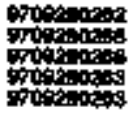 & 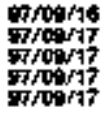 & 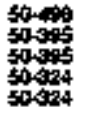 \\
\hline 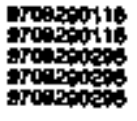 & 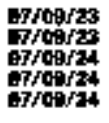 & $\begin{array}{l}50-206 \\
50=0 \\
00-454 \\
50-454 \\
50-454\end{array}$ \\
\hline 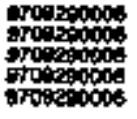 & 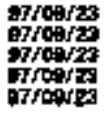 & \\
\hline 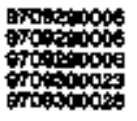 & 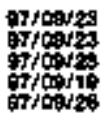 & \\
\hline 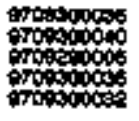 & 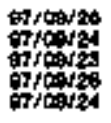 & \\
\hline 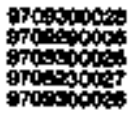 & 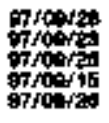 & 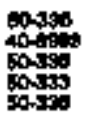 \\
\hline 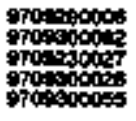 & 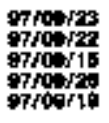 & 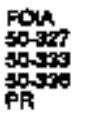 \\
\hline 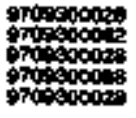 & 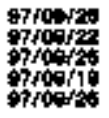 & 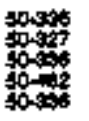 \\
\hline 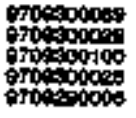 & 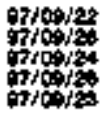 & 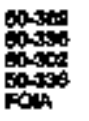 \\
\hline 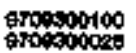 & 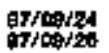 & $\begin{array}{l}\text { sosec } \\
\text { E0-sobe }\end{array}$ \\
\hline
\end{tabular}




\section{CROSS REFEAENCE OF ENCLOSURES TO PRINCIPAL DOCUIEEATS}

Actiosedn muneth

\begin{tabular}{|c|c|}
\hline 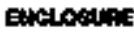 & $\frac{24}{3}$ \\
\hline 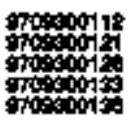 & 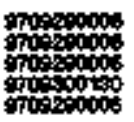 \\
\hline opoptat & 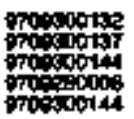 \\
\hline 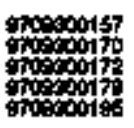 & 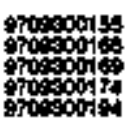 \\
\hline moposion & 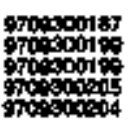 \\
\hline 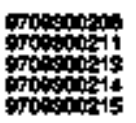 & 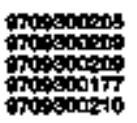 \\
\hline 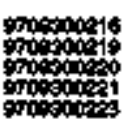 & opopont7 \\
\hline 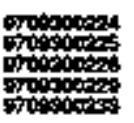 & 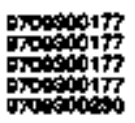 \\
\hline 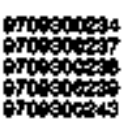 & olopost \\
\hline 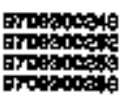 & plocosess \\
\hline
\end{tabular}

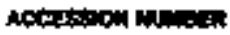

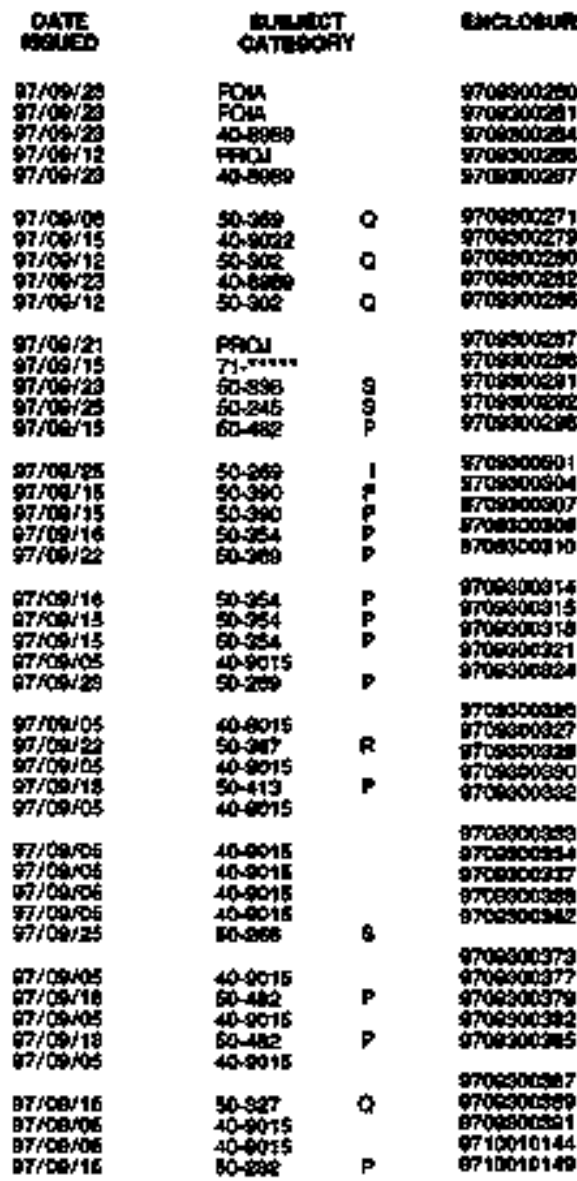

pape

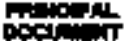

Fosogort

grasopects

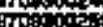

Eroopotit

moconosis

ofoction:

otesticts

moosiogrs

moesoos?

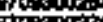

Thenctis

errasones:

orcopolon

oritopt?

Totoposon

stotocoso

$\operatorname{cocos}$

mopin:

spowoon

oncostonte

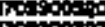

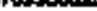

grater

choroser

propos

(1)

oriotis

orotasoit is

oruocosos

orotots

Thoos

Pivolosits

97050007

9rosonery

Prosoca

posposat

poostoit?

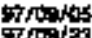

7

Trtorte

srowts

7 torts

orons

oriont

97100118

oftorito

oritio

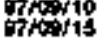

aroor 18

rroins

arion

orfopere

onforis

orrowo

orosis

orpowos

nowas

thora

ind 1

protar

mpares

propers

7 ropos

orropes

oprops

oroots.

proos.

orotis

mpoeses

00005

9700

orposes

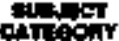

400015 ross

socia

40.9015

50.40

tot

tots

pacu

tosis

son:

5oind

50-04

40 ints

$0 \rightarrow$

0401

stose

0No15

ofin

and P

5048 P

sone

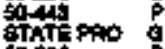

cost

60-40

res.

part

cats.

octs

this

50-40

0-60

ofeta

40015 


\section{APPENDIX A}

\section{DEFINITIONS OF DOCKET CATEGORIES}

DOCKET 27 - Low-Jevel Radioactive Waste Disposal Sites

The Docket 27 series contains documentation concerning the licensing of land disposal of low-level radionctive wastes. In the past, waste disposal licensees were assigned unique Docket 27 numbers. Unlike the other docket series, the first two digits of these docket numbers of aot correspond to the part of 10 CFR that specifies NRC's authority for issuing these licenses and the regulations that these licensees must follow. The Docket 27 licenses were issued in acoordance with Parts 30,40 , and 70 of 10 CFR in the absence of any final regulations governing the licensing of land disposal low-level radioactive wastes.

In the future, these licensing actions will be gowerned by 10 CFR Part 61, "Licensing Requitements for Land Disposal of Radioactive Waste." The prefix will then become Docket 61 followed by assigned nuenbers identifying a specific facility. Documents in these files include:
- Application for land disposal of low-level radicactive waste and supporting documents

- License and amendments

- Correspondence to and from the applicant regarding the application, license, and amendments

- Environmental report and supporting documents

- Inspection and enforcement reports and related correspondence

- Adjudicatory documents relating to proceetings before the Licensing Board and the Commission

\section{DOCKET 30 - Byproduet Material Licenses}

The Docket 39 series contains documentation on the possession or use of nuclear byproduct materials for medical, industrial, and academic purposes. These documents are maintailaed in the PDR on microfiche only.

Dowuments in this series include:
- Application for a license, license amendments, and license tenewals or modifications to the byproduct material license and related correspondence

- Denial, revocation, and suspension of a license and related correspondence 


\section{DEFINITIONS OF DOCKET CATEGORIES}

\section{DOCKET 30 - Byproduct Material Licenses (continıed)}

- Records related to the NRC inspection progran and related correspondence

- Preliminary Notifications of Occurrences (PNOs), enforcement actions, civil penalties, and related correspondence

- Event reports, misadministration reports, and related correspondence

- Correspondence to and from local and State governments
- Adjudicatory documentation telating to proceedings before the Licensing Board and the Commission

- Other techrical, legal, and licensing correspondence between NRC, the licensee, and other interested parties

- Periodic reports from licensees and related correspondence

\section{DOCKET 40 - Nuclear Source Material Facillties}

The Docket 40 series contains documentation concerring the domestic licensing of nuclear byproduct materials relating to source material licenses. Licenses issued in accordance with 10 CFR Part 40 cover the possession, use, and processing of ores (uranium and thorium) and possession and use of uranium and thorium in a refined form. Each license application is assigned a unique Docket 40 number.

Paper files are maintained in the PDR for only those Docket $\mathbf{4 0}$ licensees involved in the nuclear fuel cycle; that is, mines, mills, and research and development facitities. Material on other licensces, such as those for possession of material for medical and industrial uses, are available on microffiche only. Documents in these files include:

- Application for a license, license amendments and license renewals for source material bicense
- Source material license and license amerodments

- Licensee-issued Environmental Report (ER) for those license applications that must comply with the National Environmental Policy Act (NEPA)

- NRC-issued Draft and Final Environmental Statements (DES and FES) for license applications that must comply with NEPA

- Site visits and related correspondence

- Inspection reports and rełated correspondence

- Environmental monitoring reports

- Adjudicatory doctuments and hearing transcripts relating to proceedings before the Licensing Board and the Commission 


\section{DEFINITONS OF DOCKET CATEGORIES.}

\section{DOCKET 40 - Nuclear Source Material Facilities (contincted)}

- Other technical, kegal, and licensing correspondence between NRC, the licensee, and other interested parties

NOTE: For transcripts of Cormission meetings and papers submitted for Commission consideration on a specific docket, see the 10 CFR 9.7 and SECY nondocketed files.

\section{DOCKET 50 - Nuclear Reactors and Fuel Reprocessing Plants}

The Docket 50 series contains documentation concerting the domestic licensing of production and utilization facilities. Licenses issued in accordance with 10 CFR Part 50 cover commercial nuclear power plants; experimental, research, and test reactors; and fuel fabrication facilities. The NRC staff assigns Docket 50 number when it considers that the application and the Prelininary Safety Analysis Report (PSAR) submitted are complete for staff review. "Pre-docketed" material is assigned a project mumber and is filed in the applicant's project file.

Because of the large volume of documentation accumulated over the lifetime of a facility, each Docket 50 file is subdivided into the following categories that pertain either to different aspects of the licensing process or to document types.

\section{A. Application/Construction Stage Documents and Related Correspondence}

B. Utility Preliminary Safety Analysis Report (PSAR) and Amendments

C. Utility Environmental Report (ER), Amendments, and Related Correspondence

D. NRC Draft and Final Environmental Statements (DES and FES), Supplements, and Related Correspondence
E. NRC Safety Evaluation Report (SER), Supplements, and Related Correspondence

F. Security, Medical, Emergency, and Fire Protection Plans

G. Non-Antitrust Legal and Adjudicatory Correspondence

H. General Correspondence

I. Financial Information

J. Insurance and Indemnity Information

K. Utility Final and Updated Safety Analysis Reports (FSAR and USAR) and Amendiments

L. Limited Work Authorization (LWA) and Related Correspondence

M. Antitrust Reviews and Related Correspondence

N. Antitnust Transeripts, Testiznony, and Briefings

O. Advisory Committe on Reactor Safeguards (ACRS) Reports and Related Correspondence 


\section{DEFINIIIONS OF DOCKET CATEGORIES}

DOCKET 50 - Nuclear Reactors and Fuel Reprocessing Plants (continued)

P. Operating License Stage Doctments and Related Corresponotence

Q. Inspection Reports, Civil Penalties, Responses to Bulletins and Information Notices, and Related Correspondence

R. Periodic Operating Reports and Related Correspondence

S. Reportable Occurrences (ROs), Licensee Event Reports (LERs), Preliminary Notifications of Occurrences (PNOs), Preliminary Notifications of Safeguards Events (PNSs), and Related Correspondence

T Transcripts, Restimony, and Briefings on Non-Antitrust Matters
U. Executive, Congressional, and State Government Correspondence

V. Licensing of Operators and Related Correspondence

W. Decommissioning Stage Documents and Related Correspondence

X. Onsite Storage of Low-Level Waste and Related Correspondence

Y. Independent Spent Fuel Storage Installations (ISFSIs) and Related Correspondence

A detailed description of each category and the documents included in the files for each category follows.

\section{A. APPLICATION/CONSTRUCTION STAGE DOCUMENTS AND RELATED CORRESPONDENCE}

Documents in this category track the application from submittal to the day preceding the issuance of an operating license. It contains all correspondence and other documents with substantive technical content that are not in other specific categories. Included are:

- Application for construxtion permit

- General infornation submitted with the construction permit application

- Amendments and changes to the application
- NRC questions, Requests for Additional Information (RAIs), and applicant responses

- Early site reviews

- Correspondence and documents relating to unresolved design and construction isstes

- Meeting anmouncements and summaries of the meetings

- Correspondence relating to the utility's PTeliminary Safety Analysis Report (PSAR). 


\section{DEFINIIIONS OF DOCKET CATEGORIES}

\section{A. APPLICATION/CONSTRUCTION STAGE DOCUMENTS AND RELATED CORRESPONDENCE (CONIINUED)}

- Information on laws and changes in regula. tions or policy that affect the status of the application (National Environmental Policy Act (NEPA), Water Quality Imptovement Act, Water Pollution Control Act, etc.)

- Construction permit, amendments, related correspondence, and orders issued by the Office of Nuclear Reactor Regulation (NRR) show-cause and modification orders which do not involve the former Atomic Safety Licensing and Appesl Board

- Scheduling of license application, amendments, and reviens

- Environmental teview information (other environmental documents are filed in categories C, D, P, R, or W)

- Seismic qualification review team (SQRT) reports and related correspondence

- Application for operating license

- General information submitted with the Operating License application

- Amendments and changes to the application for Operating License
- Correspondence related to the utility's Final Safery Analysis Report (FSAR) and Updated Safety Analysis Report (USAR)

- Generic and administrative communications and related documentation that may affect the subject facility and which are not covered by o bulletin or notice

- Safety evaluation reports (SER) issued by the NRC on specific generic issues

- Reports on site visits

- Backup techrical documents (e.g, topical reports) submitted in support of the application

- Probabilistic nisk assessment (PRA) and related documentation

- Employee allegations and related documentation (other documents may be filed in categories $\mathbf{G}, \mathbf{Q}$, or $\mathbf{T}$ )

- Documents relating to possible equipment faibure and emergency instructions

- NRC's changes in internal organizational structuie

\section{B. UTLITY PRELIMINARY SAFETY ANALYSIS REPORT (PSAR) AND AMENDMENTS}

This category contains the Preliminary Safery Analysis Report (PSAR) submitted by the utility (formerty called Harards Summary report or
Safety Analysis Reports) and the following related material: 


\section{DEFINITIONS OF DOCKET CATEGORIES}

\section{B. UTLITY PRELIMRNARY SAFETY ANALYSIS REPORT (PSAR) AND AMENDMENTS (CONIINUED)}

- Amendments and changes to the PSAR

- Transmittal and acknowledgment letters
- Certificates of service to the PSAR

NOTE: All related correspondence is filed in category A.

\section{UTEITY ENVIRONMENTAL REPORT (ER), AMENDMENTS, AND RELATED} CORRESPONDENCE

The category contains the Emvironmental Report (ER) submitted by the utility and the following related matterial:

- Amendments and supplements to the ER

- Federal Register notices citing the avaitability of the $\mathrm{ER}$ and amendments

- Transmittal and acknowledgment letters

- Information on scheduling of submittal or review of $\mathbf{E R}$
- NRC questions, RAIs and applicant responses

- Correspondence retating to the review of ER

- Meeting announcements and summaries of meetings

- Correspondence relating to certification of pollution control facilities

NOTE: Other emvironmental documents are filed in categories $A, D, P, R$, or W.

\section{NRC DRAFT AND FINAL ENVIRONMENTAL STATEMENTS (DES AND FES), SUPPLEMENTS, AND CORRESPONDENCE}

This category contains Draft and Final Environmental Statements (DES and FES) prepared by $\mathrm{NRC}$, and the following related material:

- Supplements to the DES and FES
- Federal Register notices announcing the availabitity of the DES, FES, and supplements

- Correspondence relating to the DES and FES 


\section{DEFINITIONS OF DOCKET CATEGORIES}

\section{NRC DRAFT AND FINAL ENVIRONMENTAL STATEMENTS (DES AND FES), SUPPLEMENTS, AND CORRESPONDENCE (CONIINUED)}

- Comments from other Federal, State and local government agencies, organizations, and interested individuals

- Transmittal and ackrowledgment letters

- Documents related to NRC review of envirotmental issues or teview of the ER for purposes of preparing the environmental statements
- Backup technical documents submitted in support of the DES and FES

- Reports of site visits to obtain information for the DES or FES

- Meeting announcements and summaries of meeting which the primary subject of discussion is the DES or FES

NOTE: Other environmental documents and correspondeace are filed in categories $A$, C, P, R, or W

\section{E. NRC SAFETY EVALUATION REPORT (SER), SUPPLEMENTS, AND RELATED CORRESPONDENCE}

This category contains the formal Safety Evaluation Report (SER) prepared by the NRC staff (formerly called NRC-AEC hazard analysis report) in review of PSAR, and the following related material:

- Suppiements to the SER

- Federd Register notices announcing the availability of the SER and supplements

- Correspondence relating to the SER and supplements

- Information on scheduling of an SER submittal and review
- RAIs for use in preparing the SER; and responses to such requests

- Meeting announcements and meeting summaries at which the primary subject of discussion is the SER

- Transmittal and acknowledgment letters

NOTE: Other documents related to specific safety-related issues (that may be referred to as SERs) are filed in categories $A, P$ or $W$, depending on the phase of the licensing process. 


\section{DEFINITIONS OF DOCKET CATEGORIES}

\section{F. SECURITY, MEDICAL, EMERGENCY, AND FIRE PROTECTION PLANS}

All plans in this eategory are individually submitted by the applicant or licensee. Emergency and fire protection plans submitted as part of the licensing application are filed in this category.

- Fire bazard protection plan and surveys

- Plant evacuation correspondence

- Safeguard contingency plan

- Health physies program and its review

- Emergency implementing procedures

- Emergency action kevels

- Emergency plan
- Supplements, changes, and revisions to the plans

- Correspondence relating to the aforementioned plans

- Correspondence and documentation relating to guard training, emergency response centers, and emergency preparediness

- Documentation on security, non-operator fitmess procedures, employee unescorted access and related correspondence

- Transinittal and acknowledgment letters

- Meeting announcernents and meeting summaries at which the primary subject of fiscussion is related to the aforementioned plans and topics

\section{G. NON-ANTITRUST LEGAL AND ADJUDICATORY CORRESPONDENCE}

This category contains all non-antitrust legal and adjudicatory documentation, except for transcripts, briefings, and testimony, which are filed in category $T$ Show-cause and modification orders issued by the Office of Nuclear Reactor Regulation (NRR), and not before the ticensing board, are filed in categories $A$ and $P$. Exhibits to the proceedings (hearings) are maintained in the PDR on microfiche only. Paper files of the exhibits are available upon request to the PDR from the Docketing and Services Branch, Office of the Secretary.

- Atomic Safety and Licensing Board (ASLB) and the former Atomic Safety and Licensing
Appeal Board (ASLAB) hearing-related documents

- Federal Register notices regarding hearings

- Requests to make appearances at hearings

- Motions, briefs, arguments, statements, and related correspondence filed by participants for hearings

- Transmittal of supporting documents (e.g. SER, FES) to the licensing board

- Correspondence to and from intervenors 


\section{DEFINITIONS OF DOCKET CATEGORIES}

\section{G. NON-ANTTRUST LEGAL AND ADJUDICATORY CORRESPONDENCE (CONTINUED)}

- Board orders and decisions

- Formal petitions for Commission action

- Petitions filed in accordance with 10 CFR 2.206 to the Executive Director for Operations
- Legal filings, director's decisions, Commission orders and decisions

- Orders prohibiting involvement in NRClicensed activities, appeals, and related decisions

\section{H. GENERAL CORRESPONDENCE}

This category contains documents with little or no bearing on the status of the application or license. Items that have no substantive technical content and administrative documents are included.

- Change-of-address notifications (external only)

- Changes in structure of organization (external only)

- Changes in service lists

- NRC requests to applicants for additional copies of doctmnents

- Correspondence from the public requesting information about the facility, and NRC response

- Correspondence on purely administrative matters
- Letters of support, concern, or opposition to the facility

- Correspondence relating to the Local Public Document Rooms (LPDRs)

- Professional qualifications statements not pertaining to the hearing process

- Constituent, organizational, or vtility correspondence forwarded by members of Congress to NRC for response

- Resolutions passed by State and local governing bodies (e.g., town councils)

NOTE: Letters of concern, support, or opposition for all plants are inled in a General Public Comments folder and are retained for 6 months from the date of receipt in the PDR

\section{FINANCIAL INFORMATION}

This category contains all financial information except for that subinitted as part of the application or its amendments or that relate to the imposition of civil penalties. 


\section{DEFINITIONS OF DOCKET CATEGORIES}

\section{FINANCIAL INFORMATION (CONINUED)}

- Company annual reports, auditor statements, and stock prospectuses for all companies holding ownership
- Transcripts and testimony before State commissions pertinent to finarcial information requested by $\mathrm{NRC}$

- Separately submitted financial information and related correspondence

- Decommissioning funding reports

\section{J. INSURANCE AND INDEMNITY INFORMATION}

This category contains indemnity agreements and amendments as well as:

- Endorsements of insurance policies
- Other correspondence regarding indemnity and insurance matters

\section{K. UTILTY FINAL AND UPDATED SAFETY ANALYSIS REPORTS (FSAR AND USAR) AND AMENDMENTS}

This category contains the following utility documents:

- Final Safety Analysis Report (FSAR), formerly called Final Hazards Summary Report

- Updated Safety Analysis Report (USAR) periodically updated to incorporate new information or modifications to the facility

- Amendments and changes to the FSAR and USAR
- Federal Register notices announcing the availability of the FSAR and amendments

- Transmittal and acknowledgenent letters

- Certificates of service to the FSAR and USAR

NOTE: All related correspondence is filed in category A or P. Eunergency and fire protection plant submitted as part of the licensing application are filed in category $\mathrm{F}$.

\section{LIMTIED WORK AUTHORIZATION (LWA) AND REI.ATED CORRESPONDENCE}

This category contains documents pertaining to the limited work authorization (LWA) whereby applicants can conduct limited site preparation and construction before the NRC grants a construction permit.
- Limited Work Authorization (LWA) and amendments 


\section{DEFINITIONS OF DOCKET CATEGORIES}

\section{LIMTTED WORK AUTHORIZATION (LWA) AND RELATED CORRESPONDENCE (CONTINUED)}

- Correspondence pertaining to work performed under the LWA

\section{ANTITRUST REVIEWS AND RELATED CORRESPONDENCE}

This category contains documents submitted as part of the application for a construction permit and operating license that are entitled

"Information Requested by the Attomey Genera] for Antitrust Review," including:
- Correspondence in which the primary topic of discussion concerns antitrust matters

- All antitrust proceeding documents, except hearing transcripts, testimony, and briefings, which are filed in category $\mathrm{N}$

\section{N. ANTTTRUST TRANSCRIPTS, TESTIMONY, AND BRIEFINGS}

This category contains verbatim transcripts of bearings, testimany, and briefings in antitrust matters. Related comespondence is filed in category $\mathrm{M}$.

\section{O. ADVISORY COMMITITE ON REACTOR SAFEGUARDS (ACRS) REPORTS AND RELATED CORRESPONDENCE}

This category contains plant-specific documents pertaining to ACRS safety reviews. Included are:

- ACRS reports relating to facility license applications
- Amouncements of ACRS meetings relating to facility license applications, incluting descriptions of meeting agenda

- Statements and written testimony by ACRS members or presented by individuals at ACRS proceedings relating to both facility license applications and safety-related matters 


\section{DEFINTIONS OF DOCKET CATEGORIES}

\section{O. ADVISORY COMMTTIEE ON REACTOR SAFGGUARDS (ACRS) REPORTS AND RELATED CORRESPONDENCE (CONTENUED)}

- Technical correspondence and memoranda to and from ACRS, including tratsmittal and acknowledgment letters relating to facility license applications

NOTE: For generic and docurlent-specific safery-related material, transcripts of ACRS subcommittee and full committee meetings, minutes, and ACRS reports (consuitant, contract, and letter), see ACRS non-docket file.

\section{P. OPERATING LICENSE STAGE DOCUMENTS AND RELATED CORRESPONDENCE}

This category contains general technical documents dated from the issuance of the operating license until the order for termination of the license. Included are:

- Operating license, amendments, proposed amendments, changes, and related correspondence

- Show-cause and modification ordets issued by NRR (not before the licensing board) for the operating license

- Correspondence regarding the utility's Final and Updated Safety Analysis Report (FSAR and USAR)

- Fedenal Register notices announcing availability of operating license, amendments, FSAR and USAR

- Technical spectifications, proposed changes, revisions and related correspondence

- Backup technical documents (e.g, topical reports) submitted in support of the aforementioned items
- All general technical correspondence, meeting anrouncements and meeting summaries relating to the technical specifications, the operating license, or technical issues arising after the issuance of the operating license

- Correspondence relating to codes (e.g., American Society of Mechanical Engineersh, regulations, laws, and changes in policy that may affect plant operation or the operating license

- NRC questions, RAIs, and licensee responses

- Information on the Standard Review Plan (SRP)

- Results of surveys or tests conducted in accordance with the technical specifications (not periodic reports, which are filed in category $\mathbf{R}$ )

- Correspondence relating to NRC's Systematic Evaluation Program (SEP)

- Owners' group tmeeting announcements and reports 


\section{DEFINITIONS OF DOCKIT CATEGORTES}

\section{P. OPERATING LICENSE SHAGE DOCUMENTS AND RELATED CORRESPONDENCE (CONTINUED)}

- Quality assurance plan and related correspondence

- Environmental matters (technical data)

- Seismic Qualification Review Tean (SQRT) reports and related correspondence

- Emergency core cooling system documents

- Radioactive waste information exchuding onsite storage of low-level waste (filed in category $\mathrm{X}$ ) and independent spent fuel installations of high-level waste (filed in category Y)

- Correspondence relating to expansion of the spent fuel storage pool

- Startup test reports

- Generic and administrative communications and related documentation that may affect the subject facility but is not covered by $a$ Bulletin or Notice
- SERs on specific generic issues

- Doctrments relating to possible equipment failure and emergency instructions

- Documents relating to inservice testing procedures and results (not inservice inspections, which are filled in category $Q$ )

- Specific plant evaluation documents

- Probabilistic Risk Assessment (PRA) reports

- Employee allegations and related documentation (other documents may be filed in categories $\mathbf{G}, \mathbf{Q}$, or $\mathbf{T}$ )

- Reports on site visits

- Application to voluntarily terminate license and to dismantle and dispose of component parts

- Information on changes in NRC's internal organizational structure 


\section{DEFINITIONS OF DOCKET CATEGORIES}

\section{Q. INSPECTION REPORTS, CIVIL PENALTIES, RESPONSES TO BULLEIINS AND INFORMATION NOTICES, AND REILATED CORRESPONDENCE}

This category contains the following applicanthicensee material:

\section{- Inspection reports}

- Responses to Bulletins (formerly called Regulatory Operations, RO Bulletins), Circulars (discontinued in 1981) and Information Notices, and related correspondence

- Applicant/icensee determinations of the proprietary nature of inspection reports

- Letters concerning areas of noncompliance

- Preservice and inservice inspections and procedures

- Notices of violation and proposed impositions of civil penalty and Demands for Information (DFIs)

- Correspondence and orders relating to the imposition and payment of civil penalties, including Enforcement Notifications (ENs) and Significant Enforcement Actions (EAs)

- Notices of deviation and related correspondence

- Notices of enforcement conferences, meetings, and related reports

- Correspoadence relating to inspection and enforcement that does not specifically relate to incidents or events as defined in category S

- Health physics inspections and appraisals
- Emergency preparedness inspections and appraisals

- Inspections and related correspondence from the International Atomic Energy Agency (IAEA)

- Risk-based Inspection Guides (RIGs)

- Systematic Assessment of Licensee Performance (SAIP)

- Incident Investigation Team (ITT) reports

- Construction Appraisal Team/Construction Assessment Tem (CAT) reports

- Integrated Design Inspection (IDI) reports

- Independent Design Verification Program (IDVP) reports

- Notices of significant licensee meetings

- Investigation reports and related documentation, and responses to employee allegations (other documents are filed in categories A, P, G, or T)

- Performance Appraisal Team (PAT) reports

- Licensee/vendor interface inspections

- Correspondence relating to the aforeInentioned itents

NOTE: For bulletins and information notices, see non-docket I\&E file. 


\section{DEFINITIONS OF DOCKET CATEGORIES}

\section{R. PERIODIC OPERATING REPORTS AND RELATED CORRESPONDENCE}

This category contains periodic reports of a toutine nature.

- Monthy, semiannual, and anmual operating reports (other than financial) for construction, operating, and decommissioning stages

- Periodic environmental monitoring effluent testing, or similar reports

- Periodic personnel exposure reports

- Periodic fitness for duty performance data

- Periodic facility changes, tests and experiments
- Facility simulation certification four year update reports

- Periodic nonconformance reports (issued in accordance with licensing board orders)

- Operational logbooks on

- emvironmental data

- radiological data

- plant systems core

- plant status summary sheets

- Correspondence and other documents relating to the aforementioned items

\section{S. REPORTABLE OCCURRENCES (ROs), LICENSEE EVENT REPORTS (LERs), PRELMMINARY NOTIFICATIONS OF OCCURRENCES (PNOS), PRELIMINARY NOFHICATIONS OF SAFEGUARDS EVENTS (PNSS), AND RELATED CORRESPONDENCE}

This category contains all materials rekating to abtormal unusual, reportable, and non-routine occurrences or events. These include:

- All 24-hour, 10-day, 30-day, interin and final Licensee Event Reports (LFRs) and follow-up correspondence (also called incident reports, abnormal occurrences, special reports, and reportable occurrences)

- Potential or possibly reportable Deficiency Reports and related correspondence

- Individual Persomnel Exposure Reports
- Construction Deficiency Reports submitted in accordance with 10 CFR 50.55 (t)

- Documents related to the issuance of a Preliminary Notification of Occurrence (PNO), Preliminary Notification of Safeguards Events (PNS), and related correspondence

- Environbnental technical specifications violation reports and environmental event reports 


\section{DEFINITONS OF DOCKET CATEGORIES}

\section{S. REPORTABLE OCCURRENCES (ROs), LICENSEE EVENT REPORTS (PRELIMINARY NOTIFICATIONS OF OCCURRENCES (PNOS), PRELIMINARY NOTFICATIONS OF SAFEGUARDS EVENTS (PNSs), AND RELATED CORRESPONDENCE (CONTINUED)}

- Part 21 reports of defects and noncompliance

- Correspondence and other documents relating to the aforementioned items
NOTE: For PNOs and PNSs, see I\&E non-docket file.

\section{T. TRANSCRIPTS, TESTIMONY, AND BRIEFINGS ON NON-ANTITRUST MATTERS}

This category contains verbatim transcripts, testimony, and briefings except for congressional matters (filed in category $U$ ) and antitrust matters (filed in category $\mathrm{N}$ ).

- Atomic Safety and Licensing Board (ASLB) and the former Atomic Safety and Licensing Appeal Board (ASLAB) transeripts

- Testimony and statements submitted to ASLB and the former ASLAB
- Transcripts of Commission meeting

- NRC staff briefings to the Commission

- Statements and written testimony submitted at Commission metings and hearings

- Transcripts of utility briefings to NRC staff

- Corrections to the aforementioned items

NOTE: Transcripts on specific dockets are also filed in ACRS and 9.7 nondocket files.

\section{U. EXECUTIVE, CONGRESSIONAL, AND STATE GOVERNMENT CORRESPONDENCE}

This category contains documents and related correspondence that have substantive technical and administrative content, including:

- Statements and testimony before Congress or before bodies/committes appointed by the Executive or Legislative branches
- Technical reports and related information supplied for the record of congressional hearings

- Follow-up questions and answers to the aforementioned 


\section{DEFINITIONS OF DOCKET CATEGORIES}

\section{U. EXECUTIVE, CONGRESSIONAL, AND STATE GOVERNMENT CORRESPONDENCE (CONTINUED)}

- Correspondence to and from Congress and the Executive Branch on other than constituent response matters
NOTE: Routine responses to constituent inquiries are filed in category $\mathbf{H}$.

- Comespondence to or from State Legislative bodies

\section{LICENSING OF OPERATORS AND RELATED CORRESPONDENCE}

This category contains information bearing on licensing of operators and shift engineers, including:

- Power plant examination summary sheets

- Correspondence relating to operator examinations
- Documentation on operator laboratory tests or reports and related correspondence

- Docurnents relating to operator fitness for duty and related correspondence

- Orders prohibiting operators from further nuclear licensed activities, and related correspondence (other documents may be filed in category $\mathbf{G}$ )

\section{W. DECOMMLSSIONING STAGE DOCUMENTS AND RELATED CORRESPONDENCE}

This category contains general technical items dated from the order to terminate the operating license.

- Order granting authority to dismantled decommission

- Decommissioning Plan, Dismantling Plan, Emvironmental Report on dismantling and Safety Analysis Report on decommissioning
- Documents relating to the disposal of racioactive material and decontamination of the site

- Other correspondence relating to decommissioning

- All documentation relating to the ongoing operation of the plant which was formerly filed in category $P$ 


\section{DEFINITIONS OF DOCKET CATEGORIES}

\section{ONSITE STORAGE OF LOW-LEVEL WASTE AND RELATED CORRESPONDENCE}

This category contains material related to onsite storage of Low-Level Waste (LLW).

- License application, license, proposed amendments, and amendments to store LLW at a commercial reactor site
- Technical reports and related correspondence

NOTE: The documents in this category will aiso have a Nuclear Materials Safety and Safeguards (NMSS) Docket 30 license number associated with the Docket 50 number.

\section{Y. INDEPENDENT SPENT FUEL STORAGE INSTALLATIONS (ISFSIS) AND RELATED CORRESPONDENCE}

This category contains materia: related to license applications, license, atrendments and proposed amendments to possess power reactor spent fuel and other radioactive materials associated with spent fuel storage in an independent spent fuel storage installation. (Such ISFSIs may be on a reactor site or at a separate site.)
- All related correspondence and reports to the aforementioned items

NOTE: The documents in this category will have an NMSS Docket 72 license number. For these installations on a reactor site, the associated Docket 50 number also has been referenced

Docket 52 - Standard Design Certification, Eariy Site Permit, and Combined Licenses for Nuclear Power Plants

The Docket 52 series contains documentation on the domestic licensing of production facilities. Licenses issued in accordance with 10 CFR Part 52 cover preliminary and final design approval of standard desiens, early site permits, and combined ticenses for nuclear power plants. $A$ Docket 52 number is assigned when the NRC considers that the application submitted is complete for staff review. "Predocketed" material is assigned a project number and is filed in the applieant's project file.
Because of the large volume of documentation accumulated over the lifetime of an application, each Docket $\mathbf{5 2}$ file is subdivided into the folkwing categories that pertain either to different aspects of the licensing process or to document types. These categories are similar to those used for the Docket $\mathbf{5 0}$ files, but have been modified to reflect the special requirements of Docket 52 applications. 


\section{DEFINITONS OF DOCKET CATEGORIES}

A. Application for Preliminary Design Approval (PDA) Final Design Approval and Design Certification (FDAMC), Early Site Permit, Combined Construction $t$ Operating License (COL), ant Related Correspondence

B. Vendor Preliminary Standard Safety Analysis Report (PSSAR-PDA stage), Utility Preliminary Safety Analysis Report (PSAR-COL stage), and Amendments

C. Severe Accident Mitigation Design Altemative Apalysis (SAMDA-PDA andFDADDC stages), Environmental Reports (ERs-Early Site Permit and COL stages), Amendments, and Related Correspondence

D. NRC Draft and Final Environmental Statements (DES and FES), Supplements, and Related Correspondence (Early Site Permit and COL stages)

E. NRC Safety Evaluation Report (SER), Supplements, and Related Correspondence (Early Site Permit, PDA, FDAJDC, and COL stages)

E Reliability Assurance Progran (RAP), Security, Medical, Emergency, and Fire Pro tection Plans (PDA, FDA/DC, and COL stages)

G. Non-Antitrust Legal and Adjudicatory Correspondence (Early Site Permit, PDA, FDA/DC, and COL stages)

H. General Corzespondence (Early Site Permit, PDA, FDADC, and COL stages)

L Financial Information (COL stage)

J. Insurance and Indemnity Information (COL stage)
X. Vendor Final Standard Safety Analysis Report (FSSAR-FDAVDC Stage), Utility Final Safety Amalysis Report (FSAR-COL Stage), Updated Safety Analysis Repont (USAR-COL Stage), and Amendrents

L. Iimited Work Authorization (LWA) and Related Correspondence (COL Stage)

M. Antitrust Reviews and Related Correspondence (COL Stage)

N. Antitrust Hearing Transcripts, Testimony, and Briefings (COL Stage)

O. Advisory Committee on Reactor Safeguards (ACRS) Reports and Related Correspondence (Earty Site Permit, PDA, FDAVDC. and COL Stages)

P. Post-Combined Construction/Operating License Stage Documents and Related Correspondence (COL Stage)

Q. Inspection Reports, Civil Penalties, Responses to Bulletias and Information Notices, ană Related Correspondence (COL Stage)

R. Periodic Operating Reports and Related Correspondence (COL Stage)

S. Reportabte Occurrences (ROs), Licensee Event Reports (LERs), Preliminary Notifications of Occurrences (PNOs), Preliminary Notifications of Safeguards Events (PNSs), and Related Correspondence (COL Stage)

T Transcripts, Testimony, and Briefings on Non-Antitrust Matters (Earfy Site Permit, PDA, FDANDC, and COL Stages) 


\section{DEFINITIONS OF DOCKET CATEGORIES (CONTINUE)}

U. Executive, Congressional, and State Government Correspondence (Early Site Peruit, PDA, FDAIDC, and COL Stages)

V. Licensing of Operators and Retated Correspondence (COL Stage)

W. Decommisstoning Stage Documents and Related Correspondence (COL Stage)
X. Onsite Storage of Low-Level Waste and Related Correspondence (COL and Decommissioning Stages)

Y. Independent Spent Fuel Storage Installations (ISFSIs) and Related Correspondence (COL and Decommissioning Stages)

A detailed description of each category and the doctuments included in each category follows.

\section{A. APPLICATION FOR PRELIMINARY DESIGN APPROVAL (PDA), FINAL DESIGN APPROVAL AND DESIGN CERTIFICATION (FDA/DC), EARLY SITE PERMIT COMBINED CONSTRUCTION/OPERATING LICENSE (COL), AND RELATED CORRESPONDENCE}

Doctumeats in this category track the application for Preliminary Design Approval (PDA) or Final Design Approval and Design Certification (FDADC) to the issuance of the PDA or FDA, respectively, or the application of the early site permit until issuance of the permit. It contains all correspondence and documents with substantive technical content that are not in other specific categories. Inchuded are:

- Application for preliminary design approval

- Application for final design approval and design certification

- Application for earty site permit

- General information submitted with the application

- Amendments and changes submitted by applicants

- NRC questions, Requests for Additional Information (RAIs), and applicant responses
- Meeting announcements and sumumaries of the meetings

- Correspondence relating to the Prelliminary or Final Standard Safery Analysis Report (SSAR)

- Information on laws and changes in regulations or policy that affect the states of the application (National Environmental Policy Act (NEPA), Water Quality Improvement Act, Water Pollution Control Act, etc.)

- Preliminary Design Approval (PDA), and amendments and correspondence relating to the PDA

- Final Design Approval (FDA), and amendments and correspondence relating to the FDA

- Eariy site permit, amendments and related correspondence

- Scbeduling documentation

- Backup technical documents (e.g., topical reports) submitted in stupport of the application 


\section{DEFINITIONS OF DOCKET CATEGORIES}

A. APPLICATION FOR PRELIMTNARY DESIGN APPROVAL (PDA), FINAL DESIGN APPROVAL AND DESIGN CERTIFICATION (FDAJDC), EARLY SITE PERMIT, COMBINED CONSTRUCTION/OPERATTNG LCENSE (COL), AND RELATED CORRESPONDENCE (CONIINUED)

- The design-specific Probabilistic Risk Assessment (PRA), and related documentation

- Proposed tests, analyses, and acceptance criteria and related documentation

- Design Acceptance Criteria (DAC) and related correspondence

- Documents addressing policy issues raised to the Commission via Conmission papers
- Documents addressing resolution of issues for the application

- Generic and administrative communications and related documentation which may affect the subject facility and which are not covered by a Bulletin or Notice

- Employee allegations and related cocumentation (other documents may be filed in categories $\mathbf{G}, \mathbf{Q}$, or $\mathbf{T}$ )

- NRC's changes in internal organizational structure

\section{B. VENDOR PRELIMINARY STANDARD SAFETY ANALYSIS REPORT (PSSAR-PDA STAGE), UTILITY PRELIMINARY SAFETY ANALYSIS REPORT (PSAR-COL STAGE), AND AMENDMENTS}

This category contains the following documents:

- Preliminary Standard Safety Analysis Report (PSSAR) submitted by vendor

- Amendments and changes to the PSSAR
- Preliminary Safety Analysis Report (PSAR) submitted by the utility

- Amendments and changes to the PSAR

- Transmittal and acknowledgement tetters

NOTE: All related correspondence is filed in category A

C. SEVERE ACCIDENT MITIGATION DESIGN ALTERNATIVE ANALYSIS (SAMDA-PDA AND FDA/DC STAGES), ENVIRONMENTAL REPORTS (ERs-EARLY SITE PERMTT AND COL STAGES), AMENDMENTS, AND RELATED CORRESPONDENCE

This category contains the following documents:
- Severe Accident Mitigation Design Alternatives Analysis (SAMDAs) required under the National Environmental Policy Act (NEPA) submitted by vendor 


\section{DEFINITIONS OF DOCKET CATEGORIES}

C. SEVERE ACCIDENT MITIGATION DESIGN ALTERNATIVE ANALYSIS (SAMDA-PDA AND FDAVC STAGES), ENVIRONMENTAL REPOKTS (ERS-EARLY SIIE PERMIT AND COL STAGES), AMENDMENTS, AND RELATED CORROSPONDENCE (CONTENUED)

- NRC questions, RAIs, and applicant. responses relating to SAMDAS analysis review

- Correspondence relating to the SAMDAS analysis review

- Meeting announcements and summaries of metings at which the primary subject of discossion is the SAMDAS analysis

- Enviroamertal Report (ER) submitted by utility

- Amendments and supplements to the ER

- Federd Register notices anmouncing the availability of the ER and amendments
- Transmittal and acknowledgment letters of ER

- Information on scheduling of submittal or review of ER

- NRC questions, RAIs, and applicant responses relating to review of $\mathbf{E R}$

- Correspondence relating to the review of ER

- Meeting announcements and summaries of meetings at which the primary subject of discussion is the $\mathbf{E R}$

- Correspondence relating to certification of pollution control facilities

NOTE: Other environmental documents are fikd in categories $A, D, P, R$ or W.

\section{NRC DRAFT AND FINAL ENVIRONMENTAL STATEMENTS (DES AND FES), SUPPILEMENTS, AND RELATED CORRESPONDENCE (EARLY SITE PERMIT AND COL STAGES)}

This category contains the Draft and Fingl Environmental Impact Statements (DES and FES) prepared by NRC, and supplements to the DES and FES, as well as:

- Fedenal Register notices announcing the availability of the DES and FES

- Correspondence relating to the DES and FES
- Comments from other Federgl, State, and local government agencies, organizations, and interested individuals

- Transmittal and acknowledgntent letters

- Documents related to NRC review of environmental issues or review of the $\mathrm{ER}$ obtained for purposes of preparing the environmental statements 


\section{DERTNITIONS OF DOCKET CATEGORIES}

\section{NRC DRAFT AND FINAL ENVIRONMENTAL STATEMENTS (DES AND FES), SUPPLEMENTS, AND RELATED COXRESPONDENCE (EARLY SITE PERMIT AND COL STAGES) (CONINUED)}

- Backup technical documents stubmitted in support of the DES and FES

- Reports of site visits to obtain information for the DES or FES
- Meeting announcements and summaries of meetings at which the primary subject of discussion is the DES or FES

NOTE: Other environmental documents and correspondence are filed in categories A, C, P, R, or W.

\section{E. NRC SAFETY EVALUATION REPORT (SER), SUPPLEMENTS, AND RELATED CORRESPONDENCE (EARLY SITE PERMIT, PDA, FDA/DC, AND COL STAGES)}

This category contains the formal Safety Evaluation Report (SER) prepared by NRC in review of the early site permit application, PSSAR (PDA stage), FSSAR (FDA/DC stage), PSAR (COL stage), or FSAR (COL stage) and, Supplements to the SER, as well as:

- Federal Register notices announcing the availability of the SER and supplements

- Correspondence relating to the SER and supplements

- Information on scheduling of an SER submittal and review
- RAIs for use in preparing the SER and responses to such requests

- Meeting announcements and summaries of meetings at which the primary subject of discussion is the SER

- Transmittal and acknowedgment letters

NOTE: Other doctuments related to specific safety-related issues (that may be referred to as SERs) are filed in categories $A, P$, or $W$, depending on the phase of the licensing process.

\section{F. RELIABILITY ASSURANCE PROGRAM (RAP), SECURITY, MEDICAL, EMERGENCY, AND FIRE PROTECTION PLAN (PDA, FDA/DC, AND COL STAGES)}

The plans in this category may be individual submissions or may be combined under the Reliability Assurance Program. These palans may be submitted during the review of the PDA, the FDA/DC, and COL applications. Included are:
- Rejiability Assurance Program (RAP)

- Plant eracuation correspondence 


\section{DEFINITIONS OF DOCKET CATEGORJES}

\section{F. RELIABILITY ASSURANCE PROGRAM (RAP), SECURITY, MEDICAI, EMERGENCY, AND FIRE PROTECTION PLAN (PDA, FDADC, AND COL STAGES) (CONTINUED)}

- Safeguard contingency plan

- Health physics program and its review

- Emergency implementing procedures

- Emergency action levels

- Emergency plans

- Fire hazard protection plans and surveys

- Supplements, changes, and revisions to the plans
- Correspondence relating to the aforementioned plans

- Correspondence and documentation reiating to guard training, emergeacy response centers, and emergency preparedness

- Documentation on security, non-operator fitness procedures, employee unescorted access and related correspondence

- Transmittal and acknowiedgement letters

- Moeting announcements and summaries of meetings at which the primary subject of discussion is related to the aforementioned plans

\section{G. NON-ANTITRUST LEGAL AND ADJUDICATORY CORRESPONDENCE (EARLY SITE PERMIT, PDA, FDAIDC, AND COL STAGES)}

This category contains all non-antitrust legal and adjudicatory documentation, except for transcripts, briefings and testimony, which are filed in category T. Show-cause and modification orders issued by the Office of Nuclear Reactor Regulation (NRR) and not before the Licensing Board are filed in categories $A$ and P. Exhibits to the proceedings (hearings) are maintained in the PDR on microfiche only. Paper files of the exhibits are available upon rectuest to the PDR from the Docketing and Services Branch, Office of the Secretary.

- Atomic Safety and Lictusing Board (ASLB) hearing related documents
- Federal Register notices regarding hearings

- Requests to make appearances at hearings

- Motions, briefs, arguments, statements, and related correspondence filed by participants for hearings

- Transmittal of supporting documents (e.g. SER, FES) to the licensing board

- Correspondence to and from intervenors

- Board orders and decisions

- Formal petitions for Commission action 


\section{DEFINITIONS OF DOCKET CATEGORIES}

G. NON-ANTITUUST LEGAL AND ADJUDICATORY CORRESPONDENCE (EARLY SITE PERMIT, PDA, FDA/DC, AND COL STAGES) (CONTINUED)

- Petitions filed in accordance with 10 CFR 2,206 to the Executive Director for Operations

- Legal filings, director's decisions, Commission orders and decisions
- Orders prohibiting involvement in NRClicensed activities, appeals, and related decisions

\section{H. GENERAL CORRESPONDENCE (EARLY SITE PERMIT, PDA, FDADC, AND COL STAGES)}

This category contains documents with little or no bearing on the status of the application or license. Items that have no substantive technical content and administrative documents are inciuded.

- Change of address notifications (external only)

- Changes in structure of organization (external only)

- Changes in service lists

- NRC requests to applicants for additional copies of documents

- Correspondence from the public requesting information about the site, design, or factity, and NRC responses

- Correspondeace on purely administrative matters
- Letters of support, concern or opposition to the site, design, or facitity

- Correspondence relating to the Local Public Document Rooms (LPDRs)

- Professional qualifications statements not pertaining to the hearing process

- Constituent, organizational or utility correspondence forwarded by members of Congress to NRC for response

- Resolutions passed by state and local governing bodies (e.g, town councils)

NOTE: Letters of concern, suppont, or opposition for all plants are filed in a General Priblic Comments folder and are retained for 6 months from the date of receipt in the PDR.

\section{FINANCIAL INFORMATION (COL STAGE)}

This category contains all financial information except for that submitted as part of the applica- tion or its amendments or that relate to the imposition of civil penalties. 


\section{DEFINITIONS OF DOCKET CATEGORIES}

\section{FINANCIAL INFORMATION (COL STAGE) (CONTINUED)}

- Company annual reports, auditor statements and stock prospectuses for all companies holding ownership

- Separately submitted financial information and related correspondence
- Transcripts and testimony before State commissions pertinent to financial information reçuested by NRC

- Decommissioning funding reports

\section{J. INSURANCE AND INDEMNITY INFORMATION (COL STAGE)}

This category contains indemnity agreements and amendments as well as:

- Endorsements of insurance policies
- Other correspondence regarding indemnity and insurance matters

\section{K. VENDOR FINAL STANDARD SAFETY ANALYSIS REPORT (FSSAR-FDADC STAGE), UTH ITY FINAL SAFETY ANALYSIS REPORT (FSAR-COL STAGE), UPDATED FINAL SAFETY ANALYSIS REPORT (USAR-COL STAGE), AND AMENDMENTS}

This category contains the following vendor and utility documents:

- Final Standard Safety Anabysis Report (FSSAR) submitted by the vendor

- Final Safety Analysis Report (FSAR) submitted by the utility

- Updated Safety Analysis Report (USAR) submitted by the utility and perioxically updated to incorporate new information or modifications to the facility
- Amendments and changes to the FSSAR, FSAR, and USAR

- Federal Regirier notices announcing the availatility of the FSSAR and FSAR

- Transmittal and ackonodedgenent letters

- Certificates of service to the FSAR and USAR

NOTE: All related correspondence falls in category A or P. Emergency and Fire Protection Plans submitted as part of the licensing application are filed in category $F$. 


\section{DEFINITIONS OF DOCKET CATEGORIES}

\section{LMMTED WORK AUTHORIZATION (LWA) AND RELATED CORRESPONDENCE (COL STAGE)}

This categoy is limited to documents pertaining to Limited work Authorization (LWA) whereby applicants can conduct limited site preparation and construction before $\mathrm{NRC}$ grants a combined construction/operating license.
- Limited Work Authorization (LWA) and amendments

- Comespondence pertaining to work performed under LWAs

\section{ANTITRUST REVIEWS AND RELATED CORRESPONDENCE (COL STAGE)}

This category contains documents submitted as part of the application for a combined construction/operating license entitled, "Information Requested by the Attomey General for Antitrust Review," including:

- Correspondence in which the primary topic of disctission is antitrust matters
- All antitrust proceeding documents, except hearing transcripts, testimony, and briefings, which are filed in category $N$

\section{N. ANIITRUST HEARING TRANSCRIPTS, TESTIMONY, AND BRIEFINGS (COL STAGE)}

This category contains verbatim transcripts of hearings, testimony and briefings in antitrust matters. Related comespondence is filed in categary $\mathbf{M}$.

\section{O. ADVISORY COMMITTEE ON REACTOR SAFEGUARDS (ACRS) REPORTS AND RELATED CORRESPONDENCE (EARLY STITE PERMIT, PDA, FDADDC, AND COL STAGES)}

This category contains project-specific documents pertaining to ACRS safety reviews. Inchuded are:
- ACRS reports relating to a PDA or FDA DC application, early site review, or facility license application 


\section{DEFINITIONS OF DOCKET CATEGORIES}

\section{O. ADVSORY COMMITTEE ON REACTOR SAFEGUARDS (ACRS) REPORTS AND RELATED CORRESPONDENCE (EARLY SITE PERMIT, PDA, FDADC, AND COL STAGES) (CONTINUED)}

- Announcements of ACRS meetings relating to a PDA or FDA/DC application, earty site review, or facility ticense application, including descriptions of meeting agenda

- Statements and written testimony by ACRS members or presented by individuals at ACRS proceedings relating to a PDA or FDADC application, earty site review, facility license application, and safetyrelated matters
- Technical correspondence, and memoranda to and from ACRS, including transmittal and acknowledgement letters relating to a PDA or FDAVDC application, early site review, or facility license application

NOTE: For generic and docnoment-specific safety related material, transcripts of ACRS subcommittee and full committes meetings, minutes, and ACRS reports (consultant, contract and ketter), see ACRS non-docket file.

\section{P. POST-COMBINED CONSTRUCTION/OPERATING LICENSE STAGE DOCUMENTS AND RELATED CORRESPONDENCE (COL STAGE)}

This category contains general technical documents dated from the combined construction/operating license until the order for termination of the license. Included are:

- The combined construction/operating license (COL), amendments, proposed amendments, changes, and related correspondence

- Show-cause and modification orders issued by NRR (not before the licensing board) for the COL

- Technical specifications, proposed changes, revisions and related comespondence

- Backsp tecinical documents (e.g, Topical Reports) submitted in support of the aforementioned items
- Federd Register notices announcing the availability of the COL, FSAR, and amendments

- Correspondence regarding the utility Final Safety Analysis Report (FSAR) and/or the Updated Safety Anatysis Report (USAR)

- All general technical correspondence, meeting anouncements and meeting stummaries relating to the technical specifications, the COI, or technical issues arising after the issuance of the COL

- Correspondence relating to codes (e.g., American Society of Mechanical Engineers) regulations, laws, and changes in policy that may affect plant operation or bear on the COL

- NRC questions, RAIs, and licensee responses 


\section{DEFINITIONS OF DOCKET CATEGORIES}

\section{P. POST-COMBINED CONSTRUCTION/OPERATING LICENSE STAGE DOCUMENTS AND RELATED CORRESPONDENCE (COL STAGE) (CONTINUED)}

- Information on the Standard Review Plan (SRP)

- Results of surveys or tests conducted in accordance with the technical specifications (not periodic reports, which are filed in category R)

- Owners group meeting announcements and reports

- Quality assurance plan and related correspondence

- Environmental matters (technical data)

- Seismic Qualification Review Team (SQRT) reports and related correspondence

- Radioactive waste information, excluding onsite storage of low-level waste (filed category $X$ ) and independent spent fuel installations of high-level waste (filed in category $Y$ )

- Correspondence relating to expansion of spent fuel storage pool

- Startup test reports

- Generic and administrative communications and related doctuntentation that may affect the subject facility; not covered by a Bulletin or Notice

- Employee allegations and related documentation (other documents may be filed in categories $\mathrm{G}, \mathrm{Q}$, and $\mathrm{T}$ )

- SERs on specific generic issues

- Documents relating to possible equipment faiture and emergency instructions

- Documents relating to inservice testing procedures and results (not irspections which are flled in category $Q$ )

- Specific plant evaluation documents

- Probabilistic Risk Assessment (PRA) reports

- Employee allegations and related documentation (Other documents may be filed in categories $G, Q$, or $T$ )

- Reports on site visits

- Application to voluntarily temininate license, and to dismantle. and dispose of component parts

- Information on changes in NRC's internal organizational structure

\section{Q. INSPECTION REPORTS, CIVIL PENALTIES, RESPONSES TO BULLETINS AND INFORMATION NOTICES, AND RELATED CORRESPONDENCE (COL STAGE)}

This category contains the following applicant/ license material:
- Inspection reports 


\section{DEFINIMIONS OF DOCKET CATEGORIES}

\section{Q. INSPECTION REPORTS, CIVL PENALTIES, RESPONSES TO BULLETINS AND INFORMATION NOTICES, AND RELATED CORRESPONDENCE (COL STAGE) (CONTINUED)}

- Responses to Bulletins (formerly called Regulatory Operations, RO Bulletins\}, Information Notices, and related correspondence

- Applicant licensee deterrrination of the proprietary nature of Inspection Reports

- Letters concerning areas of noncompliance

- Preservice and inservice inspections and procedures

- Notices of violation and proposed impositions of civil penalty and Demand for Information (DFIs)

- Correspondence and orders relating to the opposition and payment of civil penalties, including Enforcement Notification (EN) and Significant Enforcement actions (EA)

- Notices of deviation and related correspondence

- Correspondence relating to inspection and enforcement that does not specifically relate to incidents or events as defined in category S

- Health physics inspections and appraisals

- Emergency preparedness inspections and apprásals
- Inspections and related correspondence from the International Atomic Energy Agency (IAEA)

- Risk-based Inspection Guides (RIGs)

- Systematic Assessment of Licensee Performance (SAIP)

- Incident Investigation Team (IT) reports

- Construction Appraisal Team/Construction Assessment Team (CAT) reports

- Integrated Design Inspection (III) reports

- Independent Design Verification program (DVP) reports

- Notices of significant licensee meetings

- Investigation reports and related documentation, and responses to employee allegations (ocher documents are filed in categories A, P, G, or T)

- Performance Appraisal Team (PAT) reports

- Licensee/vendor interface inspections

- Correspondence relating to the aforementioned items

NOTE: For bulletins and information notices, see nondocket I\&E file. 


\section{DEFINITIONS OF DOCKET CATEGORIES}

\section{R. PERIODIC OPERATING REPORTS AND RELATED CORRESPONDENCE (COL STAGE)}

This category contains periodic reports of a routine nature.

- Monthly, semiannual, and annual operating reports (other than financial) for construction, operating, and decommission stages

- Periodic envirommental monjtorirg, effluent testing or similar reports

- Periodic fitness for duty performance data

- Periodic personnel exposure reports

- Periodic facility changes, tests and experiments
- Facility simulation certification four year update reports

- Periodic nonconformance reports (issued in accordance with Licensing Board orders)

- Operational Logbooks on

- environmental data

- radiological data

- plant systems core

- plant status summary sheets

- Correspondence or other documents relating to the items listed

\section{S. REPORTABLE OCCURRENCES (ROS), LICENSEE EVENT REPORTS (LERs), PRELIMTNARY NOTIFICATIONS OF OCCURRENCES (PNOs), PRELIMINARY NOTIFICATTONS OF SAFEGUARDS EVENTS (PNSS), AND RELATED CORRESPONDENCE (COL STAGE)}

This category contains all materials relating to abnormal, tinusual, reportable and non-routine excurrences or events. These include:

- All 24-hour, 10-day, 30-day, interim and final Licensue Event Reports (LERs) and follow-up correspondence (also called incident reports, abnormal occurrences, and reportable occutrences)

- Potential or possibly reportable Deficiency Reports and related correspondence
- Individual Personnel Exposure Reports

- Construction Deficiency Reports submitted in accordance with 10 CFR 50.55(e)

- Documents related to the issuance of a preliminary Notification of Occurrence (PNOs) and Preliminary Notification of Safeguards Events (PNSs) and related correspondence 


\section{DEFINITIONS OF DOCKET CATEGORIES}

\section{S. REPORTABLE OCCURRENCES (ROS), LICENSEE EVENT REPORTS (LERs), PRELIMINARY NOTIFICATIONS OF OCCURRENCES (PNOs), PRELIMINARY NOTIFICATIONS OF SAFEGUARDS EVENTS (PNSS), AND RELATED CORRESPONDENCE (COL STAGE) (CONTINUED)}

- Environmental technical specifications viołation reports and environmental event reports

- Part 21 reports of defects and noncompliance
- Comespondence or other dochments relating to the aforementioned items

NOTE: For PNOs and PNSs, see I\&E nondocket files.

\section{T. TRANSCRIPTS, TESTIMONY, AND BRIEFINGS ON NON-ANTITRUST MATTERS (EARLY SITE PERMIT, PDA, FDADC, AND COL STAGES)}

This category contains verbatim transeripts, testimony, and briefings except for congressional matters (filed in category U) and antitrust matters (filed in category $\mathbf{N}$ ).

- Atomic Safety and Licensing Board (ASLB) transcripts

- Testimony and statements submitted to ASLB

- Transcripts of Commission meetings
- NRC staff briefings to the Commission

- Statements and written testimony submitted at Commission meetings and hearings

- Transcripts of vendor or utility briefings to NRC staff

- Corrections to the aforementioned items

NOTE: Transcripts on specific dockets are asso filed in the ACRS and 10 CFR 9.7 nondocket files.

\section{U. EXECUTIVE, CONGRESSIONAL, AND STATE GOVERNMENT CORRESPONDENCE (EARLY SITE PERMIT, PDA, FDAIDC, AND COL STAGES)}

This category contains documents and related correspondence that have substantive technical and administrative content, including:
- Statements and testimony before Congress or before boxies/committes appointed by the Executive or Legislative branches 


\section{DEFINITIONS OF DOCKET CATEGORIES}

\section{U. EXECUTIVE, CONGRESSIONAL, AND STATE GOVERNMENT (EARLY SITE PERMIT, PDA, FDADDC, AND COL STAGES) (CONTINUED)}

- Technical reports and related information supplied for the record of congressionat hearings

- Follow-up questions and answers to the aforementioned
- Correspondence to and from Congress and the Executive Branch on other than constituent response matters

- Coxrespondence to or from State Legislative bodies

NOTE: Routine responses to constituent inquiries are filed in category $\mathbf{H}$.

\section{LICENSING OF OPERATORS AND RELATED CORRESPONDENCE (COL STAGE)}

This category contains information bearing on licensing of operators and shift engineers, including:

- Power plant examination summary sheets

- Correspondence relating to operator exaninations
- Documentation on operator laboratory tests or reports and relsted comespondence

- Documents relating to operator fitmess for duty and related correspondence

- Orders prohibiting operators from further nuclear licensed activities, and related comespondence (other documents may be filed in category $\mathbf{G}$ )

\section{W. DECOMMISSIONING STAGE DOCUMENTS AND RELATED CORRESPONDENCE (COL STAGE)}

This category contains general technical items dated from the order for terminating the operating license.

- Order granting authority to dismantle/ deconmission

- Decommissioning Plan, Dismantling Plan, Environmental Report on dismantling, and the Safety Analysis Report on decommissioning

- Documents relating to the disposal of radioactive material ano decontamination of the site

- Other correspondence relating to decommissioning 


\section{DEFINITIONS OF DOCKET CATEGORIES}

\section{W. DECOMMISSIONING STAGE DOCUMENTS AND RELATED CORRESPONDENCE (COL STAGE) (CONTINUED)}

- All documentation relating to the ongoing operation of the plant formerly filed in crategory $\mathbf{P}$

\section{ONSITE STORAGE OF LOW-1EVEL WASTE AND REIATED CORRESPONDENCE (COL AND DECOMMISSIONING STAGES)}

This category contains material related to onsite storage of Low-Level Waste (LCW).

- Eicense application, license, proposed amendments, and amendments to store low-level waste at a commercial reactor
- All related correspondence and reports

NOTE: These documents will also bave a Nuclear Materials Safety and Safeguards (NMSS) Docket 30 license number associated with the Docket 52 number.

\section{Y. INDEPENDENT SPENT FUEL STORAGE INSTALLATIONS (ISFSIs) AND RELATED CORRESPONDENCE (COL AND DECOMMISSIONING STAGES)}

This categony contains material related to:

- License applications, license, amendments, and proposed amendments to possess power reactor spent fuel and other radioactive materials associated with spent fuel storage in an independent spent fuel storage installation. (Such spent fuel storage installations may be on a reactor site or at a separate site.)
- All related correspondence and reports to the above

NOTE: The doctiments in this category will have an NMSS Docket 72 number ticense. For these installations on a reactor site, the associated Docket $\$ 2$ number also has been referenced. 


\section{DEFINITIONS OF DOCKET CATEGORIES}

\section{DOCKET 70 - Special Nuclear Materials}

The Docket 70 files contain documentation concerning the domestic ticensing of specisy nuclear material (SNM). Each SNM license application is assigned a unique Docket 70 number. SNM licenses cover the possession and use of radioactive material, including phutoniuinenriched uranium. Paper files are maintained in the PDR only for Docket 70 licensees that possess more than 350 grams of uranium-235 or 200 grams of plutonium. Material on other licensees is available on microfiche only. Documents in these files include:

- Applications for licenses, licerse amendments, and license renewals for Special Nuclear Material licenses

- Special Nuclear Material licenses and license amendments
- Applicant technical reports concerning the facility, including Safety Anałysis Reports (SARs), Emvironmental Reports (ERs) and amendments

- NRC technical reports concerning the facility

- Emergency and radiological health plans

- Inspection reports and related correspondence

- Adjudicatory documents and hearing tzanscripts relating to proceedings before the Licensing Board

- Other techrical, legal and licensing correspondence between NRC and the licensee or other interested parties

\section{DOCKET 71 - Design of Packages for Transport of Radioactive Material and Quality Assurance Programs}

The Docket 71 files contain documentation concerning the design of radioactive material transport packages and quality assurance (QA) programs for designets, manufacturers, and users of such packages. Each package design and each QA program is individually assigned a unique Docket 71 number.

All NRC licensees who desigh, manufacture, or use radioactive material packages must comply with NRC-approved package designs and QA programs for these packages. Agreement State licensees who design, manufacture, or use radioactive material packages do not need a QA program approval, but the Department of
Transportation reguires that NRC approve the package design.

Agreement State licensees who wish to use their packages in States under NRC jurisfiction, or who design and manufacture packages for NRC licensees, must obtain approval for their QA programs. Documents in these files include:

- Application for QA program approwal and amendments to the application

- Application for package design approval, including the Safety Analysis Report (SAR), amendments, and engineering drawings 


\section{DEFINITIONS OF DOCKET CATEGORIES}

DOCKET 71 - Design of Packages for Transport of Radioactive Material and Quality Assurance Programs (continued)

- Certificate of compliance (NRC approwal) for the package design and revisions

- Approval of the QA program

- Correspondence between NRC and the designer and the manufacturer concerning particular package designs

- Requests to be registered as a user of a particular package

- Inspection reports and related correspondence

\section{DOCKET 72 - Independent Spent Fuel Storage Installations}

The Docket 72 series contains documentation concerning certification of storage casks and the licensing of independent spent fuel storage installations. These installations store reactor spent fuel and may be on a reactor site or at a separate site. Each installation is assigned a unique Docket 72 number. Documents in these files include:

- Application for license

- Safety Analysis Reports (SAR) and annual updates

- Certification of completion for vendor design of diry cask storage and related correspondence

- Licensee issued Environmental Report (ER) and amendments

- Emergency plan and amendments
- Inspection reports and related correspondence

- Reports on accidental criticality or loss of special nuclear material

- QA plan and amendments

- Conrespondence relating to the physical security plan

- Decommissioning plan and related correspondence

- Adjudicatory documents and bearing transcripts relating to proceedings between the NRC and the applicant

NOTE: Additional nondocketed information concerning spent fuel storage is avail $a b l e$ in the Project $M$ nondocket file. For installations on reactor sites, see category $Y$ in the Docket 50 or 52 series and Docket 70 for NMSS licenses. 


\section{APPENDIX B}

\section{DEFINITIONS OF NONDOCKETED CATEGORIES}

\section{NON-DOCKET FILES}

Materials that are not docket-specific are arranged according to the following classification scheme. The capitalized abbreviation is a shorthand description of each topic and corresponds to the way that it appears on the PDR Accession List.

10 CFR 9.7-Commission Meeting

Transcripts and Reports

ACRS-Advisory Committee on Reactor Safeguards Documents

ADVCM-NRC Advisory Committees

COMMISSION-NRC Commissioner

Correspondence

CONTRACT--NRC Contracts and Grants

FOIA-Freedom of Information Act

Requests and Appeals

GEN TECH IS_Generic Techunical Issues

I\&E-Inspection and Enforcement

Issuances

I\&E Manual一NRC Inspection Manual

MISC-Public Document Room

Miscellaneous
NUREG-NRC Staff and Contractor Formal Reports, Conference Proceedings, Brochures, International Agrement Reports, and Related Correspondence

ORG-Organizations

PRESS-PYess Releases

PR-Proposed and Final Rules

PRM-Petitions for Rulernaking

PROJ-Projects

PT 21-10 CFR Part 21 Deficiency Reports

QA 999-Vendor Quality Assurance Inspections

RC SSD-Registration of Certificates of Sealed Sources and Devices

REGGD-Regulatory Guides

RES-Research and Technical Assistance Reports (1974 through 1988)

REVIEW GRP -Review Groups 


\section{DEFINITIONS OF NONDOCKETED CATEGORJES}

RM-Rulemakings

SECY-Staff Position Papers prepared for the Commission

SPCH-Speeches

STATE PROG-State Programs

TOPREP-Vendor Topical Reports and

Related Correspondence
WASH-AEC-Issued Formal Reports and

Related Correspondence

WM-Waste Managernent Documents

WMRES-Waste Management Contract Research Documents

XPORTLIC-Export and Import Licenses

10 CFR 9.7 - Commission Meeting Transcripts and Reports

This file contains documents selating to open and closed Commission Meetings.

- Sunshine Federal Register notices

- Full written explanation of closed meetings

- Certification by the General Counsel on closed meetings

- Commission Determination Statements signed by the Secretary regarding the public disciosure of transcripts of Commission meetings
- Verbatim unofficial and official transcripts or portions of transeripts of open and closed Commission meetings made publicly available in accordance with the Sunshine Act and Commission policies

- Enclosures to released transcripts, including applicable viewgraphs, staff papers, and staff correspondence

- Staff Requirements Memoranca for open metings, memoranda of record, record of votes of Commissioners on items under consideration at meetings

\section{ACRS - Advisory Combittee on Reactor Safegrards Documents}

Documents in this file pertain to safety-related areas reviewed by the ACRS. ACRS serves as an independent advisory committee to the Commission.
- ACRS reports relating to safety-related matters 


\section{DEFINITIONS OF NONDOCKETED CATEGORIES}

- Arnouncements of ACRS meetings relating to safety-related matters, including meeting agenda

- Transcripts of ACRS subcommittee and full committet meetings relating to both facility license applications and safety-related matters

- Statements and written testimony of ACRS members or presented by individuals at ACRS proceedings related to both vendor or facility license applications and safety-related matters

- Minutes of ACRS subcommittee and full conmittee meetings relating to both vendor or facility license application and safety-related matters
- ACRS consultant reports, comments, and related correspondence relating to both vendor or factily license applications and safety-related matters

- Technical correspondence and memoranda to and from ACRS pertaining to safetyrelated matters

- General cotrespondence and related material about ACRS, but not prepared by ACRS or its consultants

NOTE: For docket-specific technical correspondence, memoranda and announcements of ACRS meetings, see category $O$ of Docket 50 and 52.

\section{ADVCM - NRC Advisory Committees}

Documents in this file pertain to the responsibilities of all NRC advisory comnittees other than the Advisory Committee on Reactor Safeguards, including.

- Charters of each advisory committee

- Meeting announcements and meeting agenda
- Minutes and verbatim transcripts of committee meetings

- Committee-generated reports, memoranda, and correspondence that are publicly available

- Committee-related correspondence 


\section{DEFINITIONS OF NONDOCKETED CATEGORIES}

\section{COMMISSION - NRC Commissioner Correspondence}

Documents in this file pertain to the daify conduct of Contrission business in the Office of the Commission, ircluding.

- Incoming and outgoing Commission-tevel cortespondence relating to officid NRC business, including reports and other enclosures to the aforememtioned correspondence

- Travel vouchers, telephone logs, and official appointment calendars of participating Combissioners. (These are placed in the PDR at the discretion of individual Commissioners.)
- Speeches, interviews and testimony given by Commissioners at meetings, symposia, and congressional hearings

- Vote sheets

- Weekly information reports (numbered as part of the SECY series) prepared for the Commissioners by the Executive Director for Operations (EDO) summarizing key events and items of interest undertaken by individual staff and program offices

NOTE: For trenscripts of Commission meetings, see nondocket File 10 CFR 9.7. For all other staff papers with the SECY-series designation prepared for Commission review, see the SECY nor-docket file.

\section{CONTRACT - NRC Contracts and Grants}

Documents in this file pertain to AEC and NRC contracts, grants and interagency agreements, including:

- Notification of contracts and grants exectution that specifies contract number, project title, contractor name, contract type, funding, and period of performance

- Contractual doctiments and modifications to contracts 


\section{DEFINITIONS OF NONDOCKETED CATEGORIES}

\section{FOIA - Freedom of Information Act Requests and Appeals}

Documents in this file include requests, denials, and appeals for agency records and information about records released in accordance with the provisions of the Freedom of Information Act (FOIA). Each is assigned a sequential identification number based on the year the request is made, for example, 95-110 refers to the 110th FOIA request made in 1995.

- Requests made under the FOLA for NRC records

- Correspondence between NRC and the requester relevant to the processing, granting, denial, and appeal of an FOIA regurest

- Records ot portions thereof released in response to FOLA reguests or made under FOLA appeals

\section{GEN TECH IS - Generic Technical Issues}

Documents in this file pertain to unresoived safety issues of a generic nature that are being analyzed by the NRC Office of Nuclear Reactor Regulation (NRR), including:

- Draft reports of action plans to accomplish generic tasks in the NRR program
- Correspondence with industry and within NRC discussing methodologies, analyses, the program, and technical aspects of issues

- Meeting notifications and minutes

- Interim findings and status reports on generic tasks addressing related aspects of generic technical issues

\section{I\&E - Inspection and Enforcement Issuances}

Documents in this file include bulletins and information notices that are issued by the NRC Office of Nuclear Reactor Regulation. These issuances describe some event that has occurred at a facility and that may require some follow-up action by the licensee. Also ineluded in this file are draft Bulletins, draft Information Notices, and draft Generic Communications (I\&E Misc), Preliminary Notifications of Events or Unusual
Occurrences (PNOs), Preliminary Notifications of Safeguards Events or Unusual Occurrences (PNSs), Enforcement Notifications (ENs) and significant Enforcement Actions (EAs).

- Circtlars, that describe an event at a licensed facility and are informational in nature (these were discontinued in 1981) 


\section{DEFINITIONS OF NONDOCKETED CATEGORIES}

- Information Notices, that are similar to Circulars but are preliminary in nature and are issued shortly after an event has occurred

- Bulletins describing an event at a licensed facility and requesting that all recipients petform certain actions as a result of that event

- Draft Bulletins and Information Notices that are being considered for issuance, and their related comments and evaluations

- Draft Generic Communications and related correspondence

- Preliminary Notification of Events or Unusual Occurrences (PNOs), which provide updated information of an event of safety or public interest

- Preliminary Notifications of Safeguard Events or Unustal

- Occurrences (PNSs), which provide early notice of events of possible safety significance

- Enforcement Notifications (ENs) and significant Enforcement Actions (EAs), which provide information on a notice of violation and proposed imposition of civil penalties

NOTE: For utility/vendor responses to such issuances and related correspondence, see categories $\mathrm{Q}$ and $\mathrm{S}$ in Dockets 50 and 52 .

I\&E Manual - NRC Inspection Manual

This file contains drafts, finals, revisions, Federal Register notices and related correspondence to the NRC Inspection Manual. 


\section{DEFINTIONS OF NONDOCKETED CATEGORIES}

MISC - Public Document Miscellaneous

This file contains documents about topics not included in the scope of the other non-docketed categories.

NUREG - NRC Staff and Contractor Formal Reports, Conference Proceedings, Brochures, International Agreements Reperts, and Related Correspondence

This file contains formal reports on regulatory. technical, and administrative issues of interest to staff, industry, other governmental entities, and the public which are published in the NUREG series.

- Results of licensing studies of specific plants or facilities preliminary to licensing actions

- Results of analyses of general or specific problems of a regulatory or technical nature that are of interest to a major segment of the industry

- Action and review plans, as well as guidance, for complying with NRC requirements

- Task force reports on specific topics

- Proceedings of conferences and workshops
- Management and program analysis reports

- Statistical analyses that are of interest to the staff, the industry, and the public

- Administrative reports that are of interest to the staff, the industry, and the public

- Correspondence from industry, gowernment, and the public commenting on NUREG series reports

- Internal NRC memoranda stumarizing meetings or discussions pertaining to the content of the NUREG reports series

NOTE: NUREG reports on piants or factilities are filed according to each plant's specific docket number. 


\section{DEFINTTIONS OF NONDOCKETED CATEGORIES}

\section{ORG - Organizations}

This file contains correspondence with professional organizations, government agencies, and associations with which NRC is in contact; records pertaining to specific NRC offices; and petitions not assigned Petition for Rule Making (PRM) numbers that reguest the Commission to take immediate action on a generic issue.

- Meeting summaries and technical correspondence between the $\mathrm{NRC}$ and professional associations

- Correspondence between NRC and other goverament agencies in which both parties have foint regulatory responsibility, e.g. EPA which has authority to control all rafioactive pollutants enitted into the air

- Correspondence and technical information between NRC and Congress

- Reports, technical information, and correspondence between NRC, foreign governments and international organizations

- Interral memoranda, trip teports, and scientific papers pertaining to specific NRC offices

\section{PRESS - Press Releases}

This file cortains press releases issued by the Office of Public Affairs Headquarters and Regional offices relating to the NRC's regulatory policy and programs, public hearings, and workshops. Press releases are also issued on major licensee programs, changes, proposed fines, and issues effecting the status of licenses.

\section{PR - Proposed and Final Rules}

This file contains doctments of NRC staff proposals to adopt, amend, or repeal a Commission regulation. Proposed Rules (PRs) can be initiated as a result of a Commission directive, a staff proposal, or a Commission determination that sufficient grounds exist for it to proced with a Petition for change in an NRC regulation (PRM) filed by an outside party.
- Federal Register notice stating the terms or substance of the proposed rule, or a specification of the subject and issues imvolved

- Text of the Proposed Rule (PR) 


\section{DEFINITIONS OF NONDOCKETED CATEGORIES}

- Draft and generic Proposed Rules and Federal Register notices

- Conments, staternents, information, opinions, or arguments on the proposed change to a part of the Code of Federal Regulations

- Requests from the nuclear industry, organizations, and other parties for written interpretation of the PR

- Final rules and Federal Register notices

NOTE: For staff recommendations to the Commission on whether a PR should be adopted, see the SECY noin-docket file. For Commission review of staff recommendations, see the 10 CFR 9.7 non-docket file.

\section{PRM - Petitions for Rulemaking}

This file contains documents of petitions filed with the Connission by an outside party to issue, amend, or rescind an NRC regulation.

- Petitions filed by any interested person or party to issue, amend, or rescind an NRC regulation

- Federal Register notice of the filing of the petition

- Correspondence between NRC and the petitioner relevant to the status and substance of the petition

- Comments from the nuclear industry, professional associations, public interest groups, and others in favor of or in opposition to the PRM

- Statements, motions, orders, and conespondence dealing with emergency petitions filed by parties on such topies as fire protection and a reanalysis of the capacity of operating U.S. plants to withstand earthquakes

NOTE: For staff recommendations to the Commissioners on whether the PRM should be denied or issued as a pro. posed rule, see the SECY non-docket fije. For Commission teview of staff recommendations, se the 10 CFR 9.7 non-docket file. 


\section{DEFINITIONS OF NONDOCKETED CATEGORIES}

\section{PROJ - Projects}

This file contains documents pertaining to four classes of projects: (1) commercial nuclear power plants (Docket 50) for which NRC has full review and licensing responsibilities; (2) Governmentowned reactors (also Docket 50) for which NRC provides technical expertise and occasional review; (3) design certification and combined licensing (Docket 52) for which NRC has full review and licensing responsibilities; (4) monitored retrieval storage and Topical Reports for storage casks (Project MSs) for which NRC has full review and licensing responstbilities. Each class is identified with a $\mathrm{P}$ prefix, followed by a series of numbers.

These filles contain all documentation up to the time NRC considers the application and the preliminary technical reports complete for review. The project is then assigned a docket number and the file is transferred to a docket series.

- Applications submitted by the vendors or utility

- Backup technical documents and topical reports submitted in support of the application, and related correspondence

- Preliminary Safety Analysis Report (PSAR), amendments, and related correspondence (Docket 50)
- Preliminary Standard Safety Analysis Report (PSSAR), amendments, and related correspondence (Docket 52)

- Application for Preliminary and Final Destgn Approval and design certification (Docket $\$ 2$ and Project M), and related comespondence

- Early site review information

- Meeting notices, summaries, and correspondence

- NRC's or the applicant's changes in internal organizational structure

- Reliability Asstrance Program and related correspondence (Docket 52)

- Documentation and technical correspondence between NRC and other government agencies relating to the application

- Adjudicatory correspondence

- Quality Assurance documentation, notices of deviation, and related correspondence 


\section{DEFINITIONS OF NONDOCKETED CATEGORIES}

\section{PT 21 - 10 CFR Part 21 Deficiency Reports}

This file contains deficiency reports submitted in compliance with Part 21 of Title 10 of the Code of Federol Regulations.

- Reports by firms imvolved in the nuclear industry reporting noncompliance with NRC regulations or the existence of defects that could create a substantial safety hazard
- Correspondence pertaining to the NRC review of reports to assess the possibitity of generic defects

- NRC inspection reports of defects cited

- Correspondence specifying appropriate followup actions to be taken by the agency

QA 999 - Vendor Quality Assurance Inspections

This file contains inspection reports of the vendor/licensee interface from the vendor's view, the ventior's products, and the ventor's programs including equipinent environmental qualification testing, and followup correspondence. Each vendor is assigned a tocket number and is a part of the QA 999 seties.

- Vendors are defined as companies, both U.S. and foreign, that are engaged in the design, manufacture, or testing of nuclear components or in supplying services to the nuclear industry. The controlling criteria are found in 10 CFR Part 21, 10 CFR Part 50, the PSAR and FSAR submitted by utilities for specific facilities, and vendor QA topical reports.
- NRC inspection reports of vendor facilities that cover the design, fabrication, and testing of components for use in nuclear facilities and related correspondence

- Civil penalties notices

- Notices of deviation

- Vendor corrective actions and other followup correspondence associated with Iesolution of inspection findings

NOTE: For vendor/licensee inspections from the licensee's view, see category $Q$ in Docket 50 and 52 


\section{DEFINITIONS OF NONDOCKETED CATEGORIES}

\section{RC - Registration of Certificates of Sealed Sources and Devices}

This file contains documents and related correspondence on radiation sources and device designs. Manufactures and distributors applying for registration of their products must submit for review all safety related information, design specifications, QA programs and amendments. These documents are maintained in the PDR on microfiche only.

\section{REGGD - Regulatory Gujdes}

This file contains new and revised regulatory guides and related correspondence. These guides describe to the industry and to the public methods acceptable to the NRC staff for implementing specific parts of NRC regulations. They also delineate techniques used by the staff in evaluating specific problems or postulated accidents or provide gridance to applicants.

- Federal Register notices announcing the issuance and availability of a proposed Regulatory Guide and revisions

- Revised or newly issued regulatory guides

- Drafts of proposed regulatory guides or proposed revisions
- NRC value/impact assessment

- Comments submitted by the muclear industry and interested members of the public regarding a proposed regulatory guide

- Summaries of meetings held in the NRC to discuss proposed regulatory guides

NOTE: Industry codes and standards cited in regulatory guides are available for Teference use only in the NRC's Technical Library.

RES - Research and Technical Assistance Reports (1974 through 1988)

This file contains reports and technical letters submitted by NRC contractors providing research and technical assistance to the Commission. It also includes research information letters and conference proceedings pertaining to research activities of the Commission.
For final reports of research conducted by contractors, and for conference proceedings, see the NUREG series non-docket file. See also the WMRES file for researeh reports on disposal of high-level waste. 


\section{DEFINITIONS OF NONDOCKETED CATEGORJES}

\section{REVIEW GRP - Review Groaps}

This file contains documents pertaining to the activities of internal and extermal research review groups. Such groups provide a broad base of technical expertise to aid management in reviewing research in defined technical areas. They review and comment on research plans and predicted restlts of major experiments, the validity and applicability of research results, possible redirection of research projects, new research projects, and priorities witlin the technical areas covered by the research review group.

- Agenda for quarterly review group meetings

- Summary minutes of review group meetings representing views expressed, including dissenting views
- Research plans and predicted results of major experiments and presentations by research contractors

- Comments on meetings from attendees, incluting representatives from contractors, universities, vendors, and other agencies

- Internal memoranda relating to topies under discussion

- Correspondence related to the aforementioned items

\section{RM - Rulemakings}

This file contains documents pertaining to public hearings held on proposed changes to the Commission's regulations. Hearings are instituted at the discretion of the Commission, based on the importance and the magnitude of the subject bejing considered.

- Designation of the presiding officer and any special directions for the conduct of the hearing

- Notifications of Hearing Board meetings
- Petitions, motions, testimony, asd statements submitted by parties to the hearings

- Transcripts of the proceedings

- Hearing Board orders and rulings

- Technical reports submitted as evidence is the proceedings

- Reports, if any, of the special Board to the Commission regarding the subject proceding 


\section{DEFINITIONS OF NONDOCKETED CATEGORIES}

SECY - Staff Position Papers PTepared for the Commission

This file contains staff papers (generally referred to as SECY Papers) prepared for the information or approval of the Commission. They are assigned a SECY report number.

- Policy issue papers are used to present matters relating to policy formulation

- Rulemaking issue papers are used to present rulemaking activities of the Commission

- Adjucicatory issue papers are used to present matters relating to the granting, suspending, revoking, or amending of licenses, or relating to construction permits for nuclear power plants, and activities in which the Commission has an interest

- Information Papers are used to provide information to the Commission. Some (e.g., the weekty report of the Executive Director for Operations) provides general information about agency activities without specific reference to policy formulation, rilemaking or adjudication

These categories of staff papers may be isstied as one of the following (a) a Commission meeting paper to be discussed at a Commission meeting; (b) an Affirmation paper that does not require interactive discussion among the Connmissioners in a Commission meeting but by law must be voted upon by the Commissioners in the presence of each other; (c) a notation vote paper that does not require interactive diseussion among all Commissioners or formal vote in a meeting, thereby lending itself to a written notation process; (d) a negative consent paper that contains issues which the Commission would like brought to its attention before action is taken but which does not require the fonnatity of a Commission vote; and (e) information papers that provide information to the Comr. mission and do not call for any action by the Commission.

As a general practice, papers that are regularty released to the pubtic include those that are the subject of diseussion at a public meeting of the Commission and those that address unclassified matters pertaining to the export and import of nuclear equipment and material.

NOTE: For Weekly Information Reports (WIR) prepared by the NRC Office of the Executive Director for Operations to the Commission providing a summary of key events and items of interest undertaken by staff and program offices, see the Commission Correspondence non-docket file.

\section{SPCH - Speeches}

This file contains speeches by the Chairman and Commissioners presented at National and international regulatory information conferences, industrial forums, advisory committes, symposiums, workshops, and energy policy groxps. 


\section{DEFINITIONS OF NONDOCKETED CATEGORIES}

\section{STATE PROG - State Programs}

This file contains documents that pertain to the Commission's relations with State governments. Areas covered inclute: (1) the NRC and State Agreement and its amendments, whereby States may assume certain of the NRC's regulatory authority over nuclear materials; and (2) a wide range of liaison and cooperative activities regarding such functions as licensing, inspection procedures, and waste disposal.

- Correspondence between the NRC Office of State Programs and State officials, State liaison officers and radiation control management health officials, regarding:

- Cooperative programs in such technical areas as radiological incidents, water quality, radioactive waste disposal, and transportation surveillance

- NRC reviews of Agreement State radiation control programs

- Technical assistance to Agreement States in reviewing applications, com- pliance actions, developing regulations, and other areas

- Notices prohibiting irvolvement in NRClicensed activities

- Agenda, reports, and transcripts of regional workshops

- Training programs on current matters of policy and regulations; i.e., decommissioning and radioactive waste disposal

- State legislative reports (discontinued in December 1984)

- Agreement State licensing statistics reports (discontinued in December 1984)

NOTE: For specific uranium milling and mining projects in which the NRC is providing technical assistance to States, see the Project mon-iocket file.

TOPREP - Topical Reports and Related Correspondence

This file contains Topical Reports, generally of a generic technical nature, submitted by vendors; and correspondence pertaining to the NRC review of these reports. Vendor-related corre. spondence that is not docket specific and documents geperated by vendor-related owners groups are also included in this file.

- Topical reports dealing with such technical matues as specifications, testing, and safety that are submitted by architects, engineers, and other vendors

- Revisions, modifications, and amendments to topical reports

- Correspondence between NRC and vendoss pertaining to the review of submittats 


\section{DEFINITIONS OF NONDOCKETED CATEGORIES}

- Meeting agenda, summaries, minutes and correspondence pertinent to vendor activities, programs, methodologies, and design criteria

- Vendor-related Owners' Group meeting announcements, agenda, summaries, reports and correspondence

NOTE: For reports submitted by utilities that are written by vendors on their behalf and that relate to docketed cases, see the file categories for the docket series.

WASH - AEC-Issued Formal Reports and Related Correspondence

This file contains formal reports that were issued by the U.S. Atomic Energy Commission (AEC) headquarters staff and related correspondence. The WASH reports, which consisted of administrative, scientific, and tectunical information, ended in 1975, but correspondence occasionally refers to WASH reports. Post-1975 NRC-issued formal reports earry the NUREG series designation.
This file contains documents related to the activities of the NRC Division of Waste Management. Each topic has been assigned a unique number, piefixed by the letters WM. Documents in these files include:

- NRC and DOE prelicensing activities regarding the disposal of high-kvel waste in geologic repositonies, in accordance with the Nuclear Whaste Policy Act of 1982 as follows:

- Technical high-level waste site prelicensing documentation

- Meeting agenda, minutes and backup doctuments of DOE/NRC meetings in which DOE presents information on its geologic exploration program to identify sites for nuclear waste repositories
- Briefings by DOE on cument research on muclear waste management

- NRC reviews of the site-by-site implementation of DOE's program for uranium mill tailings remedial actions, in accordance with the Uranium Mill Tailings Radiation Control Act of 1978

- Correspondence and comments between NRC and the Environmental Protection Agency (EPA) pertaining to the high-level waste standard

- NRC copical report reviews and asspciated correspondence 


\section{DEFINITIONS OF NONDOCKETED CATEGORIES}

- Correspondence from the public and other non-government organizations for the aforemention items
NOTE: For contractor research on waste management activities, see the WMRES non-docket file.

\section{WMRES - Waste Management Contract Docaments}

This file contains interim and final reports and correspondence between NRC and its contractor's regarding prelicensing activities in the area of high-jevel waste disposal in geologic repositories, including.

- Periodic contractor progress reports
- Tip reports

- Draft and final reports submitted for NRC review or information

- Correspondence between NRC stafi and contractors related to technical questions or issues to be resolved

\section{XPORTLIC - Export and Import Licenses}

This file contains records relating to the isstance of icenses to export or import nuclear facilities and materials, including:

- License applications and amendments to export or import special nuclear material, source material, byproduct material, reactors, nuclear facility components, or reactor materials

- Correspondence between NRC and applicant or licensee pertaining to the review or status of a pending application or license amendment

- Commission letters requesting Executive Branch (e.g., U.S. Department of State) views
- Review and findings of the Executive Branch on whether issuance of license would be inimical to the common defense and security of the United States

- Correspondence to and from foreign governments, international organizations, and members of the public

- Hearing records and decisions

- Memoranda from the Office of the Secretary to the Executive Director for Operations (EDO) regarding the Commission's decision on issuing a proposed license

- Licenses, license amendments, ano license renewals 


\section{DEFINITIONS OF NONDOCKETED CATEGORIES}

- Periodic report on export actions

NOTE: For transeripts of Commission meetings and papers submitted for Conmissioner consideration or information, see the 10 CFR 9.7 and the SECY non-docket file. 